\title{
AMERICAN EPILEPSY SOCIETY
}

\section{EPILEPSY URRENTS}

The Journal of the American Epilepsy Society

\section{VOLUME 13 - SUPPLEMENT 1}

\section{AES 2012 Abstract Supplement - Epilepsy Currents Online}

\section{AES 2012 Annual Meeting}

November 30 - December 4, 2012, San Diego, CA, USA

\section{Abstracts}

Poster Session 1: Saturday, December 1

Presidential Symposium: Saturday, December 1

Late Breaking Abstracts: Saturday, December 1

Investigators' Workshop: Saturday, December 1

Poster Session 2: Sunday, December 2

Investigators' Workshop: Sunday, December 2

Investigators' Workshop Noon Poster Session: Sunday, December 2 ...

Poster Session 3: Monday, December 3

Investigators Workshop: Monday, December 3

Pediatric Epilepsy Highlights Session: Monday, December 3

Platform Sessions A, B, and C: Monday, December 3

Pediatric State of the Art Symposium: Monday, December 3 
Saturday, December 1, 2012

\author{
Presidential Symposium \\ 8:30 a.m.-11:30 a.m.
}

IOM Report 2012: Epilepsy Across the Spectrum: Promoting Health and Understanding

F. E. Jensen ${ }^{1}\left({ }^{1}\right.$ Perelman School of Medicine at the University of Pennsylvania, Philadelphia, PA, United States)

Summary: This symposium will describe the process that led to the development of the 2012 IOM. There will be a report on the potential impact of IOM report on both the AES community and the health care community in general. There will be a review of the major elements of the report and how it will be implemented to change professional education, certification of epilepsy centers and specialists, and patient access to care. Actionable elements of the IOM report that will require coordinated participation of federal and nonfederal entities will be addressed. The relationship of the IOM report to the expanded NINDS and inter-institute funding programs for epilepsy research as well as how the IOM report can support the NINDS Epilepsy Benchmarks will be described. Finally, the response of patient advocacy and nongovernmental organizations will be presented, including implementation of the report recommendations and coordination with the community of professionals in epilepsy care.

\section{Saturday, December 1, 2012}

\section{Poster Session 1 \\ 11:45 a.m.-6:00 p.m.}

Translational Research: Mechanisms

\subsection{1 \\ WTIHDRAWN}

\subsection{2}

THE ENDURING EFFECTS OF EARLY-LIFE STRESS ON LIMBIC EPILEPTOGENESIS ARE MEDIATED BY HPA AXIS HYPER-REACTIVITY

A. Koe ${ }^{1}$, M. Salzberg ${ }^{2}$, T. O'Brien ${ }^{1}$, M. Morris ${ }^{3}$ and Nigel Jones ${ }^{1}$ ( ${ }^{1}$ Medicine, University of Melbourne, Parkville, VIC, Australia; ${ }^{2}$ Psychiatry, University of Melbourne, Melbourne, VIC, Australia and ${ }^{3}$ Pharmacology, University of New South Wales, Sydney, NSW, Australia)

Rationale: Enduring effects of early-life stress on the brain promotes vulnerability to the later development of experimental limbic epilepsy. This process is possibly mediated by hyper-reactivity of the hypothalamic-pituitary-adrenal (HPA) axis with increased corticosterone release. Using the maternal separation (MS) model of early-life stress in rats, this study assessed whether inhibiting corticosterone synthesis could ameliorate the adverse effects of MS stress on kindling epileptogenesis.

Methods: From postnatal days 2-14, Wistar rats were subjected to MS stress for $3 \mathrm{~h} /$ day (MS, $\mathrm{n}=17$ ) or early-handling for $15 \mathrm{~min} /$ day $(\mathrm{EH}, \mathrm{n}=21)$. At 8 weeks, female rats were assessed for seizure threshold via a bipolar electrode implanted in the left amygdala, and subsequently subjected to after-discharge threshold testing and electrical amygdala kindling. Throughout the kindling period, rats were administered either metyrapone (corticosterone synthesis inhibitor: $50 \mathrm{mg} / \mathrm{kg}$ ip) or vehicle $60 \mathrm{~min}$ before each kindling stimulation.
Results: Vehicle-treated MS rats displayed reduced seizure threshold compared to $\mathrm{EH}$ rats ( $\mathrm{p}=0.03$ ), a vulnerability attenuated by metyrapone pretreatment. During kindling, vehicle-treated MS rats also exhibited longer seizure duration compared to $\mathrm{EH}$ rats $(\mathrm{p}=0.02)$, an effect equally reversed by metyrapone treatment. Further, metyrapone treatment in MS rats retarded the progression of kindling $(\mathrm{p}=0.03)$.

Conclusions: Inhibition of corticosterone synthesis with metyrapone alleviates the enduring effects of MS stress on seizure susceptibility and severity. This suggests that HPA axis hyper-reactivity is critical to the effect of MS stress in increasing vulnerability to limbic epileptogenesis.

\subsection{3}

TRANSIENT PRENATAL HYPOXIA-ISCHEMIA IN RATS DIMINISHES CA3 HIPPOCAMPAL INHIBITORY CIRCUIT DEVELOPMENT

Lauren L. Jantzie $^{1,2}$, P. Getsy ${ }^{3,4}$, C. Yeung ${ }^{1,2}$, D. J. Firl ${ }^{1,2}$, C. G. Wilson $^{3,4}$, F. E. Jensen ${ }^{1,2}$ and S. Robinson ${ }^{1,2}$ ( ${ }^{1}$ Boston Children's Hospital, Boston, MA; ${ }^{2}$ Harvard Medical School, Boston, MA; ${ }^{3}$ Rainbow Babies \& Children's Hospital, Cleveland, $\mathrm{OH}$ and ${ }^{4} \mathrm{Case}$ Western Reserve University, Cleveland, $\mathrm{OH}$ )

Rationale: Children born preterm are prone to chronic neurological deficits including cognitive delay, epilepsy, and behavioral abnormalities. Alterations in initial inhibitory circuit development and changes in inhibitory tone have been implicated in the etiology of these disorders. The primary inhibitory neurotransmitter in the adult brain is GABA. As the cerebral cortex matures and responds to environmental cues during development, GABA responses switch from excitatory to inhibitory coincident with upregulation of the potassium chloride cotransporter KCC2. Developmental increases in $\mathrm{KCC} 2$ allows GABA receptor activation to cause hyperpolarization and aids in attenuating hyperexcitability. Previously we showed decreased cortical KCC2 protein expression in preterm infants with white matter lesions and in patients undergoing epilepsy resections. Here, we hypothesize a transient systemic hypoxic-ischemic (TSHI) insult to the developing brain during late gestation alters the spatiotemporal pattern of KCC2 upregulation, thereby disrupting essential cortical inhibitory circuit development.

Methods: Bilateral uterine artery ligation was performed on E18 rats to induce TSHI typical in early preterm birth with placental insufficiency. Erythropoietin (EPO) or vehicle (Veh) was administered following TSHI from P1-5 (2000 IU/kg/dose/ip). Changes in $\mathrm{NKCC} 1$ and $\mathrm{KCC} 2$ protein were investigated and wholecell voltage clamp of CA3 pyramidal neurons was used to determine miniature inhibitory postsynaptic currents (mIPSCs) and assess inhibitory synaptic input.

Results: Results demonstrate a developmental upregulation of $\mathrm{KCC} 2$ protein in CA3 from P7-21, concomitant with a decreased NKCC1 to $\mathrm{KCC} 2$ ratio consistent with previous reports. Following TSHI, KCC2 protein expression was significantly reduced at $\mathrm{P} 13$ by $62 \%$ compared to sham. Postnatal administration of EPO attenuated the reduction in $\mathrm{KCC} 2$ protein expression (TSHI+Veh: $39 \%$ control vs. TSHI+EPO: $155 \%$ control).TSHI ( $\mathrm{n}=12$ cells $/ 7$ rats) also decreased the mean amplitude, frequency and rise time of mIPSCs at P10-11, compared to sham $(\mathrm{n}=11$ cells $/ 6$ rats). Two distinct populations of TSHI CA3 pyramidal neurons emerged from mIPSC frequency analysis, with $67 \%$ of cells showing a frequency $<0.3 \mathrm{~Hz}$, compared to $0 \%$ of sham cells $(\mathrm{p}<0.001)$. EPO normalized the effects of TSHI and restored amplitude and frequency to baseline levels.

Conclusions: These results indicate decreased inhibitory synaptic input and $\mathrm{KCC} 2$ protein expression following prenatal HI, and may have important implications for understanding inhibitory circuit development and the effect of late gestation insults on inhibitory tone. Importantly, the postnatal administration of EPO normalized $\mathrm{TSHI}-$ induced reductions in $\mathrm{KCC} 2$ protein expression and $\mathrm{mIPSC}$ amplitude and frequency, suggesting it is possible to reverse 
abnormalities in inhibitory cortical development. Together, these data indicate that the impairments caused by prenatal insults in children born preterm, including cognitive delay and epilepsy, may be at least partially restored with a neuroprotective regimen administered in the neonatal period.

\subsection{4}

INCREASED EXPRESSION OF PAN-TRK RECEPTOR PROTEINS IN THE CORTEX OF AN ADULT RAT MODEL OF IRRADIATION-INDUCED CORTICAL DYSPLASIA Zhong Ying, A. Nemes and I. Najm (Epilepsy Center, Cleveland Clinic Foundation, Cleveland, $\mathrm{OH}$ )

Rationale: Focal Cortical Dysplasias (FCDs) are frequent causes of medically intractable epilepsy. Although the age at seizure onset is variable, the first seizure is usually the result of a brain insult known as the "second hit", such as a minor head trauma, brain infection, or cerebral ischemia. Following a latent period, seizures become medically-intractable. Neurotrophins acting via various Trk receptors have been implicated in promoting epileptogenesis in both human and animal models of epilepsy. We investigated the expression of Pan-Trk receptor proteins in an adult rat model of irradiation-induced FCD and upregulation of Pan-Trk proteins by a single subconvulsive dose of Pentylenetetrazol (PTZ) as a "2nd hit".

Methods: Timed pregnant rats were irradiated (XRT) on E17 with $145 \mathrm{cGy}$, which is the standard protocol for inducing focal cortical dysplasias in our lab. Adult rats (PND 70) that received in utero irradiation (XRT) and control rats were injected with a single dose of PTZ (40 mg/kg, i.p.). The rats were sacrificed at 2 days (2d) and 15 days (15d) following the PTZ injection for Pan-Trk

immunohistochemistry (IHC) and histological staining. All XRT rats had acquired FCD, as evidenced by histopathological examinations.

The 53 rats were divided into 6 groups:

1) -XRT/-PTZ (n=10); 2) +XRT/-PTZ (n=8); 3) -XRT/+PTZ -2d $(\mathrm{n}=9) ; 4)+X R T /+P T Z-2 d(n=8) ; 5)-X R T /+P T Z-15 d(n=10) ; 5)$ + XRT/PTZ-15d $(\mathrm{n}=8)$.

Results: Pan-Trk IHC immunoreactivates (IRs) in the cortex were qualitatively graded based on the extent of staining (percentage of stained neurons) and intensity of cellular staining. The student unpaired t- test was used for statistical analysis. A. Compared to normal rats, Pan-Trk receptor protein IRs were significantly higher in XRT rats (group 2 vs group1; group 4 vs group 3; group 6 vs group 5). B. Among non-XRT rats (group 1, group 3 and group 5), Pan-Trk IRs were significantly higher in rats of group 3 compared to rats of group 1. No significant difference was found between rats in group 5 vs rats in group 1 or rats in group 5 vs rats in group 3. C: Among XRT rats (group 2, group 4 and group 6), Pan-Trk IRs were significantly higher in rats of group 6 compared to rats in group 2. No significant difference was found between rats in group 2 vs rats in group 4; rats in group 4 and rats in group 6.

Conclusions: The Pan-Trk proteins were increased in the FCD cortex compared to normal rat cortex. Furthermore, the 2nd hit (single PTZ injection) upregulated Pan-Trk receptor proteins in both FCD and normal rat cortices, but significantly higher in FCD groups.

Moreover, the upregulated Pan-Trk receptor proteins induced by PTZ injection was temporary in normal rats but more sustained in FCD rats. It is known that neurotrophins upregulate NDMA receptors and excitatory synaptic activities. Our findings suggest that neurotrophins may have a critical role in formation and development of epileptogenicity in FCD.

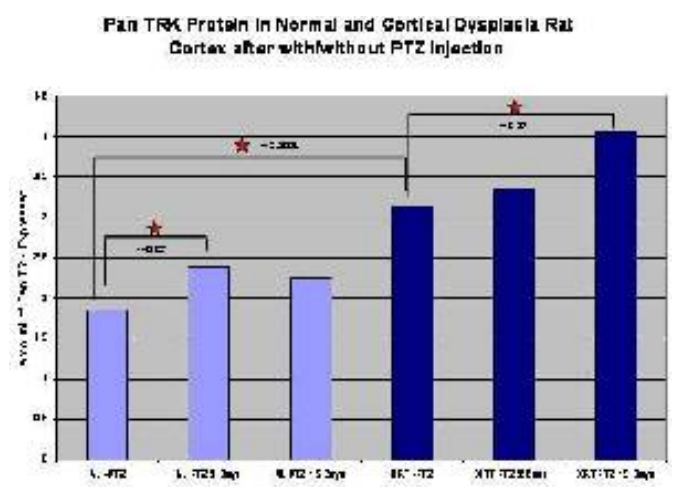

\subsection{5 \\ ALTERED SYNAPTOGENESIS AFTER ALBUMIN EXPOSURE: A MODEL FOR POSTTRAUMATIC EPILEPTOGENESIS}

Lydia Wood $^{1}$, I. Weissberg ${ }^{3}$, D. Milikovsky ${ }^{3}$, A. Friedman ${ }^{3}$ and D. Kaufer ${ }^{1,2}\left({ }^{1}\right.$ Integrative Biology, University of California, Berkeley, $\mathrm{CA} ;{ }^{2}$ Helen Wills Neuroscience Institute, University of California, Berkeley, CA and ${ }^{3}$ Physiology and Neurosurgery, Zlotowski Center for Neuroscience, Ben-Gurion University of the Negev, Israel)

Rationale: Epilepsy can develop following different precipitating events sharing blood brain barrier dysfunction as the common denominator, including traumatic brain injury. We previously demonstrated that the uptake of albumin into astrocytes is critical in the generation of perilesional hyperxcitability and seizures following blood-brain barrier compromise, and identified TGF $\beta$ signaling pathway activation as a critical step (Cacheaux et al, 2009, J

Neurosci, 29: 8927-35). However, the exact mechanims by which the astrocytic reaction to serum albumin leads to sustained hyperexcitability is largely unknown. Recent studies have shown that astrocyte-secreted factors, including thrombospondins (TSP), can control the strength and number of both excitatory and inhibitory synapses (Christopherson et al, 2005, Cell, 120: 421-433; Hughes et al, 2010, Mol Cell Neurosci, 43: 136-145). Therefore, we investigated altered excitatory synaptogenesis in primary hippocampal cultures following albumin treatment, and its dependence on TGF $\beta$ signaling and astrocyte-secreted factors. Methods: The effects of albumin exposure on synaptic connectivity were assessed using primary cultured hippocampal CNS cells. Synaptogenesis was assessed after albumin exposure using immunocytochemical labeling of pre and postsynaptic markers, Synapsin 1 and PSD-95, respectively. Gene expression levels were determined of using qRT-PCR.

Results: We exposed cultures of mixed neurons/glia hippocampal cells to albumin $(0.2 \mathrm{mM})$, or TGF $\beta 1(10 \mathrm{ng} / \mathrm{mL})$ and followed the development of excitatory synapses. The number of co-localized puncta of pre- and post-synaptic protein markers was increased 72 hours post treatment with either albumin $(169 \%$ of control, $p=0.042)$ or TGF $\beta 1$ ( $189 \%$ of control, $p=0.006)$. Interestingly, there was no change in the number of synases following the treatments in pure neuronal cultures, suggesting that the albumin/TGF $\beta$-induced increase in excitatory synapse number requires an astrocytic process. Furthermore, this albumin/ TGF $\beta$-induced increase was blocked in the presence of the TGF $\beta$ blocker SJN.

Conclusions: Our data suggest that the pathological exposure of brain cells to the serum protein albumin induces an increase in the number of excitatory synapses. This increase is dependent on the activation of TGF $\beta$ signaling and the presence of astrocytes. Overall, this aberrant synaptogenesis can lead to an excitatory/inhibitory imbalance and potentially link the proximal molecular events following the blood brain barrier failure to the delayed development of posttraumatic epilepsy. 


\subsection{6}

REDUCED PP2A ACTIVITY AND TAU

HYPERPHOSPHORYLATION IN THE AMYGDALA KINDLING RAT MODEL OF TLE: A TARGET FOR NOVEL ANTI-EPILEPTOGENIC THERAPIES?

Terence J. O'Brien ${ }^{1,2}$, S. Liu ${ }^{1}$, N. C. Jones ${ }^{1}$, T. Nguyen ${ }^{3}$, N. Corcoran ${ }^{3}$ and C. Hovens ${ }^{3}\left({ }^{1}\right.$ Medicine, The Royal Melbourne Hospital, The University of Melbourne, Parkville, VIC, Australia; ${ }^{2}$ Neurology, The Melbourne Brain Centre, Royal Melbourne Hospital, Parkville, VIC, Australia and ${ }^{3}$ Surgery, The Royal Melbounre Hospital, The University of Melbourne, Parkville, VIC, Australia)

Rationale: Protein phosphatase-2A (PP-2A) is a multi-subunit enzyme serine/threonine phosphatase and downregulation of PP-2A that plays a prominent role in a variety of pathologies, from carcinogenesis to neurodegenerative disease. One role of PP-2A is to dephosphorylate tau. Hypophosphorylated tau has been implicated in the pathogenesis of acquired forms of epilepsy, and we therefore hypothesised that down regulation of PP-2A may play a role in epileptogenesis and represent a novel target for anti-epileptogenic therapies.

Methods: We first investigated in the electrical amygdala kindled rat model of temporal lobe epilepsy (versus sham kindled rats) the level of activity of PP-2A in relevant brain regions, using a immunoprecipitation phosphatase assay kit, and using western blotting the expression levels of PP-2A subunit C (catalytic subunit), PP-2A subunit B (regulatory subunit, PR 55), total tau and phosphorylation of tau on Ser 198 and 262, residues that are dephosphated by PP-2A. Next we investigated the effect of enhancing PP-2A activity in-vivo, by chronically treating with sodium selenate, an activator of PP-2A, at $1 \mathrm{mg} / \mathrm{kg} /$ day for 28 days during the period of electrical amygdala kindling, compared with saline treatment.

Results: We found that PP-2A activities and PP-2A B subunit (PR55) expression were significantly decreased in amygdala, hippocampus and cortex of amygdala kindling rats compared with the same brain sections of sham kindled rats. Phosphorylation of tau on Ser 198 and 262 were both increased in these regions, compared with the same brain sections of sham kindled rats, consistent with a decreased activity of PP-2A. Further rats chronically treated with the PP-2A activator, sodium selenate, had significantly slower progression of the behavioural class of seizures during kindling, and of the progression of the total and primary electrographic seizure durations, compared with those treated with saline. On molecular analysis the selenate treated kindled rats had significantly increased the PP-2A activities and PP-2A B subunit expression, and decreased phosphorylation of tau on Ser 198 and 262, in amygdala, hippocampus and cortex, compared with rats treated with saline during the period of kindling.

Conclusions: These results indicated that amygdala kindling epileptogenesis is associated with a down-regulation of PP-2A activity, associated with decreased expression of the regulatory $\mathrm{B}$ subunit and an increase in tau phosphorylation, and that pharmacologically enhancing PP2A activity with sodium selenate is a potentially viable anti-epileptogenic therapeutic statergy.

\section{$\mathbf{1 . 0 0 7}$}

SODIUM SELENATE TREATMENT REDUCES

NEURODEGENERATION AND BEHAVIORAL IMPAIRMENTS IN A RAT MODEL OF POST-TRAUMATIC INJURY EPILEPSY

S. R. Shultz ${ }^{1}$, P. Zheng ${ }^{1}$, D. Wright ${ }^{4}$, L. Johnston ${ }^{4,5}$, C. Hovens ${ }^{3}$, N. C. Jones ${ }^{1}$ and Terence J. O'Brien ${ }^{1,2}\left({ }^{1}\right.$ Medicine, The Royal Melbourne Hospital, The University of Melbourne, Parkville, VIC, Australia; ${ }^{2}$ Neurology, The Melbourne Brain Centre, Royal Melbourne Hospital, Parkville, VIC, Australia; ${ }^{3}$ Surgery, The Royal Melbourne Hospital, The University of Melbourne, Parkville, VIC, Australia; ${ }^{4}$ Florey Neurosciences Institute, The University of
Melbourne, Parkville, VIC, Australia and ${ }^{5}$ Electrical and Electronic Engineering, The University of Melbourne, Parkville, VIC, Australia)

Rationale: Hyperphosphorylated tau has been implicated in the pathogenesis of a variety of neurodegenerative disorders, including traumatic brain injury (TBI) and epilepsy. Here we investigated whether treatment with sodium selenate, a drug that reduces the pathological hyperphosphorylation of tau by increasing PP2A activity, would reduce neurodegeneration, functional impairments, and post-traumatic epilepsy in a rat fluid percussion model of TBI. Methods: After a severe fluid percussion injury, or sham-injury, young-adult male Long-Evans rats were given continuous sodium selenate treatment ( $1 \mathrm{mg} / \mathrm{kg} /$ day $)$, administered via subcutaneous osmotic mini-pump, for a period of three months. Anatomical magnetic resonance imaging, diffusion weighted imaging and diffusion tensor imaging were used to assess structural damage and axonal injury one week, one month, and three months post-injury and compared to a baseline pre-injury scan. Cognitive, motor, and emotional impairments were assessed at three months post-injury. After behavioural testing rats were implanted with recording electrodes and continuously monitored for seizure activity for two weeks. Immunohistochemical and western-blot analyses were used to assess levels of PP2A, hyperphosphorylated tau, and other related pathologies.

Results: Rats treated with sodium selenate treatment had significantly reduced hyperphosphorylated tau, cortical loss on anatomical MRI, axonal injury on diffusion weighted imaging and diffusion tensor imaging (Figure 1), and behavioural impairments after a severe lateral fluid percussion injury in the rat.

Conclusions: These data indicate that sodium selenate has neuroprotective effects in a rat model of TBI, and may represent a novel approach to TBI treatment to reduce adverse neurodegenerative and neurobehavioural outcomes.

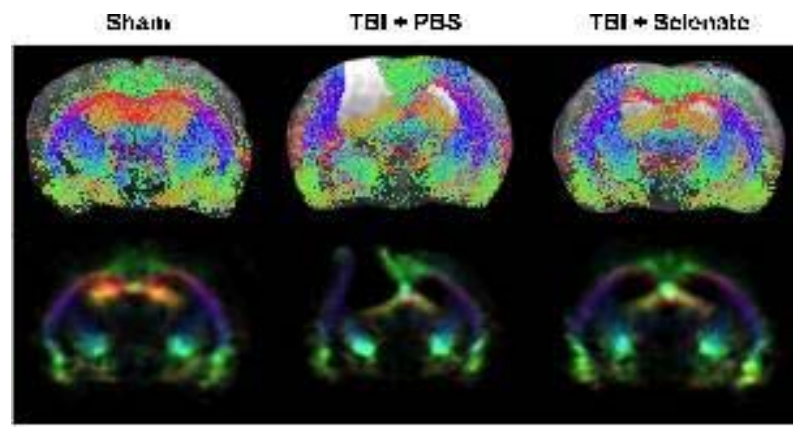

\subsection{8 \\ BIPHASIC ROLES OF INSULIN AND IGF-1 IN POST- TRAUMATIC EPILEPTOGENESIS IN ORGANOTYPIC HIPPOCAMPAL CULTURES}

Yevgeny Berdichevsky ${ }^{1,2}$, H. Mullan ${ }^{2}$, Y. Saponjian ${ }^{2}$ and K. J. Staley, ${ }^{2,3}$ ( ${ }^{1}$ ehigh University, Bethlehem, PA; ${ }^{2}$ Massachusetts General Hospital, Boston, MA and ${ }^{3}$ Harvard Medical School, Boston, MA)

Rationale: Mammalian Target of Rapamycin (mTOR) pathway is activated following trauma and during epileptogenesis, and inhibition of mTOR can reduce axonal sprouting and the number of seizures in some acquired epilepsy models. Binding of insulin and Insulin-like Growth Factor 1 (IGF-1) to their respective receptors in neurons can activate mTOR signaling through PI3K-Akt pathway in cortical impact model of brain trauma. We investigated whether insulin and IGF-1 play a role in epileptogenesis in the organotypic hippocampal culture model of post-traumatic epilepsy.

Methods: Organotypic cultures were maintained in six-well plates on a rocking platform in a $37{ }^{\circ} \mathrm{C}, 5 \% \mathrm{CO} 2$ incubator for four weeks. We maintained neurons in Neurobasal-A culture medium supplemented with bovine serum albumin (BSA), selenium, and varying 
concentrations of insulin or IGF-1. We also conducted experiments in Neurobasal-A supplemented with standard commercial culture supplement B27, with addition of different concentrations of picropodophyllotoxin (PPP), an IGF-1 receptor inhibitor. We quantified electrographic seizure activity with recordings of field potentials in CA1 and with measurements of lactate concentration in the used culture medium. Neuronal death was quantified by Nisslstaining and surviving neuron counts, and measurements of LDH release into culture medium.

Results: We found that addition of insulin or IGF-1 to culture medium was neuroprotective immediately following injury, DIV 0 3 , but caused increased spontaneous electrographic seizures and cell death between DIV 7 and DIV 28. We also found that chronic application of PPP reduced ictal-like activity and cell death between DIV 7-28.

Conclusions: Insulin and IGF-1 are neuroprotective immediately following trauma, but promote epileptogenesis in the long term. Therapy design for head trauma patients should take differential action of these hormones into account - early treatment may need to focus on neuroprotection, while later treatment can emphasize inhibition of epileptogenesis.

\subsection{9}

GENE EXPRESSION IN BRAIN AFTER ERYTHROPOETIN AND ANAKINRA TREATMENT IN A TRAUMATIC BRAIN INJURY MODEL

Gail D. Anderson ${ }^{1}$, C. Vonder Haar $^{2}$, F. M. Farin ${ }^{1}$, T. K. Bammler ${ }^{1}$, R. P. Beyer ${ }^{1}$, E. D. Kantor ${ }^{1}$ and M. R. Hoane ${ }^{2}\left({ }^{1}\right.$ University of Washington, Seattle, WA and ${ }^{2}$ Southern Illinois University, Carbondale, IL)

Rationale: Experimental studies of erythropoetin (EPO) and interleukin 1 receptor antagonist (anakinra) in models of traumatic brain injury and stroke have demonstrated a neuroprotective effect. The objective of this study was to examine the brain gene expression effects of EPO and anakinra when administered at doses selected to produce serum concentrations obtained in clinical studies in patients. Methods: 30 rats received moderate unilateral cortical contusion injury (CCI), with another 5 rats serving as intact sham controls. EPO (2500 units $/ \mathrm{kg})$, anakinra $(100 \mathrm{mg} / \mathrm{kg})$ or vehicle were administered twice daily for three days with an i.p loading dose at 2 hours post$\mathrm{CCI}$ and then s.c. doses at 12, 24, 36, 48, 60 and 72 hours post-injury. Groups of animals were euthanized at 24 hours, 72 hours and 7 days to examine gene expression in brain. RNA samples passed stringent quality control, were processed and submitted to microarray analysis using Affymetrix GeneChip Rat Gene 1.0 ST arrays. Microarray data analysis was performed with the Bioconductor packages limma and Ingenuity Pathway Analysis.

Results: The number of differentially expressed genes ( $>1.5$-fold up or down, $\mathrm{p}<0.05)$ at $24 \mathrm{hr}, 72 \mathrm{hr}$ and 7 days are given in the table 1 below. At $24 \mathrm{hrs}$ post-CCI, genes involved in the immune and inflammatory response, cell signaling, cell adhesion, metabolic processes and transporters were affected by both EPO and anakinra compared to vehicle. At $72 \mathrm{hrs}$ and 7 days post-CCI, EPO treatment, but not anakinra, altered genes involved in inflammatory and immune response. At $72 \mathrm{hrs}$ and 7 days post-CCI, EPO treatment also affected genes involved in cellular movement, cell to cell signaling and interaction and cellular growth, proliferation, function and maintenance. At $72 \mathrm{hrs}$ and 7 days post-CCI, anakinra treatment effected genes involved in carbohydrate and lipid metabolism, cell to cell signaling, molecular transport and free radical scavenging. Conclusions: EPO and anakinra treatment resulted in significant effects on brain gene expression in the CCI model of traumatic brain injury when administered at doses designed to produce serum concentrations observed clinically.

This research was supported by a grant from the NIH/NICHD (R01 HD061944-01).
Table 1: Number of differentially expressed genes ( $>1.5$ fold, $\mathrm{p}<$ $0.05)$

\begin{tabular}{|c|cc|c|c|ccc|c|c|}
\hline & & $24 \mathrm{hr}$ & & & $72 \mathrm{hr}$ & & 7 days & \\
\hline & Down & Up & Total & Down & Up & Total & Down & Up & Total \\
Vehicle/Sham & 1014 & 1457 & 2471 & 1330 & 1956 & 3286 & 318 & 1104 & 1422 \\
Г.PO/Vehiclc & 137 & 58 & 195 & 178 & 47 & 245 & 15 & 322 & 337 \\
\hline Anakinra/Vehicle & 103 & 17 & 120 & 33 & 25 & 58 & 31 & 178 & 209 \\
\hline
\end{tabular}

\subsection{0 \\ WHICH RECEPTOR SYSTEMS PLAY A ROLE IN POSTICTAL REFRACTORINESS AFTER CORTICAL EPILEPTIC AFTERDISCHARGES?}

Pavel Mares and H. Kubova (Institute of Physiology Academy of Sciences, Prague 4, Czech Republic)

Rationale: Postictal refractoriness may be taken as an expression of active mechanisms arresting seizures. This phenomenon is present not only in adult rats but also in 25-day-old animals. Therefore we started to analyze a role of neurotransmitter systems in postictal refractoriness after cortical seizures.

Methods: Cortical epileptic afterdischarges (ADs) elicited in freely moving 25-day-old rats were used as a model seizures. Stimulation of sensorimotor cortex with suprathreshold intensity elicited ADs; one minute after the end of $\mathrm{AD}$ the same stimulation was applied again. Then the drugs affecting neurotransmitter systems - inhibitory GABAergic (GABAA receptor antagonists bicuculline, picrotoxin, pentetrazol, benzodiazepine receptor inverse agonist Ro 19-4603, GABAB receptor antagonist CGP35348), adenosinergic (caffeine), opioid (mju receptor antagonist naloxone); excitatory glutamatergic (N-methyl-D-aspartate and kainate as prototypic agonists) were administered in subconvulsant doses and after ten minutes the paired stimulation was repeated.

Results: Under control conditions second stimulation 1 min after the first AD did not elicit a new AD. Antagonists of GABAA, GAGAB and adenosine receptors led to prolongation of the first AD. Drugs suppressing inhibition mediated by GABAA receptors did not affect postictal refractoriness whereas an antagonist of GABAB receptors CGP35348 and nonspecific adenosine receptor antagonist caffeine resulted in a partial block of refractoriness - second stimulation elicited AD but the duration was shorter than that of the first one. Partial block was also achieved with naloxone. On the other hand, augmentation of excitation did not lead to a block of poastictal refractoriness.

Conclusions: GABAB, adenosine and mju opioid receptors participate in postictal refractoriness after cortical ADs whereas the role of GABAA and ionotropic glutamate receptors is negligible. Refractoriness after cortical epileptic afterdischarges thus differ from that after hippocampal afterdischarges which may be abolished by a block of mju opioid receptors with naloxone.

This study was supported by a collaborative US-Czech grant

No.LH11015 and by research projects AV0Z50110509 and RVO:67985823.

\subsection{1 \\ BRAIN ALKALOSIS AND CONSEQUENT SEIZURES AFTER NEONATAL ASPHYXIA ARE CAUSED BY ACID EFFLUX ACROSS THE BLOOD-BRAIN BARRIER \\ Mohamed M. Helmy ${ }^{1,2}$, P. V. Watkins ${ }^{3}$, E. Ruusuvuori ${ }^{1,2}$, J. Voipio ${ }^{1}$, P. O. Kanold ${ }^{3,4}$ and K. Kaila ${ }^{1,2}\left({ }^{1}\right.$ Department of Biosciences, University of Helsinki, Helsinki, Finland; ${ }^{2}$ Neuroscience Center, University of Helsinki, Helsinki, Finland; ${ }^{3}$ Department of Biology, University of Maryland, College Park, MD and ${ }^{4}$ Institute for Systems Research, University of Maryland, College Park, MD)}

Rationale: Seizures are common in newborns and frequently occur 
after birth asphyxia. Increased mortality and poor outcome have been associated with a higher seizure burden. Addressing neonatal seizures is a priority, but current drugs are largely ineffective. The molecular and cellular mechanisms underlying birth-asphyxia seizures are unknown, but it is obvious that information on the seizure-triggering mechanisms is of crucial importance in the design of novel therapeutic strategies. Using 6 day-old rat pups, we have recently shown that the post-asphyxia seizure burden is linked to an increase in brain extracellular $\mathrm{pH}\left(\mathrm{pH}_{\mathrm{o}}\right)$ that consists of the recovery from the asphyxia-induced acidosis followed by a subsequent overshoot well above (0.3 pH units) normal baseline level (Helmy et al., 2011).

Methods: Our model of birth asphyxia is based on exposing neonatal rat pups to hypoxia $\left(9 \% \mathrm{O}_{2}\right)$ and hypercapnia $\left(20 \% \mathrm{CO}_{2}\right)$ for one hour. Recently (Helmy et al., 2011) we showed that the large postasphyxia seizure burden is abolished by a graded restoration of normocapnia, which is achieved by gradually reducing $\mathrm{CO}_{2}$ levels in inhaled air first to $10 \%$ for $30 \mathrm{~min}$, and then to $5 \%$ for a further 30 min. In the present study, we used 2-photon in vivo imaging to measure intraneuronal $\mathrm{pH}\left(\mathrm{pH}_{\mathrm{i}}\right)$ and $\mathrm{pH}$-sensitive electrodes to monitor $\mathrm{pH}_{\mathrm{o}}$ in both brain and body during and after asphyxia. $\mathrm{N}$ methyl-isobutyl-amiloride (MIA), a blocker of $\mathrm{Na} / \mathrm{H}$ exchange, was administered. Blood-brain barrier (BBB) permeability was assessed by sodium fluorescein.

Results: In neocortical neurons in vivo, $\mathrm{pH}_{\mathrm{i}}$ showed a biphasic acidalkaline response resulting in an alkaline plateau level about $0.3 \mathrm{pH}$ units above baseline. The alkaline overshoot of $\mathrm{pH}_{\mathrm{i}}$ was strongly suppressed by graded restoration of normocapnia. Electrode recordings of $\mathrm{pH}$ in the brain and body demonstrated net efflux of acid equivalents from the brain across the $\mathrm{BBB}$, which was abolished by MIA, or its parent molecule amiloride. Inhibiting $\mathrm{Na} / \mathrm{H}$ exchange also suppressed the seizures. No increase in BBB permeability was detected by sodium fluorescein extravasation into the brain or in the electrophysiology experiments.

Conclusions: The present work shows that post-asphyxia alkalosis in the brain and the consequent seizures are caused by a $\mathrm{Na} / \mathrm{H}$ exchange-dependent efflux of acid equivalents across the BBB. Our results raise the important and worrying question whether standard resuscitation paradigms where normocapnic conditions are established in a fast manner will, in fact, promote birth-asphyxia seizures. Graded restoration of normocapnia and/or drugs targeting the $\mathrm{Na} / \mathrm{H}$ exchange in the $\mathrm{BBB}$ offer an effective and straightforward means to functionally suppress seizures and ameliorate other neurological sequelae after birth asphyxia, and can be readily used in conjunction with other treatment modalities, such as hypothermia and optimization of blood oxygenation levels.

\section{References:}

Helmy MM, Tolner EA, Vanhatalo S, Voipio J, Kaila K. Brain alkalosis causes birth asphyxia seizures, suggesting therapeutic strategy. Ann Neurol 2011; 69: 493-500.

\subsection{2}

\section{NETWORK ACTIVITY OF CULTURED HIPPOCAMPAL NEURONS IS REDUCED FOLLOWING CHRONIC INCUBATION WITH BETA-HYDROXYBUTYRATE} C. Drummond-Main ${ }^{3,4}$, W. Afflick ${ }^{3}$, A. Farran ${ }^{3}$, L. Scott ${ }^{1}$, F. Girotto ${ }^{2}$, M. Scantlebury ${ }^{3,4}$, D. Kim ${ }^{5}$, M. Colicos ${ }^{1}$ and Jong M. Rho ${ }^{3,4}$ ( ${ }^{1}$ Physiology and Pharmacology, University of Calgary, Calgary, AB, Canada; ${ }^{2}$ Physics and Astronomy, University of Calgary, Calgary, AB, Canada; ${ }^{3}$ Pediatrics, Alberta Children's Hospital, Calgary, AB, Canada; ${ }^{4}$ Clinical Neurosciences, University of Calgary, Calgary, $\mathrm{AB}$, Canada and ${ }^{5}$ Neurology Research, Barrow Neurological Institute, Phoenix, AZ)

Rationale: Dietary interventions such as the ketogenic diet (KD) have been proven to be effective in treating medically refractory epilepsy. However, the mechanisms underlying KD action remain unclear. Earlier studies have demonstrated that acetoacetate [ACA] and acetone are anticonvulsant in several in vivo models of induced seizures, but there is yet no in vitro evidence that ketone bodies can acutely influence neuronal hyperexcitability and/or synchrony [PMID: 10668691]. Whether beta-hydroxybutyrate (BHB) is anticonvulsant remains unclear as well.

Methods: A novel photoconductive-stimulation (PCS) platform [PMID: 17406269] was used to study the chronic network effects of BHB in rat primary hippocampal cultures. PCS allows for the visualization and controlled - yet non-invasive stimulation - of a single neuron or hundreds of neurons in culture. This is accomplished by growing neuronal cultures on silicon chips and applying a beam of light to target cell(s) of interest while applying a voltage bias across the silicon wafer to induce a current, resulting in highly focused stimulation with minimal physiological manipulation. Two-week-old cultures were subsequently incubated for one week with either standard media or BHB at various concentrations $(2 \mathrm{mM}, 5 \mathrm{mM}$, and $10 \mathrm{mM}$ ), then exposed to GFP-Fluor-4 which enabled real-time visualization of intracellular calcium levels associated with neuronal membrane firing. Video data were analyzed using custom software (MatLab) and excitation of the entire field (typically 500-1000 neurons) was quantified. Firing rates were calculated as the number of firing events of the neurons per minute.

Results: We found that higher concentrations of BHB (5 \& $10 \mathrm{mM}$; $\mathrm{N}=11$ \& 12 , respectively) did not alter the average firing rate of the hippocampal neurons, whereas with $2 \mathrm{mM} \mathrm{BHB}$, there was a significant $(37 \%, \mathrm{p}<0.05)$ decrease compared to controls. The ratio of firing in the control vs. the $2 \mathrm{mM}$ cultures jumped from a baseline of 1 to $7.56, p=0.02$ (a higher ratio correlates with a decrease in firing events per minute). Interestingly, $2 \mathrm{mM}$ BHB incubation also resulted in a 5-fold reduction in the number of active cells before PCS compared to during the stimulation period $(60 \mathrm{~Hz}$ at $5 \mathrm{~V}$, for $30 \mathrm{sec})$; $\mathrm{N}=37 ; \mathrm{p}<0.05)$.

Conclusions: Our results indicate that chronic incubation of hippocampal cultures with BHB at a clinically relevant concentration leads to a significant reduction in the overall activity and excitability of neurons - effects that may contribute to an anticonvulsant effect in vivo, and may help explain in part the anticonvulsant mechanism of the KD. Our findings also suggest that a more rigorous examination of the molecular targets of BHB (and perhaps other ketone bodies) may provide novel avenues for the development of investigational compounds to treat medically refractory epilepsy.

\subsection{3}

BLOCKING MECHANISM OF THE AMPA RECEPTOR ANTAGONIST PERAMPANEL

Michael A. Rogawski ${ }^{1}$, C. Y. Chen ${ }^{2}$, L. Matt ${ }^{2}$ and J. W. Hell ${ }^{2}$

$\left({ }^{1}\right.$ Neurology, University of California, Davis, Sacramento, CA and

${ }^{2}$ Pharmacology, University of California, Davis, Davis, CA)

Rationale: Perampanel, a structurally novel pyridone, is a potent, orally active AMPA receptor antagonist currently under development for the treatment of epilepsy. Perampanel inhibits AMPA-induced calcium influx into cultured rat cortical neurons in a concentrationdependent fashion with IC50 of $93 \mathrm{nM}$ (Hanada T, et al., Epilepsia 2011;52:1331-1340). Perampanel caused complex effects on the concentration-response curve for AMPA in this assay so that its precise mode of inhibition was not defined. In the present study, we sought to characterize the mechanism of block through whole cell voltage-clamp recordings in cultured hippocampal neurons expressing native AMPA receptors.

Methods: Cultures were obtained from E19 rat embryos and recordings were carried out at 10-25 days in culture. Agonists with and without perampanel were applied using a fast perfusion system at various concentrations [kainate $(3,10$, and $100 \mu \mathrm{M})$, AMPA $(10,30$, and $100 \mu \mathrm{M})$ and NMDA (10 and $100 \mathrm{nM})]$.

Results: Perampanel caused a concentration-dependent inhibition of inward AMPA receptor currents evoked by kainate and AMPA that was unaffected by agonist concentration, indicating a noncompetitive blocking mechanism. The mean IC50 value at all kainate 
concentrations was $692 \pm 94 \mathrm{nM}$. At a concentration of $30 \mu \mathrm{M}$ that completely abolished AMPA-evoked response, perampanel had no effect on NMDA-evoked responses. Perampanel inhibited AMPAevoked responses to a similar extent regardless of the order of application (perampanel first or AMPA first). In addition, perampanel reduced the peak and steady state AMPA responses to the same extent. These observations indicate that perampanel binds with similar affinity to the open and closed states of AMPA receptors. Furthermore, perampanel had no effect on the decay time course of AMPA-evoked currents, indicating that it does not affect AMPA receptor desensitization.

Conclusions: The results demonstrate that perampanel is a selective noncompetitive AMPA receptor antagonist that does not affect NMDA receptors. The drug inhibits AMPA receptors without influencing channel kinetics.

\subsection{4 \\ SUBUNIT-SPECIFIC DEPHOSPHORYLATION OF THE PUTATIVE AP2 BINDING SITES OF THE GABA RECEPTOR DURING LITHIUM PILOCARPINED-INDUCED STATUS EPILEPTICUS}

S. Joshi, S. J. Chester, K. M. Hawk, K. Rajasekara and Howard Goodkin (University of Virginia, Charlottesville, VA)

Rationale: The activity-dependent, subunit-dependent trafficking of $\mathrm{GABA}_{\mathrm{A}}$ receptors (GABARs) is one potential mechanism to explain both the reduction in GABA-mediated inhibition and the development of benzodiazepine pharmacoresistance that occur during the prolonged seizures of status epilepticus (SE). The internalization of these receptors occurs via a clathrin-mediated process following the binding of the clathrin adaptor AP2. The intracellular domains of the GABAR $\beta$ and $\gamma$ subunits contain putative patch-binding motifs for AP2 that are accessible upon the dephosphorylation of these subunits. Prior studies have demonstrated that a decrease in the phosphorylation of the $\beta 3$ subunit occurs during SE. Whether the decrease in phosphorylation of this subunit occurs as the result of a site-selective process or whether a decrease in the phosphorylation of both sites is required for GABAR endocytosis during SE is not known. The objective of this study was to evaluate phosphorylation state of these AP2 binding sites during SE.

Methods: SE was induced in postnatal (P) 23-25 SD rats (SE-treated) either by a combination of lithium and pilocarpine (LiPilo) or kainic acid (KA). Surface expression of the $\gamma 2$ subunit of the GABAR was determined in whole hippocampal slices from SE-treated and naïve control animals using a biotinylation pull-down assay.

Phosphorylation of the $\gamma 2$ and $\beta 3$ subunits of the GABAR was determined by standard Western blotting techniques.

Results: GABAR surface expression: We confirmed our prior observation of SE model-dependent changes in the surface expression of GABARs during SE: the surface expression of the $\gamma 2$ subunit was $59 \pm 7 \%$ compared to controls $(n=5, p<0.05)$ after $1 \mathrm{hr}$ of LiPiloinduced SE and unchanged $(83 \pm 18 \%)$ compared to controls after 3 hours of KA-induced SE. The surface expression of the $\gamma 2$ subunit was also unchanged $(116 \pm 33 \%)$ compared to controls in animals in which LiPilo-induced SE was prevented by the administration of 10 $\mathrm{mg} / \mathrm{kg}$ diazepam prior to the injection of pilocarpine demonstrating that the reduced surface expression of the $\gamma 2$ subunit in this model is not a direct pharmacological effect of these agents. Changes in Phosphorylation after SE: The level of phosphorylated $\gamma 2$ subunit was unchanged $(153 \pm 24 \%, n=8 ; p>0.05)$ and phosphorylated $\beta 3$ subunit was reduced $(42 \pm 16 \%, \mathrm{n}=5 ; \mathrm{p}<0.05)$ compared to controls. Consistent with the absence of a change in the surface expression of GABARs during KA-induced SE, the level of phosphorylation of the $\gamma 2$ subunit $(115 \pm 22 \%, \mathrm{n}=5, \mathrm{p}>0.05)$ and $\beta 3$ subunit $(84 \pm 21 \%, \mathrm{n}=5$; $\mathrm{p}>0.05$ ) were similar to controls.

Conclusions: These findings suggest that the decrease in the phosphorylation of the $\beta 3$ subunit that occurs during LiPilo-induced $\mathrm{SE}$ is the result of a site-selective mechanism. Future experiments will be performed to understand this site-selective process and why it does not occur during KA-induced SE of up to 3 hours in duration.

\subsection{5 \\ HYPOTHERMIA REDUCES CALCIUM ENTRY THROUGH N-METHYL-D-ASPARTATE AND RYANODINE RECEPTOR ACTIVATED SYSTEMS IN CULTURED HIPPOCAMPAL NEURONS}

K. Phillips, Laxmikant Deshpande and R. DeLorenzo (Virginia

Commonwealth University, Richmond, VA)

Rationale: Hypothermia is neuroprotective when induced following cardiac arrest, stroke, and traumatic brain injury. The physiological effects of hypothermia are multifaceted and therefore a better knowledge of its therapeutic targets will be central to developing innovative combination therapies to augment the protective benefits of hypothermia. Development of a calcium plateau following status epilepticus has been implicated in expression of acquired epilepsy and cognitive deficits. This study was therefore initiated to evaluate the effect of hypothermia on calcium plateau. The contribution of various modes of calcium entry into a neuron following hypothermia was also evaluated.

Methods: Status epilepticus was induced into hippocampal neuronal cultures by exposing them to a solution containing no added $\mathrm{MgCl} 2$. Fluorescent calcium indicator Fura-2AM was used to compare calcium ratio under normothermic $(37 \mathrm{oC})$ and hypothermic $(31 \mathrm{oC})$ conditions. We also utilized pharmacological agents to stimulate major routes of calcium entry in hippocampal neurons.

Results: The results of this study indicate that hypothermia blocks the development of calcium plateau following status epilepticus. Hypothermia preferentially reduces calcium entry through N-methyl$\mathrm{D}$-aspartate receptors and ryanodine receptors. Hypothermia, on the other hand, did not have a significant effect on calcium entry through the voltage-dependent calcium channels or the inositol tri-phosphate receptors.

Conclusions: Altered neuronal calcium dynamics have been implicated following stroke, status epilepticus and traumatic brain injury. The ability of hypothermia to block the calcium plateau which is shown to be mediated by both N-methyl-D-aspartate receptors and ryanodine receptors-mediated calcium systems makes it an attractive pharmacological target for alleviating calcium elevations that are present following many neurological injuries.

Translational Research: Models

\subsection{6 \\ MODELING THE EFFECTIVE CONNECTIVITY OF THE VISUAL NETWORK IN HEALTHY AND PHOTOSENSITIVE, EPILEPTIC BABOONS \\ Shalini Narayana ${ }^{1}, \mathrm{~K} . \mathrm{Li}^{1}$, A. R. Laird ${ }^{1}$, C. Franklin ${ }^{1}$, F. S. Salinas ${ }^{1}$, M. M. Leland ${ }^{3}$, P. T. Fox ${ }^{1,4}$ and C. A. Szabó ${ }^{2}\left({ }^{1}\right.$ Research Imaging Institute, University of Texas Health Science Center at San Antonio, San Antonio, TX; ${ }^{2}$ South Texas Comprehensive Epilepsy Center, University of Texas Health Science Center at San Antonio, San Antonio, TX; ${ }^{3}$ laboratory Animal Research, University of Texas Health Science Center at San Antonio, San Antonio, TX and ${ }^{4}$ South Texas Veterans Administration Medical Center, San Antonio, TX)}

Rationale: The neural mechanisms of altered brain connectivity in idiopathic generalized epilepsy (IGE) remain poorly understood. A promising neuroimaging-based animal model of IGE is provided by photosensitive baboons, whose seizures can be activated by intermittent light stimulation (ILS). In this study, we compared cerebral blood flow (CBF) responses during ILS in photosensitive, epileptic (PS) and healthy control (CTL) baboons using H215O-PET. Methods: Eight photosensitive (PS) and six control (CTL) baboons matched for age, gender and weight, were classified on the basis of 
scalp EEG findings performed during the neuroimaging studies. Continuous intravenous ketamine $(5-13 \mathrm{mg} / \mathrm{kg})$ was used for sedation and the baboons were monitored for the level of sedation and epileptic discharges using scalp EEG. Five H215O-PET studies were acquired alternating between resting and activation (ILS at $25 \mathrm{~Hz}$ ) scans. PET images were acquired in 3D mode (CTI/Siemens HR+ scanner, 63 contiguous slices, $2.4 \mathrm{~mm}$ thick) and co-registered with MRI (3T Siemens Trio, T1-weighted 3D Turboflash sequence, $\mathrm{TE} / \mathrm{TR} / \mathrm{TI}=3.04 / 2100 / 785 \mathrm{msec}$, flip angle $=13$ degrees $)$. We examined the effective connectivity associated with visual stimulation in both groups using structural equation modeling (SEM), a novel, iterative model-generating procedure.

Results: Because of the differences in group size and connectivity, eleven regions of interest (ROIs) were identified in the PS baboons (Figure 2) compared to eight in the CTL baboons (Figure 1). SEM demonstrated similarities in neural connectivity of the primary visual pathways (green) between PS and CTL animals. However, second order pathways (red) diverged between the two models, with increased interhemispheric connectivity demonstrated in the PS animals. Furthermore, while the connectivity maps in the CTL animals appeared stable, third (blue) and fourth (yellow) order connections were apparent in the PS baboons, many of which were reciprocal. Even after four iterations, latent connectivity to regions outside of the PS model remained high.

Conclusions: Overall connectivity of visual pathways during ILS was different between PS and CTL baboons at the level of second order pathways. The diminished stability of the PS model compared to the CTL group may be attributed to the influence of latent connection not included in this model, as well as to the presence of reciprocating interhemispheric pathways, which may represent bidirectional feedback loops associated with the generation of generalized epileptic discharges. Evaluation of further baboons will help to expand the PS model to include further ROIs commonly connected to the epileptic networks, such as the thalamus, insula or SMA.

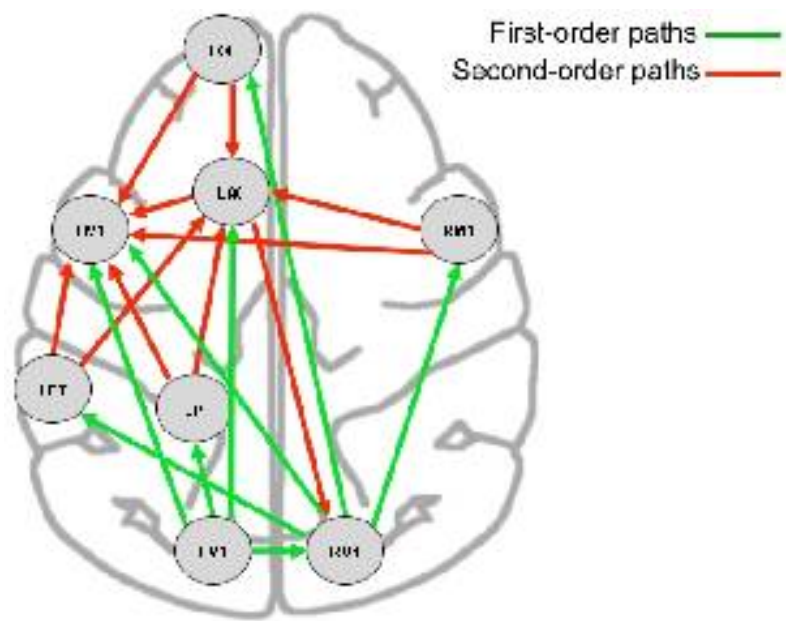

Figure 1: Final model of effective connectivity in the visual network of CTL baboons. Exploratory analysis of 8 ROIs using SEM revealed a complex network of visual connectivity that fit the data extremely well $(\chi 2=2.731, \mathrm{df}=10, \mathrm{P}=0.987$, TLI $=1.205$, GFI $=0.921$, RMSEA $=0.0000)$. Green arrows=first-order paths from visual cortices; red arrows=second-order paths. R Right, L Left, M1 primary motor, OF medial orbitofrontal, $\mathrm{PT}$ parietotemporal, $\mathrm{AC}$ anterior cingulate, $\mathrm{V} 1$ primary visual, $\mathrm{P}$ parietal

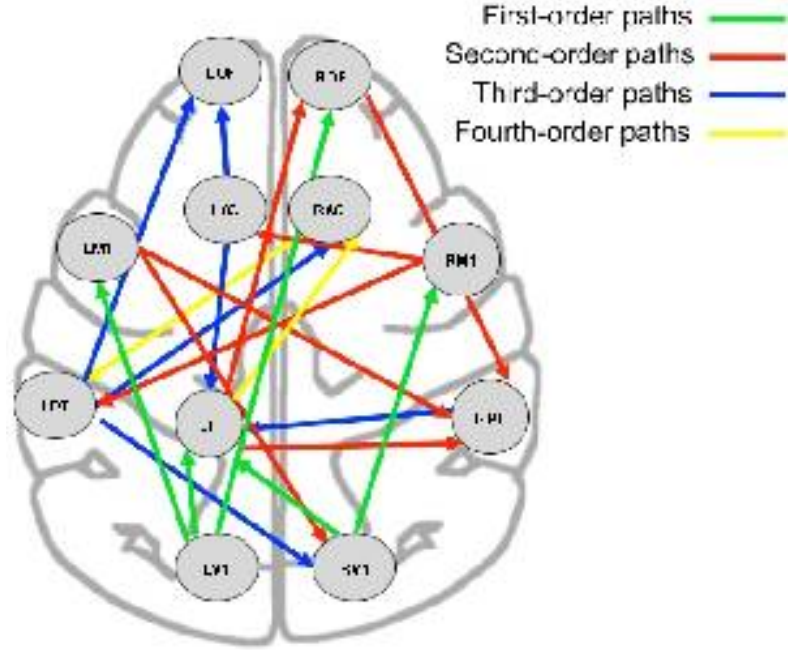

Figure 2: Final model of effective connectivity in the visual network of PS baboons. Exploratory analysis of 11 ROIs using SEM revealed a complex network of visual connectivity that fit the data extremely well $(\chi 2=23.659, \mathrm{df}=35, \mathrm{P}=0.927$, TLI $=1.385$, GFI $=0.774$, RMSEA $=0.000-0.064)$. Green arrows $=$ first-level paths from visual cortices; red arrows $=$ second-level paths; blue arrows $=$ third-level paths; and yellow arrows=fourth-level paths. For abbreviations, see legend of Figure 1.

\subsection{7 \\ BABOON MODEL OF GENERALIZED EPILEPSY: CONTINUOUS INTRACRANIAL VIDEO-EEG MONITORING WITH SUBDURAL ELECTRODES} Charles A. Szabo ${ }^{1}$, F. S. Salinas ${ }^{2}$, M. M. Leland ${ }^{3}$, J. L. Caron ${ }^{4}$, M. A. Hanes $^{3}$, K. D. Knape ${ }^{1}$, D. Xie ${ }^{5}$ and J. T. Williams ${ }^{6,7}$ ( ${ }^{1}$ South Texas Comprehensive Epilepsy Center, UTHSCSA, San Antonio, TX; ${ }^{2}$ Research Imaging Institute, UTHSCSA, San Antonio, TX; ${ }^{3}$ Laboratory Animal Research, UTHSCSA, San Antonio, TX; ${ }^{4}$ Department of Neurosurgery, UTHSCSA, San Antonio, TX; ${ }^{5}$ Department of Gynecology and Obstetrics, UTHSCSA, San Antonio, TX; ${ }^{6}$ SNPRC, TBRI, San Antonio, TX and ${ }^{7}$ Department of Genetics, TBRI, San Antonio, TX)

Rationale: The baboon provides a natural non-human primate model for photosensitive, idiopathic generalized epilepsy. This study describes an implantation procedure for the placement of subdural grid and strip electrodes for continuous video-EEG monitoring in the epileptic baboon to evaluate the generation and propagation of ictal and interictal epileptic discharges.

Methods: Subdural grid, strip and depth electrodes were implanted in six epileptic baboons and one asymptomatic animal (Table 1), targeting brain regions that were activated in functional neuroimaging studies during photoparoxysmal responses (Figure 1). Three epileptic baboons were photosensitive by previous scalp EEG studies. The asymptomatic baboon has a normal scalp EEG prior to implantation. The baboons were monitored with continuous video-EEG monitoring for 2-21 (mean 9) days. Although the animals were tethered, the EEG signal was transmitted wirelessly to optimize their mobility. Spontaneous seizures, interictal epileptic discharges (IEDs), and responses to intermittent light stimulation (ILS) were assessed. Because of cortical injuries related to the electrode implantation and their displacement in earlier studies, the procedure was modified. Histopathological examination of the three last animals monitored (11 to 21 days) determined only to reactive inflammatory changes of the meninges.

Results: Habitual myoclonic and generalized tonic-clonic seizures were recorded in four baboons. In three baboons the ictal discharge was triggered multiregionally, usually either by a discharge in the frontal, parietal or occipital cortices. In one baboon, myoclonic seizures were associated with a generalized ictal discharge. IEDs 
were recorded in all of the epileptic baboons, and similar to the ictal discharges, they were expressed multiregionally (Figure 1) and responsible for triggering most generalized spike-and-wave discharges. Generalized interictal epileptic discharges were suppressed in one baboon with a focal cortical injury due to the subdural electrodes. Generalized photoparoxysmal responses were activated only in one of three photosensitive baboons, while driving responses recorded over the occipital convexity in all three were 2.5 times the stimulus rate.

Conclusions: In contrast to previous intracranial investigations in this model, generalized ictal and interictal epileptic discharges were triggered not only by frontal, but also parietal and occipital cortices. Furthermore, targeted visual areas responded differently to ILS in photosensitive than nonphotosensitive baboons, but further studies are required before mechanisms can be implicated for ILS-induced activation of the epileptic networks.

Table 1. Demographics and Overview of Scalp/Invasive EEG Studies

\begin{tabular}{|c|c|c|c|c|c|c|c|c|c|c|c|c|}
\hline \multicolumn{4}{|c|}{$\begin{array}{l}\text { Demograplics at } \\
\text { Implantation }\end{array}$} & \multicolumn{4}{|c|}{$\begin{array}{c}\text { Results of Previous Scalp } \\
\text { EEG }\end{array}$} & \multicolumn{5}{|c|}{ Results of Invasive Video-EEG } \\
\hline Baboon & $\Lambda \mathrm{ge}$ & $\begin{array}{l}\text { Wcight } \\
(\mathrm{kg})\end{array}$ & Sex & IEDs & Seizures & Driving & PS & Days & MiDs & Seizures & Driving & PS \\
\hline $\mathrm{B} 1$ & 10 & 19 & $\mathrm{~F}$ & $N$ & $\mathrm{~N}$ & $\mathrm{~N}$ & $\mathrm{~N}$ & 7 & $\mathrm{~N}$ & $\mathrm{~N}$ & Y & $\mathrm{N}$ \\
\hline $\mathrm{B} 2$ & 7 & 27 & M & $\mathrm{Y}$ & Y & Y & $\mathrm{Y}$ & 4 & Y & $Y$ (atyp) & $\underset{(2.5 \mathrm{x})}{\mathrm{Y}}$ & $\mathrm{N}$ \\
\hline B3 & 8 & 20 & $\mathrm{~F}$ & $\mathrm{Y}$ & $\mathrm{Y}$ & Y & $\mathrm{N}$ & 3 & $\mathrm{Y}$ & $\mathrm{Y}$ & NA & $\mathrm{N} \Lambda$ \\
\hline $\mathrm{B} 4$ & 10 & 23 & M & $\mathrm{Y}$ & $\mathrm{Y}$ & Y & $\mathrm{Y}$ & 2 & $\mathrm{Y}$ & $\mathrm{N}$ & $\begin{array}{c}Y \\
(2.5 x)\end{array}$ & $\mathrm{Y}$ \\
\hline B5 & 4 & 12 & $\mathrm{~F}$ & $\mathrm{Y}$ & $\mathrm{Y}$ & Y & $\mathrm{N}$ & 14 & $\mathrm{Y}$ & $\mathrm{Y}$ & $\mathrm{Y}$ & $\mathrm{N}$ \\
\hline B6 & 7 & 15 & $\Gamma$ & $\mathrm{Y}$ & $\mathrm{Y}$ & $\mathrm{Y}$ & $\mathrm{Y}$ & 21 & $\mathrm{Y}$ & $\mathrm{Y}$ & $Y(2.5 x)$ & $\mathrm{N}$ \\
\hline B7 & 11 & 29 & M & $\mathrm{Y}$ & $\mathrm{Y}$ & $Y$ & $\mathrm{~N}$ & 11 & $\mathrm{Y}$ & $\mathrm{Y}$ & $\mathrm{Y}$ & $\mathrm{N}$ \\
\hline
\end{tabular}

IEDs interictal epileptic discharges, F(emale), M(ale), N(o), Y(es), atyp(ical), PS photosensitivity, 2.5x (2.5 times the stimulus rate)

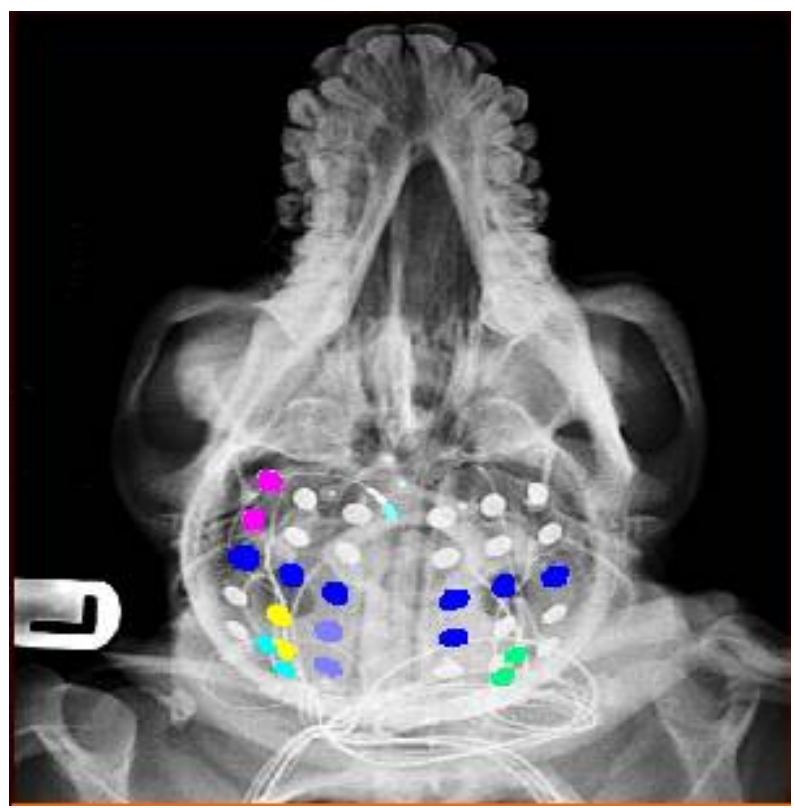

Figure 1. Distribution of IEDS (each color represents regional IED population) in B6

\subsection{8}

EFFECTIVENESS OF SSRIS OR A 5-HT 7 AGONIST ON PREVENTION OF SEIZURE-INDUCED SUDDEN DEATH IN DBA/1 MICE

Carl L. Faingold, X. Long and M. Randall (Southern Illinois University, Springfield, IL)
Rationale: The DBA/1 mouse model of sudden unexpected death in epilepsy (SUDEP) exhibits chronic susceptibility to seizure-induced cardio-respiratory death (S-ICRD). The only effective preventative therapy for sudden death in DBA/1 mice is fluoxetine, a selective serotonin (5-HT) re-uptake inhibitor (SSRI), which blocks death without blocking seizures or reducing seizure severity (Faingold et al., Epilepsy Behav., 2011). SSRIs have been shown retrospectively to reduce respiratory depression in certain types of epilepsy patients (Bateman et al., Epilepsia, 2010). Several SSRIs are currently available, but it is not clear if all of these agents are effective in blocking S-ICRD and, if so, whether there is a selectivity of effects on S-ICRD vs. anticonvulsant effects in DBA/1 mice. The present study evaluated the effects of two other SSRIs, sertraline and fluvoxamine, as well as a selective $5 \mathrm{HT}_{7}$ agonist (AS-19) to determine if these agents are effective and/or selective in blocking SICRD since $5-\mathrm{HT}_{7}$ receptors are involved in spinal control of respiration.

Methods: DBA/1 mice (8-22g, 24-65 days old) were evaluated for susceptibility to audiogenic S-ICRD, using an electrical bell (122 dB SPL). Behaviors were recorded on video, and seizure parameters were quantified visually off-line. Mice that exhibited S-ICRD were resuscitated using a rodent respirator. At least $24 \mathrm{hr}$ later mice exhibiting seizure-induced S-ICRD were given sertraline (4-80 $\mathrm{mg} / \mathrm{kg}$, i.p.), fluvoxamine (30-80 mg/kg) or AS-19 (10-60 mg/kg) and tested for S-ICRD after drug.

Results: S-ICRD was significantly reduced $(p<0.05$, Wilcoxon signed rank test) by sertraline $30 \mathrm{~min}$ after administration $(>40$ $\mathrm{mg} / \mathrm{kg}$ ), but the mice remained susceptible to audiogenic seizure at the same time. Fluvoxamine also significantly reduced S-ICRD ( $p$ $<0.05), 30 \mathrm{~min}$ after the drug $(>60 \mathrm{mg} / \mathrm{kg})$, and the mice remained susceptible to seizures at the same time. Return to S-ICRD was observed $24 \mathrm{hr}$ after drug with each SSRI. Although seizure susceptibility remained after fluvoxamine and sertraline, seizure severity was reduced, as indicated by the significantly reduced incidence of tonic seizures $(\mathrm{p}<0.05)$ at the doses that blocked SICRD. This lack of selectivity for blocking S-ICRD contrasts with relative selectivity of fluoxetine, which significantly reduced S-ICRD at doses that did not significantly reduce tonic seizures. The $5-\mathrm{HT}_{7}$ agonist was not effective in blocking S-ICRD in DBA/1 mice, and, in higher doses, was toxic and pro-convulsant.

Conclusions: Since the 5- $\mathrm{HT}_{7}$ agonist was ineffective in blocking SICRD, this suggests that this 5-HT receptor subtype and actions at the spinal level do not contribute importantly to the ability of SSRIs to block S-ICRD. The relative selectivity of fluoxetine to block S-ICRD without affecting seizure severity contrasts with the effects of sertraline and fluvoxamine and may have clinical implications for the potential prevention of SUDEP in patients that should be evaluated. (Support: EAM at SIUSM and Citizens United to Cure Epilepsy)

\subsection{9}

REVERSAL OF STRESS-INDUCED SEIZURE SEVERITY ENHANCEMENT BY GLUCOCORTICOID RECEPTORS ANTAGONISM IN A GENETIC MODEL OF AUDIOGENIC EPILEPSY

Eduardo H. Umeoka ${ }^{1}$ and N. Garcia-Cairasco ${ }^{2}\left({ }^{1}\right.$ Neuroscience and Behavioral Sciences, Ribeirao Preto School of Medicine - University of São Paulo, Ribeirão Preto, Brazil and ${ }^{2}$ Physiology, Ribeirao Preto School of Medicine - University of São Paulo, Ribeirão Preto, Brazil)

Rationale: Wistar Audiogenic Rats (WARs) develop tonic-clonic seizures (SZ) in response to acute stimulus and limbic SZ, known as Audiogenic Kindling (AK), after repeated acoustic stimuli. In addition, WARs are anxious and have a hyperactive hypothalamicpituitary-adrenal axis. Indeed, when compared to Wistars, WARs have dysplastic adrenal gland, elevated corticosterone release after ACTH injection and increased ACTH release after restraint stress (Umeoka et al., Brain Res. 2011). The aim of this study was to 
evaluate the role of glucocorticoid receptors on stress modulation of seizure susceptibility in WARs.

Methods: Male WARs $(\mathrm{n}=47)$ were submitted to AK (acoustic stimuli/twice a day/10 days). The rats were divided according to the treatment that they received before each acoustic stimulation. The control (CTR, $n=17)$ group was only submitted to AK; the stress (STR, n=7) group had 60 minutes of restraint stress before the AK. Antagonist-treated groups were injected with the mineralocorticoid receptor (MR) antagonist spironolactone $(50 \mathrm{mg} / \mathrm{kg})(\mathrm{SPI}, \mathrm{n}=6)$ or glucocorticoid receptor (GR) antagonist mifepristone $(25 \mathrm{mg} / \mathrm{kg})$ (MIF, $n=7)$ or both (SPI/MIF, $n=10)$. All drugs were diluted in propylene glycol and injected 30 minutes before the stress $(90$ minutes before acoustic stimulation). For each SZ it was attributed a severity index for mesencephalic (MSI) and limbic (LSI) SZ. Latencies for wild running (WR) and tonic convulsion (CVT) were also calculated. The MSI and the LSI were determined according to Rossetti et al., 2006 (Epilepsy Res.) and Pinel and Rovner, 1978 (Exp. Neurol.), respectively. ANOVA and Bonferroni post-tests were applied with GrahPad Prism 5. The local Ethics Committee approved this project $(012 / 2011)$.

Results: When submitted to AK CTR group developed SZ with higher MSI observed during the initial stimuli and a decrement with subsequent stimuli. In contrast, no limbic SZ were observed at the beginning of AK, but the LSI increased during its progression, in accordance with previous results from our group (Garcia-Cairasco et al., Epilepsy Res. 1996). The STR group had severe SZs from the beginning until the end of the AK and did not show the MSI decrement as observed in CTRL group. SPI, MIF and SPI/MIF groups presented a significant $(\mathrm{p}<0.05)$ reversal of the stress effect on MSI, albeit without difference among them. We did not find any significant alteration on the LSI in any of the groups. The latency for wild running $(\mathrm{WR})$ was increased $(\mathrm{p}<0.05)$ only in the SPI/MIF group. Latency for tonic convulsion (TCV) was shorter in STR group when compared with CTR, and longer in MIF and SPI/MIF groups $(p<0.05)$ when compared with and STR group and even longer than CTR groups $(\mathrm{p}<0.05)$.

Conclusions: Blockade of GR and MR, either alone or in combination, was able to revert the stress modulatory effect on the MSI. GR and MR antagonism also increased the latencies for WR and TCV, showing the crucial role of the glucocorticoids on SZ susceptibility modulation. Acknowledgments: FAPESP, FAPESPCinapce, PROEX-CAPES, CNPq, FAEPA.

\subsection{0 \\ VIDEO-EEG REVEALS SUBCLINICAL ISCHEMIC SEIZURES AND LIMITED EFFICACY OF PHENOBARBITAL IN NEONATAL MICE}

Shilpa D. Kadam ${ }^{1,2}$, S. K. Kang ${ }^{1}$, S. K. Kim ${ }^{1}$ and M. V. Johnston ${ }^{1,2}$ $\left({ }^{1}\right.$ Neuroscience, Hugo W. Moser Research Institute, Baltimore, MD and ${ }^{2}$ Neurology, Johns Hopkins University School of Medicine, Baltimore, MD)

Rationale: Ischemia in the immature brain is an important cause of neonatal seizures. The exact timing of neonatal stroke onset is usually unclear and the diagnosis delayed until presentation with seizures a few to several hours later. Optimal seizure treatment in neonates remains uncharacterized and the widely used drug phenobarbital has limited clinical efficacy. Developmental profiles of chloride cotransporter expression and function in immature brains have been proposed to dictate the age dependent anti-seizure efficacy of GABAagonists and this switch is known to occur in P8-9 mouse pups. Methods: To investigate the anti-seizure efficacy of the first line anticonvulsant and GABAA-agonist phenobarbital (PB - 25mg/kg) and NKCC1 antagonist bumetanide (BTN -0.1 to $0.2 \mathrm{mg} / \mathrm{kg}$ ) as adjunct treatment on neonatal ischemic-seizures, we utilized synchronous video-EEG and unilateral carotid-ligation to produce acute ischemic-seizures in postnatal day 7 and $10 \mathrm{CD} 1$ mice. Quantitative analyses of pre- and post-treatment EEGs were done using Pinnacle (Pinnacle technology Inc., KS) seizure scoring and Insight software (Persyst development corp., AZ) for evaluation of seizure grades (clinical vs. subclinical or electrographic) and treatment efficacy.

Results: Severity of acute ischemic seizures and incidence of subclinical seizures was higher at P7 and a gender-specific susceptibility was detected in males that correlated with a lag in KCC2 expression levels in naïve males compared to age-matched females, not detected at P10. However, the P7 brains were comparatively more resistant to infarcts unlike at P10 when they occurred frequently. PB showed a better efficacy at P10. BTN treatment $1 \mathrm{~h}$ following PB however failed to act as an efficacious adjunct therapy at the given dose and aggravated subclinical seizures in P10 females. At P7, both PB and add-on BTN treatment failed to significantly alleviate seizures. EEG power $(0.5-32 \mathrm{~Hz})$ was lowered after PB treatment at both ages. Post-ischemic down-regulation of $\mathrm{KCC} 2$ expression was detected at both ages tested compared to agematched naïve brains.

Conclusions: This study showed that 1. Quantification of subclinical seizures help better evaluate anti-seizure efficacy of treatments and 2.The down-regulation of KCC2 in ischemia may further decrease the efficacy of drug actions that are dependent on a hyperpolarizing chloride gradient for their anticonvulsant action. This decrease in efficacy failed to be rescued by blocking the immature chloride cotransporter NKCC1.

\subsection{1 \\ MECP2 PHOSPHORYLATION AT SER421 CAN BE MEDIATED VIA SEIZURE-INDUCED ACTIVATION OF CA2 + - PERMEABLE AMPA RECEPTORS IN DEVELOPING BRAIN \\ Sanjay Rakhade ${ }^{1}$, E. Rosenberg ${ }^{1}$, j. Lippman-Bell ${ }^{1}$, C. Hilario- Gomez ${ }^{1}$ and F. E. Jensen ${ }^{1,2}\left({ }^{1}\right.$ Neurology, Boston Children's Hospital and Harvard Medical School, Boston, MA and ${ }^{2}$ Program in Neuroscience, Harvard Medical School, Boston, MA)}

Rationale: Neonatal seizures (NS) are the most common type of seizures during childhood and contribute to severe long-term effects including epilepsy, cognitive impairment and behavioral deficits. Some of these phenotypic features are shared with autism spectrum disorders (ASD) which also have epileptic seizures as a comorbid condition. Genetic mutations involved in ASD have been previously implicated to be playing important role in synaptogenesis and learning. We hypothesize that in early life, seizures activate signaling pathways like MeCP2, mutations in which cause Rett's syndrome, and these pathways may provide a convergence point for comorbidities observed in early life epilepsy and ASD. Given the critical role that calcium-permeable AMPARs play in epileptogenesis, we specifically hypothesized that activate $\mathrm{CP}$ AMPARs could alter MeCP2 expression

Methods: Primary neuronal cultures generated from cortical and hippocampal tissue from E18 Long Evans rats were treated with kainate and $\mathrm{KCl}$ at $10 \mathrm{DIV}$, to induce epileptiform activity. The cultures were treated with antagonists of NMDA, AMPA, VGCCs and controls; protein lysate was prepared from cultures. P10 Long Evans rats were subjected to hypoxic induced NS and allowed to survive for varying time following NS. Cortical tissue was collected from rat pups subjected to NS, and their littermate controls at 1, 3, 6,12, 24 and 48 hours; membrane protein samples were isolated and electrophoretically separated. Expression of phosphorylated CaMKII Thr 286, total CaMKII, phosphorylated MeCP2 S 421 and Actin was analyzed using immunoblotting techniques.

Results: We observed a significant increase in CaMKII phosphorylation at Thr286 and MeCP2 phosphorylation at S421 following $\mathrm{KA}$ and $\mathrm{KCl}$-induced depolarization in cortical and hippocampal neurons. Treatment with NMDAR antagonists alone did not significantly attenuate KA-induced phosphorylation of CaMKII Thr $286(86 \pm 9 \%, \mathrm{n}=7, \mathrm{p}>0.05)$ and MeCP2 S421 $(89 \pm 13 \%, \mathrm{n}=8$, 
$\mathrm{p}>0.05$ ) in hippocampal cultures. However combination of NMDA and AMPAR antagonists significantly reduced the phosphorylation of CaMKII Thr286 $(20 \pm 3 \%, \mathrm{n}=7, \mathrm{p}<0.001)$ and MeCP2 S421 $(3 \pm 5 \%$, $\mathrm{n}=8, \mathrm{p}<0.001$ ). A significant increase in phosphorylation of CaMKII Thr 286 at 1 hour following induction of NS in P10 Long Evans rat pups $(185 \pm 24 \%, \mathrm{n}=15, \mathrm{p}<0.01)$ compared to littermate normoxic controls was observed. Similarly phosphorylation of MeCP2 S421 was significantly increased $(148 \pm 3 \%, \mathrm{n}=12, \mathrm{p}<0.001)$ at 3 hours following hypoxia induced NS. Treatment with NBQX suggested a trend towards attenuation of enhanced phosphorylation of MeCP2. Conclusions: Our data suggests that $\mathrm{Ca} 2+$ permeable AMPARs, in addition to NMDARs, play an important role in mediating seizureinduced phosphorylation of CaMKII Thr 286 and its downstream target MeCP2 S421 in the critical period of development.

Furthermore hypoxia induced NS in P10 Long Evans rats lead to early induction of CaMKII and $\mathrm{MeCP} 2$ phosphorylation, and the increase in activation of this signaling cascade may be attenuated following treatment with AMPAR antagonists.

\subsection{2 \\ WHOLE-CELL BRAIN TISSUE IMPEDANCE CHANGES AS A FUNCTION OF STATE-OF-VIGILANCE}

Madineh Sedigh-Sarvestani ${ }^{1}$, G. I. Thuku ${ }^{1}$, S. L. Weinstein ${ }^{2}$ and B. J. Gluckman ${ }^{1}$ ( ${ }^{1}$ Penn State University, University Park, PA and ${ }^{2}$ Children's National Medical Center, George Washington University, washington, DC)

Rationale: There is a focused effort on the clinical implementation of cortical implants for prediction and control of seizures. Tissue impedance, as a measure of glial swelling, has been reported as a potential feature for seizure prediction (Olsson 2006, Hochman 2012). It also mediates most other electrical measures used to quantify neural activity in chronic recordings. Furthermore, state of vigilance (SOV) is a confounding factor in seizure prediction and detection efforts (Mormann 2007). We therefore undertook to monitor tissue impedance in long-term recordings in epileptic rats and analyze its variations in time and with both SOV and seizure dynamics.

Methods: We implanted Long-Evans rats with cortical screw and hippocampal depth recording electrodes along with a pair of $250 \mu \mathrm{m}$ stainless steel stimulation electrodes. The stimulation electrode was implanted in the arc of CA3 and the current return electrode was placed $2 \mathrm{~mm}$ anterior outside the hippocampus. A single dose of tetanus toxin was injected into the ventral hippocampus to induce chronic spontaneous recurrent seizures over months. We used a current-controlled stimulator to apply polarizing low-frequency electrical field (PLEF) stimulation every $15 \pm 5$ seconds with 2 second sinusoidal packets of frequencies below $20 \mathrm{~Hz}$ and peak current of 30 $\mu$ A. Stimulation was applied for at least 24 hours in $n=3$ animals. Packet impedance was fit with a polynomial spline to reduce noise and provide a time-continuous impedance series. State of vigilance was scored and seizures detected using previously established algorithms (Sunderam 2007).

Results: In all animals, impedance rises during non-rapid-eye movement sleep (NREM), and falls during wake. The distribution of impedance slopes during NREM is significantly different than the distribution during wake, as determined by a two-sample

Kolmogorov-Smirnov test. There is a small decrease in impedance during rapid-eye-movement sleep. In all animals, impedance falls during and after generalized seizures. In one animal, in addition to changes in impedance associated with SOV, we also observed diurnal trends, with impedance lower during the subjective day (lights-off) period of the animal and higher during the subjective night (lights-on) period. Further analysis is required to determine the independence of diurnal and SOV effects.

Conclusions: We observe that the whole-cell brain impedance changes significantly over time, and has distinct variations as a function of, and on the time-scale of, sleep-wake cycles. These impedance changes should be accounted for both if impedance is used for seizure prediction as well as in the design and implementation of brain stimulation. Impedance changes may also impact other brain state measures especially used for seizure prediction. Further investigation into SOV related changes in impedance may help to shed light on the observed relationship between sleep and seizure states.

Olsson T. et al. Neuroscience 140:505-515, 2006.

Hochman D.W. Epilepsia, 53(Suppl. 1):18-25, 2012.

Mormann F et al. Brain 130(Pt 2): 314-33, 2007.

Sunderam S et al. J Neurosci Meth (163):373, 2007.

\subsection{3 \\ ANTICONVULSANT ACTIVITY OF INTRAVENOUS AND INTRAMUSCULAR ALLOPREGNENALONE}

Dorota Zolkowska $^{1}$, A. Dhir ${ }^{1}$, G. R. Cooke ${ }^{1}$, C. Wu ${ }^{1}$, L. Zhu ${ }^{2}$, H. Wulff $^{2}$ and M. A. Rogawski ${ }^{1}$ ( ${ }^{1}$ Department of Neurology, School of Medicine, University of California, Davis, Sacramento, CA and ${ }^{2}$ Department of Pharmacology, School of Medicine, University of California, Davis, Davis, CA)

Rationale: Treatment of status epilepicus requires rapid administration of antiseizure agents, which are typically delivered either by the intravenous (i.v.) or intramuscular (i.m.) routes. Allopregnanolone ( $3 \alpha$-hydroxy-5 $\alpha$-pregnan-20-one; $5 \alpha, 3 \alpha-\mathrm{P}$ ), an endogenous progesterone-derived steroid that is a positive allosteric modulator of GABAA receptors, is a powerful antiseizure agent with potential in the treatment of status epilepticus. The objective of this study was to determine the dosing of allopregnanolone to protect against seizures when delivered i.v. and i.m.

Methods: The mouse $6 \mathrm{~Hz}$ and pentylenetetrazol seizure models were used. Solutions of $5 \alpha, 3 \alpha-\mathrm{P}$ were made in $6 \%(0.5$ and 1.5 $\mathrm{mg} / \mathrm{ml}$ ) sulfobutylether- $\beta$-cyclodextrin sodium salt (Captisol $\AA$ ) in $0.9 \%$ saline. The solutions were injected i.v. or i.m. (1, 2 and $30 \mathrm{~min}$ or 2 and $30 \mathrm{~min}$, respectively) prior to administration of the $6 \mathrm{~Hz}$ electrical stimulus or PTZ ( $80 \mathrm{mg} / \mathrm{kg}$, i.p.). In case of the PTZ model, animals were observed for $30 \mathrm{~min}$ and times to myoclonic jerks and clonic and tonic seizures were recorded. Anticonvulsant activity was assessed by the delay in onset of seizure signs. Allopregnanolone plasma levels in rats were determined by LC-MS.

Results: $5 \alpha, 3 \alpha-\mathrm{P}$ exhibited protective activity in the $6 \mathrm{~Hz}$ test $1-15$ $\mathrm{min}$ after i.v. infusion $(1.5 \mathrm{mg} / \mathrm{kg})$ but was inactive at $30 \mathrm{~min}$. In contrast, with i.m. administration $(3 \mathrm{mg} / \mathrm{kg}$ ) the onset of protective activity was slower (within $2 \mathrm{~min}$ ) and lasted $<2 \mathrm{~h}$. At a dose of 0.1 $\mathrm{mg} / \mathrm{kg}$ i.v. $5 \alpha, 3 \alpha-\mathrm{P}$ failed to significantly delay seizure onset in the PTZ model at all pretreatment times (1,2 and $30 \mathrm{~min})$ whereas a dose of $0.5 \mathrm{mg} / \mathrm{kg}$ administered $1 \mathrm{~min}$ before PTZ caused a marked delay for myoclonic jerks and clonic seizures and in $62.5 \%$ of animals prevented tonic seizures and mortality that invariably accompanies tonic seizures. When injected $2 \mathrm{~min}$ before PTZ $5 \alpha, 3 \alpha-\mathrm{P}(0.5 \mathrm{mg} / \mathrm{kg})$ caused a similar increase in time to onset of seizures signs and prevented tonic seizures in $25 \%$ of animals. $5 \alpha, 3 \alpha-\mathrm{P}$ at a dose of 1.5 $\mathrm{mg} / \mathrm{kg}$ completely prevented tonic seizures and mortality when injected i.v. 1 and $2 \mathrm{~min}$ before PTZ. When injected i.m. $2 \mathrm{~min}$ before PTZ, $0.25,0.5$ and $1.5 \mathrm{mg} / \mathrm{kg} 5 \alpha, 3 \alpha-\mathrm{P}$ protected $0 \%, 50 \%$ and $100 \%$, respectively, of animals from tonic seizures. $5 \alpha, 3 \alpha-\mathrm{P}$ at the dose of $1.5 \mathrm{mg} / \mathrm{kg}$ i.m. provided significant protection against tonic seizures when injected $30 \mathrm{~min}$ before PTZ; the same dose injected i.v. $30 \mathrm{~min}$ before PTZ was inactive. In rats, an i.v. bolus dose of 0.5 and 1.0 $\mathrm{mg} / \mathrm{kg} 5 \alpha, 3 \alpha-\mathrm{P}$ caused mean peak plasma levels ( $2 \mathrm{~min}$ ) of 337 and $746 \mathrm{ng} / \mathrm{ml}$, respectively; for both doses, the pooled mean two component half-times were 2 and $22 \mathrm{~min}$.

Conclusions: Our results demonstrate that i.v. $5 \alpha, 3 \alpha-\mathrm{P}$ provides very rapid but transitory anticonvulsant activity. When injected i.m., $5 \alpha, 3 \alpha-\mathrm{P}$ acts comparably quickly and has a longer duration of action. Parenteral $5 \alpha, 3 \alpha-\mathrm{P}$ may be useful for the acute treatment of seizures. 


\subsection{4}

DECREASED SUBCORTICAL AROUSAL IN LIMBIC SEIZURES: BRAINSTEM CHOLINERGIC AND THALAMIC INHIBITION DURING CORTICAL SLOW OSCILLATIONS Joshua E. Motelow ${ }^{1}$, A. Gummadavelli ${ }^{1}$, V. Chu ${ }^{1}$, A. M. Mishra ${ }^{1}$, R. N. Sachdev ${ }^{4}$, B. G. Sanganahalli ${ }^{2}$, M. Furman ${ }^{1}$, D. Englot ${ }^{5}$, F. Hyder ${ }^{2}$ and H. Blumenfeld ${ }^{1,3}$ ( ${ }^{1}$ Neurology, Yale School of Medicine, New Haven, CT; ${ }^{2}$ Diagnostic radiology, Yale School of Medicine, New Haven, CT; ${ }^{3}$ Neurosurgery, Yale School of Medicine, New Haven, CT; ${ }^{4}$ Neurobiology, Yale School of Medicine, New Haven, CT and ${ }^{5}$ Neurosurgery, University of California, San Francisco, San Francisco, CA)

Rationale: Complex partial seizures originating in the limbic system can impair consciousness even when seizure activity does not propagate to neocortex. During these seizures, high-frequency, polyspike seizure activity dominates the temporal lobe while normal neocortical EEG is replaced with low frequency oscillations similar to those seen in non-REM sleep. We hypothesized that the transition to sleep-like cortical slow waves is due to depressed activity in subcortical arousal systems and tested this hypothesis in a rat limbic seizure model.

Methods: As described previously, limbic seizures were induced by a 2-second, $60 \mathrm{~Hz}$ electrical stimulus in the dorsal hippocampus. To map areas of increased and decreased neuronal activity we recorded blood oxygen level dependent (BOLD) fMRI data at 9.4 T. To investigate the neuronal changes underlying the BOLD signal changes, we conducted multiunit recordings from the hypothalamus, thalamus and brainstem. Within the brainstem, we recorded and labeled individual cells in the pedunculopontine tegmental nucleus using the juxtacellular method. We identified cholinergic cells using immunohistochemistry for choline acetyltransferase.

Results: During BOLD experiments, hippocampal, septal and hypothalamic BOLD increases were associated with widespread cortical, thalamic, and brainstem BOLD decreases. Multiunit electrophysiology recordings confirmed that BOLD increases represent increased neuronal activity in hypothalamus while BOLD decreases represent decreased neuronal activity. Multiunit recordings in thalamus showed decreased firing in intralaminar thalamus and spindle-like activity in thalamic relay nuclei. Our juxtacellular recordings in the pedunculopontine tegmental nucleus showed that cholinergic neurons decreased their firing dramatically during seizures during which the cortex converted to slow oscillations. Conclusions: These direct recordings in brainstem and thalamus suggest a mechanism to explain loss of consciousness during complex partial seizures: (1) seizure activity spreads from hippocampal to inhibitory structures including the lateral septal nuclei and hypothalamus, (2) increased activity in GABAergic neurons in lateral septum and anterior hypothalamus depress subcortical arousal systems, and (3) suppression of ascending arousal systems including the brainstem cholinergic nuclei leads to hyperpolarized thalamic nuclei and cortical rhythms normally present during non-REM sleep.

\subsection{5 \\ DISTRIBUTION AND FUNCTIONAL EFFECTS OF TETANUS TOXIN IN A CHRONIC MODEL OF TEMPORAL LOBE EPILEPSY \\ John G. Jefferys ${ }^{1}$, P. Jiruska ${ }^{2,1}$, L. Foss ${ }^{1}$, A. D. Powell ${ }^{1}$, W. C. Chang ${ }^{1}$, A. Ferecsko ${ }^{1}$ and A. Sik ${ }^{1}{ }^{1}$ Neuronal Networks Group, Clinical and Experimental Medicine, University of Birmingham, Birmingham, United Kingdom and ${ }^{2}$ Department of Developmental Epileptology, Institute of Physiology, Academy of Sciences of Czech Republic, Prague, Czech Republic)}

Rationale: Intrahippocampal injection of tetanus neurotoxin (TeNT) induces a chronic non-lesional model of temporal lobe epilepsy characterized by spontaneous seizures. TeNT is a protease which cleaves vesicle associated membrane protein (VAMP). A key question for this model is the spatial and temporal distribution of the effects of the toxin and how it relates to the development of the epileptic syndrome.

Methods: One microliter of TeNT or vehicle was stereotaxically injected into the right hippocampal CA3 region of Sprague-Dawley rats. Spontaneous recurrent seizures appeared after 1-2 weeks. At 8 days after injection, rats were anaesthetized, intracardially perfused with chilled protective saline and hippocampal slices prepared for in vitro recording. Whole-cell patch clamp recordings were made from pyramidal cells in CA1c (within the area exposed directly to TeNT) and CA1a (far from the injection) and from the same regions in control rats. Cells were labelled with neurobiotin and after the recording slices were fixed for subsequent immunohistochemistry (VAMP1 and VAMP2). VAMP positive terminals were counted, including around the labelled cells.

Results: VAMP1 was preferentially expressed in synaptic terminals of interneurons, while VAMP2 was expressed mainly in excitatory terminals of principal cells. The density of VAMP1 positive terminals was significantly lower in CA1c of TeNT injected rats $\left(20 \pm 3 / 100 \mu \mathrm{m}^{2}\right)$ than in controls $\left(36 \pm 2 / 100 \mu \mathrm{m}^{2}\right)$. The density of VAMP2 terminals was also lower $\left(18 \pm 5 / 100 \mu \mathrm{m}^{2}\right)$ than in controls $\left(43 \pm 3 / 100 \mu \mathrm{m}^{2}\right)$. In CA3 VAMP1 and VAMP 2 were lower in TeNT rats both ipsilateral (VAMP1: $19.8 \pm 2.6$ from $35.9 \pm 1.7 / 100 \mu \mathrm{m}^{2}$; VAMP2: $17.6 \pm 5.1$ from $43.3 \pm 2.6 / 100 \mu \mathrm{m}^{2}$ ) and, to a lesser degree, contralateral to the injection (VAMP1: $30.0 \pm 4.0$ from $37.4 \pm 1.9$ $/ 100 \mu \mathrm{m}^{2}$; VAMP2: $28.0 \pm 2.2$ from $44.8 \pm 1.7 / 100 \mu \mathrm{m}^{2}$ ).

Functionally the cleavage of VAMPs in ipsilateral CA1c was associated with decreases in frequency of spontaneous excitatory postsynaptic currents (EPSCs; $0.8 \pm 0.3 \mathrm{~Hz}$, controls $2.2 \pm 0.5 \mathrm{~Hz}$ ) and reduced maximal amplitude of evoked EPSCs (129 $\pm 23 \mathrm{pA}$, controls $594 \pm 171 \mathrm{pA})$. CA1a, far from the injection, and contralateral CA1a and $\mathrm{c}$ showed no significant changes in spontaneous or evoked EPSCs. Evoked and spontaneous inhibitory postsynaptic potentials were absent in CA1c of TeNT animals (control maximal evoked IPSCs $392.8 \pm 83.7 \mathrm{pA}$; control spontaneous IPSC frequency $5.4 \pm 1.4$ $\mathrm{Hz}$ ). Spontaneous IPSCs in CAla were reduced to $3.4 \pm 0.9 \mathrm{~Hz}$ from $6.8 \pm 1.8 \mathrm{~Hz}$. There were no changes in contralateral CA1.

Conclusions: This study demonstrates that TeNT affects both excitatory and inhibitory transmission. The impact of TeNT is stronger on inhibition, shifting the excitation-inhibition balance towards the excitation and providing plausible explanation for development of epilepsy and seizures. The localization of the loss of VAMP, to ipsilateral CA1 a and bilateral CA3 provides a plausible basis for the pathophysiology of the early stages of this model. Supported by Epilepsy Research UK and MRC

\subsection{6 PERSISTENT CARDIAC ALTERATIONS OCCUR EARLY IN A MODEL OF ACQUIRED EPILEPSY \\ Yi-Chen Lai ${ }^{1,3}$ and A. E. Anderson ${ }^{2,3}$ ( ${ }^{1}$ Pediatrics, Baylor College of Medicine, Houston, TX; ${ }^{2}$ Neurology, Baylor College of Medicine, Houston, TX and ${ }^{3}$ Cain Foundation Laboratories, Jan and Dan Duncan Neurological Research Institute, Houston, TX)}

Rationale: Clinical observations and experimental data suggest that cardiac alterations occur in epilepsy. Studies have shown that epileptic individuals exhibit altered autonomic nervous system input to the heart and intrinsic electrical properties of the heart. These changes are exacerbated during seizures, reflected in the presence of ictal and peri-ictal arrhythmias. Therefore, individuals with drugresistant epilepsy may have progressive cardiac changes resulting in propensity for fatal arrhythmias. However, little is known regarding the onset and the progression of cardiac manifestation in epilepsy. Animal data suggest that cardiac changes occur early following pilocarpine-induced status epilepticus (SE). However, whether these changes persist during early epileptogenesis remains undefined. Therefore we prospectively investigated cardiac changes in a cohort 
of epileptic and age-matched sham animals using the SE model of acquired epilepsy.

Methods: Juvenile male Sprague-Dawley rats (150-200g) underwent $1 \mathrm{~h}$ of SE by intraperitoneal kainate (16mg/kg, i.p.). At 2 and $4 \mathrm{wks}$ following SE, animals were sedated using

ketamine/xylazine/acepromazine i.p. and single channel EKG recordings were obtained. Heart rate (HR), PR, QRS and QTc intervals were manually calculated. Descriptive statistics and Student t-test were performed for each variable. Two-way ANOVA was performed to evaluate HR, PR, QRS and QTc changes over time in the sham and the SE groups. Values were expressed as mean \pm SEM. Results: At 2wks following SE, compared with the age-matched sham animals, the SE group exhibited elevated HR (281 \pm 6 vs. $311 \pm 10$ bpm, sham vs. SE, $n=10-14$ /group, $p<0.05)$, increased QRS ( $59 \pm 4$ vs. $80 \pm 5 \mathrm{msec}$, sham vs. SE, $\mathrm{n}=10-14 /$ group, $\mathrm{p}<0.01)$ and QTc ( $244 \pm 12$ vs. $307 \pm 12 \mathrm{msec}$, sham vs. $\mathrm{SE}, \mathrm{n}=10$-14/group, $\mathrm{p}<0.01$ ). At 4wks following SE, both sham and SE animals exhibited age-related decreases in HR. Additionally, the SE animals exhibited some normalization of QRS and QTc intervals. However, compared with the sham animals, the SE group still exhibited higher HR ( $259 \pm 4$ vs. $279 \pm 3$ bpm, sham vs. SE, $n=10-13$ /group, $p<0.001$ ), longer QRS ( $53 \pm 4$ vs. $69 \pm 5 \mathrm{msec}$, sham vs. $\mathrm{SE}, \mathrm{n}=10-13$ /group, $\mathrm{p}<0.05)$ and QTc ( $234 \pm 7$ vs. $297 \pm 28 \mathrm{msec}$, sham vs. $\mathrm{SE}, \mathrm{n}=10-13$ /group, $\mathrm{p}<0.01$ ). Conclusions: Our findings suggest that SE induces early cardiac changes. These alterations persist in the early epileptogenesis; raising the possibility that cardiac remodeling may occur concurrently with the development of epilepsy in this model. Studies are ongoing to examine whether the appearance of recurrent seizures later in life will exaggerate the cardiac phenotype in the epileptic animals.

\section{$\mathbf{1 . 0 2 7}$}

\section{SUBTLE SEIZURES AFTER TBI IN A UNIQUE STRAIN OF KINDLING-SUSCEPTIBLE RATS: ELECTROGRAPHIC, BEHAVIORAL, AND PHENOTYPIC FEATURES}

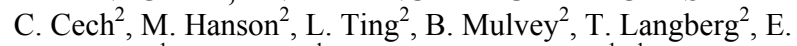
Hutchinson ${ }^{1}$, P. Rutecki ${ }^{1}$ and Thomas P. Sutula ${ }^{1}$ ( Neurology, University of Wisconsin, Madison, WI and ${ }^{2}$ University of Wisconsin, Madison)

Rationale: Kindling is implicated in adverse long-term consequences of epilepsy and has been proposed as an activity-dependent process that may contribute to delayed emergence of post-traumatic epilepsy (PTE) after traumatic brain injury (TBI). This study aimed to determine if unique strains of kindling-susceptible and kindlingresistant rats were differentially susceptible to development of seizures after TBI, and to define phenotypic differences between these strains pertinent to epileptogenesis.

Methods: Sprague Dawley (SD) rats with "fast" and "slow" rates of perforant path kindling defined as $<10$ ADs vs. $>20$ ADs to the first secondary generalized tonic-clonic (Class V) seizure were bred for 13 generations to produce strains of "fast" perforant path kindlingsusceptible (PPKS) and "slow" perforant path kindling-resistant (PPKR) rats. TBI was induced under isoflurane anesthesia by controlled cortical impact (CCI) over temporo-parietal cortex in PPKS rats $(n=9)$, PPKR rats $(n=12)$. Uninjured or "sham" injured controls without CCI served as controls ( $\mathrm{SD}, \mathrm{n}=16$; PPKS, $\mathrm{n}=12$; PPKR, n=14). Stainless steel screw epidural electrodes were placed over each hemisphere for video-EEG recording. Additional groups of PPKS, PPKR, and SD rats were also examined for differential susceptibility to seizures evoked by pentylenetetrzol (PTZ) $50 \mathrm{mg} / \mathrm{kg}$ IP.

Results: Video-EEG monitoring performed for a total of $\sim 30 \mathrm{hrs}$ in each rat for a period of $\sim 4$ mo revealed subtle recurring spontaneous electrographic and behavioral seizures consisting of trains of spike discharges increasing in frequency and amplitude with durations varying from 1-2 sec to as long as $\sim 15 \mathrm{sec}$, accompanied by behavioral arrest or "freezing" resembling Class I limbic seizures which terminated with cessation of the spike discharge. These subtle seizures were observed in 5 of 9 PPKS rats but in only 1 of 12 PPKR rats (chi square, $\mathrm{p}=0.017$ ). Generalized tonic-clonic (Class V) seizures were not observed. Similar subtle seizures were occasionally observed in uninjured controls ( 1 of 12 PPKS, 1 of 14 PPKR, 3 of 16 $\mathrm{SD})$. PPKS and PPKR rats also differed in response to PTZ, with longer seizure duration in PKKS rats compared to PPKR rats $(50 \pm 10 \mathrm{~s}$ vs. $29 \pm 4 \mathrm{~s}, \mathrm{p}=0.025$, ANOVA). PPKS rats also demonstrated slower postictal return to normal exploratory behavior $(589 \pm 64 \mathrm{~s}$ vs. $371 \pm 54 \mathrm{~s}, \mathrm{p}<0.05$, ANOVA).

Conclusions: Subtle behavioral seizures consisting of "freezing" or behavioral arrest accompanied generalized spike discharges following CCI-induced TBI in a unique strain of kindling-susceptible rats. The results identify a subtle seizure type manifesting as an outcome of TBI in this strain, suggesting that emergence of posttraumatic epilepsy is influenced by genetic back ground. Kindlingsusceptible and kindling-resistant rats may be useful for investigation of mechanisms and genetic background underlying long-term consequences of TBI including PTE and PTSD, and as a screening model for identifying therapies to prevent post-traumatic epileptogenesis.

\subsection{8 \\ NEUROPLASTIC ALTERATIONS AFTER STATUS EPILEPTICUS IN TWO EXPERIMENTAL MODELS OF TEMPORAL LOBE EPILEPSY}

Olagide W. Castro $^{1}$, E. Buriticá ${ }^{2}$, V. R. Santos ${ }^{1}$, S. S. Marroni ${ }^{1}$, M. I. Scobar $^{2}$, H. J. Pimienta ${ }^{2}$ and N. Garcia-Cairasco ${ }^{1}\left({ }^{1}\right.$ Physiology, University of São Paulo, Ribeirão Preto, Brazil and ${ }^{2}$ Centro de Estudios Cerebrales, Universidad del Valle, Cali, Colombia)

Rationale: Temporal Lobe Epilepsy (TLE) is the most common epileptic syndrome in humans. Status Epilepticus (SE), one of the primary events leading to TLE, induces neuronal loss and neurogenesis. Several studies in TLE detected alterations of subpopulations of GABAergic interneurons that express calciumbinding proteins (CaBPs). Therefore, in the present study we evaluated the expression of $\mathrm{CaBPs}$, in two experimental models of TLE induced by pilocarpine (PILO).

Methods: Adult male Wistar rats (250-300 g; Control, n=16; PILO, $\mathrm{n}=25$ ) were submitted to systemic (S-PILO) and intrahippocampal (H-PILO) (1 day of survival). Fluoro-Jade+ $(\mathrm{FJ}+)$, Caspase 3+ neurons, in H-PILO (1, 7 and 15 days of survival) were counted. SPILO (320 mg/kg; $2 \mathrm{ml} / \mathrm{kg}$, i.p); H-PILO (2.4 mg/ $\mu \mathrm{L}$ [CaBPs] $/ 1.2$ $\mathrm{mg} / \mu \mathrm{L})$ Parvalbumin $+(\mathrm{PV}+) /$ Calretinin $+(\mathrm{CR}+)$ neurons were counted in the dentate gyrus (DG), Ammon's Horn subfields (CA1, $\mathrm{CA} 2$ and CA3) and in cerebral cortex, doublecortin $+(\mathrm{DCX}+)$ neurogenesis was evaluated 15 days after SE.

Results: In the S-PILO model PV+ neurons increased in CA1 $(\mathrm{p}<0.05), \mathrm{CA} 3(\mathrm{p}<0.01)$ and cortical layers V-VI $(\mathrm{p}<0.01)$. In the HPILO model we observed significant $\mathrm{PV}+$ expression in GD $(p<0.05), C A 2(p<0.01), C A 3(p<0.01)$ and in cortical layers V-VI $(p<0.01)$. In the H-PILO model we found no changes in $\mathrm{CR}+$ cells, while in the S-PILO model, we found increases in GD ( $<<0.01)$, CA1 $(p<0.05), C A 3(p<0.01)$ and cortical subregions II-III $(p<0.05)$ and $\mathrm{V}$-VI $(\mathrm{p}<0.005)$. FJ + neurons were increased in hilus/CA3 $(\mathrm{P}<0,05) 1$ day after $\mathrm{SE}$ when compared to other time windows and in CA1 $(p<0.01) 7$ days after SE when compared with other areas. Caspase $3+$ cells increased in hilus $(p<0.0001)$ when compared with controls. DCX+ cells increased in GD 15 days after $\mathrm{SE}(\mathrm{p}<0.0001)$ when compared to controls.

Conclusions: These data indicate that in these two experimental models of TLE specific hippocampal and cortical regions are the most affected in terms of neurodegeneration/ neurogenesis processes, coupled to a differential spatial response of subpopulations of $\mathrm{PV}+$ and $\mathrm{CR}+$ interneurons.

Support: FAPESP, FAPESP-Cinapce, CNPq-PROSUL, CAPES and FAEPA. 
1.029

CHRONIC VALPROIC ACID ADMINISTRATION IMPACTS NEUROGENESIS AND WEIGHT GAIN AFTER NEONATAL STROKE

S. George ${ }^{1}$, S. Raja ${ }^{1}$, S. Yu ${ }^{1}$, A. Kwan ${ }^{1}$, d. Boothe ${ }^{3}$ and Anne Comi ${ }^{1,2}$ ( ${ }^{1}$ Neurology and Developmental Medicine, Kennedy Krieger Institute, Baltimore, MD; ${ }^{2}$ Neurology, Johns Hopkins School of Medicine, Baltimore, MD and ${ }^{3}$ Anatomy, Physiology and Pharmacology, Auburn University, Auburn, AL)

Rationale: Stroke in the immature brain presents with seizures which are treated with anticonvulsants. Post-stroke neurogenesis in the SGZ of the immature brain is decreased and may contribute to long-term cognitive impairments. Histone deacetylase (HDAC) inhibitors prevent HDAC from blocking DNA transcription and replication. Valproic acid (VPA) is a HDAC inhibitor, but also enhances GABA neurotransmission and blocks sodium channels. We aimed to determine the impact of chronic HDAC inhibition on post-stroke recovery in the immature brain.

Methods: P12 CD1 mice received right common carotid artery ligation or sham surgery and acute seizures were scored. Mice received either VPA $(200 \mathrm{mg} / \mathrm{kg})$ or vehicle twice daily from postnatal day (P)16 to P28. 5-bromo-2'-deoxyuridine (BrdU; $50 \mathrm{mg} / \mathrm{kg}$ ) was injected five times between P24 and P26 prior to sacrifice on P42. Percent of brain atrophy was quantified and immunohistochemistry was performed for Brdu/NeuN colabeling in the SGZ. In a subset of mice, serum was collected at P26 and analyzed for concentrations of VPA.

Results: Mortality was increased, but not significantly in VPAtreated ligated mice (6/21 mice; $28.5 \%$ compared to $2 / 20 ; 10.0 \%$ in saline-treated mice). VPA-treated mice exhibited significantly lower body weights than saline-treated mice (Repeated measures ANOVA, $\mathrm{p}=0.03$ injured, $\mathrm{p}=0.04$ uninjured, and $\mathrm{p}=0.003$ non-ligated controls). This difference was apparent from P17, the day after VPA was first administered, to P28 but disappeared by P42, 2 weeks after drug administration ceased.

Hemispheric and hippocampal atrophy was not significantly different in the VPA-compared to vehicle- treated stroke injured mice. Among injured VPA-treated mice, there was a $208 \%$ increase in BrdU/NeuNpositive cells in the ipsilateral dentate gyrus (DG) when compared to counts in saline treated mice $(\mathrm{p}=0.024)$; there was a $55.4 \%$ increase in contralateral DG $(\mathrm{p}=0.023)$. In uninjured VPA-treated mice, there was a 49.4 and $61.5 \%$ increase in cell counts in contralateral and ipsilateral DG versus vehicle treated $(\mathrm{p}=0.007$ and 0.004 respectively).

Peak drug levels of VPA in serum one hour after dose were: $251.7 \pm$ $13.3 \mathrm{mcg} / \mathrm{ml}$ with a maximum half-life of $1.24 \pm 0.26$ hours $(4 / 8$ trough samples were below detectable levels; the average trough level of the remaining 4 was $2.1 \pm 0.23 \mathrm{mcg} / \mathrm{ml}$ ).

Conclusions: Chronic VPA treatment increased SGZ neurogenesis. This effect may be related to HDAC inhibition given that previous studies with Trichostatin A (TSA), a strong selective HDACinhibitor, produced similar results; the impact of this increased SGZ neurogenesis requires functional assessment at about P50. The transient increased lag in weight gain in VPA- treated mice does not appear to be related to HDAC inhibition however, since this effect was not seen in TSA-compared to vehicle- treated mice; it may be related to valproate toxicity from oxidative stress or impact on mitochondrial function. Chronic administration of valproate results in a very short half-life in these juvenile mice.
Translational Research: Human Studies

\subsection{0 \\ SUDEP TISSUE DONATION PROGRAM (STOP): COLLABORATIVE NETWORK IN SUPPORT OF SUDEP REGISTRY, TISSUE REPOSITORY AND HUMAN TRANSLATIONAL RESEARCH}

Alica M. Goldman (Baylor College of Medicine, Houston, TX)

Rationale: Translational research of sudden unexplained death in epilepsy is critically dependent on the availability of quality postmortem tissue from patients that succumbed to this tragic complication of epilepsy. SUDEP registry and tissue banking are the imminent principal goals of the STOP SUDEP Program while the larger purpose rests in the development of a publicly accessible resource that will critically support and enhance the research on SUDEP molecular mechanisms and risk factors.

Methods: STOP SUDEP Program functions as a partner within a multifaceted national and international network of families of patients with epilepsy, forensic pathologists, and organizations supporting or performing epilepsy research and education. This program adheres to rules of privacy and confidentiality protection while simultaneously collecting clinical, pathological, and molecular data and postmortem tissues and organs of patients that died due to SUDEP.

Results: STOP Program has an established, IRB approved paradigm for developing a working relationship with participating families and other constituents. The collection of data and procurement of tissues have been in action since 2003 and the repository houses samples from more than 30 families of patients that dies due to SUDEP. Translational research and molecular analysis of the tissues is an inherent part of the program and detailed standardized procedures and protocols have been developed, quality tested, and applied on wide variety of conventional and alternative tissues.

Conclusions: STOP SUDEP Program is the first SUDEP registry and tissue repository concurrently involved in the analysis and research of mechanisms and molecular risk factors of SUDEP with the goal of rapid translation of scientific findings into improved diagnostic and prevention of sudden death in epilepsy. It functions as an open network for partnership, collaboration, and future scientific investigations.

Support: NINDS 3R01NS067013-02S1, 1R01 NS067013-01A1, CURE, Fiorito Fund for SUDEP Research, Emma Bursick Memorial Fund

\subsection{1 \\ BETA COHERENCE IN HUMAN TEMPORAL NEOCORTICAL MICROCIRCUITS}

R. McGinn ${ }^{2,1}$, C. Florez ${ }^{1,4}$, V. Lukankin ${ }^{1}$, I. Marwa ${ }^{1}$, S. Sugumar ${ }^{1,4}$, J. Dian $^{2}$, P. L. Carlen ${ }^{1,4}$, L. Zhang ${ }^{2,4}$ and Taufik A. Valiante ${ }^{1,3}$

( ${ }^{1}$ Fundamental Neurobiology, Toronto Western Hospital Research Institute, Toronto, ON, Canada; ${ }^{2}$ Department of Electrical and Computer Engineering, University of Toronto, Toronto, ON, Canada; ${ }^{3}$ Institute of Medical Science, University of Toronto, Toronto, ON, Canada and ${ }^{4}$ Department of Physiology, University of Toronto, Toronto, ON, Canada)

Rationale: We study, in vitro, the electrophysiology of human brain tissue from temporal neocortex removed at operation for temporal lobe epilepsy. To date, we have studied 19 patients, mainly with epilepsy emanating from the mesial temporal structures. The neocortex that we study is not directly within the most epileptogenic zone, suggesting physiological normalcy of the tissue with respect to cellular electrophysiology and neural network activity. The goal was to produce, in vitro, similar physiological rhythms that have been described in vivo in humans and in animals. Coherence between supra- and infra-granular layers was also investigated, reflecting feed-forward and feed-back integration.

Methods: Temporal neocortical slices $(500 \mu \mathrm{m})$ were made from a 
section of the resected middle temporal gyrus, and placed into a sucrose-based holding solution at $4 \mathrm{oC}$ with a glutamate receptor blocker (kynurenic acid). The slices were placed into artificial cerebrospinal fluid (ACSF) at 35oC. Extracellular field, whole cell intracellular, and cell attached recordings were performed in chambers with ACSF flow rates of $12-15 \mathrm{cc} / \mathrm{min}$ at $35 \mathrm{oC}$. Results: Normal physiological rhythms were observed with extracellular recordings as follows. In ACSF alone, only delta rhythms were noted. With the addition of a low concentration of kainate $(50 \mathrm{nM})$, and carbachol $(50 \mathrm{uM})$ to mimic endogenous activation, a number of physiological oscillations were observed. Delta rhythms were observed with higher amplitude in the deep layers, whereas the higher frequency rhythms were predominant in superficial layers. Broadband and narrowband (rhythmic) activity was observed well up into the high-gamma frequency range. Theta and beta oscillations were also observed. The rhythmic activity was correlated to the IPSCs recorded intracellularly in layer II/III pyramidal cells. "Nesting" of gamma oscillations within theta oscillations was observed within the supragranular layers. Entrainment between deep and superficial layers was found almost exclusively in the beta frequency band $(15-25 \mathrm{~Hz})$ as measured by wavelet phase coherence. Such entrainment had non-zero phase offset and was significant with an alpha-value of 0.05 using surrogate methods. Phase lag shifted directionality between measurements, possibly indicating bidirectional information transfer.

Conclusions: Physiological rhythms are demonstrated in a novel in vitro model of human in vivo oscillations. In particular, high gamma rhythms, theta-gamma modulation and beta-phase coherence have been observed and quantified.

\subsection{2}

\section{YOUNGER AGE AT SURGERY IS ASSOCIATED WITH IMPAIRED ELECTRON TRANSPORT CHAIN COMPLEX FUNCTION IN CHILDREN WITH FOCAL CORTICAL \\ DYSPLASIA}

Michael V. Miles ${ }^{1,2}$, L. Miles ${ }^{1}$, H. Greiner ${ }^{2}$, F. Mangano ${ }^{3}$, P. Horn ${ }^{5,2}$, J. Leach ${ }^{4}$, K. Lee ${ }^{8}$, T. DeGrauw ${ }^{7}$ and C. Hoppel ${ }^{6}{ }^{1}$ Pathology \& Laboratory Medicine, Cincinnati Children's Hospital Medical Center and University of Cincinnati College of Medicine, Cincinnati, OH; ${ }^{2}$ Pediatric Neurology, Cincinnati Children's Hospital Medical Center, Cincinnati, OH; ${ }^{3}$ Neurosurgery, Cincinnati Children's Hospital Medical Center, Cincinnati, OH; ${ }^{4}$ Radiology, Cincinnati Children's Hospital Medical Center, Cincinnati, OH; ${ }^{5}$ Mathematical Sciences, University of Cincinnati, Cincinnati, OH; ${ }^{6}$ Center for Mitochondrial Diseases, Case Western Reserve University, Cleveland, OH; ${ }^{7}$ Child Neurology, Children's Hospital of Atlanta and Emory University, Atlanta, GA and ${ }^{8}$ Neurology, Florida Hospital for Children, Orlando, FL)

Rationale: New strategies have been proposed to target mitochondrial dysfunction in neurological diseases. Mitochondrial dysfunction is recognized as an important contributor to many neurodegenerative diseases, but little is known about CNS mitochondria in patients with epilepsy. Young age has been associated with increased drug treatment failure and pharmacoresistance in children. Focal cortical dysplasia (FCD) is the most common pathological abnormality associated with intractable epilepsy in children. It has been postulated that epileptogenesis in patients with FCD is caused by impaired mitochondrial energy production, and is associated with local interactions between dysmature and normal postnatal neurons (Epilepsy Behav 2006;9:219). We propose to evaluate this hypothesis in tissue resected from children following epilepsy surgery.

Methods: Patients were included if they had surgery for intractable epilepsy; histopathologic confirmation of FCD based upon Palmini's criteria; electron transport chain (ETC) complex testing in resected tissue; clinical assessment by a pediatric neurologist; and age $<19 \mathrm{y}$. Patients were excluded if they had diagnosis of epilepsy secondary to tumor, hypoxic-ischemic injury, trauma, vascular malformation, tuberous sclerosis, Rasmussen's syndrome, or were $>19 \mathrm{y} .1^{\circ}$ ictal onset regions were identified by extraoperative electrocorticography. ETC activities, which were tested for complex "I" (NADH dehydrogenase or $\mathrm{CI}$ ), complex I+III [NADH-cytochrome c reductase (rotenone sensitive) or $\mathrm{CI}+\mathrm{III}]$, complex "II" (succinate dehydrogenase or CII), complex II+III [succinate-cytochrome c reductase (antimycin sensitive) or $\mathrm{CII}+\mathrm{III}]$, complex III (decylubiquinol-cytochrome c reductase or CIII), complex IV (cytochrome c oxidase or CIV), were determined by spectrophotometric methods for ETC complex assay in brain tissue. For statistical analysis data were stratified according to age at surgery, i.e. Group 1: <25\%ile; Group 2: 25th-75\% ile, and Group 3: $>75 \%$ ile). Data are expressed as mean (SD). This study was approved by the IRB.

Results: A total of 26 patients [age 9.4(5.1)y] met inclusion criteria, including 7 in Grp 1 (age range 0.5-5.3y), 12 in Grp 2 (range 5.9$13.2 y$ ), and 7 in Grp 3 (range 14.8-18.0y). Significant differences were found between study groups for ETC complexes CI, CII+III, CII, and CIV (Table). In addition, 12 patients had two specimens tested for ETC activities, i.e. one identified as $1^{\circ}$ onset region and a second as $2^{\circ}$ spread region. Comparison of ETC complex activities of paired-specimens showed that mean CIV activity was decreased $(\mathrm{P}=0.017)$ in the $1^{\circ}$ onset region of 9 patients with FCD types II (Figure), but not in 3 patients with FCD type I.

Conclusions: Decreased ETC complex activities are associated with younger age in children with FCD. Furthermore, data showing decreased CIV in $1^{\circ}$ onset regions, compared with $2^{\circ}$ spread regions, support the hypothesis that energy production is impaired in epileptogenic tissue. Mitochondrial abnormalities may be important in epileptogenesis in children with intractable epilepsy and FCD.

Table. ETC complex activity associated with age at surgery (Group 1: $<25$ th percentile; Group 2: 25-75th percentile; and Group 3: $>75$ th percentile) in brain cortex from 26 patients following epilepsy surgery. Mean (SD).

\begin{tabular}{|c|c|c|c|c|}
\hline $\begin{array}{c}\text { ETC Complex } \\
(\mu \mathrm{mol} / \mathrm{min} / \mathrm{g} \text { prot }) \\
\text { CI+III }\end{array}$ & $\begin{array}{c}\text { Group 1 } \\
(\mathrm{n}=7) \\
0.021(0.015)\end{array}$ & $\begin{array}{c}\text { Group 2 } \\
(\mathrm{n}=12) \\
0.028(0.011)\end{array}$ & $\begin{array}{c}\text { Group 3 } \\
(\mathrm{n}=7) \\
0.038(0.014)\end{array}$ & $\mathrm{P}<.05$ \\
\hline CI & $0.352(0.044)$ & $0.378(0.078)$ & $0.444(0.099)$ & $\mathrm{b}$ \\
\hline CII+III & $0.032(0.009)$ & $0.038(0.005)$ & $0.042(0.008)$ & $\mathrm{a}, \mathrm{b} . \mathrm{c}$ \\
\hline CII & $0.015(0.003)$ & $0.018(0.004)$ & $0.023(0.005)$ & $\mathrm{a}, \mathrm{b}$ \\
CII & $0.161(0.031)$ & $0.164(0.032)$ & $0.191(0.043)$ & $\mathrm{ns}$ \\
\hline CIV & $2.074(0.442)$ & $2.216(0.559)$ & $2.751(0.658)$ & $\mathrm{b}$ \\
\hline
\end{tabular}

\footnotetext{
${ }^{\text {a }}$ Groups 1 vs. 2 vs. 3.

${ }^{\mathrm{b}}$ Groups 1 vs. 3 .

${ }^{\mathrm{C}}$ Groups 1 vs. 2.
}

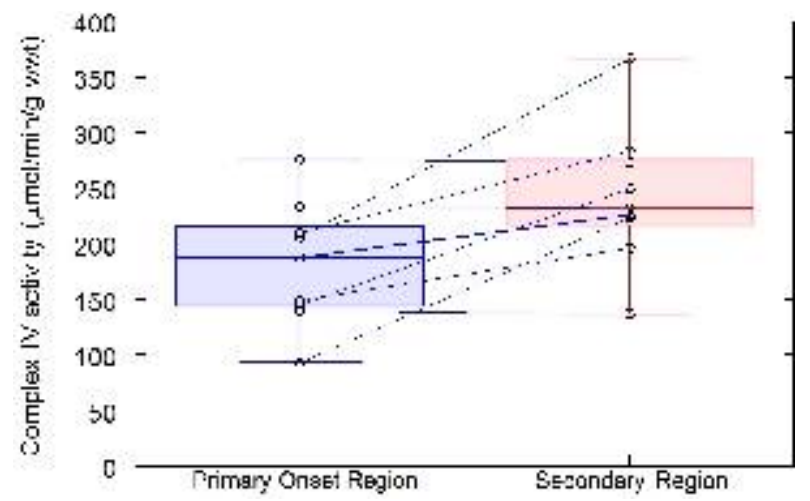

Figure. ETC complex IV activity is significantly decreased $(\mathrm{P}=0.017)$ in primary onset regions compared to matched secondary regions of 9 patients with FCD type II. 


\subsection{3}

GAP-JUNCTION IS A POTENTIAL TARGET FOR EPILEPTIC THERAPY IN HUMAN GELASTIC SEIZURES WITH HYPOTHALAMIC HAMARTOMA

Jie Wu ${ }^{1}$, S. G. Rice ${ }^{1}$, J. Beggs ${ }^{1}$ and J. F. Kerrigan ${ }^{1,2}\left({ }^{1}\right.$ Barrow Neurological Institute, Phoenix, AZ and ${ }^{2}$ Pediatric Neurology, Phoenix Children Hospital, Phoenix, AZ)

Rationale: Human hypothalamic hamartoma $(\mathrm{HH})$ is a rare developmental malformation usually characterized by gelastic seizures. Almost all $\mathrm{HH}$ patients are refractory to antiepileptic drugs (AEDs). Since the epileptogenic mechanisms of gelastic seizures are unknown, there is no available AEDs that can effectively control seizure activity. Previously, we have found that the neurons within $\mathrm{HH}$ tissue are distributed in clusters of variable size, and that most $(\sim 90 \%)$ of HH neurons are small, GABAergic neurons with pacemaker-like firing. We believe that these neuronal clusters represent the functional unit for seizure initiation within $\mathrm{HH}$ tissue, and that hypersynchrony of these small, intrinsically-firing GABAergic neurons is a mechanistic component of ictogenesis. Methods: Multiple experimental approaches were used for this study, which include electrophysiological recording from $\mathrm{HH}$ slices, immunohistochemical staining of $\mathrm{HH}$ sections with specific antibodies for gap-junction protein connexin $36(\mathrm{Cx} 36)$ and $\mathrm{Cx} 43$, Western-blot to compare $\mathrm{Cx} 36$ expression between $\mathrm{HH}$ and control tissues, and electron microscopy (EM) to show the gap-junction structures.

Results: Immunostaining and Western-blot experiments show that neuronal type of gap-junction protein connexin $36(\mathrm{Cx} 36)$ is highly expressed in human $\mathrm{HH}$ tissues freshly resected from gelastic seizure patients. EM shows a gap-junction-like structure between small $\mathrm{HH}$ neuronal pairs. Electrophysiological recordings from $\mathrm{HH}$ slices demonstrate that there are spontaneous epileptiform discharges in $\mathrm{HH}$ slice in normal artificial cerebrospinal fluid (ACFS) at $33 \pm 1{ }^{\circ} \mathrm{C}$. Bath-application of gap-junction blocker (carbenoxolone) significantly eliminates these spontaneous discharges in a concentration-dependent manner.

Conclusions: Our results suggest that the gap-junction is likely an important target for epileptogenesis within $\mathrm{HH}$ lesion, and pharmacological block of gap-junction is a novel therapeutic strategy for patients with refractory gelastic seizures and perhaps other forms of intractable epilepsy.

This work is supported by Arizona Biomedical Research Commission.

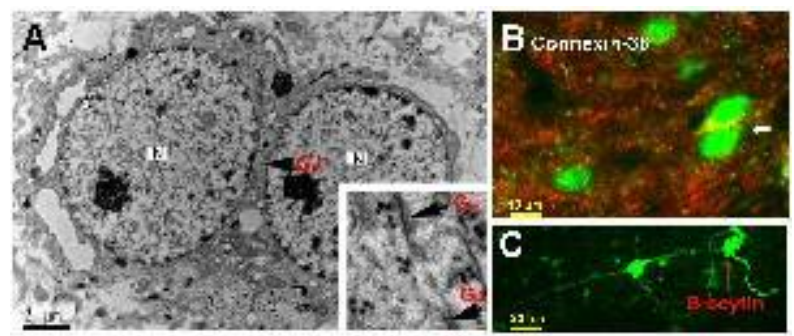

Fig. 1. Evidence of gap-junction in human $\mathrm{HH}$ tisuesA: Electron microscopy image. N: nucleus, GJ: gap junction. B: Immunohistochemical staining: labeled connexin 36 (red) surrounds $\mathrm{HH}$ neurons (green). C: An example of typical dye-coupled small $\mathrm{HH}$ neurons. Biocytin was injected into one neuron (red arrow), which results in the labeling of two $\mathrm{HH}$ neurons.

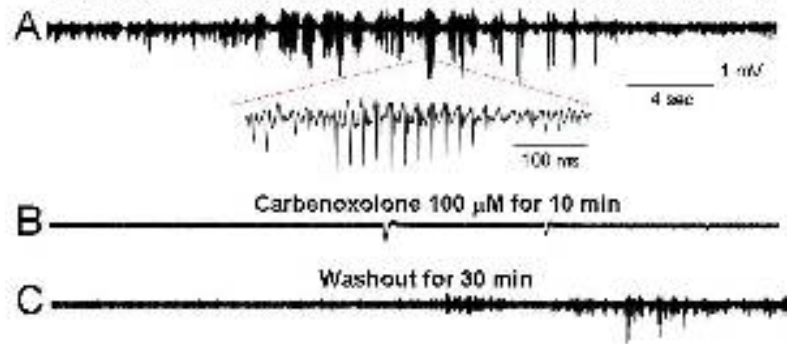

Fig. 2. A: A typical trace of spontaneous epileptiform discharges in a HH slice. Inset shows a typical spike-wave pattern of epileptic discharges. B: Gap junction blocker completely abolished epileptiform discharges. C: After washout of carbenoxolone for 30 min, the epileptiform discharges were partially recovered.

\subsection{4 \\ HUMAN INHIBITORY SINGLE NEURONS SWITCH OFF BEFORE DRAMATIC INCREASES IN SEIZURE AMPLITUDE}

Omar J. Ahmed ${ }^{1}$, W. Truccolo ${ }^{1,2}$, E. N. Eskandar ${ }^{3}$, J. R. Madsen ${ }^{4,5}$, W. S. Anderson ${ }^{5}$, G. R. Cosgrove ${ }^{6}$, N. S. Potter ${ }^{7}$, A. S. Blum ${ }^{7}$, L. R. Hochberg ${ }^{1,2}$ and S. S. Cash ${ }^{1}\left({ }^{1}\right.$ Neurology, Massachusetts General Hospital and Harvard Medical School, Boston, MA; ${ }^{2}$ Neuroscience, Brown University, Providence, RI; ${ }^{3}$ Neurosurgery, Massachusetts General Hospital and Harvard Medical School, Boston, MA; ${ }^{4}$ Neurosurgery, Children's Hospital and Harvard Medical School, Boston, MA; ${ }^{5}$ Neurosurgery, Brigham and Women's Hospital and Harvard Medical School, Boston, MA; ${ }^{6}$ Neurosurgery, Rhode Island Hospital, Providence, RI and ${ }^{7}$ Neurology, Rhode Island Hospital, Providence, RI)

Rationale: The canonical view of epilepsy is based on the notion of imbalance between excitatory and inhibitory activity. There are several theories, based on slice and animal experiments, proposing that a decrease in inhibition is a necessary prerequisite of epileptic activity. However, there are competing theories that suggest a dramatic increase in inhibition is necessary to synchronize the activity of excitatory cells into epileptiform discharges. It is also unclear what happens to the firing of inhibitory neurons as a seizure progresses. The activity of human inhibitory interneurons during seizures has never been comprehensively described. Here, we attempt to clarify the role of these neurons in human seizures by characterizing the role of human inhibitory interneuron activity during different phases of focal seizures with secondary generalization.

Methods: Four patients were implanted with intracranial grid electrodes as part of the clinical process of identifying the site of origin of their drug-resistant epilepsy. A 4x4 mm Neuroport microarray (Blackrock Microsystems) was also placed in a region of the neocortex that was expected to be in the resection site. We used these arrays to simultaneously record the activity of dozens of individual neurons during ictal activity. We then identified putative inhibitory neurons using well-established criteria, including action potential shape. These neurons most-likely correspond to the class of fast-spiking, parvalbumin-expressing inhibitory interneurons.

Results: We show that interneuron activity initially parallels that of excitatory cells as the seizure first spreads to the neocortex, a finding consistent with what is known about feedforward drive of both inhibition and excitation. Unexpectedly, however, we also find that putative inhibitory neurons switch off well before the end of a seizure. This cessation of inhibitory firing is accompanied by a dramatic increase in the amplitude of local spike-and-wave events. We present evidence based on a novel set of analyses that suggests that these inhibitory interneurons stop firing because they enter depolarization block, where most sodium channels are inactivated and incapable of sustaining another action potential. 
Conclusions: Our results show that there is some degree of inhibitory-excitatory balance during the first part of human focal seizures but a complete lack of inhibition towards the end - when seizure symptoms are sometimes at their worst. This cessation likely results from inhibitory interneurons entering depolarization block well before the termination of the seizure. Strikingly, this absence of inhibitory activity is accompanied by large increases in the amplitude of the local field potential. These results suggest that it may be possible to alter or prevent seizures by using pharmacological manipulations that prevent inhibitory interneurons from entering depolarization block. This represents a potentially novel therapeutic avenue in the treatment of human epilepsy. This research was funded by an Epilepsy Foundation Fellowship (222178) to OJA and by NIH grant NS062092 to SSC.

\subsection{5 \\ DIFFERENCES IN ENDOCRINE RESPONSE TO STRESS IN TEMPORAL LOBE EPILEPSY PATIENTS WITH AND WITHOUT SEIZURE FREEDOM}

Jerzy P. Szaflarski ${ }^{1,2}$, J. B. Allendorfer ${ }^{1,2}$, E. B. Nelson ${ }^{3}$, H. Heyse ${ }^{1}$ and L. Mendoza ${ }^{1}\left({ }^{1}\right.$ Neurology, University of Cincinnati Academic Health Center, Cincinnati, OH; ${ }^{2}$ Neurology, University of Alabama at Birmingham, Birmingham, AL and ${ }^{3}$ Psychiatry and Behavioral Neuroscience, University of Cincinnati Academic Health Center, Cincinnati, $\mathrm{OH}$ )

Rationale: Epilepsy patients report stress as a trigger for seizures. Animal models of epilepsy provide evidence for the involvement of stress in the development of epilepsy and have shown stress to be a seizure precipitant. However, the role of stress in human epilepsy is poorly understood. Our objective was to assess the endocrine response to an acute psychosocial stress in left temporal lobe epilepsy (LTLE).

Methods: Included in the study were 23 LTLE patients who either believe $(+S ; n=16)$ or do not believe $(-S ; n=7)$ that stress is an important factor in their seizure control and 17 healthy subjects (HC) matched for age and sex. Subjects underwent a stress challenge similar to the Montreal Imaging Stress Task (Dedovic et al, 2005) designed to produce moderate increases in the stress hormone cortisol (CORT). The stress challenge was performed as part of an fMRI scan session and consisted of a task in which subjects performed difficult subtraction problems and were given negative feedback regardless of their actual performance. CORT was assessed at 30 and $15 \mathrm{~min}$ before and at $0,15,30,45$ and 60 min after scanning $(t=1-7)$. 2sample t-tests assessed group differences in CORT at each of the 7 time points, with specific interest in $\mathrm{t}=3$ which is the time point directly following the stress challenge. Due to the possible interplay between stress and seizures, LTLE patients were also analyzed as those who are seizure free (i.e., no reported seizures in past 3 months; -sz) and those who are not ( + sz). Effect size and power calculations for CORT at $\mathrm{t}=3$ were performed between + sz and - sz groups. Results: There were no significant differences in CORT levels between LTLE $+\mathrm{S}$ and $-\mathrm{S}$ groups or between $\mathrm{HC}$ and either $+\mathrm{S}$ or $-\mathrm{S}$ groups (all $\mathrm{p}>$.1) indicating that chronic stress may have less effect on CORT levels than previously thought. Analysis based on seizure freedom revealed no significant differences in CORT levels between LTLE + sz and -sz groups or between $\mathrm{HC}$ and either +sz or -sz groups (all $\mathrm{p}>$.07). However, LTLE + sz showed a spike in CORT at $\mathrm{t}=3$ compared to $-\mathrm{sz}(\mathrm{p}=.13)$ and $\mathrm{HC}(\mathrm{p}=.1)$, while similar levels $(\mathrm{p}=.69)$ were observed in HC and -sz (Fig. 1). There was a medium-large effect size of CORT between + sz and -sz at $\mathrm{t}=3$ (Cohen's $\mathrm{d}=.65$ ); with larger sample sizes of 21 per group and level of significance set at $\mathrm{p}=.05$, we would have $81 \%$ power to detect group differences in poststress challenge CORT.

Conclusions: LTLE + sz show increased response to stress compared to HC and -sz groups, while HC and -sz show similar patterns of CORT throughout. The elevated stress response in + sz patients may be a factor contributing to or resulting from the continued seizures observed in this group. Given that temporal lobe regions are involved in the endocrine stress response, seizure activity may have an adverse modulatory effect. This is the first study of its kind to investigate the relationship between the endocrine stress response and human epilepsy.

\subsection{6 \\ DEVELOPMENTAL ABNORMALITIES IN EPILEPTOGENIC TUBEROUS SCLEROSIS COMPLEX LESIONS: A COMPARISON ANALYSIS OF CORTICAL TUBERS AND PERI-TUBERAL BRAIN}

Veronique Ruppe ${ }^{1}$, P. Dilsiz ${ }^{1}$, C. Shoshkes Reiss ${ }^{2}$, C. Carlson ${ }^{1}$, D. Zagzag $^{3}$, O. Devinsky ${ }^{1}$, H. Weiner ${ }^{4}$ and D. M. Talos ${ }^{1}\left({ }^{1}\right.$ Neurology, New York University School of Medicine, New York, NY; ${ }^{2}$ Biology, Microbiology and Neural Sciences, New York University, New York, NY; ${ }^{3}$ Neuropathology, New York University School of Medicine, New York, NY and ${ }^{4}$ Pediatric Neurosurgery, New York University School of Medicine, New York, NY)

Rationale: Tuberous Sclerosis Complex (TSC) is an autosomal dominant disease due to loss of TSC1 or TSC2 function and resulting in altered mTOR signaling with subsequent abnormalities in brain development. TSC manifests with cognitive dysfunction, autism and early onset epilepsy, which is frequently refractory and requires surgical therapy. While earlier studies have focused mainly on characterization of cortical tubers, in this study, we sought to analyze in greater detail the unique molecular and cellular features of the ictogenic peri-tuberal brain (PTB), as compared with cortical tuber and control specimens.

Methods: Human TSC specimens ( $\mathrm{n}=20$; ages 0 - 10 years) were collected prospectively during resective epilepsy surgery at NYUMC. Age- and region-matched post mortem samples from cases with no neurological symptoms obtained from the Maryland Brain and Tissue Bank were used as controls $(n=5)$. This study was approved by the Institutional Review Board at NYUMC. Western blotting was used to assess the expression of the mTORC1 and mTORC2 biomarkers pS6 (Ser235/236), pS6 (Ser240/244) and pAkt (Ser473). In addition, the axonal markers SMI 312 and growth associated protein (GAP)-43, as well as myelin basic protein (MBP) were quantified to characterize axonal growth and white matter development. Paraformaldehydefixed specimens were sectioned and immunolabeled with neuronal filament markers (SMI311, SMI312), glial markers (vimentin, MBP) and pS6 (Ser235/236). One-way ANOVA was used to establish statistical significance $(\mathrm{p}<0.05)$.

Results: mTORC1 activity was increased in tubers $(\mathrm{n}=4)$ and PTB $(\mathrm{n}=3)$, as demonstrated by increased $\mathrm{pS6} / \mathrm{S} 6$ ratios normalized to control: $275 \%$ in tubers and 191\% in PTB for pS6 (Ser235/236), $\mathrm{p}<0.001 ; 168 \%$ in tubers and $144 \%$ in PTB for pS6 (Ser240/244), $\mathrm{p}<0.05$. Similarly, tubers $(\mathrm{n}=4)$ and PTB $(n=3)$ showed decreased mTORC2 activity, as demonstrated by lowered pAkt/Akt ratios relative to control (57\% in tubers and $67 \%$ in PTB, $\mathrm{p}<0.0001)$. Consistent with the Western blot results, we found widespread pS6positive giant cells and dysplastic neurons in tubers and PTB. Expression of the axonal marker SMI312 and of GAP-43, a marker for active growth cones, were both increased in tubers $(n=6)$ and PTB $(\mathrm{n}=7)$ relative to control $(205 \%$ in tubers and $128 \%$ in PTB for SMI312, $\mathrm{p}<0.001$; and $178 \%$ in tubers and $146 \%$ in PTB for GAP$43, \mathrm{p}<0.001)$. This pattern correlated with reduced MBP expression relative to control ( $29 \%$ in tubers, $n=6$ and $40 \%$ in PTB, $n=7$, $\mathrm{p}<0.005)$.

Conclusions: Our results demonstrate an extension of abnormal cell types in the PTB and demonstrates, for the first time, aberrant axonal development in human TSC lesions. This data supports the hypothesis that both cellular dysplasia and altered connectivity may underlie the broad spectrum of neurological deficits in TSC patients, including seizure generation and epileptogenesis.

Acknowledgments: FACES, Tuberous Sclerosis Alliance, NICHD Brain and Tissue Bank for Developmental Disorders at the University of Maryland, Baltimore, MD, contract HHSN275200900011C. 


\section{WTIHIDAWN}

\subsection{8 \\ GAMMA-HYDROXYBUTYRIC ACID DETERMINATION FOR NEWBORN SCREENING OF SUCCINIC SEMIALDEHYDE DEHYDROGENASE DEFICIENCY, A METABOLIC EPILEPSY}

Phillip L. Pearl ${ }^{1}$, S. Forni ${ }^{2}$, K. M. Gibson ${ }^{3}$, Y. Yu ${ }^{1}$ and L. Sweetman ${ }^{2}$ ( ${ }^{1}$ Children's National Medical Center, Washington, DC; ${ }^{2}$ Institute of Metabolic Disease, Baylor Research Institute, Dallas, TX and

${ }^{3}$ Michigan Technological University, Houghton, MI)

Rationale: SSADH deficiency, an autosomal recessive disorder of GABA degradation, is characterized by elevated gammahydroxybutyric acid (GHB). Neurological outcomes may be improved with early intervention and anticipatory guidance. Morbidity has been compounded by complications, e.g. hypotonia and respiratory arrest, in undiagnosed infants with otherwise routine childhood illnesses. At least half of patients develop epilepsy, typically as myoclonic or generalized tonic-clonic seizures. SUDEP has been reported in one undiagnosed patient, identified from postmortem analysis following identification of an affected living sibling. We report methodology used to perform newborn screening, which will enable widespread and early detection.

Methods: Dried blood spots in affected patients were compared with controls. In this method, three $1 / 8^{\prime}$ dried blood spot punches are extracted with $200 \mu \mathrm{L}$ methanol containing $500 \mathrm{ng} / \mathrm{mL}$ d6-GHB deuterium-labeled internal standard. The extract is dried, reconstituted, and analyzed via ultra-performance liquidchromatography tandem mass spectrometry. GHB is completely separated from isomeric and isobaric interferences using a step gradient. Multiple selected reaction monitoring for $\mathrm{d} 6 \mathrm{GHB}$ is $\mathrm{m} / \mathrm{z}$ $109->90$ and for GHB 103->85. The same fragment is present in the spectra of the $\alpha$-hydroxy and $\beta$-hydroxy isomers. Therefore major transitions for $\alpha$-hydroxyisobutyric, $\alpha$-hydroxybutyric, $\beta$ hydroxyisobutyric, and $\beta$-hydroxybutyric acids are monitored to verify separation from GHB.

Results: The above method was validated as meeting satisfactory accuracy and reproducibility criteria, including intra-day precision and inter-day validation. Of 1,700 archival dried blood spots screened, GHB mean +/- S.D. was $8.2+5.1 \mathrm{nM}(99 \%$-tile $63 \mathrm{nM})$. The measured concentrations in blood spots of four SSADH deficiency patients were 124, 271, 609 and $715 \mathrm{nM}$.

Conclusions: GHB concentrations in all 1,700 dried blood spot cards were well below the lowest concentration of affected children. This indicates that the screening methodology for SSADH deficiency is valid, with applicability to newborn screening and enabling earlier diagnosis. Children presenting with epilepsy and metabolic disease would benefit from newborn screening for gamma-hydroxybutyric aciduria.

\subsection{9}

SERIAL EXAMINATION OF SERUM IL-8, IL-10 AND IL-1RA LEVELS IS SIGNIFICANT IN NEONATAL SEIZURES INDUCED BY HYPOXIC ISCHEMIC ENCEPHALOPATHY In-Goo Lee ${ }^{1}$, Y. Youn ${ }^{1}$, S. Kim², I. Sung ${ }^{1}$, S. Chung ${ }^{3}$, Y. Kim ${ }^{4}$ and J. $\operatorname{Han}^{1}$ ( ${ }^{1}$ Dept. of Pediatrics, Seoul St. Mary's Hospital, the Catholic University of Korea, Seoul, Republic of Korea; ${ }^{2}$ Dept of Pediatrics, St. Vincent's Hospital, the Catholic University of Korea, Suwon, Republic of Korea; ${ }^{3}$ Dept. of Pediatrics, Incheon St. Mary's Hospital, the Catholic University of Korea, Incheon, Republic of Korea and ${ }^{4}$ Dept. of Pediatrics, Uijeongbu St. Mary's Hospital, the Catholic University of Korea, Uijeongbu, Republic of Korea)

Rationale: We investigated significant changes in the levels of cytokines and the severity of brain insult in the neonatal seizure patients induced by hypoxic ischemic encephalopathy (HIE) and observed a pattern of serial cytokine concentrations

Methods: The HIE-induced seizure group consisted of 13 patients, and another 15 normal newborns were enrolled as a control group. The initial samples in the seizure groups were obtained within the first $24 \mathrm{hr}$ of seizure onset, while the samples in the control group were obtained within the first $24 \mathrm{hr}$ of admission. The second samples were obtained between 48 and $72 \mathrm{hr}$ in both groups. The third samples were taken only in the seizure group on the 5th day of seizure onset.

Results: During neonatal seizures, the levels of cytokines increased within $24 \mathrm{hr}$ and the levels of cytokines decreased after 48 to $72 \mathrm{hr}$ of seizure onset. Among the 10 interested cytokines, the levels of IL-8 significantly increased for $72 \mathrm{hr}$ in the seizure group ( $\mathrm{p}<0.05)$. IL-10 was not significant for the first $24 \mathrm{hr}$, but increased significantly between 48 to $72 \mathrm{~h}$ of seizure onset $(\mathrm{p}<0.05)$. Meanwhile, IL-1Ra decreased significantly in the 2 nd sample $(p<0.05)$. During the prognosis, one patient who was diagnosed with quadriplegic cerebral palsy at 6 months of age presented extreme elevation of IL-1beta, IL1Ra, IL-6, IL-8, IL-10 and TNF-alpha in the initial sample, reflecting the severity of brain damage

Conclusions: Our findings suggest that serial examination of serum cytokine concentrations may serve as a biomarker for brain damage of neonatal seizure when detected within $72 \mathrm{hr}$ of the seizure.

Translational Research: Devices, Technologies, Stem Cells

\subsection{0 \\ PHASE II RANDOMIZED DOUBLE-BLIND CONTROLLED TRIAL OF TRIGEMINAL NERVE STIMULATION IN 50 SUBJECTS WITH DRUG RESISTANT EPILEPSY}

Christopher DeGiorgio $^{1}$, J. Soss ${ }^{1}$, I. Cook ${ }^{2}$, D. Murray ${ }^{1}$, S. Oviedo ${ }^{3}$, G. Corralle-Leyva ${ }^{3}$, D. Markovic ${ }^{5}$, J. Gornbein ${ }^{5}$, J. Pop ${ }^{1}$, S. Gordon ${ }^{1}$, C. Kealey ${ }^{4}$ and C. Heck ${ }^{3}{ }^{1}$ Neurology, UCLA School of Medicine, Los Angeles, CA; ${ }^{2}$ Psychiatry, UCLA School of Medicine, Los Angeles, CA; ${ }^{3}$ Neurology, Keck-USC School of Medicine, Los Angeles, CA; ${ }^{4}$ NeuroSigma, Los Angeles, CA and ${ }^{5}$ Biomathematics, UCLA School of Medicine, Los Angeles, CA)

Rationale: External Trigeminal Nerve Stimulation (eTNS) is an emerging neuromodulation therapy, with positive results from openlabel trials in drug resistant epilepsy (DRE) and major depressive disorder (MDD). The purpose of this study is to explore the efficacy of eTNS on seizure frequency and mood in patients with DRE using a randomized double-blind active controlled trial (RCT) design, and to examine parameters and procedures in preparation for a phase III multicenter clinical trial.

Methods: Randomized double-blind active-control study in DRE. Subjects with two or more complex-partial or tonic-clonic seizures per month entered a 6-week baseline, and then were evaluated at 6 , 12 and 18 weeks during the acute treatment period. Subjects were randomized to active treatment (eTNS, $120 \mathrm{~Hz}$ ) or control (eTNS, 2 $\mathrm{Hz})$.

Results: At entry, subjects had highly refractory epilepsy, averaging 8.7 seizures per month in the active treatment group. Subjects randomized to the active treatment group experienced a significant improvement in the responder rate (RR) during the acute treatment period $(\mathrm{RR}=40.5 \%$ at 18 weeks versus $17.8 \%$ at 6 weeks, $\mathrm{p}=0.01$, GEE logistical model). Over the entire 18-week treatment period, the active treatment group experienced a significant reduction in seizure frequency as measured by the RRATIO ( $\mathrm{p}=0.04$, ANOVA). Furthermore, active treatment was associated with an early and significant improvement in mood as measured by the Beck Depression Inventory (BDI).

Conclusions: This phase II trial provides evidence using a RCT design that eTNS may be safe and effective in subjects with DRE. eTNS was associated with significant improvements in responder rate and mood in subjects with DRE. These results will serve as a basis to 
inform and power a large multicenter phase III clinical trial. The development of a new non-invasive therapy for DRE which significantly improves seizures and mood represents a major advance in the treatment of epilepsy.

Trigeminal Nerve Stimulation is an Investigational device. Limited by Federal (or United States) law to investigational use.

\subsection{1}

TOWARD VALIDATION OF A METHOD AND SYSTEM OF SEIZURE DETECTION USING AUDIO TRANSFORMATION M. D. Breeden ${ }^{1}$, Thomas Tcheng ${ }^{1}$, K. Cicora ${ }^{1}$, T. Skarpaas ${ }^{1}$, N. Hasulak $^{1}$, N. Nathwani ${ }^{2}$, A. Saghyan ${ }^{2}$, C. Wang ${ }^{2}$ and J. H. Goodman $^{2,3}$ ( ${ }^{1}$ NeuroPace, Inc., Mountain View, CA; ${ }^{2}$ Developmental Neurobiology, Institute for Basic Research, Staten Island, NY and ${ }^{3}$ Physiology and Pharmacology and Neurology, SUNY Downstate Medical Center, Brooklyn, NY)

Rationale: Accurately and rapidly identifying seizures in $24 / 7$ continuous EEG data is a major challenge. A rapid method of Seizure Detection using Audio Transformation (SDAT) based on listening to EEG data at high speed and visually marking seizure onsets and durations is described.

Methods: SDAT is validated by comparison with visual-only (VO) seizure scoring and measuring SDAT inter-scorer agreement. Threechannel EEG recordings (24-72 hr per data file) from two rat models of epilepsy (pilocarpine and tetanus toxin) were acquired at a sampling rate of $400 \mathrm{~Hz}$ and stored as EDF files. Notch filters were applied to each EEG channel to minimize recording noise (e.g., 60 $\mathrm{Hz}$ ) and the EDF files were converted into WAV files. The sampling rate in the WAV file header was adjusted to alter the playback speed. This made the EEG frequencies more audible to the human ear. For example, a $400 \mathrm{~Hz}$ EDF file was converted to a $32 \mathrm{kHz}$ WAV file with 80 s of EEG played in $1 \mathrm{~s}$ and $24 \mathrm{~h}$ of data played in $18 \mathrm{~min}$. WAV files were reviewed using audio editor software. Reviewers used aural and visual cues to mark the onset and duration of events of interest. These events included seizures, interictal epileptiform discharges, movement artifacts, and other artifacts. A team of nine reviewers scored a group of EEG files using SDAT. A subset of these files was scored using VO for comparison. Events scored by each reviewer were stored in a database and the results were analyzed to measure SDAT inter-scorer agreement and compare SDAT with VO. Results: Scoring a $24 \mathrm{~h}$ file using SDAT took 25-40 min, compared to 3-5 hr using VO. Over 25,000 hours of EEG data from 275 rats were analyzed using SDAT resulting in 35,566 scored seizures. A preliminary inter-scorer comparison between two expert SDAT scorers based on $247 \mathrm{hr}$ of EEG showed a total of 174 seizures identified with only one disagreement. Additional inter-scorer comparisons will be made as more EEG files are scored by multiple reviewers. A preliminary comparison between SDAT and VO scoring showed dramatic differences. In $121 \mathrm{hr}$ of EEG, more seizures were scored by VO (199) than SDAT (19). Inspection of these differences suggests that many of the VO events were artifacts and not seizures. In $217 \mathrm{hr}$ of EEG, more events were identified by SDAT (136) than VO (68). Inspection of these differences suggests that many seizures were missed by VO because of their short duration and low amplitude. In $97 \mathrm{hr}$ of EEG with high amplitude seizures and few artifacts, agreement between SDAT (17) and VO (16) is excellent. Conclusions: SDAT was developed to improve the speed and accuracy of EEG seizure scoring. Our preliminary evaluation of SDAT suggests that it is faster than VO and may allow greater accuracy in files with considerable artifacts, such as rat EEG. (Supported by NINDS 5U01NS064049)
1.042

VAGAL NERVE ACTIVATION CONTROL: A NEW APPROACH TO ELECTRICAL STIMULATION-BASED THERAPY FOR TREATMENT-RESISTANT TEMPORAL LOBE EPILEPSY

Matthew P. Ward ${ }^{1}$, G. Albors ${ }^{1}$, K. J. Otto ${ }^{1,3}$, R. M. Worth ${ }^{4}$ and P. P. Irazoqui ${ }^{1,2}\left({ }^{1}\right.$ Biomedical Engineering, Purdue University, Indianapolis, IN; ${ }^{2}$ Electrical and Computer Engineering, Purdue University, West Lafayette, IN; ${ }^{3}$ Biology, Purdue University, West Lafayette, IN and ${ }^{4}$ Neurological Surgery, Indiana University School of Medicine, Indianapolis, IN)

Rationale: Vagus nerve stimulation (VNS) is a device-based therapy for treatment-resistant temporal lobe epilepsy. Patterned electrical stimulation of the left vagus nerve mediates the therapeutic effects, but the mechanisms remain unclear. The same stimulus yields differential vagal nerve activation across patients, making efficacy comparisons between studies impossible and those within limited. If the effects of VNS are conveyed by nerve fibers activated in response to stimulation, then a more objective method of investigation and therapy is with respect to the type, proportion and rate (or pattern) of nerve fiber activation. This methodology enables comparisons of therapeutic efficacy and biomarker level changes with respect to the biological conduit of the therapy. Furthermore, it opens avenues for closed-loop control of VNS efficacy.

Methods: An Automated Nerve Control (ANC) System is presented. The closed-loop system autonomously predicts the level of nerve activation in response to any rectangular stimulus pulse. In doing so, it analogously derives a customized guide for activating $0-100 \%$ of $\mathrm{A}, \mathrm{B}$, or $\mathrm{C}$ fiber types within a nerve. A feedback signal between the predicted and measured nerve response is used to maintain any level of $\mathrm{A}, \mathrm{B}$, or $\mathrm{C}$ fiber activation. A single adjustment to the stimulus pulse amplitude or duration, as predicted by the ANC System, yields any desired A, B, or C fiber activation level.

Results: In rats, the ANC System rapidly predicts the level of vagal $\mathrm{A}, \mathrm{B}$, or $\mathrm{C}$ fiber activation in response to any rectangular stimulus pulse. Depending on the pulse repetition frequency (PRF) and ambient noise, the nerve activation prediction routine takes $0.5-5 \mathrm{~min}$ to complete. Then, the nerve activation level is maintained or adjusted in real time by entering a new activation level for one of the identifiable fiber types. Initial prediction accuracy is typically greater than $90 \%$ and improves over time as the therapy is delivered. Data demonstrate a unique ability to overcome natural and stimulusinduced changes in nerve excitability. Relative to the gold standard, constant stimulation mode of VNS therapy, the ANC System provides more consistent and reproducible control over the activity of structures innervated by the various fiber types in the vagus nerve. Conclusions: The ANC System provides a simplified dosing mechanism for electrical stimulation therapy by using the activation level of identifiable fiber types within a nerve and the PRF (or pattern) as the basis of efficacy tuning. It therefore provides a path to more consistent, efficacious and robust treatment responses as well as a less intensive stimulus adjustment period for device recipients. This enhanced treatment response stems from patient-tailored, algorithmguided control over vagal afferent and/or efferent structures via controlled vagal nerve activation therapy.

\subsection{3 \\ MICROELECTRODES PRODUCE UNRELIABLE EEG RECORDINGS William Stacey ${ }^{1,2}$, S. Kellis ${ }^{3}$, C. Butson ${ }^{4}$, P. Patil ${ }^{2}$, T. Assaf ${ }^{2}$, T. Mihaylova ${ }^{1}$ and S. Glynn ${ }^{1}$ ( ${ }^{1}$ Neurology, University of Michigan, Ann Arbor, MI; ${ }^{2}$ Biomedical Engineering, University of Michigan, Ann Arbor, MI; ${ }^{3}$ Biology, California Institute of Technology, Pasadena, $\mathrm{CA}$ and ${ }^{4}$ Neurology and Neurosurgery, Medical College of Wisconsin, Milwaukee, WI)}


Rationale: Recent work has shown that increasing the spatial resolution of intracranial EEG (iEEG) reveals signal features that may be critical in understanding epileptic networks. The work has led to several companies offering FDA-approved microelectrode grids that connect to preexisting EEG equipment. Our group recently measured the impedances several of these grids and demonstrated that, due to their large impedance, microelectrodes will attenuate EEG signals if connected to commonly-used EEG amplifiers. In this study we demonstrate the clinical implications of this attenuation. Methods: Raw iEEG data from macroelectrodes implanted on a deidentified patient were digitally filtered to simulate the signal recorded by a commercial hybrid grid ( 2 macro-, 8 microelectrodes) and a commercial EEG amplifier. The filtering method was verified by recording from the hybrid grid and EEG acquisition amplifier in a saline bath. The filtered EEG data were read by three trained epileptologists, who marked the time and location of seizures, spikes, and slow activity. A well-known automated detector for High Frequency Oscillations (HFOs) was used on each data set. Several sample hybrid grids were imaged with scanning electron microscopy (SEM).

Results: Macroelectrodes did not alter the original signal because their impedance is much lower than the amplifier's. Microelectrodes attenuated low frequencies, which led to several difficulties in clinical interpretation: theta and delta slowing were not recognized; identification of spikes and seizures was disrupted, leading to false negative as well as false positive markings; and the HFO detector produced false positives. SEM demonstrated marked variability in exposed electrode surface area, as well as frequent sharp edges that could damage tissue. In addition, during the experimental recording the higher impedances generated significantly greater noise and were especially sensitive to motion of nearby observers, which led to large baseline fluctuations and false HFOs.

Conclusions: The high impedance of microelectrodes increases recorded noise and attenuates brain signals when recorded on standard EEG amplifiers, greatly disrupting clinical interpretation. Some of these changes can be mitigated by using higher impedance amplifiers. Great care must be taken when comparing results from microelectrodes.

\subsection{4 \\ PHYSICAL AND PHARMACOLOGICAL COOLING ATTENUATES NEUROGLIAL INJURY, IMPROVES SURVIVAL AND COGNITIVE OUTCOME IN EXPERIMENTAL STATUS EPILEPTICUS}

Sandipan Pati, J. X. Yin, C. D. Oliveira, A. Deep, Y. Gan, F. D. Shi, R. Maganti, A. A. Romanovsky, M. Maalouf and D. Treiman (Neurology, Barrow Neurological Institute, Phoenix, AZ)

Rationale: Prolonged status epilepticus (SE) generally has poor long term functional outcomes. Aims of this study were to a) assess neuroprotective and anticonvulsant effects of systemic cooling in a rat model of limbic SE; b) test a novel pharmacological method of cooling by blocking cold defense mechanism (shivering); c) validate MRI/MR spectroscopy as surrogate markers for evaluating neuroprotective efficacy.

Methods: Adult male Sprague-Dawley rats $(n=96)$ were induced into SE for 4.5 hours using lithium-pilocarpine and were monitored continuously using EEG. Half of the rats were cooled (core temperature 31-33 degree C) following SE onset. Outcome was assessed at 24 hours using western blotting, bioluminescence imaging, histology and 7T MRI/MRS. Rats were tested in a Morris water maze at seventh week post SE. Anticonvulsant property of cooling was tested by injecting antiepileptic drugs (AEDs) at SE EEG Stage 3 and comparing the time to stop SE with/without AEDs. Pharmacological cooling was induced by blocking cold receptors transient receptor potential melastatin-8 (TRPM-8) using intravenous infusion of the drug M8-B (TRPM-8 antagonist) at the induction of SE.
Results: $81 \%$ of rodents treated with cooling during SE survived compared to $32 \%$ in the non-treatment SE arm at day $1(\mathrm{p}<0.05$; odds ratio of survival 9.53). At 2.5 months survival was $25 \%$ for the non-cooling and $50 \%$ for the cooling arm. Quantitative analysis of neuronal damage (H\&E, cresyl violet stain) revealed less neuronal damage in CA1,CA 2 and dentate gyrus in the cooling arm $(\mathrm{p}<0.05)$. These neuroprotective effects were confirmed by western blot analysis using: NeuN (for intact neurons) and activated caspase-3 ( apoptosis). Bioluminescence imaging in vivo detected less inflammatory response in the SE rats treated with cooling. T2 weighted imaging, ADC maps showed robust gliotic changes which were attenuated by cooling. MR spectroscopy showed lower lactate peaks following cooling. Cooling did not stop SE, but adding AEDs at SE EEG Stage 3 decreased the total duration of SE by $38 \%$, thereby suggesting a synergistic effect. Blocking TRPM- 8 receptors by M8-B decreased core body temperature by $2.5 \mathrm{C}^{\circ}$ in comparison to vehicle at a fixed ambient temperature, whereas changing the ambient temperature modulated core body temperature during and after SE. At 7 weeks post SE there was no difference in learning between cooling and non-cooling arm following 2 days of cue testing (non hippocampal learning) in water maze. However in the spatial learning paradigm (hippocampal dependent) following 4 days training the cooling arm animals performed better. This was confirmed by probe trial.

Conclusions: Hypothermia attenuates neuro-glial injury, improves survival and cognitive outcome following prolonged status epilepticus. Antiepileptic drugs together with cooling have synergistic anticonvulsant effects. Blocking TRPM-8 receptors lowers core body temperature in experimental status epilepticus, thereby demonstrating the feasibility of a novel method of cooling.
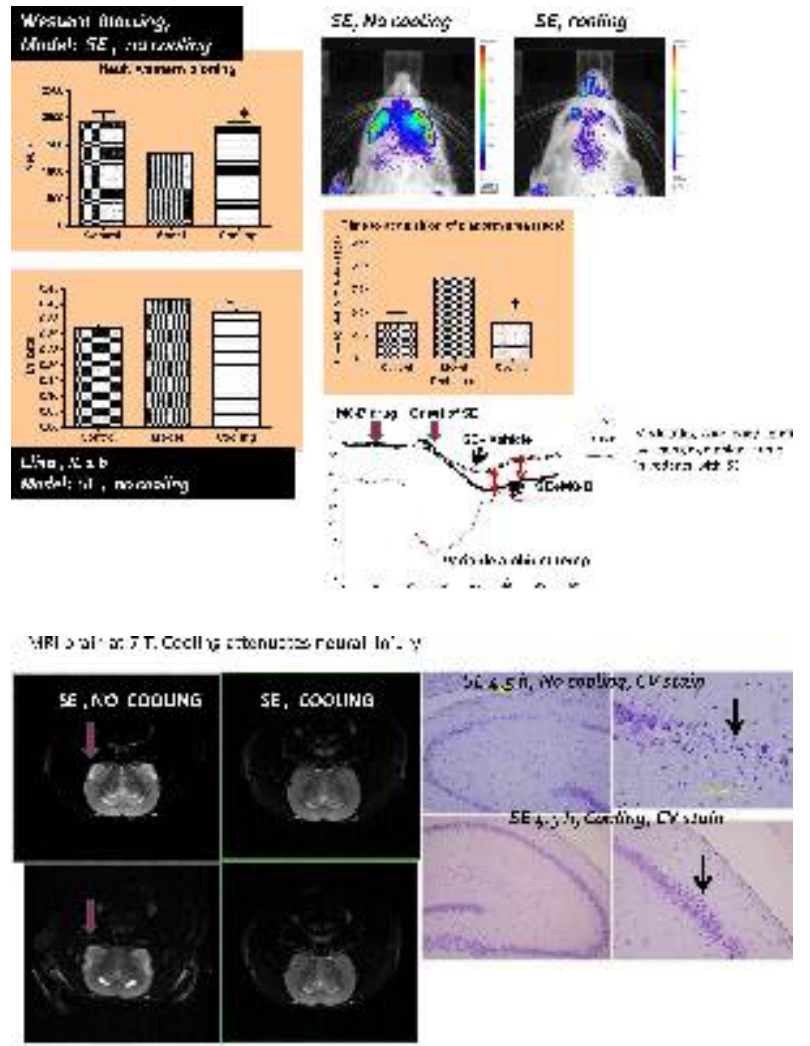


\subsection{5}

TELE-EPILEPSY: DEVELOPING A MULTI-MODAL DEVICE FOR NONEEG, EXTRAMURAL, NOCTURNAL SEIZURE MONITORING

Judith van Andel ${ }^{1}$, C. Ungureanu ${ }^{2}$ and G. Petkov ${ }^{3}$ ( ${ }^{1}$ University Medical Centre Utrecht, Utrecht, Netherlands; ${ }^{2}$ Epilepsy Centre Kempenhaeghe, Heeze, Netherlands and ${ }^{3}$ Epilepsy Centre SEIN, Zwolle, Netherlands)

Rationale: In epilepsy, $25 \%$ of patients have regular, intractable seizures, especially children with epilepsy syndromes and patients with gross brain abnormality and cognitive impairments. About half of seizures will be at night, posing problems in these vulnerable patient groups who depend on caregivers not sleeping in the same bed. A reliable seizure detection and alert system will provide a major step in patient safety, care, quality of life and disease management, however presently this is lacking. In this project a new multimodal device using an optimized combination of nonEEG sensors: audio, automated video frame analysis, ECG and 3Daccelerometry, is developed.

Methods: A diagnostic study design is used to define optimal combinations of algorithms analyzing the 4 modalities in the target population: children under 18 years of age, and mentally impaired adolescents and adults with major nocturnal seizures. The multimodal device is tested in an in-hospital setting in 100 patients, simultaneously with the gold standard of clinical video-EEG. Several methods of classification and regression analysis are explored to define optimal sets of thresholds of the separate modalities for the highest possible detection rate and lowest rate of false positive outcomes. Patient factors and inevitable dependencies due to repeated measures are taken into account.

Results: An interim-analysis of results of 50 patients will be presented in December 2012.

Conclusions: In this study, the validity of a newly developed device for home detection of epileptic seizures during sleep in children and mentally impaired adults with major nocturnal seizures is tested.

\subsection{6 \\ PROGRESS ON DEVELOPMENT OF NEUROPHYSIOLOGICALLY-BASED RESPONSIVE THERAPY IN DOGS WITH NATURALLY OCCURRING EPILEPSY}

Gregory A. Worrell ${ }^{1}$, E. Patterson ${ }^{2}$, C. Vite ${ }^{3}$, M. Bower ${ }^{1}$, V. Vasoli ${ }^{1}$, B. Sturges ${ }^{4}$, V. Ruedebusch ${ }^{5}$, L. Coles ${ }^{2}$, J. Cloyd ${ }^{2}$, B. Brinkmann ${ }^{1}$, M. Stead $^{1}$, D. Crepeau ${ }^{1}$, J. McDonnell ${ }^{2}$, J. Mavoori ${ }^{5}$, J. Howbert ${ }^{5}$, K. Leyde $^{5}$ and B. Litt ${ }^{3}\left({ }^{1}\right.$ Mayo Clinic, Rochester, MN; ${ }^{2}$ University of Minnesota, Minneapolis, MN; ${ }^{3}$ University of Pennsylvania, Philadelphia, PA; ${ }^{4}$ University of California, Davis, CA and ${ }^{5}$ NeuroVista, Seattle, WA)

Rationale: Neurophysiologically-based therapy, such as giving drugs only when needed to prevent seizures, could transform epilepsy care. Challenges for the development of devices capable of real-time seizure advisories and responsive pharmacotherapy are: 1.)Suitable animal model with spontaneous seizures 2.)Device for recording long-term intracranial EEG (iEEG) in freely behaving animals 3.)Algorithms for seizure detection and forecasting 4.)

Pharmacodynamic models of the time-course of AED effect on iEEG. Methods: Canine video-EEG monitoring units were established at 3 institutions. Intracranial EEGs (iEEGs) were monitored continuously using NeuroVista's implantable Seizure Advisory System (SAS). An automated caregiver alert system was developed to alert a caregiver (via e-mail and pager) when seizures occurred. We evaluated a multicompartment pharmacokinetic (PK) model for phenobarbital (PB) and found that it correlates to pharmacodynamic (PD) changes extracted from EEG.

Results: Ten dogs with naturally-occurring epilepsy were implanted with the SAS device. Four studies have been completed:
1.)Retrospective evaluation of the SAS seizure detection algorithm on 11,671 hrs. of iEEG data collected from six dogs. A total of 202 electrographic seizures were captured from 4 dogs. The SAS seizure detection algorithm detected $100 \%$ of the 184 clinically observed seizures and $91.1 \%$ of all detected events correlated with focal electrographic seizure activity. 2.)Retrospective evaluation of SAS seizure forecasting system was performed on 3 dogs. Over 1 year of continuous iEEG was analyzed, and 45,15 , and 83 seizures were captured from the 3 dogs, respectively. Utilizing the SAS algorithm, better than chance seizure advisories $(\mathrm{p}<5 \times 10-5)$ were obtained on all 3 dogs with 1 to 2 false-positives per day. 3.)Prospective study utilizing the care-giver alert system in 4 dogs. The SAS alerted a veterinarian via an automated message in the event of 3 or more seizures within 4 hours, or a seizure lasting longer than 5 minutes. A veterinarian confirmed the seizure activity via remote videomonitoring, and initiated diazepam or phenobarbital therapy as a single IV dose. There were 4 episodes requiring emergency therapy. 4.)PK and PD study in two epileptic dogs on a multi-week PB regimen. $\mathrm{PB}$ concentration-time data were evaluated using compartmental methods and predicted concentration-time profiles for the dogs. The quantitative iEEG features, beta-gamma band spectra and line-length, correlated with PB dosing.

Conclusions: A seizure advisory system (SAS) designed to alert patients and caregivers of seizure activity and provide real-time seizure forecasting was demonstrated in dogs with naturally occurring epilepsy. To date use of the SAS in dogs with naturally occurring epilepsy has demonstrated 1.) Ability to perform highly accurate real-time seizure detections 2.)Ability to deliver seizure alerts via e-mail and pager. 3.)Seizure forecasting at levels significantly better than chance. 4.)Feasibility of constructing PK-PD models of the effect of AEDs on iEEG.

\subsection{7 \\ GAMMA-BAND PRE-SEIZURE ACTIVITY DETECTED WITH TRIPOLAR CONCENTRIC RING ELECTRODE LAPLACIAN ELECTROENCEPHALOGRAPHY FROM SCALP}

A. V. Medvedev, Walter Besio, I. E. Martínez-Juárez, O. Makeyev, M. Fernández-González-Aragón and A. J. Moreno-Avellan (University of Rhode Island, Kingston, RI)

Rationale: Tripolar concentric ring electrode (TCRE) electroencephalography (tEEG) was first introduced by Besio et al. [1]. Two bipolar signals from the TCRE are combined to form tEEG signals derived with 16[(Middle - Disc) - (Outer - Disc)] the Laplacian algorithm first described in [1]. Where Disc is the central disc, Middle is the middle ring, and Outer is the outer ring of the TCRE. We have shown that compared with disc signals, tEEG has nearly 4 -fold (374\%) the signal to noise ratio (SNR) and less than one-tenth $(8.27 \%)$ the mutual information $[1,2]$. TCRE has also strong attenuation of common-mode artifacts [1]. Because of these advantages we sought to detect high-frequency oscillations (HFOs) during peri-seizure periods from TCRE placed on the scalp surface. Methods: We recorded electrographic activity from three patients with epilepsy by placing a set of TCREs in the prime 10-10 system locations in addition to the standard 10-20 system electrode locations used in the hospital. The conventional disk EEG (dEEG) and tEEG were both recorded at $200 \mathrm{~S} / \mathrm{s}$ (hardware filters: dEEG 1- $70 \mathrm{~Hz}$, tEEG 1-100 Hz respectively). We selected approx. 30 min segments, centered on the onset of the generalized tonic seizure, and generated spectrograms to follow the temporal dynamic of pre-seizure gammaband HFOs.

Results: Fig. 1 shows representative dEEG and tEEG signals. Panel B shows bipolar dEEG (Fp2-F4). Panel D shows tEEG recorded from location Fp2' of the 10-10 system directly behind the Fp2 conventional electrode. Panels A and $\mathrm{C}$ are the corresponding timefrequency spectrograms calculated for $2 \mathrm{sec}$ sliding windows. In the magnified traces $\mathrm{E}$ and $\mathrm{F}$ an additional $0.7 \mathrm{~Hz}$ high-pass filter was 
applied to remove a very low frequency drift component.

In two patients with generalized seizures we found specific locations where the tEEG recorded HFO activity in the gamma band $(\sim 70$ $\mathrm{Hz}$ )approximately five to ten minutes before the seizure. The electrode locations exhibiting HFOs were within the seizure onset zone determined by the neurologist (IEMJ). The red ellipse in Panel $\mathrm{C}$ highlights the HFO in the TCRE at location Fp2'. HFOs were not found in dEEG even in cases when HFOs recorded by tEEG were at frequencies $<70 \mathrm{~Hz}$ (the upper filter setting for dEEG). In the third patient who did not have a seizure, the spectrogram revealed periods of frequent interictal spikes when gamma-band HFOs were also present.

Conclusions: For the first time we report records of human ictal activity captured with tEEG. These preliminary data indicate that tEEG is capable of detecting HFOs from scalp, which are not usually seen in conventional EEG records. Thus, tEEG may provide additional tools to follow peri-seizure dynamic with greater precision especially in the high frequency range.

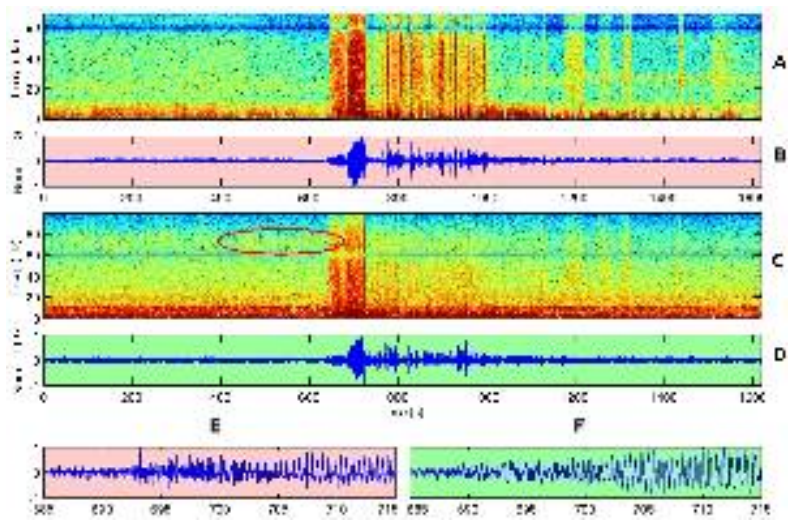

\section{Professionals in Epilepsy Care: Nursing}

\subsection{8}

\section{IMPROVING EPILEPSY AWARENESS: EDUCATION OF SAFETY ISSUES IN PATIENTS WITH EPILEPSY}

Rachel K. Ward-Mitchell, M. Philpot, W. Dotson, M. K. BensalemOwen and S. Kapoor (Kentucky Neuroscience Institute, University of Kentucky, Lexington, KY)

Rationale: The recent publication of the Institute of Medicine's report "Epilepsy Across the Spectrum: Promoting Health and Understanding" clearly outlines a gap in healthcare knowledge of epilepsy and its impact on patients. The aim of this study was to determine current knowledge among nursing staff in our institution of seizures and epilepsy, confidence in caring for and counseling patients with epilepsy with regards to safety issues.

Methods: Photographs and data were collected throughout the year from some of our clinic and hospital patients demonstrating physical injuries sustained during a seizure. A 21 question anonymous survey was administered to 50 nurses across the institution including a selection of these photographs. Additionally, we included questions to evaluate confidence of nurses in caring for patients with seizures, understanding the potential for injury with seizures, as well as overall knowledge and confidence in teaching patients about safety issues. Results: Of the 50 nurses surveyed, nursing experience ranged from less than one year to greater than 35 years, with a median of 11.2 years in practice. Eighty to $88 \%$ of the nurses were able to correctly identify the photographs as injuries that could have resulted from a seizure. Seventy-eight percent of the nurses state they feel they would respond appropriately if they were caring for a patient who experienced a seizure. Overall knowledge of seizures and epilepsy ranged from $76-98 \%$ on topics of seizure type, common misconceptions and status epilepticus. Sixty-four percent of nurses were familiar with driving restrictions in the state of Kentucky, while
$46 \%$ of the nurses state they routinely counsel their patients on safety issues. During this face-to-face survey, a variety of inquiries were made by nursing staff with regards to their specific patient population, identifying, also, a need for enhanced education regarding women with epilepsy, pregnancy and postnatal issues. Conclusions: The largest disparity identified in our institute was counseling of safety issues to people with seizures and epilepsy. Understanding these gaps will allow us to create appropriate teaching modules for both nurses and patients. Customized education modules aiming to address current gaps should impact and change current practice, enabling nurses to act as well informed lead educators and effect change across the community.

\subsection{9 \\ PARENT KNOWLEDGE ON HOME MANAGEMENT OF ACUTE SEIZURES}

Lori Cain, K. C. Nickels, E. C. Wirrell, K. K. Illg and L. C. WongKisiel (Child and Adolescent Neurology, Mayo Clinic, Rochester, $\mathrm{MN})$

Rationale: Parents of children with epilepsy acquire knowledge in first aid management of seizures and administer emergent benzodiazepine medications for prolonged or clustered seizures. The aim of this study is to identify knowledge gaps regarding home management for acute seizures among parents of children with epilepsy.

Methods: A telephone survey of parents of children with epilepsy utilizing the nursing telephone triage line at the Mayo Clinic Pediatric Epilepsy Clinic was performed. A nurse administered the telephone survey over ten working days. The survey included questions regarding access of home seizure rescue medications, parental knowledge and attitude regarding the child's seizure rescue medications, and effectiveness of home management of acute seizures.

Results: Eighteen parents (94\% mothers) of children with epilepsy were surveyed during the study period. Parents reported a history of prolonged (greater than five minutes) seizures in $10(56 \%)$ children, 3 or more seizures in an hour in $15(83 \%)$, and seizure free control without need for acute seizure rescue medication in 1 . Among the 17 with ongoing seizures, all had an active rescue plan. Diazepam Intensol and rectal diazepam (Diastat) were the two most commonly used home seizure rescue medication. Parents reported they would administer rescue medication at five minutes in $7(41 \%)$, less than 5 minutes in $4(24 \%)$ and greater than 5 minutes in $6(35 \%)$. Seven out of 12 parents $(58 \%)$ who have Diazepam Intensol and three of seven (43\%) who had Diastat at home verbalized correct administration (for Intensol - buccal administration with child lying on their side, for Diastat, Rectal administration with child lying on side using a 3 count process). Only 5/12 (42\%) families with Diazepam Intensol knew that it expired 90 days after opening the bottle. Respiratory depression was identified by $10(59 \%)$ parents as a potential side effect of benzodiazepines. All but one parent surveyed felt having the rescue medication available to them was beneficial because it kept them from calling an ambulance or going to the emergency department, and made them feel they had some control over the treatment of seizures.

Eleven of 17 parents (65\%) had used emergent benzodiazepine at least once during the past 12 months.

Conclusions: While most families have an emergency seizure rescue plan in place, knowledge gaps exist. Nearly half of responders provided could not correctly verbalize how to administer rescue medication and nearly half were not aware of respiratory depression as a side effect. A standardized training program by nursing, with regular reviews at clinic visits is needed to improve parental proficiency in the home management of acute seizures. 
1.050

HOW DO PARENTS LEARN ABOUT EPILEPSY: WRITTEN HANDOUTS OR LIVED EXPERIENCES?

Debbie Terry ${ }^{1}$, L. Hamiwka ${ }^{1,2}$ and M. Eversole ${ }^{1}\left({ }^{1}\right.$ Neurology, Nationwide Children's Hospital, Columbus, $\mathrm{OH}$ and ${ }^{2}$ Pediatrics, The Ohio State University, Columbus, $\mathrm{OH}$ )

Rationale: Previous research has shown that parents of children experiencing their first seizure or newly diagnosed with epilepsy have high needs for information and many worries about seizures and epilepsy. Although written materials are a common method of providing information to families with epilepsy the impact on knowledge and concerns has not been evaluated. The goal of this study was to determine the impact of written information on parent's knowledge and concerns about epilepsy.

Methods: Data collection is ongoing for 150 parents of children with epilepsy. To date 104 parents have been recruited and completed 3 questionnaires: Knowledge of Epilepsy (KOE), Parent Concerns About Seizures Scale (Sz Concerns) and Parent Seizure Management Concerns Scale (Management Concerns). Parents were eligible to participate if their child had at least one seizure which was not febrile or non-epileptic. Some parents had recently received a newly developed educational booklet about seizures. Data was entered into SPSS version 17. Group comparisons were made using a t-test and ANOVA analysis.

Results: Participants included: mothers (75), fathers (29), other caregivers (9). Child demographics: male (58), female (55); current age (mean $=9$ yrs., +5.28$)$; duration of epilepsy (mean $=4.58$ yrs., $+4.52)$; seizure frequency: daily (22.1\%), weekly (15\%), monthly $(13.3 \%),<$ monthly $(41.6 \%)$, seizure free $(8 \%) .19$ children were newly diagnosed and 94 had established epilepsy. 18 of 26 parents who received the educational booklet had read all or part of it. There was no significant difference between $\operatorname{KOE}(\mathrm{p}=0.350)$ and $\mathrm{Sz}$ Concerns scores $(\mathrm{p}=0.318)$ of participants who read the booklet and those who did not. Management Concern scores were lower in the group that read the booklet $(\mathrm{p}=0.010)$. Longer duration of epilepsy was associated with higher KOE scores $(\mathrm{p}=0.031)$, fewer $\mathrm{Sz}$

Concerns $(p=0.015)$ and Management Concerns $(p=0.013)$. Parents of children with newly diagnosed epilepsy showed similar scores to those with established epilepsy (KOE $\mathrm{p}=0.065$, Sz Concerns $\mathrm{p}=0.473$, Management Concerns $\mathrm{p}=0.506$ ). On visual inspection of the data we noted that knowledge and concerns did not significantly improve until children had epilepsy for at least 10 years.

Conclusions: Our results suggest that written information does not significantly improve parent's knowledge or improve their concerns about epilepsy. Duration of epilepsy does impact knowledge and concerns about epilepsy, however, these differences are only apparent several years after onset. Further investigation into how providers can impact parent's knowledge and concerns about epilepsy is needed as the current standard of providing written information is not effective. Novel ideas for engaging parents in learning about epilepsy are needed.

\subsection{1 \\ MOVING TOWARD BEST PRACTICE NURSING GUIDELINES IN THE CARE OF INTELLECTUALLY DISABLED PERSONS WITH EPILEPSY}

Theresa S. Buckley (Nursing, University of Wisconsin-Eau Claire, Colfax, WI)

Rationale: Abstract: Epilepsy occurs in one-third of individuals with intellectual disability (ID), and the rates increase as the severity of intellectual disability increases. When it occurs, epilepsy casts multiple, intense, chronic effects on the intellectually disabled person, their family and carers. Barriers to best care for this population are many, including innate skill limitations within the population and the limited preparation found in healthcare professionals' understanding of the heterogeneous needs of this vulnerable population. This capstone project reviewed the literature on intellectually disabled persons with epilepsy, assessed educational needs of healthcare professionals at a local clinical agency, developed a side-effect monitoring guide for anti-epilepsy drugs and discussed additional ways to advance nursing practice guidelines for this population.

Methods: This DNP capstone project started with a review of the literature and proceeded to assessing educational needs of the professional clinical staff of a clinical agency in northwestern Wisconsin that supports frail elders and persons with disabilities in their homes and in the community as a managed care organization. Educational needs assessment surveys were sent to 183 professional practice staff members. Educational needs were addressed in an agency-wide presentation specific to care needs of individuals with intellectual disabilities and epilepsy.

Results: The staff provided much front-line insight into the needs of healthcare professionals in providing best practice to intellectually disabled persons with epilepsy. Trends that were identified in the comments include: a need for improved, specific knowledge in this area, recognition that this is a highly complex practice area, a need for patient advocacy, difficulty identifying and classifying seizures, and treatment and management issues.

An educational presentation, entitled, "Moving toward best practice in the care of intellectually disabled persons with epilepsy" was provided to professional clinical staff of this clinical agency.

Pre-test and Post-tests demonstrated educational gains. Finally an anti-epilepsy drug adverse-effect monitoring guide was developed. Preliminary feedback from nurses who have tested the guide has been summarized and is favorable.

Conclusions: The anti-epilepsy drug adverse-effect monitoring guide was developed specifically for monitoring adverse effects in the intellectually disabled population who cannot self-report. It is a beginning practice protocol that addresses one need of a complex and specific patient population. It is an ongoing, evolving process to attain best practice guidelines which pointed this writer in ways practice can be enhanced, and in directions for improving other areas of practice.

\section{Professionals in Epilepsy Care: Psychosocial}

\subsection{2}

THE IMPACT OF NEW-ONSET EPILEPSY IN OLDER ADULTS: A QUALITATIVE STUDY

Wendy Miller and J. Buelow (Indiana University School of Nursing, Bloomington, IN)

Rationale: The incidence of new-onset epilepsy is highest after age 60 , and continues to rise steadily as the life expectancy in the U.S. increases. Despite knowledge that older adults are significantly affected by new-onset epilepsy, little research has been done with this population, and research findings generated from studies involving younger adults with epilepsy have commonly been applied to older adults. Doing so is inappropriate given unique characteristics of older adults with new-onset epilepsy (epilepsy etiologies, clinical presentation, prognosis, metabolic and cognitive changes, comorbidities, and polypharmacy). Thus, how new-onset epilepsy affects the lives of older adults, and problems they experience as a result of epilepsy, is unknown. To guide intervention development and improve epilepsy self-management outcomes, such knowledge must be generated. The purpose of this qualitative descriptive study was to describe problems and life changes experienced by older adults affected by new-onset epilepsy at or after age 60 since the time of epilepsy diagnosis.

Methods: 20 persons aged 60 or older were purposively sampled from a regional neurology office. Inclusion criteria included diagnosis of epilepsy at or after age 60 years, diagnosis of epilepsy at least six months prior to recruitment into the study, prescription of at 
least one anti-epileptic drug, community-dwelling, and able to speak and read English. Potential participants were sent a letter informing them of the study, and the researcher later called them to invite them to take part. Those who met inclusion criteria and desired to participate met with the researcher for a face-to-face, audio-recorded semi-structured interview. Participants were asked open-ended questions pertaining to problems and perceived changes experienced since being diagnosed with epilepsy. Interview data were transcribed and analyzed using content analysis.

Results: 12 women and 8 men took part in the study. The mean age was 70 years, with a range of $60-80$ years. The mean length of time since epilepsy diagnosis was 4.1 years, with a range of $0.5-10$ years. Participants reported that they had experienced problems in the following areas since being diagnosed with epilepsy: Maintaining Independence, Medications, Achieving Goals, and Memory (Table 1). Participants reported that they had experienced both negative and positive changes since diagnosis. Negative changes included those pertaining to lifestyle, perceived well-being, and physical/emotional symptoms. Positive changes were experienced regarding relationships with family and friends, perspective, and spirituality (Table 2).

Conclusions: Findings from this study demonstrate that older adults with new-onset epilepsy are at risk for experiencing problems and perceived negative life changes that are unique from those of younger adults with epilepsy. These results can inform the development of an assessment tool which can be used to guide the development and implementation of tailored epilepsy self-management interventions for this population.

Inbl: 1

\begin{tabular}{|c|c|c|}
\hline Thense & Sis-Thente $(s)$ & Fiequen: \\
\hline Maintaiuitg & Transportation & $x-8$ \\
\hline Irdependeneo & Contituing litc as nofmal & $y=16$ \\
\hline \multirow[t]{4}{*}{ Medicatious } & Financial concerns & $n=11$ \\
\hline & Side efer:L: & $n=19$ \\
\hline & Polyplanacy & 59 \\
\hline & Rememberiz! & $5-12$ \\
\hline Acbievingt Givels & & $y-12$ \\
\hline Memory & & $x-13$ \\
\hline \multicolumn{3}{|l|}{ alle: } \\
\hline There & Siththents) & $I_{x}+11+4 \%$ \\
\hline \multirow{8}{*}{ Negar ve t lianges } & Lifentyle & \\
\hline & Secint & $x=3$ \\
\hline & Re in oestigs m-h family acd fristds. & $x-2$ \\
\hline & Nuwiser of intperiers commitmatrs made & $.8-: 6$ \\
\hline & 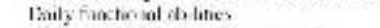 & $\therefore \quad 211$ \\
\hline & 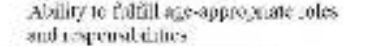 & $x-12$ \\
\hline & Chaugs if sercenter we theing & $n-9$ \\
\hline & 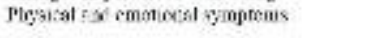 & $N-20$ \\
\hline \multirow[t]{2}{*}{ Pusilive Chariass } & Reialivenlips with acaly and treuds & $n=4$ \\
\hline & Paspective & $\begin{array}{l}x-5 \\
n=5\end{array}$ \\
\hline
\end{tabular}

\subsection{3}

THE FACTORS ASSOCIATED WITH QUALITY OF LIFE IN CAREGIVERS OF PATIENTS WITH EPILEPSY

Yichen Lee ${ }^{1}$ and $\mathrm{H}$. Yu ${ }^{2}\left({ }^{1}\right.$ Department of Neurology, National Taiwan University Hospital Hsin-Chu Branch, Hsinchu, Taiwan and ${ }^{2}$ Neurology department, Taipei Veterans General Hospital and National Yang-Ming University, Taipei, Taiwan)

Rationale: Caregivers play an important role in the physical and mental health of people with epilepsy (PWE). Our purpose is to investigate the factors associated with health related quality of life (HRQOL) of caregivers for people with epilepsy.

Methods: Adult patients who had drug resistant epilepsy and their primary caregivers were asked to complete the questionnaire of Quality of Life in Epilepsy Inventory (QOLIE-89) and the SF-36
Health Status Questionnaire respectively. Possible variables that might be associated with HRQOL of the caregivers were obtained, including patients' age of onset, seizure type, duration of epilepsy, Seizure Severity Questionnaire (SSQ), and the caregiver's gender, age, Zarit Burden Interview (ZBI), the Beck Anxiety Inventory (BAI), and the Beck Depression Inventory (BDI). Univariate analysis was used in the beginning and significant factors were analyzed in a stepwise backward multivariate linear regression to explain the variance of HRQOL of the caregivers.

Results: There were 63 PWE and their caregivers completing the questionnaires. The total scores, mental health dimension, physical health dimension of the caregivers were $75.7 \pm 15.3,71.0 \pm 16.3$, $75.2 \pm 15.3$ (mean \pm sd) respectively. The caregivers' HRQOL was associated with ZBI, BAI, BDI of themselves, and mental dimension scores of HRQOL of PWE in the univariate analysis. The caregivers' scores of SF-36 were not correlated with the patients' scores, age of onset, seizure type, epilepsy duration, or the caregivers' gender or age. In multivariate analysis, caregivers' ZBI, BAI, BDI remained in the model and accounted for $41.8 \%$ of the variance for total scores of HRQOL. The ZBI and BDI explained $46.8 \%$ of the mental dimension of HRQOL in caregivers, and the BAI and BDI accounted for $26.4 \%$ of the physical dimension of HRQOL.

Conclusions: The HRQOL in the caregivers of PWE was much affected by their burden, anxiety and depression. However, the HRQOL of the PWE had only weak association with the HRQOL of their caregivers. Reducing the burden and recognizing mood problems of the caregivers would help improve their HRQOL.

\subsection{4 \\ SEIZURES MAKE ME A LITTLE BIT LOST: CHILDREN'S DESCRIPTIONS OF THEIR EPILEPSY}

Janice Mulligan (Hospital for Sick Children, Toronto, ON, Canada)

Rationale: Children with intractable epilepsy are at increased risk of experiencing social, emotional and behavioural difficulties. The stigma and misunderstanding of epilepsy, peer difficulties including bullying and teasing and anxiety related to the unpredictability of seizures are some of the factors that can contribute to social exclusion, poor quality of life and low self esteem. Providing children with an opporutnity to make sense of their epilepsy and explore the impact of seizures in their lives is vitally important. Finding ways to assist children in expressing feelings related to seizures can offer an outlet for children as well as offer the opportunity for others to develop a better understanding of epilepsy.

Methods: As part of clinical social work services provided to patients with intractable epilepsy at the Hospital for Sick Children a psycho-social educational group was offered to children within an epilepsy classroom setting. A 6-8 week therapeutic group was established using art work, group discussion, bibliotherapy (use of books about epilepsy to increase awareness and explore feelings), role play and education. A key component of the group included Body Mapping (medical art therapy combined with children's narratives) to allow children the opportunity to draw their experiences of seizures and tell their stories.

Results: Seizure descriptions were obtained from 32 children - 16 girls and 16 boys - aged 6-13 years. Body mapping drawings were completed by 28 of these children. Children described both (1) physical feelings and (2) emotional feelings related to their seizures. Descriptions included feelings of fear, sadness, "invasion", disconnection, pain, dizziness and tiredness.

Conclusions: Children described verbally and through the use of drawings both physical and emotional feelings associated with their seizures. Children often described feelings of fear during a seizure and feelings of pain and exhaustion afterwards. The seizure descriptions provided by the children suggested intense internal experiences. These descriptions give a voice to the child's experience and can assist family, friends and helping professionals gain a better understanding of epilepsy. Body mapping can be used as a tool to (1) 
explore feelings, (2) create drawings to facilitate the sharing of the seizure experience with others and (3) engage children in conversations about their own health and quality of life. Body mapping relies on children with epilepsy being the expert about their seizures and invites them to be active participants in their own health care.

\subsection{5 \\ EXAMINING LEARNING AND ACADEMIC CHALLENGES IN PEDIATRIC NON-EPILEPTIC SEIZURES Julia Doss ${ }^{1}$, S. Plioplys ${ }^{2}$, P. Siddarth ${ }^{3}$, B. Dorwin ${ }^{3}$, B. Bursch ${ }^{3}$, T.

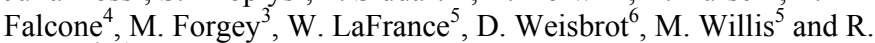 Caplan ${ }^{3}$ ( ${ }^{1}$ Psychology, Minnesota Epilepsy Group, St. Paul, MN; ${ }^{2}$ Feinberg School of Medicine at Northwestern University, Ann and H. Lurie Children's Hospital, Chicago, IL; ${ }^{3}$ Psychiatry and Biobehavioral Sciences, David Geffen School of Medicine at UCLA, Los Angeles, CA; ${ }^{4}$ Pediatric Epilespy/Child Psychiatry, Cleveland Clinic, Cleveland, $\mathrm{OH} ;{ }^{5}$ Psychiatry and Neurology (Research), Rhode Island Hospital, Warren Alpert Medical School of Brown University, Providence, RI and ${ }^{6}$ Child and Adolescent Psychiatry, Stony Brook University Medical Center, Stony Brook, NY)}

Rationale: Learning challenges have been inconsistently reported in retrospective studies of pediatric non-epileptic seizures (NES). This multisite study examined if children with NES have significantly more learning challenges involving cognitive, academic and language skill than their siblings. It also determined if within the NES and sibling groups subjects met diagnostic criteria for a learning disorder (LD) based on self-report rather than on parent report and whether LD was associated with other psychiatric diagnoses, epilepsy and coping.

Methods: NES participants aged 8-18 years had confirmed typical NES episodes on video EEG. 48 NES (33 female, 15 male) and 33 siblings (18 female, 15 male) underwent standardized cognitive, language, and achievement testing and structured psychiatric interviews. 15 NES subjects were singletons. Parents and medical charts provided information on confirmed epilepsy and psychotropic drug treatment. T-tests and chi-square analyses (Fisher's exact tests for measures with cell frequencies $<5$ ) examined between group differences in the distribution of continuous (including IQ, language and achievement scores) and categorical (including LD, presence of other psychiatric diagnoses, epilepsy) variables, respectively.

Results: Standardized cognitive, language, and achievement tests revealed average mean scores among both NES and sibling groups. However, the percentage of subjects experiencing deficits in math (NES $31.9 \%$, siblings $12.9 \%, \mathrm{p}=.05$ ) and spelling achievement scores (NES $17 \%$, siblings $3.1 \%, p=.06$ ) was significantly different. Structured psychiatric interviews identified a similar rate of LD NOS in $30(62.5 \%)$ NES and in $17(51.5 \%)$ siblings. But parents reported LD in only 7 NES and 2 siblings. Though not significantly different, both siblings (48.5\%) and NES (38.3\%) subjects reported school problems as their most bothersome stressor. Within both groups, the presence of LD was associated with multiple psychiatric diagnoses but not with a specific diagnosis. In the NES group, $83 \%$ of those with LD also had epilepsy, compared to $56 \%$ of those without LD. Among the NES group, those with LD did not differ from those with no $L D$ on a measure of coping styles. In contrast siblings with LD use significantly more monitoring (i.e., attending to or focusing on a stressor; $\mathrm{p}=.02)$, including cognitive decision-making $(\mathrm{p}=.02)$, direct problem solving $(\mathrm{p}=.0006)$, and support seeking $(\mathrm{p}=.003)$.

Conclusions: These findings highlight the importance of asking children with NES about LD even when parents and standardized testing do not confirm this diagnosis. They also demonstrate that despite multiple psychiatric diagnoses and LD in the NES and sibling groups, NES children with and without LD do not differ in the coping strategies they identify. Finally, this is the first prospective study that assessed LD in pediatric NES controlling for family variables.
Professionals in Epilepsy Care: Education

\subsection{6 \\ THE SYNTHESIS AND STRUCTURE-ACTIVITY RELATIONSHIP (SAR) STUDIES OF NOVEL ARYL ENAMINONE DERIVATIVES AS POTENTIAL ANTICONVULSANT AGENTS}

Patrice Jackson, T. Harper, C. Kellar, U. Etunnuh, A. Suleyman, M. Bratcher and L. Figgs (Pharmaceutical Sciences, University of Maryland Eastern Shore, Princess Anne, MD)

Rationale: Epilepsy is a chronic neurological disorder characterized by spontaneous, recurrent seizures that affects up to $2 \%$ of the world's population. Seizures can occur due to malfunctioning ion channels, increased excitatory neurotransmission activity or decreased inhibitory neurotransmission activity. Approximately $25-$ $30 \%$ of patients with epilepsy fail to experience adequate control of their seizures despite the optimal use of available antiepileptic drugs (AEDs), or they have control of their seizures but experience acute adverse effects. Discovery of novel agents that can correct or prevent the ionic disruption with improve side effect profiles is a key milestone in the battle against epilepsy. The primary objective for this research is to develop novel aryl enaminone analogs that have the potential to be more effective, selective, and safer anticonvulsant agents.

Methods: The 6-membered cyclic diketones of the mono-methyl, dimethyl and unsubstituted analogs underwent an amination reaction to yield the desired enaminone intermediate(s). Subsequently, selective enaminone intermediates underwent base-catalyzed sulfonylation or acylation reactions to generate the target compounds. Each compound was confirmed by melting point, proton Nuclear Magnetic Resonance (NMR), and elemental analyses.

Results: Our initial evaluation of a library of sulfonamide enaminones gave encouraging data, as 19 of the 30 compounds showed activity in the Maximal Electroshock Seizure (MES) or the 6Hertz psychomotor seizure animal models. To continue with our extensive SAR studies, seven novel aryl enaminones were successfully synthesized and six were submitted for pharmacological testing as anticonvulsants to the National Institute of Neurological Disorders and Stroke, NIH. Four compounds were found to be active in the mouse MES model at $100 \mathrm{mg} / \mathrm{kg}$ and one analog in the subcutaneous pentylenetetrazol (scPTZ) model at $100 \mathrm{mg} / \mathrm{kg}$. THA 40 showed promising activity in the rat MES model at $30 \mathrm{mg} / \mathrm{kg}$ at $0.5 \mathrm{~h}$. While THA 36 is the one analog thus far that has shown $6 \mathrm{~Hz}$ psychomotor activity at $100 \mathrm{mg} / \mathrm{kg}$ during time intervals $0.25,0.5$, and 1.0 hours in mice. The active compounds had little to no neurotoxicity.

Conclusions: To date we have a total library of 37 novel aryl enaminones. Six novel enaminone analogs were recently evaluated and all six compounds showed good to moderate protection in the mouse. All tested compounds are undergoing further screening in the 6-Hz seizure model and neuroprotection assays at NINDS.

\subsection{7 \\ IMPLEMENTATION OF SEIZURE SAFETY PRECAUTIONS IN PATIENTS ON CONTINUOUS VIDEO EEG MONITORING}

L. Schultz ${ }^{1}$, A. Zillgitt ${ }^{2}$, R. Iyengar ${ }^{2}$, J. Guanio ${ }^{3}$, C. McCloskey ${ }^{3}$, M. Kravutske $^{4}$, M. Spanaki ${ }^{2}$ and Vibhangini S. Wasade ${ }^{2}\left({ }^{1}\right.$ Public Health Services, Henry Ford Hospital, Detroit, MI; ${ }^{2}$ Henry Ford

Comprehensive Epilepsy Program, Henry Ford Hospital, Detroit, MI; ${ }^{3}$ Neurosciences, Henry Ford Hospital, Detroit, MI and ${ }^{4}$ Nursing development and Research, Henry Ford Hospital, Detroit, MI)

Rationale: Continuous portable long-term video EEG monitoring (CVEEG) is commonly used in the diagnosis of paroxysmal spells and seizures and in monitoring seizure frequency. Unlike in epilepsy monitoring unit and out-patient VEEG, CVEEG is not performed in a 
constant real-time monitored setting and brief clinical spells are likely to be missed leading to patient injury. The aim of this study was to educate nursing staff via an online educational module regarding early identification, assessment and safety measures of witnessed clinical spells and to determine assessment of spells before and after completion of the module.

Methods: Following IRB approval, an online educational module on seizure safety precautions was designed and available from 12/2011 until 02/2012 (training period) for the nurses who consented for the study. Pre and post training tests were included, and scores of only those who completed both were considered for analysis.

Independently, patients undergoing CVEEG from 9/2011 through 11/2011 (pre-training period) and from 02/2012 until 04/2012 (posttraining period), with clinical seizures, were included. CVEEG was reviewed by 2 physicians (AZ, RI) using an evaluation tool for identification and assessment of the recorded clinical spells in these period.

Results: A total of 17 complete responses of the online educational module pre and post training scores were analyzed. Mean of the pretraining scores was 76.2 (s.d. $=15.7)$ and the post-training scores (first test score) was 80.1 (s.d. $=16.0)(\mathrm{p}=0.267$, paired t-test). In addition, the correct responses for each of the six questions were investigated (Table 1).

Independent review of CVEEG identified 83 patients with 96 studies in the pre and 53 with 59 studies in the post-training period. Table 2 shows the gender, age and the rates for clinical seizures, sub-clinical seizures and non-epileptic spells on those patients within the two time periods. No significant differences were detected between these two groups. Slightly but not significantly more clinical seizures in studies were recorded during the pre than in the post training period $(20 \% \mathrm{vs}$ $9 \%, \mathrm{p}=0.057)$. The difference between the number of witnessed clinical seizures or non-epileptic spells during the pre $(73 \%)$ and the post $(56 \%)$ training period was not significant $(\mathrm{p}=0.436$, Rao-Scott chi-square test). Forty percent (10/25) of these were assessed during the pre compared to $56 \%$ ( 5 of 9 ) for the post training period, but the difference was not significant ( $\mathrm{p}=0.287$, Rao-Scott chi-square test).

Conclusions: Although there was no significant difference in the survey responses before and after training the nurses on an online educational module, adequate assessment of witnessed clinical events by the nursing staff increased implying possible improved awareness regarding seizure identification and safety precautions. This prospective study indicates that periodic delivery of educational information to nursing staff could be one step towards enhancing safety and delivery of appropriate care in patients on CVEEG.

\section{Correct responses for pre and post training scores}

\begin{tabular}{|c|c|c|}
\hline Question/[/ppropriatc answer] & $\begin{array}{c}\text { Pre } \\
\text { Response } \\
\text { \# correct/ } \\
\text { total \# } \\
(\%)\end{array}$ & $\begin{array}{c}\text { Post } \\
\text { Response } \\
\text { \# correct/ } \\
\text { total \# } \\
(\%)\end{array}$ \\
\hline $\begin{array}{l}\text { 1. During the ictal phase of a generalized all of the nursing interventions are } \\
\text { appropriate except:/ [placing tonguc blade in paticnt's mouth to protect } \\
\text { them from swallowing their tonguc] }\end{array}$ & $\begin{array}{l}13 / 17 \\
(76 \%)\end{array}$ & $\begin{array}{l}16 / 17 \\
(94 \%)\end{array}$ \\
\hline $\begin{array}{l}\text { 2. Epilepsy is a condition that causes unpredictable, unprovoked, recurrent } \\
\text { seicures, caused by abnormal excessive or synchronous neuronal activity in } \\
\text { the brain://True] }\end{array}$ & $\begin{array}{l}16 / 17 \\
(94 \%)\end{array}$ & $\begin{array}{c}17 / 17 \\
(100 \%)\end{array}$ \\
\hline $\begin{array}{l}\text { 3. One of the dependable indicators of psychogenic non-cpileptic seizures } \\
\text { includes:/[cye closure during the event] }\end{array}$ & $\begin{array}{c}4 / 17 \\
(24 \%)\end{array}$ & $\begin{array}{c}7 / 17 \\
(41 \%)\end{array}$ \\
\hline $\begin{array}{l}\text { 4. Patients experiencing partial seizures may experience a preceding aura, } \\
\text { decreased awareness of surroundings and non purposeful actions:/['True] }\end{array}$ & $\begin{array}{l}16 / 17 \\
(94 \%)\end{array}$ & $\begin{array}{r}15 / 17 \\
(88 \%)\end{array}$ \\
\hline $\begin{array}{l}\text { 5. When assessing a patient during a post ictal phase of a seizure, which of } \\
\text { the following is the most correct?/[Perform frequent ncurological } \\
\text { assessments until the patient has returned to baseline] }\end{array}$ & $\begin{array}{r}12 / 17 \\
(71 \%)\end{array}$ & $\begin{array}{l}12 / 17 \\
(71 \%)\end{array}$ \\
\hline $\begin{array}{l}\text { 6. Which of the following are indicators that a patient may need a cVEEG? } \\
\text { [A. When assessment of seizure frequency is needed. B. When the seizure } \\
\text { type is unknown. C When a critically ill patient with altered mental status is } \\
\text { suspected to have seizurcs.] }\end{array}$ & $\begin{array}{c}17 / 17 \\
(100 \%)\end{array}$ & $\begin{array}{l}15 / 17 \\
(88 \%)\end{array}$ \\
\hline
\end{tabular}

Demographic and clinical events in patients on CVEEG for pre and post training periods

\begin{tabular}{|c|c|c|c|}
\hline Variable & $\begin{array}{l}\text { Pre-training Period } \\
\mathrm{N}=83 \text { patients } \\
\text { (96 studics) }\end{array}$ & $\begin{array}{c}\text { Post-iraining Period } \\
\Lambda=52 \text { patients } \\
\text { (59 studies) }\end{array}$ & p-value \\
\hline Gender, n (\%) & & & $0.9011(1)$ \\
\hline Female & $44(53 \%)$ & $27(52 \%)$ & \\
\hline Malc & $39(47 \%)$ & $25(48 \%)$ & \\
\hline Age, Mean (s.d) & $56.5(18.4)$ & $55.6(18.6)$ & \multirow{2}{*}{$0.7852(2)$} \\
\hline Age, Median (range) & $58(18$ to 94$)$ & $56.5(161091)$ & \\
\hline Clinical seizures, $\mathrm{n}(\%)$ & $19(20 \%)$ & $5(9 \%)$ & $0.0573(3)$ \\
\hline Sub-clinical seizures, $\mathrm{u}(\%)$ & $8(8 \%)$ & $5(9 \%)$ & $0.9483(3)$ \\
\hline Non-cpilcptic spells, $\mathbf{n}(\%)$ & $13(14 \%)$ & $9(16 \%)$ & $0.7503(3)$ \\
\hline Any of the threc, n(\%) & $36(38 \%)$ & $17(29 \%)$ & $0.2803(3)$ \\
\hline Clinical scizures or non-epileptic spells, n $(\%)$ & $30(31 \%)$ & $14(24 \%)$ & $0.3213(3)$ \\
\hline
\end{tabular}

(1) p-value from chi-square test.

(2) p-value from two sample t-test.

(3) p-value from Rao-Scott chi-square test.

Witness information was missing for 4 clinical seizures/non-epileptic spells in the pre and 5 in the post training period.

\subsection{8 \\ EPILEPSY PRACTICE PATTERNS OF EPILEPTOLOGISTS AND OTHER NEUROLOGISTS BEFORE AND AFTER THE IMPLEMENTATION OF THE AAN EPILEPSY QUALITY MEASURES}

Shailaja Gaddam ${ }^{1}$, L. Schultz ${ }^{2}$, J. Snyder ${ }^{3}$, T. Howard ${ }^{4}$, M. Hawley ${ }^{4}$, V. Wasade ${ }^{1}$ and M. Spanaki ${ }^{1}$ ( ${ }^{1}$ Henry Ford Comprehensive Epilepsy Program, Henry Ford Health System, Detroit, MI; ${ }^{2}$ Public Health Services, Henry Ford Health System, Detroit, MI; ${ }^{3}$ Neurosciences, Henry Ford Health System, Detroit, MI and ${ }^{4}$ Neurology Research, Henry Ford Health System, Detroit, MI)

Rationale: A previous study by our group (T. Malone, et al. AES Meeting 2011 Abst. 3.162) showed that epileptologists have higher rate of documentation of the AAN Epilepsy Quality Measures (EQM) compared to other neurologists. The aim of this follow up study is to compare the practice patterns of epileptologists and other neurologists in our Institution before and after the publication of the EQM.

Methods: We retrospectively reviewed clinic visit data in a random sample of epilepsy patients seen by epileptologists and other neurologists before and after the implementation of EQM that included seizure type and frequency, epilepsy syndrome/etiology, EEG and MRI/CT reviewed or ordered, antiepileptic drug (AED) side effects, referral for surgical treatment, safety counseling and counseling for women with childbearing potential. The two groups were sub grouped by pre and post time period. The two groups of encounters were compared using two sample t-tests for age and duration of epilepsy and chi-square tests for categorical and binary variables. Logistic regression methods were used to assess whether the changes between the pre and post time periods were similar across the two groups of physicians. (P-values $<0.05$ )

Results: We included 407 patient visits, 207 of which occurred before the publication of the EQM (92 by an epileptologist and 115 by other neurologist) and 200 after the publication of the EQM introduction ( 100 by an epileptologist and 100 by other neurologist). There was statistically significant improvement in documentation in post compared to pre period for etiology, MRI/CT, AEDs side effects, safety counseling, counseling for women of child bearing potential and all criteria ( $\mathrm{P}$ value $<0.01)$ [Table1]. For epileptologists, documentation of EQM during the post period had significantly higher rates for etiology (pre $45 \%$, post $83 \%$ ), MRI/CT (pre $65 \%$, post $87 \%$ ), AEDs side effects (pre $65 \%$, post $87 \%$ ), safety counseling (pre $24 \%$, post $58 \%$ ), counseling for women of childbearing potential (pre $13 \%$, post $56 \%$ ) and all criteria. For neurologists, documentation 
of EQM during the post period had significantly higher rates for etiology, AEDs side effects (Table 1). The differences in pre and post periods between epileptologists and neurologists were significant for etiology $(\mathrm{p}=0.007)$, MRI/CT $(\mathrm{p}=0.013)$, AEDs side effects $(\mathrm{p}=0.023)$ and safety counseling $(\mathrm{p}=0.03)$.

Conclusions: Our study showed that both epileptologists and other neurologists improved their documentation following the publication of the EQM. Epileptologists improved greater than other neurologists in etiology, MRI/CT ordering or reviewing and safety counseling while other neurologists showed more improvement in relation to reporting AED side effects. Implementation of quality measures has an impact in the delivery of health care.

Practice patterns Pre and Post EQM implementation

\begin{tabular}{|c|c|c|c|c|c|c|c|c|c|c|}
\hline \multirow[b]{2}{*}{ Vuriable } & \multirow[b]{2}{*}{ Responice } & \multicolumn{3}{|c|}{ All Plyysicians } & \multicolumn{3}{|c|}{ I'pilepplologials } & \multicolumn{3}{|c|}{ Oher Neurologist } \\
\hline & & $\begin{array}{c}\text { Pre } \\
(\therefore-207)\end{array}$ & $\begin{array}{c}P^{P} \operatorname{lnsc} \\
(\mathbb{N}-2(00))\end{array}$ & p-val.t. & $\begin{array}{c}\text { Pree } \\
(\mathrm{V}-92)\end{array}$ & $\begin{array}{c}\text { Post } \\
(\mathrm{N}-1(0))\end{array}$ & p-value & $\begin{array}{c}\text { Pree } \\
(\mathrm{V}-115)\end{array}$ & $\begin{array}{c}\text { Post } \\
(N-100)\end{array}$ & p-valus \\
\hline \multirow{2}{*}{ Scizure lype } & Yos & $\begin{array}{c}16.5 \\
\langle 80 \%\rangle\end{array}$ & ${ }_{18151}^{161}$ & \multirow{2}{*}{0.762} & 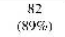 & $\begin{array}{l}91 \\
(929 ; ;\end{array}$ & \multirow{2}{*}{0.510} & $83(72 \%)$ & $\begin{array}{c}71) \\
(70 \%)\end{array}$ & \multirow{2}{*}{0.726} \\
\hline & No & $\begin{array}{l}42 \\
\text { (2015) }\end{array}$ & 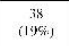 & & $\begin{array}{l}110 \\
\left(1155_{0}\right)\end{array}$ & $8(8 \%)$ & & $32(28 \%)$ & $\begin{array}{c}30 \\
(30 \%)\end{array}$ & \\
\hline \multirow{2}{*}{ Scizure tirequency } & Yo: & $\begin{array}{c}200 \\
\text { (9) }\end{array}$ & 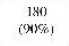 & \multirow{2}{*}{0.007} & $\begin{array}{c}\left.x^{\prime \prime}\right) \\
(97 \%)\end{array}$ & $\begin{array}{c}196 \\
(965 \% ;\end{array}$ & \multirow{2}{*}{0.785} & $\begin{array}{c}111 \\
\langle 97<\} !\end{array}$ & $\begin{array}{c}84 \\
(84 \%)\end{array}$ & \multirow{2}{*}{0.002} \\
\hline & No & $7(3 \%)$ & $20(10 \%)$ & & $3(3 \times 2)$ & $4(4 \%)$ & & $4(3 \%)$ & $\begin{array}{c}16 \\
(16 \%)\end{array}$ & \\
\hline \multirow{2}{*}{ Syndrounc elioksgy } & Yes: & $\begin{array}{c}149 \\
(72 \%)\end{array}$ & $\begin{array}{l}155 \\
(7856)\end{array}$ & \multirow{2}{*}{0.200} & $\begin{array}{c}78 \\
(8555)\end{array}$ & $\begin{array}{c}84 \\
(84 \% ;\end{array}$ & \multirow{2}{*}{0.881} & $71(62 \%)$ & $\begin{array}{c}71 \\
\left(71 w_{5}\right)\end{array}$ & \multirow{2}{*}{0.153} \\
\hline & No & $58(28 \%)$ & $45(2356)$ & & $\begin{array}{l}14 \\
(1.59 .7)\end{array}$ & $\begin{array}{l}16 \\
\text { (165; }\end{array}$ & & $44(36 \%)$ & $\begin{array}{c}29 \\
129 \%)\end{array}$ & \\
\hline \multirow{2}{*}{ Eliology } & Yos & $96\langle 46 \% 3\}$ & $\begin{array}{l}145 \\
\left(7355^{2}\right)\end{array}$ & \multirow{2}{*}{$<.001$} & $\begin{array}{c}41 \\
(45 \%)\end{array}$ & $\begin{array}{c}82 \\
(839 ;\end{array}$ & \multirow{2}{*}{$<1001$} & $55(48 \%)$ & $\begin{array}{c}63 \\
(63 \%)\end{array}$ & \multirow{2}{*}{0.026} \\
\hline & No & $\begin{array}{l}111 \\
\langle(4+\infty)\end{array}$ & $54(27 \%)$ & & $\begin{array}{c}51 \\
(555 \%)\end{array}$ & $\begin{array}{l}17 \\
(17 \% ;\end{array}$ & & $60(52 \%)$ & $\begin{array}{c}37 \\
(37 \%)\end{array}$ & \\
\hline \multirow{2}{*}{ EEG } & Yos: & $\begin{array}{l}145 \\
\text { (70)\%; }\end{array}$ & $\begin{array}{l}156 \\
1785 \times)\end{array}$ & \multirow{2}{*}{0.068} & $\begin{array}{l}70 \\
(765 \% !\end{array}$ & $\begin{array}{c}85 \\
855 ; 5 ;\end{array}$ & \multirow{2}{*}{0.118} & $75(65 \%)$ & $\begin{array}{c}71 \\
\left(71 \omega_{*}\right)\end{array}$ & \multirow{2}{*}{0.365} \\
\hline & No & $62\left(30^{\circ} c\right)$ & $44\left(22^{\infty}\right)$ & & $\begin{array}{c}22 \\
(245)\end{array}$ & $\begin{array}{l}15 \\
(15 \% ;\end{array}$ & & $40(35 \%)$ & $\begin{array}{c}29 \\
(29 \%)\end{array}$ & \\
\hline \multirow{2}{*}{ MRI/CT } & Yo: & $\begin{array}{c}1.36 \\
\langle 66 \%\rangle\end{array}$ & $\begin{array}{c}1.57 \\
\left.(799)^{6}\right)\end{array}$ & \multirow{2}{*}{ a.0.6i } & $\begin{array}{c}51) \\
(65 \%)\end{array}$ & $\begin{array}{c}87 \\
(879 ;\end{array}$ & \multirow{2}{*}{$<(1) 1$} & 77 (66s) & $\begin{array}{l}70) \\
(70 \%)\end{array}$ & \multirow{2}{*}{0.699} \\
\hline & No & $69\{(34 \%)\}$ & $43(22 \%)$ & & $\begin{array}{l}32 \\
(355 \%)\end{array}$ & $\begin{array}{l}13 \\
\text { (139; }\end{array}$ & & $37(32 \%)$ & $\begin{array}{c}30 \\
(30 \%)\end{array}$ & \\
\hline \multirow{2}{*}{ AH:DSAH } & $\mathrm{Yes}$ & $\begin{array}{c}115 \\
\langle 56 \%\rangle\end{array}$ & $\begin{array}{c}179 \\
(90 \% 5 i)\end{array}$ & \multirow{2}{*}{$<(K) 1$} & $\begin{array}{l}60 \\
(655 \%)\end{array}$ & $\begin{array}{c}87 \\
4879 ;\end{array}$ & \multirow{2}{*}{$<(1) 1$} & $55\left(48^{\prime} \%\right)$ & $\begin{array}{c}92 \\
(92 \%)\end{array}$ & \multirow{2}{*}{$<.001$} \\
\hline & No & $92(44 \%)$ & $21(10 \%)$ & & $\begin{array}{l}32 \\
(355)\end{array}$ & $\begin{array}{l}13 \\
\text { (139; }\end{array}$ & & $\cos (52 \%)$ & $818 \%)$ & \\
\hline & $\mathrm{Yes}$ & $21\left\{46^{\prime} x\right\}$ & 31 (11\%) & & $\begin{array}{c}18 \\
(444)\end{array}$ & $\stackrel{20}{20}$ & & $3(6,6)$ & $\begin{array}{c}11 \\
(65 \%)\end{array}$ & \\
\hline स & No & $25(54 \%)$ & $20(395)$ & 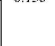 & $\begin{array}{l}23 \\
(566 \text { (x) }\end{array}$ & $\begin{array}{c}14 \\
\text { (11\%; }\end{array}$ & & $2(40 \%)$ & $6(35 \%)$ & \\
\hline Suffurcumeting & Yos & $48(23 \%)$ & $91(46 \%)$ & $x$ & $\begin{array}{c}22 \\
(245 ;)\end{array}$ & $\begin{array}{c}58 \\
\text { (1889; }\end{array}$ & 0 & $26\left(23^{\%} \%\right)$ & $\begin{array}{c}33 \\
(33 \%)\end{array}$ & 0009 \\
\hline . & No & $\begin{array}{l}159 \\
(77 \%),\end{array}$ & $\begin{array}{c}109 \\
\left(555^{2}\right)\end{array}$ & and & $\begin{array}{c}70 \\
(7665)\end{array}$ & $\begin{array}{l}12 \\
\text { (1) } 9 ;\end{array}$ & & $89(77 \%)$ & $\begin{array}{c}67 \\
(67 \%)\end{array}$ & (2) \\
\hline & Yos & $7\left(11^{\prime} k\right)$ & $18(44 \%)$ & (K) & $5(13 \%)$ & $\begin{array}{l}15 \\
156 \%:\end{array}$ & cket & $2(8 \%)$ & $\left.3\left(21^{1 \%}\right)\right\}$ & 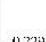 \\
\hline 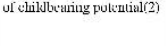 & No & $57\langle 89 \%)$ & $23(56 \%)$ & onn & $\begin{array}{c}34 \\
(87 \%)\end{array}$ & $\begin{array}{l}12 \\
(44 s ;\end{array}$ & shn & $23(92 \%)$ & $\begin{array}{c}11 \\
(79 \%)\end{array}$ & (1.2.24 \\
\hline
\end{tabular}

(1) Eligible for surgery: 46 patients pre and 51 patients post

(2) Women of childbearing potential: 64 pre and 41 post

\subsection{9}

\section{EDUCATION OF CHILDREN WITH EPILEPSY IN POLAND} - RESULTS FROM PRO-EPI SURVEY STUDY

Maria Mazurkiewicz-Beldzinska $^{1,4}$, B. Steinborn ${ }^{2,4}$, I. Bechyne-Put ${ }^{3}$ and M. Balcerzak ${ }^{3}\left({ }^{1}\right.$ Department of developmental Neurology, Medical University of Gdansk, GDANSK, Poland; ${ }^{2}$ Department of Developmental Neurology, Medical University of Poznan, POZNAN, Poland; ${ }^{3}$ UCB Pharma, Warsaw, Poland and ${ }^{4}$ Polish Society of Epileptology, Warsaw, Poland)

Rationale: Children with epilepsy are at risk of social isolation. Attending special schools or individual education programs, despite lack of contraindications, may contribute to further social discrimination.

Methods: The PRO-Epi survey study was initiated by the Polish Society of Epileptology and the UCB Pharma to investigate the facilitation of primary education system by children with epilepsy in Poland. Poles $(n=1042)$, parents of children with epilepsy $(n=313)$, school principals $(n=200)$ and neurologists $(n=179)$ were interviewed to describe the magnitude of the problem and to identify social factors associated with reluctance of children with epilepsy from the ordinary schools.
Results: Although most physicians (60\%) and school principals $(69 \%)$ agree that the ordinary school is the best choice for children with epilepsy, willingness of ordinary schools to admit these children, in the opinion of parents and physicians, is slight $(26 \%$ and $16 \%$, respectively). According to the parents of children with epilepsy, the main reasons for this reluctance are teachers' fear of seizures and their inadequate knowledge about epilepsy. 43\% of parents choose other education options for their children than the ordinary schools. Except the unarguable medical reasons, this decision is also affected by such social factors as fear of classmates' attitude $(25 \%)$, stress avoidance $(22 \%)$, other children parents' dislike $(9 \%)$, other children's exposure to the scene of seizure $(10 \%)$ and previously experienced school principals' reluctance $(26 \%)$.

Conclusions: This situation can be changed by proper education of the Polish society in general and school teachers in particular. It would improve the understanding of young patients living with epilepsy and their parents.

\section{Neurophysiology: Video EEG Epilepsy-Monitoring}

\subsection{0 \\ AMBULATORY EEG MONITORING WITH VIDEO IN ADULTS; YIELD AND CLINICAL UTILITY}

Evan Fertig, E. Feoli, M. Fleming, C. Lambrakis, O. Laban-Grant, S. Mesad, J. Politsky and M. Lancman (Northeast Regional Epilepsy Group, Hackensack, NJ)

Rationale: Inpatient video-EEG monitoring is the gold standard for differentiation of epileptic events from other phenomenon, and longer recording allows for detection of rare interictal epileptiform discharges (IEDs) and subclinical seizures. Inpatient admission has advantages, including observed AED taper, but disadvantages, such as the risk of hospital-associated infection, patient discomfort and cost. Ambulatory EEG (without video) cannot distinguish electroclinical from subclinical seizures, nor non-epileptic seizures from seizures without an ictal correlate (e.g., frontal lobe seizures). Ambulatory video-EEG (AVEEG) is now possible, but its yield and impact on management has not been examined.

Methods: A retrospective review of 307 consecutive studies in adults 18 yrs or older was performed. A 72 hour AVEEG was acquired with synchronized digital video and EEG (non-invasive, scalp, 10/20 system) with a button for subjective events. Relevant clinical data was abstracted from the chart, including the indication, and if results lead to change in AEDs or management overall (e.g., driving recommendations).

Results: Indications were 145 to assess response to treatment for epilepsy (A), 106 to assess spells without a previous epilepsy diagnosis (B), 11 to assess new spells with a previous epilepsy diagnosis (C), 39 studies to guide the decision to reduce or withdraw AEDs after prolonged seizure freedom (D), and 6 unclassified (E). Studies revealed interictal epileptiform abnormalities (IEDs) in 101 (33\%); focal slowing in $80(26 \%)$, events of any type in $56(18 \%)$. Of studies with events (some had more than one type), 12 (21\%) had electroclinical seizures, 14 (25\%) had subclinical seizures, 2 (4\%) had seizures with only clinical manifestations, $20(36 \%)$ had psychogenic non-epileptic seizures (PNES), 6 (11\%) had other types of non-epileptic events (e.g., syncope). Six had events solely off video (3 with ictal correlate) which could not be fully classified. Diagnostic studies $(B+C)$ yielded a new diagnosis in $28 \%$. Study type $\mathrm{D}$ revealed IEDs in $28 \%$ and seizures in $10 \%$ (50\% subclinical). For all studies, AEDs changed in $21 \%$ and management in $30 \%$. AED change was significantly associated with event of any type $(\mathrm{p}<$ $0.001)$ and any epileptic seizure $(p<0.001)$, but not IEDs ( $p=0.0344)$, focal slowing $(\mathrm{p}=0.7980)$ or PNES (0.77); and change in overall management was significantly associated with event of any type $(p<0.001)$, any epileptic seizure $(p<0.001)$, PNES $(p<0.001)$ but not focal slowing $(\mathrm{p}<0.5935)$ or IED $(\mathrm{p}=0.9361)$. 
Conclusions: Nearly one-third of AVEEG studies lead to a new diagnosis, a third of the time for PNES, which highlights the need for video. Events, both epileptic and non-epileptic, had a more robust effect on clinical decision-making than interictal findings, leading to management changes in one-third. Notably, $10 \%$ of reportedly seizure free candidates for AED withdrawal were in fact having seizures. In summary, for studies in adults without need for observed AED taper, AVEEG is useful, but further study is needed on patient selection and timing, and impact on clinical outcomes.

\subsection{1 \\ EVOLUTION OF SEIZURES ON CONTINUOUS VIDEO EEG IN PEDIATRIC ABUSIVE HEAD TRAUMA \\ Cynthia G. Keator ${ }^{1}$, D. Arndt ${ }^{2,1}$, N. V. Stence ${ }^{1}$, B. O'Neill ${ }^{1}$, A. Brooks-Kayal ${ }^{1}$ and K. E. Chapman ${ }^{1}$ ( ${ }^{1}$ Neurology, Children's Hospital Colorado, Aurora, CO and ${ }^{2}$ Neurology, Devos Children's Hospital, Michigan State University, Grand Rapids, MI)}

Rationale: Traumatic brain injury (TBI) is the number one cause of mortality and morbidity in the pediatric population. In moderate to severe pediatric abusive head trauma (AHT) there is a high risk of early post-traumatic seizures. However, most patients with AHT are only monitored clinically for seizures. Current treatment guidelines recommend prophylactic anti-seizure drugs in children with severe AHT.

The temporal electrographic evolution of seizures after AHT is unclear as most children are not routinely monitored with continuous video EEG. We evaluated the overall seizure burden of AHT children by incorporating prospective, standardized cEEG monitoring. Methods: Children (age 1 month-18 years), admitted to the pediatric ICU at Children's Hospital Colorado, from 8/2009 through 6/2012 with moderate to severe traumatic brain injury, both accidental and non-accidental, received an institution-wide cEEG protocol for TBI reviewed by epileptologists, and were also prospectively enrolled in an IRB approved database. Twenty-eight children were identified with AHT based on clinical history, head imaging, presence of retinal hemorrhages, fractures, and by consultation with the child abuse prevention team. Complete EEG data was available for 15/28 patients. Most children were monitored via cEEG for a minimum of 48 hours. This data was re-reviewed by the authors for details about seizure onset, duration, presence of clinical signs, and seizure burden (amount of time occupied by seizure per hour of recording).

Results: The 15 patients with AHT and complete EEG data ranged in age from 1-9 months and $10(67 \%)$ were male. All but $2(13 \%)$ were treated prophylactically with anti-seizure drugs. Nine AHT patients $(60 \%)$ were identified as having electrographic seizures on cEEG. The median number of seizures was 57 (range 6 - 479). Between 64\% and $100 \%$ of electrographic seizures were subclinical (median $93 \%$ ). Of the $6 / 15$ patients without electrographic seizures, 3 presented with clinical seizures (50\%). All but one patient survived (93\%).

Two of the 9 patients (22\%) with electrographic seizures developed seizures after 24 hours, but within 48 hours of monitoring. Five patients $(56 \%)$ continued to have seizures beyond 48 hours postinjury. Seven of 9 patients (78\%) with electrographic seizures developed at least one episode of status epilepticus (SE), and 5 (56\%) had more than 1 episode of SE which was defined as greater than $50 \%$ of ictal activity in any hour of recording. Four patients (44\%) developed SE after 24 hours of EEG recording.

Conclusions: AHT infants had a high rate of seizures and status epilepticus, most of which were subclinical, detected only by cEEG monitoring. Our data supports the importance of routine cEEG monitoring in order to identify electrographic seizures and status epilepticus in AHT patients. A minimum of 48 hours of cEEG monitoring is recommended to screen for electrographic seizures.
1.062

ONSET AND PROPAGATION OF TEMPORAL LOBE SEIZURES WITH VS WITHOUT SECONDARY GENERALIZATION: AN INTRACRANIAL EEG ANALYSIS

Ji Yeoun Yoo, M. W. Youngblood, I. Quraishi, W. Chen, H. P. Zaveri, L. J. Hirsch and H. Blumenfeld (Neurology, Yale New Haven Hospital, New Haven, CT)

Rationale: Generalized tonic-clonic seizures have devastating consequences for patient safety and quality of life. The mechanisms which prevent or permit secondary generalization of focal seizures are not well understood. Intracranial EEG (icEEG) provides a unique opportunity to study seizure propagation and areas of involvement in secondarily generalized seizures. Our goal was to study icEEG in a relatively homogeneous group of focal temporal lobe seizures to investigate the differences in onset and propagation patterns between seizures that remained focal vs. those with secondary generalization. Methods: A total of 33 seizures were analyzed in 9 patients who met the following criteria for confirmed mesial temporal lobe epilepsy and icEEG data: 1) icEEG-video monitoring with at least 1 secondarily generalized seizure, 2) pathologically proven hippocampal sclerosis, 3) no seizures for at least 1 year after anteromedial temporal lobe resection. Onset of secondary generalization was defined based on icEEG-video data demonstrating behavioral vocalization, facial clonus and tonic head/eye deviation. We obtained data from 16 focal seizures without generalization (FS), and 17 secondarily generalized seizures (GTC).

Results: Seizure onset patterns did not differ between FS and GTC, although we did observe differences in later propagation. All seizures started with low voltage fast activity except 3 seizures in one patient (2 FS, 1 GTC), which started with theta-frequency spike discharges. 15 of 33 seizures started from the hippocampus and 18 of 33 seizures (including 4 seizures without hippocampal contacts) started from the non-hippocampal anterior medial temporal lobe contacts, but did not differ between FS and GTC. We observed involvement or more prominent activation of the posterior-lateral temporal regions in GTCs prior to propagation to the other cortical regions, vs. FS which had no involvement or less prominent activation of the posterior lateral temporal cortex. Contacts placed on the occipital lobe, in those patients who had such placement, were not involved at the time of clinical secondary generalization.

Conclusions: The posterior-lateral temporal region may serve as an important "gateway" controlling propagation of temporal lobe seizures to other cortical regions. Further quantitative analysis may provide additional insight into the possible role of cortical slow or fast activity, representing inhibitory or excitatory drives, in gating seizure propagation beyond the temporal lobe. Identifying the mechanisms of seizure secondary generalization has important therapeutic implications, and may lead to improved treatments to confine seizure spread.

\subsection{3 \\ WIDESPREAD EEG CHANGES PRECEDE SEIZURES IN FOCAL EPILEPSY}

Piero Perucca, F. Dubeau and J. Gotman (Montreal Neurological Institute, Montreal, QC, Canada)

Rationale: Although seizures are defined as sudden interruptions of normal brain function, growing evidence suggests that the process by which the brain transitions into a seizure is not abrupt. We investigated EEG changes occurring at the transition to seizure in pharmacoresistant focal epilepsy, and whether these changes differ across distinct underlying pathologies.

Methods: Forty consecutive patients with pharmacoresistant lesional focal epilepsy undergoing intracranial EEG investigations were included in this study. For each patient, representative seizure types were analyzed. For each seizure, two 8-sec sections were selected from the unfiltered EEG: baseline, ending $30 \mathrm{sec}$ prior to the first 
EEG change suggestive of seizure activity; immediate preictal, ending at the first EEG change suggestive of seizure activity. In each section, standard frequency bands (delta, theta, alpha, beta and gamma) and high-frequency oscillations (ripples and fast ripples) were assessed using spectral analysis and visual inspection, respectively. Frequency band data were compared between the baseline and immediate preictal sections, in the entire set of contacts and in four contact subsets: non-lesional/non-seizure-onset zone (SOZ); lesional only; SOZ only; lesional/SOZ. These analyses were performed for the entire patient sample and for three subgroups, defined according to underlying pathology: mesial temporal sclerosis, local/regional atrophy, and malformations of cortical development. Results: In the entire sample, there was a significant increase in each EEG frequency band activity from the baseline to the immediate preictal section. For standard frequency bands, the preictal increase in spectral power was $22.1 \%$ for delta, $18.0 \%$ for theta, $12.0 \%$ for alpha, $7.7 \%$ for beta, and $6.1 \%$ for gamma (all $\mathrm{p}<0.001$ ). For highfrequency bands, the preictal increase in the percentage of time occupied by high-frequency oscillations per section was $35.7 \%$ for ripples $(p<0.001)$ and $14.6 \%$ for fast ripples $(p<0.05)$. Preictal increases in each EEG frequency band were widespread, with significant changes in lesional and/or SOZ contacts, as well as nonlesional/non-SOZ contacts. Widespread preictal changes were observed in all pathologies, although they were more pronounced in patients with mesial temporal sclerosis.

Conclusions: Changes across the entire EEG bandwidth precede the onset of focal seizures by a few sec at least, independently of the underlying pathology. The changes include the focus as well as remote regions. Considering our conservative approach to define the seizure onset (first EEG change suggestive of seizure activity), these results are unlikely due to inclusion of ongoing seizure activity in the preictal section. Rather, they could represent a facilitating state of the brain, which enables a susceptible region to generate seizures. Elucidating mechanisms underlying these changes could lead to an improved understanding of seizures and epilepsy.

This study was supported by the American Brain Foundation, the American Epilepsy Society, the Epilepsy Foundation, and the Canadian Institutes of Health Research.

\subsection{4 \\ EKG CHANGES IN PAROXYSMAL NON-EPILEPTIC SPELLS; A VIDEO-EEG STUDY}

Mubarak M. Al-Dosari, S. Sinha, O. AlSinadi, S. Hanif and K. A. Siddiqui (Epilepsy Program, Department of Neurology,, National Neuroscience Institute, King Fahad Medical City, Riyadh, Saudi Arabia)

Rationale: Paroxysmal Non-Epileptic Spells (PNES) can mimic true epileptic seizures. The key to diagnosis is a good history and a proper neurological examination, with gold standard being video EEG monitoring. Ictal arrhythmia's are hallmark of real seizures. We set out to see any EKG changes (rate and rhythm) occurred during the PNES-ictus and compared to base line.

Methods: There were about 300 patients who had video-EEG monitoring done in the Epilepsy Monitoring Unit of National Neuroscience Institute at King Fahad Medical City, between, 20072012. We found 24 patients who were diagnosed as PNES based on video-EEG evaluations. The PNES spells were characterized as 1 . Sensory, 2. Motor and 3. Behavioral. The EKG changes (rate and rhythm) during the event were noted and compared it to the baseline for each patient. We also collected the patient's demographics, presence of co-morbidities, duration of symptoms, number/type of events, history of using AEDs and MRI findings.

Results: Our cohort had 19 females (79\%) and 5 males (21\%) with mean $(+\mathrm{SD})$ age $25.3(+9)$ years. 18 patients had no co-morbidities, remaining each had a history of road traffic accident, head trauma, diabetes mellitus, bronchial asthma and mental retardation. Average duration of symptoms onset was $6.5(+7)$ years. Nine patients were on
AEDs. During EMU monitoring total number of typical spells recorded were 143, with average of 6 events per subject. Twelve $(50 \%)$ patients had motor events, $6(25 \%)$ had sensory, and $6(25 \%)$ had behavioral. Average duration of all events was 4.5 minutes with (3.9 minutes for motor, 8 minutes for sensory, 2.5 minutes for behavioral). All patients had normal ictal EEG and neuroimaging. EKG finding in all patients had baseline sinus rhythm with mean heart rate 69 (range: 60-90) beats/minute (motor: 67, sensory: 70, behavioral: 70 ). During the event, 22/24 (92\%) patients had mean increase in the heart rate to 89 beats/minute (motor: 101, sensory: 77, behavioral: 91 ) with overall increment of $22.7 \%$ compared to baseline (see table). Five patients ( 4 from motor group and one from behavior group) developed sinus tachycardia $(\mathrm{HR}>100)$ although no arrhythmias were documented. Only 2 patients, both with sensory events, had no ictal change in the heart rate and rhythm.

Conclusions: Increased heart rate without change in the rhythm was seen in majority of patients with PNES; maximal percent increment in patients with motor symptoms. However, ictal sinus tachycardia was seen in only one-quarter of patients.

Heart Rate (HR) changes

\begin{tabular}{|ccc|c|}
\hline $\begin{array}{c}\text { Type of Event } \\
\text { Motor }\end{array}$ & $\begin{array}{c}\text { Batseline HR } \\
\text { (beats/minute) }\end{array}$ & $\begin{array}{c}\text { Event HR } \\
\text { (beats/minute) }\end{array}$ & HR Increment (\%) \\
Scnsory & 67 & 101 & 33 \\
\hline Bchavioral & 70 & 77 & 9 \\
\hline All Events & 70 & 91 & 23 \\
\hline
\end{tabular}

1.065

AN EXTENSIVE EUROPEAN EEG DATABASE FOR ANALYSES OF LONG-TERM RECORDINGS

Andreas Schulze-Bonhage ${ }^{1}$, M. LeVanQuyen ${ }^{2}$, F. Sales ${ }^{3}$, B. Schelter ${ }^{4}$, M. Ihle ${ }^{1}$ and A. Dourado ${ }^{5}{ }^{1}$ Epileptology, University Hospital Freiburg, Freiburg, Germany; ${ }^{2}$ CNRS, Inserm, Paris, France; ${ }^{3}$ Neurology, University Hospital Coimbra, Coimbra, Portugal; ${ }^{4}$ Physics, University of Freiburg, Freiburg, Germany and ${ }^{5}$ Informatics, University of Coimbra, Coimbra, Portugal)

Rationale: A lack of access to high-quality long-term recordings have often hampered standardized and reproducible evaluations in the field of EEG analyses. We here present the European EPILEPSIAE database which has become available for research groups in 2012 and provides long-term EEG recordings of 275 patients with focal epilepsy

Methods: The European EEG database was designed as a relational database including raw EEG (and ECG, EMG) data from patients undergoing long-term video-EEG recordings using scalp or intracranial EEG data, standardized annotations, MR datasets, and extensive clinical metadata regarding course of the disease, histopathology, seizure semiology and imaging data. The database was complemented by software allowing for a web-based data selection and computational processing.

Results: 275 patients from the epilepsy centers of Coimbra, Paris and Freiburg with a minimum of 3 seizures were included in the database (total number of seizures: 2662), 50 of which had intracranial recordings. EEG sampling rate was $250-2500 \mathrm{~Hz}$, Areas of seizure origin, early and late propagation were annotated in a standardized manner. The inclusion of data on daily AED dosages allows to correlate the occurrence of seizures and drug exposure. In addition, derived EEG features were calculated and integrated which have been used in the context of seizure prediction.

Conclusions: The European EEG database exceeds the size of EEG databases available so far by two orders of magnitude. It can serve as a new standard for the application of algorithms to be used for spike and seizure detection as well as seizure prediction. 


\subsection{6}

PATTERNS OF INTERICTAL SPIKES AND OF ASSOCIATED HIGH-FREQUENCY OSCILLATIONS (80-500 HZ) DURING EPILEPTOGENESIS IN AN ANIMAL MODEL OF TEMPORAL LOBE EPILEPSY

Pariya Salami, R. Benini, M. Levesque, J. Gotman and M. Avoli (McGill University, Montreal, QC, Canada)

Rationale: Temporal lobe epilepsy (TLE) is one of the most common forms of partial epilepsy whereby recurrent seizures appear in early adulthood. There is often a history of an initial brain insult (such as febrile convulsions, encephalitis, or status epilepticus) years prior to the onset of seizures, which can originate from the hippocampus, the amygdala or entorhinal cortex and are often refractory to medication. The role of interictal discharges in epileptogenesis remain elusive. Using chronic rodent models of TLE, recent studies have demonstrated that interictal spikes and associated high-frequency oscillations (HFOs, 80-500 Hz) evolve during the latent and chronic periods. In this study, we used the pilocarpine-treated rat model to analyse the evolution of interictal spikes and HFOs in various limbic structures during the latent and chronic periods in an attempt to further elucidate the mechanisms leading to the establishment of epileptic networks in epilepsy.

Methods: Adult Sprague-Dawley rats $(n=3)$ were implanted with bipolar depth electrodes in the dentate gyrus, CA3 region, subiculum and entorhinal cortex, 2 days after an initial pilocarpine-induced SE (380 mg/kg, i.p.). Local field potential (LFP) recordings were then performed on a 24 -hr basis with a continuous video-EEG monitoring system starting from the 3rd to the 15 th day after SE. The morphology and the occurrence of interictal spikes were analysed at different time points during the latent (day 4) and chronic periods (day 15). Ripples $(80-200 \mathrm{~Hz})$ and fast ripples $(250-500 \mathrm{~Hz})$ were also analysed in the filtered LFPs.

Results: Pilocarpine-treated rats began to express spontaneous, recurrent seizures 5 to 7 days following the initial status epilepticus. Analysis of interictal spikes within the CA3 region revealed two morphological subtypes. Type A spikes occurred at a frequency of $0.16 \mathrm{~Hz}$, had an average duration of $766 \mathrm{~ms}( \pm 157)$ and were characterized by a spike followed by a slow wave. Type B spikes on the other hand were shorter (average duration: $285 \mathrm{~ms} \pm 38$ ), occurred at a frequency of $0.31 \mathrm{~Hz}$ and consisted of a single spike. In both the latent and chronic periods, interictal activity consisted predominantly of Type B spikes (latent period: $82 \%( \pm 7)$; chronic period: $100 \%$ $( \pm 0.6))$. However, Type A spikes, although less prominent, were present almost exclusively in the latent $(23 \%( \pm 15))$ rather than the chronic period $(0 \%( \pm 1))$. No significant changes in the duration of Type II spikes were observed between latent and chronic period. HFOs were observed in $62 \%$ of Type A and $51 \%$ of Type B spikes in the latent period. $44 \%$ of Type B spikes continued to exhibit HFOs in the chronic period.

Conclusions: Changes in the pattern of interictal spikes occurs between the latent and chronic periods within the hippocampus of pilocarpine-treated rodents. This illustrates the dynamic process underlying epileptogenesis and may serve as a biomarker for the establishment of the epileptogenic network leading to seizures.

\subsection{7 \\ PERIODIC LATERALIZED EPILEPTIFORM DISCHARGES (PLEDS) IN ADULT INPATIENTS UNDERGOING CONTINUOUS EEG (CEEG) MONITORING}

Indranil Sen-Gupta ${ }^{1}$, S. U. Schuele ${ }^{1}$, M. P. Macken ${ }^{1}$, M. J. Kwasny ${ }^{2}$ and E. E. Gerard ${ }^{1}{ }^{1}$ Department of Neurology, Northwestern University Feinberg School of Medicine, Chicago, IL and ${ }^{2}$ Department of Preventive Medicine, Northwestern University Feinberg School of Medicine, Chicago, IL)

Rationale: Prior literature has reported PLED frequencies of $0.1-1 \%$, with stroke reported as the most commonly associated lesion (Orta et al., Arch Neurol 2009; 66: 985-991, and Pohlmann-Eden et al., J Clin Neurophysiol 1996; 13: 519-530). These studies, however, principally evaluated routine EEGs in mixed inpatient/outpatient populations. Neither the frequency of PLEDs nor their underlying etiologies have been systematically assessed in an inpatient population undergoing prolonged cEEG monitoring.

Methods: Our archive of adult inpatients undergoing cEEG monitoring for non-elective indications between 2007 and 2011 was searched to identify all patients with PLEDs during this five-year period. Charts were reviewed for each patient with PLEDs, and multiple parameters pertaining to clinical demographics and cEEG monitoring were compiled.

Results: 1452 patients underwent cEEG monitoring non-electively from 2007 to 2011.90 of these patients $(6.2 \%)$ were found to have PLEDs. Within the population with PLEDs, most cerebral lesions were neoplasms $(34.4 \%)$, followed by hemorrhage $(24.4 \%)$ and ischemic stroke (14.4\%) (Table 1$) .72 .2 \%$ of patients with PLEDs had seizures detected during monitoring $(28.9 \%$ purely subclinical; $18.9 \%$ purely clinical; $24.4 \%$ clinical and subclinical). Overall, $31.1 \%$ of patients with PLEDs had independent functionality at discharge; the remainder had poor discharge outcomes $(53.3 \%$ dependent; $15.6 \%$ deceased). Median monitoring duration was 4 days. The majority of PLEDs and seizures were detected on the first day of cEEG monitoring, with greater than $90 \%$ of both detected by the second day (Figure 1). In particular, for patients with PLEDs and subclinical seizures, $71.9 \%$ of the subclinical seizures were detected on the first day of monitoring, with $91.7 \%$ detected by the second day.

Conclusions: The frequency of PLEDs was $6.2 \%$ in our inpatient series, much higher than reported for routine or mixed inpatient/outpatient EEGs. It appears that an even higher frequency of PLEDs (15.8\%) can be inferred from prior data presented by Claassen et al. in a study of critically ill patients undergoing cEEG (Neurology 2004; 62: 1743-1748). Thus, acutely ill patients appear to have much higher rates of PLEDs than commonly recognized based on previous studies involving less acute populations and routine EEGs. Underlying lesions in our PLED patients were primarily neoplastic, likely reflecting institutional trends; nonetheless, this differs from prior literature associating stroke as the most common etiology for PLEDs. More than $90 \%$ of all PLEDs, as well as both clinical and subclinical seizures in the patients whom PLEDs were ultimately found, were detected by the second day of cEEG monitoring. These results support the previous work of Claassen et al. by suggesting that patients with PLEDs should undergo cEEG monitoring for at least 2 days to detect subclinical seizures with reasonable sensitivity.

Table 1. Demographics and Clinical Parameters in Patients with PLEDs

\begin{tabular}{|c|c|}
\hline Age, median (range), y & $64(30-94)$ \\
\hline cEEG duration, median (range), d & $4(1-48)$ \\
\hline Gender, No. $(\%)$ & $\begin{array}{l}\text { Males: } 51 \text { (56.7) } \\
\text { Females: } 39(43.3)\end{array}$ \\
\hline Lesion ctiologies, No. (\%) & $\begin{array}{c}\text { Neoplasm: } 31(34.4) \\
\text { Hemorrhage: } 20(22.2) \\
\text { Stroke: } 13(14.4) \\
\text { Vascular Malformation: } 2(2.2) \\
\text { Infectious: } 2(2.2) \\
\text { Metabolic: } 1(1.1) \\
\text { Unknown: } 10(11.1) \\
\text { Other: } 11(12.2)\end{array}$ \\
\hline Seizures captured during cEEG, No. (\%) & $\begin{array}{c}\text { Clinical only: } 17(18.9) \\
\text { Subclinical only: } 26(28.9) \\
\text { Clinical and Subclinical: } 22(24.4) \\
\text { None: } 10(27.8)\end{array}$ \\
\hline Padtient Location at cEEG onsel, No. (\%) & $\begin{array}{c}\text { ICU: } 54(60) \\
\text { Non-ICU: } 36(40)\end{array}$ \\
\hline Discharge outcomes, No. (\%) & $\begin{array}{c}\text { Independent: } 28 \text { (31.1) } \\
\text { Dependent: } 48 \text { (53.3) } \\
\text { Deceased: } 14 \text { (15.6) }\end{array}$ \\
\hline
\end{tabular}




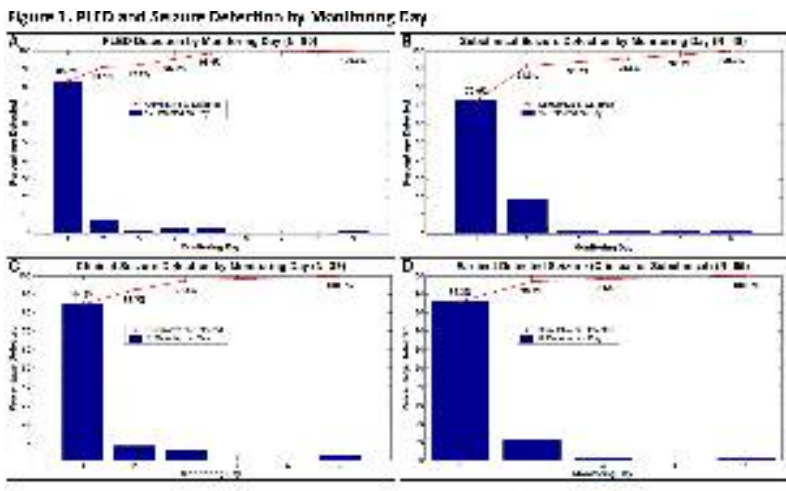

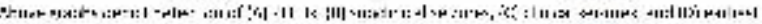

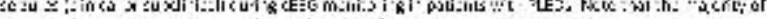

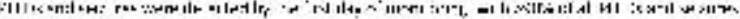
de...chet thesestdas.

\subsection{8 \\ EEG MISINTERPRETATION AND MISDIAGNOSIS OF EPILEPSY IN PSYCHOGENIC NON-EPILEPTOGENIC SEIZURES PATIENTS}

R. Lopez-Baquero ${ }^{1}$ and Ignacio Pita ${ }^{1,2}$ ( ${ }^{1}$ Neurology Section, University of Puerto Rico, San Juan, Puerto Rico and ${ }^{2}$ HIMA San Pablo, Caguas, Puerto Rico)

Rationale: EEG overinterpretation is a common cause of epilepsy misdiagnosis in patients with psychogenic non-epileptic seizures (PNES). Up to $30 \%$ of patients with PNES have been previously misdiagnosed with epilepsy given prior EEG interpretations. Errors in the diagnosis of PNES lead to overuse of health care resources, delay in diagnosis, poor outcomes, and patient stigmatization. Methods: We reviewed 20 consecutive patients with PNES admitted to the Epilepsy Monitoring Unit in 2012. All these patients had previous EEGs performed and interpreted by neurologists without formal training in clinical neurophysiology. Previous EEGs were requested and obtained in digital format for review. A board certified neurologist with added qualification in clinical neurophysiology reviewed all EEGs and created a new report.

Results: Review of initial EEG reports showed that 11 patients had all EEGs read as normal and 9 patients had at least one EEG read as abnormal with epileptogenic discharges. After review by a clinical neurophysiologist all EEGs (20) were found to be normal. Fifty-five percent of EEGs were misinterpreted. Normal activity reported as abnormal included temporal theta during drowsiness, high voltage vertex waves, wicket spikes, normal phase reversals, and artifacts. Conclusions: The study demonstrates that EEG misinterpretation is very common in patients with PNES leading to erroneous diagnosis of epilepsy in patients with PNES, unnecessary treatment with antiepileptic drugs and delay in making the correct diagnosis and offering the appropriate treatment. These findings suggest that referral of suspected PNES patients for evaluation by a neurologist with qualification in clinical neurophysiology can reduce EEG misinterpretation, and can also contribute to early diagnosis of PNES and improved patient outcomes.

\subsection{9 \\ ICTAL HIGH FREQUENCY OSCILLATIONS WITH EPILEPSY SURGICAL CANDIDATES SECONDARY TO TUBEROUS SCLEROSIS COMPLEX \\ Hisako Fujiwara $^{1}$, H. M. Greiner ${ }^{1}$, D. F. Rose ${ }^{1}$, K. Holland-Bouley ${ }^{1}$, J. Leach ${ }^{3}$, T. Arthur ${ }^{1}$ and F. T. Mangano ${ }^{2}\left({ }^{1}\right.$ Neurology, Cincinnati Children's Hospital Medical Center, Cincinnati, OH; ${ }^{2}$ Pediatric Neurosurgery, Cincinnati Children's Hospital Medical Center, Cincinnati, $\mathrm{OH}$ and ${ }^{3}$ Radiology, Cincinnati Children's Hospital Medical Center, Cincinnati, $\mathrm{OH}$ )}

Rationale: Greater than $90 \%$ of children with Tuberous Sclerosis Complex (TSC) have epilepsy, many medically refractory. The intracranial features of TSC are cortical and subcortical tubers, 'empty-gyrus', subependymal nodules/giant cell astrocytomas, and white matter radial migration lines. The presence of multiple MRI lesions creates multiple potential targets for surgical resection. Recently high frequency oscillations (HFOs) $(>80 \mathrm{~Hz})$ have been shown to have a tight relationship with seizure onset zone (SOZ); it has been shown that resecting HFO-generating cortices is critical to achieving seizure freedom in several different etiologies. The aim of this study was to investigate whether ictal HFOs recorded by intracranial EEG are specific to SOZ in children with TSC.

Methods: Children with diagnosed intractable epilepsy and TSC who were selected as surgical candidate and underwent intracranial EEG before respective surgery which were performed between January 2008 and June 2011 were retrospectively investigated. The intracranial EEG was recorded with $2000 \mathrm{~Hz}$ sampling rate. The seizure onsets were determined with conventional visual inspection at $1-70 \mathrm{~Hz}$ bandwidth filter first then analyzed with $80 \mathrm{~Hz}$ high-pass filter along with time-frequency analysis using short time fast Fourier transform. Each electrode was characterized by presence of specific findings in MR image of underlying cortex (i.e. calcification, cystic component), , SOZ, presence/characteristic of HFOs and inclusion in surgical margin. The surgical outcome was also determined and evaluated for association of the above findings using statistical analysis.

Results: Thirteen patients were identified and analyzed. HFOs were present in SOZ for all patients with at least 1 year follow-up duration. The electrodes with HFOs in SOZ were categorized as lesional vs non-lesional and further categorized based on specific imaging features. Five patients were seizure-free (ILAE class 1, 38\%). Among those patients, HFOs were identified in both lesional and non-lesional categorized electrodes; however, the ratios varied from patient to patient with different types of lesions. Six patients had poor surgical outcome (ILAE 4/5, 46\%), even though 2 patients were seizure free for at least 1 year after surgery. These latter patients had a high discordance ratio between non-lesional vs lesional HFOs and SOZ. HFO characteristics in terms of the frequency band were also not specifically associated with either lesional or non-lesional categories. Conclusions: The regions with HFOs were significantly associated with SOZ in patients with TSC. However HFO regions were not always correlated with the tubers, rather dependent on the lesion characteristics (e.g., 'empty-gyrus' vs calcifications). HFOs may be a biomarker for epileptogenicity of cortex in TSC. Further studies would be helpful to determine any other factors which predict better surgical outcome.

\subsection{0 \\ CLINICAL UTILITY OF LONG-TERM VIDEO-EEG MONITORING IN A COHORT OF PATIENTS IN A LARGE REFERRAL CENTRE IN PERú}

Jose C. Delgado Rios, L. Mija, L. Portilla, W. Zapata, W. De La Cruz and D. Chacón (Epilepsy Unit, Instituto Nacional de Ciencias Neurológicas, Lima, Peru)

Rationale: The INCN is a referral public hospital in Perú with close to 90000 neurological referrals per year, being epilepsy the main cause of morbidity, in the in- and out-patient setting. Long-term Video-EEG monitoring is an essential tool for the evaluation of patients diagnosed with refractory epilepsy, either for pre-surgical evaluation and to confirm diagnosis. Our goal was to determine the utility of this procedure in this set of patients.

Methods: This is a retrospective review of 87 cases, assessed from October 2010 to April 2012 in the newly-established epilepsy monitoring unit. VEEG recordings were obtained with an 18 channel digital EEG device (NIHON KODEN) using standard montages and monitoring of vital parameters, for the duration of 1-8 hours. The indication of sleep deprivation and/or AED withdrawal was necessary in some cases. 
Results: Of the 87 total patients assessed in the 19-month period, $53 \%(n=46)$ were male, and $55 \%(n=48)$ were between $15-25$ years. $70 \%(n=61)$ had a diagnosis of focal epilepsy (52 with symptomatic epilepsy), 16\% ( $n=14)$ generalized epilepsy (93\% idiopathic) and $14 \%(n=12)$ paroxysmal non-epileptic disorders. The most common reasons for admission were a) to classify epilepsy type or syndrome and quantification of seizure frequency $(84 \%), b)$ diagnostic purposes $(77 \%)$, and c) presurgical evaluation for medically refractory epilepsy (17\%). Diagnosis was confirmed in $72 \%(n=63)$, while $28 \%(n=24)$ were discharged with a different diagnosis. These results allowed a better patient management including medication adjustment.

Conclusions: When VEEG monitoring is used correctly, it is an invaluable tool in the diagnosis and management of epilepsy. A definite diagnosis helps to minimize the social, psychiatric, and financial burden that continued spells place on the patient and his or her family.

\subsection{1 \\ PROFITS OF NOCTURNAL EEG-POLYSOMNOGRAPHY IN EPILEPSY UNITS \\ MARTA TORRES ${ }^{1}$, L. Guzman ${ }^{2}$, R. Cambrodí ${ }^{2}$, M. Toledo ${ }^{1}$, L. Seró $^{1}$, M. Gonzalez ${ }^{1}$, M. Quintana ${ }^{1}$, E. Santamarina ${ }^{1}$, A. Ferre ${ }^{2}$, M. J. Jurado $^{2}$, X. Salas-Puig ${ }^{1}$ and O. Romero ${ }^{2}\left({ }^{1}\right.$ EPILEPSY UNIT NEUROLOGY DEPARTMENT, VALL D'HEBRON UNIVERSITY HOSPITAL, BARCELONA, Spain and ${ }^{2}$ SLEEP UNIT NEUROPHYSIOLOGY DEPARTMENT, VALL D'HEBRÓN UNIVERSITY HOSPITAL, BARCELONA, Spain)}

Rationale: We studied the influence of nocturnal EEGpolysomnography (EEG-PSG) in the management of epilepsies. Methods: We made a prospective cross-sectional study on adult patients referred from the Epilepsy Unit during the last year who underwent a 12 hours nocturnal EEG-PSG. All of them had previous neuroimaging and EEG recordings. Treatments were not modified. To evaluate the usefulness of EEG-PSG, a suspected diagnosis before the examination was then compared to the final diagnosis. We considered as variables for analysis: the epilepsy diagnostic, syndromic classification, epileptogenic source localization and slepp or cardio respiratory associated disorders. The analysis was based on the reason for request.

Results: We recruited 69 patients (58\% men; age average 47 years, range from 16 to 80 ). Epilepsy was previously diagnosed in $87 \%$ of patients. Syndromically, $53 \%$ were partials, $15 \%$ generalized and $19 \%$ were unclassifiable epilepsies. Cryptogenic epilepsies were $44 \%$. Drug-resistants were $54 \%$. Nearly $12 \%$ were considered nonepileptic spells.

EEG-PSG was request to diagnose epilepsy in 22 patients. Syndromic classification was achieved in six out of eight patients with unclassified epilepsy. Lobar localization was defined in nine out of 12 previous non-localized epilepsies $(p<0,05)$. Four seizures were recorded among all patients. SAHS were confirmed in 9 of 11 cases $(p<0,05)$. However, an incidence of $38 \%$ was observed in patients where SAHS was not previously suspected.

Conclusions: One night EEG-PSG has proved to be a useful tool to assess the diagnostic of epilepsy and to detect unsuspected SAHS, before patients undergo further continuous video-EEG monitoring.

\subsection{2}

\section{STATUS EPILEPTICUS VERSUS RECURRENT SEIZURES} IN NEWBORNS WITH HYPOXIC-ISCHEMICENCEPHALOPATHY TREATED WITH HYPOTHERMIA AND MONITORED WITH CONTINUOUS VIDEO-EEG Martina Balestri ${ }^{1}$, I. Guidotti ${ }^{3}$, S. Pro ${ }^{1}$, L. Lugli ${ }^{3}$, M. L. Lispi ${ }^{1}$, L. $\mathrm{Ori}^{3}$, D. Longo ${ }^{2}$, A. Todeschini ${ }^{4}$, F. Vigevano ${ }^{1}$, F. Ferrari ${ }^{3}$ and M. R. Cilio $^{1}\left({ }^{1}\right.$ Neurology Department, Bambino Gesú Children's Research Hospital, Rome, Italy, Neurology Unit, Rome, Italy; ${ }^{2}$ Radiology Department, Neuroradiology Unit, Rome, Italy; ${ }^{3}$ University Hospital of Modena, Division of Neonatology, Modena, Italy and ${ }^{4}$ University Hospital of Modena, Division of Radiology, Modena, Italy)

Rationale: Therapeutic hypothermia (TH) is becoming standard of care for newborns with hypoxic-ischemic encephalopathy (HIE). While neonatal seizure is considered an important risk factor for brain injury in this population, recent studies suggest that the outcome after seizures is not uniformly poor.

We describe the association between isolated or recurrent seizures and status epilepticus (SE) and outcome using brain MRI as an early outcome measure.

Methods: Consecutive newborns who underwent whole body cooling at Bambino Gesú Children's Hospital, Rome and University Hospital of Modena, Italy between March 2009 and June 2012 were studied with continuous V-EEG monitoring during TH and rewarming. Criteria for $\mathrm{TH}$ were based on those used in randomized controlled trial. SE was defined as continuous seizure activity for at least 30 minutes or recurrent seizures for over $50 \%$ of $1-3$ hours recording time. Seizures were treated with antiepileptic drugs including midazolam, phenobarbital, phenytoin and levetiracetam according to institutional guidelines. MRI was performed shortly after rewarming and injury was scored as "none-mild" or "moderatesevere" using a system strongly predictive of neurodevelopmental outcome following neonatal HIE (Barkovich et al. AJNR, 1998). Results: 44 patients ( 22 males) who completed 72 hours TH with continuous video-EEG monitoring were included in this study. Video-EEG was initiated at mean of 6.8 hours (range 2-18) and it had a mean duration of 75 hours (range 13-138). Seizures were identified in $22 / 44(50 \%)$ patients. Seizures onset varied from 2 to 78 hours of life (mean: 23.8 hours of life). While most newborns with seizures $(20 / 22,91 \%)$ had seizure onset during TH, $1(4.5 \%)$ presented with seizures during rewarming and $1(4.5 \%)$ shortly after rewarming. Among the 22 newborns with seizures, 14 (63\%) presented with isolated or recurrent seizures and $8(37 \%)$ had SE. 12 patients $(55 \%)$ never showed a clinical correlate during seizures, including 4 with subclinical SE.

1 patient with SE died shortly after TH was completed and could not be studied with MRI. 28 infants (65\%) had none-mild MRI injury while moderate-severe MRI injury was observed in 15 children $(35 \%)$. Among those neonates with isolated-recurrent seizures, 8 $(57 \%)$ had none-mild brain injury and $6(43 \%)$ had moderate-severe injury. All neonates who had SE had moderate-severe brain MRI injury. 22 neonates never had seizures. Among those, 20 (91\%) had a normal MRI and 2 had moderate-severe MRI injury.

Conclusions: While isolated or recurrent seizures were recorded in $43 \%$ of infants with moderate to severe brain injury, not all were associated with moderate to severe damage, as $57 \%$ of neonates with seizures were spared from brain injury. In contrast, SE was diagnosed only in newborns with unfavorable outcomes such as death or severely abnormal MRI. Finally, the high rate of subclinical seizures strongly suggests that continuous EEG monitoring is required for seizure detection and treatment in this population.

\subsection{3 \\ SIGNIFICANCE OF GENERALIZED PAROXYSMAL FAST ACTIVITIES IN CHILDREN WITH INTRACTABLE LOCALIZATION- RELATED EPILEPSY \\ Mahmoud Mohammadi ${ }^{1,2}$, A. Ochi ${ }^{1}$, C. Go ${ }^{1}$, T. Okanishi ${ }^{1}$ and H. Otsubo $^{1}$ ( ${ }^{1}$ Division Neurology, Hospital for Sick Children Toronto, North York, ON, Canada and ${ }^{2}$ Pediatric Neurology, Children's Medical Center, Tehran University of Medical Sciences, Tehran, Islamic Republic of Iran)}

Rationale: Generalized paroxysmal fast activity (GPFA) consists of burst of generalized rhythmic discharges; $100-200 \mu \mathrm{V} ; 8-26 \mathrm{~Hz}$; lasting 2-50 seconds; with frontal predominance; appearing during NREM sleep. We analyzed GPFA in children with intractable localization-related epilepsies, who underwent surgical resection to 
understand a significance of GPFA correlating with the epileptogenic zone.

Methods: We collected patients with GPFA among the patients who underwent scalp video EEG, MRI, MEG, intracranial video EEG and surgical resection. We analyzed characteristics of GPFA, correlating with clinical features, findings of EEG and surgical resection. Results: We found 14 (13.5\%) patients ( 7 females) with GPFA among 103 patients who underwent epilepsy surgery at the Hospital for Sick Children in Toronto (2004-2012). Age of seizure onset ranged between 2 months to 10 years old (mean, 4.1 years). Five patients presented with partial seizures, and two patients had epileptic spasms. Seven patients had multiple type of seizures. Epilepsy surgery was performed between 5 to 18 years of age (mean, 12 years).

GPFA appeared bilaterally in NREM sleep in all patients. The amplitude ranged $90-300 \mu \mathrm{V}$. Seven patients presented the predominantly higher amplitude of GPFA in one hemisphere which was surgical site. The frequencies of spikes ranged $8-16 \mathrm{~Hz}$. Rhythmic spike patterns were associated with spike and slow wave complex in 12 patients. The other 2 patients did not have spike and slow waves during and after GPFA. The duration ranged 1-6 seconds. GPFA lasting 5 seconds appeared at the seizure onset in one patient. GPFA occurred frequently (more than $60 \%$ of NREM sleep) in 7 patients, intermittently $(30 \%-60 \%)$ in 2 and occasionally $(<30 \%)$ in 5.

Conclusions: GPFA can be seen in the intractable localizationrelated epilepsy. The amplitude predominance of GPFA correlated with the epileptogenic hemisphere.

\section{Neurophysiology: Other Clinical EEG}

\subsection{4 \\ CLINICAL CHARACTERISTICS AND PROGNOSIS OF GENERALIZED SPIKE WAVE DISCHARGES WITH BENIGN FOCAL DISCHARGES OF CHILDHOOD}

T. Fangsaad ${ }^{2,1}$, L. Liu ${ }^{2}$ and N. Connolly ${ }^{2}\left({ }^{1}\right.$ Neurology, University of Rochester, Rochester, NY and ${ }^{2}$ Bhumipol Hospital, Bangkok, Thailand, Rochester, NY)

Rationale: To retrospectively evaluate the frequency of occurrence, the relevant clinical features, and the EEG evolution of combinations of generalized spike wave discharges (GSWD) and benign focal discharges of childhood (BFDC) in patients at the University of Rochester's Strong Epilepsy Center (SEC) between 2000 and 2009.

Methods: The archival EEG database and URMC's Allscripts database were the data sources and reviewed for this study. Inclusion criteria:

1) Males and females 1-20 years old, inclusive, who have been identified as having benign focal discharges and generalized spike discharges with normal EEG background

2) Normal neurological status

3) Normal brain imaging

Results: A total of 489 cases were identified. We eliminated 388 patients with benign focal discharges of childhood with centrotemporal spikes (BCECT) and 54 with benign focal discharges of childhood with occipital spike/paroxysmal (BEOP) alone. The remaining 47 patients had combined diagnoses. Age range was 218.5 years, average age was 8.2 years. Of these, $39(83 \%)$ had BCECT + GSWD and $8(17 \%)$ had BEOP+GSWD. The average age of seizure onset was 6.1 years.

There were generalized seizure in 34 patients $(72.3 \%)$ which included $28 \mathrm{BCECT}+\mathrm{GSWD}$ and $6 \mathrm{BEOP}+\mathrm{GSW}$ patients. There were focal seizures in 8 patients $(17 \%) 6$ of which had BCECT+GSWD and 2 had BEOP+GSWD. Three patients $(6.4 \%)$ with BCECT+GSWD had both generalized and focal seizures while the 2 BCECT+GSWD patients $(4.3 \%)$ had no seizures. A photoparoxysmal response was present in $8(17.2 \%)$, with $1 \mathrm{BEOP}+\mathrm{GSWD}$ and $7 \mathrm{BCECT}+\mathrm{GSWD}$ patients. There was a positive family history of seizures in 17
$(36.2 \%)$, negative in $25(53.2 \%)$ and unknown in $5(10.6 \%)$. Imaging was done in $27.7 \%$.

Normalization of the EEG during the 10 year period was $25.5 \%$ and average age to normalization 11.8 years. $10.6 \%$ resolved to only GSWD and $17 \%$ to only BCECT or BEOP. Age of first EEG with combined discharges was 8 for BCECT + GSWD and 11.6 for $\mathrm{EOP}+\mathrm{GSWD}$. On first diagnostic study, $4.26 \%$ had a normal first EEG, $12.8 \%$ had BCECT and $8.5 \%$ had GSWD. The majority of first EEGs (74.5\%) had combined discharges

Conclusions: We found the frequency of GSWD was higher in the BCECT group than in those with BEOP. However, the number of patients with BEOP was significantly smaller than that with BCECT, therefore the occurrence of BEOP in combination with GSWD was notably higher than BCECT combined with GSWD. The onset of BCECT, BEOP or GSWD can be first, followed by the second type, however, we often found a combination in the majority of first EEGs (74.47\%). Interestingly, the age of appearance of BEOP with GSWD was older than BCECT with GSWD. Clinical manifestation was categorized as generalized seizure more than focal seizure, in both groups. The few patients who had both seizure types were only in the BCECT+GSWD group, as were the few who had no detectable seizures. During the 10 years covered by the data, normalization of EEG occurred $25.53 \%$ and revealed changes in epileptiform discharges in both directions, i.e., combinations resolved to a single discharge type.

\subsection{5 \\ POST RESECTION ELECTRO-CORTICOGRAPHY PREDICTS SEIZURE OUTCOME - A MULTIVARIATE LOGISTIC ANALYSIS \\ Omotola Hope $^{1}$, P. Velur ${ }^{1}$, G. Kalamangalam ${ }^{1}$, J. Slater ${ }^{1}$ and N. Tandon $^{2}\left({ }^{1}\right.$ Neurology, UTHSC-Houston, Houston, TX and ${ }^{2}$ Neurosurgery, UTHSC-Houston, Houston, TX)}

Rationale: The utility of post resection electro-corticographic (ECoG) recordings following epilepsy surgery, in predicting outcome or guiding additional resection is unclear. We perform post-resection ECoG in patients undergoing resective surgery for epilepsy, though this is not generally used to modify the surgical plan. Thus we make predictive assessments of the inherent value of post-resection ECoG in seizure outcome. Additionally, given the relatively large numbers, we are able to control for relevant confounders such as location of the epilepsy, presence of an obvious lesion and duration of the epilepsy. Methods: From a prospectively compiled database of 195 patients undergoing epilepsy surgery by a single surgeon at the University of Texas Comprehensive Epilepsy Program, we compiled a list of all adult patients (Age $>18)$ who had undergone post-resection ECoG following respective surgery and had follow-up data for a minimum of six months post-operatively. Demographic data, clinical details such as duration of epilepsy, age at onset, the type of resection (neocortical vs. limbic), lobe of resection, etiology of epilepsy (from histo-pathology, imaging and clinical presentation) and outcome (Engel and ILAE scores) were compiled and updated based on most recent follow-up. An ordered logistic regression analysis was used to predict seizure outcome as measured by the ILAE scale was performed (Stata). Independent variables in the model were post resection ECoG $(0=$ no findings, $1=$ subtle or rare discharges $2=$ significant discharges), age of onset of epilepsy, duration of epilepsy, resection type $(1=$ mesial temporal, $2=$ temporal neocortical and $3=$ extra-temporal) and pathology type ( $1=$ cavernoma/tumor, $2=$ mesial temporal sclerosis (MTS) / focal cortical dysplasia (FCD), $3=$ gliosis / heterotopic neurons/encephalomalacia).

Results: Seventy five patients were included in the analysis (mean age $35 \mathrm{yrs}, 56 \%$ female). The mean duration of years living with epilepsy prior to surgery was 17.9 years.

There were $52(69.3 \%)$ mesial temporal lobe surgeries, 7 (9.3\%) temporal neocortical only and $16(21.3 \%)$ extratemporal neocortical cases. Pathology identified 15 cases of cavernoma/tumor (group 1), 
40 cases of mesial temporal sclerosis/ focal cortical dysplasia (group two) and 20 cases of other non-specific pathologies such as gliosis, encephalomalacia (group three).

A logistic regression model incorporating these variables revealed that more nonspecific pathologies categorized in group 3 (gliosis and heterotopias and encephalomalacias) $>\mathrm{MTS} / \mathrm{FCD}>$ defined lesions categorized in group 1 (such as tumor/cavernoma) predicted a higher i.e. worse ILAE outcome score (OR 1.65 (1.29; 5.46). Also, the presence of post resection spikes significant $>$ rare $>$ none predicts higher ILAE outcome score (OR=1.91 (1.08; 3.38).

Conclusions: The underlying pathology and the presence of post resection spike discharges alone, not the location of surgery predict worse outcomes after epilepsy surgery. This study clearly demonstrates the significant impact of no epileptiform discharges on seizure free outcome.

Table 1: Ordered logistic regression model predicting ILAE outcome

\begin{tabular}{|c|c|c|}
\hline Varriable & Odds ratio $(95 \% \mathrm{Cl})$ & p-value \\
\hline Age at onset of epilepsy & $1.02(0.98 ; 1.06)$ & 0.323 \\
Duration of epilepsy & $1.00(0.97 ; 1.04)$ & 0.88 \\
FCoGi result & $1.91(1.08 ; 3.38)$ & 0.03 \\
Type of resection & $0.95(0.56 ; 1.62)$ & 0.86 \\
\hline Path type & $2.65(1.29 ; 5.46)$ & 0.01 \\
\hline
\end{tabular}

\subsection{6}

POSTICTAL GENERALIZED EEG SUPPRESSION IN CHILDREN: A POTENTIAL MARKER OF SUDEP RISK Brian D. Moseley ${ }^{1}$, E. C. Wirrell ${ }^{2}$, J. W. Britton ${ }^{3}$, C. Nelson ${ }^{3}$, R. W. Lee $^{3}$ and E. So ${ }^{3}\left({ }^{1}\right.$ Neurology, Mayo Clinic, Rochester, MN; ${ }^{2}$ Divisions of Child and Adolescent Neurology and Epilepsy, Mayo Clinic, Rochester, $\mathrm{MN}$ and ${ }^{3}$ Division of Epilepsy and Section of Electroencephalography, Department of Neurology, Mayo Clinic, Rochester, MN)

Rationale: Although the pathophysiologic mechanism of sudden unexpected death in epilepsy (SUDEP) is unknown, autonomic dysfunction is thought to be the most likely. It has been hypothesized that respiratory depression resulting in SUDEP may be secondary to postictal generalized electroencephalography (EEG) suppression (PGES). Although PGES has been studied in adults, its significance in children has not been fully assessed. We sought to determine whether PGES is associated with ictally mediated autonomic changes and increased risk of SUDEP in children.

Methods: Children admitted to our Pediatric Epilepsy Monitoring Unit (PEMU) between 3/2009 and 10/2011 were prospectively recruited for our study. Clinical and electrophysiological data from children with PGES (an absence of electroencephalographic activity in all leads viewed at $>=10$ microvolts amplitude immediately following seizure offset) on scalp EEG were compared to those without PGES. Data collected included the occurrence of peri-ictal tachycardia, bradycardia, and hypoxemia. SUDEP risk was assessed using SUDEP-7 Inventory scores (DeGiorgio CM et al., Epilepsy \& Behavior 2010; 19(1): 78-81).

Results: Thirty seven children with 168 seizures were analyzed. PGES was observed following 27/168 (16.1\%) seizures in 12/37 (32.4\%) children. Only primary and secondarily generalized tonic clonic (GTC) seizures were marked by PGES. PGES in children was significantly associated with peri-ictal tachycardia $(\mathrm{p}=0.019)$ and hypoxemia $(\mathrm{p}=0.005)$. For partial seizures with secondary generalization, PGES was more likely to be observed following temporal versus extratemporal onset seizures $(\mathrm{p}=0.012)$. Children with PGES also had significantly higher SUDEP-7 Inventory scores than those without PGES (4.2+/-1.3 versus $2.8+/-1.4, \mathrm{p}=0.007)$. However, the scores were not significantly different between children with and children without peri-ictal tachycardia $(3.4+/-1.3$ versus $2.5+/-1.6, \mathrm{p}=0.12)$, bradycardia $(4+/-2$ versus $2.9+/-1.4, \mathrm{p}=0.45)$, or hypoxemia $(3.4+/-1.5$ versus $2.4+/-1.3, \mathrm{p}=0.051)$.

Conclusions: Children with PGES have more risk factors for SUDEP than children without PGES as measured by the SUDEP-7 Inventory. Therefore, PGES may prove to be a useful electrophysiological finding to assess SUDEP risk in children. Conversely, peri-ictal tachycardia, bradycardia, and hypoxemia are not associated with significantly higher SUDEP-7 Inventory scores.

1.077

ANALYZING THE EEGS OF FAMILY MEMBERS OF PATIENTS WITH JUVENILLE MYOCLONIC EPILEPSY Saumya Gill ${ }^{1}$, R. M. Duron ${ }^{2}$, M. Tanaka ${ }^{3,1}$, M. T. Medina ${ }^{4}$, I. E. Martinez-Juarez ${ }^{5}$ and A. Delgado-Escueta ${ }^{1,3}$ ( ${ }^{1}$ Neurology and Research Services GLA VA and David Geffen School of Medicine, UCLA, Los Angeles, CA; ${ }^{2}$ San Lucas Medical Center, Tegucigalpa, Honduras; ${ }^{3}$ Epilepsy Genetics/Genomics Laboratory, Neurology and Research Services GLA VA, Los Angeles, CA; ${ }^{4}$ Neurology, National Autonomous University of Honduras, Tegucigalpa, Honduras and ${ }^{5}$ Neurology, National Institute of Neurology \& Neurosurgery, Mexico city, Mexico)

Rationale: Practitioners often ask-- do focal spikes or sharp waves mean secondary bilateral synchrony symptomatic of a lesion in patients with diffuse spike wave complexes? In the same vein, practitioners wonder about the significance of diffuse well formed spike wave complexes found in routine EEGs of patients without epilepsy? Do these individuals have clinical epilepsy?

Methods: To address these questions, we performed EEGs in 211 members belonging to multiplex multigenerational families of four patients with JME (juvenille myoclonic epilepsy). We screened for mulations in myoclonin 1 / EFHC1 in families one and two and performed whole exome sequencing in families 3 and 4 . EEGs were 30 minutes in duration and included photic stimulation. Most were obtained in the awake and drowsy state, not all of them had hyperventilation performed. Asymptomatic members with epileptiform EEGs did not have video EEGs.

Results: 27 members of family 1 had EEGs. Family 1 was negative for myoclonin $1 / \mathrm{EFHC} 1$ mutation. The proband and 2 clinically affected members had 3-4Hz spikewave complexes. 2 other affected members had 4-6Hz PSW and 2 others had irregularly formed diffuse sharp and slow waves with spikes. 3 clinically asymptomatic members had 4-6 $\mathrm{Hz}$ polyspike waves while 2 other asymptomatics had irregularly formed $5 \mathrm{~Hz}$ spike and multispike waves. 5 clinically asymptomatic members had focal spikes while two had bifrontal spikes. 32 members of family 2 had EEGs. Four symptomatic members with myoclonin1/ EFHC1 mutations had 3-4 Hz spike waves. Seven with mutations were asymptomatic but had 3-6 Hz polyspike waves. Two asymptomatic with mutations had focal T3/T5 or F7 spikes. 33 members of family 3 had EEGs. 5 affected members with myoclonin 3 mutations had 4-6 Hz polyspike wave while 4 asymptomatic individuals with mutations had 4-6 $\mathrm{Hz}$ polyspike waves. 119 members of family 4 had EEGs. Family 4 with myoclonin 4 mutations had 11 clinically affected members, five with 4-6 $\mathrm{Hz}$ polyspike waves and six with $3 \mathrm{~Hz}$ spikewaves. Amongst the thirteen asymptomatics with myoclonin 4 mutation, 11 had 3-4 Hz polyspike waves and 2 had temporal spikes.

Conclusions: Focal spikes or sharp waves can be the expression of the JME genotype even when the individuals are clinically asymptomatic. Focal spikes or sharp waves in patients with spike wave complexes do not necessarily mean secondary bilateral synchrony. Diffuse 3-4Hz spikewave or $4-6 \mathrm{~Hz}$ polyspike waves or bifrontal spikes can also be the expression of the JME genotype in clinically asymptomatic family members. Classic JME probands and family members have $4-6 \mathrm{~Hz}$ polyspike waves, while CAE (childhood absence epilepsy) evolving into JME probands and family members have both $3 \mathrm{~Hz}$ spike wave and 4-6 Hz polyspike waves. More EEG studies should be performed in families with proven JME mutations. 
EEG of Family members of JME patients:

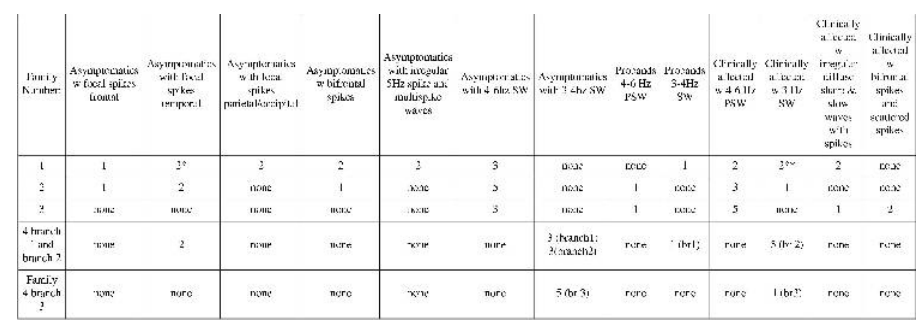

*one with focal ictal rhythm at right temporal T6

**3Hz spike and wave status in one patient

Family 4 has 3 branches as described above

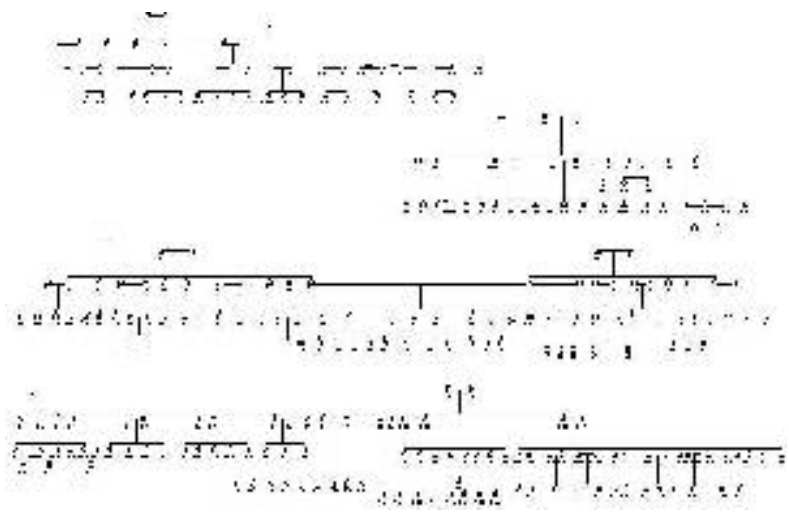

Pedigrees of Families with JME:

\subsection{8}

SLOW OSCILLATIONS IN THE MESIAL TEMPORAL LOBE PREDICT SUCCESSFUL SHORT-TERM RECALL IN A WORKING MEMORY TASK

Radhika Madhavan ${ }^{1}$, J. R. Madsen ${ }^{1}$, E. N. Eskandar ${ }^{3}$, S. S. Cash ${ }^{3}$, G. Kreiman ${ }^{1}$ and W. S. Anderson ${ }^{2}\left({ }^{1}\right.$ Neurology, Childrens Hospital Boston Harvard University, Boston, MA; ${ }^{2}$ Neurosurgery, The Johns Hopkins Hospital, Baltimore, MD and ${ }^{3}$ Neurology, Massachussetts General Hospital, Boston, MA)

Rationale: Slow oscillatory activity in the $3-10 \mathrm{~Hz}$ range in the hippocampus and the neocortex are known to play a role in the encoding of new memories and long-term recall. Intracranial electrode field potential recordings provide the temporal and spatial resolution for studying these oscillations in humans during cognitive function. In humans, theta $(3-12 \mathrm{~Hz})$ oscillations have been clearly observed in many structures, including the hippocampus during memory encoding [1] and virtual maze navigation [2]. We characterized the encoding of memory signals at multiple temporal scales and different locations in patients with epilepsy and show selective modulation of theta activity for successfully recalled memories. This effort lays the groundwork for diagnostic memory testing in epilepsy patients based on quantifying a physiologic brain rhythm.

Methods: Epileptic patients implanted with subdural electrodes for seizure localization were tested on a multi-item short-term memory task. The subjects were presented with a series of four images on a computer screen. After a 1-second delay, the patients were instructed to arrange images in the order in which they appeared using the buttons on a key-pad.

Results: Medial temporal electrodes showed transient increases in power in the $3-10 \mathrm{~Hz}$ band during the task, and these modulations were spatially localized to the temporal and prefrontal cortices. Furthermore, this increase in power in temporal lobe electrodes was significant during the correctly recalled trials $(\mathrm{p}<0.01$, Wilcoxon's rank sum test, with respect to pre-task baseline, Figure 1A), while in the incorrect trials power dropped to levels pre-trial (Figure 1B).
Conclusions: These results suggest a role of slow oscillations in the encoding of successfully

recalled memories. This spatially and temporally selective increase of theta power in the temporal structures during successful encoding of new information may be related to hippocampal theta oscillations implicated in memory formation [3]. This physiologically based quantitative assay of memory function will now be probed for utility in predicting postoperative memory outcomes in patients undergoing seizure surgery.

References

1. U. Rutishauser, I.B. Ross, A.N. Mamelak, E.M. Schuman (2010), Human memory strength is predicted by theta-frequency phaselocking of single neurons. Nature (464), 903-907.

2. M.J. Kahana, R. Sekuler, J.B. Caplan, M.Kirschen, J.R. Madsen (1999), Human theta oscillations exhibit task dependence during virtual maze navigation. Nature (399), 781-784.

3. G. Buzsaki (2002), Theta oscillations in the hippocampus. Neuron, 33(3), 325-340.

Figure 1: Temporal lobe theta activity $(3-10 \mathrm{~Hz})$ is related to task outcome. Data from an electrode on the temporal pole. A. Temporal evolution of theta for correct trials. Theta power during encoding and delay epochs was significantly higher than pre-trial baseline for successful trials $(p<0.01$, rank sum test). B. Temporal evolution of theta for incorrect trials. Theta power during encoding and delay epochs was comparable to baseline $(\mathrm{p}>0.01)$.

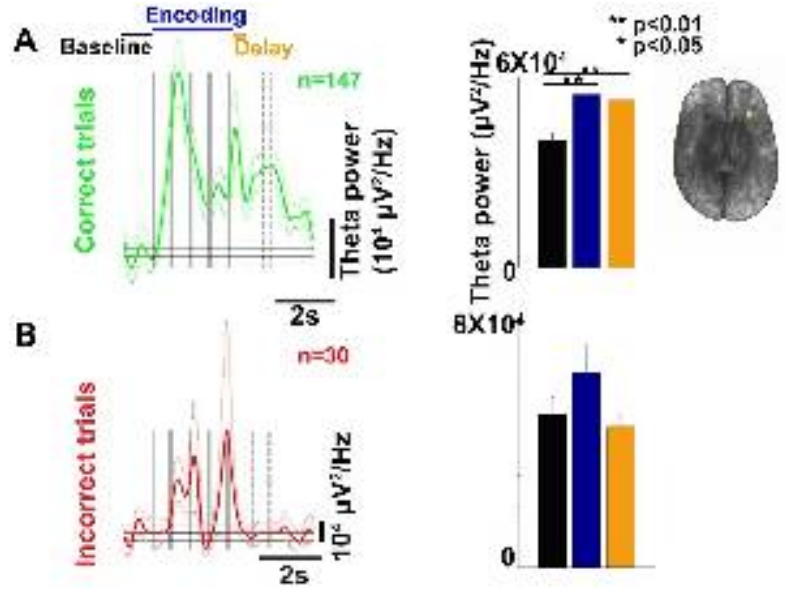

\subsection{9 \\ TIME-DOMAIN OF FOCAL INTERICTAL EPILEPTIFORM TRANSIENTS (FIET) REVISITED}

Fumisuke Matsuo (Neurology, University of Utah School of Medicine, Salt Lake City, UT)

Rationale: Systematic evaluations of polygraphic FIET analysis revealed variations in spatiotemporal characteristics at variance with conventional FIET modeling by one peak at one instant of time representing one single dominant generator. This exploratory study was designed to examine time domain of FIET in further detail with polygraphic channel overlay (PGCO) display. When baseline is controlled, PGCO can reveal subtle time-domain features prior to and following base-peak-trough-wave (BPTW) segment as well as complexity of FIET peaks (Fig). BPTW waveform template (Epilepsia 2005: 46 [Suppl 8]: 138) employed initial segment BP as reference. PGCO envelope resembles BPTW template but displays true phase reversals.

Methods: FIET samples consisted of 72 single spikes and sharp waves (SSW) from 121 mixed samples of SSW and wicket spikes (Abstract 3.117, 2011 AES Annual Meeting, www.aesnet.org). FIET were reformatted in 23 common average derivations with high and low frequency filters set at $70 \mathrm{~Hz}$ and $0.16 \mathrm{~Hz}$. PGCO were prepared manually with monitor screen images of 2-s FIET derivations with display gain controlled. PGCO were inspected with special attention 
to pre-base shift, and compared against blinded polygraphic FIET waveform ranking and BPTW scores.

Results: SSW could be differentiated against background-baseline with exception of 5 ranked at lower end. FIET ranking did not differ significantly between PGCO and blinded polygraphic review. Prebase shift was seen in 38 of 72 FIET, but its onset was not always definable.

Conclusions: SSW detection from PGCO display was more conservative, and may improve reliability of clinical EEG analysis. PGCO can reveal baseline deviation prior to BPTW segment, when display condition is favorable. Target signal magnitude and background noise-signal, of either cerebral or extracerebral origin, affected confidence of SSW detection. Display settings may have to be tailored. This method may improve clinical differentiation of SSW from FIET not predictive of epilepsy.

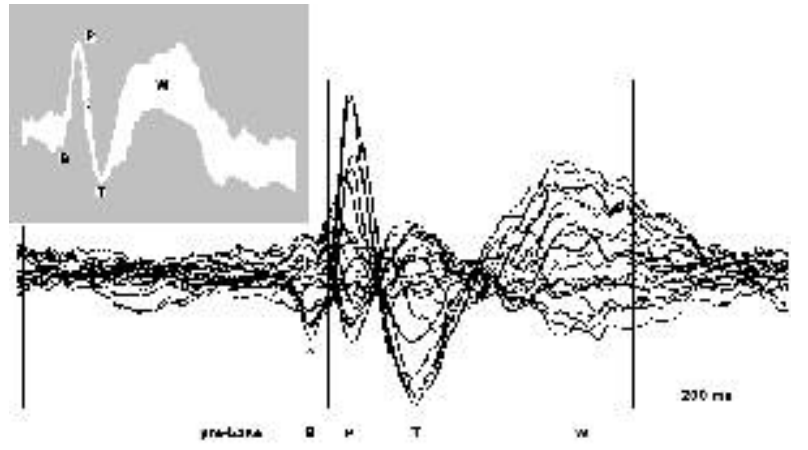

BPTW template (insert) and PGCO display. PGCO display reveals P, $\mathrm{T}$ and $\mathrm{W}$ phases consisting of multiple peaks varying in deflectionmagnitude and latency against dominant peak, resulting in variations in FIET geometry.

\subsection{0 \\ SEIZURES AND ELECTROGRAPHIC ABNORMALITIES IN PEDIATRIC MOYAMOYA DISEASE : A SINGLE CENTER STUDY}

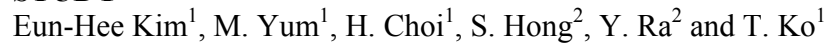
( ${ }^{1}$ Pediatric Neurology, Asan medical center, Seoul, Republic of Korea and ${ }^{2}$ Neurosurgery, Asan medical center, Seoul, Republic of Korea)

Rationale: Moyamoya disease (MMD) is an idiopathic cerebrovascular occlusive disorder and one of the main causes of pediatric stroke in Korea. Electrographically, re-build up phenomenon after hyperventilation is commonly observed. This study describes the clinical features of pediatric MMD, especially focusing on the clinical seizures as presenting symptoms or electrical abnormalities on electroencephalography (EEG).

Methods: We retrospectively analyzed the medical records of 194 patients (69 males) diagnosed as moyamoya disease, seen at the department of pediatric neurology and neurosurgery, Asan Medical Center between January 2000 and February 2012. Clinical data included age, sex, presenting symptoms, brain magnetic resonance imaging (MRI) and angiography findings, EEG findings and hospital course after surgical revascularization.

Results: Median age at diagnosis was $8.2(0.4 \sim 18)$ years and median follow-up duration after diagnosis was $6.3(1 \sim 12.2)$ years. The most common symptoms at presentation was transient ischemic attack $(150 / 194,77 \%)$, and $10(0.05 \%)$ patients presented with seizures. Brain MRI and angiography were performed in all patients, and 73 (37.6\%) patients showed cerebral infarction. Among 149 patients who performed EEG, 81 (54.4\%) showed posterior and/or centrotemporal slowings and $18(12.1 \%)$ showed epileptiform activities, which abnormalities were more frequently associated with cerebral infarction or hemorrhage $(\mathrm{p}=0.027)$. Rebuild-up phenomenon was noticed in $60(58.8 \%)$ among 102 patients who performed effective hyperventilation during EEG recording. Four patients presented clinical seizures or epileptiform activities on EEG after revascularization, caused by hypertension, intracranial hemorrhage or cerebral infarction. These postoperative seizures were also well controlled with antiepileptic medications in all four patients. Conclusions: Seizures and/or epileptiform activities on EEG were often detected in pediatric moyamoya disease. Electrographic abnormalities were significantly associated with the cerebral pathology and related seizures were well controlled with antiepileptic medication in most patients and rarely evolve into epilepsy.

\subsection{1 \\ PREDICTIVE VALUE OF THE FIRST 7 MINUTES OF 30 MINUTE EEGS IN EMERGENCY DEPARTMENT PATIENTS WITH AMS}

A. C. Grant ${ }^{1}$, J. Weedon ${ }^{1}$, Vanessa Arnedo ${ }^{1}$, G. Chari $^{1}$, E. Koziorynska $^{1}$, S. Malhotra ${ }^{2}$, D. Maus ${ }^{1}$, T. McSween ${ }^{1}$, K. A. Mortati ${ }^{1}$, A. Omurtag ${ }^{3}$, A. Reznikov ${ }^{1}$, H. Valsamis ${ }^{1}$, S. Zehtabchi ${ }^{2}$ and S. Abdel Baki ${ }^{3}\left({ }^{1}\right.$ Neurology, SUNY Downstate Medical Center, Brooklyn, NY; ${ }^{2}$ Emergency Department, SUNY Downstate Medical Center, Brooklyn, NY and ${ }^{3}$ BioSignal Group, Brooklyn, NY)

Rationale: EEG helps narrow the differential diagnosis in emergency department (ED) patients with altered mental status (AMS). We sought to determine whether a 7-minute EEG provided the same diagnostic information as a 30 -minute recording in this patient population.

Methods: As part of a study evaluating a new EEG technology, 30minute EEGs were obtained with a commercially available device and a full set of 10-20 electrodes on 261 sequential ED patients with AMS. The 227 EEGs available for review were de-identified and each one was interpreted by 2 of 6 board-certified electroencephalographers. The interpreters knew that the patients were $\geq 13$ years old and had AMS in the ED, but did not know clinical history or medications. Interpreters assigned each study to one of 6 categories: Normal (Nl), Status Epilepticus (SE), Seizure (Sz), Interictal epileptiform with or without independent slowing (Ep), Slowing only (Sl), and Uninterpretable (U). Several months later, the same EEGers blindly reinterpreted the first 7 minutes of each EEG they had read previously.

A $6 \times 6$ table of all $454(2 \times 227)$ interpretations was constructed (table). Accuracy measures of the $7 \mathrm{~m}$ interpretations as predictive of the "gold-standard" $30 \mathrm{~m}$ interpretations (predicting abnormal [including U] vs. normal) were obtained using a generalized mixed linear model with dependent variable $7 \mathrm{~m}$ interpretation, fixed effect $30 \mathrm{~m}$ interpretation, and random effects patient and rater. Similar models were used to predict SE vs. other and Sz vs. other. Bhapkar's test of homogeneity of row and column marginals was also performed.

Results: With respect to the $30 \mathrm{~m}$ recording, accuracy measures $(95 \%$ $\mathrm{CI})$ of the $7 \mathrm{~m}$ recording were: sensitivity $0.93(0.85,0.97)$, specificity 0.57 (0.36, 0.76), PPV 0.93 (0.87, 0.96), NPV 0.55 (0.36, 0.73).

Bhapkar's test was highly significant $(\mathrm{p}<0.001)$ due primarily to the finding that Ep was much more frequent in the $30 \mathrm{~m}$ recording than in the $7 \mathrm{~m}$ recording. SE was interpreted 19 times in the $30 \mathrm{~m}$ recording compared to 13 in the $7 \mathrm{~m}$ segments, with 10 interpretations in common (NPV $=0.98[0.96,0.99])$. Sz was diagnosed 8 times in both the $30 \mathrm{~m}$ and $7 \mathrm{~m}$ studies, but only 2 interpretations were common to both (NPV $=0.99[0.98,1.00])$. Two Sz interpretations in the $7 \mathrm{~m}$ segments became SE in the full study. Of $75 \mathrm{Nl}$ interpretations in the $7 \mathrm{~m}$ studies, 42 were $\mathrm{Nl}$ in the full study, and 26 of the remaining interpretations were Sl.

Conclusions: As expected, if the first $7 \mathrm{~m}$ of an EEG are interpreted as abnormal it is highly likely that the entire study will be interpreted as abnormal (PPV $=0.93$ ). Conversely, a normal first $7 \mathrm{~m}$ has low predictive value $(\mathrm{NPV}=0.55)$ for the entire study in this patient population. However, if the first $7 \mathrm{~m}$ are normal and the entire EEG abnormal, the abnormality is highly likely to be mild diffuse slowing, a relatively benign finding in ED patients with AMS. Also of clinical 
significance, if the first $7 \mathrm{~m}$ are NOT interpreted as Sz or SE, it is extremely unlikely that the entire study will be interpreted as $\mathrm{Sz}$ or SE.

\begin{tabular}{|c|c|c|c|c|c|c|c|}
\hline \multicolumn{8}{|c|}{30 minutes by 7 minutes (Frequency/Percent) } \\
\hline \multirow{2}{*}{$30 \underset{\downarrow}{\min }$} & \multicolumn{7}{|c|}{$7 \mathrm{~min} \rightarrow$} \\
\hline & Ep & N1 & S1 & SE. & Sz & $\mathbf{U}$ & Total \\
\hline \multirow{2}{*}{ Ep } & 36 & 3 & 28 & 1 & 2 & 1 & 71 \\
\hline & 7.93 & 0.66 & 6.17 & 0.22 & 0.44 & 0.22 & 15.64 \\
\hline \multirow{2}{*}{ N1 } & 0 & 42 & 26 & 0 & 0 & 3 & 71 \\
\hline & 0.00 & 9.25 & 5.73 & 0.00 & 0.00 & 0.66 & 15.64 \\
\hline \multirow{2}{*}{ S1 } & 6 & 26 & 190 & 1 & 1 & 8 & 232 \\
\hline & 1.32 & 5.73 & 41.85 & 0.22 & 0.22 & 1.76 & 51,10 \\
\hline \multirow{2}{*}{ SE } & 4 & 0 & 2 & 10 & 2 & 1 & 19 \\
\hline & .088 & 0.00 & 0.44 & 2.20 & 0.44 & 0.22 & 4.19 \\
\hline \multirow{2}{*}{$\mathrm{Sz}$} & 1 & 0 & 5 & 0 & 2 & 0 & 8 \\
\hline & 0.22 & 0.00 & 1.10 & 0.00 & 0.44 & 0.00 & 1.76 \\
\hline \multirow{2}{*}{$\mathbf{U}$} & 1 & 4 & 19 & 1 & 1 & 27 & 53 \\
\hline & 0.22 & 0.88 & 4.19 & 0.22 & 0.22 & 5.95 & 11.67 \\
\hline \multirow{2}{*}{ Total } & 48 & 75 & 270 & 13 & 8 & 40 & 454 \\
\hline & 10.57 & 16.52 & 59.47 & 2.86 & 1.76 & 8.81 & 100.00 \\
\hline
\end{tabular}

\subsection{2}

DURATION MISMATCH NEGATIVITY IN FRONTAL LOBE EPILEPSY

YUKA HIROSE $^{1}$, K. Hara ${ }^{1,2}$, S. Watanabe ${ }^{1}$, A. Matsuda ${ }^{1}$, K. Ohta ${ }^{1,3}$, M. Miyajima ${ }^{1}$, T. Maehara ${ }^{1}$, M. Hara ${ }^{2}$, E. Matsushima ${ }^{1}$ and M. Matsuura ${ }^{1}{ }^{1}$ Tokyo Medical and Dental University, Tokyo, Japan; ${ }^{2}$ Hara Clinic, Yokohama, Japan and ${ }^{3}$ Onda-daini Hospital, Matsudo, Japan)

Rationale: The mismatch negativity (MMN) is one of the most intensely investigated components of event-related potentials (ERPs). It is thought to reflect a pre-attentive auditory discrimination process and intentional switching. It has been argued that MMN has at least two main sources: the first source is located in the bilateral auditory cortex and underlies pre-perceptual sound change detection in the auditory cortex. This likely triggers the second source, the frontalcortex MMN generator, which is associated with the initiation of the attention switch to sound change. Recently, there are many MMN reports in temporal lobe epilepsy (TLE) which demonstrated the enhanced amplitudes and prolonged latencies. Conversely, there are no MMN reports in frontal lobe epilepsy (FLE) As long as we searched.

The aim of this study was to examine cognitive function in patients with FLE using duration MMN.

Methods: We examined 15 FLE patients (seven females). The mean age was $36.6 \pm 9.9$ (range 22-53 years). The mean age at epilepsy onset was $13.7 \pm 7.8$ ( 8 months to 32 years). Nine of 15 patients had been seizure free for more than 41 months. Two patients did not take AED. Four patients took 1 AED, seven patients took 2 AEDs, and two patients took $>3$ AEDs. Control data were collected from 15 agematched healthy subjects (nine females, all right handed). The stimuli were $1000 \mathrm{~Hz}$ pure tones differing in duration, such that standard stimuli had a duration of $100 \mathrm{~ms}$ and the deviant stimuli were $150 \mathrm{~ms}$ long. The MMN was recorded from the four midline ( $\mathrm{Fz}, \mathrm{Cz}, \mathrm{Pz}, \mathrm{Oz})$ sites and bilateral mastoid electrodes. The participants were instructed to watch silent cartoons during the recording session and to ignore sounds heard through their earphones. Data analysis focused on a $600 \mathrm{~ms}$ time window ranging from $100 \mathrm{~ms}$ pre-stimulus to 500 ms post-stimulus onset. At least 100 responses to deviant stimuli were analyzed for each participant. We evaluated the mean MMN amplitude and peak latency at each electrode between 190 and 340 $\mathrm{ms}$, and ERP parameters were compared by a one-way ANOVA. The study was approved by the Ethics Committee of the Tokyo Medical and Dental University. Written informed consent was obtained from all the participants after a thorough description of the study.

Results: Although the FLE patients appeared to show smaller MMN amplitudes than the controls (HC $-0.66 \pm 0.89$ vs., FLE $=-0.24 \pm-$ 0.88 micro-volts), there was no significant difference in the mean amplitude or peak latency at $\mathrm{Fz}(\mathrm{HC} 247 \pm 29$ vs. FLE $=227 \pm-30$ vs., ms).

Conclusions: We have previously demonstrated an impairment of pre-attentive processing in TLE patients. This study showed that FLE patients had a similar waveform compared to controls. The results suggest that FLE patients did not have impairments of auditory cognitive processing. Therefore, the temporal lobe, rather than the frontal lobe, may be the responsible MMN generator. Larger studies with more subjects will be necessary to investigate the MMN in FLE.

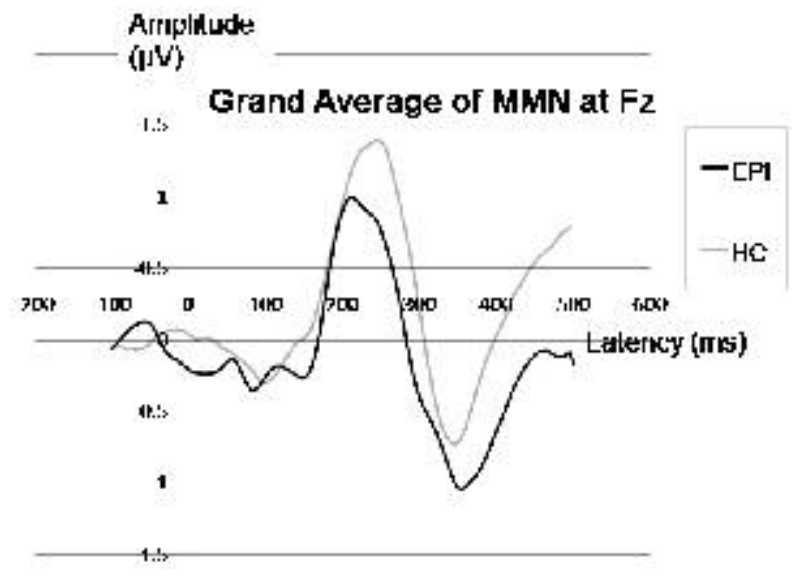

\subsection{3 \\ THE DIAGNOSTIC VALUE OF EEG IN VETERANS WITH SYNCOPE}

Firas Bannout $\left({ }^{1}\right.$ Neurology, Central Arkansas Veterans Healthcare System (CAVHS), Little Rock, AR and ${ }^{2}$ Neurology, University of Arkansas for Medical Sciences (UAMS), Little Rock, AR)

Rationale: EEG has low sensitivity in epilepsy at $25-56 \%$. Specificity is $78-98 \%$. About $50 \%$ of patients with epilepsy show Interictal Epileptiform Discharges (IEDs) in the first test (1). The incidence of IEDs in healthy population is $0.5 \%$ (2).

This study is designed to assess the EEG diagnostic value in patients with syncope, a very common symptom presenting among veterans, given the advanced age of patient population. EEG is frequently requested to rule out seizures as the leading cause of syncope. Methods: A survey on outpatient EEG demographics was conducted blindly from results. Included referral reasons were syncope, loss of consciousness, unresponsiveness, blacking out, passing out and collapsing spells without association with seizure-like activity. All inpatient EEGs and other referral reasons (episodic focal deficits, non-epileptic events and new-recurrent seizures) were excluded. A total of 639 EEGs read by an epileptologist and board certified neurophysiologist from Sep-09 to Jun-12 were researched. A total of $141(22.06 \%)$ EEGs were selected. EEGs were classified as normal vs abnormal and as having (IEDs) or not.

Results: From 141 EEGs with syncope, 103 (73.04\%) were normal and $38(26.95 \%)$ were abnormal. The presence of (IEDs) was found only in $3(2.12 \%)$ EEGs from different patients. From the 38 abnormal EEGs, 2 (5.26\%) had focal slowing alone, 19 (50\%) had diffuse slowing and $14(36.48 \%)$ had both.

In order to calculate the EEG sensitivity and specificity in this cohort, we eliminated repeated EEGs from same patients. 23/134 patients were found to have new or known diagnosis of seizures and 111 patients didn't. (IEDs) were found in $1 / 23$ and $2 / 111$ patients. Focal slowing was found in $5 / 23$ and $10 / 111$ patients. 
In veterans with syncope, the EEG sensitivity to capture (IEDs), specific markers for seizures, was only $4 \%$. The specificity, however, was $98 \%$. The positive predictive value (PPV) was $33 \%$ and the negative predictive value (NPV) was $85 \%$. However, after combining focal slowing and IEDs, the sensitivity was $26 \%$, specificity was $89 \%$, (PPV) was $33 \%$ and (NPV) was $85 \%$.

Conclusions: Our data were harmonious with other results (3) in concluding that EEG has a very low diagnostic value in screening whether seizure is the cause of syncope. The EEG sensitivity in syncope for capturing (IEDs) is low at $4 \%$ and remains low at $26 \%$ despite combining IEDs with focal slowing if the later is considered a seizure marker in certain population. In veterans, however, there is a high incidence of focal slowing due to the advanced age.

Nevertheless, the EEG provides a good probability of excluding the diagnosis of seizures as a leading cause of syncope given the reasonable high (NPV).

References:

1) S J M Smith. EEG in the diagnosis, classification, and management of patients with epilepsy. J Neurol Neurosurg Psychiatry 2005

2) Gregory RP, et al. EEG epileptiform abnormalities in candidates for aircrew training. Electroencephalogr Clin Neurophysiol1993 3) Dantas FG, et al. The role of EEG in patients with syncope. Department of Physiotherapy, State University of Paraiba, Brazil, J Clin Neurophysiol. 2012 Feb

\section{Neurophysiology: MEG}

\subsection{4 \\ INTRACRANIAL EEG POTENTIALS SIMULATED FROM MEG SOURCES: A NEW APPROACH TO EVALUATE THE SPATIAL EXTENT OF MEG SOURCES WITH IEEG MEASUREMENTS}

Christophe Grova ${ }^{1,2}$, M. Aiguabella ${ }^{1}$, J. M. Lina ${ }^{3}$, J. Hall ${ }^{1}$ and E. Kobayashi ${ }^{1}\left({ }^{1}\right.$ Montreal Neurological Institute, Montreal, QC, Canada; ${ }^{2}$ Biomedical Engineering Dpt, McGill University, Montreal, QC, Canada and ${ }^{3}$ Electrical Engineering Dpt, Ecole de Technologie Supérieure, Montreal, QC, Canada)

Rationale: Detection of epileptic discharges in MagnetoEncephaloGraphy (MEG) from background brain activity requires synchronized neuronal activity over a minimum of $4 \mathrm{~cm} 2$, as estimated when compared with ElectroCorticoGraphy data (AgirreArrizubieta Z. et al Brain 2009, Oishi et al Epilepsia 2002). We proposed and evaluated the principle of the Maximum Entropy on the Mean (MEM) (Grova et al. Neuroimage 2006, Lina et al. IEEE TBME 2012), as a distributed sources localization method able to accurately recover the generators of epileptic spikes together with their spatial extent along the cortical surface. The purpose of this study is to assess how the spatial extent recovered from MEG sources could be validated using intracranial EEG (iEEG) recordings. In order to take into account the limited spatial sampling of implanted depth electrodes, we propose to simulate iEEG potentials from MEG sources, to assess what part of MEG sources is seen by iEEG contacts.

Methods: We evaluated 4 patients with drug-resistant focal epilepsy who had a pre-operative MEG acquisition and who underwent iEEG with MRI-compatible electrodes for precise registration of recording contacts. Three patients had right orbitofrontal (ROF) spikes and one patient had spikes in the right supplementary motor area (SMA). MEG was acquired in a CTF System equipped with 275 axial gradiometers. Similar spikes were marked and averaged in both MEG and iEEG recordings. Averaged MEG spikes were localized, using MEM, along the cortical surface segmented from an anatomical MRI. This MRI was subsequently co-registered with the MRI obtained with iEEG electrodes in place, for precise identification of every iEEG contact. An iEEG forward model estimating the influence of every dipolar source of the cortical surface on each iEEG contact was then estimated. Applying this iEEG forward model to MEG sources, we simulated the iEEG potentials that would have been generated by these MEG sources estimated along the cortical surface. MEGsimulated iEEG potentials were compared with real iEEG signals at the peak of the averaged spikes.

Results: Excellent MEG/iEEG correlation was found over few lateral ROF iEEG contacts for Patient \#1 (Figure 1) and \#2, and over several contacts of three SMA electrodes for patient \#4 (Figure 2). For patient \#3, the main generator identified at a deep mesial ROF iEEG contact was not found in MEG sources. Moreover, MEG-simulated iEEG data showed that the main right frontal lateral MEG source could not have been recorded in iEEG. However, an early generator (half the peak of the spike) was identified in iEEG and MEGsimulated iEEG data over few lateral ROF contacts.

Conclusions: Precise location of active iEEG contacts allows optimization of the anatomical correlation between MEG and iEEG findings. We demonstrated that simulating iEEG potentials from MEG sources allows quantifying (1) what percentage of the cortical surface was covered by each patient-specific iEEG implantation and (2) whether the spatial extent of spike generators estimated in MEG could be validated using iEEG.

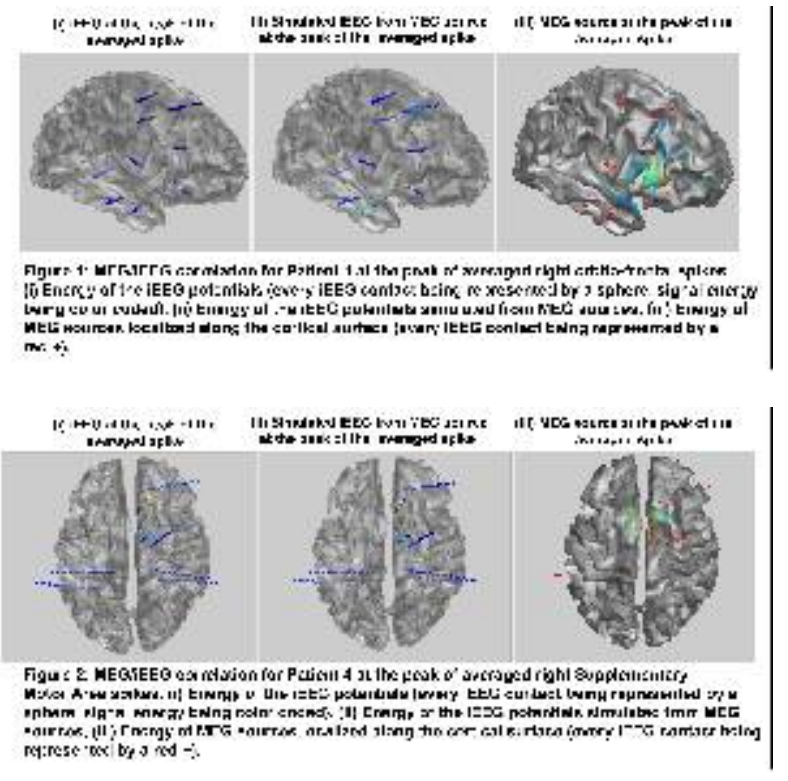

\subsection{5 \\ WHOLE-BRAIN BETA-BAND FUNCTIONAL CONNECTIVITY INCREASES WITH EXTENDED DURATION OF FOCAL EPILEPTIFORM ACTIVITY Deepak Madhavan $^{1,2}$, E. Heinrichs-Graham ${ }^{3}$ and T. W. Wilson ${ }^{1,2}$ $\left({ }^{1}\right.$ Deparment of Neurological Sciences, University of Nebraska Medical Center, Omaha, NE; ${ }^{2}$ Center for Magnetoencephalography, University of Nebraska Medical Center, Omaha, NE and ${ }^{3}$ Department of Psychology, University of Nebraska Omaha, Omaha, NE)}

Rationale: The analysis of interictal epileptiform discharges (IEDs) using magnetoencephalography (MEG) is utilized for the localization of seizure onset zones in the presurgical planning of epilepsy patients. Additionally, resting-state functional connectivity analyses using the IED area may provide novel insight into the underlying brain networks. In this study, we evaluate whether chronicity of seizures is related to whole-brain functional connectivity metrics using the area of IED generation (derived from MEG) as the seed region.

Methods: We studied 12 adults (4 females) with intractable complex partial epilepsy, who were undergoing presurgical evaluation, including MEG. All participants were scheduled for MEG after a night of sleep deprivation to increase the frequency of interictal spikes. Each identified IED was localized using data from the 204 
gradiometer-type sensors and a single equivalent-current dipole model (ECD). Phase coherence, a measure of functional connectivity that quantifies the phase relationship between two time varying signals (i.e., the seed region and any other brain area), was computed using a dense grid of regional source spaced equidistantly and the IED area as the seed region.

Results: Pearson correlational analyses indicated that the duration of epilepsy was positively correlated with the amplitude of beta-band functional connectivity between the epileptogenic zone and other brain areas as measured by the PLV, $\mathrm{r}(12)=0.61, \mathrm{p}<0.05)$. Phaselocking involving the epileptogenic zone in the delta, theta, and alpha bands was not statistically related to the duration of epilepsy. Conclusions: the significant presence of only beta band functional connectivity between regions of IEDs and other brain areas as a function of epilepsy chronicity was surprising, and raises several questions. Increases in beta activity have been seen in areas of increased GABAergic modulation (particularly with GABA-A receptor activation), thought to reflect increased inhibitory interneuron activity. Such knowledge could eventually contribute to the goal of augmenting a patient's endogenous cerebral antiepileptogenic mechanisms using targeted intracranial drug therapy and/or neurostimulation techniques, or for cellular therapy.

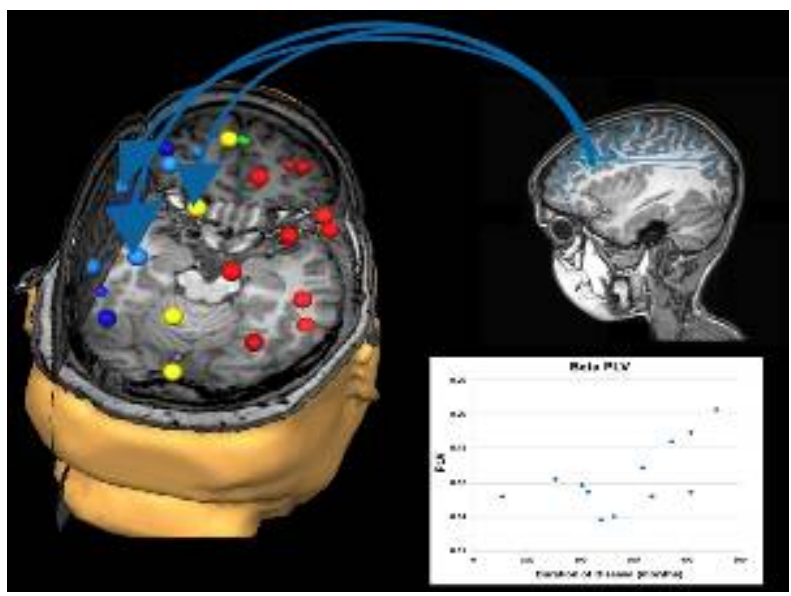

\subsection{6 \\ COMPARISON OF ONE-HOUR MEG AND MULTI-DAY SCALP VIDEO-EEG FOR PRESURGICAL EVALUATION IN 300 CONSECUTIVE EPILEPSY PATIENTS}

Susumu Ito ${ }^{1,2}$, Z. I. Wang ${ }^{1}$, J. C. Mosher ${ }^{1}$, A. V. Alexopoulos ${ }^{1}$ and R. C. Burgess ${ }^{1}$ ( ${ }^{1}$ Epilepsy Center, Cleveland Clinic, Cleveland, $\mathrm{OH}$ and ${ }^{2}$ Department of Pediatrics, Tokyo Women's Medical University, Shinjuku, Japan)

Rationale: To elucidate the utility and limitation of short-time MEG study for presurgical evaluation in epilepsy patients, supplementing the standard long-term in-patient scalp EEG (VEEG) monitoring. Methods: We reviewed the medical records of consecutive epilepsy patients who were considered candidates for epilepsy surgery, underwent VEEG and, within 6 months, also had a routine1-hour MEG, between February 2008 and January 2012. The detectability, localization and concordance of epileptiform discharges between VEEG and MEG were analyzed. Chi-square test with Bonferroni correction was used for statistics.

Results: A total of 300 consecutive patients ( 160 male, 140 female) were included. Nine patients underwent 2 MEGs, and 5 out of 9 also underwent a second VEEG. Median age was 20.3 years (range 9 months - 68 years) and duration of epilepsy was 9.0 years $(0-52$ years). The mean duration of VEEG was 4.8 days ( 1 - 13 days). Interictal epileptiform discharges were identified in 245 out of 305 $(80.3 \%)$ VEEGs and in 228 out of 309 (73.8\%) MEGs. Ictal discharges were identified in $274(89.8 \%)$ VEEGs and in $39(12.6 \%)$ MEGs. Taken together, epileptic discharges were identified more often in the 5-day VEEG than in the 1-hour MEG: 294 (96.4\%) VEEG vs $237(76.7 \%)$ MEG ( $<<0.0001)$. However, results which localized the discharges were more similar: 241 (79.0\%) 5-day VEEG vs 231 (74.8\%) 1-hour MEG, $(\mathrm{p}=0.21)$. The locations of these discharges totaled $249(28.1 \%)$ in frontal lobe, $280(31.6 \%)$ in temporal, $118(13.3 \%)$ in central, $157(17.7 \%)$ in parietal, and 83 $(9.4 \%)$ in occipital. The concordance of the discharges between VEEG and MEG was $22.9 \%$ in frontal, $38.6 \%$ in temporal, $18.6 \%$ in central, $21.7 \%$ in parietal, and $27.7 \%$ in occipital. Furthermore, the proportion of the localizable discharges only detected by MEG was $28.9 \%$ in frontal, $23.2 \%$ in temporal, $19.5 \%$ in central, $40.8 \%$ in parietal, and $49.4 \%$ in occipital. The concordance between VEEG and MEG was significant in the temporal lobe $(\mathrm{p}<0.05)$, and MEG's ability to detect and localize discharges not localized on VEEG was significantly higher in the occipital lobe $(\mathrm{p}<0.05)$. Of 111 patients who underwent lobar or multilobar resection, the resection lobe(s) was indicated by both VEEG and MEG in 35 (31.5\%), by the union of VEEG and MEG in 7 (3.3\%), by only VEEG in 27 (24.3\%), and by only MEG in $13(11.7 \%)$; and the resection was partially indicated by VEEG and MEG in $8(7.2 \%)$, by only VEEG in $1(0.9 \%)$ and by only MEG in $2(1.8 \%)$. Thusfar, 6-month surgical outcome is available in 92 patients, and $52(56.5 \%)$ were seizure free. The patients whose resected lobe(s) was indicated by both VEEG and MEG are more likely to be seizure free (19 of 27 patients, $70.4 \%$ ) $(p=0.08)$. For those patients who underwent surgery, the MEG provided unique localizing information in an additional $22(19.8 \%)$. Conclusions: Compared to a 5-day VEEG in-patient monitoring stay, a one-hour MEG study localizes the majority of the epileptiform discharges in all lobes, providing unique additional localization especially in the occipital lobes.

\subsection{7 \\ MAGNETOENCEPHALOGRAPHY FOR PRESURGICAL EVALUATION OF NONLESIONAL REFRACTORY EPILEPSY \\ Dang Nguyen ${ }^{1}$, T. Tayah $^{1}$, A. Bouthillier ${ }^{1}$, A. Bérubé ${ }^{1}$, P. Cossette ${ }^{1}$, P. FInet ${ }^{1}$, J. M. Saint-Hilaire ${ }^{1}$, M. Robert ${ }^{1}$, J. M. Leroux ${ }^{1}$, C. Grova ${ }^{2}$, M. Lassonde ${ }^{3}$ and I. Mohamed ${ }^{4}\left({ }^{1}\right.$ Notre Dame Hospital, Montreal, QC, Canada; ${ }^{2}$ Montreal Neurological Institute, Montreal, QC, Canada; ${ }^{3}$ CERNEC, Montreal, QC, Canada and ${ }^{4}$ IIWK Health Center, Halifax, NS, Canada)}

Rationale: For patients with nonlesional refractory focal epilepsy (NLRFE) drug-resistant focal epilepsy these patients, localization of the epileptogenic zone is more arduous and invasive EEG (icEEG) is frequently required. A number of studies have looked at the added value of MEG in the presurgical evaluation of refractory focal epilepsy. Most of these studies have however indiscriminately mixed patients with lesional (a subpopulation where the location of the epileptic zone can already be fairly assumed) and nonlesional epilepsies. In the present study, we report our experience with MEG in a large series of NLRFE evaluated for epilepsy surgery.

Methods: Between April 2006 and May 2012, 61 consecutive patients (mean age 34 years) with NLRFE underwent a MEG study. For the first 37 patients (group A), MEG results were not included in the preoperative consensus for several reasons (pipeline set-up, personnel recruitment and training, learning curve for interpretation of results etc.). For the remaining 24 subjects (group B), MEG findings were presented during the epilepsy surgery conference after initial review of standard presurgical work-up. To determine if MEG results would have changed patient management, anonymized results of the standard presurgical evaluation for all patients were presented randomly to a multidisciplinary epilepsy surgery team at first blinded to MEG results and then with the results. Correlation between MEG and icEEG findings and clinical outcome were also evaluated.

Results: Based on the standard presurgical evaluation, 18/61 (30\%) were presumed to have nonlesional temporal lobe epilepsy and 43/61 $(70 \%)$ nonlesional extratemporal lobe epilepsy. MEG showed spikes 
in 56/61 patients (92\%). For group A, MSI would have changed the initial management in 24/37 $(65 \%)$ had the results been available at the time of decision whether it be by (a) reducing the number of icEEG contacts (42\%); (b) increasing the number of icEEG contacts $(17 \%)$; (c) keeping the same number of icEEG contacts but changing their position (4\%); (d) allowing for direct surgery without icEEG (13\%); (e) providing enough evidence to now justify an icEEG study in previously rejected candidates $(13 \%)$ or in patients initially directed to surgery (4\%); or (f) rejecting a case previously destined for icEEG as the cluster overlaid an eloquent area (8\%). For group B, MEG results modified the treatment plan in $14 / 27(52 \%)$ by reducing icEEG contacts $(50 \%)$, or change their position $(7 \%)$, allow for direct surgery $(36 \%)$, make eligible to an icEEG someone initially though inoperable (7\%). IcEEG recordings available for 25 subjects showed good concordance with MEG results in (64\%). In 33\% of cases, MEG was localizing but ictal icEEG recordings were non-localizing. Surgery was performed in 25 subjects: 19/21 (90\%) subjects with resection of the MEG zone and 4/4 (100\%) subjects whose MEG zone was not resected had a poor outcome.

Conclusions: MEG affects patient management, icEEG planning and surgical outcome in a significant percentage of patients with NLRFE.

\subsection{8 \\ INFORMATION SOURCE IN MULTIPLE MEG SPIKE CLUSTERS CAN BE IDENTIFIED BY EFFECTIVE CONNECTIVITY IN FOCAL CORTICAL DYSPLASIA Seung-Hyun Jin $^{1}$, W. Jeong ${ }^{1}$ and C. Chung ${ }^{1,2}\left({ }^{1}\right.$ Department of Neurosurgery, Seoul National University Hospital, Seoul, Republic of Korea and ${ }^{2}$ Department of Neurosurgery, Seoul National University College of Medicine, Seoul, Republic of Korea)}

Rationale: It has been reported that patients with a single MEG spike cluster had better postoperative outcomes compared to patients with multiple clusters. However, the issue of how to determine a genuine source in the multiple MEG spike clusters remains unsolved. Therefore, we aimed to figure out where the focus of epileptic activity would be in a situation of the multiple MEG spike clusters. Methods: Six patients with intractable epilepsy who showed multiple MEG spike clusters were retrospectively investigated. All patients had pathologically proven type Ia FCD with no MRI lesion and discordant multimodal presurgical evaluations, and all of them showed a favorable postoperative outcome. With spontaneous recording of MEG signals, MEG spikes were localized and clustered using a hierarchical clustering method. Then, effective connectivity referring to the causal interaction between distant structures in the brain was applied to the source waveforms extracted from the multiple MEG spike clusters. We adopted a phase-slope index (PSI) method to estimate the direction of information flux between source waveforms. Finally, the information source, i.e., the driver location between multiple clusters could be identified.

Results: Through the present study, we found that 5 of 6 FCD patients showed that the information sources, i.e., driver regions between multiple clusters were coincident with the resection area, which was also a quite consistent at different epochs.

Conclusions: Our results suggest that the effective connectivity analysis would have a potential value in a presurgical evaluation when multiple MEG spike clusters were found. To the best of our knowledge, this is the first study that evaluated the value of the effective connectivity in discerning a real source in the multiple clusters.

\subsection{9 \\ COMPARISON OF INTERICTAL MEG WITH ELECTROCORTICOGRAM IN TEMPORAL LOBE EPILEPSY}

Naohiro Tsuyuguchi ${ }^{1}$, M. Morino ${ }^{2}$ and K. Ohata ${ }^{1}\left({ }^{1}\right.$ Osaka CIty University Graduate School of Medicine, Department of Neurosurgery, Osaka, Japan and ${ }^{2}$ neurosurgery, Tokyo Metropolitan Neurological Hospital, Tokyo, Japan)

Rationale: We compared interictal magnetoencephalography (MEG); chronically-implanted subdural electrocorticogram (ECoG) over the lateral, basal, and mesial temporal area; and intraoperative hippocampal electrogram of mesial temporal lobe epilepsy (mTLE). We evaluate the characteristics of interictal spikes, and the sensitivity of extracranial magnetoencephalography (MEG) for epileptic spikes in mesial temporal lobe epilepsy. Furthermore, we estimate the surgical prognosis of Intractable mTLE.

Methods: Twenty five patients with intractable temporal lobe epilepsy were followed up for more than one year after resection surgery. MEG and subdural ECoG were simultaneously measured at a magnetic shield room in interictal period. Additionally, hippocampal signals were also measured using intraventricular hippocampal surface electrode (IVHSE) during selective amygdalohippocampectomy or multiple subpial transaction of hippocampus.

Results: 1 . In 10 cases, MEG detected no spikes whereas intracranial EEG detected. In 2 cases, interictal MEG appeared exclusively contralateral to the ictal ECoG hemisphere. The Spike detective ratio by MEG was less than $10 \%$.

2. There was no correlation of postoperative seizure outcome (Engel's class) between cases with and without MEG.

3. Most of the subdural ECoG spikes showed negative peaks, whereas those obtained with IVHSE showed positive peaks. This may show the direction of interictal discharge is from mesial temporal surface to ventricle.

Conclusions: $1 . M E G$ hardly detects mesial temporal lobe epileptic spikes probably because their signal sources are too deep and not constantly horizontal to the scalp surface. If the back ground activity is high in comparison with MEG spikes, it becomes difficult to detect these spikes. Our results correspond to the previous reports. On the other hand, some spikes could be detect in MEG of 10 cases, it means that we may omit invasive surgery for some cases.

2.There was no correlation of seizure outcome between cases with and without MEG. In our series, MEG cannot provide the prognosis of intractable mTLE, further more studies are needed.

3.Our studies show that SE spikes showed negative peaks, and IVHSE did positive peaks. One of the reasons may come from the complicated hippocampal construction. For example, when the current occurs from the temporal base to the intraventricle, negative charge is induce on the cortical surface and positive charge on the ventricle ependymal surface.

\subsection{0 \\ MEG ABILITY TO LATERALIZE THE EPILEPTOGENIC ZONE AND TO PREDICT SURGICAL OUTCOME IN EPILEPSY PATIENTS WHO REQUIRED ICEEG Salah A. Almubarak ${ }^{1,2}$, A. Alexopoulos ${ }^{2}$, Z. Wang ${ }^{2}$, F. Schneider ${ }^{2}$, J. C. Mosher ${ }^{2}$ and R. Burgess ${ }^{2}$ ( ${ }^{1}$ Neurophysiology, King Fahad Medical City, Riyadh, Saudi Arabia and ${ }^{2}$ Epilepsy Centre, Cleveland Clinic Foundation, Cleveland, $\mathrm{OH}$ )}

Rationale: To compare MEG findings with intracranial EEG (ICEEG) and to assess MEG's predicitive abilities regarding subsequent epilepsy surgery outcome.

Methods: This is a retrospective study of 36 patients who had positive MEG findings and subsequent epilepsy surgery based on intracranial EEG findings between 2008 and 2010. Medical reports were used to compare the hemisphere implicated by MEG to the 
SPECT scan, PET scan, and side of surgical resection. Resection of the site localized by MEG was correlated to epilepsy surgery outcome.

Results: Thirty six patients had positive MEG and epilepsy surgery, of which 31 were lateralized to the side of epilepsy surgery and 5 were not. Of those 31 patients, $18(58 \%)$ had no seizures on follow up and $13(42 \%)$ had seizures reoccur $(\mathrm{P}=0.105)$. Among the 31 patients, the MEG and at least one other test (PET/SPECT) agreed on lateralizing to the side of surgery in 25, and five did not. Of those 25 patients, $15(60 \%)$ had no seizures on follow up and $10(40 \%)$ had seizures reoccur $(\mathrm{P}=0.283)$. The epileptogenic region identified by MEG was resected in 26, while in 10 there was no overlap. Of those 26 patients, $17(65 \%)$ had no seizures on follow up and $9(35 \%)$ had seizures reoccur $(\mathrm{P}=0.014)$

Conclusions: MEG predicts seizure outcome better than SPECT or PET independently. Seizure freedom was higher when lateralizing MEG was overlapping the surgical resection or was in agreement with at least one other functional imaging test.

\subsection{1}

\section{USE OF A RHYTHM-BASED VS SINGLE TIME-SLICE METHOD FOR ANALYSIS OF SEIZURE ONSET DURING MAGNETOENCEPHALOGRAPHY}

Rafeed Alkawadri ${ }^{1,2}$, R. Burgess ${ }^{1}$ and A. Alexopoulos ${ }^{1}\left({ }^{1}\right.$ Neurology, Cleveland Clinic Foundation, Cleveland Heights, $\mathrm{OH}$ and

${ }^{2}$ Neurology, Yale University, New Haven, CT)

Rationale: Literature on the yield of ictal magnetoencephalography (MEG) is limited. Use of the conventional single equivalent current dipole analysis (sECD) has been proposed in some of the published case series. However, sECD poses a conceptual challenge for certain types of ictal onset, specifically those characterized by paroxysmal fast activity. In this study we compare the standard SECD method to a tailored rhythm-oriented method that takes into account the fast activity of interest at onset and disregards other rhythms. Thus we propose the use of dynamic statistical parametric mapping dSPM estimation of sources for a narrow (seizure-specific) frequency band of interest.

Methods: 377 consecutive MEG studies performed at Cleveland Clinic between 2008 and 2011 were screened and patients who had seizures during MEG recordings were identified. Sixteen patients had seizures with onsets characterized by paroxysmal fast activity. Of these patients, 12 had subsequent epilepsy surgery with postoperative follow-up of $>6$ months. Dipoles by sECD were accepted when standard modeling parameters were met.

Our proposed method of analysis is based on identification of the ictal rhythm in a time frequency space using Morlet wavelet transformation. We then identify the dominant frequency at onset in terms of signal power. We then verify the stability in the frequency domain and evolution in time domain by mapping the band of interest onto a $2 \mathrm{D}$ helmet at the sensor level employing a bandwidth proportional to the central frequency. Once the signal was identified and accepted, we then employed 'dSPM' (Dale et al, Neuron, 2000) a conventional minimum norm-estimate (MNE) based technique that allows visualization of the data on the cortical surface weighted by signal power and statistical significance (See Figure). The frequency band was split in 4 equidistant points then averaged.

Results: sECD and dSPM analysis provided acceptable results in 8 and 9 out of 12 cases respectively. sECD and dSPM, when feasible, showed comparable results in terms of sub-lobar localization. There were two cases where dSPM provided superior localizing information as compared to SECD. Resection of the area of dSPM activation led to seizure freedom in both. SECD was not feasible in one case, while it localized to an area that was not resected in the second case. Resection of the area that contained the ictal sECD dipole appeared to correlate with Engel class Ia surgical outcome but the correlation was not statistically significant. On the other hand, this correlation was significant (RR 4.5 - 95\% 1.33-15.28) when dSPM was employed.
Conclusions: The results of this study suggest that dSPM analysis of a narrow frequency band of interest at seizure onset may provide additional and clinically relevant localizing information in patients with seizure onsets consisting of paroxysmal fast. Prospective and blinded studies will be needed to confirm the findings of this pilot study.

Figure 1: Steps of dSPM analysis (see figure) a- Identifying the ictal rhythm in time series, in this example right occipital sensors. b- Identifying the ictal rhythm in time frequency space. The box magnifies the activity at the onset to identify the dominant frequency $(30 \mathrm{~Hz}$. in this casc). c- Verifying spatial stability in frequency domain at onset. d- Verifying relative spatial stability and evolution in time domain. e-Comparisen between dSPM estimale of the Irequency of interest and single current dipole method, note that the neurological orientation is used (lelt is left). $\mathrm{f}$ - Comparison between dSPM estimate ol a narrow band (the method used in this study) and the conventional MNE/dSPM solution of the inverse problem at the same time point. The first figure represents dSPM analysis of frequency band of interest while the following 3 figures are the MNE solution at the same time point with different orientation

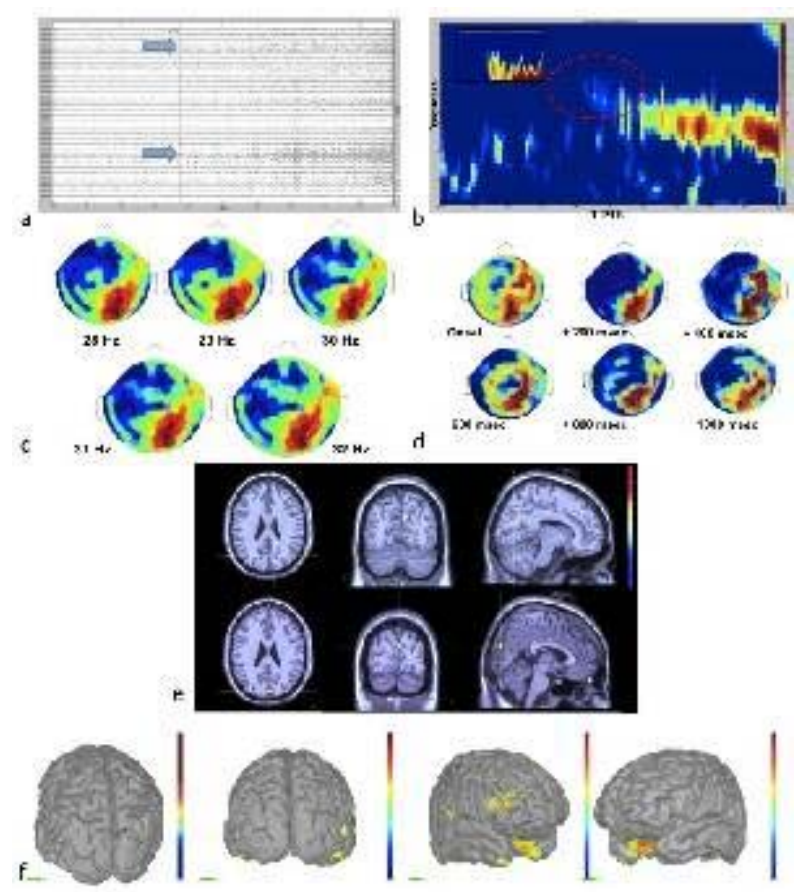

Steps of dSPM analysis. Please see Table 1 for details

\subsection{2 \\ MEG CAN DETECT SURGICAL CANDIDACY IN CHILDREN WITH GENERALIZED DISCHARGES AND INTRACTABLE FOCAL EPILEPSY} Ayako Ochi ${ }^{1}$, C. Y. Go ${ }^{1}$, E. Widjaja ${ }^{2,1}$, K. Nishioka ${ }^{1}$, S. Oba ${ }^{1}$, T. Matsui $^{1}$, J. Rutka ${ }^{3}$, J. Drake ${ }^{3}$, E. J. Donner ${ }^{1}$, S. Weiss ${ }^{1}$, C. Snead ${ }^{1}$ and H. Otsubo ${ }^{1}\left({ }^{1}\right.$ Neurology, The Hospital for Sick Children, Toronto, ON, Canada; ${ }^{2}$ Diagnostic Imaging, The Hospital for Sick Children, Toronto, ON, Canada and ${ }^{3}$ Neurosurgery, The Hospital for Sick Children, Toronto, ON, Canada)

Rationale: Scalp video EEG (SVEEG) often shows generalized spike-and-waves (GSW) in children with intractable focal epilepsy. Magnetoencephalography (MEG) can lateralize the spike dipoles of interictal GSW to assess surgical candidacy.

Methods: We retrospectively identified children who had interictal GSW during non-REM sleep on SVEEG among the children who underwent MEG and intracranial video EEG (IVEEG) at The Hospital for Sick Children (Toronto, Canada). We analyzed interictal and ictal SVEEG, the lateralization/localization of MEG dipoles and extent of resection areas.

Results: We retrospectively identified 28 children with interictal GSW during non-REM sleep on SVEEG among 103 children who 
underwent intracranial video EEG. During non-REM sleep, 21(75\%) children had frequent interictal GSW, 6 intermittent and one rare. Twenty-three (82\%) children had interictal focal discharges in addition to GSW. Lateralization of ictal SVEEG was concordant to the resective hemisphere in 15 (54\%) children. Lateralized seizure semiology was concordant to the resective hemisphere in $11(39 \%)$ children. Main seizure semiology consisted of partial seizures $(9$ children), periodic spasms (8), isolated epileptic spasms (2), tonic seizures (5), generalized tonic clonic seizures (2), myoclonic jerks (1) and atypical absence (1). Fifteen (54\%) children had more than one seizure type. MEG dipoles in the resected hemisphere ranged from 7 to 151 (mean $64 ; 53-100 \%$, mean $88 \%$ ) comparing with those in contralateral hemispheres from 0 to 31 (mean 8). Cortical resections were performed over one lobe ( 2 children), 2 lobes (9), 3 (11), and 4 (3). The remaining 3 children did not undergo resective surgery due to overlapping ictal onset and functional zone. One and two lobe resection ( 11 children) included frontal lobe in all children, but no occipital lobe involvement. The remaining 14 children underwent multilobar resection including posterior quadrant.

Conclusions: MEG can lateralize the source of interictal GSW in children with intractable focal epilepsy. Even when children show frequent interictal GSW during non-REM sleep and seizure semiology cannot be lateralized, they can still be considered for resective surgery if interictal focality on SVEEG and lateralized MEG dipoles are concordant. The resection areas, however, tend to be multi-lobar.

\subsection{3}

\section{THE VALUE OF MEG AS A PRESURGICAL EVALUATION} TOOL: ADULT NONLESIONAL NEOCORTICAL EPILEPSY Woorim Jeong, C. Chung and J. Kim (Neurosurgery, Seoul National University Hospital, Seoul, Republic of Korea)

Rationale: Only about $40 \%$ of nonlesional epilepsy patients achieve seizure-free status after surgery. Moreover, few electrophysiological studies have included magnetoencephography (MEG) when assessing the diagnostic value of presurgical modalities in a nonlesional epilepsy population. Here, we compare four electrophysiological modalities, SPECT, PET, video-EEG (VEEG), and MEG, with intracranial EEG (iEEG) to determine the value of individual modalities to surgical decisions in adult nonlesional neocortical epilepsy patients.

Methods: We retrospectively analyzed 23 adult patients $(30.7 \pm 9.0$ years; 10 females) with no abnormal MRI findings who had undergone surgical resection for intractable epilepsy between 2005 and 2011 at Seoul National University Hospital, Korea. The mean postoperative follow-up period was 2.9 years ( $\mathrm{SD}=1.3$ years). Localization of individual presurgical tests was determined for hemispheric and lobar locations based on visual analysis. For MEG, the interictal spike was visually inspected and localized using the single equivalent current dipole (ECD) method. Each localization result was compared with the ictal onset zone (IOZ) defined by using iEEG and concordance rates for each modality were calculated. Thereby, we elucidated the value of preoperative MEG ECDs in predicting surgical outcome.

Results: Postoperative seizure outcome was Engel class I in 5 (22\%) patients, class II in $3(13 \%)$, class III in $10(43 \%)$, and class IV in 5 (22\%) patients. The highest to the lowest hemispheric concordance rates, compared to the iEEG IOZ, were MEG $(83 \%)>$ ictal VEEG $(78 \%)>$ PET $(70 \%)>$ ictal SPECT $(57 \%)$. The highest to lowest lobar concordance rates, compared to the iEEG IOZ, were ictal VEEG $=$ MEG $(65 \%)>$ PET $(57 \%)>$ ictal SPECT $(52 \%)$. MEG had the highest concordance rate with IOZ at both hemispheric and lobar levels. Statistical analysis showed MEG to have a significantly higher hemispheric concordance with the IOZ than that of ictal SPECT $(\mathrm{p}<$ 0.05 ). We further analyzed the effects of MEG ECD clustered-area resection on surgical outcome. Patients who had resection of MEG ECDs showed a better surgical outcome than those without such resection $(\mathrm{p}<0.05)$.
Conclusions: Our results show that MEG-based localization had the highest concordance with the iEEG-defined IOZ. Furthermore, MEG ECD resection has prognostic significance in predicting surgical outcome.

\subsection{4 \\ FOCAL HIGH FREQUENCY OSCILLATIONS DURING CHILDHOOD ABSENCE SEIZURES}

Jeffrey Tenney, H. Fujiwara, N. Hemasilpin and D. F. Rose (Child Neurology, Division of Epilepsy, Cincinnati Children's Hospital, Cincinnati, $\mathrm{OH}$ )

Rationale: Childhood absence seizures are characterized by brief impairments of consciousness with bilaterally synchronous $3 \mathrm{~Hz}$ spike and wave discharges on EEG. High frequency oscillations (HFOs) $(80-500 \mathrm{~Hz})$ are thought to be promising biomarkers of epileptic tissue and they have been clearly linked to the seizure onset zone in patients with intractable focal epilepsies. This goal of this study was to evaluate whether HFOs occur during childhood absence seizures and to what areas does that activity localize. The corticothalamic system plays a major role in the underlying pathophysiology of CAE. The purpose of this study was to use magnetoencephalography (MEG) recordings during childhood absence seizures to complete time-frequency analyses of cortical and thalamic activity.

Methods: Children, aged 8 to 12 years old, with newly diagnosed and untreated absence seizures were recruited for the study. MEG recordings were conducted on a 275 channel CTF magnetometer and completed in 10 minute time blocks. Time-frequency analysis using short time fast Fourier transform (STFFT) was completed during absence seizures for multiple frequency bandwidths: $1-20 \mathrm{~Hz}, 20-$ $70 \mathrm{~Hz}, 70-150 \mathrm{~Hz}$, and $150-300 \mathrm{~Hz}$. Source localization for each of these frequency bandwidths was then completed using a sLORETA algorithm for sources identified with independent component analysis (ICA). The first spike-wave complex of the seizure was compared to a spike-wave complex occurring 3 seconds later but during the same seizure. Epochs of 25 milliseconds were analyzed starting 50 milliseconds prior to spike of interest, for a total time interval of 300 milliseconds.

Results: Four children, aged 8 to 12 years old, with newly diagnosed and untreated childhood absence seizures were recruited and thirteen absence seizures occurred during MEG recording. Time-frequency analysis with STFFT showed significant power density in the 1$20 \mathrm{~Hz}, 20-70 \mathrm{~Hz}$, and $70-150 \mathrm{~Hz}$ bandwidths (Figure 1A). Several components were identified with ICA between the $1-20 \mathrm{~Hz}, 20-70 \mathrm{~Hz}$, and $70-150 \mathrm{~Hz}$ but no components above the noise level were identified between $150-300 \mathrm{~Hz}$. Source localization using a sLORETA algorithm demonstrated consistent localization preferentially in the parietal region for activity at $1-20 \mathrm{~Hz}$ and localization preferentially to the lateral frontal region at $20-70 \mathrm{~Hz}$ (example, Figure 1B). Whenever a component occurred between 70$150 \mathrm{~Hz}$ it localized only to a unifocal area in the lateral frontal region. Conclusions: Using MEG, we have been able to detect focal ictal HFOs in untreated children with absence seizures. Spatial localization of activity at lower frequency bandwidths $(1-20 \mathrm{~Hz})$ tended to localize to the parietal regions whereas activity at higher bandwidths $(20-70 \mathrm{~Hz}, 70-150 \mathrm{~Hz})$ localized to a unifocal area in the lateral frontal region. Further studies will help to determine whether areas of HFOs are a critical component of the network responsible for generating childhood absence seizures and whether their presence correlates with treatment response or prognosis. 


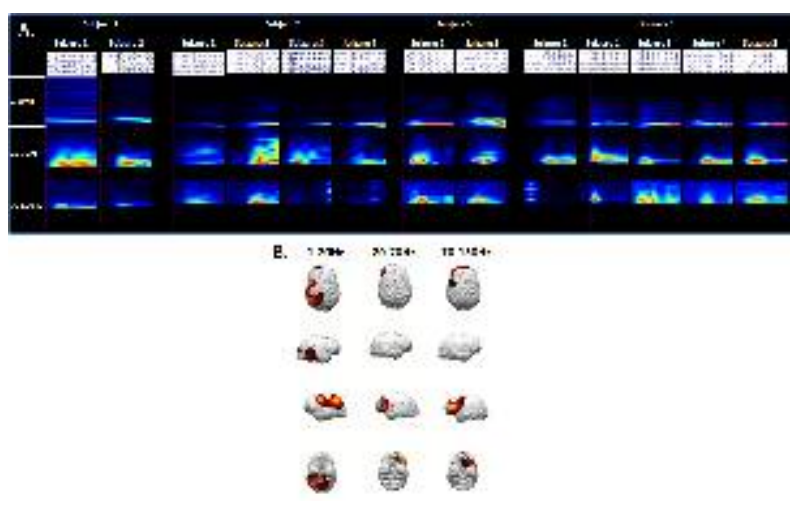

\subsection{5}

MAXIMUM RESECTION OF MEG SPIKE SOURCES ACHIEVES GOOD SEIZURE OUTCOME IN INTRACTABLE EPILEPSY PATIENTS WITH TUBEROUS SCLEROSIS COMPLEX

Tohru Okanishi, A. Ochi and H. Otsubo (Hospital for Sick Children, Toronto, Canada)

Rationale: Tuberous sclerosis complex (TSC) often causes intractable epilepsy. Epilepsy surgery is an option to control of intractable epilepsy secondary to multiple cortical tubers. Magnetoencephalography (MEG) has been applied for presurgical evaluations. We analyzed characteristics of MEG spike source (MEGSS) comparing with surgical resection and seizure outcome in TSC patients.

Methods: We retrospectively analyzed MRI, scalp video EEG (SVEEG), MEG, intracranial video EEG (IVEEG) and surgical outcome of 14 TSC patients. We used single moving dipole analysis to localize MEGSSs at an earliest phase of epileptiform discharges $(10-70 \mathrm{~Hz})$. We divided the patients into Group A (seizure free) and B (residual seizures). We analyzed number, lateralization, and removal ratio of MEGSSs.

Results: Age at surgery ranged from 2.2 to 18.1 years old (mean 8.2). MRI revealed multiple cortical tubers bilaterally in 12 patients and unilaterally in 2. SVEEG revealed hemispheric laterality of interictal discharges in all patients and laterality of ictal onsets in 12 patients. IVEEG revealed seizure onset zones in the grid areas (hemispheric in one, 3 lobes in 8, 2 in 3, one in 2 patients). Eight and 6 patients were in Group A and B, respectively, with follow-up period (3-72 months, mean 42). MEGSSs ranged 28-107 (63) in Group A and 19-82 (54) in Group B. The lateralized MEGSSs over the resection side ranged 64$100 \%$ (82) in Group A and 66-99\% (84) in Group B. The removal ratio ranged $10-92 \%$ (63) in Group A and 0-77\% (30) in Group B $(\mathrm{p}=0.013)$.

Conclusions: The removal ratio of MEGSSs in Group A was significantly higher than that in Group B. The maximum removal of MEGSSs provided the good seizure outcome in TSC patients with even lateralized MEGSSs and multiple tubers.

\subsection{6 \\ SOMATOSENSORY RESPONSES IN PATIENTS WITH INTRACTABLE EPILEPSY}

Patricia Klaas and J. Mosher (Neurological Institute, Cleveland Clinic Foundation, Cleveland, $\mathrm{OH}$ )

Rationale: Studies of evoked responses (visual and auditory) in patients with epilepsy have determined that these patients have longer cortical response latencies than other patient populations (Major, 2011). This investigation examines cortical response latencies to median nerve stimulation using magnetoencephalography. Prior research sample (Wikström et al., 1997) with a normal population identified primary somatosensory responses at 20,35 and $60 \mathrm{~ms}$. We sought to determine whether cortical response latencies would be different within a sample of patients with intractable epilepsy. Methods: Using planar gradiometers and working at the sensor level, 22 patients (mean age 26.8, 13 males) had median nerve stimulation as part of the clinical protocol for MEG. 4 members of the group had been indicated as having bilateral seizure loci while 8 were noted to have left hemisphere lateralization. Left and right median nerves were alternately stimulated at the wrist with the electrodes and voltage adjusted until a thumb twitch was elicited. Data were processed and analyzed with the BrainStorm software package and response latencies along with demographic information were entered into an SPSS database for analysis. Data from a control sample (Wikström et al., 1997) were used to compare latencies.

Results: In this pilot study, intragroup analysis found a significant negative correlation between epilepsy duration and speed of cortical response in the left hemisphere; meaning that patients who had epilepsy longer had shorter response latencies. In addition, males had longer response latencies but not significantly so. There were no significant correlations with age, but the group is small and this was not to be expected. In contrast to expected latencies in a normal population, this sample differed significantly by having significantly shorter response latencies for the 20,35 and $60 \mathrm{~ms}$ responses. Conclusions: Patients with intractable epilepsy show a difference in the speed with which information reaches the somatosensory cortex. Further research with a larger group could provide information regarding the effects of certain medications, age, gender, the degree to which seizure focus has an effect on cortical response and the influence of interictal activity on response latency.

\subsection{7 \\ MEG: CLINICAL YIELD AND LOCALIZATION ACCURACY IN 25 PATIENTS WITH PARTIAL EPILEPSY}

Chigolum C. Umeonyido-Eze ${ }^{1}$, O. Khan ${ }^{1}$, A. Bagic ${ }^{2}, \mathrm{~K}$. Zaghloul $^{1}$, J. Heiss $^{1}$, S. K. Inati ${ }^{1}$, W. H. Theodore ${ }^{1}$ and S. Sato ${ }^{1}{ }^{1}$ NINDS, National Instituite of Health, Bethesda, MD and ${ }^{2}$ University of Pittsburgh medical Center, Pittsburgh, PA)

Rationale: Epileptogenic zone localization is performed with scalp EEG recording, intracranial EEG(iEEG) and structural and functional imaging. MEG, with its temporal resolution, is a useful tool in presurgical evaluation. We evaluated MEG sensitivity in subsets of epilepsy patients, comparing MEG with MRI, iEEG, and histologically confirmed epileptogenic zone localization. Methods: 25 patients (15 males, age 13-47 years) with complete datasets were studied. 9 had mesial temporal sclerosis (MTS) defined by focal onset scalp ictal EEG findings and MTS on MRI. 8 had neocortical epilepsy ( 5 temporal, 3 extra-temporal) as defined by ictal EEG and structural MRI or FDG-PET abnormalities. 8 had ictal EEG suggestive of temporal lobe epilepsy (TLE) but normal MRI. Seventeen had iEEG and 3 intra-operative electrocorticography (ECog); 2 of these had non-localizing invasive monitoring. 23 went on to resective surgery.

MEG data was acquired with CTF 275 channel system (digitization rate $600 \mathrm{~Hz}$, digitally filtered at $1-70 \mathrm{~Hz}$, minimum 40 minutes).

Single equivalent current dipole (ECD) method was used to estimate the location of the signal source.

Results: 22 patients (88\%) had interictal MEG spikes recorded with reliable dipole fits. $2 / 3$ without MEG spikes had MRI defined MTS; one had hippocampal asymmetry.

$15 / 17$ patients with well defined MRI abnormalities had MEG spikes; 11 co-localized with MRI lesions. 3/4 with discordant MEG and MRI findings had concordant MEG and iEEG, 1 had good and 2 had poor seizure outcomes following surgical resection. All 3 were extratemporal cases. In 1 case, MEG was discordant (MEG left temporal, MRI and iEEG right temporal), with good outcome following right temporal resection. 8 patients had ambiguous or normal MRI; 6 had MEG spikes that co-localized with surface and invasive EEG; 1 patient had no MEG spikes. 
Overall, 14/18 patients showed concordance between MEG and iEEG or ECog.

18 patients had histopathologic lesions following resection (9 MTS, 3 cavernoma, 3 tumor, 2 traumatic gliosis, 1 hamartoma ). 16 had MEG spikes; 2 with MTS had none. Dipole sources for 15 with MEG spikes clustered within or close to the lesion. 1 with discordant findings had post-traumatic right frontal gliosis and right temporal MEG dipole with poor outcome.

Conclusions: Sensitivity of MEG in our series was higher than previously reported possibly due to differences in patient population and analysis methods. As reported previously, MEG spike yield is higher in neocortical versus MTLE. In our series, MEG was useful in localization in MRI lesional and non-lesional patients and was validated in $94 \%$ of pathologically proven cases

\subsection{8 \\ LOCALIZATION OF INTERICTAL ACTIVITY USING SYNTHETIC APERTURE MAGNETOMETRY IN PATIENTS WITH A VAGUS NERVE STIMULATOR}

Robert J. Kotloski ${ }^{1}$, J. R. Stapleton-Kotloski ${ }^{2}$, J. Boggs ${ }^{1}$, G. Popli ${ }^{1}$, C. A. O'Donovan ${ }^{1}$ and D. W. Godwin ${ }^{2,1}$ ( ${ }^{1}$ Department of Neurology, Wake Forest Baptist Hospital, Winston-Salem, NC and ${ }^{2}$ Department of Neurobiology and Anatomy, Wake Forest Baptist Hospital, Winston-Salem, NC)

Rationale: Magnetoencephalography (MEG) provides useful and non-redundant information in the evaluation of patients with epilepsy, and in particular in the pre-surgical evaluation of medically intractable epilepsy. Vagus nerve stimulation (VNS) is a common treatment for medically intractable epilepsy. Interpretation of MEG recordings from patients with a VNS is difficult due to the severe magnetic artifacts produced by the VNS. We present a series of eight patients with a VNS who underwent MEG recordings. While the raw MEG recordings were uninterpretable, analysis of the recordings with SAM(g2) identified foci of peak kurtosis and source signal activity that was unaffected by the VNS artifact. This case series demonstrates that SAM(g2) analysis of MEG recordings in patients with a VNS produces interpretable results and expands the use of MEG for the pre-surgical evaluation of epilepsy.

Methods: All patients were identified by a search of computerized records at Wake Forest Baptist Medical Center (IRB 15854). Selection criteria included any patient with a VNS who had a MEG recording, covering the dates from March 2006 through February 2012. All patients underwent an MEG recording in the course of an evaluation of their epilepsy. MEG recordings were performed in a CTF Systems Inc. 2005 whole-head MEG system containing 275 first-order axial gradiometers and 29 reference magnetometers. Simultaneous EEG was recorded. MEG recordings co-registered with the patient's own MRI. The total recording duration for each subject was about 40-48 minutes.

Results: Epileptiform activity was found on the EEG of 5 of 8 patients. Despite the presence of excessively large amplitude artifacts ( $>50 \mathrm{pT}$ peak to peak) due to the VNS implants, SAM(g2) analysis of the MEG data detected foci of high kurtosis in all eight patients and filtered out the VNS-induced artifact from the source signal series data for all patients. Epileptic activity in the same region was compared to other functional and structural studies. Structural abnormalities were identified in 4 cases and MEG foci clustered over these abnormalities in 2 of 4 cases. Additionally, two of the patients underwent invasive monitoring following their MEG recordings, the results of which were consistent with the $\operatorname{SAM}(\mathrm{g} 2)$ analysis.

Conclusions: The presented cases demonstrate an expanded use of MEG with the SAM(g2) beamformer, which removed the VNS artifact from the MEG recordings for all patients. In several of the cases presented, the MEG and SAM(g2) results impacted surgical decisions or surgical outcomes. We conclude that this valuable modality provides unique information that is diagnostically useful, and that with appropriate analysis MEG should not be excluded from the evaluation of patients with a vagus nerve stimulator.
Neurophysiology: Computational Analysis \& Modeling of EEG

\subsection{9}

AN AUTOMATED EVENT CLASSIFIER FOR THE DETECTION OF HIGH FREQUENCY OSCILLATIONS IN HUMAN EEG

A. Lemesiou ${ }^{1}$, K. Hashemi ${ }^{2}$, J. Heeroma ${ }^{1}$ and M. C. Walker ${ }^{1}$ $\left({ }^{1}\right.$ Clinical and Experimental Epilepsy, UCL Institute of Neurology, London, United Kingdom and ${ }^{2}$ Open Source Instruments Inc., Waltham, MA)

Rationale: The visual identification of interictal high frequency oscillations (HFO) in the intracranial EEG (iEEG) of patients with pharmacoresistant epilepsy is a laborious and time-consuming process. Moreover, it greatly relies on the use of band-pass filters for EEG analysis which introduces artifacts from filtering of epileptic transients or non-sinusoidal oscillations leading to false detection of HFO (Bénar et al 2010. Clin Neurophysiol. 121(3):301-10). Bandpass filtering is also confounded by detection of harmonics of lower frequency oscillations. The characteristics of HFO described in much of the literature are largely based on the output of such filters. Automated detection of HFO in large-scale recordings is essential for investigating their potential clinical applications as biomarkers of epileptogenicity. However, most algorithms developed to date to achieve either a supervised or an unsupervised detection of HFO work by first filtering the EEG signal in a narrow high frequency band range before performing a series of complex steps based on energy measures. We aimed to develop and validate an event classifier that automatically detects HFO by using broader band-pass filters and employing different metrics to avoid the confounders. Methods: The Event Classifier developed as an open source software aims to detect prescribed patterns in EEG recordings. A processor is used to calculate baseline power which corresponds to the minimum signal power for each channel across 1 second intervals. Three metrics are produced for each interval: power, spikeyness and intermittency. Each interval appears as a point in an N-dimensional space. The Event Classifier compares the metrics of each interval with those of a library of reference events selected from 70 channels of iEEG from subdural grids, strips and depth macroelectrodes. High frequency oscillations were visually identified by two independent reviewers in 10-minute samples of slow-wave sleep in the iEEG of four patients (total of 224 channels) sampled at $512 \mathrm{~Hz}$. The iEEG signal was displayed as raw data on a bipolar montage and with a $60 \mathrm{~Hz}$ high pass filter on both a bipolar and a referential montage. The timescale was stretched to display 1 second per screen to optimize visualization of HFO.

The same iEEG samples were processed through the event classifier which used the reference library to automatically detect HFO. Its performance was evaluated based on the number of HFO detections. Results: We report a high concordance $(\mathrm{R}=0.85)$ between the numbers of HFO identified by the reviewers across channels and the automated detections from the Event Classifier; this is similar to the concordance between the two reviewers. HFO were detected in three out of four patients and were concordant with the seizure onset zone in two.

Conclusions: We have developed a simple to use event classifier which reliably detects HFO from human intracranial EEG data and which avoids some of the confounders present using alternative methods.

\subsection{0 \\ THE SCALP EEG CAN SEE VERY SMALL CORTICAL GENERATORS OF EPILEPTIC ACTIVITY}

Rina Zelmann ${ }^{1}$, J. M. Lina ${ }^{3}$, A. Schulze-Bonhage ${ }^{2}$, J. Gotman $^{1}$ and J. Jacobs $^{2}\left({ }^{1}\right.$ Montreal Neurological Institute, Montreal, QC, Canada; ${ }^{2}$ University Hospital Freiburg, Freiburg, Germany and ${ }^{3}$ Génie Électrique, École de technologie supérieure, Montreal, QC, Canada) 
Rationale: High Frequency Oscillations (HFOs) are emerging as biomarkers of epileptogenicity. Small brain regions produce HFOs. Given the high resistivity of the skull and the assumption that a large extent of cortex is needed to observe an event on the scalp, HFOs have been mostly recorded with intracranial electrodes. Surprisingly, recent studies showed that spontaneous HFOs can be recorded from the scalp. How is it possible that these small extent events are visible on the scalp? What are the cortical correlates of scalp HFOs? Using simultaneous scalp and intracranial recordings, we studied the spatial distribution of scalp HFOs on the cortical surface. Based on simulations, we estimated the spatial extent on the scalp of focal cortical sources.

Methods: Simultaneous scalp and subdural EEGs from 11 patients with focal epilepsy were processed (low-pass filtered at $344 \mathrm{~Hz}$ and sampled at 1024Hz). Scalp HFOs and spikes were identified in 1 hour of night recording (10-20 system). Voltage maps at each event's maximum peak time were obtained and their similarity measured (cross-correlation, $\mathrm{p}<0.01$ ). We applied a linear model to relate the observations on the subdural contacts to those on the scalp (leave one out procedure). To estimate the spatial distribution of a focal source on the scalp, we simulated (using Brainstorm) 275 distributed sources of $5 \mathrm{nA} . \mathrm{m}$ situated over cortical gyri (area: $1.1 \mathrm{~cm} 2+/-0.2 \mathrm{~cm} 2$ ). Their spatial extent on the scalp was analyzed.

Results: Scalp HFOs were observed in 9 of 11 patients (115 HFOs). Voltage maps on the subdural contacts were spatially extended at the time of scalp spikes, but were focal, consisting of one or a few dipolar configurations, at the time of scalp HFOs (Figure 1). Similar scalp maps corresponded to similar maps on the grids for $50 \%$ of the spikes, but only for $15 \%$ of the HFOs. These suggest that small cortical areas generated the HFOs seen on the scalp. The linear model between subdural and scalp contacts explained the scalp topography for $83 \%$ of the spikes but only for $38 \%$ of the HFOs. Thus, the assumption that a subdural grid "sees" everything that is being seen by the nearby scalp contacts is more valid for spikes than for HFOs. When simulating 275 cortical sources of approximately $1 \mathrm{~cm} 2$, we found that we are spatially under-sampling these events with the 10 20 (17\% visible above $5 \mathrm{uV}$ ) or $10-10$ (31\%) systems (Figure 2$)$.

Conclusions: The spatial distribution on the cortical surface at the time of scalp HFOs was focal, with a spatial extent sometimes less than one square $\mathrm{cm}$. Different subdural patterns were observed for similar scalp HFO patterns. Even though the generators of HFOs are small, they can be observed on the scalp, with low amplitude and in a very focal region. A dense distribution of scalp electrodes seems therefore necessary to fully spatially sample HFOs on the scalp. A better understanding of the spatial sampling needed to observe high frequency brain activity on the scalp is important for the clinical use of scalp HFOs as biomarkers of epilepsy as well as in cognitive research.

Supported by Savoy Foundation, DAAD short-term scholarship and CIHR MOP-102710.
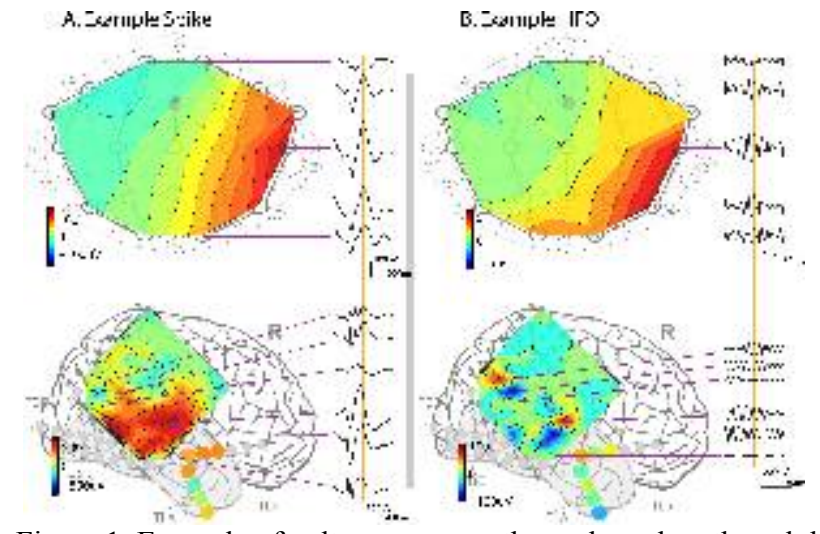

Figure 1. Example of voltage maps on the scalp and on the subdural contacts at the time of the peak of a scalp spike (A) and a scalp HFO (B) in patient 7. The subdural map at the time of a spike extends over a large cortical region, with smooth boundaries. The subdural map at the time of an HFO is focal, with a few dipolar configurations. Scalp and grid contacts are linearly interpolated. Strip contacts are depicted as circles with their amplitudes.

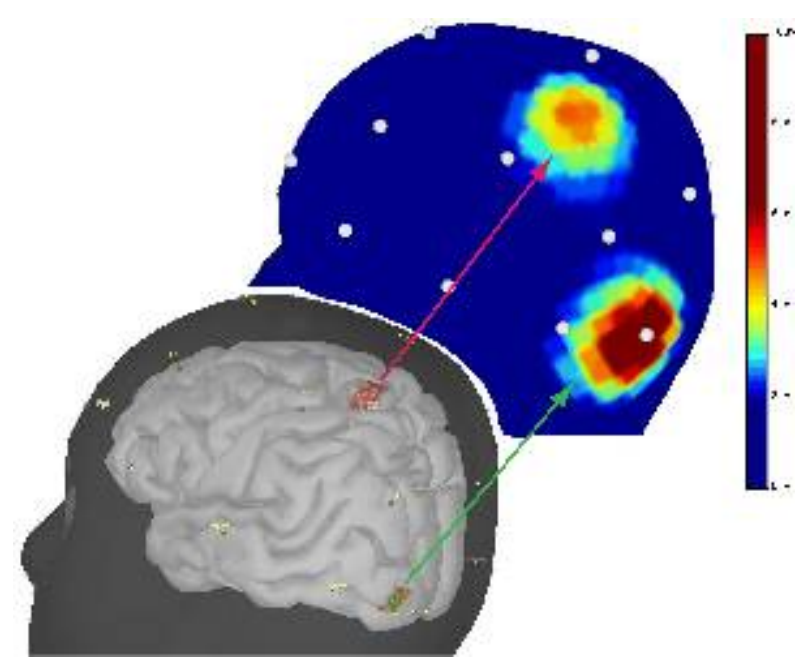

Figure 2. Example of 2 simulated focal sources and corresponding scalp spatial distribution. Green arrow indicates a source that would be visible when placing electrodes based on the 10-20 system. Magenta arrow indicates a source that corresponds to a scalp activation of similar extent, but that will not be visible when using the 10-20 system for placement of scalp electrodes. Sources intensity $=5 \mathrm{nA} . \mathrm{m}$.

\subsection{1 \\ THE ACCURACY OF ELECTRIC SOURCE IMAGING IN LOCALIZING EPILEPTIC ACTIVITY RELATIVE TO THE PREOPERATIVE GOLD STANDARD OF INTRACRANIAL EEG}

Pierre Mégevand ${ }^{1,4}$, L. Spinelli ${ }^{1}$, M. Genetti ${ }^{1}$, K. Schaller ${ }^{2}$, C. M. Michel $^{3}$, S. Vulliemoz ${ }^{1}$ and M. Seeck ${ }^{1}\left({ }^{1}\right.$ EEG and Epilepsy unit, Neurology Division, Geneva University Hospitals, Geneva, Switzerland; ${ }^{2}$ Neurosurgery Division, Geneva University Hospitals, Geneva, Switzerland; ${ }^{3}$ Basic Neuroscience Division, Geneva University Medical School, Geneva, Switzerland and ${ }^{4}$ The Sheryl and Daniel R. Tishman Cognitive Neurophysiology Laboratory, Albert Einstein College of Medicine, New York, NY)

Rationale: Electric source imaging (ESI) of interictal epileptic discharges using high-density EEG is a prospectively validated method to help localizing the epileptogenic zone in candidates for epilepsy surgery. However, its accuracy relative to the preoperative gold standard of intracranial EEG (icEEG) has not been reported. Furthermore, it is debated whether using interictal activity, as ESI does, in order to localize the source of ictal activity is adequate. Methods: Retrospective study of 35 candidates to epilepsy surgery who underwent icEEG monitoring. ESI was performed using 128- or 256-electrode EEG, a 3-shell spherical model with anatomical constraints (SMAC) and a distributed linear inverse solution (LAURA).

Results: The median distance from the ESI maximum to the nearest intracranial electrode involved in an irritative zone (IZ) was $19 \mathrm{~mm}$ (interquartile range $12-23 \mathrm{~mm}$ ), that to the most irritative electrode 30 $\mathrm{mm}(26-48 \mathrm{~mm})$, that to the nearest electrode in the seizure onset zone (SOZ) $22 \mathrm{~mm}$ (14-30 mm). The ESI-to-nearest IZ and ESI-tomaximal IZ distances were strongly correlated with the ESI-to-SOZ distance $(\mathrm{p}<0.0001$ and $\mathrm{p}<0.01$ respectively). There were no significant differences in ESI accuracy in patients with medial temporal lobe, lateral temporal lobe or extratemporal epilepsy (Figure 1). The ESI maximum was included in the resected brain volume more often in patients with favorable postoperative outcome ( 9 of 18 patients in Engel classes I and II) than in those with unfavorable 
outcome ( 1 of 8 patients in Engel classes III and IV; difference in proportions $0.375,95 \%$ confidence interval 0.0278 to 0.6667 ). Furthermore, the maximal IZ electrode was more often within the resected brain volume in the patients with favorable outcome (15 of 18 patients) than in those with unfavorable outcome ( 3 of 8 ; difference in proportions $0.4583,95 \%$ confidence interval 0.0833 to $0.8194, \mathrm{p}<0.05)$.

Conclusions: Put together, our results indicate that the localization of interictal spikes by ESI closely corresponds to the localization of irritative zones by intracranial EEG; that irritative zones are often part of the seizure onset zone or lie close to it; and that localizing interictal spikes by ESI helps localizing the seizure onset zone. Additionally, including the ESI maximum in the resected brain volume is correlated with favorable postoperative outcome. Importantly, ESI performs accurately regardless of the epilepsy subtype.

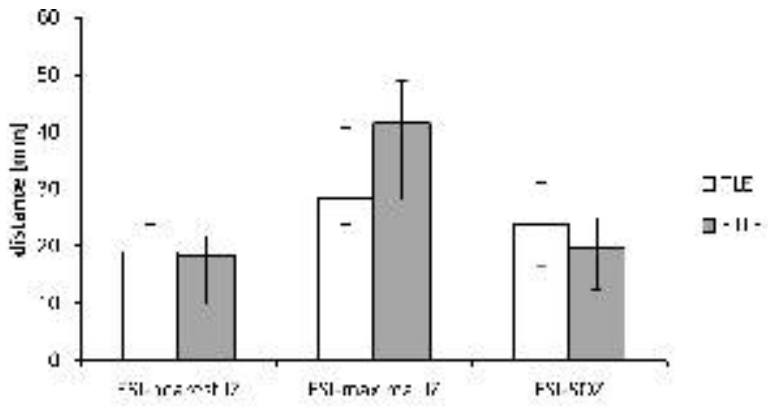

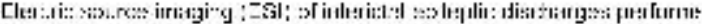
sccurately regar:less of the epilsps suzips. Predian ans in:ersuartile ange

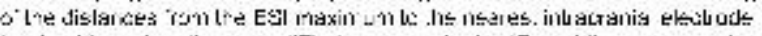

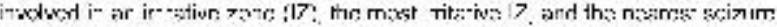

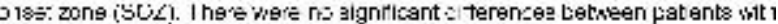
| เ:-1F"

\subsection{2 \\ DEVELOPING CORTICAL FUNCTIONAL NETWORKS ACROSS INFANCY AND CHILDHOOD}

Catherine Chu-Shore ${ }^{1}$, J. Leahy ${ }^{1}$, J. Pathmanathan ${ }^{1}$, M. B. Westover ${ }^{1}$, M. A. Kramer ${ }^{2}$ and S. S. Cash ${ }^{1}\left({ }^{1}\right.$ Neurology, Massachusetts General Hospital, Boston, MA and ${ }^{2}$ Mathematics and Statistics, Boston University, Boston, MA)

Rationale: The human brain undergoes significant age-specific anatomical and physiological changes over the course of early development. Functional connectivity network analysis provides a tractable, activity-based measure to identify dynamic relationships between developing neuronal populations (J Child Neurol, 2011;26(4):488-500). Over the last decade, these approaches have been used extensively to identify abnormalities in normal and diseased populations (J Neurosci, 2010;30(30):10076-85) . Here we characterize the developing functional brain networks in infancy and childhood using EEG. This work provides a foundation upon which to better understand normal brain development and ultimately, the impact of abnormal neurophysiology on these developing networks. Methods: We evaluated sleep rhythms and functional network connectivity patterns in 334 neuro-typical infants and children (age 010 years). 19-channel EEGs were manually reviewed, artifacts rejected, and state-specific epochs $>100$ sec were selected for analysis. Data were referenced to a bipolar and physical reference (C2) and filtered for broad band analysis (1-55 Hz). Spectra were computed using the multitaper method allowing a frequency resolution of $1 \mathrm{~Hz}$. To infer functional networks, prepared EEG data were divided into discrete $1 \mathrm{sec}$ windows for coupling analysis. The maximal cross-correlation between all electrode pairs was calculated, allowing a lag up to $100 \mathrm{~ms}$. To identify edges, we applied an analytic significance testing procedure to identify network edges with statistical confidence and a linear step-up false detection rate controlling procedure to correct for multiple comparisons (Phys Rev E, 2009;79(6 Pt 1):061916). To minimize the impact of volume conduction, edges identified with maximum correlation at zero phase lag were ignored (detailed methods in: J Neurosci 2012: 32(8)2703$13)$. For each window, significant cross-correlation values were represented as a network in the form of an undirected binary adjacency matrix. Graph metrics and similarity measures were then computed for each age-group and compared between groups.

Results: We show that the topology of functional networks changes significantly over infancy in a stereotyped fashion. These changes are most marked in the first few months of life and plateau by childhood. Conclusions: This work demonstrates that cortical functional connectivity networks change dramatically over early development, in a manner consistent with the overall arc of neurodevelopment. These changes are evident within routine EEG recordings across a time span of months and years. Functional network connectivity analysis may provide a sensitive, age-specific clinical tool to track and better understand the pathophysiological impact of altered neurophysiology during critical periods of neurodevelopment.

\subsection{3 \\ OCCIPITAL GAMMA ACTIVITY MODULATED BY SACCADIC EYE MOVEMENTS DURING WAKEFULNESS AND REM SLEEP: INTRACRANIAL RECORDING IN EPILEPTIC PATIENTS}

Mitsugu Uematsu ${ }^{1,2}$, N. Matsuzaki ${ }^{2}$, K. Kojima $^{2}$, E. C. Brown ${ }^{4,5}$ and E. Asano ${ }^{2,3}$ ( ${ }^{1}$ Pediatrics, Tohoku University Graduate School of Medicine, Sendai, Japan; ${ }^{2}$ Pediatrics, Wayne State University, Detroit, MI; ${ }^{3}$ Neurology, Wayne State University, Detroit, MI; ${ }^{4}$ Psychiatry and Behavioral Neurosciences, Wayne State University, Detroit, MI and ${ }^{5} \mathrm{MD}-\mathrm{PhD}$ Program, Wayne State University, Detroit, MI)

Rationale: Humans spontaneously perform saccades to look around during wakefulness. It has been hypothesized that the cortical processes of vision are transiently suppressed during a saccade and reactivated immediately following saccade offset. Here, we determined whether human occipital regions were indeed suppressed during saccades and subsequently reactivated, as measured by gamma activity at $80-150 \mathrm{~Hz}$. We also determined whether occipital gamma activity was likewise modulated by saccadic eye movements without external visual stimuli during rapid eye movement (REM) sleep.

Methods: We studied nine patients (age: 5-17 years) with focal epilepsy undergoing extraoperative electrocorticography (ECoG). All patients had normal intelligence and visual acuity. Using timefrequency analysis, we determined the spatiotemporal dynamics of cortical gamma activity relative to the onset and offset of spontaneous saccades during wakefulness as well as REM sleep. Results: In total, we found 725 spontaneous saccade events during wakefulness and 243 during REM sleep. No significant gammamodulation was found prior to saccade onset. During wakefulness, significant gamma-attenuation involved 43 occipital sites during saccades, 12 of which subsequently showed augmentation; an additional 47 sites exhibited only post-saccade augmentation. The polar/lateral occipital lobe showed more extensive gammaattenuation, while the medial/inferior occipital lobe showed more extensive gamma-augmentation $(\mathrm{p}<0.001$ on Fisher exact probability test). We also found that a larger saccade duration was associated with a larger gamma-attenuation in all occipital area $(\mathrm{p}<0.001$ on one-way repeated measures ANOVA). During REM sleep, the temporal profile of gamma-modulation was similar to that during wakefulness, but with smaller magnitude. Significant gammaattenuation during REM sleep involved 6 of the same sites that attenuated during wakefulness; no sites exhibited significant gammaaugmentation during REM sleep.

Conclusions: Portions of the human occipital cortex were suppressed immediately after saccade onset and throughout saccades, with 
subsequent reactivation following saccade offset, as measured by the amplitude of gamma activity at high temporal resolution. Such a transient suppression of occipital function may contribute to the maintenance of visual stability before and after saccades. Even without external visual stimuli, portions of occipital regions were modulated by saccades during REM sleep. This suggests that saccades themselves or the signals initiating saccades may induce occipital suppression regardless of visual input. The present study failed to find gamma-modulation preceding the onset of saccades.

\subsection{4}

SPATIO-TEMPORAL DYNAMICS OF INTERICTAL SPIKING AND APPLICATIONS

B. Krishnan ${ }^{1}$, I. Vlachos ${ }^{2}$, S. Mullane ${ }^{2}$, A. Faith ${ }^{2}$, K. Williams ${ }^{3}$ and L. Iasemidis ${ }^{1,2}$ ( ${ }^{1}$ Electrical Engineering, Arizona State University, Tempe, AZ; ${ }^{2}$ Biomedical Engineering, Arizona State University, Tempe, AZ and ${ }^{3}$ Neurology, Phoenix Children's Hospital, Phoenix, AZ)

Rationale: There is a continuing debate in the literature about existence of higher or lower spike rates before or after epileptic seizures. The underpinning of our study was the formulation of a mathematical framework within which spatio-temporal dynamics of epileptic spikes help elucidate clinically relevant questions. In particular, within this framework, we investigated the role of epileptic spikes in ictogenesis and the possibility to reliably localize the epileptogenic focus from interictal spiking in patients with focal epilepsy.

Methods: Long-term (5-10 days) intracranial electroencephalograms (iEEGs) from 5 patients with temporal lobe epilepsy (TLE) were analyzed in this study. Epileptic spikes were detected from the iEEGs using an in-house developed Data Adaptive Morphological Filter (DAMF) algorithm. A novel spatial synchronization measure (SSM) of spikes was then developed and applied to iEEGs. (Both DAMF and SSM algorithms were first tested on simulation data from coupled spiking neuron models.) Localization of the epileptogenic focus from interictal periods was performed on the basis of SSM and measures from graph theory (eigenvector centrality) [1].

Results: Application of SSM to interictal iEEG revealed monotonically increasing long-term (order of hours) synchronization between spike trains at critical brain sites that included the focal zone. After seizures' end, spike synchronization decreased. This behavior is in agreement with our previously postulated hypothesis that seizures occur to reset a pathologically established preictal hypersynchrony of EEG dynamics between critical brain sites [2, 3]. It is to be noted that such findings were not statistically significant when spike rates instead of individual spikes were used for analysis. With respect to focus localization, the application of DAMF to interictal spiking activity resulted to accurate localization of the focus $(p<0.05)$ in 4 out of our 5 patients. In the fifth patient, focus localization was not statistically significant $(\mathrm{p}>0.05)$ [1].

Conclusions: This study generated results that, if validated in a larger cohort of patients with focal epilepsy, could have immediate clinical applications. Preictal synchronization of individual spikes between critical brain sites and their postictal desynchronization were consistent findings across patients and seizures, as long as spiking activity could be detected in the EEG. These findings definitely render a physiological dimension to the theory of resetting of synchronized global brain dynamics at seizures [2]. Finally, the ability to localize the focus from spatio-temporal analysis of interictal spiking supports the notion that epileptogenic focus could be detected as the central node of an epileptic network at work even in interictal periods.

[1] B. Krishnan, Ph.D. Dissertation, Arizona State University, May 2012

[2] S. Sabesan et al., J. Combinatorial Optimization, 17, pp. 74-97, 2009.

[3] L. Iasemidis et al., IEEE Transactions on Biomedical

Engineering, 51, pp. 493-506, 2004.

\subsection{5}

A SEIZURE PREDICTION METHOD FOR PATIENTS WITH TEMPORAL LOBE EPILEPSY

Kais Gadhoumi ${ }^{1}$, J. M. Lina ${ }^{2}$ and J. Gotman ${ }^{1}\left({ }^{1}\right.$ Montreal

Neurological Institute, Montreal, QC, Canada and ${ }^{2}$ Electrical Engineering, École de Technologie Supérieure, Montreal, QC, Canada)

Rationale: In patients with intractable epilepsy, predicting seizures with clinically acceptable levels of sensitivity and specificity opens the doors to a variety of therapeutic solutions. This goal however, is not reached and clinically acceptable results have yet to be demonstrated. Many studies showed that preictal changes are detectable from the EEG using different measures and that seizure prediction is possible. When tested on long-term recordings, the performance consistency of the prediction algorithms and their superiority to random predictors remain however questionable. In this study, an intracranial EEG-based seizure prediction method is proposed. It is evaluated on patients with temporal lobe epilepsy (TLE).

Methods: The method is based on the study by Gadhoumi et al. (Clin Neurophysiol 2012). 1565 hours of continuous intracranial EEG data (filtered at $500 \mathrm{~Hz}$ and sampled at $2000 \mathrm{~Hz}$ ) from 17 patients with TLE were investigated. The recordings included 127 seizures. Bipolar channels from the 4 deepest contacts of bilaterally implanted electrodes in the amygdala, hippocampus and parahippocampus were analysed. In each patient the data was split into a training set and a testing set. During training, the EEG segments were analysed in 4 frequency bands (between $50 \mathrm{~Hz}$ and $450 \mathrm{~Hz}$ ) using continuous wavelet transform. A reference state was defined in the $90 \mathrm{~s}$ immediate preictal data and used to derive 3 features quantifying the discrimination between preictal and interictal data. A discriminant analysis based classifier was then trained in the feature space. Its performance was evaluated using cross validation applied to training data. The 3 channels and the frequency band yielding the best classification performance were selected for the testing procedure. The performance of the classifier is assessed for a range of Seizure Prediction Horizons (SPH) between 5 and 60 min and compared with a random Poisson predictor for statistical validation.

Results: For SPHs between 30 and $60 \mathrm{~min}$, better than random prediction performance is achieved in 7 of 17 patients (fig. 1). For this set of SPHs and for these patients, the sensitivity is higher than $85 \%$, the false prediction rate is less than $0.1 / \mathrm{h}$ and the portion of time under false alarm is less than $9.2 \%$. The median prediction time is $\sim 45 \mathrm{~min}$ (fig. 2). EEG channels selected in the training phase as best performing channels were mostly bilateral. They were not all located in the hemisphere were neurologist indicated a seizure onset predominance. Most of these patients had bilateral epilepsy however. Conclusions: A seizure prediction method has been developed and evaluated on patients with TLE. Seizures were predictable above chance $(\mathrm{p}<0.05)$ in $41 \%$ of patients. For these patients, the method yields sensitivity and specificity levels potentially interesting for applications of a closed-loop seizure control where intervention horizons above $30 \mathrm{~min}$ are desirable. The study shows that it may be possible to find a subset of patients in whom seizures can be reliably predicted.

Supported by CIHR MOP-10189, RSC-NSERC CHRPJ 323490-06. 


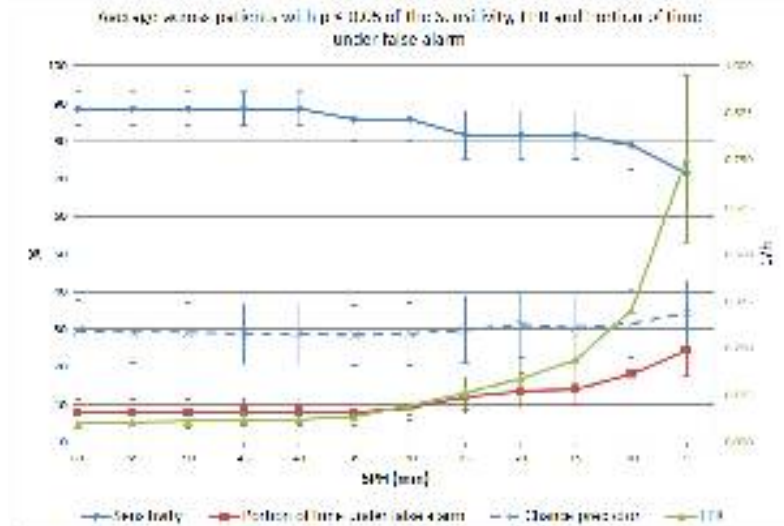

Average and standard errors of the sensitivity, portion of time under false alarm and false prediction rate for a range of SPHs for patients who showed better than random performance. Average sensitivity values of the random predictor are significantly less than those of the proposed method $(\mathrm{p} \leq 0.05)$

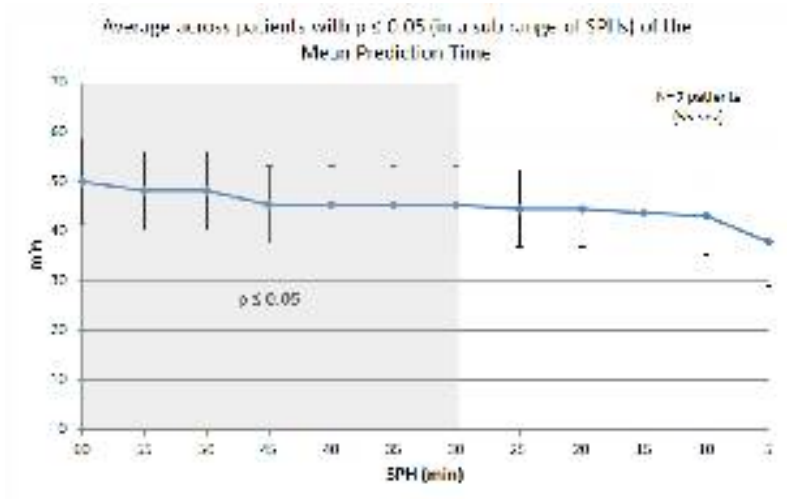

$\rightarrow$ Mtsul Hed : :van int:

Average across patients of the mean prediction time for a range of SPHs.

\subsection{6}

HIGH-RESOLUTION EEG COMBINED WITH ELECTRICAL SOURCE IMAGING: A SUBSTANTIAL AID TO LOCALIZE THE EPILEPTOGENIC ZONE IN MALFORMATIONS OF CORTICAL DEVELOPMENT

Estelle RIKIR ${ }^{1,2}$, L. Koessler ${ }^{3}$, M. Gavaret ${ }^{4}$, J. Jonas ${ }^{1}$, S. ColnatCoulbois $^{1}$, J. Vignal ${ }^{1}$, H. Vespignani ${ }^{1}$ and L. Maillard ${ }^{1}\left({ }^{1}\right.$ Neurology, CHU Nancy, Nancy, France; ${ }^{2}$ Neurology, CHU Sart-Tilman, Liège, Belgium; ${ }^{3}$ Centre de Recherche en Automatique de Nancy ( CRAN), Nancy, France and ${ }^{4}$ Neurology, CHU de la Timone, Marseille, France)

Rationale: Malformations of Cortical Development (MCD) are frequently associated with drug-resistant epilepsy. Although increasingly recognized using high-resolution MRI and scalp EEG (typical dysplastic fast activity), localizing the epileptogenic zone (EZ) associated with MCD still relies on intra-cerebral EEG recordings. Our purpose was to study the spatial correlations between the modeled electrical sources of inter-ictal events (Irritative Zone $=$ IZ) recorded with High-Resolution scalp EEG (HR-EEG) and the EZ defined by stereo-EEG (SEEG) in MCD.

Methods: This prospective, bi-centric study enrolled 26 consecutive patients (22 in Nancy and 4 in Marseille) with established or suspected MCD undergoing pre-surgical investigations between 2008 and 2012 and comprising a HR-EEG (64-channels) with electrical source imaging and SEEG recordings.

Results: Three patients were excluded from the analysis because of missing data (no SEEG seizure recording in two cases and no HREEG inter-ictal paroxysm registration in one case).
The mean age at the time of SEEG recordings was 31 years. Nine patients had temporal lobe epilepsy, ten frontal lobe epilepsy and four posterior epilepsy including occipito-temporal or posterior parietal epilepsy. According to the ILAE Task Force consensus classification, seven patients had a type 1 or 2 focal cortical dysplasia (FCD Type I or II) and four had a type 3 focal cortical dysplasia (FCD Type III). Three had polymicrogyria associated with schizencephaly and nine patients (39.1\%) were MRI negative.

HR-EEG IZ and SEEG EZ were completely or more often partially overlapping in $95 \%$. HR-EEG IZ co-localized with the EZ in 7 cases $(30.4 \%)$ and four of them were MRI negative. In seven cases, the HR-EEG IZ comprised the entire EZ and extended to the propagation zone in $3 / 7$. In six cases, HR-EEG IZ partially overlapped the EZ. In these cases, HR-EEG often identified at least a part of the propagation zone and often missed the medial part of the EZ. In 9 patients with negative MRI, HR-EEG correctly localized at least a part of the EZ. In 4/9, it allowed its precise localization and in 3/9, HR-EEG clearly influenced the SEEG electrodes position.

Conclusions: HR-EEG combined with electrical source imaging helps to localize and target the EZ associated with established or suspected MCD particularly in MRI negative cases.

\subsection{7 \\ RHYTHMIC MULTIPLE HYPERSYNCHRONIZATION IN THE CHILDHOOD ABSENCE EPILEPSY}

Eun Mi Lee ${ }^{1}$, J. Kang ${ }^{2}$ and K. Lee ${ }^{3}\left({ }^{1}\right.$ Department of Neurology, Ulsan University of Hospital, Ulsan, Republic of Korea; ${ }^{2}$ Department of Neurology, Asan Medical Center, Seoul, Republic of Korea and ${ }^{3}$ Department of Pediatrics, Ulsan University Hospital, Ulsan, Republic of Korea)

Rationale: Epileptic activity of childhood absence epilepsy (CAE) is characterized by bursts of $3-\mathrm{Hz}$ generalized spike-wave discharges (GSW), which are widely considered to be symmetric and synchronous. Synchronous oscillation produced in thalamo-cortical neuronal network and abnormal cortical hypersynchronization has been hypothesized. The purpose of this study was to investigate oscillation activity by studying the topography and spectral power of these discharges and better understanding pathologic dynamics. Methods: We studied 14 patients diagnosed with $\mathrm{CAE}(\mathrm{M}: \mathrm{F}=5: 9$, mean age: 8.3 years old). Ten second epochs of sustained $3-\mathrm{Hz}$ GSW epileptic and baseline segments showing alpha rhythm (48 epileptic and 35 baseline segment, each 10- seconds segment) were analyzed by Fast Fourier transformation using EEGLAB Toolbox for MATLAB. We compared the topography and power spectrum (0$20 \mathrm{~Hz}$ band) to epileptic and baseline segments

Results: Spectral power in epileptic epochs showed multiple rhythmic 6-7 spectral peaks (multiples of $2.5-3 \mathrm{~Hz}$ ). The first peak in epileptic epochs was observed at $2.5-3 \mathrm{~Hz}$ with maximum of the power spectrum and subsequent peaks showed gradually decremental power. Topographic map of epileptic epochs showed greatest power in diffuse frontocentral area at $-3 \mathrm{~Hz}$ and more localized frontopolar area at subsequent multiple peaks. However, baseline epochs showed maximum power at $9-10 \mathrm{~Hz}$ only at localized occipital area and no other spectral peak.

Conclusions: Characteristic epileptic hallmark of CAE (3-Hz GSW) was typical and consistent but not the sole feature in our spectral EEG analysis. We found additional rhythmic oscillatory peak power in epileptic discharges. These findings suggest that excitability in thalamocortical networks with multiple hypersynchronization and dynamic mechanism of absence epilepsy. 


\subsection{8}

\section{IDENTIFICATION OF THE ICTAL ONSET ZONE IN PATIENTS WITH TUBEROUS SCLEROSIS USING ELECTRICAL DIPOLE SOURCE ANALYSIS}

Michael Quach, M. D. Holick, B. Mudigoudar and S. Agadi (Neurology and Neurophysiology, Texas Children's Hospital, Houston, TX)

Rationale: Historically, the localization of interictal and ictal sources has primarily relied on visual inspection of scalp EEG traces. Identification of the source is based mainly on the assumption that the source underlies the most prominent negative activity. With the advent of modern computer-assisted techniques such as dipole source modeling, more quantitative methods are now available to localize EEG activities. In this study, we examined the role that electrical dipole source analysis may have in the surgical evaluation of tuberous sclerosis patients with medically refractory epilepsy. Methods: A database of tuberous sclerosis patients seen at TCH was examined to identify patients with medically refractory epilepsy who underwent epilepsy surgery after a Phase II evaluation involving invasive electrodes. Of the 129 patients in the database, six were identified meeting these criteria. Two patients were excluded due unavailability of pre-surgical scalp EEG data. The remaining four patients' presurgical scalp EEG's were retrospectively analyzed using the Brain Electrical Source Analysis (BESA) software, developed by MEGIS Software. The scalp EEG was recorded using 10-20 international system. The EEG activity was digitally recorded referentially to midline electrodes. A single neurophysiologist performed dipole source analysis on the ictal waveforms using BESA. The dipole source models were co-registered to the patient's presurgical MRI using the BrainVoyager software. The ictal dipole source models (co-registered to the patients' MRIs) were then provided independently to two neurophysiologists. The neurophysiologists were asked identify the structural lesion that they felt that the dipole source corresponded to. This information was then compared to the surgical resections that were eventually performed. Long term surgical outcomes were determined from the patients' medical record.

Results: The number of cortical structural lesions for each patient ranged from 4 to 10 , with a mean of 6.25 . The structural lesions were judged from official MRI reports to be either cortical tubers or focal cortical malformations, based on appearance. The two neurophysiologists identified the same cortical lesion in all four patients $(100 \%)$. Compared to the surgical resections eventually performed based on intracranial recordings, the resections involved all of the lesions identified by the two neurophysiologists.. Based on surgical pathology, three of the resections involved cortical tubers, the remaining case involved a cortical malformation. At five years after resection, all four patients are seizure free.

Conclusions: The results of this study suggest that dipole source modeling may accurately identify the ictal onset zone in tuberous sclerosis patients. In all patients examined, dipole source modeling identified the regions that were eventually resected based on subdural electrode recordings. Overall, this study suggests that dipole source modeling may play a valuable role in the surgical evaluation of tuberous patients with medically refractory epilepsy.

\subsection{9 \\ HOW IMPORTANT IS THE FOCUS IN EPILEPTIC BRAIN NETWORKS? \\ Christian Geier ${ }^{1,2}$, C. E. Elger ${ }^{1}$ and K. Lehnertz ${ }^{1,2}$ ( ${ }^{1}$ Department of Epileptology, University of Bonn, Bonn, Germany and ${ }^{2}$ Helmholtz Institute for Radiation and Nuclear Physics, University of Bonn, Bonn, Germany)}

Rationale: There is currently a debate whether focal epilepsies can be regarded as a solely local or as a network phenomenon. Graph theoretical approaches may help to resolve this controversy. We here address the question whether the clinically identified focus can be regarded as an important node in large scale functional brain networks. Importance is assessed via different centrality metrics (degree-, closeness-, and betweenness-centrality).

Methods: We analyzed multi-day, multi-channel EEG data recorded intercranially from up to now 13 epilepsy patients ( 8 with unifocal and 5 with multifocal epilepsies; all patients are seizure-free postoperatively). EEGs were recorded from on average 55 recording sites for a period of on average 118 hours per patient. Using a movingwindow approach (duration of each window: $20.48 \mathrm{~s}$ corresponding to 4096 data points; no overlap) we estimated the strength of interactions (via mean phase coherence) between all pairs of sampled brain regions. We then derived weighted functional brain networks by applying different rules to transform the interaction matrices to adjacency matrices, which allowed us to estimate the centralities in a time-resolved manner. We here concentrate on the inter-ictal state of the patients only and dicarded peri-ictal data $(1 \mathrm{~h}$ pre- and postictally).

Results: The temporal average of the degree-centrality indexed the epileptic focus as a node with highest importance in the epileptic brain networks from $9 / 15$ patients. In contrast, with both the betweenness- and closeness-centralities (averaged over time) we observed recording sites in the vicinity of the reference electrodes as important nodes in 14/15 patients. Findings were consistent for the networks derived by applying different transformation rules.

Conclusions: The different centrality metrics differentially indexed the clinically defined epileptic focus as an important node in epileptic brain networks during the inter-ictal state. Further work is necessary to clarify the impact of recording montages on network construction and centrality estimation.

This work was supported by the Deutsche Forschungsgemeinschaft (Grant No: LE 660/4-2)

\section{Clinical Epilepsy: Clinical Diagnosis}

\subsection{0}

CRYPTOGENIC (MRI-NEGATIVE) EPILEPSY: AUTOMATIC SEIZURE FOCUS LATERALIZATION SeokJun Hong ${ }^{1}$, H. Kim ${ }^{1}$, D. Scharder ${ }^{1,2}$, N. Bernasconi ${ }^{1}$ and A. Bernasconi ${ }^{1}\left({ }^{1}\right.$ Neurology, Montreal Neurological Institute and Hospital, Montreal, QC, Canada and ${ }^{2}$ Pediatric Neurology, University of British Columbia, Vancouver, BC, Canada)

Rationale: MRI has revolutionized the diagnosis of drug-resistant epilepsy by allowing the detection of lesions associated with the epileptogenic zone, thus leading to increased rates of successful resective surgery. However, in many patients, best-practice MRI is unable to reveal a surgical target [1]. In these MRI-negative (or cryptogenic) cases diverse seizure semiology and non-localizing EEG findings make the formulation of an a priori hypothesis about the epileptogenic zone often very difficult [2]. Indeed, surgery in the absence of an MRI-visible lesion is one of the greatest clinical challenges in many tertiary centers. There is need for alternative approaches to indentify the epileptogenic focus in these patients. We previously showed that cryptogenic epilepsy is associated with widespread bilateral cortical atrophy involving particularly the frontal lobes [3]. The purpose of this study was to automatically lateralize the seizure focus using MRI-based analysis of cortical thickness Methods: We studied 37 patients ( 22 males, $30 \pm 8$ years) with cryptogenic, drug-resistant extra-temporal lobe epilepsy. The focus was pre-central (frontal or fronto-central) in 22 and post-central (centro-parietal, parietal or parieto-occipital) in 15 (left-sided in 21 and right-sided in 16). In each region of atrophy identified by our previous analysis [3] we calculated mean cortical thickness and zscores relative to the distribution of 41 age- and sex-matched healthy controls (16 males, $31 \pm 11$ years) on 1.5T MRI (3D T1-fast field echo sequence; $\mathrm{TR}=18 \mathrm{~ms} ; \mathrm{TE}=10 \mathrm{~ms}$, voxel size $=1 \mathrm{~mm} 3$ ). We then fed into a linear discriminant classifier each cluster's mean thickness and 
the mean value of the contralateral homologous region. To avoid biases, we used iteratively various significance levels ranging from $\alpha=0.025$ to $\alpha=0.0001$. We estimated the performance of our predictive model by performing a leave-one-out cross-validation (each patient is lateralized using data of all other subjects).

Results: We correctly lateralized the focus in $77 \%$ of patients using the fronto-opercular cluster of atrophy, in $74 \%$ using the occipital cluster, and in $68 \%$ using the fronto-polar and fronto-central clusters. The frontal clusters yielded a correct classification in a higher proportion of patients with pre- than post-central foci (fronto-polar and fronto-central: $90 \%$ vs. $46 \%, \mathrm{p}<0.05$; fronto-opercular: $81 \%$ vs. $67 \%$ ). Conversely, the occipital cluster lateralized accurately postcentral foci ( $86 \%$ vs. $68 \%$ ). Combining all clusters, we lateralized $86.5 \%$ of patients (pre-central: $86 \%$; post-central: $87 \%$ ).

Conclusions: Our fully automated classifier enabled accurate and reliable lateralization of the seizure focus in the majority of patients with unremarkable conventional MRI. Higher accuracy obtained when using clusters nearby the putative seizure focus implies that, despite widespread changes, atrophy may be a biomarker for epileptogenicity. This method may substantially improve MRI analysis so that it can fulfill its role for surgical target localization in cryptogenic epilepsy.

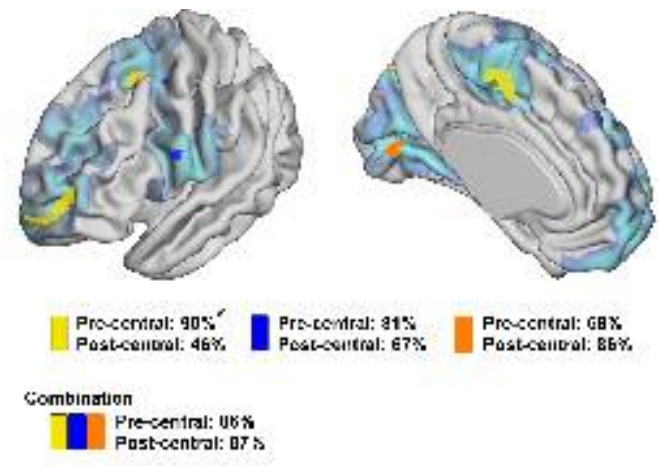

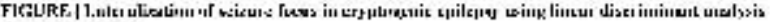
it revellest sirriphy

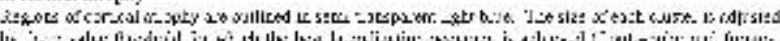

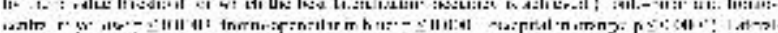
Cocen

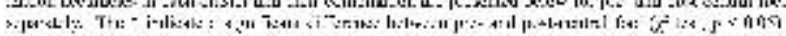

\subsection{1}

A COMPREHENSIVE MULTI-GENE SEQUENCING AND DELETION/DUPLICATION PANEL FOR THE DETECTION OF COMMON AND RARE GENETIC CAUSES OF EPILEPSY Dianalee McKnight ${ }^{1}$, E. Butler ${ }^{1}$, R. Rubenstein ${ }^{1}$, A. Gropman ${ }^{2}$, X. Ortiz-Gonzalez ${ }^{3}$, D. Hasbani ${ }^{3}$, J. Cohen ${ }^{4}$, G. Richard ${ }^{1}$ and S. Aradhya ${ }^{1}\left({ }^{1}\right.$ GeneDx, Gaithersburg, MD; ${ }^{2}$ Neurogenetics Program, Children's National Medical Center, Washington, DC; ${ }^{3}$ Division of Neurology, Children's Hospital of Philadelphia, Philadelphia, PA and ${ }^{4}$ Kennedy Krieger Institute, Baltimore, MD)

Rationale: Determining the genetic etiology of epilepsy can have important implications for treatment and management. However, primary and syndromic forms of epilepsy are genetically heterogeneous, which creates challenges for diagnostic testing. This study describes the utility of a comprehensive multi-gene testing panel for identifying mutations in genes causing epilepsy.

Methods: A targeted testing panel combining Next Generation sequencing and deletion/duplication testing by exon-level array $\mathrm{CGH}$ of 53 genes associated with epilepsy was designed and validated. Results: In 176 consecutive clinical cases with different forms of epilepsy, $20 \%$ were positive for a known or predicted disease-causing mutation that accounted for the patient's phenotype. In the largest subgroup of 51 patients with infantile epileptic encephalopathy, a mutation or likely disease-causing variant was reported in $25 \%$ (13 cases).
As expected, approximately half of the identified mutations were found in genes common for infantile epileptic encephalopathy. One patient had the two most common POLG mutations causing Alpers Syndrome. Three other patients had mutations in the SLC2A1 gene causing glucose transporter deficiency; two of these patients had mutations identified by sequence analysis while another had a partial gene deletion only detectable by exon-level array CGH. Knowledge of mutations in POLG and SLC2A1 can alter the clinical treatment and prognosis for these patients.

The remaining mutations were identified in genes that are less commonly described in infantile epilepsy. Three novel mutations in the STXBP1 gene were found. Two patients had a small deletion or duplication resulting in a premature stop codon. In the other patient, a partial STXBP1 gene deletion was identified by exon-level array $\mathrm{CGH}$, which likely would have been missed by whole genome array $\mathrm{CGH}$. A de novo mutation in KCNQ2 was found in a patient with infantile spasms. Lastly, a single patient was homozygous for a novel missense mutation in PNPO. Although this mutation has not been published thus far, the amino acid is part of a highly conserved substrate-binding domain of PNPO. This finding was of interest not only because PNPO deficiency is extremely rare and clinical testing is relatively uncommon, but also because there is available treatment. The patient had an improvement in his seizures and overall responsiveness upon treatment with pyridoxal 5' phosphate. Conclusions: These data demonstrate the clinical utility of a broad but targeted epilepsy panel that utilizes both Next generation sequencing and exon-level array CGH to analyze both common and rare genetic causes of epilepsy.

\subsection{2 \\ ELECTRICAL STIMULATION REDUCES HIGH FREQUENCY OSCILLATIONS (80-500HZ) IN PATIENTS WITH REFRACTORY NEOCORTICAL EPILEPSY Malenka A. Killmann ${ }^{1,2}$, T. Golla ${ }^{1}$, B. O. Schelter ${ }^{2,3}$, M. Dümpelmann $^{4}$, R. Korinthenberg ${ }^{1}$, A. Schulze-Bonhage ${ }^{1}$ and J. Jacobs ${ }^{1}\left({ }^{1}\right.$ Hospital of Neuropaedriatrics and Muscular Diseases, University Medical Center, Freiburg, Germany; ${ }^{2}$ Freiburg Center for Data Analysis and Modeling, University, Freiburg, Germany; ${ }^{3}$ University Department of Neurology, University Medical Center, Freiburg, Germany and ${ }^{4}$ Epilepsy Center, University Medical Center, Freiburg, Germany)}

Rationale: High frequency oscillations (HFOs, $80-500 \mathrm{~Hz}$ ) are EEG biomarkers for epileptogenic areas. HFOs predominantly occur in the seizure onset zone (SOZ) and the surgical removal of HFO generating tissue correlates with the postsurgical seizure outcome. New studies suggest that HFO can also measure the actual epileptogenicity of the underlying tissue, as HFO rates increase after reduction of antiepileptic medication. High frequency electrical stimulation (HFS) can be used for diagnostic purposes as well as therapy in patients with refractory epilepsy. This study investigates the occurrence and changes of HFOs during and after HFS performed for diagnostic purposes in patients with refractory neocortical epilepsy.

Methods: All patients which were recorded with a sampling rate of $1024 \mathrm{~Hz}$ and underwent cortical stimulation for the purpose of localizing functional or epileptic tissue at the Epilepsy Centre Freiburg were included. Ripples $(80-200 \mathrm{~Hz})$ and fast ripples $(200-$ $500 \mathrm{~Hz}$ ) were visually marked in a baseline EEG segment prior to HFS, after each period of HFS as well as after the end of HFS, in patients in whom the stimulation triggered a seizure a pre- and postictal segment was marked. Rates of HFOs were compared for the different time periods using Wilcoxon rank sum test $(\mathrm{p}<0.05)$. Areas of seizure onset were analysed separately.

Results: 12 patients with 911 EEG channels were analysed, 7 patients experienced seizures from untypical and one from typical location. Rates of ripples and fast ripples were significantly higher in the SOZ than outside $(\mathrm{p}<0.001)$. Ripple (chi-squared $=54.41 ; \mathrm{df}=9$; 
$\mathrm{p}<0.001)$ as well as fast ripple (chi-squared $=19.30 ; \mathrm{df}=9 ; \mathrm{p}=0.02$ ) rates decreased significantly over the course of stimulation. This was the case in SOZ (figure 1) as well as non-SOZ channels. Rates were reduced in patients which experienced provoked seizures (chisquared $=101.49 ; \mathrm{df}=9 ; \mathrm{p}<0,001)$ as well as those which did not (chi-squared $=33.50, \mathrm{df}=9, \mathrm{p}<0,001$ ) (figure 2).

Conclusions: HFS resulted in a gradual decrease of HFO-Rates over time in all analysed patients. This was the case for patients with provoked seizures as well as for those without. The decrease of HFOs was not limited to SOZ areas. The observed seizures mainly occurred outside the spontaneous SOZ and might result from high current stimulation. If HFOs are considered as markers of epileptogenicity the reduction in HFO-rates has to be interpreted as a reduction of epileptogenicity during the period of HFS. This observation fits well with the latest studies in whom longterm HFS results in a reduction of seizure frequency and can be used for antiepileptic therapy.
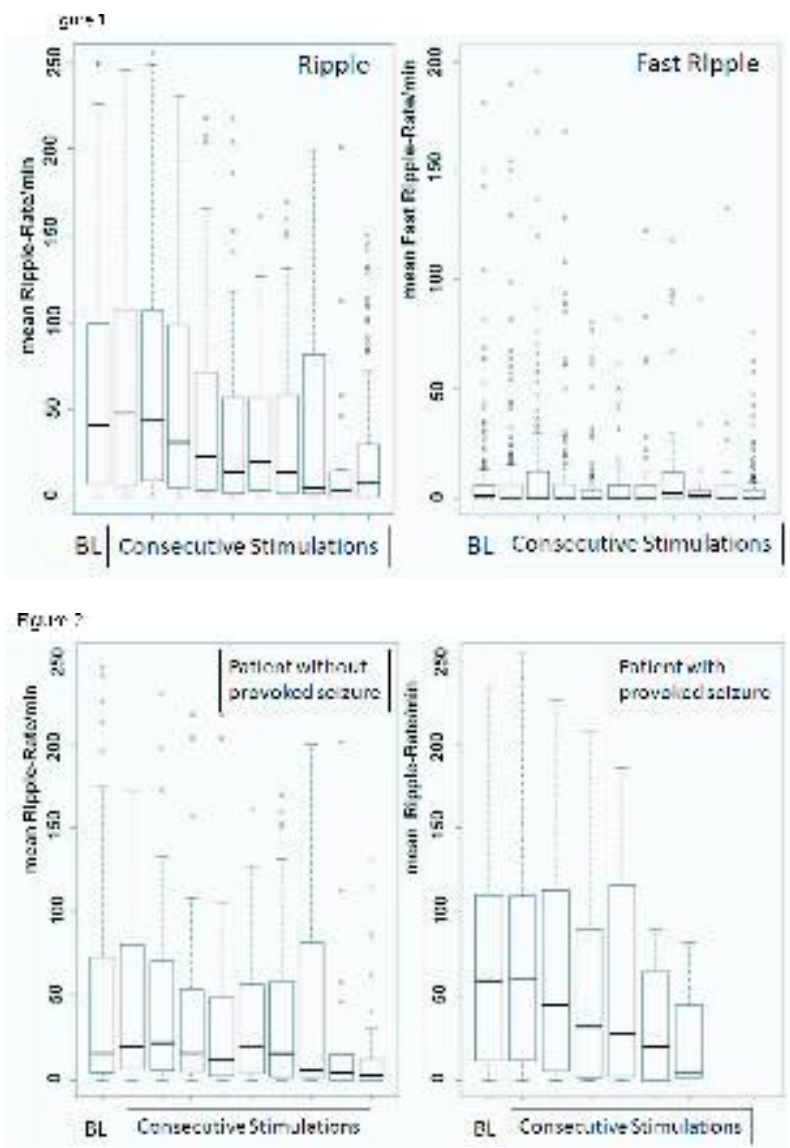

\subsection{3}

\section{CLINICAL AND ELECTROPHYSIOLOGIC RISK FACTORS} FOR SUBSEQUENT EPILEPSY IN PATIENTS WITH COMPLEX FEBRILE SEIZURES

Hunmin Kim ${ }^{1}$, S. Byun ${ }^{2}$, H. Ryu ${ }^{2}$, B. Lim $^{2}$, J. Chae ${ }^{2}$, J. Choi ${ }^{3}$, K. $\mathrm{Kim}^{2}$, Y. Hwang ${ }^{2}$ and H. Hwang ${ }^{1}\left({ }^{1}\right.$ Pediatrics, Seoul National University Bundang Hospital, Seongnam-Si, Republic of Korea; ${ }^{2}$ Pediatrics, Seoul National University Children's Hospital, Seoul, Republic of Korea and ${ }^{3}$ Pediatrics, Seoul National University Boramae Hospital, Seoul, Republic of Korea)

Rationale: To identify the risk factors for subsequent epilepsy in patients with complex febrile seizures using a single-center retrospective cohort.

Methods: The medical records of 1,091 patients discharged with a diagnosis of febrile seizures from the Seoul National University Bundang Hospital from February 2004 to October 2009 were reviewed. One hundred eighty-three patients (107 boys and 76 girls) with complex febrile seizures were included in the analysis. Clinical characteristics, including features of complex febrile seizure, initial interictal electroencephalographic findings, and subsequent epilepsy, were reviewed and the relative risk of subsequent epilepsy was estimated. The mean follow-up duration for subsequent epilepsy was 5.7 years (range, $2.15-8.0$ years).

Results: Complex febrile seizures were observed in $22.6 \%$ of all patients with febrile seizures. Among 183 patients with complex febrile seizures, 22 patients (12.0\%) developed subsequent epilepsy. The presence of multiple seizures for 24 hours was significantly more frequent in patients with subsequent epilepsy $(\mathrm{P}=0.011$; relative risk, 2.76; 95\% confidence interval, 1.22-6.24), whereas the presence of focal or prolonged seizures $(>15$ minutes) was not significantly different. Epileptiform discharges (focal in all cases) were significantly more frequent in patients with subsequent epilepsy $(50 \%$ vs $13 \%, \mathrm{P}<0.001)$, with a relative risk of 4.23 (95\% confidence interval, 1.99-8.95).

Conclusions: The presence of multiple seizures or epileptiform discharges is a significant risk factor for subsequent epilepsy in patients with complex febrile seizures. Electroencephalography should be considered in all patients with complex febrile seizures who had multiple seizures.

\subsection{4 \\ DOES HPV SHORTEN THE ADMISSION DAYS IN HOSPITAL DURING LONG TERM VIDEO EEG MONITORING?}

S. Saygi, L. Mehdikhanova, F. Irsel Tezer, N. Dericioglu and L. Dogan (Neurology, Hacettepe University Faculty of Medicine, Sihhiye/Ankara, Turkey)

Rationale: Hyperventilation is an effective and frequent method used both to trigger generalized absence seizures during routine EEG recording and facilitate different kinds of seizures during long term video-EEG monitoring. In this study, it is aimed to demonstrate the efficacy of hyperventilation in long term video-EEG monitoring unit and to evaluate the results in different kinds of seizures.

Methods: The records of 326 patients who were monitorized between January 2008 and February 2011 in HUTF video-EEG monitoring unit were reviewed retrospectively. The demographic features and the types of seizures and epilepsy syndromes of the patients who had seizures during hyperventilation were evaluated. Results: In 64 patients who have done hyperventilation, $18.75 \%$ had seizures during hyperventilation. The mean admission days of the patients with seizures during hyperventilation was 5.3 days, without seizures was 7.7 days. Furthermore, $75 \%$ of the patients had temporal lobe seizures, $16.7 \%$ of them extratemporal lobe seizures and $8.3 \%$ of them had myoclonic seizures. In patients without seizures during hyperventilation, $65.4 \%$ of them had temporal lob seizures, $19.2 \%$ had extratemporal seizures, $1.9 \%$ had generalized seizures and $1.9 \%$ had nonepileptic psychogenic seizures. Seizure type couldn't be determined in $11.6 \%$ of the patients.

Conclusions: Seizures can be induced with hyperventilation in $1 / 5$ of the patients during video-EEG monitoring. The method is more effective in patients with temporal lob epilepsy. The activation methods like hyperventilation, shortens admission days in hospital and decreases the hospital costs.

\subsection{5 \\ EFFICACY OF VIDEO-EEG IN THE DIAGNOSIS AND TREATMENT OF ALTERED MENTAL STATUS IN THE ELDERLY POPULATION}

Olgica Laban-Grant, N. R. Thakur, S. Mesad, J. Lee, T. Wojohk, C. C. Lambrakis and M. E. Lancman (Northeast Regional Epilepsy Group, White Plains, NY)

Rationale: Alteration of mental status in elderly patients is a common occurrence and frequently of multi-factorial etiology. EEG 
is frequently part of the diagnostic evaluation in patients where encephalopathy persists despite adequate treatment of the presumable underlying causation. The aim of this study was to evaluate the yield of VEEG monitoring compared to that of the routine EEG in eliciting an accurate diagnosis which would guide treatment of altered mental status in elderly patients.

Methods: A review of Northeast Regional Epilepsy Group records from a single community hospital from February 2009 until June 2012 identified 82 consecutive patients ( 32 males and 50 females), 60 years old or older (range 60-97, mean 78.7 SD 9.4), who had VEEG monitoring performed due to persistent altered mental status despite adequate treatment of the presumed underlying etiology. Patients with clinical seizures or status epilepticus at the time of onset of the VEEG monitoring were excluded from the study. The following information was obtained retrospectively: results of the VEEG, results of the previous recent routine EEG or if not available, first hour of the VEEG study, the history of seizures (19/82 patients $23.2 \%$ ), reported seizure at the onset of the altered mental status (16/82 patients $-19.5 \%$ ), and recommendations regarding initiation or changes in treatment regimens with respect to antiseizure medications.

Results: The VEEG was abnormal in 69 patients (84\%). Epileptogenic abnormalities were present in 34 of abnormal studies (49\%). In 19 patients, seizures were also recorded (27\%). The VEEG revealed dissimilar findings compared to the routine EEG or initial hour of the VEEG in 24 patients (29\%). These new findings were considered epileptogenic in 17 patients $(71 \%)$. Based on these findings, antiepileptic medications were started in 11 patients $(16 \%$ of all patients).

Fifty three patients in our group had no history of seizures in the past or at the presentation of altered mental status. In this subgroup, the VEEG was abnormal in 42 patients (79\%). Epileptogenic abnormalities were identified in 15 patients (36\%) and seizures were present in 7 patients (17\%). The VEEG revealed new findings compared to the routine/initial EEG in 14 patients in this subgroup (26\%). New epileptogenic abnormalities were identified in 10 out of 14 studies $(71 \%)$. Antiepileptic medications were started in 8 patients based solely on the VEEG study (15\% of patients who had no history of seizures in past or at the presentation of altered mental status). Conclusions: The VEEG provided valuable clinical information compared to the routine EEG in a significant number of patients whose etiology of altered mental status remained unclear. The yield of VEEG was comparable in the subgroup of patients who had no history of seizures. Our study underscores the importance of maintaining a high clinical suspicion for seizures and the usefulness of the VEEG in evaluating alteration in mental status in elderly patients.

\subsection{6 \\ ICTAL AND INTERICTAL HIGH FREQUENCY OSCILLATIONS IN TEMPORAL LOBE EPILEPSY}

Pradeep Modur and S. Miocinovic (Neurology, University of Texas Southwestern Medical Center, Dallas, TX)

Rationale: To investigate high frequency oscillations (HFOs) in temporal lobe epilepsy (TLE).

Methods: We studied patients who had temporal resection after intracranial recording with subdural grids/strips and posteroanteriorly-inserted hippocampal depths. EEG was acquired at $1000 \mathrm{~Hz}$ sampling rate. We marked seizure onset visually (bipolar montage, 50-333 Hz filter, 2-s window) based on earliest occurrence of HFOs. If no HFOs were seen, seizure onset was marked based on earliest rhythmic activity $(1-70 \mathrm{~Hz}, 10-\mathrm{s})$. Spatial extent of seizure onset was determined by the HFO channels with subsequent evolution (i.e., $\mathrm{HFO}+$, see Modur et. al., Epilepsia 2011). For this study, we defined HFO by 2 criteria: oscillation with $\geq 4$ peaks; peak frequency $\geq 70 \mathrm{~Hz}$ or total power in $70-333 \mathrm{~Hz}$ band higher than power in $1-70 \mathrm{~Hz}$ band. Using MATLAB, we developed an automated method to detect HFOs based on root mean square (RMS) of the EEG signal which was bandpass filtered at 50-333 Hz and notch filtered. We set variable thresholds based on the mean and standard deviation of the channel's RMS to identify putative HFOs. Such oscillations were rectified to count the peaks, and subjected to FFT to determine frequency and power before classifying them as definite HFOs. Consecutive HFOs ( $<200-\mathrm{ms}$ and $<20$-ms apart for ictal and interictal respectively) were combined into a single HFO. We analyzed ictal (5-s segment) and interictal (10-min per patient sampled over 2 days) HFOs in the seizure onset channels (SOC) and non-seizure onset channels (nSOC) in both amygdalo-hippocampal (AH) and neocortical temporal (NT) channels. Statistical analysis was done with GraphPad.

Results: Among 8 patients, we analyzed 22 seizures. All had ictal HFOs in at least 1 seizure. Overall, $53 \mathrm{AH}$ (16 SOC, $37 \mathrm{nSOC})$ and 478 NT (82 SOC, 396 nSOC) channels were analyzed. Interictal data were available for only 6 patients. Although HFOs ranged up to a max of $257 \mathrm{~Hz}$, the peak frequencies varied only marginally from the $70 \mathrm{~Hz}$ cutoff. Ictal HFOs had lower peak frequencies than interictal HFOs in both AH (62 vs $76 \mathrm{~Hz}, \mathrm{p}=0.001)$ and NT (68 vs $77 \mathrm{~Hz}$, $\mathrm{p}=<0.0001$ ) SOC. However, peak frequencies of interictal HFOs were similar regardless of channel type (SOC vs nSOC) or localization (AH vs NT). Peak frequencies of ictal HFOs in SOC were lower than those in nSOC in both $\mathrm{AH}$ (62 vs 77, p=0.002) and NT ( 68 vs 73, p<0.0001) onsets; in addition, peak frequencies were lower in AH than NT (62 vs 68, p=0.02) SOC. Compared to nSOC, the SOC showed lower interictal HFO firing rates in AH channels (22 vs 39 per min, $p=0.32$ ) and higher firing rates in NT channels ( 40 vs $20, \mathrm{p}<0.001)$. However, among $\mathrm{nSOC}$, the rate was higher in $\mathrm{AH}$ than NT channels ( 39 vs $20, \mathrm{p}=0.008)$. HFO durations were similar in SOC and nSOC ( 49-57 ms) regardless of localization (AH vs NT). Conclusions: Ictal and interictal HFOs appear to be generated by different network (or populations) of neurons in TLE and its subtypes. Interictal HFOs are more likely to be associated with neocortical temporal seizure foci although they seem to occur more frequently in the non-seizure generating areas of the mesial temporal structures.

\subsection{7 \\ SLEEP/WAKE AND DIURNAL OCCURRENCE OF SEIZURES IN RELATIONSHIP TO PATIENT AGE AND LOCALIZATION IN PEDIATRIC EPILEPSY}

Sriram Ramgopal $^{1}$, I. Sánchez Fernández ${ }^{1,2}$, C. Powell ${ }^{3}$, M. Vendrame $^{4}$, M. Zarowski ${ }^{1,5}$, A. V. Alexopoulos ${ }^{6}$, S. Kothare $^{1}$ and T. Loddenkemper ${ }^{1}\left({ }^{1}\right.$ Neurology, Boston Children's Hospital, Boston, MA; ${ }^{2}$ Department of Child Neurology, Hospital Sant Joan de Déu, Universidad de Barcelona, Barcelona, Spain; ${ }^{3}$ Clinical Research Program, Boston Children's Hospital, Boston, MA; ${ }^{4}$ Neurology, Boston University School of Medicine, Boston, MA; ${ }^{5}$ Polysomnography and Sleep Research Unit, Department of Developmental Neurology, Boston University School of Medicine, Poznan University of Medical Sciences, Poland and ${ }^{6}$ Epilepsy Center, Neuroscience Institute, Cleveland Clinic, Cleveland, $\mathrm{OH}$ )

Rationale: Seizures oftentimes do not occur randomly but present at certain peaks and troughs throughout the day. The propensity to have a seizure at certain times and/or during wake/sleep may be influenced by individual risk factors. We aim to identify risk factors that predict timing of seizures.

Methods: We retrospectively reviewed the charts of 955 patients that underwent video-EEG in our center. Patients $\leq 21$ years with recorded seizures were included. Seizures were evaluated based on EEG localization and their occurrence in 3-hour time bins, during the day (6AM-6PM) or night, and out of wakefulness/sleep. Patients were classified according to age: infants ( $\leq 3$ years), children (3-12 years), and adolescents ( $>12-21$ years). An analysis utilizing generalized estimating equations with a negative binomial distribution was performed. Models were expanded to test for differences in the main 
effects by age and adjusted for potential confounders (gender, semiology, MRI lesion and medications).

Results: A total of 390 patients (188 girls, mean age 9.2 years, SD $6.0)$ had 1,754 recorded seizures.

Generalized seizures (109 patients, 490 seizures) occurred more out of wakefulness $(p<0.001)$ and more during the day $(p<0.001)$. Infants had more seizures between 6AM-3PM $(\mathrm{p}<0.05)$. Children had more seizures between 6-9AM and 12-3PM $(\mathrm{p}<0.05)$. Adolescents had more seizures between 6-9AM and 6-9PM $(\mathrm{p}<0.05)$. Modeling revealed a greater occurrence of seizures at night with increasing age $(\mathrm{p}=0.046)$.

Temporal lobe seizures (62 patients, 271 seizures) occurred overall more out of wakefulness $(\mathrm{p}=0.03)$. This was specifically observed in children $(p<0.05)$ and adolescents $(p<0.01)$. In infants, seizures were more common between $3-6 \mathrm{AM}(\mathrm{p}<0.05)$. In the $>12$ age group, seizures occurred more between $12-3$ PM $(\mathrm{p}<0.01)$. There was no evidence of sleep/wake or day/night seizure variation based on age or age groups in temporal lobe seizures.

Frontal lobe seizures (41 patients, 184 seizures) occurred more out of wakefulness in infants $(\mathrm{P}<0.05)$ and more out of sleep in adolescents $(\mathrm{p}<0.0001)$. Children had more seizures between 9PM-12AM $(\mathrm{p}<0.01)$ and $12-3 \mathrm{AM}(\mathrm{p}<0.05)$ and adolescents had more seizures between 6AM-12PM $(\mathrm{p}<0.05)$. Adolescents were 3.6 times more likely to have seizures during sleep compared to other children with frontal lobe seizures (95\% confidence interval: 1.8-7.2). Modeling revealed a gradual increase in seizures during sleep with each unit increase in age $(\mathrm{p}=0.02)$. This was not affected by potential confounders.

All occipital lobe seizures ( 2 patients, 13 seizures) occurred during wakefulness. Parietal lobe seizures (11 patients, 50 seizures) did not occur in a specific pattern in relation to the sleep/wake or day/night state.

Conclusions: Seizures occur in different sleep/wake and diurnal patterns depending on patient age and EEG location. These findings are suggestive of maturational changes in circadian rhythmicity that may alter seizure susceptibility in different age groups. Results may assist in age related and EEG localization based prediction of times of greatest seizure propensity.

\subsection{8}

\section{CAN WE PREDICT EFFICACY OF TREATMENT FOR ESES} BASED ON SPECIFIC FACTORS FROM THEIR EEG?

Sonal G. Patel ${ }^{1,2}$ and A. D. Patel ${ }^{2}\left({ }^{1}\right.$ Neurology, Children's Hospital of Orange County, Orange, CA and ${ }^{2}$ Neurology, Nationwide Children's Hospital, Columbus, $\mathrm{OH}$ )

Rationale: Rationale: The goal of this study was to determine if there were certain predictive factors in the long-term video EEG (LTM) of patients with electrical status epilepticus of sleep (ESES) that could help predict patient response to treatment.

Methods: We reviewed the LTM of 26 patients that had EEG reports classified as having ESES between the years of 2005-2011 at Nationwide Children's Hospital. We reviewed whether polyspikes were present, and calculated spike wave indexes (SWI \%) and categorized them based on published criteria. In addition, we determined whether the discharges were focal or generalized and if focal, in which location. We also documented if the patient had discharges present while the patient was awake. If treatment was offered, we noted their therapeutic response to the given treatment. Results: 12 of 26 patients were essentially treated with either the high-dose valium protocol or steroids. Of the 12 treated patients, 4 patients responded well to treatment and 8 patients were refractory to treatment. Of the 8 patients that were refractory to treatment, 6 patients had polyspikes present on their LTM (one sided pvalue $=.0303$ ). Of the 4 patients that responded well to treatment, zero had polyspikes. In terms of SWI category, $5 / 8$ of the refractory patients had a higher category(level 2 or 3 ), and only $3 / 8$ of the refractory patients were category $1(\mathrm{SWI} \%<50 \%)$. Finally, of the 4 patients that responded to medication treatment, zero were category 1 and $4 / 4$ were either category 2 or 3 .

Conclusions: This study supports the hypothesis that the presence of polyspikes is a statistically significant predictor of being refractory to medication. It also supports the trend that lower percentages of SWI could be predictive of possibly being refractory to treatment and suggests the need for a larger patient population for further studies.

1.119

CLINICAL AND EEG CHARACTERISTICS OF NONCONVULSIVE STATUS EPILEPTICUS IN CRITICALLY ILL PEDIATRIC PATIENTS

Hyewon Ryu ${ }^{1}$, J. H. Lee ${ }^{1}$, S. H. Byun ${ }^{1}$, J. S. Kim ${ }^{1}$, B. C. Lim ${ }^{1}$, H. Hwang $^{1,2}$, J. H. Chae ${ }^{1,2}$, J. E. Choi ${ }^{1,2}$, K. J. Kim ${ }^{1,2}$ and Y. S. Hwang ${ }^{1,2}$ ( ${ }^{1}$ Seoul National University Children's Hospital, Seoul, Republic of Korea and ${ }^{2}$ Seoul National University College of Medicine, Seoul, Republic of Korea)

Rationale: The aim of this study was to investigate the clinical and electroencephalographic

(EEG) features and outcome in acute ill pediatric patients with nonconvulsive status

epilepticus (NCSE)

Methods: We retrospectively reviewed 22 patients (16 males and 6 females) with NCSE between May 2006 and March 2012 in the Pediatric Intensive Care Unit (PICU) of Seoul National University Children's Hospital.

Results: The patients ranged in age from 1 month old to 22 years, with a mean age of 6.8

years of age. All patients were in a lethargic or comatose state at the time of diagnosis of

NCSE. Presumed encephalitis was the most frequent etiology of NCSE in $11(50.0 \%)$,

followed by intracranial hemorrhage in $5(22.7 \%)$, multi-organ failure form septic shock in 4

$(18.1 \%)$, exacerbation of preexisting epilepsy in $3(13.6 \%)$, exacerbation of underlying

autoimmune disease in $1(4.5 \%)$. There were four patients $(18.1 \%)$ with generalized

convulsive status epilepticus (GCSE) that was followed by NCSE and 15 patients $(68.1 \%)$

developed NCSE after brief convulsive seizures. Three patients $(13.6 \%)$ presented with

NCSE as the first manifestation of seizure activity during a comatose state. EEG waveform

morphologies were quite variable and included repetitive generalized or focal spikes,

polyspike, sharp wave, spike and wave or sharp and slow wave complexes, or other

rhythmic theta or delta activity. Three patients (13.6\%) had generalized ictal discharges. Five

patients $(22.7 \%)$ died of the underlying acute medical illness.

Conclusions: In this study, we presented that the clinical and EEG features in pediatric patients with NCSE. Common etiologies of NCSE are acute CNS lesions associated with serious underlying medical condition and presumed encephalitis. Prolonged EEG monitoring to exclude NCSE may be warranted in pediatric patients in comatose state even after brief convulsive seizure.

\subsection{0 \\ CONCORDANCE OF EPILEPSY IN PATIENTS WITH POLYMICROGYRIA ENROLLED IN THE EPILEPSY PHENOME/GENOME PROJECT}

C. Shain ${ }^{1}$, Sriram Ramgopal ${ }^{1}$, I. Parulkar ${ }^{1,2}$, A. Poduri ${ }^{1}$ and .. EPGP Investigators ${ }^{1}\left({ }^{1}\right.$ Neurology, Boston Children's Hospital, Boston, MA and ${ }^{2}$ Warren Alpert Medical School, Brown University, Brown University, RI) 
Rationale: Polymicrogyria (PMG) is a heterogeneous and highly epileptogenic malformation of cortical development. The potential role of epilepsy surgery in patients with PMG has been increasingly recognized. In the PMG cohort of patients from the Epilepsy Phenome/Genome Project (EPGP), we sought to evaluate whether the predicted region of seizure onset based on seizure semiology and EEG data corresponded to the location of the PMG seen on MRI. In addition, we assessed the outcomes of epilepsy surgery in the subgroup of patients for whom this was performed.

Methods: Participants were recruited through EPGP, a multi-center collaborative including 26 sites from the United States, Argentina, Australia, and New Zealand. Detailed phenotypic data were reviewed for all patients with epilepsy and PMG. Epilepsy lateralization was assessed using EEG and seizure semiology, and then compared to MRI to establish concordance. In patients who underwent epilepsy surgery, we evaluated outcomes at last follow-up.

Results: We identified 90 patients (43 female) with PMG and epilepsy. The cohort included pediatric and adult patients with a median age of 11 years $(<1-55)$. Most patients presented with localization-related epilepsy $(64.4 \%)$; complex partial seizures were the most common seizure type (50\%). Half of the patients had bilateral PMG on MRI (50\%). Of the 45 unilateral cases, 17 (37.8\%) were located in the left hemisphere and $28(62.2 \%)$ were located in the right hemisphere.

When comparing PMG and epilepsy lateralization, $70.6 \%$ of the 17 patients with left-sided malformations had concordant data suggesting they might be potential candidates for surgery. From this group, one participant underwent epilepsy surgery. Of the 28 patients with right-sided malformations, $75 \%$ had right-sided seizure onset with 4 undergoing epilepsy surgery. One patient with bilateral/multifocal epilepsy also underwent epilepsy surgery. Among the patients with bilateral PMG, $15.6 \%$ had seizure onset on the right, $15.6 \%$ on the left, $35.6 \%$ bilateral $/ \mathrm{multifocal}$, and $11.1 \%$ generalized with 3 patients from this group undergoing epilepsy surgery (2 right, 1 left, see figure).

In total, 9 patients in our series underwent epilepsy surgery. They presented with a range of seizure semiologies and did not have strictly focal or unilateral malformations. After surgery, 7 patients had major improvement (Engel score I), 1 patient had overall worsening of seizures (Engel score IV), and 1 patient was seizurefree but had insufficient follow-up for Engel scoring.

Conclusions: The majority of the EPGP cohort with PMG and epilepsy were found to have concordance between laterality of PMG and predicted region of seizure onset based on seizure semiology and EEG data. Our data from the subset of patients who underwent epilepsy surgery suggest that surgery can be successful for carefully selected patients with PMG. Future phenotypic analysis with the addition of genotypic data may provide further insight into the prediction of surgical candidacy among patients with PMG and epilepsy.

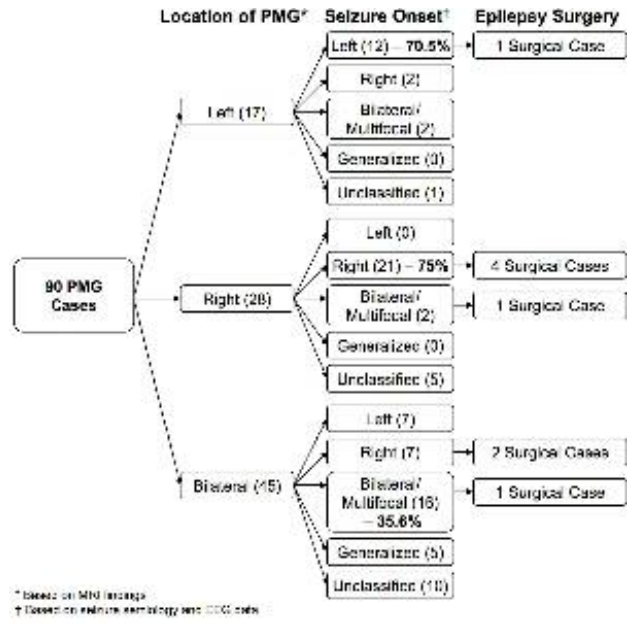

1.121

EMG-BASED SEIZURE DETECTOR: PRELIMINARY RESULTS COMPARING A GENERALIZED TONIC-CLONIC SEIZURE DETECTION ALGORITHM TO VIDEO-EEG RECORDINGS

Michael Girouard ${ }^{1}$, L. Moreno ${ }^{2}$, L. Morgan ${ }^{2}$, K. Karkar ${ }^{2}$, L. Leary ${ }^{2}$, O. $\mathrm{Lie}^{2}$ and C. Szabo ${ }^{2}{ }^{1} \mathrm{LGCH}$, Inc., San Antonio, TX and

${ }^{2}$ Neurology, South Texas Comprehensive Epilepsy Center, University of Texas Health Science Center at San Antonio, San Antonio, TX)

Rationale: Purpose: Detection of generalized tonic-clonic seizures (GTCS) at home and during activities of daily living could facilitate earlier intervention. However, to date, there are no FDA-cleared devices that provide an accurate means for GTC seizure detection during activities of daily living. This study aims to validate a GTC seizure detection algorithm to be used later in a GTC seizure detection system to be cleared by the FDA. In order to do this, we (a) compared a software algorithm analysis of EMG signals continuously acquired in an Epilepsy Monitoring Unit (EMU) to video-EEG (VEEG) recordings, and, (b) optimized settings for software algorithm using baseline measurements of maximal strength and muscle contraction.

Methods: Methods: Inpatient scalp VEEG monitoring was performed along with surface EMG recordings of unilateral biceps and triceps brachii. Twelve subjects suspected to have GTCS were admitted to the EMU for an average of 4.4 (range 3 to 6) days, providing over 372 hours of surface EMG data. The EMG data was analyzed separately by a seizure detection software algorithm (LGCH, Inc., Texas) and correlated with the determination of seizure onset and characterization by board-certified clinical neurophysiologists. The algorithm continuously compares recorded surface EMG signals to a baseline sample of muscle activity; GTCS are signaled with sustained activation of multiple frequency bands $(30-40 \mathrm{~Hz}, 130-240 \mathrm{~Hz}, 300-400 \mathrm{~Hz})$ of EMG activity. The algorithm was expected to detect GTCS within 10 seconds of arm movement during the GTCS, while minimizing false positive detections.

Results: Results: 6 of 12 subjects had a total of 7 GTCS captured by EMG recording and confirmed by VEEG. The EMG algorithm detected all 7 GTCS within 10 seconds of arm movement during the GTCS as identified by VEEG monitoring. There were no false positive detections. 69 myoclonic, 27 tonic, 12 absence and 14 complex partial seizures were recorded, but none triggered an alarm condition. No alarms were triggered during any activities of daily living or other interictal motor activities. Two subjects removed the EMG electrodes shortly before the end of their stay in the EMU due to discomfort.

Conclusions: Conclusions: This interim view of a continuing study demonstrates the feasibility of accurately detecting GTC seizures with a minimally invasive, arm-worn device analyzing EMG signals. At this early point, the sensitivity and positive predictive value of the seizure detection algorithm appears to be superior to other devices currently under investigation or currently commercialized. The ability to reliably detect a GTCS provides an alert to caregivers and facilitates a more rapid response in order to minimize seizure related complications, including injuries and SUDEP. Comfort, wearability and ensuring a quality signal for analysis will be important factors in the design of the final system.

\subsection{2 \\ UTILITY OF CONTINUOUS EEG MONITORING IN PEDIATRIC ECMO PATIENTS}

Lekha M. Rao ${ }^{1}$, R. Sankar ${ }^{1}$, J. Y. Wu ${ }^{1}$, S. A. Hussain ${ }^{1}$, C. Giza ${ }^{1,2}$ and J. H. Matsumoto ${ }^{1}\left({ }^{1}\right.$ Divison of Pediatric Neurology, Mattel Children's Hospital at UCLA, Los Angeles, CA and ${ }^{2}$ Division of Neurological Surgery, University of California Los Angeles, Los Angeles, CA) 
Rationale: Extracorporeal membrane oxygenation (ECMO) is an important life-saving technology for pediatric patients with respiratory or cardiac failure; however, it involves significant risk of neurologic sequelae, including stroke and seizures. These patients frequently receive neuromuscular blockade, precluding clinical examination. Continuous EEG monitoring therefore represents a practical method of surveillance for central nervous system (CNS) injury and can help direct management.

Methods: Twenty-eight children aged 0 days to 17 years consecutively receiving ECMO at our institution for cardiac or respiratory failure between March 2010 and June 2012 were placed on continuous EEG monitoring as part of a standard neuromonitoring protocol. All recordings utilized plastic electrodes to minimize the possibility of artifact as usually seen using standard electrodes. Results: All 28 patients had abnormal findings on EEG. 18\% (5 out of 28) had seizures, all of which had cardiac disease. $80 \%$ (4 out of 5) had subclinical seizures, with one patient found to be in nonconvulsive status epilepticus. EEG findings changed management by initiation of antiepileptics ( 5 patients), urgent neuroimaging ( 8 patients), or withdrawal of care ( 6 patients). In the 22 patients with cardiac disease, the median age of those with seizures was 17 days, and the oldest patient with seizures was 173 days old.

Conclusions: Children undergoing ECMO therapy are at risk for seizures, especially those with cardiac disease and of younger age. Continuous video EEG monitoring can significantly impact management in patients on ECMO, especially when neuroimaging and clinical exam cannot be obtained due to the patient's tenuous clinical status and neuromuscular blockade. Supported in part by the Julie \& Steve DesJardins Fellowship, CARE AND CURE, Epilepsy Foundation of Greater Los Angeles.

\subsection{3}

AN EXPLORATORY EVALUATION OF SEIZURES AND THE SUBSEQUENT DEVELOPMENT OF EPILEPSY AS AN EFFECT OF PEDIATRIC CANCER THERAPY UTILIZING CHEMOTHERAPY

M. Scott Perry ${ }^{1}$, L. Bailey ${ }^{1}$, A. Hernandez ${ }^{1}$, S. Malik ${ }^{1}$, R. Matthew ${ }^{3}$, J. Murray ${ }^{2}$ and E. Braly ${ }^{2}\left({ }^{1}\right.$ Comprehensive Epilepsy Center, Cook Children's Medical Center, Fort Worth, TX; ${ }^{2}$ Neuro-oncology, Cook Children's Medical Center, Fort Worth, TX and ${ }^{3}$ University of North Texas Health Science Center, Fort Worth, TX)

Rationale: Acute seizures occurring at diagnosis or during treatment of childhood cancer has been described. However, the subsequent development of epilepsy has not been well characterized. We sought to describe the circumstances and course of seizures encountered amongst childhood cancer survivors treated with chemotherapy at a single pediatric medical center to characterize patient risk factors, seizure types, and subsequent treatment response.

Methods: We reviewed billing records of patients $<18$ years of age treated at Cook Children's Medical Center between 2006 and 2011 with diagnosis codes for childhood cancer treated with chemotherapy and seizure, then retrospectively reviewed the medical records of this cohort. We excluded patients with nonepileptic seizures and those with a diagnosis of epilepsy prior to cancer diagnosis. Demographic data, seizure characteristics and treatment, cancer characteristics and treatment, and outcome were abstracted for descriptive analysis.

Results: Forty three patients underwent chemotherapy treatment and had seizures. Seizure types were characterized as localization-related in $20(46.5 \%)$ patients, generalized in $17(39.5 \%)$, and a combination thereof in $6(14.0 \%)$. Fifteen (35\%) had CNS tumors, $24(56 \%)$ leukemias, and $5(12 \%)$ had other cancers. Twenty five $(58 \%)$ patients had onset of seizures during chemotherapy treatment and 18 $(42 \%)$ had onset after treatment (median time to onset $=498$ days, range $=179-5714)$. Fourteen (33\%) patients had seizures only during chemotherapy treatment, while $29(67 \%)$ patients had seizures beyond chemotherapy treatment and were diagnosed with epilepsy. Six $(14 \%)$ patients developed treatment resistant epilepsy. Patients that developed epilepsy were diagnosed with cancer at an earlier mean age ( 4.32 vs $8.57 \mathrm{y}, \mathrm{F}(1,40)=8.295, \mathrm{p}=0.006)$. Patients with CNS tumors were significantly more likely to develop epilepsy compared to those with leukemia or other cancer types $(F(2,39)=9.714, p=<0.001)$. There were no significant differences in chemotherapy used or exposure to CNS radiation therapy between those that developed epilepsy and those that did not.

Conclusions: While acute seizures during chemotherapy treatment are more common, late onset seizures and epilepsy develop frequently, with a number developing treatment-resistant epilepsy. When seizures occur in patients treated with chemotherapy for childhood cancer, earlier age at cancer diagnosis and a diagnosis of CNS tumor are predictors of subsequent epilepsy development. Additional studies employing a control group of patients treated with chemotherapy without development of seizures would be useful to discover other potential risk factors.

\subsection{4 \\ DATA-DRIVEN APPROACH TO AUGMENT DECISION SUPPORT FOR PEDIATRIC NEUROLOGY IN EMR ERA Ketan Mane ${ }^{1}$, T. Loddenkemper ${ }^{2}$, P. Owen ${ }^{1}$, M. Mikati ${ }^{3}$, I. Fernández ${ }^{2}$, M. Tennison ${ }^{4}$ and A. Leviton ${ }^{2}$ ( ${ }^{1}$ RENCI, UNC-Chapel Hill, Chapel Hill, NC; ${ }^{2}$ Department of Neurology, Boston Children's Hospital, Boston, MA; ${ }^{3}$ Department of Pediatric Neurology, Duke University Medical Center, Durham, NC and ${ }^{4}$ Department of Pediatric Neurology, UNC-Chapel Hill, Chapel Hill, NC)}

Rationale: Epilepsy patient data stored in the electronic medical record (EMR) data has the potential to offer enhanced patient care, if presented in an easy to understand way. Unfortunately, the large volume of data presented in raw format (as numbers) are hard to process and interpret, thus limiting their use. Data displays and computers can aid the physician during everyday practice. Here, we describe how data displays and decision support systems can work in coordination with each other. We show how patient data and comparative population evidence can be visually displayed in a way that enables the physician to make sense of this information in a fast and intuitive manner.

Methods: Many variables are recorded in the EMR of children with epilepsy: seizure types and frequencies, medications, outcomes (measured by increase/decrease in seizure frequencies), potential medication side-effects and their severity. Patient enrollment continues at three different pediatric epilepsy centers. Computational approaches can be used to process, aggregate, and build displays of data. Visual representation help the physician better understand patient data, and aggregated evidence from comparative population. Embedded interactions empower the physician to customize the choice of comparables. The display program shows an approach to make use of the existing EMR resources without having the physician search through the patient's file.

Results: We have now collected pilot data on 20 epilepsy patients. Different data views can be built to reveal patterns in the data. Figure 1 shows dashboard with patient-level data, while Figure 2 highlights an approach to display and use comparative population treatment evidence to assist the physician in decision making. Predictive modeling approaches can be use comparable patient data to identify trend in medication response. Within a single dashboard (Figure 1 or Figure 2), different data views linked together give flexibility for data overlay, and for filter and syncing all data views. Such data displays are likely to help the physician gain a quick understanding of the characteristics of the patient data at the time of visit. Such interactive data displays have the potential to reduce information overload, free up the physician's cognition for higher level data processing, and aid informed decision making.

Conclusions: This multicenter pilot study is designed to highlight the role that data displays and a clinical decision support program can play to bridge the complementary skill sets of humans and computers (as an external aid) to rapidly derive useful information from a large 
dataset. External aid in the form of interactive data displays can be used to augment the information processing abilities of the physician and facilitate decision making.

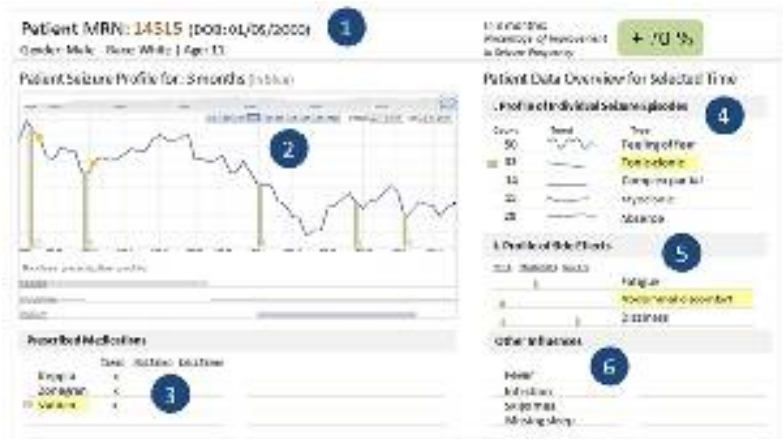

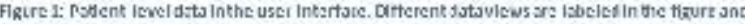

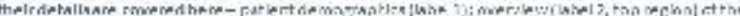

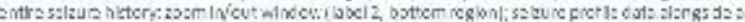

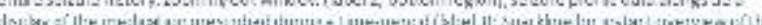
Hick a

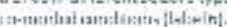

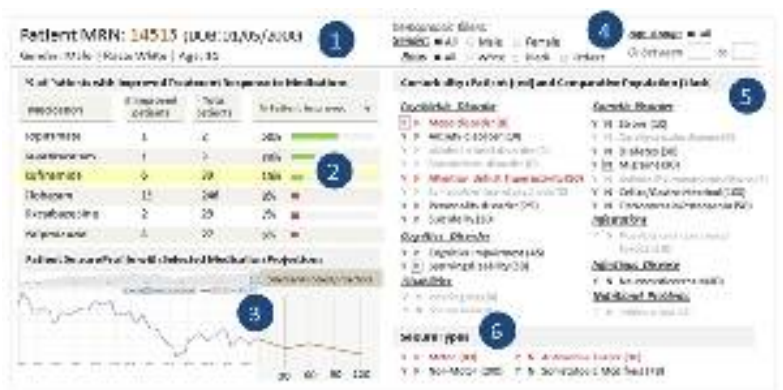

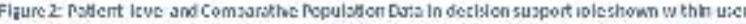

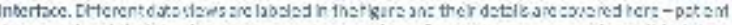

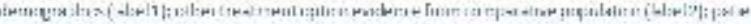

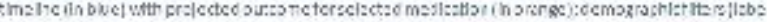

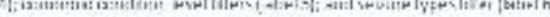

\subsection{5}

\section{AUTONOMIC NERVOUS SYSTEM (ANS) RESPONSES TO POSTICTAL GENERALIZED} ELECTROENCEPHALOGRAPHIC SUPPRESSION (PGES) IN PEDIATRIC EPILEPSY

Tesfaye Zelleke ${ }^{1}$, B. Kroner ${ }^{2}$, A. Pitruzzello ${ }^{2}$ and W. Gaillard ${ }^{1}$ $\left({ }^{1}\right.$ Children's National Medical Center, Silver Spring, MD and ${ }^{2}$ RTI international, Rockville, MD)

Rationale: PGES is relatively common $(27-40 \%)$ with generalized tonic clonic seizures (GTCs) observed on the epilepsy monitoring unit (EMU) and was significantly longer in patients that subsequently died of SUDEP than in those that survived. In addition, an increase in skin conductivity has been shown to be associated with duration of PGES. We sought to determine whether peri-ictal physiologic changes in the autonomic nervous system are correlated with PGES in order to identify additional potential risk factors for SUDEP. Methods: Six children with epilepsy, aged 3-18 and undergoing video EEG have been enrolled into the study with an enrollment target of 30 patients by November 2012. During monitoring, the patients wore unobtrusive commercially-available sensors that continuously recorded heart rate, respiration, and skin conductivity. The latter is missing on the first 2 patients enrolled.

Results: Three GTCs among 15 total seizures were observed, 2 of which were followed by PGES ( $8 \mathrm{sec}$ and $40 \mathrm{sec}$ duration). Physiological changes during the pre-ictal, ictal and PGES periods are summarized in Table 1. All 3 seizures demonstrated marked changes in heart rate and depth of breath (respiration amplitude) during the ictal period when compared with a one-minute pre-ictal interval. Heart rate and respiration amplitude peaked during the ictal period, but remained elevated throughout PGES. Sweat gland activity, quantified by the skin conductivity sensor, was measured during one GTC event that was followed by 40 seconds of PGES. Skin conductivity began to increase toward the end of the ictal period, and surged throughout the PGES period.

Conclusions: Autonomic dysfunction during the ictal and post-ictal periods may be associated with generalized seizures and is postulated to be a contributing factor to SUDEP which is a rare event. We demonstrated a robust sympathetic surge in heart rate and skin conductivity during PGES as compared to the ictal period. If this finding is replicated in our larger study of 30 patients, it may help explain the reported association between duration of PGES and SUDEP.

Physiological changes observed during three GTC seizures

\begin{tabular}{|c|c|c|c|c|}
\hline GlC event & & Event I & Event 2 & Event 3 \\
\hline latient number & & 01 & 01 & 01 \\
\hline PGISS duration (sec) & & 0 & 8 & 40 \\
\hline Maximum HR during seikzurc (bpm) & & 196 & 197 & 198 \\
\hline Hearl rate (bpm) & $\begin{array}{l}\text { Preictal } \\
\text { Ictal } \\
\text { P'GES }\end{array}$ & $\begin{array}{c}70 \pm 3 \\
148 \pm 37 \\
N / A\end{array}$ & $\begin{array}{c}65 \pm 2 \\
164 \pm 33 \\
177 \pm 2\end{array}$ & $\begin{array}{c}87 \pm 6 \\
147 \pm 53 \\
173 \pm 32\end{array}$ \\
\hline Respiration amplitude (unitless) & $\begin{array}{l}\text { Prcictal } \\
\text { Ictal } \\
\text { PGES }\end{array}$ & $\begin{array}{c}0.02 \pm 0.02 \\
0.53 \pm 0.18 \\
\mathrm{~N} / \mathrm{A}\end{array}$ & $\begin{array}{l}0.03 \pm 0.01 \\
0.46 \pm 0.20 \\
0.15 \pm 0.02\end{array}$ & $\begin{array}{c}0.08 \pm 0.02 \\
5.1 \pm 4.2 \\
1.3 \pm 0.6\end{array}$ \\
\hline Skin conductivity $(\mu \mathrm{S})$ & $\begin{array}{l}\text { Preictal } \\
\text { Ictal } \\
\text { PGiEs }\end{array}$ & $N / \wedge$ & $\mathrm{N} / \mathrm{A}$ & $\begin{array}{l}0.40 \pm 0.00 \\
0.43 \pm 0.10 \\
1.61 \pm 0.22\end{array}$ \\
\hline
\end{tabular}

\subsection{6 \\ CHARACTERIZATION OF EEG ABNORMALITIES AND SEIZURES IN MALES AND FEMALES WITH GENETICALLY DIAGNOSED FRAGILE X SYNDROME} S. V. Kothare ${ }^{1}$, S. Ramgopal ${ }^{1}$, S. Lincoln ${ }^{2}$, J. Picker ${ }^{2}$, A. Rotenberg ${ }^{1}$ and Takijah T. Heard ${ }^{1}{ }^{1}$ Neurology, Boston Children's Hospital, Boston, MA and ${ }^{2}$ Genetics, Boston Children's Hospital, Boston, MA)

Rationale: Seizures and EEG abnormalities in children with Fragile $\mathrm{X}(\mathrm{FX})$ syndrome have not been well characterized. The purpose of this study is to describe the prevalence and characteristics of seizures and EEG abnormalities in a population of children, both male and female, with known FX.

Methods: Charts of patients with genetically diagnosed FX and receiving care in a specialized clinic in a tertiary care hospital were reviewed. Patients who had at least one EEG recorded for any reason were included in the study. Patient age, sex, indication for EEG, and EEG findings were recorded. Additionally, data were collected regarding epilepsy diagnosis, seizure characteristics, antiepileptic drug (AED) use and clinical imaging.

Results: A total of 135 charts were reviewed. Eighteen patients (13.3\%, 1 female) had at least one EEG recorded. The mean patient age at EEG recorded was 6.0 years (standard deviation 3.8 years). Fourteen patients underwent outpatient EEG, three patients underwent EEG in the context of a sleep study, and one patient underwent inpatient long-term video-EEG. Indications for EEG included a history suggestive of seizure $(n=12)$, global developmental delay $(n=1)$, atypical febrile seizure $(n=1)$, and follow-up of known epilepsy $(n=1)$. Among those who had a history suggestive of seizure, six patients had a history of undiagnosed staring episodes. The three children who underwent sleep study were evaluated by polysomnography for complaint of excessive snoring. On review of EEG data, 13 patients (72\%) had abnormal findings. Eleven patients had generalized EEG findings and one patient had findings localized to the temporal lobe. The most common EEG finding was slowing of the posterior dominant rhythm for age, seen in seven patients $(53 \%)$. Other findings included epileptiform discharges $(n=6)$. Five patients $(3.7 \%$ of all patients in the studied population) were diagnosed with epilepsy on the basis of their 
clinical and EEG findings. There were insufficient females in our series to compare differences in seizures according to gender. Five patients in the series had a history of AED usage for seizure control. AEDs used in the seizure population included oxcarbazepine $(n=1)$, levetiracetam $(n=2)$, lamotrigine $(n=1)$, clonazepam $(n=1)$, valproate $(\mathrm{n}=1)$, and lacosamide $(\mathrm{n}=1)$. Of these five, four patients were seizure-free at last follow-up. Ten patients had undergone MRI of the brain. MRI abnormalities included thinning of the corpus callosum and white matter reduction ( $\mathrm{n}=1$, nonepileptic), dysplastic change of the amygdala and hippocampus ( $\mathrm{n}=1$, with epilepsy), and mesial temporal sclerosis ( $\mathrm{n}=1$, with epilepsy).

Conclusions: The prevalence of seizures in children with FX is $3.7 \%$ in our small series, and staring spells are the most common clinical symptoms prompting an EEG. Slowing of posterior dominant rhythm was seen in many of the evaluated patients. These data suggest that patients with FX may have characteristic patterns in clinical seizure presentation and electrographic abnormalities that warrant further investigation.

\subsection{7}

MISDIAGNOSIS IN JME; A JAPAN-ORIENTED CURRENT PROBLEM OF ADULT-EPILEPSY OUTPATIENT

Ichiro Takumi ${ }^{1}$, M. Noha ${ }^{2}$, J. Kadekawa ${ }^{3}$ and A. Teramoto ${ }^{4}\left({ }^{1}\right.$ Nippon Medical School, Kawasaki, Japan; ${ }^{2}$ Department of Neurosurgery, Okinawa Redcross Hospital, Okinawa, Japan; ${ }^{3}$ Neurology, Okinawa Redcross Hospital, Okinawa, Japan and ${ }^{4}$ Tokyo Rosai Hospital, Tokyo, Japan)

Rationale: JME (juvenile myoclonic epilepsy) counts $10 \%$ in the adult epilepsy population; however, its misdiagnosis still remains as a problem leading to its treatment intractable. In Japan, because of the lack of numbers of adult epileptologist, many adult with epilepsy are treated by neurosurgeons; neurosurgeons are situated in the 'neurology generalist'. To clarify this problem, we have reviewed at our JME patient.

Methods: Top author's home institution, where the neurosurgery outpatient clinic as well as referral epilepsy out-patient specialized in epilepsy surgery is on-going, was subjected to review.

Results: 11 cases of JMEs were found in our out-patient. Among them, five of them were referral from other neurosurgeons. Only 2 cases were precisely diagnosed as JME; both of them were referred from Japan board-certified epilepsy specialist. In 8 cases, some medical intervention was required for the follow up.

Conclusions: The current problem lies in "who is treating adultepilepsy patient in Japan?' We used to owe a lot to the pediatricians. An ongoing nationwide project to unite medical institutions and clinics for people with epilepsy will help to solve this problem, by clarifying to the people where they can receive proper epilepsy treatment. But in addition, the entire Japanese neurosurgeons have to be aware of this clinical entity, if we keep in relation with people with epilepsy so far.

\subsection{8}

\section{VIDEO-POLYGRAPHIC ANALYSIS OF EPILEPTIC DROP ATTACKS ASSOCIATED WITH BRIEF VOCALIZATION}

Yoshiko Hirano, H. Oguni and M. Osawa (Pediatrics, Tokyo Women's Medical University, Tokyo, Japan)

Rationale: "Drop attacks" have been observed in a number of different seizure types. However, they are remarkably brief and dramatic due not only to the seizure itself but also to gravity, such that it is often difficult to make an exact seizure diagnosis based on a witness description. Recent progress in electrophysiological devices has demonstrated that atonic seizures, myoclonic-atonic seizures, and epileptic (flexor) spasms are the main seizure types causing drop attacks. We report herein a special type of drop attack associated with an initial vocalization in children with cryptogenic myoclonic-astatic epilepsy (CMAE).
Methods: The subjects were 5 patients (male: 2, female: 3 ) whose epilepsy was ultimately diagnosed as CMAE. Drop attacks with an initial vocalization were investigated employing simultaneous video and electroencephalography-electromyography (EEG-EMG) recordings. All events were analyzed in slow motion and frame-byframe video replay mode.

Results: The video-polygraphic examination captured a total of 36 drop attacks in these 5 cases. There were 24 drop attacks associated with brief vocalizations. Detailed analyses demonstrated that all were classifiable as myoclonic-atonic seizures with or without the preceding initial vocalizations, which precisely corresponded to myoclonic EMG components of the myoclonic-atonic seizures. The ictal EEG showed generalized spike-and-wave or polyspike-andwave complexes.

Conclusions: Drop attacks associated with an initial brief vocalization were found to be myoclonic-atonic seizures whose myoclonic component appeared to momentarily involve the thorax, thereby producing the brief vocalization. Myoclonic-atonic seizures preceded by vocalizations are also the main seizure type of CMAE and should be considered in the differential diagnosis of drop attacks in childhood epilepsy.

\subsection{9 \\ DO PATIENTS WITH AMYOTROPHIC LATERAL SCLEROSIS (ALS) HAVE SEIZURES?}

Reina Roivainen ${ }^{1}$, H. Laaksovirta ${ }^{1}$, J. Toppila $^{2}$ and S. Vepsalainen ${ }^{1}$ ( ${ }^{1}$ Dept. of Neurology, Helsinki University Central Hospital, Helsinki, Finland and ${ }^{2}$ Dept. of Neurophysiology, Helsinki University Central Hospital, Helsinki, Finland)

Rationale: Calcium- and glutamate-mediated exitotoxicity is thought to be important in the pathogenesis of both ALS and epilepsy caused by structural or metabolic lesions. Increased cortical hyperexcitability in ALS may be caused by degeneration of intracortical inhibitory neurons. Cognitive impairment is found in ALS patients, implicating damage of cortical networks wider than primary motor areas alone, predisposing for epilepsy. Yet there are hardly any reports of ALS and seizures affecting the same patient.

Methods: We retrospectively identified all patients visiting Helsinki University Central Hospital neurological outpatient clinics during 2004-2011 due to ALS. Medical records were reviewed to find patients with prior diagnosis of epilepsy or in whom investigations had been carried out due to suspected seizures.

Results: Among 122 male and 121 female patients with ALS, we identified four patients $(1.6 \%)$ with an established diagnosis of epilepsy before ALS symptom onset. No change in seizure control was recorded during ALS progression in these patients. In three other patients, a single epileptiform seizure with etiology separate from ALS had been registered. In four additional patients, paroxysmal events occurring in temporal association with ALS symptom onset had been diagnosed as epilepsy. In three out of four patients, EEG had showed interictal focal disturbance, which had been classified epileptiform in two patients. In retrospective medical chart review, many features atypical for seizures were found. In one case, symptoms leading to diagnosis of epilepsy were later thought to have heralded evolving frontotemporal dementia.

Conclusions: Symptoms raising suspicion of seizures in ALS patients are fairly frequent and should be evaluated with caution. There may be a phase in ALS development during which symptomatology clinically resembling seizures occurs. The pathophysiology of these symptoms requires to be addressed in prospective follow-up studies and with ictal EEG recordings. 


\subsection{0}

POTENTIAL IMPLICATION OF EPILEPTIC ACTIVITIES IN THE PHYSIOPATHOLOGY OF THE ACUTE

\section{CONFUSIONAL STATE IN THE ELDERLY}

I. Bachir ${ }^{1}$, Naeije Gilles ${ }^{2}$, B. Legros ${ }^{2}$ and T. Pepersack ${ }^{1}\left({ }^{1}\right.$ Geriatrics, Hopital Erasme Université libre de Bruxelles, Brussels, Belgium and ${ }^{2}$ Neurology, Reference Center for the Treatment of Refractory Epilepsy, Hopital Erasme Université libre de Bruxelles, Brussels, Belgium)

Rationale: Prevalence of acute confusional state (ACS) is about 25 $\%$ in patients over 65 years old admitted to the emergency room (ER). Epilepsy and ACS share many risk factors and are both associated with confusion and fluctuating consciousness.

Nevertheless, in clinical practice, a link between epilepsy and ACS is seldom sought in the elderly.

The aim of this work was 1) to study when a link between epileptic activity (EA) and ACS was searched in an ER of an academic hospital and 2) when it was done, the prevalence of EA in elderly patients with ACS.

Methods: Charts of patients over 65 years old admitted for ACS in the ER between and were retrospectively reviewed. Risk factors for ACS and epilepsy were studied, including medical history, medications, biological and iconographic work-up, EEG results if done. Those characteristics were compared in patients who had EEG and those who hadn't. Results of EEG were classified in normal, diffuse slowing, focal slowing and the presence of EA. Clinical characteristics of patients with EA on EEG were compared to the patients without EA on EEG.

Results: Hundred and seventy seven charts were analysed. Mean age was 78 years old. There was no difference between patients who had EEG and those who hadn't. Fifty six percent (98/177) of patients had an EEG. Eighty four percent of EEGs were abnormal. Seventy four percent of EEG showed diffuse slowing, $44 \%$ focal slowing. EA were present in 15 patients : 12 with interictal epileptiform discharges (IED) and 3 with non convulsive status epilepticus (NCSE).

There was no statistical differences between patients with EA on EEG and patients without EA on EEG.

Conclusions: No clinical, biological or iconographic anomalies can distinguish a priori patients in ACS who will have EA on EEG and therefore EEGs should be performed in all elderly patients with ACS. EAs probably have a role in ACS since their prevalence is ten times higher than expected in an hospitalized population (ref). This high prevalence of EA in ACS has not been reported before and should be confirmed by prospective studies, ideally with continuous EEG monitoring since 30 minutes routine EEG may underestimates EA.

\subsection{1 \\ CLINICAL FEATURES OF CHILDREN WITH FEBRILE SEIZURE ON JEJU ISLAND, SOUTH KOREA}

Seunghyo Kim (Pediatrics, Jeju National University Hospital, Jeju, Republic of Korea)

Rationale: Febrile seizure is the most common convulsive event in children. The study for febrile seizure has not been investigated on Jeju Island, South Korea. The goal of this study was to assess the clinical and demographic characteristics of children with febrile seizure on Jeju Island, South Korea. This was the first study for febrile seizure on Jeju island.

Methods: We retrospectively reviewed patients aged 7-120 months who were admitted with febrile seizure (FS) to our pediatric department at Jeju National University Hospital between March 2007 and May 2012. Cases were identified using a computerized text search, followed by a manual chart review. We excluded patients who had evidence of intracranial infection or previous nonfebrile seizures. The variables collected included age, sex, seizure characteristics, seizure duration, recurrence within 24 hours, onset of first FS, recurrent FS after 5 years of age, family history of FS (first- degree relatives), family history of epilepsy, electroencephalographic findings, brain magnetic resonance imaging (MRI), and subsequent nonfebrile seizures.

Results: One hundred forty-three patients (94 boys and 49 girls) were enrolled in the study. The mean age at the time of the study was 48 months. The average age at the first FS was 26 months. The average total number of FS was 3.5. Family histories of FS and epilepsy were found in $42(29.4 \%)$ and 11 patients $(7.7 \%)$, respectively. Subsequent nonfebrile seizures were observed in $24(16.8 \%)$ of the children, and $46(32.2 \%)$ were diagnosed with complex FS. None of the parameters examined differed significantly between the children with and without complex FS. Forty-six (32.2\%) of the children had their first FS before 1 year of age; of these, 20 showed complex FS $(p=0.046)$. FS continued after 5 years of age in $27(18.9 \%)$ of the children, 9 of whom showed subsequent nonfebrile seizures $(p=0.019)$. We compared patients who suffered FS more than twice with patients who experienced 1 or 2 episodes. Seventy (48.9\%) of the 143 children experienced more than 2 episodes of FS, whereas the remaining children experienced 1 or 2 episodes of FS. Among these 70 patients, $29(41.4 \%)$ had a family history of FS $(p=0.002)$. Thirty of the 70 children who experienced more than 2 recurrent episodes of FS suffered their first FS before 1 year of age, whereas a smaller proportion of the children who had fewer than 3 episodes of FS had their first FS at this age $(\mathrm{p}=0.007)$.

Conclusions: Children who developed FS before 1 year of age had a tendency to experience complex FS and more than two episodes of FS. Patients whose FS persisted beyond 5 years of age tended to experience subsequent nonfebrile seizures, compared with patients whose FS did not persist beyond 5 years of age. Complex FS did not influence the risk of subsequent afebrile seizures. The recurrence rate for FS was significantly higher in children with a family history of FS.

\subsection{2 \\ HUMAN RABIES ENCEPHALITIS VIDEO EEG FINDING; A CASE REPORT}

M. Evans ${ }^{1}$, Enrique A. Feoli ${ }^{2}$ and J. Politsky ${ }^{3}\left({ }^{1}\right.$ Northeast Regional Epilepsy Group, Summit, NJ; ${ }^{2}$ Northeast Regional Epilepsy Group, Summit, NJ and ${ }^{3}$ Northeast Regional Epilepsy Group, Summit, NJ)

Rationale: There is a single report of EEG findings in human rabies encephalitis from 1981, they describe a first EEG done the second day of admission that showed diffuse slowing, and a third EEG done several days after, when the patient had a seizure and the EEG showed a high amplitude periodic discharges on the right side. We present the video EEG findings on a case of human rabies encephalitis, later confirmed (July 18,2011) by the CDC as a canine rabies virus present in Haiti.

Methods: Describe the video EEG findings of human rabies encephalitis.

Results: On June 30, a 73 y/o woman Haitian woman presented to our ER with shortness of breath, poor balance and hallucinations, she was admitted with a presumptive diagnosis of UTI. Head CT was normal. She become progressively more combative, on the next day she developed fever T 38.5, respiratory depression and tremors. Epilepsy consultation was requested; she was found combative, and restless, she was awake but could not maintain a conversation, she was having frequent episodes of jaw quivering and bilateral upper and lower extremity tremors, lasting 1 to 3 minutes. She was transferred to the ICU and intubated. CSF was unremarkable except for a slight elevation of with cells (7/ul). Video EEG was begun and she was found to be in non convulsive status epilepticus. Encephalitis was diagnosed, CSF encephalitis panel was negative. Serum, CSF, saliva and skin biopsy was sent to the CDC for rabies testing. Rabies virus antigen was detected in the skin biopsy and saliva. On July 18 she was declared brain dead.

Video EEG findings; On day 1 of monitoring, the EEG showed bilateral diffuse slowing, without proper organization. She had 
multiple seizures arising from the left temporal area, (figure 1) without generalization; the electrical changes would coincide with her clinical episodes of mouth quivering and tremor like activity of the upper and lower extremities, treatment was initiated with cerebyx $\mathrm{IV}$, on the next day she was still having frequent seizures and she was started on Versed $0.05 \mathrm{mg} \mathrm{Kg}$ hour, and keppra was added, burst suppression was reached, and attempt to lower Versed cause the reappearance of seizure activity, Vimpat was added and Versed was re-started.

On July 14th, status epilepticus had resolved and versed was tapered, however her EEG continued to show a burst suppression pattern (figure 2) 4 days after Versed was stopped, on July 19th the EEG showed electro cerebral silence, at that point the patient met criteria for brain dead.

Conclusions: There has been no documented cases of video EEG monitoring in human rabies encephalitis. Our patient had seizures that clinically were difficult to identify as such, with mouth quivering and bilateral upper more than lower extremity tremors, on EEG this were proven to be left hemispheric seizures. The evolution of the EEG, showed a progression to a burst suppression pattern (while off sedatives) and finally electro cerebral silence.

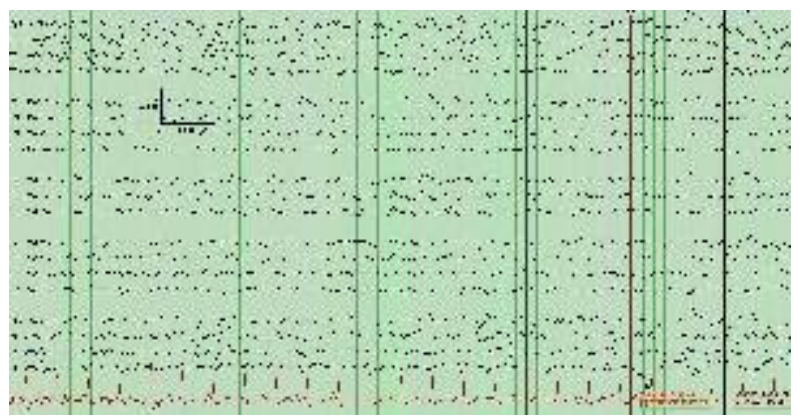

Seizure Onset

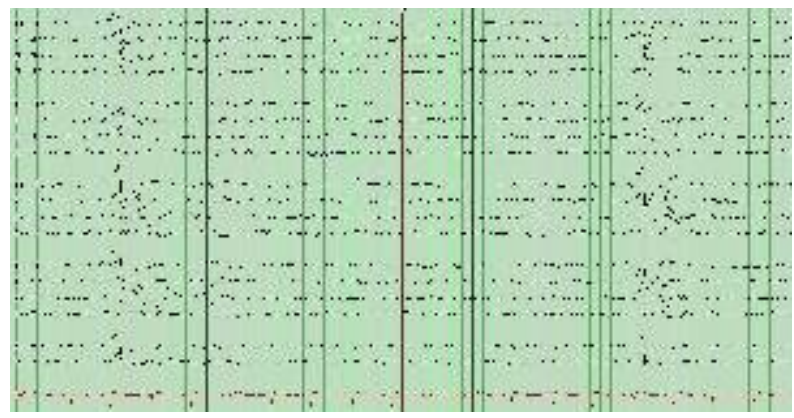

Burst Suppression

\subsection{3 \\ FIRST SEIZURE CLINIC: A PORTAL TO RE-ASSESS TERMS AND RECONSIDER CONCEPTS?}

Bernd Pohlmann-Eden ${ }^{1}$, K. T. Legg ${ }^{1}$, C. C. Crocker ${ }^{1}$, M. Blanchard ${ }^{2}$, J. J. Moeller ${ }^{1}$ and M. H. Schmidt ${ }^{2}\left({ }^{1}\right.$ Neurology, QE II Health Sciences Center, Dalhousie University, Halifax, NS, Canada and ${ }^{2}$ Radiology, QE II Health Sciences Center, Dalhousie University, Halifax, NS, Canada)

Rationale: Most of our knowledge with regard to pathophysiology, etiology and prognosis of epilepsy syndromes comes from advanced stages of epilepsy and cross-sectional studies. A paradigm shift is needed to extend this knowledge to a more dynamic longitudinal approach in early stages of epilepsy. Our First Seizure Clinic (FSC) had a particular interest in terms and classification, pathologies suggested by MRI, and treatment response.

Methods: Since May 2008, an Adult FSC at QE II Health Sciences Center, Dalhousie University Halifax has been established with a strict First Seizure Assessment Approach (FSAA): 1) Coordinated by an epileptologist and a nurse practitioner, 2) Careful triaging, 3) maximum wait-time of 2- 4 weeks (often within few days), 4) Comprehensive clinical testing (early routine and sleep-deprived EEG, early MRI, screen for psychiatric comorbidity, social and cognitive issues), 5) Clinical research protocol, 6) Genetic blood sampling, 7) Comprehensive database 8) Follow-up visits 6, 12 and 24 months.

Results: 391 new patients were seen from May 2008 to May 2012. $167(42.7 \%)$ patients had to be excluded from the database due to the diagnoses other than first seizure (syncope, psychogenic nonepileptic seizures, cerebrovascular diseases or long-lasting epilepsy). We identified 3 subgroups of all drug-naïve FSC presentations ( $\mathrm{n}=224): 1$ ) Strictly first seizure (FS), $n=113,2$ ), New-onset epilepsy $(\mathrm{NOE}=>1$ seizure within 1 year), $\mathrm{n}=85$, and 3 ) Newly-diagnosed epilepsy $(\mathrm{NDE}=$ seizure history for more than 1 year), $n=26$. MRI images (epilepsy protocol) documented "pathologies" in $72 \%$ of the FS-, $58 \%$ of the NOE, and $82 \%$ of the NDE patients. CT showed significantly fewer abnormalities in all 3 groups. White matter hyperintensities (WMH) were found in $25 \%$ of the FS, $18 \%$ of the NOE, and $20 \%$ of the NDE-patients (age range 17-79 years, average 47.7 years). Congenital malformations were found in 8 out of 224 FSC patients ( $5 \mathrm{FS}, 2 \mathrm{NOE}, 1 \mathrm{NDE}$ ) including focal cortical dysplasia $(F C D, n=4)$, heterotopia $(H T, n=2)$, polymicrogyria $(n=1)$. $87.5 \%(n=7)$ stayed seizure-free $(n=3$ without AED) over a mean follow-up of 93 weeks, $n=3$ over follow-up greater $>160$ weeks. Disease courses of patients with AED-treated NOE or NDE showed a highly variable pattern of developing pharmacoresistance with regard to primary response, initial seizure-free intervals, and secondary relapse.

Conclusions: Our preliminary experience with systematically analyzing FSC patients is very promising. Our classification approach looking into 3 different subgroups of patients diagnosed either with FS only, NOE or NDE suggests new exciting insights. It questions current concepts and terms specifically with regard to "typical time courses" of early pharmacoresistance and epileptogenicity of MRI-proven "pathologies". The high rate of seizure-free patients with congenital malformations, even without AED, including focal cortical dysplasia puts the current perception of the role of FCDs in advanced stages of refractory focal epilepsy into a totally new perspective.

\subsection{4 \\ WTIUDRAWN}

\subsection{5 \\ INTER-ICTAL SPIKE DETECTION USING SMART TEMPLATES AND THE CONCEPT OF EXPERIENCE Shaun S. Lodder ${ }^{1}$, J. Askamp ${ }^{1}$ and M. J. van Putten ${ }^{1,2}\left({ }^{1}\right.$ Clinical Neurophysiology, University of Twente, MIRA Institute for Biomedical Technology and Technical Medicine, Enschede, Netherlands and ${ }^{2}$ Dept of Neurology and Clinical Neurophysiology, Medisch Spectrum Twente, Enschede, Netherlands)}

Rationale: To diagnose patients with epilepsy, routine EEG recordings are made in which neurologists search for traces of interictal activity associated with epileptiform discharges. In practice, patients are only diagnosed with epilepsy if these traces are found. Routine EEG recordings last 15 to 25 minutes, and often no epileptiform behavior is seen within this time frame. After another seizure, the patient is required to have a second recording in which epileptiform activity can again be missed. Longer recordings such as in-home monitoring can yield higher diagnostic efficiency, but due to the time consuming nature of visual analysis, the burden is too large on the reviewer to make it practical on routine basis. With assistance from automated detection methods, review time can be reduced significantly. However, these methods are not yet reliable enough to perform this task. We present a novel approach for inter-ictal spike detection to overcome this limitation. Our method is based on smart 
templates that learn and gain experience with each classification it makes.

Methods: The method can be divided into several steps: (i) Inter-ictal spike detection is performed by cross correlating a set of templates with an EEG recording. For each template, regions are found where high correlations $(>0.9)$ exist. From these, a template can propose regions of epileptiform activity with a measure of certainty. (ii) All proposals are pooled together and grouped based on their location in time. Sufficiently large groups are considered as possible regions for epileptiform activity. (iii) A reliability measure is calculated for each group, and if it exceeds a threshold chosen by the reviewer, the region defined by the templates within that group is considered to contain epileptiform activity. A group's reliability is calculated from the weighted average of each template's track record combined with its measure of certainty for a particular event. A template's track record is found by evaluating all previous classifications made (logged by the system) and calculating the accuracy of their outcomes. By allowing the system to continuously gather information and optimize itself, sensitivity and selectivity continuously improves. Results: A database containing 714 templates was constructed from $\sim 50$ annotated EEGs. After training and optimization, a test set of $\sim 100$ EEGs was used to evaluate the algorithm. Sensitivity and the false positive rate per minute were measured given different reliability thresholds. Results show a positive outcome with sensitivities of up to 0.89 and false positive rates per minute below 0.05 , depending on the threshold chosen. More improvements can still be made. The system is capable of analyzing a $20 \mathrm{~min}$ routine EEG unsupervised in less than $15 \mathrm{~min}$, making it feasible in clinical practice.

Conclusions: An automated detection system with high sensitivity and selectivity can greatly assist in visual interpretation of the EEG. Not only does it lessen the burden on visual analysis, but it also creates practical opportunities for in-home EEG monitoring which can improve the efficiency of epilepsy diagnostics.

\subsection{6}

SEIZURES AND DYSKINESIAS IN ANTI-NMDAR ENCEPHALITIS - CAN WE TELL THE DIFFERENCE? Yee-Leng $\operatorname{Tan}^{1}$, K. Tan ${ }^{1,2}$ and N. C. Tan ${ }^{1,2}$ ( ${ }^{1}$ National Neuroscience Institute, Singapore, Singapore and ${ }^{2}$ Duke-NUS Graduate Medical School, Singapore, Singapore)

Rationale: Seizures are known to occur in anti-NMDA receptor (NMDAR) encephalitis. In the largest case series to date (Dalmau, 2008) involving 100 subjects, 76 suffered from clinical seizures, but documented epileptic activity was present in only 21 patients. At the same time, co-existence of dyskinesias in this disorder can be interpreted as seizures, leading to use of antiepileptic drug therapy which may not be completely justified. In this case series of antiNMDAR encephalitis subjects, we aimed to (a) characterize and correlate seizure semiology with electroencephalogram (EEG) and (b) determine if the dyskinesias were seizures.

Methods: A retrospective review of all anti-NMDAR encephalitis patients admitted to the National Neuroscience Institute from 2009 2012 was performed. All patients had confirmatory evidence of antiNMDAR antibodies in the serum and/or cerebrospinal fluid (assays done at Oxford Radcliffe Hospitals, UK). Their demographic profile, tumour presence / absence, seizure semiology, EEG findings, and types of dyskinesias recorded during EEG sessions are described. Results: Ten patients were identified during this study period. The mean (SD) age was $26.2( \pm 10.6)$ years; all were female. Four had ovarian teratomas; six were tumour-negative. The mean (SD) number of EEG recordings was $4.3( \pm 2.1)$ per patient, with a range from 2 to 8. Electrographic seizures were recorded in three patients on day 53, day 2 and day 6 of their disease course respectively; all EEGs fulfilled Young's criteria for EEG seizures. One EEG showed $13 \mathrm{~Hz}$ alpha activity maximum over the left frontal region evolving to $9 \mathrm{~Hz}$ alpha activity, with the patient manifesting complex partial seizures in the form of right gaze deviation and right head turn. The second patient's EEG showed $11 \mathrm{~Hz}$ alpha activity over the right frontal region, evolving to $2 \mathrm{~Hz}$ right frontal delta activity with no clinical manifestations. A third EEG showed repeated runs of generalized $2 \mathrm{~Hz}$ rhythmic delta activity lasting 10-20 seconds, with each run followed by a period of generalized attenuation; the patient was stuporous and unresponsive during the EEG. One patient in this case series with neither clinical nor EEG seizures had right occipital sharp waves. In contrast, oro-lingual-facial dyskinesias were captured during the EEG recordings of seven patients, and were not associated with any change in the EEG pattern.

Conclusions: Complex partial seizures are common in anti-NMDAR encephalitis, and can occur both early and late in the disease course. Multiple EEGs are often obtained for these patients. Unlike the faciobrachial dystonic seizures described in association with LGI1antibody limbic encephalitis, the seizures in anti-NMDA encephalitis do not appear to have a stereotypical semiology nor ictal EEG pattern, though our sample size is small. Oro-lingual-facial dyskinesias, which characterise anti-NMDAR encephalitis and which are sometimes mistaken for seizures, do not appear to be epileptiform in nature.

\section{Clinical Epilepsy: Clinical Treatments}

\subsection{7}

EFFECT OF ANTIEPILEPTIC DRUGS ON VITAMIN D LEVELS IN CHILDREN

Kay Taylor ${ }^{1,2}$, R. G. Davis ${ }^{1,2}$ and B. Peters ${ }^{1,2}$ ( ${ }^{1}$ Pediatric Neurology, P.A., Orlando, FL and ${ }^{2}$ Orlando Health, Orlando, FL)

Rationale: Rationale: Vitamin D is a neurosteroidal hormone that serves as a vital regulator for neuroprotection and antiepileptic effects. It accomplishes this via neuro-immunomodulation and interplay with neurotransmitters which directly affects the brain. Vitamin D regulates nerve growth factor (NGF) and glial cell line derived neurotrophic factor (GDNF) suggesting it may be able to provide some protection against excitatory neurotransmitters such as glutamate. It also influences apoptsosis and cell cycle in the developing brain.

Vitamin D deficiency causes abnormal brain development in children as it is associated with persistent alteration in neuronal systems. It may cause mild distortion in brain shape, increase lateral ventricle volumes and reduce brain differentiation. Antiepileptic drug (AED) use can increase the risk of vitamin D deficiency as it decreases the metabolism of Vitamin D and interferes with the re-adsorption process. This results in decreased bone quality, density, and may have significant influence with the neuronal issues such as decreasing cell proliferation and causing an increase in programmed cellular elimination.

Review of literature regarding vitamin D deficiency with AED use refers to adult patients. There are few studies measuring the effect deficiency has on pediatric neurologic children. We present 25 pediatric patients with low Vitamin D levels associated with AED treatment.

Methods: Method: Twenty five pediatric patients were selected who had low vitamin D levels on receiving AED treatment. Comparison included the effects of enzyme-inducing vs non-inducing AED's as well as single vs. multiple AED therapy. The length of time on AED's was also evaluated as well as race, age and seizure type. Results: Results: The two most frequently used AED's that caused a decrease in Vitamin D level were Keppra (7/25) and Lamictal (5/25). There was no influence due to age or weight. More Caucasians $(16 / 25)$ than non Caucasians were affected and more males (16/25) than females. Non Enzyme inducing AED's (28) caused more of a decrease in levels than enzyme inducing AED's ((9). There was more of a decrease with single use of AED's (15/25) vs multiple AED's (7). The length of time on AED's was 1 mos. to 84 mos. with the average of 47 mos. The vitamin D 25-OHD levels ranged from $14 \mathrm{ng} / \mathrm{ml}$ to 
$35 \mathrm{ng} / \mathrm{ml}$. The lowest level occurred on an $11 \mathrm{yr}$ old caucasian on 2 non-enzyme inducing AED's for 2 yrs with generalized seizures.

Conclusions: Conclusion: AED use can increase the risk of vitamin $\mathrm{D}$ deficiency in the pediatric population resulting in decreased bone density and bone mass as well as potentially altering the neuronal systems and brain development. Vitamin D levels may need to be drawn after starting all children on AED's and more closely monitored than is currently recommended. Further studies are needed to assess which AED's may be more detrimental to neuronal health in order to obtain optimization of seizure control with normal neuronal network development in children with epilepsy.

\subsection{8}

PRELIMINARY EXPERIENCE WITH A NEW SYSTEM FOR VAGUS NERVE STIMULATION

Bertil Rydenhag $^{1}$, H. Silander ${ }^{1}$ and E. Ben-Menachem ${ }^{2}$

( ${ }^{1}$ Neurosurgery, Sahlgrenska University Hospital, Goteborg, Sweden and ${ }^{2}$ Neurology, Sahlgrenska University Hospital, Goteborg,

Sweden)

Rationale: Vagus nerve stimulation (VNS) has been used to treat drug refractory epilepsy for more than 20 years; however, one of the limitations of this method is generation of side effects that limit the increase of current amplitude, hence its ultimate potential.

Methods: A new VNS system (FitNeSTM BioControl Medical Ltd, Yehud, Israel) is based on a proprietary cuff electrode, a design that facilitates implantation and reduces leakage of current to neighboring tissues. The electrode composition enables preferentially

unidirectional stimulation, while using parameters that are similar to the ones used by other commercially available VNS systems in terms of amplitude, frequency and pulse width.

Results: We have uneventfully implanted 5 patients whose condition was refractory to multiple anti-epileptic drugs. Two had previously undergone resective epilepsy surgery and the other 3 were evaluated for epilepsy surgery but deemed not operable. After implantation with FitNesTM all patients have been followed for at least 6 months, (range 6 months to 1 year). With the exception of one patient who suffers slight hoarseness, which does not correlate with stimulations, there are no other side effects that are either self-reportable or noted by relatives or friends or the treating physician. At this time, seizure frequency reduction versus baseline is $25 \%$ in one and $>50 \%$ in the other 4 subjects, at current strengths between $1.5-2.0 \mathrm{~mA}$.

Conclusions: This is the first experience with the FitNeS ${ }^{\mathrm{TM}}$ system for the treatment of epilepsy. The positive outcome in efficacy and the absence of side effects experienced by the patients even at relatively high currents is encouraging.

Further studies are recommended. Because patients do not seem to be aware of the stimulation, a double blind study of the efficacy of VNS would now be feasible.

\subsection{9 \\ NON EPILEPTIC SEIZURES (NES) ASSOCIATED WITH FUNCTIONAL (PSYCHOGENIC) PARALYSIS (FP): EFFICACY OF MULTI PULSES TMS}

D. Parain and N. Chastan (neurophysiology, university hospital, rouen, France)

Rationale: Patients with NES have a higher level at the somatization score compare with patients with epileptic seizures (1). So, NES may be associated with FP. These two functional symptoms may be due to a dysfunction of near the same neuronal network. As for functional movement disorders, a hypothesis arising from functional imaging study is that emotionally arousing events might trigger movement controlled by the supplementary motor area that is functionally disconnected from top-down control by the dorsolateral prefrontal cortex (2). In a previous study, we have shown that multi pulseTMS with circular coil on midline central and frontal area was effective in
$89 \%$ of 70 patients with FP (3). A controlled study is ongoing with the agreement of ethic committee (paralystim: ClinicalTrials.gov identifier: NCT01352910). We propose the same, well tolerated and easy to do therapy for patients with very disabling NES associated with FP.

Methods: On a population of sixty patients with EEG video recorded NES in our unit, eigth patients had disabling (out of job or school) NES and recurrent or persistent FP. For TMS, a $10 \mathrm{~cm}$ diameter coil was placed without neuronavigation, on the head midline, the posterior border the coil on the motor cortex. This coil was located upon the motor cortex, the supplementary motor area, the premotor prefrontal dorsolateral and anterior cingular cortex. A TMS session consisted of 60 pulses at motor threshold at about $0.3 \mathrm{hz}$. The sessions were repeated eventually each week, during 2 months and after, every 2 months, according to the evolution of NES frequency. We compare the baseline NES frequency during the month before the first TMS session and at 6 months.

Results: At 6 months, compared to baseline month, 5 patients had no NES, 1 patient had $90 \%$ and 2 patient $80 \%$ reduction in NES frequency. All patients had no FP and had reintegrated school or job. No adverse effect had been observed except transient headache after 1 session for 2 patients

Conclusions: In this short open trial, multi shocks TMS with circular coil seem efficient to control NES associated with FP. Controlled study is mandatory to confirm the efficacy of this type of TMS in NES.

1) Predicting psychogenic nonepileptic seizures with the Personality Assessment

Inventory and seizure variables. Stacy W. H., Shawn D. G. Epilepsy Behavior 2011 (22) 255-260

2)Aberrant supplementary motor complex and limbic activity during motor preparation in motor conversion disorder. Voon V, Brezing C, Gallea C, Hallett M. Mov Disord. 2011(13):2396-403.

3) Psychogenic paralysis and recovery after motor cortex transcranial magnetic stimulation. Chastan N., Parain D. Mov Disord. 2010; 25(10):1501-4.

1.140

FEASIBILITY OF MINDSET: A CLINIC-BASED DECISION SUPPORT SYSTEM FOR EPILEPSY SELF-MANAGEMENT R. Shegog, Charles E. Begley and A. Harding (University of Texas School of Public Health, Houston, TX)

Rationale: The Management Information \& Decision Support Epilepsy Tool ("MINDSET") is a tablet-based decision support system designed to identify patient epilepsy self-management needs, provide behavioral goals, and facilitate communication between the patient and health care provider (HCP) during a regular clinic visit. The purpose of this study was to evaluate the feasibility of MINDSET for clinical use.

Methods: A single group pre-post feasibility study was conducted during the clinic visits of adult patients at three participating neurology specialty clinics $(n=38)$. Patients interacted with MINDSET prior to and during their encounter with their HCP. Patients entered seizure, medication, and management data, then reviewed their management profile with prompts of suggested discussion points on self-management issues and needs, and then presented the tablet to their HCP. The provider and patient could choose to refer to the tablet during the encounter. Underlying behavioral theories informing MINDSET include Social Cognitive Theory, self-regulation theory, motivational enhancement strategies, and the 5A Model (Ask, Assess, Advise, Assist, Arrange). Hypotheses relating to feasibility, usability, perceived impact, correspondence and communication were investigated using written survey and interview data collection methods. 
Results: Feasibility: Time required for patients to complete their profiles in MINDSET took an average of 28 minutes (range: 12-67 min.). Patient ratings of time on task were favorable ( $88 \%)$ and MINDSET was perceived as minimally disruptive to clinic visits by patients and providers. Usability and perceived impact: Patients rated MINDSET favorably on usability parameters of helpfulness $(85 \%)$, likeability (91\%), understandability (91\%), and motivational appeal $(85 \%)$. Patients perceived MINDSET as having a positive impact on thinking about and managing their epilepsy (79\%) and communicating with their HCP (79\%). Correspondence and communication: Correspondence existed between self-management issues flagged in MINDSET and the patient-HCP discussion. The quality of communication when using MINDSET was rated positively, particularly for nonverbal attention, reinforcement, and reflective listening. Providers were positive about MINDSET indicating they would recommend it to other providers and would continue to use it in their clinic if given the opportunity.

Conclusions: Initial analysis of the results from patients and providers at three epilepsy clinics indicate that MINDSET is useful for discussing self-management issues, identifying needs, and developing an action plan as part of a clinic visit.

\subsection{1 \\ SUCCESSFUL DESENSITIZATION OF OXCARBAZEPINE AND ETHOSUXIMIDE AFTER CUTANEOUS ADVERSE REACTIONS AND HLA GENOTYPE IN KOREAN}

Bo Lyun Lee ${ }^{1}, J_{\text {. Lee }}^{2}$, M. Lee ${ }^{2}$ and H. Yu ${ }^{2}{ }^{1}$ Pediatrics, Pusan Paik Hospital, Inje University College of Medicine, Busan, Republic of Korea and ${ }^{2}$ Pediatrics, Samsung Medical Center, Sungkyunkwan University, School of Medicine, Seoul, Republic of Korea)

Rationale: Allergic reaction to specific antiepileptic drugs (AEDs) can occur in some patients and require a change of therapy. An alternative strategy is to desensitize the patients to the offending drug. This study aimed to investigate the usefulness and safety of desensitization to oxcarbazepine (OXC) and ethosuximide (ETX) in patients who have had genetic screening for the human leukocyte antigen (HLA) alleles.

Methods: We enrolled total 19 patients who previously showed positive response, but hypersensitivity reactions to OXC or ETX. They had to be discontinued the medications. Although alternative antiepileptic drugs were tried on our patients, their seizures were refractory to other drugs. Therefore, desensitization to OXC was tried in 17 children with partial seizures and one child with paroxysmal kinesigenic dyskinesia (PKD), and to ETX was attempted in one child with atypical absence seizures. High-resolution human leukocyte antigen (HLA)-A, and -B genotyping was performed to investigate the association between specific HLA allele and OXCinduced cutaneous adverse drug reactions.

Results: The mean age of desensitization was $10.5 \pm 3.6$ years (range 5.1-16.2 years) and the mean duration of follow-up after desensitization was $14.4 \pm 8.7$ months (range 3-34 months). Eighteen patients completed the desensitization protocol to a target dosage over 2-5 months. Five children developed mild itching and erythema during desensitization, but the symptoms disappeared after the next dose increasing was withheld for a short period. We did not find specific HLA genotypes associated with OXC-induced cutaneous adverse drug reactions. The frequency of seizure and attack in PKD was reduced to less than baseline in 17 patients except for one child. At last follow-up, eight patients were seizure-free, six patients showed $>90 \%$ reduction and the other three patients had $>50 \%$ reduction.

Conclusions: The desensitization protocol was well tolerated and safe without serious allergic reactions. When allergic reactions occur with OXC or ETX and there are no effective alternatives, desensitization can be a useful treatment modality.

\subsection{2 \\ LONGTERM SEIZURE FREEDOM ON ANTIEPILEPTIC DRUG MONOTHERAPY}

Martin J. Brodie, O. McGowan, V. Politi, K. Kelly and L. J. Stephen (Western Infirmary - Epilepsy Unit, Lanarkshire, United Kingdom)

Rationale: The goal of anitepileptic drug (AED) treatment is sustained seizure freedom on monotherapy with no or acceptable side effects resulting in a good quality of life. This project examined the characteristics of patients who remined seizure free on single AED at unchanged dosing for at least the previous year.

Methods: Data were acquired by database and case sheet interrogation of 6821 patients attending the epilepsy services at Western Infirmary in Glasgow, Scotland between 1982 and 2011. Results: Seizure freedom was achieved on 17 different monotherapies in 1425 patients (687 men, 738 women, aged 18-94 years [median 46 years]). Of these, 1131 (79.3\%) had partial seizures with or without secondary generalization and the remaining 294 $(20.7 \%)$ were classified as having idiopathic generalised epilepsies. Treatment with the first drug produced seizure freedom in 866 $(60.7 \%)$ of these patients. The remainder were controlled on their 2 nd $(n=309,21.7 \%), 3$ rd $(n=217,15.2 \%), 4$ th $(n=31,2.2 \%)$ or even 5 th $(\mathrm{n}=2,0.2 \%)$ AED as monotherapy. The commonest drugs taken were sodium valproate $(n=443,31.1 \%)$, carbamazepine $(n=382,26.8 \%)$, lamotrigine $(n=318,22.3 \%)$ and levetiracetam $(n=82,5.8 \%)$. Of the patients receiving sodium valproate, $67 \%$ took $\leq 1000 \mathrm{mg} /$ day $\mathrm{mg}$ (range $400-3000 \mathrm{mg}$ ), whereas $66 \%$ of carbamazepine-treated patients took $\leq 600 \mathrm{mg} /$ day

$\mathrm{mg}$ (range $200-2000 \mathrm{mg}$ ), $75 \%$ of lamotrigine-treated patients took $\leq 200 \mathrm{mg} /$ day

$\mathrm{mg}$ (range $25-700 \mathrm{mg}$ ), and $74 \%$ of levetiracetam-treated patients took $\leq 1000 \mathrm{mg} /$ day (range $500-3000 \mathrm{mg}$ ). There was no relationship between AED dosing and number of previous drug schedules. Side effects (108 [81\%] neurotoxicity, 26 [19\%] other) were reported by $134(9.4 \%)$ patients, particularly those taking carbamazepine (60 of $382,16 \%$; others 73 of $1043,7 \%$; $<<0.001$ ), all of whom continued on treatment. Patients who failed their first monotherapy due to sideeffects or lack of efficacy were more likely to fail their second AED for the same reason prior to eventually becoming seizure free on a subsequent treatment option.

Conclusions: Although the majority of patients in this analysis became seizure-free on their first AED, a substantial minority controlled on later monotherapy schedules. Doses were often modest, suggesting that good tolerability is necessary to underpin an optimal response. Some patients seemed more likely to have problems tolerating AEDs, whereas for others lack of efficacy was the major reason for treatment failure. These 2 separate populations may be worthy of further investigation.

\subsection{3 \\ TRANSCUTANEOUS VAGUS NERVE STIMULATION (T- VNS) IN PHARMACORESISTANT EPILEPSIES: AN OBJECTIVE ASSESSMENT OF EFFICACY BY COMPUTERIZED ANALYSIS}

Hermann Stefan, G. Kreiselmeyer, B. S. Kasper, T. Hammen, M. Rzonsa, W. Graf and R. Hopfengärtner (University Hospital Erlangen - FAU, Erlangen, Germany)

Rationale: Very recently we have shown in a proof of concept trial that t-VNS might be an alternative treatment option for patients with epilepsy. In this study we present detailed results concerning quantification of ictal and interictal electrophysiological activity in long term EEG recordings by means of computerized analysis. Methods: t-VNS was applied to 10 patients with pharmacoresistant epilepsy. Subjective documentation about seizure frequency was obtained by patients seizure diary. For a more reliable assessment of efficacy a continuous outpatient video EEG monitoring for one week at baseline and every three months after baseline has been performed. 
On the basis of the integrated and averaged power for special multichannel EEG montages the ictal and interictal activity have been investigated with respect to frequency and duration, taking into account an artifact rejection method. Furthermore, a visual inspection of the data by experts was done.

Results: An overall assessment of computerized EEG analysis was performed for each of the different monitoring sessions. The automated seizure detection program detected $86 \%$ of the videoEEG-documented seizures showing a corresponding EEG activity, while at the same time the number of false positive events per hour was $<0.2 / \mathrm{h}$. In one patient with a huge number of generalized spike wave complexes (GSWs) it was found that after nine months the normalized duration of GSWs with respect to recording time was reduced by approximately $30 \%$. In addition to number of seizures also the duration and intensity was analysed. The quantitative analysis of the integrated power in another patient showing all the time continuous spike wave complexes revealed a significant decrease of the interictal activity, which was in accordance with clinical findings.

Conclusions: The computerized analysis of long term EEG recordings based on multi-channel spectral methods provides important information about the ictal and interictal activity. In combination with clinical findings our approach might be interesting for a more reliable assessment of efficacy of medical treatment even in the long-term in epilepsies indicating that not only seizure frequency but also intensity (duration, power) and the quantification of interictal activity is important.

REFERENCES:

[1] Stefan H, Kreiselmeyer G, Kerling F, Kurzbuch K, Rauch C, Heers M, Kasper BS, Hammen T, Rzonsa M, Pauli E, Ellrich J, Graf W, Hopfengärtner R. Transcutaneous Vagus Nerve Stimulation (tVNS) in Pharmacoresistant Epilepsies: A Proof of Concept Trial; Epilepsia 2012 (in press).

[2] Hopfengärtner R, Kerling F, Bauer V, Stefan H. An efficient, robust and fast method for the offline detection of epileptic seizures in long-term scalp EEG recordings. Clin Neurophysiol 2007;118: 2332-43. (2007).

\subsection{4}

\section{PATIENT-CENTERED CARE IN EPILEPSY AND THE ROLE} OF SEIZURE FREQUENCY

Hyunmi Choi ${ }^{1}$, H. Mungar-Clary ${ }^{1}$, B. Wedel ${ }^{1}$, J. B. Wong ${ }^{2}$ and M. Hamberger ${ }^{1}{ }^{1}$ Neurology, Columbia Presbyterian Medical Center, New York, NY and ${ }^{2}$ Department of Medicine, Tufts Medical Center, Boston, MA)

Rationale: Epilepsy patients' valuation of their current health status is not routinely assessed in clinic settings, but is generally presumed based on measures such as seizure frequency, despite a growing national trend toward patient-centered care. Using two preferencebased quality of life (QOL) metrics that allow quantification of patients' values for their current health status (Petitti 2000), we examined preference-based QOL as a function of seizure frequency, with the primary goal to assess whether preference-based QOL is linearly related to seizure frequency.

Methods: One hundred eighty two adult patients with chronic epilepsy were consecutively sampled between 2006 and 2008 at Columbia Epilepsy Center for an in-person interview using visual analog (Bond MR 1966) and standard reference gamble metrics (Torrance 1987). Information regarding patients' epilepsy and mood were also obtained. Non-parametric test and correlation coefficients were used to examine the relationship between preference-based QOL versus ordinal categories of seizure frequency. As a sensitivity analysis, we performed a Monte-Carlo computer simulation to determine how often ordinal categories of seizure frequency failed to relate to QOL. We tested the reliability of the two metrics in a subset of patients who returned for a re-test, and also examined the validity against other proxy measures of disease. Finally, we tested significant predictors of preference-based QOL using multivariate logistic and linear regression analyses.

Results: The relationship between preference-based QOL and seizure frequency was binary, in that only the patients who had been seizure free (for $>1$ year) had significantly higher QOL than those of any patients with recurrent seizure regardless of seizure frequency $(\mathrm{p}=0.003)$. Among patients with persistent seizures, QOL and seizure frequency were not monotonically linearly related, as patients with most frequent seizures did not report lowest QOL. Among patients with similar seizure frequency, QOL varied substantially. In addition, there were large overlaps in QOL across different seizure frequency categories. In a Monte Carlo simulation using QOL values from patients, we found that seizure frequency was poorly predictive of QOL about a third of the time. In multivariate regression models to determine whether patient factors accounted for the variation in QOL, the presence of depressive symptoms $(\beta=-2.48, \mathrm{p}=.000$ for visual analog; $\beta=-.161, p=.02$ for standard reference gamble), and not seizure frequency, was the only independent predictor of quality of life, accounting for $25 \%$ to $34 \%$ of the variation of quality of life assessed through visual analog and standard reference gamble. Conclusions: Our findings underscore the singular importance of attaining complete seizure freedom. Given the lack of linear relation between QOL and seizure frequency, and a wide variation of QOL among patients experiencing similar seizure frequency, direct assessment of patients' valuation of epilepsy health status is needed in the clinic setting.

\subsection{5 \\ THE USE OF HI-FIDELITY MANNEQUIN FOR STATUS EPILEPTICUS SIMULATION TO ENHANCE MEDICAL STUDENT'S PERFORMANCE}

G. Uppal ${ }^{1}$, David V. Lardizabal ${ }^{1}$, B. Nimmana ${ }^{1}$, S. Lanigar ${ }^{1}$, C. Sanders ${ }^{2}$, R. Bell ${ }^{2}$ and P. Sahota ${ }^{1}{ }^{1}$ Neurology, University of Missouri Health Care, Columbia, MO and ${ }^{2}$ Russell D. and Mary B. Shelden Clinical Simulation Center, University of Missouri School of Medicine, Columbia, MO)

Rationale: Status epilepticus (SE) is a common neurologic emergency and it is estimated that there are about 200,000 episodes of status epilepticus in the United States annually. SE has a high mortality risk of around $22 \%$. With the acuteness of the situation and necessity to act fast and accurately, it can be frightening for the new learners. Using Hi-fidelity mannequins for simulation of SE ensures no harm during the training but definitely helps improve the skills of the trainees which we wanted to show with our two year data collection and analysis.

Methods: We used the 3 phased learning for the 3rd year Medical students in the Neurology clerkship. In academic year 2011, 20 sessions were conducted with 72 students and in academic year 2012, 24 sessions were conducted with 90 students. Students were initially provided with the Continuum article for self study (Phase 1) and then they worked in groups of 3-4 for the management of Hi-Fidelity mannequins with SE (Phase 2). The students filled a Post-Simulation questionnaire with 10 multiple choice questions (MCQs). Debriefing was done by the clerkship director (Phase 3). Students also filled a post-simulation survey.

Results: Post simulation questionnaire: The students performed excellent with scoring above $91 \%$ regarding definition of SE, refractory SE, loading dosage of fosphenytoin and lorazepam. More than $70 \%$ students answered questions about mechanism of seizure induction; securing $\mathrm{ABC}$ 's and blood glucose measurement, goal of treatment $(85 \%)$, alternative IV and non IV treatments correctly. Roughly $50 \%$ of students were aware that Myoclonic SE has highest mortality rates.

Evaluation of the exercise by students: About $95 \%$ of the students agreed that the simulation will help them manage real life patients better along with better understanding of health care practices; also they felt that this way of learning is better than the regular didactic 
session and they will be able to remember the management protocol better. Students have rated the characteristics of Realism, Quality, Organization and Duration of simulation provided as good or excellent in $90 \%$ cases. $50 \%$ of the students rated the overall experience as Excellent, $20 \%$ as Good and $30 \%$ as Adequate. Faculty observation: The groups performed Airway support, Oxygen initiation, Vitals, History, Labs, IV fluids, thiamine and glucose injection, EEG, Lorazepam, Fosphenytoin, Intubation and phenobarbital injection in $90-100 \%$ of the cases correctly. The data collected in year 2011 and 2012 were almost consistent, showing some improvement in some regards in 2012.

Conclusions: We have been doing Status Epilepticus simulation at University of Missouri, Columbia in Neurology Clerkship for 3rd year medical students for the past 2 years. The survey shows that more than $90 \%$ students' feels that this way of learning is better than the classroom teaching and helps them remember the management protocol too.

\subsection{6 \\ COMPARISON OF WEANING METHODS FOR CONTINUOUSLY INFUSED AEDS IN REFRACTORY STATUS EPILEPTICUS}

Jane G. Boggs, A. Sarwal and C. ODonovan (Wake Forest

University, Winston Salem, NC)

Rationale: Neurocritical care literature has recently addressed treatment and continuous EEG (CEEG) monitoring of refractory status epilepticus (RSE). A period of electrographic control between 24 and 48 hours is recommended before weaning continuous infusion of AEDs for RSE, and that CEEG should be continued through weaning. Subsequent "slow withdrawal" is recommended, but unfortunately, there is no guideline for methods or rate of dose reduction. Weaning protocols vary significantly among physicians, and the use of CEEG is warranted as long as the weaning is still in progress. Unneccessarily prolonged CEEG can limit availability machines for other patients, requires large amounts of tech and physician time for maintenance and interpretation, and results in scalp irritation.

Methods: We reviewed all adult ICU patients who had required at least 24 hours of continuous IV AEDs and CEEG through weaning for RSE from January 2009 through January 2012. We reviewed both the rate of weaning and time to complete weaning. Based on these observations, patients were then subcategorized into either linear, nonlinear or varying reductions. Outcomes of interest within 24 hours of weaning were recurrent seizures and death, either spontaneous or by decision to withdraw support.

Results: We identified 26 patients meeting above criteria. 12 patients had linear weaning, while 8 patients had nonlinear weaning. Six patients had varying weaning methods. While 12 patients had recurrent seizures within 24 hours of weaning, only 4 of these had recurrent SE. Seizure recurrence was noted in half of linear (6 patients) and nonlinear (4 patients) methods. Decision to withdraw support was made in 5 cases, and 2 cases spontaneously died within 24 hours of completing weaning. The time to complete weaning varied from 12 hours to 8 days. Shorter weaning times did not correlate with seizure recurrence. Scalp irritation following EEG electrode removal was mentioned in the chart for 14 patients, all of whom had EEG in place $>4$ days.

Conclusions: While much research and literature has been dedicated to optimizing treatment of RSE, there is a striking paucity of study directed toward weaning of continuous infusions in this population. Thus, highly individual methods and multiday CEEGs are often utilized. Our data indicates that both nonlinear and linear methods were equally likely to result in resolution of seizures. Weaning over a shorter time frame, however, did not result in increased risk of seizure recurrence. A longer duration of the CEEG during longer weaning was often associated with noted scalp irritation. We conclude that weaning method is less important than avoiding prolonged duration. While efforts to wean patients over shorter time frames should be encouraged, there is need for prospective study and more specific guidelines for weaning of continuous IV medication following RSE.

\subsection{7}

DOES OBJECTIVE ADHERENCE DATA MATCH WHAT PATIENTS REPORT WHEN ASKED ABOUT THEIR ANTIEPILEPTIC DRUG ADHERENCE?

James W. McAuley ${ }^{1,2}$, K. Wolowiec ${ }^{1}$, E. DeNiro ${ }^{1}$ and B. F. Shneker ${ }^{2,1}$ ( ${ }^{1}$ Ohio State University College of Pharmacy, Columbus, $\mathrm{OH}$ and ${ }^{2}$ Neurology, Ohio State University Medical Center, Columbus, $\mathrm{OH}$ )

Rationale: Adherence to the prescribed drug regimen is a major step in achieving the goal of reduced seizure burden in patients with epilepsy. Decreased antiepileptic drug (AED) adherence is associated with more than a 3 -fold increase in mortality and significantly more emergency department visits, hospital admissions, injuries and fractures (Faught 2008). An objective measure of adherence is the Medication Possession Ratio (MPR). This is a numeric value typically between 0 and 1 with a value closer to 1 indicating greater adherence. It is calculated from pharmacy refill records over a set time period. A widely accepted value for being classified as adherent is an MPR $>0.8$. Preliminary MPR data from one of our ongoing investigator-initiated projects had raised some concerns about our outpatient clinic population. The objective adherence data (MPR) was not matching what patients stated when asked about their adherence (subjective adherence). This preliminary data caused us to look at a larger patient sample to determine if a gap existed in subjective and objective measures of AED adherence.

Methods: Both subjective and objective adherence data were compared in patients with epilepsy for this cross-sectional study. During their clinic interview, patients were asked to self-assess their AED adherence (subjective measure of adherence). The objective measure of adherence was an MPR for each of their AEDs. We contacted each patient's community pharmacy and used refill records to calculate their MPR for the 6 months prior to their clinic visit. Patients provided consent for us to contact their pharmacy.

Results: Data from 27 patients (17 female) with an average age of $35.22( \pm 11.21)$ years was examined. Eighteen patients reported a seizure within the last 30 days. All patients described themselves as "adherent" when asked at their clinic visit (subjective adherence). This is contrast to the MPR data (objective adherence). Of 45 AEDs taken in the 27 patients (14 patients on AED monotherapy), the MPR was $<0.8$ for 16 of the AEDs. Ten of the 27 patients (37\%) had an MPR $<0.8$ for at least one of their AEDs. Interestingly, AED burden did not appear to impact the MPR as 6 of the 10 patients with MPRs $<0.8$ were on AED monotherapy.

Conclusions: The reason for the gap in the subjective and objective measures of adherence is unclear. One explanation could be that patients are not completely truthful in their subjective assessment of their adherence. Other factors could be associated with limitations in calculating their MPR; a single number to represent AED adherence. Limitations of the MPR include, but are not limited to 1) a lack of sensitivity to changes in doses like tapering an AED up or down and 2) patients could use more than one pharmacy. In conclusion, we identified a gap in the subjective and objective measures of AED adherence with more than one-third of the patients deemed to be nonadherent from objective data (MPR $<0.8)$. The reasons for this gap are likely multifactorial. 


\subsection{8}

\section{EFFECTS OF ADJUNCTIVE LACOSAMIDE ON MOOD IN ADULT PATIENTS WITH PARTIAL EPILEPSY}

Salvatore Mazza, G. Marano and M. Mazza (Neurosciences, Università Cattolica Del Sacro Cuore, Roma, Italy)

Rationale: Lacosamide (LCM) is a recently approved anticonvulsant which offers new mechanisms of action and favorable safety profiles. It appears to be an efficacious and cost-effective adjunctive therapy for partial-onset epileptic seizures in adult patients. This study prospectively examined whether continued add-on treatment with LCM is associated with quantitative improvement in mood and anxiety symptoms in adult patients with partial epilepsy.

Methods: Depressive symptoms and anxiety were assessed by clinical interview using the Hamilton Depression Rating Scale (HDRS), the Cornell Dysthymia Rating Scale (CDRS), the Beck Depression Inventory (BDI), and the Hamilton Anxiety Rating Scale (HARS). Thirty controls (patients with epilepsy treated with antiepileptic drugs other than LCM) and 30 LCM-treated patients were enrolled and completed the study.

Results: In our study, a significant improvement in affect, as measured by the CDRS, was demonstrated during the course of LCM treatment for 3 months. HDRS and BDI scores also declined in the LCM-treated group, but these decreases did not reach statistical significance.

Conclusions: The decline in dysthymic symptoms in LCM-treated subjects compared with controls lends support to the hypothesis that LCM improves mood.

\subsection{9 \\ KETONE STABILIZATION DURING LONG TERM FASTING IN CHILDREN ON A KETOGENIC DIET}

Yeou-Mei C. Liu, H. Lowe, M. Zak, J. Kobayashi, V. Chan and E. Donner (Neurology, The Hospital For Sick Children, Toronto, ON, Canada)

Rationale: The ketogenic diet (KD) is a medical nutrition treatment for intractable epilepsy. The KD stimulates ketosis by supplying fat (70-90\% of total calories) as the major source of calories. Patients typically require urine ketone levels in the range of $4-16 \mathrm{mmol} / \mathrm{L}$ to improve seizure control. During periods of fasting of more than 12 hours, ketone levels increase and blood glucose (BG) levels decrease. These disruptions may result in seizures. Providing continuous intravenous (IV) dextrose is not recommended for patients on the KD as it may eliminate ketosis and induce seizures. Lipid intake in parenteral nutrition is restricted to less than $3-4 \mathrm{~g} / \mathrm{kg}$ body weight (BW) per day (0.13-0.17 g/kg BW/hour) to prevent "fat overload syndrome". Typical parenteral nutrition may deliver a lower fat intake than required for children treated with a $\mathrm{KD}$ and also result in lower ketone levels and seizures. Due to these limitations, children treated with a KD may discontinue the diet prior to procedures requiring fasting, such as surgery. We employed a small continuous IV 5\% dextrose solution to maintain ketosis and normal BG levels during fasting.

Methods: Eight children, aged 3-16 years (mean $\pm \mathrm{SD}=8.4 \pm 2.3$ ) underwent a surgery or procedure that required 19-36 hours of fasting from March 2009 to May 2012. Body weights were 12.5-28.3 Kg (mean $\pm \mathrm{SD}=20.0 \pm 2.8$ ). Children received continuous IV 5\% dextrose with normal saline during the fasting period. Intravenous solution was calculated as follows:

$\mathrm{D} 5 \mathrm{~W} /$ hour rate $=($ daily carbohydrate $(\mathrm{CHO})$ allowance $\mathrm{x} 100) / 5 \div 24$ hours

Daily $\mathrm{CHO}$ allowance $=($ daily fluid intake/2) $\times 0.025$ and is the intake needed to maintain safe $\mathrm{BG}$ and urine ketones to prevent excessive seizures.

For BW (1-10 Kg): Daily CHO allowance $=(50 \times \mathrm{kg} \mathrm{BW}) \times 0.025$

For BW (11-20 Kg): Daily CHO allowance $=(\{1000+50 \times(K g B W-$ 10) $\} / 2) \times 0.025$
For BW >20 Kg: Daily CHO allowance $=(\{1500+20 x(\mathrm{Kg} \mathrm{BW}-$ 20)\}/2) $\times 0.025$

In two cases, the children's pre-procedure fluid intake was below the recommended daily intake. As such, the pre-procedure daily intake was used to calculate the IV fluid requirements.

Paired t-test was performed to compare urine ketone levels prior to and during fasting. BG levels were recorded. Seizure frequency was monitored.

Results: D5W rates ranged from $8-20 \mathrm{ml} / \mathrm{hr}($ mean $\pm \mathrm{SD}=15.9 \pm 3.1)$. Urine ketone levels were $6-16 \mathrm{mmol} / \mathrm{L}($ mean $\pm \mathrm{SD}=12.6 \pm 2.9)$ prior to fasting. Urine ketone levels were $6-16 \mathrm{mmol} / \mathrm{L}($ mean $\pm \mathrm{SD}=12.9 \pm 2.7)$ during fasting. There were no significant differences $(p=0.589)$ in ketone levels prior to and during fasting. All BG measurements during fasting were $>3.5 \mathrm{mmol} / \mathrm{L}$, within the normal range. No children had an increase in seizure frequency or intensity.

Conclusions: Careful calculation of IV fluids allows for stable BG and ketone levels during prolonged fasting in children treated with KD. No children in this cohort experienced a change in seizure frequency or intensity during prolonged fasting, using this method. Discontinuation of the KD is not necessary for children undergoing surgery.

\subsection{0 \\ IMPACT OF PERI-ICTAL INTERVENTIONS ON RESPIRATORY DYSFUNCTION, POSTICTAL EEG SUPPRESSION AND POSTICTAL IMMOBILITY}

Masud Seyal ${ }^{1}$, L. M. Bateman ${ }^{1}$ and C. S. Li ${ }^{2}\left({ }^{1}\right.$ Neurology, University of California, Davis Medical Center, Sacramento, CA and ${ }^{2}$ Public Health Sciences, Division of Biostatistics, University of California, Davis, Sacramento, CA)

Rationale: Seizure-related respiratory dysfunction (RD), duration of post-ictal generalized EEG suppression (PGES) and postictal immobility (PI) may be important in the pathophysiology of sudden unexpected death in epilepsy (SUDEP). Peri-ictal interventions may reduce this risk.

Methods: We assessed the impact of peri-ictal nursing interventions (supplemental oxygenation, oropharyngeal suction and patient repositioning) on the duration of RD, PGES and PI in patients with localization-related epilepsy and secondarily generalized convulsions (GC). Video-EEG telemetry data were reviewed retrospectively. The Wilcoxon rank-sum test was used to compare GC with and those without intervention. Robust linear regression was used to assess the association between timing of intervention and duration of hypoxemia $(\mathrm{SaO} 2<90 \%)$, PGES, immobility, and seizure.

Results: Data from 39 patients with 105 GC were analyzed. PGES $>2$ seconds occurred following $31 \mathrm{GC}$. There were $21 \mathrm{GC}$ with no intervention (NOINT) and 84 GC with interventions (INT). In the INT group, the mean duration of hypoxemia $(53.1 \pm 45.1 \mathrm{sec}(44,8-$ 158)) (mean \pm s.d.(median, range) was shorter $(\mathrm{p}=0.0014)$ when intervention occurred prior to the onset of hypoxemia compared to when intervention was delayed after hypoxemia onset (132.417 \pm $134.9 \mathrm{sec}(103,38-712))$. On regression analysis, in the INT group, earlier intervention was associated with shorter duration of hypoxemia $(p<0.0001)$, shorter duration of PGES $(p=0.0012)$, shorter duration of the seizure $(\mathrm{p}<0.0001)$ and of its convulsive component $(\mathrm{p}=0.0457)$.

The duration of postictal immobility was longer for $\mathrm{GC}$ with than $\mathrm{GC}$ without PGES $(\mathrm{p}<0.0001)$. The delay to movement following GC with PGES was $251.96 \pm 329.05 \mathrm{sec}(99.5,22-1192))$, and for GC without PGES was $66.06 \pm 150.22 \mathrm{sec}(45,0-1211))$. GC with NOINT had a shorter duration of postictal immobility than INT GC $(\mathrm{p}=0.0049)$. The duration of PI in GC with NOINT was $36.24 \pm 45.75$ sec (7, 1-131) and in GC with INT was $138.23 \pm 251.45$ (58, 01211)).

The duration of PI was associated with a lower $\mathrm{SaO} 2$ nadir $(\mathrm{p}=0.003)$ and longer duration of oxygen desaturation $(\mathrm{p}=0.0026)$. There was no association between the duration of PI and seizure duration 
$(\mathrm{p}=0.773)$, nor between the duration of PI and PGES duration $(\mathrm{p}=0.758)$.

PGES did not occur in the NOINT group. The mean duration of desaturation was longer (110.9 seconds versus 49.9 seconds) $(\mathrm{P}<0.0001)$, mean $\mathrm{SaO} 2$ nadir was lower $(72.8 \% \mathrm{v} 79.7 \%)$ $(\mathrm{p}=0.0086)$ and mean end-tidal $\mathrm{CO} 2$ was higher $(58.6 \mathrm{mmHg} \vee 50.3$ $\mathrm{mmHg})(\mathrm{p}=0.0359)$ in the INT compared with the NOINT group. The duration of the seizure or of the convulsive component was not significantly different between the INT and NOINT groups. Conclusions: In the epilepsy monitoring unit, in patients that had a peri-ictal nursing intervention, earlier intervention was associated with reduced duration of RD and PGES . The findings suggest the possibility that such interventions may be effective in reducing risk of SUDEP in the outpatient setting. Validation with a prospective study is needed before definitive conclusions can be reached.

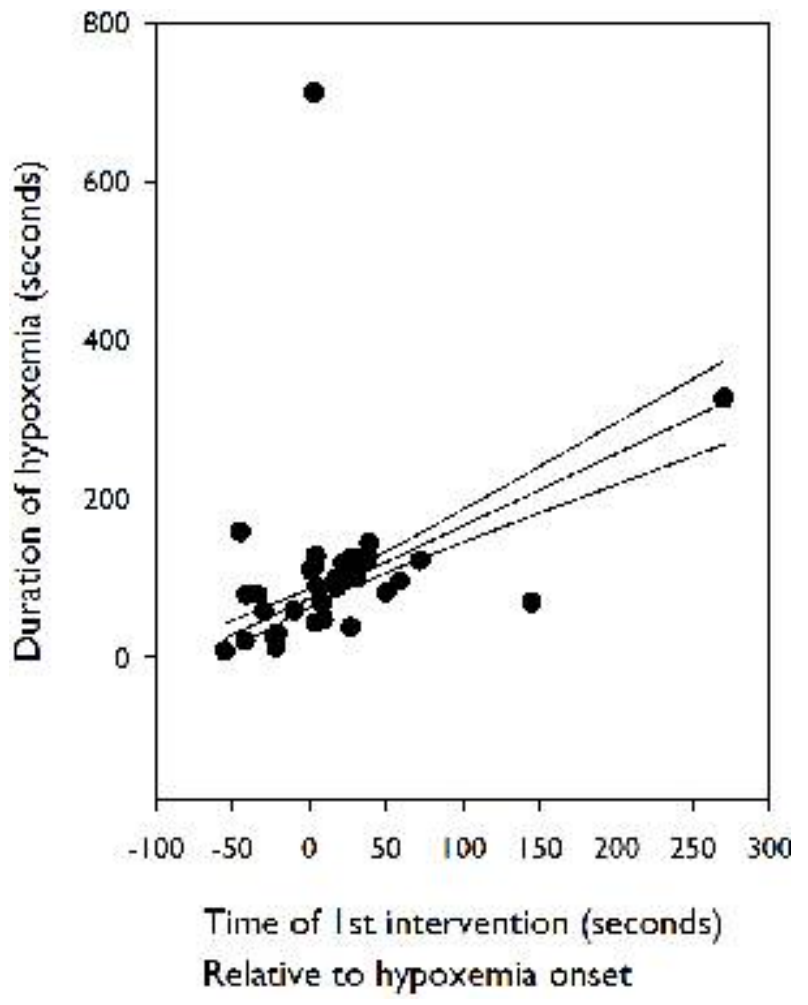

1.151

OUTCOME AND CHALLENGES IN TREATING INFANTILE SPASMS WITH HIGH DOSE ORAL PREDNISOLONE

H. Purushotham, A. Weinstock, S. Kerr and S. Finnegan (Pediatric Neurology, Womens and childrens Hospital of Buffalo,University of Buffalo, Buffalo, NY)

Rationale: Infantile spasm (IS) is a major form of severe epileptic encephalopathy of early infancy. AAN practice parameters (2004) states that ACTH is probably effective for the short term treatment of IS and resolution of hypsarrhythmia(HA). There is insufficient evidence with regards to the effectiveness of oral steroids. The U.S. consensus report (2010) suggests the high dose prednisolone (PZ) therapy to be suboptimal due to insufficient data. However it recommended further study as it appeared promising. The multicenter randomized open label UKISS study compared hormonal treatment (IM synthetic ACTH versus oral PZ) with vigabatrin for the treatment of IS. This study revealed that oral PZ was equivalent to synthetic ACTH. Kossoff et al reported good outcome on 15 patients treated with $\mathrm{PZ}$ and recommended routine use of PZ for IS. We report 5 cases of IS treated at our institution.

Methods: 5 infants were treated with high dose PZ. 4 out of 5 infants had symptomatic IS (2 had Down's syndrome), and one had cryptogenic IS. The starting dose for all infants was $40 \mathrm{mg}$ per day
.The dose of $\mathrm{PZ}$ was increased to $60 \mathrm{mg}$ per day when response was subopitmal. Age at time of diagnosis ranged from 3 to 10 months. Results: All 5 infants showed cessation of clinical spasms during therapy, while 3 had recurrence after therapy. PZ was discontinued due to reported lethargy in one patient. The duration of $\mathrm{PZ}$ treatment ranged from 3 to 8 weeks. Two patients among five required escalation of PZ dose to $60 \mathrm{mg} /$ day for non-resolution of clinical spasms. The remaining 3 patients who received $40 \mathrm{mg} /$ day of $\mathrm{PZ}$ although showed cessation of spasm by the second week of treatment, either had recurrence of IS or showed HA following treatment. Two patients on $60 \mathrm{mg}$ PZ remained spasm free, one achieved complete remission of IS with cessation of both clinical spasm and HA. The second patient had abnormal EEG with sharp waves but remained free of spasms. Side effects like lethargy, irritability and increased appetite was observed in 2 infants.One infant developed hypertension and cushingoid features and required steroid taper for HPA axis suppression. Among the 4 infants who completed PZ therapy, EEG normalized in one, revealed persistent multi-regional sharp waves in 2 and HA in 1.

Conclusions: Our study showed that PZ appeared effective mostly at $60 \mathrm{mg} /$ day and overall well tolerated for the treatment of IS. In our patient population, initial dose of $40 \mathrm{mg} /$ day either had no resolution of IS mandating higher dose or had recurrence later (clinical or EEG) after treatment completion. Side effects was seen in some patients and were consistent with effects of steroid .The outcome of this study tends to support use of PZ for IS however was limited by sample size. This warrants a prospective randomized multi center trial comparing both clinical and EEG response of ACTH vs high dose PZ.

\begin{tabular}{|c|c|c|c|c|c|c|c|c|c|c|c|}
\hline S.NO & $\begin{array}{c}\text { AGE: } \\
\text { months }\end{array}$ & SEX & Etiolegy & $\begin{array}{c}\text { HA } \\
\text { Prior } \\
\text { to } \\
\text { RX }\end{array}$ & $\Lambda \mathrm{E}$ & $\begin{array}{l}\mathrm{At} / \mathrm{P} \% \\
\mathrm{D} / \mathrm{C}\end{array}$ & DOSE & $\begin{array}{l}\text { Spasm } \\
\text { recurrence }\end{array}$ & $\begin{array}{l}\text { 2nd } \\
\text { PZ } \\
\text { dose }\end{array}$ & Other $\mathrm{Rx}$ & $\begin{array}{c}\text { Posl } \\
\text { Rx } \\
\text { EEG; }\end{array}$ \\
\hline 1 & 10 & $\mathrm{~F}$ & Down & YHS & NO & No & 40 & No & NO & $\mathrm{C} 1 \%$ & $\mathrm{HA}$ \\
\hline 2 & 5 & F & Down & YES & Fatiguc & YES & 40 & YES & no & LEV & Normal \\
\hline 3 & 3 & M & PVL & YES & Initabilly & No & 40 & YES & YES & $\begin{array}{l}\text { I.IV } \\
\text { CLLZ }\end{array}$ & $\begin{array}{l}\text { Multi } \\
\text { reg SW }\end{array}$ \\
\hline 4 & 7 & M & $\begin{array}{c}\text { Delayed } \\
\text { myelination }\end{array}$ & YES & NO & No & 60 & NO & No & PB & $\begin{array}{l}\text { Multi } \\
\text { reg SW }\end{array}$ \\
\hline 5 & 9 & M & Cryplogenic & YES & HT, Cushings & No & 60 & No & No & CLZ,LEV & Normal \\
\hline
\end{tabular}

HA Hypsarrhythmia,PZ Prednisolone,

CLZ Clonazepam, LEV-Levetiracetam

PB Phenobarbital, HT Hypertension

D/C Discontinue, AE Adverse events, RX Treatment, SW Sharp waves

\subsection{2 TREATMENT OF JUVENILE SPASMS IN TUBEROUS SCLEROSIS COMPLEX}

E. A. Thiele, David T. Hsieh and M. M. Jennesson (Pediatric Epilepsy Service, Department of Neurology, Massachusetts General Hospital, Boston, MA)

Rationale: Juvenile spasms (JS) - epileptic spasms occurring after the age of two-years-old - are not uncommon in patients with tuberous sclerosis complex (TSC). We sought to examine the efficacy of various treatments, particularly treatment response to vigabatrin (VGB), which is extremely effective for infantile spasms (IS) in TSC. Methods: The authors retrospectively reviewed the medical records of 19 patients with TSC and JS for clinical and treatment data.

Results: Of 391 patients with TSC, 19 (4.8\%) had JS. Of those with detailed clinical data, six had infantile spasms that persisted after age two-years-old, six returned later (range 2 to 24-years-old) after an initial remission of infantile spasms, and four occurred de novo over the age of two-years-old (range 2 to 20-years-old). All concurrently had other seizure types. Six patients became spasm-free with medication treatment, including four with VGB, one with VGB in combination with the low glycemic index treatment (LGIT), and one 
with felbamate. Five became spasm-free after epilepsy surgery. VGB was not effective for seven patients. Additional medications that were partially effective included clobazam (2 patients), clonazepam (1), lamotrigine (1), levetiracetam (1), oxcarbazepine (1), phenytoin (1), and rufinamide (2). Medications that had no effect included levetiracetam (1 patient), rufinamide (1), and zonisamide (2). The ketogenic diet provided partial effectiveness for one patient, and the LGIT was ineffective or not tolerated by three. The majority of patients continued to have refractory epilepsy.

Conclusions: JS in TSC are difficult to treat, and often occur in the setting of refractory epilepsy. VGB can be effective, but the success of VGB for JS in TSC is not equivalent to that of IS in TSC. Previous successful treatment with VGB for IS did not predict successful treatment with VGB upon recurrence with JS. In our series, felbamate and dietary therapy were also effective for individual patients, with other anti-convulsant medications providing only partial effectiveness or having no effect. Medication-refractory patients should be considered for surgical candidacy.

\subsection{3}

A DOUBLE-BLINDED, RANDOMIZED, HEAD-TO-HEAD TRIAL OF LEVETIRACETAM VS. SULTHIAME IN BENIGN EPILEPSY WITH CENTROTEMPORAL SPIKES

Ingo Borggraefe $^{1}$, M. Bonfert ${ }^{1}$, T. Bast ${ }^{2}$, B. A. Neubauer ${ }^{3}$, K. J. Schotten $^{6}$, K. Massmann ${ }^{1}$, S. Noachtar ${ }^{4}$, I. Tuxhorn ${ }^{7}$, T. W. May ${ }^{5}$ and F. Heinen ${ }^{1}\left({ }^{1}\right.$ Department of Pediatric Neurology, University of Munich Children's Hospital, Munich, Bavaria, Germany; ${ }^{2}$ Department of Pediatrics, Epilepsy Center Kehl-Kork, Kehl-Kork, Germany; ${ }^{3}$ Department of Pediatric Neurology, University of Giessen, Giessen, Germany; ${ }^{4}$ Department of Neurology, University of Munich, Munich, Germany; ${ }^{5}$ Pharmacological Laboratory, Society for Epilepsy Research, Bielefeld, Germany; ${ }^{6}$ Department of Medical Informatics, Biometry and Epidemiology, University of Munich, Munich, Germany and ${ }^{7}$ Department of Neurology, Case Western Reserve University, Cleveland, $\mathrm{OH}$ )

Rationale: The aim of the present study was to compare the efficacy, tolerability and safety of levetiracetam and sulthiame in benign epilepsy with centrotemporal spikes in a prospective, randomized double-blinded head to head approach.

Methods: 44 out of 89 screened patients were eligible for the study and randomly allocated to either sulthiame or levetiracetam treatment (for in- an exclusion criteria see table). Efficacy was defined as complete seizure remission during the observation period of 6 months. The dosage was $6 \mathrm{mg}^{*} \mathrm{~kg}$ and $30 \mathrm{mg}^{*} \mathrm{~kg}$ for sulthiame and levetiracetam, respectively. In addition, information of the occurrence of adverse events was collected.

Results: 43 out of 44 patients were available for final statistical analysis. One patient had to be excluded due to protocol violation. Treatment failure events (=seizure recurrence) occurred in 4 patients $(19.0 \%)$ in the levetiracetam (LEV) treatment group and in 2 patients $(9.1 \%)$ in the sulthiame (STM) treatment group, respectively, ( $\mathrm{p}=0.412$, Fig. 1a). Study termination due to adverse reactions was higher in the LEV treatment group $(n=5,23.8 \%)$ than in STM treatment group $(n=1,4.5 \%, p=0.095$, Fig. 1a). Serious adverse reactions occurred in patients treated with $\operatorname{LEV}(n=2,9.5 \%$, Fig. 1a). The total amount of dropouts due to either seizure recurrence or adverse events was significantly higher in the LEV group $(\mathrm{n}=9$, $42.9 \%$ ) compared to the STM group ( $n=3,13.6 \%, p=0.030$, Fig. $1 b)$. Conclusions: The rates of seizure free patients were high in both groups. However, the results indicate that termination of drug treatment due to seizure recurrence or adverse events occurred more frequently in the LEV group compared to STM. Behavioral disturbances were the most common adverse event causing study termination.
In- and exclusion criteria

\begin{tabular}{|cc|}
\hline Inclusion Criteria & Exclusion criteria \\
\hline Age 6-12 years & Co-incidence of other epilepsy forms \\
\hline $\begin{array}{c}\text { Diagnosis of BF.CTS* } \\
\text { Two or more seizures within six months } \\
\text { prior to study entry }\end{array}$ & $\begin{array}{c}\text { Relevant medical conditions } \\
\text { Participation in other clinical studies within } 30 \text { days } \\
\text { prior to study start }\end{array}$ \\
\hline & Mental retardation \\
\hline
\end{tabular}

*The diagnosis of BECTS was established by the history of typical seizure semiology and the presence of benign epileptic discharges in the electroencephalogram

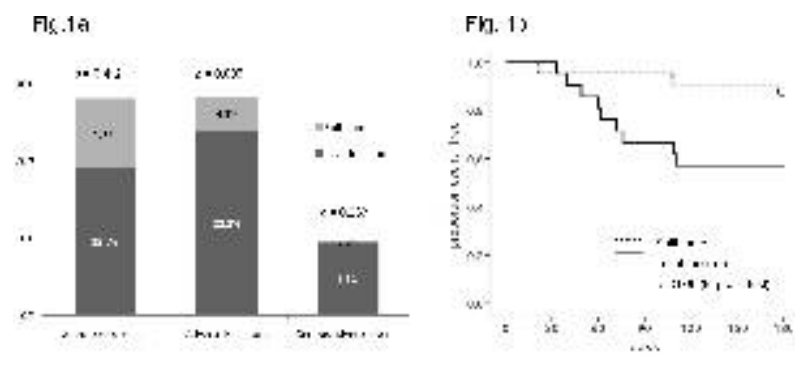

\section{Clinical Epilepsy: Prognosis}

\subsection{4}

THE RISK AND CAUSES OF DEATH IN CHILDHOODONSET EPILEPSY: A 4-STUDY COLLABORATION

Anne T. Berg ${ }^{1}$, K. Nickels ${ }^{2}$, E. C. Wirrell ${ }^{2}$, C. Rios ${ }^{3}$, A. T. Geerts ${ }^{5}$, P. M. Callenbach ${ }^{4}$, W. F. Arts ${ }^{5}$, P. R. Camfield ${ }^{6}$ and C. S. Camfield ${ }^{6}$ ( ${ }^{1}$ Epilepsy Center, Ann\&Robert H. Lurie Children's Hospital, Chicago, IL; ${ }^{2}$ Epilepsy and Child and Adolescent Neurology, Mayo Clinic, Rochester, MN; ${ }^{3}$ Neurology, Erasmus Medical Center, Rotterdam, Netherlands; ${ }^{4}$ Neurology, University Of Groningen, Groningen, Netherlands; ${ }^{5}$ Pediatrics, Yale Medical School, New Haven, CT and ${ }^{6}$ Pediatrics, Dalhousie University, Hallifax, NS, Canada)

Rationale: Young people with epilepsy experience high death rates compared to the general population. Understanding the magnitude of risk and the causes of death $(\mathrm{CoD})$ is essential for counseling and for potential prevention.

Methods: We combined the mortality experiences of four cohort studies of newly diagnosed childhood epilepsy (onset $1 \mathrm{~m}-16$ or $17 \mathrm{y}$ ). Deaths were ascertained by parent and physician report, review of national death indices and health records, and a surveillance system in place at one site. Death certificates, hospital records, and autopsy reports were obtained when available. $\mathrm{CoD}$ was categorized as SUDEP (Nasheff, 2012), other seizure-related, other natural, nonnatural, and unknown. Epilepsy was designated as complicated (neuro-deficit, intellectual impairment, brain lesion) or uncomplicated (normal exam and intellect, no identified underlying cause).

Results: 2260 subjects were followed for 30,427 person-years (PY) for an average of 13.5 years (range 9.8 to $16.4 \mathrm{y}$ across studies). 79 deaths occurred; mean age at onset $=3.9 \mathrm{y}$ (range: $1 \mathrm{~m}-15.7 \mathrm{y})$, age at death $=11$ y (range: $2 \mathrm{~m}-30 \mathrm{y}) .42(53 \%)$ were male; $71(80 \%)$ had complicated epilepsy, and $60(76 \%)$ had substantial cognitive impairment. Drug resistance was noted in $47(59 \%)$, convulsions in $52(66 \%)$, status epilepticus in $37(47 \%)$, and $61(77 \%)$ had seizures in the prior year. The crude death rate (CDR) was 260/100,000PY (95\% CI=202, 317). The CDR for complicated epilepsy was 851 $(653,1049)$ and for uncomplicated epilepsy, $36(95 \% \mathrm{CI}=11,61)$ per 100,000 PY.

Most deaths were due to natural causes and unrelated to seizures $(\mathrm{N}=58)$ with respiratory CoDs being the most common $(\mathrm{N}=43)$. These included 36 pneumonia ( 5 primary infectious, 19 aspiration, 12 
unspecified), 5 respiratory insufficiency-NOS, and 2 other. Other natural CoDs were sepsis $(\mathrm{N}=3)$, shunt failure $(\mathrm{N}=3)$, and unspecified secondary to neurometabolic disease $(\mathrm{N}=3)$. $50 / 52$ of these patients had moderate to severe cognitive impairment. Two children died of strokes occurring after the onset of seizures, and four died of medical conditions unrelated to their epilepsy (e.g. cancer).

SUDEP accounted for 10 deaths (6 definite, 2 probable, 2 possible). Two of these deaths occurred in people with uncomplicated epilepsy. The CDR for SUDEP was $32.9(\mathrm{CI}=12.5,53.2)$ overall and $9(\mathrm{CI}=0$, 21.6) for uncomplicated and $95.1(\mathrm{CI}=29.5,162.4)$ for complicated epilepsy.

The remaining CoDs were other seizure-related $(\mathrm{N}=3)$, non-natural (2 suicide, 1 homicide, 2 accidental), and unknown ( $\mathrm{N}=3)$. In all, 13 $(16 \%)$ of deaths were seizure-related (including SUDEP).

Conclusions: Deaths in young people with epilepsy are largely due to infections and complications of severe neurologic impairment. While seizure-related deaths account for $<1 / 5$ of mortality, the rate from SUDEP alone is comparable to total death rates for young people in the population. In uncomplicated epilepsy, SUDEP rates are comparable to death rates from leading, individual CoDs in the population.

Funding: NS-R37-31146, Mayo Foundation CR20 grant, The National Epilepsy Fund, Houten, The Netherlands (A72 and A85)

\subsection{5 \\ JUVENILE MYOCLONIC EPILEPSY: LONG-TERM SEIZURE AND SOCIAL OUTCOME AFTER A MEAN FOLLOW-UP OF 39.1 YEARS}

Felix Schneider ${ }^{1}$, J. Geithner ${ }^{1,2}$, Z. I. Wang ${ }^{3}$, R. Herzer ${ }^{1}$, J. Berneiser ${ }^{1}$, C. Kessler ${ }^{1}$ and U. Runge ${ }^{1}\left({ }^{1}\right.$ Neurology, Epilepsy Center, University of Greifswald, Greifswald, Germany; ${ }^{2}$ Epilepsiezentrum Berlin-Brandenburg, Berlin, Germany and ${ }^{3}$ Cleveland Clinic Epilepsy Center, Cleveland Clinic Foundation, Cleveland, $\mathrm{OH}$ )

Rationale: The long-term seizure and social outcome in juvenile myoclonic epilepsy (JME) is still controversial. The aim of this study was to investigate the long-term social outcome in relation to the seizure outcome in JME patients with a follow-up of at least 25 years. Methods: Data from 31 patients (19 women) diagnosed with JME and at least 25 years of follow-up (mean 39.1 years) were studied. All of them were re-evaluated with a review of their medial records and a face-to-face or direct telephone interview. Quality of life was assessed using the QOLIE-10 questionaire (Quality Of Life In Epilepsy).

Results: Of 31 patients, 21 (67.7\%) became seizure-free, 6 of them (28.6\%) without antiepileptic drug (AED) treatment. All patients with continuing seizures were in special medical attendance with epileptologists. The lack of factors that potentially provoke seizures, such as alcohol consumption $(\mathrm{p}=0.015)$ and sleep deprivation $(\mathrm{p}=$ 0.067), was found to be associated with a worse seizure outcome; ongoing AED treatment associates with early retirement $(p=0.002)$ and long-term unemployment $(\mathrm{p}=0.071)$. Although not significant, successful treatment decreases the chance for unemployment $(17.6 \%$ vs. $55.6 \%$ ) and family instability (divorce rate $14.3 \%$ vs. $30 \%$ ) and increases the chance for a healthier lifestyle (smoking $28.6 \%$ vs. $50 \%$, sleep disturbances $30 \%$ vs. $50 \%$ ). Epilepsy-related quality of life was significantly higher in seizure-free patients $(p=0.001)$.

Conclusions: Our study provides statistical validation that the social situation in JME patients essentially depends on the long-term seizure outcome. Successful treatment decreases the chance of unemployment and social isolation, and significantly increases the quality of life. Special medical attendence with epileptologists or specialized neurologists was shown to be essential in the treatment of patients with JME.

\subsection{6 \\ EARLY DEVELOPMENTAL AND SCHOOL PROBLEMS DO NOT PRESAGE POOR SEIZURE OUTCOME IN CHILDREN WITH UNCOMPLICATED EPILEPSY}

S. Jawed and Anne T. Berg (Epilepsy Center, Ann\&Robert H Lurie Children's Hospital of Chicago, Chicago, IL)

Rationale: Seizure prognosis in childhood onset epilepsy is largely determined by the presence of the underlying structural $/$ metabolic causes and/or presence of neuro-disability (complicated epilepsy, $\mathrm{CE}$ ). In children with uncomplicated epilepsy (UE, normal imaging, exam, IQ), have a greater burden of developmental, cognitive and school difficulties relative to peer controls. Potentially such difficulties represent the milder end of a spectrum of neurocompromise spanning $\mathrm{CE}$ and UE. If so, these difficulties should be prognostic of seizure outcomes.

Methods: In a prospective community-based cohort of 613 children with newly diagnosed epilepsy, 566 were followed $\geq 5$ years. 53 with encephalopathic epilepseis were excluded. Early difficulties were defined as Vineland Composite score $<25 \%$, being held back in school, or receipt of special services prior to the diagnosis of epilepsy. Seizure outcomes were 5-year remission at last contact (Rem-LC) and pharmacoresistance (PR, failure of $\geq 2$ AEDs fully to control seizures).

Results: Rem-LC occurred in 50/97 (52\%) of CE and in 314/416 $(75 \%)$ of UE $(p<0.0001)$. PR occurred in $34(35 \%)$ of CE and in 61 $(15 \%)$ of $U E(p<0.0001)$. Early difficulties were also more common the CE $(73,75 \%)$ than UE group $(138,33 \%, p<0.0001)$. (Table 1) In the UE group, overall, REM-LC and PR were not influenced by the presence of early difficulties $(p=0.13, p=0.72)$. Among children who were of preschool age at diagnosis, early difficulties were modestly predictive of REM-LC $(\mathrm{p}=0.03)$ but not PR $(\mathrm{p}=0.17)$. In the pre-school-aged group, early difficulties were largely based on low Vineland scores. The mean Vineland Composite scores in those with and without Rem-LC were $97.8(\mathrm{SD}=19.1)$ and $91.2(\mathrm{SD}=22.2)$, $(\mathrm{p}=0.05)$ and for those with and without $\mathrm{PR}$ were $90.3(\mathrm{SD}=21.7)$ and $97.6(\mathrm{SD}=19.5)(\mathrm{p}=0.05)$. Among children who were of school age at the time of diagnosis of epilepsy, there was no evidence that early problems were prognostic of either seizure outcome.

We considered specific epilepsy diagnoses (e.g. CAE, BECTS, etc). With the exception of some variation due to older age at onset (JME, JAE), there was no evidence that certain forms of UE were more or less likely to be associated with early difficulties, yet, these diagnoses were strongly predictive of seizure outcome (Table 2).

Conclusions: Early developmental and school difficulties do not appear to represent a continuum of brain dysfunction with clear neuro-insults that cause epilepsy in that they have little influence on long-term seizure outcomes.

Funded by NIH-NINDS-R37-31146

Table1: Associations of early developmental and school difficulties with 5 -year remission and pharmacoresistance in 416 children with uncomplicated epilepsy

\begin{tabular}{|cc|c|c|}
\hline & $\mathrm{N}(\%)$ & $\begin{array}{c}5 \text {-year remission } \\
\mathrm{N}(\%)\end{array}$ & $\begin{array}{c}\text { Pharmacoresistance } \\
\mathrm{N} 9 \%)\end{array}$ \\
\hline Tarly Difficultics & $138(33 \%)$ & $98(71 \%)$ & $19(14 \%)$ \\
\hline No Early Difficultics & $278(67 \%)$ & $216(78 \%)$ & $42(15 \%)$ \\
\hline $\begin{array}{c}\text { Pre School Aged } \\
\text { Tarly Difficultics }\end{array}$ & $42(32 \%)$ & $28(67 \%)$ & $9(21 \%)$ \\
\hline No Early Difficultics & $89(68 \%)$ & $74(83 \%)^{* *}$ & $11(12 \%)$ \\
\hline School Aged & & & $10(10 \%)$ \\
\hline Early Dilliculties & $96(34 \%)$ & $70(73 \%)$ & $31(16 \%)$ \\
\hline No Early Dilficulties & $189(66 \%)$ & $142(75 \%)$ & \\
\hline $\mathrm{p}<0.05$ & & &
\end{tabular}


Table2: Associations of epilepsy diagnosis with seizure outcomes and early developmental and school difficulties in children with uncomplicated epilepsy

\begin{tabular}{|ccc|c|c|}
\hline & $\mathrm{N}$ & $\begin{array}{c}5 \text {-year remission } \\
\mathrm{N}(\%)\end{array}$ & $\begin{array}{c}\text { Pharmacoresistance } \\
\mathrm{N}(\%)\end{array}$ & $\begin{array}{c}\text { Early dilliculties } \\
\mathrm{N}(\%)\end{array}$ \\
BFCTS & 60 & $58(97 \%)$ & $2(3 \%)$ & $15(25 \%)$ \\
Nonsyndromic-focal & 195 & $141(72 \%)$ & $38(19 \%)$ & $72(37 \%)$ \\
$\mathrm{CAF}$ & 68 & $52(76 \%)$ & $9(13 \%)$ & $18(26 \%)$ \\
\hline JAF & 18 & $13(72 \%)$ & $2(11 \%)$ & $8(44 \%)$ \\
\hline JME & 13 & $5(38 \%)$ & $2(15 \%)$ & $6(46 \%)$ \\
\hline Other GGE & 25 & $16(64 \%)$ & $3(12 \%)$ & $8(32 \%)$ \\
\hline Unclassilied & 37 & $29(78 \%)$ & $5(14 \%)$ & $11(30 \%)$ \\
\hline p-value & & $\mathrm{p}<0.0001$ & $\mathrm{p}=0.11$ & $\mathrm{p}=0.35$ \\
\hline
\end{tabular}

** This significant differences are entirely due to JAE and JME which have a later age at onset and therefore children with these disorders have a longer time in which to be identified for special services.

\subsection{7 \\ ELECTROCARDIOGRAM ORDERING PRACTICES FOR PATIENTS ADMITTED TO AN EPILEPSY MONITORING UNIT}

K. B. Krishnamurthy and Vaishnav Krishnan (Beth Israel Deaconess Medical Center, Boston, MA)

Rationale: Patients with epilepsy are at a higher risk of cardiac dysrhythmias both during the peri-ictal period (tachy- or bradyarrhythmias) and the interictal period (reduced heart rate variability, prolonged QT intervals or atrioventricular block), and the biological mechanisms underlying this vulnerability are poorly understood. Interictal electrocardiograms (ECGs) provide an inexpensive and quick screening modality to discover cardiac conduction abnormalities that may identify individuals at risk for ictal dysrhythmias, including those that may underlie Sudden Unexpected Death in Epilepsy (SUDEP). In this study, we describe the relative infrequency with which ECGs are obtained for patients admitted to our epilepsy monitoring unit (EMU).

Methods: After obtaining IRB approval, we performed a retrospective chart review of patients admitted to the EMU at the BIDMC over the past two years. Like many busy EMUs around the country, this is a resident-run clinical service supervised by a board certified attending epileptologist. We excluded patients younger than 18 or over 65 years of age. Using electronic medical records and the admission initial note, we collected demographic data, information about comorbidities and ECG results.

Results: Out of 186 patients admitted to the EMU that met eligibility criteria, only $57 \%$ (106) received 12 -lead electrocardiograms as a part of their admission testing. The two groups ("ECG" or "NO ECG") were matched in age (41.3 versus 39.7 , respectively) and sex ( $39 \%$ male versus $30 \%$ male, respectively). There was no significant difference between the groups in the incidence of thyroid abnormalities, hypertension, hyperlipidemia, asthma or anxiety/depression. Cardiac disease was not an exclusion criterion. We did identify a greater incidence of a diagnosis of migraine headaches amongst patients who did not receive an ECG $(\mathrm{p}<0.05$, Fisher's exact test).

Conclusions: Many EMU patients have medically-refractory epilepsy, and their admissions often involve withdrawal of anticonvulsants for event characterization or presurgical localization. Since previous studies have shown that seizures can be associated with both tachy- and bradyarrhythmias, the lack of baseline assessments of cardiac rhythms prior to anticonvulsant withdrawal/titration is concerning. We identified no specific practice patterns to explain why certain patients received an ECG and why others did not; the two groups were similar in age, sex and major medical comorbidities. As we continue to gather data to help us predict the likelihood of potentially lethal ictally-associated arrhythmias, common easily identifiable ECG abnormalities must be excluded in EMU patients, particularly in cases where seizures may be provoked by anticonvulsant withdrawal. These data suggest that ordering ECGs should be part of all EMU admission protocols.

\subsection{8 \\ MAINTENANCE OF SUBJECT ADHERENCE TO DAILY DIARY ENTRY FACILITATED BY USE OF A MOBILE APPLICATION IN THE WEPOD STUDY}

Nichelle Llewellyn ${ }^{1}$, C. L. Harden ${ }^{2}$, J. French ${ }^{3}$, P. B. Pennell ${ }^{1}$, E. Bartfeld $^{4}$, A. R. Davis ${ }^{5}$, C. Lau ${ }^{2}$, J. K. Lee ${ }^{3}$, A. Kirshenbaum ${ }^{1}$ and E. Bagiella ${ }^{6}\left({ }^{1}\right.$ Neurology, Brigham and Women's Hospital, Harvard Medical School, Boston, MA; ${ }^{2}$ Neurology, Hofstra North Shore-Long Island Jewish School of Medicine, Great Neck, NY; ${ }^{3}$ Neurology, New York University School of Medicine, New York, NY; ${ }^{4}$ Irody, Inc., Boston, MA; ${ }^{5}$ Obstetrics \& Gynecology, Columbia University, New York, NY and ${ }^{6}$ Health Evidence \& Policy, Mount Sinai School of Medicine, New York, NY)

Rationale: Clinical trials in epilepsy historically depended on paper diaries for reporting seizures and adverse events. Since written diary entries can be made at any time between study visits, paper diaries cannot assess the time interval between clinical event occurrence and the diary documentation, or if there is evidence of data entry fatigue. Electronic diaries may facilitate reporting by subjects and enable monitoring of the interval from occurrence of event to entry time as a marker of validity and accuracy. Electronic reporting also allows for constant monitoring of adherence, which can be particularly important in long-term clinical trials. The WEPOD (Women with Epilepsy: Pregnancy Outcomes and Deliveries) study is a 3-site prospective, observational study evaluating fertility in women with epilepsy (WWE) and healthy controls (HC) as they transition from preconception planning through pregnancy and delivery. This interim analysis assessed subjects' use of an electronic diary and whether data entry fatigue occurred over the course of subject participation in the fertility phase, which could be up to one year.

Methods: Women with epilepsy and healthy controls, ages 18-40 years, seeking pregnancy were enrolled within 6 months of discontinuing contraception. We developed a customized mobile Application (the WEPOD App ${ }^{\mathrm{TM}}$ ) for daily data tracking. The WEPOD App ${ }^{\mathrm{TM}}$ is connected to a web-based program that allows for data entry and provides central data monitoring. Subjects were given a 4th generation iPod Touch, which they could also use with the webbased program, or choose to use a paper diary. Subjects recorded menstrual bleeding and sexual activity daily (fertility diary). WWE also tracked AED compliance and seizure occurrence. The WEPOD $\mathrm{App}^{\mathrm{TM}}$ includes a "pop-up" reminder asking the subject to make their daily diary entry. Subjects track fertility data daily until conception or until 12 months elapses, creating a potentially long duration for study participation.

Results: At the time of this analysis, 119 participants (49 WWE and 70 controls) were enrolled and provided diary data for 17,191 days. Demographics are listed in Table 1. All subjects used the WEPOD app and/or web portal; none chose paper diaries. Average days of fertility data entry were $145.6 \pm 111.9$ (range $0-365$ ). The average completion of the fertility diary was $90 \%+0.21$ (minimum $0 \%$ ). Average number of days per week in the study that subjects made a data entry was 6.30, without a substantial decline over time in the study (Figure 1). A slight reduction in mean number of days diary data was recorded appeared after 44 weeks, but less than 20 subjects were still in the fertility phase of the WEPOD study at that time. Conclusions: Subjects preferred an electronic diary for monitoring of fertility-related data in the WEPOD study. Adherence with diary use was high and stable over time without showing signs of appreciable 
diary entry fatigue. This data capture tool has the potential to be used across different disease populations and clinical trials.

Demographic characteristics of participants in the WEPOD study.

\begin{tabular}{|c|c|}
\hline & $\begin{array}{l}\text { Total Subjects }(\mathrm{n}=119) \\
\%(\mathrm{n}) ; \text { mean } \pm \text { std dev }\end{array}$ \\
\hline Age (years) & $31.3 \pm 5.1$ \\
\hline \multicolumn{2}{|l|}{ Race } \\
\hline American Indian or Alaska native & $0.84(1)$ \\
\hline Asian & $14.29(17)$ \\
\hline African American/ Black & $14.29(17)$ \\
\hline White & $65.55(78)$ \\
\hline Other/Mixed & $5.04(6)$ \\
\hline \multicolumn{2}{|l|}{ Ethnicity } \\
\hline Ilispanic or Latino & $13.45(16)$ \\
\hline Not Hispanic or Latino & $86.55(103)$ \\
\hline \multicolumn{2}{|l|}{ Education } \\
\hline High School & $8.40(10)$ \\
\hline Some College & $10.08(12)$ \\
\hline Associate's Degree & $5.88(7)$ \\
\hline Bachelor's Degree & $36.13(43)$ \\
\hline Advanced Degree & $38.66(46)$ \\
\hline Missing & $0.84(1)$ \\
\hline \multicolumn{2}{|l|}{ Employment $<\mathbf{b} />$} \\
\hline Student & $7.56(9)$ \\
\hline Unemployed & $17.65(21)$ \\
\hline Part-time & $10.92(13)$ \\
\hline Full-time & $62.18(74)$ \\
\hline Missing & $1.68(2)$ \\
\hline
\end{tabular}

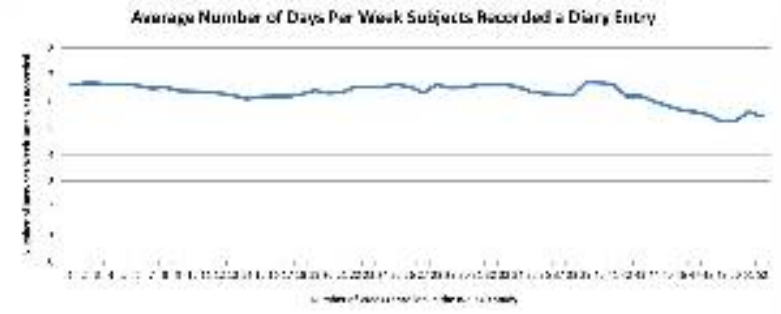

Figure 1.

\subsection{9}

ARE CANADIAN PEDIATRICIANS AWARE OF SUDEP?

Elizabeth J. Donner ${ }^{1,2}$, T. Jeffs ${ }^{1}$ and N. Jette ${ }^{3}\left({ }^{1}\right.$ Division of Neurology, Hospital for Sick Children, Toronto, ON, Canada; ${ }^{2}$ Department of Pediatrics, University of Toronto, Toronto, ON, Canada and ${ }^{3}$ Department of Clinical Neuroscience, Univeristy of Calgary, Calgary, AB, Canada)

Rationale: Mortality rates in children with epilepsy exceed those of the general population. While many deaths may be explained by the underlying cause of seizures or co-morbid conditions, a proportion of deaths in children with epilepsy remain unexplained. Sudden Unexpected Death in Epilepsy (SUDEP) refers to the death of a person with epilepsy that is sudden, unexpected and unexplained. The incidence of SUDEP in adults is estimated to be 1 death per 1000 people with epilepsy per year, with rates approaching 1 per 100 person-years in individuals with drug resistant epilepsy. The incidence of SUDEP in children has not been explored adequately; the limited literature suggests lower rates than in adults, ranging from 0.2 to 0.4 per 1000 person-years. These incidence rates are generally believed to be an underestimate, as it is widely acknowledged that poor awareness of SUDEP among health care practitioners and people with epilepsy result in low case ascertainment.

Our aims were to determine whether a knowledge gap regarding
SUDEP exists among Canadian pediatricians and to inform strategies for a prospective study of SUDEP among Canadian children. Methods: The Canadian Pediatric Surveillance Program (CPSP), operated by the Canadian Pediatric Society, gathers data from Canadian pediatricians and pediatric subspecialists each month to monitor rare diseases and conditions in Canadian children. These physicians provide health care to over seven million Canadian children and youth.

A 10 question paper survey was administered to all 2570 pediatricians enrolled in the CPSP. Content validity was completed through literature review and serial revisions achieving agreement about each questionnaire component. Variables collected included experience providing care to children with epilepsy, knowledge of mortality risk among children with epilepsy and knowledge of SUDEP. Descriptive statistics were calculated.

Results: The survey response rate was $34 \%(866 / 2570)$. Of the respondents, $78 \%$ (674) reported that they had cared for children with epilepsy in the preceding 24 months. Among these pediatricians, only $56 \%(380)$ had prior knowledge that children with epilepsy are at an increased risk of sudden unexplained death compared to children without epilepsy. Only 33\% (225) of pediatricians caring for children with epilepsy were aware of the term SUDEP.

Fourteen pediatricians reported knowledge of a case of SUDEP. Of the 11 cases for which details of the death investigation process were reported, five children (45\%) did not undergo autopsy, suggesting inadequate investigation of deaths in children with epilepsy. Survey methodology does not permit accurate incidence calculations. Conclusions: This survey identifies poor awareness of SUDEP among Canadian pediatricians, despite the finding that greater than $75 \%$ report providing care for children with epilepsy. The study highlights the need for educational initiatives to support future studies involving SUDEP case collection. Accurate incidence data will require prospective surveillance of SUDEP in children.

\subsection{0 \\ PARENTS' PERCEPTIONS OF ACTIVITY RESTRICTIONS IN CHILDREN WITH EPILEPSY: ROLE OF CHILD AND FAMILY FACTORS \\ Nathan C. King ${ }^{1,2}$, K. N. Speechley ${ }^{1,2}$ and G. Y. Zou ${ }^{1,3}$ \\ ( ${ }^{1}$ Epidemiology \& Biostatistics, Western University, London, ON, Canada; ${ }^{2}$ Children's Health Research Institute, London, ON, Canada and ${ }^{3}$ Robarts Research Institute, London, ON, Canada)}

Rationale: Children with epilepsy are less physically active and socially involved than their peers, which in turn can negatively affect children's development and health-related quality of life. As gatekeepers, parents of children with epilepsy may become overprotective and thus restrict the involvement of these children in activities. The objective of this research was to explore whether parents represent a barrier to children's physical and social activities, by examining the association between child and family factors and parents' perceptions of activity restrictions associated with epilepsy. Methods: Data were obtained from the Health Related Quality of Life in Children with Epilepsy Study (HERQULES), a multi-centre longitudinal study of children 4-12 years old with new-onset epilepsy followed for 24 months. Activity restrictions, family functioning, resources, and demands, and parental worry and concern were reported by parents. Children's seizure frequency, type, severity and timing, medication use, and co-morbid conditions were reported by neurologists, at baseline, 6, 12, and 24 months. The Physical Restrictions subscale of the Quality of Life in Childhood Epilepsy (QOLCE) questionnaire assessed parent perceived activity restrictions. The Family Inventory of Resources for Management (FIRM) measured family resources; the Family Inventory of Life Events and Changes (FILE) measured family demands; the Family Adaptability, Partnership, Growth, Affection \& Resolve (APGAR) measured family functioning; and the Parental Impact-Emotional subscale of the Child Health Questionnaire (CHQ) measured parental 
worry and concern. The Global Assessment of Severity of Epilepsy (GASE) scale was used to assess epilepsy severity. Linear mixed models were used to model the relationships among child and family factors and parents' perceptions of activity restrictions.

Results: Baseline questionnaires were completed by 374 parents (response rate $82 \%$ ) and by 282 at 24 months. There was a significant non-linear change in activity restriction scores from baseline to 24 months, on average (Time squared in weeks $=-0.0017(0.00026)$ ). Over time parents perceived fewer epilepsy-associated activity restrictions (mean (SD)): 62.9(18.5), 68.6(19.2), 73.5(18.4), and 74.1(18.6)). The improvement in activity restrictions was significant from baseline to 6 to 12 months, but not from 12 to 24 months postdiagnosis. Results from fitting the mixed model show that child factors- (age, seizure frequency, epilepsy severity and presence of a co-morbid diagnosis), and family factors- (annual household income, caregiver age, parental worry and concern, and family resources) are significantly associated with perceived activity restrictions $(\mathrm{p}<0.05)$. Conclusions: The significant association of family factors with parents' perceptions of activity restrictions associated with epilepsy in children suggests that there is an opportunity through education and discussion to remove some level of unnecessary activity restriction soon after children are diagnosed.

\subsection{1 \\ MOST CHILDREN WITH NON-SYNDROMIC, FOCAL EPILEPSY OF UNKNOWN CAUSE HAVE A GOOD SEIZURE AND SOCIAL OUTCOME 30 YEARS LATER: A POPULATION-BASED STUDY}

Peter R. Camfield and C. S. Camfield (IWK Health Care Centre, Halifax, NS, Canada)

Rationale: The prognosis is unclear for children with non-syndromic focal epilepsy with no known cause ("cryptogenic") even if intelligence and neurological function are normal. We compared the outcome of this group to otherwise normal children with focal epilepsy and cause known ("symptomatic").

Methods: Cases were selected from the population-based Nova Scotia Childhood Epilepsy cohort. All had epilepsy onset between 1977-85 at age 1 month - 16 years and were followed up for a minimum of 10 years in 2009-2012. Inclusion criteria were normal intelligence and neurological examination and only focal seizures \pm secondary generalization throughout their entire clinical course. "Benign" childhood epilepsy syndromes were excluded. Patients were divided into 2 groups. 1. Cause unknown with no recognized etiology identified from history, physical examination, neuroimaging and other investigations. 2. Cause known with a recognized cause identified any time during the clinical course. Statistical significance was $\mathrm{p}<0.05$.

Results: Of 692 children in the cohort, entry criteria were met by 117 with focal epilepsy cause unknown ( $17 \%$ of all epilepsy) and 52 with cause known $(7.5 \%)$. The two groups did not differ significantly for average age of onset (overall 7.2 $\pm 4.6 \mathrm{yrs}$ ), length of follow up $(27.8 \pm 5.2 \mathrm{yrs})$ and final age $(34.9 \pm 12 \mathrm{yrs})$. There was no difference in the proportion with complex partial seizures, focal seizures with secondary generalization, numbers of seizures throughout follow up and febrile status epilepticus. Those with unknown cause were seizure free for a significantly longer mean time ( 20 vs 14 years) and significantly less likely to have markers of severe epilepsy continuing AED treatment at the end of follow up ( $25 \%$ vs $40 \%)$, unprovoked status epilepticus ( $16 \%$ vs $30 \%)$, use of $>2$ AEDs $(28 \%$ vs $65 \%$ ), intractability ( $10 \%$ vs $21 \%)$, or epilepsy surgery (3\% vs $17 \%)$. Educational success did not differ between groups but measures of social outcome favored those with cause unknown: married or living common law ( $67 \%$ vs $42 \%$ ), having close friends (93\% vs $83 \%$ ) and employed (84\% vs 54\%).

All patients with intractable epilepsy were then removed from both groups leaving 105 with unknown cause and 42 with cause known. The differences between groups were less striking. Patients with unknown cause now had significantly fewer secondary generalized seizures ( $>100$ in $10 \%$ vs $24 \%$ ), were still less likely to use $>2$ AEDs ( $20 \%$ vs $36 \%$ ) and still had a longer mean time seizure free (22 vs 18 yrs). Socially the unknown group still had significantly higher rates of employment ( $85 \%$ vs $52 \%$ ) and a greater chance of living with a spouse (67\% vs $45 \%$ ) and not with parents or sibs (11\% vs $26 \%$ ). Conclusions: About $75 \%$ of normal children with non-syndromic, focal epilepsy and no known cause have a favorable seizure and social outcome, significantly better than those with a known cause. Much (but not all) of this difference is related to the reduced chance of developing intractable epilepsy compared to those with a known cause.

\subsection{2 \\ PSYCHOGENIC NON EPILEPTIC SEIZURES (PNES) IN PERSONS WITH DRUG REFRACTORY EPILEPSY (DRE) DUE TO A SURGICAL SUBSTRATE}

Manjari Tripathi, S. Sharma, F. U. Ahmed, A. N. Wadhawan, M. V. Padma, R. Bhatia, K. Prasad, M. B. Singh, V. Gupta and P. S. Chandra (All India Institute Of Medical Sciences, Delhi, India)

Rationale: Persons with a surgical substrate for there DRE may also have coexisting PNES. Presence of these seizures both pre and post operatively (emergent) would result in a poorer quality of life in these patients. They would also confound postoperative results and cause the outcomes to be worse if they emerge after surgery. Hence we prospectively evaluated and followed up patients with coexisting seizures and there outcomes after 5 years of follow up.

Methods: Prospective study and follow up of DRE patients who were evaluated in Unit 1 neurology, from 1999 January to 2006 January. A follow up of 5 years was done on all the patients who subsequently underwent surgery prior to 2006. All patients who had only PNES were excluded from the evaluation and further presurgical workup. All patients who had coexisting psychogenic and true epilepsy were included in the study. The demographic profile was noted. History prior to onset of both seizure types obtained. In few patients PNES emerged de novo during monitoring these were recorded. In some they emerged de novo after surgery this was recorded too. A complete profile of these patients on the NPI, and BPRS was performed . QOLIE-31 was also performed and seizure outcome documented on the Engel scale.

Results: A total of 792 recordings of 783 PWE, were review and followed up prospectively. Only 32 of these were psychogenic only seizures both by history and EMU recordings these were excluded from the study. 27 PWE demonstrated a combination seizure type. 6 had de novo appearance of PNES during the EMU recording. 15 PWE underwent surgery as true seizures were the predominant seizure type. The rest were put on follow up, and surgery deferred till the emergence of disabling seizures. 6 from this group eventually moved to the surgical arm. The rest were continued on the best medical treatment. 18 PWE with DRE who underwent surgery during this duration had emergent PNES denovo after surgery. Following surgery $11 \mathrm{PWE}$ with a combination of seizures were seizure free at the end of 5 years with 5 showing a running down phenomenon on the PNES. 4 continued to have seizures 2 both and 1 each PNES and true only. Of the PWE with post surgical burden of epilepsy denovo seizures 17 became seizure free with counseling.

Conclusions: Coexistence of both PNES and true seizures should not be a contraindication for epilepsy which should be performed depending on the predominant seizure type. Case by case management with attention to each seizure type is a must in such patients. 


\subsection{3}

INTERICTAL 12-LEAD ELECTROCARDIOGRAPHY IN PATIENTS WITH EPILEPTIC SEIZURES

Vaishnav Krishnan and K. B. Krishnamurthy (Beth Israel Deaconess Medical Center, Boston, MA)

Rationale: SUDEP (Sudden Unexpected Death in Epilepsy) is thought to occur as a consequence of peri-ictal lethal cardiac arrhythmias such as Torsades de Pointes. In this study, our objective was to identify how common ECG variables were altered in patients with recurrent epileptic seizures. Previous attempts at answering this question have produced inconsistent results, and have compared epilepsy patients to healthy controls, an approach that does not control for exposure to medications that may themselves alter ECG variables.

Methods: After obtaining IRB approval, we performed a retrospective chart review of patients admitted to the BIDMC Epilepsy Monitoring Unit in the past 2 years. We excluded patients younger than 18 or over 65 years of age, implanted with a cardiac pacemaker, displaying significant electrolyte abnormalities on admission, or who were febrile, and/or taking medications known to alter QT intervals (other than antidepressant and antipsychotic medications). Based on EEG telemetry reports, we identified patients with DEFINITE EPILEPSY / DE (those with a clinical history consistent with recurrent unprovoked seizures, at least one electroclinical seizure and interictal epileptiform discharges) and NONEPILEPTIC SEIZURES / NES (those with EEG proven nonepileptic events). In subgroup analysis, we divided DE patients into those with primary generalized epilepsy and localization-related epilepsy, and further subdivided the latter, into those with seizures arising from the left or right hemisphere. Hodges formula was used to calculate the rate corrected QT (QTc).

Results: DE $(n=51)$ and NES $(n=24)$ groups were matched in age ( 40 yo), sex ( $\sim 40 \%$ male), antidepressant ( $40 \%)$ and antipsychotic use $(\sim 15 \%)$. The average total number of antiepileptic medications taken at the time of admission was only marginally greater in DE patients (2.2 versus 1.4). DE patients had a larger QTc interval and displayed a more leftward axis deviation, while displaying similar PR intervals and ventricular rates. Preliminary subgroup analysis has revealed no differences between patients with primary generalized and localization-related epilepsy, and between left- and right-sided seizures. QTc intervals were significantly greater in women and directly proportional to age.

Conclusions: Compared to patients with nonepileptic seizures, patients with recurrent epileptic seizures displayed a greater QTc interval and leftward axis deviation, suggesting a predisposition towards cardiac arrhythmias associated with early repolarization. We continue to build on our analysis' sample size to explore how specific forms of adult epilepsy may be associated with such ECG changes, and perhaps confer an increased risk for SUDEP. These data validate this experimental approach as being able to isolate clinical features that are uniquely associated with epileptic seizures and addresses certain confounding variables such as medication exposure. Overall, these data may provide insights into the bidirectional links between epilepsy and cardiac arrhythmias.

\section{Clinical Epilepsy: Women's Issues}

\subsection{4}

\section{THE RISK OF SEIZURE RECURRENCE AFTER CHILD-} BEARING

Keiko Hara $^{1,2}$, M. Matsuura ${ }^{2}$ and M. Hara ${ }^{1}{ }^{1}$ Hara Clinic, Yokohama, Japan and ${ }^{2}$ Tokyo Medical and Dental University, Tokyo, Japan)

Rationale: Previous studies in female patients with epilepsy have largely focused on seizure status during pregnancy and on anomalies in children of patients on anti-epileptic drugs (AED). However, little is known about the influence of child-bearing on seizure recurrence after delivery in female epileptic patients in remission. We investigated the risk of seizure relapse after child-bearing. Methods: We retrospectively examined epileptic patients who had a delivery after at least two years of seizure remission. Patients had been followed for at least one year after delivery at the time of this study. We excluded patients who had a seizure recurrence following AED reduction. A total of 53 patients ( 72 deliveries) were included for further analysis. Patients were divided into two groups. The first group included 16 patients with long-lasting seizure remission group (five years or more), and the second group included 37 patients with relatively short seizure remission (2-5 years). The mean age at the time of the study was 44.5 years, and the mean follow-up duration was 8.4 years. The study was approved by the Ethics Committee at Tokyo Medical and Dental University.

Results: A total of 17 of 53 patients had seizure recurrence during follow-up. Ten of 53 patients (18.9\%) had seizure relapse within one year after delivery, and seven patients $(13.2 \%)$ had a relapse more than one year after delivery. In the long-lasting remission group, 21.6 $\%$ (8 out of 37 ) had seizure recurrence. Six of these patients $(16.2 \%)$ had a seizure within one year of delivery, and two were in the longlasting remission group (five years or more). In the group with relatively short remission, $25 \%$ (four out of 16 patients) showed seizures within one year after delivery, and $31.3 \%$ (five out of 16 patients) had seizure relapses more than one year after delivery. Short seizure remission was a risk factor for seizure relapse after childbearing $(p<0.05)$. The epilepsy classification, the gender of the child, the time between marriage and pregnancy, or number of AEDs did not show any significant correlation with the risk of seizure relapses. Conclusions: It is well known that the risk of seizure relapse is relatively low in patients who have been in remission for two years or more, without a change in AED treatment. Our result showed that seizure relapse within one year after delivery is not rare, even in patients who have been seizure free for two years or more. Seizure recurrence after delivery may pose a danger not only to the patients but also to their babies. After child-bearing, patients are at risk as a result of sleep deprivation, life style changes, and dynamic changes in hormone levels. Our results may suggest that doctors should also be aware of the risk of relapse in patients who have been seizure free for years, and that they should provide lifestyle guidance to patients and their families.

\subsection{5 \\ THE EFFECTS OF PROGESTERONE VERSUS PLACEBO TREATMENT ON QUALITY OF LIFE IN WOMEN WITH EPILEPSY}

Cynthia L. Harden ${ }^{1}$, A. G. Herzog ${ }^{2}$, S. Schaffer ${ }^{1}$, K. M. Fowler ${ }^{2}$ and B. Jobst ${ }^{3}\left({ }^{1}\right.$ Neurology, Hofstra North Shore-LIJ School of Medicine, Great Neck, NY; ${ }^{2}$ Harvard Neuroendocrine Unit, Beth Israel Deaconess Medical Center, Wellesley, MA and ${ }^{3}$ Geisel School of Medicine at Dartmouth, Dartmouth-Hitchcock Medical Center, Lebanon, NH)

Rationale: It is not known whether the use of cyclic adjunctive progesterone supplement for the treatment of intractable seizures in women with epilepsy (WWE) affects quality of life (QOL). The purpose of this analysis is to determine if progesterone therapy in the NIH Progesterone Trial affected QOL measures differently than placebo in WWE who had intractable seizures.

Methods: Two hundred ninety-four women with intractable seizures were designated to catamenial or non-catamenial strata using established criteria (Herzog et al 1997 for the Progesterone Trial Study Group) after 3 baseline months and randomized to 3 months of adjunctive treatment with progesterone or placebo in the NIH Progesterone Trial. Changes in QOL, assessed by measures of the 7 QOLIE-31 domains (dependent factors) were compared by treatment and strata (independent factors) and changes in estradiol (E2) and progesterone (P4) levels (covariates) using MANOVA. Changes in significant factors were compared by treatment and stratum using 
Wilcoxon rank-sum test and correlated using Spearman analysis. Results: One QOLIE-31 domain, seizure worry, showed a significant between-subjects effect $(p=.003)$ with treatment being a significant factor $(\mathrm{p}=.008)$ and treatment and stratum showing significant interaction $(\mathrm{p}=.007)$. Changes in seizure worry were an order of magnitude more significant with progesterone treatment in the catamenial stratum than in the non-catamenial stratum. There were no significant changes with placebo in either stratum (Table). There were significant correlations between changes in seizure worry and changes in $\mathrm{E} 2(\mathrm{r}=-.385, \mathrm{p}=.006)$ and $\mathrm{P} 4(\mathrm{r}=.318, \mathrm{p}=.026)$ levels for the catamenial stratum only. There was also a significant correlation between changes in seizure worry and seizure frequency $(\mathrm{r}=-.374, \mathrm{p}=.002)$ in progesterone treated subjects only. By way of pertinent negatives, there was no significant correlation between baseline to treatment changes in seizure worry scores and changes in measures of depression using Beck's Depression Inventory and Profile of Mood States questionnaires. Changes in depression scores showed no significant difference between progesterone and placebo treatment.

Conclusions: Seizure worry is affected favorably by progesterone treatment in WWE, especially in women with catamenially exacerbated seizures. The changes in seizure worry scores correlate with treatment related changes in hormone levels and seizure frequency.

Supported by NIH RO1 NS39466

Change in Seizure Worry by Treatment and Strata

\begin{tabular}{|c|cc|c|}
\hline $\mathrm{N}=294$ & $\begin{array}{c}\text { Baseline Phase Average } \\
\text { Seizure Worry Scores }\end{array}$ & $\begin{array}{c}\text { Treatment Phase Average } \\
\text { Seizure Worry Scores }\end{array}$ & $\mathrm{p}$-Value \\
$\begin{array}{c}\text { Progestcronc } \\
\text { Catamenial } \\
(\mathrm{N}=85)\end{array}$ & $49.0(24.7,71.1)^{*}$ & $56.0(33.0,75.3)$ & 0.003 \\
\hline $\begin{array}{c}\text { Progesterone } \\
\text { Non-catamcnial } \\
(\mathrm{N}=105)\end{array}$ & $59.3(29.5,75.0)$ & $59.8(31.1,80.0)$ & 0.023 \\
\hline $\begin{array}{c}\text { Placebo Catamenial } \\
(\mathrm{N}=45) \\
\text { Placebo }\end{array}$ & $51.0(37.2,73.0)$ & $52.5(41.1,73.6)$ & 0.669 \\
$\begin{array}{c}\text { Non-catamenial } \\
(\mathrm{N}=59)\end{array}$ & $51.7(23.4,73.6)$ & $48.0(29.6,71.4)$ & 0.921 \\
\hline
\end{tabular}

* = median (1st \& 3rd quartiles); higher scores reflect less worry

\subsection{6}

AWARENESS OF INTERACTIONS BETWEEN HORMONAL CONTRACEPTION AND ANTI-EPILEPTIC MEDICATIONS: A SURVEY OF NEUROLOGY AND OBSTETRICS AND GYNECOLOGY RESIDENTS M. Sahay ${ }^{1}$, I. Garic ${ }^{1}$, L. Gowron ${ }^{2}$, C. Hammond ${ }^{2}$, J. Kennedy ${ }^{1}$, M. Macken $^{1}$, S. Schuele ${ }^{1}$, C. Stika ${ }^{2}$, S. York ${ }^{2}$ and Elizabeth Gerard ${ }^{1}$ ( ${ }^{1}$ Department of Neurology, Northwestern University, Chicago, IL and ${ }^{2}$ Department of Obstetrics and Gynecology, Northwestern University, Chicago, IL)

Rationale: Family planning is a critical concern for women living with epilepsy (WWE). Choosing an appropriate form of contraception, however, is difficult for WWE as many anti-epileptic drugs have significant interactions with widely used forms of contraception. It is essential that healthcare providers are keenly aware of these interactions. Our aim was to assess residents' understanding of the interactions of hormonal contraception and antiepileptic medications. Previous studies have shown that physician knowledge of these drug-drug interactions, specifically the effect of enzyme-inducing AEDs (EI-AEDs) on oral contraceptive pills (OCPs), is poor (Morrell, et. al, J Womens Health Gend Based Med. 2000; 9: 959-65; Summerfield, et.al, Neurology. 1996; 46: 1534-9). Prior studies did not address the interactions EI-AEDs on other forms of contraception or the interactions of hormonal contraception with lamotrigine. Lamotrigine is becoming one of the most frequently prescribed medications for women of childbearing age and has unique interactions with synthetic hormones. Synthetic estrogens can significantly lower lamotrigine levels, increasing a patient's risk for seizures (Sidhu, et, al, Br J Clin Pharmacol. 2006; 61: 191-9). We hypothesized that residents would be aware of the interactions of EIAEDs and OCPs, but they would not be aware of other important interactions.

Methods: A survey of drug-drug interactions was developed and distributed to all twenty-two neurology residents and forty-four obstetrics and gynecology residents at our institution. For each potential interaction, respondents could indicate "yes," "no" or "I don't know." It was distributed as a paper and an online survey. Results: Nineteen neurology residents and twenty obstetrics and gynecology residents responded to the survey. Most, but not all, respondents knew that enzyme inducing antiepileptic medications, specifically carbamazepine $(76.9 \%)$, phenytoin $(71.8 \%)$, and oxcarbazepine $(59 \%)$ affect the efficacy of combined OCPs, but did not know that carbamazepine would affect the efficacy of etonogesterel implant, depot medroxyprogesterone and the contraceptive vaginal ring. Most respondents did know that levetiracetam does not have significant interactions with most forms of birth control. When asked which AEDs would be affected by estrogen containing OCPs, $46.2 \%$ indicated lamotrigine would be affected, but a similar percentage indicated the same for carbamazepine and phenytoin, suggesting they are not aware of the specific interaction.

Conclusions: Resident knowledge of interactions between EI-AED and OCPs is relatively high. Awareness regarding other important interactions between AEDs and hormonal contraception, however, is quite low. Further education through the development of guidelines and educational materials is needed and recommended during residency training.

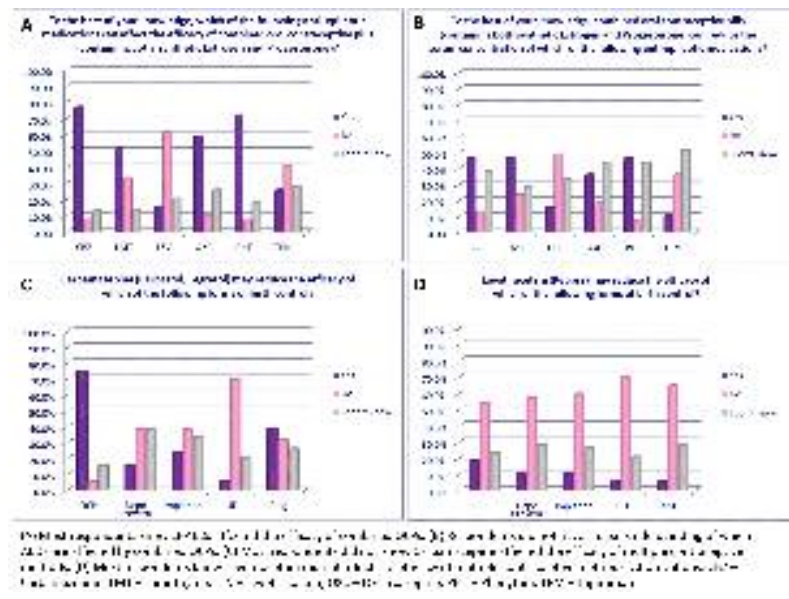

1.167

CARING FOR WOMEN WITH EPILEPSY: DOES AN EMR HELP US FOLLOW GUIDELINES?

Patricia E. Penovich ${ }^{1,2}$ and J. E. Hanna ${ }^{1,2}\left({ }^{1}\right.$ Minnesota Epilepsy Group, P.A. ${ }^{\circledR}$ of United Hospital and Children's Hospitals and Clinics of Minnesota, St. Paul, MN and ${ }^{2}$ Department of Neurology, University of Minnesota, Minneapolis, MN)

Rationale: Over the past ten years, the care of women with epilepsy (WWE) has become specialized. The AAN issued guidelines in 2003 which have been endorsed by the AES and EFA. We evaluated how one epilepsy center practices following implementation of our electronic medical record (EMR). We previously evaluated our practice in 2009, prior to EMR.

Methods: Charts of women aged 16 or older who were seen between $1 / 2012$ and 4/2012 were selected in alphabetical sequential fashion. Retrospective review included age, imaging, EEGs, cognitive status 
(IQ when available), menstrual status, epilepsy type and control, history of bone density testing, pregnancy history, and the following treatment variables: AEDs, folate use and dose, calcium and vitamin D supplementation.

Results: 112 charts were reviewed, including 18 pediatric and 94 adult patients cared for by seven different physicians. $71 \%$ are in the childbearing years (CB group, ages 16-45), while 19\% are peri- or post-menopausal. IQ is normal to mildly impaired in 96 , while 16 are moderately to profoundly cognitively impaired. $82 \%$ have partial epilepsy, $11 \%$ have JME, and $7 \%$ have other generalized epilepsies. Overall, $39 \%$ of the total sample takes folate at doses between $0.8 \mathrm{mg}$ and 5mg per day. In the $\mathrm{CB}$ group, this number is to $52 \%$. However, among patients in the $\mathrm{CB}$ group with normal or near-normal IQ (NCB subgroup), folate use is $58 \%$. Of that group, when those who had permanent sterilization or live in a closely supervised setting are excluded, folate use is 66\%. Monotherapy (MT) is employed in $41 \%$ of patients. This is consistent in the CB group and in the NCB subgroup. $15 \%$ of the $\mathrm{CB}$ group and $13 \%$ of the NCB subgroup are on valproate. $41 \%$ of patients take calcium and vitamin $\mathrm{D}$, and an additional $9 \%$ take calcium or vitamin $\mathrm{D}$ alone. There is no trend in terms of age and calcium or vitamin D supplementation. Of the women who have had DEXA scans, $63 \%$ are in the older age group ( $>45$ years). 7 patients were pregnant in the 12 months prior to their clinic visit.

Results were compared to prior analysis performed in 2009, which preceded the implementation of our EMR. There were not significant differences in the results on any of the above measures. Further data analysis will focus on practice differences between pediatric versus adult providers.

Conclusions: Despite available guidelines for care of WWE, day-today practice may lag behind present knowledge. Increasingly, the literature suggests that this care should begin in the pediatric years and extend longitudinally into the golden years. The care of WWE must involve not only the treatment of epilepsy, but also a certain degree of general medical care.

Implementation of the EMR should provide a more efficient mechanism for protocol-driven office visits and facilitate the tracking and analysis of longitudinal patient care practices. We found that, when compared to our practice before EMR, care for WWE had not changed significantly. This suggests that specific protocols or templates may be helpful in guiding care of women with epilepsy and optimizing attention to treatment recommendations.

\section{Neuro Imaging: Structural Imaging}

\subsection{8}

\section{INTERICTAL SEIZURE BURDEN ALTERS WHITE} MATTER INTEGRITY IN TEMPORAL LOBE EPILEPSY Karol Osipowicz, N. Pajor, A. Sharan, M. Sperling and J. I. Tracy (Thomas Jefferson University, Philadelphia, PA)

Rationale: Mesial temporal lobe epilepsy (mTLE) can compromise white matter (WM) integrity. Unilateral mTLE is often associated with subclinical seizure (interictal) activity in the contralateral mesial temporal lobe - the effects of this contralateral interictal seizure burden (cItSb) on WM integrity have not been investigated. We hypothesized that mTLE patients will show proximal and distal white matter deterioration relative to healthy controls, with the left TLE patients showing more pronounced deterioration; and that mTLE patients with cItSb would show more pronounced deterioration than patients with unilateral interictal activity.

Methods: We used tract based spatial statistics, an advanced registration technique, to assess WM integrity in regions proximal and distal to the epileptogenic temporal lobe, utilizing region-ofinterest and whole brain analyses, respectively. Patients were grouped based on side of ictal seizure focus, and based on the presence or absence of cItSb, based upon extensive video-EEG monitoring. GLM based statistical analyzes emphasized fractional anisotropy (FA) differences between mTLE patients and controls, and between each patient grouping.

Results: Our data demonstrates that mTLE patients, compared to controls, showed decreased FA in: (1) a large bilateral middle and inferior frontal region and (2) based on region-of-interest analyses involving the epileptogenic and contralateral/nonepileptogenic temporal lobe - the bilateral temporal lobes; both of these finding appeared independent of the side of seizure focus, or the presence of cItSb. Lastly, compared to patients with unilateral interictal activity, cItSb patients showed increased FA in a posterior cross-hemispheric region (splenium and forceps major).

Conclusions: These results suggest that unilateral mTLE compromises WM integrity within and beyond the epileptogenic temporal lobe, with extra-epileptogenic deterioration observed in the bilateral inferior/middle frontal, and contralateral anterior/mesial temporal lobes. These findings are consistent with prior literature showing that focal/unilateral mTLE has a widespread anatomical impact. Our results suggest the presence of contralateral interictal seizure burden has a very selective effect, enhancing WM integrity in a specific posterior cross-hemispheric region. This finding, while counter-intuitive in relation to the hypothesized effects of increased seizure burden, suggests that contralateral--homologous interictal seizure burden increases posterior/cross-hemispheric WM integrity. Thus, the data demonstrate the complexities in understanding the effect of seizures (propagation, interictal activity, epileptogenesis) on WM; highlighting the need for considering WM integrity and the effects of contralateral interictal seizure burden when modeling the effects of epilepsy on brain regions outside the ictal focus.

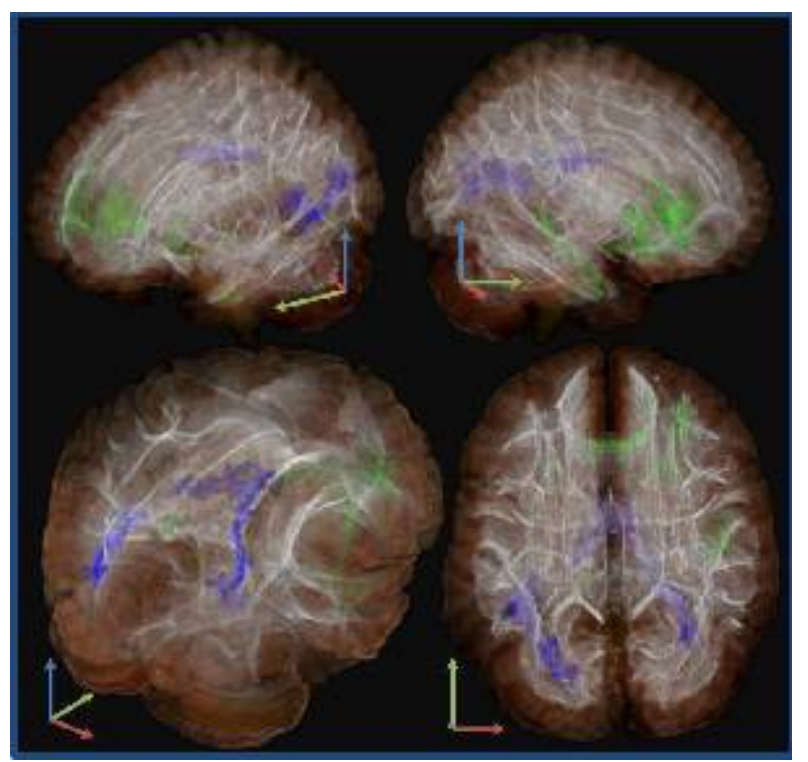

Regions with decreased FA in patients relative to controls (Green) and in patients without bilateral interictal activity relative to patients with contralateral interictal seizure burden (Blue). Orientation arrows indicate: green-anterior, blue-superior, red-right.

\subsection{9 \\ VOXEL-BASED MORPHOMETRIC MRI POST- PROCESSING IN MRI-NEGATIVE ORBITOFRONTAL EPILEPSY PATIENTS}

S. E. Jones ${ }^{2,1}$, Z. I. Wang ${ }^{1}$, A. Ristic ${ }^{3}$, C. Wong ${ }^{4}$, J. A. GonzalezMartinez ${ }^{1}$, I. M. Najm ${ }^{1}$ and A. V. Alexopoulos ${ }^{1}\left({ }^{1}\right.$ Epilepsy Center, Cleveland Clinic Foundation, Cleveland, OH; ${ }^{2}$ Diagnostic Radiology, Mellen Center, Cleveland Clinic Foundation, Cleveland, $\mathrm{OH}$; ${ }^{3}$ Epilepsy Center, Clinic of Neurology, Clinical Center of Serbia, Serbia, Serbia and ${ }^{4}$ Department of Neurology, Westmead Hospital, Sydney, VIC, Australia) 
Rationale: The orbitofrontal (OF) cortex is one of the least explored and least understood regions of the cerebral cortex. There are not many studies on patients with OF epilepsy which is electrophysiologically and surgically confirmed. Studies on OF epilepsy with a negative pre-surgical MRI are even rarer. The aim of our study is to examine the use of voxel-based MRI post-processing technique in studying surgically proven nonlesional OF epilepsy. Methods: From the surgical series of Cleveland Clinic Epilepsy Center, 10 MRI negative (MRI-) patients with OF epilepsy were identified who met the following criteria: 1) surgical resection of the OF region with or without resection of adjacent brain structure; 2) Engel class 1 outcome with minimum follow-up duration of 12 months; 3) negative pre-surgical MRI by radiology report. The postprocessing was implemented in a Morphometric Analysis Program (MAP) with methodology published by Huppertz et al. MAP study was performed on T1 high-resolution scans (MPRAGE) in Matlab SPM5. Three feature maps were evaluated: cortical junction, extension and thickness files. Z-score was determined by comparing individual feature files with a normal, scanner-specific database. Regions with z-score greater than 4 standard deviations were considered to be significant. These significant areas were then presented to the neuroradiologist (SEJ), who was the final judge of whether they present true lesions. These images were presented as part of a large ongoing retrospective study, and the reviewer was not given prior information about the type of epilepsy or whether it was a patient or control. Concordance between MAP and surgical resection area was then evaluated.

Results: Five patients had MAP+ abnormality in the OF region. Overall, there is $50 \%$ positive rate of MAP. Four patients had singlefocus lesion. Resection included area of lesion in all 4 patients. Given that all patients were seizure-free, these lesions were considered true positives. The Figure shows one patient whose MAP finding was concordant with PET, SPECT and ICEEG. We also found a patient who had multiple (3) lesions. Two of the three lesions were resected; patient has been seizure-free after surgery in 2007. The $5 \mathrm{MAP}+$ patients had pathology including FCD 1, FCD 2a, remote infarct, Rosenthal fiber formation, and nonspecific gliosis. The 5 MAPpatients all had $\mathrm{MCD} / \mathrm{CD}$.

Conclusions: We present here the largest series to date of MRIsurgically proven OF epilepsy. Conventional MRI visual analysis of OF cortex can be challenging because of the dense convexity in that area of the brain. We found that voxel-based morphometric MRI post-processing can positively indentify subtle abnormalities in 50\% of the nonlesional OF patients we studied. Lesions detected by MAP, when present, was very specific in this cohort. We hypothesize that MAP is not sensitive strictly to FCD pathology per se, but to pathological substrates (or combinations of them) causing T1 signal alteration leading to a blurred gray-white junction. Further studies on MRI post-processing in MRI- patients are ongoing in our laboratory.

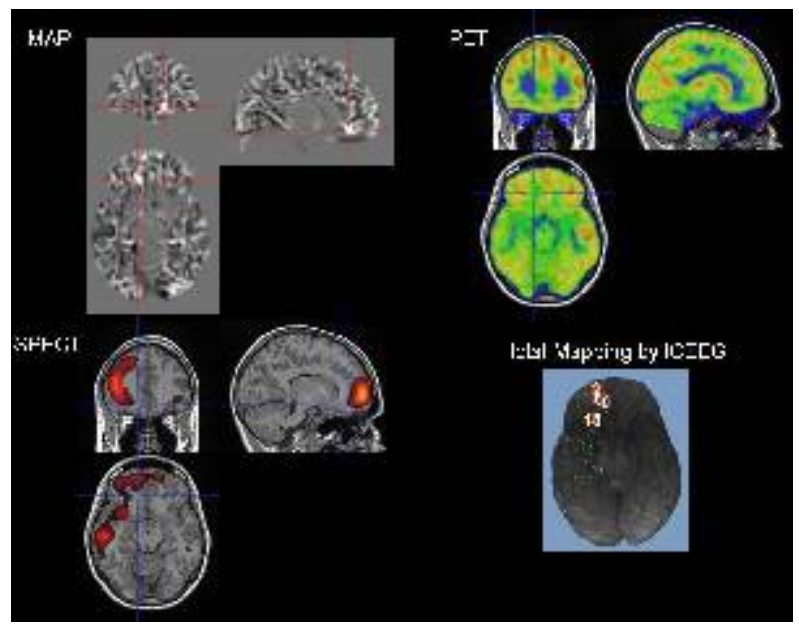

1.170

A TWO-LEVEL MULTIMODALITY IMAGING BAYESIAN NETWORK APPROACH FOR CLASSIFICATION OF PARTIAL EPILEPSY: PRELIMINARY FINDINGS

Susanne G. Mueller ${ }^{1}$, M. Hartig ${ }^{1}$, J. Barakos ${ }^{3}$, P. Garcia ${ }^{2}$ and K. D. Laxer $^{3}\left({ }^{1}\right.$ Center of Imaging of Neurodegenerative Diseases, San Francisco, CA; ${ }^{2}$ Neurology, University of California, San Francisco, San Francisco, CA and ${ }^{3}$ California Pacific's Epilepsy Program, California Pacific Medical Center, San Francisco, CA)

Rationale: Quantitative neuroimaging analyses have demonstrated characteristic gray and white matter abnormalities in group comparisons of different types of non-lesional partial epilepsy. It is unknown to what degree these type-specific patterns exist in individual patients and if they could be exploited for diagnostic purposes. In this study, a two-level multi-modality imaging Bayesian network approach is proposed that uses information about individual gray matter volume loss and white matter integrity to differentiate between patients suffering from non-lesional temporal lobe epilepsy with (TLE-MTS) or without (TLE-no) mesial-temporal sclerosis and frontal lobe epilepsy (FLE).

Methods: 25 controls, 19 TLE-MTS, 22 TLE-no and 14 FLE were studied on a 4T MRI and T1 weighted structural and DTI images acquired. Spatially normalized GM and FA abnormality maps (binary maps with voxels 1 SD below control mean) were calculated for each subject. At the first level, each group's abnormality maps were compared with those from all the other groups using GraphicalModel-based Morphometric Analysis (GAMMA). GAMMA uses a Bayesian network and a Markov random field based contextual clustering method to identify cluster of voxels that provide the maximal distinction between two groups. The result is a label map (binary image of the voxel subset associated with the function variable) a belief map (label map weighted by the confidence in the voxel/function variable association) and a probability distribution. GAMMA also determines each subject's group membership using a regional state inference algorithm. The belief maps can be used to determine the group membership of other subjects who have not been part of the initial comparison. The second level Bayesian network used a priori expert knowledge about gray and white matter abnormalities in these three epilepsy types to combine the information obtained for each individual at the first level and to calculate a probability for each subject a. to be a patient, a patient with normal imaging or a healthy control. $b$. if a patient, the probability to suffer from TLE or FLE, and c. if TLE, the probability to be TLE-MTS or TLE-no.

Results: The two-level Bayesian network classified of 48 the 55 patients. $75 \%$ of those who were classified were correctly identified 7 could not be classified because they had structural abnormalities not exceeding those observed in controls. The sensitivity of the two level Bayesian network to distinguish between the three patient groups was 0.84 for TLE-MTS, 0.72 for TLE-no and 0.64 for FLE, the corresponding specificity was 0.87 for TLE-MTS and TLE-no and 0.86 for FLE.

Conclusions: The two-level multi-modality Bayesian network approach was able to distinguish between the three epilepsy types with a reasonable high accuracy even though the majority of the images were completely normal on visual inspection. It is likely that this accuracy can be further increased, e.g. by adding additional imaging information, e.g. PET or clinical and electrophysiological information. 


\subsection{1}

BILATERAL PIRIFORM REGION ATROPHY IN REFRACTORY TEMPORAL LOBE EPILEPSY WITH UNILATERAL HIPPOCAMPAL SCLEROSIS: ULTRAHIGHFIELD MR VOLUMETRY

Thomas R. Henry $^{1,2}$, S. Maiser ${ }^{1}$, M. Chupin ${ }^{3}$, S. Lehéricy ${ }^{3}$, J. Strupp ${ }^{2}$, Z. Sha ${ }^{1}$, K. Ugurbil ${ }^{2}$ and P. Van de Moortele ${ }^{2}\left({ }^{1}\right.$ Neurology, University of Minnesota, Minneapolis, MN; ${ }^{2}$ CMRR-Radiology, University of Minnesota, Minneapolis, MN and ${ }^{3}$ Université Pierre et Marie Curie-Paris, Paris, France)

Rationale: The piriform region consists of the frontal lobe piriform cortex (FPC), temporal lobe piriform cortex (TPC), and endopiriform nucleus (EPN). These sites are particularly amenable to kindling and other experimental epileptogenesis (as the "area tempestas"), and appear also to have seizure modulatory properties. The EPN is evident in human cadaver studies, but not in current clinical MRI. The FPC and TPC have rarely been studied in temporal lobe epilepsy (TLE) patients.

Methods: We identified 8 TLE subjects who had unilateral temporal lobe ictal EEG onsets ipsilateral to hippocampal atrophy on clinical MRI. With IRB-approved consenting, we acquired 7 Tesla brain MRI with T2-weighted turbo spin echo images $(0.25 \times 0.25 \times 1.2 \mathrm{~mm} 3)$ in contiguous coronal slices of 11 healthy subjects and the TLE patients. We generated a manual segmentation protocol for FPC, TPC, and EPN, based on landmarks derived from coronal histological sections in a human brain atlas.

Results: In each healthy and TLE subject the 7T images provided the requisite landmarks for segmentation of the FPC, TPC, and EPN bilaterally (including the critical demarcation of the EPN from the adjacent amygdala, which we could not achieve with clinical $3 \mathrm{~T}$ MRI).

FPC: Healthy group mean volumes: right FPC $118 \mathrm{~mm} 3$ (standard deviation $33 \mathrm{~mm} 3$ ) and left FPC $104 \mathrm{~mm} 3$ (SD $22 \mathrm{~mm} 3$ ). TLE group mean volumes: right FPC 78 mm3 (SD 19 mm3) and left FPC 74 mm3 (SD $20 \mathrm{~mm} 3$ ).

TPC: Healthy group mean volumes: right TPC $134 \mathrm{~mm} 3$ (SD 33 $\mathrm{mm} 3$ ) and left TPC $124 \mathrm{~mm} 3$ (SD $40 \mathrm{~mm} 3)$. TLE group mean volumes: right TPC $93 \mathrm{~mm} 3$ (SD $23 \mathrm{~mm} 3$ ) and left TPC $91 \mathrm{~mm} 3$ (SD $24 \mathrm{~mm} 3)$.

EPN: Healthy group mean volumes: right EPN $76 \mathrm{~mm} 3$ (SD 22 $\mathrm{mm} 3$ ) and left EPN $68 \mathrm{~mm} 3$ (SD $21 \mathrm{~mm} 3$ ). TLE group mean volumes: right EPN $50 \mathrm{~mm} 3$ (SD $8.9 \mathrm{~mm} 3$ ) and left EPN $43 \mathrm{~mm} 3$ (SD $13 \mathrm{~mm} 3$ ).

In individual TLE patients, the FPC, TPC, and EPN ipsilateral to the sclerotic hippocampus were not relatively smaller than the contralateral homologues, compared to the mean asymmetry in healthy subjects.

Conclusions: Increased spatial and contrast resolution of 7T MRI supported manual segmentation of piriform subregions. The FPC, TPC, and EPN appeared bilaterally atrophic in a relatively symmetric fashion in individual TLE patients, despite hippocampal volume asymmetry (with hippocampal atrophy ipsilateral to ictal EEG onsets), on comparisons with piriform subregional volumes in healthy subjects. Bilateral piriform regional atrophy may be due to seizure-related injury; etiological roles of piriform sites in human epileptogenesis or ictogenesis are possible. Further study will be required to determine whether bilateral piriform regional atrophy is present in a larger group of TLE-hippocampal sclerosis patients, and whether this finding is present in non-refractory TLE and in other partial epilepsies. 7T MRI can provide improved piriform subregional anatomical correlation for functional imaging studies. This study was supported by NIH P41 RR008079 \& P30 NS057091, and the Keck Foundation.

\subsection{2}

WHITE MATTER CORRELATES OF MOTOR RECOVERY IN PATIENTS AFTER HEMISPHERECTOMY

Theodor Rüber ${ }^{1}$, J. C. Schoene-Bake ${ }^{1}$, R. Lindenberg ${ }^{2}$, M. von Lehe ${ }^{3}$, C. E. Elger ${ }^{1}$ and B. Weber ${ }^{1}\left({ }^{1}\right.$ Department of Epileptology, University Hospital of Bonn, Bonn, Germany; ${ }^{2}$ Department of Neurology, Charité University Medicine, Berlin, Germany and ${ }^{3}$ Department of Neurosurgery, University Hospital of Bonn, Bonn, Germany)

Rationale: It has been hypothesized, that motor recovery after hemispherectomy is mediated by the compensatory reinforcement of contralesional uncrossed fibers of the pyramidal tract $\left(\mathrm{PT}_{\mathrm{c}}\right)$, but also of the bilaterally organized cortico-rubro-spinal system. While the importance of so-called alternate motor fibers (aMF), which most likely comprise the cortico-rubro-spinal system, has been demonstrated in motor recovery after stroke, no white matter correlates of motor recovery in patients after hemispherectomy have been observed up to now. In the current study, we used diffusion tensor imaging (DTI) to examine $\mathrm{PT}_{\mathrm{c}}$ and aMF in these patients by relating their microstructural properties to functional motor outcome. Methods: 20 patients after functional hemispherectomy (twelve women; mean age at scan $19.6 \pm 3.0$ years; mean age at surgery $10.5 \pm 5.8$ years; etiology: porencephaly, $n=10 \mid$ neurodevelopmental disorders, $n=5$ | Rasmussen's encephalitis, $n=5$ ) and ten healthy controls (six women; mean age at scan 22.1 2 .1) underwent DTI at 3 $\mathrm{T}\left(1.72 \mathrm{~mm}^{3}\right.$ voxel size; 60 directions with $\left.b=1000 \mathrm{~s} / \mathrm{mm}^{2}, 6 b 0\right)$. Patient's motor impairment of the upper extremity was assessed with Motricity Index scores (MI). Using FSL, we applied probabilistic tractography to reconstruct $\mathrm{PT}_{\mathrm{c}}$ and aMF separately (fig. 1).

Contralesional seed regions were defined in the basis pontis (for $\mathrm{PT}_{\mathrm{c}}$ ) and in the tegmentum pontis (for aMF). Two contralesional waypoint masks were defined in the internal capsule and the primary motor cortex. Tract-specific fractional anisotropy (FA) values and measures of probabilistic connectivity were extracted for statistical analyses. To specifically assess probabilistic connectivity of cortico-rubral fibers, tractography was re-run using a seed region in the contralesional red nucleus and the waypoint masks as described above.

Results: The group mean MI was $49.3 \pm 17.1$. MI were found to be inversely related to age at surgery $(r=-0.589, p=0.006)$. aMF exhibited a trend towards higher FA values $(p=0.056)$ in patients relative to controls, whereas $\mathrm{PT}_{\mathrm{c}}$ did not show any difference $(p=0.645)$. Probabilistic connectivity of cortico-rubral fibers was found to be higher in the patient group relative to controls $(p<0.001)$. Partial correlation analyses with age at surgery as control variable yielded strong positive correlations for $\mathrm{FA}_{\mathrm{P}} \mathrm{PT}_{\mathrm{c}}(r=0.650, p=0.003)$ and $\operatorname{aMF}$ ( $r=0.597, p=0.007$; fig. 2). A final stepwise regression analysis with MI as dependent variable resulted in a significant model $(\mathrm{F}(2,19)=11.714, p=0.001)$ with FA of aMF $(p=0.007)$ and age at surgery $(p=0.014)$ as predictor variables.

Conclusions: The inverse correlation between age at surgery and MI suggests that earlier surgery is related to better motor outcome. Their associations with MI indicate that observed diffusivity alterations are functionally meaningful and may represent the result of preceding compensatory remodeling. Higher probabilistic connectivity of cortico-rubral fibers as well as results of the stepwise regression analysis suggest a crucial role of the cortico-rubro-spinal system in this plastic process. This system may be targeted in future rehabilitation trials. 

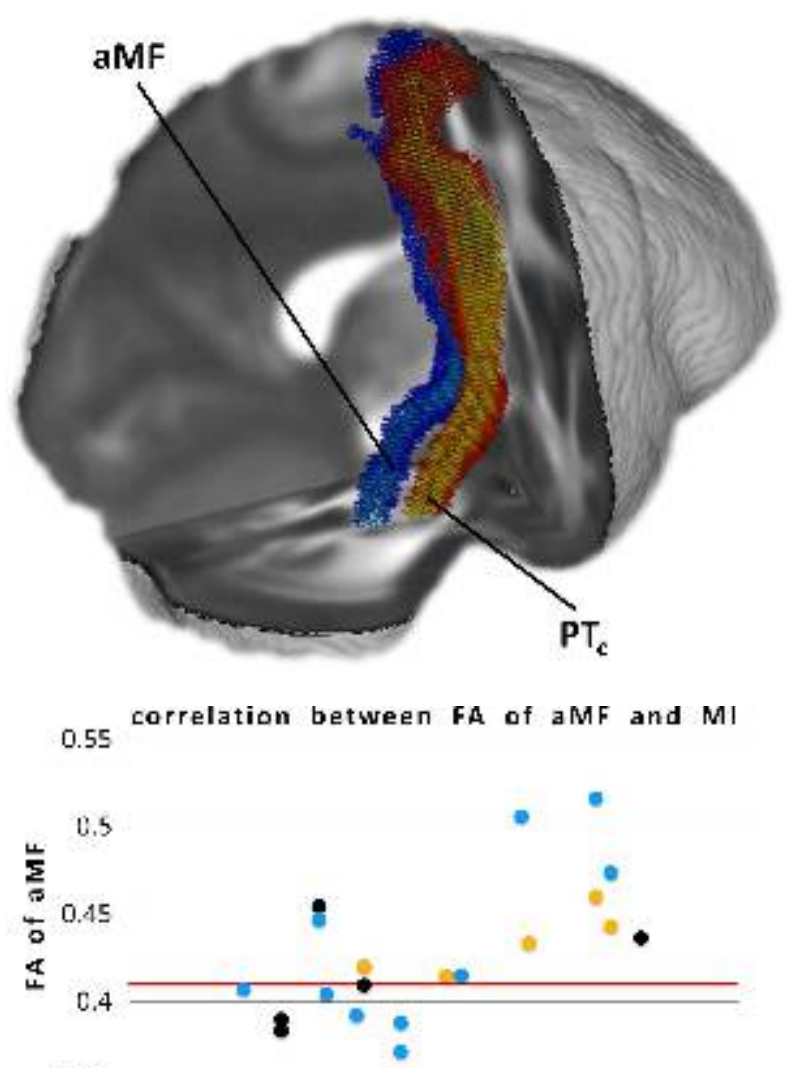

\subsection{5}

$\begin{array}{lllll}10 & 30 & 50 & 70 & 90\end{array}$

\section{MI}

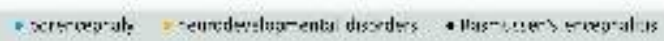

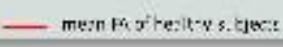

\subsection{3}

\section{REVERSIBLE AND IRREVERSIBLE CRANIAL MRI} FINDINGS ASSOCIATED WITH STATUS EPILEPTICUS Ana M. Cartagena ${ }^{1}$, G. B. Young ${ }^{1,3}$, D. H. Lee ${ }^{2}$ and S. Mirsattari ${ }^{1,2}$ ( ${ }^{1}$ Clinical Neurological Sciences, Western University, London, ON, Canada; ${ }^{2}$ Medical Imaging, Western University, London, ON, Canada and ${ }^{3}$ Critical Care Medicine, Western University, London, ON, Canada)

Rationale: Status epilepticus (SE) is a common neurological emergency for which there is limited information on resultant neuroimaging changes. The objective of this study was to characterize the abnormalities associated with SE in cranial magnetic resonance imaging (MRI) of patients with SE.

Methods: A retrospective review of our EEG database was conducted. The patients included in the EEG database were admitted between the years 2001 and 2010. In total 203 patients were identified with SE. Inclusion criteria were: (1) seizures lasting a minimum of 30 minutes or recurrent seizures without recovery in between them for at least 30 minutes including nonconvulsive electrographic seizures (2) cerebral MRI showing signal change after $\mathrm{SE}$ in sequences temporally related to seizures (3) MRI changes not attributed to an underlying primary neurological disorder including the condition that precipitated the SE. Ethics approval was obtained according to our institutional standards.

Results: Ten patients (4 female; mean age $35.4+11.96$ SD years) were suitable. MRI findings included increased T2 signal changes in the grey and/or white matter with corresponding diffusion-weighted abnormalities $(n=9)$. Apparent diffusion coefficient (ADC) values were reduced in four patients and increased in three. Findings also included changes affecting one cerebral hemisphere $(n=1)$, perilesional and homologous region $(n=1)$, hippocampi $(n=9)$, thalamus and basal ganglia $(\mathrm{n}=3)$, and brainstem and cerebellum $(n=3)$. Some signal changes were reversible and others irreversible. Conclusions: Young patients with SE showed changes on MRI that were attributable to SE and were focal, multifocal, hemispheric, and diffuse. Notably MRI changes were found beyond the hippocampi and limbic structures, involving the brainstem, cerebellum, basal ganglia and thalamus. MRI changes in the latter areas have not been previously well described and require greater attention.

Modified Results Table

\begin{tabular}{|c|c|c|c|c|c|c|c|c|}
\hline & $\begin{array}{l}\text { Age, } \\
\text { (year, } \\
\text { sexi) }\end{array}$ & Seizure type & $\begin{array}{l}\text { Duration } \\
\text { (d) }\end{array}$ & WrG lindings & Milology & $\begin{array}{l}\text { Riskevalt } \\
\text { history }\end{array}$ & $\begin{array}{l}\text { Surmunary MRI } \\
\text { findlings: }\end{array}$ & Folltwe-up MRI \\
\hline 1 & $39 \mathrm{M}$ & $\begin{array}{l}\text { GCSF, } \\
\text { NCSE }\end{array}$ & 23 & $\begin{array}{l}\text { Rilatutral kenporal } \\
\text { F'LEDS }\end{array}$ & I.nknowen & $\begin{array}{l}\text { Tnsulin } \\
\text { slepenticent } \\
\text { thabcles } \\
\text { rocllitus }\end{array}$ & 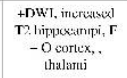 & Diftuise acriaghy \\
\hline 2 & $229 t$ & cosis & 38 & $\begin{array}{l}\text { Max lefl tempoural } \\
\text { sw, mull liple } \\
\text { independent spikes }\end{array}$ & $\begin{array}{l}\text { Unlinnown. } \\
\text { NORSE. }\end{array}$ & Voune & 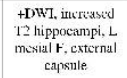 & Dillise atroghy \\
\hline 3 & $21 / \mathrm{M}$ & $\begin{array}{l}\text { Ciss: } \\
\text { NCSSI! }\end{array}$ & 21 & 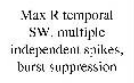 & $\begin{array}{l}\text { Unkinwn, } \\
\text { NORSI: }\end{array}$ & Nunce & 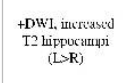 & Mininual shange \\
\hline 4 & $27 / \mathrm{M}$ & $\begin{array}{l}\text { Idlicpalthic } \\
\text { ippilicpsy }\end{array}$ & $\begin{array}{l}\text { Multiple. } \\
\text { frequent } \\
\text { episides }\end{array}$ & 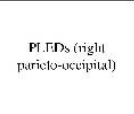 & I nknown & 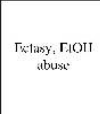 & 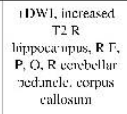 & Diffuse acroanhy \\
\hline 5 & $44 / \mathrm{M}$ & $\begin{array}{l}\text { l'IE (well } \\
\text { controlled) }\end{array}$ & 7 & - & Linknown & 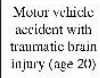 & 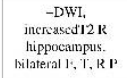 & Vol performesl \\
\hline t & $24 / \mathrm{M}$ & $\begin{array}{l}\text { PGE simlec } \\
\text { agc } 9 \text { yrs }\end{array}$ & 21 & $\begin{array}{l}\text { Rigll Licmisphenic } \\
\text { spikik:NLEDS }\end{array}$ & Linknosen & $\begin{array}{l}\text { Alcohol and } \\
\text { diug abusie } \\
\text { (hashish! }\end{array}$ & 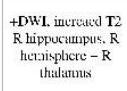 & $\begin{array}{c}\text { Initially R } \\
\text { hemispphcric } \\
\text { swelling, then R } \\
\text { WTS ant R } \\
\text { hemiatrophy }\end{array}$ \\
\hline 7 & 379 & $\begin{array}{l}\text { CIS, } \\
\text { secondarily } \\
\text { CiTes }\end{array}$ & 193 & $\begin{array}{l}\text { Weft } \\
\text { temmpron-parietal } \\
\text { seizures ant spilikes }\end{array}$ & T'apering $\mathrm{Al}: 1$ ) & $\begin{array}{c}\text { I.cet parictal } \\
\text { contical } \\
\text { dyypplasia }\end{array}$ & 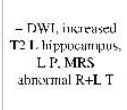 & 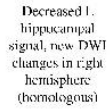 \\
\hline 8 & 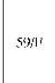 & P(I) & $<1$ & $\begin{array}{l}\text { Gencratized slowing } \\
\text { ipost-icral) }\end{array}$ & Sepsis & $\begin{array}{l}\text { ESRD (failed } \\
\text { (riansplant), } \\
\text { ('MV colitix. } \\
\text { mixed (111) }\end{array}$ & 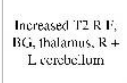 & Vol peristimed \\
\hline 9 & $41 / \mathrm{M}$ & PTE & 14 & $\begin{array}{l}\text { Righl finotial } \\
\text { polysw }\end{array}$ & Tapcrinty AED & $\begin{array}{l}\text { Irititage 15. } \\
\text { cartus } \\
\text { callositumy } \\
\text { age } 22\end{array}$ & 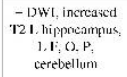 & Diffuse alroghy \\
\hline 10 & $40, \pi$ & $\begin{array}{c}\text { CPS, } \\
\text { secondarity } \\
\text { GTCS }\end{array}$ & $<1$ & $\begin{array}{l}\text { Slowing maximum } \\
\text { efl (temporal } \\
\text { (posil-ictal) }\end{array}$ & Non-compliance & $\begin{array}{c}\text { CPS age } 30, \\
\text { drug aluse in } \\
\text { past }\end{array}$ & $\begin{array}{l}\text { +Dw1, increased } \\
12 \text { hippocampi }\end{array}$ & Vol pervilimed \\
\hline
\end{tabular}

Legend: $\mathrm{EEG}=$ electroencephalogram, $\mathrm{F}=$ female, $\mathrm{M}=$ male, NCSE $=$ non-convulsive status epilepticus, GCSE $=$ generalized convulsive status epilepticus, $\mathrm{SW}=$ spike and wave, $\mathrm{PLEDS}=$ periodic lateralized epileptiform discharges, NORSE $=$ new-onset refractory status epilepticus

DWI $=$ diffusion weighted imaging

$\mathrm{PGE}=$ primary generalized epilepsy, $\mathrm{Y}=$ years, $\mathrm{PTE}=$ posttraumatic epilepsy, CPS = complex partial seizures, GTCS = generalized tonic-clonic seizures, $\mathrm{SW}=$ spike and wave, $\mathrm{AED}=$ antiepileptic drugs, PLEDS = periodic lateralized epileptiform discharges, $\mathrm{ESRD}=$ end-stage renal disease, $\mathrm{CMV}=$ cytomegalovirus, $\mathrm{CTD}=$ connective tissue disease

$\mathrm{F}=$ Frontal

$\mathrm{O}=$ Occipital

$\mathrm{T}=$ temporal

$\mathrm{P}=$ parietal

$\mathrm{R}=$ right

MTS = Mesial Temporal Sclerosis

$\mathrm{L}=$ left

MRS $=$ MR spectroscopy

$\mathrm{BG}=$ basal ganglia 


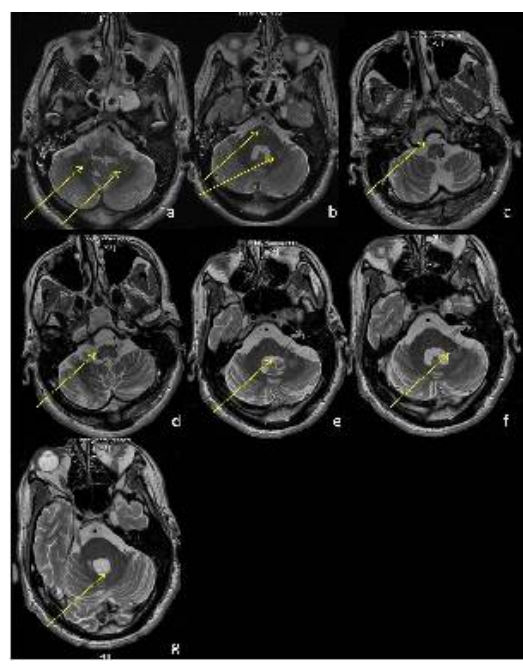

\subsection{4}

DISRUPTED ANATOMICAL WHITE MATTER NETWORK IN LEFT MESIAL TEMPORAL LOBE EPILEPSY

Min Liu ${ }^{1}$, Z. Chen ${ }^{1}$, L. Concha ${ }^{2}$, C. Beaulieu and D. W. Gross ${ }^{1}$ ( ${ }^{1}$ University of Alberta, Edmonton, AB, Canada and ${ }^{2}$ Instituto de Neurobiologia, Queretaro, Mexico)

Rationale: Recent studies on temporal lobe epilepsy (TLE) with mesial temporal sclerosis (MTS) have demonstrated widespread grey and white matter (GM/WM) abnormalities within and beyond the affected temporal lobe. However, the topological properties of the TLE anatomical brain network remain largely unknown. A recent study has shown altered network organization in TLE patients using regional cortical thickness correlation (Bernhardt BC et al, Cereb Cortex 2011, 21: 2147-57). The purpose of this study was to investigate the topological organization of the WM brain network constructed from diffusion tensor image (DTI) tractography in TLE patients with left MTS using graph theoretical analysis.

Methods: Whole brain DTI and T1 images were acquired from 15 TLE and left MTS (TLE+LMTS) patients ( $39 \pm 12$ years, 8 male) and 21 controls ( $37 \pm 12$ years, 13 male) on a $1.5 \mathrm{~T}$ Siemens scanner. Anatomical automatic labeling atlas in standard space was transformed into each individual space (SPM) and used to parcellate the cortical GM into 78 regions. Whole brain WM fibers were obtained by deterministic tractography from DTI (ExploreDTI). The number of fibers connecting any two of the 78 cortical regions and their corresponding mean fractional anisotropy was calculated to construct a weighted WM connectivity matrix $(78 \times 78)$ for each subject. Graph theoretical analysis was used to measure the network topological properties and multiple regression model was used to compare those parameters between groups controlling for age and sex. The network parameters with significant between-group differences were correlated with disease duration and age of seizure onset in patients.

Results: The WM network of the TLE+LMTS patients showed significant decreased clustering coefficient $(\mathrm{p}=0.004)$, global efficiency $(p=0.01)$ and local efficiency $(p=0.019)$ and increased characteristic shortest path length $(p=0.0004)$ versus controls. Further analysis revealed 12 cortical regions with significantly decreased regional efficiency in TLE+LMTS patients that are mainly in the left temporal lobe (inferior/middle/superior temporal gyri, temporal pole, fusiform and parahippocampal gyri) along with left inferior frontal gyrus, inferior parietal and supplementary motor area and right angular gyrus. Regional efficiency of left inferior temporal gyrus was negatively correlated with disease duration $(\mathrm{r}=-0.785, \mathrm{p}=0.002)$ and positively correlated with age of seizure onset $(\mathrm{r}=0.587, \mathrm{p}=0.045)$ and regional efficiency of left parahippocampal gyrus was positively correlated with age of seizure onset $(\mathrm{r}=0.763, \mathrm{p}=0.004)$.

Conclusions: The WM network of TLE+LMTS patients was associated with decreased local and global interconnectivity and increased cost of information propagation mainly in the ipsilateral temporal lobe as well as some areas of left frontal lobe and bilateral parietal lobes. The ipsilateral temporal lobe of patients with long disease duration and early seizure onset tend to have poorer connectivity with other brain regions. Our findings provide evidence of disrupted topological organization of the WM structural network in TLE+LMTS patients.

\subsection{5 \\ FDG-PET USING STATISTICAL ANALYSIS PROVIDES GOOD SURGICAL CANDIDATE WITH TEMPORAL LOBE EPILEPSY}

Hidenori Sugano, M. Nakajima, T. Higo, Y. Iimura and H. Arai (Juntendo University, School of Medicine, Tokyo, Japan)

Rationale: FDG-PET is one of useful evaluations to localize the epileptic areas in patients with epilepsy. In order to utilize FDG-PET more for detecting epileptic focus and predicting therapeutic outcome, statistical analysis has been indicated comparing to normal database. In this paper, we evaluate whether FDG-PET with statistical analysis is useful for predicting seizure outcome.

Methods: Patients with temporal lobe epilepsy treated at Juntendo University, Tokyo, Japan from 2006 to 2011 were enrolled in this study. They were 33, and evaluated interictal glucose metabolism using 18F-FDG-PET before surgery. After obtaining the glucose metabolism data, we performed statistical analysis (3D-SSP) using normal database. We evaluated them dividing into some areas of interest as followings, hippocampus, amygdala, uncus, parahippocampal, fusiform, T1, T2, and T3 gyruses. Areas of hypometabolism greater than 1.69 of $\mathrm{Z}$-score were accepted as statistical significant. We analyzed the relationship between the percentage of significant ROI (\%ROI) and seizure outcome (Engel's class I to class II IV) in each area. We also analyzed the correlation between \%ROI in the hippocampus to epileptic discharge (intraoperative EEG from the hippocampus) and pathological change (Watson's grading).

Results: In good surgical outcome (Engel's class I) group, \%ROIs in ipsilateral all area we interested were significantly greater than those of cotralateral side. In comparison between Engel's class I and II IV, $\%$ ROI of Engel's class I group at all area except fusiform gyrus were greater with statistical significant. We also found correlation between $\% \mathrm{ROI}$ in the hippocampus and pathological grading, but no relation between \%ROI and intraoperative IEDs.

Conclusions: FDG-PET using statistical analysis can predict the surgical outcome. Hypometabolism in mesial and lateral temporal lobe especially hippocampus and amygdala is a great predicator of good outcome. The hypometabolism in the hippocampus does not come from epileptic discharges but pathological damage of hippocampus.
1.176
TRACTOGRAPHY OF THE VISUAL PATHWAYS - CORRELATION TO AND PREDICTION OF VISUAL FIELD DEFECTS AFTER TEMPORAL LOBE RESECTION Daniel T. Nilsson ${ }^{1}$, Y. Lilja ${ }^{1}$, M. Ljungberg ${ }^{3}$, K. Malmgren ${ }^{2}$, B. Lindblom ${ }^{5}$, G. Starck ${ }^{3,4}$ and B. Rydenhag ${ }^{1}\left({ }^{1}\right.$ Dept of Neurosurgery, Institute of Neuroscience and Physiology, Epilepsy Research Group at the Sahlgrenska Academy at University of Gothenburg, Göteborg, Sweden; ${ }^{2}$ Dept of Neurology, Institute of Neuroscience and Physiology, Section of Clinical Neuroscience and Rehabilitation, Epilepsy Research Group at the Sahlgrenska Academy at University of Gothenburg, Göteborg, Sweden; ${ }^{3}$ Dept of Medical Physics and Medical Engineering, Sahlgrenska University Hospital, Göteborg, Sweden; ${ }^{4}$ Dept of Radiation Physics, Sahlgrenska Academy at University of Gothenburg, Göteborg, Sweden and ${ }^{5}$ Dept of Ophthalmology, Institute of Neuroscience and Physiology, Section of 
Clinical Neuroscience and Rehabilitation at the Sahlgrenska Academy at University of Gothenburg, Göteborg, Sweden)

Rationale: Visual field defect (VFD) occurs frequently after temporal lobe resection (TLR) for temporal lobe epilepsy (TLE), as a result of injury to the visual pathways. This study investigated if there was a correlation between perimetry results and tractography (TG) of injury to the optic radiation and whether a postoperative visual field defect could be predicted from preoperative tractography results. Methods: Diffusion tensor imaging-based probabilistic TG and perimetry was done before and after TLR in seven patients with TLE. Distances between the temporal pole and the most anterior part of the visual pathways in the temporal lobe, Meyers loop (TP-ML distance), were measured pre-and postoperatively. Visual inspection of the optic radiations pre-and postop were performed. The results were correlated to postoperative perimetry.

Results: Six of seven patients developed a postoperative VFD, 5/7 were less than a quadrant. Preoperative TP-ML varied from $19 \mathrm{~mm}$ to $40 \mathrm{~mm}$ (mean $31.7 \mathrm{~mm}$ for right side, $34.5 \mathrm{~mm}$ for the left side). Optic radiation injury could be seen in all subjects with VFD, but not in the subject with normal visual fields. Mean postoperative TP-ML was 36.9 on the operated side and $37.2 \mathrm{~mm}$ on the intact side (n.s.). No correlation between preoperative ML-TP distance and postoperative VFD was found.

Conclusions: Tractography can localize the visual pathways, and detect injury to the optic radiation causing VFD. Preoperative TG could not accurately predict VFD occurrence after standardized TLR. To minimize injury to the visual pathways, individualized mapping used intraoperatively and TLR tailored to avoid the visual pathways, including subtemporal approach, should be considered.

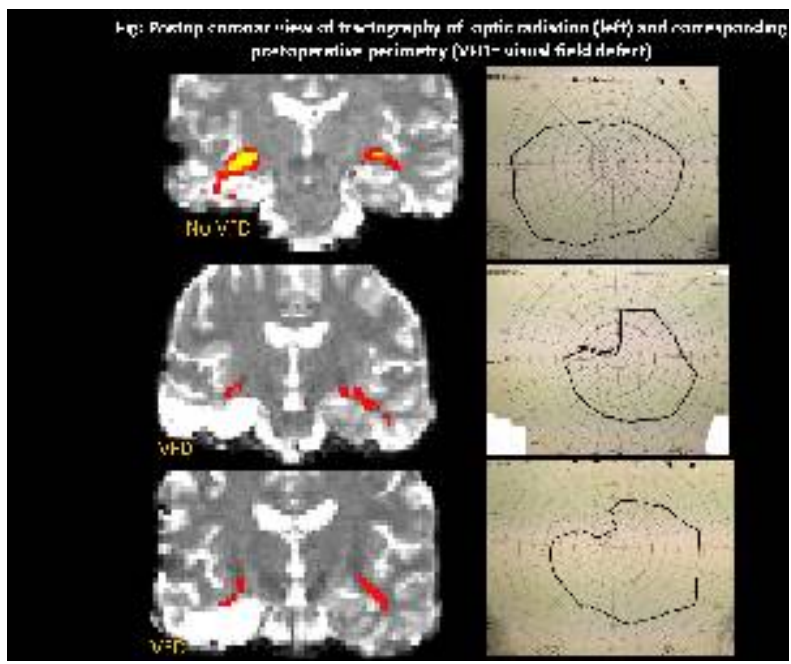

Fig: Postop coronar view of tractography of optic radiation (left) and corresponding postoperative perimetry $(\mathrm{VFD}=$ visual field defect)

\subsection{7 \\ RELAXOMETRY AND MAGNETIZATION TRANSFER IN THE HIPPOCAMPUS OF TLE PATIENTS: IN VIVO AND EX VIVO MRI INDICATE THAT FUNCTIONAL AND STRUCTURAL CHANGES INFLUENCE TISSUE MR SIGNAL \\ Jose E. Peixoto-Santos ${ }^{1}$, T. R. Velasco ${ }^{1}$, J. A. Assirati ${ }^{2}$, C. G. Carlotti $^{2}$, R. C. Scandiuzzi ${ }^{1}$, C. G. Salmon ${ }^{3}$, A. C. Santos ${ }^{4}$ and J. P. Leite $^{1}$ ( ${ }^{1}$ Neuroscience and Behavioral Sciences, University of São Paulo, Ribeirão Preto, Brazil; ${ }^{2}$ Neurosurgery, University of São Paulo, Ribeirão Preto, Brazil; ${ }^{3}$ Physics, University of São Paulo, Ribeirão Preto, Brazil and ${ }^{4}$ Internal Medicine, University of São Paulo, Ribeirão Preto, Brazil)}

Rationale: In temporal lobe epilepsy (TLE), a common type of epilepsy in adults, hippocampal changes are frequently observed in magnetic resonance imaging (MRI), such as reduced volume and increased T2-weighted signal. Ex vivo MRI studies in brain specimens have shown that the same signal alterations observed in vivo can be seen in fixed tissue. Our aim was to evaluate the hippocampus of TLE patients in vivo and ex vivo conditions using two quantitative magnetic resonance techniques ( $\mathrm{T} 2$ relaxometry and Magnetization Transfer)

Methods: Patients with TLE $(n=18)$ were scanned prior to surgical resection of the hippocampus (in vivo imaging) in a $3 \mathrm{~T}$ MRI scanner. For T2 relaxometry, spin echo images with multiples echoes ( $\mathrm{TE}=20,40,60,80$ and $100 \mathrm{~ms}$; TR $=3 \mathrm{~s}$ ) were acquired and postprocessed. Other two images, gradient echo $(\mathrm{TR}=7.3 \mathrm{~ms}, \mathrm{TE}=3.6$ $\mathrm{ms}$, with/without magnetization transfer pulse), were used to estimated the Magnetization Transfer Ratio (MTR) maps. After surgery, a $2 \mathrm{~mm}$ thick coronal section from hippocampal body was fixed for 8 days, and very similar ex vivo images were performed in the same scanner. Age-matched controls were obtained from voluntaries (radiological control, $\mathrm{RC}, \mathrm{n}=12$ ) for the in vivo measurements, and from autopsy tissues (histological and radiological control, HRC, $n=6$ ) for the ex vivo measurements. Results were considered significant at $\mathrm{p}<0.05$

Results: TLE patients presented increased T2 values (TLE $=117 \mathrm{~ms}$, $\mathrm{RC}=104 \mathrm{~ms} ;$ median, $\mathrm{p}=0.005)$ and reduced MTR $(\mathrm{TLE}=48 \pm 2$ $\%, \mathrm{RC}=50 \pm 1 \%$; mean \pm standard error, $\mathrm{p}=0.012$ ) when compared to RC. In qualitative terms, ex vivo imaging had a better anatomical definition than in vivo imaging. This better anatomical definition allowed us to identify and quantify the T2 values in hippocampal neuronal layers (NL), radiatum/oriens layers (ROL) and molecular layers of fascia dentata (ML) with great confidence. Ex vivo relaxometry revealed increased $\mathrm{T} 2$ in TLE, compared to $\mathrm{HRC}$, in the whole hippocampus (TLE $=83 \mathrm{~ms}, \mathrm{HRC}=76 \mathrm{~ms}$; median, $\mathrm{p}=$ $0.006)$ and in the subdivisions NL (TLE $=85 \mathrm{~ms}, \mathrm{HRC}=78 \mathrm{~ms}$; median, $\mathrm{p}=0.006$ ), ROL (TLE $80 \mathrm{~ms}, \mathrm{HRC}=72 \mathrm{~ms}$; median, $\mathrm{p}=$ $0.033)$ and $\mathrm{ML}(\mathrm{TLE}=84 \mathrm{~ms}, \mathrm{HRC}=78 \mathrm{~ms}$; median, $\mathrm{p}=0.008)$. No correlation was observed between in vivo and ex vivo conditions for $\mathrm{T} 2$ values in the whole hippocampus neither in the several regions Conclusions: Ex vivo acquisitions provided an excellent anatomical imaging of the hippocampus. In vivo and ex vivo T2 values were increased in TLE patients, but no correlation was observed between both conditions. While the alterations in the in vivo $\mathrm{T} 2$ are related to gliosis and tissue edema, the ex vivo alteration must be purely structural, since no edema remains after the fixation process. These differences between the substrates related to in vivo and ex vivo signals could be responsive to the lack of correlation in the relaxometry technique. Taken together, our data suggest that structural and functional changes account for MR signal, and ex vivo MRI can identify some of these alterations

\subsection{8}

TBSS ANALYSIS OF DIFFUSION TENSOR IMAGING IN CRYPTOGENIC WEST SYNDROME : COMPARISON WITH EEG AND SEIZURE OUTCOME

Jun Natsume ${ }^{1}$, T. Fukasawa ${ }^{1}$, H. Yamamoto ${ }^{1}$, N. Ishihara ${ }^{1}$, T.

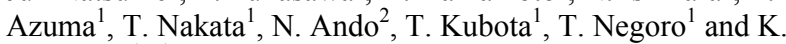
Watanabe ${ }^{1}\left({ }^{1}\right.$ Department of Pediatrics, Nagoya University Graduate School of Medicine, Nagoya, Japan and ${ }^{2}$ Department of Neonatology and Pediatrics, Nagoya City University Graduate School of Medical Sciences, Nagoya, Japan)

Rationale: Cryptogenic West syndrome (C-WS) has normal development before the onset, and no structural abnormalities are observed on conventional MRI. However, seizure and developmental outcome is very diverse. Diffusion tensor imaging (DTI) is an MRI technique that can provide information about white matter fiber orientation and integrity. We prospectively performed DTI to assess white matter abnormalities in patients with C-WS. The results were compared with EEG abnormalities and seizure outcome. 
Methods: We studied 14 consecutive patients with C-WS. DTI was acquired using 3T MRI (3.0T Trio, Siemens) at 12 months of age, and fractional anisotropy (FA) images were constructed. FA images in patients were compared with those of 12 controls. Statistical analysis of FA images was carried out using TBSS implemented in FSL (The Oxford FMRIB Software Library). TBSS projects all subjects' FA data onto a mean FA tract skeleton, before applying voxelwise cross-subject statistics. Individual FA data were then projected onto the skeleton, and then correction for multiple comparisons was performed. Regions with significant differences were identified with threshold: $\mathrm{p}<0.05$. Then we calculated the value of FA, and axial, radial, and mean diffusivity in the skeleton with significant difference on TBSS. We compared the each parameter between patients with and without EEG abnormalities at 12 months of age.

Results: The patients showed significantly decreased FA in corpus callosum, deep white matters, and fronto-temporal white matters. Radial diffusivity was increased in the skeleton with significant difference. Seven patients showed EEG abnormalities at 12 months, and 5 of them had relapse of seizures. Patients with EEG

abnormalities had lower FA and higher radial diffusion than those of patients with normal EEG at 12 months.

Conclusions: Decreased FA and increased radial diffusion were observed in C-WS, especially with EEG abnormalities and seizures after initial treatments. Increased radial diffusion may reflect delayed myelination or white matter damage by epileptic activities. Persistent EEG abnormalities and seizures may affect white matter maturation in C-WS.

\subsection{9 \\ MORE EXTENSIVE ABNORMAL WHITE MATTER THAN GRAY MATTER IN CHILDREN WITH FRONTAL LOBE EPILEPSY}

Elysa Widjaja, A. Kis, C. Go, O. C. Snead and M. L. Smith (Hospital for Sick Children, Toronto, ON, Canada)

Rationale: We hypothesized there was impaired white matter (WM) and gray matter (GM) integrity in children with frontal lobe epilepsy (FLE), with more extensive WM abnormality than GM abnormality. The aim of this study was to assess WM integrity using DTI and GM integrity using cortical thickness in children with FLE.

Methods: 43 patients with FLE, mean age 13.2 years, were recruited. All patients had normal MRI. 28 patients had left FLE and 15 had right FLE. The control group consisted of 44 age-matched healthy subjects, mean age of 12.9 years, without neurological or psychiatric disorders and had normal MRI. DTI and volumetric T1-weighted imaging were acquired on $3 \mathrm{~T}$ in both patients and controls. DTI was analyzed using FreeSurfer, corrected for multiple comparisons. Cortical thickness was also analyzed using Freesurfer, corrected for multiple comparisons using false discovery rate (FDR).

Results: (i) DTI

(a) Left FLE: There was significant reduction in fractional anisotropy (FA) in corpus callosum, right precentral and insular WM, and significant increase in apparent diffusion coefficient (ADC) in brainstem. There was a significant increase in axial diffusivity (AD) in brainstem, and decrease in $\mathrm{AD}$ in corpus callosum and right angular WM. Significant increases in radial diffusivity (RD) were identified in corpus callosum, left internal capsule, left postcentral $\mathrm{WM}$, brainstem, and right supramarginal, insular and precentral WM after correction for multiple comparisons.

(b) Right FLE: There was significant reduction in FA in corpus callosum, right posterior cingulate and angular WM, right thalamus, right and left internal capsule, left supramarginal WM, left external capsule, and brainstem. There was significant elevation in ADC in corpus callosum; right isthmus cingulate, postcentral WM and thalamus, right and left supramarginal WM, left parahippocampal WM, brainstem, and cerebellum. There was significant decrease in $\mathrm{AD}$ in right angular and precuneus WM and right internal capsule, and increase in $\mathrm{AD}$ in right posterior cingulate, right thalamus, brainstem, and cerebellum. Significant increases in RD were identified in corpus callosum, right temporal stem, right pre and post central and angular WM and right optic radiation; right and left internal capsule, anterior cingulate, superior parietal, and parahippocampal WM; left superior frontal and supramarginal WM, left external capsule, left thalamus, brainstem, and cerebellum after correction for multiple comparisons.

(ii) Cortical Thickness

(a) Left FLE: There was significant reduction in cortical thickness in the left superior frontal and precentral cortex, right superior frontal, precentral, and inferior temporal cortex (FDR corrected).

(ii) Right FLE: There was significant reduction in cortical thickness in the right middle frontal and left lateral occipital cortex (FDR corrected).

Conclusions: We have found widespread areas of impaired WM and GM integrity, with more regions of abnormal WM than GM in children with FLE. RD and AD were more sensitive markers of impaired WM integrity than FA and ADC in children with FLE.

\subsection{0 \\ THE CLINICAL AND NEUROIMAGING FINDINGS OF POSTERIOR REVERSIBLE ENCEPHALOPATHY SYNDROME IN CHILDREN}

Hiroyuki Yamamoto ${ }^{1}$, J. Natsume ${ }^{1}$, T. Takeuchi ${ }^{1}$, T. Nakata $^{1}$, N. Ishihara $^{1}$, T. Negoro ${ }^{1}$ and K. Watanabe ${ }^{2}{ }^{1}$ Pediatrics, Nagoya University Graduate School of Medicine, Nagoya, Japan and ${ }^{2}$ Faculty of Medical Welfare, Aichi Shukutoku University, Nagakute, Japan)

Rationale: Posterior reversible encephalopathy syndrome (PRES) is defined by a potentially reversible vasogenic edema with a predilection of parenchyma supplied by the posterior circulation. The major symptoms of PRES are headache, decreased alertness, cortical blindness and seizures. Although clinical and radiologic findings have been well recognized, atypical imaging findings and nonreversible clinical course were increasingly reported, including subsequent epilepsy. In order to better understand the radiological and clinical spectrum of PRES in children, we conducted a retrospective multicenter study.

Methods: We studied 30 patients who were diagnosed with PRES at pediatric departments of 13 affiliated hospitals of Nagoya university and Nagoya City University from 1999 to 2012. Child neurologists reviewed the clinical charts and neuroimaging findings. We investigated the underlying disorders, previous use of immunosuppressive agents, clinical symptoms, blood pressure at the onset, and brain MRI findings. We also reviewed the presence of neurological sequelae, epilepsy and nonreversible MRI lesions during follow-up period.

Results: The age of the patients at the onset ranged from 3 to 22 years of age (median 8 years). Underlying disorders were neoplasm in 19 patients, renal disease in 8 , collagen disease in 3 , and the others (bronchial asthma, congenital immunodeficiency, hemophagocytic syndrome). Although 29 patients had high blood pressure at the onset, the degree of the hypertension was variable. Twenty three patients had seizures, 19 had alteration of consciousness, 6 had headache and 4 had visual disturbances. The MRI lesions were observed in occipital lobes in 29 patients, parietal lobes in 22, frontal lobes in 6, and temporal lobes in 6. Five patients had MRI lesions in cerebellum and 1 had in thalamus and basal ganglia. Apparent diffusion coefficient (ADC) map was constructed in MRI studies of 26 patients, and 6 of them revealed decreased ADC values at the lesions. Twenty four patients had follow-up MRI studies, and 5 of them had focal gliosis and cortical atrophy. In 6 patients with ADC reduction during the acute period, 4 patients had follow-up studies and 3 of them had gliosis and atrophy. On the other hand, all patients with normal or increased ADC values during acute period showed no abnormalities on the follow-up MRI. During follow-up period, 4 patients had focal epilepsy. Three of them had decreased ADC values 
at the onset. Two patients had occipital lobe epilepsy, and epilepsy focus was unknown in the other 2.

Conclusions: While high ADC values suggest reversible vasogenic edema, decreased ADC values indicate irreversible cytotoxic edema. Evaluation of ADC values on MRI during acute period has a good predictive value for nonreversible MRI lesions and subsequent onset of epilepsy.

\subsection{1 \\ LATERALIZATION OF MEG-NEGATIVE MESIAL TEMPORAL LOBE EPILEPSIES USING QUANTITATIVE HIPPOCAMPAL FLAIR ANALYSIS: THE CCF EXPERIENCE}

Z. I. Wang ${ }^{1}$, Z. Jaisani ${ }^{1}$, R. Prayson ${ }^{2}$, S. E. Jones ${ }^{3,1}$, I. M. Najm ${ }^{1}$ and A. V. Alexopoulos ${ }^{1}\left({ }^{1}\right.$ Epilepsy Center, Cleveland Clinic Foundation, Cleveland, $\mathrm{OH} ;{ }^{2}$ Anatomic Pathology, Cleveland Clinic Foundation, Cleveland, $\mathrm{OH}$ and ${ }^{3}$ Diagnostic Radiology, Mellen Center, Cleveland Clinic Foundation, Cleveland, $\mathrm{OH}$ )

Rationale: High resolution MRI has revolutionized the identification and surgical treatment of patients with mesial temporal lobe epilepsy (mTLE). Hippocampal sclerosis (HS) on MRI usually presents with atrophy of the hippocampus and hyper-intensity in FLAIR. However another group of temporal lobe epilepsy patients exists, whose HS are so subtle that the MRIs are completely negative (MRI-). In these cases, an objective, quantitative MRI post-processing technique that could help lateralize the pathological hippocampus would be very desirable. The aim of our study is to apply a recently published quantitative hippocampal FLAIR analysis in a strictly defined cohort of MRI-negative, pathologically verified mTLE patients.

Methods: From the surgical series of Cleveland Clinic Epilepsy Center between 2002.1 and 2011.12, patients were included if they:

1) underwent anterior temporal lobe resection with mesial structures; 2) were considered MRI- prior to surgery, and (3) HS pathologically verified. All MRI scans had been evaluated prospectively by dedicated epilepsy neuroradiologists at the time of pre-surgical evaluation, and evaluated again with epileptologists during patient management conference, where results of all noninvasive investigations were considered. For a strictly defined MRI- cohort, we only selected patients who were still considered MRI- after the two-step reviewing process. Nine patients fulfilled all requirements and were analyzed using a novel MRI post-processing technique for automated quantitative hippocampal FLAIR analysis based on algorithms of SPM5 (Huppertz et al., Epilepsy Res. 2011). The T2 FLAIR images underwent spatial and intensity normalization, and were thresholded by a probabilistic hippocampal mask. The resulting left and right hippocampal images were then calculated for the mean voxel intensity and compared to each other. This analysis was performed on upper quartile of voxels belonging to each hippocampus. Lateralization from the analysis was then compared to histopathology findings by careful re-review of the surgical pathology slides.

Results: Out of the 9 patients analyzed, 4 had right-sided HS and 5 had left-sided HS according to histopathology. One patient had coexisting FCD Ia in the neocortical temporal structure. Actual value of the average hippocampal intensity can be quite variable among the patients. However, in all 9 patients, the side of hippocampus with higher average intensity correlates well with pathology confirmation (Figure 1).

Conclusions: Improving noninvasive lateralization of MRI-negative mTLE cases is critical to management of the epilepsy. Our experience using the automated quantitative FLAIR analysis in a strictly defined MRI-negative cohort supports that this technique can be a valuable additional tool to the presurgical evaluation of suspected mTLE patients with negative or questionable MRI findings. Further refinement of this technique would involve generation of a normative scanner-specific database so that descriptive statistics can be calculated.
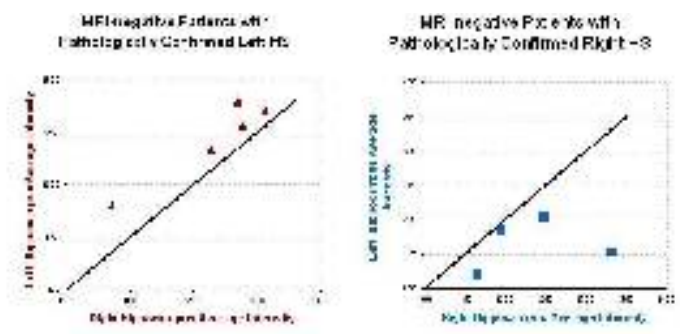

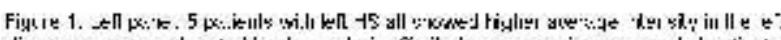

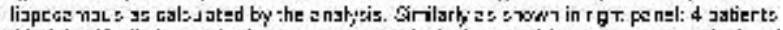

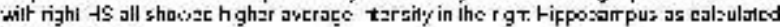
ry Ita anaky:

\section{Neuro Imaging: Functional Imaging}

\subsection{2 \\ IMPROVED LOCALIZATION OF EXTRATEMPORAL ICTAL ONSET-ASSOCIATED BLOOD FLOW CHANGES USING A 72-DETECTOR FOCUSED COLLIMATOR RING SPECT SCANNER SYSTEM \\ Marvin A. Rossi ${ }^{1}$ and K. Krug ${ }^{2}\left({ }^{1}\right.$ Rush University Medical Center, Chicago, IL and ${ }^{2}$ BluePointDX, Boston, MA)}

Rationale: High resolution (3-4 mm voxel) single-photon emission computed tomography (SPECT) has been recently developed using a 72-detector focused collimator ring scanner system (inSpira, NeuroLogica Corp). The goal of this study is to demonstrate the maximal extent of transient hyperperfusion-related changes within the ictal onset zone in extratemporal epilepsy, not seen with a conventional 2-headed detector SPECT scanner (Siemens). Methods: Eight subjects with medically refractory extratemporal epileptic sources were enrolled in this investigator-initiated IRBapproved study prior to resection. Injection of SPECT radiotrace, $99 \mathrm{mTc}-\mathrm{HMPAO}$ (Ceretec) was successfully completed at the ictal onset (mean time to injection from clinical or electrocerebral seizure onset $=32 \mathrm{sec}+/-8 \mathrm{sec}$ ). A baseline SPECT was acquired following about a 24 hour seizure-free period. Ratio ictal SPECT co-registered to MRI (RISCOM) was performed using the SPECT datasets for each patient. The data were normalized and processed using custom software utilities. These data were co-registered to each subject's gapless SPGR MRI study using the ITK co-registration algorithm and visualized (MIM Software). Subtraction ictal SPECT co-registered to MRI (SISCOM) was also performed for each subject acquired with the inSpira scanner system and a Siemens 2-detector SPECT scanner using Analyze v10 (AnalyzeDirect). For each patient, RISCOM and SISCOM analyses of the extratemporal ictal onset zones using the 72-detector scanner were compared with datasets acquired with the Siemens SPECT scanner. These data were validated utilizing chronic electrocorticography capturing stereotypic seizures prior to resection. Results: RISCOM datasets using the 72-detector system demonstrated a concordant overlap of transient regions of hyperperfusion seen with the SISCOM data obtained with the 2detector SPECT scanner. However, the InSpira RISCOM data demonstrated regions of hyperperfusion not seen with the SISCOM datasets. For 6 of the 8 patients, these new regions of hyperperfusion were concordant with chronic electrocorticography and included in the volume of resection. All 8 patients demonstrated an Engel's class I-II outcome after a mean of 9-12 months post-resection.

Conclusions: RISCOM image processing using the 72-detector ring collimator SPECT scanner facilitated identification of the ictal onset in extratemporal epileptic circuits not visualized with conventional 2detector SPECT scanner technology. Application of this novel SPECT technology can facilitate clarifying the extent of the ictal onset zone for strategic placement of chronic intracranial electrodes. 
1.183

LOCALIZING THE ICTAL ONSET ZONE WITH DATADRIVEN FMRI ANALYSIS

Borbala Hunyadi $^{1}$, S. Tousseyn ${ }^{2}$, M. De $\operatorname{Vos}^{3,1}$, S. Van Huffel ${ }^{1}$ and W. Van Paesschen ${ }^{2}\left({ }^{1}\right.$ ESAT-SISTA,Department of Electrical Eningeering, KU Leuven, Leuven, Belgium; ${ }^{2}$ Department of Neurology, University Hospital Gasthuisberg, Leuven, Belgium and ${ }^{3}$ Neuropsychology Lab, Department of Psychology, University of Oldenburg, Oldenburg, Germany)

Rationale: The spatial resolution of fMRI allows the accurate localization of functional regions in the brain. Simultaneously recorded EEG-fMRI can be used to localize hemodynamic changes corresponding to interictal epileptic discharges (IEDs) observed on EEG. However, as interictal activity is not always visible on scalp EEG due to the presence of artifacts, being generated by deep brain sources, or the limited time of the acquisition, there is an increasing demand for techniques capable of localizing epileptic activity based solely on the fMRI time series.

Methods: Our study includes 20 refractory focal epilepsy patients who underwent full presurgical evaluation including ictal single photon emission computed tomography (SPECT). The ictal onset zone (IOZ) was determined by a neurologist as the overlap between the subtraction ictal SPECT co-registered to MRI (SISCOM) hyperperfusion map $(\mathrm{Z}>1.5)$ and the effective or intended resection zone.

Functional images were acquired using a 3T MR scanner with a whole brain single-shot T2* gradient-echo Echo Planar Imaging sequence $(\mathrm{TE}=33 \mathrm{~ms}, \mathrm{TR}=2.5 \mathrm{~s} / 2.25 \mathrm{~s} / 2.2 \mathrm{~s}$, voxel size $2.6 / 3 / 2.6$ $\mathrm{mm}$ ). The images were realigned, slice-time corrected, normalized to MNI space and spatially smoothed with an isotropic Gaussian kernel of $6 \mathrm{~mm}$ FWHM using SPM8 software (Wellcome Department of Cognitive Neurology). Scalp EEG was simultaneously recorded with a 64 or 32 channel MR-compatible EEG cap (Brain Products) or a 24 channel electrode set (Yves EEG solutions inc). The EEG signals were amplified (BrainAmp amplifier, sampling rate $5000 \mathrm{~Hz}$, resolution $0.5 \mu \mathrm{V}$ ) and transmitted outside the scanning room. The EEG was filtered offline between $1-50 \mathrm{~Hz}$, gradient and pulse artifacts were removed.

2 patient groups were defined based on whether clinically concordant interictal spikes were seen on the EEG or not. Group 1 contained 10 patients with clinically concordant spikes, and Group 2 contained 10 patients where no such spikes were seen.

Spatial independent component analysis (ICA) was performed on the preprocessed fMRI time series to obtain spatially independent activation maps. The component activation maps were converted to z-scores, thresholded at $\mathrm{z}>3$ and their overlap with the IOZ was calculated.

GLM-based IED-correlated fMRI activation maps of Group 1 patients were also obtained using SPM8, and the overlap between these activation maps and the IOZ was calculated.

Results: Both the GLM-based activation map and at least one ICAbased component activation map overlapped with the IOZ in all patients in Group 1 (Figure 1). We show that the ICA-based maps overlap more with IOZ in 8 out of 10 patients than the GLM-based maps do. Moreover, at least one ICA-based component activation map overlapped with the IOZ in all patients in Group 2 as well

\section{(Figure 2).}

Conclusions: ICA is capable of deriving activation maps from the fMRI time series which overlap more precisely with the IOZ than the GLM-based activation maps. Furthermore, ICA also finds such activation maps in patients, where the traditional GLM-based analysis can not be performed due to the lack of clinically concordant IEDs on the EEG.

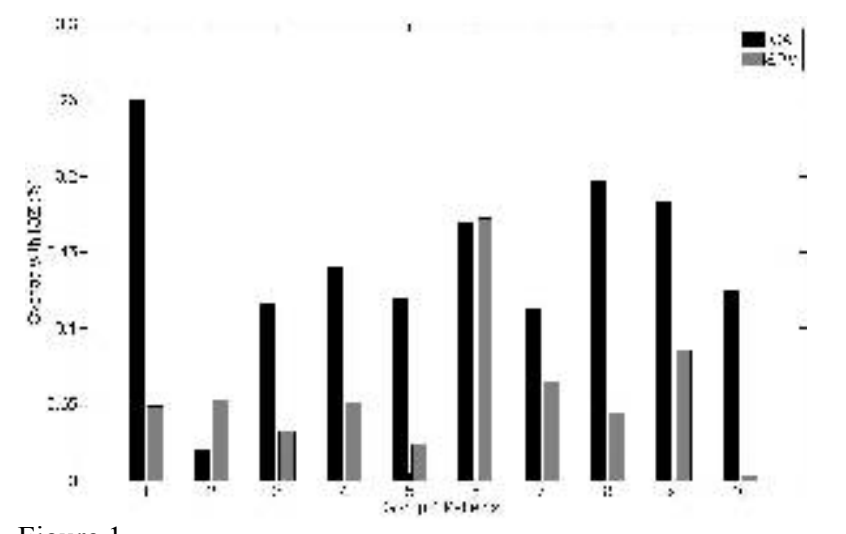

Figure 1.

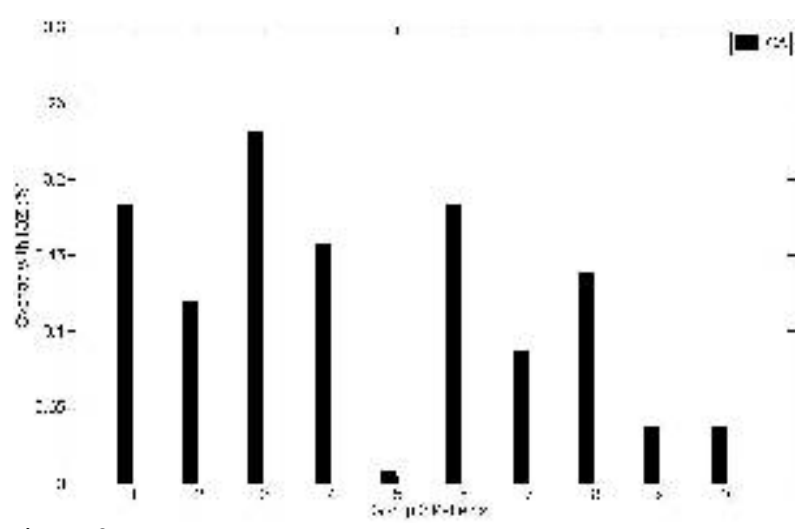

Figure 2.

\subsection{4}

INCREASED NEURAL RESPONSE TO STRESS IN TEMPORAL LOBE EPILEPSY PATIENTS WHO BELIEVE STRESS AFFECTS THEIR SEIZURE CONTROL

Jane B. Allendorfer ${ }^{1,2}$, H. Heyse ${ }^{1}$, E. B. Nelson ${ }^{3}$, L. Mendoza ${ }^{1}$ and J. P. Szaflarski ${ }^{1,2}$ ( ${ }^{1}$ Neurology, University of Cincinnati Academic Health Center, Cincinnati, OH; ${ }^{2}$ Neurology, University of Alabama at Birmingham, Birmingham, AL and ${ }^{3}$ Psychiatry and Behavioral Neuroscience, University of Cincinnati Academic Health Center, Cincinnati, OH)

Rationale: Epilepsy and stress go hand in hand, but our understanding of this relationship in humans is lacking. Our objective was to assess the neural response to psychosocial stress during fMRI in patients with left temporal lobe epilepsy (LTLE).

Methods: Included in the study were 16 LTLE patients who believe that stress is a factor in their seizure control (+S) and 7 who do not ($\mathrm{S})$. The stress paradigm is based on the Montreal Imaging Stress Task, which increased the stress hormone cortisol during fMRI in a group of healthy subjects (Dedovic et al., 2005). The paradigm consists of (1) a control task in which subjects performed simple subtraction and (2) a stress task in which subjects performed difficult subtraction. Subjects were given positive feedback during the control task and negative feedback during the stress task regardless of performance. MRI data processing (image coregistration, motion correction, spatial normalization), subject- and group-level statistics and visualization were performed using AFNI. General linear model (GLM) was used to assess each subject's neural response to stress by comparing stressful and non-stressful conditions (e.g., difficult vs. easy math problems and negative vs. positive feedback). 1-sample ttests of GLM results were performed to determine overall patterns of stress-related activation for each group.

Results: Both $+\mathrm{S}$ and $-\mathrm{S}$ groups were more accurate and responded faster for math problems during the control task compared to the stress task (all $\mathrm{p}<.0005$ ), but the groups did not differ in accuracy or response times for math problems during either task (all $\mathrm{p}>.12$ ). 
Increased activation was observed in $+\mathrm{S}$ bilaterally in the superior temporal gyrus (STG) in response to difficult compared to easy math problems (corrected $\mathrm{p}<.05$; voxelwise $\mathrm{p}<.005$, cluster $>18$ voxels). Increased activation also occurred for $+\mathrm{S}$ in the left insula and bilaterally in the STG, Brodmann area 39 and posterior cingulate in response to negative feedback compared to positive feedback (corrected $\mathrm{p}<.05$; voxelwise $\mathrm{p}<.005$, cluster $>18$ voxels). Increased activation to the stressful compared to non-stressful conditions were not observed in the $-\mathrm{S}$ group.

Conclusions: The $+\mathrm{S}$ group showed an overall increased response to stressful task conditions than the -S group despite similar behavioral performance between the two. The increased activation in the $+\mathrm{S}$ group to the stressful compared to non-stressful conditions may be part of a compensatory mechanism in order to achieve similar task performance as the $-\mathrm{S}$ group. These data support a relationship between the perceived importance of stress in seizure control and brain activation to stressful stimuli among patients with LTLE. Group differences in brain activation patterns may also be related to seizure frequency and requires further study.

\subsection{5 \\ ABNORMAL STRUCTURAL AND FUNCTIONAL CONNECTIVITY IN A SPECIFIC THALAMOCORTICAL CIRCUIT IN JUVENILE MYOCLONIC EPILEPSY}

Jonathan O'Muircheartaigh ${ }^{1,2}$, C. Vollmar ${ }^{3,4}$, G. Barker $^{1}$, V. Kumari ${ }^{5}$, M. Symms ${ }^{4}$, P. Thompson ${ }^{4}$, J. Duncan ${ }^{4}$, M. Koepp ${ }^{4}$ and M. Richardson ${ }^{6}\left({ }^{1}\right.$ Department of Neuroimaging, King's College London, Institute of Psychiatry, London, United Kingdom; ${ }^{2}$ Department of Engineering, Brown University, Providence, RI; ${ }^{3}$ Epilepsy Centre, Department of Neurology, University of Munich, Munich, Germany; ${ }^{4}$ Department of Clinical and Experimental Epilepsy, UCL Institute of Neurology, London, United Kingdom; ${ }^{5}$ Department of Psychology, Institute of Psychiatry, King's College London, London, United Kingdom and ${ }^{6}$ Department of Clinical Neuroscience, Institute of Psychiatry, King's College London, London, United Kingdom)

Rationale: Juvenile Myoclonic Epilepsy (JME) is the most common idiopathic generalised epilepsy (IGE), characterised by frequent myoclonic jerks, generalised tonic-clonic seizures and, less commonly, absences. Neuropsychological and, less consistently, anatomical studies have indicated frontal lobe dysfunction in the disease (e.g. Ronan et al, 2011). However, IGEs probably involve large-scale network phenomena rather than localised regional abnormalities (Avanzini et al, 2012). Given its assumed thalamocortical basis, here we investigated thalamo-cortical structural and functional network connectivity in JME.

Methods: Structural connectivity, as measured by diffusion tensor imaging (DTI) tractography, was assessed in a cohort of patients with JME ( $n=28, M: F 12: 16$, mean age 33.61 years) compared to a large sample of healthy controls ( $n=38, M: F 17: 21$, mean age 31.74 years). White matter bundles passing through the thalamus were parcellated using independent component analysis (ICA; O'Muircheartaigh et al, 2011) and the resulting bundles were compared. From regions of reduced thalamocortical structural connectivity, task modulated functional connectivity from the thalamus was then investigated using psychophysiological interactions (PPI, Friston et al, 1997) during a verbal fluency fMRI task to investigate the effect of abnormal structural connectivity on neuropsychological function. Results: We found reduced representation of an anterior thalamocortical bundle in JME (figure 1, $\mathrm{p}<0.05$, corrected for 27 multiple comparisons using the Bonferroni method). We then investigated task-modulated functional connectivity using fMRI from the same anterior thalamic region. We demonstrated an alteration in task-modulated connectivity in the JME group in a region of frontal cortex directly connected to the thalamus via the same anatomical bundle (figure 2; $<<0.05$ corrected for multiple comparisons across the whole brain using cluster correction). Functional connectivity with the thalamus in this medial frontal region was increased during the phonemic fluency task in JME compared with controls. Connectivity of this region correlated with the number of generalised seizures in the previous year $(\mathrm{p}<0.05)$. Connectivity also related to out-of-scanner performance in verbal fluency differentially between JME patients with and without seizures (figure 2, panels b and c). Conclusions: By integrating methods examining structural and effective inter-regional connectivity, these results provide convincing evidence for abnormalities in a specific thalamo-cortical circuit, with reduced structural and task-induced functional connectivity, which may underlie the neuropsychological changes in JME.
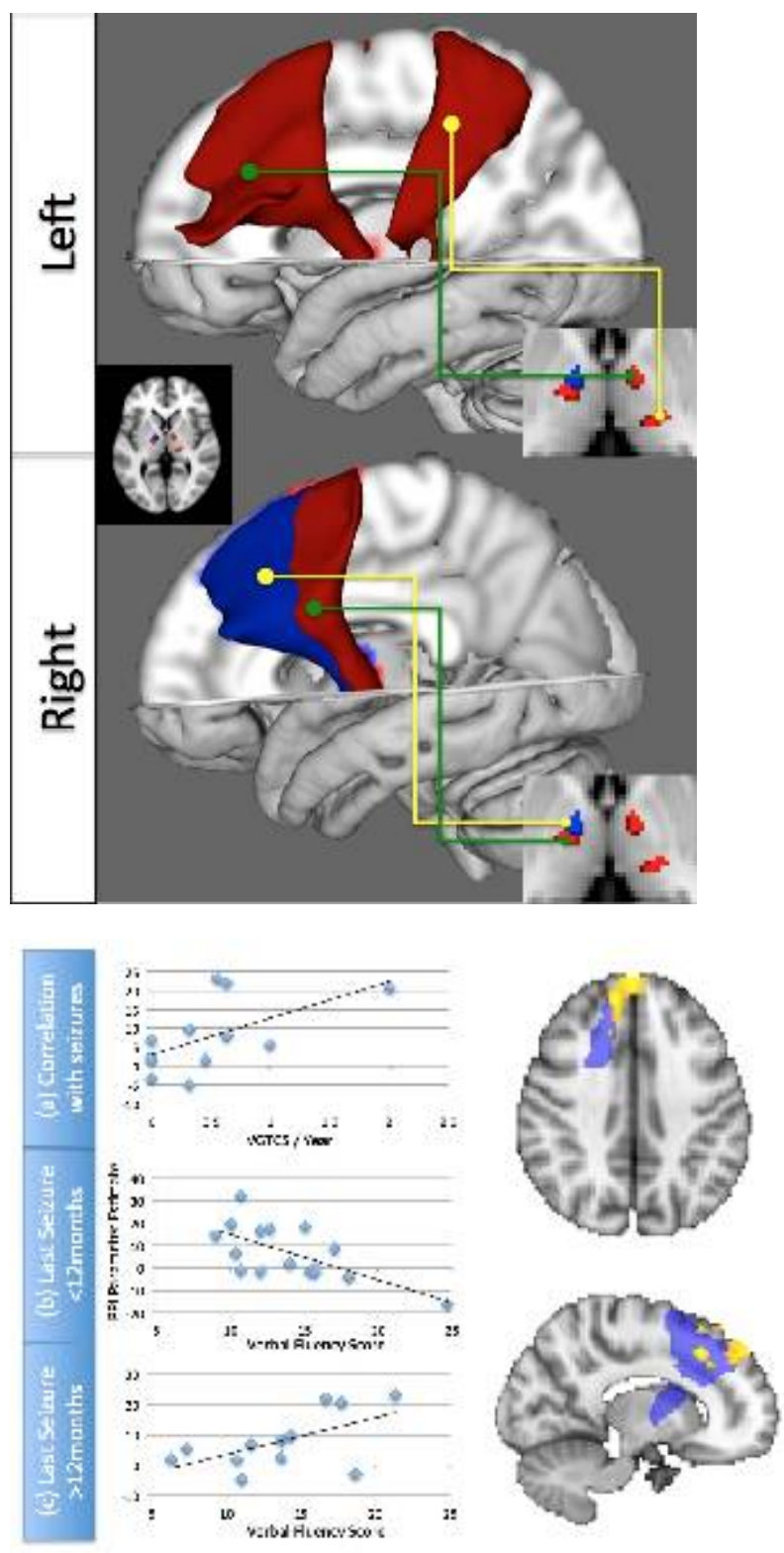

\subsection{6 \\ EEG-FMRI IN THE PRE-SURGICAL EVALUATION OF TEMPORAL LOBE EPILEPSY PATIENTS}

Ana C. Coan ${ }^{1}$, U. J. Chaudhary ${ }^{2}$, B. M. Campos ${ }^{1}$, S. Perani ${ }^{2}$, R. Thornton $^{1}$, S. Vulliemoz ${ }^{4}$, F. Grouiller ${ }^{4}$, G. C. Beltramini ${ }^{3}$, B. Diehl ${ }^{2}$, C. Scott ${ }^{2}$, R. Covolan ${ }^{3}$, F. Cendes ${ }^{1}$ and L. Lemieux ${ }^{2}$ ( ${ }^{1}$ Neuroimaging Laboratory, Department of Neurology, State University of Campinas, UNICAMP, Campinas, Brazil; ${ }^{2}$ Department of Clinical and Experimental Epilepsy, Institute of Neurology, University College London, Queen Square, Epilepsy Society, London, United Kingdom; ${ }^{3}$ Neurophysics Group, Gleb Wataghin Physics Institute, State University of Campinas, UNICAMP, Campinas, Brazil and ${ }^{4}$ Presurgical Epilepsy Evaluation Unit and Functional Brain Mapping 
Laboratory, Neurology Department, University of Geneva, Geneva, Switzerland)

Rationale: Temporal lobe epilepsy (TLE) presents with a high percentage of refractoriness and for these patients a thorough investigation is necessary to define the epileptogenic zone for surgical treatment[1]. Simultaneous EEG-fMRI has been proposed as a non-invasive pre-surgical evaluation tool for patients with focal refractory epilepsy[2,3]. However, the technique's specific role in different types of focal epilepsy remains to be determined. In this study we tried to evaluate the value of EEG-fMRI as a pre-surgical evaluation tool in patients with refractory TLE and assess its potential post-surgical outcome prediction value.

Methods: Fifty-eight patients with diagnosis of refractory TLE and investigated for epilepsy surgery were invited to undergo EEG-fMRI between July/2007 and March/2012. All patients underwent 20-48 minutes EPI sequences in 1.5 or 3T MRI scanners. EEG was acquired using a cap with $64 \mathrm{MR}$-compatible electrodes and corrected for gradient and pulse-related artifacts. fMRI data was analyzed with SPM8. Spikes were identified on the scanner EEG and used to build a model of BOLD changes in a general linear model (GLM). In addition the cross-correlation between the EEG topographic map calculated from the average of spikes recorded during long term video telemetry outside the scanner and the map at each scanner EEG time point was added as a condition[4], following convolution with a haemodynamic kernel. Thus it allowed the analysis of all data irrespective of the presence or absence of spikes on the scanner EEG. The degree of concordance of the EEG-fMRI maps was defined according to the presence of the global statistical maximum BOLD voxel in relation to the temporal lobe ipsilateral to the resected area (Concordant); the presence of a significant cluster in the temporal lobe ipsilateral to the resected area but with the global maximum in a different region (Some Concordance); or no significant cluster in the temporal lobe ipsilateral to the resected area (Discordant). Surgical outcome was accessed according to ILAE classification, ILAE criteria 1-3 being considered good surgical outcomes and ILAE 4-6, poor outcomes.

Results: From the 58 TLE patients who underwent EEG-fMRI, 23 $(40 \%)$ underwent surgery. All were submitted to unilateral temporal lobe resection of different extent according to the pathology. The mean follow-up period after surgery was 22 months (range: 1-53 months). Twelve patients undergoing surgery had spikes inside the scanner and 11 did not. Significant spike-related BOLD changes were revealed in all but one case. Four patients $(22 \%)$ were classified as Concordant results, and of those, 3 (75\%) had a good surgical outcome (ILAE 1-3). Nine patients were classified as Some Concordance and six of these $(67 \%)$ had a good surgical outcome. Two of the nine patients (22\%) classified as Discordant had a good surgical outcome.

Conclusions: EEG-fMRI can provide useful information for the presurgical evaluation of patients with TLE and resection of the temporal lobe including spike-related significant BOLD changes may lead to better postoperative outcome.

\subsection{7}

STRIKING A BALANCE: CORRELATION BETWEEN GABA-A BINDING POTENTIAL AND REGIONAL GLUCOSE METABOLIC RATE IN TEMPORAL LOBE EPILEPSY PATIENTS

Alana D'Alfonso $^{1}$, A. Nugent ${ }^{2}$, S. Thada ${ }^{3}$, I. Dustin ${ }^{1}$ and W. Theodore ${ }^{1}\left({ }^{1}\right.$ Clinical Epilepsy Section, National Institute of Neurological Disorders and Stroke, National Institutes of Health, Bethesda, MD; ${ }^{2}$ Section on Neuroimaging in Mood and Anxiety Disorders, Molecular Imaging Branch, National Institute of Mental Health, National Institutes of Health, Bethesda, MD and ${ }^{3}$ PET Imaging Department, Clinical Center, National Institutes of Health, Bethesda, MD)
Rationale: On positron emission tomography (PET), patients with temporal lobe epilepsy (TLE) have reduced GABA-A receptor binding and glucose metabolism (RMRGlu) in seizure foci. Previous studies have focused on measuring independent neurotransmitter system abnormalities rather than considering the relation between GABA receptor binding and RMRglu. PET studies in healthy controls showed strong negative coupling between GABA-A BP and RMRglu in insular and mesial temporal lobe regions. We predicted decoupling between GABA-A binding and regional glucose metabolic rate (RMRGlu) in temporal lobe epilepsy patients. Methods: We studied 9 patients and 10 healthy volunteers who underwent both [11C]Flumazenil and [18F] FDG PET on an ECAT high resolution research tomograph (full width half maximal resolution 3 millimeters). [11C]Flumazenil binding potential (BP) was calculated using the time-activity curve from the pons as the input function. Skull-stripped MRI images (3DSkullStrip, AFNI) were segmented into grey matter, white matter, and CSF binary masks (FSL FAST) and ROIs were applied to MRI images in Talairach space (infralimbic cortex, left and right interior insula, anterior hippocampus, posterior hippocampus, amygdala, anterior cingulate, posterior cingulate, superior temporal gyrus, parietaloccipital cortex). Images were converted back into MRI space, multiplied by the gray matter mask, and applied to partial volume corrected RMRGlu and GABA-A BP images. Mean RMRGlu and BP values within each region were compared between groups with independent samples T-tests. Correlations between glucose metabolism and GABA-A binding in each region were determined for both patients and controls.

Results: We found significant correlations between GABA-A BP and RMRGlu in healthy controls in ipsilateral superior temporal gyrus, as well as both ipsi- and contralateral subgenual prefrontal cortex. In contrast, significant relations were not found between RMRglu and GABA-A BP in TLE patients. One region (posterior hippocampus), showed significant correlation values in TLE patients but not healthy controls (Figs. $1 \& 2$ ).

Conclusions: These results support our previous pilot study showing high correlations between GABA-A BP and RMRGlu within areas of increased GABA transmission. This relationship was not found in TLE patients, suggesting potential decoupling of these two systems.

Fig.1

\begin{tabular}{|cc|c|cc|}
\hline \multirow{2}{*}{ ROI } & \multicolumn{2}{c}{ Epilepsy Patients } & \multicolumn{2}{c|}{ Healthy Controls } \\
\cline { 2 - 5 } Ipsilateral Superior Temporal Gyrus & .020 & .959 & .696 & .026 \\
Contralateral Subgenual Prefrontal Cortex & .285 & .456 & .658 & $.039 *$ \\
Ipsilatcral Subgenual Prefrontal Cortex & .161 & .679 & .638 & $.047^{*}$ \\
\hline Ipsilatcral Postcrior IIippocampus & .829 & $.006 *$ &. .120 & .742 \\
\hline
\end{tabular}

$*$ = significant, $\mathrm{p}<.05$ 


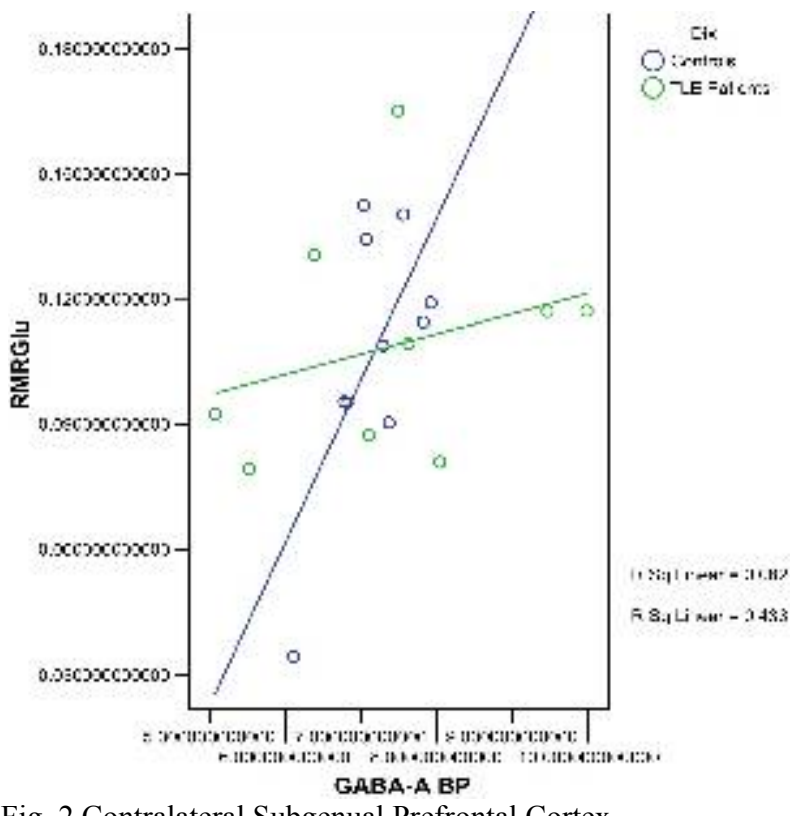

Fig. 2 Contralateral Subgenual Prefrontal Cortex

\subsection{8}

CORRELATION OF EEG/FMRI BOLD RESPONSE WITH POSTOPERATIVE OUTCOME IN FOCAL EPILEPSY

Dongmei An, F. Fahoum, J. A. Hall, A. Olivier, J. Gotman and F. Dubeau (Department of Neurology and Neurosurgery, Montreal Neurological Institute and Hospital, Montreal, QC, Canada)

Rationale: EEG/fMRI can noninvasively measure the hemodynamic response related to interictal epileptic discharges (IEDs) on scalp EEG. The clinical significance of EEG/fMRI is still under assessment. This study aims to correlate BOLD response related to IEDs with the localization of surgical resection to determine the conditions in which surgical outcome can be predicted better. Methods: All consecutive refractory focal epilepsy patients who underwent surgical treatment after EEG/fMRI from June 2006 to December 2010 and with 12 months postsurgical follow up were included. Each patient underwent a $2 \mathrm{hr} 3 \mathrm{~T}$ scan. IEDs were visually identified. A statistical $t$ map of BOLD response was generated using general linear model. EEG/fMRI results were not used for surgical decision making. BOLD response with the maximum $t$-value ( $t$-max, activation or deactivation) was correlated with the resection. The concordance between the resection and BOLD response was evaluated by co-registering individual $t$ map with postoperative MRI or, when not available, with postoperative CT or operative protocol. Surgical outcome was defined according to Engel's classification: Class $1 \& 2$ was considered a good outcome and Class $3 \& 4$ a poor outcome.

Results: Forty-six patients had surgery after EEG/fMRI study, 13 were excluded: 4 had no IEDs in the scanner and 9 showed no significant BOLD response during IEDs. Thirty-three patients were included ( 8 non-lesional) and classified in 4 groups defined by the concordance between resection and $t$-max. Group 1, fully concordant (Fig. 1): 10 patients with $t$-max concordant and response mostly confined to resection area. 8 of these 10 patients had a good surgical outcome. Group 2, partially concordant: 8 patients with $t$-max at the immediate edge of the resection, and response usually as part of a larger cluster overlapping or adjacent to the resection. 4 (50\%) with well confined cluster had a good outcome, and 4 had a poor outcome but in those the resection included only a small portion of a widespread BOLD response. Group 3, partially discordant: 6 patients with $t$-max remote from resection, but with an additional less significant BOLD cluster in the resection. Half of the patients had a good and half a poor outcome. Group 4, fully discordant (Fig. 2): 9 patients with no significant BOLD response in the resection. Only 1 patient from this group had a good outcome.
Conclusions: In patients with fully concordant or fully discordant BOLD response and resection (19/33, or 58\%), EEG/fMRI predicted the surgical outcome. These findings suggest that complete removal of the maximum BOLD response should lead to a seizure-free condition. The study also indicates that a widespread BOLD response is probably associated with a large epileptogenic zone, in which condition a small resection should not lead to a favorable outcome. Finally, the concomitant occurrence of less significant clusters but localized in the suspected epileptogenic zone should not be neglected as their removal sometimes also led to a good outcome. Support by Canadian Institutes of Health Research.
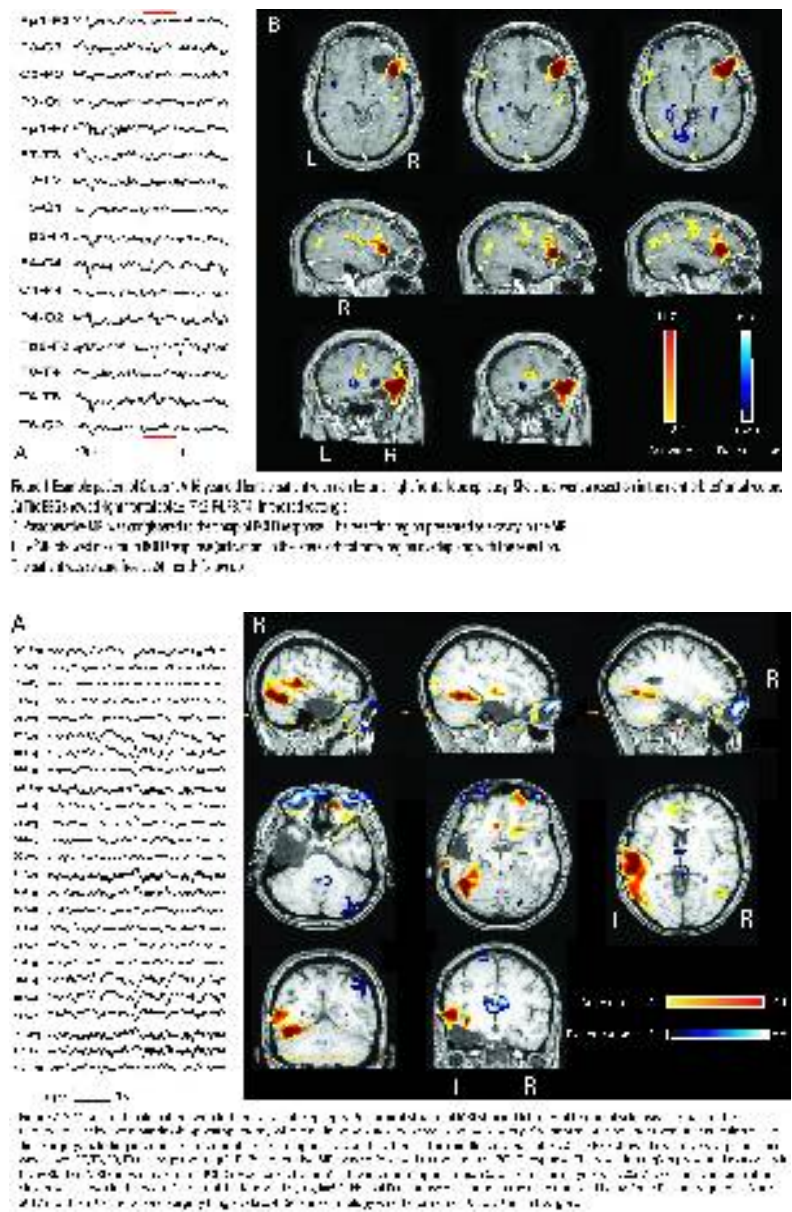

\subsection{9 \\ COMPARISON BETWEEN TASK-BASED AND RESTING- STATE FUNCTIONAL MRI FOR LANGUAGE LATERALIZATION}

Naoaki Tanaka, H. Liu, N. Suzuki and S. M. Stufflebeam (Athinoula A. Martinos, Charlestown, MA)

Rationale: Task-based functional MRI (fMRI) is commonly used for language lateralization in presurgical evaluation of epilepsy. Restingstate fMRI also demonstrates laterality by calculating intrinsic activity. We assessed concordance of laterality obtained from taskedbased, resting-state fMRIs and intracarotid amobarbital procedure (IAP) in epileptic patients.

Methods: Fifteen patients (male:7, female:10, age:15-46) were studied. MPRAGE sequences were acquired on a $3 \mathrm{~T}$ scanner $(\mathrm{TR}=2000 \mathrm{~ms}, \mathrm{TE}=2.37 \mathrm{~ms}$, flip angle $=90,1-\mathrm{mm}$ isotropic voxels $)$. Resting-state fMRI was obtained using an echo-planar imaging (EPI) gradient-echo pulse sequence $(\mathrm{TR}=3000 \mathrm{~ms}, \mathrm{TE}=30 \mathrm{~ms}$, flip angle $=85$, $3-\mathrm{mm}$ isotropic voxels, nframes $=160$ ). Patients looked at a white screen for 8 minutes run. The relative correlation strengths between seed and target region pairs were measured, deploying 200 regions in each hemisphere. For each region, laterality index (LI) was defined 
by [(LL-RL)-(RR-LR)]/[|LL|+|LR|+|RR|+|RL|], where LL/LR/RR/RL are the correlation strength between the left/right seed and the left/right target. We performed factor analysis by using principal axis factoring and obtained LIs in four independent subregions. The fourth factor includes frontal and temporal language areas in the distributed anatomy, and is highly correlated with language laterality represented by task-based fMRI in healthy population (Liu et al., 2009). We used the fourth factor LI for further analysis.

The task-based study consisted of blocked trial runs of a semantic language task. Patients decided whether each word was "abstract" or "concrete" in visually presented words. Patients performed three runs, each of which consisted of three 36-second blocks of word presentation and four 28 -second blocks of eye fixation. EPI sequences were acquired $(\mathrm{TR}=200 \mathrm{~ms}, \mathrm{TE}=30 \mathrm{~ms}$, flip angle $=90,3$ $\mathrm{mm}$ isotropic voxels) for each run. The data were analyzed using FSFAST. We computed maximum positive activation in parsopercularis, parstriangularis, superior/middle temporal, supramarginal and inferior parietal cortices on both hemispheres. LI was obtained by $(\mathrm{L}-\mathrm{R}) /(\mathrm{L}+\mathrm{R})$, where $\mathrm{L}$ and $\mathrm{R}$ is the sum of the maximum activation in each area.

For both methods, language laterality was determined based on the LI as follows; $\geq 0.1$ :left, $0.1>\mathrm{LI}>-0.1$ :bilateral, $\leq-0.1$ :right.

Results: In IAP, 13 patients showed the language laterality on the left side. One patient was right-sided and the other one was bilateral. In task-based fMRI, 12, one and two were left-, right-sided and bilateral. In resting-state fMRI, six, three and six were left-, right-sided and bilateral. We estimated the concordance of task-based and restingstate fMRI with IAP regarding the laterality in the epileptogenic hemisphere. Positive and negative predictive values were $89 \%$ and $100 \%, 70 \%$ and $80 \%$ for task-based and resting-state fMRI, respectively.

Conclusions: Resting-state fMRI showed more bilateral representation of laterality than task-based fMRI. Intrinsic functional connectivity of the language network may be altered in epileptic patients due to plasticity in the overall network induced by epilepsy.

\subsection{0 \\ MOTOR REORGANIZATION IN FRONTAL LOBE EPILEPSY}

Kristine E. Woodward ${ }^{2,3}$, I. Gaxiola ${ }^{2,3}$, D. Mainprize ${ }^{3}$, M. Grossi ${ }^{3}$, B. Goodyear $^{2,3}$ and P. Federico ${ }^{1,3}\left({ }^{1}\right.$ Clinical Neurosciences, University of Calgary, Calgary, AB, Canada; ${ }^{2}$ Hotchkiss Brain Institute, Calgary, AB, Canada and ${ }^{3}$ Seaman Family MR Center, Calgary, AB, Canada)

Rationale: Motor cortex can undergo significant reorganization in patients with a variety of neurological diseases. Reorganization of language networks has also been shown in patients with temporal lobe epilepsy (Jensen et al. 2011, Epilepsia 52:2013-2021; Jensen et al. 2011, Epilepsy Res 93:155-163), but such reorganization has not been demonstrated to date in human subjects with frontal lobe epilepsy (FLE). FLE may cause subtle changes in motor cortex organization, which could explain functional motor deficits experienced by these patients. Indeed, experimentally induced seizures in animals produce abnormal expansion of movement representations within the motor cortex (Teskey et al. 2002, Cereb Cortex 12:98-105). However, it is not known whether such reorganization occurs specifically in humans and if this would allow for the maintenance of normal motor function. Therefore, cortical motor organization in patients with FLE was studied using functional magnetic resonance imaging (fMRI).

Methods: Ten subjects with right FLE and ten control subjects were studied. All participants were right handed and between the ages of 18 and 65 . fMRI was performed at $3 \mathrm{~T}$ and participants underwent a series of hand movement sequences. The first task required participants to tap their fingers in time with a visual stimulus, both unimanually and bimanually. The second task required participants to execute a series of coordinated unimanual or bimanual hand movements as instructed by visual stimuli. Each task involved 6 runs of 12 movements per run, taking approximately one hour to complete. fMRI data was analyzed using standard methods, and first level analysis for each task was then entered into three separate group analyses with subject group (right FLE, control) and motor task (right/left unimanual, bimanual) entered as variables. Maps were generated for each task to determine brain regions exhibiting a significant difference in response magnitude between groups and tasks $(Z>2.3$, corrected cluster significance $p=0.05)$.

Results: Patients with right FLE had significantly decreased activation in the hemisphere ipsilateral to the seizure focus during the bimanual and left handed finger-tapping tasks compared to controls. Patients with right FLE had significantly increased activation in the hemisphere contralateral to the seizure focus during the bimanual and left-handed coordination tasks compared to controls. No significant difference was found between patients with right FLE and controls during the right-handed tasks.

Conclusions: Reorganization of the motor cortex occurs in patients with right FLE, therefore giving us a better understanding of the long-term effects of FLE on cortical organization. The possibility that seizures induce brain reorganization has important implications for epilepsy surgery, and supports the notion that a combination of methods, including fMRI, should be used when brain mapping is required.
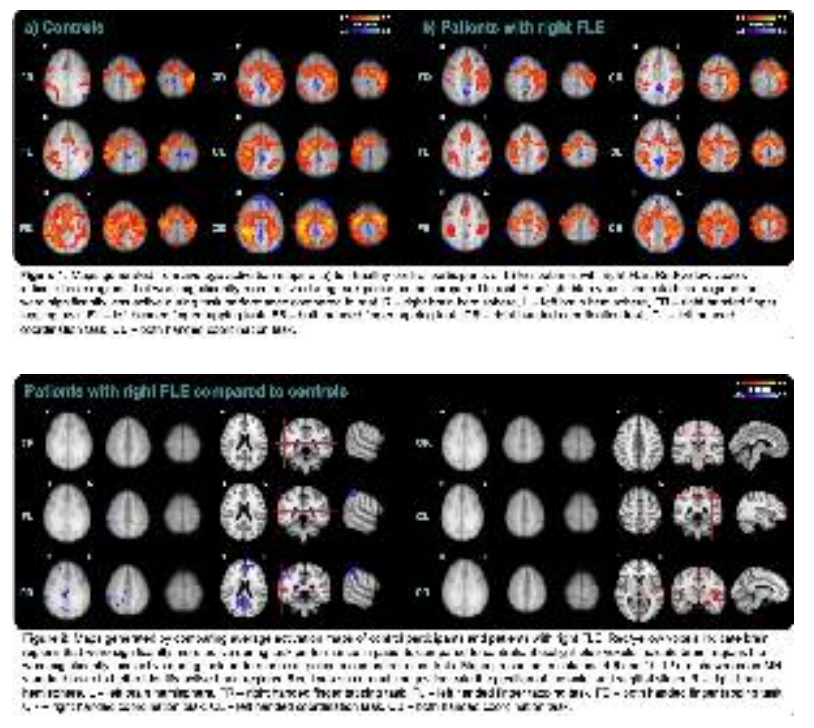

\subsection{1 \\ THE MINIMUM NUMBER OF DISCHARGES NEEDED TO DETECT BOLD SIGNALS USING INTRACRANIAL EEG- FMRI AT 3T}

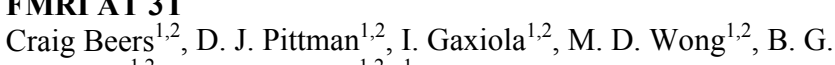
Goodyear ${ }^{1,2}$ and P. Federico ${ }^{1,2}\left({ }^{1}\right.$ Hotchkiss Brain Institute, University of Calgary, Calgary, AB, Canada and ${ }^{2}$ Seaman Family MR Research Centre, University of Calgary, Calgary, AB, Canada)

Rationale: Simultaneous scalp EEG-fMRI is a powerful tool that is quickly gaining popularity in both research and clinical settings. Combining intracranial EEG with fMRI (iEEG-fMRI) is of particular interest as it would allow interictal discharges to be recorded during fMRI with unprecedented precision. To this end, we have successfully implemented simultaneous iEEG-fMRI at 3T. In this study, we sought to determine the minimum number of epileptiform discharges required to generate consistent maps of BOLD activation during simultaneous iEEG-fMRI.

Methods: Three iEEG-fMRI datasets were selected that had a high number of interictal discharges. All subjects were connected to a MR compatible EEG system, and simultaneous fMRI was performed at 3T using a GE Signa LX scanner. Functional images were collected 
using a series of single-shot GRE-EPI volumes, providing T2*-W contrast. In addition to the functional scans, the protocol included low resolution anatomical and $\mathrm{T} 1-\mathrm{W}$ high resolution anatomical scans. BOLD fMRI responses were modelled by convolving the timing of epileptiform events with a hemodynamic response function. Statistical maps were generated for the BOLD activation every 10 discharges until all recorded epileptiform activity had been modelled (i.e. 10 discharges, 20 discharges, etc.). The fMRI maps generated by all discharges recorded during the experiment were then used to contrast each discharge interval for significant differences (e.g., for Subject 1: 676 discharges vs. 10 discharges; 676 discharges vs. 20 discharges, etc.).

Results: Subject 1 had independent bitemporal discharges that were modeled separately: 284 discharges from the left, and 194 from the right. We recorded 676 discharges from the temporal foci of Subject 2 and 820 from Subject 3's temporal foci. Upon analysis of the stratified data, it was found that the calculated locations of significant BOLD activation changed little after a certain threshold was reached for each subject: 96 discharges for Subject 1-left, 61 discharges for Subject 1-right, 90 discharges for Subject 2 and 180 for Subject 3 (Figure 1). As the number of interictal discharges included in the model increased, the statistical power of the BOLD activation increased and the changes became more localized with the subsequent elimination of noise. A contrast analysis was performed between the BOLD signal seen with the maximal number of discharges versus fewer discharges (Figure 2). There were few statistically significant differences and no large voxel cluster differences once 96 discharges were recorded for Subject 1-left, 61 for Subject 1-right, 50 for Subject 2 and 60 for Subject 3 .

Conclusions: Based on these data, a minimum of 50-100 interictal discharges need to be recorded during simultaneous iEEG-fMRI in order to produce reliable maps of BOLD activation. While as few as 10 discharges did result in localized BOLD activation, the activation was much more scattered and diffuse, with more noise. Although EEG-fMRI may produce maps of BOLD activation in subjects with relatively few discharges, these maps may not be accurate.
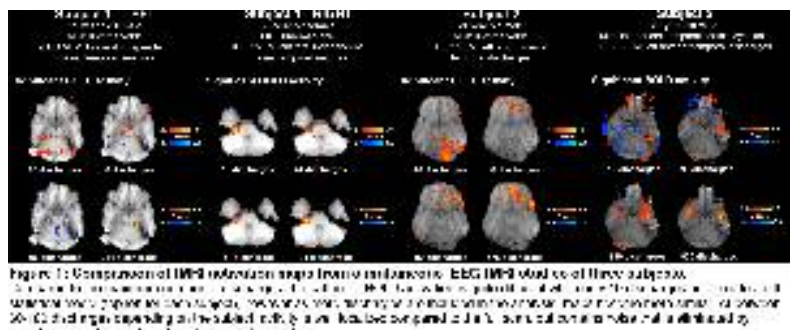

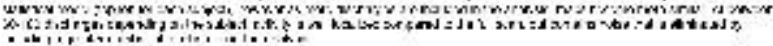

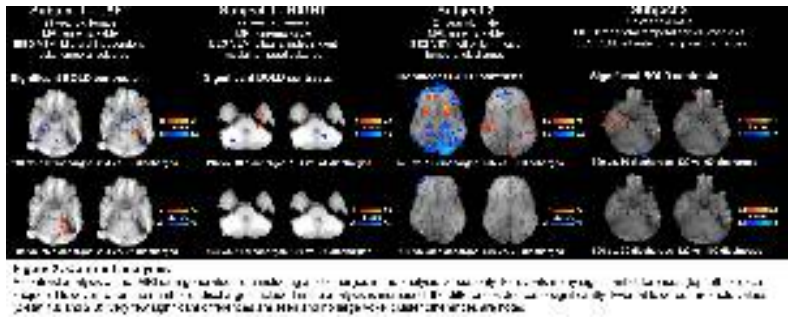

\subsection{2}

IN VIVO METABOTROPIC GLUTAMATE RECEPTOR TYPE 5 (MGLUR5) ABNORMALITIES IN PATIENTS WITH FOCAL CORTICAL DYSPLASIAS

Jonathan M. Dubois ${ }^{1,2}$, J. Rowley ${ }^{2}$, P. Rosa-Neto ${ }^{2}$ and E. Kobayashi ${ }^{1,2}\left({ }^{1}\right.$ Montreal Neurological Institute, McGill University, Montreal, QC, Canada and ${ }^{2}$ Translational Neuroimaging Laboratory, Douglas Research Institute, McGill University, Montreal, QC, Canada)
Rationale: Metabotropic glutamate receptor type 5 (mGluR5) modulates neuronal excitability and synaptic plasticity in the healthy brain. Pathological studies of surgical specimens from patients with epilepsy suggest that mGluR5 is upregulated in focal cortical dysplasias (FCD). However, resected foci provide only a limited glimpse of molecular and structural abnormalities within the epileptic brain, limited by the scope of the surgical resection. It is now possible to access in vivo whole brain mGluR5 availability with positron emission tomography (PET) using the radioligand [11C] ABP688, which binds specifically to the transmembrane allosteric site of mGluR5. This is a first study to evaluate [11C] ABP688 binding potentials (BP) in patients with FCD.

Methods: Five patients with radiologically confirmed FCD and epilepsy ( 4 female, mean age \pm SD of $41 \pm 9$ years), and 14 healthy controls ( 6 female, mean age \pm SD of $46 \pm 19$ years) were included in the study. [11C]ABP688 PET scans consisted of a 1hour dynamic acquisition following [11C]ABP688 injection, in the HR+ ECAT PET scanner. Images were reconstructed using filter-back projection. PET volumes were coregistered to each subjects's T1-weighted 3T MRI and resampled to standard space. [11C]ABP688-BP maps were generated using a simplified reference tissue method with the cerebellum as a reference region. A voxel-wise whole brain z-test of [11C]ABP688-BP was performed between each FCD patient and the control group. Multiple comparison correction was conducted separately for the lobe ipsilateral to the lesion and for the remaining cortex using a cluster-based random field theory method. Each cluster had a minimum voxel threshold of $\mathrm{p}<0.001$.

Results: Gender and age were not significantly associated with [11C]ABP688-BP. Visual inspection of [11C]ABP688-BP maps suggested reduced binding within the FCD topography. Therefore, for quantitative analysis, we manually segmented the grey matter of the dysplastic lesion, the grey matter of the lobe ipsilateral to the FCD, and the remaining cortex. Voxel-wise analysis confirmed a greater percentage of negative z-scores within the area of FCD and within the lobe ipsilateral to the FCD relative to the rest of the cortex. Positive z-scores were not found within the FCDs but were observed in a scattered pattern, unspecific to the FCD location.

Conclusions: Our findings in this small group of patients confirm abnormal mGluR5 availability in patients with FCD, as previously suggested by pathological studies. The results show additional areas of reduced binding, anatomically adjacent to the FCD lesion. Increased binding seemed less structurally specific and less related to the FCD. In vivo mGluR5 availability may aid radiological diagnosis/confirmation in patients with suspected MCDs. Supported by the Savoy Foundation

\subsection{3 \\ AGE-RELATED MORPHOLOGICAL AND FUNCTIONAL MATURATION OF THE CORPUS CALLOSUM: A QUANTITATIVE STUDY WITH MRI AND INTRAOPERATIVE EVOKED POTENTIALS IN PATIENTS WITH EPILEPSY \\ Tomonori Ono ${ }^{1}, \mathrm{H}$. Baba ${ }^{1}, \mathrm{~K}$. Toda ${ }^{1}, \mathrm{~S} . \mathrm{Baba}^{2}$ and K. Ono ${ }^{3}$ $\left({ }^{1}\right.$ Neurosurgery, National Nagasaki Medical Center, Omura, Japan; ${ }^{2}$ Neurosurgery, Nagasaki University School of Medicine, Nagasaki, Japan and ${ }^{3}$ Yokoo Hospital, Isahaya, Japan)}

Rationale: The juvenile brain is still developing with age. One developmental hallmark in the central nervous system is the maturation of neuronal fibers. Magnetic resonance imaging (MRI) visualizes age-related processes in cerebral white matter, and diffusion tensor imaging (DTI) has recently enabled more quantitative analysis. These changes are mainly believed to represent the process of neuronal fiber myelination. Myelination is not only a morphological change with formation of a myelin sheath around the fiber, but also contributes to functional development by allowing nerve impulses to propagate more rapidly. However, the relationship between these age-related changes on MRI/DTI and neurofunctional 
development has yet to be elucidated. Transcallosal response (TCR) is an evoked potential study recorded intraoperatively during epilepsy surgery and these waveforms may provide a window into the electrical conduction function of the corpus callosum. Latency of the first positive wave (P1-TCR) represents the conduction time of callosal fibers. This study investigated age-related changes in DTI of the corpus callosum and TCR in patients with epilepsy who underwent corpus callosotomy, and clarified the potential correlations.

Methods: These epilepsy surgery program and studies were approved by the institutional review board. Eighty-five patients (51 males, 34 females) between 10 and 504 months of age were reviewed. All patients underwent corpus callosotomy as a surgical option to treat drug-resistant epilepsy, including infantile spasms, Lennox-Gastaut syndrome and other symptomatic generalized epilepsies, and frontal lobe epilepsy. TCR and high-resolution DTI data were available for 77 and 29 subjects, respectively. Apparent diffusion coefficient (ADC) and fractional anisotropy (FA) from preoperative DTI and latency of P1-TCR were plotted against patient age. Correlations were statistically tested with linear or non-linear regression models.

Results: Both ADC and FA changed in an age-dependent manner. In particular, ADC decreased with age and mostly plateaued after 100 months of age $(\mathrm{R} 2=0.53)$. Similarly, latency of P1-TCR continuously decreased before 100 months of age and was significantly correlated with age (non-linear regression, $\mathrm{p}<0.001, \mathrm{R} 2$ $=0.64$ ). Finally, ADC and latency of P1-TCR correlated significantly with each other (linear regression, $\mathrm{p}<0.01, \mathrm{R} 2=0.54$ ).

Conclusions: Age-related changes to the corpus callosum as identified on MRI/DTI suggest both morphological and functional maturation of neuronal fibers and may provide a non-invasive means of estimating the developmental age of the brain.

\subsection{4 \\ THE POST-OPERATIVE CHANGES IN MAJOR WHITE MATTER FIBERS FOR CHILDREN WITH MEDICALLY INTRACTABLE FOCAL EPILEPSY: A DIFFUSION TENSOR IMAGING STUDY}

Hyunmi Kim, A. Harrison, P. Kankirawatana, C. Rozzelle and J. Blount (University of Alabama at Birmingham, Birmingham, AL)

Rationale: Previous DTI studies suggested bilaterally widespread abnormalities beyond epileptogenic zone in medically intractable focal epilepsy. This study aimed to investigate the post-operative changes in the major white matter fibers for children with medically intractable focal epilepsy secondary to focal cortical dysplasia (FCD). Methods: Thirty patients with medically intractable focal epilepsy had both pre-operative and post-operative Diffusion tensor imaging (DTI) at the Children's Hospital of Alabama. The mean age at surgery was $10.0 \pm 4.7$ years (1 year 11 months-16 year 2 months). Only thirteen (43.3\%) had visible lesions on conventional, preoperative brain MRI. Most patients (29/30) underwent intracranial EEG monitoring prior to surgery. Twenty had seizure onset zones in fronto-parietal regions including frontal lobe localization (10) while the rest had in temporo-occipital seizure onset zones including temporal lobe localization (6). The mean post-operative follow-up duration was $31.2 \pm 11.2$ months (10-51 months). Twenty-six patients (86.7\%) have favorable outcomes (Engel class I-17, II-9). The pathology was FCD in all cases: Type IA (4), Type IB (1), Type IIA (19), and Type IIB (6). One patient had also focal meningeal angiomatosis. Age at pre-operative MRI and time between pre- and post-operative MRIs were $9.3 \pm 4.6$ years and $12.5 \pm 6.3$ months, respectively. The Regions of interest (ROIs) for the major white matter tracts included the genu and splenium of the corpus callosum (CC), the posterior limb of the internal capsule (CST), the inferior fronto-occipital fasciculus (IFO), the inferior longitudinal fasciculus (ILF), the superior fronto-occipital fasciculus (SFO) and the superior longitudinal fasciculus (SLF). Fractional anisotropy (FA) and apparent diffusion coefficient (ADC) were determined in each ROI. A matched-pairs t-test was used to assess statistical significance of differences between pre- and post-operative values at each ROI.

Results: Compared to pre-operative values, post-operative FA values were significantly lower and ADC values higher in CC genu and IFO ipsilateral to the resection $(\mathrm{p}<0.05)$. In SLF ipsilateral to surgery, the post-operative FA values were decreased $(p<0.05)$ but the postoperative ADC values were not significantly different from the preoperative ones. In ipsilateral ILF, the post-operative ADC values were higher $(\mathrm{p}<0.05)$ but the post-operative FA values were not significantly different from the pre-operative ones. In CC genu, ipsilateral IFO, and ipsilateral SLF, $\lambda 1$ values showed no significant difference, while $\lambda 2$ and $\lambda 3$ values were significantly increased ( $\mathrm{p}$ $<0.05)$.

Conclusions: The post-operative pathologic changes occurred mainly in white matter fibers ipsilateral to the resection. When considering the post-operative changes were not significant in the contra-lateral side, the epilepsy surgery may block the progression of diffuse pathologic changes, which were observed in medically intractable focal epilepsy, in the contra-lateral side to operation.

FA and ADC values at each ROI

\begin{tabular}{|c|c|c|c|c|c|c|c|c|c|c|c|}
\hline & $\mathrm{N}$ & $\begin{array}{c}\text { FA } \\
\text { Mean }\end{array}$ & $p$ & $\begin{array}{l}\text { ADC } \\
\text { Mcan }\end{array}$ & $\mathrm{p}$ & $\begin{array}{c}\lambda 1 \\
\text { Mean }\end{array}$ & p & $\begin{array}{c}\lambda .2 \\
\text { Mcan }\end{array}$ & $\mathrm{p}$ & $\begin{array}{c}\lambda .3 \\
\text { Mean }\end{array}$ & p \\
\hline CC-genu-Pre & \multirow{2}{*}{28} & 0.64 & \multirow{2}{*}{$0.0086^{*}$} & 0.71 & \multirow{2}{*}{$0.0397^{*}$} & 1.31 & \multirow{3}{*}{0.5844} & 0.48 & \multirow{3}{*}{$0.0079 *$} & 0.33 & \multirow{3}{*}{$0.0265^{*}$} \\
\hline CC-genu-Post & & 0.59 & & 0.75 & & 1.30 & & 0.56 & & 0.38 & \\
\hline $\begin{array}{l}\text { CC-splcnium-Pre } \\
\text { CC-splenium-Post }\end{array}$ & 28 & $\begin{array}{l}0.72 \\
0.68\end{array}$ & 0.11 & $\begin{array}{l}0.68 \\
0.71\end{array}$ & 0.1547 & & & & & & \\
\hline $\begin{array}{l}\text { CST-contra-Pre } \\
\text { CST-contra-Post }\end{array}$ & 24 & $\begin{array}{l}0.64 \\
0.65\end{array}$ & 0.2992 & $\begin{array}{l}0.61 \\
0.62\end{array}$ & 0.5361 & & & & & & \\
\hline CST-ipsi-Pre & \multirow{2}{*}{24} & 0.65 & \multirow{2}{*}{0.5322} & 0.61 & \multirow{2}{*}{0.1099} & & & & & & \\
\hline CST-ipsi-Post & & 0.64 & & 0.63 & & & & & & & \\
\hline II:O-contra-Pre & \multirow{2}{*}{30} & 0.44 & \multirow{2}{*}{0.7918} & 0.68 & \multirow{2}{*}{0.9009} & & & & & & \\
\hline IFO-contra-Post & & 0.45 & & 0.69 & & & & & & & \\
\hline $\begin{array}{l}\text { LOO-ipsi-Pre } \\
\text { IFO-ipsi-Post }\end{array}$ & 30 & $\begin{array}{l}0.43 \\
0.39\end{array}$ & $0.0224^{*}$ & $\begin{array}{l}0.69 \\
0.74\end{array}$ & $0.0131 *$ & $\begin{array}{l}1.04 \\
1.05\end{array}$ & 0.8182 & $\begin{array}{l}0.59 \\
0.67\end{array}$ & $0.002^{*}$ & $\begin{array}{l}0.44 \\
0.49\end{array}$ & $0.0296^{2 *}$ \\
\hline $\begin{array}{l}\text { II.F-comtra-Pre } \\
\text { ILli-contra-Post }\end{array}$ & \multirow[t]{2}{*}{30} & $\begin{array}{l}0.45 \\
0.43\end{array}$ & 0.1648 & $\begin{array}{l}0.70 \\
0.73\end{array}$ & \multicolumn{2}{|l|}{0.0503} & \multirow{3}{*}{0.1499} & \multirow{3}{*}{$\begin{array}{l}0.66 \\
0.72\end{array}$} & \multirow{3}{*}{0.0626} & \multirow{3}{*}{\begin{tabular}{|l|}
0.43 \\
0.50 \\
\end{tabular}} & \multirow{3}{*}{0.0112} \\
\hline ILF-ipsi-Pre & & 0.43 & \multirow{2}{*}{0.0547} & 0.72 & \multirow{2}{*}{$0.0244 *$} & 1.07 & & & & & \\
\hline II.F-ipsi-Post & 29 & 0.39 & & 0.78 & & 1.11 & & & & & \\
\hline $\begin{array}{l}\text { SIO-contra-Pre } \\
\text { SF()-contra-Post }\end{array}$ & 30 & $\begin{array}{l}0.35 \\
0.37\end{array}$ & 0.1935 & $\begin{array}{l}0.69 \\
0.67\end{array}$ & 0.4039 & & & & & & \\
\hline $\begin{array}{l}\text { SFO-ipsi-Pre } \\
\text { SIO)-ipsi-Post }\end{array}$ & 30) & $\begin{array}{l}0.35 \\
0.38\end{array}$ & 0.5246 & $\begin{array}{l}0.71 \\
0.75\end{array}$ & 0.1499 & & & & & & \\
\hline SLF-contra-Pre & 7) & 0.46 & 0070 & 0.66 & 1) 1486 & & & & & & \\
\hline SLF-contra-Post & & 0.46 & & 0.68 & & & & & & & \\
\hline SLF-ipsi-Pre & 36 & 0.44 & $00051 *$ & 0.68 & 006 & 1.01 & 0018 & 0.61 & 00360 & 0.40 & 001240 \\
\hline SLF-ipsi-Post & & 0.40 & & 0.71 & 0.00 & 1.01 & 0.7104 & 0.65 & 0.0502 & 0.45 & 0.0124 \\
\hline
\end{tabular}

Contra: contralateral side to operation site, ipsi: ipsilateral side to operation site, Pre: preoperative, Post: postoperative

\subsection{5 \\ INCREASED CORTICAL GLUTAMATE LEVELS AND SEIZURE SEVERITY IN CHILDREN WITH STURGE- WEBER SYNDROME: A MAGNETIC RESONANCE SPECTROSCOPIC IMAGING STUDY}

Csaba Juhasz ${ }^{1,2}$, J. Hu$^{3}$, Y. Xuan ${ }^{3}$ and H. T. Chugani ${ }^{1,2}\left({ }^{1}\right.$ Pediatrics and Neurology, Wayne State University, Detroit, MI; ${ }^{2}$ Pediatric Neurology, Children's Hospital of Michigan, Detroit, MI and ${ }^{3}$ Radiology, Wayne State University, Detroit, MI)

Rationale: Impaired cortical venous drainage can lead to hypoxic brain damage in children with Sturge-Weber syndrome (SWS). Cortical hypoxia can trigger excessive release and extracellular accumulation of glutamate (Glu), thus potentially inducing seizures. 
Recent tissue studies also suggested that epileptiform activity in SWS cortex is driven by glutamatergic synapses (Tyzio et al., Ann Neurol, 2009;66:209-18). Based on these, we hypothesized that proton magnetic resonance spectroscopic imaging (MRSI) may detect elevated Glu levels in the epileptogenic hemisphere in children with SWS, and we also tested if such Glu increases are related to seizure severity and intractibility.

Methods: Using a 3-Tesla MR scanner, conventional MRI was supplemented by MRSI in 9 children (age: 1.1 - 6.5 years) with SWSassociated seizures and unilateral hemispheric involvement. A chemical shift imaging sequence, using STEAM, was employed to acquire 2D MRSI data in the interictal state (parameters: $\mathrm{TR}=1500$ $\mathrm{ms}, \mathrm{TE}=40 \mathrm{~ms}, \mathrm{TM}=8 \mathrm{~ms}$, voxel size $=20 \mathrm{~mm} \times 20 \mathrm{~mm} \times 15 \mathrm{~mm}$, $\mathrm{NA}=16$, total acquisition time $\left.=10^{\prime} 48^{\prime \prime}\right)$. Glu (separated from glutamine and gamma-aminobutyric acid; as described by Hu et al., J Magn Reson, 2007;185:204-13), N-acetyl aspartate (NAA) and Creatine $(\mathrm{Cr})$ were quantified in multiple voxels in axial brain slices positioned at levels showing typical MRI signs of SWS. Glu/Cr ratios, measured in voxels showing maximal Glu values, were compared between regions ipsi- (affected hemisphere) vs. contralateral (homotopic region in the unaffected hemisphere) to the leptomeningel angioma. NAA/Cr ratios were also measured in the same regions. Glu/Cr and NAA/Cr asymmetries (using ipsilateral/contralateral ratios) were correlated with clinical variables: age, age at epilepsy onset, duration of epilepsy and seizure frequency. Results: The highest Glu values were found in the affected hemisphere in all 9 children: the maximum ipsilateral/contralateral $\mathrm{Glu} / \mathrm{Cr}$ ratios ranged between 1.11 and 2.50 (median: 1.63). In the same regions, NAA/Cr ratios showed variable abnormalities: either decreases or mild increases (ipsilateral/contralateral ratios ranging between 0.75 to 1.19 , median: 0.99 ). High $\mathrm{Glu} / \mathrm{Cr}$ ratios in the affected hemisphere were associated with high seizure frequency scores (Spearman's rho: 0.79, $\mathrm{p}=0.01$ ). The three SWS children with the highest $\mathrm{Glu} / \mathrm{Cr}$ ratios ( $\geq 75 \%$ increase on the affected side) had the most severe, intractable seizures: one had weekly clusters of infantile spasms, while the other two had intractable partial seizures requiring resective surgery after the scan. NAA/Cr asymmetries showed no correlation with any clinical variables.

Conclusions: These data suggest a role of increased cortical Glu levels in epileptogenesis associated with SWS. The maximum level of Glu increases, detected by MRSI in the affected hemisphere, may be an imaging marker of seizure severity. Glutamate levels with $75 \%$ or greater increase, as compared to the contralateral homotopic region, may be associated with severe, intractable seizures in children with unilateral SWS.

\subsection{6 \\ EVALUATION OF INTERICTAL EEG-FMRI SENSITIVITY AND SPECIFICITY FOR DETECTION OF THE ICTAL ONSET ZONE IN REFRACTORY FOCAL EPILEPSY}

Simon Tousseyn, P. Dupont, S. Sunaert and W. Van Paesschen (University Hospitals Leuven, KU Leuven, Leuven, Belgium)

Rationale: The aim was to define sensitivity and specificity of Blood Oxygen Level Dependent (BOLD) responses to interictal epileptic spikes during EEG-correlated functional MRI (EEG-fMRI) for detecting the ictal onset zone (IOZ).

Methods: Twenty-seven consecutive adults with refractory focal epilepsy and a well delineated IOZ after a full presurgical evaluation were studied with EEG-fMRI. After standard EEG artifact removal, concordant interictal spikes could be marked visually in 10 patients. The canonical hemodynamic response to spikes was modeled using an event-related design.

We delineated the IOZ as the region of subtraction ictal SPECT coregistered to MRI (SISCOM) hyperperfusion $(\mathrm{Z}>1.5)$ within the presumed epileptogenic zone or resection zone in patients who underwent epilepsysurgery.
Areas of BOLD activations or deactivations overlapping the IOZ in patients were considered as true positives and if no overlap was found, it was considered as a false negative. To determine true negative and false positive fractions, we included 10 age- and gendermatched healthy case-controls. Each control underwent EEG-fMRI using the same parameters as the corresponding patient. The interictal spike event-related regressor of the patient was used in the design matrix of the case-control normal subject. Suprathreshold BOLD activations or deactivations in any part of the brain in healthy controls were considered as false positives, absence of BOLD responses as true negatives. Sensitivity and specificity for IOZ detection were calculated for different statistical Z-thresholds and cluster sizes (1, 5 and 50 voxels) and illustrated in ROC-curves. The commonly used statistical thresholds uncorrected $\mathrm{p}<0.001$ and family wise error (FWE) corrected $\mathrm{p}<0.05$ correspond to a Zthreshold of 3.1 respectively $Z$ in the $4.8-5.1$ interval (the exact $Z$ value depends on the search volume and the smoothness of the data). Results: Figure 1 illustrates the ROC-curve corresponding to a minimal cluster size of 5 voxels. When using an uncorrected $\mathrm{p}<$ 0.001 (combined with a 5 voxel cluster extent), sensitivity is $100 \%$ and specificity $10 \%$. In contrast, when a significance level of FWE corrected $\mathrm{p}<0.05$ was used, the sensitivity dropped to $70 \%$ but the specificity increased to $100 \%$. The latter results were the same when using no additional cluster size or when using a minimal cluster extent of 50 voxels. Interestingly, if only BOLD activations were considered with a minimum cluster extent of 5 voxels, the same sensitivity and specificity was reached at FWE corrected $\mathrm{p}<0.05$. Conclusions: This study reports that BOLD changes to interictal spikes during EEG-fMRI, corresponding to a voxel based significance level of FWE corrected $\mathrm{p}<0.05$, resulted in $70 \%$ sensitivity and $100 \%$ specificity for localizing the IOZ in 10 refractory focal epilepsy patients.

Sources of funding: IWT-TBM grant (080658)

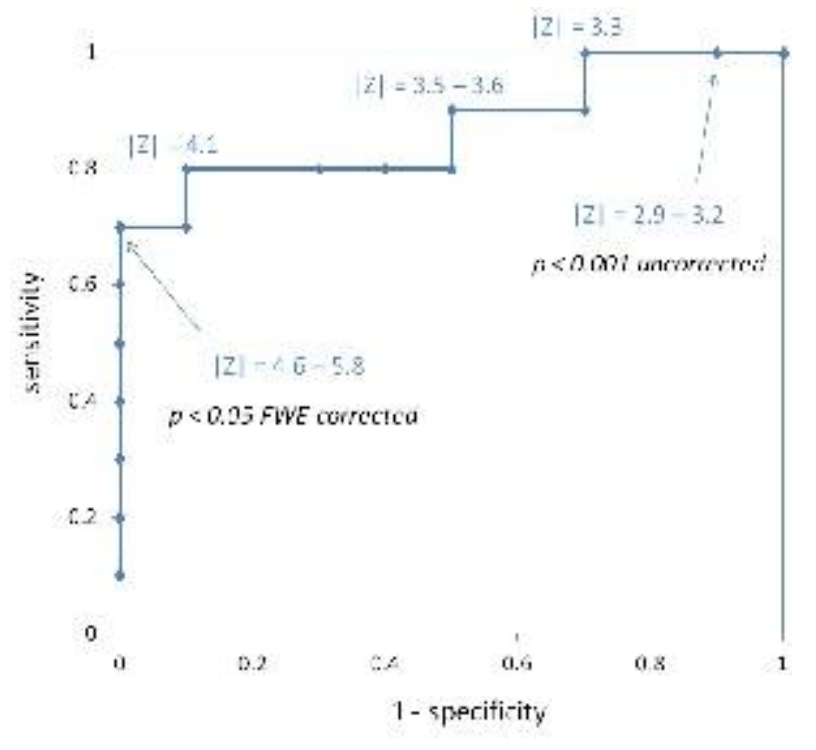

Fig 1. ROC-turve iduster extert:2 5 voxels)

\subsection{7}

FAMILIAL PATTERNS OF BOLD ACTIVATION

Patrick W. Carney ${ }^{1,2}$, R. A. Masterton ${ }^{1}$, S. F. Berkovic ${ }^{2}$ and G. D. Jackson ${ }^{1}\left({ }^{1}\right.$ Brain Research Institute, Florey Neuroscience Institutues, Melbourne Brain Centre, Heidelberg, VIC, Australia and ${ }^{2}$ Epilepsy Research Centre, University of Melbourne, Melbourne Brain Centre, Heidelberg, VIC, Australia)

Rationale: EEG-fMRI studies of generalized epileptiform activity have identified a consistent pattern of blood oxygen level dependent (BOLD) signal change in group studies (1-3). However individual 
variability is noted in BOLD change and the reasons for this are not clear. Genetic generalized epilepsy (GGE) displays complex inheritance patterns and is likely to involve multiple genetic elements (4). In this study we explored the role of genetic factors on event related BOLD signal change during EEG-fMRI by studying twins and siblings with concordant and discordant GGE syndromes. We hypothesized greater genetic and clinical heterogeneity between siblings would be reflected in greater heterogeneity in activation maps.

Methods: Three sibling pairs (including one monozygotic twin pair) with GGE were recruited through the EEG departments of the Austin Hospital and the Royal Children's Hospital in Melbourne. EEGfMRI, data pre-processing and event related analysis was performed as described in our previous study on absence epilepsy (2). All studies were performed on a 3-T GE Signa LX whole-body scanner (General Electric, Milwaukee, Wisconsin). fMRI data were preprocessed and analyzed using SPM8 (Statistical Parametric Mapping, Welcome Department of Imaging Neuroscience, London, United Kingdom).

A Pearson's correlation coefficient was calculated comparing all voxels between each sibling pair to quantify the degree of similarity. As a comparator, three subjects with GGE, who each had two EEGfMRI studies of similar duration, were studied.

Results: The Pearson's correlation coefficient for the three subjects studied twice was $0.43-0.77$. Monozyotic twins with juvenile absence epilepsy had a similarly high correlation of 0.58 . A non-twin sibling pair with same syndrome (eyelid myoclonia with absence) had a correlation of 0.4 , whereas the sibling pair with different syndromes had the lowest correlation of 0.23 .

Conclusions: Our findings demonstrate that greater phenotypic and genotypic similarity leads to a stronger correlation in BOLD change across the brain. This suggests that genetic factors may contribute to the pattern of BOLD change seen with event related analysis of epileptiform events.

References:

1. Bai X, Vestal M, Berman R, et al. Dynamic time course of typical childhood absence seizures: EEG, behavior, and functional magnetic resonance imaging. J Neurosci 2010;30:5884-5893.

2. Carney PW, Masterton RA, Harvey AS, Scheffer IE, Berkovic SF, Jackson GD. The core network in absence epilepsy. Differences in cortical and thalamic BOLD response. Neurology 2010;75:904-911. 3. Moeller F, Siebner HR, Wolff S, et al. Simultaneous EEG-fMRI in drug-naive children with newly diagnosed absence epilepsy.

Epilepsia 2008;49:1510-1519.

4. Mulley JC, Scheffer IE, Harkin LA, Berkovic SF, Dibbens LM. Susceptibility genes for complex epilepsy. Human Molecular Genetics. 2005;14 Spec No. 2:R243-249.

\subsection{8 \\ MAPPING EPILEPTIC NETWORKS IN JUVENILE MYOCLONIC EPILEPSY USING MEG AND FMRI} S. M. Bowyer ${ }^{1,2}$, H. Soltanian-Zadeh ${ }^{3}$, Q. Jiang ${ }^{1}$, K. M. Mason ${ }^{1,2}$, V. S. Wasade ${ }^{1,2}$, M. Spanaki ${ }^{1,2}$, D. E. Burdette ${ }^{1,2}$ and Andrew Zillgitt ${ }^{1,2}$ ( ${ }^{1}$ Neurology, Henry Ford Hospital, Detroit, MI; ${ }^{2}$ Henry Ford Comprehensive Epilepsy Program, Henry Ford Hospital, Detroit, MI and ${ }^{3}$ Radiology, Henry Ford Hospital, Detroit, MI)

Rationale: Juvenile myoclonic epilepsy (JME) is a common genetic generalized epilepsy accounting for approximately $18 \%$ of all genetic generalized epilepsies and $4-6 \%$ of all epilepsies. It is a

heterogeneous condition that can be broken into four subsyndromes. The two most common subsyndromes consist of patients with myoclonic and generalized tonic-clonic seizures and patients with absence, myoclonic, and generalized tonic-clonic seizures. With recent advances in functional neuroimaging, there has been an increase in the understanding of the pathophysiology underlying JME, but more studies are needed to provide insight into the pathogenesis of this condition.
Methods: Two patients with a clinical diagnosis of JME completed a brief seizure history questionnaire (Table 1 - patient's epilepsy history). Two resting-state neuroimaging studies were performed: 1) 148 channel MEG combined with 32 EEG, 2) fMRI. MEG and EEG were reviewed for interictal and ictal patterns of epilepsy. Equivalent current dipoles (ECD) and Coherence analysis were performed on the MEG data, results were coregistered and displayed on the patient's MRI. Coherence is a measure of oscillating neuronal activity and their synchronicity across the cortex, which defines the neuronal network connectivity. fMRI was performed on each patient using a 3T MRI system. Diffusion Tensor imaging (DTI) analysis was used to measure and evaluate the connectivity between 9 regions of interest by calculating power spectra and coherence measurements. Results: Both patients demonstrated a normal awake posterior dominant rhythm as well as physiologic sleep architecture seen in MEG and EEG. Patient 1 had multiple generalized polyspike-andwave discharges present on both EEG and MEG. Four of the five interictal events mapped to the right middle frontal gyrus (MFG), and one mapped to the left MFG. MEG Coherence analysis revealed scattered areas of high coherence within the right and left postcentral gyrus, precentral gyrus, as well as the left supramarginal gyrus, and right superior frontal gyrus. Patient 2 had multiple generalized spike and polyspike-and-wave discharges present on both EEG and MEG. Five interictal spike events mapped to the right precentral gyrus, MFG, and right and left inferior frontal gyri. MEG Coherence analysis showed high coherence within the left fusiform gyrus. fMRI results for Patient 1 displayed more consistent differences from controls than Patient 2. The bilateral prefrontal cortices and middle temporal gyrus showed stronger connectivity in Patient 1 and less connectivity with Patient 2.

Conclusions: Two patients with a clinical diagnosis of JME had a MEG and fMRI that demonstrated different imaging patterns. Although other reports have found involvement of the prefrontal cortex in patients with JME, no clear epileptic network was identified in these two patients. However, each patient showed different patterns of neuronal oscillations with MEG coherence as well as different connectivity patterns on fMRI. These findings may suggest different subsyndromes of JME have different epileptic networks.

Epilepsy History

\begin{tabular}{|c|c|c|}
\hline History & Patient 1 & Patient 2 \\
\hline Age & 30 years-old & 26 years-old \\
\hline $\operatorname{Sex}$ & Femalc & Female \\
\hline Age at first seizure & 12 years-old & 4 ycars-old \\
\hline First type of seizure & Absence & Febrile scizure (GTCS) \\
\hline $\begin{array}{c}\text { Age when epilepsy was } \\
\text { diagnosed }\end{array}$ & 15 years-old & 7 years-old \\
\hline Current seizure types & Absence, myoclonic, GTCS & Myoclonic and GTCS \\
\hline $\begin{array}{l}\text { Years diagnosed with } \\
\text { cpilepsy }\end{array}$ & 15 ycars & 19 ycars \\
\hline $\begin{array}{l}\text { Seizure lirequency per } \\
\text { montl }\end{array}$ & 0 & $\begin{array}{c}\text { No GTC } \\
4-5 \text { clusters of myoclonic seizures } \\
\text { (15) per month most pronounced } \\
\text { around menses }\end{array}$ \\
\hline Seicure provocation & $\begin{array}{l}\text { Visual stimulation, sleep } \\
\text { deprivation, medication } \\
\text { withdrawal or missed doses }\end{array}$ & $\begin{array}{l}\text { Sleep deprivation, alcohol, stress. } \\
\text { menses }\end{array}$ \\
\hline Scizure risk factors & None known & Febrile scizure \\
\hline $\begin{array}{l}\text { Anti-seizure medications } \\
\text { tried }\end{array}$ & VPA, LTG & $\begin{array}{c}\text { CB7, VPA, LTG, LEV, TPM, ZNS, } \\
\text { LCM, clonazepam }\end{array}$ \\
\hline $\begin{array}{l}\text { Reason anti-seizure } \\
\text { medications were } \\
\text { discontinucd }\end{array}$ & VPA - tolerability (weight gain) & $\begin{array}{l}\text { LEV - tolerability (depression), VPA } \\
\text {-tolerability (pregnancy concerns) } \\
\text { TPM, ITG, CBZ - incffectivc }\end{array}$ \\
\hline $\begin{array}{l}\text { Cument inti-seizure } \\
\text { medication }\end{array}$ & $\begin{array}{l}\text { LTG } 250 \mathrm{mg} \text { in the morning and } \\
450 \mathrm{mg} \text { QHS }\end{array}$ & $\begin{array}{l}\text { ZNS } 200 \mathrm{mg} \text { QIIS } \\
\text { LCM } 200 \mathrm{mg} \text { QHS } \\
\text { VPA } 500 \mathrm{mg} \text { QHS } \\
\text { clona7cpam } 0.5 \mathrm{mg} \text { PRN }\end{array}$ \\
\hline Last EEG & 1997 & 2011 \\
\hline
\end{tabular}


Abbreviations - Generalized tonic-clonic seizure (GTCS); valproic acid (VPA), lamotrigine (LTG), carbamazepine (CBZ), levetiracetam (LEV), topiramte (TPM), zonisamide (ZNS), lacosamide (LCM)

\subsection{9}

THE TONIC SEIZURES OF LENNOX GASTAUT PHENOTYPE: ICTAL SPECT SHOWS A CORTICORETICULAR NETWORK

U. Intusoma ${ }^{1,2}$, M. Stagnitti ${ }^{1}$, R. Masterton ${ }^{3}$, D. Abbott $^{3}$, M. Newton $^{5,3}$, G. Jackson ${ }^{3,5}$, J. Freeman ${ }^{4}$, A. S. Harvey ${ }^{4,1}$ and John S. ARCHER $^{1,3}$ ( ${ }^{1}$ The University of Melbourne, Melbourne, VIC, Australia; ${ }^{2}$ Paediatric Neurology, Prince Songkla University, Hat Yai, Thailand; ${ }^{3}$ Florey Neuroscience Institutes, Melbourne, VIC, Australia; ${ }^{4}$ Royal Children's Hospital, Melbourne, VIC, Australia and ${ }^{5}$ Austin Health, Melbourne, VIC, Australia)

Rationale: Lennox-Gastaut syndrome (LGS) is a severe epilepsy phenotype with characteristic electro-clinical features but diverse aetiologies. Key EEG features are interictal slow spike-and wave (SSW) and generalised paroxysmal fast activity during sleep (GPFA). Tonic seizures are a key clinical feature. These shared electro-clinical characteristics suggest common networks are involved in generating epileptiform activity in LGS. Our study aimed to determine the common pattern of cerebral perfusion changes during the tonic seizures by comparing ictal and interictal single photon emission computed tomography (SPECT).

Methods: From the databases of the Comprehensive Epilepsy Programmes of Austin Health and The Royal Children Hospital, Melbourne (Jan 2001 - Dec 2011) we identified patients with 'Lennox-Gastaut Phenotype' (LGP) who had ictal SPECT. The inclusion criteria were 1) interictal EEG with SSW and GPFA; 2) tonic seizures on video-EEG monitoring (VEM), with EEG features of electrodecrement, low voltage fast activity followed by high voltage SSW or notched delta; 3) ictal SPECT performed during/or immediately after the seizure. Seizure onset was marked as the start of electrodecrement and/or tonic stiffening, and seizure offset was defined as the end of low voltage fast activity. Injection latency was the time between the offset of ictal fast activity and SPECT injection. After global intensity normalisation, re-alignment and Gaussian smoothing, we performed a voxel-wise comparison of ictal and interictal studies across the group (SPM8). Spatial evolution of blood flow changes was explored by examining early and late injection groups. SPECT changes were displayed thresholded at $\mathrm{p}<0.05$ (uncorrected) and extent $\mathrm{k}>125$ voxels.

Results: We identified ten ictal-interictal SPECT pairs from 7 patients (4 males; age $20.1 \pm 14.5 \mathrm{yrs}$ ). As expected, tonic seizures were brief with a median duration of 10 seconds (range 6-29 sec). Injection latency was -8 to 48 seconds. In the early injection group ( $<10$ seconds; 3 studies) hyperperfusion was observed over bilateral association cortices (prefrontal, premotor and parietal association cortex, Fig 1A) with a significant cluster of SPECT activation in pons $(\mathrm{p}<0.05$ corrected FWE, $\mathrm{k}<125)$. Reduced perfusion was seen in primary cortical areas including sensori-motor and visual regions. In the late injection group, hyperperfusion was seen over cerebellum and bilateral parietal cortices (Fig 1B). Pooling all studies, SPECT increases were most prominent in the cerebellum.

Conclusions: This study provides confirmatory evidence that the tonic seizures of LGS involve the pons, and suggests that tonic seizures result from activity in a cortico-reticular network, containing bilateral frontal and parietal association areas, and the pontine brainstem reticular formation. This may explain why tonic seizures of LGS share similar, axial motor predominant clinical features, despite aetiologies that include cortical lesions of varied type and location. We believe these concepts help with clinical interpretation of the otherwise confusing SPECT changes of tonic seizures.

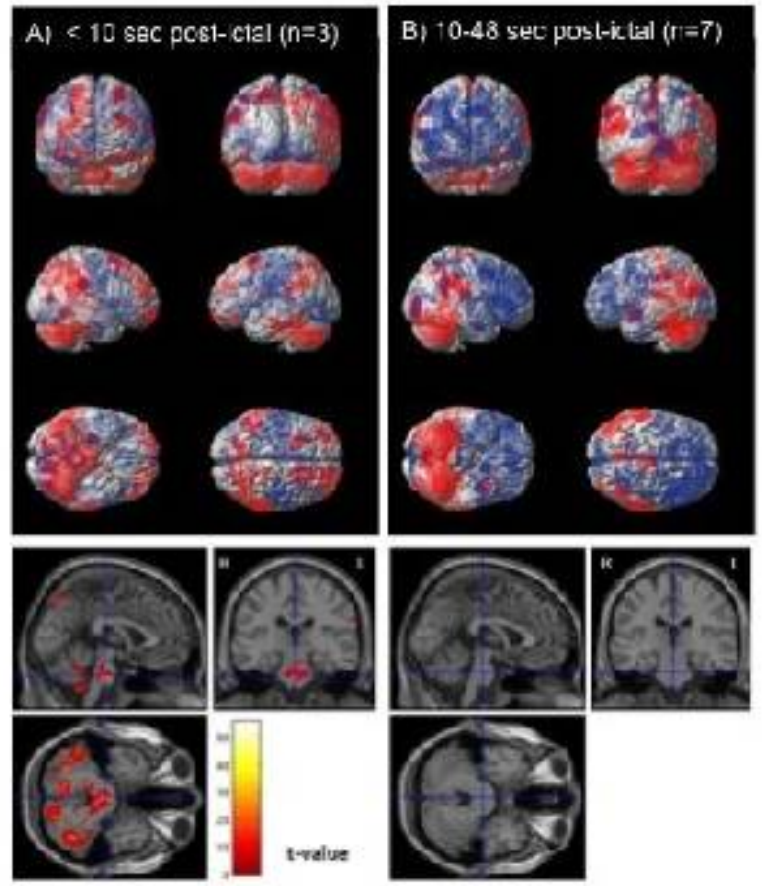

Ictal-interictal SPECT of tonic seizures - Upper panel: surface rendering, $\mathrm{p}<0.05$ (uncorrected), extent $\mathrm{k}>125$ voxels; Lower panel: T1-MRI overlay, $\mathrm{p}<0.05$ (corrected for FWE), $\mathrm{k}>125$ voxels

Cormorbidity (Somatic and Psychiatric): Medical Conditions

1.200

PREVALENCE OF COMORBIDITIES AT ONSET OF UNPROVOKED SEIZURES IN CHILDREN - A REPORT FROM STOCKHOLM INCIDENCE REGISTER OF EPILEPSY (SIRE)

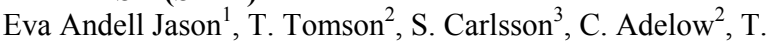
Andersson $^{3}$, E. Hellebro ${ }^{2}$ and P. Amark ${ }^{1}\left({ }^{1}\right.$ Institute of Women and Child Health, Karolinska Institute, Stockholm, Sweden; ${ }^{2}$ Department of Clinical Neurosciences, Karolinska Institute, Stockholm, Sweden and ${ }^{3}$ Institute of Environmental Medicin, Karolinska Institute, Stockholm, Sweden)

Rationale: Comorbidities influence prognosis, treatment strategies and everyday life of children with epilepsy. The present study was designed to analyze the presence of comorbidities at the time of seizure onset in a population-based cohort of children with newly diagnosed unprovoked seizures.

Methods: From September 1st 2001 a surveillance system has been in use to identify incident cases of first unprovoked seizures (neonatal seizures excluded) and epilepsy among residents of Northern Stockholm, Sweden. Inclusion is based on validation through review of relevant medical records of potential cases.

The medical records from the time of the first unprovoked seizure and the following six months of all children ( 0 -18 years) identified as cases through December 31st 2006, n=766, were analysed to identify potential comorbidities (developmental delay, language problems, mental retardation, cerebral palsy, autism spectrum disorders, attention deficit hyperactivity disorder, psychiatric diagnoses) at. We also applied the acronym ESSENCE (Early Symtomatic Syndromes Eliciting Neurodevelopmental Clinical Examinations) to describe prevalence of developmental disorders in children younger than 6 years where more specified diagnostic criteria may not have been met yet.

Comorbidities were considered to be present when a relevant defined diagnosis appeared in the medical records or when the records included a clear problem description highly suggestive of a comorbidity. 
Results: As early as at the time of the first recognised seizure or the following six months $32 \%(n=247)$ of the children in the cohort had one or more of the defined comorbidities. Out of the cases, $11 \%$ $(n=87)$ were diagnosed with, or highly suspected to have, two or more of the diagnoses: mental retardation, cerebral palsy, autism spectrum disorders, attention deficit hyperactivity disorder or psychiatric diagnoses.

We found ESSENCE highly suspected or ascertained among 34\% $(n=105)$ of the children younger than 6 years $(n=315)$ with a recently diagnosed unprovoked seizure.

All studied comorbidities were more frequent in our cohort of children with newly diagnosed unprovoked seizures compared with reported rates in the general population. Mental retardation was found in $16 \%(\mathrm{n}=119)$ in our seizure cohort vs. $3 \%$ in the general pediatric population, cerebral palsy in $9 \%(n=71)$ vs. $2.5 \%$, autism spectrum disorders in $7 \%(n=50)$ vs. $1 \%$, ADHD in $6 \%(n=48)$ vs. $5 \%$.

Conclusions: The high prevalence of neurodevelopmental problems in this group of children at onset of unprovoked seizures supports the concept that seizures often appear as part of a broader neurological problem and that in most cases comorbidities should not be considered consequences of the seizure disorder. The observations serve as an important reminder of the needs for a broader approach to the management of children with epilepsy than treatment of their seizures.

Sources of funding: Stockholm County Council, AFA

\subsection{1 \\ DOES THE AMYGDALA PLAY A ROLE IN WEIGHT GAIN AFTER EPILEPSY SURGERY? -- A TERTIARY CARE CENTER EXPERIENCE}

VIJAYALAKSHMI RAJASEKARAN, T. Weimer, P. Pergami, G. Pawar and A. Palade (NEUROLOGY, WEST VIRGINIA UNIVERSITY, MORGANTOWN, WV)

Rationale: Obesity is a major risk factor for multiple medical comorbidities and the number one health problem in the US. Recently there has been increased interest in mechanisms that regulate body weight and obesity. Multiple studies have shown that the amygdala and its associated circuits play a crucial role in regulating the behavioral and neural mechanism of feeding behavior. The purpose of this study is to investigate the effect of amygdala resection on weight gain in patients with intractable epilepsy. . Methods: This is a retrospective chart review to include patients older that 18 who underwent amygdalo-hippocampectomy at our institution between 2002 and 2012. Demographics, type of surgery, surgical site, pathology and follow up data were collected. Results: 60 patients ( 36 female, 24 male) who had either selective amygdalohippocampectomy or cortical amygdalohippocampectomy for intractable epilepsy were included in this retrospective study. 36 patients had left temporal lobe epilepsy surgery, and 24 had the same procedure on the right side. Initial and follow up weight was recorded for group comparison. In the left sided surgical patients, 19 out of 22 (86\%) females and 9 out of 14 (64\%) males gained weight. In the right sided surgical patient 9 out of 14 (64\%) female patients and 7 out of $10(70 \%)$ male patients lost weight. In total $73 \%$ showed weight changes either by gaining or losing weight depending on their surgical side. There was no significant change in their reported mood to explain the weight changes. In addition this appeared to have no relationship with seizure reduction or freedom reported by patients or antiepileptic medication being used.

Conclusions: Previous studies in humans and animal models have shown that amgydala lesions influence the weight and mood changes. Our study shows that there is a difference between left and right sided amygdala regulating mechanisms on feeding behavior and weight gain. This is significant and it cannot be attributed to normal weight gain due to aging as studies have shown that people gain weight approximately $0.23-0.39 \mathrm{~kg} / \mathrm{y}$ depending upon their age over 20 year periods. There is limited research on weight changes after amgydala surgery for temporal lobe epilepsy and and our study suggest a possible relation between surgical side and weight changes. This observation needs to be explored in the future.

\subsection{2 \\ EPILEPSY PATIENTS SHOULD RECEIVE DXA SCREENING}

L. Lee ${ }^{1}$, M. Wagner ${ }^{1}$ and B. $\mathrm{Wu}^{2}\left({ }^{1}\right.$ Rutgers University, Piscataway, $\mathrm{NJ}$ and ${ }^{2}$ University of Medicine and Dentistry of New Jersey, New Brunswick, NJ)

Rationale: Patients with epilepsy (PWE) have a higher fracture risk possibly due to vitamin D deficiency, seizure-related injuries, or antiepileptic drug therapy (AED) reducing bone mineral density (BMD). Screening of fracture risk in PWE may be necessary at a younger age. Currently, other than Dual-Energy X-Ray Absorptiometry (DXA), there are limited tools available to assess such a risk of fracture. We evaluated the cost-effectiveness of an online assessment tool FRAX in comparison with DXA on screening PWE at high risk of fracture. Methods: We used the online FRAX tool to determine a patient's risk of fracture for the next 10 years. High risk was a FRAX threshold of $\geq 3 \%$ for hip and $20 \%$ for major osteoporotic fracture (MOF). We surveyed and calculated FRAX scores for 126 patients attending the Robert Wood Johnson outpatient epilepsy clinic. Patients included were age 40-90 years with a diagnosis of epilepsy and currently taking AEDs. We observed the effect of adjusting the FRAX tool by including DXA scans or adding epilepsy as a secondary osteoporosis risk factor.

Results: The average FRAX scores for 126 patients with mean age 52.5 years and body mass index (BMI) 28.5 were $4.8 \%$ for MOF and $0.7 \%$ for hip fracture, and 8 patients $(6.4 \%)$ were above treatment threshold. Eighty-four of these patients also had a DXA scan, in which 43 (51.2\%) had low BMD (T-score between -1 and -2.5) and of those, $7(16.2 \%)$ had osteoporosis (T-score $\leq-2.5)$. Two of the 7 osteoporotic patients identified by DXA did not have a FRAX score above threshold even when including BMD or adding epilepsy as a secondary risk factor (Table 1). Of patients with low BMD, 48.8\% had poor seizure control, $83.7 \%$ were taking AEDs known to reduce BMD, and $65.1 \%$ were taking calcium and vitamin D supplements. Based on national osteoporosis guidelines, $8(9.5 \%)$ of the 84 patients with DXA scans were eligible for bone treatment. Thirty patients had a second DXA scan, and $8(26.7 \%)$ experienced an increase in both their lumbar spinal and left femoral neck BMD. All 8 were taking AEDs known to induce low BMD and $87.5 \%$ reported at least taking calcium or vitamin D supplements. This is compared to the 22 (73.3\%) patients whose BMD declined in the left femoral neck, spine or both, in which $13(59.1 \%)$ were taking medium to high-risk AED's and half were taking calcium or vitamin D supplements. Patients less than 40 years old were excluded, but of those patients ( $n=93), 8.6 \%$ had BMDs below their expected range for age and $7.5 \%$ had osteoporosis.

Conclusions: More patients were identified for treatment by using the DXA than by using the FRAX tool, even when adjusting for BMD or secondary osteoporosis. Counseling about bone protective behaviors was not a part of this study and more extensive counseling may have resulted in greater improvement in BMD. Patients taking AEDs should be evaluated for DXA screening and counseled about bone protective behaviors as part of routine care. Earlier detection of low BMD allows clinicians to implement bone protective strategies or change high-risk AEDs to minimize fracture risk. 


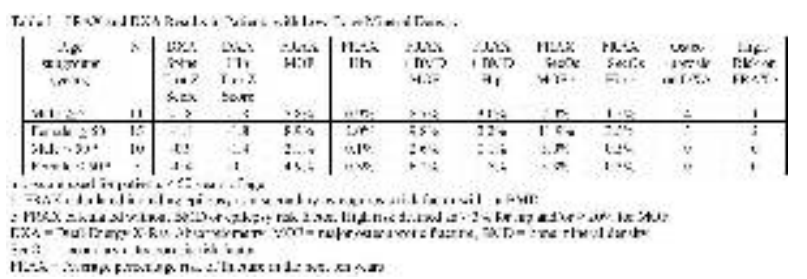

Table 1. FRAX and DXA Results in Patients with Low Bone Mineral Density

\subsection{3}

\section{WHAT IS THE WORST THING ABOUT HAVING} EPILEPSY? A CHILD AND PARENT PERSPECTIVE

A. F. VanStraten and Yu-Tze Ng (Oklahoma University Health Sciences Center, Oklahoma City, OK)

Rationale: The psychosocial burden on children with epilepsy and their families has been documented although often without specific reasons why epilepsy is so bad and the effects of the disease on the child and the parent can be vastly different. Do children and parents view epilepsy similarly?

Methods: Children with epilepsy who attended a one-week summer camp specifically for children with epilepsy and their parents were asked to participate in a voluntary survey (formal questionnaire). Full parental consent was obtained.

Results: Twenty campers, aged 9 to 15 years, and their parents responded. Thirty-eight percent of campers and $30 \%$ of parents chose being thought of as different or being teased as the worst thing about having epilepsy whereas only $37 \%$ and $33 \%$ respectively, chose the actual seizures themselves. Over half of children and parents would want to have fewer or no seizures if they could change one thing. The majority of children and parents, $65 \%$ and $93 \%$, agree that epilepsy camp is fun while the other third of children cite it as a place where they feel normal.

Conclusions: Overall, children with epilepsy and their parents tend to view the burdens of epilepsy the same. We believe that it is dramatic that despite the morbidities and potential risks of the seizures and treatments themselves, the majority of children are most concerned about the stigma attached to having epilepsy! Epilepsy camps and similar activities are beneficial for the children and their families to feel better, normal and be more independent with their diagnosis.

\begin{tabular}{|c|c|c|c|c|c|c|}
\hline & & \multicolumn{2}{|c|}{ Children } & \multicolumn{2}{|c|}{ Parents } & \multirow[b]{2}{*}{ p-value } \\
\hline & & \# & $\%$ & \# & $\%$ & \\
\hline \multicolumn{7}{|c|}{ What is the worst part about epilepsy? } \\
\hline A & Feeling helpless & 0 & 0 & 3 & 20 & 0.14 \\
\hline B & Actual seizures & 6 & 37.5 & 6 & 40 & 0.6 \\
\hline c & Thought different/Teased & 8 & 50 & 4 & 26.7 & 0.29 \\
\hline D & Meds & 2 & 12.5 & 2 & 13.3 & 0.68 \\
\hline $\mathrm{E}$ & Other & 0 & 0 & 0 & 0 & - \\
\hline \multicolumn{7}{|c|}{ What would you change about having epilepsy? } \\
\hline A & People's views/prejudices & 1 & 6.25 & 2 & 12.5 & 0.52 \\
\hline B & To not take meds & 2 & 12.5 & 4 & 25 & 0.38 \\
\hline c & To have less or no scizunes & 10 & 62.5 & 10 & 62.5 & 0.61 \\
\hline D & To not have seizures in public & 2 & 12.5 & 0 & 0 & 0.27 \\
\hline E & Other & $\mathrm{J}$ & 6.25 & 0 & 0 & 0.52 \\
\hline \multicolumn{7}{|c|}{ What do you think about Camp Candlelight? } \\
\hline A & I was made to come/hate it & 0 & 0 & 0 & 0 & - \\
\hline B & It's the greatest thing ever & 10 & 66.7 & 13 & 92.9 & 0.38 \\
\hline C & $1 \mathrm{focl}$ normal/fit in & 5 & 33.3 & 1 & 7.1 & 0.17 \\
\hline D & Don't really mind/care & 0 & 0 & 0 & 0 & - \\
\hline
\end{tabular}

1.204

ASSESSING HEALTH IN EPILEPSY AND NON EPILEPSY PATIENTS USING THE PATIENT-REPORTED OUTCOMES MEASUREMENT INFORMATION SYSTEM (PROMIS)

Marianna Spanaki-Varelas ${ }^{1}$, V. Remedio ${ }^{1}$, C. Barth $^{3}$, S. Gaddam ${ }^{1}$, V. Wasade $^{1}$, L. Pietrantoni ${ }^{1}$, D. Nerenz ${ }^{2}$, G. Barkley ${ }^{1}$ and L. Schultz ${ }^{3}$ ( ${ }^{1}$ Henry Ford Comprehensive Epilepsy Program, Henry Ford Health System, Detroit, MI; ${ }^{2}$ Health Policy and Health Services Research, Henry Ford Health System, Detroit, MI and ${ }^{3}$ Public Health Services, Henry Ford Health System, Detroit, MI)

Rationale: The Patient-Reported Outcomes Measurement Information System (PROMIS) supported by the NIH is a validated and easy to use tool for self-reported health. We used a set of PROMIS measures in patients admitted to the Henry Ford Epilepsy Monitoring Unit (EMU) and diagnosed with either epilepsy or seizure like events with psychological basis (psychogenic non epileptic seizures; PNES) or both. We aimed to determine if there are any significant differences amongst the groups. This is a follow up study on our previous preliminary study presented at the AES meeting in 2011.

Methods: A 46-item, five-domain measure set (PROMIS short forms) was used to assess general physical, social, and mental health (10 questions), pain behavior (7 questions), pain interference (6 questions), sleep disturbances (8 questions), depression (8 questions) and anxiety (7 questions). We included all EMU patients in the past 8 months except for those who declined participation, were younger than 18 or mentally challenged. The t-scores for all measures are scaled to a mean of 50 and standard deviation of 10 . The EuroQoL (EQ-5D) index score (a disease non specific quality of life instrument) was also calculated. "Epilepsy", "epilepsy and nonepilepsy", and "non-epilepsy" groups were compared using ANOVA or a Kruskal-Wallis test. If a significant difference was found, posthoc analyses were done. The post-hoc comparisons were either a two sample t-test, if ANOVA was used, or a Wilcoxon rank sum, if Kruskal-Wallis was performed. A Bonferroni adjustment was done for the post-hoc comparisons. Additional Chi-Square tests were done for race and gender comparison.

Results: We included 69 patients (50 females; mean age $40.9 \pm 12.5$; $80 \%$ Caucasian). Thirty patients had epileptic seizures, 32 psychogenic non epileptic and 7 both.

For pain interference, pain behavior, global physical health, and EQ$5 \mathrm{D}$ the "Epilepsy" patients were significantly different $(p<0.05)$ than both the "Epilepsy and Non-Epilepsy" and "Non-Epilepsy" patients. We found no significant differences between the "Epilepsy and NonEpilepsy" and "Non-Epilepsy" patients. Epilepsy patients had better pain interference $(52.7 \pm 9.9)$ and pain behavior $(52.0 \pm 10.2)$ t-scores (lower scores), global physical health $(47.4 \pm 7.0)$ and EQ-5D $(0.69 \pm$ $0.09) \mathrm{t}$-scores (higher scores) than non epilepsy $(62.4 \pm 10.9,58.4 \pm$ $9.4,39.3 \pm 10.0$ and $0.60 \pm 0.12$, respectively).

No differences were found in anxiety, depression and global mental health t-scores between the "Epilepsy" and the other two groups. Conclusions: We found no difference between "epilepsy: and "non epilepsy patients" in domains such anxiety, depression, sleep and global mental health. This finding is in agreement with a previous study from our group (Tojek et al, 2000) that showed that depression and anxiety were only marginally greater in non epilepsy when compared with epilepsy patients. In our studied groups patients with seizure like events with psychological basis have more impairment due to pain and worse global physical health and quality of life.

\subsection{5 WTTHDRAWN}




\subsection{6}

IMPACT OF MALNUTRITION IN PATIENTS WITH LENNOX GASTAUT SYNDROME

gessen salmeron gomez and E. Barragan Perez (Hospital Infantil de Mexico Dr. Federico Gomez, Distrito Federal, Mexico)

Rationale: To establish the impact of malnutrition in patients with Lennox Gastaut Syndrome.

Methods: We followed up 35 patients 5 years: the establishment of the degree of malnutrition by Gomez classification and Waterloo, EEG findings were reviewed and number of medications used. Results: Predominant male sex 55\%, with a mean age 6 years. Malnutrition was present in $77 \%$. EEG was observed increased impairment in patients with malnutrition, number of drugs in patients without malnutrition average 2 , while the malnourished 2.8

Conclusions: Malnutrition is a common phenomenon in pediatric patients with Lennox-Gastaut syndrome, and in accordance to gravity has a greater impact on crisis management by promoting refractoriness and thereby the quality of life. This gives a need to reevaluate therapeutic strategies for the management of these patients.

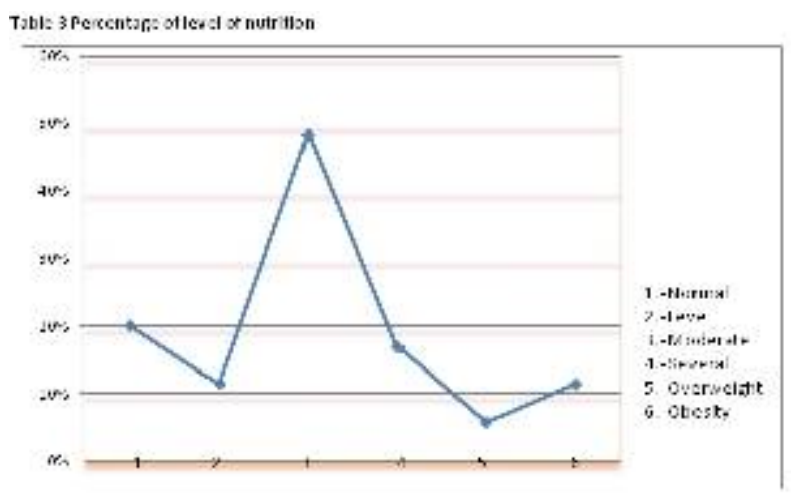

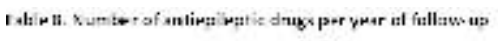

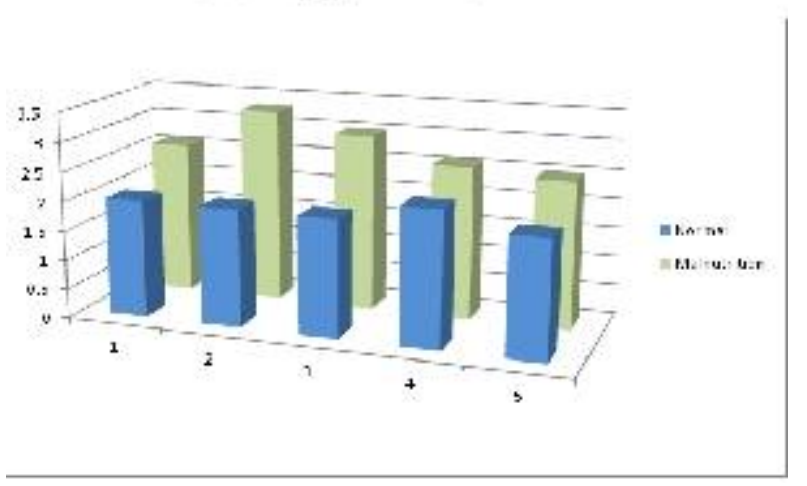

Cormorbidity (Somatic and Psychiatric): Psychiatric Conditions

\subsection{7}

COMMON COMORBIDITIES IN WOMEN AND MEN WITH EPILEPSY AND THE RELATIONSHIP BETWEEN NUMBER OF COMORBIDITIES AND HEALTH PLAN PAID COSTS IN 2010

Andrew N. Wilner ${ }^{1}$, A. Soucy ${ }^{2}$, B. Sharma ${ }^{2}$, A. Krueger ${ }^{2}$ and A. Thompson ${ }^{2}\left({ }^{1}\right.$ Neurology, Lawrence and Memorial Hospital, New London, CT and ${ }^{2}$ Disease Management, Accordant Health Services, a CVS/Caremark company, Greensboro, NC)

Rationale: Epilepsy affects more than 2 million people in the United States (U.S.). Comorbidities associated with epilepsy increase heath care costs and complicate epilepsy diagnosis and management. Identifying and treating comorbidities is one of the gaps in epilepsy care identified in the Institute of Medicine's 2012 epilepsy report. Our objectives were to determine 1) the prevalence of common comorbidities by gender, and 2) the relationship between the number of comorbidities and health plan paid costs for each gender.

Methods: Data came from eight commercial health plans located in different parts of the U.S. that contract with Accordant Health Services (AHS) for care management of members with rare and/or chronic conditions. Members with epilepsy were identified using an in-house algorithm that utilizes ICD-9 diagnosis codes and pharmacy utilization. The presence of comorbidity-specific diagnosis codes for 29 comorbidities was examined in health insurance claims for the year 2010. The top 10 most prevalent comorbidities were identified for women and men. The health plan paid amount per member per month (\$, PMPM) was summarized by gender and by number of comorbidities for each gender. The correlations between number of comorbidities and health care costs were examined separately for women and men.

Results: The study population consisted of $\mathrm{N}=6,621$ individuals with epilepsy ( $52 \%$ women; $48 \%$ men). The average age was 36 years for women and 34 years for men. More women (50\%) than men (43\%) had one or more of the 29 comorbidities specified $(\mathrm{P}<0.05)$. For women, the top 10 comorbidities and the prevalence rates were psychiatric diagnosis $(16 \%)$, hypertension $(12 \%)$, asthma (11\%), hyperlipidemia $(11 \%)$, headache $(7 \%)$, diabetes $(6 \%)$, UTI $(5 \%)$, hypothyroidism (5\%), anemia (5\%) and migraine (4\%). For men, the top 10 comorbidities and the prevalence rates were psychiatric diagnosis (15\%), hyperlipidemia (12\%), hypertension (12\%), asthma $(8 \%)$, diabetes $(5 \%)$, headache $(4 \%)$, cancer $(4 \%)$, CAD $(3 \%)$, anemia (3\%) and GERD (3\%). The health plan paid cost $(\mathrm{PMPM} \pm \mathrm{SEM}$ ) was $\$ 1,045 \pm 61$ for women and $\$ 1,052 \pm 63$ for men, but these costs were statistically similar $(\mathrm{P}>0.05)$. The correlation between comorbidity burden and the health plan paid cost PMPM was $\mathrm{r}=+0.22(\mathrm{P}=<0.001)$ for women and $\mathrm{r}=+0.26(\mathrm{P}=<0.001)$ for men. The rate of increase in the health care cost PMPM (\$) with an increasing number of comorbidities was greater for men than for women $(\mathrm{P}<0.05)$.

Conclusions: More women than men with epilepsy had one or more comorbidities $(\mathrm{P}<0.05)$. Psychiatric diagnosis, hyperlipidemia, hypertension, asthma, diabetes, headache and anemia were among the top 10 comorbidities common for women and men. The average health plan paid cost was not different by gender $(\mathrm{P}>0.05)$. The number of comorbidities was positively and significantly associated with the health plan paid cost for both women and men $(\mathrm{P}<0.05)$. The rate of increase in the health care cost PMPM (\$) with increasing comorbidity burden was greater for men than for women $(\mathrm{P}<0.05)$.

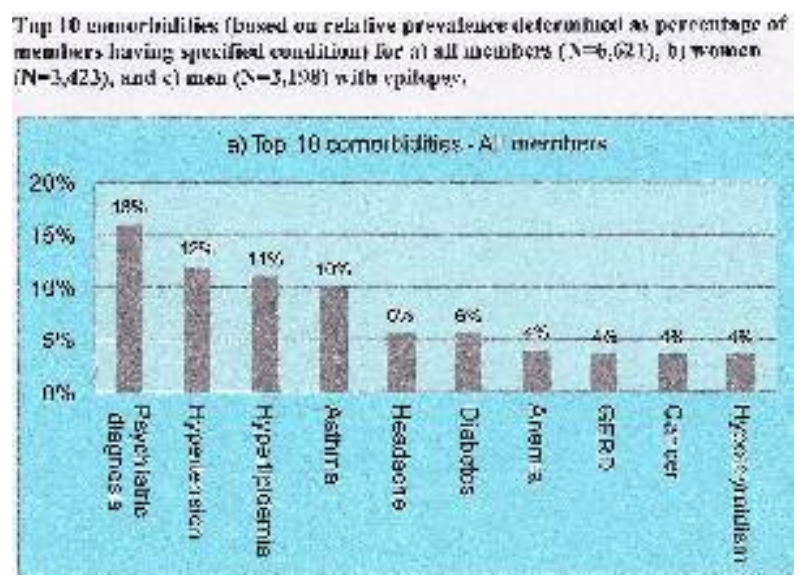

1.208

BRIGHT LIGHT THERAPY FOR SYMPTOMS OF DEPRESSION AND ANXIETY IN EPILEPSY

Sallie Baxendale, J. O'Sullivan and D. Heaney (Institute of Neurology, UCL, London, Buckinghamshire, United Kingdom) 
Rationale: Bright Light Therapy (BLT) influences the regulation of melatonin and is an established treatment for seasonal affective disorder (SAD). It is also an effective treatment for non seasonal depression in some patients. Depression and anxiety are amongst the most common psychiatric co-morbidities in epilepsy, with epidemiological studies suggesting a bi-directional relationship. This randomised control trial was designed to examine the efficacy of BLT for symptoms of anxiety and depression in adults with focal epilepsy .

Methods: 101 adults with medically intractable, focal epilepsy were recruited to a parallel design, double blind, randomized trial of BLT. Participants completed the Hospital Anxiety and Depression Scale (HADS) at the beginning (T1) and end of a 12 week baseline period (T2). 51 of the participants were allocated a high intensity light box emitting 10,000 lux using an automated permuted block randomisation grid; 50 were allocated a cosmetically identical box emitting 2,000 lux (low intensity), a sub therapeutic dose in other patient populations. Both groups were instructed to use their box for 20-30 minutes, upon waking every day for 12 weeks. Following the end of the trial the participants completed the HADS for a third time (T3).

Results: 60 participants (31 high intensity/29 low intensity) completed the trial and returned adequate data for analyses. Anxiety scores were significantly reduced following the BLT at T3 compared to the baseline measurements (T1 \& T2) in both the high and low intensity groups. The same pattern was evident in the depression data with depression scores significantly reduced following the BLT at T3 compared to the baseline measurements $(\mathrm{T} 1 \& \mathrm{~T} 2)$ in both the high and low intensity groups.

Conclusions: We did not find any differences between the high vs. low intensity bright light therapy groups on self reported symptoms of anxiety and depression. Both groups reported a significant reduction in symptoms following treatment. BLT may be an effective treatment for symptoms of low mood in epilepsy at lower intensities than those typically used to treat SAD. Further work is needed to investigate this hypothesis with an adequate placebo condition. Trial Registration: www.ClinicalTrials.gov. Registration number NCT01028456.

Source of Funding: This study was funded by a research grant number AP1202 from Action Medical Research, UK.

\subsection{9}

RELATIONSHIP BETWEEN OF DEPRESSION, ANXIETY, AND QUALITY OF LIFE OUTCOMES POST EPILEPSY SURGERY: A PROSPECTIVE MULTICENTER STUDY Hamada Hamid ${ }^{1,2}$, K. Blackmon 3 , X. Cong ${ }^{4}$, L. Atlas ${ }^{3}$, J. Dziura ${ }^{4}$, M. Sperling $^{5}$, S. Shinnar ${ }^{6}$, A. Berg ${ }^{7}$, B. G. Vickrey ${ }^{8}$, J. Langfitt ${ }^{9}$, T. S. Walczak $^{10}$, C. Bazil ${ }^{11}$ and O. Devinsky ${ }^{3}\left({ }^{1}\right.$ Neurology, Yale University, New Haven, CT; ${ }^{2}$ Neurology, VA Hospital, West Haven, CT; ${ }^{3}$ New York University School of Medicine, New York City, NY; ${ }^{4}$ School of Public Health, Yale University, New Haven, CT; ${ }^{5}$ Thomas Jefferson University Medical School, Philedelphia, PA; ${ }^{6}$ Albert Einstein College of Medicine, New York City, NY; ${ }^{7}$ Northern Illinois University, DeKalb, IL; ${ }^{8}$ University of California at Los Angeles, Los Angeles, CA; ${ }^{9}$ University of Rochester School of Medicine, Rochester, NY; ${ }^{10}$ Minnesota Comprehensive Epilepsy Program, St. Paul, MN and ${ }^{11}$ Columbia University, New York City, NY)

Rationale: People with seizures have a lower quality of life (QOL) compared to people with other chronic disease. Depression and anxiety scores strongly correlate with QOL scores in cross-sectional studies. However, the relationship between depression and anxiety symptoms and QOL outcomes after epilepsy surgery has not been explored with a prospective, longitudinal design.

Methods: The design, measures, and subject recruitment of the Epilepsy Surgery Multicenter Study has been detailed elsewhere. Briefly, 7 tertiary epilepsy centers enrolled 396 patients and completed a comprehensive diagnostic workup that included a comprehensive medical history and physical exam; neuropsychological, neuroimaging and neurophysiology testing; and a psychiatric as well as quality of life evaluation. Subjects were evaluated prior to surgery, then at 3, 6, 12, 48, and 60 months after surgery. Standardized, assessments included The Quality of Life in Epilepsy Inventory-89 (HRQOL) and Beck Depression (BDI) and Anxiety (BAI) Inventories. Seizure outcome was classified into one of four categories: "excellent" for subjects seizure free (and no auras) for all five years, "good" for two consecutive years but not all five, "fair" if subjects were seizure free for one year but never two consecutive years, and "poor" if subjects never had a one year period of seizure freedom.

A mixed-model repeated-measures analysis was used to analyze the association between depression, anxiety, seizure outcome, seizure history, and overall HRQOL scores over time. A separate analysis was completed for each of the four HRQOL subscales (cognitive distress, physical health, mental health, epilepsy-targeted). Each model included gender, race, education, duration of seizure history, laterality of seizure focus, resection location, BDI and BAI scores, and time as fixed effects, as well as random intercept and slope. Results: All four subscores of QOL improved over time $(\mathrm{p}<0.0001)$. Excellent and good seizure control had a significant positive impact on the overall QOL compared to fair and poor seizure control; interestingly, there was no difference in change in overall QOL over time between the fair and poor groups. The time and seizure control interaction was marginally significant $(\mathrm{p}=0.0606)$. Subjects who had left-sided resections showed a significantly lower rate of improvement in overall QOL score compared to those with rightsided resection. The BDI and BAI scores were strongly negatively associated with overall QOL; increases in BDI and BAI scores were associated with decreased overall QOL score. This association appeared to be driven by the cognitive subscore and the association was not significant in the other subscores. Duration of seizure history, gender, race, education and temporal versus extratemporal resection did not show significant association with overall QOL.

Conclusions: Depression and anxiety are strongly and independently associated with worse QOL post epilepsy surgery. Management of mood and anxiety is a critical component to post-surgical care.

Demographic Characteristics, Seizure History, and Surgical Outcome of Subjects

\begin{tabular}{|c|c|}
\hline Characteristics & $\begin{array}{l}\mathrm{N}(\mathscr{\%}) \mathrm{S} \text { or Mean } \\
\quad \pm \mathrm{SD}\end{array}$ \\
\hline Age at the first seizure (year) & $14.7 \pm 11.5$ \\
\hline Age at the surgery(year) & $37.7 \pm 10.4$ \\
\hline \multicolumn{2}{|l|}{ Giender } \\
\hline Female & $201(53.0)$ \\
\hline Male & $178(47.0)$ \\
\hline \multicolumn{2}{|l|}{ Fthnicity } \\
\hline Black or African-America, not of Hispanic Origin & $35(9.3)$ \\
\hline Hispanic & $20(5.3)$ \\
\hline White, not of Hispanic Origin & $306(81.0)$ \\
\hline Olher & $17(4.5)$ \\
\hline \multicolumn{2}{|l|}{ Education } \\
\hline High School or Less & $154(40.8)$ \\
\hline College or Partial College & $174(46.2)$ \\
\hline Graduate Sehool or Professional Schesol & $49(13.0)$ \\
\hline \multicolumn{2}{|l|}{ Surgery type } \\
\hline Extra-Temporal lobe Resection & $49(12.9)$ \\
\hline 'lemporal Lobe Resection & $330(87.1)$ \\
\hline \multicolumn{2}{|l|}{ Side of Surgery } \\
\hline $1 . \mathrm{eft}$ & $185(48.8)$ \\
\hline Right & $194(51.2)$ \\
\hline \multicolumn{2}{|l|}{ Seizure control status over live year period } \\
\hline Complete seizure-free for 5 year following surgery (Excellenl) & $108(35.1)$ \\
\hline $\begin{array}{l}\text { Two or more consecutive years of seizure freedom but at least one seizure withia } \\
\text { the five year period (Good) }\end{array}$ & $106(34.4)$ \\
\hline One of more year but less than two consecutive years of scizure freedom (Tair) & $36(11,7)$ \\
\hline Never one full year of seizure freedom throughout the five year peried (Poor) & $58(18.8)$ \\
\hline
\end{tabular}


Numbers may not sum to totals due to missing data, and column percentages may not sum to $100 \%$ due to rounding.
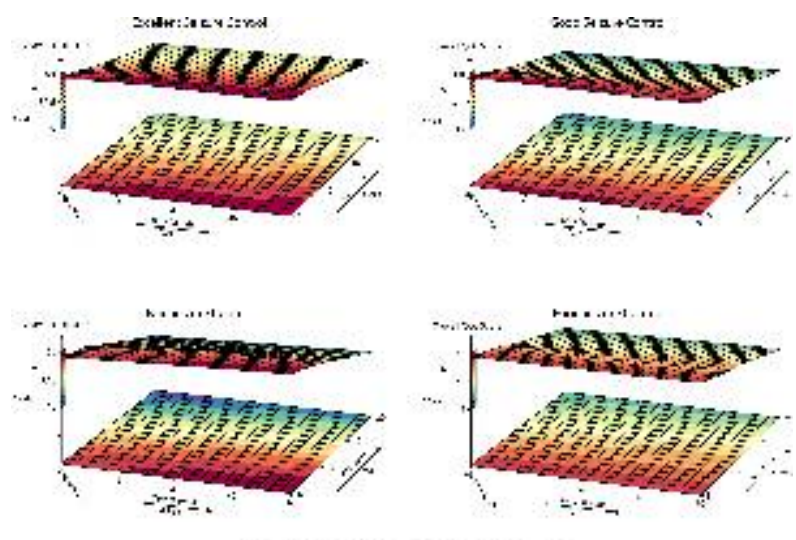

\subsection{0}

\section{DEPRESSION, SEIZURE TYPE AND POLYTHERAPY WITH} AED INFLUENCE SUBJECTIVE MEMORY IN WOMEN WITH EPILEPSY

Barbara C. Jobst ${ }^{1}$, C. L. Harden ${ }^{3}$, R. C. Scott $^{1}$, K. M. Fowler ${ }^{2}$, A. G. Herzog $^{2}$ and ,. For the progesterone study group ${ }^{4}\left({ }^{1}\right.$ Neurology, Geisel School of Medicine at Dartmouth, Hanover, NH; ${ }^{2}$ Harvard Neuroendocrine Unit, Beth Israel Deaconess Medical Center, Wellesley, MA; ${ }^{3}$ Neurology, Hofstra North Shore-LIJ School of Medicine, Great Neck, NY and ${ }^{4}$ Harvard Neuroendocrine Unit, Wellesley, MA)

Rationale: Memory impairment, a common co-morbidity of epilepsy, significantly impacts quality of life, but treatment options are few. Subjective memory complaints do not necessarily correlate with objective neuropsychological impairment. We examined which factors influence subjective memory complaints in a large well documented patient cohort of women with epilepsy (WWE). Methods: The National Institute of Health Progesterone Trial randomized 294 women, ages 11-46 years, with focal, poorly controlled epilepsy. In 249 patients, baseline data about subjective memory impairment was available as part of the quality of life assessment (QOLIE-31). Seizure frequency, AED type and epilepsy characteristics were well documented for three months in the baseline phase of the trial. Depression and mood was repeatedly measured by the Beck Depression Inventory (BDI II) and Profile of Mood States (POMS) without treatment intervention over three months. Cognitive complaints were correlated to epilepsy characteristics, AEDs, seizure frequency, BDI II, POMS mood states, body mass index (BMI) using linear regression.

Results: Cognitive complaints were highly correlated with depression $(\mathrm{p}<0.001)$ on BDI II and all mood state subscales $(p<0.001)$ on the POMS (tension, vigor, depression, anger, fatigue, confusion). Overall $25.4 \%$ of patients showed mild depressive symptoms (BDI II $>11$ ) and $14.3 \%$ showed clinically significant depression (BDI II $>15$ ). Memory complaints also correlated with seizure type and whether the patient was on mono- or polytherapy with AEDs. Patients with secondarily generalized seizures had more significant memory complaints than patients with focal seizures as their main seizure type $(\mathrm{p}<0.001)$. Polytherapy increased cognitive complaints $(\mathrm{p}<0.05)$. There was no difference in memory complaints whether patients received enzyme-inducing or non-enzyme inducing AEDs. Seizure type, type of AED therapy and depression were independently related to memory complaints in a multiple regression model.

Memory complaints did not correlate with age, BMI, catamenial seizure pattern or average seizure frequency over three months $(p>0.05)$. Side of seizure onset, unilateral or bilateral seizure onset and temporal or extratemporal seizure onset had no impact on subjective memory $(\mathrm{p}>0.05)$.
Conclusions: Depression is a major determinant for cognitive complaints in WWE. Among epilepsy related factors, secondarily generalized seizures, as most severe seizure type, and type of AED polytherapy were independently associated with subjective cognitive dysfunction. Although the relationship is not proven to be causal, the management of depression may be important in the treatment of memory impairment, a frequent co-morbidity in WWE.

\subsection{1}

ATTENTION DEFICIT DISORDER SYMPTOMS RATES AND IMPACT IN ADULTS WITH SELF-REPORTED EPILEPSY: RESULTS FROM THE NATIONAL EPIDEMIOLOGICAL SURVEY OF EPILEPSY

Alan B. Ettinger ${ }^{1,3}$, R. Ottman ${ }^{2}$, R. B. Lipton ${ }^{3}$, J. Cramer ${ }^{4}$ and M. L. $\operatorname{Reed}^{5}\left({ }^{1}\right.$ Neurological Surgery PC, Lake Success, NY; ${ }^{2}$ Columbia University, New York, NY; ${ }^{3}$ Albert Einstein College of Medicine, Bronx, NY; ${ }^{4}$ Yale University, Houston, TX and ${ }^{5}$ Vedanta Research, Chapel Hill, NC)

Rationale: Symptoms of Attention Deficit Disorder (ADD and ADHD) have been well-described in pediatric epilepsy cases(1) but little is known about rates and impact of ADD/ADHD in adults with epilepsy.

Methods: An 11-item screening survey to 340,000 households in 2008 obtained self-reported information on epilepsy and other conditions from a representative sample of 172,959 adults aged $18+(2)$. A 2009 follow-up postal survey to persons with epilepsy included the 6-item Adult ADHD Self-Report Scale (ASRS) to identify individuals at risk for Attention Deficit Disorder (ADHD). Validated measures assessing quality of life (QOLIE-10, QLESQ), social functioning (Sheehan Disability Scale, SDS), depression (PHQ-9) and anxiety (GAD-7) were included as were sociodemographic and employment status questions. Chi-square was used to assess sociodemographics differences between those at risk for ADHD (ASRS+) and those not at risk (ASRS-). Linear regression models (controlling for sociodemographics) were used to contrast ASRS+ vs. ASRS- cases on quality of life and social functioning measures. Logistic regression was used to assess differences in rates of work related disability. Depression and anxiety frequently cooccurred with ADHD risk and were included as covariates in further modeling to assess their influence on ASRS group differences. Beta $(\beta)$ or Odds Ratios (OR) and 95\% confidence intervals (CI) were calculated for each model.

Results: N=1451 individuals reported doctor-diagnosed seizure or epilepsy and were currently using prescription seizure medication. The analysis included $n=1361$ with complete ASRS data. $\mathrm{N}=251$ $(18.4 \%)$ were ASRS+ and more likely to be younger $(\mathrm{p}=.045)$, have lower incomes $(p<.001)$ and live rurally $(p=.004)$. No gender difference was observed $(\mathrm{p}=.301)$. Compared with ASRS- cases, ASRS+ cases had higher/worse QOLIE scores ( $\beta$ 8.44, CI 7.42-9.45, $\mathrm{p}=.000)$, lower QLESQ social $(\beta-0.21$, CI $-0.24--0.19, \mathrm{p}=.000)$, QLESQ physical $(\beta-0.19, \mathrm{CI}-0.22--0.17, \mathrm{p}=.000)$ and SDS global scores $(\beta 9.82$, CI 8.52-11.13, $\mathrm{p}=.000)$. They were also more likely to be on work related disability (OR 2.59 CI 1.89-3.53, $\mathrm{p}=.000$ ). PHQ-9 and GAD-7 scores above clinical cut-point occurred in $23.5 \%$ and $25.7 \%$ of ASRS + cases respectively, and in $2.0 \%$ and $5.7 \%$ of ASRScases. Regression results were attenuated but remained significant when PHQ-9 and GAD-7 scale scores were included as model covariates.

Conclusions: Attention Deficit Disorder symptoms are common in adults with epilepsy and have an adverse impact upon quality of life and psychosocial functioning, independent of comorbid depression and anxiety.

References:

1. Hamoda et al. Expert Rev Neurother. 9(12): 1747-1754

2. Ottman et al. Epilepsia, 52(2):308-315 


\subsection{2}

LONGITUDINAL MONITORING OF DEPRESSION IN AN ADULT EPILEPSY CLINIC

Robert C. Doss ${ }^{1,2}$, S. M. Mason ${ }^{1}$ and P. E. Penovich ${ }^{1,2}\left({ }^{1}\right.$ Minnesota Epilepsy Group, St. Paul, MN and ${ }^{2}$ Neurology, University of Minnesota, Twin Cities, MN)

Rationale: Epilepsy clinics serve the needs of individuals with complex medical and mental health challenges who may require more frequent and focused intervention. The monitoring of depression (DEP) and suicidal symptoms in these patients allows for broadening our understanding of the relationship between mood and epilepsy. The purpose of the current project is to explore selfreported DEP symptoms in epilepsy clinic patients evaluated over consecutive appointments.

Methods: The DEP screening questionnaires of adult patients seen at the Minnesota Epilepsy Group outpatient clinic over a 20 month period were reviewed. The questionnaire included the Neurological Disorders Depression Inventory for Epilepsy (NDDI-E) and the suicide question from the Beck Depression Inventory-II (BDI-SUI). In addition, data was collected regarding demographics, current antiepileptic drugs (AEDs), other psychotropic medications, and current involvement in mental health services. Those individuals who were identified as depressed at their first appointment were given a DEP information and resource packet.

Results: 1997 screening questionnaires were completed by 1226 patients over a 20 month time period. $155(13 \%)$ patients were identified as having at least 3 clinic appointments during this same time period. The mean NDDI-E total scores for appt1, appt2, and appt3 were 12.1, 11.7, and 10.8, respectively. Paired t-tests between appt 1 and appt 3 revealed a significant difference $(\mathrm{p}<.001)$ with the trend towards progressive symptom reduction. Additionally, $23 \%$ of this patient sub-group scored in the clinically depressed range $(>15)$ on the NDDI-E at appt 1 and $6 \%$ indicated suicidal ideation on the BDI-SUI. Further review of this DEP sub-group showed a similar trend in symptom reduction over time with mean NDDI-E scores for appt1, appt2, and appt3 of 18.1, 15.3, and 14.4, respectively. Paired ttests between appt 1 and appt 3 revealed a significant reduction in depressive symptoms $(\mathrm{p}<.001)$. The improved NDDI-E scores between appt 1 and appt 3 coincided with increased utilization of psychiatry ( $9 \%$ vs. $21 \%$ ) and counseling (17\% vs. $21 \%)$ while psychotropic medication management remained stable at 50\%. There was little change in mean number of AEDs prescribed between appt1 and appt3 (1.9 vs. 2.1).

Conclusions: Approximately $16 \%$ of adult patients presenting to an epilepsy clinic report significant DEP per the NDDI-E. This study has established that depressive symptoms improve over time in a subgroup of regularly seen patients. There was no clear relationship between the reduction in depressive symptoms and psychological or psychiatric treatment, although these services were accessed slightly more frequently over time. The number of AEDs and psychotropic medications remained constant. We suggest that the DEP screening process, minimal intervention (i.e., info about DEP), and/or regular return-to-clinic appointments likely offer therapeutic benefit through symptom acknowledgment and educational/resource support. With close monitoring and modest intervention, patients presenting with DEP are apt to experience improved quality of life.

\subsection{3 \\ ASSOCIATION OF ANXIETY SYMPTOMS WITH EPILEPSY TYPE AND SEIZURE LOCALIZATION}

Heidi Munger Clary and M. J. Hamberger (Neurology, Columbia University Medical Center, New York, NY)

Rationale: Psychiatric comorbidity, including anxiety, is increasingly recognized as an important determinant of quality of life in epilepsy. Nevertheless, little is known regarding the association between anxiety symptoms and epilepsy related factors, including lateralization and localization of epileptogenic foci.
Methods: A cohort of 500 adults with epilepsy (mean age: 39.3 years) who underwent neuropsychological evaluation at the Columbia Comprehensive Epilepsy Center between 1999 and early 2012 was identified, using a clinical database derived from medical records. Anxiety symptoms were assessed using the anxiety subscale of the Symptom Checklist 90-R (SCL-90-R), a validated self-report instrument, with $T$ score of $>60$ corresponding to the 84th centile of anxiety symptoms among an adult non-patient normative group. The cohort was divided into 2 groups by reported anxiety symptoms: high anxiety (SCL-90R anxiety T score $>=60$ ) and lower anxiety (anxiety $\mathrm{T}$ score $<60$ ). The association of anxiety group with demographic and epilepsy related factors was assessed using chi square and twosample $t$ tests. The association of anxiety group with lateralization and localization of epileptogenic foci was also assessed within focal epilepsy and temporal lobe epilepsy (TLE) subgroups.

Results: The overall prevalence of high anxiety symptoms (SCL-90R T score $>=60$ ) was $46 \%$. There was no significant association between anxiety group and age, gender, education level, seizure frequency, age of epilepsy onset, presence of epilepsy causing lesion, or presence of mesial temporal sclerosis. High anxiety was more common among noncaucasians than caucasians $(\mathrm{p}=0.025)$. Also, the number of current antiepileptic drugs (AEDs) was associated with anxiety group $(\mathrm{p}=0.018$, chi square $)$, with more patients on AED polytherapy in the high anxiety group, though test for trend was not statistically significant $(\mathrm{p}=0.075)$. Epilepsy type (focal vs. generalized) was significantly associated with anxiety $(\mathrm{p}=0.021)$, as $47 \%$ of focal epilepsy patients $(\mathrm{N}=362)$ reported high anxiety symptoms, in contrast to $31 \%$ of generalized epilepsy patients $(\mathrm{N}=58)$. TLE $(\mathrm{N}=313)$ was also associated with high anxiety symptoms compared to non-TLE $(\mathrm{N}=98)(\mathrm{p}=0.008)$. Among focal epilepsy patients, lateralization of seizure focus was not associated with anxiety group. However, among TLE patients, there was a nonsignificant trend toward patients with left foci reporting high anxiety symptoms $(\mathrm{p}=0.082)$, as $52 \%$ of patients with left TLE reported high anxiety( $\mathrm{N}=177)$, while only $41 \%$ of right TLE patients reported high anxiety $(\mathrm{N}=111)$. Among TLE patients, there was no association between anxiety group and mesial versus lateral temporal focus.

Conclusions: Anxiety symptoms are highly prevalent among epilepsy patients in this sample, particularly among those with TLE and other focal epilepsies. AED polytherapy and noncaucasian race may be risk factors for comorbid anxiety in epilepsy, though these findings require further study. Screening for anxiety symptoms may be useful in the clinical setting, especially among those with focal epilepsy.

\subsection{4 \\ PREVALENCE AND ASSOCIATES OF RECENT SUICIDAL IDEATION AND BEHAVIOR IN TREATMENT-RESISTANT FOCAL EPILEPSY WITHOUT RECENT DEPRESSION: THE ASERT STUDY}

Andres M. Kanner ${ }^{1}$, D. Hesdorffer ${ }^{2}$, J. Pollard ${ }^{3}$, M. Sperling ${ }^{4}$, G. Krauss $^{5}$, C. Harden ${ }^{6}$, K. Posner ${ }^{7}$ and J. French ${ }^{8}\left({ }^{1}\right.$ Neurological Sciences, Rush University Medical Center, Chicago, IL; ${ }^{2} \mathrm{GH}$ Sergievsky Center, College of Pysicians and Surgeons Columbia University, New York, NY; ${ }^{3}$ Neurology, University of Pennsylvania School of Medicine, Philadelphia, PA; ${ }^{4}$ Neurology, Thomas Jefferson University, Philadelphia, PA; ${ }^{5}$ Neurology, John Hopkins University, School of Medicine, Baltimore, MD; ${ }^{6}$ Neurology, Long Island Jewish Hillside Medical Center, New Hyde Park, NY; ${ }^{7}$ Psychiatry, College of Physicians and Surgeons, New York, NY and ${ }^{8}$ Neurology, New York University School of Medicine, New York, NY)

Rationale: While suicidal ideation (SI) and suicidal behavior (SB) have been typically associated with depressive disorders in patients with epilepsy (PWE), these phenomena have been observed in the absence of a mood disorder. Occasionally, SI has been identified in patients with epilepsy without "overt" psychiatric illness, but the 
frequency of this phenomenon is not known. The purpose of this study is to assess recent SI and SB in treatment-resistant focal seizures without depression and without any identifiable psychiatric disorders.

Methods: A mulitcenter, cross-sectional study at 6 epilepsy centers. Inclusion criteria were: 18-70 years; EEG-confirmed focal epilepsy for $>2$ years; $>1$ simple partial (with motor component), complex partial/secondarily generalized seizure per month for the past 6 months; currently taking 1-3 AEDs; and failed >2 AEDS, including current therapy. SI and SB were assessed with the Columbia Suicide Severity Rating Scale. The MINI International Neuropsychiatric Interview assessed current Axis I psychiatric disorders. High risk SI included intent / plan. SB included suicide attempt (SA), interrupted or aborted attempt, preparatory acts/behavior, and non-suicidal selfinjurious behavior. Analyses examined current psychiatric disorders and $\mathrm{SI} / \mathrm{SB}$ in the absence of recent depression and any psychiatric disorder, according to our findings on the MINI.

Results: Among the 204 participants, those with current depression $[\mathrm{n}=31(15.2 \%)]$ were more likely to have current SI (51.6\% vs $12.7 \% ; \mathrm{p}<0.0001)$, but were not more likely to have current SA (9.7\% vs $4.1 \%$; $\mathrm{p}=0.2)$. Among the $173(84.8 \%)$ without recent depression, 25 (14.5\%) displayed SI and/or SB: 18 (10.4\%) had recent SI only, $3(1.7 \%)$ had recent SB only and $4(2.3 \%)$ had both recent SI and SB. Among the 22 subjects with SI, 5 (22.7\%) had high risk SI. Among the 7 with SB, $3(42.9 \%)$ attempted suicide, 2 (28.6\%) engaged in non-suicidal self-injurious behavior, 1 (14.3\%) aborted the SA, and $2(28.6 \%)$ prepared for a SA. Among the 22 subjects with SI without current depression, anxiety was present in 7 $(31.8 \%)$, eating disorders in $1(4.6 \%)$, and substance abuse with a psychiatric disorder in $3(13.6 \%)$. One of these patients had anxiety and eating disorders. Among the 7 subjects with SB without current depression, anxiety was present in $1(14.3 \%)$, and eating disorders in $1(14.3 \%)$. Among the 132 who denied any symptoms of current psychiatric disorder, $11(8.3 \%)$ had SI only, $2(1.5 \%)$ had SB only and $4(3.0 \%)$ had both SI and SB.

Conclusions: In patients with treatment-resistant focal epilepsy, the occurrence of SI and SB can be associated not only with depression, but also with anxiety and eating disorders without concurrent depression. In addition, SI and /or SB can be identified in close to $13 \%$ of patients with no "apparent" psychiatric disorder. Thus, screening for depression is not sufficient to identify all patients at risk for suicidality.

\subsection{5 \\ SCREENING FOR MENTAL HEALTH SYMPTOMS IN A MULTIDISCIPLINARY PEDIATRIC EPILEPSY CLINIC} Kelly McNally ${ }^{1}$, J. Cass ${ }^{1}$, K. Robinson ${ }^{1}$ and L. Hamiwka ${ }^{2}\left({ }^{1}\right.$ Pediatric Psychology and Neuropsychology, Nationwide Children's Hospital, Columbus, $\mathrm{OH}$ and ${ }^{2}$ Neurology, Nationwide Children's Hospital, Columbus, $\mathrm{OH}$ )

Rationale: Despite the high rates of psychiatric co-morbidities that have been described in children with epilepsy, routine standardized assessment of mental health symptoms is often difficult to accomplish in a clinic setting. Furthermore, studies have suggested that there is a low rate of mental health service usage within this population. The primary aim of this study was to determine the utility of the Strengths and Difficulties Questionnaires (SDQ; Goodman, 1999) in identifying previously unmet mental health needs of children and adolescents with epilepsy presenting to a multidisciplinary clinic.

Methods: The SDQ was administered to parents of children and adolescents (age 5-17; mean age $=10.23$, S.D $=3.78 ; 52 \%$ male) presenting to a multidisciplinary pediatric epilepsy clinic $(\mathrm{n}=81$ to date; however, data collection is ongoing). This screening instrument is designed to quickly assess for mental health problems and impairments in children and has been validated in several populations. Parents also completed a brief questionnaire that indicates whether their child had received any previous mental health diagnoses or treatments.

Results: High rates of mental health problems were reported on the SDQ with $54 \%$ of the sample scoring above established clinical cutoffs on at least one subscale (Hyperactivity $=43 \%$ in clinical range; Conduct Problems $=25 \%$; Emotional Symptoms $=19 \%$ ). No gender differences were found on any subscales. Higher rates of hyperactivity symptoms were found in younger children $(r=-.23, p=$ .04 ); no other subscales were related to age. Of those patients with at least one clinical elevation, $64 \%$ were not receiving any type of mental health treatment; only $25 \%$ were participating in mental health counseling and $27 \%$ were taking a psychotropic medication. Conclusions: The SDQ is a valuable tool that can be used in an epilepsy clinic setting to identify children who have additional mental health needs. High rates of mental health symptoms, with relatively lower service usage, further highlight the need for standardized screening and psychology services within the clinic setting.

\subsection{6 \\ IMPACT OF A SCREENING TOOL ON MANAGEMENT OF PSYCHOSOCIAL COMORBIDITIES IN PERSONS WITH EPILEPSY}

Tracie Caller and B. Jobst (Dartmouth-Hitchcock Medical Center, Lebanon, NH)

Rationale: Psychosocial and behavioral problems are often neglected in the process of delivering care to persons with epilepsy (PWE). This stems from both under-recognition of these underlying problems as well as limited resources and programs with which to intervene. Unfortunately, the consequences are sometimes more disabling than the seizures themselves. Depression is the most commonly studied co-morbidity and is prevalent in an estimated $25-55 \%$ of PWE. Memory dysfunction is also prominent and can also be significantly disabling. A recently published Institute of Medicine report summarized the public health implications of co-morbidities in PWE such as depression and memory dysfunction. The aim of this study was to implement a screening tool to improve the recognition of psychosocial comorbidities such as depression and cognitive symptoms in an epilepsy clinic.

Methods: The study was conducted at Dartmouth-Hitchcock Epilepsy Center (DHEC). A screening tool was developed based on an existing tool created by Giliam et al, which quantifies symptoms of depression (NDDI-E, scored 6-24, with scores $>15$ suggestive of major depression) and a quality of life Likert scale (scale of 1-10, with 10 equaling best quality of life) along with a review of systems which includes memory and concentration. The screening tool was distributed to all patients seen by DHEC's 6 epilepsy providers over an 18 month period.

Medical records were reviewed for all patients age 18-49 completing questionnaires. Patients with severe intellectual disability and purely non-epileptic seizures were excluded. Records were reviewed to determine which providers used the data obtained by the screening tool for clinical decision making (designated as "adaptors") and whether the screening tool impacted referrals to mental health providers or new prescriptions for antidepressants.

Results: Of 140 patients who met inclusion criteria, the average NDDI-E score was 11 and average OOL 6.8; 26\% qualified for major depression based on the NDDI-E. The prevalence of memory symptoms was $55 \%$, and this correlated significantly with QOL and NDDI-E scores. Three providers reported routinely used the questionnaire in decision making and were considered "adapters". Major depression was addressed with mental health referral or medication change in $45 \%$ of cases by adapters and $36 \%$ by nonadapters $(\mathrm{p}=0.6)$. Adapters made medication changes in $36.6 \%$ $(p=0.01)$ and prescribed new antidepressants for $3.9 \%$ of cases $(\mathrm{p}=0.02)$ compared to non-adapters who made medication changes in only $10 \%$ and did not prescribe any new antidepressants. Compliance with new antidepressant at 3 months was $57 \%$. Only one patient with memory symptoms was referred for cognitive rehabilitation. 
Conclusions: Psychosocial comorbidities such as depression and cognitive dysfunction impact quality of life for PWE and contribute significantly to socioeconomic burden, yet are often not addressed routinely during clinic visits. Implementation of a screening tool can improve the recognition of these comorbidities and improve the rate at which these symptoms are addressed.

Comparison of adapters vs. non-adapters of a screening tool to address psychosocial comorbidities in PWE.

\begin{tabular}{|c|c|c|c|}
\hline & $\begin{array}{c}\text { Adapter } \\
\mathrm{n}=85\end{array}$ & $\begin{array}{c}\text { Non Adapler } \\
\mathrm{n}=55\end{array}$ & $\mathrm{p}$-value \\
\hline Average NTDDI-Г: scorc & 11.0 & 11.1 & 0.98 \\
\hline Average QOI, score & 6.8 & 7.1 & 0.4 \\
\hline Major Depression (NDDI-E >15) & $26(15 \%)$ & $29(44 \%)$ & $* 0.01$ \\
\hline Mental Health Addressed & $31 \%$ & $21 \%$ & 0.2 \\
\hline $\begin{array}{l}\text { Major Depression } \\
\text { Addressed }\end{array}$ & $45 \%$ & $36 \%$ & 0.6 \\
\hline Medication Change & $18 \%$ & $4 \%$ & $* 0.01$ \\
\hline New Antidepressant & $10 \%$ & $0 \%$ & $* 0.02$ \\
\hline mprovement in NDDI-E Score at Follow-up & 1.0 & 0.6 & 0.6 \\
\hline
\end{tabular}

*significant $(\mathrm{p}<0.05)$

\subsection{7}

\section{DEPRESSION AND QUALITY OF LIFE IN HISPANIC IMMIGRANTS AND US BORN EPILEPSY PATIENTS} Marcela A. Bonafina, M. Lancman, L. Myers, O. Laban, E. Feoli, E. Fertig and M. Lancman (Northeast Regional Epilepsy Group, New York, NY)

Rationale: Studies suggest that mood and anxiety disorders are more frequent among patients with epilepsy than in the general population. Mood and anxiety disorders are typically associated with indices of a poorer quality of life (QOL). The purpose of this study is to determine whether Hispanic immigrants with epilepsy present higher rates of depression and anxiety and reports of a lower quality of life than US-born epilepsy patients.

Methods: The sample included 28 Hispanic immigrants and 27 controls (US born patients with epilepsy) who completed the Beck Depression Inventory-II (BDI-II), Beck Anxiety inventory (BAI), Quality of Life Inventory-31 (QOLIE-31), the Woodcock-Munoz Bateria (BIA), and the Wechsler Abbreviated Scales of Intelligence (WASI). Patients with associated non-epileptic seizures were excluded. A structured chart review was performed to select epilepsy, social, and psychological variables.

Results: Of the 28 Hispanic patients, 8 were males and 20 females. For Hispanics, the mean age at the time of study was $44.28 \pm 3.029$ years, mean education was $10.00 \pm .768$, mean age of epilepsy onset was $25.90 \pm 3.687$. For the control group, 10 were males and 17 females. The mean age was $35.75 \pm 1.861(\mathrm{p}=0.021)$ and mean education was $13.15 \pm .449$. Educational attainment differed significantly between groups $(\mathrm{p}=0.001)$. Age of onset was 16.00 \pm 1.822 which is significantly younger than in the Hispanic group $(\mathrm{p}=0.026)$. The mean age at the time of testing was also significantly different (Hispanics: $44.28 \pm 3.029$ and control group: $35.75 \pm 1.981$ ). When BDI raw scores were compared, the Hispanic group mean was $24.71 \pm 3.098$ and the control group mean was $14.61 \pm 2.143$ ( $\mathrm{t}=$ 2.742, $\mathrm{p}=0.008)$. In fact, $25 / 28$ Hispanics (89\%) BDI scores fell in the moderate to severe range of depression while the control group did so in 11/27 (41\%). No significant differences were noted between the groups on QOLIE Total, Emotional Well-being, Social, Energy or Cognitive subscales. Of interest, on Emotional Well-Being, over 60 percent of respondents in both groups received a clinically impaired score on this subscale. No significant differences were noted between groups on a measure of anxiety (BAI). Intellectual functioning was significantly lower among Hispanic patients compared to the
American group (BIA SS $=79.75 \pm 2.208$, WASI SS $=91.35 \pm 2.562$, $\mathrm{t}=-3.443, \mathrm{p}=0.001)$. However, given that the tests used in both languages are different, the comparability of standard scores between the two is questionable.

Conclusions: Hispanics with epilepsy reported significantly higher levels of depression than controls. The two groups showed equally poor Emotional Well-being (QOLIE-31) but did not differ significantly on this or other indices, or on anxiety. This suggests that both groups have an equally diminished quality of life, while a significantly higher number of Hispanics also qualify for clinical depression. This supports that direct assessment for mood pathology should be regularly conducted in this subpopulation.

\subsection{8 \\ MULTI-CENTER TREATMENT TRIAL PILOT FOR PSYCHOGENIC NONEPILEPTIC SEIZURES}

W. Curt LaFrance ${ }^{1,2}$, A. Frank Webb ${ }^{1}$, A. S. Blum ${ }^{1,2}$, G. I. Keitner ${ }^{1,2}$, J. Barry ${ }^{3}$ and J. Szaflarski ${ }^{4}\left({ }^{1}\right.$ Rhode Island Hospital, Providence, RI; ${ }^{2}$ Brown University, Providence, RI; ${ }^{3}$ Stanford University, Palo Alto, $\mathrm{CA}$ and ${ }^{4}$ University of Cincinnati, Cincinnati, $\mathrm{OH}$ )

Rationale: Patients with psychogenic nonepileptic seizures (PNES) are often severely disabled, difficult to treat, and are frequently encountered in neurology, psychiatry, and emergency departments. The phenomenology and causes of PNES are well delineated, however, much less is known about effective treatments for PNES. Based on the prior single-center pilot trials suggesting benefit of cognitive behavioral therapy (CBT) for PNES (LaFrance et al, 2009) and a pharmacologic pilot randomized controlled trial (RCT) (LaFrance et al, 2010), this pilot RCT sought to evaluate different treatments for patients with PNES compared to standard medical care (SMC).

Methods: 38 patients with video EEG-confirmed lone PNES consented, and 35 completed baseline evaluations and were randomized among three sites to one of four treatment arms: Medication (flexible-dose sertraline) only, CBT only, CBT and Medication combined, or SMC. Seizure frequency, psychosocial and function variables were collected at baseline, and prospectively at week 2, week 8 (midpoint) and week 16 (treatment completion). Within group analyses for each arm were performed on primary (seizure frequency) and secondary outcomes.

Results: The CBT arm showed significant seizure reduction $(\mathrm{p}=0.03)$, improvement in functioning $(\mathrm{p}<0.001)$ and in symptoms scales $(\mathrm{p}<0.005)$. The combined arm showed significant improvements in functioning $(\mathrm{p}<0.01)$, and seizure improvement approached significance $(\mathrm{p}=0.06)$. Medication showed improvement in depression scores $(\mathrm{p}=0.04)$ and a trend in improved functioning $(\mathrm{p}=0.08)$. SMC showed no significant seizure reduction or improvement in secondary outcomes.

Conclusions: This prospective, multi-center, multi-arm, combined treatment pilot RCT for PNES revealed significant seizure reduction and improvements in symptoms and functioning with CBT for PNES. CBT+Medication also demonstrated significant improvements, and medication alone improved depression and approached significance on secondary measures. No improvements occurred in the SMC arm. This study supports the effect of CBT for PNES and demonstrates feasibility for a multi-center fully powered RCT for PNES.

Cormorbidity (Somatic and Psychiatric): Animal Studies

1.219

DEVELOPMENT OF ALLODYNIA FOLLOWING STATUS EPILEPTICUS IN TWO MODELS OF TEMPORAL LOBE EPILEPSY IN RATS

Eduardo Pineda ${ }^{1}$, S. You ${ }^{1,2}$, D. Shin ${ }^{1}$, R. Sankar ${ }^{1}$ and A. Mazarati ${ }^{1}$ ( ${ }^{1}$ University of California, Los Angeles, CA and ${ }^{2}$ SanggyePaik Hospital, Seoul, Republic of Korea) 
Rationale: Epilepsy and migraine are recognized as comorbid episodic disorders

and are believed to share common pathophysiology. Migraine is characterized by a dysfunction of pain processing and migraine sufferers often report the incidence of cutaneous allodynia, defined as hypersensitivity to touch by normally innocuous stimuli. We examined two established models of temporal lobe epilepsy (TLE), Lithium-pilocarpine (LiPC) and intrahippocampal kainic acid (iKA) to

develop and validate a model of migraine as a comorbidity of epilepsy.

Methods: Lithium-pilocarpine (LiPC) and intrahippocampal kainic acid (iKA) to

develop and validate a model of migraine as a comorbidity of epilepsy.

Status Epilepticus (SE) was induced using the Lithium-Pilocarpine model or by intrahippocampal injection of Kainate into the hippocampus. Two months after SE, epileptic subjects underwent behavioral tests for (I) cutaneous allodynia using the hot-plate test to measure acute thermal pain evident as latency to paw withdrawl and

(II) response to tonic persistent pain, using hindpaw injection of formalin (which elicits a sustained nociceptive stimuli) and measuring

total paw-licking time. The analgesic, Sumatriptan, is an agonist of serotonin 5-HT1B/5-HT1D receptors and is used as a treatment for migraine and cluster headaches. We tested the effect of Sumatriptan on

epilepsy associated allodynia. Furthermore, we examined the expression

of serotonin receptors $5-\mathrm{HT} 1 \mathrm{~B} / 5-\mathrm{HT} 1 \mathrm{D}$ in the periaqueductal gray (PAG),

which processes pain and thermal input in the midbrain.

Results: Our results

show that LiPC and iKA Post-SE animals demonstrated a reduced latency

to paw licking compared to vehicle treated animals exposed to temperatures of $47 \mathrm{C}-50 \mathrm{C}$ (i.e. at $48 \mathrm{C}$, Con:50secs vs iKA:25secs; $\mathrm{p}<0.01, \mathrm{n}=10$ ), indicative of

thermal allodynia. Intraperitoneal injection of Sumatriptan

$(300 \mathrm{ug} / \mathrm{kg})$

attenuated thermal allodyina in epileptic animals as evident by an increased latency to pawlick in the hot-plate test $(\mathrm{p}<0.01, \mathrm{n}=10)$.

Formalin tests

showed a measurable but not statistically significant difference in sensitivity to tonic sustained pain. Immunohistochemistry analyses revealed an increased expression of 5-HT1B and 5-HT1D receptors in the

PAG of epileptic animals compared to naïve animals.

Conclusions: The data presented provides evidence of the development of nociceptive impairments characteristic of allodynia, in two models of TLE in rats. Additionally, immunohistochemical analysis reveals changes in serotonin receptor expression may contribute to the development of allodynia. Together, our results show the development of allodynia in epileptic animals and

suggest that dysfunction of serotonin signaling may contribute to the establishment of epilepsy associated migraine impairments including allodynia.

\subsection{0 \\ ORIGINS OF DEPRESSION IN TEMPORAL LOBE EPILEPSY}

Céline M. Dubé ${ }^{1}$, P. Maras ${ }^{1}$, M. Choy ${ }^{1}$, S. Quddusi ${ }^{1}$, M. Look ${ }^{1}$, M. Pakhdikian $^{1}$, V. B. Risbrough ${ }^{2}$ and T. Z. Baram ${ }^{1}$

( ${ }^{1}$ Anatomy/Neurobiology and Pediatrics, University of CaliforniaIrvine, Irvine, CA and ${ }^{2}$ Psychiatry, University of California-San

Diego, La Jolla, CA)
Rationale: Temporal lobe epilepsy (TLE) is the most frequent epilepsy in adults. In addition to seizures, patients with TLE have a high prevalence of depression, estimated at up to $35 \%$ (vs $5-7 \%$ in the general population) and higher than in patients with other chronic diseases. TLE-associated depression is thus a major factor in reducing quality of life, and may result in suicide in up to an $11 \%$. Despite the clear importance of TLE-associated depression, its origins are not well understood. Whereas depression is often thought to result from the TLE, we tested the alternative hypothesis, that the insult that causes TLE might also provoke depression. We used an animal model of Febrile Status Epilepticus (FSE), a strong risk factor for TLE in humans and a direct cause of TLE in rodents. Specifically we examined if FSE in immature rats leads to depression-like behaviors.

Methods: Experimental FSE was induced in rats on post-natal day 10 $(\mathrm{n}=11)$. Rats that experienced FSE and normothermic controls $(\mathrm{n}=6)$ were implanted with cortical and bipolar electrodes one month after FSE and monitored for the presence of limbic seizures and interictal activity for 12 months. Experimental and control rats were tested for depression-like behaviors at 3 and 12 months of age, using the forced swim test.

Results: Three months after FSE, the duration of immobility, a measure of depression-like behavior, was quite similar and tightly clustered in the normal range in the normothermic control group. In contrast, duration of immobility was significantly increased in a subset of rats that experienced FSE. When compared to the mean \pm two standard deviations of the normothermic control rats, values were above this range in 4/11 FSE rats. Two out of these four rats developed spontaneous recurrent seizures (TLE) starting after the occurrence of depression-like behaviors. The depression-like behaviors at 10 months of age are under analysis.

Conclusions: These initial data suggest: (a) an insult known to cause TLE may provoke depression-like behaviors that precede TLE onset; (b) there is only a partial overlap between TLE and depression after an early-life insult that affects the limbic circuit. Still, the data suggest that in TLE, depression and epilepsy may evolve from the same insult, rather than TLE being the direct cause of depression. Supported by NIH grants NS35439, NS78279 and the Hewitt foundation.

\subsection{1 \\ EVIDENCE OF PATHOLOGY IN THE LATERAL HYPOTHALAMUS: A CONNECTION BETWEEN SLEEP DISORDER AND EPILEPSY IN THE KV1.1 KNOCK-OUT MOUSE}

Harrison M. Roundtree, K. A. Simeone, K. K. Samson and T. A. Simeone (Pharmacology, Creighton University School of Medicine, Omaha, NE)

Rationale: Common symptoms experienced by epileptic patients with sleep disorders include decreased sleep efficiency, greater sleep fragmentation, and longer sleep latency. These symptoms are seen in members of the non-epileptic population with dysregulation of the hypocretin network. Hypocretin neurons are located in the lateral hypothalamus ( $\mathrm{LH})$ and stimulate arousal pathways. Extracellular adenosine is responsible for promoting sleep by inhibiting the spontaneous activity of the hypocretin neurons via the A1 receptor (A1R). The Kcna1-null mouse, a Kv1.1 potassium channel knockout (KO) mouse, is a well defined model for temporal lobe epilepsy (TLE). We have previously shown that KO mice display the same symptoms seen in hypocretin dysregulation. We hypothesize that $\mathrm{KO}$ mice would exhibit injury in the LH, an area of the brain with multisynaptic connections to limbic structures such as the seizuregenic hippocampus. We know that astrogliosis reduces adenosine's inhibitory tone in the hippocampus by increasing expression of adenosine kinase (ADK), an enzyme responsible for regulating 
adenosine levels. Therefore we hypothesize that injury present in the LH accounts for hypocretin dysregulation.

Methods: The extent of injury in the LH was determined using immunohistochemical staining of WT and KO LH. Blood-brain barrier (BBB) permeability was measured using anti-mouse ImmunoglobulinG ( $\operatorname{IgG})$ to identify free antibodies. Glial fibrillary acidic protein (GFAP) was used as a marker to determine extent of astrogliosis. Expression of ADK was determined in LH with western blotting. Changes in A1R expression were also determined using western blot. Sensitivity of LH to the A1R agonist,

cyclopentyladenosine (CPA), was determined using multi-unit recordings acquired with an extracellular multi-electrode array and sorted based on characteristics of hypocretin neurons.

Results: KO mice show signs of injury in the lateral hypothalamus. BBB permeability in the KO LH was observed as punctate and diffuse IgG staining. Reactive astrocytes were observed in $\mathrm{KO} \mathrm{LH}$. Expression of ADK as determined by western blot did not differ $(\mathrm{n}=$ 6 per group). A1R protein expression was reduced in the KO LH $(\mathrm{n}=6$ per group, $\mathrm{p}<.05)$. Spontaneous activity decreased following application of CPA in both WT and KO LH, however KO LH was relatively less sensitive $(n=15, p<.0001)$.

Conclusions: We determined changes in the LH of a mouse model of TLE. As previously shown, KO mice also exhibit sleep disorder symptoms that are similar to those associated with a hypocretin dysregulation. We hypothesized that this dysregulation may be caused by injury in the LH affecting adenosine tone. Increase in IgG staining in KO LH suggests a compromised BBB. Injury was further evident by the presence of reactive astrocytes. Reduction in A1R expression and a decreased efficacy of an A1R-specific agonist suggests a reduction in adenosinergic regulation in $\mathrm{KO}$ LH. Together, these data suggest injury and reduced inhibitory adenosine tone in the LH of an epileptic mouse model may contribute to sleep disorder comorbidities.

\subsection{2 \\ POSSIBLE ASSOCIATION BETWEEN EPILEPSY AND ALZHEIMER'S DISEASE IN THE WISTAR AUDIOGENIC RAT (WARS) STRAIN}

Marilia G. Pereira $^{1}$, V. R. Santos ${ }^{1}$, J. A. Oliveira ${ }^{1}$, C. M. Costa-Neto ${ }^{2}$ and N. Garcia-Cairasco ${ }^{1}\left({ }^{1}\right.$ Department of Physiology, University of São Paulo, Ribeirão Preto, Brazil and ${ }^{2}$ Department of Biochemistry, University of São Paulo, Ribeirão Preto, Brazil)

Rationale: Recent studies have shown that epileptic seizures can exhibit pathophysiological processes such as cognitive impairment similar to Alzheimer's Disease (AD). Additionally, different degrees of memory problems, language and of executive functions can occur in patients with epilepsy. Despite many studies on the association between epilepsy and $\mathrm{AD}$, their mechanisms and neurobiological bases is still unclear. In this study we investigated the markers of AD in a model of epilepsy and comorbidities, the Wistar Audiogênic Rat (WAR) strain.

Methods: We used 2 years old animals divided into 4 groups: (1) Wistar submitted to no stimulus (WT2-NS); (2) WAR submitted to no stimulus (WAR2-NS); (3) WAR submitted to 28 audiogenic stimuli with 2 years old (WAR2-28S) and (4) WAR submitted to 28 audiogenic stimuli with 3 months old (WAR3m-28S). 3 months old WAR submitted to no stimulus was used as control of the experiments. We conducted immunohistochemistry for $\beta$-amyloid and phospo-tau (S396) in accordance with manufacturer protocols. $\beta$ amyloid and phospho-tau (S396) levels were assessed by Western blotting in hippocampal samples. Approved by Ethics Comittee (protocol number: 002/2011).

Results: The levels of $\beta$-amyloid into the 4 groups of senescent animals presented no difference, however preliminary data in WAR with 3 months old showed about $50 \%$ decrease of $\beta$-amyloid levels when compared to 2 years old animals (Wistar and WAR). These preliminary data suggest a possible regulation of $\beta$-amyloid in the
WAR strain, that we could not demonstrate in 2 years old animals, since at this age the Wistar rats (WT2-NS) exhibited high $\beta$-amyloid deposition, similar to the epileptic rats. For phospho-tau analysis, we found that phosphorylation levels were up-regulated 1.5 fold in WAR2-NS and WAR2-28S, but interestingly the WAR3m-28S showed no difference in the regulation when compared to WT2-NS and significant decrease in the phosphorylation when compared to WAR2-NS and WAR2-28S. Moreover, in preliminary results, the phospho-tau level was up-regulated in 2 years old WAR when compared to the 4 groups of 2 years old animals. These results suggest that WAR strain exhibit an intrinsic increased tau phosphorylation and that, when WAR were submitted to epileptic seizures at young age this was associated to a decrease of the tau phosphorylation in senescence.

Conclusions: In summary, we report for the first time, the possible association between epilepsy and AD in the WAR strain, a genetic model of epilepsy. Furthermore, early epileptic seizures were able to decrease the tau phophorylation in the senescence. Thus, plastic phenomena associated to the establishment of the epilepsy can represent neuroprotection mechanisms for the onset of AD physiopathology in this model. Financial support: FAPESP, CinapceFAPESP, Capes-PROEX, CNPq, FAEPA.

\section{Antiepileptic Drugs: Clinical Trials}

\subsection{3 \\ EFFICACY AND SAFETY OF DIAZEPAM AUTO- INJECTION FOR THE MANAGEMENT OF PATIENTS WITH EPILEPSY WHO REQUIRE INTERMITTENT MEDICAL INTERVENTION PROVIDED BY FAMILY OR CAREGIVERS TO CONTROL EPISODES OF ACUTE REPETITIVE SEIZURES}

Bassel Abou-Khali1 ${ }^{1}$, J. Wheless ${ }^{2}$, J. Rogin ${ }^{3}$, K. Wolter ${ }^{4}$, G. C. Pixton $^{5}$, R. B. Shukla ${ }^{6}$, N. A. Sherman ${ }^{4}$, C. L. Roland ${ }^{5}$, K. Sommerville ${ }^{5}$ and V. Goli ${ }^{7}{ }^{1}$ Neurology Department, Vanderbilt University Medical Center, Nashville, TN; ${ }^{2}$ Pediatric Neurology, University of Tennessee Health Science Center, Memphis, TN; ${ }^{3}$ Minneapolis Clinic of Neurology, Ltd, Golden Valley, MN; ${ }^{4}$ Pfizer Inc, New York, NY; ${ }^{5}$ Pfizer Inc, Cary, NC; ${ }^{6}$ Meridian Medical Technologies, Inc., Columbia, MD and ${ }^{7}$ Primary Care Clinical Sciences, Duke University Medical Center, Cary, NC)

Rationale: Introduction: Many patients with epilepsy experience acute repetitive seizures (ARS) which can evolve into status epilepticus if not treated promptly. An intra-muscular auto-injector of diazepam has been developed that can be administered by a patient's family or caregiver to treat ARS (Fig 1).

Rationale: To evaluate the efficacy and safety of a diazepam autoinjector administered by family or caregivers, for the management of patients with epilepsy who require intermittent treatment to control episodes of ARS.

Methods: In a Phase 3, randomized, double-blind, parallel, placebocontrolled, multicenter study, with an optional open-label continuation, patients were randomized to either placebo or diazepam auto-injector. Patients were stratified according to age group (2-5, 6$11, \geq 12 \mathrm{yrs}$ ), and assigned dose (5 mg, $10 \mathrm{mg}, 15 \mathrm{mg}$, or $20 \mathrm{mg}$ ) based on age and weight range. The primary endpoint was time to next seizure or rescue from $15 \mathrm{~min}$ to $12 \mathrm{~h}$ post-dose. Secondary endpoints included frequency of rescue events, numbers of seizures experienced post-dose, caregiver and physician treatment assessments, and safety.

Results: Patients $(n=234)$ were randomized to receive placebo or diazepam auto-injector, of whom $81 / 110$ patients in the placebo and $82 / 124$ patients in the diazepam auto-injector were included in the intent to treat group. Baseline characteristics were similar for the placebo and diazepam auto-injector groups. The time to next seizure or rescue showed statistically significant favorable results for diazepam over placebo auto-injector group (Fig 2). The 25th 
percentile for time to the next seizure was $1.18 \mathrm{hrs}$ for placebo and $2.70 \mathrm{hrs}$ for the diazepam auto-injector groups. Median time to event was inestimable for diazepam due to low number of events in the diazepam auto-injector group. Hazard ratio (diazepam vs. placebo, adjusted for age group) and 95\% CI for an endpoint was 0.55 (0.34 to $0.88, \mathrm{p}=0.012$ ). Rescue medication post-dose occurred in $30 \%$ (24/81) placebo vs. $17 \%$ (14/82) diazepam auto-injector group $(\mathrm{p}=0.066)$. A statistically significant reduction in the number of seizures for diazepam compared to placebo auto-injector group during the 12-h post-dose period was observed (median 1.0 seizures placebo, 0.0 seizures diazepam, $\mathrm{p}=0.010$ ). Treatment-emergent adverse events were reported in $43 \%$ (34/79) of placebo and $42 \%$ (34/81) diazepam auto-injector patients. The most common AEs were injection site pain, $14 \%$ placebo / $17 \%$ diazepam auto-injector group and injection site hemorrhage, $6 \%$ placebo/ $5 \%$ diazepam autoinjector group.

Conclusions: The diazepam auto-injector was significantly more effective than placebo at delaying the time to next seizure or rescue. Secondary efficacy endpoints were supportive of this primary result. Family or caregiver administered diazepam auto-injector was an effective and well tolerated treatment with AEs similar to placebo.

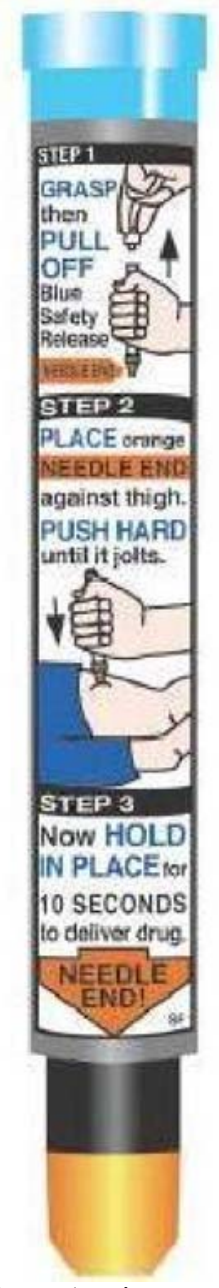

Figure 1: Diazepam Auto-injector - An Automatic, Pre-filled, Selfinjecting Syringe

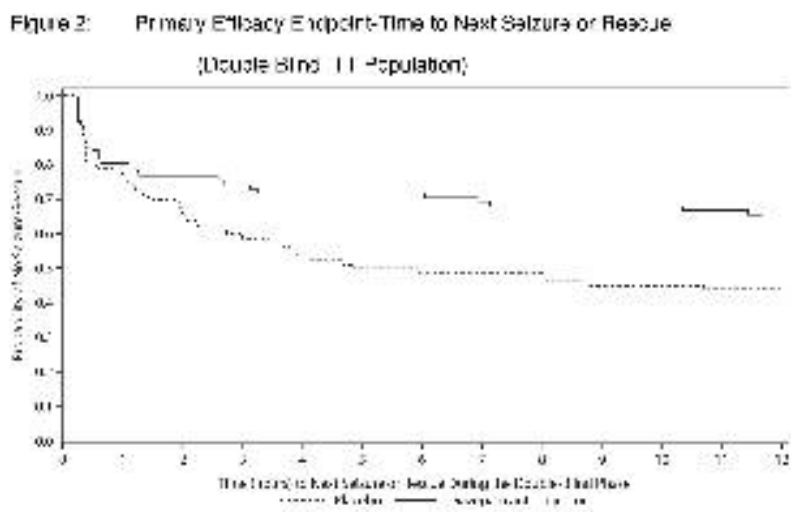

Figure 2: Primary Efficacy Endpoint-Time to Next Seizure or Rescue (Doube Blind ITT Population) Note: A patient was considered to have an event if they had a seizure or required rescue medication within the period 15 minutes to 12 hours following study drug administration. Patients without an event were censored at 12 hours if a diary was returned for the patient indicating no seizures or rescue occurred within 12 hours. If no diary was returned, or the diary did not provide answers to questions about seizures and rescue during the 12 hour follow-up period, the patient was considered censored as of 15 minutes past the treatment time, unless another contact was documented.

\subsection{4 \\ BIOEQUIVALENCE STUDIES AMONG GENERIC AND BRAND-NAME MODIFIED-RELEASE ANTIEPILEPTIC DRUGS SUPPORTS THERAPEUTIC EQUIVALENCE AND GENERIC SUBSTITUTION}

R. K. Juluru ${ }^{1}$, B. M. Davit ${ }^{1}$, C. S. Chaurasia ${ }^{1}$, Y. T. Chang ${ }^{2}$ and G. L. Krauss $^{2}\left({ }^{1}\right.$ Division of Bioequivalence II, Office of Generic Drugs, Food and Drug Administration, Rockville, MD and ${ }^{2}$ Department of Neurology, John Hopkins University, Baltimore, MD)

Rationale: Generic modified-release (MR) formulations of the antiepileptic drugs (AEDs) carbamazepine, phenytoin sodium, divalproex sodium, and levetiracetam are currently marketed in the US. MR formulations offer the advantages of less frequent dosing, improved maintenance of therapeutic drug plasma concentrations, and better patient compliance. In a previous study, we found that the smoother drug release provided by a MR AED formulation decreased serum fluctuations, leading to fewer breakthrough seizures. Thus, the objective of the present retrospective study is to evaluate the robustness of the bioequivalence (BE) measures determined in the pivotal clinical studies submitted to the FDA to support marketing approval of generic MR AEDs. BE studies, which determine whether a generic and corresponding brand drug provide the same rate and extent of absorption, are generally performed in healthy normal subjects given single doses of the drugs. The BE measures compared statistically are Cmax (peak drug plasma concentrations, to assess absorption rate), and AUC (total area under the drug plasma concentration versus sampling time profile, to assess absorption extent).

Methods: We collected Cmax and AUC data from the pivotal BE studies used to support the approvals of 25 generic AED MR formulations. Data were from BE studies under both fasting and fed conditions. We determined distributions of $90 \%$ confidence interval (CI) limits for generic/brand ratios of AUC and Cmax in the BE studies. We also obtained from the FDA-approved labeling information about the inactive ingredients in the generic and brand formulations.

Results: We evaluated $53 \mathrm{BE}$ studies, including data from 1570 subjects. The $90 \%$ CIs of the generic/brand AUC and Cmax ratios differed by $<15 \%$ in $86.8 \%$ and $77.4 \%$ of the BE studies, respectively. The $90 \% \mathrm{CIs}$ of the generic/brand AUC and Cmax ratios differed by 15 to $25 \%$ in $13.2 \%$ and $22.6 \%$ of the studies, 
respectively. In general, $\mathrm{BE}$ measures were more variable for $\mathrm{MR}$ AEDs, compared to our previous observations of immediate release approved generic and brand AEDs. However, all MR AED products surveyed were well within the BE acceptance limits of 80 to $125 \%$ (90\% CIs). Intersubject variability in Cmax and AUC was moderate and did not differ significantly between generic and brand. MR AED formulations of poorly soluble drugs demonstrated higher rates of variability irrespective of whether the formulation was generic or brand.

Conclusions: Generic MR AED formulations provided total and peak drug delivery similar to that of brand products, ensuring therapeutic equivalence. Generic formulations were bioequivalent to the corresponding brand formulations despite differences in excipients and release mechanisms. This comparison of BE measures obtained in the pivotal studies supporting the approvals of generic MR AEDs suggests that patients can switch from brand to generic formulations with little or no changes in drug plasma concentrations and thus no apparent compromise in the safety and efficacy.

\subsection{5 \\ LEVETIRACETAM IS SUPERIOR TO CARBAMAZEPINE- SR IN NEWLY DIAGNOSED EPILEPSY IN THE ELDERLY: RESULTS OF THE STEP-ONE TRIAL}

Konrad J. Werhahn ${ }^{1}$, E. Trinka ${ }^{2}$, J. Dobesberger ${ }^{2}$, C. Ruckes ${ }^{3}$ and G. Krämer $^{4}\left({ }^{1}\right.$ Department of Neurology, University Medical Centre, Mainz, Germany; ${ }^{2}$ Department of Neurology, Paracelsus Medical University, Salzburg, Austria; ${ }^{3}$ Interdisciplinary Center for Clinical Trials, University Medical Center, Mainz, Germany and ${ }^{4}$ Swiss Epilepsy Centre, Zurich, Switzerland)

Rationale: A multicentre, double-blind, randomized, head-to-head comparison of levetiracetam (LEV), slow-release carbamazepine (CBZ-SR), and lamotrigine (LTG) in newly diagnosed elderly patients with focal epilepsy (NCT00438451; EudraCT Number: 2005-003324-19).

Methods: Patients aged 60 years or above with new onset focal epilepsy (either at least one seizure and spike discharges on EEG or a relevant lesion on $\mathrm{CT} / \mathrm{MRI}$ or a total of 2 spontaneous seizures) were eligible; those with symptomatic epileptic seizures due to acute $(<2$ weeks) cerebral lesions were excluded. Patients received LEV, CBZSR, or LTG (initial target dose of $1000 \mathrm{mg}$ LEV, 400mg CBZ-SR, or $100 \mathrm{mg}$ LTG) in a parallel group design over 58 weeks (6 weeks titration and 52 weeks maintenance). Following titration patients were treated individually in a double-blind manner depending on efficacy and tolerability on doses between $500-3000 \mathrm{mg}$ for LEV, 200 - 1200mg for CBZ-SR, or 50 - 300mg for LTG.

Results: A total of $n=361$ patients were randomized and $n=359$ included in the intention-to-treat (ITT) population. Mean $( \pm \mathrm{SD})$ age was $71.8 \pm 7.5$ for LEV ( $\mathrm{n}=122,34 \%$ female), $71.7 \pm 6.7$ for CBZ-SR $(\mathrm{n}=120,46 \%)$, and $70.7 \pm 7.4$ for LTG $(\mathrm{n}=117,41 \%)$. The mean number of seizures prior randomization were $3.8 \pm 9.9,4.8 \pm 10.8$, and $2.7 \pm 3.1$ for LEV, CBZ-SR, and LTG respectively ( $p<0.05$ for CBZ-SR vs. LTG, t-test). Based on the ITT population retention rates at 58 weeks (primary endpoint) were $61 \%(95 \% \mathrm{CI}$ : 53-70) for LEV, $46 \%$ (37-55) for CBZ-SR, and 56\% for LTG (47-65) [p-values: overall 0.048; LEV vs. CBZ-SR 0.02, LEV vs. LTG 0.36, LTG vs. CBZ-SR 0.15, Fisher exact test]. Logistic regression revealed an odds ratio (OR) for a retention to treatment at 58 weeks of $1.838(95 \% \mathrm{CI}$ : 1.092-3.093) for LEV vs. CBZ-SR, of $1.169(0,689-1,984)$ for LEV vs. LTG, and of 0.636 (0.377-1.073) for CBZ-SR vs. LTG. The number of concomitant diseases significantly influenced 58-week retention (OR 0.921 ; 95\% CI 0.859-0.987), i.e. chances to remain on therapy were reduced by $7.9 \%$ with each additional concurrent disease. There were no significant differences in seizure-freedom rates at week 30 (LEV 48\%, CBZ-SR 39\%, LTG 49\%) and 58 (LEV: $43 \%$, CBZ-SR 33\%, LTG 38\%) and in time-to-first seizure from randomization or after titration (after week 6).

Conclusions: Results demonstrate evidence of superiority of LEV in monotherapy to CBZ-SR in new-onset focal epilepsy in the elderly. As the trial was not powered for the comparison of LEV and LTG there was no significant difference between these treatment arms. However, results do suggest that LEV should be among the first-line treatments in new-onset epilepsy of the elderly population. Future head-to-head trials with novel or other antiepileptic drugs in the elderly population need to consider LEV as comparator.

\subsection{6 \\ PREGABALIN MONOTHERAPY IN PATIENTS WITH PARTIAL ONSET SEIZURES: A RANDOMIZED, DOUBLE- BLIND, HISTORICAL-CONTROLLED TRIAL}

T. Fakhoury ${ }^{1}$, J. French ${ }^{2}$, P. Kwan ${ }^{3}$, V. Pitman ${ }^{4}$, J. Weaver ${ }^{4}$, L. Knapp ${ }^{4}$ and Lorraine Yurkewicz ${ }^{4}{ }^{1}$ St. Joseph Health System, Lexington, KY; ${ }^{2} \mathrm{NYU}$ Comprehensive Epilepsy Center, New York, $\mathrm{NY} ;{ }^{3}$ Prince of Wales Hospital and the Chinese University of Hong Kong, Hong Kong, China and ${ }^{4}$ Pfizer Inc, New York, NY)

Rationale: Pregabalin (PGB) is registered for adjunctive treatment of partial onset seizures (POS) in many countries. This study investigated the efficacy and safety of PGB monotherapy for POS, using a historical-controlled conversion-to-monotherapy (CTM) design recently endorsed by an advisory panel of the US Food and Drug Administration (FDA). CTM trials employing an internal control of 'pseudo-placebo', used in the past to support FDA approval of antiepileptic drug (AED) monotherapy, are not favored in contemporary epilepsy research due to ethical concerns. This is the second reported historical-controlled trial of AED monotherapy. Methods: Patients with POS and inadequate seizure control $(\geq 4$ partial seizures during an 8-week baseline phase) while on 1 or 2 AEDs were randomized to double-blind monotherapy with PGB 600 or $150 \mathrm{mg} /$ day (4:1) at 54 centers in the US, Hong Kong, Ukraine, and Czech Republic. Following an 8-week prospective baseline phase, patients entered a 20 -week double-blind phase comprising an 8-week conversion period (during which PGB dose was escalated and other AEDs tapered) and a 12-week monotherapy period. Primary endpoint was the seizure-related exit rate in the $600 \mathrm{mg} /$ day group, based on discontinuations due to predefined exit criteria. Efficacy was declared if the upper limit of the $95 \%$ confidence interval (CI) for the exit rate was below a historical-control threshold of $74 \%$. If successful, testing proceeded to step-wise evaluation using a threshold of $68 \%$. Following an interim analysis, the trial was stopped early for efficacy.

Results: Results for the interim analysis ( $\mathrm{n}=134$, with 125 evaluable for efficacy) and full study population ( $\mathrm{n}=161$, with 148 evaluable for efficacy) are shown in Table 1. Interpretation of PGB $150 \mathrm{mg}$ /day results must reflect the small sample size. For the full study population: At screening, $70.2 \%$ of patients were receiving 1 and $29.8 \% 2$ AEDs. Common seizure types during baseline included complex partial seizures (64.9\% of patients) and simple partial seizures $(62.8 \%)$. Overall, $58.3 \%$ of patients in the $600 \mathrm{mg} /$ day group, and $53.6 \%$ in the $150 \mathrm{mg}$ /day group, completed 20 weeks' double-blind treatment without experiencing an exit criterion. Seizure-related exit rate in the $600 \mathrm{mg}$ /day group $(27.5 \%$; $95 \% \mathrm{CI}$ $17.8,37.2$ ) was significantly below both the $74 \%$ and $68 \%$ thresholds. Eight patients on $600 \mathrm{mg} /$ day and $2 \mathrm{on} 150 \mathrm{mg} /$ day were seizure-free throughout the PGB monotherapy period. The overall safety profile of PGB was consistent with prior trials. One patient on PGB $600 \mathrm{mg}$ /day died of cardiorespiratory arrest (probable SUDEP) which was considered not treatment-related.

Conclusions: This historical-controlled study showed that PGB 600 $\mathrm{mg} /$ day was safe and demonstrated efficacy as monotherapy for patients with POS and inadequate seizure control while receiving 1 or 2 AEDs. 
Table 1: Patients meeting exit criteria or adjudicated as an exit by the data monitoring committee

\begin{tabular}{|c|c|c|c|c|}
\hline \multirow[b]{2}{*}{ Exit criteria } & \multicolumn{2}{|c|}{$\begin{array}{l}\text { Interim analysis; evaluable } \\
\text { for efficacy }(\mathrm{N}=125 \text { ) }\end{array}$} & \multicolumn{2}{|c|}{$\begin{array}{l}\text { Full study population: } \\
\text { evaluable for efficacy }(\mathrm{N}= \\
\qquad 148)^{a}\end{array}$} \\
\hline & $\begin{array}{c}\mathrm{P}(\mathrm{GB} 6 \mathrm{60}) \\
\mathrm{mg} / \mathrm{day}(\mathrm{a}= \\
102)\end{array}$ & $\begin{array}{l}\mathrm{PCBB} 150 \\
\text { mg/day }(\mathrm{n}= \\
23)\end{array}$ & $\begin{array}{l}\text { PGiB 600 } \\
\text { my/day (n = } \\
120)\end{array}$ & $\begin{array}{l}\mathrm{PGiB} 150 \\
\mathrm{mg} / \mathrm{day}(\mathrm{n}= \\
28)\end{array}$ \\
\hline $\begin{array}{l}\text { Unacecptable increase in } \\
\text { frequency/intensity of seizure } \\
\text { activity, } \mathrm{n} \text { (\%) }\end{array}$ & $15(14.7)$ & $4(17.4)$ & $17(14.2)$ & $4(14.3)$ \\
\hline $\begin{array}{l}\text { 28-day SR during DB }>2 \times \text { the } \\
\text { maximum 28-day SR during BL., } \\
\text { (๘) }\end{array}$ & $11(10.8)$ & $3(13.0)$ & $11(9.2)$ & $3(10.7)$ \\
\hline $\begin{array}{l}\text { 2-day SR during DB }>2 x \text { the } \\
\text { maximum 2-day SR during BL, a } \\
\text { (\%) }\end{array}$ & $8(7.8)$ & $3(13.0)$ & $7(5.8)$ & $4(14.3)$ \\
\hline Fpisode of status epilcpticus, n (\%) & $1(1,0)$ & 0 & $2(1.7)$ & 0 \\
\hline $\begin{array}{l}\text { SCiTC xcizure if not experienced } \\
\text { wilhin } 2 \text { years of entry, } \mathrm{n}(\%)\end{array}$ & $3(2.9)$ & 0 & $2(1.7)$ & 0 \\
\hline $\begin{array}{l}\text { T'ual meeting } \geq 1 \text { exil criterion, } \mathrm{n} \\
\qquad(\%)\end{array}$ & $29(28.4)$ & $9(39.1)$ & $30(25.0)$ & $10(35.7)$ \\
\hline $\begin{array}{l}\text { Scizurc-rclated exit rate, } \%(95 \%) \\
\qquad \mathrm{Cl}^{\mathrm{b}}{ }^{\mathrm{b}}\end{array}$ & $\begin{array}{l}31.9(20.7 \\
43.1)^{\mathrm{c}}\end{array}$ & $\cdot$ & $\begin{array}{l}27.5(17.8 \\
37.2)^{\mathrm{c}}\end{array}$ & $\cdot$ \\
\hline
\end{tabular}

${ }^{\mathrm{a}}$ The full study population represents a final resolved database compared to the interim analysis. One patient was removed from the interim analysis population due to a modified intent-to-treat criterion. ${ }^{b}(1$ - Kaplan-Meier product limit estimate for the survival function) $\mathrm{x}$ $100 \%$. ${ }^{\mathrm{c}} \mathrm{p}<0.001$ versus the $74 \%$ and $68 \%$ thresholds. PGB, pregabalin; SGTC, secondarily generalized tonic-clonic; DB, doubleblind treatment; SR, seizure rate; BL, baseline

\subsection{7 \\ LONG-TERM TREATMENT OF PARTIAL-ONSET SEIZURES IN ADULTS EXPOSED ONLY TO APPROVED LACOSAMIDE DOSES: POOLED ANALYSIS OF THREE OPEN-LABEL EXTENSION STUDIES}

Neil D'Cruz ${ }^{1}$, P. Doty ${ }^{1}$, C. McShea ${ }^{1}$, S. Dimova ${ }^{2}$ and M. DeBacker ${ }^{2}$ ( ${ }^{1} \mathrm{UCB}$ Pharma, Raleigh, NC and ${ }^{2} \mathrm{UCB}$ Pharma, Brussels, Belgium)

Rationale: Lacosamide is a newer antiepileptic drug approved to a maximum dose of $400 \mathrm{mg}$ /day as adjunctive treatment for partialonset seizures (POS) in adults. The safety and efficacy of adjunctive lacosamide has been assessed in double-blind, placebo-controlled trials (lacosamide dose range 200 to $600 \mathrm{mg}$ /day) and in long-term open-label extension (OLE) trials (lacosamide dose range 100 to 800 $\mathrm{mg}$ /day). This subgroup analysis evaluated the long-term efficacy and tolerability of lacosamide within the approved dose range $(\leq 400$ $\mathrm{mg} /$ day).

Methods: Data were pooled from three long-term OLE trials (SP615; SP756 [Husain et al, Epilepsia 2012]; SP774) in adults with POS who completed a Phase II or III trial of adjunctive lacosamide. During open-label (OL) treatment, dosage adjustments of lacosamide (100 to $800 \mathrm{mg} /$ day) and/or concomitant AEDs were allowed to optimize tolerability and seizure control. Outcomes were evaluated from the OLE studies for patients exposed to $\leq 400 \mathrm{mg} /$ day lacosamide during the lead-in and OLE trials.

Results: Of 1,054 patients who initiated OL lacosamide treatment, $34.4 \%(n=363)$ had been exposed only to lacosamide doses $\leq 400$ $\mathrm{mg} /$ day, with $33.3 \%$ (121/363) of those patients completing the OLE trial and $89.3 \%(108 / 121)$ of completers continuing to commercial lacosamide treatment. At Baseline (lead-in trial), 83.7\% $(n=304)$ of patients included in this subanalysis were taking 2 to 3 AEDs and $71.9 \%(\mathrm{n}=261)$ had tried $\geq 4$ lifetime AEDs. Baseline (lead-in trials) median seizure frequency per 28 days was 9.0 (range 2.5 - 404.5). A total of $66.7 \%(n=242)$ of patients discontinued prior to the end of the exposure period (up to 8 years), most commonly for lack of efficacy (24.0\%), adverse event (16.8\%), and consent withdrawal (17.6\%). The proportion of patients exposed to $\leq 400 \mathrm{mg} /$ day lacosamide for $>1,>2,>3,>4$ and $>5$ years was $64.2 \%, 50.1 \%$, $41.0 \%, 34.2 \%$, and $15.7 \%$. As all patients had an opportunity to complete at least 3 years of OL treatment, the lower rates of exposure after 3 years is attributed to study closure as well as premature discontinuations. The most commonly $(\geq 10 \%)$ reported treatmentemergent adverse events (TEAEs) were dizziness $(21.5 \%)$, headache $(14.0 \%)$, and nasopharyngitis $(10.7 \%)$; most were mild to moderate in intensity. For 1-, 2-, 3-, 4- and 5-year completer cohorts, the median percent reduction from Baseline in POS frequency per 28 days was $59.4 \%, 64.1 \%, 67.9 \%, 69.3 \%$ and $71.0 \%$; Figure 1 depicts seizure reduction by cohort over time. The $\geq 50 \%$ responder rates for the 1-, 2-, 3-, 4- and 5-year completer cohorts were $60.2 \%, 65.9 \%$, $68.0 \%, 72.6 \%$ and $70.2 \%$; the $\geq 75 \%$ responder rates were $36.8 \%$, $42.3 \%, 42.7 \%, 44.4 \%$, and $49.1 \%$ (Figure 2 ).

Conclusions: Used in the approved dose range (up to $400 \mathrm{mg} /$ day), long term adjunctive lacosamide was generally well tolerated and associated with a reduction in seizure frequency and a maintenance of efficacy.

Sponsored by UCB
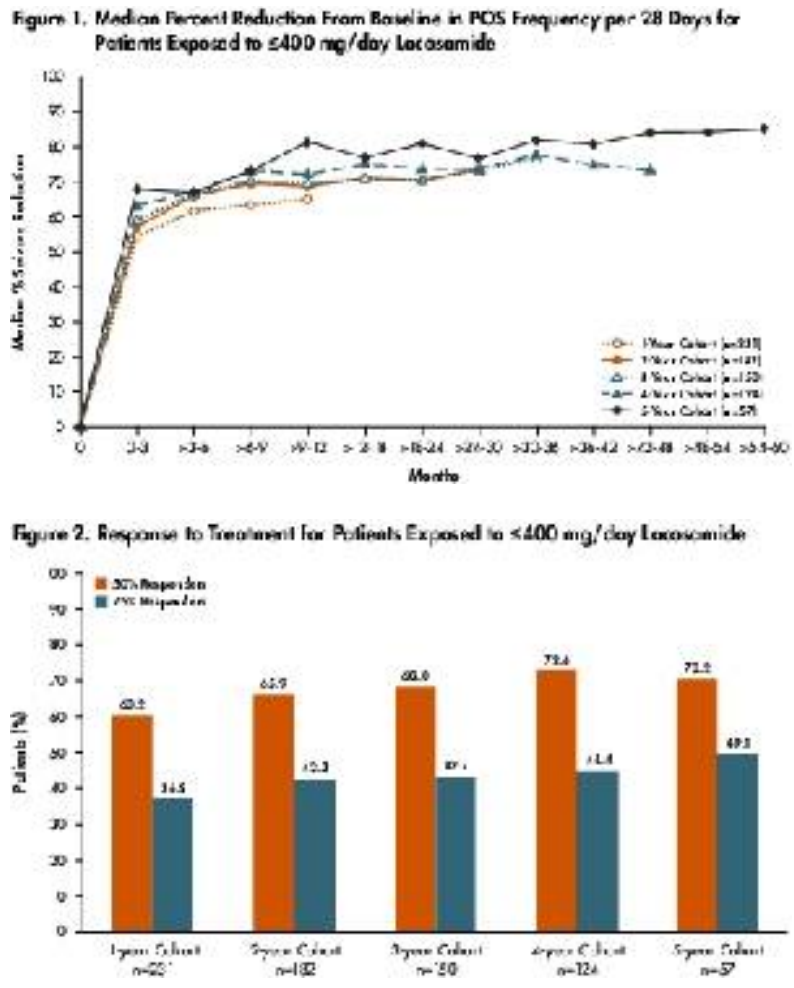

1.228

OPEN-LABEL PILOT STUDY OF ADJUNCTIVE LACOSAMIDE FOR UNCONTROLLED PRIMARY GENERALIZED TONIC-CLONIC SEIZURES R. T. Wechsler ${ }^{1}$, R. Leroy ${ }^{2}$, C. Beller ${ }^{3}$ and P. Doty ${ }^{3}\left({ }^{1}\right.$ Idaho Comprehensive Epilepsy Center, Boise, ID; ${ }^{2}$ Neurological Clinic of Texas, Dallas, TX and ${ }^{3}$ UCB Pharma, Raleigh, NC)

Rationale: Lacosamide is an antiepileptic drug approved for adjunctive treatment of partial-onset seizures (POS) in adults. This is the first assessment of the safety of adjunctive lacosamide for uncontrolled primary generalized tonic-clonic seizures (PGTCS) in adults with idiopathic generalized epilepsy.

Methods: This was a Phase 2, multicenter, open-label pilot study (SP0961) comprised of a 4-week prospective Baseline, followed by 3-week Titration and 6-week Maintenance phases. Dose titration started at $100 \mathrm{mg} /$ day $(50 \mathrm{mg}$ bid) and increased weekly to a maximum of $400 \mathrm{mg} /$ day. Primary variables were change in number of seizure days/28 days from prospective Baseline to Maintenance for absence and myoclonic seizures. Other variables were percent change from prospective Baseline in number of seizure days $/ 28$ days for absence and myoclonic seizures, percent change from combined Baseline (12week retrospective period +4 -week prospective Baseline) in 28-day 
seizure frequency for PGTC seizures, changes in count of generalized and $3 \mathrm{~Hz}$ spike-wave discharges on 24-hour ambulatory EEG, treatment-emergent adverse events (TEAEs) and withdrawals due to TEAEs.

Results: Of 49 patients enrolled (mean age 29.7 years, $73.5 \%$ female), 40 (81.6\%) completed the study. A majority $(69.4 \%)$ were taking 2-3 concomitant AEDs at baseline. All had a history of tonicclonic seizures; $69.4 \%$ and $55.1 \%$ had a history of absence and myoclonic seizures, respectively. At prospective Baseline, mean absence and myoclonic seizure days/28 days were $4.7 \pm 8.48$ and $4.7 \pm 7.98$, respectively. For absence seizures, mean change from prospective Baseline was $0.26 \pm 3.67$ to Titration and $-0.37 \pm 4.80$ to Maintenance, corresponding to a mean percent change of $10.63 \pm 76.3$ to Maintenance. For myoclonic seizures, mean change from prospective Baseline was $-1.29 \pm 6.26$ to Titration and $2.19 \pm 5.80$ to Maintenance; mean percent change to Maintenance was $-44.95 \pm 73.9$. Mean percent change (combined Baseline to Maintenance) was $-26.37 \pm 156.16$ for PGTC 28 -day seizure frequency; 29 (59.2\%) patients did not have tonic-clonic seizures during Maintenance. Frequently reported TEAEs $(>15 \%)$ were dizziness (19 patients [38.8\%]), nausea (13 [26.5\%]), headache ( 8 [16.3\%]), and somnolence (8 [16.3\%]). One patient experienced a serious TEAE of "petit mal status", though EEG indicated nonspecific diffuse cortical dysfunction with possible nonconvulsive status epilepticus. Nine (18.4\%) patients discontinued early: 5 $(10.2 \%)$ due to a TEAE, $4(8.2 \%)$ withdrew consent. There was no consistent correlation between changes in EEG discharges and clinical outcome.

Conclusions: Adjunctive lacosamide appears to be safe in this population in that it did not systematically worsen either absence or myoclonus, though a small subset of patients may have had an increase in absence seizures. Reductions in PGTC seizure frequency were observed. TEAEs were similar to those observed among patients with POS treated with adjunctive lacosamide, except for seizure-related events. Results of this pilot trial support further evaluation of lacosamide for treatment of PGTCS.

Sponsored by UCB

\subsection{9}

\section{WTTHDRAWN}

\subsection{0}

\section{A POST-HOC ANALYSIS OF THE TIME TO ONSET OF EFFICACY AFTER INITIATION OF ESLICARBAZEPINE ACETATE AS ADJUNCTIVE THERAPY IN ADULT PATIENTS WITH REFRACTORY PARTIAL-ONSET SEIZURES}

C. Elger ${ }^{1}$, B. Steinhoff ${ }^{2}$, J. Serratosa ${ }^{3}$, C. Marchal $^{4}$, F. Sales ${ }^{5}$, Rui Sousa $^{6}$, T. Nunes ${ }^{6}$ and P. Soares-da-Silva ${ }^{6,7}$ ( ${ }^{1}$ University of Bonn, Bonn, Germany; ${ }^{2}$ Epilepsiezentrum Kork, Kehl-Kork, Germany; ${ }^{3}$ Hospital Universitario Fundación Jiménez Díaz, Madrid, Spain; ${ }^{4} \mathrm{CHU}$ Bordeaux, Bordeaux, France; ${ }^{5}$ Hospitais da Universidade de Coimbra, Coimbra, Portugal; ${ }^{6}$ BIAL - Portela \& Ca, S.A., S. Mamede do Coronado, Portugal and ${ }^{7}$ Faculty of Medicine, University of Porto, Porto, Portugal)

Rationale: Eslicarbazepine acetate (ESL) is a novel once-daily (QD) anticonvulsant, extensively converted to eslicarbazepine after oral administration, which blocks voltage-gated sodium- and calciumchannels. This post-hoc analysis evaluated the efficacy, tolerability and time to onset of a clinically relevant reduction in seizure risk (RSR) after initiation of ESL as adjunctive therapy in adults with partial-onset seizures (POS).

Methods: Data from two (BIA-2093-301 and -302) phase III multicentre, double-blind, randomized, placebo-controlled studies in adult patients with $\geq 4$ partial-onset seizures per 4 weeks despite treatment with 1-3 AEDs was pooled and analysed. ESL QD was administered at doses of $400 \mathrm{mg}, 800 \mathrm{mg}$ and $1200 \mathrm{mg}$. The key efficacy endpoints were seizure frequency during the 12-week maintenance phase adjusted per 4 weeks (primary endpoint), relative reduction in seizure frequency, and responder rate ( $\geq 50 \%$ reduction in seizure frequency). To evaluate time to onset of a clinically relevant effect, mean cumulated seizure frequency over time (MCF) was calculated for the entire double-blind period. From the increments in MCF, the instantaneous daily seizure rate was estimated using an Epanechnikov kernel estimator (bandwidth $=28$ days). Relative hazard functions over time were derived comparing each of the 3 ESL dosage groups with placebo group. Tolerability was also assessed.

Results: Safety population comprised 797 patients (placebo, $n=202$; ESL 400 mg, n=196; ESL 800 mg, n=199; ESL 1200 mg, n=200) and intention-to treat population included 752 patients (placebo, $\mathrm{n}=198$; ESL 400 mg, n=192; ESL 800 mg, n=182; ESL 1200 mg, n=180). Compared with placebo adjusted seizure frequency over the 12-week maintenance period was significantly reduced with ESL $800 \mathrm{mg}$ and $1200 \mathrm{mg}$ ( $\mathrm{p}<0.0001$ for both groups); ESL $400 \mathrm{mg}$ was not significantly superior to placebo $(\mathrm{p}=0.15)$. Median relative reduction in seizure frequency was $13 \%$ with placebo and $34 \%$ and $38 \%$ with ESL $800 \mathrm{mg}$ and $1200 \mathrm{mg}$, respectively. Responder rate was $19 \%$ with placebo and $36 \%$ and $43 \%$ with ESL $800 \mathrm{mg}$ and $1200 \mathrm{mg}$, respectively. Including the titration period (up to 2 weeks), the onset of a clinically relevant effect (defined as $20 \%$ RSR compared with placebo) was observed in 28 days in ESL $1200 \mathrm{mg}$ group and in 49 days in ESL $800 \mathrm{mg}$ group. The $90 \%$ maximal effect was observed in 43 days in ESL $1200 \mathrm{mg}$ group (24.5\% RSR) and in 48 days in ESL $800 \mathrm{mg}$ group (19.9\% RSR) (figure 1). Treatment-emergent adverse events occurring in at least $10 \%$ of patients in any group were dizziness, somnolence, headache, diplopia and nausea (table 1). Conclusions: In this post-hoc analysis, once-daily ESL $800 \mathrm{mg}$ and $1200 \mathrm{mg}$ was effective as adjunctive treatment of adult patients with refractory partial-onset seizures. Including the titration period, a clinically relevant reduction in seizure risk was achieved in 28 and 48 days of therapy with once-daily ESL $1200 \mathrm{mg}$ and $800 \mathrm{mg}$, respectively. Both effective dosages were well tolerated.

Table 1. Incidence of treatment-emergent adverse events (TEAEs) in at least $10 \%$ of patients in any treatment group (safety populations).

\begin{tabular}{|ccc|cc|}
\hline MedDRA Preferred Term & $\begin{array}{c}\text { Placebo } \\
(\mathrm{n}=202)\end{array}$ & $\begin{array}{c}\text { ESL 400 mg } \\
\text { QD } \\
(\mathbf{n}=196)\end{array}$ & $\begin{array}{c}\text { ESL } 800 \mathrm{mg} \\
\text { QD } \\
(\mathrm{n}=199)\end{array}$ & $\begin{array}{c}\text { ESL 1200 mg } \\
\text { QD } \\
(\mathrm{n}=200)\end{array}$ \\
\hline $\begin{array}{c}\text { Total paticnts with TEATs, } \mathrm{n} \\
(\%)\end{array}$ & $100(49.5)$ & $119(60.7)$ & $133(66.8)$ & $140(70.0)$ \\
\hline Dizziness, $\mathbf{n}(\%)$ & $12(5.9)$ & $26(13.3)$ & $44(22.1)$ & $57(28.5)$ \\
\hline $\begin{array}{c}\text { Somnolence, } \mathbf{n}(\%) \\
\text { Headache, } \mathbf{n}(\%)\end{array}$ & $19(9.4)$ & $21(10.7)$ & $26(13.1)$ & $31(15.5)$ \\
Diplopia, $\mathbf{n}(\%)$ & $15(7.4)$ & $17(8.7)$ & $24(12.1)$ & $30(15.0)$ \\
\hline Nausca, $\mathbf{n}(\%)$ & $4(2.0)$ & $10(5.1)$ & $22(11.1)$ & $21(10.5)$ \\
\hline
\end{tabular}

Notes: QD, once-daily; TEAEs, therapeutic emergent adverse events.

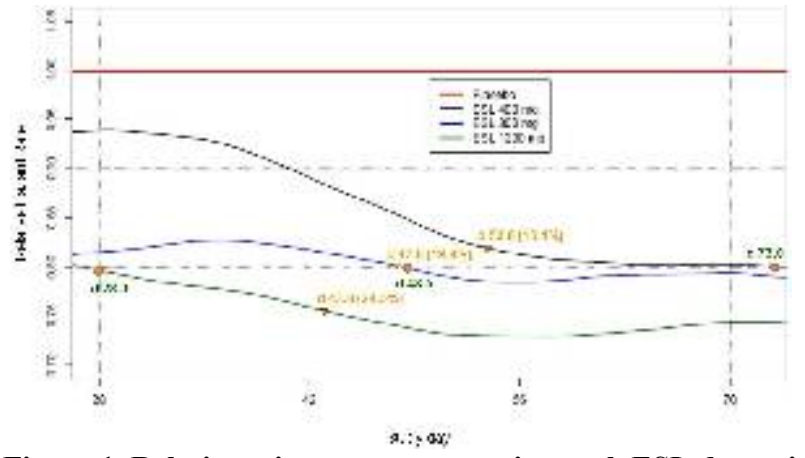

Figure 1. Relative seizure rate comparing each ESL dose with placebo. The onset of a clinically relevant effect, defined as $20 \%$ 
reduction in seizure risk (RSR) compared with placebo, is shown by green circles together with the estimated time (in days). The time when $90 \%$ of maximal RSR compared to placebo has been obtained is shown by orange triangles together with the estimated time (in days) and the percentage RSR. Notes: The seizure rates were obtained using an Epanechnikov kernel estimator. A bandwidth of 28 days was chosen so the kernel estimator uses data in an interval of 28 days before and after the time of interest. This implies that the estimates in the first 28 days of therapy are influenced by baseline data and the estimates after day 70 are influenced by the data from the tapering-off period. The estimates between days 28 and 70 are not influenced by the pre-randomization or post-maintenance period so they are considered reliable.

\subsection{1}

\section{A LIQUID CHROMATOGRAPHY-TANDEM MASS SPECTROMETRY METHOD FOR THE QUANTIFICATION OF LAMOTRIGINE IN PATIENT PLASMA}

J. M. Wong ${ }^{1}$, W. Jiang ${ }^{2}$, T. Ting ${ }^{3}$, James E. Polli ${ }^{1}$ and M. Kane ${ }^{1}$ ( ${ }^{1}$ Department of Pharmaceutical Sciences, University of Maryland, Baltimore, MD; ${ }^{2}$ Food and Drug Administration, Rockville, MD and ${ }^{3}$ Department of Neurology, University of Maryland, Baltimore, MD)

Rationale: The Bioequivalence in Epileptic Patients (BEEP) study at the University of Maryland School of Pharmacy has been initiated to address neurologists' and epileptic patients' concerns about the bioequivalence of generic lamotrigine to Lamictal. This bioequivalence study requires the quantification of lamotrigine in human plasma, including in the presence of other drugs, as this study employs epileptic patients rather than healthy volunteers. Liquid chromatography-tandem mass spectrometry (LC-MS/MS) is the bioanalytical method of choice for quantification of analytes in complex matrices. An essential aspect of method validation is the assessment of the influence of concomitant drugs and plasma components due to the possibility of ion suppression/enhancement. The objective was to develop and validate a LC-MS/MS method to measure the concentration of lamotrigine in patient plasma. A range of analytical conditions, as well as a backflush step to reduce matrix effects, were examined.

Methods: Lamotrigine was extracted from plasma through protein precipitation with acetonitrile. The supernatant of each sample was diluted in water before injection. Lamotrigine-13C3-d3, a stable isotope-labeled compound, was used as an internal standard. Samples were run utilizing a two pump system in a LC-MS/MS. The method was validated for accuracy, precision, inter- and intraday coefficient of variation, specificity, limit of detection (LOD), limit of quantitation (LOQ), linearity, range, and instrument precision. Specificity considered interference of other anti-epileptic drugs (AEDs), including levetiracetam, topiramate, phenytoin, and carbamazepine. Freeze-thaw, dilution, and sample stability tests were also performed. All calculations were based on the peak area response ratio of lamotrigine to lamotrigine-13C3-d3.

Results: A backflush step reduced matrix effects. A final method was validated for a linear range of 8 to $10,000 \mathrm{ng} / \mathrm{mL}$ of lamotrigine $(\mathrm{r} 2=$ 0.9999 ). Tests were ran at various concentration levels, including LOQ at $8 \mathrm{ng} / \mathrm{mL}, 2.5 \times$ LOQ at $20 \mathrm{ng} / \mathrm{mL}$, low quality control (QC) sample at $50 \mathrm{ng} / \mathrm{mL}$, medium QC at $500 \mathrm{ng} / \mathrm{mL}$, and high QC at 5000 $\mathrm{ng} / \mathrm{mL}$. LOD was determined to be $1 \mathrm{ng} / \mathrm{mL}$, with interday and intraday results less than $10.0 \%$ and $10.4 \%$, respectively (range: 20 $5000 \mathrm{ng} / \mathrm{mL}$ ). The percent recovery varied from 96.6 to $109.3 \%$ (range: $20-5000 \mathrm{ng} / \mathrm{mL}$ ), with no interference peak from plasma or other AEDs found. The freeze-thaw and dilution results were also similar to the standard curve. The only elevated stability result was the short-term temperature test, with peak areas increased by 7.0 to 19.5\% (range: $50-5000 \mathrm{ng} / \mathrm{mL}$ ).

Conclusions: An LC-MS/MS method was developed and fully validated to measure lamotrigine concentration in patient plasma. A backflush step allowed for consistent results and reduced matrix effects, affording a simple precipitation and dilution procedure to determine lamotrigine in patient plasma. The methods will be applied in the BEEP study to compare brand versus generic lamotrigine.

\subsection{2 \\ A PHARMACOKINETIC STUDY COMPARING ESLICARBAZEPINE ACETATE ADMINISTERED ORALLY AS CRUSHED OR INTACT TABLET IN HEALTHY VOLUNTEERS}

J. Kharidia, D. Blum and H. Cheng (Sunovion Pharmaceuticals, Inc., Marlborough, MA)

Rationale: Eslicarbazepine acetate (ESL) is a novel once-daily (QD) anticonvulsant, extensively converted to eslicarbazepine after oral administration. Eslicarbazepine is a voltage-gated sodium- and calcium-channel blocking agent. Some patients with refractory partial onset seizures may require administration of crushed tablets, thus it is important to understand the pharmacokinetic (PK) characteristics of ESL administered as crushed tablet relative to intact tablet. This study compared the relative bioavailability and bioequivalence of a crushed or intact tablet administered orally to healthy volunteers. Methods: Healthy male and female volunteers age 18 to 50 years were enrolled in a single dose (ESL $800 \mathrm{mg}$ ) open label, randomized, two-period, cross-over study, with a 5-day washout period between treatments. Following an overnight fast, subjects were randomized to two different sequences of intact or crushed tablet of ESL $800 \mathrm{mg}$. Crushed tablets were sprinkled on $4 \mathrm{oz}$ (113 g) of applesauce followed by $240 \mathrm{~mL}$ water. Blood samples were analyzed for measurements of ESL and its main metabolites; PK parameters were derived for eslicarbazepine. Bioequivalence was determined if the eslicarbazepine Least Squares (LS) Mean ratios and 90\% Confidence Interval (CI) for $\mathrm{AUC}_{0-\infty}$ and $\mathrm{C}_{\max }$ fell within $80 \%-125 \%$.

Results: A total of 27 subjects completed both arms of the study. Mean age was 27 years $(\mathrm{SD}= \pm 8$ ) with $75 \%$ males, and $68 \%$ Caucasian. The PK parameters for eslicarbazepine after oral administration of crushed or intact tablets are shown in Table 1. The LS Mean ratios and $90 \%$ CI for eslicarbazepine comparing the crushed tablet to the intact tablet were: $\mathrm{C}_{\max }(\mathrm{ng} / \mathrm{mL}) 1.030(0.971$, 1.090) and $\mathrm{AUC}_{0-\infty}(\mathrm{ng} * \mathrm{~h} / \mathrm{mL}) 0.967(0.944,0.992)$. Adverse events reported by $>1$ subject included fatigue $(n=3 ; 11 \%)$, excoriation $(n=2$; $7 \%)$, headache $(n=3 ; 11 \%)$, and somnolence $(n=2 ; 7 \%)$.

Conclusions: In this study, administration of ESL $800 \mathrm{mg}$ to healthy volunteers as a crushed or intact tablet was shown to be bioequivalent. The eslicarbazepine median $\mathrm{T}_{\max }$ and mean $\mathrm{T}_{1 / 2}$ were similar whether the ESL tablet was crushed or taken intact. This study showed that the pharmacokinetic profile of ESL is not significantly altered by crushing, indicating that ESL tablets can be administered crushed or intact.

Mean (SD) Pharmacokinetic Parameters for Eslicarbazepine Administered as ESL $800 \mathrm{mg}$ Dose as Crushed or Intact Tablet in Healthy Volunteers

\begin{tabular}{|c|c|c|}
\hline PK l'arameter & \multicolumn{2}{|c|}{ ESL 800 mg Dosage Form } \\
\hline & $\begin{array}{c}\text { Crushed } \\
(\mathbf{N}=\mathbf{2 8})\end{array}$ & $\begin{array}{c}\text { Intact } \\
(\mathbf{N}=\mathbf{2 7})\end{array}$ \\
\hline $\begin{array}{c}\mathrm{C}_{\max }(\mathrm{ng} / \mathrm{mL}) \\
\text { Mean }(\mathrm{SD}) \\
\mathrm{T}_{\max }(\mathrm{h}) \\
\text { median (min, max })\end{array}$ & $11699(2843)$ & $11522(2967)$ \\
\hline $\begin{array}{c}\mathrm{T}_{\text {I/2 }}(\mathrm{h}) \\
\mathrm{Mean},(\mathrm{SD})\end{array}$ & $3.0(1.00,5.00)$ & $3.0(0.48,6.00)$ \\
\hline $\begin{array}{c}\mathrm{AUC} \mathrm{C}_{0-\infty}(\mathrm{ng} * \mathrm{~h} / \mathrm{mL}) \\
\text { Mean }(\mathrm{SD})\end{array}$ & $10.2(0.98)$ & $10.3(1.26)$ \\
\hline
\end{tabular}




\subsection{3}

IMPACT OF DELAYED DOSE OR MISSED DOSE ON PERAMPANEL PLASMA CONCENTRATIONS B. Gidal ${ }^{1}$, O. Majid ${ }^{2}$, J. Ferry ${ }^{3}$, Z. Hussein ${ }^{2}$, H. Yang ${ }^{3}$, J. Zhu ${ }^{3}$, R. Fain $^{4}$ and A. Laurenza ${ }^{3}\left({ }^{1}\right.$ School of Pharmacy, University of Wisconsin-Madison, Madison, WI; ${ }^{2}$ Eisai Limited, Hatfield, United Kingdom; ${ }^{3}$ Neuroscience Product Creation Unit, Eisai, Inc., Woodcliff Lake, NJ and ${ }^{4}$ Medical and Scientific Affairs, Eisai, Inc., Woodcliff Lake, NJ)

Rationale: Perampanel (PER) is a selective AMPA receptor antagonist currently being evaluated as adjunctive therapy in patients with refractory partial seizures. PER is metabolized primarily via CYP3A4, yet has a relatively long elimination half-life (T1/2) of 105 $\mathrm{h}$, and as such, is given on a once-daily (QD) basis. Enzyme-inducing antiepileptic drugs (EIAEDs) may substantially reduce PER T1/2 due to an increase in oral clearance (CL/F). Carbamazepine (CBZ) increases PER CL/F 3-fold, while oxcarbazepine (OXC) or phenytoin (PHT) increases $\mathrm{CL} / \mathrm{F} \sim 2$-fold. AEDs with a long T1/2 may facilitate medication adherence by reducing administration frequency and may minimize peak to trough fluctuations, especially in the setting of erratic adherence. In addition, the impact of a delayed or missed dose of PER on plasma concentrations may be less with PER than with short T1/2 AEDs. Hence, we sought to examine the pharmacokinetics (PK) of PER following simulated missed/delayed dose paradigms.

Methods: Simulations were done using validated PER PK parameters, derived from 19 Phase I studies in 606 subjects, to study the effect on PER concentration of (1) missing a dose followed by delayed replacement of the missed dose; (2) missing a dose followed by resumption of scheduled therapy; (3) missing a dose in the presence/absence of CBZ. Simulations were done for a typical patient receiving $8 \mathrm{mg}$ PER once daily, using NONMEM v7.2 in conjunction with PDx-pop v5.

Results: Simulation results are presented in Table 1.

Following QD dosing, peak-trough fluctuation for PER is relatively flat, as would be expected from its long T1/2. Fluctuation index (FI) is greater when PER is given in the presence of EIAEDs due to the shorter T1/2. Following a missed dose and in the absence of EIAEDs, PER trough concentrations $\left(\mathrm{C}_{\mathrm{min}}\right)$ would be expected to decline mildly; while in the presence of EIAEDs, the predicted reduction in $\mathrm{C}_{\min }$ is moderate. Replacing a missed dose, even after a 6- or 12-h time period, results in a mild decline in $\mathrm{C}_{\min }$ in the induced and noninduced state. Changes in peak $\left(\mathrm{C}_{\max }\right)$ were also only minor following dose replacement.

Conclusions: While perfect medication adherence is always preferable, it is often not consistently achieved. Our results corroborate that given the PK characteristics of PER, plasma concentration fluctuation is less than might be expected in the case of a short half-life drug, and supports QD dosing. PER FI increases with EIAEDs. In that the efficacy of PER appears to be related to drug exposure (ie, plasma concentration), these data suggest that declines in trough concentrations are likely to be modest in the event of a missed dose, and that supplementing a missed dose 6-12 h later can lessen these predicted declines without resulting in excessive "spikes" in PER plasma concentrations.

Table 1. Simulated PER Missed-Dose and Delayed-Dose Concentration Profiles

\begin{tabular}{|c|c|c|c|c|c|c|c|c|c|c|}
\hline \multirow{3}{*}{$\begin{array}{l}\text { QD PER } \\
\text { Regimen }\end{array}$} & \multicolumn{5}{|c|}{ Without inducer (CBZ) } & \multicolumn{5}{|c|}{ With inducer (CBZ) } \\
\hline & $\underset{s s}{C_{\min },}$ & $\begin{array}{c}\mathrm{C}_{\text {min }} \\
\text { Change }\end{array}$ & $\mathrm{C}_{\max }$, & $\begin{array}{c}C_{\max } \\
\text { cos } \\
\text { Change }\end{array}$ & FI & $\underset{\kappa}{C_{\min },}$ & $\begin{array}{c}\mathrm{C}_{\text {min }} \\
\text { Change } \\
\text { Change }\end{array}$ & $\mathrm{C}_{\mathrm{max}}$ & $\begin{array}{l}\mathrm{C}_{\max } \\
\text { Change }\end{array}$ & FI \\
\hline & $\mathrm{ng} / \mathrm{mL}$ & $\%$ & $\mathrm{ng} / \mathrm{mL}$ & $\%$ & $\%$ & $\mathrm{ng} / \mathrm{ml}$ & & $\mathrm{ng} / \mathrm{mL}$ & & $\%$ \\
\hline Adherent & 423 & - & 634 & -. & 41.3 & 115 & - & 323 & -. & 122 \\
\hline $\begin{array}{c}\text { Missed } \\
\text { dose }\end{array}$ & $345^{\circ}$ & -18.4 & 559 & -11.8 & 51.2 & $64.5^{\circ}$ & -43.9 & 274 & -15.2 & 159) \\
\hline $\begin{array}{c}\text { 6-h } \\
\text { Delayed } \\
\text { dose }\end{array}$ & 402 & $-5,0$ & $\begin{array}{r}615 \\
(645)^{\prime}\end{array}$ & $\begin{array}{c}3.0 \\
(+1.7)^{t}\end{array}$ & -- & 99 & -13.9 & $\begin{array}{c}308 \\
(331)^{+}\end{array}$ & $\begin{array}{c}-4.6 \\
(+2.5)^{\dagger}\end{array}$ & -. \\
\hline $\begin{array}{c}\text { 12-h } \\
\text { Delayed } \\
\text { dose }\end{array}$ & 382 & -9.7 & $\begin{array}{c}595 \\
(650)^{5}\end{array}$ & $\begin{array}{c}6.2 \\
(+2.5)^{t}\end{array}$ & - & 86 & -25.2 & $\begin{array}{c}295 \\
(340)^{5}\end{array}$ & $\begin{array}{c}-8.7 \\
(+5.3)^{\prime}\end{array}$ & .. \\
\hline
\end{tabular}

$\mathrm{C}_{\min }$, trough concentration, $\mathrm{C}_{\max }$ peak concentration; $\mathrm{C}_{\mathrm{av}}$, mean concentration; ss, steady state; FI, fluctuation index or peak-to-trough fluctuation; ${ }^{*} \mathrm{C}_{\min }$ immediately preceding next scheduled dose; ${ }^{\dagger} \mathrm{C}_{\max }$ at next scheduled dose in parentheses.

\subsection{4 \\ PERAMPANEL IMPROVES RESPONDER RATES, IRRESPECTIVE OF CONCOMITANT ANTIEPILEPTIC DRUGS (AEDS), AND INCREASES SEIZURE FREEDOM: A POOLED ANALYSIS OF THREE PHASE III TRIALS Elinor Ben-Menachem ${ }^{1}$, E. Perucca ${ }^{2}$, D. Squillacote ${ }^{3}$, H. Yang ${ }^{4}$, J. $\mathrm{Zhu}^{4}$ and A. Laurenza ${ }^{4}$ ('Sahlgrenska Academy, Gothenburg, Sweden; ${ }^{2}$ University of Pavia, Pavia, Italy; ${ }^{3}$ Eisai Global Medical Affairs, Woodcliff Lake, NJ and ${ }^{4}$ Eisai Neuroscience Product Creation Unit, Woodcliff Lake, NJ)}

Rationale: Perampanel is an orally active, non-competitive AMPA receptor antagonist under review as an adjunctive therapy for refractory partial seizures. Primary data from three placebocontrolled phase III trials (studies 304 [NCT00699972]; 305 [NCT00699582]; 306 [NCT00700310]) are reported elsewhere (French et al. Annual Meeting of the AAN 2011: Abstract LBS.002; French et al. Epilepsia 2011; 52 (Suppl 6): 10; Krauss et al. Neurology 2012; 78: 1408-1415). Here we report pooled analyses of responder rates and seizure freedom from these trials. The impact of common concomitant AEDs on responder rates was also assessed. Methods: Patients ( $\geq 12$ years), with uncontrolled partial seizures despite receiving 1-3 AEDs, were randomized to once-daily placebo, perampanel 8 or $12 \mathrm{mg}$ (Studies 304 and 305), or placebo, perampanel 2, 4, or $8 \mathrm{mg}$ (Study 306). Trials included 6-week Baseline, 6-week Titration, and 13-week Maintenance Periods. Patients recorded seizures in daily diaries. Responder rates (percentages of patients with $\geq 50 \%$ or $\geq 75 \%$ reductions in the frequency of all partial seizures, complex partial plus secondary generalized seizures [CPS+SGS], or secondary generalized seizures [SGS] only; Maintenance vs Baseline) were assessed in the intent-totreat (ITT) analysis set (all randomized and treated patients with any seizure data). Seizure freedom (percentage of patients seizure free during Maintenance) was assessed for all partial seizures and SGS in the Maintenance completer set. $50 \%$ responder rates were analyzed by the most common concomitant AEDs. P-values are used to highlight effects of interest; these are multiple, non-independent, unadjusted values.

Results: Overall, 1480 patients were randomized and treated. In the pooled ITT analysis set ( $\mathrm{n}=1478$ ), perampanel 4-12 mg (effective dose range) was associated with greater $50 \%$ and $75 \%$ responder rates and greater seizure freedom rates vs placebo (Table 1). At Baseline, the mean number of concomitant AEDs was 2.2; the most common were carbamazepine $(n=491 ; 33.2 \%)$, valproic acid $(n=478$; $32.3 \%)$, lamotrigine $(n=458 ; 30.9 \%)$, and levetiracetam $(n=435$; $29.4 \%$ ). In patients receiving $\geq 1$ of these AEDs, perampanel 8 and 12 mg consistently improved $50 \%$ responder rates vs placebo (all seizure types; Table 2, statistical significance not evaluated).

Conclusions: In pooled analyses of Phase III data by randomized dose, adjunctive perampanel improved responder and seizure freedom rates vs placebo in patients with refractory partial seizures. Responder rates were improved at higher doses, for all partial seizures, including SGS, irrespective of co-administration of the most common AEDs. Previous analyses, based on actual dose and withinpatient responses, showed a benefit of perampanel $12 \mathrm{mg}$ over $8 \mathrm{mg}$ (Kramer et al. AAN 2012: Abstract P06.117), and

pharmacokinetic/pharmacodynamic data indicated a linear exposureefficacy relationship across doses 2-12 $\mathrm{mg}$, independent of concomitant AEDs (Hussein et al. AAN 2012: Abstract P06.127). Support: Eisai Inc 
Table 1. Responder rates $(\geq 50 \%$ or $\geq 75 \%$ reduction in seizure frequency, Maintenance vs Baseline) and seizure freedom by seizure type (ITT analysis set)

\begin{tabular}{|c|c|c|c|c|c|}
\hline All partial seizures<i> & $\begin{array}{l}\text { Placelo } \\
n=441\end{array}$ & $\begin{array}{c}\text { Perumpanel } \\
2 \mathrm{mg} \\
n=180\end{array}$ & $\begin{array}{c}\text { Perumpounet } \\
4 \mathrm{mg} \\
n=172\end{array}$ & $\begin{array}{c}\text { Percunponel } \\
8 \mathrm{mg} \\
n=431\end{array}$ & $\begin{array}{c}\text { Perampunet } \\
12 \mathrm{mg} \\
n=2.54\end{array}$ \\
\hline $50 \%$ responder rate. $\%$ & 19.3 & 20.6 & $28.5^{\circ}$ & $35.3^{* * 4 *}$ & $35.0^{* * * t}$ \\
\hline $75 \%$ responder rate, $\%$ & 6.1 & 10.0 & 12.2 & $17.4^{* 6 * 9}$ & $16.90 \%$ \\
\hline $\begin{array}{c}\text { Seizare freedom, \% (Maintenance } \\
\text { completers, } n \text { ) }\end{array}$ & $\begin{array}{l}1.0 \\
(392)\end{array}$ & $\begin{array}{l}1.9 \\
(154)\end{array}$ & $\begin{array}{l}4.4^{\circ} \\
(158)\end{array}$ & $\begin{array}{l}3.5^{*} \\
(367)\end{array}$ & $\begin{array}{l}4.1^{\circ} \\
(193)\end{array}$ \\
\hline$C^{P}+S G$ seizures $<$ is & $n=405$ & $n=167$ & $n=1,57$ & $n=393$ & $n=2.33$ \\
\hline $50 \%$ responder rate, $\%$ & 23.2 & 26.9 & $36.3 * \star$ & $39.4^{20.6}$ & $38.2^{252}$ \\
\hline $75 \%$ responder rate, \% & 10.9 & 15.0 & $17.8^{*}$ & 20.9464 & 20,6040 \\
\hline SG seizures $<i>$ & $n=173$ & $n=68$ & $n=7 I$ & $n=I 57$ & $n=95$ \\
\hline Sorto responder rate, $\%$ & 37.0 & $4+.1$ & 49.3 & $60.5^{60917}$ & $53.7^{* *}$ \\
\hline $75 \%$ responder rale, \% & 24.3 & 32.4 & 31.0 & $46.5^{\text {*w* }}-1-10$ & $38.9^{\circ}$ \\
\hline $\begin{array}{c}\text { Seizure freedom, } \% \text { (Maintenance } \\
\text { completers, } a)\end{array}$ & $\begin{array}{l}14.2 \\
(155)\end{array}$ & $\begin{array}{l}15.3 \\
(59)\end{array}$ & $\begin{array}{l}22.7 \\
(66)\end{array}$ & $\begin{array}{c}28.9^{\text {** }} \\
(I 35)\end{array}$ & $\begin{array}{c}27.0^{4} \\
(7.4)\end{array}$ \\
\hline
\end{tabular}

$* \mathrm{p}<0.05 ; * * \mathrm{p}<0.01 ; * * * \mathrm{p}<0.001$ vs placebo

$\mathrm{CP}+\mathrm{SG}$, complex partial plus secondary generalized; ITT, intent-totreat; SG, secondary generalized

Table 2. Responder rate ( $\geq 50 \%$ reduction in seizure frequency, Maintenance vs Baseline) by concomitant AED and seizure type (ITT analysis set)

\begin{tabular}{|c|c|c|c|c|c|}
\hline & \multicolumn{5}{|c|}{ Responder rate, \% (number of patients evaluated) } \\
\hline & Placebo & $\begin{array}{l}\text { Perampanel } \\
\quad 2 \mathrm{mg}\end{array}$ & $\begin{array}{l}\text { Perampanet } \\
\quad 4 \mathrm{ing}\end{array}$ & $\begin{array}{c}\text { Perampanet } \\
8 \mathrm{mg}\end{array}$ & $\begin{array}{l}\text { Perampanet } \\
12 \mathrm{mg}\end{array}$ \\
\hline \multicolumn{6}{|c|}{ Concomitant carbanazepine $<i>$} \\
\hline All partial seizures & $23.1(14.3)$ & $13.8(58)$ & $19.6(56)$ & $29.7(138)$ & $31.3(96)$ \\
\hline $\mathrm{CP}+S G$ seizures & $26.7(137)$ & $17.9(56)$ & $25.5(57)$ & $31.4(127)$ & $33.7(86)$ \\
\hline SG seizures & $37.3(51)$ & $38.1(21)$ & $35.0(20)$ & $51.2(43)$ & $51.5(33)$ \\
\hline \multicolumn{6}{|c|}{ Concomitant valproic acid $<i>$} \\
\hline All partial seizures & $20.0(140)$ & $18.8(80)$ & $34.7(75)$ & $38.3(120)$ & $36.5(63)$ \\
\hline$C^{P+S G}$ seizures & $24.4(127)$ & $28.2(78)$ & $43.5(69)$ & $40.5(111)$ & $48.3(58)$ \\
\hline SG seizures & $42.2(64)$ & $27.6(29)$ & $43.3(30)$ & $67.4(46)$ & $62.5(24)$ \\
\hline \multicolumn{6}{|c|}{ Concomitant lamotrigine $<$ i/ $>$} \\
\hline All partial seizures & $13.6(125)$ & $27.4(56)$ & $32.4(68)$ & $35.6(146)$ & $30.6(62)$ \\
\hline$C P+S G$ seizures & $16.1(118)$ & $28.3(53)$ & $42.6(61)$ & $42.1(133)$ & $32.7(55)$ \\
\hline SG seizures & $35.6(45)$ & $54.5(22)$ & $63.0(27)$ & $55.6(54)$ & $68.2(22)$ \\
\hline \multicolumn{6}{|c|}{ Concomitan levetiracetam $<$ is $>$} \\
\hline All partial seizures & $20.0(125)$ & $14.6(48)$ & $20.0(45)$ & $35.9(131)$ & $4.3 .0(86)$ \\
\hline$C P+S G$ seizures & $26.7(116)$ & $25.0(44)$ & $28.6(42)$ & $39.2(1 / 20)$ & $45.6(79)$ \\
\hline SG seizures & $39.5(43)$ & $45.0(20)$ & $66.7(15)$ & $67.0(47)$ & $66.7(27)$ \\
\hline
\end{tabular}

$\mathrm{CP}+\mathrm{SG}$, complex partial plus secondary generalized; ITT, intent-totreat; SG, secondary generalized

\subsection{5 \\ RELATIVE BIOAVAILABILITY OF CLOBAZAM ORAL SUSPENSION AFTER A SINGLE 20-MG DOSE IN HEALTHY STUDY VOLUNTEERS}

E. Sales ${ }^{1}$, A. Juan ${ }^{2}$, I. Bekersky ${ }^{1}$ and D. Tolbert ${ }^{1}\left({ }^{1}\right.$ Lundbeck LLC, Deerfield, IL and ${ }^{2}$ Seaview Research, Inc., Miami, FL)

Rationale: Clobazam 5-, 10-, and 20-mg tablets are available as adjunctive therapy for adult and pediatric patients with LennoxGastaut syndrome (LGS). For LGS patients who have difficulty swallowing tablets, Lundbeck LLC has developed an oral suspension formulation of clobazam. As a suspension formulation is a new dosage form, we conducted a single-dose bioavailability study vs. the commercial tablet formulation.

Methods: This Phase I study was a randomized, open-label, two-way crossover study investigating the relative bioavailability, safety and tolerability of oral suspension vs. oral tablets following a single 20$\mathrm{mg}$ dose to healthy male and female study participants $(\mathrm{N}=30)$. Doses were administered on Days 1 and 15. Each administration was separated by a washout period of 14 days. The pharmacokinetic profiles of clobazam and $N$-desmethylclobazam ( $N$-CLB) were obtained from serial plasma concentrations during 312 hours postdoses. The relative bioavailability of suspension compared with that of a clobazam tablet was evaluated using bioequivalence criteria: the $90 \%$ confidence intervals (CIs) of the ratios of the oral suspension to the oral tablet for the pharmacokinetic parameters of $\mathrm{AUC}_{0 \text {-inf }}$ and $\mathrm{C}_{\max }$ for clobazam were contained within the interval from 0.80 to 1.25 .

Results: The suspension/tablet ratio point estimate and $90 \%$ CIs for CLB were: $\mathrm{C}_{\max }=1.19$ (1.12 to 1.27); $\mathrm{AUC}_{0 \text {-last }}=0.997$ (0.977 to 1.02); and $\mathrm{AUC}_{0 \text {-inf }}=0.995$ (0.976 to 1.01). Thus, bioequivalence

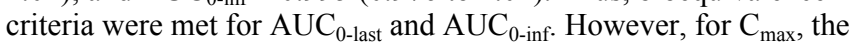
upper boundary of the $90 \%$ CI was just outside the bioequivalence limit of 1.25. All other parameters of exposure were similar. The slight increase observed in $\mathrm{C}_{\max }$ was a consequence of the physicochemical properties of the suspension dosage form (i.e., a suspension is designed to deliver the drug faster than a tablet) and were not considered clinically meaningful, as two dissimilar dosage forms were compared.

Conclusions: The 20-mg clobazam oral suspension was functionally bioequivalent to $20-\mathrm{mg}$ commercial tablets. Clobazam has a wide therapeutic window and is intended for long-term administration for patients with chronic LGS. Thus, the slight increase in $\mathrm{C}_{\max }$ is clinically insignificant. There were no clinically relevant differences in safety and tolerability between the two formulations.

\subsection{6 \\ WTIHDRAWN}

\subsection{7 \\ EVEROLIMUS IMPROVES SEIZURE CONTROL IN TUBEROUS SCLEROSIS COMPLEX}

Darcy A. Krueger ${ }^{1}$, A. A. Wilfong ${ }^{2}$, K. Holland-Bouley ${ }^{1}$, A.

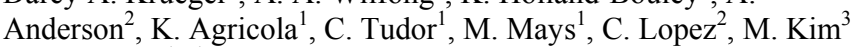
and D. Franz ${ }^{1}\left({ }^{1}\right.$ Child Neurology, Cincinnati Children's Hospital Medical Center, Cincinnati, OH; ${ }^{2}$ Pediatric Neurology, Texas Children's Hospital, Baylor College of Medicine, Houston, TX and ${ }^{3}$ Biostatistics and Epidemiology, Cincinnati Children's Hospital Medical Center, Cincinnati, OH)

Rationale: Epilepsy is a major neurological manifestation of Tuberous Sclerosis Complex (TSC), occurring in $80-90 \%$ of patients. Previous studies with everolimus to treat subependymal giant cell astrocytoma (SEGA) yielded differing results, possibly due to uncontrolled variables with potential influence on the secondary endpoint measures related to epilepsy in these studies. The current study is an open-label, multi-center combined phase I/II clinical trial where these same variables were identified and controlled to better determine the benefit of everolimus on seizure control.

Methods: Patients $\geq 2$ years of age with confirmed diagnosis of TSC and medically refractory epilepsy (defined as failure of two or more approved antiepileptic drug therapies and currently experiencing two or more seizures/week) were treated with everolimus $5 \mathrm{mg} / \mathrm{m} 2 /$ day and titrated to serum trough level between $5-15 \mathrm{ng} / \mathrm{ml}$ over a four week period. Patients then continued treatment for an additional eight weeks. Changes in concomitant antiepileptic medications were not permitted during observation, titration, and maintenance study phases. The primary efficacy endpoint was percentage of patients demonstrating a $50 \%$ or greater reduction in seizure frequency at the end of the maintenance period compared to baseline. Patients with $25 \%$ or greater reduction were considered partial responders. Secondary endpoints evaluated effect of treatment on 23-hour continuous video EEG recordings, quality of life assessments, and behavioral rating scales. Patients demonstrating beneficial effect after completing the maintenance study phase were eligible for optional extension. 
Results: Between May 2010 and August 2011, 20 patients were enrolled. Mean age was 9.0 years (range 2-21 years). Mean number of seizures/week was 14 (range 4-17) at baseline. All 20 patients completed the main study phase. We found that everolimus significantly decreases seizure frequency and shortens seizure duration in TSC patients with severe epilepsy overall (pvalue $=0.0004,0.0258$, respectively). Post-hoc analysis by ILAE classification suggests that the decreased seizure counts and shortened seizure duration was mainly driven by significant changes in complex partial seizures ( $\mathrm{p}$-value $=0.0007,0.0049$ ). Individually, 19 of the 20 patients demonstrated a reduction in seizure frequency. 14 were considered responders, including 4 becoming seizure-free. Another 4 were partial-responders and the remaining 2 were nonresponders. All responders and near-responders continued treatment in the extension phase. As of data cut-off (December 31, 2011), 17 patients in extension demonstrate continued efficacy and remain on treatment. Overall medication was well tolerated, with most common AE encountered being mouth ulcers and upper respiratory symptoms, consistent with previously published results.

Conclusions: Patients with TSC and medically refractory epilepsy demonstrated a clinically and statistically significant reduction in seizure frequency and duration following treatment with everolimus.

\subsection{8}

\section{WTIHDRAWN}

\subsection{9}

\section{ADJUNCTIVE PERAMPANEL IS EFFECTIVE AGAINST} PARTIAL SEIZURES, IRRESPECTIVE OF CONCOMITANT ANTIEPILEPTIC DRUGS (AEDS): A POOLED ANALYSIS OF THREE PHASE III TRIALS

Patrick Kwan ${ }^{1}$, M. Brodie ${ }^{2}$, D. Squillacote ${ }^{3}$, H. Yang ${ }^{4}$, J. Zhu ${ }^{4}$ and A. Laurenza ${ }^{4}{ }^{1}$ The Chinese University of Hong Kong, Prince of Wales Hospital, Hong Kong, China; ${ }^{2}$ Western Infirmary, Glasgow, United Kingdom; ${ }^{3}$ Eisai Global Medical Affairs, Woodcliff Lake, NJ and ${ }^{4}$ Eisai Neuroscience Product Creation Unit, Woodcliff Lake, NJ)

Rationale: Adjunctive perampanel, an oral, non-competitive AMPA receptor antagonist, reduced the frequency of refractory partial seizures compared with placebo in three Phase III trials (studies 304 [NCT00699972]; 305 [NCT00699582]; 306 [NCT00700310]). Primary data from these trials are reported elsewhere (French et al. Annual Meeting of the American Academy of Neurology [AAN] 2011: Abstract LBS.002; French et al. Epilepsia 2011; 52 [Suppl 6]: 10; Krauss et al. Neurology 2012; 78: 1408-1415). Here, data were pooled to explore changes in seizure frequency by seizure type and concomitant AEDs.

Methods: Patients ( $\geq 12$ years), with partial seizures despite receiving 1-3 AEDs, were randomized to once-daily placebo, perampanel 8 or $12 \mathrm{mg}$ (studies 304 and 305), or placebo, perampanel 2, 4, or $8 \mathrm{mg}$ (study 306). Trials included a 6-week Baseline Period and a Doubleblind Treatment Phase (6-week Titration; 13-week Maintenance). Patients recorded the number of partial seizures and their types (simple partial seizures with/without motor signs, complex partial $[\mathrm{CP}]$ seizures, and CP seizures with secondary generalization [SG seizures]) daily in a diary. Investigators reviewed the diaries with patients to ensure correct seizure classification. Median percentage changes in the frequencies of all partial seizures, $\mathrm{CP}$ plus SG $(\mathrm{CP}+\mathrm{SG})$ seizures, and SG seizures only (Baseline vs Double-blind Phase), were analyzed in the intent-to-treat (ITT) analysis set (all randomized and treated patients with any seizure data), and also according to the most common concomitant AEDs at Baseline. Pvalues are presented to highlight effects of interest for these analyses; they are not adjusted for multiplicity and the data for the subgroups may overlap.

Results: The ITT analysis set included 1478 patients from studies $304(\mathrm{n}=387), 305(\mathrm{n}=386)$, and $306(\mathrm{n}=705)$. At Baseline, most patients were receiving $\geq 2$ concomitant $\operatorname{AEDs}(1, n=206 ; 2, n=751 ; 3$, $\mathrm{n}=522)$ : the most common were carbamazepine $(\mathrm{n}=491 ; 33.2 \%)$, valproic acid $(n=478 ; 32.3 \%)$, lamotrigine $(n=458 ; 31.0 \%)$, and levetiracetam $(n=435 ; 29.4 \%)$. Median reductions in the frequencies of all partial seizures, $\mathrm{CP}+\mathrm{SG}$ seizures, and $\mathrm{SG}$ seizures were greater with perampanel 4-12 mg than placebo, in the overall population (Table $1 ; \mathrm{p}<0.01$, all comparisons) and in subgroups of patients receiving any of the 4 most common AEDs (Table 2; statistical significance not evaluated).

Conclusions: In this analysis of Phase III data by randomized dose, adjunctive perampanel reduced the frequency of different types of partial seizures, including SG seizures, compared with placebo; results were consistent in patients receiving any of the 4 most commonly co-administered AEDs. Additional analyses based on both actual dose and within-patient responses have shown a benefit of perampanel $12 \mathrm{mg}$ over $8 \mathrm{mg}$ (Kramer et al. AAN 2012: Abstract P06.117).

Support: Eisai Inc

Table 1. Median Baseline seizure frequency per 28 days and median percentage changes in seizure frequency per 28 days (Baseline vs Double-blind Phase), by seizure type (ITT analysis set)

\begin{tabular}{|c|c|c|c|c|c|}
\hline All parial seizures & $\begin{array}{l}\text { Placebo } \\
n=441\end{array}$ & $\begin{array}{c}\text { Perumpanel } \\
2 \mathrm{mg} \\
t \mathrm{t}=180\end{array}$ & $\begin{array}{c}\text { Perampanel } \\
4 \mathrm{mg} \\
n=172\end{array}$ & $\begin{array}{c}\text { Perampanet } \\
8 \mathrm{mg} \\
n=4.31\end{array}$ & $\begin{array}{c}\text { Perampane } \\
\qquad 12 \mathrm{mg} \\
n=2.54\end{array}$ \\
\hline $\begin{array}{c}\text { Median Baseline seizure } \\
\text { frequency, } n \\
\text { (Min, max) }\end{array}$ & $\begin{array}{l}11.1 \\
(3.3 \\
569.1)\end{array}$ & $\begin{array}{c}70.7 \\
(3.2,429.6)\end{array}$ & $\begin{array}{c}10.0 \\
(2.9 \\
4503.9)\end{array}$ & $\begin{array}{c}12.2 \\
(2.4 \\
1030.8)\end{array}$ & $\begin{array}{l}13.0 \\
(1.4 \\
1083.1)\end{array}$ \\
\hline $\begin{array}{l}\text { Median percentage change in } \\
\text { seizure frequency per } 28 \text { days. \% }\end{array}$ & -12.8 & -13.6 & $-23.3 * *$ & $-28.8^{\circ 90}$ & $-27.2^{* * * * *}$ \\
\hline$C P+S G$ seizures $<i />$ & $n=40.5$ & $f t=167$ & $n=157$ & $f=393$ & $n=2.3 .3$ \\
\hline $\begin{array}{c}\text { Median Baseline seizure } \\
\text { frequency, } n \\
\text { (Min. max) }\end{array}$ & $\begin{array}{c}7.8 \\
(0.6 \\
569.1)\end{array}$ & $\begin{array}{c}6.8 \\
(0.7,429.6)\end{array}$ & $\begin{array}{c}7.5 \\
(0.6 .303 .9)\end{array}$ & $\begin{array}{c}8.0 \\
(0.7,576.3)\end{array}$ & $\begin{array}{c}9.8 \\
(0.7,598.4)\end{array}$ \\
\hline $\begin{array}{l}\text { Median percentage change in } \\
\text { seizure frequency per } 28 \text { days, } \%\end{array}$ & -13.9 & -20.5 & $-31.2 * * *$ & $-35.6 \% \%$ & $-28.6 * * * *$ \\
\hline SG seizures & $n=173$ & $n=68$ & $n=71$ & $n=157$ & $n=95$ \\
\hline $\begin{array}{c}\text { Median Buseline seizure } \\
\text { frequency, } n \\
\text { (Min, max) }\end{array}$ & $\begin{array}{c}3.7 \\
(0.6 \\
169.4)\end{array}$ & $\begin{array}{c}3.4 \\
(0.7,31.4)\end{array}$ & $\begin{array}{c}3.7 \\
(0.7,104.5)\end{array}$ & $\begin{array}{c}3.4 \\
(0.6,158.7)\end{array}$ & $\begin{array}{c}4.1 \\
(0.6,138.6)\end{array}$ \\
\hline $\begin{array}{l}\text { Median percentage change in } \\
\text { seizure frequency per } 28 \text { days, } \%\end{array}$ & -19.4 & -28.0 & $-48.6 * *$ & $-62.9 * * *$ & $-53.3 * k * *$ \\
\hline
\end{tabular}

$* * p<0.01 ; * * * p<0.001$ vs placebo

$\mathrm{CP}+\mathrm{SG}$, complex partial plus secondary generalized; ITT, intent-totreat; SG, secondary generalized

Table 2. Median percentage changes in seizure frequency per 28 days (Baseline vs Double-blind Phase), by seizure type and concomitant AED (ITT analysis set)

\begin{tabular}{|c|c|c|c|c|c|}
\hline & \multicolumn{5}{|c|}{$\begin{array}{l}\text { Median percentage change in seizare frequency per } 28 \text { days, \% } \\
\text { (number of putients evuluated) }\end{array}$} \\
\hline & Placelon & $\begin{array}{l}\text { Peronponel } \\
2 \text { mtg }\end{array}$ & $\begin{array}{l}\text { Perampanel } \\
\quad 4 \text { mg }\end{array}$ & $\begin{array}{l}\text { Perampanel } \\
\text { \& mig }\end{array}$ & \begin{tabular}{|l} 
Perampanel \\
$\quad 12 \mathrm{mg}$
\end{tabular} \\
\hline \multicolumn{6}{|c|}{ All partial seizures } \\
\hline Concomitant carbanczepine & $-12.7\{(143)$ & $-5.9(58)$ & $.19 .7(56)$ & $-25.4(138)$ & $-20.3(96)$ \\
\hline Concomitant valproic acid & $-17.2(I+0)$ & $-16.0(80)$ & $-26.2(75)$ & $-27.7(120)$ & $-37.2(63)$ \\
\hline Concomilant lamotrigine & $-11.4(125)$ & $-21.3(56)$ & $.20 .6(68)$ & $-29.6(146)$ & $-31.2(62)$ \\
\hline Conconitant levetiracelant & $-18.2(125)$ & $-10.2(48)$ & $.19 .0(45)$ & $-25.7(131)$ & $-34.7(86)$ \\
\hline \multicolumn{6}{|c|}{$C P+S C$ seizares } \\
\hline Concomitant carbamazepine & $-13.9(|3|)$ & $-7.6(56)$ & $-28.7(5 /)$ & $-25.5(121)$ & $-20.3(86)$ \\
\hline Concomitant valproic acid & $-17.2(127)$ & $-21.5(78)$ & $-37.5(69)$ & $-36.3(m)$ & $-38.3(58)$ \\
\hline Conconitent lamotrigine & $-11.5(178)$ & $-20.5(53)$ & $-40.3(61)$ & $-38.8(133)$ & $-31.8(55)$ \\
\hline Concomitant levetiracetam & $-79.5(116)$ & $-11.1(44)$ & $-23.4(42)$ & $-36.8(120)$ & $-39.2(79)$ \\
\hline \multicolumn{6}{|c|}{$S G$ seizures $\langle i />$} \\
\hline Concomilant carbanazepine & $-12.7(5 /)$ & $-30 . /(21)$ & $-33.2(20)$ & $.53 .8(43)$ & $-52.8(33)$ \\
\hline Conconniant valproic acid & $-34.4(64)$ & $-19.7(29)$ & $-48.3(30)$ & $-65.4(46)$ & $-75.4(24)$ \\
\hline Concomitant lamorrigine & $-22.9(45)$ & $-65.4(22)$ & $60.5(27)$ & $61.1(54)$ & $-75.5(22)$ \\
\hline Concomitant levetimacetam & $.19 .1(43)$ & $-26.9(20)$ & $.63 .6(15)$ & $.61 .1(4 I)$ & $-70.7(27)$ \\
\hline
\end{tabular}

$\mathrm{CP}+\mathrm{SG}$, complex partial plus secondary generalized; ITT, intent-totreat; SG, secondary generalized 


\subsection{0}

RELATIONSHIP BETWEEN QUALITY OF LIFE AND THE FREQUENCY OF REFRACTORY PARTIAL SEIZURES: A POOLED ANALYSIS OF THREE PHASE III TRIALS OF PERAMPANEL

Gregory L. Krauss ${ }^{1}$, E. Faught ${ }^{2}$ and W. R. Simons ${ }^{3}\left({ }^{1}\right.$ Johns Hopkins School of Medicine, Baltimore, MD; ${ }^{2}$ Emory University, Atlanta, GA and ${ }^{3}$ Eisai Global Health Technology Assessment, Woodcliff Lake, NJ)

Rationale: Pooled data from Phase III trials of perampanel were analyzed to explore the relationship between quality of life and reductions in the frequency of refractory partial seizures.

Methods: Three Phase III clinical trials evaluated the efficacy and safety of adjunctive perampanel in patients with uncontrolled partial seizures (studies 304 [NCT00699972]; 305 [NCT00699582]; 306 [NCT00700310]). Trials included a 6-week Baseline Period and a Double-Blind Treatment Phase (6-week Titration; 13-week Maintenance). Percent changes in seizure frequency between Baseline and the Double-Blind Phase were recorded. Patients were classed as responders if seizure frequency was reduced by $\geq 50 \%$ between Baseline and Maintenance. Quality of Life in Epilepsy (QOLIE)-31P (questionnaire with 30 items across seven subscales [seizure-worry, overall quality of life, emotional well-being, energyfatigue, cognition, medication effects, and social function] and one item on overall health status) was administered at Baseline and End Of Treatment (EOT) in patients ( $\geq 18$ years). The minimally important difference (MID) for QOLIE was defined as an $11.8 \%$ change in overall score (Wiebe et al. J Neurol Neurosurg Psychiatry 2002; 73: 116-120). Multivariate regressions were used to estimate (a) marginal changes in QOLIE associated with a $1 \%$ reduction in seizure frequency, and the reduction in seizure frequency required to produce QOLIE MID, and (b) marginal changes in QOLIE in responders; changes greater than the MID established a direct correlation between the responder definition and QOLIE MID. Results: Of 1478 patients in the pooled intent-to-treat analysis set, 959 perampanel-treated patients from studies $304(\mathrm{n}=183), 305$ $(n=262)$ and $306(n=514)$ had QOLIE data; 742 had complete data for inclusion in the multivariate analysis. Mean (SD) QOLIE scores at Baseline were 57.4 (16.7), 55.0 (14.7) and 55.6 (15.8) for the three trials, respectively. Overall, $62.4 \%$ of patients had Baseline QOLIE scores $<50$ and mean improvement at EOT was $12.5 \%(\mathrm{p}=0.01)$. A $48.0 \%$ reduction in seizure frequency was required to produce QOLIE MID and a 1\% reduction in seizure frequency was associated with a change of 0.01 in overall QOLIE score $(\mathrm{p}=0.03)$. Compared with non-responders, responders ( $27.9 \%$ of perampanel patients) had greater mean improvements in overall QOLIE (additional 21.1\% beyond that achieved in non-responders), and the subscales for seizure-worry $(55.3 \%)$, cognition (35.1\%), and emotional well-being (38.1\%; $<<0.01$ for all); these mean improvements were greater than the MID.

Conclusions: Refractory partial seizures have a major impact on well-being, with most patients in this analysis having overall QOLIE scores $<50$ at Baseline. A $48 \%$ reduction in the frequency of these seizures was associated with a significant, meaningful improvement in overall quality of life. In addition, responder rate, defined by a $\geq 50 \%$ reduction in seizure frequency, corresponded with significant improvements in quality of life according to the QOLIE MID. Support: Eisai Inc

\subsection{1}

EFFICACY AND SAFETY OF ONCE-DAILY ADJUNCTIVE PERAMPANEL, A SELECTIVE AMPA ANTAGONIST IN PATIENTS WITH TREATMENT-RESISTANT PARTIALONSET SEIZURES: THE NORTH AMERICAN EXPERIENCE B. Vazquez ${ }^{1}$, H. Yang ${ }^{2}$, B. Williams ${ }^{3}$, S. Zhou ${ }^{3}$, A. Laurenza ${ }^{2}$ and R. Fain $^{3}$ ( ${ }^{1}$ NYU Langone Medical Center, New York, NY;

${ }^{2}$ Neuroscience Product Creation Unit, Eisai, Inc., Woodcliff Lake, NJ and ${ }^{3}$ Medical and Scientific Affairs, Eisai, Inc., Woodcliff Lake, NJ)
Rationale: Perampanel (PER), an orally active, highly selective, noncompetitive AMPA antagonist, has been evaluated in 3 pivotal Phase 3 trials. Results from the separate trials have been presented previously; here, we present the pooled efficacy and safety results from 2 of the Phase 3 studies that included the North American (NA) patient subpopulation as compared to the overall pooled Phase 3 results.

Methods: Patients $\geq 12$ years of age with refractory partial seizures (treated with 1-3 AEDs) were randomized to once-daily, double-blind placebo (PBO), or PER 2, 4, 8, $12 \mathrm{mg} /$ day. Studies 304 (N=388; conducted in North America, Central America, South America) and $305(\mathrm{~N}=386$; conducted in Europe, Asia, South Africa, North America, Australia) compared PBO with PER doses of $8 \mathrm{mg}$ /day and $12 \mathrm{mg} /$ day, and included patients from the United States $(\mathrm{N}=294)$ and Canada (N=25). Study 306 (N=706; PBO, $2 \mathrm{mg}, 4 \mathrm{mg}, 8 \mathrm{mg}$ ) was conducted in Australia, Europe, and Asia. Endpoints included median $\%$ change in seizure frequency $/ 28$ days and $50 \%$ responder rate and safety.

Results: The NA patient cohort in the Phase 3 trials had longstanding disease (mean duration, 23.9 years), often with no known etiology (53.8\%). Most had complex partial seizures (CP) (89.6\%), and had a baseline history of seizures with secondary generalization (SG) $(72.0 \%)$. The ITT population consisted of 318 patients $(n=106$, PBO; $\mathrm{n}=105,8 \mathrm{mg} ; \mathrm{n}=107,12 \mathrm{mg}$ ). The safety population consisted of 319 patients ( $n=106$, PBO; $n=105,8 \mathrm{mg} ; n=108,12 \mathrm{mg}$ ).

Median changes from baseline in seizure frequency/28 days during the double-blind period and the responder rates during maintenanceLOCF period for the NA subpopulation and the pooled Phase 3 population are presented in Table 1. For the NA patient pool, median $\%$ changes in seizure frequency/28 days in $\mathrm{CP}+\mathrm{SG}$ during the double-blind period were $-16.8(\mathrm{~N}=97),-40.1(\mathrm{~N}=102)$, and -34.9 $(\mathrm{N}=98)$ and in SG seizures were $-10.7(\mathrm{~N}=49),-60.6(\mathrm{~N}=41)$, and $67.9(\mathrm{~N}=41)$ for $\mathrm{PBO}, 8 \mathrm{mg}$, and $12 \mathrm{mg}$, respectively. In the overall Phase 3 population, these were $-13.9(\mathrm{~N}=405),-20.5(\mathrm{~N}=167),-31.2$ $(\mathrm{N}=157),-35.6(\mathrm{~N}=393)$, and $-28.6(\mathrm{~N}=233)$ for $\mathrm{CP}+\mathrm{SG}$ and -19.4 $(\mathrm{N}=173),-28.0(\mathrm{~N}=68),-48.6(\mathrm{~N}=71),-62.9(\mathrm{~N}=157),-53.3(\mathrm{~N}=95)$ for the SG seizures with PBO, $2 \mathrm{mg}, 4 \mathrm{mg}, 8 \mathrm{mg}$, and $12 \mathrm{mg}$, respectively. Study completion rates for the NA region were $86.8 \%$, $90.5 \%$, and $72.2 \%$ for $\mathrm{PBO}, 8 \mathrm{mg}$, and $12 \mathrm{mg}$, respectively.

Treatment-emergent adverse events (TEAEs), occurring in $\geq 10 \%$ of patients, for the NA population and the overall Phase 3 population are presented in Table 2.

Conclusions: PER was efficacious in the treatment of refractory partial onset seizures in the NA subpopulation, the majority of whom had $\mathrm{CP}$, with and without SG, in the Phase 3 trials. Efficacy results both in terms of improvement in seizure frequency and responder rates in the NA subpopulation were consistent with the overall Phase 3 population. PER had a favorable safety profile and tolerability that was consistent with the overall study population.

Table 1. Median Change in Seizure Frequency and Responder Rates in Phase 3 North American Subpopulation and the Pooled Phase 3 Population by Randomized Dose

\begin{tabular}{|c|c|c|c|c|}
\hline & \multicolumn{4}{|c|}{$\begin{array}{l}\text { Median Change From Baseline in Scizure Frequency/28 Days Over Double-Bline } \\
\text { Period }\end{array}$} \\
\hline & \multicolumn{2}{|c|}{ Overall Pooled Phase 3} & \multicolumn{2}{|c|}{ North American Subpopelation } \\
\hline & $\mathrm{N}$ & Change (\%) & $\mathrm{s}$ & Change (\%) \\
\hline PBO & 441 & -12.8 & 106 & -16.2 \\
\hline PER $2 \mathrm{mg}$ & 180 & -13.6 & - & - \\
\hline PER 4 mg & 172 & -23.3 & - & - \\
\hline PER $8 \mathrm{mg}$ & 431 & .288 & 105 & .34 .2 \\
\hline $\begin{array}{l}\text { PER } 12 \\
\text { mg }\end{array}$ & 254 & -27.2 & 107 & .34 .5 \\
\hline & \multicolumn{4}{|c|}{ Responder Rate During Maintenance-LOCY Period } \\
\hline & \multicolumn{2}{|c|}{ Overall Pooled Phase 3} & \multicolumn{2}{|c|}{ North American Subpopelation } \\
\hline & $\mathrm{N}$ & Responders (\%) & $\mathrm{s}$ & Responders (\%) \\
\hline PBO & 441 & 18.4 & 106 & 20.8 \\
\hline PER 2 mg & 180 & 22.4 & - &. \\
\hline PER 4 mg & 172 & 30.8 & - & -. \\
\hline PER $8 \mathrm{mg}$ & 431 & 37.6 & 105 & 41.9 \\
\hline $\begin{array}{c}\text { PER } 12 \\
\mathrm{mg}\end{array}$ & 254 & 39.5 & 107 & 40.2 \\
\hline
\end{tabular}


Table 2. Treatment-Emergent Adverse Events That Were Very Common ( $\geq \mathbf{1 0 \%}$ in Any Treatment Group) for the Pooled Phase 3 North American Sub-Population Compared With the Overall Pooled Phase 3 Population

\begin{tabular}{|cc|c|cc|c|cc|c|}
\hline \multicolumn{8}{|c|}{ Ovcrall Pooled Phase 3 (\%) } & \multicolumn{3}{c|}{ North American Subpopulation (\%) } \\
\hline & PBO & $\mathbf{2} \mathbf{~ m g}$ & $\mathbf{4 ~ m g}$ & $\mathbf{8} \mathbf{~ m g}$ & $\mathbf{1 2} \mathbf{~ m g}$ & PBO & $\mathbf{8 ~ \mathbf { m g }}$ & $\mathbf{1 2} \mathbf{~ m g}$ \\
V & 442 & 180 & 172 & 431 & 255 & 106 & 105 & 108 \\
Dizziness & 9 & 10 & 16.3 & 31.8 & 42.7 & 12.3 & 38.1 & 39.8 \\
\hline Somnolence & 7.2 & 12.2 & 9.3 & 15.5 & 17.6 & 12.3 & 16.2 & 12 \\
\hline Headache & 11.3 & 8.9 & 11 & 11.4 & 13.3 & 14.2 & 17.1 & 7.4 \\
\hline Fatigue & 4.8 & 4.4 & 7.6 & 8.4 & 12.2 & 9.4 & 11.4 & 13 \\
Irritability & 2.9 & 3.9 & 4.1 & 6.7 & 11.8 & 4.7 & 10.5 & 12 \\
\hline Fall & 3.4 & 1.1 & 1.7 & 5.1 & 10.2 & 5.7 & 13.3 & 17.6 \\
\hline
\end{tabular}

Non-AED/Non-Surgical Treatments (Hormonal, ketogenic, alternative, etc.): Adult

\subsection{2}

\section{THE USE OF SUPPLEMENTS AND COMPLEMENTARY} MEDICINE IN PATIENTS WITH EPILEPSY

Kristen M. Kelly and S. S. Chung (Neurology, Barrow Neurological Institute, St. Joseph's Hospital, Phoenix, AZ)

Rationale: Many patients who take prescription medications also take supplements or "natural" medications. Few studies have investigated the use of supplements and complementary medicine in patients with epilepsy. This is an important topic given the potential interaction between AEDs and supplements, and patient perception of safety and use of prescription and non-prescription treatments for epilepsy.

Methods: From February 2012 to June 2012, consecutive patients seen at the Barrow Neurological Institute Epilepsy clinic were given multiple choice and fill in questionnaires regarding details of their epilepsy and supplement use. 436 surveys were completed. Statistical analysis with the graphPad Instat program was used, with ANOVA to compare groups.

Results: $5.3 \%$ of patients had epilepsy for less than one year, $14.5 \%$ for $1-5$ years, $14.5 \%$ for 5 to 10 years, and $65.7 \%$ for more than 10 years. The percentage of patients taking at least one supplement was $45 \%, 40 \%, 45 \%$, and $47 \%$ for the groups of epilepsy less than one year, 1-5 years, 5-10 years, and over 10 years, respectively. $52 \%$ of the patients surveyed took at least one alternative supplement. Of the 202 patients who listed their supplements, the average number of alternative treatments was 2.7 (range 1-9, SD 1.95). Multivitamins were the most common supplement. The second most used supplement was fish oil/Omega-3 supplements. Marijuana was used by three patients. Two patients took Huperzine A.

$22.4 \%$ of responders take supplements "because it helps my seizures." $6.9 \%$ of responders take supplements to counteract AED side effects or toxicities; $64.9 \%$ for other medical reasons; $5.7 \%$ do not know why they take them. $66 \%$ of all patients surveyed would use a "natural" supplement for seizure control if it were available.

Conclusions: The use of supplement and complementary medicine is common in patients with epilepsy (52\%). The percentage of patients using these treatments did not vary significantly based on the length of time they have had epilepsy. Most patients take these treatments for other medical problems, but a significant minority (22\%) takes them as a treatment of epilepsy or because of perceived side effects of the medications $(6.9 \%)$. Many patients would like to use a "natural" supplement to treat epilepsy if it were available.

This study shows that supplements and alternative medicine use are very common in patients with epilepsy. Based on previous studies, patients may not mention these treatments to their neurologist because, very frequently, the treatments are not related to their epilepsy. About 1 out of 5 patients took alternative treatments to treat their epilepsy, such as Husperzine A and marijuana. As these supplements become more available, neurologists will be faced with patients questioning, and sometimes requesting, alternative treatments for their epilepsy. It is important for neurologists to be aware of the common use of supplements and alternative medicine in patients with epilepsy so that neurologist can better counsel and treat their patients.

\subsection{3 \\ CHARACTERISTICS OF PEOPLE WITH STRESS TRIGGERED SEIZURES}

Michael D. Privitera, M. Walters, A. Dwivedi, W. Weng, A. Fleck and D. Schwieterman (Neurology, University of Cincinnati, Cincinnati, $\mathrm{OH}$ )

Rationale: Up to $50 \%$ of people with epilepsy believe stress is a trigger for seizures yet little is known about the specific stressors, the timing of the stressors and other associated factors for stresstriggered seizures. We surveyed a large epilepsy practice to identify patients who felt seizures were sometimes triggered by stress and determined whether other epilepsy or mood factors were associated with stress-triggered seizures.

Methods: Sequential patients in the outpatient clinics of the Epilepsy Center of the University of Cincinnati Neuroscience Institute were asked: 1) were seizures sometimes triggered by stress; 2) could they identify a specific instance where stress lasting days to months made seizures more likely; 3 ) could they identify a specific instance where an acute stress lasting minutes to hours made a seizure more likely; and 4) if they had tried some type of stress reduction and whether they thought the stress reduction worked to reduce seizures. Subjects were also asked about ability to predict seizures. Excluded patients had cognitive impairment making them unable to complete the questionnaire or elected to not participate. All subjects signed informed consent. Midway through the study, data were gathered on a control population of subjects who thought stress played no role in their seizures (stress (-)). Data on seizure type, history of and treatment for depression, depression (NDDI-E) and anxiety (GAD-7) screening scores (these instruments are administered to all patients at each visit in the clinic), demographics and seizure types were gathered.

Results: This preliminary analysis is based on 203 stress $(+)$ subjects and 28 stress (-) controls. Among stress $(+)$ subjects, $85 \%$ endorsed chronic stress as a seizure trigger; $68 \%$ endorsed acute stress as a trigger. In stress $(+)$ subjects, some type of relaxation or stress reduction treatment was used by $57 \%$ and of those who tried this treatment, $88 \%$ thought it improved seizures. Interestingly, $25 \%$ of stress (-) subjects tried relaxation or stress reduction and $71 \%$ thought seizures improved. Comparing stress $(+)$ and stress (-) subjects in a univariate analysis the following characteristics were associated with stress $(+)$ subjects (odds ratio $>1$ including $95 \% \mathrm{CI}$ ): history of depression, higher NDDI-E, higher GAD-7. In a multivariable logistic regression, only GAD-7 score and seizure prediction was associated with stress $(+)$. Stress $(+)$ subjects were more likely to believe they could at least occasionally predict seizures $(51.8 \% \mathrm{vs}$. $14.3 \%$; OR 8.6, $\mathrm{p}=.0005$ ).

Conclusions: Stress-triggered seizures are common, may be associated with chronic or acute stress, and are associated with higher depression and anxiety scores. Patients who endorse stress-triggered seizures are more likely to believe their seizures can be predicted. Many patients have tried stress reduction and the large majority of those who tried believe it helped reduce seizures. A randomized, controlled trial of stress reduction methods in medication resistant epilepsy is indicated. 


\subsection{4}

REPETITIVE TRANSCRANIAL MAGNETIC STIMULATION THERAPY FOR INTRACTABLE TEMPORAL LOBE

\section{EPILEPSY}

Ta-Cheng Chen ${ }^{1}$ and M. Chang ${ }^{2}\left({ }^{1}\right.$ Neurology, Changhua Christian Hospital, Changhua, Taiwan and ${ }^{2}$ Pediatric Neurology, Changhua Christian Hospital, Changhua, Taiwan)

Rationale: Transcranial Magnetic stimulation (TMS) is a kind of non-invasive tool and had been used to study neurophysiology since 1985, TMS employed extensively in clinical neurophysiology, is an alternative approach for epilepsy treatment. Reduced seizure frequency has been reported in a small number of patients treated with rTMS (repetitive Transcranial Magnetic Stimulation) at lowfrequency stimulus rates. However, all of these studies were short period follow up and variable treatment protocol. To reassess the therapeutic potential of TMS and cortical physiological parameter in patients with epilepsy, we conducted case research of rTMS treatment trial in patient with extremely drug resistant focal onset epilepsy.

Methods: Totally 3 intractable right temporal lobe origin epilepsy patients were enrolled in this study, 2 female and 1 male, aged 23 to 44 years old. Study design incorporates 2 months baseline observational period that records seizure frequency and severity of both major and minor seizure attack and other epilepsy profile, Three months Study period that apply $0.5 \mathrm{HZ} 95 \%$ RMT sub-threshold repetitive TMS treatment with $70 \mathrm{~mm}$ double coil stimulator for $30 \mathrm{~min}$ each week and measure single pulse cortical silent period (CSP), Resting Motor Threshold (RMT) before and after treatment and finally, 2 months follow up period that record seizure frequency; severity as well. In addition, EEG studies before and just after rTMS treatment course was executed in each patient to evaluate quantities of epileptic discharge.

Results: Three impressive findings were revealed in this treatment study; first, all Neurophysiological parameters (magnetic evoked potential) following TMS appear to be absent or ill-defined waveforms; second, reduction of seizure frequency were revealed mainly during study period and up to within first month of follow up period in one case. Third, apparent reduction of epileptiform discharge of EEG study was found in one case, much reduction of spike counts and rhythmic mid-temporal theta activity

Conclusions: Motor evoked potential study in epilepsy patients as previous reports, behave attenuated waveform presentation and even further absent in our case series, which is supposed to be caused by the effect of anticonvulsants; multi-pharmacy (3- 4 categories) were prescribed in each patient of our case series. Repetitive TMS (rTMS) treatment in intractable focal temporal lobe epilepsy is of short term benefit effect. Further study including large case series and variable treatment profile of rTMS should be designed and executed to further delineate the role of rTMS in epilepsy treatment.

\section{Non-AED/Non-Surgical Treatments (Hormonal, ketogenic, alternative, etc.): Pediatrics}

\subsection{5 \\ USE OF THE KETOGENIC DIET IN INFANTILE SPASMS REFRACTORY TO FIRST-LINE TREATMENT : A PROSPECTIVE STUDY}

M. E. Pires ${ }^{1}$, A. Ilea ${ }^{1}$, E. Bourel-Ponchel ${ }^{2}$, V. Bellavoine ${ }^{1}$, P. Berquin $^{2}$ and Stephane Auvin ${ }^{1}\left({ }^{1}\right.$ Ped Neurology, Robert Debre Childrens Hospital, Paris, France and ${ }^{2}$ Ped Neurology, CHU Amiens, Amiens, France)

Rationale: We report the effect of ketogenic diet (KD) to treat infantile spasms (IS) as a third-line treatment, after vigabatrin (VGB) and oral steroid. We aimed to evaluate the efficacy and the tolerability of KD in IS using the rate of seizure-free patient at 1 month because of the prognosis of this epilepsy syndrome
Methods: We report 17 patients treated by KD for IS. The data were extracted from a prospective database of 2 French child neurology departments (Amiens \& Robert Debré-Paris, France) over a 18 month period. Follow-up clinic visits with EEG were done at 1, 3, 6 and 12 months after initiating the diet were scheduled to access efficacy and tolerability. All the patients were followed the KD for 6 months. The addiction of an antiepileptic drug was allowed after 1 month of KD Results: The KD was initiated at the mean age of $9.4 \pm 1.1$ months. After 1 month with KD 6/17 (35\%) were seizure free while 11/17 $(65 \%)$ were seizure-free after the third month. However, an additional antiepileptic drug (felbamate or topiramate) has been added in all patients that were not seizure free under KD.

Conclusions: All patients but one were treated by KD before the age of 1 year. The KD was used early in the treatment of IS (Third-line treatment). Comparing our data to previous studies, it seems that the responders rate in case of IS is similar regardless the age of the patient and the precocity of the KD in the management of IS. We also found that an additional AED may significantly improve the rate of seizure control.

\subsection{6 WTIHDRAWN}

\subsection{7 \\ TREATMENT OF INFANTILE SPASMS WITH VERY HIGH DOSE PREDNISOLONE BEFORE HIGH DOSE ACTH}

Shaun A. Hussain, G. Kwong, J. Matsumoto, J. T. Lerner, J. Y. Wu, W. D. Shields and R. Sankar (Pediatric Neurology, Mattel Children's Hospital at UCLA, Los Angeles, CA)

Rationale: The relative efficacy of hormonal therapies in the treatment of infantile spasms (IS) is unclear. Although high-dose ACTH ( $150 \mathrm{IU} / \mathrm{m} 2 /$ day $\times 2$ weeks $)$ is more effective than traditionally high-dose prednisone $(2 \mathrm{mg} / \mathrm{kg} /$ day $)$, very high-dose prednisolone (PRED) (4-8 mg/kg/day) may yield similar response rates as compared to high-dose ACTH. This study was conducted to evaluate the short-term efficacy of PRED to achieve clinical and electroencephalographic resolution of IS, and to avoid the use of $\mathrm{ACTH}$ and its requisite delay in therapy initiation (1-2 days), pain (intramuscular injections), and cost.

Methods: A standardized treatment protocol for IS was instituted based on previously reported efficacy and side effect profile. 22 subjects with video-EEG confirmed IS whose treatment conformed to the protocol were retrospectively identified. Very high dose PRED (8 $\mathrm{mg} / \mathrm{kg} /$ day divided TID, with a max dose of $60 \mathrm{mg} /$ day) was the initial therapy. After 2 weeks, all patients with cessation of spasms were admitted for video-EEG to confirm a complete response (absence of both spasms and hypsarrhythmia). Subjects with a complete response were tapered off PRED over 2 weeks. All subjects with incomplete response were immediately transitioned to high dose ACTH (150 IU/m2/day divided BID) and PRED was discontinued without tapering. Response was again evaluated by video-EEG two weeks after ACTH initiation.

Results: Mean age of IS onset was 9.4 months (SD 7.5), and mean age at PRED initiation was 15.3 months (SD 11.6). Etiology of IS was cryptogenic $(n=3)$ or symptomatic (identified cause, $n=13$; unidentified cause, $n=6$ ). Only 3 patients were completely treatmentnaive and patients failed a mean of 1.8 medications prior to PRED treatment. Only 2 patients exhibited normal development prior to onset of IS. $50 \%(11 / 22)$ of patients had a complete response to PRED. Of the remaining 11 patients, 4 exhibited a partial response to PRED (continued spasms but resolution of hypsarrhythmia). 2 weeks after transition to ACTH, 36\% (4/11) of these patients completely responded. After 4 weeks, a cumulative $68 \%(15 / 22)$ of patients completely responded to therapy. One PRED-responder and one ACTH-responder were lost to follow-up after protocol completion. Among the 20 subjects with follow-up (mean 21 months), 27\% (4/15) 
of responders exhibited a relapse of IS between 2 and 9 months after initial response. For the patients with known developmental outcome at age 2 years $(n=13)$ and 4 years $(n=6)$, all exhibited at least mild developmental delay. Although hypertension was common, there were no adverse events during treatment that required discontinuation of PRED or ACTH.

Conclusions: Among this cohort of infants with IS who were relatively old, and for whom hormonal therapy was generally delayed, very high dose oral PRED appears to be an effective alternative to intramuscular ACTH. Still, A randomized trial comparing high-dose ACTH (150 IU/m2/day) to very high-dose PRED (4-8 $\mathrm{mg} / \mathrm{kg} /$ day) is required to determine the best hormonal therapy for IS. In the absence of a definitive trial, the treatment protocol presented here is a reasonable option.

\subsection{8 \\ KETOGENIC DIET EFFICACY IN THE TREATMENT OF INTRACTABLE EPILEPTIC SPASMS}

H. Kayyali, M. Gustafson, M. Williams, L. Thompston, L. Vaughn and Ahmed Abdelmoity (Comprehensive Epilepsy Center, Children's Mercy Hospital Univ. Of Missouri at Kansas City, Kansas City, KS)

Rationale: To determine the efficacy of the ketogenic diet in controlling epileptic spasms after failing traditional medication therapy.

Epileptic spasms are considered one of the most devastating types of seizures in the pediatric age group. Besides adrenocorticotropic hormone $(\mathrm{ACTH})$ and vigabatrin, there has been limited data on the efficacy of other anti-epileptic medications for its treatment. The ketogenic diet has been shown to be an efficacious nonpharmacological method of treatment for a variety of epileptic syndromes.

Methods: We reviewed the charts of 118 pediatric patients on the ketogenic diet in a tertiary epilepsy center. 20 of them have infantile epileptic encephalopathy. All these infants continued to have epileptic spasms with evidence of severe epileptic encephalopathy on electroencephalograms (EEGs) despite treatment with antiepileptic medications. We collected data from the charts of 18 patients in regards to seizure control rates and quality of life improvement at 3,6 and 12 months visits. Quality of life improvement was charted based on the care giver's perspective.

Results: 20 patients were included in the study (16 males, 4 females). Age of spasms onset is 2-9 months (mean 4.5 months). Age of ketogenic diet initiation is 1-34 months (mean 13 months). 15 patients had cryptogenic epileptic spasms; three had perinatal hypoxic ischemic injury, one has lissencephaly and one has STXBP1 mutation. Fifteen patients failed to respond to ACTH and/or vigabatrin before going on the ketogenic diet. The remaining 5 patients tried at least 3 other antiepileptic medications before the diet was started. $67 \%$ of the patients had more than $50 \%$ seizure reduction (including two patients were seizure free) after starting the diet. This effect was maintained at 3,6 and 12 months visits. Majority of patients achieved cessation of epileptic spasms within 4-10 weeks (mean 6 weeks) after starting the ketogenic diet. All care givers reported improvement of the quality of life at 3 months visit. This ratio was $80 \%$ and $63 \%$ at 6 and 12 months visits respectively. Conclusions: The ketogenic diet is a safe and potentially effective method of treatment for patients with epileptic spasms; especially those who did not respond to customary medication therapies.

\subsection{9}

\section{CATCH-UP GROWTH AFTER LONG-TERM} IMPLEMENTATION AND WEANING FROM KETOGENIC DIET IN PEDIATRIC EPILEPTIC PATIENTS

J. Lee ${ }^{1}$, Hyo Jeong Kim ${ }^{1}$, J. Kim ${ }^{1}$, H. Kang ${ }^{1}$, J. Song ${ }^{1}$, M. Lee ${ }^{1}$, Y. $\mathrm{Lee}^{2}$, E. Lee ${ }^{3}$ J. Lee ${ }^{1}$ and H. Kim ${ }^{1}\left({ }^{1}\right.$ Pediatric Neurology, Pediatric Epilepsy Clinics, Severance Children's Hospital, Epilepsy Research
Institute, Yonsei University College of Medicine, Seoul, Republic of Korea; ${ }^{2}$ Pediatrics, Pusan National University Children's Hospital, Yangsan, Republic of Korea and ${ }^{3}$ Dietetics, Severance Hospital, Seoul, Republic of Korea)

Rationale: To assess the presence of growth retardation during longterm implementation of ketogenic diet (KD) among children with epilepsy, and verify the presence of catch-up growth in same patients 1 year after diet discontinuation.

Methods: Retrospective chart review of 20 males and 20 females was performed and $\mathrm{z}$ scores of height, weight and body mass index was obtained at baseline, 2 years into $\mathrm{KD}$, and 1 year after discontinuation of KD.

Results: Our analysis revealed significant reduction in both height and weight gain among children with epilepsy after prolonged KD. After a year of diet discontinuation, significant catch-up growth was evident in both height and weight. According to specific subsets, ambulation had favorable influence on growth during $\mathrm{KD}$ and after diet discontinuation and in addition, uncontrolled epilepsy, and younger age at the start of $\mathrm{KD}$ contributes a negative impact on a child's growth.

Conclusions: This preliminary observation into long-term growth pattern among recipients of KD will aid both health care providers and patients alike in planning for long-term care extending beyond the duration of dietary treatment.

\subsection{0 \\ HIGH FAT DIET CONTROL OF SEIZURES IN DOOSE SYNDROME \\ Elisabeth Simard-Tremblay ${ }^{2,1}$, P. Berry ${ }^{2}$, B. Cook $^{2}$, A. Owens ${ }^{2}$, M. Mazzanti $^{2}$, E. Novotny ${ }^{2,1}$ and R. Saneto ${ }^{2,1}$ ( ${ }^{1}$ University of Washington, Seattle, WA and ${ }^{2}$ Seattle Children's Hospital, Seattle, WA)}

Rationale: Myostatic astatic epilepsy (MAE) or Doose syndrome is a generalized epilepsy syndrome associated with massive myoclonic and/or myoclonic-astatic seizures inducing drop attacks. Other seizure types also occur with generalized spike waves and cognitive deterioration. Seizure freedom using medications remains unsatisfactory. We report a chart review of six patients treated with either the Ketogenic Diet (KD) or modified Atkins Diet (MAD). Methods: We retrospectively analyzed six patients who were diagnosed with MAE and experienced significant improvement in seizure control on the KD or MAD. All were males between two and four years when $\operatorname{diet}(\mathrm{s})$ was started. SCN1A gene sequencing was performed in two patients and SCLA2A1 gene sequencing was performed in three patients. All returned normal. Multiple EEG studies and MRI scans were performed on each patient.

Results: Three patients were started on the KD and three were started on the MAD. Four of the six patients were tried on at least four seizure medications before diet therapy. The remaining two patients were tried on one and two seizure medications respectively. Four of the six patients became seizure free within several weeks of dietary therapy. Seizure freedom has been between two and three years in all four patients. The last two patients went from having multiple daily seizures to having an average of two seizures per month in one case and no seizures in a 38-day period in the other. MRI scans of the brain were normal. EEG demonstrated predominantly generalized spike discharges. Clinically, all patients had drop seizures. Seizure medications were able to be discontinued in four patients. One patient was started on the diet less than a year ago and remains on two antiseizure medications and one patient was kept on monotherapy while on the diet. One patient has fully weaned off the diet and remains without seizures. Another patient was weaned off the diet because of familial preference. By family reporting, all patients have had a significant improvement in development.

Conclusions: Results suggest that a subpopulation of MAE patients can be effectively treated with high fat diets. Seizure medications 
were able to be tapered off in most patients. All six patients obtained significant improvement in seizure frequency, four of which became seizure free. In addition, all families reported cognitive improvement. Half of the patients were started on the KD suggesting that MAD diet initiation may be effective in this population.

\subsection{1 \\ QUALITY IMPROVEMENT OPPORTUNITIES IN HOSPITALIZED CHILDREN ON ESTABLISHED CLASSIC KETOGENIC DIET}

Daniel Kenney, K. Nickels, E. Wirrell and L. Wong-Kisiel (Mayo

Clinic, Rochester, MN)

Rationale: Successful seizure control in children already established on the classic ketogenic diet relies on proper diet implementation, strict adherence and laboratory monitoring. The aim of this study is to evaluate opportunities for quality improvement among hospitalized children on established ketogenic diet.

Methods: This is a retrospective chart review of children on established classic ketogenic diet admitted to the Mayo Eugenio Children's Hospital in Rochester, MN between November 2008 and February 2012. An "admission" was defined as the period during which a given hospital service was responsible for the patient's care, regardless of whether the patient was coming from home or transferred from another service. Admissions were stratified according to indication and admitting services. Inpatient records were reviewed for serum beta-hydroxybutyrate (BHB), urine ketones, dietetics and pharmacy consultations, and administration of highcarbohydrate medications or dextrose-containing fluids.

Results: Fourteen children (male 46\%; median age 75 months; range 12 - 166 months) on established classic ketogenic diet were hospitalized during the study period. A total of 46 admissions were reviewed, including 20 admissions (43\%) for seizure exacerbation. Admitting pediatric services included general hospital service (19), intensive care unit (18), pediatric epilepsy monitoring (6), surgical subspecialties (2), and physical medicine/ rehabilitation (1). Urine ketones were performed daily in 17 (37\%) of all admissions, 10/19 $(53 \%)$ admissions to general pediatric floor and 4/18 (22\%) admissions to intensive care unit. Among admissions for breakthrough seizures, urine ketones were checked less than daily in $10 / 20(50 \%)$ admissions and were never checked in 5/20 (25\%) admissions. Among admissions for reasons other than immediate seizure control, only $7 / 26$ (27\%) had daily urine ketones monitored. Fourteen admissions had BHB testing recorded, and only 3/14 (21\%) were in therapeutic range. Two of these admissions were to the Epilepsy Monitoring Unit, and one was to the General Pediatric service for medical issues unrelated to seizure. There were 3 administrations of high carbohydrate-containing medication during hospitalization and no administration of dextrose-containing fluid. All admissions but one were reviewed by a dietician within 24 hours. Medication reconciliation by a pharmacist was performed in $37 / 46$ (80\%) admissions.

Conclusions: Insufficient laboratory monitoring in hospitalized children on established classic ketogenic diet occurs in about two thirds of patients requiring hospitalization, providing an opportunity for quality improvement in the care of these patients. Particular areas for attention include critical care admissions and patients admitted for reason other than seizure.

\subsection{2 \\ A COMPARISON BETWEEN DIFFERENT SIDE EFFECT PROFILES OF KETOGENIC DIET FORMULAS} Ahmed Abdelmoity ${ }^{1}$, C. DeCock ${ }^{1}$, M. Gustafson ${ }^{1}$, M. Williams ${ }^{1}$, L. Thompson $^{1}$, L. Vaughn ${ }^{1}$, S. Abdelmoity ${ }^{2}$ and H. Kayyali ${ }^{1}$ ( ${ }^{1}$ Comprehensive Epilepsy Center, Children's Mercy Hospital. Univ. Of Missouri at Kansas City, Kansas City, MO and ${ }^{2}$ Blue Valley West High school, Overland Park, KS)
Rationale: The ketogenic diet has been proven to be an effective method in treating pharmacologically intractable epilepsy. This study, aims to assess side effect profiles associated with different ketogenic diet formulas. Having such data will help predict, and avoid different complications that might arise with ketogenic diet treatment in patients with pharmacologically intractable epilepsy. Methods: We conducted a retrospective chart review of all patients who received ketogenic diet therapy, followed from April of 2005 through January 2011 in the Comprehensive Epilepsy Center at Children's Mercy Hospital ( $\mathrm{N}=118)$ with an age ranging between 4 months, and 20 years old at the time of diet initiation. Ketogenic diet ratio ranged between 1:1 to 4:1 fat to protein and carbohydrate. Ketocal formula was used in 55 patients, RCF formula in 19 patients, and oral ketogenic menu diet in 58 patients. 14 patients had a combination of more than one formula. We monitored Gastrointestinal (GI), Renal, Neurological, and metabolic side effects in different diet formulas

Results: Data from 118 patients were collected, and was found that none of the patients had side effects serious enough to terminate the diet; however a number of patients had different side effects as outlined in this table:

Conclusions: In our study we found that side effects were minor, transient and did result in discontinuation of the ketogenic diet in any of the patients. It was also noticed that gastrointestinal side effects are the most common. Food menu diet seems to have the least metabolic and renal side effects. RCF formula resulted in significantly less gastrointestinal and neurological side effects comparing to Ketocal and food menu.

Side effects profiles with different formulas

\begin{tabular}{|c|c|c|c|c|c|}
\hline Diet lype (N) & All Side effects (\%) & GI (\%) & Renal (\%) & Neuro (\%) & Metabolic (\%) \\
\hline Ketokal (55) & $29(52.7)$ & $13(23.6)$ & $5(9)$ & $10(18)$ & $12(21.8)$ \\
RCF (19) & $10(52.6)$ & $4(7.2)$ & $2(10.5)$ & $3(5.4)$ & $4(21)$ \\
Food $(58)$ & $26(44.8)$ & $15(25.8)$ & $4(6.9)$ & $8(13.8)$ & $6(10.3)$ \\
\hline
\end{tabular}

1.253

BREASTFEEDING IS COMPATIBLE WITH THE KETOGENIC DIET AND EFFICACIOUS IN TREATING SEIZURES IN INFANTS

L. Thompston, M. Gustafson, H. Kayyali and Ahmed Abdelmoity (Comprehensive Epilepsy Center, Children's Mercy Hospital Univ. Of Missouri at Kansas City, Kansas City, MO)

Rationale: The ketogenic diet is a safe and effective treatment for intractable epilepsy in infants. Breastmilk is the optimal source of nutrition for infants less than one year of age. Breastfeeding has not been traditionally continued upon initiation of the ketogenic diet and to date, there have been few reports in the literature of successful treatment of seizures with the ketogenic diet and concurrent breastfeeding.

Methods: Retrospective chart review was conducted of all patients who received ketogenic diet therapy in the Comprehensive Epilepsy Center at Children's Mercy Hospital between May 2005 and January 2011 (N=118). Seizure reduction and quality of life were collected by means of self report. Four patients were identified who continued to receive breast milk while receiving treatment with the ketogenic diet. All mothers expressed breast milk which was subsequently mixed with ketogenic formula.

Results: Of the 4 patients that continued breastfeeding while receiving ketogenic diet therapy, all 4 experienced a $>50 \%$ reduction of seizures during the first 3 months of diet therapy. While one patient discontinued the diet, the 3 remaining patients were able to maintain $>50 \%$ reduction of seizures up to 6 months. One patient became and remained seizure free for a year. All of the patients reported improved quality of life on the ketogenic diet up to 3 months. 
Conclusions: We conclude that breastfeeding is both possible and efficacious in conjunction with the ketogenic diet for treatment of intractable seizures.

Patient Demographics
\begin{tabular}{|c|cc|cc|}
\hline Patient & Diagnosis & $\begin{array}{c}\text { Age of Seizure } \\
\text { Onset }\end{array}$ & $\begin{array}{c}\text { Age of KGD } \\
\text { Initiation }\end{array}$ & $\begin{array}{c}\text { Time on KGD while } \\
\text { breastreeding }\end{array}$ \\
\hline A & Epileptic Spasms & 5 months & 7 months & 1 months \\
B & Fpilcptic Spasms & 7 months & 9 months & 5 months \\
C & $\begin{array}{c}\text { Structural Focal } \\
\text { Epilepsy }\end{array}$ & 1 day & 12 months & 4 months \\
\hline D & Aicardi Syndrome & 1 month & 3 months & 7 months \\
\hline
\end{tabular}

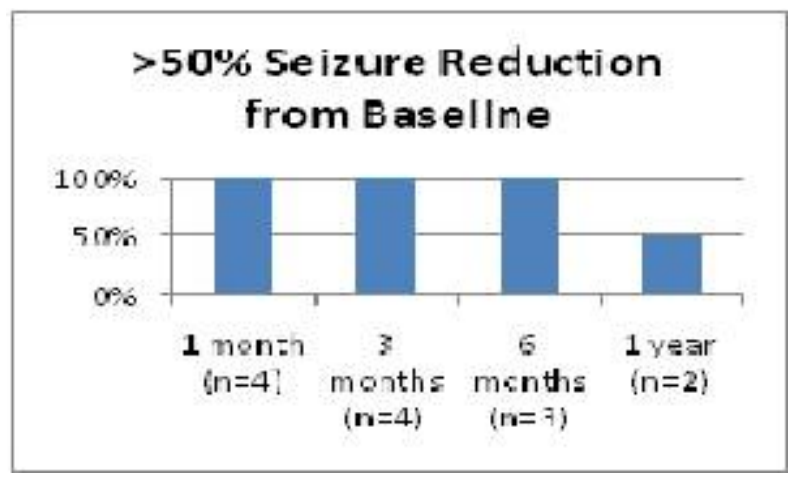

Seizure Reduction

Non-AED/Non-Surgical Treatments (Hormonal, ketogenic, alternative, etc.): All Ages

\subsection{4 \\ LONG-TERM SAFETY AND EFFICACY OF EVEROLIMUS IN PATIENTS WITH SUBEPENDYMAL GIANT CELL ASTROCYTOMA (SEGA) ASSOCIATED WITH TUBEROUS SCLEROSIS COMPLEX (TSC): 3-YEAR UPDATE}

D. N. Franz ${ }^{1}$, M. M. Care ${ }^{1}$, K. Holland-Bouley ${ }^{1}$, K. Agricola ${ }^{1}$, C. Tudor $^{1}$, J. Lebrec ${ }^{2}$, D. Lam ${ }^{3}$ and D. A. Krueger ${ }^{1}\left({ }^{1}\right.$ Cincinnati Children's Hospital Medical Center, Cincinnati, OH; ${ }^{2}$ Novartis Pharma AG, Basel, Switzerland and ${ }^{3}$ Novartis Pharmaceuticals Corporation, Florham Park, NJ)

Rationale: TSC is characterized by tumors in multiple organ systems and disabling neurological disorders. Currently the standard of care for SEGA associated with TSC is watchful waiting unless the disease progresses to symptoms. Symptomatic SEGAs, a surgical emergency, are managed with neurosurgical resection, but may not be possible in select patients due to location or size of the tumor or other underlying health issues. A prospective, open-label, phase I/II trial

(NCT00411619) of everolimus, an oral, selective mTOR inhibitor, demonstrated a significant reduction in asymptomatic SEGA tumor volume and patient-reported seizure frequency. The extension phase of this trial was undertaken to monitor the long-term safety and efficacy of ongoing everolimus treatment in patients with SEGA associated with TSC.

Methods: Patients $\geq 3$ years of age with a definitive TSC diagnosis and serial increase in SEGA lesion size on $\geq 2$ MRI scans received everolimus starting at $3 \mathrm{mg} / \mathrm{m}^{2} /$ day orally, titrated to achieve blood trough concentration of $5-15 \mathrm{ng} / \mathrm{mL}$, subject to tolerability. In the initial study, the primary endpoint was to evaluate the safety and potential side effects of everolimus. Measures of efficacy were reduction from baseline in primary SEGA volume and corresponding $\geq 30 \%$ or $\geq 50 \%$ response rate.

Results: At analysis cut-off date (14 Dec 2011), 24 of the 28 patients initially enrolled in the study remained on everolimus treatment (median dose, $5.22 \mathrm{mg} / \mathrm{m}^{2} /$ day [range, $\left.2.0-11.8\right]$ ). Median treatment duration was 45.7 months (range, 4.7-58.5). After 36 ( $n=23), 42$ $(n=16)$, and $48(n=10)$ months of everolimus treatment, there was a $\geq 30 \%$ reduction from baseline of the primary SEGA volume in $78.3 \%, 75.0 \%$, and $90 \%$ of patients, respectively, and a $\geq 50 \%$ reduction of the primary SEGA volume from baseline in $43.5 \%$, $37.5 \%$, and $50.0 \%$ of patients at the same time points. Seizure frequency (patient reported) generally decreased over time (Table 1). At 36 months $(n=22), 72.7 \%$ patients reported no seizure since their last visit compared with $38.5 \%$ at baseline, and only $4.5 \%$ had $\geq 1$ seizure per day compared with $26.9 \%$ at baseline. Adverse events (AEs) remained similar to those previously reported for everolimus, mostly grade $1 / 2$ (Table 2 ). There were no drug-related grade 4 AEs and there were 6 drug-related grade 3 AEs. No patient discontinued treatment due to an AE.

Conclusions: The results of the 3-year analysis report the latest follow-up data for these patients and confirm maintenance of reductions in SEGA volume. No new safety concerns were identified, and everolimus was well tolerated with prolonged use, which becomes important as other uses of everolimus are investigated, including epilepsy. Longer term everolimus therapy continues to be safe and effective for treatment of SEGA in patients with TSC.

Table 1. Patient-Reported Seizure Frequency by Time Report

\begin{tabular}{|c|c|c|c|c|c|c|c|c|c|}
\hline Seizure frequency & $\begin{array}{c}\text { Baseline } \\
\mathrm{n}=26 \\
\%\end{array}$ & $\begin{array}{c}\text { Month } \\
6 \\
\mathrm{n}=25 \\
\%\end{array}$ & $\begin{array}{c}\text { Month } \\
12 \\
n=25 \\
\%\end{array}$ & $\begin{array}{c}\text { Month } \\
18 \\
n=24 \\
\%\end{array}$ & $\begin{array}{c}\text { Month } \\
24 \\
\mathrm{n}=23 \\
\%\end{array}$ & $\begin{array}{c}\text { Month } \\
30 \\
\mathrm{n}=22 \\
\%\end{array}$ & $\begin{array}{c}\text { Month } \\
36 \\
\mathrm{n}=22 \\
\%\end{array}$ & $\begin{array}{c}\text { Month } \\
42 \\
\mathrm{n}=13 \\
\%\end{array}$ & $\begin{array}{c}\text { Month } \\
48 \\
n=7 \\
\%\end{array}$ \\
\hline $\begin{array}{l}>6 \text { months since } \\
\text { last seizure belore } \\
\text { baseline or no } \\
\text { scizure since last } \\
\text { visit }\end{array}$ & 38.5 & 48.0 & 60.0 & 66.7 & 65.2 & 59.1 & 72.7 & 76.9 & 71.4 \\
\hline $\begin{array}{l}\geq 1 \text { seizure since } \\
\text { last visit but }<1 \\
\text { per month }\end{array}$ & 15.4 & 16.0 & 16.0 & 12.5 & 8.7 & 22.7 & 0 & 15.4 & 14.3 \\
\hline $\begin{array}{c}\geq 1 \text { scizure per } \\
\text { month but }<1 \text { per } \\
\text { week }\end{array}$ & 3.8 & 8.0 & 8.0 & 4.2 & 13.0 & 4.5 & 0 & 0 & 14.3 \\
\hline $\begin{array}{c}\geq \text { I seizure per } \\
\text { week but }<1 \text { per } \\
\text { day }\end{array}$ & 15.4 & 20.0 & 12.0 & 12.5 & 0 & 4.5 & 22.7 & 7.7 & 0 \\
\hline$\geq 1$ seizure per day & 26.9 & 8.0 & 4.0 & 4.2 & 13.0) & 9.1 & 4.5 & 0 & 0 \\
\hline
\end{tabular}

Table 2. Adverse Events Occurring in at Least $15 \%$ of Patients Regardless of Study Drug Relationship, by Preferred Term and Year of Emergence

\begin{tabular}{|c|c|c|c|c|c|}
\hline \multirow[b]{2}{*}{ Preferned term } & \multicolumn{5}{|c|}{ Everolimus } \\
\hline & $\begin{array}{c}\leq \text { Month } \\
12 \\
\mathrm{n}=28 \\
\mathrm{n}(\%)\end{array}$ & $\begin{array}{l}\text { Month } \\
13-24 \\
\mathbf{n}=27 \\
\mathbf{n}(\%)\end{array}$ & $\begin{array}{l}\text { Month } \\
25-36 \\
\mathrm{n}=25 \\
\mathrm{n}(\%)\end{array}$ & $\begin{array}{c}\text { Month } \\
37-48 \\
n=23 \\
n(\%)\end{array}$ & $\begin{array}{c}>\text { Month } \\
48 \\
n=8 \\
n(\%)\end{array}$ \\
\hline Any preferred term & $28(100.0)$ & $26(96.3)$ & $24(96.0)$ & $19(82.6)$ & $4(50.0)$ \\
\hline Stomatitis & $19(67,9)$ & $16(59.3)$ & II $(44.0)$ & $4(17.4)$ & $2(25.0)$ \\
\hline $\begin{array}{l}\text { Lpper respiratory tract } \\
\text { infection }\end{array}$ & $16(57.1)$ & $14(51.9)$ & $13(52.0)$ & $8(34.8)$ & $1(12.5)$ \\
\hline Otitis media & $10(35.7)$ & $7(25.9)$ & $4(16.0)$ & $3(13.0)$ & 0 \\
\hline Sinusitix & $10(35.7)$ & $2(7.4)$ & $6(24.0)$ & $7(30.4)$ & 0 \\
\hline Pyrexia & $7(25.0)$ & $2(7.4)$ & 0 & $1(4.3)$ & 0 \\
\hline Diaurhea & $6(21.4)$ & $5(18.5)$ & $2(8.0)$ & 0 & 0 \\
\hline Dermatitis acneiform & $6(21.4)$ & $1(3.7)$ & 0 & 0 & 0 \\
\hline Cellulitis & $5(17.9)$ & $3(11.1)$ & $4(16.0)$ & $1(4.3)$ & 0 \\
\hline Convulsion & $5(17.9)$ & $3(11,1)$ & $1(4.0)$ & 0 & 0 \\
\hline Vomiting & $5(17.9)$ & $3(11.1)$ & 0 & $2(8.7)$ & 0 \\
\hline Body tinea & $5(17.9)$ & 0 & I $(4.0)$ & 0 & 0 \\
\hline Gastroenteritis & $4(14.3)$ & $1(3.7)$ & $6(24.0)$ & $3(13.0)$ & $1(12.5)$ \\
\hline Otitis extema & $2(7.1)$ & $5(18.5)$ & $3(12.0)$ & $1(4.3)$ & 0 \\
\hline Abnormal behavior & $1(3.6)$ & $1(3.7)$ & $4(16.0)$ & 0 & 0 \\
\hline Skin infection & $1(3.6)$ & $1(3.7)$ & $4(16.0)$ & 0 & 0 \\
\hline Headache & $1(3.6)$ & $4(14.8)$ & 0 & 0 & 0 \\
\hline Mouth ulceration & 0 & $4(14.8)$ & $3(12.0)$ & $5(21.7)$ & 0 \\
\hline Laceration & 0 & 0 & $5(20.0)$ & $1(4.3)$ & I (12.5) \\
\hline
\end{tabular}




\subsection{5}

\section{THE MODIFIED ATKINS DIET FOR THE TREATMENT OF} JUVENILE MYOCLONIC EPILEPSY

Eric Kossoff, B. Henry and M. C. Cervenka (Johns Hopkins Hospital, Baltimore, MD)

Rationale: Juvenile myoclonic epilepsy (JME) is often successfully treated with anticonvulsants; however some cases may be medically resistant. The modified Atkins diet (MAD) has been reported as effective for idiopathic generalized epilepsy (absence) and is increasingly being selected for use in adolescents and adults over the ketogenic diet due to the greater flexibility in its administration. We reviewed our experience in using the MAD for JME.

Methods: Since 2006, eight adolescents and adults were started on the MAD for JME at the Johns Hopkins Hospital. Six patients were followed prospectively and records were retrospectively reviewed for two patients to ascertain efficacy, diet duration, and adverse effects. Results: Of these 8 patients, $6(75 \%)$ were female, with a mean age of seizure onset of 10.5 years (range: 6-13 years) and MAD initiation at 24.3 years (range: $15-44$ years). Patients had tried a mean of 5.4 anticonvulsants before dietary therapy with the exception of a 15year-old female who attempted the MAD as first-line therapy. Seven achieved at least moderate ketosis; the mean diet duration to date is 11.2 months (range: 0.5-36 months). After 1 month, 6/8 (75\%) had $>50 \%$ seizure reduction and after 3 months, $5 / 8(63 \%)$ had $>50 \%$ improvement. Two patients became seizure-free for extended periods on MAD. Five reported reduction in both myoclonic and generalized tonic-clonic seizure frequency. Side effects included weight loss in 5 patients and a temporary increase in cholesterol in one. Most patients found the MAD difficult to adhere to, with 3 reporting temporarily increased seizures during periods of noncompliance.

Conclusions: In this limited experience, the modified Atkins diet was efficacious adjunctive therapy for young adults with typically very medically resistant juvenile myoclonic epilepsy, as long as patients remained motivated and compliant. It may be also helpful for patients with juvenile myoclonic epilepsy and comorbid obesity and females who wish to avoid valproate. Prospective clinical trials are warranted.

\subsection{6}

LEUCINE IS PROTECTIVE IN ACUTE SEIZURE TESTS Adam Hartman ${ }^{1,2}$, P. Santos ${ }^{1}$ and J. M. Hardwick ${ }^{2}\left({ }^{1}\right.$ Neurology, Johns Hopkins University School of Medicine, Baltimore, MD and ${ }^{2}$ Molecular Microbiology and Immunology, Johns Hopkins Bloomberg School of Public Health, Baltimore, MD)

Rationale: Leucine is a ketogenic amino acid that protects against picrotoxin- and pentylenetetrazol-induced seizures in rats (Skeie, Pharmacol Biochem Behav 1994; Dufour, Brain Res 1999) but not against hexafluorodiethyl ether seizures (Gallagher, J Neurochem 1969). Similar to rapamycin (which suppresses seizures), leucine depletion suppresses mTOR activity in cell culture (Zeng, J Neurosci 2009; Laplante, J Cell Sci 2009). Leucine also decreases synaptic glutamate concentration (Yudkoff, Prostaglandins Leukot Essent Fatty Acids 2004). It is unknown whether leucine has an anticonvulsant mechanism similar to treatments that affect metabolism, including the ketogenic diet or rapamycin. We investigated the anticonvulsant effects of leucine using acute seizure tests in normal mice.

Methods: In Cohort \#1, NIH Swiss mice aged 3 weeks received leucine via drinking water $(1.5 \% \mathrm{w} / \mathrm{v})$ for 12 days (control mice received water without leucine). Seizures were induced using the 6 $\mathrm{Hz}$, pentylenetretrazol, maximal electroshock, and kainic acid tests. Blood ketones were measured prior to seizure testing. In Cohort \#2, mice aged 5 weeks were injected intraperitoneally with leucine (300 $\mathrm{mg} / \mathrm{kg}$ in PBS) either 3 hours before or 15 minutes after kainic acid injection.

Results: In Cohort \#1, leucine-treated mice were protected against 6 Hz-induced seizures $(P=0.02)$. In the pentylenetetrazol test, mice treated with leucine had a prolonged latency to the first tail twitch ( $P$ $=0.04$ ) but did not differ in other parameters. In the kainic acid test, leucine-treated mice had lower overall seizure scores than control mice $(\mathrm{P}=0.0009)$. There was no difference in the maximal electroshock threshold test. Blood ketone levels were similar between leucine-treated and control mice. In Cohort \#2, mice treated with leucine 3 hours prior to kainic acid had significantly lower overall seizure scores $(\mathrm{P}=0.0002)$ and a shorter duration of convulsions $(\mathrm{P}=$ $0.03)$. Mice treated with leucine $15 \mathrm{~min}$ after kainic acid treatment had lower maximum seizure scores $(\mathrm{P}=0.01)$ and a borderline longer latency to onset of overt convulsions $(\mathrm{P}=0.049)$ but no differences in other parameters.

Conclusions: Leucine is protective in acute seizure tests. Leucine has an acute seizure test profile that is distinct from anticonvulsants currently used in the clinic and other metabolism-based antiseizure treatments, including the ketogenic diet and rapamycin.

Anticonvulsant effects could not be attributed to differences in blood ketone levels.

\subsection{7 \\ EFFICACY OF VAGUS NERVE STIMULATION IN PATIENTS ON UNCHANGED DOSE OF ANTIEPILEPTIC DRUG REGIMEN: 2 YEAR OUTCOME AT A COMMUNITY BASED COMPREHENSIVE EPILEPSY CENTER \\ S. Chayasirisobhon ${ }^{1}$, L. Cahan ${ }^{2}$, S. Choi ${ }^{1}$, B. Enos ${ }^{2}$, J. Hwang ${ }^{2}$, M. \\ Lin $^{2}$, J. Schweitzer ${ }^{2}$, B. Spurgeon ${ }^{1}$, E. Stuckert ${ }^{1}$ and Suresh Gurbani ${ }^{1,2}\left({ }^{1}\right.$ Kaiser Permanente, Anaheim, CA and ${ }^{2}$ Kaiser Permanente, Los Angeles, CA)}

Rationale: To establish long term persistent efficacy of vagus nerve stimulation (VNS)

for the adjunctive treatment of intractable epilepsy (failed at least 3 appropriate drugs) in the patients on unchanged antiepileptic drug (AED) regimen.

Methods: We conducted epilepsy treatment outcome analysis of clinical data of 39 consecutive patients with VNS implant whose AED regimen was unchanged for a period of 24 months following the VNS implant surgery. The efficacy of VNS therapy was evaluated at 6 months, 12 months and 24 months.

Results: Of the 39 patients, $24(61.5 \%)$ at 6 months, $25(64.1 \%)$ at 12 months and

$25(64.1 \%)$ at 24 months showed $\geq 50 \%$ reduction in seizure frequency. Nineteen

$(48.7 \%)$ patients had $\geq 50 \%$ reduction in seizure frequency in all three periods. Partial

response was seen in $10(25.6 \%)$ patients. Ten patients $(25.6 \%)$ showed no clinically significant benefit in all three periods. A complete $(100 \%)$ seizure control at 6 months, 12 months and 24 months was seen in $2(5.1 \%), 7(17.9 \%)$ and $8(20.5 \%)$ patients respectively. We analyzed the efficacy of VNS for different types of seizures in three study periods. Out of 39 patients, 23 patients had partial seizures with and without secondary generalized seizures, and 16 patients had primary generalized types of seizures. Twelve $(52.2 \%)$ of the 23 patients with partial seizures showed $\geq 50 \%$ reduction of seizure frequency. Seven (43.8\%) of the 16 patients with generalized types of seizures showed $\geq 50 \%$ reduction of seizure frequency. VNS therapy was tolerated well with no patient requiring discontinuation of therapy.

Conclusions: 1) Patients with unchanged AED regimen on VNS therapy had persistent seizure control lasting for 24 months. 2) Number of patients achieving complete seizure control increased with increasing duration of VNS therapy. 3) No major complications or side effects requiring discontinuation of therapy were noted. Our experience supports the persistent efficacy and safety of longterm adjunctive VNS therapy in treatment of intractable epilepsy. 


\subsection{8}

RASMUSSEN ENCEPHALITIS: 5 YEAR FOLLOW-UP UNDER IMMUNOADSORBTIONS AND RITUXIMAB

B. Schmalbach and Nicolas Lang (Christian-Albrechts-University Kiel, Kiel, Germany)

Rationale: Rasmussen encephalitis (RE), a chronic inflammatory disease, affects and subsequently destroys one cerebral hemisphere leading to intractable focal onset seizures and progressive neurological deficits. It is currently understood that Granzyme B mediated T-cell cytotoxicity directed at neurons plays a pivotal role in the pathogenic mechanisms of RE. Whereas anticonvulsive pharmacotherapy is poorly effective, surgical deafferentiation of the affected hemisphere can be used for seizure treatment, but is often withheld until later stages of the disease when a fixed hemiparesis has developed. Due to the assumptive pathogenesis various forms of immunotherapy are applied to prevent progressive loss of neurological function.

Methods: We describe the case of a 25 year old female patient with biopsy proven RE who was treated with a combination of immunoadsorptions and rituximab, a monoclonal antibody against the protein $\mathrm{CD} 20$, in addition to antiepileptic polytherapy for the course of five years. After diagnosis of RE seven years earlier numerous regimens of antiepileptic and anti-inflammatory drugs had not lead to control of seizures even though progression of hemiatrophy was relatively mild.

Results: After the first application of immunoadsorption followed by administration of rituximab $(375 \mathrm{mg} / \mathrm{m} 2$ intravenously once a week over four weeks) seizures ceased completely and for the first time since disease onset the patient remained seizure free for 6 months. During the following five years the patient repeatedly presented with focal status epilepticus or with increasing and daily life impairing seizures. We administered either immunoadsorptions, rituximab or a combination of both, which lead to complete cessation of seizures or to a reduction to only minor, not impairing seizures for up to six months. Antiepileptic treatment could successively be reduced down to triple therapy without sedative or cognition impairing medication. Immunoadsorptions were done when the patient presented with focal status epilepticus, which made a fast treatment response necessary. In case that seizures could not be controlled with immunoadsorptions alone a treatment with rituximab was added. Rituximab alone was administered three times in a prophylactic approach and the seizure free intervals could thereby be extended. However, seizure aggravation or status epilepticus always reoccurred after several months. Rituximab was well tolerated except for an increased occurrence of minor infections. Immunoadsorption was associated with more side effects such as infections and occlusions of the shunt and once cardio-pulmonary-reanimation was necessary after an iatrogenic uncontrolled citrate infusion.

Conclusions: This observation challenges the traditional view that non-surgical seizure treatment is largely unsuccessful and demonstrates a therapeutic approach that is able to control seizures and tissue loss. Furthermore, it indicates that therapeutic approaches other than those primarily directed at cellular immunity, such as rituximab and immunoadsorption, can be beneficial in Rasmussen encephalitis.

\section{Non-AED/Non-Surgical Treatments (Hormonal, ketogenic,} alternative, etc.): Animal

\subsection{9 \\ THE COGNITIVE EFFECTS OF LONG TERM ADMINISTRATION OF KETOGENIC DIET IN RATS} Prabhu Emmady ${ }^{1}$ and J. Harney ${ }^{2}\left({ }^{1}\right.$ Penn State-Hershey Medical Center, Hershey, PA and ${ }^{2}$ University of Hartford, Hartford, CT)

Rationale: Several complications have been reported with the administration of Ketogenic diet (KD) and the exact mechanism underlying its clinical efficacy has been largely debated. Additionally, cognitive decline in children with epilepsy on KD has been a concern, but it is unclear if cognition improves after cessation of KD.

Methods: 36 Sprague-Dawley rats at postnatal day 20 were randomly assigned into 4 groups to receive Rodent chow(Ad lib), $8 \% \mathrm{KD}, 14 \% \mathrm{KD}$ and $18 \% \mathrm{KD}$ respectively. All rats had ad libitum access to water and were housed in individual cages. Spatial Acquisition Trial (T1) was done after 22 weeks of assigned diet administration. Spatial Reversal Trial (T2) was done 1 week after T1. Trial 3 (T3) was done 6 weeks after KD cessation during which time all rats were fed on rat chow.

Results: All rat groups had 9 animals at the start of the experiment. 1 rat in the $8 \% \mathrm{KD}$ died during the diet, the cause of death was unknown. Bonferroni Post-Hoc Test for combined T1, T2 showed a mean difference of -115.44 (SE 5.5), -31.0 (SE 5.5) and -30.11 (SE 5.7) for Ad lib compared to $8 \% \mathrm{KD}, 14 \% \mathrm{KD}$ and $18 \% \mathrm{KD}$ respectively, which were statistically significant $(\mathrm{p}<0.001)$. The mean difference for same groups for T3 were -2.14 (SE 7.84), -7.25 (SE 7.8) and -11.23 (SE 8.08), which were not statistically significant (P $=1$ ). Sphericity tests were performed to measure the change in cognitive function of all KD rat groups from T1 to T3. They showed a significant difference in the performance of rats between these two periods when the diets were changed. [F=256.884 (1), $\mathrm{p}<0.001]$. All Rats fed on calorie restricted ketogenic diet had ketones, average Ad $\mathrm{lib}=0,8 \% \mathrm{KD}=1.035,14 \% \mathrm{KD}=0.881$ and $18 \% \mathrm{KD}=0.791$.

Conclusions: The highest ketone concentration was seen in $8 \% \mathrm{KD}$ Rats as they received a greater fat concentration and lower protein and carbohydrate concentration in their diet. Cognitive decline was seen with all $3 \mathrm{KD}$ diet groups. The degree of cognitive decline was greater when a severe carbohydrate and protein restricted high fat diet is used. This cognitive decline improves after cessation of KD and no significant cognitive differences existed in them compared to Ad lib diet.

\subsection{0 \\ NANOMOLAR LEVELS OF A CYCLIC ANTI- INFLAMMATORY PEPTIDE PROTECTS AGAINST ELECTRICALLY INDUCED SEIZURES IN MICE}

Karin Borges, N. K. Thomas, D. She, Y. Singh, T. M. Woodruff, M. Hodson and M. Benson (University of Queensland, St Lucia, Australia)

Rationale: Complement activation leading to inflammation appears to play a role in epilepsy and seizure development. Levels of various complement factors, including the membrane attack complex, are increased in both animal models of epilepsy and patients with human temporal epilepsy (Aronica et al., 2007; Neurobiol Dis 26:497ff), suggesting that the anaphylatoxin $\mathrm{C} 5 \mathrm{a}$ is produced in the epileptic brain. We hypothesized that activation of receptors for $\mathrm{C} 5 \mathrm{a}$, specifically CD88, promote seizure generation.

Methods: The anticonvulsant profile of the cyclic anti-inflammatory peptide PMX53, an inhibitor of CD88, was investigated in several murine seizure models. PMX53 levels were quantified in plasma and saline perfused brains at 15,30,60, and 90 min using liquid chromatography coupled to mass spectroscopy (LC-MS/MS). Results: PMX53 displayed reproducible anticonvulsant effects in both the $6 \mathrm{~Hz}$ and chronic corneal kindling models, but not in the pentylenetetrazole (i.v.) or maximal electroshock threshold tests. Statistically significant elevations in CC50 value (mean +/- SEM: 8.1 $+/-1.2 \mathrm{~mA} \mathrm{n}=5$ independent experiments) were seen in CD1 mice when PMX53 was administered 30 minutes prior to $6 \mathrm{~Hz}$ seizure induction at a dose of $3 \mathrm{mg} / \mathrm{kg}$ (s.c.). PMX53 (3 mg/ $\mathrm{kg}$ s.c.) also protected fully corneally kindled CD1 mice against stage 3-5 seizures $(\mathrm{p}=0.04$ and $\mathrm{p}=0.027 \mathrm{n}=2$ experiments). The anticonvulsant effects in the $6 \mathrm{~Hz}$ model disappeared by $60-90$ minutes after administration. Our pharmacokinetic study using quantitative LC-MS analysis revealed peak levels in plasma and brain within 30 minutes of 
PMX53 (3mg/kg s.c.) administration. Peak plasma levels were $482+/-$ $57.5 \mathrm{nM}$ (mean $+/-$ SEM, $\mathrm{n}=6$ ) at 15 minutes post administration, with a rapid decline in plasma levels following this timepoint. Brain levels were $11.5+/-1.9 \mathrm{ng} / \mathrm{g}$ and $15.6+/-4.1 \mathrm{ng} / \mathrm{g}$ (mean +/- SEM, $\mathrm{n}=6$ ) at 15 and 30 minutes, respectively, post administration indicating a 40-60 $\mathrm{nM}$ level in the extracellular fluid. These levels in the extracellular fluid are close to the IC50 of PMX53 on human CD88 of approximately 20nM (Woodruff et al., $2005 \mathrm{~J}$ Pharmacol Exp Ther 314:811-7). We are currently assessing to which extent PMX53's effects in the $6 \mathrm{~Hz}$ model are dependent on CD88 using CD88deficient and wild type mice.

Conclusions: PMX53 is anticonvulsant in the murine $6 \mathrm{~Hz}$ and corneal kindling models. The anticonvulsant time window of PMX53 matches with its brain concentration peaks early after administration. In conclusion we show blood brain barrier permeability and reproducible anticonvulsant effects of a cyclic anti-inflammatory peptide against electrically induced seizures in mice. Elucidation of its CNS target and development of more stable analogues appear to be a promising approach to find new treatments for pharmacoresistant epilepsy.

\subsection{1}

\section{ANTICONVULSANT EFFECTS OF PIOGLITAZONE ON HIGH POTASSIUM SEIZURE-LIKE EVENTS IN IN VITRO HIPPOCAMPAL SLICES}

NIRUPAMA P. RANADE, K. K. Samson, K. A. Simeone and T. A. Simeone (Pharmacology, Creighton University School of Medicine, OMAHA, NE)

Rationale: Peroxisome proliferator activated receptor gamma (PPARg) is a transcription factor that regulates genes involved in neuroprotection and regulating inflammation. As such, PPARg is under consideration as therapy for ischemic stroke, Alzheimer's disease, Parkinson's disease, multiple sclerosis and traumatic brain injury. Previous in vivo experiments using acute seizure models suggest PPARg agonists have anticonvulsant efficacy. Here, we tested the hypothesis that acute application of a PPARg agonist, pioglitazone, would reduce seizure-like events (SLEs) in an in vitro model of epilepsy.

Methods: Recordings of spontaneous and evoked extracellular field potentials were acquired using a 64 multi-electrode array. Acutely isolated hippocampal slices from wild-type mice were positioned with electrodes in the fields of CA1, CA3 and dentate gyrus. Either vehicle or pioglitazone with or without GW9662, a selective PPARg irreversible antagonist, was bath applied for 90 minutes. During drug wash-in paired pulse stimulations (50 ms ISI) of Schaffer-collaterals assessed effects on field potentials and synaptic plasticity. After drug wash-in, the aCSF was switched to a high potassium $(8 \mathrm{mM}) \mathrm{aCSF}$ that contained the appropriate drug. The high potassium aCSF induced SLEs in all slices. Analyses quantified latency to first SLE, SLE duration, intra-SLE spike frequencies and inter-SLE intervals. Results: Pioglitazone significantly decreased field potential slopes and increased paired pulse ratios. In addition, application of pioglitazone increased the latency to the first high potassium SLE by $\sim 62 \%(\mathrm{p}<0.05)$ and slowed spiking during the SLEs, as the intra-SLE frequency decreased by $\sim 50 \%(\mathrm{p}<0.05)$. These effects were attenuated by GW9662.

Conclusions: These data suggest that PPARg activation is a potential anticonvulsant target. The increased PPR indicates that these effects may involve decreased excitatory neurotransmission via a reduction in presynaptic neurotransmitter release.
1.262

Chronic Administration of Beta-hydroxybutyrate in Spontaneously Epileptic Kcna1-null Mice Results in Neuroprotective Effects and Suppression of mTOR Signaling J. Vallejo ${ }^{1}$, M. Abdelwahab ${ }^{2}$, Q. Liu ${ }^{2}$, L. Leung ${ }^{1}$, G. Turner ${ }^{2}$, Y. $\mathrm{Ahn}^{3}$, J. Rho ${ }^{3}$ and Do Young Kim ${ }^{2}\left({ }^{1}\right.$ Department of Physiology, Midwestern University, Glendale, AZ; ${ }^{2}$ Barrow Neurological Institute, St. Joseph's Hospital \& Medical Center, Phoenix, AZ and ${ }^{3}$ Alberta Children's Hospital, University of Calgary, Calgary, AB, Canada)

Rationale: The ketogenic diet (KD) is a proven treatment for medically refractory epilepsy, and results in prominent production of ketone bodies (KB) such as D- $\beta$-hydroxybutyrate (BHB), acetoacetate (ACA) and acetone. While the mechanisms of KD action remain unclear, it has long been hypothesized that $\mathrm{KB}$ might mediate the clinical effects of the KD. And although several studies have shown acute anticonvulsant properties of ACA and acetone, it remains unclear whether BHB has similar activity. Recent studies have shown that the KD can inhibit mTOR signaling and prevent hippocampal atrophy in an experimental model of multiple sclerosis (PMID: 21371020, 22567104). Here, we asked whether BHB is anticonvulsant in spontaneously epileptic Kcna1-null (KO) mice, and whether this action is associated with alterations in mTOR signaling and structural changes in hippocampus.

Methods: KO mice (P31-37) were implanted s.c. with Alzet osmotic mini-pumps containing $10 \mathrm{mM}$ BHB dissolved in saline. Seizure frequency was assessed with continuous video-EEG monitoring over 3-day periods beginning 1, 7 and 12 days after implantation. After 15 days of BHB treatment, hippocampal structural changes were studied with manganese-enhanced imaging $\left(\mathrm{MnCl}_{2}, 0.2 \mathrm{mM} / \mathrm{kg}, 1\right.$ day after i.p. injection) on a 7T MRI scanner. Hippocampal lysates were collected for Western blots from four groups of mice : saline-treated wild-type (WT); saline-treated KO; BHB-treated WT; and BHBtreated KO mice. Samples ( $\mathrm{n}=8$ per group) were probed with antibodies against upstream modulators of mTOR (e.g., Akt and AMPK) and downstream effectors of mTOR (e.g., S6 and 4EBP1). Results: Saline-treated KO expressed a mean daily tonic-clonic seizure frequency of $5.8 \pm 0.6$ at $12-15$ days, and this effect was significantly reduced by BHB treatment $(p<0.01)$. A similar change was seen in $\mathrm{KO}$ mice exposed to BHB for 7 days, but not after 1 day. Saline-treated KO mice showed a significant increase in phospho-S6 expression ( $31 \%$ increase compared to saline-treated WT), whereas $\mathrm{BHB}$ exposure led to a reduction in phospho-S6 in KO mice $(20 \%$ decrease as compared to saline-treated $\mathrm{KO}$ ). No differences in Akt, AMPK and 4EBP1 were seen amongst control and experimental groups. In saline-treated KO mice, MR imaging revealed increased signal intensities in dentate gyrus (DG) and CA3 hippocampus, but only slight increases in CA1. No significant differences were seen in DG between BHB-treated KO and saline-treated WT mice, but BHB treatment led to partial normalization of the MR signal in the CA3 subfield of KO animals.

Conclusions: Chronic in vivo administration of BHB results in a significant anticonvulsant effect in Kcna1-null mice. Moreover, BHB alone appears to exert a structural neuroprotective effect in hippocampus, and inhibits aberrant mTOR signaling in these epileptic animals. The combination of morphological and functional neuroprotective effects of BHB in this genetic model of epilepsy suggests that KB may be beneficial for neurological conditions other than epilepsy.

Supported by Midwestern University Start-Up Funds (JV), the Barrow Newrologicat Foundation (DYK), and the National Institutes of Health NINDS ROI (NSO70267) 


\subsection{3}

A NOVEL MITOCHONDRIA-TARGETED ANTICONVULSANT TREATMENT

Kristina A. Simeone, S. A. Matthews, K. K. Samson and T. A. Simeone (Pharmacology, Creighton University, Omaha, NE)

Rationale: Mitochondria promote cell viability by generating ATP and buffering intracellular calcium levels, but also can generate injurious oxidative species and trigger cell death signals. We and others have shown that mitochondrial impairment is associated with epilepsy and may be involved in mediating its pathologies. Here, we hypothesize that rescuing mitochondria function with a targeted treatment will be anticonvulsant and restore mitochondrial function. Methods: Wild-type mice were pretreated with a mitochondriatargeted cocktail, AATP $(250 \mathrm{mg} / \mathrm{kg}$ ascorbic acid; $30 \mathrm{mg} / \mathrm{kg}$ tocopherol-succinate; $500 \mathrm{mg} / \mathrm{kg}$ and sodium pyruvate) or vehicle 30 min prior to kainate $(15 \mathrm{mg} / \mathrm{kg})$ to induce status epilepticus. Seizures were monitored via video-EEG for 3 hrs. Efficacy of AATP treatment on recurrent spontaneous seizures was determined using a second model of epilepsy, Kcna1-null mice, which naturally develop seizures around P22 and model several clinical epilepsy syndromes. Seizure activity was scored during baseline ( P22-P24), 4-6 days of daily AATP treatment and $48 \mathrm{hr}$ washout period. Scores were compared within treatment and between age-matched controls using ANOVA. Mitochondria function was determined using standard oxygen polarography.

Results: Typical kainate associated EEG ictal activity and behavioral manifestations were apparent within the first 30 min following kainate treatment and increased in severity in vehicle-treated wildtype mice. In AATP-pretreated mice, seizure onset and progression were significantly prolonged $(\mathrm{p}<0.05)$ and overall seizure severity scores were reduced $(n=4-6, p<0.0005)$. In Kv1.1-/- mice, daily AATP treatment significantly reduced seizure frequency and severity when compared to pretreatment baseline scores $(n=9 ; \mathrm{p}<0.05)$ and between age-matched controls (by $\sim 45 \%$, $\mathrm{p}<0.05$ ). Following discontinuation of treatment, seizures remained reduced by $\sim 63 \%$ when compared to age-matched controls $(p<0.005)$. Mitochondrial function is impaired in Kcna1-null mice. In AATP-treated Kcna1null mice, complex I-driven state III and state $\mathrm{V}$ respiration and uncoupling function were restored to control values in isolated hippocampal and cortical mitochondria.

Conclusions: These data suggest that targeting the mitochondria may be an effective and novel anticonvulsant treatment strategy.

\subsection{4}

\section{EFFICACY OF ENVIRONMENTAL ENRICHMENT IN ATTENUATING MICROGLIAL INFLAMMATORY RESPONSE IN PEDIATRIC STATUS EPILEPTICUS MOUSE MODELS}

Kelly Liesse ${ }^{1,2}$, L. Mlsna ${ }^{1}$ and S. Koh ${ }^{1,2}\left({ }^{1}\right.$ Neurobiology, Lurie Children's Hospital, Chicago, IL and ${ }^{2}$ Neurobiology, Northwestern University, Evanston, IL)

Rationale: Currently available pharmacological anticonvulsant treatments employed to control seizures in children remain ineffective in over $30 \%$ of patients and also fail to attenuate disease progression. Additionally, these drugs produce cognitive, behavioral and mood altering side effects. Housing in an enriched environment (EE) has shown efficacy in ameliorating the detrimental effects of prolonged seizures in developing animals. Here, we investigated the ability of EE to mitigate seizure-induced microglia activation and behavioral deficits following kainate-induced status epilepticus (KA$\mathrm{SE}$ ) in young mice.

Methods: P21 CX3CR1EGFP/+ transgenic mice were injected intraperitoneally with either Kainic acid $(12 \mathrm{mg} / \mathrm{kg})$ or PBS and monitored for progression of seizures for at least one hour. After recovery, animals who experienced at least 30 minutes of continuous seizures were divided into two housing conditions: enriched environment (EE - 4 KA-injected and 4 PBS-injected mice housed in two large cages connected by tunnels and equipped with multiple toys and running wheels) and isolated (ISO - mice housed singly in standard cages). Behavior was assessed at 2 and 8 days post-KA-SE using the open field and elevated plus maze tests. Ten days after KASE (P31), mice were perfused and their brains collected for analysis. $40 \mu \mathrm{m}$ coronal sections containing the lateral septum, CA3 region of the hippocampus, and amygdala were mounted and quantified at $20 \mathrm{x}$ resolution.

Results: Fluorescence microscopy revealed striking differences in microglial activation between mice housed in EE and ISO conditions. At P31, KA-EE mice showed no significant microglial activation relative to control (PBS-EE) mice. However, KA-ISO mice exhibited residual and heightened levels of microglial activation compared to KA-EE mice $(\mathrm{p}<0.003)$, with markedly increased clumping of microglia in the CA3 region. Similarly, significantly increased activation in the KA-ISO group was present in the lateral septum $(\mathrm{p}<.014)$ and amygdale $(\mathrm{p}<.004)$. While KA-ISO mice exhibited persistent deficits in exploratory behavior - as measured by open arm time in an elevated plus maze-relative to KA-EE littermates $(\mathrm{p}<.047) 8$ days after seizure induction, EE produced a timedependent reduction in behavioral impairments.

Conclusions: We show that EE attenuates the detrimental effects of KA-induced seizure including inflammatory microglia response and behavioral impairment. Prior research has established microglia as key cellular mediators of neuroinflammatory processes, where activated microglia undergo rapid proliferation to facilitate efficient immune response and release inflammatory proteins such as cytokines and chemokines. Thus, by reducing microglia activation, the current data demonstrated the anti-inflammatory action of EE. The research revealed extensive protective effects of EE at both cellular and behavioral levels and proposes the therapeutic efficacy of EE in pediatric epilepsy patients.

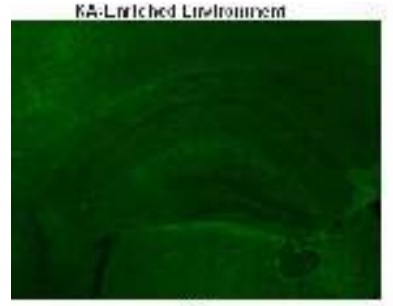

$(x)$

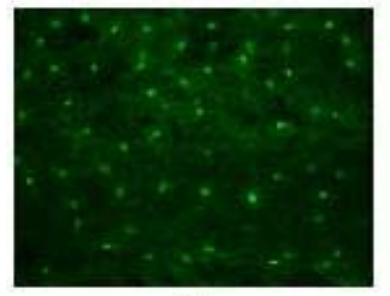

$\Leftrightarrow$

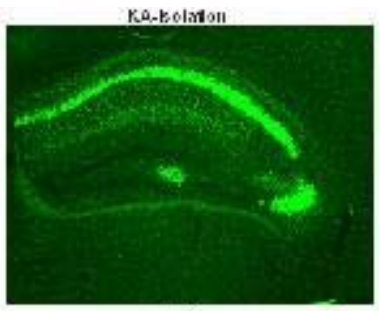

|a

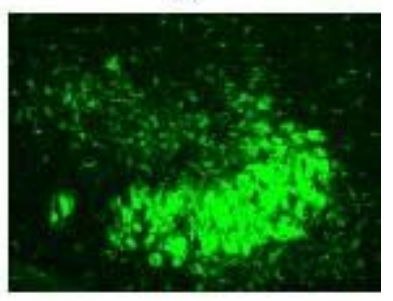

[il)
Fluorescent Microscopic images of the hippocampus at postnatal day 31 (a) microglial activation of a mouse housed in an enriched environment for 10 days after kainic acid status epilepticus induction. (b) Persistent marked microglial activation of mouse housed in isolation for 10 days after KA seizure induction. Both images are taken at 10x objective. (c) \& (d) magnified (20x objective) of the CA3 hippocampal region of images (a) and (b) respectively. The animals reared in isolation (b) \& (d) --display significantly heightened levels of microglial activation compared to those reared in EE (a) \& (c). Additionally, increased clumping of microglial in the CA3 region of isolated mice relative to the EE mice was noted (d). 


\subsection{5}

ANTICONVULSANT TRIHEPTANOIN RESULTS IN FEW CHANGES IN BRAIN METABOLISM

Tanya S. McDonald ${ }^{1}$, M. G. Hadera ${ }^{2}$, U. Sonnewald $^{2}$ and K. Borges ${ }^{1}$ ( ${ }^{1}$ School of Biomedical Sciences, University of Queensland, St Lucia, QLD, Australia and ${ }^{2}$ Department of Neuroscience, Norwegian University of Science and Technology, Trondheim, Norway)

Rationale: Triheptanoin, the triglyceride of heptanoate (C7 fatty acid), has recently been found to be anticonvulsant in several mouse models. We hypothesized that triheptanoin will alter brain metabolism of some amino acids, energy metabolites and glutathione, which may underlie its anticonvulsant effects.

Methods: The pilocarpine mouse model was used. It results in spontaneous recurrent seizures in mice that had experienced pilocarpine-induced status epileptics (SE), but not in mice without SE (no SE mice). After pretreatment with $2 \mathrm{mg} / \mathrm{kg}$ methyl-scopolamine (i.p.) 7-8 week old male CD1 mice were injected with pilocarpine (280-360 mg/kg s.c.) and $90 \mathrm{~min}$ later with $25 \mathrm{mg} / \mathrm{kg}$ pentobarbital (i.p.). All mice were immediately placed on a standard control or $35 \%$ (caloric value) triheptanoin diets with matched protein, vitamin and mineral levels. To encourage weight gain in animals with SE, mice were injected with $4 \%$ dextrose in saline (s.c) and hand fed, for the first few days after SE. Three weeks later mice were sacrificed by focal $5 \mathrm{KW}$ microwave irradiation on the head. Polar metabolites from the cerebral cortex and hippocampal formations were extracted using methanol/chloroform and then quantified with HPLC using alpha-aminobutyric acid as an internal standard. For each metabolite and brain area One-Way ANOVA's with post-hoc Bonferroni tests with selected comparisons were used to compare SE and no SE mice on standard and triheptanoin diet ( $\mathrm{n}=9-10$ mice per group) separately. Only changes that were statistically significant in the ANOVA and post test with $\mathrm{p}<0.05$ are shown.

Results: After SE, mice showed 1.5-fold increases in myo-inositol consistent with astrogliosis found in this model $(p<0.001)$. Mice on our standard diet which experienced SE showed reduced hippocampal levels of glutathione (by $20 \%, \mathrm{p}<0.01$ ), glutamate $(28 \%, \mathrm{p}<0.01)$ and alanine $(35 \%, \mathrm{p}<0.001)$ compared to those without SE. Similar changes after SE were seen in mice fed triheptanoin, except that there were no significant decreases in glutathione, indicating that triheptanoin feeding protects against loss of this important antioxidant in the "epileptic" hippocampal formation. Also, triheptanoin decreased hippocampal threonine levels in SE mice $(26 \%, \mathrm{p}<0.01)$ and in the cortex in both SE $(36 \%, \mathrm{p}<0.01)$ and no SE mice $(28 \%, \mathrm{p}<0.05)$, raising the possibility that triheptanoin interferes with threonine uptake or metabolism. Triheptanoin induced no changes in the levels of aspartate, glutamate, serine, glutamine, glycine, taurine, alanine, tyrosine, GABA, lysine and methionine. Currently, we are assessing to which extent triheptanoin alters high energy phosphate levels in the hippocampus and cortex.

Conclusions: Triheptanoin feeding did not change any of the measured amino acids in the cerebral cortex and hippocampal formation of healthy and epileptic mice, except for lowering threonine levels. Therefore, it is advisable to monitor this amino acid in our clinical trial of triheptanoin. The inhibition of the decrease of the GSH level in SE mice by triheptanoin may contribute to its protective effect against seizures.

\subsection{6}

EFFECTS OF CHRONIC METFORMIN TREATMENT AND A 35\% TRIHEPTANOIN DIET ON SEIZURE THRESHOLD OF PILOCARPINE-INDUCED EPILEPTIC MICE

Alejandra M. Fernandez ${ }^{1}$, S. Willis ${ }^{1}$, K. Borges ${ }^{2}$ and J. Stoll ${ }^{1}$ ( ${ }^{1}$ Department of Biomedical Sciences, Texas Tech Health Sciences Center, Amarillo, TX and ${ }^{2}$ School of Biomedical Sciences, University of Queensland, St. Lucia, QLD, Australia)
Rationale: Triheptanoin, a triglyceride composed of seven carbon fatty acids, has been shown to be anticonvulsant in two mouse chronic seizure models but not in acute seizure models (Epilepsy Res, 2012, 99(3):312-7). In one chronic model, triheptanoin increased the seizure threshold of pilocarpine-induced epileptic mice when given as $35 \%$ of the caloric intake (Neurobiol Dis, 2010, 40(3):565-572).

However, the mechanism by which triheptanoin exerts its protective effects is unclear. AMP-Activated protein kinase (AMPK) is an important energy sensor that regulates metabolic function. Previously, it was shown that chronic administration of metformin, an AMPK activator, is neuroprotective in an animal model of stroke. Hence, we decided to explore the effects of chronic metformin treatment in conjunction with a $35 \%$ triheptanoin diet on seizure threshold in epileptic and non-epileptic mice.

Methods: Male CF-1 mice were administered methylatropine ( $2 \mathrm{mg} / \mathrm{kg}$, i.p.) followed by pilocarpine $(310-340 \mathrm{mg} / \mathrm{kg}$, s.c.) and observed for the induction of seizures for four hours. Seizures were terminated with diazepam $(10 \mathrm{mg} / \mathrm{kg}$, i.p.). Mice that experienced status epilepticus (SE) were immediately placed on either a $35 \%$ triheptanoin diet or a control diet in combination with either metformin $(50 \mathrm{mg} / \mathrm{kg}$, s.c. $)$ or saline treatment for three weeks. Mice that did not develop SE (No SE mice) were placed on a control diet and administered saline. After three weeks, SE and No SE mice were subjected to an intravenous pentylenetetrazole (PTZ) seizure threshold test.

Results: Pilocarpine-induced SE mice had significantly lower PTZ tonic seizure threshold compared to No SE mice. The PTZ threshold for tonic extension seizures for No SE mice was $98.7 \pm 2.4 \mathrm{mg} / \mathrm{kg}$ $(n=29)$, whereas that for SE mice on control diet was $75.3 \pm 2.8$ $\mathrm{mg} / \mathrm{kg}(\mathrm{n}=29)$. Chronic metformin treatment prevented the increase in seizure threshold observed in SE mice with the 35\% triheptanoin diet alone. The PTZ tonic seizure thresholds for SE mice on triheptanoin diet alone and SE mice on triheptanoin and metformin treatment were $88.2 \pm 3.1 \mathrm{mg} / \mathrm{kg}(\mathrm{n}=25)$ and $79.9 \pm 2.3 \mathrm{mg} / \mathrm{kg}(\mathrm{n}=26)$, respectively. Conclusions: Metformin blocked the beneficial effects of the triheptanoin diet. At this time, the mechanism by which metformin blocks the effects of the triheptanoin diet remains elusive. One possibility is that the triheptanoin diet may attenuate AMPK activity in the brain. Another possibility is that metformin may interfere with the triheptanoin metabolism in the liver. Future work will be directed towards investigating these mechanisms.

\section{Surgery: Adult}

\subsection{7 \\ RECOVERY OF MEMORY FUNCTION FOLLOWING SELECTIVE AMYGDALOHIPPOCAMPECTOMY VIA THE INFERIOR TEMPORAL GYRUS}

Haruhiko Kishima $^{1}$, S. Oshino ${ }^{1}$, N. Tani ${ }^{1}$, M. Hirata ${ }^{1}$, K. Hosomi $^{1}$, T. Maruo $^{1}$, H. Khoo ${ }^{1}$, T. Yanagisawa ${ }^{1}$, S. Morris ${ }^{1}$, A. Kato ${ }^{2}$ and T. Yoshimine $^{1}\left({ }^{1}\right.$ Department of Neurosurgery,, Osaka University, Graduate School of Medicine, Suita, Japan and ${ }^{2}$ Neurosuegery, Kinki University, Faculty of Medicine, Osaka-Sayama, Japan)

Rationale: We have already reported that selective amygdalohippocampectomy (SAHT) via the inferior temporal gyrus using neuronavigation system provides in excellent epilepsy control in patients with refractory mesial temporal lobe epilepsy (MTLE). This surgical procedure is less invasive and safer. Here, we analyzed the outcome of memory function after this surgical procedure.

Methods: Nighteen patients with mesial temporal lobe epilepsy have undergone the SAHT procedure for MTLE via the inferior temporal gyrus. Among them, 12 patients utilized the Wechsler Memory Scale-Revised (WMS-R) score to evaluate memory function both just before and 6 to 12 months after SAHT. The lesions were located on verbal dominant hemispheres in 5 cases and on verbal non-dominant hemispheres in 7 cases. 
Results: The postoperative outcomes of 18 of all 19 cases were seizure free categorized as Engel's class I. The pathology of these 18 cases was hippocampal sclerosis. The other case resulted in Engel's class IIa. The pathology of this case was cortical dysplasia. The pre-operative verbal, visual, and total memory scores were $78.2 \pm 7.8$ (Ave \pm SE), 93.7 \pm 8.7 , and 79.0 \pm 7.8 , respectively. Postoperative scores were $87.6 \pm 11.0,101.2 \pm 11.5$, and $91.3 \pm 10.9$, respectively. Post-operative scores of all three scales were significantly improved compared to pre-operative scores. The scores of attention and delayed recall were also significantly improved postoperatively. The cases of verbal dominant hemispheres revealed significant improvement of visual memory. In contrast, the cases of verbal non-dominant hemispheres revealed significant improvement of verbal and total memory. More than $10 \%$ of verbal memory was lost in one case of dominant hemisphere MTLE.

Conclusions: Not only good seizure outcome but also improvement of memory function, especially the verbal memory in non-dominant and the visual memory in dominant hemisphere MTLW, can be expected with SAHT via the inferior temporal gyrus preserving the temporal stem.

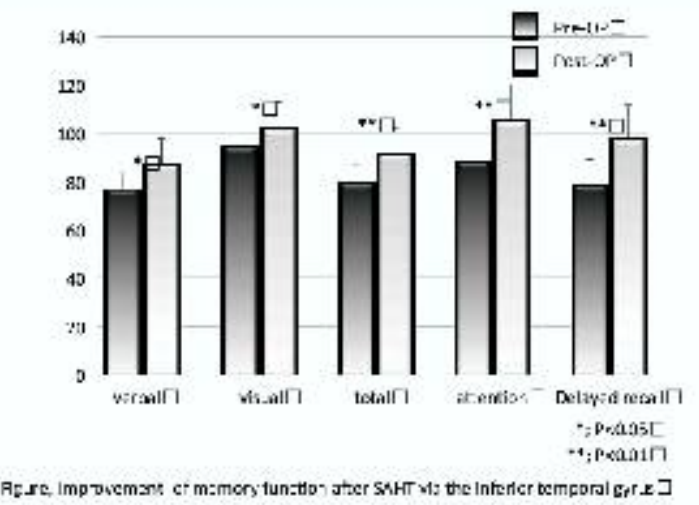

\subsection{8 \\ EXPANDED FOLLOW-UP ON OUTCOME OF MULTIPLE HIPPOCAMPAL TRANSECTIONS IN PATIENTS WITH TEMPORAL LOBE EPILEPSY AND NORMAL MEMORY} Emine S. Kahriman ${ }^{1}$, J. Miller ${ }^{1}$, R. Macinuas ${ }^{1}$, C. Bailey ${ }^{1}$, P. Fastenau $^{1}$, T. Syed ${ }^{1}$, S. Amina ${ }^{1}$, A. Tanner ${ }^{2}$, K. Karanec ${ }^{2}$, I. Tuxhorn ${ }^{1}$, H. Lüders ${ }^{1}$ and M. Koubeissi ${ }^{1}$ ( ${ }^{1}$ University Hospitals, Cleveland, $\mathrm{OH}$ and ${ }^{2}$ Saint Mary's Health Care, Grand Rapids, MI)

Rationale: To provide follow up on seizure outcome and memory function after multiple hippocampal transections (MHT) of the hippocampus in patients with intractable mesial temporal lobe epilepsy (MTLE) and normal memory scores. Hippocampal anatomy suggests that the circuits of memory processing (transverse lamellae) may be different from those of seizure propagation (longitudinal pathways). Thus, transverse cuts may interrupt seizure circuitry without interfering with memory function.

Methods: 13 patients with nonlesional MTLE had depth electrode recordings confirming hippocampal seizure onset and underwent MHT. Duration of epilepsy ranged between 5 and 55 years. Antiepileptic drugs (AEDs) were not changed postoperatively. Intraoperative monitoring was done with 5-6 hippocampal electrodes spaced at around $1 \mathrm{~cm}$ intervals, and spike counting for approximately 10 minutes before each cut. The number of transections ranged between 4 and 7. Neuropsychological assessment was completed preoperatively and at 6 months postoperatively for 5 patients and at 1 year postoperatively for 5 other patients.

Results: There were no complications. MHT resulted in marked spike reduction $(\mathrm{p}=0.003$, paired t-test) intraoperatively. 10 patients $(77 \%)$ are seizure-free (average follow-up $=25$ months, range 9-55 months) without medication changes. One of the 3 patients with persistent seizures had an MRI revealing incomplete transections, another proved to have a neocortical seizure focus, and the third had only 2 seizures in 4 years, one of which occurred during AED withdrawal. Using a $90 \%$ confidence interval for reliable change indices that were corrected for practice (RCI-P), Two of 9 dominantside MHT patients $(22.2 \%) 2$ of 10 patients $(20 \%)$ declined on auditory-verbal memory and 3 of $10(30 \%)$ declined on visual-spatial memory after MHT, compared to $38 \%$ and $28 \%$ of patients who declined in auditory-verbal and visual-spatial memory (respectively) after L temporal lobectomy (Lineweaver et al. Epilepsia 2006;47:1895-1903). One of 10 patients (10\%) improved reliably on visual-spatial memory after MHT, compared to $16 \%$ improved after L temporal lobectomy (Baxendale et al. Neurology 2008;71:131925). declined reliably on Auditory-Verbal Memory after MHT, and 3 of 9 patients (33\%) declined reliably on Visual-Spatial Memory, while one patient (10\%) improved on Visual-Spatial Memory after MHT. Right and left hippocampal volumes were not different preoperatively $(\mathrm{n}=12, \mathrm{p}=0.64$, Wilcoxon signed-rank test), but the transected hippocampal volume decreased postoperatively $(\mathrm{p}=0.0173)$.

Conclusions: MHT provides an effective intervention and a safer alternative than to temporal lobectomy regarding memory preservation in patients with MTLE. MHTand appears to spare verbal memory in about 1 of 2 more L TLE patients compared to lobectomy, while yielding similar results to left temporal lobectomy for visual memory.

\subsection{9 \\ THE EFFECT OF EPILEPSY SURGERY ON THE CAREGIVERS' QUALITY OF LIFE}

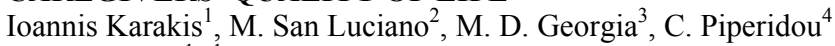
and A. J. Cole ${ }^{1}\left({ }^{1}\right.$ Massachusetts General Hospital, Harvard Medical School, Boston, MA; ${ }^{2}$ University of California, San Francisco, San Francisco, CA; ${ }^{3}$ Boston Medical Center, Boston University, Boston, MA and ${ }^{4}$ Democritus University of Thrace, Alexandroupolis, Greece)

Rationale: Epilepsy surgery has been shown to improve the patients' quality of life (QOL). Little is known though about its effect on the QOL of their caregivers.

Methods: 26 patients and 13 caregivers participated in this prospective study conducted at MGH from 09/2009 till 06/2011. All patients underwent Epilepsy surgery for medically refractory Epilepsy. The patients completed Epilepsy directed QOL (QOLIE31 ) and psychological (Beck's depression-BDI and anxiety inventoryBAI) questionnaires before and after surgery. Their participating caregivers completed generic health related QOL (SF36v2) and disease burden (Zarit burden inventory-ZBI) questionnaires before and after surgery. Demographic data for all participants and caregivers and disease/surgery related data for the patients were collected. Statistical analysis was performed to compare the patients' and caregivers' QOL before and after surgery, using Fischer's exact/paired t-test for categorical/continuous data or their non parametric equivalents.

Results: Out of 26 patients, $13(50 \%)$ were male with a mean age of 37 years. The majority were employed $(69 \%)$, of higher education $(85 \%)$ and were living with relatives $(92 \%)$. The mean age of onset of their disease was 20 years of age and the mean duration was 18 years. Pre-operatively they had on average 4 seizures/month, and they were on average on 2.2 antiepileptic drugs (AEDs). Most patients suffered from symptomatic partial epilepsy (77\%). 54\% of them underwent a left anterior temporal lobectomy (ATL), 24\% right ATL and the rest extra-temporal resections. On follow up at approximately 9 months, $69 \%$ had a surgical outcome of Engel class I, $23 \%$ of class II and $8 \%$ class IV. Postoperatively, the patients remained on average on 1.9 AEDs. There was a statistically significant improvement for both the aggregate QOLIE-31 score and all its subscales. Similarly BAI but not BDI scores were statistically 
better post surgery. $96 \%$ of the patients felt that the decision to go through surgery was worthwhile. Out of 16 caregivers who participated in the study, $11(69 \%)$ were female with a mean age of 47 years. $50 \%$ were spouses, $37.5 \%$ were parents and the rest siblings of the patients. The majority were employed $(87.5 \%)$ and of higher education $(87.5 \%) .50 \%$ of them stated that their overall time devoted to patient's care decreased after surgery and $50 \%$ that it remained unchanged. The ZBI and the physical component of their QOL (SF36v2, PCS) scores did not significantly vary before and after surgery but the mental component of their QOL (SF36v2, MCS) showed statistically significant improvement. $75 \%$ of caregivers deemed their QOL better post surgery vs 19\% similar. 94\% of the caregivers felt that the decision to go through surgery was worthwhile.

Conclusions: Successful Epilepsy surgery has a positive impact not only to the patients' QOL but also to their caregivers. To our knowledge, this is the first study to systematically address the impact of Epilepsy surgery on the caregivers providing additional leverage to Epilepsy surgery as the optimal treatment modality in carefully selected, medication refractory patients.

Patients' demographic and disease/surgery and caregivers' demographic characteristics

\begin{tabular}{|c|c|c|c|}
\hline $\begin{array}{c}\text { Patients' and caregivers' demographic } \\
\text { characteristics } \\
\text { Gender (n, \% femalc) }\end{array}$ & $\begin{array}{l}\text { Patients }(\mathrm{n}=26) \\
13(50 \%)\end{array}$ & $\begin{array}{c}\text { Caregivers }(\mathrm{n}=16) \\
11(68.75 \%)\end{array}$ & \\
\hline$\Lambda \mathrm{ge}$ in ycars (mcan $\pm \mathrm{SD}$, range) & $\begin{array}{c}37.42 \pm 11.60 \\
(19-59)\end{array}$ & $\begin{array}{c}47.69 \pm 13.02 \\
(28-68)\end{array}$ & \\
\hline Race (n, \%Calucisian) & $25(96.15 \%)$ & $15(93.75 \%)$ & \\
\hline Employed $(\mathrm{n}, \%)$ & $18(69.23)$ & $14(87.50 \%)$ & \\
\hline Education (n, \%some college and higher) & $22(84.62 \%)$ & $14(87.50 \%)$ & \\
\hline I.jving situation (n, \%living with family) & $24(92.31 \%)$ & $16(100 \%)$ & \\
\hline Marital status (n, \%marricd) & $13(50 \%)$ & $14(87.50 \%)$ & \\
\hline Patients' diseatse and surgery characteristics & Belore surgery & After surgery & $\underset{\text { value }}{\mathrm{P}}$ \\
\hline $\begin{array}{l}\text { Age of onset of epilepsy in years (mean } \mathrm{SD} \text { ), } \\
\text { range) }\end{array}$ & $19.81 \pm 13.32(1-56)$ & & \\
\hline Duration of cpilcpsy in years (mean \pm SD, rangc) & $\begin{array}{c}17.58 \pm 15.38 \\
(1.5-49)\end{array}$ & & \\
\hline Seizures per month (median, IQR) & $4(2-6)$ & $0(0-0.125)$ & $<0.001$ \\
\hline AED use (mean number of drugs $\pm \mathrm{SD}$, range) & $2.19 \pm 0.89$ & & 0.17 \\
\hline $\begin{array}{c}\text { Type of epilepsy }(\mathrm{n}, \%) \\
\text { Partial } \\
\text { Partial with secondary gencralization }\end{array}$ & $\begin{array}{c}6(23.08 \%) \\
20(76.92 \%)\end{array}$ & & \\
\hline $\begin{array}{l}\text { Epilepsy etiology }(\mathrm{n}, \%) \\
\text { Symptomatic } \\
\text { Cryptogenic }\end{array}$ & $\begin{array}{l}20(76.92 \%) \\
6(23.08 \%)\end{array}$ & & \\
\hline $\begin{array}{c}\text { Surgery type }(\mathrm{n}, \%) \\
\text { L ATI } \\
\text { R ATL } \\
\text { Extra-temporal }\end{array}$ & $\begin{array}{l}14(53.85 \%) \\
7(23.92 \%) \\
5(19.23 \%)\end{array}$ & & \\
\hline $\begin{array}{l}\text { Time since surgery in months (mean } \pm \text { SD, } \\
\text { range) }\end{array}$ & & $9.19 \pm 3.87$ & \\
\hline Post-operative delicits $(\mathrm{n}, \%)$ & & $1(3.85 \%)$ & \\
\hline $\begin{array}{l}\text { Engel Class } \\
\text { Class I } \\
\text { Class II } \\
\text { Class III } \\
\text { Class IV }\end{array}$ & & $\begin{array}{c}18(69.23 \%) \\
6(23.08 \%) \\
0(0 \%) \\
2(7.69 \%)\end{array}$ & \\
\hline & & & \\
\hline
\end{tabular}

Patients' and caregiver's QOL measures before and after surgery

\begin{tabular}{|c|c|c|c|}
\hline Patients' QOL measures & Before surgery & Afler surgery & P-value \\
\hline \multicolumn{4}{|l|}{ QOLIE-31 } \\
\hline Scizure worry & $47.75 \pm 27.65$ & $69.23 \pm 29.73$ & 0.0054 \\
\hline Overall quality of life & $63.84 \pm 21.31$ & $73.65 \pm 15.51$ & 0.041 \\
\hline Emotional & $66.19 \pm 18.79$ & $75.27 \pm 15.60$ & 0.03 \\
\hline Energy & $46.73 \pm 21.16$ & $58.27 \pm 19.59$ & 0.017 \\
\hline Cognitive & $57.29 \pm 22.82$ & $66.56 \pm 22.08$ & 0.042 \\
\hline Medication & $57.26 \pm 26.32$ & $68.91 \pm 27.29$ & 0.086 \\
\hline Social & $55.85 \pm 24.65$ & $71.04 \pm 25.10$ & 0.01 \\
\hline Overall score & $56.96 \pm 16.61$ & $68.66 \pm 14.65$ & 0.0037 \\
\hline Beck Depression Inventory & $9.81 \pm 7.30$ & $7.61 \pm 6.73$ & 0.26 .5 \\
\hline Beck Anxiety Inventory & $11.19=7.77$ & $7.54 \pm 6.36$ & 0.035 \\
\hline $\begin{array}{c}\text { QOL change (n.\%) } \\
\text { Betler } \\
\text { Worse } \\
\text { Similar }\end{array}$ & & $\begin{array}{c}20(76.92 \%) \\
2(7.69 \%) \\
4(15.38 \%)\end{array}$ & \\
\hline Worthwhile surgetery (n, \%) & & $24(96.00 \%)$ & \\
\hline Caregivers" QOL measures & Before surgery & Adter surgery & P-value \\
\hline$\$+36 \mathrm{v} 2$ & & & \\
\hline SF36v2 PCS & $53.46-9.32$ & $53.44 \pm 8.92$ & 0.98 \\
\hline $\mathrm{S} \Gamma 36 \mathrm{v} 2 \mathrm{MCS}$ & $43.22=9.54$ & $47.56 \pm 9.75$ & 0.02 \\
\hline Zarit Burden Inventory & $25.06 \pm 13.04$ & $23.62 \pm 13.37$ & 0.71 \\
\hline $\begin{array}{l}\text { Time spent with patient }(\mathrm{n}, \%) \\
\text { Increased } \\
\text { Decreased } \\
\text { Unchanged }\end{array}$ & & $\begin{array}{l}8(50.00 \%) \\
8(50.00 \%)\end{array}$ & \\
\hline $\begin{array}{l}\text { QOL Change }(n, \%) \\
\text { Betler } \\
\text { Worse } \\
\text { Similar }\end{array}$ & & $\begin{array}{c}12(75.00 \%) \\
1(6.25 \%) \\
3(18.75 \%)\end{array}$ & \\
\hline Worthwhile surgery (n, \%) & & $15(93.75 \%)$ & \\
\hline
\end{tabular}

\subsection{0}

OUTCOME OF EPILEPSY SURGERY IN OLDER PATIENTS Jane Y. Hwang ${ }^{1}$, B. Enos ${ }^{1}$, L. Cahan ${ }^{2}$, J. Schweitzer ${ }^{2}$, S.

Chayasirisobhon $^{1}$, K. Earnest ${ }^{3}$, B. Spurgeon ${ }^{1}$, S. Gurbani ${ }^{1}$, H. Sindhu ${ }^{1}$ and M. Lin ${ }^{1}\left({ }^{1}\right.$ Neurology, Southern California Kaiser Permanente, Los Angeles, CA; ${ }^{2}$ Neurosurgery, Southern California Kaiser Permanente, Los Angeles, CA and ${ }^{3}$ Psychiatry, Southern California Kaiser Permanente, Los Angeles, CA)

Rationale: Epilepsy surgery for medically refractory epilepsy can be a very effective treatment for some patients who continue to have recurrent, debilitating seizures despite maximal anti-epileptic drugs. Because epilepsy surgery is considered an elective treatment procedure, potential benefits must be weighed against risk for surgical complications. As with any surgical intervention, age is a major factor contributing to perioperative morbidity.

The purpose of this study is to assess the efficacy and the complication rate of epilepsy surgery in a cohort of patients 50 years and older.

Methods: Chart review was conducted on 132 patients aged 13 and older who underwent epilepsy surgery at Kaiser Permanente Los Angeles Medical Center from 1993 to 2006. Patients were included only if the primary purpose for the operation was to improve seizure control. Data was collected by medical paper and electronic chart review of the office notes and, when necessary, telephone or email contact with the patient or an immediate family member to obtain information. Data collected include demographic characteristics, age at time of operation, complications, and postoperative seizure outcome using the Engel rating classification.

Results: Of the 132 patients reviewed, 22 were aged 50 or older (range 50-64, average age 53.9, median age 53). 13 were female and 9 were male. 14 underwent en bloc temporal lobectomy and 8 underwent a selective amygdalohippocampectomy. There were no extratemporal or temporal neocortical resections. At 1 year, 1 patient was lost to follow up, 12/21 (57\%) had Engel Class I, 2/21 (9.5\%) had Engel Class II, and 7/21 (16\%) had Engel Class III/IV outcomes . 
At 2 years, 2 patients were lost to follow up, 13/20 (65\%) had Engel Class I, $3 / 20$ (15\%) had Engel Class II, and 4/20 (20\%) had Engel Class III/IV outcomes. 5/22 (23\%) patients had perioperative complications.

There were 110 patients age 13 to 49 in this cohort (average age 32.9, median age 34) (64 female, 46 male). 37 underwent selective AHC, 59 underwent en bloc temporal lobectomy, 10 underwent extratemporal neocortical resection and 4 underwent temporal neocortical resections. At 1 year, 7 patients were lost to follow up, 82/103 (80\%) had Engel Class I, 7/103 (7\%) had Engel Class II, 14/103 (13\%) had Engel Class III/IV outcomes. At 2 year, 13 patients were lost to follow up, 74/97 (76\%) had Engel Class I, 3/97 (3\%) had Engel Class II, 20/97 (21\%) had Engel Class III/IV outcomes. 4/110 (3.6\%) patients had perioperative complications.

Conclusions: Focal resective epilepsy surgery remains an effective treatment for medication resistant partial epilepsy in patients aged 50 and older but is associated with somewhat greater perioperative risks compared with younger patients.

\subsection{1 \\ OUTCOMES OF EPILEPSY SURGERY IN ELDERLY WITH TEMPORAL LOBE EPILEPSY}

Fawad A. Khan, L. Selwa and T. Mihaylova (University of Michigan, Ann Arbor, MI)

Rationale: Surgery for refractory temporal lobe epilepsy (TLE) has been well-supported as an effective treatment but remains underutilized in the elderly. There is skepticism about postsurgical outcomes in this group related to the presence of multiple comorbidities, preexisting cognitive dysfunction and longer seizure duration. The goal of this study is to assess the efficacy and tolerability of surgery in patients older than 55 years with a history of treatment resistant TLE.

Methods: We reviewed data from all patients who underwent epilepsy surgery from 1998 to 2011 . Only 18 met the inclusion criteria for age at surgery ( 55 or older) and history of TLE. Patients with both lesional and nonlesional imaging characteristics were included. Demographics collected were age at seizure onset, seizure semiology, frequency and duration, number of failed medications, results of standard presurgical workup, type of resection, histopathology and postsurgical seizure reduction over a follow-up period from 1 to 12 years. Fig1.

Presurgical data included results of long-term video-EEG monitoring, brain Magnetic resonance imaging (MRI), neuropsychological assessment, speech and language evaluation and Wada test. Selected patients underwent Single Photon Emission Computed Tomography (SPECT) or/and Positron Emission Tomography (PET) scan. Intracranial recording was performed in patients whose noninvasive data was inconclusive. The degree of postsurgical seizure reduction was reported using Engel outcome criteria.

We performed a bivariate analysis and found no significant predictors of outcome in type of resection, duration of epilepsy, number of failed medications, or type of MRI lesion.

Results: 18 patients aged 55 or older underwent surgery for treatment resistant TLE. All patients had localization-related epilepsy with or without secondarily generalization. The seizure frequency varied from 1-7 per week to 1 per month. The mean number of failed anticonvulsants was 6.6. The mean epilepsy duration was 34 years. 11 patients $(61 \%)$ had left temporal onset and 7 right temporal onset. 14 patients (78\%) had MRI evidence of mesial temporal sclerosis (MTS). One patient had normal MRI. Fig.2

The surgical approach included anterior temporal lobectomy in 8 patients and selective amygdalohippocampectomy in 10 . The patients with MRI evidence of MTS showed varying degree of neuronal loss and gliosis.

Engel Class I outcome was achieved in 14 patients (78\%) during average follow-up of 4.5 years.
Conclusions: Our results indicate that the epilepsy surgery is well tolerated and efficacious in patients aged 55 and above who have a longstanding history of treatment resistant TLE. As such our data appear to be in general agreement with previous reports. 78\% achieved Engel class I outcome for average of 4.5 years. The long disease duration does not serve as a negative predictor for postsurgical outcome. As such our results are in disagreement with previously suggested observations of higher incidence of seizure recurrence due to long-lasting secondary epileptogenesis and poorer outcomes.

Fig.1. Demographics.

\begin{tabular}{|cc|}
\hline Gender & $\mathrm{M}: \mathrm{F} \rightarrow 11: 7$ \\
\hline Age at surgery & $55-66$ years (mean 58 years) \\
Agc at scizurc onset & 6 months-57 ycars (mean 21.3 years) \\
Гpilcpsy duration & 6 months-65 ycars (mcan 34 ycars) \\
\hline
\end{tabular}

Fig.2. Pre-surgical brain MRI findings

\begin{tabular}{|c|c|}
\hline MTS (N) & Other Lesion (N) \\
\hline L MTS(+/-atrophy) (8) & R middle temporal gyrus cystic lesion (1) \\
R MTS(+/-atrophy) (3) & I. mesial temporal cavernous hemangioma (1) \\
bilatcral MTS (R>I) (3) & I. mesial temporal cystic encephalomalacia (1) \\
\hline
\end{tabular}

\subsection{2 \\ LONG TERM EFFICACY OF THE SANTE TRIAL (STIMULATION OF THE ANTERIOR NUCLEUS OF THALAMUS FOR EPILEPSY)}

Vicenta Salanova ${ }^{1}$, R. Fisher ${ }^{2}$ and g. Sante ${ }^{2}{ }^{1}$ neurology, indiana university, indianapolis, IN and ${ }^{2}$ stanford, stanford, CA)

Rationale: Fisher et al. (Epilepsia, 51:899,2010), reported the results of 110 patients who participated in a multicenter, double blind, randomized controlled trial of bilateral stimulation of the anterior nuclei of the thalamus for localization-related epilepsy, and found benefit that persisted through 2 years. This is a report of the efficacy for patients with at least 5 years stimulation.

Methods: Subjects were 18-65 yrs old with at least 6 partial or secondarily generalized seizures per month, who had failed at least 3 AEDs due to lack of efficacy. Patients with IQ $<70$,inability to complete neuropsychological testing or progressive neurological lesions were excluded. The trial utilized a prospective, randomized, double-blind, parallel group design. After a 3-month baseline, deep brain stimulation (DBS) electrodes were implanted in the ANT bilaterally using a stereotactic technique. One month after implantation,subjects were randomized to stimulation at $5 \mathrm{~V}$ or no stimulation. After 3 months of blinded treatment, all received stimulation;limited stimulation changes were allowed.Long term follow-up began at 13 months with stimulation parameters adjusted at the investigators 'discretion. Primary analysis was performed on subjects with at least 70 days of seizure diary data.

Results: DBS therapy reduced the number of seizures in a very refractory patient population, with a median seizure frequency reduction of $40 \%$ by the end of the blinded phase (vs. $14.5 \%$ for control). There was continuous efficacy improvement; the median percent reduction from baseline at one year was $41 \%$, at 2 years was $56 \%$ and at 5 years was $69 \%$. The responder rates $(>=50 \%$ reduction in seizure frequency) also improved over time; at one year the responder rate was $43 \%, 54 \%$ at 2 yrs and $69 \%$ at 5 years . Over the entire study $16 \%$ of patients were seizure free for at least 6 months. There were no unanticipated adverse device effects, and no symptomatic intracranial hemorrhages. The Liverpool seizure severity scale and quality of life measures (QOLIE-31) also showed statistically significant improvement over baseline by 1 year, which continued to be significant at 5 years $(p<0.001)$. 
Conclusions: Long term follow-up of bilateral stimulation of the anterior nuclei of the thalamus showed sustained efficacy and continuous improvement, with a median percent reduction in seizure frequency of $69 \%$ at 5years. Based on the results of the SANTE trial deep brain stimulation of the thalamus for medically refractory partial and secondarily generalized seizures has been approved in Europe and Canada but remains investigational in the USA

\subsection{3}

REAL-TIME MRI-GUIDED STEREOTACTIC LASER THERMAL AMYGDALOHIPPOCAMPOTOMY (SLTAH) FOR MESIAL TEMPORAL EPILEPSY

J. T. Willie ${ }^{1}$, Robert E. Gross ${ }^{1}$, N. G. Laxpati ${ }^{1}$, A. M. Saindane ${ }^{2}$ and S. G. Nour ${ }^{2}\left({ }^{1}\right.$ Neurosurgery, Emory University, Atlanta, GA and

${ }^{2}$ Radiology, Emory University, Atlanta, GA)

Rationale: Traditional open anterior temporal lobectomy and selective amygdalohippocampectomy $(\mathrm{AH})$ are effective for treating mesial temporal lobe epilepsy (MTLE), but can be associated with cognitive deficits from collateral temporal lobe injury due to the surgical approach. We describe a novel minimally invasive stereotactic technique for producing an $\mathrm{AH}$ ablation in patients with medically intractable MTLE utilizing computer-controlled laser induced interstitial thermal therapy with real-time magnetic resonance thermal image guidance, and we report initial outcomes. Methods: 8 consecutive patients (ages 18-64 y) with medically intractable MTLE were offered standard AH or SLTAH and selected the latter. 2 patients were operated prior to initiation of a prospective clinical protocol, resulting in parahippocampal without hippocampal ablation. 7 patients ( 6 de novo; 1 with previous parahippocampal ablation) enrolled in the prospective clinical trial and were examined prospectively. A saline-cooled fiber optic laser applicator targeting anterior hippocampus and terminating in the amygdala was inserted from an occipital trajectory utilizing a stereotactic frame under general anesthesia. Laser energy was delivered during continuous MR imaging (Figure 1). Temperature-sensitive phase images and estimates of thermal damage during heating were superimposed on anatomical images in real-time. Prospective baseline and postoperative seizure diaries, quality of life measures, and neuropsychometric testing are being performed.

Results: In the first 2 patients, both without mesial temporal sclerosis (MTS), ablations limited to the parahippocampal gyrus did not yield seizure-freedom. All subsequent procedures, with patients followed prospectively, produced technically successful anterior AH ablations. Of these, four patients with MTS were Engel class I at 3-6 months. Of 3 patients without MTS, 2 were Engel class II at 3-6 months (Figure2), and the third was Engel class IV (additional EEG monitoring revealed bilateral seizure onsets and non-epileptic seizures). One of original 2 patients underwent a repeat laser procedure resulting in successful amygdalohippocampotomy but also a homonymous hemianopsia possibly resulting from deflection of the introducing cannula superiorly through the thalamus .

Conclusions: SLTAH is technically feasible with ablations consistently conforming to the mesial temporal structures without involving the thalamus or midbrain. Preliminary results indicate that seizure outcomes with SLTAH might differ in patients with and without MTS. The safety and efficacy of SLTAH, an appealing minimally-invasive approach, needs to be carefully evaluated with larger cohorts over periods of at least one year.
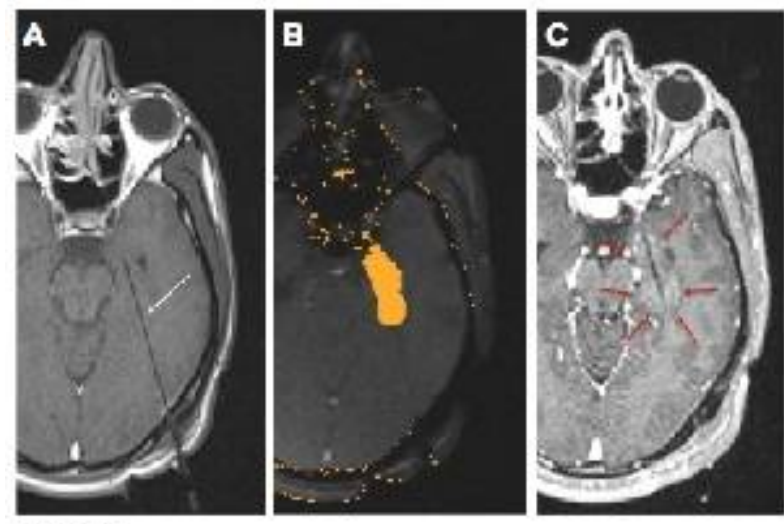

Flgure 1.

A. Pre-ablation T1 MRI show ng p acement of laser optica fiber (white arrow) in misial lempo al sil ustures.

P. MR themal imagino demcnstreting irreversib e damage estimate (crange) of entire lasion (area approximataly $40 \mathrm{~mm} \times 17 \mathrm{~mm}$ in axial cros5-section;.

C. Postaliation axnirastes. T1 MFI demonatrat ng ea on (red arrows Jerrarcate borders).

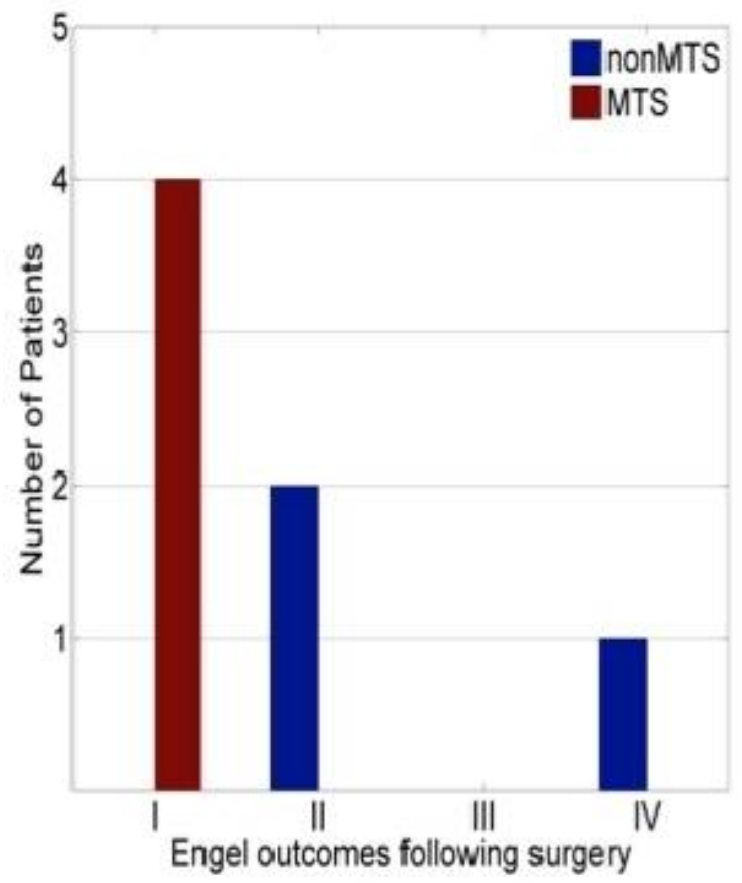

Figure 2. Following SLTAH, 4 of the 7 patients were free of disabling seizures (Engel Class I), while 2 had rare disabling seizures (Engel Class II). One patient (non-MTS, Engel Class IV) continued to experience frequent seizures.

1.274

EPILEPSY SURGERY IN THE ELDERLY: THE KORK EXPERIENCE

Bernhard J. Steinhoff, A. M. Staack and A. Wendling (Epilepsy Center Kork, Kehl-Kork, Germany)

Rationale: To collect patients olden than 60 years at the Kork Epilepsy Centre who underwent epilepsy surgery and to investigate their outcome.

Methods: Out of the series of adult patients at the Kork Epilepsy Centre who had undergone epilepsy surgery those were identified who had been at least 60 years of age at the time of surgery. Seizure 
outcome, postsurgical neuropsychological performance and quality of life were followed.

Results: Out of 383 adult patients of the Kork Epilepsy Centre who had undergone resective epilepsy surgery $12(3.1 \%)$ were at least 60 years of age. Mean age was 64.2 years with a range between 60 and 69 years. In 11 cases temporal lobe epilepsy surgery was performed with five right-sided and sic left-sided operations. The remaining operation was a fronto-parietal resection. In 7 patients hippocampal sclerosis with and without additional lesions was apparent. Five patients (42\%) became completely seizure-free (Outcome Engel Ia). Three patients $(25 \%)$ had a marked reduction of seizures or seizures without loss of consciousness. after surgery (Engel IIA). The remaining patients had some improvement (Engel IIIB).

Postoperative quality of life declined in 5 cases (42\%) with 2 seizurefree patients among them. Complications were more frequent than in younger patients.

Conclusions: In line with the literature our data show that epilepsy surgery may be still very successful in elderly patients. However, the seizure outcome tends to be less favourable and complications as well as neuropsychological impairment are more common so that an improvement of quality of life is less predictable even in case of seizure-freedom.

\subsection{5}

\section{OUTSTANDING DC CURRENT SHIFT IN THE POST-ICTAL} SUPPRESSION GUIDED ICTOGENIC TARGET

Hisanori Hasegawa (Bearumont Advanced Epilepsy Service, Grosse Pointe, MI)

Rationale: The post ictal generalized EEG suppression is seen after an event of the secondary generalized seizure. During the suppression phase, electrocortical activities are diffusely attenuated while the exact mechanism has not been completely elucidated. The observation of the post-ictal suppression is comparable with pharmacologically induced EEG suppression by mesohextal in which inhibitory GABA activity is excessively emphasized. In the mesohextal suppression, the most potent epileptogenic focus will come back first in suppression phase, and it identifes the primary epileptogenic focus. We report that observation of emerging DC current shift in post-ictal suppression phase recorded in subdural grid recording.

Methods: We encountered a case of intractable seizure with daily aura and frontal disinhibiting symptoms due to congenital right hemispheric stroke and encephalomalacia. This 23 year old woman had phase I evaluation in which there was no IID in non-invasive recording. In the phase II evaluation, subdural grid electrodes were placed in temporal neocortex, perisyluvian, prefrontal and orbitofrontal on the pathologic non-dominant hemisphere. Multiple IID foci were identified in temporal lobe but the primary epileptogenic zone was not clear. The patient experienced a secondary generalized seizure attack and post-ictal suppression, which revealed several noteworthy findings which resulted in successful surgical intervention.

Results: Multi-focal interinctal discharges is the baseline description of the interictal subdural grid recordings. The post-ictal generalized suppression was induced by a seizure originating from perisylvian posterior frontal neocortical seizure. During the suppression phase, the first time of the reemerging ictal phenomena was not sharp acivity but DC current shift in orbito-frontal cortex, which propagated posteriorly and induced high amplitude spike epileptiform discharges in the temporal neocortex with 0.7 second latency. Subsequently, resection of the orbito-frontal lobe and temporal lobe were resected. The patient subsequently became seizure free and mood swing symptom has disappeared.

Conclusions: These clinical findings suggests (1) DC current shift in the post-ictal suppression may be useful indicator of ictogenic cortex, (2) DC current shift may induce epileptiform discharges in remote cortex which suggests inerhemispheric connections, and (3) virulent focus comes back first in post-ictal suppression that is comparable with the mesohextal suppression test. The returning activity could be DC current shift from irritable cortex outside of the epileptogenic cortex. It is suspected that DC current shift is more resistant to the mechanism of post-ictal suppression than epileptiform discharges.

\subsection{6 \\ ANTERIOR LANGUAGE AND MOTOR NETWORK CONNECTIVITY WITHIN THE FRONTAL LOBE: AN EXTRA-OPERATIVE CORTICO-CORTICAL EVOKED POTENTIAL STUDY}

Victoria Wykes ${ }^{1}$, A. Miserocchi ${ }^{1}$, A. Lemisiou ${ }^{2}$, J. S. Duncan ${ }^{3,4}, \mathrm{~T}$ Wehner $^{2,3}$, A. W. McEvoy ${ }^{1,3}$ and B. Diehl ${ }^{2,3}$ ( ${ }^{1}$ Department of Neurosurgery, National Hospital Neurology and Neurosurgery, London, London, United Kingdom; ${ }^{2}$ Department of Clinical Neurophysiology, National Hospital Neurology and Neurosurgery, London, London, United Kingdom; ${ }^{3}$ Department of Clinical and Experimental Epilepsy, University College London, London, United Kingdom and ${ }^{4}$ National Hospital of Neurology and Neurosurgery, London, United Kingdom)

Rationale: To minimise surgical morbidity, knowledge of not only eloquent cortex but also its connectivity is imperative. This may be a particular challenge in patients with dyplastic lesions. A recent study used cortico-cortical evoked potentials (CCEPs) to demonstrate functional connectivity of the anterior and posterior language areas (1). Using the same technique we investigated the connectivity between the anterior language area and frontal motor areas, including primary motor cortex, premotor cortex and supplementary motor area (SMA), in patients with and without focal cortical dysplasia (FCD) within the left inferior frontal gyrus (IFG).

Methods: 5 left hemisphere language dominant patients underwent invasive monitoring with a minimum of 64 contact subdural grid electrodes, for pre-surgical evaluation of medically intractable epilepsy. 3 patients had left FCD within the IFG, 1 had a previous pilocytic astrocytoma resected from the middle frontal gyrus and 1 was non-lesional. (See table 1 for patient characteristics). All had medial, lateral and posterior frontal coverage and 3 patients had additional SMA coverage.

Electrodes associated with speech arrest were identified in the anterior language area by conventional extra-operative electrical stimulation. 15-30 bipolar single pulse electrical stimuli (SPES) were delivered to the language electrodes to investigate cortico-cortical connections, and CCEPs were obtained by averaging time locked to SPES. Time from onset of stimulus to peak depolarization (early N1, or late N2) or repolarization (early P1) was recorded.

Results: The anterior language area had a variable position in the IFG and was displaced in some patients with FCD. Early and late CCEPS occurred in close proximity to the particular language electrode demonstrating strong connectivity with surrounding cortex, and mouth and tongue motor areas (N1 latency of $\approx 20 \mathrm{~ms}$, and N2 latency of $\approx 60 \mathrm{~ms}$ ).

In patients with diffuse or middle IFG FCD, and in the non lesional patient, CCEPs were recorded from a large area over the medial and lateral frontal cortex including primary motor mouth area, and premotor area.

In patients with SMA coverage early CCEPs $(\mathrm{N} 1 \approx 15 \mathrm{~ms})$ were also seen within this area.

In the patient with a large posterior IFG FCD, stimulation resulted in a more limited field of CCEPs.

Conclusions: Our work suggests direct connections between the anterior language area, primary motor cortex, premotor areas and SMA in a widespread cortico-cortical network. Lesions within these areas may lead to a reorganisation of functional connectivity, and results need to be reproduced in a larger patient population with FCD to better understand plasticity. Anatomically the connections may be wide spread, however elucidating functionality will facilitate safe surgical planning. 
Reference

1)Matsumoto R. et al. (2004) Functional connectivity in the human language system: a cortico-cortical evoked potential study. Brain 127:2316-30.

Table 1: Patient characteristics

\begin{tabular}{|c|c|c|c|c|c|}
\hline Patient & 1 & 2 & 3 & 4 & 5 \\
\hline $\begin{array}{l}\text { Age, Sex, } \\
\text { Handedncss }\end{array}$ & $34, \mathrm{~F}, \mathrm{R}$ & $\begin{array}{c}27, \mathrm{M} . \\
\mathrm{I} .\end{array}$ & $49, \mathrm{~F}, \mathrm{R}$ & $28, M, R$ & $\mathrm{~F}, 36, \mathbf{R}$ \\
\hline $\begin{array}{l}\text { Language } \\
\text { Duminatice } \\
\text { (fMRI) }\end{array}$ & L & L & L & $L$ & L \\
\hline $\begin{array}{l}\text { Post-ictal } \\
\text { language } \\
\text { disturbance }\end{array}$ & No & Yes & No & Ycs & No \\
\hline Implantation side & $\begin{array}{c}\text { L lateral } \\
\text { and orbito- } \\
\text { FI. }\end{array}$ & $\begin{array}{l}\text { L lateral } \\
\text { I. }\end{array}$ & $\begin{array}{l}\text { L lateral and } \\
\text { mesial F. }\end{array}$ & $\begin{array}{l}\text { L lateral and mesial } \\
\text { F. }\end{array}$ & $\begin{array}{c}\text { L lateral } \\
\text { and mesial } \\
\text { ГI. }\end{array}$ \\
\hline Lesion location & $\begin{array}{l}\text { L IfFG } \\
\text { diffuse }\end{array}$ & $\begin{array}{l}\text { L IfG } \\
\text { middle }\end{array}$ & L IFG posterior & L MFG middle & $\begin{array}{c}\text { Non } \\
\text { lesional }\end{array}$ \\
\hline $\begin{array}{l}\text { Eppileptogenic } \\
\text { zone (ictal EE()) }\end{array}$ & $\begin{array}{l}\text { I. TFG and } \\
\text { MFCi, } \\
\text { anterior }\end{array}$ & $\begin{array}{l}\text { I. IFG } \\
\text { middele }\end{array}$ & I. IF. $i$ posterior & I. IF G anterior & $\begin{array}{l}\text { I. mesial } \\
\text { FL }\end{array}$ \\
\hline l'athology & $\begin{array}{c}\text { FCD } \\
\text { Type Ilb }\end{array}$ & $\begin{array}{l}\text { FCD } \\
\text { Type } \\
\text { Ib }\end{array}$ & $\begin{array}{c}\text { No resection } \\
\text { (complere overlap } \\
\text { with language) }\end{array}$ & $\begin{array}{l}\text { Scar tissue pust } \\
\text { previous resection } \\
\text { pilocytic astrocytoma }\end{array}$ & Nil \\
\hline
\end{tabular}

F: Female, M=Male, FCD: Focal Cortical Dysplasia, IFG: Inferior Frontal Gyrus, MFG: Middle Frontal Gyrus, SFG: Superior Frontal Gyrus, L: Left, R:Right, FL: Frontal Lobe

\section{Surgery: Pediatrics}

\subsection{7 \\ COST ANALYSIS OF EPILEPSY SURGERY IN PEDIATRIC DRUG-RESISTANT EPILEPSY}

Michael S. Oldham ${ }^{1}$, J. Tsevat ${ }^{2}$, P. Horn ${ }^{1}$, H. Greiner ${ }^{1}$ and S. Standridge ${ }^{1}\left({ }^{1}\right.$ Child Neurology, Cincinnati Children's Hospital Medical Center, Cincinnati, $\mathrm{OH}$ and ${ }^{2}$ Center for Clinical and Translational Science and Training, University of Cincinnati College of Medicine, Cincinnati, $\mathrm{OH}$ )

Rationale: Epilepsy surgery is an important treatment option in pediatric drug-resistant epilepsy. The associated expense of presurgical evaluation and surgical intervention is thought to be mitigated by subsequent decreased hospitalizations and improvement in quality of life, but this has not been well studied. This study compared health care costs of surgical versus medical management of children with drug-resistant epilepsy at a mid-Western US children's hospital, analyzed trends in health care costs pre- and postoperatively, and evaluated change in seizure frequency.

Methods: Included patients were evaluated for epilepsy surgery between 2008 and 2010, were 0-21 years old, and were considered surgical candidates. Cost data were derived from the institution's electronic medical records and cost accounting systems, and included: epilepsy-related outpatient clinic visits, outpatient laboratory and radiographic studies, outpatient anti-epileptic drugs (AEDs), and epilepsy-related inpatient hospitalizations. Data analysis was performed using SAS $\AA^{\circledR} \mathrm{v} 9.3$, specifically two-tailed t-tests to compare between-group demographic variables and costs, and the paired t-test to compare the change in seizure frequency. For categorical variables, Fisher's Exact Test was used to compare group frequencies.

Results: There were 93 subjects in the surgical group (48\% female); 20 in the medical group (55\% female). The average age of seizure onset and the average number of AEDs used in the past for the medical and surgical group were not significantly different (3.1 vs 3.8 years, $\mathrm{p}=0.69 ; 4.3$ vs 3.6 AEDs, $\mathrm{p}=0.4$ ). Total estimated annual cost at 1-year follow-up was not significantly different between the medical and surgical group when the cost of the surgical procedure was excluded from the surgical group (USD20123 vs 20717, $\mathrm{p}=0.938$ ). The average cost of surgery was USD226606, and the average length of stay for epilepsy surgery was 10.6 days. Therefore, when adding the total average cost of epilepsy surgery, the estimated annual cost at 1-year follow-up for the surgical group was USD247323. In the surgical group, 74\% of subjects had an ILAE seizure outcome classification of 1, 2, or 3 at 1-year follow-up. There was a significant change in daily seizure frequency in the surgical group ( 2.7 fewer, $\mathrm{p}=0.005$ ), but not in the medical group ( 1.4 fewer, NS).

Conclusions: This study is important because it is one of the only studies to systematically compare the costs of medical and surgical management of pediatric drug-resistant epilepsy in the US. Prior studies have evaluated epilepsy surgery costs, but they were conducted in Canada, which has a very different healthcare system and cost structure. The cost of epilepsy surgery is higher than medical management at 1-year follow-up. Further analysis is necessary to determine if seizure control may play a role in the cost effectiveness of surgery over a longer duration of follow-up. Also, the largest contributions to the cost of surgery are from areas with the best potential for cost reduction including room/board, EEG monitoring, AEDs, and decreasing the length of hospital stay.

\subsection{8 \\ LASER ABLATION OF NON-MESIAL TEMPORAL FOCI WITH MEG LOCALIZATION AND SURFACE EEG RECORDING DURING MRI GUIDED ABLATION: FOUR PEDIATRIC CASES WITH EEG NORMALIZATION POST- LASER ABLATION}

Michael G. Chez ${ }^{1}$, S. Ciricillo ${ }^{1}$, A. Ghasseimi ${ }^{1}$, C. Lepage ${ }^{1}$, H. E. $\mathrm{Kirsch}^{2,3}$, S. M. Honma ${ }^{3}$ and M. M. Mantle ${ }^{3}$ ( ${ }^{1}$ Sutter Neuroscience Medical Group, Sacramento, CA; ${ }^{2}$ Epilepsy, UCSF Medical Center, San Francisco, CA and ${ }^{3}$ Biomagnetic Imaging Center, University of California San Francisco Medical Center, San Francisco, CA)

Rationale: Epilepsy surgery may be the long term best solution for patients with refractive epilepsy. Current guidelines suggest that earlier surgery is better and reduces long term comorbidity. Often patients fear the process of craniotomy and surgical removal or brain tissue. The fear of procedure, longer hospital stays, and often difficult to treat extra-temporal foci lead to many not feeling they have a surgical option. We present a new less invasive technique with non-invasive localization using surface EEG,

MEG(Magnetoencephalography) and fMRI combined with Brainlab Localization. This yields minimally invasive MRI guided ablation and shortened hospital stay and recovery in less than 24 hours. We present 4 cases.

Methods: Four pediatric refractory epilepsy patients with focal origin were evaluated with video EEG, fMRI, MEG( and 3 tessla MRI using Brainlab localization (Table). Patients deemed focal in origin had 3 frontal and one superior temporal gyrus localizations with refractive EEG and seizure control affecting behavior, Patients were 2 males ( age 11,8$)$ and 2 females age (19 and age 14). Three patients had autism spectrum or cognitive disability making standard intracranial grid mapping and craniotomy less desirable. These patients elected to have laser ablation with Brainlab guided laser placement. All had MRI compatable plastic electrodes during MRI guidance of laser ablation showing pre-ablation and post-ablation spike data.as a method of intraoperative EEG monitoring. Laser system was from Visualase . MEG scan was performed at UCSF Biomagnetic iIImaging Center

Results: All 4 patients underwent SLA at Sutter Medical Center Sacramento between 11-11and 6-12 (Picture). All patients showed resolution of pre-ablation spikes on intraprocedure EEG taken immediately post ablation. . Patients were pain free, eating, and ambulatory within 6-8 hours post-ablation and discharged after less than 24 hours. .. Patients were seen after 1-2 weeks, and the first patient monitored for 6 months post-operatively, remains seizure free off medications with memory and language improvement. $19 \mathrm{yr}$ old with Noonan Syndrome and spikes along superior temporal gyrus had 
a reduction from 4 seizures per day to 2-3 seizures per week initially, then became less than 1 per week, while 3 frontal localized seizures remained seizure free with one already tapered off medications with normal EEG.

Conclusions: .SLA may offer an attractive option for deep nonmesial temporal and non-lesional epileptic foci to be surgically destroyed with minimal risk and invasiveness. This technique can be combined with MEG spike data fused to MRI, and intraprocedural MRI-compatible surface EEG recordings to monitor successful ablation of refractive focal epileptic zones. More studies showing long term efficacy and reduction in drug burden is recommended.

Pediatric Non-Mesial tTemporal Patient Data

\begin{tabular}{|c|c|c|c|c|c|c|}
\hline l'atient & Sex/age & $\begin{array}{l}\text { Medicine } \\
\mathrm{prc} / \text { post }\end{array}$ & $\begin{array}{c}\text { Ablation Silr } \\
\text { Localization } \\
\text { ГГT }\end{array}$ & $\begin{array}{l}\text { Ablation } \\
\text { Sitc }\end{array}$ & $\begin{array}{c}\text { EEG } \\
\text { Post-Iaser }\end{array}$ & $\begin{array}{c}\text { Months } \\
\text { Followed/ seizure } \\
\text { frec }\end{array}$ \\
\hline $\mathrm{JP}$ & 11 & $\begin{array}{c}\text { Kcppra } \\
\text { Vimpat } \\
\text { Depakotef } \\
\text { none post }\end{array}$ & $\begin{array}{l}\text { Left anterior } \\
\text { frontal }\end{array}$ & $\begin{array}{l}\text { Lelt Anterior } \\
\text { Frontal }\end{array}$ & Normal & 25 weeks \\
\hline $\mathrm{BO}$ & 8 & $\begin{array}{l}\text { Vimpal } \\
\text { Depakote }\end{array}$ & $\begin{array}{l}\text { Right anterior } \\
\text { frontal }\end{array}$ & $\begin{array}{l}\text { Right Anterior } \\
\text { frontal }\end{array}$ & Normal & 4 weeks \\
\hline $\mathrm{NC}$ & 19 & $\begin{array}{l}\text { Vimpat } \\
\text { Depakote } \\
\text { Keppra } \\
\text { Onfi }\end{array}$ & $\begin{array}{l}\text { Superior } \\
\text { temoral T6 } \\
\text { P4 border }\end{array}$ & $\begin{array}{l}\text { Superior } \\
\text { Mid- } \\
\text { Temporal }\end{array}$ & Normal & $\begin{array}{c}<4 \text { weeks } \\
\text { about } 12 \text { wecks } \\
\text { months } \\
\text { Reduction }>80 \%\end{array}$ \\
\hline $\mathrm{CS}$ & 14 & $\begin{array}{l}\text { Depakote } \\
\text { Vimpat }\end{array}$ & $\begin{array}{l}\text { right Frontal } \\
\text { polc }\end{array}$ & $\begin{array}{l}\text { Anterior Right } \\
\text { frontal region }\end{array}$ & Normal & $\begin{array}{l}4 \text { weeks/possible } \\
\text { bricf occasional } \\
\text { partial sciizure }\end{array}$ \\
\hline
\end{tabular}

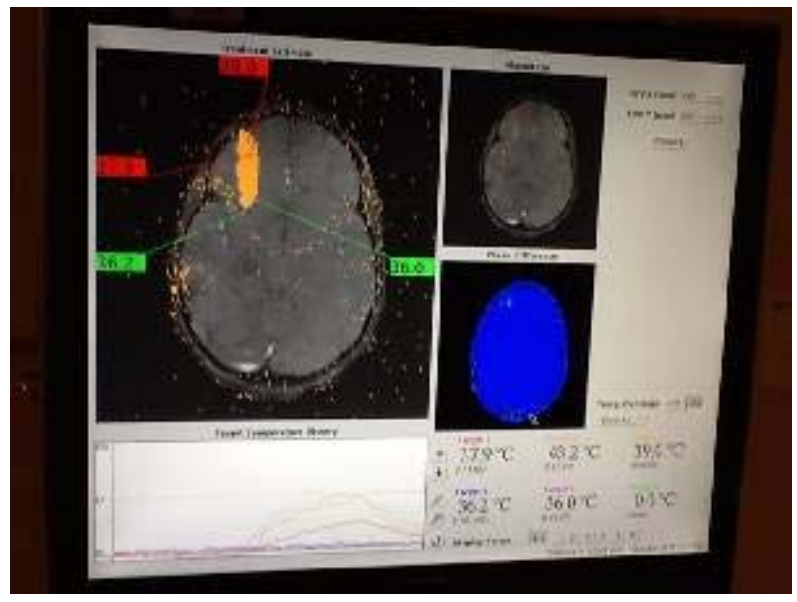

\subsection{9}

\section{A LONG-TERM FOLLOW-UP RESULTS OF CALLOSOTOMY FOR 51 PATIENTS WITH WEST SYNDROME}

Hiroshi Baba, K. Toda, T. Ono, S. Baba and K. Ono (Neurosurgery, National Nagasaki Medical Center, Omura, Japan)

Rationale: Callosotomy (CC) for patients with West syndrome was reported by Pinard (1993). However, long-term outcome of seizure and psychomotor developments has not been reported. From 2001 to 2012, we performed CC on 74 patients with West syndrome. Surgical outcome and changes of psychomotor developments (PD) were retrospectively analyzed.

Methods: 51 patients with follow-up periods more than 2 years were included. Patients with a resectable MRI lesion were excluded. Mean age at seizure onset was 4.6 months. ACTH treatment was used in 42. Number of anti-epileptic medication ranged from 1 to 4 (mean 2.7 drugs). Mean preoperative developmental quotient (DQ) was 15.6 and developmental age (DA) was 4.1 months. 11 patients $(22 \%)$ were diagnosed as EIEE at the onset of seizure and 24 (47\%) showed severe psychomotor delay before onset of seizure. During preoperative EEG monitoring, 36 patients had only tonic spasms (SP). Multiple types of seizures were observed in 15.10 had both SP and tonic seizure (TS). MRI showed no abnormality in 8, diffuse atrophy, predominantly in the frontal lobe in 41 . For the assessment of psychomotor developments, we used Kinder Infant Developmental Scale (KIDS), which was developed for Japanese children. Anterior $\mathrm{CC}$ was performed in 2 and total $\mathrm{CC}$ in 49 . Mean age at $\mathrm{CC}$ was 24.4 months. Mean follow-up period was 49 months.

Results: At final follow-up point, $33 \%$ of patients were seizure free (F). 20\% showed excellent (E) (greater than $80 \%$ reduction in seizure frequency, $26 \%$ was good $(\mathrm{G})$ (greater than $50 \%$ reduction), and $22 \%$ was poor $(\mathrm{P})$ (no significant change). Results were significantly better for SP than TS. $47 \%$ of SP spasm was F and $24 \%$ showed E after CC. As compared to SP, results for TS were significantly poor. There was no seizure free patient.

To confirm effects of surgical outcome on PD, we analyzed postoperative changes of $\mathrm{PD}$ in $\mathrm{F}+\mathrm{E}$ and $\mathrm{G}+$ Poor group. In $\mathrm{F}+\mathrm{E}$, postoperative decline of DQ was kept at the level of preoperative state. At 12 month after CC and final follow-up point, DQ and DA were significantly higher in $\mathrm{F}+\mathrm{E}$ than in $\mathrm{P}+\mathrm{G}$. Gradual decline of $\mathrm{PD}$ were observed in $\mathrm{P}+\mathrm{G}$.

Early onset of epilepsy, presence of severe developmental delay before onset of epilepsy, longer duration from onset to surgery and presence of TS was significantly related to poor outcome.

Conclusions: $\mathrm{CC}$ is an effective and important therapeutic option in West syndrome. Bilateral cortical recruitment through the corpus callosum may be important for generation of SP. CC is more effective on SP than TS suggesting that different mechanisms may be present. CC prevents decline of DQ in patients with favorable surgical outcome suggesting that early surgery is recommended before severe psychomotor delay develops for postoperative improvements of the psychomotor function.

\subsection{0 \\ FUNCTIONAL LESIONECTOMY: A MINIMALLY RESECTIVE STRATEGY EFFECTIVE IN CHILDREN WITH MRI-NEGATIVE, INTRACTABLE EPILEPSY}

Ann Hyslop ${ }^{1}$, I. Miller ${ }^{1}$, S. Bhatia ${ }^{1,2}$ and P. Jayakar ${ }^{1}\left({ }^{1}\right.$ Neurology, Miami Children's Hospital, Miami, FL and ${ }^{2}$ Neurosurgery, University of Miami Miller School of Medicine, Miami, FL)

Rationale: Excisional surgery achieves seizure-freedom in a large proportion of children with MRI negative epilepsy, but the resections based on functional data are often extensive. We explored the possibility that more restricted resections within a carefully selected MRI negative cohort could achieve comparable success.

Methods: We report a subset of 25 children with MRI-negative, intractable partial epilepsy who underwent focal corticectomy at our institution between the years of 2005-2011. The epileptogenic region was identified by integrating multimodal functional data including 3D EEG source localization, SPECT, PET, and invasive EEG data using co-registration software. Corticectomies were minimized by resecting regions that showed convergence of multimodal abnormality.

Results: The mean age of seizure onset was 5.6 years (range, 1 day to 14 years) and mean age at surgery was 11.8 years (range, 6 months to 19.9 years). Corticectomies were convergent with scalp EEG in all cases and 3-D EEG source localization of scalp interictal data in 11 of 12 analyzed. Convergent, focal hyperperfusion was seen in 10 of 15 patients who had ictal SPECT scans and, of the 17 patients that underwent PET scan, 12 had convergent regions of hypometabolism and 2 had highly localized, convergent hypermetabolic areas. Twenty-three children had convergent interictal and/or ictal discharges on intraoperative ECoG and 24 had ictal onset in corresponding regions during extraoperative subdural monitoring. Resections were considered complete in 7 and incomplete in 18 . Histopathology revealed definite cortical dysplasias in 19 patients, 7 of which were Palmini type I and 12 of which were type II. The remaining 6 showed $\mathrm{mMCD}$. 
At 1 year follow-up, 13/25 (52\%) were seizure-free, 5 (20\%) experienced persistent seizures with an $>90 \%$ reduction in frequency, and the remaining $7(28 \%)$ had no change in seizure burden. The rate of seizure freedom in children with type I FCD, type II FCD, and mMCD was $3 / 7$ (43\%), 7/12 (58\%), and 3/6 (50\%), respectively [not significant]. Outcomes were unrelated to completeness of resection and all three histopathologic classes were represented in each Engel outcome class. The majority of children with Engel class IV outcomes had data convergence of fewer functional modalities than that seen in almost all children in more favorable outcome classes.

Conclusions: Our findings demonstrate that functional lesionectomy is successful in a carefully selected subset of a MRI negative cohort. Outcomes were not related to completeness of resection and did not vary significantly between children with different histopathologies, but more favorable outcomes were associated with a greater number of convergent functional modalities. Minimizing resection based on convergent mulitmodal functional data helps avoid unnecessarily large resections and may have important implications not only for optimizing seizure control but also for improvement in functional and developmental outcomes.

\subsection{1}

RESECTIVE EPILEPSY SURGERY BEFORE THE AGE OF 2

Hyeeun Kwon, J. Kang, H. Kang, J. Lee and H. Kim (Pediatric Neurology, Severance Children's Hospital, Yonsei University College of Medicine, Seoul, Republic of Korea)

Rationale: To evaluate the surgical, developmental outcomes and safety issues in infants under 2 years undergoing epilepsy surgery. Methods: We identified 38 patients younger than ages of 2 years who underwent resective surgery at Severance Children's Hospital in Seoul, Korea, from February, 2003 to August, 2011. Etiology, postoperative seizure outcomes, pre and post-operative cognitive function, re-operation rates and complications were analyzed by retrospective examination of clinical data.

Results: All patients had intractable epilepsy and thirty-four patients had an epileptogenic lesion visible on brain MRI. Most frequent etiologies were predominantly malformation of cortical development (28), and the rest were Sturge-Weber syndrome (4), destructive encephalopathy (1), tumor (1), tuberous sclerosis complex (1), and others (1). The median age at surgery was 12 months (range $0.2-2$ years) and lead time to surgery was 0.7 years (range $0.1-1.9$ years). Surgeries were functional hemispherectomy $(53 \%)$, lobar or multilobar resection and 1 posterior quadrantectomy. In twelve children, re-operation was carried out. At last follow-up (median 3.4 years), $78 \%$ of patients were in Engel Class I and thirteen of them successfully discontinued AED without seizure relapse. There were 2 perioperative mortalities. The most common surgical complications among the 52 operations in 37 patients were hydrocephalus (6). Conclusions: Out results show that epilepsy surgery in infancy can be relatively safely performed with satisfactory long-term results.

\subsection{2 \\ EFFECTS OF CORPUS CALLOSOTOMY ON EEG IN INTRACTABLE PEDIATRIC EPILEPSY}

Joon Won Kang ${ }^{1}$, T. Khusainov ${ }^{2}$, H. Kwon ${ }^{1}$, H. Kang ${ }^{1}$, J. Lee ${ }^{1}$, Y. $\mathrm{Lee}^{3}$, D. Kim ${ }^{4}$ and H. Kim ${ }^{1}{ }^{1}$ Pediatric Neurology, Pediatric Epilepsy Clinics, Severance Children's Hospital, Epilepsy Research Institute, Yonsei University College of Medicine, SEOUL, Republic of Korea; ${ }^{2}$ Pediatric Neurology, Republic Children's Hospital Tashkent Pediatric Medical Institute, Tashkent, Uzbekistan; ${ }^{3}$ Pediatrics, Gangnam Severance Hospital, Yonsei University College of Medicine, SEOUL, Republic of Korea and ${ }^{4}$ Pediatric Neurosurgery, Severance Hospital, Yonsei University College of Medicine, SEOUL, Republic of Korea)
Rationale: Corpus callosotomy is a palliative disconnection procedure that consists of microsurgically sectioning the corpus callosum which is to disrupt the bilateral propagation of epileptic discharges, thereby decreasing frequency and severity of seizure. The overall goal of the current study is to evaluate the preoperative lateralizing values in patients of corpus callostomy.

Methods: A total 105 patients (male: 63, female 42) underwent corpus callosotomy at the Severance Children's Hospital between October 2003 and July 2011. Lennox-Gastuat syndrome was the most diagnosis, and was 86 patients $(81.9 \%)$. The medical records were retrospectively reviewed for all patients. Clinical characteristics, surgical outcomes, etiologies, and EEG profiles (background, asymmetric slowing, independent sharp wave discharges, generalized slow sharp and wave discharges, generalized paroxysmal fast activities, localized paroxysmal fast activities, subclinical seizure, clinical seizure, and dominancy) were reviewed. Statistical analyses were performed using the SAS (version 9.2, Institute, Cary, NC, USA). For analyzing differences of EEG profiles between preoperative and postoperative features, McNemar's test and logistic regression were used. The probability value less than 0.05 we defined as statistically significant.

Results: The age at seizure onset was 2.5 years (range, 0.5 month15.5 year). The age at surgery was 8.0 years (range, 3.6 month-20.7 year). The period between seizure onset and surgery was 5.5 years (range, 2.4 month-17.5 year). Total corpus callosotomy was the most common procedure, and was performed in 96 patients $(91.4 \%)$. Partial corpus callosotomy was performed in 9 patients $(8.6 \%)$. Twenty-nine patients $(27.6 \%)$ demonstrated an Engel class I outcome at least 12 months after callosotomy. The mean follow-up duration after surgery was 4.0 years (range, 1.1-9.2 years). Significant lateralization in post-callostomy patients were found in $29(27.6 \%)$ patients. Preoperative asymmetric slowing on EEG could predict lateralization (OR: 3.77, CI: 1.45-9.78). Twenty-five patients had done secondary resective surgery due to lateralization on postoperative follow-up EEG findings. Ten patients (40.0\%) demonstrated an Engel class I outcome after secondary resective surgery.

Conclusions: Corpus callosotomy could be an possible lateralizing method for patients suffering from intractable generalized pediatric epilepsy that are not candidates for resective surgery with generalized EEG features.

\subsection{3 \\ FUNCTIONAL HEMISPHERECTOMY IN YOUNG CHILDREN WITH CORTICAL DYSPLASIA AND INTRACTABLE EPILEPSY}

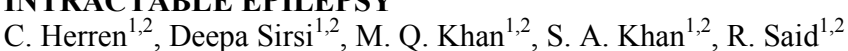
and S. T. Arnold ${ }^{1,2}$ ( ${ }^{1}$ Pediatric Neurology, University of Texas Southwestern Medical Center, Dallas, TX and ${ }^{2}$ Pediatric Neurology, Childrens Medical Center, Dallas, TX)

Rationale: Children with unilateral cortical dysplasia may present with intractable focal seizures in infancy or early childhood that are refractory to anti-epileptic drugs (AEDs). Uncontrolled seizures can be associated with stagnation or regression of neuro-cognitive development likely secondary to a combination of seizures and polytherapy with AEDs. In such children epilepsy surgery can be a successful treatment option.

Methods: The medical records of patients who underwent functional hemispherectomy for intractable epilepsy secondary to cortical dysplasia between 2006 and 2012 at a large tertiary care academic center were reviewed.

Results: Six cases were identified.

- 4 patients had focal cortical dysplasia, 1 hemimegalencephaly and 1 had diffuse dysplasia of the right hemisphere.

- Ages ranged from 8 months to 6.5 years with median age of 2.8 years.

- 4 of 6 patients had cortical dysplasia involving the left hemisphere. 
- 3 of 6 patients had undergone previous focal frontal resections prior to hemispherectomy.

- 1 patient required additional resection after functional hemispherectomy for residual tissue.

- Follow-up ranged from 2 months to 4 years, average 2 years. 4 of 6 patients had an Engel class 1a outcome and 2 of 6 patients had an Engel class $1 \mathrm{~b}$ outcome.

- 4 of 6 patients had mild to moderate hemiparesis prior to hemispherectomy. ( 2 of them subsequent to a focal frontal resection). - Post-operatively contralateral hemiplegia slowly improved in all patients with arm recovery being incomplete. All 6 were ambulating independently at follow up.

- Significant improvement in cognitive and developmental abilities was noted after surgery. 4/6 patients underwent left

hemispherectomy. 3 of these 4 patients had improved language function at follow up (1y $3 \mathrm{~m}$ and 4 years).

- EEG in 3/6 patients showed multifocal or generalized spikes, although imaging and clinical exam did not show evidence of migrational abnormality in the contralateral hemisphere.

- Pathology confirmed focal cortical dysplasia in all 6 cases.

- Complications were limited to expected hemiplegia.

Conclusions: 1 . Very early hemispherectomy has been reported in literature in children as young as 2 months. Based on our series early surgery should be considered in well selected infants and young children with severe epilepsy and unilateral hemispheric cortical dysplasia to optimize seizure control and to minimize the effect of catastrophic epilepsy on the developing brain.

2. Epilepsy surgery in the presence of multifocal or generalized epileptiform discharges in a child with unihemispheric cortical dysplasias is compatible with good outcome for seizure control. Clinical examination, MRI findings and other imaging data should however be concordant. The diffuse electrographic abnormalities may be due to the impact of the epileptogenic lesion on the developing brain.

3. Hemispherectomy may be considered after failed focal resections of cortical dysplasia.

\subsection{4 \\ FDG-PET MAXIMAL HYPOMETABOLISM OFFERS INSIGHT INTO THE SEIZURE-ONSET ZONE IN REFRACTORY NEOCORTICAL EPILEPSY \\ Larry D. Olson ( ${ }^{1}$ Pediatrics and Neurology, Emory University, Atlanta, GA and ${ }^{2}$ Neurology, Childrens Healthcare of Atlanta, Atlanta, GA)}

Rationale: Although FDG-PET imaging is recognized as important in presurgical evaluation in medically refractory focal temporal lobe and neocortical epilepsy, there has been no investigation as to whether some regions of hypometabolic zones may be of particular importance in defining the seizure-onset zone or potentially epileptogenic zone. This report illustrates with several case examples that techniques isolating the maximally hypometabolic zone may exquisitely identify the seizure-onset zone, sometimes even when not apparent with grid electrodes placed directly on overlying cortex in both MRI positive and negative cases.

Methods: Several novel techniques are presented which have proven useful in identifying maximally hypometabolic regions. These include color map LUTs, windowing PET hypometabolism, isocontour thresholding, and restriction of PET to cortex by way of masking with coregistered MRI grey matter segmentation- alone or in combination. Subtle hypometabolic regions are often restricted to a gyrus, and can be difficult to identify with conventional greyscale PET. Color maps and windowing may help identify subtle hypometabolism. Isocontours are useful in 3D lateral hypometabolism visualization. Even within larger hypometabolic regions, identification of maximally hypometabolic regions may exquisitely localize seizure onset zone in gyral depth with or without a lesion even if not apparent a few centimeters away on subdural grids. Volumetric imaging to coregister to T1 MRI helps assure that the hypometabolic region is cortical rather than white matter, and helps in precise placement of intracranial contacts. These techniques are augmented by volumetric 3D or orthographic visualizations.

Results: 6 cases are used to illustrate the methods of identifying the maximally hypometabolic regions, the potential value of these regions in iEEG planning, and the value of these localizations in identifying seizure-onset zones.

Conclusions: Although the seizure-onset zone may not represent the entire potentially epileptogenic zone, it is unquestionably a critical component of this. These methods therefore may offer important guidance in achieving successful epilepsy surgery outcomes.

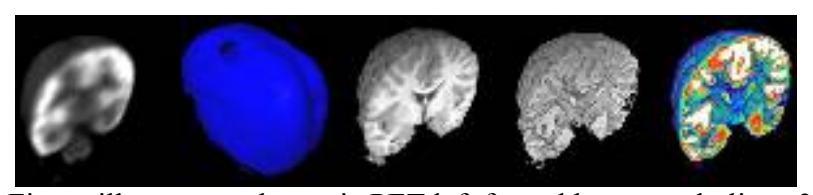

Figure illustrates volumetric PET left frontal hypometabolism, 3D isocontour representation, normal MRI, grey matter MRI segmentation, PET masked with grey matter segmentation shown with custom color map. iEEG identified hundreds of ictal onsets in the depth of the gyrus and spread to overlying cortex with clinical seizures.

\subsection{5 \\ OUTCOMES OF VAGUS NERVE STIMULATION (VNS) IN PEDIATRIC EPILEPSY \\ Camilla D. Yu ${ }^{1,2}$, I. Abdelmoumen ${ }_{5}^{2}$, S. Ramgopal ${ }^{2}$, C. Powell ${ }^{3}, \mathrm{~K}$. Remy $^{2,4}$, M. Libenson ${ }^{2}$, J. Madsen ${ }^{5}$, A. Rotenberg ${ }^{2}$ and T. Loddenkemper ${ }^{2}\left({ }^{1}\right.$ Physiology, McGill University, Montreal, QC, Canada; ${ }^{2}$ Neurology, Boston Children's Hospital, Boston, MA; ${ }^{3}$ Clinical Research Program, Boston Children's Hospital, Boston, MA; ${ }^{4}$ Psychology, Emory University, Atlanta, GA and ${ }^{5}$ Neurosurgery, Boston Children's Hospital, Boston, MA)}

Rationale: To examine the efficacy of vagus nerve stimulation (VNS) therapy in children and adolescents with medically refractory epilepsy.

Methods: We reviewed the charts of all patients implanted with a VNS at a single center between December 1997 and March 2011. All patients with complete follow-up data at 6 and 12 months were included. Patients were divided into two groups according to the median seizure frequency at baseline. A Wilcoxon Rank sum test was performed to determine if there was significant seizure reduction from baseline to month 12 across various subgroups organized by baseline characteristics.

Results: 252 patients were identified. Complete follow-up data was available for 69 patients (29 girls, mean implantation age 11.1 years, SD 5.3). Mean age of seizure onset was 28.6 months (SD 34.3). Ten patients had generalized epilepsy, 30 had focal epilepsy, and 29 had both generalized and focal features.

Forty-seven and 44 subjects had reductions in seizure frequency at months 6 and 12, respectively. The overall median percentage change (seizures/month) from baseline was $-50 \%$ [25\%, 75\% Quartiles: ($73 \%, 0 \%)]$ at 6 months $(\mathrm{p}=0.005)$ and $-40 \%[25 \%, 75 \%$ Quartiles: ($75 \%, 25 \%)]$ at 12 months $(\mathrm{p}=0.058) .4$ patients were seizure-free at 12 month follow-up.

Patients with higher baseline seizure frequency ( $>45 /$ month) experienced a greater percentage reduction in seizures, with a median reduction of $61 \%$ [25\%, 75\% Quartiles: $(-94 \%,-50 \%)](\mathrm{p}<0.001)$ and $69 \%$ [25\%, 75\% Quartiles: $(-96 \%,-30 \%)](\mathrm{p}<0.001)$ at 6 and 12 months, respectively. Patients with a lower baseline seizure frequency $(\leq 45 /$ month) experienced a median seizure reduction of $25 \%$ [25\%, 75\% Quartiles: $(-50 \%, 50 \%)]$ at 6 months $(\mathrm{p}=0.94)$ and $0 \%[25 \%, 75 \%$ Quartiles: $(-50 \%, 100 \%)]$ at 12 months $(\mathrm{p}=0.39)$. Of the excluded patients, 6 had treatment discontinued during the 12 month follow-up period. Reasons for discontinuation included 
infection $(n=3)$, nausea $(n=1)$, skin breakdown $(n=1)$, and perceived lack of efficacy $(n=1)$. Sixteen of the 69 patients experienced adverse effects which did not warrant discontinuation of therapy, including voice changes, coughing, throat tickle, difficulty sleeping, and pain over the VNS area.

Outcomes were compared across multiple subgroups including gender, age at VNS implantation, seizure semiology, previous history of SE, number of medications at baseline, presence or absence of MRI lesion, and duration of epilepsy prior to implantation. There were no significant differences in outcome in any of these subgroups $(p>0.05)$. In the low baseline group, however, there was a tendency for patients who were younger at the time of VNS implantation to experience moderately better outcomes $(\mathrm{p}=0.06)$.

Conclusions: VNS therapy resulted in a reduction in seizure frequency at both 6 and 12-month follow-up periods. These data suggest that VNS is a viable treatment for patients with medically refractory epilepsy, but may provide more consistent seizure reduction in patients with higher baseline seizure frequencies.

\subsection{6 \\ CORPUS CALLOSOTOMY VERSUS VAGUS NERVE STIMULATION IN CHILDREN WITH REFRACTORY EPILEPSY: ONE CENTER'S EXPERIENCE \\ K. Havens ${ }^{1}$, A. Yaun ${ }^{1,2}$, T. Zelleke ${ }^{1,2}$, T. N. Tsuchida ${ }^{1,2}$, P. Pearl ${ }^{1,2}$, J. Conry $^{1,2}$, A. Kao ${ }^{1,2}$, W. D. Gaillard ${ }^{1,2}$ and D. T. Depositario- Cabacar ${ }^{1,2}\left({ }^{1}\right.$ Center for Neuroscience, Children's National Medical Center, Washington, DC and ${ }^{2}$ George Washington University Medical Center, Washington, DC)}

Rationale: Corpus callosotomy and vagal nerve stimulation (VNS) are palliative and adjunctive procedures performed for patients with refractory epilepsy. Although they both reduce seizures, there is little reported data on the comparison of corpus callosotomy and VNS in children. We have retrospectively reviewed children who have undergone corpus callosotomy and VNS surgery in our center and examined their features and surgical outcomes.

Methods: We identified patients from our epilepsy surgery database who had corpus callosotomy or VNS for medically intractable epilepsy. The database and medical records were queried for seizure type, etiology, Engle outcome, morbidity and complications. Only patients with follow up of more than 6 months after both procedures were included.

Results: Fifteen out of 308 patients (4.8\%) had corpus callosotomy surgery performed. Seizure types included: complex partial seizures (cps) (1), generalized tonic clonic (GTC) (1), mixed (12), and Lennox-Gastaut syndrome (1). Etiologies included: chromosomal abnormality (1), encephalitis/infectious (1), cerebral malformation (5), tuberous sclerosis complex (1), perinatal injury (1), mesial temporal sclerosis (1), and unknown (5).

Thirty nine out of 308 patients $(12.6 \%$ ) had VNS placement with more than 6 months follow up. Seizure types included: cps (5), myoclonic (1), mixed (28), and Lennox-Gastaut syndrome (5). Etiologies included: chromosomal abnormality (3), mesial temporal sclerosis (1), encephalitis/infectious (3), nonaccidental trauma (3), cerebral malformation (4), tuberous sclerosis complex (1), Aicardi syndrome (1), Angelman syndrome (1), and unknown (22).

Surgical outcome for corpus callosotomy include $20 \%(\mathrm{n}=3)$ with Engle outcome Class II, 53\% $(n=8)$ with Class III and $26 \%(n=4)$ with Class IV outcome. One patient went on to have VNS placement. For VNS, $15 \%(n=6)$ had Class II Engle outcome, 23\% $(n=9)$ had Class III outcome, and $58 \%(\mathrm{n}=23)$ had Class IV outcome. One patient failed to follow up in clinic and had no outcome data. Six out of $40(15 \%)$ patients with VNS stimulator went on to have a corpus callosotomy.

Two complications were seen with corpus callosotomy. One had a superficial wound, and another required drainage of a

cephalhematoma, after picking on the suture site. Four complications were seen with VNS and include 1) infection that required a new pulse generator; 2) chronic infection requiring removal of the VNS; 3) hoarseness and imbalance; and 4) pain around the incision site. Conclusions: Children with intractable epilepsy can have a reduction in their seizures with both corpus callosotomy and VNS surgery. Corpus callosotomy surgery was more effective in reducing seizures compared to VNS. Children were more likely to have corpus callosotomy surgery after VNS than vice versa. Minimal complications were seen with both surgeries.

\subsection{7 \\ EARLY EXPERIENCE WITH MINIMALLY INVASIVE EPILEPSY SURGERY USING LASER ABLATION IN A PEDIATRIC COHORT}

Ian Miller, J. Ragheb, S. Bhatia and A. Hyslop (Miami Children's Hospital, Miami, FL)

Rationale: The Visualase system has been FDA-approved for thermal ablation of soft tissue in neurosurgery since 2007 . It has many theoretical advantages over conventional neurosurgical resection, including smaller exposure, shorter recovery, and less pain. The system also allows real-time MR guidance, which may further improve safety and surgical accuracy. Whether these benefits are observed in children undergoing epilepsy surgery has not yet been reported.

Methods: Miami Children's Hospital began using the Visualase system in May, 2011. We report on the clinical history, surgical approach, and outcomes for all five patients with intractable epilepsy who have been treated using Visualase since that time. Intraoperative neuronavigation included both the Leksell Stereotactic System, as well as the Brainlab navigational system.

Results: A total of five patients with intractable epilepsy underwent surgery at Miami Children's Hospital between 5/2011 and 6/2012.

The mean age at surgery was 14.9 years (range 11.5 to 18 ), with the mean age of epilepsy onset of 7.6 years (range 4-10.7). Of five patients undergoing Visualase surgery, there were six total procedures. The only complication observed was placement-related: one patient was converted to conventional resection, one patient required a second Visualase ablation, and three were satisfactory. Mean length of stay was 1.6 days (range 1-2). The were no complications from infection, bleeding, or unintended neurological injury. Two patients were Engel class I (seizure free) after Visualase surgery, one of whom required a second Visualase ablation. In the remainder, one patient was Engel class III, one patient was Engel class IV, and the fifth patient was Engel class I following conversion to conventional resection.

Conclusions: Minimally invasive epilepsy surgery in children is safe and effective. Length of stay is shorter than conventional surgery, and we experienced no unanticipated complications. The biggest factor determining success was precise placement of the laser fiber, and complete destruction of the epileptogenic zone. Visualase is a promising mode of treatment for intractable pediatric epilepsy; small, deep lesions (such as those seen in tuberous sclerosis) may be particularly amenable to this approach. 


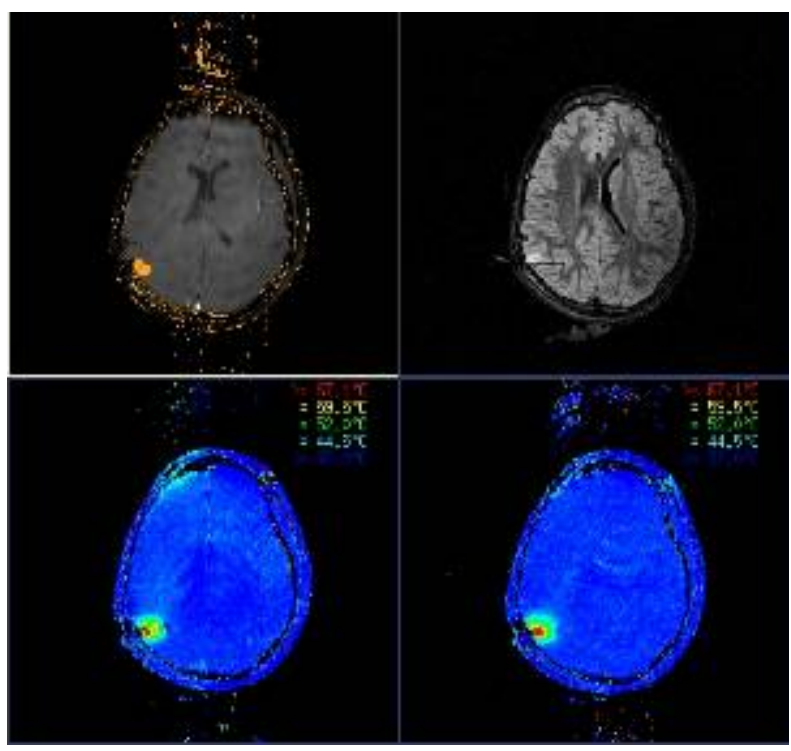

Realtime visual feedback as seen during the ablation; ablation estimate (filled orange area on top left image), anatomy (top right), and thermal maps (bottom left and bottom right).

\subsection{8 \\ RESECTIVE SURGERY IN INFANTS WITH INTRACTABLE EPILEPSY ASSOCIATED WITH FOCAL CORTICAL DYSPLASIA}

Dilek Yalnizoglu $^{1,2}$, D. C. Tarquinio ${ }^{1}$, C. Dunoyer ${ }^{1}$, A. Hyslop ${ }^{1}$, I. O. Miller ${ }^{1}$, T. Resnick ${ }^{1}$, M. Duchowny ${ }^{1}$ and P. Jayakar ${ }^{1}\left({ }^{1}\right.$ Clinical Neurophysiology, Miami Children's Hospital, Miami, FL and ${ }^{2}$ Pediatric Neurology, Hacettepe University Children's Hospital, Ankara, Turkey)

Rationale: Resective surgery is successful in alleviating seizures in a majority of children with intractable epilepsy; developmental pathology being the commonest substrate. While there is considerable experience with surgery for focal cortical dysplasia (FCD) in older children, there is little in the very young. We describe outcomes of children who had resective epilepsy surgery during the first two years of life.

Methods: We reviewed patients who underwent epilepsy surgery at Miami Children's Hospital Comprehensive Epilepsy Center between January 1997 and April 2011. We identified 22 patients who had at least one focal resective surgery during the first 2 years of life, and had a pathological diagnosis of FCD. All patients had presurgical evaluation including clinical and developmental assessment, interictal and ictal video-EEG recordings, MRI and intraoperative ECoG. Selected patients had SPECT, PET, functional MRI, chronic subdural recordings, functional mapping, and somatosensory evoked potentials with invasive electrodes. Seven patients underwent reoperation. Seizure outcome following the last operation was analyzed using modified Engel Scale.

Results: The mean age at seizure onset was 2.8 months ranging between first day of life-10 months, age at surgery was 10.7 months (1.6-21.7 months). Fourteen patients had abnormal neurological examination and 17 had developmental delay. Eighteen patients had complex partial seizures, one had secondarily generalized seizures resulting in status epilepticus, and 3 had spasms. Localizing and/or lateralizing findings were present in 17/22 interictal, and all ictal EEG. MRI showed lesions compatible with FCD in 18, normal findings in 2, and nonspecific findings in 2 . Focal convergent findings were seen in ictal SPECT (15/15), interictal SPECT (5/7), and PET (3/4). Initial surgery included 10 lobar and 12 multilobar resections, second surgery included 3 lobar and 4 multilobar resections, and third surgery included one hemispherectomy. At mean follow-up of 5.5 years, 11 patients were seizure free $(50 \%), 3$ patients had $>90 \%$ reduction in seizure frequency (14\%), 4 patients had 50 -
$90 \%$ reduction ( $18 \%$ ), and 4 had $<50 \%$ reduction. Postoperative complications included cerebrovascular insult (6), resulting in permanent neurologic deficit (3), infection (2), edema (2), excessive blood loss (1). Histopathology revealed FCD type $2 \mathrm{~B}$ in 7 patients, type 2A (6), type 1 (5), mild CD (3), and type 4D (1). Favorable outcome was associated with older age at the time of surgery, and longer time from seizure onset to surgery. Seizure free outcome was not associated with abnormal neurological exam, developmental delay, seizure type, presence of lateralizing/localizing features on scalp EEG, lesional MRI, interictal SPECT/PET, performance of subdural implantation, completeness of resection or pathology. Conclusions: Our findings demonstrate that resective surgery in the FCD substrate is successful even in very young children. However, completeness of resection which is a reliable predictor of outcome in older patients cannot be applied to young infants.

\subsection{9 \\ BROAD BILATERAL INTRACRANIAL ELECTRODES PLACEMENT CAN SAFELY IDENTIFY SEIZURE FOCUS IN CHILDREN WITH INTRACTABLE EPILEPSY}

Po Ching Chen ${ }^{1}$, D. Stephanie ${ }^{1}$, M. Korostenskaja ${ }^{1}$, J. Baumgartner ${ }^{2,3}$ and K. Lee ${ }^{3}{ }^{1}$ Functional Brain Mapping and Brain Computer Interface Lab, Comprehensive Pediatric Epilepsy Center, Center for Pediatric Research and Outcomes, Florida Hospital for Children, Orlando, FL; ${ }^{2}$ Neurosurgeon for Kids, Florida Hospital for Children, Orlando, FL and ${ }^{3}$ Comprehensive Pediatric Epilepsy Center, Florida Hospital for Children, Orlando, FL)

Rationale: Surgical resection is considered the first line option for treating intractable epilepsy. Surgical treatment is usually discouraged, when non-invasive approaches cannot provide adequate lateralization. Bilateral intracranial EEG (iEEG) survey has been proven to be a safe and effective option for addressing such cases. Conventionally, bilateral intracranial electrode placement is implemented through small bilateral burr holes or vertex craniotomy. However, it provides limited access and constrains the electrode coverage for revealing the possible seizure focus. An additional unilateral craniotomy is usually required to place more electrodes for precise localization after the initial lateralization survey. This increases the number of surgeries and the duration of $\mathrm{iEEG}$ monitoring. We present an alternative approach using bilateral craniotomy for electrode placement. This approach provides broad access to both hemispheres with an opportunity for extensive intracranial electrode coverage facilitating precise localization of seizure focus and functional mapping.

Methods: We retrospectively reviewed 10 pediatric patients with drug-resistant epilepsy who underwent bilateral craniotomy for iEEG monitoring. Because all non-invasive diagnostic methods, such as various neuroimaging modalities (MRI, PET and SPECT) and scalp video electroencephalography, did not effectively lateralize seizure focus, a bilateral survey was proposed. A bilateral craniotomy with reverse question mark incision was performed to provide access for subdural electrode placement. The wide incision allowed comprehensive subdural grid and strip coverage on both hemispheres (figure 1).

Results: The number of intracranial electrodes placed in each patient ranged from 152 to $224(171 \pm 32$ - mean \pm std). The duration of iEEG monitoring ranged from 3 to 30 days with an average $11 \pm 8.5$ (std) days. Seizure foci were found in 8 patients who underwent subsequent resective surgery. Seizure focus was not localized in the other 2 patients through the initial bilateral iEEG monitoring. These two patients subsequently underwent a complete corpus callosotmy (CC) followed by additional iEEG monitoring. Resective surgery was performed on these two patients as the post-callosotomy iEEG successfully lateralized and localized the ictal onset zone. One patient developed infection requiring IV antibiotics.

Conclusions: Bilateral craniotomy provides wide access for comprehensive bilateral subdural electrode implantation. Our 
experience with 10 pediatric patients suggests that this procedure can be carried out safely and effectively for localization of focal seizure origin with prolonged iEEG monitoring. This approach could broaden the availability of epilepsy surgery and might improve overall surgical outcomes.

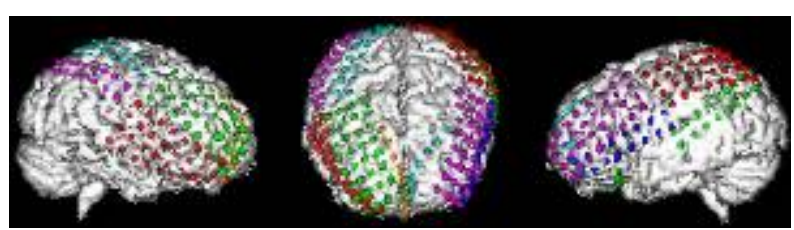

Figure 1. 3D rendering of CT images co-registered with MRI image illustrating the bilateral intracranial subdural electrode placement over the cortex.

\subsection{0 \\ THE IMPORTANCE OF INSULAR INVOLVEMENT IN EPILEPSY SURGERY}

Dave F. Clarke, D. R. Monsivais, S. Jean, K. Keough, K. L. Tindall and M. R. Lee (Dell Children's Medical Center, Austin, TX)

Rationale: Controlling seizures produced by insular cortex epilepsy can present a perplexing clinical challenge due to the ambiguity of seizure symptoms and the complex technical requirements for a definitive diagnosis. Insular involvement on subtraction SPECTS are often overshadowed by the cortical element. Failure to accurately isolate the true seizure origin may explain why some epilepsy surgeries fail to resolve seizures. We studied the utility of insular depth placement in better defining insular involvement in ictal onset or evolution.

Methods: We retrospectively reviewed our database from 2010 to present to determine which patients had insular depths placed. High resolution MRI was then used post-operatively to confirm accurate electrode placement. Once a seizure focus had been confirmed in the insular cortex after a period of 2-8 days, patients underwent insular removal or partial insular resection.

Results: Of the 74 surgical resections performed 15 had longitudinal 8 channel depths placed traversing the anterior-posterior length of the insular on the side of seizure onset using an MRI-stereotactic frameguided system. Their ages at the time of surgery ranged from 4 years 3 months to 20 years one month. Seizure semiology varied greatly. In 10 of 15 patients the insular was involved at seizure onset and was partially removed (anterior or posterior insular determined by the electrodes involved) or removed in totality from the involved hemisphere. The anterior insular was involved at seizure onset in cases where the seizure involved the anterior to mid-frontal region but those with posterior frontal, parietal or more extensive involvement, the posterior or entire insular was involved at onset. Patients were followed post-operatively for up to 23 weeks for the development of seizures. All of the patients had seizure improvement and 7/10 was seizure free during their follow up period (3-23 weeks). Conclusions: Insular involvement should be entertained in cases where seizure onset is not easily identified. Longitudinal depth placement allows for sampling of the entire length of the insular cortex. This may be instrumental in determining the full extent of involvement of ictal onset, hence allowing for resection of epileptic focus in its entirety.

\subsection{1 \\ THE UTILIZATIONS OF CORPUS CALLOSTOMY IN PEDIATRIC EPILEPSY SURGERY}

V. Baute, S. Strickland and Y. Park (Georgia Health Sciences Medical Center, Evans, GA)

Rationale: Objective: The utilization of corpus callosotomy (CC) has evolved from one of conventional palliative treatment to one of adjunctive therapy before further surgical resection and more importantly as a lateralizing tool in a select group of patients where presurgical evaluation has been non- localizing. $\mathrm{CC}$ has been well accepted as a surgical option for atonic seizures commonly associated with Lennox Gastaut Syndrome. The object of this study is to review the varying roles of $\mathrm{CC}$ and its impact on seizure outcome in pediatric patients with intractable epilepsy.

Methods: Methods: The authors retrospectively reviewed the Pediatric Epilepsy Surgery database of 63 patients who underwent CC from 1990-2010. Chart review established demographic data, race, age of surgery, EEG findings, MRI findings, concomitant syndrome or presence of infantile spasm, and pathology when available. Inclusion criteria for patients eligible for $\mathrm{CC}$ were multifocal EEG pattern or bisynchronous spike and slow wave activity with poorly lateralized scalp EEG. Outcome measures were determined by parental reporting. Post resection seizure outcomes were determined by Engel criteria.

Results: Results: Of the 63 patients who underwent CC, 37 patients had conventional palliative surgery for atonic seizures associated with Lennox Gastaut Syndrome. A reduction of greater or equal to 75 percent in atonic seizures occurred in 75 percent of patients in this cohort. Twenty patients had nonconventional $\mathrm{CC}: 7$ with adjunctive $\mathrm{CC}$ at the time of a cortical resection and 13 with $\mathrm{CC}$ for nonlocalized intractable complex partial and/or generalized tonic clonic seizures. Not surprisingly, given the baseline refractory nature of this population, the outcome of this combined cohort remained poor at 12 months (Engel class III or IV). Six patients underwent CC with the expectation of forced lateralization for focal resection. Of these 6 patients, subsequent grid placement with focal resection yielded 67 percent class I Engel outcome at 12 month follow up. Despite the lateralized findings on EEG, MRI findings in this cohort were nonlateralizing, including 4 normal MRIs, 1 lissencephaly, and 1 Dandy Walker syndrome.

Conclusions: Conclusion: Medical management often fails in this intractable group of pediatric epilepsy patients. Presurgical evaluation may prove challenging in attempting to localize an epileptic focus. This review illustrates a broad use of CC in surgical planning. We discuss $\mathrm{CC}$ as an additive benefit in presurgical evaluation for hemispheric lateralization for management of intractable epilepsy in children. Although most patients continue to have seizures following $\mathrm{CC}$, improvement in progression of an intractable disease as well as the possibility of subsequent resection of an epileptic focus proves worthwhile. In the absence of other lateralizing findings, CC should be considered as an additional therapeutic tool.

\section{Behavior/Neuropsychology/Language: Adult}

\subsection{2}

\section{WTTHDRAWN}

\subsection{3 \\ COGNITIVE OUTCOME FOLLOWING FRONTAL LOBECTOMY FOR TREATMENT OF INTRACTABLE EPILEPSY IN ADULTS \\ Robyn M. Busch ${ }^{1,2}$, D. Floden ${ }^{3,2}$, R. Sarkis ${ }^{1}$, C. K. Kenney ${ }^{2}$, L. E. Jehi $^{1}$, P. Ruggieri ${ }^{4}$, R. I. Naugle ${ }^{2,1}$, W. Bingaman ${ }^{1}$ and I. M. Najm ${ }^{1}$ $\left({ }^{1}\right.$ Epilepsy Center, Cleveland Clinic, Cleveland, OH; ${ }^{2}$ Psychiatry \& Psychology, Cleveland Clinic, Cleveland, $\mathrm{OH} ;{ }^{3}$ Center for Neurological Restoration, Cleveland Clinic, Cleveland, $\mathrm{OH}$ and ${ }^{4}$ Diagnostic Radiology, Cleveland Clinic, Cleveland, OH)}

Rationale: Frontal lobectomy has been associated with declines in executive functioning, speed/attention, language, and motor coordination in adults with frontal lobe epilepsy. However, most prior studies have been conducted on small samples with limited cognitive batteries. The goal of the current study is to characterize cognitive outcome in a large sample of adult patients who have 
undergone frontal lobectomies for the treatment of medically intractable epilepsy using objective methods to characterize meaningful cognitive change.

Methods: Adult patients who underwent frontal lobe resections at Cleveland Clinic between 1989 and 2011 were included if they had pre and postoperative neuropsychological data available for review. A total of 96 patients were identified (right-sided resection $=49$, leftsided resection $=47$; male $=52$, female $=44$ ) ranging in age from $16-59$ $(\mathrm{M}=29.3)$. Mean age at seizure onset was $13.12(\mathrm{SD}=9.85)$ and mean epilepsy duration was 16.32 years (10.76). Reliable change indices $(90 \% \mathrm{CI})$, developed on nonsurgical epilepsy patients, were used to characterize cognitive change following epilepsy surgery. Chi-square analyses were then used to 1) determine whether the base rate of cognitive change following frontal lobe resection was greater than would be expected by chance alone (i.e., $5 \%$ improvement, $5 \%$ decline) and 2) examine differences in cognitive outcome as a function of surgical side.

Results: A larger proportion of patients demonstrated declines in cognitive functioning than expected by chance alone. This was most apparent on measures of intelligence, problem-solving, and confrontation naming. However, there were also more patients than expected with meaningful postoperative improvements on these measures. In addition, a large proportion of patients demonstrated improvements in fine manual dexterity and visuomotor processing speed following surgery. Patients who underwent surgery in the nondominant hemisphere were more likely to show declines in complex visuomotor sequencing than those who had dominant resections. Although they did not reach statistical significance $(p=.06-.08)$, a higher proportion of patients who underwent dominant surgeries showed declines in auditory immediate and delayed memory and confrontation naming.

Conclusions: This study presents cognitive outcome data on a large sample of patients who underwent frontal lobectomies for treatment of epilepsy. Although surgery resulted in cognitive declines in some patients, a substantial portion of patients demonstrated cognitive improvements following surgery. Preliminary data on the role of site/extent of surgical resection and seizure outcome are presented, and results are discussed in the context of the existing literature.

\subsection{4}

\section{AN ABBREVIATED SUPPORT GROUP THERAPY FOR PSYCHOGENIC NONEPILEPTIC EVENTS: A NEUROLOGIST-INITATED PROGRAM IN AN EPILEPSY CENTER}

Atul Maheshwari ${ }^{1}$, G. Trolley ${ }^{1}$, R. Franks ${ }^{2}$ and D. K. Chen ${ }^{1,2}\left({ }^{1}\right.$ Baylor College of Medicine, Houston, TX and ${ }^{2}$ Michael E. Debakey Veteran Affairs Medical Center, Houston, TX)

Rationale: After the diagnosis of psychogenic non-epileptic events (PNEE) in the epilepsy monitoring unit (EMU), many patients continue to experience significant emotional exasperation from their attacks, and return visits to the emergency room (ER) are not uncommon. Formal psychotherapies and support programs dedicated to PNEE frequently demand significant dedication from both the patients and providers. Here, we aim to evaluate a novel intervention, initiated by the treating neurologist, with the primary goal of effecting measurable changes in the quality of life of patients with PNEE.

Methods: Patients were randomized to the control or treatment groups after video-EEG (VEEG) confirmation of PNEE at the EMU of Michael E. DeBakey VA Medical Center, Houston, Texas. Both groups received the standard of care disclosure/explanation of their diagnosis, and then completed a pre-discharge questionnaire. The control group completed a post-discharge questionnaire during a standard follow-up clinic visit after 3 months. The treatment group underwent 3 successive monthly therapy sessions led by a nurse practitioner. The first session entailed a psycho-educational seminar dedicated to enhancing the understanding of PNEE. Two subsequent support group sessions focused on specific coping strategies. Group members were encouraged to openly share their experiences. After the third therapy session, patients were given a post-discharge questionnaire analogous to the control group's post-discharge questionnaire. All patients who were unable to successfully complete the follow-up questionnaire were excluded from analysis. Comparisons were then made between the control and treatment groups using the Student's T-test and the Chi-Squared Test as appropriate.

Results: Out of 35 patients randomized into our study, 16 patients were assigned to the control group and 19 patients were assigned to the treatment group. After excluding those lost to follow-up, statistical analysis was performed on 9 control and 14 treatment group patients. Patients who completed the prescribed intervention showed significantly more affirmative endorsement of the following statements: "My attacks do not really bother me or affect my life that much anymore" $(\mathrm{p}<.001)$, and "I have some control over my attacks" $(\mathrm{p}=0.003)$. In addition, patients in the treatment group had a significantly lower post-EMU discharge visit rate to the ER $(7.14 \%$ vs $22.22 \%$ in treatment and control group, respectively, $\mathrm{p}=0.018$ ).

Conclusions: We show that among patients with VEEG confirmed PNEE, a novel abbreviated and neurologist-initiated support group program can be effective in improving the quality of life of patients with PNEE. These improvements were further supported by the observation of a significantly reduced ER visit rate in the treatment group. Future studies with greater sample size and longer follow-up can help determine the long-term efficacy of this intervention.

\section{"My attacks do not really bother me or affect my life that much any more"}

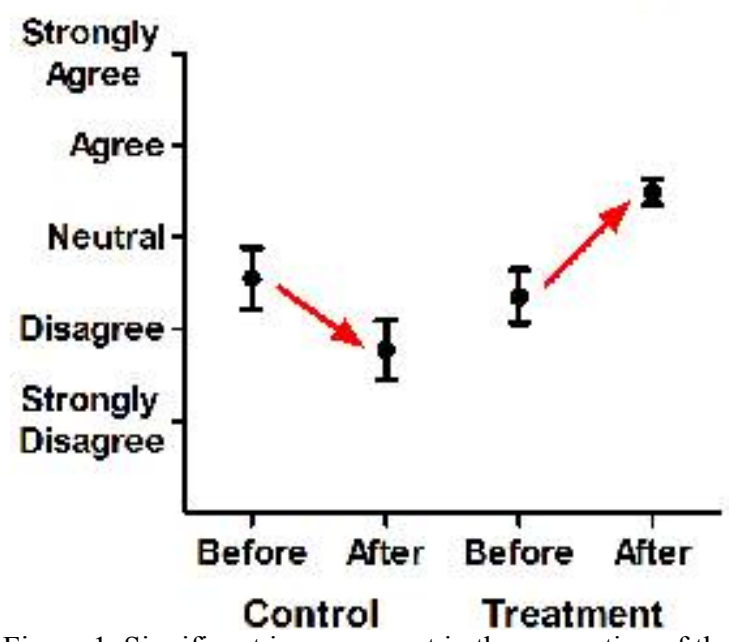

Figure 1. Significant improvement in the perception of the impact of PNES with support group therapy (showing mean with SEM, $\mathrm{p}<0.001)$. 


\section{"I have some control over my attacks"}

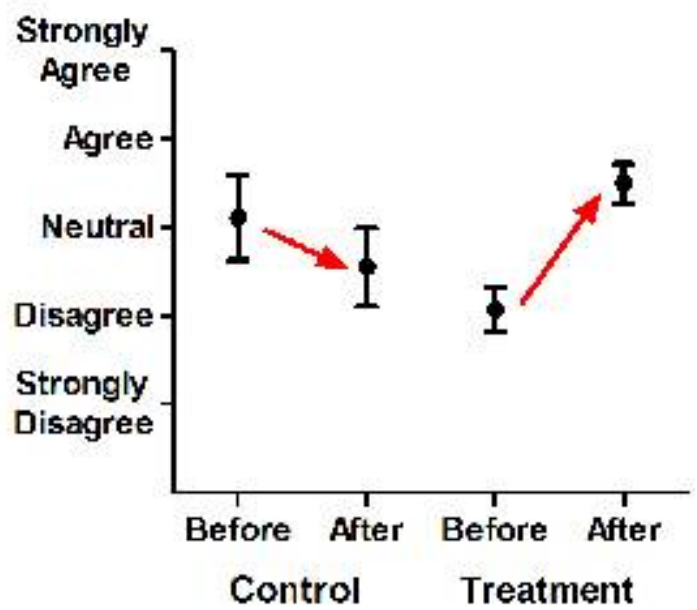

Figure 2. Significant improvement in the perception of control with support group therapy (showing mean with SEM, $\mathrm{p}=0.003$ ).

\subsection{5}

THE IMPACT OF HABITUAL SEIZURES ON AUTOBIOGRAPHIC MEMORY FUNCTIONING IN TEMPORAL LOBE EPILEPSY

Genevieve Rayner $^{1,3}$, J. M. Wrench ${ }^{1}$, G. D. Jackson ${ }^{3,4}$ and S. J. Wilson ${ }^{1,2}\left({ }^{1}\right.$ Melbourne School of Psychological Sciences, The University of Melbourne, Melbourne, VIC, Australia;

${ }^{2}$ Comprehensive Epilepsy Program, Austin Health, Melbourne, VIC, Australia; ${ }^{3}$ Brain Research Institute, Florey Neuroscience Institutes, The University of Melbourne, Melbourne, VIC, Australia and ${ }^{4}$ Department of Medicine, The University of Melbourne, Melbourne, VIC, Australia)

Rationale: Previous research suggests that patients with temporal lobe epilepsy [TLE] have difficulty recollecting personal life events i.e., autobiographic memories. These studies have largely focused on localizing different components of this deficit to various regions of the left and right temporal lobes, with limited consideration of the effects of seizures. Our aim was to investigate the rôle of epileptological factors in determining the nature of the autobiographic memory impairment commonly seen in people with TLE.

Methods: We prospectively assessed 23 patients with medicallyrefractory TLE, recruited from the Comprehensive Epilepsy Program of Austin Health, Melbourne Australia. Their performances on widely-used measures of autobiographic memory were compared to those of a sociodemographically-matched healthy control sample ( $\mathrm{n}=$ $37 ; \mathrm{N}=60$ ). Epilepsy variables of interest included International League Against Epilepsy seizure classification, duration of epilepsy, age at seizure onset, seizure frequency, surgical candidacy, presence of a lesion on neuroimaging, and pharmacotherapy. Participants also completed a screen of psychological functioning, including mood, as part of a larger behavioral study. Controls were significantly older than patients $(\mathrm{p}=.016)$, but otherwise did not differ on demographic variables $(\mathrm{p}>.05)$.

Results: When asked to recall incidences from their life that were precisely located in place and time (episodic autobiographic memory), patients with TLE recollected significantly less than controls $(\mathrm{p}=.023)$, and produced more responses that summarized multiple incidences over time $(\mathrm{p}=.035)$. Poor episodic autobiographic recall in patients was related to younger age at onset of epilepsy (especially $<20 \mathrm{yrs}, \mathrm{r}=.58 ; \mathrm{p}<.001$ ) and longer duration of epilepsy $(\mathrm{r}=.38)$. These two factors were correlated $(\mathrm{r}=.46 ; \mathrm{p}=$ .03 ) and represent the chronicity of illness. There was also a trend for poor episodic recall to relate to stronger endorsement of current mood symptoms (anxiety, $\mathrm{r}=.38$; depression, $\mathrm{r}=.33$ ). In contrast, impoverished semantic autobiographic recall (knowledge of names and other personally relevant 'facts') was associated with younger age at testing $(\mathrm{r}=.46 ; \mathrm{p}<.01)$.

Conclusions: This study provides initial evidence that habitual seizures contribute to the poor recall of autobiographic events in people with TLE, in addition to temporal lobe pathology. Our findings indicate that chronic epilepsy can undermine the episodic element of autobiographic memory recall, but may not effect semantic knowledge associated with personal life events. The results are consistent with psychiatric research suggesting that lowered mood can impair episodic autobiographic memory. We propose that habitual seizures fundamentally disturb the patients' ability to vividly recall personal life events by interrupting the encoding of autobiographic memories, as well as limiting the breadth of coherent life experiences from which autobiographic memories are drawn.

\subsection{6 \\ RISK-TAKING BEHAVIOUR IN JUVENILE MYOCLONIC EPILEPSY \\ Britta Wandschneider ${ }^{1}$, M. Centeno ${ }^{1}$, C. Vollmar ${ }^{1,3}, \mathrm{~J}_{\text {. }}$

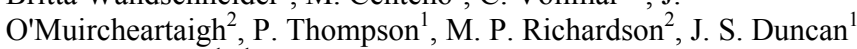 and M. J. Koepp ${ }^{1}$ ( ${ }^{1}$ Department of Clinical and Experimental Epilepsy, UCL Institute of Neurology, London, United Kingdom; ${ }^{2}$ Department of Clinical Neuroscience, King's College London, London, United Kingdom and ${ }^{3}$ Department of Neurology, University of Munich, Munich, Germany)}

Rationale: Patients with Juvenile Myoclonic Epilepsy (JME) appear to be less able to learn from previous experience. They often present with risk-taking and impulsive behaviour, suggestive of frontal lobe dysfunction. This is in keeping with findings of impaired working memory and executive functions in JME (Hommet et al. Neurosci Biobehav Rev.2006;30(1):85-96). The Iowa Gambling Task (IGT) simulates real-life decisions and is a tool to measure impulsive and risky decision-making (Bechara et al. Brain 2000;123:2189-

2202).This study investigated impulsivity in JME patients and healthy controls (HC) using the IGT to characterize decision making behaviour.

Methods: $21 \mathrm{JME}$ patients and $11 \mathrm{HC}$, comparable for gender and verbal IQ, were assessed with a computerized version of the IGT. Patients were older than HC (mean age 29 +/- 4(SD) years vs. 35 +/10). Subjects chose from four decks of cards (A to D) with a total of 100 cards split into five blocks of 20. Deck A and B were indicative of high risk, $\mathrm{C}$ and $\mathrm{D}$ of low risk behaviour. Advantageous minus disadvantageous choices $(\mathrm{C}+\mathrm{D}-[\mathrm{A}+\mathrm{B}])$ were established for the total number of cards, as well as for each block of 20 cards to analyze changes in risk taking behaviour over time.

Results: No difference in the total index of advantageous choices (JME: mean score 14.9 +/- 29.3 (SD); HC: 18.4 +/- 27.0; $p=.744$ ) was observed. Both groups learned throughout the task (last versus first block: JME: mean score difference 8.1 +/- 9.2 (SD), $\mathrm{p}=.001$; HC: $10.9+/-11.3, \mathrm{p}=.009)$. Post-hoc subgroup analysis revealed that in the seizure free group, the majority of patients improved throughout the task, whereas only half of the patients still experiencing seizures reduced their risk-taking behaviour over time (seizure-free patients: 12 learner/ 2 non-learner vs. 3/ 4 ; $\mathrm{p}=.040$ ).

Conclusions: This study provides evidence for impaired learning from experience in JME patients who still suffer from seizures. This suggests that inter-ictal network dysfunction might impair the ability to learn, while control of seizures is associated with improved functionality. 


\subsection{7}

FACIAL EMOTION RECOGNITION OF ANGER AFTER ANTEROMEDIAL TEMPORAL LOBECTOMY IN PATIENTS WITH EPILEPSY

Satsuki Watanabe ${ }^{1}$, M. Yamada ${ }^{2}$, T. Maehara ${ }^{3}$, M. Matsuura $^{4}$ and E. Matsushima ${ }^{1}{ }^{1}$ Section of Liaison Psychiatry and Palliative Medicine, Tokyo Medical and Dental University, Bunkyo-ku, Japan; ${ }^{2}$ Molecular Imaging Center, National Institute of Radiological Sciences, Chibashi, Japan; ${ }^{3}$ Department of Neurosurgery, Tokyo Medical and Dental University, Bunkyo-ku, Japan and ${ }^{4}$ Section of Life Sciences and Biofunctional Informatics, Tokyo Medical and Dental University, Bunkyo-ku, Japan)

Rationale: Recent lesion and neuroimaging studies have demonstrated the important role of the amygdale and the anteromedial temporal lobe in emotion recognition. In most of the studies, subjects were asked to select a verbal label that best described an emotion shown in each picture. This type of tasks might be insensitive to subtle deficits. Moreover, subjects were given unlimited time exposure to pictures in many studies. Therefore, we applied the rapid facial emotion recognition task which use twoalternative forced choice with time limited exposure to stimuli. In the current study, we analyzed the data using the signal detection theory (SDT) to calculate the sensitivity of anger detection in patients with temporal lobe epilepsy (TLE) who underwent the anteromedial temporal lobectomy (ATL).

Methods: Twenty-one patients (left-ATL: 11, right-ATL: 10) and 20 healthy control subjects (CTRL) participated in this study. Six faces each of anger and happiness were morphed to create single faces expressing varying intensities of anger (strong: $75 \%$, moderate: $50 \%$, weak: $25 \%$ ). These morphed faces were used as target stimuli. The procedure consisted of five blocks. In one block, the set of 96 stimuli were presented in random order. An individual face was presented for $67 \mathrm{~ms}$ as a target stimulus and immediately masked by a neutral face, followed by a response selection screen. The participants were required to make a judgment on whether the target stimulus expressed anger or happiness by clicking either of the two buttons. Hits ("angry" responses to angry faces) and false alarms ("angry" responses to happy faces) were summed for each of the three angry faces. The sensitivity $\left[\mathrm{d}^{\prime}=\mathrm{Z}\right.$ (hit)-Z(false alarm)] for anger detection was analyzed with SDT. This study was approved by the Ethics Committee of Tokyo Medical and Dental University.

Results: A three (GROUP: right-ATL vs. left-ATL vs. CTRL) $\times$ three (STIMULUS: strong vs. moderate vs. weak) repeated-measures ANOVA examining the sensitivity of anger detection showed a significant main effect of STIMULUS $(\mathrm{p}<0.01)$ and GROUP $(\mathrm{p}<$ $0.01)$ and interaction between STIMULUS and GROUP $(\mathrm{p}<0.01)$. Post-hoc tests revealed that the sensitivity of anger detection in leftATL group was significantly lower than in CTRL group for strong and moderate anger.

Conclusions: The current study revealed that left-ATL group had difficulty in detecting anger in facial expressions. The left anteromedial temporal lobe could play an important role for anger detection in accordance with previous neuroimaging studies, which indicated the increased activation of the left amygdale when processing angry facial expressions. Applying SDT to the facial emotion recognition task was useful to clarify the subtle impairment which might be overlooked in previous lesion studies.

\subsection{8}

\section{LATERALITY EFFECTS OF TEMPORAL LOBECTOMY ON} QUALITY OF LIFE AND DEPRESSION

M. R. Meager ${ }^{1}$, Gregory P. Lee ${ }^{1}$, Y. D. Park ${ }^{1}$, A. M. Murro ${ }^{1}$, S. M. Strickland $^{1}$, D. Moore-Hill ${ }^{1}$ and C. A. Giller ${ }^{2}\left({ }^{1}\right.$ Neurology, Medical College of Georgia, Georgia Health Sciences University, Augusta, GA and ${ }^{2}$ Neurosurgery, Medical College of Georgia, Georgia Health Sciences University, Augusta, GA)
Rationale: Quality of life (QOL) after epilepsy surgery can vary considerably depending on many factors (e.g., degree of seizure relief, cognitive decline, mood, seizure worry, social support). In previous studies, left temporal lobe epilepsy patients endorse higher levels of anxiety and rate QOL lower, while right TLE patients endorse lower anxiety and rate QOL higher. The current study further investigated these lateralized differences in QOL and depression in epilepsy surgery patients by examining specific QOL subdomains, viz., Overall QOL, Role Functioning, Cognition, and severity of depression before and after temporal lobectomy.

Methods: Sixty-nine temporal lobectomy patients $(\mathrm{LTL}=40$; RTL $=$ 29) underwent baseline and post-surgical neuropsychological assessment at the Medical College of Georgia with a mean age of 34 years $(\mathrm{SD}=12.5) ; 12$ years of education $(\mathrm{SD}=2)$; seizure duration of 17.5 years $(\mathrm{SD}=12.6)$; and a FSIQ of $87(\mathrm{SD}=14.6)$. Patients were administered the WAIS-III to assess intelligence; self-ratings on the QOLIE-89; and the Beck Depression Inventory (BDI) as part of a larger neuropsychological battery, both pre- (Pre TL) and posttemporal lobectomy (Post TL). There were no significant differences between the LTL and RTL groups with regard to age, education, FSIQ, seizure duration, or surgical outcome.

Results: Pre TL analyses revealed no significant differences between LTLs and RTLs on the BDI or any of the QOLIE-89 scales examined. In contrast, Post TL analyses revealed significant differences between LTLs and RTLs on the BDI $(\mathrm{p}=.003)$, which slightly worsened in LTLs and greatly improved in RTLs, and Overall Quality of Life $(p=.009)$, which stayed the same in LTLs and improved in RTLs. In addition, there were slight improvements in Work/Driving/Social Function in both LTLs and RTLs $(\mathrm{p}=.001)$. QOLIE Memory scores did not change in LTLs and improved in RTLs $(\mathrm{p}=.007)$. There were no significant differences in Attention/Concentration or Language although there were slight improvements in both groups. With regard to mood, there were no significant differences between groups on the BDI before surgery ( $p$ $=.99$ ), but after surgery significantly more LTL patients had elevated BDI scores compared to RTLs $(\mathrm{p}=.001)$.

Conclusions: LTL patients had significantly lower self-ratings on important quality of life domains (viz., overall QOL, work/driving/social function, and memory) compared with RTL patients following surgery. Examination of the performances of each group pre- to post-surgery showed that LTLs generally did not change on their QOL ratings while RTLs improved across all QOL domains examined. As previous studies have suggested, it is possible the presence of depression among LTLs are driving their lower QOL ratings.

\subsection{9 \\ TREATMENT STRATEGIES FOR PSYCHOGENIC NON- EPILEPTIC SEIZURES: A PILOT STUDY}

G. Ganesh, D. Drane, D. Loring, D. Teagarden, K. Kress and Suzette M. Laroche (Emory University, Decatur, GA)

Rationale: Psychogenic non-epileptic seizures (PNES) are typically diagnosed in long-term video EEG monitoring (LTVM) units. The practice of delivering the diagnosis and arranging follow-up for PNES varies among LTVM units and no standard treatment approach exists. The contribution of an inpatient psychiatry consultation also varies across centers, and patients who do not understand their diagnosis often return to emergency departments or seek second opinions, incurring high healthcare costs. We compared event frequency and behavioral outcomes following three different treatment approaches with the goal of identifying the best treatment option and developing a standardized approach to PNES following diagnosis.

Methods: Patients admitted to the LTVM unit for diagnostic evaluation of spells of unclear etiology at Emory University Hospital from July 2011 to May 2012 were consented for the study. All subjects completed a baseline questionnaire as well as the Beck 
Depression Index (BDI) and Quality of Life in Epilepsy-10 (QOLIE10) scale. Patients diagnosed with PNES by video EEG were randomized to one of three treatment groups: Group A received an unscripted diagnosis delivered by the epilepsy attending and no further management; Group B received a scripted discussion of the diagnosis, a formal psychiatry consultation, and an educational handout; and Group C underwent the same treatment as Group B but also received weekly phone follow-up. All groups were called at 8 weeks to determine event frequency, BDI and QOLIE-10 score. Results: A total of 75 patients were consented for the study of which 30 were randomized and completed follow-up with the following distribution: Group $\mathrm{A}=10$, Group $\mathrm{B}=9$, Group $\mathrm{C}=11$. There were no significant differences in baseline characteristics among the groups (Table 1). There was a significant decrease in event frequency at 8 week follow-up for patients in Group B and C ( $\mathrm{p}=0.011,0.004$, respectively) (Table 2). QOLIE 10 scores improved in all patient groups, particularly in group B and $C(p=0.05,0.001,0.001)$. Patients in Group B and C also endorsed fewer social and work limitations and were less afraid of having further events. There was a significant decline in BDI score seen in group C only $(p=0.004)$. Although not statistically significant, Group $\mathrm{C}$ was also more likely to seek follow-up from a mental health provider and believe their events would stop, while they were less likely to seek a second opinion.

Conclusions: A defined PNES treatment strategy that includes inpatient psychiatry consultation, scripted discussion of the diagnosis, and weekly phone contact may impact event frequency, quality of life, depression, and appropriate healthcare utilization. Further studies evaluating PNES treatment algorithms are warranted.

Baseline Characteristics For All Groups
\begin{tabular}{|c|cc|c|}
\hline & Group A & Group B & Group C $\quad$ p value between groups 1 \\
& $\mathrm{n}=10$ & $\mathrm{n}=9$ & $\mathrm{n}=11$ \\
Average Age (yrs.) & 44.0 & 37.2 & 35.7 \\
Male/Female & $2 / 8$ & $3 / 6$ & $1 / 10$ \\
Avg. Yrs. of Education & 10.0 & 9.0 & 13.7 \\
\hline Avg. Age at Event Onset (yrs.) & 35.7 & 27.4 & 32.3 \\
\hline Average \# of Current AEDs & 2.3 & 1.1 & 1.4 \\
\hline History of Abuse2 & 5 & 3 & 4 \\
\hline Mood disturbance3 & 10 & 7 & 11 \\
\hline History of Psychiatric Treatment & 6 & 5 & 3 \\
Avg. Beck Depression Index Scorc & 23.5 & 22.4 & 19.1 \\
Avg. QOL.IF-10 Scorc & 32.1 & 32.1 & 30.1 \\
\hline Event Frequency4 & 3.0 & 3.0 & 2.8 \\
\hline
\end{tabular}

1 Listed if $\mathrm{p}$ value $<0.05$

2Sexual, physical, or emotional

3Depression, anxiety, or mood swings

4Number of events in last month

Outcomes For All Groups at 8 Weeks

\begin{tabular}{|c|c|c|c|c|c|c|}
\hline & Group A & & Group & & Gruep C & \\
\hline & $\mathrm{n}=10$ & pralue* & $\mathrm{n}=9$ & pvalue & $\mathrm{a}=11$ & p value \\
\hline \multicolumn{7}{|c|}{ Reck Depression Index Score } \\
\hline Baseline & 23.5 & & 22.4 & & 19.1 & \\
\hline 8 Wecks & 22.5 & $\mathrm{Ns}$ & 18.7 & vs & 11.6 & 0.004 \\
\hline \multicolumn{7}{|l|}{ QOH.IE 10} \\
\hline Buseline & 32.1 & & 32.1 & & 30.1 & \\
\hline 8 Weeks & 27.6 & 0.05 & 19.0 & 0.001 & 22.3 & 0.001 \\
\hline \multicolumn{7}{|c|}{ QOLIE $14+5 * 1$} \\
\hline Baseline & 6.6 & & 7.6 & & 6.2 & \\
\hline \multicolumn{6}{|c|}{ QOI.IT: * * *2 } & 0.012 \\
\hline Baseline & 1.7 & & 1.9 & & 1.6 & \\
\hline 8 Weeks & 1.5 & $\mathrm{~ns}$ & 3.1 & 0.002 & 3.0 & 0.0001 \\
\hline \multicolumn{7}{|c|}{ QOLLEE $210^{73}$} \\
\hline Buseline & 3.9 & & 3.3 & & 2.6 & \\
\hline 8 Weeks & 3.8 & is & 2.9 & NS & 2.6 & NS \\
\hline \multicolumn{7}{|c|}{ Event Frequency } \\
\hline Bascline & 30 & & 3.0 & & 2.8 & \\
\hline 8 Wecks & 2.4 & Ns & 1.8 & 0.011 & 1.8 & 0.004 \\
\hline
\end{tabular}

*p value listed only if less than 0.05 for any group.

*1QOLIE-10 Questions 4 \& 5 address work and social limitations. A higher score is worse.

*2QOLIE-10 Question 9 asks how afraid are you of having a seizure in next 4 weeks. A higher score is better.

*3QOLIE-10 Question 10 asks how your quality of life has been over the past 4 weeks. A higher score is worse.

\subsection{0 \\ EDUCATION LEVELS AFFECT SCORES AND LATERALIZATION ABILITY OF NEUROPSYCHOLOGICAL TESTING IN SPANISH SPEAKING PATIENTS WITH INTRACTABLE EPILEPSY S. J. Shaw ${ }^{1,2}$, D. Millett ${ }^{1,2}$, C. Campelo Smith ${ }^{1}$, V. Chavarria ${ }^{1}$, B. Kolberg ${ }^{1}$ and Jason Smith ${ }^{1}\left({ }^{1}\right.$ Rancho Los Amigos National Rehabilitation Center, Downey, CA and ${ }^{2}$ Neurology, University of Southern California, Los Angeles, CA)}

Rationale: Neuropsychological testing assists in lateralization of seizure focus in presurgical workup of English-speaking medically intractable epilepsy (IE) patients. However, studies on a Spanishspeaker battery, Neuropsychological Screening Battery for Hispanics (NeSBHis), are limited and have shown differing results on lateralization ability (Barr et al 2009; Lancman et al 2012). These studies did not account for educational differences, but Pontón et al (1996) demonstrated the influence of educational level (Ed) on NeSBHIS scores. We postulated that Ed differences may suppress the NeSBHIS' lateralizing properties and that Ed effects would hold in a unique, southwestern USA, Hispanic Spanish-speaking sample of IE patients.

Methods: Retrospective analysis was conducted on 76 Spanish speaking subjects undergoing presurgical video-EEG telemetry and neuropsychological evaluation including NeSHBIS. Subjects were classified into Low Education (LE) ( $\leq 6$ yrs schooling, $n=33$ ) or High Education (HE) $(>6$ yrs, $n=43)$, and Left $(\mathrm{LH}, \mathrm{n}=25)$ or Right Hemisphere $(\mathrm{RH}, \mathrm{n}=27)$ seizure onset. Bilateral or inconclusive seizure lateralization was excluded from lateralization analyses. Comparisons of NeSHBIS scores and seizure lateralization between LE and HE were made with t-tests.

Results: Mean age was $35.9 \pm 10.1$, median epilepsy onset age was 9 years, and mean education was $8.1 \pm 3.5$ years.

There were no significant differences in age, gender, seizure lateralization, or epilepsy duration between LE vs HE. LE subjects performed significantly worse than $\mathrm{HE}$ on Ravens Progressive Matrices, $\mathrm{p}<.001$; Block Design, $\mathrm{p}=.02$; Digit Symbol, $\mathrm{p}<.001$; Digit Span, $\mathrm{p}=.004$; Modified Boston Naming Test, $\mathrm{p}<.001$; Controlled Oral Word Association Test, $\mathrm{p}=.006$; Color Trails $1, \mathrm{p}=$ .002; Color Trails 2, $\mathrm{p}=.009$; Rey Complex Figure (RCFT) Copy, $\mathrm{p}$ $=.007$; RCFT Delay, $\mathrm{p}=.045 ;$ WHO AVLT 1, $\mathrm{p}=.006 ; \mathrm{WHO}$ AVLT 5, $\mathrm{p}=.031$; and WHO AVLT 8, $\mathrm{p}=.013$.

When all subjects were analyzed, no differences were seen between $\mathrm{LH}$ and RH onset patient scores. LH and RH did not differ in education years, $\mathrm{p}=.22$.

In subgroup analysis, LE subjects still yielded no significant differences in LH vs RH-onset, but the HE group showed significant differences on the WHO UCLA AVLT Trial 5, wherein LH-onset patients learned fewer words than $\mathrm{RH}$-onset patients after the fifth exposure to the word list, $\mathrm{p}=.048$.

Conclusions: Our results illustrate a robust effect of education level on neuropsychological scores of Spanish speakers with IE. Similar to Barr et al, the NeSBHIS did not predict seizure lateralization in our sample. However, when divided by education group, word list recall accurately predicted seizure lateralization in those with greater than primary school education, while the NeSBHIS remained unpredictive in patients with $\leq 6$ years schooling.

This study suggests that the NeSBHIS' ability to lateralize seizure onset could be improved by accounting for educational level and that future studies should include education considerations. 


\subsection{1}

COMPARISON OF EXECUTIVE FUNCTIONS IN PATIENTS WITH PSYCHOGENIC NON-EPILEPTIC SEIZURES (PNES) AND TEMPORAL LOBE EPILEPSY (TLE) AFTER CONTROLLING FOR MALINGERING

Lorna Myers, M. E. Lancman and M. Lancman (Neuropsychology, Northeast Regional Epilepsy Group, New York, NY)

Rationale: The aim of this study was to compare executive functions in patients with psychogenic non-epileptic seizures (PNES) and temporal lobe epilepsy patients (TLE) after having excluded those who "failed" the Test of Memory Malingering.

There are a growing number of reports that functional abnormalities are not distinctly restricted to the temporal lobe in TLE; executive function and working memory is also compromised (Stretton J \& Thompson PJ, 2012).

Deficits in working memory and executive functioning have also been reported in PNES (Chapman et al., 2011).

Our hypothesis was that those with a diagnosis of PNES and TLE would exhibit deficits in executive functions and that no significant between- group difference would be evident.

Methods: The diagnosis of PNES and TLE was confirmed through video-EEG monitoring. Our standard neuropsychological battery included the Wechsler Abbreviated Sales of Intelligence (WASI), the Test of Memory Malingering (TOMM), the Trail Making, ColorWord Interference and Verbal Fluency subtests of the Delis Kaplan Executive Function System (DKEFS).

Of 110 consecutive patients with the diagnosis of PNES, 97 were included. Seven who "failed" the TOMM, 3 who earned a $<70$ Full Scale IQ, and 3 who had a dual diagnosis of PNES/ES were excluded for this analysis. Of the 105 consecutive patients with a diagnosis of TLE, 89 were included. One who "failed" the TOMM and 15 who earned a $<70$ Full Scale IQ were excluded for this analysis. Raw scores and "pass-fail" conversions from all executive measures were analyzed.

Results: Of the 97 PNES patients, 14 were males and 83 females, mean age at the time of study was $39.3 \pm 1.2$ years and mean education was 13.65 years \pm .291 . Of the 89 TLE patients, 31 were males and 58 females, mean age was $45.0 \pm 1.7$ and mean education was 12.82 years \pm .302 .

Both groups evidenced impairments in all measures. Eighteen to 23 percent of patients with PNES and 22 to 30 percent of patients with TLE earned scores $<2$ standard deviations below the mean on all three executive function subtests. No significant differences were found between PNES and TLE on any measures (trail making, color word interference, verbal fluency and switching).

Conclusions: We found that a sizable number of PNES and TLE patients exhibited weaknesses on tests of executive function. Continued assessments of this cognitive area within these two groups are warranted. Future research directions include identifying contributory factors in deficient executive skills of these two patient groups.

Comparison between PNES and TLE performances on DKEFS subtests: Impaired function percentage $(<2 \mathrm{SD})$

\begin{tabular}{|ccc|}
\hline DKEFS subtests & PNES $(\%<2$ SDs $)$ & TLE $(\%<2$ SDs $)$ \\
\hline Trails I (DKEFS) & 16 & 14 \\
\hline Trail making 4 & 18 & 30 \\
Verbal fluency 1 & 20 & 26 \\
Verbal fluency 2 & 22 & 24 \\
\hline Verbal flucncy 3 & 19 & 20 \\
\hline C-W 3 & 23 & 20 \\
\hline
\end{tabular}

1.302

NEUROPSYCHOLOGICAL OUTCOMES IN POORLY CONTROLLED IDIOPATHIC GENERALIZED EPILEPSY

Rani Sarkis, A. Cheung, A. C. Pietras and B. Dworetzky (Brigham and Women's Hospital, Brookline, MA)

Rationale: Although the majority of patients with idiopathic generalized epilepsy have well controlled seizures, a significant percentage remains refractory to antiepileptic drugs (AEDs). These patients are poorly characterized in the literature, and their neuropsychological and psychosocial profiles have rarely been described. Such data would aid in patient counseling and clinical management.

Methods: A retrospective chart review of patients admitted to the Brigham and Women's epilepsy monitoring unit (EMU), between 2003 to 2011, was performed. During the admission, patients had neuropsychological assessment. Patients with a diagnosis of an idiopathic generalized epilepsy (IGE) were selected if they failed at least 2 medications, had a full scale IQ $>70$, a nonlesional MRI, and completed neuropsychological testing. A control sample consisted of unilateral temporal lobe epilepsy (TLE) patients who underwent similar testing and did not have a history of encephalitis, traumatic brain injury, prior epilepsy surgery, or brain tumor. Epilepsy characteristics, level of education, and employment status were also obtained.

Results: A total of 17 IGE and 18 TLE patients fulfilled study criteria with a mean $( \pm \mathrm{SD})$ age of epilepsy onset of $13.5( \pm 3.7)$ and $14.6( \pm 8.8)$ years respectively, and a mean epilepsy duration of 36.5 $( \pm 15.7)$ vs. $36.1( \pm 9.9)$ years. There was an average monthly seizuredays of $10.3( \pm 13.3)$ vs. $5.9( \pm 9.0)$ years, and average yearly generalized tonic clonic seizures (GTCs) of $9.5( \pm 14.7)$ vs. $1.7( \pm 2.0)$ $(\mathrm{p}=0.001)$.

Of the IGE patients, $53 \%$ had a college education and $41 \%$ were on disability or unemployed as compared to $44 \%$ and $28 \%$ for the TLE patients. There was a trend for IGE patients to have lower scores on the beck depression inventory (BDI-II) 8.7 v.s. 15.8 ( $\mathrm{p}=0.08$ ), while the quality of life in epilepsy (QOLIE-31) scores were not significantly different (54.3 vs. 49.3).

Patients with IGE performed worse on the trails making A and B tests, $51.8( \pm 29.0)$ and $144.2( \pm 86.2)$ compared to $29.7( \pm 6.9)$ and $74.7( \pm 35.7)$ seconds respectively $(\mathrm{p}=0.0028, \mathrm{p}=0.0033)$. IGE patients also had lower scores on the Performance IQ $91.7( \pm 15.4)$ v.s. $101.3( \pm 12.5),(\mathrm{p}=0.05)$. There were no differences in full scale IQ, verbal IQ, the controlled oral word association test, or Ray auditory verbal learning test.

Conclusions: Patients with poorly controlled IGE performed worse on tests of executive functioning than patients with poorly controlled TLE. Interestingly, despite a higher burden of convulsions for the IGE patients, they had a trend of lower depression scores than patients with TLE, which suggests factors other than seizure burden in the pathophysiology of depression in TLE. Further research on patients with uncontrolled IGE may provide insights into mechanisms of cognitive dysfunction, which may in turn guide patient counseling, and treatment planning.

\section{Behavior/Neuropsychology/Language: Pediatrics}

\subsection{3 \\ ICTAL DEFICITS IN BEHAVIOR DURING CHILDHOOD ABSENCE EPILEPSY}

Stephen Jhun ${ }^{1}$, J. Rodriguez-Fernandez ${ }^{1}$, J. Guo ${ }^{1}$, J. Gonzalez ${ }^{1}$, W. $\mathrm{Xiao}^{1}, \mathrm{M}$. Negishi ${ }^{1}, \mathrm{X} . \mathrm{Bai}^{1}$, N. Danielson ${ }^{1}, \mathrm{X} . \mathrm{Han}^{1}$, R. Constable ${ }^{2}$ and H. Blumenfeld ${ }^{1,3}$ ( ${ }^{1}$ Neurology, Yale University, New Haven, CT; ${ }^{2}$ Diagnostic Radiology, Yale University, New Haven, CT and

${ }^{3}$ Neurosurgery, Yale University, New Haven, CT)

Rationale: Patients with childhood absence epilepsy (CAE) experience brief seizures characterized by $3-4 \mathrm{~Hz}$ spike and wave 
discharges on electroencephalogram (EEG). CAE represents approximately one sixth of all diagnosed childhood epilepsies, and causes impaired attention and social dysfunction in school-age children. There is increasing evidence demonstrating that behavioral impairments may persist in-between seizures, even when well controlled with medication. Our group previously examined such interictal deficits during behavioral testing using EEG-fMRI. Here we investigate relationships between variably impaired ictal attention and: (1) task difficulty; (2) seizure duration; (3) timing of task to seizure onset and end; and (4) interictal attention deficits.

Methods: We tested attentional vigilance in patients (6-18 years old) with a diagnosis of CAE. Each subject underwent either a Repetitive Tapping Task (RTT) in which a button was pressed in response to letters presented on a screen once per second, or a more difficult Continuous Performance Task (CPT) in which a button was pressed when the target letter (X) appeared in a series of non-target letters, again displayed once per second. Both tasks were administered with simultaneous EEG-fMRI data collection (not discussed here). Ictal task performance was characterized by omission rate, commission rate, reaction time, d-prime, and beta, and related to interictal performance, seizure frequency, and seizure duration.

Results: Out of a total of 90 children, 34 had absence seizures during behavioral testing (328 seizures during RTT and 323 during CPT). The mean seizure duration was $5.09 \pm 0.17 \mathrm{~s}($ mean $\pm \mathrm{SD})$. The mean ictal omission rate was $70 \%$ for CPT and $53 \%$ for RTT. For both RTT and CPT, ictal performance was weakly correlated with seizure duration; however, clear outliers exist in both tasks where longer seizures have spared performance and shorter seizures have very impaired performance. A weak positive correlation was also observed between ictal and interictal omission rates in both tasks. For RTT tasks, performance was relatively spared on average in the initial $\sim 1 \mathrm{~s}$ of seizures. For both CPT and RTT, performance deficits tended to recover towards the end of the seizure (last 1-2s).

Conclusions: It is becoming increasingly clear that CAE patients sustain residual behavioral deficits in-between seizures, even when well controlled. CAE patients make a greater number of errors on sustained attention testing in the interictal period when compared to controls. Our data suggest that task difficulty and task timing have important effects on attention performance during absence seizures. Seizure duration is less consistently related to severity of deficits. Ictal attention impairment may be related to interictal deficits, suggesting chronic dysfunction in attention networks. This investigation will help us understand the neural mechanisms of attention deficits observed in CAE patients. In addition, further studies will examine the correlation between these behavioral patterns and EEG-fMRI data in the ictal and interictal periods.

\subsection{4 \\ BEHAVIOR DISORDERS IN PEDIATRIC EPILEPSY ARE LINKED TO SUBCORTICAL STRUCTURAL ABNORMALITIES}

Jack J. Lin ${ }^{1}$, P. Siddarth ${ }^{2}$, J. Riley ${ }^{1}$, S. Gurbani ${ }^{3}$, J. G. Levitt ${ }^{2}$, A. W. Toga $^{4}$ and R. Caplan ${ }^{2}\left({ }^{1}\right.$ Department of Neurology, University of California, Irvine, Irvine, CA; ${ }^{2}$ UCLA Semel Institute for Neuroscience and Human Behavior, David Geffen School of Medicine at UCLA, Los Angeles, CA; ${ }^{3}$ Department of Pediatrics, University of California, Irvine, Irvine, CA and ${ }^{4}$ Laboratory of Neuro Imaging, Department of Neurology, David Geffen School of Medicine at UCLA, Los Angeles, CA)

Rationale: Behavior disorders are common comorbidities of pediatric epilepsy with enduring adverse effects on life performances, but their neuroanatomical underpinning is unclear. Subcortical structural abnormalities have been associated with childhood onset psychiatric disorders, suggesting that these brain regions play a role in the pathophysiology of behavior disturbances in children. We, therefore, hypothesized that subcortical volumes will be reduced in children with epilepsy, compared to healthy controls and the degree of atrophy will be linked to measures of behavior problems. Methods: T1 weighted SPGR MR images (1.5 Telsa GE Signa scanner) were obtained in 20 children with complex partial seizures (CPS, age $11.0 \pm 2.7 \mathrm{yrs}$ ), 21 children with childhood absence epilepsy (CAE, age 9.6 $\pm 2.1 \mathrm{yrs}$ ), and 27 healthy controls (HC, age $10.9 \pm 2.6$ yrs). The groups were not significantly different in age, gender, and socioeconomic status. Subcortical volumes (caudate, putamen, pallidum, thalamus, nucleus accumbens, hippocampus and amygdala) were obtained from automated segmentation using FIRST (part of FSL, http://www.fmrib.ox.ac.uk/fsl)). Mixed models, with lateralization (left, right) as within-subject factor and group (HC, CPS, CAE) as between-subject factor, were used to compare subcortical volumes, controlling for age, gender and total intracranial volumes. Specific subcortical volumes in children with epilepsy that were significantly different from controls were correlated with Childhood Behavioral Checklist (CBCL) using Spearman correlation coefficients. Asymmetries were compared using ANCOVAs.

Results: The left thalamic volumes were significantly different among the three groups $(\mathrm{p}=.03)$. Post-hoc pair-wise analyses showed left thalamic volumes were reduced in CPS $(8036.9+802.6, \mathrm{p}=0.01)$ and CAE $(8146.2+899.7, \mathrm{p}=0.05)$ compared to $\mathrm{HC}(8419.6+911.2)$. There were no significant group differences in other subcortical regions. Although a left greater than right volumetric asymmetry was found in all three groups (all $\mathrm{p}<.05$ ), the degree of asymmetry was significantly smaller in CPS and CAE, compared to HC $(p<.05)$. In CPS and CAE, the left thalamic volume was significantly correlated with social problems (CPS, $r=-0.52, \mathrm{p}=0.04$; $\mathrm{CAE}, \mathrm{r}=-0.46, \mathrm{p}=0.05$ ). In CPS, left thalamus volume was also associated with attention $(\mathrm{r}=$ $0.57, \mathrm{p}=0.02)$ and school problems $(\mathrm{r}=.56, \mathrm{p}=0.05)$. There were no correlation between left thalamus volume and CBCL scores in HC. Conclusions: The left thalamus was selectively atrophied and correlated with social problems in both CPS and CAE, suggesting that this subcortical region has a shared role in the pathophysiology of commonly found social problems in childhood onset epilepsy. However, the association of left thalamus atrophy with attention problems and school problems in CPS but not in CAE implies a syndrome specific contribution. These findings provide important new insights into the anatomical correlates of behavior problems in childhood onset epilepsy.

\subsection{5 \\ RELATION OF IQ TO CHANGES IN QUALITY OF LIFE AFTER PEDIATRIC EPILEPSY SURGERY}

Mary Lou Smith ${ }^{1,2}$ and Y. Soliman ${ }^{1}$ ( ${ }^{1}$ Psychology, University of Toronto, Mississauga, ON, Canada and ${ }^{2}$ Hospital for Sick Children, Toronto, ON, Canada)

Rationale: Epilepsy and Intellectual Disability (ID) independently affect children's quality of life (QOL). It is possible that seizure freedom is associated with fewer improvements in QOL in children with ID. This study examined QOL changes after epilepsy surgery in children with and without ID to explore the hypothesis that more improvements would be seen in children without ID.

Methods: QOL was measured using the QOLCE, a parent-report questionnaire for children with epilepsy; this questionnaire has 16 subscales reflecting different aspects of QOL. Scores were available for 37 children before and one year after surgery. Ten had an IQ of 70 or less (ID group). There were no differences between the two IQ groups on sex, handedness, age of seizure onset, and number of antiepileptic drugs. 27 of the children were seizure-free at follow-up (ID=7, non-ID=20).

Results: Children with ID had poorer QOL in the Attention/Concentration, Behavior, Social Activities and Other Cognitive subscales of the QOLCE. When examining all children irrespective of IQ and seizure outcome, improvements in QOL after surgery were found for the following scores: Energy/Fatigue, Other Cognitive, Depression, Anxiety, Control/Helplessness, Social Interactions, Social Activities, Stigma, General Health, and overall 
QOL. Better QOL was found in the seizure-free children in the Social Interactions, Social Activities, General Health, and Overall QOL subscales. Examination of change in QOL after surgery considering both IQ status and seizure freedom found significant improvement only in the Behaviour scale; this improvement was greater for the seizure free- children and for those in the non-ID group.

Conclusions: The results suggest that in the first year following epilepsy surgery, many aspects of QOL show improvements, not all of which are linked to seizure freedom. Children without ID experience improvements in limited aspects of QOL compared with children with higher IQ. Changes in behaviour appear to be linked to seizure freedom and higher IQ.

\subsection{6 \\ IMPROVED BEHAVIOR AFTER PEDIATRIC EPILEPSY SURGERY}

Lisa L. Ferguson ${ }^{1}$, J. S. Haut ${ }^{1,2}$, P. Klaas ${ }^{1,2}$, D. Lachhwani ${ }^{2}$, W. Bingaman $^{2}$ and R. M. Busch ${ }^{1,2}\left({ }^{1}\right.$ Department of Psychiatry and Psychology, The Cleveland Clinic, Cleveland, $\mathrm{OH}$ and ${ }^{2}$ Epilepsy Center, The Cleveland Clinic, Cleveland, $\mathrm{OH}$ )

Rationale: Children with epilepsy often exhibit more behavioral problems than healthy children and children with other chronic illnesses. Along with the demonstrated success of epilepsy surgery in reducing seizure burden, research indicates improved behavior after surgery. It is unclear if the reported behavioral improvements can be attributed to surgery or other factors. This study examines postoperative behavioral change in children following epilepsy surgery as compared to a control group of children with epilepsy who have not had surgery.

Methods: Using the Child Behavior Checklist for Ages 6-18 (CBCL), change in behavior was examined in 113 pediatric patients (mean age $=10.85$ ) after epilepsy surgery as compared to 42 nonsurgical pediatric epilepsy patients (mean age $=10.21$ ) taking AEDs for seizure control. The surgical and control groups did not differ significantly in demographic composition or seizure variables; however, the surgical group scored significantly higher on Social Problems than the nonsurgical group presurgically. Change in behavior was assessed with a series of repeated-measures ANOVAs. Then, using a 10-point standard deviation change in t-score to define meaningful change, subjects were classified as improved, declined, or unchanged. Chi-squares were used to analyze differences in behavior outcome between the surgical and nonsurgical groups.

Results: There was a significant interaction between time and group on Thought Problems [F(153) $=7.071, p=.009]$ as well as trends toward significant interactions on Social Problems $[\mathrm{F}(153)=3.479$, $p=.064]$ and Aggressive Behavior [ $\mathrm{F}(153)=3.388, p=.068]$. For all three scales, the control group maintained relatively stable scores over time while the surgical group showed a mean decrease in endorsed behavior problems following surgery. Significant main effects were observed on Somatic Complaints and Attention Problems, with both groups showing significant improvements over time. No significant group differences were observed on the remaining scales.

Chi-square analyses revealed that a larger percentage of surgical patients showed improvements on Somatic Complaints $\left[\chi^{2}(2)=6.057\right.$, $p=.048]$ than nonsurgical patients. A greater percent of surgical patients also reported improvements on Thought Problems, while a greater percentage of nonsurgical patients reported declines on this scale $\left[\chi^{2}(2)=6.159, p=.046\right]$. The same trend was apparent on Social Problems $\left[\chi^{2}(2)=5.408, p=.067\right]$.

Conclusions: Caregivers report fewer thought problems and social difficulties and less aggressive behavior following epilepsy surgery in children. These improvements appear to be largely attributable to surgery as caregivers of nonsurgical children do not report significant behavior changes on these scales over the same time interval. Interestingly, both surgical and control groups showed improvements on scales assessing Somatic Complaints and Attention Problems, suggesting changes in these reported symptoms over time are not specific to surgery. Further research into predictors of behavior change and examination of side and site of surgery is suggested.

\subsection{7 \\ SOCIAL SKILLS IN SIBLINGS OF CHILDREN WITH EPILEPSY \\ Suresh Gurbani ${ }^{1}$, P. Siddarth ${ }^{2}$, J. Levitt ${ }^{2}$, R. Ly ${ }^{2}$, R. Sankar ${ }^{2}$ and R. Caplan ${ }^{2}\left({ }^{1}\right.$ Kaiser Permanente, Anaheim, CA and ${ }^{2}$ UCLA, Los Angeles, CA)}

Rationale: Parents report normal social skills in siblings of children with epilepsy (S-CWE) but poor social skills in the probands. Normal or problem social behaviors in the siblings of children with a chronic illness or disability are usually attributed to psychosocial factors (e.g., coping skills, parenting, and family functioning). This is the first study to examine if social problems and poor peer interaction are related to biological factors in S-CWE. It compared parent reports of peer interaction and social problems and the relationship of these social measures with fronto-temporal volumes in S-CWE and healthy contols (HC) subjects.

Methods: : 38 S-CWE, aged 10.3 (2.67) years, and $36 \mathrm{HC}$ subjects, aged 10.5 (2.56) years, underwent MRI scans at 1.5T. Tissue was segmented and total brain, frontal lobe, frontal parcellations (inferior frontal gyrus (IFG), orbital frontal gyrus (OFG), dorsolateral prefrontal cortex (DLPFC)), and temporal lobe (TL) gray and white matter volumes (GMV, WMV) were computed. Parent Child Behavior Checklists yielded social problem and peer interaction scores. A social composite $\mathrm{Z}$ score was constructed by taking an average of the $\mathrm{z}$-transformed peer interaction and social problem scores. (Social problems scores were inverted so that increasing scores represented fewer problems.) ANCOVAs, controlling for age and gender, were used to determine group differences in social scores and volumetric measures. General linear models, with the regional volumes as the dependent variables and group, social score and interaction of group and social scores as predictors, were estimated to determine if the two groups differed in the relationships between volumetric measures and social scores.

Results: The S-CWE exhibited significantly lower peer interaction scores and social composite $\mathrm{Z}$ scores compared to the $\mathrm{HC}$ but there were no significant differences in the mean social problem scores and fronto-temporal volumes. A significant group x social score interaction was observed for TL GMV $(\mathrm{F}(1,57) 12.0, \mathrm{p}=.001)$, TL WMV $(\mathrm{F}(1,58) 5.3, \mathrm{p}=.02)$, and $\mathrm{OFG} \operatorname{WMV}(\mathrm{F}(1,55) 6.2, \mathrm{p}=.02)$. The social $\mathrm{Z}$ score was significantly and negatively related to right $(\mathrm{r}$ $=-.53, \mathrm{p}=.003)$ and left $(\mathrm{r}=-.46, \mathrm{p}=.01) \mathrm{TL}$ GMV in the HC but not in the S-CWE. The social $\mathrm{Z}$ scores were negatively related to right TL WMV $(\mathrm{r}=-.32, \mathrm{p}=.09)$ in the $\mathrm{HC}$ and positively related in the S-CWE $(r=.32, p=.08)$, but the associations were of borderline significance. The social $\mathrm{Z}$ score of the $\mathrm{S}-\mathrm{CWE}$ group also correlated significantly with right OFG WM volumes $(r=-.39, p=.04)$ but not in the HC.

Conclusions: This is the first study to identify that the social difficulties of S-CWE are related to structural abnormalities in the right OFG, a brain region involved in the integration of factual and emotional information, regulation of social decision-making, and emotional regulation.

\subsection{8 WTIHIDAWN}

1.309

COGNITIVE CHARACTERISTICS OF MITOCHONDRIAL DISEASES IN CHILDREN

Hillary Shurtleff $^{1,2}$, T. Firman ${ }^{1}$, M. Warner ${ }^{1,2}$ and R. P. Saneto ${ }^{1,2}$ ( ${ }^{1}$ Seattle Children's Hospital, Seattle, WA and ${ }^{2}$ University of Washington, Seattle, WA) 
Rationale: Little is known about the incidence and patterns of cognitive involvement in children with mitochondrial cytopathy. ${ }^{6}$ While preliminary studies have documented cognitive impairment, data are limited, with studies reporting on only a few children ${ }^{5}$ or older teenagers and adults. ${ }^{2}$ Although studies have reported lower nonverbal versus verbal intelligence $e^{2,3,5,8}$ and other effects such as cognitive slowing, ${ }^{4}$ the literature is limited. This retrospective chart review study was undertaken to define the clinical profiles of children with mitochondrial disorders.

Methods: We retrospectively identified 32 patients with definitive mitochondrial diagnoses (based on the modified Walker criteria ${ }^{1}$ ) who underwent neuropsychological evaluations at Seattle Children's Hospital. 16 had seizures (Group 1); 16 had none (Group 2). Each group had 11 males and 5 females. Mean ages were similar: Group 1 was 116 months; Group 2106 months. Only 10 Group 1 patients had Wechsler IQ testing; all of Group 2 had Wechsler IQ testing. 6 Group 1 patients could not complete IQ testing, but the Vineland Adaptive Behavior Scale (Vineland) was administered. Because topiramate has been shown to have deleterious neuropsychological impact, 3 patients on topiramate were separated out although all of these patients only had Vineland interviews.

Results: Group 2 (no seizures) obtained a mean Full Scale IQ of 93.1 $(\mathrm{SD}=18.7)$ compared to Group 1 (seizures) FSIQ $=74.5(\mathrm{SD}=$ 27.6). Vineland mean summary scores were $52.3(\mathrm{SD}=16.6)$ versus $40(\mathrm{SD}=10.8)$ for the 3 patients on topiramate.

Conclusions: Results suggest that seizures may represent a worse phenotype, as compared to no seizures, as suggested by consistently lower IQ means in conjunction with the finding that almost half of the patients with seizures were too low functioning to complete Wechsler IQ testing. Results were also consistent with previous smaller pilot studies that have identified a discrepancy between verbal and nonverbal abilities in patients with mitochondrial cytopathy.

\subsection{0 \\ KNOWLEDGE AND MANAGEMENT OF PSYCHOPATHOLOGY IN CHILDREN WITH EPILEPSY AMONG BEHAVIORAL HEALTH PROFESSIONALS} Jayne Pacheco-Phillips ${ }^{1}$, D. Terry ${ }^{1}$ and L. Hamiwka ${ }^{1,2}$ ( ${ }^{1}$ Nationwide Children's Hospital, Columbus, $\mathrm{OH}$ and ${ }^{2}$ Division of Child Neurology, The Ohio State University College of Medicine, Columbus, $\mathrm{OH}$ )

Rationale: Epidemiological and community studies conducted over the past twenty-six years have confirmed high rates of psychopathology (i.e., psychiatric diagnoses, behavior and emotional problems) in children with new onset and chronic epilepsy both with and without intellectual disability who are treated medically or by epilepsy surgery. Despite prevalent psychiatric comorbidities in pediatric epilepsy, only one-third of children with average intelligence have had a psychiatric, psychological,

neuropsychological, speech and language evaluation, or treatment of these comorbidities. The goal of this study was to assess the attitudes, general knowledge, and comfort in managing psychopathology in pediatric epilepsy among behavioral health providers.

Methods: An online questionnaire was developed for behavioral health professionals (BHP) with content validity reviewed by 3 experts in the area of psychopathology in pediatric epilepsy. A link to the questionnaire, which measured general knowledge, attitude toward and comfort in management of psychopathology in children with epilepsy, was e-mailed to clinical directors of 9 governmentfunded behavioral health agencies providing services primarily to children and youth requesting distribution to staff. 171 of $500(33 \%)$ BHP participated in this cross-sectional cohort study. 146 (85\%) completed the survey. Group comparisons were made using t-tests and ANOVA's. The BHP surveyed are included in Table 1.

Results: Significant differences in attitude, knowledge, or comfort in managing psychopathology in children with epilepsy among the disciplines $(\mathrm{p}=0.6)$ outlined in Table 1 were not identified. However BHP providing services in an institution, such as a school or hospital, possessed better attitudes $(\mathrm{p}=0.003)$ and more comfort in managing psychopathology in children with epilepsy $(p=0.002$.) than those who provided treatment in the community. BHP's who have witnessed a seizure had significantly higher comfort in management scores $(\mathrm{p}=0.001)$. Similarly BHP who knew someone with epilepsy indicated a significantly better attitude $(\mathrm{p}=0.003)$ and more comfort in managing $(\mathrm{p}=0.007)$ psychopathology in epilepsy. Of the $146 \mathrm{BHP}$ surveyed, $84 \%$ indicated a desire for training about providing behavioral health services to children with epilepsy.

Conclusions: In this first study assessing attitude, general knowledge and comfort in managing psychopathology of BHP in children with epilepsy, we found that previous exposure to seizures has a significant impact on BHP's attitudes and comfort in managing psychopathology in children with epilepsy. Additionally, communitybased BHP express a lack of confidence and the knowledge needed to adequately manage psychopathology in children with epilepsy. Our results emphasize an overwhelming need to educate and establish relationships with BHP so that they play an effective role in the multi-disciplinary care of children with epilepsy.

Professional Discipline

\begin{tabular}{|c|c|}
\hline Discipline & Completed \\
\hline Psychologists & 31 \\
Clinical Counsclors & 25 \\
\hline Clinical Social Workers & 47 \\
\hline Physicians & 5 \\
\hline Nurses & 3 \\
Unlicensed/Other & 35 \\
& 146 \\
\hline
\end{tabular}

\subsection{1}

\section{ARE INEFFECTIVE COPING SKILLS ASSOCIATED WITH} PEDIATRIC NON-EPILEPTIC SEIZURES?

Brenda Bursch $^{1}$, S. Plioplys ${ }^{2}$, J. Doss ${ }^{3}$, P. Siddarth ${ }^{1}$, D. Birt ${ }^{1}$, T. Falcone $^{4}$, M. Forgey ${ }^{1}$, W. C. LaFrance ${ }^{5}$, D. M. Weisbrot ${ }^{6}$, M. D. Willis ${ }^{5}$ and R. Caplan ${ }^{1}\left({ }^{1}\right.$ Psychiatry and Biobehavioral Sciences, David Geffen School of Medicine at UCLA, Los Angeles, CA; ${ }^{2}$ Psychiatry and Behavioral Sciences, Feinberg School of Medicine at Northwestern University, Chicago, IL; ${ }^{3}$ Minnesota Epilepsy Group, St. Paul, MN; ${ }^{4}$ Epilepsy Center, Neurological Institute, Cleveland Clinic, Cleveland, OH; ${ }^{5}$ Psychiatry and Neurology (Research), Rhode Island Hospital, Warren Alpert Medical School of Brown University, Providence, RI and ${ }^{6}$ Child and Adolescent Psychiatry, Stony Brook University Medical Center, Stony Brook, NY)

Rationale: Pediatric non-epileptic seizures (NES) are associated with significant medical and psychiatric morbidity, increased health care utilization, and decreased functioning. To examine stress and coping, we hypothesized that, compared to their siblings, youth with NES would report significantly more anxiety sensitivity, somatic symptoms, daily hassles, parental rejection and over-protection, and ineffective coping strategies.

Methods: This IRB-approved, multisite study includes 81 children (48 with NES and 33 siblings). Youth with NES were older than their siblings $(p=0.01)$. Groups did not differ by gender or race/ethnicity. See Table 1.

Participants completed several self-report scales: Childhood Anxiety Sensitivity Index (CASI) measures the tendency to view anxietyrelated bodily sensations as dangerous; Children's Somatization Inventory (CSI) measures the severity of nonspecific somatic symptoms; Children's Hassles Scale (CHS) measures the frequency and impact of daily hassles; Parker Parental Bonding Instrument (PBI) measures care-rejection and parental overprotection-autonomy 
(each child completed this measure twice; once about each parent); and, Children's Coping Questionnaire (CCQ) measures child coping strategies with 14 subscales, reflecting three factors (monitoring, blunting, and venting).

Youth with NES and siblings were compared on the measures using mixed models, with family as a random effect. Two-tailed tests of significance at the 0.05 level were used. Age was used as a covariate. Results: As predicted (Table 2), compared to siblings, youth with NES reported significantly more anxiety sensitivity and somatic symptoms (including more headaches, faintness/dizziness, body weakness, and trouble walking). Youth with NES and siblings reported their most bothersome problem was "schoolwork" $38.3 \%$ and $48.5 \%$, respectively) or "family" (38.3\% and $39.4 \%$,

respectively). Additionally $23.4 \%$ of youth with NES and $12.1 \%$ of siblings reported their most bothersome problem was "getting along with other kids." To cope with their most bothersome problem, youth with NES were more likely than siblings to report venting (coping via emotional expression, acting out, or worrying) and, specifically via solitary emotional expression (yelling, crying, hitting pillow, etc). Approaching significance, youth with NES reported more negative daily hassles than siblings. No significant differences were detected related to their reports of parental bonding. Within the NES group, no significant differences were found on any of the measures between those with epilepsy $(n=35)$ and those without epilepsy $(n=13)$. Conclusions: Consistent with research on children with other nonspecific somatic symptoms (e.g. chronic pain), youth with NES reported significantly more anxiety sensitivity, somatic symptoms, and ineffective coping. Youth with NES may benefit from development of interventions that teach them to more frequently utilize accommodative coping strategies (e.g., distraction, acceptance, positive thinking, cognitive restructuring), which has been found to be helpful for youth with other somatic symptoms.

Table 1. Demographic Features of NES and Sibling Groups

\begin{tabular}{|c|c|c|c|}
\hline & & $\begin{array}{c}\text { Youth with NES } \\
\qquad \mathrm{N}=48\end{array}$ & $\begin{array}{l}\text { Siblings } \\
. \mathrm{V}=33\end{array}$ \\
\hline Agc (ycars) & Mcan (StandardI)cviation) & $14.9(2.6)$ & $13.6(2.3)$ \\
\hline Gender & \# Males $(\%)$ & $15(31.25 \%)$ & $15(45.45 \%)$ \\
\hline Race/Ethnicily & $\begin{array}{c}\text { \# Whitc/ Caucasian }(\%) \\
\text { \# Latino (\%) } \\
\text { \# African American/ Black (\%) } \\
\text { \# Asian (\%) } \\
\text { \# Other/Missing (\%) }\end{array}$ & $\begin{array}{l}30(62.5 \%) \\
8(16.7 \%) \\
6(12.5 \%) \\
1(2.1 \%) \\
3(6.25 \%)\end{array}$ & $\begin{array}{l}19(57.6 \%) \\
6(18.2 \%) \\
4(12.1 \%) \\
1(3.0 \%) \\
3(9.1 \%)\end{array}$ \\
\hline
\end{tabular}

Table 2. Summary of Notable Results

\begin{tabular}{|c|c|c|c|c|}
\hline Anxicty Sensitivity & $\begin{array}{l}\text { Youth with } \\
\text { NES } \\
\text { Siblings }\end{array}$ & $\begin{array}{c}\text { Me:m (Standard } \\
\text { Deviation) } \\
14.2(7.5) \\
10.1(5.4)\end{array}$ & $\begin{array}{c}\mathrm{F} \\
\Gamma(1,31)=9.2\end{array}$ & $\begin{array}{c}P \\
p= \\
0.005\end{array}$ \\
\hline Somatization & $\begin{array}{l}\text { Youth with } \\
\text { NTSS } \\
\text { Siblings }\end{array}$ & $\begin{array}{l}31.4(20.0) \\
15.7(17.2)\end{array}$ & $\begin{array}{c}F(1,30)= \\
10.7\end{array}$ & $\begin{array}{c}p= \\
0.003\end{array}$ \\
\hline Daily Hassles & $\begin{array}{l}\text { Youth with } \\
\text { NES } \\
\text { Siblings }\end{array}$ & $\begin{array}{l}23.5(15.3) \\
18.2(11.7)\end{array}$ & $\mathrm{F}(1,29)=3.7$ & $p=0.06$ \\
\hline $\begin{array}{l}\text { Coping Style } \\
\text { (Factor: Venting) }\end{array}$ & $\begin{array}{l}\text { Youth with } \\
\text { NES } \\
\text { Siblings }\end{array}$ & $\begin{array}{l}8.8(4.9) \\
7.4(4.6)\end{array}$ & $\Gamma(1,30)=4.7$ & $p=0.04$ \\
\hline $\begin{array}{c}\text { Coping Style } \\
\text { (Subscale: Tixpressing } \\
\text { Feclings) }\end{array}$ & $\begin{array}{l}\text { Youth with } \\
\text { NT:S } \\
\text { Siblings }\end{array}$ & $\begin{array}{l}2.5(1.9) \\
1.8(2.1)\end{array}$ & $\Gamma(1,30)=9.0$ & $\begin{array}{c}p= \\
0.005\end{array}$ \\
\hline
\end{tabular}

\section{Genetics: Human Studies}

\subsection{2}

TARGETED RESEQUENCING OF KNOWN AND CANDIDATE EPILEPSY GENES IN 500 PATIENTS WITH EPILEPTIC ENCEPHALOPATHIES

Gemma L. Carvill ${ }^{1}$, B. J. O'Roak ${ }^{2}$, S. C. Yendle ${ }^{3}$, J. C. Cook ${ }^{1}$, S. F. Berkovic $^{3}$, J. Shendure ${ }^{2}$, I. E. Scheffer ${ }^{3}$ and H. C. Mefford ${ }^{2}$ ( ${ }^{1}$ Department of Pediatrics, University of Washington, Seattle, WA; ${ }^{2}$ Department of Genome Sciences, University of Washington, Seattle, WA and ${ }^{3}$ Epilepsy Research Center and Department of Medicine, University of Melbourne, Melbourne, VIC, Australia)

Rationale: Epilepsy is one of the most common neurological disorders, with a lifetime incidence of $3 \%$. The epileptic encephalopathies (EE) are the most severe of all the epilepsies. Patients typically present with refractory epilepsy with multiple seizure types, cognitive arrest or regression, and have a poor prognosis. Several EE genes and EE-associated CNVs have been described, but a systematic mutation screening approach across multiple EE has not been undertaken and most cases have an unknown etiology.

Methods: We screened 32 known and 40 candidate genes for disease-causing changes in a cohort of 518 patients with a diverse spectrum of EE. This was achieved by targeted resequencing of all exonic regions of these 72 genes, using molecular inversion probes for targeted capture followed by multiplexed massively parallel sequencing. This data was analyzed using two different pipelines. Firstly, standard variant calling tools were used to identify all DNA sequence changes in EE patients, and compared to large control data sets to identify rare variants. The presence of these rare mutations was then assessed in familial samples to determine pathogenicity. Secondly, using read depth data followed by normalization and a standard score analysis we were also able to detect copy number variations (CNVs) in the target genes. Putative CNVs will be validated using a custom oligonucleotide array with high-density coverage of all target genes.

Results: To date, we have analyzed data from 365 individuals for 13 known and 19 candidate EE genes with $93 \%$ of targeted nucleotides sequenced at $>25$ fold coverage required for accurate variant calling. At this coverage threshold we detected $100 \%$ of known single nucleotide variants $(n=622)$ and $86 \%$ of known CNVs $(n=15)$. Segregation analysis of rare, possibly damaging variants in known EE genes detected in $317 \mathrm{EE}$ probands revealed pathogenic changes in SCN1A ,GABRG2, CDKL5, GRIN2A and PCDH19 in five families and possibly pathogenic, inherited changes in an additional five families. The clinical manifestations of mutation carriers often differed from those previously described, broadening the clinical spectrum of phenotypes associated with known EE genes. We also observed intra-familial variability and incomplete penetrance. Rare variants within our candidate genes, comprising glutamate transporters, the $\mathrm{NA}+/ \mathrm{K}+$ transporter ATPase-interacting proteins and synaptic vesicle exocytosis proteins is ongoing.

Conclusions: In summary, we present a cost-effective, efficient method of screening multiple EE genes in large cohorts that will transform molecular testing in EE, enabling rapid early diagnosis, informing prognosis, treatment and genetic counseling.

\subsection{3 \\ MUTATIONS IN KCNT1, CODING FOR A SODIUM-GATED POTASSIUM CHANNEL, CAUSE AUTOSOMAL DOMINANT NOCTURNAL FRONTAL LOBE EPILEPSY WITH INTELLECTUAL DISABILITY AND PSYCHIATRIC FEATURES}

Leanne M. Dibbens ${ }^{1}$, K. R. Smith ${ }^{2}$, M. Bahlo ${ }^{2}$, L. Nobili ${ }^{3}$, E. Kahana $^{4}$, L. Licchetta ${ }^{5}$, K. L. Oliver ${ }^{5}$, A. Mazarib ${ }^{6}$, Z. Afawi ${ }^{7}$, A. Korczyn $^{8}$, G. Plazzi ${ }^{9}$, S. Petrou ${ }^{10}$, S. F. Berkovic ${ }^{5}$, I. E. Scheffer ${ }^{5,11}$ and S. E. Heron ${ }^{1}\left({ }^{1}\right.$ Pharmacy and Medical Sciences, University of 
South Australia, Adelaide, SA, Australia; ${ }^{2}$ Bioinformatics Division, The Walter and Eliza Hall Institute of Medical Research, Melbourne, VIC, Australia; ${ }^{3}$ Epilepsy Surgery Center, Sleep Medicine Center, Ospedale Niguarda, Milano, Italy; ${ }^{4}$ Department of Neurology, Barzilai Medical Center, Ashkelon, Israel; ${ }^{5}$ Department of Medicine, University of Melbourne, Melbourne, VIC, Australia; ${ }^{6}$ Tel-Aviv Sourasky Medical Center, Ramat Aviv, Israel; ${ }^{7}$ Sackler Faculty of Medicine, Tel-Aviv University, Ramat Aviv, Israel; ${ }^{8}$ Department of Neurology, Tel-Aviv University Medical School, Ramat Aviv, Israel; ${ }^{9}$ Department of Neurological Sciences, University of Bologna, Bologna, Italy; ${ }^{10}$ Centre for Neuroscience and Melbourne Brain Centre, Florey Neuroscience Institute, Melbourne, VIC, Australia and ${ }^{11}$ Epilepsy Research Centre, Department of Medicine, University of Melbourne, Melbourne, VIC, Australia)

Rationale: We ascertained a family with a severe form of Autosomal Dominant Nocturnal Frontal Lobe Epilepsy (ADNFLE) with major comorbidities of intellectual disability (ID) and psychiatric problems. Since the family did not have a mutation in a known ADNFLE gene we set out to identify a new gene for this form of focal epilepsy. Methods: Genotyping was carried out using the Infinium HumanHap660W-Quad BeadChip genotyping array (Illumina, San Diego, CA). Parametric multipoint whole genome linkage analysis was performed using MERLIN. Whole-exome capture and sequencing was performed on two affected family members. Coding sequences were enriched using the SureSelect Human All Exon $50 \mathrm{Mb}$ kit (Agilent Technologies, Santa Clara, CA) and sequenced using the SOLiD v4 instrument (Applied Biosystems, Carlsbad, CA). Sequence variants were obtained with SAMtools and annotated using SeattleSeq. High resolution melt curve analysis was performed using the LightScanner ${ }^{\mathrm{TM}}$ instrument (Idaho Technologies, Salt Lake City, UT).

Results: Genome-wide linkage analysis of the family identified a single peak with a maximum parametric LOD score of 2.71 on chromosome $9 \mathrm{q} 34.3$, which corresponded to a $2.35 \mathrm{Mb}$ region containing 99 genes. Exome sequencing identified a novel heterozygous variant, p.Arg928Cys, within the chromosome 9 linkage interval, in the gene KCNT1. The variant showed complete co-segregation with the ADNFLE phenotype in the family and was absent in controls. Analysis of 45 unrelated probands with either ADNFLE or NFLE by high resolution melt curve analysis identified two further KCNT1 mutations, p.Tyr796His and p.Arg398Gln, in two additional families. The three families each showed features of a more severe ADNFLE phenotype with an earlier mean (6 years compared with 10 years in classical ADNFLE associated with nicotinic receptor subunit mutations) age of onset, ID in 3 members of family A and 2 of family B, and psychiatric or behavioural problems including psychosis, catatonia and aggression in 5 individuals from family A, and 2 members from each of family B and family C. The degree of ID varied from mild to severe.

Conclusions: We have identified KCNT1, which encodes the sodium-activated potassium channel subunit KCa4.1 (also called SLACK ), as a new gene for the focal epilepsy disorder ADNFLE. Although ID and psychiatric disease have been previously reported in cases with ADNFLE, they are infrequent in families with nicotinic acetyl choline receptor (nAchR) subunit gene mutations. The penetrance observed for each of the KCNT1 mutations was $100 \%$, well above the $60-80 \%$ observed in classical ADNFLE with nAchR mutations. Thus, the phenotype associated with KCNT1 mutations is both more severe and more penetrant than that typically seen with nAchR mutations. This clinico-molecular distinction has significant implications for the genetic counselling of families with KCNT1 mutations.
1.314

RECURRENT RECIPROCAL GENOMIC

REARRANGEMENT OF 17Q12 AS A CAUSE OF GEFS+ Katia Hardies $^{1,2}$, E. Peeters ${ }^{3}$, A. Suls ${ }^{1,2}$, S. Weckhuysen ${ }^{1,2}$, P. Holmgren $^{1,2}$, W. Van Paesschen ${ }^{3}$ and P. De Jonghe ${ }^{1,4}\left({ }^{1}\right.$ Department of Molecular Genetics, VIB, Wilrijk, Belgium; ${ }^{2}$ University of Antwerp, Institute Born-Bunge, Wilrijk, Belgium; ${ }^{3}$ Departement of Neurology, University Hospital Gasthuisberg, Leuven, Belgium and ${ }^{4}$ Departement of Neurology, Antwerp University Hospital, Wilrijk, Belgium)

Rationale: A rapidly increasing number of reports associating genomic rearrangements with human diseases has established genome-wide microarrays as a valuable tool in clinical diagnostics. For patients with neurodevelopmental disorders, such as intellectual disability (ID) and autism, the detection rate of pathogenic variants amounts to $20 \%$. Despite the observation that most patients harboring such a variant experience a wider range of symptoms (which frequently includes seizures), epilepsy patients are not yet systematically investigated for genomic rearrangements. We did, however, identified a $2.12 \mathrm{Mb} 17 \mathrm{q} 12$ microduplication in a proband of a generalized epilepsy with febrile seizures plus (GEFS+) family, whom had ID, febrile, myclonic, tonic-clonic seizures and absences. The recurrent reciprocal genomic rearrangement of 17q12 is known to be associated with a combination of various renal abnormalities and neurological alterations. Only two of the 31 reported patients with this duplication and three of the 43 with the reciprocal deletion are described to have seizures. Our diagnostic finding proved that the 17 q12 duplication can occur in individuals without renal disease or diabetes. But it furthermore suggested that the 17q12 duplication may be associated with an epilepsy phenotype restricted to GEFS+. We therefore analyzed the segregation pattern in the probands family $(\mathrm{n}=8)$, and aimed to determine the frequency of $17 \mathrm{q} 12$ genomic rearrangements in our epilepsy cohorts $(n=502)$.

Methods: Initial molecular karyotyping of the proband was performed with the 180k Cytosure ISCA v2 array. Segregation analysis in the eight available family members of the four-generation GEFS+ family and all follow up studies were performed with the multiplex amplicon quantification (MAQ) technique.

Results: We determined the complete cosegregation of the $17 \mathrm{q} 12$ microduplication in the six affected family members of the fourgeneration GEFS+ family. Subsequently we identified one additional three-generation GEFS + family (three patients) segregating the $17 q 12$ duplication and found that one out of 273 control samples also carried this duplication.

Conclusions: Our results suggest that genomic rearrangements of $17 q 12$ can be associated with a phenotype restricted to GEFS+. This association, however, needs to be confirmed by studying larger samples, especially in light of the presence of the duplication in our control group. Incomplete penetrance is a common finding in molecular genetics of epilepsy, therefore the occurrence in the control population does not formally argue against the pathogenic nature. Although it is already evident that $17 \mathrm{q} 12 \mathrm{CNVs}$ are not a frequent cause of GEFS+, it remains an intriguing question how these rearrangements actually lead to epilepsy, e.g. by disrupting an important gene, dosage effect of gene(s) within the rearrangement or position effects.

\subsection{5 \\ GENOME-WIDE ASSOCIATION STUDY AND EXPRESSION ANALYSIS SUGGEST STIMULATION OF NEURITE GROWTH MAY BE A NOVEL MECHANISM OF EPILEPTOGENESIS IN FOCAL EPILEPSY}

Larry Baum $^{1}$, S. Zhang ${ }^{1}$, Y. Guo ${ }^{2}$, P. Sham ${ }^{2}$, S. Cherny ${ }^{2}$ and P. $\mathrm{Kwan}^{3,4}$ ( ${ }^{1}$ School of Pharmacy, The Chinese University of Hong Kong, Shatin, Hong Kong; ${ }^{2}$ Department of Psychiatry, The University of Hong Kong, Pokfulam, Hong Kong; ${ }^{3}$ Department of Medicine, The University of Melbourne, Royal Melbourne Hospital, 
Melbourne, VIC, Australia and ${ }^{4}$ Department of Medicine and Therapeutics, The Chinese University of Hong Kong, Prince of Wales Hospital, Shatin, Hong Kong)

Rationale: In a genome-wide association study (GWAS) of symptomatic epilepsy (Two-stage genome-wide association study identifies variants in CAMSAP1L1 as susceptibility loci for epilepsy in Chinese. Hum Mol Genet. 2012;21:1184-9), the strongest association we identified was with a polymorphism in the CAMSAP1L1 gene: $p=1.0 \times 10^{-8}$ and odds ratio $=0.63$ for the $G$ allele of rs2292096. Little is known about CAMSAP1L1. However, a homolog of this gene is known to bind microtubules and to inhibit neurite outgrowth. With double immunostaining, we demonstrated partial colocalization of CAMSAP1L1 with $\beta$-tubulin. We hypothesize that the $\mathrm{G}$ allele of rs2292096 tags a haplotype expressing increased levels of CAMSAP1L1, which inhibits neurite growth after brain insults, thus reducing the risk of forming ectopic neural circuits that lead to epilepsy.

Methods: To test the hypothesis, we performed quantitative real-time PCR to measure CAMSAP1L1 and GAPDH expression in RNA of brain samples from surgery on refractory epilepsy patients. We genotyped rs2292096 in the patients and compared expression among genotypes. To determine the effect of CAMSAP1L1 on neurite growth, we transfected SH-SY5Y human neuroblastoma cells, treated with $25 \mu \mathrm{M}$ retinoic acid (RA), with siRNA to CAMSAP1L1 or negative control siRNA. Western blotting demonstrated gene knockdown. Cells were fixed 60 hours after transfection and were stained with $\beta$-tubulin antibody to label neurites and 4'-6-diamidino2-phenylindole (DAPI) to label nuclei. Metamorph software was used to quantify total neurite outgrowth, mean outgrowth per cell (neurite length per cell), and neurite branches per cell.

Results: In 28 individuals contributing brain samples, there were 20 AA, 6 AG, and 2 GG genotypes. CAMSAP1L1 expression, corrected for GAPDH expression, tended to differ by genotype (Kruskal-Wallis test, $\mathrm{p}=0.17$ for 26 hippocampal samples and $\mathrm{p}=0.06$ for 20 temporal lobe samples), with higher levels for GG. Transfection by siRNA reduced CAMSAP1L1 expression $\sim 50 \%$. Knockdown of CAMSAP1L1 increased neurite length per cell $(\mathrm{p}<0.05)$ for both undifferentiated and differentiated cells (Figure 1A). Knockdown also increased neurite branches per cell $(p<0.05)$ for both undifferentiated ( $0 \mu \mathrm{M}$ RA) and differentiated ( $25 \mu \mathrm{M}$ RA) cells (Figure 1B). Double immunostaining (Figure 2) for CAMSAP1L1 (red) and $\beta$-tubulin (green) demonstrated partial colocalization of CAMSAP1L1 with microtubules (from our poster: CAMSAP1L1 is a potential epilepsy gene in the Chinese population. 9th Asian \& Oceanian Epilepsy Congress, Manila, Philippines, 22-25 March 2012).

Conclusions: CAMSAP1L1 inhibits neurite outgrowth, and the $\mathrm{G}$ allele of rs2292096, which is associated with decreased risk of symptomatic epilepsy, may also be associated with increased expression of CAMSAP1L1. These results are consistent with the hypothesis that CAMSAP1L1 expression inhibits neurite growth, thus reducing the risk of symptomatic epilepsy.

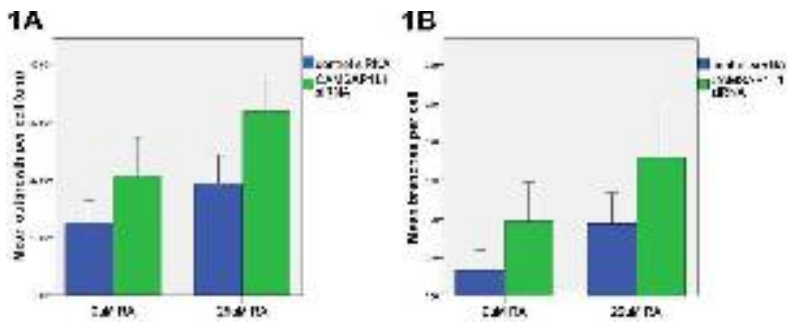

2
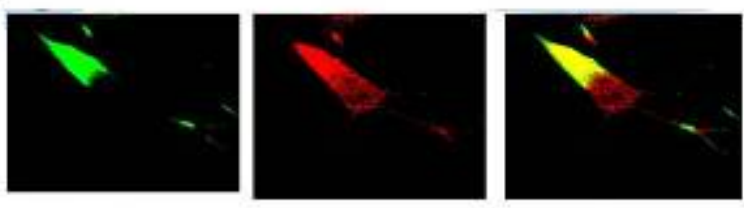

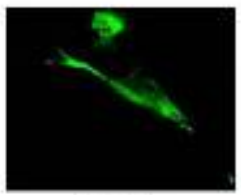

Tubuth

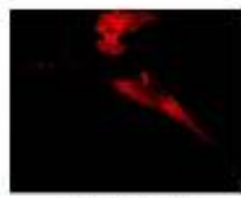

CAIASAP1L1 A2

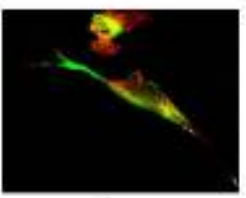

Nlerge

\subsection{6}

A POLYMORPHISM IN THE SCN1A GENE INFLUENCES THE EFFECT OF CARBAMAZEPINE ON CORTICAL EXCITABILITY - A PHARMACOGENETIC TMS-STUDY Katja Menzler $^{1}$, A. Hermsen ${ }^{1}$, K. Balkenhol ${ }^{1}$, C. Duddek ${ }^{1}, H$

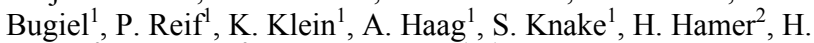
Trucks $^{3}$, T. Sander ${ }^{3}$ and F. Rosenow ${ }^{1}\left({ }^{1}\right.$ Epilepsy Center Hessen, Department of Neurology, Philipps-University Marburg, Marburg, Germany; ${ }^{2}$ Epilepsy Center Erlangen, University Hospitals Erlangen, Erlangen, Germany and ${ }^{3}$ Cologne Center for Genomics, Cologne University, Cologne, Germany)

Rationale: The SCN1A gene encodes the sodium channel alpha subunit NAv1.1. A biallelic splice-site variation in this gene (SNP IVS5N+5 G>A, rs3812718) is associated with electrophysiological properties of the channel and the effect of sodium-channel blocking antiepileptic drugs in vivo and in vitro. In the present study, we investigated the effects of the SNP genotype on cortical excitability at baseline and after administration of carbamazepine.

Methods: Paired-pulse transcranial magnetic stimulation (TMS) was applied to 49 healthy volunteers with genotype AA and 43 with genotype GG of the $S C N 1 A$ IVS5N $+5 \mathrm{G}>\mathrm{A}$ polymorphism at baseline and 5 hours after administration of $400 \mathrm{mg}$ of carbamazepine or placebo in a double-blind, randomized, cross-over design. Resting motor threshold (RMT), short interval intracortical inhibition (SICI) and facilitation (SICF) as well as cortical silent period (CSP) were determined.

Results: There was a significant influence of SNP genotype on the effect of carbamazepine. Volunteers with genotype GG had a higher increase in CSP duration compared with genotype AA after intake of carbamazepine as compared to placebo $(21.53 \pm 6.31 \mathrm{~ms}$ vs. $0.56 \pm 5.93$ $\mathrm{ms}, \mathrm{p}=.013)$. Furthermore, we observed, as other studies have, an increase in RMT, independent of genotype. At baseline, there was no significant difference in any TMS parameter between subjects with genotype AA and GG.

Conclusions: We showed a differential effect of carbamazepine on the CSP duration which depended on the SCN1A SNP genotype and reflects changes in GABAergic cortical inhibition. Our results support earlier studies which showed that alternative splicing of the SCN1A gene modulates inhibition of GABAergic cortical interneurons, possibly due to selective expression of NAv1.1 in these interneurons. In accordance with clinical observations, our results provide further evidence that this SNP affects pharmacoresponse to sodium channel blockers. Furthermore, this study shows that TMS can be used to investigate the pharmacogenetics of cortical excitability.

1.317

MUTATION OF THE CLN6 GENE IN TEENAGE-ONSET PROGRESSIVE MYOCLONUS EPILEPSY

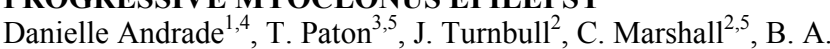
Minassian $^{2,4}$ and S. W. Scherer ${ }^{3,5}\left({ }^{1}\right.$ Neurology, University of Toronto, Toronto, ON, Canada; ${ }^{2}$ Genetics and Genome Biology, University of 
Toronto, Toronto, ON, Canada; ${ }^{3}$ The Centre for Applied Genomics, University of Toronto, Toronto, ON, Canada; ${ }^{4}$ Pediatric Neurology, University of Toronto, Toronto, ON, Canada and ${ }^{5}$ McLaughlin Centre and Department of Molecular Genetics, University of Toronto, Toronto, ON, Canada)

Rationale: Progressive Myoclonus Epilepsies (PMEs) are severe intractable neurodegenerative epilepsies that afflict patients of all ages, but more commonly adolescents, and are the main differential diagnosis of Juvenile Myoclonic Epilepsy. Genetic diagnosis is available for many teenage-onset PMEs, such as EPM1, EPM2A and EPM2B, NEU1, SCARB2 and several mitochondrial genes. However, some forms of PME may only be diagnosed through brain biopsy. We report a multiplex family with autosomal recessive teenage-onset PME that had remained undiagnosed despite extensive genetic and pathological testing.

Methods: The proband is a 24 year-old Pashtun Afghani man. Spontaneous and stimulus-induced myoclonus began at age 15 . Generalized tonic-clonic convulsions are controlled with valproate. Cognitive decline appeared. Examination shows generalized hyperreflexia, mild ataxia, and stimulus-sensitive multifocal myoclonus. Two older cousins (Fig. 1A) had had the same disease. Both had died at around age 30 . None of the three patients suffered visual decline. Investigations in all three showed cortical myoclonus and multifocal epileptiform discharges, and generalized brain atrophy. There were no urinary oligosaccharides (sialidosis). Skin and muscle biopsy and genetic testing for Lafora disease, UnverrichtLundborg disease, MERRF and NCL were negative. Rectal and brain biopsies or autopsy were declined.

We used whole exome sequencing (WES) combined with homozygosity mapping using a microarray-based SNP assay and genotyped over 600,000 SNPs in the affected proband and his male affected cousin.

Results: SNP microarray studies revealed 2 regions of shared homozygozity in the patients' two genomes: one in chromosome 10 and another in chromosome 15. Of the 41,597 variants found in the WES only two were exonic, novel, nonsynonymous, and located within the shared homozygous regions (Fig. 1C). One of these variants was in the gene encoding the NYD-SP5 testis development protein, which we did not pursue further. The other was a homozygous sequence change (c.768C $>\mathrm{G}$ ) in the CLN6 gene, which substitutes the protein product's aspartic acid residue at position 256 with glutamate (D256E). Aspartate in this position is conserved throughout studied evolution (Fig. 1D). The CLN6 (c.768C $>$ G) variant segregates with the disease in the pedigree (Fig. 1A). Sequencing for it in 268 control individuals, including 176 Pashtun, revealed it to be present in a single Pashtun individual, in heterozygote state.

Conclusions: The affected gene is the CLN6 gene previously known to underlie variant late-infantile and adult-onset neuronal ceroid lipofuscinoses. Combined with other recent work (1) our results add CLN6 to the genes mutations of which cause teenage-onset PME, expand the group of teenage-onset PME patients who can be diagnosed by genetic testing, and extend the clinical spectrum of CLN6 mutations to include teenage-onset PME. This work also exemplifies the potentiality of next generation sequencing in gene identification and in the diagnosis of patients with neurological diseases of unknown cause.

\subsection{8}

\section{FAMILIAL CONCORDANCE OF STATUS EPILEPTICUS IN} THE EPILEPSY PHENOME/GENOME PROJECT (EPGP)

\section{Judith L. Weisenberg ${ }^{1}$, L. L. Thio ${ }^{1}$, R. Fahlstrom ${ }^{2}$, D. Rabinowitz ${ }^{3}$,} M. Winawer ${ }^{4,5}$ and .. EPGP Investigators ${ }^{1}\left({ }^{1}\right.$ Washington University, St. Louis, MO; ${ }^{2}$ Neurology, University of California San Francisco, San Francisco, CA; ${ }^{3}$ Statistics, Columbia University, New York, NY; ${ }^{4}$ Neurology, Columbia University, New York, NY and ${ }^{5} \mathrm{GH}$ Sergievsky Center, Columbia University, New York, NY)
Rationale: Relatively little is known about the pathophysiology of status epilepticus (SE) despite the fact that it is the most common neurological emergency in children and a common one in adults. One approach to elucidating the underlying mechanisms is to identify predisposing genes. We have used the EPGP study population to test the hypothesis that genetic mechanisms contribute to SE.

Methods: EPGP is a multi-center collaborative effort to collect detailed phenotypic and genetic data on a large number of epilepsy patients. EPGP has enrolled approximately 2,000 sibling pairs and parent-child pairs with idiopathic generalized epilepsy (IGE) or localization-related epilepsy (LRE).

An analysis was performed on all IGE and LRE families with complete data in the EPGP database as of $12 / 31 / 2011$. We assessed familial concordance of SE $(\geq 30 \mathrm{~min})$ or prolonged seizures $(\geq 10$ min) within family units based on both the medical record abstraction (MRA), completed via formal chart review, and a detailed diagnostic interview (DI) administered to the participant or their parent at enrollment. The observed number of concordant pairs was compared to the expected number based on a binomial distribution using a chi square test. A mixed effect logistic regression analysis was performed to adjust for age, gender and broad epilepsy type.

Results: There were 1497 participants in 626 families that had 2 or more participants with completed data collection. 764 participants had IGE and 505 participants had LRE. The average length of followup from time of epilepsy diagnosis was 11.3 years. The data from the MRA and DI did not show strong agreement. For instance, on the MRA 94 participants had a history of SE versus 86 on the DI, of which only 37 were identified by both. Assuming the MRA is gold standard, the DI has a sensitivity of $39 \%$, a specificity of $97 \%$, a positive predictive value of PPV of $43 \%$, and a negative predictive value of $97 \%$. Similar discrepancies occurred when looking at the concordance for SE within families. For instance, the MRA identified 10 families in which both participants were concordant for SE, and the DI identified 5 such families. Only 2 of those families were identified by both. Therefore, we analyzed the MRA and DI data independently for familial concordance for the occurrence of SE and prolonged seizures. For both the MRA and the DI, the concordance rate for $\mathrm{SE}(\mathrm{p}<0.0001)$ and prolonged seizures $(\mathrm{p}<0.0001)$ was significantly greater than expected. To ensure that we were not assessing concordance of complex febrile seizures, an analysis was performed excluding those participants with a history of a febrile seizure $\geq 15$ min on the MRA (24 participants). The familial concordance for $\mathrm{SE}(\mathrm{p}=0.005)$ and prolonged seizures $(\mathrm{p}=0.003)$ remained greater than expected. Adjusting for age, gender and epilepsy type did not eliminate the effect.

Conclusions: The EPGP database shows greater than expected concordance for prolonged seizures and SE within families. These findings suggest that genetic mechanisms contribute to SE.

1.319

INFANTILE SPASMS OF UNKNOWN ETIOLOGY: PHENOTYPIC FEATURES OF A LARGE COHORT Maura R. Madou ${ }^{1}$, C. J. Yuskaitis ${ }^{3}$, K. B. Howell ${ }^{2}$, A. Poduri ${ }^{3,4}$, I. E. Scheffer $^{2,5}$, E. H. Sherr ${ }^{1}$, *. EPGP investigators ${ }^{1}$ and *. Epi4K investigators ${ }^{1}$ ( ${ }^{1}$ Departments of Neurology and Pediatrics, University of California San Francisco, San Francisco, CA; ${ }^{2}$ Department of Neurology, Royal Children's Hospital,, Melbourne, VIC, Australia; ${ }^{3}$ Department of Neurology, Boston Children's Hospital, Boston, MA; ${ }^{4}$ Division of Epilepsy and Clinical Neurophysiology, Boston Children's Hospital, Boston, MA and ${ }^{5}$ Florey Neurosciences Institute, Melbourne, VIC, Australia)

Rationale: The etiology of infantile spasms (IS) is unknown in one third of patients despite investigation for structural, metabolic and chromosomal conditions. IS of unknown etiology is typically thought to have a better prognosis than IS with a known etiology. We report the clinical features of the largest reported cohort of patients with IS of unknown etiology. 
Methods: Participants were enrolled in the Epilepsy

Phenome/Genome Project (EPGP) Infantile Spasms Project, a multicenter study. Inclusion criteria included history of infantile spasms of unknown etiology, and EEG showing hypsarrhythmia or a hypsarrhythmia variant. Exclusion criteria included developmental delay of greater than $50 \%$ prior to onset of IS, prematurity $(<32$ weeks gestation) or a malformation other than focal cortical dysplasia on brain imaging. The full criteria can be found at www.epgp.org. For this study, medical records were reviewed by two independent reviewers. EEG and MRI brain imaging were reviewed. Patients were excluded if an etiology (including focal cortical dysplasia) was identified.

Results: Of 107 patients with IS of unknown etiology, $61(57.0 \%, \mathrm{p}=$ $0.18)$ were male and $46(43.0 \%)$ had a family history of epilepsy. The median age at last follow-up was 2.5 years (range $0.25-20$ years). IS onset occurred at a median age of 5.3 months (range 2-14 months). Treatment was initiated within one month in $64(59.8 \%)$ patients; corticosteroids were first line therapy in $71(66.3 \%)$ patients. 31 $(29.0 \%)$ had resolution of IS (defined as seizure freedom for at least six months) with the first medication used. Seizures antecedent to the IS occurred in $10(9.4 \%) .61(57.0 \%)$ of patients had other seizure types subsequent to the IS. Developmental delay occurred prior to IS in $24(22.4 \%)$. At most recent assessment, only $20(18.7 \%)$ had normal development.

Normal developmental outcome was seen in 19/58 (32.8\%) of patients with normal development prior to the onset of IS. 17/64 $(26.6 \%)$ of patients who were treated with corticosteroids within one month and 13/31 (41.9\%) of patients who had resolution of IS with the first medication used had a normal developmental outcome. Conclusions: This large cohort of patients with IS of unknown etiology shows significant heterogeneity of developmental and seizure outcomes. The majority of patients had an unfavourable developmental outcome despite the presence of factors previously identified to predict good prognosis including normal development prior to IS and prompt initiation of and response to treatment, and despite having excluded from the cohort those with severe delay before onset of spasms. The variable clinical outcomes suggest that the causes are also likely heterogenous. Whole exome sequencing of these patients will take place under the auspices of the Epi4K Project. Identification of the genetic bases of IS will allow better prognostication in individual patients as well as a greater understanding of the mechanisms underlying the development of IS, and will be the first step in the development of targeted therapies.

\subsection{0 \\ SEVERE INFANTILE MULTI-FOCAL EPILEPSY: A SLOW PROGRESSIVE FOCAL EPILEPTIC ENCEPHALOPATHY DUE TO SCN1A \\ Young Ok Kim ${ }^{1,2}$, J. M. McMahon ${ }^{1}$, K. Kelley ${ }^{3}$, D. Gill ${ }^{4}$, S. F. Berkovic $^{1}$ and I. E. Scheffer ${ }^{1,5}\left({ }^{1}\right.$ Epilepsy Research Centre, Department of Medicine, The University of Melbourne, Austin Health, Heidelberg, Melbourne, VIC, Australia; ${ }^{2}$ Department of Pediatrics, School of Medicine, Chonnam National University, Gwangju, Republic of Korea; ${ }^{3}$ Department of Pediatrics, Northwestern University Children's Memorial Hospital, Chicago, IL; ${ }^{4}$ TY Nelson Department of Neurology, The Children's Hospital at Westmead, Sydney, NSW, Australia and ${ }^{5}$ Florey Neuroscience Institutes, Melbourne, VIC, Australia)}

Rationale: In our large study of infantile-onset epileptic encephalopathies, we identified a new genetic focal epileptic encephalopathy of severe infantile multifocal epilepsy (SIMFE) defined as infantile onset epileptic encephalopathy with multifocal seizures and subsequent developmental slowing. We aimed to confirm that SIMFE is a recognizable electroclinical syndrome due to sodium channel gene (SCN1A) mutations.

Methods: Clinical and electrophysiological findings in 7 SIMFE patients were characterized. Sanger sequencing of SCN1A was performed and copy number variations were excluded by multiplex ligation-dependent probe amplification.

Results: Median age of seizure onset was 6 mths(5-19 mths). All 7 patients were female ( 3 briefly reported previously). Initial seizures were brief and afebrile in 57\%(4/7) and comprised generalized tonicclonic (TCS)(3/7), focal(3/7), and hemiclonic seizures(1/7). The most frequent seizure type was focal in all with a median onset age of 19 mths(4.5 mths $-4.3 \mathrm{yrs}) .5(71 \%)$ had TCS and $4(57 \%)$ had tonic seizures. Myoclonic (5/7), hemiclonic (4/7), or atonic seizures (1/7) were transient. Myoclonic seizures were triggered by vigabatrin or gabapentin in two patients. All patients had multiple types of focal seizures which were often well lateralized but poorly localized. The most common semiology comprised eye and/or head deviation with focal clonic or asymmetric tonic movements with unresponsiveness $(4 / 7,57 \%)$. Focal seizures (20 sec-3 mins) occurred in clusters $(71 \%)$ and evolved to bilateral convulsive seizures or SE (median onset age $3 \mathrm{yrs} ; 9 \mathrm{mths}-7 \mathrm{yrs}$ ). All developed learning difficulties (median onset age, $3.5 \mathrm{yrs} ; 16$ mths- 8 yrs) with variable cognitive ability from severe intellectual disability to normal. There is no generalized epilepsy. Early EEGs were normal or showed slowing and 3 patients showed 1 or 2 epileptiform foci. Multifocal epileptiform activity appeared later in $5(71 \%)$ (median onset age $4.3 \mathrm{yr} ; 3 \mathrm{yrs}-9 \mathrm{yr} 10$ $\mathrm{mth}$ ). Photosensitivity was not seen. Ictal EEGs showed multifocal seizures that propagating diffusely but did show a not migrating focal pattern. 6 patients $(86 \%)$ had SCN1A mutations.

Conclusions: SIMFE is due to SCN1A mutations in most cases. SIMFE is distinct from Dravet syndrome as it has multifocal rather than generalized seizures, later developmental slowing and frequent tonic seizures.

\subsection{1 \\ COMPARISON OF SCN1A MUTATIONS IN CASES AND CONTROLS IDENTIFIES REGIONS OF HIGH AND LOW PROBABILITY FOR DISEASE-CAUSATION}

Thomas E. Callis, L. R. Susswein and J. L. Carr (Clinical Genetics, Transgenomic, New Haven, CT)

Rationale: Mutations in the sodium channel alpha-1 subunit gene, SCN1A, are a cause of familial epilepsy syndromes including Dravet syndrome and genetic epilepsy with febrile seizures plus (GEFS+). Distinguishing pathogenic mutations from benign, rare variation is a major challenge in the interpretation of clinical genetic test results, particularly for missense mutations. In this study, we compared SCN1A missense mutations in cases and controls by frequency and location to identify high and low probability regions of pathogenicity. Methods: DNA sequence analysis was performed 409 unrelated patients (52\% female; age at testing $6.6 \pm 6.6$ years) referred for $\mathrm{SCN} 1 \mathrm{~A}$ genetic testing. Case variants were interpreted with respect to published literature, Transgenomic control individuals $(\mathrm{n}=$ more than 75 individuals) and the NHLBI Exome Sequencing Project (ESP) database ( $\mathrm{n}=$ more than 5,300 individuals). Singleton missense mutations present in the NHLBI ESP database were considered to be rare, benign variants in this analysis. Mutations were characterized as being located in the $\mathrm{N}$ terminus (amino acids 1 to 123), interdomain linker (IDL I to II, amino acids 426 to 762 ; IDL II to III, amino acids 993 to 1213; and IDL III to IV, amino acids 1484 to 1536 ), transmembrane/linker (domain I, amino acids 124 to 425; domain II, amino acids 763 to 992; domain III, amino acids 1214 to 1483; and domain IV, amino acids 1537 to 1786 ), or $\mathrm{C}$ terminus (amino acids 1787 to 2009 ).

Results: Overall, rare protein-altering variants were far more common $(\mathrm{p}<0.0001)$ among cases $(15 \%)$ than in controls $(1 \%)$. Missense mutations were the most common type, accounting for $61 \%$ of unique mutations identified in cases. Misssense mutations occurred with significantly higher frequency in cases compared to controls in the N-terminal $(0.7 \%$ vs $0.1 \% ; \mathrm{p}=0.01)$ and the transmembrane domain/linker regions $(6.4 \%$ vs $0.3 \% ; p<0.0001)$. No significant differences in the frequency of mutations were observed 
between cases and controls in the interdomain linker $(0.5 \%$ vs $0.4 \%$; $\mathrm{p}=0.7)$ or the C-terminal regions $(0.7 \%$ vs $0.2 \% ; \mathrm{p}=0.09)$.

Conclusions: Our comparison of rare genetic variation seen in patients referred for SCN1A genetic testing compared to rare variation seen in ostensibly healthy individuals suggests mutation location can aid in the interpretation of novel missense variants identified in clinical genetic testing. Based on this analysis, novel variants in the interdomain linker regions should be viewed as variants of uncertain significance, whereas mutations in transmembrane/linker regions may be considered more confidently to be probable disease-causing mutations.

\subsection{2}

DE NOVO GAIN OF FUNCTION KCNT1 CHANNEL MUTATIONS CAUSE SEIZURES AND DEVELOPMENTAL DELAY IN MALIGNANT MIGRATING PARTIAL SEIZURES OF INFANCY

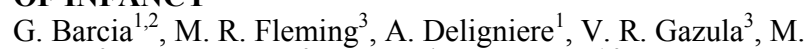
Brown $^{3}$, J. Kronengold ${ }^{3}$, R. Cilio ${ }^{4}$, P. Nitschke ${ }^{1,2}$, A. Kaminska ${ }^{1,2}$, N. Boddaert $^{1,2}$, J. L. Casanova ${ }^{5}$, I. Desguerre ${ }^{1,2}$, A. Munnich ${ }^{1,2}$, O. Dulac $^{1,2}$, L. Kaczmarek ${ }^{3}$, L. Colleaux ${ }^{1,2}$ and Rima Nabbout ${ }^{1,2}$ ( ${ }^{1}$ Necker Enfants Malades, Paris, France; ${ }^{2}$ inserm, Paris Descartes, Paris, France; ${ }^{3}$ Yale, New heaven, CT; ${ }^{4}$ ospedale bambino Gesu, Rome, Italy and ${ }^{5}$ rockefeller institute, New york, NY)

Rationale: We aimed to identify the gene causing malignant migrating partial seizures of infancy (MMPSI), a rare epileptic encephalopathy of infancy characterized by the occurrence of polymorphous focal seizures and arrest of psychomotor development in the first 6 months of life.

Methods: We collected DNA samples from 12 individuals fulfilling the criteria for MMPSI and performed exome sequencing in 3 probands and their unaffected parents. We sequenced the candidate gene and confirmed the functional effect through oovocyte expression system.

Results: A single gene, KCNT1, was affected by distinct heterozygous missence variant (p.Arg428Gln and p.Ala934Thr) in 2 unrelated probands. Both mutations were confirmed by Sanger sequencing and occured de novo. Direct sequencing of KCNT1 in the 9 remaining patients identified the same de novo p.Arg428Gln missense mutations in two further patients and 2 distinct de novo mutations p.Arg474His and p.Ile760Met in two other patients $\mathrm{KCNT} 1$ encodes a sodium-activated potassium ( $\mathrm{KNa}$ ) channel widely expressed in the nervous system. Its activity contributes to the slow hyperpolarization that follows repetitive firing. Electrophysiological studies of the identified mutations showed a gain-of-function. Conclusions: Our findings further confirm the genetic etiology of MMPSI and ascribe MMPSI to the large family of channelopathies. In addition to regulating ion flux, the KCNT1 channel interacts with the mRNA binding protein, FMRP, whose defect results in the most common form of inherited intellectual disability, namely fragile $\mathrm{X}$ syndrome. We thus propose that mutations in KCNT1 provide the first pathophysiological basis of a channelopathy linking dysfunction of firing, thus epilepsy, to impaired function of a protein involved in cognitive development, FMRP, causing arrest of psychomotor development.

\section{Neuropathology of Epilepsy: Animal Studies}

\author{
1.323 \\ ISOKETALS: PUTATIVE MEDIATORS OF LEARNING AND \\ MEMORY DEFICITS IN EXPERIMENTAL TEMPORAL \\ LOBE EPILEPSY \\ Jennifer N. Pearson ${ }^{1,2}$, L. Liang ${ }^{1}$, L. J. Roberts $\mathrm{II}^{3,4}$ and M. Patel ${ }^{1,2}$ \\ ( ${ }^{1}$ Pharmaceutical Sciences, University Of Colorado Anschutz \\ Medical Campus, Aurora, CO; ${ }^{2}$ Neuroscience Program, University \\ Of Colorado Anschutz Medical Campus, Aurora, CO; ${ }^{3}$ Division of
}

Clinical Pharmacology, Vanderbilt University, Nashville, TN and ${ }^{4}$ Department of Pharmacology, Vanderbilt University, Nashville, TN)

Rationale: Oxidative stress has been identified as a contributing factor to the cognitive decline associated with aging and neurodegenerative diseases such as Alzheimer's disease (AD). Most recently, oxidative stress has been implicated in temporal lobe epilepsy (TLE) however, to what degree oxidative stress products contribute to cognitive decline in TLE is unknown. Isoketals (IsoKs) are highly reactive gamma-ketoaldehydes formed via the isoprostane pathway of non-enzymatic, free radical catalyzed oxidation of arachidonic acid. IsoKs rapidly adduct to lysine residues and crosslink proteins.

Methods: We tested the hypothesis that IsoKs are increased in a model of experimental TLE and that scavenging of these highly reactive species would attenuate learning and memory deficits. Adult male Sprague-Dawley rats (300-325g) were treated with a single high dose of kainic acid (KA) and monitored for behavioral seizures during status epilepticus and the progression to chronic epilepsy. Brain tissue (hippocampus) was collected 24 hours and 6 weeks post$\mathrm{KA}$, corresponding to the acute and chronic phases of epileptogenesis and subjected to mass spectrometry for analysis of IsoK lysine adduct levels. Salicylamine (SA) is an orally active scavenger of gammaketoaldehydes. A separate cohort of animals was treated with KA followed by a single injection of SA $(200 \mathrm{mg} / \mathrm{kg}) 30$ minutes later and allowed free access to water supplemented with SA $(1 \mathrm{~g} / \mathrm{L})$ for 7 days. There was no difference in water consumption between groups or duration and intensity of seizures. After a 7 day medication period, animals were tested for indices of learning and memory in a novel object recognition task (NOR). The NOR task is uniquely suited for testing KA treated animals at this time point as it is minimally stressful and requires little training of the animal.

Results: At the 24 hour time point, we found increased levels of IsoK adducts in isolated hippocampus of KA treated animals in a region specific manner such that the dentate gyrus had the largest increase (60\% higher than control) followed by CA3 and CA1. At the 6 week time point, we found a global increase in levels of IsoK adducts in all areas of the hippocampus of KA treated animals (50\% higher than control), suggesting that IsoKs may be a druggable target in TLE. On the NOR behavioral task, KA treated animals that received SA performed significantly better than animals not treated with SA (KA vs $\mathrm{KA}+\mathrm{SA} \mathrm{p}=0.01$ ), at a level equivalent to control animals (KA+SA vs Control $\mathrm{p}=0.15$ ). Upon completion of the behavioral task, brain tissue and plasma was collected and subjected to HPLC analysis for levels of SA. As expected, SA was present in plasma samples at an average concentration of $0.818 \mathrm{uM}$ and in brain tissue at an average abundance of $29.445 \mathrm{nmol} / \mathrm{g}$ tissue.

Conclusions: These data suggest that IsoKs are potential mediators of cognitive dysfunction associated with experimental TLE and establish SA as a therapeutic to attenuate these deficits.

Funding: CURE Multidisciplinary Award (Patel and Roberts) and NIHRO1NS039587-S1 (Patel and Pearson)

\subsection{4 \\ APOCYNIN TREATMENT ATTENUATES SEIZURE SUSCEPTIBILITY OF THE MICE FOLLOWING SYSTEMIC INFLAMMATION}

w. Huang and Hung-Ming Wu (Department of Neurology, Changhua Christian Hospital, Changhua city, Taiwan)

Rationale: Systemic inflammation leads to a number of the mediated physiological changes in central nervous system. The underlying mechanisms and the signaling pathways involved in these phenomena remain yet well understood. We hypothesized that peripheral inflammation leads to increased neuronal excitability arising from a CNS immune response. The research aim was to investigate the role of activated microglia in seizure susceptibility of the mice following sepsis. 
Methods: We induced inflammation by administration (i.p.) of lipopolysaccharide (LPS) to adult male mice. Seven days later, the LPS-treated mice were randomly assigned to treat daily with vehicle, dextromethorphan $15 \mathrm{mg} / \mathrm{kg}$, apocynin $10 \mathrm{mg} / \mathrm{kg}$. To examine the excitability of the brain in vivo, we administered pentylenetetrazole (i.p.) to evoke clonic seizures. In vitro hippocampal slices from the mice were performed to measure spontaneous interictal burst firing. Results: The LPS-treated mice exhibited a marked, persistent inflammatory response within the hippocampus, characterized by microglial activation. Mice treated with apocynin showed decreased susceptibility to PTZ seizures compared with those with vehicle treatment. In vitro hippocampal slices from LPS-treated mice showed increased spontaneous interictal burst firing, which was attenuated in the apocynin-treated mice.

Conclusions: Peripheral inflammation induced persistent neuroinflammation and, such as microglia activation, and increased seizure susceptibility in mice. By inhibition of microglia activation, apocynin demonstrated to attenuate intrinsic excitability in hippocampus and recovery seizure threshold in LPS-treated mice. Our finding of a microglia-mediated increase in CNS excitability provides insight into potential mechanisms underlying the disparate neurological change following systemic inflammation.

\subsection{5}

\section{LONG TERM FOLLOW-UP OF THE MULTIPLE-HIT RAT} MODEL OF SYMPTOMATIC INFANTILE SPASMS Ozlem Akman ${ }^{1}$, S. W. Briggs ${ }^{1}$ and A. S. Galanopoulou ${ }^{1,2}$ ( ${ }^{1}$ Saul R. Korey Department of Neurology, Albert Einstein College of Medicine, Bronx, NY and ${ }^{2}$ Dominick P. Purpura Department of Neuroscience, Albert Einstein College of Medicine, Bronx, NY)

Rationale: Infantile spasms (IS) syndrome is a pediatric epilepsy syndrome manifesting with epileptic spasms in infancy and usually leads to other seizure types and epilepsy syndromes, including the Lennox-Gastaut Syndrome. IS due to structural/metabolic etiology are usually associated with worse prognosis. We previously characterized the early phenotype of a chronic rat model of IS due to structural lesion, the multiple hit rat model of IS, showing the presence of an early period with clusters of spasms and emergence of other types of seizures till postnatal day 21 (PN21). In this study, we evaluated whether the multiple-hit model of IS exhibits epilepsy in adulthood.

Methods: Male Sprague-Dawley rats received right intracerebral infusions of doxorubicin and lipopolysaccharide at PN3 and intraperitaneal p-chlorophenylalanine at PN5. Epidural cortical EEG electrodes were placed between PN66-89 and long-term video-EEG records were recorded (XLTEK system) till PN150-306 and were reviewed by an observer blinded to treatment allocation. Rats were monitored in 24 hour epochs, 2-3 days per week, with video on between $9 \mathrm{am}-6 \mathrm{pm}$, following a $12 \mathrm{hr} / 12 \mathrm{hr}$ light/dark cycle.

Results: Spontaneous motor seizures (stage 3-5) were detected in 3 out of 6 animals (50\%) with younger age at occurrence PN127.5 \pm 4 .1. All seizures started from the sleep state and had stereotypical appearance: initial flattening of the background with overlapping low amplitude 20-22.2 Hz fast activity, followed by rhythmic theta slowing at the right parietal area evolving into rhythmic sharp waveforms in the right parietal hemisphere and subsequent generalized 4-5 Hz continuous rhythmic spikes or spike and slow waves in the EEG. A postictal diffuse attenuation of the background was seen. In two seizure events, an initial burst of high amplitude rhythmic slowing intermixed with spike and slow waves preceded the onset. Average seizure duration was $41.6 \pm 3.7$ seconds. The interictal background demonstrated excessive sleep patterns, continuous polymorphic slowing at the right parietal region and frequent right parietal spikes. In addition, bursts of spike and slow wave discharges $(6 \mathrm{~Hz})$ were detected in 1 out of 6 animals (\% 16.6), which has not manifested motor seizures yet, with the first appearance at PN 87. Conclusions: Our results show that in the multiple-hit model of IS,
$50 \%$ of the rats manifest epilepsy with motor seizures in adulthood, all of which arise from sleep and share electrographic similarities with the nocturnal tonic seizures seen in Lennox Gastaut syndrome. In addition, 1/6 rats with prior spasms manifested bursts of spike and slow wave complexes at a slower frequency range $(6 \mathrm{~Hz})$ compared to the absence seizures seen in models of absence seizure $(\sim 8.5 \mathrm{~Hz})$. These slow spike and wave complexes are therefore reminiscent to the slow spike wave pattern of Lennox Gastaut syndrome. These findings indicate that the multiple-hit model of IS recapitulates the evolution of IS into other types of epilepsy syndromes, including Lennox-Gastaut syndrome. This study was funded by NINDS NS 20253, Autism Speaks, and the Heffer Family Foundation.

\subsection{6 \\ DECREASED HIPPOCAMPAL NEUROGENESIS AS A LONG-TERM CONSEQUENCE OF EARLY-LIFE STATUS EPILEPTICUS IN RATS}

H. Kubova, G. Tsenov and Pavel Mares (Developmental

Epileptology, Institute of Physiology Academy of Sciences, Prague 4, Czech Republic)

Rationale: Convulsive status epilepticus (SE) in adult animals leads to increased neurogenesis shortly after the insult. In later intervals, severe decline of neurogenesis was documented. Previous studies also demonstrated significant increase of neurogenesis one week after SE elicited in two-week-old rats. Nothing is however known about changes in neurogenesis in long interval after early SE. Therefore present experiment was focused on long-term changes in production of new neurons in the hippocampus of rats experiencing SE at postnatal day $(\mathrm{P}) 12$.

Methods: SE was induced by pilocarpine $(40 \mathrm{mg} / \mathrm{kg})$ in $\mathrm{LiCl}$ $(3 \mathrm{meq} / \mathrm{kg}$ ) pretreated $\mathrm{P} 12$ rats. To prevent mortality and to improve recovery rats were given a single dose of paraldehyde $(0.07 \mathrm{mg} / \mathrm{kg})$ $1.5 \mathrm{~h}$ after $\mathrm{SE}$ onset. Controls received saline instead pilocarpine. Animals were video/EEG monitored 5-6 mo after SE to detect spontaneous seizures. After the end of monitoring, animals were perfused and newborn neurons were detected using doublecortin (DCX) immunohistochemistry. Number of positive neurons in the dorsal hippocampus was assessed using stereological cell count in 5 consecutive sections. Adjacent sections were Nissl stained and hippocampal volume was morphometrically assessed.

Results: SE developed in all 19 animals. Clinically, status epilepticus was characterized by twitching of facial muscles, chewing, head bobbing, forelimb clonus, tail erection and "swimming" movements. Paraldehyde suppressed motor seizures. Mortality after SE was $36 \%$. Slight decrease of the body weight was seen $24 \mathrm{~h}$ after SE (3\% decrease whereas controls gained $17 \%$ of their weight at P12). Electrographic (subclinical) seizures were detected in subpopulatin of animals. The hippocampal volume in animals with SE at P12 reached about $93 \%$ of control value, but difference was not statistically significant. When compared to controls, number of DCX-positive neurons was about $37 \%$ lower in animals with SE.

Conclusions: Early SE leads to impaired dentate neurogenesis in adult rats. Whether these changes are related to epilepsy or development of comorbidities has to be further analyzed. This study was supported by grants No P302/10/0971 and No P304/12/G069 of the Grant Agency of the Czech Republic, and No ME08045 of the Ministry of Education of the Czech Republic.

\subsection{7 \\ PROGRESSION OF EEG PATTERN IN STATUS EPILEPTICUS CORRELATES WITH THE DEGREE OF NEURONAL DEGENERATION}

Kenneth W. Imerman, S. T. Marsh and D. M. Treiman (Neurology /

Epilepsy, Barrow Neurological Institute, Scottsdale, AZ) 
Rationale: Animal models of status epilepticus (SE) have shown a progression of glutamate-mediated neuronal destruction with increasing duration. Depending on the induction model, animals progress through SE with varying time courses, from hours to days. Therefore, duration of SE is not a reliable predictor of the degree of damage.

Treiman et al. (Epilepsy Res. 1990; 5: 49-60) described a predictable progression of EEG patterns in SE that varied little across species and across different induction models: stage I - discrete seizures; stage II - merging of seizures; stage III - continuous ictal activity; stage IV continuous ictal pattern with periods of attenuation; stage V - PLEDs. Our objective was to correlate EEG stage of SE with the degree of necrosis. A supplementary objective was to evaluate what degree of pathology, if any, corresponds with prolonged PLEDs.

Methods: 36 Male Sprague Dawley rats were implanted with EEG electrodes. SE was induced with an IP injection of lithium chloride followed by subcutaneous injection of pilocarpine. SE was terminated by IP injection of diazepam and phenobarbital after the animals' progression to their respective stage of SE. Of the 30 rats that survived, 6 were terminated at stage I (discrete seizures), 6 at stage III (continuous ictal pattern), 7 at stage V (PLEDS), and 6 at stage $\mathrm{V}$ plus 4 hours. Three pre-treatment controls and two sham controls were analyzed for comparison.

Seven days post-SE, animals were sacrificed. The brains were removed, fixed, frozen and sectioned at 40 microns in the coronal plane through the hippocampus. One set of sections was stained with H\&E and a second was stained with de Olmos amino cupric silver stain, which stains necrotic neuronal cell bodies, axons, and dendrites black.

23 brain regions were inspected. $H \& E$ sections were analyzed at $20 \mathrm{x}$ with a Zeiss light microscope. De Olmos stained sections were photographed at $2.5 \mathrm{x}$ at 3 levels corresponding to $0.20 \mathrm{~mm}, 3.30 \mathrm{~mm}$, and $5.60 \mathrm{~mm}$ from Bregma. With ImageJ software (NIH), densiometry was used to quantify the degree of necrosis in each of the 23 regions. Treatment groups were compared to one another using appropriate statistical models.

Results: pre-treatment controls and stage I rats showed sparse, nonspecific degeneration. In stage III, regional damage became apparent, particularly in the hippocampus (CA1 and CA3) as well as the striatum and cortex. Degeneration increased in stage V (PLEDs), particularly in the cortex, striatum, and thalamus. In Stage V plus 4 hours, progression of neuronal degeneration was noted in most brain regions. The quantitative analysis will be discussed further on the poster.

Conclusions: EEG stage correlates with the degree of neuronal degradation. Furthermore, additional neuronal degeneration occurred in the 4 hours after rats entered stage V (PLEDs) of SE, providing evidence that PLEDs denote a physiologic process that is not benign. This study will be repeated using different induction models to see if the correlation between EEG stage and extent of necrosis persists, independent of the duration of SE.

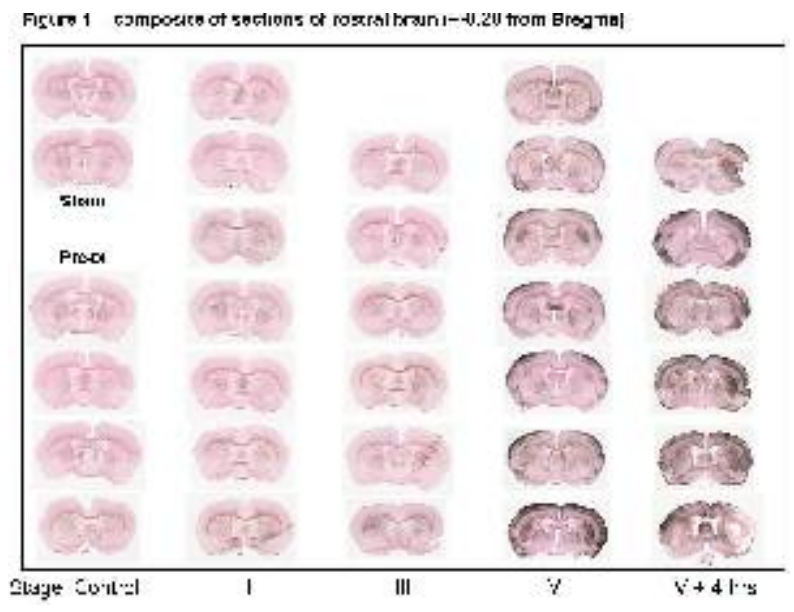

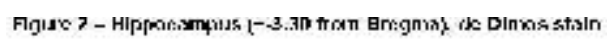

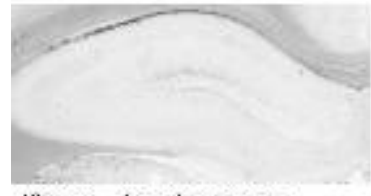

Stage - I sereta selzurse

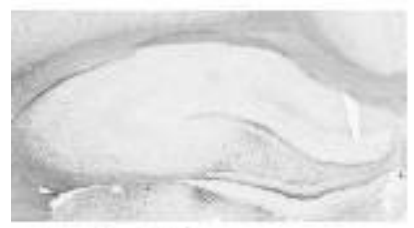

Stage $\mathrm{Y}-\mathrm{PLE} \mathrm{X}_{\mathrm{s}}$
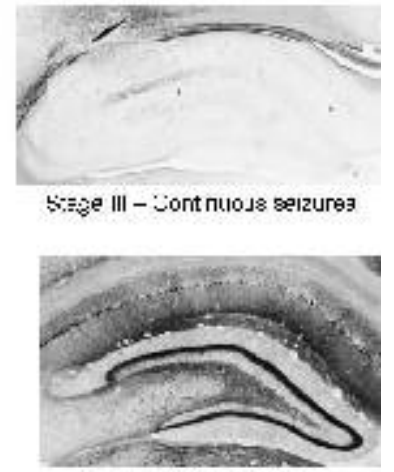

Stage $\mathrm{v}-4 \mathrm{~h}-=$ rolon- $-\mathrm{d}$ F.ED

\subsection{8 \\ MTORC1 INHIBITION RESCUES LEARNING AND MEMORY BUT NOT SOCIAL DEFICITS FOLLOWING STATUS EPILEPTICUS}

Angela Carter ${ }^{1,2}$, A. L. Brewster ${ }^{2,3}$, J. N. Lugo ${ }^{2,5}$, V. V. Patil ${ }^{2,3}$, W. L.

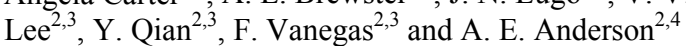

$\left({ }^{1}\right.$ Neuroscience, Baylor College of Medicine, Houston, TX; ${ }^{2}$ The Mary and Gordon Cain Pediatric Neurology Research Foundation Laboratories, Houston, TX; ${ }^{3}$ Jan and Dan Duncan Neurological Research Institute, Houston, TX; ${ }^{4}$ Neurology, Baylor College of Medicine, Houston, TX and ${ }^{5}$ Psychology and Neuroscience, Baylor University, Waco, TX)

Rationale: Status epilepticus (SE) is associated with the development of epilepsy and comorbidities, including deficits in cognition and social behavior. Previous studies have implicated the mammalian target of rapamycin (mTOR) signaling cascade in the pathophysiology of SE and epilepsy using the mTOR complex 1 (mTORC1) inhibitor rapamycin (Rap). Under basal conditions, mTORC1 regulates protein synthesis and is an important mediator of synaptic plasticity. However, whether aberrant mTOR signaling contributes to behavioral deficits following SE is unclear. In the studies reported here, we determined whether aberrant mTORC1 signaling plays a role in hippocampal-dependent learning and memory tasks, social behavior, locomotion, and anxiety. Methods: SE was induced in rats using pilocarpine (SE) while controls (Sham) received saline. Two weeks after SE induction, we administered Rap or vehicle (Veh) every other day for one week. The treatment groups were Sham + Veh, Sham + Rap, SE + Veh, and SE + Rap ( $n=7-12$ per group). We tested SE and Sham animals (from each treatment group) in the Morris Water maze (MWM), Novel Object Recognition (NOR), Social Interaction (SI), and the Open Field task (OF) tasks. After behavioral testing was completed, we performed western blotting to verify inhibition of mTORC1 (S6 phosphorylated at S240/244).

Results: SE + Veh rats exhibited significantly longer escape latencies during the acquisition phase in MWM test and decreased time spent in the target quadrant searching for a hidden platform in the probe trial compared to Sham + Veh animals $(p<0.05$ and $p<0.01$, respectively). In NOR, $\mathrm{SE}+\mathrm{Veh}$ animals displayed no preference for the novel object as compared to controls $(p<0.001)$. In the SE + Rap group, the deficits in MWM and NOR were rescued to performance levels of Sham + Veh animals. In SI task, SE + Veh animals spent less time with a social partner than Sham + Veh animals $(p<0.05)$. Locomotor activity in the OF revealed that $\mathrm{SE}+\mathrm{Veh}$ rats spent more time in the inner portion of the OF arena than Sham + Veh and displayed increased distance traveled and travel velocity $(\mathrm{p}<0.05)$. In the SE + Rap animals, neither the time spent with a social partner in SI nor the travel distance or velocity in OF were rescued. For all behavioral tasks, Rap had no effect on Sham + Rap animals. 
Conclusions: These data suggest that aberrant mTORC1 signaling contributes to deficits in hippocampal-dependent learning and memory but not aberrant social behavior following SE. Future studies are planned to evaluate whether inhibition upstream in the mTOR pathway to target both mTORC -1 and -2 will rescue both learning and memory and social behavior deficits following SE.

\subsection{9 \\ NEURONAL DEGENERATION IS OBSERVED IN MULTIPLE REGIONS OUTSIDE THE HIPPOCAMPUS AFTER LITHIUM PILOCARPINE-INDUCED STATUS EPILEPTICUS IN THE IMMATURE RAT}

E. A. Scholl ${ }^{1,2}$, F. E. Dudek ${ }^{1}$ and J. J. Ekstrand ${ }^{2}\left({ }^{1}\right.$ Neurosurgery, University of Utah School of Medicine, Salt Lake City, UT and ${ }^{2}$ Pediatrics, University of Utah School of Medicine, Salt Lake City, UT)

Rationale: Although the hippocampus is frequently identified as an epileptic focus in patients with temporal lobe epilepsy, neuropathology studies of similar patients have shown damage to other areas including parahippocampal regions (e.g. entorhinal cortex), amygdala, and areas outside the temporal lobe. Similar neuronal death occurs in most animal models of acquired epilepsy in the adult brain and, because of the traditional focus on the hippocampus, these models have been used to study how the hippocampal lesion may contribute to the development of epilepsy. In acquired epilepsy models in juvenile animals, where the brain is more resistant to neuronal loss and less likely to develop epilepsy, the evidence that hippocampal death contributes to epileptogenesis is less clear. The inability to demonstrate a relationship between hippocampal damage and the development of epilepsy raises the possibility that other cortical structures not typically examined may be important for epileptogenesis in the immature brain.

Methods: This study used the chemoconvulsant lithium pilocarpine model of status epilepticus in immature animals to assess more comprehensively which structures outside the hippocampal formation are injured acutely after prolonged status epilepticus. Sprague Dawley rat pups were implanted with surface EEG electrodes, and status epilepticus was induced at 20 days of age with the chemoconvulsant lithium pilocarpine. After $72 \mathrm{~h}$, brain tissue from 12 animals was examined with Fluoro-Jade B, a histochemical marker for degenerating neurons, and compared to control animals that did not receive lithium pilocarpine.

Results: Lithium pilocarpine provoked prolonged status epilepticus that was studied with both electrographic recording and behavior. All treated animals demonstrated Fluoro-Jade B staining in areas outside the hippocampus. The severity of neuronal degeneration was variable across animals, but still revealed a consistent pattern of damage that identified several brain regions and nuclei. The most prominent staining was seen in thalamus (mediodorsal, paratenial, reuniens, and ventral lateral geniculate nuclei), amygdala (ventral lateral, posteromedial, basomedial nuclei), ventral premammillary nucei of hypothalamus, and paralimbic cortices (perirhinal, entorhinal, and piriform) as well as parasubiculum and dorsal endopiriform nuclei. Conclusions: These results demonstrate that lithium pilocarpineinduced status epilepticus in the immature rat brain consistently results in neuronal injury outside of the hippocampus proper. These regions may be critical to the development of epilepsy in the immature brain.

\subsection{0 \\ LONG-TERM EXPRESSION OF IL-1 BETA IN THE RAT HIPPOCAMPAL REACTIVE ASTROCYTES AFTER KAINIC ACID-INDUCED STATUS EPILEPTICUS}

SATORU SAKUMA ${ }^{1,2}$, H. Otsubo ${ }^{2}$, H. Shintaku ${ }^{1}$ and T. Yamano ${ }^{1}$ ( ${ }^{1}$ Pediatrics, Osaka City University Graduate School of Medicine, Osaka, Japan and ${ }^{2}$ Neurology, The Hospital for Sick Children,

Toronto, ON, Canada)
Rationale: Status epilepticus(SE) during febrile seizure has been known as one of the cause for development of epilepsy. The inflammatory cytokine, interleukin- $1 \beta$ (IL-1 $\beta$ ) has been reported to have a neuroinvasive influence on the hippocampus after brain damage including experimental model of SE. We evaluated the expression of IL- $1 \beta$ during the time course of the epileptogenesis using rats with kainic acid-induced status epilepticus.

Methods: SE was induced on adult(8-week-old) rats by intraperitoneally administration of kainic acid $(10 \mathrm{mg} / \mathrm{kg})$. Saline was injected for the controls rats. Two hours after SE,

diazepam $(10 \mathrm{mg} / \mathrm{kg})$ was injected to abort SE. At 12 hours and days 1 , $3,7,14$ and 21 after SE, left brains specimens were fixed in $4 \%$ paraformaldehyde and sliced on coronal sections. The expressions of IL- $1 \beta$ on the hippocampus were immunohistochemically examined before and after SE. We homogenized the right hippocampus in RIPA buffer and centrifuged. We measured IL-1 $\beta$ levels of the supernatant using Luminex technology.

Results: Spontaneous generalized tonic-clonic seizures appeared on day 7 after SE. Pyramidal cell loss were seen at CA1 and CA 3 on day 7. Reactive astrocytes increased with subsequent hypertrophy from day 7 and followed by gliosis. Expressions of IL- $1 \beta$ transiently increased on the pyramidal cells at CA3 on day 1 and then decreased. IL-1 $\beta$ expressed on the reactive astrocytes forming gliosis at CA1 on day 7 . The total level of IL- $1 \beta$ in the hippocampus was significantly elevated and maintained after SE.

Conclusions: The IL-1 $\beta$ in the hippocampus after SE demonstrated the different expression between the pyramidal cells and the reactive astrocytes in the time course of spontaneous seizures. IL-1 $\beta$ on the reactive astrocytes may contribute to epileptogenesis in the rat with spontaneous seizures post SE and gliotic hippocampus.

\section{Neuropathology of Epilepsy: In Vitro Studies}

\subsection{1}

SEVERE TRAUMA DISRUPTS CATIONIC GRADIENTS IN HIPPOCAMPAL NEURONS OF THE DEVELOPING BRAIN Trevor Balena and K. Staley (Massachusetts General Hospital, Charlestown, MA)

Rationale: Severe head trauma has been shown to cause a disruption in neuronal transmembrane ion gradients and can lead to epileptic seizure activity. We have recently provided evidence supporting the idea that post-traumatic accumulation of intracellular Cl- results in a shift in the action of GABA (which mediates Cl- flux through its receptors) from hyperpolarizing to depolarizing. This leads to reduced inhibition, enhanced propagation of neuronal firing, and may contribute to early post-traumatic seizures. Charge balance dictates that traumatic increases in intracellular $\mathrm{Cl}$ - must be accompanied by either a loss of another intracellular anion (unlikely because the only other mobile anion, HCO3-, is strongly buffered) or a gain of an accompanying cation. Gain of a cation could underlie cytotoxic edema, the abnormal neuronal swelling that is known to accompany epilepsy. Our objective was therefore to test for changes in intracellular cation concentration, beginning with $\mathrm{Na}+$.

Methods: The experiments were performed on acute hippocampal slices $(\sim 300 \mu \mathrm{m}$ in thickness), which were prepared from juvenile wild-type C57BL/6J mice whose ages ranged from P13 to P26. We recently demonstrated that acute hippocampal slices are an excellent model of traumatic axonal injury. The slices were incubated with the $\mathrm{Na}+$-sensitive dye SBFI for 4 to 5 hours prior to imaging. Following calibration of the dye, two-photon imaging was used to excite SBFI at both $\mathrm{Na}+$-sensitive and -insensitive wavelengths, allowing for the ratiometric determination of the intracellular $\mathrm{Na}+$ concentration.

Results: SBFI fluorescence of neurons in the CA1 and CA3 regions of the hippocampus indicated a significantly higher $\mathrm{Na}+$ concentration than has been reported in undamaged neurons. Specifically, neuronal intracellular $\mathrm{Na}+$ was highest near the cut surface of the slice, and lowest in the deepest neurons. 
Conclusions: These data indicate that trauma results in significant increases in intracellular $\mathrm{Na}+$. Our initial correlations of intracellular $\mathrm{Na}+$ and $\mathrm{Cl}$ - support the hypothesis that $\mathrm{Na}+$ is the principal cation accompanying the traumatic increases in intraneuronal Cl-. If so, then increases in intracellular $\mathrm{NaCl}$ after brain injury could engender cerebral edema, depolarizing shifts in the reversal potential for GABA, and early epileptic seizures.

\section{Epidemiology}

\subsection{2}

NEUROLOGICAL, COGNITIVE AND NEUROIMAGING OUTCOMES WITHIN 10 YEARS AFTER CHILDHOOD STATUS EPILEPTICUS: A POPULATION-BASED STUDY R. Chin ${ }^{1,3}$, Suresh Pujar ${ }^{1,2}$, R. Scott ${ }^{1,4}$, M. Martinos ${ }^{1}$, W. Chong ${ }^{1,5}$ and B. Neville ${ }^{1,2}$ ( ${ }^{1}$ UCL Institute of Child Health, London, United Kingdom; ${ }^{2}$ Young Epilepsy, Lingfield, United Kingdom; ${ }^{3}$ The University of Edinburgh, Edinburgh, United Kingdom; ${ }^{4}$ Dartmouth Medical School, Hanover, $\mathrm{NH}$ and ${ }^{5}$ Great Ormond Street Hospital for Children NHS Trust, London, United Kingdom)

Rationale: Childhood convulsive status epilepticus (CSE) is associated with significant short-term morbidity but long-term outcomes remain unclear. Determining outcomes following CSE and predictors for a poor outcome is needed to aid prognostication and appropriate resource allocation.

We carried out the first prospective pediatric population-based study on CSE, the north London convulsive status epilepticus in childhood surveillance study (NLSTEPSS). We are now investigating this unique cohort to determine their outcomes within 10 years after CSE, and in this abstract report preliminary data on neurological, cognitive and neuroimaging outcomes.

Methods: In the current study, the status epilepticus outcomes study (STEPSOUT), enrolled children are invited to have a clinical neurological evaluation, cognitive assessment using the Wechsler Abbreviated Scale of Intelligence (WASI), and brain MRI on a 1.5T scanner. All MRIs were qualitatively assessed by an experienced pediatric neuroradiologist.

Results: 90 children (41 male) have been investigated. Median follow-up was 8.1 years (range 6.4-9.6). 53 had complete (clinical, cognitive, and MRI) assessment. 22 had prolonged febrile seizures (PFS), 8 acute symptomatic (AS), 13 remote symptomatic (RS), 6 idiopathic, and 4 unclassified CSE.

All children with PFS were neurologically normal, had full-scale IQ score $>80$, and none had epilepsy. The neuroimaging was normal in all, except findings suggestive of neurofibromatosis type 1 in one child (5\%), and unilateral reduction in hippocampal volume without signal change in another (5\%). Amongst those who had AS CSE, one developed epilepsy (12\%) that remitted after 2 years; MRI was normal in $4(50 \%)$, abnormal but with findings unlikely to be clinically significant in $3(37 \%)$, and showed unilateral mesial temporal sclerosis (MTS) in 1 child (13\%) who had CSE due to pneumococcal meningitis. All had IQ scores $>80$.

All children with RS CSE, except two, had active epilepsy, significant cognitive (IQ <70) and/or motor impairments. The MRI findings were normal in 2 , and suggestive of hypoxic ischemic injury in 2, compensated hydrocephalus in 3 , perinatal middle cerebral artery infarction in 3, hypoglycaemic injury, unilateral MTS, and unilateral reduction in hippocampal volume without signal change in one each.

Two (33\%) children with idiopathic CSE had active epilepsy, and IQ scores of $<70$. The remaining had IQ scores $>90$ and neuroimaging was normal in all. All children with unclassified CSE had normal neuroimaging, IQ scores $>80$, and none had epilepsy.

Conclusions: Children with PFS have good neurological and cognitive outcomes within 10 years after CSE but those with prior known neurological problems at the time of CSE do not. The relationship between PFS and subsequent MTS is probably not as strong as long believed and other forms of CSE can be associated with MTS

\subsection{3 \\ CARDIOVASCULAR FITNESS AND FUTURE RISK OF EPILEPSY: A PROSPECTIVE STUDY}

Jenny Nyberg 1 , M. A. Åberg ${ }^{1}$, K. Torén ${ }^{2}$, H. G. Kuhn ${ }^{1}$ and E. BenMenachem ${ }^{1}{ }^{1}$ Center for Brain Repair and Rehabilitation, Institute for Neuroscience and Physiology, University of Gothenburg, Gothenburg, Sweden and ${ }^{2}$ Occupational and Environmental Medicine, Institute of Medicine, University of Gothenburg, Gothenburg, Sweden)

Rationale: Physical exercise has a positive impact on brain structure and function and can protect the brain from neurodegenerative disorders. Studies have shown positive effects of exercise on seizure frequency and severity in patients with epilepsy. Although exercise have positive outcomes for patients who already have epilepsy, possible preventative effects of exercise on seizure susceptibility and development of epilepsy in humans is unknown. However, results from experimental animal models are encouraging where exercise influences epileptogenesis in a positive manner. There is a clear need for longitudinal prospective studies with an objective measure of physical fitness in humans. Therefore, we performed a large, prospective cohort study of men at age 18 and followed them for up to 40 years. The aim was to determine whether cardiovascular fitness measured at age 18 was associated with risk of developing epilepsy later in life. We also wanted to investigate if presence of a prior traumatic head injury influenced the observed associations. Methods: Population-based, prospective cohort study of Swedish male conscripts $(n=1,173,079)$ born in 1950-1987, who were followed for up to 40 years. Objective data of cardiovascular fitness, as measured by the cycle ergonometric test, were collected during conscription exams and linked with national hospital discharge registers and other national databases to calculate future risk of epilepsy. We used Cox proportional hazards models to assess the influence of the cardiovascular performance at age 18 on the occurrence of epilepsy later in life.

Results: The numbers of individuals who received inpatient treatment for epilepsy during the observation period was 6,796. In fully adjusted models, low cardiovascular fitness at age 18 was associated with increased risk for future epilepsy (HR 1.79, 95\%, CI 1.57-2.03). In a full-brother model, thus controlling for familial influences, low cardiovascular fitness remained a significant predictor (HR 1.66, 95\%, CI 1.39-1.98). The associations between cardiovascular fitness and later epilepsy remained in individuals who had a head injury prior to the epilepsy diagnosis (HR 1.88, 95\%, CI 1.41-2.51).

Conclusions: Low cardiovascular fitness at age 18 increases the risk for epilepsy later in life. The associations of cardiovascular fitness and epilepsy were still significant after controlling for a number of possible confounders including familial factors. This is a unique study with a large and prospective cohort, long observation period and objective measures of physical performance that correlates well with and extends other studies reporting positive effects of exercise on patients already diagnosed with epilepsy. We propose that physical activity may act as a positive disease-modifying behavior for the development of epilepsy.

\subsection{4 \\ DEFINING INCIDENT CASES OF EPILEPSY IN ADMINISTRATIVE DATA}

P. M. Bakaki ${ }^{1}$, S. M. Koroukian ${ }^{1}$, L. W. Jackson ${ }^{1}$, J. M. Albert ${ }^{1}$ and Kitti Kaiboriboon ${ }^{2}$ ( ${ }^{1}$ Department of Epidemiology \& Biostatistics, Case Western Reserve University, Cleveland, $\mathrm{OH}$ and ${ }^{2}$ Department of Neurology, University Hospitals Case Medical Center, Cleveland, $\mathrm{OH})$ 
Rationale: Algorithms to identify incident cases of epilepsy using claims data have never been investigated. This study was designed to determine optimal enrollment duration to accurately identify incident cases of epilepsy in administrative data.

Methods: We performed a retrospective dynamic cohort study using Ohio Medicaid data from 1992-2006. Individuals were identified as having epilepsy if they had $\geq 2$ claims of epilepsy (ICD-9-CM: $345 . x x$ ) or $\geq 3$ claims of convulsion (ICD-9-CM: 780.3 or 780.39), and $\geq 2$ claims of antiepileptic drugs. Each of the diagnosis or pharmacy claims had to be $>30$ days apart (Figure 1). Individuals were considered incident cases if they met the epilepsy case definition and had at least 1 year of follow-up prior to epilepsy diagnosis (epilepsy-free (EF) interval). Epilepsy incidence was then examined, extending the EF interval to a maximum of 8 years, the greatest interval that we could obtain stable estimates. To determine the optimal duration of EF period that minimizes misclassifying prevalent cases as incident cases, we computed an overestimation of incidence by comparing the incidence in each interval to the incidence for the 5-year interval. We used incidence rate of 5-year EF interval as a reference because this incidence rate among people without pre-existing disability was comparable to that obtained from a recent meta-analysis of population-based studies. The incidence overestimation was then plotted against the EF intervals to identify the optimal EF duration. We also stratified the analysis on preexisting disability status.

Results: Of the 318,123 Medicaid enrollees, 9,661 people met the epilepsy case definition during the study period. Of those, 5,037 individuals were incident cases when there was 1 year of EF interval. As the length of EF interval increased, the incidence rates decreased. Incidence rates in subjects with 1- and 8-year EF interval were $5.50 / 1,000$ and 2.65/1,000 person-years, respectively. Within each interval, the incidence among people without pre-existing disability was lower than that of people with pre-existing disability. Figure 2 shows the plot of the incidence overestimation by EF intervals ranging from 1 to 4 years. As compared to the 5-year EF interval, the 1- and 2-year interval resulted in an overestimation of the incidence by $53.6 \%$ and $26.8 \%$, respectively. The incidence overestimation declined to $13.1 \%$ and $7.4 \%$ when using 3 - and 4-year EF interval, respectively. A sharper initial decline in incidence overestimation as EF interval increased was also noted among people with and without pre-existing disability.

Conclusions: The 5-year EF interval appears to be the most appropriate to differentiate incident from prevalent cases in administrative data. If this is not possible, 3- or 4-year EF interval might be adequate. Shorter or longer EF intervals could result in over- or under-estimation of epilepsy incidence. This study was approved by the ODJFS and supported by the Epilepsy Foundation.

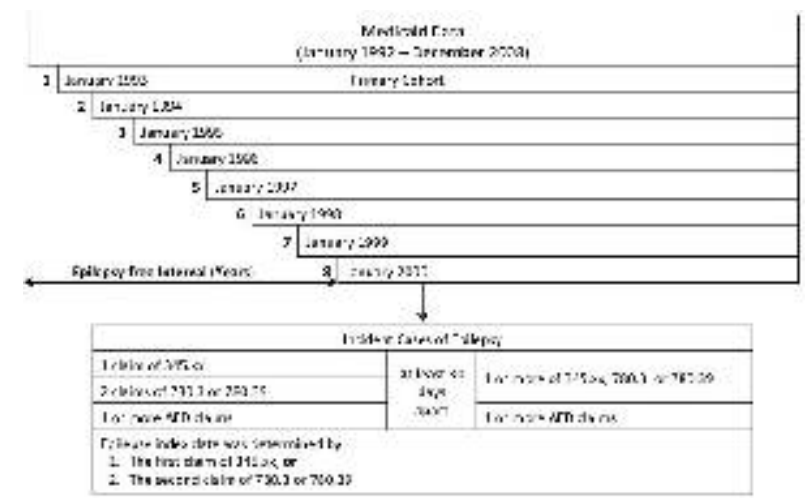

Figure 1: Algorithm to identify the study cohort and epilepsy cases. To examine the impact of epilepsy-free interval on the incidence estimation, the epilepsy-free interval was extended by 1 year at a time to each interval up to 8 years. Abbreviations: AED, antiepileptic drug; 345.xx, ICD-9-CM for epilepsy; 780.3 and 780.39, ICD-9-CM for non-febrile convulsions.

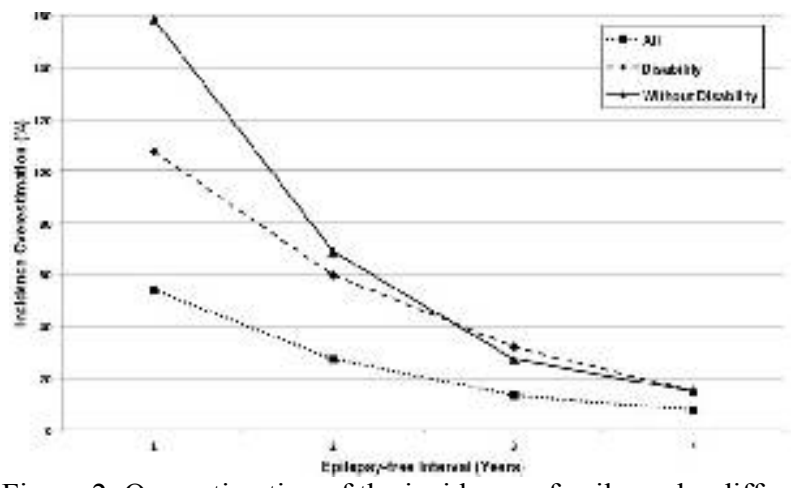

Figure 2: Overestimation of the incidence of epilepsy by different length of epilepsy-free intervals, compared to the incidence of 5-year epilepsy-free as reference.

\subsection{5 \\ WHY DO SEIZURES RECUR IN SEIZURE-FREE CHILDREN? A 30 YEAR POPULATION-BASED COHORT OF CHILDREN WITH EPILEPSY}

Katherine Nickels, L. Wong-Kisiel and E. Wirrell (Neurology, Mayo Clinic, Rochester, MN)

Rationale: To determine: 1) How often seizure-free children have seizure recurrence, 2) Potential risk factors for seizure recurrence, 3) Whether seizure recurrence and intractability occurred more often after anti-epileptic medication (AED) withdrawal.

Methods: Records of children age 0-17 years diagnosed with newonset epilepsy while resident in Olmsted County, Minnesota between 1980-2010 were reviewed. Records of all children treated with AEDs, who became seizure free for more than 1 year and followed $>2$ years after last seizure were reviewed to determine seizure recurrence and intractability. Potential risk factors for seizure recurrence were assessed, including: AED withdrawal, cognition, neurologic exam, etiology, age of onset, time between diagnosis and seizure freedom, number of AEDs failed due to lack of efficacy, seizure control prior to seizure freedom, history of status epilepticus, and EEG.

Results: 308 children fulfilled inclusion criteria, 191 withdrew AEDs. Children with normal cognition $(\mathrm{p}<0.001)$, normal exam $(\mathrm{p}=0.004)$, and genetic/unknown etiology $(\mathrm{p}=0.021)$ were more likely to discontinue AEDs after seizure freedom.

Overall, 110 had seizure recurrence. Of these, 63 (20\%) were not seizure free at last follow-up. Seizure recurrence was more likely in those with abnormal cognition ( $\mathrm{p}=0.008)$, epilepsy onset prior to age 1 year $(\mathrm{p}=0.05)$, structural $/$ metabolic etiology $(\mathrm{p}=0.001),>2$ years between epilepsy diagnosis and seizure freedom $(\mathrm{p}=0.002)$, and $>1$ AED failed due to lack of efficacy $(\mathrm{p}=0.027)$, regardless of AED withdrawal. Seizures recurred more often in those who did not discontinue AEDs $(\mathrm{p}<0.001)$.

Sixteen $(5 \%)$ patients had intractable seizures at last follow-up. Potential risk factors for intractability include: epilepsy onset prior to age 1 year $(p<0.001)$, abnormal neurologic exam $(p=0.005),>1$ AED failed due to lack of efficacy $(\mathrm{p}<0.001)$, last EEG abnormal $\mathrm{p}=0.001)$, history of status epilepticus ( $\mathrm{p}=0.001)$, structural/metabolic etiology $(p<0.001)$, and seizures at least every 3 months prior to seizure freedom $(\mathrm{p}=0.004)$.

Conclusions: : Seizure recurrence after one year of seizure freedom was common, with $20 \%$ still having seizures at last follow-up and $5 \%$ with intractable epilepsy. However, this is consistent with previous reports of seizure remission in $70-80 \%$ of children. Potential risk factors suggest those with epilepsy onset at a younger age, that is more difficult to treat, with more frequent seizures, and due to structural/metabolic etiology are more likely to have seizure recurrence and epilepsy intractability. Furthermore, these children are also more likely to continue AEDs, even after seizure freedom. This is consistent with the finding that seizures recurred more often in 
children who did not discontinue AEDs. Therefore, although seizures recur in children with epilepsy, the rate of recurrence is not greater than the overall expected rate of intractable epilepsy in children, is more likely to occur in children with complicated epilepsy, and is unlikely to occur due to AED withdrawal.

\subsection{6}

INCIDENCE AND PREVALENCE OF EPILEPSY AMONG POOR HEALTH \& LOW INCOME AMERICANS: A LONGITUDINAL COHORT STUDY (1992-2006)

Kitti Kaiboriboon ${ }^{1}$, P. M. Bakaki ${ }^{2}$, S. D. Lhatoo ${ }^{1}$ and S. M. Koroukian ${ }^{2}{ }^{1}$ Department of Neurology, University Hospitals Case Medical Center, Cleveland, $\mathrm{OH}$ and ${ }^{2}$ Department of Epidemiology \& Biostatistics, Case Western Reserve University, Cleveland, $\mathrm{OH}$ )

Rationale: Resource poor countries usually have higher incidence and prevalence of epilepsy than industrialized countries. These differences are thought to be related to inequalities of socioeconomic status (SES), access to healthcare, environmental exposures, and/or genetic factors. There are only a few studies that report incidence of epilepsy in people with low SES who live in developed countries. These studies demonstrate that people with low SES have higher incidence of epilepsy than their counterparts with high SES. No study, to our knowledge, has specifically examined the incidence and/or prevalence of epilepsy in Medicaid population. The aim of this study was to determine the incidence and prevalence of epilepsy in this educationally and socioeconomically disadvantaged population. Methods: We performed a retrospective dynamic cohort study using Ohio Medicaid claims data between 1992 and 2006. Individuals were identified as prevalent cases of epilepsy if they had $\geq 2$ claims of epilepsy (ICD-9-CM: 345.xx) or $\geq 3$ claims of convulsion (ICD-9CM: 780.3 or 780.39 ), and $\geq 2$ claims of antiepileptic drugs. Each of the diagnosis or pharmacy claims had to be $>30$ days apart. Incident cases of epilepsy were required to have no epilepsy or convulsion claims for at least 5 years before epilepsy diagnosis. In addition, subjects were determined as having pre-existing disability and/or comorbid conditions including brain tumor, depression, developmental disorders (DD), migraine, schizophrenia, stroke, and traumatic brain injury (TBI) when at least one of these conditions occurred prior to epilepsy onset. In addition to the incidence and prevalence rates, we used log-binomial Poisson regression analysis to estimate prevalence ratio (PR) and log-linear Poisson regression to estimate incidence rate ratio (IRR) controlling for gender, age, race, pre-existing disability, and co-morbid conditions. We also estimated attributable risk (AR) for each co-morbid condition.

Results: There were 9,056 prevalent cases and 1,608 incident cases of epilepsy. The prevalence of epilepsy was 13.2/1,000 (95\% confidence interval (CI), 13.0/1,000-13.5/1,000). The incidence of epilepsy was 3.6/1,000 person-years $(95 \% \mathrm{CI}, 3.4 / 1,000-3.8 / 1,000)$. The incidence and prevalence were significantly higher in men, in older people, in Blacks, and in people with pre-existing disability and/or co-morbid conditions. The most common pre-existing comorbid conditions in epilepsy subjects were depression, DD, and stroke, whereas people with brain tumor, TBI, and stroke had the highest risk of developing epilepsy.

Conclusions: Medicaid population has a high incidence and prevalence of epilepsy, in an order of magnitude greater that that reported in the U.S. general population. This indigent population carries a disproportionate amount of the epilepsy burden and deserves more attention for their health care needs and support services. This study was approved by the ODJFS and supported by the Epilepsy Foundation.

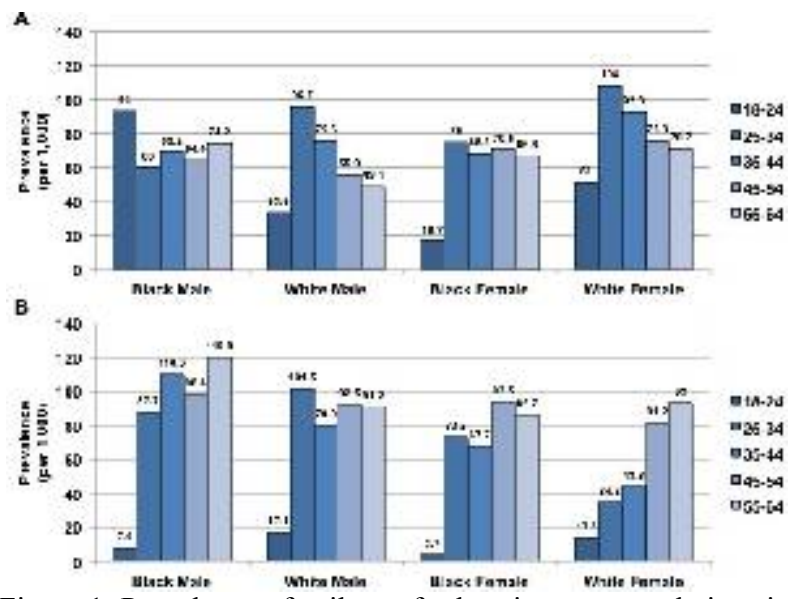

Figure 1: Prevalence of epilepsy for low-income populations in the U.S. between 1992 and 2006 by gender, race, and age at epilepsy diagnosis. (A) Prevalence of epilepsy for people with pre-existing disability. (B) Prevalence of epilepsy for people with pre-existing brain tumor, stroke, depression, developmental disorders, migraine, schizophrenia, and/or traumatic brain injury.

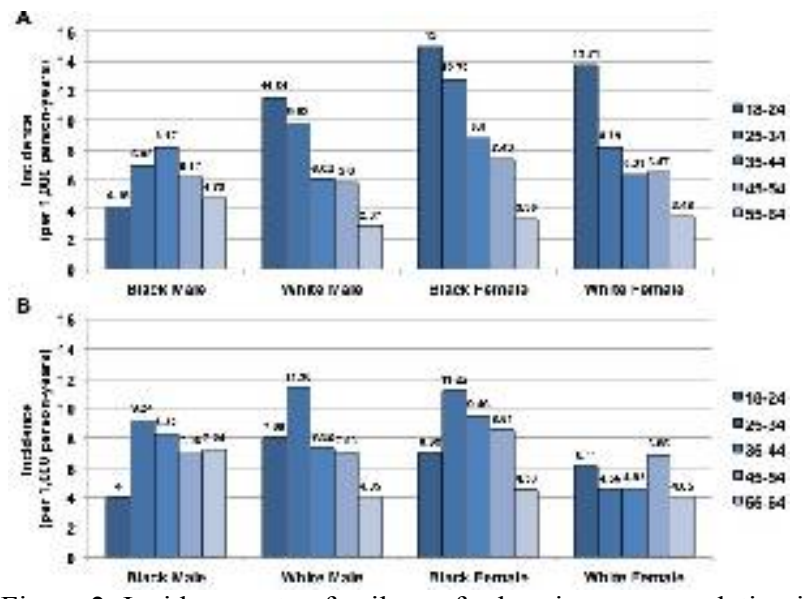

Figure 2: Incidence rate of epilepsy for low-income population in the U.S. between 1997 and 2006 by gender, race, and age of epilepsy onset. (A) Incidence rate of epilepsy for people with pre-existing disability. (B) Incidence rate of epilepsy for people with pre-existing brain tumor, stroke, depression, developmental disorders, migraine, schizophrenia, and/or traumatic brain injury.

\subsection{7 \\ ACUTE SEIZURES AND 12-MONTH OUTCOME AFTER ACUTE ISCHEMIC STROKE}

Erwin Chiquete $^{1}$, H. Sentíes-Madrid ${ }^{1}$, M. Alonso-Vanegas ${ }^{2}, \mathrm{G}$. García-Ramos ${ }^{1}$ and C. Cantú-Brito ${ }^{1}\left({ }^{1}\right.$ Neurology and Psychiatry, Instituto Nacional de Ciencias Médicas y Nutrición Salvador Zubirán, Mexico City, Distrito Federal, Mexico and ${ }^{2}$ Neurosurgery, Instituto Nacional de Neurología y Neurocirugía "Manuel Velasco Suárez", Mexico City, Distrito Federal, Mexico)

Rationale: In most populations, cerebrovascular disease represents the main cause of provoked seizures in adults. The presence of acute symptomatic seizures influences short-term outcome after stroke; however, scarce information exists on the association of seizures with long-term outcome. We aimed to describe the factors associated with symptomatic seizures after acute ischemic stroke and the impact on functional outcome at 12-month follow-up.

Methods: This is a descriptive cohort study on 1246 non-epileptic patients with acute ischemic stroke included in a multicenter Mexican registry, who received long-term follow up after a first-ever or 
recurrent brain infarction. Multivariate analyses were performed to evaluate factors associated with acute provoked seizures and the functional outcome at 12-month follow-up.

Results: The frequency of provoked seizures after acute ischemic stroke was $8.1 \%$ [ $95 \%$ confidence interval (CI): $6.7 \%$ to $9.8 \%$ ]. In a binary logistic regression model, risk factors significantly associated with provoked seizures were a scoring of the National Institutes of Health Stroke Scale (NIHSSS, US) $>10$ [odds ratio (OR): $2.21,95 \%$ CI: 1.40-3.47], recurrent ischemic stroke (OR: $2.17,95 \%$ CI: $1.34-$ 3.53 ) and age $<65$ years (OR: $1.69,95 \%$ CI: $1.09-2.62$ ). After a Cox proportional hazards model and Kaplan-Meier actuarial analyses, the presence of provoked seizures was significantly associated with the risk of important functional disability or death (a modified Rankin scale $>3$ ) at 12-month follow-up [hazard ratio (HR): 1.37, 95\% CI: 1.04-1.83], as well as NIHSS $>10$ (HR: $4.47,95 \%$ CI: $3.53-5.65$ ), age $\geq 65$ years (HR: $1.74,95 \%$ CI: 1.38-2.20), heart failure (HR: 1.61 , 95\% CI: $1.22-2.13$ ) and atrial fibrillation (HR: 1.35 , 95\% CI: $1.05-$ 1.74).

Conclusions: The frequency of provoked seizures after acute ischemic stroke in this cohort was $8 \%$. Age $<65$ years and severity of the brain infarction are the main risk factors for acute provoked seizures. In turn, provoked seizures represent a factor associated with functional disabilities or death one year after acute ischemic stroke.
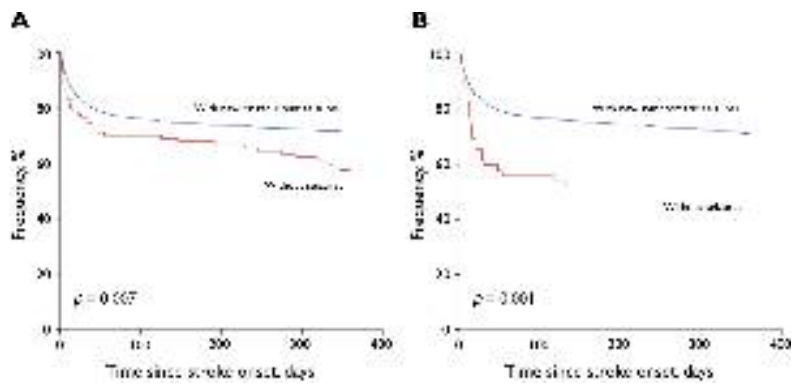

Figure. Kaplan-Meier survival analyses as a function of new or recurrent seizures (A), and new acute symptomatic seizures exclusively (B) after acute ischemic stroke $(n=1246)$.

\subsection{8 \\ THE IMPACT OF EPILEPSY ON DAILY LIFE - RESULTS FROM A CANADIAN SURVEY}

S. M. Nurse ${ }^{1}$, L. Sultan-Khan ${ }^{1}$, G. Dempsey ${ }^{1}$, Nicole Beauregard ${ }^{2}$, J. $\mathrm{Smyth}^{2}$ and R. Tam ${ }^{2}\left({ }^{1}\right.$ Canadian Epilepsy Alliance, Toronto, ON, Canada and ${ }^{2}$ UCB Canada, Oakville, Canada)

Rationale: Approximately 300,000 (1 in 100) Canadians are affected by epilepsy (Tellez-Zenteno et al 2004). Epilepsy can severely impact the independence, productivity and overall quality of life of patients. This report provides the findings of the first Canada-wide survey conducted to explore the impact of epilepsy on life and health among adults living with the disorder.

Methods: A nationwide online or paper survey was conducted August-October 2011 among adults living with epilepsy. The survey was developed in collaboration with representatives from community based epilepsy agencies in both official languages (French and English). It included questions on seizure control, the impact of epilepsy on daily life and access to healthcare and support. Subjects volunteered to participate anonymously in the survey through media advertisements.

Results: 671 respondents age 18 years and older volunteered to participate. $50 \%$ were diagnosed with epilepsy under 16 years of age and had been living with the condition on average for 23.5 years. The most commonly reported seizure types were tonic-clonic (48\%), complex partial (37\%), absence (32\%) and simple partial (28\%). Most patients $(82 \%)$ required medications to control seizures and had been treated on average with 3.9 anti-epileptic drugs (AEDs). Only $3 \%$ of participants had achieved seizure freedom (no seizures at all), while $46 \%$ had $<50$ and $16 \%$ had $>50$ seizures per year. Most (61\%) had at least one current comorbidity. Patients reported that lack of independence (56\%), impoverished social life (38\%),

stigma/discrimination (38\%), difficulties in maintaining employment $(31 \%)$ and relationships (35\%) were the most negative aspects of living with epilepsy. Medication side effects (63\%), memory impairment (60\%), injuries (44\%) and learning difficulties/cognitive decline $(40 \%)$ had the greatest negative impact on respondents' health. On average, respondents waited 4 years from their first seizure to be diagnosed with epilepsy. $40 \%$ were managed by their general practitioner or family doctor regularly, and only $30 \%$ have had a consultation with an epileptologist and waited an average of 9.8 months to see an epilepsy specialist.

Conclusions: This survey reveals that epilepsy imposes a substantial burden on the lives of Canadians with epilepsy. Lack of independence, social life, employment and relationship opportunities are most affected by continuing seizures. Better seizure control, timely diagnosis and access to specialized care and support services could improve the burden of the disorder and help remove the barriers that people with epilepsy face on a day-to-day basis

\subsection{9 \\ WTIHDRAWN}

\section{Public Health}

\subsection{0 \\ BARRIERS TO BONE HEALTH COUNSELING FOR WOMEN WITH EPILEPSY ON ANTIEPILEPTIC DRUGS Julie L. Roth ${ }^{1,2}$, C. Paniszyn ${ }^{2}$, V. Gendron ${ }^{1,2}$, C. Harini ${ }^{3,4}$ and A. S. Blum $^{1,2}$ ( ${ }^{1}$ Neurology, Rhode Island Hospital, Providence, RI; ${ }^{2}$ The Warren Alpert Medical School, Providence, RI; ${ }^{3}$ Neurology, Children's Hospital Boston, Boston, MA and ${ }^{4}$ Neurology, Harvard Medical School, Boston, MA)}

Rationale: Many medications used to treat epilepsy are known to have adverse effects on bone health - in particular, the firstgeneration, hepatic enzyme-inducing antiepileptic drugs (EIAEDs). Female patients are at particular risk for bone loss later in life; consequently, it is most important for neurologists to counsel women taking EIAEDs about bone health risks during the childbearing years. We sought to identify the prevalence of bone health counseling among neurologists treating female patients with first-generation EIAEDs, and to identify barriers to counseling if present.

Methods: We performed a retrospective, observational study to examine prevalence and potential barriers to bone health counseling among academic neurologists treating female patients with epilepsy. We identified 760 females with epilepsy, ages 11-60, by ICD-9 codes for "seizure" or "epilepsy" seen in 2010 at the outpatient academic neurology clinics affiliated with Hasbro Children's Hospital and Rhode Island Hospital (RIH). Outpatient neurology notes for subjects identified were then analyzed over a five-year span (2005-2010) and documentation of AEDs and bone health counseling was collected. We also collected demographic information, primary language, presence of a cognitive disorder, race and ethnicity when known, insurance status, number of outpatient visits over 5 years, and provider expertise (epileptologist versus general neurologist). Results: Among the group of 760 epilepsy patients, 280 were taking first-generation EIAEDs, of whom 53\% (148/280) had been counseled about bone health. First-generation EIAEDs were more commonly used by uninsured (39/59, 66\%), Medicaid (52/103, 50\%), and Medicare $(42 / 85,49 \%)$ patients compared to the privately insured $(146 / 509,29 \%)$. Counseling rates correlated most with insurance status: $31 \%(16 / 52)$ of Medicaid and 36\% (14/39) of uninsured patients had been counseled, versus $64 \%(27 / 42)$ of Medicare and $62 \%(91 / 146)$ of privately insured patients $(\mathrm{p}<0.001)$. Counseling also correlated with patient age, with $10 \%(2 / 21)$ of patients under 20 counseled versus $66 \%$ (37/56) of those aged 51-60. 
Primary language or the presence of a cognitive disability did not influence counseling rates, and race and ethnicity data were incomplete. Median age was higher among Medicare (46) and Medicaid (44) versus private (28) and uninsured (34) groups. Conclusions: Among neurologists treating female patients with firstgeneration EIAEDs for epilepsy, insurance status and age correlated most with bone health counseling rates. Uninsured or Medicaid patients were about half as likely to be counseled as privately insured or Medicare patients $(\mathrm{p}=<0.001)$. Yet, first-generation EIAEDs were more commonly prescribed to uninsured or Medicaid patients compared to privately insured patients. Age was also a barrier to counseling; patients younger than 20 years old were counseled the least. Further study into barriers to counseling is warranted, as is a streamlined quality improvement intervention to remedy these apparent disparities in counseling practices among neurologists.

\subsection{1 \\ THE QUALITY OF EPILEPSY CARE: ARE WE ON THE SAME PAGE?}

A. $\mathrm{Oh}^{2}$, D. Becker ${ }^{2}$ and Hyunmi Kim ${ }^{1}\left({ }^{1}\right.$ Pediatric Neurology, University of Alabama at Birmingham, Birmingham, AL and ${ }^{2} \mathrm{Health}$ Care Organization and Policy, School of Public Health, University of Alabama at Birmingham, Birmingham, AL)

Rationale: Specialized epilepsy centers have been developed to provide an accurate diagnosis of epilepsy and appropriate treatments. There has been emphasis on the necessity and importance of a collaborative interdisciplinary care team to achieve optimal epilepsy care. Beyond the professional knowledge and expert skills, it is indispensable that team members share a common set of beliefs regarding the delivery of optimal epilepsy care. Understanding the nature of individual and shared team-based knowledge is critical to organizational performance. This study aimed to investigate the characteristics of individual and team knowledge of epilepsy care professionals towards the quality of epilepsy care.

Methods: In this pilot study a concept mapping method was used to explore the epilepsy care professionals' knowledge. The study invited ten epilepsy team members in the UAB pediatric epilepsy center including 2 epileptologists, 2 neurosurgeons with specialization for epilepsy surgery, 3 nurse practitioners, 1 registered nurse, and 2 electroencephalogram (EEG) technologists. Participants were asked to answer the open-ended question, "What factors do you think influence the quality of epilepsy care?" They made out the lists of factors and the reasons why they considered those important. They also ranked the factors in the order of importance. With the participants' written descriptions, the author constructed individual concept maps representing knowledge structures as the relationships between concepts in a graphic form. Each concept map was revised through each participant's confirmation. Content analysis of maps was conducted and the results were used to produce a team concept map.

Results: A total of 227 concepts were found in ten participants' descriptions. The median was 25 (minimum 12 and maximum 28). Among all concepts, the total numbers of factors were 96 . The median was 9 (minimum 6 and maximum 14). Detected concepts and factors were grouped into 12 categories including access to epilepsy care, awareness and education, caregivers, compliance, diagnosis, epilepsy care professionals, patient-provider relationships, patients' quality of care, primary physicians, treatment, support groups, and others. For the concepts, 'treatment' was the most common concept with 17.6 percent share (40/227). This was followed by 'access to epilepsy care' and 'epilepsy care professionals' with 16.7 percent (38/227) and 13.7 percent (31/227), respectively. Concerning the factors, 'access to epilepsy care (23/96)' was the most common factor (24.0\%). 'Epilepsy care professionals (16/96)' was the second most common factor (16.7\%). With 14.6 percent share, 'treatment (14/96)' and 'awareness and education (14/96)' were in the third place.

Conclusions: This pilot study showed the potential use of concept mapping methods to extract individual and team knowledge. Twelve categorized concepts and eleven factors were found shared among team members. This study can be the base to compare team knowledge with the consensus of experts group.

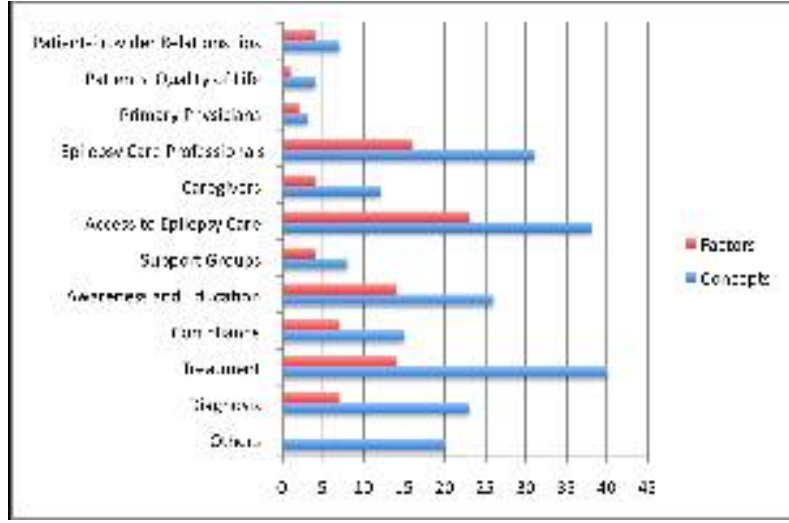

\subsection{2 PHYSICIANS PRACTICES FOR EPILEPSY SURGERY IN PEDIATRICS}

Freedom Perkins $^{1}$, K. L. Kime ${ }^{1}$, N. Bower ${ }^{1}$, C. A. Hovinga ${ }^{1}$, J. B. Titus $^{1}$ and D. F. Clarke ${ }^{1,2}\left({ }^{1}\right.$ Pediatric Neurology, Dell Children's Medical Center, Austin, TX and ${ }^{2}$ Pediatric Neurology, University of Texas Southwestern Medical Center, Dallas, TX)

Rationale: Recently there has been attention made to published guidelines regarding the diagnosis and treatment of epilepsy. Few studies have examined the awareness of physicians and their understanding of best practices in epilepsy. This study assessed the initial diagnosis and treatment practices of physicians seeing pediatric patients with epilepsy and concordance with current guidelines. Methods: A survey was constructed to assess the familiarity that healthcare providers had with guidelines pertaining to epilepsy surgery. Information including physician age, number of years of practicing medicine, subspecialty, practice affiliation, and patient population were collected in order to see if there were any associations with guidelines. The survey was to health care providers in Central Texas whose focus is in pediatrics, family medicine, neurology/neurosurgery.

Results: $146(\sim 10 \%)$ responded to the survey. Among respondents, $57.5 \%$ were from pediatrics, $12.6 \%$ from neurology/neurosurgery, and $29.9 \%$ family medicine. Approximately half the respondents were from private practice. Respondents were equally represented for gender with $48.2 \%$ being male. The majority were Caucasian (74\%). Respondents saw a median of one (range:0-100) new cases of seizures per month and a median of 2.5 (range:0-200) pediatric patients with epilepsy per month. Approximately half the clinicians had been practicing medicine for less than 15 years. Among physicians, the definition of drug refractory epilepsy was unclear with $60 \%$ of those surveyed being unsure or disagreeing that failing 2-3 anticonvulsants constituted refractoriness. Nearly $75 \%$ agreed that referral for epilepsy surgery evaluation should be considered, if the child has intractable epilepsy. 53.5\% agreed that a child should be referred after 3 years of failed AED treatment and $38 \%$ were unsure. $46.8 \%$ were unsure if a child should be referred after a failed VNS and $50 \%$ were also unsure after a failed ketogenic diet. The highest percentage of those who were unsure regarding referrals for a failed VNS and/or diet were those in family medicine. Surgery was agreed to be effective for children with partial epilepsy by $29.2 \%$ of respondents. $63.1 \%$ were unsure with family practitioners comprising the majority. $63.3 \%$ were unsure if surgery was agreed to be effective for those with generalized epilepsy and $22.7 \%$ disagreed. As for those with temporal lobe epilepsy, $64.1 \%$ were unsure whether surgery was effective and $31.3 \%$ disagreed. Family practitioners still represented the majority of those who were unsure for these types of epilepsy. 
More than half were unsure that surgery is related to good cognitive outcomes for those with intractable epilepsy. More than half, however, agreed that surgery is associated with quality of life improvement.

Conclusions: There remains significant misinformation regarding when epilepsy surgical referrals in pediatric patients should be made. This appears to be associated with what constitutes intractable epilepsy as well as for what types of seizures may be amendable to epilepsy surgical intervention.

\subsection{3}

KNOWLEDGE OF PHYSICIANS AND PRACTICE PARAMETERS PERTAINING TO THE DIAGNOSIS AND DRUG MANAGEMENT OF PEDIATRIC EPILEPSY Collin A. Hovinga ${ }^{1,2}$, K. L. Kime ${ }^{1}$, N. R. Bower ${ }^{1}$, J. B. Titus ${ }^{1}$, F. F. Perkins ${ }^{1}$ and D. F. Clarke ${ }^{1}\left({ }^{1}\right.$ Research, Dell Children's Medical Center, Austin, TX and ${ }^{2}$ Pharmacy, University of Texas, Austin, TX)

Rationale: Few studies have examined pediatric practitioner understanding of best practices in epilepsy. This study assessed the initial diagnosis and treatment practices of physicians seeing pediatric patients with epilepsy and concordance with current guidelines. Methods: A survey was constructed to assess physician familiarity with guidelines pertaining to the evaluation and drug treatment of children with epilepsy. Information including physician age, number of years of practicing medicine, subspecialty, practice affiliation, and patient population was collected in order to see if there were any associations with guidelines. The survey was to health care providers in Central Texas whose focus is in pediatrics, family medicine, neurology, and neurosurgery.

Results: $146(10 \%)$ responded to the survey. Among the total respondents $57.5 \%$ were from pediatrics, $12.6 \%$ from neurology/neurosurgery, and $29.9 \%$ were from family medicine. Approximately half the respondents were from private practice. Respondents saw a median of one (range: $0-100)$ new case of seizures per month and a median of 2.5 (range: $0-200$ ) pediatric patients with epilepsy per month. Approximately half of the surveyed clinicians had been practicing medicine for less than 15 years. Overall, $6.3 \%$ felt that a child with a first febrile seizure needed an EEG, whereas $85 \%$ felt that an EEG should be obtained after the 1st unprovoked seizure. Half of respondents felt that an EEG should be ordered after the child has failed an AED. Family medicine practitioners were more likely to obtain an EEG after an initial seizure than pediatricians or those from neurology/neurosurgery. Eighty-three percent of physicians agreed that a CT or MRI should be ordered if a child has had 2 or more unprovoked seizures. Ninety-four percent agreed that one should be ordered if the child's physical or neurological examination suggested focal impairment. Eighty-eight percent felt a CT or MRI should be ordered if the child is being considered for epilepsy surgery and $71 \%$ felt one necessary if the child has any epileptiform abnormality on their EEG. Eighteen percent of physicians were unsure if an AED should be administered after the 1st unprovoked seizure. Fourteen percent were still unsure if one should be administered after more than 2 unprovoked seizures, and $12 \%$ were unsure if it should be after more than 3 unprovoked seizures. Twenty eight percent of physicians were unsure if failing 1 AED constituted as having intractable epilepsy. Likewise, about $1 / 3$ of the respondents were unsure if children were considered as intractable if they had failed 2-3 AEDs. Thirty-five percent of physicians were still unsure if failing 6 or more AEDs meant a child had intractable epilepsy.

Conclusions: In general, physicians were in agreement with guidelines for obtaining EEGs and imaging in pediatric patients with epilepsy. However, there are significant misconceptions regarding AED management and understanding what constitutes drug resistant epilepsy.

\subsection{4}

REFRACTORY EPILEPSY IN PREGNANT WOMEN

L. M. Aenlle-Matusz ${ }^{1}$, S. E. Nadeau ${ }^{1,2}$ and Jean E. Cibula ${ }^{1}$

( ${ }^{1}$ Neurology, University of Florida HSC, Gainesville, FL and

${ }^{2}$ Neurology, Malcolm Randall Veterans Affairs Medical Center, Gainesville, FL)

Rationale: There are over 1 million adult women with epilepsy in the United States alone. One out of every 200 pregnancies involves a woman with active epilepsy. Although some small studies have associated the type of seizure (focal versus generalized) with relative refractoriness of seizures, there are no known reliable predictors of increased seizure during pregnancy. Prolonged seizures and status epilepticus are known threats to both the mother and the fetus. This study evaluates the self-discontinuation of anti-convulsant therapy as a potential cause of increased seizure frequency in pregnant women with epilepsy.

Methods: A retrospective cohort study design was applied using a tertiary care neurology department billing database from 2006-2010. To be included, the patients must have been billed for their visits under the codes epilepsy conditions (780.30 or 345.xx) and maternity (v22.xx), diagnosed with epilepsy by a neurologist or epileptologist, and seen while pregnant $(\mathrm{N}=128)$. If more than one pregnancy, only the first pregnancy was included. One physician reviewed each individual chart and collected all of the descriptive data points.

Results: 96 pregnant women with epilepsy met the inclusion criteria. The mean age was 26 years old, standard deviation 5.65 years, range 16- 40 years. The mean gestational age (GA) at which the patient had her first visit to a neurologist was 22 weeks, SD 9.53 weeks, and range 4- 40 weeks. The majority of pregnant women had less than or equal to a twelfth grade education $87.5 \%(\mathrm{~N}=21$ of 24$) ; 99 \%$ of the women had prescription drug coverage. The mean number of neurology encounters per pregnancy was 1.9, SD 1.3, and range 1 to 6 . The seizure type was predominately primary generalized $(\mathrm{N}=69$, $72 \%$ ), specifically juvenile myoclonic epilepsy (JME) (N=15), generalized $+/$ - NES $(\mathrm{N}=7)$, and generalized $+/$ - syncope $(\mathrm{N}=3)$. In the focal seizure group (27 patients, 28\%), 11 (41\%) also experienced secondary generalization. Of the 96 women, 15 self-discontinued their anti-convulsant medication without their physicians' knowledge and 15 experienced an increase in seizure frequency. 11 of the 15 women with increased seizure frequency had self-discontinued their medication (versus $4 / 81$ without an increase in seizure frequency)(p $<0.05$ ) and $11 / 15$ pregnant women who self-discontinued anticonvulsant therapy had an increase in seizure frequency (versus $4 / 81$ who had not self-discontinued their anticonvulsant $)(p<0.05)$. Six $(40 \%)$ of the pregnant women who self-discontinued their anticonvulsant medications did so prior to neural tube closure and 3 $(20 \%)$ did so because of advice given to them directly by their pharmacist.

Conclusions: Based on a sample of 96 pregnant women with epilepsy, an increase in seizure frequency was statistically significantly associated with self-discontinuation of anticonvulsants. Educating pharmacists and epileptic women of childbearing potential about the importance of continuing anti-convulsant therapy during pregnancy may yield considerable benefits.

\subsection{5}

\section{EPILEPSY RELATED ACCIDENTS IN A SAUDI COHORT}

Sadia Hanif, S. Sinha and K. A. Siddiqui (King Fahad Medical City, Riyadh, Saudi Arabia)

Rationale: Motor vehicle accidents (MVAs), burns, drowning and head injuries are among the most feared epilepsy-related injuries. Risk factors for such injuries include number of antiepileptic drugs, history of generalized/complex partial seizures, and seizure frequency. .Patients with more severe form of epilepsy are at higher risk of injuries and accidents There is currently no data regarding the frequency of accidents including MVAs in patients with Epilepsy in 
Kingdom of Saudi Arabia.. The purpose of our study was to investigate frequency of seizure-related accidents.

Methods: We interviewed 65 consecutive patients seen in Epilepsy clinic at National Neuroscience Institute, King Fahad Medical City. The questionnaire used for interview consisted of demographic details, disease related parameters including duration of epilepsy, seizure frequency, number of anti epileptic drugs, compliance to treatment, and number of accidents including MVA. We calculated the frequency of accidents in our cohort and also compared to the demographic and clinical variables.

Results: There were 38 males and 27 females, with mean age (+ SD) of 29.4 (+12.6) years. Average duration of epilepsy was 108 (range 1-480) months with mean seizure frequency of 1-3 per year. Number of AEDs per patient ranged between $0-4$. All but 2 patients were on AEDs and were complaint to the treatment. All kind of accident were seen in 23/65 (35.4\%) patients ( 6 females and 17 males). MVAs were seen in $8 / 38(21 \%)$ male patients. Besides, MVA other accidents included burns, joint dislocations, bone fractures and head injuries related to falls. No correlation of accidents was seen with the seizure type and severity, duration of epilepsy, and number of AEDs used in our cohort.

Conclusions: Seizure related accidents were seen in about one-third of our patients with epilepsy. MVAs were seen in one-fifth of the driving population. Our data highlights the importance of appropriate counseling to the patients and provides recommendation for strict epilepsy related driving laws in Saudi Arabia.

\subsection{6}

\section{MEDICATION ADHERENCE IN EPILEPSY PATIENTS} AFTER A SINGLE NEUROLOGIST VISIT IN RURAL INDIA Jeffrey Bigelow ${ }^{1}$, V. Singh ${ }^{1}$ and M. B. Singh ${ }^{2}{ }^{1}$ University of California San Francisco, San Francisco, CA and ${ }^{2}$ All India Institute of Medical Sciences, Delhi, India)

Rationale: Ninety percent of people with epilepsy live in less developed countries and more than three-fourths of affected people in developing countries do not get the treatment they need. In India, there are less than 2000 practicing neurologists for a population greater than 1.15 billion people, most of these neurologists practice predominantly in urban areas. In 2009, Dr. Mamta Singh established a mobile epilepsy clinic, as part of the Lifeline Express (LE) medical train, where she and colleagues travel to rural areas and see epilepsy patients for a single visit, recognizing a follow-up visit is impractical in a majority of cases. This approach raises the question of whether a one-time mobile clinic can be a feasible and effective model for the chronic treatment of epilepsy in rural India. This could be evaluated by measuring continued medication adherence in patients at greater than 6 months after the visit.

Methods: The researchers developed an interview tool composed of demographics and primarily close-ended questions, with limited open-ended questions. Two LE stop locations in different areas of northern India were chosen, Babrala and Morena, due to similar characteristics (seen 7-9 months previously and similar proximity to Delhi). Patients who had been seen at the LE clinic were contacted for an in-person interview where possible; otherwise the survey was completed over the phone. Data was then coded and summarized. Results: Of the 334 patients originally seen during the clinics at Babrala and Morena, 36 were excluded for various reasons (nonepileptic spells, no medications given, death, or refusal to participate), leaving 298 eligible patients, 142 of which were interviewed $(47.7 \%)$. Of those patients contacted, $72.5 \%$ of patients were still taking medications; the non-respondents $(52.3 \%)$ had an unknown adherence status. Patients who were more likely to be adherent included those who were already being treated when they were seen at the LE epilepsy clinic $(93.7 \%)$, who had previously seen a doctor regarding their seizures $(81 \%)$, who believed epilepsy was a treatable condition $(79.1 \%)$, who had the perception that medications improved their seizures $(86.8 \%)$, and who reported fewer seizures over the preceding month $(82.8 \%)$. There were no significant differences due to age, sex, marital status, education level, employment status, type of epilepsy, length of time with epilepsy, or frequency of seizures at the time of visit.

Conclusions: Most of the contacted patients seen on the Lifeline Express were still taking medications between 6-9 months after a one-time visit with a neurologist. Those patients who had previously been treated for epilepsy and who had the perception that epilepsy could be treated and that medications improved seizures were more likely to be adherent. This one-time clinic appears to be an effective model of treating epilepsy, and could be a model for epilepsy care in other countries.

\subsection{7 \\ DIRECT COSTS OF LENNOX GASTAUT SYNDROME IN A HIGHLY SPECIALIZED HOSPITAL}

gessen salmeron gomez and M. Pizarro Castellanos (Neurology, Hospital Infantil de Mexico "Dr. Federico Gomez", Distrito Federal, Mexico)

Rationale: Lennox Gastaut Syndrome (LGS) produces significant economic costs for their chronic condition, with an impact of the patient's illness, his family and society. Objective: To determine the direct cost of SLG in a tertiary care hospital in Mexico

Methods: We performed a cost analysis of the disease, using a generalized linear model from an institutional perspective of the health sector in Mexico. All costs are in Mexican pesos (MXP), updated to December de2011

Results: 58expedientes were reviewed of patients who met criteria of the International League Against Epilepsy for SLG and followed up for 5 years. The male gender predominance in $56 \%(n=34)$, mean age 5 years 7 months to diagnosis. The average total cost of the first year of monitoring a patient is \$38,974.60 SLG MxP, second year \$ 31,534.27 MxP, third year \$40,592.65 MxP, fourth year \$ 13,522.84 $\mathrm{MxP}$, fifth year $\$ 16,738.90 \mathrm{MxP}$. The greatest cost of illness is associated with hospitalizations during the first 3 years of follow up, while the last two years monitoring by the outer query is first followed by hospitalizations. Auxiliary diagnostic methods in third place and drugs in last place.

Conclusions: The direct cost per year of SLG average follow-up from the institutional perspective is $\$ 28,272.65 \mathrm{MxP}$. The cost associated with hospitalizations for the highest morbidity in our series.

It is necesary the timely management of patients with LGS to improve the quality of life and cost of illness by reducing comorbidities.

\subsection{8 \\ QUALITY OF EEG RECORDINGS OBTAINED BY NON- SPECIALIST TECHNICIANS IN RURAL HOSPITAL SETTINGS BEFORE AND AFTER A ONE-DAY INTENSIVE TRAINING COURSE}

Kristine S. Ziemba, M. T. Hoerth, J. F. Drazkowski, K. H. Noe, L. C. Helepololei, J. R. Muccioli, L. M. Tapsell, B. J. Mill and J. I. Sirven (Mayo Clinic Arizona, Scottsdale, AZ)

Rationale: At Mayo Clinic in Arizona (MCA), we are committed to improving health care in underserved populations throughout our state, through remote telemedicine consultation when appropriate. One challenge in the area of teleneurology is the need for urgent EEGs to facilitate appropriate clinical decision-making. While larger metro hospitals have on-call technologists with extensive training in EEG set-up, monitoring, troubleshooting and interpretation, the supply of such technologists is much too small to staff hospitals in remote areas. This project was designed to determine if the quality of EEGs obtained by non-specialized technicians (respiratory technicians) at a remote hospital could be improved significantly after an intensive, one-day training course. 
Methods: Respiratory technicians from a rural Arizona hospital (Kingman Regional Medical Center) were trained in the fundamentals of EEG setup and recording in a one-day session at MCA. Prior to this training, they had been applying electrodes using a template system (BraiNet $\left.{ }^{\circledR}\right)$, following only vendor-specific instructions. Afterward, they applied surface electrodes using systematic measurements according to the international 10-20 system. EEGs recorded either before or after training were reviewed in a blinded fashion by registered EEG technologists or neurologists at MCA, and various aspects of technical quality were rated using 9 statements with a Likert scale $(1=$ "strongly disagree" to $5=$ "strongly agree).

Results: Prior to our training efforts, many EEGs obtained at the remote site were deemed "non-interpretable". Qualitative evaluation was undertaken only for EEGs that were interpretable. Out of a possible total score of 45 ( 9 quality statements, with 5 points being the most positive score), the "pre-training" EEGs were scored at an average of $32.5(n=6)$ and the "post-training" EEGs at an average of $33.8(\mathrm{n}=6)$, a non-significant increase. Some individual technical aspects showed improvement, such as "Impedances were documented at less than 5kOhm" and "Artifacts were identified and minimized", but also not to a statistically significant degree in this limited sample. Conclusions: EEGs obtained in rural hospitals in Arizona may be read by experts at a distance, but only if recordings are made that meet minimal technical requirements. This project is a step in the direction of improving the quality of EEGs by providing education to non-specialist technicians in these areas. This is an ongoing effort that will be modified as more data are collected in order to improve neurologic care in rural Arizona communities.

\section{History of Epilepsy}

\subsection{9}

\section{THE PORTRAYAL OF SEIZURES AND EPILEPSY ON YOUTUBE}

Victoria Wong, M. Stevenson and L. Selwa (Neurology, University of Michigan, Ann Arbor, MI)

Rationale: New media platforms such as YouTube and Twitter are increasingly important in popular culture. Prior studies noted that nearly all forms of traditional popular media presented seizures in a dramatized or negative manner. Social media on the Internet appears to follow a similar practice. Tweets referencing seizures or epilepsy on Twitter are often derogatory. Similarly, comments on YouTube videos referencing seizures or epilepsy are frequently negative. User generated content such as YouTube videos represent an important source of public exposure to seizures. The ease of production ensures that persons with epilepsy may share their unfiltered personal accounts, in stark contrast to traditional media. We hypothesize that this user-generated format may yield content that is more sympathetic towards those with epilepsy relative to prior forms of media.

Methods: A search was performed in YouTube using the terms "epilepsy" and "seizures." Two independent reviewers analyzed 100 YouTube videos. Reviewers assigned each video to one of eight descriptive categories: Personal Experience / Anecdotal, AdviceSeeking, Informative / Educational; Advertisement, Opinion, Entertainment / Popular Culture, Joke / Ridicule, and Oblique Reference. The content of each video was also rated on scales assessing the accuracy of information regarding seizures or epilepsy, the overall positive (sympathetic) or negative (derogatory) impression suggested by the video regarding seizures or epilepsy, and the difficulty level of the video.

Results: $28 \%$ videos contained an ictal event. $25 \%$ featured a person with epilepsy recounting personal experience. The most common categories were Personal Experience/Anecdotal (44\%) and Informative/Educational (38\%). In rating accuracy, $51 \%$ of videos were judged as accurate, and only $9 \%$ inaccurate; accuracy was not an applicable attribute in the remainder of videos. In rating attitude, $85 \%$ of videos were sympathetic, $9 \%$ neutral, and only $6 \%$ derogatory. Only $2 \%$ of videos containing undefined technical language while $98 \%$ of videos were thought to be easily understood by the layperson.

Conclusions: Our study suggests a more positive portrayal of epilepsy than seen in traditional media. With the increasing prominence of user-generated content, allowing for those with epilepsy to post personal accounts by uploading videos for public consumption, sources such as YouTube appear to be more accurate and sympathetic while being more accessible than traditional media.

To our knowledge, this is the first study describing the presentation and classification of seizure and epilepsy videos on YouTube. We are optimistic that with a shifting ratio towards sympathetic content about epilepsy, the amount of stigma towards epilepsy and seizures will continue to lessen.

\subsection{0 \\ EPILEPSY AND THE HEART - A HISTORICAL REVIEW $1827-1935$}

Sanjay P. Singh, R. R. Sankaraneni and J. D. VanDerWerf (Creighton University School of Medicine, Omaha, NE)

Rationale: In recent times, the effect of epilepsy on the heart has become a subject of great interest to Neurologists, particularly since it is believed to be the main mechanism implicated in Sudden Unexpected Death in Epilepsy (SUDEP). It is interesting to note how neurologists had commented on this interaction almost 100 years ago. Methods: Extensive review of the published medical literature from 1827 to 1935 .

Results: : In 1827, Robert Adams described a seizure in association with bradycardia and cardiac arrest. In 1906 there were two seminal articles published in the Lancet. The first article authored by Russell et al. was titled "Cessation of the Pulse during the onset of Epileptic Fits". He describes a case where after the onset of a seizure, the patient was found to have no pulse. The authors also speculate on the mechanism of cardiac inhibition via the vagus nerve. The second article was authored by J.F. Munson and discusses the action of the heart in an epileptic seizure. The author has used a unique device, a kymograph, to monitor the heart rate in relation to epilepsy. He found no cardiac arrest in the 10 cases he studied, but did find a case where there was a slight slowing of the heart. In the other cases there was tachycardia. . In 1907 Fred High Clarke published an article on occurrence of tachycardia and bradycardia in relation to an epileptic attack. Notkin et al. published an article in American J. Psychiatry in 1932 which described in detail the changes in cardiovascular parameters during a seizure. In this article, three patients had an imperceptible pulse during the seizure. In 1933 W. Penfield described cases where seizures would be accompanied by fall of blood pressure, slowing and weakening of pulse; this was done as part of his Wesley Carpenter Lecture.

Conclusions: The interaction between epilepsy and cardiovascular parameters was well documented in a few seminal articles published between 1827 to 1935 . It is thus fascinating that interest in investigating this interaction has only been revived recently in light of SUDEP.

\subsection{1}

FRANCISCO GOYA AND THE IMPENITENT'S SEIZURE Enrique Carrazana ${ }^{1}$ and M. R. Lopez ${ }^{2}{ }^{1}$ Acorda Therapeutics, Inc., Hawthorne, NY and ${ }^{2}$ University of Miami Miller School of Medicine, Miami, FL)

Rationale: The biblical view that epileptic seizures were manifestations of demonic possession was prevalent during the Middle Age and continue past the Baroque period. Within this 
religious framework, epilepsy was occasionally the subject of the arts, often making reference to Christ's miracle of the epileptic child (Mark 9:14-29).

Methods: The clinical and historical angles of Francisco Goya's "St. Francis Borgia at the Deathbed of an Impenitent" are examined in detail.

Results: Painted in 1788 by Goya, "St. Francis Borgia at the Deathbed of an Impenitent" was commissioned by the Duke of Osuna for the Saint's Chapel at the Cathedral of Valencia, Spain. The painting presents a stunning mystical drama. The Saint, holding a bleeding crucifix in 1 hand and raising the other hand in a gesture of consternation, stares towards the impenitent, in the presence of a cluster of 4 laughable demonic creatures. The dying man's torment, underscored by the heaving chest, sunken eyes, gasping mouth, rigid body, and the disheveled bed sheets, is highly suggestive of an epileptic generalized seizure at its initial tonic phase, seconds before the impenitent's death. Goya based the scene on an account of the Saint's life, c. 1557, given at the canonization depositions and later included in a published biography of St. Francis. Seizures at end-oflife are a well known occurrence, although persons with epilepsy have a greater risk of death due to an underlying common cause than from the seizure itself.

Biographical accounts of the impenitent's criminal background raise possibilities such as head trauma and alcoholism as explanations for the seizure.

Conclusions: The painting could be interpreted as abetting the demonic superstitions attributed to epilepsy during Goya's time - eg, seizure as demonic punishment for a life of crime. However, Goya's comical depiction of the bedside demons keeps with his introverted and humanistic/biting satire on social attitudes of the time and transforms these symbols of darkness into those of folly and ignorance.

Support: Acorda Therapeutics, Inc.

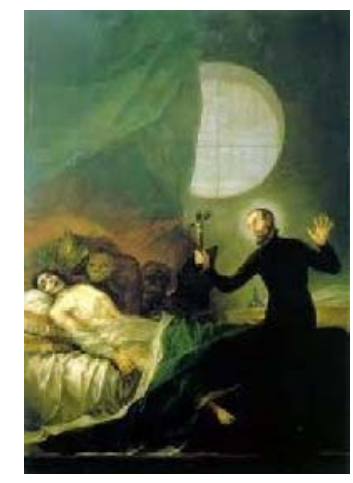

Saturday, December 1, 2012

Late Breaking Abstracts

\subsection{2}

ON THE NATURE OF SEIZURES: THEORETICAL

\section{APPROACH}

C. Bernard ${ }^{1}$, V. K. Jirsa ${ }^{1}$, A. Ivanov ${ }^{1}$, W. Stacey ${ }^{2}\left({ }^{1}\right.$ INSERM U1106, Marseille, France; ${ }^{2}$ 2Dept. Neurol. and Dept. Biomed. Engin, Ann Arbor, MI, United States)

Rationale: The synchronous, rhythmic activity of large neuronal assemblies underlies spontaneous recurrent seizures in epilepsy. This activity often takes the form of complex sequences of fast and slow oscillations. Despite all heterogeneity and variability in epilepsy, there is a remarkable invariance of certain dynamic features in seizures suggesting the existence of certain dynamic primitives. These primitives are typical features of seizures recorded in patients with temporal lobe epilepsy and include a pre-ictal state, followed by tonic and tonic-clonic discharge patterns. Mathematically primitives can be expressed as attractor states in nonlinear dynamic systems. These attractor states form equivalence classes that are unambiguously characterized by the topology of the dynamic flows. Methods: Using time scale separation techniques and bifurcation theory we demonstrate that repetitive complex sequences of seizure like events can be understood in terms of three dynamic primitives composed of a 3D-spiral flow, a 2D excitable system and a linear fixed point flow. The three primitives account for 5 metavariables characterizing the neurophysiological state of the network. They are described by coupled systems of not linear differential equations, thus constituting the model called EPILEPTOR.

Results: When properly coupled, the three primitives deconstruct the complex sequences of seizure like events and explain various phenomena including bistability and hysteresis of seizures, the associated DC shift, emergence and statistics of pre-ictal spikes, and the role of spike-wave complexes. In particular we demonstrate the causal role of the DC shift for the seizure dynamics. We demonstrate how a system can approach the bifurcation to seizure genesis using various pathways, including stimulation and noise.

Conclusions: Our results demonstrate that seizures are simple objects, which can be generated by most neuronal networks. There are multiple ways to approach seizure onset, which explain why they are unpredictable most of the time. We also demonstrate how the trigger zone can be outside the epileptogenic region. EPILEPTOR provides a framework to interpret clinical and experimental data. Our predictions are experimentally tested in the companion poster and integrate the phenomenological model of three primitives (the "epileptor") in a physiological context.

\subsection{3}

ON THE NATURE OF SEIZURES: EXPERIMENTAL APPROACH

W. C. Stacey ${ }^{1,2}$, A. Ivanov ${ }^{3}$, V. Jirsa ${ }^{3}$, C. Bernard ${ }^{3}\left({ }^{1}\right.$ Neurology, University of Michigan, Ann Arbor, MI, United States; ${ }^{2}$ Biomedical Engineering, University of Michigan, Ann Arbor, MI, United States; ${ }^{3}$ Université de la Méditerranée, Marseille, France)

Rationale: Seizures are paroxysmal episodes of abnormal synchronization that overwhelm normal brain activity. Despite decades of research, little is known about how they are formed. An intriguing characteristic of clinical epilepsy is that, despite a wide range of underlying pathologies in different patients, seizure dynamics are very similar. Likewise, seizure triggers such as sleep deprivation, stress, and drug withdrawal are common among many different forms of epilepsy. These similarities suggest that different types of seizures are governed by common, intrinsic mechanisms. Methods: In the companion poster we developed a mathematical analysis on the nature of seizures, leading to several predictions: DC shift is an integral component of seizure genesis, seizure dynamics from onset to end require the presence of slowly evolving parameters, a critical level of noise can push a system to generate seizures, and seizures terminate with logarithmic scaling. These predictions were verified in experiments with intact mouse hippocampi and zebrafish, and observed in intracranial human EEG.

Results: Lowering external magnesium triggered spontaneous seizure-like events (SLEs). All SLEs were associated with a DC shift as previously reported in numerous other studies. Synaptic noise rose prior to the spontaneous SLEs in the majority of recorded cells. We found that oxygen levels, ATP consumption and external potassium levels in the intact preparation during SLEs followed the dynamics of the slow parameter predicted by the model. We used a modified recording chamber to modulate septum excitability and send uncorrelated synaptic noise to the hippocampus, which was independently maintained in subthreshold conditions for SLE genesis. When reaching a critical value predicted by stochastic theories, this remotely-generated synaptic noise was sufficient to 
trigger SLEs in the hippocampus. Seizures terminated following logarithmic scaling in mice, zebrafish, and humans. Human seizures recorded with DC coupling demonstrated baseline shifts that were not present in the traditional recording.

Conclusions: We conclude that seizure dynamics can be described by the interaction of 5 metavariables that are present in a wide range of brain physiology. We found similar results across seizures in mice, zebrafish, and humans. Seizures constitute a generic pattern of activity that is embedded in neuronal networks, and which are expressed when appropriate conditions are met.

\subsection{4 \\ CLUSTERING OF MUTATIONS IN A NORTH AMERICAN SERIES SUPPORTS A DOMINANT-NEGATIVE MECHANISM OF KCNQ2 ENCEPHALOPATHY} E. C. Cooper ${ }^{1}$, L. Carmant ${ }^{2}$, R. Flamini ${ }^{3}$, F. D. Kendall ${ }^{4}$, P. M. Levisohn $^{5}$, J. J. Millichap ${ }^{6,7}$, X. R. Ortiz-Gonzalez ${ }^{8}$, K. Park ${ }^{5}$, B. E. Porter $^{9}$, B. T. Tran ${ }^{1}$, T. N. Tsuchida ${ }^{10}\left({ }^{1}\right.$ Neurology and Neuroscience, Baylor College of Medicine, Houston, TX, United States; ${ }^{2}$ Pediatrics, Univ. of Montreal, Montreal, QC, Canada; ${ }^{3}$ PANDA Neurology, Atlanta, GA, United States; ${ }^{4}$ Virtual Medical Practice, Alpharetta, GA, United States; ${ }^{5}$ Pediatrics and Neurology, Univ. of Colorado School of Medicine, Aurora, CO, United States; ${ }^{6}$ Epilepsy Center, Ann \& Robert H. Lurie Children's Hospital of Chicago, Chicago, IL, United States; ${ }^{7}$ Pediatrics, Northwestern Univ. School of Medicine, Chicago, IL, United States; ${ }^{8}$ Neurology, Children's Hospital of Philadelphia, Washington, PA, United States; ${ }^{9}$ Neurology, Stanford Univ. School of Medicine, Stanford, CA, United States; ${ }^{10}$ Neurology, Children's National Medical Center, Washington, DC, United States)

Rationale: Recent Austro-European and Japanese series found de novo KCNQ2 mutations in 11 cases of early onset epileptic encephalopathy and Ohtahara syndrome [1,2]. KCNQ2 variants identified were missense mutations in channel domains critical for trafficking, ion conduction, or voltage gating, suggesting a potential dominant-negative mechanism for the severe phenotype [3]. This hypothesis would be supported if additional cases were found bearing mutations clustered in the same domains.

Methods: We screened patients for known causes of early infantile epileptic encephalopathy as part of routine care, including genetic testing for $\mathrm{KCNQ}^{2} \mathrm{In}$ some, testing was extensive including analysis for mutations and copy number variation in multiple genes.

Results: KCNQ2 missense mutations were found in 13 cases. Some clinical data are available in 11 cases. In this subgroup, all had seizures beyond the neonatorum and moderate to severe delay. Seizures began early in the first week (mean 1.8 day, $n=10$ ). In 5 cases where parental testing was completed, patient KCNQ2 variants were de novo. Two patients are siblings, bearing a variant in the S1S2 loop of KCNQ2; they experienced neonatal and infantile seizures, and have moderate delay at age $8 \mathrm{mo}$. and $3 \mathrm{yr}$. Three patients bear mutations within or near the voltage-sensing S4 helix. Six patients bear mutations in the pore loop. Two patients bear mutations in the intracellular C-terminus, near calmodulin binding sites. Thus, all mutations are clustered in a small $(\sim 15 \%)$ portion of the channel polypeptide. A structural model shows the mutation clusters are functionally critical domains with high potential for dominant negative effects. Consistent with that, no copy number variations, frameshift, indel, or nonsense mutations were found.

Conclusions: The KCNQ2 variants we identified are missense mutations clustered in the same channel functional domains identified previously $[1,2]$. This supports the hypothesis that KCNQ2 encephalopathy results from dominant-negative effects [3]. If this mechanism is confirmed by in vitro and in vivo studies of recombinant mutant channels, KCNQ opener drugs may provide gene-specific treatment for KCNQ2 encephalopathy.

${ }^{1}$ Weckhuysen et al. (2012) Ann. Neurol. 71:15-2 ${ }^{52}$ Saitsu et al. (2012) Ann. Neurol. 72:298-300. ${ }^{3}$ Millichap and Cooper (2012) Epil. Curr. $12: 150-2$.
1.355

TREATMENT OF KCNQ2 ENCEPHALOPATHY WITH EZOGABINE

J. J. Millichap ${ }^{1,2}$, P. M. Levisohn ${ }^{3}$, T. N. Tsuchida ${ }^{4}$, E. C. Cooper ${ }^{5}$ ( ${ }^{1}$ Epilepsy Center, Ann \& Robert H. Lurie Children's Hospital of Chicago, Chicago, IL, United States; ${ }^{2}$ Pediatrics, Northwestern University Feinberg School of Medicine, Chicago, IL, United States; ${ }^{3}$ Pediatrics and Neurology, Univ. of Colorado School of Medicine, Aurora, CO, United States; ${ }^{4}$ Neurology, Children's National Medical Center, Washington, DC, United States; ${ }^{5}$ Neurology and Neuroscience, Baylor College of Medicine, Houston, TX, United States)

Rationale: KCNQ2 encephalopathy is a syndrome of early neonatalonset, refractory seizures with EEG suppression burst. Although seizures usually decrease in frequency over the first 1-3 years of life, patients exhibit persistent severe psychomotor disability. Ezogabine is a potent, selective activator of neuronal KCNQ channels containing combinations of subunits KCNQ2- ${ }^{5}$ Therefore, treatment of patients with KCNQ2 encephalopathy could be potentially beneficial. Methods: Three cases of neonatal onset epileptic encephalopathy with predicted pathogenic KCNQ2 mutations were treated with ezogabine. Data collected includes genotype, phenotype, EEG, and ezogabine dosing, tolerability, serum levels, and initial response. Results: All 3 patients presented with seizures before the 2 nd day of life; neonatal EEG showed suppression burst. In pt. 1 (3.5 yr. old), seizures evolved to infantile spasms. At 3 yr. old there were daily clusters of epileptic spasms, severe psychomotor impairments, and EEG showed disorganization and multifocal epileptiform discharges. A previously-described pathogenic KCNQ2 mutation was detected. Ezogabine was started, and after 5 months treatment, the EEG showed the new appearance of rudimentary sleep architecture, but continued spasms and tonic seizures. In pt. 2 (3 mo. old), seizures were refractory to anticonvulsants and evolved to multiple daily alternating asymmetric tonic spasms. EEG evolved from early suppression burst at 6 days of life to hypsarhythmia at 3 months old. A trial of ezogabine was recently started. In pt. 3 (1.5 yr. old) the EEG evolved from early suppression burst to hypsarhythmia. Seizures remitted with ACTH, and anticonvulsants were withdrawn at 10 mo., but psychomotor delay persisted. After a KCNQ2 mutation was detected, ezogabine was started with the aim of improved development. After several months, the treatment is well tolerated and qualitative improvement in motor activity has been observed by parents.

Conclusions: Ezogabine was well tolerated and mildly beneficial in initial treatment of two young children; a trial just started in one infant. This series represented 3 stages of the typical evolution pattern of KCNQ2 encephalopathy syndrome: pt. 2 was diagnosed in infancy and has frequent daily seizures, pt. 1 was diagnosed in early childhood and continues to have daily seizures, and pt. 3 was diagnosed after seizure remission but remains encephalopathic. At minimum, ezogabine treatment trials in young children provide valuable data on dosing and tolerability. We hypothesize early diagnosis and treatment may hold the greatest potential for reducing the effects of KCNQ2 dysfunction.

1.356

ACUTE AND LONG TERM SAFETY AND EFFICACY OF EXTERNAL TRIGEMINAL NERVE STIMULATION FOR DRUG RESISTANT EPILEPSY

C. M. DeGiorgio ${ }^{1,2}$, J. Soss ${ }^{1}$, I. Cook $^{5,2}$, D. Markovic ${ }^{4}$, C. Kealey ${ }^{2}$, D. Murray $^{1}$, S. Oviedo ${ }^{3}$, G. Corral-Leyva ${ }^{3}$, C. Heck $^{3}$ ( ${ }^{1}$ Neurology, UCLA School of Medicine, Los Angeles, CA, United States; ${ }^{2}$ NeuroSigma, Los Angeles, CA, United States; ${ }^{3}$ Neurology, KeckUSC School of Medicine, Los Angeles, CA, United States;

${ }^{4}$ Biomathematics, UCLA School of Medicine, Los Angeles, CA, United States; ${ }^{5}$ Psychiatry, UCLA School of Medicine, Los Angeles, CA, United States) 
Rationale: To explore the acute and long-term safety and efficacy of eTNS in patients with drug resistant epilepsy (DRE).

Methods: Fifty subjects with two or more partial onset seizures per month entered a 6-week baseline period, and were randomized to treatment or active-control parameters, then followed for 18 weeks. After completion of the acute treatment period, patients were offered continuation in long-term follow-up. Subjects randomized to low settings were crossed over to high settings at the beginning of the long-term followup period.

Results: On average, subjects failed 3.35 AED's prior to enrollment, with an average duration of epilepsy of 21.5 years (treatment group) and 23.7 years (active control group). eTNS was well tolerated. Side effects included headache $4 \%$, anxiety $4 \%$, and skin irritation $14 \%$. The treatment group experienced a significant within-groups improvement in responder rate (RR) over the 18-week treatment period (from $17.8 \%$ at 6 weeks to $40.5 \%$ at 18 -weeks, $p=0.01$, GEE). The RR was $30.2 \%$ for the treatment group versus $21.1 \%$ for the control group for the entire treatment period (not significant, $\mathrm{p}=$ 0.31 , GEE model). Subjects in the treatment group were more likely to respond than patients randomized to control $(\mathrm{OR}=1.73$, CI $0.59-$ $0.51)$. eTNS was associated with significant reductions in seizure frequency as measured by the Response Ratio (RRATIO, $\mathrm{p}=0.04$, ANOVA), and significant improvements in mood on the Beck Depression Inventory ( $\mathrm{p}=0.02$, ANOVA).

Long Term Results: 35 subjects of the original 50 continued in longterm follow-up: 19 for the treatment group and 16 for the active control group. All subjects were crossed over to effective parameters. The RR for the active treatment group was $36.8 \%$ at 6 and 12 months, versus $25 \%$ for the active control group for a total combined RR rate of $31.4 \%$. The RRATIO increased significantly over the long-term treatment period.

Conclusions: eTNS was well tolerated, and associated with a responder rate of $40.5 \%$ acutely, and $36.8 \%$ in long-term followup at 1 year. eTNS is a promising new therapy for Drug Resistant Epilepsy.

The study was supported by grants from the Epilepsy Therapy Project, Epilepsy Foundation of America, Michael Milken Family Foundation, and Boston Scientific.

In the United States, eTNS is an investigational Device. Limited by Federal (or United States) law to investigational use.

\subsection{7}

AN OPEN-SOURCE PIPELINE FOR VISUALIZATION OF INTRACRANIALLY IMPLANTED ELECTRODES USING 3D CT-MRI CO-REGISTRATION.

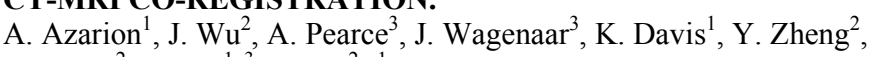
H. Wang ${ }^{2}$, B. Litt ${ }^{1,3}, \mathrm{~J}_{\text {. Gee }}{ }^{1}{ }^{1}$ Neurology, Hospital of the University of Pennsylvania - University of Pennsylvania School of Medicine, Philadelphia, PA, United States; ${ }^{2}$ Radiology, University of Pennsylvania School of Medicine, Philadelphia, PA, United States; ${ }^{3}$ Bioengineering, Univeristy of Pennsylvania, Philadelphia, PA, United States)

Rationale: Visualization of intracranial electrodes in 3D space can potentially improve the accuracy of surgical resection for epilepsy patients. The open-source algorithm we have developed allows for accurate 3D display of electrode locations on a parcellated brain. Methods: CT and MR scans were obtained on 7 patients shortly after intracranial electrode implantation. CT-MR rigid registration was employed using mutual information as the similarity metric to align the CT and MR images. The CT scan was deemed the floating image and the MR scan as the fixed image, thus allowing CT data to be transformed to the MR space. An external brain atlas with cortical annotation was used to segment the patient MR scan. This atlas was propagated to the patient MR image with non-rigid registration. To adapt the labels to the patient's anatomy, we performed prior-based segmentation to smooth the resultant brain parcellation. The three images of the patient CT scan, MR scan, and the labeled atlas were normalized in the same space as the patient MR scan. The electrodes were then merged with the parcellated brain, allowing localization and visualization of the intracranial electrodes with a 3D image (Fig. 1).

Validation of the co-registration algorithm was performed by marking 4 identical landmarks on both the post-implant CT and postimplant T1 MRI for all subjects, independent of the co-registration. The landmarks used were the pineal gland, midline inferior most point of the nasal bridge, a frontal cortical point immediately posterior to the superior most midline of the frontal sinus, and the confluence of sinuses. The 3-dimensional coordinates of the segmented masks from the T1 MR images were averaged to locate the center. Following co-registration of the post-implant $\mathrm{CT}$ with the post-implant T1 MRI, the same procedure was repeated to calculate the center of the masks on the co-registered CT image.

Results: Using this co-registration pipeline we were able to automatically super-impose the electrodes on the parcellated brain for all 7 subjects.

The validation measure on all subject images was calculating the distance between the centers of the two masks. The mean of the distances are as follows: Pineal gland (mean distance, SD) $3.46 \mathrm{~mm} \pm$ $1.00 \mathrm{~mm}$, inferior point of nasal bridge $2.08 \mathrm{~mm} \pm 0.81 \mathrm{~mm}$, frontal cortical point $2.94 \mathrm{~mm} \pm 1.35 \mathrm{~mm}$, and confluence of sinuses 2.98 $\mathrm{mm} \pm 1.21 \mathrm{~mm}$.

Conclusions: This work allows for accurate visualization of intracranial electrodes in 3D space and also demonstrates the feasibility of applying brain parcellation to epileptic brains. This imaging algorithm will be available as an open-source pipeline for worldwide use on the IEEG (International Epilepsy Electrophysiology Portal).

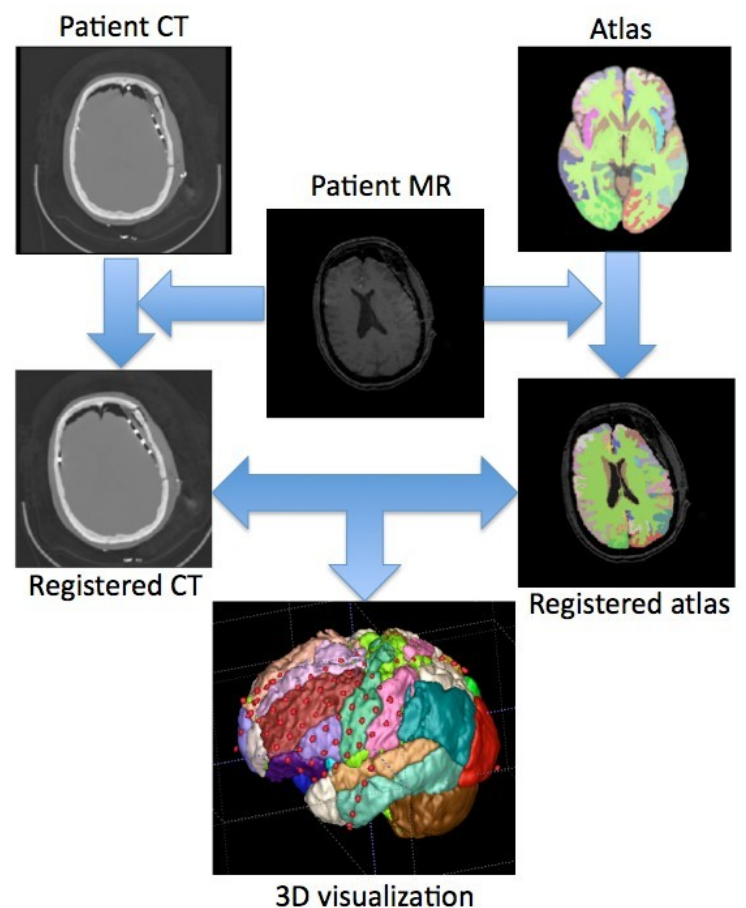

Fig. ${ }^{1}$ The flowchart of the proposed image processing pipeline. Patient CT (top left) is registered to patient MR image (middle). An external labeled atlas (top right) is also registered to patient MR image. The results of the two registrations are integrated to generate the parcellated brain overlaid with electrodes (bottom). 


\subsection{8}

\section{BILATERAL PERIVENTRICULAR NODULAR} HETEROTOPIA: THE CLINICAL PROFILE OF 84 PATIENTS

I. Parulkar ${ }^{1}$, Z. Fallil ${ }^{2}$, C. Shain ${ }^{3}$, A. Poduri ${ }^{3}\left({ }^{1}\right.$ Alpert Medical School of Brown University, Providence, RI, United States; ${ }^{2}$ Neurology, NYU Langone Medical Center, New York, NY, United States;

${ }^{3}$ Neurology, Boston Children's Hospital, Boston, MA, United States)

Rationale: Periventricular nodular heterotopia (PVNH) is a malformation of cortical development due to impaired neuronal migration resulting in the formation of nodular masses of neurons and glial cells in close proximity to the ventricular walls. There have been several large case series of patients with nodular heterotopias that have included the periventricular, subcortical and leptomeningeal varieties. We report the clinical characteristics of the largest case series of FLNA genome negative patients with seizures who have pure bilateral periventricular heterotopia

Methods: The present series includes 84 patients enrolled from 17 sites located within and outside of the United States as part of the epilepsy phenome/genome project. Included subjects had to have had at least one lifetime seizure and imaging confirmed bilateral PVNH. Participants and/or their parents were interviewed to gather comprehensive data.

Results: A total of 84 patients with seizures and bilateral periventricular heterotopia were included in this series. There was a female predominance with 52 female and 31 male patients affected. The ages ranged from 1 month to 47 years, with the mean age being 23 (SD 11.41). The cohort was predominantly Caucasian $(63 / 84$ $75 \%)$. Developmental delay was noted in $19 \%(16 / 76)$ while a learning disability was present in $9.5 \%(8 / 84)$. Sixty-nine percent $(52 / 75)$ of the patients earned a high school degree and above. An abnormal neurological examination was found in $8 \%(6 / 74)$. All $(100 \%)$ of the patient population experienced at least one lifetime seizure as this was an inclusion criteria and $84.5 \%(71 / 84)$ received a diagnosis of epilepsy. The age of onset of seizures ranged from 1 month to 27 years with a mean age of 11.8 (SD 8.21). Febrile seizures were reported in $12 \%(10 / 83)$ of the group. Eighty percent $(55 / 68)$ of the patients had localization related epilepsy, $10 \%(7 / 68)$ had generalized epilepsy and $9 \%(6 / 80)$ had both. Of the patients with localization related epilepsy $53 \%(29 / 55)$ had a bilateral ictal onsets, $16 \%(9 / 55)$ had left, and 16\% (9/55) had right sided ictal onsets. Multifocal ictal onsets were seen in $11 \%(6 / 55)$ of the population and $14 \%(8 / 55)$ had unclear yet focal ictal onsets. One or more family members carried a diagnosis of epilepsy in 39\% (29/75).

Conclusions: This series of 84 patients is the largest FLNA gene negative series of patients with purely bilateral periventricular heterotopia and seizures published to date. Findings of interest include a high incidence of febrile seizures, but more important are the finding of high prevalence of a family history of epilepsy. This study validates some of the findings of previous smaller studies in regard to development, intelligence and ictal onsets. These clinical findings will be correlated to the imaging findings of this cohort to explore further associations.

\subsection{9}

\section{EXPRESSION OF A POTASSIUM LEAK CHANNEL} INHIBITS PILOCARPINE-INDUCED SEIZURES D. Dey ${ }^{1}$, V. Eckle ${ }^{2}$, I. Vitko ${ }^{2}$, J. M. Williamson ${ }^{3}$, J. Kapur ${ }^{3}$, E. PerezReyes $^{2}$ ( ${ }^{1}$ Neuroscience Graduate Program, University of Virginia, Charlottesville, VA, United States; ${ }^{2}$ Pharmacology, University of Virginia, Charlottesville, VA, United States; ${ }^{3}$ Neurology, University of Virginia, Charlottesville, VA, United States)

Rationale: Epilepsy is characterized by recurring spontaneous seizures. In childhood absence epilepsy (CAE) patients, missense variants in the T-type calcium channel gene CACNA1H were discovered and introduced into the recombinant channel (Cav3.2). In hippocampal neurons, this mutation increased spontaneous burst firing 4-fold when compared to wild-type Cav3. ${ }^{2}$ The two-poredomain potassium channel TREK-1 (KCNK2) is expressed throughout the nervous system and plays important roles in the determining neuronal cell properties such as resting membrane potential and input resistance. Therefore, we hypothesized that overexpression of TREK-1 might silence hyperactive neurons. Methods: To reduce compensatory second messenger regulation of the channel, we generated a triple mutated TREK-1 construct (E306A, S300A, S333A; "TREK M"). This cDNA was expressed using a self-complementary adeno-associated virus (scAAV) with the following elements: CMV enhancer-GABRA4 (574 bp promoter; ref 5)-CMV minimum-TREKM-SV40 minimum polyA signal encapsulated in serotype ${ }^{5} \mathrm{scAAV}$ was injected bilaterally into the DG and EC. Seizures were induced using the $\mathrm{Li} /$ pilocarpine $(50 \mathrm{mg} / \mathrm{kg}$ ) method and recorded using video/EEG monitoring.

Results: Expression of this mutant in HEK-293 cells produces high levels of $\mathrm{K}+$ leak current and dramatically lowered the input resistance and resting membrane potential. We studied expression of TREK-M in cultured hippocampal pyramidal neurons transfected with the C456S variant. TREK-M normalized spontaneous firing by decreasing the input resistance and resting membrane potential (from -57 to $-66 \mathrm{mV}$ ). We next studied the in vivo effect of TREK-M delivered by a novel scAAV under the control of an enhanced GABRA4 promoter on pilocarpine induced seizures in rats. TrekM expression reduced the ability of pilocarpine to induce tonic-clonic seizures by $70 \%(n=6, P<0.0005)$. Similarly, TrekM expression reduced the time spent in status epilepticus measured electrographically by $50 \%(\mathrm{P}<0.005)$.

Conclusions: These findings provide proof for the concept that AAV delivery of TREK-M may be a viable treatment for mesial temporal lobe epilepsy.

\subsection{0 \\ FAILURE OF HEALTH CARE PROVIDERS TO MEET PATIENT EDUCATION NEEDS ABOUT SUDEP} C. Wright ${ }^{1}$, O. Devinsky ${ }^{4,2}$, B. Kroner ${ }^{3}$, K. Macher $^{2}\left({ }^{1}\right.$ Menlo Park, CA, United States; ${ }^{2}$ Epilepsy Therapy Project, Middleburg, VA, United States; ${ }^{3}$ Multisite Epidemiology \& Statistics Program, RTI International, Rockville, MD, United States; ${ }^{4} \mathrm{NYU}$, New York, NY, United States)

Rationale: Controversy remains as to whether or not neurologists should discuss SUDEP with all epilepsy patients or only those at high risk. We initiated a web-based survey to determine whether persons with epilepsy (PWE) and caregivers of PWE (C-PWE) want to know about SUDEP from their medical providers and when they want to learn about it.

Methods: An online survey for adult PWE and C-PWE was designed based on a previous survey of neurologists (Friedman et al, 2012; AES abstract). Respondents were solicited from an email blast and newsletter sent to Epilepsy Therapy Project (ETP) members. A survey link was also posted on epilepsy.com. The survey was IRB approved.

Results: 1,325 PWE and 574 C-PWE completed at least some of the survey. Demographic features and epilepsy-related factors are summarized in Table ${ }^{1}$ Attitudes and knowledge of SUDEP are summarized in Table ${ }^{2}$ Of the PWE respondents. 57\% worry at least a little about dying from their epilepsy and $68 \%$ heard about SUDEP before the survey. Of these $68 \%, 52 \%$ learned of SUDEP from the internet, only $9.6 \%$ from their epilepsy doctor; only $29 \%$ feel welleducated about it; $46 \%$ don't know whether they are at risk; and $69 \%$ never discussed SUDEP with their doctor. Of the $32 \%$ who had not heard of SUDEP, $50 \%$ were scared, anxious or sad after reading the definition but only $3 \%$ did not want to ever be told about SUDEP. $65 \%$ will go to their epilepsy doctor for more information. Of the responding C-PWE, 92\% worry at least a little about the PWE dying from epilepsy. $80 \%$ had heard about SUDEP before our survey and 
$55 \%$ of these learned about it from the internet and $11.8 \%$ from the epilepsy doctor. Only $28 \%$ felt well-educated about it; $64 \%$ think the PWE is at risk for SUDEP; and 55\% never discussed SUDEP with the doctor. Of the $20 \%$ that had not heard of SUDEP prior to our survey, $92 \%$ were scared, anxious or sad after reading the definition but only $1 \%$ did not want to ever be told about SUDEP. $83 \%$ will go to their epilepsy doctor for more information.

Conclusions: Over two thirds of people with epilepsy and their caregivers want to know about SUDEP regardless of the risk and less than $12 \%$ learn about it from their epilepsy doctor. Despite the majority first learning about SUDEP from the internet, people affected by epilepsy do not feel well educated about it and they want to discuss it with their epilepsy provider. Only 3\% of epilepsy patients and $1 \%$ of caregivers never want to be informed about SUDEP. Approximately half of patients and caregivers believe knowledge about SUDEP would influence epilepsy management. Determining which PWE are at high risk and how best to educate PWE and their caregivers about SUDEP remains the challenge for neurologists. There is a large gap between the need for and the delivery of SUDEP education. Sources of funding were ETP and RTI International.
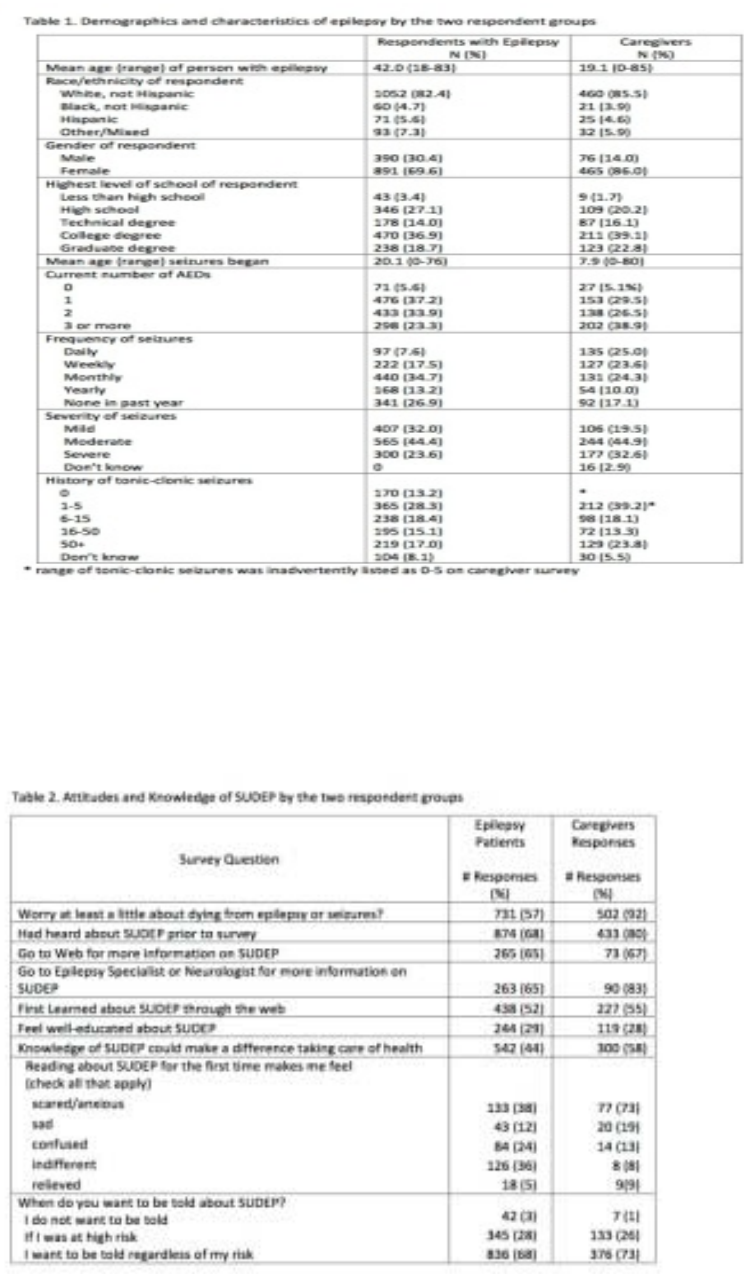

\subsection{1}

\section{FUNCTIONAL CHARACTERIZATION OF GENE} REGULATORY ELEMENTS ASSOCIATED WITH EPILEPSY R. Y. Birnbaum ${ }^{1,2}$, Y. Zhang ${ }^{3}$, C. Wei ${ }^{3}$, N. Ahituv ${ }^{1,2}$ $\left({ }^{1}\right.$ Bioengineering and Therapeutic Sciences, UCSF, San Francisco, CA, United States; ${ }^{2}$ Institute for Human Genetics, UCSF, San
Francisco, CA, United States; ${ }^{3}$ DOE Joint Genome Institute, Walnut Creek, CA, United States)

Rationale: Epilepsy is a complex and heterogeneous disease making it difficult to precisely diagnose and provide effective treatments. Infantile spasms (IS) is an uncommon epilepsy syndrome that typically begins in infancy and is associated with ventral forebrain development and forebrain synapse function. Since a major cause of complex diseases, such as epilepsy, could be mutations in gene regulatory elements, we set out to identify these elements in the mouse forebrain at embryonic day 16.5 (E16.5) which could be associated with IS.

Methods: Using chromatin immuneprecipitation followed by deep sequencing (ChIP-Seq) with enhancer marks (H3K27ac, p300, RNAPoll2), we identified active enhancer candidates in the mouse E16.5 forebrain. In addition, using chromatin interaction analysis followed by paired-end tag sequencing (ChIA-PET) on mouse E16.5 forebrain we are determining the physical interactions of these enhancer candidates and IS-associated genes. Several enhancer candidates are then tested for their enhancer activity using both zebrafish and mouse transgenic enhancer assays.

Results: Our results provide a novel dataset of neuronal enhancers that could control the spatiotemporal expression of IS-associated genes. These sequences pose as great candidates for mutation analyses in IS patients, a screen which we are currently carrying out. Conclusions: Combined, this work will shed light on neuronal gene regulation in general and identify novel genomic regions that could be involved in epilepsy pathogenesis and brain development.

\subsection{2 \\ LACK OF EFFECT OF LACOSAMIDE ON THE PHARMACOKINETIC AND PHARMACODYNAMIC PROFILES OF WARFARIN}

A. Stockis ${ }^{1}$, J. J. van Lier ${ }^{2}$, W. Cawello ${ }^{3}$, T. Kumke ${ }^{3}$, K. Eckhardt ${ }^{3}$ $\left({ }^{1}\right.$ UCB Pharma, Braine l'Alleud, Belgium; ${ }^{2} \mathrm{PRA}$ International, Zuidlaren, Netherlands; ${ }^{3}$ UCB Pharma, Monheim, Germany)

Rationale: Warfarin is a widely used anticoagulant frequently prescribed in the elderly and is prone to drug-drug interactions that can alter its anticoagulant activity. Warfarin is administered as a racemate but the anticoagulant effect is mostly due to the Senantiomer. Lacosamide is an antiepileptic drug approved for the adjunctive treatment of partial-onset seizures in adults. Coadministration of warfarin and lacosamide can be expected. The objective of this study was to evaluate the effect of lacosamide on the pharmacokinetics (PK) and pharmacodynamics (PD) of warfarin. Methods: In this Phase I, open-label, randomized, 2-way crossover study, 16 healthy adult male volunteers received a single $25 \mathrm{mg}$ dose of warfarin, either alone or co-administered with lacosamide following multiple doses of lacosamide $200 \mathrm{mg}$ twice daily. Plasma concentrations of S- and R- warfarin, prothrombin time (PT) and international normalized ratio (INR) were determined serially over time. PK and PD parameters with and without lacosamide were statistically compared, using standard bioequivalence criteria. Tolerability and safety were monitored by recording vital signs, laboratory values, electrocardiograms (ECGs) and treatmentemergent adverse events (TEAEs).

Results: All volunteers completed the study. $\mathrm{C}_{\max }$ and AUC of Sand R-warfarin, as well as peak value and AUC of PT and INR, following warfarin and lacosamide co-administration were equivalent to those after warfarin alone. In particular, the AUC ratio $(90 \%$ confidence interval) was $0.97(0.94-1.00)$ for plasma S-warfarin, and 1.04 (1.01-1.06) for INR. There were no serious TEAEs or discontinuations due to TEAEs. No clinically relevant changes from baseline in laboratory, vital signs or ECG measurements were observed.

Conclusions: Co-administration of lacosamide $400 \mathrm{mg} /$ day did not alter the PK of warfarin or the anticoagulation level. These results 
suggest there is no need for dose adjustment of warfarin when coadministered with lacosamide.

Sponsored by UCB

1.363

LONG-TERM SAFETY AND EFFICACY OF CLOBAZAM FOR LENNOX-GASTAUT SYNDROME (LGS): FINAL RESULTS OF AN OPEN-LABEL EXTENSION (OLE) STUDY Y. $\mathrm{Ng}^{1}$, J. Conry ${ }^{2}$, L. Kernitsky ${ }^{3}$, W. Mitchell ${ }^{4}, \mathrm{R}$. Veidemanis ${ }^{5}, \mathrm{R}$. Drummond $^{5}$, R. Muniz ${ }^{5}$, J. Isojarvi ${ }^{5}$, D. Lee ${ }^{5}$, J. Paolicchi ${ }^{6}$ $\left({ }^{1}\right.$ University of Oklahoma Health Sciences Center, Oklahoma City, OK, United States; ${ }^{2}$ Children's National Medical Center, Washington, DC, United States; ${ }^{3}$ Virginia Commonwealth University Medical Center, Richmond, VA, United States; ${ }^{4}$ Columbus Hospital Los Angeles, Los Angeles, CA, United States; ${ }^{5}$ Lundbeck LLC, Deerfield, IL, United States; ${ }^{6}$ Weill Cornell University, New York, NY, United States)

Rationale: Clobazam (CLB) is FDA-approved for the adjunctive treatment of seizures associated with LGS for patients (pts) $\geq 2$ years. In the longest study of an antiepileptic therapy in LGS, we assessed the safety and efficacy of open-label CLB through 6 years.

Methods: In an OLE study (OV-1004), LGS pts who had completed 1 of 2 randomized controlled trials - OV-1002 (Phase $\mathrm{II}^{1}$ ) or OV1012 (Phase III/the CONTAIN trial ${ }^{2}$ ) - received open-label CLB. Most pts initially received $0.5 \mathrm{mg} / \mathrm{kg} /$ day ( $\leq 40 \mathrm{mg} /$ day). Dosages were then adjusted based on safety and efficacy, $\leq 2.0 \mathrm{mg} / \mathrm{kg} /$ day $(80$ $\mathrm{mg}$ /day) max. Visits were at Day 1, Week 1, Months 1, 2, 3, 6, 9, and 12 , and every 6 months thereafter. Investigators could freely modify therapy (i.e., add/subtract therapies and/or adjust dosages) throughout the OLE. During the week preceding each visit, parents/caregivers maintained seizure diaries. Pts outside the United States could not continue in the study beyond 24 months, per protocol, resulting in much lower pt numbers for Year 3 and beyond. Here we report efficacy results through 3 years.

Results: 267 of 306 pts, including 61 of 68 pts from OV-1002 and 206 of 238 from OV-1012, entered the OLE. As of March 23, 2012 (study termination date), $188(70.4 \%)$ had completed the study. 251 pts had received CLB for $\geq 6$ months, 229 for $\geq 1$ year, 210 for $\geq 2$ years, 121 for $\geq 3$ years, 54 for $\geq 4$ years, 44 for $\geq 5$ years, and 11 for $\geq 6$ years. At entry, pts' mean age was 11.3 years, and their mean time since diagnosis was 6.4 years. Median \% decreases (vs. baseline, or last assessment before first dose of CLB) in average weekly drop and total seizures indicated sustained efficacy over the long term (table). Of U.S. pts in the study at the time of CLB's FDA approval (Oct. 21, 2011), $99 \%$ had transitioned to commercial product by the completion date. Dosages were adjusted, and, for those who had received CLB for $\geq 6$ months and $\geq 1$ year (up to $>6$ years), mean modal daily dosages were 0.978 and $1.001 \mathrm{mg} / \mathrm{kg}$, and mean maximum dosages were 1.263 and $1.288 \mathrm{mg} / \mathrm{kg}$. $246 \mathrm{pts}$ had $\geq 1$ treatment-emergent $\mathrm{AE}$, and $115 \mathrm{had} \geq 1 \mathrm{SAE}$ (12.2\% attributed to CLB). Most common AEs experienced in this OLE were URI (28.1\%), pyrexia (19.5\%), somnolence (17.2\%), and pneumonia $(17.2 \%)$.

Conclusions: This OLE provides long-term data on CLB for LGS. Notable findings include a high patient retention rate, stable dosages, and continued substantial seizure improvements for pts treated $\geq 3$ years.

${ }^{1}$ Conry JA, et al. Epilepsia. 2009;50:1158-66.

${ }^{2} \mathrm{Ng}$ YT, et al. Neurology. 2011;77:1473-81.

Median \% Decreases from Baseline in Average Weekly Rates of Drop and Total Seizures

\begin{tabular}{|c|c|c|c|c|}
\hline & $\begin{array}{c}\text { 3 Months } \\
(\mathbf{N}=250)\end{array}$ & $\begin{array}{c}\text { 1 Year } \\
(\mathbf{N}=216)\end{array}$ & $\begin{array}{c}\text { 2 Years } \\
(\mathbf{N}=191)\end{array}$ & $\begin{array}{c}\text { 3 Years } \\
(\mathbf{N}=113)\end{array}$ \\
\hline Drop & 73.6 & 85.1 & 87.0 & 92.3 \\
\hline
\end{tabular}

\begin{tabular}{|c|c|c|c|c|}
\hline & $(\mathrm{N}=258)$ & $(\mathrm{N}=224)$ & $(\mathrm{N}=197)$ & $(\mathrm{N}=118)$ \\
\hline \multirow[t]{3}{*}{ Total } & 66.9 & 79.2 & 79.0 & 81.6 \\
\hline & \multicolumn{4}{|c|}{$\begin{array}{l}\text { Categories of Improvement for Average Weekly Rates of } \\
\text { Drop Seizures, N (\%) }\end{array}$} \\
\hline & $(\mathrm{N}=\mathbf{2 5 0})$ & $(\mathrm{N}=216)$ & $(\mathrm{N}=191)$ & $(\mathrm{N}=113)$ \\
\hline$\geq 25 \%$ & $188(75.2)$ & $182(84.3)$ & $154(80.6)$ & $93(82.3)$ \\
\hline$\geq 50 \%$ & $156(62.4)$ & $165(76.4)$ & $144(75.4)$ & 88 (77.9) \\
\hline$\geq 75 \%$ & $123(49.2)$ & $134(62.0)$ & $118(61.8)$ & 73 (64.6) \\
\hline $100 \%$ & $74(29.6)$ & $68(31.5)$ & $67(35.1)$ & $43(38.1)$ \\
\hline
\end{tabular}

${ }^{\mathrm{a}}$ Pts outside U.S. did not continue in the study beyond 24 months.

\subsection{4 \\ LONG-TERM, OPEN-LABEL SAFETY AND TOLERABILITY STUDY OF ONCE-DAILY EXTENDED-RELEASE OXCARBAZEPINE (OXTELLAR XR ${ }^{\text {TM})}$ ) AS ADJUNCTIVE THERAPY IN PATIENTS WITH REFRACTORY PARTIAL- ONSET SEIZURES}

J. Johnson ${ }^{1}$, S. Brittain ${ }^{1}$, D. Louro ${ }^{1}{ }^{1}$ Supernus Pharmaceuticals, Inc., Rockville, MD, United States)

Rationale: Extended-release oxcarbazepine (OXC XR; Oxtellar $\mathrm{XR}^{\mathrm{TM}}$, Supernus Pharmaceuticals, Inc.) is a novel, once-daily formulation of immediate-release oxcarbazepine that employs matrix technology. OXC XR was recently approved by the FDA as adjunctive therapy for the treatment of adults and children aged $\geq 6$ years with partial-onset seizures. The efficacy and safety of OXC XR was assessed in a randomized, double-blind, placebo-controlled trial of adults with refractory partial epilepsy who were on up to 3 concomitant antiepilepsy drugs (AEDs). A 1-year, open-label followup study was conducted to determine the long-term efficacy and safety of OXC XR. Results from this trial are reported here.

Methods: Patients who elected to continue treatment after the randomized, double-blind trial were blindly titrated to once-daily OXC XR $1200 \mathrm{mg} /$ day over 2 weeks. Thereafter, adjustments for OXC XR (600-2400 mg/day) and for concomitant AEDs were allowed as clinically indicated. The primary end points were longterm safety and tolerability; the secondary end point was the percent change in partial-onset seizure frequency per 28 days $\left(\mathrm{PCH}_{\mathrm{T}}\right) \mathrm{vs}$ double-blind results. All comparisons are descriptive.

Results: Of 366 patients enrolled, 248 (67.8\%) completed the double-blind study and $214(58.5 \%)$ received OXC XR during the open-label extension (mean age: 37.4 years [range: $18-67$ years]; $51.9 \%$ female); of these, $179(83.6 \%)$ completed treatment. Mean (SD) treatment duration was 356.4 (85.21) days. Median $\mathrm{PCH}_{\mathrm{T}}$ reduction was -58.95 and -26.25 relative to the end of baseline and maintenance periods of the double-blind study, respectively. Treatment-related adverse events (AEs) occurred in 69 patients (32.2\%); 11 (5.1\%) discontinued due to AEs. The most frequent AEs were dizziness $(15.4 \%)$, headache $(11.2 \%)$, diplopia (9.3\%), nausea (7.5\%), vomiting $(6.1 \%)$, and somnolence $(5.6 \%)$. Compared with rates of AEs observed in the double-blind trial, rates decreased during the open-label extension, possibly because $64.1 \%$ of patients in the extension had already been exposed to OXC XR in the preceding double-blind study. Most AEs were mild or moderate in intensity. Serious AEs occurred in $15(7 \%)$ patients; one death due to thromboemboli was considered unrelated to OXC XR. No clinically significant changes from baseline occurred in vital signs, electrocardiogram, or laboratory values.

Conclusions: In this 1-year, open-label follow-up study where dose was allowed to be titrated to effect, adjunctive OXC XR was well tolerated and further reduced seizure frequency beyond that observed during the double-blind study. Once-daily OXC XR may provide long-term improvement in tolerability, adherence, and seizure reduction in patients with refractory epilepsy. This study was funded by Supernus Pharmaceuticals, Inc. 
OCCURRENCE OF EEG EPILEPTIFORM ABNORMALITIES AFTER SINGLE UNPROVOKED SEIZURE OR EPILEPSY DIAGNOSIS. A POPULATIONBASED STUDY.

E. Baldin ${ }^{1}$, D. C. Hesdorffer ${ }^{1}$, W. A. Hauser ${ }^{1}$, J. R. Buchhalter ${ }^{2}$, R. Ottman ${ }^{1}$ ('Sergievsky Center, Columbia University, New York, NY, United States; ${ }^{2}$ Section of Pediatric Neurology, Alberta Children's Hospital, Calgary, AB, Canada)

Rationale: A first routine EEG does not always show epileptiform abnormalities in subjects with single unprovoked seizure or incident epilepsy. The reported yield of epileptiform abnormalities on the first routine EEG is $18-56 \%$ in children and $23-49 \%$ in adults. The cumulative yield of serial EEGs is $80-90 \%$ in selected populations. No prior population-based studies have addressed these questions. We evaluated the yield and predictors of epileptiform abnormalities on routine EEGs in a population-based sample followed from incidence of epilepsy or single unprovoked seizure.

Methods: We analyzed all residents of Rochester,MN, with diagnosis of incident epilepsy or single unprovoked seizure in 19601994 who had at least one EEG. Logistic regression was used to assess the effect of demographic and clinical factors on yield of epileptiform abnormalities on the first EEG. To estimate the yield of epileptiform abnormalities across all EEGs performed, we used life table analysis. In individuals with epilepsy, we used Cox proportional hazard regression to evaluate the effect of the number of EEGs on the time from diagnosis to the first epileptiform abnormality, controlling for age at diagnosis and seizure type.

Results: We included 678 individuals, of whom 519 (76.5\%) had incident epilepsy and $159(23.5 \%)$ a single unprovoked seizure. The yield of epileptiform abnormalities on the first EEG was $50.4 \%$ in subjects with single unprovoked seizure and $62.6 \%$ in those with epilepsy. In subjects with epilepsy, the cumulative percentage of epileptiform EEGs was $84.9 \%$ by the third EEG (Fig1; $p=0.002$ ). The first EEG showed epileptiform abnormalities in $69.9 \%$ of subjects $<20$ yrs of age at diagnosis and in $53 \%$ in those $\geq 20$ (Fig2; $\mathrm{p}<0.0001)$.

Logistic regression for epileptiform abnormality on the first EEG revealed age at onset $1-19$ vs. $20-59$ yrs $(\mathrm{OR}=2.2,95 \% \mathrm{CI}$ : $1.5-3.3)$ and generalized compared to focal seizure $(2.1,1.3-3.5)$ as risk factors in epilepsy; the latter was no longer significant after stratifying by age $(<20 \mathrm{vs}$. $\geq 20)$. No factors showed an association for single unprovoked seizure.

In a Cox model for epilepsy, adjusted for age, increasing number of EEGs was not associated with shorter time from diagnosis to an epileptiform abnormality. Compared to focal seizures, generalized seizures were associated with an increased risk of epileptiform abnormalities across EEGs (Hazard Ratio=1.6, 95\%CI: 1.2-2.2). Conclusions: The cumulative yield of an epileptiform abnormality with three EEGs is $>80 \%$ for both incident epilepsy and single unprovoked seizure. After the third non-epileptiform EEG, the yield of subsequent EEGs decreases markedly. This is most evident in subjects with epilepsy, particularly younger subjects. It is important to consider the clinical characteristics of the subjects (i.e.change in seizure semiology), before ordering multiple EEGs for the detection of an epileptiform abnormality.
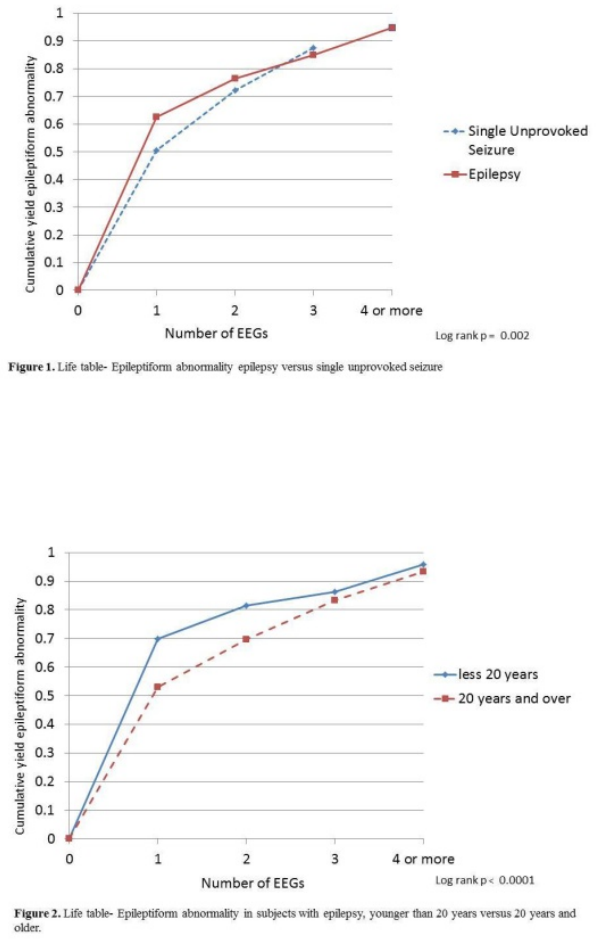

1.366

SAFETY AND PHARMACODYNAMICS OF USL261, A NOVEL FORMULATION OF MIDAZOLAM OPTIMIZED FOR INTRANASAL ADMINISTRATION, IN SUBJECTS WITH EPILEPSY

H. J. Altman ${ }^{1}$, H. A. Dworak ${ }^{1}$, M. B. Halvorsen ${ }^{2}\left({ }^{1}\right.$ Upsher Smith Laboratories, Inc., Morristown, NJ, United States; ${ }^{2}$ Upsher Smith Laboratories, Inc., Morristown, NJ, United States)

Rationale: USL261 is a formulation of midazolam (MZ) optimized for easy, rapid intranasal (IN) administration that is in development for the orphan indication of outpatient rescue treatment of seizures in patients who require control of intermittent bouts of increased seizure activity, often referred to as seizure clusters or acute repetitive seizures. The pharmacodynamics of USL261 have previously been evaluated in healthy volunteers. This study was conducted to assess the degree and duration of sedation and impairment of psychomotor performance and safety of USL261 in volunteer subjects with epilepsy on stable antiepileptic drug (AED) regimens.

Methods: This randomized, open-label, inpatient study enrolled 90 male and female subjects between the ages of 12 and 62 years old. Subjects were administered a single dose of $2.5(\mathrm{n}=18), 5.0(\mathrm{n}=36)$, or $7.5 \mathrm{mg}(\mathrm{n}=36)$ USL261 by a unit-dose nasal spray device.

Pharmacodynamic assessments of sedation (Stanford Sleepiness Scale [SSS] and Observer's Assessment of Alertness/Sedation Scale $[\mathrm{OAA} / \mathrm{S}]$ ) and psychomotor performance (Digit-Symbol Substitution Test [DSST]) were conducted before dosing and at set time points after dosing for a period of $12 \mathrm{hr}$. Safety was assessed by the incidence of treatment-emergent adverse events (TEAEs) and by monitoring respiratory rate (RR) and pulse oximetry

Results: For the SSS, mean peak scores (7-point scale; 1=fully alert, $7=$ nearly asleep) were $4.8,5.2$, and 5.0 after $2.5,5.0$, and $7.5 \mathrm{mg}$ USL261, respectively. The mean peak composite OAA/S score (5point scale; $1=$ asleep, $5=$ fully awake) was $4.3,3.7$, and 3.7 after the increasing doses of USL2 ${ }^{1}$ Signs of sedation were evident $10 \mathrm{~min}$ after dosing, and mean peak sedation scores occurred between 1 and $2 \mathrm{hr}$ for SSS and in $<1 \mathrm{hr}$ for OAA/S. The peak effect on mean $\%$ correct answers on the DSST occurred at approximately $30 \mathrm{~min}$ and increased with increasing dose, reaching $96 \%, 90 \%$, and $84 \%$ after 
$2.5,5.0$, and $7.5 \mathrm{mg}$, respectively. Scores for all 3 instruments returned to near baseline levels by $4 \mathrm{hr}$ after dosing. TEAEs were reported by 59 of 60 adults ( $98 \%$ ) and by 29 of 30 adolescents $(97 \%)$ with a similar incidence across dosing cohorts. Nearly all TEAEs were mild or moderate in severity and were considered related to treatment. The most common (reported by $>10 \%$ of subjects) TEAEs were dysgeusia, oropharyngeal pain, rhinalgia, and burning sensation. Conclusions: The onset of pharmacodynamic effects were seen as early as 10 min after dosing with USL261, and scores of each instrument returned to near baseline levels by $4 \mathrm{hr}$. USL261 at doses up to $7.5 \mathrm{mg}$ was generally well-tolerated and did not result in excessive or prolonged sedation or psychomotor impairment. Consistent with results in healthy volunteers, these results support the further development of USL261 for outpatient seizure rescue among patients with intermittent bouts of increased seizure activity.

\subsection{7 \\ SEIZURE PROPOGATION IN VIVO IS PROMOTED BY INACTIVATION OF HIPPOCAMPUS AND RETRAINED BY ACTIVATION OF HIPPOCAMPUS.}

P. A. Forcelli ${ }^{1}$, M. Proctor ${ }^{1}, K$ K. Gale ${ }^{1}$ ( ${ }^{1}$ Department of Pharmacology \& Physiology, Georgetown University, Washington, DC, United States)

Rationale: Despite the widespread belief that the hippocampus is a critical site of origin for forebrain seizures, in vivo suppression of hippocampal activity has not been directly tested for anti-seizure effects. Moreover, the effect of focal activation of hippocampus, which can restrain seizure-like activity in the combined hippocampalentorhinal slice model, has not been evaluated for effects on seizures in vivo. Finally, because theta activity in hippocampus has been previously suggested to be anticonvulsant, we also investigated treatments that activate or suppress theta rhythms.

To determine the effect of hippocampal activity on forebrain seizures, we utilized focal pharmacological manipulation (i.e., activation and inactivation) of hippocampus in rats prior to evaluating experimentally-evoked seizures.

Methods: Adult, Male Sprague-Dawley rats were stereotaxically implanted with cannulae in the dorsal hippocampus. A subset of animals were implanted with surface and depth EEG electrodes to record activity patterns in hippocampus and distal sites during intrahippocampal drug application. Pharmacological activation of hippocampus was accomplished by microinfusion of either: bicuculline methiodide (GABA-A antagonist, 236pmol), kainic acid (glutamate agonist, 234pmol), baclofen (GABAA-B agonist, 400pmol). Pharmacological inactivation of hippocampus was accomplished by microinfusion of muscimol (GABA-A agonist, $175 \mathrm{pmol}$ ), kynurenic acid (glutamate antagonist, 89nmol). The 5HT1 a receptor agonist buspirone $(10 \mathrm{nmol})$ was applied to promote theta, while the 5-HT2 agonist 2,5,dimethoxy-4-iodoamphetamine (DOI; $1.5 \mathrm{nmol}$ ) was applied to suppress theta. 10 min after drug infusion, seizures were evoked by focal application of bicuculline to area tempestas, or by intravenous bicuculline.

Results: Treatments that suppressed hippocampal activity (i.e., muscimol, kynurenic acid, 5-HT1a agonist) potentiated both focallyand systemically-evoked seizures. Treatments that activated the hippocampus (i.e., bicuculline, kainic acid, baclofen, buspirone) attenuated both focally- and systemically-evoked seizures. Intrahippocampal bicuculline administration induced electrographic seizure activity characterized by local ictal and/or interictal bursts. Local field recordings and surface EEG indicated that this activity was confined to hippocampus. Consistent with prior reports we found an increase in slow wave $(1-7 \mathrm{~Hz})$ activity occurred consistently following hippocampal activation.

Conclusions: Our data do not support the hypothesis that hippocampal activation contributes to forebrain seizure generation, and instead suggest that hippocampal activation exerts a seizuresuppressive action. Thus, there appears to be a reciprocal relationship between focal seizure-like activity within hippocampus and seizure activity that propagates through the larger limbic-forebrain network.

\subsection{8 \\ THE KETOGENIC DIET AND EPILEPSY: NUTRITIONAL ASPECTS}

J. Weinfeld ${ }^{1}$, H. Getty ${ }^{1}$, M. Durner ${ }^{1}\left({ }^{1}\right.$ Psychiatry, Mount Sinai School of Medicine, New York, NY, United States)

Rationale: The ketogenic diet is a high fat, adequate protein, low carbohydrate diet that mimics the physiological state of starvation. It has been observed that this state has anti-epileptic effects, yet the underlying mechanism is still unknown. This therapeutic form of treatment is often prescribed to patients with medically refractory epilepsy, specifically in the pediatric population. Given that the best responders to the diet are children, it is imperative to understand how a metabolic imbalance during critical growth periods can present clinically in the future. Patients that experience a reduction in seizures often remain on the ketogenic diet for an extended period of time, for as long as two or more years. There is minimal primary research investigating the long-term nutritional and neurological outcomes of adopting the ketogenic diet. We examined 18 clinical trials that investigate the influence of the ketogenic diet on seizure reduction and 12 clinical trials investigating the nutritional implications of prolonged exposure to the ketogenic diet.

Methods: We have performed literature searches in order to locate primary sources of research that measure the nutritional adequacy and neurological responses to the ketogenic diet. Each article has been evaluated based on the methods, time span, sample size, efficacy, and results.

Results: The ketogenic diet has been shown to be an effective treatment alternative especially for young children with intractable seizures. The nutritional risks that present with the diet include decreased vitamin A, selenium, magnesium, phosphorus, folate, and calcium levels. These vitamins and minerals are crucial to proper growth and development. Despite their supplementation, weight gain and height growth is very minimal on the diet.

Conclusions: : It is questionable whether or not the benefits outweigh the risks when putting a child on the ketogenic diet. Nutritional deficiencies at a young age can have major consequences in the future. Since pediatric patients have better compliance and success rates with the diet, there is a need for more prospective longterm studies that follow pediatric patients over time in order to track seizure activity and brain development into adulthood.

\subsection{9 \\ THREE-YEAR RESULTS FROM THE SABRIL® REGISTRY CONTINUE TO YIELD LOW RATE OF VISUAL FIELD DEFECTS \\ R. Sergott ${ }^{1}$, R. Foroozan ${ }^{2}$, J. Pellock ${ }^{3}$, E. Faught ${ }^{4}$, W. D. Shields ${ }^{5}$, G. Burkhart $^{6}$, G. Krauss ${ }^{7}$, V. Prabhakaran ${ }^{8}$, S. Torri ${ }^{8}$, D. Wesche ${ }^{8}$, J. Isojarvi ${ }^{8}\left({ }^{1}\right.$ Wills Eye Institute and Thomas Jefferson University Medical College, Philadelphia, PA, United States; ${ }^{2}$ Baylor College of Medicine, Houston, TX, United States; ${ }^{3}$ Virginia Commonwealth University, Richmond, VA, United States; ${ }^{4}$ Emory University Hospital, Atlanta, GA, United States; ${ }^{5}$ UCLA Medical Center, Los Angeles, CA, United States; ${ }^{6}$ Epidemiology Consultant, Reston, VA, United States; ${ }^{7}$ Johns Hopkins University, Baltimore, MD, United States; ${ }^{8}$ Lundbeck LLC, Deerfield, IL, United States)}

Rationale: An important safety issue for vigabatrin (VGB) is risk of vision loss. To manage this risk, the FDA and Lundbeck require a comprehensive Risk Evaluation and Mitigation Strategy (REMS), including an ongoing patient registry, designed to ensure regular vision monitoring, facilitate ongoing benefit-risk assessments, and detect VGB-associated peripheral visual field defects as early as possible. 
Methods: Registry participation is mandatory for all US VGB prescribers and patients (pts). Basic registry methodology has been published. ${ }^{1}$ Current analyses cover the 3-year period from Aug. 21, 2009, through Aug. 22, $201^{2}$ Two independent expert neuroophthalmologists, members of a registry steering committee, evaluated voluntarily submitted, detailed vision test findings for technical adequacy and clinical significance.

Results: As of Aug. 22, 2012, 4,292 pts were enrolled, of which 2,676 had infantile spasms (IS), 1,354 had refractory complex partial seizures (rCPS), and 251 had other diagnoses. Median duration of time in registry was 8.1 mos. Total exposure to VGB is unknown, because some pts entered after having received VGB from other sources. 2,339/4,292 (55\%) had discontinued VGB after enrolling in the registry, and 14/2,339 (0.6\%) discontinued because of reported visual field defects. 307/4,292 pts underwent perimetry evaluations, and $\sim 10 \%$ of pts eligible for vision testing at each time point both completed perimetry and had interpretable results. Six pts continuing on therapy met predefined REMS criteria for vision loss. A separate data subset of 718 pts with 1,125 sets of cumulative detailed vision results (i.e., all those for whom vision results were voluntarily submitted) was reviewed by the neuro-ophthalmologists. $259 / 1,125(\sim 23 \%)$ of all tests reviewed showed pre-existing, clinically significant pathology assessed to be not related to VGB (and independent of prior exposure to VGB), a reflection of effects of their underlying conditions and prior treatments (e.g., neurosurgery) on vision. Twenty-eight were found to have vision abnormalities possibly or probably related to VGB. Testing variability was present, especially for IS pts, because some did not undergo

electrophysiologic testing, while some clinicians repeatedly performed these tests. The chief factor governing testing was cognitive ability.

Conclusions: Registry data continue to detect afferent visual system abnormalities at baseline for some pts with IS, rCPS, or other epileptic conditions, confirming previous cross-sectional studies. No major ophthalmologic dysfunction or signs of early visual impairment $(<6$ mos) during VGB treatment have been reported. Few pts to date were reported to have had a vision deficit possibly related to VGB. Cumulative exposure at this time is relatively low, and few pts have had serial perimetry performed over $6 \mathrm{mos}$, perhaps reflecting the difficulty of conducting a set of prescribed tests across all age groups, especially when pts may not be able to cooperate because of age, cognitive ability, or neurologic status.

${ }^{1}$ Pellock JM, et al. Epilepsy Behav. 2011;22:710-7.

\subsection{0}

\section{AUTOMATING CLINICAL JUDGMENT OF SEIZURE} SIMILARITY

M. Lippmann ${ }^{1}$, E. D. Marsh ${ }^{3}$, B. E. Porter ${ }^{4}$, B. Litt ${ }^{1,2}$

( ${ }^{1}$ Bioengineering, University of Pennsylvania, Philadelphia, PA, United States; ${ }^{2}$ Neurology, University of Pennsylvania, Philadelphia, PA, United States; ${ }^{3}$ Neurology, Children's Hospital of Philadelphia, Philadelphia, PA, United States; ${ }^{4}$ Neurology, Stanford School of Medicine, Palo Alto, CA, United States)

Rationale: Increasingly complex, long-term, and high-resolution brain implants will require automated methods to augment clinician characterization of seizures. We present an automated method for seizure comparison: a seizure distance measure that is significantly and practically related to clinical judgment of seizure similarity.

Methods: Physician groupings. To establish inter- and intra-rater reliability, prior to developing an objective distance measure, two physicians partitioned 150 seizures from eight patients, in two separate rounds eight weeks apart. The adjusted rand index assessed agreement between partitions.

Log-time-spectral distance (LTSD). Analogous to the log-spectral distance used in speech processing, we develop the LTSD (Fig. 1) and compute it between all pairs of seizures for each of the eight patients from the physician grouping task, to yield eight dissimilarity matrices.

LTSD dissimilarity matrices versus physician groupings. To assess whether LTSD is related to clinical judgment, we convert physician seizure-groupings into coarse distances, yielding a second set of eight dissimilarity matrices and then compute Pearson's $r$ between each pair of matrices for the eight patients, determining significance with a Mantel test.

LTSD clustering. To assess the practical strength of these correlations, the LTSD matrices are used to create hierarchical clusters, with the number of clusters set by the number of physician groupings per patient. The adjusted rand index was used to compare LTSD clusters with the physician partitions.

Results: Physician groupings. For half of the patients, the physicians had significant intra- and inter-observer agreement $(\mathrm{p}<0.05)$. Across all patients and rounds, inter-rater agreement was significant $69 \%$ of the time, and intra-rater agreement was also significant $69 \%$ of the time $(\mathrm{p}<0.05)$.

LTSD dissimilarity matrices versus physician groupings. Across all patients and rounds, agreement between algorithm and physicians was $72 \%(\mathrm{p}<0.05)$, with a trend towards significance for patients with more recorded seizures $(\mathrm{r}=0.40, \sigma=0.1631$; Table 1$)$

LTSD clustering. Across all patients and rounds, there was $66 \%$ agreement between the LTSD-determined clusters and physician groupings $(\mathrm{p}<0.05)$.

Conclusions: The LTSD is an objective measure that can be used to characterize seizures without human intervention. Its performance is comparable to experts and it can be incorporated into automated analyses to support clinical decision-making. Methods like LTSD will become increasingly important as high resolution, broadband invasive recordings become more widely used in research and clinical care.

$$
\begin{gathered}
\operatorname{LSD}(P(w), \hat{P}(w))=\sqrt{\frac{1}{2 \pi} \int_{-\pi}^{\pi}\left[10 \log _{10} \frac{P(w)}{\hat{P}(w)}\right]^{2} d w} \\
\operatorname{LTSD}(P(t, w), \hat{P}(t, w))=\sqrt{\frac{1}{2 \pi} \frac{1}{T} \int_{-\pi}^{\pi} \int_{0}^{T}\left[10 \log _{10} \frac{P(t, w)}{\hat{P}(t, w)}\right]^{2} d t d w}
\end{gathered}
$$

Figure 1. Log-spectral distance and log-time-spectral distance

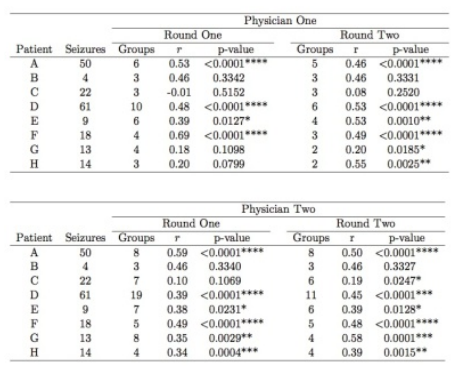

Table 1. Correlation between log-time-spectral distance seizure dissimilarity matrices and physician seizure grouping matrices $(* \mathrm{p}<$ $0.05, * * \mathrm{p}<0.01, * * * \mathrm{p}<0.001, * * * * \mathrm{p}<0.0001)$. The "Seizures" columns refer to the number of recorded clinical seizures for that patient. The "Groups" columns refer to the number of subsets in the physician's partition for that patient and round.

\subsection{1 \\ WTIHDRAWN}




\subsection{2}

MATERNAL IMMUNE ACTIVATION PRODUCES EPILEPSY AND AUTISM-LIKE BEHAVIOR IN THE OFFSPRING VIA SELECTIVE INDUCTION OF INTERLEUKINS 6 AND 1BETA

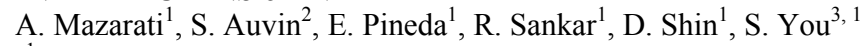
( ${ }^{1}$ Pediatrics, David Geffen School of Medicine at UCLA, Los Angeles, CA, United States; ${ }^{2}$ Hôpital Robert Debré, Service de Neurologie Pédiatrique, Paris, France; ${ }^{3}$ Sanggye Paik Hospital, Inje University College of Medicine, Seoul, Korea, Republic of)

Rationale: Maternal immune activation (MIA) triggered by infections, has been identified as a cause of autism in the offspring. Considering the involvement of perturbations in innate immunity in epilepsy, we examined whether MIA represents a risk factor for epilepsy as well. The role of specific MIA components- interleukin-6 and interleukin-1beta was also addressed.

Methods: Maternal viral infection was mimicked in C57BL/6 mice by polyinosinic-polycytidylic acid (PIC, $2.5 \mathrm{mg} / \mathrm{kg}$ ) injected intraperitoneally during embryonic days $12-1{ }^{6}$ Starting from postnatal day 40 , the propensity of the offspring to epilepsy was examined using hippocampal kindling; autism-like behavior was studied using the sociability test. The involvement of interleukin- 6 and interleukin1 beta in PIC-induced effects was studied by systemic coadministration of the cytokine antibodies $(250 \mathrm{ug} / \mathrm{kg})$ with PIC, and by delivering recombinant cytokines $(10-20 \mathrm{ug} / \mathrm{kg})$ in lieu of PIC. Results: The offspring of PIC-exposed mice exhibited increased hippocampal excitability, accelerated kindling rate, prolonged increase of seizure susceptibility after kindling, and diminished sociability. Epileptic impairments were abolished by antibodies to interleukin-6 or interleukin-1beta. Neither of the recombinant cytokines alone increased the propensity to seizures; however when administered together, they produced proepileptic effects similar to the ones induced by PIC. PIC- induced autism-like behavioral deficits were abolished by interleukin- 6 antibodies and were mimicked by recombinant interleukin-6; interleukin-1beta was not involved. Conclusions: MIA represents a risk factor for epilepsy in the offspring. While interleukin-6 alone mediates the development of autism, the concurrent involvement of interleukin- 6 and interleukin1 beta is required for priming the offspring for epilepsy. The presented data may shed light on mechanisms of comorbidity between autism and epilepsy.

Supported by the NIH research grant R01 NS065783 (AM).

\subsection{3 \\ PHARMACOKINETICS OF USL261, A NOVEL FORMULATION OF INTRANASAL MIDAZOLAM OPTIMIZED FOR INTRANASAL ADMINISTRATION, IN SUBJECTS WITH EPILEPSY}

R. J. Hayes ${ }^{1}$, L. L. Bancke ${ }^{2}$, M. B. Halvorsen ${ }^{2}\left({ }^{1}\right.$ Upsher Smith Laboratories, Inc., Morristown, NJ, United States; ${ }^{2}$ Upsher Smith Laboratories, Inc., Maple Grove, MN, United States)

Rationale: USL261 is a formulation of midazolam (MZ) optimized for easy, rapid intranasal (IN) administration that is in development for the orphan indication of outpatient rescue treatment of seizures in patients who require control of intermittent bouts of increased seizure activity, often referred to as seizure clusters or acute repetitive seizures. The pharmacokinetics (PK) of USL261 in healthy volunteers demonstrated rapid absorption and increased bioavailability compared to MZ for injection administered IN. This study was performed to assess the PK of single doses of USL261 in volunteer subjects with epilepsy on stable antiepileptic drug (AED) regimens.

Methods: This randomized, open-label, inpatient study enrolled 90 male and female subjects between the ages of 12 and 62 years. Subjects were administered a single dose of $2.5(n=18), 5.0 \quad(n=36)$, or $7.5 \mathrm{mg}(\mathrm{n}=36)$ USL261 by a unit-dose nasal spray device. Venous blood samples were collected before dosing and at set time points after dosing for a period of $12 \mathrm{hr}$ to determine plasma concentrations of $\mathrm{MZ}$ and its major metabolite, 1-hydroxymidazolam (1-OHMZ). Standard PK parameters were calculated for both compounds, including maximum plasma concentration $\left(\mathrm{C}_{\max }\right)$, time to peak plasma concentration $\left(\mathrm{T}_{\max }\right)$, area under the plasma concentrationtime curve (AUC), and half-life $\left(\mathrm{t}_{1 / 2}\right)$.

Results: The study subjects had a mean age of $31.3 \pm 14.0 \mathrm{yr}$ (mean \pm SD) and a mean body weight of $77.8 \pm 19.9 \mathrm{~kg}$. Maximal plasma MZ concentrations were rapidly achieved after dosing in each group (median $\mathrm{T}_{\max }=10-15 \mathrm{~min}$ ). Increasing doses of USL261 resulted in mean $\mathrm{MZ} \mathrm{C}_{\max }$ of $45.2,64.0$, and $66.5 \mathrm{ng} / \mathrm{mL}$ and in mean $\mathrm{MZ} \mathrm{AUC}_{0-\infty}$ of $56.6,119$, and $131 \mathrm{ng} \bullet \mathrm{hr} / \mathrm{mL}$. Mean terminal MZ $\mathrm{t}_{1 / 2}$ ranged from 2.8 to $4.2 \mathrm{hr}$. Plasma levels of 1-OHMZ peaked 45 to 60 min after dosing, with mean $\mathrm{C}_{\max }$ of $6.4,8.9$, and $13.8 \mathrm{ng} / \mathrm{mL}$ and mean $\mathrm{AUC}_{0-\infty}$ of $37.2,43.4$, and $64.3 \mathrm{ng} \bullet \mathrm{hr} / \mathrm{mL}$ in the $2.5,5.0$, and $7.5 \mathrm{mg}$ dosing groups, respectively. Plasma clearance of 1-OHMZ paralleled that of the parent compound and demonstrated similar $\mathrm{t}_{1 / 2}$ (mean $\mathrm{t}_{1 / 2}=3.3-4.3 \mathrm{hr}$ ).

Conclusions: Consistent with previous results in healthy volunteers, $M Z \mathrm{C}_{\max }$ was rapidly achieved after dosing with USL261 in subjects with epilepsy, and both MZ and 1-OHMZ were rapidly eliminated, with mean $\mathrm{t}_{1 / 2}$ between about 3 and $4 \mathrm{hr}$. In general, $\mathrm{MZ} \mathrm{C}_{\max }$ and $\mathrm{AUC}_{0-\infty}$ for both compounds increased with dose, but doseproportionality analysis was inconclusive. These results support the further development of USL261 for outpatient seizure rescue among patients with intermittent bouts of increased seizure activity.

\subsection{4 \\ THE CLINICAL IMPORTANCE OF INTRACRANIAL EEG INFRASLOW ACTIVITY}

E. Rodin ${ }^{1}$, T. Constantino ${ }^{2}$, J. Bigelow ${ }^{2}\left({ }^{1}\right.$ Neurology, University of Utah, Sandy, UT, United States; ${ }^{2}$ Neurology, Intermountain Medical Center, Murray, UT, United States)

Rationale: It has been established that ictal EEG baseline shifts (also called DC shifts) can be recorded with AC amplifiers and have a smaller electrical field than the conventional frequency band of 0.5 $70 \mathrm{~Hz}$. this has been shown to be advantageous in seizure onset localization. We have previously demonstrated that these baseline shifts consist of an increase in infraslow $(<0.1 \mathrm{~Hz})$ background activity (ISA). Since the files of long-term video-monitoring sessions are not kept in their entirety, but sampled for storage purposes, interictal information in this frequency range is usually not available. Preliminary data on the presence of episodic increase in AES in the interictal state was presented by us to the ACNS in February $201^{2}$ But since the series was small and in part beset by technical difficulties the number of patients was expanded in order to attain more robust data.

Methods: The entire intracranial video-monitoring session from eleven consecutive patients recorded between August 2011 and October 2012 were transferred, after de-identification, to a separate drive. An XLTEK EEG system had been used and the platinum electrodes had been supplied by Ad-Tech. Recording sessions ranged from 2-8 days (mean 5.1 SD 2.2) in ten cases, and for 17 days in one. With one exception all implantations had been unilateral; electrode coverage ranged from 26- 70 contacts. The data were analyzed with the BESA ${ }^{\circledR}$ software program. The highpass filter was removed and the lowpass filter was set to $0.1 \mathrm{~Hz}$. To avoid contamination by the reference electrode the data were transformed to an average common reference.

Results: Episodic focal increase in ISA amplitude was observed in all patients on every day of the recording session in the interictal state. It was not limited to the epileptogenic zone, even in patients with partial seizures, but frequently involved in addition a separate lobe and could occur in the contralateral hemisphere. Its duration ranged from a few minutes to several hours and the amplitude was at times in the milliV range. It was clearly distinguishable from 
electrode artifact not only by wave form but also because several neighboring electrodes tend to be involved. Two patients complained of subjective abnormal sensations during one or more of the sessions and although the conventional frequency band did not show pathologic activity, ISA increase was observable at that time. Conclusions: Interictal ISA provides information which is not available from the conventional frequency band and is likely to be important for our understanding of the epileptogenic process. To what extent it will influence the results of surgical resections will require long-term follow-up studies. When a patient complains of subjective discomfort, and the conventional frequency band does not show abnormalities, filter settings should be changed for viewing of ISA. The assessment of the physiology and pathophysiology of ISA will require animal studies.

\section{Saturday, December 1, 2012}

\section{Investigators' Workshop \\ fMRI Task Selection for Presurgical Mapping in Children: Goals and Challenges 6:15 p.m.-7:45 p.m.}

Madison Berl ${ }^{1}$, Leigh Sepeta ${ }^{1}$, Louise Croft $^{2}$ and Simona Ghetti ${ }^{3}$ ( ${ }^{1}$ Neuropsychology, Children's National Medical Center, Washington, DC; ${ }^{2}$ University College of London, London, United Kingdom and ${ }^{3} \mathrm{UC}$ Davis Mind Institute, Davis, CA)

Summary: fMRI is a staple in presurgical planning but conducting studies with children presents unique challenges. Moreover, although the aim of studies is to plan for surgical resection, clinical studies are also fundamental to advances in cognitive neuroscience and thus considering research aims as well as clinical aims adds another layer to the decision making process when deciding on paradigms. Language paradigms are used regularly; however, this workshop will discuss the challenges that occur including using overt/covert tasks, deciding the optimal scanning protocol when a child is not able to scan for very long or is cognitively delayed, and dealing with movement. Memory paradigms are not regularly used in children, but would be an important next step in reducing the need for invasive procedures (e.g., Wada). The final two talks will focus on what paradigms have been utilized in children thus far to target hippocampal functioning and what we know from developmental cognitive neuroscientists regarding hippocampal functioning across development to inform task selection for clinical purposes. Dr. Ghetti is a cognitive neuroscientist that is NIH funded to examine memory development in children with multiple neuroimaging techniques. As an expert in her field, she is an outside speaker that will enhance the conversation as it applies to clinical epilepsy populations.

\section{Saturday, December 1, 2012}

\author{
Translational Investigators' Workshop \\ What Do Interictal Spikes Mean - \\ Do They Have Predictive Value \\ 6:15 p.m.-8:15 p.m.
}

Christophe Bernard (INSERM UMR 1106, Marseille, France)

Summary: Interictal spikes constitute a pathological hallmark of some types of epilepsies. Although the regions generating interictal spikes may not necessarily correspond to the epileptogenic region, interictal spikes may carry clinically relevant information. This is the concept that we will develop in this workshop, which is meant to bridge basic and clinical research, and to show the potential translational value of interictal spikes.

Interictal spikes come in different shapes and flavor. Massimo Avoli will present and develop the notion that interictal spikes can have both anti- and pro-seizure action, depending upon the conditions and their region of origin.

Interictal spikes are not only linked to ictogenesis, they may also play a role in epileptogenesis, the process leading to epilepsy.

Experimental models of temporal lobe epilepsy have shown that interictal activity precedes by days the occurrence of recurrent seizures.

Kevin Staley (in collaboration with the Dudek lab) will show that interictal spikes are present prior to the onset of spontaneous seizures. However, suppression of spikes in vitro does not alter the subsequent appearance of seizures. Spikes may not be a necessary aspect of at least some forms of epilepsy after brain injury, but they appear to be reliable biomarkers of epileptogenesis.

Christophe Bernard will show that the evolution of some parameters characterizing interictal spikes during epileptogenesis is predictive of the first spontaneous seizure in rodents.

Finally, Elaine Wirrell will show how interictal spikes can predict not only the recurrence of seizures after the first seizure or after surgery in Human, but also the remission of epilepsy.

\section{Sunday, December 2, 2012}

Poster Session 2

8:00 a.m.-6:00 p.m.

Translational Research: Mechanisms

2.001

RAPAMYCIN SUPPRESSES HIPPOCAMPAL MICROGLIOSIS INDUCED BY STATUS EPILEPTICUS Amy Brewster ${ }^{1}$, W. L. Lee ${ }^{1}$, Y. C. Lai ${ }^{1}$ and A. E. Anderson ${ }^{1,2}$ ( ${ }^{1}$ Pediatrics-Neurology, Baylor College of Medicine, Houston, TX and ${ }^{2}$ Neuroscience, Baylor College of Medicine, Houston, TX)

Rationale: An episode of status epilepticus (SE) is associated with the development of unprovoked seizures later in life. At the cellular level, SE is associated with activation and proliferation of microglia (microgliosis), and hyperactivation of the mammalian target of rapamycin (mTOR) pathway in the hippocampus. Recent studies have shown that LPS- and cytokine-induced activation of microglia is mTOR dependent, and consequently microgliosis is suppressed with the mTOR pathway inhibitor rapamycin. In fact, rapamycin is a potent immunosuppressant that has been shown to decrease microgliosis following traumatic brain injury. However, whether rapamycin reduces SE-induced microgliosis has not been evaluated. Therefore, we tested the hypothesis that the SE-induced mTOR pathway dysregulation occurs in microglial cells, and that rapamycin suppresses the associated microgliosis.

Methods: Pilocarpine was used to induce SE (1hr). Two weeks later, $\mathrm{SE}$ and sham rats were treated with rapamycin (Rap) or vehicle (Veh) for 1 week. Immunohistochemistry was performed in different cohorts of rats 2 and 3 weeks after SE, before and after the treatments. Antibodies against the phosphorylated ribosomal S6 protein (P-S6), IBA1 and NeuN were used to assess mTOR pathway activation, and to stain microglia and neurons, respectively. Immunohistochemical analyses focused on the hippocampal formation.

Results: Increased P-S6 and IBA1 staining was evident 2 and 3 weeks after SE. The SE-induced increase in P-S6 signal was apparent throughout the hippocampus in the principal cell layers and in cells 
scattered throughout CA1 strata radiatum and lacunosum moleculare. In hippocampi from Sham+Veh and SE+Veh rats, P-S6 signal colocalized with NeuN, indicating neuronal activation of mTOR. However, relatively more P-S6 staining was co-localized with IBA1 in the SE+Veh group, where microglial cells were hypertrophied (an indication of microglial activation). Rapamycin decreased the P-S6 signal below basal levels in both sham and SE groups, and dramatically reduced IBA1 staining and reversed the microglial hypertrophy in the SE+Rap group.

Conclusions: Our data indicate that mTOR dysregulation occurs in neurons and reactive microglia, and that rapamycin suppresses microgliosis. These data suggest that SE-induced mTOR dysregulation may alter neuronal and glial properties, which together may contribute to the molecular and behavioral phenotypes associated with epileptogenesis.

\subsection{2 \\ DENTATE GRANULE CELL MATURATION-DEPENDENT PLASTICITY OF MOSSY FIBERS IN EXPERIMENTAL TEMPORAL LOBE EPILEPSY}

Jack M. Parent ${ }^{1,2}$, A. L. Althaus ${ }^{1,2}$, H. Zhang ${ }^{1}$, E. A. Messenger ${ }^{1}$ and H. Umemori ${ }^{2,3}$ ( ${ }^{1}$ Neurology, University of Michigan, Ann Arbor, MI; ${ }^{2}$ Neuroscience Graduate Program, University of Michigan Medical Center, Ann Arbor, MI and ${ }^{3}$ Cell \& Developmental Biology and Molecular and Behavioral Neuroscience Institute, University of MI, Ann Arbor, MI)

Rationale: Axonal reorganization of dentate granule cells (DGCs) is a prominent feature of temporal lobe epilepsy (TLE). Mossy fiber sprouting into the inner molecular layer (IML) has been investigated for decades using immunohistochemistry, Timm staining, and biocytin labeling of individual cells, but little is known about axonal changes in the hilus and CA3. In the hilus, in particular, changes in synaptic connections have been inferred, but not characterized. In CA3, recent work shows seizure-induced changes in bouton size, structure, and number, but nothing is known about the identity of DGCs responsible for these changes. Findings from our laboratory and others indicate that DGC maturity during epileptogenic insults is a critical factor in how the individual cell responds to the insult and the extent to which it participates in aberrant circuitry. We therefore examined the potential relationship between DGC age and extent of seizure-induced axonal reorganization.

Methods: To study axonal projections of age-defined cohorts of DGCs born before or after pilocarpine-induced status epilepticus (SE) we generated a retrovirus (RV) construct in which synaptophysin, a synaptic vesicle protein that is targeted to boutons, is conjugated to a yellow fluorescent protein (YFP). RV only integrates into dividing cells, which allows birthdating of DGCs with labeled axon terminals based on when the RV was introduced into the dentate gyrus. Male Sprague Dawley rats were subjected to the pilocarpine TLE model, undergoing SE at postnatal day 56 (P56). RV was injected into the dentate gyrus either at P7 (labeling cells that would be seven weeks old at the time of SE) or at P60 (labeling cells born 4 days after SE). Controls for either group received saline in place of pilocarpine. Brains were perfusion-fixed 10 weeks after SE/sham treatment and sectioned at $60 \mu \mathrm{m}$ to visualize cells. Tissues were processed for immunohistochemistry using antibodies against YFP and bassoon (pre-synaptic marker), and images were acquired on a confocal microscope. P60-injected groups were analyzed first.

Results: We found robust labeling of mossy fiber boutons in the hilus and CA3 in all animals, as well as in the IML in epileptic rats injected with RV 4 days after SE. In CA3, we observed increases in bouton size and in the number of satellite boutons in the P60 SE group. In the hilus, DGCs labeled after SE showed increased bouton density compared to controls.

Conclusions: These findings suggest that mossy fibers of DGCs generated after SE are highly susceptible to seizure-induced plasticity in the hilus, IML and CA3. Ongoing work aims to compare DGCs generated at $\mathrm{P} 7$ with those born on $\mathrm{P} 60$, and to elucidate the postsynaptic targets of remodeled axons.

Supported by an Epilepsy Foundation Predoctoral Fellowship (ALA) and NINDS NS058585 (JMP)

\subsection{3 \\ DYSREGULATION OF FMRP FOLLOWING EARLY-LIFE SEIZURES IN RATS AND HUMANS}

Jocelyn Lippman Bell $^{1,2}$, M. Lechpammer ${ }^{1,2}$, S. E. Francomacaro ${ }^{1}$, E. Rosenberg ${ }^{1}$ and F. E. Jensen ${ }^{1,2}$ ( ${ }^{1}$ Boston Children's Hospital, Boston, MA and ${ }^{2}$ Harvard Medical School, Boston, MA)

Rationale: Epilepsy is one of the most prevalent pediatric neurological disorders, and can be comorbid with autism. An example of this interaction occurs in Fragile X Syndrome (FXS), in which Fragile X Mental Retardation Protein (FMRP) is silenced, manifesting with autistic behavior, seizures, and altered synaptic plasticity. The mammalian Target of Rapamycin (mTOR) pathway has also been shown to be dysregulated in FXS. We have recently shown that early-life seizures result in later-life epilepsy and autism, with $\mathrm{mTOR}$ upregulation playing a critical role, as treatment with rapamycin blocks these consequences (Talos et al, 2012). In addition, we recently reported that early-life seizures increase dendritic spine density, similar to that observed in FXS (Lippman Bell et al, 2010 AES abstract). Given that FMRP and mTOR regulate each other, we evaluated neonatal seizure-induced changes in FMRP in cortical tissue from epilepsy patients and cortical and hippocampal tissue in rats following hypoxia-induced seizures (HS).

Methods: Seizures were induced in P10 rats by global hypoxia. Brains were collected 1, 3, and 24h post-HS and from age-matched littermates. Fresh, frozen tissue was collected from 7-14 year old patients with refractory seizures and focal cortical dysplasia (FCD), who underwent surgical resection, and compared to autopsy agematched controls. Western blots were run and probed for phospho (Ser499) and total FMRP. Data was expressed as a percent of control of either phospho/total ( $\mathrm{p} / \mathrm{t}$ ) or total FMRP normalized to actin (for rat) or adult control (for human).

Results: In rat hippocampus $1 \mathrm{~h}$ after seizures, FMRP expression and $\mathrm{p} / \mathrm{t}$ ratio began to trend upwards by $10 \%$ and $15 \%$ respectively, compared to controls $(\mathrm{p}=0.2$ for total FMRP, $\mathrm{p}=0.1$ for $\mathrm{p} / \mathrm{t} ; \mathrm{n}=13$ con, $13 \mathrm{HS}$ ). By $24 \mathrm{~h}$ post-HS, total FMRP expression significantly increased by $20 \%(\mathrm{p}=0.03, \mathrm{n}=16 \mathrm{con}, 13 \mathrm{HS}$ ). In the cortex, $\mathrm{p} / \mathrm{t}$ FMRP trended towards $20 \%$ and $15 \%$ decreases at 1 and $24 \mathrm{~h}$ postHS, respectively ( $\mathrm{p}=0.1$ for both, $\mathrm{n}=3$ /group at $1 \mathrm{~h}, 11$ con and $8 \mathrm{HS}$ at 24h), with no change in total FMRP. Phosho and total FMRP appeared unchanged $3 \mathrm{~h}$ post-HS in hippocampus and cortex $(\mathrm{n}=5$ con, $3 \mathrm{HS})$. In cortical tissue from human epilepsy $(\mathrm{n}=2)$ and FCD $(n=3)$ cases, seizures also appear to dysregulate FMRP, showing an increase in total FMRP $83 \%$ and 53\% above age-matched controls $(\mathrm{n}=2)$, respectively. In contrast, $\mathrm{p} / \mathrm{t}$ FMRP in epilepsy and FCD tissue decreases by $58 \%$ and $42 \%$, respectively, from age-matched controls. Conclusions: These results show altered FMRP expression and phosphorylation in human and rat tissue after seizures. When phosphorylated, FMRP stalls translation of many synaptic proteins, including itself. FMRP is dephosphorylated in an activity-dependent manner by p70S6 kinase, activity of which is also altered post-HS (Talos et al, 2012). Many proteins regulated by FMRP modulate important signaling pathways involved in synaptic plasticity, and thus potentially in cognitive function. Therefore, we conclude that earlylife seizures disrupt the FMRP pathway, and that this can contribute to later-life cognitive dysfunction and epileptogenesis. 


\subsection{4}

ELECTROPHYSIOLOGICAL PROPERTIES OF AGEDEFINED NORMALLY AND ABNORMALLY INTEGRATED DENTATE GRANULE CELLS IN A RODENT MODEL OF TEMPORAL LOBE EPILEPSY

Jack M. Parent ${ }^{1,2}$, A. L. Althaus ${ }^{1,2}$, H. Zhang ${ }^{1}$ and G. G. Murphy ${ }^{3,2}$ ( ${ }^{1}$ Neurology, University of Michigan, Ann Arbor, MI; ${ }^{2}$ Neuroscience Graduate Program, University of Michigan, Ann Arbor, MI and ${ }^{3}$ Physiology and Molecular and Behavioral Neuroscience Institute, University of Michigan, Ann Arbor, MI)

Rationale: Dysregulated hippocampal neurogenesis is a prominent feature of temporal lobe epilepsy (TLE), but the relationship between neurogenesis and epileptogenesis is unclear. Anatomical data indicate that most dentate granule cells (DGCs) generated during or after epileptic insults develop abnormally, including ectopic location, persistent hilar basal dendrites (HBDs) and mossy fiber sprouting, and promote increased excitability. Others appear to integrate normally and may reduce excitability. Studies that eliminate postinsult neurogenesis also show mixed results. Few studies have investigated both anatomical and physiological properties of different populations of DGCs in the same animal model. We recently used a retroviral (RV) GFP reporter to birthdate DGCs with respect to the timing of pilocarpine-induced status epilepticus (SE) in adult rats. We found that DGCs that were mature at SE were anatomically normal, while those born after SE displayed characteristic pathology of DGCs in TLE. To test whether post-SE generated DGCs promote pathological function while established DGCs retain normal function, and how morphological abnormalities influence their behavior, we examined electrophysiological characteristics of age- and morphologically-defined DGC cohorts.

Methods: Adult male Sprague-Dawley rats experienced pilocarpineinduced SE at 8 weeks of age. We birth-dated DGCs by bilateral stereotaxic injection of RV-GFP into the dentate gyrus on postnatal day 7 (P7) to label cells that would be mature at SE or on P60 to label cells that were generated after SE. Recordings were made 8-12 weeks after SE from acute hippocampal slices using whole-cell patch-clamp under IR-DIC optics. For TLE tissues, cell morphology was visualized by biocytin fills and assigned post-hoc to one of three categories: granule cell layer (GCL), GCL with HBD, and ectopic. Results: Preliminary results indicated that ectopically integrated cells showed greater repetitive action potential firing than those with HBDs or that appeared normally integrated in TLE. Interestingly, we found that even normally integrated cells in TLE were more excitable than DGCs in control tissue. Intrinsic properties that might influence excitability such as input resistance, membrane potential and action potential (AP) threshold were not different among groups.

Conclusions: These findings suggest that most adult-born, abnormally integrated DGCs generated after pilocarpine-induced SE are hyperexcitable and may contribute to recurrent seizures. Even normally integrated adult-born DGCs show abnormal excitability. Experiments are ongoing to compare these findings to DGCs that are mature at the time of SE, and to look specifically at synapticallydriven changes in network function.

Supported by an Epilepsy Foundation Predoctoral Fellowship (ALA) and NINDS NS058585 (JMP)

\section{$\mathbf{2 . 0 0 5}$ \\ ACUTE EPILEPTOGENESIS ON MICROELECTRODE SLICE ARRAYS REVEALS INDEPENDENT AND DEPENDENT LAYER-SPECIFIC MICROSCOPIC FOCI OF HYPERSYNCHRONY}

Ruggero Serafini ${ }^{1}$ and J. A. Loeb ${ }^{1,2}\left({ }^{1}\right.$ Neurology, Wayne State University, Detroit, MI and ${ }^{2}$ The Center for Molecular Medicine and Genetics, Wayne State University, Detroit, MI)

Rationale: Interictal epileptiform discharges (IEDs) originate from synaptic connectivity changes leading to neuronal hypersynchrony.
Theories on IEDs mechanisms are based on recordings of single cells and of field potentials with limited number of electrodes: the spatial distribution of IED field potentials has not been studied extensively. It remains unclear which cells in which cortical layers generate IEDs and how large cortical areas are recruited. We have previously found that human epileptic neocortex and neocortex of rats with IEDs activate key signaling pathways in the neocortex superficial layers. We aim at a spatially detailed neurophysiological characterization of IED-related field potentials to correlate IED neurophysiology with the expression of genes activated in epileptogenesis.

Methods: We used multielectrode arrays to record extracellular IEDs from neocortical slices from adult animals stimulated with zero magnesium and several concentrations of 4-Aminopyridine (4-AP). Preparation of viable acute slices from adult rats has been optimized allowing brains to cool down to 2-3 C for prolonged time intervals and by maintaining the vibratome chamber temperature constantly at 3-5 C. Through this protocol more than $90 \%$ of adult rat neocortical slices showed epileptiform activity. Electrodes were localized to specific cortical layers and those exhibiting discharges were mapped to the corresponding zone in the cortical slice.

Results: There was a 4-AP dose-dependent increase of the frequency of IEDs. IEDs were observed over several cortical layers though more often over layers I and II/III. IEDs occurred in a mosaic of multiple, circumscribed, microscopic foci. In the same slice IEDs of distinct microscopic foci were often independent from one another. At times in each slice the discharge of one microscopic focus appeared to recruit epileptiform activity in a distinct focus that was otherwise firing independently. Superficial layers could recruit deep layers but also deep layers initiated IEDs subsequently spreading to superficial layers. In long duration recordings, through time there was often a slow and gradual increase in synchronicity between these spatially distinct foci. Ictal patterns were observed more rarely and appeared over superficial layers or over deeper cortical layers but more over deeper layers.

Conclusions: IED are prevalent over the superficial cortical layers, in the same location of MAP-kinase activation: this biochemical pathway may participate to IED generation over Layers I and II/III. IEDs over the deep layers may be generated by a different pathway. Epileptogenesis may present as a mosaic of independent microscopic foci of neuronal hyper-synchrony. The gradual increase in synchronicity between different foci might result from synaptic plasticity triggered by IEDs themselves. Epileptogenesis may consist in a progressive cascade recruitment of foci generating IEDs over surfaces sufficiently large to ultimately disrupt cortical function.

\subsection{6 \\ NEONATAL SEIZURES IMPRINT ON NEURODEVELOPMENT \\ G. Hoogland ${ }^{1}$, A. Swijsen ${ }^{2}$, M. Raijmakers ${ }^{2}$, O. Schijns ${ }^{1}$, E. Clynen ${ }^{2}$, V. Visser-Vandewalle ${ }^{1}$ and J. Rigo ${ }^{2}{ }^{1}$ Neurosurgery, Maastricht University Medical Center, Maastricht, Netherlands and ${ }^{2}$ BIOMED Research Institute, Hasselt University, Hasselt, Belgium)}

Rationale: Febrile seizures (FS) are the most common type of seizures in children, affecting $4 \%$ of infants between the age of 3 months and 5 years. Retrospective studies indicate that adult patients with hippocampal sclerosis-associated temporal lobe epilepsy have a $40 \%$ incidence of FS, suggesting a causal relationship. Preclinical research suggests that FS persistently increase hippocampal excitability, resulting in enhanced seizure susceptibility. Based on these findings, it is hypothesized that FS change the expression and/or properties of neuronal ion channels. A possible mechanism to achieve this changed neuronal ion channel functioning is by neurogenesis.

Aim: To analyze the long-term impact of FS on hippocampal dentate granule cell functioning of cells that are born immediately after these seizures. 
Methods: In an established model, FS are induced in 10-day old rat pups by exposing them for $30 \mathrm{~min}$ to heated air. Control pups undergo a normothermia treatment and do not display behavioral seizures. One to eight weeks later, neurogenesis was studied by bromodeoxyuridine (BrdU) immunohistochemistry and responses to GABA were recorded in dentate granule cells by whole-cell patchclamp technique.

Results: Eight weeks after treatment, FS animals exhibit 25\% more BrdU-positive granule cells. Though more than $90 \%$ of these cells mature in adult neurons (i.e. co-localize with NeuN), they have differentiated in a different phenotype (i.e. less cells express glutamate transporters (EAAT3) and more cells express GABAA 32,3$)$. One week after treatment, GABAA receptors from FS animals are two-times more sensitive to GABA.

Conclusions: These data support the hypothesis that FS persistently alter the neuronal excitability.

\subsection{7 \\ FOCAL TRAUMATIC BRAIN DAMAGE RESULTS IN LOCALIZED GABA NEURON LOSS AND JAK/STAT ACTIVATION EARLY FOLLOWING INJURY}

Jeffery A. Boychuk ${ }^{1}$, C. R. Butler ${ }^{1}$, D. Raible ${ }^{2}$, L. Frey ${ }^{3}$, A. R. Brooks-Kayal ${ }^{4,5}$ and B. N. Smith ${ }^{1,6}\left({ }^{1}\right.$ Department of Physiology, University of Kentucky, Lexington, KY; ${ }^{2}$ Neuroscience Graduate Program, University of Colorado Denver, Aurora, CO; ${ }^{3}$ Department of Neurology, University of Colorado Denver, Aurora, CO; ${ }^{4}$ Departments of Pediatrics, Neurology, and Pharmaceutical Sciences, University of Colorado Denver, Aurora, CO; ${ }^{5}$ The Children's Hospital Colorado, Aurora, $\mathrm{CO}$ and ${ }^{6}$ Spinal Cord and Brain Injury Research Center (SCoBIRC), University of Kentucky, Lexington, $\mathrm{KY}$ )

Rationale: Traumatic brain injury (TBI) is a leading cause of acquired temporal lobe epilepsy (TLE), yet the mechanisms underlying posttraumatic epileptogenesis are not known. TBI is associated with cell loss and changes in cellular and synaptic signaling in cortical structures. Activation of the JAK/STAT3 pathway has been implicated as a participant in the reactive plasticity associated with epileptogenesis. Here, a murine model of TBI that results in spontaneous seizures was used to examine aspects of hippocampal GABA network modification shortly after brain injury. We tested the hypothesis that regional JAK/STAT3 pathway modulation and hilar GABAergic interneuron loss occurs shortly after focal brain injury.

Methods: Severe controlled cortical impact (CCI; impact depth $=1.0$ $\mathrm{mm}$ ) was administered to male mice at $6-8$ weeks of age. 24 hours post- injury, hippocampi from male CD-1 mice were isolated and processed for western blot analysis of STAT3 and pSTAT3 proteins, with the latter used as a marker of JAK/STAT3 activation. The effectiveness of the STAT3 inhibitor WP1066 (EMD Millipore; 30 and $90 \mathrm{~min}$ post-CCI; $50 \mathrm{mg} / \mathrm{kg}$; i.p.) on blocking JAK/STAT3 activation was also tested. Hilar GABA cell loss 2-3 days following CCI was examined in male mice expressing GFP in a subset of hilar interneurons (GIN mice; FVB-Tg(GadGFP)4570Swn/J). Coronal sections $(30 \mu \mathrm{m})$ were sampled in a 1 in 5 series. GFP-positive cell counts and hilus area were analyzed using a laser scanning confocal microscope (Zeiss, LSM 5 LIVE).

Results: At 24 hours following CCI an increase in hippocampal pSTAT3 protein expression ipsilateral to the injury was observed, relative to either the contralateral hemisphere or Sham-operated controls. Both Sham-operated controls and the contralateral hemisphere of injured animals exhibited low levels of hippocampal pSTAT3 protein expression. Administration of the STAT3 inhibitor WP1066 inhibited pSTAT3 protein expression in the hippocampus ipsilateral to the injury, but had little effect on the contralateral hemisphere of injured animals or Sham operated controls. Quantification of GFP positive cells from GIN mice revealed a decrease in the number of hilar interneurons within dorsal hippocampus ipsilateral to the injury relative to the contralateral hemisphere or to Sham-operated controls. Preliminary data indicate that administration of WP1066 did not inhibit hilar GABA cell loss ipsilateral to the injury.

Conclusions: Early time-points following brain injury with CCI are associated with changes in hippocampal GABA networks. These changes are prominent in the hippocampus ipsilateral to the injury and include a loss of hilar GABAergic interneurons as well as activation of the JAK/STAT signaling pathway. Previous studies have associated both of these changes with alterations in GABA signaling associated with TLE. Alterations in hippocampal GABA neuron function following head injury may support the eventual expression of spontaneous seizures in posttraumatic epilepsy.

\subsection{8 \\ ABERRANT HIPPOCAMPAL GRANULE CELL NUMBER IS CORRELATED WITH SEIZURE FREQUENCY AND DURATION}

Michael Hester ${ }^{1,2}$, R. Pun ${ }^{1}$ and S. Danzer ${ }^{1,2}\left({ }^{1}\right.$ Cincinnati Children's Medical Center, Cincinnati, $\mathrm{OH}$ and ${ }^{2}$ University of Cincinnati, Cincinnati, $\mathrm{OH}$ )

Rationale: Epilepsy is a common, debilitating disease for which there is no effective preventative. Developing novel therapies will require further understanding of the disease pathology. Here, we assessed the relationship between abnormal, newly-generated, dentate granule cells (DGCs) and epileptogenesis. DGCs have long been implicated in temporal lobe epilepsy (TLE), as they regulate excitatory signaling through the hippocampus and exhibit neuroplastic changes during epileptogenesis. Furthermore, DGCs are unusual in that they are continually generated into adulthood, with aberrant integration of newly-generated DGCs underlying the majority of restructuring in the dentate gyrus during disease progression. While it is known that these abnormal networks promote abnormal neuronal firing and hyperexcitability, it has yet to be established whether they directly contribute to seizure generation. Here, we sought to determine whether a positive correlation existed between the number of abnormal, newly-generated granule cells in an animal, and the frequency of spontaneous seizures.

Methods: In this study, we utilized mouse cre-loxP technology to selectively activate GFP in postnatally-generated hippocampal granule cells during the development of epilepsy. Mice were rendered epileptic by inducing status epilepticus with pilocarpine. Two months after status epilepticus, seizure frequency was determined by $24 / 7$ video/EEG monitoring for a period of four weeks. Following EEG recording, animals were sacrificed and percentage of newlygenerated granule cells exhibiting abnormal morphologies was determined using confocal microscopy. Abnormal structures examined included basal dendrites, ectopic somata and mossy fiber axon sprouting.

Results: Epileptic animals exhibited considerable variability in mean seizure frequencies, ranging from 0.1 to 3.0 seizures/day. When seizure frequency was correlated with the percentage of abnormal cells in each animal, significant positive correlations were found with: 1) the number of hilar ectopic DGCs, 2) the amount of mossy fiber sprouting and 3 ) the extent of mossy cell death. Basal dendrites tended to be more common in animals with high seizure rates, but this effect did not reach signficance.

Conclusions: These studies provide correlative evidence in support of the hypothesis that abnormal granule cells contribute to the development of epilepsy, but also indicate that other factors particularly mossy cell loss - are likely important as well. 


\subsection{9}

COMPARING PROPAGATION DYNAMICS BETWEEN SEIZURE AND DEFAULT-STATE ACTIVITY IN ECOG RECORDINGS

Zoltan Nadasdy $^{1,2}$, J. Shen ${ }^{1,3}$, D. E. Briggs ${ }^{1,3}$, M. R. Lee ${ }^{1,4}$, D. F. Clarke $^{4,3}$ and R. J. Buchanan ${ }^{1,2}\left({ }^{1}\right.$ Neurosurgery, Seton Brain and Spine Institute, Austin, TX; ${ }^{2}$ Psychology, University of Texas at Austin, Austin, TX; ${ }^{3}$ Neurology, University of Texas Southwestern Medical Center, San Antonio, TX and ${ }^{4}$ Comprehensive Epilepsy Program, Dell Children's Hospital, Austin, TX)

Rationale: The goal was to define the propagation dynamics during seizure activity relative to the default-state dynamics. Our hypothesis was that seizure dynamics is associated with a faster conductivity and steeper field gradient than default state is, as a result of hypersynchrony predominant during seizures. We expected to find a consistent pattern of oscillation frequencies across subjects that best discriminate between seizure and default-state dynamics.

Methods: We retrospectively analyzed ECoG data recorded from epilepsy patients $(n>10)$, diagnosed with frequent complex partial seizures, candidates for resective surgeries. We compared the propagation dynamics between seizure activity and default-state activity. We divided the oscillations into three frequency bands: theta-alpha $(1-25 \mathrm{~Hz})$, high-beta $(25-40 \mathrm{~Hz})$ and gamma $(40-80 \mathrm{~Hz})$. We reconstructed the propagation dynamics of oscillations in each frequency band by mapping the relative phases in electrode space. From the slopes of propagation gradients between electrode pairs, we computed the average field-gradient (propagation speed), and compared those between default- and seizure-states from the same subject. For computing the phase we used (a) the Hilbert-transform, (b) the relative delay of oscillation peaks between electrodes, and (c) the cross-correlation of oscillations peaks between electrodes. The three different frequency bands and the three phase definitions with the two behavioral states provided 18 conditions.

Results: The average propagation velocity was $\sim 1 \mathrm{~mm} / \mathrm{ms}$ during default-state, 6-9 times lower than expected (Nunez, 1995). More surprisingly, the seizure activity deviated from this dynamics in a frequency dependent fashion. While the theta-alpha component of seizures displayed an increasing phase asynchrony and slower propagation than during default-state, gamma oscillations displayed hyper-synchrony, faster and more anisotropic propagation across electrodes during seizures than default-state does.

Conclusions: Our preliminary result suggests a frequency dependent propagation pattern during both default-state and seizure activity. Moreover, seizures affect the macroscopic propagation dynamics within cortical tissues in a frequency dependent fashion.

\subsection{0 \\ EFFECTS OF KETOGENIC DIET AND CONSTITUENTS ON PATHOLOGIC SHARP WAVES AND HIGH FREQUENCY OSCILLATIONS}

Kaeli Samson ${ }^{1}$, K. A. Simeone ${ }^{1}$, J. M. Rho ${ }^{2}$ and T. A. Simeone ${ }^{1}$ ( ${ }^{1}$ Pharmacology, Creighton University School of Medicine, Omaha, $\mathrm{NE}$ and ${ }^{2}$ Paediatrics and Clinical Neurology, Alberta Children's Hospital, Calgary, AB, Canada)

Rationale: The ketogenic diet (KD) is a highly effective anticonvulsant treatment for pediatric refractory epilepsies. Efforts to identify the mechanism of action of the KD have focused on biochemical constituents such as ketone bodies [acetone, acetoacetate (ACA), beta-hydroxybutyrate (BHB)] and long chain omega-3 and omega-6 polyunsaturated fatty acids (LcPUFAs) [docosahexaenoic acid (DHA), eicosapentaenoic acid (EPA) and arachidonic acid (AA)]. Although, acute effects of ketone bodies on KATP channels, vesicular glutamate transporters and mitochondria have been elucidated, it is unclear whether ketone bodies have a significant effect on pathologic oscillatory activity of a hyperexcitable network. Similarly, LcPUFAs are known to inhibit sodium channels and L- type calcium channels, but whether this effect translates into dampening network activity is not known. We have found that hippocampal slices dissected from epileptic Kcna1-null mice display sharp wave-high frequency oscillation (SPW-HFO) complexes that are significantly different from wild-type mice. Kcna1-null SPWHFOs occur more frequently, have longer duration and have spectral disorganization with the emergence of pathologic fast ripples. KDtreatment reduces seizures in Kcna1-null mice; thus, we hypothesized that KD-treatment would reduce the pathologic abnormalities of in vitro Kcna1-null SPW-HFOs. We further hypothesized that acute application of ketone bodies and/or LcPUFAs would have effects on Kcna1-null network oscillations.

Methods: Kcna1-null mice were weaned (P21) onto either a standard diet or KD for 10-14 days. Recordings of spontaneous and evoked extracellular field potentials were acquired using a 64 multi-electrode array. Acutely isolated hippocampal slices from wild-type (WT) and Kcna1-null mice were positioned with electrodes in the fields of CA1, CA3 and dentate gyrus. Electrodes underlying axon fibers of the perforant path, mossy fibers and Schaffer-collaterals were used for stimulation.

Results: The Kcna1-null mossy fiber-CA3 synapse exhibited decreased paired pulse ratios (PPRs) compared to WT suggesting an increase in neurotransmitter release. Acute treatment of ketone bodies or LcPUFAs did not have effects on evoked field potentials or PPRs. Slices isolated from KD-treated Kcna1-null mice had significantly increased PPRs suggesting reduced neurotransmitter release. Application of either ketone bodies or LcPUFAs reduced the incidence of Kcnal-null SPW-HFOs by $20-40 \%$, but did not affect other parameters or fast ripples. KD-treatment normalized all Kcna1null SPW-HFO parameters to near WT values and abolished fast ripple emergence.

Conclusions: These data indicate that the KD alleviates pathologic network oscillations associated with epilepsy. This finding supports the notion that in vitro Kcna1-null SPW-HFOs are a useful measurement of anticonvulsant activity. In contrast, ketone bodies and LcPUFAs had limited effects on pathologic network activity suggesting that determining their importance in the mechanism of the $\mathrm{KD}$ may require methods of chronic exposure.

\subsection{1 NEUROSTEROIDS MODULATE HIGH-FREQUENCY OSCILLATIONS (80-500 HZ) IN THE PIRIFORM CORTEX IN VITRO}

rochelle herrington, M. Levesque, G. Panuccio and M. Avoli (Department of Neurology and Neurosurgery, Montreal Neurological Institute, Montreal, QC, Canada)

Rationale: High-frequency oscillations (HFOs, ripples: 80-200 Hz, fast ripples: $250-500 \mathrm{~Hz}$ ) are thought to be important markers of epileptogenesis and ictogenesis. They are recorded in epileptic patients, in animal models of temporal lobe epilepsy and in in vitro preparations. Here, we addressed the role of neurosteroids in the modulation of HFOs recorded in vitro in the piriform cortex, a highly excitable structure that is implicated in seizure generation and maintenance.

Methods: Epileptiform activity was induced in horizontal rat brain slices by bath application of 4-aminopyridine (4AP, $50 \mu \mathrm{M})$. Pharmacological manipulation of GABAergic signaling was performed under 4AP conditions with the application of THDOC at $100 \mathrm{nM}, 1 \mu \mathrm{M}$ and $5 \mu \mathrm{M}$ concentrations. Field potential recordings were obtained from the anterior and posterior piriform cortex $(n=6$ for each experimental condition). Traces were analyzed offline with pClamp. HFOs were detected with custom built functions in MATLAB.

Results: THDOC significantly reduced the duration of ictal-like events in both the anterior and posterior piriform cortex. Under 4AP alone, ictal events were $89.46 \pm 9.03 \mathrm{~s}( \pm \mathrm{SEM})$ in duration, but with the application of $100 \mathrm{nM}, 1 \mu \mathrm{M}$ and $5 \mu \mathrm{M}$ THDOC, the duration 
decreased by $26 \%, 15 \%$ and $27 \%$, respectively. The analysis of HFOs showed that the anterior piriform cortex contained a greater number of HFOs compared to the posterior piriform cortex. On average, 14 ripples and 45 fast ripples were detected per ictal-like event in the anterior region compared to 7 ripples and 14 fast ripples detected in the posterior piriform cortex. THDOC reduced the number of both ripples and fast ripples in the anterior piriform cortex by $70 \%$ with no significant difference between concentrations but failed in inducing significant changes in HFOs in the posterior piriform cortex. Conclusions: Our results demonstrate that enhancing extrasynaptic GABAA receptor activity with THDOC (which presumably leads to a greater tonic inhibitory current) decreases the duration of ictal events. These changes appear to influence the occurrence of HFOs in the anterior piriform cortex, possibly due to the differential expression of GABAA receptors and their respective subunits between the two regions. We propose that GABAA receptor mediated signaling can modulate 4AP-induced ictogenesis and HFOs by affecting network excitability.

\subsection{2}

\section{A MOUSE MODEL OF DRAVET SYNDROME REVEALS} ALTERATIONS IN CARDIAC ELECTROPHYSIOLOGY David S. Auerbach (Pharmacology, University of Michigan, Ann Arbor, MI)

Rationale: Dravet Syndrome (DS) is the most severe form of genetic childhood epilepsy. In addition to seizures, patients exhibit developmental delay, cognitive decline, and sudden death. Death may be due to epilepsy-related causes or SUDEP: Sudden Unexpected Death in Epilepsy. Cardiac arrhythmias are one of the proposed causes of SUDEP, however, the susceptibility to and mechanisms of arrhythmogenesis in DS remain unknown. More than $70 \%$ of DS patients have de novo mutations in SCN1A, encoding the $\alpha$-subunit of the voltage-gated sodium channel, Nav1.1, a channel that is critical for the activation of neurons and cardiac myocytes. We hypothesized that DS mutations in SCN1A that produce haploinsufficiency result in altered cardiac electrical function, arrhythmias, and a potential cardiac mechanism for SUDEP.

Methods: Single cell voltage clamp $\mathrm{Na}+$ channel and current clamp action potential recordings were acquired from acutely isolated juvenile (15-21 days old, P15-21) DS (heterozygous for R1407X) and WT mice. Radiotelemetry ECG, temperature, and activity recordings (DSI ETA-F10) were obtained from conscious free moving mice (P16-62).

Results: DS mice recapitulate the DS patient neuronal phenotype, yet their cardiac phenotype remains unknown. We observed a 2-fold increase in transient and persistent $\mathrm{Na}+$ current (INa) density in DS ventricular myocytes $(p<0.01)$. There was a leftward shift in the voltage dependence of INa availability and conductance $(\mathrm{p}<0.05)$, consistent with increased Nav1.5, the predominant cardiac $\mathrm{Na}+$ channel $\alpha$-subunit. In agreement with increased INa, DS myocytes exhibited increased excitability (assessed by minimum stimulus threshold and upstroke velocity), and prolongation of the action potential duration. In addition, we observed a 2 -fold increase in the incidence of early after-depolarizations, which are predicted to promote the initiation of arrhythmias. Twenty one percent of DS mice in our experiments died by P152, with $69 \%$ of these dying by P52. In contrast, none of the WT mice died up to 360 days $(n=75$ for each WT and DS). Finally, continuous radiotelemetric ECG recordings recapitulated the multisystem DS phenotype of seizures, cardiac triggered activity (e.g., premature ventricular complexes), cardiac arrhythmias, bradycardia, and sudden death.

Conclusions: These results shed significant insights into how changes in INa may establish a substrate for the initiation of arrhythmias, and provide a potential mechanism for SUDEP in DS.

\subsection{3}

RNAI-MEDIATED KNOCKDOWN OF NAV1.1 DISRUPTS A COGNITIVE NEURAL NETWORK

Alex C. Bender ${ }^{1}$, B. Luikart ${ }^{1}$, G. Holmes ${ }^{1}$, R. Scott ${ }^{1,2}$ and P. LenckSantini ${ }^{1}\left({ }^{1}\right.$ Neurology, Neuroscience Center at Dartmouth, Geisel School of Medicine, Lebanon, NH and ${ }^{2}$ University College London, Institute of Child Health, London, United Kingdom)

Rationale: Dravet syndrome (DS) is a childhood-onset epilepsy leading to severe and permanent cognitive impairment. In most patients, anti-epileptic treatments fail to ameliorate cognitive deficits, indicating that mechanisms other than seizures may be involved. As many as $85 \%$ of cases of DS are linked with loss-of-function mutations in the SCN1A gene, coding for the type I voltage-gated sodium channel (Nav1.1). However, the impact of Nav1.1 loss of function on neural networks involved in cognition has not been studied. Here, we develop an RNAi approach to investigate the direct effects of Nav1.1 down-regulation on specific neural networks in vivo.

Methods: We first screened siRNA constructs in vitro for efficient suppression of SCN1A expression by real-time PCR and western blot. We then examined the effects of siRNA injections in vivo by targeting the basal forebrain, a region known to be critical for learning and memory in rodents. Short-term effects on hippocampal depth EEG and spatial memory were tested in a reaction-to-novelty task. Because siRNA induces only a transient knockdown, a second approach was then developed to achieve a stable suppression of SCN1A expression. Using the same nucleotide sequence targeted to SCN1A, we constructed a lentiviral vector expressing short hairpin RNA (shRNA), and we subsequently evaluated its longer-term effects on SCN1A expression.

Results: Transfection of siRNA complexes into rat neuroblastoma cells transiently suppressed SCN1A expression by $71 \%$ compared to a negative control siRNA construct $(\mathrm{F}(2,5)=28.24, \mathrm{p}<.01, \mathrm{n}=3$ per group) with an equivalent reduction ( $73 \%$ ) at the protein level after 48 hours $(\mathrm{t}(4)=6.15, \mathrm{p}<.01, \mathrm{n}=3$ per group). When evaluated in vivo, knockdown of Nav1.1 in the basal forebrain region induced a spatial memory deficit. Notably, spatial memory performance was significantly related to the frequency of hippocampal theta oscillations in controls ( $22=0.76, \mathrm{p}<.05, \mathrm{n}=6)$. However, this relationship was abolished after Nav1.1 knockdown $(\mathrm{R} 2=0.005$, $\mathrm{p}>.05, \mathrm{n}=6$ ). Lentiviral expression of shRNA similarly suppressed SCN1A expression in rat neuroblastoma cells. Despite a relatively low infection efficiency in this cell line (less than $50 \%$ of cells revealed GFP+ fluorescence), SCN1A expression was reduced by $51 \%(\mathrm{t}(10)=3.25, \mathrm{p}<.01, \mathrm{n}=6$ per group $)$. In contrast to the transient effects of siRNA, this effect was stable and was observed after two weeks from the time of infection.

Conclusions: We describe a novel approach to investigate the effects of Nav1.1 loss of function that has precise temporal and spatial control in vivo. Secondly, we show that Nav1.1 down-regulation in the basal forebrain region causes a dysregulation of oscillations that is related to spatial cognition. New strategies employing lentiviral expression of shRNA to stably suppress Nav1.1 expression will allow further elucidation of the neural mechanisms responsible for the dysfunction of such networks. Importantly, our results to this point strongly suggest that the loss of function of Nav1.1 in Dravet syndrome may directly impact cognition through mechanisms other than seizures.

\subsection{4 \\ RAPID ONSET OF A KAINATE-INDUCED MIRROR FOCUS IN RAT HIPPOCAMPUS MEDIATED BY CONTRALATERAL AMPA RECEPTORS}

David Mogul and T. Sobayo (Biomedical Engineering, Illinois Institute of Technology, Chicago, IL) 
Rationale: The development of an independent epileptic focus in an area of the brain contralateral to the primary epileptic focus has been observed before. This mirror focus typically evolves over days in the experimental setting after status epilepticus or electrical kindling of the primary focal region. In contrast, we observed the rapid development of an apparent mirror focus in the contralateral hippocampus following microinjection of Kainic acid (KA) in the ipsilateral hippocampus in rats and sought to gain a better understanding of its underlying dynamics and neurotransmittermediated mechanisms using multisite intracranial recordings.

Methods: Local field potentials (LFPs) were recorded in anesthetized rats using electrodes implanted in the CA3 region of both hippocampi and in the anteromedial nucleus of the thalamus of adult male rats. Epileptogenesis was induced in the rats with a microinjection of KA in the ipsilateral CA3 region. Development of seizure dynamics was followed under three experimental perturbations to the contralateral hippocampus: (A) no treatment $(\mathrm{n}=18)$, (B) pre-treatment with microinjection of the AMPA/Kainate receptor antagonist CNQX $(\mathrm{n}=15)$, and $(\mathrm{C})$ pre-treatment with microinjection of the selective Kainate receptor antagonist UBP $301(\mathrm{n}=16)$.

Results: Both the control and the UBP 301 groups had seizures preferentially originate from the hippocampus contralateral to the injection site and appearing within ten minutes of KA injection. In contrast, the CNQX group had seizures preferentially originate from the ipsilateral hippocampus. By tracking the order of seizure onset between the three recording sites, it was observed that the probability that a hippocampal seizure would propagate across commissural fibers prior to any thalamic seizure activity was significantly reduced in the CNQX group when compared to the control and UBP groups suggesting that the AMPA receptor mediated component responsible for mirror focus development was also necessary for the spread of ictal activity via the commissural fibers.

Conclusions: Epilepsy is largely a network phenomenon of the brain which, because of the vast variability in which seizures arise, makes it difficult to treat. Understanding how a complex circuit in the brain develops, such as in the case of a mirror focus, may be critical to uncovering ways of either disrupting its development or treating its effects. The rapid appearance of a contralateral mirror focus via AMPA receptors in a limbic epilepsy model might be the mechanism by which a putative long-term mirror focus is established in vivo and may also underlie how secondary generalization progresses in some cases.

\subsection{5 \\ SUBCELLULAR DISTRIBUTION OF INTRANEURONAL CHLORIDE AND ITS MODIFICATION BY TONIC

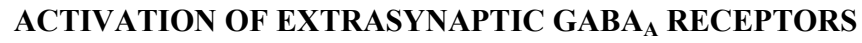
Kiyoshi Egawa, V. Dzhala and K. Staley (Department of Neurology, Massachusetts General Hospital and Harvard Medical School, Boston, MA)

Rationale: Clarification of the subcellular intraneuronal chloride $\mathrm{CCl}^{-}$ ]$_{\mathrm{i}}$ ) distribution is essential to understand the dynamics of GABAergic transmission and mechanisms of epilepsy because the driving force for $\mathrm{GABA}_{\mathrm{A}}$ receptor is largely determined by the $\left[\mathrm{Cl}^{-}\right]_{\mathrm{i}}$ in the microdomain of synaptic inputs. In this view, extrasynaptic $\mathrm{GABA}_{\mathrm{A}}$ receptor mediated-tonic $\mathrm{Cl}$ influx might play an important role for the dynamic spatial modification of the synaptic inhibition by focally changing $\left[\mathrm{Cl}^{-}\right]_{\mathrm{i}}$.

Methods: We utilized the high spatial resolution of two-photon microscopy and the transgenically-expressed fluorophore Clomeleon to image intraneuronal chloride in acute hippocampal slices and organotypic slice cultures.

Results: The value of dendritic $\left[\mathrm{Cl}^{-}\right]_{\mathrm{i}}$ was more broadly distributed and lower than somatic $\left[\mathrm{Cl}^{-}\right]_{\mathrm{i}}$ in both CA1 pyramidal neurons and interneurons. Application of $2 \mathrm{uM}$ THIP, which at this concentration is a selective agonist for $\delta$ subunit containing extrasynaptic GABAA receptors, did not alter $\left[\mathrm{Cl}^{-}\right]_{\mathrm{i}}$ of pyramidal cells. In interneurons, somatic $\left[\mathrm{Cl}^{-}\right]_{\mathrm{i}}$ was unchanged but $\left[\mathrm{Cl}^{-}\right]_{\mathrm{i}}$ was focally increased in some dendritic branches. In contrast, $2 \mathrm{uM}$ THIP increased $\left[\mathrm{Cl}^{-}\right]_{\mathrm{i}}$ more generally dentate granule cells, both at the soma and dendrites. These data do not exclude regional variance in $\mathrm{Cl}$ cotransport as the mechanism for the observed subcellular variance in THIP-induced changes in $\left[\mathrm{Cl}^{-}\right]_{\mathrm{i}}$. However, studies of dendritic $\mathrm{Cl}$ transport rates have not suggested such heterogeneity, and we will test this possibility more rigorously by repeating the current experiments in the presence of $\mathrm{Cl}$ cotransport inhibitors. We currently favor the idea that postsynaptic components of tonic $\mathrm{GABA}_{\mathrm{A}}$ recaptor-mediated inhibition, which previously have only been evaluated electrophysiologically at single subcellular recording locations, may exhibit substantial subcellular variance.

Conclusions: The subcellular localization of tonic $\mathrm{GABA}_{\mathrm{A}}$ receptormediated inhibition implies both a richer computational role for this conductance in selected cell types, and the possibility that local postsynaptic plasticity underlies the observed subcellular variance.

\section{Translational Research: Models}

\subsection{6 \\ CHARACTERIZATION OF NEONATAL SEIZURES IN AN ANIMAL MODEL OF HYPOXIC-ISCHEMIC ENCEPHALOPATHY}

Dayalan Sampath, A. White and Y. H. Raol (Pediatric Neurology, University of Colorado Denver, Aurora, CO)

Rationale: Hypoxic-ischemic encephalopathy (HIE) is one of the most common causes of seizures in full-term infants. Studies in both human neonates and animal models suggest that seizures themselves may independently contribute to brain injury and poor neurological outcome. Hypoxia-Ischemia (HI) induced injury in neonatal rats is a commonly used model to study acute as well as chronic changes caused by HIE. In the current study, we characterize HI-induced acute neonatal seizures and changes in the EEG in an animal model of HIE.

Methods: Sprague-Dawley rat pups were implanted with bilateral sub-dural electrodes on postnatal day 6 (P6). To induce HI, the right carotid artery of $\mathrm{P} 7$ pups was ligated and the pups were then exposed to hypoxia in an $8 \%$ oxygen environment for 2 hours. Rats were video EEG (VEEG) monitored before HI, during hypoxia exposure, immediately after hypoxia and 24 hours after HI. Electroclinical seizures were defined as an EEG pattern that differs from background in either / or both amplitude and frequency, evolves over time, contains spikes or sharps lasting for 10 seconds or more and is associated with a change in the rat's behavior. Electrographic seizures were those observed on EEG that were not associated with changes in rat behavior. Electrographic discharges were defined as EEG patterns similar to those in electrographic seizures, but lasting less than 10 seconds. These may or may not be associated with a change in the rat's behavior.

Results: Analysis of VEEG recordings revealed that the rat pups exhibit multiple electroclinical seizures during 2 hours of hypoxia (mean $\pm \mathrm{SD}=13.25 \pm 1.7 ; \mathrm{n}=4$ ). The first electroclinical seizure was observed $11.07+11.39$ minutes (mean $\pm \mathrm{SD}$; $\mathrm{n}=5$ ) after initiation of hypoxia. The total duration of electroclinical seizures during 2 hours of hypoxia was $9.03+4.1$ minutes (mean $\pm \mathrm{SD} ; \mathrm{n}=$ 4). Multiple electrographic discharges and myoclonic jerks were also observed during hypoxia. In only one rat, electrographic seizures (without clinical correlate) were observed during hypoxia $(n=4)$. HI rat pups have multiple acute post-hypoxia seizures and seize $26.1 \%$ of the time in the first 2 hours after hypoxia $(n=4)$. Twenty-four hours after HI, one rat was observed to have electroclinical seizures $(\mathrm{n}=2)$. Behavioral seizures included forelimb and hindlimb clonus, vibratory tonic seizures, whole body jerks, forelimb jerks, facial automatisms, tail stiffening, forelimb and hindlimb paddling, thrashing and circling. Power spectrum analysis showed suppression of EEG activity at lower frequencies $(3-20 \mathrm{~Hz})$ in both cortices 
during hypoxia ( $\mathrm{p}<0.04$; paired $\mathrm{t}$-test). The EEG power returned to baseline values after hypoxia i.e., during the reperfusion period. Conclusions: In this model of neonatal HI, we found that rats have multiple seizures during and immediately after hypoxia. We also note a decrease in power in the EEG during hypoxia, likely due to the suppression of the normal background rhythms in the rats. This is similar to the suppression noted in humans following neonatal HI.

\subsection{7}

IMPACT OF GENETIC AND PHARMACOLOGICAL MODULATION OF THE ENDOCANNABINOID SYSTEM ON KINDLING ACQUISITION

Eva-Lotta von Rüden ${ }^{1,2}$, M. Jafari ${ }^{1,2}$, R. M. Bogdanovic ${ }^{1,2}$, C. Wotjak $^{3}$ and H. Potschka ${ }^{1}$ ( Inst. of Pharmacology, Toxicology, and Pharmacy, LMU, Munich, Germany; ${ }^{2}$ Graduate School of Systemic Neurosciences, Munich, Germany and ${ }^{3}$ Max Planck Institute of Psychiatry, Munich, Germany)

Rationale: The endocannabinoid (eCB) system serves a central function in regulating neuronal plasticity. Thus, the eCB system can be considered a putative target for central nervous system diseases including epilepsies. In this study, we modulated the cascade genetically and pharmacologically and analyzed the impact on epileptogenesis and ictogenesis.

Methods: Twenty male conditional cannabinoid (CB)-1 receptor knockout $(\mathrm{KO})$-mice $\left(\mathrm{CB}^{1 \mathrm{f} / \mathrm{f} ; \mathrm{CaMKII \alpha Cre}}\right)$ along with littermate controls $\left(\mathrm{CB}^{1 \mathrm{f} / \mathrm{f}}\right)$ and twenty male NMRI mice, for the genetic and

pharmacological studies respectively, were stimulated once daily via an implanted depth electrode. NMRI mice received i.p. injections of either the selective CB1 receptor-antagonist SR-141716 (5mg/kg i.p.) or vehicle thirty minutes prior to each kindling stimulation.

Following kindling we analyzed locomotion, exploratory and anxiety-like behaviors in the Black and White Box (BWB) and the Open Field (OF). Neuronal progenitor cells were stereologically assessed based on immunohistological labeling of doublecortin. Results: Both the lack of CB1 receptors in KO mice $(n=10)$ as well as antagonism at $\mathrm{CB} 1$ receptors $(\mathrm{n}=11)$ caused no differences in the development of generalized seizures but resulted in significantly longer seizure durations. Lower initial thresholds were detected in KO-mice as compared to littermate controls $(\mathrm{p}=0.0281, \mathrm{n}=10)$. In the $\mathrm{OF}, \mathrm{KO}$ mice spent less time in the center (non-kindled $\mathrm{n}=8$, $p=0.0421$; kindled $n=9, p=0.0374$ ) and more time engaging in thigmotaxis $(\mathrm{p}=0.0111$ and $\mathrm{p}=0.012)$ as compared to littermate controls (non-kindled $n=8$, kindled $n=10$ ). The two-way ANOVA revealed a significant impact of the genotype $(\mathrm{p}<0.0001)$ on anxietyrelated behavior. In contrast, there was no inter-group difference between vehicle-treated (non-kindled $n=11$, kindled $n=9$ ) and SR141716-treated mice (kindled $\mathrm{n}=11$ ).

In the $\mathrm{BWB}$, kindled $\mathrm{KO}$ mice spent a significantly longer time in the white compartment $(\mathrm{p}=0.012)$ and had $41 \%$ more transitions $(p=0.0025)$ than non-kindled controls $(p=0.012$; kindled $n=10$, nonkindled $n=9)$. No significant inter-group differences in the pharmacological modulation of the eCB system were observed (vehicle non-kindled $n=11$, vehicle kindled $n=9$, kindled SR-141716 $\mathrm{n}=10)$.

Compared to the non-stimulated control, kindling resulted in an increased hippocampal density of neuronal progenitor cells without any genotype-dependent differences.

Conclusions: The study demonstrated that the eCB cascade affects seizure susceptibility as well as endogenous termination of seizure activity, but fails to influence kindling acquisition. Thus, eCB signaling seems to play a role in ictogenesis but not epileptogenesis. We are grateful to Prof. Dr. Beat Lutz for generating and providing the conditional knock-out mice. In addition we thank Marion Fisch, Barbara Kohler, Andrea Wemeyer and Angela Vicidomini for their excellent technical assistance. The authors are grateful for a scholarship from the Elitenetzwerk Bayern for E.-L. von Rüden.
2.018

NEUROIMAGING AND EEG DURING GENERALIZED TONIC-CLONIC SEIZURES IN FERRETS

Mark W. Youngblood ${ }^{1}$, A. M. Mishra ${ }^{1}$, S. Enamandram ${ }^{1}$, B. G. Sanganahalli ${ }^{2}$, J. E. Motelow ${ }^{1}$, H. X. Bai ${ }^{1}$, A. Gribizis ${ }^{1}$, A. Lighten ${ }^{1}$, F. Hyder ${ }^{2}$ and H. Blumenfeld ${ }^{3}{ }^{1}$ Neurology, Yale School of Medicine, New Haven, CT; ${ }^{2}$ Diagnostic Radiology, Yale School of Medicine, New Haven, CT and ${ }^{3}$ Neurology, Neurobiology and Neurosurgery, Yale School of Medicine, New Haven, CT)

Rationale: Because of their wide availability and relatively low expense, rodent models of epilepsy are by far the most common substrate for studying tonic-clonic activity in-vivo. While seizures observed in rats reliably mimic the electrographic morphology and behavioral manifestations seen in humans, the relative simple development of the rodent cortex makes neuroimaging studies difficult to interpret. This study attempts to correct this deficiency by using a bicuculline-induced, ferret model of tonic-clonic seizures. Compared to rodents, ferrets have a closer evolutionary link to humans and share many of the same anatomical structures in cortical and subcortical regions. Functional neuroimaging data during ictal periods may elucidate the networks involved in seizure propagation and provide some insight on focality of tonic-clonic activity in humans.

Methods: Simultaneous measurements of scalp EEG and Blood Oxygen Level Dependent (BOLD) signal were collected during ictal periods in ferrets. Seizures were induced through intravenous injections of bicuculline solution, which is a strong GABAA receptor antagonist. All ferrets were female and ranged in weight from 300 to 700 grams. BOLD measurements were recorded with a 9.4T Varian magnet while the animal was under ketamine / xylazine anesthesia. Recordings of scalp EEG were collected using two carbon-filament electrodes. To reduce movement artifact during fMRI recordings, each animal was paralyzed with d-tubocurarine- $\mathrm{Cl}$ and artificially ventilated through a tracheostomy. Heart rate and blood pressure were monitored through an arterial line attached to an analogue-todigital pressure transducer. Blood gas levels were periodically measured to ensure appropriate physiology, and corresponding adjustments were occasionally made to respiration rate and tidal volume.

Results: All collected data was processed with in-house fMRI analysis software used in previous studies from our lab. A twosample t-test was performed to determine significant changes in blood-oxygenation between baseline and seizure periods (as defined by EEG activity). The results revealed widespread increases in BOLD signal for both cortical and sub-cortical regions during ictal periods. A time-course analysis of neuroimaging data showed progressive spread of hemodynamic increases as the seizure propagated from its initial onset zone. Functional increases during ictal periods were followed by large post-ictal decreases in cortical and sub-cortical areas. This correlated closely with the cortical suppression seen on EEG during this time period.

Conclusions: Using simultaneous recordings of scalp EEG and functional neuroimaging, a new model of tonic-clonic seizures was investigated. Regions involved in the initiation and spread of seizures in ferrets have closer anatomical correlates to human patients, and discoveries made using this model could guide more targeted therapy in humans suffering from this disorder. Future work could further characterize the time-course of fMRI changes during the ictal period, and may include electrophysiology experiments that better describe neuronal activity.

\subsection{9 \\ CALIFORNIA SEA LIONS: A MODEL OF HUMAN TEMPORAL LOBE EPILEPSY}

Paul S. Buckmaster ${ }^{1}$, X. Wen ${ }^{1}$, I. Toyoda ${ }^{1}$, L. Kim ${ }^{1,2}$, F. Gulland ${ }^{3}$ and W. Van Bonn ${ }^{3}\left({ }^{1}\right.$ Stanford University, Stanford, CA; ${ }^{2}$ University of California, Davis, CA and ${ }^{3}$ Marine Mammal Center, Sausalito, CA) 
Rationale: Harmful algal blooms that generate the neurotoxin domoic acid (DA) are increasing in frequency and severity in coastal waters of the western United States. Previous studies discovered that DA-producing algae become concentrated in filter-feeding fish, such as anchovies, which are eaten by birds and mammals. California sea lions (Zalophus californianus) frequently are affected by DA toxicosis after feeding on these fish. Exposed sea lions display acute neurological signs including status epilepticus, and many die. A substantial fraction of those that survive develop behavioral abnormalities and epilepsy. In epileptic animals, MRI reveals hippocampal atrophy, and hematoxylin and eosin staining shows neuron loss in olfactory and limbic areas, especially hippocampus. The present study tested whether DA-exposed sea lions develop temporal lobe epilepsy.

Methods: Hippocampi were isolated immediately after euthanasia from a DA group that consisted of 7 animals in which DA had been detected in feces or urine and/or they were in status epilepticus when admitted during an algal bloom, and 6 others that displayed signs of epilepsy including spontaneous seizures, abnormal EEG, and/or small hippocampal volume. Spontaneous, recurrent seizures were observed in 9/13 of these animals. In most cases, exact time of exposure to DA was unknown, but the minimum period between exposure and euthanasia was 6-210 d (51 $\pm 14 \mathrm{~d}$, mean \pm sem $)$. Control tissue was obtained from 11 sea lions that were euthanized because of pneumonia, cancer, or shark bite wounds that were unresponsive to treatment. After immersion-fixation hippocampi were sectioned transversely from the septal pole to the temporal pole. Adjacent series of sections were processed for Nissl stain, somatostatinimmunocytochemistry, and Timm stain. Hilar neuron numbers were estimated by the optical fractionator method.

Results: Nissl staining revealed obvious hilar neuron loss in 11/13 DA sea lions with variable additional loss of other hippocampal neurons. In addition, 2 animals originally categorized above as controls displayed hippocampal sclerosis. Control sea lions without hippocampal sclerosis had 333,000 $\pm 14,000$ hilar neurons/hippocampus. Of the 13 animals with hippocampal sclerosis, 3 displayed severe bilateral hilar neuron loss, but in 10 hilar neuron numbers were reduced only unilaterally. When unilateral hilar neuron loss occurred, it was extreme, with numbers down to $4-19 \%$ of the contralateral hippocampus. In contrast, in epileptic kainate-treated rats neuron loss is more symmetrical and graded, with hilar neuron numbers ranging from $2-88 \%$ of controls. Somatostatin-

immunoreactivity showed loss of many hilar interneurons but paradoxical exuberance of axons in the dentate gyrus of DA sea lions. Timm staining demonstrated aberrant mossy fiber sprouting into the inner molecular layer of the dentate gyrus in 13/13 in the DA group and in only $1 / 11$ controls.

Conclusions: DA-exposed sea lions display neuropathological findings, including unilateral hippocampal sclerosis and mossy fiber sprouting, that are similar to those reported for patients with temporal lobe epilepsy.

\subsection{0}

\section{EPILEPSY AFTER HEAD INJURY IN DOGS: A NATURAL MODEL OF POSTTRAUMATIC EPILEPSY}

Wolfgang Loscher ${ }^{1}$, S. Steinmetz ${ }^{2}$ and A. Tipold ${ }^{2}$ ( ${ }^{1}$ Department of Pharmacology, Toxicology, and Pharmacy, University of Veterinary Medicine, Hannover, Germany and ${ }^{2}$ Department of Small Animal Medicine and Surgery, University of Veterinary Medicine, Hannover, Germany)

Rationale: In humans, traumatic brain injury (TBI) is one of the most common causes of acquired (symptomatic) epilepsy, but as yet there is no treatment to prevent the development of epilepsy after TBI. Animal models of posttraumatic epilepsy (PTE) are important to characterize epileptogenic mechanisms of TBI and to identify clinically effective antiepileptogenic treatments. The prevalence and phenomenology of naturally occurring canine epilepsy is very similar to human epilepsy. However, the risk of epilepsy after TBI has not been systemically studied in dogs. We therefore performed a large retrospective study in 1004 dogs referred to our clinical department over a period of 11.5 years with the aim to determine the incidence of early and late seizures after head trauma in this species.

Methods: Two strategies were used: in group I $(\mathrm{n}=384)$, we evaluated whether dogs referred for the treatment of a trauma developed seizures after the trauma, while in group II $(n=620)$ we evaluated whether dogs referred for the treatment of recurrent epileptic seizures had a history of head trauma. Data for this study were obtained from our clinical database, questionnaires sent to the dogs' owners, and owner interviews.

Results: In group I, $6.6 \%$ of the dogs developed PTE. The risk of PTE increased with severity of TBI; $13.3 \%$ of the dogs with skull fracture developed PTE. In group II, $15.5 \%$ of the dogs with epilepsy had a history of head injury, which was significantly higher than the incidence of PTE determined for group I. Other causes of epilepsy in group II included brain tumors (8\%), brain infections (6.1\%), and cortical dysgenesis $(2.1 \%)$, while cerebrovascular disease did not play any significant role, because it is rare in dogs. Idiopathic or cryptogenic epilepsy was diagnosed in $63 \%$ of the dogs of group II. Conclusions: Our study indicates that head trauma in dogs is associated with a high risk of developing epilepsy. Thus, dogs with severe TBI are an interesting natural model of PTE that provides a novel translational platform for studies on human PTE.

\subsection{1 \\ EFFICACY OF VIGABATRIN AS AN ANTI-STATUS DRUG IN A RAT MODEL OF STATUS EPILEPTICUS}

Hussam Seif Eddeine and D. M. Treiman (Neurology, Barrow

Neurological Institute, Phoenix, AZ)

Rationale: To determine the anticonvulsant properties of the antiepileptic drug vigabatrin (VGB) in the treatment of lithiumpilocarpine ( $\mathrm{Li}-\mathrm{Pi})$ and the cobalt-homocysteine (Co-Ho) rat models of status epilepticus (SE).

Methods: Male Sprague-Dawley rats had epidural screw electrodes implanted in the skull for EEG recording. Two models of SE were tested, the first induced by intraperitoneal (IP) injection of lithium chloride ( $3 \mathrm{mmol} / \mathrm{kg}$ ) followed by subcutaneous (SC) injection of pilocarpine $(30 \mathrm{mg} / \mathrm{kg}$ ) 20-24 hrs later, and the second created by implanting cobalt $6 \mathrm{mg}$ in the left frontal region (F3 location) then inducing status by injecting homocysteine $5.5 \mathrm{mmol} / \mathrm{kg}$ IP 96-120 hours later. Following injection of pilocarpine or homocysteine, the EEG was monitored continuously. VGB solution was made by dissolving $300 \mathrm{mg}$ in normal saline. VGB monotherapy effect was tested at early SE, as well as at late refractory SE as a tertiary treatment following $0.1 \mathrm{mg} / \mathrm{kg}$ diazepam (DZM) and $0.25 \mathrm{mg} / \mathrm{kg}$ phenobarbital (PB).

Results: Early SE (SE EEG Stage I) was induced with Li-Pi in 4 groups of 3 rats. Each of these groups was treated with either 1000 , 2000,3000 , or $4000 \mathrm{mg} / \mathrm{kg}$ of VGB . No clear alteration in the progression through the 5 stages of SE was noted in these groups. Late SE (SE EEG Stage V) was induced with Li-Pi in 2 rats. VGB, $3000 \mathrm{mg} / \mathrm{kg}$, was given IP 10 minutes after DZM and PB. DZM/PB resulted in cessation of the clinical manifestation of $\mathrm{SE}$ as expected but without a clear alteration of the EEG findings, and the addition of VGB did not show a clear effect on the EEG findings. Both animals died within the following 36 hours. SE EEG Stage I was induced with Co-Ho in 2 rats. Both received VGB $3000 \mathrm{mg} / \mathrm{kg}$ after reaching Stage I. Both animals progressed through the 5 stages of status epilepticus. In the first animal, SE stopped around 8 hours later and the rat survived, the other rat died 1 hour after the onset of the status. Further evaluation of the VGB effect of VGB in late stage Li-Piinduced SE as well as early and late stages of Co-Ho SE is currently in progress.

Conclusions: SE remains as one of the life-threatening neurological emergencies that is frequently difficult to treat. Therefore, there is a 
great need to develop new treatments for SE. VGB has been found to increase GABA concentration in the frontal cortex but not in the hippocampus. Thus we chose to study two models of SE: a "cortical" model (Co-Ho) and a "hippocampal" model (Li-Pi). The preliminary results so far show that VGB has no effect on the early or late (Li-Pi) SE. This might be due to the fact that the maximum increase in GABA concentration has been reported to occur around 3 hours following VGB IP administration. Alternatively, VGB might have less effect on the "hippocampal" model of status epilepticus given that it has been shown to have no effect on GABA concentration in the hippocampus. This could explain why in one of the Co-Ho rats SE stopped and the rat survived. Further testing of the effect of VGB on the "cortical" model of SE is necessary and is currently in progress.

This research was supported by a grant from Lundbeck, Inc.

\subsection{2}

ACUTE MORPHOLOGICAL CHANGES IN KINDLED MICE Shatrunjai P. Singh ${ }^{1}$, X. He ${ }^{3}$, J. O. McNamara ${ }^{3}$ and S. C. Danzer ${ }^{2}$ ( ${ }^{1}$ Developmental and Molecular Biology Program, Cincinnati Children's Hospital Medical Center, Cincinnati, OH; ${ }^{2}$ Department of Anesthesia, Cincinnati Children's Medical Center, Cincinnati, $\mathrm{OH}$ and ${ }^{3}$ Department of Neurobiology, Duke University Medical Center, Durham, NC)

Rationale: Seizure activity is associated with changes in both hippocampal dentate granule cell morphology and integration. These changes are evident in different models of epilepsy including status epilepticus models, genetic models and traumatic brain injury models. All of these models are associated with substantial neuron loss and the development of spontaneous recurrent seizures. By contrast, it is possible in the kindling model to administer a limited number of stimulations sufficient to produce a lifelong enhanced sensitivity to stimulus evoked seizures without associated spontaneous seizures. Here we asked whether stimulations sufficient to induce enhanced sensitivity to evoked seizures, but not frank epilepsy, will induce morphological changes similar to those observed in other models of epilepsy

Methods: Thy-1 GFP expressing transgenic mice were used to analyze granule cell morphology in kindled and control animals. Morphological parameters examined included soma area, apical dendrite number, basal dendrite number, axon varicosity density, the relative position of the cell within the granule cell layer, spine density and the thickness of the granule cell layer. Animals were examined either one day or one month after experiencing five kindling-evoked seizures.

Results: We observed an increase in the thickness of the granule cell body layer and a decrease in the number of spines in the inner and outer molecular layers one day after kindling. No differences were observed between control and kindled animals among any of the other parameters examined. However, by one month after kindling, granule cells from control and kindled animals were

indistinguishable, indicating that the acute changes observed are transient.

Conclusions: These findings demonstrate that kindling epileptogenesis does not produce the striking rearrangements of granule cell structure seen in other models of epilepsy, such as hilar projecting basal dendrites - at least for the cell populations examined. Whether the absence of robust restructuring in these animals accounts for the absence of spontaneous seizures remains to be determined.

\subsection{3}

\section{LYN AS A POTENTIAL TARGET FOR GLIOMAS RELATED} WITH EPILEPSY

Alberto E. Musto ${ }^{1,3}$, O. Prakash ${ }^{2}$, S. Bhattacharjee ${ }^{1}$, B. Brandon ${ }^{1,3}$, C. Walker $^{1,3}$, W. Lukiw ${ }^{1}$, K. Reiss ${ }^{2}$ and F. Culicchia ${ }^{1}$ ( ${ }^{1}$ Neurosurgery Department, Louisiana State University Health Science Center, New
Orleans, LA; ${ }^{2}$ Cancer Center, Louisiana State University Health Science Center, New Orleans, LA and ${ }^{3}$ Neuroscience, Louisiana State University Health Science Center, New Orleans, LA)

Rationale: Glioblastomas show high incidence of epilepsy. The tyrosine kinase family protein, Lyn plays an important role in synaptic excitability mediated neuroinflammation. Accordingly, Lyn could mediate the mechanism between inflammation excitability in acquired epileptogenesis related to glioblastoma. The goal was to examine levels of Lyn in vivo and in vitro of glioma and in synaptosome extract from experimental temporal lobe epilepsy. Methods: Glioblastoma cells (U87MG) was implanted in the right striatum in adult female nude mice. Behavior was monitoring during 6 weeks. Total Lyn and phosphorylated Lyn were analyzed in glioblastoma cell culture in clinical relevant Lyn kinase inhibitor, was administered at different doses during cell growth. Status epilepticus (SE) was induced by intraperitoneal administration of pilocarpine followed by diazepam administration 90 minutes later in adult male C57BL/6 mice. Seizures were monitored using continuous videolocal field potential recordings during 7 days and then brain samples were collected for histology and western blot analysis according established protocols. Mice with spontaneous seizures only were selected for the study. Coronal sections of the brain were stained against anti p-Lyn and co-localized with synaptic markers. Dorsal hippocampal regions were analyzed through decovo-

immunofluorescence microscopy and imaging software.

Synaptosome was extracted from the total hippocampi and homogenized in sucrose solution, then stored for assay in a buffer with a cocktail of various protease inhibitors. Western Blots were performed in glioblastoma and synaptosome samples with the phosphorylated Lyn antibody and detected using chemiluminescence western detection kit. Immunoblot images were acquired using scanner and analyzed with imaging software.

Results: Level of phosphorylated Lyn increased in glioblastoma and also in synaptosome extracts from hippocampi of epileptogenic mice. Lyn activation was effectively inhibited by bafetinib. In addition pLyn IR like profiles increase in hippocampal layers and co-localized with synaptic markers.

Conclusions: Lyn activation could mediate neuronal hyperexcitability during acquired epileptogenesis. Inhibition of Lyn kinase may provide a new therapeutic target as antiepileptogenesis mediated by gliomas.

\subsection{4 \\ CHARACTERIZATION OF STATUS EPILEPTICUS IN A RAT MODEL OF ORGANOPHOSPHATE PARAOXON TOXICITY}

Laxmikant Deshpande, D. S. Carter, R. E. Blair and R. J. DeLorenzo (Virginia Commonwealth University, Richmond, VA)

Rationale: Nerve agents are amongst the most lethal chemical weapons developed. Organophosphate (OP) compounds such as DFP and paraoxon are pesticides that act similar to nerve agents. These compounds are readily available and therefore are attractive targets for being weaponized and used against civilian population by terrorists. OP compounds are irreversible inhibitors of acetylcholinesterase. Exposure to these compounds produces severe cholinergic symptoms and status epilepticus (SE) that if left untreated are fatal. Survivors of OP poisoning often exhibit chronic neurological disorders including acquired epilepsy and cognitive deficits. In this study we describe the development of a rat survival model of OP induced SE using paraoxon (POX) in order to realistically mimic both acute and long-term effects of nerve agent intoxication.

Methods: Male Sprague-Dawley rats (250-300 g) were injected with POX (4 mg/kg, ice-cold PBS, s.c.). The ensuing cholinergic crisis was behaviorally observed and SE was recorded on EEG using surface electrodes implanted in brain. Treatment of OP poisoning 
included interventions with atropine bromide (10 mg/kg, saline, i.p.), 2-PAM (25 mg/kg, saline, i.m.) and diazepam ( $5 \mathrm{mg} / \mathrm{kg}$, i.p.). Results: OP poisoning with POX $(4 \mathrm{mg} / \mathrm{kg})$ produced a rapid cholinergic crisis characterized by increased salivation, bradypnea, bradycardia followed by tonic-clonic jerks that evolved into unremitting seizures (SE) and a rapid death. Time to death following POX exposure was 6-8 min. Mortality was $100 \%$ when no therapeutic interventions were done. Treatment with atropine alone reduced acute mortality $(30 \%)$ but the chronic mortality was high ( $90 \%$ at 72 -h post POX exposure). Addition of 2-PAM to atropine further reduced both acute mortality (15\%) but chronic mortality was still very high $(80 \%)$. Addition of diazepam to this drug combination promptly terminated SE and markedly alleviated mortality. Acute mortality was $12 \%$ and chronic mortality was reduced to $18 \%$. The EEG profile for POX induced SE was marked similar to those reported for other OP's and nerve agents. EEG alterations following POX administration were rapid and correlated with behavioral seizure manifestations. Low-voltage fast activity started to appear with 2-3 min of POX administration. High-voltage slow activity appeared within the next minute and rapidly progressed into highvoltage spiking correlating with behavioral seizure activity by approximately 5-6 minutes. By this time animals were in electrographic SE with fully developed seizures. The seizures continue unabated for one hour at the end of which they are terminated using diazepam.

Conclusions: We have developed a rat survival model of OP intoxication using POX. Markedly improved survival rates were obtained by optimizing the three drug regimen for alleviating symptoms of POX poisoning. This model is ideally suited to test effective countermeasures for OP exposure and study molecular mechanisms underlying neurological disorders following OP intoxication.

\subsection{5 \\ GAINING INSIGHTS INTO EPILEPSY- NEUROPSYCHIATRIC COMORBIDITIES AND SUDEP: LESSONS FROM THE STUDY OF THE WISTAR AUDIOGENIC RAT (WAR) STRAIN}

Norberto Garcia-Cairasco ${ }^{1}$, E. M. Granjeiro ${ }^{1}$, G. Silva ${ }^{1}$, S. S. Marroni $^{1}$, M. Pereira ${ }^{1}$, E. Umeoka ${ }^{1}$, C. A. Silva ${ }^{1}$, H. Giusti ${ }^{1}$, J. A. Oliveira $^{1}$, M. L. Glass ${ }^{1}$, R. Fazan ${ }^{1}$, N. Montano ${ }^{2}$, H. C. Salgado ${ }^{1}$ and C. Costa Neto ${ }^{1}\left({ }^{1}\right.$ Ribeirao Preto School of Medicine, Ribeirao Preto, Brazil and ${ }^{2}$ Department of Clinical Sciences "Luigi Sacco", University of MIlan, Milan, Italy)

Rationale: The Wistar Audiogenic Rat (WAR) is a genetically selected strain susceptible to audiogenic seizures (AS) and a model for epilepsy studies. We demonstrated that AS express brainstemdependent tonic-clonic seizures and audiogenic kindling (AK) is a model of temporal lobe epilepsy. Using neuroethology, EEG, cellular and molecular approaches, we showed that WARs have innate alterations or evoked after AK. WARs have hypertension, tachycardia, increased sympathetic tonus, endogenous anxiety, hyperactivity of the HPA axis and endogenous compulsive grooming. Therefore, as a step forward, the main goals of this study were to characterize WAR strain endogenous cardio-respiratory responses with potential impact for studies of sudden unexpected death in the epilepsies (SUDEP).

Methods: Besides the protocols from previous studies from our laboratories, WARs were compared to Wistar animals for cardiorespiratory characterization in the presence of hypoxia $(7 \% \mathrm{O} 2)$ and hypercapnia (7\% CO2). Behavioral analyses were performed using neuroethological methods. Body plethysmography and temperature sensors were used to obtain, respectively, pulmonary ventilation and body temperature $(\mathrm{Tb})$. Additional evaluations were made in freely moving WAR and Wistar, with long-term ECG recordings to evaluate spontaneous arrhythmias and heart rate variability. Cardiac function was also evaluated using the pressure-volume conductance system in anesthetized rats.
Results: In the current studies the ventilatory responses of WARs were attenuated relative to Wistar animals during hypercapnia and hypoxia $(\mathrm{p}<0.01)$. This attenuation was associated with behavioral responses. Although decreases in Tb were observed in both groups, the $\mathrm{Tb}$ decreases were greater in the WARs during exposure to both hypercapnia and hypoxia $(\mathrm{p}<0.05)$. Electrocardiographic analysis showed that the basal cardiac interval (CI) was similar between WAR and Wistar rats. CI variability, studied by linear and non-linear approaches, strongly suggests an autonomic imbalance with sympathetic predominance in WAR, which is an isolated risk factor for sudden death. Arterial pressure and left ventricle systolic pressure were lower in WAR than in Wistar rats. WAR also exhibited indices of systolic and diastolic dysfunction and a significantly higher number of ventricular ectopic beats when compared to Wistar rats. Conclusions: Our current data on cardio-respiratory and thermoregulatory parameters clearly demonstrate an increase in various risk factors and therefore support the view that the WAR strain is a suitable model for SUDEP. Current and previous data from our laboratories show that with the progression of genetic selection, WAR animals express, besides epilepsy susceptibility, altered brain circuits and cardio-respiratory responses. This makes the strain a reliable genetic model of epilepsy-neuropsychiatry comorbidities and SUDEP. Institutional efforts, with the leadership of our Laboratory, are under way to make the WARs available worldwide. Acknowledgments: FAPESP, FAPESP-Cinapce, PROEX-CAPES, CNPq, FAEPA.

\subsection{6 \\ TEMPORAL TRANSCRIPT PROFILE OF BDNF AND NTRK2 GENES AFTER SEIZURE INSULT IN ADULT ZEBRAFISH BRAIN \\ Claudia Maurer Morelli, P. G. Barbalho,, R. Mangolin and F. C. Reis-Pinto (Department of Medical Genetics, University of Campinas - UNICAMP, Campinas, Brazil)}

Rationale: Brain-derived neurotrophic factor (BDNF) appears to be associated with epileptogenesis. Danio rerio, popular named as zebrafish, has been recognized for epilepsy studies; however, the temporal transcript profile of the BDNF in the zebrafish brain after an acute seizure is unknown. The main aim of this study was to investigate the transcript profile of $b d n f, n t r k 2 a$ and $n t r k 2 b$ genes in adult zebrafish brain after a seizure.

Methods: Adult zebrafish were separated in control group (CG, $n=6$ ) and seizure group ( $\mathrm{SG}, \mathrm{n}=6)$; a pool of two brains were used to compose one sample. Animals from SG were individually exposed to PTZ 15mM for 2-3 minutes and observed the three phases of epileptic behavior as previous described by Baraban et al. 2005. CG was submitted to the same handling condition and time as SG, but in PTZ-free water. Animals were anesthetized and their brains removed for RNA extraction, by TRIzol ${ }^{\circledR}$ protocol at $0.05 \mathrm{~h}, 12 \mathrm{~h}, 24 \mathrm{~h}, 48 \mathrm{~h}$ and $72 \mathrm{~h}$ after seizure. Quality of RNA was assessed by spectrophotometry and agarose gel electrophoresis. Reverse transcriptase quantitative-PCR using TaqMan (Applied Biosystems) assays was performed to evaluate the transcript profile of $b d n f$ and its receptors $n t r k 2 a$ and $n t r k 2 b$ genes. Runs were carried out in triplicate using $18 S$ as endogenous control (Applied Biosystems). The relative quantification (RQ) was calculated by the equation $R Q=2-\Delta \Delta C T$. This study was approved by animal ethical committee of our Institution.

Results: Comparisons between CG and SG showed an increase of $b d n f$ mRNA levels at $0.05 \mathrm{~h}$ after seizure, $\mathrm{RQCG}=0.27 \pm 0.01$ and $\mathrm{RQSG}=0.67 \pm 0.20$. No difference was found for $n t r k 2 b \mathrm{mRNA}$ levels at the same time (RQCG $=0.40 \pm 0.03$ and $R Q S G=0.31 \pm 0.06$ ), but a slight down regulation was found to $n$ trk $2 a$ (RQCG $=0.26 \pm 0.02$ and $\mathrm{RQSG}=0.17 \pm 0.02$ ). At $24 \mathrm{~h}$ and $48 \mathrm{~h}$, an up-regulation of $b d n f$ and $n t r k 2 b$ was found in $\mathrm{SG}$ group $(b d n f 24 \mathrm{~h}$ : RQCG $=0.59 \pm 0.10$ and RQSG $=1.02 \pm 0.27$; bdnf 48h: RQCG $=0.43 \pm 0.07$ and $\mathrm{RQSG}=$ $1.03 \pm 0.16$; ntrk2b 24h: RQCG $=0.60 \pm 0.07$ and $\mathrm{RQSG}=1.16 \pm 0.26$; 
$n$ trk $2 b$ 48h: RQCG $=0.35 \pm 0.06$ and $\mathrm{RQSG}=0.83 \pm 0.18)$. There are no difference between groups at $12 \mathrm{~h}$ and $72 \mathrm{~h}$. The relationship between ligand and receptors showed that the transcript of bdnf gene is more abundant than $n t r k 2 a$ and $n$ trk $2 b$ transcripts only immediately of seizure $(b d n f \mathrm{RQSG}=0.67 \pm 0.20$; ntrk $2 a \mathrm{RQSG}=0.17 \pm 0.02$; $n t r k 2 b$ RQSG $=0.31 \pm 0.06)$.

Conclusions: It has been reported that both BDNF transcript and protein levels are increased after seizures in patients and experimental models. In rats, Bdnf transcript levels have returned close to the control levels $12 \mathrm{~h}$ after seizure. Our results have yielded insights into a different pattern of transcript profile of the bdnf gene and its receptors after seizure insult.

Support: CNPq and FAEPEX.

Reference: Baraban SC, Taylor MR, Castro PA, Baier H.

Pentylenetetrazole-Induced Changes in Zebrafish Behavior, Neural Activity, and c-fos Expression. Neuroscience 2005; 131:759-768.

\subsection{7}

\section{THE EFFECT OF LEVETIRACETAM ON STATUS} EPILEPTICUS-INDUCED NEURONAL DEATH IN THE RAT HIPPOCAMPUS

\section{H. Song ${ }^{3,4}$, Dong-Jin Shin ${ }^{1}$, D. Lee ${ }^{2,3}$, H. Ryu ${ }^{2,3}$, H. Choi ${ }^{3,4}$ and T.} Kang $^{2,4}$ ( ${ }^{1}$ Neurology, Gachon University Gil Hospital, Inchon, Republic of Korea; ${ }^{2}$ Anatomy and Neurobiology, Hallym University, Chunchon, Republic of Korea; ${ }^{3}$ Neurology, Hallym University, Chunchon, Republic of Korea and ${ }^{4}$ Institute of Epilepsy Research, Hallym University, Chunchon, Republic of Korea)

Rationale: Although levetiracetam (LEV, 2S-(oxo-1pyrrolidinyl)butanamide, Keppra ${ }^{\circledR}$, UCB Pharma) has been reported to be well tolerated and effective in SE refractory to benzodiazepine (BDZ), there was little preclinical or clinical data concerning the outcomes of LEV in comparison to DZP, and VPA in SE-induced neuronal death.

Methods: To address this relevant lack of information, we have performed the preclinical study to investigate the effect of diazepam (DZP), valproate (VPA), and LEV alone, and the efficacy of LEV as an add-on treatment with DZP on the SE-induced neuronal death.

Results: In the present study, LEV ( $\geq 50 \mathrm{mg} / \mathrm{kg}$ ) was significantly effective to protect neuronal damages from SE in comparison to DZP and VPA. However, it is noticeable that LEV as an add-on drug with DZP could not alleviate SE-induced neuronal damage as compared to LEV alone, and showed the similar effect of DZP alone. VPA ( $\geq 100$ $\mathrm{mg} / \mathrm{kg}$ ) was significantly effective to protect neuronal damages from SE, as compared to DZP. In contrast to LEV, VPA (50 and 100 $\mathrm{mg} / \mathrm{kg}$ ) as an add-on drug with DZP significantly reduced SEinduced neuronal damage as compared to DZP alone, and showed the similar effect of VPA $(150 \mathrm{mg} / \mathrm{kg})$ alone.

Conclusions: These findings indicate that, unlike VPA, LEV may negatively interact with DZP, and suggest that LEV may be more effective to prevent SE-induced neuronal death as a first line drug than as a second line therapy after BDZ treatment, and that LEV as an add-on drug with $\mathrm{BDZ}$ may not provide any additional benefit to outcome of SE.

\subsection{8 \\ COMPARATIVE CHARACTERIZATION OF ORGANOPHOSPHATE-INTOXICATION AND LITHIUM- PILOCARPINE MODELS OF REFRACTORY STATUS EPILEPTICUS AND NEURODEGENERATION}

Doodipala S. Reddy, R. Kuruba and X. Wu (Neuroscience and Experimental Therapeutics, Texas A\&M Health Science Center, College of Medicine, Bryan, TX)

Rationale: Status epilepticus (SE), an emergency condition with continuous seizure activity lasting more than 30 minutes, causes significant mortality and morbidity. SE can cause devastating damage to the limbic system leading to cognitive impairment and risk of epilepsy. Benzodiazepines are the first-line drugs for the treatment of SE. However, 35-50\% patients exhibit partial or complete resistance to standard drugs, a condition known as refractory SE. Novel therapies are desperately needed for refractory SE, which is characterized by persistent seizures, progressive internalization of GABA-A receptors, and benzodiazepine resistance. However, there is currently no comparative analysis of animal models that recapitulates neurological features of refractory SE. In this study, we did comparative characterization of the organophosphate (OP)intoxication and pilocarpine models of refractory SE using electrographic, behavioral and neuropathological approaches and by determining diazepam's ability to abort seizures and neurodegeneration.

Methods: Persistent SE was induced chemically by lithiumpilocarpine regimen. OP intoxication was induced by exposure of rats to diisopropylfluorophosphate (DFP), an OP insecticide that, like nerve agents, causes SE and neuronal damage. One minute after DFP exposure, atropine sulfate and 2-PAM were administered to mimic available field treatment regimens. Diazepam was administered at 60 min after the onset of SE to attenuate seizures and ascertain benzodiazepine resistance. The onset and termination of SE was determined by video-EEG recordings for up to $24 \mathrm{~h}$. Animals were perfused at $72 \mathrm{~h}$ for neuropathological studies. Histological assessment was made by Nissl and Fluoro-Jade-B staining. The extent of neurodegeneration of principal and interneurons was determined by the NeuN and NP-Y immunostaining, respectively. Results: Pilocarpine and DFP-treated animals exhibited electrographic and behavioral SE for over $8 \mathrm{~h}$, which represent a state of refractory SE. Diazepam partially aborted seizures but sustained suppression of either behavioral or electrographic seizure activity was not evident, a profile indicative of refractoriness. At $72 \mathrm{~h}$ following $\mathrm{SE}$ induction, there was massive neuronal death in CA1, CA3 pyramidal regions, and in the dentate hilus regions. There was extensive neuronal damage in the amygdala, but such damage was not observed in the hypothalamus. The extent of neuronal damage was similar in pilocarpine and DFP models. Diazepam produced marginal protection against the SE-induced neurodegeneration in both models.

Conclusions: Pilocarpine and DFP model are associated with refractory seizures and neurodegeneration. In the DFP model, persistent seizure activity and neurodegeneration was comparable to pilocarpine model, therefore providing a field model of refractory SE that can be used for the development of new treatment approaches. ** Supported by NIH grant NS076426 \& NS051398 **

\subsection{9}

SEIZURE PREDICTION UTILIZING STATE OF VIGILANCE B. J. Gluckman ${ }^{1}$, M. Killmann ${ }^{2}$, W. Mader ${ }^{2}$, B. Schelter ${ }^{2}$, S. J. Schiff $^{1}$, M. Sedigh-Sarvestani ${ }^{1}$, S. Sunderam ${ }^{3}$, G. I. Thuku ${ }^{1}$ and S. L. Weinstein ${ }^{4}\left({ }^{1}\right.$ Penn State University, University Park, PA; ${ }^{2}$ University of Freiburg, Freiburg, Germany; ${ }^{3}$ University of Kentucky, Lexington, $\mathrm{KY}$ and ${ }^{4}$ George Washington University, Washington, DC)

Rationale: There is an established relationship between sleep and seizure states in animal and human models of epilepsy. There has also been a significant effort to develop seizure prediction algorithms both as a warning tool for patients and as part of closed-loop control systems. To date, published methods and corresponding performance have been difficult to implement and reproduce. Although it has been noted that false-prediction rates follow a diurnal variation (Schelter 2006), the state of vigilance (SOV) has not been used as a seizure prediction feature. We therefore examined the likelihood of seizure onset as a function of SOV in the chronic tetanus toxin model of temporal lobe epilepsy. We then quantify prediction performances utilizing SOV.

Methods: Long-Evans rats were focally injected in the ventral hippocampus with tetanus toxin to induce chronic spontaneous 
seizures. We use existing algorithms to classify SOV (Sunderam 2007) and identify seizures from 380 hours of recordings in 6 rats. SOV was classified in $10 \mathrm{~s}$ windows as either wake, rapid-eyemovement sleep (REM) or non-REM sleep (NREM). We utilize a forecast seizure probability as the predictor output, and quantify its performance with a Brier score (Jachan 2009). We consider three predictor models - one based on overall seizure rate, one on seizure rate and SOV, and one on SOV, time of day (TOD) and seizure rate. We considered the prediction formalism presented in (Schelter 2006b), and optimized performance with respect to seizure prediction horizon and seizure onset period. We separated the data into training and testing sets. Means and variances were then computed from this process.

Results: We find for this model that the likelihood of REM prior to seizure dominates all other states up to $180 \mathrm{~s}$ ahead of seizure onset. Visual verification of data suggests that seizures are arising from REM and not the transition out of REM. A predictor conditioned on SOV performs significantly better than the unconditioned seizure predictor. However, the addition of TOD to a predictor already conditioned on SOV does not further improve predictive power as indicated by the Brier score.

Conclusions: Our observations are in contrast to findings that REM is an effectively anti-epileptic state (Shouse 2000), and challenge prevailing hypothesized mechanisms of sleep-related epileptogenesis (Shouse 2000, Colom 2006). Furthermore, they support the notion that $\mathrm{SOV}$ is a primary biologically relevant feature for prediction of seizure. We further conjecture that it may overwhelm in significance other seizure prediction features, although that remains to be demonstrated.

Schelter B et al. Epilepsia 47(12):2057-70, 2006.

Sunderam S et al. J Neurosci Meth (163):373, 2007.

Jachan M et al. IFMBE Proceedings, 22(11):1701-1705, 2009.

Schelter B et al. Chaos 16, 013108, 2006b.

Shouse MN et al. Clin Neurophys 111(S2):9-18, 2000.

Colom LV et al. J Neurophys 95(6):3645-53, 2006.

\section{Translational Research: Human Studies}

\subsection{0}

\section{MRNA BLOOD EXPRESSION PATTERNS IN NEW ONSET IDIOPATHIC PEDIATRIC EPILEPSY}

Hansel Greiner ${ }^{1}$, P. S. Horn ${ }^{1,2}$, K. Holland ${ }^{1}$, J. M. Collins ${ }^{1}$, A. D. Hershey ${ }^{1}$ and T. A. Glauser ${ }^{1}\left({ }^{1}\right.$ Cincinnati Children's Hospital Medical Center, Cincinnati, $\mathrm{OH}$ and ${ }^{2}$ Department of Mathematical Sciences, University of Cincinnati, Cincinnati, $\mathrm{OH}$ )

Rationale: Determine if blood mRNA expression patterns in children with newly diagnosed untreated epilepsy are different than those in healthy controls. Determine the differential expression patterns between epilepsy subjects with generalized onset or partial onset seizures compared to healthy controls.

Methods: Blood was obtained from otherwise healthy pediatric subjects with newly diagnosed untreated epilepsy along with healthy pediatric controls. mRNA was isolated and hybridized to Affymetrix HGU $1332.0+$ microarrays. Analysis was performed using Genespring. Differentially expressed gene lists resulted from comparison of i) epilepsy and control groups and ii) seizure type subgroups with controls. Tissue expression and gene ontology analysis was performed using DAVID.

Results: Thirty-seven epilepsy patients and 28 controls were included. Overall, 575 genes were differentially expressed in subjects with epilepsy compared to controls. The generalized seizure subgroup versus control (GvC) gene list and the partial seizure subgroup versus control $(\mathrm{PvC})$ gene list were different $(\mathrm{p}<0.05)$. Tissue expression analysis identified almost half of the genes in $\mathrm{GvC}$ and $\mathrm{PvC}$ as brain based. Functional group analysis identified several biologically relevant pathways. In $\mathrm{GvC}$, these included mitochondria and lymphocyte activation. In $\mathrm{PvC}$, apoptosis, inflammatory defense and cell motion pathways were identified.
Conclusions: A unique, biologically meaningful mRNA expression pattern is detectable in the blood of pediatric subjects with new onset and untreated epilepsy. This analysis finds many similar pathways to those identified in brain studies examining lesional intractable epilepsy. Blood mRNA expression patterns show promise as a target for biomarker development in pediatric epilepsy.
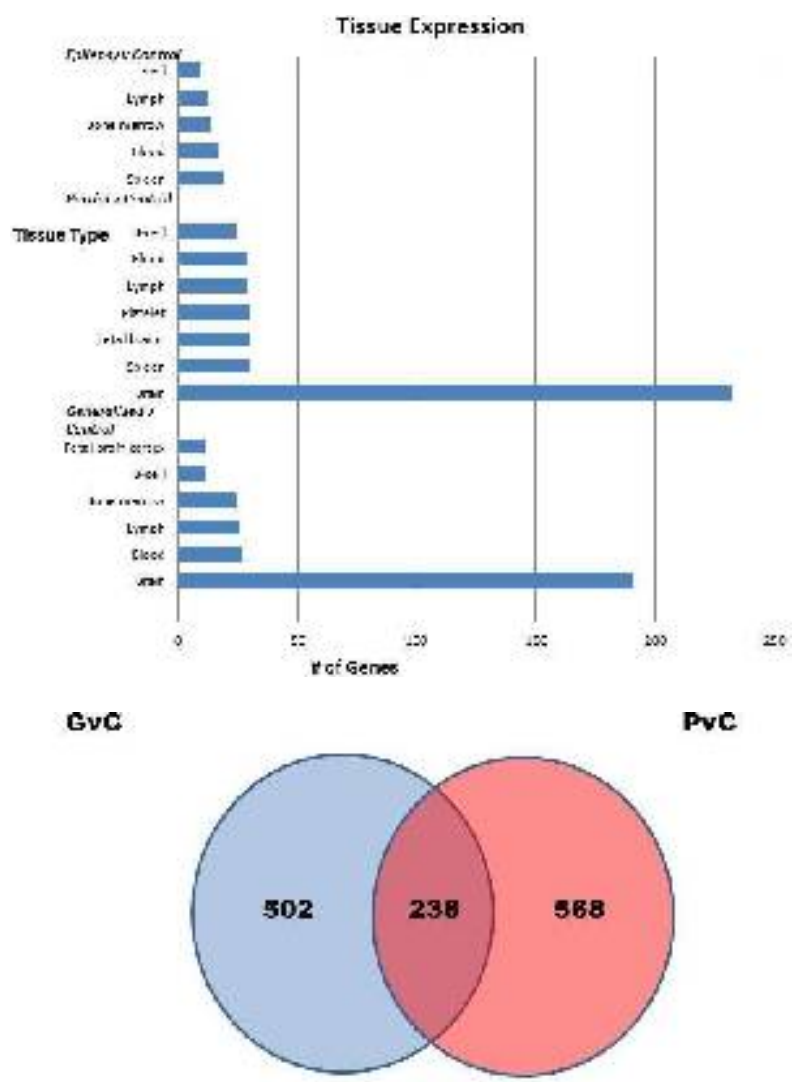

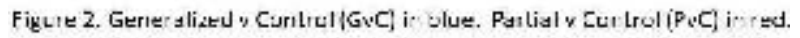

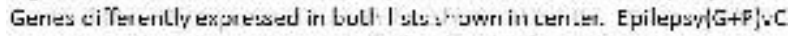
gene: not clfferently expressed ir $G W C$ or $P(n-2(n=9)$ no: snown.

\subsection{1 \\ MRI AND IMMUNOHISTOCHEMICAL EVIDENCE OF SEPTO-HIPPOCAMPAL CHOLINERGIC SYSTEM AUGMENTATION IN HUMAN TEMPORAL LOBE EPILEPSY}

Tracy Butler ${ }^{1}$, L. Zaborszky ${ }^{2}$, D. Talos ${ }^{1}$, P. Dilsiz ${ }^{1}$, X. Wang ${ }^{1}$, C. R. McDonald $^{3}$, K. Blackmon ${ }^{1}$, J. DuBois ${ }^{1}$, C. Carlson ${ }^{1}$, W. B. Barr ${ }^{1}$, J. French $^{1}$, R. Kuzniecky ${ }^{1}$, E. Halgren ${ }^{3}$, O. Devinsky ${ }^{1}$ and T. Thesen ${ }^{1,3}$ $\left({ }^{1}\right.$ New York University, New York, NY; ${ }^{2}$ Rutgers, Newark, NJ and ${ }^{3}$ UCSD, San Diego, CA)

Rationale: Basal forebrain septal nuclei provide the main cholinergic innervation to the hippocampus, and play a critical role in modulating hippocampal excitability. In animal models of epilepsy, destruction of septal cholinergic neurons facilitates seizures and epileptogenesis (e.g. Ferencz et al, Neuroscience 2001; Silveira et al, Dev. Brain Res. 2002), while septal stimulation is antiepileptic (e.g. Colom et al, J Neurophys. 2006; Miller et al, Epil. Res. 1994). Septal nuclei have received little study in human TLE.

Methods: Septal volume was measured in patients with pharmacoresistant temporal lobe epilepsy (TLE; $n=24$ ), extratemporal epilepsy (ETE; $n=23$ ) and controls $(n=89)$ using MRI and recently-developed probabilistic maps of human septal nuclei (Zaborszky et al, Neuroimage 2008.) To confirm results, an independent dataset consisting of 20 TLE patients and 25 controls was also analyzed. Expression of the high affinity choline transporter 
(HACT), a marker for septal cholinergic nerve terminals, was assessed in surgical specimens from patients who had undergone temporal lobectomy for intractable TLE and autopsy controls ( $\mathrm{n}=5$ /group) by immunocytochemistry and Western blot analysis. Results: Patients with TLE had significantly larger bilateral septal nuclei than patients with ETE and controls $(p<0.05)$. Patients with TLE demonstrated HACT upregulation in the hippocampus $(204 \pm 9 \%$ of control), but not in the temporal cortex ( $38 \pm 49 \%$ of control). Increased HACT expression was mostly observed in the in the molecular layer of the dentate gyrus.

Conclusions: These results demonstrate augmentation of the septohippocampal cholinergic system in patients with pharmacoresistant TLE. Similar results have been demonstrated in an animal model of TLE (Holtzman et al. J. Neurosci., 1995). We interpret septal enlargement in TLE as MR-detectable evidence of plasticity/augmentation of the normally-antiepileptic cholinergic septo-hippocampal system. Further investigation of this system could be relevant to development of new therapies such as septal stimulation for refractory TLE.

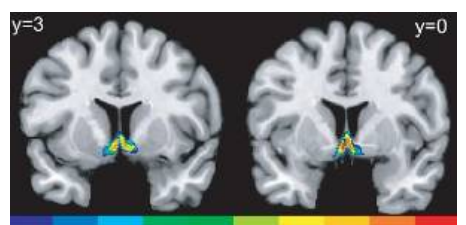

Septal nuclei probabilistic maps. Colors correspond to frequency of overlap in 10 brains (red=all 10 brains overlap; blue=1 brain).

\subsection{2}

HIGH-DENSITY EEG AND BEHAVIORAL PERFORMANCE DURING CHILDHOOD ABSENCE SEIZURES Jennifer Guo, S. Jhun, A. Kundishora, R. Kim, X. Bai, M. Negishi, G. Castellucci, J. Rodriguez-Fernandez, H. Mistry, C. Bailey, M. J. Crowley, R. T. Constable, L. C. Mayes and H. Blumenfeld (Yale University School of Medicine, New Haven, CT)

Rationale: Childhood absence epilepsy (CAE) seizures are seen as 3$4 \mathrm{~Hz}$ spike-and-wave discharges on electroencephalography (EEG). Behaviorally, seizures are characterized by temporary loss of consciousness. While CAE is considered a generalized type of epilepsy, prior studies show a frontal maximum in seizure activity on EEG and involvement of areas of the brain thought to be important for attention, including the frontoparietal network and thalamus, on fMRI. The relationship between EEG changes during absence seizures and behavioral impairment is not known.

Methods: We recorded 256-lead high density EEG in 20 pediatric patients as well as simultaneous 32-lead EEG and fMRI in 34 patients as they performed a continuous performance task (CPT) or an easier repetitive tapping task (RTT) requiring less attentional vigilance. Power across discrete frequency bands were compared for each electrode and determined for poor versus spared performance conditions. Signals during seizures from the high-density EEG were analyzed using source localization. Sources found with high-density EEG were compared to areas of activation in fMRI.

Results: Absence seizures were associated with worse performance during the continuous performance task (CPT) compared to the repetitive tapping task (RTT), though variable performance were observed across seizures and across patients. Seizures associated with poor behavioral performance ( $>75 \%$ omissions) showed greater frontal 3-4 Hz power compared to seizures with good behavioral performance ( $<25 \%$ omissions). Source localization for seizure activity using both distributed and single dipole models localized spikes to medial frontal cortex. Sources on high-density EEG showed some concordance with activated regions on fMRI.

Conclusions: Childhood absence seizures show variability in ictal task performance and power distribution on EEG. Abnormal activity in frontal cortex during absence seizures may be crucial for producing impaired attention in this disorder. Understanding electrophysiological and fMRI signals related to CAE could ultimately lead to improved treatments to prevent loss of consciousness during absence seizures.

\subsection{3 \\ UTILITY OF EEG PPR IN PREDICTING CHRONIC AED EFFICACY: A DOUBLE BLIND PLACEBO CONTROLLED STUDY OF LTG VS VPA IN JME}

Paul L. Timmings ${ }^{1}$ and D. G. Kasteleijn- Nolst Trenité ${ }^{2}\left({ }^{1}\right.$ Neurology Dept, University of Auckland, Waikato School of Medicine, Hamilton, New Zealand and ${ }^{2}$ Medical Genetics, University of Utrecht, Utrecht, Netherlands)

Rationale: JME is a common IGE but therapy is fraught with problems because optimal treatment involves VPA. Particular difficulties arise in selecting appropriate therapy for women of child bearing age because of risks of spina-bifida, other malformations and reduced IQ in their children.

For many of these patients prolonged trials of therapy to prove efficacy are not a viable option. Methods to rapidly assess likely effectiveness of alternative therapies are needed.

We propose that change (and suppression) of the EEG PhotoParoxysmal-Response (PPR) would provide an early indicator of efficacy.

Methods: We designed a 20 wk 1:1 randomised double blind 1-way cross-over parallel group study to compare VPA and LTG monotherapy in JME patients. At 4 weekly visits; LTG \& VPA doses were adjusted blindly on clinical grounds via a double dummy, tandem, dosing technique. And trough AED concentrations, seizure frequencies \& types, PPR during IPS and adverse effects were recorded. Only grade 3 or 4 PPR's were included. IPS testing was carried out and standardised to "SPR" utilising a standard protocol (Kasteleijn-Nolst Trenite et al., 2012).

Results: 74 JME patients were identified. All gave informed consent. Ethics approval was obtained prospectively. 40 were taking VPA monotherapy. 17 VPA Rx'd patients agreed to enter the study (M:F=13:4), nine of whom had PPR on previous EEG. At study entry 4 exhibited a persistent PPR.

At study end 2 VPA Rx'd patients still had PPR's and 4 LTG Rx'd patients had PPR's. All the LTG PPR's were higher than baseline. In the LTG group the PPR had risen and trended down again as dose was increased (Figure 1). In the LTG group myoclonic seizures had increased markedly but returned to baseline levels by study end associated with progressive LTG dose increases (Figure 2).

Conclusions: LTG initially suppressed PPR less effectively than VPA but a dose-response effect of LTG on PPR was identified. Efficacy of LTG for all types of JME seizures was demonstrated, also with a dose-response relationship. Increased LTG doses appeared to overcome transient worsening of myoclonus.

Change in PPR did correlate with LTG effect and may be utilised as an early indicator of efficacy.

Reference

Kasteleijn-Nolst Trenite D, Rubboli G, Hirsch E, Martins da Silva A, Seri S, Wilkins A, Parra J, Covanis A, Elia M, Capovilla G, Stephani U, Harding G. (2012) Methodology of photic stimulation revisited: Updated European algorithm for visual stimulation in the EEG laboratory. Epilepsia 53:16-24.

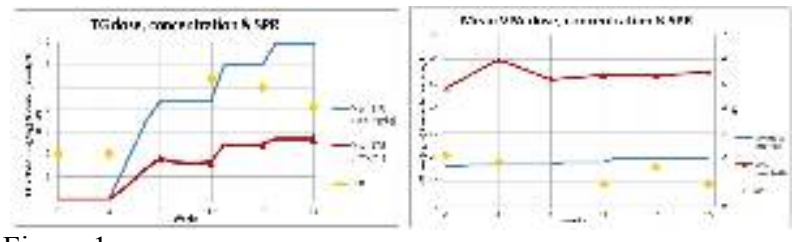

Figure 1 


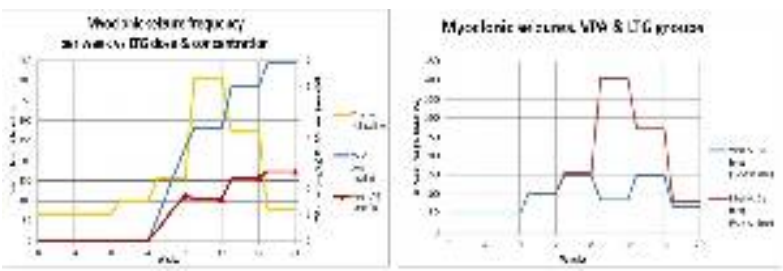

Figure 2

\subsection{4 \\ SLOW OSCILLATIONS COORDINATE FUNCTIONAL CONNECTIVITY BETWEEN RETROSPLENIAL CORTEX AND MEDIAL TEMPORAL LOBE: IMPLICATIONS FOR SEIZURE PROPAGATION AND MEMORY PROBLEMS IN PATIENTS WITH TEMPORAL LOBE EPILEPSY}

A. Kaveh, B. L. Foster and Josef Parvizi (Stanford University, Stanford, CA)

Rationale: Modern neuroimaging studies have shown the involvement of the human retrosplenial cortex (RSC) in episodic memory processing. However, due to its location deep in the brain and between the two cerebral hemispheres, the electrophysiological correlates of RSC function cannot be understood with scalp EEG. The RSC shares strong anatomical connections with regions involved in episodic memory processing, such as the medial temporal lobe (MTL). These regions both exhibit well characterized theta oscillations $(2-6 \mathrm{~Hz})$ that coordinate local neural activity and may mediate a functional interaction between RSC and MTL during memory processing.

Methods: Intracranial electrocorticography was recorded from RSC and MTL sites in 4 patients undergoing surgical evaluation for the treatment of refractory epilepsy. Responses were recorded from patients during self-paced true/false judgments of arithmetic and autobiographical stimuli.

Results: As predicted by their anatomical relationship, RSC and MTL were found to exhibit significant $(\mathrm{p}<0.05)$ functional connectivity selective to episodic memory processing. Phase locking (PLV) was found to increase with episodic memory stimuli (but not other control stimuli) in the hypothesized theta band $(2-6 \mathrm{~Hz})$ that was independently from concomitant theta band power increases. Furthermore, an increase in the RSC broadband power $(70-180 \mathrm{~Hz})$ followed the termination of theta band PLV between the RSC and MTL.

Conclusions: We have shown a role of slow oscillations in mediating memory selective communication between RSC and MTL.

Furthermore, our results suggest RSC's role in memory processing to be initiated by transitory involvement in a larger memory processing network including the MTL and hippocampus. Due to its anatomical and functional connectivity with the MTL, the RSC can be a key structure for propagation of ictal discharges and memory deficits in patients with temporal lobe epilepsy. It is also possible that seizures originating in the RSC may be clinically similar to MTL seizures.

\subsection{5 \\ OVEREXPRESSION OF ADENOSINE KINASE IN CORTICAL LESIONS FROM PATIENTS WITH TUBEROUS SCLEROSIS COMPLEX AND CORTICAL DYSPLASIA TYPE IIB}

Tianfu Li, F. Zhai, Y. Chen and G. Luan (Beijing Sanbo Brain hospital, Capital Medical University, Beijing, China)

Rationale: Tuberous sclerosis complex (TSC) and focal cortical dysplasia (FCD) type IIB, according to Palmini classification, share histopathologic similarities, specifically the presence of dysmorphiccytomegalic neurons and balloon cells, which are believed to be the epilepogenic cells and cause pharmacoresistant epilepsy. As our previous studies have demonstrated that overexpression of adenosine kinase (ADK) decreases extracellular adenosine and consequently leads to seizures and overexpression of ADK as a pathological hallmark of temporal lobe epilepsy, we hypothesized that the epileptogenic mechanisms underlying TSC and FCD type IIB is related to changes in ADK expression and that those changes might be associated with the development of epilepsy in these patients. Methods: Surgically resected human epileptic cortical specimens from TSC $(n=13)$ and FCD $(n=10)$ type IIB, we use immunohistochemistry approach to examine the expression of ADK and GFAP; double-label immunohistochemistry for coexpression of ADK and GFAP, and compared with normal control cortical tissue. Western blotting was used to quantify changes of ADK expression in TSC and FCD type IIB versus controls.

Results: We demonstrate that lesions in TSC and FCD type IIB contain a broad spectrum of abnormal cell types such as dysmorphic neurons, giant cells, balloon cells and reactive astrocytes.

Dysmorphic neurons, giant cells, balloon cells and reactive astrocytes express high levels of ADK, especially in balloon cells. In contrast, Nonplastic neurons in TSC and FCD type IIB express very low ADK levels The increased ADK expression within the dysplastic cortex of TSC and FCD type IIB was confirmed by Western blotting. Conclusions: The dysregulation of ADK in balloon cells and dysplastic neurons within cortical lesions of TSC and FCD type IIB suggested the possible role of this enzyme in the intrinsic and increased epileptogenesis of malformations of cortical development associated epilepsy. ADK may represent a potential antiepileptogenic target in the treatment of epilepsy in TSC and FCD type IIB.

\subsection{6 \\ DIFFICULTIES WITH RECRUITMENT OF TRAUMATICALLY BRAIN INJURED PATIENTS FOR A PROSPECTIVE POST-TRAUMATIC EPILEPSY STUDY}

Larsa Al-Omaishi, N. Theodore, L. J. Treiman and D. M. Treiman (Barrow Neurological Institute, Phoenix, AZ)

Rationale: Traumatic Brain Injury (TBI) is a well-known risk factor for post-traumatic epilepsy (PTE), especially among survivors of penetrating head injuries such as gun shot wounds (GSW), assaults, and blast injuries. We sought to enroll new civilian TBI patients into a prospective study designed to predict the onset of PTE through computerized analysis of electroenchalography (EEG) and microRNA biomarkers with a target enrollment of 90 patients. One critical component of a prospective study such as this is a reasonable expectation of enrollment and an understanding as to why a majority of TBI patients are poor study subjects.

Methods: This is an ongoing study. We screened all new TBI patients upon admission as a Level 1 trauma to St. Joseph's Hospital and Medical Center (SJHMC) beginning October 16, 2011. Criteria for enrollment included moderate to severe penetrating or closed head injuries (initial Glasgow Coma Score $(\mathrm{GCS})<13$ ), with no previous diagnosis of epilepsy, and not participating in another clinical trial. If the patient was unable to sign for him or herself, families were approached with information about the study and the legally authorized representative was asked to sign for consent. Results: To date (6/15/12) 111 patients who came through SJHMC as a Level 1 trauma patient who had sustained moderate to severe TBI (mean age: 38, GCS at admission: 7.1, mean lowest GCS: 5.7) have been screened for enrollment. Of these, 85 patients were ineligible for study for a variety of reasons; 33 died from their injuries (mean time from injury to death $=6.4$ days), 13 lived far from the Phoenix Metropolis and would be unable to return, 10 were not interested, 8 were chronic alcoholics, 7 had a history of seizure disorders, 4 were polysubstance abuse users with suicidal ideologies, and 12 were rejected for other reasons. Of the 26 enrolled, 3 died from their injuries, 5 suffered acute traumatic seizures (but have not yet developed PTE) and 5 were withdrawn. This means only $12.6 \%$ of all moderate to severe Level 1 TBI patients are active study participants. Mechanisms of injury were as follow: motor vehicle 
collision (30), auto vs. pedestrian (20), GSW (14), motorcycle crash (14), assault (11), fall (10), ATV accident (8), and other (4). Alcohol was a factor in $60 \%$ of all injuries; $36 \%$ of patients were positive for other substances including but not limited to cocaine, marijuana, and opiates, and amphetamines.

Conclusions: Prospective clinical studies of PTE after TBI which require long-term follow-up are difficult. Our experience has been that only 1 of 8 such patients $(12.6 \%)$ could be enrolled and continuously followed over six or more months. This experience should be considered when planning a long term follow-up study of moderate to severe TBI patients with either penetrating or closed head injuries.

\subsection{7}

FIRST UNPROVOKED SEIZURE WHILE DRIVING

Nina M. Hynick ${ }^{1}$, B. Pohlmann-Eden ${ }^{2,1}$ and K. Legg ${ }^{2}$ ( ${ }^{1}$ Dalhousie Medical School, Halifax, NS, Canada and ${ }^{2}$ Division of Neurology, QEII Health Sciences Center, Halifax, NS, Canada)

Rationale: Seizures while driving in known epilepsy is a welldocumented phenomenon with a wide range of medico-legal implications and impact on driving privileges. There is almost no data on patients who have no previous history of epilepsy and experience their first seizure while driving (FSWD).

Methods: Out of 311 patients who presented to the Halifax First Seizure Clinic (FSC) between 2008 and 2011, 158 patients were identified who strictly met the criteria of a strict first seizure (FS) or drug-naïve unknown new-onset epilepsy (NOE). A retrospective chart review was conducted. The goal of this study was to assess FSWD with regard of 1) prevalence, 2) clinical presentation, 3) coping strategies, and 4) Time of driving before accident.

Results: The prevalence of FSWD while driving was $8.2 \%$ (thirteen out of hundred and fifty-eight patients). 11 patients had generalized tonic-clonic seizures, one evolving from a complex partial seizure, three from visual auras. 2 patients had complex partial seizures, one starting with an autonomous seizure. Patients were i) able to actively stop the car ( $\mathrm{n}=4$, three of them had visual auras), ii) not able to stop the car resulting in accident ( $\mathrm{n}=7)$, and iii) passenger was able to pull the car over $(n=2)$. One accident was fatal to the other party. 11 out of 13 patients did drive for less than 1 hour, 2 more than 1 hour.

Conclusions: Our results support the theory that FSWD is a frequent and possibly unrecognized phenomenon in FS and NOE patients. It is therefore suggested to better document and evaluate this piece of history since FSWD often lead to accidents. Onset with simple partial seizures seems to give the driver some warning and to help to avoid accidents. Pathophysiological mechanisms remain speculative. While complex mental tasks have been considered to be rather seizureprotective than facilitating seizure, it cannot ruled out that critical visuo-motor tasks while driving may lower seizure threshold in predisposed individuals.

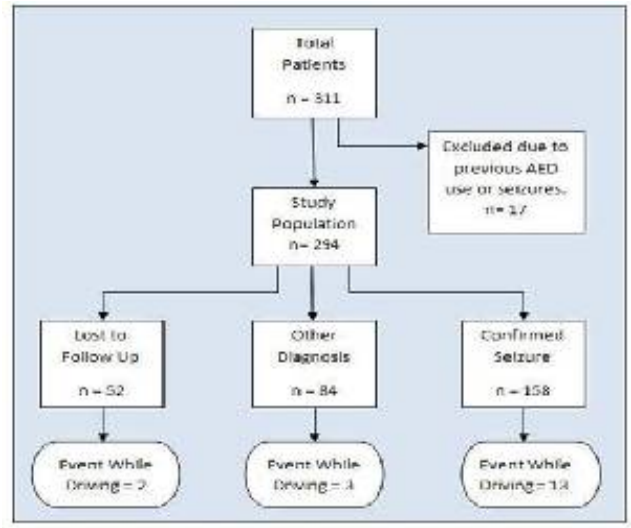

Finure 1: Allusestion of FSC pationse to Staḑ Papulation.

\subsection{8 \\ FUNCTIONAL MAPPING OF THE POSTEROMEDIAL CORTEX IN CONSCIOUS HUMAN SUBJECTS USING ELECTRICAL BRAIN STIMULATION}

B. Foster and Josef Parvizi (Stanford University, Stanford, CA)

Rationale: As a core node of the human default mode network (DMN), the posteromedial cortex (PMC) has been endowed with a wide variety of functional roles, but its unique contribution to cognition still remains unclear. Clinically, the PMC shows sensitive changes in metabolic and hemodynamic activity, as revealed by neuroimaging, during the loss or restoration of consciousness (e.g. generalized seizures, anesthesia, sleep and coma). Functional neuroimaging has also revealed the PMC, and DMN more generally, to be activated during tasks of putative 'self-referential' processing, such as autobiographical memory.

Methods: We studied the effects of direct electrical stimulation of the human PMC via intracranial electrodes over the mesial parietal and occipital cortices in 15 human subjects. All stimulations were delivered through a bipolar pair of proximal electrodes; which were classified as being within (PMC) or outside the PMC (non-PMC), based on formal anatomical boundaries within each subject using high-resolution electrode localization.

Results: From a total of 552 stimulations, we observed 277 reported effects (patient report, or observed behavior). Interestingly, the bulk of these responses could be characterized as either motor behavior (e.g. leg movement), or sensory phenomena reported by the patients as visual (e.g. phosphenes) or tactile (e.g. somatosensory tingling) sensation. Furthermore, the anatomical distribution of stimulations producing these effects conformed to an interesting functional configuration. Visual effects were observed for sites surrounding and inferior to the parietal-occipital sulcus (POS), whereas motor and sensorimotor effects were elicited from sites surrounding or superior to the marginal branch of the cingulate sulcus (mbCS). Consequently, this left a large number of stimulations $(n=128)$, which occurred within the PMC (i.e. in between POS and mbCS), which produced no reported or observed changes in perception or behavior. Whereby only $13.7 \%$ of the stimulations within the PMC, produced any reported or observed effects, however all of these were highly similar to the motor or sensory effects typically noted for regions outside of the PMC or on its boundary (indicative of charge spread). Consistent with these observations a non-parametric test of association revealed a significant difference in the occurrence of responsive stimulations between PMC and non-PMC sites (Chi-square $(2)=48.6, \mathrm{p}<.001)$. Conclusions: Although the PMC has been implicated in a host of higher order cognitive functions, including consciousness itself, direct causal perturbation of this region produced no profound changes in conscious state. Importantly, these stimulations included sites where clear functional responses were observed for autobiographical memory retrieval during experimental paradigms. Together such data promotes the notion that associative cortices, not involved in basic sensory transduction and detection, can only be functionally mapped under conditions of task related perturbation (i.e. during their associative/integration functional engagement).

\subsection{9 \\ FRONTAL LOBE EPILEPSY: THE NEUROETHOLOGY CONTRIBUTION TO SEMIOLOGY} Poliana Bertti ${ }^{1,2}$, A. P. Martins ${ }^{2}$, M. C. Dal-Cól ${ }^{2}$, V. C. Terra ${ }^{2}$, J. A. Cortes de Oliveira ${ }^{1}$, T. R. Velasco ${ }^{2}$, A. C. Sakamoto ${ }^{2}$ and N. GarciaCairasco ${ }^{1,2}$ ( ${ }^{1}$ Physiology Department, University of São Paulo, Ribeirão Preto, Brazil and ${ }^{2}$ Epilepsy Surgery Center, Neuroscience and Behavioral Science Department, University of São Paulo, Ribeirão Preto, Brazil)

Rationale: Frontal lobe epilepsy (FLE) represents the second most common form of focal epilepsies, occurring in approximately $20 \%$ of patients admitted to epilepsy surgery programs. Valuable information 
about the location of the epileptogenic zone (EZ) and seizure spread pathways can be obtained by the ictal semiology analysis. In temporal lobe epileptic patients, detailed and systematic observation of behavioral sequences (neuroethology) suggested localizing and lateralizing signs, and the coupling of neuroethology and SPECT data also contributed to the understanding of epileptic networks (Dal-Cól et al, 2006, Epilepsy Behav; Bertti et al, 2010, Epilepsy Behav). Our goal in the current study was to apply the neuroethology method to epileptic seizures in FLE.

Methods: We retrospectively studied a group of pharmacoresistant FLE patients successfully operated (Engel's class I; Engel, 1993) in our institution (CIREP/FMRP-USP). Behavioral sequences of each of the seizures, and also one minute of the preictal and postictal periods, underwent statistical analysis with ETHOMATIC software. The results were represented as flowcharts, with frequency, mean duration and statistical interaction between behaviors calculated by a chisquare test $(\mathrm{X} 2<3.84, \mathrm{P}<0.05)$. Approved by Ethics Committee (13528/2010).

Results: Eighteen patients, 9 adults (50\%), 3 teenagers (16.6\%) and 6 children older than 6 years $(33.3 \%)$ were selected. A total of 120 seizures were observed and three major types were present: tonic (supplementary motor area), focal clonic or complex partial seizures. Sleep preceded the seizure onset in 34 seizures $(28.33 \%$; $\mathrm{p}<0.001)$. Behavioral arrest was related with the seizure onset in 39 seizures $(32.5 \% ; \mathrm{p}<0.01)$. A series of behaviors were identified, such as automatisms, behavioral arrest, head deviation or version, dystonic, tonic and clonic postures, among others. Tonic postures were the most common, with unilateral or bilateral expressions, involving the extension or flexion or even abduction of the upper, lower or both upper and lower limbs. Head and eye version, unilateral tonic posture, speech arrest and vocalization had localizing or lateralizing significance. The postictal facial wiping $(\mathrm{FW})$ was observed in 11 patients $(61.1 \%)$ and with the exception of two, it was not executed exclusively ipsi or contralaterally to the EZ.

Conclusions: The neuroethology of FLS detected semiological types, evidenced behavioral sequences and showed items with lateralizing and localizing values, such as the presence of tonic postures, the head and eyes versions, in addition to complex movements. The statistical interactions, frequency and duration of signs proved to be suggestive of potential spread pathways. The pre and postictal analysis detected events previously described in literature, like sleep before the seizure onset, the rapid postictal recovery and also the lack of lateralizing value of postictal FW. Further studies involving a larger sample and also the association with electroencephalographic and neuroimaging data will be coupled by computational approach.

Financial support: CNPq, CINAPCE-FAPESP, PROEX-CAPES, FAEPA.

\subsection{0 \\ CHANGES IN NEURONAL ACTIVITY OUTSIDE OF THE SEIZURE FOCUS IN PATIENTS WITH TEMPORAL LOBE EPILEPSY}

Jason Naftulin, O. J. Ahmed and S. S. Cash (Neurology,

Massachusetts General Hospital and Harvard Medical School, Boston, MA)

Rationale: Focal seizures are considered to be focal because of their localized onset. However, our concept of the focus and even if there is a focus, is being increasingly questioned due to a variety of imaging and physiological data which suggests that there may be greater heterogeneity in activity both inside and outside of the traditionally defined seizure onset zone than expected. To further explore the behavior of areas outside of the presumptive focus we analyzed intracranial EEG data from patients with temporal lobe epilepsy.

Methods: Continuous intracranial EEG monitoring was used to localize seizure onsets for patients with longstanding pharmacoresistant epilepsy. In this study, we analyzed data from five such patients who were implanted with bilateral depth electrodes and also had at least one focal temporal lobe seizure. Fourier analysis was used to quantify changes in power over delta, theta, alpha, beta, gamma, and high gamma frequency bands in regions distant from the area of seizure onset. Seizure onset was determined by clinical electroencephalographers as the first electrographic change in particular channels.

Results: We analyzed data from 24 seizures in 5 patients. In 9 of those seizures there was an increase in power in the theta and/or alpha band in channels widely separated from the seizure onset zone which preceded the electrographically determined seizure onset by anywhere between 10 - $165 \mathrm{sec}$. As an example, in one seizure which began in the right mesial temporal structures, there was increasing activity in the left posterior temporal and subfrontal cortex $66 \mathrm{sec}$ before seizure onset.

Conclusions: Our results suggest that despite being "focal" in origin, seizure initiation may involve network interactions among disparate brain regions. However, it is also possible that there are non-specific, non-ictal increases in brain activity across a number of cortical and subcortical structures, with only the pathological region likely to convert these changes in activity into epileptic seizures. Furthermore, these changes may only reflect state changes (although obvious changes from sleep to wake or visa versa were not present at the time of seizure onset). More fine-grained analysis of the temporal ordering of these changes in neuronal activity is needed to select between these or other possibilities and help extend and refine our understanding of focality in localization related epilepsy. This work was funded by grants from the NIH (Grant NS062092) and the Epilepsy Foundation (Grant 222178).

\section{Translational Research: Devices, Technologies, Stem Cells}

\subsection{1 \\ MULTICOMPARTMENT COMPUTATIONAL MODEL OF LAMINAR NEOCORTEX EXHIBITING EPILEPTIFORM SPIRAL WAVES \\ William S. Anderson ${ }^{1}$, G. K. Bergey ${ }^{2}$ and P. J. Franaszczuk ${ }^{3,2}$ $\left({ }^{1}\right.$ Neurosurgery, The Johns Hopkins University School of Medicine, Baltimore, MD; ${ }^{2}$ Neurology, The Johns Hopkins University School of Medicine, Baltimore, MD and ${ }^{3}$ Human Research and Engineering Directorate, U.S. Army Research Laboratory, Adelphi, MD)}

Rationale: Computational simulations have been used in a variety of cellular and electrophysiological formats to explore the timefrequency and spatial properties of seizures and therapeutic interventions. We present here a multicompartment Hodgkin-Huxley based model of neocortex that exhibits realistic epileptiform behavior. The model has a three dimensional structure, and allows the user to explore local field potential recordings within it from a variety of current sources including total synaptic currents as well as specific synaptic currents between various cell classes. The model exhibits spiral wave behavior spontaneously as observed in experimental preparations.

Methods: The model represents a region of cortex of dimension 1.0 $\mathrm{mm}$ X $1.0 \mathrm{~mm}$, and includes 12 neuron classes organized by cortical layer, inhibitory or excitatory properties, and spiking characteristics. There are a total of 33,600 modeled Traub-like multicompartment neurons (range 59-137 compartments) that operate on a modified version of Hodgkin-Huxley dynamics [1]. The intercellular wiring is based on our previous modeling studies and is patterned after histological data [2]. A spatially uniform distributed Poisson background activity representing inputs from neighboring cortex affects a given percentage of the modeled neurons. Time-frequency analysis of the spontaneous bursting patterns is performed using the FFT. Local field potentials are recorded from the ongoing activity with a variety of simulated microelectrode recording elements and positions, and can be focused on specific sets of synaptic currents. The simulations are performed on a 16-node distributed 64-bit processor system in the parallel Genesis (pGenesis) environment [3]. 
Results: The activity in the model is characterized by a decreasing frequency spectrum in the LFP, with peaks at the primary network oscillation frequency and harmonics. Varying the global spatial extent of connections by all cell classes quickly alters the dynamics of the model. The LFP derived strictly from synaptic currents or subsets of synaptic currents continue to demonstrate the higher frequency components in the time-frequency spectrum indicating their likely importance in microelectrode recording efforts. Spatially evolving spiral wave dynamics are readily and spontaneously produced by the model.

Conclusions: This multicompartment simulation of neocortex demonstrating epileptiform behavior has been created in the freeware parallel Genesis (pGenesis) environment and currently runs on 16 computational nodes. It allows the user to selectively record LFPs from various synaptic current sources, or LFPs from arrays of superficially simulated microelectrodes. The time-frequency behavior of the modeled seizures is presented, and demonstrations of spiral wave activity similar to experimental preparations are shown. References: 1. Traub RD, et al. J Neurophys 2005;93:2194-2232. 2. Anderson WS, et al. Biol Cybern 2007;97:173-194. 3. Bower JM, Beeman D. The Book of GENESIS. Springer, NY, 1997. Support: Charles H. Hood Foundation (WSA), NIH-NINDS K08 (1K08NS066099-01A1) (WSA)
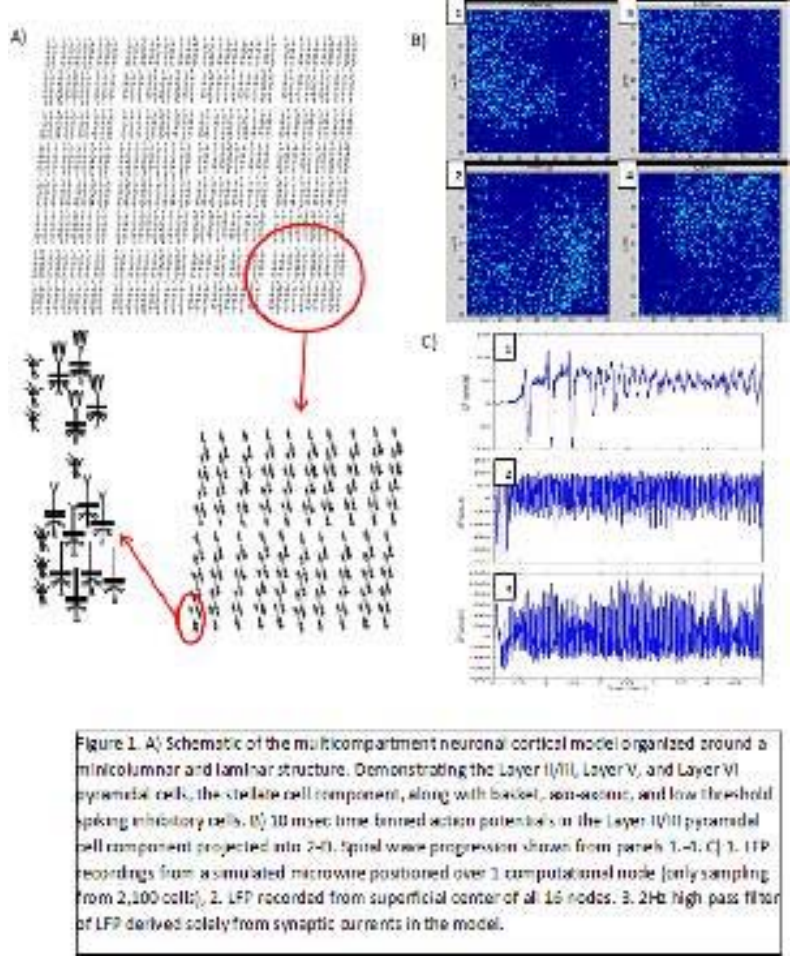

\subsection{2}

\section{A PERSONALIZED STEREOTACTIC FIXTURE FOR IMPLANTATION OF DEPTH ELECTRODES IN STEREOENCEPHALOGRAPHY (SEEG)}

Andrei Barborica ${ }^{1,2}$, R. Franklin' 1 , J. Ciurea ${ }^{3}$, I. Mindruta ${ }^{4}$, A. Rasina ${ }^{3}$, B. Balanescu ${ }^{3}$ and C. Donos ${ }^{2}\left({ }^{1}\right.$ FHC Inc, Bowdoin, ME; ${ }^{2}$ Physics Department, Bucharest University, Bucharest, Romania;

${ }^{3}$ Neurosurgery, Bagdasar-Arseni Emergency Hospital, Bucharest, Romania and ${ }^{4}$ Epilepsy Monitoring Unit, University Emergency Hospital, Bucharest, Romania)

Rationale: The SEEG implantation procedures still represent a challenge due to the intrinsic complexity of the method and the number of the depth electrodes required.

Methods: We have designed a custom stereotactic fixture based on the STarFix technology (FHC Inc, ME) that allows implantation of depth electrodes used in presurgical evaluation of patients with focal pharmacoresistant epilepsy. We used the traditional orthogonal approach, but oblique trajectories are supported as well. The fixture is built using 3D laser sintering technology based on a computer-generated model to fit each patient's anatomy. Fiducial markers that also serve as anchors for the fixture are implanted into patient's skull prior to the surgery. The 3D fixture model is designed to align with the patient's anatomy (AC-PC and mid-plane points), such that the frame coordinates are identical to the anatomical coordinates, making targeting of individual structures more intuitive and consistent across patients. A rectangular grid of guides orthogonal to the sagittal plane and centered on the mid-commissural point (MCP) can be accommodated in the platform design, allowing an arbitrary selection of orthogonal trajectories.

The frame has no adjustable parts, reducing the risk of inaccurate coordinate settings. Due to this feature, the procedure time is significantly reduced.

Results: We have used the fixture for the implantation of depth electrodes for presurgical evaluation of a patient with temporal lobe epilepsy, with an overall two-fold reduction of the time required for planning and implantation.

Conclusions: The custom stereotactic fixture design greatly simplifies the planning procedure, significantly reduces the OR time while maintaining a high accuracy.

\subsection{3 \\ BURSTS OF HIGH FREQUENCY REPETITIVE TRANSCRANIAL MAGNETIC STIMULATION SUPPRESS SEIZURES IN A RAT KAINATE STATUS EPILEPTICUS MODEL}

Roman Gersner $^{1}$, A. Zangen ${ }^{2}$, A. Pascual-Leone ${ }^{1}$ and A. Rotenberg $\left({ }^{1}\right.$ Neurobiology, Harvard Medical School, Boston, MA and ${ }^{2}$ BenGurion University, Beer-Sheva, Israel)

Rationale: Status epilepticus (SE) is characterized by frequent or prolong seizures, and pharmacologic treatment is not always effective. SE does not always respond to first or second line of anticonvulsants, and may require sedating anticonvulsants and need for intubation and intensive care support. Thus there is still a need for novel approaches to terminate the prolonged seizures of SE. Repetitive transcranial magnetic stimulation (rTMS) is a method for focal brain stimulation where small intracranial electrical currents are induced by a powerful fluctuating extracranial magnetic field. rTMS, especially when applied at low frequencies to reduce cortical excitability, is emerging as a therapeutic option in epilepsy. Yet the rTMS capacity to terminate ongoing seizures of SE, particularly with bursts of high frequency stimulation which may interrupt sustained cortical activity, has not been tested.

Methods: Nonconvulsive seizures were triggered by kainic acid (KA; $15 \mathrm{mg} / \mathrm{kg}$ ip) injection in adult Sprague Dawley rats previously anesthetized with urethane $(1.2 \mathrm{mg} / \mathrm{kg}$ ip). The urethane anesthesia enabled continuous EEG seizure monitoring and rTMS in immobilized subjects. Rats were divided into three groups to receive (1) KA and verum rTMS (ten $3-\mathrm{sec} 20 \mathrm{~Hz}$ trains, $30 \mathrm{sec}$ intertrain interval, $80 \%$ machine output intensity; $\mathrm{n}=7$ rats), (2) KA and sham rTMS ( $8 \%$ machine output intensity and otherwise identical to the verum rTMS group; $n=7$ rats), or (3) no KA and sham rTMS identical to group 2 ( $\mathrm{n}=6$ rats).

Results: Nonconvulsive seizures were reliably triggered in all KA injected rats. In the verum rTMS group spike frequency was significantly reduced during ( $46 \%$ sham; $\mathrm{p}=0.037)$ and after $(68 \%$ sham; $\mathrm{p}=0.045$ ) treatment in comparison to sham rTMS, while rats receiving no KA and sham rTMS had no EEG spikes at any stage. In addition, Fast Fourier Transformation analysis of EEG, revealed a significant reduction in the 1.6 to $3.2 \mathrm{~Hz}$ activity band in the verum rTMS group after treatment ( $\mathrm{p}=0.048$ ).

Conclusions: Our data suggest that high-frequency burst rTMS, a protocol which may be applied more rapidly, particularly in the 
emergent setting, than low frequency rTMS, has antiepileptic potential. The data also indicate that the rTMS effect outlasts the stimulation train. More research on burst stimulation rTMS modalities in epilepsy is warranted.

\subsection{4 \\ IDENTIFICATION OF SEIZURES IN PROLONGED VIDEO- EEG RECORDINGS}

J. L. Carlsen ${ }^{1}$, H. L. Grabenstatter ${ }^{1}$, R. Lewis ${ }^{2}$, C. Mello ${ }^{2}$, A. R. Brooks-Kayal $^{1}$ and Andrew M. White ${ }^{1}$ ( ${ }^{1}$ Pediatrics, Children's Hospital Colorado, Denver, CO and ${ }^{2}$ Computer Science, University of Colorado - Colorado Springs, Colorado Springs, CO)

Rationale: To determine the efficacy of different agents in the prevention or mitigation of epilepsy, it is necessary to identify the presence of seizures in prolonged (greater than 1 month) video-EEG recordings. Currently, most investigators employ expert personnel to determine the presence and frequency of seizures, resulting in both large time commitments as well as great expense. In this presentation, we describe an automated method that can be used to accurately and efficiently identify both latency period and seizure frequency.

Methods: Rats used in this study were implanted with bilateral subdural electrodes above temporolimbic cortex (1) prior to and (2) 4 weeks after pilocarpine treatment to determine the accuracy of the algorithm for detecting the initial spontaneous seizures and late spontaneous seizures $>1$ month after pilocarpine-induced status epilepticus. Both sets of rats were monitored using video-EEG for periods of approximately 1 month. The EEG recordings were then reviewed by an expert technician with Racine seizure stage being confirmed on video recording. The autonomous algorithm was implemented using subroutines written in Visual Basic, implemented on a Windows platform. The software exploits characteristics present in EEG recordings of seizures: (1) a change in frequency or amplitude, (2) an increase in coherence between the two recorded channels, (3) a decrease in dimension of the signal during a seizure, (4) characteristic evolution of the seizure, and (5) the presence of EEG changes following the seizure. The algorithm developed uses a novel, efficient technique for identifying changes in frequency and amplitude in which a local mean line is constructed. The areas above and below the line and line crossing distances are determined and averaged over a fixed time interval. A metric is then determined based on these parameters. The calculation of the metric is independent of the individual rat.

Results: A total of over 1000 hours of video-EEG were analyzed by the expert and using the computer algorithm. There were a total of 1260 seizures identified by the expert. The algorithm identified $100 \%$ of the seizures. The algorithm had a false positive rate of 0.063 per hour. False positives were more likely in the record early after status epilepticus, or at times when the amplitude and duration of seizures decreases. Signal artifact substantially increased the number of false positives.

Conclusions: These results demonstrate that we have developed an accurate algorithm for identifying seizures in prolonged video-EEG recordings employing several novel techniques. This algorithm will significantly decrease the time and cost of identification of seizures in prolonged EEG recordings. Further testing will be performed to demonstrate efficacy in other animal models and with signals containing significant artifact. Additional work will also be performed to create an adaptable network which will learn the pattern for each individual rat, thereby reducing the number of false positives.

Acknowledgements:

Work was performed using support from grants 5 K08 NS053610-05 (AMW) and NIH R01 NS051710 (ABK).
2.045

INTERNEURON PRECURSORS DISTRIBUTE IN A LAYERSPECIFIC MANNER FOLLOWING TRANSPLANTATION INTO NEOCORTEX AND PROPORTIONALLY INCREASE TONIC INHIBITION

Joy Sebe and S. C. Baraban (Neurological Surgery, University of California, San Francisco, San Francisco, CA)

Rationale: Previously, we demonstrated that transplantation of interneuron precursors into epileptic mice dramatically suppresses spontaneous seizures (Baraban et al. 2009). Precursors are obtained from a transient embryonic structure - the medial ganglionic eminence (MGE) - that produces the majority of cortical interneurons. MGE cells dissected from mouse embryos and transplanted into a postnatal mouse brain exhibit an impressive capacity to migrate and differentiate into various functional interneuron subtypes. Here, we further examined how grafted interneuron precursors anatomically and functionally integrate into the host neocortex.

Methods: Interneuron precursors from MGE were isolated from E13.5 GFP+ mouse embryos. MGE cells were injected into the neocortices of postnatal day 3 host mice and graft recipients were sacrificed 30-40 days later. Immunostaining for GAD67, parvalbumin (PV), somatostatin (SOM), neuropeptide Y (NPY), calretinin (CR), DAPI and layer-specific markers was performed. GABA-mediated tonic currents were recorded from host L2/3 pyramidal cells in the presence of GABA uptake blockers followed by bath application of SR95531 $(100 \mu \mathrm{M})$. GFP+ cell number within a 40x field of view that neighbored the recorded pyramidal neuron was counted in the living slice

Results: Grafted interneuron precursor cells preferentially migrated to GAD67-immunoreactive (IR) and cell sparse layers of the host neocortex (L1 and L5), and filled these layers with extensive neuritic processes. GFP+ cell somas and processes formed a distinct band within L1 and L5 such that grafted cells appeared to encounter a barrier repelling them from neighboring layers. Further, we are examining whether grafted interneurons subtypes, namely the CR-, PV- and SOM-IR cells, exhibit the same layer preferences as their endogenous counterparts in the host brain. Given that GFP cell density is not uniform throughout the neocortex, we recorded tonic GABA currents from host pyramidal cells surrounded by varying numbers of grafted derived GFP+ cells. We found that tonic current increases proportionally with the number of neighboring GFP+ cells. Conclusions: Grafted MGE-derived interneuron somas and their processes prefer GAD67 and cell sparse neocortical layers of the host brain. These studies suggest that grafted cells may (i) respond to endogenous GABA within these neocortical layers or (ii) prefer neocortical layers that are less densely packed and can thereby accommodate new neurons and synapses. Further, the spatial variations in interneuron density following transplantation may affect the amount of tonic inhibition in the host brain.

\subsection{6 \\ LOCAL BRAIN HYPOTHERMIA SUPPRESSES PERIFOCAL EPILEPTIFORM DISCHARGES INDUCED BY FOCAL CEREBRAL ISCHEMIA IN RATS \\ Yeting $\mathrm{He}^{1}$, M. Fujiii ${ }^{1}$, T. Inoue ${ }^{1}, \mathrm{~S}$. Nomura ${ }^{1}$, Y. Maruta ${ }^{1}$, Y. Owada $^{2}$, H. Kida ${ }^{3}$, T. J. Yamakawa ${ }^{4}$, T. Tokiwa ${ }^{5}$, T. Yamakawa ${ }^{5}$ and M. Suzuki ${ }^{1}{ }^{1}$ Neurosurgery, Yamaguchi University School of Medicine, Ube, Japan; ${ }^{2}$ Organ Anatomy, Yamaguchi University School of Medicine, Ube, Japan; ${ }^{3}$ Systems Neuroscience, Yamaguchi University School of Medicine, Ube, Japan; ${ }^{4}$ Electrical and Electronics Engineering, Faculty of Engineering, Shizuoka University, Hamamatsu, Japan and ${ }^{5}$ Life Science and Systems Engineering, Kyushu Institute of Technology, Kitakyushu, Japan)}

Rationale: In acute ischemia, high extracellular potassium and excess glutamate release from the ischemic core lead to 
depolarization and increased metabolic demand in neighboring neural tissues. These conditions induce periodic epileptiform discharges (EDs) and brain injuries. On the other hand, we have reported that focal brain cooling (FBC) terminates EDs in drug-induced seizure models. Therefore, the aim of this study was to investigate whether FBC also has a favorable effect on focal cerebral ischemia in rats. Methods: Healthy male adult Wistar rats weighing $350 \pm 50 \mathrm{~g}$ were used in the study. All surgical procedures were conducted under the guidelines of the Yamaguchi University School of Medicine. Under general anesthesia, a small craniotomy was made and focal cerebral ischemia was induced with the photothrombosis technique (illumination after intravenous injection of Rose Bengal at 1.3 $\mathrm{mg} / 100 \mathrm{~g}$ of body weight in $0.9 \%$ sterile saline) in the primary sensorimotor area (SI-MI). An additional larger craniotomy was made in the SI-MI area and FBC was performed at a temperature of $15^{\circ} \mathrm{C}$ for $5 \mathrm{~h}$. Electrocorticograms (ECoG) were recorded with needle-type electrodes placed on the cortex at the border zone of the ischemic focus for $5 \mathrm{~h}$ during cooling and for $1 \mathrm{~h}$ after cooling. After recording of the ECoG, rats were sacrificed and the infarct area was measured with triphenyltetrazolium chloride (TTC) staining. Seven rats each were used in the cooling and non-cooling groups.

Results: FBC suppressed all the ECoG frequency bands $(1-30 \mathrm{~Hz})$ during and after cooling $(\mathrm{p}<0.05)$, except for the delta frequency band in the precooling versus rewarming periods. The injured areas (sections at the ischemic core level) in the cooling and non-cooling groups were $0.99 \pm 0.30$ and $1.71 \pm 0.54 \mathrm{~mm}^{2}$, respectively. Lesion size was significantly reduced in the cooling group $(\mathrm{p}=0.026)$. Conclusions: FBC suppresses periodic EDs and has a protective effect on focal cerebral ischemia. These results suggest that FBC has potential for treatment of cerebral infarction.

\subsection{7}

\section{MAGNETICALLY INSERTED MICROELECTRODE AND CHRONIC CHARACTERIZATION IN VIVO}

Henry Zhang, I. Dryg, H. Mei, K. Qing and P. Irazoqui (Biomedical Engineering, Purdue University, West Lafayette, IN)

Rationale: The principal challenge in achieving long-term, high fidelity neural recording is maintaining long-term viability of the electrode-tissue interface. One reason for this challenge is the need for rigid, strong, large electrodes - thin, flexible electrodes buckle from the force of insertion. Using rigid, large electrodes leads to trauma from insertion, long-term damage, and encapsulation at the electrode-tissue interface due to aggravation of the foreign-body response. This damage and encapsulation results in a loss of signal fidelity over time. Previous studies showed macrophage and astrocyte clusters at the electrode interface of $165 \mu \mathrm{m}$ diameter electrodes; while $25 \mu \mathrm{m}$ diameter electrodes showed little to no scarring. By reducing the mismatch of the mechanical properties between the electrode and tissue, trauma is minimized. However, the $25 \mu \mathrm{m}$ electrodes require numerous steps in fabrication and showed variability in accuracy and depth of magnetic insertion. Thus, a new electrode design is desirable.

Methods: The procedure uses a magnetic insertion system where a microelectrode is loaded in an anti-static ejection tube inside a driver coil. A current pulse is discharged through the coil, generating a transient magnetic field in the ejection tube and accelerating the electrode through a craniotomy into brain tissue. Through a growing relationship with Fort Wayne Metals, we are in the process of testing their customized $25 \mu \mathrm{m}$ and $10 \mu \mathrm{m}$ microelectrodes. These electrodes are made from a ferromagnetic and biocompatible alloy, Fe60Pt. They are insulated with parylene $\mathrm{C}$, and magnetically accelerated into rat deep brain structures. The microelectrode accelerates at high enough speeds to prevent buckling, overcoming the disadvantage of microelectrodes. The implanted end of the electrode interfaces with brain tissue while the other end protruding from the skull is connected to circuit pins, which can connect to external circuitry. Electrochemical impedance spectroscopy (EIS) is used on awake, behaving animals to assess the reactive tissue response and infer the degree of electrode encapsulation.

Results: Long-term implantation and recording of $25 \mu \mathrm{m}$ electrodes in a rat using the magnetic insertion system is demonstrated. The new Fe60Pt microelectrodes offer advantages over stainless steel microelectrodes by being more biocompatible and therefore foregoing gold plating. This simplifies the electrode production process and may increase consistency with insertion and recording. Additionally, this new material exhibits more ferromagnetism, allowing for greater electrode accuracy and depth control. Conclusions: Chronic performance of flexible microelectrodes implanted using the magnetic insertion system and integrated with recording circuitry is supported, although additional investigation is required. This technology allows for the chronic monitoring of small neural populations in live rodent models, possibly improving implantable deep brain stimulation (DBS) devices for the treatment of many neurological diseases including epilepsy by reducing the foreign-body response at the electrode-tissue interface.

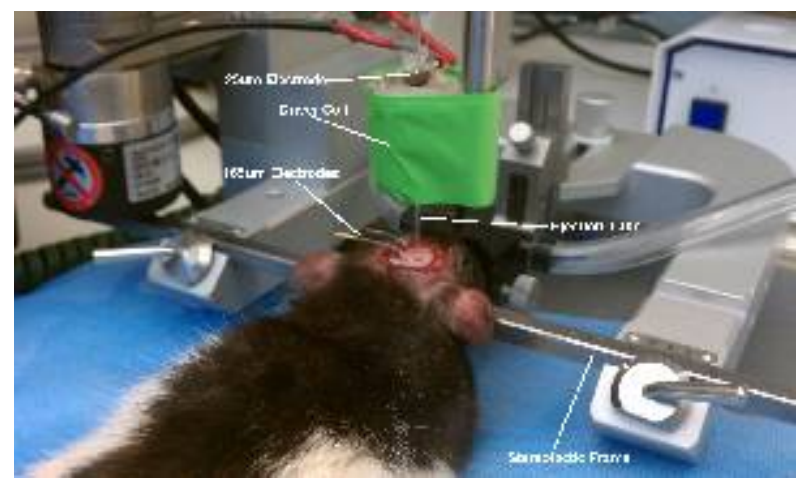

\subsection{8 \\ MAPPING EPILEPTIC NETWORKS USING THE INTERNATIONAL EPILEPSY DATA PORTAL}

Joost Wagenaar $^{1}$, W. C. Stacey ${ }^{3}$, A. Schulze-Bonhage ${ }^{4}$, A. Dümpelmann $^{4}$, I. Osorio ${ }^{5}$, A. Lyubushin ${ }^{5}$, H. P. Zaveri ${ }^{6}$, C. A. Schevon ${ }^{7}$, S. S. Cash ${ }^{8}$, Z. Ives ${ }^{1}$, M. Stead ${ }^{2}$, B. Brinkmann ${ }^{2}$, J. Echauz $^{1}$, V. Vasoli ${ }^{2}$, G. Worrel ${ }^{2}$ and B. Litt ${ }^{1}\left({ }^{1}\right.$ The University of Pennsylvania, Philadelphia, PA; ${ }^{2}$ Mayo Clinic, Rochester, MN; ${ }^{3}$ University of Michigan, Ann Arbor, MI; ${ }^{4}$ University of Freiburg, Freiburg, Germany; ${ }^{5}$ University of Kansas, Kansas City, GA; ${ }^{6}$ Yale University, New Haven, CT; ${ }^{7}$ Columbia University, New York, NY and ${ }^{8}$ Harvard University, Boston, MA)

Rationale: More than ever before, breakthroughs in neuroscience are generated by multi-center collaborations. Central to these collaborations is the ability to rapidly collect, annotate and analyze shared data across geographically dispersed centers. We present a new, cloud based, platform for this purpose, centered on epilepsy and funded by NINDS. The platform provides a web-based viewer for data and results, computational power for parallel analysis, a repository for sharing algorithms and their source code, and communication between collaborating centers. It allows the user to analyze the data by providing direct access to the data using commonly used software development platforms such as Matlab, and provides the capability to run analysis in parallel on multiple worker nodes to maximize efficiency and speed.

Methods: In a collaborative effort to demonstrate the effectiveness and features of the international EEG portal, data from a single patient was analyzed by 6 independent epilepsy research centers across the world. The analyzed dataset contained multichannel data belonging to a patient with neocortical epilepsy implanted with macro- and micro-wire electrode arrays, which were sampled at $5000 \mathrm{~Hz}(0.5-1000 \mathrm{~Hz}$ band-pass filtered). Investigators were free to use any methods they would like and were asked to upload their results to the database. The interaction with the portal was optimized 
to impose minimal changes to code the user might already be using locally. Four centers provided a high frequency oscillations (HFO) detector; one center developed a spike detector and one center provided a seizure predictor. The entire experiment was conducted over 2 weeks.

Results: HFO detections correlated with the vicinity of the clinicianmarked seizure onset zone, though there was great variability in the number of HFOs detected across different algorithms. This highlights the need for a centralized EEG platform where results from analysis can be compared on standardized datasets. Further investigation will allow quantitative comparisons between the qualities of the provided algorithms. Localization of interictal spikes did not correlate well with seizure onset zone and did not correlate well with the HFO events.

Conclusions: Sharing data and validating analysis using a standardized dataset requires significant efforts from the epilepsy research community, but has potential to greatly accelerate collaborative research. This is not uncommon in other research fields. Our results clearly identify the need for shared data sets that can validate and compare algorithms from different research centers. With this platform in place, collaborative research using these tools can be rapidly accelerated. Challenges will include aligning incentives for investigators to share data, algorithms and knowledge, as current systems for funding and promotion can discourage these endeavors.

\section{Neurophysiology: ICU EEG}

\subsection{9}

NON-CONVULSIVE SEIZURES AND NON-CONVULSIVE STATUS EPILEPTICUS IN A NEUROLOGICAL INTENSIVE CARE UNIT: RISK FACTORS AND OUTCOMES

Ikuko Laccheo $^{1}$, H. Sonmezturk ${ }^{1}$, J. Barwise ${ }^{2}$, L. Tomycz ${ }^{3}$, Y. Shi ${ }^{4}$, M. Ringel ${ }^{5}$, G. Dicarlo ${ }^{5}$, B. Abou-Khalil ${ }^{1}$ and K. Haas ${ }^{1}{ }^{1}$ Division of Epilepsy, Department of Neurology, Vanderbilt University Medical Center, Nashville, TN; ${ }^{2}$ Division of Anesthesiology Critical Care Medicine, Vanderbilt University Medical Center, Nasvhille, TN; ${ }^{3}$ Department of Neurosurgery, Vanderbilt University Medical Center, Nasvhille, TN; ${ }^{4}$ Department of Biostatistics, Vanderbilt University Medical Center, Nasvhille, TN and ${ }^{5}$ Division of Neurodiagnostics, Department of Neurology, Vanderbilt University Medical Center, Nasvhille, TN)

Rationale: Previous studies show the best estimate of non-convulsive seizure (NCS) prevalence is $8-19 \%$ in patients with altered mental status (AMS). Non-convulsive status epilepticus (NCSE) may cause permanent neuronal damage via an increase in extracellular brain glutamate leading to brain swelling and apoptosis. Delayed diagnosis and treatment of NCS may lead to an increase in mortality in patients with acute brain injury if duration exceeds 20 hours. However, there is little data on which factors influence outcome in critically ill patients with NCSE. This study seeks to better understand the risk factors, characteristics and outcome of NCS/NCSE in critically sick patients in the neurological ICU.

Methods: This is a preliminary report of our prospective observational study, recruiting patients with unexplained AMS admitted to the adult neuro ICU at Vanderbilt University Medical Center, collected from January to May 2012. Coma due to anoxic brain injury or induced hypothermia was excluded from the study. Patients underwent brain imaging (CT or/and MRI) and were placed on continuous EEG monitoring as medically indicated. NCSE is defined as continuous or recurrent ictal discharges for at least 30 minutes and subcategorized according to the EEG findings. Data was collected and analyzed for prevalence of NCSE or/and NCS, EEG patterns (lateral vs. generalized), associated risk factors (e.g. metabolic derangement, acute cerebral lesion, preexisting epilepsy), AED use, treatment response, and eventual outcome.

Results: NCS/NCSE was detected in $21 \%$ of subjects (23 of 108): 9 NCS and 14 NCSE. $34 \%$ of the NCS/NCSE group, and $28 \%$ of the
non-NCS/NCSE group were intubated. Most all subjects (87\%) had abnormal brain imaging. Risk factors for NCSE/NCS were female gender $(\mathrm{p}=0.11)$, history of epilepsy $(\mathrm{p}=0.086)$, ventriculoperitoneal shunt $(\mathrm{p}=0.3)$, CNS tumor $(\mathrm{p}=0.004)$, and acute intracerebral hemorrhage $(\mathrm{p}=0.29)($ Table 1$)$. Though patients are comatose, subtle clinical signs such as twitching of mouth or ocular muscles, eye deviations were often found on exam in $57 \%(\mathrm{p}=0.003)$ of the NCSE/NCS group (Table 2). Death rate was higher, $26 \%$ in NCSE/NCS vs. $12 \%$ in non-NCS/NCSE group $(p=0.23)$. Among NCSE/NCS patients, generalized ictal EEG pattern had worse outcome, (death rate of $43 \%$ ) compared to the unilateral (19\%). All patients (4/4) placed on drug induced burst suppression died. Conclusions: The diagnosis of NCSE is challenging and requires urgent continuous EEG availability. This study highlights the importance of subtle clinical findings in combination with brain imaging abnormalities and medical history in raising the suspicion for NCSE/NCS. Mortality is high in NCSE, and the subtype of NCSE and its underlying etiology are likely important mortality. We are continuing this prospective study to better identify risk factors for NCSE and factors that are important in determining NCSE outcome in critically ill patients.

Table 1: Patient Characteristics

\begin{tabular}{|c|c|c|c|}
\hline Total $\mathrm{n}=108$ & NCSE/NCS $n=23$ & Others n=85 & $\mathrm{P}=$ value \\
\hline Age & $56+/-18$ & $60+1-17$ & 0.48 \\
\hline Gender & - & - & \\
\hline Male & $39 \%(9 / 23)$ & $58 \%(49 / 85)$ & 0.11 \\
\hline Female & $61 \%(14 / 23)$ & $42 \%(36 / 85)$ & \\
\hline Past Medical History & - & - & - \\
\hline Epilepsy & $26 \%(6 / 23)$ & $12 \%(10 / 85)$ & 0.086 \\
\hline Ischemic stroke & $13 \%(3 / 23)$ & $14 \%(12 / 85)$ & 0.9 \\
\hline Intracerebral bemorrhage & $4 \%(1 / 23)$ & $1 \%(1 / 85)$ & 0.32 \\
\hline CNS mass & $26 \%(6 / 23)$ & $6 \%(5 / 85)$ & 0.004 \\
\hline VI'S & $9 \%(2 / 23)$ & $4 \%(3 / 85)$ & 0.3 \\
\hline Psych & $9 \%(2 / 23)$ & $15 \%(13 / 85)$ & 0.42 \\
\hline Primary Diagnosis & - & - & - \\
\hline Traumalic brain injury & $4 \%(1 / 23)$ & $5 \%(4 / 85))$ & 0.94 \\
\hline Intracerebral hemonthage & $26 \%(6 / 23)$ & $16 \%(14 / 85)$ & 0.29 \\
\hline Ischemic stroke & $13 \%(3 / 23)$ & $22 \%(19 / 85)$ & 0.33 \\
\hline SAII & $4 \%(1 / 23)$ & $7 \%(6 / 85)$ & 0.64 \\
\hline SDH & $13 \%(3 / 23)$ & $8 \%(7 / 85)$ & 0.48 \\
\hline CNS mass & $4 \%(1 / 23)$ & $11 \%(9 / 85)$ & 0.36 \\
\hline Seizures & $74 \%(17 / 23)$ & $28 \%(24 / 85)$ & $<0.001$ \\
\hline Abnormal imaging & - & - & - \\
\hline Yes & $91 \%(21 / 23)$ & $86 \%(73 / 85)$ & 0.49 \\
\hline Acule & $70 \%(16 / 23)$ & $67 \%(57 / 85)$ & 0.82 \\
\hline Chronic & $43 \cos (10 / 23)$ & $32 \%(27 / 85)$ & 0.29 \\
\hline Mass effect & $33 \%(9 / 23)$ & $27 \%(23 / 85)$ & 0.94 \\
\hline GCS & $7.2(+/-3.2)$ & $5.7(+/-2.9)$ & 0.075 \\
\hline Outcome & Lnilateral ( $n=16)$ vs. Generalized ( $n=7$ ) & - & \\
\hline Death & $19 \%(3)$ vs. $43 \%(3)$ & $12 \%(10)$ & \\
\hline Baseline & $31 \%(5)$ vs. $28 \%(2)$ & $40 \%$ (34) & \\
\hline Neuro deficit & $50 \%(8)$ vs. $28 \%(2)$ & $49 \%(42)$ & \\
\hline
\end{tabular}

Psych: psychiatric history, VPS:ventricular peritoneal shunt, $\mathrm{SAH}$; subarachnoid hemorrhage, SDH; subdural hemorrhage, GCS;glasgo coma scale

Table 2: Clinical Signs, and IV BZ or AED use

\begin{tabular}{|c|c|c|c|}
\hline & NCSE/NCSE $\mathrm{n}=23$ & Others $\mathrm{n}=85$ & p values \\
\hline Did patient have a clinical sei<ure prior to EEG? & - & - & \\
Yes & $17 \%(4 / 23)$ & $19 \%(16 / 85)$ & \\
Subtle Signs (c.g. facc, cyclid twitch ctc) & $57 \%(13 / 23)$ & $19 \%(16 / 85)$ & 0.003 \\
No & $9 \%(2 / 23)$ & $18 \%(15 / 85)$ & \\
Unknown & $17 \%(4 / 23)$ & $45 \%(38 / 85)$ & \\
\hline Did Patient receive IV BL prior to EEG? & - & - & \\
Yes & $45 \%(10 / 23)$ & $25 \%(18 / 85)$ & \multirow{2}{*}{0.056} \\
No & $55 \%(12 / 23)$ & $75 \%(64 / 85)$ & \\
Unknown & $0.5 \%(1 / 23)$ & $4 \%(3 / 85)$ & \\
\hline Did patient receive IV AED prior to EE(i? & - & - & \\
Yes & $59 \%(13 / 23)$ & $52 \%(44 / 85)$ & \multirow{2}{*}{0.57} \\
No & $41 \%(9 / 23)$ & $48 \%(40 / 85)$ & \\
Unknown & $0.5 \%(1 / 23)$ & $1 \%(1 / 85)$ & \\
\hline
\end{tabular}

BZ; benzodiazepine, AED; antiepileptic drug 


\subsection{0}

REAL-TIME SEGMENTATION AND INFERENCE OF METABOLIC STATE FROM BURST SUPPRESSION EEG PATTERNS

M. Brandon Westover ${ }^{1,2}$, S. Ching ${ }^{2,3}$, M. M. Shafi ${ }^{1}$, S. S. Cash ${ }^{1}$ and E. N. Brown ${ }^{2,3}\left({ }^{1}\right.$ Neurology, Massachusetts General Hospital, Belmont, MA; ${ }^{2}$ Department of Brain and Cognitive Sciences, Massachusetts Institute of Technology, Boston, MA and ${ }^{3}$ Department of Anaesthesia and Critical Care, Massachusetts General Hospital, Boston, MA)

Rationale: The depth of burst suppression EEG patterns is widely held to reflect the brain's global metabolic status, and recent investigations have clarified the underlying neurophysiologic mechanisms. The need to quantify burst suppression frequently arises during continuous EEG (cEEG) monitoring in neuro-ICU patients, e.g. while monitoring depth of anesthesia in pharmacologically induced coma for refractory status epilepticus, and in coma prognostication after hypoxic ischemic injury. Manual cEEG analysis is labor intensive and subjective, necessitating automated methods. We present real-time algorithms for cEEG segmentation and inference of cerebral metabolic state, and validate these across a wide spectrum of burst suppression patterns encountered in ICU cEEG.

Methods: A real-time method for segmenting cEEG into burst and suppression epochs is presented based on adaptive variance thresholding. This algorithm is validated against manual segmentations of $20 \mathrm{cEEG}$ recordings by two experienced human electroencephalographers, and robustness of the results to variations in algorithm parameters settings is analyzed. We present an inference algorithm, based on a mathematical model of the mechanisms which generate burst suppression, allowing real-time estimation of the global brain metabolic state. Finally, we present a principled method for fitting this model to burst suppression data from ICU patients undergoing cEEG monitoring.

Results: Automated segmentation of burst suppression cEEG records in all 20 cases produced agreement with each human expert that was as good or better than inter-expert agreement. These results were robust to variations in algorithm parameter settings and there was no evidence of 'overfitting' to the clinical training data. Furthermore, the method was superior to 'naïve' thresholding methods sometimes advocated in the literature. Moreover, real-time model-based inference of underlying cerebral metabolic state is shown to be feasible across a wide spectrum of ICU EEG burst suppression patterns, and to faithfully reflect key nonlinear EEG features encountered in pharmacologically-induced burst suppression, namely, the nonlinear relation between brain-compartment anesthetic concentration and duration of suppressions in burst suppression EEG. Conclusions: Automated real-time segmentation and inference of cerebral metabolic state from burst suppression cEEG patterns is feasible across a wide range of patterns encountered in neurological ICU patients. Performance was comparable or superior to that achieved by human experts. These results demonstrate that continuous, high quality, real-time computational analysis of burst suppression EEG patterns, critical to enabling optimal use of cEEG data in the neurological ICU setting, is technically achievable. Examples of clinical applications of this capacity include tracking recovery from insults such as anoxic brain injury, and EEG-based closed loop control of anesthetic delivery for the treatment of refractory status epilepticus.

\subsection{1 \\ ELECTROGRAPHIC SEIZURES ARE COMMON IN INFANTS WITH ABUSIVE HEAD TRAUMA} D. M. Hasbani ${ }^{1,2}$, A. A. Topjian ${ }^{1,2}$, J. Huh ${ }^{1,2}$, S. H. Friess ${ }^{1,2}$, T. J. Kilbaugh $^{1,2}$, C. W. Christian ${ }^{1,2}$, D. J. Dlugos ${ }^{1,2}$ and Nicholas S. Abend ${ }^{1,2}$ ( ${ }^{1}$ The Children's Hospital of Philadelphia, Philadelphia, PA and ${ }^{2}$ The University of Pennsylvania School of Medicine, Philadelphia, PA)
Rationale: Abusive head trauma is a leading cause of disability and death in children. We evaluated the prevalence of non-convulsive seizures (NCS) in children with abusive head trauma, and hypothesized that NCS would occur in at least $50 \%$ of children. We explored indications for continuous EEG monitoring (cEEG), clinical and radiographic risk factors for seizures, and clinical outcomes. Methods: A retrospective study was conducted at a tertiary care hospital. Child Protection Team consult lists from $7 / 1 / 09$ to $1 / 9 / 12$ were screened to identify infants $\leq 2$ years of age, evidence of head trauma by imaging or physical exam, determination of abuse by the Child Protection Team, and admission to the pediatric ICU (PICU). Clinical data was obtained from electronic medical records including presenting symptoms/signs, Glasgow Coma Scale (GCS), intubation status, EEG features including seizure occurrence and characteristics, imaging findings, PICU and hospital length of stay, mortality, and follow-up outcome. Data analysis was performed using Stata 10. Results: 32 abused children were identified with a median age of 4 months (interquartile range 3-5.5 months), and 21 underwent cEEG. Those monitored were more likely to have a lower admission GCS ( 8 vs. $15, \mathrm{p}=0.053)$, more likely to be intubated ( 16 vs. $2, \mathrm{p}=0.002)$, and have longer PICU ( 6 days vs. 1 day, $\mathrm{p}=0.001)$ and hospital stays $(15$ days vs. 6 days, $\mathrm{p}=0.002$ ). Electrographic seizures occurred in 12 of $21(57 \%)$. Status epilepticus occurred in 8 of $12(67 \%)$ and seizures were entirely non-convulsive in 8 of 12 (67\%). EEG background categories (discontinuous and slow/sedated sleep) were associated with presence of seizures $(p=0.034)$. Subjects with seizures on EEG monitoring were not more likely to have clinically evident seizures on admission ( $67 \%$ with seizures vs. $33 \%$ without seizures, $\mathrm{p}=0.6)$, parenchymal imaging abnormalities (61\% with seizures vs. $39 \%$ without seizures, $\mathrm{p}=0.4)$, or extra-axial imaging abnormalities $(56 \%$ with seizures vs. $44 \%$ without seizures, $\mathrm{p}=0.72)$. Four of $21(19 \%)$ patients died prior to discharge; none had seizures, but all had an attenuated or flat background. Among the 17 survivors, outcome data was available for 15 at a median duration of 10 months following PICU admission (interquartile range 6-16 months). For survivors, the presence of NCS was not associated with the Glasgow Outcome Score at follow-up $(\mathrm{p}=0.1)$.

Conclusions: NCS are common in infants with abusive head trauma. Most seizures had no clinical correlate and thus would not be identified without cEEG. The associations between seizures and a higher GCS and more moderate EEG background abnormalities likely reflects that infants with the most severe brain injury and worse outcomes do not seize. In this small sample, neither clinically evident seizures on admission or parenchymal imaging abnormalities predict seizure occurrence during monitoring, although further study of seizure risk factors is needed. Further study is also needed to determine whether seizure identification and management improves outcome.

\subsection{2 \\ DENSITY SPECTRAL ARRAY FOR SEIZURE IDENTIFICATION IN CRITICALLY ILL CHILDREN} A. Pensirikul ${ }^{1,2}$, L. A. Beslow ${ }^{1,2}$, S. K. Kessler ${ }^{1,2}$, A. A. Topjian ${ }^{1,2}$, D. J. Dlugos ${ }^{1,2}$ and Nicholas S. Abend ${ }^{1,2}$ ( ${ }^{1}$ The Children's Hospital of Philadelphia, Philadelphia, PA and ${ }^{2}$ The University of Pennsylvania School of Medicine, Philadelphia, PA)

Rationale: Electrographic seizures (ES) are common in critically ill children with acute encephalopathy. ES identification by neurophysiologist review of conventional EEG is resource intense and often is not available for use in real-time. We aimed to evaluate the validity of neurologist interpretation of density spectral array (DSA) EEG for ES identification.

Methods: We studied continuous EEG tracings from 21 consecutive critically ill children. Conventional EEG was scored for ES by a pediatric neurophysiologist, which served as the gold standard for seizure identification. EEG tracings were then converted into 8 channel DSA displays. Four DSA images, each containing two hours, 
were generated per patient. Eight neurophysiologists received brief and standardized DSA training and then circled DSA elements thought to represent seizures. Images were reviewed in random order with no clinical information (Group A) or with information regarding presence or absence of seizures in the initial 30 minutes and with patient images in order, as might occur in a clinical setting (Group B). Inter-rater reliability for accurately classifying an image as containing a seizure was assessed in both groups (kappa). Sensitivity and specificity for correctly identifying a seizure on each image and the area under the receiver operating characteristic (ROC) curve were calculated for each group. Using full array EEG, 73 ES were evaluated for duration, number of electrodes involved at maximal extent, morphology (spikes versus rhythmic), and evolution (slowly versus clearly evolving). Factors associated with DSA seizure identification were assessed (Wilcoxon rank-sums).

Results: ES prevalence was $48 \%$ by neurophysiologist review. Interrater agreement was moderate in both groups (Group A: kappa 0.51 (95\%CI 0.35-0.66), p<0.001; Group B: 0.46 (95\%CI 0.33-0.59), $\mathrm{p}<0.001)$. Sensitivity and specificity were: Group A, $62 \%(95 \% \mathrm{CI}$ 51.2-71.9\%), 92.2\% (88.1-95.2\%) and Group B, 75\% (95\%CI 64.9$83.4 \%$ ), $79.1 \%$ (95\%CI 73.5-84\%). ROC curves were not different between the groups (AUC 0.77 for both, $\mathrm{p}=0.99$ ). Positive predictive values for Groups A and B were $75 \%(95 \% \mathrm{CI} 63.7 \%-84.2 \%)$ and $57.5 \%$ (95\%CI 48.1\%-66.5\%), respectively. Negative predictive values for Groups A and B were 86.5\% (95\%CI 81.8\%-90.4\%) and $89.4 \%$ (95\% CI $84.5 \%-93.1 \%$ ), respectively. Of the 73 seizures, $53 \%$ were identified by $\geq 6$ raters. Predictors of seizure identification were seizure duration $>2$ minutes $(\mathrm{p}<0.001)$ and non-subtle evolution $(p<0.001) .10 \%$ of images were falsely classified as containing a seizure and among those, the mean ( \pm standard deviation) false positive rate per hour was $1.5 \pm 2$.

Conclusions: When interpreted by neurophysiologists, DSA may be a useful screening tool for ES identification, although it does not identify all seizures and has only moderate inter-rater reliability. False positives occur indicating that seizure confirmation by conventional EEG review is needed. Additional study is required to determine whether non-neurophysiologist raters can use DSA and whether bedside implementation of DSA leads to more rapid seizure identification.

\subsection{3}

\section{VALUE ANALYSIS OF CONTINUOUS VERSUS ROUTINE 20-40 MINUTE EEG IN PATIENTS UNDERGOING THERAPEUTIC HYPOTHERMIA AFTER CARDIAC ARREST}

Amy Crepeau $^{1}$, J. E. Fugate ${ }^{1}$, A. A. Rabinstein ${ }^{1}$, E. F. Wijdicks ${ }^{1}$, R. D. White ${ }^{2}$ and J. W. Britton ${ }^{1}\left({ }^{1}\right.$ Neurology, Mayo Clinic, Rochester, $\mathrm{MN}$ and ${ }^{2}$ Anesthesiology, Mayo Clinic, Rochester, MN)

Rationale: Therapeutic hypothermia $(\mathrm{TH})$ is the standard of care for patients after ventricular fibrillation cardiac arrest (CA). Continuous EEG monitoring (cEEG) is increasingly used to monitor for subclinical seizures during $\mathrm{TH}$ and rewarming and to provide prognostic information. The value of this practice in terms of clinical impact in relation to cost, versus a practice of selective use of clinically-directed routine 20-40 minute EEG (rEEG) has not been performed. We compared clinical outcome and charges in a TH population in which cEEG was performed routinely with an equivalent cohort in which it was not.

Methods: In December 2005, our institution implemented a protocol for $\mathrm{TH}$ after $\mathrm{CA}$ which did not include routine use of cEEG (comprising the pre-TH-cEEG cohort). In 2009, due to concerns in the literature for subclinical seizures during TH, cEEG was initiated on all CA TH patients (TH-cEEG cohort). Clinical outcome, Cerebral Performance Category (CPC) and estimated EEG charges were calculated for the pre-TH-cEEG and TH-cEEG cohorts. Clinical data was gathered from the EMR system. EEG estimated unit charges were based on the National Charge Data 50th percentile charges expressed in USD. The source of these data is the CMS 2010 Standard Analytical File as reported in Code Correct by MedAssets, Inc. CPT code 95822 was used to estimate the unit charge for rEEG, and 95951 for cEEG.

Results: 91 patients were in the pre-TH-cEEG and 47 in the THcEEG cohorts. There was no statistical difference for age, mortality, hospital duration or CPC between the cohorts. In the pre TH-cEEG cohort, 19 patients $(21 \%)$ underwent rEEG and $3(3 \%)$ were placed on cEEG. The total estimated EEG charges for the entire pre-THcEEG cohort was $\$ 29,860.25$ for a mean of $\$ 1571.59 /$ patient for the 19 patients undergoing EEG. In the TH-cEEG cohort, the total estimated EEG charge was $\$ 203,998.50$ at a mean charge of $\$ 4340.39 /$ patient $(\mathrm{p}<0.0001)$. The mean duration of cEEG in the THcEEG cohort $=33.1$ hours (range 15-102.5). Two patients in the preTH-cEEG cohort had recorded seizures, compared to five in the THcEEG cohort. All seven had a poor outcome.

Conclusions: The main impetus for using cEEG in TH is to detect subclinical seizures in order to maximize clinical outcome. However, cEEG adds to costs as reflected in the significantly higher estimated charges identified in our study. Our study did not demonstrate improved outcomes associated with these higher charges.

Implementation of cEEG increased the rate of detection of subclinical seizures, but those with seizures detected and treated by this modality had poor outcomes, thus raising questions regarding the value of routine deployment of cEEG in this setting. Future research on cEEG in TH should include critical assessments of economic value as well as overall clinical outcome in order to determine its optimal place in the care of CA TH patients.

\subsection{4 \\ THE YIELD OF CVEEG IN DETECTING SEIZURES IN A TERTIARY HOSPTIAL SETTING

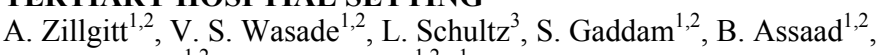 D. E. Burdette ${ }^{1,2}$ and M. Spanaki ${ }^{1,2}$ ( ${ }^{1}$ Neurology, Henry Ford Hospital, Detroit, MI; ${ }^{2}$ Henry Ford Comprehensive Epilepsy Program, Henry Ford Hospital, Detroit, MI and ${ }^{3}$ Public Health Services, Henry Ford Hospital, Detroit, MI)}

Rationale: The utility of continuous video electroencephalography (cVEEG) in detecting seizures has been well documented in the literature. The majority of these studies have focused on seizures in critically ill patients, a population in which $8-48 \%$ of patients may experience a seizure. The aim of this study was to determine the rationale and the yield of cVEEG in detecting seizures in patients admitted to general neurology and medical units in addition to critical care units.

Methods: The Henry Ford EEG laboratory database was retrospectively searched to identify patients who underwent cVEEGs from 10/1/2011 through 12/31/2011 and then from 02/11/2012 through $04 / 14 / 2012$ as part of IRB approved quality implementation study. All cVEEGs were initially reviewed by epileptologists at the Henry Ford Comprehensive Epilepsy Program. Demographic data, rationale for $\mathrm{cVEEG}$, requesting hospital unit and the presence of seizures were collected on each patient. Each cVEEG report was reviewed to determine any abnormal finding defined as periodic epileptiform discharges (PEDs), interictal epileptiform activity (IEA), triphasic waves (TW), intermittent rhythmic delta activity (IRDA), focal slowing, background slowing, excessive beta activity, or normal.

Results: A total of 135 patients who had cVEEG were included (65 males, 70 females) with ages ranging 16-94 years-old (mean 56.5). Twenty-three patients (17\%) had either one clinical seizure (12 patients), one subtle seizure (7 patients), or one clinical and one subtle seizure (4 patients). Patients with a referring diagnosis of seizure were more likely to experience a seizure when compared to patients with other referring diagnoses $(34.8 \%$ vs $7.9 \%, p<0.001$, Fisher's exact test) (See table 1). In addition, patients with PEDs on cVEEG had significantly higher rate of seizures when compared to 
the other primary diagnoses $(52.6 \%$ vs $11.2 \%$, p $<0.001$, Fisher's exact test) (Table 2). Neurology units were most likely to order cVEEG with $74.1 \%(n=100)$, with $11.1 \%(n=15)$ from other ICUs and $14.8 \%(n=20)$ from other units. Overall, the neurology units accounted for 19 of the 23 seizure patients. Although many of these patients with seizures were in critical care units ( 7 in the NICU, 5 in the stroke unit, and 2 in other ICUs), 9 patients were in general neurology and other general medical units. The difference in seizure rates among the units (neurology 19\%, other ICUs 13.3\%, other units $10 \%$ ) was not significant ( $\mathrm{p}=0.69$, Fisher's exact test).

Conclusions: This study demonstrates that seizures are common in hospitalized patients and illustrates high utility for cVEEG in patients with suspected seizures in critical care units as well as in general neurology and medical units. Seventeen percent of patients receiving cVEEG had at least one seizure. Patients with PEDs had almost 5 times the risk of having a seizure when compared to patients with other diagnoses and patients with a referring diagnosis of seizure were over 4 times as likely to experience a seizure during monitoring. cVEEGs remains a helpful diagnostic tool in detecting seizures in different hospital unit settings.

Seizure rates by referring diagnosis
\begin{tabular}{|c|c|c|}
\hline Referring diaguosis & $\begin{array}{c}\text { Tolal number } \\
\text { (cEEG N }=135) \\
\mathrm{N}(\%)\end{array}$ & Number of patients with seizures (\%) \\
\hline Scizurc & $46(34.1 \%)$ & $16(34.8 \%)$ \\
Seizures/epilepsy & 34 & $11(32.4 \%)$ \\
Status epilepticus & 12 & $5(41.7 \%)$ \\
Tincephaloputhy & $18(13.3 \%)$ & $1(5.6 \%)$ \\
Infection & $14(10.4 \%)$ & $2(14.3 \%)$ \\
CNS & 9 & $2(22.2 \%)$ \\
Other & 5 & 0 \\
Structural lesions & $27(20 \%)$ & $3(11.1 \%)$ \\
IIemorthagc & 12 & $1(8.3 \%)$ \\
Siroke & 7 & 0 \\
Tumor & 8 & $2(25 \%)$ \\
Other & $30(22.2 \%)$ & $1(3.3 \%)$ \\
Noncpilcpsy spells & 9 & 0 \\
Other neurologic condition & 7 & 0 \\
Hyperlension & 4 & 0 \\
Cardiac ctiologic & 4 & 0 \\
Miscellancous & 6 & $1(16.7 \%)^{*}$ \\
\hline
\end{tabular}

* The patient carried a diagnosis of cystic fibrosis

Seizure rates by primary cVEEG diagnosis

\begin{tabular}{|c|c|c|}
\hline $\begin{array}{c}\text { Primary cVEEG diagnosis (besides } \\
\text { seizure) }\end{array}$ & $\begin{array}{c}\text { Total number (cVEEG } N= \\
135) \\
N(\%)\end{array}$ & $\begin{array}{c}\text { Number with seizures } \\
(\%)\end{array}$ \\
\hline PIDs & $19(14.1 \%)$ & $10(52.6 \%)$ \\
\hline IEA & $26(19.3 \%)$ & $3(11.5 \%)$ \\
\hline TW & $6(4.4 \%)$ & 0 \\
\hline IRDA & $17(12.6 \%)$ & $1(5.9 \%)$ \\
\hline Focal slowing & $23(17.1 \%)$ & $7(30.4 \%)$ \\
Rackgronnd slowing and suppression & $22(16.3 \%)$ & $1(4.5 \%)$ \\
Excessive beta activity & $6(4.4 \%)$ & 0 \\
\hline Normal & $16(11.9 \%)$ & $1(6.3 \%)$ \\
\hline
\end{tabular}

Abbreviations - periodic epileptiform discharges (PEDs), interictal epileptiform activity (IEA), triphasic waves (TW), and intermittent rhythmic delta activity (IRDA).

\subsection{5}

\section{AUTOMATED DETECTION OF PERIODIC DISCHARGES IN ICU PATIENTS THROUGH ANALYSIS OF SCALP EEG} Jui-Hong Chien ${ }^{1}$, S. M. LaRoche ${ }^{2}$, J. J. Halford ${ }^{3}$, J. C. Sackellares ${ }^{1}$, K. M. Kelly ${ }^{4,5}$ and D. S. Shiau ${ }^{1}{ }^{1}$ Optima Neuroscience, Gainesville, FL; ${ }^{2}$ Neurology, Emory University School of Medicine, Atlanta, GA; ${ }^{3}$ Neuroscience, Medical University of South Carolina, Charleston, $\mathrm{SC} ;{ }^{4}$ Center for Neuroscience Research, Allegheny-Singer Research Institute and Allegheny General Hospital, Pittsburgh, PA and ${ }^{5}$ Neurology; Neurobiology and Anatomy, Drexel University College of Medicine, Philadelphia, PA)
Rationale: Periodic discharges (PDs, such as lateralized PDs - LPDs, bilateral independent PDs - BIPDs, and generalized PDs - GPDs) are important EEG findings in ICU patients because they are often associated with seizures, acute structural lesions, and CNS infections. PDs have also been associated with poor prognosis (Claassen et al., 2005) and higher mortality. Therefore, early recognition of PDs can be important in diagnostic and therapeutic management. However, continuous review and interpretation of raw EEG data to identify PDs in long-term EEG monitoring is labor intensive and therefore impractical in most clinical settings. Tools aiding in rapid and accurate detection of PDs would greatly improve efficiency of continuous EEG (cEEG) monitoring and enhance the quality of patient care in ICUs. We report a preliminary investigation of a novel automated algorithm for the detection of PDs in cEEG recordings from ICU patients.

Methods: One hundred 20-sec EEG epochs were sampled from cEEG recordings in each of 6 ICU patients, three with PDs noted in their video-EEG reports and the others without. To ensure a sufficient sample size of PD epochs in each "PD" patient, an initial sampling was conducted such that 30 epochs clearly exhibit PDs (based on the 2012 ACNS definition of PDs) and the remaining 70 were randomly sampled from segments without clear PDs. For the three "Non-PD" patients, all 100 epochs were randomly sampled from their cEEG recordings and thus include various interictal states as well as artifacts (muscles, chewing, etc.). These 600 EEG epochs were then independently reviewed by four EEG experts and an epoch was considered a PD epoch if it was marked by at least two experts. The PD detection algorithm reported herein first identified, in each channel, EEG discharges (i.e., spikes, sharp waves, or sharply contoured waves) in the epoch based on their peak values, durations, and morphology. A PD event was detected if, in any channel, the identified discharges repeated for more than five times with a deviation among the inter-discharge intervals smaller than a threshold. Sensitivity and specificity of the algorithm were evaluated based on the comparisons of the detection results against the epoch classifications determined by the EEG experts.

Results: Based on the experts' review, the proposed algorithm performed a sensitivity of $90.5 \%$ with an overall specificity of $99.4 \%$ ( $100 \%$ in "PD" subjects and $99.0 \%$ in "Non-PD" subjects). In addition, the analysis of the inter-rater variability revealed a moderate (1 out of 6 rater pairs) to substantial agreement among the four EEG experts (Cohen's kappa statistic: range $=[0.57,0.91]$, mean $=0.75$, $\mathrm{SD}=0.12$ ), which provided a sufficient confidence level on the assessment of algorithm performance.

Conclusions: These findings suggest that the proposed PD detection algorithm has potential to be the basis of clinically useful software that can assist ICU staff in timely identification of PDs. Further studies in a larger sample of patients that include more EEG patterns of PDs and background activities are warranted.

\subsection{6 \\ DYNAMICS OF SEIZURE RISK DURING CONTINUOUS EEG MONITORING IN CRITICALLY ILL PATIENTS Mouhsin Shafi ${ }^{1,2}$, M. B. Westover ${ }^{1}$, E. S. Rosenthal ${ }^{1}$, A. J. Cole ${ }^{1}$, R. D. Kilbride ${ }^{1}$, D. B. Hoch ${ }^{1}$ and S. S. Cash ${ }^{1}\left({ }^{1}\right.$ Neurology, Massachusetts General Hospital, Boston, MA and ${ }^{2}$ Neurology, Beth Israel Deaconess Medical Center, Boston, MA)}

Rationale: The purpose of this study was to determine how the risk for seizures in acutely ill patients evolves as a function of abnormalities on continuous EEG (cEEG) monitoring. Previous work has shown that in patients without epileptiform abnormalities early in the recording, the risk of seizures in the subsequent 24 hours is low. However, one concern is that the delayed seizure rate in this population is still high. In the present study we quantify the temporal evolution of seizure risk in relation to cEEG findings over a long time period by generating models of the transitions between different cEEG states. These models are used to determine the expected 
incidence of seizures over time in patients with and without epileptiform abnormalities.

Methods: We analyzed initial detection times of key EEG abnormalities (slowing, sporadic and periodic epileptiform discharges, and electrographic seizures) in 242 consecutive cases of acutely ill patients with at least 18 hours of continuous EEG (cEEG) monitoring. Baseline clinical and EEG variables were analyzed to identify predictors of subsequent seizures, and the transition rates over time for developing epileptiform abnormalities or seizures were estimated as a function of pathological features seen so far. These analyses were carried out using methods from survival analysis, including cumulative distribution functions, future event probability curves, and hazard functions.

Results: Seizures occurred in $29 \%(\mathrm{n}=70)$ of cEEG recordings. In 52 patients, the first seizure occurred within the first 30 minutes of monitoring. Of the remaining 190 patients, 63 had early epileptiform discharges, and 103 had no epileptiform abnormalities. Seizures were later detected in $22 \%(\mathrm{n}=14)$ with early epileptiform discharges, vs $3 \%(n=3)$ without early epileptiform abnormalities $(\mathrm{p}<0.001)$. Though seizure risk steadily declined throughout the course of monitoring, our projections suggest that even after a 24 hour seizurefree monitoring interval, patients with epileptiform abnormalities remain at moderate risk for seizures over the following 48 hours (nearly 4\%); in contrast, patients without epileptiform discharges at 24 hours have a very low risk of developing seizures directly $(<1 \%)$, though our analysis suggests a persistent moderate risk of developing delayed seizures indirectly via first transitioning to a higher risk group with epileptiform discharges (nearly $3 \%$ ), then subsequently developing seizures.

Conclusions: Seizure risk in acutely ill patients undergoing cEEG monitoring evolves substantially over time, and is strongly modulated by the nature of cEEG abnormalities detected early in the course of monitoring. This study presents a novel analysis of seizure temporal risk dynamics in acutely hospitalized patients undergoing cEEG monitoring. Substantial seizure risk stratification is possible on the basis of very early cEEG features. These findings provide guidance to allow more refined, patient-specific determination of the required duration of cEEG in critically ill neurological patients.

\subsection{7 \\ DIAGNOSTIC VALUE OF CONTINUOUS EEG IN THE TREATMENT OF SUB-ARACHNOID HEMORRHAGE- INDUCED VASOSPASM \\ Jeffrey M. Politsky ${ }^{1,2}$, P. Rodgers ${ }^{1,2}$ and I. Ugorec ${ }^{2}$ ( ${ }^{1}$ Atlantic}

Neuroscience Institute, Northeast Regional Epilepsy Group, Summit, NJ and ${ }^{2}$ Atlantic Neuroscience Institute, Overlook Medical Center, Summit, NJ)

Rationale: Failure to recognize and treat subarachnoid hemorrhageinduced vasospasm (SAH-V) results in significantly higher morbidity and mortality in this population of critically ill patients. We sought to determine if continuous EEG (CEEG) has predictive value for the early identification and treatment of SAH-V.

Methods: This was a retrospective review of all 108 patients admitted to our institution with a diagnosis of SAH in 2011. 56/108 patients underwent CEEG. Upon diagnosis, patients were classified as grade I-V using the Hunt Hess ( $\mathrm{HH})$ grading system. Patients underwent one (CEEG1) or two (CEEG2) phases of CEEG. CEEG was initiated within 24 hours of admission; CEEG2 was started at about day 6-7. In $46.4 \%$ of cases CEEG1 lasted $<72$ hours, and in $53.6 \%$ the study lasted $>5$ days. Abnormalities included mild background slowing (mbs), focal or diffuse slowing, polymorphic irregular sharply contoured waveforms (SAH-V pattern), spikes, electrographic seizures (Sz), periodic discharges (PD). Patient outcomes were tracked and categorized as discharge to home, rehab facility, long term care (LTC) facility, or death. Patients diagnosed with $\mathrm{SAH}-\mathrm{V}$ were treated with triple $\mathrm{H}$ therapy (hypertension, hypervolemia, hemodilution).
Results: Of the 56 patients who underwent CEEG, 15 underwent a second period of recording. CEEG2 was initiated because of clinical deterioration. Patients who underwent CEEG had the following $\mathrm{HH}$ breakdown: CEEG1: Grade I = 5; Grade II = 15; Grade III = 16; Grade IV $=15$; Grade V = 5; CEEG2: Grade I = 0; Grade II = 3; Grade III $=4$; Grade IV $=8$; Grade V $=0$.

SAH-V was diagnosed in 34/56 (60.1\%) who underwent CEEG. 19 patients had CEEG1 and 15 patients had CEEG2. 7/19 CEEG1 patients with SAH-V had prolonged CEEG studies. Of the 15 CEEG2 SAH-V patients $60 \%$ were discharged to home or rehab and $40 \%$ expired or went to long term care.

The EEG was characterized as mbs in $100 \%$ of patients with no evidence of vasospasm and was characterized as abnormal due to SAH-V pattern in $86.7 \%$ of $(13 / 15)$ CEEG 2 SAH-V patients. Electrographic Sz and/or PDs occurred in 13.3\% (2/15). Additionally, the SAH-V pattern was observed in $100 \%$ (11/11) of CEEG1 SAH-V patients in whom CEEG was prolonged. Electrographic sz and/or PDs occurred in $22.2 \%(2 / 11)$. Overall, this distinct SAH-V pattern occurred in $24 / 26$ patients $(92 \%)$ with SAH-V. The prevalence of sz and/or PDs was 4/26 (15\%).

Conclusions: We identified a distinct electrographic pattern associated with vasospasm - the SAH-V pattern. Patient outcomes appear to be more favorable when $\mathrm{HH}$ grade is I-III and when SAH$\mathrm{V}$ is identified and treated as early as possible. Our initial clinical experience in patients with SAH undergoing CEEG strongly suggests that CEEG can detect pathophysiologic changes associated with SAH-V as early or earlier than other accepted methods, such as clinical deterioration or angiogram. A prospective study comparing CEEG with CT or invasive angiogram is required to provide definitive evidence of the utility of this diagnostic tool.

\subsection{8 \\ EFFECTS OF MORPHINE ON EEG AND BEHAVIOR OF NEWBORNS TREATED WITH HYPOTHERMIA} M. Asunis ${ }^{1,5}$, M. Gardner ${ }^{1}$, S. L. Bonifacio ${ }^{2}$, K. B. Nash ${ }^{3}$, J. E. Sullivan $^{3}$, D. M. Ferriero ${ }^{4}$ and Maria R. Cilio ${ }^{1}\left({ }^{1}\right.$ Department of Neurology, University of California San Francisco, San Francisco, CA; ${ }^{2}$ Division of Neonatology, University of California, San Francisco, CA; ${ }^{3}$ Division of Epilepsy and Clinical Neurophysiology, University of California, San Francisco, CA; ${ }^{4}$ Department of Pediatrics, University of California, San Francisco, CA and ${ }^{5}$ Epilepsy Unit, Child Neuropsychiatry Department, University of Cagliari, Cagliari, Italy)

Rationale: Morphine is used to control shivering in neonates undergoing therapeutic hypothermia $(\mathrm{TH})$ for hypoxic-ischemic encephalopathy. Side effects of opiates such as hyperalgesia, myoclonus and seizures, have been reported in animals as well as in human neonates. Elevated serum morphine concentrations have been demonstrated in TH-treated newborns compared to normothermic infants at similar morphine dosage. We aimed to investigate the presence of morphine-associated EEG and behavioral changes in newborns during $\mathrm{TH}$.

Methods: Continuous video-EEGs of 115 consecutive term newborns treated with TH between November 2007 and November 2011, who received morphine per protocol, were retrospectively reviewed. Infants with abnormal MRI, subdural hemorrhage or large scalp edema, AEDs prior to onset of video-EEG were excluded. Results: 45 infants were included. Video-EEG monitoring was initiated at a mean of 9.5 hours of life for 89 hours. 13/45 infants $(30 \%)$ presented with symptoms ascribable to morphine: repetitive, intermittent, spontaneous myoclonic jerks(MJ) involving upper and lower extremities occurring during sleep and awake states, during several hours and despite escalating doses of morphine. All infants showed hyperalertness, hyperexcitability, limb hypertonia and hyperreflexia. MJ were observed 1 to 11 hours (mean 7 hours) after beginning of morphine administration, which included continuous infusion and boluses. EEG was normal or mildly discontinuous in 8 
infants, low voltage in 2 infants, and showed a severe burstsuppression pattern with interburst intervals from 25 to 40 seconds in 3 infants. MJ did not have a clear EEG counterpart or were associated with a diffuse broad slow wave. However, in 3 patients with abnormal EEG ( 2 with burst-suppression and 1 with low voltage) MJ progressed into true ictal events characterized by prolonged focal or bilateral slow discharges of high voltage sharp waves lasting 2 to 30 minutes associated with clonic activity time-locked with the EEG discharges. Six patients were treated with lorazepam and/or Phenobarbital. Mean duration of symptoms was 12 hours ( 2 to 24 hours) with shorter duration in treated patients. When abnormal, EEG completely normalized within 12 hours and it was normal at 24 hours of life. Seizures never recurred. While the rate of morphine infusion did not significantly differed between symptomatic and asymptomatic infants, (20.4 microgram $/ \mathrm{kg} / \mathrm{h}$ vs $17.8 \mathrm{microgram} / \mathrm{kg} / \mathrm{h}$ $\mathrm{p}=0.054)$, there was a significant difference in the amount of morphine received as boluses $(0.22 \mathrm{mg} / \mathrm{kg}$ vs $0.12 \mathrm{mg} / \mathrm{kg} \mathrm{p}=0.0051)$. Conclusions: Our findings suggest that morphine administration in newborns undergoing hypothermia treatment may paradoxically be associated to neuronal hyperexcitability and/or profound yet reversible EEG alterations. The spontaneous resolution of symptoms in some infants and the EEG rapid normalization could be explained by previous data showing that, differently from children and adults, morphine plasma concentrations in newborns tend to decrease with time despite unchanged dosage.

\section{Neurophysiology: Other Clinical EEG}

\subsection{9 \\ ICTAL ONSET EEG SOURCE IMAGING IN PEDIATRIC MEDICALLY REFRACTORY EPILEPSY}

Ravindra Arya, D. F. Rose, K. D. Holland, H. Fujiwara and F. T. Mangano (Cincinnati Children's Hospital, Cincinnati, $\mathrm{OH}$ )

Rationale: In the evaluation of patients with medically refractory epilepsy for resective surgery, localization of ictal onset zone (IOZ) using invasive EEG with subdural grids is conventionally considered the gold standard. However, this procedure is associated with not insignificant morbidity. In contrast, scalp EEG though much safer, has localization only to head regions and may have a poor coregistration with underlying cortex especially in patients with distorted anatomy. Further, a single electrode on scalp EEG carries dipolar source information from about $30 \mathrm{~cm} 2$ of cortical surface, which has insufficient specificity for surgical planning. We used several different algorithms for EEG source localization and coregistration with patient's segmented cortex to study the internal validity of these algorithms and agreement with localization of IOZ on invasive EEG monitoring.

Methods: All patients with medically refractory epilepsy admitted for non-invasive pre-surgical evaluation were eligible for inclusion. Inability to co-operate with electrode digitization was an exclusion criterion. After scalp EEG electrode placement, the electrode position was digitized using a Polhemus digitizer. Sections of the EEG showing seizure onset were snipped and uploaded as 'functional data' in the Curry 7 Neuroscan software, the electrode positions being obtained from the digitizer file. Patient's brain MRI was uploaded as 'image data' and boundary element head model was constructed with segmented cortex. EEG was filtered at 1-70 Hz, with additional notch filter at $50 \mathrm{~Hz}$ and its harmonics. Following source localization algorithms were then carried out: rotating equivalent current dipole (ECD), multiple signal classification (MUSIC), current density methods including minimum norm estimate (MNE), standardized low resolution electro-tomography (sLORETA) and sLORETA weighted minimum norm (SWARM). Source localizations were obtained at the point of ictal onset determined by visual analysis of scalp EEG (+/-5 $\mathrm{msec}$ ). The agreement between lobar localization thus obtained was compared with that of scalp EEG using Fleiss kappa statistic.

Results: Five patients with a total of 9 seizures have been analyzed so far. Each seizure was counted separately as an observation. All patients had extra-temporal epilepsy. In this small sample, sLORETA algorithm was found to have best agreement with visual analysis of scalp EEG at a lobar level $(\kappa=0.67,95 \%$ CI 0.54, 0.80). In addition, all 3 current density/ distributed dipole methods (MNE, sLORETA, SWARM) had high internal consistency $(\kappa=0.73,95 \%$ CI 0.59 , 0.87).

Conclusions: EEG source imaging may potentially offer a safe, noninvasive and relatively specific method to increase accuracy of localization of ictal onset zone for surgical candidates with medically refractory epilepsy. For better clinical utility we intend to compare EEG source localization with IOZ defined on prolonged video-ECoG monitoring.

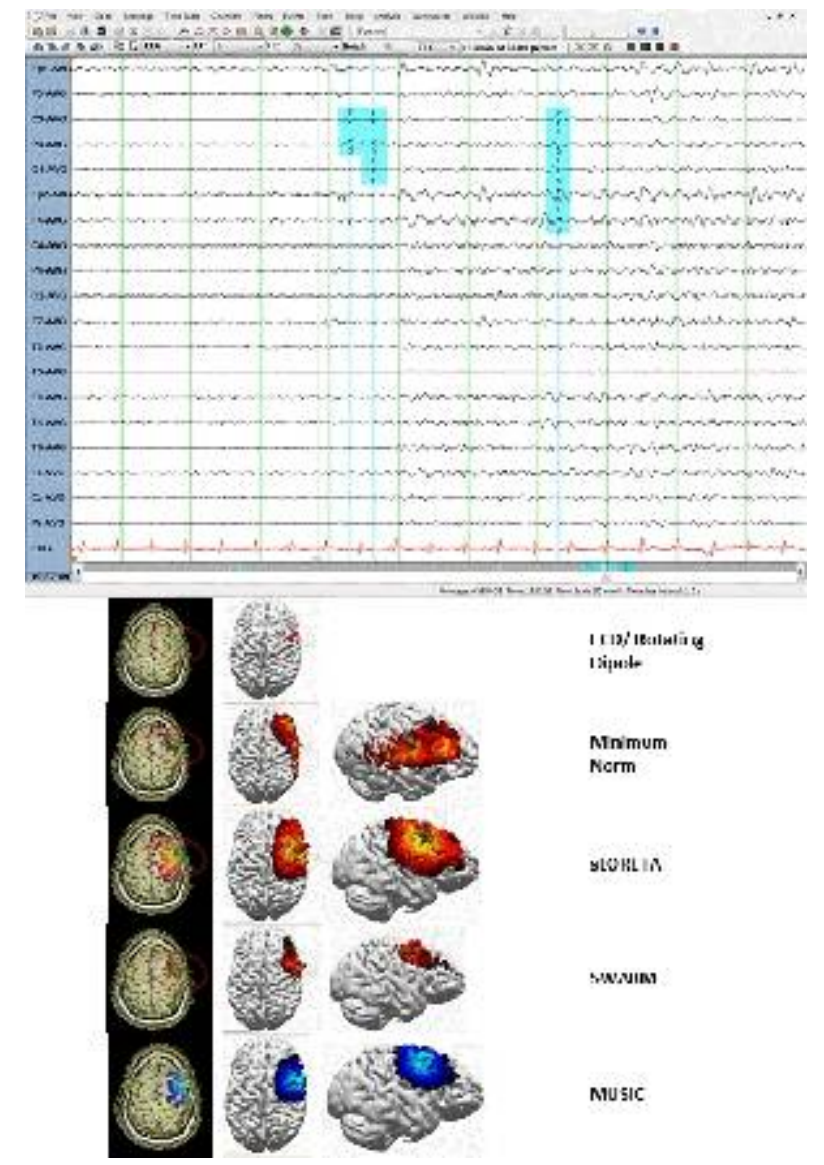

\subsection{0 \\ USE OF COLORED FILTERS FOR ASSESSMENT OF THE INHIBITION OF PHOTOPAROXYSMAL RESPONSES IN CHILDREN WITH PRIMARY GENERALIZED EPILEPSY IN THE OUTPATIENT SETTING}

A. Faris, J. Higgins, N. Bhamani, S. Kozlik and L. Bello-Espinosa (Alberta Children's Hospital, Calgary, AB, Canada)

Rationale: Photosensitivity is a common trait among children with primary paediatric epilepsies. Testing of photoparoxysmal (PPR) responses is challenging in children and EEG assisted by an optometrist is usually not readily available. Colored filters are not commonly used during EEG. The objective of this study was to assess whether the use of sequentially photic-stimulator installed color filters affected the photoparoxysmal response of children with primary epilepsy

Methods: Thirty-three children and adolescents with primary epilepsy (mean age=12.6 ) with abnormal photo-paroxysmal responses were identified among 5510 sequential EEG studies completed between August 2010 and Feb 2012 at Alberta Children's Hospital in Calgary. The sample consisted of 9 males (27.2\%) and 24 
females $(72.7 \%)$. Thirteen $(39.3 \%)$ children were already on antiepileptic medication at the time of the evaluation. 10-20 EEG montages and conventional intermittent unfiltered photic stimulation (IPS) recommended parameters were used initially. After identifying the initially unfiltered inducing photo-paroxysmal responses (PPR) on the conventional testing, to identify the potentially inhibiting effect of the filters, the photic stimulation testing was repeated applying sequentially a red, a yellow, a orange, a green and a blue filters on the photic stimulator box. All EEGs were performed by experienced and registered certified EEG Technologists.

Results: The use of the green and blue filters blocked the photoparoxymal response in $30(90.9 \%)$ and $29(87.9 \%)$ children respectively. The red filter blocked the PPR in 13 (33.9\%) children and yellow and orange filters only blocked it in $3(9.1 \%)$ and 6 $(18.1 \%)$ respectively. No photoconvulsive events were induced during the repetitive filter testing.

Conclusions: The use of strobic-photic stimulator mounted colored filters during EEG testing may be useful to detect specific inhibition of photoparoxysmal inducing frequencies in children with epilepsy. Blue and green color filters appear to be more effective blocking these epileptogenic photoparoxysmal responses. Low-cost colored filters can be safely and easily implemented for the assessment of photosensitivity during routine pediatric EEG.

\subsection{1}

\section{GUIDING PEDIATRIC EPILEPSY SURGERY TOWARDS IMPROVING LANGUAGE OUTCOMES}

\section{Milena Korostenskaja ${ }^{1}$, P. C. Cheng ${ }^{1}$, C. M. Salinas ${ }^{2}$, J. Cook ${ }^{3}$, J.} Baumgartner $^{4}$, S. Delgado ${ }^{1}$, M. Westerveld ${ }^{2}$ and K. H. Lee ${ }^{4}$ ( ${ }^{1}$ Functional Brain Mapping and Brain Computer Interface Lab, Comprehensive Pediatric Epilepsy Center, Center for Pediatric Research and Outcomes, Florida Hospital for Children, Orlando, FL; ${ }^{2}$ Neuropsychology, Walt Disney Pavilion, Florida Hospital for Children, Orlando, FL; ${ }^{3}$ Radiology, Florida Hospital, Orlando, FL and ${ }^{4}$ Comprehensive Pediatric Epilepsy Center, Florida Hospital for Children, Orlando, FL)

Rationale: Accurate language localization in children provides critical information for surgical planning, thereby expanding surgical epilepsy treatment options and allowing more aggressive resection of epileptogenic tissue while reducing the risk of post-surgical language deficit and benefiting children's quality of life. The objective of this research is to evaluate the electrocorticography (ECoG)-based realtime functional mapping (RTFM) technique (Schalk et al. 2008) for localizing language-specific regions in children. The central hypothesis of our proposed research is that that RTFM can be used as a tool for pre-surgical mapping of language function in pediatric patients.

Methods: Seven patients with intractable epilepsy underwent RTFM testing since the inception of Epilepsy Program at Florida Hospital in August 2011. Here we present the data from representative subject \#3 (13 yo right-handed female). Baseline ECoG activity was first recorded (g.USBamp, g.tec, Austria, sampling frequency $1200 \mathrm{~Hz}$ ) for about 6 minutes followed by administration of paradigms, assessing receptive and expressive language function (Fig. 1). The sensitivity and specificity of RTFM were calculated only for sites where both RTFM and ESM were tested. RTFM sensitivity was calculated as the percentage of sites that were both RTFM $(+)$ and $\operatorname{ESM}(+)$ among all $\operatorname{ESM}(+)$ sites, and RTFM specificity was calculated as the percentage of sites that were both RTFM(-) and ESM(-) among all ESM(-) sites.

Results: RTFM demonstrated low sensitivity (25\%) and high specificity (90\%) when compared to ESM. RTFM showed several areas of activation related to expressive language function that were ESM negative. The patient underwent standard temporal lobectomy that included areas that were ESM negative but RTFM positive. The majority of the posterior superior temporal gyrus was left intact in order to avoid possible postsurgical expressive language deficits predicted by ESM mapping (Fig. 1). However, two month postsurgical neuropsychological testing showed expressive language decline. The patient demonstrated very low performance in visual confrontation naming when compared to same-aged peers. A mild decline was observed in phonemic fluency. A more noticeable decline in semantic fluency was found. Maternal report also suggested a decline in language functioning within the patient's everyday environment. It is possible to speculate that this resection based only on ESM results is associated with observed post-surgical expressive language deficits. The pediatric neurosurgeon indicated that if RTFM was a validated technique, he would use information provided by it to avoid post-surgical language decline.

Conclusions: Preliminary results indicate that RTFM has the potential to make a substantial contribution to surgical planning. Post-operative language outcome showed acute decline in a patient whose surgery included areas that were ESM negative but RTFM positive. Further validation of this method is underway.

References: Schalk G et al. (2008) Real-time detection of eventrelated brain activity. NeuroImage 43: 245-9

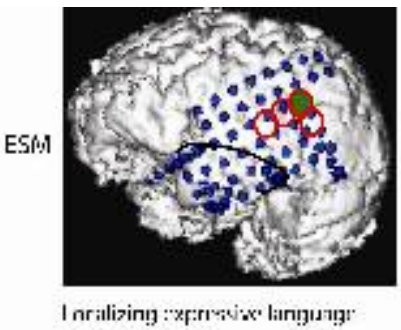

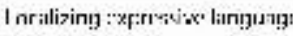
ivabilli, IASK:

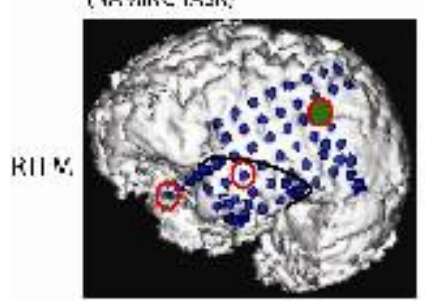

Area of overlap belween ESW and RTF:'

Q Area of distinctian between $r$ SM and HII $\mathrm{S}$.

- Surgical rescction murgin

Figure 1. Results of eloquent language cortex mapping with ESM and RTFM in patient \#3.

\subsection{2 \\ ABNORMAL MISMATCH NEGATIVITY FOR PURE-TONE SOUNDS IN FRONTAL LOBE EPILEPSY}

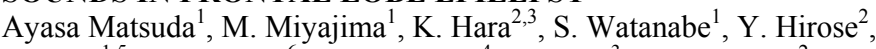

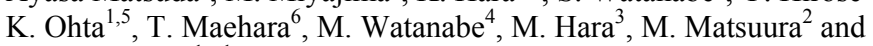
E. Matsushima ${ }^{1}\left({ }^{1}\right.$ Section of Liaison Psychiatry and Palliative Medicine, Graduate School of Medical and Dental Sciences, Tokyo Medical and Dental University, Tokyo, Japan; ${ }^{2}$ Department of Lice Sciences and Bio-informatics, Graduate School of Health Care Sciences, Tokyo Medical and Dental University, Tokyo, Japan; ${ }^{3}$ Hara Clinic, Yokohama, Japan; ${ }^{4}$ Department of Psychiatry, National Center Hospital of Neurology and Psychiatry, Tokyo, Japan; ${ }^{5}$ Ondadaini Hospital, Chiba, Japan and ${ }^{6}$ Department of Neurosurgery, Tokyo Medical and Dental University, Tokyo, Japan)

Rationale: Event-related potentials (ERPs) are averaged electroencephalographic (EEG) responses time-locked to external stimuli or internal events (Naatanen \& Kahkonen, 2009). Mismatch negativity (MMN), one of a component of ERP, usually peaks approximately $100-250 \mathrm{~ms}$ stimulus onset at the fronto-central sites. It has been shown that MMN reflects pre-attentive function and has two generators, one is in the frontal lobe which associates with attention switching and another is in the temporal lobe which associated with sensory memory mechanism. MMN is suitable to examine the existence of dysfunction in the temporal lobe or in the frontal lobe. Miyajima et al. (2011) revealed that both the temporal and frontal cortices contributing to MMN generation were affected in temporal 
lobe epilepsy (TLE) patients. In order to elucidate whether there are any pre-attentive dysfunctions in frontal lobe epilepsy patients, we performed the same paradigm as Miyajima et al.

Methods: We examined seven FLE patients (five female, six righthanded), with a mean age of $35.9 \pm 7.4$ (22-45 years). The mean age of seizure onset was $14.9 \pm 14.7$ (6 months-44 years). Six were seizure free for more than one year. Six patients took one or two antiepileptic drugs (AEDs). One patientsdid not take any AED. Control data was collected from seven age-matched healthy controls (HCs, five females, all right handed). Epileptic foci were on the left for four patients, right in two patients, and unclear for one patient. The stimuli presented were pure-tones $(1000 \mathrm{~Hz}$ as standard and $1050 \mathrm{~Hz}$ as deviant). Numbers of stimuli given were 800 standard and 200 deviant.

We evaluated the MMN (deviant minus standard) peak and mean amplitude, and peak latency separately for each subtraction waveforms at $\mathrm{Fz}, \mathrm{Cz}$, left, and right mastoids between 100-250 ms stimuli onset. The study was approved by the Ethics Committee at Tokyo Medical and Dental University, and written informed consent was obtained from each participant. There is no conflict of interest. Results: Visual inspection revealed that MMN at Fz was larger in FLE patients than HCs. MMN at the right mastoid was smaller in FLE patients than in HCs, whereas MMN at the left mastoid was similar between FLE patients and HCs.

We confirmed that the MMN difference between HC and FLE at fronto-central sites were attributed by the difference of the deviant responses rather than standard responses. Conversely, the amplitude difference between right and left mastoid sites in FLE was due to the small deviant responses at right mastoid site in FLE (Figure1 and 2). Conclusions: In the present study, MMN in the fronto-central region was larger in FLE patients than in HCs. This result might reflect hyper excitability in fronto-central region in FLE. We observed smaller MMN amplitude in the right mastoid than left mastoid in FLE patients. The limitation of this study is that the number of patients was small. A larger number of patients are needed to confirm the present asymmetry at the mastoids.

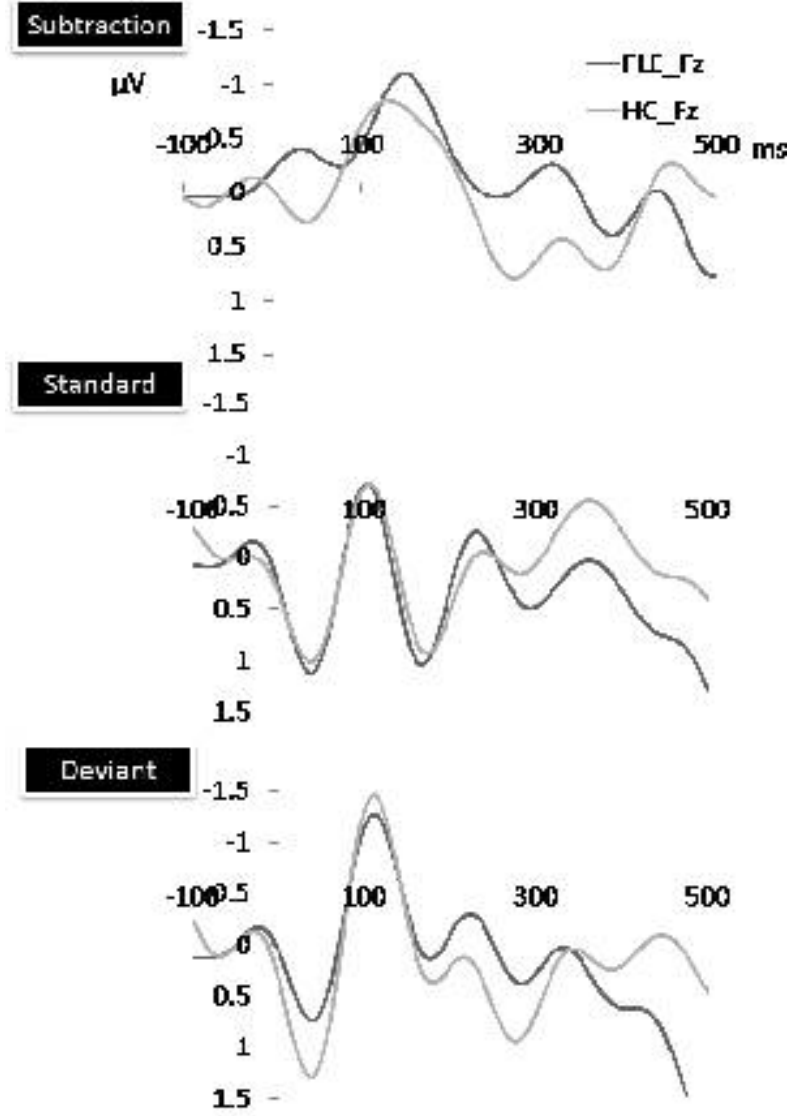

Grand average waveforms for the subtraction (MMN, upper), for standard stimuli (middle) and for the deviant stimuli (lower) in the FLE group (black) and in the control group (gray) at Fz.

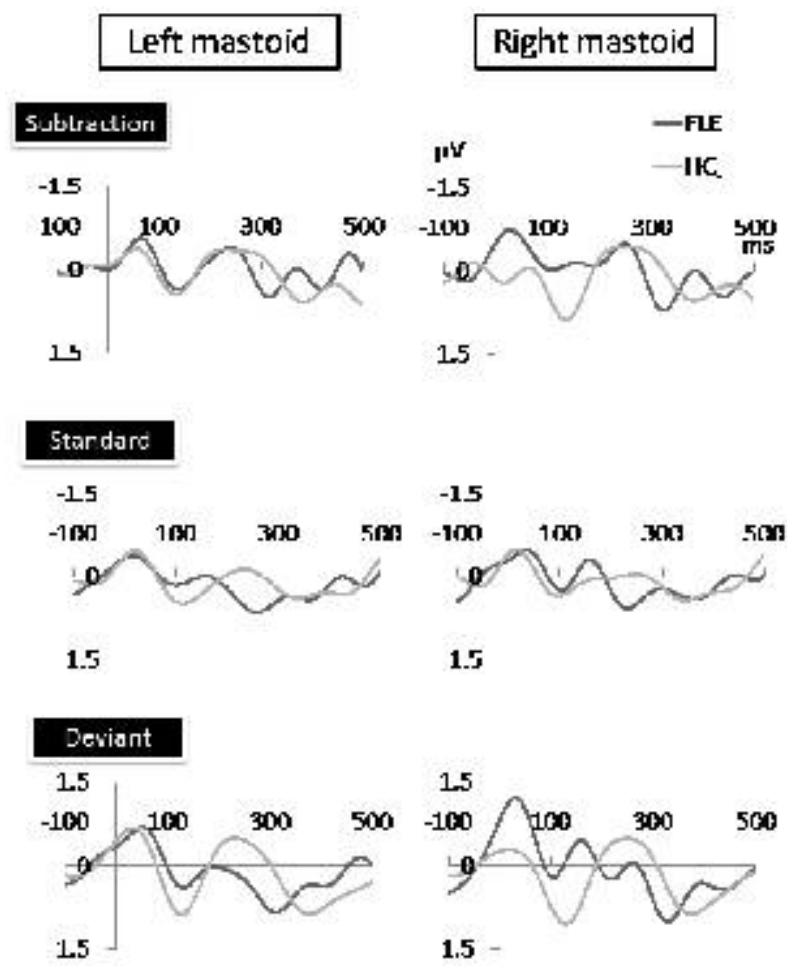

Grand average waveforms for the subtraction (MMN, upper), for standard stimuli (middle) and for the deviant stimuli (lower) in the FLE group (black) and in the control group (gray) at left and right mastoids.

\subsection{3 \\ HUMAN SLEEP SPINDLES DECREASE IN FREQUENCY WHEN THEY COINCIDE WITH K-COMPLEXES IN PATIENTS WITH EPILEPSY}

Eleftherios S. Papathanasiou and S. S. Papacostas (Cyprus Institute of Neurology \& Genetics, Nicosia, Cyprus)

Rationale: The second stage of human non-rapid eye movement (NREM) sleep is characterized by two major electroencephalographic (EEG) elements: the K-complex (KC) and the sleep spindle. Although the $\mathrm{KC}$ was first described by Loomis et al. 70 years ago, its functional role is still a debatable issue, but is considered by some to be an arousal reaction. Similarly, the function of the sleep spindle also remains undefined, but there are data supporting its role as an arousal inhibitor and thus a sleep preservation mechanism. There are also data supporting a role of spindles in memory consolidation during sleep. A recent paper by Kokkinos and Kostopoulos has shown that sleep spindles are blocked upon spontaneous K-complex coincidence and resume as higher frequency spindles afterwards in normal volunteers. This effect has been explained by a brief arousing stimulus triggering a $\mathrm{KC}$ and at the same time blocking spindles by a brainstem inhibitory action at the reticular nucleus $(\mathrm{RN})$ level, upon the depolarizing rebound of which a new spindle rhythm of higher frequency is produced. In this study we wished to determine whether this phenomenon persisted or was altered in patients with epilepsy, and if this could shed additional light on epileptic mechanisms. Methods: Routine EEG studies recorded over a 20 month period were included in this study. We included patients followed at our institution with a definite diagnosis of epilepsy. A sleep spindle had to precede and follow a $\mathrm{KC}$ to be analyzed and the $\mathrm{KC}$ had to have a minimum duration of $500 \mathrm{msec}$. Superimposed spindle and KCs followed or preceded immediately by similar patterns $(\mathrm{KC} /$ spindle trains) were not analyzed. The frequency of the sleep spindle was 
calculated with the filter bandpass set at 6-17 Hz. Statistical analysis was performed using the Student's t-test.

Results: A total of 350 patient recordings were analyzed. Six patients only were found to have the required superimposed sleep spindle and $\mathrm{KC}$ appearance. Only four of these patients had a final diagnosis of epilepsy (with 11 sleep spindles and coincident $\mathrm{KC}$ recorded in total from all four patients). Two of these patients had generalized and two had focal onset epilepsy. The mean frequency of the sleep spindles before the $\mathrm{KC}$ was $13.568 \pm 1.072$ standard deviations and $12.182 \pm$ 1.068 after the KC. The difference was found to be significant $(\mathrm{t}=2.896,0.01<\mathrm{p}<0.05)$ with a sample size of 11 .

Conclusions: In contrast to the findings in physiologically normal volunteers, we recorded a significant decrease in spindle frequency following a $\mathrm{KC}$ in patients with epilepsy. This finding may indicate a decrease in brainstem inhibitory activity at the RN level and contribute to the excitability present in epilepsy.

\subsection{4 \\ CONTINUOUS HIGH FREQUENCY ACTIVITY: A PECULIAR SEEG PATTERN RELATED TO SPECIFIC BRAIN REGIONS}

Federico Melani, R. Zelmann, F. Mari and J. Gotman (Montreal Neurological Institute, McGill University, Montreal, QC, Canada)

Rationale: By analyzing High Frequency Oscillations in the SEEGs of epileptic patients, we have observed a continuous/semi-continuous (C/SC) ripple activity in some mesial temporal structure (MTS) channels, which we defined continuous High Frequency Activity (HFA)(Mari et al., Epilepsia 2012;53:797-806). We now extend the search for this activity evaluating its potential occurrence also in neocortical (NC) areas. We systematically reviewed and classified the background of each SEEG channel of 20 patients implanted in the MTS and NC regions, defining correlations with neurophysiological and neuro-radiological features to provide an accurate definition of the clinical significance of this particular SEEG pattern.

Methods: 20 patients with intracranial electrodes placed in the MTS and $\mathrm{NC}$ areas were selected, enabling an extensive coverage of many different brain regions. The anatomical localization of the electrodes in each lobe was defined by post-implantation CT and postexplantation MRI. One minute of slow-wave sleep, low-pass filtered at $500-\mathrm{Hz}$ and sampled at $2000 \mathrm{~Hz}$, was reviewed. The background was analyzed on segments 1 second away from spikes (time resolution: $0.92 \mathrm{~s} /$ page; LF:80 Hz). The background was classified as $\mathrm{C} / \mathrm{SC}$, Irregular or Sporadic, based on the length of the fast oscillations and on the presence of a clear separation between the transient elements. Ripples and fast ripples were marked independently of background classification. Each channel was classified as inside the Seizure Onset Zone (SOZ) or in a visible lesion.

Results: 790 bipolar channels (688 NC, 102 MTS) were analyzed. The C/SC background was found in 54 channels (22 MTS, $32 \mathrm{NC}$ ). Considering the anatomical distribution, the most frequent localization in the MTS was the hippocampus $(\chi 2(2,22)=14.5$, $\mathrm{p}<0.001)$; in the $\mathrm{NC}$, this pattern was not randomly distributed $(\chi 2(4,32)=60.6, p<<0.001)$, being the most common the occipital lobe (fig. 1); this region showed $63 \%$ of all the $\mathrm{NC} \mathrm{C/SC} \mathrm{background.}$ In the occipital lobe the $\mathrm{C} / \mathrm{SC}$ background was independent from the occurrence of the alpha rhythm (no correlation between the power spectrum of alpha and ripple band during eyes open/closed). The $\mathrm{C} / \mathrm{SC}$ background showed low sensitivity $(16 \%)$ and high specificity $(96 \%)$ for the SOZ, and no correlation with lesional channels; it had the highest rates of spikes, ripples and fast ripples compared to the Irregular and Sporadic backgrounds ( $\mathrm{p}<<0.01, \mathrm{~K}-\mathrm{W}$ test).

Conclusions: This study provides a further description of a particular type of HFA as a continuous oscillatory activity, and defines its occurrence in neocortical areas as well as in MTS. This continuous HFA, quite rare ( $7 \%$ of the channels), shows a clear preferential distribution for the hippocampus and the occipital lobe. The continuous HFA characterizes 'normal' channels as well as those inside the $\mathrm{SOZ}$ or in a lesion, showing no correlation with behavioral changes (appearance of the alpha rhythm). These findings suggest that this pattern may be an intrinsic characteristic of specific brain regions. Supported by Robb fellowship and CIHR MOP-102710

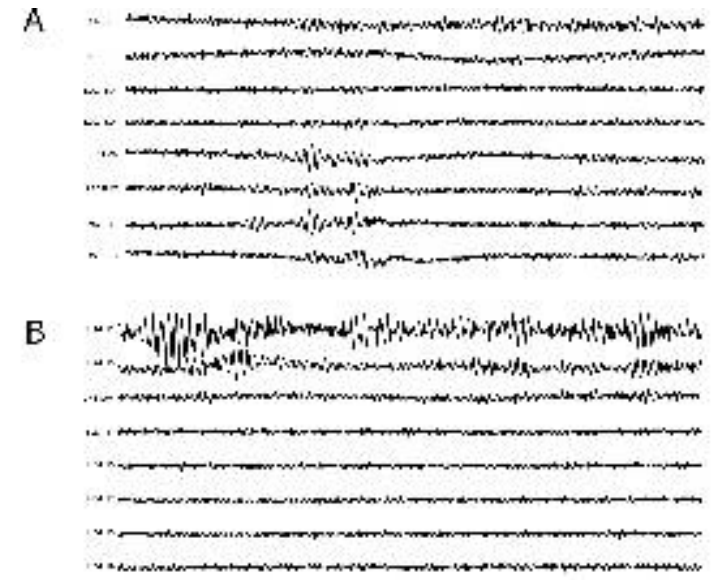

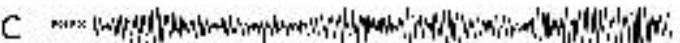

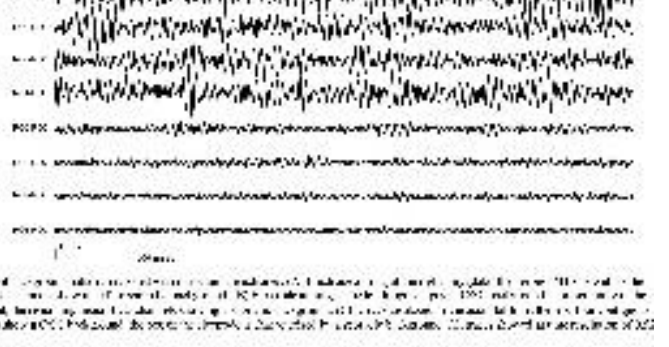

\subsection{5}

SPIKES WITH DISTINCT HIGH FREQUENCY OSCILLATIONS IDENTIFY BETTER THE SOZ THAN HF POWER CHANGES DURING SPIKES

Julia Jacobs LeVan ${ }^{1}$, C. Vogt ${ }^{1}$, R. Zelmann ${ }^{2}$, J. Gotman ${ }^{2}$ and K. Kobayashi ${ }^{3}\left({ }^{1}\right.$ Department of Neuropediatrics and Muscular Diseases, University of Freiburg, Freiburg-Baden Wurttemberg, Germany;

${ }^{2}$ Montreal Neurological Institute, Montreal, QC, Canada and ${ }^{3}$ Department of Child Neurology, Okayama University Hospital, Okayama, Japan)

Rationale: High frequency oscillations (HFOs) between $80-500 \mathrm{~Hz}$ can identify the seizure onset zone (SOZ) with high specificity. Spikes with distinct HFOs are more specific for the SOZ than spikes without HFOs. Visual or semi-automatic detection of HFOs however can be time consuming and difficult. Another method uses statistical analysis of averaged power spectra of interictal spikes to identify those spikes with large high frequency (HF) components. This study compares the value of distinct HFOs and HF components of spikes for identifying the SOZ.

Methods: 15 patients with neocortical refractory epilepsy from the Montreal Neurological Institute were included (low pass: $500 \mathrm{~Hz}$, sampling rate: $2000 \mathrm{~Hz}$ ). HFOs inside and separate of spikes were marked visually and rates of HFOs (ripple: $80-250 \mathrm{~Hz}$, fast ripple: $250-500 \mathrm{~Hz}$ ) were calculated. For the analysis of HF components, at least 50 spikes with similar morphologies were marked in all spiking channels. Time-frequency spectra of spikes were averaged and FDR corrected statistics was used to identify significant increases and decreases of frequencies between $80-250 \mathrm{~Hz}$ as well as $250-500 \mathrm{~Hz}$ (Figure 1). Specificity and sensitivity for identifying the SOZ were calculated on a single patient and group level and compared for both methods. 
Results: In 71 channels (16 in the SOZ), polyspikes were observed which did not allow the analysis of HF power within these spikes. A significantly higher rate of HFOs and spikes carrying HFOs was found in the SOZ compared to outside. At a level of $95 \%$ specificity the rate of spikes carrying ripples was the most sensitive (30\%) to identify the SOZ. When applying the rate threshold at the $95 \%$ specificity level to single patients, either spikes carrying ripple or spikes carrying fast ripple showed the highest sensitivity in 11 and the highest specificity in 14 of 15 patients. For the increase of HF power within spikes no difference was found inside or outside the $\mathrm{SOZ}$ whereas there was a significantly larger postspike decrease for frequencies between $80-250 \mathrm{~Hz}$ and $250-500 \mathrm{~Hz}$ inside the $\mathrm{SOZ}$ $(p=0.01)$. Thus, HF power increases in the spikes could not be used for identification of the SOZ.

Conclusions: In patients with neocortical epilepsy, spikes which were either carrying ripple or fast ripples were best for identifying the SOZ. Thus, it could be expected that HF power in spikes, if similar to distinct HFOs, would show the same discriminatory features. In contrast, only the postspike decrease in HF power was different for the SOZ. Thus, strong HF power changes in spikes and spikes carrying HFOs seem to represent different entities and may occur over different regions. This may be additionally influenced by methodological problems occurring in channels with very frequent spiking, which prevent HF power analysis in spikes. The observed prominent postspike decrease in $\mathrm{HF}$ power in SOZ channels may represent increase inhibition in these channels and should be investigated further.

I1]Uก:

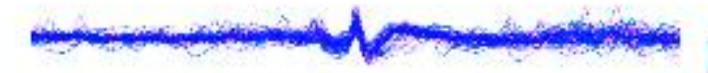

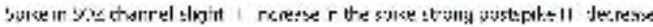
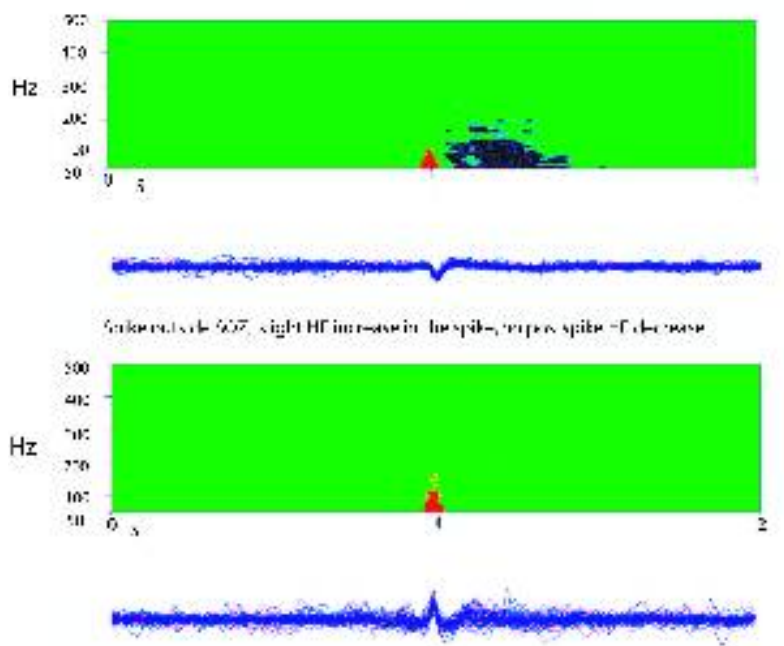

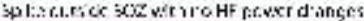

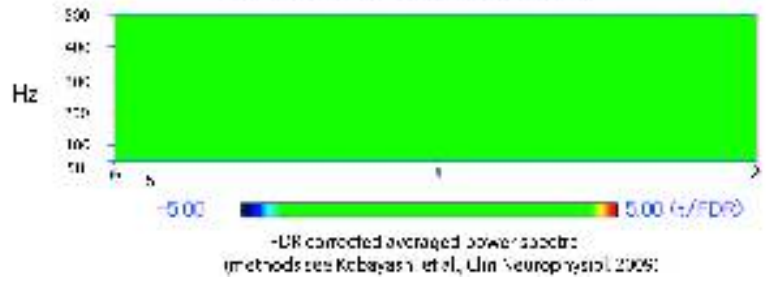

\subsection{6}

PREDICTING SUCCESS OF VAGUS NERVE STIMULATION (VNS) FROM INTERICTAL EEG

Cecile C. de $\operatorname{Vos}^{1,2}$, W. van Drongelen ${ }^{3}$, M. H. Kohrman ${ }^{3}$ and M. J. van Putten ${ }^{1,2}$ ( ${ }^{1}$ Department of Clinical Neurophysiology, Medisch Spectrum Twente, Enschede, Netherlands; ${ }^{2}$ MIRA institute for Biomedical engineering and Technical medicine, University of Twente, Enschede, Netherlands and ${ }^{3}$ Department of Pediatrics, University of Chicago, Chicago, IL)
Rationale: Vagus nerve stimulation (VNS) has shown to be an effective treatment for drug resistant epilepsy in numerous patients. Most long-term studies showed that more than 50\% seizure reduction was accomplished in 20 to $55 \%$ of the patients. Since it is still not possible to predict which patients will profit from VNS, we searched predictive interictal EEG features for seizure reduction after VNS. Quantifying EEG symmetry using the Brain Symmetry Index (BSI) showed promising results in predicting seizure frequency reduction as a result of VNS treatment in relatively healthy adults with mainly focal epilepsy [Seizure: Eur J Epilepsy 2011;20:541-545]. Pairwise derived Brain Symmetry Index (pdBSI) values [Clin Neurophysiol 2009;120:845-855] of the delta, theta and alpha band could significantly discriminate between responders and non-responders. These findings however need to be validated in different patient groups before they can be generalized.

Methods: From the Pediatrics department of the University of Chicago we included 16 young patients (age 5-20 y) with medically refractory epilepsy and an implanted VNS system. All patients suffered from (multi) focal, medically intractable epilepsy with varying focus locations. Most patients were severely cognitively impaired. Long term monitoring EEG registrations, recorded before implantation, were retrospectively analyzed. The first 20 minutes without seizures and artifacts were selected for quantitative analysis. The pdBSI was tested for correlation with VNS outcome. Reduction in seizure frequency was used to define positive response to VNS treatment.

Results: Ten patients obtained a (stable) reduction in seizure frequency of at least $75 \%$ during a follow-up period of 2 years and are defined as good responders to VNS therapy. Six patients did not show any reduction. On average, we found higher asymmetry, reflected in higher pdBSI values, in delta, theta, alpha and beta bands for non-responders compared to good responders. However, differences were not statistically significant.

Conclusions: Quantifying EEG symmetry using the pdBSI showed promising results in predicting the seizure reducing effect of VNS treatment in non-cognitively impaired adult patients, but these results were not as robust in multifocal neocortical epilepsy in young patients.

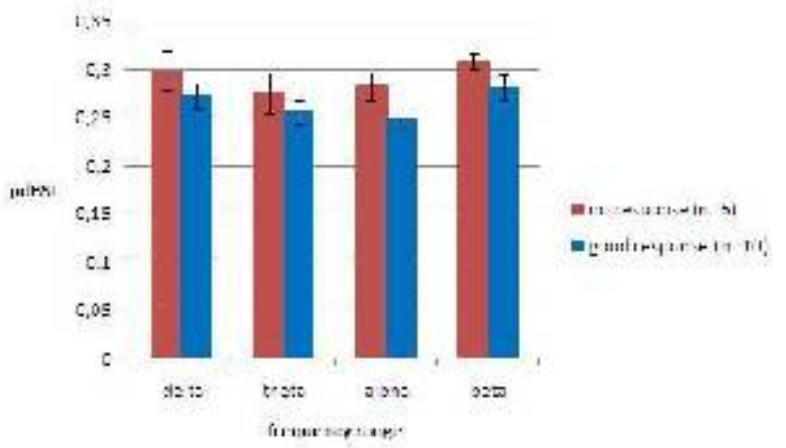

Differences between non-responders $(n=6)$ and good responders $(n=10)$ in brain symmetry (pdBSI) for 4 frequency bands, extracted from EEG recordings before onset of VNS treatment. Error bars represent the standard error of the mean.

\subsection{7}

CLINICAL YIELD OF ROUTINE EEG IN ADULT PATIENTS

AWAD A. MORTADA, J. Jirsch, S. N. Ahmed and D. W. Gross (UNIVERSITY OF ALBERTA, EDMONTON, AB, Canada)

Rationale: Electroencephalograms (EEGs) are commonly used in the investigation of patients presenting with seizures, paroxysmal events, loss of consciousness, confusion and new onset psychiatric symptoms in order to rule out the possible diagnosis of a seizure disorder. Objective: To determine the incidence of diagnostic epileptic activity on EEGs performed on adults and to determine whether the yield of EEG is different based on the reason for referral. 
Methods: Single-center retrospective study, of sequentially referred adult patients more than 17 yrs of age with clinical seizures, paroxysmal event of unknown etiology, altered mental status or new psychiatric symptoms. Patients having long-term video-EEG recording and patients who had EEG in an intensive care unit were excluded. Standard 30 minute EEGs were performed by an EEG technician using a commercially available cap and interpreted by one of three fellowship trained electroencephalographers. Between group difference in results was compared using Analysis of Variance (ANOVA).

Results: Over an 18 month period, 569 patients underwent 600 routine EEGs. Twenty-seven had two EEGs, two had three EEGs and the remaining patients had a single EEG. One hundred and eightytwo patients were referred for EEG with a diagnosis of epilepsy or seizures, 276 with paroxysmal events of unknown etiology, 75 with altered mental status and 36 with a new onset of psychiatric symptoms. Overall, epileptic activity was found in 41 of 569 patients (7.2\%). Epileptic activity was found in 20 of $182(11 \%)$ patients with seizures or epilepsy, 18 of $276(6.5 \%)$ patients with paroxysmal events, three of $75(4 \%)$ of patients with altered mental status and zero of $36(0 \%)$ patients with new onset psychiatric symptoms. Overall ANOVA demonstrated a significant difference in the incidence of epileptic activity for different reasons of referral ( $p=0.045$ ) with no specific between group comparisons reaching significance on post-hoc analysis.

Conclusions: In patients referred with paroxysmal events and altered level of consciousness, the incidence of a diagnostic finding on EEG was relatively low $(6.5 \%$ and $4 \%$ respectively). Given the fact that epileptic EEG findings were only observed in $11 \%$ of patients with a known diagnosis epilepsy or seizures, a nondiagnostic result cannot be considered to rule out epilepsy. The observation that no patients with new onset psychiatric symptoms had a positive EEG could imply that epilepsy patients are being accurately diagnosed by primary care physicians and not mistakenly referred for psychiatric evaluation.

\subsection{8 COMPARISON OF BINARY AND ORDINAL SCORING FOR EPILEPTIFORM TRANSIENT DETECTION}

Jonathan Halford ${ }^{1}$, C. G. Waters ${ }^{2}$, B. J. Wolfe ${ }^{3}$, S. R. Benbadis ${ }^{4}$, W. O. Tatum ${ }^{5}$, R. P. Turner ${ }^{1}$, A. Arain ${ }^{6}$, N. B. Fountain ${ }^{7}$, S. R. Sinha ${ }^{8}$, P. B. Pritchard ${ }^{1}$, G. U. Martz ${ }^{1}$, E. Kutluay ${ }^{1}$, J. C. Edwards ${ }^{1}$ and B. C. Dean ${ }^{2}\left({ }^{1}\right.$ Neurosciences, Medical University of South Carolina, Charleston, SC; ${ }^{2}$ Computer Science, Clemson University, Clemson, $\mathrm{SC} ;{ }^{3}$ Biostatistics, Medical University of South Carolina, Charleston, SC; ${ }^{4}$ Neurology, University of South Florida, Tampa, FL;

${ }^{5}$ Neurology, Mayo Clinic, Jacksonville, FL; ${ }^{6}$ Neurology, Vanderbilt University, Nashville, TN; ${ }^{7}$ Neurology, University of Virginia, Charlottesville, VA and ${ }^{8}$ Medicine, Neurology Division, Duke University Medical Center, Durham, NC)

Rationale: Reliable computerized detection of epileptiform transients (ETs), characterized by interictal spikes and sharp waves in the electroencephalogram (EEG), is a useful goal since this would assist physicians in reviewing scalp EEG recordings. It is our goal to create standardized datasets to help train automated ET detection algorithms. In this study, we used EEGnet, a distributed web-based platform for the analysis of scalp EEG recordings to compare the inter-rater reliability for marking ETs. Two different methods of scoring were compared. The first method involved labeling paroxysmal activity as either epileptiform or non-epileptiform (binary scoring). The second method involved labeling paroxysmal activity on a scale of $0-4$, depending on the degree of epileptiform appearance (ordinal scoring).

Methods: One hundred 30-second routine scalp EEG segments from 100 different patients were selected for analysis. Fifty of these segments were selected because they contained ETs from patients with known epilepsy and the other fifty were selected because they contained benign paroxysmal activity (exaggerated alpha activity, wicket spikes, and small sharp spikes) which could easily be misinterpreted by an inexperienced reviewer. Scoring was performed in three phases by ACNS board certified academic

neurophysiologists. In the first phase, seven scorers marked all of the paroxysmal activity in the segments (including epileptiform activity, other EEG activity, and artifacts). In the second phase, eleven scorers marked each paroxysmal event as either artifact, epileptiform activity, or non-epileptiform EEG activity. In the third phase, the eleven scorers categorized all of the events marked by at least one scorer (in phase two) as epileptiform as well as some randomlyselected non-epileptiform events as either non-epileptiform, or epileptiform on a scale of 1-4. For inter-rater analysis, we examined all pair-wise rater agreement scores using the kappa statistic and generated a composite score based on the average agreement score across all pairs for both the binary and the ordinal data. using Cicchetti-Allison weights. We also calculated the reliability coefficient described in Wilson et. al (1996) using the Spearman correlation.

Results: The average kappa value for the data with binary scores was $0.40+/-0.12$ with correlations with a range of $0.12-0.59$. The average kappa value for the data with ordinal scores was $0.42+/-0.078$ with a range of $0.30-0.62$. The kappa values were not significantly different between the binary and ordinal scoring, but the variability of the kappa values were greater for the binary in comparison to the ordinal scoring data. The reliability coefficient was not significantly different for the binary scoring method (0.89) compared to the ordinal scoring method (0.91).

Conclusions: Inter-rater agreement for categorizing paroxysmal activity as epileptiform activity was moderate. The inter-rater agreement for binary and ordinal scoring were not significantly different, although there was decreased variability for the ordinal scoring.

\section{Neurophysiology: Brain Stimulation}

\subsection{9}

\section{ABNORMAL TMS/EEG RESPONSES IN EPILEPSY}

Esther M. ter Braack ${ }^{1}$, I. Silva Santos ${ }^{1,2}$ and M. J. Van Putten ${ }^{1,3}$ $\left({ }^{1}\right.$ Clinical Neurophysiology, University of Twente, Enschede, Netherlands; ${ }^{2}$ Physics, New University of Lisbon, Lisbon, Portugal and ${ }^{3}$ Neurology \& Clinical Neurophysiology, Medisch Spectrum Twente, Enschede, Netherlands)

Rationale: The diagnostic process in epilepsy is often timeconsuming, partially due to the limited sensitivity of the routine EEG. Therefore, there is a need for additional diagnostic measures. Transcranial magnetic stimulation (TMS) enables quantification of the brain's excitability. In an earlier study, the excitability of firstseizure epilepsy patients was higher when compared to healthy controls, and this returned to normal values after anti-epileptic drugs were prescribed (Badawy 2010). When TMS is applied while recording EEG, a characteristic waveform - the TMS evoked potential (TEP) - is induced in the EEG. TMS/EEG may be useful as an additional technique for diagnosing epilepsy and monitoring the response to anti-epileptic drugs. Indeed, a previous study showed that TEP consists of an early part, which is always present, and a late part, that was present in 9 out of 11 epilepsy patients and not in healthy subjects (Valentin 2008).

Methods: We recorded 64-channel EEG in healthy controls and adult patients with epilepsy to study late TMS evoked potentials. At present, 18 healthy subjects and 8 epilepsy patients ( 2 with focal epilepsy and 6 with generalized epilepsy) have been included. At the time of the measurements, 7 patients were taking anti-epileptic drugs. TMS/EEG was recorded using a Magstim Rapid2 stimulator and a 64-channel EEG amplifier (ANT Neuro, Enschede). TMS was targeted at the left and right motor cortex. We administered 75 pulses at an intensity of $110 \%$ motor threshold for both targets. The TEP 
was obtained by averaging over all TMS pulses for a specific target. For quantification, we calculated the power for a post-stimulus time period (400 to $950 \mathrm{~ms}$ ), where the late responses would be present, and a baseline established from 800 to $200 \mathrm{~ms}$ before the TMS pulse. To evaluate if TMS induced an increase in activity for each channel, we subtracted the baseline period power from the response period power. Only increases in power with a significance level of $\mathrm{p}<0.01$ were regarded as a late response.

Results: In all healthy controls and epilepsy patients we found an early TEP. All patients showed a late response when we combined results for left and right motor cortex stimulation, with 5 out of 8 patients demonstrating this late response for both TMS targets. Only 1 out of 18 healthy subjects showed a late response for both TMS targets. However, 9 out of 18 healthy subjects showed a response for left or right motor cortex stimulation, resulting in a sensitivity of $100 \%$ (95\% confidence interval $63-100 \%)$ and a specificity of $50 \%$ (95\% confidence interval 26-74\%) for late responses in either left or right motor cortex stimulation. Table 1 summarizes these findings. Figure 1 illustrates responses from a patient and a healthy subject. Conclusions: TMS/EEG is a promising technique to evaluate the brain's response to an external perturbation, and initial results demonstrate that epilepsy patients show a late response to TMS more often than healthy volunteers.

Badawy et al, Ann Neurol 2010;67:64-73

Valentin et al, Epilepsia 2008;49(3):470-480

Table 1. Overview of presence of late responses in patients and controls.

\begin{tabular}{|cc|c|}
\hline & Epilepsy patients & Heallhy coutrols \\
\hline Late response MCL & $7 / 8$ & $5 / 18$ \\
T.ate responsc MCR & $6 / 8$ & $5 / 18$ \\
I.ate response MCI. or MCR & $8 / 8$ & $9 / 18$ \\
\hline Late response MCL and MCR & $5 / 8$ & $1 / 18$ \\
\hline
\end{tabular}

MCL: Motor cortex left hemisphere

MCR: Motor cortex right hemisphere

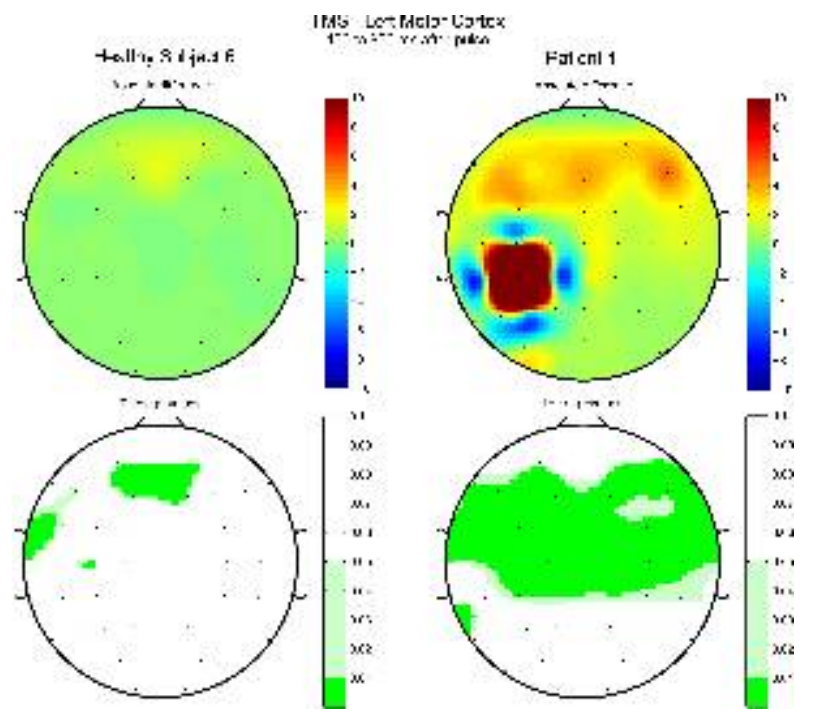

Figure 1. Top row: Example of topoplots of a control (left) and a patient (right), showing the difference in power at 400-950 ms after the TMS pulse. The patient shows a late response at electrode position CP3, while the healthy control does not show any late responses. At the bottom, significance levels are shown.

\subsection{0}

PREDICTION OF ICTAL PROPAGATION USING SINGLE PULSE ELECTROCORTICAL STIMULATION EARLY RESPONSES

D. Keizer ${ }^{1,2}$, G. J. Huiskamp ${ }^{1}$, M. A. Van 't Klooster' ${ }^{1}$, M. J. Van

Putten $^{2}$, F. S. Leijten ${ }^{1}$, C. H. Ferrier ${ }^{1}$ and G. J. Zijlmans ${ }^{1}\left({ }^{1}\right.$ Neurology and Neurosurgery, Rudolf Magnus Institute of Neuroscience, University Medical Center Utrecht, Utrecht, Netherlands and ${ }^{2}$ Clinical Neurophysiology, MIRA Institute for Biomedical Technology and Technical Medicine, Enschede, Netherlands)

Rationale: Single pulse cortical electrical stimulation (SPES) and its responses yield information about the epileptic brain. Research has mainly focused on delayed SPES responses which are assumed to be associated with the underlying pathology. However, early SPES responses can map effective brain connectivity networks. SPES can be rapidly applied during surgery and might help to predict seizure spread without the need of an actual seizure. Accurate predictions of seizure spread may be useful for decision making in focal resections and evaluating the effectiveness of surgery. This could be specifically useful in cases when subpial transsections are preferred over resection for example when the seizure onset zone lies in an eloquent area. We aim to use clinical SPES data to assess the feasibility of predicting ictal propagation.

Methods: In this study, data from pre-surgical evaluation of 5 patients suffering from focal epilepsy with a marked gamma onset on chronic electrocorticograpy were used. SPES (bipolar monophasic $0.2 \mathrm{~Hz}$ stimulation with $0.1 \mathrm{~ms}$ pulse width) was applied to electrodes in the initial seizure onset zone. An automatic detection algorithm for early responses, using Wiener filtering to correct for stimulus artifacts and response amplitude offset, was developed. Based on standardized early response amplitude thresholds we predicted which electrodes could be involved in seizure spread. The resulting electrode positions were then compared to those involved in actual seizure spread as indicated by an experienced

electroencephalographer. This was used as the reference for calculating sensitivity and specificity of predicted seizure spread. Furthermore, we assessed if the delay of the early responses was correlated to the timing of seizure spread for each individual patient using Spearman's rank correlation $(\mathrm{p}<0.05)$.

Results: The 5 patients analyzed in this study had 16-30 (mean 23) electrodes marked as being involved in seizure spread. SPES early responses were able to predict seizure spread to these electrodes with an average sensitivity of $69 \%$ (range $57-82 \%$ ) and specificity of $83 \%$ (range 80-88\%). No significant correlations between response delay and seizure spread delay were found in all but one patient (rho = $0.63, \mathrm{p}=0.02$ ).

Conclusions: Our results show that SPES early responses can be a valuable tool to peroperatively map the epileptic brain. SPES early responses were able to predict seizure spread of the patients' dominant seizure type based on stimulation of the epileptic focus. Future research should be performed to further improve this prediction and evaluate the clinical use.

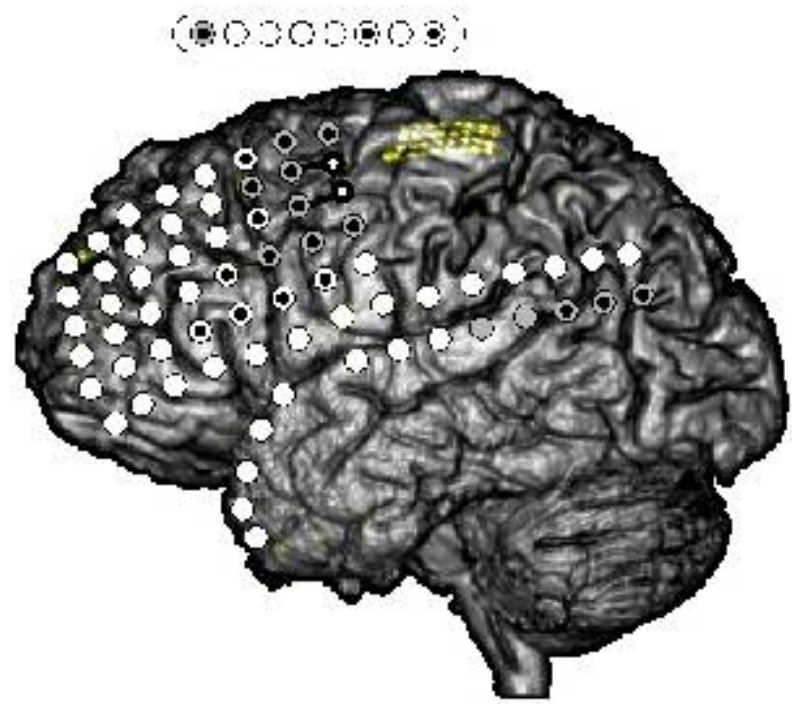

Electrode placement and overview of predicted versus actual ictal propagation. An inter-hemispheric electrode strip not visible in the 
current view is depicted above the brain. Electrodes in which initial onset was marked are shown as thick edged circles. Predicted propagation is shown in dotted electrodes whereas actual propagation is shown in gray. Stimulated electrode pairs are connected by a black line.

\subsection{1}

\section{OLFACTORY HALLUCINATIONS ELICITED BY}

ELECTRICAL STIMULATION VIA SUBDURAL ELECTRODES: EFFECTS OF DIRECT STIMULATION OF OLFACTORY BULB AND TRACT

Gogi Kumar ${ }^{1,2}$, C. Juhasz ${ }^{1}$, S. Sood ${ }^{1}$ and E. Asano ${ }^{1}$ ( ${ }^{1}$ Children's Hospital of Michigan, Detroit, MI and ${ }^{2}$ Children's Medical Center, Dayton, $\mathrm{OH}$ )

Rationale: In 1954, Penfield and Jasper briefly described that percepts of unpleasant odor were elicited by intraoperative electrical stimulation of the olfactory bulb in patients with epilepsy. Since then, few peer-reviewed studies have reported such phenomena elicited by stimulation mapping via subdural electrodes implanted on the ventral surface of frontal lobe. Here, we determined what types of olfactory hallucinations could be reproduced by such stimulation in children with focal epilepsy.

Methods: This study included 16 children (age range: 5 to 17 years), who underwent implantation of subdural electrodes to localize the presumed epileptogenic zone and eloquent areas. Pairs of electrodes were electrically stimulated and clinical responses were observed (Figure 1). In case a patient reported a perception, she/he was asked to describe its nature. We also described the stimulus parameters to elicit a given symptom.

Results: Eleven patients reported a perception of smell in response to electrical stimulation while the remaining five did not. Nine patients perceived an unpleasant smell (like bitterness, smoke, or garbage; see red bars in Figure 2), while two perceived a pleasant smell (like strawberry or good food; see blue bars in Figure 2). Such olfactory hallucinations were induced by stimulation proximal to the olfactory bulb or tract on either hemisphere but not by that of orbitofrontal gyri lateral to the medial orbital sulci. The range of stimulus parameters employed to elicit olfactory hallucinations was comparable to those for other sensorimotor symptoms.

Conclusions: Our systematic study of epileptic children replicated stimulation-induced olfactory hallucinations. We failed to provide evidence that a positive olfactory perception could be elicited by conventional stimulation of secondary olfactory cortex alone.

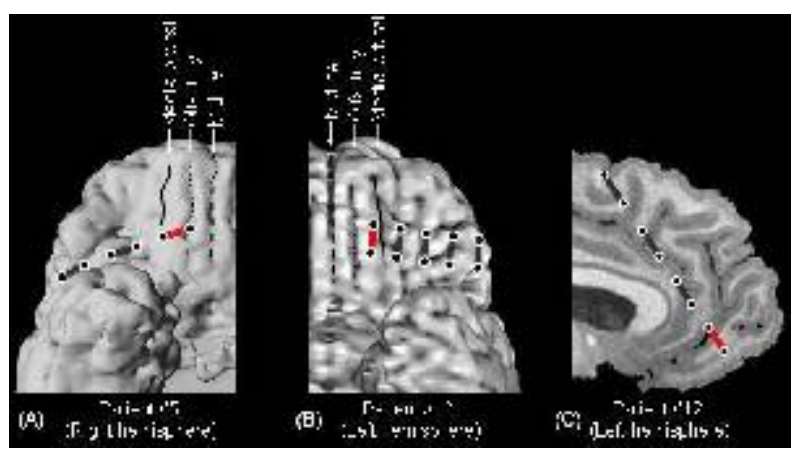

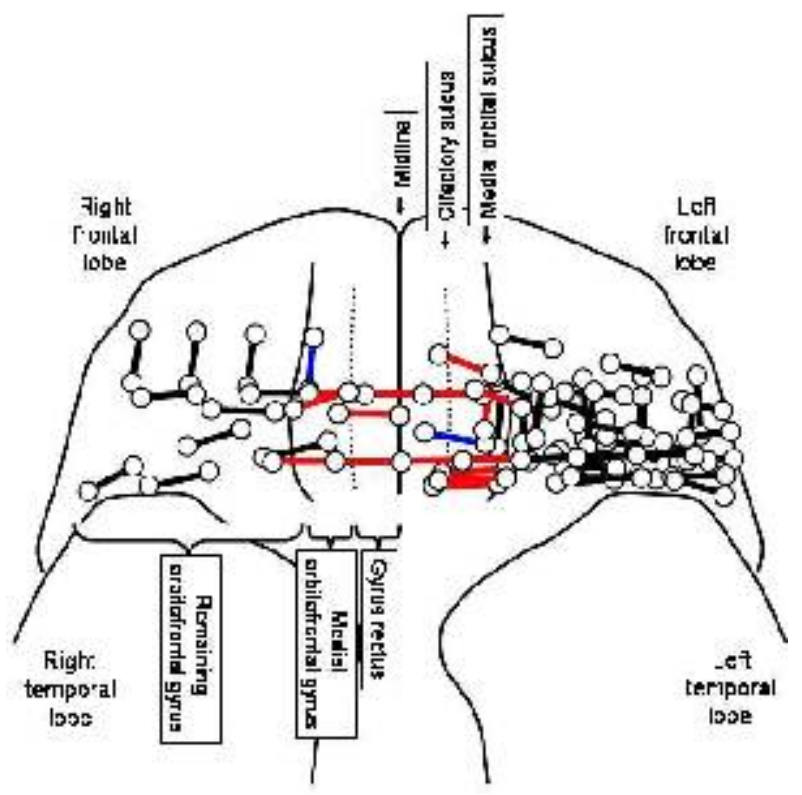

\subsection{2}

EMERGENCE OF NEURONAL SYNCHRONY DURING KINDLING

Xin Ren (chemical engineering, University of Virginia, Charlottesville, VA)

Rationale: Recurrent epileptic seizures involve the hippocampus in the brain. We hypothesized that pathological synchronous behavior of neurons occurs during the genesis of epilepsy. Further, we hypothesized that propagation of seizures takes place in both transverse and longitudinal directions in the hippocampus CA1. We tested above hypothesis by using quantitative methods to measure the synchrony and seizure propagation during electrically-kindled seizures.

Methods: Adult male Sprague-Dawley rats (250-300g) were studied. A bipolar stimulation electrode was stereotactically implanted in hippocampal CA3 region. A four-channel microelectrode array was placed to record neuronal activities in contralateral hippocampal CA1 region along the transverse (lamellar) or longitudinal (septotemporal) hippocampal axis. One channel of each microelectrode array was filtered $(0.1-5,00 \mathrm{~Hz})$ to record raw EEG, whereas, in parallel, all the channels were filtered $(300-4,000 \mathrm{~Hz})$ and sampled at $25 \mathrm{kHz}$ to record high frequency activates. Kindled seizures were initiated by using a standard stimulation protocol $(10 \mathrm{~s}, 50 \mathrm{~Hz}, 1 \mathrm{~ms}$ biphasic pulses), and behavioral seizures were scored according to Racine stages. Synchrony between simultaneously recorded activities was quantified by theta phase analyses and event synchronization (ES). Raw EEG signal was band-pass filtered between $4-10 \mathrm{~Hz}$ to extract theta oscillation. Theta phase approaches work directly with the timing of neuronal discharges by assigning phase value to the waveform. Shannon entropy was used to quantify the order of theta phase distribution of the waveform during the seizures. ES quantifies the number of times a peak occurs in two recordings within a small window. Peaks that occur in both recordings within a time interval are considered to be synchronous.

Results: We recorded 150 kindled seizures from 5 freely moving rats with transverse microelectrode array configuration. The mean Shannon entropy was $1.959 \pm 0.033$ for stage 1 seizures, and lowered to $1.509 \pm 0.097$ for stage 5 seizures, which showed neuronal synchrony increased as kindling progressed in CA1 lamellar direction. Theta phase analyses also demonstrated that CA1 pyramidal cells fired preferentially around the negative peak of theta oscillation with increasing behavioral seizure scores. Propagation pattern, revealed by calculating phase differences, showed that kindling from $\mathrm{CA} 3$ region resulted in neuronal discharges in 
CA1along lamellar from proximal to distal. Seizures $(n=145)$ recorded from longitudinally placed electrodes showed no significant change in Shannon entropy as kindling progressed. Further, no preferred timing was observed between neuronal firing and theta oscillation. Finally, neuronal firing revealed no propagation in septotemporal direction. Event synchronization showed neuronal firings along lamellar direction were more correlated (ES strength= 0.67) compared to septotemporal direction (ES strength $=0.36$ ). Conclusions: During kindled seizures, neuronal firings in CA1 are synchronized and propagate along its lamellar axis, but not in its septotemporal direction.

\subsection{3}

PROVOCATIVE TEST BY ELECTRICAL STIMULATION OF THE ENTORHINAL AREA PREVENTS MEMORY IMPAIRMENT FOLLOWING

\section{AMYGDALOHIPPOCAMPECTOMY}

Hui Ming Khoo ${ }^{1}$, H. Kishima ${ }^{1}$, N. Tani ${ }^{1}$, S. Oshino ${ }^{1}$, K. Hosomi ${ }^{1}$, T.

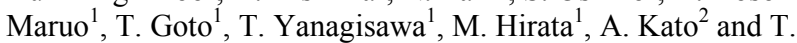
Yoshimine $^{1}\left({ }^{1}\right.$ Neurosurgery, Osaka University, Suita, Japan and ${ }^{2}$ Neurosurgery, Kinki University, Higashi-Osaka, Japan)

Rationale: Amygdalohippocampectomy (AHP) has been widely applied for medically refractory temporal lobe epilepsy (TLE). However, its indication for the verbal dominant hemisphere is limited due to the possibility of postoperative memory impairment. To prevent this disabling complication, methods with strong predictive power are highly required. Based on our preliminary experience, we describe the efficacy of provocative test by electrical stimulation of the entorhinal area in preventing postoperative memory impairment. Methods: We performed the abovementioned provocative test on ten consecutive patients with medically refractory TLE involving the dominant hemisphere. Prior to AHP, depth electrodes were inserted to the entorhinal area with stereotaxic technique. During the provocative test, verbal and visual memory functions were evaluated with delayed recall and/or recognition tasks. Electrical stimulation through the depth electrodes was performed during memorization (Figure 1). The pre and postoperative memory function were evaluated using Mini-Mental State Examination (MMSE) and Wechsler Memory Scale-Revised (WMS-R) and were compared in 6 patients who underwent AHP.

Results: Table 1 shows the results of the provocative test and postoperative memory functional outcome. Out of the 10 patients, delayed recall and recognition tasks following electrical stimulation during memorization were performed in 8 and 10 patients respectively for verbal memory. Visual memory task was performed in 7 patients.

Following electrical stimulation during memorization, delayed recall for verbal memory was affected in 6 out of 8 and recognition in 6 out of 10 patients. Among the 6 patients who underwent AHP, postoperative memory impairment developed in 2 patients in whom disturbances of both delayed recall and recognition for verbal memory were provoked. In contrast, memory function was intact in 3 without provoked verbal memory disturbance and also in one who demonstrated only provoked disturbance of delayed recall for verbal memory. None of the patients demonstrated provoked visual memory disturbance. AHP was considered not applicable to the remaining 4 patients based on the result of the provocative test.

Conclusions: Our result suggests that recognition tasks following electrical stimulation during memorization is the most predictive for the possibility of postoperative memory impairment. Provocative test using depth electrodes implanted in the entorhinal area might be promising in preventing the memory disturbance following AHP of dominant hemisphere. This technique warrants further evaluation as a predictive tool for possible post-AHP memory impairment.
Table1 Results of the provocative test and postoperative memory functional outcome

\begin{tabular}{|c|c|c|c|c|c|c|c|}
\hline \multirow{3}{*}{$\begin{array}{l}\text { Pt } \\
\text { no. }\end{array}$} & \multirow{3}{*}{$\wedge \mathrm{ge} / \mathrm{Sex}$} & \multirow{3}{*}{$\begin{array}{c}\text { MRI } \\
\text { rindings }\end{array}$} & \multicolumn{3}{|c|}{$\begin{array}{l}\text { Provocative lest by electrical } \\
\text { stimulation } \\
\text { Stimulation during memorization }\end{array}$} & \multirow[t]{3}{*}{$\Lambda H P$} & \multirow{3}{*}{$\begin{array}{l}\text { Postoperative memory } \\
\text { function outcomc }\end{array}$} \\
\hline & & & \multicolumn{2}{|c|}{ Verbal memory } & \multirow{2}{*}{$\begin{array}{l}\text { Visual } \\
\text { memory }\end{array}$} & & \\
\hline & & & $\begin{array}{l}\text { Delayed } \\
\text { recall }\end{array}$ & Recognition & & & \\
\hline 1 & $25 \mathrm{M}$ & $\begin{array}{l}\text { Arachnoid } \\
\text { cyst }\end{array}$ & $\downarrow$ & $\rightarrow$ & $\rightarrow$ & + & intact \\
\hline 2 & $43 \mathrm{M}$ & No lesion & $\downarrow$ & $\downarrow$ & $\rightarrow$ & + & $\begin{array}{c}\text { MMSГ: } 30 \rightarrow 26 \\
\text { WMS-R: } \\
\text { verbal } 79 \rightarrow 54, \\
\text { delayed recall } 82 \rightarrow 67\end{array}$ \\
\hline 3 & $66 \mathrm{M}$ & Glioma & $\downarrow$ & $\downarrow$ & $\rightarrow$ & - & \\
\hline 4 & $25 \mathrm{~F}$ & No lesion & $\downarrow$ & $\downarrow$ & $\rightarrow$ & - & \\
\hline 5 & $58 \mathrm{~F}$ & No lesion & $\downarrow$ & $\downarrow$ & $\rightarrow$ & + & $\begin{array}{l}\text { MMST: } 29 \rightarrow 25 \\
\quad \text { WMS-R: } \\
\text { verbal } 102 \rightarrow 68\end{array}$ \\
\hline 6 & $34 \mathrm{M}$ & No lesion & $\downarrow$ & $\downarrow$ & $\rightarrow$ & - & \\
\hline 7 & $28 \mathrm{~F}$ & No lesion & $\rightarrow$ & $\rightarrow$ & $\rightarrow$ & + & intact \\
\hline 8 & $16 \mathrm{M}$ & No lesion & n.a. & $>$ & n.a. & + & intact \\
\hline 9 & $18 \Gamma^{\circ}$ & No lesion & n.a. & $\downarrow$ & n.a. & - & \\
\hline 10 & $38 \mathrm{M}$ & No lesion & $\rightarrow$ & $\rightarrow$ & n.a. & + & intact \\
\hline
\end{tabular}

$\rightarrow$ no change, $\downarrow$ affected, n.a. not available,

AHP: Amygdalohippocampectomy,

MMSE: Mini-Mental State Examination,

WMS-R: Wechsler Memory Scale-Revised.

Figure 1 Functional mapping of hippocampus

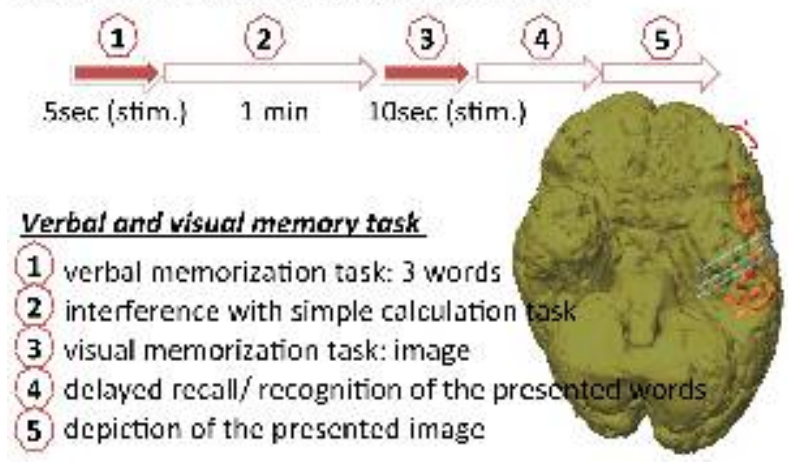

2.074

MULTIMODAL INVESTIGATION OF THE SEGREGATION OF FUNCTIONAL AND PATHOLOGICAL NETWORKS Corey Keller, ${ }^{1,2}$, L. Entz ${ }^{5}$, S. Bickel ${ }^{1}$, D. M. Groppe ${ }^{1}$, E. Toth ${ }^{6}$, P. B.

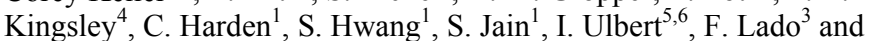
A. D. Mehta ${ }^{1}\left({ }^{1}\right.$ Neurology and Neurosurgery, Hofstra North Shore LIJ School of Medicine and Feinstein Institute for Medical Research, New Hyde Park, NY; ${ }^{2}$ Neuroscience, Albert Einstein College of Medicine, Bronx, NY; ${ }^{3}$ Neurology, Montefiore Medical Center, Bronx, NY; ${ }^{4}$ Radiology, North Shore LIJ Health System, Manhassat, NY; ${ }^{5}$ Institute for Psychology of the Hungarian Academy of Sciences, Budapest, Hungary and ${ }^{6}$ Pázmány Péter Catholic University, Faculty of Information Technology, Budapest, Hungary)

Rationale: Determining the extent of 'eloquent' cortex as well as regions involved in early seizure activity will likely benefit epilepsy surgery. However, techniques used to measure these networks involve high frequency stimulation to observe behavioral arrests and recording of ictal activity, respectively. Localization of these networks during the interictal period would provide a complementary measure to the current gold standards and possibly identify regions not implicated during clinical workup. 
Methods: We combined single pulse stimulation, resting state fMRI, and resting state electrocorticography (ECoG) from 5 patients with intractable epilepsy undergoing surgical evaluation of seizure foci. Cortical regions involved in seizure initiation and early propagation (the 'pathological network') were identified visually in the iEEG by a neurologist blind to the study. Functional networks were defined by behavioral changes in language, vision, movement, or sensation as a result of high frequency stimulation mapping. Single pulse stimulation (10mA, 100us/phase, 20 trials, $2 \mathrm{~s} \mathrm{ISI)} \mathrm{was} \mathrm{applied} \mathrm{to}$ adjacent contacts on implanted grids and strips and the evoked potentials (CCEPs) were recorded at each location. Pre-operative resting state fMRI and post-implantation 'resting state' ECoG were performed for 3-5 minutes while patients were instructed to close their eyes and let their mind wander. Correlated fluctuations of the BOLD signal (resting state functional connectivity - RSFC) as well as correlated fluctuations of the high gamma $(70-150 \mathrm{~Hz})$ power signal (HGP correlations) at each electrode pair were quantified. For each electrode, its network was identified ('functional', 'pathologic', or neither) and the connectivity at electrodes within or outside each network were compared. Additionally, graph theoretical measures were computed from each connectivity matrix (RSFC, HGP correlations, CCEPs).

Results: For each subject, connectivity measures produced similar spatial maps. Functional and pathological networks were highly segregated, exhibiting higher connectivity in all three measures within each network than outside the network. Considering that most of these networks are located in close proximity to each other, we applied a distance correction factor and found that within-network measures were still significantly higher than between-network measures. Higher path length and lower degree nodes were present in pathological networks when compared to other nodes.

Conclusions: We found functional and pathological networks to be segregated from other cortical regions by means of metabolic and neuronal connectivity measures. Localization of these networks using non-invasive methods during the interictal period would provide a complementary measure to the current gold standards and possibly identify regions not implicated during clinical workup.

\subsection{5 \\ LOW FREQUENCY ELECTRICAL STIMULATION OF WHITE MATTER TRACTS IN INTRACTABLE MESIAL TEMPORAL LOBE EPILEPSY}

M. Koubeissi ${ }^{1}$, D. Durand ${ }^{2}$, E. S. Kahriman ${ }^{1}$, T. Syed ${ }^{1}$, J. Miller ${ }^{1}$ and H. Lüders ${ }^{1}\left({ }^{1}\right.$ University Hospitals Case Medical Center, Cleveland, $\mathrm{OH}$ and ${ }^{2}$ Case Western Reserve University, Cleveland, $\mathrm{OH}$ )

Rationale: To explore the tolerability and efficacy of low-frequency stimulation (LFS) of white matter tracts (WMT) in patients with intractable mesial temporal lobe epilepsy (MTLE). Deep brain stimulation has had limited success in epilepsy. However, WMTs have not been stimulated, and low frequencies have not been adequately explored for treatment of epilepsy.

Methods: Eleven adult patients with MTLE underwent implantation of depth electrodes in WMTs: the corpus of the fornix $(n=2)$ and lateral to the splenium of the corpus callosum where fibers of the dorsal hippocampal commissure travel with the crus of the fornix $(n=9)$. All patients had hippocampal electrodes. 1-Hz stimulation of the WMT in all subjects elicited hippocampal evoked potentials (EPs), confirming connectivity. The EP duration was approximately $200 \mathrm{msec}$ suggesting that $5-\mathrm{Hz}$ stimulation would elicit continuous interference of hippocampal activity. LFS-WMT occurred in 4-hour sessions (1-3 sessions/patient at $5 \mathrm{~Hz}$, current $8 \mathrm{~mA}$, pulse width $0.2 \mathrm{msec}$ ) while in the video-EEG unit.

Results: There were no complications, and hourly mini-mental status examination scores showed a mean increase of 0.9 during LFS-WMT $(\mathrm{n}=11 ; \mathrm{P}<0.001$, Generalized estimating equations [GEE]-Identity Link Function [ILF]). Patients 8-10 proved to have an extrahippocampal seizure onset, so they were excluded from the spike and seizure analysis, but were still included in the MMSE analysis. LFS-WMT resulted in a significant reduction of hippocampal spikes ( $\mathrm{n}=8 ; \mathrm{p}=0.001$, GEE-ILF), which persisted for the subsequent 4 hours $(\mathrm{p}=0.05)$. Seizure odds were reduced by $91 \%$ in 1-2 days following each 4-hr LFS-WMT session $(n=8 ; p=0.001$, GEE- Logit Link Function), without medication changes. Conclusions: Our data show that LFS-WMT activates the hippocampus and other areas of Papez circuit, and reduces epileptiform discharges and seizures, without affecting memory. Stimulation of WMTs appears to be attractive because it interferes with spontaneous electrical activity of a relatively extensive cortical area, and requires less current injection, implying more safety and a longer battery life. Our data illustrate the need for an exploratory trial to confirm safety and tolerability of LFS-WMT using different parameters using a chronic implantable pulse generator.

\subsection{6 \\ REDUCTION OF SEIZURE FREQUENCY BY RESPONSIVE JUST-IN-TIME VNS IN AN ANIMAL MODEL OF CHRONIC EPILEPSY

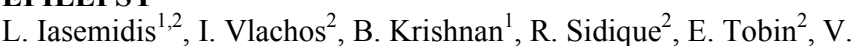 Venkataraman $^{1}$, A. Faith ${ }^{2}$, S. Prasanna ${ }^{1}$, A. Shafique ${ }^{1}$, K. Tsakalis ${ }^{1}$, S. Marsh ${ }^{3}$, D. Treiman ${ }^{3}$, S. Sabesan ${ }^{4}$ and S. Maschino ${ }^{4}\left({ }^{1}\right.$ Electrical Engineering, Arizona State University, Tempe, AZ; ${ }^{2}$ Biomedical Engineering, Arizona State University, Tempe, AZ; ${ }^{3}$ Neurology, Barrow Neurological Institute, Phoenix, AZ and ${ }^{4}$ Cyberonics, Inc., Houston, TX)}

Rationale: We developed a closed-loop responsive Vagus Nerve Stimulation (VNS) platform to investigate the just-in-time effect of VNS stimulation on seizure frequency in the lithium-pilocarpine animal model of epilepsy.

Methods: Eight male Sprague-Dawley rats were induced to chronic epilepsy by the lithium-pilocarpine model of epilepsy. Each rat was subsequently implanted with 8 Tungsten microwires (two in centromedial thalamus, two in hippocampus and four on cortex). Rats were then implanted with two helical leads made of platinum/iridium coated with silastic (Cyberonics Inc., Houston, TX) wrapped around the left vagus nerve in the ventral cervical region for VNS electrical stimulation by programmable stimulators (A-M Systems Inc., Sequim, WA). The experiment involved two phases per rat: stimulation (S) and no stimulation (NS), 4 to 5 days each in duration. During (S), VNS was administered upon warnings issued when analyzed EEG dynamics from critical pairs of brain sites were found to be significantly ( $p<0.05$, t-test) entrained over the preceding 10 min interval. VNS stimulation was delivered until the critical pairs of sites disentrained or up to a maximum of 1 hour interval after a warning. By design, the maximum rate that just-in-time (JIT) VNS stimulation could occur was once every 3 hours; in reality the mean rate of JIT VNS was a fraction of this value, depending on each rat's EEG dynamics. This closed-loop procedure was performed automatically and in real time by our online, JIT seizure prediction software [1]. During (NS), no stimulation was administered at the issued warnings (control phase).

Results: Seizures occurred in both phases of the experiment and were detected by our in-house developed seizure detection algorithms. The frequency of seizures in each phase was then estimated and compared with each other, per rat and across rats. Six of the eight rats showed a reduction in seizure frequency during the $(\mathrm{S})$ phase compared with the (NS) phase. Cumulatively, across all rats, a statistically significant ( $\mathrm{p}<0.05$, t-test) reduction of the mean seizure frequency, from 8 seizures a day without stimulation to 2 seizures a day with stimulation, was observed over the experiment's duration (see Figure $1)$.

Conclusions: Responsive, just-in-time, VNS was found to be effective in significantly reducing the seizure frequency in the lithium-pilocarpine animal model of epilepsy. These results provide an initial supporting evidence for the use of closed-loop just-in-time 
VNS stimulation in the treatment of epilepsy.

Acknowledgement: This study was funded by Cyberonics Inc. Houston, TX.

[1] Good L., Sabesan S., Marsh S., Tsakalis K., Treiman D. \& Iasemidis L.D., Int. J. Neural Systems, 19(3), 173-196, 2009.

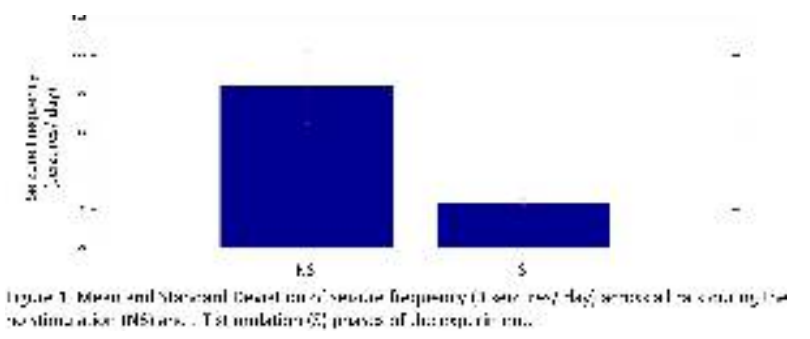

\subsection{7}

ALTERED CORTICAL EXCITABILITY IN PATIENTS WITH IDIOPATHIC GENERALISED EPILEPSY AND THEIR ASYMPTOMATIC FIRST DEGREE RELATIVES

Adam D. Pawley, F. A. Chowdhury, P. Rowe and M. P. Richardson (Clinical Neuroscience, King's College London, London, United Kingdom)

Rationale: The majority of idiopathic generalised epilepsies (IGE) have a complex genetic inheritance, (Trends Neurosci 2006; 29(7): 391-397). Although the exact mechanisms underlying the pathogenesis of epilepsy are unknown, it is thought to result from an imbalance from excitatory and inhibitory pathways. Transcranial magnetic stimulation (TMS) allows investigation of both excitatory and inhibitory functions of central motor pathways in vivo providing non -invasive clinical measurements of neuronal excitability (Clin Neurophys 2003; 114 (5): 777-798). Previous studies using TMS measures have shown altered excitability in patients with IGE including prolonged cortical silent period (CSP). (Neurology 2001; 57(4): 706-8, Seizure 2004; 13(7):481-485). CSP is thought to be mediated by intracortical inhibitory mechanisms involving GABA in the primary motor cortex (J Physiol 1999; 517(2): 591-7).

Endophenotypes are heritable traits with a simpler genetic basis than the full disorder and may be present in family members who do not have the disease (Am J Psych 2003; 160(4): 636-45).

Endophenotypes can help increase our understanding of the pathophysiology of complex genetic diseases. TMS parameters have been shown to be heritable (J Neurosci 2009; 28(6): 464-73). In this study we used TMS to investigate cortical silent period as a potential endophenotype for idiopathic generalised epilepsy by testing whether CSP is prolonged in a group of patients with IGE and their first degree relatives.

Methods: We studied thirty-two patients with IGE (7 JME, 4 JAE, 4 CAE, 11 with GTCS only, 1 with absences with eyelid myoclonia), 35 of their first-degree relatives and 37 age and sex-matched healthy controls. Magnetic stimulation was performed using two Magstim 200 stimulators connected via a MagStim BiStim Unit (Magstim, Dyfed, UK) with EMG activity measured from first dorsal interossei muscle bilaterally. Single pulse TMS was used to measure resting and active motor threshold (R/AMT) and CSP. To reduce observer bias, CSP duration was analysed using an automated method cumulative sum (Cusum) technique (J NeuroSci Meth 2006; 150(1): 96-104. Group differences were investigated using Kruskal-Wallis test.

Results: Patients with IGE showed significantly higher motor thresholds (dominant RMT $\mathrm{p}=0.006$, non-dominant $\mathrm{RMT} \mathrm{p}=0.005$, dominant AMT $\mathrm{p}=0.008$, non-dominant AMT $\mathrm{p}=0.001$ ) compared with controls). There were no significant differences in motor thresholds between relatives and controls.

First-degree relatives had significantly prolonged cortical silent periods compared with controls in both hemispheres (dominant $\mathrm{p}=0.020$, non-dominant $\mathrm{p}=0.005$ ). Patients also exhibited longer CSP than control subjects in the dominant hemisphere $(p=0.006)$. See Figure 1.

Conclusions: Higher motor threshold in patients may be related to medication effects. However, the presence of prolonged cortical silent period in IGE patients and their first-degree relatives may reflect an inherited alteration in cortical excitability ie. an endophenotype in epilepsy.

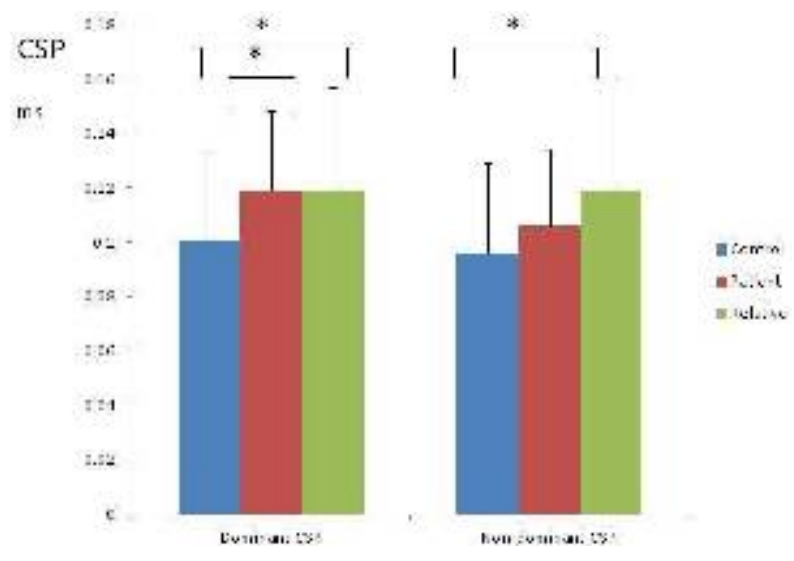

\subsection{8 \\ INCIDENCE AND TYPES OF STIMULATION-EVOKED EPILEPTIFORM ACTIVITY DURING EXTRA-OPERATIVE CORTICAL STIMULATION MAPPING}

Anna M. Bank, C. A. Schevon, A. C. Williams and M. J. Hamberger (Columbia University, New York, NY)

Rationale: Extra-operative electrical cortical stimulation mapping (ESM) is frequently used to identify language, motor, and sensory cortex prior to surgical resection, with the goal of preserving function postoperatively. Although attempts are made to avoid stimulationevoked afterdischarges (ADs) and seizures, the frequency and clinical impact of these events is unclear. By qualifying and quantifying the frequency of ADs and seizures associated with ESM, we sought to determine the extent to which these events 1) interfere with mapping, and 2) simulate the semiology and localization of patients' typical seizures.

Methods: A retrospective review of clinical documentation was conducted for 66 patients with refractory focal epilepsy (mean age 30.6 years \pm 12.1 ) who underwent subdural electrode implantation and ESM at Columbia University Comprehensive Epilepsy Center between 1997 and 2012. Regions implanted included temporal $(60.6 \%)$, frontal (31.8\%), parietal (10.6\%), and occipital cortex $(10.6 \%)$. Fifty implants $(75.8 \%)$ involved the dominant hemisphere. For each patient, we determined 1) rate and type of stimulationevoked epileptiform activity, 2) rate of evoked events that interfered with mapping, and 3) rate of evoked events that were similar to patients' typical seizures. Interference was defined as cessation of the mapping session.

Results: Of 1713 electrode pairs stimulated, afterdischarges were provoked at 695 pairs $(40.6 \%)$ in 54 patients $(81.8 \%)$. The mean number of affected pairs per patient was $11.6 \pm 9.4$, or a mean $40.4 \%$ of pairs tested. $30.6 \%$ of ADs in 21 patients $(31.8 \%)$ spread to neighboring electrodes without producing clinical seizures. These ADs interfered with ESM in only 2 patients (3\%). ADs evolved into clinical seizures at 57 pairs $(3.3 \%)$ in 23 patients $(34.8 \%)$. Seizures were classified as simple partial in 8 patients $(12.1 \%)$, complex partial in 8 patients $(12.1 \%)$, secondarily generalized in 6 patients $(9.1 \%)$, and were unspecified in 3 patients $(4.5 \%)$. Simple partial seizures did not interfere with ESM in any patients. Complex partial seizures and unspecified seizures each interfered in 3 patients $(4.5 \%)$ and generalized seizures interfered in all 6 patients $(9.1 \%)$. Of the 23 patients with stimulation-evoked seizures, $15(65 \%)$ had seizures that were clinically similar to their baseline seizures. Five patients had 
seizures originating only within their typical seizure onset zones, 4 patients had seizures originating only outside the seizure onset zone, and 14 patients had seizures originating both within and outside the seizure onset zone.

Conclusions: Stimulation-evoked afterdischarges and partial seizures occur frequently during ESM. While they may have an impact on the quality of functional localization, ADs and partial seizures were rarely noted to disrupt the mapping procedure. Secondarily generalized seizures, however, are more disruptive. Although stimulation-evoked seizures are undesirable, they may provide valuable localizing information as they often replicate patients' habitual events.

\subsection{9}

ALTERATION OF CONSCIOUSNESS DUE TO ELECTRICAL STIMULATION OF THE CLAUSTRUM IN THE HUMAN BRAIN

M. Z. Koubeissi ${ }^{1}$, Abdelrahman Beltagy ${ }^{1}$, F. Picard ${ }^{2}$ and M. Edrees ${ }^{1}$ ( ${ }^{1}$ Epilepsy center, Neurological Institute, University hospitals, Case Western Reserve University, Cleveland, $\mathrm{OH}$ and ${ }^{2}$ Neurosciences, Neurology Service, Hôpitaux Universitaires de Genève, Genève, Switzerland)

Rationale: The claustrum is a thin layer of grey matter that is separated from the insula by the extreme capsule and from the putamen by the external capsule. The function of the claustrum is unknown, although animal studies have demonstrated bidirectional connectivity between the claustrum and all neocortical regions. This extensive connectivity suggests a role in binding visual, somatosensory, auditory, and motor information, and it has been suggested that the claustrum plays a main role in integration of conscious percepts (Crick \& Koch 2004). Electrical stimulation of the claustrum in cats results in a behavioral arrest and unresponsiveness, similar to the alteration of awareness that accompanies some seizures in humans (Gobar 1964). Here, we present the results of electrical stimulation of the claustrum in humans.

Methods: A 58-year-old woman presented with intractable complex partial epilepsy and olfactory auras. Her non-invasive video-EEG monitoring confirmed left hemispheric seizure onset. She then underwent invasive monitoring with depth electrodes for identification of the seizure focus, including a 12-contact depth electrode that sampled the left insula and claustrum. Scalp electrodes were also added. She underwent electrocortical stimulation mapping at $50-\mathrm{Hz}$ with current intensities reaching $18 \mathrm{~mA}$.

Results: Stimulation of the claustrum at currents $8 \mathrm{~mA}$ or higher resulted in a behavioral arrest, blank staring, and unresponsiveness with reduction of spontaneous respiratory movements. This effect was consistently reproducible. The patient returned to her baseline as soon as the stimulation stopped with no recollection of the stimulation period. Her symptoms during stimulation could not be attributed to negative motor phenomena or aphasia. She was able to continue repetitive movements with her tongue and hands during stimulation if initiated before the stimulus train. Similarly, if she started to say a word repeatedly before stimulation, she could continue the task through stimulation, although with some dysarthria. No seizures or after discharges were seen on her depth electrode or scalp EEG. Stimulation of the Insula or the white matter surrounding the claustrum did not elicit any changes.

Conclusions: Our findings indicate that the claustrum is an essential part of the neuronal network that controls awareness and suggest that involvement of the claustrum may explain the alteration of awareness typical of temporal lobe seizures. In addition, absence seizures may be associated with bilateral claustral involvement as suggested by EEG-fMRI studies that found increased activation in the insulae and claustrums during idiopathic generalized epileptiform discharges (Gotman et al., 2005; Liu et al., 2008).

\subsection{0}

LONG-TERM EFFECT OF VNS ON SEIZURE BURDEN IN AN ANIMAL MODEL OF CHRONIC EPILEPSY

I. Vlachos ${ }^{1}$, B. Krishnan ${ }^{2}$, R. Sidique ${ }^{1}$, E. Tobin ${ }^{1}$, V. Venkataraman ${ }^{2}$, A. Faith ${ }^{1}$, S. Prasanna ${ }^{2}$, A. Shafique ${ }^{2}$, K. Tsakalis ${ }^{2}$, L. Iasemidis ${ }^{1,2}$, S. Marsh $^{3}$, D. Treiman ${ }^{3}$, S. Sabesan ${ }^{4}$ and S. Maschino ${ }^{4}\left({ }^{1}\right.$ Biomedical Engineering, Arizona State University, Tempe, AZ; ${ }^{2}$ Electrical Engineering, Arizona State University, Tempe, AZ; ${ }^{3}$ Neurology, Barrow Neurological Institute, Phoenix, $\mathrm{AZ}$ and ${ }^{4}$ Cyberonics, Inc., Houston, TX)

Rationale: In a longitudinal animal study, we investigated the effect of Vagus Nerve Stimulation (VNS) on seizure burden employing the lithium-pilocarpine model of epilepsy in rats. We tested the hypothesis that the seizure burden is reduced upon stimulation of the vagus nerve over a comparatively long time. This hypothesis was tested by utilizing a protocol with VNS alternatively ON and OFF for 2 weeks at a time. The plasticity of the effect of VNS on seizure burden also was investigated.

Methods: Three male Sprague-Dawley rats were induced to chronic epilepsy according to the lithium-pilocarpine model of epilepsy [1]. Rats were subsequently implanted with a total of 8 Tungsten microwires for long-term EEG recording from thalamus, hippocampus and cortex, and with two leads (Cyberonics Inc., Houston, TX) wrapped around the left vagus nerve in the ventral cervical region for electrical stimulation by externally positioned, programmable, stimulators (A-M Systems Inc., Sequim, WA). The VNS was delivered periodically (60 sec ON, $300 \mathrm{sec}$ OFF). During the ON phase, a $30 \mathrm{~Hz}$ train of pulses, each of $220 \mu \mathrm{sec}$ in duration and $0.5 \mathrm{~mA}$ in amplitude, was delivered. The long-term EEG was recorded and analyzed over five separate periods of the experiment that each lasted for at least 2 weeks: Baseline 1 (no stimulation), Stimulation 1 (VNS stimulation), Baseline 2 (no stimulation), Stimulation 2 (VNS stimulation), Baseline 3 (no stimulation). Seizures' occurrence and duration were detected by retrospective analysis of the continuously recorded EEG via in-house developed software based on Teager energy. Seizure burden was defined as the percentage of recording time a rat spent in ictal state.

Results: All three rats showed a statistically significant $(\mathrm{p}<0.05$, $t$ test) reduction of seizure burden during VNS stimulation periods when compared with their preceding baselines (e.g. see Figure 1). In addition, a trend towards reduction of seizure burden over time (from Baseline 1 to Baseline 3) was observed in two rats. In the third rat, increase of the seizure burden was observed from Baseline 1 to 2, and saturation from Baseline 2 to 3 .

Conclusions: VNS was found to be effective in significantly reducing the seizure burden in the limited number of rats with chronic epilepsy that participated in this longitudinal study. Furthermore, plasticity of the VNS effect was observed in the majority of the rats. These results provide supporting evidence for the longitudinal use of VNS in reducing seizure burden.

Acknowledgement: This study was funded by Cyberonics Inc., Houston, TX.

[1] Persinger, M.A., K. Makarec, and J.-C. Bradley, "Characteristics of limbic seizures evoked by peripheral injections of lithium and pilocarpine". Physiology \& Behavior. 44(1) (1988) 27-37.

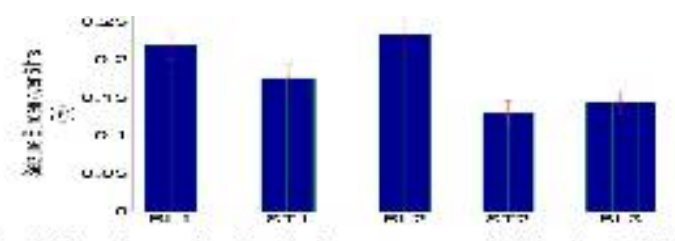

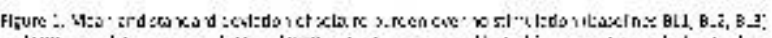

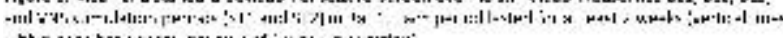

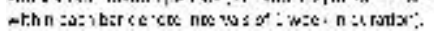




\subsection{1}

HUMAN EPILEPTOGENIC NETWORKS ARE REVEALED BY PROPAGATION OF ICTAL AND POST-ICTAL HIGH FREQUENCY ACTIVITY (70-170 HZ)

Anna Korzeniewska $^{1}$, P. J. Franaszczuk ${ }^{2}$, M. C. Cervenka ${ }^{1}$, C. C. Jouny ${ }^{1}$, G. K. Bergey ${ }^{1}$ and N. E. Crone ${ }^{1}\left({ }^{1}\right.$ Johns Hopkins School of Medicine, Baltimore, MD and ${ }^{2}$ US Army RDECOM, Army Research Laboratory, RDRL-HRS-C, Aberdeen Proving Ground, MD)

Rationale: High frequency oscillations are observed more frequently, and in closer proximity to the seizure onset zone than outside of it, suggesting that they may help identify epileptogenic networks. However, their temporal and spatial characteristics may vary by seizure type and between individuals. We hypothesize that the patterns of propagation of high frequency activity among cortical networks will help identify the epileptogenic zone, which could ultimately improve seizure localization for epilepsy surgery planning. Methods: Intracranial EEG recordings (ECoG) of six patients with focal and regional epilepsy were analyzed with a matching pursuit (MP) method to identify ECoG signals containing high frequency ictal activity. Signals were parameterized using multivariate autoregressive models (MVAR), and analyzed with event-related causality method (ERC). ERC estimates the direction, intensity, and temporal evolution of neural activity propagation among cortical sites, and statistically compares non-stationary time series of interest (e.g. seizure) with non-stationary reference time series (e.g. interictal interval). We used ERC to study the patterns of propagation of high frequency activity $(70-170 \mathrm{~Hz})$ during inter-, pre-, and ictal intervals, as well as significant differences between the patterns of inter-, pre-, and ictal activity propagation.

Results: In patients with focal epilepsy, we observed a common sequence of increases in high-frequency propagation when ictal intervals were compared with interictal or pre-ictal intervals. Immediately after seizure onset, propagations occurred from sites identified by epileptologists as ictal onset zones to all other analyzed recording sites. Next, ictal propagations were observed between ictal onset zones and sites in close proximity to these onset zones.

Interestingly, subsequent increases of ictal propagation also involved sites inferred as onset zones from pre-surgical scalp recordings. Changes in high-frequency propagation between the ictal onset zone and all other analyzed recording sites were also observed in the postictal interval (compared to inter- or preictal intervals). In patients with poorly localized (regional) ictal onsets, these patterns were not observed.

Conclusions: Comparisons of patterns of high-frequency activity propagation during inter-, pre-, ictal, and post-ictal intervals may be useful for localizing the epileptogenic zone for epilepsy surgery. The seizure onset zone may be characterized by increases in high frequency activity propagation from the onset zone to other recording sites at seizure onset, as well as by changes in propagation between the onset zone and other recording sites during post-ictal intervals. This project was funded by NINDS R01 NS40596.

\subsection{2 \\ INCLUSION OF SPHENOIDAL ELECTRODES IN REALISTIC EEG SOURCE IMAGING CHANGES LOCALIZATION RESULTS IN MESIAL TEMPORAL LOBE EPILEPSY \\ Mehdi Bagheri-Hamaneh $^{1}$, K. Kaiboriboon ${ }^{2}$, J. Turnbull ${ }^{2}$, D. Dimitriu $^{2}$, H. Luders ${ }^{2}$ and S. Lhatoo ${ }^{2}\left({ }^{1}\right.$ Case Western Reserve University, Cleveland, $\mathrm{OH}$ and ${ }^{2}$ University Hospitals Case Medical Center, Cleveland, $\mathrm{OH}$ )}

Rationale: Sphenoidal electrodes $(\mathrm{SpE})$ have been shown to be helpful in detecting mesial temporal spikes. Unfortunately, including $\mathrm{SpE}$ in realistic EEG source imaging (ESI) is difficult, because the exact positions of these electrodes are unknown, and also because the existing ESI software usually calculate the potential only on the surface of the head and not at the locations of SpE. We now report, for the first time, the feasibility to determine the locations of $\mathrm{SpE}$ and to incorporate this information into ESI. In addition, the impact of including $\mathrm{SpE}$ on source localization in mesial temporal lobe epilepsy (MTLE) is assessed.

Methods: Nine patients with refractory MTLE, based on their clinical semiology and electrophysiologic data, were included for this study. The potentials recorded at 10-20 electrodes, a pair of subtemporal electrodes (FT9/10 or F9/10) and SpE were used for ESI. The locations of the scalp electrodes were determined using a digitizer. To find the positions of SpE two orthogonal X-rays (sagittal and coronal) were taken while the scalp electrodes were attached to the head and the sphenoidal needle was inserted. An in-house computer program was then used to find the locations of the tip of the needle relative to the surface electrodes on the X-rays. This information was used to determine the $3 \mathrm{D}$ coordinates of the tip of the needle.

A finite element method (FEM) based on the patient's MRI was used for forward solution calculations. This method allows computation of the electrical potential at the true locations of SpE. For each patient, at least 10 similar spikes were selected for averaging. Then a single fixed dipole was fitted to the measured data over the time interval starting at the midway between the baseline and the peak, and ending at the peak of each averaged spike. The SpE were then turned off and the calculations were repeated. For each patient the distance between the dipoles in presence and in absence of $\mathrm{SpE}$ was calculated. T-tests (at the 5\% significance level) were then performed to determine if the observed differences between locations of the dipoles in two cases were significant.

Results: The maximum/minimum observed distance between the two dipoles (with and without $\mathrm{SpE}$ ) was $36.5 / 0.3 \mathrm{~mm}$ and the average was $17.9 \mathrm{~mm}$. The t-tests, however, showed that only the difference in the vertical direction was significant. In fact in $78 \%$ (7) of the cases the dipole location in presence of $\mathrm{SpE}$ was determined to be lower than the corresponding dipole when these electrodes were turned off. In the patient with the biggest dipole shift, inclusion of $\mathrm{SpE}$ moved the location of the source from the right inferior insula to the right anterior temporal pole.

Conclusions: We report on a technique to determine the actual locations of SpE for realistic ESI. Our results show that inclusion of SpE could significantly change ESI results in patients with MTLE, even when surface subtemporal electrodes are present.

\subsection{3}

AUDITORY-LANGUAGE-RELATED GAMMA ACTIVITY: A CONSECUTIVE SERIES OF 74 EPILEPTIC PATIENTS Katsuaki Kojima $^{1}$, E. C. Brown ${ }^{5,6}$, R. Rothermel ${ }^{4}$, A. Carlson ${ }^{1,4}$, D. Fuerst $^{2}$, N. Matsuzaki ${ }^{1}$, A. Shah ${ }^{2}$, M. Atkinson ${ }^{2}$, M. Basha ${ }^{2}$, S. Mittal $^{3}$, S. Sood ${ }^{3}$ and E. Asano ${ }^{1,2}\left({ }^{1}\right.$ Pediatrics, Wayne State University, Detroit Medical Center, Detroit, MI; ${ }^{2}$ Neurology, Wayne State University, Detroit Medical Center, Detroit, MI; ${ }^{3}$ Neurosurgery, Wayne State University, Detroit Medical Center, Detroit, MI; ${ }^{4}$ Psychology, Wayne State University, Detroit Medical Center, Detroit, MI; ${ }^{5}$ Psychiatry and Behavioral Neuroscience, Wayne State University, School of Medicine, Detroit, $\mathrm{MI}$ and ${ }^{6} \mathrm{MD}-\mathrm{PhD}$ Program, Wayne State University, School of Medicine, Detroit, MI)

Rationale: The potential complications following epilepsy surgery include temporary or permanent language deficits. Postoperative development of language deficit may require speech therapy and may delay the return to home, school or work. Surgeries of the lateral left hemisphere generally pose the greatest risk of such deficit. That is, unless essential language function is reorganized to the right hemisphere, as most commonly seen in left-handed epileptic patients with early-onset neocortical lesions on neuroimaging. To minimize the risk of postoperative deficits, investigators try to achieve accurate 
localization of eloquent cortices prior to tissue resection. Studies have shown that language-related augmentation of gamma activity on electrocorticography (ECoG) often detects language-related sites defined by electrical stimulation.

Methods: We validated the utility of auditory-language-related gamma-augmentation as a biomarker of underlying language function, by analyzing a consecutive series of 74 epileptic patients (age: 4 - 56 years). First, in left-handed patients with left-sided seizure focus and early-onset left-sided neocortical lesions, essential language function was estimated to reorganize to the right hemisphere. In the remaining patients, the left hemisphere was estimated to contain essential language function, regardless of handedness. We determined the effects of seizure onset zone, electrode location, and patient age upon gamma-augmentation elicited by an auditory-naming task. We determined if resection of sites showing gamma-augmentation predicted a language deficit. Results: Significant gamma-augmentation was less frequently elicited within seizure onset sites compared to the other sites. Regardless of age, patients showed gamma-augmentation, most often involving the $80-100 \mathrm{~Hz}$ frequency band. Gamma-augmentation initially involved the superior-temporal regions, bilaterally, followed by the middle-temporal, medial-temporal, inferior-frontal, dorsolateral-premotor, and medial-frontal, and concluded with bilateral inferior-Rolandic involvement. Sites showing gammaaugmentation outside of superior-temporal and inferior-Rolandic regions were more extensive in the left hemisphere with considerable temporal overlap. Compared to younger patients, those older than 10 years had a larger proportion of left dorsolateral-premotor sites showing gamma-augmentation. The incidence of a post-operative language deficit requiring speech therapy was best predicted by the number of resected sites with significant gamma-augmentation in the superior-temporal, inferior-frontal, dorsolateral-premotor, and inferior-Rolandic regions of the left hemisphere estimated to contain essential language function ( $\mathrm{r}$-square $=0.651 ; \mathrm{p}=0.001$; Odds Ratio: 8.9 [95\%CI: 2.6-31.0]). The prediction performance of this logistic regression model incorporating gamma-augmentation remained significant after controlling for the extent of resection or electrical stimulation data.

Conclusions: Auditory-language-related gamma-augmentation on ECoG can provide additional information useful in the localization of primary language areas.

\subsection{4 \\ TRANSIENT EFFECT OF INTERICTAL SPIKES FROM A FRONTAL FOCUS ON LANGUAGE-RELATED GAMMA ACTIVITY}

Erik Brown ${ }^{1}$, E. Asano ${ }^{2}$ and N. Matsuzaki ${ }^{2}\left({ }^{1}\right.$ Psychiatry and Behavioral Neurosciences, Wayne State University, Detroit, MI and ${ }^{2}$ Neurology, Children's Hospital of Michigan, Detroit, MI)

Rationale: The presumption of a deleterious impact of focal epileptic spike-and-wave activity is not new. Shewmon and Erwin demonstrated in 1989 that posteriorly localized spike-and-wave activity could transiently increase reaction time and error rate in a visual reaction time task. Studies combining electroencephalography and imaging have since suggested spike-related metabolic changes around the seizure focus and beyond. Use of a modality more directly measuring the dynamics of spike-induced deficits is warranted. We have amassed a sizable database of language-related electrocorticography $(\mathrm{ECoG})$ data in focal epilepsy patients. This presents an opportunity to study the effects of interictal spikes upon on-going activities engaging higher order function, such as language. We determined whether interictal spikes from a frontal seizure focus transiently suppressed language-related augmentation of gamma activity at $80-100 \mathrm{~Hz}$.

Methods: A retrospective search of our patient cohort employed the following inclusion criteria: (i) an ECoG-defined left frontal seizure onset zone (SOZ) and (ii) mapping of naming-related gamma- augmentation via ECoG using an auditory naming task. Of the result, four patients survived the following exclusion criteria: (i) multiple seizure foci, (ii) massive brain malformations, or (iii) right language dominance by Wada testing or left-handedness when Wada test results are not available. An automated spike detection algorithm was utilized (Barkmeier et al. Clin Neurophysiol, 2011). Naming task trials were grouped into 'Spike' and 'Non-Spike' trials based on whether a spike occurred during stimulus presentation; any trials with spikes occurring between stimulus-offset and response-onset were excluded. Peak gamma activity between stimulus-offset and response-onset was determined for each trial and compared between Spike and Non-Spike trials.

Results: Of all electrodes showing task-related gamma-augmentation across all patients, one site exhibited a reduction in peak gamma activity during Spike trials that survived Bonferroni correction; mean peak amplitude reduction was $58.4 \%$ of baseline ( $95 \%$ C.I.: $31.6 \%$ to $85.1 \%$; $p$-value $=0.000078)$. This site was located near to the $\mathrm{SOZ}$ in the dorsolateral frontal lobe and possessed a spike count similar to that of the SOZ. All other electrodes were located a greater distance away from or exhibited a spike count much lower than that of the respective SOZ; these sites showed no significant difference in peak gamma activity between Spike and Non-Spike trials. Neither within nor across patients was a change in response time found.

Conclusions: We have demonstrated that a negative effect of interictal spikes originating from a single frontal lobe focus upon cortical language function: (i) can occur in the absence of a behavioral effect, (ii) may be more prominent at sites nearer to the SOZ, and (iii) may be more prominent at sites associated with epileptiform activity similar to that of the SOZ. The deleterious effects of interictal spikes were local, rather than global, in our small cohort; a preliminary result requiring further validation.

\subsection{5 \\ PROSPECTIVE MULTICENTER STUDY OF AN AUTOMATIC SEIZURE DETECTION SYSTEM} M. Hartmann ${ }^{4}$, F. Fürbass ${ }^{4}$, A. J. Colon ${ }^{2}$, L. Elezi ${ }^{1}$, G. Lindinger ${ }^{3}$, T. Kluge $^{4}$, P. Ossenblok ${ }^{2}$, E. Pataraia ${ }^{3}$, H. Perko ${ }^{4}$ and C. Baumgartner ${ }^{1}$ ( ${ }^{1}$ 2nd Neurological Department, General Hospital Hietzing with Neurological Center Rosenhuegel, Vienna, Austria; ${ }^{2}$ Department of Research and Development, Epilepsy Centre Kempenhaeghe, Heeze, Netherlands; ${ }^{3}$ Department of Clinical Neurology, Medical University of Vienna, Vienna, Austria and ${ }^{4}$ Department Safety \& Security, AIT Austrian Institute of Technology GmbH, Vienna, Austria)

Rationale: A prospective multicentre study is being performed at three epilepsy monitoring units (EMU) to evaluate the automatic seizure detection system EpiScan [1]. The study will determine the detection performance on a large prospective patient group and quantify the added value of an automatic seizure detection for epilepsy diagnosis and for workflow optimization. Here we will present preliminary results of the first 43 out of 180 planned patients. Methods: 43 consecutive patients over the age of 18 undergoing long term video-EEG monitoring for epilepsy diagnostic and pre-surgical evaluation were evaluated. During recording normal clinical procedures were applied. The seizure detection system was used outof-the-box and no parameters were changed during the analysis. EEG-data were visually analysed by experienced epileptologists based on EEG and video following the routine procedures for EEG analysis. The resulting seizure markings were taken as basic truth and the results of the automatic detection were evaluated based on these seizure markers.

A marker of the automatic detection was defined as true positive (TP) when it was within 3 minutes after the marker based on visual analysis. A marker outside of this time window was defined as false positive (FP). False positives lasting for more than 30 seconds were counted multiple times. A false negative (FN) is defined as missed seizure. The sensitivity is defined as the ratio of the number of TP to the number of all seizures $(\mathrm{TP}+\mathrm{FN})$. The specificity is given in false 
alarms per hour $(\mathrm{FA} / \mathrm{h})$ calculated as the ratio of the number of FP to the number of recorded hours.

Results: Preliminarily results from the first 43 patients with a total of 3602 hours of EEG-recording are presented. Out of 43 patients 17 had seizures, 84 in total. All seizures were detected by the automatic detection system for 8 out of 18 patients. For 7 patients a sensitivity of at least $50 \%$ was achieved, for 2 patients a sensitivity of less than $50 \%$ was reached. The mean sensitivity over all patients was $73 \%$. For the false alarm rate per hour we calculated an average of 0.32 $\mathrm{FA} / \mathrm{h}$. In addition to the detection of epileptic seizures the automated system provided several markers in the EEG that contained pathologic EEG like spike-wave complexes, slowing or theta oscillations.

Conclusions: Our preliminary results show that the new seizure detection system EpiScan can be of value for a large fraction of patients reducing the time to analyse the data. The false alarm rate is low enough to be used as an online alerting system. The good performance was achieved without the need to adjust parameters on the software, which was used as shipped. In contrast to many other reports on seizure detection we compared the results of the automatic system with the result of the normal clinical workflow on consecutive patients in a prospective study. Furthermore, the automated system provided additional information by dragging attention to interictal parts of the EEG that show abnormal activity.

[1] M. Hartmann et.al. "Online seizure detection for epilepsy monitoring units"; AES 2011

\subsection{6 \\ DRIVING OR BEING DRIVEN: SHORT- AND LONG- RANGED NETWORK INTERACTIONS OF THE EPILEPTIC FOCUS}

Henning Dickten ${ }^{1,2}$, C. E. Elger ${ }^{1}$ and K. Lehnertz ${ }^{1,2}$ ( ${ }^{1}$ Epileptology, University of Bonn, Bonn, Germany and ${ }^{2}$ Helmholtz Institute for Radiation and Nuclear Physics, University of Bonn, Bonn, Germany)

Rationale: We consider the question whether the clinically defined epileptic focus drives or is driven by local or even remote areas in large scale brain networks. For this purpose we analyzed short- and long-ranged directed interactions in functional brain networks of patients suffering from focal epilepsies.

Methods: We investigated EEG recordings from up to now 20 epilepsy patients (15 with unifocal and 5 with multifocal epilepsies; all patients are seizure-free post-operatively). EEGs were recorded from on average 56 intracranial recording sites for a period of 117 hours per patient. A total of 131 seizures occurred. Directionality of interactions was assessed in a time-resolved manner by calculating the transfer entropy (TE) as well as an index based on phase interactions (DI) for all channel combinations using a moving window approach.

Results: When considering all interactions, both directionality measures indicated the epileptic focus as a driving structure in more than $50 \%$ of cases. However, in these cases the driving effect could mainly be observed for long-ranged interactions (that even extended to the contralateral hemisphere) while the focus appeared to be driven by its immediate surroundings. For the other patients the aforementioned short-ranged interactions could also be observed with TE but the effect reversed with DI. Long-ranged interactions were negligible. In all cases neither pre-ictal phenomena nor daily rhythms appeared to affect the observed driving phenomena.

Conclusions: Our findings indicate that the epileptic focus appears to be driven, however, only if short-ranged interactions are taken into account. The directionality indices used here capture different aspects of directed interactions within epileptic brain networks and can help to improve our understanding of the epileptic process.

This work was supported by the Deutsche Forschungsgemeinschaft (Grant No: LE 660/5-2)

\subsection{7}

DYNAMICS OF EEG IN A LONGITUDINAL STUDY OF POST-TRAUMATIC EPILEPSY AFTER TBI

E. Tobin ${ }^{1}$, J. White-James ${ }^{4}$, I. Vlachos ${ }^{1}$, B. Krishnan ${ }^{2}$, D. Treiman ${ }^{4}$ and L. Iasemidis ${ }^{1,3}\left({ }^{1}\right.$ School of Biological and Health Systems Engineernig, Arizona State University, Tempe, AZ; ${ }^{2}$ School of Electrical, Computer and Energy Engineering, Arizona State University, Tempe, AZ; ${ }^{3}$ Department of Neurology, Mayo Clinic, Phoenix, AZ and ${ }^{4}$ Department of Neurology, Barrow Neurolgical Institute, Phoenix, AZ)

Rationale: Development of post-traumatic epilepsy (PTE) after traumatic brain injury (TBI) is a major health concern $(5 \%$ to $50 \%$ of TBI cases). PTE is a long-term process spanning months to years after TBI. We thus postulated that quantitative analysis of continuous long-term EEG after TBI might shed light into the underlying mechanisms of PTE and epileptogenesis.

Methods: Six male Sprague-Dawley rats, weighing approximately 375 grams each, were subjected to a controlled cortical impact (CCI). A $6 \mathrm{~mm}$ piston was pneumatically driven into the right parietal (RP) cortex to a depth of $3 \mathrm{~mm}$ with velocity of $5.5 \mathrm{~m} / \mathrm{s}$. The rats were subsequently implanted with a total of six EEG electrodes in the left thalamus (LT), hippocampus (LH), parietal (LP) and frontal (LF) cortex, and right occipital (RO) and frontal (RF) cortex (no electrodes in the ipsilateral to the impact hippocampus and temporal cortex). Following a two week recovery period, long-term (14-week) continuous EEG recordings were conducted (L.P. filtered up to $40 \mathrm{~Hz}$ ). Using linear (coherence) and non-linear (Lyapunov exponents STLmax) measures of EEG dynamics, we studied the evolution over time (at a ten sec resolution) of the global as well as the local functional connectivity between brain sites.

Results: Four of the six TBI-induced rats developed PTE six to ten weeks after the initial insult to the brain. Analysis of the continuous EEG from these rats showed a gradual increase of the functional connectivity between critical brain sites in terms of their EEG dynamics, starting at least two weeks prior to their first spontaneous seizure and in some cases continuing long thereafter. In contrast, such an increase was not observed for the rats that did not develop epilepsy. The linear measure (coherence) provided evidence of an increased level of connectivity between the site closest to the impact (RO) and three distant brain sites (RF, LF, LP). These changes were observed mostly in the alpha, beta and gamma EEG frequency bands. The non-linear measure (STLmax entrainment) provided evidence of an increased level of connectivity over weeks similarly between RO and the contralateral frontal and parietal cortices. In addition, STLmax suggested an increase in functional connectivity between RO and the contralateral hippocampus. Consistent behavior of functional connectivity changes between brain sites and the "focus" (site of impact) over time was demonstrated for coherence in one out of the four epileptic and in both non-epileptic rats, while for STLmax in three out of the four epileptic and in both non-epileptic rats. Conclusions: Our preliminary results strongly support a network pathology in TBI that worsens with time. In rats that eventually developed PTE, long-term trends of increasing functional connectivity between the impaired cortex and the rest of the brain, both prior to and after the first spontaneous seizure, were observed. In those rats that did not develop PTE, no such increase was found. This research is supported by Department of Defense (DOD) PH/TBI Research Program, Office of Congressionally Directed Medical Research Programs (CDMRP) grant PT090712.

\subsection{8}

MEMORY SCORE AND SHIVERING DURING WADA TEST

C. Maideniuc ${ }^{1}$, A. Shah ${ }^{2}$, M. Basha ${ }^{3}$ and D. Fuerst ${ }^{4}$ ( ${ }^{1}$ Neurology, DMC/WSU, Detroit, MI; ${ }^{2}$ Neurology, DMC/WSU, Detroit, MI;

${ }^{3}$ Neurology, DMC/WSU, Detroit, MI and ${ }^{4}$ Neurophysiology, DMC/WSU, Detroit, MI) 
Rationale: Patients with medical intractable epilepsy undergo WADA test for evaluation of memory reserve of the contralateral temporal lobe. Transient shivering during the WADA procedure is thought to be related to perfusion of the anterior circulation, transiently removing the tonic inhibition of the anterior thermoregulatory center due to drug effect. It is also believed that hippocampus is perfused primarily by the posterior circulation and effects just on the anterior circulation of the sodium amytal may be inadequate to properly test hippocampal memory function. We hypothesize that if the shivering occurs during WADA test, it suggest only effect on the anterior circulation, and the memory score will be higher. In case the effect is on anterior and posterior circulation, then the memory score will be affected.

Methods: A retrospective chart review of the information on patients undergoing WADA test for pre surgical evaluation for epilepsy surgery was performed. Presence of shivering and memory score on each injection was recorded. Statistical analysis was done to determine the factors that influence the memory score, including dominance of the hemisphere and side of seizure onset.

Results: A total of 208 consecutive injections in 104 patients were included in our study. We created two groups: shivering and nonshivering. Shivering was noted after 62 injections. A difference between memory score in shivering group was significantly higher $(\mathrm{p}=0.02)$ compared to the non-shivering group. When we divided patients into concordant side of seizure onset (unilateral only) and shivering versus discordant side of onset and shivering, we found no effect on memory performance $(\mathrm{F}(1,68)=0.04, \mathrm{p}=0.84$, n.s. $)$.

Conclusions: Patients with shivering during the WADA suggest that posterior circulation was not affected with effects from sodium amytal and the effect on memory will be limited, memory score will be higher. Thus, shivering can be used as a surrogate for inadequate effect on the hippocampus and raises the concerns about reliability of the WADA memory score.

\subsection{9 \\ THE RELATIONSHIP BETWEEN SIMULTANEOUSLY ACQUIRED SCALP AND INTRACRANIAL EEG POWER: BACKGROUND RHYTHMS}

Ognen A. Petroff ${ }^{1}$, D. D. Spencer ${ }^{2}$, I. Goncharova ${ }^{1}$ and H. Zaveri ${ }^{1}$ $\left({ }^{1}\right.$ Neurology, Yale University School of Medicine, New Haven, CT and ${ }^{2}$ Neurosurgery, Yale University, New Haven, CT)

Rationale: The first studies recording both scalp and cortical EEG noted a significant loss of EEG power and loss of fidelity in the scalp recordings. Most of the subsequent work focused on the loss of fidelity of epileptiform activity and evoked potentials. Quantitative EEG methods applied to scalp EEG have contributed to the utility of continuous EEG monitoring in the critical care setting. The purpose of our study was to investigate the effects of the intact skull on background EEG rhythms of patients undergoing long term combined scalp and intracranial EEG monitoring.

Methods: Twenty patients (age 18-55) from the Yale University Epilepsy Surgery Program undergoing intracranial EEG monitoring for surgical evaluation were recruited. Scalp EEG recordings were made using Grass platinum needle electrodes located over the C3, $\mathrm{C} 4, \mathrm{O} 1$, and $\mathrm{O} 2$ sites. Simultaneous recordings were obtained from subjacent subdural strip electrodes ( $4 \mathrm{~mm}$ diameter platinum disks with $2.3 \mathrm{~mm}$ exposed surface diameter) as previously reported (Zaveri et al 2011). Offline analysis was performed with custom software written in a mixture of high level languages and MATLAB. Scalp and intracranial EEG epochs, 1 hour in duration, at least 6 hours removed from a seizure, 3 or 4 days after electrode implantation surgery, during wakefulness between 9-10 AM were selected for analysis. Scalp and subdural recordings were examined for artifacts and segmented with 1-second resolution. EEG power of artifact-free EEG segments was obtained for each electrode contact studied and averaged over the epoch. Total power was calculated as the signal power between 0.5 and $35 \mathrm{~Hz}$, delta $0.5-3.9 \mathrm{~Hz}$, theta $4-8.4 \mathrm{~Hz}$, alpha
8.5-13 Hz, and beta $13.1-25 \mathrm{~Hz}$ frequency bands.

Results: EEG power recorded using subdural electrodes decreased with higher frequencies: delta $\left(2.82 \mathrm{mV}^{2}, 95 \% \mathrm{CI} 1.26-6.35\right)$, theta (1.49 $\mathrm{mV}^{2}, 95 \%$ CI 0.82-2.49, alpha $\left(0.41 \mathrm{mV}^{2}, 95 \% \mathrm{CI} 0.22-0.71\right)$, beta $\left(0.28 \mathrm{mV}^{2}, 95 \% \mathrm{CI} 0.17-0.43\right)$. EEG power recorded using scalp electrodes decreased with higher frequencies: delta $\left(0.41 \mathrm{mV}^{2}\right.$, $95 \%$ CI $0.21-0.65)$, theta $\left(0.12 \mathrm{mV}^{2}, 95 \%\right.$ CI $\left.0.06-0.25\right)$, alpha $(0.02$ $\mathrm{mV}^{2}, 95 \%$ CI $\left.0.01-0.04\right)$, beta $\left(0.02 \mathrm{mV}^{2}, 95 \% \mathrm{CI} 0.01-0.03\right)$. Median power over the entire $0.5-35 \mathrm{~Hz}$ frequency band recorded from the scalp electrodes $\left(0.58 \mathrm{mV}^{2}, 95 \% \mathrm{CI} 0.30-0.99\right)$ was $11 \%$ of the EEG power recorded using the subdural electrodes $\left(5.14 \mathrm{mV}^{2}, 95 \% \mathrm{CI}\right.$ 2.53-10.19). The ratio of EEG power recorded on the scalp divided by that recorded using subdural electrodes was attenuated further at higher frequencies: delta $(0.15)$, theta $(0.08)$, alpha $(0.06)$, beta (0.06).

Conclusions: The power of the background EEG recorded using subdural electrodes decreased by about 10-fold at higher frequencies. Additionally, the intact human skull attenuated EEG power at the median of all frequencies by 9-fold from 7-fold at delta up to 18-fold at the beta frequencies. The intact human cranium acts as a low-pass filter set at about $5 \mathrm{~Hz}$.

\subsection{0 \\ CURRENT-SOURCE DISTRIBUTION OF INTERICTAL SPIKES IN SCHIZENCEPHALIC PATIENTS}

Oh-Young Kwon, D. Kim, S. Jung and E. Joo (Department of Neurology, Gyeongsang National University Hospital, Jinju, Republic of Korea)

Rationale: The epileptogenicity of schizencephalic lesions may differ from that of other cortical malformations, such as focal cortical dysplasia. The lesions may point to epileptogenic zone rather than contain it. The purpose of this study is to investigate the current source distribution of epileptiform discharges of schizencephalic patients, and correlate it with the lesions revealed by magnetic resonance imagings (MRI).

Methods: Three consecutive patients with schizencephaly in brain MRI and interictal spikes in EEG were enrolled. Seizure semiology of the patients was clinically consistent with temporal lobe epilepsy (TLE). Scalp electroencephalograms (EEGs) were taken according to International 10-20 System with 25 channels including subtemporal electrodes (F9/F10, T9/T10, P9/P10). Sampling rate was $400 \mathrm{~Hz}$ and filter set was 1.6 to $30 \mathrm{~Hz}$. We obtained current-source densities (CSDs) of the peak points of interictal spikes using low resolution brain electromagnetic tomography (LORETA).

Results: In the first patient, the schizencephalic lesions were on the parietal and perisylvian area of the right cerebral hemisphere. Interictal spikes were observed at P10 elctrode, and CSDs of them were on the temporoparietal cortex of the same hemisphere, and midline cortices of the parietal areas. In the second patient, the schizencephalic lesions were on the parietal areas of the right hemisphere, and lissencephlay was seen in the parietal area of the contralateral hemisphere. Interictal spikes were at F10 electrode, and CSDs of them were on the temporal and the parietal areas of the right hemisphere, and midline cortices of the frontal and occipital areas. In the third patient, the schizencephalic lesions were on the temporal areas of both hemispheres. Interictal spikes were at T7 and T10 electrode. Current source for T7 spikes were distributed on the temporal area of the left hemisphere. Those for T10 spikes were on the temporal area of the right hemisphere and the midline cortices of the frontal area.

Conclusions: Our results show that schizencephaly may have both of intrinsic and extrinsic epileptogenicity. The current-source distributions of epileptic discharges in schizencephalic patients indicated that epileptic focus could be localized outside the lesions. In TLE with schizencephaly, the temporal cortex of the same hemisphere with the lesions which is not structurally abnormal may be the source of epileptogenicity. 


\subsection{1}

GAMMA ACTIVITY MODULATED BY NAMING OF AMBIGUOUS AND UNAMBIGUOUS IMAGES: INTRACRANIAL RECORDING IN EPILEPTIC PATIENTS Yoshimi Cho-Hisamoto $^{1,2}$, K. Kojima ${ }^{1}$, E. C. Brown ${ }^{3}$, N. Matsuzaki ${ }^{1}$ and E. Asano ${ }^{1,2}\left({ }^{1}\right.$ Pediatrics, Children's Hospital of Michigan, Wayne State University, Detroit, MI; ${ }^{2}$ Neurology, Children's Hospital of Michigan, Wayne State University, Detroit, MI and ${ }^{3} \mathrm{MD}-\mathrm{PhD}$ Program, Wayne State University, School of Medicine, Detroit, MI)

Rationale: Among important cognitive functions to be preserved in epilepsy surgery is the naming of visible objects. It has been hypothesized that naming of a vague and ambiguous silhouette, compared to naming of an unambiguous, realistically drawn object, may require a greater utilization of cognitive resources, mediated partly by the frontal lobe. We determined whether naming of ambiguous images, compared to that of unambiguous images, would elicit augmentation of gamma activity to a larger extent in frontal regions.

Methods: We studied 340 cortical sites sampled from four patients (age: 13 - 28 years) undergoing extraoperative electrocorticography (ECoG) as part of clinical management of uncontrolled focal epilepsy. A total of 120 images (60 ambiguous images of low spatial frequency and 60 unambiguous images of high spatial frequency) were presented on a screen and each patient was asked to overtly state the name of the object as it is first perceived. We determined when and where the amplitude of gamma activity at $50-120 \mathrm{~Hz}$ was augmented compared to that during the silent resting period.

Results: The response time of naming of ambiguous images was larger than that of naming of unambiguous ones (median: 1,675 vs $976 \mathrm{~ms} ; \mathrm{p}<0.0001$ ). Both tasks commonly elicited gammaaugmentation sequentially involving occipital, occipital-temporal, lateral-frontal, and Rolandic regions bilaterally. Naming of ambiguous images elicited gamma-augmentation specifically involving the right orbitofrontal, left inferior-frontal and left supramarginal regions following the onset of image presentation but prior to vocalization. Naming of unambiguous objects elicited gamma-augmentation more intensely in the occipital regions, compared to that of ambiguous ones, immediately following the onset of image presentation.

Conclusions: Behavioral results suggest naming of ambiguous images to demand a larger workload, relative to unambiguous images. Frontal-parietal gamma-augmentation specifically elicited by naming of ambiguous images may reflect the additional cortical processing involved in generating a guess. Enhanced occipital gamma-augmentation elicited by unambiguous images can be explained by visual processing of stimuli with richer detail.

\section{Clinical Epilepsy: Classification and Syndromes}

\subsection{2 \\ TONIC PHASE OF A GENERALIZED CONVULSIVE SEIZURE IS AN INDEPENDENT PREDICTOR OF POSTICTAL GENERALIZED EEG SUPPRESSION}

James Tao, I. Yung, A. Lee, S. Rose, J. Jacobsen and J. Ebersole (University of Chicago Medical Center, Chicago, IL)

Rationale: Postictal generalized EEG suppression (PGES) is commonly observed in patients after generalized convulsive seizures (GCS). It may be a causative factor in sudden unexpected death in epilepsy (SUDEP). The objective of this study is to investigate the incidence, risk factors, and clinical correlates of PGES, in so doing, to further understand its significance in the pathogenesis of SUDEP. Methods: We retrospectively reviewed the video-EEG of 109 consecutive patients with 151 GCS during long term video-EEG monitoring. We determined the incidence, duration, and clinical correlates of PGES. We also investigated whether factors such as age, sex, seizure type (primary vs. secondarily GCS), total seizure duration, duration of tonic and clonic phases, and seizure termination (abrupt vs. gradual) influenced PGES.

Results: PGES was observed in 64 of 109 (58.7\%) patients and in 98 of $151(64.9 \%)$ GCS. Average duration of PGES was $42.4 \pm 19.1 \mathrm{~s}$. Statistical analysis showed that patients with PGES had no significant difference in age, gender, total seizure duration, total convulsive duration, clonic phase, seizure type, and seizure termination, as compared to those without PGES. However, PGES was observed in 97 of $123(78.9 \%)$ GCS with tonic phase, and was observed only in 1 of $28(3.6 \%)$ GCS without tonic phase. In addition, tonic phase was significantly prolonged in patients with (23.4 s) PGES than in those without (12.8 s) PGES ( $\mathrm{P}=0.00086)$. Furthermore, regression analysis showed that a seizure's tonic phase proved to be an independent predictor of PGES. A one second increase in tonic phase duration was associated with a 0.06 increase in log odds of PGES (odds ratio $=1.1, \mathrm{P}=0.00055$ ). Clinically, $97.3 \%$ patients were comatose during PGES, whereas only $26.7 \%$ patients without PGES were comatose immediately after seizure termination. Postictal tachycardia and tachypnea associated with increased respiratory effort and coarse breathing sounds were commonly observed in patients with GCS, and such seizure related respiratory compromise appeared more prominent in patients with PGES than in those without PGES.

Conclusions: PGES is a common EEG feature in patients with GCS. The presence and duration of a tonic phase is an independent predictor of PGES. Postictal coma, loss of brain stem autoresuscitative reflexes, and respiratory compromise accompany patients with PGES. Given that 7 of 8 patients in video-EEG recorded SUDEP cases died in prone position, it is likely that the convergence of postictal coma, respiratory compromise and peripheral hypoventilation (i.e. prone position) play an important role in patients who suffer SUDEP.

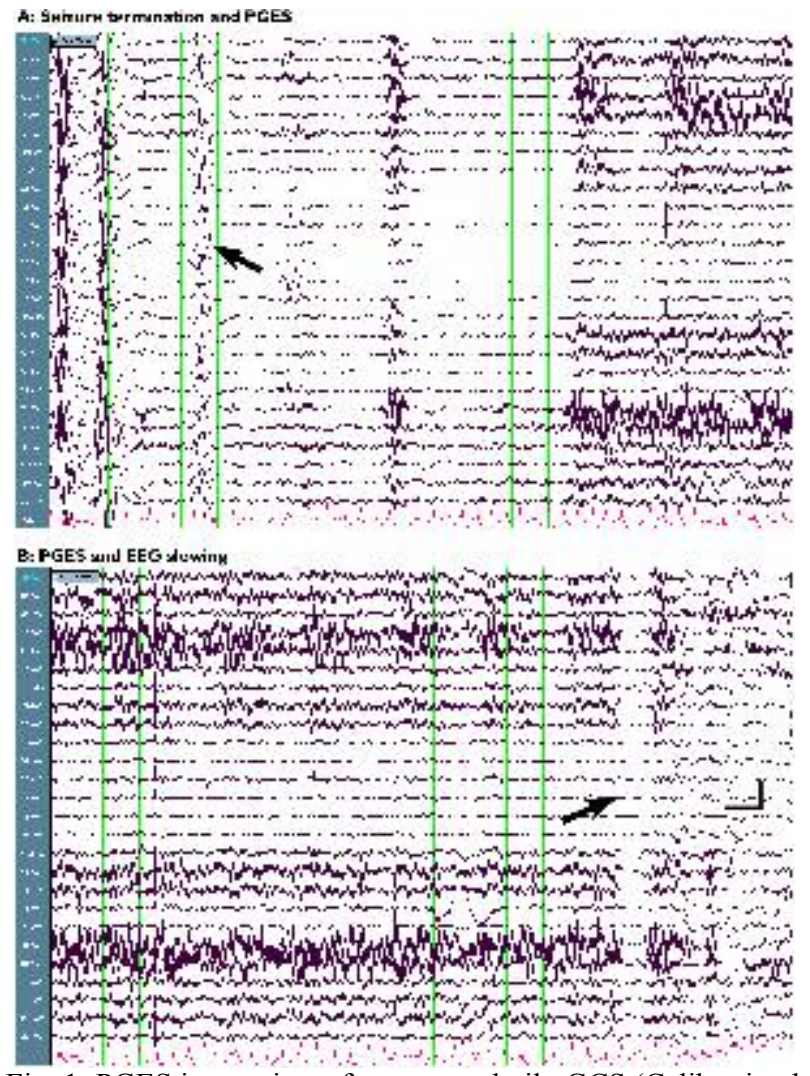

Fig. 1: PGES in a patient after a secondarily GCS (Calibration bar: $1.0 \mathrm{sec}, 50 \mu \mathrm{V}$ ). Panel A: abrupt seizure termination (as indicated by arrow) and followed by PGES. Panel B showed continued PGES subsequently evolved to postictal EEG slowing (as indicated by arrow). PGES was superimposed by prominent EEG respiratory muscle artifacts due to the increased respiratory effort. Postictal HR: $135 \mathrm{bpm}$ and RR: 24 per min. The duration of PGES (between 
arrows) was $31 \mathrm{~s}$, during which the patient was comatose as indicated by absence of spontaneous movement and responsiveness to verbal and physical stimulations.

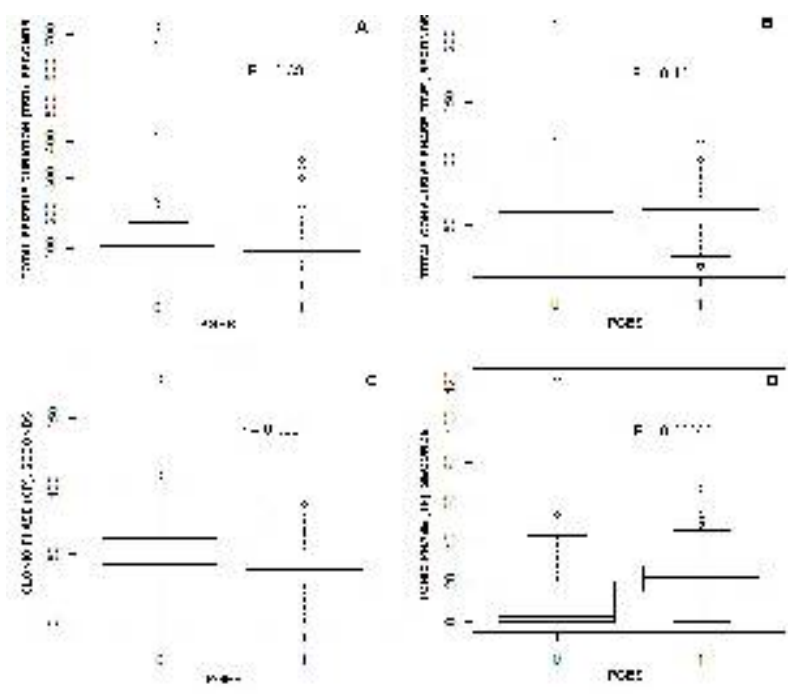

Fig. 2: Pairwise relationships between PGES and total seizure duration (TSD), total convulsive phase (TCP), clonic phase (CP) and tonic phase (TP). Wilcoxon rank sum test showed that there was no significant difference in TSD, TCP, CP in patients with (1) and without (0) PGES, whereas TP was significantly prolonged in patients with PGES than in those without PGES. Panel A: TSD in patients with and without PGES $(\mathrm{p}=0.39)$. Panel $\mathrm{B}$ : TCP in patients with and without PGES $(\mathrm{P}=0.42)$. Panel $\mathrm{C}$ : $\mathrm{CP}$ in patients with and without PGES ( $\mathrm{p}=0.02$, statistically not significant after Bonferroni correction). Panel D: TP in patients with and without PGES $(\mathrm{p}=0.00086)$.

\subsection{3 \\ RELATIONSHIP BETWEEN INITIAL EEG CHARACTERISTICS AND SEIZURE OUTCOMES IN THE CHILDREN OF INFANTILE SPASMS WITHOUT MRI ABNORMALITIES \\ Yun Jin Lee ${ }^{1}$, G. Yeon ${ }^{1}$, Y. Kim ${ }^{2}$ and S. Nam ${ }^{1}\left({ }^{1}\right.$ Pusan National University Children's Hospital, Yangsan, Republic of Korea and ${ }^{2}$ Pusan National University Hospital, Busan, Republic of Korea)}

Rationale: To characterize the initial interictal electroencephalography (EEG) activities associated with seizure outcomes in children of infantile spasms (IS) with normal MRI finding and their worth in predicting the outcomes of seizure-free (SF) vs no seizure-free (NoSF).

Methods: We retrospectively reviewed the initial scalp EEGs of at least 30 minutes duration and medical records of 58 children who were diagnosed as IS without MRI lesion in our hospital and were followed-up for $4.2 \pm 2.1$ years. We assessed the following clinical and EEG findings; seizures onset, development, underlying etiologies, initial interictal EEGs, and seizure evolution. These variables were compared between two groups of SF vs NoSF.

Results: Thirty (51.7\%) children had SF outcomes and 28 (48.3\%) had NoSF outcomes during long-term follow-up (4.1 \pm 2.3 vs $4.2 \pm 2.0$ years). Age at seizure onset was similar in SF and NoSF groups (5.0 \pm 3.0 vs $6.4 \pm 3.2$ months). Delayed development before spasms was similarly observed in two groups (33.3\% vs 53.6\%). Preceding other seizures were more frequently seen in NoSF than SF group $(25.0 \%$ vs $6.7 \%, p=0.054)$. Initial EEG findings significantly differed with posterior-dominant generalized epileptiform discharges (ED) $(50.0 \%$ vs $3.6 \%, \mathrm{p}=0.0001)$ and normal or borderline sleep spindle (SS) $(60.0 \%$ vs $28.6 \%, \mathrm{p}=0.016)$ being involved more often in SF group, and typical hypsarrhythmia $(78.6 \%$ vs $50.0 \%, \mathrm{p}=0.024)$, multiple independent spike foci $(75.0 \%$ vs $46.7 \%$, $\mathrm{p}=0.028)$, frontal- dominant generalized ED $(17.9 \%$ vs $0.0 \%, \mathrm{p}=0.008)$ or frontaldominant focal ED ( $7.1 \%$ vs $0.0 \%, p=0.023)$, and no normal SS (71.4\% vs $40.0 \%, \mathrm{p}=0.016)$ involved more often in NoSF group. Conclusions: Initial interictal EEG findings may predict seizure outcomes in patients of IS without MRI lesion. Patients with SF outcomes showed the posterior-dominant generalized ED and normal or borderline SS, and NoSF group had more often typical hypsarrhythmia, frontal-dominant generalized or focal ED and no normal SS.

\subsection{4 \\ THE ROLE OF BRAIN NODES AND BRAIN NETWORKS IN THE DYNAMIC EMERGENCE OF SEIZURES Mark P. Richardson ${ }^{1}$, O. Benjamin ${ }^{2}$ and J. R. Terry ${ }^{3}\left({ }^{1}\right.$ Clinical Neuroscience, King's College London, London, United Kingdom; ${ }^{2}$ Engineering Mathematics, University of Bristol, Bristol, United Kingdom and ${ }^{3}$ College of Engineering, Mathematics and Physical Sciences, University of Exeter, Exeter, United Kingdom)}

Rationale: Observations suggest that focal epilepsies may involve widespread brain networks, and generalised epilepsies may have focal cortical regions that drive seizure onset (J Neurosci 2002;22(4):1480-95). These observations have contributed to a reappraisal of the classification of epilepsies, and seizures, and underpinned the concept that seizures may emerge from network dynamics (Epilepsia 2010; 51(4): 676-685 \& Epilepsia 2012; 53(5): 771-778). We hypothesise that whether an EEG discharge appears focal or generalised is driven by the pattern of connections in brain networks, irrespective of the presence of focal brain abnormality. Here, we explore this question using a computational brain model. Methods: We have previously described a brain model consisting of grey-matter "nodes" and white matter "connections" (J Math Neurosci 2012; 2(1):1). Dynamics in the nodes are governed by mathematical equations which allow a bistable state; one state represents normal low-amplitude EEG activity, the other state highamplitude EEG ictal discharges. Transitions between the states are driven by intrinsic noise in each node and the synchronising influences felt from other nodes via the connections in the network. Nodes were modelled with "normal" dynamics, in which transition from normal to pathological activity had a low probability; in some instances we modelled nodes with "hyperexcitable" dynamics, in which the transition from normal to pathological activity had a higher probability. We explored the effects of altering connectivity structure versus the effects of introducing an "abnormal" brain region, and the interactions between these factors.

Results: Computer simulations demonstrated that EEG discharges representing either generalized or focal seizures arose purely as a consequence of subtle changes in network structure, without the requirement for any localized pathological brain region. Further we found that introducing a pathological region gave rise to focal, secondary generalized or primary generalized seizures depending on the network structure.

We illustrate this in the Figure: (a) and (b) show patterns of connectivity between normal nodes which result in normal EEG activity. (c) and (d) show the consequence of introducing a hyperexcitable node (in red) to the networks in (a) and (b) respectively: the pattern of connectivity in (c) results in generalised discharges, but the pattern of connectivity in (d) results in focal discharges. (e) and (f) show that a set of normal brain nodes can generate generalised (seen in (e)) or focal discharges (seen in (f)) entirely as a result of network connectivity structure, without an abnormal node.

Counter-intuitively, we found that decreasing connectivity between regions of the brain increased the frequency of seizure-like activity. Conclusions: Our findings may enlighten current controversies surrounding the concepts of focal and generalized epilepsy, and help to explain recent observations in genetic animal models and human epilepsies, where loss of white matter pathways was associated with 
the occurrence of seizures

a
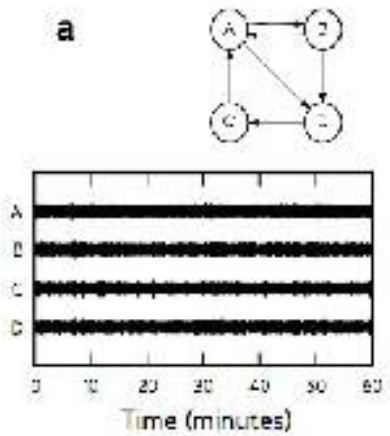

c
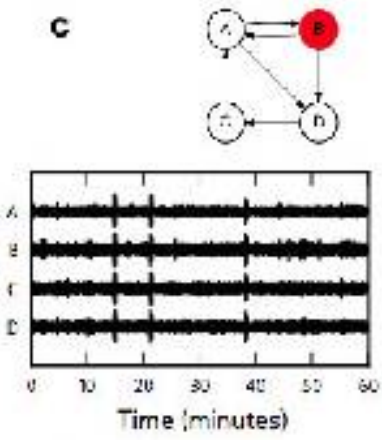

e
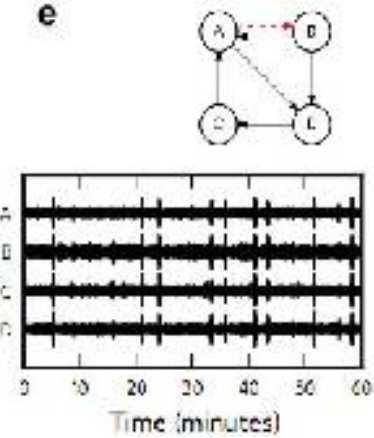

2.095

SURVEY ON TERMINOLOGY ON ELECTRICAL STATUS EPILEPTICUS IN SLEEP AND CONTINUOUS SPIKES AND WAVES DURING SLEEP

J. M. Peters ${ }^{1}$, Iván Sánchez Fernández ${ }^{1}$, K. E. Chapman ${ }^{2}$, A. T. Berg ${ }^{3}$ and T. Loddenkemper ${ }^{1}\left({ }^{1}\right.$ Department of Neurology, Boston Children's Hospital, Boston, MA; ${ }^{2}$ Children's Hospital Colorado, Denver, $\mathrm{CO}$ and ${ }^{3}$ Lurie Children's Hospital, Chicago, IL)

Rationale: The terms "Electrical status epilepticus in sleep (ESES)" and "Continuous spikes and waves during sleep (CSWS)" are variably used in the literature. The aim of this study was to collect the current views of clinical epileptologists regarding the meaning and use of these terms.

Methods: We administered an online survey asking the members of the American Epilepsy Society about their views on terminology and conceptualization of ESES and CSWS. The survey was administered through an online survey website (www.surveymonkey.com) and distributed through the "Professional Connection" website of the American Epilepsy Society. All responses were automatically registered by the online survey website.

Results: We received 78 responses ( 72 complete, 6 had some missing data). The demographic features of the respondents are summarized in Table 1. The terms ESES and CSWS were considered synonymous by $39(54 \%)$ respondents, not synonymous by $28(39 \%)$, $5(7 \%)$ respondents stated that they did not know the answer, and 6 respondents skipped this question. The minimum EEG cut-off value of epileptiform activity during sleep for ESES was considered: at least $85 \%$ by $37(52 \%)$ respondents, at least $50 \%$ by $27(38 \%)$ and any amount of spike-waves by $1(1 \%) ; 6(9 \%)$ were not sure about the cut-off value, and 6 respondents skipped this question. The cutoff value was considered mandatory by 32 (44\%), typical but not required by $32(44 \%), 8(11 \%)$ did not know the answer and 6 respondents skipped this question. The diagnosis of CSWS was performed based on both clinical and EEG assessment together by 47 $(65 \%)$ respondents, and based on EEG assessment alone by 21 (29\%). The segment used to quantify the epileptiform activity was: all phases of sleep during the whole night by $17(26 \%)$, all non-REM phases of sleep during the whole night by 32 (49\%), comparison of epileptiform activity during wakefulness and sleep by $10(15 \%)$, and variable segments for every patient by $3(5 \%) ; 8$ respondents used other segments, and 13 respondents skipped this question. Regarding the extent of discharges, $11(15 \%)$ respondents only quantified bilateral and synchronous spike-waves, 22 (31\%) quantified bilateral spike-waves even if not synchronous, and 30 (42\%) quantified unilateral spike-waves as equivalent to bilateral spike-waves; $9(13 \%)$ respondents did not know the answer and 6 skipped the question. The quantification of epileptiform activity was performed as the percentage of one-second bins occupied by spike-waves by $42(64 \%)$ respondents and as the total number of spike-waves per unit of time in $24(36 \%) ; 4$ respondents used other methods and 12 skipped this question. The understanding of the nature and prognosis of CSWS is graphically represented in Figure 1.

Conclusions: Our data show that the terms ESES and CSWS are used differently by epileptologists. Variability may impact clinical care, communication and clinical research. A common terminology and diagnostic criteria are urgently needed.

Table 1. Demographic features of the respondents

\begin{tabular}{|c|c|c|}
\hline Feature & Options & Number (percentuge) \\
\hline \multirow{3}{*}{ Typc of practice } & General neurology & $9(12)$ \\
& Tpilcptology & $50(64)$ \\
& Both & $19(24)$ \\
\hline \multirow{3}{*}{ Patient population } & Adults & $25(32)$ \\
& Children & $44(56)$ \\
& Mixcd & $9(12)$ \\
& $50-100$ & $3(4)$ \\
Number ol patients per year & $100-200$ & $8(10)$ \\
& $200-300$ & $11(14)$ \\
& $>300$ & $55(71)$ \\
\hline \multirow{2}{*}{ Board certification in clinical neurophysiology } & Yes & $46(61)$ \\
& No & $30(40)$ \\
\hline & USA & $65(83)$ \\
& Furope & $10(13)$ \\
& Canada & $4(5)$ \\
& Latin America & $2(3)$ \\
& Asia & $1(1)$ \\
& Occania & $1(1)$ \\
& Elscwhere & $1(1)$ \\
\hline \multirow{2}{*}{ Clinical training } & $0-5$ & $24(31)$ \\
& $5-10$ & $15(19)$ \\
& $10-20$ & $22(28)$ \\
& $>20$ & $17(22)$ \\
\hline
\end{tabular}

Based on your experience, continuous spikes and waves during sleep is:

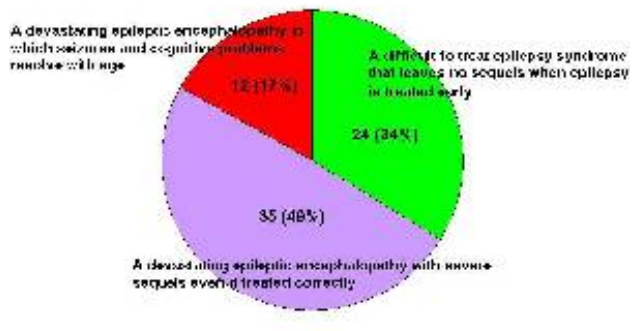

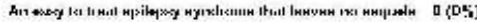

skipped giexion 3 


\subsection{6}

SEMIOLOGIC STRATIFICATION OF GENERALIZED TONIC CLONIC SEIZURES

Thomas Berk, D. Friedman, D. Gazzola, P. Dugan, C. Carlson, R. Kuzniecky and J. French (Neurology, NYU School of Medicine, New York, NY)

Rationale: The Generalized Tonic-Clonic Convulsion (GTCC) has been described as a stereotyped seizure consisting of a symmetric tonic posture, followed by vibratory and clonic phases - defined as movements at a frequency of $>5 \mathrm{~Hz}$ and $<5 \mathrm{~Hz}$ respectively. We examined how frequently the classic GTCC occurs in a population and what factors, if any, contributed to deviations from this pattern. Methods: We reviewed the video EEG of 100 consecutive inpatients of the NYU Comprehensive Epilepsy Center that had bilateral limb movements as part of their seizure semiology. Each seizure was reviewed by 2 reviewers; any records in which the patient was obscured on the video were excluded from further analysis. Any seizure with bilateral symmetric tonic, vibratory and clonic phases in that order was categorized as "typical GTCC" (tGTCC), if one phase was absent, asymmetric or in the wrong order of progression it was considered "atypical GTCC" (aGTCC), if two phases were absent it was not a GTCC (nGTCC). All aGTCC were reviewed by at least 3 reviewers.

Results: 104 seizures (41 from women) from 100 patients were reviewed, 2 patients were excluded due to obscured video. 45 had a tGTCC while 15 were aGTCC, and 42 were nGTCC - either being a tonic, myoclonic-tonic, or partial seizure with dystonic, clonic, or complex motor features. $40 \%$ of nGTCCs were reported as a GTCC by physicians/nurses and $96 \%$ of tGTCCs and $93 \%$ of aGTCCs were reported as a GTCC. The median duration (seconds) of seizures was 110.5 for tGTCC, 109.5 for aGTCC, and 95.5 for nGTCC (KruskalWallis $\mathrm{p}=0.061$ ). Table 1 shows epilepsy classifications for each of the three groups; $89 \%$ of idiopathic generalized epilepsy was associated with tGTCC and none with aGTCC. Excluding patients with no imaging data, 15/34 (44\%) of tGTCC, $6 / 10(60 \%)$ of aGTCC had lesional MRIs including post-operative changes (Fisher's Exact $\mathrm{p}=0.48$ ).

Conclusions: These data demonstrate that the GTCC does not always present with the classic progression from tonic to vibratory and then clonic activity; in $15 \%$ of patients overall, atypical features were observed. Of all GTCCs recorded in patients with focal seizures $28 \%$ were atypical. No patients with IGE had aGTCCs. These data demonstrate a trend for shorter seizure durations for nGTCCs versus a/tGTCC. Furthermore, tGTCCs are more frequently associated with non-lesional structural imaging. GTCCs are associated with an increased risk of SUDEP, a worse surgical outcome, and a higher risk for seizure-related injury. The association between aGTCC and these findings is unclear and requires further investigation.

Imaging Findings Across All Groups

\begin{tabular}{|c|c|c|c|}
\hline & Focal Epilepsy & Idiopathic Generalized & symplomalic Generalized \\
\hline tGICC & 37 & 8 & 0 \\
aGTCC & 15 & 0 & 0 \\
nGTCC & 39 & 1 & 2 \\
\hline
\end{tabular}

\subsection{7}

GENETIC EPILEPSY WITH FEBRILE SEIZURES PLUS: THE FULL SPECTRUM ENCOMPASSES GGE AND FOCAL EPILEPSIES

Ingrid E. Scheffer ${ }^{1,8}$, Y. H. Zhang ${ }^{1}$, J. P. Malone ${ }^{1}$, G. C. Glubb ${ }^{1}$, K. Helbig $^{1}$, L. Dibbens ${ }^{2}$, L. Vadlamudi ${ }^{1}$, A. Bleasel ${ }^{3}$, R. Burgess ${ }^{1}$, B. E. Grinton $^{1}$, D. Vears ${ }^{1}$, Z. Afawi ${ }^{4}$, H. Goldberg-Stern ${ }^{5}$, S. Kivity ${ }^{5}$, A. D. Korczyn $^{6}$, J. C. Mulley ${ }^{2,7}$ and S. F. Berkovic ${ }^{1}{ }^{1}$ University of Melbourne - Austin Health, Heidelberg, Australia; ${ }^{2}$ University of Adelaide - Women's and Children's Hospital, Adelaide, SA,
Australia; ${ }^{3}$ Westmead Hospital, Sydney, NSW, Australia; ${ }^{4}$ Tel-Aviv Sourasky Medical Center, Tel Aviv, Israel; ${ }^{5}$ Schneider Children's Medical Center of Israel, Petach Tikvah, Israel; ${ }^{6}$ Tel Aviv University, Tel Aviv, Israel; ${ }^{7}$ University of Adelaide, Adelaide, SA, Australia and ${ }^{8}$ Florey Neuroscience Institue and Royal Children's Hospital, Melbourne, VIC, Australia)

Rationale: GEFS+ (generalized epilepsy with febrile seizures plus) is a familial epilepsy syndrome characterized by phenotypic heterogeneity that we described in 1997. The GEFS+ spectrum includes Febrile Seizures (FS), Febrile Seizures Plus (FS+), FS/FS+ with generalized and focal seizures, and a range of epileptic encephalopathies including Dravet syndrome and epilepsy with myoclonic-atonic seizures. GEFS + is infrequently associated with mutations of sodium channel mutations including SCN1A and SCN1B. Less frequently GABA receptor subunit gene mutations have been identified. In addition, susceptibility genes have been found in smaller families. We studied 31 new families and analyzed the phenotypic spectrum in these families and our previously published families.

Methods: We performed detailed electro-clinical phenotyping on all available affected family members and reviewed EEG and neuroimaging studies. We report the phenotypic findings on 408 affected individuals in a total of 60 families and compare this data to other GEFS+ studies.

Results: New phenotypes in GEFS+ families included focal seizures without preceding FS (16/408, 4\%), classical genetic generalized epilepsies $(22 / 408,5 \%)$ most commonly childhood absence epilepsy, and afebrile generalized tonic-clonic seizures $(9 / 408,2 \%)$. FS remains the most frequent phenotype in GEFS + $(178 / 408,44 \%)$ followed by FS+ (110/408, 27\%). Large GEFS+ families are suggestive of autosomal dominant inheritance. Many smaller families exist where the inheritance pattern is more suggestive of complex inheritance.

Conclusions: Initially GEFS + was conceived as comprising generalized epilepsy phenotypes and identified through large autosomal dominant families. Our data, show that the spectrum of phenotypes within GEFS+ is greater than originally conceived. Focal epilepsies and classical GGE are seen in GEFS+ families. We propose that GEFS + be renamed "genetic epilepsy with febrile seizures plus" in view of the number of individuals with focal epilepsies. The overlap between GEFS+ and the classical GGE is considerably greater than first thought and suggests that the two major groups of generalized epilepsies have shared genetic determinants.

\subsection{8 \\ SIGNIFICANCE OF RAPID BILATERAL EYE BLINKING DURING PARTIAL SEIZURES}

Prasuna Velur and G. K. Kalamangalam (Neurology, University of Texas, Houston, TX)

Rationale: Rapid bilateral eye blinking (RBEB) is commonly seen during generalized absence seizures, and is the defining character of seizures in the Jeavons syndrome. Complex partial seizures (CPSs) in focal epilepsy are usually associated with the opposite semiology fixed staring - though we have also observed RBEB in CPSs. In this pilot study we surveyed the electroclinical associations of RBEB in a cohort of focal epilepsy patients with CPSs.

Methods: We retrospectively reviewed scalp video-EEG monitoring seizure data on all patients with proven partial epilepsy and CPSs encountered over a 12-month period (year 2009). Seizures with RBEB were identified by obvious excessive eyeblink artifact on the EEG that lasted $\geq 10$ seconds at any time during the seizure. The total number of eye blinks for the duration of the seizure, as well as the 10 -second epoch with the highest number of eye blinks (peak blink rate) was noted.The ictal EEG of each seizure (whether with RBEB or not) was classified as having a lateralized or nonlateralized onset pattern. 
Results: 156 partial seizures $(n=156)$ were recorded in 41 patients $(\mathrm{N}=41)$ that fit the above criteria. Ninety-one seizures $(\mathrm{n} 1=91)$ had clearly lateralized ictal EEG onsets; the remainder $(\mathrm{n} 2=65)$ had nonlateralized onsets. Four patients had 8 seizures $(m=8)$ that showed RBEB; in each such seizure, RBEB was confirmed by peak eye blink rates during the seizure significantly higher than at baseline $(\mathrm{p}<$ 0.001 ). Assuming $\mathrm{N}$ and $\mathrm{n}$ as population figures, the probabilities of a random seizure having lateralized versus nonlateralized onset were $\mathrm{n} 1 / \mathrm{N} \approx 0.58$ versus $\mathrm{n} 2 / \mathrm{N} \approx 0.42$. Thus, the probability of $\mathrm{m}$ seizures with RBEB all having nonlateralized onset was $p=(0.42) 8<0.001$

Conclusions: (i) RBEB, a semiological feature often seen in seizures arising in generalized epilepsy syndromes, may occur rarely in seizures in focal epilepsy $(4 / 41 \approx 10 \%$ of patients and $8 / 156 \approx 5 \%$ of seizures, in this series). (ii) There is a statistically significant association between seizures with RBEB and a diffuse (nonlateralized) ictal EEG pattern at onset. This observation argues for direct bihemispheric propagation of seizures with RBEB, or alternatively, preferential spread of such seizures to regions (e.g. the orbital and medial frontal cortices) that secondarily project to widespread brain areas. Functional imaging studies demonstrate medial and orbital frontal activation with spontaneous eye blinking (Tsubota et al, Exp Eye Res 69(1):1-7, 1999; Yoon et al, Neurosc Lett 381(1-2):26-30, 2005). These brain areas are also among those that display an early increase in the BOLD signal during generalized spike-wave paroxysms in simultaneous EEG-fMRI studies (Benuzzi et al, Epilepsia 53(4):622-30, 2012). (iii) It is therefore plausible that in CPSs with RBEB, seizure spread occurs early to 'eye-blink eloquent' brain areas that results in both rapid eye blinking as well as a diffuse ictal EEG. The further associations of RBEB with epilepsy syndrome and treatment responsiveness will be investigated in a larger study that confirms these preliminary findings.

\subsection{9 \\ EYELID MIOCLONIA WITH AND WITHOUT ABSENCES: CLINICAL FORMS AND THEIR PROGNOSIS}

Priscila O. Conceição, F. P. Safatle, C. G. Uchida, K. Carvalho, I. S. Tudesco, R. Nojoza, G. M. Araújo Filho, L. M. Guilhoto and E. M. Yacubian (Universidade Federal de São Paulo, são Paulo, Brazil)

Rationale: Eyelid myoclonia and absence or Jeavons Syndrome is considered a form of idiopathic generalized epilepsy, with eyelid myoclonia, childhood onset, photosensitivity and eye closure-induced seizures/EEG paroxysms. However, the main clinical features of Jeavons Syndrome, eyelid myoclonia, can be observed in different epileptic syndromes. This study aims to review electroclinical features of patients with idiopathic generalized epilepsy, presenting eyelid myoclonia with and without absences and classify them according different proposals.

Methods: Clinical and video-EEG data of patients whith eyelid myoclonia or eyelid mioclonia and absences were analyzed and classified in electroclinical groups according to the following authors: a) Covanis et al. (2005): 1) early onset eyelid myoclonia (<4yrs.), normal MRI and intellectual disability; 2) generalized tonic-clonic seizures (GTCS) at onset, normal MRI and intellectual disability; 3) benign course with infrequent limb myoclonia; 4) classical form with eyelid myoclonia, onset in childhood, photosensitivity and eye closure-induced seizures/EEG paroxysms; 5) juvenile myoclonic epilepsy and later onset eyelid myoclonia; 6) eyelid myoclonia associated with frequent non convulsive status; b) Yalçin et al. (2006): overlap cases of EMA and JME; c) Caraballo et al. (2009): 1) classical JS; 2) eyelid mioclonia and absences with ID; 3) eyelid myoclonia with frequent GTCS and limb myoclonia; and d) Capovilla et al. (2009): eyelid myoclonia with intellectual disability and polyspike / polyspike and waves in ictal EEG.

Results: Seventeen female patients aged 10-35 years (mean 23.6) were included. Applying the Covanis' classification, 12 (70.6\%) could be classified, 5 in group 1; 5 in group 5; and 2 in group 4. Four patients $(23.5 \%)$ were included in Yalçin group. When Caraballo's proposal was applied, 14 patients $(82.3 \%)$ were classified, 9 in group $3 ; 3$ in group 1 and 2 in group 2. Finally, one patient (5.9\%) was included in Capovilla's group. All groups, but Covanis's fifth and Yalçin group, had more than $50 \%$ of patients drug resistant. Conclusions: The present data demonstrated that the proposed classifications are not able to include all clinical features of patients with eyelid myoclonia in idiopathic generalized epilepsy. Although using Caraballo's proposal the majority of patients could be classified, Covanis's classification was the only one which delineated difference among the 6 groups related to prognosis.

\subsection{0 \\ CONCOMITANT BENIGN ROLANDIC AND CHILDHOOD ABSENCE EPILEPSY: A REPORT OF THREE CASES}

Anita Datta, L. Bello-Espinosa, M. Scantlebury and J. M. Rho (University of Calgary, Calgary, AB, Canada)

Rationale: Childhood absence epilepsy (CAE) and benign rolandic epilepsy (BRE) are distinct age-dependent epileptic syndromes, and are not considered entities within an electroclinical continuum (PMID: 10487473). However, generalized slow spike-wave (S-W) discharges are not uncommonly seen in patients with BRE. When present, these are thought to be due to secondary bilateral synchrony. In contrast, generalized $3 \mathrm{~Hz} \mathrm{~S}-\mathrm{W}$ discharges are only rarely seen in BRE. Here, we describe three patients who were diagnosed with both $\mathrm{CAE}$ and BRE at different time-points. Two of these patients first presented with CAE and subsequently developed BRE.

Methods: We conducted a retrospective review of clinical and EEG records of patients diagnosed with either CAE or BRE at the Alberta Children's hospital from 2007 to 2012. Three patients at our institution were identified as having both CAE and BRE. All were female and age at initial presentation ranged from 6 to 9 years.

Results: The first patient had a history of absence epilepsy successfully treated with valproic acid (VPA). She developed staring spells associated with generalized $3 \mathrm{~Hz} \mathrm{S-W}$ discharges. After two years of seizure freedom, a follow-up EEG was obtained which showed independent left and right central temporal spikes with a tangential dipole consistent with BRE. The patient was not experiencing any clinical seizure activity and it was recommended that she be weaned off VPA. A second patient was diagnosed with $\mathrm{CAE}$ at 7 years of age. Her EEG showed generalized $3 \mathrm{~Hz} \mathrm{S-W}$ discharges. Seizures were well-controlled on VPA. A follow-up EEG one year later showed electrographic features that were consistent with both BRE and CAE. A third patient was diagnosed at age 9 with BRE confirmed with a characteristic EEG pattern on initial EEGs. Clinical seizures were also consistent with BRE (nocturnal with sensory changes involving the face and mouth followed by secondary generalization). The patient was treated successfully with oxcarbazepine. On a follow-up EEG 2 years later, there was evidence of generalized $3 \mathrm{~Hz} \mathrm{~S}-\mathrm{W}$ discharges.

Conclusions: Our cases suggest that in some patients with idiopathic epilepsies, there may be an atypical course or perhaps a continuum between CAE and BRE. Another possibility is that electroclinical conversion from one phenotype to another may be induced by antiepileptic drugs such as ethosuximide and phenobarbital (PMID: 22467741 and 12018965). It is possible that oxcarbazepine may have induced $3 \mathrm{~Hz}$ S-W discharges in our third patient. Based on published reports, $\mathrm{CAE}$ is thought to develop rarely in patients who were initially diagnosed with BRE; however, two of our patients had a reverse presentation. It is important for clinicians recognize that patients may evolve from BRE to CAE and vice-versa. The mechanisms underlying this unusual ontogeny remain unclear and require further study. 


\subsection{1}

\section{CHILDREN WITH POSTERIOR SPIKES - A DETAILED} EEG, SEIZURE AND MRI ANALYSIS

Tugba Hirfanoglu ${ }^{1,2}$ and I. Tuxhorn ${ }^{2}$ ( ${ }^{1}$ Division of Pediatric Epilepsy, Department of Pediatric Neurology, Gazi University, Faculty of Medicine, Ankara, Turkey and ${ }^{2}$ Division of Pediatric Epilepsy, Pediatric Department, Rainbow Babies and Children's Hospital, University Hospitals Case Medical Center, Cleveland, $\mathrm{OH}$ )

Rationale: Epilepsy in children is common and may be associated with considerable medical and social morbidity. Focal seizures may arise from any region of the brain and accurate identification opens up medical and surgical treatment options that may cure or remit seizures. Occipital spike activity is more common in children compared to adults and may reflect a very heterogeneous group of epilepsies including benign focal epilepsies of childhood (BFEC) which are age dependent electroclinical syndromes. The two most commonly described BFECs of childhood are benign childhood epilepsy with centrotemporal spikes (BCECTS) and benign childhood epilepsy with occipital paroxysms (BEOP). In addition occipital spikes may be seen in symptomatic epilepsies due to brain metabolic disorders or lesions. There are limited detailed studies of seizure semiology, the scalp EEG and MRI imaging to define specific pediatric etiologies in pediatric occipital lobe epilepsy. We aimed to study detailed clinical semiology and scalp EEG findings of both benign and symptomatic variants in children with occipital lobe epilepsy to provide diagnostic and therapeutic approaches. Methods: All pediatric patients ( $<16$ years) with occipital spikes recorded between January 2008 to October 2011 in the neurophysiology department Rainbow Babies and Children's University Hospital were selected. Spikes were analysed according to morphology, localization, changes with eye closure, sleep, photic stimulation. Where available ictal patterns were also analysed. Seizure semiology was analysed with regard to visual auras, lateralizing signs, motor, autonomic and other features. The benign group was defined based on published spike characteristics. The symptomatic group was defined by the presence of MRI pathology. Results: 32 patients were studied, $50 \%(16 / 32)$ were symptomatic, $50 \%(16 / 32)$ had BEOPs studied at age $5.89( \pm 4.83)$ and $7.8( \pm 6.6)$ years of age retrospectively. Autonomic symptoms were predominantly seen in the benign goup (2/16), versive (7/16) and tonic clonic seizures $(6 / 16)$ were more frequent in the symptomatic patients. ESES was seen in both symptomatic (4/16) and benign $(2 / 16)$ patients. MRI pathologies found in the symptomatic ischemic and developmental lesions such as heterotopia, tubers, focal cortical dysplasias Focal slowing (7/16), more extensive spike localization and bilateral spikes $(16 / 16)$ were frequent in the symptomatic cases. Conclusions: Seizures and EEG findings in children with BEOPs and occipital spikes associated with MRI pathology occured equally in 32 pts studied. ESES was seen in both groups. Motor features and more widespread spike localization and focal dysfunction were more prominent in the symptomatic group suggesting involvement of a more widespread epileptogenic network.

\section{Clinical Epilepsy: Clinical Diagnosis}

\subsection{2}

APNEIC SEIZURES IN NEONATES AND INFANTS: SUBTLE SEIZURES OF TEMPORAL LOBE ORIGIN

Richard P. Morse ${ }^{1}$, M. Otero ${ }^{2}$ and J. Filiano ${ }^{1}\left({ }^{1}\right.$ Pediatrics and Neurology, Dartmouth-Hitchcock Medical Center, Lebanon, NH and ${ }^{2}$ Neurology, Dartmouth-Hitchcock Medical Center, Lebanon, NH)

Rationale: Apnea is a common event in babies and occurs for multiple reasons. As a manifestation of a seizure, apnea is rare and most often is associated with other signs (change in heart rate, blood pressure, pupillary dilatation) that raise clinical suspicion. The clinical seizures may be subtle and easily overlooked, and video-EEG may be required to demonstrate them. In infants, apnea is a common presentation of an apparent life threatening event (ALTE), and even less commonly results from a seizure than in neonates. Previous reports have proposed a temporal lobe/limbic system origin for ictal events associated with apnea, and have described hemorrhagic stroke as the most common finding in neonates with this presentation. Methods: We present four children, three neonates and one infant (two months old) referred to our center because of apneic spells. In all four, V-EEGs were performed for evaluation of apneic spells and demonstrated an ictal basis. Subtle clinical seizures accompanying the desaturation and apnea in the neonates were identified on review of the video and had been overlooked by the intensive care nursing staff. All four patients had temporal lobe-based seizures.

Results: In all cases V-EEG demonstrated ictal events associated with apnea. All three neonates had sufficiently subtle seizures as to have been overlooked by intensive care nursing staff, and the apnea/desaturations were detected at or near the end of the seizures in $2 / 3$ instances. Two neonates had a change in the heart rate (one increased, one decreased) associated with the seizures. The infant had oral automatisms and a characteristic cry at the onset of seizures. The three neonates were found to have hemorrhage (2) or infarct (1) involving the temporal lobe, and the infant had normal neuroimaging. Conclusions: When apnea is reported in a neonate or infant, a seizure should be considered if there is not another obvious explanation. The seizure associated with apnea may be subtle and overlooked, especially given the dramatic nature of the apnea, which may be clinically recognized as the seizure is ending. The temporal lobe/limbic system is most often involved in apneic seizures in the neonate and infant, and this helps explain the subtle nature of the seizures. V-EEG is a useful modality to determine the ictal nature of apnea in neonates and infants.

\begin{tabular}{|c|c|c|c|c|}
\hline $\begin{array}{c}\text { Subject } \\
\#\end{array}$ & $\begin{array}{c}\text { Age at } \\
\text { Presentation }\end{array}$ & Clinical Description & $\mathrm{V}$-EEG results & Neuroimaging \\
\hline $1-B R$ & FT-I day & $\begin{array}{l}\text { non-traumatic delivery. } \\
\text { appe:/bradyeardia and } \\
\text { desaturations at } 2 \text { hours }\end{array}$ & $\begin{array}{l}\text { electrographic seizures, } R \\
\text { temporal, some with } \\
\text { desaturations }\end{array}$ & $\begin{array}{l}\text { R temporal } \\
\text { hemonorlage }\end{array}$ \\
\hline 2-AK & FT-I day & $\begin{array}{l}\text { cyanosis, dcsaturations. } \\
\text { tachycardia at } 3 \text { hours after } \\
\text { atraumatic birlh. } \\
\text { progressed to apnea }\end{array}$ & $\begin{array}{l}\text { L temporal lobe seizures, } \\
\text { desaturations to } 30 \% \\
\text { worsening towards end of } \\
\text { event. subule behavioral } \\
\text { arrest as seizure preceding }\end{array}$ & $\begin{array}{l}\text { 1. temperal } \\
\text { hemorraage }\end{array}$ \\
\hline 3-JD & FT-2 days & $\begin{array}{l}\text { mom with GBS, received } \\
\text { penicillin, otherwise } \\
\text { atraumatic birth apneic } \\
\text { events noted in first } 24 \\
\text { hours }\end{array}$ & $\begin{array}{l}\text { I. temporal seizures, apnea } \\
\text { noted at end, xeizure subtle } \\
\text { oral automatisms, staring }\end{array}$ & $\begin{array}{l}\text { infanct I. } \\
\text { temporal, part ot } \\
\text { Larger stroke }\end{array}$ \\
\hline $4-A C$ & 2 months & $\begin{array}{l}\text { apnea with perioral } \\
\text { cyanosis diagnosed with } \\
\text { GERD and breathholding } \\
\text { spells (reported out of slecp } \\
\text { by parents) }\end{array}$ & $\begin{array}{c}\text { L anterior temporal seizure, } \\
\text { oral autoanausms mimic } \\
\text { numinationsubrte behavioral } \\
\text { arrest }\end{array}$ & $\begin{array}{l}\text { normal brain } \\
\text { MRI }\end{array}$ \\
\hline
\end{tabular}

\subsection{3 \\ SEVERE DRUG-RESISTANT EPILEPSY IN PATIENTS PREVIOUSLY TREATED FOR CHILDHOOD ACUTE LYMPHOCYTIC LEUKEMIA (ALL)}

Mercedes Alvarez Blanca ${ }^{1}$ and F. Dubeau ${ }^{2}\left({ }^{1}\right.$ Hospital Puerta de Hierro Majadahonda, Madrid, Spain and ${ }^{2}$ Montreal Neurological Institute, McGill University, Montreal, QC, Canada)

Rationale: Seizures are seen in $8-13 \%$ of the children with ALL. In the great majority of the cases, they suffer of acute symptomatic seizures occuring during the induction and consolidation phases of the therapy. More rarely, patients develop a chronic seizure disorder. We describe four adult patients treated during childhood for ALL who later developed drug-resistant refractory epilepsies. Methods: This is a retrospective study of four patients with severe epilepsy refered to the outpatient clinic of the Montreal Neurological Hospital between 1996 and 2012. Patients' epilepsy history and clinical and laboratory features were reviewed and compared to similar cases described in the literature. 
Results: The four patients (all females; mean age, 25.7 yrs) were diagnosed with ALL before age 5 (range, $18 \mathrm{mo}$ to $4.5 \mathrm{yr}$ ). They received standard chemotherapy protocols including intrathecal methotrexate in all, and two also received prophylatic whole-brain radiation. None of the patients experienced CNS complications including acute symptomatic seizures during induction or consolidation therapy, nor they developed leukemic CNS infiltration. There was no family history for seizures and, except for the hematological disease, personal antecedants were negative. One patient, who received whole-brain radiation, developed a drugresistant temporal lobe epilepsy 8 years after the cessation of the ALL treatment, due to a left temporal lobe cavernous angioma. She became seizure-free after the lesion was removed. A second patient developed a severe epileptic disorder 6 years after the chemotherapy ended with EEG showing bilateral, diffuse or multifocal epileptic discharges. Brain MR and CT were normal. The two remaining patients presented with an intractable generalized epilepsy and clinical and EEG features of Lennox-Gastaut Syndrome, one starting at the end of a 3-yr maintenance therapy and the other 4 years after therapy (received prophylactic whole-brain radiation). They both had multiple calcifications on imaging studies (CT and MR) consistent with mineralizing microangiopathy. In these 3 patients, the seizures remained severe in spite of different antiepileptic drug polytherapies $(n=3)$, VNS $(n=2)$ and anterior callosotomy $(n=1)$. The four patients developed cognitive decline preceding or following the epilepsy. Conclusions: Remote intractable seizures are a rare complication of pediatric ALL In many instances, chronic seizures are delayed sequelae resulting from a focal CNS damage (explained either by the disease or the treatment), leading to focal epilepsy. In other cases, seizures appear to result from a diffuse brain insult due to leukemic treatment given during a crucial phase of brain development, and leading to catastrophic multifocal or generalized epilepsy. Factors contributing to the appearance of such secondary severe generalized or diffuse seizure disorders are still unclear.

\subsection{4}

\section{CLINICAL SEMIOLOGY AND SURGERY OUTCOME OF} SEIZURES ORIGINATING IN THE PERISYLVIAN OPERCULUM BASED ON STEREO-

\section{ELECTROENCEPHALOGRAFIC (SEEG) STUDIES} Ioana Mindruta $^{1}$, L. Minotti ${ }^{2,6}$, A. Montavont ${ }^{3}$, D. Hoffmann ${ }^{4}$, S. Chabardes $^{4,6}$, M. Guenot ${ }^{5}$, P. Ryvlin ${ }^{3}$ and P. Kahane ${ }^{2,6}\left({ }^{1}\right.$ Neurology, University Emergency Hospital, Bucharest, Romania; ${ }^{2}$ Neurology, University Hospital, Grenoble, France; ${ }^{3}$ Head Epilepsy, Sleep and Pediatric Neurophysiology, University Hospital, Lyon, France; ${ }^{4}$ Neurosurgery, University Hospital, Grenoble, France;

${ }^{5}$ Neurosurgery, University Hospital, Lyon, France and ${ }^{6}$ Institute of Neuroscience - Inserm U 836, Grenoble, France)

Rationale: The cerebral operculum harbors areas essential for motor, sensory, auditory and language processing.

Most of the evidence about opercular cortex ictal involvement emerged from the studies on temporal lobe and insular seizures propagation (Kahane et al., Avanzini G, Beaumanoir A, Mira L.Limbic seizures in children.John Libbey;2001;115-27; Isnard et al.,Epilepsia 2004;45:1079-90).

Specific semiology as well as surgery outcome of epilepsies with seizures that start distinctly in the perisylvian operculum and secondarily involve insula or other structures are scarcely documented in literature.

Methods: We retrospectively analyzed clinical semiology of opercular seizures based on video analysis correlated with SEEG ictal recordings and direct electrical stimulation (DES) of opercular cortex.

Fifteen patients were included, based on the following criteria: opercular and insular structures were appropriately sampled, SEEG recordings demonstrated that the ictal discharge had origin in the opercular structures sparing the insular cortex at seizure onset and the follow up period after surgery comprised at least one year. We also compared semiology of seizures and general characteristics of patients suffering from seizures with suprasylvian (SSY) versus infrasylvian (ISY) opercular origin with focus on the symptom clustering, anatomo-electro-clinical correlations and results of surgery.

Results: Hierarchical cluster analysis revealed that association of facial motor involvement, autonomic and somatosensory symptoms characterize the semiology of seizures originating in the perisylvian operculum.

In the SSY group, significantly more patients had somatosensory auras and experienced early during their seizures facial motor involvement and speech arrest $(\mathrm{p}=0.001)$. In this group, seizures were significantly shorter in duration and occurred more often during sleep $(\mathrm{p}=0.001)$. The propagation of ictal discharge in the SSY group involved the insular structures but also the motor, premotor mesial cortex and anterior cingulate gyrus.

In the ISY group, significantly more patients were able to warn at seizure onset $(\mathrm{p}=0.04)$ and had auditory auras $(\mathrm{p}=0.001)$. During the ictal period, they displayed more frequently staring and/or activity arrest and experienced loss of contact $(\mathrm{p}=0.04)$. SEEG study documented a multidirectional spreading of ictal discharges involving the insular cortex, suprasylvian operculum and temporal structures. Surgical resections were performed in 10 out of 15 patients due to functional restraints. Five patients $(50 \%)$ were seizure free after a follow-up period of at least 24 months.

Conclusions: Our study showed that a particular cluster of symptoms and signs characterizes perisylvian opercular seizures. Moreover, we pointed to a specific semiology that differentiates seizures starting in the SSY versus ISI border.

Surgery could be successful in patients with seizures originating in the perisylvian operculum providing that complete removal of the epileptogenic zone is not interfering with the language eloquent network.

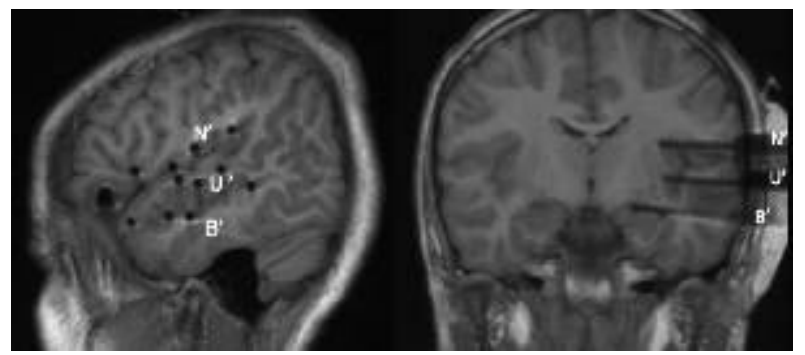

Postimplantation MRI images (lateral and frontal view) showing the orthogonal approach of intracerebral electrodes, based on SEEG method, over the suprasylvian and infrasylvian opercula, with internal contacts reaching the insular cortex. Apart from opercular structures, intracerebral electrodes targeted temporal mesial structures, lateral neorcortex (anterior and posterior) and temporal pole.

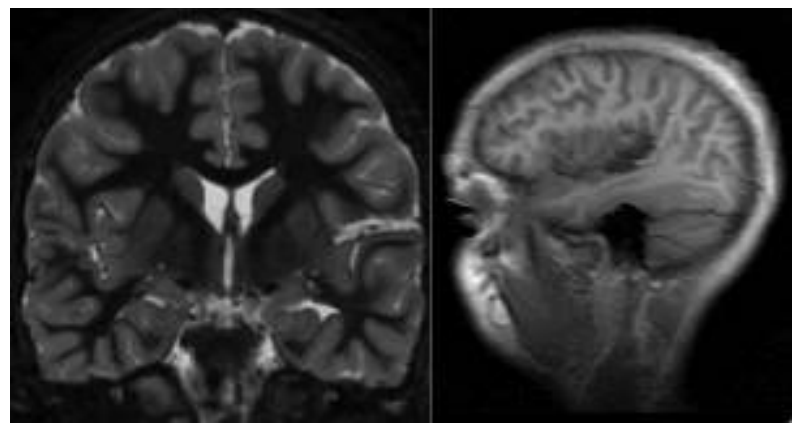

Cerebral MRI images post surgery displaying limited resections in the operculo-insular cortex, in the centro-parietal division (left side) and superior temporal gyrus (right side) in two cases that are seizure free after at least a 24 months follow up period. 


\subsection{5}

STATNET EEGS DECREASE THE DELAY TO DIAGNOSIS IN THE EMERGENCY DEPARTMENT

F. Moien-Afshari, S. T. Herman, M. Barmard and T. D. Pang (Neurology, Beth Israel Deaconess Medical Center, Boston, MA)

Rationale: Delays inidentification and treatment of non-convulsive status epilepticus (NCSE) may result in increased morbidity and mortality. An emergency electroencephalogram (EEG) is often difficult to obtain due to lack of EEG technologists and readily available electroencephalographers for immediate interpretation. The conventional method of EEG lead application is time consuming and can only be performed by specially-trained personnel. The StatNet electrode set is a system that could be applied by non-EEG personnel after minimal training. We have previously shown that EEGs recorded using the StatNets are comparable to conventional recordings. This study is designed to assess 1 . The feasibility of using StatNet electrodes for stat EEGs in the ED, 2. Compare the time delay to EEG acquisition and interpretation between the StatNet and conventional EEG group, 3. Evaluate the impact on flow of patient care in the ED.

Methods: Our hypothesis is that using StatNet electrodes will decrease the delay to EEG interpretation and facilitate patient care in the ED. For urgent EEG requests from the ED, minimally trained ED staff applied StatNet electrodes to record EEGs. We compared the following parameters between patients receiving StatNet EEGs with those receiving conventional EEGs in the historical control group: 1 . Time from EEG request to start of EEG acquisition, 2. Time from EEG start to time of communication of results to ED staff, and 3. Time from EEG start to time of ED disposition. The nonparametric Mann-Whitney two sample T-test was used for comparisons, and results are expressed in mean minutes $+/$ - standard error of the mean. Results: The delay from the time of EEG requisition to the start of EEG acquisition in the StatNet group was significantly shorter at $72.6+/-9.3(\mathrm{~N}=25)$ than the historical control group which was $164.6+/-32(\mathrm{~N}=23), \mathrm{P}=0.02$ (Figure 1A). The time delay from EEG acquisition to communication of results to ordering physicians was also significantly shorter in the StatNet group, 54.6+/-3.6 $(\mathrm{N}=25)$ compared to historical controls, $158+/-47.5(\mathrm{~N}=25), \mathrm{P}=0.008$ (Figure 1B). Finally, the time from EEG start to ED disposition was significantly shorter in the StatNet group, 205.5+/-21.2 ( $\mathrm{N}=23)$ versus historical controls $374+/-7.2(\mathrm{~N}=25), \mathrm{P}=0.0005$ (Figure 1C). Conclusions: This study demonstrates feasibility of using the StatNet electrodes for emergency EEGs by non-EEG trained personnel. The StatNet EEGs were associated with significantly shorter delays in obtaining the study and communication of results to the responsible physician. It also facilitated patient management by reducing the time spent in the ED, reflecting timely treatment or transfer to the relevant services for further treatment. Thus, it is a desirable method to obtain urgent EEGs to facilitate rapid diagnosis of NCSE. Furthermore, it has the potential for widespread use and may improve the care of patients in the ED, by reducing time spent in the ED while reducing health care costs.
A

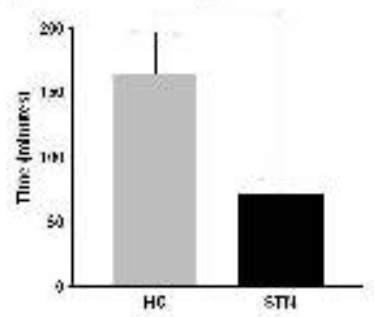

B

$c$

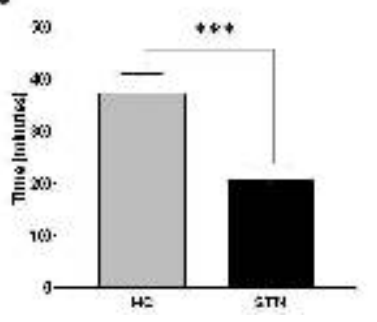

Figure 1. A: T ne tom EEG crde to EEG :tart i ${ }^{*} p=0.23 ;: 8$ : TIme from [LG sta t to I interpretatlor comnur cation $\{* \beta=0.005\}: \mathrm{C}$ : TIme trom [CG start to $[D$ dispus $L 0 . I^{+* p^{2}}=0.0005$;

\subsection{6 \\ INCIDENCE OF EPILEPTIFORM ACTIVITY IN ADULT PATIENTS TREATED WITH THERAPEUTIC HYPOTHERMIA AFTER CARDIAC ARREST}

J. J. Asconape, R. Kumar, A. H. Iglesias and T. L. McKiernan (Loyola University Medical Center, Maywood, IL)

Rationale: Therapeutic hypothermia (TH) has become a standard procedure for the management of cardiac arrest. An increased incidence of seizures or epileptiform discharges, especially during the rewarming period, has been observed. The actual incidence of epileptiform activity, however, has not been adequately studied. Methods: We retrospectively reviewed the continuous EEG monitoring (c-EEG) findings of 24 patients undergoing TH between June, 2009 and June, 2012. EEG monitoring was started at the onset of TH and continued for at least 24 hours after the rewarming process was started. All the patients were 18 years or older and hemodynamically stable. Patients were maintained at a core body temperature of $32-34^{\circ} \mathrm{C}$ for twenty-four hours, and received midazolam, fentanyl and vancuronium.

Results: Six of the 24 patients (25\%) presented some type of epileptiform activity during the c-EEG. Three patients (12.5\%) were diagnosed with post-anoxic myoclonic status epilepticus, with overt myoclonic activity and generalized epileptiform discharges on EEG. One patient (4.2\%) developed generalized electrographic status epilepticus during the rewarming period. Two patients (8.3\%) had interictal epileptiform discharges: one with generalized spike-wave discharges and bursts of diffuse attenuation, the other with transient multifocal epileptiform discharges.

Conclusions: In our series the incidence of epileptiform activity, if cases of myoclonic status epilepticus are excluded, was relatively low, but still clinically significant. C-EEG monitoring should be considered an essential component of the therapeutic hypothermia protocol and extend throughout the rewarming process. Further studies are necessary to properly assess the incidence of epileptiform activity in this population.

\subsection{7}

\section{UNIHEMISPHERIC BURST-SUPPRESSION: AN} ELECTROCLINICAL STUDY OF TWO CASES

Cornel Rogers $^{1}$, F. Torres-Delgado ${ }^{1}$, R. Juarbe-Arroyo ${ }^{1}$, E. C. Mader $^{1,2}$, N. R. Villemarette-Pittman ${ }^{1,2}$ and P. W. Olejniczak ${ }^{1,2}$ ( ${ }^{1}$ Department of Neurology, Louisiana State University Health Sciences Center, New Orleans, LA and ${ }^{2}$ Epilepsy Center of Excellence, Louisiana State University Health Sciences Center, New Orleans, LA) 
Rationale: Burst-suppression (BS) consists of periodic bursts of high-voltage slow waves and sharp waves in the

electroencephalogram (EEG), alternating with attenuation of the background activity. Typical bihemispheric BS, whether pharmacologically-induced or secondary to encephalopathy, is often viewed as a non-epileptic phenomenon. On the other hand, unihemispheric BS is rare and its clinical significance is poorly understood.

Methods: Two patients whose initial EEG tracings showed unihemispheric BS underwent continuous EEG monitoring in the intensive care unit. Patient-1 is a 56-year-old woman with a left temporoparietal tumor who presented in convulsive status epilepticus. Her EEG showed left hemispheric BS after clinical seizure termination with lorazepam and propofol. Patient-2 is a 39 -year-old woman with multiple medical problems and a vague history of seizure disorder. After undergoing abdominal surgery, she experienced a convulsive seizure prompting treatment with propofol. Her EEG showed left hemispheric BS.

Results: In both cases, titration of propofol resulted in disappearance of unihemispheric BS. The brain MRI of Patient-1 showed a mass lesion in the left anterior parietal area predominantly involving the white matter and the deep diencephalic structures with mass effect. The brain CT of Patient-2 showed left hemispheric encephalomalacia predominantly involving the left posterior parietal lobe. Both patients improved and were discharged from the hospital.

Conclusions: The prevailing view that typical bihemispheric BS is a non-epileptic phenomenon should not be automatically extrapolated to unihemispheric BS. In the two cases presented, clinical seizures occurred and unihemispheric BS resolved easily with propofol, suggesting that an epileptic mechanism may be responsible for unihemispheric BS.

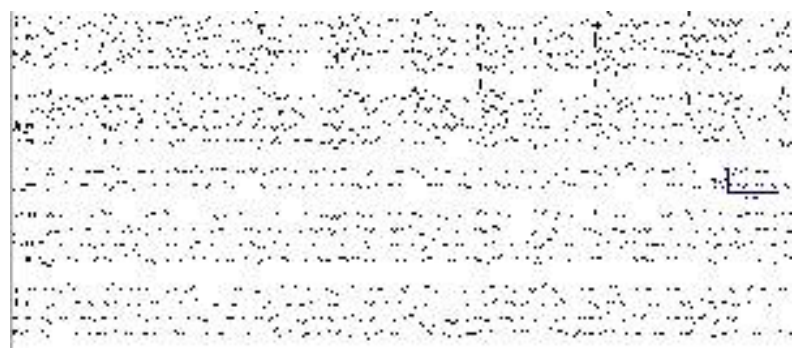

EEG from patient 1 showing unilateral burst suppression.

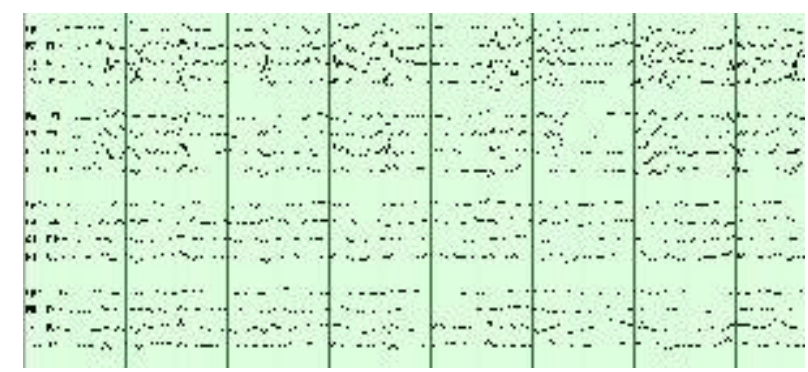

EEG from patient 2 showing unilateral burst suppression.

\subsection{8 \\ SCN1A MUTATIONS IN A POPULATION OF CHILDREN WITH STATUS EPILEPTICUS}

Christian Korff ${ }^{1}$, S. Lebon ${ }^{2}$, G. Ramelli ${ }^{3}$, A. Datta ${ }^{4}$, D. GubserMercati $^{5}$, O. Maier ${ }^{6}$, C. Combescure ${ }^{1}$, M. Rodriguez ${ }^{1}$, M. Seeck ${ }^{1}$, E. Roulet-Perez ${ }^{2}$ and F. Le Gal ${ }^{1}$ ( ${ }^{1}$ University Hospitals Geneva, Geneva, Switzerland; ${ }^{2} \mathrm{CHUV}$, Lausanne, Switzerland; ${ }^{3}$ Cantonal Hospital, Bellinzona, Switzerland; ${ }^{4}$ UKBB, Basel, Switzerland; ${ }^{5}$ Cantonal Hospital, Neuchâtel, Switzerland and ${ }^{6}$ Cantonal Hospital, St Gallen, Switzerland)

Rationale: Status epilepticus (SE) may present as an isolated episode in normal infants during a febrile illness. On the other side, it may be repeatedly observed in severe epileptic syndromes of infancy, such as Dravet syndrome, with which the differential diagnosis is difficult initially. Abnormalities on the gene that encodes SCN1a, are found in $70-80 \%$ of patients with classic Dravet syndrome. The objective of this study was to analyze the frequency and nature of SCN1a abnormalities and to identify potential predictive factors for the development of Dravet syndrome in a population of children who presented at least one episode of SE.

Methods: Children who presented with at least one episode of SE between 1 month and 16 years, were included. These children were selected in a larger group of children with seizures in which SCN1a was analyzed. To detect any partial or complete deletion/duplication on SCN1a, multiplex ligation-dependent probe amplification was performed. Prescreening for potential DNA point mutations was carried out by high-resolution melting curve analysis (HRMCA). SCN1a was divided in 40 fragments. DNA from patients was tested in parallel with DNA from control patients. Normalized and temperature-shifted difference plots were compared between samples. Fragments showing abnormal HRMCA profiles were amplified by pcr. Direct sequencing of the purified products was performed and further analyzed on capillary electrophoresis using primers designed to cover all exons and their flanking sequences. Blood samples from the parents of children with abnormal genetic results were analyzed.

Results: 110 children were tested for SCN1a mutations. 73 of them who presented with at least one episode of status epilepticus were included in this study. SCN1a abnormalities were found in a total of 14 patients and in $11(15 \%)$ of those with SE. Nine $(82 \%)$ of the latter had Dravet syndrome, and 2 (18\%) were on the GEFS+ spectrum. All of those with abnormal SCN1a results who later developed Dravet syndrome were boys; their mean age at initial SE was 9 months. Abnormalities were de novo in 6 Dravet patients, unknown in 2 (parents not tested, and inherited in one (mutation unlikely to be causal per se). The initial SE was hemiclonic in four, and five had apparently generalized tonic-clonic convulsions. One girl with Dravet phenotype but negative SCN1a analyses presented with SE at 36 months.

Conclusions: In our study, the risk of developing Dravet syndrome after an initial SE episode was particularly high in boys whose initial SE episode was observed between 6-18 months. One girl with Dravet phenotype and negative SCN1a analysis presented with SE at 36 months. These results indicate that performing SCN1a analysis may be warranted in every child who presents with an initial episode of $\mathrm{SE}$ at less than 18 months. This may help in the early diagnosis and management of Dravet syndrome. Our results also carry fundamental prognostic value, and may optimize genetic counseling. In addition, SCN1a testing is unlikely to be useful if the initial SE episode is observed after 36 months.

\subsection{9 \\ PREDICTIVE VALUE OF ICTAL SCALP EEG FOR THE DIAGNOSIS OF BILATERAL INDEPENDENT TEMPORAL LOBE EPILEPSY}

J. Fatehi, B. AlJafen, W. Hader, N. Jette, S. Wiebe and Y. Aghakhani (Department of Clinical Neurosciences, University of Calgary, Calgary, AB, Canada)

Rationale: Bilateral interictal epileptiform discharges, reported in 8$70 \%$ of patients with unilateral temporal lobe epilepsy (TLE), have low predictive value for the diagnosis of bilateral independent TLE (BiTLE). However, the predictive value of the ictal EEG is not well known. We explored the likelihood of true BiTLE in patients whose ictal scalp EEG suggested BiTLE.

Methods: We reviewed our epilepsy monitoring unit database from July 2003 to Dec 2010 to identify patients with an initial diagnosis of presumed BiTLE based on ictal scalp EEG, who subsequently underwent intracranial EEG (IEEG) monitoring. Variables of interest included demographic variables, clinical variables (e.g. epilepsy 
duration, seizure types, etc), MRI variables, scalp EEG variables (percent lateralization, etc), intracranial EEG variables (ictal onset, etc) and surgical outcomes (Engel classification for seizures, etc). Descriptive statistics were calculated.

Results: Of 917 patients admitted to the epilepsy monitoring unit, 35 patients had an initial diagnosis of presumed BiTLE based on ictal scalp EEG. Eighteen of them (50\%) (11 males) underwent IEEG with bilateral temporal and some extra-temporal coverage (our study population). Average age and duration of epilepsy were 28.7 and 14.2 years, respectively.

Ten patients $(56 \%)$ had true BiTLE on IEEG, of which three underwent a temporal resection. One of these patients had a tumour (Engel Class I) and the other two had no MRI lesions (1 Engel Class I, 1 Engel Class IV) at $>3$ year follow up.

Eight patients did not have BiTLE on IEEG. Seven of them had unilateral TLE while one had frontal epilepsy. Of the seven with unilateral TLE, five had a temporal resection. Two of these patients had an MRI lesion (Engel class I) and the remaining three had no MRI lesion (Engel Class I in one, Engel Class IV in two).

Conclusions: About $40 \%$ of patients with ictal scalp EEG patterns simulating BiTLE turn out to have unilateral TLE. IEEG is crucial to determine if patients have BiTLE or even extratemporal epilepsy. However, in those with BiTLE and no lesion on MRI, the role of IEEG to determine surgical candidacy (and laterality) requires further exploration.

\subsection{0 \\ SEIZURE ENDING SIGNS IN FOCAL SEIZURES WITH LOSS OF AWARENESS AND/OR RESPONSIVENESS}

J. Gavvala, J. Kennedy, I. Garic, I. Sen-Gupta, M. Macken, E. Gerard and Stephan Schuele (Department of Neurology, Northwestern University, Chicago, IL)

Rationale: Signs indicating the end of a focal seizures characterized by loss of awareness and/or responsiveness are (LOA/R) are poorly defined and can be difficult to detect. Not recognizing changes between ictal and postictal behavior can affect accuracy of seizure reporting by family members and may lead to erroneous nursing response or seizure classification during video EEG review. Methods: Our epilepsy monitoring unit database was searched for focal seizures without secondary generalization for the period from 2007 to 2011. The first focal seizure in a patient with LOA/R and/or behavioral arrest and/or automatisms were included. Seizures without objective symptoms or inadequate video-EEG quality were excluded. Seizure onset was defined by the first clinical or EEG change indicating seizure onset. Seizure end was determined by resolution of the ictal EEG pattern.

Results: A total of 41 patients were included, 10 men, 31 women, average age 43.1 (19-80), 30 with temporal, 11 with extra-temporal focal epilepsy; 23 from the left and 17 from the right hemisphere. Average clinical duration 1:18 min (0:14 - 10:36), electrographic duration 1:21 min (0:05 - 10:38). 21 events occurred out of wakefulness and 20 out of sleep (stage II or deeper).

In the 21 awake patients, four went untested. 20 showed abrupt change in motor activity and contact with the environment. Specific signs were seen in 10 out of 21 events: nose wiping in 5, cough in 3 , throat clearing or deep sigh in 2.

Eleven of 20 events out of sleep went untested. Four out of the 11 patients went back to sleep without awareness of the event, one of them showed nose wiping. Seven of 11 were awake at the end of the seizure, a sigh or cough was seen in 3 . None of them alerted the nursing staff about having had a seizure. Of the nine tested seizures out of sleep, 8 showed an abrupt change in behavioral activity and contact at the end of the event. Nose wiping was seen in 3 , sigh and spontaneous laughter were seen each in 1 patient.

Specific ending signs (nose wiping, cough, sigh or throat clearing, laughter) were seen in 19 out of 25 temporal lobe seizures and 8 out of 16 extra-temporal lobe seizures (Fisher exact, $\mathrm{p}=0.11$ ).
Conclusions: Seizure ending signs are often subtle and the most common finding is a sudden change in motor activity level and resumption of contact with the environment. More specific signs, nose wiping, coughing or throat clearing, or a deep sigh, are seen in around half of patients. There was a trend of those signs being more frequent in temporal vs. extra-temporal seizures, which would be consistent with an insular, autonomic mechanism.

A significantly higher proportion of seizures out of sleep went untested as compared to those out of wakefulness ( 11 out of 20 compared to 4 out $21, \mathrm{p}=0.025$, OR 5.2, 95\% CI: 1.1-27.3), despite 24 hour supervision. None of the 11 patients with untested seizures out of sleep were aware of their events afterwards. This demonstrates that seizure semiology can be very subtle and arousals during video monitoring should alert the monitoring staff.

\subsection{1 \\ AUTONOMIC PHYSIOLOGICAL CHANGES DURING PARTIAL AND GENERALIZED SEIZURES IN CHILDREN WITH EPILEPSY ON THE EMU}

Barbara Kroner ${ }^{1}$, A. M. Pitruzzello ${ }^{3}$, W. D. Gaillard ${ }^{2}$ and D. Strube ( ${ }^{1}$ Statistics and Epidemiology, RTI International, Rockville, MD; ${ }^{2}$ Comprehensive Pediatric Epilepsy Program, Children's National Medical Center, Washington, DC and ${ }^{3}$ Engineering and Technology, RTI International, Research Triangle Park, NC)

Rationale: Some seizures cause injury and even death, but there are no reliable detection methods that can alert a caregiver when a seizure occurs. We measured physiological responses caused by changes in autonomic nervous system activity in children with refractory epilepsy to identify patterns that correlate with seizures but not with non-seizure behavior. This pattern recognition algorithm will be used to develop a non-invasive wearable seizure alert system that detects generalized seizures with high sensitivity and precision. Methods: Six children with epilepsy, aged 3-18 and undergoing video EEG have been enrolled into the study with an enrollment target of 30 patients. During monitoring, the patients wore unobtrusive sensors that continuously recorded heart rate, respiration, torso orientation, surface electromyography (EMG), and skin conductance. A preliminary seizure detection algorithm based solely upon cardiac changes (\% maximum heart rate, rate of change in heart rate) was developed and tested to detect generalized seizures and minimize false positives. Algorithms incorporating the full sensor array will be developed from the first 10 patients enrolled and tested on the data from the second set of 10 patients enrolled.

Results: Fifteen seizures ( 3 generalized tonic clonic [GTC], 12 partial) were observed during 242 hours of monitoring. Significant changes in cardiac activity, respiration, muscle activity, and sweat gland activity were observed during the seizures, the most prominent being a rapid increase in heart rate (Figure 1). Maximum heart rate measured during GTC seizures was $197 \pm 1 \mathrm{bpm}$, and $154 \pm 35 \mathrm{bpm}$ during partial seizures. For all seizures, heart rate elevation was observed within 5 to 20 seconds of electrographic onset, and it preceded or occurred simultaneous with the first clinical sign. Using the most stringent detection criterion, 3 out of 3 GTC seizures were detected, along with 2 false positives (FPs) or on average one every 5 days. Other physiological changes consistently observed during seizure events included a rapid change in respiration rate and amplitude, a characteristic rhythmic increase in muscle activity (EMG), and a rapid increase in sweat gland activity (skin conductivity). These findings confirm the physiologic changes we reported previously (Kroner et al, AES 2010) from seizures observed in the residential setting.

Conclusions: Results support the hypothesis that generalized and some partial seizures can be detected by autonomic physiological changes. Detection of these changes using nonobtrusive sensors will form the basis of a seizure monitoring device for daily, non-clinical use. Use of multiple sensors, in addition to cardiac, will improve seizure detection sensitivity and specificity. Such a device could have 
impact on the potential prevention of SUDEP, status epilepticus, and seizure-related injury, as well as improvement in quality of life and increased independence for both caregivers and persons with epilepsy. Source of funding was RTI International.

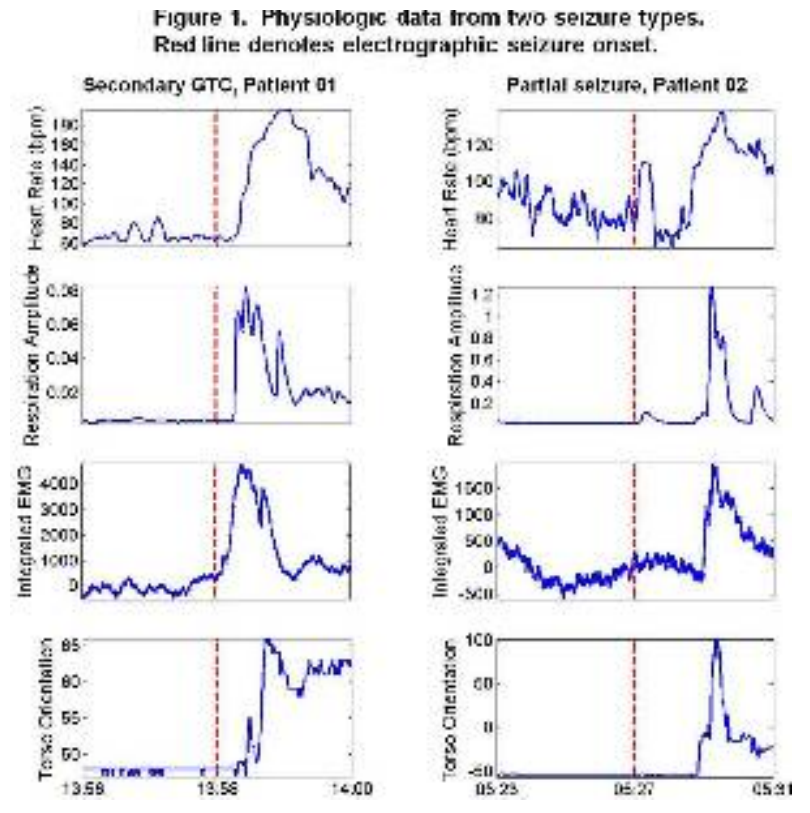

\subsection{2}

\section{WTIHDRAWN}

\subsection{3 \\ COMBINATION OF VIDEO-EEG AND MEG LOCALIZATIONIN FRONTAL LOBE EPILEPSY: CORRELATION TO OUTCOME}

Xintong $\mathrm{Wu}^{1,2}, \mathrm{~S}$. Rampp ${ }^{1}$, R. Hopfengärtner ${ }^{1}$, M. Buchfelder ${ }^{3}$, D. Zhou ${ }^{2}$ and H. Stefan ${ }^{1}{ }^{1}$ Neurology Clinic, University Hospital Erlangen, Erlangen, Germany; ${ }^{2}$ Neurology, West China Hospital of Sichuan University, Chengdu, Sichuan, China and ${ }^{3}$ Neurosurgery, University Hospital Erlangen, Erlangen, Germany)

Rationale: Localization of epileptogenic area of frontal lobe and preservation of the functional area are critical points for frontal lobe epilepsy (FLE), which are important factors for favorable postoperative outcome without or with fewer complications. Both of video-EEG (VEEG) and MEG alone can provide informative clues for localization of epileptogenic areas in FLE, but not for all the cases. This study aims to investigate combination use of MEG and VEEG in FLE.

Methods: Thirty patients with pharmaco-resistant FLE who underwent epilepsy surgery were retrospectively enrolled. Video EEG was recorded using an IT-med system using 10/20 system. Regional localization of spikes in VEEG was defined as spikes discharged from adjacent electrodes and no further propagation to a large and/or contralateral area. Magnetoencephalography was recorded for the purpose of focus assessment.

Magnetoencephalography spikes were detected for dipole localization of the epileptogenic cortex and the epileptogenic area was classified as mono- or multi-focal.

Results: Regional spike discharges were identified in the interictal VEEG of 20 patients and in the ictal VEEG of 17 patients. Thirteen patients had regional spikes in both interictal and ictal VEEG. Monofocal localization was identified in the MEG of 20 patients. Fourteen of these patients had regional spike discharges in VEEG. In the remaining six patients, sources localization was only identified by MEG and there were no regional spike discharges either interictal or ictal VEEG. In $53.3 \%$ patients (16/30), the combination of VEEG and MEG provided localization of epileptic activities, including 11 patients had Engel 1 outcome.

Conclusions: In clinical practice, VEEG is the routine procedure in the presurgical evaluation of FLE. However, we found six cases in which VEEG failed to locate the epileptogenic area that was identified by MEG. We therefore propose that combining VEEG and MEG will optimize the noninvasive presurgical evaluation of epileptiform activities in FLE.

[This study was supported by the Deutsche Forschungsgemeinschaft (DFG, STE-380/14-1) ].

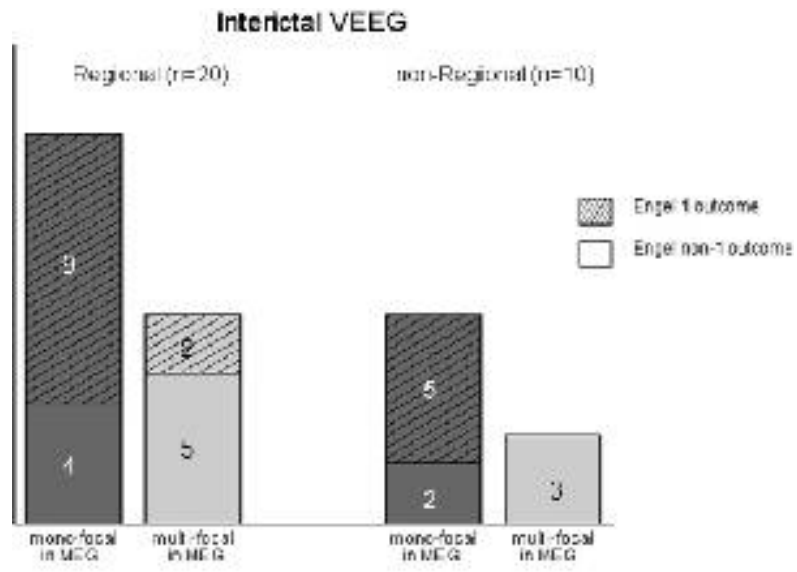

Fig 1: The results of interictal VEEG combining with MEG. 20/30 patients had regional spikes and 10/30 had non-regional spikes (including bi-lateral discharge and no spikes). Totally, 20/30 patients had mono-focal localization in MEG (dark gray), consisting of 13 in regional VEEG group and 7 in non-regional VEEG group. 10/30 patients had multi-focal localization in MEG (light gray), consisting of 7 in regional VEEG group and 3 in non-regional VEEG group. The columns with oblique lines mean patients with Engel 1 outcome; in contrary, without oblique lines mean patients with non-Engel 1 outcome.

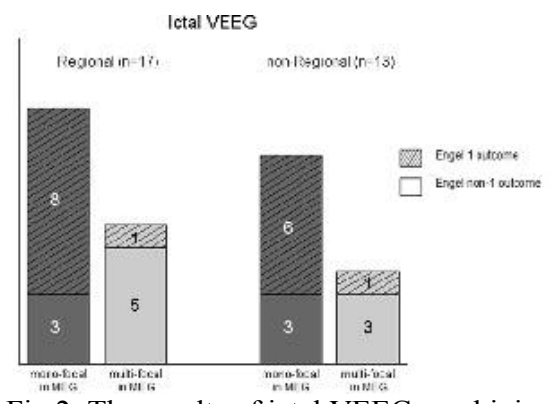

Fig 2: The results of ictal VEEG combining with MEG. 17/30 patients had regional spikes and 13/30 had non-regional spikes (including bilateral discharge and no spikes). Totally, 20/30 patients had mono-focal localization in MEG (dark gray), consisting of 11 in regional VEEG group and 9 in non-regional VEEG group. 10/30 patients had multi-focal localization in MEG (light gray), consisting of 6 in regional VEEG group and 4 in non-regional VEEG group. The columns with oblique lines mean patients with Engel 1 outcome; in contrary, without oblique lines mean patients with non-Engel 1 outcome.

\subsection{4 \\ CAN THE MEDICATION PROFILE HELP DISTINGUISH BETWEEN PATIENTS WITH PSYCHOGENIC NONEPILEPTIC SEIZURES VS. EPILEPTIC SEIZURES?} G. Ro and J. Cohen (Beth Israel Medical Center, New York, NY)

Rationale: The gold standard for identification of patients with psychogenic nonepileptic seizures (PNES) is video/eeg monitoring (VEEG). Other potentially useful adjuncts include history and 
prolactin levels. When confronted with a new patient with unexplained spells, the physician often relies on certain risk factors (e.g. physical or sexual abuse) to suggest the possibility of a PNES diagnosis. However, it would be useful to have other easily obtainable data at the time of the visit to support or refute one's suspicion of PNES. We decided to look at the patient's medication profile in detail, to determine whether there were features in the list that help distinguish between a diagnoses of PNES vs. epileptic seizure (ES).

Methods: Consecutive cases of patients with PNES ( $\mathrm{n}=17)$ and ES $(n=16)$, confirmed by VEEG monitoring were retrospectively identified from the Epilepsy Monitoring Unit database. Patient charts were reviewed and analyzed for demographics, medication lists (past and present) and co-morbidities. Preliminary review suggested that benzodiazepines were used for anxiety, and not for seizure. Data were analyzed descriptively. IRB approval was obtained.

Results: The mean age was: PNES: 38 (26-66), ES: 34 (24-66). M/F ratio: PNES: 4/13; ES:7/9. Duration of 'SZ' in years: PNES: 5; ES: 7. Total Rxs: PNES: 6.5; ES: 7.7. All patients were taking antiepileptic medications (AEDs), though the average number was slightly higher for ES (2.2) vs. PNES (1.6). More patients with PNES (35\%) vs. ES $(12.5 \%)$ were taking benzodiazepines. More patients with PNES $(29.4 \%)$ than ES (18.8\%) were taking antipsychotics. The same trend was true for antidepressants: PNES (35\%) vs. ES (12.5\%). Also, PNES (29.4\%) vs. ES (18.8\%) were more likely to be taking antihypertensives and antidiabetic agents (PNES: $11.7 \%$ vs. $0 \%$ ). Asthma (PNES: $23.5 \%$ vs. ES: $6.3 \%$ ) and pain (PNES: $11.7 \%$ vs. ES:6.3\%) medications were more common in PNES patients. GI meds were slightly more common in PNES (17.6\%) vs. ES (12.5\%). While homeopathic medications appeared infrequently in both groups, they were slightly more common in PNES (6\%) vs. ES (0\%). Vitamin use (ES: $81.3 \%$ vs. PNES: $29.4 \%$ ) and thyroid medications (ES: $100 \%$ vs. PNES: $66 \%$ ) were more common in ES.

Conclusions: This study suggests that there are differences in the medication profile of patients with PNES vs. ES. While the total number of medications was slightly greater for ES patients, with several exceptions, PNES patients were taking medications for more co-morbidities. The greater number of medications in the ES group most likely reflected a greater number of AEDs. More PNES patients were taking various CNS psychiatric medications. They were also taking more asthma, pain antihypertenisives and antidiabetic agents. Limitations of the study include retrospective nature, small number of subjects, single center, and selection bias (referral for VEEG). Nonetheless, attention to a patient's medication list may provide an additional clue to help distinguish among patients with PNES vs. ES.

\subsection{5}

\section{MARIJUANA USE IN FIRST SEIZURE PATIENTS: THE} HALIFAX ADULT FIRST SEIZURE CLINIC EXPERIENCE Karen Legg ${ }^{1}$, C. E. Crocker ${ }^{1}$, J. J. Moeller ${ }^{21}$ and B. PohlmannEden $^{2,1}$ ( ${ }^{1}$ Division of Neurology, QEII Health Sciences Centre, Halifax, NS, Canada and ${ }^{2}$ Dalhousie University, Halifax, NS, Canada)

Rationale: Marijuana (cannabis) is one of the most commonly consumed illegal social drugs in Canada. The prevalence of past-year cannabis use among Canadians 15 years of age and older is $10.7 \%$ according to Health Canada. Nova Scotia's rate of consumption is $13.2 \%$, statistically significantly higher than the national average. There is a paucity of valid clinical and experimental information available in regards to the role of cannabis in preventing or promoting seizures and its impact on seizure threshold. According to a review of the literature describing the effects of marijuana on epileptic symptoms in humans, cannabis use can reduce seizure frequency in some cases, provoke seizures in others, but in the majority of cases it probably has no effect. No one has systematically examined the role of regular cannabis use in persons presenting with first seizure.
Methods: In our Adult First Seizure Clinic (FSC) at the QEII Health Sciences Centre, Halifax, N.S. we looked at self reported cannabis use in all patients referred for suspected first seizure. All patients who were assessed in the First Seizure Clinic were asked about consumption of marijuana. 395 new patients were assessed between May 2008 and June 2012. This information was then correlated with initial diagnosis; specifically First Seizure (FS), New Onset Epilepsy (NOE), Newly Diagnosed Epilepsy (NDE) or Other (O) diagnosis. NOE was defined as evidence of a preceding seizure(s) within 12 months of the index seizure. NDE was defined as evidence of a preceding seizure(s) beyond one year from the index seizure. Further information was collected in regards to age, gender, level of education, and employment status. We further analyzed marijuana usage in regards to occasional, regular, and heavy use. As part of our detailed assessment and counselling, we considered any other potentially provoking or triggering factors.

Results: 170 patients were excluded from the data set for diagnosis other than FS, NDE or NOE. The remaining 228 patients were analyzed. None of these were felt to have experienced an acutely provoked seizure, according to ILAE criteria, as a result of either acute withdrawal or excessive use of marijuana. 112 were diagnosed with FS, 87 with NOE, and 26 with NDE. We found that $25.4 \%$ (58) of patients reported regularly using marijuana. $24.1 \%$ (27 patients) of those with FS, $31.0 \%$ (87 patients) with NOE and 34.6\% (9 patients) with NDE reported regular use of marijuana.

Conclusions: Our approach, which has been entirely epidemiological and descriptive at this stage, shows that self reported cannabis use in the Halifax First Seizure Clinic population is $25.4 \%$; significantly more frequent than the $13.2 \%$ use reported in the general population for Nova Scotia. The numbers were similar regardless of initial diagnosis (FS, NOE, NDE). The population referred to the Halifax First Seizure Clinic reported unusually high regular use of marijuana. It is unclear exactly what role regular cannabis use has in this population in regards to impacting the individual seizure threshold.

\subsection{6 WTIHDRAWN}

\section{Clinical Epilepsy: Clinical Treatments}

\subsection{7}

\section{LONG-TERM OUTCOME AFTER HIGH-DOSE DIAZEPAM} TREATMENT FOR ESES

Iván Sánchez Fernández ${ }^{1}$, S. V. Kothare ${ }^{1}$, S. Hadjiloizou ${ }^{1}$, A. Bergin $^{1}$, A. Rotenberg ${ }^{1}$, J. M. Peters ${ }^{1}$, I. Abdelmoumen ${ }^{1}$, S. An ${ }^{1}$, S. Ramgopal $^{1}$, M. Takeoka ${ }^{1}$, J. Riviello ${ }^{2}$, B. Bourgeois ${ }^{1}$ and T. Loddenkemper ${ }^{1}\left({ }^{1}\right.$ Boston Children's Hospital, Boston, MA and ${ }^{2} \mathrm{New}$ York University, New York, NY)

Rationale: Treatment strategies in patients with the electroencephalographic (EEG) pattern of electrical status epilepticus in sleep (ESES) are based on expert opinions and small case series. The objective of this study was to review our long-term experience with high-dose diazepam treatment.

Methods: We retrospectively reviewed patients who were monitored by overnight inpatient video-EEG at Boston Children's Hospital with: 1) presence of ESES considered as at least $50 \%$ of the non-REM sleep tracing occupied by spike-waves, 2) on high-dose diazepam treatment $(1 \mathrm{mg} / \mathrm{Kg}$ the first night, followed by $0.5 \mathrm{mg} / \mathrm{Kg} /$ day for a variable time subsequently and eventually a tapering schedule), 3) during the period from 2000 to 2007, and 4) had at least 4 years of clinical and EEG follow-up data after the last treatment with highdose diazepam. We investigated their electro-clinical features and recorded the age of occurrence of the significant clinical events. Per patient, we recorded the number and timing of treatments with highdose diazepam, any adverse effects associated with this treatment protocol, and the timing of any other anti-epileptic treatments needed 
after the last high-dose diazepam treatment.

Results: 25 patients were identified. Demographic features are presented in Table 1. Treatment with high-dose diazepam led to improvement in clinical (seizure frequency and severity, cognitive and behavioral function) and EEG features in all patients, but its effect slowly wore off over time. Five patients received two trials of high-dose diazepam treatment and four patients received three trials of high-dose diazepam (Table 2). 20/25 patients required other antiepileptic treatments after high-dose diazepam, including epilepsy surgery (functional hemispherectomy and corpus callosotomy) in two cases. Adverse effects of the high-dose diazepam treatment protocol included daytime somnolence (two patients), agitation (two patients), and ataxia (two patients); these adverse effects led to treatment discontinuation in one patient. There were no respiratory complications related to the treatment administration.

Conclusions: Children with ESES experienced improvement in clinical and EEG features after high-dose diazepam treatment. Beneficial effects dissipated and further trials of high-dose diazepam and/or other treatments were needed. Infrequent and mild adverse effects were found with the high-dose diazepam treatment protocol. No patients suffered from respiratory complications related to the treatment.

Table 1. Demographic features of our study population

\begin{tabular}{|c|c|c|c|c|c|c|c|}
\hline Patient & Gender & $\begin{array}{c}\text { Age at } \\
\text { seizure } \\
\text { onsct }\end{array}$ & $\begin{array}{c}\text { Age at } \\
\text { regression }\end{array}$ & $\begin{array}{c}\text { Age at } \\
\text { recogaition of } \\
\text { the FSES } \\
\text { pattem in EFG }\end{array}$ & $\begin{array}{c}\text { Age at } \\
\text { disappearance of } \\
\text { the TSSS pattern }\end{array}$ & $\begin{array}{l}\text { Age at last } \\
\text { follow-up }\end{array}$ & $\begin{array}{l}\text { Age at } \\
\text { seizure } \\
\text { freedom }\end{array}$ \\
\hline 1 & Male & 0.08 & 8.2 & 8.5 & No & 15.4 & No \\
\hline 2 & Male & 0.5 & No & 9.5 & 10.9 & 17 & 10.9 \\
\hline 3 & Female & 6 & 6.3 & 6 & 8.5 & 12.9 & 6.5 \\
\hline 4 & Male & 5 & 4.5 & 5 & 6.9 & 11.5 & 9.5 \\
\hline 5 & Temale & 4.3 & 4.6 & 5.8 & No & 6.6 & No \\
\hline 6 & Female & 3.1 & 4.5 & 4.3 & 9.2 & 13.8 & 9.8 \\
\hline 7 & Ficmalc & 11.2 & 9.2 & 11.8 & No & 19.5 & No \\
\hline 8 & Male & 0.7 & No & 10.7 & 12.5 & 16.7 & No \\
\hline 9 & Male & 3.9 & 4.6 & 4 & No & 10.4 & 5.8 \\
\hline 10 & Malc & 4 & 4.5 & 4 & 5.9 & 10.3 & No \\
\hline 11 & Malc & 5.5 & 1.5 & 4.4 & 6.5 & 10.3 & 7.2 \\
\hline 12 & Femalc & 0.006 & No & 6.8 & 10.4 & 11.6 & No \\
\hline 13 & Male & 4.8 & No & 8.5 & 15.3 & 15.5 & 12 \\
\hline 14 & Female & 7.9 & 4.5 & 6.6 & 9.3 & 13.6 & No \\
\hline 15 & Male & 4.4 & 5 & 4.8 & 6.8 & 16.8 & 6.6 \\
\hline 16 & Female & 5.7 & No & 7.4 & 12.3 & 15.1 & 10.3 \\
\hline 17 & Temale & 6 & 6.7 & 6 & No & 17.9 & 7 \\
\hline 18 & Malc & 5.4 & 5.4 & 6.4 & No & 16.6 & 15.5 \\
\hline 19 & Malc & 2.5 & 3.6 & 3.2 & 8.2 & 13.6 & 4.3 \\
\hline 20 & Male & 1 & 3 & 3 & 6.5 & 15.8 & 7.1 \\
\hline 21 & Male & 0.2 & 1 & 4 & 6.8 & 7.1 & 6.8 \\
\hline 22 & Female & 3.8 & 4.4 & 4.3 & 10.2 & 13.4 & 9.6 \\
\hline 23 & Male & 1 & No & 17.3 & 17.5 & 18 & No \\
\hline 24 & Malc & 3.4 & 9.3 & 9.3 & 13 & 13.4 & 11.4 \\
\hline 25 & Male & 5 & 9.3 & 9.9 & No & 12.8 & 12.1 \\
\hline
\end{tabular}

All ages are given in years
Table 2. Information on treatments in our patient population

\begin{tabular}{|c|c|c|c|c|c|}
\hline Palien1 & 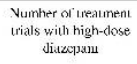 & $\begin{array}{l}\text { Age at trea.ment will } \\
\text { higa-dose diazcapam }\end{array}$ & 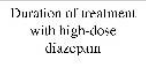 & Adverse events & 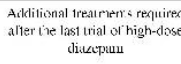 \\
\hline 1 & 1 & 8.5 & 0.4 & None & $1: \mathrm{TG}, \mathrm{Cl} \%$ \\
\hline 2 & 3 & 9.6.9.9.10.4 & $0.08 .0 .08,0.8$ & $\begin{array}{l}\text { Sümmolence, } \\
\text { nonke, norric }\end{array}$ & LFH \\
\hline 3 & 1 & 6.3 & 0.4 & Nione & $\mathrm{IIV}, \mathrm{ITG}$ \\
\hline 4 & 1 & 5.1 & 2.8 & None & $P R I D_{2}|V| K i, 1, I N, I . T C_{i}$ \\
\hline 5 & 1 & 5.8 & 0.2 & Nonc & L.EV \\
\hline ti & 1 & $7 . x$ & 0.004 & Ageication & 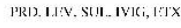 \\
\hline 7 & 3 & $11.8 .12,3,13$ & $0.3 .0 .5,1$ & $\begin{array}{l}\text { Nonc, nome. } \\
\text { nons }\end{array}$ & IFV, 7NS, PRT, VNS, TPM \\
\hline 8 & 1 & 10.8 & 0.8 & Nonc & LEV, MIIX, RFM. CC \\
\hline 9 & 1 & 4.6 & $0.6 i$ & Nonc & I.T.V. LICG. P'B, P'RI, ETXX \\
\hline 10 & 1 & 4.9 & 3.3 & Mild ataxia & LTG, PRD, SLI \\
\hline 11 & 1 & 5.9 & 1.8 & Nesule & OXC, TPM, LEV \\
\hline 12 & 1 & 6.8 & 0.3 & Nivile & RFM \\
\hline 13 & 1 & 9.9 & 0.3 & Nisil & LTG. LEV. CLZ. PRD \\
\hline 14 & 3 & 6.7.7.4.8.5 & 0.3 .0 .3 .0 .5 & $\begin{array}{l}\text { None, name, } \\
\text { nowe }\end{array}$ & Nonc \\
\hline is & 2 & $5.1,6.8$ & $0.1,1.1$ & Vunce, nune: & IFV, IVIG \\
\hline 16 & 1 & 7.4 & 1.6 & None & IISY \\
\hline 17 & 3 & $6.8 .7 .7,14.3$ & 0.08 .0 .3 .3 .2 & $\begin{array}{l}\text { None, mild } \\
\text { atnxia, none }\end{array}$ & Nunc \\
\hline 18 & 2 & 9,10 & $0.5,0.6$ & Nome. norte & IVIKG. I.IIV \\
\hline 19 & 2 & 7.3 .4 .8 & $0.5,4.9$ & Nane. nnone & None \\
\hline 20 & 1 & 8.4 & 3.2 & Nonc & Nonc \\
\hline 21 & 2 & 4.2 .6 .8 & 0.08 .0 .3 & Yone, nunc & LEV \\
\hline 22 & 1 & 9.8 & 0.4 & Nonc & None \\
\hline 23 & 1 & 17.3 & 0.08 & Surtuolentec & LEV \\
\hline 2.1 & 2 & $9.2,10.1$ & $0.9,0.1$ & Nonc, nowe & $\mathrm{KI}$ \\
\hline 25 & 1 & 9.9 & 2.3 & Aggisatium & TPY, ITG \\
\hline
\end{tabular}

All ages are given in years

Legend: CC: corpus callosotomy. CLZ: clonazepam. ETX: ethosuximide. IVIG: intravenous immunoglobulin. KD: ketogenic diet. LEV: levetiracetam. LFH: left functional hemispherectomy. LTG: lamotrigine. MTX: methosuximide. PB: phenobarbital. PRD: prednisone. RFM: rufinamide. SUL: sulthiame. TPM: topiramate. VNS: vagal nerve stimulator. ZNS: zonisamide.

\subsection{8}

VAGAL NERVE STIMULATION; EFFECTIVENESS IN CHILDREN WITH DRAVET SYNDROME

Deepa Sirsi ${ }^{1,2}$ and S. T. Arnold ${ }^{1,2}$ ( ${ }^{1}$ Pediatric Neurology, University of Texas Southwestern Medical Center, Dallas, TX and ${ }^{2}$ Pediatric Neurology, Childrens Medical Center, Dallas, TX)

Rationale: Treatment of Dravet syndrome is challenging and frequently disappointing in early childhood. Non-pharmacologic treatments for epilepsy including ketogenic diet, corpus callosotomy and Vagal Nerve Stimulation are considered and used for management of medically refractory epilepsy. In this series we report on the efficacy and tolerability of Vagal Nerve Stimulation in 6 children with Dravet syndrome.

Methods: A retrospective chart review of patients with Dravet syndrome implanted with Vagal Nerve Stimulator (VNS) for treatment of intractable epilepsy between 2005 and 2012 at tertiary care academic epilepsy center was performed. Efficacy for seizure control, alertness, cognition was evaluated. Other information sought included number of anti-epileptic medications added after VNS implantation as a measure of efficacy of VNS.

Results: 6 children with Dravet syndrome received VNS for intractable epilepsy. All were girls. Age at VNS implantation ranged from 2-7 years. Mean age was 5.3 years and median age was 6.5 years. Average duration of treatment with VNS ranged from 0.5 to 11 years with an average of 4.9 years.

When compared to baseline 3 of 6 children had a $50-75 \%$ \%reduction in seizures. 1 child had no change in seizure frequency but had decreased duration of seizures which resulted in reduced use of rectal valium and emergency room visits. 2 children had no significant improvement in seizures after VNS.

In 1 child VNS was turned off around the time of anticipated battery 
replacement. Complex partial seizures recurred and VNS was turned back on. Battery was replaced after 8-9 years in 2 patients. In 1 child significant improvement in alertness and interaction was observed after VNS. The device was well tolerated in all patients without significant complications.

Conclusions: 1 . VNS is well tolerated in young children with Dravet syndrome.

2. 4 of 6 children had a significant improvement in seizure control . 3. 1 child had an improvement in cognitive function in addition to reduction in seizures.

4. Response to VNS in children with Dravet syndrome is similar to that reported in other intractable childhood epilepsies such as Lennox- Gastaut syndrome.

5. VNS should be considered as an option for children with Dravet syndrome who have medically intractable epilepsy and/or cannot tolerate medications.

\subsection{9}

\section{SUPER-REFRACTORY STATUS EPILEPTICUS (SRSE): OUR EXPERIENCE AT A PEDIATRIC ACADEMIC TERTIARY CARE CENTER}

L. Shah, C. Herren and Rana Said (University of Texas Southwestern Medical Center, Dallas, TX)

Rationale: Refractory status epilepticus (SE) is defined as status epilepticus lasting $>60$ minutes. It occurs in $12-43 \%$ of SE cases. However, in a subset of patients, seizures can last $>24$ hours and may require further treatment. Given the paucity of published data in this area, we present our experience in a pediatric population.

Methods: A retrospective review of our EEG database and the medical record was performed on patients seen at Children's Medical Center Dallas/UTSW from 2003 and 2012, who were in superrefractory status epilepticus. This was defined as refractory SE lasting longer than 24 hours despite treatment. We reviewed age, gender, number of days in SE, duration of ICU stay, etiology, and outcome. We also reviewed number of antiepileptic drugs (AEDs), including anesthetic agents and/or immune therapy.

Results: 22 patients were identified, 7 girls and 15 boys, ranging from 2 weeks to 16 years old. The duration of status epilepticus ranged from 24 hours to 88 days with an average ICU stay of 17.4 days. Etiologies associated with SRSE included infection $(8 ; 36.4 \%)$, autoimmune disease $(4 ; 18.2 \%)$, known epilepsy $(3 ; 13.6 \%)$, stroke $(2 ; 9 \%)$, trauma $(2 ; 9 \%)$, unknown $(2 ; 9 \%)$, and malignancy $(1 ;$ $4.5 \%$ ). The average number of AEDs used was 3.1 (range 1 to 7 ). Treatment also included at least one general anesthetic agent in $9 / 15$, specifically pentobarbital (7), thiopental (1), and midazolam (6). In patients with a suspected autoimmune process, nonbacterial encephalitis, and those with unknown etiology despite extensive workup, immunotherapy was administered. This included high-dose steroids (8), IVIG (3), plasmapheresis (5), and chemotherapy (1). In one case, ketogenic diet was attempted but child could not achieve adequate ketosis in the ICU. Vagal nerve stimulator was placed in two children

Conclusions: Super-refractory status epilepticus in the pediatric population is associated with a high morbidity and mortality. In our experience, several conclusions were identified, including: (1)In our patients whose SRSE resolved and survived, the majority had significant morbidity including subsequent development of epilepsy, new learning disabilities, developmental regression, and feeding difficulties. (2) There was a $38.3 \%$ mortality ( $8 / 22$ patients). Of note, in 7 of these 8 patients, the underlying medical cause for the status epilepticus likely resulted in their death. (3) In addition, careful consideration needs to be given regarding the potential adverse effects of our interventions. (4)Despite standardization in initial treatment protocols for early and evolving status epilepticus, in patients who progress to SRSE, no universally agreed upon treatment approach exists. Given the diverse etiologies encountered, protocol driven therapy may not be feasible. However, lack of standardization can make treatment decisions difficult in these multidisciplinary care settings. (5) Given significant adverse effects of medications, nonmedication alternative therapies such as Ketogenic diet or VNS should be considered for SRSE.

\subsection{0 \\ KETAMINE USE IN THE TREATMENT OF REFRACTORY STATUS EPILEPTICUS}

Andrea Synowiec $^{1}$, D. S. Singh ${ }^{1}$, V. Yenugadhati ${ }^{1}$, C. J. Schramke ${ }^{1,2}$, J. P. Valeriano ${ }^{1,3}$ and K. M. Kelly ${ }^{1,3}\left({ }^{1}\right.$ Neurology, Allegheny General Hospital, Pittsburgh, PA; ${ }^{2}$ Neurology, Drexel University College of Medicine, Philadelphia, PA and ${ }^{3}$ Neurology, Temple University College of Medicine, Philadelphia, PA)

Rationale: Refractory status epilepticus (RSE) has been well characterized as a serious neurological emergency with a high morbidity and mortality. Data regarding treatment options for patients who have failed traditional therapies are limited. A small number of case reports exists regarding use of ketamine in RSE in the literature. We describe our experience with ketamine in treatment of RSE over an eight-year period.

Methods: Eleven adults with RSE who were treated with ketamine in addition to other antiepileptic drugs (AEDs) between 2003 and 2011 were identified using billing codes at discharge. All patients had failed initial treatment with benzodiazepines and weight-based intravenous loading doses of standard AEDs. Ketamine was given intravenously as a continuous infusion, typically after an initial bolus dose. Data were collected on age, gender, history of epilepsy, etiology of RSE, daily total dosing of ketamine, co-therapeutic agents, treatment response, and disposition.

Results: RSE was successfully terminated in all 11 patients. Coinfused continuous anesthetic agents were able to be discontinued in $8 / 11(73 \%)$ of patients within 72 hours. Ketamine was the last AED used prior to resolution of RSE in 7/11 (64\%) cases. In the remaining four cases, one other AED was added after ketamine infusion had begun. While 7/11 (64\%) of our patients required pharmacological vasopressor support prior to the initiation of ketamine, administration of ketamine was uniformly associated with decreased pressor support, and 6/7 (85\%) patients were weaned from pressors during ketamine infusion. Favorable outcome as defined by discharge to home or inpatient rehabilitation facility was attained in 5/11 (45\%) patients. No acute adverse effects were noted with either loading doses or continuous infusion of ketamine.

Conclusions: These findings suggest that ketamine may be a useful adjunctive agent in the treatment of RSE, and may be specifically considered in patients whose treatment options for RSE are limited by hypotension.

\subsection{1 \\ SUCCESSFUL FUNCTIONAL HEMISPHERECTOMY IN TWO REFRACTORY EPILEPTIC PATIENTS WITH CEREBRAL HEMIATROPHY AND PREDOMINANT- CONTRALATERAL EEG FINDINGS}

Rumiko Takayama, K. Imai, K. Baba, Y. Takahashi and Y. Inoue (National Epilepsy Center, Shizuoka, Japan)

Rationale: Epilepsy surgery has been reported to be successful in children with refractory epilepsy caused by congenital or earlyacquired brain lesions, regardless of generalized or contralateral EEG findings (Neurology 2007; 69: 389). We report two cases of refractory epilepsy with cerebral hemiatrophy and predominantcontralateral EEG findings, in which functional hemispherectomy was effective to control seizures.

Methods: We investigated EEG, MRI, SPECT and clinical charts of two epileptic patients with cerebral hemiatrophy and predominantcontralateral EEG findings in our hospital. 
Results: Case 1: A 5-year-1-month-old girl was admitted to our hospital for pre-surgical evaluation. She had incomplete paresis of right extremities and severe mental retardation due to porencephaly after congenital left middle cerebral artery infarction. From age 1year-7-month, she had refractory tonic seizures with right arm predominant tonic component. MRI showed left porencephaly corresponding to left middle cerebral artery area. Interictal discharges showed right-predominant diffuse spikes and waves and independently left frontal sharp waves. Ictal discharges showed rightpredominant diffuse fast wave bursts. The first ictal SPECT showed hyperperfusion in left occipital region and the second depicted hyperperfusion in left frontal region. Left functional hemispherectomy was performed at 5-year-4-month of age. She remained seizure-free for 18 months thereafter. Right-predominant diffuse spikes and waves on EEG disappeared, and right incomplete paresis was unchanged.

Case 2: A 3-year-8-month-old boy was admitted to our hospital for pre-surgical evaluation. He had incomplete paresis of right extremities and mild mental retardation after septic meningitis due to H. influenzae at 1-year-7-month of age. From age 2-year-2-month, he developed right-predominant tonic seizure. MRI FLAIR image showed left cerebral hemiatrophy with hyperintense signals. Interictal discharges showed right-predominant diffuse spikes and waves. Ictal discharges showed right-predominant diffuse fast wave bursts followed by diffuse rhythmic spikes and waves. Ictal SPECT was performed twice; hyperperfusion area was (1) left basal ganglia and insular cortex and (2) left frontal region, each. Left functional hemispherectomy was performed at 4-year-1-month of age. He remained seizure-free for 15 months thereafter. Right-predominant diffuse spikes and waves on EEG disappeared. Right incomplete paresis was worsened transiently but improved soon afterwards. Conclusions: Cerebral hemiatrophic lesion can cause functional disability and epileptic seizures, and interictal discharges and ictal discharges may be contralateral. Ictal SPECT findings were effective to confirm the epileptogenic side.

\subsection{2}

EFFECTIVENESS OF ANTIEPILEPTIC THERAPY IN PATIENTS WITH PCDH19-MUTATIONS

Jan Lotte ${ }^{1,3}$, T. Bast ${ }^{2}$, A. Müller ${ }^{3}$, P. Borusiak ${ }^{4}$, I. Fernández ${ }^{5}$, A. Fogarasi $^{6}$, R. Guerrini ${ }^{7}$, H. Hjalgrim ${ }^{8}$, S. Leiz, M. Linder-Lucht ${ }^{10}$, T. Loddenkemper $^{5}$, S. Pellacani ${ }^{7}$, S. G. Philip ${ }^{11}$, S. Ruf ${ }^{12}$, K. Schlachter $^{13}$, P. Striano ${ }^{14}$, R. J. Vermeulen ${ }^{15}$, M. Staudt ${ }^{3}$ and G. J. Kluger ${ }^{3,16}\left({ }^{1}\right.$ Kinderzentrum Maulbronn, Maulbronn, Germany; ${ }^{2}$ Epilepsieklinik für Kinder und Jugendliche, Epilepsiezentrum Kork, Kork, Germany; ${ }^{3}$ Neuropediatric Clinic and Clinic for Neurorehabilitation, Epilepsy Center for Children and Adolescents, Schön Klinik Vogtareuth, Vogtareuth, Germany; ${ }^{4}$ Department of Pediatrics, HELIOS Hospital Wuppertal, Witten/Herdecke University, Wuppertal, Germany; ${ }^{5}$ Division of Epilepsy and Clinical Neurophysiology, Boston Children's Hospital, Boston, MA; ${ }^{6}$ Neurology Department, Bethesda Children's Hospital, Budapest, Hungary; ${ }^{7}$ Child Neurology Unit, A. Meyer Children's Hospital, University of Florence, Florence, Italy; ${ }^{8}$ Epilepsihospitalet Filadelfia, Danish Epilepsie Center, Dianalund, Denmark; ${ }^{9}$ Neuropädiatrie, Kinderklinik Dritter Orden, München, Germany; ${ }^{10}$ Servicio de Pediatría y Unidad de Epilepsia, Hospital del Mar, Barcelona, Spain; ${ }^{11}$ Children's Hospital Birmingham, Birmingham, United Kingdom;

${ }^{12}$ Neuropädiatrie, Universitätskinderklinik Tübingen, Tübingen, Germany; ${ }^{13}$ Department of Pediatrics, Landeskrankenhaus Bregenz, Bregenz, Austria; ${ }^{14}$ Pediatric Neurology, Institute Gaslini, University of Genova, Genova, Italy; ${ }^{15}$ Child Neurology, VU University Medical Center, Amsterdam, Netherlands and ${ }^{16}$ Paracelsus Medical University, Salzburg, Austria)

Rationale: Mutations in the protocadherin 19 (PCDH19) gene cause epilepsy and mental retardation limited to females (EFMR) or Dravet-like syndromes. Especially in the first years of life epilepsy is known to be highly pharmaco-resistant, still there is no concept how to treat these patients. In this retrospective study we analyzed effectiveness of antiepileptic therapy in patients with PCDH19mutations.

Methods: Retrospective analysis of 32 patients aged 2-19 years (mean age: 9.3 years) with PCDH19-mutations. All patients were female and suffered from epilepsy with variable seizure types, mostly generalized tonic-clonic seizures (84\%), with clusters of seizures (97\%), starting at the mean age of 9.5 months (range: 4-20). Most of them showed a mild to severe mental retardation (88\%) with predominantly autistic behavioral problems (53\%).

Results: Antiepileptic drugs administered were carbamazepine (CBZ, n=19), clobazam (CLB, n=16), clonazepam (CZP, n=7), ethosuximide (ESM, $n=1)$, gabapentin (GBP, $n=1)$, lacosamide (LCM, n=2), lamotrigine (LTG, $n=16$ ), levetiracetam (LEV, $n=25$ ), lorazepam (LZP, $n=1)$, nitrazepam (NZP, $n=1)$, oxcarbazepine (OXC, $n=12)$, phenobarbital (PB, $n=16)$, phenytoin (PHT, $n=9)$, pregabalin (PGB, $n=1)$, rufinamide (RFN, $n=5)$, sulthiame (STM, n=6), stiripentol (STP, $n=4)$, topiramate (TPM, $n=16$ ), valproate (VPA, $n=25$ ), vigabatrin (VGB, $n=5$ ) and zonisamide (ZNS, $n=2$ ). Patients received overall an average of six different antiepileptic drugs (range: 1-11). In addition, three girls received steroids, two girls ketogenic diet (KD), and one girl received a vagus nerv stimulator (VNS). Seizure reduction lasting for at least 3 months was achieved with CBZ (32\%), CLB (94\%), CZP (43\%), LTG (31\%), LEV (44\%), LZP (1/1), OXC (33\%), PB (50\%), PHT (11\%), RFN (40\%), STM (17\%), STP (50\%), TPM (31\%), VPA (44\%), VGB (20\%) and ZNS (2/2), as well as with ketogenic diet (1/3) and VNS (1/1). 12 patients $(38 \%)$ became seizure free for at least 3 months, two of them for almost four years. Aggravation was observed with CBZ (5\%), LCM (1/2), LTG $(6 \%)$ and $\mathrm{OXC}(8 \%)$.

Conclusions: The most effective drug in patients with PCDH19mutations resulted to be CLB, followed by PB, STP, VPA, LEV, CZP and RFN. Although epilepsy in PCDH19 mutations is known to be pharmaco-resistant, more than a third of the patients became seizure free at least for 3 months, some up to almost four years, although a possible age-dependent spontaneous seizure remission has to be considered.

Few cases of aggravation are reported for CBZ, LCM, LTG and OXC, and none for PHT. Although PCDH19 is the second frequent Dravet-associated gene, therapy concept in patients with PCDH19 mutations might be different than in classical Dravet syndrome, where sodium channel blockers are known to cause frequent aggravation. Further data are needed to evaluate the effect of specific antiepileptic drugs to define rational treatment concepts in patients with PCDH19 mutations. 


\begin{tabular}{|c|c|c|c|c|}
\hline & total & proteclive & aggravaling & indiflerent \\
\hline $\mathrm{CB} \angle$ & 19 & $6(32 \%)$ & $1(5 \%)$ & $12(63 \%)$ \\
\hline CBI. & 16 & $15(94 \%)$ & 0 & $1(6 \%)$ \\
\hline $\mathrm{C} 7 \mathrm{P}$ & 7 & $3(43 \%)$ & 0 & $4(57 \%)$ \\
\hline ESM & 1 & 0 & 0 & $1(1 / 1)$ \\
\hline GBP & 1 & 0 & 0 & $I(1 / 1)$ \\
\hline LCM & 2 & 0 & $1(1 / 2)$ & $1(1 / 2)$ \\
\hline LIG & 16 & $5(31 \%)$ & $1(6 \%)$ & $10(63 \%)$ \\
\hline LEV & 25 & $11(44 \%)$ & 0 & $14(56 \%)$ \\
\hline I.7.P & 1 & $1(1 / 1)$ & 0 & 0 \\
\hline N7P & 1 & 0 & 0 & $1(1 / 1)$ \\
\hline OXC & 12 & $4(33 \%)$ & $1(8 \%)$ & $7(58 \%)$ \\
\hline $\mathrm{PB}$ & 16 & $8(50 \%)$ & 0 & $8(50 \%)$ \\
\hline PGB & 1 & 0 & 0 & $1(1 / 1)$ \\
\hline PHT & 9 & $1(11 \%)$ & 0 & $8(89 \%)$ \\
\hline RFN & 5 & $2(40 \%)$ & 0 & $3(60 \%)$ \\
\hline STM & 6 & $1(17 \%)$ & 0 & $5(83 \%)$ \\
\hline STP & 4 & $2(50 \%)$ & 0 & $2(50 \%)$ \\
\hline TPM & 16 & $5(31 \%)$ & 0 & $11(69 \%)$ \\
\hline VPA & 25 & $11(44 \%)$ & 0 & $14(56 \%)$ \\
\hline VGB & 5 & $1(20 \%)$ & 0 & $4(80 \%)$ \\
\hline $7 \mathrm{NS}$ & 2 & $2(2 / 2)$ & 0 & 0 \\
\hline Steroids & 1 & 0 & 0 & $1(1 / 1)$ \\
\hline $\mathrm{KD}$ & 3 & $1(1 / 3)$ & 0 & $2(2 / 3)$ \\
\hline VNS & 1 & $1(1 / 1)$ & 0 & 0 \\
\hline
\end{tabular}

Results: 155 patients were recruited ( 80 women; mean age: 45.8 years; mean duration of epilepsy: 18.1 . years). $80 \%$ of the patients had tried 2-12 previous antiepileptic drugs (AEDs). Currently, 124 and 66 patients have been assessed at 6 and 12-month follow-up. By the 12 -month assessment, $34.8 \%$ of patients $(23 / 66)$ had been free of complex partial and secondarily generalized seizures for at least the preceding 3 months.

Nineteen patients $(28.8 \%)$ were seizure free for more than 6 months. Mean monthly seizure frequency at 12 months was significantly reduced from seizure frequency at baseline $(12.3 \pm 4.8$ to $1.5 \pm 0.4$, $\mathrm{p}<0.0001, \mathrm{~K}-\mathrm{S}$ test) with median LCM dose of $375 \mathrm{mg}$ (range $50-500$ $\mathrm{mg})$. Seventeen patients discontinued LCM ( $70 \%$ in the first trimester) due to side effects $(n=10)$, lack of response $(n=4)$ or both $(n=3)$. No association was found between good response to LCM and drug load of sodium-channel-blockers and non-sodium channelblockers AEDs.

Conclusions: LCM maintains an efficacious and well-tolerated profile in the long-term treatment of focal epilepsy in combination with different AEDs regardless its mechanism of action.

\subsection{4}

\section{TUMOR ASSOCIATED EPILEPSY AT THE UNIVERSITY OF} MINNESOTA

Miguel E. Fiol ${ }^{1}$, M. Qureshi ${ }^{1}$, H. Schendell ${ }^{1}$, M. Hunt ${ }^{2}$ and M. M. $\operatorname{Adil}^{1}\left({ }^{1}\right.$ Neurology, University of Minnesota, Minneapolis, $\mathrm{MN}$ and ${ }^{2}$ Neurosurgery, Univ of Minnesota, Minneapolis, MN)

Rationale: Seizures are presenting sign in $25-50 \%$ of gliomas. Tumor Associated Epilepsy "TAE" has significant morbidity and loss of quality of life (QOL). Improving control of TAE may improve QOL and Karnofsky Performance Status (KPS).

Impact of "tumor associated epilepsy" (TAE) on morbidity of gliomas is significant.. In TAE seizure outcome using Engel's Classification and KPS scores may be related to multiple variables, e.g age, gender, location, WHO classification of glioma, radiation treatment, extent of resection by MRI, anti-epileptic drugs (AED), EEG findings and others.

Methods: 145 patients with gliomas and TAE seen between 20062012 at the University of Minnesota Neurosurgery/Neurology Departments were reviewed.

We investigated the association of seizure outcome at last visit using Engel's classification I-IV and KPS scores with multiple clinical variables described above..

Chi square analysis was done.

Results: On the 145 subjects, age was $43 \%$ at $40-59$ years, males $62 \%, 41 \%$ had WHO grade 2 , and $27 \%$ Grade $4.52 \%$ had frontal location and $53 \%$ presented with seizures. $20 \%$ had oligodendroglioma with $1 \mathrm{p} 10 \mathrm{q}$ deletions, $3 \%$ had positive family history of glioma.

$52 \%$ responded to one anti convulsant, namely Levetiracetam. KPS scores of 80-100 was seen at last visit in $48 \%$ and Engel's seizure outcome was I -II for 118 of the subjects.

All these variables were correlated with KPS score and older age $>60$ yrs $(P=.07)$, higher malignancy grades 3 and $4(\mathrm{p}=.09)$ and abnormal EEG $(\mathrm{P}=, 03)$ had lower KPS scores at $<80$.,

Seizure control (Engel's) was better in males $(\mathrm{p}=, 01)$ only and no other variables significanlty correlated.

Conclusions: Some clinical variables traditionally used to predict outcome, i.e KPS score and seizure control, in gliomas in this serie of 145 patients had marginal, not statistically siginficant association. Further studies are needed looking at other clinical parameters, perhaps molecular genotypes, that may better correlate with outcome in so far as seizure control and functionability of glioma patients. 


\subsection{5}

SURVEY OF THE CURRENT APPROACH TO INFANTILE SPASMS: NEED FOR STANDARDIZATION

John R. Mytinger ${ }^{1}$, S. Joshi ${ }^{2}$, K. Knupp ${ }^{4}$ and E. Wirrel ${ }^{3}$ ( ${ }^{1}$ Pediatrics, Ohio State University, Columbus, OH; ${ }^{2}$ Pediatrics, University of Michigan, Ann Arbor, MI; ${ }^{3}$ Neurology, Mayo Clinic, Rochester, MN and ${ }^{4}$ Pediatrics, Colorado Children's Hospital, Aurora, CO)

Rationale: Infantile spasms are age-specific seizures commonly associated with an epileptic encephalopathy. There is limited evidence to guide treatment and the approach to infantile spasms varies among clinicians. The aim of this survey was to assess how clinicians currently evaluate and treat infantile spasms.

Methods: To determine common practice among clinicians who treat infantile spasms, a survey was distributed via the Child Neurology Society to its members. Anonymous responses were collected over a two week period.

Results: The response rate was $18.5 \%$ with 222 responses. Ninetyfour percent of responders were from the United States. Seventy percent of responders reported seeing 10 or fewer new-onset cases of infantile spasms annually. For infantile spasms of unknown etiology, the most commonly used first-line treatments were adrenocorticotropic hormone (ACTH) (67\%), oral corticosteroids $(15 \%)$, and vigabatrin $(9 \%)$. For spasms of structural/metabolic etiology, excluding tuberous sclerosis, the most commonly used firstline treatments were ACTH (44\%), oral corticosteroids (23\%), vigabatrin (14\%), and topiramate (12\%). Most responders (86\%) use vigabatrin as the first-line treatment for infantile spasms due to tuberous sclerosis. Widely divergent doses of ACTH are used, with just over half of responders starting with high dose $(150 \mathrm{U} / \mathrm{m} 2 /$ day $)$ and others using variably lower doses. The preferred dosing of oral corticosteroid likewise varied significantly. We found that the diagnostic evaluation of infantile spasms also differed among clinicians.

Conclusions: There is significant variation among clinicians in both first-line treatments as well as the initial evaluation of infantile spasms. While ACTH is still the most commonly used first-line treatment for infantile spasms not due to tuberous sclerosis, a wide range of dosing is used. Although a significant number of clinicians used corticosteroids first-line for infantile spasms, there is also a wide range of preferred dosing. Well-designed comparative effectiveness studies are needed to determine the optimal medication and dosing regimen in this devastating epileptic encephalopathy of infancy. However, collaboration within and between centers with agreement upon standardized protocols will be required to answer these important questions.

\subsection{6 \\ ALTERNATIVE THERAPY AND INFLUENCE OF RELIGION IN PEDIATRIC EPILEPSY}

Angie Elia ${ }^{1}$ and M. Goyal ${ }^{1,2}$ ( ${ }^{1}$ Children's of Alabama, Birmingham, $\mathrm{AL}$ and ${ }^{2}$ University of Alabama at Birmingham, Birmingham, $\mathrm{AL}$ )

Rationale: Despite much discussion of complementary and alternative therapies in multimedia, there are few reports in the literature on its utilization in children with epilepsy. The wide range of alternative therapies and religious practices may potentially have an impact on treatment outcome of children.

The purpose of this study is to assess the types, frequency, and determinants of complementary and alternative medicine (CAM) use as well as religious practices in children with epilepsy followed at Children's of Alabama.

We hypothesized that the use of alternative therapy in the South is low and that religious beliefs play a larger role in our patient population.

Methods: This study was approved by the IRB at the University of Alabama. The primary caregivers of children with epilepsy were asked to complete a questionnaire in the clinic setting at Children's of
Alabama. Data gathered included use and frequency of alternative therapies such as herbs, vitamins, yoga/meditation, and religious practices used to influence epilepsy outcome.

Results: To date 20 surveys have been analyzed.

Ten caregivers $(50 \%)$ reported using CAM either in the past or currently. These therapies included aromatherapy $(n=2)$, chiropractic manipulation $(n=2)$, herbs $(n=4)$, massage $(n=3)$, vitamins $(n=6)$, and yoga $(n=1)$.

Eighteen caregivers $(75 \%)$ reported that they had an interest in trying various alternative therapies although they had not actually used them. These therapies included acupuncture $(n=2)$, aromatherapy $(n=4)$, chiropractic manipulation $(n=4)$, marijuana $(n=1)$, massage $(n=2)$, magnetic therapy $(n=1)$, music therapy $(n=2)$, oxygen therapy $(n=1)$, yoga $(n=3)$, and visiting a shaman in Ecuador $(n=1)$.

Amongst religious practices, prayer was the most common. Seventeen $(85 \%)$ caregivers reported using prayer specifically for the purpose of influencing their child's health. Sixty percent reported that prayer was of great benefit, while $40 \%$ reported that prayer was of some benefit.

Conclusions: While data is still being collected and analyzed, the survey shows that most caregivers have entertained utilization of CAM.

Prayer is commonly used and felt to be of some benefit to seizure outcome.

Our study has several limitations. It is currently limited to the outpatient setting, with a bias towards a subpopulation with new onset seizures and perhaps milder forms of epilepsy. It is also sampling families evaluated in a children's hospital, not a common setting.

\subsection{7 \\ TREATMENT AFTER ACUTE SEIZURE IN THE EMERGENCY ROOM}

Juan G. Ochoa and E. Gore (University of South Alabama, Mobile, AL)

Rationale: We currently don't have a standardized protocol to treat patients who go to the emergency room after an acute seizure. The evaluation and treatment of the acute seizures may have significant implications for the patients and for the health system.

This study is performed to assess the current practice at a university hospital to treat those patients.

Methods: We performed a retrospective study at the University of South Alabama Medical Center in Mobile, AL. We reviewed the ED medical records from January 1-May 9, 2010 with an ICD-9 code of convulsion, seizure, or epilepsy of any type $(780,345)$. We included only those patients with a history of prior seizures who present to the ED after a breakthrough seizure. We excluded any patient with documented evidence of recent anoxic brain injury, acute stroke, new tumor, meningoencephalitis, ethanol withdrawal, benzodiazepine withdrawal, amphetamine intoxication, and suspected pseudoseizures. We suspected pseudoseizures if bilateral convulsions but no altered level of consciousness were described or witnessed or no antiepileptic treatment was given despite a convulsion was witnessed in the ED. We reviewed each patient's available history and physical, laboratory evaluation, and imaging performed in the emergency department.. Patients who met the inclusion criteria were enrolled in this study. We reviewed the demographic data and the description of seizure type, number of seizures prior ED admission, prior AED therapy, type, route, dose and administration time of any antiepileptics or benzodiazepines given in the ED. We also reviewed the imaging and laboratory data, length of stay, discharge disposition, and record of recurrent seizures within 24 hours after discharge from the ED.

Results: 66 patients met the inclusion criteria. Of these, four patients who left against medical advice and one patient with incomplete records, were excluded from this analysis. $60 \%$ were male and the mean age was 37 years. Seizure types were not clearly documented 
but majority of them were described as convulsive (92\%). Neurology was consulted in $21 \%$ of these cases. $40 \%$ were evaluated with a CT scan and none of these imaging studies demonstrated an acute abnormality. Five different treatment groups were identified. 25 patients received no initial treatment in the ED for the seizure, and 3 of these patient had a second seizure in the ED (12\%). 18 patients received IV phenytoin load without benzodiazepine, 6 patients received IV lorazepam without further AED therapy, 2 patients received IV lorazepam followed by IV AED, 10 patients received oral AED while in the ED, and 2 patients received oral AED after IV lorazepam. The patients who recieved IV phenytoin loading had a longer ED stay (5.6 hours) compared to patients treated with either IV lorazepam or oral AED (3.5 hours).

Conclusions: This study demonstrated a lack of uniform criteria from ED physicians when treating patients after a seizure event. Seizure recurrence in the ED is a risk for untreated patients but IV AED loading may not be necessary and it appears to be associated with a longer ED stay.

\subsection{8}

EFFECTIVE ANTIEPILEPTIC DRUGS FOR INTRACTABLE PARTIAL EPILEPSIES IN CHILDREN AND YOUNG ADULTS ARE DIFFERENT AMONG ACTUAL SEIZURE SYMPTOMS

Kenji Sugai $^{1}$, E. Nakagawa ${ }^{1}$, H. Komaki ${ }^{1}$, Y. Saito ${ }^{1}$, T. Saito ${ }^{1}$, A. Ishiyama ${ }^{1}$, M. Sasaki ${ }^{1}$ and T. Otsuki ${ }^{2}{ }^{1}$ Child Neurology, National Center Hospital, National Center of Neurology and Psychiatry, Kodaira, Japan and ${ }^{2}$ Epilepsy Center, National Center of Neurology and Psychiatry, Kodaira, Japan)

Rationale: More and more antiepileptic drugs (AEDs) become available, however, considerable numbers of epilepsies are still intractable. Most of the reports on the efficacy of a particular AED are based on the treatment for patients with newly diagnosed epilepsy, and a few have been reported regarding its efficacy for intractable epilepsies. Partial seizures are traditionally classified as simple partial, complex partial and secondarily generalized, however, this classification is not useful for the treatment of intractable epilepsies. We studied whether effective AEDs for intractable partial epilepsies are different among seizure symptoms.

Methods: Based on a widely accepted definition of intractability that the epilepsy is intractable when it has failed to two appropriate AEDs, we analyzed the efficacy of AEDs for actual seizure symptoms (ASS) described below in 289 cases of chronic nonidiopathic partial epilepsies, aged 2 and 35 years, that had not achieved seizure freedom by one year or longer treatment with two AEDs and treated with the third or further AEDs. They consisted of 240 cases of frontal lobe epilepsy, 29 cases of temporal lobe epilepsy, and 20 cases of parietal or occipital lobe epilepsy. There were one ASS in 146 cases, two ASSs in 123 cases and three ASSs in 20 cases. $\geqq 50 \%$ responder rate (RR) were obtained for each AED administered in $\geqq 10$ cases in each ASS, and RRs were classified as excellent (ERR: $R R \geqq 75 \%$ ), good (G-RR: $R R=50-74 \%$ ), fair (F-RR: $R R=25-$ $49 \%$ ) and poor RR (P-RR: $R R<25 \%$ ).

Results: AEDs with E-RR, G-RR, F-RR and P-RR for tonic Sz after a total of 855 trials were bromide/ZNS, PB/ CLZ/LTG/PHT, TPM/CZP/AZM, and CLB/LEV/VPA/CBZ/GBP, respectively. AEDs with these were: PHT/CZP/ZNS/PB, CLB/TPM/CBZ, LTG/VPA, and none, for secondarily generalized tonic-clonic $\mathrm{Sz}$ (234 trials); CBZ/CLB, CZP/PHT, none, and VPA, for clonic Sz (103 trials); PHT, CBZ, LTG/CZP, and ZNS/VPA, for hypermotor Sz (107 trials) ; ZNS, PB, none, and CBZ/CLB/VPA/CZP for atonic or negative myoclonic seizure of frontal origin or negative motor area Sz (172 trials); CLB, CBZ/CLZ, CZP, and VPA/ZNS, for altered consciousness and/or motion arrest (156 trials); and none, $\mathrm{CBZ}$, none, and none, for sensory or autonomic seizures (71 trials).

Conclusions: CBZ and VPA are first and commonly used for partial seizures, however, they do not respond to all partial seizures, and particularly VPA does not respond to intractable partial epilepsies. Although more cases are needed to make the present results firmly, the present study indicates that effective AEDs for intractable partial epilepsies are different among ASS, and suggests that AEDs choice, depended on not merely partial seizure but ASS, is effective way for the treatment of intractable partial epilepsies.

\subsection{9}

VAGUS NERVE STIMULATION AS TREATMENT FOR REFRACTORY STATUS EPILEPTICUS

David J. Donahue, L. Bailey, A. Hernandez, S. Malik, J. Honeycutt and M. S. Perry (Cook Children's Medical Center, Fort Worth, TX)

Rationale: Background/Rationale: Status epilepticus (SE) is a lifethreatening condition, requiring immediate medical therapy. Refractory status epilepticus (RSE) is defined as status epilepticus persisting beyond 60 minutes despite appropriate treatment. Several case reports have suggested that vagus nerve stimulation (VNS) appeared to result in cessation of RSE not responding to conventional medical treatment. Since published reports of children so managed remain few, we retrospectively reviewed children with RSE deemed candidates for VNS in our institution from 2003 through 2011 to describe our experience.

Methods: We retrospectively reviewed charts of all patients who underwent VNS implantation as treatment of RSE at Cook Chidren's Medical Center between January 2003 and January 2011. For the purpose of this study, RSE is defined as seizure $>60$ minutes not responding to appropriate treatment or the re-emergence of SE following discontinuation of high-dose suppressive therapy.. For each patient, we recorded precipitating factors for SE, duration of RSE before VNS, number of anticonvulsants administered prior to institution of VNS, duration of RSE after implantation, VNS stimulation parameters, and outcome.

Results: Five patients met inclusion criteria. Patients ranged in age from 0.7 to 9 years (mean 5.3 years). Presumed precipitating factors for SE included: anticonvulsant withdrawal (1); metabolic complications associated with Leigh's Disease (1); and viral encephalitis (3). None were considered candidates for focal cortical resection. Patients received a median of 10 AEDs (range 5-11) and had been in RSE a median 26 days (range 4-50) prior to institution of VNS. Initial stimulation parameters for each patient were: output current $0.25 \mathrm{~mA} ; 30 \mathrm{~Hz}$ frequency; Pulse width 250 us; signal 'on \& off' time: $30 \mathrm{~s}$ 'on,' 3 min 'off.'. All 5 children required aggressive increment in output current and duty-cycles [on/(on + off times)] before RSE ceased. Two patients were considered responders to treatment with cessation of RSE within 7 days, while the others had persistent RSE for 21-84 days following implantation. Three children have continued VNS for over six years, achieving better seizure control, but none are seizure free.

Conclusions: VNS may be associated with interruption of RSE in patients who fail standard medical treatment. Aggressive adjustment of stimulation parameters may contribute to response, though the contribution of individual parameters to outcome is unknown. In patients with RSE who have failed standard treatments, VNS may be considered as an alternative treatment option.

\subsection{0 \\ LONG TERM FOLLOW-UP IN THE OUTCOMES OF A NEW ONSET SEIZURE CLINIC AT A TERTIARY REFERRAL CENTER}

Ravinda Samaraweera and D. M. Ficker (University of Cincinnati, Cincinnati, $\mathrm{OH}$ )

Rationale: Tertiary epilepsy centers were founded for the evaluation and treatment of refractory epilepsy. Current data suggests that patients with refractory epilepsy are not referred for subspecialty epilepsy care for an average of 15-20 years. A new onset seizure 
clinic (NOSC) was established in November 2005 to promptly evaluate and treat patients with new onset or suspected seizures. The goals of this clinic were to provide expert clinical care for patients with new onset or suspected seizures. The goal of this research project is to review the long term outcomes of those who were seen in our clinic initially with newly treated epilepsy.

Methods: We reviewed the charts of patients seen in the new onset seizure clinic in the calendar year of 2007. The University of Cincinnati IRB approved this study.

Results: 121 patients were initially seen in 2007 in new onset seizure clinic for suspected seizures. Of those, 53 patients $(43 \%)$ were treated with antiepileptic drugs. By 2011, however, only 38 patients continued to be seen in our clinic and were on antiepileptic drug therapy at that time. Most patients were on monotherapy with Keppra or Lamictal being the most often prescribed drug. Of the 38 patients on an AED seen in our clinic, $68 \%$ were seizure free.

Conclusions: The benefit of a new onset seizure clinic allows for expert care to those with suspected seizures. It also allows for prompt access to an epilepsy monitoring unit and expert care for those with non-epileptic events. The seizure free rate in subjects seen for epilepsy was $68 \%$ which is similar to prior reported outcomes in newly diagnosed epilepsy studies. Our clinic continues today and offers prompt evaluation for those with suspected seizures.

\subsection{1}

VAGUS NERVE STIMULATION FOR THE TREATMENT OF EPILEPSY IN CHILDREN: A REVIEW BASED ON EPILEPSY SYNDROME AND SEIZURE TYPE Deborah L. Holder ${ }^{1}$, M. Tamber ${ }^{2}$ and P. D. Adelson ${ }^{3}$

( ${ }^{1}$ NeuroDevelopmental Science Center, Akron Children's Hospital, Akron, OH; ${ }^{2}$ Neurosurgery, Children's Hospital of Pittsburgh, Pittsburgh, PA and ${ }^{3}$ Neurosurgery, Phoenix Children's Hospital, Phoenix, AZ)

Rationale: Despite appropriate treatment $30 \%$ of children will develop pharmacoresistant epilepsy. For some of these patients epilepsy surgery will be the best treatment option; however, many patients will not be candidates for surgery. Patients who have primary generalized epilepsy, multifocal seizure onset, mixed epilepsy with both focal and generalized seizures will not be candidates for a surgical resection. Vagus Nerve Stimulation (VNS) is an option for pharmacoresistant patients who are not surgical candidates. VNS has been available for use for over ten years but there is limited pediatric data available to help physicians determine which patients are the best candidates and which seizure types or syndromes will respond the best.

Methods: An IRB approved data base was created and all patients who met entrance criteria were added to the database. Entrance criteria included video/EEG proof of seizure type, MRI, seizure frequency documentation for at least 3 months prior to implant and follow-up data available for at least 1 year post implant. Seizure frequency, side effects, quality of life improvements and VNS settings were collected at each visit. All patients had their VNS programmed following the same protocol. Based on history, EEG findings, neurologic exam and imaging findings patients were classified for analysis by seizure type, epilepsy syndrome and etiology when possible.

Results: At time of review, the database had 114 patients who met entrance criteria and had at least 1 year follow-up data. Results by syndrome and seizure type are included in Table 1. Overall, patients with primary generalized epilepsy, especially those with a symptomatic etiology, had a better response to VNS than patients with focal onset seizures. Patients with Lennox-Gastaut Syndrome also responded well to VNS with significant reductions in drop seizures, atypical absence seizures and tonic seizures. Seizure reductions improved from the 6 month visit to the 1 year visit and continued for those patients with longer than 2 years follow-up. Side effects were minimal, improved over time and were often decreased by changes to the VNS settings. Overall $58 \%$ of patients were able to decrease or discontinue one or more medications.

Conclusions: VNS is a safe and effective treatment for children with pharmacoresistant epilepsy who are not candidates for epilepsy surgery. All of the patients in our study had video/EEG proof of seizure type resulting in outcomes that are based on specific seizure type, epilepsy syndrome and etiology when possible. We believe this data will help physicians chose patients who would be good candidates for VNS therapy and better advise patients of expected results based on their specific seizure type, epilepsy syndrome or etiology.

Table 1: Results

\begin{tabular}{|c|c|c|}
\hline Syndrome & Seizure Type & Median Seizure Reduction \\
\hline Lennox-Gastaut & Generalized tonic-clonic & $38 \%$ \\
& Tonic & $55 \%$ \\
& Myoclonic & $75 \%$ \\
& Atypical absence & $94 \%$ \\
\hline Childhood Absence & Drop & $85 \%$ \\
\hline & Absence & $53 \%$ \\
\hline Mulilocal partial & Simple/complex partial & $63 \%$ \\
\hline Symptomatic Generalized & Atypical absence & $42 \%$ \\
& Gencralizcd tonic & $94 \%$ \\
& Myoclonic & $47 \%$ \\
& Simple/complex partial & $50 \%$ \\
\hline Tuberous Sclerosis Complex & Secondarily generalized & $79 \%$ \\
\hline
\end{tabular}

\subsection{2}

\section{LACOSAMIDE EFFICACY IN REFRACTORY EPILEPSY: A} PROSPECTIVE OPEN LABEL STUDY

Jaromir Janousek, G. Mathews, L. Goldman, A. Barber and P. Klein (Mid Atlantic Epilepsy and Sleep Center, Bethesda, MD)

Rationale: In spite of the introduction new AEDs between 19932006 there has been no significant reduction in the rate of pharmacoresistant epilepsy. Lacosamide is a new anticonvulsant approved for treatment of refractory localization-related (LRE) epilepsy in 2009. In the present study, we sought to evaluate efficacy of lacosamide as adjunctive treatment refractory LRE in adults at a single epilepsy center.

Methods: Patients with refractory LRE with partial complex seizures evaluated and treated between 2010-2011 at a single epilepsy center in whom lacosamide treatment was initiated were followed prospectively in an open label study. Patients were followed prospectively from the time of initiation of lacosamide treatment. Seizure diaries were reviewed at regular intervals by epileptologists in face to face interviews with the patients/caregivers. Lacosamide dosing was titrated up to either seizure freedom or clinical toxicity. Background medications were chosen on clinical grounds and were adjusted according to best clinical judgment of treating physicians. Only patients with lacosamide treatment of $\geq 6$ months lacosamide treatment or with lacosamide treatment failure before 6 months of treatment were included in the analysis. Seizure freedom was defined as 0 seizures for $\geq 6$ months.

Results: 70 patients were treated with lacosamide (30 M, $40 \mathrm{~F})$. Average age was 38.5 years, duration of epilepsy was 20.7 years, average number of previous AEDs 5.9, average number of concomitant AEDs 2.1, and average pre-treatment seizure frequency 24.4 per month. Average treatment duration was 12.85 months. Mean monthly seizure frequency decreased by $37.64 \%$ to $9.2 /$ month $(62.3 \%) .11$ patients $(15.7 \%)$ became seizure free. 19 patients $(27.1 \%)$ had $\geq 75 \%$ seizure frequency reduction. Average final dose was $385 \mathrm{mg}$ (range 200-850 mg). Side effects occurred in 
49.2\% patients during treatment initiation, and declined to $19.7 \%$ patients at final treatment dose, following dose adjustment. 25/50 (35.7\%) patients stopped lacosamide, $7(10 \%)$ because of adverse events, $18(25.7 \%)$ because of lack of efficacy.

Conclusions: Lacosamide is effective treatment in patients with refractory localization-related epilepsy. Unlike in the fixed setting of the pivotal Phase 2/3 studies, a significant proportion of previously refractory patients may achieve seizure freedom with adjunctive lacosamide treatment in the clinical setting of adjustable dose and concomitant medications.

\subsection{3}

\section{DOES A SEIZURE-FREE INTERVAL (HONEYMOON PERIOD) OCCUR AFTER ABRUPT WITHDRAWAL OF AEDS IN PATIENTS WITH DAILY SEIZURES?}

Lindsey L. Benefield, J. Pimentel, A. C. Childers, L. Bailey, A. Hernandez-Mulero, S. Malik, D. Donahue and M. S. Perry (Cook Children's Medical Center, Fort Worth, TX)

Rationale: A seizure free interval or "honeymoon period" after initiation of antiepileptic drugs (AEDs) has been described.1 Likewise, an increased seizure free interval has been described following reinstitution of AEDs following discontinuation or reduction during admission for video encephalography (EEG). We sought to examine whether a similar "honeymoon period" was present after abrupt withdrawal of AEDs in patients reporting daily seizures prior to admission for video EEG.

Methods: We reviewed all patients admitted to the Cook Children's Epilepsy Monitoring Unit for an epilepsy surgery evaluation since January 2010 that reported at least one seizure type that occurred daily (target seizure) prior to admission. Patients not on AEDs or with nonepileptic seizures were excluded. Demographic data, epilepsy characteristics, and treatment history were abstracted from the medical record. We compared time to target seizure occurrence between patients with complete discontinuation of AEDs, those with partial wean of AEDs, and those remaining on baseline AEDs throughout their video EEG. We also analyzed for effect of patient characteristics on time to target seizure.

Results: One hundred and fourteen patients with daily seizures were identified. Thirty-one were excluded for lack of consent and 12 met exclusion criteria; thus, 71 patients were included in the sample (mean age 8.3years). Eleven (15\%) had generalized epilepsy, 43 (61\%) localization-related epilepsy, and 17 (24\%) mixed-type epilepsy. Thirteen (18\%) were on 1 AED, $30(42 \%)$ were on 2, and $28(40 \%)$ were on $>3$ AEDs at the time of admission. Thirty seven (52\%) patients had AEDs completely discontinued, 14 (20\%) had a partial AED changes, and $20(28 \%)$ had no change. There were no significant differences in patient characteristics between groups. Between group comparisons indicated a significant difference between the percent of patients with discontinued AEDs that had a seizure on day $1(62.2 \%)$ and those with no change to AEDs at all $(95.0 \%)$. While the median days to seizure in all groups was 0 , the mean time to seizure occurrence was 0.51 for those with complete AED withdrawal, 0.86 for those with partial changes, and 0.05 for those with no change $(\mathrm{F}(2,68)=3.714, \mathrm{p}=.029)$.

Conclusions: This study demonstrates abrupt withdrawal of AEDs in patients with daily seizures results in a period of seizure freedom. The time to seizure occurrence was delayed by approximately one day in some patients when AEDs were discontinued upon admission. In patients with daily seizures undergoing video EEG, alteration of AED regimens is likely unnecessary and may result in prolongation of time until seizures occur, unless less frequently experienced seizure types are targeted for evaluation. The presence of a "honeymoon period" following initiation and withdrawal of AEDs presents the possibility that intermittent dosing of AEDs may be a method to improve efficacy for some patients with frequent seizures. Additional studies examining this phenomenon are warranted.
Clinical Epilepsy: Prognosis

\subsection{4}

SEIZURES IN NEWBORNS WITH CONGENITAL HEART DISEASE

Marina N. Aberastury, W. H. Silva, C. Puga, M. M. Vaccarezza, C. Maxit and G. Agosta (Child Neurology, Hospital Italiano de Buenos Aires, CABA, Argentina)

Rationale: Newborns with congenital heart disease have a high risk of seizures, associated to developmental delay, cerebral palsy, and epilepsy.

Methods: Observational retrospective descriptive study of newborns with CHD with seizures admitted between June 2005-November 2011 at the Italian Hospital Neonatology Unit.

Results: 116 consecutive newborns with seizures were included. We analyzed $29(25 \%)$ with CHD. Median gestational age was 38 weeks (30-40wk.) and birth weight 3100gr (1200 - 4375).

All patients had symptomatic seizures, associated to: hemodynamic decompensation $51.7 \%(\mathrm{n}=15)$, sepsis $17,2 \%(\mathrm{n}=5)$ and stroke, el $6,9 \%(\mathrm{n}=2)$.

Neurological exam was normal in $24.3 \%(n=7) .48 .3 \%(n=14)$ had only one seizures, and $4 p$. (14.3\%) had status epilepticus.

EEGs were abnormal in 21 p. (72.4\%). $10(35,7 \%)$ had focal paroxysms, $6(21.4 \%)$ low amplitud, $4(13.8 \%)$ minimal alterations and $1 \mathrm{p}$. had burst suppression.

9p. 31\% died, due to hemodynamic decompensation. 9/9 had an abnormal EEG, (p: 0.027) and an abnormal neurological exam, p:0.05. 4/9, 44\% presented status epilepticus. P: 0.0053

At 6 months, 2 more newborns died and 2 had refractory epilepsy. $6 / 18$ patients had a normal neurological exam. $6 / 18$ had a normal neonatal neurological exam, $4(66 \%)$ had had only one seizure and 5 $(83.3 \%)$ a normal EEG p:0.025 .

Conclusions: In this study population we observed a high mortality and adverse neurological outcome. The strongest predictors were abnormal EEG, abnormal neurological exam and status epilepticus. Future studies of neurologic outcome and neuroprotective strategies are needed to improve neurodevelopmental outcomes.

\subsection{5}

LATER-LIFE COGNITION AND BRAIN STRUCTURE IN PERSONS WITH CHILDHOOD ONSET EPILEPSY M. Sillanpää ${ }^{1}$, M. Karrasch ${ }^{2}$, J. Rinne ${ }^{3}$, K. Dabbs ${ }^{5}$, P. Tiitta ${ }^{3}$, K. Anttinen $^{3}$, P. Sonninen ${ }^{3}$, M. Erkinjuntti ${ }^{3}$, S. Shinnar ${ }^{4}$ and Bruce P. Hermann ${ }^{5}\left({ }^{1}\right.$ Child Neurology and Public Health, University of Turku, Turku, Finland; ${ }^{2}$ Psychology and Logopedics, Abo Akademi University, Turku, Finland; ${ }^{3}$ Turku PET Center and Department of Neurology, University of Turku, Turku, Finland; ${ }^{4}$ Neurology, Montefiore and Albert Einstein College of Medicine, Bronx, NY and ${ }^{5}$ Neurology, University of Wisconsin School of Medicine and Public Health, Madison, WI)

Rationale: The implications of childhood onset epilepsy for later life cognition and brain structure remain uncertain. Here we present preliminary findings from the very long-term follow-up of a population-based cohort of persons with childhood onset (<age 16) epilepsy and non-affected population-based controls. Participants underwent neuropsychological assessment and quantitative neuroimaging in order to characterize the very long term cognitive and neuroanatomic course of childhood onset epilepsy.

Methods: The population-based cohort of persons with childhood onset epilepsy and healthy controls of Sillanpää et al. (1998) are returning for assessment of cognition and brain structure in later life. Here we report findings from the participants seen and assessed to date including persons with childhood onset epilepsy $(n=26$, mean age $=56.8$ [4.7]) and healthy controls $(n=31$, mean age $=58.2$ [2.9] . Participants underwent comprehensive neuropsychological assessment (general mental status, language, verbal/visual learning 
and memory, executive function, psychomotor speed) and quantitative MRI using Freesurfer (version 5.1) for image postprocessing, focusing on analysis of cortical thickness and volumes of targeted subcortical structures. Cognitive data were transformed as indicated.

Results: Analysis of cortical thickness showed no difference between the epilepsy and control groups after correction for multiple comparisons. There was a significant increase in the white matter hypointensity index in the epilepsy group as well as a trend $(<.10)$ toward larger left and right lateral ventricles, the later finding depending on the covariate used. Analysis of cognitive data showed significantly worse $(\mathrm{p}<.05)$ performances across measures of visuomotor function (WAIS-R Digit Symbol), verbal fluency (Phonemic and Semantic Fluency) and memory consolidation (WMS-R Logical memory percent savings) in the epilepsy group. Secondary analyses revealed that subjects with an abnormal EEG and either active epilepsy/seizures into adulthood were more adversely affected compared to those who remitted.

Conclusions: Persons with childhood onset epilepsy show evidence of age-accelerated changes in aspects of cognition (psychomotor speed, fluency, aspects of memory performance) compared to controls. They also exhibit subtle accelerated abnormalities in brain structure compared to controls. While interesting in their own right, these findings will provide the base upon which to track cognition and brain structure into elder years in order to characterize, for the first time, the life span impact of childhood epilepsy on cognition and brain.

\subsection{6}

SEIZURE FREQUENCY, HIPPOCAMPAL VOLUMES AND MEMORY IMPAIRMENT IN PATIENTS WITH MESIAL TEMPORAL LOBE EPILEPSY

Denise Pacagnella ${ }^{1}$, T. M. Lopes ${ }^{1}$, C. L. Yasuda ${ }^{1}$, F. M. Cappabianco $^{2}$, A. C. Coan ${ }^{1}$, M. E. Morita ${ }^{1}$ and F. Cendes ${ }^{1}$

( ${ }^{1}$ Neurology, State University of Campinas - UNICAMP, Campinas, Brazil and ${ }^{2}$ Science and Technology Institute, Federal University of São Paulo - UNIFESP, São Paulo, Brazil)

Rationale: Mesial temporal lobe epilepsy (MTLE) consists of a syndrome that is often refractory to clinical treatment, with up to 50$60 \%$ of patients not achieving optimal seizure control despite adequate antiepileptic drugs (AEDs) according to hospital series. However, recently it has been described a subgroup of MTLE patients with excellent response to AEDs, referred to as "benign MTLE". It is well established that MTLE patients have memory deficits due to hippocampal system damage, however, little is known about the impact of seizure frequency on memory in these patients. Therefore, the main purpose of this study was to compare memory performance between patients with refractory MTLE and patients with benign MTLE and to investigate the association between memory deficits, hippocampal volumes (HV) and the clinical response to AEDs.

Methods: MRIs were acquired in a 3-T scanner (Philips Medical Systems) from 25 patients with refractory MTLE (mean age 47.76 \pm 9.68 years, 14 women), 21 patients with benign MTLE (mean age $44.00 \pm 13.31$ years, 14 women) and 30 healthy subjects (mean age $40.03 \pm 13.19$ years, 15 women) for comparison of volumetric data. Hippocampus manual segmentation was performed by a single observer using the Display software

(http://www.bic.mni.mcgill.ca/ServicesSoftware/MINC) without prior knowledge of disease history and participant's identification. The neuropsychological assessment included: the Edinburgh Handedness Inventory; subtests of the Wechsler Memory ScaleRevised (WMS-R); Rey Auditory Verbal Learning Test (RAVLT) and subtests of the Wechsler Adult Intelligence Scale-III. Statistical analysis was performed using Systat 9 software.

Results: The groups were homogeneous regarding demographic and clinical features as shown in Table 1. However, there was a difference between the HA laterality which was most frequently on the right side in the refractory group $(72 \%$ versus $38.1 \%$ in the benign group). We observed a reduction of ipsilateral and contralateral $\mathrm{HV}$ in patients when compared to the controls $(p<0.001)$, as shown in Figure 1. Both patients' groups showed memory impairment, without significant differences between groups. We also observed a correlation between duration of epilepsy and estimated IQ $(r=-0.28, p<0.05)$ and with performance on the WMS-R delayed recall $(\mathrm{r}=-0.3, \mathrm{p}=0.04)$, between seizure frequency and ipsilateral HV $(r=0.33, p<0.05)$ and with contralateral HV $(r=0.32$, $\mathrm{p}<0.05)$, as well as between the right HV and RAVLT recognition $(\mathrm{r}=0.3, \mathrm{p}=0.04)$ and between the left HV and RAVLT A6 $(\mathrm{r}=0.28$, $\mathrm{p}<0.05)$.

Conclusions: We conclude that patients with MTLE exhibit memory deficits related to the presence of HA and duration of epilepsy, but independent of seizure frequency.

Table 1. Demographic and clinical features of refractory and benign groups

\begin{tabular}{|c|c|c|c|c|c|c|c|}
\hline & \multicolumn{3}{|c|}{ Refractory } & \multicolumn{3}{|c|}{ Benign } & \multirow[b]{2}{*}{$\mathrm{p}$} \\
\hline & Mean (SD) & Maximum & Minimum & Mean (SD) & Maximum & Minimum & \\
\hline$\wedge \mathrm{gc} *$ & $\begin{array}{l}47.76 \\
(9.68)\end{array}$ & 71 & 30 & $44(13.31)$ & 74 & 23 & 0.55 \\
\hline$\Lambda \mathrm{ge}$ at onset & $\begin{array}{c}19.32 \\
(13.13)\end{array}$ & 51 & 2 & $\begin{array}{c}16.95 \\
(10.68)\end{array}$ & 39 & 2 & 0.57 \\
\hline $\begin{array}{l}\text { Duration of } \\
\text { epilepsy* }\end{array}$ & $\begin{array}{c}28.44 \\
(12.32)\end{array}$ & 58 & 10 & $\begin{array}{c}27.05 \\
(13.06)\end{array}$ & 49 & 8 & 0.71 \\
\hline Fducation *** & $7.72(4.13)$ & 16 & 1 & $8(4.54)$ & 16 & 1 & 0.95 \\
\hline Estimated IQ & $\begin{array}{c}70.8 \\
(13.47)\end{array}$ & 103 & 57 & $\begin{array}{c}70.33 \\
(14.03)\end{array}$ & 100 & 51 & 0.99 \\
\hline
\end{tabular}

* in years; ** years of education; IQ, Intelligence Quotient; SD, Standard Deviation

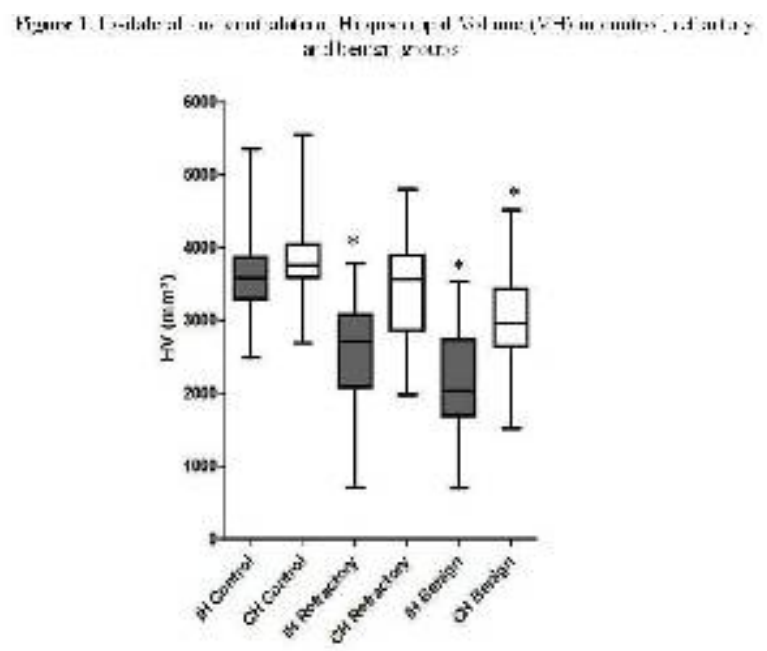

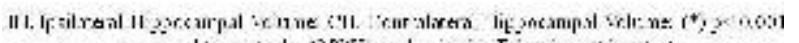

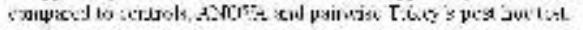

\subsection{7 \\ SURGICAL TREATMENTS FOR MTLE AND SEIZURE FREQUENCY CAN INTERFERE WITH MEMORY PERFORMANCE}

Daniela A. Fernandes, T. M. Lopes, C. L. Yasuda, B. P. Damasceno, E. Ghizoni, H. Tedeschi, E. Oliveira and F. Cendes (Neurology, State University of Campinas- UNICAMP, Campinas, Brazil)

Rationale: Mesial temporal lobe epilepsy (MTLE) is the most common focal epilepsy in adults and frequently do not respond well to antiepileptic drugs. Patients with refractory MTLE may present progressive atrophy of limbic structures and cognitive deficits. 
Neuropsychological assessment (NPA) allows the measurement of intensity of cognitive impairment and magnetic resonance imaging (MRI) can assess the presence of structural damage. We proposed to investigate the cognitive performance of patients undergoing surgery for MTLE and associate it with lateralization, seizure frequency and volume of the surgical lacunae (VSL).

Methods: We evaluated 49 patients (26 operated on the left side; 23 on the right), using the Engel classification; 21 were in Engel IA (seizure-free) and 28 in other Engel classifications (IB-III). They were submitted to pre/postoperative NPA, including the Wechsler Memory Scale-Revised, Rey auditory Verbal Learning Test (RAVLT) and Wechsler Adult Intelligence Scale-Revised. We used a 3D-T1-weighted MRI acquisition for volumetry. The manual volumetry of VSL was performed using software DISPLAY (David McDonald, www.bic.mni.mcgill.ca/software). We used statistical tests: t-Student test or Wilcoxon test for comparing the performance of NPA between groups pre/postoperatively, unpaired t-test to compare the performance NPA between patients groups operated on the right and left side and to access the differences in cognitive performance between groups Engel IA and Others; Pearson correlation between NPA and VSL. We considered $p<0.05$. Results: Statistical difference between groups pre/postoperative subtests: intelligence coefficient- IQ, general, verbal and visual memory, delayed recall, indicating better performance in preoperative period (table 1). There were statistical differences between patients operated on the right and on the left side: general $(\mathrm{t}=3.3, \mathrm{p}=0.002)$ and verbal memory $(\mathrm{t}=3.92, \mathrm{p}=0.001)$, delayed recall $(\mathrm{t}=2.73, \mathrm{p}=0.009)$, RAVLT-A7 $(\mathrm{t}=4.95, \mathrm{p}=0.001)$ and RAVLTrecognition $(t=2.72, p=0.009)$, showing the worst performance for left-sided surgery. Among the patients, $43 \%$ was allocated the Engel IA scale, $8 \%$ Engel IB, 21\% Engel IC, 10\% Engel ID, 4\% Engel IIA, $6 \%$ Engel IIB and IID and 2\% Engel IIIA. There were significant differences between groups Engel IA and Others for performance tests: IQ $(\mathrm{t}=2.33, \mathrm{p}=0.025)$, general $(\mathrm{t}=2.18, \mathrm{p}=0.035)$ and visual memory $(\mathrm{t}=2.35, \mathrm{p}=0.024)$. These results indicate better performance of Engel IA. In other subtests, although not statistically significant, there was a better performance of patients classified in Engel IA. We found association between VSL right with IQ $(r=0.55, \mathrm{p}=0.006)$ and visual memory $(\mathrm{r}=0.45, \mathrm{p}=0.03)$.

Conclusions: The data suggest that surgical resection accentuate the cognitive deficits in patients with MTLE, being more pronounced in the left-sided resection and in those with persistent seizures postoperatively. Paradoxically, the greater the VSL the lesser cognitive impairment, probably due to the relationship between more extensive surgical resection and better seizure control.

Table 1: Average, standard deviation (SD) and values $\mathrm{p}$ of tests used in pre/post-operative neuropsychological assessment (NA).

\begin{tabular}{|c|cc|c|c|c|}
\hline NA & Pre-operative & Post-operative & SD & Test & $p$ \\
\hline IQ estimated & 89.6 & 82.7 & \pm 8.5 & $\mathrm{t}=5.74$ & $0.0001^{*}$ \\
WMS-R general mem. & -0.56 & -2.25 & \pm 0.98 & $\mathrm{t}=11.99$ & $0.0001^{*}$ \\
WMS-R verbal mem. & -0.37 & -1.64 & \pm 0.85 & $\mathrm{z}=-5.35$ & $0.0001^{*}$ \\
WMS-R visual mem. & -0.64 & -1.87 & \pm 0.97 & $\mathrm{t}=8.82$ & $0.0001^{*}$ \\
\hline WMS-R delaycd recall & -0.67 & -1.84 & \pm 1.07 & $\mathrm{z}=-4.47$ & $0.001^{*}$ \\
\hline
\end{tabular}

IQ: estimated intelligence coefficient; WMS-R: Wechsler Memory Scale-revised; mem.: memory; ${ }^{*}$ significant $\mathrm{p}<0.05$.

\subsection{8 \\ NREM SLEEP STABILITY UNAFFECTED BY REFRACTORINESS OF EPILEPSY: A CASE-CONTROLLED CAP ANALYSIS STUDY ON REFRACTORY VERSUS CONTROLLED EPILEPSY}

Garima Shukla ${ }^{1}$, A. Gupta ${ }^{1}$, P. Zanzmera ${ }^{1}$, R. Thomas ${ }^{2}$, V. Goyal ${ }^{1}$, A. Srivastava ${ }^{1}$ and M. Behari ${ }^{1}\left({ }^{1}\right.$ Department of Neurology, All India Institute of Medical Sciences, New Delhi, India and ${ }^{2}$ Division of
Pulmonary, Critical Care \& Sleep, Dept. of Medicine, Beth Israel Deaconess Medical Center, Boston, MA)

Rationale: We recently demonstrated significant differences in selfreported as well as polysomnographically confirmed sleep quality parameters among patients with refractory versus controlled epilepsy. Some reports suggest high cyclic alternating pattern (CAP) rates among patients who have seizures during sleep.

Objective: To evaluate NREM sleep stability (CAP parameters) among patients with refractory epilepsy versus controlled epilepsy. Methods: Single night polysomnography (PSG) recordings scored according to AASM guidelines, for consecutive prospectively enrolled patients with medically refractory epilepsy (group-1) versus those with controlled epilepsy (group-2), were analyzed for various CAP parameters.

Results: PSG recordings of 20 patients in Group-1 (mean age $19.3+6.7]$ and of 15 patients in Group-2(mean age $21.2+7$ ), were analyzed in this study. The mean duration of epilepsy was $12.4+8$ years in group- 1 and $5.4+7.7$ years in group-2. Seizure frequency and number of patients on $>2$ AEDs were significantly different between the 2 groups. Significant differences were observed between the two groups in sleep efficiency (means $77+15] \& 95+3$ ), WASO (mean 21.8+13.6] \& 5.4+3.5) and arousal indices (mean $12.9+8 \&$ $5.5+3.7)(\mathrm{p}<0.05)$. However, on CAP analysis, no significant difference was found between the 2 groups in terms of mean CAP time $(121.8+82 \& 116.2+118 \mathrm{~min})$, CAP rate $(29.2+16.5 \& 33.2$ $+41.3)$ and number of CAP cycles $(298+199 \& 278+188)$ respectively. No differences were also observed in terms of rates and indices of different phases (A\&B) and phase subtypes (A1, A2, A3) between the 2 groups. (Table 1 )

Conclusions: Despite significant differences in number of awakenings and arousals between the two groups, CAP rates and other CAP parameters were found to be similar among patients with refractory versus controlled epilepsy. The non-occurrence of seizures during any of the PSG recordings as well as the blunting effect of anti-epileptic drugs may be responsible for this similarity in NREM stability.

Table 1 Polysomnographic conventional sleep and CAP parameters among patients with refractory epilepsy (Group 1) versus those among patients with controlled epilepsy (Group 2).

\begin{tabular}{|c|c|c|c|}
\hline $\begin{array}{l}\text { Conventional Sleep parameters } \\
\text { [mean (SD)] }\end{array}$ & Group 1 & Group 2 & P'value \\
\hline Sleep I.atency (min) & $22.6(25.4)$ & $11.4(13.3)$ & 0.01 \\
\hline Sleep efficiency (\%) & $77.2(15.4)$ & $95.2(2.9)$ & 0.00 \\
\hline WASO & $21.8(13.6)$ & $5.4(3.5)$ & 0.00 \\
\hline Arousals nu. & $66(46)$ & $42.7(26.6)$ & 0.08 \\
\hline Arousal Index & $12.9(8.6)$ & $5.5(3.7)$ & 0.003 \\
\hline $\mathrm{AHI}$ (events/hour) & $2.4(3.0)$ & $0.9(0.6)$ & 0.09 \\
\hline PLCM index & $0.6(0.07)$ & $1.005(0.9)$ & 0.11 \\
\hline CAI' l'aramelers [mean (SD)] & Group 1 & Group 2 & P'value \\
\hline CAPTime & $121.9(81.9)$ & $116.2(118.6)$ & 0.35 \\
\hline CAP Rate & $29.2(16.6)$ & $33.2(25.1)$ & 0.06 \\
\hline No. of CAP cycles & $298(199)$ & $278(188)$ & 0.69 \\
\hline CAP Index & $43.05(34.7)$ & $48.7(37.6)$ & 0.56 \\
\hline A] CAP rate & $22.3(13)$ & $28.1(21)$ & 0.29 \\
\hline Al CAI' Index & $32.1(18.8)$ & $40.3(30.8)$ & 0.34 \\
\hline A2 CAI' rate & $2.9(3.0)$ & 2.6(3.5) & 0.35 \\
\hline A2 CAP index & $4.5(5.0)$ & $3.8(5.3)$ & 0.38 \\
\hline A3 CAP rate & $3.9(5.6)$ & $2.5(2.6)$ & 0.58 \\
\hline$\wedge 3 \mathrm{CAP}$ index & $6.4(8.4)$ & $4.5(4.0)$ & 0.78 \\
\hline
\end{tabular}

CAP - cyclic alternating pattern, WASO - wake after sleep onset periods, AHI - apnea hypopnea index, PLM - periodic limb movement 
2.139

EPILEPSY RELATED DEATH IN SCN1A MUTATION POSITIVE DRAVET SYNDROME

Andreas Brunklaus ${ }^{1}$, R. Ellis ${ }^{2}$, E. Reavey ${ }^{2}$, G. Forbes ${ }^{2}$ and S. M. Zuberi ${ }^{1}{ }^{1}$ The Paediatric Neurosciences Research Group, Royal Hospital for Sick Children, Glasgow, United Kingdom and ${ }^{2}$ Duncan Guthrie Institute of Medical Genetics, Royal Hospital for Sick Children, Glasgow, United Kingdom)

Rationale: Dravet syndrome is a severe infantile onset epileptic encephalopathy associated with mutations in the SCN1A gene encoding the alpha subunit of the voltage gated sodium channel Nav1.1. There is a well-recognised excess mortality in children with the syndrome. Our aim was to analyse electro-clinical characteristics within a large cohort of SCN1A positive cases to determine whether any disease characteristics are associated with sudden unexpected death in epilepsy (SUDEP) or death following status epilepticus.

Methods: We prospectively collected data on 207 SCN1A mutation positive Dravet syndrome cases over a five year period. All genetic diagnostic studies were performed in our centre. From structured referral data we examined a comprehensive range of clinical characteristics including epilepsy phenotype, seizure precipitants, vaccination history, EEG data, imaging studies, mutation type and neuro-cognitive sequelae. Differences between deceased and nondeceased patients were determined using Mann-Whitney U test for continuous variables and chi-square statistics for categorical differences. Where assumptions for a Chi-square test are not fulfilled, Fisher's exact probability is given.

Results: In our cohort of 207 patients with classical Dravet syndrome we have had 8 reported epilepsy-related deaths (4\%). 5 cases were designated SUDEP and 3 deaths were associated with status epilepticus. Epilepsy related death was significantly more common in infants that had abnormal interictal EEG findings in the first year of life $(6 / 48,13 \%)$ compared to those with a normal interictal EEG in the first year of life $\left(1 / 58,2 \% ; X^{2}=4.9, d f=1, p=0.045\right)$. One or more previous episodes of status epilepticus were more common in the deceased patients $(7 / 8,88 \%)$ compared to the non-deceased patients $(154 / 195,79 \%)$, however this difference did not reach statistical significance. None of the remaining factors including mutation type (truncating vs missense), vaccination history, age at onset of seizures, seizure types, imaging studies or neuro-cognitive sequelae could be associated with epilepsy related death.

Conclusions: Identifying factors that are associated with SUDEP or death due to status epilepticus are important to inform counselling. Identifying risk factors will encourage early syndrome specific therapy. Prevention of status epilepticus with regular medication and emergency protocols is important.

\subsection{0 \\ PREDICTORS FOR LONG-TERM SEIZURE OUTCOME IN JUVENILE MYOCLONIC EPILEPSY: 25 - 63 YEARS OF FOLLOW-UP \\ J. Geithner ${ }^{1,2}$, Felix Schneider ${ }^{1}$, Z. I. Wang ${ }^{3}$, R. Herzer ${ }^{1}$, J. Berneiser ${ }^{1}$, C. Kessler ${ }^{1}$ and U. Runge ${ }^{1}\left({ }^{1}\right.$ Neurology, Epilepsy Center, University of Greifswald, Greifswald, Germany; ${ }^{2}$ Epilepsiezentrum Berlin-Brandenburg, Berlin, Germany and ${ }^{3}$ Cleveland Clinic Epilepsy Center, Cleveland Clinic Foundation, Cleveland, $\mathrm{OH}$ )}

Rationale: The long-term seizure outcome of Juvenile Myoclonic Epilepsy (JME) is still controversial; the value of factors that are potentially predictive for seizure outcome remains unclear. The aim of this study was both to investigate the long-term seizure outcome in patients with JME after a follow-up of at least 25 years and to identify factors that are predictive for the seizure outcome.

Methods: Data from 31 patients (19 women) with JME were studied. All of them had a follow-up for at least 25 years (mean 39.1 years) and were re-evaluated with a review of their medical records and direct telephone or face-to-face interview.
Results: Of 31 patients $21(67.7 \%)$ became seizure-free, in 6 of them $(28.6 \%)$ antiepileptic drug (AED) treatment was stopped due to seizure-freedom. The occurrence of generalized tonic-clonic seizures (GTC) preceded by bilateral myoclonic seizures (BMS) $(\mathrm{p}=0.03)$, a long duration of epilepsy with unsuccessful treatment $(p=0.022)$, and AED polytherapy $(\mathrm{p}=0.023)$ were identified as significant predictors for a poor long-term seizure outcome, whereas complete remission of GTC under AED significantly increases the chance for complete seizure-freedom $(\mathrm{p}=0.012)$. The occurrence of photoparoxysmal responses significantly increases the risk of seizure recurrence after AED discontinuation $(\mathrm{p}=0.05)$.

Conclusions: This study shows conclusively that JME is a heterogeneous epilepsy syndrome. Life-long AED treatment is not necessarily required to maintain the patients seizure-free. Several long-term outcome predictors that can potentially increase the clinicians' ability and confidence to recommend different treatment options to patients with JME were identified.

\subsection{1 \\ COMPARISON OF CLINICAL CHARACTERISTICS BETWEEN CNS INFECTION-RELATED AND CONVENTIONAL MEDIAL TEMPORAL LOBE EPILEPSY WITH HIPPOCAMPAL ATROPHY; FOCUSED ON PROGNOSTIC FACTORS

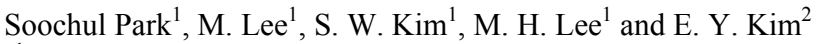 $\left({ }^{1}\right.$ Neurology, Yonsei University, College of Medicine, Seoul, Republic of Korea and ${ }^{2}$ Radilogy, Yonsei University, College of Medicine, Seoul, Republic of Korea)}

Rationale: Clinical characteristics of medial temporal lobe epilepsy (MTLE) with hippocampal atrophy (HA) by CNS infection as epileptic substrate might be different from those of conventional MTLE. The clinical variables affecting the prognosis between CNS infection-related and conventional MTLE with HS were analyzed. Methods: Thirty seven MTLE patients with antecedent history of CNS infection and 117 patients with conventional MTLE were recruited. All patients showed isolated HS on brain MRI. Clinical profiles such as demographic data, antecedent etiologies, semiologic features of TLE including characteristics of seizure (accompanied tonic-clonic attacks, clustering nature, nocturnal preponderance), laterality of HA on MRI, and prognosis were compared between the 2 groups. The minimal follow up duration for analysis of prognosis was two years. The prognosis of patients who had undergone epilepsy surgery was based on a 12-month follow-up before the surgery. Statistically significant prognostic variants were identified using multiple regression analysis.

Results: Simple comparison showed statistically difference in the onset age of epilepsy, laterality of HA on MRI, and prognosis. Clinical covariates affecting prognosis with statistical significance were CNS infection, automatism, and nocturnal preponderance. Multiple logistic regression analysis showed the presence of CNS infection and automatism worked as negative prognostic factor with 5.5 and 2.5 folds respectively. In contrast, nocturnal preponderance did as positive prognostic factor with 3.4 folds.

Conclusions: These results showed that there were some clinical differences, especially in the prognosis between CNS infectionrelated MTLE and conventional MTLE with HA. The presence of CNS infection and automatism were associated with a poor prognosis, while nocturnal preponderance as a good prognosis between two groups.

\subsection{2 \\ TRAJECTORIES OF HEALTH-RELATED QUALITY OF LIFE IN CHILDREN WITH NEW-ONSET EPILEPSY}

K. Speechley ${ }^{1,2}$, Mark Ferro ${ }^{3,4}$, C. Camfield ${ }^{5,6}$, S. Levin ${ }^{1}$, M. Smith ${ }^{7}$, S. Wiebe ${ }^{8}$ and G. Zou ${ }^{2,9}$ ( ${ }^{1}$ Paediatrics, Western University, London, ON, Canada; ${ }^{2}$ Epidemiology and Biostatistics, Western University, 
London, ON, Canada; ${ }^{3}$ Psychiatry and Behavioural Neurosciences, McMaster University, Hamilton, ON, Canada; ${ }^{4}$ Offord Centre for Child Studies, McMaster University, Hamilton, ON, Canada; ${ }^{5}$ Child Neurology, IWK Health Centre, Halifax, NS, Canada; ${ }^{6}$ Paediatrics, Dalhousie University, Halifax, NS, Canada; ${ }^{7}$ Psychology, University of Toronto, Toronto, ON, Canada; ${ }^{8}$ Clinical Neuroscience, University of Calgary, Calgary, AB, Canada and ${ }^{9}$ Robarts Research Institute, Western University, London, ON, Canada)

Rationale: Previous research suggests that, on average, the healthrelated quality of life (HRQL) of children with epilepsy improves during the first 24 months after diagnosis. However, less is known about subgroups of children who may experience less favourable outcomes over time. The objectives of the current study were to: 1) document trajectories of HRQL and 2) identify predictors of trajectory group in children with new-onset epilepsy.

Methods: Data were obtained from the Health Related Quality of Life in Children with Epilepsy Study (HERQULES), a prospective multisite study of children 4-12 years old with new-onset epilepsy followed for 24 months. Child (age, sex, family history, seizure type, epilepsy classification, duration, AEDs, severity, HRQL), parent (age, marital status, education, employment, depressive symptoms), and family (functioning, resources, demands, income) factors were examined using parent and neurologist report at baseline (postdiagnosis), 6, 12, and 24 months. Health-related quality of life was measured using the Quality of Life in Childhood Epilepsy (QOLCE). Trajectories of HRQL were investigated using latent class trajectory modeling. Multinomial logistic regression using backward selection was used to identify child, parent, and family predictors of HRQL trajectories.

Results: A total of 374 families responded at baseline and 283 completed the study. Five HRQL trajectories were observed: low increasing (4\%), moderate decreasing (12\%), moderate increasing $(22 \%)$, high increasing (32\%), and high stable (30\%). Using the high stable group as the reference, children in the low increasing group were prescribed more AEDs $(\mathrm{OR}=2.7)$, had more behavior $(\mathrm{OR}=18.7)$ and cognitive problems $(\mathrm{OR}=5.7)$, parents with elevated depressive symptoms (1.1), and families with worse functioning $(\mathrm{OR}=0.8)$ and more demands $(\mathrm{OR}=1.1)$. Children in the moderate decreasing group were also prescribed more AEDs $(\mathrm{OR}=2.2)$, had more cognitive problems $(\mathrm{OR}=10.2)$, and families with worse functioning $(\mathrm{OR}=0.8)$ and more demands $(\mathrm{OR}=1.1)$. Children in the moderate increasing group had more behavior $(\mathrm{OR}=5.6)$ and cognitive problems $(\mathrm{OR}=3.3)$, parents with elevated depressive symptoms $(\mathrm{OR}=1.1)$, and families with worse functioning $(\mathrm{OR}=0.9)$ and more demands $(\mathrm{OR}=1.1)$. Finally, children in the high increasing group had parents with elevated depressive symptoms $(\mathrm{OR}=1.1)$. Conclusions: While the HRQL of children with new-onset epilepsy generally improves during the first 24 months after diagnosis, results suggested that children are not homogenous, but consist of groups with different trajectories and predictors of HRQL. Importantly, 10\% of children experienced clinically important declines in HRQL. Problems associated with child behavior and cognition were the strongest predictors identified. Given that several risk factors are modifiable, it is important to examine these as potential targets to improve HRQL of children with new-onset epilepsy. Health care professionals should consider routinely assessing HRQL during clinic visits for pediatric epilepsy.

\subsection{3}

\section{FACTOR STRUCTURE OF THE CHILD HEALTH QUESTIONNAIRE AND PREDICTORS OF HEALTH- RELATED QUALITY OF LIFE IN CHILDREN WITH NEW- ONSET EPILEPSY}

Mark Ferro ${ }^{1,2}$ and K. Speechley ${ }^{3,4}\left({ }^{1}\right.$ Psychiatry and Behavioural Neurosciences, McMaster University, Hamilton, ON, Canada; ${ }^{2}$ Offord Centre for Child Studies, McMaster University, Hamilton, ON, Canada; ${ }^{3}$ Paediatrics, Western University, London, ON, Canada and ${ }^{4}$ Epidemiology and Biostatistics, Western University, London, ON, Canada)

Rationale: Health-related quality of life is an important outcome in understanding the illness course and treatment effects in children with epilepsy. Few health-related quality of life instruments have undergone rigorous psychometric testing in paediatric epilepsy, however. In a large sample of children with new-onset epilepsy, the objectives of the current study were to: 1) model the factor structure of the Child Health Questionnaire using confirmatory factor analysis and 2) identify post-diagnosis predictors of health-related quality of life at 24 months.

Methods: Data were obtained from the Health Related Quality of Life in Children with Epilepsy Study (HERQULES), a prospective multisite study of children 4-12 years old with new-onset epilepsy followed for 24 months. Child (age, sex, family history, seizure type, epilepsy classification, duration, AEDs, severity, health-related quality of life), parent (age, marital status, education, employment, depressive symptoms), and family (functioning, resources, demands, income) factors were examined using parent and neurologist report at baseline (post-diagnosis), 6, 12, and 24 months. Health-related quality of life was measured using the Child Health Questionnaire (CHQ). Child, parent, and family predictors of health-related quality of life were investigated with multiple regression using a backward selection procedure.

Results: A total of 374 families were included in the analysis. The original higher-order factor structure of the CHQ with physical (PHS, four factors) and psychosocial (PSS, six factors) summary scores was replicated and the confirmatory model had adequate fit: chisquare $=1743.46(\mathrm{df}=768), \mathrm{CFI}=0.945, \mathrm{TLI}=0.941, \mathrm{RMSEA}=0.058$ ( $90 \%$ CI: $0.055,0.062)$. Controlling for post-diagnosis PHS, factors predicting better 24-month PHS were: older age at seizure onset, fewer cognitive problems, parent with fewer depressive symptoms, and families with fewer demands $(\mathrm{p}<0.0001$ for all). Controlling for post-diagnosis PSS, factors predicting better 24-month PSS were: older age at seizure onset, fewer behavior problems, and families with better functioning and fewer demands ( $p<0.0001$ for all).

Conclusions: This is the first study to confirm the factor structure of the $\mathrm{CHQ}$, a commonly used generic measure of health-related quality of life in a sample of composed exclusively of children with epilepsy. Clinicians and researchers can be confident in using the CHQ to capture important domains of health-related quality of life paediatric epilepsy. From the predictive models, problems associated with child behavior and cognition were the strongest predictors identified. Given that several risk factors include modifiable parent and family characteristics, it is important to examine these more closely as potential targets for intervention to improve HRQL of children newly diagnosed with epilepsy. Health care professionals should continue to routinely assess health-related quality of life and consider adopting a family-centered care approach during clinic visits to optimize health outcomes.

\section{Neuro Imaging: Structural Imaging}

\subsection{4 \\ HIPPOCAMPAL AND ITS ADJACENT REGIONAL ATROPHY IN MESIAL TEMPORAL LOBE EPILEPSY: MANUAL VS. AUTOMATIC SEGMENTATION} Sung C. Lim ${ }^{1}$, Y. I. Kim ${ }^{3}$ and Y. M. Shon ${ }^{2}\left({ }^{1}\right.$ Department of Neurology, The Catholic University of Korea, St. Vincent's Hospital, Suwon, Republic of Korea; ${ }^{2}$ Department of Neurology, The Catholic University of Korea, Seoul St. Mary's Hospital, Seoul, Republic of Korea and ${ }^{3}$ Department of Neurology, The Catholic University of Korea, St. Paul's Hospital, Seoul, Republic of Korea)

Rationale: Hippocampal(HP) and its related structural(amygdala;AMG, entorhinal cortex;EC, thalamus; $\mathrm{TH}$ ) volumetry is a widely used clinical tool for the detection and 
lateralization of mTLE. Traditionally, the volume of these structures is measured by manually segmenting on serial sections of a T1weighted MRI. The manual approach requires a trained operator in a reliable and consistent manner. Automated software-based segmentation now allow us to use MRI to obtain estimates of these structural volume without requiring manual input. However, few of these studies explicitly compared automated volume estimates with the corresponding manual estimate in the same subject.

In the studies in which a direct comparison of manual and automated techniques was made, an automated segmentation technique was used Freesurfer. The aim of this study is to determine if automated volumetric methods are suitable for detection of hippocampal and its adjacent regional atrophy in a mTLE cohort with unilateral hippocampal sclerosis.

The outcomes of this study will help determine whether automated techniques should replace the manual technique as the standard volumetric methodology for the detection and lateralization of hippocampal atrophy in a clinical setting.

Methods: Volumetric MRIs were measured on 26 patients with mTLE (16 left, 10 right) and 20 age- and gender-matched healthy controls. Volumes of hippocampus, subcortical regions (thalamus, amygdala) and entorhinal cortex were measured manually, and using the automated reconstruction software (FreeSurfer). The sensitivities of two techniques were compared by determining the effect size for average volume reduction in patients with MTLE compared to controls.

Results: Significant volume reduction in affected hippocampi in mTLE compared to controls was detected by manual hippocampal volume measurement ( $\mathrm{p}<1.0 \times 10-6$, effect size 16.0\%), FreeSurfer $(\mathrm{p}<0.05$, effect size $5.5 \%)$ after correction forbrain volume. FreeSurfer correlated reasonably with this manual segmentation on pathological HP in left $(\mathrm{r}=0.69, \mathrm{p}<0.01)$ and right $(\mathrm{r}=0.63, \mathrm{p}<0.01)$ mTLE patients. Contrary to previous reports, the spatial correlation between manual and automated segmentation was reduced in affected hippocampi, suggesting the accuracy of automated segmentation was increased in pathologic brains. The discriminant function analysis revealed that patients with mTLE were best distinguished from one another using a combination of its hippocampus and amygdala volumes by manual volumetry, but not by FreeSurfer.

Conclusions: Expert manual volumetry is more sensitive than automated methods for the detection of hippocampal atrophy associated with mTLE. Volumetric data obtained with automated segmentation of HP and AMG approximate data from manual tracings. Our data suggest that automated segmentation may suggest to be clinically useful for evaluating the nature and extent of structural damage in patients with mTLE and may increase diagnostic classification of patients. Further studies with more patients should be warranted.

\subsection{5 \\ CORTICAL COMPLEXITY IS INCREASED IN PATIENTS WITH CRYPTOGENIC EPILEPSY}

Dewi V. Schrader, S. J. Hong, N. Bernasconi and A. Bernasconi (Neuroimaging of Epilepsy Laboratory, Montreal Neurological Institute, McGill University, Montreal, QC, Canada)

Rationale: Although epileptogenic lesions are often detected by MRI, in many patients with drug-resistant seizures visual inspection of conventional imaging fails to pinpoint a surgical target ${ }^{1}$. Growing evidence, however, suggests that cryptogenic (MRI-negative) epilepsy is not necessarily non-lesional, the primary histopathological substrate being subtle cortical dysplasia ${ }^{2,3}$. Histologically, subtle dysplasia (ILAE Type I) is characterized by cortical thinning, and persistence of developmental radial microcolumns and loss of horizontal hexalaminar structure ${ }^{4}$. These post-migrational cortical organization defects likely arise from abnormalities in neuronal rearrangement ${ }^{5}$ and cortical network maturation ${ }^{4}$. Importantly, during the dynamic process of cortical organization, differential growth of cortical layers and viscoelastic forces from axonal tension between interconnected areas ${ }^{6,7}$ are thought to lead to the formation of sulci and gyri. As sulco-gyral patterns are thought to be a footprint of cortical development, we set out to quantitatively map folding complexity across the neocortex in cryptogenic epilepsy.

Methods: We studied 37 patients ( 22 males, $30 \pm 8$ years) with cryptogenic, drug-resistant extra-temporal epilepsy. Based on clinical and EEG data, the focus was pre-central (frontal or fronto-central) in 22 and post-central (centro-parietal, parietal or parieto-occipital) in 15 (left-sided in 21 and right-sided in 16). To quantify folding complexity, we obtained whole-brain surface-based measures of absolute mean cortical curvature ${ }^{8}$ from 1.5 T MRI scans (3D T1-fast field echo sequence, $\mathrm{TR}=18 \mathrm{~ms}$; $\mathrm{TE}=10 \mathrm{~ms}$, voxel size $\left.=1 \mathrm{~mm}^{3}\right)$. Patients were compared to 41 age- and sex-matched healthy controls (males 16, 31 \pm 11 ) using vertex-wise t-tests. Prior to analysis, curvature measurements were blurred using a $20 \mathrm{~mm}$ surface-based diffusion kernel. In order to rule out potential confounds of cortical atrophy, we statistically corrected the curvature value at each vertex by the corresponding thickness measure.

Results: Compared to controls, patients with cryptogenic epilepsy displayed increased folding complexity in the orbitofrontal cortex, bilaterally ( $<<0.04$; Figure 1). We found no association between curvature changes and the location of seizure focus, or epilepsy duration.

Conclusions: Cryptogenic epilepsy is associated with increased sulco-gyral complexity. These anomalies are independent of cortical atrophy and seizure focus location. Their distinct location suggests altered loco-regional connectivity. Increased folding complexity suggests morphological commonality with polymicrogyrias and reinforces the notion that cryptogenic epilepsy may belong to the recently-described group of cortical malformations that are secondary to abnormal post-migrational development ${ }^{9}$.

\section{Figure 1}

A

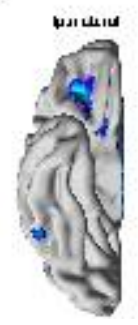

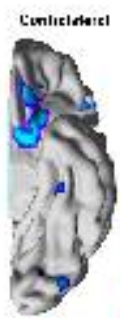

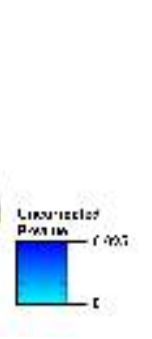

B

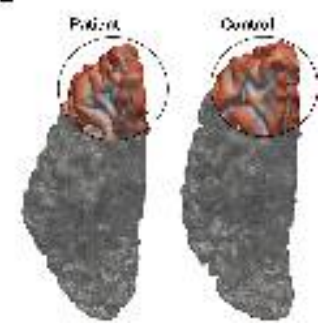

Figure | Cortical folding complexity in cryptogenic epilepsy. A) Regions of increased cortical complexity are outlined in purple. Data are corrected using random field theory for non-isotropic images (cluster level). Trends are displayed at an uncorrected threshold of $p$ $<0.025$. B) A patient with obvious increased complexity is shown in comparison to a control (fronto-basal cortices highlighted in red within the circle).

\subsection{6 \\ ALTERED GLOBAL STRUCTURAL BRAIN NETWORKS IN NEW-ONSET PEDIATRIC EPILEPSY: INCREASED SEGREGATION AND IMPAIRED INTEGRATION \\ K. Dabbs ${ }^{2}$, A. Tabesh ${ }^{1}$, Leonardo Bonilha ${ }^{1}$, B. P. Hermann ${ }^{2}$, D. Hsu ${ }^{2}$, C. E. Stafstrom ${ }^{2}$ and J. J. Lin ${ }^{3}$ ( ${ }^{1}$ Medical University of South Carolina, Charleston, SC; ${ }^{2}$ University of Wisconsin School of Medicine and Public Health, Madison, WI and ${ }^{3}$ University of California, Irvine, CA)}

Rationale: We previously demonstrated that widespread structural and cognitive abnormalities are present in children at the time of epilepsy onset. It is unknown whether these abnormalities are accompanied by disruptions of large-scale brain structural networks. Specially, new-onset pediatric epilepsy may have a significant neurodevelopmental impact in the remodeling of the brain organizational networks. In this study, we aimed to investigate global 
structural brain network metrics in patients with localization related epilepsy (LRE) and idiopathic generalized epilepsy (IGE), compared with healthy controls.

Methods: We studied sixty-seven subjects: 28 healthy controls (mean age $13.3 \pm 3.28$ years, 11 males), 39 children with new-onset epilepsy (21 LRE (11.6 \pm 2.68 years, 12 males) and 18 IGE $(15 \pm 3.3$ years, 7 males). Patients and controls were similar in age $(\mathrm{Chi}=1.9$, $\mathrm{p}=0.4$ ) and gender distribution (one way ANOVA $\mathrm{F}=5.72$, with significant group effect $\mathrm{p}=0.005$, with Tukey HSD demonstrating younger age in IGE compared to LRE but not controls). All subjects underwent MRI scanning yielding SPGR images (1.5 Tesla GE Signa MR scanner, $\mathrm{TR}=24 \mathrm{~ms}, \mathrm{TE}=5 \mathrm{~ms}$, flip angle $=40^{\circ}$, Slice thickness $1.5 \mathrm{~mm}$ ). Images underwent volumetric segmentation utilizing FreeSurfer according to the 'Destrieux Atlas'. Data from 171 regions of interest were utilized for the construction of group-wise adjacency matrices (controls, LRE and IGE) where each one of the $171 \times 171$ entries represented the partial correlation between the volumes from each pair of regions, controlled for age, bootstrapped 100 times. The adjacency matrices were then utilized for the evaluation of global graphical metric properties (Global Efficiency, Mean Betweenness Centrality, and Mean Clustering Coefficient) across fixed density threshold binary matrices (ranging from 0.05 to 0.95) through the use of the Brain Connectivity Toolbox. The level of significance for statistical comparison was set a False Discovery Rate corrected $\mathrm{q}=0.05$.

Results: The density matrices demonstrated an overall increase pairwise correlation between regions. The upper row in Figure 1 demonstrates the adjacency matrices for each group of patients, and the location of significant increase in pairwise correlation is shown in the bottom row of Figure 1. Global network metrics demonstrated a significant increase in Mean Clustering Coefficient in patients, while Global Efficiency was significantly reduced in patients compared with controls (Figure2).

Conclusions: Children with new-onset epilepsy showed abnormal configuration of structural neural networks in the developing brain. Compared with controls, a more segregated (increased clustering coefficient) and less integrated (decreased efficiency) global network was evident in children with epilepsy, indicating a less optimal topological organization. These findings indicate an abnormal global network brain configuration early in the course of epilepsy, which may have significant implications for neurocognitive development.
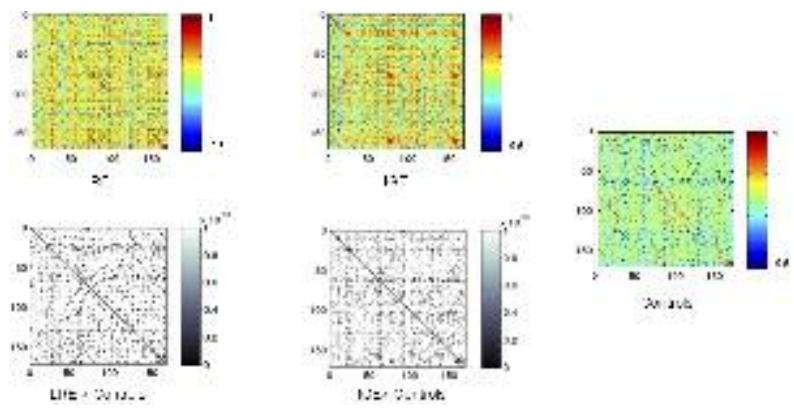

Figure 1 demonstrates the average bootstrapped adjacency matrices for patients with LRE, IGE (upper row) and controls. The regional differences are demonstrated by an equivalent plot of $\mathrm{p}$ values (bottom row), where dark cells represent significant differences between groups.
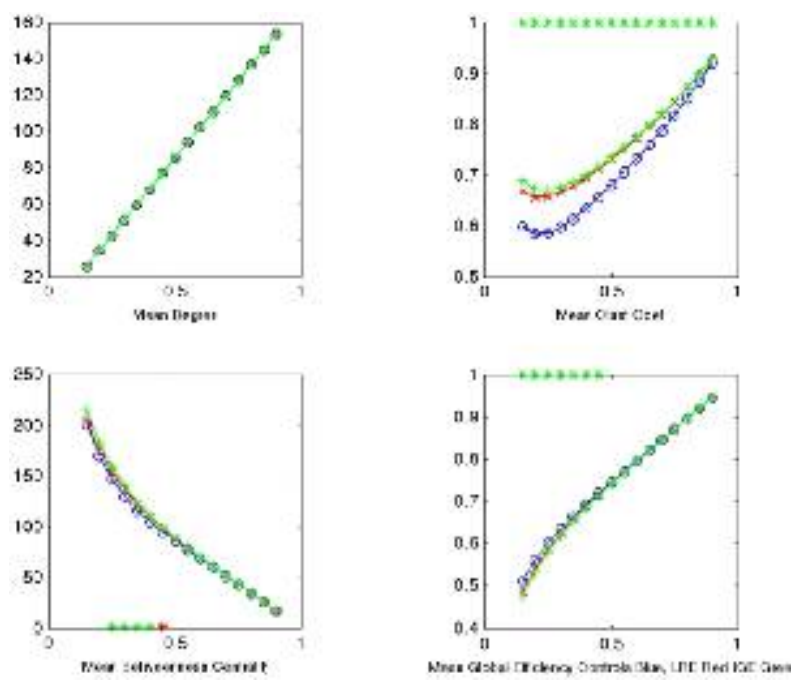

Figure 2 demonstrates the distribution of global network measures (yaxis) across fixed density binarized network thresholds (x-axis) for patients (LRE - red, IGE - green) and controls (blue). Green stars represent thresholds where the measure is significantly different between IGE and LRE versus controls. Red stars represent thresholds where IGE is different compared with LRE.

\subsection{7 \\ CONTRALATERAL SEIZURE ONSET OR ICTAL PROPAGATION DOES NOT AFFECT CONTRALATERAL HIPPOCAMPAL VOLUME IN UNILATERAL MESIAL TEMPORAL SCLEROSIS}

M. L. Yamaki ${ }^{3}$, K. Lyra $^{2}$, M. G. Otaduy ${ }^{2}$, V. Passarelli ${ }^{1}$, H. CastroLima $^{1}$, C. L. Jorge ${ }^{1}$, R. M. Valerio ${ }^{1}$ and Luiz Henrique Castro ${ }^{1}$ $\left({ }^{1}\right.$ Neurology, Universidade de Sao Paulo, Sao Paulo, Brazil; ${ }^{2}$ Radiology - LIM 44, Universidade de Sao Paulo, Sao Paulo, Brazil and ${ }^{3}$ Universidade de Sao Paulo, Sao Paulo, Brazil)

Rationale: Ictal seizure onset in mesial temporal sclerosis (MTS) associated epilepsy may occur ipsi or contralaterally to the lesion. Seizures may also initiate on the MTS side and propagate to involve contralateral mesial temporal structures. It is not known whether contralateral seizure onset or involvement is associated with structural lesion in the contralateral hippocampus. Presence of a structural lesion on the contralateral hippocampus may negatively impact on seizure outcome after epilepsy surgery or on cognition. We investigated hippocampal volume in patients with concordant $(\mathrm{C})$ or discordant (D) ictal onset in relation to the lesion in unilateral MTS patients.

Methods: Adult patients with unilateral MTS underwent video-EEG monitoring with registration of at least three seizures with an EEG correlate. Patients also underwent a 3T MRI study, with whole brain and hippocampal volumetrics, with Freesurfer. Cases were classified as concordant if all seizures EEG onset were ipsilateral to the MTS or discordant if seizure onset occurred contralaterally to the MTS or if seizure onset was ipsilateral to the MTS, with later propagation of ictal discharges to the contralateral side. Corrected hippocampal volumes (hippocampus/whole brain volume) were calculated for right (R) and left (L) C and D cases.

Results: We studied 36 patients: 26 were $\mathrm{C}$ (16 left; 10 right) and 10 were $\mathrm{D}(5 \mathrm{~L} ; 5 \mathrm{R})$ and 36 healthy controls. Groups did not differ in relation to age $(\mathrm{LC}=32.8+/-9.3 ; \mathrm{LD}=34.8+/-10.8 ; \mathrm{RC}=33.9+/-10.5$; $\mathrm{RD}=30.6+/-9.1$ years $)$, gender $(\%$ women $=\mathrm{LC}=62.5 \% \mathrm{LD}=40 \%$ $\mathrm{RC}=40 \% \mathrm{RD}=60 \%$ )

education $(\mathrm{LC}=10.1+/-1.5 \mathrm{LD}=12.6+/-2.2 \mathrm{RC}=11.1+/-2.5$ $\mathrm{RD}=10.8+/-0.4)$ and epilepsy duration $(\mathrm{LC}=25.2+/-11.8 \mathrm{LD}=25.0+/-$ 16.5 $\mathrm{RC}=26.3+/-12.6 \mathrm{RD}=29.0+/-8.2)$. Mean Hippocampal volume was significantly lower on the MTS side $(\mathrm{LC}=0.250 ; \mathrm{LD}=0.238$; $\mathrm{RC}=0.256 ; \mathrm{RD}=0.271$, compared with the unaffected side $\mathrm{LC}=0.339$ $\mathrm{LD}=0.346 ; \mathrm{RC}=0.355 ; \mathrm{RD}=0.348$ and controls $\mathrm{L}=0.342 \mathrm{R}=0.355$ 
$\mathrm{p}<0.001)$. Considering contralateral hippocampal volumes, we did not find any statistically significant differences between $\mathrm{L}$ and $\mathrm{R}$ concordant and $\mathrm{L}$ and $\mathrm{R}$ discordant cases.

Conclusions: Seizure onset or ictal discharge propagation to the contralateral hippocampus did not affect hippocampus volume. This finding speaks in favor of lack of structural damage in the contralateral hippocampus in unilateral MTS cases that present contralateral seizure onset or ictal involvement of the contralateral mesial temporal structures. This finding is in agreement with other studies that have indicated a favorable seizure outcome after epilepsy surgery in discordant cases.

\subsection{8 \\ PROGRESSIVE FIBER TRACT AFFECTIONS AFTER TEMPORAL LOBE SURGERY}

Jennifer Faber ${ }^{1,2}$, J. C. Schoene-Bake ${ }^{1,2}$, P. Trautner ${ }^{2}$, M. von Lehe ${ }^{3}$, C. E. Elger ${ }^{1,2}$ and B. Weber ${ }^{1,2}\left({ }^{1}\right.$ Dept. of Epileptology, University of Bonn, Bonn, Germany; ${ }^{2}$ Life\&Brain Center, University of Bonn, Bonn, Germany and ${ }^{3}$ Dept. of Neurosurgery, University of Bonn, Bonn, Germany)

Rationale: Temporal lobe resection is an effective treatment for pharmacoresistant forms of temporal lobe epilepsy (TLE) with unilateral hippocampal sclerosis. Several studies reported changes in the white matter architecture following temporal lobe resections in patients with TLE at short intervals after surgery. To investigate how the Fractional Anisotropy, as a marker for the integrity and strength of white matter fiber tracts, develops after surgery, we included leftsided TLE patients before and at two different times after surgery (36 months and about 12 months) and investigated the diffusion weighted MR images applying Tract-based spatial statistics (TBSS). Methods: We investigated 20 patients with intractable left-sided TLE by applying TBSS on diffusion weighted MR images aquired before and after temporal lobe resection. The patients were scanned postoperative at an early (about 4 months, range 3-6 months; N13) and a late (about 12 months, range 10-23 months; N13) interval after surgery to investigate the postsurgical plasticity.

Results: We observed an ongoing loss of fiber tract integrity mainly in the ipsilateral temporal fiber tracts: hippocampal part of the cingulum, stria terminalis of the fornix and in the late group also the uncinate fasciculus. In the late postoperative outcome group a significantly more widespread pattern of FA reduction in comparison to the preoperative data was observed than in the early postoperative scans.

Conclusions: Our results suggests that the decline of white matter tracts directly associated to the resected epileptogenic focus is continuing over at least one year after surgery. Thus, our data shows that the remodeling of brain connectivity after surgical interventions continues and that structural alterations and modifications of the white matter architecture are progressive and long lasting. The remodelling seems to be essentially but not solely characterized by the loss of fiber tract integrity. Further longitudinal studies including larger sample sizes investigated over prolonged time periods after surgery are needed to identify the time course of white matter remodelling after TLE surgery in detail and its association to seizure and cognitive outcome.

\subsection{9}

\section{WHITE MATTER PATHWAYS IN EPILEPTIC PATIENTS}

Jeremy E. Lankford ${ }^{1}$, J. Juranek ${ }^{1}$, M. B. Bhattacharjee ${ }^{2}$ and G. K. Von Allmen ${ }^{1}\left({ }^{1}\right.$ Pediatrics, University of Texas at Houston, Houston, TX and ${ }^{2}$ Pathology, University of Texas at Houston, Houston, TX)

Rationale: Language deficits are common in epilepsy and have led to increased investigation in associated white matter tracts. The aim of this series is to evaluate white matter (WM) integrity before and after surgical resection of a seizure focus targeting the arcuate fasiculus
(AF), a major WM pathway connecting frontal and parietal association areas important for language reception and production. We investigate how the structural connectivity between AF pathways ipsilateral and contralateral to the seizure focus measured by fractional anisotropy (FA) changes following focal topectomy in pediatric patients with intractable epilepsy.

Methods: Using a 3T scanner, MRI data were acquired on 3 pediatric patients with intractable seizures before surgery and 6-12 months after surgery. DTI data was processed and analyzed using FSL v4.1.7. FSL was used to conduct probabilistic tractography of the AF in each hemisphere. FA values were recorded for each AF which correlates with structural connectivity. Pathology was also performed on resected specimens.

Results: In all patients, AF pathways in each hemisphere crossed through the splenium of the corpus callosum before surgery. Following surgery, the spatial extent and strength of structural connectivity and FA values between AF pathways changed. In two patients where focal cortical dysplasia was found to underlie the epileptic focus, FA values were increased in both hemispheres. In the other patient, the epileptic focus pathology was consistent with heterotopic neurons and FA values increased in the contralateral hemisphere but were reduced in ipsilateral hemisphere following resective surgery.

Conclusions: Patients with focal cortical dysplasia tend to have better prognosis and less related neurologic compromise than patients with heterotopic neurons which may be more widely distributed. Finding increased FA values in the contralateral hemispheres after resection were consistent with previous reports suggesting an influence from the ipsilateral focus. Increases in ipsilateral FA value in resected focal dysplasia suggest removal of factors prohibiting typical connectivity. The lack of an ipsilateral increase in the patient with heterotopia may indicate persistence of anomalies (apart from the seizure focus) that affect normal neurologic function.

\subsection{0 \\ POTENTIAL ROLE OF NON-NEOPLASTIC MESIAL TEMPORAL CYSTS IN TEMPORAL LOBE EPILEPSY Mohamad Haykal $^{1}$, C. Wushensky ${ }^{2}$ and B. Abou-Khalil ${ }^{1}$ ( ${ }^{1}$ Department of Neurology, Vanderbilt University Medical Center, Nashville, TN and ${ }^{2}$ Department of Radiology, Vanderbilt University Medical Center, Nashville, TN)}

Rationale: Non-neoplastic cystic lesions in the mesial temporal lobe are occasionally encountered on magnetic resonance imaging (MRI) of patients with epilepsy. These cysts are most commonly located in the choroidal fissure above the hippocampus. The role of these lesions in epileptogenesis is unknown. We studied the localization of the epileptogenic zone in relation to mesial temporal cysts in patients evaluated for epilepsy surgery.

Methods: We searched the medical records and multidisciplinary surgery conference notes for any reference to a cystic lesion in the mesial temporal lobe in patients with drug-resistant partial epilepsy evaluated for epilepsy surgery at Vanderbilt University Medical Center between 1989 and 2011. Neoplastic lesions with cystic component were excluded. The MRI scans with a mesial temporal cystic lesion were mixed with control MRI scans and reviewed by a blinded neuroradiologist who confirmed the presence of cysts. Volumetric measurements of the cysts were obtained using manual tracing in Analyze 10.0 software. We noted the age of epilepsy onset, duration of epilepsy, results of pre-surgical workup, and for patients who had epilepsy surgery, the type and location of surgery and postoperative outcome.

Results: We identified 13 patients with MRI evidence of mesial temporal cystic lesions. The majority of lesions were choroidal fissure cysts (10/13). In 9 patients, there were no other abnormalities on MRI. Hippocampal sclerosis was seen in 3 patients, ipsilateral to the lesion in one and contralateral in two patients. Mild increased T2 signal was seen around an anterior mesial temporal cyst in one case. 
Presurgical localization of the epileptogenic zone corresponded to the cystic lesion in $9 / 13$ patients $(64 \%)$; choroidal fissure cysts in these patients (6/9) had a relatively large volume $(285-1328 \mathrm{~mm} 3$; mean $789 \pm 465 \mathrm{~mm} 3)$. Six of these patients underwent amygdalohippocampectomy or anterior temporal lobectomy: two patients achieved long-term seizure freedom at 4 and 6 years of follow-up; two were seizure-free for 2-3 years before seizures recurred; one had only rare residual seizures but died one year after surgery due to an unrelated illness; and one patient did not have any improvement in seizure control. In the four cases where the epileptogenic zone had a discordant localization, the lesions were choroidal fissure cysts that were relatively small (75-267 mm3, mean $165 \pm 95 \mathrm{~mm} 3)$. Two of these patients had contralateral hippocampal sclerosis.

Conclusions: Our results demonstrate that non-neoplastic mesial temporal lobe cysts may represent epileptogenic lesions in some patients. The clinical significance of these lesions, specifically choroidal fissure cysts, appears to be related to their size- cysts smaller than $270 \mathrm{~mm} 3$ were not clinically relevant. While more studies are needed to confirm these findings, large mesial temporal cystic lesions should be considered potential epileptogenic lesions in patients with drug-resistant partial epilepsy.

\subsection{1}

\section{SURFACE BASED FRACTIONATION OF CORTICAL} THICKNESS AND IDENTIFICATION OF DIFFUSION CHANGES IN THE EPILEPTIC CORTEX

Rajkumar Munian Govindan, E. Asano, C. Juhasz, J. Jeong and H. T. Chugani (Pediatrics and Neurology, Wayne State University, Detroit, MI)

Rationale: Microstructural alterations seen in the epileptic cortex have been associated as a cause and also as a result of multiple seizure activity. In the present study, we attempted to evaluate the diffusion changes of water at different cortical thickness fractions of the epileptic cortex and compared them with electrographically normal cortex and also with corresponding cortical regions of controls.

Methods: We selected 18 children (mean age: 7.8 years \pm 4.46 ) who had normal MRI findings and underwent two stage epilepsy surgery for control of their seizures. 18 normal age-matched volunteers (mean age: $7.8 \pm 4.6$ ) were used as controls. Structural and diffusion MRI images were acquired using a 3-Tesla GE MR Scanner. Delineation of the grey-white and grey-pial intersection surfaces were performed using the structural (T1) image (Freesurfer). Mean diffusivity values were calculated and the diffusion images were spatially aligned with the structural image using rigorous non-linear registration (FNIRT, FSL). Using the delineated surfaces as reference, the whole cortex was fractionated along the cortical thickness into Grey-White zone, inner-, middle-, outer-fractions and Grey-Pial zone. Cortical regions representing seizure onset and ipsilateral electrographically normal (IPSI) cortex were differentiated using the electrocorticography and mean diffusivity (MD) values for these regions were noted for all cortical fractions in the patients as well in the controls. Statistical analysis involved repeated measures with group, electrographic type and cortical fractions as factors.

Results: Three-way interaction between the group, electrographic type and cortical fractions was significant $(p=0.024)$. In seizure onset regions, the $\mathrm{MD}$ of all five cortical fractions were significantly $(p<0.01)$ higher compared to the IPSI regions within the patient group (Figure). A specific and significantly $(\mathrm{p}=0.001)$ marked increase in MD was noted in the outer-fraction (and middle-fraction: less marked but significant; $\mathrm{p}=0.047$ ) of the onset regions compared to the corresponding regions of the controls as well as IPSI region $(p=0.001)$ (Figure). In IPSI regions, the MD values were significantly $(\mathrm{p}<0.022)$ lower in the inner-, middle-fraction and Grey-Pial zone (and apparently lower in the grey-white zone and outer-fraction) as compared to the controls.
Conclusions: Our analysis suggests specific patterns of diffusion changes in the outer cortical fractions of the seizure onset zone compared to IPSI and control regions. This abnormal increase in MD of the outer and middle cortex might be associated with microstructural abnormalities commonly seen in layer II through IV of the epileptogenic cortex or to alterations caused by repeated seizure activity. Furthermore, the MD values were lower in electrographically normal regions compared to seizure onset and control regions; this decrease in diffusivity might be a common feature of the epileptic brain.

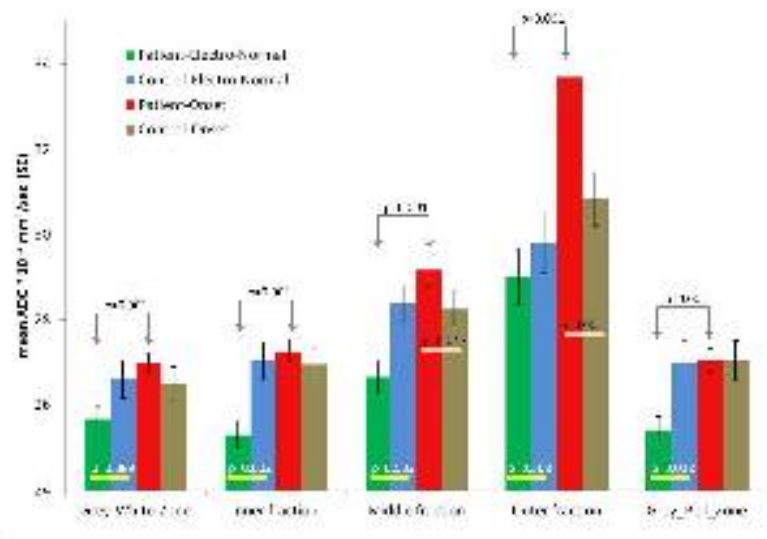

\subsection{2 \\ DIFFUSIONAL KURTOSIS IMAGING REVEALS WIDESPREAD STRUCTURAL ABNORMALITIES IN MEDIAL TEMPORAL LOBE EPILEPSY}

A. Tabesh, J. H. Jensen, J. A. Helpern, M. V. Spampinato, J. C. Edwards and Leonardo Bonilha (Medical University of South Carolina, Charleston, SC)

Rationale: Medial temporal lobe epilepsy (MTLE) is the most common form of treatment-refractory epilepsy, affecting approximately one million Americans. Despite its prevalence, there is a lack of reliable biomarkers for monitoring the clinical course and treatment response of MTLE. Diffusion MRI (dMRI) offers a unique and powerful method to characterize structural abnormalities in MTLE and as such may provide an accurate marker to better inform treatment decisions. This study aims to investigate MTLE-related microstructural deficits with diffusional kurtosis imaging (DKI), an extension of the commonly used dMRI technique of diffusion tensor imaging (DTI) that provides complementary information to DTI.

Methods: Ten patients with left MTLE and 20 age- and sex-matched control subjects underwent DKI and T1-weighted (T1w) scans on a 3T clinical MRI system. The MTLE diagnosis was based on a history of partial epilepsy and the presence of unilateral hippocampal sclerosis on conventional diagnostic MRI or unilateral seizure onset confirmed by ictal video-electroencephalography. DTI and diffusional kurtosis parametric maps of mean diffisuvity (MD), fractional anisotropy (FA) and mean kurtosis (MK) were obtained from the DKI scans, and were then submitted to voxel-wise analysis yielding maps of microstructural deficits. T1w scans were submitted to voxel-based morphometry yielding maps of tissue volume (TV) loss.

Results: Significant differences in MK were observed between patients and controls, suggesting extensive microstructural abnormalities in patients, including significant MK reductions in bilateral hippocampi, perihippocampal WM, pericingulate regions, frontal lobes, orbitofrontal regions, and occipitoparietal areas. The MD in patients was significantly increased in the ipsilateral perihippocampal area and bilateral orbitofrontal regions. The FA showed significant reductions in bilateral perihippocampal WM and the ipsilateral thalamus. The TV was significantly reduced in the ipsilateral hippocampus, contralateral insula, bilateral thalami, cerebellum, and occipital cortex. 
Conclusions: This preliminary study suggests that DKI may provide substantially more sensitive markers of structural abnormalities in MTLE than DTI and TV measures. The extent of abnormalities observed with MK is consistent with DTI and TV studies with significantly larger cohorts.

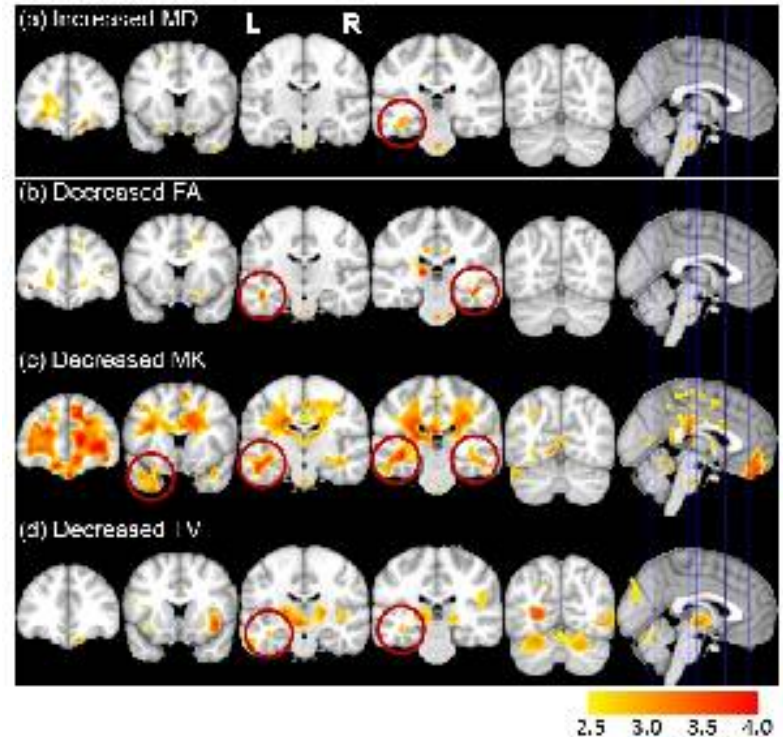

Voxelwise analysis of DTI, diffusional kurtosis, and TV metrics in 10 patients with left MTLE and 20 age- and sex-matched healthy volunteers. (a) Increased MD; (b) decreased FA; (c) decreased MK; and (d) decreased TV in patients. FA was compared in the WM, whereas other metrics were compared in the brain parenchyma. Images are in neurological convention.

\subsection{3}

SAFETY OF MRIS FOLLOWING DISCONNECTION OF VNS DEVICE FROM IMPLANTED LEADS

V. Woodard, Jane G. Boggs and C. ODonovan (Wake Forest University, Winston Salem, NC)

Rationale: 2012 updated labeling for the use of MRI in patients treated with VNS includes a precautionary statement not to perform MRI when the generator has been explanted, but all or part of the lead remains. Potential tissue damage has been a concern due to the risk for radio-frequency heating of the lead, a property which is a function of the lead length, Tesla of the MRI, and whether a head or body coil system is used. Prior to this advisory statement, patients who had disconnected or nonfunctioning VNS devices but retained partial or full length leads could have had subsequent MRIs. We retrospectively reviewed our cases of MRIs after VNS explants, or end of battery function in order to determine whether our clinical experience is concordant with the postulated risk.

Methods: We reviewed all cases of VNS patients followed at WFBH who had any known or possible disconnection of lead from the VNS battery. This included patients in whom the battery was explanted and the lead remained (isolated), those who had original lead problems and the lead was cut and new lead and battery were implanted(partial), and those who had an intact lead and battery, but the battery was nonfunctional, thus lead integrity was untestable, and thus potentially disrupted (dead battery).We identified patients within this population who had any MRI and reviewed by strength of magnet, type of coil and anatomic site of imaging. Charts were reviewed for evidence of adverse effects of MRIs.

Results: We identified a total of 22 VNS patients with lead-device disconnections, twelve of whom had MRIs (which would have been prohibited by current labeling change). Of these, 5 patients had surgically disconnected VNS leads. Of these, 2 had MRIs since explant. An additional 7 patients had expired VNS batteries which were not replaced prior to an MRI being done. Four of these patients underwent subsequent MRIs. Nine patients had the VNS turned off, and 6 had subsequent MRIs. All patients underwent 1.0 or $1.5 \mathrm{~T}$ head coil studies, predominantly of the brain for further evaluation of medically refractory epilepsy. Several patients had multiple MRIs. There were no reports of any complaints or complications following the MRIs.

Conclusions: The current change in labeling of VNS safety in MRI poses practical problems, especially for medical centers that serve as tertiary referral centers for medically refractory epilepsy management. Patients who had previously failed VNS therapy may have the device explanted or the device may simply run down. In these instances, the current labeling change may prohibit some centers from performing MRIs of any type, including those needed for anatomic localization for epilepsy surgery. Evidence from our cases indicates that MRIs can be performed in patients with disconnected VNS leads. We conclude that further definition of specific circumstances in which MRIs are safer need to be excluded from this restriction.

\subsection{4 \\ THE PROBABILITY DENSITY CHARACTERISTICS OF DIFFUSION TENSOR IMAGE IN MAJOR NEURAL PATHWAYS OF PATIENTS WITH NONLESIONAL EPILEPSY}

Yue-Loong Hsin and T. Harnod (Buddhist Tzu-Chi General Hospital, Hualien, Taiwan)

Rationale: By diffusion tensor imaging (DTI), reduced apparent diffusion coefficient (ADC) and increased fractional anisotropy (FA) have been noticed in epilepsy patients with hippocampal atrophy, cortical dysplasia or other visible brain abnormality. In limited researches from small numbers of patients with MRI-negative epilepsy, similar changes in ADC and FA were also reported. We collected patients with MRI-negative extratemporal lobe epilepsy to evaluate the integrity of white matter. Our aim was to identify the probability density characteristics of ADC and FA in different neural pathways.

Methods: 29 healthy volunteers ( 16 males, mean age $=27 \pm 4)$ and 24 patients with neocortical epilepsy ( 9 males, mean age $=26 \pm 13$ ) were included. DTI data and High-resolution T1-weight fast spoiled gradient recalled echo images (FSPGR) were.

Images were first corrected for eddy-current distortion and head motion using FMRIB's diffusion toolbox. Using Analyze 10.0 software, ADC and FA maps were produced. We further extracted $\mathrm{ADC}$ and FA and generated nerve fibers for white-matter regions of interest (WM ROIs) separately, each of which was defined in ICBM probabilistic atlas. By DARTEL tool in SPM8, we deformed each ROI from ICBM template to individual with reference to personal SPGR. Then we produced neural fibers by each ROI to calculate lengths of created fibers.

Furthermore, we depicted corresponding histograms of FA, ADC and mean fiber length of patient and health groups.

Results: Diffusely increased ADC and reduced FA in different pathways were found in the individual analyses and group comparison. All the histograms of ADC, FA and fiber length showed similar shape. The shape of ADC distribution was symmetrical. The $\mathrm{ADC}$ frequency peak of patient group was lower and shift to right. The shape of FA distribution was right skewed. The FA frequency peak was higher and shift to left. The shape of fiber length was right skewed. The fiber length frequency peak was higher and shift to left. The differences of distribution shapes were obvious in the posterior thalamic radiation and the sagittal stratum (patient vs. control groups by two-tailed t test, $\mathrm{p}<0.001$ ).

Conclusions: In this study, we not only confirmed the white matter changes in epilepsy patients without visible brain anomaly but also investigate the probability density of FA, ADC value and mean fiber lengths in detail. Regardless of the epileptogenic zones were 
determined by EEG in patients with extratemporal lobe epilepsy, common features exist in the major neural pathways. The results indicate that the major neural networks were aberrant and morphological changes in patients with nonlesional epilepsy. The network changes could correspond to specified propagation of epileptic activities and cognitive disorders.
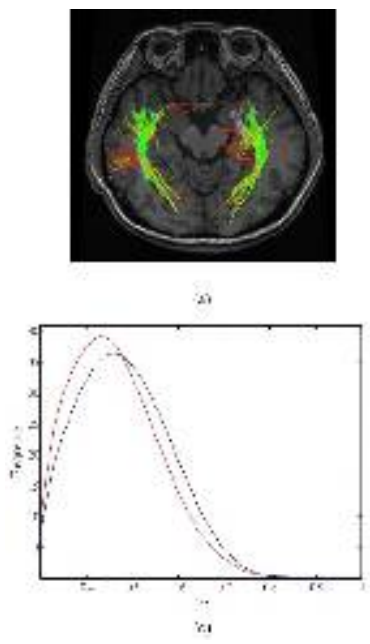
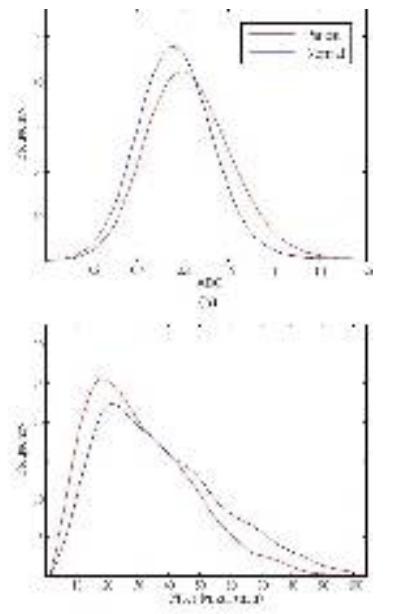

iı

\subsection{5}

MEASURE OF THE THALAMIC APPARENT DIFFUSION COEFFICIENT IN JUVENILE MYOCLONIC EPILEPSY

W. P. Blasque, A. S. Braga, F. F. Bellentani, M. C. Libardi, D. R. Kirchner and Luiz E. Betting (Faculdade de Medicina de Botucatu / UNESP, Botucatu, Brazil)

Rationale: Routine magnetic resonance imaging (MRI) of patients with juvenile myoclonic epilepsy (JME) is normal or with minor abnormalities. Quantitative techniques of neuroimaging showed subtle abnormalities involving the thalamo-cortical network. Until now there are no typical abnormalities and routine imaging is useless for diagnosis and follow-up of these patients. This study investigates apparent diffusion coefficient (ADC) features in the thalamus of patients with JME.

Methods: 10 patients ( 7 women, mean age $26 \pm 7$ years) with JME and 7 controls ( 3 women, $35 \pm 10$ years) were submitted to $1.5 \mathrm{~T}$ MRI. Routine DWI sequence was acquired. Apparent diffusion coefficient (ADC) maps were derived from this acquisition. A 2D pixel-based approach was used for analysis. Regions of interest (ROI) with $0.4 \mathrm{~cm} 2$ were selected in the anterior, posterior and superior portion of the right and left thalamus and the mean ADC was extract. ANOVA test was used for statistical analysis after observation of normal distribution (Kolmogorov-Smirnov test).

Results: The values of the mean ADC were: a) patients $0.73 \pm 0.04$ $103 \mathrm{~mm} 2 / \mathrm{sec}$ and $0.73 \pm 0.04$ (posterior), $0.71 \pm 0.07$ and $0.69 \pm 0.07$ (anterior), $0.73 \pm 0.04$ and $0.72 \pm 0.06$ (superior) right and left thalamus respectively; b) controls $0.72 \pm 0.05$ and $0.72 \pm 0.03$ (posterior), $0.73 \pm 0.03$ and $0.73 \pm 0.02$ (anterior), $0.70 \pm 0.04$ and $0.73 \pm 0.07$ (superior). Statistical analyses did not revealed differences between the means of patients and controls but showed greater variance in JME group.

Conclusions: In this preliminary evaluation there were no differences between the thalamic ADC of patients with JME and controls. However, a great variability was disclosed in patients with JME. Further investigations with increased number of patients are needed.
2.156

NEUROANATOMICAL ABNORMALITIES IN JUVENILE MYOCLONIC EPILEPSY: A VOXEL-BASED MORPHOMETRY STUDY

Antonia Villagran ${ }^{1,2}$, F. Woermann ${ }^{1}$ and B. Pohlmann-Eden ${ }^{1,3}$ ( ${ }^{1}$ Epilepsy Center Bethel, EVKB, Bielefeld, Germany; ${ }^{2}$ Soerlandet sykehus HF, Arendal, Norway and ${ }^{3}$ Division of Neurology, Dalhousie University of Halifax, Halifax, NS, Canada)

Rationale: Concepts and classifications of epilepsy syndromes are currently under debate. This is particular true for the dichotomy of focal versus generalized epilepsy syndromes. According to the latest report of the ILAE commission on Classification and Terminology these terms should be abandoned, but clinically well-defined subsyndromes as the Juvenile myoclonic epilepsy (JME) are still used both by clinicians and scientifics. By definition, JME is characterized by the absence of structural brain abnormalities and considered to be a functional disturbance. Advanced magnetic resonance imaging (MRI) studies of JME have reavealed subtle focal changes predominantly in the frontal lobe and the thalamus. Our study aimed to demonstrate further evidence for "focality" in JME patients by using Voxel based morphometry (VBM), an operatorindependent method for whole-brain analysis.

Methods: All patients were diagnosed and treated at one center, at the Epilepsy Center Bethel, Bielefeld, Germany. 41 patients (19 women, mean age $30,2 \pm 15,5$ ) with the confirmed diagnosis of JME were identified. Data were compared with 44 healthy controls (21 women, mean age $36,0 \pm 11,3$ ). All subjects underwent 1,5 T MRIscanning. Subsequently magnetic resonance voxel-based morphometry analysis (VBM) was performed using SPM5 in order to identify abnormalities in gray matter (GM) volume between patients and controls. Image postprocessing consisted of spatial normalization, automated grey matter segmentation, modulation, smoothing, and voxel-wise statistical analysis between the 2 groups was performed. Statistical level selected was a $\mathrm{p}<0.05$ with correction for multiple comparisons (family-wise error).

Results: VBM in this JME patient group revealed significantly increased grey matter $(\mathrm{GM})$ volume $(\mathrm{p}<0 ; 05)$ in the bilateral medial frontal gyrus (coordinates $\mathrm{x}=-13, \mathrm{y}=60, \mathrm{z}=0, \mathrm{Z}$-score 5,17, voxelcluster $125 ; x=15, y=58, z=3, Z$-score 4,85 , voxelcluster 32 ) and significantly reduced (GM) volume bilateral in the insula (coordinates $\mathrm{x}=-33, \mathrm{y}=21, \mathrm{z}=-9, \mathrm{Z}$-score 4,68, voxelcluster $12 ; \mathrm{x}=$ $42, y=-6, z=13, Z$-score 4,93 , voxelcluster $255 ; x=41, y=2, z=2, Z$ score 4,79 , voxelcluster $128 ; x=37, y=19, z=1, Z$-score 4,72 , voxelcluster 40$)$ compared to controls $(\mathrm{p}<0.05)$. This particular group of JME patient was further characterized by a rather unfavorable clinic course showing pharmacoresistence in 34\%. Conclusions: Our results suggest the enforced implication of the frontal and insular cortex in JME patients and support the concept of an epileptogenic- thalamo-cortical network in JME. We assume that the pronounced abnormalities are a reflection of this specific JME cohort with unfavorable disease course. We did not find any clearly lateralizing abnormalities in our study population. Our data detecting subtle structural changes in JME are encouraging to perform further studies focusing on functional-structural relationships in patients diagnosed with idiopathic "generalized" epilepsies.

\subsection{7}

NEUROIMAGING FINDINGS IN STATUS EPILEPTICUS ASSOCIATED WITH PARANEOPLASTIC ENCEPHALITIS Silvana I. Sarria ${ }^{1}$, M. Toledo ${ }^{2}$, C. Lorenzo i Bosquet ${ }^{3}$, E. Lainez ${ }^{4}$, S. Siurana $^{1}$, C. Auger ${ }^{1}$, E. Santamarina ${ }^{2}$, X. Salas-Puig ${ }^{2}$ and A. Rovira ${ }^{1}$ ( ${ }^{1}$ MR Unit. Radiology, Vall d'hebron University Hospital, Barcelona, Spain; ${ }^{2}$ Epilepsy Unit. Neurology, Vall d'Hebron University Hospital, Barcelona, Spain; ${ }^{3}$ Nuclear Medicine, Vall d'hebron University Hospital, Barcelona, Spain and ${ }^{4}$ Epilepsy Unit. Neurophysiology, Vall d'hebron University Hospital, Barcelona, Spain) 
Rationale: Patients with systemic cancer may have new onset status epilepticus (SE) as the main clinical feature of a paraneoplastic encephalitis. Five such patients who underwent MRI during the SE or immediately after recovering are described.

Methods: We retrospectively reviewed the MRI of five patients who suffered a SE as the onset of a paraneoplastic encephalitis during the last 6 years in our hospital. All of them fulfilled criteria for paraneoplastic syndrome. Two out of three patients with small-cell lung carcinoma had Anti-Hu antibodies. Two more cases had intestinal and lung adenocarcinoma. SE was convulsive for two patients and none-convulsive for three.

Results: All patients showed hyperintense lesions in T2-weighted images (T2WI) involving the limbic structures, specifically the hippocampus. Three of them showed further scattered extralimbic areas of encephalitis. The T2WI hyperintensities were related to the electroclinical onset of the seizures. Perfusion obtained by arterial spin labeling (ASL) in one patient showed hyperperfusion overlapping the inflammatory lesions. When performed, SPECT and PET observed increased metabolism limited to the areas of encephalitis. Mild to moderate contrast enhancement was seen in some patients. After recovering we observed atrophy in the hippocampus involved. Two patients had recurrences of the paraneoplastic encephalitis as SE, showing new T2WI lesions with a different topography.

Conclusions: The presence of limbic and extralimbic encephalitis can be suggestive of paraneoplastc syndrome in new onset SE.

Lesions are constantly seen as hyperintense in T2WI, can show mild contrast enhancement and increased perfusion or metabolism as seen by ASL or PET/SPECT.

\begin{tabular}{|c|c|c|c|c|}
\hline Patient & Cerebral $\mathrm{Cl}^{\prime}$ & Brain-MRI & $\begin{array}{l}\text { Follow-up } \\
\text { MRI }\end{array}$ & $\begin{array}{c}\text { Cerebral } \\
\text { HDG-PET/SPECI' }\end{array}$ \\
\hline 1 & $\begin{array}{l}\text { Non-contrast } \\
\text { enhanced low } \\
\text { densily area in the } \\
\text { temporal lobes } \\
\text { and parictal } \\
\text { cortex. }\end{array}$ & $\begin{array}{l}\text { Increased ГI.ATR/T2WI } \\
\text { signal in both mesial } \\
\text { temporal lobes and left } \\
\text { superior temporal gyrus and } \\
\text { adjacent parictal cortex. } \\
\text { DWI: Negative. } \\
\text { Contrast enhancement: } \\
\text { Negative }\end{array}$ & Not done & $\begin{array}{l}\text { FDG-PET and SPECT } \\
\text { showed increased } \\
\text { metabolism in the left } \\
\text { superior temporal gyrus }\end{array}$ \\
\hline 2 & Normal & $\begin{array}{c}\text { Increased FI.ATR/T2WI } \\
\text { signal of the hippocampus. } \\
\text { DWI: Negative. } \\
\text { Contrast enhancement: } \\
\text { Negative }\end{array}$ & Nol done & Nol done \\
\hline 3 & $\begin{array}{l}\text { Subtle contrast } \\
\text { enhaced low } \\
\text { density area in the } \\
\text { left insular region }\end{array}$ & $\begin{array}{l}\text { Increased FI.ATR/T2WI } \\
\text { signal in both amygdalas. } \\
\text { hippocampii, and anterior } \\
\text { temporal cortex, as well as in } \\
\text { subcortical areas of both } \\
\text { parictal lobes and left frontal } \\
\text { lobe. } \\
\text { DWI: Negative. } \\
\text { Contrast enhancement: } \\
\text { Negative } \\
\text { ASL: Increased perlusion } \\
\text { within the affected areas. }\end{array}$ & $\begin{array}{l}\text { Right } \\
\text { hippocampal } \\
\text { sclerosis }\end{array}$ & $\begin{array}{c}\text { Ictal and interictal } \\
\text { SPECI showed } \\
\text { increased metabolism in } \\
\text { the same areas that } \Lambda \mathrm{SL} \\
\text { did. } \\
\text { follow-up SI'EC'I was } \\
\text { normal. }\end{array}$ \\
\hline 4 & $\begin{array}{c}\text { Diffuse } \\
\text { hypodensity of } \\
\text { right hemisphere }\end{array}$ & $\begin{array}{l}\text { Increased FLAIR/T2WI } \\
\text { signal in the right } \\
\text { temporo-parietal lobe. } \\
\text { DWI: Negative. } \\
\text { Contrast enhancement: } \\
\text { Negative. }\end{array}$ & $\begin{array}{l}\text { Right } \\
\text { hippocampal } \\
\text { atrophy }\end{array}$ & $\begin{array}{c}\text { Follow-up TDG-PET } \\
\text { showed hypometabolim } \\
\text { in the right temporal } \\
\text { lobe }\end{array}$ \\
\hline 5 & $\begin{array}{l}\text { Bilateral } \\
\text { hippocampal } \\
\text { hypodensity. }\end{array}$ & $\begin{array}{l}\text { Increased } \Gamma L \Lambda I R / T 2 W I \\
\text { signal in both hippocampii. } \\
\text { DWI: Negative. } \\
\text { Contrast enhancement: } \\
\text { Negative. }\end{array}$ & $\begin{array}{l}\text { Bilateral } \\
\text { hippocampal } \\
\text { atrophy }\end{array}$ & Not done \\
\hline
\end{tabular}

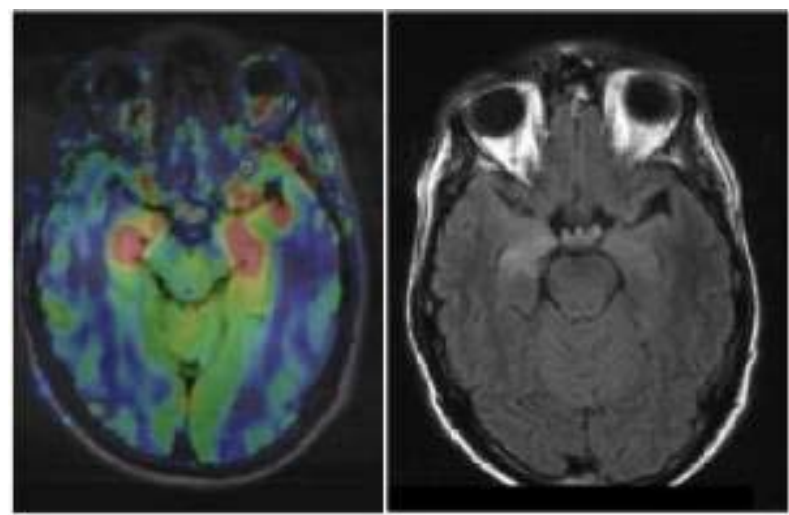

Neuro Imaging: Functional Imaging

\subsection{8}

EPILEPTIFORM NETWORK IDENTIFICATION IN THE PREOPERATIVE PLANNING OF EPILEPSY SURGERY: A MODEL- AND DATA-DRIVEN APPROACH

P. Ossenblok ${ }^{1}$, Petra van Houdt ${ }^{2,3}$, A. Colon ${ }^{4}$, F. Leijten ${ }^{5}$, P. Boon ${ }^{2}$ and J. C. de Munck ${ }^{3}\left({ }^{1}\right.$ Clinical Physics, Kempenhaeghe, Heeze, Netherlands; ${ }^{2}$ Research \& Development, Kempenhaeghe, Heeze, Netherlands; ${ }^{3}$ Physics and Medical Technology, VU University Medical Center, Amsterdam, Netherlands; ${ }^{4}$ Neurology, Kempenhaeghe, Heeze, Netherlands and ${ }^{5}$ Clinical Neurophysiology, University Medical Center Utrecht, Utrecht, Netherlands)

Rationale: EEG-correlated functional MRI (EEG-fMRI) detects the distribution of brain regions involved with interictal epileptic discharges (IEDs). The goal of this study was to cross-validate EEGfMRI correlation patterns with intracranial EEG recordings and outcome of surgery as gold standards. We also assessed the added value of Independent Component Analysis (ICA), a data-driven technique applied to fMRI alone, for those cases where no IEDs are present in the EEG.

Methods: EEG and fMRI data were acquired of 21 patients who were later implanted with depth electrodes $(n=5)$ or subdural grids $(n=16)$. EEG was corrected for gradient and pulse artifacts and annotated for the presence of IEDs. These events were used as a predictor in the general linear model framework, yielding a correlation pattern indicating the brain regions that were significantly associated with the IEDs. For the validation of these results, a quantitative approach was developed to reveal the spatiotemporal patterns of the IEDs present in the invasive EEG (Van Houdt PJ, Ossenblok PPW, Colon AJ, Boon PAJM, de Munck JC (2012) A framework to integrate EEG-correlated fMRI and intracerebral recordings. NeuroImage 60: 2042-2053). The EEG-fMRI results were also compared to the location of the seizure onset zone and resection area. The fMRI data of those patients in whom EEG-fMRI overlapped with the resection area and who were seizure free after surgery (Engel score 1, n=9), were also analyzed with ICA (Beckmann CF, Smith SM (2004) Probabilistic independent component analysis for functional magnetic resonance imaging. IEEE Trans Med Imaging 23:137-152) for data selections with and without IEDs.

Results: Table 1 summarizes the results of the comparison of EEGfMRI and invasive EEG patterns. At least one EEG-fMRI cluster overlapped with active invasive electrodes (i.e. electrodes reflecting high correlations) in all data sets, whereas EEG-fMRI overlapped with all active ECoG/SEEG electrodes in $50 \%$ of the datasets. In 11 of the $16 \mathrm{ECoG}$ datasets, more than one BOLD region was concordant with active electrodes; some regions were related to the onset of spike activity and others to propagation. The EEG-fMRI clusters included the complete seizure onset zone in $83 \%$ of the data sets and the resection area in $90 \%$ of the data sets. These values are similar to those obtained by comparing interictal invasive patterns to 
the seizure onset zone (83\%) and resection area (95\%). Finally, for the 9 datasets in which ICA was performed, one component was concordant with the seizure onset zone and resembled the EEG-fMRI pattern regardless of whether IEDs were present in the corresponding EEG or not.

Conclusions: This study shows that EEG-fMRI has substantial predictive value regarding the seizure onset zone and resection area. In addition with the finding that ICA may reveal epileptic components without the presence of IEDs, this suggests that restingstate fMRI could play an important role in the presurgical planning of invasive recordings.

Summary of comparison between EEG-fMRI and invasive EEG patterns, seizure onset zone and resection area.

\begin{tabular}{|c|c|c|c|}
\hline & Invasive EEG & $\begin{array}{c}\text { Seizure Onset } \\
\text { Zone }\end{array}$ & $\begin{array}{c}\text { Resection } \\
\text { area }\end{array}$ \\
$\begin{array}{c}\text { TE:G-fMRI } \\
\text { clusters }\end{array}$ & $\begin{array}{c}(\mathrm{n}=21) \\
(\mathrm{n}=17)\end{array}$ & $(\mathrm{n}=20)$ \\
\hline invarive TEG & $50 \%$ & $83 \%$ & $90 \%$ \\
\hline seizure onset zone & - & $83 \%$ & $95 \%$ \\
\hline
\end{tabular}

\subsection{9}

LESION-NEGATIVE TEMPORAL LOBE EPILEPSY SHOWS DECREASED HIPPOCAMPAL FUNCTIONAL CONNECTIVITY IN THE DEFAULT MODE NETWORK

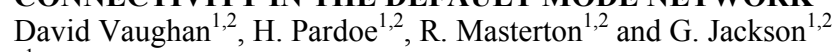
( ${ }^{1}$ Brain Research Institute, Florey Neuroscience Institutes, Melbourne, VIC, Australia and ${ }^{2}$ University of Melbourne, Melbourne, VIC, Australia)

Rationale: Resting state functional connectivity identifies broad networks of correlated brain activity, which have been shown to be affected in MTLE. We investigated whether adult temporal lobe epilepsy patients with no anatomic abnormality of the hippocampus showed similar alterations to functional connectivity of the hippocampus.

Methods: 14 adult patients with lesion-negative temporal lobe epilepsy (11 males, age range 27-57 years) were compared to 12 patients with mesial temporal lobe epilepsy with hippocampal sclerosis ( 2 males, age range $24-55$ years), and to 16 healthy nonepileptic controls ( 8 males, age range $23-52$ years). The lesionnegative group showed no abnormality of the hippocampus on routine MRI. Diagnosis was confirmed on video-EEG monitoring and the side of ictal onset ascertained (lesion-negative: 7 left, 5 right, 2 bilateral; hippocampal sclerosis: 5 left, 7 right). A seed based connectivity analysis was performed using resting state BOLD data, acquired on a 3 T MRI scanner over 10 minutes $(3 \times 3 \times 3 \mathrm{~mm}$ voxels, TE $30 \mathrm{~ms}$, TR $3000 \mathrm{~ms}, 210$ volumes). Standard data pre-processing techniques were used including band-pass filtering of $0.1 \mathrm{~Hz}$ to 0.005 $\mathrm{Hz}$, and regression of the mean CSF and white matter signals.

Participants with right sided ictal onset had their images flipped rightto-left, before non-linear registration to a symmetric brain template. Individual hippocampal seeds were generated using an automated approach, and the mean time-course from this region was correlated against all brain voxels. The resulting statistic maps of hippocampal connectivity were compared between groups using voxel-wise t-tests (threshold $\mathrm{p}<0.01$ uncorrected), and significant clusters reported after false-discovery rate cluster correction at $\mathrm{p}<0.05$.

Results: The lesion-negative group (LNTLE) showed regions of reduced connectivity compared to controls. The regions affected were the bilateral anterior cingulate cortex, mesial frontal cortex, bilateral superior frontal gyrus (peak t-value 5.6) and bilateral precuneus/posterior cingulate gyrus (peak t-value 5.2). Comparison of the hippocampal sclerosis group (MTLE-HS) to controls also showed reduced connectivity in the bilateral precuneus/posterior cingulate cortex and in the right superior frontal gyrus. Direct comparison of lesion-negative versus hippocampal sclerosis showed reduced connectivity to the mesial frontal cortex in the lesionnegative group.

Conclusions: Patients with lesion-negative temporal lobe epilepsy show reduced resting state functional connectivity between the hippocampus and regions involved in the anterior and posterior parts of the default mode network. Alteration of large scale brain networks may occur in LNTLE, notably in the absence of structural changes, and in a pattern that is similar to, but also distinct from, that seen in MTLE with hippocampal sclerosis.

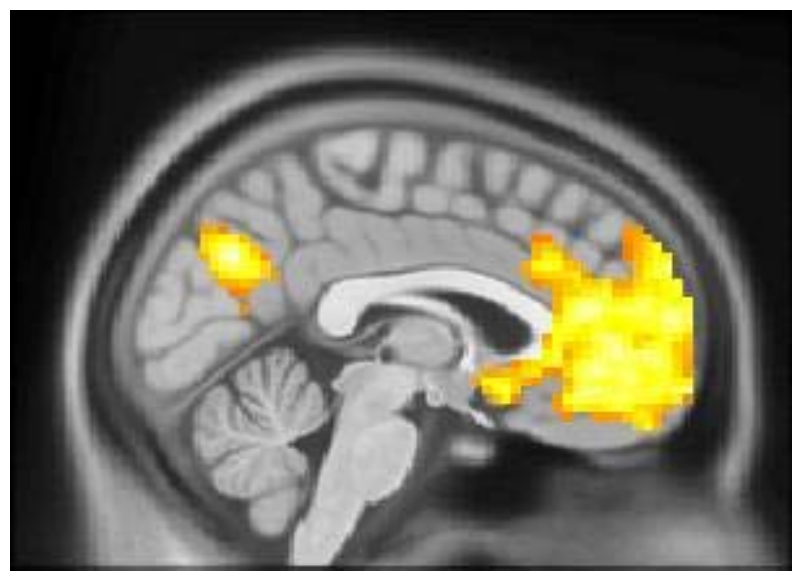

Figure 1: Decreased hippocampal connectivity in lesion-negative temporal lobe epilepsy versus controls $(\mathrm{p}<0.05$ FDR cluster corrected). Slice $\mathrm{x}=-2 \mathrm{~mm}$.

\subsection{0 \\ A METHOD TO DISPLAY MEG RESULTS IN 3 DIMENSIONS}

J. Paugh, Mark H. McManis, A. Hernandez and S. Perry

(Neuroscience, Cook Children's Medical Center, Fort Worth, TX)

Rationale: Data from multiple imaging modalities contribute to the successful localization of the epileptogenic zone in patients with treatment-resistant epilepsy. Each imaging modality has inherent strengths and weaknesses with regards to spatial and temporal resolution. Multimodal coregistration of pre-surgical imaging modalities may overcome the weaknesses of individual imaging datasets, and is generally accepted to improve outcome after surgical therapy. In order to maximize the utility of multimodal neuroimaging for surgical planning, it is important to be able to present any combination of modalities in a 3-dimensional model. We present a standardized process for coregistration of magneto-encephalography (MEG) data for presentation in a three-dimensional model concurrently with other neuroimaging modalities.

Methods: MEG source estimates for epileptiform and functional localization are coregistered to a T1 thin-sliced 3T MRI to create the magnetic source images. This MRI serves as the anatomic basis for coregistration of all other pre-surgical imaging modalities (eg PET, Ictal SPECT subtraction). MEG source estimate representations are extracted using a histogram analysis to produce a dataset which may be overlaid with additional coregistered imaging modalities. A 3dimensional anatomic brain model is created from the MRI using MeVisLab. Isocontours of the MEG dipoles and other coregistered modalities can then be overlaid in varying combinations and manipulated to provide a more complete picture of the anatomical structure, function, and epileptogenic localization in relation to each other.

Results: The process was developed and applied to 6 cases in which epileptiform MEG dipoles and either Ictal SPECT Subtraction or PET data were available. The resultant 3-dimensional models were then compared with the original 2-dimensional studies to confirm that they are accurate representations. The model (Figure 1) can be oriented and clipped in all 3 orthogonal planes to maximize 
appreciation for the epileptogenic zone localization as identified by MEG and its relationship to functional cortex. This model can be expanded to include epileptogenic localization based on other modalities and functional response MEG dipoles. The typical 2dimensional presentation of the data is shown in Figure 1 for comparison.

Conclusions: This standardized protocol for displaying MEG data in 3-dimensions provides improved visual presentation. The overall relationship between modalities is more readily apparent and can be easily manipulated between the axial, coronal, and sagittal planes. In addition, this protocol allows assessment of congruence amongst complementary anatomic and functional imaging studies with regards to epileptogenic zone localization and the proximity to functional cortex, potentially contributing to improved subdural grid placement and surgical outcomes. Subsequent research validating the value of 3dimensional multimodal modeling in regards to surgical planning and outcome is planned.

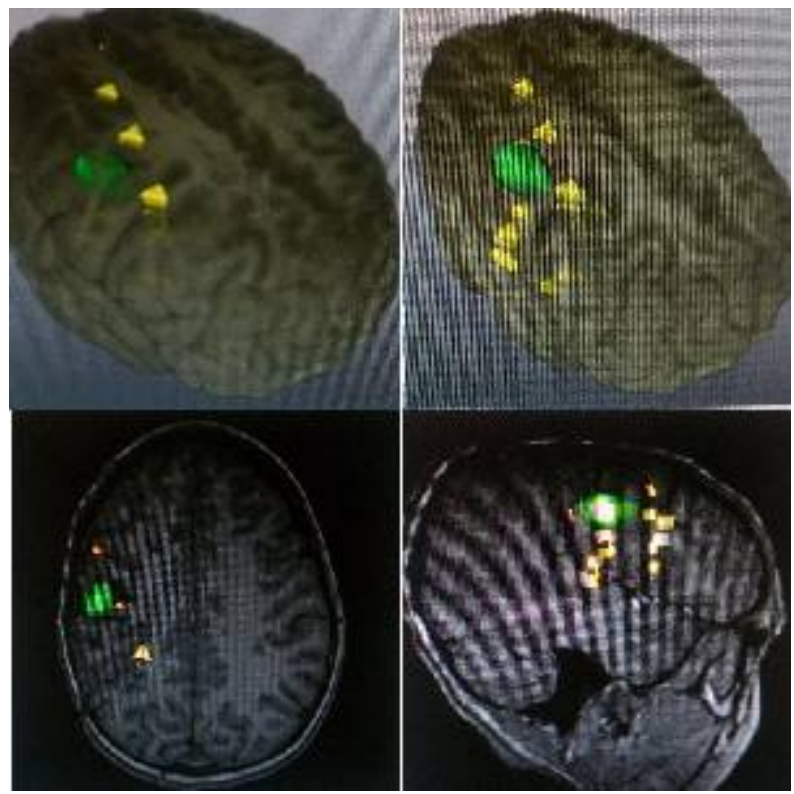

Fig 1 Top Row: 3D Model - Coregistered MEG Epileptiform discharges (yellow) and Ictal SPECT Subtraction (green). Bottom Row: Equivalent 2-dimensional view of the coregistered MRI, MEG dipoles, and ictal SPECT subtraction.

\subsection{1}

\section{THE USE OF PASSIVE FUNCTIONAL MRI IN PRESURGICAL PLANNING FOR PAEDITRIC PATIENTS WITH EPILEPSY}

M. Watson ${ }^{2}$, Dave F. Clarke ${ }^{1}$, C. Hovinga ${ }^{1}$ and N. Nussbaum ${ }^{1}\left({ }^{1}\right.$ Dell Chilldren's Comprehensive Epilepsy Program, Dell Children's Medical Center, Austin, TX and ${ }^{2}$ The University of the West Indies, Bridgetown, Barbados)

Rationale: To assess the usefulness of passive functional magnetic resonance imaging for localizing the language cortex and motor cortex in children with epilepsy who are surgical candidate. To compare fMRI language acquisition data for the sedated and unsedated child with epilepsy.

Methods: Data was gathered from 31 children with refractory epilepsy between the ages of 3 years to 22 years. The study looked at two groups of the sedated and unsedated patients. The unsedated group consisted of 17 patients between the ages of 7 to 22 years. The sedated group consisted of 14 patients between the ages of 3 to 18 years. fMRI was done in possible candidates for epilepsy surgery. The study used various paradigms to asses motor, language and sensory cortex. Not all patients were administered the same paradigms.
Propofol, sevoflurane, precedex or a combination of previous were used in the sedated group. Propofol was the most commonly used sedating agent. Motor paradigms used were finger tapping, wrist movements, foot movement and tactile hand for both groups. Language paradigms included word listening, story listening and maternal story listening sedated group. Word listening, story listening and maternal story listening were conducted through prerecorded stories presented to the patient via headphones during the scan. Language paradigms for the unsedated group included the above task, as well as word generation, verb generation, sentence completion and object naming. A tactile stimulation task was used to assess the sensory cortex.

Results: Sedate Group: Successful motor activation was seen in 13 of 14 patients (bilateral predominance). 11 of the 13 patients in whom motor activation was seen went onto have epilepsy surgery.

Successful sensory activation was seen in 7 of 8 patients and 5 out 7 patients went on to surgery.

Successful language activation via 1 or more language paradigm was seen in 11 of 12 patients. 8 out of the 11 patients went on to surgery. Story Listening via their mother's recorded voice was attempted for 4 patients and 3 out of the 4 patients had successful activation. All 3 patients went on to surgery.

Nonsedated Group: Successful motor activation was seen in 16 of 16 patients with bilateral predominance. $15 / 16$ went onto to have surgery.

There was successful sensory activation in both patients with whom it was attempted.

Activation for the language cortex was seen in 10 of the 12 patients and it was predominantly bilateral. $10 / 12$ patients went on to have surgery. All 4 patients showed successful activation for maternal story reading. All activation was bilateral with left side predominance in 2 out of the 4 patients.

When comparing the two groups there was no statistical difference the sedated and unsedated group for activation of motor $(p$ value $=0.47$ ) sensory ( $p$ value $=1)$ and language $(p$ value $=1)$ cortex. Conclusions: There was similar activation of the sedated and unsedated samples for motor, auditory and sensory cortex using various paradigms for fMRI studies with no statistical difference between the two groups.

\subsection{2 \\ DYNAMIC SEIZURE IMAGING IN PEDIATRIC PATIENTS WITH PARTIAL EPILEPSY}

Yunfeng Lu ${ }^{1}$, L. Yang ${ }^{1}$, B. Brinkmann ${ }^{2}$, C. Nelson ${ }^{2}$, G. Worrell ${ }^{2}$ and B. $\mathrm{He}^{1}$ ( ${ }^{1}$ University of Minnesota, Minneapolis, $\mathrm{MN}$ and ${ }^{2}$ Mayo Clinic, Rochester, MN)

Rationale: Focal epilepsy in children is often refractory to medications and associated with significant developmental problems. Diagnosis and determination of epileptogenic zone is important for understanding the mechanism of epilepsy and developing effective treatment plans. EEG has been widely used in clinical to capture the electrophysiological neuronal activity and to identify the pathologically abnormal brain. Recent progress in source imaging has advanced the usage of EEG in clinical settings. However, the source imaging of seizures remains challenging due to the dynamic characteristics of seizures and the EEG contamination of motion artifacts. In this study, we investigate the feasibility of using a dynamic seizure imaging approach to help noninvasively identify the seizure sources.

Methods: Nine pediatric patients with medically intractable partial epilepsy were studied. The pre-operative scalp EEG was recorded during a long-term video EEG monitoring. All the patients had preoperative high-resolution MRI scans and the MRI images were used to build patient-specific boundary element head models. A dynamic seizure imaging approach was utilized to extract the spatiotemporal ictal features from independent component analysis and to image the seizure sources in the cortex. All patients had resective surgery and 
the post-operative MRI images were acquired from the patients. Seven of the patients underwent intracranial EEG (iEEG) monitoring before the surgery. The seizure source imaging results were evaluated by comparing them with the surgically resected region and the seizure onset zone (SOZ) of intracranial recording.

Results: The seizure source imaging results in the nine patients are concordant with the surgical resection. The estimated source maxima in 7 out of the 9 patients are within the surgically resected regions and the results in the other 2 patients are in close vicinity to the resection boundary. Six months after the surgery, five patients have been seizure free and four patients have seizure reduction outcome. In the 7 patients with intracranial recording available, the seizure imaging results are concordant with the seizure onset zone marked from iEEG. The estimated source maxima in 5 patients are around 1 electrode distance to the SOZ and the sources in the remaining 2 patients are 2 3 electrode distances to the iEEG-SOZ.

Conclusions: Noninvasive imaging of seizure activity shows promise for the epilepsy treatment. Our study suggests that the spatiotemporal ictal features can be extracted and used to image the dynamics of seizure activity. The imaged seizure sources of the patients are colocalized with the surgically resected regions and seizure onset zones determined by intracranial monitoring. These results show the feasibility of utilizing EEG seizure imaging to noninvasively localize seizure sources and suggest its potential application for the presurgical planning of epilepsy patients.

\subsection{3}

\section{STATISTICAL PARAMETRIC MAPPING BASED SPECT PROCESSING IN NONLESIONAL TEMPORAL LOBE EPILEPSY}

Vlastimil Sulc $^{1,2}$, D. Hanson ${ }^{3}$, S. Stykel ${ }^{3}$, B. Brinkmann ${ }^{1}$, D. Jones ${ }^{1}$, D. Holmes ${ }^{3}$, R. Robb ${ }^{3}$, M. Senjem ${ }^{4}$ A. Larson ${ }^{3}$, B. Mullan ${ }^{4}$, D. Horinek $^{2}$, G. Cascino ${ }^{1}$, E. So ${ }^{1}$ and G. Worrell ${ }^{1}\left({ }^{1}\right.$ Department of Neurology, Mayo Clinic, Rochester, MN; ${ }^{2}$ International Center for Clinical Research, St. Anne's University Hospital, Brno, Czech Republic; ${ }^{3}$ Biomedical Imaging Resource, Mayo Clinic, Rochester, $\mathrm{MN}$ and ${ }^{4}$ Department of Radiology, Mayo Clinic, Rochester, MN)

Rationale: Ictal SPECT has proven to be a useful tool in the presurgical evaluation of epilepsy surgery candidates, and subtraction of ictal and interictal SPECT with coregistration to MRI (SISCOM, O'Brien et al, 1998) has become a standard in seizure onset localization. The specificity of SISCOM, however, is likely reduced by the physiological variability of interictal perfusion. To overcome this issue, methods using statistical parametric mapping (SPM) applied to scans from a population of control subjects were developed. To evaluate the benefit of SPM based methods over traditional SISCOM in nonlesional temporal lobe epilepsy we compared Ictal-Interictal SPECT Analyzed by SPM (ISAS, McNally et al., 2005) and Statistical Ictal SPECT coregistered to MRI (STATISCOM, Kazemi et al., 2010) to SISCOM.

Methods: We retrospectively identified 21 cases of nonlesional temporal lobe epilepsy that had a normal preoperative "seizure protocol" MRI, and underwent a standard anterior temporal lobectomy between January 1997 and December 2005 at Mayo Clinic, Rochester, MN. Interictal and ictal SPECT scans were analyzed using SISCOM, ISAS and STATISCOM to create hyperperfusion and hypoperfusion maps for each patient. The SPM based analysis was performed with a group of 30 control subjects. Perfusion maps were created with thresholds $p=0.001$ for ISAS, $p$ $=0.027$ for STATISCOM, and 2 standard deviations for SISCOM. A training set of 5 cases with strong temporal lobe activation were used to create consistent perfusion maps and to optimize thresholds. Mean ictal injection time (as determined by scalp EEG) was $26 \mathrm{~s} \pm 8.7$ seconds, and all were ictal injections. Hyperperfusion and hypoperfusion maps were co-registered to the patient's MRI. We created a custom software tool for displaying, reviewing, and annotating the thresholded hyperperfusion and hypoperfusion images in sagittal, axial a coronal planes. In this preliminary study, a single reviewer (VS) blinded to clinical data and analysis method (ISAS, STATISCOM or SISCOM) marked the lobe of probable seizure origin and rated his confidence (scale of 1 to 10) in the localization. Results: Out of 21 nonlesional temporal lobe cases ISAS correctly determined resection site in $19(90 \%)$, STATISCOM in $18(86 \%)$, and SISCOM in $13(62 \%)$. Comparison of the three methods show a significant difference in localization between the SPM based methods to standard SISCOM, with $\mathrm{p}=0.005$ (ISAS vs SISCOM); $\mathrm{p}=0.011$ (STATISCOM vs SISCOM).

Mean confidence rating in ISAS cases was $7.26(\mathrm{SD} \pm 0.96)$, in STATISCOM 7.30 (SD \pm 1.43$)$, SISCOM $4.43(\mathrm{SD} \pm 2.5)$.

Conclusions: Both SPM based methods of ictal and interictal SPECT processing were superior to the standard SISCOM analysis in terms of localization and confidence rating. There was no significant difference between ISAS and STATISCOM results. In the future we plan to: 1) Perform a multi-reviewer study to investigate the concordance between blinded expert reviewers for each analysis method. 2) Investigate the association between seizure outcome in temporal and extratemporal lobe epilepsy.

\subsection{4 \\ CONCORDANCE BETWEEN FUNCTIONAL MAGNETIC RESONANCE IMAGING AND SUBDURAL ELECTRICAL STIMULATION LANGUAGE MAPPING IN PEDIATRIC EPILEPSY}

Christine M. Salinas ${ }^{1}$, M. Korostenskaja ${ }^{2}$, P. Chen ${ }^{2}$, J. Cook ${ }^{3}$, J. Baumgartner ${ }^{4}$, K. Lee ${ }^{4}$ and M. Westerveld ${ }^{1}\left({ }^{1}\right.$ Neuropsychology, Florida Hospital for Children, Orlando, FL; ${ }^{2}$ Functional Brain Mapping and Brain Computer Interface Lab, Comprehensive Pediatric Epilepsy Center, Florida Hospital for Children, Orlando, FL; ${ }^{3}$ Radiology, Florida Hospital, Orlando, FL and ${ }^{4}$ Comprehensive Pediatric Epilepsy Center, Florida Hospital for Children, Orlando, FL)

Rationale: The goal of improving seizures with early surgical treatment is closely tied to improving cognitive and quality of life outcomes. Avoiding or minimizing deficits following surgery depends on identification of critical cortical regions prior to resection. Functional MRI is emerging as a noninvasive alternative for localization of language, but poses challenges in children with cognitive and behavioral impairments and stimulation of subdural grid electrodes remains the gold standard for precise localization of language areas. There have been very few studies that have validated fMRI by comparison with electrical cortical stimulation mapping (ESM), and none in children undergoing epilepsy surgery. We report preliminary data on concordance of fMRI and ESM in children using a novel quantitative approach.

Methods: Three children (3 girls, 11-15 years, 2 right-handed) underwent language mapping with fMRI and ESM in the left hemisphere within the first year of our epilepsy surgery program. fMRI language mapping was accomplished using a covert Verb Generation (VG) task performed on a 3-Tesla GE scanner. Children were presented with concrete nouns at different rates $(0.2 \mathrm{~Hz}, 0.3 \mathrm{~Hz}$, $0.5 \mathrm{~Hz}$ ) in $30 \mathrm{~s}$ activation blocks alternating with $30 \mathrm{~s}$ blocks of finger tapping. ESM was conducted using an Ojemann stimulator with amperages ranging from 4-10 milliamps ( $50 \mathrm{hz} ; 5 \mathrm{sec}$. duration) during expressive language tasks. Fusion of fMRI and postimplantation CT images was performed to allow for direct comparison.

Results: fMRI during VG elicited robust activation in the left inferior frontal gyrus in all 3 patients. Bilateral activation was observed in the left-handed patient. ESM identified eloquent cortex in all three patients. A total of 67 contacts were stimulated across patients; 15 produced a positive response. In the whole series, $8 / 15$ positives contacts were located within an active fMRI cluster using a threhold of $\mathrm{p}<.01$ (Bonferroni). Among the 52 negative contacts, 6 were located within an active fMRI cluster and 46 were not. The overall 
sensitivity of language fMRI was .53 and the specificity was .88 . The false positive rate (+fMRI activation; - ESM finding) was .46, and the false negative rate was .11.

Conclusions: To our knowledge, we are the first to compare subdural ESM and fMRI in children with intractable epilepsy, and employ a novel co-registration technique allowing for objective analysis of concordance. In our small case series, language fMRI did not activate all critical regions displayed in ESM, and there was significant interpatient variability $(0 \%-100 \%$ for temporal and frontal lobe, respectively). fMRI VG failed to activate left temporal language areas in patients who are at high risk for post-surgical language deficit. Concordance was higher in frontal language areas, but fell short of acceptable levels. Our preliminary results suggest that fMRI may be used as a complementary tool to ESM. Development of comparable fMRI and ESM paradigms may improve concordance.

\subsection{5}

\section{STRUCTURAL SUBSTRATES OF RESTING NETWORK ALTERATIONS IN TEMPORAL LOBE EPILEPSY}

Neda Bernasconi ${ }^{1}$, N. L. Voets ${ }^{2,1}$ and A. Bernasconi ${ }^{1}{ }^{1}$ McConnell Brain Imaging Centre, Montreal, QC, Canada and ${ }^{2}$ FMRIB Centre, Oxford, United Kingdom)

Rationale: MRI methods measuring inter-regional brain signaling at rest are powerful tools to probe organizational properties of functional networks. In temporal lobe epilepsy (TLE), abnormal mesiotemporal functional connectivity has been attributed to epileptogenic or functionally compensatory processes (1). Our aim was to determine the extent to which resting connectivity in TLE is associated with co-localized structural pathology. In line with the distributed nature of structural damage in this condition, we used independent component analysis that optimizes sensitivity to widescale resting signaling, correcting for voxel-wise grey-matter density. In addition, we assessed the relationship between resting signaling and white matter microstructure using tract-based spatial statistics. Methods: We acquired resting state fMRI, diffusion tensor imaging and high-resolution anatomical MRI data in 35 patients with drugresistant TLE (mean age: $34 \pm 9$ years) and 20 healthy subjects (mean age: $29 \pm 7$ years) on a 3Tesla scanner. Using Melodic Independent Component Analysis (2) applied to our healthy controls, we identified 10 resting state networks based on visual neuroanatomical correspondence and cross-correlation with consistently identified resting state networks described in the literature. A dual regression approach (3) was used to derive the matching spatial networks, and their time-courses, in every patient and control. Subsequently, functional connectivity within each network was compared between groups using 'randomize' permutation testing. A voxel-wise grey matter covariate was generated using automated tissue class segmentation to determine the association between resting fMRI connectivity and grey matter volume. Finally, resting functional connectivity values from clusters showing between-group differences were used as covariates in Tract-Based Spatial Statistics analyses (4) to assess relationships between functional connectivity and voxelwise fractional anisotropy.

Results: Compared to controls, patients showed altered functional connectivity between the hippocampus, anterior temporal, precentral cortices and the default mode and sensorimotor networks $(\mathrm{p}<0.05)$ (Fig 1). Reduced network integration of the hippocampus was explained by variations in grey matter density (Fig1), while functional connectivity of the parahippocampus, and frontal and temporal neocortices showed atypical associations with white matter coherence within pathways carrying connections of these regions $(\mathrm{p}<0.05)$ (Fig 2).

Conclusions: Our multimodal MRI study provides strong indication that in TLE hippocampal atrophy and microstructural white matter damage impact functional resting connectivity. Structural damage may explain previously reported discrepancy between increased interictal EEG synchronicity and decreased mesiotemporal fMRI signaling (5).
References:

(1) Bettus G., J Neurol Neurosurg Psychiatry, 2010

(2) Beckmann CF., Phil. Trans. Roy. Soc. Lond., 2005

(3) Khalili-Mahani N., HBM, 2011

(4) Smith S., Neuroimage, 2006

(5) Bettus G., HBM, 2009

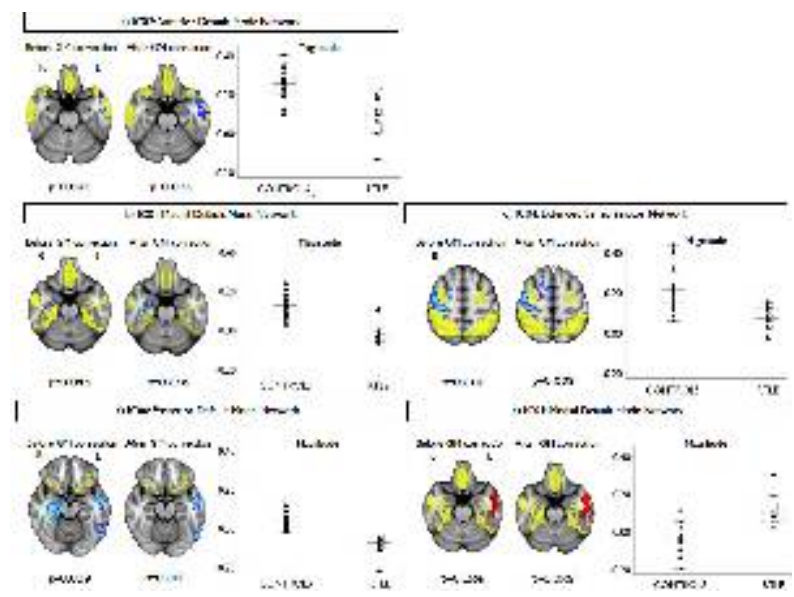

Figure 1. Group-wise results conducted within each of the 10 resting state networks in LTLE (a) and RTLE (b-e). T1-MRI overlaid with the resting network (yellow) and clusters showing reduced (blue) or increased (red) functional connectivity to this network. Plots of the connectivity effect magnitude extracted from each cluster are also shown.
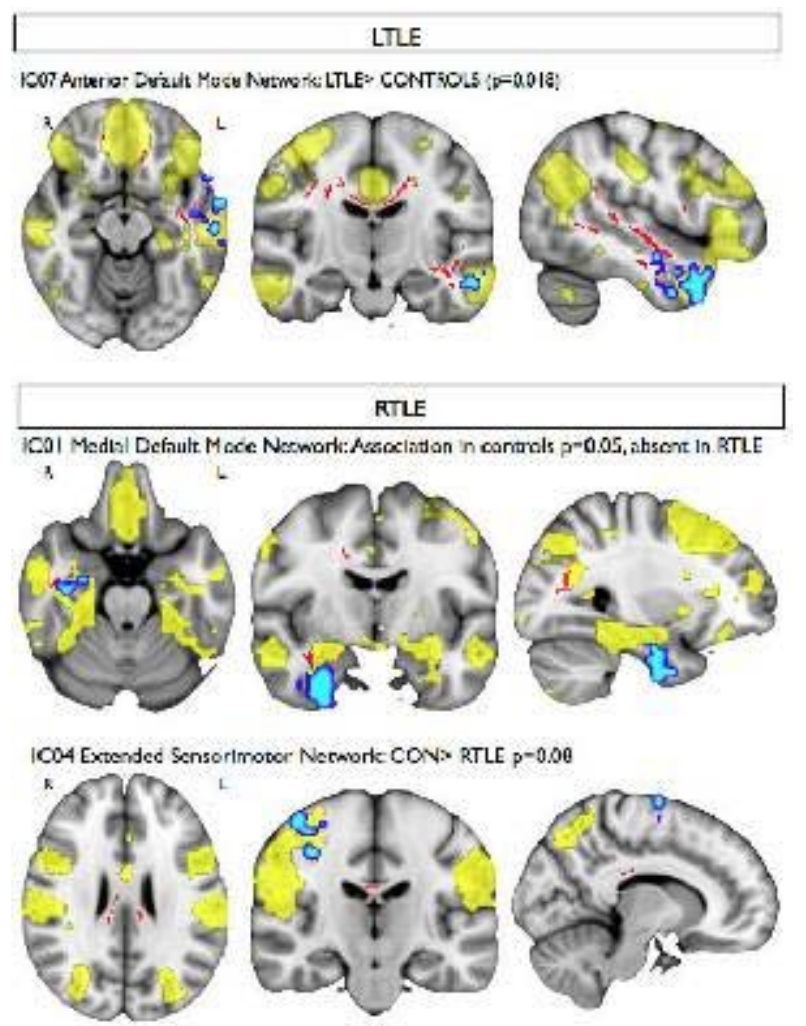

Figure 2. Whole-brain tract-based spatial statistics results depict voxels (red) showing correlations between fractional anisotropy and resting functional connectivity (blue) to resting state networks (yellow) for clusters showing altered resting connectivity that were not explained by grey matter density. 


\subsection{6}

DECREASED REGIONAL HOMOGENEITY, A MEASURE OF LOCAL FUNCTIONAL CONNECTIVITY, IN INTRACTABLE FOCAL EPILEPSY

K. Weaver ${ }^{2}$, Jeffrey G. Ojemann ${ }^{1,4}$, A. Poliakov ${ }^{4}$, N. Kleinhans ${ }^{2}$, G. Pauley $^{2}$, T. Grabowski ${ }^{2}$ and E. Novotny ${ }^{2,3}\left({ }^{1}\right.$ Neurological Surgery, University of Washington, Seattle, WA; ${ }^{2}$ Radiology - Integrative Brain Imaging Center, University of Washington, Seattle, WA; ${ }^{3}$ Neurology, Seattle Children's Hospital, Seattle, WA and

${ }^{4}$ Neurosurgery, Seattle Children's Hospital, Seattle, WA)

Rationale: Functional connectivity (fc) imaging identifies patterns of brain connectivity by correlating intrinsic, low-frequency fluctuations of BOLD signal during task-free, resting state fMRI. Regional homogeneity ( $\mathrm{ReHo}$ ) is a new fc approach that was developed to estimate the magnitude of connectivity within a local environment (i.e. the degree to which the time-course within a given voxel is associated with nearest neighbors). In this pilot study, we employed ReHo to examine the degree to which fc is altered within the local cortical environment immediately adjacent to and surrounding the seizure focus

Methods: Four pre-surgical patients with intractable, focal epilepsy (EP; age range 18-37, 2 females) and 4 matched controls (MC) underwent resting state fMRI scans (3T, TR/TE: $2 \mathrm{~s} / 21 \mathrm{~ms}$, volumes: 160). Standard pre-processing pipelines were employed to remove non-neuronal sources of variance including 1) motion realignment, 2) spatial smoothing 3) grand-mean intensity normalization, 4) linear drift and CSF signal removal and low-pass temporal filtering of frequencies below $0.01 \mathrm{~Hz}$ (cf Power et al., NeuroImage

59:2142,2011). Data were then passed through a Kendal's coefficient of concordance ReHo (KCC-ReHo) analysis, computing an average correlation coefficient across the time domain for each (center) voxel with its surrounding 27 neighboring voxels. Each 3D ReHo data set was then normalized by the global mean KCC-ReHo value within the brain mask. Finally, images were spatially registered and sub-divided into regions-of-interest (ROIs) as outlined by the MNI atlas (fig 1a). A mean KCC-ReHo value was calculated for each ROI and compared across groups using a paired-sample student's T test for three regions: 1) seizure focus, 2) an uninvolved region located in the occipital pole and 3) whole brain value. Seizure focus was confirmed from the postoperative resection following invasive monitoring.

Results: The Figure (a) shows 5 ROIs surrounding the seizure focus for one patient. The difference between KCC ReHo values between the patient and control are greatest in the ROI that houses the focus (basal temporal, *-p<.05). The KCC ReHo differed significantly between patients and controls for both the whole brain and the focus (Figure 1b; *-p<.05). For the seizure focus ROI, mean KCC-ReHo was lower for EP than MC individuals (EP: $2.47+/-0.29$; MC: $3.54+/-$ $0.27, \mathrm{p}<0004)$, and whole-brain normalized KCC-ReHo was also lower (EP: $3.83+/-0.29$; MC: $4.07+/-.25, \mathrm{p}=0.02$ ) but with no difference in the uninvolved (occipital lobe) region (EP: $3.73+/-.27$, MC: $4.07+/-.14, \mathrm{p}=0.24$ ).

Conclusions: These preliminary results suggest a disruption in local functional connectivity and potentially a desychronization of the regional low frequency, resting state fMRI signal at the seizure focus in focal epilepsy. Future work will explore the degree to which ReHo is associated with post-operative outcomes and its capacity to facilitate identification of the seizure foci in focal epilepsy.

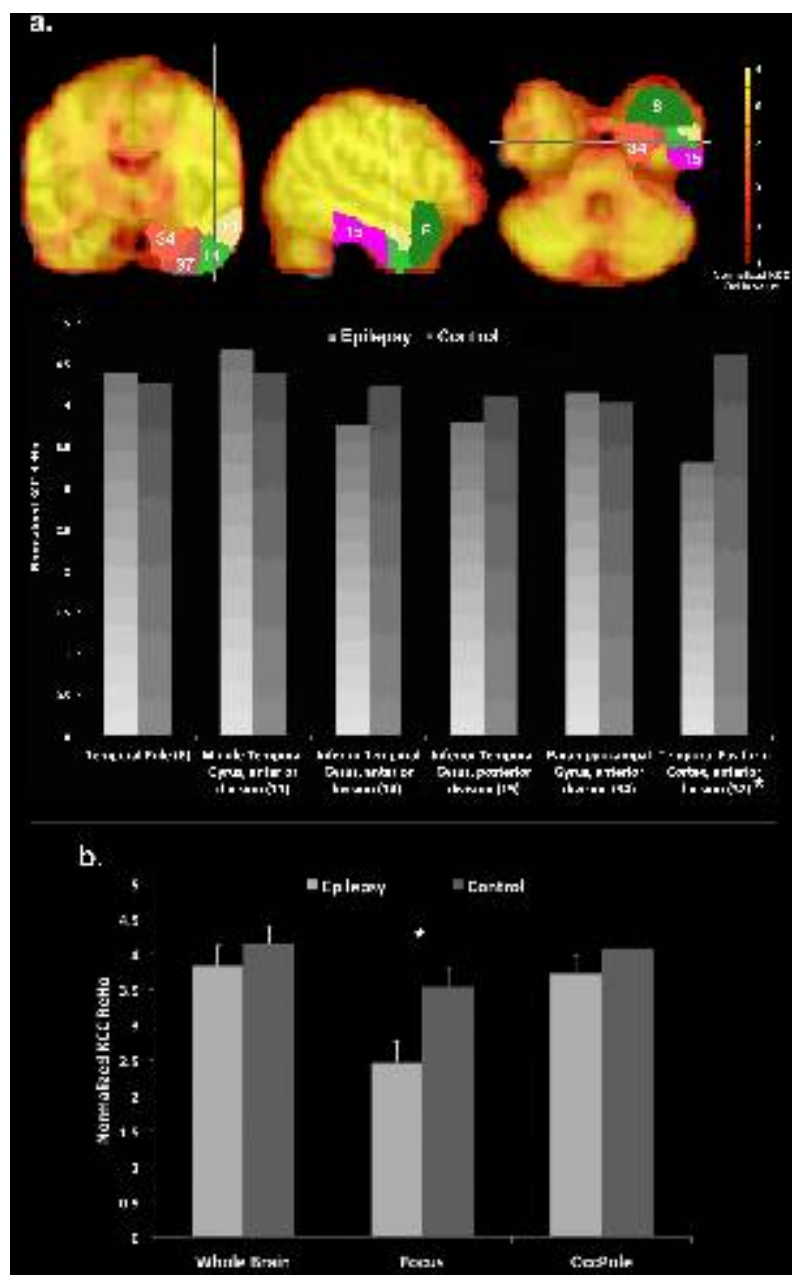

\subsection{7}

GRAPH THEORETICAL ANALYSIS OF SPIKE AND WAVE DISCHARGE IN PATIENTS WITH JME

Ki-Young Jung ${ }^{1}$, G. Lee ${ }^{1}$, C. Lee ${ }^{1}$, Y. Koo ${ }^{1}$ and Y. Cho ${ }^{2}\left({ }^{1}\right.$ Korea University College of Medicine, Seoul, Republic of Korea and ${ }^{2}$ Neurology, Keimyung University School of Medicine, Daegu, Republic of Korea)

Rationale: Generalized Spike and Wave Discharge (GSWD) is an important characteristic of juvenile myoclonic epilepsy (JME) which is a kind of idiopathic generalized epilepsy. Hence, investigating GSWD may lead to understand underlying pathophysiology of JME. To study characteristics of neuronal network during GSWD, graph theoretical analysis of connectivity network was conducted in present study.

Methods: 64-channel EEG data were acquired from 3 patients with JME. Representative epochs during spike free baseline period and GSWD of $1 \mathrm{~s}$ were selected. The coherence in each pairwise combination of two electrodes was calculated for each frequency band (i.e., delta, theta, alpha, beta, gamma, and ripple). Graphs were constructed from this coherence matrix by applying upper $95 \%$ threshold, and characterized by a characteristics path length and a clustering coefficient. The graph measures were compared between baseline and GSWD.

Results: During GSWD, coherence was increased over all frequency bands, and increasing in gamma and ripple band was noticeable, especially. Furthermore, spatial characteristic of connectivity shows that bilateral frontal region was highly involved. The clustering coefficient was increased during GSWD in the beta, gamma, and ripple bands, whereas the shortest path length showed only slight changes through all frequency bands. 
Conclusions: Our results showed the changes of neural synchronization during GSWD were more related to high frequency oscillations (HFO) than low band, which suggests HFO plays an important role for GSWD. The clustering coefficient is a measure of the local interconnectedness of the graph. Thus, increasing clustering coefficient of neural network in high frequency band during GSWD may imply that specific regions are highly involved in onset and propagation of GSWD, which is consistent with previous studies.

\subsection{8}

HIPPOCAMPAL FUNCTIONING IN CHILDREN AND ADULTS WITH EPILEPSY

Leigh Sepeta $^{1,2}$, L. Zimmaro ${ }^{1,2}$, M. M. Berl ${ }^{2,3}$, B. Xu ${ }^{1}$, A. D'Alfonso ${ }^{1}$, W. Theodore ${ }^{1}$ and W. D. Gaillard ${ }^{1,2}\left({ }^{1}\right.$ National Institute of Neurological Disorders \& Stroke, NIH, Bethesda, MD; ${ }^{2}$ Children's National Medical Center, Washington, DC and ${ }^{3}$ Georgetown University, Washington, DC)

Rationale: Memory impairments are a common comorbidity in adults and children with epilepsy; however, few studies have used functional magnetic resonance imaging (fMRI) to assess memory functioning in adults and children with epilepsy. Therefore, we sought to develop a paired association learning fMRI task to directly probe verbal memory and elicit hippocampal activation on an individual basis. We hypothesized that activation would be lateralized and would be related to task memory performance. Methods: We examined hippocampal activation during an EPI BOLD 3 T block design task of paired association learning with word pair stimuli. Six blocks were presented with each block consisting of three trials [learning, distraction (baseline), and recall]. Eleven typically developing controls (age range: 11-29 years) and 5 patients with focal epilepsy (age range: 12-52 years) have been piloted on the memory paradigm. Imaging processing and statistical analyses were conducted in SPM8. The region of interest (ROI) for bilateral hippocampi was based on the Anatomical Atlas Library in the Wake Forest PickAtlas. We calculated a laterality index (LI) for the hippocampal ROI using the LI Toolbox.

Results: On an individual basis, 91\% (10 of 11) of the TD controls and $100 \%$ ( 5 of 5 ) of the patients demonstrated activation in the hippocampus during the learning trial. During the recall block, $73 \%$ ( 8 of 11 ) of the TD controls and $80 \%$ (4 of 5) of patients demonstrated hippocampal activation (all analyses thresholded at $\mathrm{p}=0.05$, uncorrected). A group map ( $\mathrm{p}=\mathrm{FDR})$ for the controls rendered a cluster of 13 voxels in the hippocampus for the learning trial, and 292 voxels for the recall trial. The pattern of categorical distribution of laterality was left lateralized for adult controls, particularly during the learning block (adult controls: 7 left, 1 bilateral) and more bilateral for adult patients ( 2 bilateral). In the pediatric group, both controls and patients with epilepsy showed similar laterality patterns during the learning condition (pediatric controls: 2 left, 1 right; pediatric patients: 2 left, 1 right).

Conclusions: The preliminary results demonstrate that this verbal learning paradigm reliably activates the hippocampus on an individual basis. Further, adult controls showed primarily leftlateralized hippocampal activation during the learning block, as expected. We plan to investigate the effect of age, duration of epilepsy, and other clinical variables, as well as task performance on memory activation patterns.

\subsection{9}

USING MEG TO DEVELOP A PASSIVE MOTOR MAPPING PROTOCOL FOR PEDIATRIC EPILEPSY PATIENTS Mark H. McManis ${ }^{1}$, E. Darrow ${ }^{2}$, A. Hernandez ${ }^{1}$, S. Malik ${ }^{1}$, S. Perry ${ }^{1}$, A. Childers ${ }^{1}$, R. Ross ${ }^{1}$, E. Guevara ${ }^{1}$ and K. Mooneyham ${ }^{1}$ ( ${ }^{1}$ Neuroscience, Cook Children's Medical Center, Fort Worth, TX and ${ }^{2}$ Anesthesiology, Cook Children's Medical Center, Fort Worth, TX)
Rationale: Magnetoencephalography (MEG) has become a regular tool for functional mapping in cases of epilepsy requiring surgery. However, in cases involving patients who need sedation for the MEG procedures, functional mapping is limited by the patient's inability to perform the necessary tasks. For these patients, there is a need to develop tasks that activate the necessary brain regions associated with the function using passive tasks that don't require any voluntary activities on the part of the patient.

There is growing evidence that passive peripheral motor flexion induces activation of the motor cortex, thus making it possible passively map the motor cortex. As part of our clinical practice, we have developed a protocol for sedating children using intravenous (IV) dexmedetomidine sedation in conjunction with a passive flexion of the patient's index fingers.

Methods: Three children with intractable epilepsy were seen in the Cook Children's Medical Center MEG laboratory as part of their presurgical evaluation. The children ranged in age from $4-11$ years old. Sedation was required for the patients to complete the MEG scans. Motor mapping was done as part of a larger set of MEG scans for presurgical evaluation. Sedation: The patients were brought to the radiology preparation area and received the anxiolytic agent midazolam, orally or intravenously. Patients were then brought to the MEG scanner anteroom where they received inhalational nitrous oxide $70 \%$ and an IV catheter was placed. Patients were then given a bolus dose of dexmedetomidine $(1 \mathrm{mcg} / \mathrm{kg})$ over ten minutes. Following the bolus, patients received a continuous infusion of dexmedetomidine $(0.2-0.4 \mathrm{mcg} / \mathrm{kg} / \mathrm{hr})$ for the duration of the MEG scan. Motor activation: Motor cortex activation was achieved by having a technician manually manipulate the index fingers on the left and right hands of the patient, using a fiber optic device to indicate each movement to the recording computer. Approximately 125 individual movements were made on each index finger at a rate averaging 2 movements every 3 seconds. Data were filtered and averaged and the resulting evoked magnetic field waveform was analyzed using the equivalent current dipole model. The peak of the response was localized and plotted on an MRI image.

Results: For all cases, the sedation procedure was well tolerated and we were able to localize the motor cortex in both hemispheres. Analysis of the passive flexion responses shows that the peak in the motor cortex does not appear to be inhibited by dexmedetomidine, allowing for the mapping of the motor cortex in patients requiring sedation to complete their MEG scans.

Conclusions: We conclude that a sedation protocol using dexmedetomidine makes it possible to do functional mapping of the motor cortex in patients who require sedation to complete their MEG scans. The present case series shows that children undergoing evaluation for epilepsy surgery can receive the benefits of MEG functional mapping, when appropriately sedated. Additional discussion includes the use of sedation in a pediatric MEG center for other functional modalities.

\subsection{0}

LANGUAGE IMPAIRMENT AND LANGUAGE NETWORK REORGANIZATION IN NON-DOMINANT MESIAL TEMPORAL SCLEROSIS

J. P. Almeida ${ }^{1,2}$, Bettina Martins-Castro ${ }^{1,2}$, C. T. Khallil ${ }^{2}$, P. R. Arantes $^{2}$, C. S. Messas ${ }^{1}$, C. L. Jorge ${ }^{1}$, R. M. Valerio ${ }^{1}$, E. Amaro $\mathrm{Jr}^{2}$ and H. M. Castro L ${ }^{1}\left({ }^{1}\right.$ Department of Neurology, Faculdade de Medicina - Universidade de São Paulo, São Paulo, Brazil and ${ }^{2}$ Department of Radiology (LIM 44), Faculdade de Medicina Universidade de São Paulo, São Paulo, Brazil)

Rationale: INTRODUCTION: Functional MRI allows preoperative brain mapping in patients with refractory epilepsy secondary to mesial temporal sclerosis (MTS). Atypical language lateralization occurs more often in left MTS patients. Although language difficulties are well recognized in left MTS patients, language impairment in patients with right MTS is still poorly understood. We 
compared fMRI brain activation patterns on language tasks in patients with temporal lobe epilepsy with right and left HS with a visual confrontation naming paradigm.

Methods: METHODS: We studied 22 left (L) and 15 right (R) patients with epilepsy associated with right and left MTS, and 18 healthy controls (C). Patients and controls were 18 or older, had at least 8 years of education, and were all right handed. Patients and controls underwent a language battery test that included phonemic and semantic word list generation, confrontation naming (proper nouns, objects and verbs), responsive naming and comprehension tests. Patients underwent WADA testing that documented left hemisphere language dominance. Subjects underwent an fMRI study with a visual confrontation naming paradigm. Data were acquired using 3T MRI scanner with compressed GRE EPI BOLD images, (40ACPC oriented slices, $3.3 \mathrm{~mm}$ isometric voxels, TR4s, TE30ms FA90), and normalization to MNI152 space. Paradigms were block designed, each with a five minutes' duration, and stimuli had balanced randomization. Data analysis was performed with FSL, with a nonparametric statistical inference approach, and motion correction. A between groups ANOVA comparison (patients

leftXrightXcontrols) was performed with a significance level of $\mathrm{p}<0.05$.

Results: RESULTS: Patients and controls did not differ in terms of age (LMTS 34.0 +/- 8.9; RMTS 34.4 +/- 9.8; and C 32.5 +/-10.8yrs), and education (LMTS $10.7+/-2.0$; RMTS $10.8+/-2.0$; and C $11.7+/-$ $2.1 \mathrm{yrs})$. R and LMTS patients had comparable age at epilepsy onset (L $11.4+/-6.7$ vs. R 14.4 +/- 7.2yrs), epilepsy duration (L 23.0 +/-9.4 vs. R $20.1+/-1.4$ yrs) and history of an initial precipitating event (L50\% vs. R73\%). Both LMTS and RMTS performed worse than C in semantic fluency (fruit and supermarket items) and naming of proper nouns. RMTS patients performed worse than LMTS and controls in naming of verbs. On fMRI studies left and right MTS displayed increased BOLD effect in the right hemisphere on visual confrontation naming paradigms compared to $\mathrm{C}$. There was no difference of BOLD effect patterns when we compared right and left MTS patients.

Conclusions: CONCLUSIONS: Our findings indicate that language impairment and functional reorganization of language networks is not limited to dominant language hemisphere MTS, but it also occurs in non-dominant MTS. Rather than a lesion laterality effect, a more widespread disease effect appears to influence language function and reorganization in epilepsy associated with MTS. Factors related to disease process and, possibly treatment, other than lesion laterality or hemispheric dominance for language appears to play a role in these aspects of language dysfunction in these patient groups.

\subsection{1}

\section{ABNORMAL FUNCTIONAL NETWORKS IN TEMPORAL NEOCORTEX}

Luigi Maccotta, L. Eisenman and R. E. Hogan (Neurology, Washington University, St. Louis, MO)

Rationale: Mounting evidence continues to demonstrate abnormal functional connections in patients with temporal lobe epilepsy (TLE). Focus has largely been on the medial temporal region, as this is the typical seizure onset region and the most common site of pathology observed with the disease. However, there is increasing evidence that the medial temporal region forms a functional network with other brain regions, suggesting that other nodes in this network may be affected and function as disease markers for TLE. Here we used resting state BOLD fMRI to characterize the functional network of the medial temporal region with the inferior, medial and superior temporal neocortical regions and demonstrated abnormal connectivity that has lateralizing value with regards to seizure onset.

Methods: Thirty-two patients with TLE were consecutively enrolled in a resting-state BOLD fMRI study in a $3 \mathrm{~T}$ scanner. Each patient had video-EEG localization of seizures. Only patients with unilateral seizure onset were included. A group of age-, gender-, and handedness matched healthy subjects $(\mathrm{n}=32)$ served as controls. Regions of interest (ROIs) were defined a priori based on anatomical segmentation, and functioned as seed in a functional connectivity analysis using typical methods (e.g. Fox et al., J. Neurophysiol., 2009, 101:3270-83).

Results: Neocortical temporal regions exhibited significantly altered functional connectivity in TLE compared to healthy controls. Specifically there was a significant decrease in the strength of the functional connections between homologous temporal regions in the inferior, medial and superior temporal gyri, mirroring the decoupling between medial temporal regions also seen with the disease $(p<.01$, post-hoc paired t-test, after cluster-level correction for multiple comparisons). Moreover, in the hemisphere ipsilateral to seizure onset, neocortical temporal regions showed increased connectivity with the ipsilateral insula and regions immediately medial to it, such as the basal ganglia $(\mathrm{p}<.01$, post-hoc paired t-test, after cluster-level correction for multiple comparisons).

Conclusions: TLE is associated with strong functional network changes that go beyond the medial temporal region and involve the temporal neocortex. The observed increased coupling with the insula and basal ganglia exhibited by temporal neocortex ipsilateral to the seizure focus is of particular interest, and may be a correlate of a seizure propagation pathway or speculatively reflect an increased propensity of the network to generate and/or propagate seizures. The laterality it provides may have a clinical role in seizure localization and presurgical planning.

\subsection{2}

THE EFFECT OF CLINICAL FACTORS ON LISTENING AND READING LANGUAGE FMRI PARADIGMS IN PATIENTS WITH LEFT HEMISPHERE FOCAL EPILEPSY K. Miller ${ }^{2,1}$, M. M. Berl ${ }^{2,1}$, L. A. Zimmaro, ${ }^{2,1}$, W. H. Theodore ${ }^{2}$ and W. D. Gaillard ${ }^{2,1}\left({ }^{1}\right.$ Neuroscience, Children's National Medical Center, Washington, DC and ${ }^{2}$ Clinical Epilepsy Section, NIH, NIH, Bethesda, MD)

Rationale: Functional magnetic resonance imaging (fMRI) is an effective and noninvasive tool that can be used to determine localization of language networks in the frontal and temporal regions. We aimed to determine the differences in lateralization induced by reading and listening paradigms, and whether early seizure onset would cause a higher incidence of atypical reading dominance due to the late development of this skill.

Methods: 101 patients with left focal epilepsy (46 females, 55 males; mean age 23.2 yrs, range 8-50 years; mean age of seizure onset 11.6 yrs, range $0-46$ yrs; mean duration of epilepsy 11.6 yrs, range $0-47.5$ yrs; $80.2 \%$ right-handed, $15.8 \%$ left-handed, $4 \%$ ambidextrous) and 24 right handed normal controls (10 females, 14 males; mean age 29.2 yrs, range 21-56 yrs) performed reading and listening paradigms using $3 \mathrm{~T}$ BOLD fMRI. Language activation was characterized by region of interest (ROI) laterality indices: Wernicke's area (WA; BA 21, 22, 39), inferior frontal gyrus (IFG; BA 44, 45, 47), and middle frontal gyrus (MFG; BA 9, 46). Language dominance was deemed left or atypical ( $\mathrm{LI} \leq 0.20$ atypical).

Results: The reading task exhibited more left lateralization than the listening task for MFG $(\mathrm{t}=-2.99, \mathrm{p}=.003)$. There was a trend of weaker left lateralization in patients than controls $(p=0.061)$; More patients had atypical lateralization than controls. Patients and controls LIs differed from each other (main effect), but this is not due to different ROIs or tasks $(\mathrm{F}=6.59, \mathrm{p}=0.011)$. Differences in LI between patients and controls were significant in WA $(\mathrm{t}=2.38, \mathrm{p}=.021)$ in the reading task and WA $(\mathrm{t}=4.41, \mathrm{p}=0.00 ; \chi 2=5.19, \mathrm{p}=0.023)$ and $\mathrm{MFG}$ $(\mathrm{t}=3.25, \mathrm{p}=0.002 ; \chi 2=8.29, \mathrm{p}=0.004)$ in the listening task. To determine that epilepsy rather than pathological tissue caused the atypical LI, LIs of patients with normal MRIs were compared to controls. Patients had weaker left LI during the listening task in WA $(\mathrm{t}=3.46, \mathrm{p}=0.001)$. Both findings were confirmed when separating LIs into two categories (atypical and typical): listening in WA $(\chi 2=5.50$, 
$\mathrm{p}=0.019)$ and listening in MFG $(\chi 2=3.98, \mathrm{p}=0.046)$. Unlike the listening task, patients with early seizure onset $(<6 \mathrm{yrs})$ had a higher LIs than patients with late seizure onset during the reading task, significant for WA $\left(\mathrm{t}=-2.03, \mathrm{p}=.045 ; \chi^{2}=6.77, \mathrm{p}=0.009\right)$ and lower LI $(\mathrm{p}<0.045)$.

Conclusions: We found modest differences across language tasks. Patients had weaker left lateralization than controls, not dependent on MRI pathology, task, or region. Older age of seizure onset was associated lower lateralization in WA during the reading task and provides modest evidence for reorganization of reading networks associated with late development of reading skills.

\subsection{3 \\ RESTING STATE FUNCTIONAL CONNECTIVITY ABNORMALITIES EMERGING FROM THE LEFT AMYGDALA ARE RELATED TO ANXIETY AND DEPRESSION IN RIGHT BUT NOT LEFT MESIAL TEMPORAL LOBE EPILEPSY}

G. Doucet, Karol Osipowicz, A. Sharan, M. Sperling and J. Tracy (Thomas Jefferson University, Philadelphia, PA)

Rationale: Mood disorders are a common psychiatric comorbidity of mesial temporal lobe epilepsy (MTLE). This comorbidity has led to suggestions that certain forms of epilepsy and psychiatric symptoms share a common neurobiologic substrate involving the amygdala. It is well-known that the amygdala is composed of functionally distinct nuclei that contribute to emotion processing in specific ways through interactions with other brain structures. To date, no studies have investigated functional connectivity (FC) emerging from amygdala subdivisions in MTLE to determine if these networks differ from normal controls (NC), and are associated with psychiatric symptoms, and perhaps bear preferential relationships with anxiety or depression.

Methods: We collected resting-state in fMRI in 11 left and 11 right MTLE patients, and 19 age-matched NCs. We tested if distinct FC patterns can be detected across 3 amygdala subdivisions: laterobasal (LB), centromedial (CM), and superficial (SF). Using each of them as a seed, we compared the associated functional network between each group, using 2-sample t-tests. Then, we investigated if these FC abnormalities were correlated to the anxiety and depression levels in the patients. The 'Anxiety' (ANX), 'Anxiety-Related Disorders' (ARD) and 'Depression' (DEP) scales of the Personality Assessment Inventory were used to assess these symptoms.

Results: No significant differences emerged between the left MTLE and the NCs. In contrast, and regardless of the side of the seed, we observed increased FC between each seed and contralateral regions in the right MTLE group, compared with NCs. The NCs showed increased FC in the hemisphere ipsilateral to the seed compared to the RMTLE group. Based on these results, we revealed 3 significant relations with psychiatric symptoms in the RMTLE group. We found that higher level of ARD was associated with increased FC between the left $\mathrm{CM}$ amygdala and a region of the left cerebellum ( $\mathrm{p}=0.008$, Spearman $r=0.86)$. In contrast, higher levels of both DEP $(p=0.001)$ and ANX $(p=0.002)$ were associated with reduced FC between the left LB amygdala and a right inferior temporal region (Fig. 1). Conclusions: Overall, these results indicate that MTLE alters FC in areas outside the epileptogenic temporal lobe, in ways that correlate with key psychiatric symptoms common in these patients. We highlight that right MTLE differentially affects the FC emerging from each amygdala's subdivision, indicating these distinct FC patterns mediate different emotional networks. Our data show that seizure pathology, and its effect on FC, impacts psychiatric symptoms in important ways that vary with the pathologic hemisphere. More generally, pathology in the emotion-dominant right hemisphere appears more closely related for the expression of emotion networks. To our knowledge, this is the first description of FC alterations in MTLE involving a specific limbic structure that have relevance to the emotional problems of these patients.

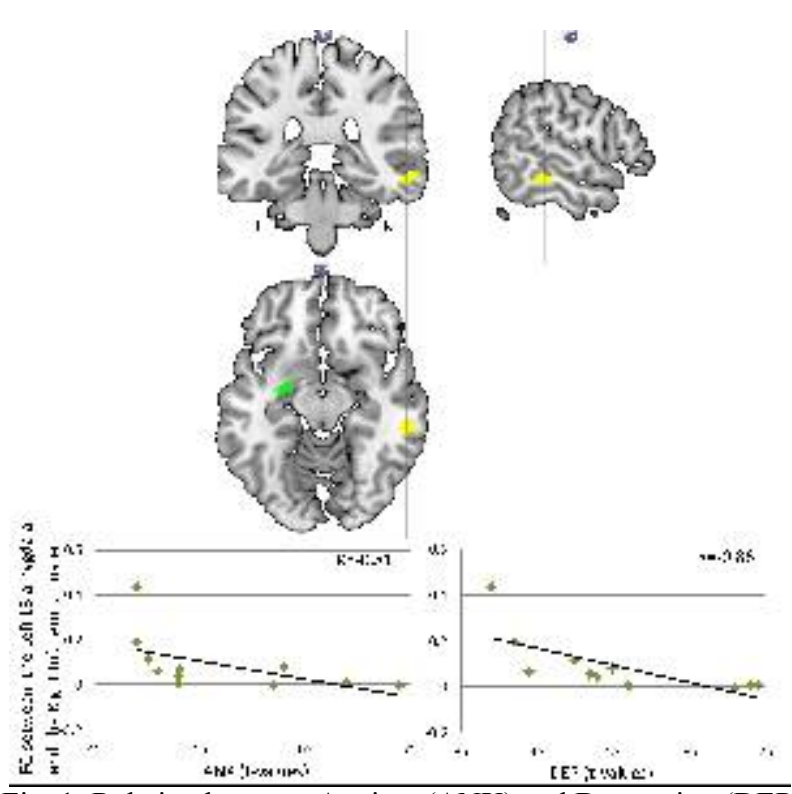

Fig. 1: Relation between Anxiety (ANX) and Depression (DEP) levels in right MTLE patients and their abnormal FC between the left laterobasal (LB) amygdala and a right inferior temporal region.

\subsection{4 \\ SPONTANEOUS BLOOD OXYGEN LEVEL DEPENDENT SIGNAL (BOLD) DIFFERENCES IN TEMPORAL LOBE (TLE) AND IDIOPATHIC GENERALIZED EPILEPSY (IGE) Jennifer L. Robinson ${ }^{1,2}$, R. Rister ${ }^{1}$, B. Bohannon ${ }^{1}$, A. Clark ${ }^{1}$, D. Barron $^{3}$, P. Fox ${ }^{3}$ and B. Kirmani ${ }^{1,2}{ }^{1}$ Scott \& White Memorial Hospital, Temple, TX; ${ }^{2}$ Neurosurgery, Texas A\&M Health Science Center College of Medicine, Temple, TX and ${ }^{3}$ Research Imaging Institute, University of Texas Health Science Center at San Antonio, San Antonio, TX)}

Rationale: A strong driving force in epilepsy research is the desire to delineate differences in spontaneous BOLD signal as measured by resting state fMRI (rsfMRI). Understanding these differences may lead to the establishment of a biomarker for disease severity, a predictor of treatment response, and/or could facilitate new drug developments. To date, multiple rsfMRI studies have been conducted. However, no study to our knowledge has examined different epileptic groups using a voxel-to-voxel rsfMRI connectivity analysis. This approach may be more robust than region-of-interest motivated analyses given the evidence for extratemporal involvement.

Methods: Participants were recruited from the Scott \& White Healthcare Epilepsy Center. 14 healthy controls (HCs), 12 TLE patients (7 LTLE/5 RTLE, the latter were flipped left-right prior to processing to be consistent with LTLEs), and 8 IGE patients underwent a 5-minute rsfMRI study (TR/TE: 2500/30ms; flip angle $90^{\circ}$, slice thickness $3 \mathrm{~mm}, 38$ axial slices), on a Siemen's $3 \mathrm{~T}$ Tim Trio. A high-resolution 3D volume was also collected for anatomical considerations, and to register functional data. RsfMRI data were first preprocessed in SPM8 using standard steps including slice-time correction, realignment, reslicing, normalization, and smoothing. In preparation for functional connectivity analyses, physiological and other potentially confounding sources of noise were removed using the CompCor method. This step included regressing out the BOLD signal contributions from white matter and cerebrospinal fluid masks as well as head motion parameters. A temporal band pass filter of $0.01 \mathrm{~Hz}$ to $0.1 \mathrm{~Hz}$ was applied to the data. Voxel-to-voxel intrinsic global connectivity analyses were performed.

Results: We found that HCs had greater intrinsic connectivity (voxel threshold $\mathrm{p}<0.001$ uncorrected; extent threshold $\mathrm{p}<0.05$ FDRcorrected) compared to TLE in 4 distinct clusters encompassing the 
left inferior and middle temporal gyri, bilateral somatosensory association cortex, bilateral posterior cingulate cortex, and bilateral angular gyrus. Compared to IGE, HCs had greater connectivity in the right inferior and middle temporal gyri, and the right fusiform gyrus. TLEs had greater connectivity (voxel $p<0.01$ uncorrected; extent threshold $\mathrm{p}<0.05$ FDR-corrected) throughout the visual cortex. TLEs had greater connectivity to the right premotor and primary motor cortices, as well as the right dorsolateral prefrontal cortex compared to the IGEs. IGEs had greater connectivity (voxel $\mathrm{p}<0.01$ uncorrected; extent threshold $\mathrm{p}<0.05$ FDR-corrected) in the left dorsolateral prefrontal cortex, premotor cortex, and anterior cingulate compared to TLEs and evidence of greater connectivity throughout the visual cortices compared to HCs.

Conclusions: These results suggest that whole-brain rsfMRI connectivity analyses may yield important insights into the neuropathophysiological mechanisms of epilepsy. Our data suggest that extratemporal differences may be present in both forms of epilepsy.

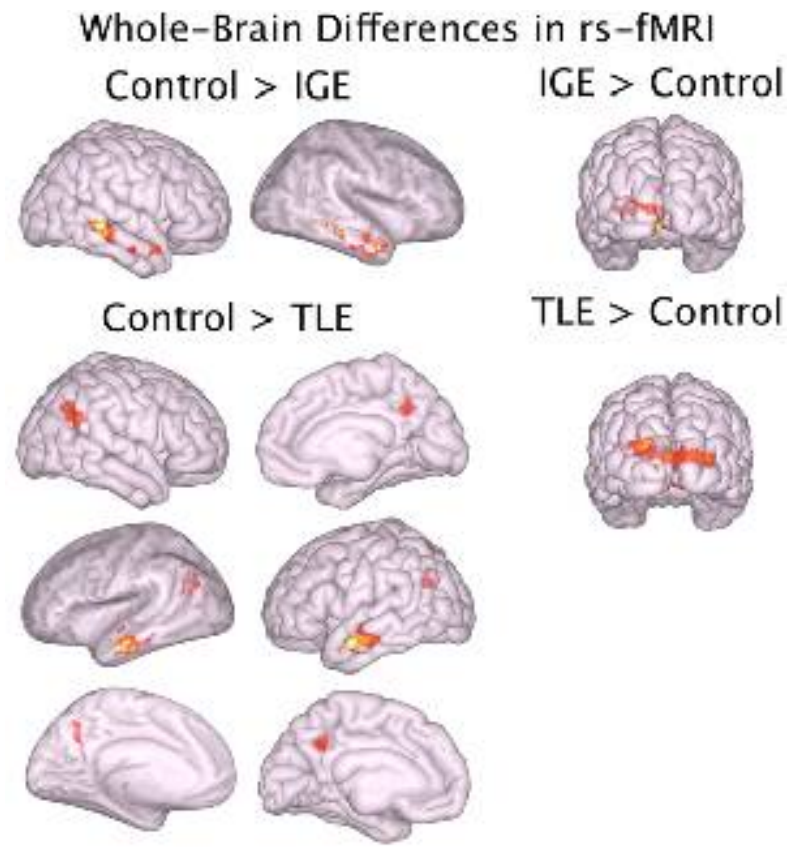

\subsection{5}

\section{CEREBRAL HEMISPHERECTOMY: THE EFFECTS OF REPEAT PULSE OF INTENSIVE THERAPY}

Stella de Bode (UCLA, Santa Monica, CA)

Rationale: The effects of continuous therapy in individuals with chronic disability is unknown. In case of cerebral hemispherectomy there is no prospect of recruiting additional areas in the affected hemisphere and contralateral corticospinal tract is not available. This limits recovery to what the remaining ipsilateral tract can do together with the mechanisms of spinal cord that are poorly understood. We evaluated the effects of intensive therapy on motor outcomes and cortical representations in 20 individuals after right and left hemispherectomy. Age-appropriate gait, balance and walking speed scores were calculated and varied between 65 and $85 \%$ of respective scores for neurologically-intact controls. Brain imaging showed that all participants had partial overlap between M1S1 of both feet that increased following therapy. Six years later a subset of the same group has repeated therapy to investigate the resiliency of cortical remodeling noted in therapy round 1 .

Methods: Twenty individuals post cerebral hemispherectomy (right resection, $\mathrm{n}=8$, age range 6-25 years) underwent intensive Body Weight Supported therapy to improve gait, balance and walking speed. Each participant had $30 \mathrm{hrs}$ of individual therapy that included treadmill and overground training with brain imaging performed before and after therapy. The subset of this cohort underwent the repeat therapy pulse 6 years later to investigate both motor development and resiliency of cortical remodeling we documented during the first round of intervention.

Results: Age-appropriate normalized scores were calculated and showed that all participants either remained on the same functional level or showed motor regression. Participants presented with significant pain issues related to uneven gait. The improvements following therapy Round 2 were much more modest than what we saw during Round 1. Motor maps seen pre-therapy showed very little overlap between affected and non-affected feet similar to what we saw during first session. In the same vein, following intervention there was more overlap between the maps and stronger activations. Conclusions: During 6 years between therapy pulses and with no physical therapy offered to our participants they regressed in their motor functional presentation. Similar to individuals with chronic disabilities our participants developed pain and emotional disturbances associated with motor activity necessary for everyday functioning. Motor representations did not show resiliency and "migrated" into their old places. We were able to see improvements and cortical remodeling following repeat therapy pulse, but results were less pronounced than what we documented the first time. We suggest that repeat therapy pulses are necessary to arrest the loss of therapy-induced gains and cortical remodeling associated with it.
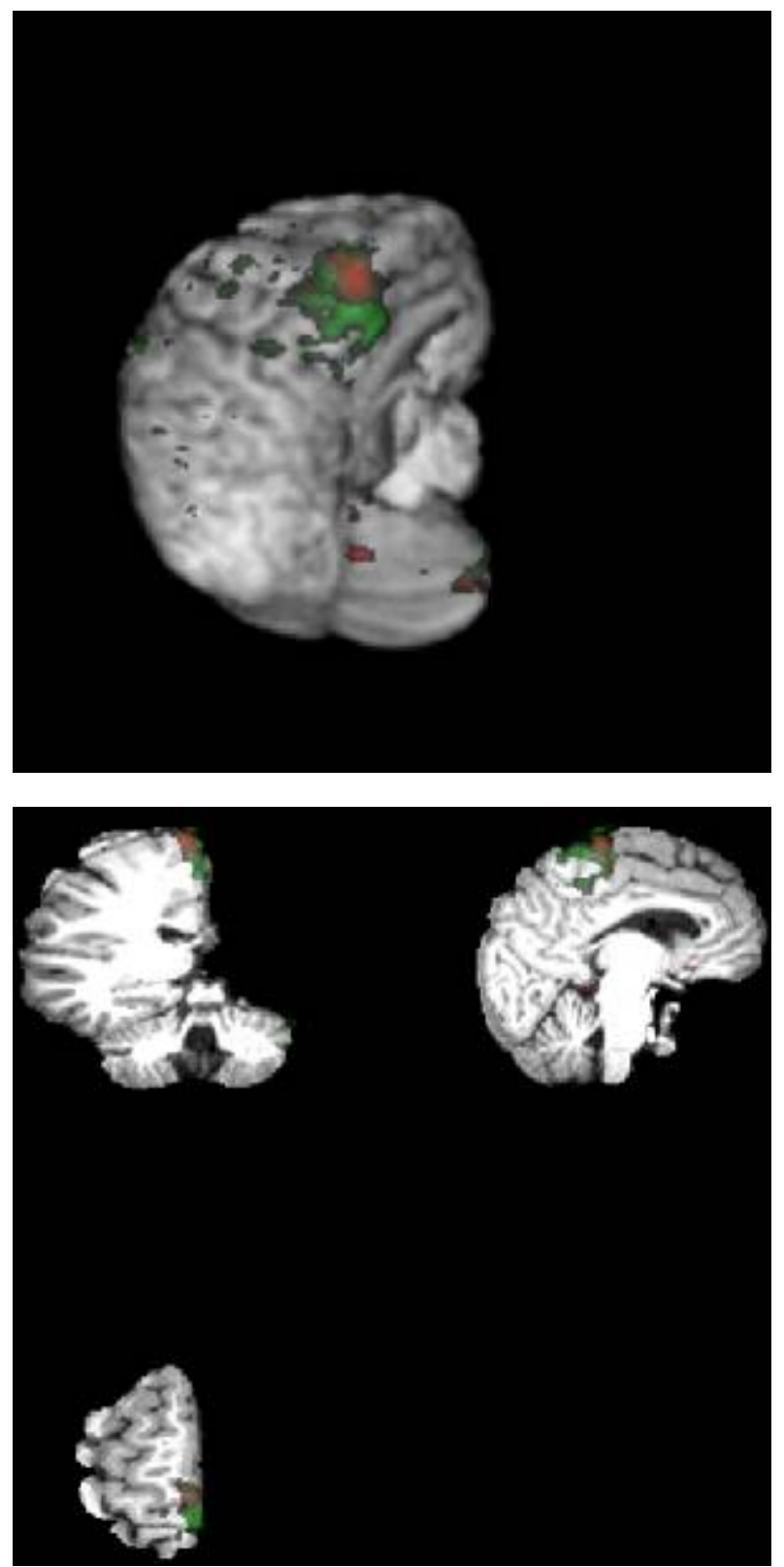


\section{Neuro Imaging: Other Emerging Techniques}

2.176

FEASIBILITY OF AN INTRACRANIAL EEG-FMRI PROTOCOL AT 3T: RISK ASSESSMENT Ismael Gaxiola $^{1,5}$, C. A. Beers ${ }^{2,5}$, D. J. Pittman ${ }^{2,5}$, B. Goodyear ${ }^{3,5}$ and P. Federico ${ }^{2,4}\left({ }^{1}\right.$ Electrical and Computer Engineering, University of Calgary, Calgary, AB, Canada; ${ }^{2}$ Clinical Neurosciences, University of Calgary, Calgary, AB, Canada; ${ }^{3}$ Radiology, University of Calgary, Calgary, AB, Canada; ${ }^{4}$ Hothckiss Brain Institute, Calgary, AB, Canada and ${ }^{5}$ Seaman Family MR Research Centre, Calgary, AB, Canada)

Rationale: Simultaneous acquisition of intracranial EEG (iEEG) and functional MRI (fMRI) data could provide the temporal and spatial resolution necessary to reveal mechanisms underlying seizure generation. However, the introduction of iEEG electrodes in the MR environment has inherent risk and data quality implications that require consideration prior to clinical use. We aimed to assess three health risks associated with the presence of iEEG electrodes during MRI scanning using a 3T scanner: RF-induced heating, gradient switching-induced voltage on the electrode circuit, and magnetic field-induced torque on the electrodes. Additionally, we evaluated the feasibility of running a continuous iEEG-fMRI scanning protocol.

Methods: Three head phantoms were constructed to mimic shape, size and conductivity of the human head. The first phantom contained a depth electrode, the second a grid electrode and the third a combination of depth, grid and strip electrodes that are commonly used for intracranial monitoring (Ad-Tech) in our institution. A body phantom was designed to model the human torso. MR scans were performed using a GE Discovery MR750 3T scanner. Temperature data were collected from MR sequences typically performed during an EEG-fMRI experiment using a fiber-optic thermometry system (Fiso Technologies), including alternative anatomical sequences. Voltage data were collected using an MR-compatible EEG system (Compumedics Neuroscan). EEG cable locations and electrode orientations were varied to mimic typical intracranial electrode implantations in patients. Torque induced on electrodes was assessed using the Deflection Angle Method developed by the American Society for Testing Materials. A 70-min iEEG-fMRI protocol including anatomical and functional scans was employed to assess heat accumulation.

Results: No significant temperature increases were detected $(<1.0 \mathrm{C})$ when using the individual MR sequences common to an iEEG-fMRI protocol (Table 1). Additionally, repeated measures of a continuous 70 minute iEEG-fMRI protocol did not induce temperature changes $>$ 1.0 C (Fig. 1). These results were consistent across different cable locations and electrode orientations. MR sequences with high Specific Absorption Rate (SAR), such as FLAIR and Fast-Spin Echo (FSE), generated significant temperature increase $(>1.5 \mathrm{C}$ and $>4.9$ C). Harmful voltages that could induce neuronal stimulation $(<10$ $\mathrm{KHz}$ and $>100 \mathrm{mV}$ ) were not observed during any test condition. Deflections induced on electrodes were not significant ( $<1$ degree). Conclusions: Simultaneous iEEG-fMRI poses low risk and is feasible at 3T for the conditions reported. High SAR sequences (typically FSE sequences) can potentially result in clinically significant tissue heating and should be avoided. The settings described in our study produced no inadvertent induced voltages, which could result in neuronal stimulation. Simultaneous iEEG-fMRI at $3 \mathrm{~T}$ is a promising tool for epilepsy research that may help elucidate the mechanisms of seizure generation and ultimately improve patient outcomes.

Maximum temperature changes for different pulse sequences run in isolation for a grid and depth electrode in standard orientation.

\begin{tabular}{|c|c|c|c|c|c|}
\hline \multirow{2}{*}{ Sequence } & \multirow{2}{*}{ Estimated SAR (W/Kg) Avg|Max } & \multicolumn{2}{|c|}{ Depth Max $\Delta \mathrm{T}^{\prime}\left({ }^{\circ} \mathrm{C}\right)$} & \multicolumn{2}{|c|}{ Grid Max $\Delta \mathrm{T}\left({ }^{\circ} \mathrm{C}\right)$} \\
\hline & & $\mathrm{EI}$ & $\mathrm{E} 4$ & E1 & E14 \\
\hline \multicolumn{6}{|c|}{ Typical F.FG-fMRI sequences } \\
\hline 3P. & $0.51 \mid 1.02$ & 0.3 & 0.1 & 0.3 & 0.2 \\
\hline 3D & 0.2410 .48 & 0.4 & 0.2 & 0.5 & 0.3 \\
\hline $2 \mathrm{D}$ & $0.17 \mid 0.35$ & 0.2 & 0.1 & 0.2 & 0.1 \\
\hline HOS & $0.19 \mid 0.39$ & 0.1 & 0.1 & 0.2 & 0.1 \\
\hline IMRI & 0.2010 .40 & 0.2 & 0.1 & 0.3 & 0.3 \\
\hline \multicolumn{6}{|c|}{ Other sequences } \\
\hline ASSET & 1.9013 .79 & 0.2 & 0.1 & 0.5 & 0.3 \\
\hline DWT & $0.34 \mid 0.67$ & 0.6 & 0.3 & 0.5 & 0.3 \\
\hline ГL_AIR & 1.0912 .17 & 1.9 & 0.6 & 1.5 & 0.9 \\
\hline FSE & 2.9715 .94 & 4.9 & 2.4 & 4.9 & 4.3 \\
\hline
\end{tabular}

Abbreviations: 2D - 2D anatomical, 3D - 3D anatomical, 3PL - 3 plane localizer, ASSET - coil sensitive calibration scan for parallel imaging, DWI - diffusion weighted imaging, FLAIR - fluid attenuated inversion recovery, fMRI - functional MRI, HOS - high order shim.

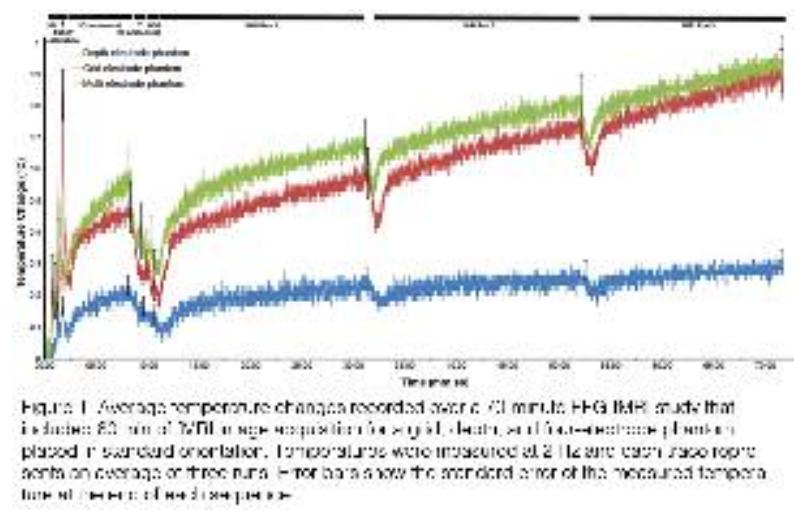

2.177

THALAMIC MEDIAL DORSAL NUCLEUS ATROPHY IN MEDIAL TEMPORAL LOBE EPILEPSY: A VBM METAANALYSIS

Daniel S. Barron ${ }^{1}$, P. M. Fox ${ }^{1}$, A. R. Laird ${ }^{1}$, J. L. Robinson ${ }^{2}$ and P. T. Fox ${ }^{1}\left({ }^{1}\right.$ UTHSCSA, San Antonio, TX and ${ }^{2}$ Scott \& White, Temple, TX)

Rationale: Medial temporal lobe epilepsy (MTLE) is a common form of adult focal epilepsy. Hippocampal sclerosis (HS) is an important diagnostic marker for MTLE and, when resected, is associated with good surgical outcome. Extra-hippocampal brain damage is often more subtle and, when detected, is associated with poorer outcome. Voxel-based morphometry (VBM) is a group-wise neuroimaging analysis tool capable of detecting subtle brain pathology and has often been used in MTLE to survey extrahippocampal pathology. Prior MTLE VBM studies have reported pathology in a large number of inconsistently distributed areas and could not derive a focused consensus on where extra-hippocampal pathology is most common, limiting VBM's impact on MTLE research and treatment.

Recently refined coordinate-based meta-analysis methods offer unprecedented analytic rigor and could identify which regions and pathways demonstrate the most consistent MTLE pathology. To this end, we applied anatomic likelihood estimation (ALE) to 22 wholebrain VBM experiments representing 562 MTLE patients with unilateral HS. We then tested whether the results of this ALE analysis were functional and anatomic constituents of the epileptogenic network using meta-analytic connectivity modeling and preliminary single-subject diffusion tractography. 
Methods: We adopted standard coordinate-based meta-analysis, MTLE-HS specific, and data non-redundancy inclusion filters in our paper selection. Paper retrieval included an internet search and a bibliographic search of relevant retrieved papers. Using the latestreleased Eickhoff (2012) ALE method, we meta-analyzed 300 VBM foci in 3 groups: left MTLE, right MTLE, and rectified (left MTLE rectified to right + right MTLE). We created a meta-analytic connectivity model (MACM) according to the Robinson (2010) method and used ALE-reported, hand-painted tissue labels to create a diffusion tractography map in one healthy subject within 3D Slicer. Results: The three VBM ALE analyses yielded similar Results: spatial convergence across studies exists in the epileptogenic hippocampus and the bilateral thalamus (voxel-level FWE $\mathrm{p}>0.05$ ). Within the thalamus, the medial dorsal nucleus (MDN) represented the greatest consensus from each group.

Confirmatory analyses of hippocampal-MDN connectivity confirmed functional (MACM) and anatomical (diffusion tractography) connectivity. Importantly, anatomical connectivity was validated in a single subject study.

Conclusions: In unilateral MTLE-HS, we found consistent gray matter pathology only within the epileptogenic hippocampus and bilateral thalami. Our ALE meta-analysis yields significant dividends from VBM studies: the MDN thalamus represented the most significant thalamic pathology, or $76 \%$ of included studies - nearly equal to the epileptogenic hippocampus. We demonstrated hippocampo-MDN functional and anatomic connectivity, aligning with other studies that report MDN pathology as part of an MTLE epileptogenic network. Our observation of hippocampo-MDN anatomic connectivity in a single subject further suggests the thalamus as a clinically useful indicator in MTLE.
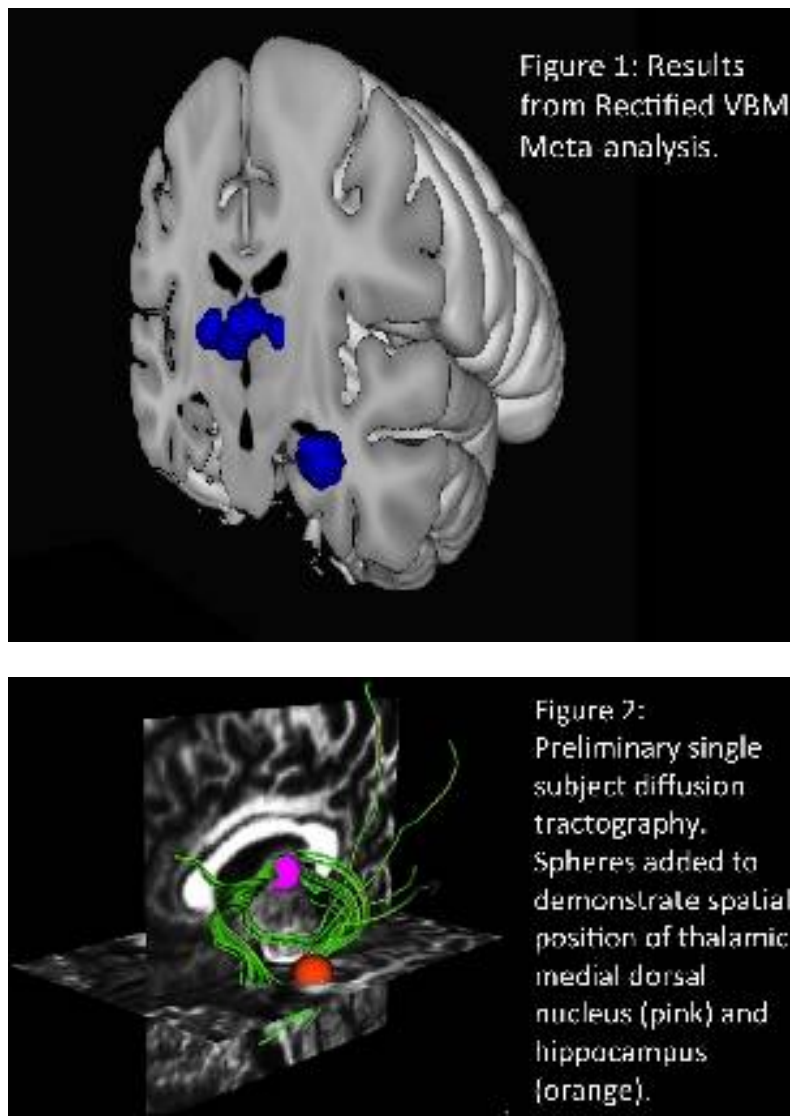

2.178

STRUCTURAL AND FUNCTIONAL CONNECTIVITY OF HIPPOCAMPAL NETWORKS IN TEMPORAL LOBE EPILEPSY

Nuri Erkut Kucukboyaci ${ }^{1,3}$, N. Kemmotsu ${ }^{2,3}$, H. M. Girard ${ }^{3}$, C. Cheng $^{3}$, E. Tecoma ${ }^{4}$, V. Iragui ${ }^{4}$ and C. R. McDonald ${ }^{2,3}\left({ }^{1}\right.$ JDP in Clinical Psychology, SDSU/UCSD, San Diego, CA; ${ }^{2}$ Psychiatry, UCSD, San Diego, CA; ${ }^{3}$ Multimodal Imaging Laboratory, UCSD, San Diego, CA and ${ }^{4}$ Neurosciences, UCSD, San Diego, CA)

Rationale: Increasing evidence has shown that structural damage in temporal lobe epilepsy (TLE) is not limited to the temporal lobes, but is often distributed across multiple brain networks. The functional organization of these networks can be observed in vivo by functional connectivity MRI (fcMRI) analysis. FcMRI evidence regarding the direction and the extent of changes in the hippocampal networks has been equivocal. There is also limited information available on the colocalization of structural and functional connectivity impairments in epilepsy despite the evidence that combining these two modalities can enrich our understanding of canonical brain networks.

Methods: T1- and diffusion-weighted (DW) MRI data were collected on 15 healthy controls and 14 patients with refractory TLE. Diffusion tensor imaging (DTI) data were used to obtain information on fiber tract structure using a probabilistic DTI atlas. Functional connectivity data were derived from a task-regressed event-related semantic decision task. Bilateral hippocampal seed regions were used to generate whole-brain correlation maps, which were then subjected to group-level statistical analyses.

Results: Compared to healthy controls, we observed a reduced correlation between bilateral hippocampi and the posterior cingulate region in patients with TLE ( $p<0.0005$; cluster size $\geq 5$ voxels). The reduction extended towards the precuneus and was more extensive for the left-hippocampal network. Patients with TLE also exhibited an increased correlation between the anterior superior frontal gyrus and right hippocampus. A group difference trend in structural connectivity was also observed in the right hemisphere for the superior longitudinal fasciculus (SLF), inferior longitudinal fasciculus (ILF), and the cingulum fiber fractional anisotropy (FA; all p-values $<.05)$. The former appeared to be driven by control vs. left TLE FA differences while the latter two were driven by control vs. right TLE FA differences. We also observed an association between the strength of the functional connectivity in the posterior cingulate and the FA of the cingulum.

Conclusions: Our fcMRI data reveal bilateral reductions in connectivity between the hippocampus and the posterior cingulate region, known to be part of the default mode network (DMN). These reductions in functional connectivity co-localize with reductions in structural integrity of the SLF, ILF and the cingulum. While these results agree with previous findings of reduced DMN functional connectivity in patients with TLE, hippocampal specificity of the findings and the directionality of the relationship between colocalizing structural and functional connectivity changes remains uncertain. 


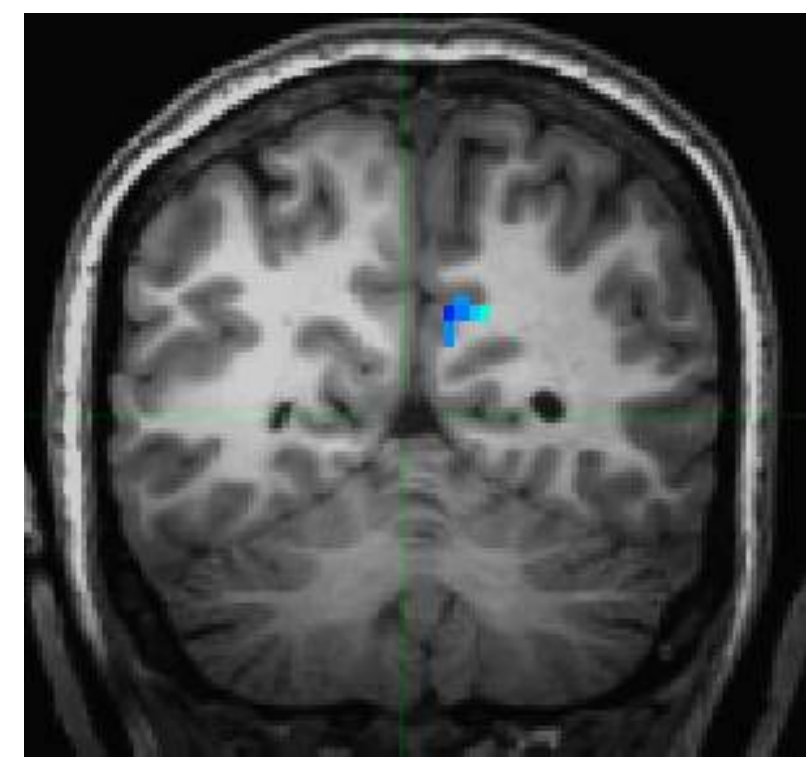

Image 1: Our task-regressed fcMRI analysis showed a significantly reduced correlation between left hemisphere hippocampus and the left posterior cingulate area in patients with TLE $(\mathrm{p}<0.0005$; cluster size $\geq 5$ voxels)

\subsection{9}

THREE-DIMENSIONAL REAL-TIME ELECTROPHYSIOLOGICAL FUNCTIONAL MAPPING OF ELOQUENT CORTEX

Anthony L. Ritaccio ${ }^{1}$, P. Brunner ${ }^{1,2}$, A. Gunduz ${ }^{3}$, M. Adamo ${ }^{1}$, D. Gupta $^{1}$ and G. Schalk ${ }^{2,1}$ ( ${ }^{1}$ Neurology, Albany Medical Center, Albany, NY; ${ }^{2}$ Wadsworth Center, NYS Department of Health, Albany, NY and ${ }^{3}$ Biomedical Engineering, University of Florida, Gainesville, FL)

Rationale: Functional mapping of eloquent cortex is typically performed prior to resective brain surgery to minimize post-surgical morbidity. Electrical cortical stimulation (ECS) and functional magnetic resonance imaging (fMRI) are the major two clinical techniques to perform this mapping. ECS is based on a lesional effect and provides two-dimensional maps, while fMRI is based on a metabolic effect and provides three-dimensional maps of eloquent cortex. While ECS and fMRI are widely accepted to provide clinical value, they have some or all of the following three major limitations: (1) they depend on experts and/or post-hoc analysis; (2) they are based on an indirect measure; (3) they produce results in anatomical space (fMRI) or electrode space (ECS), but not both at the same time. We had previously described (Brunner et al., 2009) the development and validation of a new functional mapping technique (SIGFRIED) that uses task-related electrocorticographic (ECoG) signals in the gamma (i.e., $70-110 \mathrm{~Hz}$ ) band to provide a map of eloquent cortex in real time. In the present study, we are extending the capacities of this method to three-dimensional mapping.

Methods: We evaluated the clinical feasibility of this procedure in a study with 5 human epilepsy patients that underwent surgical implantation of 64-128 subdural electrocorticographic electrodes. For each patient, we co-registered the cortical anatomy defined by a preoperative MRI with the electrode locations defined by a postoperative CT, which resulted in a three-dimensional surface cortical model and the locations of the electrodes. We then visualized the output of our SIGFRIED technique during language- and motor-tasks directly on the three-dimensional cortical model in real time. Specifically, the patients were asked to perform an average of five 10 -sec trials of cued motor (e.g., manipulating a rubik's cube) and receptive language tasks (e.g., listening to voices and tones). While the subjects performed these tasks, our SIGFRIED procedure provided two- and three-dimensional maps of cortical areas whose
ECoG activity changed with the task. We then validated our results against the results derived using ECS.

Results: Validation showed high concordance with ECS, it also revealed two shortcomings of our procedure: (1) task-relevant eloquent cortex may not be essential to function, e.g., in the case of bi-lateral language function; (2) ictal activity may obscure taskrelevant changes.

Conclusions: Despite the discussed shortcomings, the extension of our established SIGFRIED technique to three-dimensional real-time mapping further increases the potential value of this new mapping technique in invasive surgery protocols.

\subsection{0 \\ POST-ICTAL EEG SUPPRESSION AND 5HT-1A RECEPTOR BINDING IN PATIENTS WITH MEDICALLY-REFRACTORY LOCALIZATION RELATED EPILEPSY}

John M. Schreiber ${ }^{1}$, S. Inati ${ }^{2}$, I. Dustin ${ }^{1}$, S. Sato ${ }^{2}$, P. Reeves-Tyer ${ }^{1}$ and W. H. Theodore ${ }^{1}\left({ }^{1}\right.$ Clinical Epilepsy Section, NINDS, National Institutes of Health, Bethesda, MD and ${ }^{2}$ EEG section, NINDS, National Institutes of Health, Bethesda, MD)

Rationale: Sudden unexpected death in epilepsy (SUDEP) is an important cause of mortality in epilepsy, but little is known about its pathophysiology. A recent case-control study (Lhatoo, et al. 2010) prompted by several case reports found SUDEP was associated with post-ictal generalized EEG suppression (PGES). Evidence from animal models and human subjects prescribed SSRIs indicate that serotonin may play a critical role in respiratory function during the post-ictal period. We aimed to explore the relationship between serotonin and PGES, to evaluate occurrence of PGES in our patient population, and to document any associated findings.

Methods: PET was performed with 18F-FCWAY for 5HT1A receptor binding estimation and $18 \mathrm{~F}-\mathrm{FDG}$ for glucose metabolism (CMRglc). After partial volume correction, 5HT1A receptor binding and CMRglc were measured in regions drawn on co-registered MRI. $18 \mathrm{~F}-\mathrm{FCWAY}$ PET binding was determined in various regions of interest (ROI) for each patient and asymmetry indices (AI) were calculated for ROIs in the cerebral hemispheres. Patients with medically refractory localization-related epilepsy who had at least one secondarily generalized convulsive seizure recorded on video EEG from 2002-2010 were identified from the 18F-FCWAY PET database. The first such seizure of each patient was reviewed by an author blinded to PET results (JMS) and analyzed for seizure duration (focal/ generalized), PGES duration, return of spontaneous movement, EKG abnormality, and apnea. PGES was defined as generalized absence of electroencephalographic activity $>10 \mu \mathrm{V}$ in amplitude, allowing for muscle, movement, breathing, and electrode artifacts. We analyzed $18 \mathrm{~F}-\mathrm{FCWAY}$ PET binding in the median raphe nucleus and the ROI corresponding to a patient's seizure focus, then compared values in groups with and without PGES using Student's t-test.

Results: Twelve patients who fulfilled the above criteria were identified. $42 \%$ were female with mean age 34 years at time of video EEG. Five had right temporal lobe epilepsy, six had left temporal lobe epilepsy, and one had left frontal lobe epilepsy. Seven (58\%) had PGES with mean duration 58 seconds. Patient demographics were similar between groups with and without PGES. PGES was not associated with seizure duration (total $[\mathrm{p}=0.46]$ and generalized $[\mathrm{p}=0.44])$ or latency to return of spontaneous movement $(\mathrm{p}=0.85)$. No patients had apnea or EKG abnormalities other than sinus tachycardia in the post-ictal period. There were no group differences in $18 \mathrm{~F}$ FCWAY binding in the median raphe nucleus $(\mathrm{p}=0.5)$ and the $\mathrm{AI}$ in $\mathrm{ROI}(\mathrm{s})$ corresponding to a patient's seizure focus $(\mathrm{p}=0.77)$.

Conclusions: PGES is common in individuals with medically refractory localization-related epilepsy. It is not known whether a link between PGES and dysfunctional serotonergic neurotransmission exists, or if they represent two separate mechanisms that may contribute to SUDEP. Our study was not sufficiently powered to 
detect differences in 18F-FCWAY binding. Further study into SUDEP pathophysiology is crucial to guide prevention strategies.

\subsection{1}

\section{ATYPICAL LANGUAGE REPRESENTATION IN EPILEPSY} PATIENTS

Dawn S. Eliashiv ${ }^{1,4}$, j. Chung ${ }^{1,4}$, S. Otis ${ }^{2}$ and N. Gage ${ }^{3}\left({ }^{1}\right.$ Cedars-Sinai Medical Center, Beverly Hills, CA; ${ }^{2}$ scripps clinic, La Jolla, CA; ${ }^{3} \mathrm{UCI}$, Irvine, CA and ${ }^{4} \mathrm{UCLA}$, los angeles, CA)

Rationale: Patients with medically refractory partial seizures who are candidates for Resective Epilepsy Surgery frequently undergo language mapping as to minimize resection of eloquent cortex. Unique representation of language in Epilepsy patients especially these with childhood onset of seizures may alter the surgical approac Methods: We used a large array 148 channel Magnetometer (MEG, 4D Neuroimaging) and multiple tasks (Auditory Word Recognition, Visual Verb Generation and Picture Naming) in 16 candidates for resective epilepsy surgery to investigate cortical organization for receptive and productive language function.Laterality indices were calculated (R-L)/(R+L) after Galaburda et al., 1978 for left and right hemisphere late fields $\sim 150-400 \mathrm{~ms}$. Values fell between $+/-1.0$, with negative values reflecting left-biased processing.Laterality indices were calculated (R-L)/(R+L) after Galaburda et al., 1978 for left and right hemisphere late fields $\sim 150-400 \mathrm{~ms}$. Values fell between $+/-1.0$, with negative values reflecting left-biased processing.

Results: $5 / 16$ patients showed leftward laterality for all three tasks $4 / 16$ patients showed rightward laterality all three tasks. $6 / 16$ of those patients had laterally discordant receptive and productive language function.

$1 / 16$ showed bilateral receptive language and leftward expressive language

Conclusions: Receptive and Expressive language may have divergent hemispheric dominance in patients with Medically Refractory Epilepsy with childhood and adolescent onset of seizures. The right sided receptive language hemispheric dominance in epilepsy patients may have implications for surgical planning as well as emphasize the need to separately assess both receptive and expressive language in these patients. MEG with multiple language tasks can be used non-invasively to assess language in both hemispheres. This group larger than our previously reported cohort and shows that atypical representation is frequent

\subsection{2}

INTRA OPERATIVE 3T MRI WITH DIFFUSION TENSOR IMAGING (DTI) SEQUENCES IMPROVES THE EXTENT OF DISCONNECTION DURING CORPUS CALLOSOTOMY AND MODIFIED FUNCTIONAL HEMISPHERECTOMY

Jane C. Cook, P. Chen, M. Korostenskaja, J. Baumgartner and K. Lee (Florida Hospital, Orlando, FL)

Rationale: Corpus callosotomy and modified functional hemispherectomy are surgical procedures for seizure control in pediatric epilepsy patients whom depend upon division of white matter tracts to be effective. Success rate of corpus callosotomy (up to $70 \%$ ) and functional hemispherectomy (up to $78 \%$ ) can be improved with intraoperative anatomic $1.5 \mathrm{~T}$ MRI scan (up to $90 \%$ ) by identifying residual crossing white matter tracts. To date, the authors are not aware of other studies evaluating the effectiveness of DTI in improving this post surgical outcome. We chose to evaluate intraoperative 3T MRI scans with DTI images for these complicated patients.

Methods: Unenhanced MRI's of the brain ( $n=21)$ were retrospectively reviewed in 17 pediatric epilepsy patients. Intraoperative MRI's immediately after first resection attempt were obtained in 10 patients receiving corpus callosotomy and 7 patients receiving modified functional hemispherectomy. A total of six patients receiving intraoperative DTI evaluation showed incomplete white matter tract disconnection which prompted immediate second attempt surgery. These patients received subsequent second look intraoperative MRI showing complete disconnection on DTI. All intraoperative MRI scans included axial and coronal T2 weighted images as well as 20 directional DTI. The cases were all scanned on the same intra operative Siemens 3T MR scanner. Two CAQ Pediatric Radiologists and one CAQ Neuroradiologist read all of the scans.

Results: 2 out of 10 corpus callosotomy patients had incomplete disconnection of white matter fiber tracts based on DTI images. These patients proceeded to immediate second attempt surgery which showed complete disconnection on subsequent intraoperative DTI. 4 out of 7 modified functional hemispherectomy patients had incomplete disconnection with as little as $2 \mathrm{~mm}$ of white matter fibers remaining on DTI. All of these patients underwent immediate second surgery for complete fiber disconnection proven on second intraoperative DTI.

In summary, $100 \%$ of patients $(n=6)$ with incomplete disconnection on first intraoperative DTI proceeded immediately to complete disconnection proven by second intraoperative DTI.

Conclusions: Preliminary data suggests that adding DTI sequences (less than 10 minute scan) to intraoperative MRI, may improve outcome in these complicated patients whom depend upon complete disconnection of their white matter tracts for optimal post surgical results.

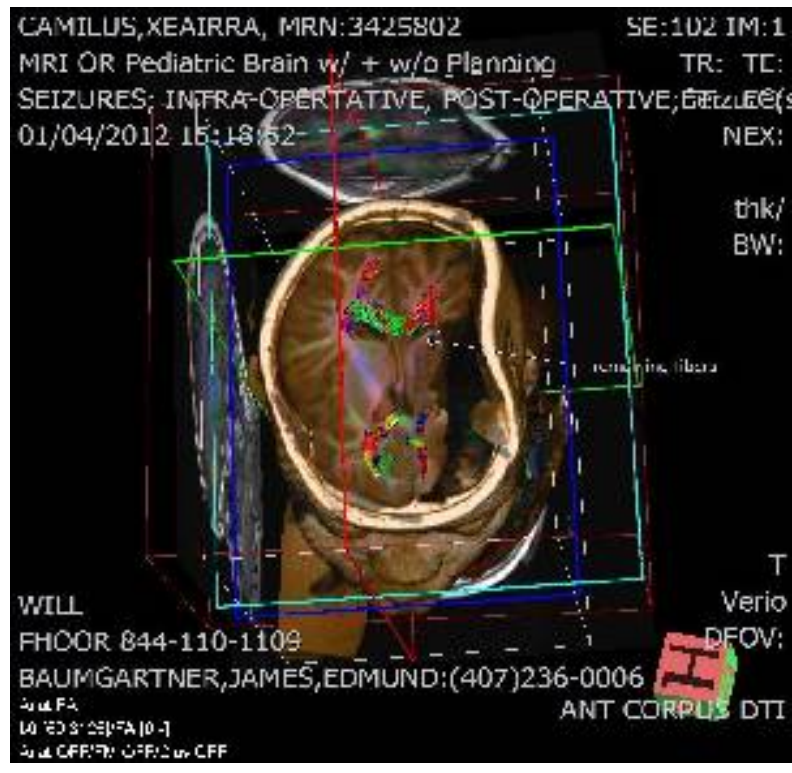

2.183

7 TESLA MAGNETIC RESONANCE SPECTROSCOPY IN THE ASSESSMENT OF PATIENTS WITH NORMAL 1.5 TESLA MRI TEMPORAL LOBE EPILEPSY

Simona Nikolova ${ }^{1}$, D. A. Steven ${ }^{2}$, J. Penner ${ }^{1}$, J. G. Burneo ${ }^{2}$ and R. Bartha ${ }^{1}\left({ }^{1}\right.$ Imaging Research Laboratories, Robarts Research Institute, Western University, London, ON, Canada and ${ }^{2}$ Clinical Neurological Sciences, Epilepsy Program, London Health Sciences Centre, London, ON, Canada)

Rationale: It has been demonstrated that MRS is a useful and sensitive technique for identification of hippocampal pathology in patients with temporal lobe epilepsy, particularly when associated with structural changes such as mesial temporal sclerosis. However, its role in patients with normal structural studies is still controversial. Most studies have been retrospective and included small numbers of patients with normal 1.5 or $3 \mathrm{~T}$ MRI, but the utility of $1 \mathrm{H}-\mathrm{MRS}$ in idiopathic localization-related temporal lobe epilepsy has not been prospectively studied with a high magnetic field (7T) MRI. 
The purpose of this study is to determine whether $\mathrm{N}$-acetylaspartate (NAA) is decreased and glutamate (Glu) is increased on the side of seizure onset in patients with cryptogenic (normal MRI) localizationrelated temporal lobe epilepsy.

Methods: 1H spectroscopy data were acquired on a 7.0 Tesla head only Agilent MRI using the LASER localization sequence $(\mathrm{TE}=38$ $\mathrm{ms}$ ), from a $2.7 \times 1.3 \times 1.3 \mathrm{~cm} 3 \mathrm{voxel}$ in the left and right hippocampi of three subjects. Levels of NAA, choline (Cho), Myo-inositol (Myo), taurine (Tau), and Glu were measured using the fitMAN software (v1.7) and normalized to creatine (Cr). Lateralization of epileptogenic focus was determined by clinical assessment, interictal and ictal video-EEG recordings, and neuropsychological evaluation. PET data was included when available. Metabolite levels were compared between ipsilateral and contralateral hippocampi using a ttest.

Results: Figure 1 shows all metabolite ratios in patients (ipsilateral to seizure onset hemisphere in blue, contralateral side in red). In this preliminary study, there were no significant differences in metabolite levels between the ipsilateral and contralateral side.

Conclusions: The identification of metabolic abnormalities not seen with conventional/standard MRI, will allow a better surgical selection of patients with temporal lobe epilepsy. Better identification of the epileptogenic focus will improve outcomes from seizure surgery. Recruitment of additional subjects is needed to confirm these findings.

Funding for this project was provided by the Ontario Brain Institute Epilepsy Discovery Project.

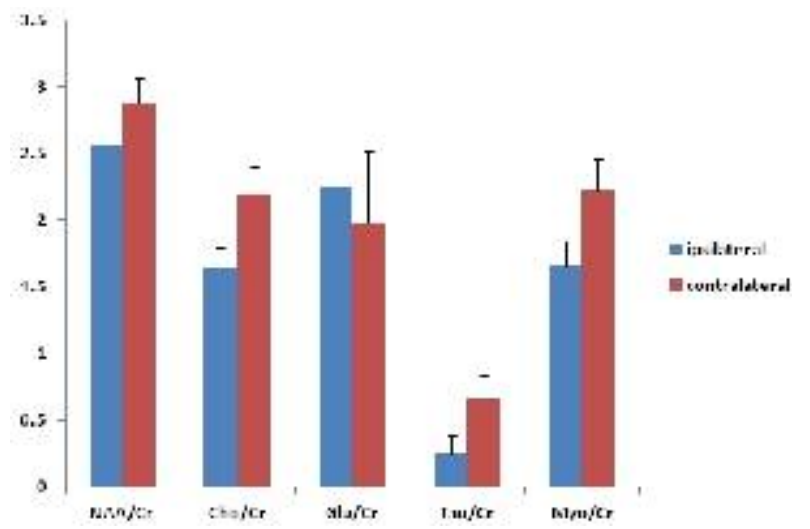

Figure 1: Metabolite ratios in patients and controls (ipsilateral to seizure onset hemisphere in blue, contralateral side in red). Error bars represent standard error of the mean.

\subsection{4}

QUANTIFYING METAL DISTRIBUTIONS USING SYNCHROTRON X-RAY FLUORESCENCE IMAGING OF NEOCORTEX RESECTED IN HUMAN EPILEPSY SURGERY Ann Lam ${ }^{1,2}$, C. M. Florez ${ }^{2,3}$, S. M. Webb ${ }^{4}$, B. Kocar ${ }^{4}$, S. Mylvaganam $^{2,3}$, T. A. Valiante ${ }^{2,3}$, P. L. Carlen ${ }^{2,3}$ and E. L. Ohayon ${ }^{1,3}$ ( ${ }^{1}$ Green Neuroscience Laboratory, Neurolinx Research Institute, La Jolla, CA; ${ }^{2}$ Toronto Western Research Institute, Toronto, ON, Canada; ${ }^{3}$ University of Toronto Epilepsy Research Program, Toronto, ON, Canada and ${ }^{4}$ Stanford Synchrotron Radiation Lightsource, Menlo Park, CA)

Rationale: Metals have been shown to elicit seizures in several experimental models of epilepsy and have been implicated in clinical conditions. Although metals have many essential roles in healthy brain function, their precise connection to epilepsy is not well understood. The study of the relation between metals and epilepsy has been limited, in part, by challenges in quantifying the levels of multiple brain metals, while also ascertaining their spatial distribution. Here we show that synchrotron X-ray fluorescence imaging (SXRF) can be used to reveal information about multiple metal co-localization in human resected brain tissue.
Methods: Resected cortical tissue from surgical cases of patients $(n=12)$ with intractable mesial temporal lobe epilepsy (MTLE) were imaged using rapid-scanning SXRF and microprobe imaging (beamlines 10-2 and 2-3) at the Stanford Synchrotron Radiation Lightsource. Nissl stained and unstained tissues were compared for gross and microscale differences in endogenous metals. Fluorescence maps of metals previously implicated in epileptogenesis (e.g., Fe, Zn, $\mathrm{Ca}, \mathrm{Cu}, \mathrm{Mn}$ ) were generated by windowing the $\mathrm{K}$ - or L- edges of these elements. However, the complete spectra were also captured for later analysis of additional metals. Images were analyzed using Sam's Microprobe Toolkit.

Results: Using SXRF imaging we were able to co-localize and quantify multiple metals in sequential cross sections. This included the co-localized imaging of $\mathrm{Fe}, \mathrm{Zn}, \mathrm{Ca}, \mathrm{Cu}, \mathrm{Mn}$ at resolutions as high as $1 \mu \mathrm{m}$. The resected tissue showed large variability in metal distribution both between subjects and within the local cortical area. Results also showed that Nissl staining affected the levels of some metals (e.g., $\mathrm{Cu}, \mathrm{S}, \mathrm{K}$ ) more than others (e.g., $\mathrm{Fe}, \mathrm{Zn}$ ).

Conclusions: Our study demonstrates the surveying ability of SXRF to detect low but biologically significant levels of endogenous metals. Although increases in sample size will help determine if multiple metal-based subgroups of epilepsies exist, the method has allowed for the first quantification of multiple metals at high resolutions in the human epileptic brain. Imaging distribution and co-localization of metals in the brain may be critical for identifying mechanisms of epileptogenesis in certain types of intractable epilepsy as well as identifying novel therapeutic approaches.

Cormorbidity (Somatic and Psychiatric): Psychiatric Conditions

\subsection{5}

PRE-SURGICAL MOOD DISORDER IS ASSOCIATED TO

WORSE POST-SURGICAL SEIZURE OUTCOME IN PATIENTS WITH REFRACTORY TEMPORAL LOBE EPILEPSY AND MESIAL TEMPORAL SCLEROSIS FRANCINALDO L. GOMES ${ }^{2}$, G. M. Filho ${ }^{1,2}$, L. Mazetto ${ }^{1,2}$, M. M. Marinho $^{2}$, I. M. Tavares ${ }^{2}$, L. O. Caboclo ${ }^{2}$, E. M. Yacubian ${ }^{2}$ and R. S. Centeno ${ }^{2}\left({ }^{1}\right.$ PSYCHIATRY, UNIVERSIDADE FEDERAL DE SÃO PAULO UNIFESP/EPM, SÃO PAULO, Brazil and ${ }^{2}$ NEUROLOGY AND NEUROSURGERY, UNIVERSIDADE FEDERAL DE SÃO PAULO, SÃO PAULO, Brazil)

Rationale: The prevalence of comorbid psychiatric disturbs (PD) in patients with temporal lobe epilepsy and mesial temporal sclerosis (TLE-MTS) reaches $20-40 \%$, rising to $70 \%$ in patients with refractory forms of epilepsy. Mood disorders are the most common (24-74\%). The association between pre-surgical PD and a worse postsurgical seizure outcome in patients with refractory epilepsy submitted to epilepsy surgery has been also increasingly recognized. This study aims at verifying the impact of pre-surgical mood disorder, specially depression, on seizure outcome in surgically treated patients with refractory temporal lobe epilepsy and mesial temporal sclerosis (TLE-MTS).

Methods: After previous consent, retrospective data from 115 surgically treated (corticoamygdalohyppocampectomy) TLE-MTS patients (65 females; $56.5 \%$ ) were reviewed. Clinical, sociodemographic, and electrophysiological variables were analyzed. Psychiatric evaluations were performed by the same psychiatrist and through DSM-IV or ILAE criteria. The mean follow-up interval after surgery was of $4.7 \pm 1.66$ (one to eight) years. Engel IA was established as a favorable post-surgical outcome. Univariate statistical analyses was performed firstly, followed by multivariate statistical analysis (logistic regression model) to identify predictors of a non-favorable seizure outcome, and the odds-ratio (OR) was calculated for significant risk factors. $\mathrm{P}$ value of $<0.05$ was considered significant.

Results: Forty-five patients (41.6\%) were classified as Engel IA; 47 patients $(40.8 \%)$ presented pre-surgical PD. Depression $(\mathrm{OR}=5.11$; 
$\mathrm{p}=0.004)$ appeared as a risk factor associated to a non-favorable seizure outcome.

Conclusions: In patients with refractory TLE-MTS, the presence of depression predicts an unfavorable surgical outcome. The findings of the present paper are of great value and reinforce the importance of performing a detailed psychiatric pre-surgical evaluation in all epilepsy patients candidates for surgical treatment.

\subsection{6 \\ SOCIAL RELATIONSHIPS AND FEELING OF LONELINESS IN YOUTH WITH EPILEPSY: RESULTS FROM A POPULATION-BASED STUDY}

Kristin . Alfstad ${ }^{1}$, J. Clench-Aas ${ }^{2}$ and M. I. Lossius ${ }^{1}\left({ }^{1}\right.$ Oslo

Unioversity Hospital, The National Centre for Epilepsy, Sandvika, Oslo, Norway and ${ }^{2}$ Norwegian Institute of Public Health, Division of Mental Health, Oslo, Norway)

Rationale: We have previously published data showing a higher prevalence of symptoms of psychological distress and increased risktaking behaviour in youth with epilepsy compared to controls. The aim of this presentation was to investigate how youth with epilepsy (YWE) aged 16-19 years perceive their social relationships with emphasis on loneliness, compared to controls.

Methods: Data were cross-sectional and based on questionnaires from 10.571 youths (response rate $79 \%$ ).

The youths were asked to describe their feelings concerning social relationships. Questions could be answered as correct, partly correct or not correct. Answers were then grouped as correct or partly correct vs. not correct. Chi square was used for testing categorical variables. A multivariate analysis was conducted with feeling lonely as the dependant variable and having/having had epilepsy, gender, low family income and living with a single parent as independent factors. Results: 114 youths reported having or having had epilepsy (i.e. a life time prevalence of 1.2\%). Significant differences were found between YWE and controls in social relationships: "I cannot choose whom I want to be friends with" was reported by $13.2 \% \mathrm{vs} .7 .1 \%$ in controls ( $\mathrm{p}=0.012$ ), "I have a feeling that nobody knows me well" in $11.6 \%$ in YWE vs. $6.8 \%$ in controls ( $\mathrm{p}=0.045)$, and $10.7 \%$ of YWE stated that they felt lonely ( $5.5 \%$ in controls, $\mathrm{p}=0.016)$. The multivariate statistical analysis showed that low family income was the strongest predictor of feeling lonely (OR 2.3; CI 1.8-2.9; $\mathrm{p}<0.001$ ), followed by having/having had epilepsy (OR 2.0; CI 1.13.7; $\mathrm{p}=0.026)$, female gender (OR 1.4; CI 1.2-1.7; $\mathrm{p}<0.001)$ and living with a single parent (OR 1.3; CI 1.0-1.6; $\mathrm{p}=0.019$ ). Other chronic diseases (asthma or diabetes) were not found to be a predictor.

Conclusions: Youth with epilepsy report more loneliness and problems in social relationships than their healthy peers. The feeling of being different, poor self-esteem and stigma reported in other studies could all be contributing factors. The resulting isolation at an age nearing adulthood could be a risk factor for continued problems with psychological and somatic health.

\subsection{7 \\ CLINICAL AND DEMOGRAPHICS CHARACTERISTICS OF A BRAZILIAN POPULATION WITH PSYCHOGENIC NON- EPILEPTIC SEIZURES}

R. Alessi and Kette D. Valente (University of Sao Paulo, San Paulo, Brazil)

Rationale: Most of our knowledge on Psychogenic Non-Epileptic Seizures (PNES) is based on studies conducted with populations from United States or Europe. The authors believe that socioeconomic and cultural background affect the characteristics of PNES. However, there is scarce data on PNES on South-American countries, usually with small sample sizes and without video-EEG. The aim of the present study was to describe the demographics and clinical characteristics considering stress factors, psychiatric diagnosis and social functioning in a large Brazilian series with PNES.

Methods: We reviewed videos and medical records of 102 patients (mean age 35.27 years; $76.4 \%$ female) with PNES, documented by video- EEG (mean 7.82 days) from 2006 to 2011. All patients evaluated were over 18 years of age. Patients with epilepsy were not excluded. Diagnosis of malingering (with clear panic attacks) was a criterion of exclusion. Patients who presented PNES, during videoEEG, that were not recognized by their families as typical of those presented at home were also excluded. We collected data on age; gender; years of schooling; marital status; living situation; employment; psychiatric diagnoses or other acute or chronic somatic illnesses; concomitant epilepsy and stress factors. Previous use of antiepileptic drugs (AEDs), use of medical services and side effects were also documented.

Results: Mean age at PNES onset was 27.85 years; mean time to diagnosis was 7.89 years; 89 patients $(87.25 \%)$ lived with their families; 58 (56.86\%) were single; 41 (40.19\%) had finished high school; and 33 (39.75\%) worked on regular basis. Eighteen patients $(21.68 \%)$ were on permanent disability, seven $(38.88 \%)$ out of these with the diagnosis of refractory epilepsy. Depression was diagnosed in 49 patients $(48.03 \%)$, anxiety in $28(27.45 \%)$ and $32(31.37 \%)$ had somatic symptoms. Thirty-three patients misdiagnosed with epilepsy (49.25\%) were using antiepileptic drugs. Fifteen patients $(14.70 \%)$ had previous hospitalization (ER/ICU). All patients had performed ancillary diagnostic testing and had frequent physician visits. Stress factors were identified in 59 patients $(57.84 \%)$, the most commons being family related (15.68\%). Physical abuse was reported by $17.64 \%$ and sexual abuse by $5.88 \%$.

Conclusions: Most of our findings are in line with those of studies conducted in the United States and Western Europe. However, we observed major differences in comparison with studies conducted in China and Eastern Europe. These findings indicate that cultural and socioeconomic differences may affect the characteristics of PNES.

\subsection{8 \\ EVALUATING THE YIELD AND REFERRAL FOLLOW- THROUGH OF ROUTINE BEHAVIORAL AND PSYCHOLOGICAL SCREENING IN EPILEPSY CARE SETTINGS}

Breanne Fisher ${ }^{1}$, C. Dezort ${ }^{1}$ and A. Berg ${ }^{1,2}\left({ }^{1}\right.$ Ann and Robert H. Lurie Children's Hospital of Chicago, Chicago, IL and ${ }^{2}$ Northwestern University, Chicago, IL)

Rationale: Behavioral and psychological co-morbidities are common in children with epilepsy. The yield and referral follow- through of systematic screening for such co-morbidities in the epilepsy clinic has not been previously assessed. We evaluated the yield of systematic screening for behavioral and psychological concerns using the Strengths and Difficulties Questionnaire (SDQ) administered by allied health professionals in a hospital-based epilepsy care program. Methods: Parents of pediatric patients evaluated in the epilepsy monitoring unit (EMU), including established and new onset patients, those and seen in Ketogenic Diet clinic were asked to complete the SDQ, a behavioral and psychological screening instrument. A retrospective chart review of patients whose parents had already completed the screening instrument as standard clinical care was performed for patients seen from November 1st, 2010 until May 15th ,2011 and prospectively from May 16th,2011 until April 1st, 2012. The number and percent of children ages 4-17 years who screened positive were tabulated.

Screening was conducted by the unit's advance practice nurses (APNs) who were also responsible for scoring and discussing results with parents, and arranging for referrals when necessary. The screening results were considered "actionable" if the SDQ identified a child with behavioral or psychological concerns who was not already receiving adequate services in those areas; "confirmatory" if they had a positive screening result but were already receiving 
services; and negative for children who screened negative.

Results: From November 1, 2010 to April 1, 2012, a total of 71 children age 4-17 were screened using the SDQ. The average age of the children was 9 years; 41 (58\%) were boys. $53(75 \% \%)$ were established epilepsy patients and $18(25 \%)$ were patients seen in a new-onset epilepsy clinic. Behavioral or psychological concerns were identified in 41children (58\%). Concerns were found in one or more areas including: overall stress $(n=26)$, emotional distress $(n=18)$, behavioral difficulties $(\mathrm{n}=15)$, difficulties getting along with others $(n=21)$, hyperactivity and attentional difficulties $(n=22)$, and kind and helpful behavior $(n=25)$. Of the 41 with positive screening findings,, results were confirmatory in $29(71 \%)$ who were receiving adequate services already and actionable in $12(29 \%)$ for whom services were either inadequate or not yet being offered.

Appropriate referrals for further evaluations were made for all 12 children with actionable findings to one or more of the following services: psychiatry, social work, educational specialist, neuro psychological testing, pediatrician. The family followed through with the referral in $8(67 \%)$ or the cases.

Conclusions: The yield of routine screening of children in the EMU and ketogenic diet settings for behavioral and psychological concerns using the SDQ is sufficiently high to support its use both in new onset and in established patients and to consider whether such screening should be extended to children seen in all epilepsy care settings.

\subsection{9}

EVALUATING THE EFFECTIVENESS OF A PARENT COMPLETED CHECKLIST VERSUS A COMPREHENSIVE SCREENING PROGRAM FOR CHILDREN WITH EPILEPSY Catherine L. Dezort ${ }^{1}$, B. Fisher ${ }^{1}$, F. Zelko ${ }^{1,2}$ and A. Berg ${ }^{1,2}$ ( ${ }^{1}$ Ann \& Robert H. Lurie Children's Hospital of Chicago, Chicago, IL and ${ }^{2}$ Northwestern University, Chicago, IL)

Rationale: Developmental delay, cognitive deficits, autism, behavioral and psychiatric comorbidities are common in children with epilepsy. A systematic screening program looking at these comorbidities individually can be informative, but time consuming for parent and provider. We looked at the effectiveness of a parentcompleted checklist to guide further screening needs versus a comprehensive screening program with specific screening forms evaluating for difficulty in development, autism, the psychosocial arena, cognition, and schooling.

Methods: Parents of patients evaluated in the epilepsy monitoring unit, including established and new onset patients, and the ketogenic diet clinic of a tertiary pediatric medical center completed the Epilepsy Center Developmental and Educational History Questionnaire (ECQ) which queries concerns regarding development, cognition, and behavior. In addition, parents completed ageappropriate screening batteries as follows: ages birth to 4 years, Ages and Stages Questionnaire (ASQ) for development and the M-CHAT for autism; ages 4 - 6 years, ASQ, the Social Communication Questionnaire (SCQ) for autism, and the Strengths and Difficulties Questionnaire (SDQ) for behavior and psychiatric concerns; ages $\geq 6$ years, SCQ, SDQ, and the Perceived Cognitive Functions Questionnaire (PCF-43) to evaluate cognitive function. Data were obtained retrospectively from 11/1/2010 until 5/15/2011 and prospectively from $5 / 16 / 2011$ through $4 / 1 / 2012$. Screening was conducted by the unit's advance practice nurses who scored, interpreted, and relayed results to parents. We compared positive screening results found through the comprehensive screening program to results based on the ECQ alone.

Results: 194 children birth-17y were screened. The average age was 6y; 146 (75\%) were established epilepsy patients and 49 (25\%) were seen in the new-onset epilepsy clinic. One or more areas of concern was noted on the ECQ checklist in $148(76 \%)$, and $120(62 \%)$ scored positive on one or more formal screeners: ASQ:73/85 (86\%); mCHAT: 31/62 (50\%); SCQ:18/112 (16\%); and SDQ: 41/71 (58\%).
A positive ECQ-checklist result was highly sensitive to positive results on these four screeners overall and individually: sensitivities were $89 \%$ for a finding on any screener, $86 \%$-ASQ, $97 \%$-mCHAT, 95\% SCQ, and 95\% SDQ. Specificity of the ECQ checklist for negative screening results was $55 \%$ overall. With the exception of the ASQ, specificity was low: $82 \%$-ASQ, 29\%-mCHAT, 29\%-SCQ, $37 \%-S D Q$.

Conclusions: A structured parent checklist can be sensitive to developmental and behavioral problems requiring further evaluation, although in this study its specificity was low. In a high risk population such as seen in a tertiary center, formal screening is likely the most justifiable and efficient approach to detecting problems. In a less high-risk setting and with further development, a simple checklist may serve as a first step and guide further, formal screening. This approach could facilitate routine screening for comorbidities of epilepsy in an out-patient and non-specialty setting.

\subsection{0 \\ ADULTS VERSUS TEENAGERS WITH PSYCHOGENIC NON-EPILEPTIC SPELLS (PNES): TIME FROM ADMISSION TO FIRST EVENT AND ANNUAL PRESENTATION}

J. Loplumlert ${ }^{1}$, C. Munoz ${ }^{1}$, T. Hirfanoglu ${ }^{1}$, B. Zonjy ${ }^{1}$, J. Freitas ${ }^{1}$, S.

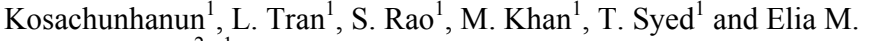
Pestana Knight ${ }^{2}\left({ }^{1}\right.$ Epilepsy Center, University Hospital Case Medical Center, Cleveland, $\mathrm{OH}$ and ${ }^{2}$ Pediatric Epilepsy, Rainbow Babies and Children's Hospital, Cleveland, $\mathrm{OH}$ )

Rationale: Over the last decades, adults and children with PNES have substantially been studied by VEEG monitoring to determine the clinical features of PNES (Brown RJ et al., 2011; Hema Patel et al., 2007). However, a small number of studies have focused on annual presentation of PNES and how soon, after VEEG recording initiation, the first PNES occurred (Parra J et al., 1998; Lobello K et al., 2006; Friedman DE et al., 2009; Margaret W et al., 2010). Our aims are: (1) to compare time from admission to first PNES in adults and teenagers and (2) to identify annual variations in the presentation of PNES in adults and teenagers.

Methods: We retrospectively reviewed charts adults and teenagers diagnosed with PNES between 7/1/2010 and 6/30/2011 in the Epilepsy Monitoring Unit of University Hospitals Case Medical Center and Rainbow Babies and Children's Hospital. Forty patients (27 adults and 13 teenagers) were eligible for analysis. Time from onset of EEG recording to first PNES and month of the first PNES were the variables studied.

Results: $66.7 \%$ (18 of 27) of adults and 92.3\% (12 of 13) of teenagers were able to report the month of PNES onset. Peak months of presentation for teenagers were March and October (23\% each) followed by July and September (15\% each). For adults, peak of presentation were June (22\%) followed by August, September and December (16\% each).

Time from admission to first PNES recorded was different between adults and teenagers (Figure 1). 59\% of the adults have first event after 6 hours of admission whereas $50 \%$ of teenagers have the first event in less than 5 minutes from recording and $100 \%$ had the event before 6 hours.

Conclusions: In our study, the PNES in adults peaked around approaching summer months (June) and remained high at the onset of fall (August) and remained at the same level until the end of the year. We do not know what life events provoked these changes in adults. One explanation could be the onset of fall season as it has been described in other psychiatric conditions. PNES in teenagers peaked around spring break (March) as well as the end (June) and onset (September-October) of academic year. It seems that onset and end of the academic year are obvious stressor in teenagers.

We found a significant difference regarding time to admission to first event in teenager compared to adults. Half of the teenager group had the first PNES within the first 5 minutes of VEEG recording whereas more than half had of the adults had it after 6 hours of recording. 
More data regarding the nature of the stressors will be needed to interpret this data.

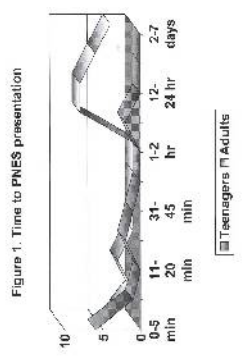

\subsection{1}

AN EXPLORATORY QUALITATIVE ANALYSIS OF THE PSYCHOLOGICAL PROCESSES UNDERLYING PNES Linda Macleod ${ }^{1,2}$, A. M. McIntosh ${ }^{2}$, S. F. Berkovic ${ }^{2}$, I. E. Scheffer ${ }^{2}$ and S. J. Wilson ${ }^{1,2}\left({ }^{1}\right.$ Psychological Sciences, University of Melbourne, Parkville, VIC, Australia and ${ }^{2}$ Comprehensive Epilepsy Program, Austin Health, Heidelberg, VIC, Australia)

Rationale: Many psychological and social variables have been correlated with Psychogenic Non-epileptic Seizures (PNES), but an understanding of the underlying psychological processes remains elusive. We aimed to qualitatively explore the psychological processes underlying PNES, with an emphasis on the formation of personal and social identity or self.

Methods: 20 patients (13 female; mean age $43.6 \pm 15.3$ years) with PNES were recruited through Austin Health (Melbourne, Australia) for an in-depth semi-structured interview (90 minutes) covering themes about individual development, emotional experiences, and the social milieu. Interpretative Phenomenological Analysis was used to analyze the interview transcripts. Key themes present in at least $50 \%$ of the sample were extracted and clustered using Nvivo9 qualitative analysis software.

Results: Overall, 38 themes were identified and grouped into four broad categories; maladaptive childhood experiences, poor development of personal and social identity, difficulty reflecting on one's thoughts and emotions, and poor understanding of the behaviour of others. More frequently reported themes indicating adverse childhood experiences included childhood abuse (100\%) and a lack of parental attention or care $(95 \%)$. Poor adaptation to stress $(100 \%)$ and avoidant and dependent coping styles (95\%) represented poor regulation of the patient's emotions and thought processes, and chronic acrimonious relationships with partners, family, and friends $(100 \%)$ highlighted the difficulties these patients have relating to other people. Most striking, poor formation of personal and social identity were strongly represented by $95 \%$ of patients reporting their PNES was integral to the way they defined themselves, yet out of their control. Themes related to poor identity or self-development were common to $95 \%$ of patients reporting themes covering maladaptive childhood experiences, difficulty reflecting on their own thoughts and emotions, and a poor understanding of the behaviour of others.

Conclusions: This initial analysis highlights a new framework for understanding PNES, in which poor identity or self-development is critical for its expression. Themes representing poor identity or selfdevelopment provide a basis for understanding the association between poor mental and social outcomes in these patients and the emergence of PNES. A more comprehensive understanding of the psychological processes underlying PNES and their development across the lifespan will enhance treatment specificity and efficacy.
2.192

DOES HIPPOCAMPAL SCLEROSIS CORRELATES WITH DEPRESSION IN PATIENTS WITH MEDICALLY REFRACTORY EPILEPSY?

Angela M. Velez, E. Gherman and C. A. Szabo (University of Texas, San Antonio, TX)

Rationale: Hippocampal sclerosis (HS) is a common cause of medically refractory mesial temporal lobe epilepsy (MTLE, Quigg et al., 1997). Hippocampal injury is also implicated in the pathophysiology of major depression; unilateral and bilateral hippocampal atrophy have been reported in patients with major depressive disorder (Campbell et al., 2004; Videbech and Ravnkilde, 2004). As psychiatric conditions are prevalent in patients with epilepsy mainly mood and anxiety disorders (Ertekin et al., 2009; Guarnieri et al., 2009; Sperli et al., 2009; Tellez-Zenteno et al., 2007), we evaluated whether mood disorders were associated with hippocampal sclerosis in a cohort of patients with MTLE.

Methods: This prospective cohort analysis included 13 patients who underwent neurosurgical intervention for intractable epilepsy at the South Texas Comprehensive Epilepsy Center (STCEC), between 2010 and 2012. The study was approved by the UTHSCSA IRB office.

Demographic variables were evaluated such as age, gender, marital status, education level, and employment; while clinical variables included age at onset of epilepsy, etiology of epilepsy, presence of Axis I Disorders (assess using the Mini International Neuropsychiatric Interview MINI PLUS), age at surgery, area of resection, and MRI findings.

Results: The majority of patients were male (54\%), single (54\%), with a high school degree (69\%). Mean age at time of resection was 31 years. MRI lesions were found in twelve (92\%) patients. Ten (8\%) patients had temporal lobe epilepsy, seven of whom had right-sided foci. HS was identified in 7 (54\%) of patients, while the remaining three had cortical dysplasia and encephalomalacia. Preoperative depression mood was diagnosed in $46 \%$, followed by anxiety in $31 \%$. All were on psychotropic medications either antidepressant or atypical antipsychotic. Depression was diagnosed preoperatively in $46 \%$ of patients with temporal lobe epilepsy and $31 \%$ of patients with HS. While all were seizure free and maintained on psychotropic medications at three and six months follow-up, none of the patients suffered an exacerbation of the mood symptoms after surgery. The same was found for three patients $(23 \%)$ with extratemporal foci, $67 \%$ of them had presurgical diagnosis of depression, and were taking psychotropic medications at time of surgery, none of them had postsurgical exacerbation of depressive symptoms at three and six months follow up and all of them were seizure free.

Conclusions: This study presents preliminary data on psychiatric outcome in a prospective cohort undergoing epilepsy surgery. While the numbers are still small, this cohort confirms the association of HS with depression. With adequate psychotropic treatment there is no evidence of worsening of depression in patients with HS posoperatively, particularly when they are seizure free.

\begin{tabular}{l} 
Demographic Data \\
\begin{tabular}{|c|c|}
\hline Gender & Femule $46 \%(\mathrm{n}=6)$ \\
Male $54 \%(\mathrm{n}=7)$ \\
Age at onsct of epilcpsy (ycars) & 16 \\
\hline Age at surgery (ycars) & 31 \\
\hline Marital Slatus & Single $54 \%(\mathrm{n}=7)$ \\
& $\begin{array}{c}\text { Married } 31 \%(\mathrm{n}=4) \\
\text { Divorced } 15 \%(\mathrm{n}=2)\end{array}$ \\
Tducation 1.evel & High School $69 \%(\mathrm{n}=9)$ \\
College Degrec $23 \%(\mathrm{n}=3)$
\end{tabular} \\
\hline
\end{tabular}


Clinical Data

\begin{tabular}{|c|c|}
\hline Resection & $\begin{array}{l}\text { Right Temporal Lobe } 54 \%(n=7) \\
\text { Left Temporal Lobe } 23 \%(n=3) \\
\text { Right Frontal T.obe } 7.6 \%(n=1) \\
\text { Left Frontal Lobe } 7.6 \%(n=1) \\
\text { Right Fronto-Temporal } 7.6 \%(n=1)\end{array}$ \\
\hline MRI Finding $x$ & $\begin{array}{c}\text { Hippocampal Sclerosis } 54 \%(n=7) \\
\text { Venous Cavemous hemangioma } 7.6 \%(n=1) \\
\text { Ilemorrhage } 7.6 \%(n=1) \\
\text { Cyst } 7.6 \%(n=1) \\
\text { Encephalomalacia } 7.6 \%(n=1) \\
\text { Cortical Dysplasia 15\% }(n=2)\end{array}$ \\
\hline Axis 1 Dagnosis & $\begin{array}{c}\text { Major Depressive Disorder } 23 \%(n=3) \\
\text { Depression NOS } 31 \%(n=4) \\
\text { Generalized Auxiety Disorder } 15 \%(n=2) \\
\text { Anxiety Disorder NOS } 7.6 \%(n=1) \\
\text { Panic Disorder } 7.6 \%(n-1)\end{array}$ \\
\hline
\end{tabular}

\subsection{3}

COGNITIVE PROFILE OF PERSONS WITH TEMPORAL LOBE EPILEPSY AND COMORBID DEPRESSION IN AN URBAN COMMUNITY

Ainie Soetanto ${ }^{1}$, J. J. McGinley ${ }^{2}$ and S. R. Haut ${ }^{2}\left({ }^{1}\right.$ Albert Einstein College of Medicine, Bronx, NY and ${ }^{2}$ Department of Neurology, Montefiore Medical Center, Albert Einstein College of Medicine, Bronx, NY)

Rationale: Patients with temporal lobe epilepsy (TLE) may suffer from significant neuropsychological/neuropsychiatric complications, including cognitive impairment and depression. Depression can affect up to one half of all TLE patients, and is itself associated with cognitive impairment. Thus, patients with TLE and comorbid depression may suffer from a double cognitive burden. The existence and nature of this double burden have been inconsistently reported (Paradiso et al., J Neurol Neurosurg Psychiatry 2001; 70:180-185; Dulay et al., Epilepsy Behav 2004; 5:522-531; Helmstaedter et al., Epilepsy Behav 2004; 5:696-701). The purpose of this study was to explore the relationship of depression and cognition in TLE in an urban population, where the prevalence of depression is high and potentially more severe than that of previously studied cohorts. Methods: A retrospective study was conducted on 42 adult presurgical patients diagnosed with medically intractable unilateral TLE. Subjects had electrographically confirmed left or right unilateral TLE, had failed treatment with at least 2 anti-epileptic drugs (AEDs), and were 18 years or older. Exclusion criteria included premorbid mental retardation or developmental disorder, comorbid neurodegenerative disease, and poor test effort as confirmed by formal symptom validity assessment. All subjects underwent comprehensive pre-surgical evaluation which included MRI/PET imaging, EEG monitoring, and neuropsychological testing. Cognition was assessed with the Wechsler Adult Intelligence Scale (WAIS-III or IV) or Wechsler Abbreviated Scale of Intelligence (WASI), and depressive symptoms were assessed with the Beck Depression Inventory (BDI-II). The BDI-II is a 21-item questionnaire with a total score range of 0 to 63 . Scores of 14, 20, and 29 represent thresholds for mild, moderate, and severe depression, respectively. The following variables were evaluated: age, sex, education, lateralization of seizure focus, AED usage, age of onset of epilepsy, and duration of epilepsy. A multivariate linear regression analysis was performed to assess the relationship between BDI score and cognitive scores. Results: Thirty-one patients were included. Mean age was 38.6 (range 18-70), 61\% were female, and 55\% and 45\% had left TLE and right TLE, respectively. Mean Full Scale IQ score was 86.1 (range 65-112), and mean BDI-II score was 14.7 (range 0-38). Seventeen patients were minimally depressed, 4 were mildly depressed, 6 were moderately depressed, and 4 were severely depressed. Regression analyses demonstrated a significant association between depression and cognition $(\mathrm{p}<0.01)$, especially in those with left-sided seizure foci.

Conclusions: These results suggest that depression increases the cognitive burden experienced by patients with TLE, most profoundly left TLE. Therefore, it is imperative that depression be recognized and treated concurrently with the treatment of epilepsy.
2.194

[5 HT] IN HIPPOCAMPAL TISSUE OF PATIENTS WITH MESIAL TEMPORAL SCLEROSIS (MTS) IS NOT RELATED TO THE PRESENCE OF PSYCHIATRIC DISORDERS Natascha Fonseca ${ }^{1}$, H. P. Joaquim ${ }^{2}$, L. L. Talib ${ }^{2}$, S. Vincentiis ${ }^{1}$, W. F. Gattaz $^{2}$ and K. D. Valente ${ }^{1}{ }^{1}$ Laboratory of Neurophysiology, Institute of Psychiatry, University of Sao Paulo, Sao Paulo, Brazil and ${ }^{2}$ Laboratory of Neurosciences - LIM 27, Institute of Psychiatry, University of Sao Paulo, Sao Paulo, Brazil)

Rationale: Temporal lobe epilepsy determined by MTS have a higher incidence of depression. Studies using animal models and neuroimaging in humans suggest a common serotonergic mechanism. There is a lack of studies in human brain. Measurement of serotonin in brain is a research strategy commonly used in animal models. We compared the concentration of 5HT in hippocampus of patients with TLE-MTS with and without psychiatric disorders (PD). We also compared [5HT] with the severity of epilepsy.

Methods: Thirty-five surgically treated patients with TLE-MTS, 16 $(45,7 \%)$ with PD were included. [5HT] was measured using High Pressure Liquid Chromatography with fluorescence detection. Results: No differences were found between groups regarding the dose of carbamazepine or oxcarbazepine, monotherapy or polytherapy. The mean [5HT] was $0,3359 \mathrm{ng} / \mathrm{mg}$. There was a correlation between lower levels of $[5 \mathrm{HT}]$ and the presence of generalized tonic clonic seizures $(\mathrm{GTC})(\mathrm{p}=0,48)$, but not frequency $(\mathrm{p}=0.138)$ of GTC. There was also a correlation between the use of SSRIs and lower frequency of GTC $(p=0,028)$. There was no difference among [5HT] and the presence of PD, age of onset and duration of epilepsy, seizure frequency and history of febrile seizures or status epilepticus. However, the use of antipsychotic drug was correlated with lower [5HT] $(p=0.029)$. No significant differences were found between the presence of psychosis and lower [5HT]. Conclusions: This is the first study measuring the concentration of serotonin in human brain and comparing groups with TLE-MTS with and without PD. In our study, we showed that [5HT] is related to the severity of epilepsy, but not to the presence of PD. Patients with and without PD, as well as patients who did or did not had GTC seizures, were similar regarding AEDs, considering dose and monotherapy/polytherapy. AEDs effect is unlikely to interfere with the results of the [5HT] levels. We demonstrated a correlation between a greater presence of GTC seizures in patients who did not use SSRIs. The hypothesis that patients with depression have more GTC seizures is in contradicted by the lack of correlation between presence of GTC seizures with PD. Another possibility is an antiepileptic effect of the SSRI providing an adjuvant effect in the treatment of these patients. We found a correlation between antipsychotic use and lower levels of serotonin. Although a relationship between serotonin levels and PD was not found, this finding can be explained by a lower concentration of serotonin in patients with a more severe presentation of psychosis. However this sample was too small for this assumption. In conclusion, the relationship between epilepsy and PD probably involve more intricate mechanisms than serotonin concentration. We also suggest that SSRIs may have an antiepileptic effect, which has been recently documented in literature. 


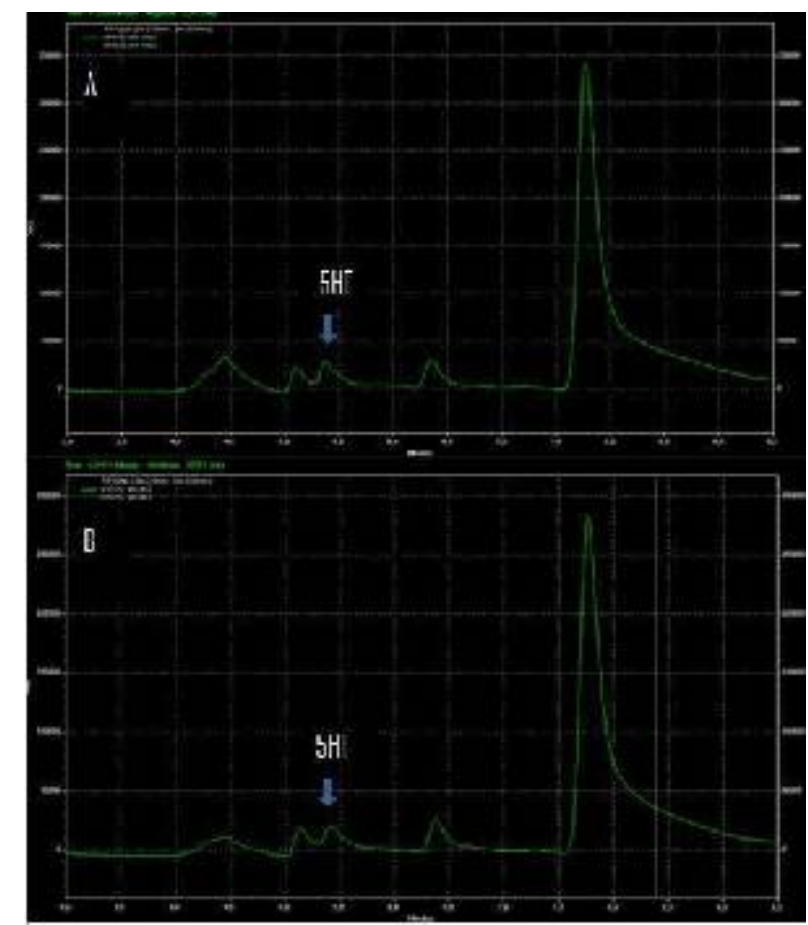

Chromatograms of patients with (A) and without (B) GTC seizures history showing peak of $5 \mathrm{HT}$ at 5,4 minutes and of external standard at 7,6 minutes. In the first case the $[5 \mathrm{HT}]$ is $0,30522 \mathrm{ng} / \mathrm{mg}$ and the second case is $0,25678 \mathrm{ng} / \mathrm{mg}$.

\subsection{5}

CORRELATIONS OF PHQ-9 WITH NDDI-E IN EPILEPSY PATIENTS: A PILOT STUDY

Jaivir S. Rathore, G. E. Tesar, N. Obuchowski, R. Busch, D. Humbel and L. E. Jehi (Epilepsy Center, Neurological Institute, Cleveland

Clinic Foundation, Cleveland, $\mathrm{OH}$ )

Rationale: Depression is the most common, yet under-recognized \& under-treated psychiatric co-morbidity in patients with epilepsy. One of the most significant obstacles to treatment may be the failure of adequate screening $\&$ diagnosis. The purpose of our study is to validate a widely used depression screening tool, the Patient Health Questionnaire (PHQ-9) by comparing it with the Neurological Disorders Depression Inventory for Epilepsy (NDDI-E) developed and validated specifically for patients with epilepsy. The PHQ-9 total score ranges from $0-27$ and scores of 10 or greater have been found to correlate with major depression of at least a moderate degree. However, the PHQ-9 has not been specifically validated in epilepsy patients, where multiple somatic, cognitive or AED related side effects \& epilepsy manifestations could falsely elevate the total PHQ9 score.

Methods: This is a prospective study where 60 clinically/EEG diagnosed epilepsy patients were interviewed to complete the PHQ-9 $\&$ NDDI-E in random order within a 24 hours period, mostly in the same sitting. The sensitivity \& specificity of the PHQ-9 at cut-points defined in the literature was estimated \& 95\% C.I constructed. Various PHQ-9 score cut-points for a "PHQ-9 positive" screen of 10 thru 15 were compared to NDDIE score of $>=15$ (considered as "NDDI-E-positive"). Clinical, demographic, imaging, and EEG patient characteristics were collected.

Results: There were only 20 patients $(33.3 \%)$ who were on antidepressant medications (ADM), including 9 who still had active depressive symptomatology (NDDIE score $>=15$ ). The mean number of ADM was 0.42, (range 0-3, median 0, standard deviation 0.7). Similarly for the anti-epileptic drugs (AEDs), the mean was 2.28 (range 0-5, median 2, standard deviation 1.2). The PHQ-9 mean score was 8.5 (range $0-26$, median 7 ). Twenty seven patients $(45 \%)$ had a PHQ-9 score of 10 or higher; while only 16 patients $(27 \%)$ had a
PHQ-9 score of 15 or higher. NDDIE mean score was 12, (range 622 , median 12). A quarter of our cohort displayed active depressive symptomatology (NDDI-E score $>=15$ ) with only half of those being on any ADM. Details of other results are depicted in the attached table and charts.

Conclusions: The prevalence of depression in epilepsy patients in our study is similar to the prior reported prevalence in the literature. Close to half the patients with active depression were not on any ADMs, and close to half the patients who were on depression treatment were not controlled suggesting challenges both with disease recognition and treatment. The PHQ-9 may be an alternative depression screening tool for patients with epilepsy, with optimal sensitivity and specificity achieved using cut-points of 11 or 12 rather than 10 . This is significant as the validation of this widely used scale in the specific population of epilepsy patients will allow cross comparisons and larger scale cohort studies evaluating depression across the variety of neurological disorders.
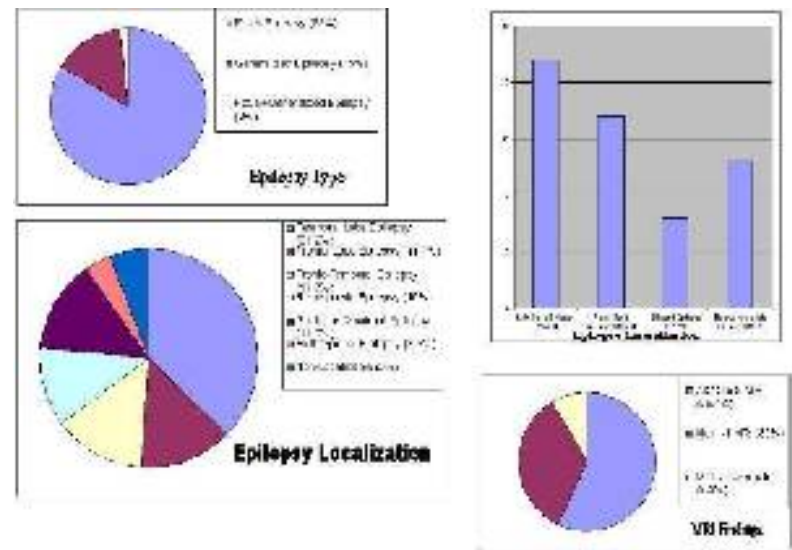

Epilepsy Type, Localization, Lateralization \& MRI Findings.

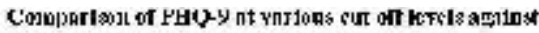
3 DUD if postrie cut of exore of 15 or Jupter.

\begin{tabular}{|c|c|c|c|c|}
\hline${ }^{-7} \mathrm{H}: 9$ & 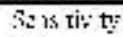 & $\Sigma x e$ ii & P.ritive & senarec \\
\hline Cul :Ir: & is/s; & f"...i & I'uccicions & I I cidictise \\
\hline Sicto & & & Valve i’ó & 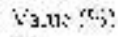 \\
\hline$\therefore-\mid$ Ir. & $\overline{8} 7 \bar{z}$ & 7 ic... & 52 & $\therefore 4$ \\
\hline$\because \quad 11$ & $7^{c}$ & 3it:- & $6: .7$ & ن.:5 \\
\hline$\therefore-17$ & $f \geq x$ & whis & $f x:$ & 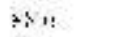 \\
\hline$\therefore-13$ & $2=$ & $\because$ & 7.4 & $\bar{Y} \bar{i}$ \\
\hline$\therefore \quad 11$ & $x_{-}=5$ & 9.3.2 & 77 & $\div 2.2$ \\
\hline$\therefore \quad 1:$ & $\mathrm{f}: \therefore$ & 544 & \&i: & $\therefore: 1$ \\
\hline
\end{tabular}

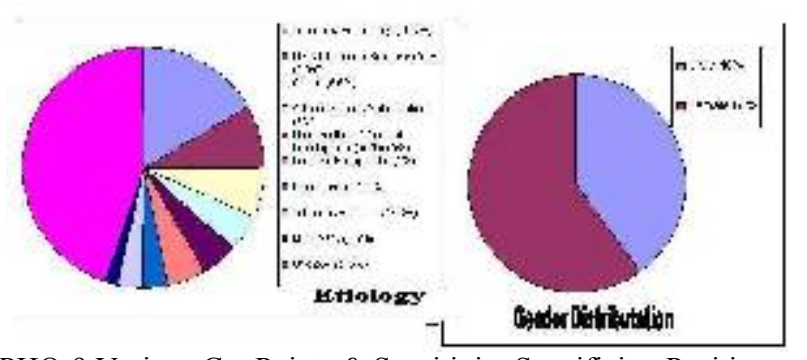

PHQ-9 Various Cut Points \& Sensitivity,Specificity, Positive and Negative Predictive Values Table.

\subsection{6}

MULTIFACTORIAL ORIGIN OF INTERICTAL BEHAVIOUR IN FRONTAL AND TEMPORAL LOBE EPILEPSY

Christoph Helmstaedter and J. A. Witt (Epileptology, University of Bonn, Bonn, Germany)

Rationale: Based on discussions of the of the so called "epileptic personality" in epilepsy patients, interictal behavioural impairments 
in frontal and temporal lobe epilepsies were examined in a multivariate approach which took subject variables, clinical and neuropsychological determinants into consideration.

Methods: A total of 428 patients epilepsies originating from the temporal $(84 \%)$ or frontal $(16 \%)$ lobes were examined in regard to personality (FPZ personality questionnaire) and mood (BDI I). Prevalence of impaired behavioural domains was determined. Etiologically relevant determinants of behavioural problems were identified via multiple regression analyses.

Results: Elevated depression scores (BDI) were evident in 38\% of the patients, and not different in TLE and FLE. As for personality, introversion, poorer mood, sociability, and self-determination as well as problems with interpersonal communication were frequent. The TLE group tended to show greater neuroticism and introversion, while FLE appeared more associated with aspects of an organic psycho-syndrome. Multivariate analyses indicate demographic (age, gender, education), clinical aspects (psychiatric history, affected hemisphere, mesial pathology, seizure frequency, cognitive functions), and treatment (antiepileptic drugs) as relevant determinants, explaining, however, maximally $30 \%$ of the behaviour. Conclusions: Behavioural abnormalities in patients with frontal or temporal lobe epilepsy are common but mostly mild. Within a multivariate etiological model, localization (mesial yes/no) and lateralization (left >right) dependent behavioural problems in TLE and FLE are overshadowed by other variables, of which patients and their families psychiatric history, person variables and pharmacological treatment are of major importance. Education and cognitive capabilities may be discussed as protective features.

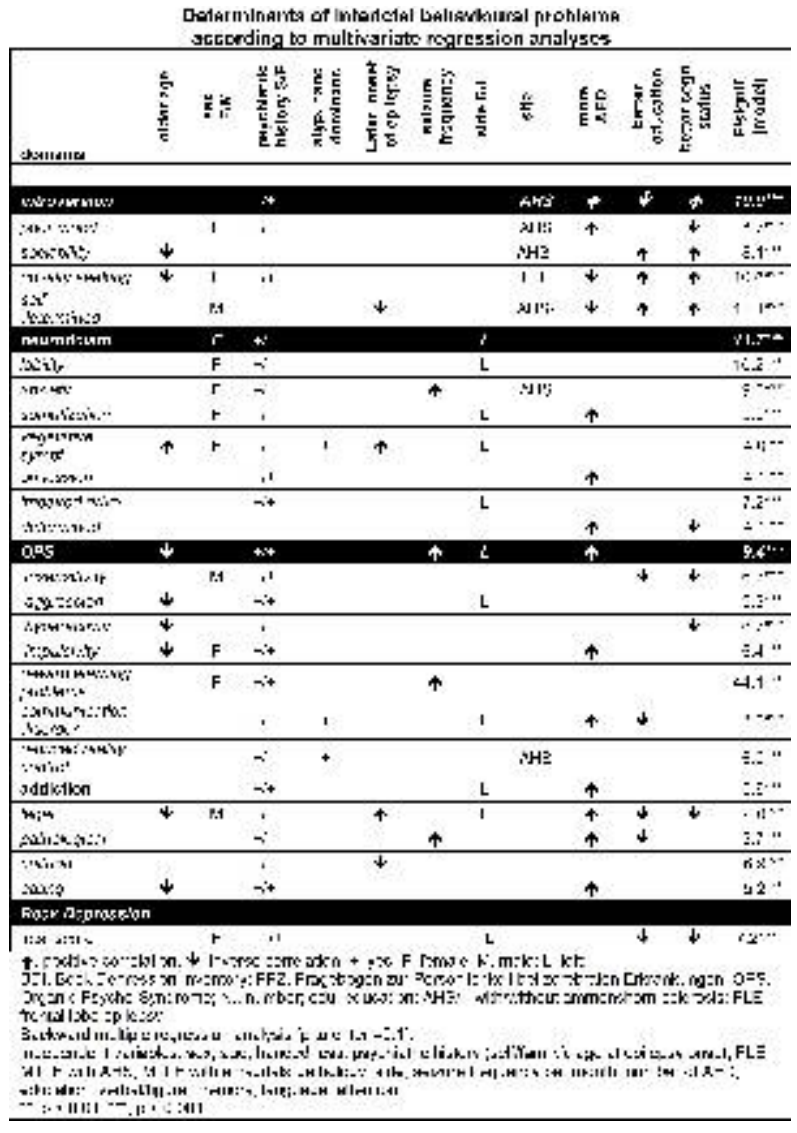

\subsection{7}

\section{WHAT IS SPECIFIC ABOUT THE PSYCHOLOGICAL} PROFILE OF PEDIATRIC PATIENTS WITH PNES/PNEE? M. Ransby ${ }^{1,2}$, K. Frampton ${ }^{1}$, K. Nash ${ }^{1}$, A. Ho ${ }^{2}$, M. Connolly ${ }^{1,2}$ and D. Kingdon ${ }^{3}\left({ }^{1}\right.$ Neurosciences Programme, BC Children's and Women's Hospital, Vancouver, BC, Canada; ${ }^{2}$ Departments of Pediatrics and Psychology, University of British Columbia, Vancouver, BC, Canada and ${ }^{3}$ Department of Psychology, Concordia University, Montreal, QC, Canada)

Rationale: Little is known about specific features of psychological functioning amongst pediatric samples with Psychological Non Epileptic Seizures (PNES) and Non Epileptic Events (PNEE). Comorbid psychopathology, including depression and anxiety, is one of the best predictors of prognosis in children with PNES, but this is not a distinguishing feature. Adult PNES patients have unique psychological characteristics, including higher rates of alexithymia, or the propensity to not express feelings, and less effective coping mechanisms in response to stress. Family dysfunction is also more frequently experienced. We sought to contribute to the question of what is specific about the psychological profile of PNES/PNEE patients compared to Epileptic (ES) and symptom-free patients in a pediatric sample. We hypothesized that despite comparable levels of depression and anxiety, the PNES/PNEE children would be (1) more alexithymic, (2) have weaker coping abilities, and (3) demonstrate more disrupted family relations.

Methods: We present data from a sample of 30 children (aged 7 to $19 ; 70 \%$ girls), including 16 PNES/PNEE patients and 9 ES patients, who were referred for psychological treatment by their neurologists. PNES/PNEE diagnoses were made following Video-EEG and/or imaging to rule out organic etiologies. Five symptom-free siblings of PNES/PNEE patients were also included. No differences in variables of interest emerged between the ES patients and the siblings so they were collapsed as controls. We administered a battery of standardized and experimental tests including parent- and/or child-report objective rating scales (e.g., Achenbach and Millon inventories), the Children's Alexithymia Measure using clinician ratings, standardized projective measures (Rorschach), and a projective measure of family relations (Family Relations Button Sort ${ }^{\mathrm{TM}}$ ).

Results: High proportions of the overall sample received clinically significant ratings of depression $(64 \%)$ or anxiety $(57 \%)$

symptomatology from either parent or child report; however, these psychiatric diagnoses did not distinguish between groups. Despite comparable ratings of internalizing psychopathology, the

PNES/PNEE group was rated as more alexithymic than controls $(\mathrm{p}<$ $0.01)$. In addition, clinically and statistically significant differences emerged on Rorschach indices, whereby the PNES/PNEE group showed greater sensitivity to others' emotions $(\mathrm{p}<0.02$; Affective Ratio, Afr) and poorer coping ability ( $\mathrm{p}<0.05$; Coping Deficit Index, $\mathrm{CDI}$ ). Contrary to predictions, no differences were found regarding child perceptions of family relations.

Conclusions: Symptoms of anxiety and depression do not distinguish PNES/PNEE from ES or symptom-free patients in a pediatric sample. Rather, their psychological profile is characterized uniquely by greater alexithymia, sensitivity to others' emotional expression, and poorer coping. Thus, when planning optimal psychotherapy interventions for pediatric patients with PNES/PNEE, the therapist must consider their non-specific psychiatric diagnoses as well as their more unique alexithymia, emotional sensitivity, and coping deficits.

\section{Antiepileptic Drugs: Cohort Studies}

\subsection{8 \\ SODIUM VALPROATE IS ASSOCIATED WITH PARIETAL LOBE CORTICAL THINNING AND REDUCED BRAIN VOLUME}

Heath R. Pardoe ${ }^{1}$, A. T. Berg ${ }^{2}$ and G. D. Jackson ${ }^{1,3}$ ( ${ }^{1}$ Brain Research Insitute, Florey Neuroscience Institutes, Melbourne, VIC, Australia; ${ }^{2}$ Department of Pediatrics, Ann \& Robert H. Lurie Children's Hospital of Chicago, Chicago, IL and ${ }^{3}$ Department of Medicine, University of Melbourne, Melbourne, VIC, Australia)

Rationale: Sodium valproate is one of the most widely used antiepileptic medications. Mental deterioration with reversible brain pseudoatrophy has been reported as a rare adverse effect associated 
with valproate [1]. The aim of this study was to determine if there are group-level structural brain changes associated with valproate use for seizure control in epilepsy. We used quantitative analysis of structural MRI to investigate cortical thickness and brain volume differences in epilepsy patients currently taking valproate, compared with epilepsy patients not currently taking valproate and controls. Methods: Cohort A: Intractable epilepsy subjects referred for 3T imaging as part of the Comprehensive

Epilepsy Program, Austin Hospital, Melbourne.

Cohort B: Community-based childhood-onset epilepsy subjects recruited as part of the Connecticut

Study of Epilepsy in Children.

Age-matched controls were included for each cohort. Whole brain T1-weighted MRI was acquired. MRI scans were processed using Freesurfer 5.1. Cortical thickness values were averaged over each major brain lobe (frontal, parietal, occipital and temporal). Total brain (TBV) and white matter volume (WMV) was measured. Thickness and volume differences were investigated in epilepsy subjects currently taking valproate relative to epilepsy subjects not currently taking valproate and controls. Age was included as a covariate in all analyses. Cohort A was the primary cohort; Cohort B was used to confirm findings from Cohort A.

Results: Cohort A: Nine epilepsy subjects currently taking valproate (age $33 \pm 8.1$ years), 27 epilepsy subjects not taking valproate $(34.7 \pm$ 10.3 years), 45 controls (age $29.5 \pm 10.2$ years) were included. All subjects were male. Parietal lobe thickness was reduced in the valproate group relative to controls $(0.1 \mathrm{~mm}, \mathrm{p}<0.01)$. No thickness differences were observed in other lobes. White matter and whole brain volumes were reduced in the valproate group relative to controls (WMV \& TBV: $\mathrm{p}<0.05$ ). No changes were observed in the non-valproate epilepsy group.

Cohort B: Eleven epilepsy subjects taking valproate (age 24.6 \pm 3.7 years, 4 female), 122 epilepsy subjects not taking valproate $(23 \pm 3.1$ years, 63 female) and 38 controls (age $23.3 \pm 3.3$ years, 19 female) were included. The finding of reduced parietal thickness in the valproate group was replicated in Cohort B $(0.07 \mathrm{~mm}, \mathrm{p}<0.05)$. White matter and brain volumes were reduced in the valproate group (WMV: $\mathrm{p}<0.05$, TBV: $\mathrm{p}<0.1$ ). No thickness or volume reductions were observed in the non-valproate epilepsy group or other cortical lobes.

Conclusions: Valproate treatment for seizure control in epilepsy is associated with subtle cortical thinning in the parietal lobes, and white matter and whole brain volume reductions. Because a significant proportion of the non-valproate groups would have taken valproate in the past, the observed effect is likely transient. Funding: NHMRC program grant 628952 (Australia), NIH-NINDS R37-NS31146

References:[1] Guerrini, R. et al (1998).Epilepsia 39(1):27-32.

\subsection{9}

COMPARATIVE EFFECTIVENESS OF LEVETIRACETAM AND OXCARBAZEPINE AS FIRST DRUG MONOTHERAPY FOR CHILDREN WITH FOCAL EPILEPSY

Sudha K. Kessler ${ }^{1,2}$ and J. Wilson ${ }^{1,2}\left({ }^{1}\right.$ Neurology and Pediatrics, Perelman School of Medicine at the University of Pennsylvania, Philadelphia, PA and ${ }^{2}$ Children's Hospital of Philadelphia, Philadelphia, PA)

Rationale: To assess effectiveness of two commonly used antiepileptic drugs (AED) for new onset focal epilepsy in children, using drug failure rate as the primary outcome measure.

Methods: We conducted a retrospective cohort study of children ages 1 to 17 years with new onset focal epilepsy presenting to a single referral center between January 2008 and June 2010. Potential subjects were identified through billing codes and eligibility was confirmed by subsequent review of electronic medical records. Exclusion criteria included status epilepticus or hospitalization for greater than 48 hours at presentation, and less than 6 months of follow up information available. Differences in baseline characteristics between treatment groups were evaluated using t-tests and chi-square tests. Kaplan-Meier methods were used to estimate the rates of drug failure, defined as discontinuation of first AED or addition of a second AED. Factors potentially influencing time to failure (including reason for failure, age at presentation, presence of co-morbid conditions, and epilepsy etiology) were evaluated using Cox proportional hazards models.

Results: Of 291 patients meeting inclusion criteria, 220 were initiated either on levetiracetam (LEV, $\mathrm{n}=93$ ) or on oxcarbazepine $(\mathrm{OXC}, \mathrm{n}=127)$. Median age at first clinic visit was 7.3 years (range $1.2-17$ years), and $53.6 \%$ of subjects were male. Treatment groups were similar with respect to age, sex, presentation to ER or hospitalization at time of diagnosis, presence of co-morbid conditions, and presence of EEG abnormalities. The study comprised 1429 person-treatment months for LEV and 2140 person-treatment months for OXC. Amongst all subjects, probability of remaining on the first AED was $76 \%$ at 6 months, and $58 \%$ at 40 months. Drug failures due to lack of tolerability occurred sooner (median time 1.7 months) compared to failures due to lack of efficacy (median time 4.56 months). Time to drug failure was shorter in patients on LEV $(\mathrm{p}=0.04, \log$ rank test), and this was accounted for by the higher number of tolerability failures for LEV (19 of 93) compared to OXC (16 of 127). Rate of drug failure was 2.8 per 100 person months for LEV and 1.7 per 100 person months for OXC (hazard ratio 1.6, $\mathrm{p}=0.04$ ). No other factors influenced differences in drug failure rates. Conclusions: Overall, LEV and OXC are effective medications for treatment of new onset pediatric epilepsy, with the majority of patients continuing on monotherapy with the first drug for two years. Most drug failures occurred within the first six months, and tolerability failures occurred earlier than efficacy failures. OXC had a lower failure rate than LEV, a finding primarily related to tolerability. These data may help guide AED choice for children with new onset focal epilepsy.

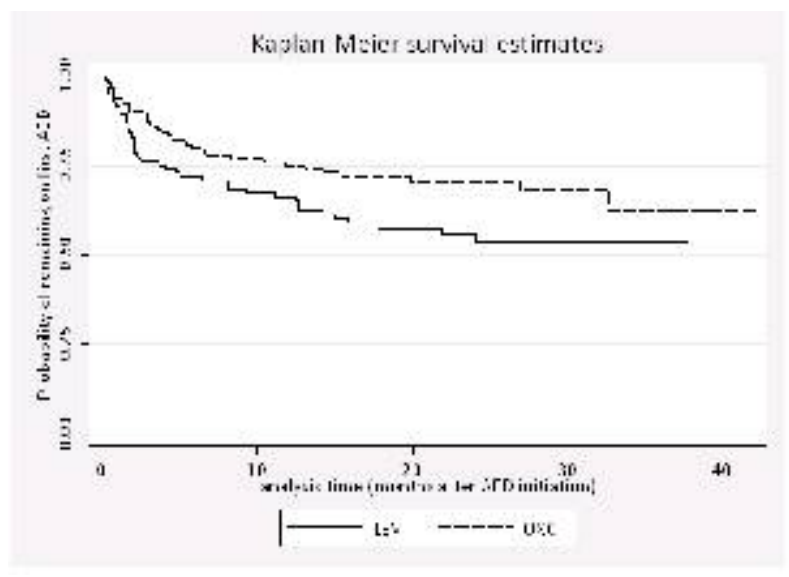

\subsection{0 \\ PROSPECTIVE POST-AUTHORIZATION OBSERVATIONAL STUDY OF ESLICARBAZEPINE (ESL) IN THE TREATMENT OF PHARMACORESISTANT EPILEPSIES}

Rodrigo A. Rocamora ${ }^{1}$, A. Massot ${ }^{1}$, R. Vivanco ${ }^{1}$, A. Principe ${ }^{2}$ and J. Roquer ${ }^{1}\left({ }^{1}\right.$ Neurology, Hospital del Mar, Barcelona, Spain and ${ }^{2}$ Neurophysiology, Hospital del Mar, Barcelona, Spain)

Rationale: Eslicarbazepine acetate (ESL) is a new antiepileptic drug (AED) analogue to carbamazepine (CBZ) and oxcarbazepine (OXC) authorized as add-on therapy in focal pharmacoresistant epilepsies. ESL was introduced in Spain in January 2011. The aim of this study is to analyze the efficacy, adverse-events profile, used dosage, early retention rate and combinations with other AEDs. Furthermore, in a subgroup of patients, the overnight switch from CBZ and OXC to ESL was evaluated. 
Methods: 61 patients with a broad spectrum of pharmacoresistant epilepsies were prospectively evaluated after the beginning of ESL therapy. The initiation of ESL was performed both in an in-patient fashion and in the outpatient's clinic. The overnight switch from $\mathrm{CBZ}$ and $\mathrm{OXC}$ was carried-out in an equivalence of $1: 1,3$ and $1: 1$ respectively during a VEEG monitoring.

Results: 19 male and 42 female patients were included in the observational study. The average age was 43 y.o. (SD 14.4). The median follow-up period was 4 months (range 1,5-8). The most frequent epilepsy class was temporal lobe epilepsy (TLE) $(62,3 \%)$, malformations of cortical development $(13,1 \%)$ and secondary to traumatic brain injury $(9,8 \%)$. The average seizure frequency was 18,9 seizures/month. 12 patients were treated with $400 \mathrm{mg}$ ESL $(19,7 \%) ; 26$ with $800 \mathrm{mg}(42,6 \%) ; 15$ with $1200 \mathrm{mg}(24,6 \%)$ and 8 with $1600 \mathrm{mg}(13,1 \%)$. The seizure frequency variation was evaluated at 3 months in a subgroup of 40 patients. The average seizure frequency dropped from 22 to 9 seizures/month. 5 patients were seizure-free $(12,5 \%), 12(30 \%)$ had a reduction $\geq 80 \%, 6$ patients (15\%) showed a reduction of 50\%, $5(12,5 \%)$ presented a reduction of $30 \%, 5$ patients $(12,5 \%)$ reported no changes and 7 got worse $(17,5 \%)$. The most important AEs were unsteadiness, dizziness and nausea $(31,1 \%)$, cognitive and mood complaints $(9,8 \%)$ and sleep disorders $(16,4 \%)$. Of the AEs, $54,3 \%$ appeared only during the titration phase. Two skin allergic reactions were reported and in 8 cases a mild hyponatremia were detected. The early retention rate at 3 months was $75,4 \%$. The switch from CBZ or OXC to ESL was conducted in 25 cases. In all cases, AEs were well tolerated and transitory ( $61,5 \%$ and $33 \%$ respectively). 3 months after the switch, 9 out of 19 (47\%) showed an improvement in seizure frequency.

Conclusions: ESL shows a positive efficacy in the treatment of focal epilepsies with a early retention rate $>70 \%$. The AE profile occurs during the titration phase and is modulated by the associated AEDs. The overnight switch from CBZ and OXC to ESL could be performed in a safe manner.

\subsection{1}

SAFETY PROFILE OF ESLICARBAZEPINE ACETATE: TWO YEAR EXPERIENCE IN A TERTIARY HOSPITAL Joel Freitas $^{1}$, F. Correia ${ }^{2}$, R. Loureiro ${ }^{3}$, R. Magalhaes ${ }^{4}$, J. Lopes ${ }^{5}$, J. Ramalheira $^{6}$, J. Lopes-Lima ${ }^{7}$ and J. Chaves ${ }^{8}\left({ }^{1}\right.$ Neurology, Hospital Santo Antonio - CHP, Oporto, Portugal; ${ }^{2}$ Neurology, Hospital Santo Antonio - CHP, Oporto, Portugal; ${ }^{3}$ Neurology, Hospital Santo Antonio - CHP, Oporto, Portugal; ${ }^{4}$ UNIFAI, ICBAS - UP, Oporto, Portugal; ${ }^{5}$ Neurophysiology, Hospital Santo Antonio - CHP, Oporto, Portugal; ${ }^{6}$ Neurophysiology, Hospital Santo Antonio - CHP, Oporto, Portugal; ${ }^{7}$ Neurology, Hospital Santo Antonio - CHP, Oporto, Portugal and ${ }^{8}$ Neurology, Hospital Santo Antonio - CHP, Oporto, Portugal)

Rationale: Eslicarbazepine acetate (ESL) is a voltage-gated sodium channel blocker approved in Europe since 2009. It is available in Portugal since April 2010. In spite of the good safety and efficacy shown in clinical trials, little is known about its safety and effectiveness in clinical practice.

Our aim was to assess the safety profile and retention rates of ESL in the outpatient clinic patients, from our tertiary epilepsy center. Methods: All outpatients who initiated treatment with ESL between 04/01/2010 and 03/31/2012 were consecutively included. We retrospectively collected data on demographics, clinical features, neurophysiology, imaging, treatment response, compliance and side effects. Only patients with at least one follow-up visit were included in the safety analysis. Follow-up time was defined as the time between ESL initiation and the last follow-up visit.

Results: We included 148 patients in the safety analysis, $72(48.6 \%)$ were women. Mean age at ESL initiation was 38.5 years-old $(\mathrm{sd}=14.4)$, mean epilepsy duration was 26.0 years $(\mathrm{sd}=13.3)$ and median seizure frequency was 10 seizures/month. At the baseline, $58.8 \%$ were taking $\leq 2$ antiepileptic drugs (AEDs).
The median follow-up time was 12.3 months $(\mathrm{IQR}=5.4-20.1)$. The retention rates at 6 months, 12 months and 18 months, were $80.1 \%$ (109/136 patients), 65.8\% (75/114) and 52.7\% (48/91), respectively. Forty five patients (30.4\%) discontinued ESL during the follow-up; 2 after 18 months. Therapy discontinuation was due to side effects in 28 patients $(62.2 \%)$, lack of efficacy in 14 patients $(31.1 \%)$ and other causes in 3 patients $(6.7 \%)$.

Total adverse event rate was $39.2 \%$ (58 patients); in 51.7\% (30 patients) the adverse events did not lead to treatment discontinuation. The most frequent adverse events were somnolence/cognitive slowness (16 patients), dizziness (15) and gastrointestinal symptoms (7).

Conclusions: In our series, ESL appears to be well-tolerated. Adverse events were present in approximately 2 out of 5 patients, but only in half led to the discontinuation of medication.

\subsection{2 \\ HEALTH OUTCOMES ASSOCIATED WITH SEQUENTIAL MONOTHERAPY AND COMBINATION THERAPY WITH ANTIEPILEPTIC DRUGS IN PATIENTS WITH PARTIAL ONSET SEIZURES}

J. E. Cavazos ${ }^{1}$, R. Simons ${ }^{2}$, R. Fain ${ }^{2}$, A. Powers ${ }^{2}$ and Zhixiao Wang ${ }^{2}$ $\left({ }^{1}\right.$ University of Texas Health Science Center, San Antonio, TX and ${ }^{2}$ Eisai Inc, Woodcliff Lake, NJ)

Rationale: Traditionally, for patients with partial onset seizures (POS) who fail initial antiepileptic drug (AED) monotherapy, physicians try sequential monotherapies (SM) before considering combination therapy (CT). However, relatively fewer of those patients (potentially refractory) achieve complete control with SM trials, which may have significant cost implications. This study compared seizure-related hospitalizations (SRH) and overall healthcare costs in POS patients receiving SM versus CT.

Methods: POS patients (ICD-9 codes 345.4 and 345.5) during the calendar year 2009 were identified from the MarketScan Commercial and Medicare Database (32 million covered lives) and followed into 2010. 2008 data were used to ensure that patients had no previous epilepsy diagnoses or AED use. Patients were assigned to one of three treatment algorithms: SM, CT at onset (CT), and initial SM followed by CT (SMCT). Patients on SM received several single agents while patients on $\mathrm{CT}$ were treated with two or more agents within 29 days following initial AED prescription and maintained combination therapy for at least 60 days. Patients on only one AED or diagnosed with bipolar disorder, migraines or headache were excluded. SRH were limited to those with a primary or secondary diagnosis of POS. Costs were accrued for inpatient and outpatient services with a diagnosis of epilepsy and AED pharmacy costs. SRH and costs were adjusted for time on treatment. Mean and standard errors for average monthly costs were evaluated for total health care as well as by type of service.

Results: 34,311 patients with newly diagnosed POS that received AEDs were initially identified. Patients receiving only one AED $(\mathrm{n}=20,035)$ were excluded, while patients receiving SM $(n=3,112)$, CT $(n=7,728)$, and SMCT $(n=3,426)$ comprised the analysis population. Mean age was approximately 37 years with $45 \%$ male. At baseline, $6 \%$ of patients had diabetes, $13 \%$ hypertension, and $3 \%$ depression. There was no statistical difference in baseline demographics and comorbidities across treatment algorithms. The marginal cost per seizure-related hospitalization was $\$ 29,602$ (STDERR: 271.78). Patients treated with SM had SRH of 0.055 per month compared to $0.028(\mathrm{P}<0.01)$ for patients treated with $\mathrm{CT}$. Patients treated with SMCT had SRH of 0.085 and 0.001 per month during the pre- and post-combination period respectively. Mean total monthly reimbursed costs were $\$ 4,547$ (202.56) for SM, $\$ 2,209$ (201.02) for CT, and $\$ 3,464$ (207.93) for those who received SMCT. Outpatient pharmacy consisted of $\$ 2,620$ (93.84), \$1,206 (59.74) and $\$ 2,340$ (126.44) of total costs, respectively. 
Conclusions: Our study showed that for patients with potentially refractory epilepsy, combination therapy led to fewer seizure-related hospitalizations than sequential monotherapy, and consequently, lower overall healthcare costs. Therefore, early identification of these potentially refractory patients and appropriate AED management may help those patients achieve earlier and improved seizure control and also reduce healthcare costs for payers.

\subsection{3 \\ LACOSAMIDE AND DECREASED MORTALITY IN REFRACTORY STATUS EPILEPTICUS: A COMPARATIVE COHORT STUDY}

Stephan Rueegg ${ }^{1}$, S. Marsch ${ }^{2}$ and R. Sutter ${ }^{3}$ ( ${ }^{1}$ Neurology, University Hospital Basel, Basel, Switzerland; ${ }^{2}$ Intensive Care Medicine, University Hospital Basel, Basel, Switzerland and ${ }^{3}$ Division of Neuroscience Critical Care, Department of Anesthesiology, Critical Care Medicine and Neurology, Johns Hopkins University School of Medicine, Baltimore, MD)

Rationale: Refractory status epilepticus (RSE) is a life-threatening neurologic emergency with high morbidity and mortality requiring prompt neurointensive care. To date, established treatment paradigms include first generation antiepileptic drugs (AED) and narcotics. Lacosamide (LCM) is a new broad spectrum AED with a dual mode of action, holding promises as a potent treatment option for RSE. However, high-class evidence regarding safety and efficacy in treatment of RSE is lacking. The objective of the study was to evaluate the safety and efficacy of i.v. administered LCM as an addon treatment in patients with RSE

Methods: To evaluate the safety and efficacy of i.v. administered LCM as an add-on treatment in patients with RSE. Medical chart review was performed of all consecutive patients treated for RSE between January 2005 and December 2011 at the intensive care units of the University Hospital Basel (Switzerland).

Results: 111 of $260(42.7 \%)$ patients with SE developed RSE of which 59 (53.2\% of RSE) consecutive patients were treated with i.v. LCM. In patients with LCM RSE could be terminated significantly more often than in patients without LCM (OR 3.91, p=0.015) and mortality was significant lower (OR $0.37, p=0.014$ ), even when adjusted for age, resuscitation and stroke (OR 3.73, $\mathrm{p}=0.04$; OR 0.33, $\mathrm{p}=0.018$ ). There were no serious adverse events due to antiepileptic treatment.

Conclusions: Patients with i.v. LCM had significant decreased mortality and more frequent cessation of RSE compared to patients without LCM after adjusting for potential confounders. LCM was well tolerated and safe in this cohort of patients with severe RSE. Prospective studies are warranted to further strengthen evidence of efficacy, safety and tolerability of LCM for the treatment of RSE.

\subsection{4 \\ INTRAVENOUS KETAMINE FOR REFRACTORY STATUS EPILEPTICUS: A RETROSPECTIVE MULTICENTER STUDY FROM THE CRITICAL CARE EEG RESEARCH CONSORTIUM}

Nicolas Gaspard ${ }^{1}$, L. M. Judd ${ }^{2}$, B. $\mathrm{McCoy}^{3}$, A. Al-Otaibi ${ }^{3}$, C. D. $\mathrm{Hahn}^{3}$, R. Kilbride ${ }^{4}$, I. Sanchez Fernandez ${ }^{5}$, T. Loddenkemper ${ }^{5}$, L. C. Mendoza $^{6}$, J. P. Szaflarski ${ }^{6}$, L. J. Hirsch ${ }^{1}$ and S. M. LaRoche ${ }^{2}$ $\left({ }^{1}\right.$ Neurology and Comprehensive Epilepsy Center, Yale Comprehensive Epilepsy Center, New Haven, CT; ${ }^{2}$ Neurology and Comprehensive Epilepsy Center, Emory University University, Atlanta, GA; ${ }^{3}$ Paediatrics - Division of Neurology, The Hospital for Sick Children - University of Toronto, Toronto, ON, Canada; ${ }^{4}$ Neurology - Epilepsy and Clinical Neurophysiology Services, Massachussets General Hospital, Boston, MA; ${ }^{5}$ Neurology - Division of Epilepsy and Clinical Neurophysiology, Boston Children's Hospital, Boston, MA and ${ }^{6}$ Neurology and the Neuroscience Institute, University of Cincinnati Academic Health Center,
Cincinnati, $\mathrm{OH}$ )

Rationale: The treatment of refractory status epilepticus (RSE) is notoriously difficult. Glutamate antagonists might be more efficient than GABA agonists in the late stage of status epilepticus. Ketamine is an NMDA receptor antagonist with an attractive safety profile because it does not cause hypotension. It is occasionally used for this indication although current evidence to support its use is weak. We reviewed the safety and efficacy of ketamine for RSE.

Methods: We conducted a retrospective multicenter study involving 6 academic centers. Medical records and EEG reports were reviewed of all patients receiving IV ketamine for RSE from 2004 to 2012. We included children and adults. Continuous EEG was used in all but one case.

Results: We identified 22 patients and 23 episodes of RSE treated with ketamine. Mean age was $28+/-4$ years ( 7 months- 74 years). The most common etiologies were CNS infection (5), autoimmune disease (4), malformation of cortical development (4) and genetic disorder (3). Five cases had purely electrographic status epilepticus while the remaining patients had associated clinical manifestations, including generalized (4), partial (3) and subtle (7) motor activity. In most cases, ketamine was introduced at least 1 week after the onset of SE (median time: 12 days; range: 1-44 days). An initial loading dose was commonly used $(1.5-2.5 \mathrm{mg} / \mathrm{kg})$, followed by continuous infusion (usual initial rate of $0.1-0.5 \mathrm{mg} / \mathrm{kg} / \mathrm{h}$ ). The maximal infusion rate was typically $1-2 \mathrm{mg} / \mathrm{kg} / \mathrm{h}$, although in 4 patients it was greater than $5 \mathrm{mg} / \mathrm{kg} / \mathrm{h}$. The duration of ketamine treatment ranged from 2 to 24 days. Vasopressors were increased in 8 patients but only one developed new onset hypotension. Three patients developed severe acidosis that was attributed to shock and acute kidney injury in 2 cases and a syndrome similar to propofol infusion syndrome (PRIS) in the third patient. SE was permanently controlled within $72 \mathrm{~h}$ of ketamine initiation in 7 patients. However, ketamine was the last drug to be used in only 1 of these patients; the others received at least one additional drug, most commonly benzodiazepine (7) and phenytoin/fosphenytoin (6). Overall, status epilepticus was temporarily controlled in 14 cases and permanently controlled in 10 cases.

Conclusions: Assessing the efficacy of ketamine in RSE is difficult as it is used in the late stage of refractory SE and with a wide range of doses. This study indicates that ketamine is associated with a low incidence of identifiable serious adverse events, although we describe for the first time its association with a syndrome similar to PRIS. It is often transiently effective, and can be associated with permanent cessation of RSE in some patients. Further study is needed before recommendation can be made to extend its usage, but its hemodynamic profile, mechanism of action and preliminary results are promising. It should be included in future studies on RSE.

\subsection{5 \\ IMPACT OF CONCOMITANT USE OF ANTIEPILEPTIC DRUGS AND STATINS ON RISK OF CARDIOVASCULAR EVENTS}

Sudeep Karve ${ }^{1}$, D. Mitra ${ }^{1}$, K. Rajagopalan ${ }^{2}$, D. Blum ${ }^{2}$, T. Grinnell ${ }^{2}$ and V. Bollu ${ }^{2}\left({ }^{1}\right.$ RTI Health Solutions, Research Triangle Park, NC and ${ }^{2}$ Sunovion Pharmaceuticals Inc, Marlborough, MA)

Rationale: Studies suggest that patients taking enzyme inducing (cytochrome P450) antiepileptic drugs (EIAEDs) and hepatically metabolized statins (HMS) show an increased clearance of statins, which may lower statin efficacy and subsequently increase cardiovascular event risk (Murphy, 1999; Khandwala, 2006). The objective of the current study was to evaluate whether concomitant use of EIAEDs and HMS is associated with increased cardiovascular events in epilepsy patients compared to patients on enzyme neutral AEDs (ENAEDs) and non hepatically metabolized statins (NHMS). Methods: A retrospective longitudinal analysis of a large electronic medical records (EMR) database for the years 1996 - 2009 was conducted. Patients (aged $\geq 12$ years) diagnosed with epilepsy and 
using AEDs were selected and classified into 6 mutually exclusive groups (EIAED+HMS; EIAED+ NHMS; ENAED+HMS;

ENAED+NHMS; EIAED only; ENAED only) based on concomitant statin use. Patients receiving multiple AEDs or switched from their index AED were removed. Using covariate-adjusted cox proportional hazard regression analyses, incidence and relative risk of a CVD event (composite measure based on: myocardial infarction, stroke, transient ischemic attack, congestive heart failure and angina) during the follow-up period among patients in the $6 \mathrm{AED}$ /statin groups were assessed.

Results: A total of 21,963 epilepsy patients using AEDs of which $19.5 \%$ also had evidence of statin use were included. Overall, the mean (SD) age of the cohort was 46 (18) years and over 55\% were male. Patients receiving statins concomitantly were older (58 vs 42) and had higher prevalence of other risk factors for CVD like diabetes and hypertension. The mean follow-up time for the cohort was 2.1 years and ranged from 1.5 years for ENAED+NHMS to 2.4 years EIAED users. Of those patients receiving AEDs and statins concomitantly, any CVD event was observed among $11.3 \%$ of EIAED+HMS, $9.6 \%$ of ENAED+NHMS, $8.8 \%$ of EIAED+NHMS, and $8.7 \%$ of ENAED+NHMS users. Congestive heart failure was the most commonly observed CVD event among EIAED+HMS (4.7\%), EIAED+NHMS (3.9\%) and ENAED+ HMS (3.1\%). The likelihood of a CVD event did not differ significantly between EIAED+NHMS (hazard ratio [HR]: 0.83; 95\% confidence interval [CI]: 0.59-1.17), ENAED+HMS (HR:1.02; 95\% CI: 0.82-1.27), ENAED+NHMS (HR:1.00; 95\% CI: 0.58-1.71) and EIAED+HMS group (Reference group).

Conclusions: The analysis findings suggest that concomitant use of enzyme inducing AEDs and hepatically metabolized statins was not associated with increased risk of CVD events during the follow-up period. Studies with longer follow-up duration are required to determine the impact of long-term concomitant use of enzyme inducing AEDs and statins on CVD events.

\subsection{6 \\ ADJUNCTIVE RETIGABINE IN DRUG-RESISTANT LOCALISATION-RELATED EPILEPSIES}

Kevin Kelly, L. J. Stephen, P. Parker and M. J. Brodie (Epilepsy

Unit, Western Infirmary, Glasgow, United Kingdom)

Rationale: When a new drug is licensed, the only available information on its use comes from placebo-controlled, dose-ranging, randomized clinical trials. This prospective audit explored outcomes with the recently introduced retigabine (RTG), a novel first-in-class neuronal $\mathrm{K}+$ channel opener, as adjunctive treatment in everyday clinical practice.

Methods: After 12 weeks on stable AED dosing, RTG was introduced in a three times daily schedule and titrated as necessary in patients with drug-resistant localization-related epilepsy. Review took place every 6-8 weeks until 1 of 4 end-points was reached: seizure freedom for $>6$ months on a given RTG dose; $>50 \%$ (responder) or $<50 \%$ (marginal benefit) seizure reduction over 6 months compared with baseline on the highest tolerated RTG dose; withdrawal of RTG due to lack of efficacy, side effects or, or both. Results: To date, 31 of 51 recruited patients $(23 \mathrm{M} ; 28 \mathrm{~F}$, age range 20-67 years [median 45 years]) with uncontrolled partial-onset seizures with or without secondary generalisation (monthly frequency 1-60; [median 3]) have reached an endpoint. They received a median of one (range 1-4) antiepileptic drug (AED), having previously tried 1-14 schedules (median 3). Seizure freedom has been achieved in 3 (9.7\%) patients (150mg, 300mg, 600mg RTG daily) so far. All took RTG in combination with lamotrigine. Three $(9.7 \%)$ other patients could be classified as responders, $(600 \mathrm{mg}, 600 \mathrm{mg}$, $900 \mathrm{mg}$ RTG daily) with $11(35.5 \%)$ more demonstrating marginal benefit and remaining on the drug (300 - 1200mg RTG daily). RTG was withdrawn in $14(28 \%)$ patients, all due to side-effects ( 8 taking 150mg, 3 taking 300mg, 1 taking 450mg, 1 taking 600mg, 1 taking 900mg all in a thrice daily schedule). Problems resulting in drug withdrawal included headache, dizziness, diplopia, ataxia, agitation, sedation, tiredness, depression, parasthaesia and poor sleep pattern. Three of these patients developed urinary hesitancy and/or frequency. There were no differences in mean World Health Organisation Defined Daily Doses (http:/www.whocc.no/atc_ddd_index/.) of adjunctive AEDs in patients reaching a positive endpoint $(\mathrm{n}=17$; 1.97) compared with those not tolerating RTG $(n=14 ; 1.94)$.

Conclusions: Adjunctive RTG was effective and well-tolerated in some patients with drug-resistant localization-related epilepsy. Others discontinued treatment due to a range of side-effects, often at low dosage. These patients did not have a higher baseline drug burden than those tolerating RTG administration. No patients have come off treatment due to lack of efficacy alone at this time.

\subsection{7 \\ TREATMENT OF REFRACTORY SEIZURE CLUSTERS AND STATUS EPILEPTICUS WITH INTRAVENOUS LACOSAMIDE: SINGLE CENTRE RETROSPECTIVE STUDY COMPARING TWO INITIAL DOSES: 200 AND 400} MG

Benjamin Legros ${ }^{1}$, C. Depondt ${ }^{1}$, M. Levy-Nogueira ${ }^{1}$, N. Ligot ${ }^{1}, N$. Mavroudakis $^{1}$, G. Naeije ${ }^{1}$ and N. Gaspard ${ }^{2,1}$ ( Neurology, Hôpital Erasme, Brussels, Belgium and ${ }^{2}$ Yale University Comprehensive Epilepsy Center and Computational Neurophysiology Laboratory, Yale University, New Haven, CT)

Rationale: The treatment of refractory status epilepticus (RSE) or seizure clusters remains empirical because of the lack of evidencebased data. The place of new antiepileptic drugs (AEDs) remains largely unknown. Lacosamide (LCM) is a new AED available in intravenous (IV) form with a good safety profile. Small series suggested that it might be useful in RSE. However, the relationship between dose, efficacy and safety has not been studied. We report here our experience with IV LCM, comparing 2 IV initial doses, 200 and $400 \mathrm{mg}$.

Methods: We retrospectively reviewed the charts of all consecutive patients who received IV LCM for status epilepticus or seizure clusters between October 2010 and June 2012. The first patients received an IV load of $200 \mathrm{mg}$ of LCM, followed 12 hours later by another dose of $200 \mathrm{mg}$ (group 1). Due to poor initial results with this dosage, we decided to increase the loading dose to $400 \mathrm{mg}$ of LCM followed by $200 \mathrm{mg} 12$ hours later (group 2). Seizure cessation after LCM, seizure cessation after another AED treatment, and mortality were studied. Continuous video EEG monitoring was used in all patients.

Results: Group 1 included 11 patients (mean age 51; range 32-74). Six patients were in non convulsive status epilepticus (NCSE), 3 were in post convulsive NCSE, and 2 had seizure clusters. LCM was used in third line in 5 patients, fourth in 4, fifth in 1 and sixth in 1. Intravenous LCM was the last drug added in 2 patients (18\%). Six patients needed further AEDs to stop seizing; in 1 patient, seizure cluster worsened and 2 patients died uncontrolled. One patient with renal failure developed confusion and myoclonus, which disappeared after LCM dose reduction.

Group 2 included 12 patients (mean age 56; range 17-84). Eight patients were in NCSE, 2 were in post convulsive NCSE, 1 was in convulsive status epilepticus and 1 had seizure cluster. LCM was used in second line in 1 patient, third in 1, fourth in 7, and fifth in 3. LCM was the last drug added in 6 patients $(50 \%)$. Two patients needed another AED treatment to stop seizing, and 4 patients died uncontrolled. No adverse event occurred.

Conclusions: In this small retrospective study, an initial dose of 400 $\mathrm{mg}$ of IV LCM seemed to be superior to $200 \mathrm{mg}$, stopping RSE or seizure clusters in $50 \%$ of the patients. It was not associated with a higher rate of identifiable serious adverse events, including cardiac arrhythmia. This study also further suggests that LCM should be considered for the treatment of RSE and seizure clusters. Further 
prospective studies are needed to confirm these findings and define the place of LCM in the therapeutic algorithm of SE.

\subsection{8 \\ PREVALENCE OF BASELINE HYPONATREMIA AND ASSOCIATION WITH INITIAL ANTIEPILEPSY TREATMENT SELECTION AMONG EPILEPSY PATIENTS}

V. Bollu ${ }^{2}$, Sudeep Karve ${ }^{1}$ and D. Mitra ${ }^{1}\left({ }^{1}\right.$ RTI Health Solutions, Research Triangle Park, NC and ${ }^{2}$ Sunovion Pharmaceuticals Inc, Marlborough, MA)

Rationale: Prior research suggests the use of sodium channel blocker antiepileptic drugs (AEDs) is associated with higher risk of hyponatremia among epilepsy patients (Asconapé, 2002; Dong et al., 2005; Lin et al., 2010). Patients with acute hyponatremia may experience symptoms such as headache, confusion, and in some cases seizures and coma may occur. The objective of this analysis was to estimate the prevalence of hyponatremia among epilepsy patients prior to the initiation of AED therapy and assess differences in treatment selection between patients with and without baseline hyponatremia.

Methods: A retrospective, longitudinal analysis of patients (aged $\geq$ 12 years) diagnosed with epilepsy in a large electronic medical records database from 1996 - 2009 was conducted. Patients were required to have $\geq 1$ prescription for AEDs following their initial epilepsy diagnosis. The date of first evidence of AED use defined each patient's index AED date. Selected patients were required to have at least 1 sodium measurement during the 6-month pre-index period. Using the most recent sodium measurement prior to the index AED date (among patients with multiple sodium measurements), patients were categorized as with (sodium measurement $\leq 135$ $\mathrm{mmol} / \mathrm{L}$ ) or without (sodium measurement $>135 \mathrm{mmol} / \mathrm{L}$ ) hyponatremia. Among the two patient groups, differences in preindex period demographic (i.e., age, sex, race, payer, provider) and clinical (i.e., comorbidity burden using Charlson Comorbidty Index $[\mathrm{CCI}]$ score) characteristics and type of AED initiated (i.e., sodium channel blockers and non-sodium channel blockers) were assessed using univariate (Student's t-test and chi-square test) and multivariate logistic regression models.

Results: A total of 3,850 patients with epilepsy who initiated AED therapy were identified. Among the selected patients, $13.5 \%(\mathrm{n}=521)$ had hyponatremia prior to their index AED date. Patients with hyponatremia were older (mean [std dev]: 57.95 [17.59] vs. 52.40 [19.34] years; $\mathrm{P}<0.001$ ) and had greater comorbidity burden (mean CCI [std dev]: 0.73 [1.38] vs. 0.55 [1.15]; $\mathrm{P}=0.001$ ) compared with patients without hyponatremia. The presence of hyponatremia did not differ by other demographic characteristics, including sex $(\mathrm{P}=0.289)$ or race $(\mathrm{P}=0.071)$. Significant differences were observed in the distribution of provider $(\mathrm{P}=0.004)$ and payer $(\mathrm{P}<0.001)$ type among patients with and without hyponatremia. The proportion of patients initiating a sodium channel blocker AED did not differ significantly between patients with and without $(60.1 \%$ vs. $59.1 \%$; $\mathrm{P}=0.155)$ hyponatremia.

Conclusions: In this retrospective, longitudinal analysis of an electronic records database, about 1 in 10 epilepsy patients had baseline sodium levels $\leq 135 \mathrm{mmol} / \mathrm{L}$. Older age and greater comorbidity burden were associated with higher prevalence of baseline hyponatremia. Receiving initial treatment of sodium channel blocker AED is not influenced by the presence of baseline hyponatremia. Future analysis may assess differences in hyponatremia events by treatment post-baseline.

\subsection{9 \\ PATIENTSLIKEME ${ }^{\circledR}$ EPILEPSY COMMUNITY: FACTORS AFFECTING QUALITY OF LIFE}

C. de la Loge ${ }^{1}$, S. Dimova ${ }^{1}$, T. Durgin ${ }^{2}$, G. Phillips ${ }^{3}$, K. Mueller ${ }^{4}$, C. Lafosse $^{5}$ and P. Wicks ${ }^{6}\left({ }^{1}\right.$ UCB Pharma, Brussels, Belgium; ${ }^{2} \mathrm{UCB}$
Pharma, Smyrna, GA; ${ }^{3}$ UCB Pharma, Raleigh, NC; ${ }^{4}$ CB Pharma, Monheim, Germany; ${ }^{5}$ Keyrus Biopharma, Levallois-Perret, France and ${ }^{6}$ PatientsLikeMe Inc, Cambridge, MA)

Rationale: The PatientsLikeMe Epilepsy Community is a free online platform developed in partnership with UCB and launched in January 2010. Analyses of previous data exports (March $2010^{1}$, September $2010^{2}$, and May $2011^{3}$ ) provided insights into users' characteristics and experiences with epilepsy. This November 2011 data export analysis assessed the impact of epilepsy on patients' lives by describing symptoms and perceived side effects and identifying predictors of poor health-related quality of life (HRQoL).

Methods: The PatientsLikeMe platform allows patients with epilepsy to record, monitor, and share their sociodemographic and clinical characteristics (including seizure frequency, symptoms, treatments, and perceived side effects) and to complete Patient Reported Outcomes (PRO) instruments (e.g. QOLIE-31/P). Multivariate logistic regressions were performed to identify variables associated with poor HRQoL (<Quartile 1 of QOLIE-31/P total score).

Results: By November 2011, 3073 patients had joined the

PatientsLikeMe Epilepsy Community and recorded a diagnosis of epilepsy and multiple seizures.

$2135(69.5 \%)$ patients completed the pre-defined symptom checklist. Moderate/severe symptoms most frequently reported (Figure 1) were memory problems $(60.2 \%)$, problems concentrating $(53.8 \%)$, fatigue $(50.0 \%)$, and somnolence (41.4\%). 639 (20.8\%) reported side effect(s) they perceived as associated with treatment; most frequently somnolence, fatigue, and memory impairment. 1121 (36.5\%) completed the QOLIE-31/P. Multivariate logistic regression (Figure 2) indicated that poor HRQoL was more likely in patients reporting (Odds Ratio [95\% Confidence Interval]): i) moderate/severe problems concentrating (2.96 [1.91-4.59], depression (2.53 [1.75$3.66])$, memory problems (2.14 [1.39-3.30]), anxiety (1.62 [1.12$2.35])$, fatigue (1.60 [1.05-2.44]); ii) moderate/severe side effects (2.11 [1.25-3.59]); iii) $\geq 1$ tonic-clonic [TC] seizure (2.87 [1.654.99]); $\geq 1$ non-TC seizure 1.61 [1.07-2.42]) during the 4 weeks preceding QOLIE-31/P completion; iv) shorter epilepsy duration $(\leq 1$ year 2.51 [1.41-4.45]; $>1-10$ years 1.71 [1.15-2.53]; both vs $>10$ years). Patients on newer AEDs as monotherapy (0.32 [0.13-0.80]) or polytherapy $(0.24$ [0.10-0.61]) were less likely to report poor HRQoL than patients on polytherapy with older AEDs, as were patients not reporting AED treatment (0.26 [0.08-0.80]).

Conclusions: Cognitive problems, fatigue, and somnolence were the most frequently recorded symptoms and perceived side effects by users of the PatientsLikeMe Epilepsy Community. The most predictive factors of poor HRQoL were moderate/severe problems concentrating, depression, memory problems, and side effects; occurrence of generalized TC seizures and epilepsy duration $\leq 1$ year. These results suggest that a holistic approach beyond seizure control should be considered when treating people with epilepsy. UCB-sponsored

References

${ }^{1}$ de la Loge et al. AES 2010, Abstract 1.305, www.aes.org

${ }^{2}$ de la Loge et al. AES 2011, Abstract 2.271, www.aes.org

${ }^{3}$ Dimova et al. Abstract P01.062, AAN 2012, Neurology 2012;78(Suppl.1); www.neurology.org

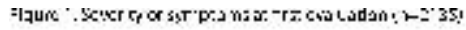

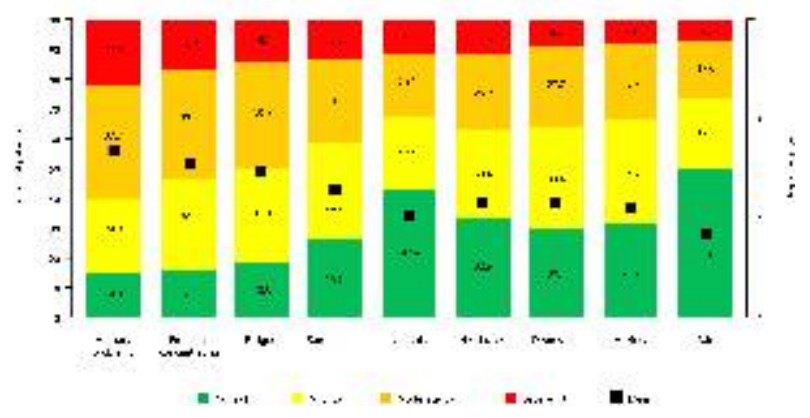




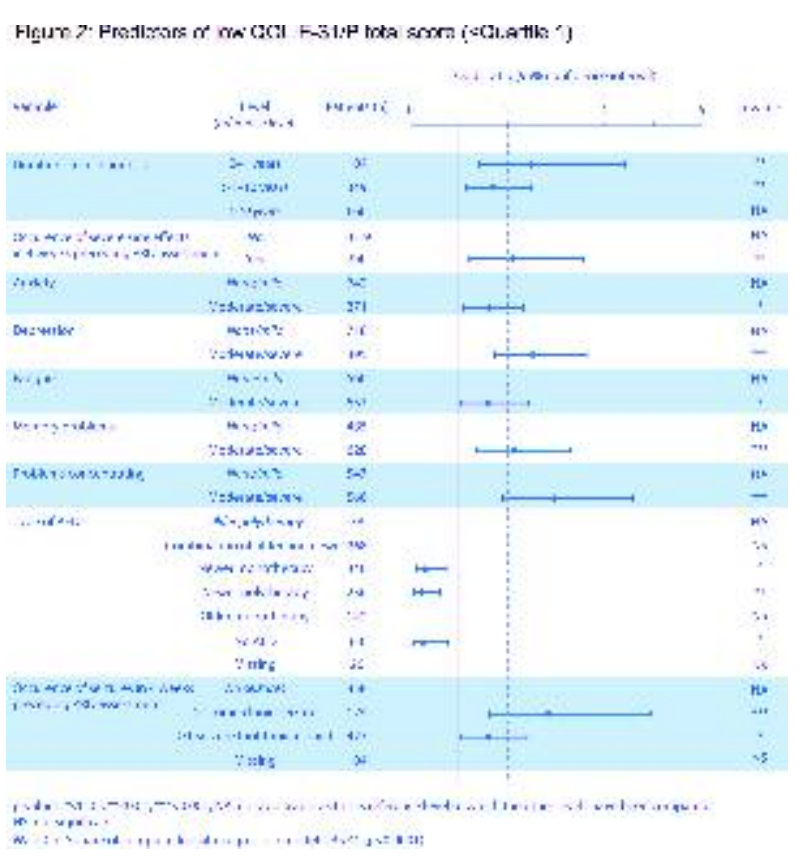

Antiepileptic Drugs: Drug Interaction

\subsection{0 \\ EFFECTS OF ENZYME-INDUCING AEDS ON LIPID CONTROL AND STATIN USE IN ADULT PATIENTS WITH EPILEPSY}

Ted Grabarczyk ${ }^{1,2}$, B. E. Gidal ${ }^{3}$, A. Schuna ${ }^{1}$ and A. Margolis ${ }^{1}\left({ }^{1} \mathrm{Wm}\right.$. S. Middleton Memorial VA Hospital, Madison, WI; ${ }^{2}$ Johns Hopkins Hospital, Baltimore, MD and ${ }^{3}$ Pharmacy Practice, University of Wisconsin - Madison, Madison, WI)

Rationale: Many traditional, older antiepileptic drugs (AEDs) are broad spectrum inducers of hepatic and intestinal CYP enzymes. Concomitant administration with enzyme-inducing AEDs (EIAED) may result in kinetic interactions that may compromise the efficacy of other medications a patient may be receiving, such as a statin. While PK interactions between EIAEDs and several statins are well recognized, clinical outcomes have yet to be studied beyond a small cohort of patients. Our objective therefore was to characterize the effect of EIAEDs, versus non-enzyme inducing anti-epileptic medications (NEIAED) on lipid control and required statin dose in patients with epilepsy.

Methods: A retrospective chart review of patients concurrently prescribed an AED along with a statin was performed. Patients were divided by those prescribed an EIAED versus solely NEIAEDs. Clinical endpoints included most recent LDL level on concurrent therapy, as well as proportion of patients with LDL reaching goal and the required atorvastatin-normalized statin dose. Eligible patients included those receiving a stable dose of an AED, at least one LDL value at least 3 months after the first instance of concurrent AED/statin co-prescription. Patients were excluded if receiving any other CYP450 inducers or inhibitors or pravastatin.

Statistical analysis included T-tests and chi-square for continuous and categorical variables, respectively. Linear regression was used to evaluate the difference in LDL values while minimizing the effects of confounding variables (age, sex, BMI, number of concomitant LDL lowering medications, and adherence to both statins and AEDs).

Results: Of 219 records reviewed, 82 were excluded based on the pre-defined exclusion criteria. Of those receiving EIAED $(n=64)$ phenytoin was the most frequently prescribed $(62.5 \%)$, followed by carbamazepine $(34.4 \%)$. In the NEAED $(n=73)$, levetiracetam $(69.9 \%)$ followed by lamotrigine $(30.1 \%)$ were the most commonly used agents.
Baseline characteristics of these groups were similar (sex, age, BMI, co-morbidities diabetes, $\mathrm{CAD}, \mathrm{CAD}$ risk equivalent, medication adherence).

Treatment with an EIAED was associated with a $12.6 \mathrm{mg} / \mathrm{dl}$ higher mean LDL after controlling for the aforementioned covariates $(\mathrm{p}<0.003)$. The EIAED group also had higher total cholesterol (170 $\mathrm{mg} / \mathrm{dl}$ vs. $156 \mathrm{mg} / \mathrm{dl}, \mathrm{p}<0.02$ ), and higher HDL (54 mg/dl vs. 48 $\mathrm{mg} / \mathrm{dl}, \mathrm{p}<0.04)$ ).

Interestingly, although both groups were similar in achieving LDL targets (EIAED: $79.7 \%$ vs NIAED: $83.6 \%, \mathrm{p}=\mathrm{NS}$ ); as compared to patients prescribed NEIAEDs, patients prescribed an EIAED were receiving significantly higher atorvastatin-normalized statin doses (44.8 mg/d vs. $28.9 / \mathrm{d} \mathrm{mg,} \mathrm{p<0.04).}$

Conclusions: These data support the notion that PK interactions with enzyme inducers may likely result in less than optimal therapeutic outcomes. Clinicians should be mindful that in the presence of EIAEDs, more attention to routine dosages of concomitant medication may be required.

\subsection{1 \\ EVALUATION OF EFFICACY AND SAFETY OF PERAMPANEL IN THE PRESENCE OF CONCOMITANT CYP3A4-INDUCING AEDS: ANALYSES FROM THE PERAMPANEL PHASE 3 CLINICAL TRIALS

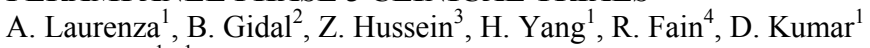 and J. Ferry ${ }^{1}\left({ }^{1}\right.$ Neuroscience Product Creation Unit, Eisai, Inc., Woodcliff Lake, NJ; ${ }^{2}$ School of Pharmacy, University of Wisconsin- Madison, Madison, WI; ${ }^{3}$ Eisai Limited, Hatfield, United Kingdom and ${ }^{4}$ Medical and Scientific Affairs, Eisai, Inc., Woodcliff Lake, NJ)}

Rationale: Perampanel (PER), a selective, noncompetitive AMPA receptor antagonist, has been evaluated as adjunctive treatment for partial-onset seizures (POS) in patients older than 12 years of age. Following oral administration, PER is rapidly and completely absorbed. PER is mainly eliminated by oxidative metabolism, primarily mediated by the CYP3A4 isozyme. PER absorption and elimination pharmacokinetics (PKs) are linear, regardless of concomitant antiepileptic drugs (AEDs). Population PK analyses has shown that 3 known CYP3A4-inducing AEDs (EIAEDs) increased PER oral clearance $(\mathrm{CL} / \mathrm{F})$. Coadministration of carbamazepine (CBZ) increased $\mathrm{CL} / \mathrm{F} \sim 3$-fold, while oxcarbazepine (OXC) or phenytoin (PHT) increased CL/F 2-fold. Given that PER efficacy appears to be related to systemic exposure (as assessed here by average steady-state concentration), it is clinically relevant to evaluate the impact of concomitant EIAEDs on both efficacy and safety.

Methods: Patients with refractory POS enrolled in the 3 phase 3 trials were aged $\geq 12$ years and were receiving 1 to 3 concomitant AEDs. Following a 6-week baseline, patients were randomized to once-daily double-blind treatment (6-week titration, 13-week maintenance) with placebo, PER $8 \mathrm{mg}$ or $12 \mathrm{mg}$ (studies 304 or 305); and placebo, PER $2 \mathrm{mg}, 4 \mathrm{mg}$, or $8 \mathrm{mg}$ (study 306). Study endpoints included change in seizure frequency $/ 28$ days, $50 \%$ responder rate, and safety.

Results: Population PK analyses indicate that PER plasma concentrations increase proportionally with doses between 2-12 mg. As expected, in the presence of EIAEDs, PER average steady-state plasma concentrations $\left(\mathrm{C}_{\mathrm{avss}}\right)$ are reduced but also increase linearly in a dose-dependent manner (Table 1). Median \% change in seizure frequency as well as responder rates was more robust when PER was given concomitantly with non-EIAEDs, but reduced in the presence of EIAEDs (Table 2).

With regard to safety, the overall incidence of treatment-emergent adverse events was similar regardless of the presence of EIAEDs. The probability of occurrence of some AEs such as fatigue, somnolence, gait disturbances, dizziness, weight increase, irritability, dysarthria, and euphoric mood increased with an increase in PER plasma concentration. 
Conclusions: Perampanel demonstrates efficacy in the presence of, as well as absence of EIAEDs. In particular, efficacy appears to be related to systemic exposure, with increased efficacy rates being observed with higher average steady-state plasma concentrations. As expected, concomitant administration of PHT, CBZ or OXC reduces plasma PER concentrations. Taken together, these observations support the important concept of dosing to clinical effect. Clinicians should recognize that they may need to use higher doses of perampanel when patients are also receiving inducing drugs. Clearly, recognition of these pharmacokinetic interactions will be important in the optimization of this novel medication.

Table 1. Median of Observed Perampanel Concentrations During Maintenance by Last Dose

\begin{tabular}{|c|c|c|c|c|c|c|}
\hline Median $C_{\text {avss }} \mathrm{ng} / \mathrm{mL}$ & $\underset{n=137}{2 \mathrm{mg}}$ & $\begin{array}{c}4 \mathrm{mg} \\
\mathrm{n}=136\end{array}$ & $\begin{array}{l}6 \mathrm{mg} \\
\mathrm{n}=42\end{array}$ & $\begin{array}{c}8 \mathrm{mg} \\
\mathrm{n}=282\end{array}$ & $\begin{array}{l}10 \mathrm{mg} \\
\mathrm{n}=21\end{array}$ & $\begin{array}{l}12 \mathrm{mg} \\
\mathrm{n}=127\end{array}$ \\
\hline With ГIАГD & 54.1 & 83.8 & 208 & 190 & 290.1 & 277.4 \\
\hline Without EIAED & 124.3 & 251.8 & 425.9 & 511.2 & 710.8 & 714.4 \\
\hline
\end{tabular}

Table 2. Median \% Change From Baseline in Seizure Frequency/28 Days Over Double-Blind Period and Responder Rate During Maintenance Period in Completers by Last Dose (Studies 304, 305, and 306)

\begin{tabular}{|c|c|c|c|c|c|c|c|}
\hline & Placebo & $2 \mathrm{mg}$ & $4 \mathrm{mg}$ & $6 \mathrm{mg}$ & $8 \mathrm{mg}$ & $10 \mathrm{mg}$ & $12 \mathrm{mg}$ \\
\hline & $N=392$ & $N=162$ & $N=161$ & $N=56$ & $\mathrm{~N}=329$ & $\mathrm{~N}=\mathbf{2 4}$ & $\mathrm{N}=140$ \\
\hline \multicolumn{8}{|c|}{ Number of patients } \\
\hline Concomitant $\Gamma \mathrm{I} \Lambda \Gamma \mathrm{D}$ & 229 & 92 & 85 & 29 & 197 & 17 & 101 \\
\hline No concomitant EIAED & 163 & 70 & 76 & 27 & 132 & 7 & 39 \\
\hline \multicolumn{8}{|c|}{ Median Change From Bascline in Scizure Frequency/28 Days Over Double-Blind Period } \\
\hline Concomitant ELAED & -12.7 & -10.33 & -29.04 & -30.16 & -26.64 & -17.75 & -17.57 \\
\hline No concomitant ELAED & -16.42 & -23.01 & -20.12 & -49.91 & -35.87 & -38.95 & -43.5 \\
\hline \multicolumn{8}{|c|}{ Responder Rate During Maintenance Period } \\
\hline Concomitant ГI $\Lambda$ ГD & 21.8 & 20.7 & 27.1 & 13.8 & 33.5 & 35.3 & 30.7 \\
\hline No concomitant EI $\triangle \mathrm{ED}$ & 17.2 & 25.7 & 35.5 & 51.9 & 44.7 & 28.6 & 51.3 \\
\hline
\end{tabular}

\subsection{2}

\section{MAINTENANCE OF VALPROATE AND LAMOTRIGINE EFFICACY DURING ONE YEAR IN A LARGE SERIES OF PATIENTS WITH DROP ATTACKS}

Maria S. Thome-Souza and K. D. Valente (Psychiatry Department, University of Sao Paulo, Sao Paulo, Brazil)

Rationale: Valproate and lamotrigine has been seen as an effective association in refractory epilepsy. Two recent studies (Machado et al. 2011; Thome-Souza \& Valente 2011) demonstrated the efficacy of this association in drop attacks. Larger series are important in order to corroborate these findings. Herein, we describe a large series of patients with drop attacks and evaluate the efficacy in one-year follow up.

Methods: We followed 60 patients with drop attacks, 39 (65\%) were male and 21 (35\%) females, ranged for 3 to 48 years (mean 13.3 years; median 10 years), $46976.7 \%$ ) patients under the age 18 years. Related to syndromic diagnosis: 26 had symptomatic generalized epilepsy (43.3\%); 11 with probably symptomatic generalized epilepsy (18.3\%); 16 with symptomatic partial epilepsy (26.7\%); six with probably symptomatic partial epilepsy $(10 \%)$, and one with idiopathic generalized epilepsy (1.7\%). Fifty-six (93.3\%) patients were taking valproate, lamotrigine and one benzodiazepine.

Results: In the first year of follow up, we observed that out of 22 $(36.7 \%)$ patients with focal epilepsy, six $(27.3 \%)$ were seizure-free for drop attack, $14(63.6 \%)$ had control $>75 \%$, and two $(9.1 \%)$ discontinued treatment due to adverse effects (skin rash). In the group of $38(63.3 \%)$ patients with generalized epilepsy, $18(47.4 \%)$ were seizure-free, nine $(23.7 \%)$ had control $>75 \%$., six $(15.8 \%)$ control between $50-75 \%$, one $(2.6 \%)<50 \%$, and four $(10.5 \%)$ discontinued treatment. There was no significant difference in efficacy related to the type of epilepsy (focal or generalized). Eight (13.3\%) patients had Lennox-Gastaut Syndrome, and seven (87.5\%) of these had superior efficacy to $75 \%$ in the control of drop attacks. Six (10\%) patients discontinued treatment due to adverse effects $(83.3 \%)$ or abandoned treatment (16.7\%). (Figure 1)

Conclusions: The association of valproate, lamotrigine, and one benzodiazepine is effective in controlling of drop attacks seizure, regardless of epilepsy syndrome.

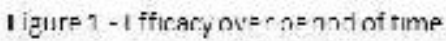

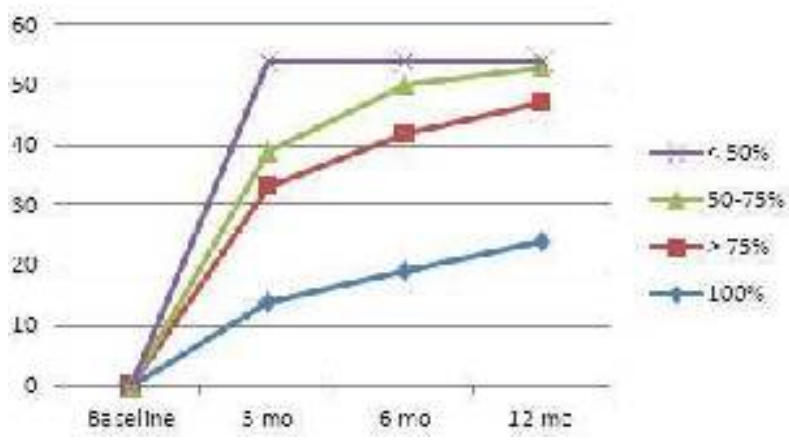

Antiepileptic Drugs: Drug Side Effects

\subsection{3}

\section{AGGRESSION IN LGS PATIENTS TREATED WITH CLOBAZAM DURING THE CONTAIN TRIAL}

J. Paolicchi ${ }^{1}$, J. Isojarvi $^{2}$ and D. Lee ${ }^{2}\left({ }^{1}\right.$ Cornell University, Ithaca, NY and ${ }^{2}$ Lundbeck LLC, Deerfield, IL)

Rationale: Aggression and irritability frequently occur in patients diagnosed with Lennox-Gastaut syndrome (LGS). ${ }^{1}$ Benzodiazepines are often used to treat seizures in LGS, but have been associated with aggression. $^{2}$ This can lead to discontinuation of an effective therapy. Clobazam, a 1,5-benzodiazepine, was FDA-approved in October 2011 for the treatment of seizures associated with LGS. With data from a Phase III, randomized controlled trial of clobazam in LGS, we conducted post-hoc analyses of aggression-related adverse events, with emphasis on time to onset and resolution.

Methods: The CONTAIN trial, ${ }^{3}$ a prospective, double-blind, placebo-controlled study, compared 3 oral dosages of clobazam with placebo as adjunctive therapy for LGS. Patients 2 to 60 years of age with LGS (documented by both clinical and electroencephalographic criteria) enrolled. Following a 4-week baseline phase, patients who had $\geq 2$ drop seizures per week were randomized to placebo or 1 of 3 dosages of clobazam $(0.25,0.5$, and $1.0 \mathrm{mg} / \mathrm{kg} /$ day $)$, up to a maximum daily dosage of $40 \mathrm{mg}$. Treatment included a 3-week titration phase, followed by a 12 -week maintenance phase. The safety population included all patients who received $\geq 1$ dose of study drug or placebo. We searched the adverse event database for the preferred terms of aggression, irritability, abnormal behavior, perserveration, and negativism, using MedDRA terms as a guide (identified as aggression-related events).

Results: As previously reported, ${ }^{3} 301$ patients were screened, 238 were randomized, 217 comprised the modified, intention-to-treat population (efficacy analyses), and 177 completed the study. Four patients discontinued because of aggression (1 medium- and 3 highdosage patients). A total of $37 / 238$ patients $(15.5 \%)$ experienced a total of 43 aggression-related events. As anticipated, occurrence of aggression- related events was dosage-related. Time to onset for the 
majority of events in the medium- and high-dosage groups was during the 3-week titration period, while in the placebo- and lowdosage groups, time to onset was distributed throughout the course of the 15 -week study. Resolution occurred for $43 \%, 40 \%, 63 \%$, and $73 \%$ of events in the placebo, and low-, medium-, and high-dosage groups respectively. More than $50 \%$ of events resolved within 40 days, and all patients who received high-dosage clobazam who resolved did so within $\sim 60$ days.

Conclusions: From these subanalyses, we determined that 1) onset of aggression-related events was dosage-related, 2) onset of the majority of the events was during titration, and 3) the majority resolved, contrary to standard belief about aggression outcomes, especially in the medium- and high-dosage groups. These data provide important information for counseling patients and their families who are experiencing aggression with clobazam therapy.

References:

${ }^{1}$ Glauser TA. Epilepsia. 2004;45(Suppl. 5):23-6.

${ }^{2}$ Weisman AM, et al. Int Clin Psychopharmacol. 1998;13:183-8.

${ }^{3} \mathrm{Ng}$ YT, et al. Neurology. 2011;77:1473-81.

\subsection{4}

\section{EFFECTS OF NEW GENERATION ANTIEPILEPTIC DRUGS} ON VASCULAR RISK FACTORS IN NEWLY DIAGNOSED EPILEPSY PATIENTS

\section{Dong Wook Kim ${ }^{1}$ and J. H. KIm ${ }^{2}\left({ }^{1}\right.$ Konkuk University Medical} Center, Seoul, Republic of Korea and ${ }^{2}$ Department of neurology, Korea University Guro Hospital, Seoul, Republic of Korea)

Rationale: Treatment of epilepsy frequently lasts for years, so minor metabolic derangements have the potential to become a concern because of cumulative adverse effects. While it is well documented that long-term therapy with older generation antiepileptic drugs (AEDs) can cause metabolic consequences that lead to an increase in risk of atherosclerosis, there is only limited information on the metabolic effect of newer generation AEDs. We performed a multicenter, open-label observational study to assess the metabolic effects of newer generation AEDs in newly diagnosed epilepsy patients. Methods: We recruited adult epilepsy patients who began to receive monotherapy with one of the newer generation AEDs, including levetiracetam (LEV), oxcarbazepine (OXC) and topiramate (TPM). We excluded patients from the analysis when the patients were under AED polytherapy or co-administration of lipid-lowering agents. Circulatory markers of vascular risk including lipid battery, homocysteine, apolipoprotein B, and apolipoprotein B/apolipoprotein A1 ratio were measured before and after six months' AED treatment. Results: A total of 133 patients were included. Fifty-nine patients received LEV, and OXC and TPM were administered in 45 and 29 patients respectively. Six months' treatment of newer-generation AEDs caused significant increase in the circulatory markers of vascular risk including total cholesterol, low-density lipoprotein (LDL), homocysteine, apolipoprotein B, and apolipoprotein $\mathrm{B}$ /apolipoprotein $\mathrm{A} 1$ ratio $(\mathrm{p}<0.001$ by paired $\mathrm{T}$ test), while there was no significant change in the levels of folate and vitamin B12. There was no remarkable difference in the circulatory markers of vascular risk among the three AED groups ( $p>0.05$ by one way ANOVA).

Conclusions: Our study shows that monotherapy with newer generation AEDs can also exhibited altered circulatory markers of vascular risk that may contribute to the acceleration of the atherosclerotic process. Therefore, our study suggests that the longterm metabolic effects should be considered even in epilepsy patients with newer generation AEDs.

\subsection{5 \\ SOMNOLENCE AND SEDATION WERE TRANSIENT ADVERSE EVENTS FOR MOST PATIENTS RECEIVING CLOBAZAM THERAPY DURING THE CONTAIN STUDY IN LGS}

B. Renfroe ${ }^{1}$, J. Isojarvi ${ }^{2}$ and D. Lee ${ }^{2}\left({ }^{1}\right.$ Child Neurology Center of NW FL, Gulf Breeze, FL and ${ }^{2}$ Lundbeck LLC, Deerfield, IL)

Rationale: Somnolence and sedation are common adverse events related to treatment with benzodiazepines. It has been hypothesized that treatment with clobazam, a 1,5-benzodiazepine, may be less frequently associated with these types of adverse events than treatment with 1,4- benzodiazepines. This post-hoc subanalysis of the Phase III CONTAIN trial, ${ }^{1}$ evaluated the incidence and time to resolution of somnolence and sedation for patients with LGS. Methods: The CONTAIN trial, ${ }^{1}$ a prospective, double-blind, placebo-controlled study, compared 3 oral dosages of clobazam with placebo as adjunctive therapy for LGS. Patients 2 to 60 years of age with LGS (documented by both clinical and electroencephalographic criteria) enrolled. Following a 4-week baseline phase, patients who had $\geq 2$ drop seizures per week were randomized to placebo or 1 of 3 dosages of clobazam $(0.25,0.5$, and $1.0 \mathrm{mg} / \mathrm{kg} /$ day $)$, up to a maximum daily dosage of $40 \mathrm{mg}$. Treatment included a 3-week titration phase, followed by a 12 -week maintenance phase. The mITT analysis included all patients who had $\geq 1$ daily seizure measurement during the maintenance phase. Incidence, time to onset, duration, and time to resolution of somnolence-related AEs (defined as somnolence and sedation) were analyzed for patients treated with placebo, or low, medium-, or high-dosage clobazam.

Results: The incidence of somnolence and/or sedation was greater for patients treated with clobazam (26\%) than patients treated with placebo (15\%). Further, the incidence increased with greater dosages of clobazam (low-dosage, 17\%; medium-dosage, 27\%; and highdosage, $32 \%$ ). For most patients, onset of these events was within the first 3 weeks of treatment (i.e., during titration). The majority of these events resolved (placebo, 73\%; low-dosage, $82 \%$; medium-dosage, $63 \%$; and high-dosage, $83 \%$ ). The median duration of these somnolence-related adverse events was within 2-3 weeks for all clobazam treatment groups.

Conclusions: Somnolence and sedation were relatively common adverse events observed during clobazam treatment in CONTAIN, and were dosage-related. However, most of these events were transient and resolved within a few weeks.

References:

${ }^{1} \mathrm{Ng}$ YT, et al. Neurology. 2011;77:1473-81.

\subsection{6 \\ FETAL ANTIEPILEPTIC DRUG EXPOSURE AND CEREBRAL LATERALIZATION AT AGE 6 YEARS} Kimford J. Meador ${ }^{1}$, G. Baker ${ }^{2}$, N. Browning ${ }^{3}$, M. Cohen ${ }^{4}$, R. Bromley $^{5}$, J. Clayton-Smith ${ }^{6}$, L. Kalayjian ${ }^{7}$, A. Kanner ${ }^{8}$, J. Liporace ${ }^{9}$, P. Pennell ${ }^{10}$, M. Privitera ${ }^{11}$, R. May ${ }^{2}$ and D. Loring ${ }^{1}\left({ }^{1}\right.$ Neurology, Emory University, Atlanta, GA; ${ }^{2}$ Walton Centre for Neurology \& Neurosurgery, University of Liverpool, Merseyside, United Kingdom; ${ }^{3}$ EMMES Corporation, Rockville, MD; ${ }^{4}$ Neurology \& Pediatrics, Georgia Health Sciences University, Augusta, GA; ${ }^{5}$ Clinical Psychology, University of Manchester, Manchester, United Kingdom; ${ }^{6}$ St. Mary's Hospital, Manchester, United Kingdom; ${ }^{7}$ Neurology, University of Southern California, Los Angeles, CA; ${ }^{8}$ Neurology, Rush University Medical Center, Chicago, IL; ${ }^{9}$ Neurology, Riddle Health Care, Media, PA; ${ }^{10}$ Neurology, Brigham and Women's Hospital, Boston, MA and ${ }^{11}$ Neurology, University of Cincinnati, Cincinnati, $\mathrm{OH}$ )

Rationale: In a previous report (Meador et al., Brain 2011), we noted greater impairment of verbal vs. non-verbal abilities at age 3 in children exposed in utero to antiepileptic drugs (AEDs). We postulated that this performance discrepancy might be due to AED 
effects on cerebral lateralization during neurodevelopment. Here, we test this hypothesis by examining handedness and verbal/non-verbal functions in our cohort at age 6 years.

Methods: The NEAD Study is a prospective observational multicenter study in the USA and UK, which enrolled pregnant women with epilepsy on AED monotherapy from 1999 to 2004. The purpose of the investigation is to determine if differential long-term neurodevelopmental effects exist across four commonly used AEDs (carbamazepine, lamotrigine, phenytoin, or valproate). 225 children were assessed at age 6 , and handedness information was available for 215 of them. Verbal and Non-verbal Indexes were derived from subscores of the Differential Abilities Scales (DAS) and the Developmental Neuropsychological Assessment (NEPSY), and also the Expressive One-Word Picture Vocabulary Test, and the Developmental Test of Visual Motor Integration (DTVMI). Handedness was assessed in 167 children by a 7-item modification of the Edinburgh Handedness Inventory. Hand performance was assessed for writing, drawing, ball throwing, scissor cutting, brushing teeth, eating with a spoon, and opening a box. A handedness index was calculated using $((\mathrm{R}-\mathrm{L})$ / $(\mathrm{R}+\mathrm{L}) \mathrm{X} 100$. In another 48 children, handedness was determined by hand use on the Grooved Pegboard or DTVMI. Handedness results were compared to handedness information from 187 normal six year old children in the standardization sample of the Wechsler Intelligence Scale for Children (WISC-4).

Results: The Verbal Index score was less than the Non-verbal Index score across all AEDs combined $(\mathrm{p}<.017)$ and for lamotrigine $(\mathrm{p}<.028)$ and valproate $(\mathrm{p}<.006)$ groups. In the WISC sample, $93 \%$ of children are right-handed. Compared to this normative sample using a logistic regression analysis, right-handedness is reduced in the NEAD children overall ( $86 \%$ right-handed, $\mathrm{p}=.04)$ and for lamotrigine $(83 \%$, $\mathrm{p}=.03)$ and for valproate $(79 \%, \mathrm{p}=.009)$ groups. Right-handedness was reduced for valproate compared to carbamazepine $(\mathrm{p}=.028)$ and a trend compared to phenytoin $(\mathrm{p}=.064)$. NEAD children exhibited an overall AED dose $(\mathrm{p}=.033)$ effect for reduced right-handedness. Conclusions: The reduced frequency of right-handedness and the relatively greater impairment of verbal vs. non-verbal abilities for some AEDs are observations consistent with reduced cerebral lateralization. We postulate that an adverse impact of fetal AED exposure on immature neurons alter normal cerebral lateralization during development. Further research is needed to confirm these findings and delineate the mechanisms.

\subsection{7}

\section{METABOLIC SYNDROME IN ADULT EPILEPTIC} PATIENTS WITH VALPROIC ACID MONOTHERAPY Aleksei Rakitin ${ }^{1}$, T. Eglit ${ }^{2}$, T. Rajasalu ${ }^{2}$, M. Lember ${ }^{2}$, S. Kõks ${ }^{3}$ and S. Haldre ${ }^{1}{ }^{1}$ Department of Neurology and Neurosurgery, Tartu University Clinics, Tartu, Estonia; ${ }^{2}$ Department of Internal Medicine, Tartu University Clinics, Tartu, Estonia and ${ }^{3}$ Department of Physiology, Tartu University, Tartu, Estonia)

Rationale: Valproic acid (VPA) is an extensively used broadspectrum antiepileptic drug. One of the most common side effects of the treatment with VPA is weight gain which occurs in about half of the patients and is associated with important metabolic and endocrine abnormalities. VPA treatment has been reported to be associated with high fasting serum insulin concentrations, insulin resistance in parallel with an unfavorable serum lipid profile and hyperandrogenism and polycystic ovaries in woman. Nevertheless, there are no studies that explored the prevalence of metabolic syndrome (MS) in patients treated with VPA, compared to the general population. The aim of our study was to compare the prevalence of MS and its components in VPA-treated patients to the general population.

Methods: 75 adult patients (40 male and 35 female) from Southern Estonia receiving VPA monotherapy for at least 3 months were included in this study. These participants were interviewed, clinically examined, and blood samples for VPA, fasting plasma glucose, serum insulin, C-peptide concentrations were taken after an overnight fast. The homeostasis model assessment (HOMA) index was calculated. MS was diagnosed according to Adult Treatment Panel III criteria. Data was compared to the results of a population-based cross-sectional multicentric study on the prevalence of metabolic disorders $(n=495)$ conducted in Southern Estonia two years earlier. Results: The occurence of MS in VPA-treated patients was not increased comparing to the general population $\mathrm{IR}=0.99(95 \% \mathrm{CI}$ $0.52-1.63)$. The weighted prevalence of MS in Estonian population was $25.9 \%$. The body mass index (BMI) also did not significantly differed between both groups: $26.6 \pm 5.7$ in VPA-treated patients, $28.2 \pm 6.2$ in controls. Nevertheless, the VPA-treated patients had significantly lower fasting plasma glucose concentrations $5.09 \pm 0.56$ $\mathrm{mmol} / \mathrm{l}(\mathrm{p}=0.002)$ and higher fasting serum insulin levels $10.10 \pm 8.30$ $\mathrm{mU} / 1$ ( $\mathrm{p}<0.0001)$ comparing to the controls: $5.43 \pm 0,82 \mathrm{mmol} / 1$ and $6.34 \pm 5.5 \mathrm{mU} / 1$, respectively. In addition, the HOMA index $(\mathrm{p}=0,004)$ was higher in patients than in control subjects. Interestingly, the normal-weight VPA-treated patients $(\mathrm{BMI}<25 \mathrm{~kg} / \mathrm{m} 2)$ had also higher serum insulin $(\mathrm{p}<0.0001)$ concentrations than the normal-weight control subjects. HOMA index was also higher $(p<0.0001)$ in the VPA-treated normal-weight patients than in normal-weight controls. Conclusions: Metabolic syndrome is not more common in VPAtreated adult epilepsy patients than in the general population. However, VPA-treated patients have lower serum glucose, higher insulin concentration in the peripherial circulation and increased insulin resistance independently of the presence of obesity.

\subsection{8}

OXCARBAZEPINE-INDUCED HYPONATREMIA

Young-Soo Kim, S. Lee, K. Chu, J. Byun, J. Lim, B. Park, J. Shin and J. Moon (Neurology, Seoul National University Hospital, Seoul, Republic of Korea)

Rationale: Oxcarbazepine (OXC) is a new-generation antiepileptic drug (AED) and, recently the usage has increased. Hyponatremia, side effect for OXC treatment is well known. But, the prevalence of hyponatremia in OXC-treated patients varied greatly (23\% to $73.3 \%)$ and the results were derived from relatively small studies. The purposes of this study are to evaluate the frequency and determine the risk factors for hyponatremia in patients with epilepsy treated with oxcarbazepine (OXC).

Methods: One-hundred eighty four adult patients with epilepsy who received OXC therapy were enrolled in this study. We used crosssection study design. Inclusion criteria were 1) epilepsy patient with OXC treatment, 2) age 16 years or older, 3) active out-patients in the period 2010 to 2012,4 ) at least one sodium level documented in that period. Demographic and clinical data were collected by the review of medical records. In statistical analysis, to compare serum sodium level and continuous variables (age, OXC dose, and the number of current AEDs being used) were assessed using univariate linear regression. Using ANOVA, normal sodium group, hyponatremia $(\mathrm{Na}+\leq 134 \mathrm{mEq} / \mathrm{L})$ group, and severe hyponatremia group $(\mathrm{Na}+\leq$ $128 \mathrm{mEq} / \mathrm{L}$ ) were analyzed to compare the age, sex, other medication.

Results: A total of 184 patients with epilepsy 16 year and older and exposed to OXC with sodium documented were included in the analysis. The mean age of patients was $56 \pm 19.1$ (16-95), the mean OXC daily dose was $960.76 \pm 517.28 \mathrm{mg}(150-4200)$, the mean number of other AEDs was $2.0 \pm 1.12(1-8)$, and the mean serum sodium concentration was $136.3 \pm 6.44 \mathrm{mEq} / \mathrm{L}$. Univariate linear regression showed that two variable were significantly associated with the serum level of sodium concentration. They were older age $(\mathrm{p}$ $=0.003)$ and higher number of other AEDs $(p=0.021)$. Multivariate analysis using linear regression analysis was performed with the variable which had been significant $(\mathrm{p}<0.05)$ in univariate analysis. Multivariate linear regression analysis revealed that older age and higher number of other AEDs were independently associated with 
lower serum sodium level. The comparative results of the a few clinical features between patient without hyponatremia, with hyponatremia, and with severe hyponatremia using by ANOVA showed that two features were significantly associated. They were older age $(\mathrm{p}<0.0001)$ and other non-AEDs medications (antiplatelet, NSAID, diuretics, opiate, and TCA, $\mathrm{p}<0.0001$ ).

Conclusions: Older age, the number of combination AEDs, and other non-AEDs medications may increase the risk of OXC-induced hyponatremia in patient with epilepsy.

\subsection{9 \\ PARADOXICAL SEIZURES DURING TREATMENT WITH RUFINAMIDE}

Pina Patel, M. Andriola and R. Spiegel (Stony Brook University Hospital, Stony Brook, NY)

Rationale: Rufinamide (Banzel), a triazole derivative, is an antiepileptic drug (AED) approved by the United States Food and Drug Administration in 2009 for the treatment Lennox-Gastaut syndrome (LGS) in patients aged $\geq 4$ years. Clinical trials have shown that rufinamide is effective in lowering seizure frequency in LGS and refractory partial onset epilepsy. In this retrospective study, we analyzed patients treated with rufinamide as an adjunctive therapy. Our data reveals that although rufinamide has efficacy in reduction of seizures, in some individuals it might be associated with exacerbating seizures.

Methods: We completed a retrospective chart review of patients with intractable seizures treated with rufinamide from 2010 to 2012. Data variables included gender, age, epileptic syndrome, etiology, EEG findings, AED dosage, and adverse effects. Seizure frequency prior to and after introducing rufinamide was recorded.

Results: We identified 24 patients on rufinamide, 17 males and 7 females. Rufinamide dosage was 200mg BID to $1600 \mathrm{mg}$ BID. Fifteen had LGS, 6 had partial onset epilepsy, and 3 had primary generalized epilepsy. Of the total 24 patients, 14 remained on rufinamide and had decreased seizure frequencies. Eight patients discontinued rufinamide due to adverse effects, which included increased seizure frequency (5/8), anorexia (1/8), rash (1/8), and dizziness $(1 / 8)$. Two patients did not have any improvement and were weaned off. Out of the 5 patients with paradoxical effects, 4 carried the diagnosis of LGS as follows: three were symptomatic and one was cryptogenic. One of the 5 patients had partial onset epilepsy. Patients' ages with a paradoxical effect ranged from 19-45 years old and were all male. All were on polytherapy and treated with other AEDs, including valproic acid (3/5), lamotrigine (3/5), topiramate, $(2 / 5)$, carbamazepine $(1 / 5)$, phenobarbital $(1 / 5)$, levetiracetam $(1 / 5)$, zonegran (1/5), and pregabalin (1/5). In all of these patients rufinamide was the last AED that was introduced prior to the increase in seizure frequency. All four patients with LGS had frequent generalized spike/polyspike with slow wave discharges and significant background slowing on EEG. All five patients had increased frequency of seizures, respective of their usual seizure types, from baseline. Among our total patient population an increase in seizure frequency was seen in $21 \%$ of patients.

Conclusions: This retrospective study reports a paradoxical effect on five males prescribed rufinamide as an adjunctive treatment. During the clinical course, patients experienced more seizures with initiation and increase in dose. Although, rufinamide appears to be a safe and effective treatment for many individuals with intractable epilepsy, we found it had a paradoxical effect in approximately one- fifth of our population. Despite the already published studies our data calls for more prospective studies in the future with larger sample size to determine the probability of a paradoxical effect with rufinamide to better educate both health care providers and patients.

\subsection{0 \\ BONE HEALTH IN EPILEPTIC PEDIATRIC PATIENTS AT KFSH-D}

Raidah S. Al-Baradie (neurscience, King Fahad University Hospital, Dammam, Saudi Arabia)

Rationale: Long-term antiepileptic drug (AED) use causes multiple abnormalities in calcium and bone metabolism that have been most extensively described in children. The objective is to determine if the vitamin D3 (vit D3) dose (800 unit/day) is appropriate prophylactic to children on long- term (AED).

Methods: A retrospective study was conducted of 63 patients (35 males and 28 males between $0-16$ years old) on anticonvulsant therapy and vit D3 800 unit per day for at least 6 months who presented to neurologists at a tertiary referral center. Bone mineral density (BMD) as well as serum 25 hydroxyl-vitamins D (25-OHD), serum calcium, parathyroid hormone, and alkaline phosphatase were measured. Signs of vitamin D deficiency were recorded more frequently ere. A detailed questionnaire assessing calcium intake as well as previous and current intake of antiepileptic medications was administered to all patients.

Results: Over $90 \%$ of children had normal 25-OHD levels, and this finding did correlate with BMD. Subjects on enzyme-inducing drugs such as phenytoin, phenobarbital, carbamazepine, and primidone tended to have lower BMD than those on noninducers such as valproic acid, lamotrigine, clonazepam, gabapentin, topamirate, and ethosuximide.

Conclusions: Epilepsy and its therapy, including the newer drugs, are risk factors for low bone density, irrespective of vitamin D levels. It is suggested that a dose 800 unit/day of vitamin D might be most suitable to avoid biochemical signs of vitamin D deficiency in children on antiepileptic drugs but monitoring of 25-OHD levels level is still indicated to adjust the dose in patients on chronic antiepileptic therapy.

\subsection{1 \\ SEVERE ADVERSE EFFECTS OF ANTIEPILEPTIC DRUGS: DESCRIPTION OF A PEDIATRIC POPULATION IN COLOMBIA \\ Oscar M. Espitia Segura ${ }^{1}$, J. D. Ramos-Guevara ${ }^{1}$, M. D. Herrera ${ }^{2}$, Y. C. Zuñiga ${ }^{1}$, N. E. Penagos ${ }^{1}$, D. C. Benitez ${ }^{1}$, N. A. Pardo ${ }^{1}$, S. M. Ramirez $^{1}$, A. C. Vargas ${ }^{1}$, M. J. Villarreal ${ }^{2}$, R. A. Naranjo ${ }^{1}$ and A. H. Izquierdo ${ }^{1}\left({ }^{1}\right.$ Pediatric Neurology, Universidad Nacional de Colombia, Bogota, Colombia and ${ }^{2}$ Medicine, Universidad Nacional de Colombia, Bogota, Colombia)}

Rationale: An adverse effect to medication is defined as a noxious effect when drug is used with therapeutic purpose. Among the adverse effects using anti epileptic drugs (AED), severe dermatological effects, hematological disorders, hepatic or pancreatic lesion and neurotoxicity can be seen. The adverse effects, even milder ones, can cause up to $40 \%$ of AED failure and poor adherence and control of epilepsy

Methods: Descriptive statistical analysis and retrospective study of 15 patients under 18 years old is carried out in this study. Charts of patients hospitalized because of severe adverse effects to AED (SAEAED) in the Hospital de la Misericordia, a pediatric hospital in Bogota, Colombia, were reviewed from 2009 to 2011. The statistical analysis was performed using SPSS 17

Results: In this study, 15 patients with SAEAED were reported, the mean age was $6.5 y$ (2-13y), $60 \%$ female, all with partial epilepsies: idiopathic $6 \%$, cryptogenic $40 \%$, symptomatic $53 \%$. Treated on monotherapy $67 \%$, bitherapy $20 \%$ and three AED in $13 \%$. The AED used were carbamazepine (CBZ) 53\%, valproate (VPA) 46,6\%, clobazam (CLB) 20\%, oxcarbazepine (OCBZ) 13,3\% and vigabatrine (VGB) and lamotrigine (LTG) 6,6\% each. 5 patients had associations such as VPA-LTG-CLB, CBZ-VPA, VGB-VPA. Systems involved were dermatological 66,7\%; hematological 13,3\%(bicytopenia, 
thrombocitopenia); gastrointestinal 6,7\%(hemorrhagic pancreatitis), central nervous system (CNS) (ataxia, impaired consciousness) in $13,3 \% .26 \%$ of the cases required intensive care because of multiorganic failure, with a mean stay of 9,5 days and also had the longest total inpatient time 13-38 days; two of them because of toxic epidermal necrolysis (TEN), one thrombocytopenia and one pancreatitis.

Among the dermatological lesions (10 patients), we found severe $\operatorname{rash}(3)$, TEN(2), Stevens Johnson(2), DRESS syndrome(2) and serum-like sickness(1). Most received iminodibenzyl AED (CBZ(5) or OCBZ(1)) $\mathrm{p}=\mathrm{NS}$. Also, CBZ was related to CNS symptoms. The treatment to all skin reactions was antihistaminic, and some of them required immunomodulatory agents (corticosteroids(4), immunoglobulin(2), cyclosporine(2)); it was observed also that AED withdrawal improved the hematologic SAEAED, as well as the CNS's ones

Conclusions: This study found that most of SAEAED were dermatological, and a tendency to be associated with iminodibenzyl usage as reported elsewhere.

We neither find association of SAEAED with age, titration rate, length of treatment nor AED number, confirming its idiosincratic appearance, thus to date they are unpredictable. Also most of them required aggressive management as well as longer stay in hospital in patients sufering dermatological or gastrointestinal effects. Characterizing most frequent and severe effects let physicians to develop a high risk clinical profile which could help in perform earlier diagnosis and better treatment possibilities; however, given its unpredictable nature on clinical basis, can be helpful to develop biomarkers in selected populations to infer further recomendations in order to improve diagnostic yield and outcome

\subsection{2 \\ EVALUATION OF THE BODY COMPOSITION WITH BIOELECTRICAL IMPEDANCE ANALYSIS IN EPILEPTIC PATIENTS TREATED WITH VALPROATE}

Semra Hiz ${ }^{1}$, E. Bayram ${ }^{1}$, Y. Topcu ${ }^{1}$, P. Karakaya ${ }^{1}$, M. Bayram ${ }^{2}$ and U. Yis ${ }^{1}\left({ }^{1}\right.$ Pediatric Neurology, Dokuz Eylül University School of Medicine, Izmir, Turkey and ${ }^{2}$ Pediatric Nephrology, Dokuz Eylül University School of Medicine, Izmir, Turkey)

Rationale: Valproate is an effective antiepileptic agent for generalized and partial seizures. One of the major side effects of the treatment with valproate is weight gain. Here, we aimed to describe the changes in anthropometric measurements, body composition and laboratory results in epileptic children treated with valproate.

Methods: Non-obese children diagnosed with idiopathic epilepsy were included in this study. Anthropometric measurements (body weight, height, waist and hip circumferences) were measured. Total body water (TBW), intracellular water (ICW), extracellular water (ECW), fat free mass index (FFMI), body fat mass index (BFMI), fat percentage and basal metabolic rate (BMR) were calculated with bioelectrical impedance analysis (BIA) at the beginning, third month and sixth month of valproate treatment. Before the treatment and at the control visits fasting serum glucose, lipids, insulin levels and thyroid hormone values were recorded. Results were compared with pretreatment values.

Results: There were 25 ( 17 boys, 8 girls) non-obese children with idiopathic epilepsy with a mean age of $8.21 \pm 3.96$ years. Treatment related side effects were seen in $3 / 25(12 \%)$ of the patients, one patient had hair loss and other two patients developed elevated liver enzymes during the treatment. Mean values of anthropometric measurements, BIA and laboratory results of the patients were summarized in tables 1 and 2. BIA showed similar values of fat percentage and TBW between 0., 3. and 6.months follow up of the patients $(\mathrm{p}>0.05)$. However, ECW and ICW percentages of the patients were significantly decreased between $0 .-3$. months and 0 .6.months of the treatment $(\mathrm{p}<0.05)$. In addition, BMI, BMR, BFMI and FFMI values of the patients were significantly increased when we correlate the $0 .-3$. months and 0.-6.months of the treatment $(p<0.05)$. In the laboratory results, similar values of fasting glucose, insulin, cholesterol, triglycerides, LDL, HDL and fT3 were detected at the 6 . month follow up ( $>0.05)$. Free T4 levels were significantly decreased and TSH levels were significantly increased between the 0 . and 3 . months results. In addition, TSH levels were significantly increased between the 0 . and 6 . months results of the treatment $(\mathrm{p}<0.05)$.

Conclusions: BIA may be a useful technique to assess the effects of the valproate treatment. More investigations are needed to explain the effect of valproate on body composition.

\subsection{3 \\ TREATMENT-EMERGENT ADVERSE EVENTS BY AGE FOR PATIENTS WITH LGS TREATED WITH CLOBAZAM DURING PHASE II/III TRIALS}

J. Isojarvi ${ }^{1}$, D. Lee ${ }^{1}$ and J. Buchhalter ${ }^{2}\left({ }^{1}\right.$ Lundbeck LLC, Deerfield, IL and ${ }^{2}$ Phoenix Children's Hospital, Phoenix, AZ)

Rationale: In Phase II (OV-1002 $\left.{ }^{1}\right)$ and Phase III (CONTAIN $\left.{ }^{2}\right)$ studies, clobazam, a 1,5-benzodiazepine, significantly decreased the weekly frequencies of drop and total seizures associated with Lennox-Gastaut syndrome (LGS). Adverse events were as expected, given what is known about clobazam from extensive previous clinical development efforts. ${ }^{2}$ Following an earlier analysis indicating that clobazam was generally consistently efficacious in treating LGS across all age groups, ${ }^{3}$ we conducted a pooled analysis of both trials to determine if safety events varied by age.

Methods: In the Phase II trial, ${ }^{1}$ LGS patients, following a 4-week baseline phase, were randomized to 1 of 2 dosages of clobazam $(0.25$ and $1.0 \mathrm{mg} / \mathrm{kg} /$ day). Treatment included a 3 -week titration period, followed by a 4-week maintenance period. The Phase III CONTAIN trial $^{2}$ compared 3 oral dosages of clobazam with placebo as adjunctive therapy for LGS. Following a 4-week baseline phase, patients who had $\geq 2$ drop seizures per week were randomized to placebo or 1 of 3 dosages of clobazam $(0.25,0.5$, and $1.0 \mathrm{mg} / \mathrm{kg} /$ day $)$. Treatment included a 3 -week titration phase, followed by a 12 -week maintenance phase. The safety population included all patients who received $\geq 1$ dose of study drug or placebo. We evaluated safety results from the baseline period to the maintenance phase in each trial for patients $\geq 2$ to $<12$ years of age; patients $\geq 12$ to $<16$ years; and patients $\geq 16$ years.

Results: As previously reported, ${ }^{2} 301$ patients were screened, 238 were randomized, 217 comprised the mITT population, and 177 completed the study. For overall incidence of serious adverse events, no notable differences between age groups were observed (table). Incidences of low hemoglobin and hematocrit concentrations were lower for older vs. younger patients. In addition, the percentages of patients with $\geq 1$ elevated alkaline phosphatase concentration were notably different between groups. None of these differences were considered clinically important by the study investigators.

Conclusions: From pooled Phase II/III results for clobazam in LGS, we observed no clinically important differences in treatmentemergent adverse events and laboratory values when patients were categorized by age.

References:

${ }^{1}$ Conry JA, et al. Epilepsia. 2009;50:1158-66.

${ }^{2} \mathrm{Ng}$ YT, et al. Neurology. 2011;77:1473-81.

${ }^{3}$ Mitchell W, et al. Epilepsia. 2011;Abstract \#B.04. 
TEAEs by Age for LGS Patients Receiving Clobazam During Phase II/III Trials

\begin{tabular}{|c|c|c|c|}
\hline & \multicolumn{3}{|c|}{$\begin{array}{c}\text { Clobazam-Treated Patients } \\
N(\%)\end{array}$} \\
\hline & $\begin{array}{c}\geq 2 \text { to }<12 \text { Years of Age } \\
\qquad(\mathrm{N}=192)\end{array}$ & $\begin{array}{c}\geq 12 \text { to }<16 \text { Years of } \\
\text { Age } \\
(\mathrm{N}=51)\end{array}$ & $\begin{array}{c}216 \text { Years of } \\
\text { Age } \\
(\mathrm{X}=56)\end{array}$ \\
\hline Patients with $\geq 1 \mathrm{TEAE}$ & $174(90.6)$ & $43(84.3)$ & $53(94.6)$ \\
\hline Constipation & $25(13.0)$ & $5(9.8)$ & $8(14.3)$ \\
\hline Pyrexia & $39(20.3)$ & $7(13.7)$ & $3(5,4)$ \\
\hline Nasopharyngitis & $23(12.0)$ & $9(17.6)$ & $7(12.5)$ \\
\hline Olitis media & $31(16.1)$ & $4(7.8)$ & 0 \\
\hline Pneumonia & $33(17.2)$ & $6(11.8)$ & $2(3.6)$ \\
\hline $\begin{array}{l}\text { Lpper resp. tract } \\
\text { infection }\end{array}$ & $55(28.6)$ & $6(11.8)$ & $6(10.7)$ \\
\hline Fall & $21(10.9)$ & $8(15.7)$ & $10(17.9)$ \\
\hline Drooling & $15(7.8)$ & $6(11.8)$ & $9(16.1)$ \\
\hline Lethargy & $18(9.4)$ & $13(25.5)$ & $10(17.9)$ \\
\hline Sedation & $17(8.9)$ & $4(7.8)$ & $9(16.1)$ \\
\hline Somnolence & $44(22.9)$ & $12(23.5)$ & $16(28.6)$ \\
\hline Aggression & $21(10.9)$ & $4(7.8)$ & $13(23.2)$ \\
\hline Insomnia & $20(10.4)$ & $5(9.8)$ & $6(10.7)$ \\
\hline
\end{tabular}

TEAE=treatment-emergent adverse event.

\subsection{4}

COGNITIVE EFFECTS OF LACOSAMIDE

Dominique IJff $^{1,2}$, M. Majoie ${ }^{1,2}$ and A. Aldenkamp ${ }^{1,2}$

( ${ }^{1}$ Kempenhaeghe, Heeze, Netherlands and ${ }^{2}$ Maastricht University

Medical Centre, Maastricht, Netherlands)

Rationale: Lacosamide (LCS) is a novel antiepileptic drug (AED) with potential benefit as adjunctive treatment in patients with partialonset seizures1-3. The cognitive effects have, as yet, not been studied. In this open clinical prospective study the cognitive effects of LCS when used as adjunctive antiepileptic therapy in patients with epilepsy was evaluated.

Methods: We included 33 patients ( 24 females, 9 males) aged between 16-74 years (mean 37 years sd:14.5). All patients had a localisation-related epilepsy ( $42 \%$ cryptogenic; $58 \%$ symptomatic). Patients were assessed at baseline before starting treatment and at follow-up when the optimal clinical dose was achieved. Patients were using LCS treatment with a daily dose between 100 to $600 \mathrm{mg}$ (mean daily dose of $298.2 \mathrm{mg}$ /day). Mean follow-up time was 7 months (range 1-24). Patients were treated with up to four concomitant AEDs (mean: 1.9). Subjective complaints were evaluated using the SIDAED (subjective list of 46-items with possible AED-related complaints); effects on cognition were evaluated using the CVST, a test measuring speed of central information processing ('mental speed').

Results: The CVST showed significant faster information processing reaction times at the second evaluation $(\mathrm{p}=.013)$. On the SIDAED, patients complained more about their cognitive function at the second evaluation $(\mathrm{p}=.002)$.

Conclusions: Lacosamide has a positive effect on the information processing speed. As this is the most sensitive function for effects of AEDs, LCS does not seem to induce negative cognitive effects. Remarkably patients complained more, especially about their cognitive function. This 'doing better, feeling worse' phenomenon is well described in e.g. mild cognitive impairment and in traumatic brain injury. Here this discrepancy can be caused by the deepened worries of patients when ending a trial (losing extra attention).

\subsection{5}

UTILITY OF A SPECIFIC INSTRUMENT TO EVALUATE ANTIEPILEPTIC DRUGS TOXICITY

Nahuel F. Pereira de Silva, P. Caballero Murguia, M. T. Morello, S. S. Kochen and P. Saidon (Epilepsy Center - Ramos Mejia Hospital, Capital federal, Argentina)
Rationale: The toxicity associated with antiepileptic drugs (AEDs) is one of the parameters to consider assessing the response to treatment (ILAE Classification 2010). Perucca described that $61 \%$ of patients treated with AEDs have at least one adverse event (AE). However, the evaluation of toxicity depends on the AE patients and report of physicians. The underreporting from physicians may limit the usefulness of the new classification.

Methods: Forthe report of physicianst we use an AE report form (CIOMS). In a database was recorded, AEDs exposure, casualty analysis and outcome. AE were classified according to the mechanism of production classification proposed by Perucca. For patients reporting we use a validated Spanish version survey (Liverpool Adverse Event Profile).

Results: Year 2007 was taken to estimate the real incidence of AE reported by physicians (1.44\%). 320 surveys were conducted, where patients reported $4800 \mathrm{AE}$, leading to a $\mathrm{AE}$ patients report incidence of $15 \%$. AE were classified according to the mechanism of production in type A (dose dependent), B (idiosyncratic) and C (chronic).

Conclusions: There is a clear unbalance between the report of AE by physicians and spontaneous reporting of patients conducted with a specific instrument. The use of a specific method facilitates the classification of response to treatment with AEDs.

\section{Antiepileptic Drugs: Other}

\subsection{6 \\ PHARMACOKINETICS OF $N$-DESMETHYLCLOBAZAM, THE ACTIVE AND PRIMARY METABOLITE OF CLOBAZAM}

D. Tolbert and I. Bekersky (Lundbeck LLC, Deerfield, IL)

Rationale: Following oral administration, clobazam is rapidly and quantitatively absorbed into systemic circulation. It is metabolized by the liver via CYP3A4, and, to some extent, by CYP2C19 and CYB2B6, into more than 20 metabolites. The primary route of metabolism is by oxidative demethylation to form $\mathrm{N}$ desmethylclobazam, the active, primary metabolite in systemic circulation.

Methods: The pharmacologic activity of $N$-desmethylclobazam has been demonstrated by several in vitro and in vivo studies. In vitro and ex vivo protein binding studies were conducted via spiked plasma samples and blood samples collected from study participants who were dosed to steady state with clobazam. Plasma concentration data from single-dose trials and multiple-dosage trials in healthy volunteers were used to calculate absorptive and post-absorptive parameters, as well as accumulation factors for $N$-desmethylclobazam at steady state. Population pharmacokinetic analyses were used to calculate elimination rate constants and clearance values for patient populations. With these results, we pooled pharmacokinetic data from all studies to provide $N$-desmethylclobazam's pharmacokinetic profile following administration of clobazam.

Results: The in vitro plasma protein binding of clobazam and $\mathrm{N}$ desmethylclobazam ranged from $78 \%$ to $89 \%$ and was concentrationindependent. The ex vivo plasma protein binding of both compounds was approximately $90 \%$. In vitro, $N$-desmethylclobazam was found to be a substrate (but not an inhibitor) of the P-glycoprotein transport system and unlikely to be a substrate for the organic cation or anion transport systems. After an initial dose, clobazam was the predominant moiety (mean $\mathrm{AUC}_{0 \text {-inf }}$ range of 8,890-11,600 hr*ng/mL and $\mathrm{C}_{\max }$ range of $310-402 \mathrm{ng} / \mathrm{mL}$ ). Following repeated administrations, $N$-desmethylclobazam becomes the major circulating moiety. Further, steady-state concentrations of $N$-desmethylclobazam were attained within 2 weeks of dosing. At steady state, the mean AUC and $\mathrm{C}_{\max }$ of clobazam were $10,350 \mathrm{hr} * \mathrm{ng} / \mathrm{mL}$ and $1,076 \mathrm{ng} / \mathrm{mL}$ respectively. The corresponding parameters for $N$ desmethylclobazam were $30,350 \mathrm{hr} * \mathrm{ng} / \mathrm{mL}$ and $2783 \mathrm{ng} / \mathrm{mL}$, respectively. 
Conclusions: Based on this population pharmacokinetic analysis, the median half-lives of both clobazam and $N$-desmethylclobazam were approximately 36 hours and 79 hours, respectively. At steady state, $\mathrm{N}$-desmethylclobazam exposures are approximately 3-5 times greater than clobazam. Renal insufficiency, hepatic dysfunction, or stable dosages of other antiepileptic drugs had no effect on $\mathrm{N}$ desmethylclobazam disposition. Moreover, $N$-desmethylclobazam had no effect on cardiac conduction, age, weight, race, and sex did not significantly affect $N$-desmethylclobazam as well.

\subsection{7}

INTRAVENOUS LACOSAMIDE IS SAFE AND EFFECTIVE IN TREATING REFRACTORY STATUS EPILEPTICUS IN A CRITICALLY-ILL POPULATION: A LARGE RETROSPECTIVE CASE SERIES

Christopher R. Newey ${ }^{1}$ and S. Hantus ${ }^{1,2}\left({ }^{1}\right.$ Epilepsy, Cleveland Clinic, Cleveland, $\mathrm{OH}$ and ${ }^{2}$ Cerebrovascular, Cleveland Clinic, Cleveland, $\mathrm{OH})$

Rationale: Status epilepticus (SE) is a neurological emergency and often does not respond to initial treatment. Algorithms to treat SE with well established medications such as phenytoin (PHT) and phenobarbital exist, but treatment of SE when these medications fail is less clear. Several newer antiepileptics (AEDs) are available in intravenous (IV) formulations (such as levitiracetam (LEV) and lacosamide (LCM)), but their safety profiles and efficacies are not well established. Several case reports, small case series, and animal models have suggested efficacy for LCM in SE. The purpose of this study was to examine the safety of IV LCM in a critically-ill population and obtain an estimate of efficacy in patients with refractory SE on continuous video EEG (cEEG) in a large, retrospective case series.

Methods: Retrospective review of prospectively collected data of patients in SE on cEEG treated with IV LCM located in the electronic medical record and epilepsy database of the Cleveland Clinic from June 2009 to April 2011. GraphPad InStat (San Diego, CA) was used for statistical analyses.

Results: Eighty-four patients in SE (59.6 yrs, 43F/41M ) were identified over a two-year period treated with LCM of which $59.5 \%$ had nonconvulsive SE. 38.1\% had prior history of seizures with $8.3 \%$ having prior SE. Suspected etiologies of the SE included ischemic stroke $(16.7 \%)$, hemorrhagic strokes $(15.5 \%)$, tumor $(14.3 \%)$, epilepsy (14.3\%), anoxia (9.5\%), and other (29.7\%).

Safety parameters included measurement of blood pressure change, PR interval on EKG, LFTs, and renal function. There was no significant change in serial blood pressure monitoring prior to LCM and 1, 4 and 24 hrs after LCM; 19 patients were on pressors prior to LCM and four patients required pressors following LCM. No significant change in PR interval was seen before or after LCM on EKG, but 11 patients with non-symptomatic PR prolongations were noted. Serum creatinine was used to follow renal function pre-LCM and 1 and 7 days post-LCM with no significant change. There was no significant change in AST/ALT at 1 and 7 days although there was a lot of variability in this population.

Fifty one patients were treated with LCM after the onset of SE on cEEG and the time to seizure termination after LCM dose was used as an estimate of efficacy in this retrospective series. Cessation of SE after LCM occurred in $15.7 \%$ (8) by 4 hrs, $25.5 \%$ (13) by 12 hours, $58.8 \%$ (30) by 24 hours and $82.4 \%(42)$ by $48 \mathrm{hrs}$. The mean length of SE until cessation was $31.1 \mathrm{hrs}$. Cessation of SE after PHT and levetiracetam (LEV) were also analyzed. Mean number of IV AEDs prior to LCM was 2.4 (range $0-4$ ). An average of 0.9 AEDs were required after LCM.

Conclusions: IV LCM appears safe in our series of 84 critically-ill patients with SE. The retrospective estimate of efficacy for LCM appears promising for SE and comparable to PHT and LEV. Prospective, randomized controlled studies are needed to better determine the role of LCM in treating SE.
2.228

SATISFACTION WITH EPILEPSY TREATMENT IN PATIENTS WITH EPILEPSY: A SYSTEMATIC REVIEW K. M. Fiest ${ }^{1}$, Jonathan Dykeman ${ }^{1}$, X. R. Liu ${ }^{1,2}$, S. Patten ${ }^{1}$, N. Jette ${ }^{1}$ and S. Wiebe ${ }^{1}{ }^{1}$ University of Calgary, Calgary, AB, Canada and ${ }^{2}$ Institute of Neuroscience and the Second Affiliated Hospital of Guangzhou Medical University, Guangzhou, China)

Rationale: In epilepsy research, outcomes of treatment, rather than satisfaction with treatment, have been the primary focus of measurement. However, patient satisfaction influences health-related decision-making, medical adherence, and follow through with treatment planning. Our aim was to describe the literature surrounding the measurement of patient satisfaction with epilepsy therapy.

Methods: Medline, PsychINFO, CINAHL, Cochrane Central Register of Controlled, and Embase were searched using subheadings and keywords related to patient, client, and consumer satisfaction, and treatment outcomes. Two independent reviewers screened abstracts and full-text and abstracted data from eligible studies. Included studies measured patient satisfaction with epilepsy therapy, were original research, and described how satisfaction was assessed. Analyses were carried out to 1) assess the purpose and sample characteristics, 2) summarize satisfaction scale development and content, and 3 ) assess reported outcomes.

Results: Of 6368 abstracts identified by the search, 125 were reviewed in full-text, with 16 meeting all eligibility criteria. The median number of patients included in the studies was 112 (range: 18-1960). Assessments were primarily completed in adult patients and satisfaction in children was measured using parental responses. Two studies used validated scales for assessing general treatment satisfaction, which address any current medications. These were the Satisfaction with Medicines Questionnaire (SATMED-Q) and the Treatment Satisfaction Questionnaire for Medication (TSQM 1.4). Six studies used a single question to measure satisfaction using Likert scale ratings. The remaining 10 studies commonly included domains related to adverse events $(8 / 10)$ and effectiveness of the therapy (7/10). Others included preference, contentedness, compliance, and the number of tablets. Six studies assessed satisfaction at multiple time points and all reported an increase in satisfaction over time. One study on satisfaction with current medication use found that over $90 \%$ of patients would prefer to take antiepileptic drugs (AEDs) only once per day, with only $10 \%$ believing that taking them once a day only means they are less effective, and $44 \%$ believing that more side effects will be experienced with more frequent AED administrations. Another study found that $63.8 \%$ of participants were satisfied with the ease and convenience of their treatment regimen, with $62.9 \%$ reporting undesirable medication effects. Another study found that well-controlled patients were more highly satisfied (79.1\%) with the effectiveness of their epilepsy treatment than those patients who were poorly controlled $(67.0 \%)$.

Conclusions: We did not identify any systematically developed and validated tools for assessing satisfaction with epilepsy-specific treatment. Whether a disease-treatment specific tool or general measure of treatment satisfaction is superior should be investigated. Such a tool would aid in clinical management of patients with epilepsy and serve as an important outcome measure in clinical research.

\subsection{9}

SAFETY AND EFFICACY OF OXCARBAZEPINE IN NEONATES IN THE NEONATAL INTENSIVE CARE UNIT Kaitlin James and K. Velayudam (Vanderbilt University, Nashville, $\mathrm{TN})$

Rationale: Seizures are commonly seen in the neonatal intensive care unit, but there is clear lack of evidence regarding anticonvulsant use in the neonatal population. Phenobarbital and phenytoin have 
long been the standard of care, despite studies suggesting that they are less effective or even harmful in this age group. The objective of our study was to review efficacy and safety of oxcarbazepine in neonates in our Neonatal Intensive Care Unit (NICU).

Methods: We identified 22 neonates who received Oxcarbazepine in our NICU from 2006-2011. A retrospective chart review was performed, and data was collected including gestational age at birth; corrected gestational age at seizure onset; semiology; etiology; EEG, imaging and genetic test results; prior anticonvulsants used; initial and target Oxcarbazepine dose; efficacy and adverse effects. Continuation of Oxcarbazepine upon discharge from the NICU was also evaluated as a surrogate marker of tolerability.

Results: Twenty of twenty-two neonates were term. The majority (90\%) developed seizures between 37-43 weeks corrected gestational age. The most common semiology was clonic (10/22), followed by tonic $(5 / 22)$, eye movements or gaze deviation $(5 / 22)$, apnea with desaturation $(3 / 22)$, lip smacking or tongue thrusting $(3 / 22)$, or bicycling movements $(2 / 22)$. A minority of seizures were confirmed electrographically but were not associated with clinical signs $(3 / 22)$. Ictal EEG was captured in 17/22 neonates. Almost 94\% (16/17) had focal electrographic seizures. The most common etiologies included hypoxic ischemic encephalopathy, infarct, or hemorrhage (32\%), or genetic abnormalities (32\%). All neonates had previously failed Phenobarbital, phenytoin, Keppra, or a combination of the three. The most common starting dose of Oxcarbazepine was $10-20 \mathrm{mg} / \mathrm{kg} /$ day, although four neonates were started on $30 \mathrm{mg} / \mathrm{kg} / \mathrm{day}$. Sixty percent were titrated to a goal dose of $40-60 \mathrm{mg} / \mathrm{kg} /$ day. Complete seizure control was seen in 13/22 (60\%) of neonates. Partial seizure control with clinically significant decrease in seizure frequency or duration was seen in $6 / 22(27 \%)$. Three of 22 neonates had no change or worsening of their seizures (14\%). The majority of the neonates $(10 / 13)$ who had complete seizure control had focal semiology or localized ictal EEG onset. Of the three neonates who did not respond to Oxcarbazepine, one had bilateral grade IV IVH, one had bilateral hemorrhagic PCA strokes and the third had a lethal genetic mutation. Somnolence was the most common reported adverse effect, occurring in $32 \%$ of neonates. However, all were able to continue treatment. No life-threatening adverse effects were reported. All neonates continued Oxcarbazepine treatment upon discharge from the NICU.

Conclusions: Our data suggests that Oxcarbazepine is a safe and effective adjunctive anticonvulsant medication in neonates with focal seizure semiology or clearly localized ictal EEG patterns. The most common adverse effect was somnolence, which tended to be mild and did not require discontinuation of therapy.

\subsection{0 \\ TREATING STATUS EPILEPTICUS WITH LACOSAMIDE: A SYSTEMATIC REVIEW}

M. V. Villafuerte ${ }^{1}$ and J. G. Burneo ${ }^{2}\left({ }^{1}\right.$ Epilepsy Programme, Instituto Nacional de Ciencias Neurológicas, Lima, Peru and ${ }^{2}$ Epilepsy Programme, Department of Clinical Neurological Sciences, London Health Sciences Center, University of Western Ontario, London, ON, Canada)

Rationale: Lacosamide (LCM) has been approved as adjuntive treatment in the treatment of Partial Epilepsy. There have also been reports about the use of this new drug in the management of status epilepticus. We performed a systematic review to assess the use of LCM in status epilepticus.

Methods: We included all clinical reports of patients with status epilepticus (convulsive and non-convulsive), in who LCM was used alone or in combination with other agents. We interrogated MEDLINE and LILACS, and found 137 abstracts. Of them we selected 16. We excluded review articles, letters to editor (without clinical cases), editorials, and duplicate studies. We also included 8 abstracts from epilepsy meetings. 16 were case series.

Results: Of these 24 papers, there were 222 patients (Age: 56.8 years), and 56 had convulsive status. 208 had refractory status epilepticus. The LCM dose ranged from 50 to $400 \mathrm{mf}$ (median dose: $200 \mathrm{mg}$ ) as an initial dose, followed by $50-200 \mathrm{mg}$ bid IV. MRI and CT scan information was available in 125 patients; abnormal findings were present in 86 patients, being ischemic stroke the most common pathology. Focal discharges were found in 73 patients, and generalized discharges in 23. Response to lacosamide was successful in 133 cases $(60 \%)$. A meta-analysis was not performed given the heterogeneity and the fact that most of the papers were single case reports.

Conclusions: There is some evidence supporting the use of LCM for the management of Status epilepticus, although studies with stringent methodology are needed.

\subsection{1 \\ THE EFFECTS OF TOPIRAMATE AND VAGAL NERVE STIMULATOR ON SEIZURE CONTROL AND WEIGHT IN PEOPLE WITH DIFFICULT TO TREAT EPILEPSY}

Claudia A. Murton ${ }^{2,1}$, R. Shankar ${ }^{2,5}$, M. Walker ${ }^{3,4}$ and H. Sullivan ${ }^{2}$ ( ${ }^{1}$ Plymouth Community Heathcare, Plymouth, United Kingdom; ${ }^{2}$ Cornwall Partnership Foundation Trust, Truro, United Kingdom; ${ }^{3}$ Institute for Neurology, UCL, London, United Kingdom; ${ }^{4}$ National Hospital for Neurology and Neurosurgery, London, United Kingdom and ${ }^{5}$ Peninsula College of Medicine and Dentistry, Truro, United Kingdom)

Rationale: Vagal nerve stimulation (VNS) can help improve epileptic control in clients who have had insufficient epileptic control despite many different antiepileptic medications (AED's) or combinations of AED's. VNS is often used in conjunction with AED's in difficult to treat epilepsy.

Topiramate is an AED with a common side effect of weight loss Vagal nerve stimulation with parameters typically used for the treatment of epilepsy is not usually associated with clinically significant weight loss. A series of nine clients' case notes with four controls were reviewed to examine the effect of the combination of topiramate and VNS on epileptic control and weight. The goal of this case series was to assess the effect of topiramate in combination with VNS on weight and epileptic control.

Methods: Case notes of nine clients with difficult to treat epilepsy were reviewed and divided into those who had a vagal nerve stimulator and were on topiramate and clients with a vagal nerve stimulator and pharmacotherapy other than topiramate.

The first group contained five clients who were taking topiramate with inadequate effect, having previously been on other antiepileptic drugs (AED's) or combinations of AED's. Topiramate therapy continued after VNS was fitted.

In the second group were four clients who had been treated with pharmacotherapy and did not gain satisfactory control of their epilepsy. They were fitted with a vagal nerve stimulator but were not on topiramate at the same time. They continued with pharmacotherapy other than topiramate in combination with their vagal nerve stimulators.

Literature search was completed.

Results: In the first group topiramate did not cause its well known side effect of weight loss when used alone and did not have an adequate effect on seizure reduction. However when clients on topiramate were fitted with a vagal nerve stimulator all of the clients gained better seizure control $(100 \%)$ and four out of the five clients $(80 \%)$ lost weight in conjunction with improved seizure control. Furthermore one client put weight back on when the battery of the VNS failed and suffered worsened seizure control at the same time. When the battery was replaced they began to lose weight again and regained better seizure control. The client who did not lose weight had co morbid hypothyroidism.

In the second group, who were treated with vagal nerve stimulator and medication other than topiramate, all four had improved seizure control after VNS was inserted (100\%), although weight loss was noticed in only one out of the four clients $(25 \%)$. 
Conclusions: If topiramate has been unsuccessful it could be revisited with VNS in those with difficult to treat epilepsy, and could be a viable treatment option here.

If topiramate has not been successful and not caused weight loss when used alone or as part of a medical regime, it may be worth revisiting after VNS insertion in people who are overweight with difficult to treat epilepsy.

\subsection{2}

CLOBAZAM AS AN ADJUNCTIVE TREATMENT IN SEIZURES ASSOCIATED WITH LENNOX-GASTAUT SYNDROME: EFFECT ON REDUCING SEIZURE FREQUENCY AND MEDICATION BURDEN IN THE CLINICAL SETTING

Gina Deck and G. Montouris (Neurology, Boston University School of Medicine, Boston, MA)

Rationale: Clobazam is a 1,5 benzodiazepine approved for use in the adjunctive treatment of seizures associated with Lennox-Gastaut syndrome (LGS). Patients with LGS or LGS-like seizures typically have refractory tonic/atonic epilepsy. Previously approved therapies, in addition to VNS, in this cohort of patients have failed to control these seizure types. The adjunctive use of clobazam was initiated in this population for the purpose of reducing frequency of atonic/tonic seizures.

Methods: Patients with cognitive delay, intellectual disability, and refractory tonic/atonic epilepsy (LGS or LGS-like syndromes) were seen in routine outpatient clinic visits. Clobazam was initiated, typically at $5 \mathrm{mg}$ twice daily, which was increased to $5 \mathrm{mg}$ qAM and $10 \mathrm{mg}$ qPM after 1 week. Clobazam dose was increased subsequently only if the patient had breakthrough seizures. The primary outcome was reduction in seizure frequency after initiation of clobazam. Secondary outcomes included the decreased need for rescue benzodiazepines and a goal of reduction in concomitant anti-epileptic medications.

Results: A total of seven patients were initiated on treatment with clobazam with a minimum 4-week follow-up. Additional patients with $<4$ week follow-up are also being followed, but not reported at time of abstract. All patients had tonic or atonic seizures requiring 25 daily drug regimens. 6 of 7 patients had vagal nerve stimulators implanted. Monthly seizure frequency decreased from an average of 8.7 seizures (range 1-24) to an average of 0.77 seizures (range 0-6) after initiation of clobazam therapy (see table 2). Seizure frequency was measured 8 weeks prior to clobazam initiation. Seizure frequency after clobazam initiation ranged 4-16 weeks. 3 of 7 patients were seizure free on clobazam $15 \mathrm{mg}$ daily. One patient was seizure free on that dose with the exception of 1 seizure in the setting of an infection. Overall, 5 of 7 patients achieved $>90 \%$ seizure frequency reduction after clobazam initiation; the remaining 2 patients achieved $>75 \%$ seizure frequency reduction. With reduction in seizure frequency, use of rescue benzodiazepines was no longer required. All patients were able to be down titrated on one AED in their regimen.

Conclusions: Clobazam demonstrated significant efficacy as an adjunctive treatment in tonic/atonic seizures resulting in dramatic seizure reduction. As a result, there was a decreased need for rescue benzodiazepines as well as a reduction in medication burden. Followup is ongoing.
Table 1: Patient Demographics

\begin{tabular}{|c|c|c|c|c|c|c|c|}
\hline Patient & Age & Sex & VNS & $\begin{array}{c}\text { Total } \\
\text { clobazam } \\
\text { dose (mg) }\end{array}$ & $\begin{array}{l}\text { Other } \\
\text { AEDs** } \\
\text { (\#) }\end{array}$ & $\begin{array}{c}\text { Rescue } \\
\text { benzodiazepines }\end{array}$ & Seizure etiology \\
\hline 1 & 26 & F & Yes & 15 & 3 & No & $\begin{array}{l}\text { Medulloblastomas s/p } \\
\text { resection \& XRT }\end{array}$ \\
\hline 2 & 40 & F & Yes & 20 & 2 & Yes & $\begin{array}{c}\text { Lemnox-Gastaut-like } \\
\text { syndiome }\end{array}$ \\
\hline 3 & 61 & $\Gamma$ & Yes & 15 & 4 & Yes & s/p infantile encephalitis \\
\hline 4 & 46 & M & Yes & 25 & 3 & No & $\begin{array}{l}\text { Gencralized, } \\
\text { Lennox-Gastaul-like } \\
\text { syndrome }\end{array}$ \\
\hline 5 & 57 & M & Yes & 15 & 5 & No & I.ennox-Gastaut syndrome \\
\hline 6 & 51 & M & Yes & 15 & 4 & No & $s / p$ meningitis \\
\hline 7 & 49 & $\Gamma$ & No & 20 & 3 & Yes & Ccrebral palsy \\
\hline
\end{tabular}

* AEDs included Banzel, Carbatrol, Depakote, Dilantin, Felbetol, Keppra, Lamictal, Lyrica, Phenobarbital, Topamax, Trileptal, Vimpat, Zonegran

Table 2: Seizure Frequency Pre- \& Post-clobazam Initiation

\begin{tabular}{|c|c|c|}
\hline Patient & $\begin{array}{c}\text { Seizure frequency prior to } \\
\text { clobazam (monthly) }\end{array}$ & $\begin{array}{c}\text { Seizure frequency after initiation ol clobazam } \\
12 \text { weck followup: seizure frec }\end{array}$ \\
\hline 2 & 10 & $\begin{array}{c}16 \text { weck followup: } 1 \text { breakthrough scizurc on low dosc, } \\
\text { seizure free for } 2 \text { months on increased dose }\end{array}$ \\
\hline 3 & $1-8$ & 8 week followup: 1 seizure in setting of infection \\
\hline 4 & 5 & $\begin{array}{c}12 \text { week lollowup: } 2 \text { seizures on low dose, seizure lree on } \\
\text { increased dose }\end{array}$ \\
\hline 5 & 5 & 4 wcck followup: scizurc frec \\
\hline 7 & 5 & 4 wcck followup: scizurc frec \\
\hline
\end{tabular}

2.233

ROLE OF INTRAVENOUS LEVETIRACETAM IN ACUTE SEIZURE MANAGEMENT IN PRETERM NEONATES O. A. Khan ${ }^{1}$, C. Cipriani ${ }^{1}$, C. Wright ${ }^{1}$, R. Castillo ${ }^{2}$, E. D. Crisp ${ }^{2}$ and Batool Kirmani ${ }^{2}\left({ }^{1}\right.$ Pediatrics, Scott and White Hospital, Temple, TX and ${ }^{2}$ Neurology, Scott and White Epilepsy Center/ Texas A \& M HSC College of Medicine, Temple, TX)

Rationale: Due to the treatment of neonatal seizures having limited efficacy and leading to adverse effects, off-label use of antiepileptic drugs in children and neonates is increasing. A pressing need exists to study newer antiepileptic drugs in the neonatal population.

Intravenous levetiracetam became available in August 2006 for use in patients above 16 years of age. There are insufficient data about the efficacy and safety of intravenous levetiracetam in preterm neonates. Methods: We retrospectively analyzed data from preterm neonates that were treated with intravenous levetiracetam at our institution between January 2007 and December 2011. Data was acquired from review of our institution's electronic medical record regarding patients who were treated with intravenous levetiracetam during the neonatal period ( 0 -28 days) and were born at preterm gestational age $(<37$ weeks).

Results: Twelve patients met our inclusion criteria and had received levetiracetam for neonatal seizures. There were 8 females $(67 \%)$ and 4 males (33\%) with gestational ages ranging from 23.3 to 36 weeks. Our primary objective was to assess response to treatment based on clinical and/or electroencephalographic chart documentation. Secondary objectives included indication for initiation of levetiracetam, adverse events, and prevalence of seizure freedom at well child follow-up visits. Response to intravenous levetiracetam was favorable.

Nine patients $(75 \%)$ reached seizure cessation while receiving levetiracetam. No serious side effects were evident. Seven patients $(59 \%)$ were discharged on oral levetiracetam alone, four patients 
(33\%) were discharged on no oral AED and one patient (8\%) was discharged on levetiracetam and phenobarbital. Ten of out of twelve patients were followed up to 6 months after receiving intravenous levetiracetam. Of these, six patients $(60 \%)$ had achieved seizure freedom and been completely weaned off of all AEDs. Two (20\%) had achieved seizure freedom while still on oral levetiracetam. Two $(20 \%)$ continued to experience seizures on oral levetiracetam. Conclusions: Levetiracetam appears to be efficacious in seizure management in preterm neonates.

\subsection{4 \\ ANTIEPILEPTIC DRUG TRANSPORT AT THE BLOOD- BRAIN BARRIER BY MONOCARBOXYLATE TRANSPORTERS}

H. L. Jones, A. Owen, M. Pirmohamed and G. J. Sills (Molecular and Clinical Pharmacology, University of Liverpool, Liverpool, United Kingdom)

Rationale: Changes in expression of drug transporters at the bloodbrain barrier (BBB) may impact on brain penetration of antiepileptic drugs (AEDs) and contribute to the phenomenon of drug resistant epilepsy. Monocarboxylate transporters (MCTs) are members of the solute carrier (SLC) transporter family. Three isoforms, MCT1, MCT4 and MCT8, are expressed in cerebrovascular endothelial cells and are believed to contribute to the functionality of the BBB. This study employed an immortalised human cerebral microvascular endothelial cell line (hCMEC/D3) and a panel of compounds proposed to inhibit MCT transporters and aimed to determine whether these MCTs are involved in the active transport of commonly used AEDs.

Methods: Transport of $\left[{ }^{3} \mathrm{H}\right]$-phenytoin $(25 \mu \mathrm{M}),\left[{ }^{3} \mathrm{H}\right]$-carbamazepine $(20 \mu \mathrm{M}),\left[{ }^{3} \mathrm{H}\right]$-sodium valproate $(300 \mu \mathrm{M}),\left[{ }^{3} \mathrm{H}\right]$-lamotrigine $(10 \mu \mathrm{M})$, $\left[{ }^{3} \mathrm{H}\right]$-gabapentin $(20 \mu \mathrm{M}),\left[{ }^{3} \mathrm{H}\right]$-topiramate $(10 \mu \mathrm{M})$, and $\left[{ }^{3} \mathrm{H}\right]$ levetiracetam $(6 \mu \mathrm{M})$ was assessed in cultures of hCMEC/D3 cells, with $\left[{ }^{14} \mathrm{C}\right]$-lactic acid $(2 \mu \mathrm{M})$ used as a positive control. Cells were exposed to AED plus inhibitor or vehicle (1\% DMSO) for 60 minutes at $37^{\circ} \mathrm{C}$. MCT inhibitors comprised salicylic acid (1mM), 4, $4^{\prime}-$ diisothiocyanatostilbene-2,2'-disulfonic acid (DIDS; $1 \mathrm{mM}$ ), imatinib $(100 \mu \mathrm{M})$, simvastatin $(10 \mu \mathrm{M}), 4$-acetamido-4'-isothiocyanato-2,2'stilbenedisulfonic acid (SITS; 1mM), 5-nitro-2-(3-

phenylpropylamino)benzoic acid (NPPB; $100 \mu \mathrm{M})$, and 4(hydroxymercuri)benzoic acid $(4-\mathrm{MBA} ; 100 \mu \mathrm{M})$. Transport was determined by scintillation counting of cellular radioactive content in comparison to standards of known concentration, with experiments performed in triplicate on at least three occasions $(n \geq 9)$. Results were expressed as mean percentage of control transport ( \pm SEM), with statistical analysis performed by ANOVA with Dunnett correction for multiple comparisons.

Results: Transport of gabapentin was decreased to $3.0 \%( \pm 0.3 \%$; $\mathrm{p}<0.001)$ of control in the presence of 4-MBA (MCT1/MCT4 inhibitor) and increased to $255 \%( \pm 14.5 \% ; \mathrm{p}<0.001)$ and $159 \%$ $( \pm 13.8 \% ; \mathrm{p}<0.005)$ of control with NPPB (MCT4 inhibitor) and DIDS (non-specific MCT inhibitor), respectively. Transport of phenytoin was decreased to $57 \%( \pm 5.2 \% ; \mathrm{p}<0.005)$ of control in the presence of simvastatin (MCT4 inhibitor), whereas transport of lamotrigine was decreased to $33 \%( \pm 2.5 \% ; \mathrm{p}<0.05)$ of control with imatinib (MCT8 inhibitor). Changes in relative transport of AEDs for all other drug and inhibitor combinations failed to reach statistical significance.

Conclusions: This study suggests that the contribution of MCTs to the brain penetration of AEDs is modest, at best. Gabapentin appears to undergo active transport in the hCMEC/D3 cell line, possibly mediated by MCT4, but a lack of inhibitor selectivity and contradictory findings prevented identification of the transporter involved. Phenytoin and lamotrigine were similarly subject to transport, potentially by MCT4 and MCT8 respectively, but again the identity of the MCT isoform could not be confirmed. Further investigation is required to clarify the contribution of individual MCT transporters to the brain penetration of these AEDs.

\subsection{5 \\ EFFICACY OF LACOSAMIDE INTRAVENOUS IN SE (STATUS EPILEPTICUS)}

ESTEVO SANTAMARINA, M. Toledo, M. Sueiras, M. Raspall, E. Lainez, J. Durà and X. Salas Puig (HOSPITAL VALL HEBRON, BARCELONA, Spain)

Rationale: Lacosamide(LCM) has an intravenous formulation with favorable side effect profile and a low potential for drug interaction; furthermore it exhibits linear pharmacokinetics. For these reasons, it is a possibility for use in SE. Our aim is describe the efficacy of LCM in SE in both situations of refractory and non-refractory status Methods: We analyzed all SE in which intravenous LCM was used from September-2010 to June-2012. Demographic variables, status etiology, AEDs used, loading dose, infusion rate, treatment response and time of resolution were collected. We initially analyzed patients in which LCM was used as a 4/5th option(refractory); subsequently we analyzed all patients where LCM was used as $2 \mathrm{nd} / 3 \mathrm{rd}$ option (non-refractory).

Results: We evaluated a total of 31 patients: 19 were refractory to other AEDs. $63 \%$ of them responded to LCM. Median dose was 400mg (200-600). When comparing responders and non-responders, those patients with convulsive or minor motor symptoms $(\mathrm{p}=0.05)$, or without previous epilepy $(\mathrm{p}=0.024)$ had a worse response. We found no differences in either the dose or infusion rate.

In a second group of 12 patients, LCM was used earlier ( $2 / 3$ option). In this case the efficiency was $70 \%$. Regarding the etiology, all nonresponders were acute symptomatic. Median dose was $400 \mathrm{mg}$ (200600 ), and the infusion rate of $40 \mathrm{mg} / \mathrm{min}$.

There were no major side effects.

Conclusions: Despite its limitations, LCM is an alternative and useful therapeutic tool for treatment of SE, mainly in patients with previous epilepsy and non-convulsive status. The effectiveness seems to increase slightly when it is administered earlier.

\subsection{6 \\ ABSORPTION OF GABAPENTIN IN NURSING HOME ELDERLY PATIENTS}

Ghada F. Ahmed $^{1}$, R. C. Brundage ${ }^{1}$, I. L. Leppik ${ }^{1}$ and A. K. Birnbaum ${ }^{1,2}\left({ }^{1}\right.$ Experimental and Clinical Pharmacology, University of Minnesota, Minneapolis, MN and ${ }^{2}$ Center for Clinical and Cognitive Neuropharmacology, University of Minnesota, Minneapolis, MN)

Rationale: Gabapentin (GBP) is indicated for both epilepsy and pain. Dosing based on GBP pharmacokinetic (PK) information in elderly nursing home $(\mathrm{NH})$ patients is not available due to lack of GBP PK studies in this population. This study characterized the saturable absorption profile of GBP in NH elderly patients

Methods: The study was approved by the University of Minnesota's Institutional Review Board. A prospective PK study was conducted in NH elderly patients receiving GBP for chronic pain. Residents who did not have a change in GBP dose for at least 4 weeks (steadystate), in facility for at least 2 months, and on a stable-dose of comedications were included. Comatose subjects or those with unstable medical conditions were excluded. The study included multiple observation visits per subject (range 4- 6). At each visit, a blood sample was collected for measurement of drug level and time after dose recorded. PK analysis consisted of nonlinear mixed effects modeling (NONMEM 7). A one-compartment model fit the data. Covariates (estimated glomerular filtration rate (EGFR), age, weight, and sex) were tested for hypothesized effect on apparent drug clearance $(C L)$ through forward inclusion $(\chi 2, p \leq 0.05$,

$\mathrm{df}=1)$ /backward elimination $(\chi 2, \mathrm{p} \leq 0.01, \mathrm{df}=1)$. In addition, the effect of increasing GBP dose on the extent of absorption was evaluated by testing for the significance of a saturable absorption profile. The final model was qualified through a standardized visual predictive check (SVPC) and precision of parameter estimates determined using bootstrap analysis 
Results: Data from 30 patients ( 7 men: 23 women) were available for analysis. Mean weight and age were $85 \mathrm{~kg}$ and 76 years (range 6195), respectively. Median (range) EGFR was estimated to be 62.4 $\mathrm{mL} / \mathrm{min} / 1.73 \mathrm{~m} 2$ (13.8-109.9). Patients received a median (range) daily GBP dose of $500 \mathrm{mg}$ (100-2400) divided into 3 (range 1-4) doses/day. GBP CL was significantly dependent on EGFR (change in $\mathrm{OFV}=50, \mathrm{p}<0.0001$ ), while the bioavailability (F) decreased in an inhibitory sigmoidal Emax manner with increasing dose of GBP (change in $\mathrm{OFV}=11.3, \mathrm{p}<0.01$ ). The model estimated a maximum absolute reduction (IMAX) in F of 50\% $(24 \%, 76 \%)$ and a dose that produces $50 \%$ of the maximum saturation (ID50) of $\sim 400 \mathrm{mg}(95 \%$ CI 50, 700). These estimates are lower than the ID50 of $1020 \mathrm{mg}$ and $1120 \mathrm{mg}$ reported for healthy volunteers (Bockbrader, et al. Epilepsia 1996, 37, 159; Gidal, et al. Epilepsy Research 1998, 31, 91-99) and younger adult neuropathic pain patients (Carlsson, et al. Ther Drug Monit 2009, 31, 86-94), respectively. Visual inspection of SVPC plots demonstrated an adequate performance of the model Conclusions: A dose-dependent bioavailability and a strong association of CL on EGFR were demonstrated in NH elderly patients receiving GBP. The ID50 of saturation was estimated to be $400 \mathrm{mg}$ and found to be markedly less than the reported ID50 in younger adults indicating a clinically meaningful effect of aging on the saturable absorption profile of GBP. Smaller doses given more often may be warranted in this population when using GBP for seizure reduction

Funded by NIH NIA R01AG026390

\subsection{7}

\section{USE OF LACOSAMIDE AS ADJUVANT THERAPY IN REFRACTORY STATUS EPILEPTICUS AND RECURRENT SEIZURES}

Khondoker P. Alam ${ }^{1}$, P. Mullin ${ }^{1}$, S. Park ${ }^{1}$, K. Berger ${ }^{2}$ and A. J. Rosengart ${ }^{1}\left({ }^{1}\right.$ Weill Cornell Medical College, New York, NY and ${ }^{2}$ Pharmacy, Weill Cornell Medical College, New York, NY)

Rationale: Status epilepticus (SE) and repetitive seizures (RS) are commonly reported sequelae after brain injury occurring in up to $34 \%$ of patients depending on cause and comorbidity. Although the treatment of SE and RS has been standardized to include an escalating approach of benzodiazepines, phenytoin and valproic acid, up to $30 \%$ of SE/RS patients remain refractory to initial treatment. The purpose of this study was to evaluate the effectiveness of lacosamide (LCM), a recently approved slow sodium channel blocking agent, as de novo therapy in terminating refractory SE/RS, an approach that has only been studied in small case series throughout the literature.

Methods: We retrospectively reviewed all pharmacy dispensing records at Weill Cornell Medical College, a tertiary academic referral center, for LCM usage between 2009 and 2011. Only patients with the diagnosis of SE/RS and concurrent video EEG monitoring $(n=67)$ were selected and of those, 41 patients were excluded because of LCM therapy prior to SE/RS occurrence. The remaining 26 patients were analyzed with respect to clinical presentation, EEG findings as well as LCM dosing and effectiveness (i.e., EEG-proven termination of SE/RS). Successful LCM therapy was defined when: 1) LCM was the only anticonvulsant actively titrated immediately prior to seizure resolution; 2) epileptiform activities on vEEG remained suppressed for at least 24 hours in patients without evidence of burst suppression; and 3) lack of seizure recurrence for at least 48 hours after all sedatives were weaned. All diagnostic criteria were applied by 2 independent examiners.

Results: Of the 26 patients, 19 (73\%) received the diagnosis of nonconvulsive SE (NCSE) and 7 (27\%) nonconvulsive RS (NCRS) refractory to standard SE treatment approach. The median age of all patients was 68 (range 6 to 90) years and (15) 58\% were female. The most common admission diagnoses leading to the seizures were brain tumor in 11 patients $(42 \%)$, intracerebral hemorrhage $7(27 \%)$, ischemic stroke $2(8 \%)$, cardiac arrest $2(8 \%)$, toxic metabolic encephalopathy $2(8 \%)$, cortical dysplasia $1(4 \%)$ and static encephalopathy $1(4 \%)$. Median LCM initiation (first) dose was of $200 \mathrm{mg}$ while the 24-hour total dose ranged from 70 to $600 \mathrm{mg}$ with a median dose of $300 \mathrm{mg}$. The most commonly employed LCM maintenance dose was $400 \mathrm{mg}$ every 24 hours in $11(42 \%)$ patients. In $11(42 \%)$ patients LCM led to seizure termination; in $3(12 \%)$ seizures were terminated but not within our time interval criteria; in 1 seizure frequency decreased significantly; and 11 patients experienced no observable treatment benefit. Side effects included P$\mathrm{R}$ interval increase in 1and mild increase of liver enzymes in 10 who were also concomitantly on other medications.

Conclusions: In this retrospective case series of refractory SE/RS adjuvant LCM led to seizure termination in 42\% (11 of 26 patients) within 24 hours and to seizure improvements in an additional 15\% (4 patients). These results point to a potential usefulness of LCM in refractory SE/RS; a prospectively study seems warranted.

\section{Surgery: Adult}

\subsection{8 \\ SURGICAL OUTCOME FOLLOWING SELECTIVE AMYGDALOHIPPOCAMPECTOMY FOR MEDICALLY REFRACTORY TEMPORAL LOBE EPILEPSY \\ David Spencer ${ }^{1}$, M. C. Salinsky ${ }^{1}$, R. Hoffenberg ${ }^{1}$ and K. Burchiel ${ }^{2}$ \\ ( ${ }^{1}$ Oregon Health \& Science University, Portland, OR and \\ ${ }^{2}$ Neurosurgery, Oregon Health \& Science University, Portland, OR)}

Rationale: Selective amygdalohippocampectomy (S-AH) has been employed as a tissue-sparing alternative to standard anterior temporal lobectomy (ATL) for treatment of well-defined medically refractory mesial temporal lobe epilepsy. Several studies suggest that postoperative seizure control following this procedure may equal that following anterior temporal lobectomy, with less risk of postoperative neuropsychological deficits. The present study reports surgical outcome at 1 and 3 years follow-up in a consecutive series of patients treated with S-AH.

Methods: A retrospective chart review was performed of 96 consecutive patients who underwent selective amygdalohippocampectomy at our institution since 1993 and who were at least one year postoperative. Known lesional cases other than hippocampal sclerosis were excluded. Patients ranged in age from 1557 years at surgery (mean 37.2, SD 10.8). Preoperative MRI findings were: normal (5), hippocampal atrophy (29), hippocampal atrophy + increased signal on T2 weighted/FLAIR images (52), increased signal without atrophy (3) bilateral hippocampal atrophy (3), and abnormal hippocampal morphology (4). Surgical outcome is reported at 1 year and 3-year follow-up using modified Engel's classification. Results: 96 patients were identified who met the inclusion criteria; outcome data were available on 91 at 1 year $(95 \%) .82$ patients were identified who met the inclusion criteria and were least 3 years postoperative. Three year follow-up data were available on 72/82 (88\%). The postoperative seizure outcome results are listed in table 1 . A separate analysis of the cohort of 72 patients who had both 1 year and 3 year follow-up is listed in table 2

Conclusions: In a selected population of patients with medial temporal lobe epilepsy excellent surgical outcome (Engel class I, II) was achieved in $93 \%$ of patients at 1 year, and $89 \%$ at 3 year followup. In the cohort of patients with both 1 year and 3-year follow-up, excellent outcome was mostly sustained over the 3 -year period. Surgical outcome following S-AH appears comparable to reported outcomes following standard anterior temporal lobectomy. 
Table 1

\begin{tabular}{|c|cccc|}
\hline Follow-up & Class I & Class II & Class IIl & Class IV \\
\hline 1 year & $76 / 91(84 \%)$ & $8 / 91(9 \%)$ & $2 / 91(2 \%)$ & $5 / 91(5 \%)$ \\
\hline 3 years & $52 / 72(74 \%$ & $11 / 72(15 \%)$ & $4 / 72(6 \%)$ & $5 / 72(7 \%)$ \\
\hline
\end{tabular}

Table 2

\begin{tabular}{|c|cccc|}
\hline Follow-up & Class I & Class Il & Class IIl & Class IV \\
\hline 1 year & $59 / 72(82 \%)$ & $8 / 72(11 \%)$ & $1 / 72(1 \%)$ & $4 / 72(6 \%)$ \\
\hline 3 ycars & $52 / 72(72 \%)$ & $11 / 72(15 \%)$ & $4 / 72(6 \%)$ & $5 / 72(7 \%)$ \\
\hline
\end{tabular}

\subsection{9}

VAGUS NERVE STIMULATION REDUCES

\section{HOSPITALIZATION AND EMERGENCY ASSISTANCE IN} PATIENTS WITH REFRACTORY EPILEPSY

Xiana Rodriguez Osorio ${ }^{1}$, T. García Sobrino ${ }^{1}$, A. López Ferreiro ${ }^{1}$, E. Corredera García $^{1}$, M. Peleteiro ${ }^{3}$, A. Prieto ${ }^{2}$ and J. López-González ${ }^{1}$ ( ${ }^{1}$ Neurology, Hospital Clinico Universitario de Santiago, Santiago de Compostela, Spain; ${ }^{2}$ Neurosurgery, Hospital Clinico Universitario de Santiago, Santiago de Compostela, Spain and ${ }^{3}$ Neurophysiology, Hospital Clinico Universitario de Santiago, Santiago de Compostela, Spain)

Rationale: Vagus nerve stimulation (VNS) is an effective method (with $\geq 50 \%$ of seizures reduction) for patients with refractory epilepsy that are not suitable candidates for resective surgery. We aim to evaluate its additional benefit for reducing the need of medical assistance in these patients.

Methods: Retrospective study of adult epilepsy patients implanted with VNS and $\geq 1$ year follow up. We evaluated number of days of hospitalization, number of emergency department attendances and number of consultations regarding their epilepsy, comparing every year after surgery, up to four years, with the year prior to the intervention.

Results: We included 44 patients (55\% women) aged $40[30,51]$ years old and a median time of evolution of epilepsy of $25[17,37]$ years. $52 \%$ had $>20$ seizures/month. Good response to VNS was achieved in $44 \%(\mathrm{n}=44,1$ year), $51 \%(\mathrm{n}=41,2$ years $), 53 \%(\mathrm{n}=$ $34,3$ years $)$ and $52 \%(n=29 ; 4$ years $)$ of patients. Days of hospitalization (preVNS $=5.66 \pm 15.80)$ decreased at 1 year $(1.42 \pm$ $9.15, \mathrm{p}=0.03), 2$ years $(0.02 \pm 0.16, \mathrm{p}=0.01), 3$ years $(0.76 \pm 3.48$, $\mathrm{p}=0.008)$ and 4 years $(1.00 \pm 3.18, \mathrm{p}=0.03)$. Attendances to the emergency room were also reduced from $0.86 \pm 1.37$ days (prior to VNS) to $0.44 \pm 0.76$ ( 1 year, $p=0.076), 0.32 \pm 0.65$ ( 2 years, $\mathrm{p}=0.005), 0.24 \pm 0.61$ ( 3 years, $\mathrm{p}=0.005)$ and $0.24 \pm 0.61$ ( 4 years, $\mathrm{p}=0.02)$. Follow-up visits initially increased from $5.39 \pm 2.29$ (preVNS) visits to $5.95 \pm 2.03$ ( 1 year, $\mathrm{p}=0.166)$ and $4.51 \pm 1.79$ (2 years, $\mathrm{p}=0.158)$ and then reduced to $4.9 \pm 1.97$ ( 3 years, $\mathrm{p}=0.008)$ and $3.93 \pm 1.62$ ( 4 years, $p=0.004)$.

Conclusions: VNS increases its efficacy over time and it reduces the need for urgent care and hospitalization days in patients with refractory epilepsy.

\subsection{0}

VISUAL FIELD DEFECTS AFTER RADIOSURGERY FOR MESIAL TEMPORAL LOBE EPILEPSY

H. Hensley-Judge ${ }^{1}$, N. M. Barbaro ${ }^{2}$, S. A. Newman ${ }^{4}$, E. Chang ${ }^{3}$, M. Ward $^{3}$ and Mark S. Quigg ${ }^{1}\left({ }^{1}\right.$ Neurology, University of Virginia, Charlottesville, VA; ${ }^{2}$ Neurosurgery, Indiana University, Indianapolis, IN; ${ }^{3}$ Neurosurgery, University of California at San Francisco, San Francisco, CA and ${ }^{4}$ Ophthalmology, University of Virginia, Charlottesville, VA)

Rationale: Gamma Knife radiosurgery (RS) may be an alternative to open surgery for mesial temporal lobe epilepsy (MTLE), but the anticonvulsant mechanisms of RS are unclear, with some data suggesting a necrotic lesion at the target and some suggesting neuromodulatory effects. Examination of the visual field defects (VFD) after RS may provide evidence of the extent of a postoperative fixed lesion. VFD occur in $\sim 50 \%$ of patients undergoing open surgery for MTLE.

Methods: This multicenter prospective trial of RS enrolled patients with unilateral hippocampal sclerosis and concordant video-EEG findings. Main results of this US Pilot Study have been published before. An exclusion criterion was presence of pre-operative VFD. Patients were randomized to low (20Gy) or high (24Gy) volumes delivered to the amygdala, hippocampal head, and parahippocampal gyrus. Exposure to optic nerve was limited to $<8 \mathrm{~Gy}$. Postoperative perimetry (Goldman or Humphries) were obtained at $24 \mathrm{~m}$ after RS. Perimetry VFD were classified by two independent reviewers blinded to other study data. Results were contrasted with RS dose (20Gy/24Gy), 50\% isodose volume, seizure remission (1 year seizure free between postoperative $24 \mathrm{~m}-36 \mathrm{~m}$ ), volume of peak effect (at 12 $\mathrm{m})$ radiation-induced change at the surgical target, and side of RS (language-dominant/nondominant).

Results: Postoperative VF results were available for 26 of the 30 patient sample. No patients complained of VFD or poor acuity, and confrontation examinations were normal. None had scotoma or arcuate defects. 17 (65\%) had postoperative VFD. VFD were homonymous superior quadrantanopias reaching to the horizontal midline $[7 / 26(27 \%)]$ or sparing the horizontal midline $[n=10 / 26$ $(38 \%)]$. No VFD reached below the horizontal midline. Although the proportion of those with VFD was higher in the high dose [10/12 $(83 \%]$ than the low dose arm [7/14 (50\%)], the difference, probably because of the sample size, was not statistically significant (Fisher's exact test $\mathrm{p}=0.08$ ). Volumes of $\mathrm{T} 2$-weighted radiation-induced change at $12 \mathrm{~m}$ were higher in those with VFD $(403 \pm 350 \mathrm{ml}$ vs $182 \pm 250, p=0.11$ t-test). The side of RS, isodose volumes, and seizure remission were not associated with presence of or severity of VFD.

Conclusions: VFD appeared after RS in proportions similar to those published for open surgery for MTLE. The nature of VFD was consistent with lesions of the optic radiations, sparing optic nerve. These findings, along with previously published findings on spectroscopic changes following RS, support the hypothesis that the anti-epileptic effects of RS are destructive rather than modulatory.

\subsection{1 \\ LONG-TERM PSYCHOSOCIAL OUTCOMES OF ANTERIOR TEMPORAL LOBECTOMY: FIVE, TEN, AND FIFTEEN YEARS LATER}

Jana E. Jones ${ }^{1}$, J. Blocher ${ }^{2}$, D. Jackson ${ }^{1}$ and B. P. Hermann ${ }^{1}$ ( ${ }^{1}$ Neurology, University of Wisconsin School of Medicine and Public Health, Madison, WI and ${ }^{2}$ Psychology, Long Island University, Brookville, NY)

Rationale: 57 participants (surgical: $\mathrm{M}$ age $=31.3$ years, $\mathrm{SD}=8.9$ ) who underwent ATL and completed 5- and 10-year follow-up interviews; 41 participants also completed a 15-year follow-up interview. 18 TLE individuals who did not undergo ATL (medicallymanaged control group: $\mathrm{M}$ age $=34.7$ years, $\mathrm{SD}=10.3$ ) were followed at 5- and 10-years; 9 participants were available for interview at 15-year follow-up. Assessment of psychosocial status focused on full-time employment, driving, independent living, and financial independence.

Methods: 57 participants (surgical group: $\mathrm{M}$ age $=31.3$ years, $\mathrm{SD}=$ 8.9) who underwent ATL and completed 5- and 10-year follow-up interviews; 41 participants also completed a 15 -year follow-up interview. 18 TLE individuals who did not undergo ATL (medicallymanaged control group: $\mathrm{M}$ age $=34.7$ years, $\mathrm{SD}=10.3$ ) were followed at 5- and 10-years; 9 participants were available for interview at 15-year follow-up. Assessment of psychosocial status focused on full-time employment, driving, independent living, and financial independence. 
Results: At 5-years post-surgery, surgical participants were more likely to be living independently $(p<0.001)$ and driving $(p=0.004)$ than were their medically-managed counterparts. Additionally, surgical participants were more likely to be financially independent $(\mathrm{p}=0.032)$ and have full-time employment $(\mathrm{p}=0.025)$. The same was true at 10 -year follow-up: independent living $(\mathrm{p}=0.022)$; driving $(\mathrm{p}=0.003)$; financial independence $(\mathrm{p}=0.003)$; full-time employment $(p=0.037)$. At 15 -years post-surgery, surgical participants were more likely to be living independently $(p=0.014)$ and driving $(\mathrm{p}=0.020)$ than were medically-managed controls. There was a trend noted between the groups on financial independence $(\mathrm{p}=$ $0.065)$ but no significant difference for full-time employment rates ( $p$ $=0.209$ ). Within-group analyses showed that the surgical participants made significant gains in ability to live independently and drive from pre-surgery to 5-years post-surgery, and these gains were maintained across both 10- and 15-year follow-ups (all p's $<0.05$ ). Nonsignificant gains were seen relative to baseline rates of full-time employment and financial independence. At the 15-year follow-up $68 \%$ of the sample was seizure free in the previous 12 months compared to $11 \%$ in the medically managed group $(\mathrm{p}=0.0001)$, and $47 \%$ of those in the surgery group had no seizures or auras since surgery. Additionally, $37 \%$ of the surgical group was no longer taking medications compared to $11 \%$ in the medically-managed group.

Conclusions: Individuals undergoing ATL demonstrated improvement across several psychosocial domains relative to a medically-managed group. Within-group gains were observed in rates of independent living and driving across all follow-up intervals with smaller gains maintained in financial independence and full time employment. By examining these psychosocial outcomes, and by the inclusion of a control group, these data show the persisting benefit of ATL beyond decreases in seizure frequency and severity.

\subsection{2 \\ SUBCLINICAL SEIZURES: WHAT IS THEIR CLINICAL SIGNIFICANCE?}

Pue Farooque and R. Duckrow (Yale University School of Medicine, New Haven, CT)

Rationale: Subclinical seizures have been reported to be clinically significant with their characteristics influencing surgical outcome. There have been few studies done evaluating the significance of subclinical seizures and the vast majority of seizures studied have been temporal in origin. The purpose of this study was to evaluate the clinical significance, characteristics and prognostic value of subclinical seizures of temporal and extra temporal origin. Methods: We reviewed our epilepsy surgical database from 20032011 for patients that underwent intracranial EEG recording, surgical resection and had subclinical seizures during their intracranial EEG recording. After those patients were identified we then evaluated seizure onset zone, electrographic pattern at onset, seizure type, seizure spread, presence or absence of MRI lesion, and surgical outcome at least one year post-surgery. Patients were grouped into two groups. Group 1 where both the clinical and subclinical seizures had the same seizure onset region, and group 2 where some or all of the clinical and subclinical seizures originated from different regions. Results: A total of 27 patients were found to have had subclinical seizures during their intracranial EEG recording. Of those, 14 patients had temporal lobe epilepsy(12 mesial and 2 lateral) and 15 were extra temporal( 5 frontal, 8 parietal/occipital). There were a total of 791 seizures with 310 of those seizures being subclinical in nature. Among the clinical and subclinical seizures that were co-localized, $33 \%$ had poor surgical outcome, and $67 \%$ had excellent surgical outcome. For those that were partially co-localized or not colocalized $91 \%$ had poor outcome while $9 \%$ had excellent outcome. Eleven patients were found to have subclinical seizures that propagated to a region beyond their seizure onset zone. Of those 11 patients, 6 patients had subclinical seizures propagate to a different anatomical region than the clinical seizures. All six of these patients had poor surgical outcome.

Conclusions: Our study like others has found that subclinical seizures are clinically significant and if are included with the region of surgical resection will predict good surgical outcome(Fisher's exact test $\mathrm{p}=0.011$ ). An observation from our study is that subclinical seizures may represent a distinct epileptic network from clinical seizures as they can propagate to different anatomic regions from clinical seizures. Propagation of subclinical seizures to a different region than clinical seizures can affect surgical outcome(Fisher's exact test $p=0.06$ ). Further studies with a larger sample size will need to be conducted to fully assess the effect on surgical outcome and determine its statistical significance.

\subsection{3 \\ FRAMELESS STEREOTAXIC LASER THERMAL ABLATION OF THE HIPPOCAMPUS IN MESIAL TEMPORAL LOBE EPILEPSY DUE TO HIPPOCAMPAL SCLEROSIS: REPORT OF TWO PATIENTS}

S. F. Danish and Stephen Wong (UMDNJ - Robert Wood Johnson Medical School, New Brunswick, NJ)

Rationale: In patients with refractory mesial temporal lobe epilepsy due to hippocampal sclerosis (MTLE-HS), surgery with anterior temporal lobectomy and amygdalohippocampectomy (ATL-AH) or selective amygdalohippocampectomy (SelAH) has been associated with seizure-freedom in up to $70 \%$. These procedures may not be options for patients with a less favorable risk-benefit ratio due to medical comorbidity or a lower predicted surgical benefit. Recently, MRI-guided stereotaxic laser thermal tissue ablation (Visualase, Inc.) has been FDA-approved and is being applied towards epilepsy surgery. We report our experience with this procedure in two cases of refractory MTLE-HS.

Methods: Patient A is a 61 year-old right-handed male with refractory epilepsy from childhood, with monthly clusters of partial complex seizures and annual secondarily generalized convulsions. Routine EEG revealed left fronto-temporal spike-and-wave complexes. Brain MRI revealed left hippocampal sclerosis. Brain $\mathrm{PET} / \mathrm{CT}$ revealed mild hypometabolism of the left medial temporal region. Video-EEG revealed left temporal interictal spikes and ictal onsets. Wada testing revealed right hemispheric language and memory dominance. Because of numerous medical comorbidities, he was offered laser ablation of the left hippocampus.

Patient B is a 26 year-old right-handed female with refractory epilepsy since age 6 , with monthly clusters of 2-3 partial complex seizures and secondarily generalized convulsions every few months. Routine EEG was unremarkable. Brain MRI revealed right hippocampal sclerosis. Brain PET/CT revealed right posterior temporal hypometabolism. Video-EEG revealed 24 temporal seizures, 21 from the right, 2 from the left, and one non-localized. Wada testing showed left hemispheric language and memory dominance. Because of the lower chance for seizure-freedom, she was offered laser ablation of the right hippocampus.

Results: Laser ablation of the hippocampus was achieved with frameless stereotaxy in both cases. Both patients tolerated the procedure well without postoperative neurological deficits and were discharged on postoperative day 1. Patient A had an Engel Class Ib / ILAE Class II (auras only) outcome 7 months later (last followup). Patient $\mathrm{B}$ experienced complex partial seizures with her typical right hemispheric semiology on postoperative days 1 and 4 . She experienced recurrent seizures with her typical left hemispheric semiology 2 months after the procedure, though the electrographic lateralization of her seizures remains unknown.

Conclusions: Laser thermal ablation of the hippocampus is welltolerated procedure and warrants consideration for patients with MTLE-HS who may have a less favorable risk-benefit profile for craniotomy. Because a smaller extent of the epileptic network is removed, residual auras and acute postoperative seizures resembling 
the typical pre-operative seizures may be more common. Though the procedure appears to be associated with less morbidity than ATL-AH and SelAH, it remains to be seen whether the seizure outcome is equivalent in MTLE-HS.

\subsection{4}

SEIZURE FREEDOM FOLLOWING STANDARD ANTERIOR TEMPORAL LOBECTOMY COMPARED TO SELECTIVE AMYGDALOHIPPOCAMPECTOMY: A SYSTEMATIC REVIEW AND META-ANALYSIS

C. B. Josephson ${ }^{2}$, Jonathan Dykeman ${ }^{1}$, K. M. Fiest ${ }^{1}$, X. R. Liu ${ }^{1,3}$, R. M. Sadler ${ }^{2}$, N. Jette ${ }^{1}$ and S. Wiebe ${ }^{1}{ }^{1}{ }^{1}$ University of Calgary, Calgary, AB, Canada; ${ }^{2}$ Dalhousie University, Halifax, NS, Canada and ${ }^{3}$ Institute of Neuroscience and the Second Affiliated Hospital of Guangzhou Medical University, Guangzhou, China)

Rationale: Temporal lobe surgery is of benefit for patients with medically refractory mesial temporal lobe epilepsy. The superior operative procedure for seizure control, a standard anterior temporal lobectomy (ATL) or a selective amygdalohippocampectomy (SAH), has yet to be established. The objective of this study was to compare seizure outcome between ATL and SAH.

Methods: We searched Medline and Embase using Medical Subject Headings and keywords related to ATL and SAH. Two reviewers independently screened abstracts and full-texts and abstracted data from eligible studies. We resolved any disagreement through consensus discussion. We included original research that directly compared quantified seizure outcomes in patients undergoing SAH or ATL for temporal lobe epilepsy. We did not restrict studies on the basis of sample size but did exclude those with less than 15 participants in any arm in a pre-specified sensitivity analysis. We investigated heterogeneity using the $\mathrm{I} 2$ statistic and the Cochrane $\mathrm{Q}$ statistic. Publication bias was assessed through evaluation of funnel plots. In the absence of heterogeneity, a fixed effect model was used to derive a pooled odds ratio (OR) for either an Engel class I (free of disabling seizures) or a composite of an Engel class I and II (rare disabling seizures) outcome.

Results: Of 4675 abstracts initially identified by the search 65 were reviewed in full-text. Fifteen studies met all eligibility criteria. We excluded two studies from the meta-analysis that reported data from the same population of patients that were included in more recent studies. The remaining 13 studies contained data from 8 countries $(5$ continents) and included patients of all age ranges. Eleven studies comprising 1203 patients demonstrated that participants were statistically more likely to achieve an Engel class I outcome following ATL compared to SAH (OR 1.56, 95\% confidence interval $[95 \% \mathrm{CI}] 1.18$ to $2.05, \mathrm{p}=0.002)$. The result remained significant in a sensitivity analysis in which two studies that contained $<15$ participants in at least one arm were excluded (OR 1.57, 95\%CI 1.18 to $2.09, \mathrm{p}=0.002$ ). Eight studies comprising 983 patients demonstrated that participants had a statistically similar chance of achieving an Engel class I or II outcome following ATL or SAH (OR $1.12,95 \%$ CI 0.78 to $1.61, p=0.53)$. Studies were robust to potential publication and reporting bias.

Conclusions: Standard ATL confers an improved chance of achieving freedom from disabling seizures in patients with temporal lobe epilepsy according to this pooled analysis of available studies. Improved seizure freedom must be balanced against the neuropsychological impact of each procedure. A randomised controlled trial is justified.

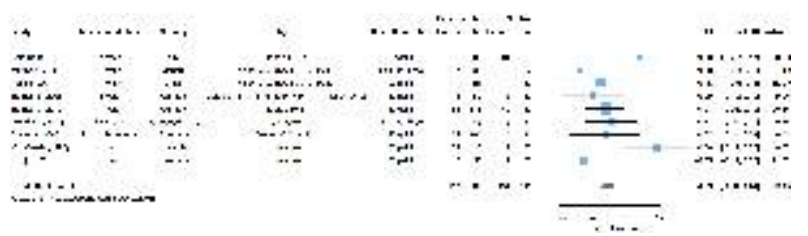

Figure 1. Meta-analysis using a fixed effect model of an Engel class I outcome (free from disabling seizures) in studies comparing selective amygdalohippocampectomy to standard anterior temporal lobectomy in patients with temporal lobe epilepsy.

\subsection{5 \\ LONG-TERM OUTCOME AND PROGNOSTIC FACTORS OF EPILEPSY SURGERY IN MESIAL TEMPORAL LOBE EPILEPSY}

Jiyoung Kim, D. Koo, E. Joo, D. Seo and S. Hong ()

Rationale: Postsurgical outcome studies of patients with mesial temporal lobe epilepsy (MTLE) showed considerable amount of seizure recurrence in a long-term follow-up. Thus identification of predictive factors of long-term surgical outcome is important and useful to improve surgical outcome of MTLE.

Methods: We enrolled 315 consecutive MTLE patients who had at least 2 years of postsurgical follow-up (ranged 2 to 17 years). Clinical characteristics and results of diagnostic tests were included as prognostic factors. Univariate and multiple logistic regression models were used to identify presurgical, surgical, and postsurgical factors that are independently predictive of the long-term outcome of our patients. And Kaplan-Meier survival curves were applied to define long-term seizure outcome.

Results: Two conditions were significantly associated with poor seizure outcome at the last follow-up according to univariate analyses: the presence of cortical dysplasia in lateral temporal lobe as not detected on MRI $(\mathrm{p}=0.032)$ and the seizure recurrence during first postoperative year $(\mathrm{p}<0.001)$. Multivariate logistic regression analysis revealed the independent factors for prediction of unfavorable seizure outcome: seizure recurrence within first postoperative year $(\mathrm{p}<0.001)$, and existence of cortical dysplasia in lateral temporal lobe that was confirmed by histopathology $(\mathrm{p}=0.021)$.

Conclusions: The absence of cortical dysplasia in lateral temporal lobe was an independent prognostic factor for favorable surgical outcome in our study. We also observed that the absence of seizure recurrence within first postoperative year was the predictor for good surgical outcome.

\subsection{6 \\ INFECTIVE COMPLICATIONS COULD BE LESSENED WITH LONG TERM INTRACRANIAL ELECTRODE IMPLANTATION BEFORE SURGERY IN PATIENTS WITH MEDICALLY INTRACTABLE PARTIAL EPILEPSY \\ Sumiya Shibata ${ }^{1}$, T. Kunieda ${ }^{1}$, R. Inano ${ }^{1}$, M. Sawada ${ }^{1}$, Y. Yamao ${ }^{1}$, T. Kikuchi $^{1}$, R. Matsumoto ${ }^{2}$, A. Ikeda ${ }^{2}$, N. Mikuni ${ }^{3}$ and S. Miyamoto ${ }^{1}$ ( ${ }^{1}$ Neurosurgery, Kyoto University Graduate School of Medicine, Kyoto, Japan; ${ }^{2}$ Neurology, Kyoto University Graduate School of Medicine, Kyoto, Japan and ${ }^{3}$ Neurosurgery, Sapporo Medical University Graduate School of Medicine, Sapporo, Japan)}

Rationale: When epileptogenic foci can't be determined noninvasively in epilepsy surgery candidates, intracranial EEG recording is considered as the next presurgical evaluation. Such invasive recording provides invaluable information about accurate epileptogenic foci and eloquent cortices and it also carries the risk of several complications. We aimed at evaluating the infective complications with intracranial EEG recording in order to lessen them.

Methods: We retrospectively reviewed our database of epilepsy surgery cases between May 1992 and March 2012. We have 53 cases who underwent intracranial electrode implantation. All of the patients needed intracranial monitoring because they were candidates for epilepsy surgery and noninvasive results didn't reveal localizing information enough in detail to delineate a resection procedure. The number and location of the subdural electrodes to be implanted was carefully individualized based on the noninvasive evaluation. The 
following clinical information were analyzed: onset age of seizures, age at surgery, gender, the time interval between disease onset and surgery, duration of invasive monitoring, season of monitoring, side of electrode implantation, the number of electrodes (excluding depth and cavernous sinus electrodes), pathological diagnosis, state of associated infections and seizure outcome. Univariate analysis was conducted. Chi-squared test was used to test for association between the categorical variables and the presence of infective complications. T-test was used to assess associations between the continuous variables and the presence of them. Statistical significance was adopted as $P$ value $<0.01$.

Results: Infective complications related to intracranial electrode implantation occurred in 4 cases. Causative agents were identified as Staphylococcus aureus in 3 cases and Staphylococcus epidermidis in 1. On univariate analysis, the significant risk factor was only season of monitoring. Monitoring performed in the fall was significantly associated with infective complications.

Complication occurred associated with a larger number of electrodes, longer duration of monitoring, older age of patient, and the location of implanted electrode in the past report (Neurology 2002;58(1):97103). In our study, age, gender, duration of monitoring, location and the number of electrodes and pathological diagnosis didn't seem to be an increased risk for infective complications. Infective complications had no significant influence on seizure outcome.

Conclusions: Invasive monitor in the fall was a risk factor for infective complications. S. aureus was a common pathogen. Nasal carriers of $S$. aureus was reportedly an risk of infection with this pathogen(Lancet Infect Dis 2005;5(12):751-62). Previous study of the nasopharyngeal bacterial flora in infancy suggested that $S$. aureus was more common in the fall and winter months(FEMS Immunol Med Microbiol 1999;25(1-2):19-28). Decolonization of the nose before surgery may decrease the occurrence of infection especially during this season.

\subsection{7 \\ SURGICAL MANAGEMENT OF EPILEPSY DUE TO CEREBRAL CAVERNOMAS USING NEURONAVIGATION AND INTRAOPERATIVE MR IMAGING}

B. Sommer ${ }^{1}$, B. Kasper ${ }^{2}$, M. Coras ${ }^{3}$, I. Bluemcke ${ }^{3}$, H. M. Hamer ${ }^{2}$, M. Buchfelder ${ }^{1}$ and Karl Roessler ${ }^{1}\left({ }^{1}\right.$ Neurosurgery, University Erlangen Nuremberg, Erlangen, Germany; ${ }^{2}$ Neurology, University Erlangen Nuremberg, Erlangen, Germany and ${ }^{3}$ Neuropathology, University Erlangen Nuremberg, Erlangen, Germany)

Rationale: Cure from epilepsy due to a cerebral cavernoma may be surgically achieved depending on both, the complete removal of the cavernoma, as well as the hemosiderotic rim around the lesion. Intraoperative MR imaging and neuronavigation may play a crucial role to achieve both goals. Thus, we retrospectively investigated the impact of neuronavigation combined with intraoperative MR imaging on the seizure outcome of such patients.

Methods: Altogether, 34 patients ( 17 female, 17 male, mean age 40.8 yrs, range: 17 to 65 yrs) suffering from epilepsy due to a cerebral cerebral cavernoma ( 25 temporal, 9 extra- temporal), were operated on at the Epilepsy Center, University Erlangen Nuremberg between 2002 and 2012. Mean duration of epilepsy was 9.3 years (range: 1 month to 38 years). Three patients suffered from multiple cavernomas and 3 from dual pathologies. Twenty-nine selective lesionectomies and 5 lesionectomies combined with partial temporal lobe resections were performed.

Results: The use of neuronavigation and intraoperative MR lead to complete cavernoma removal in all patients as documented by delayed postoperative imaging. In 5 patients (14.7\%), an intraoperative second look procedure according to intraoperative MRI findings was necessary to complete resection. The removal of the hemosiderotic rim was confirmed in $31 / 34$ (91\%) patients by intraoperative MRI imaging. Futher resection of hemosiderin in 3/34 ( $9 \%$ ) patients was abandoned, due to intended preservation of the optic radiation. No severe complications occured. In 3 patients mild visual field defects occurred (upper quadrantanopsia). Excellent seizure outcome (Engel Classification Grade I) was achieved in $79.3 \%$ of the patients (mean FU 26.6 month, range 2-83). No correlation between the duration of epilepsy and seizure control was found.

Conclusions: In a retrospective analysis of surgically treated patients suffering from temporal lobe epilepsy due to temporal cavernomas, resection of the lesion and the hemosiderotic rim was performed safely and sufficiently using neuronavigation and intraoperative MR imaging, leading to an excellent seizure outcome.

\section{Surgery: Pediatrics}

\subsection{8 \\ EPILEPSY SURGERY IN CHILDREN WITH BILATERAL MRI LESIONS}

Monisha Goyal, C. Rozzelle and J. Blount (University of Alabama at Birmingham, Birmingham, AL)

Rationale: Epilepsy surgery outcomes are best when a well demarcated lesion is visible on MRI, enabling a complete resection such as with malformations of cortical development.

Although MRI can delineate characteristic structural abnormities in many patients, functional imaging and physiological recordings may indicate a more widespread epileptogenic region than is evident on MRI.

Patients with bilateral MRI lesions (with the exception of Tuberous Sclerosis) may not be considered optimal surgery candidates.

The purpose of this study was to assess surgical outcomes in children with bilateral lesions and intractable epilepsy.

Methods: We describe the history and clinical course of 3 consecutive children with bilateral lesions on MRI who underwent epilepsy surgery at Children's of Alabama between 2010 and 2011. Children with Tuberous Sclerosis were excluded.

Results: Patient 1 is an 18 year old female with panhypopituitarism, $\mathrm{S} / \mathrm{P}$ craniopharyngioma resection at 7 and 11 years old with intractable epilepsy beginning at 16 years old. MRI showed bifrontal encephalomalacia, maximal on the right but EEG was more localizing to the left. After an initial bilateral subdural strip evaluation, left frontal grid evaluation revealed seizure onset from the left anterior mesial frontal and pre-frontal region.

Patient 2 is a 14 year old female with congenital right hemiparesis and intractable seizures since 1 year old. She had a known perinatal lesion involving the frontal and parietal lobes, maximal left, with bilateral cystic dilatation of the lateral ventricles and numerous areas of cortical thickening and heterotopia. Scalp and invasive physiologic studies, however, indicated a left frontal epileptogenic zone.

Patient 3 is a 5 year old male with intractable seizures since 8 months old. MRI showed multiple areas of mass-like grey matter gyral thickening and asymmetry within the left temporal lobe and right occipital lobe with periventricular heterotopia in bilateral occipital horns of the lateral ventricles. Invasive EEG revealed a right posterior epileptogenic zone. With an average follow up of 1.5 years, all 3 patients are seizure free since epilepsy surgery.

Conclusions: Our results show that perinatal or acquired bilateral lesions may be amenable to epilepsy surgery.

While the epileptogenic zone may extend beyond the visible margins such as in certain types of cortical dysplasia, our patients' results show that the epileptogenic zone may also be confined to a smaller substrate well within the visible lesion(s) in one hemisphere despite bilateral MRI lesions.

Bilateral lesions are not a contraindication to epilepsy surgery. Successful seizure outcomes can safely be achieved in these children with careful pre-surgical evaluation and planning. 


\subsection{9}

\section{DEPTH ELECTRODES USE IN PEDIATRIC EPILEPSY SURGERY}

J. Kassiri, M. Wheatley, J. Pugh, L. Jurasek, S. Carline, T. Snyder and D. B. Sinclair (Comprehensive Epilepsy Program;, University of Alberta, Edmonton, AB, Canada)

Rationale: The surgical removal of the epileptogenic zone in medically intractable seizures depends on accurate localization to minimize the neurological sequelae and ensure against future seizures. To date, few studies have demonstrated the use of depth electrodes in a pediatric population. Here, we report our study of pediatric patients at our epilepsy center who were successfully operated for medically intractable seizures following the use of intracranial depth electrodes. In addition, we detail three individuals with distinct clinical scenarios in which depth electrodes are helpful and describe our technical approach to surgery.

Methods: We retrospectively reviewed 18 pediatric epilepsy patients requiring depth electrode studies who presented at the University of Alberta Comprehensive Epilepsy Program between 1999 and 2010 with medically intractable epilepsy. Patients underwent cortical resection following depth electrode placement according to the Comprehensive Epilepsy Program surgical protocols after failure of surface electroencephalogram and magnetic resonance imaging to localize ictal onset zone.

Results: The ictal onset zone was successfully identified in all 18 patients. Treatment of all surgical patients resulted in successful seizure freedom (Engel class I ) without neurological complications. Conclusions: Intracranial depth electrode use is safe and able to provide sufficient information for the identification of epileptogenic zone in pediatric patients previously not considered for epilepsy surgery.

\subsection{0 \\ POST-OPERATIVE EPILEPSY SURGERY SEIZURE MEDICATION WITHDRAWAL: WHO NOT WHEN THAT MATTERS \\ Pongkiat Kankirawatana ${ }^{1}$, H. Kim ${ }^{1}, \mathrm{~A} . ~ \mathrm{Oh}^{3}$, C. J. Rozzelle ${ }^{2}$ and J. Blount ${ }^{2}\left({ }^{1}\right.$ Pediatrics, University of Alabama at Birmingham, Birmingham, AL; ${ }^{2}$ Neurosurgery, University of Alabama at Birmingham, Birmingham, AL and ${ }^{3}$ School of Public Health, University of Alabama at Birmingham, Birmingham, AL)}

Rationale: The ideal goal of successful epilepsy surgery would be life without seizures and free of seizure medication. However, few literature reports describe long term epilepsy surgery outcomes, especially in terms of seizure medication reduction post-surgery. Furthermore, there is no clear guideline on how and when to reduce seizure medications after epilepsy surgery. The aim of this study is to report our own experience on these issues.

Methods: We retrospectively reviewed our pediatric epilepsy surgery database to identify all resective epilepsy surgery patients at the Children's of Alabama Hospital between December 2003 and June 2010 with at least 2 years follow up information. Individual seizure medication regimens before and after surgery were collected as well as the onset of seizure medication weaning after operation and seizure relapse date (if any). Clinical characteristics, magnetic resonance imaging (MRI) and surgical resection margin were analyzed for their potential impact on seizure medication reduction and seizure relapse. Statistical significance of differences was assessed using Chi-square, Fischer Exact test and Kaplan-Meier survival analysis for each group.

Results: A total of 72 patients were identified. Forty-eight had an attempt to wean off seizure medications after epilepsy surgery and twenty-four patients remained on their seizure medications with no attempt to wean. There was no significant difference in demographic data between the groups. For the wean group, the seizure relapse rate was $31.25 \%(15 / 48)$ and for the no wean group, the seizure relapse rate was $62.5 \%(15 / 24)$. The mean onset of wean of the whole cohort was 8.33 month after operation (SD 11.5 months) and the median onset of wean of the whole cohort was 5 months after operation. The timing of medication wean after surgery did not affect the seizure relapse. The duration of epilepsy was significantly longer in the no wean group than the wean group. Even though the overall class-I outcome rate for the whole cohort was $66.67 \%$, only four patients (5.55\% of 72 patients) were seizure-free without medication.

Conclusions: The decision to wean off seizure medications after epilepsy surgery should be considered on a case-by-case basis. When to wean the medication may not be as important as who should be weaned.

\subsection{1 \\ DIAGNOSIS AND TREATMENT OF EPILEPSY ARISING FROM CEREBELLAR LESIONS}

J. Wheless, Amy McGregor and R. Boop (University of Tennessee, Memphis, TN)

Rationale: To describe the diagnosis and treatment of patients who were found to have seizures arising from the cerebellum.

Methods: The records of two patients with cerebellar lesions who underwent continuous video-EEG monitoring with depth electrodes in place were reviewed.

Results: The first patient was an 8-year-old male who began having seizures at age 3 years. He was found to have a lesion in the right cerebellar peduncle, which was biopsied and found to be gliosis. However, he continued to have seizures despite multiple medications. During his Phase I evaluation in the epilepsy monitoring unit (EMU), he had seizures with secondary generalization that had maximum involvement of the right body with motor activity followed by right leg weakness. SPECT showed decreased metabolism in the area of the abnormality. It was thought that he would benefit from a Phase II evaluation with intracranial electrodes in the lesion and repeat biopsy at that time. Video-EEG monitoring was performed using scalp electrodes and depth electrodes within the lesion. His secondarily generalized tonic-clonic seizures began from a right cerebellar depth electrode. Biopsy from the time of placement of the depth electrodes confirmed that the lesion was gliosis. Medication controlled his seizures, so his parents opted not to pursue surgery.

The second patient was a 3-year-old male who underwent partial resection of a juvenile pilocytic astrocytoma at an outside hospital. At the time of presentation, he was having as many as 25 myoclonictonic seizures per hour, which did not respond to treatment. He underwent a Phase I evaluation in the EMU, and it was felt that his seizures were likely of cerebellar origin. He had generalized EEG changes at the time of his seizures and was noted to have an apparent deep inspiration in a myoclonic-tonic manner. He was readmitted for Phase II epilepsy surgery evaluation, including placement of 2 cerebellar depth electrodes. He then underwent continuous videoEEG monitoring. Myoclonic-tonic seizures were again recorded. The onset was first seen in one of the depth electrodes in the cerebellum and then changes were seen on the scalp recording. He returned to the operating for resection of the lesion. Electrocorticography performed in the cerebellar lesion cavity following resection showed the absence of epileptiform discharges. Intraoperative MRI showed a gross total resection of the complete lesion. Pathology findings were consistent with pilocytic astrocytoma. Medication changes were made postoperatively. Currently, he is seizure-free on one medication. Conclusions: In patients with cerebellar structural lesions and seizures, one should consider a causative relationship. Surface EEG may not be helpful. However, continuous video-EEG monitoring using simultaneous scalp recording and intracranial monitoring with depth electrodes within a cerebellar lesion can determine whether seizures arise from the lesion. If seizures are intractable, resection of epileptogenic cerebellar lesions should be considered, and complete resection should be pursued whenever possible. 
2.252

INCIDENCE OF DISABLING HEADACHE POST HEMISPHERECTOMY IN CHILDREN WITH RASMUSSEN SYNDROME

Siobhan J. Hannan, P. Prabhaker, W. Harkness, J. H. Cross and S. Varadkar (Great Ormond Street Hospital for Children, London, United Kingdom)

Rationale: To examine the incidence, characteristics and morbidity of headache in children who have undergone hemispherectomy at a tertiary centre for Rasmussen Syndrome.

Methods: Data were collected retrospectively and prospectively on 20 children (10 female), age 4-16 years (mean 10 years) with drug resistant epilepsy who underwent hemispherectomy for Rasmussen Syndrome, 1994-2011. At follow up parents were asked to complete the Pediatric Migraine Disability Assessment Score questionnaire (PEDI MIDAS) which measures headache disability (score of 0-10 none or mild disability; 11-30 mild; 31-50 moderate and above 51 severe disability). We also recorded incidence, frequency, characteristics, treatment and family history of headache.

Results: At surgery all children had drug resistant epilepsy, mild to moderate hemi-paresis and cognitive decline. None reported troublesome headache prior to surgery, 1 reported a family history of headache. Mean duration of follow up was 7.4 years (range 0.5-17.0). Following surgery, almost all were seizure free (Engel grade I (17/20); II (2/20) and III (1/20). Nearly half (9/20) reported episodes of severe disabling headache (PEDI MIDAS score $>51$ ), commencing at 1-24 (median 10) months post hemispherectomy, impacting on ability to function, with days off school and missed social activities. All children with headache met the International Headache Society Classification for migraine (with or without aura) with bilateral or unilateral (fronto or temporal) headache and associated symptoms of nausea, vomiting and/or sound and light sensitivity: 1/9 developed severe headache related to hydrocephalus requiring VP shunt; 2/9 were investigated with ICP monitoring but pressures found to be normal. No clear correlation between AED wean and emergence of headache was determined: 4/9 with headache were taking AEDs compared to 3/11 without headache. Symptom management was disappointing. In all children over-the-counter non-steroidal antiinflammatory drugs were unsuccessful. Oral triptans for acute headache and pizotifen or propranolol prophylaxis showed some symptom improvement for $3 / 9$.

Conclusions: Seizure freedom is likely following hemispherectomy for Rasmussen Syndrome. However a significant number of children have chronic postoperative headache with significant impact on daily life. Incidence of headache in children post epilepsy surgery is not well documented. Headache semiology in this group was migrainous suggesting potential for better symptomatic management (both pharmacological and non-pharmacological). Though numbers are small, the incidence here is greater than expected in the normal population. Further investigation is required specifically whether chronic headache is related to the underlying disease process rather than as a result of surgery.

\subsection{3 \\ FOCAL CORTICAL RESECTION FOR ELECTRICAL STATUS EPILEPTICUS: A CASE SERIES}

Amy Martyanov, E. Wirrell and K. Nickels (Child and Adolescent Neurology, Mayo Clinic, Rochester, MN)

Rationale: Report on outcomes of three patients who underwent focal cortical resection for Electrical Status Epilepticus in Sleep (ESES).

Methods: Through a comprehensive search of the Medical Index and EEG reports, all patients with ESES seen at the Mayo Clinic from 2007-2009 were identified. Their charts were reviewed to identify patients who underwent focal cortical resection and the outcome thereof.
Results: Three patients were identified. Patient \#1 had a history of perinatal intraventricular hemorrhage, shunted hydrocephalus, right hemiparesis and global delay. She developed focal and secondarily generalized epilepsy at age 2 years. Overnight EEG revealed ESES with multifocal discharges maximal over the parieto-occipital regions. Regression occurred at age 5 years and she was diagnosed with Continuous Spike Wave in Sleep Syndrome (CSWS). She did not respond to numerous medication trials and deep brain stimulation. She also had seizures recorded from the left frontotemporal region. She underwent left temporal lobectomy at age 7 . She continues to take levetiracetam and diazepam with remission of seizures, but she is still in ESES.

Patient \#2 had a history of perinatal infarct, hydrocephalus, VP shunt, right mesial temporal sclerosis, and global developmental delay. $\mathrm{He}$ developed focal and secondarily generalized epilepsy at age 2 years arising from the right mesial temporal lobe. Developmental regression occurred at age 10 years. Overnight EEG revealed ESES with generalized and focal right frontal discharges and he was diagnosed with CSWS. He did not respond to diazepam. He underwent right temporal lobectomy at age 11 with remission of ESES. He is on lamotrigine with rare breakthrough seizures. Patient \#3 had no prior epilepsy risk factors. She developed focal epilepsy including focal status epilepticus at age 3 years arising from the right temporal region. Developmental regression occurred at age 4 years. Overnight EEG revealed ESES with generalized discharges maximal over the right temporal region and she was diagnosed with Landau Kleffner Syndrome (LKS). She did not respond to multiple medical therapies. SISCOM and intracranial monitoring revealed right temporal ictal focus. She underwent right parietal resection at age 6. She is currently in remission of ESES and seizures on levetiracetam.

Conclusions: CSWS and LKS are rare but treatable seizure syndromes of childhood characterized by developmental regression and electrical status epilepticus in slow wave sleep (ESES). At present, the pathophysiology of ESES is unknown. However, in some cases, ESES may be caused by a focal cortical lesion. Focal cortical resection should be considered in CSWS or LKS patients with focal seizures of known or unknown etiology who have not responded to medications.

\subsection{4 \\ PARENTAL SATISFACTION AFTER TOTAL CORPUS CALLOSOTOMY IN PATIENTS WITH INFANTILE OR EARLY CHILDHOOD ONSET EPILEPSY}

Masaki Iwasaki $^{1}$, M. Uematsu ${ }^{2}$, T. Nakayama ${ }^{2}$, K. Haginoya ${ }^{2}$, S. Shin-ichiro ${ }^{1}$, K. Jin $^{3}$, N. Nakasato ${ }^{3}$ and T. Tominaga ${ }^{1}{ }^{1}$ Neurosurgery, Tohoku University Graduate School of Medicine, Sendai, Japan; ${ }^{2}$ Pediatrics, Tohoku University Graduate School of Medicine, Sendai, Japan and ${ }^{3}$ Epileptology, Tohoku University Graduate School of Medicine, Sendai, Japan)

Rationale: Corpus callosotomy is usually intended to alleviate epileptic seizures. The chance of seizure freedom after corpus callosotomy is generally $10 \%$ or less, so that the efficacy of surgery has been assessed with several different measures. However, it is difficult to assess the benefit of surgery, because of low chance of post-operative seizure freedom, the presence of severe developmental delay, and seizure related risk of injury as a goal of surgery. Here, we investigated parental satisfaction after corpus callosotomy.

Methods: Questionnaires were sent anonymously to parents of 16 patients with infantile or early childhood onset epilepsy. All patients underwent total corpus callosotomy and had minimum 6 months follow-up. The questionnaire included items about relative changes in seizure frequency and parental satisfaction.

Results: Questionnaires were returned from 15 patients. Parental satisfaction was categorized as "quite satisfied" in 5, "satisfied" in 4, "satisfied little" in 2, "neither satisfied or unsatisfied" in 3 and "unsatisfied little" in 1 patient. Freedom from any seizures and from 
drop attacks was reported from 4 and 6 patients, respectively, all associated with satisfactory results. Four-tier grading system (1. Complete seizure freedom including drop attacks, 2. Seizure reduction and free from drop attacks, 3. Only seizure reduction, 4. No worthwhile reduction) was highly correlated with parental satisfaction (Spearman's rank-order correlation coefficient, rho $=$ 0.92 ).

Conclusions: Parental satisfaction is achieved after corpus callosotomy in more than half of patients. Complete seizure freedom and/or free from drop attacks are associated with greater parental satisfaction. A new measure incorporating those factors was proposed for assessing post-operative outcome after corpus callosotomy.

\subsection{5}

FUNCTIONAL HEMISPHERECTOMY FOR INTRACTABLE EPILEPSY IN CHILDREN

Rita M. Yu ${ }^{1,4}$, H. Kwon ${ }^{1,4}$, J. Kang ${ }^{1,4}$, Y. Lee ${ }^{2,4}$, H. Kang ${ }^{1,4}$, J. Lee ${ }^{1,4}$, D. Kim ${ }^{3,4}$ and H. Kim ${ }^{1,4}$ ( ${ }^{1}$ Department of Pediatric Neurology, Severance Children's Hospital, Seoul, Republic of Korea; ${ }^{2}$ Department of Pediatrics, Gangnam Severance Hospital, Seoul, Republic of Korea; ${ }^{3}$ Department of Pediatric Neurosurgery, Severance Hospital, Seoul, Republic of Korea and ${ }^{4}$ Yonsei Uiversity, College of Medicine, Seoul, Republic of Korea)

Rationale: To analyze the outcomes of functional hemispherectomies(FH) performed at Severance Children's Hospital in Seoul, Korea, from October 2003 to December 2011, among 468 epilepsy surgeries carried out in 388 patients

Methods: A total of 52 patients have underwent functional hemispherectomies during the specified period and retrospective study of their medical charts was done. Two of the patients died due to surgical complications, one had expired within the initial three months after surgery, and one was lost to immediate follow up and a total of 48 cases were reviewed according to individual pre-operative conditions such as etiology of epilepsy, initial epilepsy classification, lead time to surgery, and post-operative outcomes were also reviewed to investigate improvement in seizure frequency, post-operative Engel and ILAE class, changes in antiepileptic medications, and more. To supplement the follow up information on the patients' latest status, additional questionnaire was asked to the patients' families by phone, which focused on changes in cognition, motor function, and quality of life for the patients.

Results: The most common etiology of the patients was malformation of cortical development $(23,47.9 \%)$, with destructive encephalopathy(20,41.7\%), Sturge-Weber syndrome(5, 10.4\%) next in order, with one case each for Rasmussen encephalitis, and Tuberous Sclerosis complex. For 40 out of the total 48 patients(83.3\%), functional hemispherectomy was performed as final option as they have experienced seizure relapse after other epilepsy surgeries. Complications such hydrocephalus or hemorrhage have occurred 17 patients $(35.4 \%$ ). As only $22.9 \%$ (11 patients) were followed up to five years after $\mathrm{FH}$, we have reviewed the percentage for each Engel and ILAE class among the total of patients followed up at each designated period, which were 3 months, and each year up to the 5 th year, and have found that more than $50 \%$ of those followed up at each period were able to maintain Engel class 1A status. 34 out of 48 patients $(70.8 \%)$ were able to reduce their initial antiepileptic medications and $15(31.3 \%)$ actually have completely discontinued the medications altogether. $60.4 \%$ of the patients have replied to our questionnaire, which reported much improvement in $37.9 \%$ to $72.4 \%$ of the patients according to different area of cognition, such as control of hyperactivity to concentration. While $65.5 \%$ of the patients and families have answered to have motor deficit after the procedure, 93.1\% have expressed overall satisfaction in improved seizure control and better quality of life.

Conclusions: This study shows high seizure free rate and excellent cognitive outcome despite of inevitable motor deficit in functional hemispheretomy patients with less complications. Also for those epilepsy surgery patients who have relapsed afterwards, this procedure can be very viable option.

\subsection{6 \\ ON THE RELATIONSHIP BETWEEN CORPUS CALLOSOTOMY AND VNS IN MEDICALLY RESISTANT GENERALIZED EPILEPSY IN CHILDREN \\ Jeffrey P. Blount ${ }^{1,2}$, P. Kankirawatana ${ }^{1,3}$, H. Kim ${ }^{1,3}$, M. Goya ${ }^{1,2}$ and C. J. Rozzelle ${ }^{1,2}{ }^{1}$ Children's Hospital of Alabama, Birmingham, AL; ${ }^{2}$ Surgery-Neurosurgery, UAB, Birmingham, AL and ${ }^{3}$ Pediatrics- Neurology, UAB, Birmingham, AL)}

Rationale: Generalized medically resistant epilepsy remains a major burden in pediatric epilepsy. Surgical options are limited to palliative interventions of VNS (vagus nerve stimulator) implantation and corpus callosotomy(CC). Candidacy criteria for each intervention have never been critically and systematically evaluated and there exists a wide range of utilization of each procedure between experienced pediatric surgical centers. We review here our experience in a cohort of surgically treated patients with generalized epilepsy to discern relative contributions, risks and outcomes from each procedure.

Methods: Pediatric patients undergoing surgical intervention (either VNS or CC) for generalized epilepsy were identified from our Pediatric Epilepsy Center database and retrospectively reviewed. All patients had at least 2 years of follow up. Data related to pre and post operative seizure type, pattern, frequency, burden of falls and injuries, severity of associated encephalopathy were summarized and tabulated. Uni-variate and multi-variate analysis was performed. Results: Between 2004 and 201023 patients underwent CC and 67 patients underwent VNS implantation.. For CC there were 17 complete callosotomies and 6 anterior 2/3rds callosotomies that resulted in seizure freedom in 4 patients $(17 \%)$, elimination/marked reduction of drop events in $19(83 \%)$ and $4(12 \%)$ patients respectively and 2 transient neurologic deficits $(8 \%)$. VNS resulted in a $76 \%$ reduction in drop events in 14 patients who had pre-operative drops, a $14 \%$ rate of marked reduction/elimination of seizures and a $65 \%$ rate of overall improvement in seizure control.

Conclusions: Callosotomy and VNS implantation are both safe, effective interventions that may contribute toward improving outcomes in pediatric generalized epilepsy

\subsection{7 \\ MR GUIDED STEREOTACTIC LASER ABLATION OF HYPOTHALAMIC HAMARTOMA (HH)} Angus A. Wilfong ${ }^{1}$, M. M. Quach ${ }^{1}$, A. Shetty ${ }^{2}$ and D. J. Curry ${ }^{1}$ ( ${ }^{1}$ Baylor College of Medicine, Houston, TX and ${ }^{2}$ Visualase, Inc., Houston, TX)

Rationale: Surgical intervention for $\mathrm{HH}$ has been limited due to modest outcomes (37- 50\% seizure freedom), difficult location, and associated surgical morbidity (7-10\% permanent). Seizures are primarily gelastic and may occur every few minutes, often progressing to intellectual deterioration, and behavioral impairment. The seizures are often not recognized early as they typically consist of mirthless laughter beginning early in infancy. Most patients fail appropriate trials with antiepileptic medications. We report seizure outcomes after completion of minimally invasive stereotactic laser ablation (SLA) procedure in five patients.

Methods: After diagnosis, the parents were offered epilepsy surgery with a transcallosal craniotomy, a subfrontal endoscopic resection, stereotactic radiotherapy, or laser ablation. In all cases, they elected laser ablation and informed consent was obtained. An MRcompatible laser catheter $(1.6 \mathrm{~mm}$ dia) was navigated to the deep seated midline target with frame-based stereotaxy and a $3.2 \mathrm{~mm}$ twist drill hole. An FDA-cleared laser surgery system (Visualase; Visualase, Inc., Houston, TX) was utilized to monitor the ablation 
process with real-time MRI thermometry. After confirmation test at $3 \mathrm{~W}$, higher doses of $6-10 \mathrm{~W}$ for $50-120$ seconds were used to ablate the stalk and body. Temperature limits were set to protect the hypothalamus (above) and basilar artery and optic tract (below). Results: At last follow-up, continued seizure freedom was observed in all 5 patients. The average OR time was $4.1 \mathrm{hrs}$ and average MR time was 1.4 hrs. No surgical complications, neurological or vision deficits, or endocrine disturbances including diabetes insipidus occurred.

Conclusions: SLA was demonstrated to be a safe and effective minimally invasive tool to destroy epileptogenic HH. Seizure freedom was achieved without surgical morbidity. Short ablation times (60-120 seconds) and real-time MRI thermometry enabled protection of adjacent critical structures. Early intervention with SLA provides a precise minimally invasive tool to achieve seizure freedom in $\mathrm{HH}$. Stereotactic laser procedures for minimally invasive treatment of epilepsy enables destruction of deep seated seizure foci like $\mathrm{HH}$ with the potential for higher rates of seizure freedom, in comparison to traditional surgeries.

Patient characteristics and outcomes after 3-15 months of follow-up

\begin{tabular}{|c|c|c|c|c|c|}
\hline Palient & Age & Sex & Type & Initial Seizure Frequency & Engel Class after SLA \\
\hline A & 10 & M & II & 30 per day & $1 \mathrm{~A}$ \\
B & 15 & M & III & 10 per day & IA \\
C & 6 & M & III & 20 per day & 1 A \\
D & 8 & M & II & 5 per day & Scizurc-frec $<6$ months FC \\
\hline E & 8 & M & II & 5 per day & Scizure-frec $<6$ months FL \\
\hline
\end{tabular}

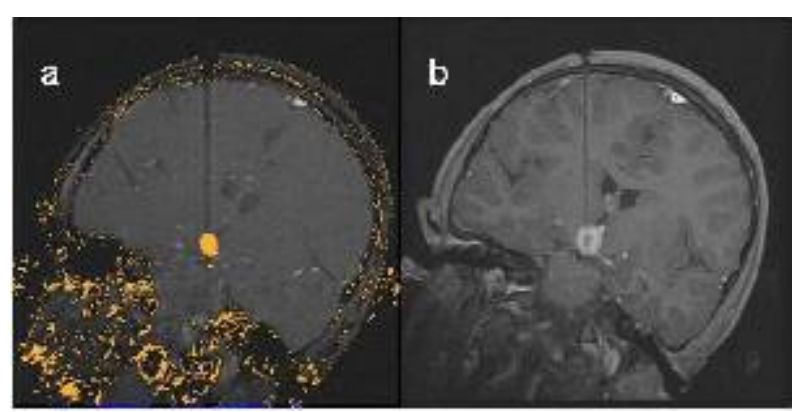

a) The heat induced damage predicted (orange color on right image) based on the temperature and time history of the ablated region. b) The post-contrast imaging done after the laser ablation confirms the targeted heating.

\subsection{8}

\section{WTTHDRAWN}

\subsection{9 \\ VENTRICULO-PERITONEAL SHUNTING AFTER FUNCTIONAL HEMISPHERECTOMY}

Arthur Cukiert, J. Burattini and C. Cukiert (Neurolopgy and Neurosurgery, Clinica de Epilepsia de Sao Paulo, Sao Paulo, Brazil)

Rationale: Hemispherectomy is an extremely useful technique for treatment of refractory epilepsy associated with hemiplegic syndromes, Rasmussen syndrome, Sturge-Weber and hemispheric cortical dysplasia, among other etiologies. Different techniques (anatomical, functional, hemispherotomy) have been employed. Anatomical resections were shown to carry higher early and late complication rates; functional hemispherectomy and hemispherotomy have roughly the same complication rate. Intracranial hypertension due to hydrocephalus is one of the possible complications after hemispherectomy. We describe the frequency, type and treatment of post-functional hemispherectomy hydrocephalus in our series.
Methods: Seventy-three patients were studied. All patients were submitted to functional hemispherectomy. Thirty-six had hemiplegic syndrome, 24 had Rasmussen encephalitis, 4 had hemimegaloencephaly, 4 cortical dysplasia, 1 had post-encephalitic syndrome and 4 had Sturge -Weber. All patients were followed-up for at least one year.

Results: Three patients developed post-hemispherectomy hydrocephalus. One patient had a hemiplegic syndrome, one had Rasmussen syndrome and one post-encephalitic syndrome. The patient with Rasmussen syndrome received an ipsilateral VP shunt 1 month after hemispherectomy; the patient with hemiplegic syndrome received an ipsilateral VP shunt 4 months after hemispherectomy and the patient with post-encephalitic syndrome (who was previously shunt-dependent), received a contralateral VP shunt 2 days after hemispherectomy. All patients fully recovered after shunting. Conclusions: Hypertensive hydrocephalus might occur in around 4\% of the patients submitted to functional hemispherectomy, and its ocurrance is apparently not related to a specific etiology. Patients who would develop hypertensive hydrocephalus did so within the first months post-operatively, or very early. Although lifethreatening, adequate treatment led to full resolution in all patients.

\subsection{0 \\ PREDICTORS OF POSTSURGICAL SEIZURE OUTCOME IN PEDIATRIC PATIENTS WITH TUBEROUS SCLEROSIS Pavel Krsek ${ }^{1}$, A. Jahodova ${ }^{1}$, M. Kyncl ${ }^{2}$, M. Kudr ${ }^{1}$, V. Komarek ${ }^{1}$, P. Jayakar $^{3}$, I. Miller ${ }^{3}$, B. Korman ${ }^{4}$, G. Rey ${ }^{4}$, T. Resnick ${ }^{3,5}$ and M. S. Duchowny ${ }^{3,5}$ ( ${ }^{1}$ Department of Pediatric Neurology, FN Motol University Hospital, Prague 5, Czech Republic; ${ }^{2}$ Department of Radiology, FN Motol University Hospital, Prague 5, Czech Republic; ${ }^{3}$ Department of Neurology and Comprehensive Epilepsy Program, Brain Institute, Miami Children's Hospital, Miami, FL; \\ ${ }^{4}$ Neuropsychology Section, Brain Institute and Behavioral Medicine, Miami Children's Hospital, Miami, FL and ${ }^{5}$ Department of Neurology, University of Miami Miller School of Medicine, Miami, $\mathrm{FL})$}

Rationale: Epilepsy is a leading cause of morbidity in children with Tuberous Sclerosis Complex (TSC). Despite several specific challenges to epilepsy surgery planning in TSC population, a significant proportion of patients $(>60 \%)$ could become seizure-free with proper surgical candidate selection. Variable predictors of favorable/unfavorable postsurgical outcome have been reported. We analyzed a large surgical series of pediatric TSC patients in order to identify prognostic factors crucial for selection of these subjects for epilepsy surgery.

Methods: 33 children with TSC who underwent resective epilepsy surgery at Miami Children's Hospital between 1994 and 2010 were retrospectively reviewed. Only patients who had a definite diagnosis of TSC and known seizure outcome at two years after surgery were selected. A total of 29 clinical, neuropsychological, EEG, MRI and surgical variables were analyzed and related to surgical outcomes. Fisher's exact test was used to examine the significance of associations between the variables and seizure outcomes. The association strength was expressed by Quetet-Yule coefficients. Results: 18 patients (55\%) have been seizure-free two years after the (final) surgery. Seizure outcome in remaining subjects was classified as follows: Engel II in five patients (15\%), Engel III in four patients $(12 \%)$ and Engel IV in six patients $(18 \%)$. For the purpose of the study, these 15 subjects were pooled into "non-seizure-free" group. The following predictors of seizure-free outcome were demonstrated: (1) complete removal of the epileptogenic tissue detected by both MRI (i.e., one large tuber or a region with several tubers supposed to represent the epileptogenic zone) and intracranial EEG (i.e., brain tissue exhibiting significant intracranial EEG abnormalities as defined previously); (2) occurrence of regional scalp interictal and ictal EEG patterns; (3) agreement of interictal and ictal EEG localizations; (4) incidence of simple partial seizures; (5) presence of 
preoperative hemiparesis; and (6) one-stage surgery (without previous long-term invasive EEG). Other factors such as age at seizure onset, incidence of infantile spasms, duration of epilepsy, seizure frequency, mental retardation, number of brain regions affected by tubers as well as extent of resections did not influence outcome.

Conclusions: The size of the data set, all collected at one institution, enabled to distinguish clinically important variables influencing surgical outcome in children with intractable epilepsy due to TSC. Our findings may assist in the surgical management of these patients.

\subsection{1}

\section{EPILEPSY SURGERY FOR PATIENTS WITH AUTISM}

Saadi Ghatan $^{1,2}$, P. E. McGoldrick ${ }^{2}$, C. A. Palmese ${ }^{2}$, M. LaVega ${ }^{2}$, H. Raynes $^{2}$, R. Goodman ${ }^{2}$ and S. Wolf ${ }^{2}$ ( ${ }^{1}$ Neurological Surgery, Columbia University Neurological Institute, New York, NY and

${ }^{2}$ Neurology, Beth Israel Medical Center, New York, NY)

Rationale: Autism is frequently associated with epilepsy, and seizures are medically refractory in approximately $1 / 3$ of cases. Limited data and experience have been reported to support surgical intervention in patients with autism and treatment resistant epilepsy (TRE), despite evidence that epilepsy surgery is safe, effective, and improves quality of life in children and adults with both normal and impaired development. We report a series of children with autism and TRE who underwent surgery.

Methods: Between 2004 and 2012, among a group of approximately 4400 patients evaluated for epilepsy, 1297 presented with the comorbidity of autism. Amongst these patients, 250 were found to have TRE and 33 underwent evaluation and surgery for epilepsy.

Results: 47 surgeries were performed, including 13 staged resections with invasive monitoring (grid, strip, +/- depth electrodes), 2 singlestage lesionectomies, 2 hemispherotomies, 14 corpus callosotomies, and 3 vagus nerve stimulator placements. Two patients underwent invasive monitoring without a secondary resection. With a mean follow-up of 34 months, Engel outcomes were as follows: Engel I, 16 patients; Engel II, 7 patients, Engel III, 7 patients, Engel IV, 3 patients. Disruptive behavior decreased in 29 children and there were improvements in social interactive abilities and psychosocial functioning in 22. Invasive monitoring was well tolerated in all patients who underwent staged resections in the EMU/ICU. Conclusions: Epilepsy surgery is feasible, safe, and effective in carefully selected children with autism and TRE. Marked improvements in behavior and quality of life accompanied a cessation or significant reduction in seizures.

\subsection{2}

\section{WTTHDRAWN}

\subsection{3 \\ OUTCOME AFTER CALLOSOTOMY OR VAGUS NERVE STIMULATION IN CONSECUTIVE PROSPECTIVE COHORTS OF PATIENTS WITH SECONDARY GENERALIZED EPILEPSY AND NON-SPECIFIC MRI FINDINGS}

J. Burattini ${ }^{2,1}$, Arthur Cukiert ${ }^{1}$, A. Lima ${ }^{2}$, C. Cukiert ${ }^{1}$, M. ArgentoniBaldochi $^{2}$, C. Baise ${ }^{2}$ and C. Forster ${ }^{2}\left({ }^{1}\right.$ Neurology and Neurosurgery, Clinica de Epilepsia de Sao Paulo, Sao Paulo, Brazil and ${ }^{2}$ Neurology and Neurosurgery, Hospital Brigadeiro, Sao Paulo, Brazil)

Rationale: There is currently no resective (potentially curative) surgical option useful in this patient population. Palliative procedures such as callosotomy $(\mathrm{Cx})$, vagus nerve stimulation (VNS) or deep brain stimulation have been offered. We compared the outcome after $\mathrm{Cx}$ or VNS in two consecutive prospective cohorts of patients with secondary generalized epilepsy.
Methods: Twenty patients were submitted to callosotomy from 2006 to 2007 (Group 1); 20 additional patients were submitted to VNS from 2008 to 2009 (Group 2). They had secondary generalized epilepsy of the Lennox-Gastaut or Lennox-like type. They were submitted to a neurological interview and examination, interictal and ictal video-EEG, high resolution 1.5T MRI, and cognitive and quality of life evaluations. Two-year post-operative follow-up results were considered for each patient.

Results: Final mean stimuli intensity was $3.0 \mathrm{~mA}$ in Group 2 patients. There were $10 \%$ of seizure-free patients in Group 1, and none in Group 2. Ten percent of Group 1 patients were non-responders, while $16 \%$ of Group 2 did so. Improvement in attention and quality of life was noted in $85 \%$ of both Group 1 and 2 patients. Rupture of secondary bilateral synchrony was noted in $85 \%$ of Group 1 patients; there was no EEG modification after VNS in group 2. Both procedures were effective regarding the control of atypical absences and generalized tonic-clonic seizures. Both procedures were not effective in controlling tonic seizures. Callosotomy was very effective in reducing atonic seizure's frequency, while VNS was ineffective. On the other hand, callosotomy was not effective in reducing myoclonic seizures, while VNS did so.

Conclusions: Callosotomy might be preferred as primary treatment in kids with Lennox-Gastaut syndrome and no specific findings on MRI if atonic seizures prevail in the patient's clinical picture; when myoclonic seizures prevail this might hold true in favor of VNS. When atypical absence or generalized tonic-clonic seizures are the main concern, although both procedures carry similar effectiveness, VNS should be considered as an initial approach taking into account the adverse event profile. Patients should be advised that both procedures are not very effective in the treatment of tonic seizures.

\section{Surgery: All Ages}

\subsection{4 \\ BONE FLAP EXPLANTATION, STEROID USE AND RATES OF INFECTION DURING CRANIOTOMY FOR IMPLANTATION OF SUBDURAL ELECTRODES FOR EPILEPSY}

E. H. Hersh, M. S. Virk, H. Shao, A. J. Tsiouris and Theodore H. Schwartz (New York Presbyterian Hospital, New York, NY)

Rationale: Subdural implantation of electrodes is commonly performed to localize an epileptic focus in preparation for resective surgery. Although generally safe, complications are not uncommon and techniques vary between centers. Whether to temporarily explant or leave implanted the bone plate and whether to employ perioperative steroids is unclear.

Methods: We retrospectively reviewed records on all unilateral intracranial implants performed between November 2001 and June 2011. Patients were divided into three groups: Group 1: $(n=24)$ Bone explanted, no peri-operative steroids; Group 2: $(n=42)$ Bone implanted, no peri-operative steroids; Group 3: $(n=25)$ Bone implanted, given peri-operative steroids. Rates of infection, time to first seizure, number of seizures in first three days, severity of headache in first three days, midline shift and size of subdural hematoma on post-operative CT scan, and rate of emergency reoperation were correlated with each group using ANOVA, Fisher's exact and Generalized Estimating Equation (GEE) statistics.

Results: Out of a series of 324 cranial epilepsy surgeries, there were 91 unilateral implants, of which 86 met inclusion criteria. Average (SD) number of seizures in the entire monitoring sessions was 4.7 (8.3), and $66.3 \%$ of patients had a seizure within the first three days. Headaches were severe in $33.7 \%$, and $18.7 \%$ had $>4 \mathrm{~mm}$ midline shift. $41.8 \%$ of patients had a subdural hematoma $>10 \mathrm{~mm}$ in thickness, the rate of early re-operation was $7.7 \%$, and the rate of infection was $12.1 \%$. There was a statistically higher rate of infection when the bone was explanted (33.3\%) compared with when the bone was left in place $(4.5 \% ; \mathrm{p}<0.01)$. Leaving the bone in place also 
increased the number of seizures and decreased the time to first seizure but also increased the size of the subdural hematoma, although there was no increase in midline shift, severity of headache or emergency re-operation. The use of steroids did not appear to have any effect on any of our outcome measures.

Conclusions: Temporary bone flap explantation during craniotomy for implantation of subdural electrodes can result in high rates of infection, possibly due to the frequent change of hands in transferring the bone to the bone bank. Leaving the bone in place may increase the size of subdural hematoma and frequency of seizures but does not increase the rate of complications. These results may be institutiondependent.

\subsection{5}

RELATIONSHIP BETWEEN HOSPITAL SURGICAL VOLUME, LOBECTOMY RATES, AND ADVERSE PERIOPERATIVE EVENTS AT U.S. EPILEPSY CENTERS Dario J. Englot ${ }^{1}$, D. Ouyang ${ }^{1}$, J. D. Rolston ${ }^{1}$, D. D. Wang ${ }^{1}$, P. A. Garcia $^{2}$ and E. F. Chang ${ }^{1}$ ( ${ }^{1}$ Neurosurgery, University of California, San Francisco, San Francisco, CA and ${ }^{2}$ Neurology, University of California, San Francisco, San Francisco, CA)

Rationale: Epilepsy surgery remains significantly underutilized. We recently reported that the number of lobectomies for localized intractable epilepsy in the U.S. has not changed despite the implementation of clear evidence-based guidelines ten years ago supporting early referral for surgery. To better understand why epilepsy surgery continues to be under-utilized, our objective was to carefully examine hospital-related factors related to: 1) where patients are being admitted for the evaluation of epilepsy, 2) rates of utilization for surgery across hospitals, and 3) peri-operative morbidity between hospitals with low vs. high volume of epilepsy surgery.

Methods: We performed a population-based cohort study of U.S. hospitals from 1990 to 2008 using the Nationwide Inpatient Sample (NIS), stratifying epilepsy surgery rates and trends as well as perioperative morbidity rates by hospital surgical volume.

Results: The number of lobectomies for epilepsy performed at high volume centers ( $>15$ lobectomies/year) significantly decreased from 1990 to $2008(\mathrm{~F}=20.4, \mathrm{p}<0.001)$, while significantly more procedures were performed at middle volume hospitals (5-15 lobectomies/year) over time $(\mathrm{F}=16.1, \mathrm{p}<0.001)$ (Fig 1A). No time trend was observed for hospitals performing $<5$ procedures per year (Fig 1A). However, patients admitted to high volume centers were significantly more likely to receive lobectomy than those at low volume hospitals (RR 1.05, 95\% CI 1.03-1.08, p < 0.001) (Fig 1B). Also, the incidence of perioperative adverse events was significantly higher at low volume hospitals $(12.9 \%)$ than at high volume centers (6.1\%) (RR 1.08, 95\% CI 1.03-1.07, p < 0.001).

Conclusions: Hospital volume is an important predictor of epilepsy surgery utilization and peri-operative morbidity. Patients with refractory epilepsy should be referred to a comprehensive epilepsy treatment center for surgical evaluation by an experienced clinical team.
A

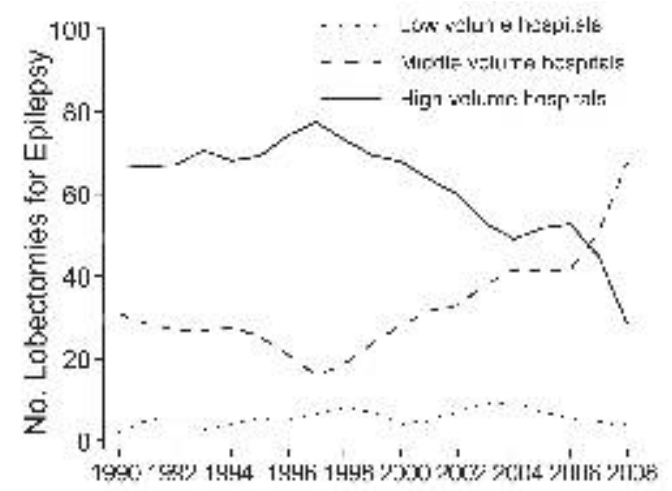

Year

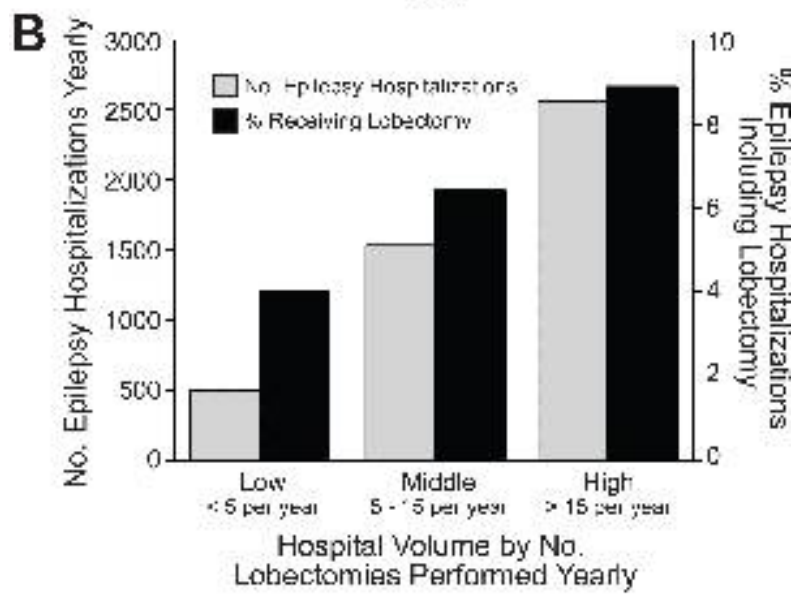

2.266

DIAGNOSTIC OUTCOME OF SURGICAL REVISION OF INTRACRANIAL ELECTRODE PLACEMENTS FOR SEIZURE LOCALIZATION

Ricky Lee ${ }^{1}$, G. A. Worrell ${ }^{1}$, G. D. Cascino ${ }^{1}$, W. R. Marsh ${ }^{2}$, N. M. Wetjen $^{2}$, E. C. Wirrell ${ }^{3}$ and E. L. So ${ }^{1}{ }^{1}$ Neurology, Division of Epilepsy, Mayo Clinic, Rochester, MN; ${ }^{2}$ Neurologic Surgery, Mayo Clinic, Rochester, $\mathrm{MN}$ and ${ }^{3}$ Neurology, Divisions of Child and Adolescent Neurology and Epilepsy, Mayo Clinic, Rochester, MN)

Rationale: Intracranial EEG (iEEG) recording is considered to be the gold standard for seizure localization in many patients with epilepsies that are intractable and complex. Due to inherent morbidities associated with invasive neurosurgery and intracranially indwelling foreign substance, the extent of iEEG has to be judiciously determined and limited to reduce the risk of complications. Even though iEEG implantation is guided by thorough presurgical evaluations, including scalp EEG and imaging studies, there are still situations in which iEEG is not able to localize the seizure-onset zone. Repeat surgery is sometimes required to revise the placement of the intracranial electrodes. Given the increased risk of repeat brain surgery, it is important to evaluate the diagnostic yield of repeat surgeries for revising intracranially implanted electrodes.

Methods: We retrospectively reviewed our epilepsy surgery database between 1997 and 2010. We identified patients who had revision of intracranial electrode placements during one episode of inpatient evaluation, because the initial implantation did not adequately localize seizure onset. Data collected included seizure semiology, interictal and ictal scalp EEG discharges, MRI head, and initial iEEG findings. Fisher's exact test was used to evaluate for statistical significance of each factor.

Results: Twenty patients consecutively fit our inclusion criteria. Two patients did not have another seizure after the revision of the electrode placements. Revised implantation and recording localized seizure onset in 10 of the remaining 18 patients $(55.6 \%)$. Factors that were statistically significant in localizing a seizure focus after 
implantation revision are: 1) lateralizing seizure semiology $(71.4 \%$ in lateralizing vs. $0 \%$ in non-lateralizing; $\mathrm{p}=0.023) ; 2$ ) focal scalp interictal discharges (100\% in focal vs. $38.5 \%$ in non-focal; $p=$ $0.036)$; and 3) initial iEEG showing ictal onset at the edge of the electrode grid $(100 \%$ in ictal onset at the edge of the electrode grid vs. $20 \%$ in indeterminate ictal onset; $p=0.001)$. Focal scalp ictal EEG onset could be a prognostic factor, because it nearly reached statistical significance ( $80 \%$ in focal vs. $25 \%$ in non-focal; $p=$ 0.054 ). MRI head findings (normal vs. abnormal) did not show an association in this study. There was no permanent complication associated with revised implantation but one patient experienced transient right foot apraxia. 6/10 patients who underwent resective surgery after localizing revised implantation were seizure- free during a limited period of follow-up (1 month to 4 years).

Conclusions: Our study shows that the diagnostic yield of repeat surgeries for revising intracranially implanted electrodes is about $55 \%$. However, the yield is improved by a focal ictal onset at the edge of the original implanted grid, lateralizing seizure semiology, or focal scalp interictal discharges.

\subsection{7}

\section{PRELIMINARY EXPERIENCE WITH MAGNETIC RESONANCE TEMPERATURE IMAGING (MRTI) AND STEREOTACTIC LASER ABLATION (SLA) FOR HIPPOCAMPAL SCLEROSIS (HS)}

A. D. Sharan ${ }^{1}$, C. $\mathrm{Wu}^{1}$, A. Shetty ${ }^{2}$, C. T. Skidmore ${ }^{1}$, D. J. Curry ${ }^{4}$, A. A. Wilfong ${ }^{4}$, W. R. Marsh ${ }^{3}$, G. A. Worrell ${ }^{3}$, R. E. Watson ${ }^{3}$, J. J. VanGompel $^{3}$ and M. R. Sperling ${ }^{1}{ }^{1}$ Thomas Jefferson University Hospitals, Philadelphia, PA; ${ }^{2}$ Visualase, Inc., Houston, TX; ${ }^{3}$ Mayo Clinic, Rochester, $\mathrm{MN}$ and ${ }^{4}$ Baylor College of Medicine, Houston, TX)

Rationale: Patients with unilateral hippocampal sclerosis (HS) represent the most suitable candidates for epilepsy surgery; however, the risk of cognitive decline and the invasiveness of an open craniotomy is a limiting concern - particularly with HS of the dominant-hemisphere. Minimally invasive stereotactic laser ablation (SLA) using a 980nm diode laser may enable precise ablation of seizure foci with sparing of eloquent neocortical structures.

Methods: Patients were candidates for SLA if EEG revealed seizures originating in the temporal lobe while MRI showed concordant mesial temporal sclerosis. Preoperative evlauation also included neuropsychological evaluation. Frame-based navigation was used to introduce a $1.6 \mathrm{~mm}$ diameter MR-compatible laser applicator housing a $1 \mathrm{~cm}$ long diffusing tip optical fiber into the amygdalohippocampal complex (AHC) from a temporo-occipital trajectory. The nearinfrared laser ablation system (Visualase, Inc, Houston, TX) used consists of a 15W 980nm diode laser, a light-diffusing tip, and magnetic-resonance thermal imaging (MRTI) software. This laser produces a cylindrical to ellipsoid light distribution in the tissue along the axis of the diffusing element. AHC ablation was performed with 2 exposures of 10-12W for 90-130sec. MRTI was performed using proton resonance frequency (PRF) phase difference imaging techniques using a fast field echo (FFE) sequence. Temperature was monitored and safety limits $(>50 \mathrm{degC})$ were placed near critical structures such as the optic tract, cerebral arteries, and hypothalamus. Temperatures at these pre-designated locations were set to terminate laser delivery if heating was excessive. Post-ablation T1- weighted gadolinium series confirmed areas of thermal ablation.

Results: Five patients at 3 institutions ( 2 females and 3 males), ages 16-65, presented with 2-20 seizures/month. Preoperative MRI demonstrated HS alone in 4 patients, and HS in addition to a second lesion in 1 patient. Trajectories used were appropriate for controlled thermal ablation of desired structures and volumes as confirmed by MRI. Post-ablation T1-weighted gadolinium series demonstrated a mean ablation volume of $3.65+/-0.93 \mathrm{cc}(2.18-4.91 \mathrm{cc})$. Average length of stay was 1 day. Asymptomatic delayed hemorrhage within the ablation site was observed in 1 patient. There were no other surgical related complications. After follow-up of 3-13 months, all patients were improved with regard to seizure control with some experiencing seizure remission. Poorer outcomes were associated with smaller volumes of ablation, the presence of another radiographic lesion or seizure focus, or abrupt discontinuation of AEDs.

Conclusions: SLA has promise as a treatment for refractory epilepsy secondary to HS. Seizures appear to respond to this minimally invasive therapy; and the procedure is associated with much less discomfort and a shorter hospital stay than conventional resective surgery. A larger study appears warranted by these preliminary results.

\begin{tabular}{|c|c|c|c|c|c|c|}
\hline Patient & Age & Sex & $\begin{array}{l}\text { Side of } \\
\text { MTS }\end{array}$ & $\begin{array}{l}\text { Initial Seizure } \\
\text { Frequency } \\
\text { [per month] }\end{array}$ & $\begin{array}{l}\text { Volume of } \\
\text { Ablation } \\
\text { [cc] }\end{array}$ & Outcome after SLA \\
\hline$\Lambda$ & 56 & $\Gamma$ & Left & 20 & 4.91 & Fingel $\mathrm{IA}$ \\
\hline B & 49 & M & Leli & 3 & 2.18 & $\begin{array}{l}\text { Persistent seizures but reduction in } \\
\quad \text { frequency and severity }\end{array}$ \\
\hline C & 16 & $\mathrm{H}$ & Right & 4 & 3.97 & Engel $\amalg$ \\
\hline D & 17 & M & Right & 12 & 4.21 & $\begin{array}{l}\text { Seizure-free for } 3 \text { months followed } \\
\text { by recurrence of rare scizures }\end{array}$ \\
\hline E & 65 & M & Right & 2 & 3.94 & Engel IA \\
\hline
\end{tabular}

Patient characteristics and outcomes after 3-13 months of follow-up. Engel

Classification of Outcomes are provided only if at least 6 months of follow-up were available.

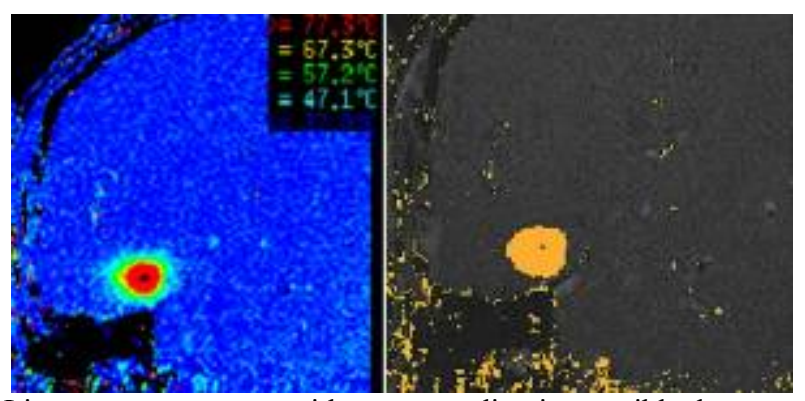

Live temperature map with corresponding irreversible damage estimate for Patient C. Slices acquired perpendicular to the long-axis of the laser fiber.

\subsection{8 \\ HIGH FREQUENCY OSCILLATIONS IN THE INTRA- OPERATIVE ELECTROCORTICOGRAM: THE EFFECT OF PROPOFOL}

Maeike Zijlmans, G. J. Huiskamp, O. Cremer, C. H. Ferrier, A. C. van Huffelen and F. S. Leijten (UMC Utrecht, Amsterdam, Netherlands)

Rationale: Epileptic high frequency oscillations (HFOs: 80-500 Hz) may be used to guide neurosurgeons during epilepsy surgery to identify epileptogenic tissue. We studied the effect of the anesthetic agent propofol on the occurrence of HFOs in the intra-operative electrocorticogram (ECoG).

Methods: We selected patients undergoing surgery for temporal lobe epilepsy with a standardized electrode grid placement. Intra-operative ECoG was recorded at $2048 \mathrm{~Hz}$ following cessation of propofol. The number and distribution of interictal spikes, ripples $(80-250 \mathrm{~Hz})$ and fast ripples (FRs: 250-500 Hz) were analyzed. The amounts of events on mesiotemporal channels and lateral neocortical channels were compared between patients with a suspected mesiotemporal and lateral epileptogenic area (t-test) and HFOs were compared to the irritative zone (correlation between amounts of events per channel) to provide evidence for the epileptic nature of the HFOs. Then, the 
amount of events within the first minute and the last minute were compared to each other and the change in events over the whole epochs were analyzed using correlation analyses of ten epochs during the emergence periods (Spearman rank test). It was studied whether the duration of HFOs changed over time. The change in events within presumed epileptogenic area was compared to the change outside this area. Periods of burst-suppression and continuous background activity were compared (t-tests).

Results: 12 patients were included, 5 with suspected mesiotemporal epileptogenic area and three with suspected lateral epileptogenic area (and 4 other). Spikes, ripples and FRs were related to the epileptogenic areas. Figure 1 shows that ripples and FRs increased during emergence from propofol anesthesia (mean number of ripples: first minute to last minute: 61.5 to $73.0, \mathrm{R}=0.46 \mathrm{p}<0.01$; FRs: 3.1 to 5.7, $\mathrm{R}=0.30, \mathrm{p}<0.01)$ and spikes remained unchanged (80.1 to 79.9, $\mathrm{R}=-0.05, \mathrm{p}=0.59)$. There was a decrease in number of channels with spikes $(\mathrm{R}=-0.18, \mathrm{p}=0.05)$, no change in ripples $(\mathrm{R}=-0.13, \mathrm{p}=0.16)$ or FRs $(\mathrm{R}=0.11, \mathrm{p}=0.45)$. There was no change in the durations of HFOs. The amount of HFOs in the presumed epileptogenic areas did not change more than the amount outside the presumed epileptogenic area, while spikes paradoxically decreased more within the suspected epileptogenic area. Six patients showing burst-suppression had lower rates of ripples than six other patients with continuous background activity $(\mathrm{p}=0.02)$. No significant difference was found between burstsuppression and continuous background activity in four patients, but there was a trend towards showing more ripples during continuous background activity $(\mathrm{p}=0.16)$.

Conclusions: Propofol, known for its anti-epileptic effects, reduces the number of epileptic HFOs, but has no effect on spikes. This enforces the hypothesis that in epilepsy HFOs mirror the disease activity and HFOs might be useful to monitor anti-epileptic drug treatment. It is feasible to record HFOs during surgery, but propofol infusion should be interrupted some minutes to improve the detection.

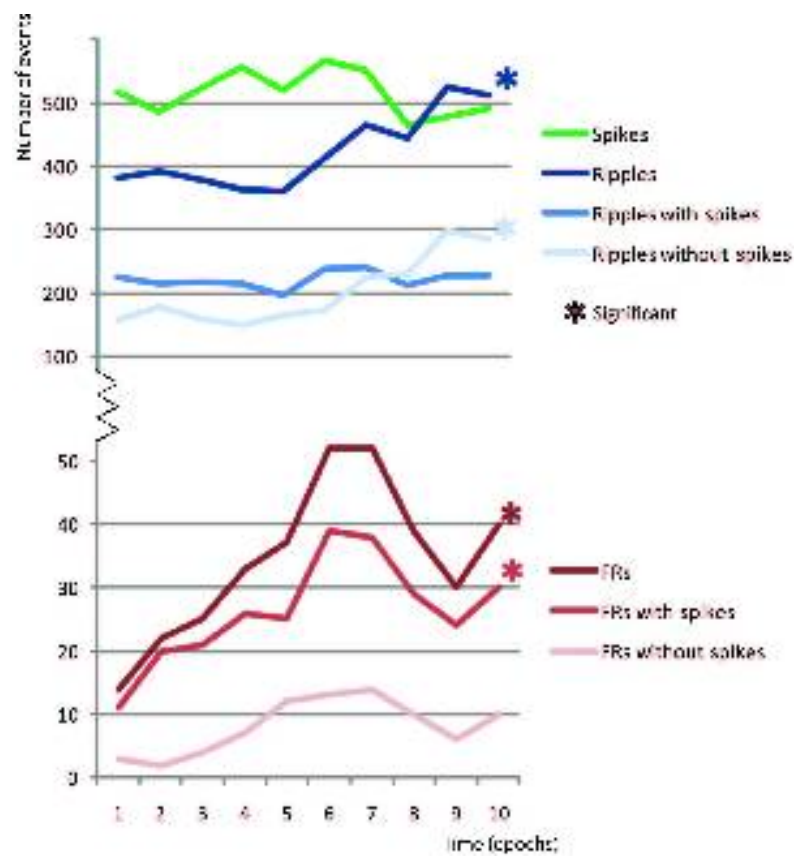

\subsection{9}

NEUROLOGISTS' KNOWLEDGE AND ATTITUDES TOWARDS EPILEPSY SURGERY: A CANADIAN SURVEY

Chantelle Hrazdil, J. I. Roberts, S. Wiebe and N. Jette (University of Calgary, Calgary, AB, Canada)
Rationale: Practice guidelines stipulate that epilepsy surgery should be considered in patients with drug resistant focal epilepsy, yet referral patterns have not changed and epilepsy surgery continues to be underutilized. Patients still wait on average 10-20 years before surgical referral. One recent study shows that non referral by neurologists is even higher than that by family physicians. We aimed to determine neurologists' knowledge and attitudes towards epilepsy surgery in addition to their perception of local barriers to appropriate and timely surgical evaluation.

Methods: A 5-minute nationwide paper or electronic questionnaire was administered to all Canadian neurologists registered with the Canadian Medical Directory. Content validity was completed through literature review and serial face-to-face revisions achieving agreement about each questionnaire component. Face validity was achieved by administering it to ten neurologists from across Canada. Descriptive statistics were calculated.

Results: Of 825 listed Canadian adult and pediatric neurologists who were contacted, 332 responded after the second reminder (response rate $40 \%$ ). Of the respondents, 294 saw epilepsy patients and were asked to complete the questionnaire in full. Over half (58\%) practiced general neurology, while $11 \%$ specialized in epilepsy/EEG. Most were affiliated with an academic institution (78\%) and had access to an epilepsy program in their city (77\%).

Respondents were generally well informed about epilepsy surgery. Just under $90 \%$ reported feeling quite knowledgeable about the indications for epilepsy surgery. Almost all (96\%) felt that epilepsy surgery is safe when performed in carefully selected patients. The risk of clinically significant (disabling) and permanent adverse effects from anterior temporal lobectomy was appropriately estimated at under $10 \%$ in $96 \%$ of respondents.

While $87 \%$ of those surveyed felt that they had access to adequate expertise, technology and resources to allow for appropriate selection of epilepsy surgical candidates, the majority reported concerns about temporal and physical barriers to access; $20 \%$ estimated their waitlists for surgical evaluation at over 1 year, and there was a frequent need to refer patients to better-equipped centers in other jurisdictions. Geographic variations in responses were explored. Areas for improvement included poor familiarity with epilepsy surgery clinical practice guidelines $(56 \%)$ and the recent definition of drug-resistant epilepsy (66\%); $43 \%$ believed that at least 3

antiepileptic drugs must fail in order for a patient to be drug-resistant. Conclusions: While most Canadian neurologists are well-educated about epilepsy surgery, there remains a need for better knowledge dissemination tools amongst neurologists. Resource limitations are identified as the largest nationwide barrier to optimization of surgical availability for those with refractory epilepsy. The association between neurologists' responses and actual practice requires exploration.

\subsection{0 \\ SURGICAL TREATMENT OF THE PATIENTS WITH RASMUSSEN'S ENCEPHALITIS-20 CASES Guoming Luan ${ }^{1}$, Y. Guan ${ }^{1}$, J. Zhou ${ }^{1}$ and X. Liu ${ }^{2}$ \\ ( ${ }^{1}$ Neurosurgery,Epilepsy Center, Beijing Sanbo Brain Hospital, Capital Medical University, Beijing, China and ${ }^{2}$ Neurology, Epilepsy Center, Beijing Sanbo Brain Hospital, Capital Medical University, Beijing, China)}

Rationale: To describe the clinical, electrophysiologic, neuroradiologic, and histologic findings of patients with Rasmussen encephalitis (RE) and to evaluate the outcome of their surgical treatment.

Methods: 20 RE patients were confirmed by presurgical evaluation including semiology, magnetic resonance imaging (MRI), interictal/ictal scalp video-electroencephalography (VEEG), intracranial recording and biopsy. Presurgical evaluation modalities and surgical treatment were analyzed retrospectively. 
Results: 11 functional hemispherectomy (FH), 5 anatomical hemispherectomy (AH), 5 hemispherotomy, 3 tailored resection, 1 multilobar resection and one bipolar electro-coagulation on functional cortexes (BEFC) procedures were undertaken, of which 14 were right-sided and 6 were left-sided. 3 patients underwent two surgical procedures, 2 patients underwent three surgical procedures. According to Engel's criteria, 6 patients $(80 \%)$ achieved an Engel Class I status, and 1 patient (5\%) had an Engel Class II outcome, 1 patients $(5 \%)$ had a significant decrease in seizure frequency (Engel Class III) and 2 patients (10\%) had no change in seizure frequency (Engel Class IV). 1 patient showed contralateral seizure after $\mathrm{AH}$ and be diagnosed as bilateral RE. All of the patients excepting one bilateral RE had increases in cognitive abilities, behavior, and quality of life after the surgery. After the surgery, most of the patients can walk independently, but the fine movement of the hands was lost. The main early complications were fever (35\%) after hemispheric surgeries. Postoperative hydrocephalus was observed one and a half years after $\mathrm{AH}$ in one patient and no death in of this series. Conclusions: Hemispherectomy and hemispherotomy were confirmed as both beneficial procedures in controlling seizures and improving quality of the life in cases with RE.

Surgical technique and outcome

\begin{tabular}{|c|c|c|c|c|c|}
\hline Surgical technique & Patients & Engel I & Engel II & Engel IIl & Engel IV \\
\hline $\mathrm{I}^{\prime} \mathrm{B}+\mathrm{I}^{\prime} \mathrm{I} R$ & 1 & 0 & 0 & 1 & 0 \\
\hline $\mathrm{PT}+$ biopsy $+\mathrm{B}$ ताए & 1 & 0 & 0 & 0 & 1 \\
\hline 1 MI.R; 2 TH & 1 & 1 & 0 & 0 & 0 \\
\hline 1 PB+hiopsy+ВГГГC; 2 ГТТ: $3 \wedge \mathrm{II}$ & 1 & 0 & 0 & 0 & 1 \\
\hline $1 \mathrm{TR} ; 2 \mathrm{TR}: 3 \mathrm{FH}$ & $\mathrm{I}$ & I & 0 & 0 & 0 \\
\hline $1 \mathrm{HH}: 2 \mathrm{AH}$ & 2 & 2 & 0 & 0 & 0 \\
\hline $\mathrm{AH}$ & 2 & 2 & 0 & 0 & 0 \\
\hline $\mathrm{FH}$ & 6 & 6 & 0 & 0 & 0 \\
\hline Hemispherectomy & 5 & 4 & 1 & 0 & 0 \\
\hline Total & 20 & $16(80 \%)$ & $\mathrm{I}(5 \%)$ & $1(5 \%)$ & $2(10 \%)$ \\
\hline
\end{tabular}

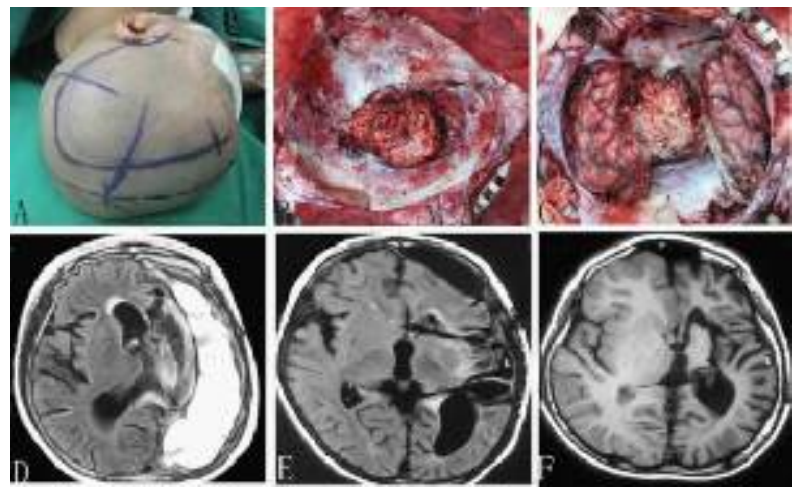

A. The picture of the seventh case showed the surgical incision of left cerebral hemisphere. B. The picture of the seventh case with left anatomical hemispherectomy. C. The picture of twelfth case with functional hemispherectomy. D. Postoperative MRI (axial T1weighted image) showed changes after anatomical hemispherectomy in the the seventh case. E. Postoperative MRI (axial T1- weighted image) showed changes after functional hemispherectomy in the the twelfth case. F. Postoperative MRI (axial T1- weighted image) showed changes after hemispherotomy in the fourteenth case.
MEDICAL VS ELECTRICAL THERAPY FOR MESIAL TEMPORAL LOBE EPILEPSY: A MULTICENTER RANDOMIZED TRIAL

S. Wiebe ${ }^{1}$, Z. Kiss ${ }^{1}$, N. Ahmed ${ }^{4}$, D. Andrade ${ }^{5}$, R. Brownstone ${ }^{3}$, M. Del Campo ${ }^{5}$, Jonathan Dykeman ${ }^{1}$, K. M. Fiest ${ }^{1}$, D. Gross ${ }^{4}$, N. Jette ${ }^{1}$, M. W. Lowerison ${ }^{1}$, R. McLachlan ${ }^{2}$, A. Parrent ${ }^{2}$, N. Pillay ${ }^{1}$, R. M. Sadler $^{3}$, S. Save ${ }^{1}$, E. Sherman ${ }^{1}$, T. Valiante ${ }^{5}$, R. Wennberg ${ }^{5}, M$. Wheatley ${ }^{4}$ and M. Eliasziw ${ }^{6}$ ( ${ }^{1}$ University of Calgary, Calgary, AB, Canada; ${ }^{2}$ Western University, London, ON, Canada; ${ }^{3}$ Dalhousie University, Halifax, NS, Canada; ${ }^{4}$ University of Alberta, Edmonton, $\mathrm{AB}$, Canada; ${ }^{5}$ University of Toronto, Toronto, ON, Canada and ${ }^{6}$ Tufts University, Boston, MA)

Rationale: Although surgical resection is the treatment of choice for patients with drug-resistant mesial temporal lobe epilepsy (MTLE), a small fraction of patients are not candidates for this intervention. Hippocampal electrical stimulation (HS) may benefit these patients, but the evidence is inconclusive. In a double blind randomized controlled trial (RCT), we aimed to determine whether (HS) is safe and more effective than simply implanting an electrode in the hippocampus without electrical stimulation (HI). The trial was stopped due to poor recruitment. We present results of the data collected.

Methods: A five-centre, parallel-group, double blind RCT allocated patients with MTLE to HI or HS (ratio 1:1 initially, then 1:2). Unilateral or bilateral HS was delivered using a 4-contact electrode implanted along the hippocampal longitudinal axis, and $135 \mathrm{~Hz}$ continuous cathodal stimulation of all electrodes involved in seizure generation. Outcomes were assessed during a seven-month period post-randomization. The primary outcome was change in seizure frequency between baseline and six months of follow-up. Secondary outcomes included safety, cognitive function, quality of life (QOLIE89 ), and proportion with $>50 \%$ seizure reduction. Non-parametric tests compared differences between groups $(p<.05)$.

Results: The target sample of 57 patients could not be recruited. The RCT was stopped after only six patients were randomized. Four patients were randomized to HI (ages 35 to 46 years, 2 left, 2 right), and two to HS (age 30 years, both bilateral). HI patients (median monthly baseline seizure frequency $=10$ ) had a median seizure increase of $60 \%$. HS patients (median monthly baseline seizure frequency $=12$ ) had a mean seizure decrease of $45 \%$. For convulsive (GTCS) and dyscognitive (CPS) seizures the baseline median monthly seizure frequencies were 1 in $\mathrm{HI}$ and 2 in HS patients, and the median change was $31.3 \%$ and $50 \%$ increase, respectively. None of the between-group differences was statistically significant. Only one patient (HS group) achieved a $>50 \%$ in seizure reduction. The overall QOLIE-89 score at 7 months worsened by 13 points with $\mathrm{HI}$ and improved 3 points with HS (not significant). QOLIE-89 attention/concentration scores decreased by 4 points with $\mathrm{HI}$ and increased by 20 points with HS (borderline significant, $\mathrm{p}<0.06$ ). Subjective memory scores using QOLIE- 89 memory scales decreased by 34 points with $\mathrm{HI}$ and increased by 10 points with HS (not significant). Scores of cognitive scales assessing recall (RAVLT, RCFT) were generally lower in the HS group compared to the HI group (not significant).

Conclusions: Despite collective efforts and multicentre participation, the feasibility of adequately powered RCTs of HS for patients with MTLE remains enormously challenging. The HS paradigm used in this RCT in a few patients resulted in: 1) non-significant trend towards improvement with HS in the frequency of all seizures (but not of GTC or CPS), and in subjective memory function; 2) a borderline significant improvement in attention/concentration; 3) a non-significant worsening in recall function. 


\subsection{2}

A FRAMEWORK FOR MULTIMODAL DATA REPRESENTATION IN THE PLANNING OF SUBDURAL GRID PLACEMENT

Maryse A. van 't Klooster ${ }^{1}$, J. L. Veelenturf ${ }^{2,1}$, G. J. Huiskamp ${ }^{1}$ and F. S. Leijten ${ }^{1}\left({ }^{1}\right.$ Neurology and Neurosurgery, University Medical Center Utrecht, Utrecht, Netherlands and ${ }^{2}$ Technical Medicine, University of Twente, Enschede, Netherlands)

Rationale: When invasive monitoring is required in epilepsy surgery patients it is important that subdural grids are optimally placed. Essentially, the seizure onset zone needs complete coverage. However, the more grids placed the higher the complication risk. For planning non-invasive techniques are available: PET, MEG, SPECT, EEG-fMRI. All of these typically produce results as $3 \mathrm{D}$ volumes that indicate regions involved in epilepsy. The challenge for the clinician is to integrate all 3D data, often presented independently, into a single strategy for placing electrode grids on the $2 \mathrm{D}$ cortical surface that is accessible to the surgeon. Here we present a single framework for integrating 3D presurgical data on the 2D cortical surface. This allows the clinician to plan grid placement in a consistent way. It also allows quantitative evaluation of the performance of noninvasive methods when ECoG recorded in grid electrodes can be considered the gold standard.

Methods: Retrospectively, for a number of patients 3D MRI, interictal MEG spikes, interictal FDG-PET, post-implantation CT and (inter)ictal ECoG were available. All modalities were co-registered using CURRY NeuroImaging suite. A triangular mesh (edge $3 \mathrm{~mm}$ ) of the surface enveloping the cortex was set up. Based on this mesh a volume conductor model for MEG was defined, and interictal spikes were modeled using the MUSIC algorithm. Resulting 3D points (resolution $3 \mathrm{~mm}$ ) around the maximum of the MUSIC metric were stored. The PET hypometabolic areas were marked and enclosed 3D points $(3 \mathrm{~mm})$ stored. Grid electrode positions were extracted from $\mathrm{CT}$ and projected on the surface mesh. Electrodes containing (inter)ictal activity in ECoG were marked. Subsequently, all data was processed in MATLAB. Pre-implantation processing steps included: (1) determination of direction of center-of-mass (CM) of 3D points (PET/MEG) to nearest point on the surface mesh, (2) projection on the mesh of 3D points along this direction and marking of triangles involved, (3) computation of distance between projected CM's and overlap between marked triangles for PET/MEG. All results were plotted on the surface mesh and presented to the clinician with the computed measures. Post-implantation steps were: (1) triangles that represent sampling areas of grid electrodes containing (inter)ictal ECoG activity are marked and CM is computed, (2) calculation of distance and overlap between marked areas for CM PET/MEG and CM ECoG.

Results: Results indicate that for some patients grid placement strategy would have changed if pre-implantation results were presented according to the framework introduced here. A subfrontal grid would, e.g., now be favored over a temporal pole position based on MEG results which were initially regarded as ambiguous. Postimplantation results show that interictal PET and MEG can indicate separate regions with different distances and overlap with ECoG. Conclusions: The framework presented here supports decision making in subdural grid placement. The proposed method allows quantification of the relation between PET, MEG, and (inter)ictal ECoG in future studies.

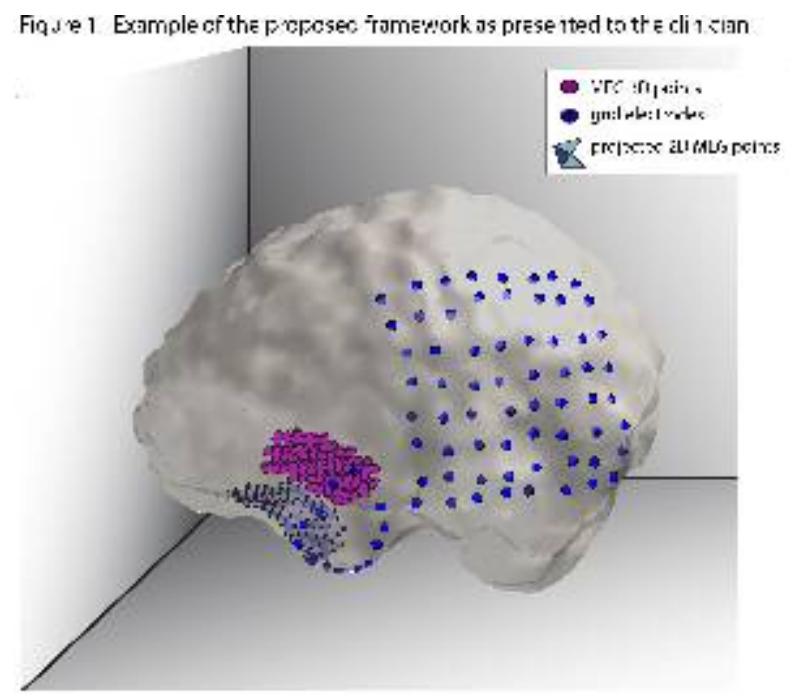

Example of the proposed framework as presented to the clinician

\subsection{3 \\ SUBTEMPORAL VS. TRANSSYLVIAN APPROACH FOR SELECTIVE AMYGDALAHIPPOCAMPECTOMY (SAH) IN MESIAL TEMPORAL LOBE EPILEPSY (MTLE) - AVOIDING VISUAL FIELD DEFECTS (VFD) AND THE ROLE OF DIFFUSION TENSOR IMAGING IN THE VISUALIZATION OF THE OPTIC RADIATION (MEYER'S LOOP)}

Daniel Delev ${ }^{1}$, M. von Lehe ${ }^{1}$, J. Schramm ${ }^{1}$, B. Weber ${ }^{2}$, C. Elger ${ }^{2}$ and J. Schoene-Bake ${ }^{2}\left({ }^{1}\right.$ Department of Neurosurgery, University of Bonn, University Medical Center, Bonn, Germany and ${ }^{2}$ Department of Epileptology and Life\&Brain Center, University of Bonn, University Medical Center, Bonn, Germany)

Rationale: MTLE is the most common form of pharmacoresistant focal epilepsy in adults. SAH is a safe and well-recognized surgical procedure for the treatment of MTLE. One of the procedural risks are visual field deficits due to damage of the optic radiation (OR), the socalled Meyer's Loop. Over the years, several approaches for SAH have been introduced - here we compare the transsylvian and the subtemporal approach with regard to VFDs both clinically and with Diffusion Tensor Imaging (DTI).

Methods: Overall, 65 consecutive patients with MTLE (right:left 22:43, male:female 37:28, mean age 43 yrs.) underwent temporal resections. Visual fields were determined using Goldmann visual field kinetic perimetry test performed on OCULUS Twinfield2 perimeter both before and after surgery. Conventional MRI is regularly performed as part of the presurgical workup on a Siemens 3T scanner with 60 gradient directions and a resolution of $1.7 \mathrm{~mm} 3$. DTI data was processed with FSL tools (FMRIB, Oxford, UK) and probabilistic tractography of the OR was carried out using previously described ROIs (Winston, et al., 2011, 2012).

Results: A total of 56 patients with SAH had pre- and postoperative perimetry data and were evaluated for this study (33 with transsylvian, 23 with subtemporal approach. Eight patients $(24.2 \%)$ from the transsylvian approach group showed no VFDs and $3(10 \%)$ had unspecific changes, whereas the remaining 22 exhibited some form of visual field impairment related to surgery (66.7\%).

Interestingly, only 12 patients $(52.1 \%)$ with subtemporal approach showed surgery related VFDs (unspecific changes in 1 (4\%) patient). Thus, 10 patients (43.2\%) from the latter group had no VFD (Figure 1). Visualization of the OR with DTI of good quality (Figure 2) was successfully performed in 49 of 65 patients. The quantitative analysis of the DTI-data is not yet included and further correlation with VFDs is in process. There was no significant difference in seizure outcome between the two groups. 
Conclusions: The subtemporal approach reduces the risk for VFDs in patients, who undergo SAH. DTI-based tractography can accurately delineate the optic radiation and may help to avoid VFDs in the future by choosing the most appropriate surgical approach. Acknowledgements

The study was part of the project A1 of transregional research cluster SFB TR3 "Mesial temporal lobe epilepsies" of the Deutsche

Forschungsgemeinschaft.

References

Winston, G. P., Yogarajah, M., Symms, M. R., McEvoy, A. W., Micallef, C., \& Duncan, J. S. (2011). Diffusion tensor imaging tractography to visualize the relationship of the optic radiation to epileptogenic lesions prior to neurosurgery. Epilepsia, 52(8), 14301438.

Winston, G. P., Daga, P., Stretton, J., Modat, M., Symms, M. R., McEvoy, A. W., Ourselin, S., et al. (2012). Optic radiation tractography and vision in anterior temporal lobe resection. Annals of Neurology, 71(3), 334-341.
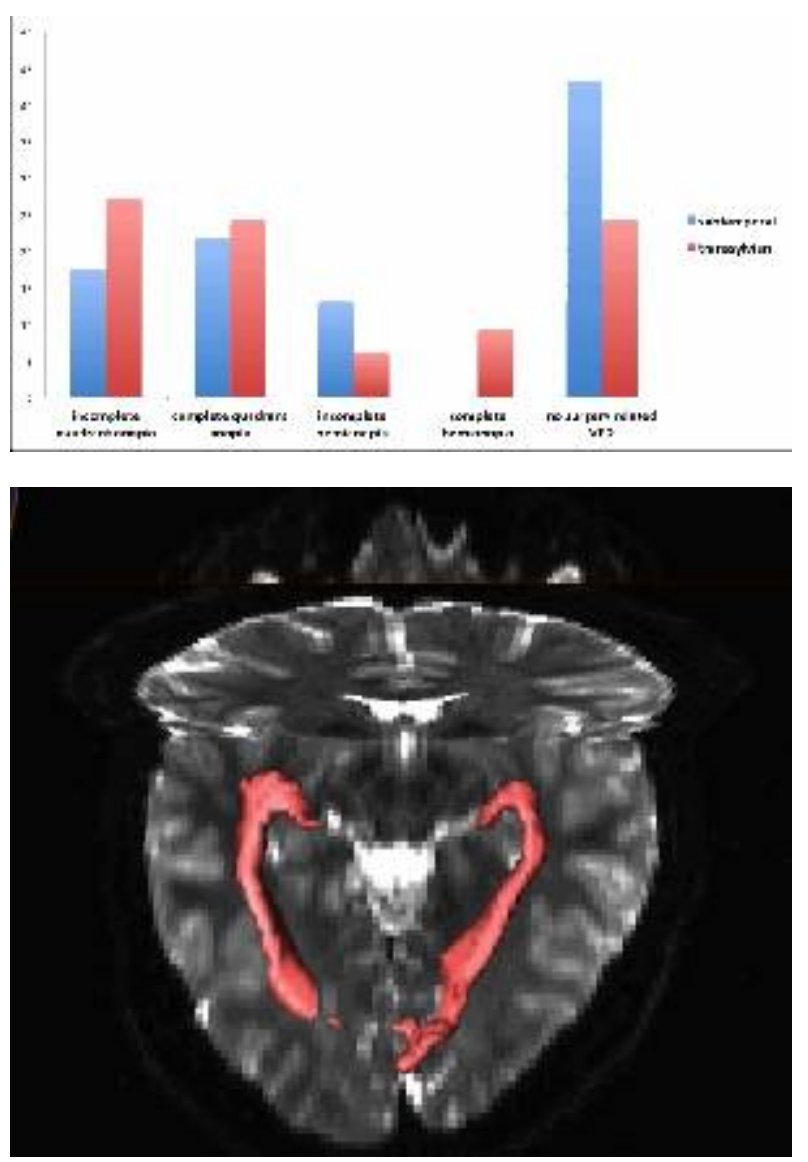

\subsection{4}

\section{EEG FINDINGS AFTER EPILEPSY SURGERY AND ITS} RELATION WITH OUTCOME

\section{Ana G. Besocke ${ }^{1}$, D. Benech ${ }^{1,2}$, S. Scalise ${ }^{1}$, E. Cristiano ${ }^{1}$, M.} Aberastury ${ }^{1}$, M. García ${ }^{1}$ and W. Silva ${ }^{1,2}\left({ }^{1}\right.$ Neurología, Hospital Italiano Buenos Aires, Buenos Aires, Argentina and ${ }^{2}$ Neurología, Instituto Argentino de Investigación Neurológica (IADIN), Buenos Aires, Argentina)

Rationale: Describe pos surgical EEG findings and try to establish its prognostic value with regard to epilepsy surgery outcome. Methods: We included patients suffering from pharmaco resistant epilepsy who had undergone epilepsy surgery between April 2005 and July 2010 and completed 1 year of postoperative follow-up. Epilepsy surgery subtypes were classified into: Temporal Resections (TR): standard anterior temporal lobectomies (ATL) and ATL associated with adjacent neocortical resections; Neocortical Resections (CORT), hemispherectomy and hemispherotomy (HEM). We classified outcome according to Engel Score: Favorable outcome (Engel I), unfavorable outcome (Engel $>$ I). EEG findings were further classified in two groups: Non epileptiform EEG (NE-EEG) and epileptiform EEG (E-EEG).

We calculated the sensitivity, specificity, predictive values and accuracy for the presence of IED with respect to seizure outcome (Engel I vs Engel $>$ I). We used Fisher exact tests to compare the patients with favorable and unfavorable seizure outcomes with respect to the presence of E-EEG or NE-EEG in the different resection subtypes; a $p$ value of $<0.05$ was considered significant. Results: Fourty four patients were included, median time of follow up was 37 months (12-75). Twenty five patients had favorable outcome: 13 had undergone TR, of them 12 (92\%) had NE-EEG, and 1 E-EEG; 12 had undergone CORT, $6(50 \%)$ had E-EEG. The difference between EEG findings in both types of resection was statistically significant, $(\mathrm{p}<0,01)$.

Thirteen patients had unfavorable outcome: in 3 TR was performed, 1 patient had E-EEG and 2 NE-EEG; 10 had undergone CORT, 7 of them had E-EEG (70\%), and 3 NE-EEG.

In 6 patients HEM was performed, 5 had favorable outcome, 4 of whom had E-EEG.

In TR post-surgical EEG sensitivity is 33\%, specificity: $92 \%$, negative predictive value: $85 \%$, positive predictive value: $50 \%$ and accuracy: $81 \%$. In CORT, post-surgical EEG sensitivity was: $70 \%$, specificity: $50 \%$, negative predictive value: $66 \%$, positive predictive value: $53 \%$ and accuracy: $59 \%$.

Conclusions: A NE-EEG was statistically significant associated with favorable outcome in patients who had undergone TR. This finding was also supported by post surgical EEG high specificity and negative predictive value in this type of resections. We observed a trend toward more frequent E-EEG in patients with unfavorable outcome in whom CORT was performed. No relationship was found between postsurgical EEG and outcome in patients who had undergone HEM.

\begin{tabular}{|c|c|c|c|}
\hline & RT(CI) & CORT(CI) & HEM (CI) \\
\hline Sensitivity & $33,3 \%(6,1-79,2)$ & $70 \%(39,7-89,2)$ & $100 \%(20-100)$ \\
Specificity & $92,3 \%(66,7-98,6)$ & $50 \%(25,4-74,6)$ & $40 \%(11,8-76,9)$ \\
Positive Predictive Value & $50 \%(9,5-90,5)$ & $53,8 \%(29,1-76,8)$ & $25 \%(4,6-69)$ \\
Negative Predictive Value & $85,7 \%(60,1-96)$ & $66,7 \%(35,4-87,9)$ & $100 \%(34-100)$ \\
\hline Accuracy & $81,2 \%(57-93,4)$ & $59.1 \%(38,7-76.7)$ & $50 \%(18-81)$ \\
\hline
\end{tabular}

\subsection{5 \\ INTRACAROTID SODIUM AMOBARBITAL INACTIVATES HIPPOCAMPAL STRUCTURES}

Myrta I. Otero, R. M. Roth, S. Guerin and B. Jobst (Geisel School of Medicine at Dartmouth Medical School, Lebanon, NH)

Rationale: The relevance of IAP has been a topic of debate over the years and the use of this procedure has significantly decreased, mainly because it poses risk factors and concerns with reliability and validity. Another controversy has been the anatomical cerebrovascular variability among patients putting in question the intracranial brain structures affected during IAP since the internal carotid artery only supplies the anterior hippocampus via the anterior choroidal artery. The hippocampus is mainly irrigated by the vertebrobasilar system supplied by the posterior cerebral artery. Thus the hippocampus may potentially be unaffected by an ICA injection. Memory impairment is hypothesized to be due to lateral cortical inactivation only. We demonstrate in two patients that sodium amytal injection does inactivate hippocampal structures as injection occurred with intracranial EEG monitoring.

Methods: Two medically refractory patients with focal seizure and secondary generalization had IAP with intracranial electrodes 
implanted along the axis of the hippocampus, as well as over the lateral temporal cortex. One patient had left and the other right-sided mesial temporal sclerosis as well as hippocampus atrophy. Both patients had bilateral hippocampal and temporal lateral electrodes implanted. IAP was performed on both sides in patient one, and only on the left side in patient two according to clinical requirements. Results: In both patients there was clear suppression patterns in the hippocampal structures, that also involved the anterior portion of the hippocampus. Slow wave activity was increased. Slowing and burst suppression also occurred over the lateral temporal structures, implicating that both medial and lateral temporal structures are involved during IAP. This indicates that large memory networks are inactivated during the test and that IAP testing is valid for hippocampal function.

Conclusions: IAP definitely inactivates the hippocampal structures, however inactivation is widespread and also involves large areas of the cortex. Our case series shows that IAP is valid for memory testing.

\subsection{6}

EXPANDING THE INTRACRANIAL MONTAGE DOES NOT INCREASE ICEEG MORBIDITY

Jason Gerrard ${ }^{1}$, H. P. Zavari ${ }^{2}$, W. S. Kasoff ${ }^{1}$, K. P. Vives ${ }^{1}$, L. R. Hirsch $^{2}$, B. R. Duckrow ${ }^{2,1}$ and D. D. Spencer ${ }^{1}\left({ }^{1}\right.$ Neurosurgery, Yale School of Medicine, New Haven, CT and ${ }^{2}$ Neurology, Yale School of Medicine, New Haven, CT)

Rationale: Many years of experience at our institution and emerging evidence elsewhere support the presence of epileptogenic networks. While these networks tend to involve regions normally strongly interconnected (ie. temporal-occipital network) the epileptogenic network often extends beyond focal abnormalities and may be larger than predicted. Furthermore, a recent study involving the placement of several "microelectrodes" between typical macroelectrodes revealed seizure activity within microelectrodes that was not detected on the typical clinical macroelectrodes (Stead etal. 2010). Thus we have more to learn about the spatial and temporal scales of epilepsy and epileptic networks. Together, these observations suggest that intracranial EEG (icEEG) recordings that are focused only on areas of small structural or metabolic abnormalities are likely to undersample a given patient's epileptogenic network. In order to facilitate increased data acquisition, high density recording systems that are widespread in the neurosciences are becoming more commonly used in epilepsy centers. Accordingly, our practice in the Yale Epilepsy Surgery Program (YESP) has been one of increasingly broad EEG coverage in the evaluation of most forms of LRE. As we increase the number, density and types of contacts used to improve icEEG monitoring, patient safety must remain paramount.

Methods: The Yale Epilepsy Surgery Program has pro-spectively maintained a database of all patients undergoing icEEG monitoring. We are retrospectively reviewing this database from 1991 to 2011 and reviewing morbidity data when available. The number of icEEG contacts placed per patient as well as the types of electrodes used were analyzed. Surgical outcomes were reviewed with attention to morbidity during icEEG studies.

Results: Previously, we have reported a steady increase in the number of icEEG contacts used during icEEG monitoring sessions, with average numbers of contacts rising from $80-100$ per patient in the early 1990 s to 200 per patient in the mid-2000s. This trend has continued in recent years as our technical capabilities have increased $(\mathrm{p}<.01, \mathrm{R} 2=.81)$. Despite the significant increase in the number and types of electrodes, the morbidity of icEEG implantation and monitoring has not changed significantly $(p>0.20)$ and infection has remained the most common morbidity.

Conclusions: The widescale ongoing review of the Yale Epilepsy Surgery Database has not revealed any significant change in patient morbidity, despite the significant increase in electrodes used for icEEG. However, the mass effect, bleeding and infection risks of intracranial electrodes are well known and new approaches to improve these devices should be an ongoing process. We believe that broad, multimodal icEEG monitoring is needed to fully understand the cases of LRE requiring icEEG recordings and to provide the best treatment strategies.

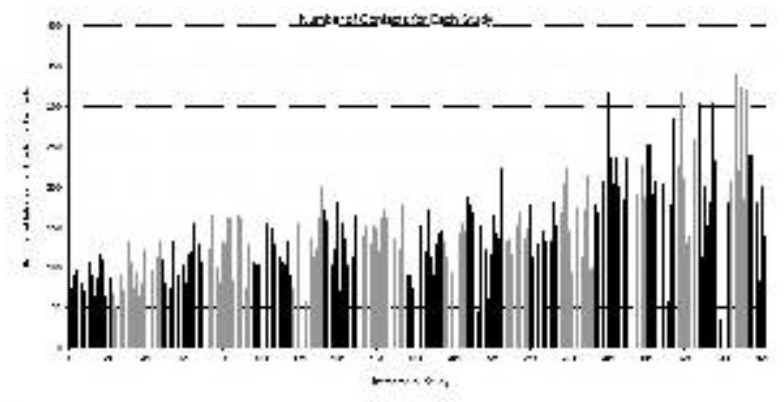

2.277

PRE- AND POST-OP ACTIVATION OF THE MOTOR CORTEX IN EPILEPSY WITH HEMIPARESIS AND ROLANDIC ISCHEMIA

Romulo severino, A. Palmini, E. Paglioli, D. Crestani, J. Hoefel, R. Nunes and J. Costa (Neurology, São Lucas Hospital, Porto Alegre, Brazil)

Rationale: We want to study the role of the primary motor cortex (PMC) in patients with vascular destructive lesions in perirolandic regions. Neuroplastic mechanisms operate and these patients have some motor functionality. This picture is more complex when occur seizures arousing from the scar and eventually when this area is resected. We report pre-and post-operative motor functional magnetic resonance (fMRI) findings in 12 patients with refractory epilepsy. Methods: Twelve patients with congenital hemiparesis underwent pre-surgical fMRI. All had cortical resections and were re-scanned 12 to 36 months after surgery. Activation maps were superimposed over anatomical images; analysis using Student $t$ test with a $\mathrm{P}<0.0001$. Limbs were evaluated with neurological exam in 12 to 36 months. Results: Pre-operatively, fMRI showed 3 main patterns. The first showed activation of PMC adjacent to the destructive lesion. The second was characterized by absence of activation of the PMC ipsilateral to the lesion; and activation in the normal hemisphere. A third displayed bilateral activation of PMC. Postoperatively, patients who did not have activation of the motor cortex adjacent to the lesion regained activation, and that was accompanied by improved motor function.

Conclusions: These findings suggest that the PMC in the lesioned hemisphere may play a role in the motor function of the paretic limbs. Furthemore, the post-operative activation of previously silent $\mathrm{PMC}$ in some patients suggest that recurrent seizures and epileptogenic discharges may inhibit potentially preserved function. The improved motor function accompanied these positive fMRI changes adds credibility and raises the issue of new therapeutic.

\subsection{8 \\ EVOLUTION UP TO 18 YEARS AFTER SURGERY FOR TEMPORAL EPILEPSY WITH HIPPOCAMPAL SCLEROSIS: IMPACT OF TECHNIQUE, MEDICATION MANAGEMENT AND PRESURGICAL VARIABLES}

D. Crestani, A. Palmini, M. Hemb, E. Paglioli, R. Severino, E. Paglioli, J. Costa, N. Azambuja, M. Portuguez, V. Viuniski and M. Nunes (SAO LUCAS HOSPITAL (PUCRS), Porto Alegre, Brazil)

Rationale: The chances of remaining seizure free for very long periods are still unclear for patients undergoing surgery for mesial temporal lobe epilepsy and unilateral hippocampal sclerosis (MTLE/HS). Likewise, the impact of practical variables such as 
surgical technique and post-operative reduction of medication needs clarification.

Methods: We followed 108 patients with unilateral MTLE/HS for 8 to 18 years and generated Kaplan-Maier survival curves for the probability of remaining seizure free over the years and for the modulating effect of medication management and surgical technique. Univariate and multivariate regression analyses were then perfomed to determine the impact of these and other variables on the long-term evolution.

Results: A history of generalized tonic clonic seizures (GTCS) was present in $10 \%$ of the patients and only $16 \%$ needed intracranial EEG. The probability of remaining completely seizure-free at 12 and 18 years was $65 \%$ and $62 \%$. Around $75 \%$ of patients have discontinued or significantly reduced antiepileptic drugs (AEDs). The type of surgical technique did not impact on outcome. Multivariate analysis showed that both a history of GTCS and remaining on full or almost full doses of AEDs significantly diminished the probability of remaining seizure free (Cox regression: $\mathrm{p}=0.003 \mathrm{CI}$ : $1.84-22.41$ for GTCS; $\mathrm{p}=0.048$, CI: 0.07-5.27 for AED management).

Conclusions: Patient selection may be the most important determinant of the chances of remaining seizure free over the years. When the epileptologic profile indicates more restricted disease, removal of neocortical structures does not lead to better chances of seizure control and the reduction or discontinuation of AED does not prevent favorable results.

\section{Behavior/Neuropsychology/Language: Adult}

\subsection{9 \\ CORRELATION OF NEUROPSYCHOLOGICAL FUNCTIONING AND PET HYPOMETABOLISM IN INTRACTABLE MESIAL TEMPORAL LOBE EPILEPSY: EXPLORING THE CONCEPT OF THE "FUNCTIONAL DEFICIT ZONE"}

Alex Knopman ${ }^{1,2}$, C. H. Wong ${ }^{3}$, R. J. Stevenson ${ }^{2}$, J. Homewood ${ }^{2}$, A. Mohamed $^{4}$, E. Somerville ${ }^{5}$ L. Wen ${ }^{4}$, S. Eberl ${ }^{4}$, M. Fulham ${ }^{4}$ and A. Bleasel $^{3}\left({ }^{1}\right.$ Dept of Medical Psychology, Westmead Hospital, Sydney, NSW, Australia; ${ }^{2}$ Dept of Psychology, Macquarie University, Sydney, NSW, Australia; ${ }^{3}$ Dept of Neurology, Westmead Hospital, Sydney, NSW, Australia; ${ }^{4}$ Dept of PET and Nuclear Medicine, Royal Prince Alfred Hospital, Sydney, NSW, Australia and ${ }^{5}$ Dept of Neurology, Prince of Wales Hospital, Sydney, NSW, Australia)

Rationale: This study explored the concept of the "functional deficit zone" (the brain area showing abnormal functioning in the interictal period) by correlating neuropsychology measures and PET hypometabolism. It was hypothesised that (a) verbal memory is associated with dominant temporal lobe (TL) hypometabolism, (b) nonverbal memory is associated with nondominant TL hypometabolism, (c) FSIQ, executive, attention/working memory and/or speed of thinking is associated with prefrontal hypometabolism. Additionally, we examined whether any of these neuropsychology variables could predict poor outcome following temporal lobectomy.

Methods: Thirty-four patients (23 females, mean age $34.7 \pm 10.8$ ) with FSIQ $>69$ were included in the study, all of whom had medically refractory mesial temporal lobe epilepsy (TLE), had undergone temporal lobectomy (14 dominant) and were followed up for at least one year. Presurgical FDG-PET was analysed voxel-by-voxel using SPM to identify significant regions of glucose hypometabolism relative to normal controls. Presurgical neuropsychology variables included FSIQ (WAIS-R/III), delayed verbal and nonverbal memory indices (WMS-R/III), executive functioning (Wisconsin Card Sorting Task perseverative errors (WCSTpe) or a composite of WAIS-R/III Similarities, Block Design, Matrix Reasoning and COWAT), attention/working memory (composite of WAIS-R/III Digit Span, LNS and Spatial Span) and speed (composite of WAIS-R/III Coding, Symbol Search and TMT-A).
Results: Nondominant TLE patients demonstrated poorer visual memory than dominant patients $(\mathrm{t}=2.29, \mathrm{p}<0.05)$. Dominant TLE patients demonstrated weaker verbal memory relative to visual memory $(\mathrm{t}=2.85, \mathrm{p}<0.05)$. Poor verbal memory was associated with dominant anteromesial TL hypometabolism $(\mathrm{r}=-0.36, \mathrm{p}<0.05)$, whereas poor nonverbal memory was associated with nondominant TL hypometabolism $(\mathrm{r}=-0.39, \mathrm{p}<0.05)$. FSIQ was not associated with prefrontal hypometabolism $(\mathrm{r}=0.23, \mathrm{p}=0.19)$. There was no association between prefrontal hypometabolism and executive (WCSTpe: $\mathrm{r}=0.2, \mathrm{p}=0.32$; composite: $\mathrm{r}=0.26, \mathrm{p}=0.14$ ), attention/working memory $(\mathrm{r}=0.21, \mathrm{p}=0.23)$ or speed $(\mathrm{r}=0.10$, $\mathrm{p}=0.58$ ) measures. No neuropsychology variables were able to predict surgical outcome (Engel class 1).

Conclusions: Standard neuropsychological memory indices correlate with unilateral temporal lobe hypometabolism in the expected material-specific way, a relationship not previously identified for both verbal and nonverbal memory. FSIQ is not correlated with prefrontal hypometabolism, in contrast with the findings reported by Jokeit et al. (Brain 120:2283,1997). Furthermore, other specific neuropsychological indices do not correlate with prefrontal hypometabolism, an unexpected finding given the widely held view that the prefrontal region broadly subserves cognition. This suggests remote PET hypometabolism (Wong et al. Epilepsia 51:1365,2010) is not concordant with the functional deficit zone. Neuropsychological measures do not predict seizure outcome following temporal lobectomy.

\subsection{0 \\ QUALITY OF LIFE AND PERCEIVED SOCIAL SUPPORT IN PATIENTS WITH GENERALIZED VERSUS TEMPORAL LOBE EPILEPSY}

Jesse Thon and M. J. Hamberger (Neurology, Columbia University, New York, NY)

Rationale: It is well established that people with epilepsy report poorer overall quality of life than their healthy peers. Although some studies have additionally shown QOL to be poorer in patients with generalized seizures relative to those with focal seizures, others have not found this pattern. We hypothesized that although overall quality of life might not consistently differ between people with generalized versus focal epilepsy, specific subcomponents of quality of life might distinguish the two groups. Using the QOLIE-89, we compared QOL subscale scores between generalized and focal (temporal lobe epilepsy "TLE") epilepsy patients. We also assessed the potential influence of mood on quality of life via standardized self-report mood evaluation.

Methods: Subjects were 167 adult epilepsy patients (generalized: 36, TLE: 131 , women: 150 ; mean age $39.4(\mathrm{SD}=13.8)$; mean years education $15.1(\mathrm{SD}=2.87))$ referred for neuropsychological testing at the Columbia Comprehensive Epilepsy Center from 2003 to 2012 who completed QOLIE-89 for assessment of quality of life, and the SCL-90-R for assessment of mood. T-tests were used to compare groups.

Results: Compared to the TLE group, patients with generalized epilepsy exhibited lower scores on the social support subscale (45.2 $+/-9.80$ vs. $50.3+/-9.64, \mathrm{p}=.008$ ) of the QOLIE-89. However, there were no significant differences between groups on subscale scores of social functioning $(48.2+/-10.9$ vs. $46.3+/-11.7, \mathrm{p}=.357)$, social isolation $(48.2+/-8.38$ vs. $50.6+/-9.48, \mathrm{p}=.154)$, any other subscales, or in overall quality of life index scores $(47.2+/-11.8$ vs. $47.4+/-11.3, p=.920)$. Further, there were no differences between groups in self-reported mood (all subscale and index scores: $\mathrm{P}>.10$ ). Conclusions: These results suggest that patients with generalized epilepsy experience reduced social support relative to patients with TLE. This finding did not extend to overall quality of life. More specifically, this was distinct from social functioning and feelings of social isolation, and appeared unrelated to mood. We speculate that this difference could potentially reflect generalized epilepsy patients' 
perception or experience of greater social stigma due to the dramatic nature of their events. The absence of group differences in social functioning and social isolation suggests similar self-perception of outward behavior between TLE and generalized epilepsy patients, yet different perceived responsiveness from others. Generalized epilepsy patients might benefit from clinicians' attention to issues regarding social support.

\subsection{1}

\section{INCREASING AGE AND STIMULATION IDENTIFIED} NAMING SITES

Marla J. Hamberger ${ }^{1}$, A. C. Williams ${ }^{1}$, G. M. McKhann ${ }^{2}$ and C. A. Schevon ${ }^{1}\left({ }^{1}\right.$ Neurology, Columbia University Medical Center, New York, NY and ${ }^{2}$ Neurological Surgery, Columbia University Medical Center, New York, NY)

Rationale: Electrical stimulation mapping to identify essential language cortex is typically performed prior to cortical resection involving the language dominant hemisphere. Within the past decade, several studies have shown that in childhood, the number of language sites identified via ESM increases with age. This has been interpreted to reflect the establishment of language areas associated with maturation. However, beyond adolescence, the influence of age on the number and topographical distribution of language sites has not been explored. Behavioral studies of naming across the age span have shown that naming is relatively unchanged until approximately the seventh decade of life. Given the relative stability of naming ability, we hypothesized that the number and distribution of naming sites identified by ESM would also show no significant change across the adult age span, below age 70 .

Methods: Subjects were 49 pharmacologically refractory temporal lobe epilepsy patients (age range: $19-64$ years, mean age $=35.3$, SD $=10.6$ ) who underwent ESM for language mapping prior to surgical resection for seizure control (21 intraoperative, 28 extraoperative). Naming was assessed during stimulation using both visual picture naming and auditory description naming at each site. A mean of 26 cortical sites per patient was tested (range: 15-55). Preoperative baseline naming tasks included the Boston Naming Test and the Visual and Auditory Naming tests. Pearson correlations assessed the relations between age, number of naming sites, and naming performance. Independent sample t-tests assessed differences between groups.

Results: Age and number of visual naming sites were not correlated $(\mathrm{r}=-.02, \mathrm{P}=.85)$; however, increasing age was significantly associated with a greater number of auditory naming sites $(\mathrm{r}=.33, \mathrm{P}$ $=.03$ ). Restricting this analysis to extraoperative cases, which received more extensive mapping, the correlation between auditory naming sites and age was stronger $(\mathrm{r}=-.49, \mathrm{P}=.01)$, whereas the correlation between visual naming sites and age remained nonsignificant $(\mathrm{r}=.21, \mathrm{P}=.27)$. Similarly, mean number of auditory naming sites per patient was greater in patients over 30 years relative to those below age $30(<$ age 30 mean $=.62, \mathrm{SD}=.96 ;>$ age 30 : mean $=1.76, \mathrm{SD}=1.5 ; \mathrm{P}=.02)$, whereas there was no group difference for number of visual naming sites $(\mathrm{P}=.12)$. Importantly, there was no relation between age and total number of sites tested $(\mathrm{r}$ $=-.09, \mathrm{P}=.63$ ). Spatially, older patients had significantly more auditory naming sites in the anterior temporal region and more diffuse representation of visual naming sites relative to younger patients.

Conclusions: At odds with our hypothesis, these results suggest an increase in stimulation-identified auditory naming sites with increasing age. It is unlikely this increase represents the establishment of new naming areas in older adults. Rather, we speculate that language, and perhaps, auditory naming in particular, becomes more susceptible to disruption over the adult age span.

\subsection{2 \\ MESIAL TEMPORAL ACTIVATION IN MAGNETIC SOURCE IMAGING: RELATIONSHIP TO WORD RECOGNITION AND DELAYED RECALL IN A RECEPTIVE LANGUAGE PARADIGM}

Gail L. Risse, R. C. Doss and W. Zhang (Minnesota Epilepsy Group, St. Paul, MN)

Rationale: Activation of the mesial temporal region is frequently observed in response to a Magnetic Source Imaging (MSI) auditory language paradigm involving recognition of abstract nouns that are repeated over trials. We have previously reported that this activation may be associated with Wada memory scores, atypical language classification and may be more likely in the right hemisphere of patients with left MTS. The current study examines mesial temporal activation (MTA) in relation to word recognition over trials and 30 minute delayed recall of the same target word list.

Methods: The subjects were 29 epilepsy or brain tumor surgery candidates ranging in age from 16 to 66 years who underwent MSI language mapping. The MEG unit consisted of a 148-channel magnes $2500 \mathrm{WH}$ system and data were analyzed using the single equivalent dipole (ECD) model across each whole hemisphere. The activation test was an auditory word recognition task in which subjects were instructed to raise the right (or left) index finger whenever they heard a word from the original target list. Responses were scored for accuracy via video camera for six presentation trials. Delayed recall of target items was elicited 30 minutes following completion of the language task. The presence or absence of MTA was recorded for each subject during the language analysis.

Results: Sixteen of 29 subjects (55\%) demonstrated MTA during the receptive language task, typically in the language dominant hemisphere. The incidence of MTA was significantly higher (78\%) for patients whose trial one recognition score was less than 20 (out of 30) compared to those with higher recognition scores (MTA incidence of $33 \%$; Fisher exact test $\mathrm{p}=.038$ ). A similar trend was noted for 30-minute delayed recall scores and for "best trial" scores, although these difference did not reach statistical significance. Conclusions: These results suggest an inverse relationship between MTA in the language dominant hemisphere and accuracy of verbal memory processing. The possibility that MSI activation (at least within the mesial temporal region) may represent underlying impairment rather than efficient functioning should be considered.

\subsection{3 \\ SKILLS FOR BETTER SUPPORT: DEVELOPING SELF- REGULATION SKILLS AMONG FAMILY MEMBERS TO IMPROVE THE QUALITY OF INFORMAL HELP PROVIDED TO ADULTS WITH EPILEPSY}

Shelley C. Stoll ${ }^{1}$, A. Gorelick ${ }^{2}$, R. Derry ${ }^{2}$, L. M. Selwa ${ }^{1}$, R. P. Kelly ${ }^{1}$ and N. M. Clark ${ }^{1}{ }^{1}$ University of Michigan, Ann Arbor, MI and ${ }^{2}$ Epilepsy Foundation of Michigan, Southfield, MI)

Rationale: Informal and psychosocial support along with material assistance in day to day management of epilepsy is thought to be an important adjunct to conventional treatment. Evaluation of interventions to provide such support is recommended as a high priority in research. The recently piloted FOCUS on Epilepsy intervention is designed to enhance informal support provided to people with epilepsy (PWE) through developing self-regulation skills of a family member or close friend ("supporters").

Methods: The content and processes of "FOCUS" were informed by 1) the peer-reviewed literature, 2) the needs of PWEs, their supporters, and epilepsy professionals as studied by the Epilepsy Foundation of Michigan and the University of Michigan MEW Network team. The program consists of a day-long workshop for PWE and their supporters, followed by telephone sessions for each participant with a coach. In these calls $(n=3)$, supporters develop and practice self-regulation skills. Through the process, they identify how 
to give stronger and more relevant assistance. Coaches help supporters develop strategies to meet their specific goals for improving the way in which they provide support; for example, using less directive language when communicating with the PWE. Evaluation data were collected from 17 of 19 participating PWEs and 14 of 19 participating supporters via telephone survey. The survey employed closed and open ended items at baseline and four months following program completion. Qualitative data were examined by paired t-test comparing mean scores from baseline to follow-up. Qualitative data were coded for themes and common responses. Results: Supporters were: spouses/partners (9), parents (3), siblings (3), adult children (2), and friends (3). Ages ranged from 23 to 82 $($ mean $=47) ; 15(71 \%)$ were women; 5 were African American and 15 Caucasian; 5 had a high school education or less; 8 had some college; 8 had a college degree or higher.

At follow-up, supporters reported more frequently using selfregulation skills to change how they provided support to their PWE $(\mathrm{p}=0.01)$, and most $(86 \%)$ reported meeting their self regulation goal in full or in part. In qualitative data, consistent themes among supporters were "greater understanding of epilepsy" and "awareness of more effective ways to support their PWE."

PWEs also reported improvements, for example, of the 11 PWE who cited something at baseline they would like to change about how their loved one provided support, $9(82 \%)$ indicated positive change at follow-up.

Of the 16 supporters interviewed at the completion of the program, 15 (94\%) would recommend the program to others.

Conclusions: Pilot findings suggest that an intervention focusing on self-regulation can generate important improvements in the ways family members and friends support a PWE. Further evaluation with a larger sample and longer follow-up period is warranted.

\subsection{4 \\ PREVALENCE AND PREDICTORS OF MOOD DISORDERS, ANXIETY DISORDERS, AND SUICIDE RISK IN WOMEN WITH EPILEPSY}

Sima Patel, N. Foldvary - Schaefer, L. Jehi, G. Tesar and A. Viguera (Neurology, Cleveland Clinic, Cleveland, $\mathrm{OH}$ )

Rationale: The prevalence of major depressive disorder (MDD), generalized anxiety disorder (GAD), and suicide risk (SR) is high in epilepsy patients. Studies suggest a higher prevalence of depression and anxiety in women with epilepsy (WWE). Our aim was to determine prevalence and predictors for MDD, GAD, and SR in WWE utilizing the Mini International Neuropsychiatry Interview (MINI).

Methods: 87 WWE in Cleveland Clinic Epilepsy Monitoring Unit were interviewed with the MINI. The MINI is a validated psychiatric diagnostic interview designed to assess for Axis I disorders based on the DSM-IV. Subjects also completed a menopause questionnaire, Quality of Life in Epilepsy-10 (QOLIE-10), European Quality of Life 5 (EQ-5), Liverpool Seizure Severity Scale (LSSS), Patient Health Questionnaire-9 (PHQ-9), and Generalized Anxiety Disorder-7 (GAD-7). Menopause was defined as cessation of menses for $>12$ months and $>60$ days of amenorrhea regardless of cause.

Results: Mean age was $40 \pm 14$ and 46\% (41) were employed. Mean age of epilepsy onset was $21 \pm 17$, monthly seizure frequency was 12 \pm 25 , number AEDs was $2.4 \pm 1$, and $93 \%$ (83) had focal epilepsy including 33\% (29) with temporal lobe epilepsy (TLE). Based on the MINI, the prevalence of Axis I disorders (lifetime or current) were: $31 \%$ (27) MDD, 25\% (22) anxiety disorder, 11\% (10) GAD, and 34\% (30) SR. Predictors of MDD were: Older age of epilepsy onset ( $p=$ 0.01 ), worse QOL on EQ-5 ( $\mathrm{p}=0.04)$, and more depressive symptoms on the PHQ-9 $(\mathrm{p}=0.001)$ and anxiety symptoms on GAD$7(\mathrm{p}=0.002)$ were associated with MDD. Predictors GAD were: Poor QOL on the QOLIE-10 (p=0.002) and EQ-5 $(\mathrm{p}=0.02)$, increased seizure severity on LSSS ( $\mathrm{p}=0.001)$, and more depressive symptoms on PHQ-9 $(p=0.02)$ as well anxiety symptoms on GAD-7 ( $p=$
0.007). Predictors of SR were: More than 2 AEDs $(p=0.04)$, poor QOL on QOLIE-10 $(\mathrm{p}=0.001)$ and EQ-5 $(\mathrm{p}=0.001)$, more depressive and anxiety symptoms [PHQ-9 $(\mathrm{p}=0.001)$; GAD-7 ( $\mathrm{p}=$ $0.002)]$, and a comorbid diagnosis of any anxiety disorder on the MINI $(p=0.001)$. There was no significant difference in the prevalence of MDD, GAD, or SR between premenopausal and menopausal WWE. However, premenopausal WWE were more likely to have a MDD diagnosis if unemployed $(p=0.03)$ or had TLE $(p=0.01)$, while for menopausal WWE, older age of epilepsy onset $(\mathrm{p}=0.03)$ was a significant predictor of MDD.

Conclusions: To our knowledge, this is the first study to investigate prevalence and predictors of Axis I disorders and suicide risk in WWE using a structured diagnostic interview. While the prevalence of MDD, GAD, and SR is high in WWE, menopausal status does not seem to be a significant predictor of Axis I disorders, although this requires confirmation in larger series. Predictors associated with MDD, GAD, and SR included poor QOL and high severity scores of current depression and anxiety symptoms. Additionally, older age of epilepsy onset and higher number of AEDs are important predictors of MDD and SR, respectively, in WWE. Larger studies exploring causative factors and burden of psychiatric co-morbidities in WWE are warranted.

\subsection{5 \\ CONCORDANCE OF NEUROPSYCHOLOGICAL DATA WITH VEEG AND NEUROIMAGING IN PRESURGICAL EVALUATION}

Y. Cukier, Sarah Schaffer and C. Harden (North Shore LIJ - Hofstra Medical School, Great Neck, NY)

Rationale: Neuropsychological data has been used in conjunction with VEEG recordings and neuroimaging to help lateralize seizure focus, and it has been previously demonstrated to increase presurgical predictive value with regard to lateralization. However, when the neurologic data are not strongly lateralized, the extent to which this is also reflected in the neuropsychological data has not been studied. The aim of this study was to examine the concordance between neuropsychological data and measures used to lateralize seizure focus, specifically ictal EEG, interictal EEG abnormalities, and brain MRI.

Methods: Thirty seven patients (mean age: 31.92 , median age: 31.00 , range: $45, \%$ female: $48.6 \%$ ) with a diagnosis of epilepsy who were being considered for resective surgery completed comprehensive presurgical evaluations that included results from 1) ictal VEEG, 2) interictal VEEG (electrographic abnormalities), 3) MRI, and 4) neuropsychological assessment. Results of each of these four diagnostic modalities were coded as "left lateralized," "right lateralized," or "nonlateralizing," regardless of localization. Frequency of concordance was examined using Chi square analyses. Results: Lateralization through neuropsychological data was most strongly concordant with interictal epileptiform abnormalities $(\chi 2$ (4, $37)=16.75, p<.01)$. In contrast, neuropsychological data was not found to be significantly concordant with either ictal VEEG or neuroimaging, although the former approached significance.

Conclusions: These results suggest that interictal activity is exerting a greater effect on neurocognitive functioning than actual seizures and/or structural abnormalities in the brain. The primary goal of epileptologists is to stop seizures in patients with epilepsy, while less emphasis is placed on identification and/or reduction of interictal epileptiform activity. However, given the current findings demonstrating a lateralized association between EEG interictal physiologic dysfunction and decreased performance on neuropsychological testing, epileptologists should consider whether reducing interictal activity should be added to the treatment goals. 


\subsection{6}

THE UTILITY OF THE MEYERS NEUROPSYCHOLOGICAL SYSTEM FOR USE WITH SPANISH SPEAKING PRESURGICAL EPILEPSY PATIENTS

Yolanda Leon ${ }^{1,2}$, S. R. Benbadis ${ }^{2}$ and J. J. Sesta ${ }^{3}\left({ }^{1}\right.$ Neuropsychology, Pediatric Eiplepsy \& Neurology Specialists, PA, Tampa, FL; ${ }^{2}$ Neurosciences, USF/TGH Epilepsy Treatment Program, Tampa, FL and ${ }^{3}$ Medical Psychology, Suncoast Neuropsychology, LLC, Baton Rouge, LA)

Rationale: Neuropsychologists strive to ensure that their patients receive a linguistically, culturally, and clinically competent evaluation (Judd et.al, 2009). The estimated Hispanic/Latino population of the United States as of 2010 is 50.5 million, and these speakers of Spanish constitute $16 \%$ of the total U.S. population(U.S. Census Bureau, 2010). To date, there are only few, limited instruments available for administration of a comprehensive neuropsychological test battery that can be administered in Spanish with Spanish Speaking patients. Consequently, Spanish speaking patients with Epilepsy have presented a challenge when referred for presurgical neuropsychological evaluation. The neuropsychologist faces the challenge of determining what available measures in Spanish should comprise the battery of tests for presurgical assessment. The Meyers Neuropsychological System (MNS) is a system of neuropsychological assessment that utilizes the integration of a modified Rohling Interpretive Method and a profile matching approach, allowing comparison of patient group profiles with one's individual patient. The battery of tests that comprise the comparison groups are well validated and widely accepted instruments that are translated into Spanish. The present case review aims to demonstrate the utility of the MNS Spanish Form for presurgical neuropsychological assessment of monolingual Spanish-Speaking patients with documented temporal lobe epilepsy (TLE).

Methods: The MNS was administered as part of a comprehensive battery for Spanish Speaking presurgical Epilepsy patients who were non-English, monolingual speakers of Spanish. The patients included one male patient and one Female patient. Both patients had TLE confirmed by EEG-video monitoring, MRI, and PET scan. One was a patient with Right TLE, whereas the other was a patient with Left TLE. Mean age was 35. Both patients were administered a comprehensive neuropsychological assessment as part of their presurgical evaluation and the MNS was used to generate a comparison data base with which to compare each patient's profiles to profiles of patient groups that had right TLE or left TLE. Results: The MNS was used to compare the domain profiles of each patient to the comparison groups of patients with, right TLE, left TLE, Left learning disorder, and Right learning disorder. There were strong correlations between the patient's profiles and that of the normative samples for patients with these disorders.

Conclusions: The results of the present pilot study indicate that the MNS is a viable system for use as part of a comprehensive battery for assessing presurgical epilepsy patients who are monolingual speakers of Spanish. The scoring system provides for the assessment of many of the domains of behavior and cognition that are dependent on adequate brain functioning, uses regression based norms, has built in symptom validity check measures, and has comparison groups based on more than 8,000 patients with a variety of central nervous system disorders including epilepsy.

\subsection{7 \\ GENDER DIFFERENCES IN NON-EPILEPTIC SEIZURE SEMIOLOGY AND RISK FACTORS}

Alissa A. Thomas and K. A. Bujarski (Neurology, Dartmouth Hitchcock Medical Center, Lebanon, NH)

Rationale: Non-epileptic seizures (NES) are paroxysmal events of altered behavior that outwardly resemble epilepsy but are caused by psychiatric disease. Diagnosis of suspected NES can be made in the outpatient clinic prior to video EEG monitoring by identification of specific NES risk factors and specific elements of seizure semiology from the clinical history. Since psychiatric disease often presents differently in women and men, we questioned whether genderspecific differences exist in NES risk factors and semiology. Such differences can be used to improve the outpatient diagnosis of suspected NES.

Methods: Male and female patients were identified from a video EEG monitoring database. Seizure semiology for each patient was analyzed de novo and categorized into one of three established semiology clusters. In addition, patients' medical records were reviewed and data on possible risk factors was obtained.

Results: 42 patients were included in the analysis ( 26 women, 16 men) who ranged in age from 19 to 78 (mean 40.5 years). Among female patients, seizure semiology was classified as major motor in $30.7 \%$, minor motor in $30.7 \%$, and non-motor in $23.8 \%$. Among male patients, seizure semiology was classified as major motor in $43.8 \%$, minor motor in $31.3 \%$, and non-motor in $25 \%$. There were no significant differences between men and women in any of the semiology clusters (major motor X2 $=0.27, \mathrm{p}=0.60$; minor motor $\mathrm{X} 2=0.1, \mathrm{p}=0.75$; and non-motor $\mathrm{X} 2=0.32, \mathrm{p}=0.57$ ). Analysis of NES risk factors showed significantly higher rate of reported physical/emotional/sexual abuse $(\mathrm{p}=0.002)$, pre-ictal headache $(p=0.02)$, and history of prior suicide attempt $(p=0.004)$ in female patients as compared to male.

Conclusions: Our results support the use of the same "semiology criteria" for the outpatient diagnosis of suspected NES in both female and male patients. In contract, our results support the use of variable "risk factor criteria" for making a diagnosis of suspected NES based on gender.

\subsection{8 \\ NUMBERS AND WORDS IN HUMAN BROCA'S AREA: ELECTRICAL BRAIN STIMULATION AND ELECTROCORTICOGRAPHY STUDY}

D. Banerjee, L. Demetri and Josef Parvizi (Stanford University, Stanford, CA)

Rationale: Broca's Area (BA) has been the focus of extensive research for its role in speech production. To gain new insight into its functional heterogeneity, we performed a combination of electrical brain stimulation (EBS) and electrocorticography (ECoG) on patients implanted with intracranial electrodes. Fusion of high resolution preop MR and post-op CT scans allowed us to precisely localize intracranial electrodes over different BA subregions in multiple subjects.

Methods: We used EBS to deliver electrical pulses between two electrodes while the patient read or repeated given sentences. Additionally, we recorded ECoG signals during a task in which patients read numbers, number-words, or non-number words aloud. For each electrode in BA, we compared the ECoG responses with the effect of EBS on the patient's ability to produce language.

Results: Our findings revealed that only a subset of stimulations within the anatomical boundaries of BA caused speech arrest, and only a subset of electrodes located within BA had broadband activity (i.e., $30-180 \mathrm{~Hz}$ ) activity during speech production. The EBS and ECoG results were significantly correlated. Our findings also revealed a heterogeneous pattern of electrophysiological responses in various parts of BA during articulation of different classes of stimuli. While an increase in broadband gamma activity preceded speech onset in some but not all BA subregions, the power of this response was greatest during word production and lowest during number production in most of the active BA electrodes.

Conclusions: These results suggest that 1) certain regions of BA are more essential for speech production than others; 2) there is significant overlap between EBS and ECoG data; and 3) distinct neural populations in BA may be semantically selective to the numerical vs. non-numerical content of speech. 


\subsection{9}

CO-EXISTING PSYCHOGENIC NON-EPILEPTIC SEIZURES AND EPILEPTIC SEIZURES IN AN EPILEPSY MONITORING UNIT POPULATION: INCIDENCE AND

\section{RELEVANCE}

Diane F. Thomas and A. Krumholz (University of Maryland Medical Center, Baltimore, MD)

Rationale: It is well described that psychogenic non-epileptic seizures (PNES) and epileptic seizures (ES) may co-exist in the same patient, but how to best define this co-existence and its incidence are debated.

Methods: We reviewed all epilepsy monitoring patients at the University of Maryland Medical Center over an 18 month period to assess the incidence of both PNES and ES co-existing in the same patient as documented by capturing both PNES and ES during the same admission. Patients who had more than one admission were counted only once. Patients who were characterized in our database as having both PNES and ES were identified and those cases were reviewed.

Results: Of our 256 total epilepsy monitoring patients, 167 (65\%) were female, and the mean age was 41 years (range 16-88, standard deviation 14.7). Of the total patients, $70(27 \%)$ had documented PNES during their admission, and 11 (15.7\%) of these PNES patients had co-existing epileptic seizures determined by video EEG during that same admission.

Conclusions: The percentage of co-existing PNES and ES captured on video EEG monitoring in our patients $(15.7 \%$ of all PNES patients) was higher than expected, and may relate in part to more aggressive seizure medication tapers to provoke events, thereby unmasking more epileptic seizures in our patients. In addition, embellished auras mimicking PNES cannot absolutely be ruled out in a small number of cases. Still, the incidence of co-existing PNES and ES in such a high percentage of patients is notable and suggests that caution is warranted when tapering medication in some patients with PNES.

\section{Behavior/Neuropsychology/Language: Pediatrics}

\subsection{0}

\section{HIGH GAMMA MAPPING OF COGNITIVE TASKS WITH} ECOG IN CHILDREN

Douglas F. Rose ${ }^{1}$, H. Fujiwara ${ }^{1}$, J. A. Wilson ${ }^{3}$, H. Greiner ${ }^{1}$, L. Rozhkov ${ }^{1}$, J. Buroker ${ }^{1}$, R. Arya ${ }^{1}$ and F. T. Mangano ${ }^{2}\left({ }^{1}\right.$ Neurology, Cincinnati Children's Hospital Medical Ctr, Cincinnati, OH; ${ }^{2}$ Neurosurgery, Cincinnati Children's Hospital Medical Ctr, Cincinnati, $\mathrm{OH}$ and ${ }^{3}$ Neurosurgery, University of Cincinnati, Cincinnati, $\mathrm{OH}$ )

Rationale: Direct cortical electrical stimulation (DCS) during electrocorticographic $(\mathrm{ECoG})$ recordings has been the gold standard for functional mapping during Phase II epilepsy surgery evaluations in adults. In children, the procedure duration may exceed their ability to cooperate. Recent studies have shown utility in mapping highgamma frequency $(70-150 \mathrm{~Hz})$ cortical activity changes during functional tasks. We hypothesized that measuring changes in highgamma ECoG during functional tasks would show results similar to DCS.

Methods: Eleven patients, 6-20 years old, had subdural ECoG recorded during Phase II epilepsy surgery evaluation. Grid placements were based on Phase I presurgical evaluation, but in all patients this included coverage of the posterior frontal lobe, temporal lobe, and parietal lobe. Between 64 and 128 channels were recorded; grids were placed on the left hemisphere for 6 subjects and right for 5. ECoG was recorded simultaneously with Stellate amplifiers for clinical analysis and g.USBamp amplifiers (g.tec, Austria) sampled at
$1200 \mathrm{~Hz}$ with no filtering applied. BCI2000 with the SIGFRIED system (Brunner et. al, 2009) was used at the bedside to analyze realtime changes in cortical activity in the $70-150 \mathrm{~Hz}$ power band. A resting baseline of $6 \mathrm{~min}$. was compared to finger movement, tongue movement, lateral eye movements, passive stroking of the hand, listening to stories, music, and white noise, and picture naming, performed by the patients both overtly and covertly. Ten of the patients subsequently had conventional DCS with stimulation across pairs of adjacent electrodes to elicit motor movements and to inhibit picture naming to map language critical regions. Median nerve somatosensory evoked responses (MNSSEP) were done to map central sulcus. The electrodes showing activation with BCI were compared to DCS and MNSSEP findings.

Results: Individual electrodes showed high-gamma changes during specific tasks, whereas DCS stimulation was between pairs of electrodes. MNSSEP showed phase reversal at pairs of electrodes, whereas hand sensation activation and motor activation were compared to arrive at central sulcus. Active tasks had specific instructions (finger movement, tongue movement, or lateral eye movement) and therefore elicited changes on a small subset of electrodes, compared to DCS which often elicited combined motor movements including primarily arm, hand, and face movement, and at times fingers, tongue, or visual gaze. Real-time mapping detected changes in cortical activity at common electrodes to DCS in $6 / 8$ patients for hand motor, $5 / 6$ patients for tongue/face, $2 / 2$ patients for language production, $2 / 2$ patients for lateral eye movements and $2 / 2$ for central sulcus.

Conclusions: Changes in high gamma power during functional tasks may be useful for pediatric patients particularly for localizing somatomotor regions. The current results for language concordance, though favorable, did not involve enough patients who also had language DCS for comparison.

\subsection{1 PHYSICIANS' PERSPECTIVES IN UTILIZING NEUROPSYCHOLOGICAL SERVICES IN PEDIATRIC PATIENTS WITH EPILEPSY \\ Jeffrey B. Titus, C. Hovinga, K. Kime, N. Bower, F. Perkins and D. Clarke (Dell Children's Comprehensive Epilepsy Program, Dell Children's Medical Center of Central Texas, Austin, TX)}

Rationale: Neuropsychology is a critical tool in managing pediatric patients with epilepsy, and awareness of the indications for neuropsychology is important for appropriate patient referrals. However, there is currently little information on physician understanding of the benefits of neuropsychology for this population. This study assessed physician perspectives on the benefits of neuropsychology in managing pediatric patients with epilepsy. Methods: A survey was constructed to assess the familiarity of healthcare providers with the indications for neuropsychological assessment in the management of pediatric patients with epilepsy. Information including physician age, number of years of practicing medicine, subspecialty, practice affiliation, and patient population were also collected. The survey was sent to health care providers in Central Texas specializing in pediatrics, family medicine, neurology, and neurosurgery. Data was summarized using descriptive statistics. Results: $146(\sim 10 \%)$ responded to the survey. $57.5 \%$ were pediatric, $12.6 \%$ were neurology/neurosurgery, and $29.9 \%$ were family medicine physicians. Gender was equally represented ( $48.2 \%$ male) and the majority were Caucasian (74\%). Respondents saw a median of 1 new seizure case per month and a median of 2.5 pediatric epilepsy patients per month. Half had been practicing medicine for $<15$ years. Most clinicians indicated that neuropsychology was useful for planning educational services $(90 \%)$, and a majority agreed that neuropsychology was helpful in determining how seizures affect functioning (83\%). Only $67 \%$ reported that neuropsychology was helpful in managing the medical treatment of epilepsy, and only $65 \%$ indicated neuropsychology was essential to epilepsy surgery workup. 
On most items, physicians either agreed or were unsure about the benefits of neuropsychology, with few disagreeing that it is helpful. A higher rate of neurologists/neurosurgeons disagreed with neuropsychology's role in helping manage the medical treatment of epilepsy (29\%), and pediatricians/family medicine physicians were much less sure of neuropsychology's role in surgery planning $(36.4 \%$ and $34.3 \%$, respectively). Pediatricians/family medicine physicians reported higher rates of uncertainty about the benefits of neuropsychology for improving quality of life and functional outcomes (21.7\% and $22.2 \%$, respectively).

Conclusions: Physicians generally agree that neuropsychology is helpful for planning educational services, and their responses suggest general agreement that neuropsychology can help identify the impact of seizures on level of functioning and help improve quality of life. Disagreement and uncertainty exists about neuropsychology's role in treatment planning, with neurologists/neurosurgeons expressing more disagreement that neuropsychology can help in guiding medical decision making. Pediatricians/family medicine physicians are more likely to endorse uncertainty about the role of neuropsychology in surgical planning. These results identify opportunities to educate subspecialists about the benefits of neuropsychology for children with epilepsy.

\subsection{2 \\ RISK FACTORS FOR PEDIATRIC NON-EPILEPTIC SEIZURES (NES): PSYCHIATRIC AND MEDICAL COMORBIDITIES}

Sigita Plioplys ${ }^{1}$, J. Doss ${ }^{2}$, P. Siddarth ${ }^{3}$, D. Birt ${ }^{3}$, B. Bursch ${ }^{3}$, T. Falcone $^{4}$, M. Forgey ${ }^{3}$, C. LaFrance ${ }^{5}$, D. Weisbrot ${ }^{6}$, M. Willis ${ }^{5}$ and R. Caplan ${ }^{3}\left({ }^{1}\right.$ Child and Adolescent Psychiatry, Anne and Robert H.Lurie Children's Hospital, Chicago, IL; ${ }^{2}$ Minnesota Epilepsy Group, St. Paul, MN; ${ }^{3}$ David Gefen School of Medicine, UCLA, Los Angeles, $\mathrm{CA} ;{ }^{4}$ Neurological Institute, Cleveland Clinic, Cleveland, OH; ${ }^{5}$ Rhode Island Hospital, Warren Alpert Medical School of Brown University, Providence, RI and ${ }^{6}$ Stony Brook University Medical Center, Stony Brook, NY)

Rationale: Previous research demonstrated high rates of psychopathology in NES youth, but related epilepsy, medical illness, and trauma risk factors have not been systematically studied. This study examined the specificity of psychiatric, epilepsy, medical, and trauma variables and the age of their occurrence in NES youth by comparing to their siblings.

Methods: This multisite study included 48 NES youth and 33 siblings, aged 8-18 years. A video EEG confirmed an NES diagnosis. A demographic questionnaire on the child's medical/epilepsy, psychiatric, family, and educational history was completed by parents. Each child and parent underwent a structured psychiatric interview about the child that yielded DSM-IV diagnoses and information on traumatic experiences. Each child had standardized cognitive, language, and achievement testing. Probands and siblings were compared using a mixed model with family as a random effect for continuous variables. T-tests and chi-square analyses (Fisher's exact tests for measures with cell frequencies $<5$ ) examined between group differences in the distribution of continuous and categorical variables (deficit groups, psychiatric diagnosis, epilepsy), respectively.

Results: NES youth were older than siblings (14.9 years (SD 2.6) vs. 13.6 years (SD 2.3). DSM-IV diagnoses were more prevalent in probands $(100 \%)$ than siblings $(81.8 \%)(p=.002)$ with significantly higher rates of Conversion $(\mathrm{p}<.0001)$, Anxiety $(\mathrm{p}<.0001)$, Depression $(\mathrm{p}=.007)$, PTSD $(\mathrm{p}=.02)$, Bipolar $(\mathrm{p}=.03)$, and Tic $(\mathrm{p}=.03)$ disorders. Compared to siblings, significantly more probands had multiple psychiatric diagnoses $(\mathrm{p}<.0001)$, experienced crime/violence $(p=.04)$ and sexual abuse $(p=.05)$. Within the NES group, those with epilepsy as compared to those without epilepsy had a greater number of psychiatric diagnoses $(\mathrm{p}=.05)$, learning disorder $(\mathrm{p}=.04)$, and past traumatic experiences $(\mathrm{p}=.04)$. No differences were found between these two groups on child's age, age of NES onset, cognitive or achievement scores, age of first psychiatric diagnosis, or presence of other types of psychiatric diagnosis.

Significantly more NES probands than siblings had epilepsy $(72.9 \%$ vs. $0 \%, p<.0001)$; additional central nervous system illnesses $(\mathrm{p}<$ $.0001)$, general medical problems $(\mathrm{p}=.02)$; and were taking medications $(\mathrm{p}=.0009)$. Probands also had significantly more life time emergency room (ER) visits $(\mathrm{p}<.0001)$ and hospitalizations $(p<.0001)$ compared to siblings; the number of NES ER visits was negatively correlated with the age of NES onset $(\mathrm{p}=.0005)$. Although probands missed significantly more days of school $(p=.0005)$, there was no significant difference in the rates of special education. Conclusions: This is the first study to identify risk factors for pediatric NES in a large sample of NES youth compared and control subjects. Our findings reflect a biopsychosocial model of psychopathology and show that multiple psychiatric diagnoses, increased past and present use of intensive medical services, epilepsy and other CNS disorders, violent trauma, and missed school days are risk factors for pediatric NES.

\subsection{3 \\ MAGNETIC SOURCE IMAGING AND LANGUAGE OUTCOME AFTER PEDIATRIC EPILEPSY SURGERY Elizabeth Adams, F. J. Ritter and W. Zhang (Minnesota Epilepsy Group, St Paul, MN)}

Rationale: Children seem to be at less risk than adults for measurable and lasting language decline after epilepsy surgery. This may be due to increased plasticity of the young brain, relatively shorter duration of epilepsy, improved accuracy of pre-surgical diagnostics, and a variety of other contributing factors.

Magnetoencephalography/magnetic source imaging (MEG/MSI) is one such pre-surgical diagnostic technique that facilitates prediction of post-surgical language outcome. This study describes patterns of relevant clinical features among patients with non-left language dominance on MSI, and reports post-surgical language outcome in relation to MSI findings.

Methods: Data from 34 pediatric epilepsy patients (ages 3-17 years) was collected via retrospective record review. All patients were being considered for epilepsy surgery, due to focal intractable seizure disorder. All patients produced valid language lateralization data during the receptive language (CRM) task of the MSI protocol. Twenty patients had primarily left-hemisphere seizure focus; 14 patients had primarily right-hemisphere seizure focus. A subset of 20 patients from this sample underwent focal resective epilepsy surgery (10 left, 10 right) and post-surgical neuropsychological evaluation. Patients who underwent $\mathrm{CC}$ or hemispherectomy were excluded from the post-surgical outcome portion of the analysis.

Results: MSI data demonstrated left language dominance for 16 patients (47\%), right language dominance for 6 patients $(18 \%)$, and bilateral language for 12 patients (35\%). Of the 18 patients who showed atypical (non-left) language dominance, $15(83 \%)$ had seizure onset $<5$ years of age; $14(78 \%)$ had duration of epilepsy $>4$ years; $11(61 \%)$ had primarily left hemisphere seizure focus. Among the subset of 20 patients who underwent focal epilepsy surgery, only 3 demonstrated $>15$ point decline in Verbal Comprehension Index (VCI) after surgery. Given significant confounding factors (multiple surgeries for 2 and psychosis for 1), these 3 cases were excluded from the subsequent analyses. Duration of epilepsy and age of seizure onset were significantly correlated with pre and post-surgical VCI. 12 of the remaining 17 patients were classified as bilateral or right hemisphere dominant for language on MSI. 4 of the 17 had focal resection in the MSI-classified language-dominant hemisphere and none of these patients demonstrated language decline after surgery. Conclusions: Preliminary data analysis implicates younger age at seizure onset, longer duration of epilepsy, and left hemisphere seizure focus as prominent characteristics in the majority of patients with bilateral or right-dominant language representation on MSI. Analysis 
of post-surgical outcome measures confirms previously documented findings to suggest that only a relatively low proportion of a pediatric sample demonstrates significant language decline after focal resective epilepsy surgery, regardless of side of surgery, and in spite of presurgical diagnostic findings to suggest at least some language representation in the to-be-resected hemisphere.

\subsection{4 \\ THE IMPACT OF MATERNAL ANXIETY ABOUT EPILEPSY ON RESTRICTIONS IN CHILDHOOD ACTIVITIES \\ Karen Evankovich ${ }^{1}$, R. J. Schultz ${ }^{2}$ and M. L. Chapieski ${ }^{1}$ ( ${ }^{1}$ Psychology-Neurology, Baylor College of Medicine, Houston, TX and ${ }^{2}$ Neurology, Baylor College of Medicine, Houston, TX)}

Rationale: Parental anxiety has been reported to affect the quality of life and behavioral development of children with epilepsy. The purpose of this study was to examine the relationship between maternal anxiety about epilepsy and restrictions on childhood activities.

Methods: The subjects were seventy-seven English-speaking mothers and their children with epilepsy who were at least 6 years of age and had Full Scale IQ scores of $\geq 70$. Mothers provided demographic and completed the Hague Restrictions in Childhood Epilepsy Scale (HARCES). Maternal anxiety about epilepsy was assessed with the Parental Anxiety About Epilepsy Questionnaire (PAE). Intellectual potential (FSIQ) was assessed with an ageappropriate Wechsler Intelligence Scale. Data were analyzed using descriptive statistics and stepwise multiple regression. The Baylor College of Medicine Institutional Review approved this study. Results: The children with epilepsy were $48 \%$ female with a mean age of $12.25(\mathrm{SD}=3.2)$ and mean intelligence of $92.36(\mathrm{SD}=12.74)$. Higher HARCES scores, indicating increased restriction in childhood activities, were associated with higher levels of maternal anxiety about epilepsy $(\mathrm{r}=0.513, \mathrm{p}<.0001)$ and shorter duration of seizure disorder $(r=-.345, p=.001)$. Maternal anxiety accounted for $26.3 \%$ of the variance $(\mathrm{p}<.0001)$ in restriction in childhood activities with duration of seizure disorder accounting for an additional $7.4 \%$ variance $(p=.006)$. Maternal anxiety about epilepsy was associated with the number of anticonvulsant medications $(r=.313, p<.006)$ and lower maternal education $(\mathrm{r}=-.289, \mathrm{p}=.005)$.

Conclusions: Maternal anxiety about epilepsy is associated with greater maternal restrictions on the activities of children, particularly in the early stages of the diagnosis. The number of medications appears to affect a mother's perception of the seriousness of the disorder and, thereby, her anxiety. This finding suggests that improved and, perhaps, ongoing education about epilepsy for mothers of newly diagnosed patients has the potential to reduce maternal anxiety and the ensuing restrictions on childrens' activities, thereby promoting quality of life for both the children and their mothers.

\subsection{5 \\ ARITHMETIC SKILLS IN CHILDREN WITH EPILEPSY: THE ROLE OF ATTENTION, INHIBITION, AND MEDICATION}

Lynn Chapieski $^{1}$, K. Hopkins ${ }^{1}$, C. Johnson ${ }^{2}$ and M. Hiscock ${ }^{2}$

( ${ }^{1}$ Department of Pediatrics, Baylor College of Medicine, Houston, TX and ${ }^{2}$ Department of Psychology, University of Houston, Houston, TX)

Rationale: Children with epilepsy are at risk for poor academic performance. Math may be a particularly problematic subject because good performance is dependent on normal attention and executive control, abilities that are weak in many children with seizure disorders. We investigated the impact of sustained attention and inhibition on arithmetic performance. We also examined the contribution of epilepsy-specific factors to specific calculation errors as well as overall calculation performance.
Methods: 77 patients with intractable seizures, ages 8 to 18 years, were administered the Applications and Math Computations subtests from the Kaufman Test of Educational Achievement (K-TEA). The patients were also administered a test of intellectual functioning (WISC or WAIS), a continuous performance test (CPT) and the Color-Word Interference-Inhibition (DKEF-IN) and Color-Word Interference /Switching (DKEF-SW) subtests of the Delis Kaplan Executive Functions System. Each error on the computation test was coded as a knowledge error, a procedural slip, a skipped problem or misread operation. Errors were only coded if they occurred on items within the patient's range of age-expected competence.

Results: The two KTEA math tests were highly correlated; consequently, only the computation subtest (Comp) scores were used in the analyses. Using multiple regression analysis, KTEA Comp scores were predicted with variables from the CPT and DKEF. Several CPT and DKEF variables were significantly correlated with math performance, but only DKEF-IN met the criteria for inclusion in the regression model, and it accounted for $24 \%$ of the variance in Comp scores. When IQ was forced into the model, it accounted for $39 \%$ of the variance, but inhibition continued to explain $6 \%$ of the variance. COMP errors were primarily due to lack of knowledge $(62 \%)$. Inattention also contributed in the form of procedural slips (24\%), misread operators (7\%) and skipped problems (7\%). Epilepsyspecific factors-- duration of disorder, seizure frequency over the past 3 months, and number of AEDs--were not directly predictive of math performance. However, the number of AEDs was related to the number of procedural slips $(\mathrm{r}=.23)$ and the number of misread operators, $(\mathrm{r}=.21)$.

Conclusions: The biggest contributor to poor math performance in children with epilepsy is level of intellectual functioning. Ability to inhibit a competing response makes an additional but small contribution. Error analysis, similarly, revealed that the predominant error in math calculations is knowledge-based rather than attentional. Nevertheless, $38 \%$ of the errors were attentional, and patients who were taking higher numbers of AEDs were more likely to make those errors.

\subsection{6 \\ HOME-BASED COMPUTERIZED INTERVENTION FOR WORKING MEMORY IN PEDIATRIC EPILEPSY}

Madison Berl, E. Fisher, L. Sepeta, L. Zimmaro and T. Tsuchida

(Children's National Medical Center, Germantown, MD)

Rationale: Executive function impairments are common comorbidities in children with epilepsy. These impairments have been associated with decreased quality of life. A computerized cognitive training program was developed to improve working memory skills and has demonstrated efficacy in children with ADHD. We present preliminary results using this computerized cognitive training program in children with epilepsy.

Methods: Twenty-one children with well controlled epilepsy (ages 815; mean $=11.7$; 6 female) completed training and received pre- and post-neuropsychological assessment. Successful completion criteria is a minimum of 20 sessions within an 8-week timeframe. Entry criteria was elevated parent rating of working memory skills $(T>60)$ but IQ $>80$. Patients had relatively "smooth sailing" epilepsy being well controlled but experiencing cognitive problems; epilepsy was type was localization-related, absence, or benign rolandic with no major abnormality on MRI and one AED. Patients were randomized to a "hi" or "low" dose. Hi dose was the standard Cogmed RM program of 12 game-like activities with success-adapted trials. Low dose includes all components of hi dose but, difficulty is consistently low intensity (span of two items). Outcome measures included parent ratings and direct measures of working memory skills. Descriptive and repeated measures MANOVA were conducted to examine differences in treatment group across measures of working memory skills. Effect sizes were also calculated given the small sample sizes. 
Results: There were no differences between treatment groups for age or IQ. There was a main effect of Time $(p<.001)$ with improved parent ratings of working memory (Hi Dose mean fell below clinical significance) and better performance on direct measures of verbal and visual short term memory. There was an interaction of Time by Condition $(\mathrm{p}<.05)$ such that improvements for verbal and spatial short term memory were greatest for the Hi Dose condition (See Figure). Effect sizes consistently were larger for Hi Dose compared to Low Dose, and ranged from small to large (See Figure). Conclusions: Preliminary results demonstrate that Cogmed significantly improved verbal and visual short term memory and everyday working memory skills in children with pediatric epilepsy. Although the Hi Dose was most effective, it appears that the Low Dose also had moderate treatment effects. Future work will investigate further which patients demonstrate the greatest gains to elucidate what factors may be important for optimizing treatment. In addition, another follow-up assessment at 3 months post treatment is underway and reliable change indices will be calculated to determine more rigorously if observed changes are more than might be expected on test-retest. This is the first study in children with epilepsy to demonstrate the effects of a non-pharmacological treatment for a cognitive comorbidity associated with epilepsy. It is feasible and may be effective at both hi and low dose. A low dose option may be beneficial to maintain a child's motivation related to a greater number of success trials.
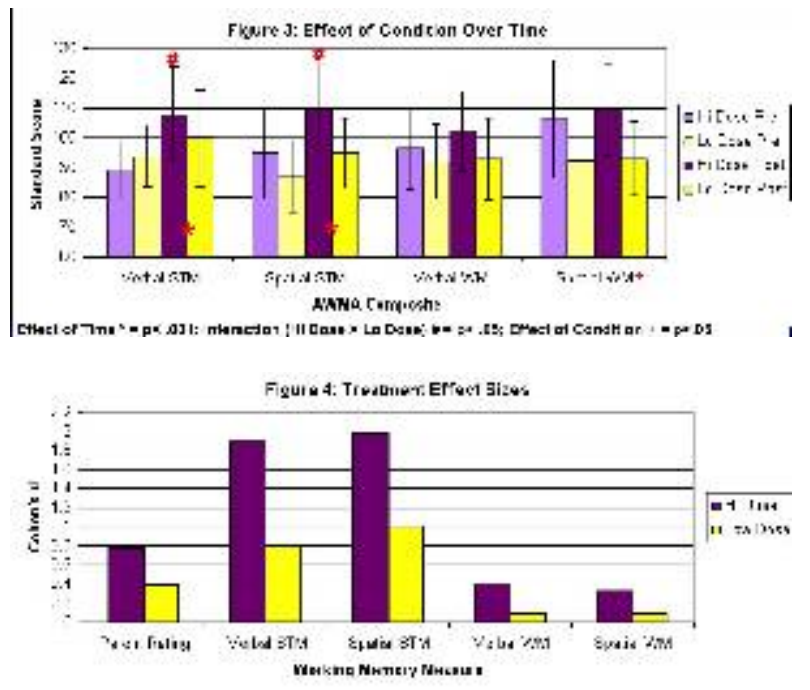

\subsection{7 \\ BIOLOGICAL UNDERPINNINGS OF SOCIAL SKILL DEFICITS IN CHILDREN WITH EPILEPSY}

Prabha Siddarth $^{1}$, J. Levitt ${ }^{2}$, R. Ly ${ }^{2}$, S. Gurbani ${ }^{3}$, R. Sankar ${ }^{4}$ and R. Caplan ${ }^{1}\left({ }^{1}\right.$ Semel Institute for Neuroscience and Human Behavior, UCLA, Los Angeles, CA; ${ }^{2}$ Child Psychiatry, UCLA, Los Angeles, $\mathrm{CA} ;{ }^{3}$ Neurology, Kaiser Permanente, Anaheim, CA and ${ }^{4}$ Pediatric Neurology, UCLA, Los Angeles, CA)

Rationale: Poor social skills are consistently found in pediatric epilepsy. Subtle to mild cognitive, academic, and linguistic/ communication deficits; stigma; and bullying are associated with these social difficulties. Similar findings in pediatric central nervous system disorders (head trauma, neurofibromatosis, and cancer) imply a role for biological factors. Therefore, this study compared the relationship between social skills and fronto-temporal volumes (FTC) in children with epilepsy to matched healthy controls (HC).

Methods: 47 children with localization related epilepsy with complex partial seizures (CPS), 24 children with absence epilepsy (CAE), and $36 \mathrm{HC}$ subjects underwent MRI scans at 1.5T. Tissue was segmented and frontal parcellations of inferior frontal gyrus (IFG), orbital frontal gyrus (OFG), dorsolateral prefrontal cortex (DLPFC)), and temporal lobe (TL) gray and white volumes (GMV, WMV) were computed. Parent Child Behavior Checklist provided social problems and peer interaction scores. A social composite $\mathrm{Z}$ score was constructed by taking an average of the $\mathrm{z}$-transformed peer interaction and social problems scores. Social problems scores were inverted so that increasing scores represented fewer problems. ANCOVAs, controlling for age and gender, determined group differences in social scores and FTV. General linear models, with FTV as the dependent variables and group, social score, and interaction of group and social scores as predictors, were estimated to determine group differences in the relationships between FTV and social scores.

Results: Significantly higher mean social problem scores $(\mathrm{p}<.0001)$ and lower peer interaction scores $(\mathrm{p}=.0001)$ were observed in the epilepsy groups compared to the $\mathrm{HC}$ group but there were no significant differences on these scores between the epilepsy groups and on FTV between the three groups. The social $\mathrm{Z}$ score correlated significantly and negatively with DLPFC GMV $(r=-.45, \mathrm{p}<.02)$ and TL GMV $(\mathrm{r}=-.51, \mathrm{p}<.006)$ in the HC but not in the CPS and CAE groups. There was a significant group $\mathrm{x}$ social score interaction for TL GMV $(\mathrm{F}(2,89)=4.19, \mathrm{p}<.02)$ and a trend for DLPFC GMV $(\mathrm{F}(2$, $89)=2.66, \mathrm{p}<.08)$. Smaller left $(\mathrm{t}=2.4, \mathrm{p}<.03)$ and right TL GMV $(\mathrm{t}=2.9, \mathrm{p}<.005)$ were significantly associated with higher social $\mathrm{Z}$ scores in the HC. In the CPS group, larger left $(\mathrm{t}=2.32, \mathrm{p}<.03)$ and right DLPFC GMV $(\mathrm{t}=2.78, \mathrm{p}<.02)$ were significantly related to higher social $\mathrm{Z}$ scores. Although not statistically significant, like the CPS group, the CAE group showed a positive association of social scores with DLPFC and TL GMV. The peer interaction score alone accounted for these findings.

Conclusions: This is the first study to demonstrate that poor peer interaction in children with epilepsy with average intelligence reflects structural abnormalities in brain regions involved in executive function, language, and social communication. Studies on larger samples are needed to determine if the deficits in executive function, language, and social communication in children with epilepsy drive this finding.

\subsection{8 \\ HIPPOCAMPUS INTEGRITY MAY NOT BE NECESSARY FOR SEMANTIC MEMORY: EVIDENCES FROM CHILDREN WITH TEMPORAL LOBE SCLEROSIS}

Patricia Rzezak $^{1}$, C. A. Guimarães ${ }^{2}$, D. Fuentes ${ }^{1}$, M. M. Guerreiro ${ }^{2}$ and K. D. Valente ${ }^{1}$ ( ${ }^{1}$ Psychiatry Institute - HCFMUSP, Sao Paulo, Brazil and ${ }^{2}$ Neurology Department - UNICAMP, Campinas, Brazil)

Rationale: In one of the first attempts to investigate the semantic memory of children with TLE, our group demonstrated that children with mesial temporal sclerosis (MTS) had worse performance in this cognitive function than healthy children (Rzezak et al., 2011). The role of the hippocampus in episodic memory is well established. It remains to be described whether the hippocampus also have an essential part in the semantic memory of children with TLE. Our hypothesis is that patients with MTS would not have worse performance than non-MTS patients in semantic memory tests. In order to test this hypothesis, we evaluated semantic and episodic memory deficits in children with mesial temporal sclerosis (MTS) compared to non-MTS children) Thus, the aim of this study was to analyze semantic and episodic memory deficits in children with mesial temporal sclerosis (MTS) compared to non-MTS children. Methods: For this purpose, 19 consecutive children with MTS and adolescents, mean age of 11.46 years (range: $8-16, \mathrm{SD}: \pm 2.06$ ) and a mean estimated IQ of 97.79 (range: $80-135$, SD: 13.16) were evaluated and their performances in five tests for episodic memory (evaluating short and long-term memory and learning for verbal and visual stimuli) and in four tests for semantic memory was compared to those of 17 non-MTS children (temporal dysplasia, temporal tumor, temporal tuber, temporal cysts and temporal cavernoma) with a mean age of 12.12 (range: $8-16, \mathrm{SD}: \pm 2.47$ ) and a mean estimated 
IQ of 101.47 (range: $83-122$, SD: \pm 13.48 ). Patients' performances on memory tests were compared with ANCOVA using age as a covariate.

Results: Patients with MTS had worse performances in immediate verbal episodic contextualized memory (Story Memory I, p:0.04 and $\mathrm{d}=0.75$ ), immediate visual episodic contextualized memory (Design Memory, p:0.012 and $\mathrm{d}=0.95)$, and in verbal and visual learning (Verbal and Visual Learning I, p:0.010, $\mathrm{d}=0.97$ and p:0.040, $\mathrm{d}=0.78$ ). Patients with MTS and non-MTS did not differ in any semantic memory test.

Conclusions: Our data confirm the role of the hippocampus in contextualized immediate verbal and visual memory and in learning abilities as patients with MTS had a worse performance than nonMTS patients in these neuropsychological testes. By the other hand, it does not speak in favor of the role of hippocampus in semantic memory. As previously demonstrated, children with MTS had worse performance in semantic memory compared to healthy controls. It may be hypothesized that other variables, besides the structural damage to the hippocampus, can influence the presence of this type of deficits in childhood MTS. One possible explanation of our findings is the participation of the lateral temporal structures in semantic memory.

\section{Behavior/Neuropsychology/Language: All Ages}

\subsection{9 \\ EXPLORING MECHANISMS OF SELF-CONTROL IN EPILEPSY - MODULATION OF EPILEPTIC SPIKES BY EMOTION AND RELAXATION \\ Siegward M. Elsas ${ }^{1,2}$, G. White ${ }^{1}$, G. Navarro ${ }^{1}$ and B. Oken ${ }^{1}$ $\left({ }^{1}\right.$ Neurology, Oregon Health and Science University, Portland, OR and ${ }^{2}$ Neurology and Research, Ita Wegman Klinik, Arlesheim, Switzerland)}

Rationale: Various behavioral interventions have shown promise in improving seizure control for epilepsy patients. Frontotemporal cortex is the most frequent focus of epileptic seizures and serves in emotion processing and decision making. A potential role of stress or emotions as seizure triggers has often been proposed with little evidence.

Methods: The potential of emotions to function as seizure triggers, and the effect of relaxation on epileptic spikes were explored in 14 patients with focal epilepsy. 32 EEG recordings were obtained under conditions of evoked fear, anger or neutral emotion. Each emotional condition was followed by relaxation and a washout of 5 minutes each. Epileptic spikes in each segment were manually counted under blinded conditions. In addition to subjective reports of stress and drowsiness, physiologic measurements of continuous noninvasive blood pressure, ECG heart rate, respiration rate, temporal EEG theta power and facial EMG were obtained to assess physiologic changes during emotional stress and relaxation states.

Results: Epileptic spikes increased during the fear condition as compared to neutral emotion $(\mathrm{p}=0.013)$, and also increased during the relaxation technique as compared to baseline $(p=0.05)$.

Decreases in blood pressure z scores, respiration rate and facial EMG all confirmed an effective relaxation. Physiologic changes during the fear condition included a drop in blood pressure $\mathrm{z}$ score, a marginal increase in heart rate and a marginal decrease in temporal EEG theta power z score.

Conclusions: These preliminary findings suggest that stressful emotions such as fear can modulate epileptic spikes, which would support the notion that stressful emotions may function as triggers for partial seizures. The observation that epileptic spikes are increased during a state of relaxation is consistent with the known increase of spikes at the onset of sleep and may suggest that any beneficial effects of meditative relaxation on seizures may be similar to the effects of sufficient sleep: both temporarily increase epileptic spikes and may decrease seizures in the long term.
This study was made possible with support from the Medical Research Foundation (MRF) grant number 0425 to S.M.E., NIH K23 AT01993-01 to S.M.E., NIH AT002656 and AT005121 to B.O., and the Oregon Clinical and Translational Research Institute (OCTRI), grant number UL1 RR024140 from the National Center for Research Resources (NCRR).

\subsection{0 \\ TRANSIENT COGNITIVE IMPAIRMENT DUE HIPPOCAMPAL INTERICTAL EPILEPTIFORM ACTIVITY IN HUMANS}

Jonathan Kleen, P. P. Lenck-Santini, R. Scott, G. L. Holmes and B. Jobst (Dartmouth-Hitchcock Medical Center, Hanover, NH)

Rationale: Whether interictal epileptiform discharges (IEDs) can cause cognitive impairment is controversial. We recently used a rat model to show that IEDs in the hippocampus, a common focus for seizures, produce a disruption to ongoing cognition that is processspecific. We wanted to investigate whether these findings translate to humans, and in particular which hippocampal IED characteristics increase vulnerability to transient memory impairment.

Methods: Ten patients had depth electrodes implanted into their hippocampi for preoperative seizure localization. EEG was recorded during 2070 trials of a working memory task that required delayed recall to elicit hippocampal function. Eight of ten patients had left sided seizure onset. Hippocampal IEDs were manually and blindly detected offline and classified into three major subtype groups and according to laterality. The impact of IEDs on short-term memory processes was analyzed and adjusted for individual differences between patients.

Results: Hippocampal IEDs during in the memory retrieval period increased the likelihood of task errors when they occurred contralateral to the seizure onset zone $(\mathrm{p}<0.05)$ or bilaterally $(\mathrm{p}<$ 0.001 ). IEDs also increased task errors during memory maintenance when they were bilateral $(\mathrm{p}<0.01)$ and particularly with longer repetitive spike-wave complexes $(p<0.01)$. These memory impairments occurred independently of reaction time, which was not affected by hippocampal IEDs.

Conclusions: Hippocampal IEDs disrupt short-term memory retrieval, but not memory encoding as with cortical IEDs in previous literature. Repetitive spike-wave complexes can also disrupt memory maintenance when they are sufficiently long or bilateral. The preferential effect of IEDs contralateral to the seizure focus may relate to neural compensation which may have redistributed function to the more functional hemisphere over time. This study provides biological validity to the use of a rodent model in the study of IEDinduced transient cognitive impairment. Moreover, it strengthens the argument that IEDs contribute to a proportion of cognitive impairment in epilepsy that is dependent upon when and where they occur.

Funding was provided by NIH Grants F30NS064624 (J.K.K.), R21MH086833 (P.-P.L.-S.), R01NS044295 and R01NS073083 (G.L.H.)

\subsection{1 \\ COMPARISON OF PRE- VERSUS POST-SURGICAL COGNITIVE FUNCTIONING IN PATIENTS WITH HYPOTHALAMIC HAMARTOMA AND REFRACTORY EPILEPSY}

Jennifer Wethe, G. P. Prigatano, J. Gray, K. Chapple, H. Rekate and J. F. Kerrigan (Neuropsychology, Neurology \& Neurosurgery, Barrow Neurological Institute, Phoenix, AZ)

Rationale: Patients with hypothalamic hamartoma $(\mathrm{HH})$ and refractory epilepsy typically demonstrate cognitive impairments, but the nature and level of impairments are quite variable. We wish to determine if these patients improve in their cognitive functioning post 
neurosurgical resection of their $\mathrm{HH}$, and explore what variables correlate with cognitive outcome.

Methods: Thirty-two (32) patients underwent pre- and post-operative neuropsychological testing. The age range of patients was between 3.3 to 39.3 years $($ mean $=12.2$ years, S.D. $=7.0)$. The average time interval between surgery and post-operative neuropsychological testing was 23.4 months (range between 5.1 and 47.2 months). Tests administered varied on the basis of the patient's age and clinical condition.

Results: As a group, measures of overall intelligence showed improvement post surgery with associated improvement in attention and processing speed. Memory scores did not demonstrate consistent improvement or decline (See Table 1). Duration of epilepsy, age at surgery, neurological complications of surgery, and level of neurocognitive functioning prior to surgery were correlated with post-surgical cognitive status (See Table 2). Multiple regression using duration of epilepsy and an estimate of pre-surgical intellectual functioning as the independent variables and change in estimated intellectual functioning as the dependent variable was performed and accounted for $39 \%$ of the variance in intellectual functioning $[\mathrm{F}(2,22)=6.92, \mathrm{p}<.01]$ such that patients with the lowest pre-surgical intellectual functioning and shortest duration of epilepsy showed the greatest improvement in intellectual functioning post-surgery. Conclusions: Despite the great variability in level of cognitive impairment in patients with $\mathrm{HH}$ and refractory epilepsy, level of intelligence may show mild to moderate improvements post surgery if no surgical complications occur. The variables that predict cognitive outcome are not fully delineated, but testable individuals with the greatest pre-surgical cognitive impairment and those with the shortest duration of epilepsy (and younger age at surgery) appear to make the greatest gains in intellectual functioning. Findings have clinical implications for surgical decision making (e.g., patient selection and timing of surgery) and providing education to patients and families prior to surgery.
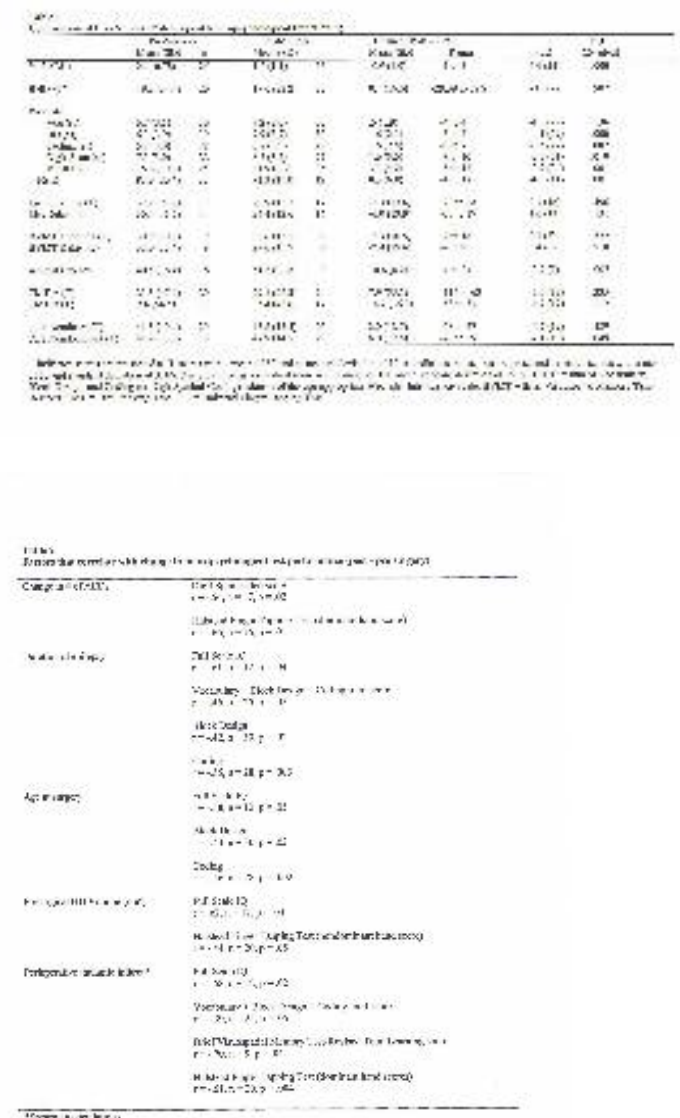

2.302

THE REVISED RESPONSIVENESS IN EPILEPSY SCALE (RES-II): AN IMPROVED TOOL FOR ASSESSMENT OF ICTAL IMPAIRMENT

Andrew Bauerschmidt ${ }^{1}$, N. Koshkelashvili ${ }^{1}$, B. T. Kiely ${ }^{1}$, C. C. Ezeani $^{1}$, J. Yoo ${ }^{1}$, Y. Zhang ${ }^{1}$, L. N. Manganas ${ }^{1}$, Z. Kratochvil ${ }^{1}$, L. Rojas $^{1}$, A. McPherson ${ }^{1}$, K. Kapadia ${ }^{1}$, D. Palenzuela ${ }^{1}$, C. P. Schmidt ${ }^{1}$, R. Lief ${ }^{1}$, L. J. Hirsch ${ }^{1}$, K. Detyniecki ${ }^{1}$, J. T. Giacino ${ }^{2}$ and H. Blumenfeld ${ }^{1}\left({ }^{1}\right.$ Neurology, Yale University School of Medicine, New Haven, CT and ${ }^{2}$ Physical Medicine and Rehabilitation, Harvard Medical School, Boston, MA)

Rationale: Impaired consciousness in epilepsy is challenging to study using objective measures. We previously devised a prospective testing battery which provides objective data on patient responsiveness and cognition during seizures, the Responsiveness in Epilepsy Scale (RES). However, since the RES was cumbersome to administer, we recently developed a simpler, revised version (RESII). This revised scale still evaluates a wide range of cognitive and sensorimotor functions, yet was designed to be faster and less errorprone.

Methods: To validate the new scale, we compared RES and RES-II in an epilepsy monitoring unit. Patients undergoing continuous video/EEG monitoring (VEEG) for seizure evaluation at Yale New Haven Hospital were tested with RES or RES-II during the ictal and immediate postictal periods. We compared data from RES (75 seizures in 24 patients) with RES-II (34 seizures in 11 patients). Results: With the new scale, more questions were asked per ictal period on average (13.4 vs 7.1). There was a trend towards a decrease in the average time required to ask one question during the ictal period, decreasing from a mean of 16.4 to 10.9 seconds $(p=0.08)$. In spite of this quicker pace, and an identical administrator training process, there was a nearly 10 -fold reduction in the average number of errors during administration of the revised scale, decreasing from 2.01 to 0.24 errors per seizure $(p<0.01)$. All errors consisted of the tester asking a question out of sequence or asking the wrong question. Performance on the initial cycle of questions asked upon onset of partial seizures showed a bimodal distribution, similar to the pattern of impairment observed using RES. On these initial questions, patients tended to be either entirely unimpaired or completely unresponsive in most seizures. This distribution of impairment was typically sustained until seizure termination. These distinct patterns of impairment correlate with those seen in the traditional categories of "simple partial" and "complex partial" seizures.

Conclusions: Our results demonstrate that prospective testing of responsiveness during seizures can be reliably performed using the revised RES-II scale. RES-II is substantially less error-prone and simpler to administer, yet patterns of impairment observed using the revised scale were similar to those using the prior scale. Improved objective testing of impaired consciousness in epilepsy may lead to better physiological understanding of ictal cognitive deficits.

\subsection{3}

\section{ELECTROCORTICOGRAPHIC MAPPING OF AUDITORY} DESCRIPTIVE NAMING AND VISUAL OBJECT NAMING Mackenzie Cervenka $^{1}$, J. Corines ${ }^{1}$, V. Parekh ${ }^{1}$, D. Boatman-Reich ${ }^{1}$, P. Franaszczuk ${ }^{2}$, A. Korzeniewska ${ }^{1}$ and N. Crone ${ }^{1}\left({ }^{1}\right.$ Department of Neurology, Johns Hopkins School of Medicine, Baltimore, MD and ${ }^{2}$ Army Research Laboratory, US Army RDECOM, Aberdeen Proving Ground, MD)

Rationale: Planning for dominant hemisphere epilepsy surgery often requires functional language mapping for surgical planning and patients frequently report lanugage deficits despite standard mapping procedures, suggesting the need for more sensitive mapping techniques. Visual object naming is most often tested, however, no standard language battery is currently administered. Subdural electrodes are implanted for seizure localization, and task-related 
increases in electrocorticographic (ECoG) high gamma $(65-150 \mathrm{~Hz})$ activity recorded from these electrodes during language task performance has been used to detect cortical activation with excellent spatial and temporal resolution. We hypothesized that ECoG mapping would discriminate the cortical representations of auditory descriptive naming (AN) and visual object naming (VN), and that areas activated during $\mathrm{AN}$, but not during $\mathrm{VN}$, would lie within the boundaries of standard dominant resections.

Methods: We studied 16 epilepsy patients (mean age 32.6, range 1450 ) in whom subdural electrodes were implanted over dominant cortex for epilepsy surgery planning. Patients underwent ECoG mapping of visual object naming and auditory descriptive naming. We performed time-frequency analyses of ECoG signals using a matching pursuit algorithm, and detected statistically significant increases in event-related high gamma activity with respect to a prestimulus baseline (defined as $\mathrm{ECoG}+$ sites). $\mathrm{ECoG}+$ sites were localized by co-registration of post-surgical CTs to pre-surgical volumetric MRIs and normalized onto a Montreal Neurologic Institute brain atlas, then assigned appropriate Brodmann areas. The number of $\mathrm{AN}$ and $\mathrm{VN}$ ECoG+ sites were compared in anterior temporal (3.5-4.5 $\mathrm{cm}$ posterior to the dominant temporal tip) and frontal (anterior to the primary motor cortex, excluding BA 44 and 45) regions of interest, that represented the boundaries of potential dominant temporal and frontal resections.

Results: Of the 932 total electrodes tested, 230 were ECoG+, 162 during AN (97 during AN only), 133 during VN (68 during VN only), and of these, 65 during both AN and VN. AN-specific regions were identified in all patients, mainly over dominant anterior temporal and frontal cortices, and nearly half (29) of VN-specific regions were identified over dominant basal temporal-occipital cortex. Over half (35) of sites that were ECoG + during both tasks localized to perisylvian, primary motor (BA4) or somatosensory (BA1-3) cortices. A significantly larger number of AN than VN electrodes were located within the boundaries of a potential dominant anterior temporal or frontal lobectomy.

Conclusions: ECoG spectral mapping in a relatively large sample of patients revealed distinct and shared $\mathrm{ECoG}+$ regions during auditory descriptive naming and visual object naming. AN sites were more likely than $\mathrm{VN}$ sites to be located within the potential boundaries of dominant anterior temporal or frontal lobe resections, suggesting that functional anatomy supporting AN may be in jeopardy when planning dominant resections on the basis of $\mathrm{VN}$ mapping alone.

\subsection{4}

\section{TYPOGRAPHIC LEGIBILITY FACTORS ASSOCIATED TO} READING EPILEPSY

M. G. Cano-Celestino ${ }^{2,1}$ and Ildefonso Rodriguez-Leyva ${ }^{2,1}$

( ${ }^{1}$ Universidad Autonoma de San Luis Potosi, San Luis Potosi, Mexico and ${ }^{2}$ Neurology, Universidad Autonoma de San Luis Potosi, San Luis Potosi, Mexico)

Rationale: The causes that lead to the manifestation of a seizure caused by the reading of printed texts in Reading Epilepsy (RE) are not entirely clear. Since its definition by Bickford and collaborators in 1956, theories have contributed from the Neurological, Ophthalmological, Linguistic and Psychological point of view, but studies of diseases related to Graphic Design and possible typographic legibility factors involved are almost null.This study tries to demonstrate that the factors associated with family, size and length of line in printed texts, are related to the facilitation of abnormal electroencephalographic activity when reading aloud.

Methods: Two populations were selected (control and experimental), of both genders with ages between 10 and 25 years (median 16 years), each one of 10 subjects, to demonstrate the difference between brain electrical activity in apparently healthy persons, and the affected with Absence epilepsy (AE) and Juvenile myoclonic epilepsy (JME), two types of epilepsies with important index of relation in RE, according to the medical literature. General data was recorded, pathological history inherited-family, personal history nonpathological, perinatal history and current condition. In the reading test's design was applied typographic legibility factors for optimal and minimal text, printed in six stories of myths and legends. The survey was recorded in three moments (Baseline, Hyperventilation and Reading) through a Grass Model 8-channel electroencephalograph; the electrodes were placed according to the International 10-20 System.

Results: The changes produced in two different periods were analyzed: Baseline and Hyperventilation; Baseline and Reading. A significant difference was obtained on the second time (p. 0.007), attributing its cause to the legibility factors studied. Of the three registered moments, reading caused in 13 patients ( 9 experimental, 4 control), a higher rate of electrical brain abnormality, which proves a highly significant statistical association. The electroencephalographic abnormalities were the sharp wave, spike, spike-slow wave and slow wave, predominantly in the left temporal lobe and of minimal percentage in the right; of focal onset but also bilateral and generalized.

Conclusions: The short column width which was between 20 and 28 characters per line, integrated by letters and spaces of interwords, was a factor related to the increase of abnormal brain electrical activity in the sample studied, with a frequency of restarts (p. 0.019) and repeats (p. 0.005) during the reading aloud. This research concludes that the bad reading aloud, and the ocular fatigue, could be subject to the employment of certain typographic legibility factors. The results should be corroborated in future studies.

\subsection{5 \\ ACCURACY OF PATIENTS' SEIZURE REPORTING DURING VIDEO EEG MONITORING}

Celestine C. Ezeani ${ }^{1}$, K. Detyniecki ${ }^{1}$, A. Bauerschmidt ${ }^{1}$, F. S. Winstanley $^{1}$, R. B. Duckrow ${ }^{1,2}$, L. J. Hirsch ${ }^{1}$ and H. Blumenfeld ${ }^{1,2}$ ( ${ }^{1}$ Neurology, Yale University School of Medicine, New Haven, CT and ${ }^{2}$ Neurosurgery, Yale University School of Medicine, New Haven, CT)

Rationale: Seizures are the main symptoms of epilepsy and the focus of treatment. Thus, accurate knowledge of seizure frequency is important for evaluating therapeutic efficacy. Information about seizure frequency is usually gathered from patients although evidence from previous studies show that this could be unreliable. The goal of this study was to assess patients' reporting of their seizures. We also looked at predictors that could influence reporting of seizures. Further, we examined the impact that deficits in memory and consciousness could have on patients' seizure reporting.

Methods: We recruited patients undergoing continuous VEEG evaluation at the Yale Comprehensive Epilepsy Center over a 2-year period. We compared their subjective response to daily questionnaires with a more objective measure by analyzing the video recordings of their seizures. We determined statistical significance using Chi-squared or Fisher's exact tests where appropriate to evaluate relationships between patients' characteristics and seizure reporting. To determine which factors impacted seizure reporting, a multivariate binary logistic regression was performed.

Results: 115 patients were recruited, of which 65 met all criteria for analysis (confirmed epilepsy, completed questionnaires, seizures captured during VEEG monitoring). For these 65 patients, 51\% were female and $49 \%$ were male. The mean age was 34 years at time of admission, with 59 adults ( $\geq 18$ years) and 6 pediatric patients. Overall, we captured a total of 295 partial and 42 sGTC seizures. $30 \%$ of all seizures were not reported while $70 \%$ were reported. On the patient level, $80 \%$ had partial seizures (SPS and GTC) only while $20 \%$ had only sGTC. Also, $54 \%$ of patients always reported their seizures while $46 \%$ did not always report their seizures. We found that SPS were more often reported than both CPS and sGTC in pairwise comparisons, with p-values of 0.008 and $<0.001$ respectively (Chi-square). This was consistent with our finding on patient level, 
where patients with only SPS more often reported their seizures. We also found that loss of consciousness (OR $0.2595 \%$ CI $0.07-0.92$ $\mathrm{p}=0.037)$ and temporal lobe onset (OR $0.1795 \%$ CI 0.08-0.37 $\mathrm{p}<0.001)$ had a negative influence on seizure reporting. Although patients who were awake at seizure onset reported more of their seizures (Chi-square $17.34 \mathrm{p}<0.0001$ ), sleep state was not a significant independent predictor of seizure reporting. We didn't observe an effect of seizure lateralization on seizure reporting. Conclusions: This study demonstrates that seizures with impaired consciousness were more likely to go unreported suggesting that consciousness may be a factor influencing the ability of patients to recognize and accurately report their seizures. Temporal lobe onset significantly influenced seizure reporting. The impact of altered memory function and language impairment on seizure reporting needs to be further assessed. Relying exclusively on patients' seizure reporting may lead to inadequate treatment of seizures. Additional measures should be used clinically to determine accurate seizure frequency.

\subsection{6 \\ COGNITIVE REHABILITATION AFTER EPILEPSY SURGERY: WHAT IS THE EVIDENCE?}

A. Mazur-Mosiewicz ${ }^{1}$, H. Carlson ${ }^{1}$, Jonathan Dykeman ${ }^{2,3}$, B. L. Brooks ${ }^{1,2}$ and S. Wiebe ${ }^{2,3}$ ( ${ }^{1}$ Alberta Children's Hospital, Calgary, AB, Canada; ${ }^{2}$ Department of Clinical Neurosciences, University of Calgary, Calgary, AB, Canada and ${ }^{3}$ Hotchkiss Brain Institute Clinical Research Unit, University of Calgary, Calgary, AB, Canada)

Rationale: Up to $40 \%$ of patients undergoing dominant temporal lobe resection for epilepsy experience reliable declines in verbal memory and naming function. Cognitive rehabilitation (CR) is a well established therapeutic option for memory and other cognitive problems resulting from neurological disorders or insults. CR offers mnemonics and compensation strategy training aimed at improving daily functional performance in selected groups of patients. Although $\mathrm{CR}$ has been advocated in many reviews for epilepsy surgery, there is no notion regarding specific forms of CR applicable to this population, about its impact, or how often it is used. This systematic review of the literature aimed at (1) identifying the proportion of epilepsy patients in the literature who are offered CR as an element of post-surgical treatment, and (2) exploring the most commonly used $\mathrm{CR}$ approaches in epilepsy surgery patients.

Methods: A comprehensive literature search using PubMed, EmBase, and the Cochrane databases identified articles published between 1991 and 2010, related to surgical outcomes of focal resective epilepsy surgery. All articles meeting inclusion and exclusion criteria were reviewed for further selection. All full-length articles published in English and containing pre-surgical and postsurgical neurocognitive performance were included. Additional references were identified from experts and from references of relevant articles.

Results: Of 5254 citations related to epilepsy surgery, 148 included longitudinal cognitive outcome variables in patients with left and right operations. Twenty three studies (16\%) described cognitive outcomes in detail, but none reported any form of CR, memory compensation strategies training, or any other form of CR. Nine additional references found from other sources described CR interventions as follows: internal compensatory strategies, external memory aids, psychoeducation, verbal and visual memory training, as well as exercises of attention and executive functions. The results of these interventions suggest that $\mathrm{CR}$ has a significant positive impact on verbal learning and memory performance. However, improvements seem also to be significantly related to the site of the surgery, attentional skills, mood, age, and education.

Conclusions: Reliable cognitive declines in specific domains occur frequently after temporal lobe epilepsy surgery. Although memory rehabilitation is a well established therapy, it is not included as a standard element of post-surgical care, and the literature rarely mentions it. Sparse reports suggest that CR may benefit patients with left temporal lobe epilepsy who suffer from verbal memory decline, and that improvement is more likely in younger patients with less depressed mood, relatively strong concentration skills, and who are less educated. An expanded comprehensive search is underway focusing on these elements. CR should be given greater attention after epilepsy surgery to determine its efficacy and role in managing these patients.

\subsection{7 \\ SAME PLACE 11 YEARS LATER: A CASE OF TEST- RETEST RELIABILITY OF CORTICAL LANGUAGE MAPPING}

Kirsty E. Bortnik ${ }^{1}$, G. M. McKhann $\mathrm{II}^{2}$ and M. J. Hamberger ${ }^{1}$ ( ${ }^{1}$ Department of Neurology, Columbia University, New York, NY and ${ }^{2}$ Department of Neurological Surgery, Columbia University, New York, NY)

Rationale: Electrical stimulation mapping (ESM) is often used to identify essential language cortex prior to resection of epileptogenic tissue in the language dominant hemisphere. Although considered the "gold standard" for language localization, there is limited information concerning the reliability of the procedure, particularly over long time intervals. We present a case report of a young man with refractory left temporal lobe epilepsy who underwent intraoperative language mapping at age 14 years and again at age 25 years. Methods: This patient developed focal seizures at age 13 and was found to have a left temporal dysembryoplastic neuroepithelial tumor (DNET), consistent with scalp vEEG evidence of left (dominant) temporal seizure onset. He underwent surgical resection of the tumor and epileptogenic cortex at age 14, with poor seizure outcome. Following multiple medication trials he underwent further resection at age 25. Intraoperative stimulation mapping was conducted prior to both resections. Tasks included visual object naming and auditory description naming. For both mapping procedures the neuropsychologist administering language tasks was blind to the location of the stimulation. Sixteen sites were tested during the first procedure, 10 sites were tested during the second procedure.

Results: Results from the first mapping identified a positive auditory naming site approximately $5 \mathrm{~cm}$ from the temporal pole on the superior temporal gyrus, just at the anterior aspect of the tumor. No reliable visual naming areas were identified. Stimulation over the tumor was not associated with any language difficulties. Results from the second intraoperative mapping again identified a positive auditory naming site on the superior temporal gyrus approximately $5 \mathrm{~cm}$ from the temporal pole. Stimulation at other tested sites was not associated with changes in speech or language. The location of the positive language (auditory naming) site initially identified at age 14 was replicated 11 years later at age 25 .

Conclusions: Repeat ESM following an 11-year interval provided a unique opportunity to investigate the reliability of ESM-identified language areas. Although with caveat given the limited ability to generalize from a single case study, this finding of the same type of language site in the same location supports the reliability of the procedure and suggests intra-individual reliability in the location of language cortex over a prolonged time interval.

\subsection{8 \\ FACTORS UNDERLYING MSI BILATERAL LANGUAGE CLASSIFICATION IN WADA-CONFIRMED LEFT DOMINANT PATIENTS}

Ann M. Hempel and G. L. Risse (Minnesota Epilepsy Group, St. Paul, MN)

Rationale: A previous study suggested that patients misclassified on magnetic source imaging (MSI) as right hemisphere dominant are more likely than those corcordant with Wada-confirmed left language 
dominance to evidence compromise of language functioning on baseline neuropsychological testing and experience an earlier age of seizure onset. The aim of this study was to examine language functioning or other factors that might account for bilateral activation on MSI in those who display uneviquocal left hemispheric dominance on the Wada.

Methods: The sample included nineteen temporal lobe epilepsy and/or tumor patients ages 18-60 years who underwent both MSI for language lateralization and demonstrated unequivocal left hemisphere dominance on the intracarotid amobarbital procedure (IAP). Those who demonstrated MSI left dominance (MSI-L, $n=13$ ) were compared to those who demonstated MSI bilateral language (MSI-B, $\mathrm{n}=6$ ) on tests of language (Boston Naming Test, Wechsler VC index), verbal memory (delayed recall on a list learning task), age of seizure onset, IAP hemispheric memory capacity, side of seizure onset, and presence of a temporal lobe structural lesion. Results were analyzed using the Fisher Exact Test and t-test.

Results: Although short of statistical significance, there was a trend toward younger age at seizure onset among MSI-B (12.2 years) than MSI-L (23.7 years). Patients in the MSI-B group (67\%) were somewhat more likely than MSI-L (23\%) to display below normal language on BNT or Wechsler VC (Fisher exact Test $\mathrm{p}=.12$ ). MSI-B and MSI-L did not differ significantly on presence or absence of a temporal lobe structural abnormality, IAP hemispheric memory capacity, or side of seizure onset, although a slightly larger proportion of MSI-B (100\%) than MSI-L (69\%) had a left temporal seizure origin.

Conclusions: Although differences were not clinically significant, there was a trend toward greater likelihood of language compromise and earlier age of seizure onset among Wada-confirmed left dominant patients who displayed bilateral language activation on MSI. These data are consistent with previous findings suggesting that greater compromise of the left hemisphere may be associated with the appearance of increased right hemisphere activation on MSI.

\subsection{9}

\section{ATTENTION IN PEOPLE WITH EPILEPSY: FACTOR} STRUCTURE OF THE CONNERS' CPT Thomas J. Snyder ${ }^{1,2}$, C. Wong ${ }^{1}$, S. N. Ahmed ${ }^{1,2}$, D. Gross ${ }^{1,2}$, J. Jirsch $^{1,2}$, D. B. Sinclair ${ }^{1,2}$, R. Tang-Wai ${ }^{1,2}$, B. M. Wheatley ${ }^{1,2}$ and J. Edgerton ${ }^{2}\left({ }^{1}\right.$ University of Alberta, Edmonton, AB, Canada and ${ }^{2}$ Alberta Health Services, Edmonton, AB, Canada)

Rationale: Attention is an essential function that is often impaired in people with epilepsy (PWE) regardless of seizure type. Computerized continuous performance tests (CPTs) are frequently used as measures of attention, including the Conners' CPT (CCPT) which provides 15 variables that purportedly measure sustained attention. Factor analysis of adult CCPT performance, however, has shown that four dimensions of attention are measured by the CCPT: Focused Attention, Sustained Attention, Hyperactivity/Impulsivity, and Vigilance (Egeland \& Kovalik-Gran, 2010a). Three of these dimensions have been shown to discriminate between clinical groups (Egland et al, 2010b). The factor structure of CCPT performance was therefore analyzed to determine what dimensions of attention are evident in PWE.

Methods: A mixed clinical sample of patients $(n=322)$, including 248 PWE, were administered the CCPT-II as part of standard neuropsychological assessment. Age ranged from 6 to 61 years. PWE included 138 adults and 110 children/adolescents (6 to 17 years of age). The majority of PWE were being investigated for medically intractable focal seizures.

Results: Principal component factor analyses followed by varimax rotation were done on CCPT performance (age and sex-corrected Tscores) of the total sample, PWE, and separately on PWE grouped according to age: child/adolescent (CWE) and adult. The results are presented in Tables 1 and 2 and showed that for the total sample and PWE four factors accounted for $74 \%$ of the variance. These factors were identical to the dimensions of attention described by Egeland et al (2010a). Among PWE, separate analysis of child/adolescent CCPT performance showed three component factors accounting for $71 \%$ of the variance, two identical to adults (hyperactive/impulsive, sustain) and one unique to CWE. This last factor was more complex than for adults with epilepsy and children with ADHD and primarily consisted of a combination of focused attention and vigilance. Conclusions: In adults with mixed clinical diagnoses or epilepsy, CCPT performance captures four dimensions of attention: Focus, Sustain, Hyperactive/Impulsive, and Vigilance. In CWE, three dimensions are measurable, two identical to those in adults but one unique and potentially valuable for discriminating between CWE and children with other attention deficits, e.g., ADHD, as well as between CWE with different types of seizures, e.g., focal vs. generalized. Further study of the clinical usefulness of CCPT indices of attention in PWE is merited.

Factor Structure of Conner' CPT From a Mixed Clinical Sample (N = 322)

\begin{tabular}{|c|c|c|c|c|}
\hline \multirow{2}{*}{} & \multicolumn{4}{|c|}{ Allention Component } \\
\cline { 2 - 5 } Perseverations & 1 Focus & 2 Vigilance & $3 \mathrm{H} / 1$ & 4 Sustain \\
Hit RT SF & .819 & -.061 & -.085 & .085 \\
Variability & .787 & .488 & .216 & .139 \\
\hline Omissions & .745 & .446 & .040 & .166 \\
\hline ISI Change SE & .675 & -.104 & .152 & .020 \\
\hline HRT' ISI Change & .005 & .887 & -.020 & .089 \\
\hline Commissions & .087 & .858 & .065 & .039 \\
\hline Response Style & .175 & .044 & -.844 & .146 \\
Hit RT & .441 & .001 & .735 & .071 \\
\hline HRT Block Change & .050 & .047 & .679 & .000 \\
\hline Block Change SE & .147 & .100 & .004 & .903 \\
\hline
\end{tabular}

Note: $\mathrm{CPT}=$ Continuous Performance Test; $\mathrm{H} / \mathrm{I}=$

hyperactivity/impulsivity; $\mathrm{SE}=$ standard error; $\mathrm{RT}=$ reaction time; ISI $=$ inter-stimulus interval. Extraction method $=$ principal component analysis; rotation method = varimax with Kaiser normalization.

Factor Structure of Conners' CPT From Children/Adolescents with Epilepsy $(\mathrm{N}=110)$

\begin{tabular}{|c|c|cc|}
\hline & \multicolumn{3}{|c|}{ Altention Component } \\
\cline { 2 - 4 } & 1 Unique & 2 Sustain & $3 \mathrm{H} / 1$ \\
Hit RT SF & .948 & .172 & .103 \\
Variability & .854 & .330 & -.074 \\
HRT ISI Change & .837 & -.001 & .123 \\
\hline Ilit RT & .698 & -.047 & .514 \\
\hline Perseverations & .693 & -.049 & -.412 \\
\hline ISI Change SE & .684 & .270 & .068 \\
\hline Omissions & .509 & .098 & .103 \\
\hline HRT Block Change & .079 & .924 & .095 \\
\hline Block Change SE & .232 & .863 & -.152 \\
\hline Commissions & .087 & .111 & -.863 \\
\hline Response Style & .235 & .075 & .741 \\
\hline
\end{tabular}

Note: $\mathrm{CPT}=$ Continuous Performance Test; $\mathrm{H} / \mathrm{I}=$

hyperactivity/impulsivity; $\mathrm{SE}=$ standard error; $\mathrm{RT}=$ reaction time; ISI $=$ inter-stimulus interval. Extraction method $=$ principal component analysis; rotation method = varimax with Kaiser normalization. 


\subsection{0}

STAND UP FOR EPILEPSY SAN DIEGO PHOTO-SHOOT: A PERSONAL ODYSSEY

Kenneth R. Kaufman ${ }^{1}$ and N. D. Kaufman ${ }^{2}\left({ }^{1}\right.$ Psychiatry, UMDNJRobert Wood Johnson Medical School, New Brunswick, NJ and ${ }^{2}$ West Windsor-Plainsboro High School South, Princeton Junction, $\mathrm{NJ})$

Rationale: Elite athletes, as celebrity-icons, assist in the social wellbeing of patients. Athlete-patient interactions destigmatize illness while ennobling athletes/patients/society. Athletes can bring those with illnesses out of the shadows. ILAE Sports and Epilepsy Task Force directed Stand Up For Epilepsy (SUFE), an international project involving action photographs of persons with epilepsy (PWE) with elite athletes. The North American photo-shoots served as a personal odyssey.

Methods: SUFE San Diego photo-essay with commentary.

Results: Photo-shoots involved 7000+ miles travelled/7 photographers/10 PWE/33 athletes/11 national teams. Each photoshoot held personal significance for the senior author culminating in the San Diego photo-shoot: 1300+ photographs/24 athletes/9 PWE. Parents' comments summarized the significance of SUFE and the senior author's role: "You don't know how special it is for these children...they are playing with Olympians as people; no one cares about their epilepsy... but look at you, you have epilepsy and look how far you have gone - you are a doctor/psychiatrist, on an international Task Force, competed internationally, have a gold medal...do you have any idea what a representative and role model you are for these children about how far a PWE can go...you have proven there is no limit." The odyssey was completed when one parent simply stated "Do you know how proud your mother is of you? Today you really made your mother proud to be a mother." Conclusions: SUFE initiated athlete-PWE interactions with positive PWE/athlete/parent responses. Expansion of SUFE is recommended to further destigmatize epilepsy. Participation in SUFE can serve as a personal odyssey for PWE.

\section{Genetics: Human Studies}

\subsection{1}

OHTAHARA SYNDROME AND OTHER NEONATAL-ONSET EPILEPTIC ENCEPHALOPATHIES CAUSED BY MISSENSE MUTATIONS OF THE KCNQ2 GENE

Mitsuhiro Kato ${ }^{1}$, H. Saitsu ${ }^{2}$, T. Yamagata ${ }^{5}$, R. Kusano ${ }^{6}$, H. Arai ${ }^{9}$, T. Fujii $^{10}$, Y. Hirata ${ }^{11}$, Y. Kusama ${ }^{11}$, S. Yamashita ${ }^{7}$, T. Nakagawa ${ }^{8}$, A.

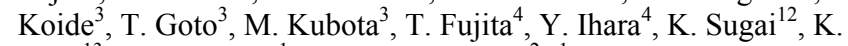
Saito $^{13}$, K. Hayasaka ${ }^{1}$ and N. Matsumoto ${ }^{2}$ (' ${ }^{1}$ Yamagata University, Yamagata, Japan; ${ }^{2}$ Yokohama City University, Yokohama, Japan; ${ }^{3}$ Tokyo Metropolitan Children's Medical Center, Fuchu, Japan; ${ }^{4}$ Fukuoka University, Fukuoka, Japan; ${ }^{5}$ Jichi Medical University, Shimotsuke, Japan; ${ }^{6}$ Ota Memorial Hospital, Ota, Japan; ${ }^{7}$ Kanagawa Children's Medical Center, Yokohama, Japan; ${ }^{8}$ Kobe University, Kobe, Japan; ${ }^{9}$ Morinomiya Hospital, Osaka, Japan; ${ }^{10}$ Showa University, Tokyo, Japan; ${ }^{11}$ Jikei University Kashiwa Hospital, Kashiwa, Japan; ${ }^{12}$ National Center of Neurology and Psychiatry, Kodaira, Japan and ${ }^{13}$ Tokyo Women's Medical University, Tokyo, Japan)

Rationale: Neonatal epilepsies are divided into two forms, benign (familial) seizures and epileptic encephalopathies (EE). Ohtahara syndrome is a representative form of neonatal-onset EEs showing frequent tonic seizures or epileptic spasms with suppression-burst pattern on EEG and severe psychomotor developmental delay. Mutations of two genes, $A R X$ and $S T X B P 1$, are known to be responsible for Ohtahara syndrome, while the etiology of more than half cases remains unknown. We recently performed whole-exome sequencing in 12 patients with Ohtahara syndrome and found 3 missense mutations in KCNQ2 [Saitsu et al. Ann Neurol 2012 online]. KCNQ2 was first identified as the causative gene for benign familial neonatal convulsions and then a few case reports confirmed that KCNQ2 mutations are associated with EE. A recent study by Weckhuysen et al [Ann Neurol 2012; 71(1):15-25] revealed KCNQ2 mutations in 8 patients with neonatal-onset EEs. Some patients demonstrated tonic seizures and suppression-burst suggesting Ohtahara syndrome.

To unveil the clinical features of EEs caused by KCNQ2 mutations, we carried out mutation screening of KCNQ2 in patients with Ohtahara syndrome and other early-onset EEs.

Methods: We performed whole-exome sequencing or high-resolution melting (HRM) analysis for KCNQ2 mutation screening in patients with Ohtahara syndrome and other early-onset EEs. Patients with brain malformations or metabolic disorders were excluded. Patients' blood samples were mainly recruited from Japanese patients and their parents following informed consent. Clinical information including EEG findings and brain MRI were also collected. The study was approved by IRB for Ethical Issues at Yokohama City University School of Medicine and Yamagata University Faculty of Medicine. Results: Whole-exome sequencing revealed additional 3 heterozygous non-synonymous KCNQ2 mutations in 3 patients and HRM analysis disclosed 5 heterozygous mutations in 6 patients. All mutations were missense and de novo. Detailed clinical information was obtained from 9 patients with KCNQ2 mutation including 3 patients described elsewhere in brief [Saitsu et al. Ann Neurol 2012 online] was analyzed. Six patients had Ohtahara syndrome characterized by tonic seizures and suppression-burst pattern on EEG. Three patients showed unclassified EE with tonic seizures in 3 and multifocal spikes in 2 . All patients showed initial seizures at neonatal period, particularly within a week in 8 patients. Only 3 patients showed hypsarrhythmia on EEG and 3 patients were seizurefree treated with CBZ or PHT or TPM in each. All patients showed profound to moderate mental retardation and 5 patients were bedridden.

Conclusions: Mutations of KCNQ2 are recurrently found in patients with Ohtahara syndrome. Patients with KCNQ2 mutation show distinct features with very early neonatal-onset, tonic seizures, suppression-burst pattern, relatively infrequent evolution to West syndrome, and good response to sodium channel blockers.

\subsection{2 \\ THE IMPACT OF GENOMIC STRUCTURAL VARIATIONS ON DRUG RESISTANT EPILEPSY: AN ASSOCIATION STUDY OF COPY NUMBER VARIATIONS AND REFRACTORY EPILEPSY}

HON CHUNG P. FUNG (Department of Neurology, Section of Epilepsy, Chang Gung Memorial Hospital, Taiwan, Taoyuan County, Taiwan)

Rationale: Epilepsy is one of the most common disorders of the brain. One of every ten people will have at least one epileptic seizure during a normal lifespan, and a third of these will develop epilepsy. Medical treatment with one or more antiepileptic drugs (AEDs) will render a majority of patient seizure free. However, despite long term treatment with various AEDs, about $30 \%$ of patients with epilepsy remain medically intractable. Recently, several genetic factors have been found to be associated with the occurrence of refractory epilepsy.

The discovery of CNVs has had far-reaching implications for disease research, enabling scientists to correlate genetic alterations with disease. For epilepsy, altered CNV has been found to be related to the different types of seizure disorders. Furthermore, evolving data showed that CNV may also play an important role in pathogenesis of epilepsy. Though we have accumulating data showing that seizure patterns of epilepsy, the occurrence of RE are associated with different level of genetic variations, CNV has not yet been well explored for describing the mechanisms of RE occurrence. CNVs, as inter-individual significant minor variants, should be good candidates 
for the association study between phenotypes and genotypes in RE. Methods: This project will be a three-year prospective observational study in a naturalistic out-patient clinical setting. In total, 200 epilepsy patients (100 drug resistance epilepsy patients and 100 drug responsive epilepsy patients) will be recruited. They will be assigned into different groups throughout the study according to their clinical manifestation and the responsiveness of AEDs. The copy number variations in the whole genome of each individual will be determined by using the DNA microarray copy number variation analysis. ANOVA and chi-square test will be used for testing the association between the copy number variations and the occurrence of refractory epilepsy. Meanwhile, multivariate logistic regression model will be performed for analyzing the strength of association between those genetic factors and the disease progression after adjustment of the covariates.

Results: Totally 405 patients were recruited in our study. The mean age of all our patients were 42.9 year (SD 15.336); 244 (60.2\%) were male, and 161 (39.8\%) were female. Frou our finding, we have found 9 loci upon the whole human genome, related to the occurence of RE. The result has been summarized in the table.

Conclusions: From our result, we will understand the impact of the copy number variations and its magnitude on the occurrence of refractory epilepsy. Our results will not only provide the information of basic research about the influence of genomic structural variations and gene-environment interaction on the RE, but also set up a foundation for future research on the epidemiological and genetic study of RE in our Taiwanese population.

Candidate Genes in RE from Taiwanese cohort

\begin{tabular}{|c|c|c|}
\hline Chromosomal Lociation & Gene & Encoding Protein \\
\hline $1 \mathrm{p} 35.2$ & MATN1 & MATRILIN 1 \\
\hline $2 q 12.3$ & EDAR & ECTODYSPI.ASIN A RF.CF.PTOR \\
\hline $8 \mathrm{p} 21.1$ & EI.P3 & FI ONGATION PROTEIN 3 \\
\hline $11 p 13$ & DCDC! & DOUBLLCORTIN DOMAIN-CONTAINING PROTEIN 1 \\
\hline $18 \mathrm{q} 21.33$ & CDII20 & CADIIERIN 20 \\
\hline
\end{tabular}

\subsection{3}

\section{ABNORMAL MICRORNA REGULATION IDENTIFIED IN FOCAL CORTICAL DYSPLASIAS}

S. H. Avancini ${ }^{1}$, Danyella B. Dogini ${ }^{1}$, F. R. Torres ${ }^{1}$, F. Rogerio ${ }^{3}$, A. C. Coan ${ }^{2}$, R. Secolin ${ }^{1}$, C. S. Rocha ${ }^{1}$, A. F. Costa ${ }^{3}$, A. L. Costa ${ }^{2}$, A. S. Piaza $^{3}$, L. A. Reis ${ }^{3}$, E. P. Oliveira ${ }^{2}$, H. Tedeschi ${ }^{2}$, L. S. Queiroz ${ }^{3}$, F. Cendes ${ }^{2}$ and I. Lopes-Cendes ${ }^{1}\left({ }^{1}\right.$ Medical Genetics, University of Campinas, Campinas, Brazil; ${ }^{2}$ Neurology, University of Campinas, Campinas, Brazil and ${ }^{3}$ Anatomical Pathology, University of Campinas, Campinas, Brazil)

Rationale: MiRNAs are small noncoding RNAs which regulate posttranscriptional gene expression. Focal cortical dysplasia (FCD) is a malformation of cortical development which affects up to $36 \%$ of patients with drug-resistant epilepsy. Our goal is to investigate differences in microRNA (miRNAs) regulation in FCDs.

Methods: We used brain tissue obtained after surgery for the treatment of medically refractory seizures from nine patients with FCD (four patients with FCD type IIa and five patients with FCD type IIb). In addition, we used cortical tissue from autopsy as controls $(\mathrm{n}=5)$. Total RNA was isolated with RecoverAllTM kit (Ambion) and RNA integrity was assessed by Agilent RNA Pico Chip Kit and BioAnalyzer 2100.MiRNA expression profile was assessed by Affymetrix GeneChip platform miRNA array. Background correction, summarization and normalization were performed by RMA function. MiRNA expression was analyzed using RankProd ( $\mathrm{p}$ $<0.05)$.

Results: We identified 23 miRNAs with significant different expression when patients and control group were compared.
Furthermore, when FCD type IIa and FCD type IIb groups were compared we found six differentially expressed miRNA types. Among them, we observed a significant down-regulation of several elements belonging to the miR-17 92 cluster. This cluster is known to contribute to transcriptional regulation of stem cell differentiation, aging, as well as fine-tuning of pathways involved in neuronal differentiation.

Conclusions: Our results clearly show that neurodevelopment pathways are indeed involved in the pathophysiology of FCD. In addition, we identified a different miRNA expression signature in different FCD subtypes which may contribute to improve classification.

\subsection{4}

AUTOSOMAL DOMINANT VASOVAGAL SYNCOPE: CLINICAL FEATURES AND LINKAGE TO CHROMOSOME 15Q26

Karl M. Klein ${ }^{1,2}$, C. J. Bromhead ${ }^{3}$, K. R. Smith ${ }^{3,4}$, C. J. O'Callaghan ${ }^{5}$, S. J. Corcoran ${ }^{6}$, S. E. Heron ${ }^{7}$, X. Iona ${ }^{7}$, B. L. Hodgson ${ }^{7}$, J. M. McMahon $^{1}$, K. M. Lawrence ${ }^{1}$, I. E. Scheffer ${ }^{1}$, L. M. Dibbens ${ }^{7}$, M. Bahlo $^{3,8}$ and S. F. Berkovic ${ }^{1}$ ( ${ }^{1}$ Epilepsy Research Centre, Department of Medicine, The University of Melbourne, Austin Health,

Melbourne, VIC, Australia; ${ }^{2}$ Epilepsy Center Hessen, Department of Neurology, University Hospitals Giessen \& Marburg, and PhilippsUniversity Marburg, Marburg, Germany; ${ }^{3}$ The Walter and Eliza Hall Institute of Medical Research, Parkville, VIC, Australia; ${ }^{4}$ Department of Medical Biology, The University of Melbourne, Parkville, VIC, Australia; ${ }^{5}$ Department of Clinical Pharmacology, Department of Medicine, University of Melbourne, Austin Health, Heidelberg, VIC, Australia; ${ }^{6}$ Baker IDI Heart and Diabetes Institute, Melbourne, VIC, Australia; ${ }^{7}$ Epilepsy Research Program, School of Pharmacy and Medical Sciences, Division of Health Sciences, University of South Australia, Adelaide, SA, Australia and ${ }^{8}$ Department of Mathematics and Statistics, The University of Melbourne, Parkville, VIC, Australia)

Rationale: Vasovagal syncope (VVS) is the most frequent type of syncope and a common differential diagnosis of epilepsy. Using a twin-family design, we recently confirmed that VVS has a genetic etiology and showed that complex inheritance is usual (Klein et al. Neurology 2012 in press). Families consistent with autosomal dominant inheritance are also described in the literature. We previously reported a family with suggestive linkage to chromosome 15 (Klein et al. Epilepsia 2009;50(S11):149). Here, we describe an updated analysis of this family as well as five other families with autosomal dominant VVS, characterize the phenotype, refine the causative locus through linkage analyses and establish genetic heterogeneity.

Methods: We recruited patients with VVS and a family history of syncope. A standardized questionnaire addressing features differentiating syncope from epilepsy was administered to all available family members. Medical records were obtained and additional diagnostic tests performed in selected individuals. Linkage analysis was performed in the largest family using SNP genotyping microarrays and in four other families with microsatellite markers for chromosome 15q26. Candidate genes within the linkage interval were analyzed by sequence analysis.

Results: The largest family A contained 30 affected individuals over three generations with a median onset of 8-9 years. Five other families comprised between four and 14 affected individuals. The affected family members reported typical triggers of VVS such as sight of blood, injury, medical procedures, prolonged standing, pain and frightening thoughts. There was considerable variation of the triggers within the families. Linkage analysis revealed significant linkage to chromosome $15 \mathrm{q} 26$ in family A (LOD score 3.28) with two peaks, one at chromosome $15 \mathrm{q} 26.1$ spanning $6.28 \mathrm{cM}$ or $1.7 \mathrm{Mb}$, and the other at chromosome $15 \mathrm{q} 26.2$ spanning $1.19 \mathrm{cM}$ or $0.5 \mathrm{Mb}$. Linkage to chromosome $15 \mathrm{q} 26$ was excluded in two medium-sized 
families but not in two smaller families. No mutation was found in the candidate genes SLCO3A1, ST8SIA2 and NR2F2.

Conclusions: The presented families demonstrate that familial VVS following autosomal dominant inheritance is not rare and has similar features to sporadic VVS. The refined genetic locus on chromosome $15 q 26$ in the largest family increases the susceptibility to fainting but does not predispose to a particular vasovagal trigger. Genetic heterogeneity was established by linkage analyses in the other families. Identification of the causal mutations will help to further understanding of pathophysiology and guide further genetic research.

\subsection{5}

\section{INFANTILE SPASMS IS ASSOCIATED WITH ABNORMAL} COPY NUMBER VARIANTS

Vijay N. Tiwari ${ }^{1,2}$, S. K. Sundaram ${ }^{1,2}$, H. T. Chugani ${ }^{1,2}$ and A. M. Huq ${ }^{1}\left({ }^{1}\right.$ Pediatrics and Neurology, Wayne State University, Detroit, MI and ${ }^{2}$ PET Center, CHM, Wayne State University, Detroit, MI)

Rationale: We tested the hypothesis that de novo copy number variants (CNVs) or the CNVs implicated in known genomic disorders ("pathogenic CNVs") as described in online mendelian inheritance in man (OMIM) morbid map are significant predisposing factors for infantile spasms.

Methods: We performed a genome-wide analysis of single nucleotide polymorphism (SNP) genotyping microarray data to identify the role of de novo/known pathogenic large CNVs in 13 trios of children affected by infantile spasms using the Illumina 610Quad platform.

Results: A rare large (4.8 MB) de novo duplication was detected in 15q11-13 region of one patient (Figure $1 \mathrm{~A}$ ). In addition, 3 known pathogenic CNVs (present in patient as well as one of the parents) were detected in total. In one patient, a known pathogenic deletion was detected in the region of 2q32.3 (Figure 1 B). Similarly, in another patient two known pathogenic deletions in the region of 16p11.2 (Figure 2 A) and Xp22.13 (containing CDKL5) (Figure 2 B) were detected. These two patients were phenotypically different from the patient where the de novo CNV was detected.

Conclusions: These findings suggest that some specific pathogenic CNVs predispose to infantile spasms and may be associated with different phenotypes. However, additional modifying factors (genetic and environmental) likely also play an important role in determining the overall phenotype.

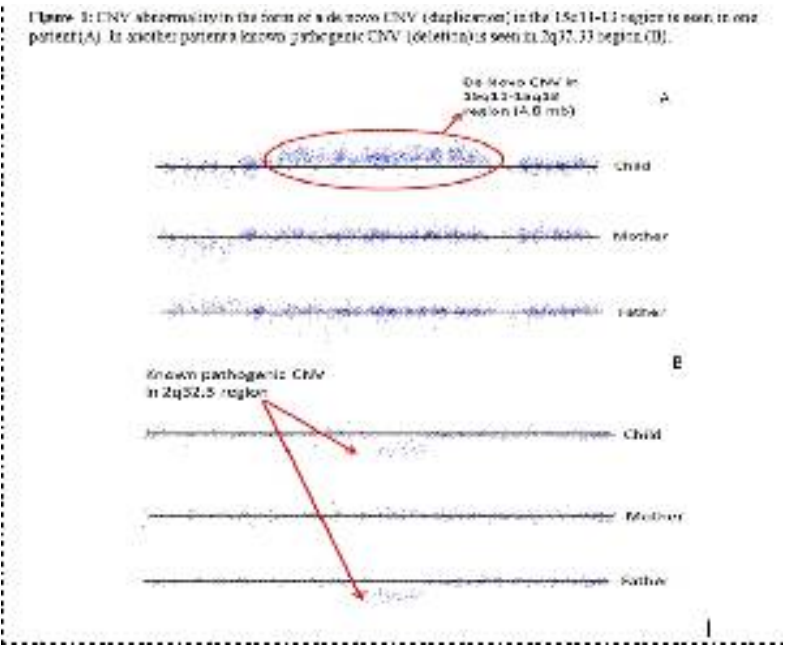

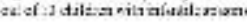
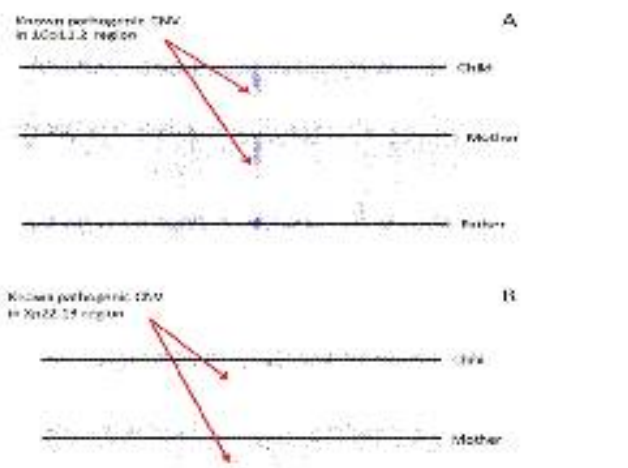

2.316

DNA METHYLATION PROFILES OF SEIZUREASSOCIATED NEURODEVELOPMENTAL DISORDERS IDENTIFIES TAC1 AS A MECP2 TARGET GENE Kimberly Aldinger ${ }^{1}$, J. Plummer ${ }^{1}$ and P. Levitt ${ }^{1,2}\left({ }^{1}\right.$ Zilkha Neurogenetic Institute, Keck School of Medicine of USC, Los Angeles, CA and ${ }^{2}$ Department of Cell \& Neurobiology, Keck School of Medicine of USC, Los Angeles, CA)

Rationale: Several proteins involved in epigenetic regulation cause seizure-associated syndromic neurodevelopmental disorders when the human genes are mutated [e.g. MeCP2 in Rett syndrome (RTT)]. However, more general involvement of epigenetic mechanisms in these phenotypes is unclear. We investigated whether DNA methylation profiles of lymphoblastoid cell lines (LCLs) could be used to differentiate related neurodevelopmental disorders into etiological categories and identify specific genes that might be relevant to pathophysiology.

Methods: DNA methylation profiling was performed on bisulfate converted DNA from LCLs in discovery $(n=20)$ and replication cohorts $(n=40)$ of females with Rett syndrome (RTT), autism (AUT), seizure disorder (SEZ), and controls (CTL) using Illumina HumanMethylation27 arrays. DNA methylation within the TAC1 promoter was confirmed using a Sequenom EpiTYPER assay, and gene expression by qRT-PCR was assessed in postmortem brain from RTT and AUT+SEZ females. Chromatin immunoprecipitation was performed in HEK cells using an anti-MeCP2 antibody, followed by qPCR at the TAC1 promoter.

Results: In the discovery cohort, hierarchical clustering and principal component analysis using $40 \mathrm{CpG}$ sites with significant differential methylation between etiological groups $(\mathrm{P}<0.01, \mathrm{FDR}<0.10)$ distinguished RTT and AUT from CTL, but not from each other. These $40 \mathrm{CpG}$ sites did not differentiate etiological groups in the replication cohort, but a pooled analysis identified four significant differentially methylated $\mathrm{CpG}$ sites $(\mathrm{P}<0.0005$, FDR $<0.005)$, including a $\mathrm{CpG}$ site in the TAC1 promoter. TAC1 hypermethylation in AUT and SEZ $(\mathrm{P}<0.005)$ was independently confirmed. In postmortem brain from RTT and AUT + SEZ females, TAC1 expression was significantly reduced in cingulate cortex $(\mathrm{P}=0.003)$, but not in striatum or temporal cortex $(\mathrm{P}>0.05)$. Chromatin immunoprecipitation in HEK cells confirmed MeCP2 binding at the TAC1 promoter, which was abolished following acute valproic acid treatment.

Conclusions: These data newly identify TAC1 as a MeCP2 target gene and suggest underlying seizure disorder and/or antiepileptic treatment affects DNA methylation peripherally, which is associated with region-specific gene expression changes in the brain. 


\subsection{7}

\section{CLINICAL GENETICS OF EYELID MYOCLONIA WITH} ABSENCES

Lynette Sadleir $^{1}$, D. F. Vears ${ }^{2}$, B. Regan ${ }^{2}$, N. Redshaw ${ }^{1}$, A. F. Bleasel $^{3,4}$ and I. E. Scheffer ${ }^{2,5}\left({ }^{1}\right.$ Department of Paediatrics, University of Otago, Wellington, New Zealand; ${ }^{2}$ Department of Medicine, University of Melbourne, Austin Health, Melbourne, VIC, Australia; ${ }^{3}$ Faculty of Medicine, University of Sydney, Sydney, NSW, Australia; ${ }^{4}$ Department of Neurology, Westmead Hospital, Sydney, NSW, Australia and ${ }^{5}$ Florey Neuroscience Institutes, Melbourne, VIC, Australia)

Rationale: Eyelid Myoclonia with Absences (EMA) is an uncommon absence epilepsy syndrome that is often refractory to treatment. The clinical genetics of EMA has not been well characterized although a family history of seizures is not infrequent.

Methods: Individuals with EMA were ascertained by referral and through the investigators' clinical practices. All available family members were assessed for seizures using a validated seizure questionnaire. Electroclinical data were obtained on each proband and all affected family members; pedigrees were constructed. Families were analyzed for phenotypic patterns.

Results: Eighteen individuals with EMA were recruited. A history of seizures was found in 34 relatives in 15/18 (83\%) families. In terms of epilepsy syndromes, nine relatives from $7 / 15$ families had febrile seizures. Two relatives had EMA. Classical Genetic Generalized Epilepsy (GGE) syndromes were seen in five relatives: two Generalized Tonic-Clonic Seizures alone, two Childhood Absence Epilepsy (CAE) and one Juvenile Myoclonic Epilepsy. Genetic Epilepsy with Febrile Seizures Plus (GEFS+) phenotypes occurred in 16 relatives. On review of the epilepsy syndromes within each family, seven families had a pattern consistent with GEFS+ while three families had classical GGE.

Conclusions: The clinical genetics of EMA is suggestive of complex inheritance with shared genetic determinants overlapping with both classical GGE and GEFS+. The epilepsy syndromes in relatives of probands with EMA differ from those found in families of probands with CAE supporting the concept that EMA is a distinct syndrome from CAE. This presumably reflects different genetic components contributing to their genetic architecture.

\subsection{8}

PRRT2 MUTATION IN JAPANESE CHILDREN WITH BENIGN INFANTILE EPILEPSY

Akihisa Okumura $^{1}$, K. Shimojima ${ }^{2}$, T. Kubota ${ }^{3}$, S. Abe ${ }^{1}$, S. Yamashita $^{4}$, K. Imai ${ }^{5}$, T. Okanishi ${ }^{6}$, H. Enoki ${ }^{6}$, T. Fukasawa ${ }^{3}$, T. Tanabe $^{7}$, T. Shimizu ${ }^{1}$ and T. Yamamoto ${ }^{2}{ }^{1}$ Juntendo University Faculty of Medicine, Tokyo, Japan; ${ }^{2}$ Tokyo Womens' Medical University Institute for Integrated Medical Sciences, Tokyo, Japan; ${ }^{3}$ Anjo Kosei Hospital, Anjo, Japan; ${ }^{4}$ Juntendo Nerima Hospital, Tokyo, Japan; ${ }^{5}$ National Epilepsy Center, Shizuoka Institute of Epilepsy and Neurological Disorders, Shizuoka, Japan; ${ }^{6}$ Seirei Hamamatsu General Hospital, Hamamatsu, Japan and ${ }^{7}$ TanabeKadobayashi Children's Clinic, Hirakata, Japan)

Rationale: Mutations in PRRT2 genes have been identified in patients with benign infantile epilepsy and/or paroxysmal kinesigenic dyskinesia. However, the rate of PRRT2 mutations in Japanese children with benign infantile epilepsy and/or paroxysmal kinesigenic dyskinesia has not been determined.

Methods: We explored PRRT2 mutations in Japanese children who had had unprovoked infantile seizures or convulsion with mild gastroenteritis. The probands included 16 children with benign infantile epilepsy, 6 children with convulsions with mild gastroenteritis, and 2 siblings with benign early infantile epilepsy. In addition, we recruited samples from family members when PRRT2 mutation was identified in the proband. Statistical analyses were performed to identify differences in probands with benign infantile epilepsy according to the presence or absence of PRRT2 mutation. Results: Among a total of 24 probands, PRRT2 mutations was identified only in 6 probands with benign infantile epilepsy. A common insertion mutation, c.649_650insC, was found in 5 families and a novel missense mutation, c. $981 \mathrm{C}>\mathrm{G}(\mathrm{I} 327 \mathrm{M})$, in one. The family history of paroxysmal kinesigenic dyskinesia was more common in probands with PRRT2 mutations than in those without mutations.

Conclusions: Our study revealed that PRRT2 mutations are common in Japanese patients with benign infantile epilepsy as well as Han Chinese or European-Caucasian patients.

Comparison of the probands with BPEI according to the presence or absence of PRRT2 mutation

\begin{tabular}{|c|c|c|c|}
\hline & $\begin{array}{l}\text { Probands with } \\
\text { PRR'2 mutation } \\
(\mathrm{n}=6)\end{array}$ & $\begin{array}{l}\text { Probands with } \\
\text { no mutation } \\
(n=10)\end{array}$ & \\
\hline $\operatorname{Sex}(\mathrm{M}: \Gamma)$ & $3: 3$ & $3: 7$ & NS \\
\hline Age at $S_{z}$ onsel $(\mathrm{m})$ & $7.5(3-11)$ & $4.5(3-10)$ & NS \\
\hline Age at the last $S z(\mathrm{~m})$ & $8(3-21)$ & $10(3-11)$ & NS \\
\hline Cluster of Szs & $4(67 \%)$ & $9(90 \%)$ & NS \\
\hline PKD & $1(17 \%)$ & 0 & NS \\
\hline Febrile $S 7$ & $1(17 \%)$ & $1(10 \%)$ & NS \\
\hline CwG & 0 & $2(20 \%)$ & NS \\
\hline Developmental delay & 0 & $2(20 \%)$ & NS \\
\hline \multicolumn{4}{|l|}{ Family history } \\
\hline Linprovoked infantile Sz & $3(50 \%)$ & $4(40 \%)$ & NS \\
\hline PKD & $3(50 \%)$ & 0 & $\mathrm{p}<0.05$ \\
\hline Febrile $\$ z$ & $2(33 \%)$ & $3(30 \%)$ & NS \\
\hline CwG & 0 & 0 & NS \\
\hline Developmental delay & 0 & 0 & NS \\
\hline
\end{tabular}

Sz: seizure, PKD: paroxysmal kinesigenic dyskinesia, $\mathrm{CwG}$ convulsion with mild gastroenteritis, NS: not significant

\subsection{9 \\ GLUT1-DEFICIENCY IN THE GENETIC GENERALISED EPILEPSIES}

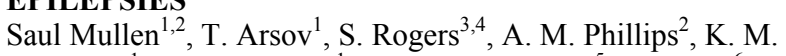
Lawrence $^{1}$, J. A. Damiano ${ }^{1}$, H. Goldberg-Stern ${ }^{5}$, Z. Afawi ${ }^{6}$, S. Kivity $^{5}$, C. Trager ${ }^{2}$, S. Petrou ${ }^{2,7}$, S. F. Berkovic ${ }^{1}$ and I. E. Scheffer ${ }^{2,8}$ ( ${ }^{1}$ Epilepsy Research Centre, University of Melbourne, Melbourne, VIC, Australia; ${ }^{2}$ Florey Neurscience Institute, University of Melbourne, Melbourne, VIC, Australia; ${ }^{3}$ St Vincents Institute of Medical Research, Melbourne, VIC, Australia; ${ }^{4}$ Dept. of Genetics, University of Melbourne, Melbourne, VIC, Australia; ${ }^{5}$ Epilepsy Centre, Schneider Children's Medical Center of Israel, Petah Tiqwa, Israel; ${ }^{6}$ Department of Neurology, Sourasky Medical Center, Tel Aviv, Israel; ${ }^{7}$ Centre for Neuroscience, University of Melbourne, Melbourne, VIC, Australia and ${ }^{8}$ Department of Paediatrics, Royal Children's Hospital, University of Melbourne, Melbourne, VIC, Australia)

Rationale: The genetic generalized epilepsies (GGEs) are common, heritable epilepsies which usually follow complex inheritance; currently little is known about their genetic architecture. Previously considered rare, GLUT1-deficiency, due to mutations in SLC2A1, leads to failure of glucose transport across the blood brain barrier and inadequate glucose for brain metabolism. GLUT1-deficiency was first associated with an encephalopathy and more recently found in rare dominant families with epilepsy and paroxysmal exertional dyskinesia (PED).

Methods: We examined whether GLUT1-deficiency causes common GGEs. 504 probands with GGEs and 470 controls underwent SLC2A1 sequencing. Glucose transport was assayed following expression of SLC2A1 variants in Xenopus oocytes. All available relatives were phenotyped and $S L C 2 A 1$ sequenced 
Results: Functionally validated mutations in SLC2A1were present in $7 / 504(1.4 \%)$ probands and 0/470 controls. PED, undiagnosed prior to study, occurred in one proband and 3/13 relatives with mutations. The GGEs in probands and relatives were indistinguishable from typical GGE. Three cases $(0.6 \%)$ had mutations of large functional effect and showed autosomal dominant inheritance or were de novo. Four $(0.8 \%)$ cases had a subtle functional effect; two showed possible dominant inheritance, two did not. These alleles leading to subtle functional impairment may contribute to complex, polygenic inheritance of GGE.

Conclusions: SLC2A1 mutations contribute to approximately $1 \%$ of GGE both as a dominant gene and as a susceptibility allele in complex inheritance. Diagnosis of GLUT1-deficiency has important treatment (ketogenic diet) and genetic counselling implications. The mechanism of restricted glucose delivery differs from the current focus on GGEs as ion channels disorders.

\subsection{0}

\section{DELETIONS OF MITOCHONDRIAL DNA IN HIPPOCAMPAL SUBFIELDS OF PATIENTS WITH HIPPOCAMPAL SCLEROSIS}

Wolfram Kunz, V. Peeva, E. Taherzadeh-Fard and G. Zsurka (Department of Epileptology, University of Bonn, Bonn, Germany)

Rationale: In this work we investigated large deletions of mitochondrial DNA as potential molecular cause for mitochondrial dysfunction in hippocampal subfields of patients with hippocampal sclerosis, one of the most common forms of therapy-resistant temporal lobe epilepsy. These mitochondrial DNA rearrangements are the accepted cause for mitochondrial dysfunction in various neurological disorders and aging.

Methods: Applying long-range PCR, single molecule PCR and multiplex PCR we identified, quantified and mapped mitochondrial DNA deletions in hippocampal subfields of 63 patients with hippocampal sclerosis and of 20 patients with lesional temporal lobe epilepsy, who underwent epilepsy surgery. Using a next generation sequencing approach we performed in a limited number of patients deep sequencing of the mitochondrial genome to determine the complete deletional spectrum.

Results: The deletion loads approached in the CA3 hippocampal subfield and the dentate gyrus of patients with hippocampal sclerosis 2-8 \% of total mitochondrial DNA content. A major fraction of the detected deletions had a prominent 3 ' breakpoint hot spot at the end of the D-loop (around np 16,070) (Fig. 1, cf. red vertical bar at $16,000)$. That location of 3' breakpoints strongly implies reactive oxygen species-caused mitochondrial DNA double strand breaks as potential mechanism of deletion formation. High levels of deleted mitochondrial DNA molecules appear to correlate with the presence of cytochrome c oxidase-negative succinate dehydrogenate positive neurons in the CA3 and CA4 hippocampal subfields of patients with hippocampal sclerosis. Hippocampal samples from age-matched patients with epilepsy due to lesions in the temporal lobe did contain substantially lower levels of deleted mitochondrial DNA (Fig. 2, please note the approximately 10 -fold lower amouts of deletion species). Additionally, these deletions observed in lesional epilepsy showed the standard distribution of deletional breakpoints (Fig. 2, cf. low vertical bar at 16,000), which has been reported for the aging brain (Guo X., Popadin K.Y., Markuzon N., Orlov Y.L., Kraytsberg Y., Krishnan K.J., Zsurka G., Turnbull D.M., Kunz W.S., Khrapko K. Repeats, longevity and the sources of mtDNA deletions: evidence from 'deletional spectra'. Trends Genet. 2010; 26:340-343).

Conclusions: Dysfunction of mitochondrial oxidative phosphorylation in the epileptic focus of patients with Ammon's horn sclerosis appears to be related to clonal expansion of specific types of deleted molecules of mitochondrial DNA generated by increased oxidative stress. This mechanism is proposed to be relevant for epileptogenesis and seizure generation in the hippocampus of patients with temporal lobe epilepsy and hippocampal sclerosis.

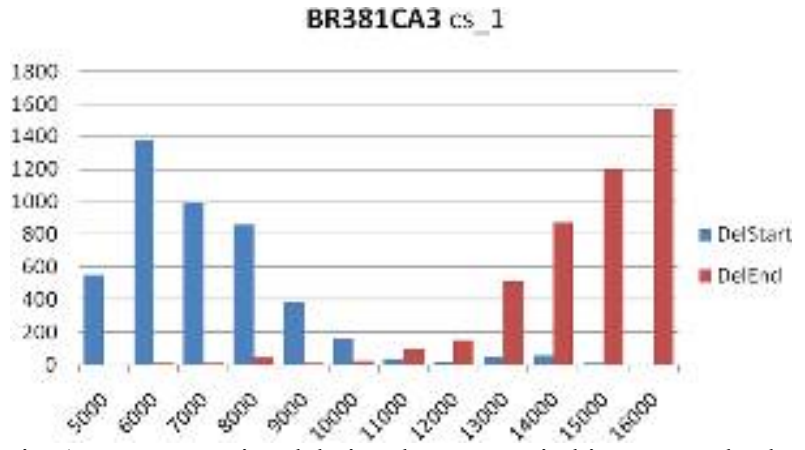

Fig. 1: Representative deletional spectrum in hippocampal sclerosis obtained by deep sequencing

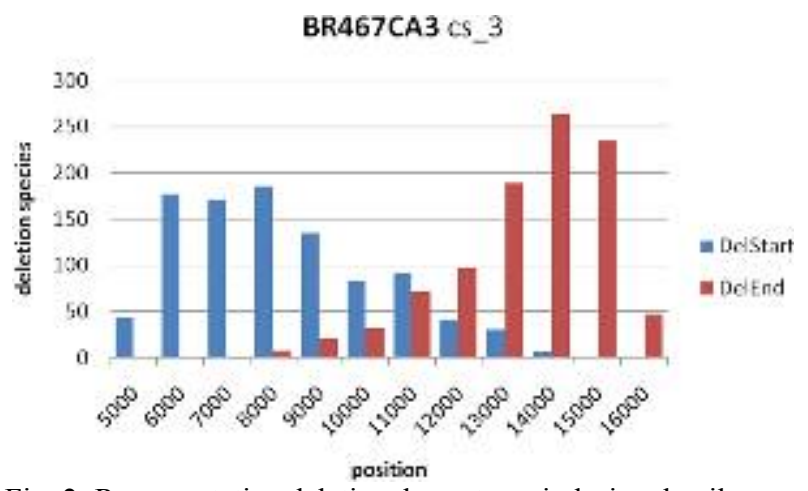

Fig. 2: Representative deletional spectrum in lesional epilepsy obtained by deep sequencing

\section{Health Services: Delivery of Care}

\subsection{1 \\ BATHROOM SAFETY AND FALLS IN THE EPILEPSY MONITORING UNIT}

Katherine C. Riordan ${ }^{1}$, K. H. Noe ${ }^{1}$, B. M. Corbett ${ }^{2}$, M. T. Hoerth ${ }^{1}$, J. I. Sirven ${ }^{1}$ and J. F. Drazkowski ${ }^{1}{ }^{1}{ }^{1}$ Neurology, Mayo Cilnic Arizona, Phoenix, AZ and ${ }^{2}$ Nursing Quality Management, Mayo Clinic Arizona, Phoenix, AZ)

Rationale: Rationale: Falls are a major safety issue in the epilepsy monitoring unit (EMU). Falls in the bathroom are a particular concern as patient supervision must be balanced with privacy concerns. In the bathroom lack of video monitoring, hard surfaces, and limited space could all compromise the ability of staff to recognize and respond to events. Little is known about the absolute fall risk in the EMU bathroom and current safety practices vary widely among epilepsy centers. The purpose of this study was to quantify the frequency of falls in the bathroom and the risk of injury, as well as to determine if certain event types are more likely to occur in the bathroom.

Methods: Methods: A retrospective review of 616 consecutive adult admissions to the Mayo Clinic Arizona EMU from January 1, 2008 to December 31, 2010 was performed. Any clinical event described by the patient or witnessed by EMU staff was included. Data was collected on event type, location (in bathroom or not), and whether or not there was an associated fall.

Results: Results: There were 1767 recorded events resulting in 10 falls. 140 patients had 675 cumulative clinical seizures: $4(0.6 \%)$ were associated with a fall and 4(0.6\%) were in the bathroom. 157 patients had 651 cumulative psychogenic non-epileptic events (PNES): $4(0.6 \%)$ were associated with a fall and $8(1.2 \%)$ were in the bathroom. 2 of 135 non-epileptic physiologic events $(1.5 \%)$ resulted in a fall (both from syncope), and $2(1.5 \%)$ were in the bathroom. Differences in fall frequency or percentage of events occurring in the bathroom by spell type were not statistically 
significant. 4/10 falls (40\%) occurred in the bathroom, 2 with seizure and 2 with PNES. The only 2 falls resulting in injury were from seizures in the bathroom, both injuries were minor. No major injuries occurred. Seizures in the bathroom were significantly more likely to result in fall $(2 / 4,50 \%$ vs. $2 / 671,0.3 \%$; $=0.0002)$. PNES in the bathroom were also significantly more likely to result in fall $(2 / 10$, $20 \%$ vs. $2 / 641,0.3 \%$; $\mathrm{p}=0.0013$ ).

Conclusions: Conclusions: With safety precautions including limited mobility and close 1:1 supervision of patients when out of bed, falls occurred in $1.6 \%$ of EMU admissions and were associated with minor injury in $0.3 \%$ of admissions. Seizures, PNES, and other physiologic events were no more likely to occur in than out of the bathroom. Seizures and PNES occurring in the bathroom were more significantly more likely to result in falls. Bathroom safety precautions are justified given the risk of fall and injury.

\subsection{2 \\ MULTIDISCIPLINARY SAFETY ROUNDS IN THE EPILEPSY MONITORING UNIT: OUTCOMES FROM A QUALITY IMPROVEMENT INITIATIVE}

Katherine Noe, J. Drazkowski, S. Zarkou, J. Gerke, M. Hoerth, J. Sirven and R. Zimmerman (Mayo Clinic, Phoenix, AZ)

Rationale: Patient safety is an important focus for all health care environments. Patients in epilepsy monitoring units (EMU) can be potentially harmed by provoked seizures, medication changes, medical co-morbidities, and falls. Strategies for improving patient safety include surveillance; analysis of contributing technical, process or human factors; and corrective actions to prevent future harm. Patient safety rounds are one intervention that has been successfully employed to improve inpatient care, but safety rounds specific to the EMU setting have not previously been reported.

Methods: In January of 2011 we initiated a program of active prospective surveillance for safety issues in patients admitted to the EMU at Mayo Clinic Arizona. Multidisciplinary safety rounds were incorporated into the regularly scheduled epilepsy surgery conference which included epilepsy neurology, neurosurgery, neuropsychology, EEG technology, radiology, and nursing. Events and recommended responsive actions based on multidisciplinary discussion were tracked. Findings from Jan 1, 2011 through May 31, 2012 are reviewed.

Results: During the 17 month time frame there were 345 adults monitored for a total of 1475 days. 17 safety events were identified: 1 prolonged focal motor seizure requiring IV medication, 1 fall, 2 shoulder dislocations from convulsions in patients with history of same, 3 potentially serious cardiac arrhythmias, 1 ICU transfer for complication of intracranial monitoring, 2 potential medication errors, 4 technical issues with EEG monitoring, 1 missed pregnancy test in a non-pregnant patient undergoing nuclear imaging, 1 issue with nursing seizure assessment, 1 patient with a weapon (gun) in bed. $5 / 17$ events resulted in harm to the patient, and $12 / 17$ were near miss events. 10/17 (59\%) events were considered preventable. 11/17 $(65 \%)$ events resulted in changes in policies or procedures after discussion in safety rounds.

Conclusions: With prospective surveillance potential safety issues were identified in 5\% (17/345) of EMU admissions and lead to harm in $1.5 \%(5 / 245)$. Multidisciplinary safety rounds provided opportunity for inter-professional education and a safe, effective environment for dissemination of information on safety risks. It resulted in root cause analyses and formulation of responsive actions to reduce preventable harm. Safety rounds facilitated quality improvement through changes in policies and procedures to improve future outcomes in the majority of events reviewed.

\subsection{3}

A MULTIDISCIPLINARY PROCESS TO KEEP KIDS AND STAFF SAFE IN A PEDIATRIC EPILEPSY SURGERY PROGRAM

Cornelia H. Gonsalves ${ }^{1}$, P. Pilcher ${ }^{2}$ and S. Fletcher ${ }^{3}\left({ }^{1}\right.$ Neurology, Cincinnati Children's Hospital, Cincinnati, OH; ${ }^{2}$ Nursing, Cincinnati Children's Hospital Medical Center, Cincinnati, OH and ${ }^{3}$ Social Work, Cincinnati Children's Hospital Medical Center, Cincinnati, $\mathrm{OH})$

Rationale: Pediatric epilepsy surgery patients frequently have behaviors or mental health issues that impact the safety of the child, family or staff. An interdisciplinary process was developed, at a large midwestern pediatric medical center, to enhance safety during epilepsy surgery inpatient admissions or related outpatient visits. The aim of this new approach was to, first, identify safety problems for children going through the multistaged surgical process and, then, to develop a strategic plan to lessen serious safety events and assist families in having more positive hospital experiences.

Methods: Formulation of this approach included 24 of 43 children with medically-resistant epilepsy who had a history of aggression, threatening behavior, self-destructive behavior, significant anxiety or a psychotic mental health disorder. All children that had been referred to the epilepsy surgery program from June 2011 through March 2012 were included, regardless of the reason for referral. Chidlren were scheduled for new-patient visits, involved in Phase I testing, undergoing surgical procedures or being evaluated in clinic for pre and post-operative visits. The hospital's serious safety data was reviewed and safety events involving epilepsy surgery patients tracked. Hospital resources, interdisciplinary colleagues and components of the safety process were identified. An algorithm was created to outline the steps and flow of the safety process which would meet child, family and staff needs.

Results: We identified 11 out of 24 children who would benefit from a multidisciplinary safety approach during evaluation or surgery. These children had a history of aggression to people or animals, schizophrenia, obsessive compulsive disorder, anxiety, pervasive developmental disorder or autism. Prior to implementation of the safety process, two staff members and one parent were injured in clinic, one staff member was hurt in the emergency department and two staff were injured on the epilepsy monitoring unit. Since implementing this process, there have been no serious safety events. Increased satisfaction with the safety process and inpatient hospital stays was reported by careproviders and parents

Conclusions: This new approach, described in the algorithm, demonstrates the feasibility of reducing the number and severity of serious safety events and improving family satisfaction with early identification of at-risk patients, advance planning and notification of disciplines in respective areas, use of multi-disciplinary research, and implementation of the safety process, along with continual evaluation to maintain safety and improve the process. Secondary outcomes included use of this approach as a model for developing a hospital-wide Behavior Safety Team with the goal to decrease serious safety events hospital-wide and increasing patient-family satisfaction with the hospital experience. In addidtion, children who may not have been epilepsy surgery candidates for surgery because of mental health issues are now able to fully evaluated and considered for surgical intervention. 

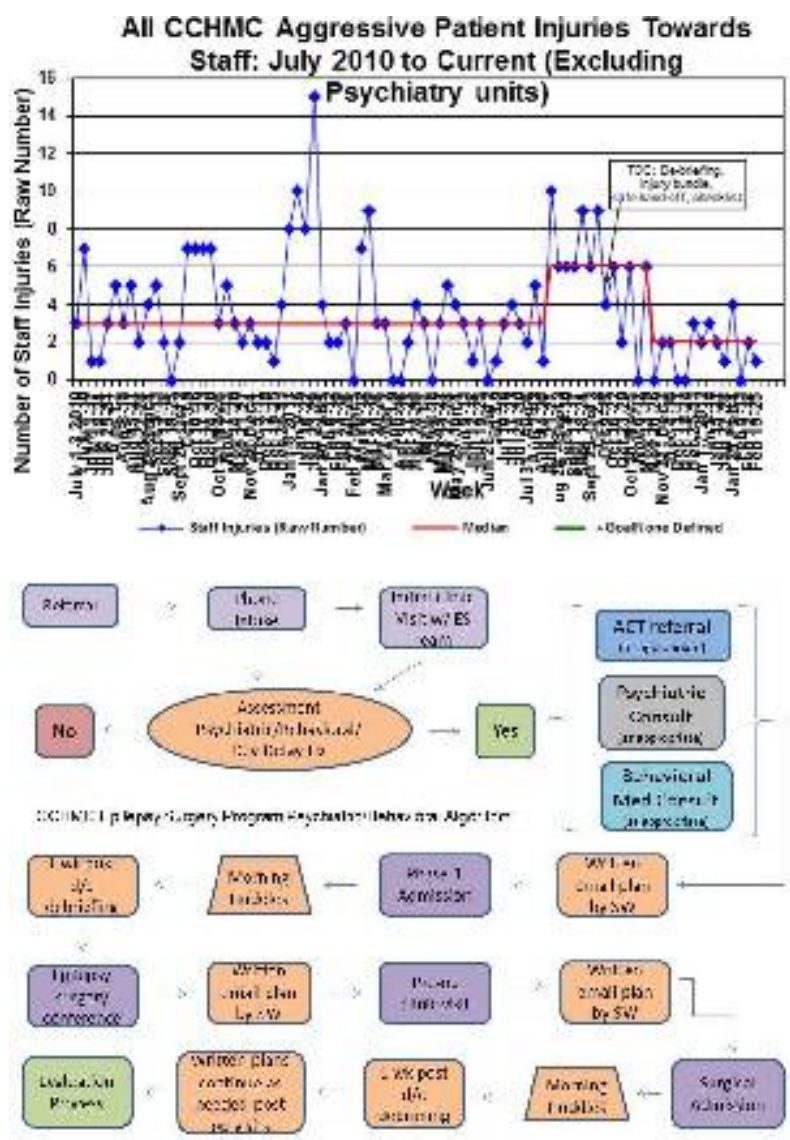

\subsection{4}

SUDDEN UNEXPECTED DEATH IN EPILEPSY: KNOWLEDGE AND EXPERIENCE AMONG US AND CANADIAN NEUROLOGISTS

Daniel Friedman ${ }^{1}$, E. J. Donner ${ }^{2}$, D. Stephens ${ }^{2}$, C. Wright ${ }^{3}$ and O. Devinsky ${ }^{1}{ }^{1}$ Neurology, NYU Langone Medical Center, New York, NY; ${ }^{2}$ SickKids Hospital, Toronto, ON, Canada and ${ }^{3}$ Stormlab Consulting, Boulder, CO)

Rationale: Several groups have suggested that all patients with seizure disorders and their caregivers be counseled about sudden unexpected death in epilepsy (SUDEP) though some authors have expressed concern about universal discussions due to fear of creating unnecessary distress for those who are at low risk. Little is known about the current SUDEP discussion practices of neurologists in the U.S. and Canada.

Methods: An electronic, web-based survey to assess knowledge and experience with SUDEP and discussing SUDEP with patients/caregivers was sent to 17558 neurologists in the US and Canada. Survey questions included years in practice, type of practice, training experience and average number of epilepsy followed annually, number of SUDEP cases in past the 24 months, frequency of SUDEP discussion, reasons for discussing/not discussing SUDEP, timing of SUDEP discussions, and perceived patient reactions. We examined factors which influence the frequency of SUDEP discussion and a perceived negative patient response using multivariate logistic regression.

Results: There were 1200 completed surveys. Only $6.8 \%$ of respondents discussed SUDEP with nearly all ( $>90 \%$ of the time) of their patients with epilepsy/caregivers while $11.6 \%$ never discussed it. Factors which independently predicted whether SUDEP was discussed nearly all of the time were number of epilepsy patients seen annually (OR 1.27, 1.01-1.59 95\% CI, p = 0.043) and if the respondent had a SUDEP case in the past 24 months (OR 2.33, 1.42$3.81, \mathrm{p}=0.0008$ ). The most common reported patient reaction to SUDEP discussion was "anxiety." Negative reactions were reported as most common by $59.5 \%$ of respondents. Frequency of SUDEP discussion was independently associated with an increased likelihood of a negative perceived reaction $(\mathrm{OR} 1.19,1.04-1.39, \mathrm{p}=0.015)$ while years in practice was associated with a decreased likelihood of negative reaction (OR 0.41, 0.24-0.70, p = 0.0011).

Conclusions: U.S. and Canadian neurologists rarely discuss SUDEP with all of their patients with epilepsy/caregivers though discussions are more common among neurologists who see frequently see epilepsy patients and have had a recent SUDEP in their practice. Perceived negative reactions to SUDEP discussions are common but not universal; more experienced neurologists are less likely to encounter negative reactions suggesting there may be ways to frame the discussion of this important but thankfully rare outcome of epilepsy in a way that minimizes patient/caregiver distress.

\subsection{5}

ARE PATIENTS SAFE WHEN THEY SEIZE IN HOSPITAL? Khara M. Sauro, ${ }^{1,3}$, H. Dhaliwal ${ }^{1}$, F. Abdulla ${ }^{1}$, M. Suddes ${ }^{4}$, S. Macrodimitris $^{4,5}$, C. Krassman ${ }^{4}$, S. Wiebe ${ }^{1,3}$, N. Pillay ${ }^{1}$, P. Federico $^{1,2}$, W. Murphy ${ }^{1}$ and N. Jette ${ }^{1,3}\left({ }^{1}\right.$ Clinical Neurosciences, University of Calgary, Calgary, AB, Canada; ${ }^{2}$ Radiology, University of Calgary, Calgary, AB, Canada; ${ }^{3}$ Community Health Sciences, University of Calgary, Calgary, AB, Canada; ${ }^{4}$ Clinical Neurosciences, Alberta Health Services, Calgary, AB, Canada and ${ }^{5}$ Psychology, University of Calgary, Calgary, AB, Canada)

Rationale: Quality and safety of care in hospitals is being increasingly scrutinized. Because of the methods used to induce seizures (e.g. antiepileptic drug withdrawal), patients in the SMU are more likely to experience generalized tonic-clonic seizures (GTCs), which may increase their risk of injury. In order to mitigate the risk of injury to patients, the proper management of seizures in the SMU is essential. The objectives of this study were to: 1) develop a tool to evaluate the management of GTCs, and 2) evaluate the management of GTCs in a SMU using the newly developed tool.

Methods: (1) Tool development: Key steps to the management of GTCs were first identified through a literature search. The list of items was refined using SMU expert opinion (epileptologists, neuroscience nurses, EEG technologists, quality/safety personnel). Once consensus was reached, a tool was created which examined Safety, Assessment, and Management (SAM) of GTCs in the SMU. (2) Tool implementation: The tool was applied retrospectively on two separate samples to all GTCs captured on video-EEG in the SMU between January 2008-December 2009 and again between January 2011-May 2012 after the implementation of general education sessions (not specifically targeting the tool or the management of GTCs) for SMU nursing staff. An overall completion rate for every step and percent of steps completed was calculated for each "SAM" category. Descriptive statistics (means and frequencies) were generated and student t-test and correlation analysis were performed to examine differences between tests administrations.

Results: A 17-item GTC management rating tool was created. The tool consisted of three categories: safety (6 items), assessment (4 items) and management ( 7 items). All items on the tool were forced choice (complete/NA or incomplete). There were significant improvements overall, and in each category $(\mathrm{p}<0.001)$ from the first administration to the second. The safety category improved over time by $6.6 \%(\mathrm{p}<0.01)$, the assessment category by $7.7 \%(\mathrm{p}<0.01)$ and the management category by $19.5 \%(\mathrm{p}<0.001)$. Overall, the mean number of steps completed was $67 \%$. Of the three components of the rating tool, the management category was on average the most completed category (mean steps completed $=75.3 \%$ ) while the assessment one was the least completed (mean steps completed $=$ $45.4 \%)$.

Conclusions: The results of the present study revealed areas for potential improvement in the management of GTCs in a SMU. Despite this, it is encouraging that a large proportion of the safety and management aspects of caring for patients during a GTC, are being 
executed. A significant improvement was observed in the management of GTCs over time, possibly as a result of the implementation of a general training course introduced for staff in our SMU. We therefore plan to create a training module specific to GTC management, to further improve the management of GTCs in the SMU. The tool will be implemented prospectively after the SMU staff has completed the GTC management training module.

\subsection{6 \\ PROVIDER AND PATIENT PERSPECTIVES OF EPILEPSY SELF-MANAGEMENT NEEDS}

Erica K. Johnson ${ }^{1,2}$, R. T. Fraser ${ }^{3,4}$, J. W. Miller ${ }^{4}$, N. Temkin ${ }^{5,6}$, J. Barber $^{5,6}$, L. Caylor ${ }^{7}$, P. Ciechanowski ${ }^{8}$ and N. Chaytor ${ }^{4}\left({ }^{1}\right.$ Health Promotion Research Center, School of Public Health, University of Washington, Seattle, WA; ${ }^{2}$ Human Services \& Rehabilitation, Western Washington University, Seattle, WA; ${ }^{3}$ Rehabilitation Medicine, University of Washington, Seattle, WA; ${ }^{4}$ Neurology, University of Washington, Seattle, WA; ${ }^{5}$ Neurological Surgery, University of Washington, Seattle, WA; ${ }^{6}$ Biostatistics, University of Washington, Seattle, WA; ${ }^{7}$ Neurosciences Institute, Swedish Medical Center, Seattle, WA and ${ }^{8}$ Psychiatry \& Behavioral Sciences, University of Washington, Seattle, WA)

Rationale: Epilepsy management issues for patients include poor knowledge about the disorder, treatment, and consequences; seizure frequency and medical adherence; and psychosocial aspects (anxiety, depression, social stigma, cognition, and family adjustment). Issues are often reported separately in studies by either health care professionals or patients, but rarely by both sources at the same time, using a similar metric. Methodological challenges in epilepsy selfmanagement trials are apparent: lack of power estimates; recruitment issues; high dropout; and questions of engagement in online programs where attendance behavior is difficult to monitor. The challenge of participant engagement in research trials is salient. To clarify the relevance of patient-centered planning in epilepsy selfmanagement programs, epilepsy patients and clinicians were surveyed regarding self-management problem areas and program design preferences (leadership, session format, session approach/content).

Methods: Respondents came from two populations: patients and providers. The patient sample was adults age 18 and older with chronic epilepsy $(\mathrm{n}=165)$. The provider sample was medical and allied health workers who treat people with epilepsy in specialty epilepsy centers $(n=20)$. Both groups completed a mail survey that was similar, but not identical. The patients rated their life problems related to work; independent living; socializing; epilepsy; emotions; cognition; health and well-being; and medical care, and rank ordered or otherwise selected preferences for a self-management program (format, content, leadership, duration). The providers rated the same life problem areas based on their typical patients over the past 6 months. For the program items, they responded based on their average patient.

Results: Results indicated two patient subgroups: one with "epilepsyonly" and one with "epilepsy-plus" marked by probable depression and/or cognitive problems. The subgroups differed in responses to problem areas. Their responses were examined separately against provider responses. Providers rated patient problems more severe in all domains: Work, Independent Living, Socializing, Life with Epilepsy, Mood, Cognition, General Health and Well-Being, and Medical Care (all $\mathrm{p}<0.001$ ). Pairwise analyses indicated providers rated all of the eight domains higher (worse) than the "epilepsy-only" subgroup (all $\mathrm{p}<0.001$ ). Providers rated four of eight domains higher (worse) than the more impaired "epilepsy-plus" subgroup: Work $(\mathrm{p}=$ 0.035), Independent Living ( $\mathrm{p}=0.005)$, Life with Epilepsy ( $\mathrm{p}=$ 0.029), and Medical Care $(\mathrm{p}=0.027)$. With the Bonferroni correction, only the Independent Living comparison was significant. Although differences were seen between groups regarding program preferences, these were largely not significant after correcting for multiple comparisons.
Conclusions: Findings demonstrate dissonance between patient and clinician perceptions of self-management problem areas. This has implications for engaging patients in self-management programs. Efforts to address problems patients endorse, in a context they prefer, should be made.

\subsection{7}

\section{SATISFACTION WITH EPILEPSY CARE: A SYSTEMATIC REVIEW}

N. K. Wiebe ${ }^{1}$, K. M. Fiest ${ }^{1}$, Jonathan Dykeman ${ }^{1}$, X. R. Liu ${ }^{1,2}$, N. Jette $^{1}$, S. Patten ${ }^{1}$ and S. Wiebe ${ }^{1}\left({ }^{1}\right.$ University of Calgary, Calgary, AB, Canada and ${ }^{2}$ Institute of Neuroscience and the Second Affiliated Hospital of Guangzhou Medical University, Guangzhou, China)

Rationale: Satisfaction with epilepsy care is related to more than simply the delivery of management and services. It is related to expectations, attitudes, disease course, and many other factors. Our aim was to describe the current literature surrounding the measurement of patient satisfaction with epilepsy care.

Methods: Medline, PsychINFO, CINAHL, Cochrane Central Register of Controlled Trials, and Embase were searched using subheadings and keywords related to patient satisfaction,

client/consumer satisfaction, and treatment outcomes. Two independent reviewers screened abstracts and full-text and abstracted data from eligible studies. Included studies measured satisfaction with epilepsy care, were original research, and described how satisfaction was assessed. Analyses were carried out to 1) assess the purpose and sample characteristics, 2) summarize satisfaction scale development and content, and 3) assess reported outcomes.

Results: Of 6368 abstracts identified by the search, 125 were reviewed in full-text with 28 studies meeting all eligibility criteria. The median number of patients included in the studies was 143 (range: 18-1275). Assessments were primarily completed in adult patients and satisfaction in children was measured using parental responses.

All studies used some form of parent, patient or physician self-report. Eight studies used a previously published scale to measure satisfaction, with two being specific to epilepsy. Satisfaction domains can be broadly categorized into disease course,

communication/information, access to care, and physician competence. Studies conflicted in whether patients were more satisfied with specialist or general practitioner (GP) care. Four reported patients preferring GP care, while 10 studies reported preference with specialist care.

Most (9/13) studies addressing information provision reported high levels of patient satisfaction, except for information related to medication side effects. However, one study found that $90 \%$ of respondents wanted more information about epilepsy, and only $20 \%$ felt they were given enough information on medication side-effects. Twelve studies assessed patient physician communication and most people were satisfied with the level of communication. In one study, $78.2 \%$ felt that epilepsy was explained adequately and adequate information was provided. However, in one study $48 \%$ of patients describing their care as excellent or good believed that they did not receive enough information from their specialist, though $81 \%$ felt that their doctor knew enough about epilepsy.

Conclusions: Though measures of satisfaction with epilepsy-care exist they have not been extensively validated nor do they contain many of the important domains identified by this review. It is also unclear whether epilepsy-specific scales are superior to generic satisfaction scales in persons with epilepsy. Satisfaction is a patientcentered concept, and it is important to ensure this perspective is maintained in any scale employed in epilepsy research. 


\subsection{8}

\section{ADHERENCE TO QUALITY INDICATORS IN EPILEPSY} CARE AT A COMPREHENSIVE EPILEPSY CENTER

Roxana Pourdeyhimi, C. Tennant, B. Wolf and G. Martz (Medical University of South Carolina, Charleston, SC)

Rationale: The American Academy of Neurology published a set of 8 quality indicators for epilepsy management, intended for use in provider self-assessment and for guiding reimbursement. Pugh et al reported similar data showing $44 \%$ adherence to a related set of quality indicators at a tertiary center. We assessed the degree to which specific measures [(1) characterization of seizure type and frequency, (2) specific epilepsy diagnosis, and (3) availability of EEG information] are met in the comprehensive epilepsy clinics at the tertiary referral center at the Medical University of South Carolina (MUSC).

Methods: We retrospectively identified outpatients seen by MUSC epilepsy providers between 1/1/2011 - 12/31/2011 that received an epilepsy ICD code (345.xx). From this population, we randomly selected subjects for chart abstraction. We determined the number of patients for whom there was adherence with quality indicators 1-3. Adherence was defined based on the descriptions published by the AAN. Subjects met criteria for indicator (1) when at least one seizure type was described AND seizure frequency was documented either quantitatively or qualitatively. Positive criteria for indicator (2) required documentation of etiology or specific epilepsy syndrome. Adherence with indicator (3) required documentation of results from a prior EEG or plans to obtain a future EEG. The number of patients meeting each indicator was identified.

Results: 45 epilepsy patients ( 25 female) were identified with 69 visits. Mean age was 36 (range 7-75). 28 subjects had a single visit during the review period (range 1-3). Of 69 visits, 5 were new-patient appointments. Mean duration of epilepsy was 16.6 years (range 2 weeks -51 years). Eight providers were represented, with a mean of 8.5 visits per provider (range 1-20). INDICATOR 1: 33 of 45 subjects had at least one visit with both seizure type and frequency documented. Of 15 subjects with $>1$ visit, 7 had these documented at every visit. 12 subjects never had a visit with both seizure type and frequency documented. These 12 subjects also never had a documented reason for lacking this information. INDICATOR 2: 9 of 45 subjects had at least one visit that met positive criteria. Of 15 subjects with $>1$ visit, 1 met this at every visit. All 9 subjects had a specific epilepsy syndrome listed, and none referred to a specific etiology. INDICATOR 3: 33 of 45 subjects had either a prior EEG documented or orders for a future EEG. Of 15 subjects with $>1$ visit, 7 had these documented at every visit. While 12 of 45 subjects never had an EEG documented. Of these 12, none had a reason explaining the absence of EEG.

Conclusions: Level of adherence with quality indicators at MUSC was in the range expected based on prior published reports. Further analysis with additional subjects is needed to determine the impact of these measures on the quality of care provided.

\subsection{9}

\section{NURSES' PERCEPTION OF KNOWLEDGE RELATED TO THE NEUROPSYCHOLOGICAL EVALUATION IN EPILEPSY}

Linda S. Allen $^{1}$, m. Paulson-Conger ${ }^{1}$, S. Jansen ${ }^{1}$, K. Schroeter ${ }^{1}$, D. Sabsevitz ${ }^{1}$, J. Bergholte ${ }^{1}$ and M. Plueger ${ }^{1,2}\left({ }^{1}\right.$ Neuroscience, Froedtert and Medical College of Wisconsin, Milwaukee, WI and ${ }^{2}$ Neurology, Barrow Neurological Institute, Phoenix, AZ)

Rationale: Nurses are key providers of patient/family education. Neuroscience nurses with a sound knowledge-base may be better able to improve the overall understanding of patients regarding their illness and what implications epilepsy has on their lifestyle.: The purpose of this study is to determine nurses' perception of their knowledge related to neuropsychological testing in epilepsy. A secondary purpose of this project is to determine if an educational intervention on neuropsychological testing in epilepsy can increase the knowledge and confidence level of neuroscience nurses. Methods: A convenience sample of full/part-time registered nurses and graduate nurses (potential $n=119$ ) from a 34-bed inpatient neuroscience unit and a 12-bed neuroscience intensive care unit in an accredited Epilepsy Center at an academic medical center located in the Midwest was used for this study. This IRB-approved descriptive study used a 25 question pre/post survey, which focused on the perceived confidence and knowledge of the subjects related to neuropsychological evaluation. There were 14 questions focusing on nurses' knowledge and confidence and 6 questions that referred to their beliefs. A 30 minute educational presentation was viewed prior to the post-survey. All descriptive and statistical tests were analyzed using PASW Statistics 19, Release Version 19.0.0.

Results: All data was analyzed using Predictive Analytics SoftWare Statistics software. Descriptive statistics, including Chi Square Tests, Independent-Samples T-test or Non-parametric tests will be utilized for data analysis. $\mathrm{N}=38$ (Pre-survey $\mathrm{n}=74$ ) Measures of confidence questions improved significantly following the education, but beliefs did not change.

Conclusions: Implications may include that improvement in selfreported nurse confidence in practice competencies and knowledge promotes better overall care. Also neuroscience nursing education, understanding, and confidence levels may improve nurses' abilities to enhance patient outcomes.

\subsection{0}

\section{VISITS FOR SEIZURES TO AN EMERGENCY ROOM IN A LARGE COMMUNITY HOSPITAL: DEMOGRAPHIC AND CLINICAL CHARACTERISTICS}

David E. Friedman $^{1,2}$, S. Ye ${ }^{1,2}$, P. A. Patrick ${ }^{1,2}$ and B. Rosenthal ${ }^{1,2}$ $\left({ }^{1}\right.$ Winthrop University Hospital, Mineola, NY and ${ }^{2}$ SUNY Stony Brook School of Medicine, Stony Brook, NY)

Rationale: Optimal control of seizures is accomplished through consistent and regular outpatient management. However, many people with epilepsy make use of the emergency room (ER) for each breakthrough seizures. In fact, current literature provides evidence that poor seizure control is associated with regular use of the ER and inconsistent follow up with outpatient neurologists. The aim of this study, therefore, was to describe patients using the ER for management of epileptic seizures so that targeted education care can be recommended.

Methods: This retrospective cohort study collected data on adult patients presenting at the emergency room of Winthrop University Hospital between June 1, 2011 and December 31, 2011 with a first unprovoked, recurrent unprovoked, or a provoked seizure. For patients presenting more than once during the study period, the most recent visit was included in these analyses.

Results: A total of 155 patients presented with seizures as follows: recurrent unprovoked (epileptic) seizures, $71.0 \%$; first unprovoked seizures, $16.8 \%$; and, provoked seizures, $12.3 \%$. Subjects with epileptic seizures $(n=110)$ were $44.9 \pm 21.8$ years of age on average (range: 18 - 96) and $58.2 \%$ were male. Almost $60 \%$ of subjects were Caucasian and $31.8 \%$ were African-American. Most patients experienced generalized seizures $(93.6 \%)$. Sixty percent of subjects were under the care of a neurologist; primary care physician, $20.9 \%$; no provider, $15.5 \%$; and, epileptologist, $3.5 \%$. For patients presenting at the ER multiple times $(n=22,20 \%)$, these preliminary analyses did not reveal any differences in patient demographic characteristics. Acute seizure related injuries were present among $31.6 \%$ of patients $(\mathrm{n}=49)$. Though definitive causes for most seizures $(59.1 \%)$ could not be determined based on evaluation in the ER, others were thought to be secondary to medication noncompliance $(18.4 \%)$, toxic intake (8.7\%), and metabolic abnormalities (6.2\%).

Conclusions: Most acute care evaluations for seizures in the ER were for breakthrough epileptic seizures. Unlike prior studies, our initial 
analyses revealed no differences in patient characteristics between patients visiting the ER once versus those visiting multiple times. Acute seizure related injuries were commonly encountered. Investigators will continue to evaluate and compare these groups as more patients are enrolled in our study. Further study indentifying potential factors associated with ER care for seizures in our patient population could lead to ways to possible minimize ER visits, and shift most care to the outpatient setting, if appropriate.

\section{Health Services: Access to Care}

\subsection{1}

\section{DISPARITIES IN ACCESS TO SPECIALIZED EPILEPSY} CARE AMONG PEOPLE WITH EPILEPSY

Nicholas K. Schiltz $^{1}$, S. M. Koroukian ${ }^{1}$, T. E. Love ${ }^{1,3}$, M. E. Singer ${ }^{1}$ and K. Kaiboriboon ${ }^{2}\left({ }^{1}\right.$ Epidemiology \& Biostatistics, Case Western Reserve University, Cleveland, OH; ${ }^{2}$ Department of Neurology, University Hospitals Case Medical Center, Cleveland, $\mathrm{OH}$ and ${ }^{3}$ Center for Health Care Research \& Policy, MetroHealth Medical Center, Cleveland, $\mathrm{OH}$ )

Rationale: Disparities in access to specialized epilepsy care, especially epilepsy surgery have long been suspected, but available studies have focused only on the impact of individual characteristics such as age, race, and income. The impact of community characteristics, such as socioeconomic status of the neighborhood and health system resources, on access to specialized care in persons with epilepsy has never been investigated. We evaluate the association between individual-level and contextual-level characteristics and access to video-EEG monitoring (VEEG) and epilepsy surgery, with specific emphasis on insurance status and proximity to an epilepsy center.

Methods: This study is a retrospective cross-sectional study using data between 2005 and 2009 from the California State Inpatient Sample (SID), the State Ambulatory Surgery Database (SASD), and the State Emergency Department Database (SEDD), which provided information on all hospital discharges, ambulatory surgeries, and ER visits. These datasets were linked with 2009 Area Resource File, which provided health resource information and socioeconomic characteristics at the county level. A two-level hierarchical logistic regression model was used to model the probability that an individual would receive VEEG or surgery. Individual-level predictors included insurance status, age, race/ethnicity, gender, and comorbidities, while county level predictors included proximity to a comprehensive epilepsy center (EC) and social and economic characteristics.

Results: Our study included 195,166 adults with epilepsy, among whom 4,707 had VEEG monitoring and 779 underwent surgery during the study period. The uninsured individuals were less likely to have VEEG (Adjusted odds ratio (AOR), 0.16; 95\% Confidence Interval (CI), 0.13-0.19) and surgery (AOR, 0.05; 95\%CI, 0.04-0.08). Similarly, those with Medicaid had lower odds of receiving VEEG (AOR, 0.65; 95\% CI, 0.58-0.73) and surgery (AOR, 0.38; 95\%CI, 0.29-0.50) compared to private insurance. In addition, Blacks, Hispanics, older age, and the presence of comorbid conditions were also associated with low likelihood of VEEG and surgery. These individual characteristics remained significant after adjusting for community characteristics. Moreover, individuals who routinely received their services in the area where ECs are located were more likely than those who had regular source of care elsewhere to undergo VEEG (AOR, 1.61; 95\%CI, 1.02-2.54) and surgery (AOR, 2.64; 95\%CI, 1.23-5.67).

Conclusions: Our findings show clear disparities in access to specialized epilepsy care. While almost half of the study population had public insurance, this group had significantly less access to a treatment modality that has proven to be highly effective. Both individual-level (e.g., age, race, and insurance status) and community-level characteristics (e.g., the availability of ECs) play important roles in determining access to high quality epilepsy care.
Policy interventions that incorporate strategies to address disparities at both levels are necessary to improve access to specialized care for people with epilepsy.

\subsection{2}

MEDICAL INFORMATION FRAGMENTATION FOR PEOPLE WITH EPILEPSY IN NEW YORK CITY DIFFERS BY TYPE OF VISIT

Zachary M. Grinspan $^{1,2}$, L. Berg ${ }^{4}$, A. Onyile ${ }^{4}$, R. Kaushal ${ }^{1,2}$ and J. Shapiro ${ }^{3,4}$ ( ${ }^{1}$ Pediatrics, Weill Cornell Medical College, New York, $\mathrm{NY} ;{ }^{2}$ Center for Healthcare Informatics and Policy, Weill Cornell Medical College, New York, NY; ${ }^{3}$ Emergency Medicine, Mount Sinai School of Medicine, New York, NY and ${ }^{4}$ Healthix, Inc, New York, NY)

Rationale: Medical information fragmentation occurs when patients seek care at multiple hospitals that do not have common medical record systems, and places patients at risk for medical errors, duplicate testing, and increased costs. Among patients with epilepsy, the degree of medical information fragmentation and the affected visit types are not well understood. We used a novel modification of a medical information fragmentation measure (Bourgeois et al 2010) to assess the degree of information fragmentation for three visit types (outpatient, emergency department, and inpatient).

Methods: We extracted a de-identified data set from NYCLIX (New York Clinical Information Exchange), a regional health information organization (RHIO) that draws clinical data from several New York City hospitals. We defined a cohort of 8660 people with epilepsy as patients with an ICD9 code of 345.x during the one-year period April 2009 - March 2010. We extracted the year and location of three visit types (outpatient, emergency department, and inpatient) over a threeyear period (April 2009 - March 2012). We created a visit type specific fragmentation index to answer the following question: among patients who had a specific visit type at least twice, what percentage made that visit type at multiple hospitals? For each visit type, we calculated the fragmentation index as the number of patients with that visit type at multiple hospitals divided by the number of patients with at least two of that visit type. To assess how many patients may have benefited from electronic health information exchange, we reviewed the NYCLIX usage logs. NYCLIX was only accessed by a pilot group of emergency department users during the study period.

Results: The fragmentation index was $36 \%$ for outpatient visits (i.e. 1615 patients made outpatient visits at multiple hospitals out of 4440 patients with at least two outpatient visits), $20 \%$ for emergency department visits (593 of 2993 patients), and 13\% for inpatient admissions (397 of 3152 patients). The fragmentation index for outpatient visits was significantly higher than for emergency department visits and inpatient admissions (test of proportions; $\mathrm{p}<$ $10^{-15}$ for both comparisons). Clinical information was accessed via NYCLIX for $0.5 \%$ of the cohort (44 of 8660 patients) during 72 of their emergency department visits.

Conclusions: We used a novel information fragmentation index to show that people with epilepsy frequently seek care at multiple sites for outpatient, emergency, and inpatient care. We found that information fragmentation for outpatient visits is significantly worse than for emergency and inpatient visits, both in terms of the number of patients affected and the degree of fragmentation. These data suggest that to improve care continuity for patients with epilepsy, health information exchange networks should prioritize efforts to share clinical information in the outpatient setting.

\subsection{3 \\ EPILEPSY CLINIC HOTLINE AUDIT: EXPECTATIONS, PERFORMANCE, SATISFACTION AND IMPACT}

Anny Laforme, P. Cossette, D. K. Nguyen and A. A. Bérubé (CHUM Hospital Notre-Dame, Montreal, QC, Canada) 
Rationale: Several adjustments of antiepileptic drug (AED) treatment may be required before one can attain seizure-freedom with no or minimal side effects, especially with drug-resistant epilepsies. A telephone nursing line in a hospital-based epilepsy clinic can aid in the provision of care. To identify areas for improvement, a prospective audit of our epilepsy clinic hotline was carried out to determine patient expectations, delivery of care, patient satisfaction and impact (on use of healthcare resources).

Methods: Between March 2011 and May 2012, patients calling the epilepsy clinic hotline for notification of seizures or side effects were asked to participate in this survey. The survey included 9 questions evaluating the patient's expectations regarding acceptable delays to return calls and modify their treatment, level of satisfaction and what they would have done were the hotline was unavailable. A Likert scale was used to determine patient satisfaction. Call time, call motive, call return time and medication modification time, if applicable, was tabulated for patients.

Results: In the study period, 79 patients who used the epilepsy hotline agreed to complete the survey. Sixteen percent of patients expected to have their call returned within 8 hours and more than half of patients $(57 \%)$ within 24 hours. In reality, while the majority of calls (78\%) were answered within 48 hours, only 54\% were called back within 24 hours. Despite this slightly longer than desired delay, $91 \%$ of patients strongly agreed $(68 \%)$ or agreed $(23 \%)$ that the actual response time was satisfactory. With regards to treatment modification, patients generally expected a medical prescription within $24(42 \%)$ or 48 hours (33\%), followed by 72 hours $(16 \%)$, less than a week (5\%) and more than a week (5\%). Only a minority found that a one-week delay was acceptable for a medication modification (5\%). Would the hotline service not exist, $87 \%$ said they would have tried to have their medical appointment moved up. If the latter was not possible, more than half (69\%) would have gone to the E.R and $18 \%$ to the family physician. A few patients would have changed their medication themselves $(6 \%)$ or wait for the next medical appointment (5\%). Finally, most agreed (88\% strongly agree, $7 \%$ agreed) that a telephone nursing line greatly contributed to the overall satisfaction of the epilepsy clinic.

Conclusions: Patients using the epilepsy clinic hotline generally expect an answer within a day and treatment modification within 2 days. While these expectations are not fully met in time, the hotline is still well appreciated and can potentially reduce the number of ER visits.

\subsection{4}

SHORTER TIME TO PEDIATRIC EPILEPSY SURGERY IS RELATED TO INCREASED EPILEPSY SEVERITY, OLDER AGE AT ONSET, MRI PRIOR TO REFERRAL, PRIVATE INSURANCE AND HISPANIC ETHNICITY Christine Bower Baca ${ }^{1,2}$, B. G. Vickrey ${ }^{1,2}$, S. Vassar ${ }^{1,2}$, J. S. Hauptman $^{3}$, A. Dadour ${ }^{3}$, T. Oh ${ }^{3}$ and G. W. Mathern ${ }^{3}$ ( ${ }^{1}$ University of California Los Angeles, Los Angeles, CA; ${ }^{2}$ Neurology, VA Greater Los Angeles, Los Angeles, CA and ${ }^{3}$ Neurosurgery, University of California Los Angeles, Los Angeles, CA)

Rationale: Despite evidence of the benefits of pediatric epilepsy surgery, a substantial proportion of children with medically intractable epilepsy fail to receive this care in a timely manner. We examined the relative contribution of clinical, diagnostic and sociodemographic factors with time to resective surgery in a large cohort of children over the past 25 years.

Methods: Clinical (age of onset, mean 2.5 years); seizure frequency, (79.4\% $\geq$ daily seizures); history of infantile spasms, (33.5\%); etiology, (51.2\% neurodevelopmental); diagnostic (magnetic resonance imaging (MRI) prior to referral, 76.0\%) and sociodemographic (gender, male 53\%; race/ethnicity, 76.9\% nonHispanic white, $19.2 \%$ Hispanic; $78.3 \%$ private insurance) data were retrospectively abstracted from medical records of 430 children who had resective epilepsy surgery at UCLA $(<18$ years at time of surgery) from 1986 to 2010. Multivariable Cox proportional hazards models were used to measure the association of clinical, diagnostic and sociodemographic variables with 'time to surgery' (age of onset to age of surgery, years; mean 4.5 years, $S D=3.9$ ) controlling for year of surgery.

Results: In multivariate analysis, older age of epilepsy onset (HR 1.09; 95\% CI 1.05-1.14); active (HR 5.67; 95\% CI 3.74-8.60) and successfully treated infantile spasms (HR 2.20; 95\% CI 1.63-2.96); daily seizures (HR 2.09; 95\% CI 1.58-2.76); MRI prior to referral (HR 1.95; 95\% CI 1.47-2.58); Hispanic ethnicity (HR 1.38; $95 \%$ CI 1.01-1.87); and private insurance (HR 1.54; 95\% CI 1.14-2.09) (vs. Medicaid) were significantly associated with a shorter 'time to surgery' (Table $1 ; \mathrm{p}<0.04$ ). There were significant race-insurance group differences in 'time to surgery' (Log-rank $\mathrm{p}=0.049$ ) with shortest 'time to surgery' amongst those of Hispanic ethnicity with private insurance (Figure 1).

Conclusions: While shorter time to pediatric resective epilepsy surgery is most strongly associated with greater disease severity (history of infantile spasms, daily seizures) and older age at epilepsy onset, other non-clinical diagnostic and sociodemographic factors also play a role. The finding that having an MRI prior to referral shortened time to surgery may reflect unmeasured factors such as referring physician and parent knowledge and advocacy.

Furthermore, the observation that Hispanic (vs. non-Hispanic white) children with private insurance experienced a shorter time to surgery was unanticipated and warrants further investigation, but may reflect regional race/ethnicity differences and outreach efforts. Identifying the clinical and non-clinical impediments to timely surgery is important for developing interventions designed to improve access and outcomes.

Table 1: Associations of time to surgery with clinical, diagnostic, and demographic variables

\begin{tabular}{|c|c|c|}
\hline & \multicolumn{2}{|c|}{ Time to surgery (years) } \\
\hline & Hazard Ratio $(95 \% \mathrm{Cl})$ & p-value \\
\hline Clinical Variables & & \\
\hline Age of epilepsy onset. & $1.09(1.05-1.14)$ & $<0.001$ \\
\hline Infantile Spasms (Reference: No history of spasms) & & \\
\hline Active spasms & $5.67(3.74-8.60)$ & $<0.001$ \\
\hline Successfully treated spasms & $2.20(1.63-2.96)$ & $<0.001$ \\
\hline Daily Seizure (Relerence: < daily) & $2.09(1.58-2.76)$ & $<0.001$ \\
\hline Etiology Neurodevelopmental (ND) (Reference: non-ND) & $1.22(0.95-1.56)$ & 0.11 \\
\hline \multicolumn{3}{|l|}{ Number of AEDs (Reference: 0 - 1) } \\
\hline $2 \wedge \Gamma \mathrm{Ds}$ & $0.80(0.59-1.09)$ & 0.17 \\
\hline$\geq 3 \wedge \Gamma \mathrm{T})_{\mathrm{s}}$ & $0.98(0.72-1.34)$ & 0.90 \\
\hline \multicolumn{3}{|l|}{ Diagnostic Variables } \\
\hline MRI Prior to Referral (Reference: No/Unknown) & $1.95(1.47-2.58)$ & $<0.001$ \\
\hline \multicolumn{3}{|l|}{ Demographic Variables } \\
\hline $\begin{array}{l}\text { Male } \\
\text { Race (Reference: non-Hispanic White) }\end{array}$ & $1.20(0.97-1.49)$ & 0.09 \\
\hline Hispanic & $1.38(1.01-1.87)$ & 0.04 \\
\hline Other ( $\wedge$ frican American, Middle Eastern, Asian) & $0.77(0.51-1.16)$ & 0.21 \\
\hline Private Insurance (Reference: Medicaid) & $1.54(1.14-2.09)$ & 0.005 \\
\hline
\end{tabular}

Cox proportional hazard (CPH) model. Increased Hazard Ratio means shorter time to surgery (years) compared to reference group. $\mathrm{CPH}$ model includes time to surgery (years) as dependent variable. Independent variables include clinical, diagnostic and demographic variables as described above, all adjusted for year of cohort. Abbreviations: AEDs = anti-epileptic drugs; MRI = Magnetic resonance imaging 
Figure 1: Kaplan-Heier for Time to Surgery by Race and Insurance

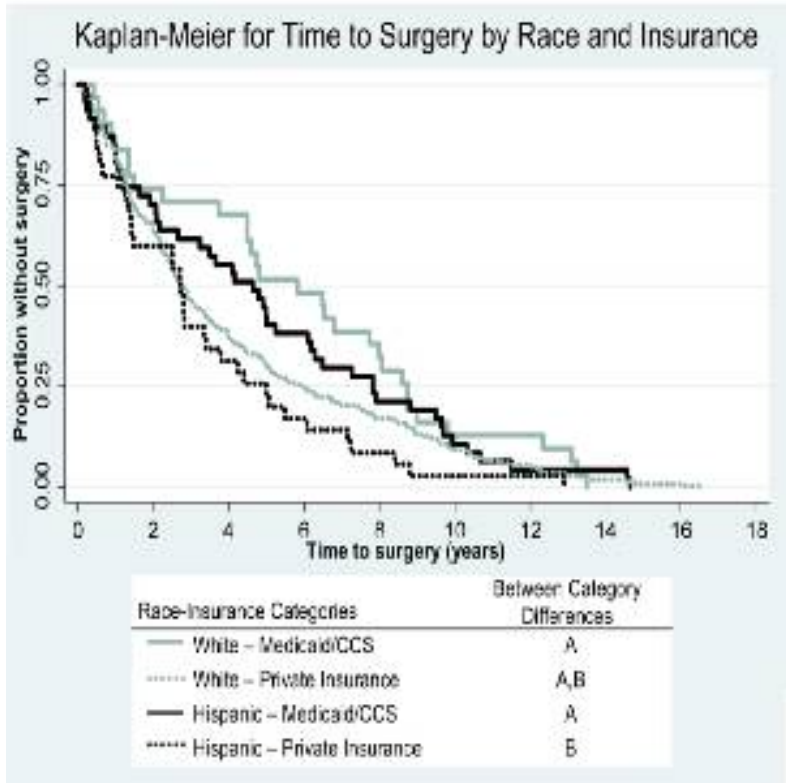

Figure 1 Legend: Race-Insurance categories with different letters are significantly different from one another using Cox proportional hazard test ( $\mathrm{p} \leq 0.05)$. Log-rank test across all four categories for 'time to surgery' $(\mathrm{p}=0.049) * \mathrm{~N}=389$ based on total sample $\mathrm{n}=430$ minus missing ( $\mathrm{n}=12$; Race/ethnicity, $\mathrm{n}=2$ and Insurance type, $\mathrm{n}=10)$ and those with of other race/ethnicity $(n=29)$

\section{Health Services: Health Care Models}

\subsection{5 \\ PATTERNS OF CARE FOR EPILEPSY IN A VERTICALLY INTEGRATED HEALTH SYSTEM}

G. Barkley ${ }^{1}$, D. Nerenz ${ }^{2}$, A. Li ${ }^{2}$ and M. Spanaki ${ }^{1}$ ( ${ }^{1}$ Henry Ford Comprehensive Epilepsy Program, Henry Ford Health System, Detroit, MI and ${ }^{2}$ Center for Health Policy and Health Services Research, Henry Ford Health System, Detroit, MI)

Rationale: Epilepsy outcomes depend on the extent to which, and the speed with which, patients who benefit from referrals to specialists get those referrals. At the invitation of an Institute of Medicine Committee, we collected data on epilepsy referrals from the Emergency Department (ED) or Primary Care (PC) to Neurology. Our goal was to describe "pathways of epilepsy care" in a vertically integrated health care system.

Methods: We identified individuals with a primary or secondary ICD-9 diagnostic code of epilepsy or seizure between 2009 and 2010 at Henry Ford Health System (HFHS), a large, vertically integrated system with a health plan, a multispecialty group practice, 6 hospitals, and a tertiary Epilepsy Center in Southeast Michigan. We used an administrative HFHS patient encounter database (Corporate Data Store:CDS) to collect data on the site of care for the first encounter. To validate the accuracy of the ICD-9 codes, we completed a review of a randomly selected sample of electronic charts. We calculated time intervals between initial presentation for epilepsy and consult with a neurologist and "flow patterns" between the Emergency Department, other sites of care (i.e. PC) and Neurology.

Results: Of 9,588 patients with epilepsy, the first known point of contact was Neurology for 4,269 (44\%). At some time point, 7,107 (74\%) saw a Neurologist at HFHS. The majority of epilepsy patients seen in Neurology $(4,269$ of 7,$107 ; 61 \%)$ were referrals outside the HFHS. Of the patients who had a first point of contact for epilepsy other than Neurology, 2,838 saw a Neurologist in the system at some point; 2,481 did not see a Neurologist in the system. The Emergency Department (ED) was the second most common first point of known contact other than Neurology $(n=2,199)$. Of those patients, 52\% did not have a subsequent visit in Neurology, $32 \%(n=697)$ had a visit in some other HFHS department before being seen by Neurology and $16 \%$ referred from the ED to Neurology $(n=349)$. For the patients who had a first point of contact other than Neurology, the average time from first contact to a Neurology visit was 15.3 months (range : 0 - 192 months). The average time was longer for African American patients than Caucasians (16.4 vs. 15.4 months). Of the 4,269 patients seen first in Neurology, 3,303 had subsequent epilepsy care provided in HFHS by both PC and neurologists; the remainder were either onetime consults $(\mathrm{n}=939)$ or were subsequently seen exclusively by primary care $(\mathrm{n}=27)$.

Conclusions: Our study showed that even in communities with large integrated health systems with tertiary epilepsy care, the ED is the first point of contact for the diagnosis of epilepsy for a significant number of epilepsy patients. However, the great majority of epilepsy patients received care by a neurologist in our system at some time point, and most patients who had a first system contact in Neurology continued to receive care in the system rather than having just the one visit. Using administrative data validated by electronic patient charts, we can obtain important information about "patterns" and "flow" of care for epilepsy patients.

\section{Health Services: Special Populations}

\subsection{6 \\ IS RISK FACTOR OF INTELLECTUAL DISABILITY (ID) FOR SUDDEN UNEXPECTED DEATH IN EPILEPSY (SUDEP) DEPENDENT ON GEOGRAPHY? R. Shankar ${ }^{1,2}$, C. Young ${ }^{1}$, D. Cox ${ }^{1,2}$ and B. Maclean ${ }^{3}\left({ }^{1}\right.$ Learning Disability epilepsy, cornwall Partnership NHS Foundation Trust, Truro, United Kingdom; ${ }^{2}$ Peninsula Medical and Dental School, Truro, United Kingdom and ${ }^{3}$ department of Neurology, Royal Cornwall Hospital Trust, Truro, United Kingdom)}

Rationale: SUDEP occurs in $0.001 \%$ of people with epilepsy. A literature search was done to highlight current risk factors for SUDEP to develop a communication tool. The search identified ID as a risk in some reviews. Studies appeared to vary in their interest in exploring the role of ID. Further investigation was done to see if ID was a risk or a protective factor. The search was expanded to see if the type of health care system and country had a role in considering ID as a risk factor.

Methods: A literature search was undertaken in pubmed using search terms such as 'sudden' 'death', 'mortality', 'epilepsy', 'risk factor', 'protective factor', 'learning disability', 'mental retardation', 'intelligence' and 'intellectual disability' in different permutations and combinations. Papers were excluded if they explored one (non ID related) risk factor or non-SUDEP deaths in epilepsy. Each paper was then examined to see if ID was investigated as a risk factor, noted to be either a risk or a protective factor and for the country of investigation. We researched the type of health care utilized in the country of investigation.

The literature search found over 300 studies. After the exclusion criteria were applied, 50 papers were identified to be suitable. None of the papers found ID was a protective factor. Of the 50 papers 23 $(46 \%)$ investigated ID as a risk factor and $12(24 \%)$ identified it as a risk factor.

Results: We compared ID risk factor studies against the country of origin and the health care system of the country. 35 (70\%) studies were conducted in Europe compared to 12 (24\%) in Northern America. Australasia produced 3.31 (62\%) of the 50 papers were written from countries having a public health care system. Of these 31,14 explored and 9 identified LD as a risk. $10(20 \%)$ of the 50 papers came from countries with a predominantly private health care system of which 4 had explored and 2 identified ID as a risk. 9 of the 50 papers arose from countries having mixed health funding of varying proportions of private and public liaison. Of these 9 (18\%), 5 
explored ID and 1 identified ID as a risk.

$62 \%$ of the papers were researched in countries where the health care systems are publicly funded (UK, Sweden and Canada). The remaining papers were researched in nearly equally between private and dual health care (USA, Australia).

Of the 23 studies investigating ID as a risk factor, 13 of them were from publicly funded heath care systems compared to 4 from private. Conclusions: ID is looked into as a risk factor by predominantly papers emerging from countries with public health systems. There could be a discrepancy in the level of attention paid to ID as a risk factor based on the place an individual lives. There needs to be more work done to neutralize the skew currently existing regarding the understanding of ID as a risk factor for SUDEP. It is important that research is done to estimate ID as a risk factor in all health systems to help identify if a difference exists in national care models to private health insurance led models towards epilepsy care for people with ID.

\subsection{7}

\section{CHARACTERISTICS AND BURDEN OF SEIZURES IN} PATIENTS WITH TUBEROUS SCLEROSIS COMPLEX: RESULTS OF A PATIENT AND CAREGIVER SURVEY IN THE US

M. D. Frost ${ }^{1}$, A. M. Rentz ${ }^{2}$, C. L. Pashos ${ }^{2}$, J. Liu ${ }^{3}$, C. Pelletier ${ }^{3}$, J. Prestifilippo $^{3}$, J. A. Nakagawa ${ }^{4}$, J. W. Wheless ${ }^{5}$ and D. W. Dunn ${ }^{6}$ $\left({ }^{1}\right.$ Minnesota Epilepsy Group, PA, St. Paul, MN; ${ }^{2}$ United BioSource Corporation, Bethesda, MD; ${ }^{3}$ Novartis, Corporation, East Hanover, $\mathrm{NJ} ;{ }^{4}$ TS Alliance, Silver Spring, MD; ${ }^{5}$ Le Bonheur Children's Hospital, Memphis, TN and ${ }^{6}$ Riley Hospital, Indianapolis, IN)

Rationale: Patients with tuberous sclerosis complex (TSC), a genetic disorder characterized by benign tumor growth in multiple organs, may experience neurological disease progression stemming from severe and early-onset epilepsy. Many patients with TSC and epilepsy begin having seizures in infancy, continuing with increased frequency and severity throughout childhood. Epilepsy is a significant source of morbidity and mortality among TSC patients. This analysis aimed to assess the prevalence, presentation, and associated impact of seizures in patients with TSC in the United States (US).

Methods: An Institutional Review Board-approved Web-based survey of US TSC patients and their caregivers obtained information on prevalence of different TSC manifestations, their treatment and management, and epidemiological, clinical, economic and healthrelated quality of life (HRQoL) burden on patients and caregivers. This analysis focused on the prevalence, presentation, outcomes, and management of seizures.

Results: The online survey is on-going. Of 380 TSC patients whose data have been collected to date, $59 \%$ were female and mean age was 30.4 years (median: 32.5 , range: $0-70$ ). Two hundred respondents (53\%) were patients with TSC and $180(47 \%)$ were caregivers of patients with TSC. Of the 380 TSC patients $176(46 \%)$ had been diagnosed with seizures. Patients with seizures also experienced skin lesions (78\%), cognitive concerns (66\%), kidney complications $(51 \%)$, and SEGAs (30\%). Seventy-seven percent of seizure patients reported taking anti-epileptic drugs (AED) in the past 6 months, $27 \%$, $31 \%, 13 \%$, and $6 \%$ of seizure patients have taken $1,2,3$, or 4 or more in the past 6 months. Employed patients with seizures $(n=28)$ report missing approximately $18 \%$ of their working week and experiencing $40 \%$ impairment while working. Employed caregivers of patients with seizures $(n=69)$ report missing approximately $9 \%$ of their working week and experiencing 30\% impairment while working. Conclusions: Initial survey data have demonstrated that seizures due to TSC are associated with significant epidemiological, economic, and clinical burden. The complete survey data will provide further insights on the clinical profile and economic and HRQoL impact of seizures due to TSC.

\section{Practice Resources}

\subsection{8}

CLINICAL PREDICTORS OF AN ABNORMAL BRAIN MRI IN CHILDREN WITH EPILEPSY

S. N. Steski ${ }^{1}$, K. M. Stannard ${ }^{2,3}$, B. Dufault ${ }^{4}$ and J. P. Appendino ${ }^{2}$ ( ${ }^{1}$ Faculty of Medicine, University of Manitoba, Winnipeg, MB, Canada; ${ }^{2}$ Section of Pediatric Neurosciences, Children's Hospital of Winnipeg at HSC, Winnipeg, MB, Canada; ${ }^{3}$ Section of Pediatric Neurosciences, Mass General for Children at NSMC, Salem, MA and ${ }^{4}$ Department of Community, Faculty of Medicine, University of Manitoba, Winnipeg, MB, Canada)

Rationale: To date, children with epileptic seizures undergo brain MRI to determine a structural etiology. The indication for brain MRI in these children is largely based on expert opinion rather than objective criteria and algorithms.

The aim of this study was to identify clinical factors that predict an abnormal brain MRI in a retrospective cohort of children with epilepsy at our pediatric medical center.

Methods: An 18 months retrospective chart review was conducted in all children age 1 month to 18 years of age diagnosed with epilepsy, who underwent a brain MRI from January 1, 2010 through July 30, 2011. Each child's medical chart was reviewed and specific and detailed clinical markers including patient history, physical examination, EEG and MRI findings were identified. This information was entered into a clinical database Multivariable Poisson regression with robust variance estimates were used to calculate adjusted relative risks using the GEE capability of SAS PROC GENMOD. To assess model predictive capability, ROC curves were also estimated.

Results: A total of 228 patients were identified, $54 \%$ males. The average age of the children at diagnosis of epilepsy was 5.52 years old. When all components of the physical exam were combined, $39.9 \%$ of patients had an abnormality. A total of 225 EEG's were reviewed, of which $92 \%$ were abnormal. The majority of abnormalities found were focal epileptiform discharges in 125 patients' EEG's (55.6\%), whereas diffuse epileptiform discharges were only found in 55 EEG's (24.4\%). EEG slowing was also analyzed, with the majority having diffuse changes in 102 EEG's (45.3\%), and focal slowing in 66 EEG's $(29.3 \%)$. Of the $228 \mathrm{MRI} s$ reviewed, 91 were abnormal $(39.9 \%)$. In the multivariate analysis, developmental delay was found to have a relative risk of 1.4995 (CI: $1.0648-2.1118, \mathrm{p}=0.0204$ ) for an abnormal brain MRI; in addition, patients with a diffusely abnormal physical exam had a relative risk of 1.5753 (CI: $1.1279-2.2002, p=0.0077$ ). Interestingly, focal findings on physical exam were not found to be predictive (OR: 1.147; CI: 0.494 - 2.666, $\mathrm{p}=0.7494$ ).

Conclusions: : The expected role of unilateral focal findings on clinical examination and/or EEG was not found to be predictors of an abnormal MRI of brain. This study found that diffuse abnormal physical examination findings and developmental delay are independent predictors of an abnormal brain MRI in children with epilepsy. The results from this study are not statistically robust to build a diagnostic algorithm; however, to our knowledge this is the first study of its kind to assist physicians in their clinical decision to obtain MRI imaging in children with epilepsy. Since this is a retrospective study with its known limitations, further prospective research is needed to create a diagnostic algorithm for clinicians to aid in the management of children with epilepsy.

\subsection{9 \\ "TELE-EEG": CAN THE IPAD IMPROVE ACCESIBILITY FOR A NEW FRONTIER OF EEG INTREPRETATION?} Matthew T. Hoerth, J. F. Drazkowski, K. H. Noe and J. I. Sirven (Mayo Clinic Arizona, Phoenix, AZ) 
Rationale: Although the fundamental principles of recording an EEG have been unchanged for decades, the technology in that surrounds the acquisition and interpretation has greatly advanced the field. The greatest example of this is the development of digitized EEG allowing for post-recording manipulation of the EEG, magnifying interpretation abilities. The next frontier of technology has been the invention of wireless technology and mobile devices. Like digitization, "going wireless" will change the way in which we are able to practice clinical EEG.

Methods: Mayo Clinic Department of Neurology has recently implemented EEG reading services at three other institutions across the state of Arizona, termed "tele-EEG." The epilepsy division then has four institutions where they have remote access to EEG interpretation. There are two different EEG acquisition systems (Natus and Nihon-Koden) and two different methods to provide remote access (VMWare and Citrix). Please see table 2 for details. Ease of access is instrumental in managing the complexities of multiple systems, therefore the iPad devices were compared to a standard laptop (HP EliteBook 2540p) and on-campus desktop access.

Results: Table 1 summarizes the technical specification of each of the devices. Table 2 summarizes the speed of access from each type of system that is used. The laptop used in this study is a relatively small laptop; however it is still larger and heavier than the iPads, with a higher cost. The advantage is that it has a larger screen. The time it takes to open an EEG measured showed no significant difference between the devices, with a trend toward being slightly slower with the laptop. The boot-up time is significantly longer for the laptop and desktop. In addition, the $4 \mathrm{G}$ access appears essentially no different than wifi.

Subjectively consultants who are using the iPad as compared to the standard laptop feel that it has the advantage of the larger screen and easier to input data. However, the iPad has significant advantages as it gives the reader more freedom to leave their home/hospital despite being on call, as well as feeling that it is faster and easier to use. Cost is also significantly less. There have been times that were noted that the laptop would loose connection, where this would not happen with the iPad. Testing this in alternate conditions, such as in a moving vehicle, did not decrease the ability to access remote systems.

Conclusions: With high volumes of EEGs to read and multiple systems and facilities to read from, the efficiency of technology is essential to many physician practices. Despite the marginally smaller screen size; the ease of use, accessibility, and reliability make the use of the iPad a viable option for its integration into the tele-EEG practice. At this time, iPads can be best used as a complementary to the laptop that EEG readers are using at their home wifi networks. It is reassuring, however, that with an increasingly busy practice, tabletstyle devices can keep electroencephalographers connected wherever they may be.

Table 1: Technical Specifications

\begin{tabular}{|c|c|c|c|c|}
\hline & Laplop (wili) & ilad 2 (wili) & ipad 3 (wili) & iPad 3 (4G) \\
\hline Cost & S1099-S1629 & $\$ 399$ & $\$ 499-\$ 699$ & $\$ 629-\$ 829$ \\
\hline Dimensions & $11.1 \mathrm{in} \times 8.4$ in & $\begin{array}{c}9.50 \text { in } x \\
7.31 \text { in }\end{array}$ & $9.50 \mathrm{in} \times 7.31 \mathrm{in}$ & 9.50 in $\times 7.31$ in \\
\hline Weight & 3.97 pounds & 1.33 pounds & 1.44 pounds & 1.46 pounds \\
\hline $\begin{array}{c}\text { Screen Size } \\
\text { (diagonal) }\end{array}$ & 12.1 in & 9.7 in & 9.7 in & 9.7 in \\
\hline $\begin{array}{c}\text { Screen } \\
\text { Resolution }\end{array}$ & $1280 \times 800$ & $1024 \times 768$ & $2048 \times 1536$ & $2048 \times 1536$ \\
\hline Processor & $\begin{array}{l}\text { Intel Core } \\
2.13 \mathrm{GH} 7 \\
\text { Dual-Corc }\end{array}$ & $\begin{array}{l}\text { Dual-core } \\
\text { Apple } \triangle 5\end{array}$ & $\begin{array}{c}\text { Dual-core Apple A5X } \\
\text { with quad-core } \\
\text { graphics }\end{array}$ & $\begin{array}{c}\text { Dual-core Apple A5X } \\
\text { with quad-core } \\
\text { graphics }\end{array}$ \\
\hline
\end{tabular}

Table 2: Time to Open EEG Software

\begin{tabular}{|c|c|cc|cc|}
\hline & $\begin{array}{c}\text { Standard } \\
\text { Desktop } \\
\text { Boot-up time }\end{array}$ & $\begin{array}{c}\text { Laptop } \\
\text { (wifi) }\end{array}$ & $\begin{array}{c}\text { il'ad 2 } \\
\text { (wifi) }\end{array}$ & $\begin{array}{c}\text { iPad 3 } \\
\text { (wifi) }\end{array}$ & $\begin{array}{c}\text { iP'ad 3 } \\
\text { (4G) }\end{array}$ \\
$\begin{array}{c}\text { MCII } \\
\text { (VPN+VMW:are+Natus) }\end{array}$ & $0: 22$ & $1: 58$ & $1: 56$ & $1: 35$ & $1: 32$ \\
\hline $\begin{array}{c}\text { KRMC (VMWare+NK) } \\
\text { BGSMC (Citrix+Natus) }\end{array}$ & $1: 10$ & $1: 07$ & $0: 58$ & $0: 50$ & $0: 57$ \\
$\begin{array}{c}\text { FMC } \\
\text { (Citrix+NK) }\end{array}$ & $1: 16$ & $1: 10$ & $1: 04$ & $0: 58$ & $1: 00$ \\
\hline
\end{tabular}

Times noted in mm:ss; boot-up time is time from off to login screen; Time to Open EEG includes login to remote computer + open EEG software + open EEG for review; VPN is Virtual Private Network; $\mathrm{MCH}$ is Mayo Clinic Hospital; KRMC is Kingman Regional Medical Center; BGSMC is Banner Good Samaritan Medical Center; FMC is Flagstaff Medical Center; NK is Nihon-Koden

\subsection{0 \\ THE PEDIATRIC STATUS EPILEPTICUS RESEARCH GROUP (PSERG): ESTABLISHMENT OF A MULTICENTER NORTH AMERICAN RESEARCH NETWORK}

T. Loddenkemper ${ }^{1}$, Iván Sánchez Fernández ${ }^{1}, \mathrm{~S} . \mathrm{An}^{1}, \mathrm{~N}$. Abend ${ }^{5}, \mathrm{~S}$. Agadi $^{3}$, J. Carpenter ${ }^{2}$, K. Chapman ${ }^{8}$, W. Gaillard ${ }^{2}$, H. Goodkin ${ }^{6}$, C.

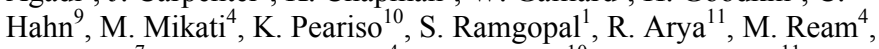
J. Riviello ${ }^{7}$, A. Veerapandiyan ${ }^{4}, \mathrm{~K}$. Williams ${ }^{10}$ and T. Glauser ${ }^{11}$ $\left({ }^{1}\right.$ Children's Hospital Boston, Boston, MA; ${ }^{2}$ Children's National Medical Center, Washington, DC; ${ }^{3}$ Baylor College of Medicine, Houston, TX; ${ }^{4}$ Duke Children's Hospital, Durham, NC; ${ }^{5}$ Children's Hospital Philadelphia, Philadelphia, PA; ${ }^{6}$ University of Virginia health system, Charlottesville, VA; ${ }^{7}$ New York University Langone Medical Center, New York, NY; ${ }^{8}$ Children's Hospital Colorado, Aurora, CO; ${ }^{9}$ Sickkids, Toronto, ON, Canada; ${ }^{10}$ Phoenix Children's Hospital, Phoenix, AZ and ${ }^{11}$ Cincinnati Children's Hospital, Cincinnati, $\mathrm{OH}$ )

Rationale: Management of refractory convulsive status epilepticus (RCSE) varies at different centers and from patient to patient within a given center. We aimed to establish a North-American multicenter network of tertiary referral hospitals to analyze RCSE management which could provide useful information on outcome and outcome predictors.

Methods: Pediatric epileptologists frequently caring for children with RCSE first met at the Child Neurology Society Meeting and American Epilepsy Society meetings in 2010, and subsequently established monthly phone conferences in order to establish a research group on pediatric RCSE. Common inclusion criteria for RCSE included: 1) age 1 month to 21 years; 2) convulsive seizures at onset; and 3) failure of two or more antiepileptic drugs (AEDs) or requirement of any kind of continuous infusion of AEDs to abort seizures. We excluded patients with: 1) non-convulsive SE detected on EEG (without convulsive seizures at onset); and 2) nonconvulsive status epilepticus and infrequent myoclonic jerks. Results: The network began with 8 centers in 2010 and presently comprises 11 tertiary epilepsy centers in the United States and Canada. The group meets monthly in highly-focused 30 minute phone conferences, and biannually in person. These meetings are attended by $>75 \%$ of participants regularly. IRB approval was obtained from all sites. Funding was obtained from the Epilepsy Foundation of America. Data elements for status epilepticus were developed based on NINDS common data elements. A web-based interface based on the NINDS childhood absence epilepsy trial was developed to permit multi-center anonymized data entry. Data on demographics, comorbidities, semiology of the status epilepticus, AEDs required to stop the seizures, EEG features and complications of the status epilepticus and/or its treatment are being collected for every patient. 
Since October 2011 we prospectively enrolled 25 children with RCSE. We expect subject recruitment to continue until January 2013 to yield 50 subjects. We plan to perform detailed analysis on the use, timing, efficacy and adverse effects of AEDs in RCSE. Future collaboration may allow for the collection of biologic samples to determine genetic factors and provide data for interventional treatment trials.

Conclusions: We have developed a North-American multicenter network of tertiary hospitals that care for children with RCSE. We are successfully enrolling patients prospectively. With further development and continued enrollment, this registry will allow us to compare differences across the 11 institutions. This will yield valuable data regarding the most effective RCSE treatment and monitoring strategies which can then be studied by the network as interventional treatment trials. Additionally, newly developing consortium links will permit investigation of genetic markers of SE susceptibility and outcome. This study may help in setting up prospective comparative efficacy studies on therapy of status epilepticus.

\subsection{1 \\ CLINICAL FACTORS AFFECTING LENGTH OF STAY IN THE EPILEPSY MONITORING UNIT}

E. Lampe, E. Herbst and L. C. Frey (Neurology, University of Colorado, Aurora, CO)

Rationale: Long-term continuous video EEG monitoring in the Epilepsy Monitoring Unit (EMU), an essential clinical tool for the diagnosis of patients with spells, is a limited clinical resource. Knowledge of the predicting factors for length of stay (LOS) in the EMU may allow clinicians and administrators to more efficiently utilize bed space in the EMU.

Methods: The records for all consecutive admissions to the EMU at the University of Colorado Hospital between December 1, 2010 and May 31, 2011 ( $n=142)$ were retrospectively reviewed. Data on patient demographics, seizure types and frequencies, antiepileptic therapies, comorbidities, EMU LOS, time to first diagnostic event and event descriptions were abstracted and analyzed. Patients younger than 18 or older than 80 years, patients with intracranial electrodes, and patients admitted to the EMU for reasons other than spell capture and characterization were not included.

Results: Data from 114 patients were ultimately included in the analysis. The mean LOS for all patients was $83.52+/-4.24$ hours. The mean time to first diagnostic event in those patients who had events was $30.93+/-3.37$ hours. Overall, patients with nonepileptic seizures had a statistically significantly shorter mean LOS than those patients with epileptic seizures or nondiagnostic studies. Patients with a normal EEG background had a statistically significant shorter mean LOS than patients with abnormal EEG backgrounds (slowing or epileptiform discharges). EMU LOS (in hours) was not significantly correlated with patient age, age at event onset, number of event types, or number of antiepileptic drugs at admission. EMU LOS (in hours) was modestly, but significantly, correlated with self-reported event frequency prior to admission $(\mathrm{r}=-0.350, \mathrm{p}=0.0003)$. When analyzed separately, those patients that were diagnosed with nonepileptic seizures in the EMU $(n=43)$ also showed a statistically significant modest correlation between LOS and self-reported event frequency $(r=-0.436, p=0.0055)$, while the correlation was not significant for those patients diagnosed with epileptic seizures $(n=35)$.

Conclusions: Pre-admission clinical features, such as self-reported event frequency, may predict EMU LOS. After confirmation with further prospective analyses, these factors could be used to schedule EMU admissions for maximum resource utilization.

\subsection{2}

\section{SUDEP: WHAT DO PARENTS WANT TO KNOW?}

Rajesh Ramachandran Nair, S. M. Jack, B. F. Meaney and G. M. Ronen (McMaster University, Hamilton, Canada)
Rationale: There is neither empirical evidence nor consensus on the question of whether to inform

patients and family members about the possibility of SUDEP.

Objectives: To understand the range of parental views on whether

and how to approach the issue of SUDEP with families, to clarify the optimal timing and formulation of the information, and to learn from the true experts (the primary caregivers) the optimal counseling strategies to minimize the inherent emotional burden.

Methods: The principles of fundamental qualitative description were used to guide this study of parent experiences and perceptions.

Stratified purposeful sampling included (i) parents who lost children to SUDEP and, parents of children with (ii) moderate to severe (iii) mild and, (iv) new onset epilepsy. Data were collected through 1:1 interviews and focus groups.

The principles of content analysis were used to code and categorize data. Line by line coding of the transcripts followed. Second level coding was then conducted to collapse the codes into categories and themes.

Results: 42 parents participated in the study. Focus group interview were conducted with 36 parents (M:F- 15:21) of 21 children. 6 parents (M:F- 2:4) of 4 children who died due to SUDEP participated in 1 to 1 interviews. $63 \%$ of parents had previously heard about SUDEP, but most of could not describe the meaning of SUDEP but overestimated the risk. There was full consensus, across both genders and regardless of seizure severity, that routine counseling about SUDEP should be provided by pediatric neurologists, during the appointment when the diagnosis of epilepsy is shared with parents, with opportunities for follow-up and discussions with clinic nurses or social workers.

There was consensus among all participants that it is the responsibility of the pediatric neurologist to provide routine SUDEP counseling. Parents were emphatic in explaining that they should not first learn about SUDEP from a pamphlet or an Internet website. It was identified that SUDEP counselling should occur in a face-toface interaction between the neurologist and the parents, and not be provided in a phone consultation. Parents expressed a need to be informed of the risk of SUDEP. However, some parents expressed that it is important for neurologists to stress that SUDEP is rare and to balance the message of risk, with one of hope. There was group endorsement for also receiving written information about SUDEP to reinforce the content shared by the neurologist. Participants identified that both parents (as appropriate) should be present at the appointment to receive routine SUDEP counseling. There was consensus that it is the parents' decision as to whether or not, and when to inform the child about the risk of SUDEP.

Conclusions: Parents expressed their clear preferences on whether, when, where and how to deliver routine counseling on SUDEP. Over all credibility of the data was enhanced by data type (focus groups and 1:1 interviews) and data source (purposeful stratified sampling) triangulation. Results of this study will help create a practise guideline on how to counsel parents of children with epilepsy on the risk of SUDEP.

\subsection{3}

OUTCOME OF VIDEO EEG MONITORING IN A TERTIARY CARE EPILEPSY CENTER

Nurkia Mihu, M. Oller-Cramsie, M. Kumar-Pelayo, S. T. Hwang and C. Harden (Hofstra North Shore-LIJ School of Medicine, Manhasset, NY)

Rationale: Video-EEG (VEEG) monitoring is a diagnostic tool that is used to help confirm the diagnosis of epilepsy, characterize seizure type, and localize epileptic foci. Within the literature, the utility of VEEG is variable and dependent on patient demographics as well as purpose and length of monitoring. To investigate this within our population, we analyzed VEEG outcomes in consecutive adult patients referred to our metropolitan tertiary care epilepsy center. 
Methods: The outcome of 100 consecutive adult elective VEEG admissions at Long Island Jewish Hospital from July 2011 to January 2012 was reviewed. These patients were referred for admission from both clinic and faculty private practice. The questions to be answered by the admission were documented in the clinic note prior to admission. Our analysis included:

1. What was the question to be answered by VEEG?

2. Was the question answered by VEEG monitoring?

3 . When the question was not answered, why?

4. Did the outcome of monitoring alter medical or surgical management?

Results: The admission question was answered in 77 patients (see table for admission question). Of the 23 patients whom the question remained unanswered, 14 did not have events, 4 did not wish to continue monitoring, and 4 had events suspicious for simple partial seizures or auras without EEG correlate. In 1 patient, the reason the question was not answered was unclear (see chart). The mean length of stay was 3.08 days (SD 1.93) in the patients for whom the question was answered. The mean length of stay was 2.74 days (SD 1.60) in those for whom the question was not $(p=0.218)$. In the patients whom the admission question was answered, 48 had medications adjusted upon discharge: 11 had medications solely increased, 2 had medications solely decreased, 11 had a new medication added, 8 had one medication stopped and 16 had multiple medication adjustments. Default practice was to resume baseline anti-epileptic drug regimen, which occurred in 29 patients. Fifteen of 23 patients admitted for seizure localization proceeded with surgical evaluation. Of these patients, 5 proceeded with epilepsy surgery (craniotomy) to date. Conclusions: We demonstrated in our population that VEEG is a useful tool to aid in seizure localization, diagnosis and characterization since the question of admission was answered in the majority of patients (77\%). The main reason for not answering the admission question was lack of events. We also demonstrated that results of VEEG monitoring affect outcome both medically and surgically. Medications were altered upon discharge in approximately $2 / 3$ of patients whom the question was answered. Fifteen percent of total admissions proceeded with epilepsy surgical evaluation and 5\% underwent craniotomy for epilepsy surgery.

Table: Questions for Admission-Overview

\begin{tabular}{|c|c|c|c|}
\hline Question Lor Admission & $\begin{array}{c}\text { Number } \\
\text { N=100 }\end{array}$ & $\begin{array}{c}\text { Was Question } \\
\text { Answered? } \\
\text { Ycs }\end{array}$ & $\begin{array}{c}\text { Was Question } \\
\text { Answered? } \\
\text { No }\end{array}$ \\
\hline Fpilcpsy vs. Non Epilepsy? & 41 & 29 & 12 \\
\hline Where is the epileptic focus? & 23 & 23 & 0 \\
\hline What is the Epilepsy Syndrome? & 32 & 22 & 10 \\
\hline $\begin{array}{c}\text { Can medications be adjusted safely as an } \\
\text { inpaticnt? }\end{array}$ & 4 & 3 & $23 \%$ \\
\hline TOTAL & 100 & $77 \%$ & \\
\hline
\end{tabular}

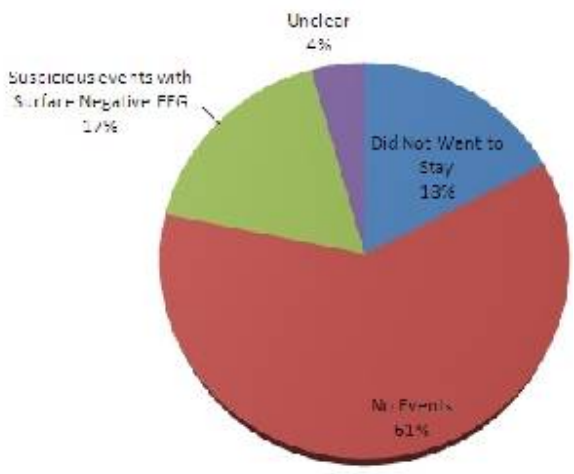

Chart: Reasons Why the Question Was Not Answered

\author{
2.345 \\ THE ESTABLISHMENT OF PROFESSIONAL EPILEPSY \\ PRACTICE, CALLED "THEATRICAL EPILEPSY \\ PRACTICE", WHERE THERE IS NO FULL-TIME EPILEPSY \\ SPECIALIST IN THE OKINAWA ISLAND AREA, JAPAN \\ M. Noha ${ }^{1}$, I. Takumi ${ }^{2}$, J. Kadekawa ${ }^{3}$, E. Takara ${ }^{1}$ and A. Teramoto ${ }^{4}$ \\ ( ${ }^{1}$ Neurosurgery, Okinawa Red Cross Hospital, Naha, okinawa, Japan; \\ ${ }^{2}$ Neurosurgery, Nippon Medical School Musashi Kosugi Hospital, \\ Kawasaki, Japan; ${ }^{3}$ Neurology, Okinawa Red Cross Hospital, Naha, \\ Okinawa, Japan and ${ }^{4}$ Neurosurgery, Tokyo Rosai Hospital, Tokyo, \\ Japan)
}

Rationale: Okinawa mainland holds almost one million populations, where there is no epilepsy center. There is no full-time adult epilepsy specialist. This means that any epilepsy patient could not be benefited from long term video EEG monitoring (LT-vEEG), intracranial electrodes recordings or conventional epilepsy surgery ourselves. In addition, Okinawa area is geographically as well as philosophically a typical archipelago locality, where most epilepsy patient would hesitate to travel out to other areas for further treatment. Okinawa is such an isolated area for epilepsy treatment. What can we do? This was the initial motif of our project. This report mentions the proper treatment of epilepsy in the lower epilepsy resource area in Japan, and also discusses the minimum requirement for the modern epilepsy therapy.

Methods: We have started up by inviting a part-time epilepsy specialist and opened the epilepsy out-patient clinic in our institute. Results: This out-patient could serve not only as to provide conventional epilepsy therapy to our area but also as for the source of the epilepsy education to our medical staff. Three medical specialists, neurosurgeon, neurologist, and the epilepsy specialist sit together in one booth at the time of out-patient, facing one patient and her/his family members. Two out of three clinicians are full-time neurology/neurosurgery specialists in our institute. Our epilepsy outpatient are held on Thursday afternoon every other week, but the part-time epilepsy specialist could join to us only once in every other months. Therefore, after patients are examined, diagnosed, and plotted for therapeutic strategy with the epilepsy specialist, the rest of the two clinicians had to follow the policy by ourselves until the next chance when epilepsy specialist is coming. Eventually, the clinical skills for the two local clinicians are elevated. The roles for each of us are like three actors on theater. We called our practice as "Theatrical Epilepsy Practice" in a friendly manner.

Conclusions: The minimum requirement for intractable epilepsy treatment would be EEG, MRI, and LT-vEEG in general; but out of our successful launch of our out-patient, we would address our novel minimum requirements are EEG, MRI, and the experienced epilepsy specialist with the Theatrical Epilepsy Out-Patient.

Epidemiology

\subsection{6 \\ PERI-ICTAL ELECTROPHYSIOLOGICAL CHARACTERISTICS OF NOCTURNAL GENERALIZED CONVULSIVE SEIZURES}

Anthony Lee, I. Yung, X. Zhou, S. Rose, J. Liebenthal and J. X. Tao (Neurology, University of Chicago, Chicago, IL)

Rationale: To determine the peri-ictal electrophysiological characteristics of nocturnal and diurnal generalized convulsive seizures (GCS) and to further understand the implications of sleep in sudden unexpected death in epilepsy (SUDEP).

Methods: We retrospectively reviewed the video-EEG of 109 consecutive patients with $150 \mathrm{GCS}$ during long-term video-EEG 
monitoring. We determined the heart rate (HR), respiratory rate (RR), total seizure duration, total convulsive phase, tonic phase, and clonic phase, as well as the presence and duration of postictal generalized EEG suppression (PGES) in patients with nocturnal GCS and diurnal GCS.

Results: Forty patients had 67 nocturnal GCS, 61 patients had 83 diurnal GCS, and 8 patients with both nocturnal and diurnal GCS. Pre-ictal HR was significantly lower in patients with nocturnal GCS than in those with diurnal GCS $(\mathrm{p}=0.0016)$. However, there was no significant difference in total seizure duration, total convulsive phase, tonic phase and clonic phase as well as postictal HR and RR in the two patient groups. Forty-five of $67(67.2 \%)$ nocturnal GCS were associated with PGES, whereas 49 of 83 (59\%) diurnal GCS were associated with PGES. The duration of PGES was prolonged in patients with nocturnal GCS $(46.7 \pm 21.7 \mathrm{~s})$ than in those with diurnal GCS $(38.5 \pm 15.6 \mathrm{~s})$, however, it was not statistically significant $(\mathrm{p}=0.63)$.

Conclusions: Nocturnal GCS is associated with a higher pre-ictal parasympathetic tone comparing to diurnal GCS. However, postictal autonomic dysfunction as reflected by postictal HR and RR, and the duration of PGES were comparable in two patient groups. Future studies involving factors such as sleep environment and position may be necessary to further understand the implications of sleep in the pathogenesis of SUDEP.

\begin{tabular}{|c|c|c|c|}
\hline & $N$ :x_z<ny & scound G:S & Lunel ics \\
\hline Fitere & \multicolumn{3}{|c|}{ 3 $7=0$ a } \\
\hline 30 & $35, y$ & $\Rightarrow 1 \cdot \dot{r}$ & स1. $\% 1111$. \\
\hline 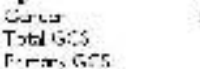 & 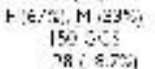 & 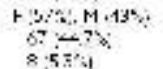 & 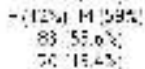 \\
\hline Strate-cos & IXffI $3 \times$ & $=539.3$ & a3: $: 21$ \\
\hline 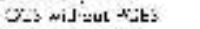 & SSIB: $=1$ & $\because 1 \ldots$ & -121 \\
\hline 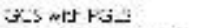 & $4<1 \leqslant 2 \leqslant 1$ & 45,351 & $472 \%$ \\
\hline FradlRneisusd & $130 \% 7 \%$ & (j) $<\times 1$ & $\because-1)$ \\
\hline 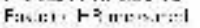 & $1>\mathrm{F}: \mathrm{W}$ & $\because 35 \%$ & 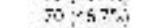 \\
\hline 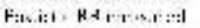 & $\$ 3,694$ & 16:3:1: & 6315 \\
\hline
\end{tabular}

\begin{tabular}{|c|c|c|c|}
\hline & 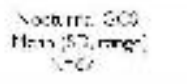 & 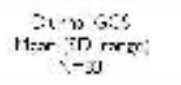 & $\begin{array}{c}\text { Pulds } \\
\text { inirsior }\end{array}$ \\
\hline$x_{2} y+1<1$ & $7:|1 \leqslant|-73 \mid$ & $=-15,1 C, 1,1$-5: & as \\
\hline Lis & 101 $18.23 \%-56:$ & Jills fors. & us \\
\hline SFi: & SE. 1s: IU-11\%, & $6 ! 1$ 15.3 IS-2 1 & $a j$ \\
\hline$-p: ?$ & $19: 14.35<1$ & $9+16012$ & $\therefore 6$ \\
\hline 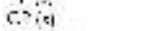 & $\therefore: 155$ 5.2514 & $=37-1, n-173$ & $6 \div 1$ \\
\hline 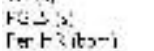 & $\begin{array}{l}\text { s. } 1.2 \mathrm{csc} \\
79.3: 17.5+213 \%\end{array}$ & 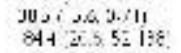 & a:s \\
\hline$F::$ - & $17=515-10$ & 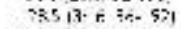 & $a=7$ \\
\hline r-e or irentrol & $\because c ; 4<, 4=1$ & 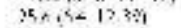 & OH \\
\hline
\end{tabular}

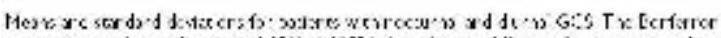

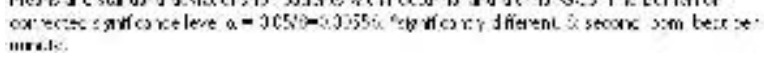

\subsection{7}

THE WORLDWIDE PREVALENCE OF EPILEPSY: A SYSTEMATIC REVIEW AND META-ANALYSIS J. Dykeman ${ }^{1}$, C. S. Kwon ${ }^{2}$, H. Dhaliwal ${ }^{1}$, K. Fiest ${ }^{1}$, S. Wiebe ${ }^{1}$, S. Patten $^{1}$, T. Pringsheim ${ }^{1}$ and Nathalie Jette ${ }^{1}\left({ }^{1}\right.$ University of Calgary, Calgary, AB, Canada and ${ }^{2}$ Harvard University, Boston, MA)

Rationale: Epilepsy is one of the most common neurological conditions worldwide. The purpose of this study was to estimate the international prevalence of epilepsy and explore sources of heterogeneity between studies. This study was part of a larger initiative funded by the Public Health Agency of Canada, the National Population Study of Neurological Conditions, to facilitate a better understanding of the burden of 15 neurological conditions in Canada and worldwide.

Methods: MEDLINE and EMBASE databases (1985 to Dec 13, 2010) were searched using pre-specified MeSH terms and keywords related to seizures, epilepsy, convulsions, incidence, prevalence and epidemiology. Population-based studies reporting the incidence and/or prevalence of epilepsy were included. Studies focusing solely on febrile convulsions or seizures (not epilepsy) were excluded. Two reviewers independently reviewed all abstracts, full text articles and abstracted data. A third reviewer was consulted to resolve any disagreement. Reported prevalence was abstracted for each study. Median estimates were calculated. A random effects model was also used to pool the prevalence [ $95 \%$ confidence interval] per 1,000 people (after testing for heterogeneity) where feasible.

Results: Embase and Medline identified 8061 and 5191 abstracts respectively, with 442 abstracts selected for full text review and 170 articles meeting all eligibility criteria. Globally the prevalence ranged from 1.55 per 1,000 in Hong Kong to 105 per 1,000 in Cameroon. Most studies used some variation of two or more clinical recurrent unprovoked seizures as their definition of epilepsy. An important source of heterogeneity was geographic location, and specifically developed vs. underdeveloped regions. The median estimates of prevalence for each continent were: 7.85 per 1,000 (range: $2-105)$ in Africa ( $\mathrm{n}=30$ studies), 4.88 per 1,000 (range: $1.54-77)$ in Asia ( $\mathrm{n}=50$ studies), 5.25 per 1,000 (range: $2.26-15.4$ ) in Europe ( $\mathrm{n}=50$ studies), 7.15 per 1,000 (range: $3.4-28$ ) in North America ( $\mathrm{n}=28$ studies) and 9.8 per 1,000 (range: $3.8-32$ ) in South America ( $\mathrm{n}=12$ studies). There were no population-based studies examining the prevalence of epilepsy in Australia. The results of the meta-analysis and exploration of additional sources of heterogeneity will be presented.

Conclusions: Epilepsy is common, particularly in developing countries, although gaps still exist regarding the global prevalence of epilepsy. Specifically, there was a noticeable lack of evidence from Australia and South America. The higher prevalence found in developing countries is consistent with prior studies, and is believed to be multifactorial, and likely due in part to possible genetic factors, but more significantly to the higher incidence of infections and head trauma in resource poor countries. In continents where much evidence exists, the focus should be shifted towards the investigation of sources of heterogeneity and the identification of modifiable risk factors and approaches to reduce the burden of epilepsy.

\subsection{8}

ASSOCIATION OF BREATHING AND CARDIAC COMPLICATIONS WITH EPILEPTIC SEIZURES IN CHILDREN: A PRELUDE TO UNDERSTANDING SUDEP KANWALJIT SINGH ${ }^{1,2}$, E. Katz ${ }^{4,5}$, M. Zarowski ${ }^{2,3}$, T. Loddenkemper $^{2,3}$, N. Llewellyn 6 , S. Manganaro ${ }^{2}$, M. Gregas ${ }^{2,3}$, M. Pavlova $^{6,3}$ and S. Kothare ${ }^{2,3}$ ( ${ }^{1}$ Pediatrics, MASSACHUSETTS GENERAL HOSPITAL, LEXINGTON, MA; ${ }^{2}$ Neurology, Children's Hospital, Boston, MA; ${ }^{3}$ Neurology, Harvard Medical School, Boston, MA; ${ }^{4}$ Medicine, Children's Hospital, Boston, MA; ${ }^{5}$ Medicine, Harvard Medical School, Boston, MA and ${ }^{6}$ Neurology, Brigham and Women's Hospital, Boston, MA)

Rationale: Sudden Unexpected Death in Epilepsy Patients (SUDEP) is an important cause of death in epilepsy patients. Role of cardiopulmonary abnormalities in SUDEP is unclear in pediatric population. This study was done to assess cardio-pulmonary abnormalities during epileptic seizures in children, with the long-term goal of identifying potential mechanisms of SUDEP.

Methods: We prospectively recorded and analyzed cardio-pulmonary abnormalities by pulse-oximetry, EKG and respiratory-inductanceplethysmography (RIP) during 101 seizures in 26 children. Logistic regression was done to evaluate association of these abnormalities with patient characteristics.

Results: RIP provided data in $78 \%$ and pulse-oximetry in $63 \%$ seizures. Ictal-apnea increased with younger-age $(\mathrm{p}=0.01)$, malegender $(p=0.08)$ temporal-lobe $(p=0.0005)$, left-sided $(p=0.003)$, symptomatic-generalized (vs. primary-generalized and complexpartial) $(\mathrm{p}=0.01$ and 0.08$)$, complex-partial (vs. primary-generalized) $(\mathrm{p}=0.08)$, longer-duration seizures $(\mathrm{p}=0.0002)$, desaturation $(\mathrm{p}<0.0001)$, ictal-bradycardia $(\mathrm{p}=0.02)$, more antiepileptic-drugs (AEDs) $(\mathrm{p}=0.006)$ and decreased in frontal-lobe seizures $(\mathrm{p}=0.004)$. 
Ictal-bradypnea increased in temporal-lobe $(\mathrm{p}=0.07)$, left-sided $(\mathrm{p}=0.04)$, lesional (on MRI-brain) $(\mathrm{p}=0.09)$, symptomatic-generalized seizures (vs. complex-partial and primary-generalized) $(\mathrm{p}=0.005$, $0.005)$, desaturation $(\mathrm{p}=0.07)$, more AEDs $(\mathrm{p}=0.07)$ and decreased in frontal-lobe seizures $(\mathrm{p}=0.07)$. Ictal-tachypnea increased in older-age $(\mathrm{p}=0.01)$, female-gender $(\mathrm{p}=0.05)$, frontal-lobe (vs. temporal and other foci) $(\mathrm{p}=0.09,0.02)$, right-sided seizures $(\mathrm{p}=0.0008)$ less AEDs $(p=0.005)$ and decreased in lesional $(p=0.03)$ symptomaticgeneralized seizures (vs. complex-partial and primary-generalized) $(p=0.02,0.03)$. Ictal-bradycardia increased in younger-age $(p=0.09)$ male-gender $(\mathrm{p}=0.03)$ longer-duration seizures $(\mathrm{p}=0.03)$, desaturation $(p=0.001)$, more AEDs $(p=0.04)$, and decreased in frontal-lobe seizures $(\mathrm{p}=0.01)$. Ictal and post-ictal bradycardia were directly correlated $(\mathrm{p}=0.02)$. Ictal tachycardia increased in older-age $(\mathrm{p}=0.08)$, female-gender $(\mathrm{p}=0.001)$, frontal-lobe $(\mathrm{p}=0.06)$ seizures, and decreased with lesional-seizures $(\mathrm{p}=0.01)$ and more AEDs $(\mathrm{p}=0.02)$. Desaturation increased with longer-duration seizures $(\mathrm{p}<0.0001)$, ictal-apnea $(\mathrm{p}<0.0001)$, ictal-bradycardia $(\mathrm{p}=0.001)$, and more AEDs $(\mathrm{p}=0.001)$.

Conclusions: Potentially life-threatening cardio-pulmonary abnormalities like bradycardia, apnea and hypoxemia in pediatric epileptic seizures are associated with predictable patient and seizure characteristics, including seizure sub-type and duration.

Understanding epidemiology of cardio-pulmonary compromise in epilepsy patients may lead to preventative strategies for patients at high-risk for SUDEP.

Bivariate relationships between cardiopulmonary disturbances and patient characteristics (significant $(\mathrm{p} \leq 0.05)$ and borderline $(0.05<\mathrm{p}$ $<1.0)$ relationships in

bold.

\begin{tabular}{|c|c|c|c|c|c|c|c|c|c|c|c|c|}
\hline \multirow{2}{*}{$\begin{array}{l}\text { Oulcestic } \\
\text { Preclictor }\end{array}$} & \multicolumn{2}{|c|}{ Iclat Apputa } & \multicolumn{2}{|c|}{ Ictal Tacthypnes } & \multicolumn{2}{|c|}{ ktal Bradypoca } & \multicolumn{2}{|c|}{$\begin{array}{c}\text { Ieta } \\
\text { Bracyeardia }\end{array}$} & \multicolumn{2}{|c|}{$\begin{array}{c}\text { lecal } \\
\text { Tachycardia }\end{array}$} & \multicolumn{2}{|c|}{ Desaturation } \\
\hline & $\begin{array}{l}\text { Odds } \\
\text { Ratio }\end{array}$ & $\mathrm{p}$-value & $\begin{array}{l}\text { Odds } \\
\text { Ralio }\end{array}$ & p-value & $\begin{array}{l}\text { Odds } \\
\text { Ralic }\end{array}$ & p-valuc: & $\begin{array}{l}\text { Odds } \\
\text { Ratio }\end{array}$ & p-viluc: & $\begin{array}{l}\text { Odds } \\
\text { Raties }\end{array}$ & $p$-value & $\begin{array}{l}\text { Odds } \\
\text { Rativi }\end{array}$ & p-viluo \\
\hline Ager & 0.85 & 0.01 & 1,19 & 0.01 & 1.03 & 0.75 & 0,89 & $0 .(19)$ & 1.12 & 0.08 & 0.96 & 0.49 \\
\hline Gender (ficmalc ws. malc) & 0.39 & 0.08 & 2.79 & 0.05 & 0.44 & 0.31 & 0.21 & 0.028 & 1.99 & 0.001 & 0.45 & 0.12 \\
\hline $\begin{array}{l}\text { (ieneralization fyes of } \\
\text { nit) }\end{array}$ & 1.11 & 0.82 & 0.97 & 0.95 & 0.94 & 0.92 & 0.45 & 0.19 & 1.08 & 0.89 & 0.91 & 0.85 \\
\hline $\begin{array}{l}\text { I.scalizacion (temporal } \\
\text { vs, liontal) }\end{array}$ & 8.04 & 0.0005 & 0.22 & 0.009 & 4.89 & 0.07 & 1.95 & 0.43 & 0.80 & 0.75 & 3.36 & 0.78 \\
\hline $\begin{array}{l}\text { 1. coal iratitum Ifromal is. } \\
\text { mothers! }\end{array}$ & 1.38 & 0.61 & 0.91 & 0.88 & 0.95 & 0.94 & 0.30 & 0.15 & 2.67 & 0.20 & 0.86 & 0.84 \\
\hline $\begin{array}{l}\text { Localization f(temporal } \\
\text { vs. ofhers? }\end{array}$ & 0.17 & 0.004 & 4.08 & 0.02 & 0.19 & 0.07 & 0.15 & 0.01 & 3.33 & 0.06 & 0.26 & 0.25 \\
\hline $\begin{array}{l}\text { Taleralization flefi vs. } \\
\text { niglll) }\end{array}$ & 2.91 & 0.116 & 0.12 & 0.10018 & 10.11 & 0.04 & 1.03 & 0.97 & 1.29 & 0.72 & 1.94 & 0.31 \\
\hline MRI L.csions (ycs ps. no) & 1.81 & 0.25 & 0.34 & 0.03 & 6.0 & $0.09)$ & 1.97 & 0.35 & 0.19 & 0.01 & 2.08 & 0.18 \\
\hline 1.cog Duratiom & 3.51 & 0.00002 & 1.30 & 0.24 & 1.20 & 0.53 & 2.20 & 0.13 & (0.) & 1).6. 6 & 9.35 & $<0.0001$ \\
\hline $\begin{array}{l}\text { Seizure rype (focal vs. } \\
\text { primary generalizedl) }\end{array}$ & 3.45 & 0.08 & 0.77 & 0.67 & 2.79 & 0.35 & 0.56 & 0.12 & 0.94 & 0.92 & 1.39 & 0.61 \\
\hline 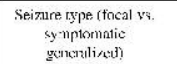 & 0.15 & 13.0.18 & 9.44 & 0.12 & 0.1 & 0.01015 & 0.25 & 0.13 & 3.0 & 0.2 .3 & $0.2^{4}$ & (0.16 \\
\hline 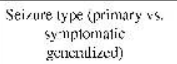 & 0.04 & 0.01 & 10.28 & 0.03 & 0,05 & 0.005 & 0.44 & 0.42 & 3.20 & 0.26 & 0.21 & 0.12 \\
\hline $\begin{array}{l}\text { Sleep state Gattake ws. } \\
\text { slicp) }\end{array}$ & 1.24 & 8..65 & $0(x)$ & 0.27 & $0.7 \mathrm{~s}$ & $0 .(19)$ & 1.14 & 0.8 .3 & 0.613 & 0.4 & 0.79 & 0.64 \\
\hline Desalturz:iun (yes vs, $\mathrm{nw})$ & 55.00 & <0.0001 & 0.31 & 0.07 & 7.67 & 0.07 & 33.38 & 0.001 & 0.19 & 0.18 & & \\
\hline Iatal Appea (yes v8. mo) & & & & & & & 13.85 & 0.02 & 1.08 & 0.90 & 55.00 & $<0.0601$ \\
\hline $\begin{array}{l}\text { lekal bradyeardia (yes vs, } \\
\text { noi) }\end{array}$ & 13.85 & 0.02 & $0.50)$ & 0.43 & 2.50 & 0.36 & & & & & 33.38 & 0.001 \\
\hline 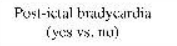 & & & & & & & 7.99 & 0.02 & & & 2.55 & 0.31 \\
\hline $\begin{array}{l}\text { Ictal tachycardia ives ws. } \\
\text { tuo) }\end{array}$ & 1.08 & 0.9 & 1.68 & 0.90 & 1.97 & 0.34 & 0.44 & 0.39 & & & $0.4 \%$ & 0.18 \\
\hline AIDM & 1.80 & 0.006 & 0.56 & 0.0015 & 1.93 & 0.07 & 1.90 & 0.04 & 0.56 & 0.01 & 2.34 & 0.001 \\
\hline
\end{tabular}

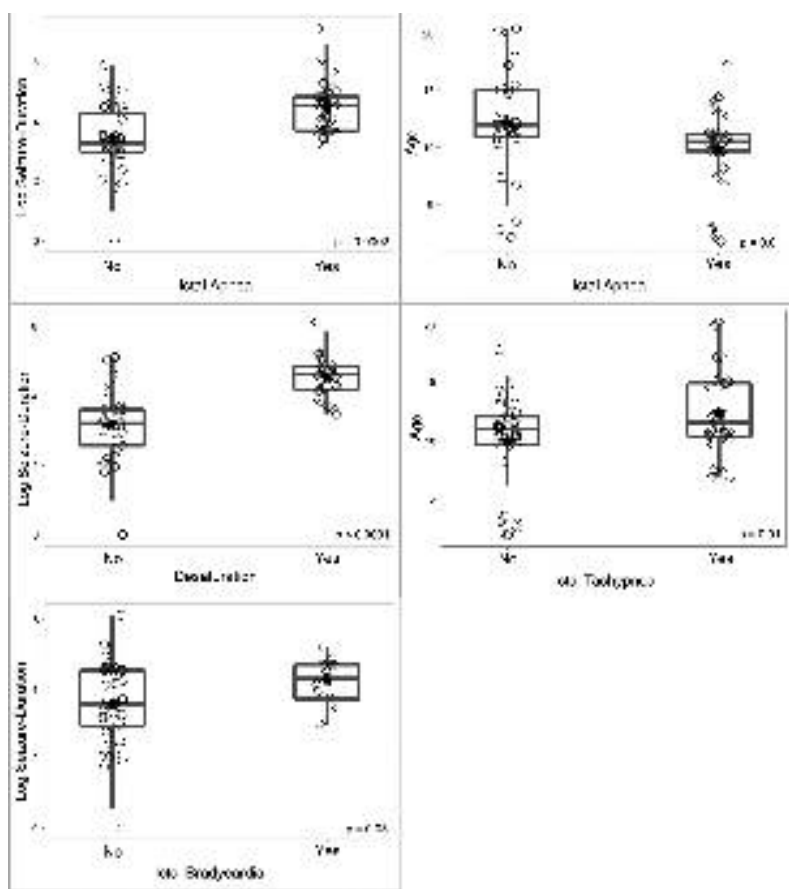

Scatter and box plots showing distribution of cardiopulmonary associations (apnea, tachypnea, bradycardia, and desaturation) in relationship to seizure-duration (plotted as $\log$ ) and age. Patients with ictal apnea, desaturation, and ictal bradycardia had longer seizures. Patients with ictal apnea were younger, and patients with ictal tachypnea were older.

\subsection{9 \\ PREVALENCE OF EPILEPSY AND SOCIOECONOMIC FACTORS IN SOUTH CAROLINA, 2006-2010}

Dulaney A. Wilson, G. M. Smith and A. W. Selassie (Medicine /

Biostatistics \& Epidemiology, Medical University of South Carolina, Charleston, SC)

Rationale: Epilepsy is a spectrum disorder with impact on quality of life. Social stigma, economic challenges and comorbidities can further degrade quality of life. The disproportionate burden of epilepsy in minorities and the economically disadvantaged suggest an association between epilepsy and socioeconomic status. Relatively few population based surveillance systems for epilepsy exist. The South Carolina Epilepsy Surveillance System (SCESS) uses South Carolina's Integrated Data System to collect, analyze, and monitor the incidence and prevalence and identify characteristics that impact quality of life. This analysis describes prevalence of epilepsy in the South Carolina population according to socioeconomic status. Methods: The SCESS receives data on all patient encounters with a diagnosis of epilepsy and/or unspecified seizure. As of 1995, a unique identifier allows linkage across multiple providers and services. Cases of epilepsy are confirmed as definite and probable based on a confirmatory diagnosis of epilepsy or an algorithm incorporating procedure codes, medication usage, and repeat visits. The annual prevalence of epilepsy is described by socioeconomic factors including insurance status, poverty level by county, and rural/ urban residency.

Results: Between 2006 and 2010, 89,936 individuals were diagnosed with epilepsy in South Carolina. Annual prevalence in children (18 and under) was $0.54 \%$, in adults (19 to 64 ) was $0.37 \%$ and was $0.24 \%$ in seniors (65 and over). Annual prevalence in females was $0.47 \%$ and in males was $0.33 \%$. Annual prevalence in AfricanAmericans was $0.63 \%$, in whites was $0.32 \%$ and in Hispanics was $0.22 \%$. Annual prevalence in persons with Medicaid was $1.67 \%$, with Medicare was $0.30 \%$, and private insurance was $0.15 \%$ while the annual prevalence in the uninsured was $0.22 \%$. The prevalence in counties with high poverty was $0.40 \%$, moderate poverty was $0.47 \%$, 
and in counties with low poverty was $0.37 \%$. The prevalence in residents of rural counties was $0.59 \%$ compared to $0.32 \%$ in residents of urban counties.

Conclusions: Epilepsy prevalence was twice as high in AfricanAmericans compared to whites. The prevalence in persons with Medicaid was nearly 7 times that of persons covered by private insurance; those insured by Medicare had 2 times the prevalence of those with private insurance. The prevalence was not very different by poverty status of counties; however, the ranges of incomes in a given county may decrease the impact of poverty on epilepsy. Rural residents have high prevalence of epilepsy and face difficulties in accessing health care because of limited availability of public transportation in the state. Our findings suggest the discordant distribution of living with epilepsy is disproportionately tilted towards vulnerable population groups.

\subsection{0 \\ CHILDREN WITH EPILEPSY AND ACADEMIC PERFORMANCE - ARE WE DOING ENOUGH?}

C. Prasad ${ }^{2}$, B. Corbet ${ }^{3}$ and Asuri N. Prasad ${ }^{1}\left({ }^{1}\right.$ Pediatrics and Clinical Neurosciences, Western University \& Schulich School of Medicine \& Dentistry, London, ON, Canada; ${ }^{2}$ Schulich School of Medicine \& Dentistry, Western University, London, ON, Canada and ${ }^{3}$ Statistics Canada Research Data Centre, Western University, London, ON, Canada)

Rationale: Epilepsy co-exists with other developmental disabilities, likely resulting in functional impairment in school-age children. We examined the school readiness, sense of general self and math scores in Canadian children and compared children with self-reported epilepsy to the national cohort using Canada's National Longitudinal Survey of Children and Youth (NLSCY).

Methods: Using data from Cycles 1 to 5 of the NLSCY, standard scores on the Peabody Picture Vocabulary Test - Revised (PPVT-R), administere to children (4-5 years) were employed in a regression analysis to compare school readiness in children with and without epilepsy. The general self-score (Marsh Self Description Questionnaire) and a standardized mathematics score were included in a multi-level growth model with individual measures at level 1 and between person measures at level 2 using HLM 6.3. The intercept was centred at 6 years.

Results: A total of 31000 children were included in the analysis and 68 responders reported epilepsy. Children with epilepsy

underperformed on the school readiness test (PPVT-R) in comparison to the rest of their cohort $(\beta=0.05, \mathrm{se}=0.01, \mathrm{p}=0.000)$. The Male cohort also underperformed in comparison to females $(\beta=0.033$, $\mathrm{se}=0.001, \mathrm{p}=0.000$ ). Using a multi-level growth model, we found general self scores were not significantly different between children with or without epilepsy. While females scored significantly lower on the general self score at the intercept compared to males $(\beta=.313$, se $=0.039, \mathrm{p}=0.000$ ), there was no statistically significantly difference in the math scores between the genders or for children with epilepsy at the intercept or slope. There was a higher proportion of missing data among students with epilepsy, indicating that students with more severe disabilities may not have been included in the data collection. Conclusions: Children with epilepsy display lower school readiness, however, this does not necessarily translate into poorer performance measures in the general self and math scores, for students that are able to complete the tests in later school years.

\subsection{1 \\ CLINICIAN ADHERENCE TO PRACTICE PARAMETERS ON FIRST NONFEBRILE SEIZURE IN CHILDREN}

Jennifer M. Avallone, A. Patel, N. Baumer, A. Sansevere, L. Doyle, N. Mehta, S. Choi, A. Pinto and T. Loddenkemper (Boston Children's Hospital, Boston, MA)
Rationale: Monitoring of practice parameter adherence and assessment on the effect on outcome in pediatric patients is limited. This study aims to assess adherence to American Academy of Neurology (AAN) practice parameters in children with first nonfebrile seizure at our center.

Methods: We retrospectively reviewed charts of pediatric patients age 1 month - 21 years who presented to our Emergency Department for first time convulsive seizure between January 2010 and December 2011. Patients with ICD 9 codes 345.1, 345.9, and 780.39 were included. Patients who presented with a first time febrile seizure or had a known brain tumor were excluded. Using a standardized assessment tool, we reviewed adherence to the AAN practice parameters related to the recommended ordering of routine electrolytes, toxicology screen, EEG, lumbar puncture, urgent head $\mathrm{CT}$, and brain MRI imaging. When a diagnostic study was ordered that did not adhere to guidelines, we determined if the test was abnormal and if the abnormality could explain the seizure or required urgent management.

Results: One hundred ten patients met inclusion criteria. Chart review showed that physicians followed AAN guidelines on ordering of toxicology screens (88\%; 95\% C.I. 82-94\%), EEG (96\%; 95\% C.I. 93-100\%), lumbar puncture (96\%; 95\% C.I. $93-100 \%)$, and nonurgent brain MRI imaging (95\%; 95\% C.I. 95-99\%) well. Although the majority of physicians followed the guidelines regarding routine electrolytes (79\%; 95\% C.I. 71-87\%) and emergent head CT imaging (75\%; 95\% C.I. $67-84 \%$ ), adherence to these parameters was lower. There was non-adherence to the parameter on ordering of routine electrolytes in $21 \%$ of the patients. Electrolyte results in these patients revealed mild abnormalities in 56\% (95\% C.I. $34-77 \%)$ including hyponatremia, hyperkalemia, and hyperglycemia. Electrolyte findings did not explain the etiology of the seizure or require intervention. There was non-adherence to the parameter on obtaining emergent head CT imaging in $25 \%$ of the patients. Of those, $77 \%$ had head CT imaging that was not indicated, while emergent imaging was not performed when indicated in $23 \%$ of the patients. $65 \%$ of the emergent head CTs performed when not indicated were ordered by outside emergency departments prior to transfer to our center. Of the patients who underwent unnecessary head CT imaging, $80 \%$ were normal and 20\% (95\% C.I. 6-44\%) showed an abnormal result, which did not require urgent intervention (i.e. chronic changes, PVL).

Conclusions: Child neurologists and pediatricians adhere to the majority of AAN practice parameters on children with first nonfebrile seizure. Occasional cases of non-adherence to guidelines including ordering of electrolytes or emergent head CTs within our and referring emergency departments did not alter urgent management supporting available guidelines. As a result of this review, we will implement a hospital wide standardized clinical management and assessment plan for first time nonfebrile seizure in children and continuously assess adherence. Additional efforts to include referring centers into this treatment plan are underway.

\subsection{2 \\ THE WORLDWIDE INCIDENCE OF EPILEPSY: A SYSTEMATIC REVIEW AND META-ANALYSIS} Nathalie Jette $^{1}$, C. S. Kwon ${ }^{2}$, H. Dhaliwal ${ }^{1}$, K. Sauro ${ }^{1}$, K. Fiest ${ }^{1}$, S. Wiebe $^{1}$, S. B. Patten ${ }^{1}$, T. Pringsheim ${ }^{1}$ and J. Dykeman ${ }^{1}{ }^{1}$ University of Calgary, Calgary, $\mathrm{AB}$, Canada and ${ }^{2}$ Harvard University, Boston, MA)

Rationale: Epilepsy is one of the most common neurological conditions worldwide. The purpose of this study was to estimate the international incidence of epilepsy and explore sources of heterogeneity between studies. This study was part of a larger initiative funded by the Public Health Agency of Canada, the National Population Study of Neurological Conditions, to facilitate a better understanding of the burden of 15 neurological conditions in Canada and worldwide. 
Methods: MEDLINE and EMBASE databases (1985 to Dec 13, 2010) were searched for this systematic review using pre-specified $\mathrm{MeSH}$ terms and keywords related to seizures, epilepsy, convulsions, incidence, prevalence and epidemiology. Population-based studies reporting the incidence and/or prevalence of epilepsy were included. Studies focusing solely on febrile convulsions or seizures (not epilepsy) were excluded. Two reviewers independently reviewed all abstracts, full text articles and abstracted data. A third reviewer was consulted to resolve any disagreement. Reported prevalence and/or incidence were abstracted for each study. A random effects model was used to pool the average annual incidence (AAI) $[95 \%$ confidence interval] per 100,000 people as well and the incidence rate per 100,000 person-years of epilepsy (after testing for heterogeneity).

Results: Embase and Medline identified 8061 and 5191 abstracts respectively, with 442 abstracts selected for full text review with 33 articles meeting all eligibility criteria. Incidence studies were identified in Europe $(n=20)$, North America $(n=4)$, Africa $(n=4)$, South America $(n=3)$ and Asia $(n=2)$, with most studies originating from Iceland $(n=3)$, the UK $(n=3)$, and the USA $(n=3)$. No Canadian studies addressing the incidence of epilepsy were identified. The most important source of heterogeneity was whether the examined region was developed or developing, with higher average annual incidence and incidence rate in developing countries. The highest AAI was reported in the Kenya pediatric study at 187.36 (114.12$307.61)$ per 100,000 children per year, with the lowest AAI reported in the Netherlands at $29.50(22.17-39.236)$ per 100,000 people per year. The highest incidence rate was reported in Uganda at 215 (157.76-293.01) per 100,000 person-years while the lowest incidence rate was reported in the USA at 15.15 (12.20-18.81) per 100,000 person-years.

Conclusions: Epilepsy is common, particularly in developing countries, although significant gaps still exist regarding the incidence of epilepsy worldwide, even in developed countries such as Canada. The higher incidence found in developing countries is consistent with prior studies, and is believed to be multifactorial, and likely due in part to possible genetic factors, but more significantly to the higher incidence of infections and head trauma in resource poor countries. Stratified AAI and incidence rate will be discussed, along with various sources of heterogeneity. There is a need for more uniform definitions of epilepsy and comparable methods of ascertainment for future epidemiological studies.

\subsection{3}

SEIZURES AT STROKE PRESENTATION VERSUS SEIZURES DURING HOSPITALIZATION IN ISCHEMIC STROKES: CLINICAL CHARACTERISTICS, RISK FACTORS AND IMPACTS ON OUTCOME

Chin-Wei Huang ${ }^{1}$, G. Saposnik ${ }^{2}$, J. Fang ${ }^{3}$ and J. Burneo ${ }^{1}$ ( ${ }^{1}$ Epilepsy Programme, Department of Clinical Neurological Sciences, University of Western Ontario, London, ON, Canada; ${ }^{2}$ University of Toronto, St Michael's Hospital, Toronto, ON, Canada and ${ }^{3}$ Institute for Clinical Evaluative Sciences, Toronto, ON, Canada)

Rationale: Seizures in stroke are not uncommon in clinical practice. But little is known about the differences between seizures at stroke presentation (SSP) and seizures during hospitalization (SDH), and their impacts on outcome.

Methods: This cohort study included consecutive patients with acute ischemic stroke between July 2003 and March 2008 from the Registry of the Canadian Stroke Network. Main outcome measures included morbidity (modified Rankin Scale), mortality with 30 days and one year, length-of-stay, and discharge disposition. Stroke outcome in SDH stratified by stroke severity was analyzed. Logistic regression analysis was used.

Results: A total of 10,261 patients with ischemic strokes were recruited; 157 (1.53\%) patients had SSP and 208 (2.02\%) patients had SDH. Compared to their counterparts without seizures, both SSP and SDH had significantly lower Canadian Neurological Scale (CNS) score $(\mathrm{p}<0.00001)$, were less common in lacunar syndrome $(8.3 \%$ vs $17.3 \%, 8.2 \%$ vs $17.3 \%, \mathrm{p}<0.00001)$, higher admission rate to ICU $(21.7 \%$ vs $8.0 \%, 20.7 \%$ vs $8.0 \%, \mathrm{p}<0.00001)$, higher morbidity $(\mathrm{p}=0.0439)$ and mortality $(\mathrm{p}<0.00001)$, and more dispositions to acute care $(p<0.0017)$. Importantly, patients with less severe strokes (CNS score $>5$ ) had significantly higher morbidity and mortality within 30 days and 1 year $(p=0.00001$ to 0.0049$)$, if SDH occurred. Variables predicting overall mortality were SDH, older age, higher Charlson index, lower CNS score and non-alert status on arrival (all $\mathrm{p}<0.0001$ ). Conclusions: Although both SSP and SDH in ischemic strokes are associated with younger age, severe strokes, and higher morbidity and mortality, they show different characteristics. SDH aggravate prognosis in patients, especially in those with less severe strokes. Increased awareness of SSP and prevention of SDH would be important in clinical stroke care.

\section{Sunday, December 2, 2012}

\author{
Investigators' Workshop \\ 8:45 a.m.-5:15 a.m.
}

\section{MULTIMODAL NEUROIMAGING TO DIRECT EPILEPSY SURGERY}

John S. Duncan ${ }^{1}$, Andrew Mcevoy ${ }^{2}$, William Bingaman ${ }^{3}$ and Christian Vollmar ${ }^{4}\left({ }^{1}\right.$ Epilepsy, UCL Institute of Neurology, London, United Kingdom; ${ }^{2}$ Neurosurgery, NHNN, London, United Kingdom; ${ }^{3}$ Neurosurgery, Cleveland Clinic, Cleveland, $\mathrm{OH}$ and ${ }^{4}$ Neurology, Groshadern Clinic, Munchen, Germany)

Summary: Implantation of intracranial electrodes and neurosurgical resection for refractory focal epilepsy carries risk of significant morbidity, particularly if carried out close to critical white matter tracks and eloquent cortex. The location of the target for implantation and/or resection will determined by consideration of multiple imaging data including structural MRI, functional MRI, PET, SPECT, MEG, electrical source imaging, tractography, arteriography and venography.

This workshop will review current approaches to the 3D display of multimodality brain imaging for planning these procedures and consider the strengths and limitations of different methods, the development roadmap the future applicability and utility of the techniques.

This workshop will be of interest to neurosurgeons, neurophysiologists and neuroimaging specialists interested in optimizing their surgical practice and outcome.

\section{THE EXTRACELLULAR MATRIX IN EPILEPSY}

Brenda E. Porter ${ }^{1,2}$, Alexander Dityatev ${ }^{4}$, Chrysanthy Ikonomidou ${ }^{3}$ and Paulette $\mathrm{McRae}^{2}\left({ }^{1}\right.$ Neurology and Pediatrics, The Children's Hospital of Philadelphia, Philadelphia, PA; ${ }^{2}$ Neurology, The Perelman School of Medicine at the University of Pennsylvania, Philadelphia, PA; ${ }^{3}$ Neurology, University of Wisconsin, Madison, WI and ${ }^{4}$ Neuroscience and Brain Technologies, Italian Institute of Technology, Genoa, Italy)

Summary: The extracellular matrix is crucial to the proper formation and functioning of the mature nervous system. A complex structure, the matrix appears to stabilize, neuron glial interactions and synaptic connectivity. A large number of proteases are present in the nervous system allowing for ongoing remodeling of the matrix in normal and pathologic conditions. There has been growing evidence that the extracellular matrix plays a role in epilepsy. Mutations in the 
extracellular matrix protein LGI have been associated with a rare epilepsy syndrome. Abnormal expression of extracellular matrix proteins and complex carbohydrates have been identified in CSF, hippocampus, and focal cortical dysplasia specimens from epilepsy patients. Destruction, as well as, inappropriate expression of extracellular matrix proteins has also been found in acquired rodent epilepsy models. This Investigator's Workshop will educate the audience on the current state of knowledge of how changes in the matrix, either during development, in cortical malformations, or in models of acquired epilepsy may play an important role in the progression and/or maintenance of the epileptic state.

\section{SUDEP RESEARCH CONSORTIUM: A NEW COLLABORATIVE NETWORK TO DISCOVER PREDICTIVE GENES, MECHANISMS AND BIOMARKERS OF SUDEP}

Alica M. Goldman ${ }^{1}$, George Richerson ${ }^{2}$, Jack M. Parent ${ }^{3}$, Lisa Bateman ${ }^{4}$, Douglas R. Nordli ${ }^{5}$ and Jeffrey L. Noebels ${ }^{1}\left({ }^{1}\right.$ Neurology, Baylor College of Medicine, Houston, TX; ${ }^{2}$ Neurology, University of Iowa, Iowa, IL; ${ }^{3}$ Neurology, University of Michigan, Ann Arbor, MI; ${ }^{4}$ Neurology, UC Davis, Sacramento, CA and ${ }^{5}$ Epilepsy Center Childrens Memorial Hospital, Chicago, IL)

Summary: This translational workshop will explore, from the bench to the bassinet, and from the stem cell to the EMU bedside, ways to measure relevant genetic, cellular, and clinical parameters in epilepsy patients that may predict the risk of SUDEP, and raise questions to identify the gaps in our understanding. Where are the therapeutic opportunities to predict and prevent SUDEP? What is the evidence that it is fundamentally a deficiency of a post-ictal respiratory drive arising in the brainstem? An inherited fatal cardiac arrhythmia? A primary dysautonomia? An idiosyncratic antiepileptic drug withdrawal reaction? Can a latent deficiency be identified in a patient before it is too late? Discussion will center on specific experimental data emerging from laboratories analyzing mouse models and human patients that display premature death in epilepsy, and possible clinical trials with existing medicines to prevent respiratory depression or cardiac arrhythmia.

Alica Goldman moderator

Jeff Noebels, MD, PhD (Baylor) will describe human cases and mouse models illustrating the genes that predispose to combined seizure and cardiac arrhythmia syndromes, and highlight the challenges to evaluating cardiac arrhythmia risk in epilepsy patients. George Richerson, MD, PhD (U. Iowa) will present data on brainstem mechanisms of respiratory depression and experiments in serotonergic mouse mutants and extracellular serotonin levels in epilepsy patients.

Lisa Bateman, MD (UC Davis) will outline the obstacles, current findings, and practical solutions to accurate measurement of peri-ictal cardiorespiratory biomarkers in the adult EMU. Dr. Douglas Nordli (Northwestern) will provide pediatric perspecives in the follow-up discussion.

\section{VALIDATION OF EPILEPSY BIOMARKERS IN HUMANS: GOALS, CHALLENGES, OPPORTUNITIES}

Jeffrey Loeb ${ }^{1}$, Laura A. Jansen ${ }^{2}$ and Gilles Huberfeld ${ }^{3}\left({ }^{1}\right.$ Neurology $/$ Ctr Mol Med and Genetics, Wayne State University, Detroit, MI; ${ }^{2}$ Neurology, University of Virginia, Charlottesville, VA and ${ }^{3}$ Cortex and Epilepsy, Centre de Recherche de l'Institut du Cerveau et de la Moelle Epinière, Université Pierre et Marie Curie (UPMC), Paris, France)

Summary: The availability of fresh human brain regions removed for the treatment of medically refractory epilepsy or epileptogenic lesions produces an unparalleled opportunity to probe epilepsy in humans. By the time brain tissue has been surgically resected extensive imaging and electrical mapping studies are done to improve surgical outcome, but also provide detailed in vivo data that can be linked to specific biomarkers of epilepsy within tissues removed. These biomarkers can be genes, proteins, or signaling pathways, but could also be changes in neuronal circuitry that makes a specific brain region prone to seizures. In vitro, neuronal networks are preserved so that these tissues may still generate spontaneously epileptic activities. In this workshop, the speakers will present three diverse types of studies that can be done on resected human tissues. These range from probing the developmental pattern of GABAergic properties in children, to human slice recordings of neocortex and hippocampus to identify altered circuitry in epilepsy, to highthroughput genomics and systems biology studies to identify new pathways in human epilepsy. For each, the key advantages and opportunities as well as challenges and potential pitfalls will be discussed.

Dr. Laura Jansen will present studies of post-surgical and postmortem human tissue, focusing on the normal developmental acquisition of mature GABAergic function and how it may be disrupted in epilepsy.

Dr. Gilles Huberfeld will summarize elegant in vitro studies of human epileptic tissues to unravel in situ mechanisms of genesis of epileptic discharges with a focus on local networks and on excitatory effects of glutamatergic but also GABAergic signaling.

Dr. Jeffrey Loeb will present an approach to identify novel biomarkers, cellular and histological abnormalities, and drug targets using high-throughput systems biology on electrically mapped human neocortical tissues.

\section{SWIMMING TOWARD NEW PATHS FOR DRUG DISCOVERY IN EPILEPSY: AN OPEN DISCUSSION OF TRADITIONAL AND NOVEL APPROACHES, INCLUDING ZEBRAFISH, ORGANOTYPIC SLICES AND MORE}

F. Edward Dudek ${ }^{1}$, H. Steve White ${ }^{2}$, Scott C. Baraban ${ }^{3}$ and Yevgeny Berdichevsky ${ }^{4,5}$ ( ${ }^{1}$ Physiology, University of Utah, Salt Lake City, UT; ${ }^{2}$ Pharmacology and Toxicology, University of Utah, Salt Lake City, UT; ${ }^{3}$ Neurological Surgery, University of California San Francisco, San Francisco, CA; ${ }^{4}$ Neurology, Massachusetts General Hospital, Boston, MA and ${ }^{5}$ Electrical and Computer Engineering, Lehigh University, Bethlehem, PA)

Summary: The epilepsy community is currently involved in an active discussion of how best to identify new drugs to treat - and possibly even prevent - intractable epilepsy. Thirty years of traditional acute seizure models in rodents have generated more than a dozen new AEDs that have clearly helped a population of patients with epilepsy. The merits of this approach continue to be actively debated. However, $30-40 \%$ of patients remain pharmacoresistant, and this has not substantially improved with time. This workshop seeks to explore novel methods that could contribute to and expand upon traditional approaches. Specifically, a critical need persists (1) to rapidly screen for new AEDs targeted against the seizures associated with rare genetic diseases of childhood and (2) to devise novel strategies to efficiently and reliably suppress or block acquired epileptogenesis after brain injuries. Steve White (White and Wilcox, Univ. Utah) will begin the workshop with a discussion of recent modifications to the protocols of the Anticonvulsant Screening Project using established and emerging rodent models of pharmacoresistant epilepsy, which may continue to lead to the discovery of more effective therapeutics. This will be compared with newly emerging high-throughput programs using larval zebrafish (Baraban, UCSF) and a novel medium-throughput strategy focused on anti-epileptogenesis tests in organotypic culture, followed by and coupled with chronic recordings in animal models of acquired epilepsy (Berdichevsky/Staley, MGH/Harvard Med. Sch.; Dudek, Univ. Utah). Scott Baraban will describe how zebrafish, a vertebrate model system that could significantly alter and advance the drug discovery process can be employed in a rapid and cost-effective manner to identify new AEDs. A two-tiered drug-screening process 
will be discussed using a zebrafish mutant for Nav1.1, a voltagegated sodium channel mutated in children with Dravet Syndrome and/or Severe Myoclonic Epilepsy of Infancy. These high-throughput assays are based on protocols developed in the Baraban laboratory for automated locomotion-tracking in a 96-well format and in vivo electrophysiology in individual mutant fish. Yevgeny Berdichevsky will describe novel medium-throughput strategies focused on antiepileptogenesis tests in organotypic culture. This approach exploits the rapid epileptogenesis exhibited by organotypic hippocampal slices over 4 weeks in culture. Periodic lactate and lactate dehydrogenase assays are used to test whether agents alter seizure activity or seizure-induced neuronal death, respectively, and to separate anti-epileptogenic from antiseizure effects (Berdichevsky and Staley). This is followed by in vivo chronic EEG monitoring and computerized seizure detection during epileptogenesis after experimental status epilepticus or other brain injuries (Dudek and Staley). These approaches each have advantages and limitations, and this workshop will provide a forum for a lively discussion of these important issues.

\section{SEARCHING FOR LESIONS IN "NON-LESIONAL" EPILEPSY}

Neda Bernasconi (Montreal Neurological Institute, Neuroimaging of Epilepsy Laboratory, McGill University, Montreal, QC, Canada)

Summary: Drug-resistant epilepsy is potentially curable by surgery. Although lesions associated with the epileptic focus can often be accurately detected by MRI, in many patients conventional imaging based on visual evaluation is unable to pinpoint the surgical target. These subjects with so-called cryptogenic or non-lesional epilepsy represent one of the greatest clinical challenges in tertiary centers. Importantly, it has become increasingly clear that epilepsies that are considered cryptogenic are not necessarily nonlesional, the primary histopathological substrate being subtle cortical dysplasia, as shown by surgical series.

This workshop will assemble a panel of experts to discuss state-ofthe-art evaluation methods aimed at unveiling epileptogenic lesions and propose new cohesive approaches to evaluate cryptogenic ("nonlesional") epilepsy, with particular emphasis on the extra-temporal type.

F. Cendes (University of Campinas, Brazil) will present effective strategies to increase the sensitivity of MRI visual evaluation.

A. Bernasconi (Montreal Neurological Institute, McGill University, Canada) will discuss the diagnostic yield of image processing to unveil subtle cortical dysplasias.

F. Chassoux (Sainte-Anne Hospital, Paris, France) will present data on FDG-PET and its contribution to the understanding of metabolic characteristics related to dysplastic lesions.

This workshop will provide participants with: a) strategies to optimize the evaluation of conventional and metabolic imaging; b) principles of novel structural image analysis methods; c) multidisciplinary discussions on new integrative approaches aimed at improving the understanding, diagnosis and management of cryptogenic epilepsy.

\section{WHAT'S NEXT? A WORKSHOP FEATURING RESEARCH TALKS BY YOUNG INVESTIGATORS}

Scott Baraban (Neurological Surgery, UCSF, San Francisco, CA)

Summary: Each year the American Epilepsy Society and Epilepsy Foundation of America award pre-, postdoctoral and young investigator awards grants to innovative, timely and cutting-edge research projects. Historically, many of these funded projects result in top-tier publications and several of these awardees have moved on to successful careers in epilepsy research. To highlight this cuttingedge research and provide a glimpse into what "may be next", this workshop brings together four recent awardees. Heidi Grabenstatter
(University of Colorado, Denver; Mentor, Amy Brooks-Kayal) is investigating signaling pathways in early and late epileptogenesis. Her talk will discuss whether signal pathways that are activated following acute, induced seizures may also undergo altered activation following spontaneous seizures seen in a chronic model of epilepsy. Potential therapeutic interventions will be considered. Tara Klassen (Baylor College of Medicine; Mentor, Jeff Noebels) will present data on the extensive and complex patterns of genetic variation in a large candidate gene set from individuals with sporadic idiopathic (genetic) epilepsy. Comprising over 400 functionally related members, ion channels make up over $1 \%$ of the entire human genome and 17 of the known familial epilepsy genes encode ion channel subunits; implications for personal risk prediction in epilepsy will also be discussed. Jeanne Paz (Stanford; Mentor, John Huguenard) will present evidence that cortical photo-thrombotic stroke leads, over time, to thalamic hyperexcitability and epileptic seizures. Using a novel technical approach to detect seizures within less than one second and to reduce using optogenetics, in real-time, the thalamocortical output her work shows that the thalamus is strictly required for seizure expression in a cortical stroke model. Csaba Varga (UC Irvine; Mentor, Ivan Soltesz) is studying cell type-specific modulation of CA1 pyramidal cell discharges by distinct interneuronal subtypes during behaviorally relevant, state-dependent hippocampal network oscillations in awake control and epileptic mice. His talk will highlight new insights into mechanisms underlying the temporal organization of normal chronocircuit behaviors and their dysfunction in epilepsy. Scott C. Baraban (UCSF) will introduce and moderate this session. Together these research talks should stimulate discussion on emerging topics and techniques in epilepsy research while providing a forum for a new generation of investigators.

\section{DYSFUNCTIONAL PHOSPHORYLATION SIGNALING IN EPILEPSY}

Anne Anderson ${ }^{1}$, James Trimmer ${ }^{2}$, Nicholas Poolos ${ }^{4}$ and Amy Brewster ${ }^{3}\left({ }^{1}\right.$ Pediatrics, Neurology, and Neuroscience, Baylor College of Medicine, Houston, TX; ${ }^{2}$ Neurobiology, Physiology and Behavior, UC Davis, Davis, CA; ${ }^{3}$ Pediatrics, Baylor College of Medicine, Houston, TX and ${ }^{4}$ Neurology, Physiology \& Biophysics, University of Washington, Seattle, WA)

Summary: Post-translational modification of proteins by phosphorylation is a ubiquitous cellular signaling mechanism. There is increasing evidence that altered phosphorylation signaling occurs in epilepsy and may underlie both epileptogenesis and altered neural excitability in chronic epilepsy. In this session we will discuss how phosphorylation-dependent mechanisms underlie some forms of both genetic and acquired epilepsy, and what therapeutic possibilities exist for pharmacological targeting of phosphorylation. James Trimmer, $\mathrm{PhD}$ will review the regulation of ion channel properties by phosphorylation and how recent technical advances in mass spectrometry now allow large-scale measurement of ion channel phosphorylation state. Nicholas Poolos, MD, PhD will discuss how altered phosphorylation signaling in an acquired epilepsy model underlies the loss of HCN channel expression and function, a key player in epileptogenesis. Amy Brewster, $\mathrm{PhD}$ will describe recent research in the Pten model of cortical dysplasia, and how modulation of phosphorylation activity can alter the course of epileptogenesis. Anne Anderson, MD will serve as a discussant.

\section{MASSIVELY PARALLEL SEQUENCING IN EPILEPSY}

Samuel Berkovic ${ }^{1}$ and Peter De Jonghe ${ }^{2}\left({ }^{1}\right.$ Epilepsy Research Centre, University of Melbourne, Melbourne, VIC, Australia and ${ }^{2}$ Molecular Genetics, University of Antwerp, Antwerp, Belgium)

Summary: Massively Parallel Sequencing ("Next Generation Sequencing") is a very new technology that allows whole exomes 
(entire coding sequence) and whole genomes to be efficiently sequenced. In 2011, two large long-term projects in epilepsy were launched - an NIH funded Centre without Walls project called "Epi4K" aimed at sequencing 4,000 epilepsy patients in 5 years and a European project with sequencing largely at the Sanger Center called "EuroEPINOMICS". The two groups have agreed to work collaboratively. This Investigators Workshop will present data from both groups.

Lead projects from both groups involve early childhood encephalopathies and this is the focus of the Workshop. The technology will be explained and it is important that clinicians have a grasp of this revolutionary change in genetics that will enter clinical practice soon. Preliminary results on early childhood epileptic encephalopathies will be presented. Early data suggest that this technology will markedly change our understanding of the biology and mechanism of this devastating group of epilepsies.

\section{NEOCORTICAL FOCAL SEIZURES IN ETIOLOGICALLY REALISTIC MODELS OF ACQUIRED EPILEPSY}

Raimondo D'Ambrosio ${ }^{3}$, Harry Sontheimer ${ }^{2}$ and Ramon DiazArrastia ${ }^{1}{ }^{1}$ Uniformed Services University of the Health Sciences, Bathesda, MD; ${ }^{2}$ University of Alabama Birmingham, Birmingham, AL and ${ }^{3}$ University of Washington, Seattle, WA)

Summary: Several models of acquired epilepsy based on realistic etiologies have been introduced in the last decade. These include, but are not limited to, epilepsy induced by head injury, stroke, febrile status, perinatal hypoxia, encephalitis, glioma. A consistent finding in these models is that the overall pathology induced by the insult differs in many ways from that commonly induced by agents such as pilocarpine or kainate in classic status epilepticus-based models of epilepsy. Consistent with the different pathology, the ensuing epilepsy syndromes are also different, including seizures that do not originate from the hippocampus but from the neocortex. Most basic scientists are more familiar with hippocampal seizures, but the human neocortex is highly epileptogenic and neocortical seizures are often difficult to treat.

The specific aims of the session are:

1) Familiarize basic scientists with the semiology of acquired human neocortical focal seizures, and clinicians with rodent neocortical seizures.

2) Present neocortical focal seizures in the rat in two different realistic models in which the neocortical origin of some of the chronic seizures has been verified.

3) Discuss similarities/differences between the realistic animal models and the human data, and between neocortical seizures and temporal lobe seizures.

\section{BRAIN PH IN THE GENERATION AND SUPPRESSION OF SEIZURES}

Kai Kaila ${ }^{1}$, Patrick W. Carney ${ }^{2,3}$, Steven Petrou ${ }^{2,4}$ and Saul Mullen ${ }^{2,4}$ ( ${ }^{1}$ Department of Biosciences and Neuroscience Center, University of Helsinki, Helsinki, Finland; ${ }^{2}$ Florey Neuroscience Institutes,

University of Melbourne, Melbourne, VIC, Australia; ${ }^{3}$ Department of Neurology, Austin Health, University of Melbourne, Melbourne, VIC, Australia and ${ }^{4}$ Department of Anatomy and Neuroscience, University of Melbourne, Melbourne, VIC, Australia)

Summary: Changes in $\mathrm{pH}$ exert a strong modulatory effect on neuronal signaling under normal and pathophysiological conditions, whereby alkalosis enhances and acidosis reduces brain excitability. Convulsive seizures are known to produce an acidosis due to reduced ventilation and build-up of lactic acid. This acidosis is not a mere bystander but contributes to seizure termination. Similarly, respiratory alkalosis produced by hyperventilation has been a standard way to provoke absence seizures. Identifying the $\mathrm{pH}$ dependent mechanisms does not only shed light on the process of epileptogenesis but, importantly, provides insights for the development of novel anticonvulsant therapies. Using a model of birth asphyxia based on 6-7 day old rats exposed for $1 \mathrm{~h}$ to hypercarbia $\left(20 \% \mathrm{CO}_{2}\right)$ and hypoxia $\left(9 \% \mathrm{O}_{2}\right)$, we found that postasphyxia seizures are triggered by a brain-confined alkalosis which takes place both in neurons (in vivo imaging) and in the extracellular space ( $\mathrm{pH}$ microelectrodes). This alkalosis is caused by enhanced extrusion of acid via $\mathrm{Na} / \mathrm{H}$ exchange across the blood-brain barrier. Both the alkalosis and seizures are suppressed by blockers of $\mathrm{Na} / \mathrm{H}$ exchange and by graded restoration of normocapnia after the period of asphyxia. The latter finding suggests that standard resuscitation paradigms in the clinic may, paradoxically, promote birth-asphyxia seizures. The effects of breathing carbogen containing $5 \% \mathrm{CO}_{2}$ on seizure susceptibility were tested in two mouse seizure models. In the first we employed a model of hyperthermic seizures where $\mathrm{SCN} 1 \mathrm{~B}(\mathrm{C} 121 \mathrm{~W})$ heterozygous mice, a mutation based on a family with genetic febrile seizures, were placed in a pre-heated $(42 \mathrm{C})$ chamber and the time to first tonic-clonic seizure was measured. Increasing chamber $\mathrm{CO}_{2}$ from atmospheric to $5 \%$ resulted in a significantly enhanced time to first seizure, and in some cases, the mice did not experience a seizure. Consistent with this, in an in vitro model of network oscillations in brain slices from C57B1/6 mice decreasing $\mathrm{pH}$ from 7.2 to 6.8 abolished oscillations as effectively as carbamazepine. In a second series of in vivo experiments we tested the effect of $5 \% \mathrm{CO}_{2}$ on spontaneous seizures using the GABRG2(R43Q) "absence" mouse model. Somewhat surprisingly, $5 \% \mathrm{CO}_{2}$ significantly worsened the number of SWD discharges and the behavioral arrest events suggesting that the anti-seizure mechanism of $\mathrm{CO}_{2}$ acts on specific seizure pathways. This will be discussed in light of recent findings showing that thermogenic seizure and SWD seizure phenotypes can emerge from a single genetic change but with presumed unique functional mechanisms. Finally, we will present preliminary data from a clinical trial evaluating $5 \% \mathrm{CO}_{2}$ as an acute anticonvulsant. Patients with focal seizures received either carbogen or oxygen in a blinded crossover trial where the primary outcome was seizure duration. We aim to develop mild respiratory acidosis as an acute antiepileptic, avoiding the sedation and depression of respiration seen with conventional antiepileptic drugs.

\section{COMORBIDITY BETWEEN EPILEPSY AND AUTISM SPECTRUM DISORDER: CHALLENGES IN THE DIAGNOSIS}

Stephane Auvin ${ }^{1}$, Rochelle Caplan ${ }^{2}$, Anne T. Berg ${ }^{3}$ and Elizabeth Thiele $^{4}\left({ }^{1}\right.$ Ped Neurology Dpt \& INSERM U676, Robert Debré Hospital, Paris, France; ${ }^{2}$ UCLA Semel Institute for Neuroscience and Human Behavior, UCLA, Los Angeles, CA; ${ }^{3}$ Epilepsy Center Children's Memorial Hospital, Northwestern University, Chicago, IL and ${ }^{4}$ Pediatric Epilepsy Program \& Herscot Center for Tuberous Sclerosis Complex, Massachusetts General Hospital, Boston, MA)

Summary: There has been a growing interest in neuropsychiatric comorbidities of epilepsy. Autism and Epilepsy is an area of major concern and one of the hardest issues that pediatric specialists have to deal with. This workshop will advance participants' understanding of the relation between autism and epilepsy by addressing three primary areas: 1) The definition and diagnostic criteria for autism, the errors in diagnosing autism, and the prevalent use of the diagnosis of autism to obtain services for children who, while having special needs often do not have autism per se. 2) The role of intellectual disability which may be the primary if not the sole factor linking autism and epilepsy. 3 ) The nature and appearance of autistic features and autism in genetically defined forms of epilepsy.

Stéphane Auvin, MD, PhD, Child Epileptologist in Robert Debré Hospital in Paris, as the organizer, will stress the lack of knowledge in patients with epilepsy and autism.

The CIW faculty will provide the background by presenting different aspects of Autism and Epilepsy: 
1. Rochelle Caplan, M.D., Professor, UCLA Semel Institute for Neuroscience and Human Behavior, will review the diagnostic categories included in autism spectrum disorder (ASD), the criteria for diagnosis, and the differential diagnosis. In discussing the change over time in who is making this diagnosis and how it is being done, she will focus on the diagnostic pitfalls related to ASD in early onset intractable epilepsy and in Landau-Kleffner Syndrome, Electrical Status Epilepticus in Sleep, and Continuous Spikes and Waves during slow Sleep. She will conclude with the need to avoid both overdiagnosis and under-diagnosis of ASD in children with epilepsy. 2. Anne T. Berg, PhD, Professor, Epilepsy Center Children's Memorial Hospital, Northwestern University, will be the second speaker. She will first describe two common clinical scenarios: patients with epilepsy that develop autism and patients with autism that start epilepsy. She will then describe clinical data such as incidence, age of the onset, type(s) of seizure, risk factors in epilepsy which predispose to the development of autism, and risk factors in primary autism patients which predispose to the evolvement of epilepsy. She will finish by discussing the data on EEG findings in patients with autism.

3. Elizabeth Thiele, M.D. Ph.D., Director of the Pediatric Epilepsy Program and of the Herscot Center for Tuberous Sclerosis Complex, Massachusetts General Hospital, Boston, will be the third speaker. She will discuss our knowledge about epilepsy syndromes observed in autism patients and epilepsy syndromes leading to autism behavior. She will describe the frequency of autistic features versus autism proper in several genetic disorders and the relation to intellectual disability She will also stress how challenging it is to differentiate certain types of seizure from autistic behaviors. She will conclude by mentioning the treatment options for these patients.

\section{Sunday, December 2, 2012}

\section{Investigators' Workshop - Poster Session 12:00 p.m.-2:00 p.m.}

For abstract content, please refer to the following poster numbers listed below in Poster Session 3 on Monday, December 3, 2012.

3.003, 3.004, 3.005, 3.006, 3.007, 3.008, 3.010, 3.011, 3.013, 3.015, 3.016, 3.017, 3.027, 3.029, 3.032, 3.033, 3.034, 3.035, 3.036, 3.037, 3.045, 3.046, 3.056, 3.064, 3.074, 3.097, 3.175, 3.186, 3.189, 3.192, 3.193, 3.194, 3.195, 3.196, 3.213, 3.312, 3.321, 3.337, 3.338, 3.341

\section{Monday, December 3, 2012}

\section{Poster Session 3}

8:00 a.m.-3:00 p.m.

\section{Translational Research: Mechanisms}

\subsection{1}

EXPLORING THE STRATEGIES THAT GUIDE THE REBUILDING OF NEURAL NETWORKS AFTER INJURY Waldemar B. Swiercz ${ }^{1,2}$, K. Lillis ${ }^{1,2}$ and K. J. Staley ${ }^{1,2}\left({ }^{1}\right.$ Neurology, Massachusetts General Hospital, Boston, MA and ${ }^{2}$ Neurology, Harvard Medical School, Boston, MA)

Rationale: Severe brain trauma changes neural network structure by altering neuronal connectivity. It is followed by self repair including replacment of lost connections. Despite the efficacy of this process, post traumatic epileptogenesis is a frequent consequence. It is generally assumed epileptogenesis consists of series of changes in the neural network including: axonal sprouting, an imbalance between inhibition and excitation, and homeostatic adjustments within neurons. However, the timing, extent, and strategies for execution of these adaptive changes are unknown, and we do not know how they are influenced by the nature of the initial circuit compromise / injury. By modeling this process, we hope to understand the range of changes that lead to epilepsy, as well as determine whether rebuilding circuits produce patterns of activity that could serve as early biomarkers for pro-epileptic changes. Such biomarkers would make feasible the administration of yet-to-be-discovered effective treatments that allow network repair but prevent epileptogenesis. Methods: We used a large-scale computer model of the CA3 region of the hippocampus to study the impact of network changes on the spatial and temporal patterns of synchronous activity propagation. Our experiments were designed using two network topologies, one with a Gaussian distribution of the length of synaptic connections, and a scale-free network where the number of neurons with a specific radius of connectivity was inversely proportional to the radius length. Each type of network was subjected to neuronal injury using following strategies of cell death: a) random, or preferential death of the following classes of neurons: b) highly connected cells c) most active d) interneurons. Afterwards each network was rewired using the following strategies: a) restoring damaged pathways, b) random with constant radii of connectivity c) random with expanding radii of connectivity.

Results: The results of our computational simulations were compared with multiphoton imaging of neuronal activity recorded in organotypic slice cultures of rat hippocampus over a period of 3 weeks in vitro, during which time the cultures became uniformly epileptic. Comparisons of model output and physiological recordings were based on measurements of cell activity correlations at different synaptic path lengths and correlation time windows. We also compared networks' synaptic structures by comparing log-log plots of the number of connections per neuron. We present the results of these simulations including the sequence of network changes that most closely reproduced the changes observed in-vitro.

Conclusions: Modeling studies provide useful information regarding the types of circuit changes that lead to spontaneous epileptiform activity. Ensemble recordings of real recovering neural networks constrain the models sufficiently that the importance of broad recovery strategies can be robustly evaluated; these evaluations will be presented.

\subsection{2 \\ EFFECTS OF FEBRILE SEIZURES AND EXPERIMENTAL TRAUMATIC BRAIN INJURY IN INFANT RATS}

Lucy J. Treiman ${ }^{1}$, R. Perez ${ }^{1}$, D. Schooley ${ }^{1}$, J. McDorman ${ }^{1}$, J. Tierney ${ }^{1}$, D. Treiman ${ }^{1}$ and P. D. Adelson ${ }^{2}{ }^{1}$ Neurology Research, Barrow Neurological Institute, Phoenix, AZ and ${ }^{2}$ Pediatric Neurosurgery/ Children's Neurosciences, Phoenix Children's Hospital, Phoenix, AZ)

Rationale: Five to $50 \%$ of people who suffer traumatic brain injury (TBI) eventually develop post-traumatic epilepsy (PTE). TBI is a leading cause of death and disability in children. Factors that influence the development of PTE, in addition to the severity of the brain insult, are poorly understood. This is a report of an ongoing study to test the hypothesis that prior insult (febrile seizures) increases the likelihood of mortality associated with TBI and the probability of subsequently developing PTE.

Methods: Offspring of timed-pregnant Sprague-Dawley rats (Charles River Laboratories) were used in these experiments. Litters were reduced to 8 pups ( 4 males, 4 females) on post-natal day (PND) 1. On PND 10 febrile seizures were induced in half of each litter using a hyperthermia chamber. One week later (PND 17) TBI was created by controlled cortical impact (CCI) using a pneumatically controlled 
impactor with a $6 \mathrm{~mm}$ tip traveling at $4 \mathrm{~m} / \mathrm{s}$ to compresses the exposed cortex $2.0 \mathrm{~mm}$. Sham animals were subjected to all procedures except impact. The bone flap was replaced. Four weeks later cortical screw electrodes were implanted into the skull for EEG recording at three and six months post TBI. Death within 72 hours of TBI-induction was included in the determination of mortality. Epileptiform activity and seizure frequency were determined by visual inspection of the EEG. Some animals were sacrificed at 2 weeks and 6 weeks for histology

Results: There were four experimental groups: Group 1 - Febrile seizures (FS) plus CCI; Group 2 - FS plus craniotomy only; Group 3 - no-FS plus CCI; Group 4 - no-FS plus craniotomy only. There were clear differences in mortality associated with surgery. Mortality rates were Group 1: 29.4\% (10/34), Group 2: 19.2\% (5/26), Group 3: $13.3 \%(4 / 30)$, Group 4: $6.1 \%$ (2/33). Overall, mortality was greater for FS animals: $25 \%(15 / 60)$ compared to no-FS animals: $9.5 \%$ (6/63).

Post-traumatic epilepsy after CCI or craniotomy was surprisingly infrequent: Group 1: 28.6\% (2/7), Group 2: 20\% (1/5), Group 3: $14.2 \%(1 / 7)$, Group 4: $0 \%(0 / 5)$. None-the-less, the incidence of PTE was greater in FS rats: $25 \%$ (3/12) than for no-FS animals: $8.3 \%$ (1/12). A number of animals exhibited epileptiform discharges without definite seizures.

Histology studies for extent of injury (hematoxylin and eosin) and mossy fiber sprouting (Timm stain), and studies of RNA expression from selected areas of the brain, harvested by using laser capture microscopy are underway and will be presented.

Conclusions: Febrile seizures one week before traumatic brain injury in immature rats markedly increases mortality associated with TBI and increases the likelihood of PTE developing after TBI. These observations support the hypothesis that prior insult (febrile seizures) increase the likelihood of mortality associated with TBI and the probability of subsequently developing PTE.

\subsection{3}

FUNCTIONAL RE-WIRING OF HIPPOCAMPAL NEURONS DURING POST-TRAUMATIC EPILEPTOGENESIS K. P. Lillis ${ }^{1,2}$, W. B. Swiercz ${ }^{1,2}$, M. A. Kramer ${ }^{3}$, G. Q. Zhao ${ }^{4}$, J. L. Raymond $^{4}$, B. J. Bacskai ${ }^{1,2}$ and K. J. Staley ${ }^{1,2}\left({ }^{1}\right.$ Neurology, Massachusetts General Hospital, Charlestown, MA; ${ }^{2}$ Neurology, Harvard Medical School, Boston, MA; ${ }^{3}$ Mathematics and Statistics, Boston University, Boston, MA and ${ }^{4}$ Neurobiology, Stanford University School of Medicine, Stanford, CA)

Rationale: $20 \%$ of symptomatic epilepsy is post-traumatic. In infants, severe traumatic brain injury involving extensive axonal shearing leads to epilepsy in up to $60 \%$ of patients. Although we presume that sprouting of axons and dendrites in injured tissues leads to local hyper-connectivity and recurrent excitation, very little is known about the rules governing the process of neural reorganization following injury.

Methods: Here, we model post-traumatic epilepsy using organotypic slices, which are prepared from neonatal mice (P5-P8) using shearing trauma (slicing). Slices become severely epileptic over the course of 3 weeks in culture. The mice used for these experiments continuously express in all neurons the calcium-sensitive protein Yellow Cameleon 3.6 (YC3.6). Brain slices were cultured using the membrane insert technique in which the sample rests on a piece of filter paper at the interface between culture media and air. The samples were imaged at intervals of 2-4 days-in-vitro (DIV) beginning the day after slicing (1 DIV). Using two-photon, targeted path scan (TPS) imaging through a dry objective (to allow the slice to remain at an interface), we recorded 15 minute epochs of spontaneous activity from at least 30 cells at a sampling rate of $25-50 \mathrm{~Hz}$ (sampling rate with TPS depends on the path selected). These imaging sessions were repeated at consecutive weeks in vitro, as the slices underwent epileptogenesis. Results: TPS calcium imaging revealed a pattern of epileptogenesis consistent with previously published electrophysiological measurements, with epileptiform activity appearing as large, rapid, spatially correlated increases in calcium (manifest as increases in the YC3.6 ratio). Activity began at 1DIV as brief bouts of synchronous activity in subpopulations of the imaged neurons. The activity transitioned to population-wide synchronous bursting, followed by intense seizure-like activity during the second week in vitro. The seizures then became longer and less intense (lower peak calcium for individual neurons) during the third week in vitro. Correlation analyses reveal a spatial distribution of highly correlated activity that was local during the first week, uniform (local and long-distance correlations) during the second week, and preferentially local during the third week. We are currently using network analysis techniques to quantify changes in network structure revealed by calcium imaging of neural activity.

Conclusions: Our results support a model in which initial sprouting following injury targets nearby neurons. We hypothesize that, when the sprouting extends to include long-distance connections, intense seizure activity leads to death of the most heavily connected neurons. This initial wave of status epilepticus-induced cell death leaves behind an epileptic network that consists of predominantly local connections. While the organotypic slice culture represents an extreme model of injury (resulting in epilepsy in $100 \%$ of samples), we propose that similar principles of neural re-wiring following traumatic injury lead to epilepsy in vivo.

\subsection{4 \\ THE RECEPTOR FOR ADVANCED GLYCATION END PRODUCTS (RAGE) IS OVEREXPRESSED IN MESIAL TEMPORAL LOBE EPILEPSY (MTLE) AND CONTRIBUTES TO EXPERIMENTAL SEIZURES AND EPILEPTOGENESIS Annamaria Vezzani ${ }^{1}$, V. Iori $^{1}$, M. Carli ${ }^{1}$, R. Vertemara ${ }^{1}$, T. Ravizza ${ }^{1}$, E. Aronica ${ }^{2}$ and M. Maroso ${ }^{1}\left({ }^{1}\right.$ Dept. Neuroscience, Mario Negri Institute for Pharmacological Research, Milano, Italy and ${ }^{2}$ Dept. of (Neuro)Pathology and Epilepsy Institute, Academisch Medisch Centrum and The Netherlands Foundation, Amsterdam and Heemstede, Netherlands)}

Rationale: We discovered a novel proinflammatory/proconvulsant pathway involving the release of the danger signal High Mobility Group Box 1 (HMGB1) from neurons and glia and its interaction with Toll-like receptor 4 (TLR4), a key receptor of innate immunity, overexpressed by neurons and astrocytes following proconvulsant challenges. We now explore the role in ictogenesis and epileptogenesis of RAGE, a receptor also stimulated by HMGB1 and $\mathrm{S} 100 \beta$, a protein released by astrocytes.

Methods: C57BL/6 adult male wild-type (WT) or RAGE-/- mice were injected unilaterally in the hippocampus either with $7 \mathrm{ng}$ of kainic acid to induce acute recurrent seizures for about $90 \mathrm{~min}$, or with $200 \mathrm{ng}$ of kainate to induce status epilepticus (SE) followed by spontaneous recurrent (drug-resistant) epileptic activity ( $n=6-8$ each exp group). We studied in WT and RAGE-/- mice ictogenesis and epilepsy development by EEG analysis, cognitive performance, neuropathology and neurogenesis.

Results: RAGE-/- mice displayed an average 30\% reduction in EEG seizure activity vs WT mice following intrahippocampal injection of $7 \mathrm{ng}$ kainic acid. Intrahippocampal injection of $10 \mu \mathrm{g}$ HMGB1 in WT mice increased the number and duration of kainate seizures by $2.5-$ fold on average, and reduced their onset time by $50 \%$. These proconvulsant effects were reduced by 2 -fold in RAGE-/- mice, except for seizures onset which remained anticipated as in WT mice. SE developed similarly in WT and RAGE-/- mice, as assessed by EEG analysis of frequency and total duration of spike activity. The time to onset of spontaneous epileptic activity following SE was similar in both mouse strains. However, the time spent in epileptic activity and the number of paroxysmal episodes were both significantly decreased $(\mathrm{p}<0.05)$ in RAGE-/- vs WT mice. Both strains of epileptic mice showed similar cognitive deficits in the novel object recognition test. Histological brain evaluation in 
epileptic RAGE-/- mice showed increased neurodegeneration specifically in CA1 pyramidal cells while cell loss was similar to WT in the other hippocampal regions. Granule cell dispersion and neurogenesis were similarly affected in both strains of epileptic mice. The number of S100 $\beta$-positive astrocytes in the hippocampus was increased in epileptic WT mice, like in human mTLE tissue, but not in epileptic RAGE-/- mice. Immunohistochemical studies showed evidence of RAGE induction in both astrocytes and microvessels in human and experimental mTLE hippocampi.

Conclusions: RAGE signaling is activated in human and experimental epileptic tissue. RAGE contributes to the mechanisms of ictogenesis, and mediates the proconvulsant effects of HMGB1 in concert with TLR4. RAGE activation concurs both to spontaneous epileptic activity and CA1 cell loss developing after SE. Our findings unveil a new molecular mechanism of ictogenesis and epileptogenesis, supporting its involvement in human epilepsy.

\subsection{5 \\ A REORGANIZED GABAERGIC CIRCUIT IN A MODEL OF EPILEPSY: EVIDENCE FROM CRE-DEPENDENT LABELING IN A SOMATOSTATIN-CRE MOUSE} Zechun Peng ${ }^{1}$, C. S. Huang ${ }^{1}$, Y. Cetina ${ }^{1}$, N. Zhang ${ }^{1}$ and C. R. Houser ${ }^{1,2}$ ( ${ }^{1}$ Neurobiology, David Geffen School of Medicine at UCLA, Los Angeles, CA and ${ }^{2}$ Research Service, VA Greater Los Angeles Healthcare System, Los Angeles, CA)

Rationale: Somatostatin (SS) neurons in the dentate hilus provide a major GABAergic innervation to the outer molecular layer of the dentate gyrus, and these hilar GABA neurons are among the most vulnerable neurons in temporal lobe epilepsy. Despite persistent loss of these neurons, a substantial SS/GABAergic innervation gradually reappears in the dentate molecular layer during the chronic stage. The goal of this study was to determine the source of this innervation and test the hypothesis that the axons could originate from SS neurons in stratum (s.) oriens of CA1 rather than the dentate gyrus.

Methods: Sustained seizures were induced in SS-Cre mice by the administration of pilocarpine (Peng et al., J. Neurosci. 24:8629-8639, 2004). Following status epilepticus, the mice recovered and then developed spontaneous seizures within 1-2 weeks. At 5 weeks following pilocarpine-treatment, a Cre-dependent viral vector expressing channelrhodopsin 2 and yellow fluorescent protein (YFP) was injected stereotaxically in s. oriens of CA1 to selectively label SS neurons in this region that expressed Cre recombinase. Age-matched control SS-Cre mice received similar injections. At 3 weeks following these injections, the mice were prepared for histological study. Cre-dependent labeling of SS neurons at the injection site in s. oriens was determined by analysis of YFP localization in neuronal cell bodies and their axonal projections.

Results: Cre-activated YFP labeling of SS neurons was confined to s. oriens of CA1 in both control and pilocarpine-treated mice. In control animals, a prominent band of YFP-labeled axon terminals was present in s. lacunosum-moleculare of CA1, consistent with the normal innervation of this region by SS neurons in s. oriens, but only a small number of GFP-labeled axons projected into the molecular layer of the dentate gyrus. In contrast, in the pilocarpine-treated mice, a dense plexus of YFP-labeled axons extended into the dentate molecular layer, and many of the labeled axons were continuous with those in s. lacunosum-moleculare. Immunohistochemistry confirmed that SS neurons in the hilus were severely depleted in the pilocarpinetreated mice, but that SS-labeled terminals were present in the molecular layer in a pattern resembling the YFP labeling.

Conclusions: The findings demonstrate that remaining GABA neurons in the hippocampus are capable of extensive axonal sprouting in response to a decrease in GABAergic innervation in an adjacent region that they normally do not innervate. A unique GABAergic circuit appears to have been created between GABA neurons in s. oriens of the hippocampus and granule cells of the dentate gyrus. While the reorganized axons are expected to be functional, they are unlikely to control excitability of the granule cells in the epileptic animals in response to the normal inputs to the dentate gyrus. The findings are significant for they demonstrate that the changes in GABA neurons in temporal lobe epilepsy include not only neuronal loss and local sprouting but also the creation of novel, aberrant GABAergic circuitry.

\subsection{6 \\ IS THE LOSS OF ASTROCYTIC GLUTAMATE REUPTAKE IN THE DEVELOPING CORTEX EPILEPTOGENIC?}

Chris Dulla, L. Andresen, A. Taylor, E. Hanson, M. Freeman and D. Cantu (Tufts University School of Medicine, Boston, MA)

Rationale: Glutamate reuptake is critical to brain development and function. Decreased glutamate reuptake by reactive astrocytes is thought to contribute to pathological network activity by increasing extracellular glutamate levels. We have investigated the maturation of glutamate reuptake in the developing rat neocortex and hippocampus and in a model of cortical brain malformation associated with network hyperexcitability. We hypothesize that loss of astrocytic glutamate reuptake during neonatal cortical development may directly contribute to pathological synaptogenesis and may be an important component of neonatal epileptogenesis.

Methods: Cortical malformations were created by briefly placing a freezing probe on the skulls of neonatal rat pups. Neocortical brain slices from sham operated and freeze lesioned rats were prepared 360 days later. Brain slices were then loaded with glutamate FRET biosensor and MNI-glutamate was photolysed using UV laser light. Glutamate biosensor images were collected using a high-speed RedShirt NeuroCCD camera. Glutamate transporter currents were recorded by patch-clamping astrocytes at $-80 \mathrm{mV}$ and photolysing MNI-glutamate.

Results: We have found that protein expression of GLT-1, GLAST, and glutamine synthetase reach adult levels earlier in the hippocampus as compared to neocortex. We next used a combination of FRET-based glutamate biosensor imaging and UV laser photolysis of MNI-glutamate to assay glutamate reuptake in acute brain slices. Recovery from an exogenous glutamate challenge was faster in the immature hippocampus as compared to neocortex. We patch clamped astrocytes in the developing neocortex and hippocampus and recorded glutamate transporter currents evoked by UV laser photolysis of MNI-glutamate. Glutamate transporter decay kinetics were significantly slower in the neocortex as compared to the hippocampus until P21. We next examined how neonatal brain insult affected glutamate transport by astrocytes. Rat pups were anesthetized and a cortical freeze lesion was performed. We examined glutamate reuptake in freeze lesioned animals at P7, a time when reactive astrocytosis is high. We found that in the area surrounding the freeze lesion GLT-1 protein expression was decreased. Combined FRET-based glutamate biosensor imaging and UV laser photolysis of MNI-glutamate revealed a decreased ability to recover from exogenous glutamate challenges. Surprisingly, however, glutamate transporter currents recorded form individual astrocytes had faster decay kinetics in the freeze lesioned neocortex. Conclusions: Our results suggest that while global glutamate transport capacity may be compromised, individual astrocytes have robust glutamate transport in the neonatal brain following injury. Future studies will examine this apparent disconnect between individual astrocyte function and global glutamate handling and its role in increasing synaptogenesis. Overall, these studies indicate that there is a developmental delay in the maturation of neocortical glutamate reuptake compared to hippocampus and that insult has complex effects on glutamate reuptake. 


\subsection{7}

P75 NEUROTROPHIN RECEPTOR MODULATION AND JAK/STAT INHIBITION: ROLE IN THE PROGRESSION OF EPILEPSY IN THE PILOCARPINE RAT MODEL

Heidi Grabenstatter ${ }^{1}$, Y. CruzDelAngel ${ }^{1}$, J. Carlsen ${ }^{1}$, T. Yang ${ }^{2}$, A. M. White $^{1}$, F. M. Longo ${ }^{2}$, S. J. Russek ${ }^{3}$ and A. R. Brooks-Kayal ${ }^{1}$ ( ${ }^{1}$ Pediatrics, Division of Neurology, University of Colorado, Aurora, $\mathrm{CO} ;{ }^{2}$ Neurology and Neurological Sciences, Stanford University, Palo Alto, CA and ${ }^{3}$ Pharmacology and Experimental Therapeutics, Boston University, Boston, MA)

Rationale: BDNF is initially expressed in a precursor form (proBDNF) and cleaved to form mature BDNF (mBDNF). Stimulation of primary neurons in cell culture with either recombinant proBDNF or mBDNF induces JAK/STAT activation, specifically phosphorylation of STAT3. Following status epilepticus (SE), increases in BDNF regulate a wide variety of cell signaling pathways including pro-survival and cell death machinery in a receptor-specific manner. ProBDNF preferentially binds to the $\mathrm{p} 75$ neurotrophin receptor (NTR), while mBDNF is the major ligand of the tropomysin related kinase receptor (TrkB). LM11A-31, a small molecule ligand that binds to $\mathrm{p} 75 \mathrm{NTR}$ promotes survival signaling and inhibits cell death. Recent studies have shown that inhibition of p75NTR prevents BDNF-induced activation of the JAK/STAT pathway in cultured hippocampal neurons. In the current study, we examined the effect of upstream regulation of p75NTR signaling (using LM11A-31) vs. a direct inhibitor of STAT3 phosphorylation (using WP-1066) on SE-induced STAT3 phosphorylation and subsequent progression of epileptogenesis.

Methods: Rats were injected with $200 \mathrm{mg} / \mathrm{kg}$ of LM11A-31 or vehicle at time of pilocarpine injection and again at onset of SE then sacrificed $1 \mathrm{~h}$ and $3 \mathrm{~h}$ later for tissue and plasma harvesting. A second set of animals received $50 \mathrm{mg} / \mathrm{kg}$ WP1066 at SE onset and the same dose 45 minutes later. Continuous video-EEG was collected from animals treated with LM11A-31, WP1066, and vehicle using the same drug administration protocols to evaluate effects on daily seizure frequency for one month following SE.

Results: LM11A-31 produces a transient decrease in STAT3 phosphorylation in hippocampus after SE. There was significantly less LM11A-31 present in the brain and plasma $3 \mathrm{~h}$ after SE when compared to levels $1 \mathrm{~h}$ after SE. A similarly rapid pharmacokinetic clearance was observed with the pSTAT3 inhibitor. LM11A-31 reduced SE-induced phosphorylation of STAT3 by $\sim 75 \% 1 \mathrm{~h}$ after $\mathrm{SE}(\mathrm{SE}, \mathrm{n}=5 ; \mathrm{SE}+\mathrm{LM} 11 \mathrm{~A}, \mathrm{n}=5 ; \mathrm{p}=0.002)$, but not $3 \mathrm{~h}$ after $\mathrm{SE}$ (SE, $\mathrm{n}=4$; SE+LM11A, $\mathrm{n}=4$ ). Similar to LM11A-31, the pSTAT3 inhibitor significantly decreased pSTAT3 immunoreactivity by $\sim 58 \%$ at $1 \mathrm{~h}$ after SE (SE, n=4; SE+WP1066, $n=4 ; \mathrm{p}<0.0001$ ). Neither treatment affected electrophysiological parameters of SE or seizure latency. The pSTAT3 inhibitor slowed the progression of epileptogenesis (i.e., reduced the daily seizure frequency over time; $\mathrm{SE}+$ vehicle, $\mathrm{n}=14$; SE+WP1066, n=8). LM11A-31 treated rats had cumulative seizure frequency similar to vehicle-treated rats ( $\mathrm{SE}+$ vehicle, $\mathrm{n}=6$; SE+LM11A-31, $\mathrm{n}=6$ ).

Conclusions: Despite early inhibition of STAT3 phosphorylation by both LM11A-31 and the pSTAT3 inhibitor, upstream modulation of the JAK/STAT pathway using LM11A-31 did not inhibit the progression of epileptogenesis, while direct block of STAT3 phosphorylation successfully inhibits epilepsy progression. Further studies are required to understand the role of p75NTR activation in epileptogenesis and identify potential cell-specific pSTAT3-related mechanisms responsible for seizure generation.

\subsection{8 \\ FORMATION AND REGULATION OF HETEROMERIC HCN CHANNELS IN LIVE CELLS: INSIGHTS FROM TIRF/FRET IMAGING \\ Yoav Noam ${ }^{1}$, L. Regev ${ }^{1}$, A. Koh ${ }^{1}$, N. Hoshi ${ }^{2}$ and T. Z. Baram ${ }^{1,3}$ \\ ( ${ }^{1}$ Anatomy \& Neurobiology, University of California-Irvine, Irvine,}

CA; ${ }^{2}$ Pharmacology, University of California-Irvine, Irvine, CA and ${ }^{3}$ Pediatrics, Neurology, University of California-Irvine, Irvine, CA)

Rationale: Altered neuronal excitability in the epileptic brain is often associated with changes in the molecular composition and surface expression of ion channels. Among these, the Hyperpolarizationactivated Cyclic Nucleotide-gated (HCN) channels mediate the subthreshold current Ih and therefore contribute significantly to the regulation of intrinsic excitability of neurons. Four $\mathrm{HCN}$ isoforms (HCN1-4) can assemble in different combinations to yield homo- or hetero- tetrameric channels. Importantly, the molecular composition of the channels determines their biophysical properties and therefore influences their precise roles in specific brain regions and subcellular compartments. Seizure-induced heteromerization of $\mathrm{HCN} 1 / 2$ is a likely contributing mechanism to altered Ih in epilepsy (Brewster et al. 2005; Zha et al. 2008), yet very little is known about the cellular and molecular pathways that govern the assembly and membrane expression of heteromric HCN channels.

Methods: To directly study heteromeric $\mathrm{HCN} 1 / 2$ channels on the cell surface, we employed the sensitized-emission Förster Resonance Energy Transfer (FRET) technique, combined with Total Internal Reflection Fluorescence (TIRF) microscopy in live cells. This was achieved by heterologous co-expression of $\mathrm{HCN} 1$ and $\mathrm{HCN} 2$ channel constructs, fused to either the YFP variant mCitrine or the CFP variant mCerulean at their $\mathrm{N}^{\prime}$ terminus.

Results: Co-expression of fluorophore-fused HCN1 and HCN2 channels in HEK293 and CHO cells resulted in a positive FRET signal $(\mathrm{p}<0.001, \mathrm{n}=38 ; \mathrm{p}<0.01, \mathrm{n}=31)$, indicating $\mathrm{HCN} 1 / 2$ heteromerization in these cells. In contrast, a control group where mCitrine-fused HCN channels were co-expressed with un-fused mCerulean $(n=66,32)$ resulted only in negligible signal, supporting the specificity of the HCN1/2 FRET signal. To study HCN channel heteromerization in neurons, we utilized TIRF/FRET imaging in primary hippocampal neurons. In these pilot experiments, positive FRET signal was detected along the somata and dendrites of neurons, indicating the presence of heteromeric $\mathrm{HCN} 1 / 2$ channels. Simultaneous TIRF imaging of HCN1 and HCN2 channels was accomplished, thus providing for the first time a tool to study isoform-specific dynamic properties of $\mathrm{HCN}$ channel surface expression.

Conclusions: These results provide a first-time visualization of heteromeric HCN1/2 channels in live cells, demonstrating both intracellular and surface expression patterns. The FRET/TIRF approach emerges as a beneficial tool for further inquiries into the cellular pathways that govern heteromerization and isoform-specific targeting of HCN channels to the cell membrane, which is tightly controlled in a variety of physiological and pathological contexts. Supported by NIH grants R37 NS35439; T32 NS045540

\subsection{9 \\ ACTIVATION OF MITOGEN-ACTIVATED PROTEIN KINASES AND ELEVATED PHOSPHORYLATION SIGNALING IN HUMAN HYPOTHALAMIC HAMARTOMA Yao Huang, S. Semaan, Q. Liu, Y. Chang and J. Wu (St. Joseph's Hospital and Medical Center, Phoenix, AZ)}

Rationale: Human hypothalamic hamartoma $(\mathrm{HH})$ is a developmental malformation often characterized by gelastic seizures that are refractory to antiepileptic drugs. Our recent study using surgically-resected $\mathrm{HH}$ tissues has revealed that a proportion of $\mathrm{HH}$ neurons are non-GABAergic neurons with an immature phenotype. This suggests that these $\mathrm{HH}$ neurons may have relatively low expression of the outwardly-directed $\mathrm{K}+-\mathrm{Cl}$ - co-transporter (KCC2) and a relatively high intracellular $\mathrm{Cl}$ - concentration. As a result, the inhibitory neurotransmitter GABA rather plays an excitatory role in these immature $\mathrm{HH}$ neurons, potentially leading to gelastic seizures. Thus, to elucidate the molecular mechanisms underlying GABAinduced neuronal excitation and epileptogenesis in $\mathrm{HH}$ will aid the 
selection of superior strategies for drug development to better manage this disease.

Methods: Frozen human hypothalamic tissues (controls) were acquired through Harvard Brain Tissue Resource Center. HH tissues were obtained from patients who were surgically treated through the Barrow HH Program in our hospital. Total proteins were extracted from these tissues, quantitated, and resolved by SDS-PAGE. Immunoblotting (Western blot) was performed with commercially available antibodies using standard methods. Immunoblotting signals were detected with chemiluminescent substrates, and images were captured using a Kodak Molecular Imager. Total RNAs were extracted from tissues and used for cDNA synthesis. Quantitative PCR was performed using Platinum SYBR Green qPCR Supermix UDG Kit (Invitrogen) on an iQ5 Real-Time PCR Detection System (Bio-Rad). Beta-actin was used as an internal control.

Results: Proteins including KCC2, extracellular signal-regulated protein kinases 1 and 2 (ERK1/2) (p44 and p42 mitogen-activated protein kinases (MAPKs)) were expressed in both human hypothalamic and $\mathrm{HH}$ tissues, which were readily detected by immunoblotting with anti-KCC2 and anti-ERK antibodies, respectively. When assessing the phosphorylation signals by immunoblotting with anti-phosphotyrosine (pTyr) and anti-phosphoERK (pERK) antibodies, elevated overall tyrosine phosphorylation and activation of ERK1/2 were found in HH tissues compared to normal hypothalamic control tissues. Furthermore, both real-time RT-PCR and immunoblotting results showed that KCC2 expression (mRNA and protein) levels were significantly lower in $\mathrm{HH}$ than those in control tissues $(\mathrm{P}<0.05)$.

Conclusions: Our findings suggest that reduced $\mathrm{KCC} 2$ expression in $\mathrm{HH}$ could result in an elevated intracellular $\mathrm{Cl}$ - concentration and consequent GABA-mediated neuronal excitation in $\mathrm{HH}$. Activation of the Ras/Raf/MEK/ERK pathway and other phosphorylation signaling cascades could be one of the mechanisms underlying $\mathrm{KCC} 2$ down-regulation in $\mathrm{HH}$, which is currently under investigation in our laboratories.

\subsection{0 \\ SELECTIVE DELETION OF PTEN FROM HIPPOCAMPAL GRANULE CELLS PRODUCES FOCAL HIPPOCAMPAL SEIZURES}

Isaiah J. Rolle ${ }^{1,2}$, R. Y. Pun ${ }^{2}$, K. Holland ${ }^{2}$ and S. C. Danzer ${ }^{2,1}$ ( ${ }^{1}$ Neuroscience, University of Cincinnati, Cincinnati, $\mathrm{OH}$ and ${ }^{2}$ Anesthesia, Cincinnati Children's Research Center, Cincinnati, OH)

Rationale: Hippocampal granule cells with aberrant neuronal connections are hypothesized to contribute to the development of temporal lobe epilepsy. To test this hypothesis, we have developed a transgenic mouse model of epilepsy in which PTEN is deleted from hippocampal granule cells by crossing loxP flanked PTEN mutants to a Gli1-CreERT2 driver line. Treatment of these animals with tamoxifen at P14 leads to the selective deletion of PTEN from hippocampal granule cells and cortical glial cells. PTEN KO granule cells in these animals develop abnormally, exhibiting basal dendrites and mossy fiber axon sprouting. PTEN KO cortical glial cells appear morphologically normal. Animals develop spontaneous seizures around 8 weeks of age. We have hypothesized that abnormal granule cells drive seizures in this model; however, it is conceivable that PTEN KO glial cells contribute to this phenotype. Therefore, to localize the source of seizures, dual channel EEG recordings were conducted from hippocampus and overlying neocortex.

Methods: At P14, Gli1-CreERT2 hemizygous, PTENfl/fl, GFP+/reporter mice received a subcutaneous injection of $250 \mathrm{mg} / \mathrm{kg}$ of tamoxifen. At 6 weeks of age, mice were wirelessly monitored 24/7 by video/EEG via electrodes placed in neocortex and hippocampus. Following continuous EEG monitoring, mice were sacrificed for histological studies. Triple immunostaining of brain sections was conducted for GFP, PTEN, and NeuN to assess neuronal morphology and determine the percentage of PTEN KO cells in cortex and dentate gyrus. ZnT-3 antibodies were used to reveal sprouted mossy fiber axons, and antibodies to phospho-S6 were used to assess activation of the mTOR pathway. Sections were analyzed using laser confocal microscopy. Following continuous EEG monitoring, mice were sacrificed and the percentage of newly-generated granule cells lacking PTEN was determined using confocal microscopy. Mossy fiber axonal sprouting, basal dendrites, and ps6 expression were all assessed in control and epileptic animals.

Results: PTEN knock out animals exhibited spontaneous seizures within 6 weeks after tamoxifen treatment, while no seizures were observed in littermate controls. Epileptic animals exhibited a significant increase in pS6 expression, indicative of mTOR signaling, and a significant increase in mossy fiber sprouting, demonstrating the formation of recurrent excitatory circuits. Dual lead recordings to monitor hippocampus and cortex revealed robust seizure activity in hippocampus, with no spread of seizure activity to overlying cortex at earlier time points. As the epileptic syndrome progressed in these animals, hippocampal seizure activity was associated with epileptiform bursting in cortex.

Conclusions: The present findings strongly suggest that seizures in the Gli1-CreERT2::PTEN model of epilepsy originate from hippocampus. This implies that epileptogenesis is driven in these animals by aberrantly integrated PTEN KO granule cells. Aberrantly integrated granule cells are common in temporal lobe epilepsy, and the current results support a pro-epileptogenic role for these neurons.

\subsection{1}

\section{AN EPILEPSY-CAUSING MUTATION IN SCN1A CAUSES} GAIN-OF-FUNCTION IN GABAERGIC INTERNEURONS Eric Velazquez ${ }^{1}$, A. Escayg ${ }^{2}$ and A. Goldin ${ }^{1}\left({ }^{1}\right.$ Microbiology and Molecular Genetics, University of California Irvine, Irvine, CA and ${ }^{2}$ Human Genetics, Emory University, Atlanta, GA)

Rationale: Voltage-gated sodium channels (VGSC) are the primary molecules responsible for the initiation and propagation of action potentials in neurons. There are nine different genes encoding VGSC in mammals, and mutations in one of these genes (SCN1A) have been identified as the main cause of Genetic Epilepsy with Febrile Seizures Plus (GEFS+) and Dravet Syndrome (DS). Previous studies in mice with VGSC mutations causing GEFS+ or DS have demonstrated loss-of-function in inhibitory interneurons with only minor effects in pyramidal neurons. In this study, we examined the effects in mice of the D1866Y SCN1A mutation that causes GEFS+ in human. Previous studies demonstrated that this mutation increases persistent sodium channel current in Xenopus oocytes (Spampanato et al., 2004, J. Neuroscience 24:10022), but the effects in oocytes may not reflect those in neurons. To understand the physiological effects of the Scn1a D1866Y mutation, we studied the sodium channel properties in a specific population of interneurons from D1866Y knock-in mice.

Methods: We isolated and recorded sodium current from dissociated interneurons from the hippocampus of knock-in mice expressing the D1866Y mutation. To specifically examine parvalbumin-positive $(\mathrm{PV}+)$ interneurons, $\mathrm{D} 1866 \mathrm{Y}$ mice were crossed to transgenic mice in which PV+ neurons were fluorescently labeled. The properties of the sodium channels in this specific neuronal type were determined by voltage-clamping the fluorescently labeled neurons.

Results: Voltage clamp of PV+ interneurons from

Scn1aD1866Y/D1866Y P16-P17 mice showed slower kinetics of inactivation compared to wild-type mice. In addition, there was a reduction in use-dependent inactivation at $20 \mathrm{~Hz}$ compared to that in neurons from wild-type mice, and there was an increased in the level of persistent current. There was a small positive shift in the voltagedependence of activation, and no significant difference in the voltagedependence of inactivation.

Conclusions: These data suggest that the Scn1a D1866Y mutation causes a gain-of-function in hippocampal PV+ interneurons, which may be the cause of the increased seizure susceptibility in these mice. 
Because other Scn1a sodium channel mutations causing GEFS+ have been shown to cause a loss-of-function in interneurons from knock-in mice, these results suggest that different mutations in the same gene can cause seizures by different mechanisms.

\subsection{2}

\section{ENVIRONMENTAL ENRICHMENT HAS ANTI-} EPILEPTOGENIC EFFECTS IN ACQUIRED AND GENETIC MODELS OF EPILEPSY

Nigel Jones ${ }^{1}$, M. Yang ${ }^{1}$, G. Dezsi ${ }^{1}$, M. Salzberg ${ }^{2}$ and T. O'Brien ${ }^{1}$

$\left({ }^{1}\right.$ Medicine, University of Melbourne, Parkville, VIC, Australia and

${ }^{2}$ Psychiatry, University of Melbourne, Parkville, VIC, Australia)

Rationale: Increasing evidence implicates environmental influences as modulators of epileptogenesis. Environmental enrichment (EE), whereby laboratory animals are housed with running wheels, tunnels, and other stimulating objects, has been shown to be neuroprotective in several models of neurological disorders. Here we assessed whether EE affected epileptogenesis using the amygdala kindling model of acquired epilepsy, and the Genetic Absence Epilepsy Rats from Strasbourg (GAERS) model of genetic generalized epilepsy. Methods: At 3 weeks of age, male Wistar rats or GAERS were housed in either enriched (EE) or impoverished housing (IH) conditions, which were maintained for the duration of the study. For the kindling study, rats were implanted with a bipolar stimulating electrode into the left amygdala, and following recovery, were kindled twice daily. Seizure class (Racine scale) and duration were measured for each seizure until animals experienced 5 x Class V seizures, when they were deemed fully kindled. Afterdischarge threshold, and kindling rates were compared between EE and IH. For GAERS, at 8 weeks of age, rats were implanted with EEG recording electrodes, and underwent 24hour recordings each week until 20 weeks of age. Seizures were assessed offline using Spike-and-Wave Finder, and seizure frequency and duration compared over time between $\mathrm{EE}$ and $\mathrm{IH}$.

Results: In both models, EE induced an anti-epileptogenic effect. For kindling, EE increased the number of stimulations required to reach the different stages of kindling $(\mathrm{p}=0.05)$ but did not affect seizure threshold or duration $(p>0.05)$. EE also reduced mossy fibre sprouting induced by kindling. In GAERS, EE delayed the onset of seizures, such that, at 9 weeks when the first recordings were performed, only 3 out of 8 rats had developed seizures, whereas in the IH group, all rats had seizures. As time progressed, the number of seizures experienced was less in the EE group, and at the end of the study (ie: 20 weeks) EE rats were having 50\% less seizures than $\mathrm{IH}$ $(p<0.001)$. No effect was observed on seizure duration.

Conclusions: These studies show that environmental stimulation, in the form of enrichment, can retard the development of both acquired (kindling) and genetic (GAERS) forms of epilepsy. The fact that contrasting types of epilepsy are similarly influenced by enrichment may indicate that the mechanisms underlying this neuroprotection are common effects of the environment, and may be translatable to human epilepsy.

\subsection{3 \\ REDUCED CORTICAL GABA-A RECEPTOR ENDOCYTOSIS IN A MOUSE MODEL OF ABSENCE EPILEPSY}

Martin J. Gallagher and C. Zhou (Vanderbilt University Medical Center, Nashville, TN)

Rationale: Autosomal dominant mutations (S326fs328X, A322D) in the $\mathrm{GABA}_{\mathrm{A}}$ receptor $\left(\mathrm{GABA}_{\mathrm{A}} \mathrm{R}\right) \alpha 1$ subunit are associated with human absence epilepsy and juvenile myoclonic epilepsy, respectively, and both these mutations substantially reduce $\alpha 1$ subunit protein expression in vitro. Recently, we reported that heterozygous deletion of the $\alpha 1$ subunit $\left(\right.$ Het $\left._{\alpha 1} \mathrm{KO}\right)$ caused electrographic and behavioral absence seizures in mice. In addition, we reported that although $\mathrm{Het}_{\alpha 1} \mathrm{KO}$ reduced total $\alpha 1$ subunit protein by approximately $50 \%$, it also increased $\alpha 3$ subunit expression as well as the surface fraction of total $\alpha 1$ subunit and thereby altered the amplitude and kinetic time course of miniature inhibitory postsynaptic currents (mIPSCs). Here, we determined whether these changes in $\mathrm{GABA}_{\mathrm{A}} \mathrm{R}$ surface expression resulted from $\mathrm{GABA}_{\mathrm{A}} \mathrm{R}$ recruitment from the endoplasmic reticulum (ER), or from reduced endocytosis from the cell surface.

Methods: We first determined if $\mathrm{Het}_{\alpha 1} \mathrm{KO}$ altered the fraction of total $\alpha 1$ subunit localized in the ER. We prepared protein lysates from wild type and $\mathrm{Het}_{\alpha 1} \mathrm{KO}$ cortices, and incubated them either with endoglycosdiase- $\mathrm{H}$, an enzyme that selectively cleaves high mannose $\mathrm{N}$-linked glycans associated with ER-resident proteins, PNGase F, an enzyme that cleaves all N-linked glycans, or no glycosidase (control), and analyzed the digestion products on Western blot. To elucidate the effect of $\mathrm{Het}_{\alpha 1} \mathrm{KO}$ on $\mathrm{GABA}_{\mathrm{A}} \mathrm{R}$ endocytosis, we performed patchclamp electrophysiology studies on cortical layer VI pyramidal neurons and recorded mIPSCs. After a five minute baseline period, we added Dynasore, an inhibitor of clathrin-mediated endocytosis, and determined whether endocytosis inhibition altered mIPSCs differently in wild type and $\mathrm{Het}_{\alpha 1} \mathrm{KO}$ neurons.

Results: Surprisingly, in contrast to recombinant $\mathrm{GABA}_{\mathrm{A}} \mathrm{R}$ overexpressed in heterologous cells, neither wild type nor Het ${ }_{\alpha 1} \mathrm{KO}$ cortices possessed any detectable ER-resident $\alpha 1$ subunit, a result demonstrating that , $\mathrm{Het}_{\alpha 1} \mathrm{KO}$ does not recruit ER receptors to increase the fraction of total $\alpha 1$ subunit on the cell surface. Dynasore caused a time-dependent increase in mIPSC amplitudes in wild type neurons that reached $28 \%$ at 30 minutes. In contrast, Dynasore had no effect on the mIPSCs from $\mathrm{Het}_{\alpha 1} \mathrm{KO}$ neurons, a result consistent with tonically reduced $\mathrm{GABA}_{\mathrm{A}} \mathrm{R}$ endocytosis.

Conclusions: Het ${ }_{\alpha 1} \mathrm{KO}$ does not recruit ER-resident receptors to increase the surface fraction of total $\alpha 1$ subunit. However, $\mathrm{Het}_{\alpha 1} \mathrm{KO}$ does alter surface $\mathrm{GABA}_{\mathrm{A}} \mathrm{R}$ expression, at least in part, by reducing endocytosis. A tonic reduction of the rate of $\mathrm{GABA}_{\mathrm{A}} \mathrm{R}$ endocytosis would prevent $\mathrm{Het}_{\alpha 1} \mathrm{KO}$ neurons from dynamically making further reductions in endocytosis to increase synaptic inhibition on-demand. A deficit in this dynamic mechanism to fine-tune synaptic inhibition could contribute to the development of paroxysmal hyperexcitability and seizures.

\subsection{4}

EPILEPTOGENESIS IN TSC: CONTRIBUTION FROM GABA $_{\mathbf{A}}$ RECEPTOR MEDIATED EXCITATION? Hongyu Sun ${ }^{1,3}$, J. Goto $^{2,3}$, B. Kosaras ${ }^{1,3}$, P. M. Klein ${ }^{1}$, D. J. Kwiatkowski ${ }^{2,3}$ and F. E. Jensen ${ }^{1,3}\left({ }^{1}\right.$ Neurology, Children's Hospital Boston, Boston, MA; ${ }^{2}$ Brigham and Women's Hospital, Boston, MA and ${ }^{3}$ Harvard Medical School, Boston, MA)

Rationale: Tuberous sclerosis complex (TSC), an autosomal dominant disorder due to inactivating mutations in TSC1 or TSC2, frequently manifests with a constellation of neurological deficits including early life epilepsy, mental retardation and autism. The mechanism of epileptogenesis and refractory epilepsy in TSC is poorly understood. Our recent study shows that NKCC1 is overexpressed relative to $\mathrm{KCC} 2$, and depolarizing $\mathrm{GABA}_{\mathrm{A}} \mathrm{Rs}$ are present in biopsy samples from human TSC patients (Talos et al., 2012). The present work is a follow up study in a TSC mouse model for purposes of preclinical trail.

Methods: We used the Tsc1cc Nes-rtTA++ mouse model that can uniquely replicate development of giant cells with multi-organelle dysfunction including severe clinical seizures (Goto et al., 2011), to investigate the functional changes of $\mathrm{GABA}_{\mathrm{A}} \mathrm{Rs}$. Spontaneous and evoked field potential activity was recorded using extracellular recordings in ex vivo neocortical slices removed at P28-P40 and compared among groups. We also used gramicidin perforated patch clamp recordings to examine the reversal potential of GABA evoked currents. Western blot was performed to test NKCC1 and KCC2 
protein expression in TSC mutant mice and wild-type controls. Results: We first demonstrated epileptiform activity in neocortical slices from TSC1 mutant. Spontaneous epileptiform discharges in layer $\mathrm{V}$ were detected in 5 of 12 neocortical slices from TSC mutant mice, but 0 of 15 slices from wild-type mice. In slices from TSC mutant mice, evoked field potentials were often associated with polyphasic activity and/or bursts of action potentials, suggesting an overall hyperexcitability in cortical slices of TSC mutant mice. The polarity of neurotransmission mediated by $\mathrm{GABA}_{\mathrm{A}} \mathrm{Rs}$ is dependent on the transmembrane $\mathrm{Cl}$ - gradient mainly regulated by the NKCC1 and the KCC2. Quantitative western blot analysis from cortical samples demonstrated a significant upregulation of NKCC1 expression $(\mathrm{p}<0.05)$ and low KCC2 expression $(\mathrm{p}<0.05)$ in TSC mutant compared to wild-type controls, suggesting a compromised Cl- homeostasis. These changes can be seen as early as P7. To further examine if increased expression of $\mathrm{NKCC} 1$ reversed the polarity of $\mathrm{GABA}_{\mathrm{A}} \mathrm{R}$ mediated inhibition in TSC mutant mice, we measured reversal potentials of GABA evoked currents $\left(\mathrm{E}_{\mathrm{GABA}}\right)$ in layer $\mathrm{V}$ dysplastic pyramidal cells. The mean $\mathrm{E}_{\mathrm{GABA}}$ in TSC mutant mice ($52.7 \pm 3.1 \mathrm{mV}, \mathrm{n}=7)$ was significantly higher than wild-type controls ($69.2 \pm 5.2 \mathrm{mV}, \mathrm{n}=6, \mathrm{p}<0.05$ ), confirming that $\mathrm{GABA}_{\mathrm{A}} \mathrm{R}$ mediated synaptic transmission may be excitatory in TSC mutant mice.

Conclusions: These results suggest an important role of $\mathrm{GABA}_{\mathrm{A}} \mathrm{R}$ mediated excitation in tonic seizure activity in TSC mice, which may represent a new therapeutic target for seizure control in TSC. In addition, these data suggest that this mouse model will provide a suitable substrate for preclinical testing of bumetanide to determine its efficacy in increasing the efficacy of conventional GABA agonist anticonvulsant drugs.

\subsection{5 \\ SEIZURES IN MICE OVEREXPRESSING THE CALCIUM CHANNEL RECEPTOR ALPHA2-DELTA1}

Leonardo Faria ${ }^{1}$, I. Parada ${ }^{1}$, Z. D. Lou ${ }^{2}$, B. Barres ${ }^{3}$ and D. A. Prince $\left({ }^{1}\right.$ Department of Neurology and Neurological Sciences, Stanford University, Stanford, CA; ${ }^{2}$ Department of Anesthesiology and Perioperative Care, Department of Pharmacology, University of California, Irvine, Irvine, CA and ${ }^{3}$ Department of Neurobiology, Stanford University, Stanford, CA)

Rationale: Thrombospondins are astrocyte-secreted proteins that promote developmental synaptogenesis in the central nervous system by binding to the alpha2-delta1 subunit of voltage-gated calcium channels. We hypothesize that overexpression of alpha2-delta1 receptors in transgenic (TG) mice, would result in increased excitatory connectivity, hyperexcitable cortical networks and seizures.

Methods: To test this hypothesis, we used behavioral observations, whole cell patch clamp and extracellular field potential recordings from in vitro brain slices, and video/EEG monitoring of mice with chronically implanted epidural electrodes. In addition, we assessed the colocalization of Vglut1- and Vglut2-IR profiles with PSD95-IR in control and TG mice.

Results: Epileptiform field potentials were evoked in layers II/III and $\mathrm{V}$ of brain slices from TG mice $(\mathrm{n}=6$ mice) but not control $(\mathrm{n}=6$ mice). Whole cell recordings from layer $\mathrm{V}$ pyramidal cells in somatosensory cortical slices showed increased frequency and amplitude of miniature $(\mathrm{m})$ and spontaneous (s) EPSCs. Mean peak amplitude of sEPSCs was $32.6 \pm 1.2 \mathrm{pA}(\mathrm{n}=14$ cells $)$ in TG mice and $26.1 \pm 1.5 \mathrm{pA}$ in control $(\mathrm{n}=11$; unpaired t-test: $\mathrm{P}<0.005)$. Mean frequency of sEPSCs was $6.5 \pm 1.6 \mathrm{~Hz}$ in TG mice and $2 \pm$ $0.3 \mathrm{~Hz}$ in control (unpaired t-test: $\mathrm{P}<0.05$ ). In addition, recurrent spontaneous polysynaptic "burst-like" discharges, were recorded in 10/14 layer V Pyr cells from TG mice vs. 1/11 Pyr cells from control $(\chi 2$ test; $\mathrm{df}=1 ; \chi 2=9.71, \mathrm{P}<0.005)$. Dual immunoreactivity for Vglut2 and PSD95 showed an increased density of close appositions in TG mice compared to control, suggesting an increased number of excitatory synapses.
Video-EEG monitoring of chronically implanted mice aged $>$ P21 showed that TG mice, but not control had frequent abnormal spontaneous electrographic events, consisting of variable duration bihemispheric irregular bursts with a peak frequency of approximately $8-10 \mathrm{~Hz}$, without obvious behavioral arrest. In addition, behavioral tonic seizures occurred in adult TG mice during recovery from isoflurane-induced anesthesia. These consisted of $15 \mathrm{~s}$ duration episodes of rigid arched tail and head extension, frequently followed by loss of balance and falling.

Conclusions: These results suggest that overexpression of alpha2delta1 receptors increases cortical excitatory connectivity and triggers epileptogenesis and seizures in adult mice.

\subsection{6 \\ CLOSED-LOOP OPTOGENETIC CONTROL OF SPONTANEOUS SEIZURES}

Caren Armstrong, E. Krook-Magnuson, M. Oijala and I. Soltesz (University of California, Irvine, Irvine, CA)

Rationale: The inherently unpredictable nature of seizures in epilepsy poses an obvious challenge to the development of ideal therapeutic strategies that act on an 'as-needed' basis without disrupting normal behaviors. It has recently become possible to control specific subsets of neurons utilizing light-sensitive channels or pumps (opsins), allowing unprecedented specific and immediate control of cell populations of interest in behaving animals. However, the widespread use of these novel optogenetic technologies has so far been difficult to apply to epilepsy, in part because of the unpredictable nature of the seizures and difficulty in anticipating the location of seizure onset with many of the available epilepsy models in rodents.

Methods: Mice genetically expressing cre in specific neuronal populations provided selective opsin expression for these experiments. Specifically, either inhibitory opsins (halorhodopsin or archaerhodopsin) were expressed in glutamatergic cells, or excitatory opsins (channelrhodopsin) were expressed in parvalbumin-expressing GABAergic cells. Both viral vector and genetic expression of credependent opsins were tested. Kainic acid was injected unilaterally into the hippocampus to induce chronic epilepsy with recurrent behavioral seizures, and depth electrodes and optical fibers were implanted to allow both EEG recording and opsin activation. We then developed a closed-loop system for detecting and responding to spontaneous electrographic and behavioral seizures, tuned to the EEG signal from individual animals, providing on-line, real-time, seizure detection and laser triggering. In order to determine the effect of opsin activation on seizures, electrographic seizure detection triggered the delivery of light for only $50 \%$ of the events, in random sequence. Analysis of the duration of electrographic seizures was performed off-line, blinded to the presence or absence of light. Duration of electrographic events during light-on and light-off conditions was then compared in each animal using a Mann-Whitney test with a level of significance of $p<0.05$.

Results: Both silencing principal cells with inhibitory opsins ( $n=4$ of 5 animals; $p<0.05$ in each animal) and exciting GABAergic PV cell populations with excitatory opsins ( $n=3$ of 3 animals; $p<0.05$ in each animal) had an effect on the duration of electrographic seizures. For a substantial portion of events (up to $80 \%$ in individual animals), light delivery stopped the electrographic seizure within 5 seconds.

Conclusions: Diverse strategies utilizing both genetic and viralbased expression of opsins can be effective methods of modulating electrographic seizure activity. By investigating the control of electrographic and behavioral seizures with different specific populations of neurons, the potential for targeting these populations for future pharmacological or even optogenetic interventions in patients can be explored.

This work is supported by the US National Institutes of Health grant NS074702 (to IS), the Epilepsy Foundation (to CA), and the George E. Hewitt Foundation for Medical Research (to EKM). 


\subsection{7}

EFFECT OF 532 NM LOW-POWER LASER IRRADIATION ON THE MURINE HIPPOCAMPAL PYRAMIDAL CELLS : EXAMINATION BY PATCH CLAMP TECHNIQUE

Kohmei Tsuchiya, A. Kobayashi, N. Kuwahara, K. Tsuchida, H. Tegushi, T. Tachibana, H. Kawai and S. Kogure (Bio engineering, Soka University, Hachiouzi, Japan)

Rationale: We have demonstrated that $532 \mathrm{~nm}$ low-power laser irradiation (LLI) increased the paroxysmal discharge threshold in the rabbit hippocampus, and almost the same effect was obtained by application of hyperpolarization-activated current (Ih) blocker (S. Kogure et al. Lasers Med Sci, 2010). Since the underlying mechanism of such LLI effect has not been well known at cellular level, the present study was designed to reveal LLI effect using whole-cell patch-clamp recording technique.

Methods: The experiments were performed under appropriate conditions in accordance with the Declaration of Helsinki and the Bioethical Standards of Animal Experiments at Soka University. Four week old mice (FVB mouse) were used and their hippocampal slices were prepared according to the conventional methods. Pulsed LLI (532 nm, 1 ns pulse, $15 \mathrm{kHz}$, Average power: $90 \mathrm{~mW}$ ) was introduced to the pyramidal cell layer of hippocampal CA1 region for $200 \mathrm{~s}$. With a microelectrode of 4-5 M 2 , whole-cell current clampings at $-400,-300,-200$, and $+200 \mathrm{pA}$ with an interval of $5 \mathrm{~s}$ were performed for $500 \mathrm{~ms}$ each. Electrophysiological characteristics including amplitude, width and onset time of the action potential (AP) were measured before, during and after LLI.

Results: Any differences between data before and during LLI, and between data during and after LLI were not observed in the resting membrane potential, AP amplitude, AP onset time, afterhyperpolarization and hyperpolarization-induced rebound depolarization ("sag"). During LLI, AP width measured at $50 \%$ of the AP peak decreased $1.6 \pm 0.9 \%$ (mean \pm SEM) in the pyramidal neurons $(\mathrm{p}<0.01)$, but it increased $2.0 \pm 1.0 \%$ after LLI $(\mathrm{p}<0.01)$, compared with data before LLI. After LLI, inter-spike intervals of 1st-2nd, 2nd$3 \mathrm{rd}$, and 3rd-4th increased $8.7 \pm 3.9 \%(\mathrm{p}<0.01), 6.5 \pm 2.9 \%$, and $7.1 \pm 3.2 \%$, respectively.

Conclusions: These data suggest that $532 \mathrm{~nm}$ LLI directly activates potassium channels during the irradiation, but indirectly induces a delayed recovery of those channel after the irradiation. Since the temperature of the medium showed almost no changes, such effects are seemed to be based on the photochemical influence, not on the thermal effect.

\subsection{8}

\section{WTTHDRAWN}

\subsection{9}

\section{INVOLVEMENT OF NEURONAL PHOSPHOTYROSINE} SIGNAL ADAPTOR N-SHC IN THE KAINIC ACID-INDUCED EPILEPTIFORM ACTIVITY

Shiro Baba $\left({ }^{1}\right.$ Neurosurgery, Nagasaki University, Sakamoto,Nagasaki, Japan and ${ }^{2}$ Anatomy and Neurobiology, Nagasaki University, Sakamoto,Nagasaki, Japan)

Rationale: BDNF-TrkB signaling is implicated in experimental seizure and epilepsy. However, the downstream signaling mechanism of epileptogenesis from TrkB receptor activation is still controversial and whether it progresses through the PLC $\gamma$, NShc, or both is unclear.Therefore, the aim of this study is to examine whether N-Shc is involved in kainic acid (KA)-induced epileptiform activity. Methods: We used 4 to 8 weeks old homozygous N-Shc deficient mice (N-Shc -/-) and C57BL6 mice as a wild type control (N-Shc $+/+)$. (1) $5 \mu \mathrm{l}$ of K252a $(1 \mu \mathrm{M})$ or vehicle $(0.1 \%$ DMSO) were injected to the lateral ventricle of wild type mice stereotactically(i.c.v). 30 minutes after the K252a or DMSO injection, $\mathrm{KA}(30 \mathrm{mg} / \mathrm{kg}$, i.p.) was administered, and the behavior seizures were monitored and were scored by Racine seizure scale. (2) The mice (N-Shc +/+, N-Shc-/-) received a administration of KA (30 $\mathrm{mg} / \mathrm{kg}$, i.p.). Then the behavior seizures were monitored. At 1 day and 7 days after the seizure induction by KA, the brain was fixed with $4 \%$ paraformaldehyde and cut into $10-\mu \mathrm{m}$ coronal sections with a cryostat. And Nissl stein was performed for evaluate neurotoxicity induced by KA. Neuronal cell death was quantified in the CA1 and CA3 regions of the dorsal hippocampus. (3) The mice were anaesthetized and the hippocampal electrodes were implanted stereotacticaly. 7 days after surgery, the mice were connected through a cable to a digital EEG system. The baseline hippocampal EEG was recorded for at least $30 \mathrm{~min}$. Thereafter, behavior seizures were induced by KA administration $(30 \mathrm{mg} / \mathrm{kg}$, i.p.) in each mouse. EEGs were registered continuously for 2 hours after the KA treatment. The epileptiform discharge, spikes and sharp waves (highamplitude $>2 \times$ baseline, frequency; $>5 \mathrm{~Hz}$ ) were calculated and expressed as a mean frequency.

Results: We show that pre-treating wild type mice with the TrkB inhibitor K252a significantly reduced the severity of KA-induced seizures(maximam seizure scale: K252a group $1.67 \pm 2.08 ; 0.1 \%$ DMSO group 5.0 $\pm 1.0, \mathrm{p}=0.04)$, suggesting that TrkB-mediated signaling is crucial for the KA-induced seizure. Moreover, we show that a significant reduction of both the seizure scale(maximum seizure scale: NShc +/+ mice, 5.21 \pm 0.98 ; NShc-/- mice, 3.27 \pm 1.79 , $\mathrm{p}=0.001$ ) and the frequency of epileptiform discharge in the hippocampus(epileptiform discharge: NShc $+/+$ mice, $5642.8 \pm 1210$ times/h; NShc-/- mice, $1046 \pm 971$ times $/ \mathrm{h}, \mathrm{p}<0.001$ ) of N-Shc deficient mice as compared with the control mice. The KA-induced selective neuronal cell loss in the CA3 area of the hippocampus was also inhibited in the N-Shc deficient mice. These results suggest that $\mathrm{N}$-Shc is essentially involved in the KA-induced epileptiform activity.

Conclusions: We propose that the N-Shc-mediated signaling pathway would provide a potential target for the development of novel therapeutic drugs and/or approaches in the treatment of epilepsy.

\subsection{0 \\ RAPAMYCIN HAS PARADOXICAL EFFECTS ON S6 PHOSPHORYLATION IN RATS WITH AND WITHOUT SEIZURES}

Linghui Zeng ${ }^{1,2}$, J. Dong ${ }^{1}$, M. Wong ${ }^{2}$ and L. Chen ${ }^{1}\left({ }^{1}\right.$ Zhejiang University City College, Hangzhou, China and ${ }^{2}$ Washingtong University in St. Louis, St. Louis, MO)

Rationale: Accumulating data have demonstrated that seizures induced by kainate or pilocarpine activate the mammalian target of rapamycin (mTOR) pathway and mTOR inhibitor rapamycin can inhibit mTOR activation which subsequently has potential antiepileptic effects. However, a preliminary study showed a paradoxical exacerbation of increased mTOR pathway activity reflected by S6 phosphorylation when rapamycin was administrated within a short period before kainate injection. In the present study, we examined this paradoxical effect of rapamycin in more detail, both in normal rats and kainate-injected animals.

Methods: Normal Rats or kainite-treated rats pretreated with rapamycin at different time interval were sacrificed at various time points $(1 \mathrm{~h}, 3 \mathrm{~h}, 6 \mathrm{~h}, 10 \mathrm{~h}, 15 \mathrm{~h}$ and $24 \mathrm{~h})$ after rapamycin administration or seizure onset for Western blotting analysis. Phosphorylation of mTOR signaling target of Akt, mTOR, Rictor, Raptor, S6K and S6 were analyzed. Seizure activity was monitored behaviorally and graded according to a modified Racine scale ( $\mathrm{n}=6$ for each time point). Neuronal cell death was detected by Fluoro-Jade B staining in kainite-treated rats.

Results: In normal rats, we found that rapamycin showed the expected dose-dependent inhibition of S6 phosphorylation 3-24 h 
after injection, while a paradoxical elevation of S6 phosphorylation was observed 1 hour after rapamycin. Similarly, pretreatment with rapamycin over $10 \mathrm{~h}$ prior to kainate inhibits the kainate-induced mTOR activation. In contrast, rapamycin administered 1 to 6 hours before kainate causes a paradoxical increase in the kainate-induced mTOR activation. Rats pretreated with rapamycin $1 \mathrm{~h}$ prior to kainate showed an increase in severity and duration of seizures and more neuronal cell death as compared to vehicle treated groups. In contrast, rapamycin pretreated $10 \mathrm{~h}$ prior to KA had no effect on the seizures and decreased neuronal cell death. The paradoxical effect of rapamycin on S6 phosphorylation was correlated with upstream mTOR signaling and reversed by pre-treatment of perifosine, an akt inhibitor.

Conclusions: These data indicate the complexity of S6 regulation and its effect on epilepsy. Paradoxical effects of rapamycin need to be considered in clinical applications, such as potential treatments for epilepsy and other neurological disorders.
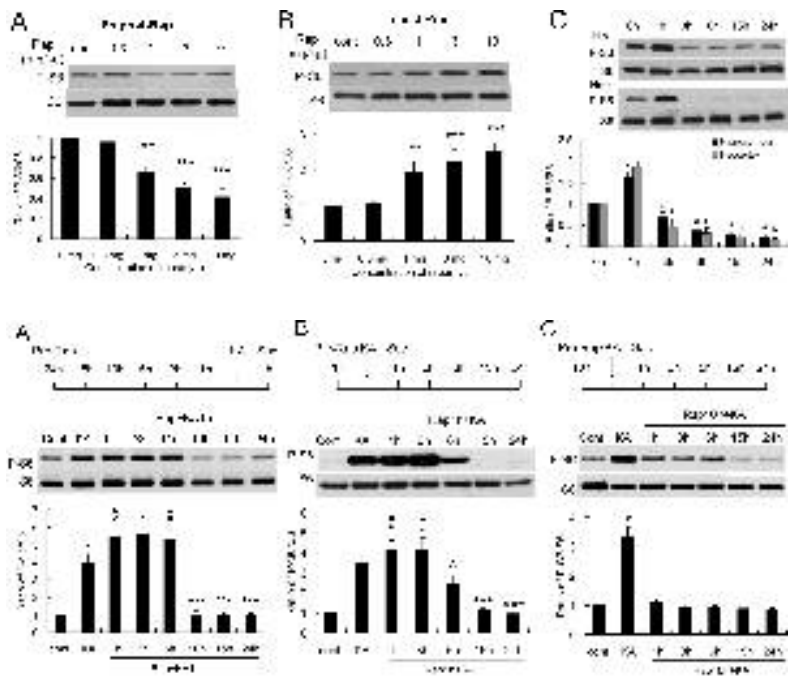

\subsection{1}

PROGRESSIVE ANTICONVULSANT RESISTANCE DURING POST-TRAUMATIC EPILEPTOGENESIS IN VITRO

Volodymyr Dzhala, Y. Berdichevsky, Y. Saponjian, M. Mail and K. Staley (Massachusetts General Hospital, Charlestown, MA)

Rationale: Traumatic brain injury is a major cause of acquired epilepsy. Seizures may occur because of the acute injury or progressive post-traumatic changes in brain metabolism, blood flow and homeostasis. Phenytoin is effective in treatment of acute seizures but failed to prevent post-traumatic epilepsy (PTE). Anticonvulsant efficacy of Phenobarbital is low with a greater risk of developing PTE. Understanding the mechanisms of anticonvulsant resistance and development of PTE is a critical for understanding the epilepsy and development of more efficient therapy for prevention and prophylaxis of PTE.

Methods: Extracellular field potential recordings, two-photon imaging of Clomeleon, lactate and lactate dehydrogenase production assays were used to monitor neuronal network activity, intracellular chloride concentration([Cl-]i) and neuronal cell death.

Results: Hippocampal slices were incubated for six-seven weeks, during which a latent one-two week period was followed by spontaneous seizure activity and electrical status epilepticus. Phenytoin exerted powerful acute concentration-dependent anticonvulsive effects (Berdichevsky et al., 2012). Chronic anticonvulsive efficacy of phenytoin progressively decreased demonstrating no antiepileptic action in this model. Washout of phenytoin was followed by a sharp increase in recurrent seizure activity. Phenobarbital was much effective at controlling posttraumatic seizures at the earlier stage of epileptogenesis.
Anticonvulsive efficacy of phenobarbital decreased from $60-70 \%$ to $0-10 \%$ during four-five weeks of epileptogenesis. Anticonvulsive efficacy of phenobarbital was correlated with intracellular chloride concentration ([Cl-]i) and GABA action. We found a correlation between seizure severity and frequency, increases in [Cl-]i and phenobarbital efficacy. Bumetanide, a sodium-potassium-chloride (NKCC1) co-transporter blocker, significantly increased anticonvulsive efficacy of phenobarbital. High concentration of furosemide ( $\mathrm{NKCC} 1$ and potassium-chloride ( $\mathrm{KCC} 2)$ co-transporter blocker) controlled acute seizures at all stages of epileptogenesis, but failed to reduce interictal epileptiform discharges and prevent epilepsy.

Conclusions: In conclusion, our data suggest a complex mechanism of anticonvulsant resistance that involves chronic post-traumatic chloride accumulation and alterations in GABA-mediated inhibition. More effective prevention of post-traumatic and seizure-induced neuronal chloride accumulation may comprise a new strategy for both anticonvulsive and antiepileptogenic therapy.

\subsection{2 \\ UNEXPECTED NEUROPROTECTIVE EFFECTS OF REACTIVE OXYGEN SPECIES IN THE CENTRAL NERVOUS SYSTEM: THE ROLE OF THE NEUROTROPHIN RECEPTOR, TRKB}

Yangzhong Huang and J. O. McNamara (Department of Neurobiology, Duke University Medical Center, Durham, NC)

Rationale: Hippocampal sclerosis is a pathological hallmark of mesial temporal lobe epilepsy. Excessive levels of reactive oxygen species (ROS) are produced during status epilepticus and thought to contribute to seizure induced hippocampal neuronal injury and sclerosis. Therefore, understanding the molecular signaling mechanism by which excess ROS impact life and death of cortical neurons may provide novel targets that could be exploited to protect neurons from seizure insults. While the neurotoxic consequences of ROS in the central nervous system are widely recognized, neuroprotective consequences of ROS are less well appreciated.Our laboratory has been studying the role of the brain derived neurotrophic factor (BDNF) receptor tyrosine kinase, TrkB, in limbic epileptogenesis. In the course of these studies, we discovered that the divalent cation, zinc, can activate TrkB in the absence of BDNF. Several facts led us to explore the effects of zinc on neuronal survival and how such effects might be related to ROS and TrkB. Enhanced TrkB activation promotes survival of CNS neurons. Vesicular zinc is present in axon terminals of hippocampal granule cells and thought to contribute to death of CA3 pyramids during status epilepticus. Methods: Using cultured embryonic rat cortical neurons as a model, we performed a series of biochemical, live cell imaging, and cell death assays.

Results: We discovered that: 1) addition of ROS in the form of exogenous $\mathrm{H} 2 \mathrm{O} 2$ to cortical neurons enhances cytosolic zinc content by promoting its release from a zinc binding protein, metallothionin-3 (MT-3); 2) both exogenous and endogenous ROS-induced release of zinc activates TrkB signaling by a Src family kinase dependent but BDNF-independent mechanism; 3) to our surprise, while ROS result in neurotoxicity, the ROS-triggered activation of TrkB exerts neuroprotective effects because inhibition of TrkB kinase activity or uncoupling She signaling from TrkB exacerbates neuronal cell death induced by $\mathrm{H} 2 \mathrm{O} 2$.

Conclusions: Thus we propose a molecular signaling event whereby ROS induce release of zinc from cytosolic MT-3, the increased cytosolic zinc promotes activation of TrkB, and the enhanced TrkB signaling promotes pro-survival effects. We suggest that such neuroprotective effects are operative in diverse acute and chronic neurological disorders, including temporal lobe epilepsy. 
Translational Research: Models

\subsection{3}

ERK AND MTOR CROSSTALK AND ITS EFFECT ON NEURONAL MORPHOLOGY IN VITRO

V. V. Patil ${ }^{1,2}$, J. W. Swann ${ }^{1,2}$ and A. E. Anderson ${ }^{1,2}$ ( ${ }^{1}$ Baylor College of Medicine, Houston, TX and ${ }^{2}$ Texas Children's Hospital, Houston, TX)

Rationale: Tuberous sclerosis (TS) and cortical dysplasia (CD) are associated with hyperexcitable cytomegalic neurons which are implicated in the drug resistant epilepsy. Microarray studies on cytomegalic neurons found aberrant regulation of the extracellular regulated kinase (ERK) and mammalian target of rapamycin (mTOR) growth pathways. The interaction between ERK and mTOR is well characterized in cancer biology, however it is not well-understood in the central nervous system. In the study presented, we wanted to evaluate the ERK and mTOR pathway crosstalk in the normal and cytomegalic mouse cortical neurons using various genetic and pharmacologic techniques.

Methods: Wildtype C57BL6/J mouse cortical neurons were transfected with constitutively active mitogen activated protein kinase kinase 1 / green fluorescent protein (caMEK1/GFP) to increase ERK activation. Immunocytochemistry was performed to evaluate phosphorylation ( $\mathrm{p}$ ) and activation of ERK and the ribosomal protein S6 (pS6), an mTOR-downstream target. Neurons and dendrites were traced using the Neurolucida software to assess morphology. In parallel, (tuberous sclerosis complex 1) TSC1fl/fl neurons were transduced with iCre-RFP lentivirus to knock out TSC1 in mouse cortical neurons to increase mTOR activation and generate an in vitro model of cytomegalic neurons. The TSC 1 and $\mathrm{pS} 6$ protein staining was used to identify TSC1 knockout (TSC1-/-) neurons. Map2 staining was used to assess morphology in these cells. Rapamycin, an mTOR inhibitor, was used to manipulate mTOR activity. Studies are underway to evaluate the effects of ERK inhibitors in these models.

Results: Wildtype C57B16/J mouse cortical neurons transfected with caMEK1/GFP showed an increase in pERK and pS6 staining, indicating increased ERK and mTOR signaling, respectively. Rapamycin decreased the levels of pS6 in caMEK1 transfected cells. The number of dendritic branches and cell size significantly decreased in the transfected neurons compared to controls $(\mathrm{p}<0.05$, $\mathrm{n}=5$ ). The soma area of TSC1-/- mouse cortical neurons significantly increased as compared to wildtype neurons $(\mathrm{p}<0.001, \mathrm{n}=4)$. The effects of increased ERK and mTOR activation on morphology were reversed by rapamycin.

Conclusions: ERK pathway activation in caMEK1/GFP transfected cortical neurons is associated with increased mTOR activation and decreased somatic size and dendritic branching. mTOR inhibition in these neurons reverses the aberrant phenotypes, suggesting an interplay between the ERK and mTOR signaling cascades in neurons. In addition, knocking out TSC1 in mouse cortical neurons increases mTOR activation and cell size as expected. Thus, this culture system will provide a model for screening ERK inhibitors and other compounds targeting these pathways.

\subsection{4}

\section{SEIZURE OCCURRENCE WITH ANTIPSYCHOTIC DRUG TREATMENT MAY BE RELATED TO INHIBITION OF OUTWARD K+ CURRENTS - ELECTROPHYSIOLOGICAL AND COMPUTATIONAL STUDIES}

Christopher French $\left({ }^{1}\right.$ Medicine, University of Melbourne, Melbourne, VIC, Australia and ${ }^{2}$ Royal Melbourne Hospital, Melbourne, Australia)

Rationale: Antipsychotic drugs are known to lower seizure threshold, and at high therapeutic doses seizure occurence is as high as $10 \%$. Antipsychotic drugs are generally considered to act through inhibition of dopamine type 2 (D2) receptors, but are also known to inhibit potassium channels in cardiac tissue. The hypothesis that seizure threshold reduction by these drugs might be due to inhibition of "membrane stabilising" outward potassium currents was examined electrophysiologically in hippocampal neurons and with computer simulations.

Methods: Whole-cell voltage clamp recordings of outward currents in acutely dissociated 3-6 week old rat CA1 neurons $(n=50)$ were performed. With sodium channel blockade, inhibition of delayed rectifier-like currents as well as inactivating "A currents" was examined at different concentrations $(10 \mathrm{~nm}-50 \mathrm{uM})$ haloperidol or clozapine. Effects on firing threshold, repetitive firing and network excitability were examined by computer simulations of single neurons and conductance-based neural networks with teh "NEURON" modelling environment.

Results: 50uM haloperidol caused a remarkable suppression of all outward currents, with $100 \%$ of peak and persistent outward current suppressed at test potential $+20 \mathrm{mV}$; at concentrations corresponding to therapeutic levels $(10 \mathrm{nM})$, peak and persistent components were reduced by at least $10 \%$; subtraction of current traces before and after drug application revealed a non-inactivating "delayed rectifier"-like current. Computer simulations of this effect revealed reduced threshold and increased repetitive firing in single neurons and reduced threshold for epileptiform activity in a neuron-network. Conclusions: The reduction of seizure threshold and seizure occurence in patients treated with antipsychotic drugs can be explained by suppression of membrane-stabilising outward currents. These channels may be related to the cardiac ERG group known to be inhibited by low concentrations of antipsychotic drugs.

\subsection{5}

\section{ALTERED INHIBITION AND NETWORK ACTIVITY IN THE HIPPOCAMPUS IN THE THEILER'S VIRUS ENCEPHALITIS MODEL OF EPILEPSY}

Roy Smeal $^{1}$, P. J. West ${ }^{1}$, E. J. Dahle ${ }^{1}$, R. S. Fujinami ${ }^{2}$, H. S. White ${ }^{1}$ and K. S. Wilcox ${ }^{1}\left({ }^{1}\right.$ Pharmacology and Toxicology, University of Utah, Salt Lake City, UT and ${ }^{2}$ Pathology, University of Utah, Salt Lake City, UT)

Rationale: CNS infections are a major risk factor for the development of temporal lobe epilepsy, a form of epilepsy that is often drug resistant and not well understood. In the Theiler's virus mouse model of infection-induced epilepsy, mice exhibit acute postinfection seizures and develop spontaneous seizures by 2 months after infection. Recent work from our laboratory has shown that the hippocampal CA3 excitatory circuit exhibits different characteristics during the acute infection period versus chronic time points. The current study tests the hypothesis that inhibition is also altered and effects network activity in the CA3 region of the hippocampus as a consequence of infection and during epileptogenesis.

Methods: Mice were intracerebrally injected with either PBS or Theiler's virus ( 2 - 3.5 x 107 PFU) and were sacrificed on days 3-7 post-injection for the acute period or left to recover for at least 2 months before sacrifice. Hippocampal slices including CA3 were cut in ice-cold sucrose. CA3 hippocampal neurons were patch clamped in Ringer's solution containing CNQX $(10 \mu \mathrm{M})$ and APV $(100 \mu \mathrm{M})$ for recording IPSCs. After baseline activity of sIPSCs was established, TTX $(1 \mu \mathrm{M})$, was washed on for 15 min to record mIPSCs. Amplitude and frequency of both sIPSCs and mIPSCs were measured. Field potentials in CA3 were evoked via stimulation of afferents for 30 minutes $(0.03 \mathrm{~Hz})$ in normal ACSF and for 30 minutes following blockade of inhibition with picrotoxin $(100 \mu \mathrm{M})$. Results: All TMEV infected mice used in the study were observed to have seizures during the acute infection period. In brains slices prepared during the acute infection period, recordings of sIPSCs revealed a significant decrease in frequency $(\mathrm{p}=.015)$ and amplitude $(\mathrm{p}=.019)$. In addition, there was a significant decrease of the mIPSC amplitudes $(p=.015)$. Surprisingly, no decreases in either frequency 
or amplitude were observed in sIPSCs or mIPSCs in brain slices prepared at the 2 month time point. Field potentials elicited in CA3 by stimulation of afferent input were significantly smaller in TMEVinfected mice relative to controls at both time points as measured by coastline burst analysis. Additionally, the coastline burst index (CBI) following blockade of inhibition was unchanged during the acute period. However, the CBI was unexpectedly smaller at the chronic time point $(\mathrm{p}=.035)$ in TMEV-infected mice.

Conclusions: The CA3 inhibitory network is differentially altered by TMEV infection during the acute infection period relative to the chronic epilepsy time point as evidenced by SIPSCs and mIPSCs. While there was an initial reduction of inhibition during the acute infection period, there was an apparent recovery of inhibition at later time points. However, the reduction of field potentials following infection at both time points and the decreased CBI following blockade of inhibition at the chronic time point suggest a complicated network phenomenon.[Supported by NINDS grants R01NS065434 (KSW \& HSW) and 1R01NS065714 (RSF), CURE, Margolis Foundation, and Robert and Joyce Rice]

\subsection{6 \\ DENTATE GYRUS-PROJECTING CA3 NEURONS IN THE SEIZURE-PRONE NAKED MOLE-RAT}

M. Zions ${ }^{1}, X$. Geoffroy ${ }^{2}$ and Daniel McCloskey ${ }^{1,2}\left({ }^{1}\right.$ Neuroscience Doctoral Subprogram iin Biology, The Graduate Center at CUNY, New York, NY and ${ }^{2}$ Center for Developmental Neuroscience, College of Staten Island/ City University of New York, Staten Island, NY)

Rationale: The unique ecological niche and social organization of naked mole-rats (Heterocephalus Glaber) make them a useful model to study how environmental, hormonal, and social factors relate to epilepsy. We have found that naked mole-rat hippocampal slices exhibit spontaneous epileptiform burst discharges in area CA3 with a morphology and frequency similar to those shown previously in chronic pilocarpine-treated rats. The purpose of the present study was to test whether abnormal hippocampal circuitry may help explain this pathophysiology.

Methods: Naked mole-rats were deeply anesthetized with $\mathrm{CO} 2$ and decapitated. Horizontal $300 \mu \mathrm{m}$ hippocampal slices were maintained in an interface chamber with humidified $95 \% \mathrm{CO} 2 / 5 \% \mathrm{O} 2$ at $32 \pm 1^{\circ} \mathrm{C}$. Slices were equilibrated in a sucrose-based artificial cerebrospinal fluid (ACSF) and perfused with a saline-based ACSF. Recording began more than 60 minutes after perfusion of saline-based solution. Slices were first recorded in the CA3 pyramidal cell layer using a glass extracellular electrode. A stimulating electrode was placed in the subgranular zone of the lower blade of the dentate gyrus to activate the mossy fiber pathway. Stimuli used were always less than half of the maximal stimulus used to evoke an othodromic population spike in the CA3 PCL (100-300 $\mu \mathrm{A}, 100 \mathrm{msec})$. Slices with biocytin iontophoretically injected at the site of stimulation were incubated overnight in streptavidin conjugated quantum dots $(705 \mathrm{~nm})$ and imaged on a confocal microscope.

Results: When the stimulating electrode was placed in the subgranular zone and the recording electrode placed in the CA3b-c region, each of 9 slices from 4 animals demonstrated an early, negative-going potential that exceeded the amplitude of the typical mossy fiber-evoked population spike that followed.This putative antidromic signal was absent when the stimulating electrode was placed at an equal distance in the oriens-lacunosum moleculare axis, ruling out current spread artifacts. The morphology of the negativegoing potential was similar when antidromic responses were elicited from the fimbria or Schaffer Collateral pathway. Bath application of the AMPA/kainate antagonist CNQX $(10 \mu \mathrm{M}, \mathrm{n}=2)$ abolished the orthodromic population spike but not the putative antidromic spike. In slices where biocytin was injected at the mossy fiber stimulation site $(n=3)$, clearly labeled pyramidal somata could be detected near the recording site.
Conclusions: This study demonstrates that there is an excitatory feedback pathway from the CA3 pyramidal cells to the dentate gyrus in the naked mole-rat. This pathway may play a role in the epileptiform burst discharges which are common in area CA3 of the naked mole-rat, and are very similar to those we have previously characterized in chronic pilocarpine-treated rats. In fact, a recent study (Zhang et al. 2012, J Neurosci. 32(4)1183-96) demonstrated a similar pathway in chronic pilocarpine-treated rats. Although the termination zones and functional consequences of these projections remain to be determined, this pathway presents an exciting new potential mechanism for hippocampal pathophysiology

\subsection{7 \\ GABAA RECEPTOR MUTANT MICE WITH ABSENCE EPILEPSY DISPLAY LOSS OF INHIBITORY TONIC CURRENTS, INCREASED CORTICAL EXCITABILITY, AND DECREASED THALAMIC BURSTING}

Kile P. Mangan ${ }^{1}$, S. Petrou ${ }^{2}$, S. Johnson ${ }^{3}$ and M. V. Jones ${ }^{1}$ ( ${ }^{1}$ Neuroscience, University of Wisconsin, Madison, WI; ${ }^{2}$ Ion Channels and Disease, Florey Neuroscience Institutes, Melbourne, VIC, Australia and ${ }^{3}$ Veterinary Medicine, University of Wisconsin, Madison, WI)

Rationale: The GABAA $\gamma$ 2R43Q mutation confers Generalized Epilepsy with Febrile Seizures Plus (GEFS+) in humans (Wallace et al, 2001) and absence-like seizures in knock-in (RQ) mice (Tan et al, 2007). Humans harboring the $\gamma 2 \mathrm{R} 43 \mathrm{Q}$ mutation show evidence of a hyperexcitable cortex compared to unaffected family members (Fedi et al., 2008), a condition hypothesized to contribute to the spike-andwave discharges (SWDs) seen with absence seizures (Marini et al., 2003). The traditional first-line clinical anti-absence medication, ethosuxamide, works by decreasing T-type $\mathrm{Ca} 2+$ conductance in neurons (Hugeunard, 1999). This T-type Ca2+ conductance promotes 'burst-firing' in thalamic relay neurons; a behavior inversely mediated by membrane potential and believed to be critical for SWD etiology (Cope et al., 2005, 2009). Interestingly, up to $40 \%$ of humans that suffer absence seizures are non-responsive to ethosuxamide (Tringham et al., 2012). Previous work in our lab shows RQ mice lack inhibitory tonic currents in both cortex and thalamus and, thus, may display altered neuronal firing and bursting behaviors.

Methods: Thalamocortical slices $(400 \mu \mathrm{m})$ (Krook-Magnuson et al., 2008) were cut from wild-type (RR) and RQ mouse brains and placed on an interface chamber perfused with low-Mg2+ artificial cerebral spinal fluid (aCSF). Multichannel extracellular recordings were made with two NeuroNexus 16-channel recording arrays placed in thalamus and cortex. Neurons were isolated using a principal components-based clustering and spike sorting algorithm. Homewritten matlab code analyzed individual neuron spike-times for Ttype $\mathrm{Ca} 2+$ 'bursts', which included a $100 \mathrm{msec}$ hyperpolarizing silence of activity followed by inter-spike-intervals of $<8 \mathrm{msec}$ (Weyand et al., 2001). Each cell's tendency to fire in bursts was defined by a t-burst fraction: \# of burst events divided by total \# of events (bursts and lone spikes). T-burst fraction, spikes-per-burst, burst duration, and over-all firing rates were calculated and compared for each area with a Kruskal-Wallis examination of medians.

Results: RQ cortical firing rates were increased compared to RR (Hz: median of means, IQR; RR: 0.04, 0.08, $\mathrm{n}=76$; RQ: 0.06, 0.12, $\mathrm{n}=81$; $\mathrm{p}<0.05$ ), while $R Q$ thalamic firing rates were decreased (RR: 0.12 , $0.51, \mathrm{n}=112$; RQ: $0.04,0.15, \mathrm{n}=75 ; \mathrm{p}<0.05$ ). RQ mice thalamus showed a decreased t-burst fraction compared to RR (median, IQR; RR: 0.20, 0.25; RQ: 0.05, 0.15; $<<0.05$ ), a decreased number of spikes-per-burst (median spikes: RR: 3, 3; RQ: 2, 0; $<0.05$ ), but no change in burst duration time (median milliseconds: RR: 6.2, 4.1; RQ: 6.1, 5.4). Similarly, RQ cortex also displayed a decreased t-burst fraction (RR: 0.14, 0.23; RQ: 0.05, 0.14; $<<0.05$ ), decreased number of spikes-per-burst (RR: 3, 3; RQ: 2, 0 ; $<<0.05$ ), and no change in burst duration time (RR: 5.4, 4.1; RQ: 3.9, 6.0). 
Conclusions: These results show that the $\gamma 2 \mathrm{R} 43 \mathrm{Q}$ mutation increases firing rates in cortex and decreases $\mathrm{T}$-type $\mathrm{Ca} 2+$ bursting in thalamus, findings consistent with a loss of inhibitory tonic currents in these neurons.

\subsection{8 \\ IMPACT OF THE CNTF-DERIVED PEPTIDE CINTROFIN ON NEUROGENESIS AND BEHAVIORAL ALTERATIONS IN THE BLA-KINDLING MODEL \\ Josephine D. Salvamoser ${ }^{1}$, M. Hadamitzky ${ }^{1}$, E. Bock ${ }^{2}$, V. Berezin ${ }^{2}$ and H. Potschka ${ }^{1}{ }^{1}$ Ludwig-Maximilians-University, Institut of Pharmacology, Pharmacy and Toxicology, Munich, Germany and ${ }^{2}$ Department of Neuroscience and Pharmacology, Protein Laboratory, Panum Institute, Copenhagen, Denmark)}

Rationale: The ciliary neurotrophic growth factor (CNTF) is a neurotrophic cytokine which plays an important role in the development of neuronal cells. CNTF protects neurons from glutamate excitotoxicity and improves learning and memory. Therefore, it is of specific interest to determine whether the CNTFderived peptide Cintrofin can influence epileptogenesis, neuronal alterations and associated behavioral deficits in a chronic epilepsy model.

Methods: Male NMRI mice received either Cintrofin or vehicleinjections $(2 \mathrm{mg} / \mathrm{kg}, 10 \mathrm{mg} / \mathrm{kg}$ s.c. $)$ once daily for 3 weeks in the BLA-kindling model. The impact of Cintrofin on seizure severity was investigated. Furthermore, the development of kindling-associated alterations in behavior and on neuronal alterations was analyzed Results: Cintrofin did not affect the progression of seizure severity in the amygdala-kindling model. As a consequence of kindling, locomotion in the open field and the elevated-plus-maze were significantly increased. Cintrofin did not attenuate these behavioral alterations. On the other hand, time spent in the inner zone of the open field was significantly increased in Cintrofin-treated control animals. In addition, Cintrofin significantly reduced the number of persistent basal dendrites in kindled animals.

Conclusions: In summary, the results argue against a diseasemodifying and antiepileptogenic effect of the CNTF-derived peptide mimetic Cintrofin. However, Cintrofin decreases seizure-associated alterations in hippocampal neurogenesis such as persistence of basal dendrites.

Acknowledgement: We thank Marion Fisch and Angela Vicidomini for their excellent technical assistance. The authors are grateful for support by a grant from the Deutsche Forschungsgemeinschaft (DFG FOR1103;PO-681/5-1)

\subsection{9}

\section{TYROSINE PHOSPHORYLATION OF VOLTAGE-GATED} SODIUM CHANNEL $\beta 1$ REGULATES NEURITE OUTGROWTH

Jeffery Calhoun ${ }^{1,2}$ and L. Isom ${ }^{1,2}\left({ }^{1}\right.$ Pharmacology, University of Michigan, Ann Arbor, MI and ${ }^{2}$ Cell and Molecular Biology, University of Michigan, Ann Arbor, MI)

Rationale: Voltage-gated $\mathrm{Na}+$ channels (VGSCs) are responsible for the initiation and propagation of action potentials in neurons. VGSC $\beta 1$ subunits (encoded by SCN1B) modulate VGSC cell surface expression, voltage dependence, and kinetics, while also functioning as immunoglobulin superfamily cell adhesion molecules (CAMs) that regulate cellular aggregation, migration, neurite outgrowth and fasciculation. Mutations in SCN1B result in the Genetic Epilepsy with Febrile Seizures plus (GEFS+) spectrum of epilepsies, including Dravet Syndrome, in human patients. Scn1b null mice exhibit a phenotype that includes spontaneous severe seizures and ataxia. These mice have defects in hippocampal and cerebellar microorganization prior to the onset of hyperexcitability, suggesting that VGSC $\beta 1$ subunits contribute to the regulation of cell-cell adhesion in vivo, further, that defects in $\beta 1$-mediated neuronal patterning may lead to hyperexcitability rather than result from it. We demonstrated, using cerebellar granule neurons (CGNs), that $\beta 1-\beta 1$ trans homophilic cell adhesion promotes neurite outgrowth via a mechanism that requires fyn kinase. We postulated that tyrosine phosphorylation of $\beta 1$ may act as a molecular switch for downstream signal transduction in response to $\beta 1-\beta 1$ trans homophilic adhesion. Previous studies provided support for this hypothesis with the observations that both $\beta 1$-mediated anykrin recruitment in response to cell-cell adhesion and $\beta 1$-mediated regulation of $\mathrm{Na}+$ current are modulated by tyrosine phosphorylation.

Methods: Here, we used CGNs as a model system to investigate the role of $\beta 1$ tyrosine phosphorylation in $\beta 1$-mediated neurite outgrowth. In this study, a BacMam baculovirus system was used to express $\beta 1 \mathrm{WT}$ or $\beta 1 \mathrm{Y} 181 \mathrm{E}$ (a phosphomimetic substitution) in Scn1b null CGNs to ask if the tyrosine phosphomimetic mutation affected neurite outgrowth. In addition, a cell aggregation model of $\beta 1-\beta 1$ trans homophilic adhesion was used to examine the effect of aggregation on the extent of tyrosine phosphorylation of $\beta 1$.

Results: Expression of $\beta 1 \mathrm{Y} 181 \mathrm{E}$ in Scn $1 b$ null cerebellar granule neurons resulted in significantly increased neurite length relative to the expression of $\beta 1 \mathrm{WT}$. Using a cell aggregation model of $\beta 1-\beta 1$ trans homophilic adhesion, we demonstrated that transfection of $\beta 1 \mathrm{WT}$ induced cellular aggregation at $37 \mathrm{C}$ while GFP-transfected cells did not aggregate. Subsequent immunoprecipitation of aggregated cells with anti-pY20 antibody demonstrated that $\beta 1 \mathrm{WT}$ is tyrosine phosphorylated following 30 minutes of aggregation. Conclusions: These results suggest a model in which $\beta 1$ subunits become tyrosine phosphorylated as a result of of $\beta 1-\beta 1$ trans homophilic adhesion. Further, the tyrosine phosphorylated form of $\beta 1$ drives neurite outgrowth.

\subsection{0 \\ PERIVENTRICULAR NODULAR HETEROTOPIA C-FOS ACTIVATION IN ORGANOTYPIC HIPPOCAMPAL SLICE CULTURES \\ E. Doisy ${ }^{1}$, J. Wenzel ${ }^{1}$, D. Nguyen ${ }^{2}$ and P. Schwartzkroin ${ }^{1}$ $\left({ }^{1}\right.$ Neurological Surgery, University of California, Davis, CA and ${ }^{2}$ Biostatistics, Public Health Sciences, University of California, Davis, CA)}

Rationale: Periventricular nodular heterotopia (PNH) is highly correlated with early and difficult to control seizure activity; however, we have little understanding of if, why, or how these PNH abnormalities affect seizure activity. We have used a rat model of $\mathrm{PNH}$, in which injection of methylazoxymethanol (MAM) into pregnant rat dams produces offspring with nodular heterotopia-like brain abnormalities, to assess nodule interactions with surrounding neocortex and hippocampus. However, our acute slice and intact animal studies are limited by interruption of connectivity (slices) and inadequate spatial coverage (intact brain). We have therefore employed organotypic hippocampal slice cultures (OHSCs) to determine baseline levels of neuronal activity and activity levels in response to treatment with a seizure-inducing agent (bicuculline methiodide (BMI)), as reflected in c-fos activation.

Methods: OHSCs from postnatal day 7-8 rat pups (from dams treated with MAM) were grown to 8-10 days in vitro and then exposed for 60 minutes to either artificial cerebrospinal fluid (ACSF) or $40 \mu \mathrm{M}$ BMI (in ACSF). OHSCs were then fixed and stained for c-fos immunoreactivity; photomicrographs of representative regions of the cortex (CTX), hippocampus (CA1) and nodule (PNH) were taken at $40 x$. For each region, the number of activated neurons and the number of pixels within each neuron expressing c-fos were quantified using ImageMeterPro (software kindly supplied by Joachim Schnier see http://flashscript.biz/AIR/imagemeter/ImageMeterPro.html), and neuronal cell density calculated based on NeuN staining.

Results: Multivariate statistical analysis showed a significant difference in number of neurons activated and average activated 
pixels per activated neuron between BMI- and ACSF-treated cultures, for all three regions. Within BMI-treated cultures, the CTX showed a significantly higher number of activated neurons than the PNH and CA1. Neuron counts based on NeuN labeling showed no significant difference in cell density (within the counting frame) across regions, confirming that a higher percentage of neurons in CTX showed c-fos activation. While the CA1 was not significantly different from the PNH in the number of neurons activated, it did show a statistically significant higher number of activated pixels per activated neuron (compared to both the PNH and CTX), suggesting a greater intensity of activation in the hippocampus.

Conclusions: These results show that neuronal activation, as measured with c-fos, can be quantified in OHSCs, and confirm that BMI does cause broad neuronal activation. At least at the time point sampled, PNH activation was not higher than activation in CTX or CA1, in either number of activated neurons or in average activated pixels per activated neuron. These data are consistent with our slice and intact animal results (Tschuluun et al., 2011, Epilepsia 52:230414 ), suggesting that the PNH is not more easily or more intensely activated than CTX or CA1.

Supported by grant NS057209 from NIH

\subsection{1 \\ TRPC3-MEDIATED HYPEREXCITABILITY AND EPILEPTIFORM ACTIVITY IN EXPERIMENTAL CORTICAL DYSPLASIA}

F. Zhou ${ }^{1,2}$ and S. Roper ${ }^{1,2}\left({ }^{1}\right.$ Neurosurgery, University of Florida, Gainesville, FL and ${ }^{2}$ McKnight Brain Institute, University of Florida, Gainesville, FL)

Rationale: Neuronal hyperexcitability plays a central role in the generation of epileptiform activity; however, cellular mechanisms underlying hyperexcitability in dysplastic cortical neurons are not clear. Low $[\mathrm{Ca} 2+]$ o or $[\mathrm{Mg} 2+]$ o conditions/models can induce hyperexcitability. Transient receptor potential canonical type 3 (TRPC3) channels are developmentally expressed. They play a pivotal role in neuronal excitability and they are activated in low $\mathrm{Ca}$ and/or $\mathrm{Mg}$ to depolarize neurons. We determined whether $0.4 \mathrm{mM} \mathrm{Ca}$ and $0.4 \mathrm{mM} \mathrm{Mg}(0.4 \mathrm{Ca}-\mathrm{Mg})$ can induce differential depolarization in an animal model cortical dysplasia (compared to controls) and examined the role of TRPC 3 channels in this process.

Methods: Pregnant rats on E17 were sham-exposed or exposed to 2.25 Gy of external irradiation. Male offspring from pregnant rats were subjected to slice electrophysiology (at P29-P31) and histology (at P30). For comparisons of electrophysiological data, significance was set at $\mathrm{P}<0.05$.

Results: TRPC3 staining was heavy in dysplastic cortex but weak in controls. Perfusion of $0.4 \mathrm{Ca}-\mathrm{Mg}$ induced a larger depolarization in pyramidal neurons from rat dysplastic cortex than in controls (Tab. 1, $\mathrm{P}<0.01$ ). Intracellular application of anti-TRPC3 antibody (to block TRPC3 channels) and bath application of Pyr3 (a selective TRPC3 inhibitor) almost completely abolished $0.4 \mathrm{Ca}-\mathrm{Mg}$-induced depolarization in dysplastic cortex (Tab. 1, $\mathrm{P}<0.01$ ). The residual depolarization was not different between the two groups after blocking TRPC 3 channels (Tab. 1, P> 0.05). 0.4 Ca-Mg induced epileptiform activity only in dysplastic cortex and it was completely suppressed 10 - 15 min after the addition of $10 \mu \mathrm{M}$ Pyr3.

Conclusions: TRPC 3 channels primarily mediate $0.4 \mathrm{Ca}-\mathrm{Mg}$-induced depolarization and epileptiform activity in dysplastic cortex. In 0.4 $\mathrm{Ca}-\mathrm{Mg}$, the dysplastic cortex is more hyperexcitable and more susceptible to epileptiform activity due to higher TRPC3 channel function.

Table 1: Depolarization $(\mathrm{mV})$

\begin{tabular}{|cc|c|}
\hline & Control cortex & Dysplustic correx \\
\hline $0.4 \mathrm{Ca}-\mathrm{Mg}$ & $2.79 \pm 0.31(7)$ & $9.57 \pm 1.01(7)$ \\
+ anti-TRPC3 $(8 \mu \mathrm{g} / \mathrm{m})$ & $1.86 \pm 0.20(5)$ & $1.75 \pm 0.18(6)$ \\
+ Pyrs $(10 \mu \mathrm{M})$ & $1.89 \pm 0.23(5)$ & $1.83 \pm 0.21(5)$ \\
\hline
\end{tabular}

mean \pm S.E.M. $(\mathrm{N})$
3.032

DYSREGULATION OF VOLTAGE-GATED ION CHANNEL EXPRESSION IN A MOUSE MODEL OF CORTICAL DYSPLASIA

Lena H. Nguyen ${ }^{1}$, A. L. Brewster ${ }^{1}$ and A. E. Anderson ${ }^{1,2}\left({ }^{1}\right.$ Baylor College of Medicine, Houston, TX and ${ }^{2}$ Texas Children's Hospital, Houston, TX)

Rationale: Cortical dysplasia (CD) is a malformation of cortical development that is often associated with drug-resistant epilepsy and aberrant signaling of the PI3K-mTOR growth pathway. Previous studies in our lab using a mouse model of CD [the neuronal subsetspecific conditional knockout of PTEN (NS-PTEN KO)] with hyperactive PI3K-mTOR signaling revealed abnormal epileptiform activity in these mice. Pharmacological inhibition of mTOR with rapamycin attenuated the epileptiform activity, suggesting a role for the mTOR pathway in the regulation of neuronal excitability. However, the underlying molecular mechanism for how aberrant PI3K-mTOR signaling contributes to the epilepsy phenotype in CD is unknown. Voltage-gated ion channel dysregulation is frequently associated with epilepsy, and more recently, evidence for mTORdependent regulation of ion channels under physiological conditions has been shown. In the current study, we evaluated whether aberrant PI3K-mTOR signaling in a model of CD contributes to altered expression of voltage-gated ion channels.

Methods: NS-PTEN KO and wildtype (WT) mice at postnatal week 6 were used for evaluation of ion channel protein levels. In parallel, a subset of animals was treated with rapamycin $(10 \mathrm{mg} / \mathrm{kg})$ or vehicle by intraperitoneal injections for 5 days/week during postnatal weeks 4 and 5. We used Western blotting to measure protein levels of Kv1.1, Kv1.2, Kv1.4, Kv4.2, Kv4.3, and HCN1 in whole hippocampal homogenates.

Results: Our results reveal a significant increase in the protein levels of Kv1.1 and a significant decrease in the levels of Kv1.2, Kv4.2, and HCN1 in the NS-PTEN KO compared littermate WT mice. There were no significant changes in the levels of Kv1.4 and Kv4.3. Rapamycin restored Kv1.1 protein levels in the NS-PTEN KO mice back to WT basal levels, but had no effect on the other ion channels that we evaluated ( $\mathrm{p}<0.05, \mathrm{n}=7-8$ per group).

Conclusions: Our findings reveal altered expression of voltage-gated ion channels in NS-PTEN KO mice, which may contribute to the epilepsy phenotype. Furthermore, our findings reveal a novel link between PI3K-mTOR dysregulation and Kv1.1 remodeling in the NS-PTEN KO model of CD. Future studies will evaluate cellular and subcellular localization of Kv1.1 dysregulation in this model.

\subsection{3 \\ MAP KINASE INHIBITION AS A NEW THERAPEUTIC TARGET FOR INTERICTAL SPIKING IN THE RAT}

Danielle Senador, D. T. Barkmeier, S. Dattloff and J. A. Loeb

(Neurology, Wayne State University, Detroit, MI)

Rationale: While seizures are infrequent, long term intracranial recordings demonstrate far more frequent interictal discharges between seizures. Previous studies from our laboratory on human epileptic brain tissues have shown a 'final common pathway' of specific genes that are upregulated at human epileptic foci. A large proportion of these genes belong to the mitogen-activated protein kinase (MAPK) and cAMP Response Element Binding Protein (CREB) pathways. Surprisingly, the expression levels of these genes do not correlate with seizure frequency, but correlate precisely with interictal spiking, suggesting that theses genes either produce or result from ongoing, electrical discharges. In order to understand interictal spike generation and their roles in epileptogenesis animal models and drugs that specifically modulate interictal spiking are needed.

Methods: A neocortical spiking focus was induced in rats using a single tetanus toxin injection into somatosensory cortex. Following 
injection, six epidural electrodes ( 3 on each hemisphere) were implanted to monitor epileptic activities through long-term video/EEG recordings using an automated spike detection program. Regions of spiking and non-spiking cortex were analyzed for MAPK/CREB activation and downstream gene activations. A selective MEK inhibitor that blocks the MAPK pathway was tested on interictal spiking.

Results: Progressive interictal spiking can be produced in localized brain regions using a single tetanus toxin injection. The system was optimized using a conical, rather than a beveled needle to concentrate the toxin in more superficial cortical layers. Long term EEG quantification was achieved using a spike detection algorithm that removes artifact channels and identifies spikes in each channel using a combination of two differed frequency filters and block scaling. In this model, focal spikes were produced with increasing frequency and amplitude over time. In addition, the spike field expanded over time, both anterior to and contralateral to the injection site. Virtually no seizures were detected. Interictal spiking was associated with a similar pattern of phosphoCREB and downstream gene inductions seen in human epileptic cortex, mostly in superficial cortical layers. The use of a selective MEK inhibitor, early on, blocked both CREB activation and also prevented the development of interictal spiking. Conclusions: This animal model recreates both the electrophysiology and molecular mechanisms of human neocortical epilepsy and therefore could be a valuable model for preclinical drug development. Our results show that interictal spiking is sufficient to induce persistent changes in the MAPK/CREB pathway that parallels observations in human epileptic neocortex. MAPK/CREB activation is required for the development of a chronic interictal state and therefore could represent a new anti-epileptogenic target.

\subsection{4 \\ SPONTANEOUS TEMPORAL LOBE SEIZURES IN A PRENATAL FREEZE LESION RAT MODEL}

Takashi Kamada $^{1}$, W. Sun ${ }^{1}$, T. Uehara ${ }^{1}$, K. Takase ${ }^{1}$, H. Shigeto ${ }^{1}$, S. O. Suzuki ${ }^{2}$, Y. Ohyagi ${ }^{1}$ and J. Kira ${ }^{1}\left({ }^{1}\right.$ Neurology, Kyushu University, Fukuoka, Japan and ${ }^{2}$ Neuropathology, Kyushu University, Fukuoka, Japan)

Rationale: Focal cortical dysplasia (FCD) is an important cause of intractable epilepsy. Because neonatal freeze lesion rat models do not show spontaneous seizures without seizure-provoking events, we created a rat model with multiple FCD, produced during embryonic development, and revealed that some of them have spontaneous seizures arising from the hippocampus. In this study, we generated more rats with prenatal freeze lesions to confirm the occurrence of spontaneous seizure. Furthermore, we examined the relationship between FCD and epileptogenesis using an immunohistochemical procedure to investigate the mechanism underlying epilepsy with FCD.

Methods: At 18 days postconception, a frozen metal probe was placed bilaterally on the scalps of Sprague-Dawley rat embryos through the uterus wall to produce multiple FCD. Rats were divided into groups receiving bilateral lesions with two lesions in each hemisphere (bilateral multiple lesion rats), bilateral lesions with a single lesion in each hemisphere (bilateral single lesion rats), and bilateral sham operations (bilateral sham-operated rats). EEG was performed intermittently from postnatal day 35 to postnatal day 77 in the bilateral frontal cortices and hippocampi. Video recording was used to monitor behavior. Brain tissues were examined immunohistochemically at postnatal days 28 and 78 using semiquantitative densitometry.

Results: Eleven of 16 bilateral multiple lesion rats (68.8\%) showed spontaneous electroencephalographic hippocampal seizures from postnatal day 47. No seizures were observed in bilateral single lesion or sham-operated animals. Movement cessation followed by sniffing and mastication, culminating in wet-dog shaking, was seen during the hippocampal discharges. FCD was observed in the bilateral frontoparietal lobes. Although spontaneous seizures were not seen at FCD lesions, the expression levels of $N$-methyl-D-aspartate receptor (NMDAR) subunits 1, 2A, 2B, the glutamate/aspartate transporter and the glial glutamate transporter 1 at FCD lesions were increased at postnatal days 28 and 78. There were no major histological abnormalities in the hippocampi compared with those in the cortex. However, the expression levels of $N$-methyl-D-aspartate receptor (NMDAR) subunits 2A and 2B were increased in the hippocampi at postnatal day 28. Also, levels of NMDAR1, 2A and 2B, the glutamate/aspartate transporter and the glial glutamate transporter 1 were increased in the hippocampi at postnatal day 78 .

Conclusions: We confirmed the reproducibility of spontaneous temporal lobe seizures in a rat model with multiple FCD produced during embryonic development. Increased excitability via glutamatergic receptors was observed both in FCD lesions and the hippocampus. This model should help clarify the mechanisms underlying temporal lobe epilepsy.

\subsection{5 \\ A NOVEL HIPPOCAMPAL SEIZURE MODEL USING OPTOGENETICS}

Shin-ichiro Osawa ${ }^{1}$, M. Iwasaki ${ }^{1}$, R. Hosaka ${ }^{2}$, Y. Matsuzaka ${ }^{3}, H$. Tomita $^{4}$, T. Ishizuka ${ }^{5,6}$, E. Sugano ${ }^{4}$, E. Okumura ${ }^{7}, \mathrm{H}_{\text {. Yawo }}{ }^{5,6}, \mathrm{~N}$. Nakasato $^{7}$, T. Tominaga ${ }^{1}$ and H. Mushiake ${ }^{3}\left({ }^{1}\right.$ Neurosurgery, Tohoku University Graduate School of Medicine, Sendai, Japan; ${ }^{2}$ Applied Mathematics, Fukuoka University, Fukuoka, Japan; ${ }^{3}$ Physiology, Tohoku University Graduate School of Medicine, Sendai, Japan; ${ }^{4}$ Chemistry and Bioengineering, Iwate University, Morioka, Japan; ${ }^{5}$ Basic and Translational Research Center for Global Brain Science, Tohoku University, Sendai, Japan; ${ }^{6}$ Developmental Biology and Neuroscience, Tohoku University Graduate School of Life Sciences, Sendai, Japan and ${ }^{7}$ Epileptology, Tohoku University Graduate School of Life Sciences, Sendai, Japan)

Rationale: Epileptic seizure is characterized by an excessive synchrony of neurons which causes transient loss of normal brain function. The mechanism of icto-genesis, i.e. how seizure develops in normal neural network, is poorly understood because it is difficult to reproduce and observe seizures using conventional animal models. Recent development of optogenetics enabled us to intervene functions of specific cells via optical control of ion channels. Although this technique facilitated our understanding on mechanism of disease at functional circuit level, applications to epilepsy research are still scarce.

Here we developed a novel in vivo model of epileptic seizures using optogenetics as a potential tool for studying icto-genesis.

Methods: Repetitive pulse or continuous photo stimuli were delivered to the hippocampus of anesthetized Thy1.2 -

Channelrhodopsin 2 - Venus (ChR2V) transgenic rat under simultaneous recording of local field potentials (LFPs). Stimulations with various parameters including pulse frequency and duty ratio were tested to induce epileptic seizures. The experiment was also performed to the hippocampus of wild Wister rats transfected with adeno-associate viral vector carrying ChR2V. The relation of c-fos expression and epileptic seizures were investigated.

Results: Epileptic seizures were induced by pulse photo-stimulation of the hippocampus in both transgenic rats and transfected wild Wister rats. The LFP during the icto-genesis was observed with no interference of electrical artifacts. Rhythmic activities emerged during stimulation and became self-sustained afterwards for 39.8 \pm 1.9 seconds with peak frequency around $10 \mathrm{~Hz}$, reminiscent of epileptic seizures in human. The seizure was most efficiently induced by stimulation with 10 and $20 \mathrm{~Hz}$ pulse frequency and smaller duty ratio. No rats were died during and after the experiment. C-Fos expression was increased in all subregions along septo-temporal axis of the hippocampus.

Conclusions: This novel hippocampal seizure model is advantageous for its high reproducibility, artifact-less observation and low 
mortality compared with conventional animal models of seizures. It is promising tool to study the mechanism of icto-genesis.

\subsection{6 \\ REGIONAL EXPRESSION PATTERNS OF CANDIDATE GENES LINKED TO LANDAU-KLEFFNER SYNDROME}

L. V. Long and Elizabeth Powell (University of Maryland School Of Medicine, Baltimore, MD)

Rationale: Landau-Kleffner syndrome (LKS) is a rare childhood disorder characterized as a language disability, abnormal electroencephalogram (EEG) and often rolandic epilepsy. Onset occurs after the development of speech, in children from $4-7$ years, with up to $80 \%$ of patients experiencing seizures and nearly all have abnormal EEG recording. LKS presents with language disorder, sleep disturbances, and possible autoimmune dysfunction Recently, SRPX2 (sushi repeat protein X-linked 2) has been identified in patients with rolandic seizures, language disability and intellectual disability. A screen for candidate molecules that interact with SRPX2 identified six molecules, including cathepsin B, ADAMTS4 (aggrecanase 1), protocadherin 10, and uPAR Previous studies with mice lacking uPAR (also known as Plaur) have shown abnormal EEG and seizures, with cognitive impairment. The Srpx2 expression is largely unknown, as is the interaction between Srpx 2 and Plaur in the developing and adult brains.

Methods: In situ hybridization and immunohistochemistry were used to reveal the expression patterns of Plaur and Srpx2 during embryonic and postnatal development. Biochemical analysis examined the changes in Srpx2 expression in the absence of Plaur. Results: Sprx 2 is selectively expressed in neuronal progenitors and neurons in the developing forebrain. The embryonic patterns of Plaur and Srpx 2 are overlapping within the emerging cerebral wall. The expression patterns of Srpx 2 in the adult mouse correspond to a role in sleep regulation, motor control, and attention. Finally, our data suggests Srpx 2 colocalizes with white matter astrocytes, which will be explored further; inflammation and brain injury may be another way in which Srpx2 is involved in language and epileptic disorders. Conclusions: The expression pattern of Sprx 2 in the mouse corresponds to structures involved in motor planning and the initiation of speech, as well as areas necessary for proper sleep regulation.

\subsection{7}

\section{CARDIAC, RESPIRATORY, AND CORTICAL FUNCTION} AFTER ELECTRICALLY-INDUCED SEIZURES IN WILDTYPE AND 5-HT NEURON DEFICIENT MICE

Gordon F. Buchanan ${ }^{1,2}$ and G. B. Richerson ${ }^{3,4}\left({ }^{1}\right.$ Neurology, Yale University School of Medicine, New Haven, CT; ${ }^{2}$ Neurology, VA Medical Center, West Haven, CT; ${ }^{3}$ Neurology and Molecular Physiology and Biophysicis, University of Iowa College of Medicine, Iowa City, IA and ${ }^{4}$ Neurology, VA Medical Center, Iowa City, IA)

Rationale: Sudden unexplained death in epilepsy (SUDEP) is a devastating condition in which epilepsy patients die for no apparent reason. It is the leading cause of death among epilepsy patients. Though this has been a long recognized syndrome, there is much to be learned about the pathophysiology of this disease. Cardiac and respiratory etiologies have been implicated to underlie SUDEP. Serotonin (5-HT) is a key regulator of breathing control. 5-HT dysfunction has been implicated in the pathophysiology of SUDEP. Here we employ a mouse model in which nearly all 5-HT neurons have been genetically deleted to determine whether 5-HT neuron absence contributes to seizure-related respiratory dysfunction and death.

Methods: EEG, EMG, EKG, body temperature, locomotor activity and breathing were recorded in adult male and female wild type (WT) mice and mice lacking 5-HT neurons $\left(L m \times 1 b^{f / f / p}\right)$ before, during and after seizure induction via electroshock (1-50 mA, $0.2 \mathrm{~s}, 60 \mathrm{~Hz}$ stimulation via ear electrodes). Seizure susceptibility and mortality were assessed. Seizure severity was scored using the extensionflexion ratio.

Results: Seizures induced by electroshock were relatively short (14$33 \mathrm{~s}$ ) in duration and were comprised of a characteristic brief hind limb flexion followed by hind limb extension as part of the tonic component. The tonic phase was typically followed by a brief clonic phase. $L m \times 1 b^{f / f / p}$ mice experienced seizures at slightly lower stimulus intensity $(5 \mathrm{~mA}, 0.2 \mathrm{~s}, 60 \mathrm{~Hz})$ than WT $(10 \mathrm{~mA}, 0.2 \mathrm{~s}, 60 \mathrm{~Hz})$.

Seizures were more severe, as assessed by the extension-flexion ratio, across stimulus intensities in $L m \times 1 b^{f / f / p}$ mice compared to WT mice and in males compared to females. In both genotypes, the tonic phase was always accompanied by respiratory arrest. Seizure-related mortality increased with increasing stimulus intensity and was increased in $L m \times 1 b^{f / f / p}$ mice compared to WT mice. There was only a sex difference in mortality at the highest stimulus intensity.

Following seizures that ultimately resulted in death, there was an immediate flattening of EEG activity, lack of recovery of spontaneous respirations, and persistence of cardiac activity for up to 6 min following terminal respiration. Cardiac activity became progressively slower, less intense, and more irregular until it ultimately ceased.

Conclusions: These results indicate that elimination of central 5-HT neurons renders mice more susceptible to seizure induction and more prone to seizure-related sudden death. These data further indicate that respiratory mechanisms are more directly responsible for death than cardiac arrest and that the 5-HT neuron deficit is responsible for the post-ictal breathing abnormalities

\subsection{8 \\ WHAT IS THE IMPACT OF ELECTROMAGNETIC WAVES ON EPILEPTIC SEIZURES?}

Nilgun Cinar ${ }^{1}$, S. Sahin ${ }^{1}$ and O. O. Erdinc ${ }^{2}\left({ }^{1}\right.$ Department of Neurology, Maltepe University Faculty Of Medicine, Istanbul, Turkey and ${ }^{2}$ Department of Neurology, Eskisehir Osmangazi University, Faculty of Medicine, Eskisehir, Turkey)

Rationale: The effects of electromagnetic waves (EMWs) on humans and their relationship with various disorders have been investigated. We aimed to investigate the effects of exposure to different frequencies of EMWs in various durations in a mouse epilepsy model induced by pentylenetetrazole (PTZ).

Methods: Total 180, four-week-old male mice weighing 25-30 g were used in this study. Each experimental group consisted of 10 mice. They were exposed to 900, 700, 500, 300 and $100 \mathrm{MHz}$ EMWs in 20 hours, 12 hours and 2 hours. Following electromagnetic radiation exposure, $60 \mathrm{mg} / \mathrm{kg}$ of PTZ was injected intraperitoneally to all mice. Each control was also injected PTZ without any exposure to EMW. The latency of initial seizure and most severe seizure onset were compared with controls.

Results: The shortest initial seizure latency was noted in the 500 $\mathrm{MHz}$ and the longest in the $300 \mathrm{MHz}$ in 2 hours group. The shortest initial seizure latency was noted in the $700 \mathrm{MHz}$ and the longest in the control group, followed by the $100 \mathrm{MHz}$ in 12 hours group. The shortest initial seizure latency was noted in the $300 \mathrm{MHz}$ and the longest in the $500 \mathrm{MHz}$ in 20 hours group. No statistically significant difference was noted in terms of the latency of the most severe seizure following 20,12 and 2 hours of EMW exposure.

Conclusions: Our results suggested that exposure to different frequencies and different duration of EMWs had different effects on epileptic seizure latency. Further studies are needed. 


\subsection{9}

\section{THE EFFECT OF FOCAL INTERICTAL SPIKES DURING DEVELOPMENT ON SHORT-TERM SYNAPTIC PLASTICITY IN THE PREFRONTAL CORTEX}

Amanda Hernan ${ }^{1}$, J. Barry ${ }^{1}$, R. Scott ${ }^{1,2}$ and G. Holmes ${ }^{1}$ ( ${ }^{1}$ DartmouthHitchcock Medical Center, Lebanon, NH and ${ }^{2}$ Institute of Child Health, University College London, London, United Kingdom)

Rationale: There is a well-described association between autism and epilepsy, but the mechanisms underlying this association remain unclear. It is hypothesized that the epileptiform discharges that occur in approximately $50 \%$ of children with autism are important for understanding the disorder but the precise role of these discharges has yet to be explored.

Methods: In order to understand the relationship between frequent epileptic discharges during the neurodevelopmental period and cognition later in life, a robust model of focal prefrontal cortical interictal spikes without overt seizures is required. No model of this type has yet been reported. To this end, we developed a model in which we gave $\mathrm{p} 21$ rat pups intracortical injections $(0.5 \mu \mathrm{L})$ of 175 $\mu \mathrm{M}$ bicuculline methiodine in normal saline whilst continuously recording EEG. Within seconds of injection, robust focal spikes were recorded at the injection site without behavioral seizures. The injections were repeated in order to achieve 5 days of epileptic activity in the absence of seizures, mimicking the prolonged aberrant activity seen in $50 \%$ of autistic children.

With this model in place, we were able to test the effects of early life epileptiform activity (ELEA) on network-level changes in short-term synaptic plasticity in the PFC that are associated with PFC function. Extracellular field potentials evoked in LII or LV of the prelimbic region of the $\mathrm{mPFC}$ were recorded in layer V in ELEA and littermate control animals at P27-P36. Short-term post-tetanic potentiation (PTP) in LII-LV circuits and LV-LV circuits was probed using a baseline single test pulse stimulation delivered repeatedly at $0.1 \mathrm{~Hz}$ and a $50 \mathrm{~Hz}$ tetanus, 15 pulses, delivered one time. Paired pulse facilitation was assessed at interpulse intervals varying from $10 \mathrm{~ms}$ $(100 \mathrm{~Hz})$ to $400 \mathrm{~ms}(2.5 \mathrm{~Hz})$. Stimulus number plasticity was also assessed through examination of the fEPSP during $50-\mathrm{Hz}$ stimulation. Results: In ELEA animals ( $\mathrm{N}=5, \mathrm{~N}=5$ controls), we found a significant increase in PTP in the LII-LV network, with a small but not significant increase in the LV-LV network. Facilitation and stimulus number dependent plasticity were not significantly different in ELEA animals than controls.

Conclusions: These changes mimic alterations that we have recently observed after early life seizures and may represent a network-level mechanism for cognitive deficits seen in children with a history of epileptiform activity. Studies are ongoing to test whether or not ELEA animals also have behavioral deficits that mimic early life seizure animals.

\subsection{0}

MODELING ELECTROCORTICAL SOURCE DYNAMICS OF INTRACRANIAL EEG DATA IN EPILEPSY

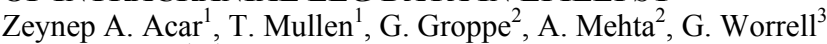
and S. Makeig ${ }^{1}{ }^{1}$ Institute for Neural Computation, University of California, La Jolla, CA; ${ }^{2}$ Center for Neuroscience, Feinstein Institute for Medical Research, Manhasset, NY and ${ }^{3}$ Department of Neurology, Mayo Clinic, Rochester, MN)

Rationale: Epilepsy is one of the most prevalent neurological diseases, affecting an estimated 50 million people worldwide. Seizures on one third of patients cannot be controlled with medications alone. For these individuals, the next best course of treatment is to surgically remove the parts of the brain responsible for the seizures. The current clinical gold standard for identifying the areas to resect relies on qualitative expert visual inspection of intracranial EEG (iEEG) data which is only successful in $40-80 \%$ of cases. Here, we report a method for analyzing and visualizing electrocortical source dynamics in epilepsy patients (Akalin Acar et al; Mullen et al, 2011, EMBC). By identifying sources of seizure activity and modeling dynamic relationships between seizure zones, this methodology can generate information and insight into seizure generation that can help experts make more accurate and objective clinical assessments and surgical plans.

Methods: The main steps of our analysis methodology can be itemized as follows:

1. Adaptive mixture independent component analysis (AMICA) (Palmer et al, 2006) is applied to iEEG data to identify and separate seizure sources. When applied to epilepsy data, AMICA can adapt to the differences in spatio-temporal source structure during pre-seizure, seizure, post-seizure and non-seizure periods (Figure 1).

2. An electrical forward head model of the patient is generated from MR and CT images of the patient's head (Akalin Acar \& Makeig, 2010, http://sccn.ucsd.edu/nft/). A sparse Bayesian method (SBL) including multi-scale patch-based source model is used to estimate the anatomical region of each iEEG independent component. 3. A pairwise mutual information (PMI) matrix is calculated to understand how the time courses differ across components. The component clusters showing high dependencies are identified and mapped on the patient's brain image.

4. Dynamics of ICA source clusters are analyzed by fitting a suitable time-varying vector autoregressive model (VAR) that captures distributed patterns of spectral dynamics in the data (Mullen et al, www.sccn.ucsd.edu/wiki/SIFT). Graph theoretic metrics are then used to summarize and visualize the complex network structure. These metrics are combined with the SBL component inverse maps to identify regions significantly implicated in seizure propagation. Results: These methods are tested on datasets collected from two patients at the Mayo Clinic (Rochester, MN) and at the Hoftsra North Shore LIJ (NY), respectively. Initial results suggest that spatiotemporal network dynamics, modeled in the absence of seizure, may provide a novel source of predictive information regarding the location and likelihood of later ictal dynamics. In both datasets, our localization of epileptogenic zone was confirmed by the (mainly) positive surgical outcomes.

Conclusions: Our work to date suggests that this method a) may improve the spatial resolution of iEEG information, and b) may provide new insights into seizure genesis and propagation by revealing seizure network interactions too complex to be readily identified by visual inspection.

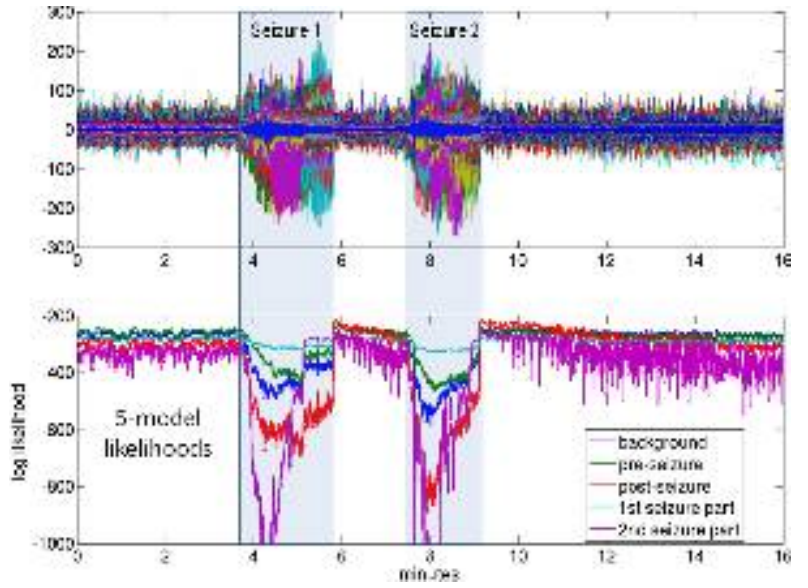

(upper) 16 minutes of iEEG data. All channels are plotted on the same axis. The seizure periods are highlighted. (Lower) Likelihood graphs for one-model and five-model Amica decomposition. 


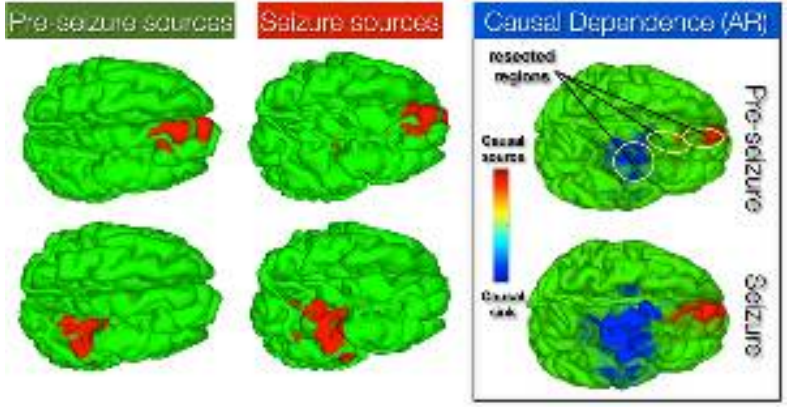

(left panels) Spatial overlap between pre-ictal and ictal dependent source areas. (right panel) Similarity in causal dependence maps during the pre-ictal and ictal periods (total source outflow-inflow; positive values indicate a net causal source area, negative values a net sink) from separate AMICA source models for these data periods.

\subsection{1}

EVALUATION OF EPILEPTOGENESIS IN TWO DIFFERENT TYPES OF STATUS EPILEPTICUS INDUCED BY ELECTRICAL STIMULATION OF AMYGDALA

Victor R. Santos, C. Q. Tilelli, O. W. Castro, A. Fernandes, F. Del

Vecchio and N. Garcia-Cairasco (Physiology, University of São Paulo, Ribeirão Preto, Brazil)

Rationale: Temporal Lobe Epilepsy (TLE) is the most common type of epilepsy. In general around one third of all epilepsies are classified as TLE, which usually settles after an initial insult. After a latent period, when neuroplastic alteration occurs, the spontaneous recurrent seizures (SRSs) emerge. To better study the plastic mechanisms involved in epileptogenenesis, we used the animal model of electrical stimulation of the amygdala to induce selfsustained status epilepticus (SSSE). Our aims were to evaluate circuitry activation, neurodegeneration and neurogenesis processes and their possible relationship with the behavior during the SSSE. Methods: Adult male Wistar rats were implanted with electrodes in the left amygdaloid complex (AmyC) and hippocampal formation (HF). Video-EEG was recorded. AmyC was electrically stimulated for $30 \mathrm{~min}$ and the animals developed SSSE for $1.5 \mathrm{~h}$, after what they received $5 \mathrm{mg} / \mathrm{kg}$ diazepam. Rats were perfused after $3 \mathrm{~h}(\mathrm{n}=7 \mathrm{SE} ; 4$ controls), $24 \mathrm{~h}(\mathrm{n}=7 \mathrm{SE} ; 3$ controls) and 14 days ( $\mathrm{n}=8 \mathrm{SE} ; 5$ controls). Control animals were implanted but were not stimulated. Brain slices were stained for Fos and Ki-67 ( 3 h group), FluoroJade (FJ) and Ki-67 (24 h group) and Doublecourtin (DCX) (14 days group). Analyzed areas were neocortex, entorhinal cortex, pyriform cortex, AmyC and HF. For the analyses of Fos and FJ each area was classified according to the relative number of stained cells based on an arbitrary scale validated by three blinded observers to the animal's group. For the analyses of cell proliferation (Ki-67) and neurogenesis (DCX), each stained cell in the dentate gyrus was counted in 4 slices. All experiments were conducted according to Ethics on Animal Experimentation.

Results: The rats were divided based on the behavioral pattern during SSSE: type I with most severe seizures (limb myoclonus, falling, running and jumping) and type II with less severe seizures (facial automatisms and neck myoclonus). Epileptiform EEG was recorded in AmyC and HF channels and was qualitatively similar for both types of SSSE. None of the control rats presented behavior or EEG alterations. There was a stronger Fos staining in the SE type I in neocortex $(p<0.05)$ and amygdala $(p<0.001)$. There was a strong neurodegeneration in all analyzed areas, and FJ staining was significantly higher in HF of SE type I than in SE type II $(\mathrm{p}<0.05)$. SE type II presented mostly unilateral neurodegeneration in all analyzed areas $(p<0.001)$. Both group of SE animals (Type I and II) show a significant increase in the number of Ki-67 cells at $3 \mathrm{~h}$ $(\mathrm{P}<0.05)$ and $24 \mathrm{~h}(\mathrm{P}<0.001)$ and DCX was also increased at 14 days $(\mathrm{P}<0.001)$ in both HF.

Conclusions: This study shows that behavioral expression of SE is associated to specific circuitry activation (Fos) and neurodegeneration (FJ) patterns. Widespread activation and neurodegeneration are seen in SE type I and they are more restrict in SE type II. However, cell proliferation (Ki-67) and neurogenesis (DCX) were equally increased in both types of SSSE in contrast with control animals. Supported by FAPESP, PROEX-CAPES, CNPq.

\subsection{2 \\ IMPROVING THE DEVELOPMENT OF PROMISING DRUG CANDIDATES: PRECLINICAL SOLUTIONS DEVELOPED IN CHRONIC MODELS OF EPILEPSY}

Melanie Langlois, C. Bouyssieres, V. Duveau, C. Dumont and C. Roucard (SynapCell, Grenoble, France)

Rationale: Despite the development of new compounds, more than $30 \%$ of patients with epilepsy are still resistant to antiepileptic drugs (AEDs). Pharmaceutical industry clearly needs new preclinical strategies to improve AED discovery and development. Accordingly, chronic animal models of epilepsy that are resistant to one or several AEDs appear of great interest.

Mesiotemporal lobe epilepsy (MTLE) is the most common form of epilepsy that is refractory to antiepileptic drugs (AEDs) and several strategies are being developed to better treat this syndrome. To this aim, the development of new animal models with similar features of MTLE is mandatory.

Methods: During the past ten years, a chronic model of MTLE in adult mice has emerged where spontaneous recurrent focal seizures with mild behavioural expression are observed. In this model, a unilateral injection of kainate (KA) into the dorsal hippocampus induces ipsilateral cell loss in CA1, CA3 and hilus areas, as well as gliosis, sprouting of mossy fibres and a progressive dispersion of the granule cells of the dentate gyrus. In addition, spontaneous recurrent hippocampal paroxysmal discharges (HPD) develop during the first 3 weeks post KA and then remain stable and stereotyped for the whole life of the animals. These HPDs occur spontaneously about 45 times per hour when the animals are in a state of quiet wakefulness, generally last $15-20 \mathrm{sec}$ and are associated with behavioural arrest and/or mild motor automatisms.

Results: By using this MTLE mouse model, SynapCell has developed a broad range of preclinical solutions to better identify promising drug candidates targeting partial drug-resistant epilepsies. First, the in vivo screening protocol allows testing of small libraries of compounds and relies on quantitative EEG evaluation of the efficacy of new AEDs on highly recurrent HPDs, using a latin-square protocol on a limited number of MTLE mice.

Then, promising drug candidates can be further studied for their dose-response effects with acute treatments, their efficacy over time with chronic protocols as well as their efficacy when co-administered with other AEDs in add-on protocols.

Finally, this model also allows the assessment of disease modifying potential of compounds on the appearance of chronic seizures during the epileptogenic period.

Conclusions: Altogether, this chronic model of MTLE reproduces some of the behavioral, electroclinical and histopathological features of human MTLE and represents a unique tool to identify new AEDs that could be efficient in drug-resistant MTLE patients.

\subsection{3 \\ EFFECT OF IH CHANNEL BLOCKER ZD 7288 ON THE AFTERDISCHARGE INDUCED BY ACUTE KINDLING OF THE RABBIT HIPPOCAMPUS}

Akira Kobayashi, K. Tsuchiya, K. Tsuchida, H. Tekushi, T. Tachibana and S. Kogure (Soka university, Hachioji, Tokyo, Japan)

Rationale: Kindling is a widely used animal model of intractable temporal lobe epilepsy. We have performed spectral analysis on the afterdischarge (AD) induced by chronic hippocampal kindling of the 
rabbit and revealed that enhancement of the higher frequency band (HFB: 12-30 Hz) component is associated with kindled stage, while decrement of it is associated with incomplete kindling stage (Tsuchiya et al., Epilepsy Res, 2011; 2012). Meanwhile, we previously demonstrated that the Ih blockers $\mathrm{CsCl}$ and ZD7288 can significantly increase the threshold of electrically induced paroxysmal discharge in the rabbit hippocampus (Kitayama et al., Epilepsia, 2003). However, the role of Ih channel in epileptogenesis is controversial. In the present study, we examined the effects of ZD7288 on ADs induced by acute kindling of the rabbit hippocampus to reveal the contribution of Ih channel in epileptogenesis with using spectral analysis.

Methods: We performed all experiments under appropriate conditions in accordance with the Declaration of Helsinki and the Guide for Animal Experimentation at Soka University. Seven adult rabbits were used. Under deeply anesthesia, we delivered acutely kindling stimulation ( $1 \mathrm{~ms}$ biphasic pulses, $50 \mathrm{~Hz}, 1 \mathrm{~s}$ ) with suprathreshold intensity for AD at 20-min intervals to the right hippocampus. Kindling stimulations were performed at least 30 times unless spectral changes occur that we have observed in chronic experiments. When HFB in AD increased to the kindled level, $1 \mathrm{mM}$ ZD7288 was injected with a rate of $1 \mu \mathrm{l} / \mathrm{min} 5 \mathrm{~min}$ before stimulating the ipsilateral side. Spectral analysis on each AD was performed with sampling frequency of $1 \mathrm{kHz}$ by Power Lab (Chart, ADInstrument).

Results: The power spectral density (PSD) ratios of the lower frequency band (LFB: 0-9 Hz), the middle frequency band (MFB: 9$12 \mathrm{~Hz}$ ) and HFB components against total PSD were calculated. During the acute kindling, PSD ratio of HFB component against total PSD changed from $15.4 \pm 9.5 \%$ (mean $\pm \mathrm{SD})$ at the initial stage to $44.2 \pm 18.2 \%(\mathrm{p}<0.001)$ at the final stage. On the other hand PSD ratio of LFB component altered from $73.2 \pm 16.2 \%$ to $36.8 \pm 17.3 \%$. ZD7288 suppressed kindling-induced ADs or retarded them, and decreased spontaneous seizure occurrence. In addition, even ADs after ZD7288 injection showed higher PSD ratio of LFB component . Conclusions: An enhancement of HFB component and decrement of LFB component in ADs also occurred in the acute kindling. Since ZD7288 suppressed seizure occurrence and enhancement of HFB component, it is suggested that Ih channels contribute to the generation of high frequency seizure discharge.

\subsection{4 \\ LOOKING FOR COMPLEXITY IN EPILEPTOGENIC CIRCUITS: CAN WE BUILD A COMMON FRAMEWORK FOR COMPUTATIONAL MODELS?}

Julian Tejada ${ }^{1,2}$, K. M. Costa ${ }^{1}$, P. Bertti ${ }^{1}$, A. C. Roque ${ }^{2}$ and N. Garcia-Cairasco ${ }^{1}\left({ }^{1}\right.$ Departamento de Fisiologia, FMRP, Universidade de São Paulo, Ribeirão Preto, Brazil and ${ }^{2}$ Departamento de Física, FFCLRP, Universidade de São Paulo, Ribeirão Preto, Brazil)

Rationale: It is widely accepted that epilepsies are complex syndromes, due to their multi-factorial cellular origins and their intricate behavioral and electrophysiological manifestations. These qualitative and intuitive appreciations are consistent with different mathematical and computational descriptions of these diseases. We can divide these theoretical approaches in two major categories: stochastic and deterministic. Both approaches can help understand the manifold underlying characteristics of epilepsy. Here we present two models, each based on one of these approaches, which shed light on the quantitative aspects of the complexity inherent to epilepsy. Methods: For the stochastic approach, we used flowchart and graph theory to characterize the neuroethological and functional neuroimaging manifestations of seizures in temporal-lobe epileptic patients. Analyzed variables were the duration, frequency and interactions of observed behaviors and the intensity and localization of brain SPECT signals. For the deterministic method, we used a compartment model that reproduced specific membrane features, in this case the electrophysiological properties of newborn dentate gyrus granular cells after pilocarpine-induced status epilepticus.
Results: The stochastic analysis, an up-down approach, was used to establish indexes that allowed to characterize seizures, and identify important factors related with the neural substrates of the epileptic seizures, including the anatomical epileptogenic zone and recruited spread pathways.

The bottom-up deterministic approach demonstrated how subtle changes in the form and function of specific cells affect network responses to exogenous stimulation. At the same time, we evaluated possible effects produced by different changes that have not yet been observed in experimental conditions, but that are plausible to occur. Conclusions: While both models offer insights into epilepsy, integration of our results with the current literature is challenging. Considering this, we point out the necessity of a common framework for analyzing and modeling pathophysiological phenomena in the context of epilepsy research. For stochastic techniques, we propose graph theory as a potential common frame of reference, due to its wide user base and relatively simple mathematical foundation. There are currently various open source software projects that can be adapted for the graphical representation of multiple variables and thus be applied to this purpose. Finding a common framework for deterministic modeling is much more challenging, as current models are being constructed under a huge variety of theoretical guidelines and computational languages. At the very least, we encourage the publication of the code behind each model in an open online database and the usage, as far as possible, of open source software, which will permit the development of global standards and potentially offer a common theoretical structure for the sharing and combination of models.

Financial support: FAPESP, FAPESP-Cinapce, PROEX-CAPES, CNPQ and FAEPA.

\section{Translational Research: Human Studies}

\subsection{5}

FROM RATS TO MEN: A VIRTUAL WATER-MAZE TASK SHOWS COGNITIVE IMPAIRMENTS IN PATIENTS WITH EPILEPSY

A. S. Titiz ${ }^{1}$, G. L. Holmes ${ }^{1}$, R. C. Scott ${ }^{1,2}$ and P. P. Lenck-Santini ${ }^{1}$ ( ${ }^{1}$ Neurology, Geisel School of Medicine at Dartmouth, Lebanon, NH and ${ }^{2}$ Dept of Neurosciences \& Mental Health, UCL Institute of Child Health, London, United Kingdom)

Rationale: In the Morris water-maze (MWM) task, rodents are trained to find a submerged platform in a pool of opaque water using the configuration of external cues present in the room. This task is widely used to assess spatial memory performance as a surrogate for episodic memory capabilities. The performance of rat models of temporal lobe epilepsy (TLE) is dramatically impaired in the MWM task, a finding that parallels the memory impairments observed in patients with TLE. Here, we developed a virtual reality analogue to the MWM task and asked whether TLE patients were impaired in the task, as observed in rodents. In parallel we tested whether intrahippocampal local field potentials could be recorded as patients performed the task.

Methods: The Human MWM task was developed using the "Source Engine" (Valve ${ }^{\mathrm{TM}}$ ). The virtual WM was presented on a laptop computer and a gamepad controller was provided. Eleven healthy volunteers and six patients with epilepsy participated in the study. The human WM environment consisted of a rectangular room ( $10240 \times 10240$ pixels, $\mathrm{h}=512$ pixels; $1 \mathrm{ft}=16$ pixels) with a cylindrical arena ( $2 \mathrm{r}=1024$ pixels, $\mathrm{h}=64$ pixels) at the center with opaque water $(\mathrm{h}=12$ pixels), surrounded by multiple visual cues. A platform $(64 \times 64$ pixels, $\mathrm{h}=12$ pixels) was submerged $\mathrm{r} / 3$ away from the edge. After habituation to the control scheme, subjects were dropped in the arena and asked to reach the platform as quickly as possible. Survival analysis was performed to assess performance. Electrophysiological recordings were made in the patients with epilepsy to assess oscillatory and single-unit activity during the task. 
Results: The Human MWM task was developed using the "Source Engine" (Valve ${ }^{\mathrm{TM}}$ ). This software is widely used to create custom video-game environments. The virtual WM was presented on a laptop computer and a gamepad controller was provided. Movements were restricted to forward/back and left/right panning. Eleven healthy volunteers and six patients with epilepsy participated in the study. The human WM environment consisted of a rectangular room ( $10240 \times 10240$ pixels, $\mathrm{h}=512$ pixels; $1 \mathrm{ft}=16$ pixels $)$ with a cylindrical arena ( $2 \mathrm{r}=1024$ pixels, $\mathrm{h}=64$ pixels) at the center with opaque water $(\mathrm{h}=12$ pixels), surrounded by multiple visual cues. A platform $(64 \mathrm{x} 64$ pixels, $\mathrm{h}=12$ pixels) was submerged $\mathrm{r} / 3$ away from the edge. After habituation to the control scheme, subjects were dropped in the arena and asked to reach the platform as quickly as possible. This process was repeated 4 times per session for 3 sessions with a minute of rest in between followed by a probe trial. Survival analysis was performed to assess performance. Electrophysiological recordings were made in the patients with epilepsy to assess oscillatory and single-unit activity during the task.

Conclusions: These preliminary results show a deficit in the MWM task in patients with epilepsy and present a striking parallel to previous rodent data. The task is easy to learn and performance improved within a few trials. The virtual MWM holds great promise as a translational research tool.

\subsection{6}

\section{EVIDENCE OF INCREASED NEUROINFLAMMATION IN HUMAN TUBEROUS SCLEROSIS COMPLEX POTENTIAL IMPLICATIONS FOR NEUROLOGICAL DYSFUNCTION} Pelin Dilsiz ${ }^{1}$, V. Ruppe ${ }^{1}$, H. Weiner ${ }^{2}$, C. Shoshkes Reiss ${ }^{3}$, S. Najjar ${ }^{1}$, J. French ${ }^{1}$, O. Devinsky ${ }^{1}$ and D. M. Talos ${ }^{1}\left({ }^{1}\right.$ Neurology, New York University School of Medicine, New York, NY; ${ }^{2}$ Pediatric Neurosurgery, New York University School of Medicine, New York, $\mathrm{NY}$ and ${ }^{3}$ Biology, Microbiology and Neural Science, New York University, New York, NY)

Rationale: Tuberous Sclerosis Complex (TSC) is a multisystem autosomal dominant disease caused by inactivating mutations in TSC 1 or TSC 2 genes. The most common neurological symptoms in TSC include treatment-resistant epilepsy, mental retardation and autism. There is now accumulating evidence that pro-inflammatory cytokines, produced and released mainly by reactive astrocytes and activated microglia, are implicated in a wide range of chronic neurological disorders, including refractory epilepsy. The purpose of this study was to evaluate the degree of inflammation in TSC brain lesions in human, with the hypothesis that activated glial cells and pro-inflammatory cytokines will be increased in both cortical tubers and peri-tuberal brain (PTB), possibly contributing to widespread network dysfunction.

Methods: Human TSC specimens ( $\mathrm{n}=10$; ages 0-7 years) were prospectively collected following epilepsy surgery at NYULMC Region-matched control autopsy brain samples from cases with normal neurological history were obtained from the Maryland Brain and Tissue Bank ( $\mathrm{n}=7$; ages 0-8 years). Tissue samples were collected and handled in accordance with the NYULMC Institutional Review Board. Fresh frozen specimens were used to prepare protein extracts for Western blotting. Blots were probed with the astrocytic and microglial markers GFAP and Iba1, as well as with the proinflammatory cytokines interleukin $1 \beta$ (IL-1 $\beta$ ) and tumor necrosis factor $\alpha(T N F-\alpha)$. To determine statistical significance $(p<0.05)$, oneway ANOVA followed by Student's t-test were used.

Results: GFAP and Iba1 levels were significantly elevated in both tubers $(343 \pm 115 \%$ of control, $n=5 ; p<0.05$ for GFAP, and $346 \pm 35 \%$ of control, $\mathrm{n}=5 ; \mathrm{p}<0.0001$ for Iba1) and PTB (225 $\pm 37 \%$ of control, $\mathrm{n}=5$; $\mathrm{p}<0.001$ for GFAP, and $247 \pm 37 \%$ of control, $\mathrm{n}=5$; $\mathrm{p}<0.001$ for Iba1). The inactive pro-IL-1 $\beta$ was highly upregulated in tubers $(319 \pm 114 \%$ of control, $n=6 ; p<0.05)$ and in PTB $(228 \pm 70 \%$ of control, $\mathrm{n}=4 ; \mathrm{p}<0.001$ ), and this was accompanied by a commensurate increase in the secreted, active form of IL-1 $\beta$
(192 $\pm 28 \%$ of control in tubers, $n=6 ; p<0.05$, and $159 \pm 17 \%$ of control in PTB, $n=4 ; p<0.05$ ). Pro-TNF- $\alpha$ expression was similarly upregulated in both tubers and PTB $(289 \pm 34 \%$ of control, $n=6$, and $204 \pm 4 \%$ of control $n=4$, respectively; $p<0.05$ ), but the levels of secreted TNF- $\alpha$ were only moderately increased $(149 \pm 24 \%$ of control in tubers, $n=6$, and $117 \pm 4 \%$ of control in PTB, $n=4 ; p>0.05$ ). Conclusions: Our data demonstrate robust increases in proinflammatory markers in TSC lesions, which extend beyond the cortical tuber borders, suggesting a potential pathogenic role of neuroinflammation in epileptogenesis, cognitive dysfunction and altered social behavior in TSC patients. Therapies that suppress glial activation, or directly block cytokine signaling, might be useful to ameliorate the complex neurological deficits in these patients. Acknowledgments: Shaw Foundation, FACES, Tuberous Sclerosis Alliance, NICHD Brain and Tissue Bank for Developmental Disorders at the University of Maryland, Baltimore, MD, contract HHSN275200900011C.

\subsection{7 \\ PATTERNS OF PI3K/AKT/MTOR PATHWAY ACTIVATION DIFFERENTIATE GENETICALLY DISTINCT FORMS OF HEMIMEGALENCEPHALY}

Laura A. Jansen $^{1,2}$, J. B. Rivière ${ }^{1}$, W. H. Roden ${ }^{1}$, J. St-Onge ${ }^{1}$, G. E. Ishak $^{4}$, R. F. Hevner ${ }^{1,3}$, J. G. Ojemann ${ }^{1,3}$ and W. B. Dobyns ${ }^{1,5}$ ( ${ }^{1}$ Center for Integrative Brain Research, Seattle Children's Research Institute, Seattle, WA; ${ }^{2}$ Pediatric Neurology, University of Washington, Seattle, WA; ${ }^{3}$ Neurological Surgery, University of Washington, Seattle, WA; ${ }^{4}$ Radiology, University of Washington, Seattle, WA and ${ }^{5}$ Genetics, University of Washington, Seattle, WA)

Rationale: Hemimegalencephaly (HMEG) is a highly epileptogenic developmental brain malformation associated with enlargement of an entire cerebral hemisphere. Pathologically, cytomegalic and dysmorphic neurons, astrocytosis, dyslamination, and polymicrogyria are seen. Previous studies have suggested abnormalities in the phosphatidylinositol-3-kinase (PI3K)/AKT/mTOR pathway in HMEG. In the present study we performed detailed immunohistochemical and genetic analyses of human HMEG tissue to further delineate involvement of this pathway.

Methods: Five children with HMEG were evaluated by standard protocols and underwent resective surgery for intractable epilepsy. Double-label fluorescence immunohistochemistry studies were performed using formalin-fixed, paraffin-embedded sections $(4 \mu \mathrm{M})$ of resected cortex. Antibodies directed against the phosphorylated forms of PDK1, AKT, tuberin, and ribosomal protein S6 were analyzed in conjunction with neuronal (MAP2) and glial (GFAP) cellular markers. DNA was isolated from specimens frozen at the time of surgery and subjected to sequence analysis using methods sensitive for detection of genetic mosaicism.

Results: All specimens demonstrated increased phospho-S6 immunofluorescence in a subset of neurons, which ranged from less than $10 \%$ to nearly $100 \%$ of the total neuronal population. Varying degrees of phospho-S6 immunofluorescence were also identified in glial cells in all specimens. However, neuronal labeling with antibodies against phosphorylated proteins upstream of S6 in the $\mathrm{PI} 3 \mathrm{~K} / \mathrm{AKT} / \mathrm{mTOR}$ pathway was present in some patients but not others, suggesting the presence of abnormal activity at different levels of the pathway. Indeed, activating mosaic mutations in two different pathway components, the catalytic subunit of PI3K and of AKT3, were identified in patients with different immunohistochemical patterns.

Conclusions: Our studies indicate that HMEG is a heterogenous condition that may be caused by mosaic activating mutations of different members of the PI3K/AKT/mTOR pathway. Different genetic forms of this malformation may also be identified by patterns of immunohistochemical labeling. These findings suggest that therapies targeting abnormal activity at different levels of the $\mathrm{PI} 3 \mathrm{~K} / \mathrm{AKT} / \mathrm{mTOR}$ pathway could be beneficial for children with intractable epilepsy due to HMEG. 


\subsection{8}

IDENTIFICATION OF MIRNAS DIFFERENTIALLY EXPRESSED IN TEMPORAL LOBE EPILEPSY PATIENTS WITH AND WITHOUT DENTATE GRANULE CELL DISPERSION

Michele Simonato $^{1,2}$, S. Zucchini ${ }^{1,3}$, B. Paradiso ${ }^{1,4}$, P. Cifelli ${ }^{1,5}$, M. Ferracin $^{3}$, M. Giulioni ${ }^{6}$, G. Marucci ${ }^{7}$, R. Michelucci ${ }^{8}$ and G. Rubboli ${ }^{8}$ ( ${ }^{1}$ Dept. Clin. Exp. Med. (Pharmacology), University of Ferrara, Ferrara, Italy; ${ }^{2}$ Natl. Inst. Neuroscience, Italy, Ferrara, Italy; ${ }^{3}$ Laboratory for Technologies of Advanced Therapies (LTTA), University of Ferrara, Ferrara, Italy; ${ }^{4}$ Dept. Exp. Diagn. Med. (Pathology), University of Ferrara, Ferrara, Italy; ${ }^{5}$ RiMED Foundation, Palermo, Italy; ${ }^{6}$ IRCCS Institute of Neurological Sciences (Neurosurgery), Bellaria Hospital, Bologna, Italy; ${ }^{7}$ Sect. Pathology, Bellaria Hospital, University of Bologna, Bologna, Italy and ${ }^{8}$ IRCCS Institute of Neurological Sciences (Neurology), Bellaria Hospital, Bologna, Italy)

Rationale: The microRNAs (miRNAs) are small size endogenous non-coding RNAs that regulate the expression of target mRNAs at post-transcriptional level. To date, more than 1000 human miRNAs have been identified. miRNAs have been demonstrated to be involved in many cell functions implicated in epilepsy and epileptogenesis, including cell death, neurogenesis, synaptic plasticity. Thus, understanding which specific miRNAs are differentially expressed in epilepsy may help to identify the mechanisms underlying the disease. Moreover, these miRNAs may represent biomarkers that identify specific subpopulations of epileptic patients, holding a prognostic value.

Methods: In the present study, we examined the expression of more than 1,000 human miRNAs in the dentate gyrus granular layer. The granule cell layer of 10 patients who underwent surgery for intractable temporal lobe epilepsy was laser-microdissected from hippocampal paraffin-embedded sections. Total RNA was extracted from tissues and the miRNAome profile was obtained using an Agilent miRNA microarray. The study was performed in accordance with the recommendations of the international guidelines for human research and approved by the local Ethical Committee.

Results: All patients had type 1a-1b mesial temporal sclerosis (Blümcke et al., Acta Neuropathol.,2007, 113:235-244), which was associated with no granule cell pathology (no GCP) in 5 patients and with granule cell dispersion (GCP type 2) in the other 5 (Blümcke et al., Acta Neuropathol., 2009, 117:535-544). The two groups of patients were similar for age, gender, clinical features of the disease. We identified 12 miRNAs that were differentially expressed in the two subgroups. Five of these are currently verified in an extended cohort of patients using RT-qPCR.

Conclusions: This miRNA signature may be useful for a prognostic evaluation and forms the basis for further mechanistic studies that may lead to the identification of new therapeutic targets.

\subsection{9}

EPILEPSY AND THE IMMUNE SYSTEM "... IS THERE ANTIBODY THERE?..."

Sukhvir K. Wright ${ }^{1}$, C. M. . Jol-van der Zijde ${ }^{2}$, M. J. D van Tol ${ }^{2}$, P. Waters $^{1}$, B. Lang ${ }^{1}$, O. F. Brouwer ${ }^{3}$ and A. Vincent ${ }^{1}\left({ }^{1}\right.$ Nuffield Department of Clinical Neurosciences, University of Oxford, Oxford, United Kingdom; ${ }^{2}$ Department of Paediatrics, Leiden University Medical Centre, Leiden, Netherlands and ${ }^{3}$ Department of Neurology, University Medical Centre Groningen, University of Groningen, Groningen, Netherlands)

Rationale: Immune mechanisms are thought to be involved in the pathological disease process in a significant number of childhood and adult epileptic syndromes, and in other seizure related disorders (e.g. encephalitis). Of particular interest is the occurrence of highly specific autoantibodies to important neuronal proteins (VGKCcomplex proteins, NMDAR, AMPAR, GABAbRs) in the blood and spinal fluid in some of these patients. The aim of this study is to examine the sera of newly diagnosed paediatric epilepsy patients for the presence of specific neuronal autoantibodies.

Methods: This Dutch cohort consists of 178 paediatric patients aged between 1 month and 16 years with newly diagnosed epilepsy. The sera are being tested for antibodies to the NMDA receptor, VGKCcomplex and associated proteins (LGI1, CASPR2, contactin-2), GAD and glycine receptor. The presence of antibodies are detected visually using antigen specific cell-based assays (NMDAR, LGI1, CASPR2, contactin-2, glycine receptor), and the levels of antibodies against the VGKC-complex and GAD are quantitated by

radioimmunoprecipitation assays.

Results: In total, $25 / 178$ patients (14\%) have been found to have a positive antibody test so far. The most commonly found antibody is to the VGKC-complex.

Conclusions: Preliminary testing of a newly diagnosed paediatric epilepsy cohort has revealed a significant number of patients with potentially pathogenic neuronal autoantibodies in the serum. Further work will include completion of testing for antibodies to the CNS antigens described, a coded comparison with age-matched controls, a search for novel antigenic targets and the correlation of patient clinical features with the presence of a positive antibody.

\subsection{0 \\ LINEAR PAIRWISE GRANGER CAUSALITY IDENTIFIES ICTAL PROPAGATION IN PATIENTS WITH PARTIAL EPILEPSY}

Edgard Andrade and Z. Liu (Pediatrics, University of Florida College of Medicine, Gainesville, FL)

Rationale: Extensive compelling evidence has demonstrated the presence of a cortical network for the genesis and propagation of partial seizures. Thus, we evaluated the ictal findings in patients with partial epilepsy (PE) and its correlates with a structurally connected network and possible ipsilateral or contra-lateral propagation using Pairwise Granger Causality (PGC) analysis of raw data EEG recordings in a retrospective case-series study.

Methods: First, we studied ictal onset (IO) of fifty-six partial complex seizures (PCS) . Then, we defined seizure directionality using PGC of EEG raw data. Last, we compared the findings against the contra-lateral, non-involved, control, frontal and temporal networks defined by visual analysis of EEG epochs and obtained 113 different PGC data points.

Results: PGC was statistically significant, using one way analysis of variance (ANOVA), for intra-hemispheric directionality when the IO was over the right temporal lobe $(\mathrm{p}=0.0257)$. PGC showed a trend for a significant intra-hemispheric directionality when the IO was over the left temporal $(p=0.0548)$ and right frontal lobe $(p=0.0558)$. Conclusions: These preliminary findings indicate possible intrahemispheric ictal propagation for subjects with PE of frontal or temporal ictal onset. It is unclear whether inter-hemispheric ictal propagation is not identifiable with PGC on human scalp EEG recordings. Further studies are indicated to evaluate alternatives methodologies in larger cohorts of individual affected with epilepsy.

\author{
3.051 \\ DIFFERENTIAL RECORDING OF HIGH FREQUENCY \\ OSCILLATIONS BY ADJACENT MICRO AND MACRO \\ CONTACTS IN THE HUMAN HIPPOCAMPUS \\ Behnaz Esmaeili ${ }^{1}$, V. J. DeStefino ${ }^{1}$, V. K. Raghu ${ }^{1}$, W. Wang ${ }^{3}$, A. \\ Popescu $^{2}$, G. R. Ghearing ${ }^{2}$, A. Bagic ${ }^{2}$ and R. M. Richardson ${ }^{1}$ \\ $\left({ }^{1}\right.$ Neurological Surgery, University of Pittsburgh, Pittsburgh, PA; \\ ${ }^{2}$ Neurology, University of Pittsburgh Medical Center, Pittsburgh, PA \\ and ${ }^{3}$ Physical Medicine and Rehabilitation, University of Pittsburgh, \\ Pittsburgh, PA)
}


Rationale: In the hippocampus, high frequency oscillations (HFOs) of $80-200 \mathrm{~Hz}$ play an important role in memory consolidation, whereas oscillations with higher spectral frequencies in the 200-500 $\mathrm{Hz}$ range may be biomarkers of epileptogenicity (pathological HFOs). It is not yet clear, however, whether standard macrocontacts or embedded microwires have a similar ability to detect HFOs in different distinct frequency bands.

Methods: We addressed this question by examining wideband intrahippocampal ECoG recordings from readily available clinical depth electrodes containing embedded micro wires interspersed between macrocontacts, implanted in patients undergoing chronic intracranial monitoring for seizure mapping. HFOs were identified using a wavelet time-frequency decomposition-based automatic detection algorithm and subsequent visual confirmation.

Results: As expected, the total number of the oscillations recorded by either contact type was enhanced in seizure onset-related brain areas compared to other areas. The number of HFOs and pHFOs recorded by any single microcontact was greater than those recorded by adjacent macrocontacts. pHFOs were detected by microcontacts at a significantly higher rate than by macrocontacts. The difference between the duration of HFOs and pHFOs recorded by either of the contact types was not significant.

Conclusions: These data demonstrate that microcontacts are more effective than macrocontacts for detecting HFOs and especially pHFOs, suggesting that choice of depth-electrode type may determine the resolution with which pHFOs can be characterized and utilized as electrophysiological biomarkers. These data also inform hypotheses regarding the spatial extent of the neural substrate generating pHFOs.

\subsection{2}

\section{ALTERED NMDA AND AMPA RECEPTOR SUBUNIT EXPRESSION IN CORTICAL TISSUE FROM AUTISM SPECTRUM DISORDERS AND TREATMENT-RESISTANT TEMPORAL LOBE EPILEPSY}

Alejandro Salah ${ }^{1,4}$, P. Dilsiz, ${ }^{2,4}$, L. Frecska-Horvath ${ }^{2,4}$, V. Ruppe ${ }^{2,4}$, C. Shoshkes Reiss ${ }^{5}$, C. Carlson ${ }^{2,4}$, W. Doyle ${ }^{3,4}$, O. Devinsky ${ }^{2,4}$ and D. M. Talos ${ }^{2,4}\left({ }^{1}\right.$ Physiology and Neuroscience, NYU School of Medicine, New York, NY; ${ }^{2}$ Neurology, NYU School of Medicine, New York, NY; ${ }^{3}$ Neurosurgery, NYU School of Medicine, New York, NY; ${ }^{4}$ NYU Neuroscience Institute, NYU School of Medicine, New York, NY and ${ }^{5}$ Biology, Microbiology and Neural Sciences, New York University, New York, NY)

Rationale: Autism spectrum disorders (ASDs), characterized by impaired social interactions, impaired communication and stereotyped behaviors, are highly associated with epilepsy (up to $38 \%$ ). A shift in cortical excitatory/inhibitory balance towards increased excitation and/or decreased inhibition may play a key role in the pathophysiology of both ASDs and epilepsy. We hypothesized that differences in AMPA receptor (AMPAR) and NMDA receptor (NMDAR) subunit expression in ASD and epilepsy patients may represent a basis for distinct clinical neurological manifestations. Identifying specific synaptic mechanisms for autism and epilepsy will allow development of targeted therapies.

Methods: Brain specimens from treatment-resistant temporal lobe epilepsy cases were collected prospectively during resective surgery at NYULMC ( $\mathrm{n}=5$; ages 21-37 years). Autopsy temporal and frontal lobe samples from ASD patients ( $n=5$; ages 4-22 years) and regionmatched control specimens from cases with normal neurological history ( $\mathrm{n}=10$; ages 5-48 years) were obtained from Maryland Brain and Tissue Bank. All ASD cases met the standard diagnostic criteria (Autism Diagnostic Interview-Revised) and had no evidence of epilepsy, while none of the epilepsy patients were diagnosed with autism. The levels of the NMDAR and AMPAR subunits (NR1, NR2A, NR2B, GluR1 and GluR2) were quantified by Western blot and compared among groups (one-way ANOVA and t-tests).

Results: Temporal lobe cortex analysis demonstrated a significant increase in NR1 expression in autism patients (288\% of control; $\mathrm{p}<0.0001)$, but no significant change in epilepsy cases $(96 \%$ of control; $\mathrm{p}>0.05$ ). In both groups, NR2A was significantly decreased (ca. $40 \%$ of control; $\mathrm{p}<0.0001$ ), while NR2B demonstrated a significant upregulation $(255 \%$ of control in autism and $511 \%$ of control in epilepsy; $p<0.0001)$. NR2B levels were significantly higher in epilepsy, relative to autism patients $(p<0.001)$. GluR1 expression was increased in both autism $(388 \%$ of control; $p<0.0001)$ and epilepsy specimens ( $514 \%$ of control; $p<0.0001)$, in contrast to GluR2 which was significantly reduced (40\% of control in autism and $32 \%$ of control in epilepsy; $p<0.0001$ ), consistent with the presence of $\mathrm{Ca} 2+-$ permeable AMPARs. There were no significant differences $(\mathrm{p}>0.05)$ in NMDAR and AMPAR subunits between temporal and frontal lobe specimens from autism cases.

Conclusions: Our results suggest increased NMDAR function in ASD patients (increased NR1) and provide a basis for novel therapeutic strategies targeting these receptors. Enhanced intracellular $\mathrm{Ca} 2+$ signaling due to altered NMDAR and AMPAR subunit composition (increased NR2B and decreased GluR2) may impair synaptic function and operate as a feed-forward loop for both autism and epilepsy. Therefore, drugs specifically targeting Ca2+permeable AMPARs or NR2B- containing NMDARs may be beneficial for both ASD and epilepsy patients.

Acknowledgments: FACES, NICHD Brain and Tissue Bank for Developmental Disorders at the University of Maryland, Baltimore, MD, contract HHSN275200900011C.

\subsection{3 \\ MOLECULAR PHENOTYPE IN THE MALFORMATIONS OF CORTICAL DEVELOPMENT ASSOCIATED WITH PEDIATRIC EPILEPSY}

Anne E. Anderson ${ }^{1,2}$, V. V. Patil ${ }^{1,2}$, S. Agadi ${ }^{1,2}$, D. Yoshor ${ }^{1,2}$, D. Curry $^{1,2}$, A. L. Brewster ${ }^{1,2}$, J. Riviello ${ }^{1,2}$, M. Quach ${ }^{1,2}$, A. Malphrus ${ }^{1,2}$, J. Owens ${ }^{1,2}$, L. Masters ${ }^{1,2}$, J. Hunter ${ }^{1,2}$, M. Chapieski ${ }^{1,2}$, J. Swann $^{1}{ }^{1}$ and A. Wilfong ${ }^{1,2}$ ( ${ }^{1}$ Baylor College of Medicine, Houston, TX and ${ }^{2}$ Texas Children's Hospital, Houston, TX)

Rationale: Malformations of cortical development such as cortical dysplasia (CD) and tuberous sclerosis (TSC) are commonly associated with drug resistant epilepsy. There are two major types of isolated cortical dysplasia - cortical dysplasia type I (CD type I) and cortical dysplasia II (CD type II). Both are characterized by cortical dyslamination with ectopic and dysplastic neurons, while only CD type II is associated with cytomegalic neurons. Electrophysiological studies in resected tissue have shown that cytomegalic neurons found in dysplastic tissue are hyperexcitable. Molecular studies performed to identify the cause of dysplasia and hyperexcitability have shown activation of mammalian target of rapamycin (mTOR) pathway. The cause of mTOR pathway hyperactivity in $\mathrm{CD}$ is unknown. One potential explanation for hyperactivation of the mTOR pathway is phosphorylation of the upstream mTOR regulator, TSC2 at the S664 site by extracellular regulated kinase (ERK). In the studies presented we sought to characterize ERK and mTOR pathway activation and interaction in malformations of cortical development such as CD and TSC.

Methods: Human brain tissue sections obtained after resection from epilepsy surgery were stained with antibodies against phosphorylated ERK (pERK), TSC2 (ERK phosphorylation site S664; pTSC2), S6K1 (phosphorylation site T389; pS6K1), and S6 (phosphorylation site S240/244; pS6). Tissue staining with these antibodies was correlated with the neuropathological and clinical history.

Results: IHC analysis of brain sections obtained from individuals with CD and TSC undergoing epilepsy surgery showed increased pS6K1 and pS6 labeling of dysplastic neurons and cytomegalic neurons. However, increased staining for pERK and pTSC2 was evident only in the cytomegalic neurons. Correlation studies confirmed previous reports of positive correlation between MRI lesion representative of CD type II and better surgical outcome with pERK staining. 
Conclusions: Our findings suggest that the mTOR pathway is aberrantly activated in CD type I and II and TSC, while the ERK pathway is activated only in CD type II and TSC. These findings provide a molecular as well as the previously described anatomical distinction for $\mathrm{CD}$. Our findings reveal ERK and mTOR pathway cross-talk at the level of TSC2 in cytomegalic neurons in epilepsy, thereby identifying providing a potential mechanism for mTOR upregulation in CD type II.

\subsection{4 \\ IMPAIRED EXPRESSION OF ANTIQUITIN (ALDH7A1) IN RADIAL GLIA AND CORTICAL ASTROCYTES IS ASSOCIATED WITH NEURONAL MIGRATION DEFECTS IN PYRIDOXINE-DEPENDENT EPILEPSY} S. M. Gospe ${ }^{1,2}$, R. F. Hevner ${ }^{2,3}$, W. H. Roden ${ }^{2}$ and Laura A. Jansen ${ }^{1,2}$ ( ${ }^{1}$ Pediatric Neurology, University of Washington, Seattle, WA; ${ }^{2}$ Center for Integrative Brain Research, Seattle Children's Research Institute, Seattle, WA and ${ }^{3}$ Neurological Surgery, University of Washington, Seattle, WA)

Rationale: Structural brain abnormalities, including cortical dysplasia and neuronal heterotopias, have been reported in individuals with pyridoxine-dependent epilepsy (PDE). PDE is caused by mutations in aldehyde dehydrogenase 7A1 (ALDH7A1), also known as antiquitin. How antiquitin dysfunction leads to neuronal migration defects is unknown. In this study, we analyzed control developing human and murine brain, as well as tissue from a child with PDE, to determine the normal distribution of antiquitin expression, its distribution in PDE, and associated neuronal migration abnormalities.

Methods: Formalin-fixed human control and PDE brain sections were subjected to routine histopathology as well as fluorescence immunohistochemistry studies. Brain tissue frozen at autopsy was utilized for Western blot analysis. Comparative studies of antiquitin distribution were performed in mouse brain sections obtained at E14.5, P0, and P20.

Results: Histologic analysis of PDE cortex revealed areas of abnormal tangential neuronal organization with microcolumns suggestive of type Ia focal cortical dysplasia. Neuronal heterotopias were identified in subcortical white matter, as well as cortical astrogliosis, hippocampal sclerosis with severe neuronal loss, and patchy gliosis of the basal ganglia. Interneuron density and distribution in PDE cortex was not altered, although total GAD-67 expression was reduced. In control human and mouse cortex, cytoplasmic antiquitin immunofluorescence was identified in astrocytes, ependyma, and choroid plexus, but not in neurons. In PDE cortex, antiquitin immunofluorescence was greatly attenuated, with perinuclear accumulation in astrocytes. Co-expression of antiquitin with the radial glial marker Sox2, but not the immature neuronal marker DCX, was identified in the developing mouse brain. Conclusions: Antiquitin (ALDH7A1) is expressed in glial cells in the brain, and its dysfunction in PDE is associated with neuronal migration defects. These structural brain abnormalities persist despite postnatal pyridoxine supplementation and may contribute to seizures and neurodevelopmental impairments in this disorder.

\subsection{5 \\ CLUSTERING SEIZURES WITHIN AND BETWEEN PATIENTS USING HIERARCHICAL BAYESIAN MODELS Drausin Wulsin ${ }^{1}$, E. Marsh ${ }^{2}$, B. Porter ${ }^{2}$ and B. Litt ${ }^{3,1}$ \\ ( ${ }^{1}$ Bioengineering, University of Pennsylvania, Philadelphia, PA; ${ }^{2}$ Neurology, Children's Hospital of Philadelphia, Philadelphia, PA and ${ }^{3}$ Neurology, University of Pennsylvania, Philadelphia, PA)}

Rationale: Current clinical practice involves clinicians examining the dynamics of seizures to ascertain important clinical factors like how similar (or dissimilar) an individual patient's seizures are to each other. Statistical models can offer decision support for clinical questions like "what are the types of seizures that a patient has" and "which other patients is this patient similar to." A challenge of the data is that every seizure of every patient is unique, though there are similarities between seizures and patients. Currently, most approaches create models in space of a single seizure. Comparing seizures between patients is challenging because the number and placement of the iEEG channels is unique for every patient. One approach to this problem is to use features of the data that generalize across an arbitrary number of channels. While this approach is attractive because it is so straightforward, we believe that it is likely to miss the important dynamics that can occur in just a few channels out of a hundred. In many cases, it is just a few channels that are of most clinical interest to physicians.

Methods: We use a recently introduced model, the multi-level clustering hierarchical Dirichlet process (MLC-HDP), to build models of individual seizures that make use of information from other seizures of that patient and the seizures of other patients. 193 seizures across 10 different patients from the Children's Hospital of Philadelphia were analyzed. On a simple level, this model can be thought of as performing clustering analysis on individual channelactivities within a seizure, on types of seizures a particular patient manifests, and on different types of patients. The seizure clusterings yielded by this model were compared to manual clusterings performed by two trained epileptologist and also to clusterings by a simpler automated method.

Results: The MLC-HDP clusterings were indeed fairly close to those of the human. In some patients, the model's clustering was more similar to the first doctor than the second doctor's. This multi-level clustering analysis yields insights on many levels of seizures: what channels tend to behave similarly within a seizure, how similar or different are a patient's seizures to each other, and what patients display similar patterns of seizures to other patients. All of these results stem from models requiring minimal assumptions, e.g. how many clusters to use, contrary to conventional clustering techniques. Conclusions: This work introduces the use of sophisticated nonparametric Bayesian models in modeling and understanding large datasets of seizures across a number of different patients. We believe that this work is an important first step in building analysis paradigms that leverage large amount of datas from many patients. Such models have more promise in providing useful clinical decision support than myopic models based on individual seizures or patients.

\section{Translational Research: Devices, Technologies, Stem Cells}

\subsection{6 \\ EPIDURAL FOCAL BRAIN COOLING SUPPRESSES NEOCORTICAL SEIZURES IN CATS AND NON-HUMAN PRIMATES}

Takao Inoue $^{1}$, M. Fujii ${ }^{1}$, H. Kida ${ }^{2}$, T. Yamakawa ${ }^{5}$, T. Tokiwa ${ }^{4}$, Y.

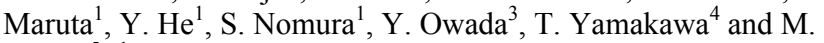
Suzuki ${ }^{5}$ ( ${ }^{1}$ Department of Neurosurgery, Yamaguchi University School of Medicine, Ube, Japan; ${ }^{2}$ Department of Systems Neuroscience, Yamaguchi University School of Medicine, Ube, Japan; ${ }^{3}$ Department of Organ Anatomy, Yamaguchi University School of Medicine, Ube, Japan; ${ }^{4}$ Department of Brain Science and Engineering, Kyushu Institute of Technology, Kitakyushu, Japan and ${ }^{5}$ Department of Electrical and Electronics Engineering, Shizuoka University, Hamamatsu, Japan)

Rationale: Focal brain cooling (FBC) is well established as an effective method for suppressing epileptiform discharges and is under investigation in preclinical of intractable epilepsy (IE) including status epileptics (SE). This method has been studied intensively in rodents, but more evidence from large animal and human studies is required prior to performance of clinical trials in drug-resistant epilepsy. To provide evidence that focal brain cooling is a safe and effective therapeutic intervention for intractable focal epilepsy, we 
investigated the placement of a titanium cooling plate over the epidural cortical surface and investigated whether focal brain cooling can prevent and/or terminate focal neocortical seizures without having a significant impact on brain functions.

Methods: In this study, two cats and two macaque monkeys were chronically implanted with an epidural focal brain cooling device over the somatosensory and motor cortex, with adjacent Electrocorticography (ECoG) electrodes, a thermo sensor and a micro-injection tube. Seizures were induced by injection of penicillin $\mathrm{G}$ in the motor cortex. Penicillin $\mathrm{G}$ was diluted to $250 \mathrm{IU} / \mu \mathrm{l}$, and 2000 IU was carefully applied to the brain surface using an injection cannula. Recordings were performed under the awake condition. After the end of the experiments, the animals were sacrificed for examination of the localization of the implanted subdural device. Results: In awake condition, no apparent changes in ECoG were caused by cooling of the cortex when the temperature of the cortical surface decreased to $15^{\circ} \mathrm{C}$. Epileptiform discharges were also significantly suppressed at $15^{\circ} \mathrm{C}$. Power spectra of low beta frequency bands, which include epileptiform discharges, were compared during the pre-cooling, cooling, and rewarming periods. A posthoc Tukey test was significant (in cat: pre-cooling vs. cooling, P $<0.01$, pre-cooling vs. rewarming, $\mathrm{P}>0.1$, cooling vs. rewarming, $\mathrm{P}$ $<0.01$; in monkey, pre-cooling vs. cooling, $\mathrm{P}<0.05$, pre-cooling vs. rewarming, $\mathrm{P}>0.1$, cooling vs. rewarming, $\mathrm{P}<0.05$ ). Implantation of the device for at least five months did not result in detrimental changes based on HE staining, compared with the ipsilateral hemisphere.

Conclusions: The results of this study suggest that focal brain cooling has a strong effect to suppress the epileptiform seizures under the awake condition. FBC has a safe and effective potential treatment modality for IE including SE that might be suitable for clinical practice in the near future.

\subsection{7}

\section{VALIDATION AND OPTIMIZATION OF DEVELOPING MICROELECTRODE ARRAY BY AN ACUTE EX-VIVO BRAIN NEURAL RECORDING SYSTEM}

Chien-Chun Huang ${ }^{1,2}$, Y. L. Hsin ${ }^{3}$, M. F. Chen ${ }^{4}$, C. W. Chang ${ }^{2}$, J. C. Chiou $^{2,5}$ and T. Harnod ${ }^{6}\left({ }^{1}\right.$ Research Center for Adaptive Data Analysis, National Central University, Jhongli, Taiwan; ${ }^{2}$ Biomedical Electronics Translational Research Center, National Chiao Tung University, Hsinchu, Taiwan; ${ }^{3}$ Neurology, Buddhist Tzu-Chi General Hospital, Hualien, Taiwan; ${ }^{4}$ Department of Medical Research, Buddhist Tzu-Chi General Hospital, Haulien, Taiwan; ${ }^{5}$ China Medical University, Taichung, Taiwan and ${ }^{6}$ Neurosurgery, Buddhist Tzu-Chi General Hospital, Hualien, Taiwan)

Rationale: Technological progress in intracranial electroencephalography has not only extended the research submission from macroscale depiction of regional brain functions to microscale analysis of neural activity, but also extended clinical application from epilepsy surgery to neural prostheses. The sticking point is the availability of sub-millimeter microelectrode for neural recording. Although validation and optimization of the different material and structure microelectrodes are basically prior to listing, it is difficult to have in vivo tests on human brain repeatedly.

Methods: Our first-generation microelectrode array is consisted of 4 silicon shafts and 4 platinum recording sites on each shaft. We maintain the neural activity of mice brain or resected human brain block in constant temperature, oxygenated artificial cerebrospinal fluid. Mice brain was obtained after induction of convulsive seizure by intraperitoneal injection of pentylenetetrazol. Human brain tissue was obtained from patient underwent epilepsy surgery. The microelectrode was inserted into the brain tissue via a controlled compact linear actuator. Responsive pressure curve of microelectrode during penetrating pia mater was recorded for adjusting the optimal insertion speed to reduce tissue damage. Impedance of each recording sites was recorded before and after insertion process. After that, brain tissue was stained to verify the studied areas.
Results: Clear near field potentials and spikes could be recorded at various recording sites. By the obtained information, we redesign following generation microelectrode array to achieve improvement of electrode attaching stability and higher signal to noise ratio.

Conclusions: Here, we report the development of an acute ex vivo human brain neural recording system and demonstrate the preliminary neural recordings of mice and resected human brain tissue via the microelectrode array. Our work represents one translation template for research uses and testing feasibility of developing biomedical devices before in vivo human brain study. The acute ex vivo brain neural recording system enable emerging implantable microelectrode array capable of addressing important issues in analysis of epileptic neural signals.

Supported: NSC 101-2220-E-303-001-Tzu-Chi Hospital Matching Grants 1, NSC 101-2220-E-303-001, NSC support for the Center for Dynamical Biomarkers and Translational Medicine, National Central University, Taiwan (NSC 100-2911-I-008-001) and Aim for the Top University Plan of the National Chiao Tung University and Ministry of Education, Taiwan.

\subsection{8 \\ SIDE EFFECTS OF VAGUS NERVE STIMULATION DURING PHYSICAL EXERCISE}

Daphne M. Mulders ${ }^{1}$, C. C. de Vos ${ }^{1,2}$, I. Vosman $^{3}$, M. J. Driesse ${ }^{4}$ and M. J. van Putten ${ }^{1,2}\left({ }^{1}\right.$ MIRA, Institute for Biomedical Technology and Technical Medicine, University of Twente, Enschede, Netherlands; ${ }^{2}$ Department of clinical neurophysiology, Medisch Spectrum Twente, Enschede, Netherlands; ${ }^{3}$ SEIN Epilepsie Instellingen, Zwolle, Netherlands and ${ }^{4}$ Department of neurosurgery, Medisch Spectrum Twente, Enschede, Netherlands)

Rationale: Vagus nerve stimulation (VNS) is a treatment option in the case of refractory epilepsy. However, several side effects have been reported, including dyspnea, coughing and bradycardias [JCA 2010: 22;213-222]. Although some patients experience hardly any side effects from the stimulation during rest, they mention a decrease in physical condition during exercise is.

The experience of a decrease in physical condition during exercise is reported as shortness of breath when the stimulator is on. It is unclear whether this actually has a laryngeal or respiratory cause, or whether there are cardiac or combined causes as well. The aim of this study is to explore the contribution of these various potential causes to the exercise intolerance reported by several patients treated with a VNS device.

Methods: In a case controlled observational study, 5 epilepsy patients who report side effects during exercise are compared to 5 patients without side effects and 5 healthy subjects. All subjects are measured during rest and during a non-maximal ergometry test. During these tests, which both take 20 minutes, respiratory parameters (tidal volume, breathing frequency and minute volume), ECG, 32-channel EEG, pulse oximetry and periodic blood pressure (BP) are measured. Epilepsy patients with VNS are asked to activate the device 3 times during the tests, to study the effects of the stimulation on the aforementioned parameters.

Results: At present, 1 patient with and 1 patient without side effects have been included, as well as 4 healthy subjects.

In healthy subjects, stable values for the parameters during rest are observed. During the first few minutes of exercise, heart rate and systolic BP increase, while diastolic BP decreases slightly. Thereafter, a steady state is reached. All values are as expected during exercise.

In the patients, the same effect of exercise as in healthy subjects occurs. The stimulation does not influence the BP, saturation and respiratory parameters. Heart rate analysis in both patients showed a small but significant increase in inter-beat intervals during stimulation, indicating a slight decrease in heart rate. This happened during both rest and exercise. 
Conclusions: Preliminary results show that during stimulation of the vagus nerve, small but reproducible slowing of heart rate occurs. No robust conclusion can yet be drawn about the effect of stimulation on respiratory parameters.

\subsection{9 \\ EVALUATION OF VOLTAGE CONTROLLED LOW FREQUENCY ELECTRICAL STIMULATION IN RAT MODELS OF MESIAL TEMPORAL LOBE EPILEPSY} Jeffrey H. Goodman ${ }^{1,2}$, N. Nathwani ${ }^{1}$, N. Hasulak ${ }^{3}$, A. Saghyan ${ }^{1}$, C. Wang ${ }^{1}$, M. Breeden ${ }^{3}$, K. Cicora ${ }^{3}$, T. Skarpaas ${ }^{3}$ and T. Tcheng ${ }^{3}$ ( ${ }^{1}$ Developmental Neurobiology, Institute For Basic Research, Staten Island, NY; ${ }^{2}$ Physiology and Pharmacology and Neurology, SUNY Downstate Medical Center, Brooklyn, NY and ${ }^{3}$ NeuroPace, Inc., Mountain View, CA)

Rationale: Prior research from our laboratory and others indicated that current controlled low frequency sine-wave stimulation reduced the likelihood of evoked seizures by $>50 \%$ and increased the afterdischarge threshold by $>200 \%$ in the kindling model of epilepsy. These initial data suggested that this type of stimulation may be effective for treating epilepsy. This stimulation has the following core features that distinguish it from stimulation strategies being tested clinically: 1) low frequency oscillation, 2) long pulse widths, 3) no interpulse delay, and 4) longer burst durations. In this study, we used a 12-cage, 24-hour EEG monitoring system to evaluate the efficacy of voltage controlled low frequency stimulation (VCLFS) on spontaneous electrographic seizures and interictal events in the rat tetanus toxin ( $\mathrm{Tx}$ ) and pilocarpine (Pilo) models of mesial temporal lobe epilepsy (MTLE). Electrographic seizures were identified using a new audio transformation method (see Breeden et al. poster at this meeting).

Methods: Bipolar, platinum-iridium electrodes were implanted bilaterally into the hippocampus of male, Sprague-Dawley rats. For Tx rats, the Tx was injected into the right ventral hippocampus (50ng, $0.5 \mu 1$ ) at the same time as electrode implantation. For Pilo rats, status epilepticus (SE) was induced $(365 \mathrm{mg} / \mathrm{kg} / \mathrm{s.c}$.) one week after electrode implantation. Tx rats were allowed to recover 2 weeks and Pilo rats 4 weeks before being placed in individual recording chambers and connected to a cable-commutator system. Electrographic seizure rates were measured during Baseline and Treatment periods using a 24-hour EEG monitoring system. Hippocampal EEG data were collected and used to determine electrographic seizure rates. Baseline seizure rates were determined for 5 days (Tx) or 10 days (Pilo). Then therapy was initiated and Treatment seizure rates were measured during an equal period of time. For both models, control animals were not stimulated. Treatment animals received VCLFS $(1 \mathrm{~Hz}, 500 \mathrm{~ms} /$ phase, $\pm 1 \mathrm{~V})$. VCLFS was delivered continuously for $60 \mathrm{~s}$ of each hour. Results: Significant decreases in electrographic seizure rates ( $p$ $<0.05$ ) were observed in response to VCLFS $(n=16)$ compared to no therapy controls $(n=7)$ in Tx model. Decreases were also observed in electrographic seizure rates in the Pilo spontaneous seizure model, however the sample sizes for this model are currently too small for statistical comparison. Data collection for the Pilo model is ongoing. Conclusions: These data indicate that scheduled VCLFS focal stimulation can decrease electrographic seizure rates in animal models of MTLE. These data also demonstrate that the Tx and Pilo spontaneous seizure models can be used to evaluate the effect of stimulation. (Supported by NINDS 5U01NS064049)

\subsection{0}

\section{A COMPARISON OF AUTOMATED VS. MANUAL DETECTION OF INTERICTAL EPILEPTIFORM ACTIVITY USING 256-CHANNEL EEG}

Tara T. Gilbert ${ }^{1}$, G. Lantz ${ }^{1}$, J. Hou ${ }^{1}$, M. Holmes ${ }^{2}$ and D. Tucker ${ }^{1}$

( ${ }^{1}$ Brain Electrophysiology Laboratory, Electrical Geodesics, Inc.,
Eugene, OR and ${ }^{2} \mathrm{UW}$ Department of Neurology, Regional Epilepsy Center at Harborview Medical Center, Seattle, WA)

Rationale: The purpose of this study is to compare the results of using an automated detection method of identifying interictal epileptiform activity against the standard method of human (manual) visual identification of this activity using dense-array EEG. An automated detection method should use information from all channels in order to identify this activity. During manual identification of interictal epileptiform activity it is likely that some EEG channel's information will not be observed and thus provide inconsistent results or lost information.

Methods: We acquired EEG from five epileptic patients using a 256channel sensor configuration. Portions of the file that were artifactfree were selected to be included in this analysis. The EEG recordings were then processed manually for interictal epileptiform activity and processed through an automated detection algorithm that identified interictal epileptiform activity. A neurologist then verified the detection rates of the manual and automated techniques for validity.

Results: We found that the automated detection algorithm identified interictal epileptiform activity more consistently then the manual visual identification method. We also found that the time of processing was less with the automated detection method then with manual detection.

Conclusions: Automated detection of interictal epileptiform activity can be a valuable tool for working with this patient population. Results of processing with this technique are more consistent and require less human processing time. This decrease in time of processing could be very valuable in the pre-surgical planning for this patient population and provide an avenue to quickly understand the epileptiform activity following an EEG exam.

\subsection{1 \\ DESIGN OF APPLICATION SPECIFIC INTEGRATED CIRCUITS FOR RF POWERED NEURAL RECORDING, AND CLOSED-LOOP ELECTRICAL OR OPTICAL STIMULATION}

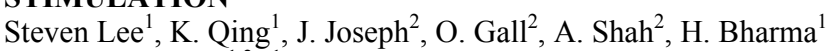
and P. P. Irazoqui ${ }^{1,2}\left({ }^{1}\right.$ Weldon School of Biomedical Engineering, Purdue University, West Lafayette, IN and ${ }^{2}$ Electrical Engineering, Purdue University, West Lafayette, IN)

Rationale: Deep brain stimulation and optical stimulation in optogenetics are two unique methods to control seizure activity. Currently, in the scientific community, devices to implement electrical or optical modulation are either tethered or wirelessly powered with a radiofrequency (RF) inductive link or battery. The tether is disadvantageous due to the restrictive environment, increased risk of infection from the percutaneous connector, and impractical implementation of large-group, longitudinal monitoring protocols. For inductive links, the internal coil (device antenna) prohibits full implantation, and the external coil limits freedom of movement. This technique is highly spatially dependent on an unpredictable alignment between the internal and external powering coils in a freely moving animal. Further, the range is limited to the reactive near-field. RF powering devices with the radiative far field, however, would allow more free ranging experiments with lower profile antennas. Custom circuitry with the far field powering would enable the epilepsy community to investigate untethered DBS and optical stimulation.

Methods: Two custom application specific integrated circuits (ASICs) have been designed to be RF powered through electromagnetic radiation. The architecture contains a front-end fully differential neural amplifier, ADC, clock, and transmitter. A biphasic constant current stimulator or charge pump is also included for electrical and optical stimulation, respectively. The ASIC designs were sent to the X-FAB mixed-signal foundry to be fabricated under the $180 \mathrm{~nm}$ CMOS process. 
Results: From Cadence software simulations, the ASICs operate at $1.8 \mathrm{~V}$ and on average, consume under $700 \mathrm{uW}$ during non-stimulating conditions. The constant current stimulator drives $\sim 170 \mathrm{uA}$ across a $10 \mathrm{kOhm}$ load, and the neural amplifier has $58 \mathrm{~dB}$ gain with approximately $1.5 \mathrm{kHz}$ bandwidth. Empirical results from the ASICs are presented.

Conclusions: Previously, our group has demonstrated LED based optical coupling, far field RF powering, and an ultralow power seizure detection ASIC. Integration of all these technologies into a singular device results in a first generation RF powered, closed-loop electrical or optical stimulator.

\subsection{2}

XENOTRANSPLANTATION OF PORCINE FETAL NEURONAL STEM CELLS (PNSCS) IN EPILEPSY - A FEASIBILITY STUDY IN AN ACUTE SEIZURE RAT MODEL Bianca Backofen-Wehrhahn ${ }^{1}$, B. Petersen ${ }^{3}$, S. Bröer ${ }^{1,2}$, M. Gernert ${ }^{1,2}$, H. Niemann ${ }^{3}$ and W. Löscher ${ }^{1,2}$ ( ${ }^{1}$ Department of Pharmacology, University of Veterinary Medicine Hannover, Hannover, Germany; ${ }^{2}$ Center for Systems Neuroscience, Hannover, Germany and ${ }^{3}$ Institute of Farm Animal Genetics, Friedrich-Loeffler-Institut (FLI), Mariensee, Neustadt a.Rbge., Germany)

Rationale: Xenotransplantation from genetically modified ("humanized") pigs provides a possible solution to the shortage of human organs for allotransplantation. Porcine fetal neuronal stem cells (PNSCs) are promising for treatment of neurological diseases, including pharmacoresistent epilepsy to overcome the shortage of human fetal neuronal stem cells and the ethical restrictions associated herewith. Clinical trials employing PNSCs grafted in patients suffering from different neurological disorders revealed repair of synaptic connections and restoration of behavioral deficits albeit the cells died shortly after transplantation. Further clinical studies were stopped because of potential risks of zoonotic infections emanating from porcine endogenous retroviruses (PERVs) that are integrated into the pig genome. However, recently the interest in porcine xenografts resumed after PERV-free pigs became available and cells or organs from transgenic pigs for xenotransplantation increasingly became available. Here, we compare wild-type versus transgenic PNSCs in their anticonvulsant efficacy in an acute seizure model, their survival rate after grafting, and integration into the host tissue. Methods: Wild-type and transgenic PNSCs were isolated from the medial ganglionic eminence and were cultivated as neurospheres. They were transplanted bilaterally into the substantia nigra pars reticulata ( $\mathrm{SNr}$ ) of adult rats. The $\mathrm{SNr}$ is a key structure in seizure propagation and modulation in several experimentally induced seizure types. The putative anticonvulsant efficacy was evaluated by the intravenous pentylenetetrazol seizure threshold test before and at different time-points after grafting (from 10 days up to 3 months). Survival rate, differentiation, and integration of grafted cells were histologically analyzed after termination of the experiments.

Results: Initial results revealed that wild-type PNSCs survived, at least in part, until the end of the experiment. However, grafting of the cells into the $\mathrm{SNr}$ failed to induce anticonvulsant effects at any investigated time point. Wild-type PNSCs differentiated into neurons and astrocytes and showed expression of the GABA-synthesizing enzyme glutamic acid decarboxylase after grafting. In future studies we will be using different types of transgenic porcine cells (hA20, hHO-1), and hope to increase the amount of surviving GABAproducing cells to induce anticonvulsant effects.

Conclusions: These preliminary results show the feasibility of grafting PNSCs into the $\mathrm{SNr}$ in an acute seizure rat model. Future studies will include cells from humanized PERV-free PNSCs to minimize the risk of cross-species infections and rejection.
3.063

POSSIBLE EFFECT OF LOW CURRENT TRANSCRANIAL FOCAL STIMULATION VIA TRIPOLAR CONCENTRIC RING ELECTRODES ON BEHAVIORAL SEIZURE ACTIVITY INDUCED BY PENTYLENETETRAZOLE IN RATS

Walter Besio, O. Makeyev and X. Liu (University of Rhode Island, Kingston, RI)

Rationale: Recently we demonstrated the effectiveness of noninvasive transcranial focal electrical stimulation (TFS) via tripolar concentric ring electrodes (TCRE) to control pentylenetetrazole (PTZ) induced seizures in rats. In [1] we showed TFS lessens behavioral seizure activity of recurrent PTZ administrations. In [2] a noninvasive seizure control system based on automatic seizure detection triggering the TFS reduced electrographic seizure activity power in the treated group. In our previous work the TFS current intensity was $50 \mathrm{~mA}$, now we report on the potential effect of $5 \mathrm{~mA}$ TFS on PTZ-induced seizure activity which is comparable to the current intensity of transcranial direct current stimulation (tDCS) that can be applied to humans.

Methods: Naive male Sprague-Dawley rats were used in this study. The TCREs were placed on the scalp with conductive paste and adhered with dental cement $24 \mathrm{~h}$ before the experimental procedure (Fig. 1A). The animals were divided into control $(\mathrm{n}=7)$ and TFStreated $(n=7)$ groups based solely on skin-to-electrode impedance. If impedances between the three recording surfaces of TCRE (s) and the isolated ground TCRE $(\mathrm{g})$ were less than $10 \mathrm{~K} \Omega$ the rat was administered TFS. PTZ ( $45 \mathrm{mg} / \mathrm{kg}$ ip) was given to both groups and TFS ( $5 \mathrm{~mA}, 200 \mu \mathrm{s}, 300 \mathrm{~Hz}, 2 \mathrm{~min}$, biphasic, charge-balanced pulses) was triggered manually in the TFS-treated group only when the first myoclonic jerk (MJ) was observed. To score behavioral seizure activity we adapted the revised Racine's scale from [1] (Table 1). The latency of initial myoclonic activity was defined as the time to the first MJ. The maximal score value and the total number of MJs were counted (first to last) for each animal as well as the duration of myoclonic activity (time elapsed between the first and the last MJs). The non-parametric Mann-Whitney U test was used to make comparisons between the control and TFS-treated groups due to nonnormality.

Results: The latency of myoclonic activity was significantly different ( $p=0.02$; Fig. 1B) with medians of $169 \mathrm{~s}$ and $74 \mathrm{~s}$ for the control and the TFS-treated group, respectively. At the same time there was no significant difference in maximal behavioral seizure activity score ( $p$ $=0.41$; both medians equal to 3 ), number of myoclonic jerks ( $\mathrm{p}=$ 0.28 ; medians of 12 and 10; Fig. 1C), and duration of myoclonic activity $(\mathrm{p}=0.41$; medians of $165 \mathrm{~s}$ and $100 \mathrm{~s}$; Fig. 1D).

Conclusions: The latency of myoclonic activity could not have been affected by TFS in either group since TFS was not turned on until the first MJ was observed. Statistically longer latency in the control group stems from lower initial susceptibility of this group to PTZ. Combined with an absence of any statistically significant differences in the other three metrics affected by TFS this suggests an anticonvulsant effect of TFS at low currents.

Stages of the revised Racine's scale for PTZ-induced seizures in rats used to score seizure-related behavioral activity

\begin{tabular}{|cc|}
\hline Calegory & Behavioral expression \\
\hline 0 & No seizure activity \\
1 & Sudden behavioral arrest and/or motionless staring \\
\hline 2 & Myoclonic jerk (sudden and fast neck jerk) \\
\hline 3 & Clonic seizure in a silling position \\
\hline 4 & Clonic seizures while lying on the belly \\
5 & Tonic-clonic seizure while lying on the belly \\
6 & Tonic-clonic scizurcs while lying on the side and/or wild jumping \\
\hline
\end{tabular}




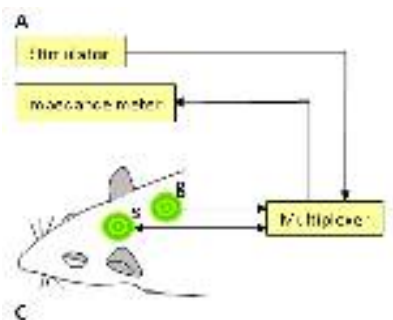

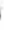
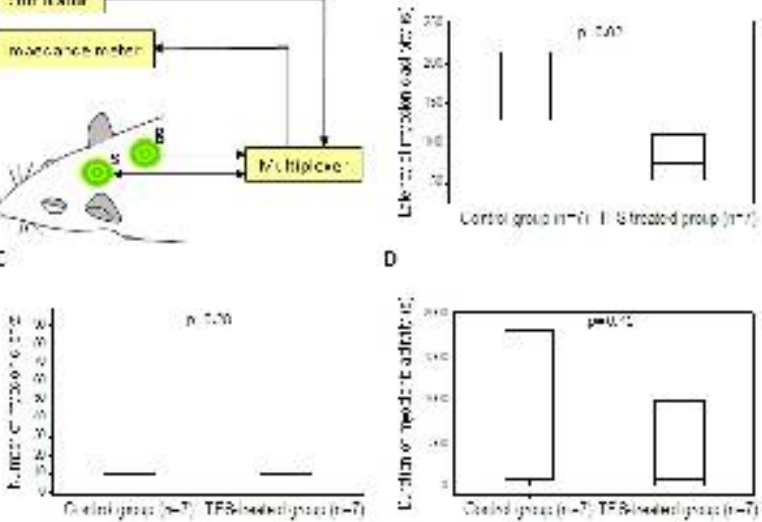

D

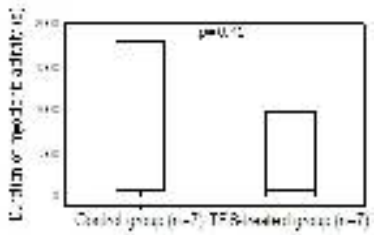

Translational Research: Biomarkers

3.064

MAGNETIC RESONANCE IMAGING WITHIN HOURS OF EXPERIMENTAL FEBRILE STATUS EPILEPTICUS PREDICTS SUBSEQUENT EPILEPSY

Man Kin Choy ${ }^{1}$, C. M. Dubé ${ }^{1}$, P. Maras ${ }^{1}$, K. Ambadipudi ${ }^{3}$, A. Blood $^{3}$, M. Hashemian ${ }^{1}$, Z. Baqai ${ }^{1}$, S. F. Quddusi ${ }^{1}$, M. Pakhdikian ${ }^{1}$, A. $\mathrm{Hasso}^{2}$, A. Obenaus ${ }^{3}$ and T. Z. Baram ${ }^{1}\left({ }^{1}\right.$ Departments of Anatomy $\&$ Neurobiology and Pediatrics, University of California-Irvine, Irvine, CA; ${ }^{2}$ Radiology University of California-Irvine, Irvine, California, University of California-Irvine, Irvine, CA and ${ }^{3}$ Department of Radiation Medicine, Loma Linda University School of Medicine, Loma Linda, CA)

Rationale: A subset of children with febrile status epilepticus (FSE) develop temporal lobe epilepsy, however, it is not possible to predict who will be affected. If a prognostic biomarker can be identified, it will facilitate the development of preventative therapies and enable targeted intervention. In both children and rats, magnetic resonance imaging (MRI) has identified acute hippocampal abnormalities after FSE. However, it is not known if the increased T2 in children is a marker of epileptogenesis, and late (1 month after FSE) changes in rats were not predictive. We used an animal model of FSE in which $\sim 45 \%$ of rats develop temporal lobe epilepsy, and a high-field $11.7 \mathrm{~T}$ MRI scanner to search for a clinically relevant early marker of epileptogenesis.

Methods: FSE-experiencing rats $(n=19)$, normothermic $(n=14)$ and hyperthermic controls $(\mathrm{n}=14)$ underwent MR imaging using an 11.7T Bruker MRI system. Rats were imaged at 2, 18, and 48 hours following FSE. Multi-slice multi-echo T2-weighted images were acquired, $\mathrm{T} 2$ relaxation time maps were generated, and $\mathrm{T} 2$ values were measured in several regions of interest (ROI), including the hippocampus and the amygdala. The development of spontaneous seizures was monitored over the following eight months using continuous video EEG recordings. ROC curves was used to assess potential biomarkers. Youden's index (sensitivity+specificity-1) was used to indicate maximum potential effectiveness of a biomarker. In a separate group of rats, the physiological basis of the T2 changes was investigated. To test the possibility that they were generated by a paramagnetic effect of deoxyhaemoglobin $(\mathrm{dHb}, \mathrm{FSE}(\mathrm{n}=13)$ and normothermic controls $(n=8)$ were imaged at the 2-hour time point, followed by blood analysis to quantify the $\mathrm{dHb}$ levels (by Radiometry; OSM-3 co-oximeter).

Results: T2 signal abnormalities were present in MRI scans obtained at 2 hours after FSE in a subset of the FSE rats. Specifically, T2 decreases were found, unlike previous studies performed at later time points and with lower magnetic fields. At high magnetic fields, paramagnetic substances such as $\mathrm{dHb}$ are known to reduce $\mathrm{T} 2$. Therefore, we examined the relationship between $\mathrm{T} 2$ decreases and
$\mathrm{dHb}$ levels following FSE, and found a significant correlation between $\mathrm{T} 2$ and $\mathrm{dHb}$ levels (Pearson correlation=-0.54, $\mathrm{p}=0.014$ ). Thus, T2 decreases may indicate increased oxygen consumption (and hence more deoxygenated hemoglobin) following FSE. Five of nineteen FSE rats developed spontaneous seizures. Reduced T2 in the amygdala (AUC $=0.83 \pm 0.2195 \% \mathrm{CI}, \mathrm{p}=0.033$ ) was predictive of epilepsy with an optimal threshold yielding $80 \%$ sensitivity and $14.3 \%$ false positive rate. At this threshold there is a positive predictive value of $66.7 \%$ and a negative predictive value of $92.3 \%$. Conclusions: Decreased MRI T2 relaxation time in the amygdala $2 \mathrm{~h}$ following FSE differentiate rats that eventually become epileptic from those that do not. Furthermore, these decreases suggest that increased metabolic demand persisting beyond the FSE may contribute to the epileptogenesis.

Supported by NIH grant NS35439 and NS 078279

\subsection{5 \\ EFFECT OF VALPROIC ACID AND MTHFR C677T POLYMORPHISMS ON PLASMA HOMOCYSTEINE CONCENTRATIONS: IMPLICATIONS FOR VASCULAR DISEASE}

Asuri N. Prasad ${ }^{1,7}$, L. Cheng ${ }^{3}$, D. Freeman ${ }^{3,6}$, F. Mahmud ${ }^{4,5}$ and M. J. Rieder ${ }^{2,3}\left({ }^{1}\right.$ Pediatrics and Clinical Neurosciences, Schulich Medicine \& Dentistry, Western University, London, ON, Canada; ${ }^{2}$ Pediatrics, Childrens Hospital, London Health Sciences Centre, London, ON, Canada; ${ }^{3}$ Physiology and Pharmacology, Schulich Medicine \& Dentistry, Western University, London, ON, Canada; ${ }^{4}$ Division of Endocrinology, Hospital for Sick Children, Toronto, ON, Canada; ${ }^{5}$ Pediatrics, Hospital for Sick Children, Toronto, ON, Canada; ${ }^{6}$ Lawson Health Research Insititute, London, ON, Canada and ${ }^{7}$ Child Health Research Institute, London, ON, Canada)

Rationale: Elevated plasma total homocysteine (tHcy) is an independent risk factor for developing vascular disease. We investigated the relationship between elevated tHcy concentrations, caused by both valproic acid (VPA) therapy, 5-

methylenetetrahydrofolate reductase (MTHFR) genotype, and indirect measures of endothelial function in epileptic children and adolescents.

Methods: Patients with epilepsy treated with VPA and controls without epilepsy (VPA naïve) were recruited. Endothelial function was assessed via peripheral arterial tonometry (PAT) in all participants at baseline. Overnight fasting blood samples were obtained from the epileptic group to assess plasma tHcy, erythrocyte folic acid and serum vitamin B12 concentrations via highperformance liquid chromatography (HPLC) and core lab procedures, while MTHFR genotypes were determined via Genotype-specific Approaches to Therapy in Childhood (GATC) protocol. Serum samples provided from controls were used to assess serum tHcy concentration via HPLC. Within 6 months the same parameters were assessed again in the epileptic group.

Results: Nine epileptic patients (78\% male; ages 7-16 years) and 31 controls (39\% male; ages 11-16 years) were recruited. tHcy concentration significantly increased in epileptic patients after follow-up compared to controls $(+29 \% ; \mathrm{P}=0.016)$ while vitamin $\mathrm{B} 12$ concentration demonstrated a trend towards a gradual decline. Epileptic patients expressing the 677CT genotype had elevated tHcy and reduced folic acid concentrations compared to patients expressing the $677 \mathrm{CC}$ genotype. Endothelial function was affected in epileptic patients, indicated by a significant reduction in the reactive hyperemia-PAT index at baseline and follow-up in epileptic patients compared to controls $(-20 \% ; \mathrm{P}=0.018$ and $-23 \% ; \mathrm{P}=0.012$, respectively), with a corresponding increase in tHcy and decrease in vitamin B12 concentrations.

Conclusions: This pilot study prospectively documents an association between elevated tHcy concentration and endothelial dysfunction assessed by PAT in children with diagnosed epilepsy expressing the MTHFR 677CT polymorphism when treated with 
VPA. Further studies are required to confirm these findings and evaluate long-term cardiovascular risk for children and adolescence with epilepsy on long-term VPA treatment.

\subsection{6 \\ PLASMA CONCENTRATIONS OF SICAM5 AND OTHER T- CELL IMMUNOMODULATORS AS BIOMARKERS OF LESIONAL EPILEPSY}

John Pollard $^{1}$, O. Eidelman ${ }^{2}$, G. P. Mueller ${ }^{2}$, P. B. Crino ${ }^{1}$, C. T. Anderson $^{1}$, E. J. Brand ${ }^{1}$, E. Burakgazi ${ }^{3}$, S. Ivaturi ${ }^{1}$ and H. B. Pollard ${ }^{2}$ $\left({ }^{1}\right.$ Neurology, University of Pennsylvania, Philadelphia, PA; ${ }^{2}$ Anatomy, Physiology and Genetics, Uniformed Services University School of Medicine, Bethesda, MD and ${ }^{3}$ Cooper Medical Center, UMDNJ, Camden, NJ)

Rationale: Inflammation has been implicated in the underlying pathophysiology of drug resistant epilepsy. Elements of innate immunity such as IL-6 have been reported as elevated in epilepsy patient plasma. We hypothesized that sICAM5, a telencephalonspecific immunomodulator, as well as other chemokines and cytokines, would differentiate between epilepsy patient plasma and non-epileptic seizure patient plasma (controls).

Methods: After obtaining IRB approval, patients in the UPenn EMU who consented had a single plasma sample drawn. The citrated sample was spun in a refrigerated centrifuge for 10 minutes and the supernatant was then stored at -80 degrees $\mathrm{C}$ until analyzed. The samples were analyzed using a MesoScale Sector 6000 electrochemiluminescent multiplexed ELISA platform. Analytes included not only sICAM5, but also GM-CSF, IFN- $\gamma$, IL-10, IL12 p70, IL-1 $\beta$, IL-2, IL-6, IL-8, TNF- $\alpha$. We compared the epilepsy patient samples to the control samples using a T-test.

Results: Two epilepsy patient samples and three psychogenic seizure patient samples were collected. The epilepsy patients averaged 33 years of age. One of the epilepsy patients had a history of oligodendroglioma with a seizure frequency in the Epilepsy Monitoring Unit (EMU) of 0-3 seizures per day, who was treated with lamotrigine, levetiracetam, and pregabalin. The other patient has tuberous sclerosis and averaged 28 seizures per day in the EMU, treated with levetiracetam, felbamate, lamotrigine and phenobarbital. The non-epileptic controls averaged 36 years old; treated with levetiracetam, lamotrigine, or no medication; and averaged less than 1 non-epileptic event per day. Three T-cell related analytes were strongly positive, while differences among the rest of the analytes were not significant. In epilepsy patients, sICAM5, an antiinflammatory modulator, was present, but below the lower limit of detection (LLOD) relative to the standard curve. We estimated the concentration of sICAM5 in these epilepsy patients to be $0.1 \mathrm{ng} / \mathrm{ml}$. By contrast, the controls had an average value of $11.87 \mathrm{ng} / \mathrm{ml}$, the difference being very significant $(\mathrm{p}=0.0017)$. IL-2 levels in epilepsy patients were elevated: $4.24 \mathrm{pg} / \mathrm{ml}$, versus $0.47 \mathrm{pg} / \mathrm{ml}$ in controls $(\mathrm{P}=0.0012)$. INF-gamma levels in epilepsy patients werealso elevated: $22.19 \mathrm{pg} / \mathrm{ml}$, versus $2.09 \mathrm{pg} / \mathrm{ml}$ in controls $(\mathrm{p}=0.0040)$. Conclusions: The lesional drug resistant epilepsy patients show a pattern of plasma inflammatory markers consistent with a proinflammatory condition, supporting the inflammation hypothesis of drug resistant epilepsy. The pattern shows that the anti-inflammatory$\mathrm{T}$ cell modulator sICAM5 is low, while the proinflammatory T-cell modulator IL-2 is high. In addition IFN-gamma, a typical product of $\mathrm{T}$-cell activation is high. This pattern is consistent with persistent involvement of T-cells in the epileptic inflammatory process. At least in lesional drug resistant epilepsy, sICAM5 and other T-cell immunomodulators may be biomarkers of disease.

\begin{tabular}{|c|c|c|c|c|c|c|}
\hline \multirow{2}{*}{\multicolumn{2}{|c|}{ Analyte }} & \multicolumn{2}{|c|}{$\begin{array}{l}\text { Epilepsy } \\
(\mathrm{n}=2)\end{array}$} & \multicolumn{2}{|c|}{$\begin{array}{l}\text { Non-epileptic } \\
\quad(\mathrm{n}=3)\end{array}$} & \multirow{3}{*}{$\begin{array}{l}\text { p valu } \\
0.0040\end{array}$} \\
\hline & & Mean & Range & Mcan & range & \\
\hline$\Pi N \mathrm{~N}-\gamma$ & $\mathrm{pg} / \mathrm{ml}$ & 22.19 & $(20.24-22.19)$ & 2.09 & $(0.31-5.21)$ & \\
\hline$\Pi,-2$ & $\mathrm{pg} / \mathrm{ml}$ & 4.24 & $(3.89-4.60)$ & 0.47 & $(0.24-0.60)$ & 0.0012 \\
\hline sIC $A M 5$ & $\mathrm{ng} / \mathrm{ml}$ & 0.10 & $(0.1)$ & 11.87 & $(10.40-13.34)$ & 0.0017 \\
\hline
\end{tabular}

\subsection{7 \\ FUNCTIONAL CONNECTIVITY IN INTRACRANIAL EEG PREDICTS SURGICAL OUTCOME IN INTRACTABLE TEMPORAL LOBE EPILEPSY}

Arun Antony ${ }^{1}$, R. C. Burgess ${ }^{1}$, L. Jehi ${ }^{1}$ and R. F. Galan ${ }^{2}\left({ }^{1}\right.$ Division of Epilepsy, Cleveland Clinic Foundation, Cleveland, $\mathrm{OH}$ and ${ }^{2}$ Department of Neurosciences,School of Medicine, Case Western Reserve University, Cleveland, $\mathrm{OH}$ )

Rationale: Despite the advances in imaging and electrophysiology, surgical outcome in non-lesional temporal lobe epilepsy remains modest. We reasoned that an analysis of functional connectivity from intracranial EEG would improve the localization of the epileptogenic zone and hence, the surgical outcome. Measures of functional connectivity have not yet been directly applied in predicting seizure outcome after epilepsy surgery

Methods: All patients with intractable temporal lobe epilepsy who underwent temporal lobectomy between January 2009 and January 2012 after an invasive EEG evaluation using stereo EEG with at least 6 months of follow up $(n=15)$ were included in the study. The temporal lobe was parcellated into 9 standardized regions and one electrode contact from each region was selected for analysis of EEG signals. EEG was sampled at $0.5 \mathrm{kHz}$ and filtered into 7 different bands: alpha, beta, theta, delta, gamma, and High-FrequencyOscillations (HFO). Functional connectivity was determined using a novel covariance-based measure, defined as follows. For each pair of recording channels, the maximum absolute value of the crosscorrelogram was taken as the value of functional connectivity between the pair. The time lags of the cross-correlogram ranged from -100 to $+100 \mathrm{~ms}$. Each cross-correlogram was normalized so that at zero lag its value equals the cross-correlation coefficient of the pair.

The functional connectivity values are thus bounded between 0 and 1 . We investigated the entire matrix of channel pairs over all of the frequency bands and compared them with seizure recurrence after surgery. A favorable outcome was defined as complete seizurefreedom since surgery

Results: Eight patients (53.3 \%) had recurrence of seizures within 6 months of temporal lobectomy, including two whose seizures occurred in the setting of missed antiepileptic medications and sleep deprivation, and two who had persistence of their typical preoperative auras. The high rate of seizure recurrence reflects the expected low rates of seizure-freedom in patients whose non-lesional, poorly localized, intractable epilepsy necessitates evaluation using invasive electrodes. Functional connectivity as defined above was higher in the temporal regions in 5/7 patients who were seizure free after surgery, while four of the 8 patients who had recurrence of seizures had lower functional connectivity among temporal regions. This suggests that our measure of functional connectivity may be a good parameter to find the epileptogenic zone. Indeed, a connectivity value greater than 0.5 in the beta band predicted well the surgical outcome with a sensitivity and specificity of $71.4 \%$ and $50 \%$ respectively for prediction of favorable seizure outcomes.

Conclusions: These preliminary results support the potential for our novel measure of intracranial EEG functional connectivity in predicting surgical outcomes after temporal lobe epilepsy surgery. Prospective studies with a larger patient sample are needed to determine the applicability of this measure as a diagnostic tool 

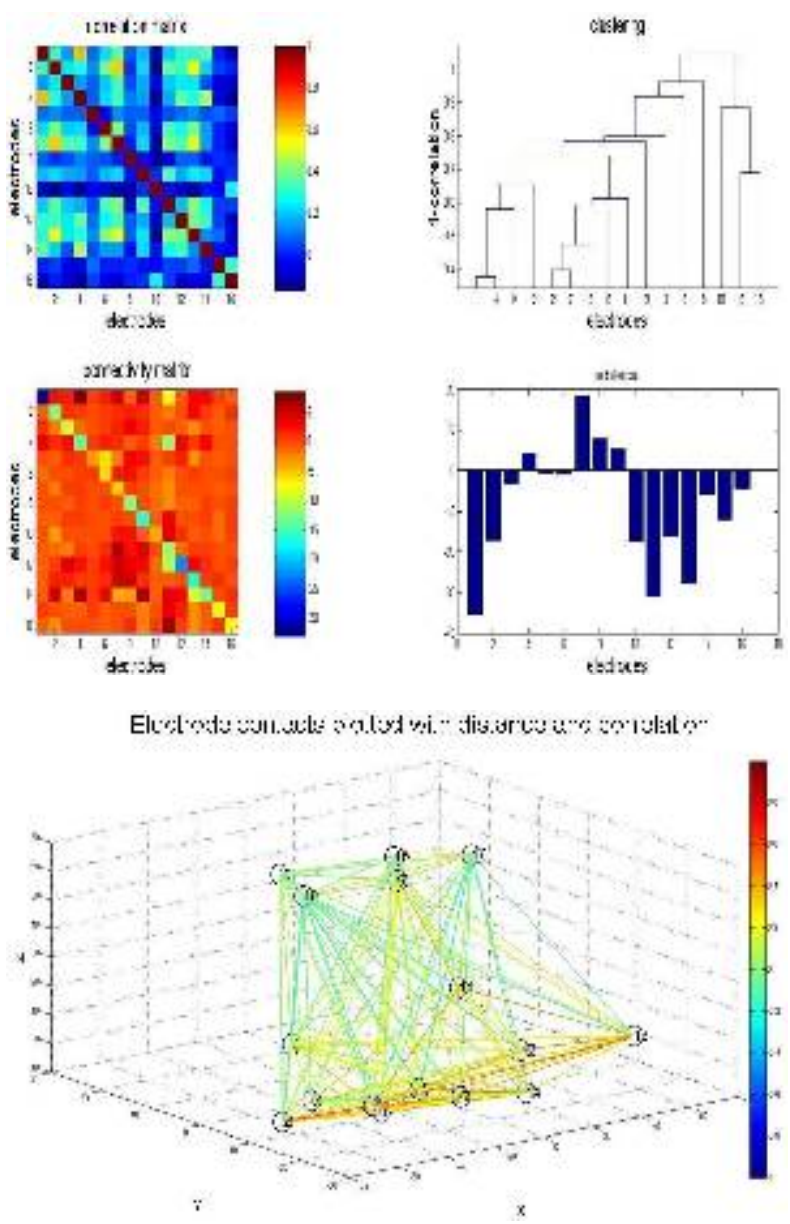

3.068

ASSESSMENT OF STATUS EPILEPTICUS SEVERITY USING EEG PARAMETERS

Andrew M. White, H. L. Grabenstatter, J. L. Carlsen, A. R. BrooksKayal and D. L. Hund (Pediatrics, Children's Hospital Colorado, Denver, CO)

Rationale: Rodent models of induced status epilepticus (SE) are commonly employed to understand mechanisms of epileptogenesis and to develop potential therapeutics for preventing the development of epilepsy or for decreasing seizure frequency. Outcomes measures resulting from SE include cell death, latency time to chronic seizures, and seizure frequency. There is significant variability in the impact of the SE, even when the same dose of the chemoconvulsant or type of injury is administered. To account for this variability it is desirable to develop an objective measure of the severity of SE. Here, we consider the use of several EEG measures as surrogates for the severity of SE, correlating these with resultant cell death, seizure latency and spontaneous seizure totals over time.

Methods: Video-EEG was obtained from a total of 19 pilocarpinetreated animals. Twelve of the animals were used to determine potential correlations between SE surrogate measures and seizure latency correlations and 7 were used to determine cell death (FluoroJade staining) correlations. Fluoro-Jade counts from CA1, CA3, and the dentate hilus of the hippocampus were averaged using 10 sections from each animal. Cortical EEG recordings were collected from both hemispheres using Pinnacle Technology systems. Sampling rates were either $200 \mathrm{~Hz}(\mathrm{n}=7)$ or $1000 \mathrm{~Hz}(\mathrm{n}=5)$. Metrics used to quantify SE included: maximum power, integrated power over the 24 hour status period, relative (to baseline) integrated power, and duration of elevated EEG power. The power was also determined in the delta, theta, alpha, $15-30 \mathrm{~Hz}, 30-50 \mathrm{~Hz}, 50-70 \mathrm{~Hz}$ and $70-100 \mathrm{~Hz}$ frequency bands. Software for these calculations was written using Visual Basic subroutines.
Results: Moderate strength correlations (0.3-0.4) were found at multiple frequencies between the integrated EEG power and cumulative seizure total at 1, 2 and 4 weeks following SE. Strong correlations $(>0.5)$ were found between relative integrated power during SE and the cumulative seizure total in multiple frequency bands. Strong correlations were also noted between time with elevated EEG power and cumulative seizure total for multiple frequency bands. Moderate strength correlations were noted at multiple frequencies between time with elevated EEG power and the Flouro-Jade counts. Correlations were greatest in the CA3 and hilar regions.

Conclusions: There were moderate correlations between both relative integrated power during SE, duration of elevated EEG power and cumulative seizure totals over time. Correlations were not consistently found between latency or cell counts and EEG characteristics during SE, possibly as a result of an insufficient number of animals and/or many additional factors impacting SE other than the EEG characteristics of the SE.

Acknowledgements:

Work was performed using support from NINDS grants 5 K08 NS053610-05 (AMW) and NIH R01 NS 051710 (ABK).

\subsection{9 \\ DETECTION OF EPILEPTOGENICITY WITH NON-LINEAR ANALYSIS OF ELECTROENCEPHALOGRAPHIC SIGNALS W. Bosl, Iván Sánchez Fernández and T. Loddenkemper (Boston Children's Hospital, Boston, MA)}

Rationale: The diagnosis of epilepsy relies on surrogate markers for epileptogenicity such as the presence of repeated seizures or spikes. The aim of this study is to test the usefulness of a type of non-linear mathematical analysis: recurrence quantitative analysis (RQA) of electroencephalogram (EEG) signals as a direct biomarker of epileptogenicity in the brain.

Methods: We enrolled 76 patients that met the following inclusion criteria: 1) age 1 month to 21 years, 2) normal neuropsychological development. 38 patients (cases) had several episodes of electroclinically documented absence seizures. The remaining 38 patients (controls) met the following criteria: 1) at least one EEG study performed because of the clinical suspicion of seizures, 2) normal EEG study results, 3) an electro-clinical presentation that was not consistent with epilepsy based on a thorough evaluation. We identified and collected sample EEG segments of 10-30 seconds duration each: 1) in the cases, three segments were collected: baseline without spikes, hyperventilation without spikes, and spikes; 2) in controls, two segments were collected: baseline without spikes, and hyperventilation without spikes. RQA was used to compute 19 different nonlinear measures for each of the 19 EEG channels, resulting in 19X19 or 361 features. From these, 20 of the most statistically relevant values were automatically selected and used for classification. These nonlinear features were used in two different machine learning algorithms to classify the EEG segments as belonging to cases (epileptic patients) or controls (non-epileptic patients). The concordance of this diagnostic classification was compared to the classification made by the clinical epileptologist (gold standard).

Results: The results of the RQA and of the human epileptologist were highly correlated as shown in table 1 . Hyperventilation increases the levels of epileptogenicity of both cases and controls, making them more difficult to differentiate. In order to control for this potential confounder, we performed a subgroup analysis eliminating the tracings during hyperventilation in both cases and controls. The resulting classification of patients into cases and controls was even more correlated with the diagnosis of the human epileptologist (Table 2).

Conclusions: Analysis of EEG signals with RQA was highly correlated with the classification of the human epileptologist (gold standard). Non-linear analysis of short EEG signals of patients with 
epilepsy, even in epilepsy patients with normal EEG on visual inspection, may be a useful method to monitor and diagnose epilepsy.

Table 1. Comparison of the automated method with the human gold standard using 10 -fold cross validation

\begin{tabular}{|c|ccc|c|}
\hline Classification method & $\begin{array}{c}\text { 10-fold cross validation } \\
\text { accuracy }\end{array}$ & Sensitivity & Specilicity & $\begin{array}{c}\text { Area under the } \\
\text { curve } \\
\text { k-ncarcst ncighbors }\end{array}$ \\
\hline $\begin{array}{c}\text { Support vector } \\
\text { machine }\end{array}$ & 0.71 & 0.74 & 0.68 & 0.77 \\
$\begin{array}{c}\text { Naive Bayes } \\
\text { Classificr }\end{array}$ & 0.77 & 0.72 & 0.84 & 0.84 \\
\hline
\end{tabular}

Table 2. Comparison of the automated method with the human gold standard after considering only the EEG samples without hyperventilation

\begin{tabular}{|c|ccc|c|}
\hline Classification Method & $\begin{array}{c}\text { 10-fold cross validation } \\
\text { accuracy }\end{array}$ & Sensitivity & Specilicity & $\begin{array}{c}\text { Area under the } \\
\text { curve } \\
\text { k-ncarest ncighbors }\end{array}$ \\
\hline $\begin{array}{c}\text { Support vector } \\
\text { machine }\end{array}$ & 0.80 & 0.87 & 0.69 & 0.74 \\
$\begin{array}{c}\text { Naive Bayes } \\
\text { Classifier }\end{array}$ & 0.78 & 0.87 & 0.66 & 0.80 \\
\hline
\end{tabular}

\subsection{0}

\section{OSCILLATIONS CHARACTERISTIC OF NON-} EPILEPTOGENIC NEOCORTEX IN A RESTING STATE David Groppe ${ }^{1}$, S. Bickel ${ }^{4}$, C. Keller ${ }^{5}$, S. K. Jain ${ }^{3}$, S. Hwang ${ }^{3}$, S. Stevens ${ }^{3}$, C. Harden ${ }^{3}$ and A. D. Mehta ${ }^{2}\left({ }^{1}\right.$ Feinstein Institute for Medical Research, Manhasset, NY; ${ }^{2}$ Neurosurgery, North Shore University Hospital, Manhasset, NY; ${ }^{3}$ Neurology, North Shore University Hospital, Manhasset, NY; ${ }^{4}$ Neurology, Albert Einstein College of Medicine, Bronx, NY and ${ }^{5}$ Neuroscience, Albert Einstein College of Medicine, Bronx, NY)

Rationale: Abnormalities in resting neocortical oscillations can be indicative of associated pathology. For example, focal attenuation of fast frequencies and focal slowing may be indicative of a lesion such as gliosis or a tumor. The ability to identify such abnormalities requires the characterization normal patterns of oscillatory activity. However, comprehensive, quantitative standards for such patterns have not been established. Towards creating such standards, we have quantified the types of oscillations that are normally observed in different healthy cortical areas when an individual is at rest.

Methods: Approximately 4 minute periods of intracranial electroencephalogram (iEEG) data were acquired from 18 individuals undergoing evaluation for surgical treatment of epilepsy while the individuals were awake but resting. Data from electrodes exhibiting epileptiform activity or near anatomical abnormalities were discarded from analysis. The cortical area under each electrode was estimated by co-registering postimplant CT scans to a preimplant MRI, and parsing the cortex into a set of 35 areas using the FreeSurfer software package and the Desikan-Killiany atlas. iEEG was whitened to highlight power spectrum peaks and divided into one second epochs with 0.5 second overlap. The power spectrum density (PSD) in each epoch was estimated using the discrete Fourier transform with two Slepian tapers and averaged across epochs. The 5\% trimmed mean PSD for each channel was normalized to unit power to control for differences in overall power across electrodes and individuals. No attempt was made to control for possible effects of analgesic or antiepileptic medications. Frequencies corresponding to line noise (60 Hz and harmonics) were ignored from analysis.

Results: Across all cortical areas, the most commonly found peaks in PSD were $\sim 7 \mathrm{~Hz}$, on the border of conventional definitions of theta and alpha. Theta peaks were most clearly present in several temporal lobe gyri, and beta peaks were evident in paracentral and inferior frontal gyri. The only region to show reliable gamma peaks was entorhinal cortex.

Conclusions: Contrary to what might be inferred from scalp EEG, which is dominated by $\sim 10 \mathrm{~Hz}$ activity, the most prominent oscillatory activity seen across much of the brain is $\sim 7 \mathrm{~Hz}$. Slower activity appears in the inferior temporal areas and is accompanied by beta activity in the pre- and post-central gyri. Surprisingly, beta activity also extends into inferior frontal areas, and gamma activity can often be quite prominent over entorhinal cortex. The lack of prominent alpha activity in these data is surely at least partially due to limited coverage of occipital cortex in these patients. Future work will investigate how these oscillatory patterns differ when these cortical regions exhibit epileptiform activity, and how they are affected by antiepileptic and analgesic medications.

3.071

AEDS REDUCE THE UPPER PHOTOSENSITIVITY LIMIT MORE THAN THE LOWER PHOTOSENSITIVITY LIMIT IN PHOTOSENSITIVE PATIENTS: IMPLICATIONS FOR THE DESIGN OF THE PHOTOSENSITIVITY MODEL

Dorothee G. Kasteleijn-Nolst Trenite ${ }^{1}$ and R. C. Reed ${ }^{2}$

$\left({ }^{1}\right.$ Neuroscience, Sapienza University II Faculty, Rome, Italy and

${ }^{2}$ Pharmacy Practice, Husson University, Bangor, ME)

Rationale: The photosensitivity model is a well known human model to determine antiepileptic properties of new AEDs as proof of principle (POP). This Photosensitivity Model still has not been thoroughly explored.

Methods: Photic induced EEG responses (Photoparoxysmal responses, PPRs) of 236 regular photosensitive patients (163 F; $69 \%)$, mean age $=20+10 \mathrm{yr}(\min 7, \max 60 \mathrm{yrs})$ were analyzed. In all patients, standardized photic stimulation, 2 - $60 \mathrm{~Hz}$, using a Grass PS 33plus had been performed with determination of upper and lower sensitivity ranges in 3 eye conditions. The PPRs for patients with vs. without AEDs were compared (t-test; Mann-Whitney; Anova regression analysis).

Results: Of the 236 patients with a PPR, 233 had a PPR on eye closure, 206 with eyes closed and 149 with eyes open. Most patients were on stable AEDs (152; 64\%) and monotherapy (104; 44\%), with VPA in 62 cases, CBZ in 18 and other AEDs in 24. Forty eight patients $(20 \%)$ took 2-4 AEDs, with PB as most often prescribed drug in combination (18), followed by ESM (14), LTG (10), one of the the benzodiazepines DZP, CNZ or CLB (9), PHT (4) and VGB (1). The upper sensitivity limit had a greater spreading and at eye closure and eyes closed they were higher in women $(p=0.002$ and 0.018$)$ than in man. Patients on AEDs had significant changes (lowering of) upper limit while the lower sensitivity limit did not change significantly (see Table I).

Conclusions: The upper sensitivity limits of photosensitive patients are lower in patients with AEDs, compared to those without. This phenomenon is also seen in individual cases participating in POP studies, in which the same patient is investigated with and without trial medication.

Determination of the upper limit may be sufficient in POP new AED trials, which reduces the total number of photic stimulations by half. This has advantages for the patient and will reduce the costs of the POP-trial.

Comparison of upper and lower sensitivity limits in 152 photosensitive patients with AEDs and 84 without AEDs

\begin{tabular}{|c|c|c|c|c|c|c|}
\hline $\begin{array}{l}\text { 'Threshold of } \\
\text { photosensitivity }\end{array}$ & $\begin{array}{l}\text { Lower } \\
\text { Limil }\end{array}$ & $\begin{array}{l}\text { Lower } \\
\text { Limil }\end{array}$ & $\begin{array}{l}\text { Lower } \\
\text { Limil }\end{array}$ & $\begin{array}{l}\text { Upper } \\
\text { Limil }\end{array}$ & $\begin{array}{l}\text { Upper } \\
\text { Limil }\end{array}$ & $\begin{array}{l}\text { Upper } \\
\text { Limit }\end{array}$ \\
\hline Fye condition & Closure & Closed & Open & Closure & Closed & Open \\
\hline $\begin{array}{c}\text { t-test } \\
\text { p value }\end{array}$ & 0.4 & 0.5 & 0.2 & 0.002 & 0.008 & 0.04 \\
\hline
\end{tabular}

No sex differences were found 


\subsection{2}

AUTOANTIBODIES TO NEURONAL PROTEINS IN PATIENTS WITH EPILEPSY

Bethan Lang ${ }^{1}$, T. Brenner ${ }^{1}$, G. Sills ${ }^{2}$, Y. Hart ${ }^{3,1}$, S. Howell ${ }^{4}$, J. Adcock $^{1}$, M. Brodie ${ }^{5}$, P. Waters ${ }^{1}$, S. Irani ${ }^{1}$ and A. Vincent ${ }^{1}\left({ }^{1}\right.$ Nuffield Department of Clinical Neurosciences, University of Oxford, Oxford, United Kingdom; ${ }^{2}$ Department of Molecular and Clinical Pharmacology, University of Liverpool, Liverpool, United Kingdom; ${ }^{3}$ Dept Neurology, Newcastle upon Tyne NHS Foundation Trust, Newcastle, United Kingdom; ${ }^{4}$ Dept Neurology, Royal Hallamshire Hospital, Sheffield, United Kingdom and ${ }^{5}$ Epilepsy Unit, Western Infirmary, Glasgow, United Kingdom)

Rationale: Autoantibodies to neuronal proteins contribute to the pathological process in an increasing number of childhood and adult epileptic syndromes and in encephalitic seizure-related disorders. Recently, antibodies to leucine-rich glioma inactivated 1 (LGI1), a protein within the voltage-gated potassium channel complex (VGKC), were identified in patients with faciobrachial dystonic seizures (FBDS). This represents the first clinically-distinctive immunotherapy-responsive adult-onset epilepsy syndrome. In this study we examined the prevalence of these and other antibodies to neuronal proteins in cohorts of patients with recent and with long standing epilepsies.

Methods: Serum samples were collected from newly-diagnosed epilepsy patients, taken before the start of a clinical treatment trial $(n=181)$ and from a cohort of consecutive epilepsy patients attending specialist clinics $(n=235)$. All were tested for antibodies to the NMDA receptor, VGKC-complex, GAD and the glycine receptor using cell-based or radioimmunoprecipitation assays

Results: Antibodies were detected in 46/416 (11\%) of patients, compared to less than $0.5 \%$ in controls. The most common antibody was to the VGKC-complex in 21 patients (5\% of all patients). Antibodies to the NMDA-receptor (1.7\%), glutamic acid decarboxylase (1.7\%) and the glycine receptor $(2.8 \%)$ were also detected. Antibodies were slightly more frequent in patients with duration of diagnosis of less than one year (13.4\%) compared to patients of duration over 1 year $(10.1 \%)$. There was a significantly higher incidence of positive antibodies in patients with cryptogenic rather than symptomatic LRE (15 vs 7\%) Of the recent-onset patients, 10 antibody positive patients $(50 \%)$ were nonresponsive to their first ever AED compared with 51/161 (31.6\%) of patients without detected antibodies.

Conclusions: The results suggest an immune-mediated component in some forms of epilepsy, although further studies will be required to establish whether the antibodies are directly pathogenic or merely markers of a secondary immune response.

\subsection{3}

\section{CARDIAC BIOMARKERS IN EPILEPSY CLINIC}

Shobhit Sinha, s. Hanif, M. Alhameed, M. M. Aldosari, S. R. Siddiqui, E. Khalid, O. Alsinaidi and K. A. Siddiqui (Epilepsy Program, Department of Neurology, National Neuroscience Institute, King Fahad Medical City, Riyadh, Saudi Arabia)

Rationale: Incidence of sudden unexpected death in epilepsy (SUDEP) is 24 times higher than in age matched controls. Multiple risk factors are duration of epilepsy, generalized seizures, cumulative effect of seizures overtime, poor seizure control and multiple antiepileptic drugs. Possible mechanisms include arrhythmias, increased myocardial oxygen consumption, excess catecholamine release during seizures and effect of AEDs on cardiac rhythm. Cardiac biomarkers (Troponin I, CK-MB and pro-BNP) are sensitive for myocardial damage, and if positive may help in detection of such patients at risk for SUDEP. Our objective was to see presence of ongoing myocardial damage in patients who attended epilepsy clinic. Methods: We evaluated 65 consecutive patients who presented at Epilepsy Clinic, National Neuroscience Institute at King Fahad
Medical City. Cardiac Biomarkers (Troponin I, CK-MB and pro$\mathrm{BNP}$ ) and EKG were requested in all patients. The questionnaire used for interview consisted of demographic details, disease related parameters (duration of epilepsy, seizure frequency, number of antiepileptic drugs, compliance to treatment), history of cardiac disease, peri-ictal heart related symptoms.

Results: Thirty nine out of 65 patients were included in the study. The excluded patient did not consent to investigations. There were 18 females and 21 males, with mean age $(+\mathrm{SD})$ of $28.2(+12.1)$ years. Average duration of epilepsy was $118(+110)$ months with mean seizure frequency of 1-3 per year. Except for 1 patient, all were on AEDs (range 0-3 drugs/patient). All but 3 patients were complaint to the treatment. Mean biomarker levels were; Troponin $\mathrm{I}=0.01(+$ $0.01)$, proBNP $=4.3(+5.06)$, and $\mathrm{CK}-\mathrm{MB}=1.1(+0.81)$.

Peri-ictal symptoms were documented in 7 patients and were shortness of breath, chest pain and palpitation. Abnormal EKGs were found in 11 (28\%) patients ( 2 had bradycardia, prolong QT interval, $\mathrm{T}$ wave abnormalities, and left ventricular hypertrophy each; 1 each with short PR interval, fusion complexes and right bundle branch block. No correlation was seen between biomarkers and EKG, or with other clinical/ demographic variables.

Conclusions: There was no detectable ongoing myocardial damage seen in patients who attended epilepsy clinic. Nonspecific EKG abnormalities were seen in $28 \%$ patient that needs further study.

\subsection{4 \\ IDENTIFICATION OF POTENTIAL GENES OF ABSENCE EPILEPSY BY MALDI IMAGING USING TWO BIDIRECTIONALLY SELECTED MOUSE LINES \\ Benoit Martin $^{1,2}$, M. Lagarrigue ${ }^{3,2}$, T. Alexandrov 4 , R. Lavigne ${ }^{3,2}, \mathrm{G}$. Dieuset $^{1,2}$, S. Baulac ${ }^{5,6}$ and C. Pineau ${ }^{3,2}$ ( ${ }^{1}$ LTSI, Inserm U1099, Rennes, France; ${ }^{2}$ University of Rennes 1, Rennes, France; ${ }^{3}$ Proteomics Core Facility Biogenouest, IRSET, Rennes, France; ${ }^{4}$ Center for Industrial Mathematics, University of Bremen, Bremen, Germany; ${ }^{5}$ Institut du Cerveau et de la Moëlle épinière, Paris, France and ${ }^{6}$ Inserm UMR S975, Paris, France)}

Rationale: MALDI imaging mass spectrometry has a great potential for the discovery of disease markers. Unfortunately this approach is unable to point out the most significant markers among a list a markers which can be long. Here we used an innovative statistical method to identify the most relevant protein markers in a mouse model for absence epilepsy.

Methods: Our study is based on the use of two inbred mouse lines, $\mathrm{BS} / \mathrm{Orl}$ and BR/Orl, issued from a bidirectional genetic selection and representing a dual genetic model for Childhood Absence Epilepsy (CAE). The BS/Orl strain displays spontaneous and recurrent spikeand-wave discharges (SWD) in both the cortex and the ventrolateral thalamus. These SWD are suppressed by the anti-absence seizure drugs ethosuximide and sodium valproate. In contrast, seizures are exacerbated by the carbamazepine, known to aggravate CAE. By contrast, the BR/Orl strain does not display any SWD either spontaneously or after injection of pentylenetetrazol known to induce SWD.

Protein mass spectra were acquired on whole brain sections using MALDI-TOF mass spectrometer. Identification of potential protein markers ( $\mathrm{m} / \mathrm{z}$ values) was performed by top-down analysis. A new cross-classification method specifically adapted to large MALDI imaging datasets was thus developed for the identification of the most significant markers and applied on our mouse model of absenceepilepsy. Finally, a top-down approach based on the analysis of intact proteins was used to identify which proteins are responsible for the significant markers that we observed.

Results: Seven proteins were differentially identified between BS/Orl and BR/Orl. One of them, Synapsin-1 was chosen to validate our method. The localization and differential expression of Synapsin1 observed by MALDI imaging were confirmed by both Western blot and immunohistochemistry experiments. Functional assays are being 
performed to explore the involvement of Synapsin-1 in the mechanisms underlying absence epilepsy.

Conclusions: The Western blot and immunohistochemistry experiments have confirmed the validity of our approach to identify the most relevant protein markers. Applied to an experimental model for absence epilepsy, it has permitted to suggest the Synapsin-1 as a protein of great interest in absence epilepsy.

\section{Neurophysiology: ICU EEG}

\subsection{5 \\ DE NOVO GENERALIZED PERIODIC DISCHARGES IN ASSOCIATION WITH PENTOBARBITAL AND PROPOFOL WITHDRAWAL}

Amar B. Bhatt ${ }^{1}$, A. Popescu ${ }^{2}$ and B. W. Abou-Khalil ${ }^{1}$ ( ${ }^{1}$ Neurology, Vanderbilt University Medical Center, Nashville, TN and

${ }^{2}$ Neurology, University of Pittsburgh Medical Center, Pittsburgh, PA)

Rationale: Anesthetic medications such as pentobarbital and propofol are widely used for the treatment of refractory status epilepticus. Continuous EEG is generally used to verify that seizure activity has been suppressed and does not recur during anesthetic withdrawal. We report a series of patients in whom anesthetic withdrawal was associated with a pattern of generalized periodic discharges, different from prior seizure activity. At times, this pattern was misinterpreted as recurrent seizure activity, leading to reinstitution of drug-induced coma, but it resolved spontaneously. We report our experience with these EEG patterns and their course. Methods: We identified five patients who developed a de novo generalized periodic pattern during pentobarbital or propofol withdrawal. Two patients received pentobarbital for increased intracranial pressure and had no prior history of seizure activity. One patient received pentobarbital and propofol for encephalopathy accompanied by a rhythmic EEG pattern that was erroneously thought to be ictal. Two patients received pentobarbital for refractory partial status epilepticus. In one of these, partial status epilepticus was not defined on EEG and was diagnosed with SPECT. In another, ictal discharges were right occipital and left frontal. The anesthetic agents were withdrawn 36-72 hours after reaching a burstsuppression pattern. We reviewed the EEGs and analyzed the course of the periodic EEG patterns and their outcome.

Results: All patients developed a de novo pattern of periodic $1-2 \mathrm{~Hz}$ generalized sharp triphasic activity, not previously seen on EEG prior to drug-induced coma. In all cases, the pattern eventually resolved spontaneously without further treatment, over 8-48 hours. However, in three cases the pattern was initially thought to represent ictal activity, and drug-induced coma was reinitiated. The pattern recurred during repeated anesthetic withdrawal, at which point it was recognized as a non-ictal pattern. In all cases but one, the patients exhibited improvement to near baseline mentation in association with clearance of anesthesia and resolution of the EEG pattern.

Conclusions: A de novo generalized periodic EEG pattern may develop after pentobarbital or propofol withdrawal. This pattern is expected to resolve spontaneously without treatment, and without recurrence of clinical seizure activity. This pattern is unlikely to represent status epilepticus and should not prompt resumption of drug-induced coma, unless there is reappearance of original electrographic seizure activity.

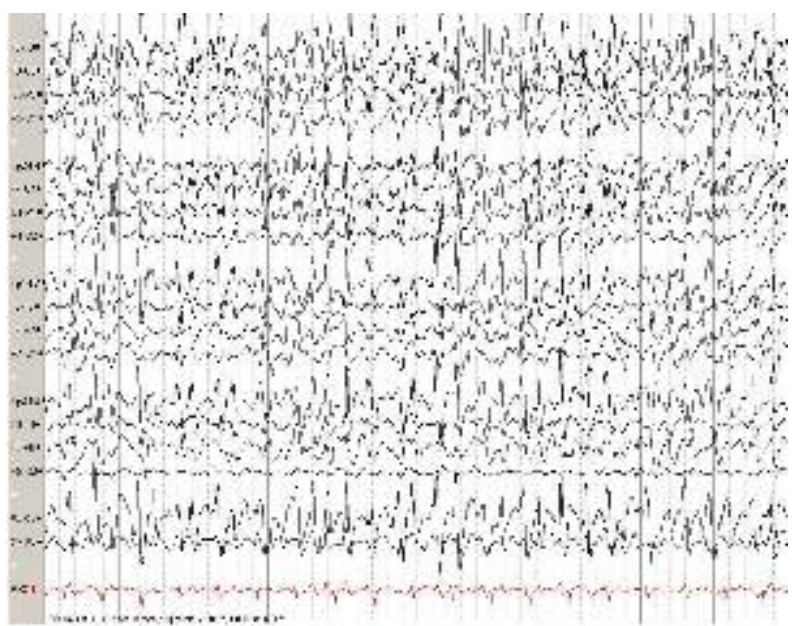

De novo generalized periodic discharges after pentobarbital and propofol withdrawal. This pattern resolved spontaneously.

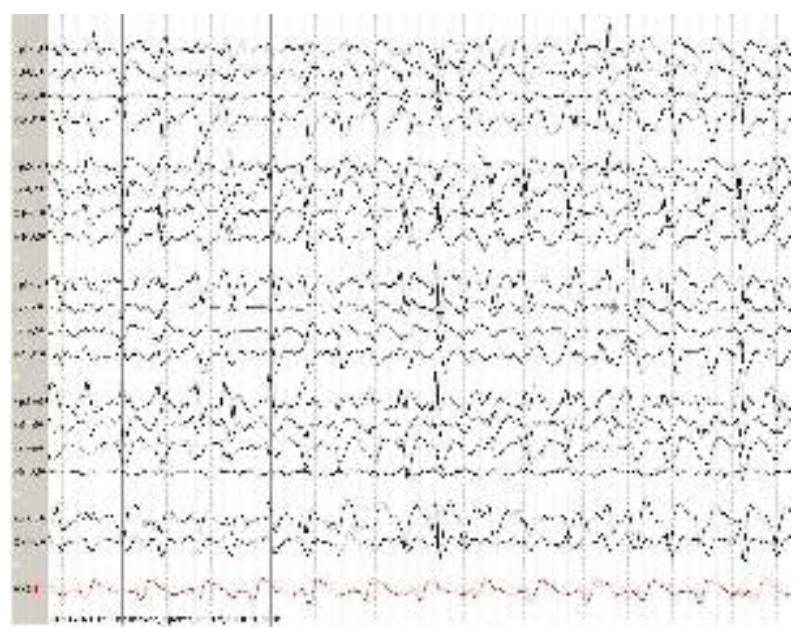

De novo generalized periodic discharges after pentobarbital withdrawal. These also resolved without treatment, and the patient made a complete neurologic recovery.

\subsection{6 \\ LATERALIZED RHYTHMIC DELTA ACTIVITY (LRDA) ON EEG IN THE CRITICALLY ILL HAS THE SAME SIGNIFICANCE AS PLEDS}

L. J. Hirsch, L. N. Manganas, N. Rampal, O. A. Petroff and Nicolas Gaspard (Neurology and Comprehensive Epilepsy Center, Yale University, New Haven, CT)

Rationale: To describe a variant of rhythmic delta activity encountered in the critically ill and its clinical correlates; and to compare it with focal polymorphic (i.e. arrhythmic) delta activity (PDA) and lateralized periodic discharges (LPDs, also known as PLEDs).

Methods: Retrospective review of urgent EEG and continuous EEG reports from $05 / 01 / 2011$ to $04 / 30 / 2012$ to identify patients with lateralized rhythmic delta activity (LRDA), LPDs and PDA. Recordings, medical charts and imaging studies were reviewed. Statistical comparisons were performed with the Chi2-test or the Fisher Exact t-test. EEG readers were all familiar with the new ACNS ICU-EEG nomenclature, including use of the term "LRDA". Results: We identified 27 patients (4.7\% of all patients) with LRDA. Mean age was 52+/-3.5years (4mo-87years) and 11 were female. Twenty patients were admitted to an ICU and 17 were stuporous or comatose. Nineteen had a lateralizing neurological examination, always consistent with the side of LRDA.

Twenty-four had a single unilateral focus, 2 had bilateral independent RDA (BIRDA) and 1 had two ipsilateral independent foci; thus, 30 
different foci of RDA were identified in these 27 patients. Eighteen were located in the frontal region, eight in the temporal region and four in the posterior region; this distribution was similar for LPDs. Most commonly, runs of LRDA consisted in $1-2 \mathrm{c} / \mathrm{s}, 50-200 \mu \mathrm{V}$, sinusoidal or saw-tooth monomorphic non-evolving waves and lasted less than $10 \mathrm{~s}$, although faster frequencies (2-3/s) and longer duration (up to $1 \mathrm{~min}$ ) were encountered.

All patients except one had an acute or remote focal brain lesion. The most common etiologies were intracranial hemorrhage, ischemic stroke, subarachnoid hemorrhage and subdural hematoma and CNS inflammatory/infectious disorder. This was overall similar to LPDs and focal PDA except that acute intracerebral hemorrhage was more frequent in the LRDA group than in the PDA group $(\mathrm{p}<0.01)$. Major systemic factors (infection, metabolic imbalance) were present in half of the cases.

Twenty-four patients had abnormal brain imaging. Lesions involved the cortex or subcortical white matter (18), the deep white matter (9) or deep gray structures (5). In case of a single focal lesion, it colocalized in the same region as LRDA in all but two cases (17/19); one had an ipsilateral thalamic hemorrhage and another had an extensive upper brainstem hemorrhage. Focal periodic and sporadic epileptiform discharges were found in 12/27 and 7/27 patients respectively and always co-localized with LRDA. Seventeen patients with LRDA (63\%) had acute seizures during their stay; this proportion was similar to that observed with LPDs/BIPDs (28/49 or $57 \%$ ) but significantly higher than with PDA (14/105 or $13 \%$; $\mathrm{p}=0.001$ ).

Conclusions: Lateralized rhythmic delta activity encountered on the EEG in critically ill patients appears to have the same clinical significance as LPDs/PLEDs, as more than half of the patients had acute seizures. Lateralized polymorphic delta has a much lower association with seizures.

\subsection{7 \\ PROGNOSTIC IMPLICATIONS OF ELECTROENCEPHALOGRAM [EEG] PATTERNS IN POST ANOXIC HYPOTHERMIC PATIENTS}

Soundarya N. Gowda, V. H. Gonzalez-Montoya, L. Kernitsky and L. D. Morton (Neurolgy, Virginia Commonwealth University, Henrico, VA)

Rationale: To determine if we can predict outcomes based on EEG patterns in patients with anoxic brain injury who are on therapeutic hypothermia.

Targeted hypothermia after cardiac arrest is now standard of care at most institutions across the country and Europe. The current AAN guidelines regarding prognostication of neurological recovery after cardiac arrest at times do not apply to the hypothermic patient populations in entirety.

Methods: A retrospective chart analysis of all patients 18 years and older, who were part of the hypothermia after cardiac arrest study was performed. Continuous EEG monitoring was performed and we gathered data on their EEG, neurological examination findings and clinical performance score [CPC] at discharge.

Results: We tested the association between presence or absence of brain stem reflexes and discharge CPC score. There was not a statistically significant association; however there was a trend towards significance: patients who had preserved brain stem reflexes had a 1.72 times higher odds of having a good CPC outcome. In patients with EEG patterns considered to be normal to benign on day 1 , the crude odds ratio for a good outcome was 31.38 [ 95\% CI (0.89$3.36)$ with $\mathrm{p}<0.0001]$. Similarly, for day 2 the crude odds ratio was 42.85 (95\% C.I. [14.49, 126.76]) and for day 3 , the crude odds ratio was $24.36(95 \%$ C.I. [8.00, 74.20])

Conclusions: There are multiple factors that affect the neurological examination and hence relying on these for prognostication might have a high false positive rate. The EEG characteristics can help predict $\mathrm{CPC}$ score and outcomes at discharge with good reliability.

\subsection{8 \\ DETERIORATION IN THE EEG FOLLOWING REWARMING IN CARDIAC ARREST PATIENTS AFTER MILD THERAPEUTIC HYPOTHERMIA}

Archana Shrestha, T. Larabee, L. Frey, K. Polovitz and J. Campbell (University of Colorado, Aurora, CO)

Rationale: Mild Therapeutic Hypothermia (MTH) is thought to provide neuroprotection after cardio-pulmonary arrest (CPA). At our institution a MTH cooling protocol is instituted following CPA. A Neurology consult and continuous video EEG (cVEEG) are often obtained in these patients. Initial clinical trials defined a 24 hour duration of $\mathrm{MTH}$, followed by a rewarming period, as the appropriate clinical treatment. However, some inflammatory processes and cerebral edema related to cerebral anoxic injury occur or persist beyond a 24 hour window, which could result in clinical and neurophysiological deterioration after rewarming in some patients.

Methods: We reviewed consecutive cases from the University of Colorado Hospital between April, 2011 and March, 2012 and identified patients who underwent MTH after CPA and had cVEEG's done. Patients were cooled for 24 hours and rewarmed over approximately 12 hours. CVEEG's were recorded during the cooling phase and continued for at least 12 hours post warming.

Results: There were a total of 27 patients with cVEEG's who underwent MTH following CPA. Three patients were identified who had deterioration in their EEG's following rewarming. During cooling, the EEG's showed generalized suppression or burst suppression in these patients.

Case 1: 79 year old female whose cVEEG initially improved after rewarming, demonstrating a continuous pattern with moderate slowing. At 64 hours post-arrest, the cVEEG deteriorated to generalized periodic epileptiform discharges followed by burstsuppression.

Case 2: 52 year old male whose cVEEG initially improved after rewarming demonstrating moderate slowing with continuity and reactivity. At 48 hours post-arrest, his cVEEG background deteriorated to burst suppression then severe suppression. Case 3: 55 year old male whose cVEEG initially improved after rewarming and was continuous and reactive, but at 66 hours postarrest deteriorated to burst-suppression with subsequent progressive suppression.

There were no clear medical changes or complications to account for the deterioration in these patients' EEG's. All 3 patients died.

Conclusions: In these 3 cases, there was improvement in the EEG after rewarming followed by deterioration to a malignant EEG pattern. This deterioration may indicate further progression of underlying secondary injury processes following anoxic brain injury. Our results highlight the potential importance of the cVEEG during MTH and the benefit of continuing it following the rewarming phase. Further neurophysiologically based studies may also help determine if MTH applied longer than 24 hours may offer an additional neuroprotective effect in some CPA patients.

\subsection{9 \\ THE TEN MINUTES EEG USING SUBDERMAL ELECTRODES IN THE ICU}

Karine Abou Khaled and S. Farhat (Hotel Dieu de France Hospital, Beirut, Lebanon)

Rationale: EEGs completed in the intensive care units (ICU) are generally time-consuming and practically not easy in a busy hospital setting. Moreover a lot of environmental factors and artifacts interfere with the quality of the recording. We report our experience using shorter recording EEG time in critically ill patients.

Methods: All adult patients admitted to the ICU at our university hospital in year 2011 and who required EEG were included. Modified brief duration EEG with 8 subdermal electrodes was done in emergency settings for the majority of the cases. Electrodes used 
were Fp1/2, C3/4, T3/4 and O1/2. Some patients had continuous EEG done after initial EEG or additional daily EEGs.

Charts were reviewed for patients' demographics, diagnosis, medications and complications.

All initial EEGs per patient were reviewed for background

characterization and identification of periodic patterns, discharges or seizures. Repeated EEGs were excluded for this analysis.

Results: The initial EEGs of 62 patients were analyzed. The mean age was 59(range 19-87). Seventy percent were males. Average EEG duration was 10.95 minutes (range 5-18).

Regarding background characterization: $74 \%$ had diffuse slowing, the majority being moderate or severe and $6.5 \%$ had electrocerebral silence. Only 3\% were within normal limits.

Seizures and epileptic discharges were seen in $9.7 \%$ of cases.

Triphasics were identified in $6.5 \%$.

Technically, impedances were perfect, with less $60 \mathrm{~Hz}$ artifacts and motion artifacts compared to disk electrodes. Notch was used at times and each recording occupied the technician for less than 20 minutes totally including EEG hookup.

No local skin complications were observed.

Conclusions: In a hospital with limited resources a 10 minutes EEG using subdermal electrodes can be an easy, efficient and practical alternative to traditional prolonged EEG in critically ill patients. Further comparative studies to prolonged EEG are needed to assess impact on management and prognosis.

\subsection{0}

\section{INCIDENCE OF STATUS EPILEPTICUS DIAGNOSED BY} EEG IN A GENERAL HOSPITAL

Taíssa Ferrari Marinho ${ }^{1}$, A. P. Hamad ${ }^{1}$, L. B. Sampaio ${ }^{1}$, R. S. Neves ${ }^{1}$, D. D. Araújo ${ }^{1}$, P. R. Sanches ${ }^{2}$ and L. O. Caboclo ${ }^{1}{ }^{1}$ Neurophysiology, Hospital Israelita Albert Einstein, São Paulo, Brazil and ${ }^{2}$ Intensive Care Unit, Hospital Israelita Albert Einstein, São Paulo, Brazil)

Rationale: Status epilepticus (SE) is a major neurological and medical emergency that requires acute medical management. SE is routinely classified in convulsive status epilepticus (CSE) and nonconvulsive status epilepticus (NCSE), when impairment of consciousness is present with no overt signs of seizure activity. NCSE is diagnosed in up to $8 \%$ of patients with coma of unknown cause (Towne et al., 2000). Confirmation of NCSE requires EEG showing seizure activity. We evaluated the frequency of CSE and NCSE in patients in whom EEG was performed in order to confirm or exclude diagnosis of SE. For diagnosis of NCSE strict EEG criteria were applied (Kaplan, 2007).

Methods: Patients older than one year submitted to EEG exams at the Neurophysiology Department of Hospital Israelita Albert Einstein, São Paulo, Brazil, between 2011 and 2012, were included in this study. EEG exams were ordered to investigate the diagnosis of SE in patients with clinically suspected SE, or in patients with impairment of consciousness of unknown cause. EEG records were reviewed by two independent observers. Diagnosis of CSE was firmed based on clinical and EEG findings. Diagnosis of NCSE, defined according to EEG characteristics, was confirmed only if 1) the criteria for SE were fulfilled, defined as continuous or nearly continuous electrographic seizure activity during the EEG record, without clinical seizure activity; and 2) at least two EEG interpreters independently agreed that the pattern represented seizure activity. Results: Four hundred and forty-eight EEG exams of 293 consecutive patients (128 women) were included in the study. Age varied from 1 to 98 years (mean 63.38 years, SD 25.30). Clinical diagnoses included impairment of consciousness with no apparent cause (132 patients), epilepsy and seizures (76), stroke (42), traumatic brain injury (11), encephalopathy (10) and others (22). A total of nine cases $(2 \%)$ of SE were identified: 7 of them had CSE and 2 NCSE. Four patients presented focal SE and 5 generalized. Conclusions: Diagnosis of CSE can usually be confirmed based on clinical characteristics. NCSE, on the other hand, requires EEG for the confirmation of diagnosis. In patients with clinically suspected SE and in those with unexplained impairment of consciousness, EEG is mandatory for confirmation of this diagnosis. In this series, the use of strict criteria for definition of NCSE might have led to the low number of confirmed cases.

\subsection{1 \\ WTIHDRAWNS}

\subsection{2 \\ NONMETABOLIC TRIPHASIC WAVES AND FRONTAL RHYTHMIC SLOW ACTIVITY IN PROGNOSIS OF SUBARACHNOID HEMORRHAGE}

Tyson Burghardt, W. Mohamed, A. Solaiman, M. Basha and A. Shah (Wayne State University School of Medicine, Detroit, MI)

Rationale: Patients with subarachnoid hemorrhage (SAH) undergo continuous electroencephalography (cEEG) monitoring in the ICU for the diagnosis of nonconvulsive status epilepticus (NCSE) in patients with impaired consciousness and for the identification of prognostic information in patients with poor-grade SAH. During prolonged monitoring, EEG patterns of unclear significance are often encountered. We describe and characterize commonly encountered EEG patterns in SAH patients.

Methods: Patients with SAH admitted to two academic institutions between July 2009 and May 2012 were identified. SAH secondary to trauma, surgery, intraparenchymal bleeds or resulting from arteriovenous malformation rupture were excluded. Of 154 such patients, 52 (34\%) received a routine EEG or cEEG monitoring during their hospitalization. EEG reports were reviewed and classified as to the presence of diffuse slowing, epileptiform activity, and frontally-predominant rhythmic and periodic slow activity. The presence of triphasic morphology in the latter category was also noted.

Results: Seventeen of 52 (33\%) exhibited frontally-predominant rhythmic and periodic slow activity, 15 of which had triphasic wave (TW) morphology without metabolic derangement (29\%). In addition 21 of 52, (40\%) exhibited diffuse arrhythmic slowing and 15 (29\%) demonstrated focal or multifocal epileptiform discharges. One patient had severe diffuse suppression and $2(4 \%)$ had a normal EEG. The average length of stay was 39 days in those with TW morphology and 24 in those without. Outcome upon discharge, however, was similar with good outcome (discharge to home or acute rehab) seen in 50\% of patients with TW morphology and $47 \%$ of patients in those without.

Conclusions: Nonmetabolic TW are scantly reported in the literature and typically associated with diencephalic and brainstem lesion. We find that nonmetabolic TW and frontally-predominant, rhythmic slow wave activity is a common EEG finding in patients with SAH. It may serve as a prognostic indicator of longer length of stay; however, further analysis is needed.

\subsection{3 \\ PECULIAR ELECTROGRAPHIC PATTERN ASSOCIATED WITH ANTI-NMDAR ENCEPHALITIS. CASE SERIES REPORT \\ Carla Bastos, E. Garzon, C. H. Moreira, C. L. Jorge, V. Passarelli, J. Q. Andrade, P. E. Marchiori, P. R. Nobrega, F. V. Silva, M. M. Simabukuro, S. E. Senaha, R. G. Watanabe, R. M. Valerio, M. L. Martyn, F. Freua, N. P. Novaes, H. Castro-Lima, A. S. Bossoni, R. Nitrini and L. H. Castro (Department of Neurology, University of São Paulo, São Paulo, Brazil)}

Rationale: Anti-N-methyl-D-aspartate receptor (anti-NMDAr) encephalitis is an autoimune disorder that presents with psychiatric manifestations, abnormal movements, seizures and status epilepticus. 
Early recognition of the cardinal clinical features and aggressive treatment with immunomodulatory agents improves outcome. We describe a peculiar, and, possibly, characteristic electrographic pattern associated with anti-NMDAR encephalitis.

Methods: Case series report of three young women that presented with cerebral spinal fluid (CSF) and serum anti-NMDAR antibodies in the setting of status epilepticus, coma, abnormal movements and psychiatric symptoms. Electroencephalogram (EEG) showed a unique pattern: monomorphic rhythmic delta activity.

Results: Three young women (ages 13 to 34 years) were admitted to the Intensive Care Unit with new onset status epilepticus, that followed a subacute course of behavioral changes, confusion, abnormal movements and seizures that quickly evolved to status epilepticus. Magnetic resonance imaging exam was normal in all cases. CSF analysis showed a mild lymphomonocytic pleocytosis. Anti-NMDAR antibodies were present in the CSF and serum in all cases. None of the patients presented an underlying malignancy. In two cases status epilepticus was refractory to antiepileptic drugs, high dose intravenous steroids, and immunosuppressive agents. In all cases, a characteristic and unique EEG pattern was noted: monomorphic rhythmic theta-delta activity. Despite aggressive treatment with immunomodulatory agentes, one patient died and another patient evolved to a minimally conscious state.

Conclusions: We describe a previously recognized characteristic EEG pattern associated with anti-NMDAR encephalitis: monomorphic rhythmic theta-delta activity. Awareness of this peculiar EEG pattern may allow early recognition of this condition, and lead early initiation of aggressive immunomodulatory therapy, with positive impact on prognosis.

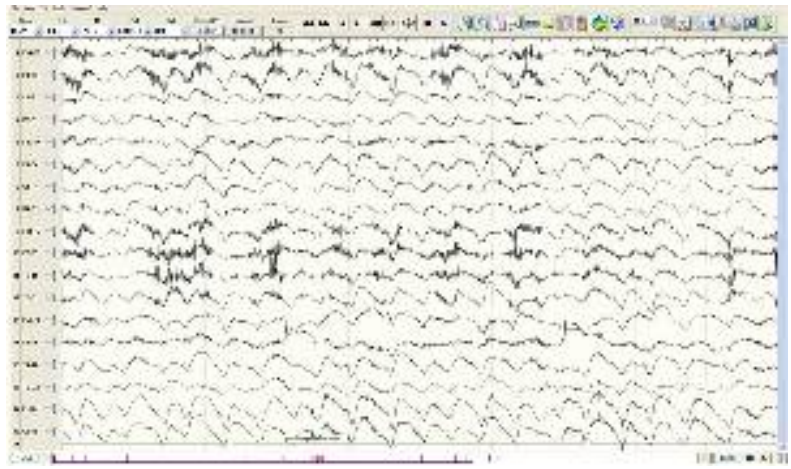

Figure 1: EEG with monomorphic rhythmic delta activity, clinically the patient showed stereotyped facial movements.

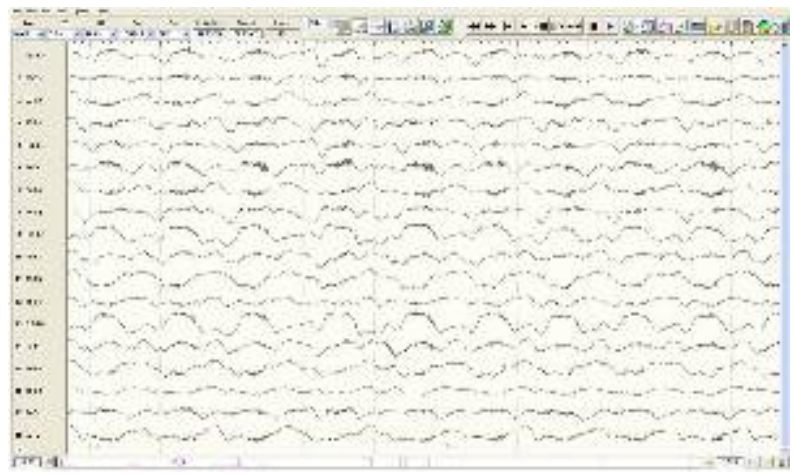

Figure2: EEG with monomorphic rhythmic delta activity, clinically patient in coma.

\section{Neurophysiology: Other Clinical EEG}

\subsection{4 \\ POLARIZED AND LENS COLOR EFFECTS ON PHOTOPAROXYSMAL RESPONSE}

E. J. Kobylarz, Myrta I. Otero, S. Rydjeski and R. P. Morse (Dartmouth Hitchcock Medical Center, Lebanon, NH)

Rationale: Approximately 5\% of epilepsy patients' photosensitivity can be detected on EEG recordings. This photoparoxysmal response (PPR) may prevent patients from performing their daily activities and also result in anxiety. The objective of this study is to optimize the use of Z1 and other polarized and colored lenses for reducing PPR in epileptics. We propose that by covering central and temporal visual fields with either $\mathrm{Z} 1$ lenses plus side guards or elongated polarized lenses may suppress the PPR by altering the luminance and/or wavelength.

Methods: 15 pediatric patients with Type 4 PPR (Waltz et al.) were tested with and without $\mathrm{Z} 1$ lenses using photic stimulation from 1 $21 \mathrm{~Hz} .10$ patients had primary generalized epilepsy (4 JME \& 6 Absence), 2 had reflex epilepsy and 2 had Dravet's Syndrome. Results: Responses were classified into 3 groups: PPR disappearance, persistence or attenuation. 9 patients had reduced PPR $(60 \%)$ and in 5 patients PPR disappeared (33\%). 1 patient $(7 \%)$ demonstrated persistence of the PPR with Z1 lenses. One patient tested with side guards plus cobalt blue tint polarized lens sunglasses showed marked PPR reduction. A patient tested with elongated polarized sunglasses covering the temporal visual field showed PPR attenuation. Placing a red lens over the photic stimulation lamp augmented the PPR from 1-19 Hz stimulation in 1 patient. A blue lens over the photic lamp resulted in disappearance of the PPR from $1-13 \mathrm{~Hz}$ and attenuation from $15-19 \mathrm{~Hz}$ in 1 patient.

Conclusions: Z1 lenses reduced the PPR in nearly all patients. Possible mechanisms include polarization, reduction of luminence, and filtering of red light. Further testing of photosensitive patients will be performed. Comparisons will be made between Z1, elongated polarized, cobalt blue, red and green lenses.

\subsection{5 \\ ELECTROENCEPHALOGRAPHIC CHANGES AND SEIZURE OUTCOME OF PRES}

Zhiyi Sha, B. Moran, A. McKinney and T. Henry (University of Minnesota, Minneapolis, MN)

Rationale: Seizure is one of the most common clinical presentations in patients with posterior reversible encephalopathy syndrome (PRES). This syndrome is usually considered to be fully reversible and not a cause for chronic epilepsy or persistent cortical dysfunction. To date, the data of EEG findings in patients with PRES is limited. In this study, we retrospectively evaluated EEG characteristics in PRES patients in a single medical center. We also evaluated the long term outcome regarding seizures occurrence beyond the acute phase in these patients

Methods: We searched the radiology database at the University of Minnesota from 1997-2011. We identified patients with radiologically diagnosed PRES. Among the patients with PRES, we reviewed MRI images, EEG finding, clinical manifestations including seizure occurrences and clinical outcome beyond the acute phase.

Results: 75 patients were included in the study. 58/75 (77.3\%) of patients with PRES had seizures. A total of 48 EEG studies were performed in 38 patients. 38/48 (79.1\%) of EEG studies during PRES had generalized slowing of the background activity and other abnormalities, 25/48 (52.0\%) had generalized slowing alone. 13/48 (27.1\%) of EEG studies had sharps and/or spikes. 4/75 (5.3\%) of these patients had seizures later than one month after the onset of PRES. None of these 4 patients had seizures before the episode of PRES. Two patients developed chronic epilepsy with seizures occurring later than one year after the PRES. 
Conclusions: The most common EEG pattern in patients with PRES was generalized slowing. Many patients had sharp waves and/or spikes. Most patients who had spikes during PRES did not subsequently develop chronic epilepsy. No patient developed chronic epilepsy in the absence of spikes during PRES. PRES may infrequently cause partial epilepsy.

\subsection{6 \\ HOW ACCURATELY CAN DENSE ARRAY EEG ESTIMATE INTERICTAL SPIKE SOURCE?}

Madoka Yamazaki $^{1,2}$, M. Terrill ${ }^{4}$, A. Fujimoto ${ }^{2}$, T. Yamamoto $^{2}$ and D. M. Tucker ${ }^{3,4}\left({ }^{1}\right.$ Daito Bunka University, Higashimatsuyama, Japan; ${ }^{2}$ Comprehensive Epilepsy Center, Seirei Hamamatsu General Hospital, Hamamatsu, Japan; ${ }^{3}$ Department of Psychology, University of Oregon, Eugene, OR and ${ }^{4}$ Electrical Geodesics, Inc., Eugene, OR)

Rationale: Dense array EEG (dEEG) consisted of over 100 channels has been focused in clinical Epilepsy field. The purpose of this study is to evaluate the clinical usefulness of dEEG for the detection rate and localization of interictal spikes in temporal lobe epilepsy.

Methods: We conducted simultaneous recording 1) 128channel dEEG and sphenoidal electrodes in 3 patients and 2) 256 channel dEEG and intracranial EEG (icEEG) implanted over the lateral and mesial temporal lobe in patients with intractable epilepsy in another 6 patients. We calculated the dEEG spike detection rate and applied source estimation to dEEG to compare noninvasive localization to the invasive recordings.

Results: 1) $90.4 \%$ of the interictal spikes detected by the dEEG were outside of the 10/20 electrode array and therefore not detected in the $10 / 20$ montage. All spikes were localized in temporal lobe and $91 \%$ spikes were well localized in medial temporal lobe.

2) 316 of 719 interictal spikes (44\%) were detected by dEEG by examining the 256 channel head surface array. The average icEEG amplitude of dEEG detectable spikes was $1036 \mu \mathrm{V}$, and that of dEEG undetectable spikes was $794 \mu \mathrm{V}(\mathrm{P}<0.05)$. All spikes detected in dEEG were localized to the temporal lobe. 262 of 316 spikes $(83 \%)$ were well localized in mesial temporal lobe, close to the position confirmed by subdural electrodes.

Conclusions: Dense array EEG may have a potential for providing an alternative to sphenoidal electrode monitoring in patients with temporal lobe epilepsy and be clinically useful in the presurgical work-up for epilepsy, providing accurate noninvasive guidance for the placement of intracranial electrodes.

\subsection{7 \\ THE RELATIONSHIP BETWEEN SEIZURE ONSET ZONE AND ICTAL TACHYCARDIA: AN INTRACRANIAL EEG STUDY}

Maria Stefanidou, C. Carlson and D. Friedman (New York University, New York, NY)

Rationale: Many seizures are associated with significant ictal tachycardia, which has been associated with increased risk of SUDEP. Prior studies using scalp EEG have suggested that the site and lateralization of seizure onset influences the degree of ictal HR changes, but precise anatomical localization of these regions is poorly understood. We retrospectively examined the relationship between the seizure onset location and spread and ictal tachycardia in patients undergoing intracranial EEG monitoring as part of their evaluation for epilepsy surgery.

Methods: We reviewed the NYU Comprehensive Epilepsy Center database to identify patients who underwent bilateral intracranial EEG recordings between January 2010 and June 2011. Children below 12 years of age and patients with prior brain resection, uninterpretable EKG at seizure onset, and those post brain radiation therapy or in status epilepticus were excluded. Nineteen patients were identified. Three consecutive, electrographically similar seizures were reviewed for each patient. For patients with fewer than 3 similar seizures and if more seizure types were present, those were also included for a total of 3-4 seizures per person. HR was calculated based on the computer detected R-R interval (CareFusion Nicolet, Madison, WI) and the EKG was visually reviewed for accuracy. Only parts of seizures correlating to accurate HR readings were included. Ictal tachycardia was defined as an elevation for HR $>20 \%$ of preictal baseline. Ictal localization was divided into frontal $(\mathrm{F})$, orbitofrontal (O-F), temporal (T), mesial temporal (MT), parietal (P) and occipital $(\mathrm{O})$ regions for each second of the seizure. Comparisons were made using Chi-square or Fischer's exact test.

Results: Fifty-nine seizures were reviewed from 19 patients (mean age 29.7, range 15-51) and 9 seizures were excluded due to uninterpretable EKG. Of the remaining seizures, 40 (80\%) were associated with ictal tachycardia. Lateralization of the seizure onset zone did not predict occurrence of ictal tachycardia $(\mathrm{p}=0.17)$. However, bilateral seizure onsets were associated with early $(<10$ $\mathrm{sec})$ ictal tachycardia $(\mathrm{p}=0.021)$. Among seizures that were unilateral at ictal onset (28), 46\% developed tachycardia only upon spread to the contralateral hemisphere. There was no association seen between lobar onset zone (F, O-F, T, MT, P, O) and development of tachycardia $(\mathrm{p}=0.35)$. There was no difference seen in development of tachycardia between genders $(\mathrm{p}=1.0)$.

Conclusions: Neither the localization or lateralization of the seizure onset zone predicts the occurrence of ictal tachycardia. However, bilateral involvement at seizure onset is associated with early ictal tachycardia ( $<10$ secs). These findings suggest that ictal tachycardia is probably linked to the ictal spread and possible involvement of subcortical networks, rather than the seizure onset zone.

\subsection{8}

ICTAL EEG RECORDING IN NEONATAL PATHOLOGY Hitoshi Yamamoto ${ }^{1}$ and A. Okumura ${ }^{2}\left({ }^{1}\right.$ Pediatrics, St. Marianna University School of Medicine, Kawasaki, Japan and ${ }^{2}$ Juntendo University School of Medicine, Tokyo, Japan)

Rationale: In order to diagnose neonatal seizures correctly and objectively, we must understand ictal EEG findings on neonatal EEG. Neonatal seizures have generally been identified in most NICUs only by direct clinical observation. However, there is usually a lack of objectivity as to whether seizures are categorized as epileptic or nonepileptic. A major characteristic of neonatal seizures is electroclinical dissociation and some of the electrographic seizures are not associated with clinical symptoms. These facts strongly suggest that ictal EEG recording is essential for the accurate identification of neonatal seizures of cortical origin and distinction from non-epileptic paroxysmal events of non-cortical origin. We described ictal and background EEG findings, and a variety of EEG patterns in infants with neonatal seizures of acute symptomatic origin.

Methods: We recorded over 10 ictal EEGs from the neonates at 1 to 10 days of age associated with several pathologies in our NICU using the digital EEG recording system (NicoletOne TM monitor). The underlying disorders included hypoxic-ischemic encephalopathy, acute metabolic disorders such as hypoglycemia and hypocalcemia, central nervous system infections, cerebrovascular disorders and inborn errors of metabolism.

Results: There were electrographic features of seizures in the neonates that were unique to this period. Ictal EEG changes of neonatal seizures were characterized by rhythmic, repetitive, and stereotyped discharges lasting for at least 10 seconds on two or more EEG channels. EEG seizure discharges were principally focal and restricted to relatively circumscribed regions of the brain regardless of underlying etiologies. All ictal EEG changes in neonates began focally, except for the more generalized activities associated with myoclonic jerks or epileptic spasms. Ictal EEG changes were unifocal or multifocal. Even in an individual infant, ictal EEG changes could arise from different foci at different times. EEG foci often migrated from one to another even during a single sizure, within one cerebral hemisphere or from one hemisphere to the other. 
Conclusions: Ictal EEG recording is essential for the accurate identification of neonatal seizures of cortical origin and distinction from non-epileptic paroxysmal events of non-cortical origin for appropriate treatments with anticonvulsants.

\subsection{9 \\ REORGANIZATION OF THE BACKGROUND ECOG UNDERLIES STIMULATION-INDUCED AFTERDISCHARGE \\ Giridhar P. Kalamangalam (University of Texas, Houston, TX)}

Rationale: First described by Adrian in 1936, afterdischarges (ADs) are runs of rhythmic focal epileptiform activity induced by cortical electrical stimulation (CES). Observations in experimental preparations (e.g. Pinsky \& Burns, J Neurophysiol 25:359-79, 1962) and humans undergoing extraoperative mapping (e.g. Blume et al, Clin Neurophysiol 115(4):982-9, 2004) document sufficient stimulus intensity for ADs to occur. Other factors predictive of ADs are brain location and low-frequency power in the background electrocorticogram (ECoG) at the stimulus site (Lesser et al, Brain 131(6):1528-39, 2008). However, it remains unclear why and how ADs occur following CES. We propose a mechanism here, based on human ECoG data.

Methods: Extraoperative CES ECoG data over the left temporal lobe were reviewed on four $(\mathrm{N}=4)$ patients undergoing presurgical subdural grid evaluation. 1079 bipolar stimuli ranging from 2-10 mA were delivered intermittently over several hours in each patient during standardized motor and language testing. Forty-two ADs were observed, 32 of which lasted $\geq 8$ seconds and were analyzed $(n=32)$. Power spectra of AD segments were compared to those from lengthmatched segments of baseline ECoG and following subthreshold stimuli.

Results: All four morphologies of AD described by Blume et al (2004) were observed: rhythmic waves, spike-waves, polyspike bursts, and sequential spikes; 12/32 (37.5\%) of ADs had features of more than one type. Power spectra of background ECoG epochs from all tested channels - including those subsequently exhibiting ADs were broadband and multimodal, with peaks in one or more of the classical $(\alpha, \beta, \delta$, etc.) EEG bands. Power spectra of ADs bore a consistent relation to background ECoG spectra from the same channel, in one of three ways that depended on Blume type: (i) a 'condensing' or 'clumping' relation, comprising lessening of the breadth of the spectrum and AD spectral peaks appearing at the same locations, but less dispersed than corresponding peaks from the background ECoG; (ii) condensation plus harmonics, where in addition, peaks appeared at selected harmonics of condensed peaks and (iii) harmonic-dominant, where there was minimal condensed peak spectral power, with harmonic content dominating. Moreover, power spectra of epochs following subthreshold stimuli differed from baseline spectra in a similar though less prominent fashion.

Conclusions: Power spectral analysis of ADs following CES indicate that ADs, rather than representing de novo change, arise fundamentally from a 'reorganization' of background brain rhythms. Reorganization takes the form of 'condensation' of pre-existing peaks, as well as a process that generates higher harmonics of the condensed spectral clusters. Together, these features synchronize the baseline multifrequency ECoG into the coherent rhythm of an $A D$, with their relative contributions determining Blume type. Putative underlying neurophysiological mechanisms will be discussed; a larger study including quantitative modeling of the phenomena seen here is under way.
3.090

THE LOCALIZATION AND DISTRIBUTION OF INTERICTAL DISCHARGES ON THE MESIAL TEMPORAL LOBE EPILEPSY -THE COMPARISON OF 256 CHANNEL DENSE ARRAY EEG WITH 10-20 INTERNATIONAL SCALP EEG-

Yuki Sasaki, A. Fujimoto, T. Yokota, H. Enoki, M. Nishimura and T. Yamamoto (Seirei Hamamatsu General Hospital, Hamamatsu, Japan)

Rationale: The mesial temporal lobe epilepsy tends to be medically refractory, however, it is considered that the seizure can be inhibited by surgery and thus the diagnosis of focus of the seizure is important. Usually 10-20 international EEG is performed and it is known that it is sometimes hard to obtain interictal discharges from the recording. We investigated how the interictal discharges are shown in 256 channel dense array EEG and compared to them in 10-20 EEG. Methods: There were four patients ( 2 male, 2 female, age 17-38; mean 30.2 years old) with the mesial temporal lobe epilepsy, who have been free from seizure after surgery. 256ch dEEG were performed before surgery. We picked up five interictal spikes recorded during light sleep each and examine which cannel's amplitude is the highest of all and investigated the distribution of the spikes, which were compared to scalp EEG recording.

Results: The channel which has the highest amplitude is located out of scalp in 18 out of 20 spikes. The highest amplitude is about 1.5 times greater than that of 10-20 at average. Mostly it was shown on forehead, zygomatic area and posterior auricle area. The channels on face detected spikes widely, including that in the opposite side of the focus of seizure.

Conclusions: On the case of the mesial temporal epilepsy, the channels on forehead, zygomatic area and posterior auricle area showed spikes well. The channels which had maximum negativities were located in that area mostly and it was much greater than that of 10-20 EEG recording. This is suggested that the channels on face can be more useful to detect interictal discharges on the mesial temporal lobe epilepsy.

\subsection{1 \\ DRUG-INDUCED EEG PATTERN PREDICTS EFFECTIVENESS OF KETAMINE IN TREATING REFRACTORY STATUS EPILEPTICUS}

Abdulradha Alqallaf, A. Shah and M. Basha (Neurology Dept., DMC, Wayne State University School of Medicine, Detroit, MI)

Rationale: Continuous electroencephalography (cEEG) is a valuable tool in the management of status epilepticus (SE) and guides treatment with antiepileptics (AEDs) and anesthetics. Ketamine is an anesthetic that has been recently employed in the treatment of SE. In patients undergoing anesthesia, Ketamine induces an alpha frontocentral rhythm that resembles EEG spindle oscillations generated by the reticular thalamus. We describe characteristic EEG rhythms induced by Ketamine in the status epilepticus population and its efficacy in control of ongoing seizures in refractory SE.

Methods: Records of 24 patients with SE requiring cEEG monitoring between July 2011 and June 2012 were reviewed. Ten received Ketamine as part of AED regimen at doses 8 to $92 \mathrm{microgram} / \mathrm{kg} / \mathrm{min}$ for 2 to 14 days. EEGs were reviewed prior to initiation, during administration, and after cessation of Ketamine.

Results: Ten of $24(41 \%)$ received Ketamine as part of the treatment for SE. No clear adverse effects were reported with prolonged use of Ketamine.

Characteristic EEG changes described as generalized frontally predominant archiform theta and alpha rhythms ( 7 to $13 \mathrm{~Hz}$ ) of 25 to 35 microvolts in amplitude were seen in 4 patients. The appearance of such EEG changes was not dependent on dose (range 8 to 67 $\mathrm{mcg} / \mathrm{kg} / \mathrm{min}$ ) and occurred regardless of co-administration of other anesthetics. 
All four patients with ketamine EEG effect had temporal or frontotemporal SE.

In 3 of 4 (75\%), Ketamine introduction resulted in termination of SE without burst suppression. In all six patients without characteristic EEG changes, Ketamine did not provide resolution of status. In 3 of 6 , the cessation of status was eventually achieved with the addition of pentobarbital (2), and with focal brain resection (1). Three of these patients died while no deaths were reported in the group where Ketamine successfully aborted status epilepticus. Five of the six patients had extratemporal SE.

Conclusions: Ketamine causes a characteristic fronto-central archiform theta and alpha rhythms in some patients being treated for SE. The underlying mechanism that generates this surface EEG pattern is unknown, but it appears to correlate with success of Ketamine in aborting SE. This may be related to the underlying pathophysiology of the patient's SE, localization of the seizure activity and patient's potential susceptibility to Ketamine.

Table 1.

\begin{tabular}{|c|c|c|c|c|c|c|c|c|c|}
\hline Piticnt & $\begin{array}{c}A g c \\
/ \operatorname{sex}\end{array}$ & $\begin{array}{l}\text { SE } \\
\text { Semiology \& } \\
\text { EEG } \\
\text { SPSH,CPSH } \\
\text { I. I. ICmp } \\
\text { PLED }\end{array}$ & $\begin{array}{l}\text { Intraparenchymal } \\
\text { hemorchage }\end{array}$ & $\begin{array}{c}\begin{array}{c}\mathrm{Max} / \mathrm{min} \\
\mathrm{d} / \mathrm{si} \\
\text { (m)ceg } / \mathrm{kg} / \mathrm{min})\end{array} \\
\\
74 / 74\end{array}$ & $\begin{array}{l}\text { to. } \\
\text { of } \\
4\end{array}$ & $\begin{array}{l}\text { Cuncomitimt } \\
\text { ancshlhetics } \\
\text { Midarolam/ } \\
\text { Pentobarbital }\end{array}$ & $\begin{array}{c}\text { Ketamine } \\
\text { tifGi } \\
\text { ellect } \\
\text { None }\end{array}$ & $\begin{array}{l}\text { Ketamine } \\
\text { cllect un } \\
\text { SE } \\
\text { No }\end{array}$ & $\begin{array}{l}\text { Paticnl } \\
\text { outcome } \\
\text { Death brain } \\
\text { stem } \\
\text { herniation }\end{array}$ \\
\hline 2 & $62 / \mathrm{m}$ & XCSE/GPED & $\begin{array}{l}\text { Anoxic Brain } \\
\text { Injury }\end{array}$ & $84 / 8$ & 1 & Midazolam & None & No & $\begin{array}{c}\text { Death } \\
\text { Withdrawal } \\
\text { ul carc }\end{array}$ \\
\hline 3 & $60 / \mathrm{m}$ & $\begin{array}{l}\text { SPSE. CPSE/ } \\
\text { I. } \\
\text { frontolemp } \\
\$ 7 .\end{array}$ & $\begin{array}{l}\text { Finepphalomialaciat. } \\
\text { scpsis }\end{array}$ & $19 / 19$ & 4 & Midlazolam & $\gamma_{c s}$ & No & $\begin{array}{l}\text { Subaculc } \\
\text { facility }\end{array}$ \\
\hline 4 & $65 / \mathrm{m}$ & $\begin{array}{l}\text { CPSH.SG / } \\
\text { independent } \\
\text { Lt and Rt } \\
\text { postcrior sL }\end{array}$ & $\begin{array}{l}\text { Linknown,? } \\
\text { medication non } \\
\text { compliamo: }\end{array}$ & $78 / 34$ & 3 & $\begin{array}{l}\text { Midazolam/ } \\
\text { Pentobarbital }\end{array}$ & None & No & $\begin{array}{l}\text { Subacute } \\
\text { facility. } \\
\text { recurrence } \\
\text { of } s z \text {. dcall }\end{array}$ \\
\hline 5 & $68 / \pi$ & $\begin{array}{c}\mathrm{SG} / \mathrm{Lt} \\
\text { frontal s/ }\end{array}$ & Muecocte & $92 / 17$ & 3 & Mielazolal:urm & Nunce & No & $\begin{array}{l}\text { Nursing } \\
\text { home }\end{array}$ \\
\hline 6 & $56 / \pi 1$ & $\begin{array}{c}\mathrm{SG} / \mathrm{Rt} \\
\text { frontukmip } \\
\text { Ss. }\end{array}$ & $\begin{array}{l}\text { Metaslatic brain } \\
\text { lesions }\end{array}$ & $67 / 15$ & 4 & Mielatallatrm & $\gamma_{\mathrm{ess}}$ & Yes & Hume \\
\hline 7 & $50 / 6$ & $\begin{array}{l}\text { Independent. } \\
\text { LI \& RL } \\
\text { frontal sz }\end{array}$ & $\begin{array}{l}\text { l.inknown, new } \\
\text { onset, hx of } \\
\text { alcohol abuse }\end{array}$ & $67 / 17$ & 1 & $\begin{array}{l}\text { Midlazolany/ } \\
\text { Propofol }\end{array}$ & None & No & Inome \\
\hline 8 & $42 / \mathrm{f}$ & $\begin{array}{l}\text { CPSE/Left } \\
\text { LCMPP l'LEDS }\end{array}$ & $\begin{array}{l}\text { Lnknown, new } \\
\text { unscl }\end{array}$ & $24 / 8$ & 4 & $\begin{array}{l}\text { Propofol/ } \\
\text { Midazolam } \\
\text { or } \\
\text { 1.orarcpan }\end{array}$ & Ycs & Ycs & Rchab \\
\hline 9 & $57 / f$ & $\begin{array}{l}\text { CPSH, SG/RI } \\
\text { frontotemp } \\
\text { sz and } \\
\text { Pl.t.lls }\end{array}$ & $\begin{array}{l}\text { RI Lionlotcmp } \\
\text { epidural \& } \\
\text { Intraparenchymal } \\
\text { hematoma; prior } \\
\text { hx of ss }\end{array}$ & $601 / 12$ & 5 & $\begin{array}{c}\text { Propofonl/ } \\
\text { Pentobarbital }\end{array}$ & None & No & $\begin{array}{l}\text { Death } \\
\text { cardiac } \\
\text { arrest }\end{array}$ \\
\hline 10 & $52 / 6$ & $\begin{array}{l}\text { ClSE. SG/ } \\
\text { RI Icmi } \\
\text { PI.tDs, I.1. } \\
\text { posterior sz }\end{array}$ & $\begin{array}{c}\text { Ru medial } \\
\text { Lemporal selcrosis }\end{array}$ & $50 / 16.6$ & 3 & Midlazolam & $\gamma_{\mathrm{es}}$ & Yes & $\begin{array}{l}\text { Still gelling } \\
\text { Ircaitment }\end{array}$ \\
\hline
\end{tabular}

Patients receiving Ketamine as part of the treatment regimen for status epilepticus. NCSE - Nonconvulsive SE, SPSE - Simple Partial SE, CPSE - Complex Partial SE, SG - Secondary Generalization, GPED- Generalized Periodic Epileptiform Discharges, PLED Periodic Lateralized Epileptiform Discharges, Lt- Left, Rt- Right, szseizures, hx- History. Patient no.4 had epilepsy surgery.

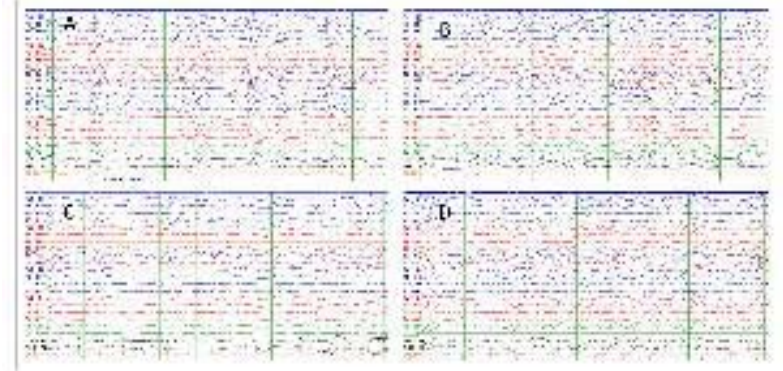

Figure 1. EEGs of patient 8. A) No anesthetic. Patient with alteration of consciousness and waxing and waning exam found to have left temporal PLEDs. B) On Propofol. PLEDs and clinical picture persisted despite addition of 2 AEDs and then Propofol was added. C) Addition of Midazolam up to $2 \mathrm{mg} / \mathrm{kg} / \mathrm{h}$. Persistence of PLEDs. D) Addition of Ketamine (after Propofol was weaned off). Resolution of PLEDs and appearance of characteristic diffuse archiform theta rhythms.

\subsection{2}

\section{H IN-HOME EEG AFTER FIRST SEIZURE IN ADULTS} M. van Putten ${ }^{1,2}$ and Jessica Askamp ${ }^{1}{ }^{1}$ University of Twente, Enschede, Netherlands and ${ }^{2}$ Medisch Spectrum Twente, Enschede, Netherlands)

Rationale: After a first seizure, patients may be erroneously diagnosed as suffering from epilepsy, or the diagnosis may be missed, which can have serious psychosocial and socioeconomic consequences. In addition to the description of the first seizure, EEG findings may be useful in the diagnosis, but may also be misleading (Epilepsia 2006;47:9-13). The first EEG shows epileptiform abnormalities in only $25-56 \%$ of the patients clinically diagnosed with epilepsy, whereas repeating EEG measurements increases the likelihood of finding epileptiform abnormalities (J Neurol Neurosurg Psychiatry 2005;76:ii2-ii7). Particularly in patients with new onset seizures and normal initial EEGs, a recording after sleep-deprivation is employed, even though there is controversy about the actual efficacy of this activation procedure (Seizure 2000;9:580-584). In this study, we evaluate the diagnostic value and feasibility of $24 \mathrm{~h}$ inhome EEG in patients evaluated for epilepsy, as compared to the standard procedure of a routine EEG- recording followed by an EEG after sleep-deprivation.

Methods: In total, 100 first-seizure patients, aged 18 years or older, will be randomized to either a group who will have a standard EEG eventually followed by a sleep-deprived EEG, or a group having a standard EEG followed by an $24 \mathrm{~h}$ in-home EEG. In-home EEGs are recorded using a dedicated portable amplifier (TMS-international). The diagnostic value, time-to-diagnosis, number of hospital visits, costs and satisfaction will be assessed. Patients will be followed for up to one year after the first seizure.

Results: At present, fifteen first-seizure patients have been included, of which eight were assigned to the in-home group. None of the standard EEGs contained epileptiform discharges, whereas one of the sleep-deprived and one of the in-home EEGs contained epileptiform discharges. The number of hospital visits ranged from three to six and from four to five visits for the standard and in-home group respectively, whereas the time-to-diagnosis varied between 13 and 93 and 59 and 107 days. All patients were satisfied with the in-home EEG recording.

Conclusions: In-home EEG in first-seizure patients seems feasible. However, the percentage of first-seizure patients with epileptiform discharges is low. Follow-up should demonstrate whether this is caused by the low sensitivity of the EEGs or by the low percentage of first-seizure patients eventually diagnosed with epilepsy. At present, the number of included patients is too limited to draw any other conclusions on the diagnostic value of $24 \mathrm{~h}$ in-home EEG in first seizure patients. However, we expect to be able to draw further conclusions on the clinical relevance of $24 \mathrm{~h}$ in-home EEG in first seizure patients when all patients are included.

\subsection{3 \\ FORENSIC IMPLICATIONS OF ICTAL OR PERI-ICTAL GRABBING BEHAVIORS}

Mark C. Spitz, M. Friedman, L. Frey, C. Drees and S. Pearson (Neurology, University of Colorado Anschutz Medical Campus, Aurora, CO)

Rationale: Grabbing is an automatism associated with epileptic seizures that is mentioned in the literature but without any quantification. The potential forensic implications of this seizure manifestation are not well-studied. 
Methods: We reviewed the clinical database for the University of Colorado Epilepsy Center, a tertiary care referral center, searching for patients with grabbing automatisms and subsequent legal consequences.

Results: Case one: A 44-year-old man developed cryptogenic seizures in elementary school. According to witnesses, he would wander at night into his mother's room and grab her in a nonspecific way, often hugging her. He would be unresponsive with lip smacking. At age 31, he came to medical attention after a secondarily generalized convulsion and was started on phenytoin. Work up included left temporal spikes on EEG and a normal MRI. His legal problem occurred at age 41 years when he was watching television on a sofa with a friend's 10 year old daughter. He allegedly began groping her; was unresponsive and she easily escaped. He did not pursue her and has no memory of the event. He had no previous criminal record. He was found guilty of sexual assault on a minor. Case two: A 40-year-old man began having seizures at the age of 34 following a closed head injury. He initially presented with a generalized tonic-clonic seizure. His seizures are believed to be right temporal onset complex partial seizures. He has a normal brain MRI scan. He averages about one seizure every 10 days. His seizures are stereotyped with chewing behaviors and an urge to wander and he frequently will grab, usually the arm of a bystander. "The event" occurred while he was in a shopping mall with his girlfriend. He had one of his typical complex partial seizures and grabbed the arm of a woman who was a stranger. The shopping mall security and local police became involved. He was first transported to a hospital where he was evaluated and released. Criminal charges have been filed against him.

Conclusions: We report two cases of patients who have temporal lobe onset complex partial seizures. Both of them had seizure-related automatisms involving grabbing people which resulted in legal complications. Defining grabbing as a clear ictal behavior may help mitigate possible legal consequences for future patients.

\subsection{4 \\ EPILEPTIFORM ENCEPHALOPATHY AS A RESULT OF PSYCHOTROPIC MEDICATIONS}

Tania B. Beltran Papsdorf, H. W. Shin and N. Chahin (University of North Carolina at Chapel Hill, Chapel Hill, NC)

Rationale: Psychotropic medications are often used in various psychiatric and neurological conditions. Potential risks of seizures have been reported with these medications. However, increased doses of these medications were not previously known to predispose to epileptiform encephalopathy. We sought to report denovo epileptiform encephalopathy from increased doses of mirtazapine and donepezil. We illustrate the effect of psychotropic medication changes on the electroencephalogram (EEG) and coorelate this with the effect of withdrawal of the medications both clinically and neurophysiologically.

Methods: A 79 year old female with a history of mild dementia, major depressive disorder, anxiety and no previous history of seizures was brought in by her family for a two week history of worsening depression and anxiety, superimposed on acute psychosis after psychotropic medication changes. The patient had been stable on mirtazapine 30 milligrams (mg) per day and donepezil $5 \mathrm{mg}$ for several months. Due to worsening depression and anxiety, mirtazapine was increased to $45 \mathrm{mg}$ per day. Donepezil was increased to $10 \mathrm{mg}$ and eszopiclone $2 \mathrm{mg}$ was started. After these changes were made, the patient had acute psychosis with increased confusion, delusions and incoherent speech. She was admitted to the psychiatric inpatient unit for treatment and evaluation. On admission, eszopiclone was discontinued but mirtazapine and donepezil were continued. Initial metabolic and infectious work up did not reveal any abnormalities. Subsequently, neurology was consulted for her encephalopathy on hospital day 3. Her neurological exam showed only moderate encephalopathy.
Results: A routine electroencephalogram (EEG) was performed with findings of frequent bifrontally predominant spikes and polyspike and slow wave discharges as well as mild to moderate background slowing (Figure 1). Subsequently, inpatient ambulatory EEG was done for 48 hours. Initial findings were similar to the previous routine EEG results (Figure 2-a). Due to the concern of epileptiform encephalopathy from psychotropic medications, mirtazapine and donepezil were discontinued on the second day of ambulatory monitoring. After discontinuation of these medications, the EEG showed significant improvement with resolution of interictal epileptiform discharges and faster background with alpha posterior dominant rhythm (Figure 2-b). During these two days of monitoring, there were multiple patient events with confusion, agitation, and psychosis without clear electrographic seizures. Over the next several days, her mental status gradually improved. Psychosis resolved and the patient was able to return home on hospital day 15 .

Conclusions: In elderly patients, even minor changes of psychotropic medications may predispose them to potential epileptiform encephalopathy and even seizures. New onset of psychosis and encephalopathy in these patients should prompt evaluation with EEG.

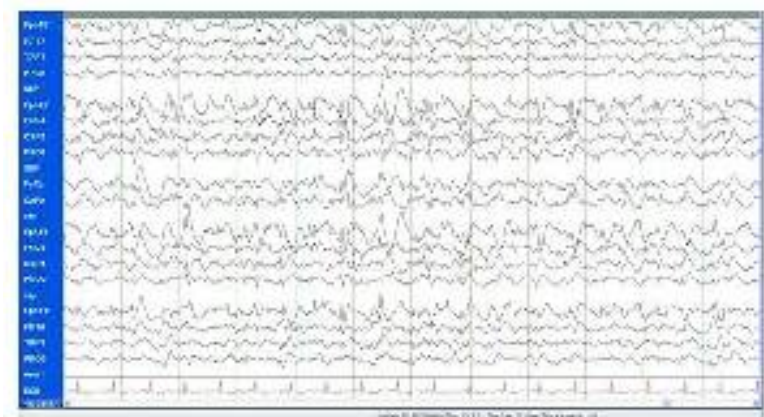

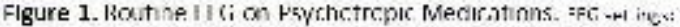

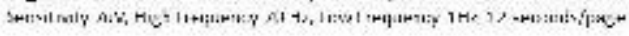

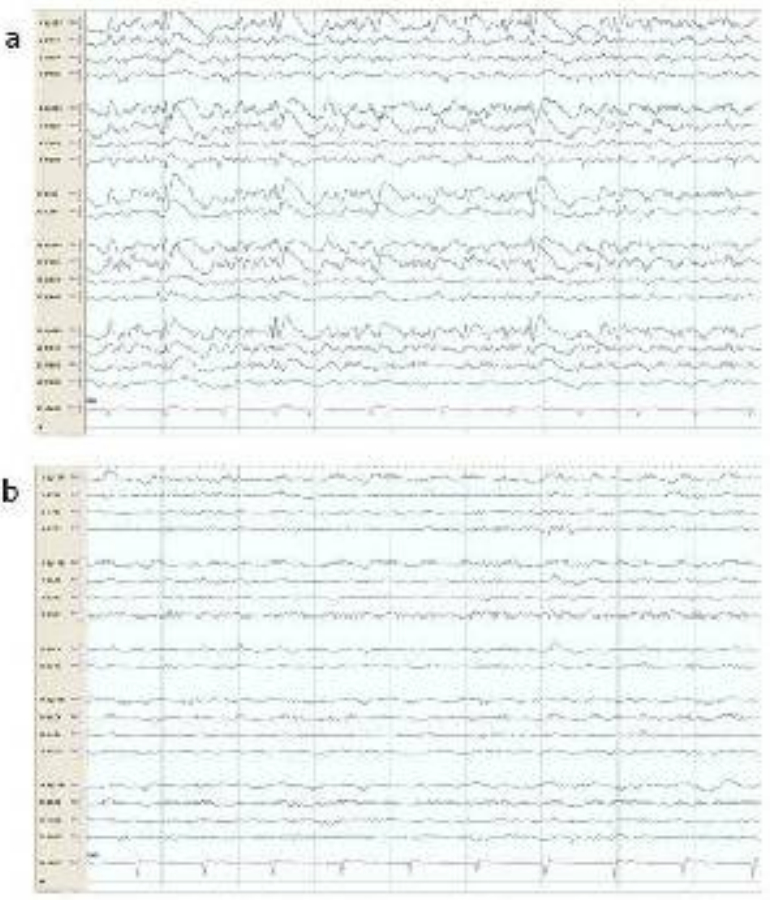

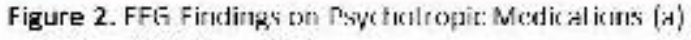
and Atter Withdrawal (b). EEC zellingsi 5ensi livily 7wis, High

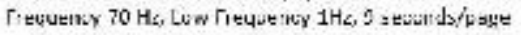




\subsection{5}

NOVEL EEG PATTERN ASSOCIATED WITH TRANSIENT REDUCTION OF CEREBRAL BLOOD FLOW IN MOYA MOYA DISEASE (MMD)

Suvendrini Lena ${ }^{1,2}$, M. Boulos ${ }^{1}$, J. Han ${ }^{1}$, D. Mikulis ${ }^{1,3}$, M. Tymianski ${ }^{1,3}$, C. Jaigobin ${ }^{1,3}$ and R. Wennberg ${ }^{1,3}$ ( ${ }^{1}$ University of Toronto, Toronto, Canada; ${ }^{2}$ Center for Addiction and Mental Health, Toronto, ON, Canada and ${ }^{3}$ University Health Network, Toronto, ON, Canada)

Rationale: We report unique EEG changes in a patient with MMD, referred for investigation of possible seizure, ultimately found to be experiencing reversible cerebral hypoperfusion. To our knowledge, this is the first report on EEG findings associated with clearly defined reversible cerebral hypoperfusion due to decreased autoregulatory capacity.

Methods: A 20 yr old woman presented with an episode of loss of consciousness, followed by new dysarthria persisting for 4 months. She had suffered bilateral fronto-parietal strokes at $1 \mathrm{yr}$. Seizures had been present in early childhood, remitting by age 10, when MMD was treated with revascularization surgery (bilateral EDAS). She was subsequently stable on Aspirin $180 \mathrm{mg}$ OD.

An EEG demonstrated normal background with frequent mediumhigh amplitude slow sharp waves recorded with bilateral synchrony over the temporo-parieto-occipital watershed regions, in slow periodic trains $(0.5-0.75 \mathrm{~Hz})$, the slow sharp waves showed maximal amplitude at $\mathrm{P} 3>\mathrm{T} 5>\mathrm{O} 1$ (with a similar distribution on the right side, of slightly lower amplitude). The trains of slow periodic discharges at times lasted $>20$ seconds, without clinical correlate.

An MRI showed no new ischemic lesions. Conventional angiography showed abnormally dilated vessels, moyamoya collateral vessels and stenoses of the supraclinoid ICAs bilaterally. CVR study showed severe bilateral reduction in vascular reserve compared to 3 years earlier; specifically, paradoxical reactivity (steal) in the left MCA and PCA territories, with corresponding, less severe, right hemispheric watershed loss of reserve. The distribution of maximal CVR loss colocalized with the left-sided amplitude predominance of the PLEDlike watershed region EEG discharges.

Given the high risk of recurrent ischemic events with impaired CVR, an omental transplant was performed to improve cerebral hemodynamics in the left hemisphere. Three months post-op bilateral hemispheric improvement in CVR correlated with resolution of the periodic slow sharp waves seen on the initial EEG.

Results: In MMD cerebral autoregulation maintains normal cerebral blood flow despite reductions in cerebral perfusion pressure (CPP) through vasodilation of resistance vessels downstream of stenotic lesions. This vasodilatory capacity is finite: reduction in CPP beyond a certain limit will lead to maximal dilation of resistance vessels and exhaustion of CVR. This patient's EEG showed a slow PLED-like pattern with maximal amplitude correlating with impaired CVR between the MCA and PCA watershed territories.

Conclusions: The patient's presentation with suspected seizure may represent syncope due to cerebral hypoperfusion or an ictal epileptic phenomenon arising from the slow periodic PLED-like pattern. Revascularization improved CVR and clearly led to resolution of the EEG pattern, demonstrating a distinct association between the slow PLED-like phenomenon and reversible ischemia. This may have important diagnostic and therapeutic implications: the EEG changes may point to salvageable brain tissue experiencing compromised blood flow.

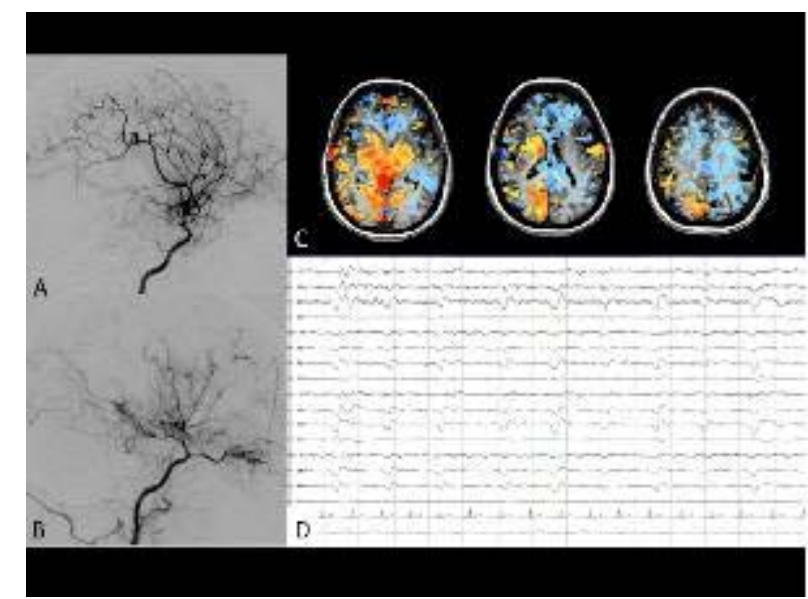

Figure 1. A) Lateral left internal carotid angiogram. Demonstrates abnomally dilated vessels, moyamoya collateral vessels and a stenotic supraclinoid ICA. B) Lateral right internal carotid angiogram. Demonstrates moyamoya type collaterals. C) Cerebrovascular vascular reserve (CVR) is a measure of cerebral hemodynamic impairment. Quantification of cerebrovascular reserve by measurement of $\%$ change BOLD MR signal intensity per $\mathrm{mmHg}$ change PetCO2 (end tidal CO2) has been validated as a means of quantifying residual autoregulatory capacity in pediatric and adult patients with Moya Moya. Here BOLD MR CVR demonstrates steal phenomenon (Blue) in the both the right and left ACA territories as well as the left MCA territory. D) Pre-operative EEG demonstrating periodic slow-sharp waves (see text for details).

\section{Neurophysiology: Animal Studies}

\subsection{6 \\ CELF4 REGULATES A VAST SET OF MRNAS, INCLUDING MANY ASSOCIATED WITH SYNAPTIC FUNCTION AND HOMEOSTATIC PLASTICITY

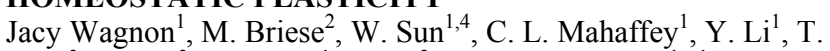 Curk $^{3}$, G. Rot ${ }^{3}$, D. T. Lin ${ }^{1}$, J. Ule $^{2}$ and W. N. Frankel ${ }^{1}{ }^{1}$ The Jackson Laboratory, Bar Harbor, ME; ${ }^{2}$ MRC Laboratory of Molecular Biology, Cambridge, United Kingdom; ${ }^{3}$ Faculty of Computer and Information Science, University of Ljubljana, Ljubljana, Slovenia and ${ }^{4}$ Janelia Farm Research Campus, Howard Hughes Medical Institute, Ashburn, VA)}

Rationale: RNA-binding proteins (RBPs) are emerging as causal agents of complex neurological diseases, including epilepsy. Mice deficient for the neuronal RBP CELF4 have a complex seizure disorder, are hyperactive, and have late-onset obesity. Recently, human CELF4 was associated with clinical features similar to that of mutant mice, indicating that CELF4 is important for mammalian neurological function. We previously showed that CELF4 is expressed mainly in excitatory neurons of the cerebral cortex and hippocampus and that it regulates excitatory, but not inhibitory, neurotransmission.

Methods: Here we further examine the mechanism underlying neuronal excitability and seizures of Celf4 mutants by identifying CELF4 target mRNAs using iCLIP, assessing their fate in Celf4 mutants using transcriptome analysis and validation by $\mathrm{qPCR}$ and immunostaining, and evaluating neuronal function in Celf4 mutant mice with electrophysiology.

Results: We find that CELF4 selectively binds $15-20 \%$ of the transcriptome, with striking enrichment for the 3' UTR. Many CELF4-bound mRNAs encode proteins involved in neuron development and function. Global changes in steady-state levels of CELF4 targets are generally modest, however transcriptome analysis of polysome fractions as well as cell body-neuropil dissections suggests CELF4 is critical for maintaining mRNA stability and availability for translation. Patch-clamp recordings of wildtype and 
Celf4 mutant cortical neurons revealed that Celf4 heterozygotes and homozygotes are more likely to generate action potentials $(p<0.01)$ and have higher persistent sodium current $\left(I_{N a P}, p<0.01\right)$, for which SCN8A $\left(\mathrm{Na}_{\mathrm{v}} 1.6\right)$ is a major determinant. Indeed, Scn8a mRNA is a direct CELF4 target, and immunostaining showed a dramatic increase in SCN8A level at the axon initial segment of Celf4 mutants $(p<0.0001)$. This increase appears to be critical for seizure generation in Celf4 mutant mice, as lowering the gene dosage of normal Scn8a leads to a remarkable elevation of seizure threshold in Celf4 mutants $(p<0.001)$. Last, Celf4 mutant neurons display diminished homeostatic plasticity following bicuculline treatment, suggesting that the overarching role of CELF4 may be as an important regulator of homeostasis in excitatory neurons.

Conclusions: Overall, these results strongly suggest that dysregulation of CELF4 target mRNAs increases neuronal excitability - at least in part by upregulating SCN8A at the AIS - and impairs homeostatic response, together leading to a complex seizure disorder. Significantly, these studies support and expand the vital roles RBPs play in regulating and fine-tuning activity of neural circuits and highlight the importance of RBP function in neurological disease.

\subsection{7 \\ T-TYPE CALCIUM CHANNELS FACILITATE NEURONAL HYPER-EXCITABILITY IN EPILEPSY}

Manoj K. Patel ${ }^{1}$, D. Gryder ${ }^{1}$, N. Hargus ${ }^{1}$, J. Renger ${ }^{4}$, V. Uebele ${ }^{4}$, E. Perez-Reyes ${ }^{3}$, E. Bertram ${ }^{2}$ and A. Nigam ${ }^{1}\left({ }^{1}\right.$ Anesthesiology, University of Virginia, Charlottesville, VA; ${ }^{2}$ Neurology, University of Virginia, Charlottesville, VA; ${ }^{3}$ Pharmacology, University of Virginia, Charlottesville, VA and ${ }^{4}$ Merck Research Labs, West Point, PA)

Rationale: Temporal lobe epilepsy (TLE) is a common form of adult epilepsy involving the limbic structures of the temporal lobe. Layer II neurons of the medial entorhinal cortex $(\mathrm{mEC})$ are spared and hyperexcitable in TLE. Recent studies have suggested a role for T-type $\mathrm{Ca} 2+$ channels in facilitating increases in neuronal activity associated with TLE. We sought to determine if T-type $\mathrm{Ca} 2+$ channels are involved in maintaining neuronal hyperexcitability of layer II stellate and non-stellate neurons in TLE.

Methods: TLE in rats was induced by electrical stimulation of the hippocampus for 90 mins to induce status epilepticus (SE). Rats having two or more spontaneous seizures per day by EEG, 3 months after SE were used in the study. Brain slices were prepared and membrane properties recorded from visually identified $\mathrm{mEC}$ layer II neurons. To establish a role for T-type $\mathrm{Ca} 2+$ channels we used TTA$\mathrm{P} 2$ at $1 \mu \mathrm{M}$, a selective T-type $\mathrm{Ca} 2+$ channel antagonist.

Results: Action potentials (APs) were evoked by a series of depolarizing current injection steps under whole cell current clamp conditions. Both $\mathrm{mEC}$ layer II stellate and non-stellate neurons from TLE rat brain slices had higher AP firing frequencies than control. At a current injection step of $470 \mathrm{pA}$, stellate frequencies were increased $(\mathrm{P}<0.05)$ from $28.0 \pm 1.4 \mathrm{~Hz}(\mathrm{n}=15)$ in control to $32.5 \pm 2.6 \mathrm{~Hz}(\mathrm{n}=$ $8)$ in TLE. Bath application of TTA-P2 significantly $(\mathrm{P}<0.01)$ reduced firing frequencies in TLE neurons by $30 \%$ (to $22.6 \pm 2.9 \mathrm{~Hz}$; $\mathrm{n}=8)$. In contrast, TTA-P2 had little effect on firing rates in control neurons $(24.6 \pm 0.9 \mathrm{~Hz} ; \mathrm{n}=15)$. Non-stellate neurons were also hyperexcitable in TLE $(21.9 \pm 0.7 \mathrm{~Hz} ; \mathrm{n}=7$ in control to $33.3 \pm 1.6$ $\mathrm{Hz} ; \mathrm{n}=13$ in TLE, $\mathrm{P}<0.001$ ). In contrast to stellate neurons, TTA-P2 had little effect on non-stellate firing rates reducing them to $15.9 \pm$ $2.4 \mathrm{~Hz}(\mathrm{n}=7)$ in control and $28.3 \pm 1.6 \mathrm{~Hz}(\mathrm{n}=2)$ in TLE. Stimulation of mEC layer III evoked AP bursts in TLE mEC stellate and non-stellate neurons, but single APs in control neurons. Application of TTA-P2 reduced evoked AP burst firing in TLE stellate and non-stellate neurons. TTA-P2 had little effect on evoked AP's in both types of control neurons. Rebound APs evoked by increasing amplitudes of hyperpolarizing steps evoked single APs in control stellate neurons, but no APs in non-stellate neurons. In TLE neurons hyperpolarizing steps evoked AP bursts in both subtypes of neurons. TTA-P2 had a more pronounced effect on APs in both subtypes of TLE neurons than control neurons, delaying and inhibiting rebound AP bursts. Quantitative RT-PCR (qPCR) of mEC layer II tissue revealed an almost 3-fold increase in Cav3.1 mRNA in TLE tissue as compared to controls. Cav3.2 or Cav3.3 levels were unchanged.

Conclusions: These findings suggest that $\mathrm{T}$-type $\mathrm{Ca} 2+$ channels, in part, contribute to the increased hyper-excitability of $\mathrm{mEC}$ layer II neurons in TLE. Increased expression of the Cav3.1 isoform, in particular, may be important for facilitating and maintaining neuronal hyper-excitability of mEC layer II neurons in TLE.

\subsection{8 \\ EARLY INVOLVEMENT OF THE CONTRALATERAL CORTEX AS A SPATIAL ORGANIZER OF THE INTERICTAL SPIKES}

Daniel Vitantonio, W. Xu, J. Wu and G. Motamedi (Georgetown University Medical Center, Washington, DC)

Rationale: Epileptiform activity propagates to different brain areas, and mirror epileptic foci on the contralateral hemisphere can be sustained over long periods. However, before a noticeable mirror focus is formed, the early involvement of contralateral cortex (CC) during primary epileptogenesis remains largely unknown. Under in vivo conditions where long range connections are preserved, we found that initiation sites of interictal spikes (ISs) exhibit spatial clustering dependent on the activity history [J Neurophysiol. 2004;91(2):934-45]. This implies that remote areas may be involved in the spatial organization of ISs. ISs may play fundamental roles during the early stages of epileptogenesis, and their spatial consolidation may promote long-term changes that give rise to seizure as disease progresses.

Methods: We use voltage-sensitive dye (VSD) imaging to map IS initiation sites in rat visual cortex in vivo. Surgical and imaging methods were described in detail in our methodology paper [ $\mathrm{J}$ Neurophysiol. 2007;98(1):502-12]. Craniotomy was created over both hemispheres, and one hemisphere was stained with VSD RH1691 solution. Each detector of a 464-channel photodiode array received light from a cortical area $\sim 160 \mu \mathrm{m}$ diameter. ISs were induced on the imaging hemisphere with bicuculline $(1 \mathrm{mM}$, epidural). For each IS the timing of the VSD signal $(\lambda \sim 700 \mathrm{~nm})$ reaching 50\% peak amplitude was measured, and the detector with earliest half-peak amplitude time is defined as the initiation site. In some experiments, $\mathrm{CC}$ was either inactivated by muscimol $(3 \mathrm{mM}$, epidural) or received point electrical stimulation $(10 \mu \mathrm{A} ; 0.5 \mathrm{~Hz})$ prior to inducing the ISs.

Results: CC facilitates the spatial consolidation of IS initiation sites. During the first $5 \mathrm{~min}$ after the earliest IS, initiation sites are widely distributed over the imaging field (mean spatial entropy $\sim 3$ bits). If $\mathrm{CC}$ is unaltered, then within $10 \mathrm{~min}$ ( $\sim 450 \mathrm{ISs})$ the initiation sites began to consolidate into a single dominant site which initiated $>80 \%$ of ISs throughout the remaining time ( $80 \mathrm{~min} ; \sim 3,000 \mathrm{ISs} ; \mathrm{n}=12$ animals; mean entropy $\sim 0.5$ bits). Consolidation is dependent on the activity of $\mathrm{CC}$ in the following ways: 1) $\mathrm{CC}$ must be active during the consolidation process. If $\mathrm{CC}$ is inactivated by muscimol, the consolidation can be completely blocked ( $\mathrm{n}=5$; mean spatial entropy $\sim 3.5$ bits; $160 \mathrm{~min}$ ). 2) CC is necessary to sustain the dominant site at least during the first hour of ISs emergence. If $\mathrm{CC}$ inactivation is timed 30-50 min after consolidation, the developed dominant site can be obliterated $(n=3)$. 3) Moderate CC stimulation is sufficient to generate a dominant IS initiation site before bicuculline treatment $(n=2)$, but this effect is blocked when muscimol is applied $(n=1)$. Conclusions: If the IS-generating area is connected with another area of normal excitation/inhibition balance, a dominant initiation site should develop. Accumulated synaptic reorganization following IS consolidation may be important for epileptogenesis. Manipulating the dominant sites may become a new strategy for intervention in epilepsy. 


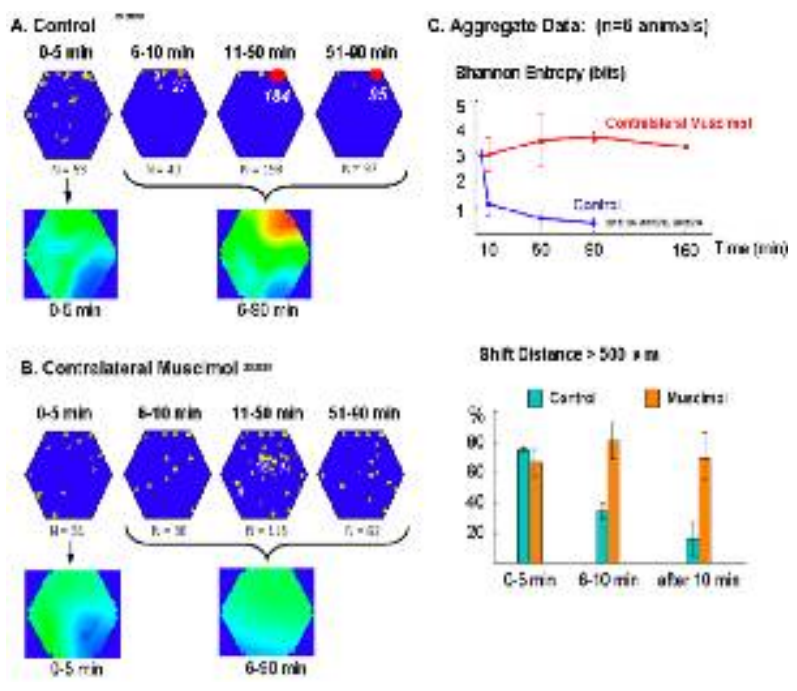

D. Dominant site obliteration

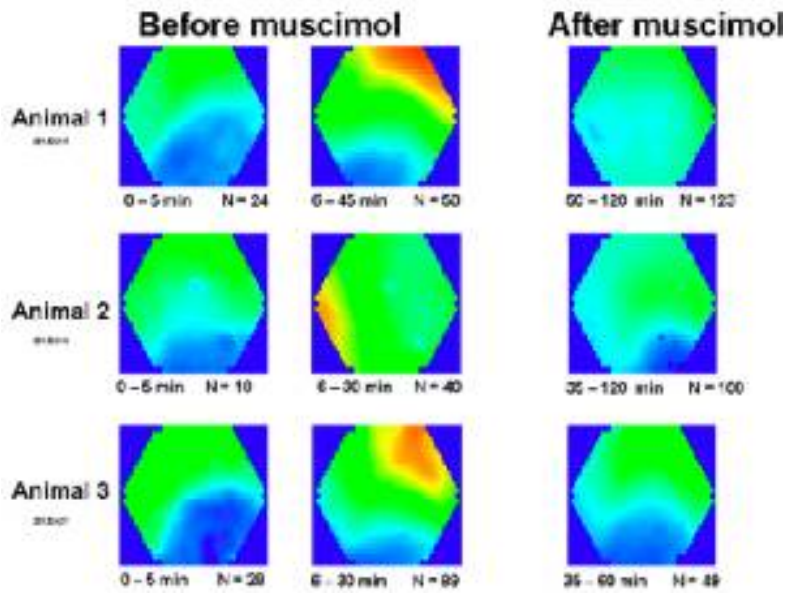

\subsection{9 \\ PATTERNS OF UP-DOWN STATE IN EPILEPTIC BRAIN AS AN INDICATOR OF EPILEPTOGENICITY AND PATHOLOGICAL FUNCTIONAL CONNECTIVITY IN EPILEPTIC BRAIN}

Anatol Bragin, F. Kheiri, S. Benassi and J. Engel (Neurology, David Geffen School of Medicine at UCLA, Los Angeles, CA)

Rationale: The UP-DOWN state (UDS) pattern occurs during sleep and anesthesia when the flow of afferent signals to the brain decreases. The electrographic pattern generated in these conditions is mostly determined by the properties of internal connections of brain areas and it may reflect a strong or weak balance between excitatory and inhibitory networks. The goal of these experiments is to investigate whether organizational changes that exist in the epileptic brain are reflected in the pattern of the UDS.

Methods: Naive and epileptic mice and rats were used in these experiments. Status Epilepticus (SE) was induced with pilocarpine hydrochloride injection. Field potentials were recorded with chronically implanted microelectrodes and single unit activity was recorded with glass microelectrodes. Recorded neurons were labeled by neurobiotine and identified later as granular cells or interneurons in histological sections. The following parameters of the UP-Down State were analyzed and compared between different groups of animals. 1) The frequency of BGA; 2) The duration of UP and Down phases; 3) Excitability changes after termination of the UP phase; 4) The existence of UP-spikes; 5) Temporal relation between areas generating UP-spikes. 6) Temporal relation between neuronal discharges and field potentials. Statistical analysis was performed using Prizm 4 (GraphPad, San Diego, CA).

Results: We did not find any difference in the frequency of BGA between normal and epileptic mice. The differences between these two groups were observed in the following parameters of the UDS pattern. In epileptic animals: a) The duration of both the UP and Down phases is significantly longer; b) Recovery of network excitability after termination of the UP phase is longer. c) UP-spikes occur during the UP phase, which transiently interrupt the development of the normal electrographic pattern of UP phase. Our findings suggest that UP-spikes result from gigantic EPSPs generated in response to afferent activity. UP-spikes in different brain areas occur in close temporal relationship indicating the existence of pathological functional connections between these areas.

Conclusions: Our data show that several parameters of the UDS are different in normal and epileptic brain. Changes in the duration of the UP- and Down phases as well increased time of recovery of excitability of epileptic brain after termination of UP phase could reflect alterations in the homeostatic properties of the neuronal network in epileptic brain. The existence of UP-spikes in epileptic brain is an additional electrographic pattern indicating epileptogenicity. They could be mediated by a network of epileptogenic pathologically interconnected neuronal clusters (PINclusters) postulated to be responsible for the occurrence and propagation of epileptiform activity between brain areas.

\subsection{0 \\ ADAPTIVE WILDTYPE GABAA RECEPTOR EXPRESSION, DISTRIBUTION AND MOBILITY IN GABRB3 KNOCK OUT MICE \\ J. Kang, O. Magvanjav, Q. Zhang, R. L. Macdonald and W. Shen (Vanderbilt University Medical Center, Nashville, TN)}

Rationale: : Gamma-aminobutyric acid type A (GABAA) receptors mediate the majority of rapid inhibitory synaptic transmission in the CNS. Mutations in the GABAA receptor $\beta 3$ subunit and deletions of chromosome $15 \mathrm{q}$, which contain the $\beta 3$ subunit gene GABRB3 are frequently associated with epilepsy as well as other neurodevelopmental abnormalities like autism and Angelman syndrome. Deletion of $\beta 3$ subunits in GABRB3 knockout mice results in $\sim 90 \%$ neonatal lethality, and the surviving GABRB3 null mice have been proposed to be both human Angelman syndrome and autism models due to their neurodevelopmental abnormalities and phenotypic presentations. The GABAA receptor GABRB3 subunit is essential for pentameric GABAA receptor assembly and trafficking. Any alteration of GABRB3 subunit expression would change GABAA receptor expression, composition, distribution and membrane diffusion in a neuronal milieu during brain development when multiple GABAA receptor subunits coexists.

Methods: We have characterized GABAA receptor expression, distribution and mobility in GABRB3 knockout mice. Considering all patients reportedly carrying GABRB3 subunit mutations are heterozygous, we decided to focus on the expression of GABAA receptor subunits in both mouse brain and cultured neurons from heterozygous GABRB3 KO mice. To do so, we employed live brain slice surface biotinylation, single Quantum dot imaging and patchclamp recording in live hippocampal neurons.

Results: We demonstrated that both surface and total expression of GABRB3 subunits were reduced in heterozygous mice. Moreover, $\gamma 2$ subunit expression was reduced and $\beta 2$ subunits had altered glycosylation in heterozygous mice. Using single quantum dot imaging, we found that surface GABAA receptor in heterozygous GABRB3 KO mice had slowed mobility and a larger fraction of them located outside of synapses. Whole-cell patch-clamp recording of hippocampal GABAergic interneurons from both heterozygous and homozygous mutant GABRB3 knockout mice showed reduced peak current amplitudes and increased zinc inhibition. The heterozygous GABRB3 knockout mice exhibited absence-like seizures in both electrographical and behavioral examination. Intriguingly, GABRB3 
knockout mice also had reduced latency to fall during rotarod testing, suggesting impaired cerebellar function.

Conclusions: Our study suggests that impairment in GABRB3 subunit alters expression, post-translational modification, distribution and mobility of other wildtype GABAA receptor subunits, which significantly impacts GABAA receptor function and synaptic transmission, resulting in a complex neurobehavioral phenotype

\subsection{1 \\ PILOCARPINE INDUCED STATUS EPILEPTICUS MODIFIES INHIBITORY AND ELECTRICAL SYNAPSE TO DENTATE FAST-SPIKING BASKET CELLS}

Jiandong Yu, A. Proddutur, F. S. Elgammal and V. Santhakumar (University of Medicine and Dentistry, Newark, NJ)

Rationale: Epilepsy is a disorder of network excitability and synchrony. In the dentate gyrus, excitability and network rhythms are regulated by GABAergic fast-spiking basket cells (FS-BCs), which express parvalbumin and underlie granule cell perisomatic inhibition. While synaptic transmission from FS-BCs to granule cells appears compromised after pilocarpine-induced status epilepticus (SE), whether GABAergic and electrical synapses to FS-BCs are modified after SE is unknown. Identifying how inhibitory and electrical synapses to FS-BC are altered after SE is important for evaluating dentate inhibitory circuit function during development of epilepsy. Methods: Whole-cell, patch-clamp recordings were conducted in hippocampal slices from male rats obtained 1 week after pilocarpine induced status epilepticus (SE) and in saline-injected controls. A combination of intrinsic physiology and morphological analysis following post-hoc immunohistochemistry was used for cell identification.

Results: The frequency of spontaneous inhibitory postsynaptic currents (sIPSCs) in morphologically and physiologically identified FS-BCs was reduced one week after SE (sIPSC frequency in $\mathrm{Hz}$, control: median $=6.9, \mathrm{IQR}=3.7-13.5, \mathrm{n}=10$ cells; post-SE, median $=5.0, \mathrm{IQR}=2.6-10.4, \mathrm{n}=9$ cells, $\mathrm{p}<0.05 \mathrm{~K}-\mathrm{S}$ test $)$. However, FS-BC sIPSC amplitude was not altered after SE. Paired interneuronal recordings demonstrated that FS-BCs in control and post-SE rats received GABAergic synaptic inputs from both FS-BCs and non-fast-spiking interneurons in the hilar-granule cell layer border. While the probability of synaptic connections between FS$\mathrm{BCs}$ were not different in slices from control and post-SE rats (connection probability, control: $8 \%, 4$ of 50 pairs, post-SE: $8.6 \%, 5$ of 60 pairs), preliminary data revealed a reduction in the release probability at inhibitory synapses between FS-BCs which did not reach statistical significance. Similarly, the probability of synaptic connections from non-fast-spiking interneurons to FS-BCs was not altered after SE (control: $6.0 \%, 5$ of 85 pairs tested; post-SE: $6.8 \%, 5$ of 74 pairs). However, consistent with a potential decrease in the probability of release, short-term depression of GABAergic synapses from non-fast-spiking interneurons to FS-BCs was reduced one week after SE (IPSC3/IPSC1 at $50 \mathrm{~Hz}$, control: $0.64 \pm 0.16, \mathrm{n}=4$; post-SE: $0.98 \pm 0.08, \mathrm{n}=3, \mathrm{p}<0.05)$. Unlike inhibitory synaptic connections between FS-BCs, the probability of electrical contacts between FSBCs was decreased after SE (probability of electrical coupling, control: $53.3 \%, 16$ of 30 pairs, post-SE: $16 \%, 4$ of 25 pairs, $p<0.05$ by chi-square test). The coupling coefficient of electrically coupled FS-BCs was not altered after SE (control: $0.05 \pm 0.02, \mathrm{n}=11$; post-SE: $0.05 \pm 0.02, \mathrm{n}=4, \mathrm{p}>0.05$ ).

Conclusions: These results demonstrate a reduction in synaptic inhibition to FS-BCs following status epilepticus. The post-SE reduction in electrical coupling among FS-BCs could undermine their role in network oscillations and synchrony. The impact of these changes on dentate network function and excitability is under investigation.

\subsection{2}

ANTISEIZURE DRUGS DIFFERENTIALLY MODULATE THETA-BURST INDUCED LONG-TERM POTENTIATION IN C57BL/6 MICE

Peter J. West ${ }^{1}$, G. W. Saunders ${ }^{1}$, G. J. Remigio ${ }^{2}$ and H. S. White ${ }^{1}$ ( ${ }^{1}$ Pharmacology and Toxicology, University of Utah Anticonvulsant Drug Development (ADD) Program, Salt Lake City, UT and ${ }^{2}$ Interdepartmental Program in Neuroscience, University of Utah, Salt Lake City, UT)

Rationale: Cognitive comorbidities are increasingly recognized as an equal (or even more disabling) aspect of epilepsy. Additionally, the side effects of some antiseizure drugs (ASDs) can impact learning and memory. Accordingly, the NINDS epilepsy research benchmarks call for the implementation of standardized protocols for screening ASDs for their amelioration or exacerbation of cognitive comorbidities. Long-term potentiation (LTP) is a widely used model for investigating synaptic plasticity and its relationship to learning and memory. While the effects of some (but not all) ASDs on LTP have been examined, none of these studies employed physiologically relevant induction stimuli such as theta-burst stimulation (TBS) or examined the effects of ASDs on brain regions beyond area CA1 of the rodent hippocampus. To address this paucity of data, we examined the effects of a broad representation of existing ASDs on TBS-induced LTP in both area CA1 and the dentate gyrus of C57BL/6 mice.

Methods: Coronal brain slices $(350 \mu \mathrm{m})$ containing the dorsal hippocampus were made. Recordings were obtained from four slices at a time using the Scientifica Slicemaster high throughput recording system. Slices exposed to ASDs were paired with slices from the opposite hemisphere; these served as controls. Field excitatory postsynaptic potentials (fEPSPs) were recorded in response to stimuli set to $50 \%$ of their maximum. All ASDs were applied to slices by bath perfusion for 20 minutes prior to the induction stimulus. LTP was induced by TBS ( 4 trains of 4 pulses at $100 \mathrm{~Hz}$ separated by $200 \mathrm{~ms}$ and repeated once with a $20 \mathrm{~s}$ interval) or by high-frequency stimulation (HFS: $100 \mathrm{~Hz} / 1 \mathrm{sec})$. The following ASDs were examined: phenobarbital (PB), phenytoin (PHT), carbamazepine (CBZ), valproate (VPA), topiramate (TPM), lamotrigine (LTG), levetiracetam (LEV), retigabine (RTG), and tiagabine (TGB). Results: Among known voltage-gated sodium channel inhibitors, $60 \mu \mathrm{M}$ LTG failed to affect plasticity, $50 \mu \mathrm{M} \mathrm{CBZ}$ significantly attenuated TBS-induced LTP $(39.4 \pm 12.3 \%$ of matched controls, $\mathrm{p}<0.01$ ), and $80 \mu \mathrm{M}$ PHT attenuated both TBS-induced LTP $(54.6 \pm 16.3 \%$ of matched controls, $\mathrm{p}<0.05)$ and post-tetanic potentiation (PTP). 10 $\mu \mathrm{M}$ RTG failed to affect LTP but did significantly affect PTP. $600 \mu \mathrm{M}$ VPA failed to affect LTP in area CA1, both in C57BL/6 mice and Sprague Dawley rats, using TBS or HFS. However, $600 \mu \mathrm{M}$ VPA did attenuate TBS-induced LTP in the dentate gyrus $(46.4 \pm 11.2 \%$ of matched controls, $\mathrm{p}<0.05)$.

Conclusions: The experiments performed here comprehensively examined the effects of the most commonly used ASDs on the preeminent in-vitro model of learning and memory while, for the first time, using physiologically relevant protocols. These results establish a foundational knowledge of ASD effects on LTP in the hippocampus and will ultimately be used to validate the predictive value of TBS induced LTP for cognitive impairments associated with novel ASDs. Supported by NINDS (Contract \# 271201100029C) and the Interdepartmental Neuroscience Program at the University of Utah.

\subsection{3 \\ COMPUTATIONAL MODELING OF A DROSOPHILA MOTONEURON FOR STUDYING ACTIVITY-DEPENDENT SPLICING CHANGES OF SODIUM CHANNELS THAT PROMOTE SEIZURE}

Cengiz Gunay $^{1}$, R. A. Baines ${ }^{2}$ and A. A. Prinz ${ }^{1}\left({ }^{1}\right.$ Biology, Emory University, Atlanta, GA and ${ }^{2}$ Life Sciences, University of Manchester, Manchester, United Kingdom) 
Rationale: Mutations of voltage-gated sodium channels (Nav) are implicated in neurological disorders like epilepsy. It is not well known how much post-transcriptional and translational modifications of Nav genes contribute to these channelopathies. In the slamdance epileptic mutant of the fruit fly, Drosophila melanogaster, an increase in the Nav alternative splicing of one exon $L$ results in an increased persistent sodium (NaP) current (Lin, Günay, Marley, Prinz, Baines, 2012. J Neurosci. 32(21):7267-7277). The change in splicing and increase in the NaP current can be partially reversed using the anticonvulsive drug phenytoin and other activity-modulation therapies in the fly. However, measuring the effect of splicing changes on neuronal activity is more difficult in genetic mutants because of compensatory regulation. Because of this, the biological experiments can be complemented by using a computational model for testing isolated manipulations of Nav parameters.

Methods: Individual Nav splice variants can be heterologously expressed and their biophysical properties measured in Xenopus oocytes (Lin, Wright, Muraro, Baines, 2009. J Neurophysiol 102:1994-2006). We previously constructed a single-compartment computational model of an identified larval Drosophila abdominal dorsomedial motoneuron called aCC or MN1-Ib that innervates the dorsal muscles (Gunay C et al., 2011. Program No. 708.01. Society for Neuroscience abstract). This model reproduces the firing rate and membrane potential response characteristics recorded from aCC motoneurons and allows simulating splicing changes in Nav properties. However, the smallness of the fly motoneurons causes filtering and offset responses observed in recordings, which cannot be replicated using a single-compartment model. By adding a second compartment in the model motoneuron to separate the active channels, we replicated these recorded responses.

Results: Using the two-compartment model motoneuron, we manipulated model parameters to mimic splicing changes observed in epileptic fly mutants. The increase in the NaP magnitude was confirmed to have an excitatory effect (Lin et al 2012, see above). The decrease in Nav activation voltage also had excitatory effect that we quantified. Furthermore, the modulatory effect of the altered presynaptic activity properties on the model motoneuron activity was characterized.

Conclusions: Although similar splicing patterns have not yet been observed in the mammalian Nav, the extensive splicing in the fly Nav promises a tractable testbed for examining the effects of genetic changes in Nav on neuronal activity. Here, we present a computational motoneuron model that provides mechanistic explanations and predictions of increased excitability in epileptic fly mutants.

\subsection{4 \\ PROGESTERONE SUPPRESSES ENTORHINAL CORTICAL EPILEPTIFORM ACTIVITY IN VITRO}

Edwin Chow $^{1}$, M. Jeffrey ${ }^{1,2}$, W. M. Burnham ${ }^{2}$ and L. Zhang ${ }^{1}$ ( ${ }^{1}$ Fundamental Neurobiology, Toronto Western Hospital, University Health Network, Toronto, ON, Canada and ${ }^{2}$ Pharmacology and Toxicology, University of Toronto, Toronto, ON, Canada)

Rationale: Clinical evidence has long shown that progesterone exhibits anticonvulsant effects. Unlike many other anticonvulsant drugs, progesterone is endogenous, making it desirable in control of epileptic seizures. It is generally believed that progesterone acts by enhancing the activity of GABAa receptors. Experimental studies, however, suggest that progesterone can affect other receptors /channels. Moreover, metabolites of progesterone have also been shown to possess anticonvulsant effects in animal models. Therefore, the aim of our present study is to explore the effects of progesterone and its metabolites on in vitro epileptiform activities in both GABAa receptor dependent and independent mechanisms.

Methods: Horizontal brain slices $(0.5 \mathrm{~mm}$ thick) were obtained from young adult male mice. The slices were perfused with a standard ACSF in a submerged chamber at $35^{\circ} \mathrm{C}$. Extracellular recordings were made from entorhinal cortical areas. 4-aminopyridine $(100 \mu \mathrm{M})$, picrotoxin $(100 \mu \mathrm{M})$ and progesterone $(100 \mathrm{nM})$ were added to the ACSF at desired concentrations.

Results: Prior to adding 4-AP and PTX, the slices showed stable synaptic field potentials following afferent stimulation, and no epileptiform potentials were recognizable under basal conditions. Upon perfusion of 4-AP and PTX, repetitive epileptiform field potentials similar tor interictal discharges were observed, with durations of 300-500 ms and incidences of 2-5s. These epileptiform field potentials were sensitive to suppression by phenytoin $(50 \mu \mathrm{M}$, $\mathrm{n}=4$ ). The application of progesterone did not abolish these epileptiform potentials nor significantly alter their amplitude and incidence. Of 10 slices in which progesterone effects were reliably assessed, 8 were shown to have a decrease in area of the epileptiform field potentials ( $\mathrm{p}=0.035$, paired test test). Such suppression by progesterone was reversible upon washing.

Conclusions: Our pilot data suggest that progesterone can inhibit epileptiform activity in the entorhinal cortex via GABAa receptorindependent actions. Works are in progression to examine the concentration-dependence of progesterone, effects of progesterone in the absence of GABAa antagonists, and the role of metabolites in suppressing epileptiform activity.

\subsection{5 \\ GABAERGIC CONTROL OF THE HPA AXIS AND SEIZURE SUSCEPTIBILITY}

Jamie L. Maguire and M. Panessiti (Tufts University School of Medicine, Boston, MA)

Rationale: Stress is a common precipitating factor for seizures and stress hormones are known to be proconvulsant. Given that cortisol levels are elevated in epileptic patients and are further increased following seizures suggests that the stress hormones may contribute to acute seizure susceptibility and that modulation of the hypothalamic-pituitary-adrenal (HPA) axis may be a therapeutic target for seizure control. The major output of the HPA axis is the parvocellular, corticotropin-releasing hormone $(\mathrm{CRH})$ neurons of the paraventricular nucleus (PVN) of the hypothalamus, which are largely under GABAergic control. We hypothesize that seizureinduced alterations in the GABAergic control of CRH neurons may underlie activation of the HPA axis following seizures and potentially contribute to future seizure susceptibility.

Methods: We recently demonstrated that excitatory actions of GABA on CRH neurons are critical to mount the physiological response to stress. We have identified that the excitatory actions of GABA on CRH neurons are due to a collapse of the chloride gradient resulting from dephosphorylation of KCC2 residue Ser940, resulting in downregulation of KCC2. Further, excitatory actions of GABA on $\delta$ subunit-containing GABAA receptors (GABAARs) play a role in mediating the stress response. In order to determine whether activation of the HPA axis plays a role in acute seizure susceptibility, we generated a mouse model in which the GABAAR $\delta$ subunit is knocked out specifically in CRH neurons (Gabrd/CRH mice).

Results: These mice exhibit blunted corticosterone levels in response to seizures induced with kainic acid. The blunted corticosterone response in Gabrd/CRH mice is associated with decreased seizure susceptibility in these animals compared to Cre-/- littermates. Administration of exogenous corticosterone restores seizure susceptibility to Cre-/- levels.

Conclusions: These data suggest that activation of the stress response plays a role in acute seizure susceptibility and HPA axis modulation may be a target for seizure control. 
3.106

TEMPORAL LOBE EPILEPTIFORM ACTIVITY FOLLOWING SYSTEMIC ADMINISTRATION OF 4AMINOPYRIDINE IN RATS

Maxime Lévesque, P. Salami, C. Behr and M. Avoli (Department of Neurology and Neurosurgery, Montreal Neurological Institute, Montreal, QC, Canada)

Rationale: Bath application of the $\mathrm{K}+$ channel blocker 4aminopyridine (4AP) can induce epileptiform synchronization in the in vitro brain slice preparation without interfering with GABAA receptor-mediated inhibition and, actually, even enhancing it. Here we tested the hypothesis that similar electrographic epileptiform patterns occur in vivo following systemic 4AP injection.

Methods: Sprague-Dawley rats $(\mathrm{n}=13)$ were implanted with bipolar electrodes aimed at the hippocampal CA3 region, entorhinal cortex, subiculum, dentate gyrus and amygdala. Animals were then injected with a single dose of 4AP (4-5 mg/kg, i.p.) and EEG recordings and video-monitoring were obtained.

Results: 4AP induced convulsive or non-convulsive seizures in 12 out of 13 rats along with generalised fascicular twitching, wet-dog shakes and myoclonic jerks. On the EEG, we observed in 7/12 animals $(58.3 \%)$ long-lasting interictal spikes from the subiculum before the occurrence of the first seizure. Once seizures had started, interictal spikes occurred in all regions and had no fixed site of origin. Seizures induced by 4AP often initiated in the entorhinal cortex and CA3 and were often convulsive (48/59, 81.3\%). Finally, 4AP also induced highly rhythmic theta $(6-11 \mathrm{~Hz})$ oscillations in CA3 and entorhinal cortex, before seizure occurrence.

Conclusions: Our findings indicate that $4 \mathrm{AP}$ administration in vivo leads to a specific type of interictal activity, behavioral symptoms and network oscillations known to be linked to enhanced GABAergic inhibition. We propose that 4AP-induced ictogenesis reflects the interaction between excitatory and inhibitory processes.

\subsection{7 \\ A SOFTWARE TOOLSET FOR RAPID ANALYSIS OF EEG SEIZURE AND VIDEO DATA}

David Johnson $^{1}$, H. Harmon ${ }^{1}$, E. Akers ${ }^{1}$, E. Naylor ${ }^{1}$, J. Clasadonte ${ }^{2}$ and P. Haydon ${ }^{2}\left({ }^{1}\right.$ Pinnacle Technology, Inc., Lawrence, KS and ${ }^{2}$ Tufts University, Boston, MA)

Rationale: Recording of electroencephalograph (EEG) activity and video recording of animal behavior is necessary for the accurate assessment and quantification of seizure activity. In rodent models, visual analysis of seizure activity paired with EEG confirmation is the gold standard for determination of seizure events as a result of kindling models, genetic alteration, or drug effects. To help streamline the collection, identification and analysis of seizure events, we have developed an integrated software toolset for scoring and analysis of EEG-based seizure data and associated video records. Methods: Five mice (adult male wild type C57BL/6J) were injected with pilocarpine to induce status epilepticus (Pilocarpine model of epilepsy in mice). Five days after the pilocarpine-induced status epilepticus, mice were continuously monitored with video-EEG (Pinnacle Technology Model 8200) for at least 2 months to monitor the occurrence of spontaneous recurrent seizures. The data samples used for preliminary testing were 24 hours long and were taken 30 days after the status epilepticus. Rapid seizure identification within large datasets is accomplished using user-defined tools based on parameters such as seizure length, specific frequency, root mean squared (RMS) power and line length. Identified seizure events can further be classified using embedded video and rated using standard Racine Scale parameters. Individual seizure events can be isolated and exported as .avi video clips for use in presentations and paper submission. The software accepts input from a variety of data formats including .pvfs and .edf while analyzed data can be outputted to formatted ASCII text files for use with 3rd party statistical and spreadsheet programs.
Results: The datasets were manually scored by an expert, and the data were then automatically scored by two different methods using the automated seizure analysis toolkit. Method 1: RMS power in the 1 to $100 \mathrm{~Hz}$ band. The results are presented as Seizures automatically found / Seizures found by the expert scorer. Mouse A: $3 / 3$, Mouse B: $5 / 5+1$ false positive, Mouse C: $3 / 3$, Mouse D: $5 / 5+1$ false positive, Mouse E: 5/5. Method 2: Line Length; Mouse A: 3/3, Mouse B: 5/5, Mouse C: $3 / 3$, Mouse D: $5 / 5$, Mouse E: $5 / 5$. In these preliminary studies, some amount of manual threshold adjustment was necessary. In the next several months, the thresholds will be chosen for each dataset either automatically or via a training algorithm.

Conclusions: Use of this type of integrated toolkit can aid with rapid scoring and analysis of large volumes of EEG-based seizure data.

\section{Neurophysiology: Computational Analysis \& Modeling of EEG}

\subsection{8 \\ SPATIO-TEMPORAL CORRELATION OF HFO DYNAMICS FOR SEIZURE ONSET LOCALIZATION}

Urszula Malinowska, G. K. Bergey, M. C. Cervenka and C. C. Jouny (Johns Hopkins University School of Medicine, Baltimore, MD)

Rationale: High frequency oscillations (HFO) are spontaneous activity observed in EEG whose descriptions and mechanisms are still evolving. Their characteristics span a wide range in frequency, amplitude, and duration. Their occurrence often correlates with epileptogenicity, although it remains unclear if HFO reflect pathophysiology or are epiphenomena. Recent works have demonstrated that fast activity progressively increases before seizure onset - suggesting the potential use as a marker for a preictal state. Removal of the areas generating HFO is associated with better postsurgical outcomes. To investigate HFO as biomarkers of epileptogenic tissue, we have examined changes of HFO activity before and during complex partial seizures in humans.

Methods: Intracranial recordings from ten patients diagnosed with mesial temporal and neocortical onset partial seizures undergoing presurgical evaluations were analyzed. For HFO detection we applied an automated method that consists of identifying at least 4 consecutive oscillations with amplitudes above $10 \mu \mathrm{V}$ and at least two times larger than the average amplitude of oscillations of the surrounding background. The analysis was performed on bipolar filtered EEG $(80-200 \mathrm{~Hz})$ signals. To detect short, transient HFO events rather than long continuous high frequency activity, a limitation of a maximum of $100 \mathrm{~ms}$ was applied for each event duration. The results of detection were compared between channels and for 2-minutes periods (interictal: 2 hours, 1 hour and 30 minute before onset; preictal: 2 minute before onset; ictal: immediately after seizure onset).

Results: During interictal periods, distributions of the rate of HFO across electrodes remains consistent, and is similar for interictal periods taken relative to consecutive recorded seizures for each patient. In some instances the distribution of the interictal activity differs from the distribution of HFO during the preictal periods. The distribution of HFO during preictal periods is also consistent between consecutive seizures.

During precictal periods, the number of HFO is increased compared to interictal levels. These levels increase further during ictal periods. The maximum number of HFO during the ictal period often correlates with the area of seizure onset as indicated by visual analysis of experienced neurologists. Consistent distributions of HFO activity between interictal and ictal periods corresponds to the better postsurgical outcomes.

Conclusions: Significantly different distributions of HFO across elecrodes during interictal and ictal periods correlating with suboptimal surgical outcomes could indicate that in some instances different regions can be responsible for generation of preictal and ictal HFO. 
It remains to be determined whether interictal/preictal and ictal HFO are different processes; however the consistency across time suggests similar substrates. Whether or not localized HFO represent seizure onset zones or irritative secondary regions remains. Detailed investigation of spatiotemporal dynamics of fast activity could help elucidate the role of HFO in ictogenesis.

Funded by NIH NS075020

\subsection{9 \\ AUTOMATED DETECTION OF HIGH FREQUENCY OSCILLATIONS USING THE DAMPED-OSCILLATOR OSCILLATOR DETECTOR: CORRELATION WITH SEIZURE-ONSET ZONE}

David Hsu ${ }^{1}$, M. Hsu ${ }^{1}$, B. H. Brinkmann ${ }^{2}$ and G. A. Worrell ${ }^{2}$ $\left({ }^{1}\right.$ Department of Neurology, University of Wisconsin, Madison, WI and ${ }^{2}$ Department of Neurology, Mayo Clinic, Rochester, MN)

Rationale: Transient high frequency oscillations (HFOs) in the human electroencephalogram (EEG) in the ripple $(80-200 \mathrm{~Hz})$ and fast ripple $(250-600 \mathrm{~Hz})$ ranges have attracted increasing interest because HFOs appear to localize to seizure onset zones (SOZs), and the resection of brain tissue with high rates of HFO activity appears to be correlated with better seizure outcomes. Expert visual inspection remains the gold standard for the identification of HFOs. However, expert visual inspection is an extremely laborious process and impractical for long-term EEG data as acquired for the patient undergoing pre-surgical localization. Here we present a computer algorithm for the detection of HFOs that may help facilitate this analysis.

Methods: We have developed a high resolution pseudo-wavelet time-frequency analyzer called the damped-oscillator oscillator detector (DOOD). This method uses a set of damped mathematical oscillators to detect oscillations in EEG data. The DOOD spectral density is first Z-normalized by subtracting out the mean and dividing by the standard deviation. Events with spectral density greater than a threshold S are flagged as candidate HFOs. Test data consist of 900s of 17-channel intracranial microwire EEG collected from a patient undergoing epilepsy surgery evaluation. The first 8 channels extend in a tuft from the tip of the electrode shaft. Channels 9-17 are spaced along the shaft of the electrode. Channels 1-11 are in the putative SOZ. Institutional review board authorization for this study was obtained.

Results: Figure 1 shows the sensitivities (Sens) and positive predictive values (PPVs) of the DOOD HFO detection algorithm as a function of the threshold S. For comparison, sensitivities and positive predictive values using the line length method are also shown. The DOOD method is capable of higher positive predictive values than the line length method. Figure 2 shows HFO event rates using DOOD with $\mathrm{S}=4$. HFO event rates from the SOZ (channels 1-11) are higher than from non-SOZ (unpaired 2-tailed T-test, $\mathrm{p}=0.00045$ ). HFO event rates with a lower threshold $\mathrm{S}=2$ fail to pick out the $\mathrm{SOZ}$ (not shown, $\mathrm{p}=0.31$ ).

Conclusions: DOOD may be useful for quantifying HFO event rates. A higher detection threshold $\mathrm{S}$ yields higher positive predictive values of HFOs and better identification of $\mathrm{SOZ}$

Funding: CURE, NIH RO1-NS63039.

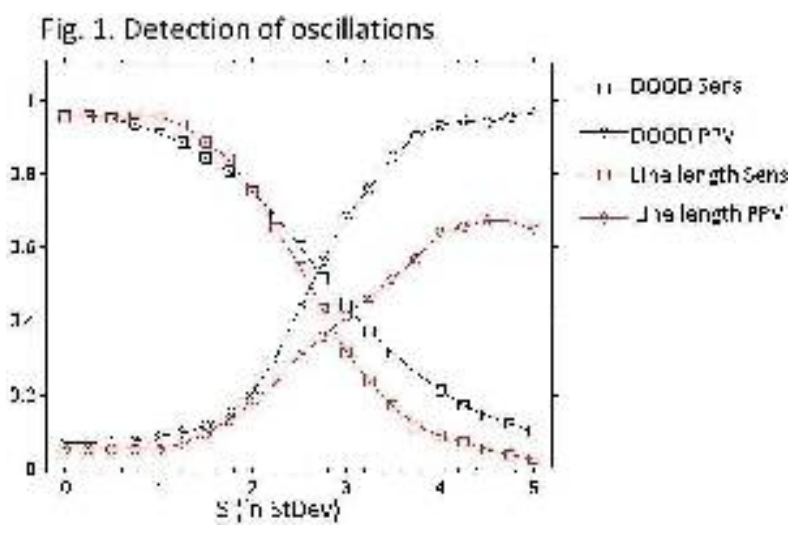

Fig. 2. HFO event rate vs EEG channel

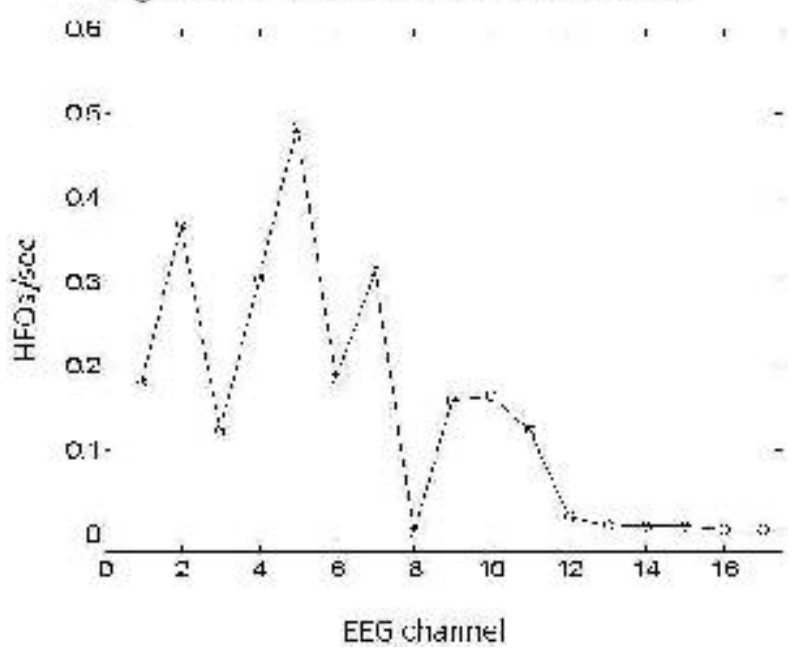

\subsection{0 \\ DIFFERENTIATING INTER-ICTAL AND PRE-ICTAL STATES: IMPACT OF WEIGHTED AND DIRECTED SHORT- AND LONG-RANGED INTERACTIONS IN EPILEPTIC BRAIN NETWORKS}

Stephan Porz ${ }^{1,2}$, C. E. Elger ${ }^{1}$ and K. Lehnertz ${ }^{1,2}$ ('Epileptology, University of Bonn, Bonn, Germany and ${ }^{2}$ Helmholtz Institute for Radiation and Nuclear Physics, University of Bonn, Bonn, Germany)

Rationale: Previous studies provided first evidence that an improved detection of pre-ictal states can be achieved through a quantification of mutual relationships between different functional subsystems that compose an epileptic brain network. We here investigate how weighted and directed short- and long-ranged interactions influence the ability to differentiate between inter-ictal and pre-ictal states. Methods: We analyzed intracranial multi-channel (mean: 54 sites), multi-day (on average 110 hours per patient) EEG recordings from up to now 15 epilepsy patients ( 8 with unifocal and 7 with multifocal epilepsies; all patients are seizure-free post-operatively). A total of 68 seizures occurred. Between the clinically defined epileptic focus and other sampled brain regions the strength and the direction of interactions were estimated in a time-resolved manner each via a phase-based (strength: R, direction: D) and an information theoretical approach (strength: $\mathrm{G}$, direction: $\mathrm{T}$ ). Of these interactions those between the focus and its vicinity were defined as short-ranged. Results: We observed the distributions of measures for the pre-ictal (assumed duration 4 hours) and the inter-ictal state (remaining data, excluding seizures and a post-ictal period of 30 minutes) to differ significantly, at least in a number of patients. However, differentiation depended on daily rhythms and on the approach used to characterize interaction properties (day: R 67\%, D 33\%, G 42\%, T $67 \%$; night: R 58\%, D 58\%, G 33\%, T 75\% of cases). Moreover, separability of states appeared to be influenced by contributions of 
short- and long-ranged interactions, which differently affected strength and direction of interactions.

Conclusions: Our findings indicate that considering differentially the contributions of weighted and directed short- and long-ranged interactions can improve the ability to distinguish between pre-ictal and inter-ictal states. This approach could improve our understanding of ictogenesis in epileptic brain networks.

This work was supported by the Deutsche Forschungsgemeinschaft (Grant No: LE 660/5-2)

\subsection{1 \\ QUANTITATIVE EEG (QEEG) ANALYSIS OF THE SLOW WAVE PHASE OF SPIKE AND WAVE ACTIVITY IN TREATED AND UNTREATED PRIMARY GENERALIZED EPILEPSY (PGE)}

Zianka Fallil and S. V. Pacia (NYU Langone Medical Center, New York, NY)

Rationale: QEEG analysis in PGE has focused primarily on spike and wave frequency, spike counts and burst duration. After-going slow waves occur when neurons do not fire, representing a period of diffuse cortical inhibition. The effects of antiepileptic medications (AEDs) on these slow waves have not been well characterized. Methods: The EEGs of 5 patients with non-absence, untreated PGE were compared to those of 5 patients, on AEDs, seizure free for a minimum of one year. A commercially available QEEG analysis tool was used to analyze spike and wave bursts from each patient, recorded while awake. After- going slow wave durations in a run of SWDs were measured and averaged. The first and subsequent slow wave durations were calculated as a percentage of the duration of the terminating wave in a burst.

Results: In a spike and wave run each successive wave progressively increased in duration in both the untreated and treated groups. While the terminating slow wave durations did not differ between groups, the first after-going slow wave in the treated group was significantly longer ( $\mathrm{p}<.05$ unpaired two tailed T-test). Additionally, slow waves in isolated spike and wave discharges did not differ between groups Conclusions: First after going slow wave durations are significantly longer in AED controlled PGE patients compared with those from drug naive patients. Further studies are needed to determine whether this quantitative EEG finding predicts seizure control and whether it differs among AEDs.

\subsection{2 \\ STOCHASTIC BEHAVIOR OF PHASE SYNCHRONIZATION AND CROSS-FREQUENCY COUPLINGS IN THE EPILEPTOGENIC ZONE ON DIFFERENT DAYS MEASURED WITH 256 CHANNEL INTERICTAL SCALP EEG}

Mark D. Holmes ${ }^{1}$ and C. Ramon ${ }^{2}{ }^{1}$ Neurology, University of Washington, Seattle, WA and ${ }^{2}$ Electrical Engineering, University of Washington, Seattle, WA)

Rationale: To determine if distinguishable spatial patterns are present in the stochastic behavior of phase synchronization and crossfrequency couplings on separate days in the interictal, high density scalp EEG, and if these patterns correspond to the proven epileptogenic zone.

Methods: We studied one patient with refractory epilepsy who underwent intracranial EEG to establish the localization of seizure onset. Prior to invasive EEG studies the subject underwent dense array 256 channel EEG (dEEG) recordings. Three minutes of interictal dEEG data from first day, second day and on the eleventh day was randomly selected for analysis. The selected segment in each day was at least two hours temporally from an epilepic seizure and, based on visual analysis, and free of interictal epileptiform patterns. Excessively noisy channels were removed and replaced with averages of surrounding electrodes. Data were imported into MATLAB for analysis. The EEG data was filtered in the appropriate EEG band. The analysis was performed in theta $(3-7 \mathrm{~Hz})$, alpha $(7-12 \mathrm{~Hz})$, beta $(12-30 \mathrm{~Hz})$ and low gamma $(30-50 \mathrm{~Hz})$ ranges. The phase synchronization index (SI) was computed after taking Hilbert transform of the EEG data. The SI for each electrode was averaged over with the nearby six electrodes. A detrended fluctuation analysis (DFA) was used to find the stochastic behavior of the SI. The stochastic behavior of the cross-frequency coupling in theta-gamma, beta-gamma and alpha-gamma bands was also examined. Contour plots with $10 \mathrm{sec}$ intervals were constructed using a montage of the layout of 256 electrode positions.

Results: Contour plots displayed over the scalp show that the stochastic behavior of the SI becomes stronger with time in the proven epileptogenic area while in other areas it becomes fragmented and scattered. The low gamma band was found to be the best indicator frequency band for localization. In contrast the crossfrequency couplings decreased in the epileptogenic area for all comparisons. For this subject, we were able to identify stable patterns of stochastic behavior in the epileptogenic area within 120 seconds of analysis. Similar findings were observed in all three days of the data. Conclusions: Stochastic behavior of SI increased and the crossfrequency coupling decreased in the epileptogenic zones on all three days for this subject. These findings have the potential to assist in localizing epileptic sites in subjects with proven epilepsy.

\subsection{3}

\section{A HYBRID WAVELET TRANSFORM AND MACHINE} LEARNING ALGORITHM FOR EEG SPIKE DETECTION Vamsidhar Chavakula $^{1}$, I. Sánchez Fernández ${ }^{2}$, J. Peters ${ }^{1,2}$, W. Bosl ${ }^{2}$, A. Rotenberg ${ }^{1,2}$ and T. Loddenkemper ${ }^{1,2}\left({ }^{1}\right.$ Harvard Medical School, Boston, MA and ${ }^{2}$ Division of Epilepsy and Clinical Neurophysiology, Department of Neurology, Children's Hospital Boston, Boston, MA)

Rationale: Manual EEG spike quantification is frequently a subjective and time-consuming process. We have previously developed a double threshold wavelet algorithm for quantification of epileptic spikes, and now report improvements to this automated algorithm via addition of a machine learning component in order to further enhance sensitivity while substantially decreasing the rate of false positives.

Methods: We applied our algorithm to EEG recordings from eleven pediatric patients (average age of 8 years) with documented ESES (Electrical Status Epilepticus in Sleep). For each patient, one segment of three to eight minutes duration was first analyzed using a double threshold wavelet method to highlight time points corresponding to potential spike events. For each detected spike, the calculated features were the amplitude, slope, and spike angle in every channel. These features were input to a machine learning algorithm to determine whether the time point was indeed a spike. Machine learning classifier training and testing for each set was performed using a 10-fold cross-validation technique. Results were compared against gold-standard visual review by two board certified clinical neurophysiologists.

Results: Two different machine learning modalities were testedSupport vector machine (SVM) and Bayesian learning. When SVM was used, the overall sensitivity was $69.6 \%(+/-15.3 \%)$ and the overall specificity was $95.7 \%(+/-10.4 \%)$. The kappa statistic was 0.878 and 0.854 respectively for reviewers A and B. When Bayesian learning was utilized, the overall sensitivity was $77.6 \%(+/-9.1 \%)$ and the overall specificity was $87.6 \%(+/-8.3 \%)$, and the kappa statistic was 0.780 and 0.771 respectively for reviewers A and $\mathrm{B}$. The detailed results are provided in Table 1, and a graphic summary is shown in Figure 1.

Conclusions: Automated spike quantification by wavelet transform is feasible and can be improved by machine learning. Machine learning improved specificity from $64 \%$ to as much as $95 \%$, while 
allowing for maintenance of higher sensitivities. Overall, there was good agreement with the gold-standard of EEG review by human visual inspection. In certain data sets, low sensitivity was noted, which was most likely due to presence of artifact, further highlighted by the fact that these sets displayed the highest amount of disagreement between the two human reviewers. We anticipate that upon further validation, the reported spike detection protocol method may be useful in quantification of epileptiform activity in research and in clinical practice.

Sensitivity and specificity results for our population

\begin{tabular}{|c|cc|c|c|}
\hline & \multicolumn{2}{|c|}{ SVM } & \multicolumn{2}{c|}{ Bayes } \\
\hline Set & Sensilivity & Specilicity & Sensitivity & Specilicity \\
\hline 1 & 0.844 & 0.622 & 0.816 & 0.693 \\
2 & 0.864 & 0.980 & 0.864 & 0.896 \\
3 & 0.729 & 0.967 & 0.785 & 0.890 \\
\hline 4 & 0.724 & 0.969 & 0.796 & 0.923 \\
\hline 5 & 0.474 & 0.975 & 0.720 & 0.913 \\
\hline 6 & 0.692 & 0.971 & 0.820 & 0.929 \\
\hline 7 & 0.828 & 0.887 & 0.611 & 0.792 \\
\hline 8 & 0.685 & 0.977 & 0.861 & 0.936 \\
9 & 0.845 & 0.953 & 0.950 & 0.893 \\
10 & 0.465 & 0.938 & 0.746 & 0.763 \\
\hline 11 & 0.499 & 0.953 & 0.717 & 0.784 \\
\hline Overall & 0.696 & 0.957 & 0.776 & 0.876 \\
\hline
\end{tabular}

$\mathrm{SVM}=$ Support Vector Machine

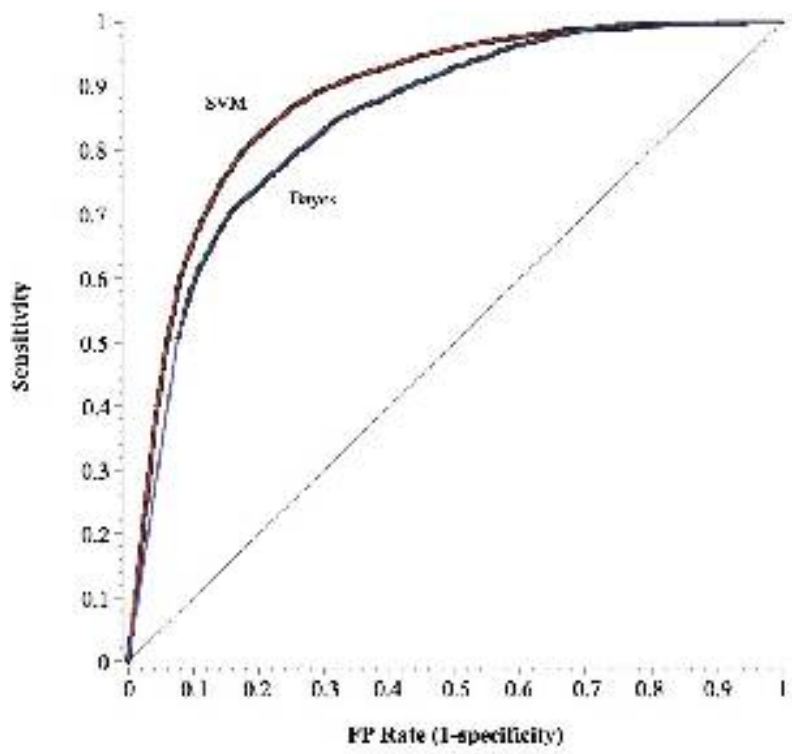

Relation of sensitivity and specificity in our algorithm when compared to the gold-standard human reviewer when using both support vector machine and Bayesian learning.

\subsection{4}

COMPARISON OF DIPOLE SOURCE ANALYSIS WITH INTRACRANIAL ELECTROENCEPHALOGRAM FOR LOCALIZATION OF THE EPILEPTOGENIC ZONE IN INTRACTABLE NON-LESIONAL EPILEPSY PATIENTS Basanagoud Mudigoudar ${ }^{1,2}$, M. Quach ${ }^{2,1}$, H. Tsoi ${ }^{2}$ and S. Agadi ${ }^{2,1}$ ( ${ }^{1}$ Clinical Neurophysiology, Baylor College of Medicine, Houston, TX and ${ }^{2}$ Pediatric Neurology, Texas Children's Hospital, Houston, TX)
Rationale: According to the existing literature, absence of a lesion in the magnetic resonance imaging (MRI) is associated with poorer prognosis after resective epilepsy surgery. Delineation of the epileptic zone by invasive intracranial recording is required in such patients. Invasive monitoring has potential complications and is perceived to be relatively more difficult due to lack of a clear radiographic target to implant the recording electrodes in patients with non-lesional epilepsy. Electroencephalogram (EEG) dipole source analysis is based on noninvasive scalp EEG data and allows estimation of the underlying generator of a given EEG potential. Its clinical utility has not been well studied in non lesional epilepsy. Here we compare the ability of dipole source analysis using scalp EEG with intracranial recording to identify the epileptic focus in these patients.

Methods: All patients with intractable epilepsy and no detectable lesion on MRI, who underwent resection of the epileptogenic zone after intracranial recording, were included in the study. Data regarding patient characteristics, scalp EEG findings, brain MRI, intracranial electroencephalogram recordings, surgical details, pathology findings and the results of dipole source analysis were collected and analyzed. Non-invasive scalp EEG's were analyzed using the Brain Electrical Source Analysis (BESA) software, developed by MEGIS Software. We performed the dipole source analysis on averaged ictal waveforms and co-registered the EEG source dipole on the patient's brain MRI. We compared the effectiveness of EEG dipole source analysis in localizing the seizure focus with intracranial recordings.

Results: Twelve patients fulfilling the inclusion criteria were identified. Three patients, who failed the resective surgery and one with incomplete follow up were excluded. Eight patients $(66 \%)$ who underwent resection of the epileptogenic zone after intracranial recording and became completely seizure free were included in the final analysis. We correctly lateralized the seizures in 7 (87\%) patients using the dipole source analysis. The dipole source localization results matched the intracranial recordings in exact localization of the epileptogenic zone in $4(50 \%)$ patients. In remaining three patients, the dipole source localization using BESA was able to correctly localize the seizure onset zone to different lobar regions of the brain.

Conclusions: Dipole source analysis using scalp EEG was able to correctly lateralize the seizure onset zone in $87 \%$ of the patients with non-lesional epilepsy. Furthermore, it precisely localized the epileptogenic zone in at least half of these patients. Estimating the EEG dipole source before invasive electrode evaluation will potentially help in guiding the clinicians in placement of intracranial electrodes and also will limit the extensiveness of the coverage, thereby potentially decreasing the complication rate, as well as financial burden.

\subsection{5}

AUTOMATED ARTIFACT DETECTION IN INTRACRANIAL EEG

Benjamin Brinkmann ${ }^{1,2}$, V. Vasoli ${ }^{2}$, J. Echauz $^{3}$, B. Litt ${ }^{4}$ and G. Worrell $^{2}\left({ }^{1}\right.$ 3D Medical Imaging, Byron, MN; ${ }^{2}$ Mayo Systems Electrophysiology Lab, Mayo Foundation, Rochester, MN; ${ }^{3} \mathrm{JE}$ Research, Atlanta, GA and ${ }^{4}$ University of Pennsylvania, Philadelphia, PA)

Rationale: Long-term, continuous, wide-bandwidth recording of human electrophysiology from hundreds of electrode channels shows promise for delineating the epileptogenic zone in focal epilepsy via detection and mapping of high frequency oscillations, microseizures, unit action potentials, and interelectrode synchrony. Data artifacts due to human motion, electromagnetic induction, and poor electrical contact are inevitable in recordings that can last over a week. The large amount of data generated by this approach precludes comprehensive human review for data quality, and regions of artifact must be excluded from automated analysis by electrophysiological detection algorithms. 
Methods: Data condition number (DCN), independent component analysis (ICA), and line length have been proposed as automatic methods for detecting time segments of poor data quality in EEG recordings. However, no existing method is universally accepted, and some current methods do not differentiate between recorded data segments where signal is uniformly poor versus segments where artifacts are limited to only a few channels, or one particular electrode. We describe and validate a modification to the DCN data quality metric that can be applied to single channels or groups of channels to provide a better understanding of data quality issues. The relative data condition number $(\mathrm{rDCN})$ for a given channel or group of channels is calculated by subtracting the DCN with the channel(s) in question omitted from the DCN calculated on the entire group. Results: The rDCN is validated and demonstrated on data from the NIH International Epilepsy Electrophysiology Database (www.ieeg.org) and is shown to successfully differentiate between data segments with global artifacts (affecting all channels) and artifacts confined to single channels or groups of channels. Its performance is characterized in comparison to the performance of other existing data quality algorithms.

Conclusions: Automated detection and analysis of EEG recording artifact is an important component to automated detection and analysis in long-term EEG recordings and ambulatory EEG monitoring systems. The rDCN has the potential to accurately and reliably identify segments and channels with poor data quality without user intervention.

\subsection{6 \\ RETROSPECTIVE SUBDURAL EEG SOURCE LOCALIZATION OF THE ICTAL ONSET ZONE IN PEDIATRIC EPILEPSY SURGERY}

Daniel C. Tarquinio, A. Salah-Eddin, Marianne Diaz, T. Stewart and P. Jayakar (Miami Children's Hospital, Miami, FL)

Rationale: Epilepsy surgery fails to effect a cure in $30-40 \%$ of children despite optimal evaluation. Visual interpretation of subdural electrodes may be confounded by complex fields and the overpowering influence of source components close to certain electrodes. Although computer simulations have demonstrated that subdural ESL provides more precise localization of deep sources than scalp ESL, no study has evaluated the relationship between subdural ESL and the epileptogenic zone as defined by successful epilepsy surgery. We aimed to develop a methodology for evaluating ESL with respect to the epileptogenic zone, hypothesizing that in successful epilepsy surgery, interictal spikes localized using subdural ESL should be within the surgical resection zone.

Methods: Retrospective data on surgical patients were reviewed, preoperative and postoperative MRIs were coregistered with intraoperative CTs of subdural electrodes, and coregistration quality was compared with surgical outcome using Spearman's rho. Patients with accurate coregistration and good surgical outcome were selected for ESL. Representative interictal spikes from subdural EEG were selected, and sources were reconstructed using a boundary element model. Five reconstruction methods were applied: one single dipole, one scanning model (Multiple Signal Classification, or MUSIC), and three distributed source models (minimum norm estimation [MNE], standardized low-resolution electromagnetic tomography

[sLORETA], and sLORETA-weighted accurate minimum norm [SWARM]). Dipoles or maximal regions of charge density were categorized as within or outside the surgical resection zone (SRZ) using a 3-dimensional volume resection mask and compared using Chi-square, and euclidean distances to surgical mask epicenter were compared among the reconstruction methods using ANOVA, and location within the resection zone was compared to patients.

Results: Coregistration on 19 individuals trended toward association with seizure freedom $(\mathrm{p}=0.07)$. Coregistration was adequate in 3 , all of whom were seizure free after surgery. In each patient 14 to 15 spikes were reconstructed (total 215 solutions), of which 59 dipoles
$(27 \%)$ lay within the SRZ. The single dipole method performed best (40\%) and SWARM worst (19\%). Considering subcategories, $41 \%$ were localized for the case with left temporal corticectomy, $37 \%$ for right parietal corticectomy, and 5\% for right frontal/interhemispheric corticectomy. Considering model, dipole performed better in the parietal case $(86 \%)$, and MNE and sLORETA performed better in the temporal case $(50 \%, \mathrm{p}<.001$ for all $)$.

Conclusions: Spikes were localized within the resection zone best using dipole model in parietal epilepsy. Success in image coregistration was weakly associated with outcome, suggesting that improved coregistration modalities should be investigated to allow ESL in patients with worse prognosis. These data support the use of subdural ESL, and the prospective evaluation of ESL to identify models suited to specific regions for improved planning of surgical resection.

\subsection{7 \\ ELECTRIC SOURCE IMAGING (ESI) IN PRESURGICAL EPILEPSY INVESTIGATIONS: BOTH A SUFFICIENT NUMBER OF RECORDING ELECTRODES AND COVERAGE OF ALL BRAIN AREAS IS IMPORTANT FOR CORRECT SOURCE IMAGING RESULTS}

Göran Lantz, M. Terrill, T. Gilbert, P. Luu, A. Bunnenberg and D. Tucker (Electrical Geodesics Inc, Eugene, OR)

Rationale: With Electric Source Imaging (ESI) the source of interictal epileptiform activity in EEG can be localized with high accuracy. There are two different aspects required, however, in order to achieve high accuracy ESI; 1) a sufficient number of recording electrodes, and 2) adequate spatial coverage of electrodes over the recording surface. Recently developed EEG systems now allow recording with up to 256 surface electrodes. The purpose of this study was to evaluate the localization accuracy of epileptic spikes recorded with 256 electrodes compared to sparser electrode arrays and compared to incomplete, or uneven, spatial electrode coverage on the head surface.

Methods: In four patients with intracranially verified temporal lobe epilepsy, the 3D location of the ictal onset in the intracranial electrodes was determined. To look at the importance of the number of recording electrodes, interictal spikes recorded with 256 electrodes were averaged, and a regular downsampling of the averaged spike was performed to 128,64 and 32 electrodes. To look at the importance the spatial distribution of electrodes, another kind of electrode reduction was used where the electrodes covering the lower temporal areas were taken out. Then, a distributed source model (LAURA) was used to localize the source of the original 256 channel spike data sets and also of both types of downsampled spikes (decreased number of electrodes and more limited spatial coverage). The source maximum was compared to the intracranial ictal onset location for validation of results.

Results: Both regular downsampling and reduction of temporal lobe coverage resulted in a clear deterioration of the ESI results. This difference was particularly clear when analyzing the early phase of the spike where the signal to noise ratio was poor. The degree to which the downsampling affected the results varied between the patients, but the most correct source localizations with respect to the intracranial ictal onset were always obtained with the 256 channel setup.

Conclusions: Both a dense sampling on the head and a sufficient coverage of the lower temporal areas is necessary for correct source localization results. Improvement of the results can be obtained at least up to 256 electrodes 


\subsection{8}

\section{ONSET FEATURES OF GENERALIZED SPIKE AND WAVE DISCHARGES}

A. S. Braga, W. P. Blasque and Luiz E. Betting (Universidade Estadual Paulista, Bauru, Brazil)

Rationale: Generalized spike-wave (GSW) discharges are the main electroencephalographic characteristics of idiopathic generalized epilepsies (IGE). Event-related spectral perturbation (ERSP) analysis quantifies changes triggered by events in the spectral power plot of the electroencephalogram (EEG). The aim of this study was to evaluate the presence of focal activity in generalized discharges of patients with IGE using the ERSP analysis.

Methods: 32 patients (14 women, mean age $32 \pm 14$ years) with IGE were investigated. The patients were submitted to routine EEG which was performed in a 22 channels recorder, with 20 minutes duration, 10-20 electrode placement system, photostimulation and hyperventilation. EEGLAB software was used for EEG processing and analysis. GSW discharges were visually selected and excerpts of 4 seconds centered in the onset of generalized discharge were stored. Then, a spectral plot was generated for each electrode. Finally, a mean of all electrodes was calculated and analyzed.

Results: 253 discharges (mean $8 \pm 12$ per patient) were evaluated. The spectral responses obtained in the evaluation of the mean of all channels in the moment of discharges showed changes in electrodes: Fp1, Fp2, F3 e F4 (4.7, 5, 4.6 and 5dB respectively). After $127 \mathrm{~ms}$, similar changes were noticed corresponding to the slow component of the discharge in these same electrodes $(5.2,4.8,5.1$ e $4.9 \mathrm{~dB})$. Conclusions: This investigation supports that frontal regions constitute the initial source of generalized discharges. Subsequently, there is fast spread and involvement of greater projection networks.

\section{Clinical Epilepsy: Classification and Syndromes}

\subsection{9}

\section{AURAS IN GENERALIZED EPILEPSY}

Patricia Dugan $^{1}$, O. Devinsky ${ }^{1}$, R. Kuzniecky ${ }^{1}$, R. Fahlstrom ${ }^{2}$ and .. EPGP Investigators ${ }^{1,2}\left({ }^{1}\right.$ Neurology, New York University Langone Medical Center, New York, NY and ${ }^{2}$ Neurology, University of California, San Francisco, San Francisco, CA)

Rationale: Auras or lateralized clinical features are conventionally considered indications that a seizure is focal, rather than generalized, in nature. However, prior research questions the validity of the conventional interpretation. Making the distinction between partial and generalized seizure disorders occurs early in diagnostic evaluation andhas significant implications for therapy. We studied this hypothesis by prospectively recording reports of auras utilizing a systematic, validated questionnaire.

Methods: Subjects were extracted from the Idiopathic Generalized Epilepsy (IGE) arm of the Epilepsy Phenome Genome Project (EPGP). All subjects who had typical clinical histories and EEG features of IGE but did not have focal epileptiform abnormalities on EEG were included. Responses to the standard diagnostic interview regarding grand mal seizures were then examined. This questionnaire initially required subjects to provide their own description of any subjective phenomena prior to their grand mal seizures. Subjects who provided answers to these questions were considered to have an aura. All subjects were then systematically queried regarding a variety of subjective phenomena prior to their grand mal seizures and were required to answer either "yes," "no," or "don't know," and were permitted to provide multiple affirmative answers.

Results: 807 participants with IGE were identified. Among these, 537 reported grand mal seizures. Of these, 185 (34.45\%) selfreported auras, with significantly higher rates of auras in female respondents than males $(p=0.0001)$. The most commonly selfreported auras were dizziness, confusion, and conscious appreciation of increasing jerks (Table 1). Analysis of responses to the systematic query showed that $389(72.44 \%)$ experienced at least one form of aura. The most common auras reported were changes in speech and comprehension, version of the head or other body parts, and a brief lapse of awareness (Table 2).

Conclusions: Auras occur frequently in generalized epilepsy. Auras typically associated with focal epilepsy, such as gustatory, visual, auditory phenomena and déjà vu were also reported in this cohort, which may support existing theories of cortical generators of IGE with variable spread patterns. Differences between self-reported and systematically queried responses may also reflect clinically relevant variances in patient responses to history-taking and surveys.

Most common self-reported signs or symptoms before a grand mal seizure

\begin{tabular}{|c|c|c|}
\hline & Number & Percentage \\
\hline Anxiety & 4 & 2.16 \\
\hline Auditory disturbance (tinnitus) & 2 & 1.08 \\
\hline Confusion & 28 & 15.14 \\
\hline Dissociative sensation & 2 & 1.08 \\
\hline Dicziness & 37 & 20.00 \\
\hline Epigastric rising sensation & 1 & 0.54 \\
\hline Eye movements & 4 & 2.16 \\
\hline Fatigue & 6 & 3.24 \\
\hline Fear & 2 & 1.08 \\
\hline Focal head turn & 5 & 2.70 \\
\hline Focal numbness & 3 & 1.62 \\
\hline Focal pain & 2 & 1.08 \\
\hline Headache & 14 & 7.57 \\
\hline Heart racing & 1 & 0.54 \\
\hline Hiccups & 1 & 0.54 \\
\hline Increasing myoclonic jerks & 26 & 14.05 \\
\hline Increasing petit ma] & 7 & 3.78 \\
\hline Nausea & 6 & 3.24 \\
\hline Prodrome & 5 & 2.70 \\
\hline Stomachache & 5 & 2.70 \\
\hline Unusual taste & 1 & 0.54 \\
\hline Vague premonitory fecling & 15 & 8.10 \\
\hline \multirow[t]{2}{*}{ Visual disturbance } & 8 & 4.32 \\
\hline & 185 & 100.00 \\
\hline
\end{tabular}

Before the seizures start, or at the beginning of the seizures, do you have:

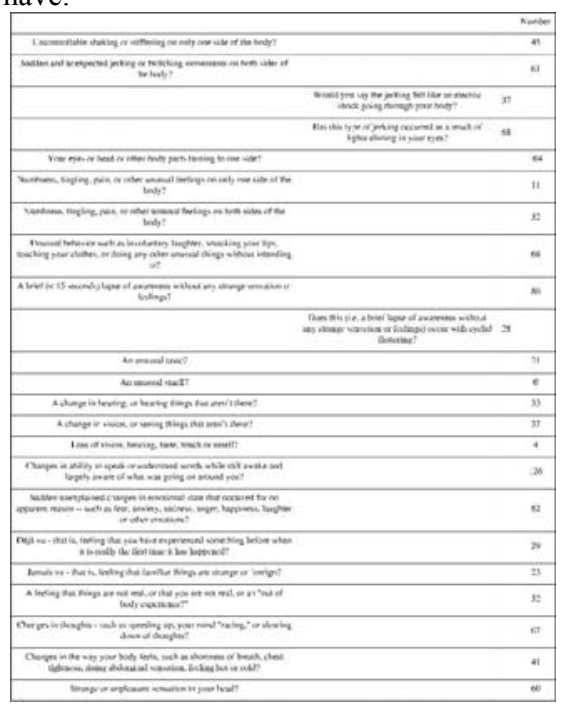


3.120

THE RELATIONSHIP BETWEEN TEMPORAL LOBE EPILEPSY AND RESTLESS LEGS SYNDROME

James D. Geyer ${ }^{1,3}$ and P. R. Carney ${ }^{2}\left({ }^{1}\right.$ Epilepsy and Sleep Medicine, Alabama Neurology and Sleep Medicine, Tuscaloosa, AL; ${ }^{2}$ Department of Pediatrics, Neurology, Neuroscience, and Bioengineering, University of Florida, Gainesville, FL and ${ }^{3}$ Department of Neurology and Sleep Medicine, University of Alabama, Tuscaloosa, AL)

Rationale: Restless legs syndrome (RLS) is a common neurological movement disorder occurring in approximately $10 \%$ of the general population. The prevalence of the moderately severe RLS is $2.7 \%$ overall (3.7\% for women and $1.7 \%$ for men). The prevalence of RLS increases with advancing age and is more common in several neurological disorders. Epilepsy is also a common neurological disorder with significant associated morbidity and impact on quality of life. We report the severity and frequency of primary RLS in patients with temporal lobe epilepsy.

Methods: All epilepsy patients seen in the outpatient clinic were screened for movement disorders. Those patients with severe language impairment, cognitive impairment and stroke were excluded from this study population. The epilepsy diagnoses were based on EEG, MRI, functional imaging (in pre-surgical evaluations only), and semiological features. Forty consecutive patients with localizationrelated temporal lobe epilepsy (TLE) (20 right TLE and 20 left TLE) who met inclusion criteria were seen in the outpatient clinic.

Each patient was evaluated with the International Restless Legs Study Group (IRLSSG) questionnaire, NIH RLS diagnostic criteria, ferritin level, and comprehensive sleep screening including polysomnography. Furthermore, patients with obstructive sleep apnea or a definite cause of secondary restless legs syndrome such as low serum ferritin or serum iron levels were also excluded from the study. Results: Restless legs occurred in 11 of 40 (27.5\%) these temporal lobe localization-related epilepsy patients overall. RLS occurred more frequently in the right TLE group $(8 / 20,40 \%)$ than the left $(3 / 20,15 \%)(p<0.05)$. The results are summarized in the table. A number of anti-epileptic agents were used in this study population creating an insufficient population for reliable statistical analysis of medication effects, both positive and negative.

Conclusions: We identified frequent, moderate to severe RLS in patients with epilepsy. The frequency of restless legs syndrome was much more common than would typically be seen in patients of similar age. The restlessness was typically described as moderately severe. The RLS symptoms were more common and somewhat more severe in the right TLE group than the left TLE group. Interestingly, it is the right TLE group that more frequently exhibits hypermobility in the seizure semiology.

It is likely that there are profound differences in the relationships between RLS and various anti-epileptic medications. Several anticonvulsants have been found effective for treating RLS in doubleblind, placebo-controlled trials including carbamazepine, gabapentin, pregabalin and clonazepam. Patients being treated with these medications may have reported lower RLS severity levels. More detailed analysis of the impact on quality of life parameters related to RLS on perceived quality of life related to epilepsy is needed.

Summary of results

\begin{tabular}{|cc|c|}
\hline & Right TLE & Lefl TLE \\
\hline Patients & 20 & 20 \\
Patients with moderate to severe RIS & $8(40 \%)$ & $3(15 \%)$ \\
Paticnts reporting RIS prior to the onset of scizures & $3(15 \%)$ & $1(5 \%)$ \\
\hline
\end{tabular}

3.121

INFANTILE SPASMS WIHTOUT HYPSARRHYTHMIAWEST SYNDROME VARIANT OR A DIFFERENT ENTITY? S. A. Khan ${ }^{1,2}$ and Deepa Sirsi ${ }^{1,2}\left({ }^{1}\right.$ Pediatric Neurology, University of Texas Southwestern Medical Center, Dallas, TX and ${ }^{2}$ Pediatric Neurology, University of Texas Southwestern Medical Center, Dallas, TX)

Rationale: West syndrome is an epileptic encephalopathy syndrome characterized by infantile spasms (IS), developmental regression and hypsarrhythmia. In this series we review 4 patients who presented to a tertiary care academic center with IS, without hypsarrhythmia and with or without developmental impairment.

Methods: A retrospective chart review of 4 children who presented with IS without hypsarrhythmia was performed. Information sought included age at onset of IS, EEG, neuro imaging, genetic and metabolic investigations. Treatments used included antiepileptic medications (AEDs), Adreno Cortico Trophic Hormone (ACTH) and vitamins (folic acid and folinic acid). Outcome measures were response of IS to treatment, developmental status and follow up EEGs.

Results: 2 of 4 children were girls. Age at onset of spasms ranged from 1 to 21 months (median age was 3.7 months). 2 children had normal development and 2 were delayed at the time of diagnosis and the developmental profile was unchanged at follow-up. None had other types of seizures prior to onset of IS. None had hypsarrhythmia on EEG. 1 had normal interictal EEG and 3 had either focal spikes or multifocal spikes that did not meet criteria for hypsarrhythmia. Ictal EEG findings consisted of electrodecremental segments (ED) with or without superimposed fast activity in 3 . No definitive ictal EEG change was observed in 1 patient. MRI brain was normal in 2 and abnormal in 2 ( 1 had abnormal signal in the left temporal white matter and the other had cerebellar and brain stem hypoplasia). Genetic and metabolic investigations were unrevealing of etiology. One child was treated with ACTH but IS recurred after ACTH taper and Topiramate (TPM) was added. All of them received TPM as first or second line treatment. 1 child received Vigabatrin as the second AED. 3 of 4 responded well with resolution of IS. Follow up interictal EEG was not significantly different and did not show hypsarrhythmia.

Conclusions: 1. IS without hypsarrhytmia may be considered an atypical form of West syndrome, rather than a separate epileptic syndrome.

2. Delayed diagnosis and treatment of IS without hypsarrhythmia could result in poor prognosis for neuro-developmental outcome and epilepsy.

3. Although this series had limited subjects and duration of follow up, this atypical presentation of IS was not a precursor to development of hypsarrhythmia including the child whose IS persisted after treatment initiation.

4. Hypsarrhythmia has been considered a cause of neuro-

developmental regression seen in West syndrome. In children with IS who do not have hypsarrhythmia it remains unclear if IS and developmental impairment are symptomatic of an underlying epileptogenic disease that is independent of hypsarrhythmia. A larger patient population and longer follow up would be helpful in improving our understanding of IS without hypsarrhythmia.

\subsection{2 \\ SEMIOLOGIC STRATIFICATION OF GENERALIZED TONIC CLONIC SEIZURES AND POST-ICTAL ELECTROGRAPHIC FINDINGS}

C. Carlson, Thomas Berk, J. French, R. Kuzniecky, P. Dugan, D. Gazzola and D. Friedman (Neurology, NYU School of Medicine, New York, NY)

Rationale: The Generalized Tonic-Clonic Convulsion (GTCC) is often associated with post-ictal electrographic slowing, and at times 
suppression. The mechanism of post-ictal EEG suppression is not known but may reflect involvement of bilateral subcortical networks. We examined the electrographic activity occurring after seizures with bilateral movement to determine if there are post-ictal features unique to the GTCC.

Methods: We reviewed the video EEG of 100 consecutive inpatients of the NYU Comprehensive Epilepsy Center that had bilateral movement as part of their seizure semiology. Each seizure was reviewed by 2 reviewers; any records in which the patient was obscured on the video were excluded from further analysis. Any seizure with bilateral symmetric tonic, vibratory and clonic phases (defined as bilateral movement $>$ and $<5 \mathrm{~Hz}$ respectively) in that order was categorized as "typical GTCC" (tGTCC). If one phase was absent, asymmetric or the progression was different, it was considered an "atypical GTCC" (aGTCC). If two phases were absent it was not a GTCC (nGTCC). All aGTCC were reviewed by at least 3 reviewers. The post-ictal EEG was categorized as: "suppression", defined as background voltage $<10 \mathrm{uV}$; "slowing" defined as decreased amplitude and/or frequency compared to baseline while still $>10 \mathrm{uV}$; or "no change from baseline."

Results: 104 seizures from 100 patients were reviewed, 5 patients were excluded due to obscured video or EEG, leaving 97 seizures reviewed. 41 were tGTCC, 14 were aGTCC and 42 were nGTCC either being a tonic, myoclonic-tonic, or dystonic complex partial seizure. Table 1 shows the post-ictal EEG findings across groups. A significant difference was seen $(\mathrm{P}<0.0001)$ with $68 \%$ of tGTCCs demonstrating post-ictal EEG suppression compared with $28 \%$ of aGTCCs and $11 \%$ of nGTCCs.

Conclusions: Although post-ictal EEG suppression is seen in all three groups, it is markedly more frequent in patients with the "typical" progression of a GTCC. The significant differences between these clinically defined and distinguishable groups suggest that future studies to identify risk factors for SUDEP may benefit from a more detailed semiologic stratification.

Post-Ictal EEG Findings Across All Groups

\begin{tabular}{|cc|c|c|}
\hline & IGTCC & aGICC & nGTCC \\
\hline Suppression & 28 & 4 & 5 \\
Slowing & 13 & 10 & 24 \\
No Changc & 0 & 0 & 13 \\
\hline
\end{tabular}

\subsection{3}

CHARACTERISTICS OF EPILEPSY IN NEUROFIBROMATOSIS TYPE I AND ASSOCIATION WITH MESIAL TEMPORAL SCLEROSIS

Anthony R. Pecoraro, W. Gallentine, E. Smith, C. Pizoli, A. Veerapandiyan and M. Mikati (Duke University, Durham, NC)

Rationale: Previous studies have reported a 3-7\% incidence of epilepsy in neurofibromatosis type 1 . The underlying causative lesion is often a supratentorial non-optic glioma tumor or unknown. To our knowledge only one case of mesial temporal sclerosis has been reported concurrent with NF 1 . Our goal was to identify the characteristics and the associated potentially causative MRI lesions in patients with epilepsy and NF 1 .

Methods: We performed a retrospective review of 175 consecutive patients seen in the NF clinic at Duke University over a period of 1 year ending in November 2010. Two groups of patients were compared - those who manifested seizures at some time in their history ( 30 patients, 27 of whom had MRIs) and those who did not (145 patients, 110 with MRIs). Additional analyses of the clinical findings of the seizure group were also performed.

Results: $27 / 175$ had epilepsy (15.4\%) and 3 additional patients had provoked seizures only; 2 had febrile seizures and 1 had a single postoperative non-febrile seizure. In the epilepsy group mean age at first seizure (available in 23 of them) was 6.6 years (range $=1$ month
- 30 yrs). The age breakdown was: 0-12 months (6/23), 13-36 months (4/23), 3-10 years $(7 / 23), 11-18$ years $(4 / 23), 18+$ years $(2 / 23, p=$ $0.51)$. In the 27 epilepsy patients focal (complex partial) seizures were the most common seizure type (12/27, $44.4 \%)$, followed by absences $(6 / 27,22.2 \%)$, generalized tonic-clonic $(3 / 27,11.1 \%)$, simple partial $(2 / 27,7.4 \%)$, and tonic seizure $(1 / 27,3.7 \%) .3 / 27$ patients had the MRI findings of mesial temporal sclerosis (MTS, $1 / 3$ ) or MTS-like findings (increased signal in the hippocampus and medial temporal lobe without associated volume loss, 2/3), Only $1 / 110$ non-seizure patients had MRI findings of MTS ( $\mathrm{p}$-value = $0.008, \mathrm{X} 2=7.019$ ). None of the 3 MTS patients had history of febrile seizures. $8 / 27$ patients with epilepsy and 11/101 without epilepsy had supratentorial non-optic glioma tumors likely to represent the epileptogenic lesion $(p=0.0126)$. Of the 27 epilepsy patients, 1 had an infarct, 1 a malformation of cortical development, and $9(33.35 \%)$ had neurofibromatosis bright objects (NBOs). 65/110 (50.7\%) patients without epilepsy had NBOs $(\mathrm{p}=0.016, \mathrm{X} 2=5.79$, indicating a negative relation of epilepsy and NBOs).

Conclusions: In our series of consecutive neurofibromatosis patients we demonstrated the novel finding of a statistically significant association with MTS related epilepsy. We also demonstrated a positive association of NF related epilepsy with tumors and no association with NBOs. In fact, NBOs appeared to be negatively correlated with the occurrence of epilepsy.

\subsection{4 \\ EARLY-ONSET VERSUS TYPICAL CHILDHOOD ABSENCE EPILEPSY: A RETROSPECTIVE STUDY CHARACTERIZING A PATIENT COHORT \\ Erick Sell and E. Lewis (Children's Hospital of Eastern Ontario, Ottawa, Canada)}

Rationale: Early onset Childhood absence epilepsy (ECAE), before the age of 3 years, has been considered a more heterogenous and often severe condition, compared to the typical presentation between ages 4-10 years. However, recent cohort study found no significant differences in outcome when compared with typical Childhood absence epilepsy. We decided to report our experience when comparing these groups of patients.

Methods: This was a retrospective study at the Division of Neurology, Children's Hospital of Eastern Ontario. Chart review of patients with an electro-clinical diagnosis of CAE diagnosed between January 2010 and January 2012 were reviewed. Statistical analyses were performed using Chi square and Fisher's Exact tests.

Results: 52 patients were diagnosed as having Absence epilepsy. 4 patients $(8 \%)$ had early-onset and $43(82 \%)$ patients had typical CAE and 5, Juvenile absence epilepsy (JAE). Generalized tonic-clonic were reported in $1(25 \%)$ male patient with early-onset and $5(11 \%)$ classical CAE. The EEG findings were similar in both groups. There were no significant differences in seizure control, anticonvulsant medications between CAE and ECAE groups. Poor seizure control was reported in 1 patient with ECAE and in $4(10 \%)$ patients with CAE. Psychiatric co morbidities occurred in $50 \%$ of patients with CAE and in 2 patients with ECAE predominantly ADHD symptoms. 2 Patients with refractory CAE seizures and 1 patient with refractory ECAE were tested for glucose transporter defect type 1 with all 3 patients testing negative

Conclusions: We did not observe significant differences with respect to psychiatric co morbidities, seizure control, anticonvulsants used or electroencephalographic findings between early-onset and classical childhood absence epilepsies. 


\subsection{5}

ANALYSIS OF PREGABALIN AND LACOSAMIDE USING THE POST-MARKETING ANTIEPILEPTIC DRUG/DEVICE SURVEY

George L. Morris (Regional Epilepsy Center, Milwaukee, WI)

Rationale: To assess the use of new antiepileptic drugs (AEDs) in everyday clinical practice outside of the research setting using an industry-independent design. Patient characteristics, titration rates, comorbidity of diseases, and other factors during clinical trials may differ from those in a clinical practice. By reporting a retrospectiveusage of new antiepileptic medications of Pregabalin and Lacosamide, we will be able to determine drug efficacy and response across a large population.

Methods: Patients, who have recently started Pregabalin or Lacosamide at the Regional Epilepsy Center, will be identified and deemed eligible by the treating physician and/or clinic staff. Eligible subjects will be assigned a subject profile entailing the subject's name, medical record number and the clinical site of treatment. Data collection can be completed using either the PADS paper forms, available as PDF files on the PADS website, or by entering information directly into the PADS webpage. Forms include an intial assessment, where subjects were first prescribed the drug, followed by a six-month follow-up form. Study personnel will complete the study forms by compiling clinically relevant information from the subject's medical chart. Data analysis will occur retrospectively (i.e.after both the initial and follow-up clinic visits have taken place) and will not affect the subject's treatment course. Information will be entered into the PADS database only after the six-month follow-up forms are concluded, after which the subject is considered complete. A review of 250 subjects taking each medication was made. A total of 342 subjects met all study enrollment criteria.

Results: A total of 342 patients were entered into the database: 170 Pregabalin; 172 Lacosamide with the average age of the patient being 43 and an average age of onset of 17 years old. Most of the patients treated had localization related epilespy syndromes (267) with a sparse number of primary generalized syndromes (28). The average intial seizure frequency of patients surveyed on pregabalin and lacosamide were 5.7 complex partial seizures (CPS) and 5 CPS per month, respectively . Average dose intake for pregabalin and lacosamide AEDs were $320 \mathrm{mg}$ and $363 \mathrm{mg}$ per day respectively. The final CPS frequency after 6 months were 3.7 and 3.4 for pregabalin and lacosamide respectively. Seizure and global assessment of patients surveyed after their 6 month follow-up varied slightly from one another. The "better" or "much better" seizure assessments occurred in $34 \%$ of pregabalin patients and $55 \%$ of lacosamide patients. However, global assessments of "better" or "much better" were $15 \%$ for pregabalin and $29 \%$ for lacosamide. Interestingly, the retention rate for pregabalin and lacosamide were 92 and 86 percent respectively.

Conclusions: Pregabalin and lacosamide were effective in reducing seizures and well tolerated based on high retention rates. Improved seizure assessments may suggest why patients remained on the drugs while tolerating some adverse experiences that lower their overall global assessment.

\subsection{6}

\section{LATE ONSET RASMUSSEN'S ENCEPHALITIS: A REPORT} OF 4 CASES

Alendia Hartshorn, C. Guardia III, V. Thadani, G. Holmes and B. Jobst (Neurology, Dartmouth-Hitchcock Medical Center, Lebanon, $\mathrm{NH})$

Rationale: Rasmussen's encephalitis (RE) is a rare, progressive form of chronic encephalitis characterized by seizures and hemiparesis. About $10 \%$ of cases are late onset, typically occurring in adolescence or early adulthood. Symptoms are often milder with slower progression. We present four cases of late onset RE, with symptoms beginning at age 13 to 23 .
Methods: Information was gathered by chart review and included age of symptom onset, initial presenting complaint, MRI results, EEG findings, age of diagnosis, and treatment.

Results: Mean onset of symptoms was 18.3 years (range 13-23). In three cases, seizure was the presenting complaint. In one patient hemiparesis was followed by seizures in one year. Two out of four patients experienced epilepsia partialis continua during the course of illness. In two cases the initial brain MRI showed inflammatory changes and atrophy. The other two cases had initially normal MRI scans and developed hemi-atrophy and diffuse unilateral T2 prolongation consistent with inflammation (16 years from onset of symptoms in both cases). All had EEG findings consistent with partial onset epilepsy. Mean age of diagnosis was 26.5 years (range 20-31) and average time from symptom onset to diagnosis was 8.8 years (range 0-16). Treatment included intravenous immunoglobulin (IVIg) and/or corticosteroids in all four patients. One of the four patients in our report progressed rapidly and was diagnosed in the first year of symptom onset. This patient is currently undergoing treatment with rituximab. To date, none required hemispherectomy. Conclusions: Late onset RE can present a diagnostic challenge, especially early in the disease. The initial MRI may be normal. Seizures may be suggestive of a partial onset epilepsy. As the disease progresses over decades, the diagnosis is established. The impact of immunosuppressive therapy in RE still needs to be determined in larger studies as compared to the natural course of the disease.

\subsection{7 \\ INCIDENCE OF STATUS EPILEPTICUS IS INCREASED IN PATIENTS WITH PARKINSON'S DISEASE}

Berend Feddersen, J. Rémi, M. Einhellig, C. Stoyke and S. Noachtar (University of Munich, Muenchen, Germany)

Rationale: To determine the rate of patients with Parkinson's Disease (PD) who developed status epilepticus, as it has been shown in animal models that a dopaminergic deficit may aggravate epileptic seizures.

Methods: The medical reports for all in- and outpatients of the Department of Neurology, University of Munich of the years 20002006 were searched for the terms "Parkinsons`s Disease", "dopaminergic deficit", "epilepsy", "epileptic seizure", and "status epilepticus". All resulting reports were reviewed and the diagnoses of PD and epilepsy were reviewed according to clinical criteria from the records. We compared the rate of epilepsy and status epilepticus in patients with and patients without PD.

Results: We identified 1398 patients with PD, 31 with concomitant epilepsy (2.2\%) and 2537 patients with epilepsy that did not have concomitant PD. Of the 31 PD patients 19 had had a status epilepticus (61.3\%), as compared to 298 patients with status epilepticus of the epilepsy group without concomitant PD (11.7\%; $\mathrm{p}<0.001)$.

Of the 19 patients with PD and status epilepticus, 9 of them had nonconvulsive status epilepticus, 8 convulsive status epilepticus and in 2 patients convulsive status epilepticus evolved into non-convulsive status epilepticus.

Conclusions: Although patients with PD are not likely to develop epileptic seizures, their risk of status epilepticus is higher than in epilepsy patients. Thus, dopaminaergic defict seems to contribute to development of status epilepticus.

\subsection{8 \\ IMPROVING SEMIOLOGICAL SEIZURE CLASSIFICATION THROUGH STRUCTURED INTERVIEWS AND VIDEO TUTORIALS \\ Jan Remi ${ }^{1}$, V. Thiel ${ }^{1}$, A. de Marinis ${ }^{2}$ and S. Noachtar ${ }^{1}\left({ }^{1}\right.$ Department of Neurology, University of Munich, Munich, Germany and ${ }^{2}$ Department of Neurology, Clínica Alemana de Santiago, Santiago, Chile)}


Rationale: The analysis of seizure semiology is well established in presurgical epilepsy monitoring. Classifying seizure semiology from patient history may be more difficult, because the recollection by the patient and next of kin may be inconsistent and flawed by subjectivity. Possibly, though, seizure semiology could be an important piece of information to triage patients whether to undergo epilepsy monitoring or not, especially in less affluent societies. We therefore studied whether history taking of seizure semiology can be improved by structured interviews and by demonstrating seizure semiology through video examples. We grouped semiological signs into signs for focal epilepsy and a group of lateralizing and localizing signs.

Methods: In a first step, we compared the seizure semiology of 208 consecutive patients as described in the patients' history and compared it to the seizure semiology recorded in the epilepsy monitoring unit of the University of Munich. In a second step we compared the yield of seizure semiology information of 72 patients, when taken from patients' records, after structured interviews and after showing seizure videos to patients and their next of kin at the Epilepsy Center of the University Del Desarollo in Santiago de Chile. Results: Of the 208 patients of the first part of the study, 138 $(66.3 \%)$ could correctly report signs for focal epilepsy as compared to 54 patients $(26.0 \%)$ that did not report any signs of focal epilepsy but had them recorded in the epilepsy monitoring unit. Six patients had generalized epilepsies and five of them had incorrectly reported focal semiology. Lateralizing signs could correctly be reported by $15.3 \%$ of patients. $7.4 \%$ reported lateralizing signs, but we could not reproduce them in the epilepsy monitoring unit and $44.1 \%$ of patients did not report lateralizing signs but we recorded them in epilepsy monitoring. Lastly, $26.2 \%$ of patients did not have lateralizing signs in either history or recording.

In the second part of the study, only 6 patients out of $56(10.7 \%)$ with focal epilepsy had lateralizing signs on record (4 with localizing signs). After a structured interview, these numbers increased to 31 $(55.4 \%)$ for lateralizing signs and $28(50.0 \%)$ for localizing signs. After showing exemplary videos to the patients, these numbers increased even more, to 49 patients $(87.5)$ for lateralizing signs and 33 patients $(58.9 \%)$ for localizing signs.

Conclusions: Our study demonstrates that the analysis of seizure semiology from patient history may be incomplete, but that it may be improved by structured interviews and even more so by demonstrating video examples of typical semiology to patients. Taken together with other information, this may improve the triage of patients towards presurgical epilepsy monitoring.

\subsection{9}

\section{SEIZURE DURATION DIFFERS IN FOCAL EPILEPSIES}

C. Stoyke, Jan Remi, M. Fritsch and S. Noachtar (University of Munich, Munich, Germany)

Rationale: We studied the duration of seizures arising from different lobes in focal epilepsy to better understand spread and inhibitory mechanisms involved. It has been noted that seizure duration may be different in different focal epilepsy syndromes, but actual duration has not been studied systematically.

Methods: We investigated the seizure duration as measured by ictal EEG and seizure semiology in 623 seizures of 84 patients with temporal (TLE), frontal (FLE) and parieto-occipital lobe epilepsies (POLE). Seizure onset was taken from EEG or seizure semiology only in case the respective activity was sustained. Seizure end was mostly considered by the end of ictal EEG, as postictal behaviour may be difficult to differentiate from ictal semiology. Subclinical ictal EEG discharges or seizures limited clinically to auras were not included in the comparisons. Since duration was not normally distributed we report median values.

Results: Seizure duration differed significantly between the syndromes. Focal seizures in FLE patients were shortest with 43 seconds, followed by seizures in POLE with $56 \mathrm{~s}$ and TLE with $68 \mathrm{~s}$ $(\mathrm{p}<0.05)$. Seizures that generalized secondarily also differed significantly between FLE (97s) and POLE (107s) on one hand and TLE on the other hand $(130 \mathrm{~s} ; \mathrm{p}<0.05)$. The prolongation of seizure duration through secondary generalization did not differ significantly between the syndromes.

Conclusions: We conclude that exhaustion and the activation of inhibitory mechanisms differ between focal epilepsies. Reasons may be different connectivity for example to the basal ganglia and differences in the cortical structures involved.

\section{Clinical Epilepsy: Clinical Diagnosis}

\subsection{0}

FUNCTIONAL ANALYSIS OF EPILETIC SPIKE AT FOCAL CORTICAL DYSPLASIA WITH SPATIAL FILTERING ANALYSIS

MIDORI NAKAJIMA ${ }^{1}$, F. Takeuchi ${ }^{2}$, Y. Ueda ${ }^{1}$, K. Otsuka $^{1}$, T. Ito ${ }^{1}$, H. Shiraishi ${ }^{1}$, S. Nakane ${ }^{3}$ and K. Takahashi ${ }^{3}\left({ }^{1}\right.$ Graduate School of Medicine, Hokkaido University, Sapporo, Japan; ${ }^{2}$ Department of Health Science, Hokkaido University Graduate School of Medicine, Sapporo, Japan and ${ }^{3}$ Division of Magnetoencephalography, Hokkaido University Hospital, Sapporo, Japan)

Rationale: Focal Cortical Dysplasia (FCD) is a major cause of intractable epilepsy of infants and children which usually results in catastrophic prognosis and severe developmental delay. Accurate diagnosis of FCDs are highest priority for child neurologist to provide patients the opportunity of surgical resection which can lead freedom from catastrophic seizures and normal development. Some of the FCDs should be found easily in MRI but the others are not and these cases are likely to have medication therapy which usually results in miserable prognosis. Our aim is to detect FCD directly by analyzing the shape of MEG spikes, using spatial filtering analysis: dynamic statistical parametric mapping (dSPM) which enables to show epileptiform activity with spatiotemporal source distribution with millisecond temporal resolution (Dale,2000).

Methods: We studied 10 patients (mean \pm DS; $17.6 \pm 10.5$ years old) with symptomatic localization-related epilepsy using a 204-channel, whole-head gradiometer system (Vecterview, Elektra Neuromag Inc., Stockholm, Sweden). We divided FCD group and Non-FCD group according to the pathologic diagnosis and existence of FCD in Magnetic resonance imaging (MRI). We selected 10 epileptic spikes from each patient and analyzed by dSPM. We projected the results to normal standard brain model to minimize the differences in shapes between the patients' brain. Thus, we investigated characteristic features of epileptic spikes by analyzing strength and temporal activity of epileptiform.

Results: The form of epileptic spike in FCD group is single and sharp in peak, emerging and vanishing in a same location of the brain without expanding its area. In contrast, that of Non-FCD group is multiple and diverse, expanding the area to the circumference and vanishing toward different lobe. A case categorized as Non-FCD group with no lesion in MRI initially revealed to have the same epileptic activity as FCD group which indicates the existence of FCD. This patient underwent cortical resection and became seizure free.

Conclusions: Functional analysis of MEG with dSPM can disclose the characteristic features of epileptic spikes with high spatial and temporal resolution. This method will help diagnose the existence of FCD as well as MRI invisible cases. 


\subsection{1}

\section{AMBULATORY EEG MONITORING WITH VIDEO IN CHILDREN; YIELD AND CLINICAL UTILITY}

Eric Segal, M. Lancman, J. Politsky, O. Laban-Grant, S. Berry and E. Fertig (Northeast Regional Epilepsy Group, Hackensack, NJ)

Rationale: Ambulatory EEG is an effective diagnostic test to determine seizure type and location of onset. While the ambulatory EEG can make testing more convenient for children and their families, it does not replace the utility of inpatient video EEG monitoring because characterization with video of event semiology is important for diagnosis and seizure localization. Ambulatory videoEEG (AVEEG) is now possible, but its diagnostic yield and impact on subsequent clinical care has not previously been examined. Methods: A retrospective review of 80 consecutive studies in patients 18 years old and younger was performed. A 72 hour AVEEG was acquired with synchronized digital video and EEG (noninvasive, scalp, 10/20 system) with a button provided to mark subjective events. Relevant clinical data was abstracted from the chart, including the indication, and if results lead to change in AEDs or management overall (e.g. withdrawal of medication).

Results: The age of the patients at the time of study ranged between 9-18 years old, with a mean of 15.35 years of age. Forty-two AVEEGs were ordered to assess spells without a previous epilepsy diagnosis (A), 20 to assess response to treatment for epilepsy (B), 4 to assess new spells with a previous epilepsy diagnosis (C), 7 studies to guide the decision to reduce or withdraw AEDs in pts with prolonged seizure freedom (D), and 7 unclassified (E). Studies revealed: interictal epileptiform abnormalities (IEDs) in $33(41 \%)$; focal slowing in $4(5 \%)$, events of any type in $34(43 \%)$. Of studies with events, $4(12 \%)$ had electroclinical seizures, $1(3 \%)$ had syncope, and $13(38 \%)$ had behavioral events. Seven studies had events solely off video (21\%). None of the AVEEGs captured subclinical seizures, clinical-only seizures, or psychogenic nonepileptic seizures (PNES). 41\% of patients sent for a diagnostic study $(\mathrm{A}+\mathrm{C})$ captured an event. In addition, 39\% of all the diagnostic studies yielded a new diagnosis. For all studies, AED changed in $24 \%$ and management in $29 \%$. There was a trend demonstrating AED change associated with the presence of IED $(\mathrm{p}=0.0127)$ and electroclinical seizures $(\mathrm{p}=0.0312)$.

Conclusions: Almost one-third of AVEEG studies lead to a change in management. There is a trend demonstrating the presence of IEDs and electroclinical seizures as predictors for change to AED regimen. AVEEG should be could be considered a useful diagnostic tool to assist the clinician in management decisions.

\subsection{2 \\ VALPROATE ENCEPHALOPATHY MIMICKING PROGRESSIVE MYOCLONUS EPILEPSY}

Danielle Andrade (University of Toronto, Toronto, Canada)

Rationale: Discuss the effects of valproate encephalopathy in a patient with juvenile myoclonic epilepsy (JME) and its differential diagnosis.

Methods: This 22 year old patient presented with myoclonic jerks at 10 years of age. Ate the age of 12 years she started having generalized tonic clonic (GTC) seizures. An EEG at the time showed the presence of generalized $4 \mathrm{~Hz}$ spike and wave discharges recorded with generalized distribution and bilateral synchrony, in addition to a normal background activity. These findings confirmed the clinical suspicion of JME. Seizures were initially well controlled on lamotrigine. However after 6 years she started having breakthrough seizures. At that point valproate was added. Her seizure frequency was not changed initially, therefore valproate dosage was gradually increased. Concomitantly, she started showing cognitive decline, reflected by poor school performance. She had to stop school due to the frequency of seizures (myoclonic and GTC) and the severity of cognitive decline. At the age of 20 she was admitted to the Epilepsy
Monitoring Unit (EMU) for diagnostic reevaluation. Continuous video-EEG recordings revealed the presence of myoclonic seizures, generalized tonic clonic seizures with prolonged duration and generalized as well as multifocal interictal epileptiform discharges (IEDs). Interestingly, some of the GTC seizures would appear to stop but a focal (usually right posterior quadrant) ictal discharge would then appear at the end of the clonic phase, and this focal activity would last for several minutes before the seizure came to a complete stop. The background activity was abnormal, usually in the low theta frequency range.

This admission to the EMU led to a new diagnosis of progressive myoclonus epilepsy (PME). No mutations were identified in the EPM1 (unverricht-Ludborg), EPM2A, EPM2B (Lafora disease), or EFHC1 (juvenile myoclonic epilepsy) genes. Valproate was substituted for lacosamide. Seizures immediately stopped. The patient has been on lacosamide for 18 months now. She continues seizure-free and her cognitive dysfunction disappeared. She is back to school and is able to obtain good grades.

Results: This case illustrates a severe encephalopathy in response to valproate. Interestingly, it showed that focal onset seizures and IEDs could be generated during this period of encephalopathy. Valproateinduced encephalopathy is not new. However, to the best of the author's knowledge it has not been associated with new, focal ictal or interictal abnormalities in patients with a previous diagnosis of JME, mimicking PME.

Conclusions: Worsening of symptoms in patients previously diagnosed with JME can be seen in early stages of PME. However, this case shows that valproate encephalopathy is an important cause of worsening of symptoms, mimicking the often fatal teenage onset PMEs. In that valproate is commonly used both for JME and PMEs, it is important to consider toxicity to this drug and in some cases a trial off valproate may prove useful.

\subsection{3}

\section{ICTAL SWEARING: A CASE SERIES}

Veronica Birca, T. Tayah, J. Saint-Hilaire and D. K. Nguyen (Neurology, Notre-Dame Hospital, Montreal, QC, Canada)

Rationale: A variety of speech automatisms have been described. Ictal swearing has not been well characterized and may provide clinical information and insight into the mechanisms of stereotyped ictal behavior.

Methods: Retrospective chart and video analysis of 8 patients featuring ictal swearing. Seizure localization was determined based on clinical (8/8), structural (7/8) and electrophysiological (8/8 scalp EEG; 4/8 intracranial EEG) data.

Results: All patients were male and right-handed except one (still with left-hemisphere dominance determined by amobarbital test). Ictal swearing occurred inconsistently during seizures and generally co-existed by other verbal or motor automatisms. All had right-sided seizures confirmed by video-EEG but focus localization was varied: orbitofrontal (1), inferior frontal gyrus (2), mesiotemporal (3), mesioparietal (1) and mesio-occipital (1).

Conclusions: Ictal swearing is more commonly seen in male subjects, lateralizes to the non-dominant hemisphere but has poor localization value.

\subsection{4 \\ RASMUSSEN ENCEPHALITIS ASSOCIATED WITH MYASTHENIA GRAVIS}

Saima N. Kayani, S. T. Arnold, M. Dowling, M. Khan and S. T. Iannaccone (UT Southwestern Medical Center, Dallas, TX)

Rationale: Rasmussen encephalitis (RE) is a rare inflammatory brain disease in children. It presents with intractable epilepsy, often associated with epilepsia partialis continua and unilateral motor and speech deficits. EEG changes are seen in contralateral hemisphere 
along with unilateral cerebral atrophy on Neuroimaging. RE leads to progressive hemispheric damage and surgical excision of the affected hemisphere is needed to control the symptoms of the disease. The underlying pathogenic mechanism is poorly understood. However proposed mechanisms include viral infection, humoral and cytotoxic T-cell mediated auto-immunity.

Methods: Case report with EMG, MRI and acetlycholine receptor antibody studies

Results: An 11 year-old girl had a history of intractable epilepsy since 3 years of age and was later diagnosed with Rasmussen's encephalitis. She had intractable epilepsy requiring three antiepileptic drugs and immunomodulation with IVIG every four weeks. She developed generalized muscle weakness, bulbar weakness with dysphonia. Further diagnostic testing was pursued and included an EMG with repetitive stimulation. Results revealed a postsynaptic decrement. A thymoma panel revealed elevated acetylcholine receptor binding and modulation antibodies. MRI of the mediastinum revealed a cystic lesion within the right aspect of the thymus. Conclusions: The coexistence of Rasmussen encephalitis and myasthenia gravis has not previously been reported. Myasthenia gravis is commonly associated with other immune-mediated diseases such as thyroiditis and multiple sclerosis. The association here suggests that in this case RE and myasthenia gravis may share a common autoimmune etiology.

\subsection{5 \\ IMPORTANCE OF INTENSIVE VIDEO EEG MONITORING IN THE EVALUATION OF CHANGE IN SEIZURE SEMIOLOGY IN INTRACTABLE EPILEPSY PATIENTS Batool Kirmani ${ }^{1,2}$ and D. Mungall ${ }^{1}\left({ }^{1}\right.$ TEXAS A\& M HSC COLLEGE OF MEDICINE, TEMPLE, TX and ${ }^{2}$ Scott \& White Neuroscience Institute, Temple, TX)}

Rationale: The objective of our study is to emphasize the importance of intensive video EEG monitoring in special circumstances in patients with well-established diagnosis of epilepsy. The idea is to diagnose new onset frequent events associated with change in ictal semiology prompting the need for frequent emergency room visits and hospital admissions.

Methods: Retrospective chart reviews were conducted on patients with intractable epilepsy who had increased incidence of new onset episodes different from the baseline ictal semiology. Patients were admitted to Scott \& White Hospital/Texas A\&M Health Science Center, Temple, TX for intensive video EEG monitoring from 2008 to 2011. Data were acquired from electronic medical records. Approval for this retrospective analysis of patient records was given by the hospital's Institutional Review Board.

Results: We retrospectively analyzed 14 patients with an established diagnosis of intractable epilepsy. Extensive chart reviews were performed with emphasis on type and duration of epilepsy, extent of interictal epileptiform abnormalities on EEG, failed and current treatment regimens, baseline frequency and semiology of seizures. The study population consisted of 14 patients (mean age: 43 years, range: $21-67$ years). There were 10 women $(71.5 \%)$ and 4 men $(28.5$ $\%)$. Eleven patients $(78.6 \%)$ had partial and $3(28.4 \%)$ had generalized epilepsy. The patients were on an average of three antiepileptic medicines (range 1-5). The duration of follow up in our neurology clinic ranges from 9 months to 9 years. The occurrence of increased frequency from the baseline associated with change in semiology of the seizures, despite therapeutic anticonvulsant levels, prompted the need for 5-day intensive video EEG monitoring. Atypical spells were documented in all patients and were without any ictal correlate.

Conclusions: Our data analysis showed that intensive video EEG monitoring is an important tool to evaluate change in frequency and ictal semiology of seizures even in patients with an established diagnosis of intractable epilepsy.

\subsection{6}

CLINICAL AND ICTAL CHARACTERISTICS IN INFANTS LESS THAN TWO YEARS OF AGE; EEG CORRELATION VIA LONG-TERM VIDEO EEG MONITORING

J. Lee, Hee Joon Yu, H. Kim, Y. Jang, J. Lee and M. Lee (Pediatrics, Samsung Medical center, Gangnam Gu, Republic of Korea)

Rationale: The semiology of infants less than two years of age is known to be different from that of adult. We executed this study to describe clinical and ictal characteristics in infants with epilepsy. Methods: The retrospective study was done for the infants with epilepsy (ages: 1-24 months) who were underwent the long-term video EEG monitoring at Samsung medical center from November 1994 to February 2012. Total 56 studies were included. We analyzed the clinical and ictal characteristics of the patients.

Results: The seizure occurred prior to 6months of age in $69 \%$ of the patients and the etiology was symptomatic in a third of the patients. Twelve seizure types were identified and the frequent seizure semiologies were as follows; infantile spasms $(25 \%)$, partial seizure (54\%), generalized seizures except spasms (21\%).

Infantile spasms were well correlated with generalized electrodecrement. The myoclonic seizure or atonic seizure was related with electrographically generalized patterns. However, generalized tonic seizure was frequently related to the primary generalized seizure or partial seizure. Generalized tonic seizure with focal onset was mainly arising from frontal areas. The hypomotor seizure showing cyanosis or decreased activities revealed their ictal origins mainly in temporal areas. The hypermotor seizure arose from frontal areas. Unilateral motor seizures or hemi-spasms showed lateralized ictal EEGs.

Conclusions: Most of the seizure semiologies in infants were well correlated with the ictal EEGs as in adults. The frontal onset seizures frequently exhibited generalized seizure. The video EEG monitoring was useful to detect and categorize seizures in infants.

\subsection{7}

AN UNUSUAL CAUSE OF EPC

R. Fabris and Jeffrey W. Britton (Neurology, Mayo Clinic, Rochester, MN)

Rationale: To present an unusual case of CJD presenting as EPC; CJD does not usually present in this manner

Methods: Case report

Results: CJD is a rapidly progressive prion-mediated neurodegenerative disorder. Although often presenting with diffuse clinical features, focal presentations are well known. Although cortical myoclonus is a hallmark, other seizure types are less common, generally occurring in later stages. In $3 \%$ of cases, seizures are the presenting symptom, CJD presenting as epilepsia partialis continua (EPC) is limited to six prior case reports.

Case:

A 66-year-old woman was transferred to the Mayo Clinic St. Mary's Hospital neurology service for a 3-month history of progressive gait instability, chorea, left upper extremity alien limb phenomenon and continuous left upper extremity jerking. Treatment with levetiracetam was unsuccessful. Video-EEG monitoring showed continuous focal seizure activity originating from the right centroparietal region. Focal periodic sharp wave discharges corresponded to left upper extremity clonic jerks recorded using a surface EMG electrode. Outside MRI showed subtle increased diffusion signal in the caudate heads bilaterally, cortex of the superior right cerebral hemisphere, and bilateral lentiform nuclei. Repeat MRI 1 month later showed progression of the increased diffusion signal most prominent in the right parietal lobe and right insular cortex. Examination of cerebrospinal fluid showed normal cell count, glucose and protein and negative inflammatory markers. Neuron specific enolase was significantly elevated at $256 \mathrm{ng} / \mathrm{mL}$ (normal $<15 \mathrm{ng} / \mathrm{mL}$ ) and 14-3-3 protein was elevated at $2.0 \mathrm{ng} / \mathrm{mL}$ (normal $<1.5 \mathrm{ng} / \mathrm{mL}$ ). Treatment with high dose phenytoin was unsuccessful. Initiation of clonazepam 
led to a decrease in myoclonic jerking. The patient passed away 2 weeks later. Autopsy findings were consistent with CJD.

Conclusions: CJD is an uncommon cause of EPC but should be included in the differential diagnosis. Recognition of this diagnostic possibility in this particular case led to diagnostic verification through CSF, which prevented fruitless trials of additional AEDs and potentially an unnecessary brain biopsy. In this case and in all previously reported cases, the patients' disease progressed especially rapidly, suggesting that EPC may predict an especially poor prognosis for this disease.

\subsection{8 \\ THE IMPORTANCE OF SIMULTANOUS ECG RECORDING DURING ROUTINE EEG}

F. Irsel Tezer, H. Onder and S. Saygi (Hacettepe University Faculty of Medicine Department of Neurology, Sihhiye/Ankara, Turkey)

Rationale: Ictal and interictal ECG abnormalities are not rare in epilepsy patients. The diagnosis of these arrhythmias can be important during follow up, especially in video-EEG monitoring unit, like; prediction of lateralization-localization of epileptic origin and choosing of antiepileptic drugs. Furthermore cardiac arrhytmias and epileptic seizure may have same etiology like channelopathy. The aim of our study was definition of arrhythmias during simultaneous routine EEG- ECG recordings and the effect of this on follow up of the patients.

Methods: The keywords related to cardiac arrhythmia have been searched in all EEG recording reports between January 2011February 2012. The demographic features, diagnosis or pre-diagnosis of patients during routine EEG recordings have been obtained from Hospital Data System. We have also evaluated the EEG-ECG recordings of patients having arrhythmia and searched for long QT interval.

Results: In all 2136 EEG reports, 48 (2\%) of them included keywords related to cardiac arrhytmias. 25 of 48 patients were female and the mean age of all was 53.18 of 48 patients had exstrasystole, 14 had tacycardia. 13 reports had no detail about type of arrhythmia, only noted as arrhythmia and 3 patients had bloc or bradiarrhythmia. After routine EEG recordings, 6 patients underwent to cardiology follow up in our center. 1 patient with an asystole during EEG recording had treated via a pace-maker. Furthermore, 5 of 48 patients had also long QT interval.

Conclusions: ECG electrodes and chanelles should be included routine EEG recordings. Cardiac arrhytmias can easily be diagnosed and change the management of the patients.

\subsection{9 \\ OUTCOMES OF LONG-TERM MONITORING FOR EPILEPSY AMONG DIFFERENT ADULT AGE GROUPS \\ J. M. Laine, Amar B. Bhatt, Y. Shi and N. J. Azar (Neurology, Vanderbilt University Medical Center, Nashville, TN)}

Rationale: When admitting patients for long term monitoring for epilepsy (LTME), neurologists often have a preconceived level of diagnostic suspicion based on many factors including age, gender, clinical event description, epilepsy risk factors, psychiatric comorbidities, and neuroimaging. The diagnostic yield of LTME has been previously studied, though not across different age groups. The purpose of this study is to determine the diagnostic outcome of LTME studies of adults in different age groups. The study also aims to evaluate the nature of the diagnoses, EEG findings, and neuroimaging results.

Methods: We reviewed all adult admissions for LTME over a period of three months. We divided the patients into three age groups (18-40 years, 41-65 years, and $>65$ years). We only included LTME data on patients admitted for diagnostic purposes. We compared gender, epilepsy risk factors, duration of presumed epilepsy, comorbid psychiatric conditions, number of event types, and diagnostic outcome (epileptic, non-epileptic, or non-diagnostic). We also compared the interictal EEG abnormalities (epileptiform or nonepileptiform) during LTME and any significant MRI abnormalities. Results: A total of forty-two records were reviewed, four of which were excluded because LTME was not performed for diagnostic reasons. Of the 38 patients included in analysis, 17 patients were 1840 years of age $(28.5 \pm 6.4 \mathrm{yrs})$, while 21 patients were $41-65$ years of age ( $49.8 \pm 5.8 \mathrm{yrs})$, with $\mathrm{p}<0.001$. There were no patients over 65 years of age admitted during this three month period. There was no significant difference in gender, epilepsy risk factors, psychiatric comorbidities, presumed epilepsy duration, or number of event types between age groups. The percentage of patients with recorded typical events during LTME was not different in the younger (12 patients, 71 $\%$ ) and older age groups (13 patients, $62 \%)$. In the younger age group, four patients $(33 \%)$ had non-epileptic diagnoses while eight patients $(67 \%)$ had epileptic diagnoses. In the older age group, seven patients $(54 \%)$ had non-epileptic diagnoses while six patients $(46 \%)$ had epileptic diagnoses. All patients with non-epileptic seizures were given a psychogenic diagnosis, except two patients in the older age group, who were given non-psychogenic diagnoses (migraine or syncope). Patients in the younger age group tended to have more epileptiform abnormalities on EEG ( $41 \%$ vs. $14 \%$ ), whereas patients in the older age group tended to have more non-epileptiform abnormalities ( $29 \%$ vs. $18 \%$ ). None of the patients with non-epileptic diagnoses had significant MRI abnormalities. There was no difference in presence of significant MRI abnormalities among the two groups.

Conclusions: Based on our preliminary analysis, the diagnostic outcomes of LTME may differ by age group. Younger adults with non-epileptic seizures tend to have a psychogenic etiology, while more of the older adults tend to have a non-epileptic, nonpsychogenic diagnosis. In younger adults, the interictal EEG during LTME may be more helpful in supporting a diagnosis of epilepsy.

\subsection{0 \\ EPILEPTOGENIC EEG PATTERNS IN PATIENTS WITH GLIOBLASTOMA MULTIFORME}

Thomas Wychowski, M. Berg and N. Mohile (University of Rochester, Rochester, NY)

Rationale: TAE occurs in more than half of patients with GBM. Potential risk factors include temporal location, hemorrhage and incomplete resection. The diagnostic and potentially predictive role of EEG has not been defined in this population, including which tumor characteristics may be associated with epileptiform abnormalities.

Methods: We performed an IRB-approved retrospective study of all newly diagnosed GBM patients who had obtained a scalp EEG at the University of Rochester between 1/1/05 and 5/13/11. EEGs were classified as epileptiform or non-epileptiform, based on the presence of discharges and paroxysmal rhythmic slowing. Records were reviewed to describe: EEG indication, tumor location, hemorrhage, resection status, and diagnosis of TAE. Characteristics of patients with epileptiform abnormalities were compared to those without. Results: 60 patients with newly diagnosed GBM and EEG were identified, 48 were diagnosed with TAE. 21/48 patients with TAE demonstrated epileptiform abnormalities on EEG. 8/21 had a mixture of electrographic seizure patterns including: 7 with PLEDs, 4 with status epilepticus, and 6 had paroxysmal rhythmic slowing. 52\% of EEGs with epileptiform abnormalities were seen in temporal tumors. In patients who achieved a complete resection, $12 / 17$ had persistent epileptiform abnormalities; only $2 / 10$ of biopsy patients had epileptiform features. Within this group, 84 EEGs were performed as indicated for new symptoms. Indications were available for all EEGs requested and only $27 \%$ of EEGs performed for AMS and $14 \%$ for resolved convulsions demonstrated epileptiform abnormalities, while $71 \%$ of studies performed for convulsions with prolonged AMS were epileptiform. 
Conclusions: Electrographic ictal patterns (i.e. SE, PLEDs) are frequent findings in patients with GBM. Epileptiform patterns on EEG were associated with temporal tumor location. Greater extent of resection was associated with more epileptiform abnormalities suggesting that tumor location (cortical vs. deep) may be a more important predictor than the surgery itself.

\subsection{1}

EEG AND MEG SPIKE SOURCE LOCALIZATION IN PATIENTS WITH SMALL FOCAL CORTICAL DYSPLASIA Hisashi Itabashi ${ }^{1}$, K. Jin ${ }^{1}$, M. Iwasaki ${ }^{2}$, E. Okumura ${ }^{1}$, A. Kanno ${ }^{3}$, K. Kato $^{1}$, R. Kawashima ${ }^{3}$ and N. Nakasato ${ }^{1}{ }^{1}$ Department of Epileptology Tohoku University Graduate School of Medicine, Sendai, Japan; ${ }^{2}$ Department of Neurosurgery, Tohoku University Graduate School of Medicine, Sendai, Japan and ${ }^{3}$ Department of Functional Brain Imaging, Institute of Development, Aging and Cancer, Tohoku University, Sendai, Japan)

Rationale: Cortical dysplasia is a major cause of pharmacoresistant epilepsy. However, small focal cortical dysplasia (FCD) is known to be often overlooked by MRI. In this study, we reviewed patients with small FCD to test whether EEG or MEG spike source localization help to assure the presence of the lesion.

Methods: The study included 5 patients with leg sensori-motor seizures. In all patients, MRIs had been reported as normal before referral, while high-field MRI in our hospital showed subtle abnormalities suggesting small FCD. EEG was measured by $42-$ channel electrodes with approximately double density of standard 1020 system. MEG was simultaneously recorded using a 160- or 200channel axial gradiometer system (MEG vision PQ1160C, Yokogawa Electric, Tokyo, Japan). Typical interictal EEG and MEG spikes were identified visually and used as templates to search for similar spatiotemporal spike patterns. Using a spike detection software (BESA Research 5.3, BESA GmbH, Germany), similar spikes were detected, averaged, and high-pass filtered $(1.6 \mathrm{~Hz})$ to enhance the spike onset. Single equivalent current dipole model was used to localize the onset-related source. We measured the distance between the center of MRI lesion and the source location of EEG and MEG spikes, respectively.

Results: In one patient, the EEG dipole was close to the lesion, while the MEG far from it but correlated with seizure semiology. In another patient, the MEG was close to the lesion as well as correlated with semiology, but the EEG far from the lesion. In the rest 3 patients, both EEG and MEG were close to the lesion and correlated with semiology.

Conclusions: The source localization of MEG and EEG spikes was helpful in the detection of small FCD. Either EEG or MEG localized small FCD in all cases, suggesting combination efficacy of EEG and MEG. Mis-localization observed in two cases implies several cautions: 1) EEG and MEG could only detect propagated spike activities; 2) MEG detects only tangential current to the scalp; and 3) EEG predominantly detects radial current.

\subsection{2 \\ VOLTAGE GATED CALCIUM CHANNEL ANTIBODIES ASSOCIATED WITH SUBACUTE LIMBIC ENCEPHALITIS AND SEIZURES}

Marissa A. Oller-Cramsie, S. T. Hwang, N. Mihu, S. Schaffer and C. Harden (Hofstra North Shore-LIJ School of Medicine, Manhasset, NY)

Rationale: The connection between paraneoplastic autoantibodies and limbic encephalitis with seizures is well established since there are several autoantibodies known to be associated with these symptoms. However, there are only rare reported cases in the literature of voltage gated calcium channel antibodies (VGCCAbs), typically associated with Lambert-Eaton myasthenic syndrome, occurring in the setting of limbic encephalitis and epilepsy. We report such an individual, with compelling improvements occurring after an aggressive search and definitive treatment for an underlying malignancy.

Methods: We describe a 63-year-old male with a history significant for smoking and a recent diagnosis of seizures, presenting with psychiatric and cognitive deterioration over the course of a year. His symptoms consisted of progressively worsening short-term memory, poor recall, severe anxiety, insomnia, depressive symptoms, and increasing frequency of seizures, culminating in an urgent admission for status epilepticus. A search for an autoimmune basis for his symptoms revealed positive VGCCAbs.

Results: Video-EEG during episode of nonconvulsive status epilepticus revealed frequent independent left and right temporal seizures manifested by an aura of anxiety, staring, behavioral arrest, and decreased responsiveness. Interictal sharp wave discharges were seen independently over the temporal regions. Neuroimaging showed microvascular changes but no evidence for vasculitis, and basic CSF analysis was unrevealing. ANA was elevated (1:320) with elevated double-stranded DNA titers. Paraneoplastic panel was positive for voltage gated calcium channel antibodies (P/Q and $\mathrm{N}$ type). Neuromuscular evaluation, previously conducted for evaluation of known cervical stenosis, did not demonstrate abnormalities on EMG or with repetitive stimulation. The presence of VGCCAbs along with patient's smoking history prompted further neoplastic evaluation including CT of the chest, abdomen, and pelvis and a full body PET scan. Work-up revealed enlarged mediastinal lymph nodes and a hypermetabolic right lower paratrachial lymph node measuring $2.3 \times 2.1 \mathrm{~cm}$. Histopathology confirmed the diagnosis of metastatic small cell lung cancer. The patient was treated with steroids, IVIG, resection of his tumor, and chemotherapy with improvement in his both his seizure frequency, cognitive, and psychological symptoms. Conclusions: VGCCAbs associated with subacute limbic encephalitis and seizures is a rarely described occurrence. It is important to consider this diagnosis in patients presenting with these symptoms as it may facilitate early diagnosis and treatment of an underlying malignancy. Early treatment may also yield better neurologic outcomes.

\subsection{3}

CASE SERIES OF NEUROCARDIOGENIC SYNCOPE WITH AN INITIAL DIAGNOSIS OF EPILEPSY

Uma Menon ${ }^{1,2}$ and E. Ramsay ${ }^{2,1}\left({ }^{1}\right.$ Neurology, Tulane University, New Orleans, LA and ${ }^{2}$ Tulane University, New Orleans, LA)

Rationale: Sudden loss of consciousness especially with a history of jerking commonly leads to the diagnosis of epilepsy. Epilepsy is one cause of transient loss of consciousness and thereby needs to be excluded as a cause. However, seizures constitute only a small percentage of cases with syncope

Methods: We present a consecutive series of 44 adult patients referred for evaluation of epilepsy in which the initial diagnosis was syncope, not epilepsy. Ages at time of referral was 24-72 years, with the duration of symptoms ranging from $0-65$ years since onset. Of these, only 3 also had a diagnosis of syncope.

Results: After extensive evaluation, only 2 patients had a definite diagnosis of epilepsy/seizures, 1 patient had a mixture of seizures and neurocardiogenic syncope (NCS), 3 patients had psychogenic non epileptic events, 1 patient had chronic fatigue and the diagnosis was inconclusive in another patient. Prodromal symptoms in these patients included dizziness (45\%), diaphoresis (15\%), and pallor $(15 \%)$.

It was also noteworthy that $38(86 \%)$ of these patients were on antiepileptic medications (multiple agents: $2-7$, in most cases) at the time of referral and the agents used included Felbamate in 2 patients, both of whom had the final diagnosis of neurocardiogenic syncope. Cardiac evaluation at time of referral had not included tilt test in any patient and when performed was abnormal in $75 \%$ of the patients. 
Conclusions: Prodromal symptoms including visual, sensory or motor symptoms were not predictive of a final diagnosis of seizures/epilepsy. Obtaining a good history is imperative to identifying patients with possible syncope. Neurocardiogenic syncope is the most common cause of reflex or neurally mediated syncope with an estimated lifetime prevalence of $30-40 \%$. The prognosis of patients with this diagnosis is benign and treatment involves lifestyle modifications and/or medications but not antiepileptic drugs.

\subsection{4 \\ NOCTURNAL FRONTAL LOBE EPILEPSY PRESENTING AS EXCESSIVE DAYTIME SLEEPINESS}

Jocelyn Cheng, D. M. Wallace, M. R. Lopez and E. Carrazana

(Neurology, University of Miami, Miami, FL)

Rationale: Excessive daytime sleepiness (EDS) is common in the general population. Etiologies include insufficient sleep, primary sleep disorders and medications. Due to its high prevalence, EDS is often overlooked by physicians. However, EDS may also be the presenting symptom of seizures. We report a series of three patients with nocturnal frontal lobe epilepsy (NFLE) who presented with a primary complaint of EDS.

Methods: Retrospective review of clinical data, including polysomnography (PSG) and EEG.

Results: Case 1: A 23 year old man presented with EDS and an elevated Epworth Sleepiness Scale (ESS) score of 13. His roommate had observed abnormal movements in sleep, including sitting up in bed and looking around the room, and bicycling movements with posturing of the left arm and leg. The patient was amnestic of these events. A sleep diary excluded insufficient sleep time. PSG was performed with a full set of EEG channels. Except for sleep fragmentation, no primary sleep disorders were seen. Multiple sleep latency testing (MSLT) was consistent with objective hypersomnia. EEG showed rhythmic, frontally predominant sharp wave activity corresponding to clinical seizures, which consisted of blinking, head turning and left arm elevation.

Case 2: A 33 year old woman presented with EDS and an elevated ESS score of 18. Her husband had observed 6 episodes of sleeprelated movements, which began with a moan and extension of both arms overhead, followed by confusion without event recall. Prior routine and ambulatory EEGs were nondiagnostic. A sleep diary excluded insufficient sleep time. PSG showed sleep fragmentation without evidence for other primary sleep disorders. MSLT was significant for shortened mean sleep latency. One day after PSG, the patient had an episode where she turned pale, with eyes rolling into the back of the head, followed by tonic-clonic activity and impaired consciousness. EEG during hospital admission showed interictal left frontal sharp waves.

Case 3: A 23 year old man presented with EDS and an elevated ESS score of 10. During sleep, his roommates reported events consisting of limb movements associated with vocalization, during which the patient was unable to respond, and for which he had no recall. He reported a daily sleep duration of 7.5 hours. Except for EDS, there were no features suggestive of sleep disordered breathing. Inpatient video-EEG monitoring was performed. Interictal sharp waves were present in the left frontotemporal and right temporal region, and several seizures were recorded. A focal seizure of left frontotemporal onset correlated with right leg movements. In the second event, the patient's eyes opened from sleep, followed by bicycling movements, which corresponded to electrographic seizures of left frontocentrotemporal onset with secondary generalization. A third seizure of right temporal onset with rapid generalization did not exhibit clear clinical manifestations.

Conclusions: EDS may present as the primary symptom of seizures. In patients with EDS and a history of stereotyped nocturnal events, clinical suspicion should be high for NFLE.
Clinical Epilepsy: Clinical Treatments

3.145

OUTCOME OF KETOGENIC DIET EXPERINCE AT KUWAIT ASMA A. AL TAWARI1, NAWAL AL QAOUD2, LADA HEBERLE CINDRO1, HAMIDA AL SHEMMERI2 1PEDIATRIC NEUROLOGY UNIT,AL SABAH HOSPITAL, MINISTRY OF HEALTH,2 FOOD AND NUTRITION ADMINISTRATION DEPARTMENT, MINISTRY OF HEALTH, STATE OF KUWAIT

Asma A. Al Tawari (Pediatric Neurology Unit, Ministry of Health, Kuwait, Kuwait)

Rationale: The Ketogenic $\operatorname{diet}(\mathrm{KD})$ is an established, effective nonpharmacologic treatment for intractable childhood epilepsy. It was observed that fasting decrease seizure frequency and the KD was designed to mimic a similar metabolic response. By providing a large quantity of fat and a small quantity of carbohydrate in the diet.

Interest in the Ketogenic diet (KD) has been renewed in recent years even though the diet has been existence for over 80 years. Our aim is to report health outcomes after treatment with the ketogenic diet experience at Kuwait.

Methods: Eight children under the care of Pediatric Neurology Unit with refractory epilepsy failed medical treatment, were offered MCT ketogenic diet treatment They were all continued a 2 year followup and management. Improvement assessment based on seizure reduction, psychosocial evaluation and neurophysiologica;studies. Results: the group age range between $4 \mathrm{~m}-11 \mathrm{y}$, gender 2 females and 6 males, Nationality 6 kuwaities, 1 lebanese and 1 saudi. They have variable underlying etiologies.the seizures onset at birth in 6 of them the other 2 after neonatal period. There were dramatic seizure control and psychosocial improvement in 5 of the group members. the other 3 were showing seizure reduction more than $50 \%$. Parents satisfaction achieved in all of the cases. One case has hyperlipidemia which was transient and managed by dietary modification.

Conclusions: This is the first successful ketogenic experience at Kuwait inspite of the small group size, the reduction of seizures control and psychosocial improvement encourage the continuation of this modality to be offered to other children with refractory epilepsy. The diet has few side effects and is generally well tolerated

\subsection{6}

USE OF MAGNESIUM IN TREATMENT OF REFRACTORY EPILEPSY

Evren Burakgazi-Dalkilic ${ }^{1}$, M. Carran ${ }^{1}$ and J. Pollard ${ }^{2}\left({ }^{1}\right.$ Neurology, Cooper University Medical Center, Cherry Hill, NJ and ${ }^{2}$ Neurology, University of Pennsylvania, Philadelphia, PA)

Rationale: Rationale: Magnesium is a potential modulator of seizure activity because of its ability to antagonize excitation through the $\mathrm{N}$ methyl-d-aspartate receptor. It has been shown that magnesium can control seizures in animal models.(1) Magnesium has been the mainstay treatment for eclampsia. (2)

There is report of refractory status epilepticus seizures being controlled with magnesium supplementation.(3)

It was shown to subside infantile spasms when combined with adrenorticotropic hormone (ACTH)(4). Recently a retrospective study reported decreased seizure frequency in patients who received magnesium oxide as add-on treatment(5).

We hypothesise that magnesium supplementation can reduce seizures in people with epilepsy.

Methods: This is an observational study of five patients with refractory epilepsy who received magnesium oxide $400-800 \mathrm{mg}$ daily as add-on to their baseline antiepileptic regimen(AED). The age of patients varied from 25 to 65 years old. There were three female and two male patients. The epilepsy syndrome was localization related epilepsy in all. The patients were followed for three months. 
Patients were given monthly seizure calendar to follow up their seizure frequency. Patients with renal failure were excluded.

Results: The patients reported decreased seizure frequency as in table 1.

The response to magnesium oxide treatment varied from being seizure free to $30 \%$ seizure reduction compared to baseline. The patients did not report any side effects of being on magnesium oxide. Magnesium levels did not change after starting magnesium oxide supplement.

Conclusions: Magnesium is important for many critical enzymatic processes in human physiology especially in central nervous system. This case series showed that magnesium supplement may have a potential role in decreasing seizure frequency in epilepsy patients. Serum magnesium levels may not reflect brain magnesium concentrations. It is possible that the detected effect is simply regression to the mean so further randomized controlled double blinded studies are needed to assess the effect of magnesium oxide in seizure control in epilepsy patients.

References:

1.Anderson, W.W., Lewis, D.V., Swartzwelder, H.S., Wilson, W.A.,1986. Magnesium-free medium activates seizure-like events in the rat hippocampal slice. Brain Res. 398, 215-219.

2Chien, P.F., Khan, K.S., Arnott, N., 1996. Magnesium sulphate in the treatment of eclampsia and pre-eclampsia: an overview of the evidence from randomised trials. Br. J. Obstet. Gynaecol. 103, 1085-1091.

3.Pandey, M., Gupta, A., Baduni, N., Vijfdar, H., Sinha, S., Jain, A., 2010. Refractory status epilepticus --magnesium as rescue therapy. Anaesth. Intensive Care 38, 962.

4.Zou, L.P., Wang, X., Dong, C.H., Chen, C.H., Zhao, W., Zhao, R.Y., 2010. Three-week combination treatment with ACTH + magnesium sulfate versus ACTH monotherapy for infantile spasms: a 24-week, randomized, open-label, follow-up study in China. Clin. Ther. 32, 692-700.

5.Abdelmalik PA, Politzer N, Carlen PL.Magnesium as an effective adjunct therapy for drug resistant seizures.Can J Neurol Sci. 2012 May;39(3):323-7

Table 1: Demographics, seizure types and \% decreased seizure frequency on magnesium oxide

\begin{tabular}{|c|c|c|c|c|c|}
\hline Patients & Gender & Age & Seizure Type & Baseline AED regimen & $\begin{array}{c}\text { Decreased seizure } \\
\text { frequency compared to } \\
\text { basclinc }\end{array}$ \\
\hline TW & Female & 28 & $\begin{array}{l}\text { Complex partial } \\
\text { seizure (CPS) }\end{array}$ & $\begin{array}{l}\text { Phenobarbital, } \\
\text { Lacosamide }\end{array}$ & $30 \%$ \\
\hline SS & Male & 45 & $\mathrm{Cl}$ 'S & $\begin{array}{l}\text { Oxcarbazepine, } \\
\text { Lamotrigine }\end{array}$ & $60 \%$ \\
\hline $\mathrm{AE}$ & Female & 65 & CPS & Valproate, Oxcarbacepine & $40 \%$ \\
\hline DD & Male & 24 & CPS & Oxcarbazepine & Seizure free \\
\hline $\mathrm{TM}$ & Female & 35 & CPS & $\begin{array}{c}\text { Oxcarbazepinc, } \\
\text { Pregabalin, levetiracetam }\end{array}$ & $30 \%$ \\
\hline
\end{tabular}

\subsection{7 \\ ESTABLISHMENT OF SEOUL NATIONAL UNIVERSITY HOSPITAL EPILEPSY REGISTRY (SNUHER)}

Young-Soo Kim, S. Lee, K. Chu, J. Byun, J. Lim, B. Park, J. Shin and J. Moon (Neurology, Seoul National University Hospital, Seoul, Republic of Korea)

Rationale: We have set up a epilepsy registry for identifying the epilepsy diagnostic patterns, trial of various AEDs medication, the treatment efficacy, and adverse events of AEDs by using an our hospital-based, prospective registry with a computerized database, named by The Seoul National University Hospital Epilepsy Registry (SNUHER).

Methods: SNUHER study has registered patients (18-80 years of age) with epilepsy, which was confirmed by clinical symptoms, electroencephalography, and brain imaging studies. The patient record consists of demographic factors, clinical features, diagnosis, treatment, and clinical outcome. We have measured the serum concentrations of current AEDs and obtained the samples for analysis of variable pharmacogenetic factors, including variation in genes encoding drug target, drug transport, drug metabolizing, and human leucocyte antigen proteins.

Results: The database collection was started in February 2011, 1492 patients were eligible for this study. $50.7 \%$ were males and median age was 38.56 years old. Diagnostic epilepsy classification included generalized epilepsy $14.9 \%$, localization-related epilepsy $82.9 \%$, and unknown cause $2.2 \%$. In generalized epilepsy, $50.5 \%$ were maintained on monotherapy. The most commonly used drugs were lamotrigine $(40.3 \%)$ and levetiracetam(37.5\%). In localization-related epilepsy, $28.9 \%$ were maintained on monotherapy and most commonly used drugs were oxcarbazepine $(45.7 \%)$. Seizure free rate were $77.9 \%$ in generalized epilepsy and $61.7 \%$ in localization-related epilepsy.

Conclusions: We have successfully established the web-based clinical database, named SNUHER. This prospective hospital-based registry allows us to identify and quantify the various clinical outcomes. At the same time, we obtained the current AEDs serum concentration and the samples for analysis of variable pharmacogenetic factors. Through this, we are presented the basis of individualized therapeutic strategies.

\subsection{8 \\ SAFETY AND EFFICACY OF HIGH-DOSE DIAZEPAM FOR TREATMENT OF ELECTRICAL STATUS EPILEPTICUS DURING SLOW WAVE SLEEP}

Emily A. Gertsch, K. E. Chapman, P. Laoprasert, K. Park, K. Knupp and S. Koh (Pediatric Neurology, Children's Hospital Colorado, Aurora, $\mathrm{CO}$ )

Rationale: Electrical status epilepticus during sleep (ESES) is an electroencephalographic pattern demonstrating significant activation of epileptiform discharges in sleep. The standard of treatment for ESES is high-dose oral diazepam. However, there have been only a few studies in the literature with the largest series being twenty-nine children. We examined the safety and efficacy of treatment with high-dose diazepam for children diagnosed with ESES.

Methods: We conducted an IRB approved retrospective review of 35 children diagnosed with ESES and subsequently treated with highdose oral diazepam at Children's Hospital Colorado from 2010 to 2011. Response to treatment as evidenced by decreased epileptiform discharges during sleep, and clinical improvement in development or language skills was assessed. Complications associated with treatment were evaluated.

Results: Thirty-five children ages 2 to 12 years (mean $=6.97$, median=7) were diagnosed with ESES and treated with high-dose oral diazepam at Children's Hospital Colorado from 2010 to 2011. Standard treatment dose initiated during hospitalization was $1 \mathrm{mg} / \mathrm{kg} /$ dose with a maximum dose of $60 \mathrm{mg}$. Eleven subjects $(31 \%)$ had a history of regular benzodiazepine use as part of their seizure treatment or sleep regimen prior to diagnosis of ESES. Two (6\%) had a history of respiratory issues prior to treatment with high-dose diazepam. Thirty-four (97\%) had a history of seizures prior to diagnosis of ESES with all but one having clinical seizures. Twentynine $(83 \%)$ had developmental delay, and nineteen $(54 \%)$ had language regression prior to diagnosis of ESES. Twenty-one $(60 \%)$ showed short term of three months improvement after treatment with high-dose diazepam manifested by reduction in epileptiform discharges during sleep or clinical improvement in language or other developmental skills. Only two children (6\%) experienced complications with high-dose diazepam, both of which were respiratory in nature. One had a brief drop in oxygen saturations to $84 \%$ requiring blow-by oxygen after administration of the first dose but was able to tolerate continued treatment. One patient on 
scheduled clonazepam at baseline had a history of significant medication related sedation prior to treatment with high-dose diazepam; he experienced significant respiratory depression with increasing oxygen requirement within two hours of first dose administration, followed by development of an aspiration pneumonia and persistent fatigue resulting in prolonged hospitalization. Treatment with high-dose diazepam was discontinued with subsequent improvement in mental status and oxygen requirement. Conclusions: 1) High dose diazepam has few side effects with respiratory depression occurring in only two patients, one of which had a previous history of severe sedation with medications. 2) $60 \%$ of patients showed short term improvement after treatment. 3) Highdose oral diazepam is a safe and effective treatment for patients with ESES although a prospective study with more patients would be helpful to verify these findings long term.

\subsection{9 \\ REPORTING AND PUBLICATION OF RANDOMIZED TRIAL ABSTRACTS AT THE AMERICAN EPILEPSY SOCIETY MEETING}

Rohit R. Das ${ }^{1}$ and K. K. Mehta ${ }^{2}\left({ }^{1}\right.$ Neurology, Indiana University, Indianapolis, IN and ${ }^{2}$ Public Health, Indiana University, Indianapolis, IN)

Rationale: The double blind placebo controlled randomized controlled trial is the gold standard for evidence for interventions in medicine. We aim to determine the rates of publication of randomized clinical trial abstracts submitted to multiple annual meetings of the American Epilepsy Society between 2000 and 2008. We also assessed characteristics of reporting of these abstracts per the CONSORT statement.

Methods: Using the online search engine available at the abstracts website of the American Epilepsy Society, we searched for all abstracts with the key words "randomized controlled". A total of 57 abstracts were found between 2000 and 2008. Abstracts that were systematic reviews of RCTs $(n=3)$ and studies pooled data from more than two different RCTs $(n=2)$ were excluded. Publication information was obtained by using the National Library of Medicine's website: pubmed.com. Publication status was established by searching the website using first author name, last author name and key words taken from abstract title. The cut-off date for publication searches was June 2012.

Results: Of the 52 abstracts analyzed, $62.7 \%$ were published at this time. Six abstracts did not state a clear objective and only 34 defined a distinct primary outcome within the abstract. $13 \%$ of the abstracts were conducted in more than 2 centers and $33 \%$ were designed to study a clinical endpoint. The median duration of these trials were 26 weeks $(\mathrm{IQR}=21)$. The median time to publication of abstracts was $15+15$ weeks. Clinical endpoints, clarity of stating of objectives or results, funding status or duration of trials were not associated with publication success.

Conclusions: There is a paucity of RCTs in the field of epilepsy, atleast as presented at this national meeting. However once presented in abstract form, it is encouraging that the majority of studies are published fully. Word restrictions impose difficulties in reporting abstracts in detail, as recommended by the CONSORT guideline. Unfortunately, a majority of RCTs presented at this meeting are designed to study surrogate endpoints and there are no clear signals indicating the fate of these abstracts with respect to future publication.

\subsection{0 \\ REPORTED MANAGEMENT DECISIONS IN (SUSPECTED) EPILEPSY}

Jessica Askamp ${ }^{1}$ and M. van Putten ${ }^{1,2}$ ( ${ }^{1}$ University of Twente, Enschede, Netherlands and ${ }^{2}$ Medisch Spectrum Twente, Enschede, Netherlands)
Rationale: The interrater agreement on the diagnosis of a first seizure in childhood is just moderate ( $\mathrm{J}$ Neurol Neurosurg Psychiatry 2004;75:241-245), but when it comes to the decision of when to start anti-epileptic drugs in children, evidence in literature and the majority opinion are well aligned: after a second unprovoked seizure (J Child Neurol 2008;23:507-515). However, in individuals with single, few or infrequent seizures, there will be more doubt and uncertainty about starting and stopping treatment. For most adults, the decision to withdraw treatment also falls into a grey area of uncertainty (Epilepsia 2006;47:58-61). This study investigates neurologists' reported decisions about treatment of adults with (suspected) epilepsy, for better understanding of the uncertainty in current practice in (suspected) epilepsy.

Methods: An online survey was developed. 1100 members of the Dutch Neurological Society were invited to participate. Currently, almost 10\% $(\mathrm{n}=98)$ has responded, among which were neurologistsin-training (29\%), general neurologists $(31 \%)$ and neurologists specialized as clinical neurophysiologists $(27 \%)$, pediatric neurologists $(9 \%)$, or epileptologists $(4 \%)$.

Results: None of the neurologists would start medication in an adult after a first seizure when the MRI and EEG are both normal, but $49 \%$ would start medication when 2 seizures occur on one day, $70 \%$ when the MRI is normal but the EEG contains epileptiform discharges, and $63 \%$ when the MRI is abnormal and the EEG is normal. When a recurrent seizure occurs after 6,12 or 24 months, $87 \%, 54 \%$ and $14 \%$ of the neurologists would start medication respectively. Figure 1 illustrates when neurologists would prefer to stop treatment (if no seizures occurred during that time), in three different cases: when the EEG and MRI are normal (1), when there are epileptiform discharges in the EEG (2), and when there is an underlying MRI abnormality (3). After two years of seizure freedom, $44 \%$ of the neurologists would immediately discontinue medication, $25 \%$ would make an EEG, and $30 \%$ would stop medication immediately if the patient wishes so.

Conclusions: There still seems to be a lack of consensus about when to start treatment in (suspected) epilepsy, mainly when two 'first' seizures occur on one day and when a recurrent seizure occurs at 12 months after the first seizure. Also, there is no consensus on when to stop medication and whether or not to make an EEG before stopping medication. More knowledge about the influence of patient factors and the use of different diagnostic tools (EEGs in particular) on management decisions may lead to better guidelines to enhance consensus on when to start or stop treatment.

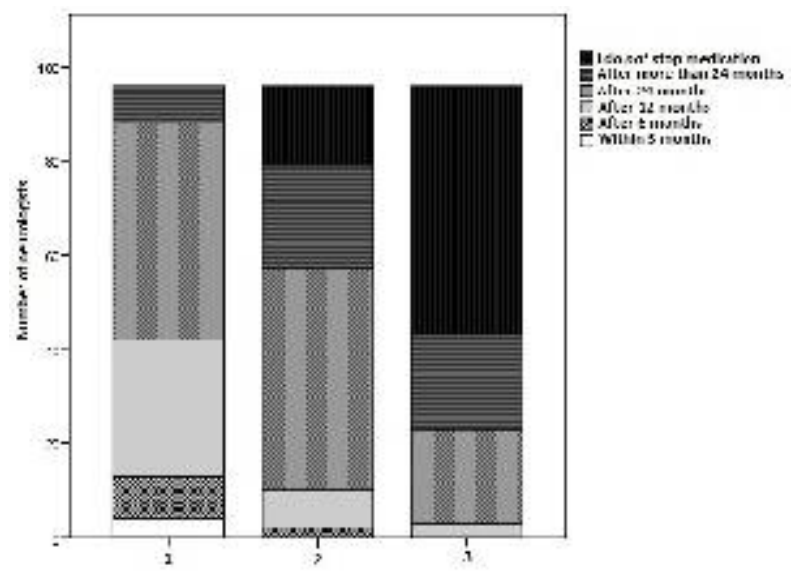

Figure 1: Period of seizure freedom after which neurologists would stop medication in three different cases: when MRI and EEG findings are normal (1), when the EEG shows epileptiform discharges (2) and when there is an underlying MRI abnormality (3). 
3.151

CASE REPORT: VAGAL NERVE STIMULATION (VNS) AND LATE ONSET ASYSTOLE

Victor O. Olotu ${ }^{1}$, R. Shankar ${ }^{1}$, N. Coles ${ }^{2}$, H. Sullivan ${ }^{3}$ and C. Jory ${ }^{4}$ $\left({ }^{1}\right.$ Nueropsychiatry, Epilepsy and Memory Service, Cornwall Partnership NHS Foundation Trust, Cornwall United Kingdom, TRURO, United Kingdom; ${ }^{2}$ Care of the Elderly, Royal Cornwall Hospital Trust, TRURO, United Kingdom; ${ }^{3}$ West Adult Learning Disabilities Service, Cornwall Partnership NHS Foundation Trust, West Resource Centre, Redruth, United Kingdom and ${ }^{4}$ East Adult Learning Disabilities Service, Cornwall Partnership NHS Foundation Trust, East Resource Centre, Bodmin, United Kingdom)

Rationale: VNS is a treatment option in patients with refractory epilepsy unsuitable for other surgical options. VNS helps in intractable cases with an average decrease in seizure frequency by $40 \%$. It involves an operation to implant under the skin a generator with coils wrapped around the left Vagus. VNS is well tolerated and safe though there have been cases reported of bradycardia occurring intraoperatively and clinically. Parasympathetic hyper stimulation of the right Vagus predisposes to bradycardia. The left Vagus when hyper stimulated predisposes to atrioventricular (AV) blocks. There has been no cases that we are aware of reporting AV blocks post VNS. We report a case of iatrogenic ventricular asystole presenting as drop attacks following VNS which initially was misdiagnosed as seizure worsening.

Methods: A 55 year old man with intellectual disability \& treatment resistant intractable epilepsy (generalised seizures including drop attacks) had a VNS fitted. There were no intraoperative complications. The VNS workup did not reveal any pre cardiac problems. His seizures improved. Two years later there was an idiopathic increase in drop attacks which was, given his past seizure history considered to be seizure worsening. There was no improvement to anti-epileptic medication and VNS titration leading to a review of his cardiac status.

Results: A 24hr ECG showed recurrent episodes of ventricular standstills, pauses and runs of complete heart block corresponding with VNS stimulation. No other cause could be found to explain this occurrence. VNS was turned off. Subsequent ECG was unremarkable. Two months later all forms of his epilepsy worsened prompting a patient choice of switching on the VNS under 24hr ECG monitoring. The ECG after switch on showed continued P wave activities but runs of ventricular asystole after VNS stimulation. The longest asystole was 9.79 seconds. This led to the VNS being turned off again.

Conclusions: VNS is considered a safe option in epilepsy management. Unfortunately it can cause side effects of iatrogenic asystole and misdiagnosis of seizure worsening. Awareness of this rare but late onset, life threatening side effect of VNS is important.

\subsection{2}

\section{AUTISM, SEIZURES AND EPILEPSY}

E. Obringer ${ }^{2}$, A. Francis ${ }^{2,1}$, C. Allen ${ }^{2}$, K. Kelley ${ }^{1,2}$ and Joseph Hageman ${ }^{1,2}$ ( ${ }^{1}$ Northshore University Health System, Evanston, IL and ${ }^{2}$ Pediatrics, University of Chicago, Chicago, IL)

Rationale: To characterize the frequency of first and second seizures in a group of children and adolescents with a clinical diagnosis of autism spectrum disorder (ASD). We also evaluated the mean age of initial seizure, occurrence of a second seizure, and choice of antiepileptic medication (AED) therapy. Note that this is a non-selected clinical group and includes all symptomatic and idiopathic epilepsies. Methods: A retrospective search of the NorthShore University patient database was carried out by clinical informatics using the ICD-9 codes for autism, seizures and epilepsy after approval was obtained from the NorthShore Institutional Review Board. An Excel spreadsheet was prepared by the investigators. Data were abstracted from patient records and included: patient demographics, age of initial and second seizure, seizure description, initial and secondary AED and effectiveness, EEG, and neuroimaging with results (CT, MRI, and PET Scan). We classified the type of autism as unknown, idiopathic, symptomatic/genetic, or autistic regression. We also reviewed daily functionality (toilet trained, mobility, current language, feeding, and sleep) of each patient.

Results: A total of 99 patients were identified; 21 patients did not meet inclusion criteria. Seventy-eight charts were reviewed. Mean age at time of chart review was 15.2 years. A total of $81 \%$ of patients are male; $91 \%$ are classified as Non-Hispanic. The majority of patients were diagnosed with autistic disorder, pervasive developmental disorder NOS, or autism spectrum disorder. Six patients were described as having autistic regression. The mean age of the initial seizure was 7.9 years; most frequent description was tonic-clonic. Seventy-two patients experienced a second seizure (72/78) occurring within a mean of 10.3 months. The choice of initial AED varied; the most frequently used were valproic acid, phenobarbital and lamotrigine.

Conclusions: In this clinical cohort of children with ASD, first seizures were commonly seen before 10 years of age with a second seizure documented in $92 \%$ of patients. The choice of initial AED was variable.

\subsection{3 \\ HIGH DOSE ORAL STEROID AS SUCCESSFUL INITIAL TREATMENT FOR INFANTILE SPASMS: FOLLOW-UP OF SEVEN CASES}

Seema Adhami ${ }^{1}$ and C. Harini ${ }^{2}{ }^{1}$ University of Massachusetts Medical Center, Worcester, MA and ${ }^{2}$ Boston Children's Hospital, Boston, MA)

Rationale: Infantile spasms (IS) constitute an age dependent epileptic encephalopathy with a high risk for later epilepsy and adverse developmental outcome. At present ACTH is considered first line treatment for IS in the United States (1). In the last few years high dose oral prednisolone has also been used as first line treatment $(2,3)$. Last year we presented seven cases of IS treated with high dose oral prednisolone and noted cessation of spasms in all seven. This is a report on follow-up of these cases with regards to seizure recurrence and development (as reported by parents $+/$ - reports from community child development services).

Methods: Details of the seven infants treated with high dose oral prednisolone for IS are shown in table 1. Each infant was treated for a total of four weeks, two weeks on full dose (minimum $4 \mathrm{mg} / \mathrm{kg} /$ day divided tid, to a max of $60 \mathrm{mg} /$ day, ) followed by a two week taper. These patients were followed with special attention to recurrence of spasms, occurrence of other seizure types, EEG, and developmental progress.

Results: Short term response with cessation of spasms was seen in all patients (one patient, \# 7, with early relapse became spasm free on day 2 of a second course, the rest had a single course of high dose oral prednisolone). Follow-up (f/u) ranged from 9 months to 32 months (mean 17 months). During this period there was no recurrence of IS or hypsarrhythmia on EEG, and no other seizure type occurred. Three patients are on no anti-epileptic medication (AED) and four are on one AED. All patients were seizure free at the time of last f/u. One patient (\#5) died at 21 months. Cause of death unclear. She was seizure free after initial treatment. Table 2 shows follow-up details.

Conclusions: This report on the follow-up of infants treated successfully with high dose oral steroids as initial treatment for IS shows no recurrence of spasms and no hypsarrythmia on EEG on follow-up ranging from 9 - 32 months. Although standardized developmental assessment was not done, parental history and developmental reports from community child development services showed developmental progress in most cases. Developmental delay was related to etiology. 
References:

1. Pellock JM, Hrachovy R, Shinnar S, Baram TZ, Bettis D, et al. Infantile Spasms: A U.S. consensus report. Epilepsia 2010;51(10):2175-2189.

2. Lux AL, Edwards SW, Hancock E, Johnson AL, Kennedy CR, et al. The United Kingdom Infantile Spasms Study comparing vigabatrin with prednisolone or tetracosactide at 14 days: a multicentre randomized controlled trial. Lancet 2004;364:1773-1778. 3. Kossoff EH, Hartman AL, Rubenstein JE, Vining EP. High-dose oral prednisolone for infantile spasms: An effective and less expensive alternative to ACTH.

Table: 1

\begin{tabular}{|c|c|c|c|c|c|c|c|}
\hline $\begin{array}{l}\mathrm{Pt} \\
\#\end{array}$ & $\begin{array}{c}\text { Age } \\
\text { (mon) at } \\
\text { onset / } \\
\text { sex }\end{array}$ & $\begin{array}{c}\text { Development } \\
\text { at onset }\end{array}$ & $\begin{array}{c}\text { Etiology of } \\
\text { IS }\end{array}$ & $\begin{array}{c}\text { Duration of IS } \\
\text { before } \\
\text { treatment }\end{array}$ & $\begin{array}{l}\text { 'lime } \\
\text { to IS } \\
\text { frec }\end{array}$ & $\begin{array}{c}\text { Post treatment } \\
\text { एTS }\end{array}$ & $\begin{array}{c}\text { Relapse } \\
\text { of IS }\end{array}$ \\
\hline 1 & $6 / \mathrm{M}$ & Delayed & $\begin{array}{l}\text { P'erinatal } \\
\text { stroke }\end{array}$ & 15 days & 5 days & $\begin{array}{c}\text { No } \\
\text { Hypsarthythmia }\end{array}$ & No \\
\hline 2 & $7 / \mathrm{M}$ & Normal & Unknown & 8 days & 5 days & Normal & No \\
\hline 3 & $10 / \Gamma$ & Delayed & $\begin{array}{l}\text { Perinatal } \\
\text { stroke }\end{array}$ & 11 days & 3 days & $\begin{array}{c}\text { No } \\
\text { Hypsarrlyythmia }\end{array}$ & No \\
\hline 4 & $7 / \mathrm{F}$ & Normal & Unknown & 14 days & 5 days & Normal & No \\
\hline 5 & $8 / \Gamma$ & Delayed & $\begin{array}{l}\text { Stroke in } \\
\text { infancy }\end{array}$ & 10 days & 3 days & $\begin{array}{c}\text { No } \\
\text { IIypsarrhythmia }\end{array}$ & No \\
\hline 6 & $6 / \mathrm{F}$ & Severe Delay & $\mathrm{HIE}$ & 12 months & 5 days & $\begin{array}{c}\text { No } \\
\text { Hypsarrhythmia }\end{array}$ & No \\
\hline 7 & $5 / \mathrm{F}$ & Normal & Unknown & 14 days & 3 days & Normal & $\begin{array}{c}\text { Yes, } 7 \\
\text { wks later }\end{array}$ \\
\hline
\end{tabular}

Table: 2

\begin{tabular}{|c|c|c|c|c|c|c|}
\hline $\begin{array}{l}\text { Pl } \\
\#\end{array}$ & $\begin{array}{l}\text { Age at } \mathrm{l} / \mathrm{u} \\
\text { in months }\end{array}$ & $\begin{array}{l}\text { Last I/u EEG } \\
\text { Left parictal } \\
\text { spike-wave }\end{array}$ & $\begin{array}{c}\begin{array}{c}\text { Seizures } \\
\text { at } \mathrm{f} / \mathrm{u}\end{array} \\
\text { No }\end{array}$ & Topiramatc & $\begin{array}{l}\text { Etiology of } \\
\qquad \text { IS } \\
\text { Perinatal } \\
\text { stroke }\end{array}$ & $\begin{array}{c}\text { Development } \\
\text { Delayed - R } \\
\text { hemiparesis specch } \\
\text { delay }\end{array}$ \\
\hline 2 & 24 & Normal & No & None & Unknown & Normal \\
\hline 3 & 41 & $\begin{array}{l}\text { Left frontal } \\
\text { spikc-wave }\end{array}$ & No & None & $\begin{array}{l}\text { I'erinatal } \\
\text { stroke }\end{array}$ & $\begin{array}{c}\text { Motor delay - R } \\
\text { hemiparesis }\end{array}$ \\
\hline 4 & 20 & Normal & No & Nonc & Unknown & Normal \\
\hline 5 & 20 & $\begin{array}{l}\text { Lelt posterior } \\
\text { spike-wave }\end{array}$ & No & Topiramate & $\begin{array}{l}\text { Stroke in } \\
\text { inlancy }\end{array}$ & Severe global delay \\
\hline 6 & 31 & Normal & No & Topiramate & HIE & Severe global delay \\
\hline 7 & 15 & $\begin{array}{l}\text { Left temporal } \\
\text { spikc-wave }\end{array}$ & No & Oxcarbazepine & Unknown & Normal \\
\hline
\end{tabular}

\subsection{4 \\ EPILEPSY SURGERY IN MRI-NEGATIVE SOUND- INDUCED STARTLE EPILEPSY}

Nina Y. Riggins, P. Li, V. Li, O. Farooq and A. Weinstock (Woman's and Children's Hospital, Buffalo, NY)

Rationale: Startle epilepsy is associated with seizures reported to closely involve the supplementary sensorimotor area (SSMA). Epilepsy surgery in such cases were reported when structural lesions were identified by magnetic resonance imaging (MRI). In a patient with debilitating, MRI-negative startle epilepsy, we sought to define the epileptogenic zones by subdural mapping to allow for epilepsy surgery.

Methods: A 23 year-old man with a history of sound-induced startle epilepsy resistant to anti-epileptic drugs and VNS, and who showed no MRI abnormalities, was evaluated with long term video EEG (LTM), PET, ictal SPECT and magneto-encephalography (MEG). LTM and MEG evaluation pointed towards epileptogenc activity at the right frontal and fronto-mesial regions. Workup included subdural grids with a total of 128 electrodes. Sampling rate was available at $250 \mathrm{~Hz}$. Review of the subdural EEG was performed with referential and bipolar montages, with a display of up to $5 \mathrm{sec}$ per page. Guided by subdural EEG recordings and cortical mapping, a resection of an area of the superior right frontal cortex was performed.

Results: Startle and spontaneous seizures were identified. Startle seizures revealed widespread ictal onset at the right frontal mesial electrodes, almost simultaneously with electrodes at the right superior frontal region. There was extreme fast spread to other frontal electrodes. About half of the seizures occurred spontaneously with ictal EEG activity, originating at the right frontal electrodes, with spreading within $200-\mathrm{msec}$ to the right frontal mesial electrodes. High-frequency oscillations (HFO) bursts were also seen interictally, predominantly over 2 frontal electrodes, and allowed localization of a definite epileptogenic area. The HFO pattern was readily identified when a bipolar montage with a limited amount of frontal electrodes was analyzed, and was difficult to appreciate on referential montages. Due to the proximity of an identified frontal eye field zone to a presumed mesial frontal seizure onset region, only resection of areas containing and surrounding the HFO pattern on the right superior frontal electrodes was performed. After resection, the patient's daily tonic seizures subsided, while subtle startle seizures remained, but without tonic progression. The patient reported significant improvement in his quality of life. The pathology report findings were consistent with focal cortical dysplasia.

Conclusions: Previous reports have indicated that epilepsy surgery is contemplated in patients with startle epilepsy when a discernible lesion is identified on MRI. Our case report reveals successful identification of the epileptogenic zone in a case of MRI-negative startle epilepsy, and that HFO could be a useful biomarker for the epileptogenic region. To our knowledge, this is the first case of a patient with MRI-negative startle epilepsy in which the epileptogenic zone was localized with HFO, leading to successful epilepsy surgery.

\subsection{5 \\ OUTCOMES IN EPILEPSY PATIENTS ON HIGH DOSE LEVETIRACETAM}

Katharine S. Dempsey ${ }^{1}$, L. J. Hirsch ${ }^{2}$, C. C. Ezeani ${ }^{2}$, R. Buchsbaum ${ }^{3}$ and H. Choi ${ }^{1}\left({ }^{1}\right.$ Comprehensive Epilepsy Center, Columbia University, New York, NY; ${ }^{2}$ Comprehensive Epilepsy Center, Yale University, New Haven, CT and ${ }^{3}$ Mailman School of Public Health, Columbia University, New York, NY)

Rationale: The FDA-recommended maximum dose for levetiracetam (LEV), a commonly used antiepileptic drug, is $3000 \mathrm{mg} /$ day. Limited data exist regarding the efficacy of using AED doses at higher than FDA-approved doses. One small, double-blind, randomized parallel group study suggests that LEV dose of $4000 \mathrm{mg}$ /day may be safe and effective (Betts et al., Seizure 2000; 9: 80-87). We examined the rate of efficacy and intolerable side effects in patients who were "pushed" to higher than FDA dose in attempts to control seizures.

Methods: We reviewed the records of 750 adults newly started on LEV at Columbia between $1 / 1 / 00$ and $1 / 1 / 10$. We examined the efficacy outcome (defined as $\geq 12$ month seizure freedom) and intolerability (defined as adverse effect [AE] requiring dose decrease) stratified by two types of doses: (1) a HIGH LEV dose (3001$4000 \mathrm{mg} /$ day), and (2) a VERY HIGH dose (>4000mg/day). Analysis was done using IBM SPSS Statistics 19.

Results: As seen in Figure 1, of 750 adults who newly started LEV, $107(14.3 \%)$ failed the FDA-approved dose and were placed on either HIGH (95 patients) or VERY HIGH (23 patients) doses. Of the 95 patients that tried HIGH dose, 4 out of 80 (5.0\%) attained $\geq 12$ month seizure remission. The remaining 15 patients had fewer than 12 months of follow up and were still on HIGH dose at last visit. In those who could tolerate HIGH dose, 4 out of $43(9.3 \%)$ attained $\geq 12$ month seizure remission. Among the 23 patients who tried VERY HIGH dose, 1 out of $20(5.0 \%)$ attained $\geq 12$ month seizure remission. The remaining 3 patients had fewer than 12 months of follow up and 
were still on VERY HIGH dose at last visit. In those who could tolerate VERY HIGH dose, 1 out of $10(10.0 \%)$ attained $\geq 12$ month seizure remission. Twenty-three patients had used HIGH dose for a period $\geq 12$ months. Three patients had used a VERY HIGH dose for a period $\geq 12$ months. Of note, mean duration of HIGH or VERY HIGH doses was 12.9 months (SD 24.2).

The most common intolerable AE on HIGH dose was drowsiness $(12.6 \%)(n=95)$. The most common intolerable AEs on VERY HIGH dose were psychiatric AEs (13.0\%), cognitive AEs (8.7\%), and drowsiness $(8.7 \%)(n=23)$. Among patients who discontinued HIGH dose $(n=63), 33.3 \%$ discontinued due to intolerability. Among patients who discontinued VERY HIGH dose $(n=19), 63.3 \%$ discontinued due to intolerability.

Conclusions: Our findings suggest that using LEV at higher than FDA-recommended dose can lead to long-term seizure freedom in about $5 \%$ of patients who failed the FDA-recommended dose, and about $10 \%$ of patients if the patient does not discontinue the high dose for intolerability. At these high doses, sedation, psychiatric and cognitive effects were common.

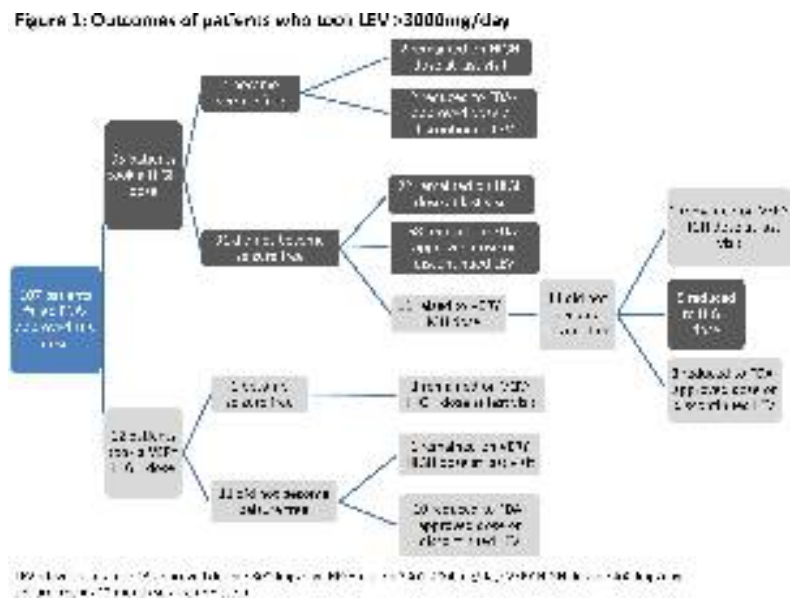

\subsection{6}

EPISODIC AUTOBIOGRAPHICAL DYSFUNCTION IN TRANSIENT EPILEPTIC AMNESIA: EFFECTIVENESS OF ANTIEPILEPTIC DRUGS

Paul B. Pritchard, M. T. Wagner and D. L. Bachman (Medical University of South Carolina, Charleston, SC)

Rationale: Transient epileptic amnesia (TEA, Kapur) is a syndrome which has variously been termed epileptic amnesic attacks (Pritchard, Holmstrom, et al.), epileptic amnesic syndrome (Gallassi, Morreale et al.), pure amnestic seizures (Palmini, Gloor, et al.), and epileptic transient amnesia (Stracciari, Ciucci, et al.). The central feature is recurrent attacks during which there is anterograde memory malfunction while consciousness is preserved.

We describe three patients with episodic amnesia who also recounted discrete episodes of disordered autobiographical memory during which they behaved as though they had resumed their life circumstances of 20-30 years in the past. For example, one woman excitedly called her husband at work to report "we've got to pack up fast because the owners are on their way to move back into the apartment." Each time her husband rushed back home from work to remind her that they no longer lived in the rented home of 20 years ago and that they were now homeowners and not compelled to move out!

Methods: We performed a retrospective analysis of clinical features, EEG, and VEEG in each case, as well as neuropsychological evaluations in two cases. We also reviewed brain imaging and responses to antiepilepic drugs in each case.

Results: All three patients experienced complex partial seizures (CPS) and episodic amnesia. In addition, each of them experienced episodes in which they believed themselves to be at an earlier point in their lives, as long as 20-30 years in their past. Patient 1 also had a more pervasive memory disorder consistent with mild cognitive impairment, as reflected in neuropsychological testing. Historical characteristics of the group are depicted in Table 1.

Each of them underwent EEG and MRI brain scans, and two out of the three were monitored with video-EEG (VEEG) recording as outline in Table 2. For patients 1 and 2, who had bilateral temporal spikes, spike counts demonstrated a preponderance of left temporal spikes for patient 1 and disproportionate right temporal spikes for patient 2. A 24 hour spike count in patient 1 showed that maximum temporal lobe spike frequency occurred during the time when she was most prone to have auto-biographical memory attacks. All three patients attained complete control of autobiographical memory attacks and transient epileptic amnesia episodes with antiepileptic drugs (two with oxcarbazepine and one with a combination of lamotrigine and levetiracetam. Followup neuropsychological testing in Patient 1 failed to demonstrate any significant improvement in her mild cognitive impairment. Conclusions: 1 . Episodic autobiographical memory dysfunction may occur in patients who have transient epileptic amnesia (TEA). 2. Antiepileptic drugs are affective treatment of transient epileptic amnesia and autobiographic memory attacks.

Historical characteristics

\begin{tabular}{|c|c|c|ccc|}
\hline Patient & Age & Gender & CPS & $\begin{array}{c}\text { Transient amnesia } \\
\text { altacks }\end{array}$ & $\begin{array}{c}\text { Episodic autobiographical memory } \\
\text { dysfunction }\end{array}$ \\
1 & 64 & Femalc & Ycs & Yes & Ycs \\
\hline 2 & 47 & Malc & Yes & Yes & Ycs \\
\hline 3 & 65 & Malc & Yes & Yes & Yes \\
\hline
\end{tabular}

3.157

TREATMENT OF SEIZURES IN SCHIZENCEPHALY MAY IMPROVE CLINICAL OUTCOME

Mirret M. El-Hagrassy ${ }^{1,2}$, K. Werner ${ }^{1,2}$ and Y. Eksioglu ${ }^{1,2}$

( ${ }^{1}$ Neurology, SUNY Upstate Medical University, Syracuse, NY and

${ }^{2}$ Neurology, Golisano Children's Hospital, Syracuse, NY)

Rationale: Information regarding MRI findings, clinical presentation, and outcomes of different treatment approaches in patients with schizencephaly is limited.

Methods: A retrospective review of patients with schizencephaly in the academic year 2011-2012 included 4 patients (1 male, 3 female). Three had a history of seizures including 1 with status epilepticus. Age of seizure onset ranged from 9 months to 14 years, all presenting with partial and/or generalized seizures, including drop attacks in one. EEG in all 3 showed multifocal and/or generalized epileptiform abnormalities.

Neuroimaging revealed left open-lipped schizencephaly in 2, right open-lipped schizencephaly in 1 and right frontal closed lip schizencephaly in another patient. The patient whose seizure onset was at 14 years of age was autistic. The other 3 patients had additional cortical malformations including heterotopia, polymicrogyria and septo-optic dysplasia. Genetic analysis was negative in 2 patients; 2 patients had never been tested.

Various antiepileptic drugs (AEDs) were used. The 3 epileptic patients were on at least 2 AEDs and received rehabilitation services. Despite moderate to severe neurocognitive delays in all 3 epileptic patients, 2 were enrolled in mainstream classes at school following appropriate management. 
Results: Only a few small studies have investigated schizencephaly with respect to MRI findings and clinical correlation. In this center, partial seizures dominated seizure semiology with all epileptic patients requiring multiple AEDs. The patients in this study did not show correlation between the severity of cortical malformations, age of seizure onset and neurocognitive status. One patient was significantly restricted by autism whereas 2 were in mainstream classes at school after receiving appropriate antiepileptic regimen and rehabilitation services.

Conclusions: Schizencephaly may present with various types of seizures and neurocognitive delays. The patients may benefit significantly from a multidisciplinary approach including appropriate AED therapy, physical/occupational/speech therapy and special education.

\subsection{8}

\section{SEIZURE PROPHYLAXIS IN BENIGN FOCAL EPILEPSY OF CHILDHOOD WITH CENTROTEMPORAL SPIKES}

Takijah T. Heard, C. Harini, J. Girard, K. Boyer and M. Takeoka (Children's Hospital Boston, Boston, MA)

Rationale: Benign focal epilepsy of childhood with centrotemporal spikes (BECTS) or benign rolandic epilepsy (BRE) is a common type of focal epilepsy in childhood; the prevalence is as high as $23-24 \%$ of school children with epilepsy. The mean age of onset of about 7 years, and $75 \%$ of seizures occur after children fall asleep, or just before they wake up. Other seizure types occur in $20-54 \%$, with only $16 \%$ having secondarily generalized seizures. Many children will have spontaneous resolution of seizures during the second decade of life, and the overall long-term seizure outcome is considered as benign; however secondary generalized seizures can be seen, and still could impose risks for safety. For such reasons, many clinicians will weigh risks and benefits of treatments with antiepileptic drugs (AEDs). There is no guideline or consensus in when to consider prophylactic AEDs in BECTS. The goal of this study is to identify factors that appear relevant to decide prophylactic treatment with AEDs.

Methods: Over a period of 2 years, we continuously retrospectively reviewed patients with benign focal epilepsy of childhood with centrotemporal spikes (BECTS) from July 2010 to June 2012. All patients were identified based on clinical seizure semiology and EEG findings with sleep potentiated centrotemporal spikes. Children who had structural brain abnormalities, focal neurological deficits, known other neurological disorders and pre-existing developmental delay were excluded, as such may suggest underlying encephalopathy beyond typical BECTS. Clinical information was assessed for demographics, unilateral or bilateral centrotemporal spikes, presence of secondary generalized seizures. These data were correlated with treatment with prophylactic antiepileptic medications.

Results: We reviewed the identified 114 patients with BECTS (63 Boys, 51 Girls, mean age $=7.4+/-2.1$ years). 64 had secondary generalized seizures (group A) while 50 had only simple partial seizures (groups B). In group A, 44 out of $64(68.8 \% \%)$ were treated with AEDs. $20(31.2 \%)$ were not treated with AEDs, but 12 patients had 1 seizure, 4 had only 2 seizures, 1 had 3 seizures, 1 had 4 seizures, 1 was lost for follow up and 1 had 10 seizures but was not treated due to parental preference. In group B, 17 out of 50 (34\%) were treated with AEDs, and $33(66 \%)$ were not. AEDs used in the following number of patients: oxcarbazepine (29), levetiracetam (28), carbamazepine (3), valproate (6), lamotrigine (2) gabapentin (2), sulthiame (1). A total of 8 patients were on multiple antiepileptic medications during the clinical course.

Conclusions: While no consensus exists in guidelines for when to start prophylactic AEDs in BECTS, our study shows that AEDs are started when patients have multiple secondary generalized seizures ( 60 out of 64 patients with more than 2 secondary generalized seizures). AEDs were started on 1/3 even when seizures are not secondary generalized, which may be potentially associated with seizure frequency and family preference. Further prospective studies will be necessary to confirm such trend.

Table of BECTS patient with treatment and semiologies
\begin{tabular}{|cccc|}
\hline & AEDS & No AEDS & Total \\
\hline With Secondarily Generalized Seizures & 44 & 20 & 64 \\
\hline Partial Scizures Only & 17 & 33 & 505 \\
\hline
\end{tabular}

\subsection{9 \\ VAGUS NERVE STIMULATION IN TREATMENT- RESISTANT EPILEPSY; LONG-TERM OUTCOME}

Joan C. Dean, R. Pavelock and C. Allen (Epilepsy Institute of North Carolina, Winston Salem, NC)

Rationale: In 2010 we presented a 10-year experience after VNS implant in 550 epilepsy patients. 220 are mentally challenged (MC), 330 have normal IQs $(\mathrm{N})$.

Methods: 10-year experience after VNS implant in 550 epilepsy patients. 220 are mentally challenged (MC), 330 have normal IQs (N) Results: 90\% (MC) 95\% (N) were able to taper at least one medication successfully after VNS implantation at 1 year. Seizure frequency reduction remains at $68 \%(\mathrm{~N})$ and $59 \%(\mathrm{MC})$. A non-responder group (treatment-resistant) emerged from this study. 15 patients (MC) and 16 patients $(\mathrm{N})$ had an average of 15 clusters or prolonged seizures per year requiring hospitalization despite continuous VNS stimulation protocols. All EEGs showed continuous spikes, spike and wave, or baseline studies. EEGs after baseline were performed at 3,6, and 12 months. Demographics were similar for both groups. Etiology of seizures include migration disorder, encephalitis, and birth defects.

Conclusions: In 10 of 15 (MC) non-reponders and 2 of 16 (N) nonresponders correlate with: some neuronal migration disorder; EEGs at baseline have a significantly longer duration of spike wave activity with eventual appearance of spike and wave intervals after 6 months and a decrease in duration of spike and wave; clinically this small group of patients do not recover functional and ultimately remain in various stages of sub-clinical status epilepticus.

\subsection{0 \\ CEREBRAL HEMATOMA AND SPONTANEOUS OBLITERATION OF CEREBRAL ANGIOMATOSIS STURGE-WEBER SYNDROME}

Yasushi Iimura, M. Nakajima, H. Sugano, T. Higo and H. Arai (Juntendo University, Bunkyo-ku, Japan)

Rationale: Sturge-Weber syndrome (SWS) is a neurocutaneous syndrome characterized by facial port wine nevus and leptomeningeal angiomatosis. It is commonly complicated by intractable epilepsy and mental retardation. In spite of broadly affected angiomatosis, spontaneous intracerebral bleeding have never been reported in childhood SWS. In this paper, we report our experience of a patient with SWS who bled in the right thalamus.

Methods: We report a girl with SWS who had two epileptic seizures and appropriate development before intracerebral bleeding. At 3years-old of age, she suddenly developed impairment of consciousness and left hemiplegia. Computed tomography showed intraventricular hemorrhage and acute hydrocephalus. We carried out emergency ventricular drainage, however she had rebleeding in the right thalamus. After continuous ventricular drainage, she gradually recovered consciousness, and rehabilitation induced motor power improvement. Magnetic resonance digital subtraction angiography which performed on day 24 showed poor development of superficial venous drainages, occluded superior sagittal sinus (SSS), and irregular shaped straight sinus. After clearing the intraventricular hematoma, we carried out ventriculoperitoneal shunting with fenestration of the septum pellucidum. Conventional angiography which performed on day 70 showed also poor drainage of the 
superficial cortical venous system, complete occlusion of the SSS, and prolonged perfusion time. Two years later, she experienced several seizures originated from temporal lobe. Magnetic resonance imaging showed remarkable atrophy in the right temporal lobe. Her psychomotor development also gradually deteriorated. Consequently, we performed the posterior quadrantectomy for her seizure control and maintaining her mental development.

Results: We supposed that the hemorrhage may have been caused by venous congestion that occurred in the deep venous system. Additional compromise of the outflow was probably related to the bleedings. We speculate that functional impairment and obtaining epileptogenesis in SWS is due to ischemia mismatched the functional demand. Epileptic seizures also require high cerebral blood flow, therefore induce relative ischemia in SWS with congestive venous drainage, and consequently vicious cycle is formed.

Conclusions: The progressive venous restriction leads to bleeding in the congested area and impairs cerebral perfusion with consequent epilepsy deterioration. Surgery can be a promissing way to control this deterioration.

\subsection{1}

\section{EFFECTIVENESS OF POLYTHERAPY OF VPA PLUS LTG IN CHILDHOOD ABSENCE EPILEPSY}

Joonghyun Bin ${ }^{1}$ and T. Eom ${ }^{2}{ }^{1}$ pediatrics, Incheon St. Mary's hospital, Incheon-si, Republic of Korea and ${ }^{2}$ pediatrics, Uijeongbu St. Mary's hospital, Uijeongbu-si, Republic of Korea)

Rationale: Ethosuximide (ESX), valproic acid (VPA), and lamotrigine (LTG) are used as antiepileptic drug (AED) for childhood absence epilepsy (CAE). In Korea, domestic pharmaceutical companies have not produced ESX for a problem of the charge for the medicine since 2010.

Accordingly, doctors in Korea had to change the medicine to LTG or VPA to treat patients.

We performed this study to compare the efficacty of monotherapy and polytherapy of LTG and VPA for CAE.

Methods: Medical records of 12 patients ( 7 girls, 5 boys) diagnosed with CAE at Bucheon St. mary's hospital between 2009 and 2011 were reviewed retrospectively.

Results: Among them, four patients (33\%) had been treated with ESX at treatment onset, six patients $(50 \%)$ had been treated with LTG, and the remaining two patients $(17 \%)$ were first treated with VPA. All of patients treated with ESX achieved seizure freedom. But ESX was replaced by LTG for ESX treatment group for the halt in production. One patient $(25 \%)$ of them achieved seizure freedom, and the rest three patients $(75 \%)$ experienced more than $50 \%$ reduction of seizure frequency after four weeks of initial titration. VPA was added on for the rest three patients, and then all of them achieved seizure freedom. One of two patients treated with VPA achieved seizure freedom and another experienced more than $50 \%$ reduction of seizure frequency after four weeks of initial titration. LTG was added on for another patient, and then she achieved seizure freedom. One patient $(17 \%)$ of patients treated with LTG achieved seizure freedom, and the rest five patients (83\%) experienced more than $50 \%$ reduction of seizure frequency after four weeks of initial titration. But one patient who achieved seizure freedom with LTG had severe headache, then we reduced dose of LTG and add on with VPA. VPA was added on for the rest five patients, and then all of them achieved seizure freedom.

Conclusions: ESX is excellent AED for CAE. But doctors in Korea treat CAE patients only with VPA and LTG. In absence of ESX, polytherapy of VPA plus LTG is better than monotherapy of VPA and LTG for CAE.

\subsection{2 \\ PROGRESSION TO INFANTILE SPASMS IN AN INFANT WITH TUBEROUS SCLEROSIS ON VIGABATRIN AND EVEROLIMUS: A CASE REPORT AND REVIEW OF LITERATURE}

S. Verma and C. J. Marcuccilli (Neurology, Medical College of Wisconsin, Milwaukee, WI)

Rationale: Vigabatrin is the treatment of choice for the treatment of infantile spasms in tuberous sclerosis (TS). However, there is little data demonstrating its role in the prevention of infantile spasms. Furthermore, everolimus has been approved for use in TS to reduce subependymal giant cell astrocytoma (SEGA) volume. It is not currently known if this translates to improved seizure control. Here we report an eight month old infant with tuberous sclerosis who developed infantile spasms during treatment with vigabatrin and everolimus.

Methods: This is a case report of an infant with prenatal diagnosis of TS that presented to the Children's Hospital of Wisconsin for evaluation of seizures at 3 weeks of life. Video

electroencephalography (EEG), MRI brain with and without contrast, and genetic testing were employed for characterization of the case. Results: MRI of the brain showed a left lateral ventricle SEGA (Fig. 1A) and multiple tubers (Fig. 1B). At presentation, The diagnosis of TS was further confirmed by genetic testing (frameshift mutation in TSC2 gene). Video EEG monitoring demonstrated clinical and electrographic right central focal epileptiform activity (Fig. 2A). Seizures were refractory to first line antiepileptic therapies, including Phenobarbital, phenytoin and topiramate. As a result, levetiracetam (LEV) and vigabatrin (VGB) were used with minimal success to decrease seizure frequency. Given the refractory nature of the patient's seizures, everolimus was initiated with the goal of reducing tuber volume and possibly decrease seizure frequency. Focal seizures evolved into flexor spasms on maximal doses of VGB, LEV and everolimus at 7 months of age. This coincided with addition of lacosamide, given persistent focal seizures. EEG confirmed hypsarrythmia (Fig. 2B). Lacosamide was discontinued, as was everolimus, 2 weeks prior to starting ACTH therapy to prevent excessive immunosuppression. Chest X-ray demonstrated asymptomatic pneumonitis. On ACTH, VGA, LVT and valproic acid, hypsarrythmia resolved (Fig. 2C). The infant has developmental delay with rare refractory seizures consistent with left upper extremity focal seizures.

Conclusions: To the best of our knowledge, this is the first example of a patient with TS developing infantile spasms on both VGB and everolimus. Focal electrographic abnormality progressed to hypsarrythmia on VGB and everolimus demonstrating the failure of 2 first line drugs in the treatment of TS. It is also unclear if there was activation of seizures with lacosamide, although abrupt discontinuation of the drug did not result in improvement of EEG. We also believe he is the youngest patient at age 5 months to be treated with everolimus with development of asymptomatic pneumonitis posing a unique challenge of immunosuppresion in infants with TS.

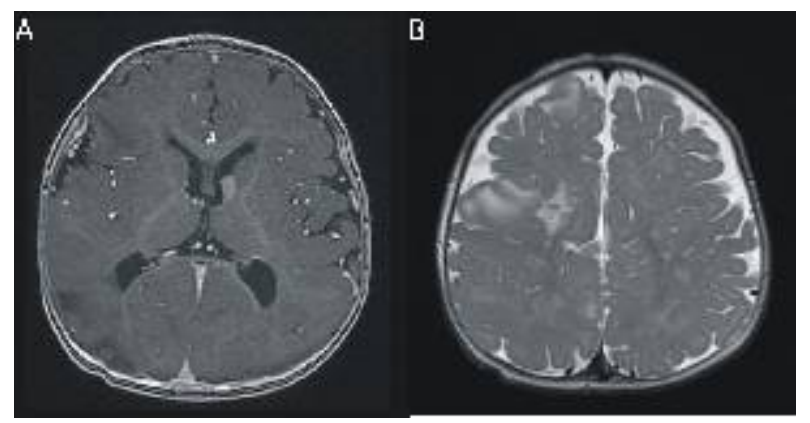

Figure 1: (A) Axial T1 weighted image post-contrast demonstrating SEGA (B) Axial T2 weighted image demonstrating multiple tubers. 


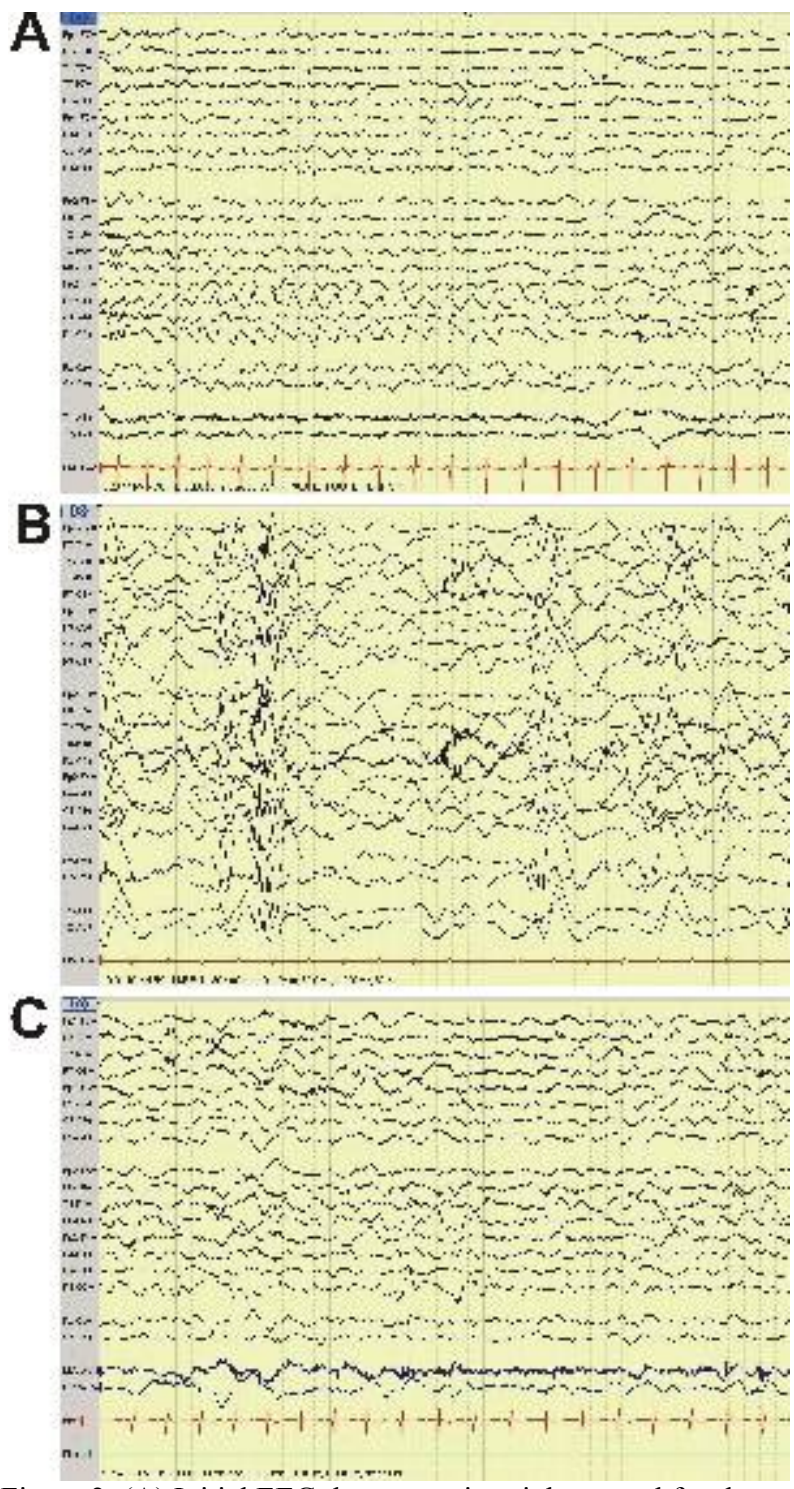

Figure 2: (A) Initial EEG demonstrating right central focal epileptiform discharges (B) Hypsarrythmia (C) EEG post ACTH and antiepileptic drug treatment.

\subsection{3 \\ RASMUSSEN'S ENCEPHALITIS: A WOBBLING GLASS, HALF FULL}

Y. Eksioglu ${ }^{1,2}$ and M. M. El-Hagrassy ${ }^{1,2}\left({ }^{1}\right.$ Neurology, SUNY Upstate Medical University, Syracuse, NY and ${ }^{2}$ Neurology, Golisano Children's Hospital, Syracuse, NY)

Rationale: Rasmussen's encephalitis (RE) is a rare acquired inflammatory encephalopathy characterized by cerebral hemispheric dysfunction and hemiatrophy, refractory partial epilepsy, cognitive deterioration and hemiparesis. Since the current data on optimum treatment modalities is inadequate, a retrospective review of patients with Rasmussen's encephalitis was carried out.

Methods: A retrospective review of SUNY Upstate Medical University records for 2011 and 2012 revealed 2 cases of brain biopsy-diagnosed and 1 case of possible Rasmussen's encephalitis. Results: Patients were in their 20's and 30's, and all had intractable epilepsy. Two of them had refractory complex partial status epilepticus and neurocognitive disability. All had brain MRIs, while functional imaging in the form of SPECT was performed in 2 cases, and $\mathrm{fMRI}$ was performed in one. All had repeated video-EEG monitoring.
Two patients underwent epilepsy surgery: 1 had a temporal lobectomy at 10 years of age, eventually necessitating a right periinsular hemispherectomy, with postoperative left-sided hemiparesis and nonobstructive hydrocephalus. One had right parietal and posterior temporal resection as well as VNS, under consideration for further surgery. One had cerebral palsy, autism and possible RE; her seizures were controlled medically.

All were on 3 antiepileptic medications. One needed an immune modulator, Imuran and her seizures improved after the addition of Coenzyme Q10. None of the patients had a family history of epilepsy.

Rasmussen's encephalitis must be diagnosed and managed appropriately. Although a biopsy would diagnose RE definitively, the European consensus statement proposed diagnostic criteria focusing on clinical, electroencephalographic, and neuroimaging features. The pathology remains unclear. One case study suggests a reduction in benzodiazepine receptors in the affected hemisphere while others suggest an immune-mediated process with auto-antibodies against GluR3. The use of corticosteroids, IVIG and plasma exchange has been suggested as an alternative therapy for refractory status epilepticus by the Neurocritical Care Society Status Epilepticus Guideline Writing Committee. Tailored AED therapy is essential. Functional and anatomic hemispherectomy interventions may contribute greatly to seizure control, but the risks and benefits must be thoroughly assessed.

Conclusions: RE can be highly disabling, and diagnosis with appropriate pharmacological and surgical management is imperative to control seizures, reduce morbidity and improve quality of life. Given the low prevalence of RE, multi center clinical studies are needed.

\section{Clinical Epilepsy: Prognosis}

\subsection{4 \\ EARLY EXPOSURE TO CARBAMAZEPINE, OXCARBAZEPINE, PHENYTOIN ,AND LAMOTRIGINE IN EPILEPSY WITH MYOCLONIC-ATONIC SEIZURES IS ASSOCIATED WITH WORSE OUTCOME}

Srishti Nangia, J. J. Millichap, A. T. Berg and D. R. Nordli (Children's Memorial Hospital, Chicago, IL)

Rationale: Epilepsy with myoclonic-atonic seizures (MAE), or Doose Syndrome, is an electroclinical syndrome in which development, neurological examination, and imaging is normal prior to the onset of seizures of generalized onset between 7 months and 6 years of age. The EEG shows theta activity in the parietal regions with interictal generalized or multifocal polyspike/spike and slow wave discharges. Valproic acid, zonisamide, levetiracetam, felbamate and ketogenic diet are considered effective treatments for MAE whereas carbamazepine (CBZ), oxcarbazepine (OXC), phenytoin (PHT), and lamotrigine (LTG) are often thought to exacerbate seizures. We hypothesized that early exposure to CBZ, OXC, PHT, or LTG might have a negative impact on the ability of later appropriate therapies to control seizures and preserve development. Methods: Patients with MAE were identified by a keyword search for MAE in the database of all video-EEG recordings performed at our center from 2002-2012. The diagnosis of MAE was confirmed by a review of all clinical information. Bad outcome was defined as either or both persistent seizures or poor cognition despite multiple appropriate trials of AEDs or ketogenic diet. Good outcome was defined as complete control of seizures and normal developmental course or mild delays only.

Results: Retrospective chart review identified 73 patients with confirmed MAE (43 boys and 30 girls). The ages of onset of epilepsy ranged from 3 months to 5 years. 20 patients (14 boys; 6 girls) had a "bad" and 53 had "good" outcomes. "Bad" outcomes occurred in $16 / 29(55 \%)$ of children previously treated with one or more of CBZ, OXC, PHT or LTG and in 4/44 (9\%) of those who had never been 
treated with any of those four drugs $(\mathrm{p}<0.0001)$. In the "bad" outcome group, 3 of the children continued to have only persistent seizures, 1 had significant cognitive impairment alone and 16 had both persistent seizures and poor developmental course. The children in this " bad" outcome group with persistent seizures had either multiple seizures per week or multiple daily seizures, ranging from 50 absence seizures per day to six convulsions per night.

Conclusions: Our retrospective review suggests that worse clinical outcome in MAE is associated with early exposure to CBZ, OXC, PHT, and LTG. These findings raise the possibility that these drugs may actually worsen the prognosis in MAE and underscore the importance of early and accurate diagnosis of childhood electroclinical syndromes and of selecting appropriate and effective treatments early in the course in order to improve long-term outcomes.

\subsection{5 \\ POSTOPERATIVE COGNITIVE PERFORMANCE AND SEIZURE CONTROL ARE SIMILAR AFTER SELECTIVE AMYGDALOHIPOCAMPECTOMY OR ANTERIOR TEMPORAL LOBE RESECTION IN PATIENTS WITH MTLE AND HIPPOCAMPAL SCLEROSIS}

Tátila M. Lopes, D. A. Fernandes, C. L. Yasuda, E. Ghizoni, H. Tedeschi, E. Oliveira and F. Cendes (Neurology, State University of Campinas- UNICAMP, Campinas, Brazil)

Rationale: Mesial temporal lobe epilepsy (MTLE) is the most common type of focal epilepsy and $30 \%$ to $40 \%$ of these patients are refractory to antiepileptic drugs (AEDs). The surgical removal of the epileptogenic focus is indicated for this subgroup of MTLE patients. Nevertheless, it is still unclear how different surgical approaches influence on postoperative cognition and seizure control in MTLE. Our aim was to compare selective amygdalohipocampectomy (SelAH) and anterior temporal lobe resection (ATL) in memory performance and seizure control in MTLE patients with long term follow up.

Methods: We evaluated 25 patients with MTLE and MRI evidence of hippocampal sclerosis (HS) who underwent SelAH and 22 who had ATL surgery. All patients underwent a comprehensive clinicalEEG evaluations and the same protocol for MRI acquisition (2 Tesla; 3D- T1-weighted images) and neuropsychological assessment (NPs). The volumetry of the surgical lacune (VSL) was performed with the Display software (David McDonald,

www.bic.mni.mcgill.ca/software) and NPs used the Wechsler memory scale- R subtests: logical memory I and II, verbal and visual paired associated I and II, figural memory and visual reproduction I and II; Rey auditory verbal learning test (RAVLT); Wechsler adult intelligence scale- III subtests: vocabulary and block design to estimate the intelligence quotient (IQ- estimated). This evaluation was performed within a mean interval of 8.5 years after surgery. We performed Mann-Whitney test to compare the cognitive performance between groups. The correlation between VSL and NPs was performed using Spearman test. Seizure outcomes were evaluated using Engel classification and we conducted a two-sided Fischer exact test to evaluate the differences between the proportions of patients classified as Engel IA (seizure-free) and other outcomes according to the type of surgical approach. We considered only $\mathrm{p}<0.05$ corrected for multiple comparisons.

Results: There was no significant differences between SelAH and ATL groups related to cognitive performance in all NPs tests ( $p>0.05$ ). In addition, the proportions of patients classified as Engel IA and other outcomes was not different in the SelAH and ATL groups ( $\mathrm{p}=0.076)$. We found a positive correlation between QIestimated and VSL for ATL group ( $\mathrm{r}=0.479$ ).

Conclusions: These findings indicate that the postoperative outcome of memory performance and seizure control is similar for both SelAH and ATL in patients with MTLE and HS. The relationship between more extensive resections (VSL) and better estimated IQ needs further investigations and may be related to better seizure control in those with more extensive surgical resections.

\subsection{6 \\ SEVERITY OF EPILEPSY AND COGNITIVE FUNCTIONING IN CHILDREN WITH EPILEPSY: A PROSPECTIVE STUDY OF FAMILY FACTORS AS MEDIATORS AND MODERATORS}

Anna I. Lambrinos ${ }^{1,4}$, W. R. Avison ${ }^{1,3}$, G. Y. Zou ${ }^{1,5}$ and K. N. Speechley ${ }^{1,2}\left({ }^{1}\right.$ Epidemiology and Biostatistics, The University of Western Ontario, London, ON, Canada; ${ }^{2}$ Pediatrics, The University of Western Ontario, London, ON, Canada; ${ }^{3}$ Sociology, The University of Western Ontario, London, ON, Canada; ${ }^{4}$ Children's Health Research Institute, London, ON, Canada and ${ }^{5}$ Robarts Research Institute, London, ON, Canada)

Rationale: Previous studies have reported that children with epilepsy are at risk for cognitive impairments. In addition to severity of epilepsy, family factors have also recently been cited as influencing cognitive functioning in children. The two objectives were to assess the: (1) relationship between severity of epilepsy and cognitive functioning in children with new-onset epilepsy at baseline, 6, 12 and 24-months; and (2) moderating and mediating effects of family factors on the relationship between severity of epilepsy and cognitive functioning at baseline, 6, 12 and 24-months.

Methods: Data came from the Health-related Quality of Life of Children with Epilepsy Study (HERQULES), a multi-centre prospective cohort study of children aged 4-12 years with new onset epilepsy that were followed for 2 years. From eligible families, 374 parents completed the mailed questionnaire at baseline (response rate $82 \%$ ) and 282 at 24-months. The Global Assessment of Severity of Epilepsy (GASE) scale measured severity of epilepsy and the cognitive functioning subscale on the Quality of Life in Childhood Epilepsy (QOLCE) scale assessed cognitive functioning in children. Three family factors were assessed. The Family Inventory of Life Events and Changes (FILE) measured family demands; Family Adaptability, Partnership, Growth, Affection \& Resolve (APGAR) measured family functioning; and the Family Inventory of Resources for Management (FIRM) measured family resources. Multiple linear regression models were used to identify the relationship between severity of epilepsy and cognitive functioning, while generalized estimating equations approach was adapted to statistically assess mediating and moderating effects of family factors on the relationship between severity of epilepsy and cognitive functioning. Results: The mean age of the sample was $7.4( \pm 2.4)$ years; approximately half $(52 \%)$ of the children in the sample were males; and $61 \%$ had partial seizures. The majority of children (59\%) had "a little severe" or "somewhat severe" epilepsy. There was a statistically significant negative association between severity of epilepsy and cognitive functioning, controlling for seizure type and antiepileptic drugs at the 6,12 and 24-month follow-up ( $p=0.0006$ to $<0.0001$ ). Family demands $(\mathrm{p}=0.02)$ and family functioning $(\mathrm{p}=0.03)$ were both found to significantly mediate the relationship between severity of epilepsy and cognitive functioning at the 12-month follow-up. Family resources was not found to be a moderator in this relationship at any time-point ( $\mathrm{p}=0.51$ to 0.81 ).

Conclusions: Children with more severe epilepsy have worse cognitive functioning. The relationship between severity of epilepsy and cognitive functioning does not depend on the level of family resources. The association between severity of epilepsy and cognitive functioning is partially mediated by family demands and family functioning at one-year post diagnosis. These findings suggest opportunities for intervention in reducing the impact of epilepsy on cognitive functioning. 


\subsection{7}

\section{PROGNOSIS IN PARTIAL STATUS EPILEPTICUS}

Makoto Kawai, B. N. McQueen and A. Verma (Neurology, The Methodist Neurological Institute, Houston, TX)

Rationale: It is well known that generalized status epilepticus (GSE) is a neurological emergency that warrants aggressive and sometimes invasive treatment. However, for partial status epilepticus (PSE), it is not clear how aggressive treatment should be. That is one reason why the duration of PSE tends to be longer than that of GSE. There is evidence that prolonged PSE results in neuronal injury; however the correlation between the duration of PSE and overall prognosis has not been established.

Unlike GSE, clinical symptoms are frequently insufficient to monitor PSE. Continuous bedside EEG monitoring is necessary for consistent evaluation of PSE.

Methods: Retrospective chart review was performed from January 2008 to January 2012 of all patients who underwent bedside EEG monitoring in our hospital. Prognosis, at the time of discharge, was classified by utilizing Glasgow-Pittsburgh cerebral performance categories (CPC), dividing performance into five categories (1: conscious and alert with a normal function or slight disabilities, 2: conscious and alert with the moderate disabilities, 3 : conscious with the severe disabilities, 4: comatose or persistent vegetative state, 5: brain death or death from other causes). All parameters of status epilepticus were obtained through EEG findings and medical records. Results: During this investigation period, 710 bedside EEG monitoring studies were performed. We identified 98 patients with electrographic seizures, and 60 patients were diagnosed as status epilepticus.

52 patients had PSE. Mean age was 60.2. Mean duration of EEG monitoring was 14 hours 27 minutes. Mean CPC score was 2.57 . Mean duration of status epilepticus was 56 hours 55 minutes. 8 patients had GSE. Mean age was 59.4. Mean duration of EEG monitoring was 250 hours 35 minutes. Mean CPC score was 4.4. Mean duration of status epilepticus was 41 hours 31 minutes. The prognosis of PSE was found to be better than that of GSE $(p=0.0007)$, but the duration of PSE did not show a statistically significant correlation with prognosis (correlation coefficient -0.12 , $\mathrm{p}=0.35$ ).

Conclusions: PSE has better prognosis than GSE. Duration of PSE alone is not correlated with prognosis.

\subsection{8 \\ MORTALITY AND RECOVERY FROM REFRACTORY STATUS EPILEPTICUS: A 7-YEAR OBSERVATION} R. Sutter ${ }^{1}$, S. Marsch ${ }^{2}$ and Stephan Rueegg ${ }^{3}$ ( Division of Neurosciences Critical Care, Departments of Neurology, Neurosurgery, and, Johns Hopkins University School of Medicine and, Baltimore, MD; ${ }^{2}$ Intensive Care Medicine, University Hospital Basel, Basel, Switzerland and ${ }^{3}$ Neurology, University Hospital Basel, Basel, Switzerland)

Rationale: Refractory status epilepticus (RSE) is one of the most life-threatening neurological emergencies with high mortality and morbidity. The aim of this study was to elucidate and quantify associations of clinical characteristics of RSE patients with outcome. Methods: Comprehensive medical chart review was performed of all consecutive patients treated for RSE between 2005 and 2011 on the ICUs of the University Hospital Basel (Switzerland). Patients' demographics, RSE etiologies and duration, comorbidities as well as outcomes were assessed. Associations of clinical characteristics and outcomes were quantified.

Results: Of 260 patients with status epilepticus 111 developed RSE with a mean duration of 101 hours. Hypoxic-ischemic encephalopathy (23\%), brain tumors (14\%), known epilepsy (10\%), and ischemic stroke (8\%) were the main etiologies. Cessation of RSE was achieved in $82 \%$. Short-term outcome was favorable in $45 \%$, unfavorable in $55 \%$, and $38 \%$ of patients died. Coronary artery disease, brain tumors, metabolic/toxic problems, and cardiopulmonary resuscitation (CPR) had high odds ratios for death. These associations remained significant after multiple adjustments for brain tumors (OR 6.3; $\mathrm{p}=0.004)$ and cardiopulmonary resuscitation (OR 18.3; $<<0.0001$ ), indicating an independence of these associations. Corresponding, the estimated hazard ratio of death for patients with CPR was $3.1(\mathrm{p}=0.001)$ as compared to patients without CPR while it was $1.1(\mathrm{p}=0.745)$ for patients with brain tumors as compared to patients without.

Conclusions: Brain tumors and CPR were distinct and independent associations with death in our cohort of critically ill patients with RSE. Prospective studies are warranted to strengthen our findings and elucidate underlying mechanisms.

\subsection{9 \\ INVESTIGATION FOR INCREASED RISK OF SEIZURES IN POST-SURGICAL ROUX-EN-Y GASTRIC BYPASS PATIENTS}

Scott Spritzer ${ }^{1}$, M. Hoerth ${ }^{1}$, J. Hentz ${ }^{2}$, J. Madura ${ }^{3}$ and J. Drazkowski ${ }^{1}$ $\left({ }^{1}\right.$ Neurology, Mayo Clinic, Scottsdale, AZ; ${ }^{2}$ Biostatistics, Mayo Clinic, Scottsdale, AZ and ${ }^{3}$ General Surgery, Mayo Clinic, Scottsdale, AZ)

Rationale: The rates of gastric bypass procedures have been increasing in the United States due to the increasing prevalence of obesity. Because of this, in recent years, neurologic complications associated with the surgery have been described. These include encephalopathy, myelopathy, and neuropathy. These complications have been felt to be related to nutritional abnormalities. Seizures and epilepsy have been considered as a potential complication, but have not yet been described. Our goal was to determine if there was an increased post-operative incidence of epilepsy, seizures, or abnormal spells in patients who had undergone Roux-en-Y Gastric Bypass surgery at the Mayo Clinic in Arizona (MCA).

Methods: After IRB approval was obtained, we compiled a general list of specific diagnoses that were commonly used within the epilepsy division at MCA to identify seizures, epilepsy, or atypical spells. We then conducted a search of the database involving all patients having undergone Roux-en-Y Gastric Bypass $(\mathrm{N}=608)$ at Mayo Clinic in Arizona between 2001 and 2010 for the ICD-9 codes of interest. Independent chart review of those patients who had received the diagnoses of interest was then conducted to get a detailed history regarding that diagnosis.

Results: Two patients out of 608 had a diagnosis involving one of the nine ICD-9 codes we identified of interest. Both of these patients had a previous diagnosis of seizures prior to their surgery, and chart review of follow-up visits indicated no exacerbation of previously existing seizure disorder. There were no new diagnoses of epilepsy, seizures, or spells in follow up of the post-operative patients over a period of two years.

Conclusions: There was no apparent increase in the incidence of epilepsy, seizures, and/or spells after Roux-en-Y Gastric Bypass. In addition, Roux-en-Ydid not exacerbate pre-existing seizure disorders. Close post-operative follow up to ensure that nutritional and metabolic abnormalities do not occur is beneficial in this vulnerable population. With an appropriate Bariatric Surgery program, the risk of developing epilepsy is not elevated.

\subsection{0}

INFANTILE SPASMS: ELECTROPHYSIOLOGICAL DESCRIPTION AND RELATION WITH PROGNOSIS Yenny C. Zuñiga $^{1,2}$, J. D. Ramos ${ }^{1,2}$, O. M. Espitia ${ }^{1,2}$, N. E. Penagos ${ }^{1,2}$, D. C. Benitez ${ }^{1,2}$, N. A. Pardo ${ }^{1,2}$, A. C. Vargas ${ }^{1,2}$, R. A. Naranjo ${ }^{2}$ and

A. H. Izquierdo ${ }^{1,2}$ ( ${ }^{1}$ Pediatric Neurology, National University of Colombia, Bogotá D.C., Colombia and ${ }^{2}$ Pediatric Neurology, Fundación Hospital de la Misericordia, Bogotá D.C., Colombia) 
Rationale: Infantile Spasms is one of the most frequent epileptic encephalopathy (incidence of 2 to 3 per 10000 live births), most with a catastrophic prognosis that has been associated with hypsarrhythmia in the electroencephalogram (EEG).

Methods: Longitudinal descriptive study. Medical charts of hospitalized infants with diagnosis of symptomatic/idiopathic epilepsy, west syndrome, focal or generalized seizures and epileptic spasms in the Hospital de la Misericordia, a pediatric hospital in Bogota, Colombia, were reviewed from 2007 to 2012. Patients with epileptic spasms clinically observed or by videoEEG were selected. Charts of outpatient follow up were also reviewed. The mean follow up was 7 months ( 1 to 49months) The Kramer scale (1997) was used to score the EEG, and define the hypsarrhythmia and no hypsarrhythmia patterns. The statistic analysis was performed using SPSS17.0.

Results: 251 patients were initially selected. Of them, 39 had epileptic spasms. 4 patients were excluded because of the lack of EEG or videoEEG. 63\% were male, with mean age 7.6 months (121 months). $71 \%$ of patients had another type of seizure, most of them focal $(68 \%)$. The symptomatic etiology was the most frequent with hypoxic ischemic encephalopathy (32\%), malformation of cortical development (28.6\%), other CNS malformations (10.7\%), meningitis $(10.7 \%)$, inborn error of metabolism $7 \%$ (Leigh disease and nonketotic hyperglycinemia) and $10.7 \%$ had symptomatic unknown etiology. Hypsarrhythmia was documented in $57 \%$ (mean score in Kramer scale: 10.8 points vs 5.2 points in patients without hypsarrhythmia). There were no differences between groups in age of onset of spasms (5.7 vs 4.6 - with hypsarrhythmia/without hypsarrhythmia, $\mathrm{p}=\mathrm{NS})$, symptomatic etiology $(75 \% \mathrm{vs} 86 \% \mathrm{p}=\mathrm{NS})$, type of spasms (symmetric $80 \%$ vs $87 \%$, in flexion $60 \%$ vs $66 \%$ ), nor abnormal magnetic resonance imaging ( 75 vs $86 \% \mathrm{p}=\mathrm{NS}$ ). There were no differences in control of spasms and hypsarrhythmia or etiology. The no hypsarrhythmia patterns were paroxysmal delta waves $(80 \%)$, generalized slowing $(73 \%)$, focal spikes $(40 \%)$, multifocal spikes (33\%), interhemyspheric asymmetry (26\%) and normal EEG (7\%). There was significant relation between poor control of spasms and other seizures with focal spikes in EEG (uncontrolled spasms with focal spikes $83.3 \%$ vs $16 \%$; uncontrolled seizures $100 \%$ vs $0 \%, \mathrm{p}=0.05$ ).

Conclusions: In this study there was no relation between neurological outcome, seizures control and hypsarrhythmia. However, we report an association between other EEG patterns and control of spasms as well as other seizures. It is very important to describe that in these patients the infantile spasms are an encephalopathy with neurological prognosis and control of seizures influenced also by EEG patterns other than hypsarrhythmia. So, we should focus the treatment on control of spasms, hypsarrhythmia and other epileptic discharges too. Although it is necessary include the patients in a prospective study.

\subsection{1 \\ ARE PATIENTS WITH POSTICTAL PSYCHIATRIC EPISODES AT INCREASED RISK FOR DEVELOPING PSYCHIATRIC CO-MORBIDITIES AFTER EPILEPSY SURGERY?

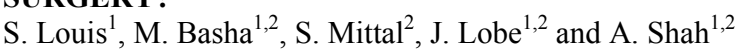 \\ $\left({ }^{1}\right.$ Neurology, DMC/wayne state university, Detroit, MI and \\ ${ }^{2}$ Neurosurgery, DMC/wayne state university, Detroit, MI)}

Rationale: Postictal psychosis (PIP) and postictal affective episodes (PAE) are psychiatric manifestations that arise within one week after a seizure, last from hours to days, and occur after a lucid interval. It is unclear how epilepsy surgery will alter the unknown underlying etiology of this postictal state, however, psychosis and de novo postictal psychiatric symptoms have been reported after epilepsy surgery. There is no known reported psychiatric outcome in patients with postictal psychiatric symptoms who undergo epilepsy surgery. We evaluate effect of epilepsy surgery on the postictal psychiatric state.

Methods: 90 adults with intractable epilepsy underwent epilepsy resective surgery between 2007 and 2011.29 patient had extra temporal lesion, 23 patient had right temporal lesion and 38 patient had left temporal lesion. Three of the 90 patient (3.3\%) were identified as having postictal psychiatric symptoms prior to surgery, all of which had left MTS. Language dominance was left in 2 of them and bilateral in one as determined by intravenous sodium amobarbital test. All three patients had left temporal lobe epilepsy, with clinical manifestation of complex partial seizures with secondary generalization. Postictal symptoms were psychotic in two patients and affective in one. Symptom onset ranged from two to 24 hours after the seizures.

Results: Three patients with postictal psychiatric symptoms (2 with PIP, and 1 with PAE) underwent epilepsy surgery with removal of left temporal lobe with Engle Class I seizure control in all patients. None of the three patients developed psychiatric symptoms postoperatively.

Conclusions: Having preoperative postictal psychiatric symptoms did not result in poor psychiatric outcome after left temporal lobe resection.

\subsection{2 \\ EPIDEMIOLOGY, AETHIOLOGY AND TREATMENT OUTCOMES OF STATUS EPILEPTICUS IN LITHUANIA Ruta Mameniskiene ${ }^{1,2}$, A. Jasionis ${ }^{1}$ and V. Budrys ${ }^{1,2}\left({ }^{1}\right.$ Clinic of Neurology and Neurosurgery, Faculty of Medicine, Vilnius University, Vilnius, Lithuania and ${ }^{2}$ Epilepsy Centre, Department of Neurology, Vilnius University Hospital "Santariskiu klinikos", Vilnius, Lithuania)}

Rationale: Although status epilepticus (SE) is an urgent, lifethreatening condition, studies concerning this topic are scarce, especially in countries, where different composition of SE causes is observed and different treatment guidelines are used. The goal of our study was to assess the causes, epidemiological rates (incidence and mortality) and treatment outcomes (case fatality, duration of SE and hospitalization) of SE in Lithuania - the country with gene-pool homogeneity.

Methods: We collected all case histories of patients, who experienced SE between 2005-2009 and were admitted to Vilnius hospitals with intensive care unit. Data regarding sociodemographic variables, previous medical history and concomittant treatment were collected. Cause, type and duration of SE, latency from onset to treatment start, diagnostic procedures $(\mathrm{CT}, \mathrm{EEG}$, biochemistry at the time of admission), treatment (medications, dosage, other measures) and outcomes (fatality, duration of hospitalization)were assessed. We used Student's T, Mann-Whitney U, binomial,chi square, Pearson's and Spearman's correlation tests for our statistical analysis.

Results: We studied 591 SE cases with clear male predominance $(67.5 \%)$. The age of patients ranged from 18 to 106 years (mean $52.75 \pm 16.05)$. Metabolic cause of SE was found in $244(41.29 \%)$ and structural CNS lesions in $143(24.2 \%)$ patients. 402 had epilepsy before (group SE1) and 189 - had not (SE2).The incidence of SE was 21.66 cases per $100^{\circ} 000$ per year. SE2 patients were older $(\mathrm{t}=-3.78$; $\mathrm{p}<0.001)$. Mean duration of SE was $8.34 \pm 29.9 \mathrm{~h}$. Average duration of hospitalization was $6.75 \pm 4.87$ days. SE caused by structural CNS lesions lasted longer while caused by metabolic factors - shorter. Mean latency from onset to treatment start was $2.73 \pm 9.52 \mathrm{~h}$.

Furthermore, it was longer for older patients and people with epilepsy. We found strong correlation between duration of SE and: a) time to treatment initiation $(\mathrm{r}=0.552 ; \mathrm{p}<0.001), \mathrm{b})$ duration of hospitalization $(\mathrm{r}=0.313 ; \mathrm{p}<0.001)$, c) blood glucose $(\rho=0.11$; $\mathrm{p}=0.007)$ and potassium $(\rho=-0.09 ; \mathrm{p}=0.03)$ levels. Mortality rate was $1.6 / 100^{\circ} 000 / \mathrm{yr}$, case fatality $-7.1 \%$. Higher fatality was among older patients $(t=3.79 ; \mathrm{p}<0.001)$, SE2 $(\chi 2=15.77 ; \mathrm{p}<0.001)$ and those with structural SE cause $(\chi 2=72.88 ; \mathrm{p}<0.001)$. Those with metabolic SE cause had better outcomes $(\chi 2=21.74 ; p<0.001)$. Death rate was 
associated with treatment delay $(\mathrm{Z}=-1.94 ; \mathrm{p}=0.05)$. Duration of hospitalization correlated with patient's age $(r=0.215 ; \mathrm{p}<0.001)$ and was longer for structural SE $(Z=-2.33 ; p=0.02)$.

Conclusions: Status epilepticus can be terminated successfully and with low in-hospital mortality in the vast majority of the patients. Delayed initiation of treatment determine longer duration of SE and hospitalization as well as higher fatality rate. Patients without epilepsy history who developed SE have higher fatality rate.

\subsection{3}

\section{THE CLINICAL CHARACTERISTICS OF CRYPTOGENIC NORSE (NEW-ONSET REFRACTORY STATUS EPILEPTICUS) SYNDROME}

Bomi Kim and S. Lee (Department of Neurology, Asan Medical Center, University Of Ulsan College Of Medicine, Seoul, Republic of Korea)

Rationale: Refractory status epilepticus is a life-threatening condition, which is characterized by the failure to respond to firstand second-line anticonvulsant therapy. The patients who previouslyhealthy adult with refractory status epilepticus as the first symptom is called new-onset refractory status epilepticus syndrome(NORSE syndrome). The etiology of NORSE syndrome include infection of central nervous system, metabolic encephalopathy and hypoxia. In some cases of these, the etiology is not found despite of extensive evaluation. It is called cryptogenic NORSE syndrome. We describe the clinical characteristics of our 11 patients with cryptogenic NORSE syndrome.

Methods: Patients admitted to the neurological intensive care unit at Asan medical center for management of status epilepticus which was diagnosed by clinical history and electroencephalogram(EEG) between Jun2006 and Mar 2012 were eligible for inclusion. We included the patients who has highly refractory to lorazepam, intravenous phenytoin(initial bolus of $20 \mathrm{mg}$ per kilogram). They also has no clear cause of status epilepticus that proved by the previous medical history, CSF analysis, PCR of viral marker, antigen to parasite, autoimmune antibody, thyroid function test, serum glucose, chemical battery, electrolyte battery and imaging study including MRI or CT(Table 1). Also the patients with CSF leukocytosis above 10 were not included to except the meningoencephalitis.

Results: A total of 11 patients ( 7 male, 4 female) were included. The mean age of onset was 29.8 years(range 19 to 49 years). All of them has no previous medical problem and risk factor of epilepsy. Antecedent febrile illness was observed in 8 patients(72.7\%). But Fever was not observed at the admission and the abnormality of general and cerebral inflammatory marker were not found. All of them presented with convulsive status epilepticus. Multifocal or generalized seizure activity were detected on EEG. Abnormal findings were observed on 4 patients, all of them were associated with ictal discharge on EEG. For the control of seizure, intravenous anesthetic agent was used in all patients. Two of them treated by inhalational anesthetic agent to control seizure. The mean duration of stay in the intensive care unit was 61 days (range 2-175 days). The 4 patients $(36.4 \%$ ) died on 2 to 20 days after intravenous phentobarbital therapy. And the others survived with frequent seizures with more than 3 anti-epileptic drugs and cognitive impairment, except 1 patient (Table 2).

Conclusions: In this study, the characteristics of cryptogenic NORSE syndrome were young age, previous good health, antecedent febrile illness, extraordinarily prolonged status epilepticus and catastrophic outcome like other literatures. But male was more prominent in gender compared with other literatures. So further study of this poorly understood form of status epilepticus and aggressive management are needed.
Table1. Diagnostic investigations considered in patients

\begin{tabular}{|c|c|}
\hline Screen & Disease or agent \\
\hline Infectious & $\begin{array}{c}\text { HSV I, HSV } \Perp, \text { VZV, HZV, EBV, CMV, Enterovirus, Mycoplasma peumoniae, HIV, } \\
\text { VDRT, Mycobacterium TB, Cryptococcus, } A \mathrm{DA} \\
\text { CSF AFB, Fungus culture, Gram staining and culture, parasite(Cysticcrcus, } \\
\text { Paragnimus, Sparganum, Clonorchis) }\end{array}$ \\
\hline Immunology & ANA, ANCA, Lupus anticoagulant, Anti-SSA $/ S S B$, Anti-dsDNA, Kheumatoid factor \\
\hline $\begin{array}{l}\text { Metabolic \& } \\
\text { Hematologic }\end{array}$ & $\begin{array}{l}\text { CBC, LFT, BCN, Creatinine, electrolyte, } \mathrm{CA} / \mathrm{Mg} \text {, lactate level, thyroid function, } \\
\text { glucose lcvel }\end{array}$ \\
\hline
\end{tabular}

Table2. Management and Clinical outcome, PHT phenytoin; VPA valproate; PHB phenobarbital; TPM topiramate; LEV levetiracetam

\begin{tabular}{|c|c|c|c|c|}
\hline Patient & Anti-epileptic drug & Anesthetic agent & $\begin{array}{l}\text { Stay in } \\
\text { ICU }\end{array}$ & Outcome \\
\hline 1 & PHT, VPA, I.FV & phentobarbitalpropofol & 20 & Death \\
\hline 2 & $\begin{array}{l}\text { PIIT, VPA, PIIB, } \\
\text { LEV }\end{array}$ & Phentobarbital, isollurane & 2 & Death \\
\hline 3 & PHI, VPA, LEV & phentobarbitalmidazolam & 175 & $\begin{array}{l}\text { Frequent seizure } \\
\text { Congnitive impairment }\end{array}$ \\
\hline 4 & PIT, VPA, PIIB & phentobarbital & 134 & $\begin{array}{c}\text { Trequent seizure } \\
\text { Congnitive impairment }\end{array}$ \\
\hline 5 & $\begin{array}{l}\text { PHI, VPA, PHB, } \\
\text { IPM, LEV }\end{array}$ & phentobarbitalmidazolam & 107 & $\begin{array}{l}\text { Frequent seizure } \\
\text { Congnitive impairment }\end{array}$ \\
\hline 6 & $\begin{array}{l}\text { PHT,VPA, PHB, } \\
\text { TPM }\end{array}$ & phentobarhitalmidazolam & 79 & $\begin{array}{l}\text { Trequent scizure } \\
\text { Congnitive impairment }\end{array}$ \\
\hline 7 & $\begin{array}{l}\text { PIIT, VPA, PIIB, } \\
\text { TPM }\end{array}$ & phentobarbitalmidazolam & 39 & $\begin{array}{c}\text { Frequent seizure } \\
\text { Congnitive impairment }\end{array}$ \\
\hline 8 & $\begin{array}{l}\text { PHI, VPA, P'HB, } \\
\text { TPM, I.TV }\end{array}$ & phentobarbital & 14 & Seizure frec \\
\hline 9 & PIIT, VP $\Lambda$, PIIB & phentobarbitalmidazolam & 3 & Death \\
\hline 10 & P'HI, VI'A, P'HB & $\begin{array}{c}\text { phentobarbilalmidazolam, } \\
\text { isoflurane }\end{array}$ & 29 & Death \\
\hline 11 & $\begin{array}{l}\text { PHT, VPA, PHB, } \\
\text { TPM, LTV }\end{array}$ & propofol & 74 & $\begin{array}{c}\text { Frequent seizure } \\
\text { Congnitive impairment }\end{array}$ \\
\hline
\end{tabular}

\subsection{4 \\ LONG TERM OUTCOMES OF INFANTILE SPASMS IN KOREA}

Jeong A Kim, J. Kang, Y. Lee, H. Kang, H. Kim and J. Lee (Department of Pediatric Neurology, Severance Children's Hospital, Yonsei University College of Medicine, Seoul, Korea, shinchondong, seodaemun gu, Republic of Korea)

Rationale: Infantile spasms is severe form of epileptic syndrome characterized by specific semiology, clustered spasms and electroencephalography patterns so called hypsarrhythmia. The prognosis of this disease is poor despite treatment. $70-80 \%$ of patients are mentally retarded and $23-54 \%$ of this disease evolves to Lennox-Gastaut syndrome. The aims of this study is to investigate the long term outcomes of infantile spasms.

Methods: Of 356 patients who were diagnosed with infantile spasms at Severance children's hospital, Seoul, Korea, from 2006 to 2011, 98 patients with 5-year follow-up were qualified for evlauation.

Retrospective electronic medical records review were conducted of 98 patients. Data were analyzed using IBM SPSS stastics version 19. Results: There were 39 female (39.8\%) and 59 male (60.2\%) patients. Mean age at the start of spasms was $7.82( \pm 5.68)$ month old. During 5-year follow-up, infantile spasms evoled to Lennox-Gastaut syndrome in 28 patients $(28.5 \%)$, at the age of evolving state was $37.79( \pm 17.71)$ month old. The proportion of seizure freedom in 5 year follow-up was 66 of $98(67.3 \%)$, and 32 of 98 patients couldn't achieve seizure freedom $(32.7 \%)$.

We applyed ketogenic diet treatment in 51 patients (52.0\%), epileptic surgery in 13 patients ( 9 total corpus callosotomies, 3 lobectomies, 1 quadrantectomy, $12.2 \%)$, steroid therapies in 35 patients $(35.7 \%)$. In 
5-year seizure free group, 39 of 63 patients $(61.9 \%)$ achieved seizure freedom on antiepileptic drug medication alone. 23 of 63 patients (36.5\%) received ketogenic diet and medication treatment. 4 epileptic surgery $(6.0 \%)$ were performed. In 5-year non-seizure free group, only 9 of $32(28.1 \%)$ received medication treatment alone, 14 of 32 $(43.7 \%)$ treated with medication and ketogenic diet, 9 of 32 patient $(28.1 \%)$ received epileptic surgery. There were significant stastical differences in proportion of treatment modlaities between two group $(p=0.02)$ but no definite stastical differences between seizure free and non-seizure free group in the etiology $(\mathrm{p}=0.3)$.

Conclusions: In this study, we reported our treatment results in infantile spasms. We can understand that differences of the proportion of treatment modalities between two groups. But there were no significant differences in etiology between two groups. This is priliminary date, so we need further researches about outcomes of infantile spasms.

\section{Neuro Imaging: Animal Studies}

\subsection{5}

SUB-SURFACE, FEMTOSECOND LASER INCISIONS AS A THERAPY FOR PARTIAL EPILEPSY

Robert N. Fetcho ${ }^{1}$, J. Nguyen ${ }^{1}$, M. Zhao ${ }^{2}$, N. Nishimura ${ }^{1}$, T. H. Schwartz ${ }^{2}$ and C. B. Schaffer ${ }^{1}\left({ }^{1}\right.$ Department of Biomedical Engineering, Cornell University, Ithaca, NY and ${ }^{2}$ Department Neurology and Neuroscience, Weill Cornell Medical College, New York, NY)

Rationale: For many patients, focally initiated cortical epilepsy is largely intractable, leaving surgical treatment as a last option. Resection of the seizure focus will stop seizures but frequently leads to other neurological deficits such as stroke. A less invasive technique, multiple subpial transections (MST), uses a mechanical hook to produce a series of incisions in the epileptic tissue, with the goal of disrupting neural connections that facilitate seizure propagation. This procedure is performed manually and, as a result, cuts are difficult to control and can often lead to collateral damage. A more controlled and reliable solution to preventing seizure propagation is desired in order to surgically treat focal epilepsy with few undesired consequences. The development of femtosecond laser technology allows for extremely precise and localized cuts below the cortical surface, all while producing minimal collateral damage. We propose the use of femtosecond laser pulses as a light scalpel to produce incisions around the epileptic focus and stop seizure propagation.

Methods: We use a rat model of focal epilepsy in which 4aminopyridine (4- AP), a seizure-inducing drug, is locally injected into the cortical tissue. First, femtosecond laser pulses are tightly focused into the brain to produce multiple 750 by $750 \mu \mathrm{m}$ box cuts at depths ranging from $200 \mu \mathrm{m}$ to $800 \mu \mathrm{m}$ below the brain surface. Through a glass micropipette, 4-AP is injected within the box of cuts to induce seizures and record local field potential (LFP), while another distant electrode is implanted outside the box to record LFP. If the laser cuts are effective, we expect seizure activity to be contained to the focal region within the cuts and not propagate outwards to the distant electrode.

Results: In sham experiments where no box cuts were made, seizures were seen to propagate to the distant electrode $100 \%$ of the time $(\mathrm{n}=$ 2 rats; 18 seizures). When box cuts were produced, the number of seizures seen to propagate was significantly reduced, with approximately $54 \%$ of seizures reaching the distant electrode $(n=13$ rats; 90 seizures; $p<0.0005$ ). Additionally, in those seizures that continued to propagate after cuts, we observed a significant delay in arrival of seizure activity at the distant electrode. For sham experiments, mean seizure delay was found to be $0.3390 \pm 0.0875$ seconds $(\mathrm{n}=15$ seizures). Cuts increased the mean delay of seizure onset at the distant electrode to $9.505 \pm 1.985$ seconds $(n=49$ seizures; $\mathrm{p}<0.02$ ). This indicates that cuts that did not completely block seizure propagation were still able to delay the spread of activity beyond the focus.

Conclusions: This work provides evidence that femtosecond laser incisions are capable of blocking seizure propagation beyond a focal region. Preliminary evidence also suggests that these cuts do not disrupt normal neural functionality. While much work remains, these results provide strong initial support for the potential of femtosecond laser incisions as a precise and controlled therapy for partial epilepsy.

\subsection{6 \\ IN VIVO SIMULTANEOUS CALCIUM AND INTRINSIC SIGNAL IMAGING OF 4-AP ICTAL AND INTERICTAL EVENTS DEMOSNTRATE DYNAMIC NEUROVASCULAR COUPLING DURING ICTAL BUT NOT INTERICTAL EVENTS}

Hongtao Ma, S. Harris, M. Zhao and T. H. Schwartz (Cornell

University Weill Medical College, Forest Hills, NY)

Rationale: The traditional models of ictal propagation involve the concept of an initiation site and a progressive outward march of activation. Using voltage sensitive dyes, we have shown widespread propagation of excitatory subthreshold membrane potential change from the onset of the seizure, however, suprathreshold activation (action potentials) were not recorded.

Methods: We now used bulk loading of Oregon Green 488 BAPTA1 , a calcium dye, to image the propagation of calcium spikes during this same model, namely 4 -aminopyridine (4-AP, $15 \mathrm{mM}, 0.5 \mu \mathrm{l}$ ) induced focal neocortical seizures. Adult SD rats were anaesthetized with $1.5-2.5 \%$ isoflurane in $30 \% \mathrm{~N} 2-70 \% \mathrm{O} 2$. A craniotomy window was created over the left hemisphere. Calcium dye was injected into the cortex at multiple sites, avoiding major blood vessels. The brain was alternatively illuminated with $530 \mathrm{~nm}$ (intrinsic optical imaging for local blood volume change) and 470nm (excitation for Calcium dye) at 200 frames/sec. The reflection and florescence change was recorded with a CCD camera in Imager 3001.

Results: Interictal events exhibit widespread and transient simultaneous calcium increases. Ictal events, on the other hand, rapidly propagate to a similarly widespread area at the onset of the seizure. As the seizure evolves, a rapid oscillation of intracellular calcium occurs initially over a widespread area which then contracts over the course of the seizure. The local blood volume change shows a similar monophasic increase in the whole field of view and does not oscillate like the calcium waves. Local blood volume increases last much longer than the calcium signal recorded from the same location. Conclusions: Our data showed that the calcium oscillations and blood volume changes are two separate but parallel behaviors of neuronal tissue during epileptiform activity further illustrating dynamic neurovascular coupling during the ictal event.

\subsection{7 \\ OPTICAL COHERENCE TOMOGRAPHY DETECTS CORTICAL OPTICAL CHANGES ASSOCIATED WITH PTZ- INDUCED SEIZURE ACTIVITY \\ Devin K. Binder ${ }^{1}$, M. M. Eberle ${ }^{2}$, C. L. Reynolds ${ }^{2}$, J. I. Szu ${ }^{1}$, Y. $\mathrm{Wang}^{2}$, M. S. Hsu ${ }^{1}$ and B. H. Park ${ }^{2}{ }^{1}$ Biomedical Sciences, University of California Riverside, Riverside, CA and ${ }^{2}$ Bioengineering, University of California Riverside, Riverside, CA)}

Rationale: Previous research has suggested that optical changes, specifically changes in near-infrared optical scattering, may precede electrographic seizure onset in in vivo models. However, previous techniques have required craniectomy for direct cortical imaging or the use of invasive implantable fiberoptic probes. In this work, we describe the first adaptation of in vivo optical coherence tomography (OCT) imaging through a thinned-skull preparation to optically assess rodent cortical seizures. 
Methods: 6-8 week old mice were anesthetized with ketamine/xylazine and a $4 \times 4 \mathrm{~mm}$ thinned-skull cortical window was created with a dental bur over the cerebral cortex. The cerebral cortex was then imaged continuously with our OCT system. The spectral domain OCT system we developed contains a broadband, lowcoherence light source consisting of two superluminescent diodes (SLD) centered at $1295 \mathrm{~nm}$ with a bandwidth of $97 \mathrm{~nm}$ resulting in an axial and lateral resolution of $8 \mu \mathrm{m}$ and $20 \mu \mathrm{m}$ respectively. The brain was continuously scanned creating a $3 \mathrm{~mm}$ imaging plane with an imaging depth of $2 \mathrm{~mm}$. Axial line scans (A-lines) were acquired at $15 \mathrm{kHz}$ and each cross-sectional area consisted of 2048 A-lines. A 10 min. imaging baseline was collected before a saline injection was administered. After 35 to $40 \mathrm{~min}$, pentylenetetrazol (PTZ)

$(100 \mathrm{mg} / \mathrm{kg}$, i.p.) was injected to induce cortical seizure activity. Mice were observed for whisker twitches/myoclonic jerks and until a stage 5 seizure occurred with fore- and hindlimb clonus.

Results: To analyze the changes in backscatter intensity, we calculated average intensities from cortical regions of interest (ROIs) over time. Two controls were used: the first was a 10 min baseline before any injection was administered to assess optical stability and the second was an injection of saline with a volume equivalent to the PTZ dosage. We observed no significant change in intensity $(95 \%$ confidence interval) from baseline after saline injection. However, in all experiments $(n=4)$, decrease in backscattered intensity after PTZ injection was observed. Latency to two standard-deviation decrease in optical scattering (empirically defined as optical "threshold") occurred $<10$ min after PTZ injection, whereas latency to whisker twitches/myoclonic jerks was $>10$ min and latency to generalized seizure activity was $>20$ min.

Conclusions: We have adapted the technique of OCT for in vivo imaging of the rodent cortex before and during PTZ-induced seizure activity. Stable baseline images through the thinned-skull preparation were uniformly obtained, and no significant changes were seen following saline injection. Reduction in backscatter intensity was observed following PTZ injection but prior to generalized seizure activity. These results provide proof-of-principle for the use of OCT through a thinned-skull preparation to monitor cortical optical changes with high spatial and temporal resolution in vivo.

\subsection{8}

MULTIPHOTON IMAGING OF NEURONAL PROJECTIONS DEEP WITHIN THE INTACT MOUSE HIPPOCAMPUS

Bethany E. Hosford ${ }^{1,2}$, J. M. Krofon ${ }^{2}$ and S. C. Danzer ${ }^{1,2}$ ( ${ }^{1}$ University of Cincinnati, Cincinnati, $\mathrm{OH}$ and ${ }^{2}$ Cincinnati Children's Hospital

Medical Center, Cincinnati, $\mathrm{OH}$ )

Rationale: Temporal lobe epilepsy is associated with a variety of structural abnormalities of the hippocampus. The organization of the hippocampus, with distinct lamina, compact cell layers, and welldefined fiber pathways, has made identifying and characterizing structural changes in this region a relatively easy task for the neuroanatomist. One aspect of hippocampal anatomy that has continued to be less tractable, however, has been assessing the extensive longitudinal fiber projections of some important cell types, such as mossy cells. Traditional methods for examining hippocampal structure require sectioning the hippocampus in a plane perpendicular to the longitudinal axis. While this is ideal for revealing the hippocampal principle cell layers, it unavoidably severs the longitudinal fibers. Here we describe a protocol which allows researchers to image deep within the intact hippocampus at micronlevel resolution, thereby allowing for analysis of fibers traversing all hippocampal axes.

Methods: C57/Blk6 transgenic mice expressing Thy1-GFP were treated with pilocarpine to induce status epilepticus. At various time points after status epilepticus treatment, animals were sacrificed and whole brains were excised, paraformaldehyde fixed and placed in the newly developed SCALE tissue clearing reagent (Nature

Neuroscience 2011, 14(11): 1481-88). Hippocampi were dissected to improve clearing efficiency. Two-six weeks later, hippocampi were imaged using a Nikon A1R MP upright confocal microscope in both single and multi-photon modes. Imaris Imaging software was used for data analysis and quantification.

Results: Within two-weeks, samples were sufficiently cleared for deep imaging. Endogenous GFP expression was not adversely affected. This methodology allowed us to image all the way through the hippocampus, from granule cells at the interior surface of the hippocampus, to CA1 stratum oriens at the exterior surface, a depth of greater than $1.0 \mathrm{~mm}$. From this data, neurons from all hippocampal axes may be reconstructed with micron-level precision allowing for quantification of cell density, spine number, mossy fiber sprouting, and other potentially important structural changes that have been observed during epileptogenesis.

Conclusions: While the numerous anatomical changes already described in the epileptic hippocampus are undeniably important, there may be significant - yet to be discovered - changes among longitudinal and laminar circuits that have resisted identification with traditional methods. With sufficient computing power, it should be possible to reconstruct the entire hippocampus with micron-level resolution. This approach should greatly facilitate assessing longprojection circuit changes in temporal lobe epilepsy.

\section{Neuro Imaging: Structural Imaging}

\subsection{9}

EVIDENCE OF MORPHOLOGIC DIFFERENCES IN THE HIPPOCAMPI OF CHILDREN WITH DOWN SYNDROME WHO DEVELOP INFANTILE SPASMS

Nicholas Phillips $^{1,2}$, A. Choudhri ${ }^{1,3}$, J. Wheless ${ }^{1,2}$, N. Shah ${ }^{1,2}$ and Z. Patay ${ }^{4}\left({ }^{1}\right.$ University of Tennessee Health Science Center, Memphis, TN; ${ }^{2}$ Child Neurology, Le Bonheur Children's Hosptial, Memphis, $\mathrm{TN} ;{ }^{3}$ Neuroradiology, Le Bonheur Children's Hospital, Memphis, TN and ${ }^{4}$ Neuroradiology, St. Jude Children's Research Hospital, Memphis, TN)

Rationale: Down syndrome (DS) is the most common genetic cause of mental retardation. The prevalence of epilepsy in children with DS is higher than the general population, but lower than in other causes of mental retardation. Seizure onset is reported to occur within a year of birth in $40 \%$ of epileptic DS. A majority of these patients will suffer from Infantile Spasms (IS). Often IS with DS can be attributed to secondary lesions however many lack any identifiable risk factors. Most neuroimaging studies have demonstrated global brain volume reduction and disproportionate reduction in the cerebellum, brainstem, frontal lobe and hippocampus. Interestingly, among mental retardation syndromes, only DS exhibits hippocampal dysfuntion. Additionally, studies of DS children, demonstrate that these low hippocampal volumes are not attributed to neurodegenerative changes, but likely are due to early developmental differences. However no definitive study of hippocampal structures of DS with IS patients has been conducted to date. We hypothesized that DS with IS patients would have abnormal hippocampal volumes and morphology compared to IS patients without DS.

Methods: An IRB approved retrospective cohort study was conducted to evaluate hippocampal rotation and volumes in patient with DS with IS, and symptomatic and cryptogenic IS without DS. Controls were matched for age and gender. Axis of rotation and volume was assessed on coronal thin section STIR, with secondary confirmation using sagittal T1 and coronal FLAIR by a board certified pediatric neuroradiologist blinded to the diagnosis. Volume loss and axis of rotation were scored in using a 4 point Likert scale $(0$ normal, 1 mild, 2 moderate, 3 severe). Intrasubject asymmetry in shape, volume and signal intensity was also evaluated. Continuous variables were compared with a 2-tailed t-test. Discrete variables were compared using a Fisher's exact test.

Results: Twelve subjects were included in this study. Six patients had diagnosis of DS with IS (age 1.07 years +/- 0.65) and six 
patients had a diagnosis of IS without DS (age 1.05 years +/- 1.00). Four IS without DS patients were classified as cryptogenic and two as symptomatic. Each group contained 3 males and 3 females. Hippocampal volumes were significantly lower in the DS with IS group $(\mathrm{p}=0.0046)$. And hippocampal malrotation was more likely to be present in DS with IS group ( $\mathrm{p}=0.0361$ ).

Conclusions: This study reviewed the hippocampal structures of 12 subjects diagnosed with Infantile Spasms. We were able to demonstrate in this retrospective cohort study, that children with Down Syndrome and Infantile Spasms have morphologicially different hippocampi compared to children with Infantile Spasms without Down Syndrome. These findings may be a demonstration of reported immunohistological evidence of pathologic changes in the GABAergic interneurons in all hippocampal subregions in Down Syndrome patients who suffer from Infantile Spasms.

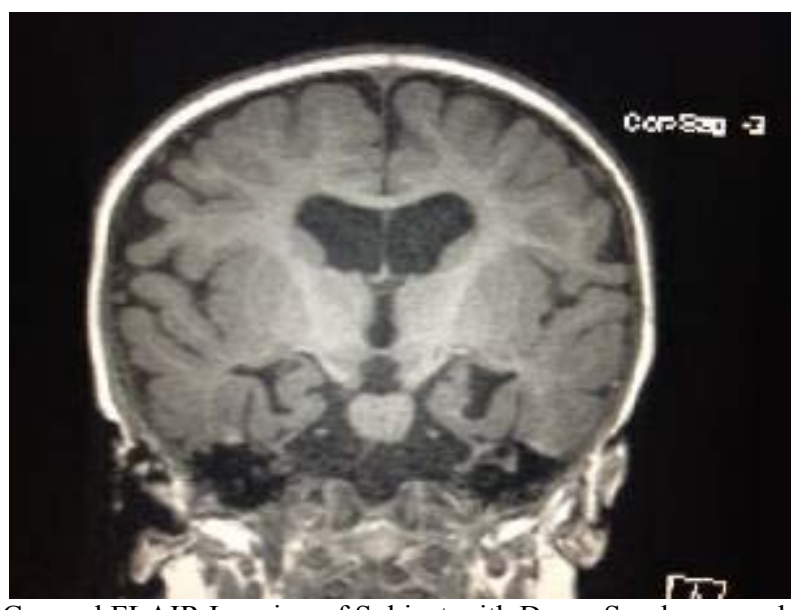

Coronal FLAIR Imaging of Subject with Down Syndrome and Infantile Spasms, Demonstrating Hippocampal Malrotation.

\subsection{0 \\ IMAGING GRAY MATTER MICROSTRUCTURE IN VIVO PREDICTS NEUROPATHOLOGY IN EPILEPSY}

Jenifer Juranek $^{1}$, M. B. Bhattacharjee ${ }^{2}$, J. E. Lankford ${ }^{1}$ and G. Von Allmen ${ }^{1}$ ( ${ }^{1}$ Pediatrics, UT- Health, Houston, TX and ${ }^{2}$ Pathology \& Laboratory Medicine, UT Health, Houston, TX)

Rationale: The objective of our research is to evaluate non-invasive Diffusion Tensor Imaging (DTI) for probing gray matter (GM) microstructure to reliably and accurately delineate malformations of cortical development (MCDs) in children with intractable seizures. Previous studies have shown that the most consistent predictor of seizure-free outcome is complete resection of aberrantly developed cortical architecture, cytoarchitecture, and myeloarchitecture underlying epileptiform activity. Thus, non-invasive procedures which reliably and accurately delineate epileptogenic MCDs for surgical resection have the potential to significantly reduce the devastating effects of chronic seizures on neurodevelopment in young children.

Methods: Prior to resective surgery, a 3T GE Signa MRI scanner was used to acquire high-resolution structural images with a $3 \mathrm{~d}-$ T1weighted sequence. In the same imaging session, a 21-direction DTI dataset was also acquired. FSL, a freely available software package, was used for conducting quantitative analyses of macro- and micro-structure throughout the cerebral cortex. Following resective surgery, neuropathology of the resected tissue was determined using standard histological procedures. Neuroimaging analyses were conducted blind to all clinical data.

Results: In our preliminary case series $(n=3)$, distinctive features of DTI metrics were observed in cortical GM regions of each research subject. Case \#1 exhibited elevated MD values ( 160\%) ipsilateral to the seizure focus in left orbitofrontal GM (1447 x10-6 mm2/s) compared to values in right orbitofrontal GM (891 x10-6 mm2/s). FA values in the same GM regions were modestly elevated $\sim 17 \%$ in the left relative to the right hemisphere. In Case \#2, elevated FA values were observed ipsilateral to the seizure focus in frontal (LH: $0.56+/-$ 0.06; RH: $0.36+/-0.05$ ) and temporal (LH: $0.51+/-0.05$; RH: 0.30 $+/-0.03)$ GM regions. MD values were not different between the same homologous regions. In Case \#3, elevated MD values were observed ipsilateral to the seizure focus in temporal GM (RH: 1169 $\mathrm{x} 10-6 \mathrm{~mm} 2 / \mathrm{s}$; LH: $1024 \times 10-6 \mathrm{~mm} 2 / \mathrm{s})$. FA values in the same GM regions were not different. Neuropathology reported that Chaslin's gliosis and variable cortical and white matter gliosis is present in all three brain resections; another common feature in all three is increased white matter neurons, singly dispersed. The left frontal lobe (\#1) cortical dysplasia lacks balloon cells, or giant cytomegalic neurons. The left temporo-parietal resection (\#2) has similar features. The right temporal lobe (\#3) also shows similar cortical dysplasia; the right hippocampal resection has atypical Ammon's horn sclerosis. Conclusions: All 3 cases converged on a common neuropathological finding (cortical dysplasia). However, DTI findings varied across subjects, thus providing additional information about GM microstructure which could be useful. Additional cases will provide us with the opportunity to determine the sensitivity and specificity of DTI metrics in GM to inform us about MCDs, including their extent, boundaries, and connectivity with other GM regions.

\subsection{1}

\section{THE COMPLEMENTARY ROLE OF MRI IMAGING AND} EEG IN FIRST SEIZURE AND NEW ONSET EPILEPSY Candice E. Crocker ${ }^{1}$, M. H. Schmidt ${ }^{2}$, K. T. Legg ${ }^{1}$, M. Blanchard ${ }^{2}$ and B. Pohlmann-Eden ${ }^{1,3}\left({ }^{1}\right.$ Neurology, Dalhousie University, Halifax, NS, Canada; ${ }^{2}$ Radiology, Dalhousie University, Halifax, NS, Canada and ${ }^{3}$ Pharmacology, Dalhousie University, Halifax, NS, Canada)

Rationale: Algorithms for timely diagnosis of a first seizure (FS) require a comprehensive clinical history, standard diagnostic testing with computerized tomography (CT), subsequent MRI imaging, routine electroencephalography (EEG) and sleep-deprived EEG. MRI and EEG are widely accepted as gold standards in investigation of all stages of epilepsy including FS presentations. Currently, we do not know the exact correlation between pathological findings on MRI and those identified on EEG.

Methods: We retrospectively collected the MRI imaging and EEG data on 172 adult patients with confirmed seizures seen from May, 2008 to August, 2011 at the Adult First Seizure Clinic at the QE II Health Sciences Center, Dalhousie University (Halifax, Nova Scotia, Canada). The patients that had a confirmed seizure were given a diagnosis category of First Seizure (FS), if the index seizure was their only seizure. Alternatively, the patients were classed as New Onset Epilepsy (NOE) if the patient had had more than one seizure in the past 12 months; or Newly Diagnosed Epilepsy (NDE) if the patient had more than one seizure in a greater than 12 month time frame. EEG recordings were performed on a Stellate Harmony digital EEG system. MRI imaging was performed according to a specialized epilepsy protocol on a $1.5 \mathrm{~T}$ GE Scanner.

Results: $51 \%$ ( 87 of 172) of patients were FS, NOE was diagnosed in $41 \%$ (71 of 172), and NDE in 8\% (14 of 172). We focused on patients who had undergone both an EEG and MRI (142 of 172). Almost a third (32\%) of the patients presented with MRI findings which we considered clinically relevant (46 of 142). Similarly, 33\% of the patients had abnormal EEG findings (47 of 142), out of which 15 were found to have generalized epileptiform discharges (GED). Interestingly, only $36 \%$ of patients with abnormal EEG findings also had an abnormal MRI ( $64 \%$ had a normal MRI), conversely $39 \%$ of the MRI positive patients had abnormal EEG findings (61\% had a normal EEG). Only $12 \%$ of all patients showed pathologies on both modalities, MRI and EEG. 4 out of the 15 patients with GED had a pathological MRI. Statistical analysis over the whole group of this data ( $2 \times 2$ contingency table) showed that MRI findings and EEG findings were not linked $(\mathrm{P}=0.497)$. 
Conclusions: Our results suggest that MRI and EEG gave a similar percentage of diagnostic yield in identification of underlying morphological or functional pathology in patients presenting with FS, NOE or NDE. The significant lack of association between an abnormal EEG finding and an abnormal MRI finding suggested that they reflect complementary, but different aspects of pathologies in these subgroup of seizure patients. Thus, from a clinical practice perspective, both tests have to be performed to capture the full range of pathologies.

\subsection{2}

VOXEL-BASED MRI POST-PROCESSING IN MRI NEGATIVE REFRACTORY EPILEPSIES WITH PATHOLOGICALLY CONFIRMED FOCAL CORTICAL DYSPLASIA: PILOT DATA ON 20 PATIENTS AND 20 CONTROLS

Zeenat Jaisani, I. Wang, S. Jones, I. Najm and A. Alexopoulos (Epilepsy Center, Cleveland Clinic, Cleveland, $\mathrm{OH}$ )

Rationale: Focal Cortical dysplasia (FCD) is a common cause of medically refractory focal epilepsy. Identification of FCD lesion on conventional MRI is challenging as up to $30 \%$ of histologically proven FCD cases are negative on conventional MRI. The entire presurgical planning can be strongly affected by presence of lesion on the MRI, and surgical outcome can be affected by completeness of lesion resection. In this study we aim to retrospectively test a voxelbased MRI post-processing technique that can help the pre-surgical evaluation by reinforcing the areas of suspicion during patient management conference (PMC).

Methods: We retrospectively studied 20 medically refractory focal epilepsy patients ( 10 frontal, 8 temporal and 1 frontotemporal and 1 temporo-occipital) and 20 age-matched controls with normal MRI. The analysis was blinded by de-identifying and randomizing the patient group and the normal controls. All selected patients (pts) underwent scalp video-EEG and had negative reported MRI and histology proven FCD. During multidisciplinary PMC the neuroradiologist/epileptologist/neurogurgeon re-assessed the MRI, in light of all other pre-surgical data, and noted a probable/questionable lesion that may be indicative of an epileptic focus. MRI postprocessing was performed with methodology consistent with Huppertz et al., in a Morphometric Analysis Program (MAP) using Matlab SPM5. In both the patient and the control group, the gray/white junction file generated by MAP was analyzed by two readers for possible abnormalities with $\mathrm{z}$ score $>4$. Final analysis to account a lesion as significant (i.e., MAP+) was completed by a neuroradiologist in a blinded fashion.

Results: Mean age was $31.35 \pm 11.16$ years and $31.55 \pm 14.64$ years for control and patient group respectively. In the control group, no areas of MAP abnormality were detected with z-score $>4$ and therefore were MAP negative. Of the 20 epilepsy pts; 10 pts had clear $\mathrm{MAP}+$ areas $(\mathrm{z}>4)$ with corresponding abnormality included in the area of resection, indicating that MAP detected subtle dysplasia $50 \%$ of the time. 14/20 (70\%) pts were seizure free (mean duration of follow-up was 25.1 month) of which $8 / 14$ were MAP positive (57\%). In the remaining 6/20 (30\%) pts seizure recurred (mean time to seizure recurrence 7.21 months). Only 2 of these 6 pts were MAP positive. However, in both pts, resection did not include the MAP+ abnormality in its entirety, and therefore incomplete resection of the epileptogenic FCD lesion may have played a role in seizure recurrence.

Conclusions: MAP is a promising and easily applicable MRI postprocessing tool to identify cryptogenic FCD lesion in up to $50 \%$ of refractory epilepsy pts with presumed normal MRI in this cohort. MAP cannot detect FCD in some pts who attain seizure-freedom following surgical resection. At the same time incomplete resection of an MAP positive area may be associated with postoperative seizure recurrence. This study is part of a large-scale ongoing study on the utility of MAP in MRI negative focal epilepsies.
3.183

QUANTIFYING THE CONTRIBUTION OF THALAMIC VOLUME AND WHITE MATTER MICROSTRUCTURE TO REGIONAL CORTICAL THINNING IN MESIAL TEMPORAL LOBE EPILEPSY (MTLE)

H. M. Girard ${ }^{1,3}$, N. Kemmotsu, ${ }^{1,3}$, N. E. Kucukboyaci, ${ }^{1,3}$, E. S. Tecoma $^{2}$, V. J. Iragui ${ }^{2}$ and C. R. McDonald ${ }^{1,3}$ ( ${ }^{1}$ Psychiatry, University of California, San Diego, La Jolla, CA; ${ }^{2}$ Neurosciences, University of California, San Diego, La Jolla, CA and ${ }^{3}$ Multimodal Imaging Laboratory, University of California, San Diego, La Jolla, CA)

Rationale: The anterior thalamic radiations (ATR) are a fiber bundle that course from the mediodorsal and anterior thalamic nuclei to the frontal and cingulate cortices. These thalamocortical fibers play an essential role in seizure spread and, along with the thalamus, may be involved in network pathology that contributes to cortical damage in patients with MTLE. In this study, we aimed to investigate the relative contributions of thalamic volume (TV) and microstructure of the ATR, as measured by fractional anisotropy (FA), to explaining patterns of gray matter cortical thinning (GMT) in patients with left and right MTLE.

Methods: Diffusion tensor imaging (DTI) and volumetric MRI were performed in 27 patients with MTLE (15 left; 12 right) who all showed evidence of mesial temporal sclerosis (MTS) on MRI, and 27 age and gender-matched healthy controls. FA of the ATR, cortical thickness, and thalamic and hippocampal volume were calculated for all subjects. Surface-based correlational analysis was used to determine the vertex-wise relationship between volumes or FA of the ATR and GMT.

Results: Patients with left and right MTLE both showed significant ipsilateral hippocampal and thalamic volume loss, and the magnitude of ipsilateral volume loss did not differ between left and right MTLE. However, patients with left MTLE showed more widespread cortical thinning relative to patients with right MTLE in both hemispheres. Patients with left MTLE also had lower FA of the ATR bilaterally, whereas patients with right MTLE demonstrated no difference in FA of the ATR compared to controls. In left MTLE, the volume of the thalamus and the FA of the ATR correlated with GMT in bilateral superior frontal areas. Where TV alone was correlated with bilateral supramarginal and ipsilateral middle temporal areas, and FA of ATR alone was correlated with contralateral temporal and precentral regions. In contrast in right MTLE, only the FA of ATR correlated with GMT in ipsilateral frontal regions. Volume and FA were not correlated with clinical-seizure related variables of reported seizure frequency, age of onset, or years of disease duration in either group. Conclusions: Patients with MTLE show pathology within thalamocortical networks, but the pattern of pathology differs between left and right MTLE. These findings may reflect differences in structural connectivity between patients with left and right MTLE that complement previous findings of functional connectivity differences between these groups. Whether or not thalamocortical seizure spread contributes to neocortical atrophy or whether cortical atrophy is the result of an aberrant developmental process or some other disease-related mechanism remains unknown. 


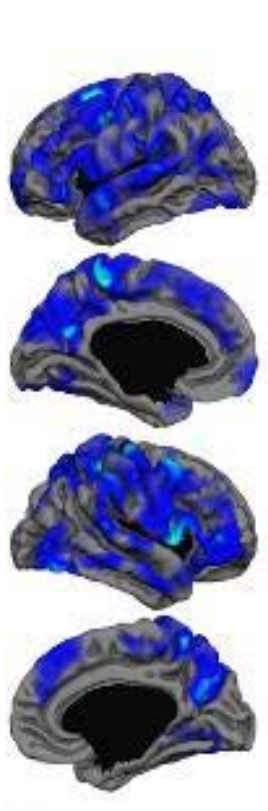

Gray Matter Thickness compared to Controls

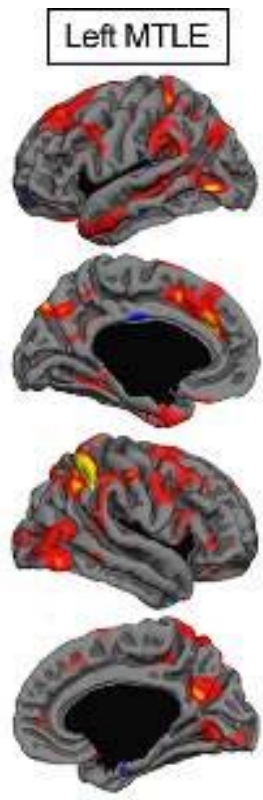

Thickness - Thalamic volume correlation
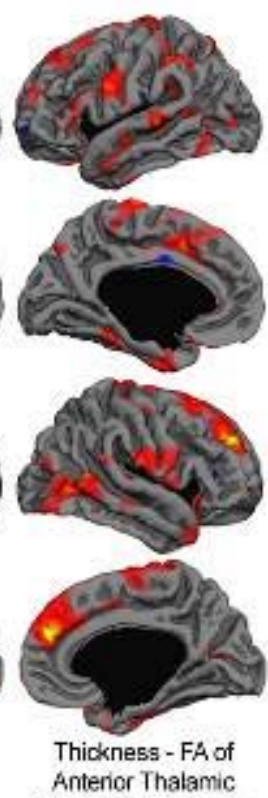

Radation correlation

\subsection{4}

SEIZURE-ONSET AGE AND DRUG-RESISTANCE ARE THE VARIABLES OF WHITE MATTER CHANGES IN PATIENTS WITH MRI-NEGATIVE EPILEPSY

Syu-Jyun Peng ${ }^{1}$, Y. Hsin ${ }^{2}$ and T. Harnod ${ }^{3}$ ( ${ }^{1}$ National Central University, Jhongli, Taiwan; ${ }^{2}$ Neurology, Hualient Tzu Chi Medical Center, Hualien, Taiwan and ${ }^{3}$ Neurosurgery, Hualient Tzu Chi Medical Center, Hualien, Taiwan)

Rationale: Increase white-matter diffusivity and decreased anisotropy have been commonly found in patients with brain structural lesion including hippocampal atrophy, cortical dysplasia, et al. We used diffusion-tensor imaging to study the integrity of different neural pathways in patients with chronic partial epilepsy and without visible lesion in brain images. Our aims are not only to find whether there is same white matter change but also to explore the possible variables which are responsible for the microstructural abnormality.

Methods: Twenty-nine healthy volunteers (16 males, mean age $=$ $27 \pm 4)$ and 24 patients with neocortical epilepsy $(9$ males, mean age $=$ $26 \pm 13$ ) were included in this study. Diffusion tensor imaging (DTI) data were acquired on a 3T GE Sigma scanner High-resolution T1weight fast spoiled gradient recalled echo images (FSPGR) were also acquired.

Diffusion-weighted images were first corrected for eddy-current distortion and head motion using FMRIB's diffusion toolbox. Using Analyze 10.0 software, maps of apparent diffusion coefficient (ADC) and fractional anisotropy (FA) were produced. We further extracted $\mathrm{ADC}$ and FA and generated nerve fibers for white-matter regions of interest (WM ROIs) separately, each of which was defined in ICBM probabilistic atlas. By DARTEL tool in SPM8, we deformed each ROI from ICBM template to individual with reference to personal SPGR.

We tested the difference of ADC and FA between the patient and health groups. Then we examined the contribution of seizure-onset age (early-onset group: 12 patients $\leqq 10$ years; and late-onset group $: 10$ patients $>10$ years), pharmacoresistance, and disease duration to the change of diffusivity and isotropy by comparing the ADC and FA to control group.

Results: Diffusely increased ADC and reduced FA in different pathways were found in the individual analyses and group comparison. Many neural pathways (anterior corona radiate, posterior thalamic radiation, splenium of corpus callosum, sagittal stratum and uncinate fasciculus) were found to have statistical difference of ADC and significantly reduced FA in the sagittal stratum was showed in the early-onset group. No significant change in ADC and FA was found in later-onset group.

More neural pathways were found to be different in the drug-resistant patient group (significantly high $\mathrm{ADC}$ in the anterior corona radiate, posterior thalamic radiation and sagittal stratum) rather than the drugresponsive patient group (only the sagittal stratum showing significant changes in ADC and FA).

Conclusions: Our data suggest that patients with MRI-negative neocortical epilepsy showed a reduction in white matter integrity relative to healthy controls particularly in sagittal striatum that is a major corticosubcortical white matter bundle. In addition, we observed widespread structural abnormality in the group of early onset and the group with pharmacoresistance.

\subsection{5}

RELATIONSHIP BETWEEN WADA MEMORY LATERALIZATION AND TEMPORAL LOBE VOLUME Kan Ding ${ }^{1}$, C. Marquez de la plata ${ }^{2}$, Y. Gong ${ }^{3}$, R. McColl ${ }^{4}$, P. Van

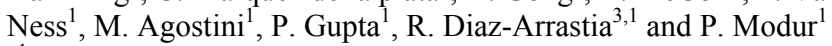
$\left({ }^{1}\right.$ Neurology and Neurotherapeutics, UT Southwestern Medical Center at Dallas, Dallas, TX; ${ }^{2}$ Center for Brain Health, The University of Texas as Dallas, Richardson, TX; ${ }^{3}$ Center for Neuroscience and Regenerative Medicine, Uniformed Services University of the Health Sciences, Bethesda, MD and ${ }^{4}$ Radiology, UT Southwestern Medical Center, Dallas, TX)

Rationale: The reason for nonlateralized Wada memory in patients with intractable temporal lobe epilepsy (TLE) remains unclear. Since quantitative morphometric MRI studies in such patients have shown bilateral regional atrophy in temporal and extratemporal structures, we hypothesized that volume loss in contralateral temporal structures could contribute to nonlateralized Wada memory results.

Methods: We investigated patients with TLE who underwent standard presurgical evaluation during 2005-10. Patients with focal lesions other than mesial temporal sclerosis (MTS) were excluded. Seizure onset was determined by video-EEG monitoring. Wada test was done with a fixed dose of $120 \mathrm{mg}$ of sodium amytal. Memory was assessed with 17 or 18 verbal and nonverbal items. Memory was considered nonlateralized if the difference in $\%$ correct recall scores between ipsilateral and contralateral injections was $\leq 11 \%$ (i.e., 2 items). Volumetric data obtained from 3T MRI using FreeSurfer software were compared against a pre-existing database of 22 age/gender-matched controls to determine the extent of abnormalities. Between-group differences in volume/cortical thickness of temporal structures was assessed by t-test. Relationship between volume and Wada memory scores was evaluated by Spearman's rank correlation coefficient. Statistical significance was declared for $\mathrm{p}$ value $<0.05$.

Results: Thirty-six patients (34 right-handed, 2 left-handed) met the inclusion/exclusion criteria. Of these, 22 had left TLE (14 MTS, 8 nonlesional) and 14 with right TLE (7 MTS, 7 nonlesional). All patients had higher Wada memory scores with injections ipsilateral to the surgical hemisphere. Ipsilateral scores were lower in patients with left TLE than right TLE (55 \pm 21 vs $67 \pm 26 \%, \mathrm{p}=0.06)$. Wada memory was lateralized in $27(75 \%)$ and nonlateralized in 9 patients $(25 \%)$. All nonlateralized scores were seen in left TLE. In left TLE, the ipsilateral scores were significantly lower in the nonlateralized than the lateralized group ( $33 \pm 16$ vs $55 \pm 21 \%$, $p=0.007)$ but the contralateral scores were similar in the two groups ( $30 \pm 22$ vs $37 \pm 19$, $\mathrm{p}=0.7)$. Among all patients, right injection scores were significantly correlated with volume/cortical thickness of left hippocampus $(\mathrm{r}=$ $0.49)$, amygdala $(\mathrm{r}=0.36)$, parahippocampal gyrus $(\mathrm{r}=0.38)$, fusiform gyrus $(\mathrm{r}=0.45)$ and entorhinal cortex $(\mathrm{r}=0.32)$. Left injection scores were significantly correlated with the volume of right hippocampus $(r=0.39)$ and left transverse temporal gyrus $(r=0.45)$. Regardless of the side of seizure onset, the volumes of temporal lobe structures 
were reduced bilaterally although more pronounced ipsilaterally. The contralateral temporal lobe volume loss was significant in patients with nonlateralized than lateralized Wada test $(\mathrm{p}<0.05)$.

Conclusions: Patients with TLE have significant volume loss in bilateral temporal structures. The observed volume loss in the nonsurgical temporal structures may contribute to the nonlateralized Wada memory in these patients.

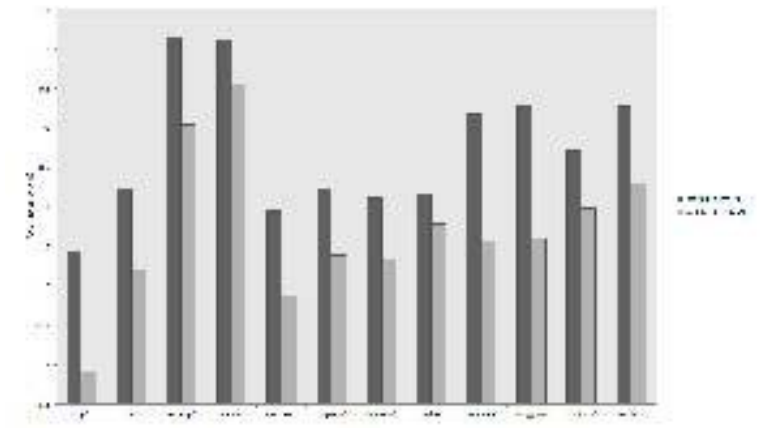

Non-lateralized Wada test was defined as the difference in $\%$ correct recall scores between ipsilateral and contralateral injection was $<$ 11\%. i: ipsilateral; c: contralateral; Hip: hippocampus; Parahip: parahippocampal gyrus; SupTem: Superior temporal gyrus; MidTem: Middle temporal gyrus. ${ }^{*}: \mathrm{p}<0.05$ with independent sample t-test.

\subsection{6 \\ THALAMOCORTICAL ALTERATIONS IN TEMPORAL LOBE EPILEPSY REVEALED USING CONNECTIVITY- BASED SEGMENTATION}

Simon S. Keller ${ }^{1}$, J. O'Muircheartaigh ${ }^{1}$, C. Traynor ${ }^{1}$, K. Towgood ${ }^{1}$, G. J. Barker ${ }^{2}$, W. R. Crum ${ }^{2}$ and M. R. Richardson ${ }^{1}$ ( ${ }^{1}$ Department of Clinical Neuroscience, King's College London, London, United Kingdom and ${ }^{2}$ Department of Neuroimaging, King's College London, London, United Kingdom)

Rationale: On radiological MRI assessment, mesial temporal lobe epilepsy (TLE) is most commonly associated with ipsilateral hippocampal sclerosis. Quantitative analysis of structural volumes has also revealed global atrophy of other structures, most commonly of the thalamus, striatum and cortex [1]. Thalamocortical and striatocortical mechanisms play important roles in the expression of seizures [2], but the vast majority of MRI research to date has concentrated on global volume loss of subcortical structures without consideration of the specific connectivity profiles of these regions. In the present study, we combined T1-weighted magnetic resonance (MR) imaging and MR diffusion tensor imaging (DTI) to investigate cortical connection probability within the thalamus and striatum in patients with TLE relative to healthy controls using connectivitybased segmentation (CBS).

Methods: Thirty-five participants were enrolled into this study, including 14 healthy controls and 21 patients with TLE (9 left, 12 right) and underwent structural and DTI scans during the same scanning session. CBS was performed using the FSL FDT pipeline (www.fmrib.ox.ac.uk/fsl/fdt) for probabilistic tractography from seeds (left / right / thalamus / striatum separately) to classification targets (ipsilateral prefrontal, precentral, postcentral, temporal (TL), parietal and occipital cortices). Seeds and targets were obtained using FreeSurfer software (http://surfer.nmr.mgh.harvard.edu) from T1weighted MRIs. A 'hard segmentation' of the intra-thalamic and intra-striatal representations of the cortical targets was performed using the 'find_the_biggest' function in FSL, and fslstats was used to determine the volume of these cortical representations within the thalamus and striatum. Quantitative analyses of between-group differences in CBS were performed in native (subject-specific) and normalized (MNI) space.

Results: In native space, patients with right TLE had significantly reduced volume of thalamic-TL connections in the left $(\mathrm{p}=0.03)$ and right ( $\mathrm{p}=0.004)$ hemisphere relative to controls. Patients with left TLE had a borderline significant reduction of left thalamic-TL connections $(\mathrm{p}=0.053)$ compared to controls. When structures were treated as ipsilateral / contralateral to TLE relative to the left/right hemisphere average in controls, significantly reduced ipsilateral thalamic-TL connections were observed for both patient groups (Figure). Findings in MNI space were consistent with native space thalamic-TL data, but only at borderline statistical levels $(\mathrm{p}<0.05)$. There were no other statistically significant differences between patients and controls for the striatum or other thalamocortical connections.

Conclusions: Here we provide preliminary evidence indicating disrupted thalamic-TL connectivity ipsilateral to the epileptogenic focus in patients with TLE. This disruption may be due to either deafferentation or excitotoxic effects generated by the connected medial temporal lobe.

References

[1] Keller, S. S., Roberts, N. 2008, Epilepsia, 49(5):741-57.

[2] Driefuss, S. et al. 2001, Neurology, 57(9):1636-41.
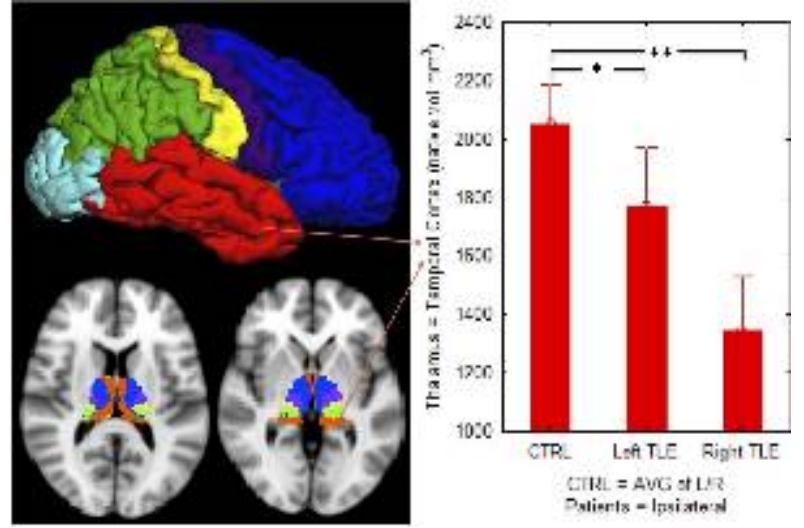

Left: Average intra-thalamic expression of cortical regions (bottom) in controls (MNI space). Results are colour-coded according to cortical targets (top). Right: Significant $\left({ }^{*} \mathrm{p}<0.05,{ }^{* *} \mathrm{p}<0.001\right)$ volume reduction of temporal lobe cortical representation in the thalamus ipsilateral to TLE compared to the left/right average in controls.

\subsection{7 \\ DECREASED CONNECTIVITY OF MAJOR CORTICAL NODES IS MORE PRONOUNCED AND LATERALIZED IN LEFT VERSUS RIGHT MESIAL TEMPORAL SCLEROSIS Vera Dinkelacker ${ }^{1,3}$, P. Besson ${ }^{2}$, R. Valabrègue ${ }^{3}$, S. Samson ${ }^{4}$, L. Thivard $^{1,3}$, M. Baulac ${ }^{1}$, D. Sammler ${ }^{5}$, S. Lehéricy ${ }^{3}$ and S. Dupont ${ }^{1,3}$ $\left({ }^{1}\right.$ Epileptology Unit, Pitié-Salpêtrière Hospital, Paris, France; ${ }^{2}$ Centre Hospitalier Régional Universitaire, Lille, France; ${ }^{3} \mathrm{CENIR}$, Institut du Cerveau et de la Moelle, Paris, France; ${ }^{4}$ Neuropsychology and Auditory Cognition, Université de Lille, Lille, France and ${ }^{5} \mathrm{Max}$ Planck Institute for Human Cognitive and Brain Sciences, Leipzig, Germany)}

Rationale: Rationale :

Temporal lobe epilepsy with hippocampal sclerosis is a chronic disease which affects neural networks way beyond hippocampal sclerosis. The latter changes have received much recent interest with the advent of diffusion tensor imaging (DTI). Several DTI-studies showed alterations of large white matter tracts, most notably in left mesial temporal sclerosis (LMTS). The way in which this affects deafferentation of specific cortical structures has so far only indirectly been examined.

Our study intended to compare whole brain connectomes of patients with left and right MTS with healthy control subjects in order to delineate deafferentation of major cortical and subcortical structures. 
Methods: We have gathered a large data base on a homogeneous group of patients with unilateral sclerosis, comprising 20 patients with right and 19 patients with left sided lesions and 28 matched control subjects. We recorded DTI sequences with 50 directions and performed whole-brain probabilistic fiber tracking using the MRtrix software package. Based on the Destrieux atlas implemented in freesurfer, 164 regions of interest in cortical and subcortical gray matter were segmented and coregistered with the DTI maps. We then computed individual graphs based on these 164 nodes. The edges between two regions of the graph were weighted by the average FA along the selected tracts, the latter weight representing the connectivity strength. Finally, we determined the connectivity strength in the patient's groups as compared to control subjects were determined with three complementary analyses : individual comparison, group permutation tests and Network Based Statistics. Results: The three lines of analysis yielded a homogeneous picture of deafferentation in both left and right MTS. The decrease of connectivity with respect to control subjects was much more pronounced in left than in right MTS. Furthermore, the left MTS pattern was strongly lateralized to the ipsilateral temporal lobe with a special impact on temporal cortex and temporal pole, hippocampus and collateral sulcus. Conversely, connectivity in right MTS was globally less affected and essentially focused on bilateral hippocampus and collateral sulcus with a right-sided predominance. Conclusions: Connectome analysis in left and right MTS corroborates a clear cut decrease in connectivity strength in left MTS, which is moreover strongly lateralized and focused on temporal lobe and hippocampus. Right MTS shows lesser and more bilateral effects. Yet, deafferentation in both patients groups targets hippocampus and collateral sulcus, a subregion often altered in MTS (Kim et al., 2008, Bernhardt et al., 2008).

\subsection{8 \\ GREY AND WHITE MATTER BRAIN VOLUMES AND INTELLIGENCE: DIFFERENCES ACROSS EPILEPSY SYNDROMES}

Daren C. Jackson ${ }^{1}$, K. Dabbs ${ }^{1}$, J. Jones ${ }^{1}$, D. Hsu ${ }^{1}$, C. Stafstrom ${ }^{1}$, M. Seidenberg ${ }^{2}$ and B. Hermann ${ }^{1}$ ( ${ }^{1}$ Neurology, University of Wisconsin, Madison, WI and ${ }^{2}$ Psychology, Rosalind Franklin University of Medicine and Science, North Chicago, IL)

Rationale: While the cognitive consequences of epilepsy are appreciated, the degree to which they are related to underlying neuroanatomy remains to be fully understood. Relatively few studies have examined and compared structure-function relations across epilepsy syndromes. Here we examined associations between brain volumes and full-scale intelligence quotient (FSIQ) in children with idiopathic generalized epilepsy (IGE), idiopathic localization-related epilepsy (ILRE), and healthy controls (HC). In particular, we focused on the degree to which children with IGE or ILRE differ in structurefunction relations, relative to $\mathrm{HC}$.

Methods: Research participants were children ages 8-18 with IGE $(n=31)$, ILRE $(n=35)$, and HC $(n=52)$. All epilepsy participants were seen and assessed within 12 months of diagnosis, and had normal neurological examinations and no identifiable lesions on clinical MRI. The HC group consisted of age- and gender-matched firstdegree cousin controls. All participants completed a test battery assessing broad neuropsychological domains, in addition to undergoing T1 volumetric MRI scans. Grey and white matter volumes were computed using the FreeSurfer image analysis suite. Analyses focused on relations between FSIQ and whole-head measures of cortical grey and white matter volume, as well as caudate and thalamus volume.

Results: Children with IGE and ILRE exhibited comparable scaled IQ within the normal range (IGE: $M=100.94, S D=14.16$; ILRE: $M$ $=100.97, \mathrm{SD}=12.80)$, although both groups were significantly lower than the $\mathrm{HC}$ group $(\mathrm{M}=107.25, \mathrm{SD}=12.22$; $\mathrm{p}$ 's $<0.05)$. Total intracranial volume did not differ across groups, and thus was not used as a covariate in further analyses. HC showed significant positive associations between FSIQ and every age-adjusted volume of interest (r's $.37-.43$; p's < 0.05). IGE participants showed a similar pattern, with r's ranging from $.37-.49 ; \mathrm{p}$ 's $<0.05$. In contrast, ILRE participants did not show this linkage between brain volumes and FSIQ, with the only significant association being that of right caudate and FSIQ $(r=.45, \mathrm{p}<0.05)$. Other correlations within the ILRE group ranged from $.018-.297$.

Conclusions: Children with new- and recent-onset ILRE differed from a $\mathrm{HC}$ group in terms of the relations between grey and white matter brain volumes and IQ in ways that IGE participants did not. This is particularly striking given that the ILRE and IGE groups did not differ in intelligence. Given that IQ is known to be associated with distributed neural systems, it appears that the brains of children with ILRE may rely on different and more diffusely associated networks to achieve similar functional outcomes (in this case, normal-range IQ). In future analyses, we plan to interrogate the nature of these networks in children with ILRE by examining performance in specific neuropsychological domains, as well as by targeting more specific grey and white matter brain regions of interest.

\subsection{9 \\ WHITE MATTER ABNORMALITIES IN PATIENTS OF JUVENILE MYOCLONIC EPILEPSY AS DETECTED BY DIFFUSION TENSOR IMAGING}

Sanjib Sinha, J. Saini, B. S. Bagepally, C. T. Ramachandraiah, K. Thennarasu, C. Prasad, A. B. Taly and P. Satishchandra (National Institute of Mental Health and NeuroSciences, Bangalore, India)

Rationale: Imaging studies in Juvenile myoclonic epilepsy (JME) had shown abnormalities of thalamus and frontal cortex. However, alterations in white matter structures have not been well studied. The purpose of this study was to systematically investigate the morphological changes in the brain white matter using diffusion tensor imaging and to correlate these changes with theseizure control and disease duration.

Methods: The study included 37(M:F=18:19; age $=22.57 \pm 5.53$ years) patients with JME and 19 (M:F=11:8; age $24.5 \pm 4.2$ years) matched controls. There were 21 patients with adequate seizure control and 16 with uncontrolled seizures. All subjects underwent MRI using standard protocol which included T1-3D TFE images with $1 \mathrm{~mm}$ thickness and DTI with 15 gradient directions and $b$ value of 1000. TBSS [Tract based spatial statistics] analysis was performed to compare between the patients and controls. Further statistical comparisons were made between the DTI metrics of the corpus callosum and anterior thalamic radiation to confirm the changes observed using TBSS.

Results: Significant changes in the FA and RD were observed in the cerebral WM affecting primarily the Corpus callosum, fronto-parietal WM, internal capsule, external capsule, brainstem and cerebellar $\mathrm{WM}$ in the JME patients $(\mathrm{P}<0.05)$ after adjusting for age, gender and ICV. Corpus callosum and anterior thalamic radiation WM indices were significantly different in the JME patients when compared to the controls. Further comparison subgroup analysis in patients with uncontrolled seizures revealed widespread WM changes while patients with controlled seizures showed no significant degenerative changes in the WM. FA of the corpus callosum and left anterior limb of internal capsule showed negative correlation with disease duration while $\mathrm{MD}$ and $\mathrm{RD}$ of the corpus callosum correlated positively with the disease duration.

Conclusions: The limitations were small sample size, lack of drug naïve subjects and small number of control subjects. This study confirms the presence of cerebral, cerebellar and brainstem WM and further raises the possibility of reversible nature of these WM changes with control of seizures. 


\subsection{0}

CORRELATIVE DISINTEGRATION IN WHITE MATTER TO STRUCTURAL VOLUME LOSS OF DEEP GRAY MATTER IN PATIENTS WITH JUVENILE MYOCLONIC EPILEPSY

seong-hoon kim, Y. M. Shon, S. C. Lim, Y. I. Kim and W. J. Kim (Neurology department, The catholic medical school of korea, seocho-gu, Republic of Korea)

Rationale: We studied to investigate the anatomical and pathophysiological nature of juvenile myoclonic epilepsy (JME) using a multimodal neuroimaging approach including structural (T1 image) and connectional (diffusion tensor imaging, DTI) imaging data.

Methods: High-resolution T1 and DTI images were acquired on a 1.5T MRI in twenty-four patients with JME and 26 controls.

Thalamic and hippocampal volumes were manually obtained from each subject's brain to identify the white matter regions which are correlated to their volume loss. We used FreeSurfer for cortical thickness measurements and Tract-based spatial statistics (TBSS) processing to assess the white matter integrity of patients.

Results: JME patients showed that hippocampal volume loss was significantly correlated with increasedMD value in anteroinferiorfrontal WM, both uncinate fasciculus, and temporal WM by TBSS analysis. Thalamic volume loss was also well correlated with both antero-inferior, dorsolateral frontal area, and deep WM of parietal and posterior cingulate area.

Conclusions: JME patients showed that structuralthalamic and hippocampal volume loss was explicitly correlatedwith the derangement of water diffusivity of white matter along brain areas that connected not only thalamic but also hippocampal regions. Further large population-based studies with not only structural T1 image but DTI may elucidate not only the underlying structural \& connectional nature, but the heterogenous clinical features of JME patients.

\subsection{1}

\section{IS THERE A CAUSAL LINK BETWEEN TUMEFACTIVE} VIRCHOW-ROBIN (VR) SPACES AND EPILEPSY?

Poonam Bhatia ${ }^{1}$, B. Sitwat ${ }^{1}$ and K. Petropoulou ${ }^{2}\left({ }^{1}\right.$ Child neurology, Childrens Hospital of Pittsburgh, Pittsburgh, PA and

${ }^{2}$ Neuroradiology, University of Pittsburgh Medical Center,

Pittsburgh, PA)

Rationale: There have been isolated case reports and very few retrospective analyses on clinical correlation of tumefactive VR spaces. There have been rare case reports of VR spaces associated with seizures but to date no clear correlation with its location has been identified.

Methods: We collected data on 4 patients with tumefactive VR spaces. Tumefactive VR spaces were identified based on their morphology, size, surrounding mass effect, contrast response, and appearance on magnetic resonance spectroscopy. We analyzed the charts for clinical and laboratory data and magnetic resonance imaging of brain for details about location and characteristics of lesions.

Results: One case had tumefactive VR space in temporal lobe (cortical and subcortical region), clinically manifesting as temporal lobe epilepsy with abnormal EEG correlate (Figs 1 and 2). Another case had tumefactive frontal lobe lesion, which was an incidental finding post concussion, currently being evaluated for staring spells. Two other cases, presenting with headache had tumefactive VR spaces in thalamus and brain stem each.

Conclusions: We describe four cases of tumefactive VR spaces. Two out of these four cases had confirmed or suspected epilepsy. These cases had lesions in known epileptogenic locations i.e. temporal or frontal lobe. One of these two cases had a cortical location of VR space, which has been reported only once in literature. Only one reported case in literature with seizures associated with tumefactive VR spaces had showed a location in temporal lobe.

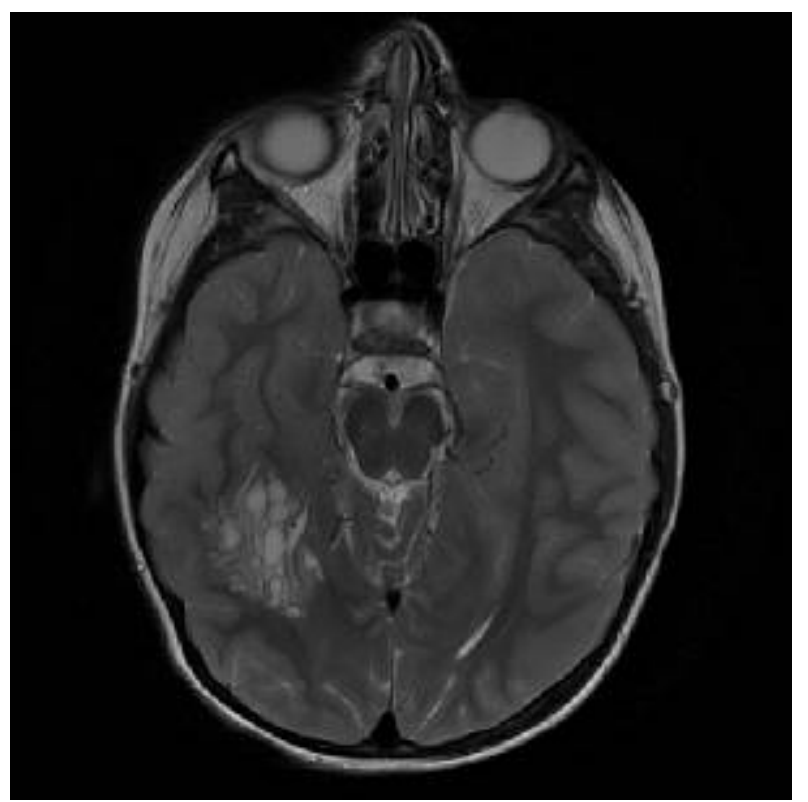

Fig 1: Tumefactive VR spaces in right temporal lobe in a patient

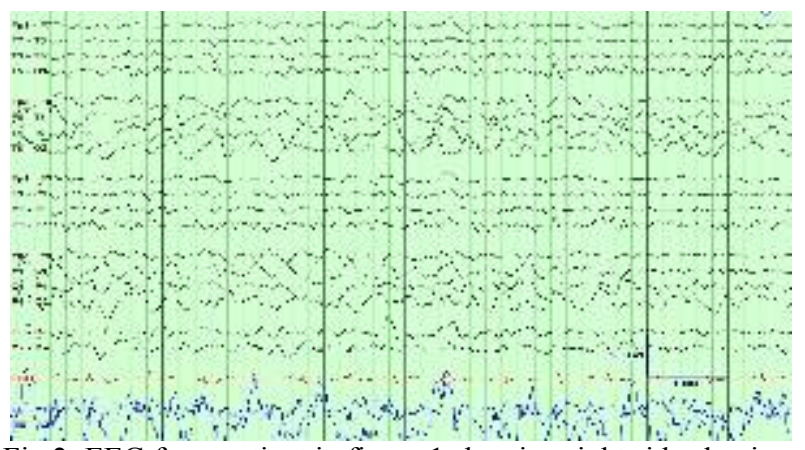

Fig 2: EEG from patient in figure 1 showing right side slowing

\section{Neuro Imaging: Functional Imaging}

\subsection{2 \\ SCALP RIPPLES ARE ASSOCIATED WITH THALAMIC BOLD CHANGES}

Firas Fahoum, F. Melani, L. P. Andrade-Valença, F. Dubeau and J. Gotman (Montreal Neurological Institute, Montreal, QC, Canada)

Rationale: Interictal high frequency oscillations $(40-200 \mathrm{~Hz})$ measured from scalp EEG of focal epilepsy patients (sHFOs) usually co-occur with spikes (Epilepsia 2011,52:1812), and were shown to be a more specific and accurate marker than spikes for delineating the seizure onset zone (Neurology 2011,77:524). The metabolic correlates of sHFOs as measured by blood oxygenation leveldependent (BOLD) signal are unknown. Measuring sHFOs in EEG acquired inside the MR scanner still poses a remarkable technical challenge because of their very low amplitude. We addressed this issue by comparing BOLD responses to spikes in patients in whom a separate sHFO investigation has demonstrated spikes with frequent sHFOs to responses in patients with spikes having rare sHFOs (gamma 40-80Hz and ripples 80-200Hz).

Methods: 28 focal epilepsy patients were studied. 6 were excluded due to negative EEG-fMRI evaluation (4 without spikes, 1 with spikes but no BOLD changes and 1 with persistent artifacts preventing the analysis). The remaining 22 patients were divided into two equal groups based on the frequency of co-occurrence of gamma or ripples with their spikes outside the scanner: high vs. low gamma 
(HG/LG) and high vs. low ripples (HR/LR), each group having 11 subjects. We looked for significant differences in spatial distribution of cortical and subcortical BOLD changes, spike field- concordant BOLD cluster volume and maximal t-value between the high and the low groups. We took into consideration the number of spikes, occurrence of spike runs and generalized spike and wave (GSW) in each group.

Results: The HR group had significantly more thalamic BOLD changes than the LR group (11/11 vs. 2/11, examples in Fig1-2). The difference remained after discarding the three patients with GSW in the HR group. In HR, 5/11 had only activation in the thalamus, 4/11 had only deactivation and $2 / 11$ had activation and deactivation. In the LR group 2/11 had thalamic activation. The lateralization of thalamic BOLD responses was concordant with the lateralization of ripples in $12 / 13$ patients.

HR had a larger spike field-concordant BOLD cluster and higher maximal t-value than LR, but these changes could be attributed to higher number of GSW and spike runs in the HR than in the LR group.

No significant changes where found between the BOLD characteristics of the HG and LG groups.

Conclusions: Interictal spikes measured on scalp EEG, when they are accompanied by high rate of HFOs in the ripple but not in the gamma band, are associated with thalamic metabolic changes that are lateralized to the side of the cortical ripples. This result could be explained by the animal data connecting HFOs generated in the cortex with spike and wave discharges and the thalamo-cortical circuitry [Neuroscience 1997, 17:6783; Neuroscience 2004, 123:299].

This work was supported by grant MOP-38079 of the Canadian Institute of Health Research.

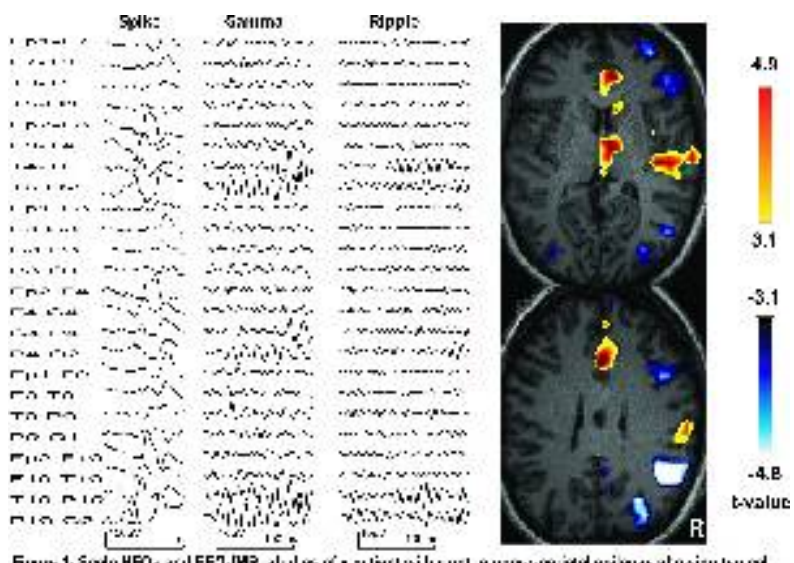

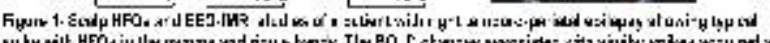

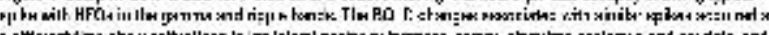

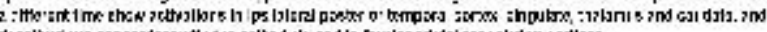

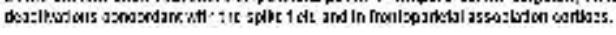

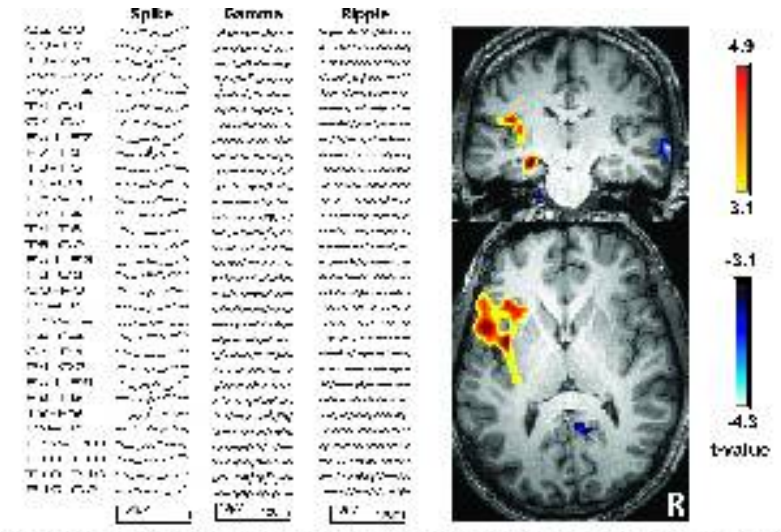

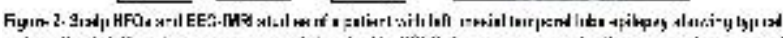

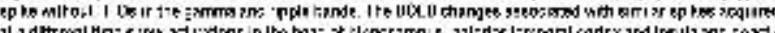

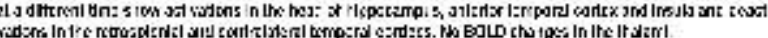

3.193

PATTERNS OF FMRI HIPPOCAMPAL LAMELLAR ACTIVATION INDUCED BY PERFORANT PATH STIMULATION IN THE KAINIC ACID RAT MODEL OF EPILEPSY

Saul Jaime ${ }^{1}$, T. Q. Duong ${ }^{3}$ and J. E. Cavazos ${ }^{1,2}\left({ }^{1}\right.$ Neurology, Pharmacology, and Physiology, Univ. Texas Health Science CenterSan Antonio, San Antonio, TX; ${ }^{2}$ San Antonio VA Epilepsy Center of Excellence, South Texas Veterans Health Care System, San Antonio, TX and ${ }^{3}$ Research Imaging Institute, Univ. Texas Health Science Center-San Antonio, San Antonio, TX)

Rationale: The hippocampus is organized into a sausage-like structure of several thin transverse slices containing tri-synaptic circuitry, also known as the lamellar organization. Our lab has been interested in the functional rearrangement of epileptic hippocampal circuitry in models and humans with intractable temporal lobe epilepsy. Using anatomical techniques, we demonstrated seizureinduced sprouting interconnecting adjacent hippocampal lamellas in several epileptic models (Cavazos et al, 2004, 2006). More recently, using physiological techniques, we showed translamellar hippocampal hyperexcitability in the Kainic Acid-Status Epilepticus (KA-SE) model. Our studies have shown that seizure-induced sprouting has a latent period followed by progressive and permanent aberrant interconnectivity between hippocampal lamellas, which precedes occurrence of spontaneous seizures of increasing frequency. We extend these observations using functional MRI (fMRI)-BOLD analysis from perforant path (PP) stimulation examining translamellar hyperexcitability in repeated observations in the same KA-SE animal.

Methods: Sprague Dawley male rats (200g) were anesthetized (1.5\% Isoflurane) and prepared for electrode implantation with a MRI compatible bipolar electrode lowered into PP (-8.0mm,4.2 mm,3.5 $\mathrm{mm})$. Electrodes were anchored with 4 nylon screws and dental cement. Rats had 7 day postsurgical recovery and given analgesics and topical antibiotics. fMRI experiments were performed on a Bruker Biospec 11.7T scanner with surface coil. T2 weighted spin echo images were obtained using rapid acquisition relaxation enhanced (RARE) and echo planar imaging (EPI) sequences. Baseline fMRI scan was triggered by hippocampal stimulation with a stimulation block of 7 trains with $10 \mathrm{sec}$ stimulation and $50 \mathrm{sec}$ rest per train. The block was repeated at $2.5,5,10$, and $20 \mathrm{~Hz}$ with $1.0 \mathrm{~mA}$ current. Images were saved and analyzed with Stimulate software. Kainic Acid (KA), 5mg/kg i.p. every $1 \mathrm{hr}$ (x3) was injected into rats. They were observed for $5 \mathrm{hrs}$ post-KA to verify status epilepticus (SE). Only rats with SE for $30+$ min were used in study. Rats were re-scanned at 7, 21, 60, and 90 days after KA-SE.

Results: Ten rats were implanted and are undergoing experimentation. At baseline scans, PP stimulation induced BOLD response signal was observed at 5,10 and $20 \mathrm{~Hz}$ in a $2-4 \mathrm{~mm}$ region of the dorsal hippocampus. At 7 days after KA-SE, during the latency period, there was no additional BOLD response recruitment in hippocampal lamellas above or below the initial activation observed in the baseline fMRI scans.

Conclusions: PP stimulation is able to trigger a pattern of hippocampal lamellar fMRI activation. At 7 days after KA-SE, no translamellar hyperexcitability was observed in agreement with previously described anatomical and physiological observations of a latency period. Patterns of fMRI hippocampal activation might be useful neuroimaging surrogate markers that might help define phenomenon of translamellar sprouting, a potential mechanism for epileptogenesis. 


\subsection{4}

REDUCED DEFAULT MODE NETWORK CONNECTIVITY IN IDIOPATHIC GENERALIZED EPILEPSY WITH UNCONTROLLED SEIZURES

Benjamin P. Kay ${ }^{1,2}$, M. W. DiFrancesco ${ }^{2,1}$, S. K. Holland ${ }^{2,1}$ and J. P. Szaflarski ${ }^{1,2}$ ( ${ }^{1}$ University of Cincinnati Academic Health Center, Cincinnati, $\mathrm{OH}$ and ${ }^{2}$ Cincinnati Children's Hospital Medical Center, Cincinnati, $\mathrm{OH}$ )

Rationale: The association between idiopathic generalized epilepsy (IGE) and generalized spike and wave discharges (GSWD) is well established, but the long-term effects of epileptogenic activity on the brain are not well understood. Simultaneous EEG/fMRI studies of IGE reveal deactivation in brain regions corresponding to the default mode network (DMN) during GSWD. Recent resting state EEG/fMRI studies of IGE have found that DMN functional connectivity is reduced even during periods of normal EEG commensurate with duration of disease. In this EEG/fMRI study we investigate the effect of uncontrolled seizures on DMN connectivity in IGE patients.

Methods: 78 IGE patients and 38 healthy control subjects underwent 1-3 consecutive 20-minute EEG/fMRI scans at 4 Tesla with $\mathrm{TR}=3$ seconds. 35 scans from 18 IGE patients out of 224 total scans were excluded because they contained GSWD. Group independent component analysis (ICA) was used to identify the DMN, and dual regression was used for group-level comparisons.

Results: Duration was correlated with a significant reduction in DMN connectivity in the cohort of all IGE patients consistent with previous studies. However, when patients with controlled and uncontrolled seizures were considered separately, the effect of duration remained significant in uncontrolled seizures but not controlled seizures. When patients were compared to healthy controls, connectivity was found to be significantly reduced in uncontrolled but not controlled seizures. Patients with uncontrolled seizures had significantly lower connectivity than those with controlled seizures, even after accounting for the effect of duration. Conclusions: DMN functional connectivity is reduced in IGE patients even during periods of normal EEG and can distinguish between patients with controlled and uncontrolled seizures. Connectivity in patients with uncontrolled seizures declines with duration of disease. DMN abnormalities may present a new biomarker for poorly controlled IGE.

\subsection{5 \\ EVALUATION OF AMYGDALA ENLARGEMENT IN SYMPTOMATIC LOCALIZATION-RELATED EPILEPSY PATIENTS USING 11C-METHIONINE-PET \\ Go Taniguchi ${ }^{1}$, K. Ito ${ }^{2}$, Y. Murata ${ }^{1}$, D. Sone ${ }^{1}$, Y. Watanabe ${ }^{1}$, M.} Okazaki $^{1}$, M. Watanabe ${ }^{1}$ and N. Sato ${ }^{2}\left({ }^{1}\right.$ Psychiatry, National Center of Neurology and Psychiatry, Kodaira-shi, Japan and ${ }^{2}$ Radiology, National Center of Neurology and Psychiatry, Kodaira-shi, Japan)

Rationale: In recent years, an increasing number of reports have indicated an association between epileptogenicity and amygdala enlargement, rather than sclerosis or atrophy. Since the seizures of these cases are relatively well controlled, few cases received surgical treatment. For this reason also, the physiopathology of this disease remains poorly understood. 11 Cmethionine-PET (Met-PET) is considered a useful measure for identifying brain tumors cause epilepsy. Then, we performed non-invasive diagnostic imaging studies including Met-PET, 18F-fluorodeoxyglucose-PET (FDGPET) and SPECT, in an attempt to gain insight into the physiopathology of this epilepsy.
Methods: Nine patients with symptomatic localization-related epilepsy, in whom amygdala enlargement was found on MRI were studied. Met-PET was performed in all patients, and SPECT and FDG-PET were conducted whenever possible. The imaging findings, clinical characteristics and EEG findings were evaluated, and the pathological implications of the findings for epilepsy were discussed. Results: Except 1 case that showed no definitive EEG abnormalities, the laterality of epileptiform EEG abnormalities was the same as that of amygdala enlargement in the other 8 cases. On Met-PET, increased uptake in the amygdala was observed in 1 case, while no increased uptake was detected in the other 8 cases. The same case also showed decreased uptake on SPECT and FDG-PET at the medial side of left temporal lobe, ipsilateral to amygdala enlargement. The 8 cases that had no increased uptake on Met-PET were divided into a group showing decreased uptake on FDG-PET and/or SPECT in the temporal lobe ipsilateral to amygdala enlargement $(n=5)$, and a group with no decreased uptake $(\mathrm{n}=3)$. Compared to the group with decreased uptake, the group showing no decreased uptake tended to have older epilepsy onset and very good seizure outcome. Four of nine patients had elderly onset epilepsy (above 60 years of age); seizures were not controlled in 1 patient with increased amygdala uptake on Met-PET, while seizures were controlled in all 4 patients who had no uptake.

Conclusions: In almost all patients, both interictal and ictal discharges on EEG showed the same laterality as amygdala enlargement, suggesting a strong association between amygdala enlargement and epileptogenicity. In the patient showing uptake on Met-PET, the etiology was probably gliomatosis cerebri based on MRI and other findings. The 5 patients showing no uptake on MetPET but decreased uptake on FDG-PET and/or SPECT probably had focal cortical dysplasia. In 3 patients with elderly onset epilepsy and no abnormal uptake in all functional imaging studies, the pathology remains unknown. However, because of the high probability of an association between amygdala enlargement and epileptogenicity, amygdala enlargement is important for understanding the pathology of epilepsy, especially in elderly patients. From the viewpoint of differentiating from tumors also, Met-PET study is effective in patients with elderly onset epilepsy showing amygdala enlargement.

\subsection{6 PROPAGATION OF INTERICTAL ACTIVITY IN FRONTAL LOBE EPILEPSY: A MEG-DTI STUDY}

E. M. Castillo ${ }^{1}$, Z. Li ${ }^{2}$, G. Von Allmen ${ }^{3}$, J. I. Slater ${ }^{4}$ and A. C. Papanicolaou ${ }^{5}{ }^{1}$ Pediatric Epilepsy Program, Florida Hospital, Orlando, FL; ${ }^{2}$ Neurology, Medical College of Wisconsin, Milwaukee, WI; ${ }^{3}$ Pediatrics, University of Texas-Houston, Houston, TX; ${ }^{4}$ Neurology, University of Texas-Houston, Houston, TX and ${ }^{5}$ Pediatrics, UTHSC College of Medicine, Memphis, TN)

Rationale: To study, in patients with frontal lobe epilepsy, the relationship between interictal epileptiform discharges and the microstructural integrity of the anterior aspects of the corpus callosum (CC). Specifically, we tested whether the interhemispheric propagation of frontal lobe discharges is correlated with changes in the functional integrity of the genu of the CC.

Methods: We analyzed, retrospectively, data from 17 right-handed patients diagnosed with refractory frontal lobe epilepsy that underwent MEG and MRI assessments, including Diffusion Tensor Imaging (DTI) sequence, as part of their presurgical evaluation. Three of the 17 cases were excluded: two due to inconclusive MEG results and one due to poor quality of DTI sequences. During the 
review of the MEG recordings, interictal discharge sources localized in the frontal lobe were further analyzed to extract the patterns of propagation (intrahemispheric or interhemispheric).Values of regional fractional anisotropy (FA) of the genu of the $\mathrm{CC}$ were extracted from DTI sequences (mid-sagittal slice) following subdivisions into seven regions, using the "Witelson" technique. Results: In the 14 cases analyzed, the patterns of interictal MEG activity was classified in two sets: The first included 6 cases showing intrahemispheric (intralobar or interlobar) and the second 8 cases showing interhemispheric propagation. Patients with interhemispheric patterns of spike propagation showed reduced FA values at the genu of the $\mathrm{CC}$ as compared to those with intrahemispheric propagation $(\mathrm{p}<0.05)$. Surgical outcome was available in 11 patients with no significant difference between the two groups.

Conclusions: Microstructural white matter alterations occur in the genu of the corpus callosum in patients with frontal epilepsy showing interhemispheric patterns of propagation of epileptic activity. The significance of this finding and its value in predicting surgical outcome should be further investigated.

\subsection{7}

\section{H-MAGNETIC RESONANCE SPECTROSCOPY IN THE ASSESSMENT OF EPILEPTIC MALFORMATIONS OF CORTICAL DEVELOPMENT}

Jorge G. Burneo ${ }^{2,3}$, S. Nikolova ${ }^{1,3}$ and R. Bartha ${ }^{1,3}$ ( ${ }^{1}$ Imaging, Robarts Research Institute, London, ON, Canada; ${ }^{2}$ Epilepsy Program, Western University, London, ON, Canada and ${ }^{3}$ Schulich School of Medicine and Dentistry, Western University, London, ON, Canada)

Rationale: Malformations of cortical development (MCDs) are disturbances in brain formation that arise from abnormalities affecting the processes of cortical development and involve cells that under normal circumstances would participate in formation of the cerebral cortex. Medically-intractable Epilepsy has been increasingly associated with MCDs. Proton (1H) MR spectroscopy (MRS) may have the potential to detect abnormalities in patients with MCDs. MRS can measure N-acetylaspartate (NAA), a marker of functional neuronal integrity, as well as creatine and phosphocreatine $(\mathrm{Cr})$, choline (Cho), glutamate (Glu) and myo-inositol (Myo). The purpose of this study is to evaluate the metabolic profile of MCDs using $1 \mathrm{H}$ MRS.

Methods: Data were acquired on a 3.0T Siemens Magnetom Tim Trio MRI, using a 12 channel head RF coil. Water suppressed spectroscopy data were acquired using PRESS (point resolved spectroscopy), from a $1.5 \times 1.2 \times 1.2 \mathrm{~cm} 3$ voxel centered on the cortex containing the malformation in each patient (repetition time $(\mathrm{TR}) /$ Echo time $(\mathrm{TE})=3200 / 35 \mathrm{~ms}$ ), and in similar cortical regions in controls. Metabolite levels were measured from spectra using the fitMAN (v1.7) software, and were normalized to Cr. 1H-MRS data were obtained for seven controls and five patients (two with cortical dysplasia and three with polymicrogyria) and compared using a t-test. Results: Figure 1 shows pooled metabolite ratios in patients (red) and controls (blue). There was a significant decrease in NAA/ $\mathrm{Cr}(\mathrm{p}<0.05)$ and a significant increase in $\mathrm{Glu} / \mathrm{Cr}(\mathrm{p}<0.05)$ in patients compared to controls. Additional patients will be recruited to validate these results. Evidence of neuronal dysfunction in patients with MCD may have relevance for the mechanisms of seizure generation in these forms of epilepsy.

Conclusions: This preliminary study demonstrates some metabolite differences between patients with MCDs and control subjects, although the small sample size did not allow specific analysis of each group of malformations. Further recruitment is necessary as there may be some particular findings pertaining to each different type of MCDs.

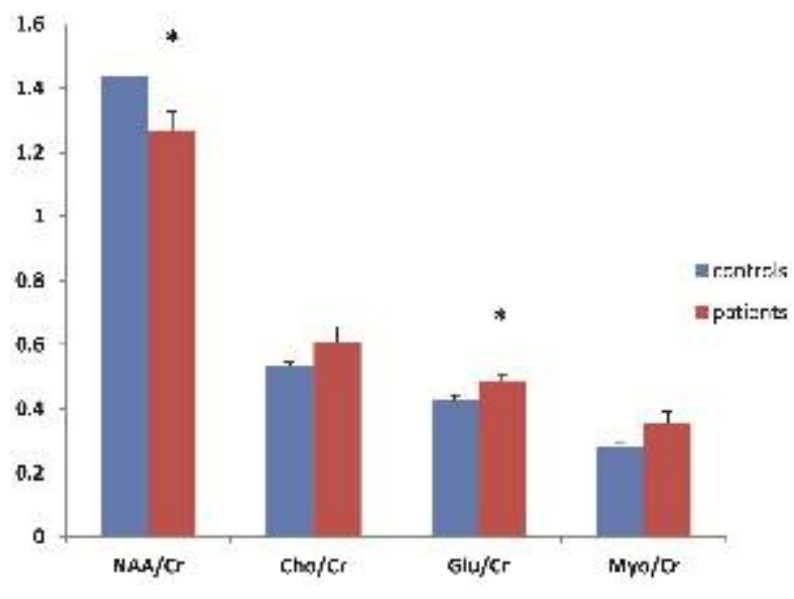

\subsection{8 \\ EEG-FMRI STUDY IN EPILEPTIC PATIENTS WITH PERIVENTRICULAR NODULAR HETEROTOPIA} Joong Koo Kang ${ }^{1}$, E. Lee ${ }^{2}$, S. Oh ${ }^{3}$ and H. Park ${ }^{3}\left({ }^{1}\right.$ Neurology, Asan Medical Center, Seoul, Republic of Korea; ${ }^{2}$ Neurology, Ulsan University Hospital, Ulsan, Republic of Korea and ${ }^{3}$ Electrical Engineering, Korean Advanced Institute of Science and Technology (KAIST), Daejeon, Republic of Korea)

Rationale: Patients with periventricular nodular heterotopia (PVNH) have epilepsy, and there is likely to have a complex interaction between epileptogenic nodular tissue and overlying cortex. However, it is unclear whether the epileptic focus is the nodule, overlying cortex, or both. Combined scalp EEG and functional MRI (EEGfMRI) may provide an interesting non-invasive alternative tool to understand those mechanisms in PVNH. The aim of this study was to reveal the cerebral structures active during interictal epileptiform discharges (IEDs) in PVNH using EEG-fMRI.

Methods: We investigated 8 epileptic patients with PVNH (6 females, mean age, $29.3 \pm 5.2$ years; bilateral PVNH in 7 and unilateral in 1) using simultaneous recording of EEG-fMRI inside a 3 T MRI scan. EEG was continuously acquired from inside the scanner. IEDs were visually identified in the filtered EEG. Imaging data were analyzed using SPM8 with canonical hemodynamic response function to detect PMRI BOLD activation and correlated BOLD changes with location of PVNH in each patient.

Results: Among 8 patients, two patients were not analyzed because of the absence or rare number of IEDs during recording. In remaining 6 patients, 5 of 6 patients showed BOLD activation. In five patients with BOLD activations, 3 patients only showed BOLD activations around PVNH, 1 showed only overlying cortex without PVNH and 1 showed both PVNH and overlying cortex area.

Conclusions: This study revealed that heterotopia and/or overlying cortical areas were simultaneously or predominantly involved in BOLD changes during IED depending on the patients.

\subsection{9 \\ IS SISCOM (SUBTRACTION ICTAL SPECT CO- REGISTERED TO MRI) HELPFUL TO THE SUCCESSFUL SURGERY IN NONLESIONAL EXTRATEMPORAL EPILEPSY?}

Pamela Song ${ }^{1}$, E. Joo ${ }^{2}$, D. Seo ${ }^{2}$, D. Koo ${ }^{2}$ and S. Hong ${ }^{2}\left({ }^{1}\right.$ Neurolgoy, Ilsan Paik Hospital, Inje University College of Medicine, Goyang, Republic of Korea and ${ }^{2}$ Neurology, Samsung Medical Center, Sungkyunkwan University School of Medicine, Seoul, Republic of Korea)

Rationale: Nonlesional extratemporal seizures are challenging to plan respective epilepsy surgery. Surgical outcomes have been reported to be worse than those for temporal lobe epilepsy. The aim 
of this study was to evaluate the overall surgical outcomes of nonlesional extratemporal epilepsy and to determine the usefulness of SISCOM (Subtraction Ictal SPECT Co-registered to MRI) in the prediction of successful postsurgical outcome.

Methods: From January 2007 to April 2010 at Samsung Epilepsy Clinic in Samsung Medical Hospital, 25patients with nonlesional extratemporal lobe seizures ( 17 female) were enrolled who had undergone comprehensive presurgical evaluation and subsequent intracranial EEG monitoring for epilepsy surgery (a postoperative period of $\geq 1$ year). Their clinical data including SISCOM analysis were all reviewed and postsurgical outcome was determined using Engel classification.

Results: Mean age at surgery was 26.8 years, mean disease duration before surgery was 13.7 years, and mean follow up was 35.9 months. There were 12 Frontal lobe epilepsy, 6 Temporo-parietal lobe epilepsy, 4 Parietal lobe epilepsy, and 3 Occipital lobe epilepsy. In 12 cases, hyperperfused areas in SISCOM were congruent to surgical resection margin, and were noncongruent in 13 cases. Hypometabolic areas in FDG-PET were congruent in 11, and were non congruent in 14 cases. Post surgically $10(40 \%)$ were seizure free (Engel class $1 \mathrm{~A})$, and $14(60 \%)$ were not seizure free. Pathology was all focal cortical dysplasia.

Seizure free outcome was predicted 6 of $11(54.5 \%)$ in congruent SISCOM, but only 4 out of 11 (36.4\%) in noncongruent SISCOM. Conclusions: Our results suggest that seizure free surgical outcome were seen in $40 \%$ of cases in nonlesional extratemporal epilepsy. It seems that SISCOM analysis is helpful to replace limited role of MRI as image study for nonlesional extratemporal epilepsy.

\subsection{0 \\ SEIZURES ARE ASSOCIATED WITH A FUNCTIONAL REORGANIZATION OF THE LIMBIC SYSTEM IN MEDIAL TEMPORAL LOBE EPILEPSY}

K. M. Sequeira, R. K. Sainju, A. Tabesh, M. A. Ahlman, K. M. Spicer, J. C. Edwards and Leonardo Bonilha (Medical University of South Carolina, Charleston, SC)

Rationale: Structural imaging studies have demonstrated that medial temporal lobe epilepsy (MTLE) is associated with limbic atrophy involving not only the hippocampus but also peri-hippocampal and extra-temporal structures. While MTLE is related to static structural limbic compromise, it is unknown whether the limbic system undergoes a dynamic functional reconfiguration during seizures. In this study, we aimed to investigate state specific (i.e. ictal versus interictal) configuration of functional limbic networks in patients with MTLE.

Methods: We studied the clinical information and single positron computed tomography (SPECT) images obtained with intravenous infusion of the radioactive tracer Technetium Tc 99m Hexamethylpropyleneamine Oxime (Tc-99m HMPAO) during ictal and interctal state in 20 patients with unilateral MTLE (12 left and 8 right MTLE). The SPECT data was normalized for whole brain radiotracer uptake, and spatially normalized using linear and nonlinear parameters using SPM8. Pair-wise voxel-based analyses were used to define global changes in tracer between states. Regional uptake was calculated for anatomical areas, and state specific adjacency matrices were constructed based on regional correlation of uptake across subjects. Graph theoretical measures were then applied to investigate global and regional state specific network reconfigurations.

Results: A significant increase in tracer uptake was observed in the medial temporal region, cerebellum, thalamus, insula and putamen, in the ictal state compared with the interictal state (Figure 1). A mild increase in whole network clustering coefficient was observed in the ictal state. Regional clustering coefficient and local efficiency were increased over the ipsilateral and contralateral hippocampus, contralateral anterior cingulate, and ipsilateral fusiform gyrus(Figure 2). Compared to whole brain analyses, graph measures unveiled a significant change in network parameters of the fusiform gyrus during the ictal state.

Conclusions: These results suggest that MTLE is associated with a functional state specific limbic system organization. Increased ictal tracer uptake suggests an increase metabolic demand of limbic regions associated with epileptogenesis, and the reorganization of the functional network during a seizure suggests higher connectivity and synchrony of limbic areas, notably leading to an increase local participation of the fusiform region.
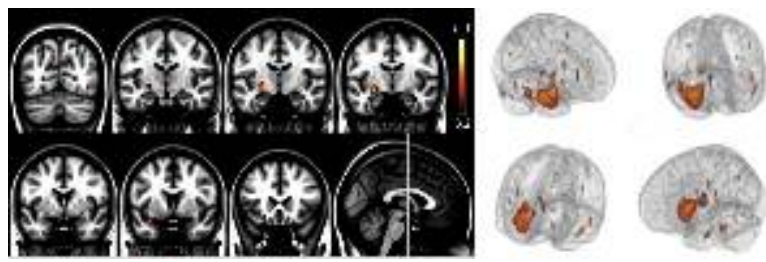

Figure 1 -Voxel wise differences in radiotracer uptake (paired $t$ test ictal $>$ interictal, FDRq $<0.05$ ).
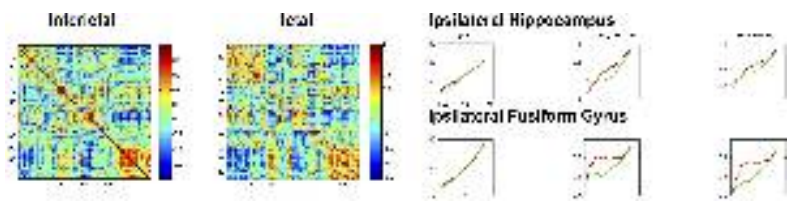

Figure 2- The panels on the left show the correlation coefficient matrices for ictal and interictal states. On the right, node graphical measures are displayed for representative regions of interest.

\subsection{1 \\ ATTENUATED AND AUGMENTED EMOTIONAL FACE PROCESSING NETWORKS IN TEMPORAL LOBE EPILEPSY}

Brett W. Fling, J. D. Riley and J. J. Lin (Neurology, University of California, Irvine, CA)

Rationale: Although deficits in social cognition and emotional processing are significant comorbidities in people with temporal lobe epilepsy (TLE), the functional underpinnings remain unclear. The amygdala is a pivotal component for processing stimuli with emotional and social significance and has been implicated in the impaired ability to evaluate emotional situations in patients with TLE. Further, an anterior-posterior gradient exists within the temporal-occipital facial processing network in which the temporal pole (TP) and superior temporal sulcus (STS) are important for integrating autobiographical and emotional information, while the fusiform gyrus (FFA), and the lateral inferior occipital lobe (OFA) are involved in the core visual analysis of faces. Given that people with TLE have impaired facial emotional recognition, we hypothesize that functional MRI signals in the emotional integration regions (TP, STS) in TLE would be reduced compared to controls, while signals in the posterior face regions (FFA, OFA) would show no group difference. We further hypothesized that a greater magnitude of signal reduction would be seen on the temporal lobe regions ipsilateral to the side of seizure onset.

Methods: Functional MRI data were obtained using a standard block design to map face sensitive regions using a fear-face paradigm in 24 patients with medically intractable TLE (Left TLE $=15$; Right TLE $=$ 9; Age $=37.4$ years; female: 13 ) and 19 healthy controls (HC, 31.8 years; female: 10). The face-responsive regions of interest were identified in each individual using the peak voxel of the activation clusters identified by the contrast "faces $>$ places" within the amygdala, TP, STS, FFA and OFA. We used a RMANOVA to identify group differences in percent signal change for these areas. Analyses were performed according to the side of seizure onset (ipsilateral vs. contralateral). Finally, we calculated a laterality index for number of significantly activated voxels within the amygdala. 
Results: TLE patients demonstrated significantly decreased activation in the ipsilateral OFA $(\mathrm{P}<0.02)$, and showed a trend toward decreased activation in the ipsilateral STS $(\mathrm{P}=0.06)$ compared to HC. Conversely, TLE subjects activated the contralateral TP significantly more than HC $(\mathrm{P}<0.02)$. No group differences were noted for activation in either the amygdala or FFA. Lastly, in patients with TLE the amygdala laterality index showed lateralized activation contralateral to the side of seizure onset (LTLE $=0.49$; RTLE $=-0.43$ ).

Conclusions: Cortical activity in face integration regions was both attenuated and augmented in people with TLE. We also confirm previous work showing clear hemispheric asymmetries in amygdala activation. The attenuated fMRI signals in the ipsilateral hemisphere may reflect local network disturbances within the epileptogenic zone, while the augmented fMRI signals in the contralateral hemisphere may suggest a compensatory mechanism, providing important insights into the pathophysiology of impaired emotional process in TLE.

NINDS Funding: T32-NS45540 (PI: Baram) and K23-NS060993 (PI: Lin)

\subsection{2 \\ LANGUAGE ACTIVITY IN EPILEPTIC PATIENTS REPRESENTED BY MAGNETOENCEPHALOGRAPHY USING AUDITORY WORD PRESENTATION}

Nao Suzuki, N. Tanaka and S. M. Stufflebeam (Athinoula A. Martinos Center, Charlestown, MA)

Rationale: Evaluation of language activity of the brain is important for planning epilepsy surgery. Magnetoencephalography, a noninvasive neuroimaging procedure, maps verbal function by using language tasks such as semantic decision, picture naming and verb generation. However, some patients cannot perform these tasks due to their limited verbal skills. The purpose of this study is to assess the feasibility of passive auditory language task for evaluating neuronal activity of language.

Methods: Four intractable epilepsy patients (male: 3, female: 1, age: 4-16, right-handed: 2 , left-handed: 2 ) were retrospectively studied. MEG was recorded with a 306-channel whole-head system at a sampling rate of $600 \mathrm{~Hz}$. In all patients, high-resolution $3 \mathrm{~T}$ anatomical MRI data were acquired with magnetization-prepared rapid acquisition gradient-echo (MPRAGE). During MEG recording, patients performed a passive word listening task with 160 English words. Interstimulus interval was $3000 \mathrm{~ms}$. MEG data epochs from $500 \mathrm{~ms}$ pre-stimulus and $1000 \mathrm{~ms}$ post-stimulus period were averaged after preprocessing. We calculated spatiotemporal source distribution of the averaged data by using dynamic statistical parametric maps (dSPMs). DSPMs were visually mapped on the MRI-derived cortical surface reconstructed by Freesurfer. We also calculated laterality index (LI) by using regions of interest (ROIs) based on the cortical parcellation obtained by Freesurfer. We selected superior temporal, middle temporal, supramarginal, inferior parietal cortices, opecular and triangular parts of the inferior frontal lobe on both hemispheres for ROI analysis, based on a priori knowledge of Wernicke and Broca areas. $\mathrm{LI}$ was obtained by $\mathrm{LI}=(\mathrm{L}-\mathrm{R}) /(\mathrm{L}+\mathrm{R})$, where $\mathrm{L}$ and $\mathrm{R}$ is the number of unit dipoles with an $\mathrm{F}$ value higher than the threshold in the ROIs within a time window of $250-550 \mathrm{~ms}$ post stimuli. The threshold was determined as the half of the maximum value over all ROIs. Language laterality was estimated based on the LI as follows; $\geq 0.1$ :left, $0.1>$ LI $>-0.1$ :bilateral, $\leq-0.1$ :right.

Results: Primary auditory response was observed at $100 \mathrm{~ms}$ post stimuli. Possible language activity was observed around $400 \mathrm{~ms}$ poststimuli. In right-handed patients, one showed activation in the left posterior temporoparietal and inferior frontal cortex $(\mathrm{LI}=0.70)$. The other one showed right temporal activation $(\mathrm{LI}=-0.63)$. Two lefthanded patients showed right temporoparietal and inferior frontal activation ( $\mathrm{LI}=-0.28,-0.14)$.

Conclusions: The results of our study suggest that the passive auditory word task activates the language function of the brain. MEG recorded during the task performance may provide information for estimating the language laterality in presurgical evaluation.

\subsection{3 \\ ENHANCED MULTIMODAL IMAGING ASSESSMENT FOR EPILEPSY}

Sandra Poliachik ${ }^{1}$, E. J. Novotny ${ }^{1,2}$, A. V. Poliakov ${ }^{1}$, G. E. Ishak ${ }^{1,2}$, S. S. McDaniel ${ }^{1,2}$, E. Simard-Tremblay ${ }^{1,2}$, J. Kuratani ${ }^{1,2}$, R. Saneto ${ }^{1,2}{ }^{\text {, }}$ and J. Ojemann ${ }^{1,2}\left({ }^{1}\right.$ Seattle Children's Hospital, Seattle, WA and ${ }^{2}$ University of Washington, Seattle, WA)

Rationale: In addition to electroencephalographic (EEG) data, neuropsychological testing and neurological exam, presurgical epilepsy evaluations typically include acquisition of imaging data that assess anatomy, metabolism and functional measures. Synthesizing a large amount of imaging data into a coherent set of images to correlate with clinical and electrophysiological data can be a challenge. Our data integration technique involves utilizing three dimensional (3D) volume renderings to enhance visualization of anatomy, metabolism and function.

Methods: We retrospectively examined the imaging performed as part of a standard epilepsy protocol for pediatric epilepsy patients. Inclusion criteria required that the patient had three imaging modalities: an epilepsy protocol MRI, PET or SPECT abnormality, and a tasked-based fMRI. Some subjects also had functional connectivity MRI (fcMRI) and postoperative MRI. Data from each patient were processed according to modality. In Biolmage Suite (1), $3 \mathrm{D}$ renderings of the linear co-registration of PET and structural T1 MRI were overlaid to identify the location of any abnormalities in metabolism. Functional MRI scans were post-processed using FSL, and a 3D rendering was linearly co-registered to the structural $\mathrm{T} 1$ and PET-T1 volumes. Data from SPECT scans were normalized and processed using BioImage Suite, and were linearly co-registered to the structural T1. Utilizing the metabolic and functional 3D renderings, the brain anatomy was assessed for abnormalities that are often more clearly observable in a $3 \mathrm{D}$ volume rendering than on two dimensional MRI views. These images also provide a more clear relationship to other anatomical landmarks. When available, postoperative MRI imaging was non-linearly co-registered with BioImage Suite to the preoperative MRI such that the metabolic and functional preoperative results could be compared to specific resection margins.

Results: For a two year period, data from 16 patients $(11 \mathrm{M} / 5 \mathrm{~F}$; range $1-19 \mathrm{yr}$ ) were evaluated using $3 \mathrm{D}$ volume renderings of imaging data. Of the 16 patients, metabolic abnormalities occurred in the temporal lobe (4), parietal lobe (6), frontal lobe (4) and a mix of parietal and occipital lobe (2). Six of the patients also had postoperative MRI data. These retrospective reviews of patient data allowed neurologists and surgeons to visualize metabolic and functional data in relation to structural MRI data.

Conclusions: Using 3D volume renderings allows the care team to incorporate results from different study modalities in an intuitive fashion. In addition, EEG data can be used to determine the relationship of the ictal and interictal epileptiform abnormalities to the location of the abnormal metabolic and functional data. The imaging is used to guide intracranial electrode studies and tailor resective surgery to increase success and decrease morbidity. Including 3D renderings as part of a standard presurgical epilepsy evaluation may enhance diagnoses from an imaging perspective. 


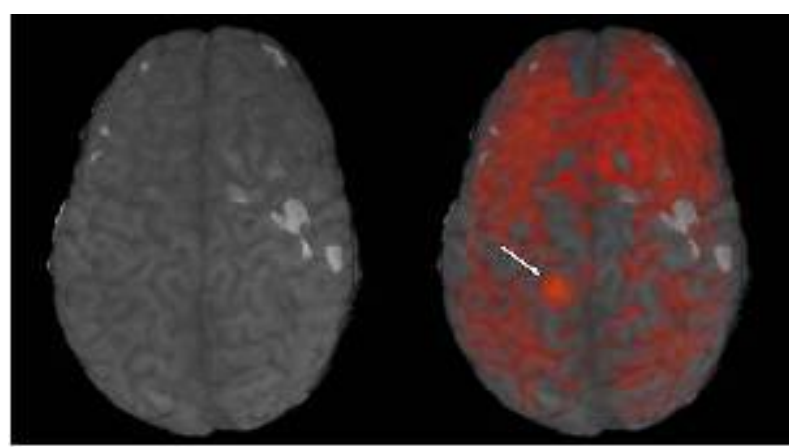

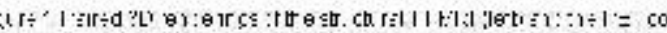

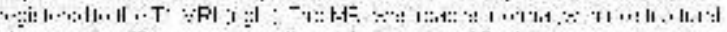

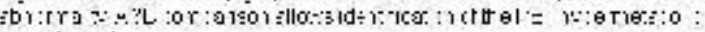
$\because 11: 1: 1: 24$

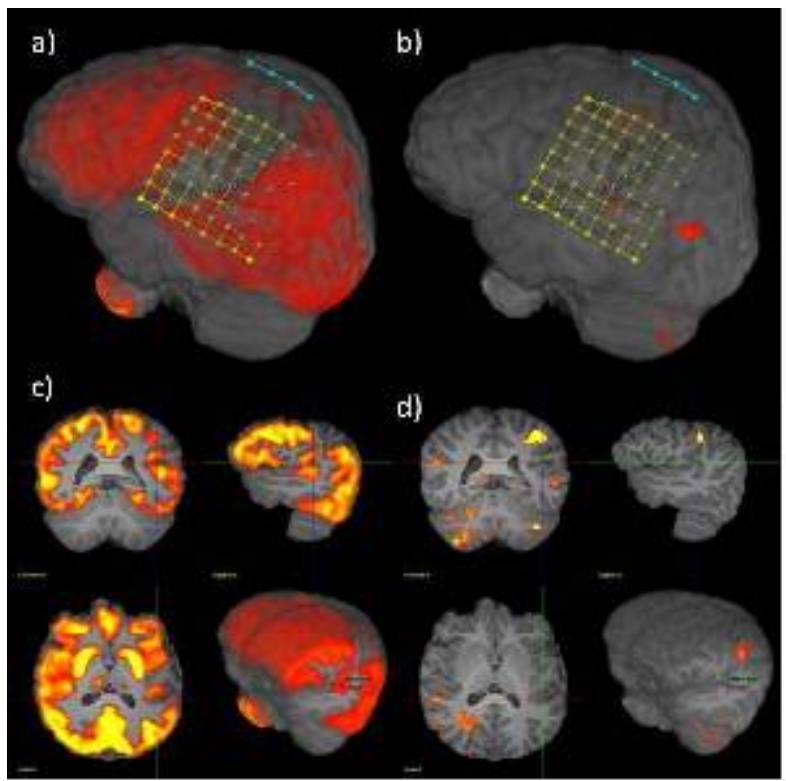

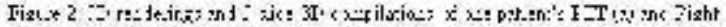

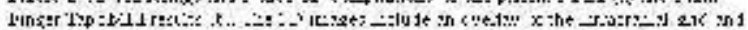

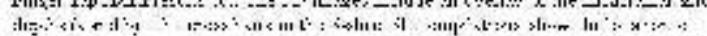
:

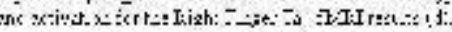

\subsection{4}

PRE AND POST OPERATIVE LATERALITY OF LANGUAGE REGIONS ASSESSED BY FMRI IN TEMPORAL LOBE EPILEPSY

Lauren A. Zimmaro ${ }^{1,2}$, A. D'Alfonso ${ }^{1}$, M. M. Berl ${ }^{2,3}$, L. N. Sepeta ${ }^{2}$, I. Dustin $^{1}$, A. L. Yaun ${ }^{4}$ J. D. Heiss ${ }^{5}$, S. Sato ${ }^{6}$, W. H. Theodore ${ }^{1}$ and W. D. Gaillard ${ }^{1,2}\left({ }^{1}\right.$ Clinical Epilepsy Section, National Institutes of Neurological Disorders and Stroke, National Institutes of Health, Bethesda, MD; ${ }^{2}$ Center for Neuroscience, Children's National Medical Center, Washington, DC; ${ }^{3}$ Pediatric Imaging and Tissue Sciences Section on Tissue Biophysics and Biomimetics, Eunice Kennedy Shriver National Institute of Child Health and Human Development, National Institutes of Health, Bethesda, MD; ${ }^{4}$ Department of Neurosurgery, Children's National Medical Center, Washington, DC; ${ }^{5}$ Surgical Neurology Branch, National Institutes of Neurological Disorders and Stroke, National Institutes of Health, Bethesda, MD and ${ }^{6}$ EEG Laboratory, National Institutes of Neurological Disorders and Stroke, National Institutes of Health, Bethesda, MD)

Rationale: Patients with intractable epilepsy may undergo surgical intervention to remove temporal lobe seizure foci. Functional MRI is an informative presurgical tool for identifying laterality of language regions and avoiding postoperative deficits. Moreover, it can provide data on the effects of persistent seizures on the functional organization of language. In the current study, we aimed to measure post-operative changes in lateralization of language related regions after temporal lobectomy in patients with focal epilepsy.

Methods: 15 normal controls (mean age $=22.6$ years, $\mathrm{SD}=3.1$ ) and 19 patients (mean age $=26.2, \mathrm{SD}=12.7)$ with temporal lobe epilepsy $(13$ left TLE, 6 right TLE) performed an auditory description decision task as part of presurgical evaluation for a temporal lobectomy and then again at least six months post-surgery (average 5.4 years). Controls performed the task with at least a six month time difference between scans (average 1.7 years). Images were processed in SPM8. A laterality index (LI) was calculated using a bootstrap method for anatomical regions of interest (ROI): inferior frontal gyrus (IFG), Wernicke's area (WA), and middle frontal gyrus (MFG).

Lateralization was left if $L I>0.20$, bilateral if $L I<0.20$, right if $\mathrm{LI}<0.20$. We conducted a repeated measures ( $2 \times 2 \times 4$ way) ANOVA to evaluate effects of group (patient or control), time (as repeated scans), and ROI. Exploratory analyses (ANOVA) directed toward the patient group examined the effect of location of resection on LI. Results: Results showed no main effect of time but main effects of group [patients had lower LI in all ROIs $(\mathrm{p}<0.001)$ ] and ROI [WA was the most lateralized and MFG was the least $(p<0.001)]$. There were no changes in LI from pre- to post-operative conditions, and this did not differ for group or ROI. Patient-directed analyses indicated that there was no effect of TLE on LI, nor was there an effect whether the surgery was in the dominant hemisphere $(n=10)$. Clinically, there were no cases of aphasia or significant naming deficits.

Conclusions: Postsurgical assessment of LI did not differ following resection - remaining lower than controls - and no significant language deficits were observed. This provides evidence that following resection, there are no substantial changes in lateralization which is likely due to the information provided by the presurgical fMRI results, and also indicates that despite the cessation of seizures and reducing of medications, LI does not normalize toward control levels. This may be because networks are no longer plastic, changes occur over a longer timeframe than were measured in this study, or greater bilateral activation is compensatory.

\subsection{5}

REORGANIZATION OF MEMORY CIRCUITS IN TEMPORAL LOBE EPILEPSY DEMONSTRATED ON PREAND POST-OPERATIVE FUNCTIONAL MRI STUDY CHUSAK LIMOTAI, R. McLachlan, S. Hayman-Abello, B. Hayman-Abello, S. Brown, F. Bihari and S. Mirsattari (Clinical Neurological Sciences, Western University, London, ON, Canada)

Rationale: Functional MRI (fMRI) has been increasing used to study the impact of temporal lobe epilepsy (TLE) over the past decade. Effect of seizures in medically refractory TLE causing reorganization of memory circuits, prior to anterior temporal lobectomy (ATL), has been observed in some previous fMRI studies. However, demonstration of potential reorganization after ATL has been rarely studied.

Methods: Nineteen patients with medically intractable TLE and 15 healthy controls were enrolled in this study. Ten patients underwent left ATL and 9 underwent right ATL. Group analyses of a novelty scene-encoding paradigm comparing areas of blood oxygen-leveldependent (BOLD) signal activation pre- and post-ATL were performed and separately analyzed between left (LTLE) and right TLE (RTLE) patients . Among these 19 patients, a time period of performing post-operative fMRI varied from 2 to 26 months after the surgery, with a mean period of 12 months. Effect of time after the surgery on memory circuits reorganization was further assessed by comparison in each group between the patients who performed the post-ATL fMRI study at 6 months or less and after 6 months. Results: Patient's age was between 20 and 63 years (mean $40.4 \pm$ 13.0) and age onset of epilepsy was between 2 and 55 years (mean 
$21.9 \pm 13.5)$. Fifteen, three, and one patient achieved Engel Class I, II and III surgical outcome, respectively. Pathology revealed hippocampal sclerosis in 12, gliosis in 5, and benign tumors in 2 patients. Pre-operative fMRI study showed smaller hippocampal and parahippocampal activation ipsilateral to the side of epileptogenic focus in both LTLE and RTLE patients. Also, contralateral extratemporal either frontal or parietal lobe signal activation was observed in both groups, but statistically significant on the RTLE group ( $\mathrm{p}=$ 0.04). In comparison to pre-operative study, shifting areas of activation at the post-operative study were disclosed over temporal region, with statistically significance in post-right ATL $(\mathrm{p}=0.01)$. This reorganization was accentuated appearing to be more robust and wider activation over time after the surgery (greater over 6 months), once again statistically significant in post-right ATL group.

Conclusions: Disturbance of normal memory circuits prior to the surgery in TLE demonstrating extratemporal recruitment was observed. Reorganization after ATL localized to the temporal area was seen in both LTLE and RTLE patients. Post-operative greater activation over time particularly at after 6 months was noted. However, statistically significant areas of fMRI activation were appreciated in RTLE group.

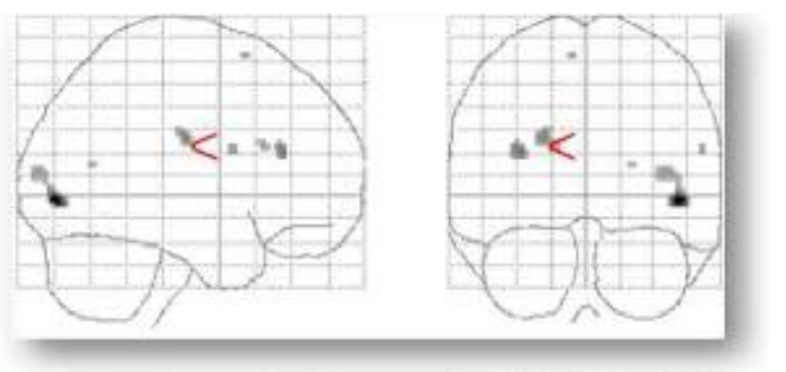

Extratemporal actlyetton at the pre-operettwe MARI study in RTLE

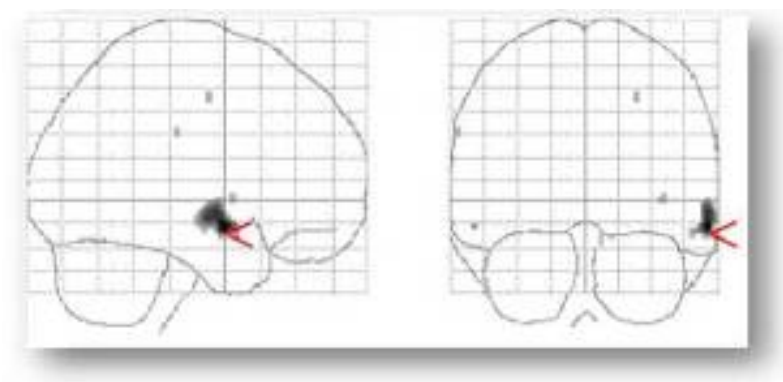

IDsilateral temporal activation after right ATL in the same group of patlents as the first pleture

\subsection{6 \\ PROXIMITY BETWEEN FMRI ACTIVATION AND STRUCTURAL LANGUAGE PATHWAYS PREDICTS VERBAL FLUENCY PERFORMANCE IN LEFT FRONTAL LOBE EPILEPSY \\ Christian Vollmar ${ }^{1,2}$, J. O'Muircheartaigh ${ }^{3}$, M. Symms ${ }^{1}$, G. Barker ${ }^{3}$, P. Thompson ${ }^{1}$, V. Kumari ${ }^{3}$, J. Duncan ${ }^{1}$, M. Richardson ${ }^{3}$ and M. Koepp ${ }^{1}\left({ }^{1}\right.$ UCL Institute of Neurology, London, United Kingdom; ${ }^{2}$ Dept. of Neurology, University of Munich, Munich, Germany and ${ }^{3}$ King's College London, London, United Kingdom)}

Rationale: Language function is frequently reorganized in epilepsy patients, particularly in left frontal lobe epilepsy (FLE). Not only is the right frontal lobe increasingly recruited, there is also a significant reorganization within the left hemisphere. The degree of functional MRI (fMRI) activation, or measures such as the laterality index are often not correlated with the neuropsychologically measured language capabilities of patients. We hypothesize, that the proximity of functional cortex to the underlying language pathways is crucial for the language performance in left frontal lobe epilepsy. We investigate this with a combined fMRI and diffusion tensor imaging
(DTI) pilot study in 16 patients with FLE.

Methods: Patients were examined on a 3T MRI scanner. They performed a verbal fluency fMRI paradigm, comprising 5 blocks of 30 seconds covert word generation, alternating with rest. fMRI was recorded using a whole brain acquisition of 50 axial slices, $2.5 \mathrm{~mm}$ slice thickness with a TR of $2.5 \mathrm{sec}$ and was analyzed with SPM 5 software. DTI was acquired with a total of 52 diffusion directions, a slice thickness of $2.5 \mathrm{~mm}$ and $2.5 \mathrm{~mm}$ in plane resolution. DTI data was processed using FSL 4.1.9 software. DTI data was normalized to a template in standard space and the inverse normalization was used to project seed and mask regions for probabilistic tracking of the arcuate fasciculus in each subjects individual space. The resulting tracts were also normalized to MNI space and superimposed with the coregistered fMRI statistical parametric maps. Both images were smoothed and thresholded, and the overlapping volume within the left hemisphere was determined.

Results: One patient failed to activate during the fMRI paradigm, two showed a right language dominance and were also excluded. For the remaining patients, the peak fMRI z-score ranged from 3.2 to 7.4. The volume of overlap between arcuate fasciculus and fMRI activation ranged from 0.8 to $31.4 \mathrm{ml}$, not normally distributed. The Verbal fluency score from neuropsycholgical assessment ranged from 4 to 22 words per minute. This was not correlation with peak fMRI activation (Spearman correlation 0.22 , but it was correlated with the overlapping volume of fMRI and DTI (Spearmann correlation 0.81). Conclusions: We present a processing pipeline for combined analysis of functional activation in relation to underlying structural pathways. In patients with left FLE, this measure correlated with their neuropsychological verbal fluency scores, whereas the fMRI activation alone showed no relation to cognitive performance. This provides evidence, that the efficiency of cortical reorganization of language depends on the proximity to major structural pathways.

\section{Antiepileptic Drugs: Mechanisms of Action}

\subsection{7 \\ BRIVARACETAM HAS A TIME- AND STIMULATION- DEPENDENT EFFECT ON SYNAPTIC TRANSMISSION} Xiao-Feng Yang ${ }^{1}$, S. M. Rothman ${ }^{1}$ and J. M. Dubinsky ${ }^{2}\left({ }^{1}\right.$ Neurology, University of Minnesota, Minneapolis, MN and ${ }^{2}$ Neuroscience, University of Minnesota, Minneapolis, MN)

Rationale: Brivaracetam (BRV) is a novel synaptic vesicle protein 2A (SV2A) ligand, 10 fold more potent than levetiracetam (LEV) in animal models of epilepsy. Previously, we demonstrated that LEV decreases synaptic transmission in a time- and stimulation frequencydependent manner and vesicular entry may mediate the effect of LEV. In the present study, we examine if the effect of LEV on the synaptic response is reproducible by BRV and if BRV.

Methods: Hippocampal slices (500 $\mu \mathrm{m}, 3-4 \mathrm{wk}$ old SD male rats) were maintained in a submerged perfusion chamber with bipolar stimulation of Schaffer collateral fibers and extracellular recordings from the dendritic layer of CA1. We examined BRV action at 1, 3, 10 and $30 \mu \mathrm{M}$ concentrations applied for either 30 minutes or 3 hours prior to stimulation ( $\mathrm{N}=8$ each group). Three trains of stimuli ( 20 pulses at 5, 10, 20, and $40 \mathrm{~Hz}$ ) were delivered, each 6 min apart, and the effect of BRV on the ratio of EPSPn/EPSP1 was measured. To determine if the effect of BRV is mediated by getting into the synaptic vesicle and binding to SV2A, we used a load-unload protocol. After $3 \mathrm{hr}$ incubation (loading), hippocampal slices were rinsed for 15 minutes with artificial cerebrospinal fluid (ACSF) to remove extracellular BRV (washout). To unload BRV from presynaptic vesicles, train stimulation (4 Min, $10 \mathrm{~Hz}$ ) was then delivered to the slices followed by another $15 \mathrm{~min}$ ACSF washout (unloading). After each stage (loading, washout, unloading) the ratio of EPSPn/EPSP1 was measured using three trains of stimuli (20 pulses at $40 \mathrm{~Hz}$ ). Statistical analyses were performed using two way repeated-measures ANOVA. 
Results: BRV decreases synaptic transmission in a time-, stimulation frequency-, and concentration-dependent manner. At $40 \mathrm{~Hz}$ train stimulation, 3 hours incubation with $1-30 \mu \mathrm{M}$ BRV and 30 minute incubation with $\geq 10 \mu \mathrm{M}$ BRV significantly decreased the ratio of EPSPn/EPSP1 compared to control beginning at pulse 2 or $3(\mathrm{P}<$ 0.05 ). At $20 \mathrm{~Hz}$ train stimulation, 3-hour incubation with $\geq 10 \mu \mathrm{M}$ BRV incubation and 30 minute incubation with $30 \mu \mathrm{M}$ BRV reduced the ratio of EPSPn/EPSP1 compared to control beginning at pulse 3 or $4(\mathrm{P}<0.05)$. At $10 \mathrm{~Hz}$ train stimulation, only 3-hour $30 \mu \mathrm{M} \mathrm{BRV}$ incubation significantly decreased the EPSPn/EPSP1 ratio compared to control starting at pulse $13(\mathrm{P}<0.05)$. The remaining conditions, we did not see any significant decrease in EPSPn/EPSP1 ratio. Previous studies with LEV showed that the effect of 3-hour 300 $\mu \mathrm{M} \mathrm{LEV}$ incubation on decreasing synaptic responses could not be altered after a 15-minute washout to remove the drug from the medium, but it could be eliminated by unloading the drug using train stimulation (4 Min, $10 \mathrm{~Hz}$ ) plus 15-minute washout. But BRV affect cannot be eliminated by either washout or unloading stimulation followed by subsequent washout.

Conclusions: Our data suggest that BRV resembles LEV in its effect on synaptic transmission, but is more potent in decreasing synaptic transmission than LEV. The load-unload results are consistent with our hypothesis that these drugs reduce synaptic transmission by getting into synaptic vesicles and binding to SV2A.

\subsection{8 \\ SCREENING ANTICONVULSANT DRUGS FOR ANTI- INFLAMMATORY EFFECT USING ACTIVATED MICROGLIA IN VITRO \\ Songtao Yu, J. Ott, J. Abraham, D. Xu and S. Koh (Neurology, Children's Hospital of Chicago Research Center, Chicago, IL)}

Rationale: Inflammatory responses by brain have been shown to be involved in both seizure occurrence and epileptogenesis, implying that the mediators of inflammatory pathway may form new molecular targets for antiepileptic drugs. Anticonvulsant drugs are a diverse group of compounds widely used in the treatment of epileptic seizures. ; their anti-inflammatory ability has, however, rarely been studied. Therefore, we used in vitro tissue culture system to screen anticonvulsant drugs that not only inhibit the symptoms of epilepsy, but also prevent or abrogate the inflammatory wing of disease pathogenesis.

Methods: Mouse microglia cell line BV-2 and mixed primary mouse glial culture ( containing more $50 \%$ of microglia cells as determined by flow cytometry) were used in this study. After determining the time course of drug effects, cells were pretreated with corresponding anticonvulsant drugs for 1-2 $\mathrm{h}$ and then stimulated with various amount of lipopolysaccharides (LPS) for $24 \mathrm{~h}$ in the presence of anticonvulsant drugs. After $24 \mathrm{~h}$ of treatment, the culture supernatants were harvested for cytokine assays with ELISA, and the total RNAs extracted from the cells were subjected to quantitative real-time PCR assay of cytokine gene expressions. The cell viability was also detected with MTS assay to measure the cytotoxicity of anticonvulsant drugs at various dosages. The anticonvulsant drugs tested so far included gabapentin, valproate, carbamazepine, topiramate, and carisbamate; the synthetic immunosuppressant dexamethasone was used as positive control.

Results: While dexamethasone exhibits potent inhibitory effects on LPS-stimulated inflammatory cytokine secretion from BV-2 cells and primary glial cultures, gabapentin, valproate, carbamazepine, and topiramate fail to show significant anti-inflammatory effect on both cell line and primary culture. However, the experimental anticonvulsant drug, carisbamate, displays a significant inhibition of LPS-induced TNF- $\alpha$ and IL-6 production in BV- 2 cells, and such inhibitory effect on IL-6 secretion is confirmed in primary glial culture too. The cell viability test indicates that all the cells are viable under the dosages applied.

Conclusions: The results of our anti-inflammation screening of anticonvulsant drugs indicates that most conventional drugs show no such effect as expected, , because the generation of anticonvulsant drugs was irrelevant with anti-inflammation. Carisbamate, on the other hand, demonstrated the anti-inflammatory effect in our in vitro system.. our data indicate that although anti-inflammation is overlooked during the development of anticonvulsant drugs as an irrelevant trait, they may accidentally exert such effect, like carisbamate; therefore, to expand our screening with more candidate drugs and to integrate the anti-inflammatory screening into the future research and development of anticonvulsant drugs may have the potential to discover existing drugs or obtain novel compounds that not only inhibit the epileptic symptoms, but also prevent the inflammatory pathogenesis of the epilepsy.

\subsection{9 \\ EVALUATION OF N-1'- N-3'- DISUBSTITUTED TRIONE DERIVATIVES OF SPIROHYDANTOIN AS ANTICONVULSANTS COMPARED TO DIPHENYLHYDANTOIN USING THE HIPPOCAMPAL PRE- SYNAPTIC NERVE TERMINAL MODEL OF GLUTAMATE RELEASE}

Reshma S. Therala and F. A. Schanne (St. John's University, Ozone Park, NY)

Rationale: Glutamate is the most abundant neurotransmitter in the brain mediating excitatory neurotransmission. Together with the inhibitory neurotransmitter GABA, it plays a crucial role in the pathophysiology of epilepsy. Psychomotor seizures are a type of focal seizures, which involves parts of the limbic portion such as hippocampus, resulting from a lesion producing localized reverberating circuits which gradually recruit adjacent cortical regions. Antiepileptic drugs (AEDs) act on various molecular targets such as voltage gated $\mathrm{Na}+, \mathrm{Ca} 2+$ and $\mathrm{K}+$ channels to reduce the seizure activity. In this study, we measured the effect of N-1'- N-3'disubstituted trione derivatives of spirohydantoin on the basal, $\mathrm{K}+-$ and veratridine - evoked endogenous glutamate release from hippocampal synaptosomes.

Methods: Glutamate release was measured using a glutamate dehydrogenase dependant NADP+ reduction assay using a highthroughput fluorescence 96-well plate method. As stoichiometry exists between the glutamate and the NADPH produced in the reaction, the amount of glutamate can be estimated. Hippocampi were dissected from 14-21 day old Sprague-Dawley rat pups and synaptosomes were isolated using percoll density gradients.

Results: Phenytoin at $500 \mu \mathrm{M}$, carbamazepine at $166 \mu \mathrm{M}$, gabapentin at $100 \mu \mathrm{M}$ and the N-1'-p-nitrophenyl, N-3'-ethyl-2'H, 3H, 5'H-spiro(2-benzofuran-1,4'- imidazolidine)-2',3,5'- trione at $133 \mu \mathrm{M}$ significantly reduced the basal and veratridine-evoked glutamate release from the presynaptic nerve terminals of CA 1 and $\mathrm{CA} 3$ regions of hippocampus. Neither phenytoin at $500 \mu \mathrm{M}$, nor carbamazepine at $166 \mu \mathrm{M}$, was able to significantly reduce the potassium-evoked glutamate release, whereas they significantly reduced veratridineevoked glutamate release. On the other hand, gabapentin at $100 \mu \mathrm{M}$, and levetiracetam at $50 \mathrm{nM}$, were able to significantly reduce the potassium-evoked glutamate release.

Conclusions: This approach should enable us to evaluate the relative efficacy and difference in the potential mechanism of action of the $\mathrm{N}$ 1'- N-3'- disubstituted trione derivatives of spirohydantoin compared with the common AEDs.
3.210
SLOW AND FAST INACTIVATION OF VOLTAGE-GATED SODIUM CHANNELS BY ESLICARBAZEPINE, CARBAMAZEPINE, OXCARBAZEPINE AND LACOSAMIDE S. Hebeisen ${ }^{1}$ and P. Soares-da-Silva ${ }^{2,3}$ ( ${ }^{1}$ B'SYS, GmbH, Witterswil, Switzerland; ${ }^{2}$ BIAL - Portela \& Ca, S.A., S. Mamede do Coronado, Portugal and ${ }^{3}$ Faculty of Medicine, University of Porto, Porto, Portugal) 
Rationale: The efficacy of adjunctive eslicarbazepine acetate (ESL) in reducing the frequency of partial-onset seizures and tolerability in adults receiving carbamazepine (CBZ) or another antiepileptic drug (AED) was similar (Epilepsia, 50, 454-463, 2009; Epilepsy Res., 89, $278-285,2010)$. On the other hand, a large number of subjects experienced neurotoxicity when lacosamide (LCM) was added to another voltage-gated sodium channel (VGSC) blocking AED (Epilepsy Behav, 16, 423-425, 2009; Epilepsy Behav, 20, 20-23, 2011). Advice was given to reduce concomitantly the conventional VGSC-blocking AEDs during LCS uptitration in cases of neurotoxicity (Epilepsy Behav, 20, 20-23, 2011). This study was aimed to determine the effects of eslicarbazepine, the major active metabolite of ESL, CBZ, oxcarbazepine (OXC) and LCM on the fast and slow inactivated sates of VGSC.

Methods: The whole-cell patch-clamp technique was used to investigate the effects of eslicarbazepine CBZ, OXC and LCM (at $250 \mu \mathrm{M}$ ) on sodium channels endogenously expressed in N1E-115 cells, in conditions of fast and slow inactivation of sodium currents. Results: At a concentration of $250 \mu \mathrm{M}$, the voltage dependence of the fast inactivation was not influenced by eslicarbazepine, whereas LCM, CBZ and OXC shifted the V0.5 value by $-4.8 \mathrm{mV},-12.0 \mathrm{mV}$ and $-16.6 \mathrm{mV}$, respectively. Eslicarbazepine- and LCM-treated fastinactivated channels recovered similarly to control conditions $(\Delta \mathrm{t}=$ $11 \mathrm{~ms}$ ), whereas CBZ- and $\mathrm{OXC}$-treated channels required longer pulses to recover $(\Delta \mathrm{t}=391 \mathrm{~ms})$. CBZ and OXC shifted by $-4.6 \mathrm{mV}$ and $-28.1 \mathrm{mV}$ the voltage dependence of the slow inactivation, whereas for eslicarbazepine and LCM the shift of the voltage dependence of the slow inactivation (V0.5) was $-31.2 \mathrm{mV}$ and -53.3 $\mathrm{mV}$, respectively. For eslicarbazepine, LCM, CBZ and OXC, the affinity to the slow inactivated state was $5.9,10.4,1.7$ and 1.8 times higher than to the channels in the resting state, respectively. For eslicarbazepine, LCM, CBZ and OXC, the time constants for entering the slow inactivated state were 7.00, 4.54, 14.49 and $12.36 \mathrm{~s}$, respectively.

Conclusions: Eslicarbazepine does not share with $\mathrm{CBZ}$ and $\mathrm{OXC}$ the ability to alter fast inactivation of VGSC, but rather appears to modify the kinetics and voltage-dependence of slow inactivation states. Both eslicarbazepine and LCM reduce VGSC availability through enhancement of slow inactivation, but LCM demonstrated higher interaction with VGSC in the resting state, with fast inactivation gating and shorter time to enter in the slow inactivated state.

\subsection{1}

\section{EFFECTS OF ESLICARBAZEPINE, R-LICARBAZEPINE AND OXCARBAZEPINE ON ION TRANSMISSION THROUGH CA 2.1 AND CA, 3.2 CHANNELS}

M. J. Bonifácio ${ }^{1}$, K. Brady ${ }^{2}$, S. Hebeisen ${ }^{2}$ and P. Soares-da-Silva ${ }^{1,3}$ $\left({ }^{1}\right.$ BIAL - Portela \& Ca, S.A., S. Mamede do Coronado, Portugal; ${ }^{2}$ B'SYS, GmbH, Witterswil, Switzerland and ${ }^{3}$ Faculty of Medicine, University of Porto, Porto, Portugal)

Rationale: $\mathrm{Ca}_{\mathrm{v}} 2.1$ calcium (P/Q-type) channels play a prominent role in initiating action-potential-evoked neurotransmitter release at central nervous system synapses and impaired function of brain $\mathrm{Ca}_{\mathrm{v}} 2.1$ may have a central role in the pathogenesis of certain cases of primary generalized epilepsy. T-type calcium channels $\left(\mathrm{Ca}_{\mathrm{v}} 3.2\right)$ are critically important in controlling the excitability of the postsynaptic compartment of neurons, both in normal and epileptic neurons. Transcriptional induction of $\mathrm{Ca}_{\mathrm{v}} 3.2$ is a critical step in epileptogenesis and neuronal vulnerability. The present study determined the effect of eslicarbazepine acetate's (ESL) major active metabolite eslicarbazepine, R-licarbazepine (a minor metabolite of ESL) and oxcarbazepine (OXC) on human $\mathrm{hCa}_{\mathrm{v}} 2.1$ and $\mathrm{hCa}_{\mathrm{v}} 3.2$ currents.

Methods: The whole-cell patch-clamp technique was used to investigate the effects of eslicarbazepine, R-licarbazepine and OXC on $\mathrm{hCa}_{\mathrm{v}} 2.1$ and $\mathrm{hCa}_{\mathrm{v}} 3.2$ calcium channels stably expressed in $\mathrm{CHO}$ and HEK 293 cells, respectively. Compounds were tested ( 0.3 to $1000 \mu \mathrm{M} ; \mathrm{n}=3-5$ cells) on $\mathrm{hCa}_{\mathrm{v}} 2.1$ and $\mathrm{hCa}_{\mathrm{v}} 3.2$ currents upon depolarisation of the cell membrane to $-10 \mathrm{mV}$ or $-25 \mathrm{mV}$ for $500 \mathrm{~ms}$ or $50 \mathrm{~ms}$ from a holding potential of $-80 \mathrm{mV}$, respectively. $\mathrm{Ca}_{\mathrm{v}} 2.1$ and Cav3.2 blockers cobalt chloride, valproic acid and mibefradil were used as reference.

Results: Eslicarbazepine, R-licarbazepine and OXC did not markedly affect hCa 2 2.1 calcium peak currents, but eslicarbazepine and Rlicarbazepine potently inhibited $\mathrm{hCa}_{\mathrm{v}} 3.2$ calcium peak currents (figure 1, table 1). The inhibition curves for blockade of $\mathrm{hCa}_{\mathrm{v}} 3.2$ calcium peak currents were best fitted with a two site binding model and a constant remaining current amplitude. A block of high affinity occurs with an IC50 of $0.43 \mu \mathrm{M}$ and $6.54 \mu \mathrm{M}$ for eslicarbazepine and R-licarbazepine, respectively (table 1). A further block occurs at higher concentrations of the test agents, with an IC50 of $62.61 \mu \mathrm{M}$ for eslicarbazepine and $883.10 \mu \mathrm{M}$ for R-licarbazepine (figure 1, table 1). OXC, up to $30 \mu \mathrm{M}$, was devoid of effect on $\mathrm{hCa}_{\mathrm{v}} 3.2$ currents. Regarding the reference compounds, $1 \mathrm{mM}$ valproic acid blocked $\mathrm{hCa}_{\mathrm{v}} 3.2$ calcium peak currents by $66.15 \pm 2.05 \%$ and mibefradil dose dependently inhibited $\mathrm{hCa}_{\mathrm{v}} 3.2$ calcium peak currents with an IC50 of $143.7 \mathrm{nM}$. Cobalt chloride $(5 \mathrm{mM})$ blocked hCa 2.1 calcium peak currents by $5.02 \pm 2.24 \%$.

Conclusions: These results demonstrated that eslicarbazepine effectively inhibits high and low affinity $\mathrm{Ca}_{\mathrm{v}} 3.2$ inward currents with higher affinity than R-licarbazepine or OXC. These findings may have implications for the clinical activity of ESL.

Table 1. IC50 values $(\mu \mathrm{M})$ determined from inhibition curves of $\mathrm{hCa}_{\mathrm{v}} 2.1$ and $\mathrm{hCa}_{\mathrm{v}} 3.2$ currents determined for eslicarbazepine, $\mathrm{R}$ licarbazepine and oxcarbazepine.

\begin{tabular}{|c|c|cc|}
\hline & $\mathrm{hCa}_{\mathrm{v}} 2.1$ & \multicolumn{2}{|c|}{$\mathrm{hCa}_{\mathrm{v}} 3.2$} \\
\hline & & High affinity & Low affinity \\
\hline Eslicarbazepine & 7836.80 & 0.43 & 62.61 \\
\hline R-licarbazepine & 6266.71 & 6.54 & 883.10 \\
Oxcarbazcpinc & 767.92 & $\mathrm{nc}$ & $\mathrm{nc}$ \\
\hline Mibcfradil & $\mathrm{nt}$ & 0.14 & $\mathrm{nc}$ \\
\hline
\end{tabular}

Notes: nc - not calculated; nt - not tested

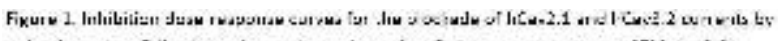

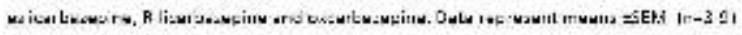
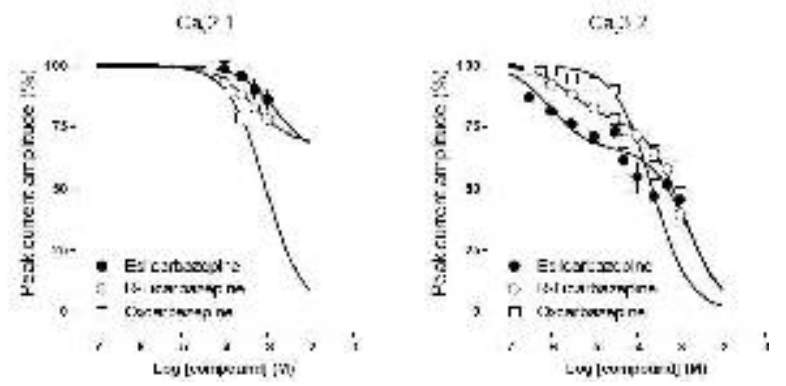

Figure 1. Inhibition dose-response curves for the blockade of $\mathrm{hCa}_{\mathrm{v}} 2.1$ and $\mathrm{hCa}_{\mathrm{v}} 3.2$ currents by eslicarbazepine, $\mathrm{R}$-licarbazepine and oxcarbazepine. Data represent means $\pm \operatorname{SEM}(n=3-9)$

\section{Antiepileptic Drugs: Animal Studies}

\subsection{2}

NP260, A GABA-A RECEPTOR ANTAGONIST WITH NOVEL SUBTYPE SELECTIVITY, FACILITATES SPONTANEOUS SYNAPTIC RELEASE OF GABA AND INHIBITS SEIZURES IN THE MTLE MODEL OF REFRACTIVE EPILEPSY

Stephen P. Wanaski ${ }^{1}$, J. Spampanato ${ }^{2}$, L. R. Shao ${ }^{2}$, T. A. Verdoorn ${ }^{1}$, C. Roucard ${ }^{3}$, F. E. Dudek ${ }^{2}$ and S. Collins ${ }^{1}$ ( ${ }^{1}$ NeuroTherapeutics Pharma, Inc., Chicago, IL; ${ }^{2}$ Department of Physiology, University of Utah, Salt Lake City, UT and ${ }^{3}$ SynapCell, SAS, Grenoble, France) 
Rationale: Imbalances of neuronal excitation and inhibition are considered a significant pathological feature of many seizure disorders. Many anticonvulsant medications such as barbiturates, benzodiazepines, and gabapentin markedly increase inhibitory transmission, thus generating therapeutic benefit along with sedative side effects. NeuroTherapeutics Pharma (NTP) has developed compounds with novel mechanisms that are hypothesized to enhance presynaptic inhibition. Specifically, these compounds increase spontaneous release of GABA in hippocampus, leading to robust anti-seizure activity.

Methods: Patch-clamp recording of hippocampal CA1 pyramidal cells was conducted to assess the ability of NTP compounds to increase the frequency of miniature inhibitory post-synaptic currents (mIPSCs). Excitatory synaptic transmission and $\mathrm{Na}+$-dependent action potentials were blocked by kynurenic acid and TTX respectively. NTP compounds were also assessed for their ability to decrease epileptiform discharges in a mouse model of mesial temporal lobe epilepsy.

Results: The NTP compound, NP260 $(30 \mu \mathrm{M})$ significantly increased the frequency of mIPSCs from $4.85 \pm 0.72 \mathrm{~Hz}$ to $5.94 \pm$ $0.88 \mathrm{~Hz}$ (Mean $\pm \mathrm{SEM}, \mathrm{n}=12$ cells, $\mathrm{p}<0.01$ compared to pre-drug baseline, paired t-test). NP260 also showed robust activity after intrahippocampal injection of kainate, a mouse model of refractory mesial temporal lobe epilepsy (MTLE) (Riban et al, 2002 Neuroscience 112(1):101-111; Langlois et al, 2010 J. Neurosci 30(49):16523-35). Single acute intraperitoneal (IP) doses of NP260 inhibited both the frequency and total duration of epileptiform discharges when measured for 60 minutes after administration. The effect of NP260 was dose-dependent, with $15 \mathrm{mg} / \mathrm{kg}$ reducing the frequency of epileptiform discharges by $52 \%$ and $30 \mathrm{mg} / \mathrm{kg}$ by $86 \%$ in these animals. NP260 showed no sedative effects based on rotarod performance or modified Irwin Test (CNS behavioral battery) at these IP doses and oral doses up to and including $1000 \mathrm{mg} / \mathrm{kg}$. Conclusions: Tests of NP260 and a series of structural analogs demonstrated a correlation between increased mIPSC frequency and decreased epileptiform discharges in the MTLE model, suggesting that the increased spontaneous release of GABA may be a critical component of the compounds' mechanism of action. The novel mechanism(s) combined with the robust in vivo efficacy of NP260 suggests that modulating GABA release with NTP compounds may represent a promising strategy for treating seizure disorders.

\subsection{3 \\ CEFTRIAXONE TREATMENT AFTER TRAUMATIC BRAIN INJURY INCREASES GLT-1 EXPRESSION, REDUCES THE GFAP MARKER OF GLIOSIS, AND REDUCES POSTTRAUMATIC SEIZURES IN RATS}

Grant S. Goodrich $^{1}$, A. Y. Kabakov ${ }^{1}$, M. Q. Hameed ${ }^{1,2}$, S. C. Dhamne ${ }^{1}$, P. A. Rosenberg ${ }^{1}$ and A. Rotenberg ${ }^{1}\left({ }^{1}\right.$ Neurology, Boston Children's Hospital, Boston, MA and ${ }^{2}$ Neurosurgery, Boston

Children's Hospital, Boston, MA)

Rationale: Excess of extracellular glutamate after traumatic brain injury (TBI) contributes to excitotoxic cell death and posttraumatic epilepsy. The only known mechanism of extracellular glutamate clearance is glutamate transport, which is mediated by glutamate transporters. GLT-1, the analog of human EAAT2, is the major glutamate transporter in the rat brain and expressed in astrocytes, axon terminals and oligodendrocytes. We previously identified that GLT-1 expression is suppressed focally after TBI, and is is restored after treatment with ceftriaxone, a well-tolerated $\beta$-lactam antibiotic previously shown to enhance GLT-1 expression. Here, we test whether ceftriaxone treatment reduces the extent of gliosis and mitigates posttraumatic epilepsy.

Methods: Under isoflurane-inhaled anesthesia, rats were exposed to moderate lateral fluid percussion injury (LFPI, $2.26 \pm 0.12 \mathrm{~atm}$ ) via a $4 \mathrm{~mm}$ diameter craniectomy over the left parietal region. Ceftriaxone was injected acutely after TBI (200 mg/kg, ip), and then daily for 7 days. 7 days after TBI, left lesioned and right nonlesioned cortex tissue was dissected separately for analysis in each rat. GLT-1 and GFAP expression was quantified by immunoblot; $\beta$-actin was used as a loading control. Rats were implanted with wireless EEG transmitters to record seizure activity 12 weeks after TBI. Seizures were detected by an automatic seizure detection algorithm and validated by visual inspection.

Results: Seven days after TBI, relative to saline control, daily ceftriaxone treatments restored lesioned cortex $(n=7)$ expression of GLT-1 to near control levels and increased its expression in the nonlesioned cortex by $29 \%(p=0.048)$. The increase in GFAP after TBI is reduced with ceftriaxone. There is a $43 \%(p=0.031)$ decrease in GFAP expression in the lesioned cortex of ceftriaxone treated rats compared to the saline control group. In addition, seizure frequency per 24 hours of recording, with seizures defined as runs of spikes 10 sec or longer in duration, was significantly reduced to $46.8 \pm 15.4$ (mean \pm SEM) seizures in the ceftriaxone group $(n=6)$ relative to $151.3 \pm 43.6$ seizures $(p=0.038)$ in the saline control group $(n=5)$. Conclusions: We conclude that one week of ceftriaxone administration can restore GLT-1 expression to near normal levels in lesioned cortex and cause an over expression in the nonlesioned cortex. Our data also imply that improved glutamate clearance after ceftriaxone treatment may contribute to reduced regional gliosis and reduced frequency of posttraumatic seizures.

\subsection{4}

INITIAL POLYTHERAPY FOR STATUS EPILEPTICUS Claude G. Wasterlain ${ }^{1,2}$, R. Baldwin ${ }^{1}$, L. Suchomelova ${ }^{1,2}$ and J. Niquet ${ }^{1,2}\left({ }^{1}\right.$ Neurology, VA Medical Center, West Los Angeles, CA and ${ }^{2}$ Neurology, UCLA, Los Angeles, CA)

Rationale: Status Epilepticus (SE) induces internalization of synaptic GABAA receptors and trafficking to the synaptic membrane of NMDA receptors, resulting in both a failure of GABAergic inhibition and an increase in glutamatergic excitation. Monotherapy with benzodiazepines, the gold standard in the treatment of SE, can only partially restore GABAergic inhibition, since the number of GABAA receptors per synapse remains reduced. It also fails to treat increased glutamatergic excitation. A combination therapy was tested. It targeted both the loss of inhibition (by stimulating the remaining synaptic GABAA receptors with a benzodiazepine and adding the anticonvulsant valproate to enhance inhibition at non-benzodiazepine sites. Glutamatergic excitation was also targeted by adding an NMDA channel blocker (ketamine).

Methods: We used a rat standard model of SE induced by lithium (3 $\mathrm{mEq} / \mathrm{kg} 20$ hours pre) and pilocarpine (60 mg/kg ip), plus methylscopolamine to reduce pulmonary secretions. We added atropine $(10 \mathrm{mg} / \mathrm{kg})$ in all groups after SE was established, to reduce the cholinergic component which could re-initiate seizures. The EEG was recorded for $24 \mathrm{hrs}$. Treatment toxicity used measures of mobility and consciousness. We administered treatment intraperitoneally after benzodiazepine pharmacoresistance was established. Triple therapy was compared to triple-dose monotherapy. Results: EEG seizure load before injection was a better predictor of therapeutic response than time elapsed since pilocarpine injection, suggesting that pharmacoresistance was seizure-induced . Diazepam monotherapy (up to $5 \mathrm{mg} / \mathrm{kg}$, Dz5) reduced mortality but did not stop SE. Linear regression of EEG power per minute(EEG AUC) over the $4 \mathrm{hrs}$ after treatment correlated with EEG AUC over the $15 \mathrm{~min}$ before treatment $(\mathrm{p}<.0001)$. Monotherapy with ketamine (up to 30 $\mathrm{mg} / \mathrm{kg}, \mathrm{K} 30$ ) had similar results. Monotherapy with valproate (up to $90 \mathrm{mg} / \mathrm{kg}$, V90) did not modify EEG seizures at all. Combinations of low-dose diazepam $(1 \mathrm{mg} / \mathrm{kg})$, ketamine $(10 \mathrm{mg} / \mathrm{kg})$ and valproate $(30 \mathrm{mg} / \mathrm{kg})(\mathrm{DKV})$ reduced the slope of the linear regression of EEG AUC compared to all triple-dose monotherapies $(\mathrm{p}<0.001)$. Low-dose triple therapy preserved the righting reflex (toxicity score $1.4 \pm 0.9$ ), while Dz5 impaired it (toxicity score $2.2 \pm 0.2$ ). 
Conclusions: Triple-therapy was significantly more effective than triple-dose monotherapy in this model, suggesting synergism between drugs. Toxicity was simply additive among drugs and dosages tested. Pharmacoresistance developed significantly more slowly with triple compared to mono-therapy, but was not completely prevented.

\subsection{5}

EFFECTS OF THE NKCC1-INHIBITOR BUMETANIDE IN THE KINDLING MODEL OF TEMPORAL LOBE EPILEPSY IN RATS

Manuel Toepfer ${ }^{1,2}, \mathrm{~K}$. Toellner ${ }^{1,2}$, C. Brandt ${ }^{1,2}$ and W. Loescher ${ }^{1,2}$ ( ${ }^{1}$ Department of Pharmacology, Toxicology, and Pharmacy, University of Veterinary Medicine, Hannover, Germany and ${ }^{2}$ Center for Systems Neuroscience, Hannover, Germany)

Rationale: A variety of brain insults have the potential to induce the development of epilepsy, particularly temporal lobe epilepsy (TLE) in humans and rodent models of TLE. The mechanisms of this process, named epileptogenesis, are still poorly understood. Accumulating evidence suggests that expression changes of the cation-chloride-cotransporters $\mathrm{KCC} 2$ and $\mathrm{NKCC} 1$ lead to a GABAshift from an inhibitory to an excitatory action. Several studies indicate that NKCC1 is upregulated in models of TLE, and that the loop diuretic bumetanide seems to be a useful tool to counteract this upregulation. Furthermore, as our group described recently, coadministration with the GABA-agonist phenobarbital leads to disease-modifying effects in the pilocarpine model of TLE. The problem of bumetanide's rapid metabolization in rats and the fact that it only hardly crosses the blood-brain-barrier prompted us to investigate several strategies to enhance brain penetration, as well as to prolong the half-life. The aim of this study was to evaluate bumetanide's antiepileptogenic and anticonvulsive properties in rats after intracerebral or systemic administration, either alone or in combination with other agents in the kindling model of TLE. Methods: Rats were kindled by once daily electrical stimulation of the basolateral amygdala. In fully kindled rats the afterdischarge threshold (ADT) was determined and repeated until all rats exhibited a reproducible ADT. Intravenous (i.v.) administration of bumetanide and, in order to prevent rapid metabolization, intraperitoneal pretreatment with the monooxygenase-inhibitor piperonyl butoxide were chosen to investigate antiepileptogenic properties on kindling development. Furthermore, to determine anticonvulsant effects, fully kindled rats were treated by either intracerebroventricular or i.v. administration of bumetanide $+/$ - the GABA-potentiating drug phenobarbital prior to ADT determination.

Results: Bumetanide alone did not exert any antiepileptogenic or anticonvulsant effects, even when administered at high doses.

However, when bumetanide was administered before a low dose of phenobarbital, which was ineffective alone, bumetanide significantly potentiated the anticonvulsant effect of phenobarbital in fully kindled rats.

Conclusions: Although alterations in $\mathrm{NKCC} 1$ and $\mathrm{GABA}$ functions have been shown previously in the kindling model, neither systemic nor intracerebral injection of bumetanide exerted any significant effects on kindling development or fully kindled seizures. However, combined administration of bumetanide and phenobarbital exerted anticonvulsant effects in fully kindled rats. It may be suggested that NKCC1 inhibition was involved in the potentiation of phenobarbital by bumetanide. These results indicate that the use of bumetanide might be beneficial as add-on treatment together with GABAmimetic antiepileptic drugs in patients with TLE. Further kindling studies with prodrugs of bumetanide are currently under way to evaluate possibly stronger anticonvulsant properties of these derivatives.
3.216

SYNERGISTIC EFFECTS OF PERAMPANEL COMBINED WITH DIAZEPAM IN THE LITHIUM-PILOCARPINE RAT MODEL OF STATUS EPILEPTICUS

T. Hanada and K. Ido (Global Biopharmacology Tsukuba, Neuroscience Product Creation System, Eisai Co. Ltd., Tsukuba, Japan)

Rationale: Perampanel, a structurally novel, potent, orally active AMPA receptor antagonist, is currently under development for the treatment of epilepsy. Perampanel has previously been shown to have anti-seizure effects in animal models (Hanada T, et al. Epilepsia 2011;52:1331-1340). In experimental models of status epilepticus, the ability of agents (including other AMPA receptor antagonists) to terminate seizures typically declines as the duration of status epilepticus increases. Perampanel has been shown to terminate established, diazepam-resistant status epilepticus in the lithiumpilocarpine rat model of status epilepticus. Here, we examined pharmacodynamic interactions between perampanel and diazepam in this same model.

Methods: Male Sprague Dawley rats weighing 250-450 g were implanted with an electroencephalography (EEG) electrode on the surface of the primary somatosensory cortex (AP: $-2.5 \mathrm{~mm}$; L: $3 \mathrm{~mm}$ according to coordinates of Paxinos and Watson [1997]). After at least 1 week recovery, $3 \mathrm{mEq} / \mathrm{kg}$ of lithium chloride wasadministered to the rats (intraperitoneal). This was followed 18-26 hours later by 5 $\mathrm{mg} / \mathrm{kg}$ scopolamine methyl bromide and $30 \mathrm{mg} / \mathrm{kg}$ of pilocarpine. Seizure initiation was documented when the first spike train was observed on EEG. Thirty minutes after seizure initiation, perampanel alone, diazepam alone, or the combination, was administered intravenously.

Results: Perampanel at $1 \mathrm{mg} / \mathrm{kg}$ failed to terminate EEG seizures, and $5 \mathrm{mg} / \mathrm{kg}$ perampanel terminated seizures in some rats (Table 1). Diazepam alone at $5 \mathrm{mg} / \mathrm{kg}$ and $20 \mathrm{mg} / \mathrm{kg}$ failed to terminate EEG seizure. Combination of perampanel $(1 \mathrm{mg} / \mathrm{kg})$ and diazepam $(5$ $\mathrm{mg} / \mathrm{kg}$ ), doses that were individually ineffective, terminated seizures in all treated rats.

Conclusions: Our data demonstrate that, after 30 minutes of status epilepticus (at which point it is typically considered to be selfsustaining and resistant), seizures were terminated by combination treatment with perampanel and diazepam at doses that were ineffective alone. Thus, perampanel shows synergistic pharmacodynamic interaction with diazepam in diazepam-resistant and established status epilepticus in the rat.

Supported by Eisai Co., Ltd.

Table 1

\begin{tabular}{|c|cr|c|}
\hline Treatment & Dose & $\mathbf{N}$ & $\begin{array}{c}\text { Response Rate (\% of animals in which scizures } \\
\text { were terminated) }\end{array}$ \\
Perampancl alonc & $1 \mathrm{mg} / \mathrm{kg}$ & 1 & 0 \\
\hline Perampancl alone & $5 \mathrm{mg} / \mathrm{kg}$ & 6 & $33 \%$ \\
\hline Diazcparm alonc & $5 \mathrm{mg} / \mathrm{kg}$ & 2 & 0 \\
\hline Diazepam alone & $20 \mathrm{mg} / \mathrm{kg}$ & 6 & 0 \\
\hline $\begin{array}{c}\text { Perampanel }+ \\
\text { diazepam } \\
\begin{array}{c}\text { Perampancl }+ \\
\text { diazcpam }\end{array}\end{array}$ & $\begin{array}{c}0.5 \mathrm{mg} / \mathrm{kg}+5 \\
\mathrm{mg} / \mathrm{kg} \\
1 \mathrm{mg} / \mathrm{kg}+5 \\
\mathrm{mg} / \mathrm{kg}\end{array}$ & 6 & $33 \%$ \\
\hline
\end{tabular}

3.217

NEUROPROTECTIVE EFFECT OF LACOSAMIDE ON HYPOXIC-ISCHEMIC BRAIN INJURY IN NEONATAL RATS Gun Ha Kim, J. Byeon, S. Eun and B. Eun (Pediatrics, Korea University Medical Center, Seoul, Republic of Korea)

Rationale: Lacosamide is an antiepileptic drug which enhances slow inactivation of sodium channels. The neuroprotective effect of 
lacosamide was investigated in a neonatal rat model of hypoxicischemic encephalopathy (HIE).

Methods: HIE was induced in rats at day 7 of postnatal age by cauterization of the right common carotid artery, followed by $2 \mathrm{~h} 30$ min of hypoxia using $8 \%$ oxygen. Immediately before and after the hypoxia-ischemia (pre-treatment group), and immediately after and 2 hours later (post-treatment group), the rats were fed with lacosamide $(100 \mathrm{mg} / \mathrm{kg} / \mathrm{dose})$ through an orogastric tube. Controls received saline only. On postnatal day 12 , brains were removed and gross morphological damage was evaluated. To quantify the severity of brain injury, bilateral cross-sectional areas of the anterior commissural and posterior hippocampal levels were analyzed using ImageJ software. Assessments of the open field activity levels at 2, 4, 6 and 8 weeks and, the Morris water maze test at 8 weeks after hypoxia-ischemia were carried out according to standard methods to see the motor activity and cognitive function.

Results: Lacosamide pre-treatment decreased the incidence of liquefactive cerebral infarction, at a dose of $100 \mathrm{mg} / \mathrm{kg} / \mathrm{dose}$ $(\mathrm{P}<0.05)$. However, no benefit was observed in post-treatment group. In the Morris water maze test, the group pretreated with lacosamide at $100 \mathrm{mg} / \mathrm{kg} / \mathrm{dose}$ showed shorter escape latencies than controls $(\mathrm{P}<0.05)$

Conclusions: Our results suggest that lacosamide pre-treatment is neuroprotective. Pre-treatment regimens could be clinically relevant in settings in which an increased risk of cerebral ischemia can be anticipated, such as in infants undergoing surgery to correct congenital heart disease.

\subsection{8 \\ A MODERATE-THROUGHPUT IN VITRO SCREEN FOR ANTIEPILEPTOGENIC COMPOUNDS}

Yero Saponjian ${ }^{1}$, Y. Berdichevsky ${ }^{2}$ and K. Staley ${ }^{1,3}$ ( ${ }^{1}$ Neurology, Massachusetts General Hospital, Boston, MA; ${ }^{2}$ Electrical \& Computer Engineering, Lehigh University, Bethlehem, PA and ${ }^{3}$ Harvard Medical School, Boston, MA)

Rationale: The antiepileptic and neuroprotective effects of an array of drugs were studied in a moderate-throughput screen utilizing the accelerated course of epileptogenesis in the in vitro organotypic hippocampal slice culture model of post-traumatic epilepsy. Methods: Organotypic hippocampal slices cultures were prepared from postnatal day 6 or 7 Sprague-Dawley rats and were maintained for 28 days at $37^{\circ} \mathrm{C}$ on a rocking platform in a culture medium consisting of Neurobasal-A, B27, $0.5 \mathrm{mM}$ GlutaMAX, and $30 \mu \mathrm{g} / \mathrm{ml}$ Gentamicin. Spent culture media was collected at media changes every 3-4 days and stored at $-80^{\circ} \mathrm{C}$ until analysis. Drugs, obtained primarily from the NINDS Custom Collection of compounds, were dissolved in DMSO (final concentration $0.1 \%$ ) and added to the media starting on DIV 3. All experiments included DMSO control slice cultures derived from the same animal. Lactate and lactate dehydrogenase (LDH) concentrations were measured in collected culture media and plotted as cumulative values over 28 days in vitro. Results: Lactate and LDH concentration measurements in the collected culture medium were strongly correlated with electrographic seizure activity and propidium iodide assays for cell death, respectively, and were used in subsequent experiments as assays for seizure activity and neuroprotection. We have screened over 100 drugs, with most drugs screened at multiple concentrations. Several drugs exhibited dose-dependent anticonvulsive or proconvulsive effects, as well as neuroprotective or neurotoxic effects.

Conclusions: The correlation between anticonvulsant and neuroprotective effects was likely due to reductions in ictal cell death. Drugs with significant antiepileptic and neuroprotective effects are being retested including 10-day wash-out experiments to differentiate anticonvulsant from antiepileptogenic effects.
3.219

INFLUENCE OF THE FIRST LINE TREATMENT ON THE PROGNOSIS OF EPILEPSY IN A MOUSE MODEL OF ABSENCE EPILEPSY

Arnaud Biraben ${ }^{1,2}$, L. Javaudin ${ }^{1,3}$, F. Wendling ${ }^{2,4}$ and B. Martin ${ }^{2,4}$ ( ${ }^{1}$ neurologie unité épilepsie, CHU Pontchaillou, Rennes, Ille-etVilaine, France; ${ }^{2}$ U642, INSERM, Rennes, Ille-et-Vilaine, France; ${ }^{3}$ Unité de Pharmacologie, CHU Pontchaillou, Rennes, Ille-et-Vilaine, France and ${ }^{4}$ Laboratoire de Traitement du Signal et de l'Image, Université Rennes1, Rennes, Ille-et-Vilaine, France)

Rationale: Some anti-epileptic drugs (AED) may aggravate some epilepsies. This is mainly the case of generalized epileptic syndromes with GABAergic AED. The aim of this work is to test the hypothesis that this bad adaptation of the initial AED to the epileptic syndrome could aggravate epilepsy and have some deleterious long term effects particularly on the therapeutic results of the next well adapted AED. This should be particularly true during infancy, on a maturing brain. Methods: We used an inbred mouse model of childhood absence epilepsy, BS/Orl, manifesting spontaneous and recurrent spike-wave discharges. Four groups of BS/Orl mice were constituted. The first treatment started for every group at the age of 5 weeks during brain maturation. The two first weeks, the first group was treated with valproate (VPA); the second group with vigabatrin (VGB) that is known to aggravate absence epilepsy in childhood; the third group received ethosuximide (ESM) that is known as the best treatment in human for this epileptic syndrome and a fourth control group received saline solution (PHY). During the 42 next days, every group except the control group, received VPA that is one of the reference treatment for this syndrome in France. Overall, the mice were tested at 5 different periods of time, and 3 times of one hour each period: before any treatment, after the first-line treatment and 3 times during the second-line treatment.

Results: During the two first weeks of treatment the four groups were significantly different. As expected, the comparison treatments vs PHY has given a significant result $(\mathrm{p}<0.006)$ with VGB worsening seizures whereas VPA and ESM reducing seizures with a much greater effect for ESM.

During the next 6 weeks (until the mice turn adults) when every group received VPA, the groups treated initially with ESM (ESMVPA) or VPA (VPA-VPA) tended to do the same; in the aggravated group VGB stopping VGB and introducing VPA (VGB-VPA group) failed to reverse the tendency of an aggravation of the seizure level and the mice never did as well as in the other groups $(p<0.008)$.

Conclusions: As observed in this mouse model, the first treatment in this syndrome seems to influence not only the short term but also the long term prognosis and the drug sensitivity of this epilepsy. This may be linked to a direct deleterious effect of the inadequate first line drug, but also of the seizures by itself on a developing brain.

\subsection{0 \\ ALLOPREGNANOLONE TREATMENT IN A RAT PEDIATRIC STATUS EPILEPTICUS MODEL: COMPARISON WITH DIAZEPAM}

Christoph Lossin, S. S. Shahangian and M. A. Rogawski (Neurology, University of California - Davis, Sacramento, CA)

Rationale: Status epilepticus (SE) is the most common neurological emergency of childhood and a cause of high morbidity and mortality. The standard of care for the treatment of pediatric SE is an intravenous benzodiazepine followed immediately by a secondary antiepileptic drug. This treatment regimen provides seizure control in only two-thirds of cases and poorer performance in prolonged SE. Here we investigated whether allopregnanolone $(5 \alpha, 3 \alpha-P)$, an alternative positive allosteric positive modulator of $\mathrm{GABA}(\mathrm{A})$ receptors, could provide equal or better SE control with fewer side effects.

Methods: Young rats (P9) were injected with kainic acid (2 mg/kg, 
i.p.), which reproducibly induced continuous tonic-clonic seizures. Ten or 40 min after the kainic acid injection, the animals received $5 \alpha, 3 \alpha-\mathrm{P}(30 \mathrm{mg} / \mathrm{kg}$, i.p.) or diazepam (DZP; $5 \mathrm{mg} / \mathrm{kg}$, i.p.). Treatment outcome (absence vs. presence of ongoing seizure activity) was recorded at 30 and 70 min after kainic acid administration.

Results: When administered early (10 $\mathrm{min}$ ) both $5 \alpha, 3 \alpha-\mathrm{P}$ and DZP caused freedom from status epilepticus at the 30 and $70 \mathrm{~min}$ evaluation time points. When administered at the late time point (40 min) DZP caused seizure freedom in $25 \%$ of animals whereas $5 \alpha, 3 \alpha$ $P$ eliminated status epilepticus in $100 \%$ of animals at the $70 \mathrm{~min}$ time point. With vehicle, an average of $67 \%$ of animals were status-free at 30 min following kainic acid versus $0 \%$ at $70 \mathrm{~min}$

Conclusions: Both treatments, DZP and $5 \alpha, 3 \alpha-\mathrm{P}$, were fully effective in our pediatric SE animal model when administered early in the course of SE. However, $5 \alpha, 3 \alpha-\mathrm{P}$ was significantly more effective than DZP when administered late. This suggests that $5 \alpha, 3 \alpha-\mathrm{P}$ may represent a superior initial treatment for children with SE, especially in cases where seizure activity is prolonged. This work was funded by a research grant from People Against Childhood Epilepsy (P.A.C.E.), New York.

\subsection{1}

\section{PERAMPANEL TERMINATES DIAZEPAM-RESISTANT STATUS EPILEPTICUS IN A LITHIUM-PILOCARPINE RAT MODEL}

K. Ido and T. Hanada (Global Biopharmacology Tsukuba, Neuroscience Product Creation System, Eisai Co. Ltd., Tsukuba, Japan)

Rationale: Perampanel, a structurally novel, potent, orally active AMPA receptor antagonist, is currently under development for the treatment of epilepsy. Perampanel has previously been shown to have anti-seizure effects in several animal models (Hanada T, et al. Epilepsia 2011;52:1331-1340). In experimental models of status epilepticus, the ability of agents (including other AMPA receptor antagonists) to terminate seizures typically declines as the duration of status epilepticus increases (e.g. Fritsch B, et al. Epilepsia 2010;51:108-117). In the present study, we explored the ability of perampanel and of diazepam to terminate seizures in the lithiumpilocarpine model of status epilepticus.

Methods: Male Sprague Dawley rats weighing 250-450 g were implanted with an electroencephalography (EEG) electrode on the surface of the primary somatosensory cortex (AP: $-2.5 \mathrm{~mm}$; L: $3 \mathrm{~mm}$ according to coordinates of Paxinos and Watson (1997)). A reference electrode was placed on the cerebellum. After at least 1 week recovery, $3 \mathrm{mEq} / \mathrm{kg}$ of lithium chloride was administered to the rats (intraperitoneal), followed $18-26$ hours later by $5 \mathrm{mg} / \mathrm{kg}$ scopolamine methyl bromide and $30 \mathrm{mg} / \mathrm{kg}$ pilocarpine. Seizure initiation was documented when the first spike train was observed on the EEG. Drugs (perampanel or diazepam) were administered intravenously 10 minutes or 30 minutes after seizure initiation. Electrographic effects were evaluated for 60 minutes after drug dosing. Seizures were considered 'terminated' if status epilepticus-type high-frequency EEG spike activity was abolished, with only occasional isolated spikes, and EEGs were spike-free at 60 minutes after drug dosing.

Results: Diazepam $(20 \mathrm{mg} / \mathrm{kg}$ ) terminated seizures when administered 10 minutes after seizure initiation in all animals, but failed to terminate seizures in any animals when administered 30 minutes after seizure initiation (Table 1). Perampanel, administered 30 minutes after seizure initiation, terminated seizures in one-third of animals at $5 \mathrm{mg} / \mathrm{kg}$ and in all animals at $8 \mathrm{mg} / \mathrm{kg}$ (Table 1). At this dose $(8 \mathrm{mg} / \mathrm{kg})$, perampanel terminated electrographic status epilepticus seizures within 1-5 minutes, with occasional spikes thereafter, and all animals were spike-free at 60 minutes after dosing. Conclusions: In the present study, both perampanel and diazepam were able to terminate status epilepticus seizures, but the therapeutic time window of perampanel is wider than that of diazepam.

Perampanel terminated seizures in animals with established status epilepticus of 30 minutes duration, a point at which it is usually considered to be self-sustaining.

Supported by Eisai Co., Ltd.

Table 1
\begin{tabular}{|c|cc|cc|}
\hline Agent & $\begin{array}{c}\text { Dose } \\
20\end{array}$ & $\begin{array}{c}\text { Status cpilepticus } \\
\text { duration (minutes) }\end{array}$ & N & $\begin{array}{c}\text { Responsc ratc (\% of animals in which } \\
\text { seizures were terminated) }\end{array}$ \\
\hline Diazcpam & 10 & 2 & $100 \%$ \\
\hline Diazepam & $\begin{array}{c}20 \\
\mathrm{mg} / \mathrm{kg}\end{array}$ & 30 & 6 & 0 \\
\hline Perampanel & $\begin{array}{c}5 \\
\mathrm{~m} / \mathrm{kg} \\
8\end{array}$ & 30 & 6 & $33 \%$ \\
Pcrampancl & $\begin{array}{c}8 \\
\mathrm{mg} / \mathrm{kg}\end{array}$ & 30 & 6 & $100 \%$ \\
\hline
\end{tabular}

\subsection{2}

EFFECT OF PERAMPANEL IN COMBINATION WITH AEDS IN AMYGDALA KINDLING MODEL

T. Wu and T. Hanada (Global Biopharmacology Tsukuba, Neuroscience Product Creation System, Eisai Co. Ltd., Tsukuba, Japan)

Rationale: Perampanel (PER), a structurally novel, potent, orally active AMPA receptor antagonist is currently under development for the treatment of epilepsy. Anti-seizure effects have been seen in several preclinical models (Hanada T, et al. Epilepsia 2011;52:13311340). Phase III clinical studies demonstrated efficacy in partialonset seizures when used as add-on to 1-3 other AEDs, but were not able to explore specific combinations in detail. Here, we explored the potential synergistic effects of PER with the second generation AEDs lamotrigine (LAM) and levetiracetam (LEV) in the amygdala-kindled rat.

Methods: Male Wistar-Kyoto rats weighing 250-400 g were used for the amygdala kindling study. A tripolar electrode was implanted into the basolateral amygdala (AP: $-2.5 \mathrm{~mm}$; L: $4.8 \mathrm{~mm}$; D: $7.5 \mathrm{~mm}$ according to coordinates of Paxinos and Watson [1997]), with a reference electrode placed on the contralateral cortex. After at least 1 week recovery, electrical stimulation of the amygdala was initiated (500 $\mu \mathrm{A}, 1 \mathrm{msec}$, monophasic square-wave pulses, 50/sec for $1 \mathrm{sec}$ ). Stimulation continued until at least 3 consecutive seizures were recorded with a score of 5 on the Racine seizure severity scale (Racine RJ. Electroencephalograph Clin Neurophysiol 1972;32:281294). The after discharge threshold (ADT) for each rat was determined, and doses of LAM and LEV which increased the ADT were selected (LAM: $20 \mathrm{mg} / \mathrm{kg}$; LEV: $50 \mathrm{mg} / \mathrm{kg}$ ). To determine drug effects on kindled seizures, a stimulation intensity of 3 times the ADT was used, and drugs were administered intraperitoneally either alone or in combination. Seizure severity, motor seizure duration (duration of score 4-5 seizures), and EEG seizure duration were measured. Data are presented as \% reduction from baseline (predrug) values.

Results: At a stimulation intensity of 3 times the ADT, PER $(0.75$ $\mathrm{mg} / \mathrm{kg})$, LAM $(20 \mathrm{mg} / \mathrm{kg})$ and LEV $(50 \mathrm{mg} / \mathrm{kg})$ achieved modest reductions in seizure score, EEG seizure duration, and motor seizure duration (Tables 1 and 2). Co-administration of PER with LAM and PER with LEV achieved reductions from baseline in seizure severity and duration that were more than additive (i.e. greater than the sum of the effects of each agent alone) in all but 1 instance (EEG seizure duration with PER + LEV [Tables 1 and 2]). A higher dose of PER $(1.5 \mathrm{mg} / \mathrm{kg})$ achieved pronounced reductions in seizure duration (6394\%), and addition of LAM or LEV had no additional effect on seizures. PER alone at $1.5 \mathrm{mg} / \mathrm{kg}$ reduced seizure severity by approximately $50 \%$, and addition of LAM or LEV achieved $70-74 \%$ reduction from baseline.

Conclusions: Here, we demonstrate that the combination of PER with LAM or LEV reduced seizure severity and duration in the rat amygdala kindling model, at doses that had minimal effects on seizures when administered alone. Supported by Eisai Co., Ltd. 
Table 1

\begin{tabular}{|cc|c|c|c|c|}
\hline & & \multicolumn{3}{|c|}{$\%$ Reduction from Basclinc } \\
\hline Agent & Dose & N & $\begin{array}{c}\text { Seizure } \\
\text { Score }\end{array}$ & $\begin{array}{c}\text { Motor Seizure } \\
\text { Duration }\end{array}$ & $\begin{array}{c}\text { EEG Seizure } \\
\text { Duration }\end{array}$ \\
\hline Vehicle & & 6 & 0 & $3 \%$ & $4 \%$ \\
\hline PER & $0.75 \mathrm{mg} / \mathrm{kg}$ & 7 & $11 \%$ & $19 \%$ & $17 \%$ \\
\hline $\mathrm{LAM}$ & $20 \mathrm{mg} / \mathrm{hg}$ & 7 & 0 & $12 \%$ & $26 \%$ \\
$\begin{array}{c}\mathrm{P}: \mathrm{R}+ \\
\mathrm{I} / \mathrm{AM}\end{array}$ & $\begin{array}{c}0.75+20 \\
\mathrm{mg} / \mathrm{kg}\end{array}$ & 8 & $45 \%$ & $72 \%$ & $56 \%$ \\
\hline
\end{tabular}

Table 2

\begin{tabular}{|c|c|c|c|c|c|}
\hline & & & \multicolumn{3}{|c|}{$\%$ Reduction from Baseline $<>>$} \\
\hline Agent $<>>$ & Dose $\langle>>$ & $N\langle>$ & $\begin{array}{r}\text { Seizure } \\
\text { Score }<>>\end{array}$ & $\begin{array}{c}\text { Motor Seizure } \\
\text { Duration< }<>>\end{array}$ & $\begin{array}{l}\text { EEG Seizure } \\
\text { Duration }<>>\end{array}$ \\
\hline Vehicle & & 8 & $\mathbf{0}$ & $21 \%$ & $4 \%$ \\
\hline PERR & $0.75 \mathrm{mg} / \mathrm{kg}$ & 8 & $20 \%$ & $38 \%$ & $31 \%$ \\
\hline LEV & $50 \mathrm{mg} / \mathrm{kg}$ & 8 & $\mathbf{0}$ & $-28 \%$ & $14 \%$ \\
\hline $\begin{array}{l}\text { PER + } \\
\text { LEV }\end{array}$ & $\begin{array}{c}0.75+50 \\
\mathrm{mg} / \mathrm{kg}\end{array}$ & 8 & $38 \%$ & $70 \%$ & $45 \%$ \\
\hline
\end{tabular}

\subsection{3}

\section{A RETROSPECTIVE DATA ANALYSIS OF LEVETIRACETAM AS INITIAL MONOTHERAPY IN PEDIATRIC EPILEPSY PATIENTS}

Addie S. Hunnicutt, A. Morales, R. R. Blouin, E. T. Foster and M. S. Patel (Pediatric Neurology, Greenville Hospital System University Medical Group, Greenville, SC)

Rationale: Levetiracetam is a pyrolidone derivative that has high oral bioavailability, rapid absorption, and no interactions with other drugs. For these reasons, it is frequently prescribed as an initial antiepileptic medication, although it is only approved as an adjunctive therapy.

Methods: We performed a retrospective chart review of new onset pediatric seizure patients. Patients were captured over a 2 year period from 9/1/2008-9/1/2010 and followed until 5/31/2012 in a general neurology practice in a community based academic children's hospital. A total of 301 patients up to age 17 years with new onset seizures were evaluated for efficacy judged by responder rate and seizure freedom, side effects, and retention. Neonates and patients with acute symptomatic seizures were excluded.

Results: $66 \%(198 / 301)$ of the patients were initially placed on levetiracetam. $70 \%$ (139) of the patients were determined to have seizures focal in onset and $28 \%$ (56) were primarily generalized. 3 patients were undetermined in seizure onset. Of the 198 patients on levetiracetam, $65 \%$ had $>50 \%$ reduction in seizures $(63 \%$ of focal and $70 \%$ of generalized) and $32 \%$ became seizure free (35\% of focal and $25 \%$ of generalized). In patients with focal onset seizure, responder rate was similar between levetiracetam and oxcarbazepine, as $63 \%(88 / 139)$ of patients responded on levetiracetam and $54 \%$ $(25 / 46)$ on oxcarbazepine $(\mathrm{p}=0.26)$. Seizure freedom rate was also similar with $35 \%(48 / 139)$ of patients on levetiracetam and $30 \%$ $(14 / 46)$ of patients on oxcarbazepine $(\mathrm{p}=0.66)$. Patients with multiple seizures per day were less likely to become seizure free $(15 \%$ with multiple seizures per day vs. $41 \%$ without multiple seizures per day) on levetiracetam monotherapy ( $\mathrm{p}<0.01$ ), but there was no difference in responder rate of $50 \%$ reduction in seizures $(67 \%$ vs. $65 \%$, $\mathrm{p}=0.84) .59 \%(116 / 198)$ continued on levetiracetam as monotherapy throughout the study period. The mean retention time on levetiracetam as monotherapy was 21.6 months (1-39). 44 patients (24\%) discontinued levetiracetam. 41 (21\%) switched to another medication and $3(1.5 \%)$ elected no medication. $61 \%$ discontinued due to side effects and 39\% discontinued for ineffectiveness of levetiracetam as monotherapy. 34 patients $(17 \%)$ continued levetiracetam but added another antiepileptic medication. Of those not started on levetiracetam as initial monotherapy, $30 \%$ switched to levetiracetam as second treatment. Side effects occurred in 65 patients $(32 \%)$ on levetiracetam. The majority of these were related to behavior problems $(95 \%)$, with 13 patients suffering severe enough side effects to discontinue the medication.

Conclusions: Levetiracetam was safe and effective as initial monotherapy in pediatric patients with new onset seizures.

\subsection{4}

USE OF RESCUE MEDICATIONS BY LGS PATIENTS TREATED WITH CLOBAZAM DURING THE CONTAIN TRIAL

D. Lee ${ }^{1}$, W. Rosenfeld ${ }^{2}$ and J. Isojarvi ${ }^{1}\left({ }^{1}\right.$ Lundbeck LLC, Deerfield, IL and ${ }^{2}$ The Comprehensive Epilepsy Care Center for Children \& Adults, St. Louis, MO)

Rationale: Clobazam, a 1,5-benzodiazepine, was FDA-approved in October 2011 for the treatment of seizures associated with LGS. With data from a Phase III, randomized controlled trial of clobazam in LGS (the CONTAIN trial ${ }^{1}$ ), we conducted post-hoc analyses to evaluate the degree to which patients employed rescue medications during the maintenance period.

Methods: CONTAIN, ${ }^{1}$ a prospective, double-blind, placebocontrolled study, compared 3 oral dosages of clobazam with placebo as adjunctive therapy for LGS. Patients 2 to 60 years of age with LGS (documented by both clinical and electroencephalographic criteria) enrolled. Following a 4 -week baseline phase, patients who had $\geq 2$ drop seizures per week were randomized to placebo or 1 of 3 dosages of clobazam $(0.25,0.5$, and $1.0 \mathrm{mg} / \mathrm{kg} / \mathrm{day})$, up to a maximum daily dosage of $40 \mathrm{mg}$. Treatment included a 3 -week titration phase, followed by a 12 -week maintenance phase. The primary endpoint was the percentage decrease in mean weekly frequency of drop seizures during the maintenance vs. baseline phases for the modified intention-to-treat (mITT) population. The mITT analysis included all patients who had $\geq 1$ daily seizure measurement during the maintenance phase. Patients were permitted rescue therapy of 1 rescue per day, with no more than a total of 4 days in 4 weeks (i.e., average of 1 rescue dosage per week). If more rescue treatments were required, the patient was considered a treatment failure, discontinued from the study, and tapered off study drug or enrolled in an openlabel extension study. Treatment options included, but were not limited to, medications such as rectal diazepam and lorazepam. Clobazam was not used as a rescue therapy.

Results: As previously reported, ${ }^{1} 301$ patients were screened, 238 were randomized, 217 comprised the mITT population (efficacy analyses), and 177 completed the study. The percentage of patients who received rescue medications at least once during the study was $<20 \%$ in all treatment groups and lower in each clobazam group than in the placebo group (differences not statistically significant) (table) Conclusions: Rescue medications were used infrequently during the maintenance period of CONTAIN. In addition, there were no clinically important or statistically significant differences between treatment groups in the percentage of patients who employed rescue medications on multiple occasions. However, there was a trend toward less frequent use of rescue medications in the low-, mediumand high-dosage clobazam groups.

References:

${ }^{1} \mathrm{Ng}$ YT, et al. Neurology. 2011;77:1473-81. 
Use of Rescue Medications During the Maintenance Therapy Period in the CONTAIN Trial (mITT Population)

\begin{tabular}{|c|c|c|c|c|}
\hline & $\begin{array}{l}\text { Placebo } \\
N=57 \\
n(\%)\end{array}$ & $\begin{array}{c}\text { Low-Dosage } \\
\text { CLB } \\
(0.25 \\
\text { mg/kg/day }) \\
N=53 \\
\text { n }(\%)\end{array}$ & $\begin{array}{c}\text { Medium-Dosage } \\
\text { CLB } \\
(0.5 \mathrm{mg} / \mathrm{kg} / \text { day }) \\
\mathrm{N}=58 \\
\mathrm{n}(\%)\end{array}$ & $\begin{array}{c}\text { High-Dosagc } \\
\text { CLB } \\
(1.0 \\
\text { mg/kg/day) } \\
\text { V=49 } \\
\text { n }(\%)\end{array}$ \\
\hline \multicolumn{5}{|c|}{$\begin{array}{l}\text { Patients receiving rescue } \\
\text { medication }\end{array}$} \\
\hline Yes & $\begin{array}{c}11 \\
(19.3)\end{array}$ & $7(13.2)$ & $5(8.6)$ & $4(8.2)$ \\
\hline No & $\begin{array}{c}46 \\
(80.7)\end{array}$ & $46(86.8)$ & $53(91.4)$ & $45(91.8)$ \\
\hline $\begin{array}{l}\text { Odds ratio } \\
(95 \% \mathrm{CI})\end{array}$ & NA & $\begin{array}{c}0.6 \\
(0.23,1.78)\end{array}$ & $\begin{array}{c}0.4 \\
(0.12,1.19)\end{array}$ & $\begin{array}{c}0.4 \\
(0.11,1.26)\end{array}$ \\
\hline \multicolumn{5}{|c|}{$\begin{array}{l}\text { Number of times rescue } \\
\text { medication was cmployed during } \\
\text { maintenance period }\end{array}$} \\
\hline 1 & $1(1.8)$ & $3(5.7)$ & $1(1.7)$ & $1(2,0)$ \\
\hline 2 & $4(7.0)$ & $2(3.8)$ & $2(3.4)$ & $2(4,1)$ \\
\hline 3 & $2(3.5)$ & 0 & 0 & 0 \\
\hline $4+$ & $4(7.0)$ & $2(3.8)$ & $2(3.4)$ & $1(2.0)$ \\
\hline
\end{tabular}

$\mathrm{CLB}=$ clobazam; $\mathrm{CI}=$ confidence interval; $\mathrm{NA}=$ not applicable.

\subsection{5}

\section{WTIHDRAWN}

\subsection{6}

RETROSPECTIVE EVALUATION OF ANTIEPILEPTIC DRUGS IN PATIENTS WITH CDKL5 MUTATIONS

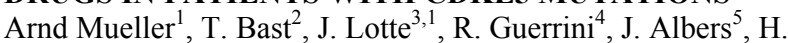
Muhle $^{5}$, S. Auvin ${ }^{6}$, C. Korenke ${ }^{7}$, S. Philip ${ }^{8}$, R. Keimer ${ }^{9}$, P. Striano ${ }^{10}$ N. I. Wolf ${ }^{11}$, B. Püst ${ }^{12}$, C. H. Thiels ${ }^{13}$, A. Fogarasi ${ }^{14}, \mathrm{~S}$. Waltz ${ }^{15}, \mathrm{M}$. Staudt ${ }^{1,16}$ and G. Kluger ${ }^{1,17}\left({ }^{1}\right.$ Clinic for Neuropediatrics and Neurological Rehabilitation, Epilepsy Center for children and Adolescents, Schoen Klinik Vogtareuth, Vogtareuth, Germany; ${ }^{2}$ Epilepsy Clinic for children and adolescents, Epilepsy Center, Kork, Germany; ${ }^{3}$ Child Center, Maulbronn, Germany; ${ }^{4}$ Child Neurology Unit, A. Meyer Children's Hospital, University of Florence, Florence, Italy; ${ }^{5}$ Dept. of Neuropediatrics, UKSH Kiel, Kiel, Germany; ${ }^{6}$ Department for child neurology and epilepsy, Robert Debré Hospital, Paris, France; ${ }^{7}$ Department of Neuropediatrics, Children's Hospital, Oldenburg, Germany; ${ }^{8}$ Children's Hospital, Birmingham, United Kingdom; ${ }^{9}$ Department of neuropediatrics, Pediatric clinic 1, Stuttgart, Germany; ${ }^{10}$ Pediatric Neurology, Institute Gaslini, University of Genova, Genova, Italy; ${ }^{11}$ Child Neurology, VU University Medical Center, Amsterdam, Netherlands; ${ }^{12}$ Department of Neuropediatrics, Catholic Children's Hospital Wilhelmstift, Hamburg, Germany; ${ }^{13}$ Department of Neuropediatrics, children's hospital, Ruhr University Bochum, Bochum, Germany; ${ }^{14}$ Neurology Department, Bethesda Children's Hospital, Budapest, Hungary; ${ }^{15}$ Department of Neuropediatrics, City hospital Cologne, Cologne, Germany; ${ }^{16}$ Department of Neuropediatrics and Child Development, University Children's Hospital, Tübingen, Germany and ${ }^{17}$ Department of Paediatrics, Paracelsus Medical University of Salzburg, Salzburg, Austria)

Rationale: Mutations of or deletions in the X-linked cyclindependent kinase-like 5 gene (CDKL5) cause an epileptic encephalopathy with early-onset severe neurological impairment and intractable seizures. So far there is no concept how to treat these rare patients.

In this retrospective study we evaluated the efficacy of antiepileptic drugs in patients with CDKL5 mutations.

Methods: 29 patients (25 female; 4 male; age: 1.6-22.4 years; mean 7.5) with CDKL5 mutations were included. All of them showed a severe global retardation. We retrospectively analyzed the efficacy of AED after 3 and 6 months. Responder rate was defined as a $50 \%$ seizure reduction.
Results: The patients were treated with 4 to 21 (mean: 9) different AED: The five most frequently used drugs were LEV (100\%), VPA (93\%), TPM (79\%), PB (69\%) and steroids (69\%).

26 patients $(90 \%)$ showed initial response to at least one AED for several weeks. In most of them, loss of efficacy occurred in the following weeks. One patient became (and still remains) seizure free with CBZ for 9 years. In the other patients seizures recurred after weeks to months. The responder rate to at least one AED after 3 months was 69\% (20/29) and 24\% (7/29) after 6 months. The highest rate of seizure reduction after 3 months was reported during treatment with FBM (2/3), CLB (5/13), steroids (6/20), VPA (6/27) and LEV (6/29). 12 patients $(41 \%)$ experienced a seizure aggravation to at least one AED. LEV (5/29), CBZ (4/12) and LTG (3/16) were described most frequently as aggravating, which lead to discontinuation. One patient $(1 / 1)$ responded to intravenous immunoglobulines and $2 / 11$ to ketogenic diet.

Conclusions: Most patients showed some but only initial response to various AEDs with different modes of actions. Seizure aggravation was frequently reported. Because of age-related and spontaneous fluctuation in seizure frequency and types, overall benefit of different antiepileptic drugs remains unclear. Collaborated clinical long-term observations might help clinicians to define treatment stretegies in patients with this rare and refractory epileptic encephalopathy.

\subsection{7 \\ EXPERIENCE OF EFFICACY AND TOLERABILITY WITH LACOSAMIDE AS MONOTHERAPY IN PEDIATRIC PATIENTS WITH PARTIAL EPILEPSY}

Eduardo Barragan and M. Hernandez (Hospital Infantil de México Federico Gómez, Mexico City, Mexico)

Rationale: we report our experience with lacosamide, a new antiepileptic drug for partial epilepsy, with a good response and tolerance as adjuntive therapy. For the minimum side effects and the efficacy, we try in children with new onset partial epilepsy. Methods: We treated 19 patients with partial epilepsy, with or without secondary generalization, after getting conventional EEG and MRI. Lacosamide was administered orally, and final dose, after a slow titration each week, ranged between 1 to $10 \mathrm{mg} / \mathrm{kg} /$ day (median dose $200 \mathrm{mg} /$ day). We follow for at least 6 months measuring seizure frequency and tolerability.

Results: Nineteen patients (12 boys) were treated. Mean treatment duration was 7 months (range 4 weeks to 12 months). Treatment efficacy and tolerability was asseessed each month, with seizure frequency and a chart of side effects. 11 patients maintain seizure free $(57 \%)$ and 4 more having a control of more than $75 \%$ of the seizures. 4 patients $(21 \%)$ dropp off, two for lack of efficacy and two for side effects.

Conclusions: Lacosamide seems to be effective and well tolerated in pediatric patients with partial epilepsy as monotherapy treatment, but studies in larger series are needed to validate these findings

\subsection{8}

A POST-HOC EXPLORATORY ANALYSIS OF THE EFFECT OF ESLICARBAZEPINE ACETATE AS ADJUNCTIVE TREATMENT IN ADULT PATIENTS WITH PARTIALONSET SEIZURES REFRACTORY TO CARBAMAZEPINE A. Gil-Nagel ${ }^{1}$, E. Trinka ${ }^{2}$, J. Chaves ${ }^{3}$, E. Hirsch ${ }^{4}$, Rui Sousa ${ }^{5}$, T. Nunes ${ }^{5}$ and P. Soares-da-Silva ${ }^{5,6}{ }^{1}$ Hospital Ruber Internacional, Madrid, Spain; ${ }^{2}$ Paracelsus Medical University, Salzburg, Austria; ${ }^{3}$ Hospital de Santo António (CHP, EPE), Porto, Portugal; ${ }^{4}$ Hôpitaux Universitaires de Strasbourg, Strasbourg, France; ${ }^{5}$ BIAL - Portela \& Ca, S.A., S. Mamede do Coronado, Portugal and ${ }^{6}$ Faculty of Medicine, University of Porto, Porto, Portugal)

Rationale: Eslicarbazepine acetate (ESL) is a novel once-daily (QD) anticonvulsant, extensively converted to eslicarbazepine after oral 
administration, which blocks voltage-gated sodium- and calciumchannels. Although ESL and carbamazepine (CBZ) do not share any common metabolites they are chemically related. Furthermore, CBZ is one of the most frequently used antiepileptic drugs (AEDs). It is important to assess the effect of ESL in patients not achieving complete seizure control under treatment with CBZ. This post-hoc analysis evaluated the efficacy and tolerability of adjunctive ESL therapy in adult patients with partial-onset seizures (POS) refractory to lower and higher dosages of CBZ.

Methods: Data from two phase III multicentre, double-blind, randomized, placebo-controlled studies in adult patients with $\geq 4$ partial-onset seizures per 4 weeks despite treatment with 1-3 AEDs was pooled and analysed. ESL was administered at QD doses of 400 $\mathrm{mg}, 800 \mathrm{mg}$ and $1200 \mathrm{mg}$. Efficacy and tolerability was evaluated in patients not co-treated with CBZ (noCBZ) and patients co-treated with $\mathrm{CBZ} \leq 800 \mathrm{mg}$ daily $(\mathrm{CBZ} \leq 800)$ or with $\mathrm{CBZ}>800 \mathrm{mg}$ daily (CBZ $>800)$, as part of their baseline AEDs.

Results: Safety population comprised 797 patients (noCBZ, $n=328$; $\mathrm{CBZ} \leq 800, \mathrm{n}=193 ; \mathrm{CBZ}>800, \mathrm{n}=276)$ and intention-to treat population included 752 patients (noCBZ, $n=314 ; C B Z \leq 800, n=183$; $\mathrm{CBZ}>800, \mathrm{n}=255$ ). Compared with placebo seizure frequency over the 12-week maintenance period (primary end point) was significantly reduced with ESL $800 \mathrm{mg}$ and $1200 \mathrm{mg}$ in all three patient subgroups $(\mathrm{p} \leq 0.05)$ (table 1); ESL $400 \mathrm{mg}$ was not significantly superior to placebo. Responder rate $(\geq 50 \%$ reduction in seizure frequency) was: noCBZ group $=23 \%$ with placebo, $40 \%$ with ESL $800 \mathrm{mg}$ and 39\% with ESL $1200 \mathrm{mg}$; CBZ $\leq 800$ group $=18 \%$ with placebo, $37 \%$ with ESL $800 \mathrm{mg}$ and $50 \%$ with ESL $1200 \mathrm{mg}$; CBZ $>800$ group $=16 \%$ with placebo, $31 \%$ with ESL $800 \mathrm{mg}$ and $44 \%$ with ESL $1200 \mathrm{mg}$. Median relative reduction in seizure frequency was: noCBZ group $=-13 \%$ with placebo, $-39 \%$ with ESL $800 \mathrm{mg}$ and $-33 \%$ with ESL $1200 \mathrm{mg}$; CBZ $\leq 800$ group $=-14 \%$ with placebo, -34\% with ESL $800 \mathrm{mg}$ and $-50 \%$ with ESL $1200 \mathrm{mg}$; $\mathrm{CBZ}>800$ group $=-9 \%$ with placebo, $-29 \%$ with ESL $800 \mathrm{mg}$ and $38 \%$ with ESL $1200 \mathrm{mg}$. Incidence of treatment-emergent adverse events (TEAEs) was higher in CBZ>800 group (54\% with placebo, $74 \%$ with ESL $800 \mathrm{mg}$ and $78 \%$ with ESL $1200 \mathrm{mg}$ ) than in CBZ $\leq 800$ group (49\% with placebo, $71 \%$ with ESL $800 \mathrm{mg}$ and $71 \%$ with ESL $1200 \mathrm{mg}$ ) and noCBZ group (46\% with placebo, 57\% with ESL $800 \mathrm{mg}$ and 64\% with ESL $1200 \mathrm{mg}$ ) (table 2).

Conclusions: In this post-hoc analysis, the efficacy of adjunctive ESL treatment was independent from concomitant CBZ therapy. Patients with POS refractory to CBZ dosages higher than $800 \mathrm{mg}$ daily achieved a significant improvement in seizure control with adjunctive once-daily ESL $800 \mathrm{mg}$ and $1200 \mathrm{mg}$. The incidence of TEAEs was higher in patients treated concomitantly with CBZ, especially if CBZ dosage was higher than $800 \mathrm{mg}$ daily.

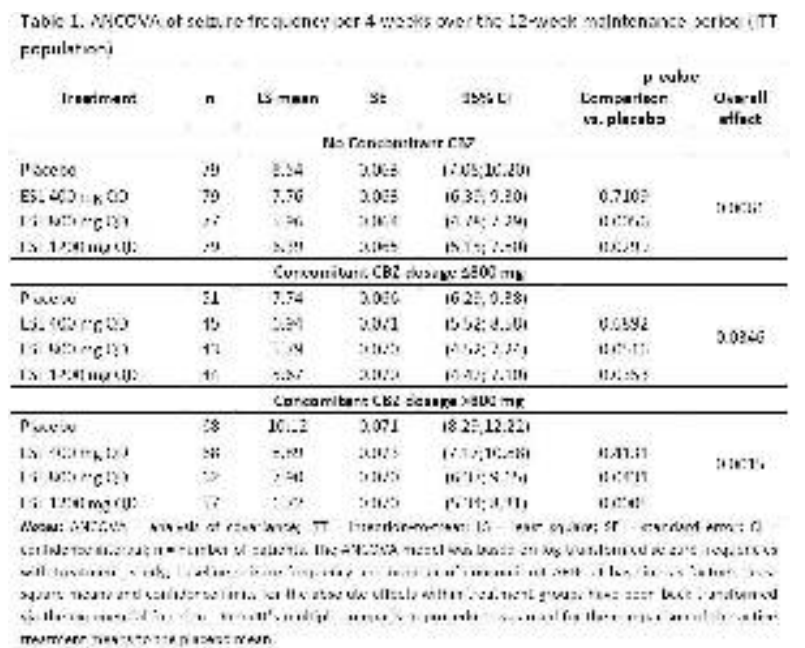

Table 1. ANCOVA of seizure frequency per 4 weeks over the 12week maintenance period (ITT population)

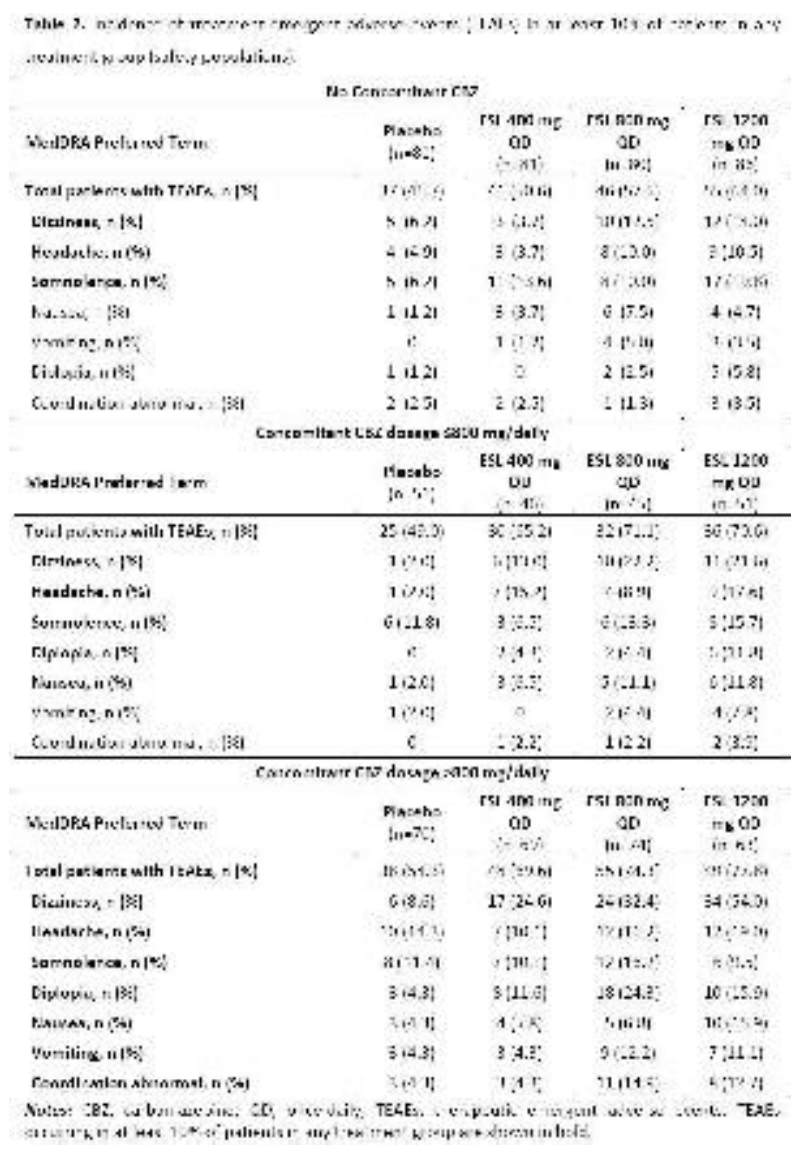

Table 2. Incidence of treatment-emergent adverse events (TEAEs) in at least $10 \%$ of patients in any treatment group (safety populations).

\subsection{9 \\ A POST-HOC EXPLORATORY ANALYSIS OF THE EFFECT OF ESLICARBAZEPINE ACETATE AS ADJUNCTIVE TREATMENT IN ADULT PATIENTS WITH PARTIAL- ONSET SEIZURES AND COMORBID CLINICALLY RELEVANT DEPRESSIVE SYMPTOMS}

M. Carreño ${ }^{1}$, E. Ben-Menachem ${ }^{2}$, T. J. O'Brien ${ }^{3}$, B. Schmitz ${ }^{4}$, J.

Pimentel $^{5}$, Rui Sousa ${ }^{6}$, T. Nunes ${ }^{6}$ and P. Soares-da-Silva ${ }^{6,7}{ }^{1}$ Hospital Clínic, Barcelona, Spain; ${ }^{2}$ Sahlgrenska Academy, Göteborg University, Goteborg, Sweden; ${ }^{3}$ The Royal Melbourne Hospital, The University of Melbourne, Melbourne, VIC, Australia; ${ }^{4}$ Vivantes Humboldt-Klinikum, Berlin, Germany; ${ }^{5}$ Hospital de Santa Maria (CHLN, EPE), Lisbon, Portugal; ${ }^{6}$ BIAL - Portela \& Ca, S.A., S. Mamede do Coronado, Portugal and ${ }^{7}$ Faculty of Medicine, University of Porto, Porto, Portugal)

Rationale: Eslicarbazepine acetate (ESL) is a novel once-daily (QD) anticonvulsant, extensively converted to eslicarbazepine after oral administration, which blocks voltage-gated sodium- and calciumchannels. Depressive disorders are common comorbidities of epilepsy and they have been associated with a poor response to antiepileptic drug therapy. This post-hoc analysis evaluated the efficacy and tolerability of ESL as adjunctive treatment in adult patients with partial-onset seizures (POS) and co-morbid clinically relevant depressive symptoms.

Methods: Data from two (BIA-2093-301 and -302) phase III multicentre, double-blind, randomized, placebo-controlled studies in adult patients with $\geq 4$ partial-onset seizures per 4 weeks despite treatment with 1-3 AEDs was pooled and analysed. ESL was administered at QD doses of $400 \mathrm{mg}, 800 \mathrm{mg}$ and $1200 \mathrm{mg}$. ESL efficacy and tolerability was evaluated in subjects with and without clinically relevant depressive symptoms at baseline, as defined by Montgomery-Asberg depression rating scale score (MADRS) $\geq 10$ and $<10$, respectively. MADRS score $<10$ have been previously 
shown to define remission from major depression (Affective Dis 2002; 72:177-184.)

Results: Safety population comprised 796 patients (MADRS $\geq 10$, $\mathrm{n}=325$; MADRS $<10, \mathrm{n}=471$ ) and intention-to treat population included 751 patients (MADRS $\geq 10, n=303$; MADRS $<10, n=448$ ). Compared with placebo seizure frequency over the 12-week maintenance period (primary endpoint) was significantly reduced with ESL $800 \mathrm{mg}$ and $1200 \mathrm{mg}$ both in patients with MADRS score $\geq 10$ ( $\mathrm{p}=0.03$ and $\mathrm{p}=0.004$, respectively) and with MADRS score $<10$ ( $\mathrm{p}=0.0007$ and $\mathrm{p}=0.0003$, respectively) (table 1 ). Responder rate $(\geq 50 \%$ reduction in seizure frequency) was: MADRS score $\geq 10$ group $=13 \%$ with placebo, $32 \%$ with ESL $800 \mathrm{mg}$ and $35 \%$ with ESL $1200 \mathrm{mg}$; MADRS score $<10$ group $=23 \%$ with placebo, $39 \%$ with ESL $800 \mathrm{mg}$ and $49 \%$ with ESL $1200 \mathrm{mg}$. Median relative reduction in seizure frequency was: MADRS score $\geq 10$ group $=-4 \%$ with placebo, -31\% with ESL $800 \mathrm{mg}$ and -30\% with ESL $1200 \mathrm{mg}$; MADRS score $<10$ group $=-20 \%$ with placebo, $-38 \%$ with ESL 800 $\mathrm{mg}$ and $-48 \%$ with ESL $1200 \mathrm{mg}$. Incidence of treatment-emergent adverse events (TEAEs) was similar in MADRS score $\geq 10$ group (55.8\% with placebo, $63.4 \%$ with ESL $800 \mathrm{mg}$ and $73.8 \%$ with ESL $1200 \mathrm{mg}$ ) and in MADRS score $<10$ group (45.6\% with placebo, $69.2 \%$ with ESL $800 \mathrm{mg}$ and $67.0 \%$ with ESL $1200 \mathrm{mg}$ ) (table 2).

Conclusions: In this post-hoc exploratory analysis, once-daily ESL $800 \mathrm{mg}$ and $1200 \mathrm{mg}$ adjunctive therapy was significantly superior to placebo in reducing POS in adult patients with co-morbid clinically relevant depressive symptoms. This subgroup of patients with MADRS scores $\geq 10$ at baseline was particularly drug resistant as compared to patients without clinically relevant depressive symptoms (MADRS scores $<10$ ) as shown by a worse seizure control either in placebo and ESL treated groups.

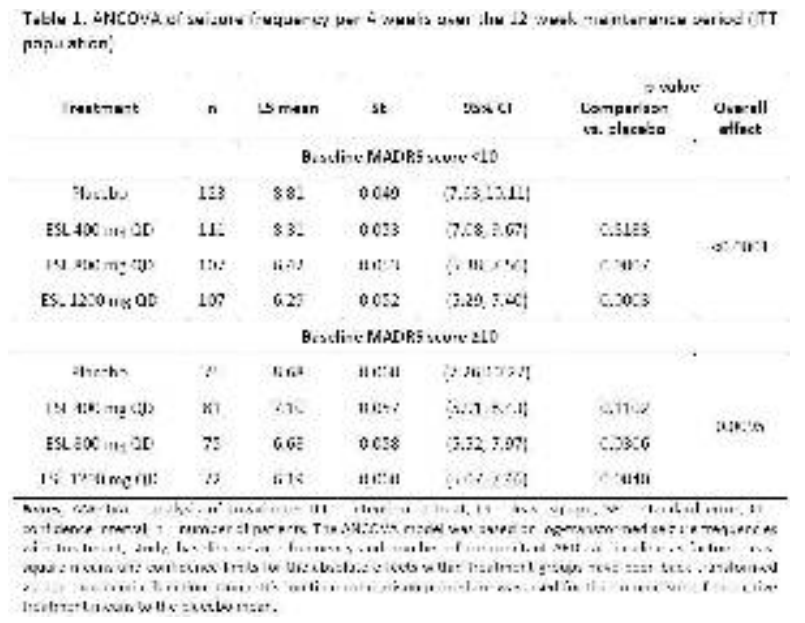

Table 1. ANCOVA of seizure frequency per 4 weeks over the 12week maintenance period (ITT population)

\begin{tabular}{|c|c|c|c|c|}
\hline \multicolumn{5}{|c|}{ BJSellns MADES scors $<10$} \\
\hline 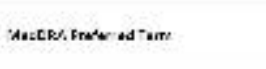 & arin & 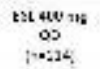 & $\begin{array}{c}\text { t. tw } 11 ; \\
\infty \\
n=2 ;\end{array}$ & $\begin{array}{c}\text { tat jogzins } \\
\infty \\
10: 2 t ;\end{array}$ \\
\hline 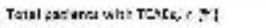 & का1:- & 7a:es & f1::8, & 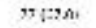 \\
\hline Veiksa tis & 014.3 & 15: $1: 60$, & $20 ; 40,0$ & $22 ; 242$ \\
\hline Hista:-test & $21: 2$ & $21+6 \mathrm{t}^{2}$ & $28: 23$ & 2t:?:2.; \\
\hline $\operatorname{sen} 20 \sec x+\pi$ & MU: & 11.96 & (7) 4.9 & I \\
\hline No.che v1.ie & An,? & $s \mid 1$ & 11,$81 ;$ & $10, \mathrm{n} n$ \\
\hline Wulte 715 & 212.8 & 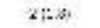 & $512=2$ & $=$ it \\
\hline 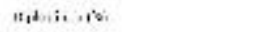 & 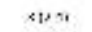 & $\therefore$ rit & weted & $\times 12 \mathbf{1 6}$ \\
\hline 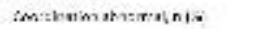 & $1,3 \%$ & $\therefore \mathrm{L}=\mathrm{H}$ & 12:3 & , tal. \\
\hline \multicolumn{5}{|c|}{ BJSClln: MADRS scors 210} \\
\hline 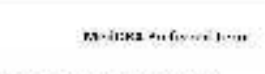 & $\begin{array}{l}\text { resento } \\
\text { in } 731\end{array}$ & 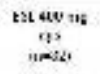 & 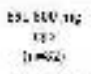 & 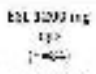 \\
\hline 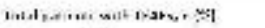 & 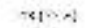 & $2 t: B$ & when & wos $x$ \\
\hline Vansa the & $01: B 1$ & $w:=: 2$ & $24:-: 5$ & $16 ; 42,0$. \\
\hline 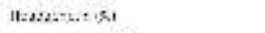 & Q1:st & sस: & yill: & $22(: 4)$ \\
\hline ו & $.1 \times 2$ & wat, & ויום & $12: 1,12$ \\
\hline 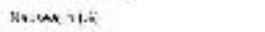 & औit & slat, & $7: 1.1$ & is: \& \\
\hline 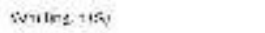 & 212.01 & $212=$ & its: & $y: 1 u \div$ \\
\hline$n, d, i, i_{i}$ & $11<1$ & sis: : & th: $: 5$ & $7+1+\cdots$ \\
\hline im:bumaknerrain|.: & 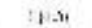 & $s y=4$ & $1: 3$ & $\therefore D$ \\
\hline
\end{tabular}

Table 2. Incidence of treatment-emergent adverse events (TEAEs) in at least $10 \%$ of patients in any treatment group (safety populations).

\subsection{0 \\ EXPERIENCE OF THE ADULT DEPARTMENT OF THE KORK EPILEPSY CENTRE (GERMANY) WITH RETIGABINE IN A HIGHLY DRUG RESISTANT POPULATION OF 40 EPILEPTIC PATIENTS}

Christoph Kurth and B. J. Steinhoff (Epilepsy Centre Kork, Kork, Germany)

Rationale: Retigabine (RTG) is a new antiepileptic drug (AED) for add-on-treatment of partial epilepsies with and without secondary generalization in adults, which is available in Germany since May 2011. Its mechanism of action, the activation of voltage gated potassium channels, is unique among AEDs. The aim of this study was the evaluation of patients under treatment with RTG with regard to seizure control and side effects.

Methods: 40 consecutive patients suffering from focal epilepsies who started add-on-therapy with RTG since May 2011 were analyzed. Patients suffering from a progressive disease or psychogenic seizures, taking part in another treatment study or undergoing epilepsy surgery or implantation of a vagal nerve stimulator during the treatment with RTG were not included. Demographic data, aetiology of epilepsy, treatment response, comedication and side effects were recorded.

Results: 23 men and 17 women of median age 36 years (range: 1972 ) and a median duration of epilepsy of 27 years (range: 4-65) were included. Median seizure rate before treatment with RTG was 16 per month (range: 2-80), number of AEDs over lifetime was between 4 and 23.

Titration was performed according to the product information up to an initial dose of $600 \mathrm{mg} /$ day. Median final dosage was $600 \mathrm{mg} /$ day (range: $200 \mathrm{mg}-1200 \mathrm{mg}$ ).

Main aetiologies: $45 \%$ cryptogenic, $17.5 \%$ focal cortical dysplasia, $10 \%$ complex cerebral malformation. $30 \%$ underwent unsuccessful epilepsy surgery or implantation of VNS before treatment with RTG. Responder rates: $15 \%$ of the patients were responders with a seizure reduction (SR) between 50\% and 100\%: 2.5\% became seizure free. In $5 \%$ of the patients SR was between $75 \%$ and $99 \%$, in $7.5 \%$ SR was between $50 \%$ and $74 \% .85 \%$ showed a SR below $50 \%$. In one patient $(2.5 \%)$ seizure situation worsened. In one patient seizure frequency did not change but non-convulsive status epileptici, occurring each week until treatment with RTG was initiated, stopped.

Main co-medication: oxcarbazepine, levetiracetam and lacosamide. Median number of AEDs in co-medication was 2 (range: 1-4). 
Side effects were seen in $40 \%$ of the patients. Main adverse reactions: dizziness and tiredness $17.5 \%$ each, $7.5 \%$ diplopia.

Medication was tapered in $60 \%$ of the patients (in $30 \%$ due to lack of effect, in $12.5 \%$ due to side effects, in $17.5 \%$ due to lack of effect and side effects).

Conclusions: Compared to pivotal trails responder rate in our patients is lower: $15.0 \%$ versus $28-56 \%$ (depending on the dosage). This may be due to our highly therapy resistant population. Main side effects and rate of tapering of RTG due to side effects seen in our patients are similar to those described in the other trials.

\subsection{1}

\section{STEADY-STATE PHARMACOKINETICS AND} TOLERABILITY OF ONCE-DAILY AND TWICE-DAILY REGIMENS OF ESLICARBAZEPINE ACETATE AND TWICE-DAILY REGIMENS OF OXCARBAZEPINE IN HEALTHY VOLUNTEERS

M. Vaz-da-Silva ${ }^{1,2}$, A. Falcão ${ }^{3}$, M. Bialer ${ }^{4}$, T. Nunes ${ }^{1}$, L. Almeida ${ }^{5}$, C. Elger ${ }^{6}$ and P. Soares-da-Silva ${ }^{1,2}\left({ }^{1}\right.$ BIAL - Portela \& Ca, S.A., S. Mamede do Coronado, Portugal; ${ }^{2}$ Faculty of Medicine, University of Porto, Porto, Portugal; ${ }^{3}$ Faculty of Pharmacy, University of Coimbra, Coimbra, Germany; ${ }^{4}$ School of Pharmacy, Faculty of Medicine, Hebrew University of Jerusalem, Jerusalem, Israel; ${ }^{5}$ Health Sciences Section, University of Aveiro, Aveiro, Portugal and ${ }^{6}$ University of Bonn, Bonn, Germany)

Rationale: In a phase II placebo-controlled, adjunctive therapy study in adult patients with partial-onset seizures eslicarbazepine acetate (ESL) 800 and $1200 \mathrm{mg} /$ day once-daily (QD) was found to be efficacious and well tolerated. However, the same dose given twicedaily (BID) was not significantly more efficacious than placebo (Epilepsia, 48, 497-504, 2007). The present study investigated the steady-state pharmacokinetics of QD and BID regimens of ESL and BID regimen of oxcarbazepine (Trileptal ${ }^{\circledR}$; OXC) in healthy volunteers. Tolerability was also assessed.

Methods: Single centre, open-label, randomised, three-way crossover study in 12 healthy volunteers. The study consisted of three 8-day treatment periods separated by a washout period of 10-15 days. In each treatment period the volunteers received a daily dose of either ESL (900 mg QD or $450 \mathrm{mg}$ BID) or $450 \mathrm{mg}$ of OXC BID. A chiral LC-MS bioanalytical method was used to quantify eslicarbazepine, (R)-licarbazepine and oxcarbazepine.

Results: Eslicarbazepine was the major active entity in plasma, accounting for $94.6 \%, 93.9 \%$ and $81.5 \%$ of total exposure within the corresponding dosing interval $(\tau)$ at steady state (AUCss, $0-\tau)$ with ESL QD, ESL BID and OXC BID, respectively. Eslicarbazepine Cmax,ss (in $\mu \mathrm{mol} / \mathrm{L}$ ) following ESL QD (87.3) was 33\% higher compared to ESL BID (65.5) and $82 \%$ higher compared to OXC BID (48.0) (Figure 1). The AUCss, $0-\tau$ (in $\mu \mathrm{mol} * \mathrm{~h} / \mathrm{L}$ ) following the last dose of an 8-day repetitive dosing was 1156, 1118 and 968 for ESL QD, ESL BID and OXC BID, respectively (Table 1). Trough plasma eslicarbazepine before the last dose was 31\% lower in the ESL QD in relation to ESL BID and OXC BID $(22,32$ and $32 \mu \mathrm{mol} / \mathrm{L}$, respectively). The ratio eslicarbazepine plasma exposure $(\mu \mathrm{mol} * \mathrm{~h} / \mathrm{L})$ to daily dose ( $\mu \mathrm{mol})$ was 0.38 (1156:3037), 0.37 (1118:037) and 0.27 (968:3567) for ESL QD, ESL BID and OXC BID, respectively, which translates in a $40 \%$ increase in efficiency of ESL QD (and ESL BID) versus OXC BID to deliver their major active entity eslicarbazepine. The extent of plasma exposure to drug entities (R)licarbazepine and oxcarbazepine after ESL QD was $72 \%$ and $61 \%$ lower than after OXC BID, respectively. Thirty, 18 and 18 treatmentemergent adverse events were reported in the OXC BID, ESL BID and ESL QD, respectively.

Conclusions: In comparison to ESL BID, administration of ESL QD resulted in 33\% higher peak plasma concentration (Cmax,ss) of eslicarbazepine, which may correlate with the efficacy profile reported with ESL. In comparison to OXC BID, administration of ESL QD resulted in $40 \%$ increase in the efficiency to deliver eslicarbazepine, less R-licarbazepine and less oxcarbazepine. which may relate to differences in the clinical profiles of ESL and OXC.

Table 1. Mean eslicarbazepine pharmacokinetic parameters following the last dose of an 8-day treatment with ESL $900 \mathrm{mg}$ QD, ESL 450 $\mathrm{mg}$ BID and OXC $450 \mathrm{mg}$ BID to 12 healthy subjects.

\begin{tabular}{|c|c|c|c|c|}
\hline Treatment & $\underset{(\mu \operatorname{mol} / \mathrm{T})}{\operatorname{Cmax}}$ & $\begin{array}{l}\operatorname{tmax} \\
\text { (h) }\end{array}$ & $\begin{array}{c}\mathrm{AUC}_{\mathrm{ss}, 0-\tau} \\
\left(\mu \mathrm{mol}^{* \mathrm{~h} .} / \mathrm{L}\right)\end{array}$ & $\begin{array}{c}\text { Apparent (terminal) } t^{1 / 2} \\
\text { (h) }\end{array}$ \\
\hline ГSSI. $900 \mathrm{mg}$ QD & $87.3(32.7)$ & $2.45(35.8)$ & $1156(19.9)$ & $9.12(13.1)$ \\
\hline ESL $450 \mathrm{mg}$ BID & $65.5(23.9)$ & $2.09(31.8)$ & $1113(18.3)$ & $9.17(16.2)$ \\
\hline OXC $450 \mathrm{mg}$ BID & $48.0(16.8)$ & $3.77(42.5)$ & $968(13.5)$ & $9.24(35.2)$ \\
\hline
\end{tabular}

Values are means (coefficient variation, \%).

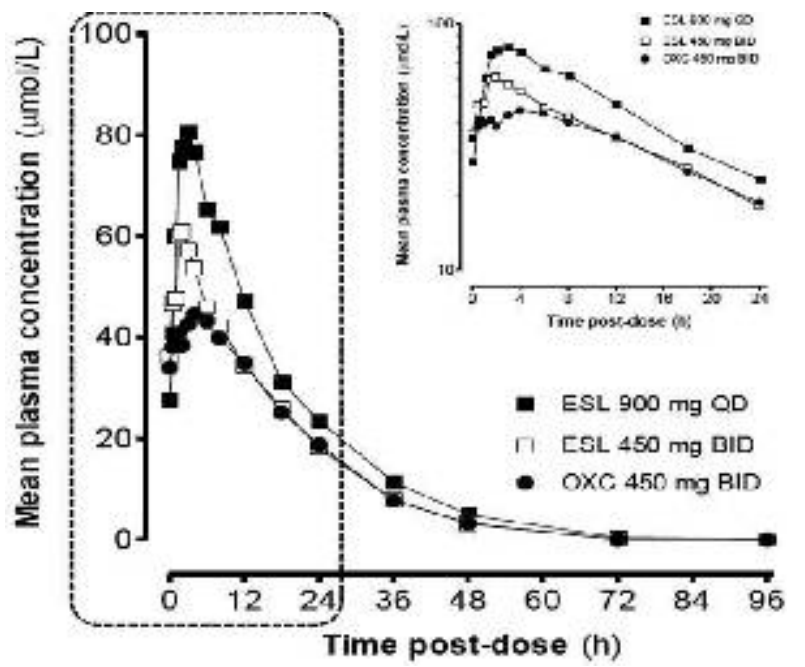

Figure 1. Mean plasma concentration-time profile of eslicarbazepine following the last dose of a 8-day repeated dosing with ESL $900 \mathrm{mg}$ QD, ESL $450 \mathrm{mg}$ BID and OXC $450 \mathrm{mg}$ BID to 12 healthy subjects. The inset shows log transformation of concentration-time profiles up to $24 \mathrm{~h}$ post-dose.

\subsection{2}

ASSESSMENT OF PHARMACEUTICAL QUALITY OF BRAND AND GENERIC LAMOTRIGINE TABLETS

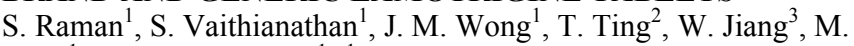
Kane $^{1}$ and James E. Polli ${ }^{1}$ ( ${ }^{1}$ Department of Pharmaceutical Sciences, University of Maryland, Baltimore, MD; ${ }^{2}$ Department of Neurology, University of Maryland, Baltimore, MD and ${ }^{3}$ Food and Drug Administration, Rockville, MD)

Rationale: The therapeutic equivalence of FDA approved generics, including lamotrigine, has been questioned by neurologists and patients with epilepsy. A potential contributor to such concerns is pharmaceutical quality. In the case of lamotrigine tablets, attributes of pharmaceutical quality include tablet appearance, presence of lamotrigine in the tablet at the correct dose and without excessive variability, lack of excessive impurities, and sufficiently rapid drug dissolution from tablet. Tablets with poor pharmaceutical quality can be expected to pose potential bioinequivalence. The objective was to assess the pharmaceutical quality of three lots of LAMICTAL $100 \mathrm{mg}$ tablets and three lots of Teva lamotrigine $100 \mathrm{mg}$ tablets.

Methods: LAMICTAL and Teva tablets were selected since they represent the brand and the most commonly dispensed lamotrigine generic, respectively. Three different lots of each brand and generic tablets were purchased commercially. The following pharmaceutical quality tests were performed: appearance, identity, assay, impurity, uniformity of dosage units, disintegration, dissolution (only lots A and D), friability, and loss on drying. Identity, assay, impurity, uniformity of dosage units, and dissolution were performed as 
specified in the USP monograph for lamotrigine tablets. Passing criteria for each test, except disintegration and loss on drying, were identified prior to testing.

Results: Each lot passed each test. Assay results of LAMICTAL lots $\mathrm{A}, \mathrm{B}$, and $\mathrm{C}$ and Teva lots $\mathrm{D}, \mathrm{E}$, and $\mathrm{F}$ were $98.9 \%, 94.1 \%, 97.3 \%$, $97.3 \%, 100.0 \%$, and $96.3 \%$, respectively. From impurity testing, lamotrigine related compound B in LAMICTAL lots A, B, and C and Teva lots D, E, and F were $0.05 \%, 0.05 \%, 0.05 \%, 0.02 \%, 0.03 \%$, and $0.02 \%$, respectively. Lamotrigine related compound $\mathrm{C}$ in LAMICTAL lots A, B, and C and Teva lots $\mathrm{D}, \mathrm{E}$, and $\mathrm{F}$ were $0.00 \%$, $0.08 \%, 0.03 \%, 0.02 \%, 0.04 \%$, and $0.05 \%$, respectively. No individual unspecified impurity exceeded $0.2 \%$. Total impurities in LAMICTAL lots $\mathrm{A}, \mathrm{B}$, and $\mathrm{C}$ and Teva lots $\mathrm{D}, \mathrm{E}$, and $\mathrm{F}$ were $0.16 \%, 0.33 \%$, $0.16 \%, 0.28 \%, 0.27 \%$, and $0.39 \%$, respectively. Regarding uniformity of dosage units, the $\% \mathrm{CV}$ from tablet weight variation for LAMICTAL lots A, B, and C and Teva lots $\mathrm{D}, \mathrm{E}$, and $\mathrm{F}$ were $1.48 \%$, $1.15 \%, 2.65 \%, 1.01 \%, 0.67 \%$, and $1.46 \%$, respectively. Dissolution at $5 \mathrm{~min}$ of lot A and D was $93.2 \%$ and $100.9 \%$, respectively. Dissolution at 10 and 15 min of lot A was $96.1 \%$ and $99.6 \%$, respectively. Disintegration from LAMICTAL lots A, B, and C and Teva lots D, E, and F were $21 \mathrm{~s}, 19 \mathrm{~s}, 16 \mathrm{~s}, 61 \mathrm{~s}, 83 \mathrm{~s}$, and $75 \mathrm{~s}$, respectively. Friability results from LAMICTAL lots A, B, and C and Teva lots D, E, and F were $0.001 \%, 0.004 \%, 0.005 \%, 0.006 \%$, $0.003 \%$, and $0.008 \%$, respectively. Loss on drying results from LAMICTAL lots A, B, and C and Teva lots D, E, and F were $4.34 \%$, $3.36 \%, 3.48 \%, 3.61 \%, 3.47 \%$, and $2.85 \%$, respectively.

Conclusions: LAMICTAL and Teva generic lamotrigine $100 \mathrm{mg}$ tablets showed acceptable pharmaceutical quality.

\subsection{3 \\ ESTABLISHING MAXIMUM TOLERATED DOSE AND DOSE-PROPORTIONALITY IN EXTENDED-RELEASE TOPIRAMATE (USL255)}

L. J. Lambrecht, T. L. Braun, W. M. Todd and M. B. Halvorsen (Upsher-Smith Laboratories, Inc., Maple Grove, MN)

Rationale: Establishing pharmacokinetic (PK) and safety characteristics of a new drug, including dose proportionality and maximum tolerated dose (MTD), are important steps in determining the drug's optimal dosing regimen. Upsher-Smith Laboratories, Inc. is developing USL255, a once-daily extended-release formulation of topiramate (TPM) for the treatment of seizures. USL255 (25 - 400 $\mathrm{mg}$ ) displays dose proportional TPM exposure with maximum peak exposure $\left(\mathrm{C}_{\max }\right)$ appearing dose proportional at higher doses (100 $400 \mathrm{mg}$ ). The objective of this study was to evaluate single-ascending doses of USL255 over a larger dose range $(600-1600 \mathrm{mg})$ to determine single-dose MTD and examine the PK profile in healthy individuals.

Methods: In this Phase 1, randomized, placebo-controlled, doubleblind, single-center, single-dose, MTD study, healthy subjects were planned for enrollment into 6 separate cohorts of 10 subjects each for up to 60 total subjects. Subjects in each cohort were randomly assigned 1:4 to placebo or a single dose of USL255 (600, 800, 1000, 1200,1400 , or $1600 \mathrm{mg}$ ). Vital sign measurements, clinical laboratory tests, ECG results, and adverse events (AEs) were collected and reviewed for 14 days post-dose. If safety and tolerability were demonstrated after dosing, the study would continue to the next ascending dose until all 6 cohorts were completed. However, if USL255 did not meet $a$ priori safety and tolerability criteria, the previous dose would be named MTD and dose escalation would cease. Blood samples were collected for PK assessments up to 14 days after dosing. Evaluation of dose proportionality was determined using a power model approach, and area under the plasma concentration-time curve (AUC) and $\mathrm{C}_{\max }$ were considered doseproportional if the $90 \%$ confidence interval (CI) for the ratio of dosenormalized geometric mean values was between $0.8-1.25$. Deviation from dose linearity was tested using the type I F test $(\alpha=.05)$.

Results: Single doses of USL255 were safe and generally well tolerated from 600 - $1200 \mathrm{mg}$, and no serious or severe AEs were observed. MTD was declared at $1200 \mathrm{mg}$ after a single subject experienced 16 mild-to-moderate treatment-related AEs, 6 of which began within the first $6 \mathrm{hr}$ following a single administration of USL255 $1400 \mathrm{mg}$. Total (AUC $\mathrm{Ainf}_{\text {inf }}$ and $\left.\mathrm{AUC}_{0-\mathrm{t}}\right)$ and peak $\left(\mathrm{C}_{\max }\right) \mathrm{TPM}$ exposures exhibited dose proportionality over the entire $600-1400$ mg dose range, as the $90 \%$ CIs were completely contained within the 0.8 - 1.25 limit. These PK variables were also linear between 600 and $1400 \mathrm{mg}$. Time to peak exposure $\left(\mathrm{T}_{\max }\right)$ ranged between 17 and $20 \mathrm{hr}$, and elimination half life $\left(\mathrm{t}_{1 / 2}\right)$ decreased with increasing doses.

Conclusions: USL255 was well tolerated up to $1200 \mathrm{mg}$, which was named the single-dose MTD after a subject experienced multiple AEs at $1400 \mathrm{mg}$. Additionally, USL255 exhibited a TPM exposure profile that was both linear and proportional to dose over the entire 600 $1400 \mathrm{mg}$ range. These data further support the predictable PK and favorable tolerability profile of once-daily USL255 and aid in determining its optimal dosing regimen.

\subsection{4 \\ EVALUATION OF LONG-TERM TREATMENT WITH LACOSAMIDE FOR PARTIAL-ONSET SEIZURES IN THE ELDERLY}

William Rosenfeld ${ }^{1}$, C. McShea ${ }^{2}$ and P. Doty $^{2}\left({ }^{1}\right.$ The Comprehensive Epilepsy Care Center for Children and Adults, St. Louis, MO and ${ }^{2}$ UCB Pharma, Raleigh, NC)

Rationale: Lacosamide is approved for the adjunctive treatment of adults with partial-onset seizures (POS), and the safety and efficacy of long-term, open-label lacosamide treatment up to 8 years have been reported previously. The prevalence of new-onset seizures in the elderly is the highest among any age group and approximately twice that of younger adults. As the elderly often take concomitant drugs for other chronic conditions, treatment decisions for epilepsy in this patient population can be challenging. To determine any potential differences in lacosamide treatment effect or safety profile among the elderly compared with the overall OLE lacosamide pool, a subgroup analysis was performed. In this analysis, the safety and efficacy of lacosamide was examined in patients $\geq 65$ years who participated in a lacosamide open-label extension (OLE) trial.

Methods: Patients entered one of three OLEs (SP615, SP756, SP774) following completion of a corresponding phase II or III trial of adjunctive lacosamide for POS. Safety and efficacy data for patients who were $\geq 65$ years at OLE study entry or by OLE study end were pooled from the three trials.

Results: Of 1,054 patients who initiated OL lacosamide treatment, 13 were $\geq 65$ years at study entry and 33 were $\geq 65$ years by study end. Of the 33 elderly patients by study end, $21(63.6 \%)$ completed the trial and 18 of the $21(85.7 \%)$ continued to commercial lacosamide use. The mean and maximum duration of lacosamide treatment was 1563.2 and 2790.0 days, respectively, and the median modal lacosamide dose was $400 \mathrm{mg} /$ day. At $>1,>3$, and $>5$ years $\mathrm{OL}$ treatment, $90.9 \%, 75.8 \%$, and $42.4 \%$ of the elderly patients remained in the trial. As all patients had an opportunity to complete approximately 3 years of exposure, the lower rates of exposure after 3 years is attributed to premature discontinuations and study closure. The primary reasons for discontinuation were adverse events $(n=4$, $12.1 \%)$, lack of efficacy $(n=3,9.1 \%)$, and consent withdrawal $(n=$ $2,6.1 \%$ ). Common treatment-emergent adverse events (TEAEs; $n \geq 5$ $[\geq 15 \%])$ were dizziness $(n=12,36.4 \%)$, fall $(n=9,27.3 \%)$, contusion or sinusitis ( $\mathrm{n}=7$ each, $21.2 \%$ ), cognitive disorder, tremor, headache, depression, or cough $(\mathrm{n}=6$ each, $18.2 \%)$, and urinary tract infection, nausea, diplopia, vision blurred, convulsion, balance disorder, or pain in extremity $(\mathrm{n}=5$ each, $15.2 \%)$. The median percent reduction in seizure frequency from Baseline for 1-, 3- and 5year completers was $62.5 \%, 58.2 \%$ and $66.6 \%$. The $\geq 50 \%$ responder rate was $63.3 \%, 68.0 \%$ and $64.3 \%$ for $1-, 3$ - and 5-year completers. Conclusions: In this small population of elderly patients with POS, long-term OL treatment with lacosamide up to 8 years was generally 
well tolerated and was associated with a reduction in seizure frequency and maintenance of efficacy similar to the overall OLE patient pool.

Sponsored by UCB

\subsection{5}

\section{RESPONSE TO CLOBAZAM IN VNS VS. NON-VNS PATIENTS: POST-HOC SUBGROUP ANALYSIS OF CONTAIN}

S. Benbadis ${ }^{1}$, J. Isojarvi ${ }^{2}$ and D. Lee ${ }^{2}{ }^{1}$ University of South Florida, Tampa, FL and ${ }^{2}$ Lundbeck LLC, Deerfield, IL)

Rationale: Patients with Lennox-Gastaut syndrome (LGS) differ by disease severity. Vagal nerve stimulation (VNS) is one of the treatment options for LGS patients. Clobazam, a 1,5-benzodiazepine, was approved in October 2011 for the treatment of seizures associated with LGS. We conducted a post-hoc subgroup analysis of the pivotal Phase III trial, CONTAIN, ${ }^{1}$ to evaluate responses to clobazam therapy for patients who were undergoing VNS during the trial vs. those who were not.

Methods: The CONTAIN trial, ${ }^{1}$ a prospective, double-blind, placebo-controlled study, compared 3 oral dosages of clobazam with placebo as adjunctive therapy for LGS. Patients 2 to 60 years of age with LGS (documented by both clinical and EEG criteria) enrolled. Following a 4-week baseline phase, patients who had $\geq 2$ drop seizures per week were randomized to placebo or 1 of 3 dosages of clobazam $(0.25,0.5$, and $1.0 \mathrm{mg} / \mathrm{kg} /$ day $)$, up to a maximum daily dosage of $40 \mathrm{mg}$. Treatment included a 3 -week titration phase, followed by a 12 -week maintenance phase. The mITT analysis included all patients who had $\geq 1$ daily seizure measurement during the maintenance phase. Patients were grouped based on whether they were receiving or not receiving VNS treatment during the study. All clobazam dosage groups (low-, medium-, and high-dosage) were combined in these analyses.

Results: Both groups experienced statistically significant mean percentage decreases in average weekly seizures for both drop and total seizures (drop plus non-drop) vs. the placebo group (table). More than $50 \%$ of patients demonstrated $\geq 50 \%$ decreases in average weekly seizure frequencies in both groups, for both drop and total seizures, and $>10 \%$ of patients achieved drop-seizure-freedom in both groups.

Conclusions: Clobazam therapy provided similar efficacy in improving rates of drop and non-drop seizures for patients with LGS, regardless of whether they were receiving VNS treatment or not. These results suggest that clobazam is efficacious not only for less severe LGS patients, but also for more refractory patients with LGS (as those receiving VNS therapy tend to be).

References:

${ }^{1} \mathrm{Ng}$ YT, et al. Neurology. 2011;77:1473-81.

Least-Square Mean Percentage Decreases in Average Weekly Rate of Drop and Total Seizures for VNS vs. Non-VNS Patients (mITT Population)

\begin{tabular}{|l|c|cc|c|}
\hline & \multicolumn{2}{|c|}{ VNS Patients } & \multicolumn{2}{c|}{ Non-VNS Paticnts } \\
\hline & $\begin{array}{c}\text { Placebo } \\
\mathbf{N = 1 6}\end{array}$ & $\begin{array}{c}\text { All Clobazam } \\
\mathbf{V = 3 8}\end{array}$ & $\begin{array}{c}\text { Placebo } \\
\mathbf{N = 4 3}\end{array}$ & $\begin{array}{c}\text { All Clobazam } \\
\mathbf{V}=\mathbf{1 4 1}\end{array}$ \\
\hline Drop Seizures & -18.7 & $52.8^{\mathrm{a}}$ & 24.7 & $52.2^{\mathrm{a}}$ \\
\hline Total Seizures & -5.8 & $51.0^{\mathrm{a}}$ & 16.3 & $46.9^{\mathrm{a}}$ \\
\hline
\end{tabular}

${ }^{\mathrm{a}} \mathrm{p}<0.01$ for all comparisons vs. placebo. Two-sided, pairwise comparison of clobazam vs. placebo (ANCOVA) with treatment, pooled center, and baseline seizure rate as model effects.
3.237

LONG-TERM SAFETY AND EFFICACY OF PREGABALIN MONOTHERAPY IN PATIENTS WITH PARTIAL ONSET SEIZURES: AN OPEN-LABEL, EXTENSION STUDY

Lorraine Yurkewicz ${ }^{1}$, P. Kwan ${ }^{2}$, T. Fakhoury ${ }^{3}$, V. Pitman ${ }^{1}$ and L. Knapp ${ }^{1}\left({ }^{1}\right.$ Pfizer Inc, New York, NY; ${ }^{2}$ Prince of Wales Hospital and the Chinese University of Hong Kong, Hong Kong, China and ${ }^{3} \mathrm{St}$. Joseph Health System, Lexington, KY)

Rationale: The efficacy and safety of pregabalin monotherapy for the treatment of partial onset seizures was demonstrated in a recent randomized, double-blind, conversion-to-monotherapy, historicalcontrolled trial. This open-label extension evaluated the long-term safety and efficacy of pregabalin monotherapy in patients completing the double-blind trial.

Methods: Patients completing the 20-week double-blind phase of the preceding trial transitioned from pregabalin 150 or $600 \mathrm{mg} /$ day to open-label pregabalin $300 \mathrm{mg} /$ day over 7 days. Patients received open-label pregabalin monotherapy for 24 weeks, with dose adjustment over the range 150-600 $\mathrm{mg}$ /day (based on investigator judgment). Safety was evaluated by monitoring of adverse events (AEs), laboratory measures and vital signs. Seizure rates were also reported based on daily seizure diaries.

Results: A total of 73 patients received $\geq 1$ dose of open-label pregabalin; 58 (79.5\%) completed 24 weeks of treatment. The most frequent reasons for discontinuation were insufficient clinical response (5 patients) and AEs (4). Median duration of treatment was 174 days (range 27-200) and the median average daily dose of pregabalin was $473.7 \mathrm{mg}$ /day (range 275-600). Overall, 31 (42.5\%) patients had $\geq 1$ treatment-emergent $\mathrm{AE}$ (all causalities); the majority were mild or moderate in intensity. The most common treatmentemergent AEs were convulsion (increase in seizure frequency or intensity; 5 patients), headache (4), nausea (4), arthralgia (3) and increased weight (3). In the preceding double-blind trial, the most common AEs were somnolence and dizziness. No new AEs of somnolence were observed during open-label treatment, but 3 patients experienced ongoing somnolence that began during the double-blind trial. 2 patients had treatment-emergent AEs of dizziness and 2 had ongoing dizziness that began during the doubleblind trial. 3 patients had a total of 4 serious AEs, 1 of which (convulsion) was considered treatment-related. 2 patients withdrew due to treatment-emergent AEs of convulsion (1 of which was considered treatment-related) and 2 due to AEs of increased weight that began during double-blind treatment (both treatment-related). 3 patients had a total of 4 treatment-emergent AEs leading to dose reduction (nausea, hypertension, dizziness, and headache), all of which were considered treatment-related. Changes in vital signs and laboratory measures were generally minor and not considered clinically significant. $63(86.3 \%)$ patients experienced $\geq 1$ seizure during open-label treatment; the most frequent seizure types were complex partial and simple partial seizures. $10(13.7 \%)$ patients were seizure-free for the duration of open-label treatment, 9 of whom completed the study.

Conclusions: In this 24-week, open-label study, pregabalin was generally safe and well tolerated, with a safety profile consistent with previous clinical trials. Almost $80 \%$ of patients completed 6 months of open-label pregabalin monotherapy, and 10 were seizure-free for the duration of the study.

\subsection{8}

PHARMACOKINETICS OF USL261, A NOVEL FORMULATION OF INTRANASAL MIDAZOLAM Lindy L. Bancke ${ }^{1}$, H. Dworak ${ }^{2}$ and M. Halvorsen ${ }^{1}{ }^{1}$ Upsher-Smith Laboratories, Inc., Maple Grove, $\mathrm{MN}$ and ${ }^{2}$ Upsher-Smith Laboratories, Inc., Morristown, NJ)

Rationale: USL261 is a proprietary intranasal (IN) formulation of midazolam (MZ) being developed for the orphan indication of rescue

\subsection{6}

\section{WTTHDRAWN}


treatment of seizures in patients who require control of intermittent bouts of increased seizure activity, often referred to as seizure clusters or acute repetitive seizures. The only product approved in the US for this indication is rectal diazepam gel which has a route of delivery that may be undesirable. MZ for injection (MZ-inj) given IN has been reported to be an effective alternative treatment but is not optimized for the IN route of administration. USL261 has been formulated for easy and rapid administration of MZ by healthcare providers and in an outpatient setting. This study was designed to assess the pharmacokinetics (PK) of USL261 in healthy volunteers and compare these results with MZ-inj given IN (MZ-inj IN) and by IV infusion.

Methods: This single-dose, open-label, 5-way crossover study randomly assigned healthy adult subjects $(\mathrm{N}=25)$ to 1 of 5 sequences of $\mathrm{MZ}$ with 5 subjects per dosing sequence. The dosing regimens included 2.5, 5.0, and $7.5 \mathrm{mg}$ USL261 delivered in a single actuation by a unit-dose nasal spray device; $2.5 \mathrm{mg} \mathrm{MZ-inj}(5 \mathrm{mg} / \mathrm{mL})$ administered via 15-min IV infusion; and 5.0 mg MZ-inj IN (0.5 mL in each nostril given by needleless syringe). The interval between dose regimens was $\geq 3$ days. Venous blood samples were collected before and periodically after dosing for a period of $12 \mathrm{hr}$ to determine plasma concentrations of MZ. Standard PK parameters were calculated, including maximum plasma concentration $\left(\mathrm{C}_{\max }\right)$, time to peak plasma concentration $\left(\mathrm{T}_{\max }\right)$, area under the plasma concentration-time curve (AUC), and half-life $\left(t_{1 / 2}\right)$.

Results: The three increasing doses of USL261 provided increasing mean $\mathrm{C}_{\max }$ values of $58.8(2.5 \mathrm{mg}), 73.5(5 \mathrm{mg})$, and $92.7 \mathrm{ng} / \mathrm{mL}(7.5$ $\mathrm{mg}$ ) and increasing mean $\mathrm{AUC}_{0-\infty}$ values of $93.4(2.5 \mathrm{mg}), 170(5 \mathrm{mg})$ and $238 \mathrm{ng} \bullet \mathrm{hr} / \mathrm{mL}$ (7.5 mg). Maximum plasma $\mathrm{MZ}$ concentrations were rapidly achieved in all USL261 dose groups (mean $\mathrm{T}_{\max }=11.5$ $16.0 \mathrm{~min}$ ). Despite identical IN doses, the $5.0 \mathrm{mg}$ dose of USL261 resulted in a higher mean $\mathrm{C}_{\max }(73.5 \mathrm{vs} 55.2 \mathrm{ng} / \mathrm{mL})$, higher mean $\mathrm{AUC}_{0-\infty}(170.1 \mathrm{vs} 128.1 \mathrm{ng} \bullet \mathrm{hr} / \mathrm{mL})$ and an earlier $\mathrm{T}_{\max }(13.4 \mathrm{vs} 18.4$ min) than the $5.0 \mathrm{mg}$ dose of MZ-inj IN. The mean relative bioavailability of $5 \mathrm{mg}$ USL261 was $134 \%$ compared with an equal dose of MZ-inj IN. The mean absolute bioavailability of USL261 2.5 $\mathrm{mg}$ (vs $2.5 \mathrm{mg} \mathrm{MZ}$-inj administered IV) was $73 \%$. The mean terminal $\mathrm{t}_{1 / 2}$ was similar for each dosing regimen, ranging from 3.61 to 3.98 hr.

Conclusions: $\mathrm{C}_{\max }$ and $\mathrm{AUC}_{0-\infty}$ increased linearly with increasing doses of USL261. USL261 provided rapid delivery of MZ with a greater $\mathrm{C}_{\max }$ and $\mathrm{AUC}$ and an earlier $\mathrm{T}_{\max }$ compared to an equal dose of MZ-inj IN, thus demonstrating improved bioavailability of this new IN formulation compared with MZ-inj IN. Based on its PK profile, USL261 demonstrated the characteristics of an optimal formulation for the outpatient delivery of MZ intranasally and supports the further development of USL261 for the rescue treatment of patients with intermittent bouts of increased seizure activity.

\subsection{9}

\section{SAFETY AND PHARMACODYNAMICS OF USL261, A} NOVEL FORMULATION OF INTRANASAL MIDAZOLAM Heather Dworak ${ }^{1}$, L. Bancke ${ }^{2}$ and M. Halvorsen ${ }^{2}{ }^{1}{ }^{1}$ Upsher-Smith Laboratories, Morristown, NJ and ${ }^{2}$ Upsher-Smith Laboratories, Maple Grove, MN)

Rationale: USL261 is a proprietary intranasal (IN) formulation of midazolam (MZ) in development for the orphan indication of rescue treatment of seizures in patients who require control of intermittent bouts of increased seizure activity, often referred to as seizure clusters or acute repetitive seizures. MZ for injection (MZ-inj) given IN has been reported in the literature to be a safe and effective treatment for this use. USL261 has been formulated for easy and rapid administration of $\mathrm{MZ}$ by healthcare providers and in an outpatient setting. This study was conducted to assess the degree and duration of sedation, impairment of psychomotor performance, and safety of USL261 in healthy volunteers and compare the results with MZ-inj dosed IN and by IV infusion.
Methods: This single-dose, open-label, 5-way crossover study randomly assigned healthy adult subjects $(\mathrm{N}=25)$ to 1 of 5 sequences of $\mathrm{MZ}$ with 5 subjects per dosing sequence. The dosing regimens included $2.5,5.0$, and $7.5 \mathrm{mg}$ USL261 delivered by a single actuation of a unit-dose nasal spray device; $2.5 \mathrm{mg} \mathrm{MZ-inj} \mathrm{(} 5 \mathrm{mg} / \mathrm{mL})$ administered via $15-\mathrm{min}$ IV infusion; and $5.0 \mathrm{mg}$ MZ-inj administered IN ( $0.5 \mathrm{~mL}$ in each nostril given by needleless syringe). The interval between dose regimens was $\geq 3$ days. Pharmacodynamic assessments of sedation (Stanford Sleepiness Scale [SSS] and Observer's Assessment of Alertness/Sedation Scale [OAA/S]) and psychomotor performance (Digit-Symbol Substitution Test [DSST]) were conducted at predetermined times before and after study drug administration. Safety was assessed by the incidence of treatmentemergent adverse events (TEAEs) and by monitoring respiratory rate (RR) and pulse oximetry.

Results: Sedation (SSS, OAA/S) and psychomotor impairment (DSST) increased with increasing doses of USL261. Mean peak changes from baseline scores occurred 30 to 75 min after dosing for SSS and OAA/S and 10 to 20 min after dosing for the DSST. MZ-inj $(5 \mathrm{mg})$ dosed IN resulted in similar times to peak response for each of the assessments with peak changes from baseline similar to USL261 $5 \mathrm{mg}$. Mean peak changes from baseline in the SSS were 5.1 vs 5.3 units (on a 7-point scale) for MZ-inj ( $5 \mathrm{mg}$ ) dosed IN and USL261 5 $\mathrm{mg}$, respectively. In all groups, scores for each assessment returned to near baseline values by $4 \mathrm{hr}$. All subjects reported at least one TEAE; all were mild in severity and occurred only in the IN dosing groups. Nearly all were considered probably related to treatment and occurred with similar frequency in the 4 IN dosing groups. The most common (reported by $>20 \%$ of subjects) TEAEs were dysgeusia, increased lacrimation, nasal discomfort, and throat irritation. No subjects had $\mathrm{RR}<8$ breaths $/ \mathrm{min}$ or $\mathrm{O}_{2}$ saturation $<90 \%$.

Conclusions: USL261 dosed up to $7.5 \mathrm{mg}$ in healthy volunteers was well-tolerated and did not result in excessive or prolonged sedation or psychomotor impairment. The safety profile of USL261 was consistent with literature reports of MZ-inj dosed IN. These results support the further development of USL261 for the rescue treatment of patients with intermittent bouts of increased seizure activity.

\subsection{0 \\ CLINICAL OUTCOME OF LEVETIRACETAM FOR EPILEPSY PATIENTS}

Tomohiro Yamazoe ${ }^{1,2}$, A. Fujimoto ${ }^{1}$, H. Enoki $^{1}$, T. Yokota ${ }^{1}$ and T. Yamamoto ${ }^{1}{ }^{1}$ Comprehensive Epilepsy Center, Seirei-Hamamatsu General Hospital, Hamamatsu, Japan and ${ }^{2}$ Neurosurgery, Hamamatsu University School of Medicine, Hamamatsu, Japan)

Rationale: Levetiracetam (LEV) was released as anti-epileptic drug (AED) in North America around 2000. In Japan, LEV was available through October 2010 as adjunctive therapy for localized-related epilepsy. We evaluated safety and efficacy of LEV as an AED in our hospital database from the release.

Methods: The record of 132 patients aged $\geq 16$ years receiving LEV as an AED for epilepsy were retrospectively evaluated with respect to classification of epilepsy, seizure outcome, adverse events and discontinuation of LEV. The rate of the adverse event and discontinuation of LEV according to starting dose $(1000 \mathrm{mg} /$ day or less) were also evaluated in 126 patients of them.

Results: The populations were constituted of localization-related epilepsy $(n=112)$ and generalized epilepsy $(n=19)$. Compared with pretreatment baseline, $53.6 \%$ of patients with localization-related epilepsy reduced $\geq 50 \%$ in seizure frequency and $28.6 \%$ of them became seizure free during the treatment period. In generalized epilepsy, 4 of 6 patients with idiopathic generalized epilepsy and 2 of 13 patients with symptomatic generalized epilepsy had a $\geq 50 \%$ reduction in seizure frequency. Adverse events were reported in $27.3 \%$ of patients and caused discontinuation of LEV in $10.6 \%$. The most frequent adverse events were somnolence (14.4\%), irritability or aggressiveness (6.1\%) and depression (4.5\%). In 67 patients of 
standard starting dose, 15 patients $(22.4 \%)$ had adverse events and 8 patients $(11.9 \%)$ discontinued. In 59 patients of lower starting dose $(<1000 \mathrm{mg} /$ day), 20 patients $(33.8 \%)$ had adverse events and 17 patients $(28.8 \%)$ discontinued.

Conclusions: LEV was well tolerated and efficacious as an adjunctive therapy for localization-related epilepsy and idiopathic generalized epilepsy. However, LEV was not so effective for symptomatic generalized epilepsy. The frequency of adverse events and discontinuation rate after LEV treatment was irrelevant to its starting dose.

\subsection{1}

EFFICACY AND SAFETY OF PERAMPANEL IN PATIENTS WITH TREATMENT-RESISTANT, PARTIAL-ONSET SEIZURES AFTER CONVERSION FROM DOUBLE-BLIND PLACEBO TO OPEN-LABEL PERAMPANEL

G. Montouris ${ }^{1}$, H. Yang ${ }^{2}$, B. Williams ${ }^{3}$, S. Zhou ${ }^{3}$, A. Laurenza ${ }^{2}$ and R. Fain ${ }^{3}\left({ }^{1}\right.$ Boston University School of Medicine, Boston, MA; ${ }^{2}$ Neuroscience Product Creation Unit, Eisai, Inc., Woodcliff Lake, NJ and ${ }^{3}$ Medical and Scientific Affairs, Eisai, Inc., Woodcliff Lake, NJ)

Rationale: Perampanel (PER), an orally active, highly selective, noncompetitive AMPA antagonist, has been evaluated as adjunctive treatment for partial-onset seizures (POS) in 3 double-blind (DB), randomized, placebo-controlled Phase 3 trials. Completers from these studies were eligible to participate in the open-label extension (OLE) study. The OLE allowed physicians a greater flexibility in the titration of PER dosage compared to the DB studies. Hence, we examined the impact of the rate of titration on the safety and efficacy of PER in patients who received placebo (PBO) in the Phase $3 \mathrm{DB}$ trials and transitioned to PER in the OLE.

Methods: Patients ( $\geq 12$ y, receiving 1-3 AEDs) with uncontrolled POS were randomized to once-daily, PBO or PER (2, 4, 8, $12 \mathrm{mg}$ ). The DB study design included a 6-wk titration (weekly titration schedule), followed by the maintenance period. The OLE began with a 16-wk blinded conversion phase (biweekly titration schedule) and was followed by the maintenance phase. In the OLE patients previously assigned to $\mathrm{PBO}$ (DB-PBO, $n=380$ ) as well as those assigned to PER (DB-PER, $n=838$ ) during DB phase were titrated up to the maximum tolerated dose ( $\leq 12 \mathrm{mg} /$ day) from $2 \mathrm{mg}$ /day and last received PER dose, respectively. Efficacy endpoints were median \% change in seizure frequency $/ 28$ days and $50 \%$ responder rate. Results: A total of 1218 patients entered the OLE (96.3\% of DB completers). By the OLE conversion period, subjects in the DB-PBO group had achieved similar reductions in seizure frequency and responder rates as those in the DB-PER group, and these rates were maintained in both groups through 52 weeks of the OLE (Table 1). A comparison of DB-PBO patients converting to the OLE and patients randomized to $12 \mathrm{mg}$ (PER-12 $\mathrm{mg}$ ) during the DB studies showed that $72.4 \%$ DB-PBO patients in the OLE, compared to $78.8 \%$ PER$12 \mathrm{mg}$ patients in the DB studies, achieved doses of 10 or $12 \mathrm{mg}$ PER at the end of the conversion and titration periods, respectively (16 wks in OLE vs 6 wks in DB). Irrespective of the rate of titration, discontinuation due to AEs in the DB-PBO patients during OLE conversion period was $10.1 \%$ compared to $9.8 \%$ for PER- $12 \mathrm{mg}$ patients in the DB titration period. Rates of the most common treatment-emergent AEs (TEAEs) for DB-PBO were similar to DBPER during OLE (Table 2).

Conclusions: By the end of the conversion period, DB-PBO patients transitioning to PER during OLE achieved seizure reduction and responder rates comparable to DB-PER patients. Tolerability and safety profiles, as indicated by the discontinuation rates due to AEs and ability to achieve MTD during the titration and conversion periods, were similar between the DB-PBO during the OLE conversion and PER-12 mg group during the DB titration. Regardless of the rate of titration (weekly in DB vs biweekly in OLE), PER demonstrates a favorable risk-benefit ratio in this study population.
Table 1. Median Change in Seizure Frequency/28 Days and Responder Analysis

\begin{tabular}{|c|c|c|c|c|}
\hline \multicolumn{5}{|c|}{ \% Change From DB Baseline of Seizure Frequency $/ 28$ Days During Treatment Duration } \\
\hline \multirow[b]{2}{*}{ Study period } & \multicolumn{2}{|c|}{ DB-PBO } & \multicolumn{2}{|c|}{ DB-PER } \\
\hline & $\mathbf{N}$ & $\%$ & $\mathbf{v}$ & $\%$ \\
\hline $\mathbf{D B}^{*}$ & 369 & -18.6 & 838 & -31.7 \\
\hline OLE conversion & 369 & -42.4 & 817 & -41.5 \\
\hline OLE wk 1-13 & 237 & -49.3 & 589 & -46.5 \\
\hline OLE wk 14-26 & 164 & -49.6 & 422 & -48.6 \\
\hline OLE wk 27-39 & 119 & -56.4 & 291 & -53.7 \\
\hline OLE wk 40-52 & 64 & -49.2 & 175 & -48.8 \\
\hline \multicolumn{5}{|c|}{ Responder Analysis Summary During Study Drug Treatment Duration } \\
\hline & \multicolumn{2}{|c|}{ DB-PBO } & \multicolumn{2}{|c|}{ DB-PER } \\
\hline Study period & $\mathbf{N}$ & $\%$ & $\mathbf{N}$ & $\%$ \\
\hline DB $^{+}$ & 369 & 20.6 & 838 & 34.0 \\
\hline OLE conversion & 369 & 44.2 & 817 & 43.3 \\
\hline OLE wk 1-13 & 237 & 49.4 & 589 & 47.4 \\
\hline OI.F. wk 14-26 & 164 & 49.4 & 422 & 49.1 \\
\hline OLE wk 27-39 & 119 & 56.3 & 291 & 52.9 \\
\hline OLE wk 40-52 & 64 & 46.9 & 175 & 47.4 \\
\hline
\end{tabular}

* Percent change from prerandomization baseline of seizure frequency $/ 28$ days during the double-blind period.

${ }^{\dagger}$ Responder rate during maintenance period.

Table 2. Rates of the Most Common Treatment-Emergent AEs for DB-PBO and DB-PER During OLE,

\begin{tabular}{|c|c|c|}
\hline & DB-PBO & DB-PER \\
\hline Study period & $\%$ & $\%$ \\
\hline Dizziness & 41.0 & 45.2 \\
\hline Somnolence & 16.7 & 21.8 \\
\hline Fatiguc & 9.3 & 13.3 \\
\hline Headache & 8.2 & 20.5 \\
\hline Ataxia & 7.9 & 5.4 \\
\hline Irritability & 7.9 & 10.6 \\
\hline
\end{tabular}

\section{Antiepileptic Drugs: Cohort Studies}

\subsection{2}

RUFINAMIDE DOSING PATTERNS IN COMMERCIALLYINSURED PEDIATRIC AND ADULT PATIENTS E. Silva ${ }^{1}$, J. M. Margolis ${ }^{2}$, Zhixiao Wang ${ }^{1}$, R. Copher ${ }^{1}$ and D. Labiner $^{3}\left({ }^{1}\right.$ Eisai Inc, Woodcliff Lake, NJ; ${ }^{2}$ Truven Health Analytics, Bala Cynwyd, PA and ${ }^{3}$ Department of Neurology, University of Arizona Colleges of Medicine and Pharmacy, Tucson, AZ)

Rationale: Rufinamide has been approved by FDA for the adjunctive treatment of seizures associated with Lennox-Gastaut Syndrome in children 4 years and older and adults since 2008. The objective of this study was to examine rufinamide dosing patterns in commercially insured patients.

Methods: Using administrative claims from the MarketScan Commercial Database, patients initiating rufinamide (index event) during 11/1/2008 - 9/30/2010 with at least 6 months pre- and postindex eligibility were selected. Patients may have received other antiepileptic drugs (AEDs) during this observation period. Demographics and clinical characteristics, including AED therapies, were measured in the 6-month pre-index period. Rufinamide dosing was measured in patients with a minimum of 3 rufinamide prescription claims during the post-index period until discontinuation of rufinamide, end of the patient's available data, or March 31, 2011. Rufinamide daily dose was computed per prescription claim as the total amount dispensed divided by the days of supply. The initial dose, modal (most common) dose, time to modal dose, maximum dose, and last dose were analyzed by age category. Discontinuation, 
defined when there was a prescription supply gap of at least 30 days, was modeled using Cox proportional hazards analysis.

Results: A total of 661 patients were included in the study, with mean age 16.9 years (Ys) $(13.8 \% \leq 5 \mathrm{Ys}, 27.0 \% 6-11 \mathrm{Ys}, 35.6 \% 12$ $21 \mathrm{Ys}, 23.8 \%>21 \mathrm{Ys}$ ), $53 \%$ male, and mean follow-up duration of 491 [SD 180] days. Pre-index comorbidities included mental retardation (14.1\%), developmental delay (19.7\%), and growth delays (18.0\%). In the 90 days prior to rufinamide, $29.6 \%$ of patients were receiving 2 AEDs and $50.8 \%$ were receiving 3 or more AEDs. The most common pre-index AEDs were lamotrigine (33.1\%), levetiracetam (32.7\%), and divalproex sodium (28.6\%). However, no specific AED combination was used in more than $2 \%$ of patients in the pre-index period. Daily doses of rufinamide are shown in Table 1 . The median days to modal dose were 33.0 [IQR 120] with mean days 72.4 [SD 91.6]. Cox regression analysis showed a hazard ratio of 0.593 for discontinuation of rufinamide when time to modal dose exceeded 30 days $(\mathrm{p}<0.001)$.

Conclusions: Rufinamide was initiated in commercially insured patients who were on highly individualized AED polytherapy regimens and had commonly received 3 or more AEDs. Dosing of rufinamide varied by age category, and for adult patients, the observed daily dose appeared to be lower than the recommended maximum dose of 3200 milligrams. The data suggest the hypothesis that time to modal dose exceeding 30 days may be associated with a lower likelihood of discontinuation.

Daily Dose of Rufinamide by Age Category

\begin{tabular}{|c|ccc|c|}
\hline All doses in milligrams & Age 0-5 & Age 6-11 & Age 12-21 & Age 22+ \\
\hline Index Dose (Mean, SD) & $471(221)$ & $708(442)$ & $1,118(752)$ & $1,142(838)$ \\
Index Dose (Median, IQR) & $400(200)$ & $600(400)$ & $800(1,186)$ & $800(1,200)$ \\
Modal Dose (Mcan, SD) & $634(270)$ & $1,018(626)$ & $1,516(900)$ & $1,712(972)$ \\
\hline Modal Dose (Median, IQR) & $600(400)$ & $800(600)$ & $1,200(1,200)$ & $1,600(1,600)$ \\
\hline Maximum Dose (Mean, SD) & $801(375)$ & $1,248(717)$ & $2,061(1,567)$ & $2,071(1,169)$ \\
\hline Maximum Dose (Median, IQR) & $800(279)$ & $1,200(800)$ & $1,600(1,600)$ & $1,867(2,000)$ \\
Final Dose (Mcan, SD) & $711(291)$ & $1,052(656)$ & $1,602(920)$ & $1,751(994)$ \\
\hline Final Dose (Median, IQR) & $700(200)$ & $800(600)$ & $1,600(1,600)$ & $1,600(1,600)$ \\
\hline
\end{tabular}

\subsection{3}

OBSTETRICAL OUTCOMES IN WOMEN WITH EPILEPSY ENROLLED IN THE NORTH AMERICAN ANTIEPILEPTIC DRUG PREGNANCY REGISTRY (NAAPR)

Autumn Klein ${ }^{1}$, T. F. McElrath ${ }^{3}$, H. A. Keenan ${ }^{6}$, N. G. Llewelyn ${ }^{2}$, C. R. Smith ${ }^{4}$, S. Hernandez-Diaz ${ }^{4}$, P. B. Pennell ${ }^{2}$ and L. B. Holmes ${ }^{5}$ ( ${ }^{1}$ Neurology, University of Pittsburgh, Pittsburgh, PA; ${ }^{2}$ Neurology, Harvard Medical School, Boston, MA; ${ }^{3}$ Obstetrics and Gynecology, Harvard Medical School, Boston, MA; ${ }^{4}$ Epidemiology, Harvard School of Public Health, Boston, MA; ${ }^{5}$ Pediatrics, Harvard Medical School, Boston, MA and ${ }^{6}$ Genetics and Epidemiology, Joslin Diabetes Center, Boston, MA)

Rationale: Little is known about obstetrical and neonatal outcomes in women with epilepsy [WWE] taking AEDs. The AAN guidelines stated that a moderately increased risk $(>1.5 \mathrm{X})$ of $\mathrm{C}$-section $(\mathrm{CS})$ is possible in WWE. CS poses a high morbidity, and it is unknown how seizures influence perinatal obstetrical management. This study aims to determine the rate of CS in WWE on AED as compared to women without epilepsy not taking an AED [WWoE]. Our hypothesis is that WWE have a higher rate of CS than WWoE. This study explores if specific $\mathrm{AED}(\mathrm{s})$ in WWE have a higher association with CS, and if there is a specific etiology for CS, including seizure.

Methods: The NAAPR is a voluntary call-in registry of women who are taking AEDs. Participants are asked a series of questions twice during pregnancy and once postpartum. Using self-reported answers, we determined how many WWE had a CS. An internal control group of WWoE on no AEDs was used in comparison.
Results: There were 6,253 WWE in the analysis as compared to 469 WWoE. WWE were slightly younger $(29.5 \pm 5.4$ v. $31.5 \pm 4.1$ years $)$ $(p<0.001)$ and were less likely to have had a previous delivery (parity $0.8 \pm 0.9$ v. $1.0 \pm 0.9, p<0.001)$, but were likely to have had an equal number of pregnancies (gravidas $2.2 \pm 1.3$ v. $2.3 \pm 1.3, \mathrm{p}=0.1$ ). WWE were more likely to report smoking during the first trimester $(14.1 \%$ v. $6.8 \%, p<0.05)$. There was a lower proportion of non-whites among WWoE $(\mathrm{p}<0.001)$, and the most common AEDs were lamotrigine (26.1\%) followed by carbamazepine $(22.9 \%)$. A total of $34.5 \%$ of WWE had a CS as compared to $29.8 \%$ of WWoE , $p=0.05$. The increased odds of receiving a CS for WWE loses significance with adjustment for age, parity and pre-existing hypertension (OR: 1.03 95\% CI: $0.54,2.0, \mathrm{p}=0.9)$. Independent of disease and drug status, age (OR: $1.1,95 \% \mathrm{CI}: 1.0-1.1$ ), parity OR: $0.895 \% \mathrm{CI}=0.7-0.9$ ) and pre-existing hypertension (OR: $2.2,95 \% \mathrm{CI}=1.5-3.3)$ are variables significantly associated with reporting a CS delivery in both WWE and WWoE.

Conclusions: There is borderline difference in the rate of CS between WWE and WWoE when adjusted for the confounders of age, parity, and pre-existing hypertension. This study is limited by retrospective, patient-reported outcomes, and information on confounders. Future Directions: These findings will be examined by etiology of CS and by drug. Future studies will include other perinatal outcomes. Future NAAPR participants will be asked detailed questions on obstetrical and neonatal outcomes to enhance these findings.

\subsection{4 \\ OUTCOME OF STATUS EPILEPTICUS IN PATIENTS TREATED WITH LACOSAMIDE}

Neelima R. Thakur, O. Laban-Grant, C. C. Lambrakis, s. Mesad, J. M. Politsky, E. Feoli, M. Evans, s. JeanBaptiste-Berry, E. Segal, E. Fertig and M. Lancman (Northeast Regional Epilepsy Group, White Plains, NY)

Rationale: Status epilepticus (SE) is a neurological emergency that results in significant patient morbidity and mortality. In recent years, newer antiepileptic drugs (AEDs) are being used in early stages of management of status epilepticus. Lacosamide is one of the newer AEDs that is being used in SE management. However, most of the available data is limited to case reports, reviews and a very few retrospective studies. The aim of this study is to present the data about our experiences regarding outcome of status epilepticus with the administration of lacosamide.

Methods: Review of Northeast Regional Epilepsy Group database from March, 2007 to May, 2012 identified 36 patients (16 males and 20 females) in multiple medical centers with diagnosis of status epilepticus that were treated with lacosamide. Status epilepticus (SE) diagnosis was confirmed in all cases with VEEG monitoring. Only patients age 18 and above were included in study (range 18-91y,mean age-60y,SD-20). Status Epilepticus outcome was identified as controlled if SE resolved (seizure freedom), or improved (decrease in seizure frequency and clinical improvement), and refractory if no clinical or electrographic improvement was achieved. Data was retrospectively collected on the SE outcome when lacosamide alone was added to other AED's, or lacosamide and another AED(s) were added.

Results: Etiology: Intracranial Hemorrhage-10 (28\%), Neoplasm7(19\%), Toxic/Infectious/inflammatory-8(22\%), Hypoxia/anoxia-5 (14\%), Stroke-3(8\%), Epilepsy-1(3\%), Other-2(6\%).

SE outcome: Resolved SE-22/36(61\%), Improved SE-6/36(17\%), Controlled SE-28/36(78\%), Refractory SE-8/36 (22\%)

SE was controlled on addition of lacosamide in 11 cases (31\%), and remained uncontrolled on addition of lacosamide in 18 cases $(49 \%)$. In 7 patients (19\%) SE was controlled on addition of lacosamide and other AED's.

Conclusions: This study shows that status epilepticus was controlled on the addition of lacosamide in a significant number of patients 
(31\%). Our study concludes that lacosamide is efficacious in treatment of different types of status epilepticus. It can be used as an adjuvant drug in management of refractory status epileptics. However, large multicenter randomized controlled studies are required to further support our findings.

\begin{tabular}{|c|cc|c|}
\hline $\begin{array}{c}\text { Type of Status epilepticus } \\
\text { based on seizure semiology }\end{array}$ & $\begin{array}{c}\text { No. of } \\
\text { patients (\%) }\end{array}$ & $\begin{array}{c}\text { No. of patients with } \\
\text { controlled SE (\%) }\end{array}$ & $\begin{array}{c}\text { No. of patients controlled on } \\
\text { addition of Lacosamide }(\%)\end{array}$ \\
\hline Partial SE & $6 / 36(17 \%)$ & $6 / 6(100 \%)$ & $3 / 6(50 \%))$ \\
$\begin{array}{c}\text { Non convulsive ST: (NCSE) } \\
\text { Generalized convulsive SF } \\
\text { (GSCE) }\end{array}$ & $24 / 36(67 \%)$ & $20 / 24(83 \%)$ & $7 / 20(35 \%)$ \\
\hline Myoclonic SE & $4 / 36(11 \%)$ & $1 / 2(50 \%)$ & $0 / 1(0 \%)$ \\
\hline
\end{tabular}

\begin{tabular}{|c|c|c|c|}
\hline VEEG trindings & $\begin{array}{c}\text { No. of } \\
\text { patients }(\%)\end{array}$ & $\begin{array}{c}\text { No. ol patients with } \\
\text { controlled SE }(\%)\end{array}$ & $\begin{array}{c}\text { No of patients controlled on } \\
\text { addition Lacosamide }(\%)\end{array}$ \\
\hline $\begin{array}{c}\text { Focal ictal } \\
\text { discharges }\end{array}$ & $26 / 36(72 \%)$ & $23 / 36(64 \%)$ & $9 / 23(39 \%)$ \\
\hline $\begin{array}{c}\text { Gencralized ictal } \\
\text { discharges }\end{array}$ & $10 / 36(28 \%)$ & $5 / 36(14 \%)$ & $2 / 5(40 \%)$ \\
\hline
\end{tabular}

\subsection{5 \\ CURRENT USAGE OF PERIOPERATIVE ANTIEPILEPTIC DRUGS FOR SURGICAL CASES OF SUPRATENTORIAL TUMOR \\ Takeharu Kunieda $^{1}$, Y. Arakawa ${ }^{1}$, T. Kikuchi ${ }^{1}$, Y. Yamao ${ }^{1}$, S.

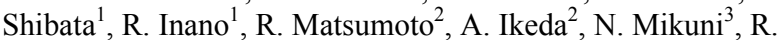 Takahashi ${ }^{2}$ and S. Miyamoto ${ }^{1}\left({ }^{1}\right.$ Neurosurgery, Kyoto University, Kyoto, Japan; ${ }^{2}$ Neurology, Kyoto University, Kyoto, Japan and ${ }^{3}$ Neurosurgery, Sapporo Medical University, Sapporo, Japan)}

Rationale: Epilepsy is common in patients with brain tumor and can substantially affect daily life, even if the tumor is under control. Its negative impact on quality of life could not be negligible, due to its unpredictability and psychological influence leading to loss of own body control. Treatment with antiepileptic drugs (AEDs) is clearly indicated for patients with preoperative tumor-related seizures. Moreover, surgical treatment itself is also associated with a high risk of postoperative seizures. Approximately, one-third of patients have seizures after resection. Despite the increasing awareness of the difficulties in controlling seizures, clinical studies on epilepsy with tumors and effects of anti-seizure therapy are limited. The goal of this retrospective cohort study was to delineate the frequency of seizures and the effect of AEDs in tumor cases.

Methods: Between April 2008 and March 2011, 254 surgeries of supratentorial brain tumor have been undergone in our department. The clinical profiles, i.e., histological diagnosis, anti-tumor treatment, seizure type and frequency, applied AEDs, seizure control and tolerability of AEDs, were retrospectively analyzed.

Results: We here reported results from 220 patients (113 males and 107 females: aged 8-83 years, mean 53), excluding patients with medically intractable epilepsy. 55 patients $(25 \%)$ were not perioperatively treated with any AEDs. Besides them, 95 cases were treated as monotherapy with zonisamide, 17 with valproic acid, 12 with carbamazepine, 12 with phenytoin, 2 each with clobazam or gabapentine. The remaining 25 patients were treated with various combinations of multiple AEDs. While $75 \%$ of 165 treated patients eventually achieved seizure control within 6 months after surgery, only 2 patients ( $4 \%$ ) of 55 non-treated patients developed seizures during that period. These pathological findings result in glioblastoma multiforme and meningioma. Initial AEDs were eventually changed in 63 cases ( $38 \%$ of the patients treated with AEDs), due to adverse or insufficient effects. Especially, the group of patients treated with multiple AEDs showed highest incidence of recurrent seizures within 6 months $(52 \%)$ and 12 months $(60 \%)$ after operation.

Conclusions: Generally, prophylactic use of AEDs is not recommended, and potential interactions between antiepileptic and chemotherapeutic agents are documented for the use of enzyme- inducing AEDs. However, routine use of AED in tumor cases still remains the prevailing practice pattern. Optimal AEDs would be applied preoperatively to control tumor-related seizures, provided that fewer side effects, lesser neuropsychological effects, and little interaction to adjunctive chemotherapeutic agents are warranted.

\subsection{6 \\ TREATING EPILEPSY IN CANADA: A OBSERVATIONAL STUDY OF ANTIEPILEPTIC DRUG USE}

Marni A. Freeman, D. M. Dhalla and J. Alfonso Ross Terres

(GlaxoSmithKline Inc., Mississauga, Canada)

Rationale: Numerous medications are currently available for the treatment of epilepsy. However, variability in patient response and tolerance contribute to complex disease management. Data demonstrating how antiepileptic drugs (AEDs) are being used is necessary to better understand the diversity of epilepsy treatment in Canada. This observational study followed a Canadian cohort of drug naïve epilepsy patients over 3 years to evaluate the treatment pathway of AEDs.

Methods: A national claims database, IMS Brogan, was utilized to identify a cohort of epilepsy patients, $\geq 18$ years, followed from 2008 to 2011. A pre-specified list of inclusion criteria, driven largely by medication history and prescriber's specialty, over the study period, was used to select the epilepsy patient cohort. In order to track up to 5 lines of therapy over 3 years, patients must have been active in their insurance plan throughout the study, and had their first claim for an AED between 2007 and 2008. Changes were grouped into 3 categories: addition of a new medication, removal of existing medication or switch to a different medication. If a removal occurred within 90 days of an addition, the change in treatment was categorized as a switch as the overlapping treatment is necessary for up and down titration. Details of all lines of therapy, including mono versus combination treatments, and the average time to each change, were assessed over the study.

Results: A treatment naïve cohort of 3600 epilepsy patients was identified and followed over 3 years. Of these patients, $2736(76 \%)$ progressed to 2nd line therapy, $2016(56 \%)$ to $3 \mathrm{rd}, 1188$ (33\%) to 4th and $576(16 \%)$ to 5 th. The average days to change in treatment were $300,271,232$, and 212 for lines 2 through 5, respectively. First line therapy was largely made up of patients on monotherapy (91\%), with the majority taking carbamazepine $(28 \%)$ or phenytoin $(22 \%)$. A reduced $60 \%$ were on monotherapy by 5 th line, driven by clonazapam (29\%), valproic acid (15\%) and gabapentin (11\%). Of the 2736 patients who progressed to the 2 nd line, $47 \%$ added, $49 \%$ switched and $4 \%$ reduced a therapy. In contrast, of the 576 patients who progressed to 5 th line, $20 \%$ added, $16 \%$ switched, and $64 \%$ removed a therapy.

Conclusions: Much of the epilepsy cohort progressed through multiple lines of therapy over 3 years. As lines of therapy increased, a reduction was observed in average days to change and monotherapy use. More variability in treatment was observed in later therapy. Additions and switches were common in earlier therapy, whereas, removals were more frequent later. The cohort was limited to the drug utilization inclusion criteria. Out-of-pocket payments for medications and reasons for changes in treatment were not captured. Further research is warranted to understand the challenges physicians face with decisions surrounding the treatment of epilepsy in Canada.

\subsection{7 \\ CLINICAL EFFECTIVENESS OF ESLICARBAZEPINE ACETATE (ZEBINEX) AS AN ADD-ON THERAPY IN LOCALIZATION RELATED EPILEPSY OVER 12 MONTHS D. Damodaran ${ }^{1}$, J. Rigby $^{2}$, P. Cooper ${ }^{1,2}$ and Rajiv Mohanraj ${ }^{1}$ ( ${ }^{1}$ Department of Neurology, Greater Manchester Neurosciences Centre, Salford, United Kingdom and ${ }^{2}$ Long term Conditions Service, Breightmet Helath Centre, Bolton, United Kingdom)}


Rationale: Eslicarbazepine acetate is a single daily dose voltagegated sodium channel blocker approved as add-on-therapy therapy in adults with partial onset seizures, with or without secondary generalization. It has demonstrated efficacy in patients with refractory focal epilepsy in randomized control trials in doses of 800 $\mathrm{mg}$ and $1200 \mathrm{mg}$. However, experience in clinical practice often varies from the results of regulatory trials, and observational outcome data in the real life setting, over longer periods of time, can help inform clinical practice.

We conducted a retrospective study to determine the clinical efficacy and tolerability of eslicarbazepine over a 12 -month period in patients with refractory focal epilepsy attending a regional neuroscience centre and a community epilepsy clinic.

Methods: 43 adult patients ( 27 female) on eslicarbazepine were identified from the clinical database at the Greater Manchester Neurosciences Centre and Breightmet Health Centre in the UK. Demographic and epilepsy details as well as clinical outcomes at 3, 6 and 12 months after initiation of eslicarbazepine was collected using a structured proforma by case note review.

Results: 42 patients had focal epilepsy, and 1 patient had unclassified epilepsy. $31(72.09 \%)$ had received treatment with 5 or more AEDs in the past.

Of the 43 patients starting on eslicarbazepine, 31 (72.09\%) took the drug for a minimum period of 12 months. Those discontinuing eslicarbazepine $(12,27.91 \%)$ did so within 3 months of initiation. 6 $(50 \%)$ patients discontinued eslicarbazepine because of lack of efficacy / worsening or seizures, and the rest due to adverse effects. Adverse effects leading to withdrawal were tiredness, feeling drunk, unsteadiness and double vision. Significant hyponatraemia was not observed in any patient in this study.

Of the 31 patients continuing eslicarbazepine, 19 (61.29\%) experienced at least $50 \%$ reduction in seizure frequency: $4(12.90 \%)$ were seizure free. $11(35.48 \%)$ found no significant change in seizure frequency. Seizure outcome was not known for one patient. 3 of the 31 patients who continued eslicarbazepine for 12 months were on $400 \mathrm{mg} /$ day, $19(44.19 \%)$ were on $800 \mathrm{mg} /$ day and 18 (41.86\%) were on $1200 \mathrm{mg}$. $3(6.98 \%)$ took $1600 \mathrm{mg} /$ day.

Eslicarbazepine was most frequently used in conjunction with levetiracetam $(n=11)$ and lamotrigine $(n=10)$.

Conclusions: Our data confirms the efficacy of eslicarbazepine in patients with refractory focal epilepsy in the 'real-world' setting. The majority of patients tolerated the drug well, with over $70 \%$ retention rate at 12 months. Of those continuing on the drug, $61 \%$ were responders, and $12.9 \%$ were seizure free in a population of patients with refractory epilepsy.

\subsection{8 \\ COMPARISON OF TREATMENT DURATION OF ANTIEPILEPTIC DRUG COMBINATION THERAPIES BASED ON MECHANISM OF ACTION IN PARTIAL ONSET} SEIZURES

Zhixiao Wang ${ }^{1}$, J. M. Margolis ${ }^{2}$, R. Copher ${ }^{1}$ and J. E. Cavazos ${ }^{3}$ ( ${ }^{1}$ Eisai Inc, Woodcliff Lake, NJ; ${ }^{2}$ Truven Health Analytics, Bala Cynwyd, PA and ${ }^{3}$ University of Texas Health Science Center, San Antonio, TX)

Rationale: Patients with refractory epilepsy often require antiepileptic drug (AED) combination therapy. It has been hypothesized that AED combination therapy with different mechanisms of action (MOA) could produce synergistic effects and therefore, lead to potentially better outcomes than AED combination therapy with similar MOAs. However, real-world evidence thus far has been limited. The objective of this study was to analyze treatment patterns, compare treatment duration and other health outcomes associated with combination therapies categorized by MOA in patients diagnosed with partial onset seizures (POS).

Methods: Using administrative claims from the MarketScan Commercial Database with approximately 96 million covered lives,
POS patients ( $\geq$ one diagnosis of ICD-9-CM code $345.4 x$ or $345.5 x$ ) who initiated AED combination therapy between 1/1/2005 and $3 / 31 / 2010$ were identified. Patients whose combination therapy treatment lasted $\geq 90$ days with at least 6 months pre-index and 12 months post-index were included in the study. Index date was defined as the first date of AED combination therapy use. Combination therapies were categorized based on MOA of each drug, including sodium channel blockers (SC), GABA analogs (G), SV2A binding (SV2), and broad (multiple) mechanism (B). Baseline demographics and clinical characteristics, including the Deyo-Charlson Comorbidity Index (DCI) and unique 3-digit ICD-9-CM diagnoses (3DDx), were measured in the 6-month pre-index period. Treatment duration was measured in the post-index period from index date to the time of treatment regimen change (switching, addition or discontinuation) or end of patient follow up.

Results: 8,615 patients were included in the study. Mean ages (Table 1) ranged from 41.7 years $(\mathrm{SC}+\mathrm{B})$ to 48.4 years $(\mathrm{G}+\mathrm{G})(\mathrm{p}<0.001)$. There were also significant gender variation across combination therapy categories $(\mathrm{p}<0.001)$ from $48.8 \%$ male $(\mathrm{SC}+\mathrm{SV} 2)$ to $32.1 \%$ male $(\mathrm{G}+\mathrm{B})$. Pre-index comorbidities varied significantly across combination therapies, evidenced by DCI and 3DDx $(p<0.001)$. Mean duration of therapy was highest for $\mathrm{SC}+\mathrm{SV} 2$ combinations at 526.7 days and lowest for $\mathrm{G}+\mathrm{G}$ combinations at 344.3 days $(\mathrm{p}<0.001)$ (Table 2).

Conclusions: In this study, patients diagnosed with POS receiving AED combination therapy exhibited different treatment durations according to the MOA of the combination, with SC+SV2 combinations the longest and $\mathrm{G}+\mathrm{G}$ combinations the shortest. However, there were also substantial differences in baseline demographics and clinical characteristics across combination categories, which may have contributed to the observed difference in duration on therapy. It also implies that there may be differential practice patterns for the selection of AED combinations based on patients' comorbidities and characteristics. Multivariate analyses on duration of therapy are being performed to account for the differences in baseline characteristics. Further analyses will focus on the comparison of healthcare utilization and comorbidities across different MOA-based combination therapies.

Baseline Patient Characteristics

\begin{tabular}{|c|cc|c|cc|}
\hline Combination & $\%$ of Pts & Age (Mean,SD) & $\%$ Male & DCI (Mean,SD) & 3DDx (Mean,SD) \\
\hline SC + SC & $13.9 \%$ & $43.7(12.3)$ & $48.0 \%$ & $0.33(0.86)$ & $6.0(5.5)$ \\
SC + SV2 & $26.3 \%$ & $43.2(13.1)$ & $48.8 \%$ & $0.67(1.47)$ & $7.7(7.5)$ \\
\hline SC + B & $21.5 \%$ & $41.7(12.7)$ & $40.7 \%$ & $0.36(0.95)$ & $6.4(6.0)$ \\
\hline G + G & $3.3 \%$ & $48.4(11.2)$ & $28.4 \%$ & $0.78(1.36)$ & $11.9(9.6)$ \\
\hline G + SV2 & $7.5 \%$ & $47.2(12.1)$ & $34.8 \%$ & $1.32(2.03)$ & $13.0(10.3)$ \\
G+ B & $8.6 \%$ & $45.0(12.2)$ & $32.1 \%$ & $0.59(1.17)$ & $9.7(7.6)$ \\
G + SC & $19.0 \%$ & $47.5(11.6)$ & $38.9 \%$ & $0.50(1.16)$ & $7.8(7.1)$ \\
\hline
\end{tabular}

Duration (Days) of Combination Therapy

\begin{tabular}{|c|c|c|}
\hline Combination & Mean Days (SD) & Median Days \\
\hline SC + SC & $512.7(530.4)$ & 313.0 \\
SC + SV2 & $526.7(505.8)$ & 364.0 \\
\hline SC+ B & $504.5(511.0)$ & 335.0 \\
\hline G+ G & $344.3(344.7)$ & 217.5 \\
\hline G + SV2 & $390.7(405.6)$ & 245.0 \\
G+ B & $384.5(394.9)$ & 240.0 \\
G+ SC & $514.3(526.0)$ & 315.0 \\
\hline
\end{tabular}




\subsection{9}

\section{NEW ANTIEPILEPTIC DRUGS IN NEWBORNS}

C. Maxit, Marina N. Aberastury, C. Vazquez Dusefante, I. Denzler, M. Vaccarezza, W. H. Silva and G. Agosta (Hospital Italiano de Buenos Aires, CABA, Argentina)

Rationale: New antiepileptic drugs (AEDs) are being indicated in newborns without enough clinical evidence.

Purpose: To analyze the efficacy and safety of new AEDs in newborns with refractory seizures.

Methods: Observational prospective study of newborns with seizures admitted between June 2005 and May 2012 at the Italian Hospital Neonatology Unit.

Results: 116 consecutive newborns with seizures were included. We analyzed $17(14.6 \%)$ with refractory seizures that receives new AEDs, $70 \%$ term and $30 \%$ premature. $70.58 \%$ had a symptomatic partial epilepsy and $29.42 \%$ presented a Neonatal Epileptic encephalopathy. Overall in-hospital mortality was $11.7 \%$.

The new AEDs indicated as second-line anticonvulsant treatment were: Vigabatrine in 2p., Oxcarbacepine 4p., Levetiracetam 1p., and Topiramate in 1p. As a 3rd or 4th AED: Vigabatrine in $7 \mathrm{p}$., Levetiracetam 4p., Topiramate 3p., Oxcarbacepine 2p. and Valproate in $1 \mathrm{p}$.

We observed a $50 \%$ or more seizure reduction in $10 \mathrm{p}$. (58\%) and $4 \mathrm{p}$. $(23.5 \%)$ with complete cessation of seizures. We noticed a $50 \%$ or more seizure reduction with Oxcarbacepine in $4 / 5$ p., Levetiracetam $3 / 5 \mathrm{p}$. and Vigabatrine in 4/9p. No adverse events were reported during treatment with any anticonvulsant.

Conclusions: In our study, we observed a favorable seizure control in newborns with refractory seizures with new AEDs.

The development of new prospective studies are needed, including more newborns, to assess the efficacy, safety and neuroprotective effect of new AEDs as first-line treatment.

\subsection{0}

EARLY EFFICACY WITH ADJUNCTIVE LACOSAMIDE TREATMENT IN PATIENTS WITH UNCONTROLLED PARTIAL SEIZURES: ANALYSIS OF MEAN PERCENTAGE OF SEIZURE-FREE DAYS PER WEEK

Cindy McShea ${ }^{1}$, M. Polinkovsky ${ }^{2}$, S. Dimova ${ }^{3}$, P. Doty ${ }^{1}$, M. DeBacker ${ }^{3}$ and S. Chung ${ }^{4}\left({ }^{1}\right.$ UCB Pharma, Raleigh, NC; ${ }^{2}$ University of North Carolina - Chapel Hill, Chapel Hill, NC; ${ }^{3} \mathrm{UCB}$ Pharma, Brussels, Belgium and ${ }^{4}$ Barrow Neurological Institute, Phoenix, AZ)

Rationale: Lacosamide is approved for adjunctive treatment of adults with partial-onset seizures. Analyses have shown a significant difference vs. placebo in median percent reduction in seizure frequency and $50 \%$ responder rate during the first $(100 \mathrm{mg} /$ day $)$ and second ( $200 \mathrm{mg} /$ day) week of actual lacosamide exposure (Chung et al, CNS Drugs, 2010). This post hoc analysis evaluated efficacy based on percentage seizure-free days during the entire Treatment Period (Titration and Maintenance).

Methods: Data were pooled from 3 Phase II/III, randomized, doubleblind, placebo-controlled trials (SP667, SP754, SP755), evaluating fixed doses of lacosamide 200, 400 and $600 \mathrm{mg}$ /day. Patients received $100 \mathrm{mg}$ /day during the initial week of lacosamide exposure, followed by weekly titration in $100 \mathrm{mg}$ increments to the assigned dose. Percentage of seizure-free days averaged over the entire Treatment Period and for each week of the Treatment Period was evaluated for the lacosamide group (all doses combined), individual dose groups and placebo. Generalized estimating equation models were used to perform pairwise comparisons of lacosamide group(s) versus placebo.

Results: Overall 935 patients randomized to lacosamide (200 $\mathrm{mg} /$ day, $\mathrm{n}=267$; $400 \mathrm{mg}$ /day, $\mathrm{n}=466$;

$600 \mathrm{mg} /$ day, $\mathrm{n}=202$ ) and 359 to placebo with at least one seizure diary entry were included in the analysis. Patients had long-standing epilepsy (mean time since diagnosis of $>20$ years for placebo and lacosamide groups) with $77 \%$ reporting $\geq 4$ lifetime AEDs. Median baseline seizure frequency per 28 days was 11.5 for the total population. A significant difference in the percentage of seizure-free days averaged over the Treatment Period was observed from the model (all doses combined versus placebo; $P<0.001$ ). During the first week of actual lacosamide exposure, when patients in all three lacosamide treatment groups were receiving $100 \mathrm{mg} /$ day, there was a significantly greater percentage of seizure-free days/week with lacosamide (all doses combined) relative to placebo $(P=0.014)$. A significant difference between lacosamide (all doses combined) and placebo was observed for all subsequent weeks in the Treatment Period (range $P<0.001$ to 0.020 ; Figure 1). Results for individual dose groups versus placebo were generally similar to the lacosamide (all doses combined) findings, with the exception of lacosamide 200 $\mathrm{mg}$ /day, which did not show a consistent difference versus placebo for all weeks in the Treatment Period. Individual dose analyses for lacosamide $200 \mathrm{mg} /$ day and $600 \mathrm{mg}$ /day versus placebo are limited by smaller sample size.

Conclusions: This post hoc analysis of pooled data from doubleblind, placebo-controlled trials further supports lacosamide efficacy starting from the first week of actual exposure (100 mg/day). Mean percentage of seizure-free days/week on lacosamide (all doses combined) increased during dose Titration and remained relatively constant during the 12-week Maintenance period.

Sponsored by UCB.

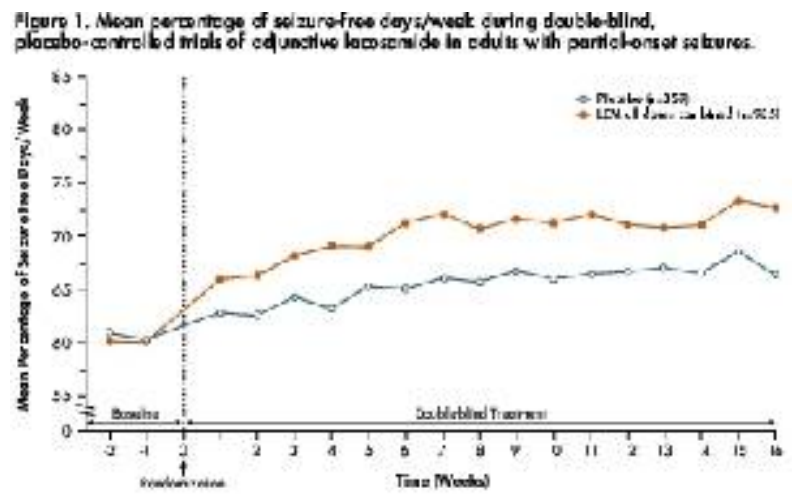

\subsection{1}

POSTMARKETING EXPERIENCE WITH LACOSAMIDE AS ADD-ON THERAPY IN REFRACTORY PARTIAL ONSET EPILEPSY IN SLOVAKIA

Gabriela Timarova ${ }^{1}$, S. Mehesova ${ }^{1}$ and E. Feketeova ${ }^{2}\left({ }^{1} 2\right.$ nd Clinic of Neurology, University Hospital, Bratislava, Slovakia and ${ }^{2}$ University Hospital, Kosice, Slovakia)

Rationale: Though big effort was done in last 20 years in developping new drugs, $20-30 \%$ of patients with epilepsy still remain refractory to medical treatment. The need of new antiepileptic drugs (AEDs) is still declared es actuall, efficacy and tolerability data about the newest AEDs are still incomplete. Lacosamide (LCS) was introduced into the clinical praxis in Slovakia in April 2009.

Methods: We analysed data in clinical cohort of 70 outpatients (29 men and 41 women) with refractory partial epilepsy visiting tertiary epilepsy centres in University Hospitals in Bratislava and Kosice in Slovakia after 12 month treatment with LCS as add-on therapy. Mean age in this group of patients was 35,6years (20-84y), mean duration of epilepsy 18,4 years(2-47y). 42/70 patients (60\%) used before LCS 5 or more AEDs, which is known risk factor for farmacoresistance. The mean used therapeutic dose in our patients was $280,6 \mathrm{mg}$ of LCS per day. The efficacy was calculated as RR (responder rate, at minimum $50 \%$ reduction in seizure frequency), the portion of seizure free (SF) patients was also calculated.

Results: RR in our group of 70 patients was $51,42 \%$ (36/70), SF patients in that $7,14 \%(5 / 70)$. SF patients were treated before LCS with less than 4 AEDs, in the patients treated with more than 5 AEDs before LCS none become seizure free. In 20\% (14/70) of patients LCS was withdrawn due to intolerable side effects or lack of efficacy. 
The most often seen adverse events were dizziness and instabile gate in $10 \%$, seizure aggravation, diplopia and headache -each $5,7 \%$. Less than $3 \%$ of patients complained about fatigue, tremor, diarrhoe, depression, one patient with suicidal ideations.

Conclusions: LCS showed efficacy and adverse event profile comparable with other new antiepileptic drugs, still seizure freedom was achieved only in patients treated with less than 4 antiepileptics before LCS administration. The results in our cohort of patients are in aggreement with other preclinical and clinical studies, which have been published yet.

\subsection{2 \\ PRESCRIPTION PATTERNS AND SELF-REPORTED SIDE EFFECTS OF ANTIEPILEPTIC DRUGS IN PATIENTS WITH EPILEPSY AT TERTIARY REFERRAL CENTER IN AUSTRIA}

Ekaterina Pataraia, R. Jung, S. Bonelli-Nauer, K. Trimmel and S. Aull-Watschinger (Medical University Department of Neurology, Vienna, Austria)

Rationale: With the marketing of a number of new antiepileptic drugs (AEDs) in recent years the pattern of prescription of AEDs has changed. The choice of optimal polytherapy poses difficulty for several reasons: there are limited data regarding favorable or unfavorable combinations and there is little systematic evidence that any two combinations are any more or less effective as any other. The clinician's choice of polytherapy is, in part, determined by his comfort with the AED chosen first for monotherapy. This may result in combinations of AEDs that are idiosyncratic for each practitioner, and not necessarily optimally selected for the patient requiring polytherapy. The aim of the present study was to examine the trends in the prescribing of AEDs, seizure frequency and self reported side effects in patients with drug resistant epilepsy at the tertiary referral center in Austria.

Methods: 530 consecutive patients with epilepsy who where examined at the specialized epilepsy outpatient clinic of Department of Neurology, Medical University of Vienna, Austria, from June 2010 until October 2011 were enrolled in the study. Demographic data, current AEDs and seizure frequency were evaluated. All patients filled in the Hospital Anxiety and Depression Scale (HADS, German version) and a brief 19-item self-report instrument - the Adverse Events Profile (AEP), before attending clinician's office. Most common used AEDs and AED combinations were correlated with seizure frequency, most frequent reported side effects and anxiety and depression scores.

Results: Monotherapy was used in $55,3 \%$ of patients, whereas $30,9 \%$ patients had 2 AEDs, $12,5 \% 3$ AEDs and 1,3\% of patients $>3$ AEDs. $32,6 \%$ of patients were seizure free, $67,4 \%$ still had seizures. $32,3 \%$ of not-seizure-free patients had monotherapy, and the remaining patients had AED combination. The most frequent used drug was LVT in focal epilepsies (37\%) and VPA in generalized epilepsies (44\%). The most frequent used AEDs in patients with focal epilepsies who had monotherapy and were seizure-free were LVT (30\%), CBZ (33\%), and in patients with generalized epilepsies - LTG (29\%) and VPA (50\%). Most frequent reported side effects of AEDs were ataxia $(p=0,048)$, aggression $(p=0,014)$, nausea $(p=0,052)$, cognitive impairment $(\mathrm{p}=0,059)$. Most common combination of AEDs were LVT + CBZ $(25,3 \%)$, almost $31 \%$ of these patients were completely seizure free. The less common combinations were LVT $+\mathrm{LTG}(9,7 \%)$, LVT + LCS $(9,3 \%)$ and LVT+OXC (8,0\%). The patients who were seizure free had scored better in HADS (anxiety 8,9\%, depression $5,8 \%$ ) compared to patients who were not seizure-free (anxiety $16,6 \%$, depression $12,5 \%$ ).

Conclusions: the present retrospective study provides updated information on the patterns of prescription of AEDs in al large population of patients with drug resistant epilepsies. The new AEDs were widely prescribed. There was a high portion of patients with more than one AED, which can be explained by increasing number of new AEDs, many of which are approved only for add on therapy.

\section{Antiepileptic Drugs: Other}

\subsection{3}

EFFICACY AND TOLERABILITY OF LACOSAMIDE IN NOCTURNAL SEIZURES

Beatriz Gonzalez Giraldez, S. Bellido Cuellar, C. Alarcon Morcillo and J. M. Serratosa (Neurology, Hospital Universitario Fundacion Jimenez Diaz, Madrid, Spain)

Rationale: Postmarketing experience suggests that lacosamide (LCM) may be particularly useful in controlling seizures emerging from sleep. The objective of this study was to evaluate the efficacy and safety of LCM in the treatment of nocturnal seizures.

Methods: We reviewed the clinical charts of all patients with epilepsy presenting seizures emerging exclusively or almost exclusively $(>90 \%)$ from sleep that had been treated with at least one dose of LCM. Only patients with a reliable account of their seizures (self-reported or witnessed) were included. Efficacy was assessed at months 6 and 12 by comparing pre-LCM monthly seizure frequency with post-LCM treatment seizure frequency. Pre-treatment monthly seizure frequency was assessed during a 12-month retrospective baseline prior to introduction of LCM. A 3-month dose-adjustment period was excluded for seizure frequency analysis. Response was defined as $>50 \%$ reduction in seizure frequency and included seizure freedom.

Results: Forty patients were included in the study. All patients presented partial epilepsy: 23 symptomatic, 13 cryptogenic, and 4 idiopathic. Reasons for adding LCM were either lack of efficacy $(n=37)$ or intolerable side effects of previous AED regimen $(n=3)$. Patients were taking a mean of 1,7 concomitant AEDs (range 0-4) prior to introduction of LCM. More than half of the patients $(62 \%)$ had previously tried three or more AEDs. At month 6, 21 of 35 patients $(60 \%)$ were responders, 9 of which $(25 \%)$ were seizure-free. Mean daily dose of LCM in this group was $290 \mathrm{mg}$ (range 100-400 $\mathrm{mg})$. At month 12, 21 of 31 patients $(67.7 \%)$ were responders, 6 of which $(19 \%)$ were seizure-free. Mean daily dose of LCM in this group was $356 \mathrm{mg}$ (range 100-600 mg). Adverse events were recorded in 12 patients (30\%) and leaded to withdrawal of LCM in 3 (7.5\%).

Conclusions: In this retrospective study LCM was well tolerated and showed high and sustained efficacy in epilepsies with exclusively or almost exclusively nocturnal seizures.

\subsection{4 \\ VITAMIN D STATUS IN A PEDIATRIC OUTPATIENT NEUROLOGY SETTING}

Joan A. Conry, J. Reese and D. Kassaye (Neurology, Childrens National Medical Center, Washington, DC)

Rationale: Patients who are on chronic antiepileptic drugs (AED) and/or nonambulatory are a vulnerable population for vitamin D deficiency and comprise a large portion of a general pediatric neurology outpatient clinic. In 2008, the American Academy of Pediatrics (AAP) recommended all children and adolescents receive $400 \mathrm{IU}$ vitamin $\mathrm{D}$ or $1000 \mathrm{ml}$ vitamin $\mathrm{D}$ fortified milk daily, with serum $25 \mathrm{OH}$ vitamin D level $>50 \mathrm{nmol} / 1(20 \mathrm{ng} / \mathrm{mL})$. AAP recommendations regarding "at risk" populations were to monitor serum $25-\mathrm{OH}$ vitamin D level, and if the $25-\mathrm{OH}$ vitamin $\mathrm{D}$ level is low, measure PTH (parathyroid hormone) and bone mineral status. Because we treat a high risk population, we chose to monitor our current practice.

Methods: With IRB approval, charts of 787 consecutive patients seen in the outpatient neurology clinic were reviewed between March 9 and April 22, 2011We collected data to characterize the at risk population, adequacy of vitamin D supplement, and bone health monitoring. Data collected included documentation of use of supplemental vitamin D (either an independent supplement or as part of a multivitamin), antiepileptic drugs (AED), ketogenic diet status, 
ambulatory status, laboratory monitoring during that visit or within the past two years, and fractures. Not all charts contained all desired information. 778 of the 787 charts contained adequate information to analyze.

Results: Of the 778 patients with available information, 592 (76\%) had previously been seen in our clinic. Over $50 \%$ of patients take AED, and $15 \%$ are non-ambulatory. Any form of vitamin D supplement is taken by less than $20 \%$ of the 592 recurrent patients, $318(54 \%)$ are on at least one AED, and $89(15 \%)$ are nonambulatory.102 (17\%) were on some form of vitamin D supplement. $46(8 \%)$ had a vitamin D level checked in the past 2 years. 29 were on AED and non ambulatory. 20 of 29 nonambulatory patients on AED were not on vitamin D. supplements.

Conclusions: The majority of at-risk pts (on AED and nonambulatory) are not receiving vitamin D supplementation or monitoring for hypovitaminosis D. This study demonstrates the need for more rigorous vitamin D supplementation and monitoring of bone health status in vulnerable populations. Neurologists may not be as aware of guidelines published by the AAP than AAN. Studies have yet to prove that intervention with the AAP guidelines will lead to decreased fracture risk. However, neurologists care for a strongly atrisk population and may be able to affect positive change for their patients through increased awareness and action concerning bone health.

Our current algorithm for monitoring bone health is:

1. Measure $25(\mathrm{OH})$ vitamin $\mathrm{D}$ in all ongoing patients on $\mathrm{AED}>6$ months and/or nonambulatory.

2. If vitamin $\mathrm{D}$ is $<20 \mathrm{ng} / \mathrm{mL}$ measure PTH and increase vitamin $\mathrm{D}$ supplement to $800 \mathrm{IU} /$ day.

3. If PTH is normal recheck vitamin D level in 6 months.

4. If PTH is elevated order Dexascan and recheck vitamin D level and PTH in 3 months.

A vitamin level of $20 \mathrm{ng} / \mathrm{mL}$ is probably too low. We will collect laboratrry results with our new alogitthm.

\subsection{5 \\ BENEFITS OF CONVERSION FROM IMMEDIATE RELEASE LAMOTRIGINE TO EXTENDED RELEASE LAMOTRIGINE IN INDIVIDUALS WITH DRUG- RESISTANT EPILEPSY OR ADVERSE EFFECTS}

Melissa R. Osborn, P. Ramey and B. Abou-Khalil (Neurology Epilepsy, Vanderbilt University Medical Center, Nashville, TN)

Rationale: Immediate release lamotrigine (LTG-IR) dosing can be limited by peak toxicity. It is thought that peak levels are responsible for some adverse effects such as dizziness, blurred vision, double vision and unsteadiness. At the same time, trough levels may be associated with reduced seizure threshold. The use of extended release lamotrigine (LTG-XR) to replace LTG-IR will be associated with lower peak levels, thus reducing adverse effects and higher trough levels, thus elevating the seizure threshold, which is expected to improve seizure control. We tested this hypothesis by analyzing seizure control and adverse effects before and after conversion from LTG-IR to LTG-XR in patients who underwent such conversion in 2009-2011.

Methods: We searched our patient database to identify patients converted from LTG-IR to LTG-XR for persistent seizures or adverse effects until December 31, 2011. We excluded patients with nonepileptic seizures, progressive cause of epilepsy, or not keeping a seizure record. We collected the following parameters: age at switch, LTG-IR dose and dosing schedule, duration on that dose, LTG-XR dose and dosing schedule, LTG serum level before and after conversion, duration of LTG-XR treatment, seizure frequency before and after conversion, and change in adverse experience profile. We also recorded baseline AEDs and any AED change during the course of the analysis.

Results: 55 patients (26 female) satisfied the inclusion/exclusion criteria. Their mean age was 45 years (range 23 to 86). 9 were on lamotrigine IR monotherapy and 24 took one other AED, most commonly levetiracetam. The mean dose was $544 \mathrm{mg} /$ day (range $150-1100 \mathrm{mg} /$ day). The mean LTG-IR serum level was 11.6 (available in 53 patients- range $4.6-21 \mathrm{mcg} / \mathrm{ml}$ ). 19 patients were converted to the same dose, while 21 had their dose slightly increased and 7 had their dose slightly decreased due to adverse effects. The mean serum level after conversion was 11.8 (available in 49 patientsrange 2.6-21.2). As a result of the switch, 25 patients experienced $\geq 50 \%$ reduction in seizure frequency, with $46 \%$ median reduction in seizure frequency. Seven patients reported improvement in adverse effects, while three reported no change.

Conclusions: A switch from LTG-IR to LTG-XR can help improve seizure control in some individuals with drug-resistant epilepsy, in addition to improving tolerability. While it is indicated in individuals experiencing peak adverse effects, it should also be considered in patients who have received incomplete seizure control from LTG-IR.

\subsection{6 \\ DETERMINING MINIMAL IMPORTANT CHANGE THRESHOLDS FOR THE SEIZURE SEVERITY QUESTIONNAIRE (SSQ) IN CLINICAL TRIALS} Joyce Cramer ${ }^{1}$, C. de la Loge ${ }^{2}$, Y. Brabant ${ }^{3}$ and S. Borghs ${ }^{2}\left({ }^{1}\right.$ Yale University, Houston, TX; ${ }^{2} \mathrm{UCB}$ Pharma, Brussels, Belgium and ${ }^{3}$ Business and Decision, Brussels, Belgium)

Rationale: The SSQ provides a rich source of information for understanding changes in specific components of seizure severity and bothersomeness. In order to interpret SSQ results in a population of patients with refractory epilepsy, this analysis aimed at defining thresholds of minimal important change (MIC), allowing classification of patients by clinically meaningful response. Methods: Data from two placebo-controlled double-blind phase III trials of adjunctive lacosamide (SP754, SP755) in partial onset seizures were pooled. Patients completed the SSQ at baseline, and the SSQ and the Patient's Global Impression of Change (PGIC) at last assessment (end of maintenance [16/18 weeks] or early discontinuation). The SSQ is a 22 -item questionnaire (addressing Aura/Warning, Ictal, and Post-Ictal events) that is scored as 3 composite scores and a total score. Responses range from 0 to 7 with higher scores indicating greater seizure severity. The PGIC is a 1item instrument with seven ratings from 'very much improved' $(+3)$ to 'very much worsened' (-3). To determine responsiveness of SSQ scores to the PGIC, mean SSQ score change from baseline to last assessment by PGIC rating was calculated. Standard anchor-based MIC thresholds were calculated, for each composite score, as the average of the absolute values of the mean score changes from baseline to last assessment associated with the PGIC categories of 'much improved,' 'minimally improved,' 'much worsened,' and 'minimally worsened' combined $(-2,-1,+1$ and +2$)$.

Results: A total of 776 patients had both SSQ and PGIC data at last assessment. Mean SSQ score changes differentiated among the PGIC ratings. Patients with a PGIC rating of 'very much improved' $(n=70)$ showed an average SSQ score change of -1 ; those with 'much improved' $(\mathrm{n}=208)$ changed to -0.7 ; and those with 'minimally improved' ( $\mathrm{n}=183$ ) changed to -0.4 . The MIC thresholds were 0.51 , $0.39,0.46$, and 0.48 points for the 3 subscales (Activity during seizures, recovery, overall severity/recovery) and Total score, respectively; and $0.34,0.48$, and 0.42 for the emotional, physical and cognitive components of Recovery, respectively. The overall item, representing general impressions of severity and bother, had an MIC of 0.84 .

Conclusions: The SSQ showed clear differentiation among PGIC improvement ratings. The estimated MIC thresholds for subscales ranged from 0.34 to 0.84 , with 0.48 points for the Total score, which averages all seizure components. These MIC data reflect the degree of change in SSQ scores that represent clinically meaningful change from the patients' perspectives, and which can be used in refractory patients to define treatment responders. Sponsored by UCB. 


\subsection{7}

THE RESPONSIVENESS OF SEIZURE SEVERITY QUESTIONNAIRE (SSQ) ITEMS TO CHANGE IN SEIZURE FREQUENCY BY TYPE

Simon Borghs ${ }^{1}$, C. de la Loge ${ }^{1}$, Y. Brabant ${ }^{2}$ and J. Cramer ${ }^{3}\left({ }^{1}\right.$ UCB

Pharma, Brussels, Belgium; ${ }^{2}$ Business and Decision, Brussels, Belgium and ${ }^{3}$ Yale University, Houston, TX)

Rationale: The SSQ has been used in large clinical trials to assess the severity of seizures and as such should differentiate between seizure types. This analysis evaluated the responsiveness of individual items to clinical response according to seizure type as further validation of the instrument.

Methods: Data from open-label extensions (OLEs) of two phase III trials of lacosamide in focal epilepsy (SP756, SP774) were pooled. Patients completed the SSQ at double-blind baseline and every 24 weeks during OL treatment. The SSQ is a 22-item questionnaire (addressing Aura/Warning, Ictal, and Post-Ictal events) that is scored as 3 composite scores and a total score, and includes questions for frequency and helpfulness of warning signs as well as severity and/or bothersomeness of ictal movement and loss of consciousness. Also included are 9 items measuring the frequency, severity, and bothersomeness of post-ictal cognitive, emotional, and physical effects. Item scores range from 1-7, with higher scores indicating greater severity, except the warning item. Mean change from baseline to week 48 of the OLE was calculated for each item by seizure type response for those with only complex partial seizures (CPS) or only secondarily generalized partial seizures (SGPS) at baseline. Responders were defined as subjects with a $\geq 50 \%$ reduction in seizure frequency in the 24 weeks before week 48 , compared to baseline. Differences between responders and non-responders were compared between seizure-type groups.

Results: SSQ data were available for 166 CPS responders and 127 non-responders; and for 26 SGPS responders and 24 non-responders. The warning item showed little difference between responders and non-responders in both seizure type groups. On the ictal movement and consciousness items, responders showed large improvements with larger differences observed in the SGPS group: $>1.5$ points compared with 0.71 to 0.96 points among CPS responders. Scores for non-responders remained stable in both groups of patients. On postictal recovery questions for cognitive, emotional or physical effects, the SGPS group showed much larger responder to non-responder differences than the CPS group for each of the 3 bothersomeness items, with less differentiation for the frequency and severity items. SGPS responders showed mean improvements of more than 2 points on frequency, severity, and bothersomeness items for after-seizure physical effects.

Conclusions: SSQ items showed excellent differentiation between seizure type responders related to ictal movement and consciousness, and related to bothersomeness of post-ictal effects. The frequency, severity, and bothersomeness of post-ictal physical effects were markedly improved for SGPS responders. These data support the usefulness of the SSQ in describing treatment effects.

Sponsored by UCB.

\subsection{8 \\ TOLERABILITY OF OVERNIGHT SWITCH FROM OXCARBAZEPINE TO ESLICARBAZEPINE ACETATE Julia Höfler, J. Dobesberger, M. Kirschner, M. Leitinger, C. Granbichler, T. Moroder and E. Trinka (Christian Doppler Klinik, Paracelsus Medical University of Salzburg, Salzburg, Austria)}

Rationale: Eslicarbazepine acetate (ESL) is a dibenzazepine derivative, like carbamazepine (CBZ) and oxcarbazepine (OXC) with a structural difference at the 10,11-position. ESL was licensed for add-on treatment of adults with focal epilepsies by the EMA in 2009.
The active metabolite of ESL enhances the slow inactivation of the fast voltage gated sodium channel, whereas CBZ inactivates it. Though both leading to ESL as the active metabolite metabolisation differs, with ESL being directly converted, while OXC-peaks in plasma and CSF about 1-2 hours after intake. This OXC peak may cause intolerable dose dependent adverse events such as dizziness, diplopia, headache or nausea, which could resolve after switching to ESL. Therefore we performed a study to investigate the safety and tolerability of an overnight switch from OXC to ESL.

Methods: We included 10 patients (6 women) with focal epilepsy, median age 38 years (yrs) (range 21-85), had median of five previously failed AEDs (range 1-14), and were not seizure free, had dose limiting adverse events under $\mathrm{OXC}$, or both. Median age at epilepsy onset was 12 yrs (range 1-74), aetiology was symptomatic in eight and unknown in two, 8 patients had temporal lobe epilepsy (6 left, 3 bilateral, 1 right), 4 patients received polytherapy of three additional AEDs, 5 of two AEDs, and 1 was on monotherapy. We applied the Adverse Event Profile (AEP), standardized test for alertness, and the QOLIE 10 immediately before and 5 days after the switch. Serum levels of MHD, eslicarbazepine and OXC were measured on the day of the neuropsychological assessments. Results: Median Dose of OXC before the switch was 900 (range 600-1800) $\mathrm{mg}$. The individual conversion ratio between OXC and ESL ranged from 1:1.1 to 1:1.9. After conversion the median dose of ESL was $800 \mathrm{mg}$ (range $800-1600$ ); $2 / 10$ pts had a reduction of seizure frequency after the switch (FU 3 to 10 months); Serum level of eslicarbazepine was median $15.8 \mathrm{mg} / \mathrm{l}$ (range 7.3-31.3). After switch to ESL patients had significantly lower sum scores on the AEP $(\mathrm{p}=0.005)$. The reduction was most prominent in the following domains: unsteadiness, problems with the stomach, weight gain, dizziness, diplopia or blurred vision, difficulties in concentration. Seven pts. continued treatment with ESL. One discontinued due to lack of efficacy, one because of concentration difficulties (which were also present before the switch, and one due to increased thirst, probably unrelated to ESL).

Alertness improved, but did not reach significant levels $(\mathrm{p}=0.09)$. QOLIE 10 showed no significant difference $(\mathrm{p}=0.08)$.

Conclusions: Overnight switch from OXC to ESL was safe and easy to perform. Clinical improvements were seen immediately after the switch. Most pts. continued treatment with ESL.

\subsection{9 \\ VALPROATE BUT NOT LEVETIRACETAM ALTERS SYSTEMIC IMMUNE PARAMATERS IN EPILEPTIC PATIENTS}

Sabina Guenther ${ }^{1}$, S. Bauer ${ }^{1}$, M. Nowak ${ }^{1}$, B. Tackenberg ${ }^{1}$, W. H. Oertel $^{1}$, F. Rosenow ${ }^{1}$ and H. M. Hamer ${ }^{2}\left({ }^{1}\right.$ Department of Neurology, Philipps-University of Marburg, Marburg, Germany and ${ }^{2}$ Department of Neurology, University of Erlangen-Nuernberg, Erlangen, Germany)

Rationale: There is growing evidence that epileptic seizures can result in changes in immunologic parameters and that immunologic processes may influence the course of epilepsies vice versa. Along with these findings, there are several hints that antiepileptic drugs could influence the immune system. Therefore, we performed a prospective study on the influence of valproate and levetiracetam on immune parameters.

Methods: 36 patients were included. 15 patients were started on valproate (10 (66,7\%) male, $5(33,3 \%)$ female, age 53,5 $\pm 27,2$ years), 21 were started on levetiracetam $(11(52,4 \%)$ male, 10 $(47,6 \%)$ female, age $44,7 \pm 19,4$ years $)$ at the discretion of the treating physician. Immunologic parameters were examined before first intake of the new drug and 6 months later. We performed a differential blood count and analyzed the distribution of CD3+CD4+and $\mathrm{CD} 3+\mathrm{CD} 8+$-leukocyte subsets using flow cytometry. Levels of IL-1 $\beta$, IL-6, TNF- $\alpha$ and MCP-1 were studied using ELISAs. Results: Valproate intake resulted in a significant decrease of the 
total number of leukocytes $(6.96 \pm 1.23 / \mathrm{nl}$ versus $6.13 \pm 1.57 / \mathrm{nl}$, $\mathrm{p}=0.026)$. The absolute count $(4.60 \pm 1.05 / \mathrm{nl}$ versus $3.69 \pm 1.30 / \mathrm{nl}$, $\mathrm{p}=0.01)$ and percentage $(65.39 \pm 7.86 \%$ versus $59.54 \pm 11.47 \%$ $\mathrm{p}=0.01)$ of neutrophil granulocytes dropped significantly. Lymphocyte subset analyses revealed a significant decrease of the percentage of CD3+CD4+ -lymphocytes from $50.93 \pm 10.23 \%$ to $45.72 \pm 12.15 \%(\mathrm{p}=0.003)$. These changes resulted in a nonsignificantly lowered CD4/CD8 ratio $(3.54 \pm 2.00$ versus $3.06 \pm 2.28$ $\mathrm{p}=0.1$ ). Comparison of cytokine-levels at baseline- and follow-up conditions did not show any significant changes.

Intake of levetiracetam did not go along with any significant changes in leukocytes, neutrophil granulocytes, lymphocytes or lymphocyte subsets. We did not see any significant alterations in cytokine concentrations although there was a trend towards decreased MCP-1 levels $(\mathrm{p}=0.09)$.

Conclusions: Valproate reduced levels of leukocytes and lowered CD3+CD4+-lymphocytes in this prospective study, confirming previous retrospective reports. Lowered counts of CD3+CD4+lymphocytes could be due to an increase of apoptosis in these cells. We did not see any significant changes associated with intake of levetiracetam.

\subsection{0}

COST-UTILITY ANALYSIS OF LACOSAMIDE ADJUNCTIVE THERAPY IN THE TREATMENT OF PATIENTS WITH REFRACTORY EPILEPSY IN CANADA

Hicham Benhaddi ${ }^{1}$, C. Vicente ${ }^{2}$ and R. Tam ${ }^{3}\left({ }^{1}\right.$ UCB Pharma, Brussels, Belgium; ${ }^{2}$ PIVINA Consulting Inc., Mississauga, ON, Canada and ${ }^{3}$ UCB Canada Inc., Oakville, ON, Canada)

Rationale: To calculate and compare the incremental cost-utility ratios for standard antiepileptic drug (AED) therapy with and without adjunctive lacosamide in patients with uncontrolled partial-onset seizures in Canada.

Methods: The economic model was based on a decision analytic approach that simulates the treatment pathway of a hypothetical patient over a period of two years from the Ministry of Health $(\mathrm{MoH})$ and Societal perspectives in Canada in 2011. The decision tree was split into four phases of six months each during which patients could become seizure free, experience a seizure reduction, or withdraw due to non-response (responder defined as $\geq 50 \%$ reduction in seizures). The Standard Therapy arm included five AEDs: carbamazepine, lamotrigine, levetiracetam, topiramate, and phenytoin. The likelihood of being in a particular health state was estimated from pooled clinical trial data. Resource utilization associated with AEDs, General practitioner visits, Specialist visits, Hospitalizations, Emergency Room visits, Pre-surgical evaluation and surgery were included. Costs and utility values attached to various health states were taken from standard reference lists or the published literature.

Results: Over a two year period, treating patients with lacosamide plus Standard Care would result in a total cost of \$12,611 CAD per patient as compared with $\$ 11,144$ for Standard Care (+placebo). Initial treatment with lacosamide + Standard Therapy and Standard Therapy (+placebo) resulted in 1.24 quality adjusted life-years (QALYs) gained and 1.20 QALYs gained, respectively. From the MoH perspective, the incremental cost was $\$ 39,156$ per incremental QALY gained versus Standard Care (+placebo). From the societal perspective, the incremental cost was $\$ 32,334$ per incremental QALY gained versus Standard Care (+placebo). Results of the probabilistic analysis revealed that at a willingness-to-pay threshold of $\$ 50,000$ per QALY, lacosamide + Standard Therapy is cost effective relative to Standard Therapy (+placebo) in approximately $90 \%$ of the scenarios.

Conclusions: Lacosamide was shown to be a cost-effective adjunctive treatment in patients with uncontrolled partial-onset epilepsy in Canada.

Sponsored by UCB

\subsection{1}

EQUIVALENCE AMONG BENZODIAZEPINES INCLUDING CLOBAZAM: A SURVEY OF EPILEPTOLOGISTS

Noemi Rincon Flores ${ }^{1,2}$ and S. R. Benbadis ${ }^{1,2}\left({ }^{1}\right.$ Neurology, University of South Florida, Tampa, FL and ${ }^{2}$ Tampa General Hospital, Tampa, FL)

Rationale: Benzodiazepines are often used in the chronic management of refractory epilepsy, and specifically clobazam now has a formal indication for "seizures associated with the LennoxGastaut syndrome." Because patients are often transitioned from one benzodiazepine to another (including to clobazam), the question of dose equivalence among various benzodiazepines is frequently asked. Methods: Using diazepam $5 \mathrm{mg}$ as the arbitrary benchmark, we asked each epileptologist for their estimated equivalent dosage of lorazepam, clonazepam, and clobazam. For each drug, mean, median, standard deviation, standard error, and coefficient of variability were calculated.

Results: See table.

Conclusions: Variability among experts is largest for clobazam, whereas it is mild to moderate for lorazepam and clonazepam. This is not unexpected since clobazam was only recently made available in the U.S. Studies on the dosage equivalence among benzodiazepines are needed in the future.

Acknowledgement:

Biostatistics Core at Clinical \& Translational Science Institute (University of South Florida) performed the statistical analysis.

\begin{tabular}{|c|c|c|c|c|}
\hline Epilepiologist & Diazepam & Loracepam & Clonsazepam & Clobuzam \\
\hline A & 5 & 1 & 0.5 & 2.5 \\
\hline$\wedge$ & 5 & 2 & 1 & 10 \\
\hline$\wedge$ & 5 & 1 & 1 & 10 \\
\hline$\wedge$ & 5 & 0.5 & 0.25 & 10 \\
\hline A & 5 & 1 & 0.5 & 10 \\
\hline A & 5 & 1 & 0.5 & 10 \\
\hline A & 5 & 1 & 0.5 & 5 \\
\hline A & 5 & 1 & 1 & 5 \\
\hline$\wedge$ & 5 & 0.5 & 0.5 & 5 \\
\hline$\wedge$ & 5 & 1 & 1 & 10 \\
\hline$P$ & 5 & 1 & 0.5 & 10 \\
\hline $\mathbf{P}$ & 5 & 0.5 & 1 & 10 \\
\hline P & 5 & 1 & 0.25 & 2.5 \\
\hline$P$ & 5 & 1 & 1.5 & 30 \\
\hline $\mathbf{P}$ & 5 & 1 & 0.25 & 2.5 \\
\hline $\mathbf{P}$ & 5 & 1 & 0.5 & 5 \\
\hline$P$ & 5 & 0.75 & 0.25 & 7.5 \\
\hline \multicolumn{5}{|l|}{ Statistics } \\
\hline Mean & 5.0000 & 0.9559 & 0.6471 & 8.5294 \\
\hline Median & 5.0000 & 1.0000 & 0.5000 & 10.0000 \\
\hline $\begin{array}{l}\text { Standar } \\
\text { Deviation }\end{array}$ & 0.0000 & 0.3335 & 0.3651 & 6.3158 \\
\hline $\begin{array}{l}\text { Standlar } \\
\text { Error }\end{array}$ & 0.0000 & 0.0809 & 0.0885 & 1.5318 \\
\hline $\begin{array}{c}\text { Coefficient of variability } \\
(\%)\end{array}$ & 0.0000 & 34.89 & 56,42 & 74.05 \\
\hline
\end{tabular}

Abbreviations: A =Adult Epileptologist

$\mathrm{P}=$ Pediatric Epileptologist

\subsection{2 \\ ANTIEPILEPTIC DRUGS AND ADHERENCE: A CRITICAL REVIEW}

A. Economos, Jocelyn Cheng and E. Carrazana (University of Miami, Miami, FL)

Rationale: Maintenance of an optimal antiepileptic drug (AED) regimen remains challenging in epilepsy patients. Suboptimal adherence to AEDs results in increased risk of seizures, injury, 
hospitalization and mortality. In order to optimize medical therapy in epilepsy patients, it is important to identify patient and AED characteristics which affect adherence.

Methods: Literature review of the Cochrane Library, MEDLINE, EMBASE and CINAHL databases. After screening, 23 studies which assessed AED adherence in epilepsy patients were identified. Results: Rates of non-adherence ranged from 25-79\%. Multiple measures of adherence were used, limiting direct comparison of studies. Variability in adherence rates was affected by demographic group, monotherapy versus polytherapy, AED dosing schedule and AED side effects. Elderly patients consistently showed lower adherence rates due to causes including cognitive or language impairment, misconceptions regarding side effects, and AED cost, with a robust negative relationship between out-of-pocket prescription costs and adherence in elderly epileptic patients. In children, adherence rates were positively correlated with married status of parents and higher socioeconomic status, and no association was found between adherence and seizure severity/frequency across multiple studies. Adolescents showed poor adherence rates secondary to social stigma, lack of social support, and the transition of medication responsibility from parent to adolescent. Compared to Caucasians, decreased adherence rates were demonstrated in lowincome ethnic minorities and African Americans. In third-world countries, adherence is adversely affected by inadequate education, lack of access to affordable AEDs and cultural beliefs. Newer AEDs generally result in better adherence due to fewer side effects and decreased drug interaction potential, although one study showed that adherence was associated with patient satisfaction with therapy efficacy despite AED side effect profile. Less frequent dosing has been associated with improved adherence in most studies, although patients on monotherapy may also demonstrate poorer adherence than patients on polytherapy. This may be due to the fact that polytherapy patients have poorly controlled seizures, which provides a greater impetus to maintain good adherence.

Conclusions: Suboptimal adherence to AEDs among epilepsy patients has serious consequences, including increased morbidity, mortality, and healthcare cost utilization. Adherence is affected by features unique to each demographic group, as well as to AED characteristics including monotherapy versus polytherapy, side effect profile, and dosing schedule. Strategies for improved adherence should account for the individual characteristics of each patient. However, there is a significant need for further studies to better understand non-adherence patterns among patients, and to develop effective interventions in order to optimize medical management.

\subsection{3}

\section{ARE PLASMA LEVELS OF LACOSAMIDE OF ANY VALUE?}

Birthe Pedersen and J. B. Rasmussen (The Danish Epilepsy Centre, Dianalund, Denmark)

Rationale: Lacosamide (LCM) is a novel antiepileptic drug approved for use as add-on therapy in partial epilepsy and works as a slow acting sodium channel blocker.

Does plasma levels of LCM correlate to doses of LCM.

Methods: To determinate LCM a high performance liquid chromatography (HPLC) rutine method with UV detection was used. Ninety-three measurements in 70 patients with patial epilepsy and on LCM were performed.

Results: Despite the limited number of determinations the plasmalevels of LCM showed a clear correlation and a therapeutic level of LCM can be proposed to be $12-35$ micromoles $/ \mathrm{ml}$ with a $80 \%$ cut off level

Conclusions: The suggested therapeutic interval for LCM needs to be validated by measurements in more patients

\subsection{4 \\ EFFICACY AND TOLERABILITY OF ADJUNCTIVE ESLICARBAZEPINE ACETATE IN ADULTS WITH DRUG- RESISTANT FOCAL EPILEPSIES IN A PORTUGUESE EPILEPSY CENTER}

A. P. Breia Neves and N. Ferreira (Neurology, Hospital Garcia de Orta, Almada, Portugal)

Rationale: Eslicarbazepine Acetate (ESL) is a voltage-gated sodium and calcium channel blocker approved by the European Medicines Agency in 2009 and available in Portugal since February 2010, indicated as adjunctive therapy in adults with partial onset seizures (POS) with or without secondary generalization. Observational studies can be useful adjuncts to randomized, controlled trials to determine whether the demonstrated efficacy translates into effective treatment in routine clinical practice. The aim of this retrospective study was to evaluate the efficacy and tolerability of adjunctive ESL in adults with refractory POS treated in our epilepsy center. Methods: All patients treated in our center that started ESL from February 2010 till December 2012 were included. All subjects underwent periodic clinical and instrumental assessments. Retrospectively we collected information regarding demographics, epilepsy history and response to ESL treatment, including seizure frequency, tolerability and ESL withdrawal.

Results: Our analysis included 34 patients: 14 (41.2\%) were male, mean age was 40.0 years. At the start of ESL therapy mean epilepsy duration was 21.7 years and mean seizure frequency per 4 weeks was 34.6. Most frequently used concomitant AEDs (conAEDs) were: valproic acid $(18 ; 52.9 \%)$, levetiracetam $(14 ; 41.2 \%)$, lamotrigine (12; 35.3\%) and carbamazepine (9; 26.5\%). Sixteen (47.1\%) patients were treated with 1-2 conAEDs and the remaining with more than 2. Most frequent etiologies were: idiopathic $(9 ; 26.5 \%)$, mesial sclerosis $(6 ; 17.6 \%)$, head trauma $(4 ; 11.8 \%)$ and cerebrovascular disease $(4$; $11.8 \%$ ). The main reasons to start therapy with ESL were refractoriness without epilepsy surgery indication $(20 ; 58,8 \%)$ or refractoriness and adverse effects with previous drugs (14, 41,2\%). At the last follow-up (FU) visit, 23 (67.6\%) patients were still on therapy (mean FU duration was 496 days, range 152 - 791). Patients were taking the following ESL daily dosages: $800 \mathrm{mg}(16 ; 69.6 \%)$, $1200 \mathrm{mg}(4 ; 17.4 \%), 400 \mathrm{mg}(2 ; 5.9 \%)$ or $1600 \mathrm{mg}(1 ; 2.9 \%)$. Main reasons for ESL withdrawal were: lack of efficacy $(5 ; 14.7 \%)$, adverse events $(2 ; 5.9 \%)$ or both $(3 ; 8.8 \%)$. Reduction in seizure frequency was $\geq 50 \%$ in $21(61.8 \%)$ patients and $\geq 75 \%$ in $16(47.1 \%)$. Three $(8.8 \%)$ patients were seizure free for more than 6 months. In patients still on ESL therapy $(n=23)$ median relative reduction in seizure frequency was $88.0 \%$; physicians rated "Clinical global impression of change - Global Improvement" as "very much improved" or "much improved" in 21 (91.3\%) patients.

Conclusions: ESL showed to be effective and well tolerated in this refractory population of adult patients with partial-onset seizures with or without secondary generalization treated at our center.

Nevertheless the conclusions are limited by the retrospective nature of the study and also by the reduced number of subjects included.

\subsection{5 \\ RECRUITING ELDERLY NURSING HOME SUBJECTS FOR ANTIEPILEPTIC DRUG STUDIES}

A. K. Birnbaum ${ }^{1}$, J. O. Rarick ${ }^{1}$, T. K. Pettus ${ }^{2}$, J. W. Mielke ${ }^{3}$, T. C. McCarthy ${ }^{2}$ and I. E. Leppik ${ }^{1,4}$ ( ${ }^{1}$ Experimental and Clinical Phamacology, University of Minnesota, Minneapolis, MN; ${ }^{2}$ Walker Methodist Health Care Center, Minneapolis, MN; ${ }^{3}$ North Ridge Care Center, Minneapolis, MN and ${ }^{4} \mathrm{MINCEP} \AA$ Epilepsy Care, Minneapolis, MN)

Rationale: There are over 1.6 million elderly nursing home residents in the US with approximately $10 \%$ being treated with antiepileptic drugs (AED). Our long-term objective was to create a viable recruiting strategy in a network of nursing homes that requires a 
minimal amount of nursing home staff time. This report provides an updated estimate of recruitment rates under the current HIPAA rules. Methods: The Institutional Review Board at the University of Minnesota and the equivalent entity at each nursing home approved this prospective study of the causes and consequences of fluctuating AED levels (Birnbaum, A., et al. Neurology 2003;60:555-559; Birnbaum, A.K., et al. Epilepsy Research 2012 e published). Inclusion criteria are nursing home residents who are taking one of four AEDs (phenytoin, levetiracetam, lamotrigine, gabapentin) as part of current clinical care and who are age greater than or equal to 60 years. Due to current HIPAA rules and limited access of study personnel, a recruitment procedure was created. The current strategy consists of a pharmacist or other employee with access to medication dispensing records in the nursing home, screening for patients meeting the above criteria and sending an unidentified list for our review. Upon our approval, potential subjects or guardians were approached by nursing home staff and asked if our study team could speak with them concerning a study. If subject/guardian agreed, a pre-consent form was signed to indicate that our team could contact them. Their names and contact information were then forwarded to our research coordinator at the University of Minnesota. Eleven nursing homes in Minnesota, New York and California are participating in this study; however, only those homes in Minnesota $(n=9)$ are included in this analysis since the recruiting strategy was identical in those cases.

Results: A total of 524 subjects were identified via pharmacy records as potential subjects. Of these, $275(53 \%)$ were approached by nursing home staff and 183 (35\%) signed pre-consent forms. Due to factors such as not meeting inclusion criteria, becoming deceased, or refusal of resident after guardian permission $113(22 \%)$ residents and/or guardians signed full consent forms and entered the study. The percent of residents recruited from a particular nursing home ranged from 16.3 to $50.0 \%$ with the average being $29.2 \%$.

Conclusions: There is variability in recruitment among nursing homes. A major barrier to recruiting subjects in a nursing home is the need to have nursing home staff approach potential subjects. This creates a system that is dependent on enthusiasm and time availability of personnel.

Funded by NIH NIA 1R01AG026390-0

\section{Surgery: Adult}

\subsection{6}

\section{ANTERIOR TEMPORAL LOBE DISCONNECTION IN} MESIAL TEMPORAL LOBE EPILEPSY

Salman Abdul Qayyum, K. A. Siddiqui, E. Khalid, A. J. Sabbagh, L. Soualmi and S. Sinha (Epilepsy Program, Department of Neurology, National Neuroscience Institute, King Fahad Medical City, Riyadh, Saudi Arabia)

Rationale: Temporal lobe epilepsy (TLE) is the most common form of refractory epilepsy. Studies have shown superiority of temporal lobectomy over medical therapy in terms of the control of seizures and the quality of life in TLE. Standard technique of Anterior Temporal Lobectomy can lead to complications such as brain shift, hematoma into the post operative cavity, superior quadrantanopia, cranial nerve palsies and stroke. In addition, this procedure requires a larger craniectomy, is more traumatic and prolongs hospital stay. We report our experience of selective Amygdalo-hippocampectomy (sAH) followed by anterior temporal lobe disconnection in patients with lesional mesial temporal lobe epilepsy.

Methods: We retrospectively carried out an observational cohort study at National Neuroscience Institute, King Fahad Medical City. The patients recruited went through a comprehensive presurgical epilepsy evaluation and were concluded as good candidate for temporal lobe resective surgery. Surgeries were done under MRI neuronavigation in Brain Suite ${ }^{\mathrm{TM}}$. Small craniotomy $3.5 \mathrm{~cm} \times 3.5 \mathrm{~cm}$ was made in the temporal area on the diseased side, (figure A).
Electrocorticography (ECoG) was done pre-resection from the anterior lateral temporal neocortex. Following that sAH was done via middle temporal gyrus approach. Post sAH, ECoG was done to see any residual epileptiform discharges, if still firing, then the anterior temporal disconnection was carried out. The disconnection was done along the imaginary line drawn from the central point (point of intersection between Central Sulcus to the Sylvian Fissure) to the base of temporal lobe,(figure B\& C).

Results: We found 6 patients who underwent sAH along with anterior Temporal lobe disconnection between Nov 2008 and May 2012. There were 4 male and 2 females with mean $( \pm$ SD) age of 27.8 $( \pm 4.8)$ years and duration of epilepsy as $19.7( \pm 9.6)$ years, (table). Five procedures were done on the left side. No intraoperative and immediate post operative complications were seen. Histopathology showed mesial temporal sclerosis in 4 , focal cortical dysplasia in 1 and pilocytic astrocytoma in 1 patient. Average follow up duration was 14.3 months (range 1- 43 months). All patients have been seizure free post op, with two patients being seizure free for more than 24 months.

Conclusions: Selective Amygdalo-hippocampectomy and temporal lobe disconnection under MRI neuronavigation and electrocorticography guidance is safe and effective surgical treatment modality for refractory mesial temporal lobe epilepsy, and may be a possible alternate for standard procedure.

Patient Characteristics

\begin{tabular}{|c|c|c|c|c|}
\hline & $\begin{array}{l}\text { Age(years) } f \\
\text { Gender }\end{array}$ & $\begin{array}{c}\text { Duration of } \\
\text { epilepsy (years) }\end{array}$ & $\begin{array}{l}\text { Follow-up } \\
\text { (months) }\end{array}$ & Histopathology \\
\hline 1 & $26 / \mathrm{M}$ & 20 & 43 & Mesial temporal sclerosis \\
\hline 2 & $32 / \mathrm{F}$ & 26 & 32 & Pilocytic astrocytoma \\
\hline 3 & $22 / \mathrm{M}$ & 2 & 7 & Mesial temporal sclerosis \\
\hline 4 & $23 / \mathrm{M}$ & 19 & 2 & Focal cortical dysplasia \\
\hline 5 & $33 / \mathrm{M}$ & 30 & 1 & Mesial temporal sclerosis \\
\hline 6 & $31 / \mathrm{F}$ & 21 & 1 & Mesial temporal selerosis \\
\hline
\end{tabular}
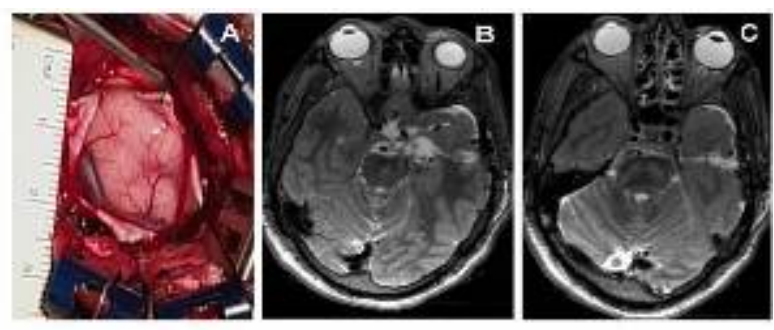

Figure: Craniectomy incision (A), Axial T2W MRI showing selective Amygdalo-hippocampectomy (B) and Axial T2W MRI showing anterior temporal lobe disconnection (C)

\subsection{7 \\ LONG-TERM OUTCOME AFTER MULTIPLE HIPPOCAMPAL TRANSECTION FOR TEMPORAL LOBE EPILEPSY}

Kenichi Usami, K. Kawai, M. Kubota and N. Saito (Graduate School of Medicine, the University of Tokyo, Tokyo, Japan)

Rationale: Mesial temporal lobectomy for drug resistant intractable mesial temporal lobe epilepsy (mTLE) can achieve favorable seizure outcome but may conduce to memory decline especially in the patients in whom hippocampal sclerosis is not apparent in verbal dominant side. Multiple hippocampal transection (MHT) is a novel surgical procedure to avoid memory decline after mesial temporal lobe surgery. The seizure outcome of MHT at one year after surgery is equivalent to that of mesial temporal lobectomy, and the memory indices were preserved at the preoperative level. However, the longterm outcome after MHT has not been clarified. In this study, we report long-term outcome of our patients after MHT in terms of seizure control and cognitive function, and discuss the significance of MHT in the surgical treatment for mTLE. 
Methods: 24 patients who underwent MHT for drug resistant intractable mTLE were followed for longer than 5 years, including 6 patients with hippocampal sclerosis, 5 with other lesions, and 13 without lesion in preoperative MRI. All patients underwent MRI, rCBF-SPECT, FDG-PET, and video EEG monitoring before surgery. When the side of epileptic focus could not be determined from noninvasive evaluations, intracranial electrodes were implanted bilaterally. Seizure outcome was evaluated using Engel's classification at one year after surgery and at the last visit. Cognitive functions were evaluated with Wechsler Memory Scale-Revised (WMS-R) and Wechsler Adult Intelligence Scale-III (WAIS-III) before surgery and one month, 6 months, and 5 years after surgery. Results: Mean follow-up period was 77 months. Seizure outcomes were Engel I/II/III in $71 \% / 25 \% / 4 \%$ of patients at one year, and $67 \% / 21 \% / 13 \%$ at the last visit. Four $(23.5 \%)$ of 17 patients who were Engel I at one year had seizure recurrence at the last visit. Memory indices were at the preoperative level at 6 months after transient decline at one month postoperatively, and were kept at the level at 5 years after surgery.

Conclusions: Long-term seizure outcome after MHT was considered slightly worse than that of temporal lobectomy, while cognitive function was well preserved even after dominant-sided MHT. MHT is a significant option for patients with mTLE in whom respective surgery carries a risk of postoperative cognitive decline.

\subsection{8}

EXECUTIVE FUNCTIONS ARE PROCESSED IN THE AREA OF ANTERIOR NUCLEUS OF THALAMUS. AN INTRACEREBRAL RECORDING STUDY

I. Rektor ${ }^{1}$, M. Bockova ${ }^{1}$, J. Chladek ${ }^{2}$, P. Jurak ${ }^{2}$, J. Halamek ${ }^{2}$, J. Stillova ${ }^{1}$, M. Balaz ${ }^{1}$ and J. Chrastina ${ }^{1}\left({ }^{1}\right.$ First Department of Neurology and Department of Neurosurgery, Central European Institute of Technology (CEITEC); Masaryk University, St. Anne's Hospital, Brno,, Czech Republic and ${ }^{2}$ Institute of Scientific Instruments of the Academy of Sciences of the Czech Republic, Brno, Czech Republic)

Rationale: This is to our knowledge the first study reporting the cognitive- related local field potentials recorded directly in the AN. The anterior nucleus of thalamus (AN) is a new target for deep brain stimulation (DBS) in epilepsy. The aim of this work was to inquire whether the AN area might participate in executive functions. Methods: Three pharmacoresistant epilepsy patients implanted with AN - DBS electrodes performed two tasks involving the writing of single letters: 1 . copying letters from a monitor; 2 . writing of any other letter than that appearing on a monitor.

Results: The cognitive load of the second task was increased by larger involvement of the executive functions. The task-related potentials (ERPs) and oscillatory changes were assesed. The local field ERPs and significant increase in lower gamma activity were elicited by the two tasks. Local event-related alpha and beta desynchronizations were more expressed during the second task. Conclusions: The AN together with frontal and temporal areas participate in cognitive networks processing complex visuomotor tasks. AN participates in network processing the executive functions. Therefore the AN stimulation could influence performance of higher mental cognitive operations. More attention should be paid to complex cognitive and particularly executive functions in subject undergoing AN DBS.

\subsection{9 \\ EPILEPSY SURGERY IN PATIENTS WITH EPILEPSY AND NEUROCYSTICERCOSIS}

Alejandro L. Escalaya, D. A. Steven and J. G. Burneo (Clinical Neurological Sciences, University of Western Ontario, London, ON, Canada)

Rationale: The majority of patients with neurocystircercosis (NCC)related epilepsy are responsive to antiepileptic treatment, however refractory cases do occur. Although cases of NCC with refractory epilepsy have been reported sporadically in the medical literature, surgical treatment has rarely been reported. We present our experience in two patients with medically refractory NCC-related epilepsy who underwent surgery.

Methods: The database of the Western Epilepsy program was interrogated and 2 cases were identified. Both cases underwent complete presurgical evaluation after it was established that more than 2 antiepileptic drugs were not successful controlling their seizures.

Results: The first case was a 25 year-old man who had right MTS as well as 3 extratemporal calcified cysticerci. He was a Canadian Mennonite with frequent travels to Mexico. Initial presurgical evaluation indicated possible frontal epilepsy, but subsequent placement of intracranial electrodes identified that seizures arose from the right mesial temporal lobe. He underwent a right temporal lobectomy and has been seizure free for over 2 years. The second case was a 30 year-old female, originally from Colombia, who had a cystic lesion (NCC) in the left hippocampus. A lesionectomy and left anterior temporal lobectomy significantly improved her seizures, as she only has occasional auras.

Conclusions: Both cases are examples that in some situations NCCrelated epilepsy can be refractory to antiepileptic treatment, hence surgical treatment should be considered. In the first case, it was unclear if NCC had a causative role in the development of MTS.

\subsection{0}

\section{SMALLER GRID SIZE REDUCES COMPLICATION} DURING INTRACRANIAL ELECTRODE RECORDING Z. Rahman ${ }^{1}$, C. Wong ${ }^{1,3}$, J. Birkett $^{1}$, M. Bartley ${ }^{1}$, T. Galea ${ }^{3}$, S. Soe ${ }^{3}$, M. Dexter ${ }^{2}$, D. Gill ${ }^{3}$, K. Byth ${ }^{4}$ and A. F. Bleasel ${ }^{1,3}$ ( ${ }^{1}$ Neurology, Westmead Hospital, Sydney, NSW, Australia; ${ }^{2}$ Neurosurgery, Westmead Hospital, Sydney, NSW, Australia; ${ }^{3}$ Neurology, Children's Hospital at Westmead, Sydney, NSW, Australia and ${ }^{4}$ Biostatistics, Millenium Institute, Sydney, NSW, Australia)

Rationale: Epilepsy surgery is increasingly offered to patients with extra-temporal non-lesional epilepsy. Inracranial EEG monitoring plays an important role in determining the epileptogenic zone and adjacent eloquent cortex. Intracranial EEG monitoring may be associated with significant morbidity. Following a study of the risk factors associated with complications during intracranial EEG monitoring at the Westmead campus (Acta Neurochirurgica 2009; 151:37-50) we created a revised protocol for implantation. Including: one to one nursing ratio, hourly observations for the initial 48 hour postoperatively, continuous heart rate and blood pressure, oxygen saturation and intracranial pressure monitoring, An external ventricular drain provides continuous ICP monitoring. PICC lines inserted in all patients. Post-operative MRI preformed 2-4h following implantation of the electrodes before transfer to the monitoring ward. An epilepsy trained registered nurse replaced the patient's relative as the sitter. Subdural electrode arrays not larger than $4 \times 8$ grid arrays were used.

Methods: All patients who underwent invasive monitoring at Westmead Hospital and the Children's Hospital at Westmead between 1988 and 2004 (Gp A) were compared with those done after the protocol changes; between 2005 and 2012 (Gp B).

Complications were categorised according to the relationship to the electrodes, outcome and the need for intervention. In those time periods a total of 296 patients (Gp A period) and 254 patients (Gp B period) underwent epilepsy surgery. All patients had intracranial monitoring because non-invasive investigations did not produce adequate localising information or the focus was adjacent to eloquent cortex.

Results: 71 (median age: 24 years) patients in Gp A and 52 patients in $\mathrm{Gp} \mathrm{B}$ (median age: 24 years) underwent intracranial electrode implantation. Transient complications requiring no treatment; Gp A $13 \%$ vs Gp B 2\%. Transient complications requiring treatment; Gp A 
$10 \%$ vs Gp B 8\%. Death occurred in Gp A ( $n=2)$ but not in Gp B. Indirect systemic complications in Gp A were $11 \%$ with none in $\mathrm{Gp}$ B. Complications directly related to intracranial EEG monitoring were significantly reduced using the revised protocol $(p=0.001)$. Infection rate was increased in Gp B (5.7\%) compared with Gp A $(2.8 \%)$, not statistically significant $(\mathrm{p}=0.90)$. Monitoring of continuous ICP prevented catastrophe in one case. Raised ICP prompted an urgent CT scan showing a $11.7 \mathrm{~mm}$ subdural collection and a new area of left frontal lobe hypodensity. Urgent removal of the subdural grids and resection of epileptogenic focus was followed by recovery. Histology confirmed venous infarction.

Conclusions: Complication rates following intracranial implantation have been reduced after the introduction of our new protocol. Intracranial infection was increased in $\mathrm{Gp} \mathrm{B}$ in comparison to $\mathrm{Gp} \mathrm{A}$. This maybe related to routine use of external ventricular drainage and further review is warranted.

\subsection{1}

\section{PSYCHOGENIC NONEPILEPTIC SEIZURES AFTER ADULT} EPILEPSY SURGERY

Sofia Markoula ${ }^{1,2}$, J. de Tisi ${ }^{1}$, J. Foong ${ }^{1}$ and J. S. Duncan ${ }^{1}$ $\left({ }^{1}\right.$ Department of Clinical and Experimental Epilepsy, University College London Institute of Neurology, National Hospital for Neurology and Neurosurgery, London, United Kingdom and ${ }^{2}$ Neurology, Medical School, University of Ioannina, Ioannina, Greece)

Rationale: Surgery is increasingly used as treatment for refractory focal epilepsy. Only a few studies have examined the development of psychogenic nonepileptic seizures (PNES) after epilepsy surgery. Our aim was to identify the factors associated with occurrence of PNES after adult epilepsy surgery.

Methods: We identified patients diagnosed with PNES from the long-term outcome data of 805 patients who underwent epilepsy surgery between 1990 and 2012, at the National Hospital for Neurology and Neurosurgery (NHNN), London, UK.

The diagnosis was clinical in all cases, further confirmed by videoEEG monitoring in doubtful cases.

Multivariate analysis was performed comparing the characteristics of the PNES patients to the other epilepsy surgery patients.

Patients' gender, the patients' age and duration of epilepsy at the time the surgery was performed, pre-surgical psychiatric co-morbidities including depression and seizure outcome after surgery were used as covariates.

Results: The frequency of patients developing PNES after epilepsy surgery in our series was $3.23 \%$. Specifically, twenty six patients $(20$ women, $76.9 \%$ ) were identified and recruited in the study, with 20 anterior temporal resections, 2 temporal lesionectomies, 3

extratemporal lesionectomies and 1 multiple subpial transaction were performed (one patient had undergone anterior temporal resection twice).

Patients had undergone surgery at a median age of $35 \mathrm{y}$ (range 18-50 $y)$ and there was a wide interval between the date of surgery and development of PNES ( 6 weeks - 10 years). The frequency of PNES was also variable, and the semiology differed from the occasional epileptic seizures in most patients.

Fourteen of the PNES patients had comorbid depression or psychosis, whereas in the remaining 12 patients, PNES developed independently of other psychiatric disorders.

Statistical analysis revealed that female gender and the pre-surgical presence of depression were significantly associated with PNES. After multivariate analysis, with all the aforementioned parameters in the model, the female gender was the only parameter independently associated with dissociative seizures $(\mathrm{p}=0.025, \mathrm{OR}=2.89)$.

Conclusions: PNES can occur after epilepsy surgery and do not appear to be related to postsurgical seizure outcome.

In patients who continue to have epileptic seizures, the semiology of the PNES tends to differ from that of their epileptic seizures.
Female patients are at greater risk of developing PNES after epilepsy surgery.

\subsection{2 \\ PET FINDINGS AFTER HIPPOCAMPAL DEEP BRAIN STIMULATION (HIP-DBS)}

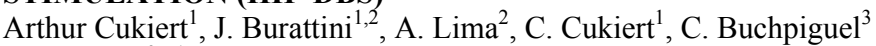
and C. Ono ${ }^{3}{ }^{1}$ Neurology and Neurosurgery, Clinica de Epilepsia de Sao Paulo, Sao Paulo, Brazil; ${ }^{2}$ Neurology and Neurosurgery, Hospital Brigadeiro, Sao Paulo, Brazil and ${ }^{3}$ Nuclear Medicine Institute, University of Sao Paulo, Sao Paulo, Brazil)

Rationale: Deep brain stimulation has been used in an increasing frequency for treatment of selected patients with refractory epilepsy who were not adequate candidates for conventional resective surgery. Hippocampal DBS (Hip-DBS) has been offered in the treatment of refractory temporal lobe epilepsy. Although there were reports showing that Hip-DBS was indeed efficacious, little is known about the mechanism of action of Hip-DBS. We studied patients submitted to Hip-DBS in the pre- and post-electrode activation periods. Methods: Three adult patients with refractory temporal lobe epilepsy were studied. Patient 1 had refractory temporal lobe epilepsy (r-TLE) associated with left mesial temporal sclerosis (MTS) and was submitted to left Hip-DBS using a Soletra device. Patient 2 had rTLE associated with post-herpetic encephalitis bilateral temporal atrophy. His video-EEG showed bitemporal interictal discharged, and ictal onset prevailing on the left side. He was submitted to bilateral Hip-DBS using a Kinetra device, although only left Hip-DBS was activated during this study. Patient 3 had r-TLE and normal MRI. His video-EEG showed bitemporal interictal discharged, and ictal onset prevailing on the left side. He was submitted to bilateral Hip-DBS using a Kinetra device, although only left Hip-DBS was activated during this study. All patients were submitted to pre- and (3 months) post-activation PET studies. PET findings were blindly reported. All patients had their electrode's location confirmed by post-operative CT scanning.

Results: Seizure frequency was reduced in all patients: in Patient 1 from 6 to 0.5/month; in Patient 2 from 16 to 4 / month and in Patient 3 from 8 to $1 /$ month. Final parameters were $2.7 \mathrm{~V}, 1.0 \mathrm{~V}$ and $3.0 \mathrm{~V}$ for patients 1,2 and 3 , respectively ( $300 \mathrm{usec}, 130 \mathrm{~Hz}$, continuous stimulation). In Patient 1, post-activation PET showed marked additional hypometabolism in both temporal lobes, mesial frontal cortices and thalamus. In Patient 2, post-activation PET showed additional hypometabolism within the stimulated temporal lobe. In Patient 3, post-activation PET was similar to the pre-activation one. Conclusions: This is very likely the first description of PET findings after Hip-DBS. Although the number of patients was very small and the etiology was heterogeneous, this study showed that Hip-DBS was able to generate additional hypometabolism within the stimulated temporal lobe and Papez circuit in some patients. At this point, we were not able to correlate these findings with clinical outcome regarding seizures, electrode positioning or stimuli intensity.

\subsection{3 \\ SURGICAL OUTCOMES FOR REFRACTORY PARTIAL EPILEPSY: A REVIEW}

D. R. Vannan, E. J. Bubrick and Barbara Dworetzky (Brigham and Women's Hospital, Boston, MA)

Rationale: Surgical treatment of medically-refractory partial epilepsy has been shown to be associated with better outcomes than medication alone in appropriately-selected patients. With advances in pre-surgical procedures, we sought to determine our outcomes following epilepsy surgery for refractory partial epilepsy.

Methods: Combined data from noninvasive measures including interictal/ictal scalp EEG, MRI, and PET were utilized to identify epileptogenic zones. When noninvasive methods produced 
insufficient or inconclusive data, intracranial EEG monitoring wer performed. This data was prospectively entered into a clinical database. We queried this database, and reviewed all epilepsy surgeries from 1993 - 2011 at our tertiary care facility. Data from 155 patients who underwent surgical treatment were included in the study. Outcome data was reported from a period at or beyond 1 3 years after surgery, using the Engel criteria. Patients with malignant neoplasms and lack of sufficient outcomes were excluded. For our review we collected surgical outcomes and pathologies of our patients.

Results: Of the 150 patients, $110(73.3 \%)$ were temporal lobe resections (TLR), 25 (16.7\%) were extratemporal resections (ETR), 6 $(4.0 \%)$ were callosotomies, and $9(6.0 \%)$ were multiple subpial transections (MSR). Of the 110 temporal lobe epilepsy cases $75(68.2 \%)$ were seizure free (Engel 1), and 21 (19.1\%) showed improvement (Engel 2, 3). Of the 25 extratemporal cases, 12 (48.0\%) were seizure free (Engel 1), while $13(52.0 \%)$ showed improvement (Engel 2, 3).Of the 155 patients $31(20 \%)$ of the patients had definite mesial temporal sclerosis, $51(32.9 \%)$ had mild gliosis, $26(17.0 \%)$ had a cortical dysplasia or heterotropia, and $14(9.0 \%)$ had a benign neoplasm.

Conclusions: Our data confirms that in appropriately-selected patients with medically-refractory partial epilepsy, surgical treatment is associated with good outcomes in a majority of cases. Our data concludes that surgical intervention for refractory partial epilepsy effective.

\subsection{4}

\section{SURGICAL TREATMENT FOR GENERALIZED EPILEPSY: EXPERIENCE FROM A LARGE COMPREHENSIVE} CANADIAN EPILEPSY PROGRAM

David A. Steven, J. G. Burneo, R. S. McLachlan, S. Mirsattari, D. Diosy, A. G. Parrent, W. T. Blume, S. de Ribaupierre and K. W. MacDougall (Clinical Neurological Sciences, Western University, London, ON, Canada)

Rationale: Although usually reserved for focal epilepsies, surgical treatment is occasionally utilized in cases of therapy-resistant generalized epilepsy. Surgery for generalized epilepsy is infrequently reported. We present our experience with surgical treatment of generalized epilepsy, including long-term outcomes.

Methods: The Western Epilepsy Program Surgery Database was interrogated for all cases of surgically-treated generalized epilepsy. Consecutive cases, since the inception of the Epilepsy Program in 1977 until December of 2005, were included. The determination of the type of epilepsy was based on history as well as interictal and ictal findings on the EEG. MRI findings, type of surgery and outcome after surgery was analyzed. Patients were followed after surgery for up to 3 years.

Results: A total of 1066 epilepsy surgeries were performed. We identified 31 cases of surgically-treated generalized epilepsy (15 females). The average age at seizure onset was 14 (range: $5 \mathrm{~m}$ to 20 y), the mean age at surgery was 30.1 years (range: $10-42 y$ ). Eleven cases were non-lesional. 8 cases required implantation of intracranial electrodes as there was concern about co-existence of focal epilepsy. Concomitant focal epilepsy was found in six. Twenty-six patients underwent corpus callosotomy only, 3 had corpus callosotomy and focal resections, 2 had hemispherectomy, and 3 cases had a focal resection. Three $(10 \%)$ became seizure free after surgery (one focal resection and two callosotomies) at the last follow up (mean $=13.2 \mathrm{~m}$, range $1-36 \mathrm{~m}$ ). Data about seizure types and their response to the surgical treatment will be presented.

Conclusions: Although uncommonly performed, our experience indicates that surgical treatment for should also be considered for the treatment of medically-refractory generalized epilepsy in specific cases.

\subsection{5}

EFFICACY OF VAGUS NERVE STIMULATION IN BRAINTUMOR ASSOCIATED INTRACTABLE EPILEPSY AND THE IMPORTANCE OF TUMOR STABILITY

Kunal S. Patel ${ }^{1}$, N. Moussazadeh ${ }^{1}$, C. Gordon ${ }^{3}$, K. Hassnain ${ }^{3}$, D. Labar $^{1}$, W. Doyle ${ }^{2}$ and T. Schwartz ${ }^{1}\left({ }^{1}\right.$ Weill Cornell Medical College, New York, NY; ${ }^{2}$ New York University Langone Medical Center, New York, NY and ${ }^{3}$ Cyberonics Inc., Houston, TX)

Rationale: There is no literature or data on the efficacy of vagus nerve stimulation (VNS) in patients with brain tumor associated medically intractable epilepsy, a group which would benefit from this therapy.

Methods: The epilepsy surgery databases at two separate epilepsy centers were reviewed to identify patients in whom a VNS was placed for tumor-related intractable epilepsy between January 1999 and February 2011. In addition, data from the VNS Therapy Patient Outcome Registry maintained by the manufacturer of the device, Cyberonics Inc. (Houston, TX), was queried to compare patients with and without a history and/or etiology of brain tumors. Pre-operative and post-operative seizure frequency and type as well as antiepileptic drug (AED) regimens and degree of tumor progression were evaluated. A case control analysis was implemented and patient outcome was measured by Engel classification, median seizure response, and responder rate (i.e. percentage of patients with at least $50 \%$ seizure reduction or equivalently Engel I, II, or III). Univariate and multivariate statistical analysis was performed using odds ratio and $t$ tests to examine efficacy.

Results: Sixteen patients from the two epilepsy centers were included in the study. Seven patients (43.8\%) had an improved outcome (Engel I, II, or III) after an average follow-up of 39 months. The mean reduction in seizure frequency was $35.3 \%(\mathrm{p}=0.005)$. After eliminating four patients who went on to have further surgery for tumor progression, mean seizure reduction was $48.4 \%(\mathrm{p}=0.003)$. There was no significant change in AEDs. Seizure frequency decreased by $10.9 \%$ in patients in whom tumor progressed and $56.1 \%$ in patients with a stable tumor $(\mathrm{p}=0.034)$. The Outcome Registry analysis showed that in 196 brain tumor patients, VNS therapy reduced seizure frequency by $71 \%$ at 24 months with a $70 \%$ responder rate ( $>50 \%$ seizure reduction). In a case control study comparing patients with and without tumors, there was no statistical difference in response rates.

Conclusions: VNS therapy in individuals with brain tumor associated medically intractable epilepsy was shown to be equally effective at reducing seizure burden as in non-brain tumor patients through the first two years of treatment. Outcomes were notably better in patients with stable as opposed to progressing tumors. Therefore, these findings support the recommendation of VNS therapy in patients with brain tumor associated intractable epilepsy, especially in cases where imminent tumor progression is not expected.

\section{Surgery: All Ages}

\subsection{6}

CLINICAL OUTCOME OF A SERIES OF CALLOSOTOMIES Jose Pimentel ${ }^{1}$, C. Romero ${ }^{2}$, J. Ferreira ${ }^{2}$, R. Pinto ${ }^{2}$, A. Campos ${ }^{3}$, S. Quintas $^{4}$, C. Bentes ${ }^{5,1}$, N. Canas ${ }^{2}$, A. Ferreira ${ }^{3}$, J. Cabral ${ }^{2}$ and P. $\mathrm{Cabral}^{2}$ ( ${ }^{1}$ Department of Neurology, Hospital de Santa Maria (CHLN, EPE), Lisbon, Portugal; ${ }^{2}$ Deparment of Neuroscienses, Hospital de Egas Moniz (CHLO, EPE) (CHLN, EPE), Lisbon, Portugal; ${ }^{3}$ Department of Neurosurgery, Hospital de Santa Maria (CHLN, EPE) (CHLN, EPE), Lisbon, Portugal; ${ }^{4}$ Department of Pediatrics, Hospital de Santa Maria (CHLN, EPE) (CHLN, EPE), Lisbon, Portugal and ${ }^{5}$ Laboratory of EEG/Sleep (Department of Neurology), Hospital de Santa Maria (CHLN, EPE) (CHLN, EPE), Lisbon, Portugal) 
Rationale: As a palliative epilepsy surgery, callosotomy is mainly indicated in those patients with drop attacks and bilateral synchronous EEG discharges. However, as a less invasive procedure, vagus nerve stimulation (VNS) dramatically decreased its choice. Although in the absence of controlled studies comparing the two procedures, there is evidence for a more effective seizure outcome after callosotomy as compared with VNS (Nei M at al., Epilepsia 2006; 47:115-122; Cukiert A et al., Epilepsia 2009; 50:1377-1380). We aim to show callosotomy keep being a rational first strategy whenever properly indicated.

Methods: Retrospective, observational study of patients submitted to callosotomy in two hospitals, after pre-surgical evaluation in the scope of the respective epilepsy surgery groups. Seizures outcome assessment by using the follow scale: decreased $>75 \%, 50-75 \%, 25$ $-50 \%,<25 \%$. Post- surgery quality of live assessment by asking relatives whether surgery has been worse while or not. Comparison of the results with those of the VNS related literature.

Results: Twenty one patients, 17 males; epilepsy aetiology: LennoxGastaut syndrome (8), peri or postnatal injury (5), cortical development malformation (3), other or no identified causes (5); median age of surgery of 21.1 years; median time from epilepsy onset to surgery of 17.3 years; most frequent seizure types: atonics/ myoclonics (dropp- attacks- 22), atypical absences/ (10), tonics (7), focal (6), tonic-clonics (TC- 4); interictal EEG: multifocal (13), bilateral (13) and focal (3); extension of callosotomy: total in 12 and anterior $2 / 3$ in 9 ; surgery morbidity: transient disconnection syndrome (4), hydrocephalus (1), CSF fistula (1), transient focal deficits (3); post-surgery men time of follow-up: 4.0 years; decreased seizures (n=19): > 75\% (8/42.1\%), 50 - 75\% (4/21.0\%), 25 - 50\% (1), $<25 \%$ (6); most frequent decreased/suppression seizure types $(\mathrm{n}=19)$ : dropp- attacks (13/59\%), TC (4); seventeen $(89.4 \%)$ relatives $(n=19)$ considered surgery worse while (would not mind to repeat it). Conclusions: We confirm the efficacy of callosotomy in treating dropp-attacks, a mainly transient morbidity of this procedure, and a fair better clinical outcome when compared with the VNS relatedone.

\subsection{7 \\ NOVEL INSTRUMENTAL PLATFORM FOR PLANNING IMPLANTATION OF INTRACRANIAL ELECTRODES IN PATIENTS WITH EPILEPSY}

Roman Rodionov $^{1,2}$, A. Miserocchi ${ }^{2}$, C. Vollmar $^{3}$, C. Micallef ${ }^{1,2}, T$. Wehner $^{1,2}$, B. Diehl ${ }^{1,2}$, A. W. McEvoy ${ }^{1,2}$ and J. S. Duncan ${ }^{1,2}\left({ }^{1}\right.$ UCL Institute of Neurology, London, United Kingdom; ${ }^{2}$ National Hospital for Neurology and Neurosurgery, London, United Kingdom and ${ }^{3}$ Department of Neurology, University of Munich, Munich, Germany)

Rationale: We implemented a workflow and instrumental platform supporting the decision making process through clinical pathway of epilepsy patients undergoing surgical treatment. The platform is based on multimodal neuroimaging integration providing spatial landmarks ("aim" and "no go" areas) utilised during the key decision making stages: (1) generation of hypothesis about ictal onset zone, (2) decision making about implantation strategy, (3) developing implantation plan, (4) decision making and plan for resective surgery, (5) analysing post-surgical outcome. The crucial characteristic of the software tool in the core of our pipeline is high quality $3 \mathrm{D}$ visualisation of the brain and the landmarks. Here we present utilisation of our image processing pipeline for planning implantation of intracranial EEG (icEEG) electrodes.

Methods: Starting point: (1) multimodal landmarks (e.g. reconstructed $\mathrm{MEG} / \mathrm{EEG}$ source of epileptiform activity, white matter tracts, eloquent areas, lesions, defined as important for formulation of implantation strategy; (2) confirmed implantation strategy represented as set of brain areas that need to be sampled by icEEG and are accessible surgically, decision about dimensions and type of intracranial electrodes chosen for implantation. Procedure: The neurophysiologist and the neurosurgeon operate in the $3 \mathrm{D}$ environment carrying all neuroimaging information (landmarks for epileptogenic areas and eloquent cortex, crucial white matter tracts, brain anatomy, blood vessels, scalp) required to develop the implantation plan. The depth electrodes are modelled as 3D objects by specifying entry point on the scalp and target point in the suspected epileptogenic cortex (Figure 1a). The grid electrodes are modelled as segments of 3D surfaces shaped to fit the cortical surface and sized to the dimensions of the real grid electrode (Figure 1b). The resulted 3D models of the electrodes are uploaded on the neuronavigation system (Stealth, Medtronic ltd) to be available intraoperatively for guidance.

Results: This pipeline was utilised for planning depth electrode placement in four patients. The post-operative comparison of the location of the implanted electrodes showed that the intended cortical areas were sampled. The observed deviation of the electrode placement from the plan was due to lack of imaging information carrying details important for surgical decision making at the stage of pre-operative planning (for example, skull).

Conclusions: The utilisation of our instrumental pipeline for planning placement of intracranial electrodes demonstrated high potential for pre- and intra-operative use through high quality visualisation and variety of presented multimodal neuroimaging information available during surgery. Our experience showed that the pipeline presents an environment which is highly valuable for collaboration between epileptologists and neurosurgeons.
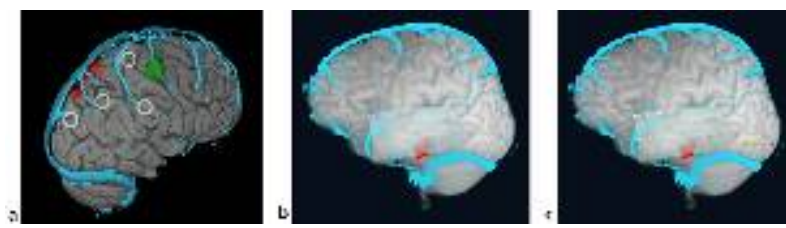

Figure 1. (a) Patient 1. 3D models of depth electrodes. Entry points circled, red - lesion, green - hand motor area, cyan - cortical veins. (b) Patient 2. Pre-implantation 3D model of the subdural grid electrode over temporal lobe. Red - lesion. (c) Patient 2. Postimplantation 3D model of actually implanted electrodes and 3D model of the planned subdural grid electrode location.

\subsection{8 \\ IMPROVED OUTCOMES WITH EARLIER SURGERY FOR INTRACTABLE FRONTAL LOBE EPILEPSY}

Thitiwan Simasathien, S. Vadera, W. Bingaman and L. Jehi (Cleveland Clinic Foundation, Cleveland hts, $\mathrm{OH}$ )

Rationale: Attention has traditionally been focused on the potential negative implications of long epilepsy duration prior to temporal lobe epilepsy surgery, but little is known about this question outside of the temporal lobe. Here, we investigate whether seizure outcomes following resective surgery for medically intractable frontal lobe epilepsy (FLE) also differ depending on age and disease duration. Methods: We reviewed patients undergoing FLE surgery for medically intractable epilepsy from January 1995 to October 2010 at Cleveland Clinic. At least 6 months of follow-up were required.

Traditional seizure outcome determinants were collected and results were analyzed using Cox-proportional hazard modeling controlling for surgical technique and extent of resection.

Results: 158 patients fulfilled study criteria and were analyzed. Half were children, and 53\% were male. As expected with FLE surgery, etiology was a major outcome determinant with tumor cases having the highest rates of seizure-freedom $(73 \%$ seizure-free at 5 years and beyond) and stroke and malformations of cortical development having the worst results (14\% and $41 \%$ respectively seizure-free at 5 years). Other expected outcome determinants were:1)- side of surgery (higher rates of seizure-freedom with nondominant resections; $\mathrm{p}=0.02$ ), and 2)- and presence of acute postoperative seizures ( $\mathrm{p}$ value $<0.0001$ ). On univariate analysis, age was also a significant seizure outcome determinant: $69 \%$ of children were seizure-free at 1 
year, $64 \%$ at 2 years, $52 \%$ at 5 years and beyond as opposed to $60 \%$ of adults at 1 year, $49 \%$ at 2 years, and $26 \%$ at 5 years and beyond $(\mathrm{p}=0.01)$, as was epilepsy duration with $64 \%$ seizure-free at 10 years if operated with less than 5 years epilepsy duration as opposed to $19 \%$ otherwise at the same time-point $(\mathrm{p}=0.0004)$. Following multivariate analysis, the following independent outcome predictors retained significance: epilepsy duration, side of surgery and presence of acute postoperative seizures (Whole model Log-Rank test $<0.0001)$. In addition, there was a tendency for earlier seizure recurrences in patients with longer epilepsy duration $(\mathrm{p}=0.06)$. Conclusions: This study suggests a possible epileptogenic effect of prolonged disease duration and recurrent seizures with extratemporal lobe epilepsy. The findings provide preliminary data to encourage and support a more aggressive and earlier practice of resective epilepsy surgery in medically intractable FLE patients.

\subsection{9 \\ SURGICAL INDICATION, PROCEDURE AND POSTOPERATIVE COURSE OF HIPPOCAMPAL \\ TRANSECTION}

Takehiro Uda and M. Morino (Neurosurgery, Tokyo Metropolitan Neurological Hospital, Fuchu, Japan)

Rationale: Although amygdalo-hippocampectomy is associated with good seizure outcome for the mesial temporal lobe epilepsy (MTLE), postoperative memory impairment has been a longstanding unresolved problem. Especially, in the case of MTLE without hippocampal atrophy or sclerosis, postoperative worsening of memory function is inevitable. To resolve this problem, hippocampal transection (HT) was developed and has conducted in some special institutions. In this report, we present the surgical indication, procedure and postoperative seizure and memory outcome of HT. Methods: Surgical indication of HT is determined considering following factors; (1) bilateral onset of seizures (2) lack of hippocampal atrophy or sclerosis in preoperative MRI (3) lack of memory score decline. From 2005 to 2012 , we performed HT in 38 cases (aged from 9 to 63, 19 men and 19 women, 19 in dominant side and 19 in non-dominant side). Of 38 cases, seven cases were associated with other intracranial lesion (tumor, cavernoma or cortical dysplasia)

HT was conducted with trans-sylvian approach aimed for less invasiveness. After approaching the hippocampal formation via temporal stem, we cut the alveus perpendicular to long axis of hippocampus in $5 \mathrm{~mm}$ interval and transect the pyramidal cell layer along to the incision of alveus. Seizure outcome was evaluated with Engel's classification. Memory outcome was evaluated with Wechsler Memory Scale-Revised (WMS-R) comparing pre and postoperative state.

Results: There were no surgical complications. Engel's class I (free of disabling seizures) was achieved in 74\% (28/38). In the nondominant side group, all indices (verbal memory, non-verbal memory, general memory, delayed recall and attention/concentration) were preserved postoperatively. In the dominant side group, verbal memory and delayed recall were temporarily declined (one month postoperatively), but recovered in one year, while another indices including non-verbal memory were preserved postoperatively.

Conclusions: Favorable seizure outcome and memory outcome were achieved with HT. We concluded that HT is an effective surgical procedure especially in temporal lobe epilepsy without hippocampal atrophy or sclerosis.

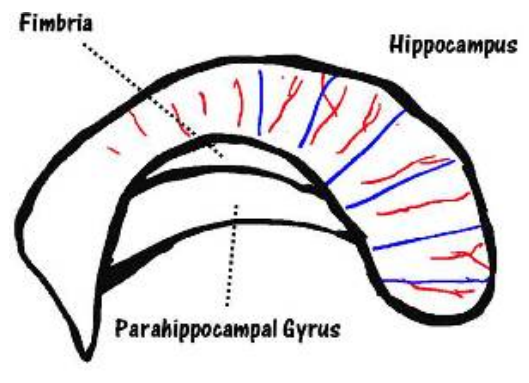

Blue : Transection lines

Shematic drawing illustrates transection lines being placed perpendicular to long axis of hippocampus ( $5 \mathrm{~mm}$ interval).

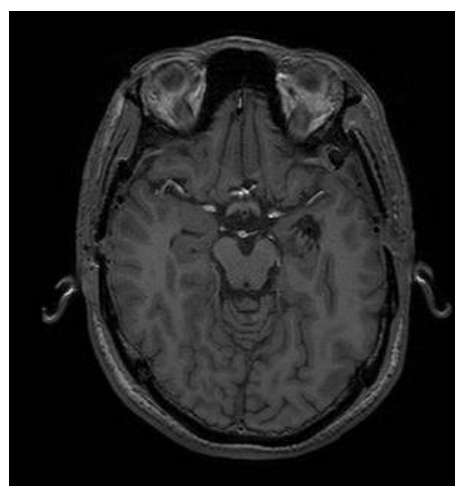

MRI after hippocampal transection showing transected hippocampus on the left side.

\subsection{0 \\ EPILEPSY SURGERY IN PATIENTS WITH MALFORMATIONS OF CORTICAL DEVELOPMENT AND HIPPOCAMPAL ABNORMALITIES}

Giorgi Kuchukhidze $^{1,2}$, I. Unterberger ${ }^{1}$, J. Dobesberger ${ }^{1,9}$, G. Walser ${ }^{1}$, E. Haberlandt ${ }^{3}$, F. Koppelstaetter ${ }^{4}$, H. Maier ${ }^{5}$, M. Ortler ${ }^{6}$, T. Czech ${ }^{7}$, M. Feucht ${ }^{8}$, G. Bauer ${ }^{1}$ and E. Trinka ${ }^{1,9}\left({ }^{1}\right.$ Neurology, Medical University of Innsbruck, Innsbruck, Tyrol, Austria; ${ }^{2}$ Neuroradiology, Medical University of Innsbruck, Innsbruck, Austria; ${ }^{3}$ Pediatrics, Medical University of Innsbruck, Innsbruck, Austria; ${ }^{4}$ Radiology, Medical University of Innsbruck, Innsbruck, Austria;

${ }^{5}$ Neuropathology, Medical University of Innsbruck, Innsbruck, Austria; ${ }^{6}$ Neurosurgery, Medical University of Innsbruck, Innsbruck, Austria; ${ }^{7}$ Neurosurgery, Medical University of Vienna, Vienna, Austria; ${ }^{8}$ Pediatrics, Medical University of Vienna, Vienna, Austria and ${ }^{9}$ Neurology, Paracelsus Medical University of Salzburg, Salzburg, Austria)

Rationale: Hippocampal abnormalities (HA) may coexist with malformations of cortical development (MCD), constituting dual pathology. This study aimed to explore the rate of epilepsy surgery and post-surgical outcome in patients with solely MCD compared to those with "dual pathology".

Methods: We identified 228 patients $(120 \mathrm{w} / 108 \mathrm{~m})$ aged $2-75$ years (mean 29.5 years) with MCD and epilepsy. MCD without HA were seen in $149 / 228(65 \%)$ of patients (group I). HA were identified in $79 / 228$ (35\%) patients (group II). All patients were clinically examined, underwent EEG and MRI.

Results: In group I, 66/149 (44\%) patients underwent presurgical assessment, 33/66 (50\%) - underwent epilepsy surgery for refractory seizures and 17/33 (51\%) have been seizure free for more than 12 months following surgery (Engel, Ia; Wieser,1a). The spectrum of MCD in patients of group I, who underwent surgery, was the following: dysplastic tumours - 17, focal cortical dysplasia - 12, tuberous sclerosis - 4. The majority of these MCD (26/33, 79\%) was located in temporal lobe. Surgery types in group I were the following: Lesionectomy (sparing the mesial temporal structures) -15/33 patients, standard resection of anterior two-third of temporal lobe 
(including the mesial temporal structures) - in 9/33 and modified resection of temporal lobe (including the mesial temporal structures) - in 9/33 patients. In group I, seizure freedom correlated neither with performed surgery type nor MCD type.

In group II, 31/79 (39\%) patients underwent presurgical assessment, yet only $7 / 31(23 \%)$ had epilepsy surgery and 6/7 (86\%) have been seizure free for more than 12 months following surgery (Engel, Ia; Wieser, 1a). In group II, 3/7 patients underwent resection of anterior two-third of temporal lobe (including mesial temporal structures); other 2/7 patients - hemispherotomy, 1/7 - resection of anterior twothird of temporal lobe (including mesial temporal structures) with parieto-occipital disconnection and 1/7 - selective amygdalohippocampectomy.

Conclusions: Recognition of dual pathology is important for the presurgical evaluation of patients with pharmacoresistant seizures as meticulously selected patients may have a favourable outcome following epilepsy surgery.

\subsection{1 \\ ELECTRICAL STIMULATION OF THE ANTERIOR AND CENTROMDIAN NUCLEUS OF THALAMUS FOR TREATMENT OF REFRACTORY EPILEPSY}

Wei Hu, B. Klassen, K. Lee, D. Clayton and M. Stead (Mayo Clinic, Rochester, MN)

Rationale: Experimental and clinical studies have suggested that electrical deep brain stimulation (DBS) of subcortical structures is a promising therapy for patients with refractory epilepsy over the last decade. Bilateral stimulation of the anterior nuclei (AN) of the thalamus is useful for some patients with medically refractory partial seizure, while the efficacy of bilateral centromedian (CM) nucleus stimulation in generalized forms of epilepsy has been recently identified. We report on the outcome after thalamic AN and CM DBS in five patients with medically refractory seizures.

Methods: Four patients with medically refractory partial and generalized seizures and one patient with continuous spike wave status (CSWS) during sleep were studied. All patients were either not candidates for resective surgery or refractory to the treatment of vagus nerve stimulation and/or resection surgery. All cases underwent bilateral AN and CM thalamic DBS. In order to optimize the DBS programming for seizure management, three patients were admitted to the Epilepsy Monitoring Unit (EMU) for quantification of seizures after DBS implantation and additional DBS programming based on their EEG recording results.

Results: Post-operative MRI documented that all electrodes were correctly located. There was no morbidity or mortality. The average seizure frequency reduction in the four epilepsy patients was 63 percent (range $50-80 \%$ ). EEG revealed that the spike-wave index was decreased from $90 \%$ to $50 \%$ in the patient with CSWS. Increased attention level was seen in all patients. Additionally, cognition and mood were stable or improved in all cases.

Conclusions: These findings suggest that Bilateral stimulation of the $\mathrm{AN}$ and $\mathrm{CM}$ of the thalamus reduces seizures. DBS programming based on EEG recording results is useful for seizure management.

\subsection{2 \\ EARLY SLEEP ONSET RELATED SEIZURES IN PATIENTS AFTER TEMPORAL LOBECTOMY WITH INITIAL SEIZURE FREE POSTOPERATIVE EVOLUTION Rafael Villalobos-Nieto ${ }^{1,2}$, J. Torres-Corzo ${ }^{2}$, F. Guzman-Palacios ${ }^{2}$, R. Rodriguez De La Veccia ${ }^{2}$ and V. Zertuche ${ }^{1,3}$ ( ${ }^{1}$ Pediatrics, University of Texas Health Science Center at San Antonio, Regional Academic Center, Harlingen, TX; ${ }^{2}$ Neurophysiology, Instituto Potosino de Neurociencias, San Luis Potosi, Mexico and ${ }^{3}$ Pediatric Neurology, Pediatric Neurology of South Texas, Brownsville, TX)}

Rationale: Temporal lobe epilepsy is a frequent diagnostic challenge since recurrence of events is a well known fact after epilepsy surgery. We wanted to identify certain recurrence patterns as a tool for prognostic indicators.

Methods: We evaluated patients after temporal lobectomy that had seizure recurrence despite having had a complete epileptogenic resection evidenced by postoperative electrocorticography (EcoG) and or neuroimmaging. Cases had history of refractory epilepsy that became seizure free after temporal lobectomy with at least one year of seizure freedom. We selected a population with a very distinct pattern of recurrence; early sleep onset events. We analyzed the different variables involved in the patient's general pre and post surgical features.

Results: A total of 5 patients out of 63 procedures performed in an epilepsy center. The age at the surgery was 17 to 28 years old. 3 cases right temporal lobe, 2 cases on the left. All the cases of grater than $90 \%$ improvement in seizure reduction. The seizure pattern was a partial seizure with similar characteristics to the presurgical events, but milder and shorter in duration, all of the events occurring in the first 90 minutes after sleep onset. The cases were treated with low dose topiramate, clonazepam or gabapentin, all but one of them are seizure free, the recurrent case has very rare seizures.

Conclusions: Epilepsy surgery is a well recognized option for patients with refractory epilepsy, a fraction of patients operated will have a distinct pattern of early sleep onset induced events, even after being seizure free for more than 1 year, possibly related to stage 1 and 2 of slow wave sleep, and will respond favorably to a low dose or short acting anticonvulsant, if that is the only seizure pattern found.

\subsection{3 \\ LOCALIZATION OF AN EXTRATEMPORAL LESION- RELATED EPILEPTOGENIC ZONE USING MAGNETOENCEPHALOGRAPHY IN PATIENTS WITH DUAL PATHOLOGY}

Koji Iida, K. Kagawa, M. Katagiri, A. Hashizume and K. Kurisu (Hiroshima University Hospital, Hiroshima, Japan)

Rationale: Dual pathology has been defined as the coexistence of both extrahippocampal lesion and hippocampal sclerosis in a pathological state. Numerous studies have revealed that a better seizure outcome can be established by the removal of both abnormalities than lesionectomy or mesial temporal resection alone. However, it is necessary to demarcate the precise extratemporal lesion-related epileptogenic zone (ETL-EZ) for optimal seizure outcome. This study is to elucidate whether magnetoencephalography (MEG) can identify the extent of ETL-EZ for presurgical evaluation in patients with dual pathology.

Methods: We retrospectively analyzed MEG data in 5 patients (A-E) who underwent resective surgery based on intracranial video-EEG (IVEEG). MEG was performed with 36-channel whole-head type system which included 204 channels of planar gradiometers. We classified distributions of an equivalent current dipole (ECD) into clusters and scatters as previously reported (Iida K et al., 2005), and compared the ECD-based distribution of epileptic areas, IVEEG results, and seizure outcome.

Results: Single-cluster ECDs were located over the middle perisylvian (A), posterior temporal (B), and posterior temporal to inferior parietal (C) regions in 3 patients (A-C). Two independent clusters were observed in the inferior-temporal and inferior parietal to lateral occipital regions in 1 patient (D). MEG localized clusters within or extended from ETL. The remaining patient (E) had no epileptic spikes on MEG recording. All single-clusters and two independent clusters were colocalized to IVEEG- or ECoG-defined epileptic zones demarcating the ETL-EZ in 4 patients (A-D: 3 single-clusters and both of 2 clusters). All patients underwent anterior temporal lobectomy (including hippocampectomy) and lesionectomy (total: $\mathrm{n}=5$; A-E; partial: $\mathrm{n}=1$; A) with $(\mathrm{n}=3$ : BDE) or without $(\mathrm{n}=1: \mathrm{C})$ additional cortical resection of ETL-EZ on IVEEG. One patient (C) 
underwent additional multiple subpial transection of the ETL-EZ due to language-associated area. Four patients had excellent seizure outcome (Engel class I), and the patient with partial lesionectomy indicated more than $90 \%$ seizure reduction (Engel class II). Conclusions: MEG can identify the extent of ETL-EZ in patients with dual pathology, as useful information for planning of IVEEG implantation.

\subsection{4}

\section{SAFETY AND VASCULAR CONSEQUENCES OF} OPERCULO-INSULAR CORTECTOMIES FOR EPILEPSY Patrice G. Finet $^{1}$, L. Crevier ${ }^{2}$, D. K. Nguyen ${ }^{3}$ and A. Bouthillier ${ }^{1}$ ( ${ }^{1}$ Neurosurgry, Notre-Dame Hospital, University of Montreal Medical Center (CHUM), Montreal, QC, Canada; ${ }^{2}$ Neurosurgery, Sainte-Justine Hospital, Montreal, QC, Canada and ${ }^{3}$ Neurology, Notre-Dame Hospital, University of Montreal Medical Center (CHUM), Montreal, QC, Canada)

Rationale: Surgery in the insular region is considered challenging because of its vascular relationships, its deep location in the sylvian fissure and the close proximity to functional areas. This study was undertaken to establish safety and vascular consequences of operculo-insular cortectomies for refractory epilepsy.

Methods: We retrospectively reviewed all our cases of operculoinsular cortectomies for refractory epilepsy between 1998 and 2012. Tumors and vascular lesions were excluded. All patients had postoperative MRI to document the location and extent of resections, and the occurrence and types of ischemic lesions (IL). Neurological exams were performed during the follow-up visits.

Results: Seventeen patients ( 9 males, 4 dominant hemispheres, mean age 29) had insular cortex resections. This was combined with opercular/lobar resections in 15 cases. No IL from the lenticulo-sriate perforators was identified. IL occurred in 12 patients (70\%). Eleven IL were related to perforating branches from the M2 segments of the middle cerebral artery (MCA) at the surface of the insula. Two IL were related to perforating branches from the M3-4 segments of the MCA contouring the opercula. One IL was related to a distal temporal M4 branch after a combined temporal lobectomy. The IL from perforating branches were small and located in the corona radiata, running from the operculo-insular to the peri-ventricular region. Excluding a superior quadranopia related to a temporal lobectomy, 8 patients $(47 \%$ ) had a postoperative neurological deficit: 7 hemiparesis and 1 dysphasia. All deficits were transient. Conclusions: In our series, operculo-insular cortectomies were associated with IL in $70 \%$ of patients. However, this finding had limited clinical consequences given that no patient had a permanent postoperative deficit. We therefore consider that operculo-insular cortex resection is a safe procedure for the treatment of refractory epilepsy.

\subsection{5 \\ SURGERY FOR OF TEMPORAL LOBE EPILEPSY SURGERY IN PATIENTS WITH NORMAL MR IMAGING: SEIZURE OUTCOME, YIELD OF RE-EVALUATION, AND OUTCOME FOLLOWING REOPERATION}

Mohamed M. Ali, S. Wiebe, N. Pillay, P. Federico, N. Jettee, L. Bello-Espinosa and W. J. Hader (Mansura University, Calgary, AB, Canada)

Rationale: Surgical failure of non-lesional TLE might be due to a seizure focus in contralateral temporal lobe, extratemporal or in complete resection of epileptogenic zone. Few studies have evaluated the relative frequency of these proposed mechanisms. The purpose of this study was to analyze the surgical failure of temporal lobe epilepsy with nMRI at the Calgary Epilepsy Program.

Methods: We retrospectively evaluated the surgical outcome of all patients who underwent temporal lobe surgery with nMRI between
2000 and 2010. All patients underwent comprehensive presurgical evaluation. Failed patient had re-evaluation and repeat surgery in selected cases

Results: A total of 43 patients underwent TLE surgery with nMRI. They were 18 women and 25 men. The mean age at surgery was 29.0 years (with 7 patients younger than 18 years). Patients underwent anteromesial temporal lobe resection and $(n=30$ trans cortical selective amygdalo-hippocampectomy $(\mathrm{n}=8)$ or neocortiectomy $(n=5)$. Of the 43 patients, 15 underwent intracranial electrodes implantation prior to the resection. At the final follow-up (average 51 months), 27 out of the 43 patients (62\%) were seizure free. Out of the 16 patients who failed surgery 15 patients $(94 \%)$ had reevaluation. The focus of recurrence was identified as follows: Posterior neocortical $(n=5)$, contralateral temporal lobe $(n=4)$, extra temporal $(n=3)$, and residual mesial structures $(n=3)$. Out of the 6 patients who had repeat surgery only 1 patient (14\%) had a favorable outcome.

Conclusions: Temporal lobe resection in well-selected patients with nMRI can provide favorable rates of seizure freedom. The majority of surgical failures are due to reasons that might be irremediable with another surgery such as bitemporal, extra temporal, or posterior neocortical foci. This may explain the low chance of favorable outcome following repeat surgery.

\subsection{6 \\ EPILEPSY SURGERY IN PATIENTS WITH BILATERAL INDEPENDENT TEMPORAL LOBE EPILEPSY: CAN WE PREDICT OUTCOME?}

Y. Aghakhani ${ }^{1}$, X. R. Liu ${ }^{1,2}$, J. Dykeman $^{1}$, M. Lowerison ${ }^{1}$, N. Jette ${ }^{1}$ and S. Wiebe ${ }^{1}{ }^{1}$ University of Calgary, Calgary, AB, Canada and ${ }^{2}$ Institute of Neuroscience and the Second Affiliated Hospital of Guangzhou Medical University, Guangzhou, China)

Rationale: In patients with bilateral independent temporal lobe epilepsy (BiTLE), the decision to perform a temporal lobe (TL) resection is fraught with uncertainty because the relative importance of various investigations has not been systematically evaluated. We explored the association between seizure outcomes, presence of a lesion and seizure laterality on intracranial stereo EEG (SEEG) in patients with BiTLE.

Methods: We searched Embase and Medline up to May 31, 2012, using sub-headings and keywords related to bilateral temporal lobe seizures, EEG, surgery and seizure outcomes. Eligible studies included 1) independent bitemporal seizures diagnosed by SEEG, 2) quantified seizure laterality, 3) description of lesion/no lesion, 4) surgical description, and 5) quantification of seizure outcome stratified by subgroups of interest. We assessed the association of independent variables in patients with a good outcome (Engel Class I and II) and in those with poor outcome (Engel Class III and IV). Inidvidual patient data were pooled where appropriate and proportions were compared using exact tests.

Results: 290 abstracts were independently reviewed by two individuals who achieved consensus on 36 articles selected for full text review. Six articles fulfilled all eligibility criteria. The studies yielded 40 patients with a mean follow-up of 4.7 years for 29 patients, and $>1$ year for the remaining 11 .

Twenty-eight patients (70\%) had good outcome (27 Class I), of whom $20(71.4 \%)$ had a TL lesion identified on MRI (13) or on pathology (7). Eighteen had mesial temporal sclerosis (MTS), and two had a hamartoma. Outcome was poor in 12 patients $(30 \%)$; of these, $5(42 \%)$ had a lesion (all MTS, 3 on MRI and 2 on pathology). The presence of a lesion was significantly associated with good outcome $(\mathrm{p}=0.05)$. All patients had surgery on the side of the lesion. The pooled proportion of seizures on SEEG that were ipsilateral to the resection did not differ between lesional (80\%, range 50\%-97\%) and nonlesional cases $(74 \%$, range $61-88 \%)(\mathrm{p}=0.14)$. The pooled proportion of seizures on SEEG that were ipsilateral to the resection did not differ between patients with good $(78 \%)$ or bad $(82 \%)$ 
outcome $(\mathrm{p}=0.68)$. In patients with good outcome, the proportion of seizures ipsilateral to the resection in individual patients ranged from $42 \%-95 \%$ (average $78 \%$ ) for lesional cases, and from $50 \%-95 \%$ (average 75\%) for non-lesional cases ( $\mathrm{p}=0.86$ ).

Conclusions: Good outcomes can be achieved in $70 \%$ of patients with BiTLE and this is significantly more likely when a lesion is present on MRI or pathology. Although clinicians pay much attention to the seizure lateralization ratio on SEEG in BiTLE, this had no relation with surgical outcome. Therefore, in the presence of a clear MRI lesion, SEEG may not be necessary. The available evidence suggests that seizure lateralization on SEEG in BiTLE in general is of questionable importance.

\subsection{7}

A METHOD TO CO-REGISTER INTEROPERATIVELY PLACED ECOG GRIDS WITH THE CORTICAL ANATOMY Disha Gupta $^{1,2}$, M. A. Adamo ${ }^{3}$, A. L. Ritaccio ${ }^{1}$ and G. Schalk ${ }^{1,2}$ ( ${ }^{1}$ Neurology, Albany Medical College, Albany, NY; ${ }^{2}$ Brain Computer Interfacing, Wadsworth Center, New York State Dept. of Health, Albany, NY and ${ }^{3}$ Neurosurgery, Albany Medical College, Albany, NY)

Rationale: Electrocorticographic $(\mathrm{ECoG})$ grids are routinely placed subdurally on the cortex in people with epilepsy to delineate cortical areas involved in seizures or function. These grids provide exceptional opportunities for neuroscientific research, because they detect signals from the brain at high spatial and temporal resolution. Relating these signals to the underlying anatomy requires coregistering the location of the electrodes to the location of the anatomy. Existing methods that support this co-registration use preoperative magnetic resonance imaging (MRI), which captures the cortical anatomy, and post-operative computerized tomography (CT), which captures the electrode locations. However, when ECoG grids are placed during a surgery, no CT that includes the grid is available. Other existing methods make use of intraoperative photographs, but they require that the electrodes can be visually related to the underlying anatomy, which is difficult or impossible with highresolution grids or when electrodes are invisible. These limitations of existing localization techniques limit the potential value of the interpretation of ECoG signals collected during surgeries. To address this problem, we developed a method to co-register ECoG grids using only a pre-operative MRI, a clinical neuro-navigation device (such as BrainLab Vector Vision), and fiducials.

Methods: The patient undergoes imaging with fiducial markers on the day of surgery. During surgery, the patient's anatomy is registered to the pre-operative images in the navigation system using laserbased surface registration. The fiducial points are then traversed with the navigator tip and the coordinates for each point are acquired and saved. The same pre-operative MRI is read into CURRY software and the fiducial points are detected. A rigid transformation matrix is estimated from these and the acquired points on the neuronavigator. Results: To validate our new technique, we collected data from four subjects that had post-operative CTs also available, and compared the resulting electrode coordinates to results using an existing coregistration technique that required a post-operative CT. We found that the electrode locations determined using the two methods were in generally close proximity (average error: $8.13 \mathrm{~mm}$ ) even in the limited dataset.

Conclusions: We developed and validated a method for coregistering ECoG electrodes to the underlying cortical anatomy when a post-operative CT is not available, such as during single-stage, intra-operative studies. Thus our technique should prove useful for research in intra-operative scenarios. More generally, it may also reduce the need for post-operative CTs and thus help to reduce radiographic imaging exposures.

\subsection{8 \\ EFFICACY AND SAFETY OF VAGAL NERVE STIMULATION FOR INTRACTABLE EPILEPSY AT RIYADH MILITARY HOSPITAL}

SONIA A. KHAN, A. Osman, K. Hussein, F. Al Ghareeb, M. Santos and D. Al Shammari (RIYADH MILITARY HOSPITAL, Riyadh, Saudi Arabia)

Rationale: Vagal Nerve Stimulation (VNS) therapy is an adjunctive treatment for patients with intractable epilepsy (IE). Evaluation of efficacy and safety of VNS in treatment of patients with intractable epilepsy not candidates for epilepsy surgery.

Methods: Prospective clinical follow-up of patients treated with VNS for IE. Inclusion criteria includes patients with intractable epilepsy not candidates for epilepsy surgery i.e. those with more than 1 disabling seizure/month despite adequate antiepileptic drugs AEDs for at least 2 years. The patients should have failed at least 2 AED trials. Each patient should have documented seizure frequency before VNS implantation and every 3 months after VNS implantation. The minimum follow up period is 6 months. The safety is measured by number and type of adverse events appeared each visit during the post implantation follow-up. The efficacy is measured by the number and percentage of patients that show significant seizure reduction post implantation.

Results: A total of 44 patients with IE were implanted with VNS from December 2006 to December 2011. Only 40 patients were included in this evaluation, 12 males and 28 females. Age of the patients ranged from 10 to 37 years with average age onset at 4 years. Average epilepsy duration before VNS implantation was 10 years. Epilepsy type included 28 patients with focal epilepsy and 12 patients with generalized epilepsy. In the focal epilepsy group 8 patients had frontal lobe epilepsy, 2 had temporal lobe epilepsy, 8 had parietooccipital epilepsy, 7 had multilobar partial epilepsy and 3 had unclassified partial epilepsy. In the generalized epilepsy group 8 patients had Lennox Gastaut Syndrome, 3 had Unverricht Lundborg Disease and 1 had Juvenile Neuronal Ceroid Lipofuscinosis. The average seizure frequency was 1 generalized tonic clonic seizure per week before VNS. At the time of VNS implantation 16 patients were on 3 AEDs, 16 patients on 2 AEDs and 8 patients on 4 AEDs. At latest follow-up, 32 patient ( $80 \%$ ) showed significant improvement ( $>50 \%$ seizure reduction). Four patients $(10 \%)$ showed less improvement ( $<50 \%$ seizure reduction) and 4 patients $(10 \%)$ showed no improvement. A trend towards better seizure control with low current output - high frequency stimulation. No serious adverse event encountered. All 40 patients had transient hoarseness and throat irritation and 35 patients had cough. Less common adverse events in 6 patients $(15 \%)$ with refractory focal status epilepticus post operative (1) patient, superficial infection (1) patient, sagging of the device (1) patient, malfunction of the wire (1) patient, transient recurrent aspiration (1) patient and aspiration pneumonia and adult respiratory distress syndrome post operative (1) patient.

Conclusions: VNS is efficient and safe adjunctive antiepileptic therapy in patients with intractable epilepsy not candidates for epilepsy surgery.

\subsection{9 \\ PRESURGICAL EVALUATION OF EPILEPSY SURGERY IN ADULT PATIENTS WITH TUBEROUS SCLEROSIS COMPLEX}

Danielle McDermott, L. A. Strom and L. C. Frey (University of Colorado, Aurora, CO)

Rationale: Epilepsy affects approximately $80 \%$ of patients with Tuberous Sclerosis Complex (TSC). Many are pharmacologically refractory. Successful epilepsy surgeries have been achieved in patients with TSC and medically intractable epilepsy, with remission rates estimated at $57 \%$. In the past this was done simply by correlating surface EEG recordings with an identifiable lesion on CT 
or MRI. More sophisticated techniques have been developed, including EEG combined MRI and ictal SPECT, allowing for better identification of epileptogenic tubers.

Methods: We present two illustrative cases from patients with TSC cared for at the University of Colorado Epilepsy Center. We chose one case with clear localization of seizure onset and one without clear localization on Phase II invasive monitoring. Their case histories, examination findings, and presurgical evaluation results are compared for potential predictors of surgical feasibility. These clinical features are then compared to the published literature for further understanding of the complexities of epilepsy surgery in this highly variable disorder.

Results: Our case series contrasts the presurgical evaluation of two well-matched, adult patients with TSC, BH and NL. Both men have a history of medically-intractable epilepsy since infancy. Both men are high-functioning intellectually. Both patients have numerous cortical hamartomas on CT and MRI. Patient BH presented with a distinct semiology which correlated well with findings on surface EEG recordings. Patient NL's seizure description was more bland. Phase I monitoring failed to localize seizures. BH did not have SPECT as part of his preoperative evaluation. NL's SPECT reiterated this diagnostic uncertainty. BH's intracranial EEG recordings clearly demonstrated a single primary epileptogenic tuber, thus making him an ideal candidate for surgical resection (see Image 1). In contrast, NL's intracranial EEG monitoring revealed multifocal and nonlocalizable areas of seizure onset. He was not a resective candidate, but went forward with vagal nerve stimulator placement. $\mathrm{BH}$ had no post operative complications. At one year follow up BH reports 4 identical complex partial seizures. He is now able to work, whereas he was disabled by his seizures prior to the surgery.

Conclusions: Our cases demonstrate the diagnostic challenges epileptogists face when confronted with presurgical evaluation of patients with TSC and medically-intractable epilepsy. Advances in functional neuroimaging and neurosurgical techniques have expanded epilepsy surgery to a group of patients previously thought to be poor surgical candidates. These advancements have shown to improve behavior, cognition, and quality of life in pediatric cohorts. However, long-term surgical outcomes data is necessary in adult populations. Future prospective studies may help determine which clinical and neurodiagnostic features predict surgical feasibility and outcomes in adult patients.

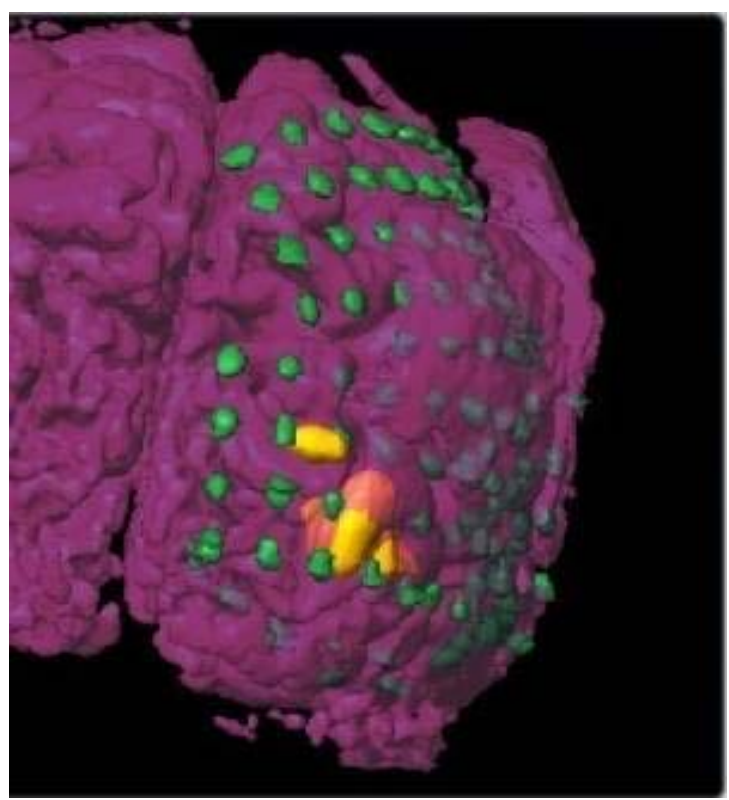

IMAGE 1. Patient BH. Coregistration Image of Preimplantation MRI wth Postimplantion CT. Epileptogenic tuber is demonstrated in right occipital temporal region.
THE OUTCOME OF 32 VAGUS NERVE STIMULATION CASES (VNS)

ayataka fujimoto, T. Yamazoe, T. Yokota, H. Enoki, Y. Sasaki, M. Nishimura and T. Yamamoto (seirei hamamatsu general hospital epilepsy center, Hamamatsu, Japan)

Rationale: As a palliative option considered for patients who are not good candidates for cranial surgery, many patients have been treated by vagus nerve stimulation (VNS). In Japan VNS is available for any types of epilepsy if patient is not open surgery candidate. The purpose of this study is to evaluate VNS efficacy on epilepsy syndromes, seizure types and VNS comorbidity.

Methods: There are 39 patients underwent VNS in our facility. 32 patients ( 20 males: 12 females) were followed up more than 6 months. The age ranged from 3 through 56 years old. Post-VNS follow-up periods are 6-18 months.

Results: There were 13 SGEs, 11 SLREs, 6 SGE/SLREs and two PMEs.

There were five seizure freedoms, 17 good responders with more than $50 \%$ seizure reduction, six moderate responders with less than $50 \%$ seizure reduction and four non-responders. Among the 5 seizure free patients there were 4 SLREs and one SGE. These 4 SLREs showed normal intelligence. There were ten patients with Status Epilepticus(SE). Nine have been free from SE. Twenty four patients had no comorbidity. Six had transient hoarseness. Three had transient cough. One has stimulation induced hoarseness.

Conclusions: VNS is effective for any types of epilepsy and SE. VNS is also harmless therapy.

\subsection{1}

CEREBRAL EDEMA AND HERNIATION DURING INVASIVE EEG MONITORING MAY BE ASSOCIATED WITH GOOD OUTCOME AND AMELIORATION OF SEIZURES

Joel M. Oster ( ${ }^{1}$ Lahey Clinic, Burlington, MA and ${ }^{2}$ Tufts University School of Medicine, Boston, MA)

Rationale: A paucity of data exists on long term sequelae of adverse events during invasive EEG monitoring. A series of 3 patients with intractable and disabling epilepsy were studied with invasive EEG monitoring to identify ictal onsets. These patients postoperatively with invasive recording electrodes in place experienced idiopathic cerebral edema and herniation to varied degrees and EEG recordings were continued during these times. Ultimately, in 1 case, the patient had an outcome of longterm seizure freedom, and in the other 2 cases, there were significant seizure reductions after focal resections were done and the cerebral edema resolved. Therefore all patients ultimately achieved a good clinical baseline without significant additional long term neurologic signs and overall improved seizure control. This article reviews the clinicopathologic details of these cases in this unique series.

Methods: In a suburban Epilepsy center, 3 disabled and intractable epilepsy patients required invasive EEG monitoring and management to delineate ictal onsets to guide possible focal resections since they had failed numerous anti-seizure medications and/or VNS. The patients had MRI neuroimgaging, metabolic imaging, Interictal surface EEG and invasive interictal and ictal recordings for data sets. Two (2) different surgeons performed surgical procedures in these cases. Retrospectively, those with resultant herniation or cerebral edema were descriptively analyzed.

Results: While the patients developed lethargy or focal neurologic signs or acute neurological defecits and clinical and radiological evidence of cerebral edema or midline shift/herniation syndromes, the decision was made in all cases $(100 \%)$ to persist with invasive EEG monitoring. Long term follow up after multiple years showed a favorable outcome. 
Conclusions: Our case series delineates that in a series of intractable epilepsy cases, invasive monitoring performed even after development of cerebral edema and clinicoradiologic herniation syndromes after invasive electrode placement or ultimately focal resection may be associated with amelioration of seizure control and favorable long term outcome.

\section{Behavior/Neuropsychology/Language: Adult}

\subsection{2}

\section{NEUROTICISM AND EXECUTIVE FUNCTION IN DRUG} REFRACTORY JUVENILE MYOCLONIC EPILEPSY Rhys H. Thomas ${ }^{1}$, A. G. Marson ${ }^{2}$, P. E. Smith ${ }^{3}$, M. I. Rees ${ }^{1}$, G. A. Baker $^{2}$ and J. Walsh ${ }^{2}\left({ }^{1}\right.$ Wales Epilepsy Research Network, Swansea University, Swansea, United Kingdom; ${ }^{2}$ Molecular and Clinical Pharmacology, University of Liverpool, Liverpool, United Kingdom and ${ }^{3}$ Neurology, University Hospital of Wales, Cardiff, United Kingdom)

Rationale: Up to $20 \%$ of people with juvenile myoclonic epilepsy (JME) do not achieve adequate seizure control despite taking sodium valproate. It is established that people with drug sensitive JME score less well on neuropsychological tests of executive function. We wanted to describe pattern of deficits in drug refractory JME; focussing on what may most affect daily life.

Methods: As part of the MRC funded ReJUMeC study a cohort had extended neuropsychological testing including elements from the BADS ( $\mathrm{n}=31$ ) (Behavioural Assessment of the Dysexecutive Syndrome) and the Eysenck personality questionnaire $(n=12)$. Alongside the main cohort they also had a semi-structured clinical interview and sat the WAIS-III, WMS, DKEFS and the Boston naming test.

Results: $12 / 31$ scored in the impaired range on the BADS; those that did were also significantly $(\mathrm{p}<0.05)$ impaired on $15 / 21$ other cognitive domains (compared to $4 / 21$ for those who scored in the average or superior range on the BADS). Lower IQ was correlated with a lower BADS score; however not with the zoo map task. The zoo map is an executive function test with good ecological validity, and impairment on this was found to be significantly correlated with having a neurotic personality. Regression analysis revealed no other factors that were significant predictors of impairment on the zoo map. Conclusions: People with drug resistant JME are not only impaired on tests of executive function but across a wide variety of cognitive domains. JME is a heterogeneous disorder: we hypothesise that the subgroups identified by neuropsychological testing will also show differences when studied using advanced imaging and genetic techniques.

\subsection{3 \\ THE EXPERIENCE OF ANGER IN PATIENTS WITH EPILEPTIC SEIZURES: THE STATE-TRAIT ANGER EXPRESSION INVENTORY-2}

Keren I. Lebeau, L. Myers, M. Evans, M. Lancman, M. Lancman and B. Matzner (Northeast Regional Epilepsy Group, Summit, NJ)

Rationale: The relationship between epileptic seizures (ES) and aggressive behavior is a controversial issue that has been widely examined in the literature (Geschwind, 1975). While some studies have found an increased prevalence of aggression in patients with ES (Bach-Y-Rita et al., 1971; Elliott, 1982), others have not demonstrated this relationship (Kligman \& Goldberg, 1975; Lishman, 1998). The purpose of this study was to determine the frequency of anger expression in ES and to examine differential aspects of anger in patients with ES. The study compared temporal lobe epilepsy (TLE) versus generalized epilepsy (GE) patients with regard to the following components: state versus trait anger and anger expression versus anger control.
Methods: All consecutive patients with ES who completed our standard epilepsy neuropsychological evaluation between January and May 15, 2012 were initially included $(n=27)$. Three patients who had earned a Full Scale IQ of less than 70 (potentially impacting their ability to respond to the STAXI-2) were excluded $(n=24)$. Patients had undergone video-EEG monitoring and had a confirmed diagnosis of TLE $(n=17)$ or GE $(n=7)$. The mean age of the sample was 44.54 (18.24), with a range of 17 to 71 . The mean education level was $13.76(2.81)$ years, with a range of 9 to 18 years. All subjects completed measures of State Trait Anger Expression Inventory-2 (STAXI-2) as part of a comprehensive neuropsychological battery. The STAXI-2 is a self-report questionnaire that assesses anger expression along four dimensions (state, trait, anger control and anger expression). Additional variables collected through chart review included epilepsy duration, lateralization, number of anti-epileptic drugs (AEDs) taken at the time of testing, and refractory epilepsy classification.

Results: No significant correlation was found between anger scores and gender, epilepsy duration, refractoriness, number of AEDs or lateralization. However, in the combined sample of GE and TLE patients, $41.7 \%$ endorsed abnormally high Anger Expression Out (AX-O) ratings $(\mathrm{T}>70)$. AXO is defined as "anger expressed outwardly towards persons or objects in the environment." In addition, higher AXO scores correlated significantly with lower Full Scale IQ $(\mathrm{p}<0.05)$.

Conclusions: The most obvious limitation in this study is that the sample size was small. However, the results suggest a possible trend towards increased symptoms of anger in epilepsy patients as a whole. The current report demonstrates a notable elevation of one STAXI-2 scale. This scale is an important one as it is an indicator of a tendency toward expression of anger directed at persons or objects in the environment. This finding has substantial implications for the mental health professionals who are part of the epilepsy treatment team. Anger can have a profound impact on interpersonal functioning and well-being. Therefore there is value to assessing this variable in ES. Future directions for research should include further exploring potential relations between anger and epilepsy variables in a larger sample.

\subsection{4 \\ LONG-TERM EFFECTS OF TEMPORAL LOBECTOMY ON ACCELERATED LONG-TERM FORGETTING IN MESIAL TEMPORAL LOBE EPILEPSY}

Cigdem Ozkara $^{1}$, L. Hanoglu ${ }^{2}, \mathrm{~S}$. Gonenc ${ }^{2}$, E. Ozmansur ${ }^{1}$ and M. $\mathrm{Uzan}^{3}\left({ }^{1}\right.$ Neurology, Cerrahpasa Medical Faculty, Istanbul, Turkey; ${ }^{2}$ Neurology, Medipol University, Istanbul, Turkey and

${ }^{3}$ Neurosurgery, Cerrahpasa Medical Faculty, Istanbul, Turkey)

Rationale: Mesial temporal lobe epilepsy (MTLE) has been associated with the phenomenon of accelerated long-term forgetting $(\mathrm{ALF})$, in which memories are retained normally over short delays but are then lost at an accelerated rate over days or weeks. The causes of ALF, and whether it represents a consolidation deficit distinct from the one associated with forgetting over short delays, remain unclear. It could be due to prevention of consolidation process by the ongoing epileptic activity or the damage developed already in TLE. In this study we aimed to demonstrate the effect of surgery on the ALF phenomena which may provide an explanation to the cause. Methods: We have evaluated 43 patients (24F/19M), mean age 37.2 years (23-56yrs) with MTLE related to hippocampal sclerosis who had anterior temporal lobectomy (ATL) and followed for at least 5 years (mean11 years, 6 -16 years). All of them were seizure free after surgery. Control group was consisted of 9 healthy controls . All were given Turkish verbal learning test (SBST) and were tested for learning, immediate recall and recognition at $30 \mathrm{~min}, 1$ week and 6 week intervals. Afterwards, accelerated forgetting rate was calculated and the scores were analysed statistically. 
Results: The results of of SBST test after 30 minutes and difference scores estimated for delayed recall did not show statistically significant difference in neither patient nor control groups.

Conclusions: Patients with favorable outcome and without consolidation deficit after ATL may provide a further evidence for the relationship between the effect of ongoing epileptic activity and accelerated long-term forgetting.

\subsection{5 \\ GENDER, SUBJECTIVE MEMORY, AND OBJECTIVE MEMORY AMONG PATIENTS WITH EPILEPSY}

Robert Trobliger, E. Feoli, M. Lancman and M. Lancman (Northeast Regional Epilepsy Group, Hackensack, NJ)

Rationale: Seizure activity is commonly associated with the onset, and then often worsening, of cognitive deficits. Most cognitive complaints in adult patients involve attention, processing speed, and memory. Previous research on the relationship between subjective memory complaints and objective memory performance has found variable results, including many findings of no to weak relationships. Multiple studies have suggested that significant perceived memory difficulties are instead strongly related to elevated levels of mood or anxiety symptoms, although does not preclude an organic component. Other research has suggested that everyday memory complaints do not tap into the same areas as neuropsychological testing measures. Studies on the differences between men and women with epilepsy have shown differences in terms of memory functioning on objective tasks, including female superiority on verbal memory tasks and inconsistent findings for visual memory. No research has yet focused on gender differences for the relationship between subjective and objective memory. This study examined such, with an eye towards implications for treatment.

Methods: Neuropsychological testing results for 105 patients with epilepsy were reviewed. Subjective measures included aspects of the Memory Complaints Inventory (MCI). Objective measures included: the California Verbal Learning Test-Second Edition (CVLT-II), the Rey Auditory Verbal Learning Test (RAVLT) and the Wechsler Memory Scale - Third and Fourth Editions (WMS-III/WMS-IV) Logical Memory I and II (LMI/LMII) subtests. Measures of mood and anxiety symptoms included the Achebach Adult Self-Report Anxiety and Depression scales, Beck Anxiety Inventory (BAI), and Beck Depression Inventory (BDI-II). Correlations between objective and subjective measures and between subjective measures and measures of mood/anxiety symptoms were computed using SPSS 16.0. Differences in significance between groups were computed by hand.

Results: The results did not suggest any differences between men and women in terms of strength of relationship between subjective and objective or mood/anxiety measures. The results were consistent with many previous studies finding no significant relationships between all objective and subjective measures of memory among patients with epilepsy. Results were also consistent with studies demonstrating significant relationships between self-reported measures of memory difficulty and self-reported levels of depression and anxiety symptoms.

Conclusions: These results point to the need for exploration of patient complaints regarding functioning in generating rehabilitation strategies, as for many patients, objective measures may not necessarily indicate deficits.

\subsection{6 \\ PREDICTION OF COGNITIVE RESPONSE BY MODEL- BASED SIMULATION OF GENERATIVE VERBAL FLUENCY SCORES AFTER A SINGLE DOSE OF TOPIRAMATE IN HEALTHY VOLUNTEERS}

Susan E. Marino ${ }^{1,2}$, G. F. Ahmed ${ }^{2}$, R. C. Brundage ${ }^{2}$, S. V. Pakhomov $^{1}$, I. E. Leppik ${ }^{2}$ and A. K. Birnbaum ${ }^{1,2}\left({ }^{1} \mathrm{Ctr}\right.$ for Clinical \&
Cognitive Neuropharmacology, University of Minnesota, Minneapolis, $\mathrm{MN}$ and ${ }^{2}$ Experimental and Clinical Pharmacology, University of Minnesota, Minneapolis, MN)

Rationale: Topiramate (TPM) is indicated for several conditions including partial onset seizures. Patients on TPM manifest varying degrees of cognitive impairment including deficits in generative (phonemic) verbal fluency as measured by the Controlled Oral Word Association (COWA) test. We explored the utility of a pharmacokinetic/pharmacodynamic (PK/PD) model derived from previously collected $100 \mathrm{mg}$ TPM data to simulate subjects' cognitive response to $200 \mathrm{mg}$. The simulation results are compared to empirical data from individuals given a single $200 \mathrm{mg}$ TPM dose to assess the validity of this approach.

Methods: We previously developed linear and exponential decline PK/PD link models to describe the effect of TPM (100 mg) exposure on COWA performance (Ahmed et al, 2012, Abstract LBI-1, ASCPT). In the current study, we administered the COWA test to healthy volunteers $(n=9,18-50$ years old) randomized to a $200 \mathrm{mg}$ TPM or placebo arm. COWA was assessed at pre- and post-treatment baselines and at $\sim 2.5$ hours after dose. Blood samples were collected immediately after the COWA for measurement of TPM levels. Model-based simulation analyses were conducted where the individual COWA-time profiles were simulated for up to 4 hours after a single dose of $200 \mathrm{mg}$ of TPM. 1000 datasets were simulated and the median, 2.5th, and 97.5th percentiles of the simulated PD profiles were overlaid on the actual observations from the current study. Plots were visually examined to assess the predictability of both link models to the PD profiles, and the percentage of observations falling inside the 95 percentile confidence intervals (CI) was calculated.

Results: Overall 25 observations for COWA were collected. The TPM PK after a single $200 \mathrm{mg}$ dose was simulated using a twocompartment linear model. Sources of variability that influenced the simulated PD (i.e., COWA) profiles were: 1) interindividual variability in PK parameters; 2) interindividual variability in parameters of the exposure-response models; and 3) practice effect; an estimated $12 \%$ increase in baseline COWA scores after the $3 \mathrm{rd}$ time the test was administered. Both models were predictive of the PD profiles after a $200 \mathrm{mg}$ TPM dose. Of the observed COWA scores, $80 \%$ and $88 \%$ fell within the 95 percentile CI of the simulated COWA-time profiles using the exponential and linear decline models, respectively. Both models simulated a comparable median COWA-time profile. The linear model resulted in a higher simulated variability in PD profiles than the exponential decline model. Conclusions: The developed PK/PD models were useful in simulating both median and interindividual variability of the COWAtime profiles after a single $200 \mathrm{mg}$ TPM dose. These models can be useful in predicting subpopulations of patients who would be more susceptible to impairment of COWA while providing PK/PD evidence-based dose optimization of TPM in such patients with epilepsy.

\subsection{7 \\ DRIVING AND EPILEPSY: PERSPECTIVES OF PATIENTS WITH EPILEPSY IN SOUTHWESTERN NIGERIA Taofiki A. Sunmonu ${ }^{1}$, M. A. Komolafe ${ }^{2}$, O. T. Afolabi ${ }^{3}$ and O. A. Ogunrin ${ }^{4}\left({ }^{1}\right.$ Medicine, Federal Mediical Centre, Owo, Ondo state, Nigeria, Owo, Ondo state, Nigeria; ${ }^{2}$ Medicine, Obafemi Awolowo University Teaching Hospitals Complex, Ile - Ife, Nigeria; ${ }^{3}$ Community Medicine, Obafemi Awolowo University, Ile - Ife, Nigeria and ${ }^{4}$ Medicine, University of Benin Teaching Hospital, Benin, Nigeria)}

Rationale: Patients with epilepsy (PWE) with poorly controlled seizures are restricted from driving in most developed countries but despite this many PWE still continue to drive 
Methods: This is a case-control study in which 42 patients with epilepsy (PWE) were studied along with 45 age and sex-matched normal control subjects. The adapted format of Driver perceptions and practices questionnaire (DPPQ) were verbally administered to the participants. DPPQ is a 46-item questionnaire that assesses the domains of safety concerns, Attitudes towards driving, Perceived susceptibility/severity, Perceived barriers, Helping relationship and Self-efficacy. The data obtained were collated and analyzed with the aid of SPSS version 15.0 statistical software.

Results: Twelve patients were driving regularly among PWE while 15 normal controls were driving regularly. The controls thought of ways to improve their safety and were less likely to have confidence to ask family or friends to drive them when compared to PWE. The PWE that were students or unemployed and those who have driving licence have lower scores on "self-efficacy" domains" when compared PWE that were employed or possessed driving licence. PWE who had a high "Perceived barriers "to changing driving behavior and "Attitude towards driving" scores were more likely to drive when compared to PWE with lower scores.

Conclusions: There were minor differences in driving behavior of PWE when compared to normal control subjects and PWE with high perceived barriers to changing driving behavior and poor attitude towards driving were more likely to drive.

\subsection{8 \\ EPILEPTIC APHASIA: AN UNUSUAL PRESENTATION OF EPILEPSY IN 10 PATIENTS}

Ana Lopez Ferreiro ${ }^{1}$, X. Rodríguez-Osorio ${ }^{1}$, J. C. Fernández-Ferro ${ }^{2}$, M. Santamaría-Cadavid ${ }^{1}$, E. Costa-Arpín ${ }^{1}$, J. Pardo ${ }^{1}$, E. Corredera ${ }^{1}$ and F. J. López-González ${ }^{1}{ }^{1}$ Neurology, Hospital Clínico Universitario de Santiago de Compostela, Santiago de Compostela, A Coruña, Spain and ${ }^{2}$ Neurology, Hospital Capio Sur Alcorcón, Madrid, Spain)

Rationale: Aphasia as sole clinical manifestation of epilepsy is a rare phenomenon. We aim to study the epileptic aphasias diagnosed in our hospital.

Methods: Ictal aphasia was considered as language production with aphasic features in conscious patients. Episodes were classified as status epilepticus or simple partial seizures according to ILAE guidelines, and may present as Broca, Wernicke or mixed aphasia. All patients were diagnosed based on clinical features plus EEG findings or clear response to antiepileptic drugs (AED), with exclusion of acute stroke on MRI. We analyzed demographic, clinical and EEG features, response to treatment and evolution.

Results: Ten women $(100 \%)$, mean age of 66.6 years old $( \pm 12,84)$. Three had history of epilepsy. Five patients presented with mixed aphasia, 4 with Broca and 1 with Wernicke aphasia. In 9 cases an EEG was obtained during the episode: 4 demonstrated left electroencephalographic seizure activity and other 4 , left hemisphere slowing. 6 patients met the clinical definition of status epilepticus. MRI was normal in $40 \%$. Benzodiazepines were used as first-line AED in all cases, with clinical response in 4, EEG response in 1 and both in 2. All patients clinically improved with AEDs (LEV 70\%). In the follow-up, 7 cases achieved an excellent control and 3 recurred. Conclusions: The diagnosis of epileptic aphasia is challenging. An epileptic etiology should be considered in the differential diagnosis of episodic speech errors. Ictal EEG findings and response to AEDs are useful in the diagnosis and management

\subsection{9 \\ COGNITIVE FUNCTIONING IN PATIENTS WITH MEDICALLY REFRACTORY TEMPORAL LOBE EPILEPSY CANDIDATES FOR EPILEPSY SURGERY}

Jose C. Delgado Rios, J. Cuenca Alfaro, R. Velasco, C. Castro, C. Vasquez and I. Tagle (Epilepsy surgery committee, Instituto Nacional de Ciencias Neurologicas, Lima, Peru)
Rationale: Patients with Temporal Lobe Epilepsy are at significant risk for cognitive impairment. The aim of this study was to assess the cognitive functions in patients with intractable symptomatic temporal lobe epilepsy. Their performance of in cognitive testing was compared to a group of non-refractory epilepsy patients. Methods: 20 patients with medically refractory temporal lobe epilepsy (MRTLE) (Group I) and 20 patients with non refractory temporal lobe epilepsy (NRTLE) (Group II), seen between September 2011 and May 2012 at the INCN-Epilepsy Surgery Program, undertook the neuropsychological test battery NEUROPSI, which includes items that are relevant for native Spanish-speaking individuals.

Results: $80 \%(n=16)$ of MRTLE patients showed cognitive impairment. There were significant differences between the two groups, as those with MRTLE had a mean score of 85.45, while those with NRTLE, had a mean score of $101.69(\mathrm{p}=0.007)$. Although there were no significant differences with respect to memory scores, there was a significant decline in the MRTLE group. In the subgroup analysis of those patients with MRTLE, a much more severe decline in non-verbal memory was noticed in those with right- compared to left- temporal lobe epilepsy ( $\mathrm{p}=0.05$ ).

Conclusions: Medically refractory temporal lobe epilepsy can lead to cognitive decline. On the individual NEUROPSI test scores, memory was more deteriorated in the MRTLE group compared to NRTLE.

\subsection{0 \\ USE OF CAFFEINE DURING INTRA-ARTERIAL AMOBARBITAL PROCEDURE (IAP) \\ Lara M. Schrader ${ }^{1}$, J. Horsfall ${ }^{2}$ and J. M. Stern ${ }^{1}\left({ }^{1}\right.$ Neurology, University of California Los Angeles, Los Angeles, CA and \\ ${ }^{2}$ Psychiatry, University of California Los Angeles, Los Angeles, CA)}

Rationale: Accurate cognitive testing during the IAP requires full patient participation. Amobarbital-induced drowsiness can preclude adequate testing. Various factors may contribute to such sedation, like posterior circulation filling and individual differences in susceptibility to amobarbital sedative side effects. Psychosocial factors may also be important. Patients may sleep less than usual the preceding night due an early morning wake-up for an early morning IAP. Anxiety over the IAP may cause sleep disruption. Lastly, instructions for no food after midnight may cause patients to miss their usual morning coffee. We present two patients with medically refractory left temporal lobe epilepsy who required IAPs as part of their epilepsy surgery evaluation. Both had IAP sleepiness that prevented adequate testing. IV caffeine was administered to combat IAP drowsiness and allow adequate testing.

Methods: A left IAP was planned for the first patient, a 34 year old man. His left internal carotid artery (ICA) angiogram showed perfusion of the left anterior cerebral artery (ACA) and middle cerebral artery (MCA) with a small amount of posterior cerebral artery (PCA) but no basilar artery filling. Injection speed was slowed to 6 seconds to successfully reduce posterior filling. $125 \mathrm{mg}$ amobarbital in $10 \mathrm{ml}$ saline was injected into the left ICA over 6 seconds. The patient became densely obtunded, non-responsive to pain and unable to be awakened. The patient recovered, and the team decided to wait at least 30 minutes and repeat the injection at a lower dose. The patient was sleeping between injections, and it was discovered he normally drinks caffeinated coffee in the morning. To avoid similar issues of drowsiness with the second injection, $500 \mathrm{mg}$ IV caffeine was administered prior to the second IAP. A left IAP was planned for the second patient, a 53 year old female. Her left ICA angiogram showed perfusion of the left ACA and MCA. The PCA was minimally perfused but reduced with a slower injection. $125 \mathrm{mg}$ amobarbital in $10 \mathrm{ml}$ saline was injected into the left ICA over 9 seconds. Cognitive testing was limited due to extreme drowsiness, requiring continual arousal with loud auditory and physical stimulation. Due to results of baseline memory scores and results of the left IAP, a right IAP was then planned. The right ICA angiogram 
showed perfusion of the right ACA and MCA with no perfusion of the PCA. To avoid a similar degree of drowsiness during the second IAP, $500 \mathrm{mg}$ IV caffeine was given.

Results: Upon receiving IV caffeine injection, the first patient went from asleep and snoring to completely awake. Then, $110 \mathrm{mg}$ amobarbital in $10 \mathrm{ml}$ saline was injected into the left ICA over 6 seconds. No sedation occurred, and cognitive testing was successfully completed. The second patient had a right IAP 45 minutes after the left IAP. $125 \mathrm{mg}$ amobarbital in $10 \mathrm{ml}$ saline was injected into the right ICA over 7 seconds. Sedation did not interfere with cognitive testing.

Conclusions: IV caffeine may be useful in select IAP cases in which drowsiness precludes adequate cognitve testing.

\subsection{1}

\section{SCENE ENCODING AND RECOGNITION FMRI PARADIGM REVEALS IPSILATERAL ACTIVATION AFTER PARTIAL RIGHT ANTERIOR TEMPORAL LOBECTOMY}

S. M. Delgado ${ }^{1}$, M. Korostenskaja ${ }^{1}$, C. Salinas ${ }^{2}$, P. C. Chen ${ }^{1}$, J. Cook $^{3}$, J. Baumgartner ${ }^{4,5}$, M. Westerveld ${ }^{2}$ and K. Lee ${ }^{4}\left({ }^{1}\right.$ Functional Brain Mapping and Brain Computer Interface Lab, Center for Pediatric Research and Outcomes, Florida Hospital for Children, Orlando, FL; ${ }^{2}$ Neuropsychology, Florida Hospital for Children, Orlando, FL; ${ }^{3}$ Florida Radiology Imaging, Florida Hospital, Orlando, FL; ${ }^{4}$ Comprehensive Pediatric Epilepsy Center, Florida Hospital for Children, Orlando, FL and ${ }^{5}$ Neurosurgeons for Kids, Florida Hospital for Children, Orlando, FL)

Rationale: Anterior temporal lobectomy (ATL) is the most common surgical procedure for adults with drug-resistant epilepsy. ATL approaches involve removal of varying portions of essential memory structures- hippocampus and parahippocampus. Resection extent and its effect on seizure and memory outcomes remains a topic of debate. Literature reports no different memory deficits for partial vs. complete resection. The results are most consistent for verbal memory decline in left temporal lobe epilepsy (TLE) patients and less so for right TLE patients. Recent studies have focused on the use of fMRI for predicting post-surgical memory outcomes. In the current study, we aimed to evaluate the effect of partial hippocampal resection on memory encoding and recognition in a young adult male with intractable right TLE.

Methods: A 19 yo (EP) with TLE 3 years post-partial right ATL and a healthy 41 yo control (HC) were given the same fMRI paradigm on a GE 3T MRI. Pre-and post cognitive and Wada results were available for EP. We adapted a paradigm described by Szaflarski et al. (2004) that involved scene encoding (indoor and outdoor) alternated with rest, and implemented a delayed recognition task. Behavioral responses during the memory paradigms were recorded and $\%$ accuracy was compared between the 2 participants using onetailed t-test for both encoding and recognition. Active voxels according to threshold (pbonf $<0.01$ ) in the hippocampus region were used to calculate laterality index (LI).

Results: EP's pre-operative Wada indicated bilateral memory representation. Neuropsychology suggested decrease in visual-spatial learning, verbal memory and stable IQ. MRI revealed volume loss in residual hippocampal body, tail and amygdala. EP's fMRI results showed right sided activation during both encoding and recognition blocks ( $\mathrm{LI}=-0.81$ and -0.36 respectively). Behavioral comparisons between $\mathrm{EP}$ and $\mathrm{HC}$ revealed no difference $(\mathrm{p}=0.23)$ during encoding and significant difference $(\mathrm{p}<0.01)$ during recognition blocks regarding \% accuracy. FMRI image analysis indicated an increase in voxel activation between recognition and encoding blocks- $311 \%$ (EP) and 679\% (HC) increase.

Conclusions: This study is unique in that it is the first to show postoperative memory fMRI activation in the hippocampal remnant. Literature has reported minimal difference in memory outcome following partial vs. complete hippocampal resection suggesting that the hippocampus functions as a single unit for memory. Thus, even partial resection impairs function. Ipsilateral activation during memory tasks following partial resection raises questions about the functional process. Behavioral data showed expected findings; right TLE patients are expected to show decline during recognition tasks yet less so during encoding. Preliminary data suggests that a block design encoding and recognition paradigm can be used to predict post-surgical outcomes.

Acknowledgements: Authors express their gratitude to Drs. Szaflarski and Vannest for sharing their paradigm. Second and 3rd authors contributed equally.
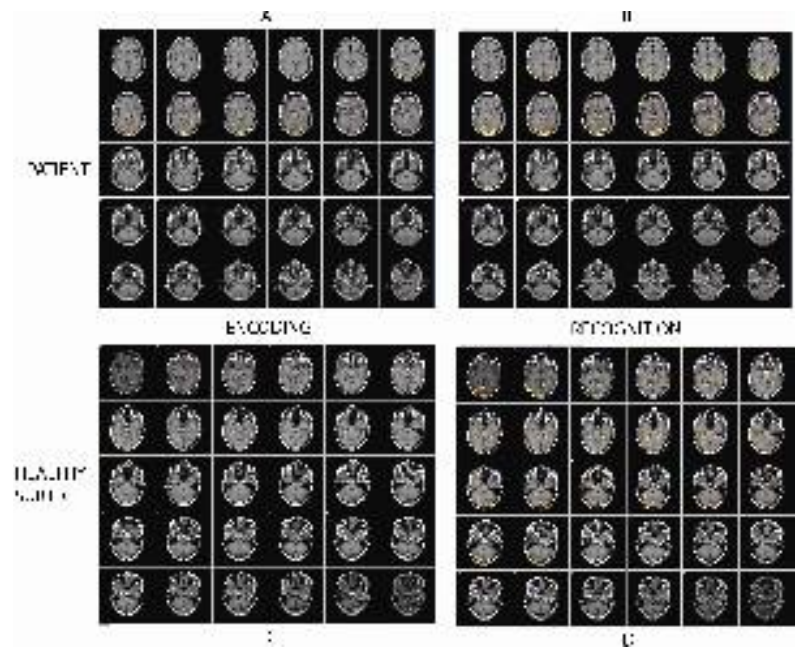

\subsection{2}

\section{UNVEILING THE MYSTERY OF DéJà VU}

M. Brazdil ${ }^{1,2}$, R. Marecek ${ }^{1,2}$, T. Urbanek ${ }^{1,3}$, T. Kasparek ${ }^{1,4}$, M. Mikl $^{1}$, I. Rektor ${ }^{1,2}$ and A. Zeman ${ }^{5}\left({ }^{1}\right.$ Behavioral and Social Neuroscience Research Group, CEITEC - Central European Institute of Technology, Masaryk University, Brno, Czech Republic; ${ }^{2}$ Brno Epilepsy Center, Department of Neurology, St. Anne's University Hospital and Medical Faculty of Masaryk University, Brno, Czech Republic; ${ }^{3}$ Institute of Psychology, Academy of Sciences of the Czech Republic, Brno, Czech Republic; ${ }^{4}$ Department of Psychiatry, Faculty Hospital Brno and Medical Faculty of Masaryk University, Brno, Czech Republic and ${ }^{5}$ Peninsula College of Medicine and Dentistry, University of Exeter, Exeter, United Kingdom)

Rationale: Déjà vu is an eerie experience that is characterized by the recognition of a situation concurrent with the awareness that this recognition is inappropriate. This feeling of irrelevant familiarity is a common phenomenon occurring both in clinical (mainly epileptic) and nonclinical population. Despite numerous theories have been suggested as to what nonpathological déjà vu is and what causes it, until now no ultimate explanation has been generally accepted. Methods: We investigated differences in brain morphology between healthy subjects with and without déjà vu using a novel multivariate neuroimaging technique, Source-Based Morphometry.

The analysis revealed a set of cortical (predominantly mesiotemporal) and subcortical regions in which there was significantly less gray matter in subjects reporting déjà vu. In these regions gray matter volume was inversely correlated with the frequency of déjà vu. Results: Our results demonstrate for the first time a structural correlate of déjà vu in healthy individuals and implicate a direct pathogenetic link between nonpathological and epileptic déjà vu. Conclusions: We hypothesize our findings reflect an alteration of hippocampal function and postnatal neurogenesis in subjects with déjà vu. 
Behavior/Neuropsychology/Language: Pediatrics

\subsection{3 \\ HIPPOCAMPAL ASYMMETRY AND MEMORY PERFORMANCE IN CHILDREN FOLLOWING PROLONGED FEBRILE SEIZURES}

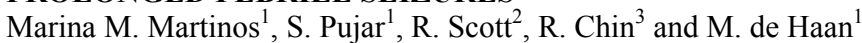
( ${ }^{1}$ University College London, London, United Kingdom; ${ }^{2}$ Dartmouth Medical School Lebanon, Lebanon, $\mathrm{NH}$ and ${ }^{3}$ The Muir Maxwell Epilepsy centre, University of Edinburgh, Edinburgh, United Kingdom)

Rationale: Retrospective studies highlight an association between hippocampal sclerosis and a childhood history of prolonged febrile seizures (PFS). Our own work has shown that despite the lack of mean hippocampal volume (HV) differences between children following PFS and controls, a larger proportion of children following PFS have hippocampal asymmetry (Pujar et al., AES 2012 abstract; Scott et al., 2002). Given the established segregation of memory roles within the hippocampus, i.e. visual memory is more dependent on the right hippocampus and verbal memory is more dependent on the left hippocampus, we wanted to investigate whether these roles were preserved in children following PFS or whether plasticity interfered with these normal functional divisions post-PFS.

Methods: We have recruited children with a history of PFS originally identified by the population-based North London Convulsive Status Epilepticus in childhood surveillance study (NLSTEPSS). Recruited children underwent detailed investigations to determine their outcome 6-10 years following their episode. Memory abilities were assessed using the Children's Memory Scale (CMS) which provides a proxy for both visual delayed and verbal delayed memory scores. To arrive at our discrepancy score we subtracted the verbal from visual memory scores. Hippocampal volumetry was performed on 3D FLASH images using FSL version 4.1.9. The HV was measured for each side and right-left asymmetry was calculated using the asymmetry index $(\mathrm{AI}=(\mathrm{HV}$ Right- HV Left $)$ / 0.5x (HV Right+ HV Left)). Pearson's correlations were used to investigate relationships between AI and memory in SPSS version 18.

Results: Nineteen children (mean age: 9.1 years; range: 7-13 years) have completed MR and neuropsychological investigations a mean of 7.6 years following their episode. Their general memory quotient falls within the normal range (mean: 102.7), and is highly correlated with their FSIQ ( $r=0.676, p=0.001)$. One PFS case (patient with highest AI) was excluded from the correlational analysis due to the presence of neurological comorbidities (neurofibromatosis), which may have affected his developmental trajectory. There is a positive relationship between AI and memory discrepancy scores in PFS patients ( $\mathrm{r}=0.492, \mathrm{p}=0.038$ ) (Figure) suggesting the presence of normative structural-functional relationships in this group. Children with larger right compared to left hippocampi were shown to do better on visual compared to verbal memory materials and the opposite was true of children with larger left compared to right hippocampi.

Conclusions: Despite the higher incidence of hippocampal asymmetry in children following PFS, our findings suggest that experiencing a PFS during childhood does not alter the functional roles undertaken by the hippocampus during development.

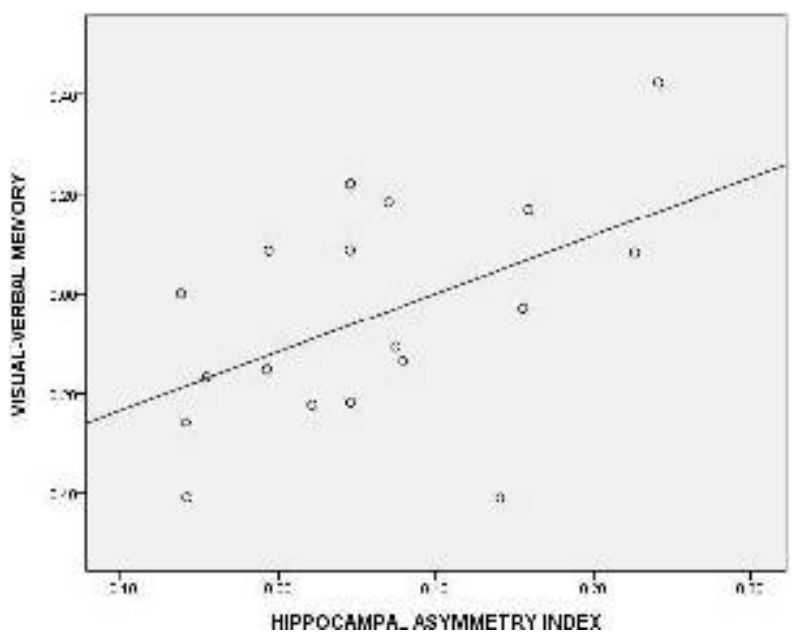

\subsection{4 \\ CHILDHOOD ABSENCE EPILEPSY: CORRELATION BETWEEN SEIZURE FREQUENCY AND NEUROPSYCHOLOGICAL PROFILE}

Luis E. Bello-Espinosa ${ }^{1,2}$, J. Rho ${ }^{1,2}$, A. Datta ${ }^{1,2}$, B. Brooks ${ }^{1,2}$ and M. Scantlebury ${ }^{1,2}$ ( ${ }^{1}$ Paediatric Neurology, Alberta Children's Hospital, Calgary, AB, Canada and ${ }^{2}$ Paediatric Neurology, University of Calgary, Calgary, AB, Canada)

Rationale: Children with childhood absence epilepsy (CAE) are often initially suspected of having attention deficit hyperactivity disorder (ADHD) given that both conditions share some common semiological features. Not surprisingly, the neuropsychological profile of children with CAE is presumed to be similar to those with ADHD. However, given the perceived benign prognosis of CAE, very few children with CAE are referred for formal neuropsychological testing. The objective of this study was to determine whether the frequency of absence seizures correlates with neuropsychological impairment.

Methods: Eighteen children and adolescents (mean age $=10.7$, $\mathrm{SD}=4.5$, range $=4.6-17.5$ ) with an established diagnosis of CAE were referred for neuropsychological assessment. Mean levels of parental education were 13.8 years $(\mathrm{SD}=2.4)$ for mothers and 14.0 years $(\mathrm{SD}=2.5)$ for fathers. The sample was mostly Caucasian $(77.8 \%)$ and right-handed $(88.9 \%)$, with slightly more females $(55.6 \%)$ than males (44.4\%). Correlations between the number of absence seizures and standardized neuropsychological measures of attention, perception, as well as motor, linguistic and academic abilities were determined. Additionally, scores on the Parental ADHD Rating Scale, Impact of Impact of Childhood Neurologic Disorder rating of QOL and Teacher ADHD Rating Scale Teacher questionnaires were obtained.

Results: Patients had a median of 45 absence seizures per month (range $=0-3000$, mean=304.5, $\mathrm{SD}=719.4$ ). Spearman's rho correlations between number of absence seizures per month and ratings of inattention, hyperactivity, internalizing or externalizing behaviours, adaptive functioning, daily executive abilities, and quality of life were not significant. Several correlations did exceed rho $>0.30$ for parental (i.e., ADHD Rating Scale Parent Total Percentile, rho=0.317; Impact of Childhood Neurologic Disorder, rho $=0.444$; Impact of Childhood Neurologic Disorder rating of QOL, rho=0.402) and teacher questionnaires (ADHD Rating Scale Teacher Total Percentile, rho $=0.397$ ). There was no statistically significant difference in the number of absence seizures over the course of one month between those with and those without an ADHD diagnosis (ADHD diagnosis, mean seizures $=76.14, \mathrm{SD}=129.69$; No ADHD diagnosis, mean seizures $=464.4,50=916.51$; Mann-Whitney $U$ $\mathrm{p}=0.161$, Cohen's $\mathrm{d}=0.66$ ).

Conclusions: In our cohort of children with CAE, only $39 \%(n=7)$ received a formal diagnosis of ADHD by a neuropsychologist. There was no statistically significant difference in the number of absence 
seizures over a given month between those with and without an ADHD diagnosis. However, despite a lack of a significant correlation, our results suggest that parents report fewer problems with inattention and quality of life in children with a greater frequency of absence seizures, whereas teachers tend to report more problems with inattention in these same children.

\subsection{5}

\section{AGE OF SEIZURE ONSET PREDICTS REDUCED} ADAPTIVE FUNCTIONING IN PEDIATRIC EPILEPSY

A. Carbonell, Michael Westerveld and C. M. Salinas (Florida Hospital, Orlando, FL)

Rationale: Rationale: Adaptive behavior in children with epilepsy is an area that has received limited attention in the literature compared to other cognitive and emotional domains. However, adaptive behavior (the child's ability to function independently and effectively in multiple settings) is closely related to quality of life in children with epilepsy as well as for their caregivers. Better understanding of how epilepsy affects a child's adaptive behavior may lead to earlier and more specific interventions, improving quality of life over time. Methods: Methods: A consecutive sample of children referred for neuropsychological testing due to seizures was included in the present study. The sample consisted of 64 children with epilepsy (37 boys; 27 girls) who ranged from 5-16 years old $(\mathrm{M}=9.78)$. All parents completed the Behavior Assessment Scales for Children (BASC) as part of a comprehensive neuropsychological examination. Information about seizure history and treatment was obtained in a clinical interview with the parents, and retrospective chart review. The mean age of seizure onset was 5.92; average duration of seizure disorder was 4.04 years.

Results: Results: Linear regression was performed, with epilepsy (age of onset, seizure status within previous year) and treatment variables (number of AEDs) as predictors of the Adaptive Skills Composite, and Social, Leadership, and Adaptability subscales on the BASC. Seizure variables accounted for $23 \%$ of the variance $(p=.043)$ on the Adaptive Skills Composite. Age of onset $(\mathrm{p}=.034)$ was the best single predictor. For the social skills subscale, only age of onset and number of AED's contributed to the model, which accounted for $32 \%$ of the variance $(p=.009)$. Seizure variables did not predict the adaptability or leadership subscales of the BASC adaptive skills composite.

Conclusions: Conclusions: Adaptive behavior deficits were associated with earlier age at seizure onset, particularly social skills, suggesting that seizures interfere with a child's acquisition of basic skills for daily living. Deficits in adaptive behavior that impact quality of life begin early in the course of seizure disorder due to the disruption of normal function during critical periods for acquisition of social skills, as well as developing independence in basic activities of daily living. These findings provide further support for social and behavior interventions in children with seizures as soon as seizures are diagnosed.

\subsection{6 \\ ASSOCIATED FACTORS WITH POOR ADHERENCE TO TREATMENT IN ADOLESCENT PATIENTS WITH \\ EPILEPSY \\ Michel Gutierrez Ceniseros and E. Barragan Perez (Pediatric Neurology, Hospital Infantil de Mexico, Mexico, Mexico)}

Rationale: During adolescence various types of epilepsy emerge, coexisting with physical, hormonal, sexual, and psychosocial changes, which can interfere with adherence to treatment. There are few studies in adolescents describing the variables that affect therapeutic adherence in this age group.

Objective. Describe the associated factors with poor adherence to treatment in adolescent patients with epilepsy
Methods: We included 100 patients with epilepsy, between eleven and eighteen years old, who attend to the Pediatric Neurology Department, between October 2011 and May 2012.Through direct interviews and a questionnaire of 35 questions geared towards physical, social, academic and personal expectations, where responses by type stickler established the presence or absence of factors of poor adherence to treatment.

Results: We evaluated 100 patients showing a clear tendency of having poor adherence to treatment $(>80 \%)$ because of psychosocial factors. The economic situation seems to be less important in the decision to continue treatment. There were no statistically significant differences between gender. Interestingly, one third of adolescents suspended treatment for reasons of fun, such as alcohol or drugs. Conclusions: Psychosocial factors are associated with poor adherence to treatment in adolescent patients with epilepsy. This establishes the need of better educational guidance which will enhance the knowledge of the patient for their treatment adherence reducing the impact of social stigma of epilepsy.

\subsection{7 \\ NEUROCOGNITIVE TRENDS IN BILINGUAL VERSUS MONOLINGUAL PEDIATRIC EPILEPSY PATIENTS} M. Connolly ${ }^{1}$, A. Hanratty ${ }^{1}$, G. Mucci ${ }^{1}$, A. Martinez ${ }^{1}$, M. Zupanc ${ }^{2,3}$ and J. Lin ${ }^{3,2}$ ( ${ }^{1}$ Pediatric Psychology, CHOC Children's Hospital, Orange, CA; ${ }^{2}$ Pediatric Neurology, CHOC Children's Hospital, Orange, $\mathrm{CA}$ and ${ }^{3} \mathrm{Neurology}$, University of California, Irvine, Irvine, CA)

Rationale: It is widely understood that various neuropsychological domains may be impacted in children with seizure disorders, depending on variables such as seizure location, age of onset, medication, and severity of seizures. However, there is little known regarding the impact that bilingualism has on the neurocognitive profile of pediatric epilepsy patients. Previous research has demonstrated that bilingual individuals tend to differ from their monolingual peers on various measures of intellectual ability. Bilingualism may also mitigate the deleterious impact that neurodegenerative illnesses have on a variety of domains due to the recruitment of different brain regions during cognitive tasks and increased opportunities to practice cognitive control. Given these findings, the goal of the present study was to elucidate the neurocognitive differences between bilingual and monolingual pediatric epilepsy patients in the domains of intellectual ability. Methods: The sample included 24 (11 bilingual; 13 monolingual) pediatric patients diagnosed with epilepsy. Nine of the bilingual participants were fluent in English and Spanish, one was fluent in English and Korean, and one was fluent in English and Tagalog. All participants were English-language dominant at the time of evaluation. There were no significant differences in age at, or time since diagnosis between the two groups. As part of a routine comprehensive neuropsychological evaluation, the participants were administered measures of intellectual ability such as the StanfordBinet, the Wechsler Intelligence Scale for Children, and the Differential Abilities Scale. Full scale IQ, verbal comprehension, perceptual reasoning, processing speed, and working memory results between the two groups were compared and differences were analyzed using ANCOVA, t-tests, and simple linear regression. Analyses were controlled for socioeconomic status (SES), which was determined using the Barratt Simplified Measure of Social Status. Results: A significant differences was observed between the groups on processing speed $(\mathrm{p}<.05)$ and approaching significance on working memory $(\mathrm{p}=.06)$ with monolinguals scoring higher on both measures. Verbal comprehension was found to be significantly lower than perceptual reasoning in the bilingual group $(\mathrm{p}<.01)$, while verbal comprehension was higher (though not significantly) than perceptual reasoning in the monolingual group. Additionally, earlier age of onset appeared to affect bilinguals more adversely than monolinguals with regards to full scale IQ, perceptual reasoning, and processing speed. 
Conclusions: Results indicate that there are noteworthy differences between the neurocognitive profiles of monolingual versus bilingual children with epilepsy. Specifically, bilingual patients demonstrate increased risk for declines in certain aspects of intellectual functioning as a function of age at onset. Due to the small sample size of this study, further research is needed to better clarify the role that bilingualism plays in the neurocognitive outcome of pediatric epilepsy patients.

\subsection{8 \\ FACTORS ON EMOTIONAL AND BEHAVIORAL PROBLEMS IN PEDIATRIC EPILEPSY}

Soyong Eom ${ }^{1}, \mathrm{~K} . \mathrm{Oh}^{2}$ and H. Kim ${ }^{3}\left({ }^{1}\right.$ Pediatric Psychiatry, Yonsei University College of Medicine, Severance Children's Hospital, Seoul, Republic of Korea; ${ }^{2}$ Psychology, Yonsei University, Seoul, Republic of Korea and ${ }^{3}$ Pediatric Neurology, Pediatric Epilepsy Clinics, Severance Children's Hospital, Brain Research Institute, Yonsei University College of Medicine, Seoul, Republic of Korea)

Rationale: to investigate the factors of demographic, epilepsy-related clinical, and psychological variables influencing on the psychosocial functions in pediatric epilepsy.

Methods: On a hospital-based and retrospective cross-sectional study, patients were recruited for the evaluations and patients' parents were established scales for their children with epilepsy. Subjects were children and adolescents $(n=311)$ between the ages of 4 and 18 years (boys $=171$, girls $=140$ ), who had been diagnosed for epilepsy. To investigate the psychosocial function in pediatric epilepsy as the dependent variables, intelligence, general adaptive function, emotional/social behavior problem, and quality of life were evaluated. As the independent variables, influencing factors, demographic variables such as age and sex, epilepsy-related clinical variables such as the age of onset, seizure duration, seizure frequency, the number of medication were included. Furthermore for psychological factors, the evaluation of parent's depression, parenting stress, and parenting attitude were included as the parenting environmental factors. Multiple linear regression analysis was performed to determine the influence of each independent variable(demographic, epilepsy-related clinical, and psychological variables) on dependent variables(cognitive/academic and emotional/social competence, general adaptive function, quality of life).

Results: Analyses on the effects of epilepsy-related clinical variables and psychological variables on general adaptive function of pediatric epilepsy showed a major difference in competence between cognitive/academic and emotional/social, demonstrating that epilepsy-related clinical variables have comparatively large influence on cognitive/academic competence such as intelligence and school competence, while psychological variables on emotional/social such as social competence and behavior problems. Whereas the results for effect of epilepsy-related clinical variables showed a relatively high influence on general adaptive function compared to psychological variables, the psychological variables as parenting environment showed a relatively high influence on quality of life compared to clinical variables.

Based on analyses on the significance of each independent variable, the number of medication and seizure duration within epilepsyrelated clinical variables were significant compared to other variables. Furthermore, parenting stress(child variable), expectation/supervision parenting attitude, parenting stress(parentchild relationship), negative and positive parenting attitude showed significant effects on the psychosocial function of pediatric epilepsy. Conclusions: Comprehensive management of children with epilepsy requires entire evaluation and understandings of clinical and psychological related variables for better therapeutic and psychosocial outcomes.

\subsection{9 \\ THE ACCURATE DIAGNOSIS AND THE CLINICAL OUTCOMES OF NONEPILEPTIC PAROXYSMAL EVENTS IN THE PEDIATRIC PATIENTS}

Yi Yoon Young, K. Hyo Jung, K. Heung Dong, L. Joon Soo, L. Young Mock and K. Hoon-Chul (Pediatrics, Pediatric epilepsy clinic, Severance Children's Hospital, Yonsei University College of Medicine, Seodaemun-gu, Republic of Korea)

Rationale: Psychogenic nonepileptic seizures (PNES) are the major causes of nonepileptic paroxysmal events (NPEs). However, there are many other reasons of NPEs in the pediatric population. And moreover, actual epileptic seizures are occasionally mistaken for PNES. The aim of our study was to review the final diagnosis and clinical outcomes, of the pediatric patients who was diagnosed with NPEs

Methods: We retrospectively reviewed the hospital chart of 48 patients who diagnosed with NPEs from January, 2005 to December, 2011.

Results: The mean age of patients at diagnosis was $11.13 \pm 5.04$ years and the male to female ratio was 1.8:1. Video

electroencephalography was performed in 33 patients. The results were normal in $93.9 \%$ (31 of 33), and epilepsy in 2 patients.

Provocation test was performed in 15 patients, 10 patients had seizures in response to suggestion and intravenous saline injection. The final diagnosis were as follows: PNES( $\mathrm{n}=25,52.1 \%$ ), epilepsy $(n=4,8.3 \%)$, psychosis $(n=3,6.2 \%)$, chorea $(n=2,4.2 \%)$, tic $(n=2,4.2 \%)$, and others (migraine, parasomina, side effect of drugs, autonomic symptoms, infantile masturbation, shudder attack, and jitterness). Before the final diagnosis, 27 patients had taken antiepileptic drugs. Twenty-three patients diagnosed with NPEs(23 of $44,52.2 \%$ ) were exposed to unnecessary antiepileptic drugs. High percentage of patients(n=20,41.7\%) were lost at follow-up after accurate diagnosis, and $60 \%$ of these patients were PNES. After a mean period of 14 months of follow-up in 28 cases, 21 cases $(75 \%)$ were symptom free.

Conclusions: In our pediatric patients, there are various causes of NPEs. Video electroencephalography was helpful to differentiate these mimics from epilepsy. Although the overall prognosis of NPEs in the follow-up groups was good however, there were many lost patient after diagnosis.

\subsection{0 \\ RECEPTIVE AND EXPRESSIVE LANGUAGE FUNCTIONING IN BILINGUAL AND MONOLINGUAL PEDIATRIC EPILEPSY PATIENTS}

Grace A. Mucci ${ }^{1}$, A. Hanratty ${ }^{1}$, M. Connolly ${ }^{1}$, A. Martinez ${ }^{1}$, M. Zupanc $^{2,3}$ and J. Lin ${ }^{3,2}\left({ }^{1}\right.$ Pediatric Psychology, CHOC Children's Hospital, Orange, CA; ${ }^{2}$ Pediatric Neurology, CHOC Children's Hospital, Orange, CA and ${ }^{3}$ Neurology, University of California, Irvine, Orange, CA)

Rationale: It is known that children with epilepsy are at risk for neurocognitive deficits, and those with temporal/frontal lobe epilepsy are at increased risk for language impairment. Furthermore, early age of onset in the dominant hemisphere may result in atypical language lateralization (Helmstaedter et al, 1997), which can have significant implications for surgical outcome. Clinical experience in our setting suggests that bilingual children with epilepsy experience greater language deficits compared to their monolingual counterparts. Several studies have found that the functional brain organization of bilinguals and monolinguals have distinct and shared sites that support both languages (Lucas et al., 2004). However, little is known about the role that bilingualism plays with respect to the nature and degree of language difficulties in children with epilepsy. This retrospective study aims to examine the differences in monolingual and bilingual children diagnosed with epilepsy on language tasks. 
Methods: The sample included 24 children diagnosed with temporal or frontal lobe epilepsy who completed comprehensive evaluations. Two children were eliminated from the analyses due to comorbid diagnoses of Autism Spectrum Disorders, leaving 11 bilingual and 11 monolingual patients. Nine of the bilingual participants were bilingual English/Spanish, one was bilingual English/Korean, and one was bilingual English/Tagalog. There were no significant differences in age at, or time since diagnosis between the two groups. Expressive and receptive language abilities were assessed with standard validated language measures. Analyses were controlled for seizure foci.

Results: Hierarchical regression analyses were conducted to evaluate whether bilingualism significantly predicted performance on language assessments above what is predicted by seizure foci. Bilingualism accounted for a significant proportion in variance of the standard scores on measures of expressive language, $\mathrm{R} 2$ change $=$ $.461, \mathrm{~F}(1,7)=6.967, \mathrm{p}=.0165$, suggesting that patients with similar seizure foci are likely to perform better on tests of expressive language if they are monolingual rather than bilingual. Bilingualism did not significantly predict performance on receptive language assessments.

Conclusions: Results suggest that based on our small study sample, pediatric bilingual epilepsy patients demonstrate increased risk for language difficulties, particularly expressive language. As found in previous studies, age of second language acquisition may be an important mediator, particularly for receptive language (Dehaene et al., 1997). The functional neural organization of language in bilingual children with epilepsy needs to be examined to aid in pre-surgical evaluations and help predict post-surgical outcome. Gaining significance with such a small sample size in the present study is promising, and future research is indicated to better understand the neurocognitive, neurodevelopmental, and neuropathological processes involved in the developing bilingual child with epilepsy.

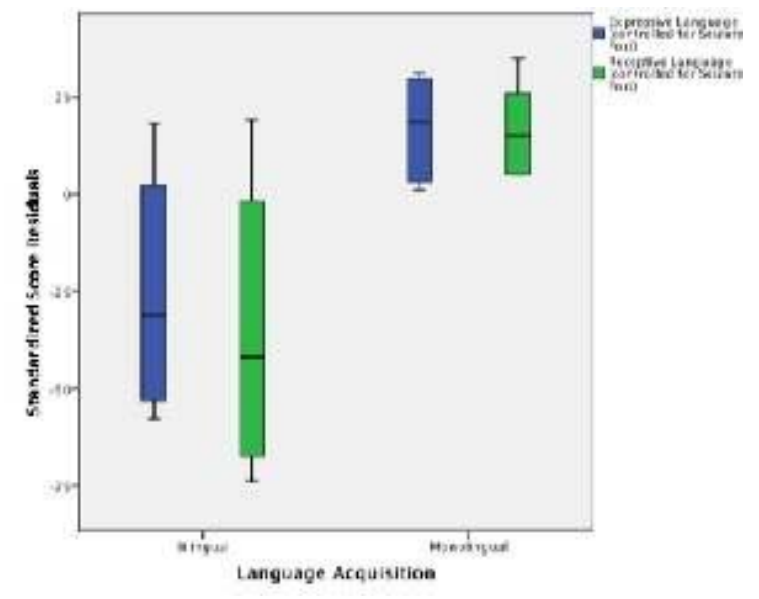

\subsection{1}

SIGNIFICANT IMPACT OF BEHAVIORAL PROBLEMS ON QUALITY OF LIFE IN HISPANIC URBAN CHILDREN WITH EPILEPSY

Arthur Partikian $^{2,1}$, A. Sandoval ${ }^{2}$, L. Hoang ${ }^{2}$ and S. Stewart ${ }^{2}\left({ }^{1}\right.$ Los Angeles County and USC Medical Center, Los Angeles, CA and ${ }^{2}$ Neurology \& Pediatrics, University of Southern California, Los Angeles, CA)

Rationale: It is well known that both biological and psychosocial variables influence the behaviors of epileptic children. We have been conducting an observational study to better understand the extent, types, possible determinants, and impact on quality of life of behavioral problems noted by parents of epileptic Hispanic children from predominantly Spanish-speaking households.

Methods: We recruited subjects between 2 to 18 years of age clinically diagnosed with epilepsy receiving ongoing treatment at Los
Angeles County + University of Southern California Medical Center. Sociodemographic and epilepsy information were collected, and caregivers completed the Short Acculturation Scale for Hispanics (SASH), developmentally and language appropriate versions of the Child Behavior Checklist (CBCL), Adaptive Behavior Assessment System II (ABAS), Pediatric Quality of Life Inventory (PedsQL), and Impact of Childhood Neurologic Disease Scale (ICNDS). Minitab Release 14.20 software was utilized for statistical analyses.

Results: We have enrolled 130 subjects with an age of $10.9 \pm 4.7$ years (mean \pm standard deviation), with $55 \%$ being males and $95 \%$ identifying themselves as Hispanic or Latino. $73 \%$ stated that they have an annual household income of less than $\$ 20,000$, with $69 \%$ overall receiving some form of government aid. Etiologically, $14 \%$ of subjects have genetic forms of epilepsy, $59 \%$ structural $/$ metabolic causes, and $27 \%$ unknown. In terms of seizure control, $52 \%$ were experiencing less than one seizure over the past three months, while the remainder had variable but higher frequency of seizures. Patients with low ABAS scores had significantly worse epilepsy control $(\mathrm{P}=$ $0.001)$ and more structural/metabolic etiologies, with poorer quality of life scores (P values $<0.001$ for both PedsQL and ICNDS). We found that $32 \%$ versus $30 \%$ score in the clinically significant range for internalizing versus externalizing behavioral problems on CBCL, respectively, with $17 \%$ having significant behavioral dysfunction in both domains. We found no correlation between clinically-significant CBCL T-scores and age, gender, or seizure characteristics (etiology, frequency, and number of antiepileptic medications). However, CBCL internalizing but not externalizing T-scores negatively correlated with ABAS scores (P value $<0.01$ ) and with two healthrelated quality of life scales (PedsQL and ICNDS, both P values $<$ 0.001 ). While we found a statistically significant negative correlation between acculturation scores and CBCL internalizing T-scores, the relatively low acculturation score of $1.8 \pm 0.97$ (mean \pm standard deviation) of our culturally-homogenous cohort prevents clinically meaningful interpretation. Moreover, acculturation scores alone were not associated with quality of life scores.

Conclusions: Even amongst poor Hispanic children from predominantly Spanish-speaking households, internalizing behavioral problems appear to be predictive of health-related quality of life, independent of seizure control, number of anticonvulsant drugs, and underlying epilepsy etiology.

\section{Genetics: Human Studies}

\subsection{2 \\ MYOTONIA IN BRAIN: 'SKELETAL' CHLORIDE CHANNEL CLC-1 LINKED TO IDIOPATHIC GENERALIZED EPILEPSY WITH FOCAL MYOTONIA Tara L. Klassen ${ }^{1}$, T. T. Chen ${ }^{1}$, J. G. Reed ${ }^{1}$, M. J. Kole ${ }^{1}$, A. M. Goldman $^{1}$, C. Marini ${ }^{2,3}$, R. Guerrini ${ }^{2,3}$ and J. L. Noebels ${ }^{1}$ ( ${ }^{1}$ Baylor College of Medicine, Houston, TX; ${ }^{2}$ Children's Hospital A. Meyer- University of Florence, Florence, Italy and ${ }^{3}$ IRCCS Stella Maris Foundation, Pisa, Italy)}

Rationale: The human $C L C N 1$ gene encodes the ClC-1 voltage-gated chloride channel that regulates excitability in skeletal muscle, where missense and nonsense mutations cause non-dystrophic myotonia congenita. Unlike family members CLCN2 (ClC-2) and CLCN3 (ClC-3), its presence and pathogenic potential in human brain have not been reported. We analyzed ion channel gene variant profiles in individuals with complex idiopathic epilepsy syndromes and identify a novel de novo CLCN1 human truncation mutation, demonstrate regional brain expression, and explore $C L C N 1$ as a candidate gene for comorbid brain phenotypes in a mouse model of myotonia. Methods: We used parallel exomic sequencing of 237 ion channel subunit genes to screen individuals with a clinical diagnosis of epilepsy including those with a muscle/movement disorder comorbidity. We examined regional expression of CLCN1 in human and mouse brain using RT-PCR, in situ hybridization, and Western 
immunoblotting. The truncated Clcn1-adr ${ }^{\mathrm{mto} 2 \mathrm{~J}}$ mouse model of myotonia was evaluated for epilepsy by videoEEG.

Results: We report a novel de novo CLCN1 truncation mutation in a patient with pharmacoresistant convulsive and absence seizures and a dystonic writer's cramp without evidence of other known genomic channelopathy for epilepsy. Molecular localization of CLCN1 and its mouse homologue Clcn1 revealed wide expression of mRNA and subunit protein in human and murine brain. Cortical EEG recordings in the myotonic Clcn1 truncation mouse demonstrated generalized synchronous discharges associated with absence-like behavioral arrest. Epileptiform discharges in littermate controls did not display behavioral arrest.

Conclusions: Disruption of the 'muscle' voltage-gated chloride channel gene reveals a candidate role for CLCN1 in human CNS excitability disorders. This is the first membrane ion channel gene linking a muscle and brain excitability phenotype. Our study illustrates how genetic screening can lead to the discovery of shared CNS expression of voltage-gated ion channels with disparate tissues, pointing to interesting and sometimes occult co-morbid neurological syndromes. Elucidating molecular links for these co-morbid syndromes may enhance their clinical detection as well as provide a more efficient therapeutic target in the treatment of multiple pathologies caused by single ion channel gene defects. Supported by Epilepsy Foundation Postdoctoral Fellowship (TLK), HHMI Medical Student Fellowship (MJK), and NINDS (JLN).

\subsection{3}

\section{CHROMOSOMAL MICROARRAY IS HIGH YIELD FOR} IDENTIFYING COPY NUMBER VARIANTS IN EPILEPSY PATIENTS

Heather Olson ${ }^{1,2}$, J. M. Avallone ${ }^{1,2}$, Y. Shen ${ }^{2,3}$, B. Wu $\mathrm{Wu}^{2,3}$ and A. Poduri ${ }^{1,2}\left({ }^{1}\right.$ Department of Neurology, Division of Epilepsy and Clinical Neurophysiology, Boston Children's Hospital, Boston, MA; ${ }^{2}$ Harvard Medical School, Boston, MA and ${ }^{3}$ Department of Laboratory Medicine, Boston Children's Hospital, Boston, MA)

Rationale: The objective was evaluate the yield of clinical chromosomal microarray (CMA) testing in a retrospective series of patients with epilepsy, and to identify novel or rarely reported overlapping copy number variants $(\mathrm{CNVs})$ in this population. Methods: We identified 977 patients with an ICD9 code for epilepsy or seizures who had a clinical CMA performed between October 2006 and February 2011 in the Children's Hospital Boston system. We retrospectively reviewed medical records to identify if the patients met clinical criteria for epilepsy and define abnormalities on CMA.

Results: On review of records, 4 patients were excluded because CMA results were not in the chart and 167 patients were excluded because they did not have clinical epilepsy. Some excluded patients had febrile seizures only or evaluation for spells which were not thought to be seizures by the treating neurologist. Of the remaining 806 patients, the CMA showed at least one CNV in 324 patients $(40 \%)$ and was normal in $482(60 \%)$. The total number of CNVs was 437 (1-4 per patient) of which there were 183 deletions (42\%) and 254 (58\%) duplications. Thirty-five were confirmed as de novo, 183 were inherited, and inheritance was unknown in 219 . Size ranged between $18 \mathrm{~kb}$ and $19 \mathrm{Mb}$, except one $142 \mathrm{Mb}$ duplication and 3 chromosomal trisomies (X, 21, and 9). One hundred forty-seven CNVs were over $500 \mathrm{~kb}$ in size (33\%). This series includes at least 5 deletions and 1 duplication involving known epilepsy related genes (KCNQ2, SCN1A, PLCB1, and CACNB4), and 24 CNVs in known hotspots for CNVs in idiopathic epilepsy (15q11.2, 15q13.3, 15q11$\mathrm{q} 13,16 \mathrm{p} 11.2,16 \mathrm{p} 13.11)$. It also includes CNVs associated with known syndromes that are associated with epilepsy such as PotockiLupski syndrome, Mowat-Wilson syndrome, Angelman syndrome, Williams-Beuren region reciprocal syndrome, MECP2 duplication syndrome, Phelan-McDermid syndrome, 22q11 duplication syndrome, 1 p36 deletion syndrome, and $6 \mathrm{q}$ deletion syndrome. In addition, there are numerous CNVs that occur multiple times with overlap. These may have novel associations with epilepsy, but require further investigation and comparison with controls. For example there are three overlapping deletions involving Xp22.31 ( $859 \mathrm{~kb}, 1.6 \mathrm{Mb}$, and $9.6 \mathrm{Mb}$ in size), one de novo and two of unknown inheritance. There are two case reports in the literature of patients with deletions in this region and associated epilepsy and intellectual disability, one with cortical heterotopias. There are 5 overlapping duplications in the same region, ranging from $311 \mathrm{~kb}$ to $1.6 \mathrm{Mb}$ in size.

Conclusions: The yield of CMA testing in epilepsy patients is high, and not infrequently identifies known abnormalities associated with epilepsy. Further phenotypic evaluation of rare or novel repetitive CNVs is warranted and may identify new genotype- phenotype correlations or strengthen rarely reported genotype-phenotype associations. For example, data support the association of CNVs at Xp22.31 with epilepsy.

\subsection{4 \\ EVIDENCE FOR A SHARED GENETIC SUSCEPTIBILITY TO MIGRAINE AND EPILEPSY IN THE EPILEPSY PHENOME/GENOME PROJECT}

Melodie R. Winawer ${ }^{1,2}$, R. Connors ${ }^{3}$ and .. The EPGP Investigators ${ }^{1,2}$

( ${ }^{1}$ G.H. Sergievsky Center, Columbia University, New York, NY; ${ }^{2}$ Neurology, Columbia University, New York, NY and ${ }^{3}$ Neurology, New York University, New York, NY)

Rationale: The comorbidity of migraine and epilepsy is well established but the genetics of this comorbidity remains unclear. Here we investigate the shared genetic effects on migraine and epilepsy in the Epilepsy Phenome/Genome Project (EPGP) cohort by examining the relationship of migraine prevalence to family history of epilepsy.

Methods: EPGP is an ongoing multi-site epilepsy genetics consortium whose goal is to recruit, perform detailed phenotyping on, and collect DNA from 3,750 participants with epilepsy and 1,500 parent controls. The analysis presented here is restricted to a subset of the cohort consisting of sibling and parent-child pairs with nonacquired focal epilepsy (NAFE) and idiopathic generalized epilepsy (IGE). Information on headache was collected in EPGP using a validated instrument. We restricted our analyses to individuals over 12 years of age because the instrument is validated for respondents $\geq 12$ years. We analyzed the predictors of migraine in current EPGP data. According to the current International Headache Society diagnostic criteria, migraine with aura (MA) and migraine without aura (MO) are not mutually exclusive; hence to distinguish the predictors of MA and MO, we defined a new category consisting of MO without MA (MO "only"). In the EPGP screening interviews, participants were asked to report additional relatives (beyond the affected sibling or parent-child pair) affected with epilepsy or a seizure disorder. We identified 1,155 additional relatives reported to have seizure disorders who were not enrolled in EPGP; 380 of these were first degree relatives of enrolled participants. We examined migraine prevalence and subtypes in enrolled participants in relation to family history of seizure disorders in these additional relatives for 917 enrolled EPGP participants with IGE or NAFE in 570 EPGP families.

Results: Prevalence of a history of migraine in EPGP was much higher than that in population-based series: for females $33 \%$ vs. $18 \%$, for males $16 \%$ vs. $6 \%$, respectively. Prevalence did not differ by epilepsy type. Prevalence of migraine in EPGP enrolled participants increased with increasing number of additional relatives reported to have seizure disorders in the family. This increase was most pronounced for the number of affected first-degree relatives with epilepsy, and most pronounced when $\geq 2$ additional relatives (beyond the enrolled pair) were reported to have seizure disorders. The increase in prevalence of migraine with increasing number of additional reported affected relatives with epilepsy was seen in both MA and MO but was more prominent for MA than MO (Figure 1). 
The relationship between number of additional individuals with seizure disorders and migraine prevalence in the enrolled relatives persisted in both IGE and NAFE.

Conclusions: These findings support a shared genetic susceptibility for epilepsy and migraine that is stronger for MA, and present in both IGE and NAFE.

\section{Figure 1. Lifetime Prevalence of Migraine in EPGP Participants, by History of Seizure Disorders in Non-Enrolled First-Degree Relatives}

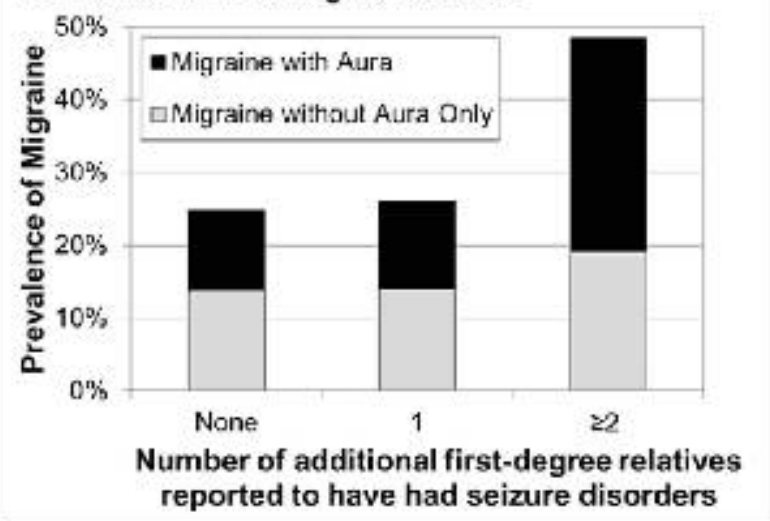

\subsection{5}

BIALLELIC DELETION OF THE NRXN1 GENE CAUSING AUTISM, DEVELOPMENTAL DELAY AND EPILEPSY IN FRATERNAL TWINS

J. Imitola ${ }^{1,3}$, C. E. Anderson ${ }^{1,2}$, K. Carvalho ${ }^{1,2}$, A. Legido ${ }^{1,2}$ and Divya S. Khurana ${ }^{1,2}\left({ }^{1}\right.$ St. Christopher's Hospital for Children, Philadelphia, PA; ${ }^{2}$ Pediatrics, Drexel University College of Medicine, Philadelphia, PA and ${ }^{3}$ Neurology, Temple University Medical Center, Philadelphia, PA)

Rationale: Neurexin 1 (NRXN1) signalling pathway is critical for activity-dependent synaptic transmision and has been associated with autism and neurodevelopmental delay in a few instances. We report on a pair of fraternal twins with biallelic NRXN1 deletions identified by array $\mathrm{CGH}$.

Methods: Both children have autism and mental retardation. In addition, one child has epilepsy. Genomic DNA was isolated from blood for whole genome array $\mathrm{CGH}$ in both children. Genomic analysis was done by using the UCSC genome browser and Esembl ingenuity pathway was used for network analysis.

Results: Whole-genome array $\mathrm{CGH}$ revealed a novel biallelic deletion of neurexin 1 in both patients. One was a $282 \mathrm{~Kb}$ deletion at band 2 p16.3 in one allele and a smaller $174 \mathrm{~Kb}$ deletion on the second allele. Both mutations affect the promoter region and exons 15 in the alpha neurexin isoform that has a role in the calcium dependent release of neurotransmitters in the CNS

Conclusions: Our report confirms that biallellic neurexin 1 mutations in the alpha neurexin 1 gene result in autism and developmental delay. NRXN1 mutations can cause mental retardation syndromes and epilepsy and broadens the range of phenotypes associated with this gene.

\subsection{6}

\section{DRAVET SYNDROME: EFFECT OF DIFFERENT SCN1A} MUTATIONS IN SEIZURE CONTROL AND GAIT IN ADULTS

Danielle Andrade ${ }^{1,2}$, J. Rilstone ${ }^{3}$, F. M. Coelho ${ }^{1,4}$ and B. A. Minassian ${ }^{2,3}$ ( ${ }^{1}$ Neurology, University of Toronto, Toronto, ON, Canada; ${ }^{2}$ Pediatric Neurology, University of Toronto, Toronto, ON, Canada; ${ }^{3}$ Genetics and Genomic Biology, University of Toronto, Toronto, ON, Canada and ${ }^{4}$ Neurology, Hospital Israelita Albert Einstein, Sao Paulo, Brazil)
Rationale: Rationale: Dravet syndrome (DS) is an aggressive epileptic encephalopathy. Pharmacoresistant seizures of several types plague most DS patients throughout life. Gait difficulties are a common, but inconsistent finding. The majority of cases are caused by mutations in the SCN1A gene, but little information is available about how particular mutations influence the adult phenotype. The purpose of this study is to correlate different types of SCN1A mutations and (1) seizure control, (2) occurrence of convulsive status epilepticus (cSE), and (3) the presence of crouch gait in adult patients.

Methods: Methods: In a cohort of 10 adult patients with DS caused by SCN1A mutations, we investigated seizure frequency, history of cSE and gait. All patients were identified in the epilepsy clinic, between 2009 and 2011. SCN1A mutations were divided in 4 different groups based on location or effect of the mutation. Retrospective chart review and recent physical examination were completed in all cases.

Results: Results: All patients had a pathogenic mutation in the SCN1A gene. Four SCN1A mutations have not been previously described. Greater than $90 \%$ seizure reduction was observed (compared to childhood frequency) in 6/7 patients with missense mutations in the pore forming region (PFR) of the Nav1.1 protein (group A) and nonsense mutations (group B). One patient with a splice-site mutation (group C) and another with a mutation outside the PFR (group D) became free of all types of seizures. cSE after the age of 19 years was observed in only 1 patient. Crouch gait, without spasticity, is identified as an element of the adult DS phenotype. However, only half of our adult DS cohort demonstrated crouch gait. This feature was observed in 5/7 patients from groups A and B and in no patients from groups $\mathrm{C}$ and $\mathrm{D}$.

Conclusions: Conclusion: This study shows that seizure control improves and cSE become less frequent in DS as patients age, independently of their SCN1A mutation type. Complete seizure freedom was seen in 2 patients (groups $C$ and D). Finally, this study shows that in DS, crouch gait can be observed in up to $50 \%$ of adults with SCN1A mutation. Although no definite statistical correlations could be drawn due to the small number of patients, it is interesting to note that crouch gait was only observed in those patients with nonsense mutations or mutations in the PRF. Future studies with larger cohorts will be required to formally assess an association of gait abnormalities with particular SCN1A mutations. 


\begin{tabular}{|c|c|c|c|c|c|c|c|c|c|c|c|}
\hline Patilint & Agerliber & Mutation & 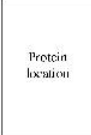 & 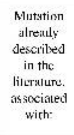 & 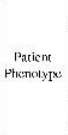 & 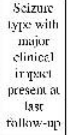 & 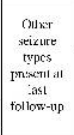 & 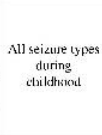 & Gair & $\begin{array}{c}\text { cecrbollar } \\
\text { signer }\end{array}$ & 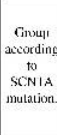 \\
\hline 1 & A페: & Misens: R:I:Y; & 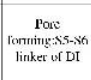 & sM:1 & sul: & (i) & AA.CPS. & 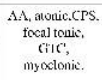 & 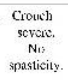 & 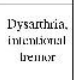 & (ixismy A \\
\hline 2 & $20 \mathrm{M}$ & Missense v v896] & 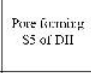 & $\begin{array}{l}\text { Nol } \\
\text { descrailedt" }\end{array}$ & SМсI & 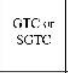 & $\begin{array}{c}\text { Alsuic: } \\
\text { (ravis) }\end{array}$ & 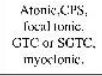 & $\begin{array}{c}\text { Nourtul. } \\
\text { no } \\
\text { spenstichty. }\end{array}$ & Sone & Group A \\
\hline 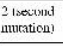 & & $\begin{array}{c}\text { Misisense } \\
\text { D158Yy }\end{array}$ & $\begin{array}{c}52.53 \text { linker } \\
\text { of DIA }\end{array}$ & Gerst & & & & & & & \\
\hline 3 & 3 3.t & 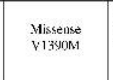 & 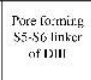 & SWFI & SWFI & Gitce & & $\begin{array}{l}\text { AM.GTC, } \\
\text { myyclonic: }\end{array}$ & 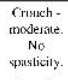 & Dyssantrix & Grove A \\
\hline 1 & $3 \mathrm{MM}$ & Missense KN:11 & 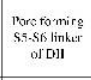 & SMLI: & sul: & stite' & 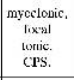 & 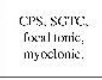 & 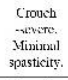 & 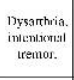 & Girme A \\
\hline 5 & $21 / F$ & 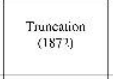 & $c$ cemmins & SMEI & SMEI & SGTC & Focal tonic & 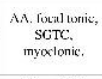 & 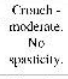 & $\begin{array}{c}\text { Intentionas } \\
\text { trmmor }\end{array}$ & Group B \\
\hline 6 & Alit: & 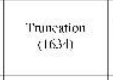 & 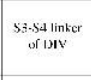 & $\begin{array}{l}\text { Not } \\
\text { allestikest }\end{array}$ & sw: & 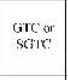 & $\begin{array}{c}\text { focal } \\
\text { woricic } \\
\text { myycolonic. }\end{array}$ & 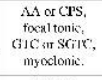 & 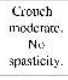 & 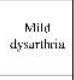 & Girvum 13 \\
\hline 7 & $19 \%$ & 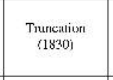 & $\mathrm{c}$ cemminus & SMII & БмГІ & sGTe & Nonc & 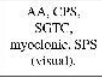 & 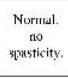 & $\sin x$ & Group B \\
\hline 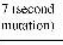 & & INSA+7__VNS4+8 & 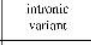 & & & & & & & & \\
\hline s & $25 \mathrm{M}$ & NVS17-2 TX & 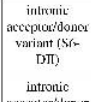 & Nivi & SHEI & Sono & Nonc & 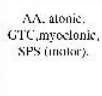 & 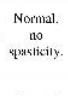 & 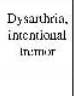 & Group C \\
\hline 9 & IS:M & $\therefore 2416-2 \mathrm{~A} \times \mathrm{C}$ & 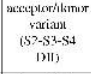 & $\begin{array}{l}\text { nol } \\
\text { descritied }\end{array}$ & Амсг & SGTC: & $\operatorname{crs}$ & $\begin{array}{l}\text { CPS, SSTIY, } \\
\text { myoclonic. }\end{array}$ & $\begin{array}{c}\text { Numrual. } \\
\text { no } \\
\text { spasticity. }\end{array}$ & $\begin{array}{c}\text { Milut } \\
\text { dysartiria }\end{array}$ & Group 0 \\
\hline 11) & 21:1: & 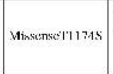 & 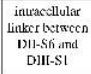 & $\begin{array}{c}\text { SMEl and } \\
\text { heming } \\
\text { vajizant } \\
\text { valiant }\end{array}$ & SMI:3 & Xowe & Nim: & CPS, SFiYK: & 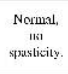 & Xane & Gimurn: \\
\hline
\end{tabular}

\subsection{7}

\section{COPY NUMBER VARIATION ANALYSIS IN PATIENTS WITH SCN1A-RELATED EPILEPSIES}

Corinna Hartmann ${ }^{1,2}$, S. von Spiczak ${ }^{1}$, A. Suls ${ }^{3}$, S. Weckhuysen ${ }^{3}$, G. Buyse $^{4}$, C. Vilain ${ }^{5}$, P. Van Bogaert ${ }^{6}$, P. De Jonghe ${ }^{3}$, H. Muhle ${ }^{1}$, U. Stephani ${ }^{1}$, I. Helbig ${ }^{1}$ and H. Mefford ${ }^{2}\left({ }^{1}\right.$ Department of Neuropediatrics, University Medical Center Schleswig-Holstein, Christian-Albrechts University, Kiel, Germany; ${ }^{2}$ Department of Pediatrics, Division of Genetic Medicine, University of Washington, Seattle, WA; ${ }^{3}$ Neurogenetics Group, VIB Department of Molecular Genetics, University of Antwerp, Antwerp, Belgium; ${ }^{4}$ Department of Pediatric Neurology, University Hospitals Leuven, Leuven, Belgium; ${ }^{5}$ Department of Medical Genetics, Hôpital Erasme, Université Libre de Bruxelles, Brussels, Belgium and ${ }^{6}$ Department of Pediatric Neurology, Hôpital Erasme, Université Libre de Bruxelles, Brussels, Belgium)

Rationale: Severe myoclonic epilepsy of infancy (SMEI), severe idiopathic generalized epilepsy of infancy (SIGEI) and genetic epilepsy with febrile seizures plus (GEFS + ) are associated with mutations in the SCN1A gene. However, SCN1A mutations only occur in a fraction of patients and therefore partially explain these disorders. Previous studies highlighted the importance of rare CNVs in the development of numerous epilepsy phenotypes, but CNV data for patients with SCN1A-related epilepsies is limited. In this study, we report rare CNVs in patients with diagnosed or suspected SMEI, SIGEI and GEFS + . We hypothesize that rare CNVs contain genes contributing to the etiology of SCN1A-related epilepsies.

Methods: We selected 38 patients with SMEI, SIGEI, GEFS+ and unclassified generalized epilepsy of infancy with febrile, myoclonic and generalized tonic-clonic seizures; 14 subjects had previous negative SCN1A testing. SCN1A sequencing is in progress for all subjects. All patients were evaluated for rare CNVs using genomewide array CGH. Arrays contained 180.000 probes covering the whole genome with an overall median probe spacing of $13 \mathrm{~kb}$. CNVs spanning at least 5 probes, containing RefSeq genes and with less than $50 \%$ overlap with a CNV detected in 4519 published controls were reported. The last criterium was not applied to established hotspot CNVs known to occur rarely in healthy controls.
Results: We identified 13 rare CNVs in 8 of 38 patients, some of which contain promising candidate genes. Two individuals had hotspot CNVs: a SCN1A-mutation-negative SMEI patient presented with a duplication of 1q21.1, a locus associated with autism and intellectual disability (ID), and a GEFS+ patient carried a distal $16 \mathrm{p} 11.2$ deletion previously reported in patients with neurodevelopmental phenotypes. Additionally, we found a 1q44 deletion in a girl with an unclassified epilepsy of infancy, ID and short stature. This rearrangement is linked to the core phenotype of corpus callosum anomalies and microcephaly. However, these features were absent in the proband at the time of examination. Examples of novel CNVs are a $217 \mathrm{~kb}$ duplication containing 5 zinc finger genes and a $191 \mathrm{~kb}$ duplication partially including ELMO1 in a SMEI patient with negative SCN1A testing. ELMO1 is highly expressed throughout the brain and encodes for a protein involved in phagocytosis and cell motility. Furthermore, we identified two deletions, one deleting the complete SLC7A11 gene and one interrupting GPHN, in a GEFS+ patient. SLC7A11 encodes for a cysteine/glutamate transporter while mutations in GPHN are associated with hyperekplexia. Both genes could contribute to the proband's phenotype. Parental DNA is being evaluated when available.

Conclusions: We report rare CNVs in 8 of 38 patients with diagnosed or suspected SMEI, SIGEI and GEFS+. Our observations illustrate the importance of rare CNVs for these epilepsies, though clinical interpretation is still difficult. Genes included in these CNV regions can be considered candidate genes. Evaluation of candidates in larger cohorts of patients with epilepsy may identify additional mutations in a subset of genes.

\subsection{8}

NOVEL PHENOTYPIC PRESENTATIONS OF EPILEPSY GENE MUTATIONS UNCOVERED USING MUTLIPLE GENE SEQUENCING

Margie A. Ream ${ }^{1}$, E. E. Davis ${ }^{3,4}$, M. McDonald ${ }^{2}$, N. Katsanis ${ }^{3,4}$ and M. Mikati ${ }^{1}\left({ }^{1}\right.$ Pediatrics - Neurology, Duke University Medical Center, Durham, NC; ${ }^{2}$ Pediatrics - Medical Genetics, Duke University Medical Center, Durham, NC; ${ }^{3}$ Center for Human Disease Modeling, Duke University Medical Center, Durham, NC and ${ }^{4}$ Pediatrics and Cell Biology, Duke University Medical Center, Durham, NC)

Rationale: Gene panels and advanced sequencing can potentially identify pathologic mutations that may not have been suspected based on previously reported manifestations of known disease-causing genes. We have identified over a period of 6 months (July December, 2011) three patients with mutations of known epilepsy genes who had novel and unexpected presentations. Here we present these cases and discuss the potential implications of our observations. Methods: Retrospective chart review of the above three patients observed over a 6 month period. Investigations for the underlying etiology of refractory seizures were done as clinically indicated. Genetic testing included the Infantile Epilepsy Panel (GeneDx, Gaithersburg, MD; patient 1), the Myoclonus Epilepsy Evaluation and Female Febrile Seizures Evaluation (Athena Diagnostics, Inc., Worcester, MA; patient 2), and whole exome sequencing as part of a research protocol (Katsanis lab Duke University) with subsequent confirmation of the detected mutation in a CLIA approved laboratory (patient 3).

Results: Patient 1 presented with agenesis of the corpus callosum, microcephaly, optic nerve hypoplasia, cerebellar hypoplasia and infantile spasms as of the age of 4 months. Extensive workup for possible underlying etiologies was negative except for a disease causing mutation in SLC2A1 which codes for glucose transporter 1. Patient 2 presented with generalized tonic clonic febrile seizure as of the age of 10 months and subsequently developed a clinical picture of progressive myoclonic epilepsy. Extensive work up revealed a disease causing mutations in EFHC1, associated with Juvenile 
Myoclonic Epilepsy, and in SCN1A. The third presented with severe developmental delay, retinal pigmentary changes, and infantile onset of drug resistant tonic seizures. Testing showed a disease causing mutation in SCN2A and increased lactate peak on MRS but mitochondrial enzyme assays from fibroblast cultures were all normal.

Conclusions: (1) Use of gene testing panels and/or exome sequencing can uncover disease causing mutations that would have otherwise not been suspected and can identify novel phenotypic presentations of abnormalities in these genes. (2) Such complex novel phenotypes are likely to be caused by coexistence of other factors, such as other gene mutations, separate from a single epilepsy causing gene mutation.

\subsection{9}

\section{SOMATIC AND GERMLINE MUTATIONS IN AKT3 CAUSE} EPILEPTIC CORTICAL MALFORMATIONS Annapurna Poduri $^{1,2}$, G. D. Evrony ${ }^{1,2}$, X. Cai ${ }^{1,2}$, P. C. Elhosary ${ }^{1}$, R. Beroukhim $^{3,4}$, M. K. Lehtinen ${ }^{1,2}$, L. B. Hills ${ }^{1}$, E. L. Heinzen ${ }^{5}$, A. Hill $^{1}$, R. S. Hill ${ }^{1,6}$, B. J. Barry ${ }^{1,6}$, B. F. Bourgeois ${ }^{1,2}$, J. J. Riviello ${ }^{7}$, E. Vining $^{8}$, B. Carson ${ }^{8}$, A. J. Barkovich ${ }^{9}$, P. M. Black ${ }^{10}$, K. L. Ligon ${ }^{1,2}$ and C. A. Walsh ${ }^{1,6}\left({ }^{1}\right.$ Boston Children's Hospital, Boston, MA; ${ }^{2}$ Harvard Medical School, Boston, MA; ${ }^{3}$ Dana Farber Cancer Institute, Boston, MA; ${ }^{4}$ Broad Insitute, Cambridge, MA; ${ }^{5}$ Duke University, Durham, NC; ${ }^{6} \mathrm{HHMI}$, Chevy Chase, MD; ${ }^{7}$ New York University, New York, NY; ${ }^{8}$ Johns Hopkins University, Baltimore, MD; ${ }^{9}$ University of California, San Francisco, San Francisco, CA and ${ }^{10}$ World Federation of Neurology, Vaud, Switzerland)

Rationale: Hemimegalencephaly (HMG) is a congenital brain malformation characterized by an enlarged, malformed cerebral hemisphere that often requires hemispherectomy for treatment of refractory epilepsy. We recently identified somatic mosaic mutations involving the gene encoding the serine threonine kinase AKT3: an activating point mutation $(\mathrm{c} .49 \mathrm{G} \rightarrow \mathrm{A}$, creating E17K) and two trisomies of chromosome 1q containing AKT3 (Poduri et al., Neuron, 2012). Here we further characterize the E17K mutation using single cell sequencing of neurons and glia, and we report a novel germline truncating variant in AKT3 in a patient with HMG.

Methods: We have identified 29 cases of HMG from which DNA from brain, leukocytes, and/or buccal cells was available. All research was conducted in accordance with the IRB of Boston Children's Hospital. We used copy number analysis of SNP data, quantitative PCR, and candidate gene sequencing. We further studied the AKT3 E17K mosaic mutation case using single cell sorting (NeuN+ vs NeuN-) and single cell sequencing to establish the relative proportions of neurons and glia that were mutation-positive. Results: (1) Having previously identified the AKT3 mosaic E17K mutation in DNA from brain tissue (and not leukocytes) in an individual with HMG, we sequenced AKT3 in our other cases. We have identified a novel AKT3 variant, W330X, in DNA obtained from a buccal cell sample in one case. This truncation does not disrupt a critical activation site (T305) but is predicted to result in the loss of the C-terminal regulatory domain, which could potentially cause activation of AKT3. Functional studies of the W330X variant are underway.

(2) We further characterized the AKT3 E17K somatic mosaic mutation using single cell sorting and sequencing. Sequencing of neurons $(\mathrm{NeuN}+)$ and glial cells $(\mathrm{NeuN}-)$ confirmed the mutation is present in both cell types, at a rate of $39 \pm 7 \%$ (SE) in single NeuN positive cells (neurons) and $27 \pm 8 \%$ in single NeuN negative cells (consisting of glial and other non-neuronal cells), suggesting a de novo mutation in a progenitor cell that generates neuronal and nonneuronal cells.

Conclusions: We now provide a genetic explanation for four cases of HMG involving activation of AKT3 in brain, either by duplication or activating point mutation leading to hemispheric overgrowth. Somatic mutations arising de novo in neuronal-glial progenitors in the developing brain can be responsible for HMG. Together, our data suggest that AKT3 is an important regulator of brain development and that mutations of this gene, whether germline or post-zygotic and affecting a subset of neurons and glia in one hemisphere, lead to HMG. These mutations in HMG present two models, somatic and germline, for the genetics of epileptic cortical malformations.

\subsection{0 \\ IDENTIFICATION OF ABNORMAL MICRORNA GENE REGULATION IN MESIAL TEMPORAL SCLEROSIS} Danyella B. Dogini ${ }^{1}$, C. S. Souza ${ }^{1}$, C. L. Yassuda ${ }^{2}$, H. Tedeschi ${ }^{2}$, E. Oliveira $^{2}$, C. V. Maurer-Morelli ${ }^{1}$, F. Cendes ${ }^{2}$ and I. Lopes-Cendes ${ }^{1}$ $\left({ }^{1}\right.$ Medical Genetics, University of Campinas, Campinas, Brazil and ${ }^{2}$ Neurology, University of Campinas, Campinas, Brazil)

Rationale: MicroRNAs are a new class of small RNA molecules (2124 nucleotide-long) that negatively regulate gene expression either by translational repression or target mRNA degradation. It is believed that about $30 \%$ of all human genes are regulated by these molecules. MiRNAs are involved in many important biological processes including cell differentiation, embryonic development and central nervous system development. The main purpose of this study was to investigate the existence of differences in microRNA expression in mesial temporal sclerosis (MTS) and to identify the regulated targetgenes.

Methods: Total RNA was isolated from hippocampal tissue of 4 patients who underwent selective resection of the mesial temporal structures for the treatment of clinically refractory seizures. In addition, we used control samples from autopsy $(n=4)$ for comparison. RNA samples were used in real-time PCR reactions with TaqMan ${ }^{\mathrm{TM}}$ microRNA assays (Applied Biosystems) to quantify 157 different miRNAs.

Results: Bioinformatic analyzes identified three miRNAs, which were differently expressed in patients as compared to controls: let-7d and miR-29b were over expressed in patients; whereas, miR-30d was down-regulated in patients. A possible target gene for let-7d is Nme6 which we also found to be down-regulated in patients. In addition, Mcl-1, the putative target gene of miR-29b was also down-regulated in patients. Mcl-1 is an anti-apoptotic protein of the Bcl-2 family and its tight regulation of protein levels is necessary, because insufficient Mcl-1 can result in inappropriate cell death. Nme6 belongs to NME (nm23 /nucleoside diphosphate kinase) gene family in humans and act as inhibitor of $\mathrm{p} 53$-induced apoptosis.

Conclusions: We have identified three different miRNA species differently expressed in MTS and its target genes: let-7d - NME6, miR-30d - SON and miR29b - Mcl-1. Biologic functions related to the possible miRNA gene-targets are mainly neurogenesis, and apoptosis. Our results point to interesting potential molecular targets which should be explored further in additional studies of MTS.

\section{Genetics: Animal Studies}

\subsection{1}

\section{LONG-TERM MTOR HYPERACTIVATION LEADS TO SOCIAL BEHAVIOR AND LEARNING AND MEMORY DEFICITS}

E. Arbuckle ${ }^{2}$, G. Smith ${ }^{1}$, j. Morrison ${ }^{1}$, C. Floruta ${ }^{1}$, O. Okonkwo ${ }^{1}$ and Joaquin N. Lugo ${ }^{1,2}\left({ }^{1}\right.$ Psychology and Neuroscience, Baylor University, Waco, TX and ${ }^{2}$ Biomedical Studies, Baylor University, Waco, TX)

Rationale: There is increasing evidence of a strong comorbidity between autism and epilepsy. Recent studies have shown that genetic deletion of genes that modulate the mTOR signaling pathway result in an autistic phenotype. These genes have also been demonstrated to play a critical role in cortical dysplasia and tuberous sclerosis complex. Furthermore, hypereractivation of the mTOR pathway has 
been reported in several animal models of epilepsy. However, there has been less emphasis on behavioral consequences due to hyperactivation of mTOR signaling. Here, we evaluated the behavioral consequences of mTOR hyperactivation by using neuron subset-specific (NS-Pten) conditional knockout mice.

Methods: Multiple cohorts of NS-Pten knockouts and wildtype mice were examined through a battery of behavioral tests. We first examined isolation-induced ultrasonic vocalizations in postnatal day 9 and 10 pups. We examined their locomotion and anxiety in an open field test. We examined their learning and memory through a standard fear conditioning test and a separate cohort was examined in a trace-fear conditioning test. We examined their social behavior in a three chamber test and a social partition test. We examined their repetitive behavior in a marble burying test.

Results: The NS-Pten KO mice displayed hyperactivity in the open field test compared to controls, $\mathrm{p}<0.001$; and spent significantly less time in the center of the open field, $p<0.05$. They showed deficits in social behavior by interacting less with a mouse in the social chamber test, $\mathrm{p}<0.05$; and spent less time at a partition with a novel mouse in the social partition test, $p<0.05$. The NS-Pten knockout pups did not show a change in the number or duration of ultrasonic vocalizations when removed from their mother. The NS-Pten mice demonstrate alterations in repetitive behavior, as measured in the marble burying test. In addition to deficits in the behavioral features that describe autism, they have learning and memory deficits in contextual learning in the conditioned fear test, but did not show a difference in their freezing to a tone (conditioned stimulus) compared to wildtype mice. Conclusions: These findings demonstrate that hyperactivation due to genetic deletion of Pten results in long-term alterations in social behavior, anxiety, repetitive behavior, and learning and memory. It has previously been demonstrated that NS-Pten mice develop seizures at 4 weeks of age that increase in duration as they age. Additionally, inhibition of $\mathrm{mTOR}$ prevents the progression of epilepsy in NS-PTEN mice. Our data demonstrates that NS-Pten mice are a valuable tool to examine the behavioral consequences due to mTOR hyperactivation. Future studies could examine whether the behavioral deficits are reversed with mTOR inhibitors. In addition, future studies could correlate the epileptogenic changes that occur in the NS-Pten mice along with the behavioral changes.

\subsection{2}

MULTIPLE MOLECULAR MECHANISMS FOR A SINGLE GABAA MUTATION IN EPILEPSY

Tae H. Kim ${ }^{1,2}$, A. Phillips ${ }^{1}$, J. Low ${ }^{1}$, S. Berkovic ${ }^{4}$, B. Luscher ${ }^{5}$, S. Petrou ${ }^{1,3}$ and C. Reid ${ }^{1}\left({ }^{1}\right.$ Florey Neuroscience Institute, The University of Melbourne, Parkville, VIC, Australia; ${ }^{2}$ Department of Anatomy and Neuroscience, The University of Melbourne, Parkville, VIC, Australia; ${ }^{3}$ Centre for Neural Engineering, The University of Melbourne, Parkville, VIC, Australia; ${ }^{4}$ Epilepsy Research Centre, The University of Melbourne, Austin Health, Melbourne, VIC, Australia and ${ }^{5}$ Department of Biology and Department of Biochemistry and Molecular Biology, Pennsylvania State University, University Park, PA)

Rationale: Predicting clinical outcomes for patients based on their personal genomes is an emerging challenge. Members of a family segregating a mutation (R43Q) in the $\gamma 2$ subunit of the GABAA receptor display febrile seizures and typical absence seizures with varying and incomplete penetrance. Here we analyse three different mouse models of GABAA $\gamma 2$ gene dysfunction to investigate the basis of the phenotypic heterogeneity seen in the family.

Methods: Spike and wave discharges (SWDs) and thermal seizure susceptibility were measured in heterozygous GABAA $\gamma 2$ knock-out and GABAA $\gamma 2$ (R43Q) knock-in mice models crossed to different mouse strains. SWDs were measured on electroencephalograms recorded from freely moving mice for $4 \mathrm{~h}$. Thermal seizure susceptibility was determined by measuring the latency to the first tonic-clonic seizure of mice subjected to a constant $42 \mathrm{oC}$ temperature.
Results: GABAA $\gamma 2$ knock-out mice spent an identical total time having SWDs compared to the GABAA $\gamma 2(\mathrm{R} 43 \mathrm{Q})$ knock-in mice $(2.07 \pm 0.16$ vs $1.96 \pm 0.22 \%, \mathrm{n}=5$ and $8, \mathrm{P}=0.74)$. In the thermal stress assay, GABAA $\gamma 2(\mathrm{R} 43 \mathrm{Q})$ knock-in mice developed seizures with a shorter latency than wild-type controls $(\mathrm{P}=0.0003)$. In contrast, there was no difference in the latency to seizures of GABAA $\gamma 2$ knock-out compared to the wild-type littermates $(\mathrm{P}=0.9492)$. To test the influence of background genetics on seizure susceptibility we crossed the GABAA $\gamma 2$ (R43Q) knock-in mice into the seizure-prone $\mathrm{DBA} / 2 \mathrm{~J}$ and relatively seizure resistant $\mathrm{C} 57 \mathrm{Bl} / 6$ strains. SWD expression was robust in the DBA/2J $(n=40 / 40)$ but this seizure phenotype was never observed in the $\mathrm{C} 57 \mathrm{Bl} / 6$ strain $(\mathrm{n}=0 / 10)$. In contrast, thermal seizure susceptibility was equally robust for the GABAA $\gamma 2(\mathrm{R} 43 \mathrm{Q})$ knock-in in both strains $(\mathrm{P}<0.05$ for both).

Conclusions: Our results show that a single gene mutation can cause distinct seizure phenotypes through independent molecular mechanisms. Haploinsufficiency underlies the genesis of absence seizures, but cannot account for the thermal seizure susceptibility. A lack of effect of genetic background on thermal seizure susceptibility is consistent with the higher penetrance of febrile seizures compared to absence seizures seen in family members with the mutation. These mouse studies help to provide a conceptual framework within which clinical heterogeneity seen in genetic epilepsy can be explained.

\subsection{3 \\ IN VIVO ANALYSIS OF CANDIDATE MODIFIER GENES FOR AN EPILEPSY MODIFIER LOCUS ON MOUSE CHROMOSOME 11}

Nicole Hawkins ${ }^{1}$ and J. Kearney ${ }^{2}\left({ }^{1}\right.$ Neuroscience, Vanderbilt University School of Medicine, Nashville, TN and ${ }^{2}$ Genetic Medicine, Vanderbilt University School of Medicine, Nashville, TN)

Rationale: Over 800 mutations have been identified in the voltagegated sodium channel genes SCN1A and SCN2A in human epilepsies, including Genetic Epilepsy with Febrile Seizures Plus (GEFS+), Dravet Syndrome and Benign Familial Neonatal-Infantile Seizures. Family members with the same mutation frequently display variation in the clinical severity of the disease, suggesting that other factors modify the primary mutation. Several mouse models have been generated to study the genetic basis of epilepsy. A common feature of these epilepsy models is that genetic strain background alters the disease phenotype, suggestive of genetic modifiers in epilepsy. The Scn2aQ54 transgenic mouse model has an epilepsy phenotype that varies depending on the genetic strain background. Scn2aQ54 mice congenic on the C57B1/6J (B6.Q54) strain exhibit delayed seizure onset and improved survival compared to F1(B6xSJL/J).Q54 mice. Two dominant modifier loci of Scn2aQ54 seizure susceptibility were mapped and designated Moe1 and Moe2. We previously fine mapped the Moe1 locus using interval-specific congenic mouse strains and identified candidate modifier genes by RNA-Seq analysis. Consideration of gene function and RNA-Seq expression data identified Cacnalg $(\alpha 1 \mathrm{G})$, encoding Cav3.1, as a strong candidate modifier gene. We are using a transgenic transfer approach to assess the modifier potential of $\alpha 1 \mathrm{G}$. Alteration of the Scn 2aQ54 phenotype by $\alpha 1 \mathrm{G}$ in double mutant mice will support its involvement as an epilepsy modifier gene.

Methods: $\alpha 1 \mathrm{G} \mathrm{BAC}$ transgenic mice were generated by microinjection of BAC RP23-65I14 into SJL/J oocytes. $\alpha 1 \mathrm{G} \mathrm{BAC}$ transgenic hemizygotes were crossed with B6.Q54 mice to generate double transgenic offspring and Scn2aQ54 single transgenic littermate controls. Spontaneous seizure frequency was measured during 30 minute observations at 3 and 6 weeks of age. Seizure severity was assessed with a modified Racine scale and survival was monitored until 12 weeks of age.

Results: The $\alpha 1 \mathrm{G} \mathrm{BAC}$ transgenic mice carry $8-10$ copies of the transgene and overexpress $\alpha 1 \mathrm{G}$ transcript and protein. Double transgenic $\alpha 1 \mathrm{G}$;Q54 male mice exhibited more spontaneous seizures than Q54 single transgenic littermates $(\mathrm{p}<0.0048)$. There was no 
significant difference in female seizure frequency $(\mathrm{p}<0.62)$. Double transgenic $\alpha 1 \mathrm{G}$;Q54 mice of both sexes exhibited increased seizure severity, as assessed by the Racine scale, compared to littermate controls. Survival analysis of double transgenic $\alpha 1 \mathrm{G}$; Q54 mice and littermate controls is in progress.

Conclusions: These experiments evaluate a potential modifier gene that may influence the clinical course of epilepsy caused by a sodium channel mutation. The interplay between various ion channels contributes to overall neuronal excitability. Understanding gene interactions that contribute to epilepsy penetrance and severity will improve the utility of molecular diagnostics and may suggest novel therapeutic strategies.

\subsection{4 \\ MOLECULAR REGULATION OF AN EPILEPSY MODIFIER GENE}

Benjamin S. Jorge and J. A. Kearney (Vanderbilt University Medical Center, Nashville, TN)

Rationale: A growing number of the genes responsible for heritable epilepsy syndromes have been identified in recent years. Mutations in voltage-gated ion channels are a common cause of monogenic epilepsies. Families that inherit mutations in these genes frequently exhibit a surprising degree of phenotype heterogeneity. This suggests that genetic modifiers may have a strong influence on the epilepsy phenotype. Identifying genetic modifiers can help elucidate the molecular pathways that are involved in epilepsy, and these pathways may contain novel therapeutic targets that could aid in the treatment of epilepsy.

We previously identified Kcnv2 as a genetic modifier of epilepsy in the Scn2a $a^{\mathrm{Q} 5}$ (Q54) transgenic mouse model of epilepsy (Bergren et al., Mamm Genome 2009; 20:359-366). Kcnv2 encodes the silent modulatory voltage-gated potassium channel subunit (Kv8.2). A screen of pediatric epilepsy patients revealed two novel KCNV2 variants. Both variants altered potassium current in a heterologous expression system, and neither variant was present in neurologically normal control chromosomes. This suggests that KCNV2 may also contribute to human epilepsy (Jorge et al., PNAS; 20(13):54435448).

Our data suggests that Kcnv2 is a quantitative modifier of the Q54 phenotype. We hypothesize that regulatory sequence variation in the promoter and/or 3'UTR of Kcnv2 influences seizure susceptibility by altering steady-state expression levels. In order to better understand how Kcnv2 influences seizure susceptibility, we are working to identify and characterize cis-regulatory elements that influence steady-state expression levels of KCNV2 in both mouse and human. This work will advance our knowledge of potassium channel regulation and the mechanisms by which genetic modifiers influence epilepsy susceptibility.

Methods: We used rapid amplification of cDNA ends (RACE) and RNase protection assays (RPA) as complementary approaches to identify the Kcnv2 transcription start site (tss) region. Luciferasebased promoter assays are currently in progress to identify the minimal promoter region of Kcnv2.

Results: The RACE and RPA results both suggest the use of multiple Kcnv2 transcription start sites that lie within an 100 bp region $130 \mathrm{bp}$ upstream of the Kcnv2 start codon.

Conclusions: Our results suggest that the tss region lies $\sim 50 \mathrm{bp}$ upstream of the tss predicted in genomic databases [Ensembl database (NCBI37/mm9), UCSC genome browser (NCBI37/mm9)]. Kcnv2 does not have a TATA box. The use of multiple transcription start sites within a tss region is consistent with what has been observed in other TATA-less genes. Determining the location of the tss region has facilitated the development of promoter assays to determine the minimal promoter region of Kcnv2. These are now in progress.

\subsection{5 \\ TEMPORAL TRANSCRIPT PROFILE OF INTERLEUKIN-1 BETA GENE IN IMMATURE AND ADULT ZEBRAFISH BRAIN AFTER SEIZURE}

Patricia G. Barbalho, F. C. Pinto, R. Mangolin and C. V. MaurerMorelli (UNICAMP, Campinas, Brazil)

Rationale: Proinflammatory cytokines, such as interleukin-1beta (illb), have shown to be up-regulated in surgical specimens of pharmacoresistent patients with epilepsy as well as in experimental rodent models of seizure; however, the expression profile of this cytokine in zebrafish brain after seizure is unknown. In this study, we investigated the illb transcript profile in immature and adult zebrafish brain after a seizure induced by Pentylenetratazole (PTZ). Methods: Zebrafish were maintained according to standard procedures and all experiments were approved by animal ethical committee/UNICAMP. Adult and larvae (7 days post fertilization) zebrafish were separated in Seizure (SG) and Control (CG) groups. Animals from SG group were individually exposed to $15 \mathrm{mM}$ PTZ (2$3 \mathrm{~min}$ for adults and 20min for larvae). Animals from CG were exposed to same handling condition, but in normal bath water. A total of 6 samples were used for each group and age. A single adult sample was composed by pooling two brains and 20 heads were used to compose each larvae sample. At $12 \mathrm{~h}, 24 \mathrm{~h}$ and $48 \mathrm{~h}$ after a seizure, animals were anesthetized and the brains were immediately isolated, frozen in N2(L) and total RNA was extracted by Trizol (Invitrogen). Reverse transcriptase quantitative-PCR amplifications were carried out in triplicates with efIa as endogenous control (TaqMan ${ }^{\circledR}$, Applied Biosystems). The relative quantification (RQ) was calculated by the equation $\mathrm{RQ}=2-\Delta \Delta \mathrm{CT}$. Statistical analysis was performed by Kruskall-Wallis test.

Results: The mean \pm Standard Deviation results obtained were: (i) adult: CG12h 0.95 \pm 0.31 ; SG12h 0.84 \pm 0.19 ; CG24h: 0.75 \pm 0.23 ; SG24h: 0.76 \pm 0.27 ; CG48h 0.85 \pm 0.16 ; SG48h 0.99 \pm 0.41 ; (ii) immature: CG12h 1.84 \pm 0.46 ; SG12h 2.00 \pm 0.61 ; CG24h: 2.18 \pm 1.07 ; SG24h: $1.15 \pm 0.51$; CG48h 2.5 \pm 0.58 ; SG48h $2.02 \pm 0.63$. No statistical significance was found between CG and SG for both, adult and larvae $(\mathrm{p} \geq 0.05)$.

Conclusions: This is the first study investigating illb transcript profile in zebrafish brain after a PTZ-induced seizure.The results indicated that $i 11 b$ transcript levels have a different pattern in zebrafish as compared to those described in rodent models of epilepsy. To better characterize the illb transcript profile pattern in mature and immature zebrafish brain, studies are under way to explore the illb expression pattern at $0.05 \mathrm{~h}, 6 \mathrm{~h}$ and $72 \mathrm{~h}$ after PTZ induced-seizure.

\subsection{6 \\ DO DIFFERENT GENETIC RAT MODELS OF EPILEPSY SHARE SIMILAR MOLECULAR MECHANISMS? Alexandre Matos ${ }^{1}$, V. Pascoal ${ }^{1,4}$, D. Nascimento ${ }^{2}$, S. Martins ${ }^{2}$, C.

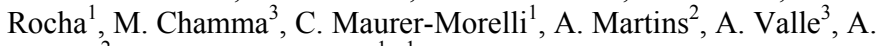 Godard $^{2}$ and I. Lopes-Cendes ${ }^{1}\left({ }^{1}\right.$ UNICAMP, Campinas, Brazil; ${ }^{2}$ UFMG, Belo Horizonte, Brazil; ${ }^{3}$ USP, Sao Paulo, Brazil and ${ }^{4}$ UFF, Nova Friburgo, Brazil)}

Rationale: Wistar audiogenic rat (WAR) is a genetic epilepsy model susceptible to audiogenic seizures. Another genetic model we have recently identified is the generalized epilepsy with absence seizures (GEAS) rat. The aim of the present study was to characterize and compare gene expression profile of these two strains in order to determine whether they share a common genetic susceptibility to seizures.

Methods: We obtained total RNA from five susceptible WAR (hippocampus and corpora quadrigemina), and five control Wistar, as well as from hippocampus of three GEAS rats and three control Wistar. Gene expression analysis was performed using the GeneChip ${ }^{\circledR}$ Rat Genome 2302.0 Array (Affymetrix ${ }^{\mathrm{TM}}$ ), and 
analyzed in R environment using the Affy and RankProd packages from Bioconductor. Overrepresented gene ontology categories were identified with DAVID software, and gene interactions and correlation networks were identified with MetaCore software. Results: Enriched gene ontology classes identified in WAR were mainly involved in neuronal development and differentiation, regulation of synaptic transmission and neuron projection, as well as cell-cell signaling. The most active signaling pathways were, signal transduction and calcium signaling. The main genes with differential expression and a possible biological role in epileptogenesis were potassium and sodium channel, glutamate receptor and GABA receptor. In the GEAS rats we found differential expression of genes related to central nervous system development, activation of MAPK transcription factors, neuronal migration and apoptosis, such as Nrsn1, Hspb1, Fos, Twist1 and Krt18. The top enriched gene ontology categories included signal peptide, extracellular region and antigen processing and presentation. Among the most activated signaling pathways was neurosystem development.

Conclusions: Our results clearly show a different molecular signature in the two genetic rat models analyzed. Our results are the first step to clarify the underlying molecular mechanism that leads to predisposition to seizures in these genetic animal models.

\section{Neuropathology of Epilepsy: Human Studies}

\subsection{7 \\ SURGICAL PATHOLOGY OF INTRACTABLE NONLESIONAL FOCAL EPILEPSIES. WHICH BOAT ARE WE MISSING?}

Andreas Alexopoulos, I. Z. Wang, Z. Jaisani, Z. Ying, I. M. Najm and R. Prayson (Cleveland Clinic, Cleveland, $\mathrm{OH}$ )

Rationale: Patients (pts) with intractable nonlesional focal epilepsies (NLFE) constitute a most challenging group undergoing presurgical evaluation, and are often denied epilepsy surgery. Yet surgery can be effective in well-selected pts with no visible MRI lesion.

Literature on pathology of intractable NLFE is limited. These pts suffer from a heterogeneous group of epileptic pathologies that are not well understood. We sought to characterize the underlying histopathology and correlate this with seizure outcome in a large series of strictly-defined MRI-negative pts undergoing epilepsy surgery.

Methods: All pts with NLFE who had surgery over a 10-year period (2002-2011) were identified. High-res 1.5 or 3T preop MRI was performed using a uniform epilepsy protocol. We included pts who had a preop MRI between 6-65 years of age, and further restricted our study to pts with strictly-defined normal MRI: Preop MRIs were visually analyzed by dedicated neuroradiologists and re-reviewed by neuroradiology and neurosurgery during pt management conference guided by results of noninvasive testing. Pts with MRIs that were initially interpreted as normal were excluded, if a

probable/questionable lesion was identified during focused re-review of the MRI. Pathological specimens were carefully re-reviewed by a single neuropathologist and characterized as focal cortical dysplasia (FCD) type I (Palmini) or balloon-cell type, hippocampal sclerosis (HS), gliosis, normal tissue and other. Resections were classified as frontal, parieto-occipital, neocortical temporal $(\mathrm{T})$, standard $\mathrm{T}$ with removal of mesial T structures and multilobar.

Results: 94 consecutive pts (mean age 30.4yrs, range 7-64yrs; 50\% females) fulfilled the above criteria. 34 underwent hippocampalsparing partial T resections, 32 standard T lobectomies, 20 frontal, 6 parieto-occipital and 2 bilobar resections. The majority had type I FCD $(n=36 ; 38.3 \%)$. Balloon-cell FCD was identified in 3; HS in 10 ( 3 with FCD; dual pathology); gliosis in 34 and others in 4 . Lastly 7 had no identifiable pathological changes. Follow-up data for $>6$ months postop were available in 87.43 (or $46 \%$ of the entire cohort) attained Engel class 1 outcome. Among seizure-free pts 19 (44.2\%) harbored type I FCD, and 2 balloon-cell FCD, 7 (16.2\%) had HS, 2 other pathologies, 11 (25.5\%) gliosis and 2 had no abnormalities. Conclusions: We present results of surgical pathology in a large single-center series of pts with strictly-defined NLFE undergoing epilepsy surgery. The most common pathological correlate not visualized by MRI was FCD, identified in 39 pts of whom only 3 had balloon-cell FCD. Pts with identifiable pathology appeared to have better chance for seizure-free outcome compared to pts without specific epileptic pathology (gliosis or normal). At the same time paucity of pathological changes in the resected specimen did not preclude favorable seizure outcome. These results highlight the heterogeneity of epileptic pathologies that cannot be visualized with available MRI technology, and the limitations of microscopic histopathological examination of resected human epileptic tissue.

\subsection{8 \\ INCREASED NUMBER OF MTOR POSITIVE NEURONS IN RESECTED CORTEX IS A PREDICTOR OF SEIZURE-FREE SURGICAL OUTCOME IN YOUNG PATIENTS WITH FOCAL CORTICAL DYSPLASIA} L. Miles ${ }^{1}$, M. Miles ${ }^{1,2}$, H. Greiner ${ }^{2}$, F. Mangano ${ }^{3}$, P. Horn ${ }^{6,2}$, J. Leach $^{4}$, K. Lee ${ }^{5}$ and T. DeGrauw ${ }^{7}\left({ }^{1}\right.$ Pathology \& Laboratory Medicine, Cincinnati Children's Hospital Medical Center and Univ. of Cincinnati College of Medicine, Cincinnati, OH; ${ }^{2}$ Pediatric Neurology, Cincinnati Children's Hospital Medical Center, Cincinnati, OH; ${ }^{3}$ Neurosurgery, Cincinnati Children's Hospital Medical Center, Cincinnati, OH; ${ }^{4}$ Radiology, Cincinnati Children's Hospital Medical Center, Cincinnati, OH; ${ }^{5}$ Neurology, Florida Hospital for Children, Orlando, FL; ${ }^{6}$ Mathematical Sciences, Univ. of Cincinnati, Cincinnati, $\mathrm{OH}$ and ${ }^{7} \mathrm{Child}$ Neurology, Children's Healthcare of Atlanta and Emory University, Atlanta, GA)

Rationale: Focal cortical dysplasia (FCD) is a common cause of treatment-resistant epilepsy in children. Improved outcome is associated with complete resection of FCD in epileptogenic tissue. The mammalian target of rapamycin (mTOR) pathway appears to be up-regulated in FCD type IIb, hemimegalencephaly, and gangliogliomas. However, detailed investigations of mTOR in children with FCD are very limited. The aims of this study are: 1) to identify the pathological features of FCD in tissue resected during epilepsy surgery, and 2) to define clinical and pathological characteristics which predict improved surgical outcome.

Methods: IRB approval was obtained. Inclusion criteria were: 1) age $<21 \mathrm{y}$; 2) medically-refractory epilepsy determined by pediatric neurologists; 3) epilepsy surgery at CCHMC between Sep. 2007-Sep. 2011 ; 4) no known lesions (e.g. tumor, vascular malformation, hemimegalencephaly, or Rasmussen's encephalitis) other than cortical anomalies and mesial hippocampal sclerosis; and 5) at least one follow-up visit not $<6$ months postsurgery. Pathological evaluations included FCD classification (based on Palmini's criteria), H\&E, mTOR, and Neu-N slides. Cortical gliosis, determined by glial fibrillary acidic protein (GFAP) stain, was reported as: superficial (sGFAP) (involving only subpial and first layer); diffuse mild (mGFAP) (sGFAP plus minimal involvement of other layers); or diffuse (dGFAP) involving all 6 layers. One high power field (hpf) at $\mathrm{x} 40$ with the highest number of $\mathrm{mTOR}$ positive neurons $\left(\mathrm{mTOR}_{\mathrm{tot}}\right)$ was identified and counted. Logistic regression with backward elimination on seizure-free outcome, included GFAP group, cortical MRI abnormality, age at epilepsy onset, age at surgery, and $\mathrm{mTOR}_{\text {tot }} /$ hpf.

Results: Fifty-one patients (median age at surgery 10.6, range 0.5$20.3 y$ ) met the inclusion criteria. Four, 13, 27 and 7 patients had FCD $\mathrm{Ia}, \mathrm{Ib}$, IIa, and IIb, respectively. Cortical gliosis was seen in all patients, including 13 sGFAP, 5 mGFAP and 33 dGFAP. The $\mathrm{mTOR}_{\text {tot }}$ ranged from $0-25 / \mathrm{hpf}$, including 17 who had no mTOR positive neurons. Clinical, neurological, and neuroimaging characteristics were similar in FCD I ( Ia+Ib) and FCD II (IIa+IIb) groups, but dGFAP and $\mathrm{mTOR}_{\text {tot }}$ were significantly increased in FCD II $(\mathrm{P} \leq 0.001)$. The univariate model with seizure free outcome vs. 
$\mathrm{mTOR}_{\text {tot }}$ was significant (Table). Logistic regression with backward elimination resulted in retaining two variables, $\mathrm{mTOR}_{\text {tot }}$ and age at surgery (Table). Because of improved goodness-of-fit, the final model included $\mathrm{mTOR}_{\text {tot }}$ and "mTOR ${ }_{\text {tot }}{ }^{*}$ age at surgery", but not the first-order term "age at surgery". Higher mTOR $_{\text {tot }}$ improves the probability of a seizure-free outcome in younger patients (Figure). Conclusions: Younger patients with high $\mathrm{mTOR}_{\text {tot }}$ have increased probability of seizure-free surgical outcome than older individuals with low $\mathrm{mTOR}_{\text {tot }}$. Quantitation of mTOR positive neurons in relation to age at surgery may predict seizure-free outcome for patients with FCD. Also activation of the mTOR pathway may be an important factor in epileptogenesis associated with FCD.

Table. Results of univariate regression and multivariate logistic regression with backward stepwise elimination for seizure-free outcome in pediatric patients following epilepsy surgery.

\begin{tabular}{|c|c|c|c|c|}
\hline & OR & $95 \% \mathrm{Cl}$ & $\mathbf{P}$ & AUR \\
\hline \multicolumn{5}{|l|}{ Univariate model $(n=5 f)$} \\
\hline mTOR positive neurons per $(x 40)$ field & 1.14 & 1.04 .1 .25 & 0.005 & 0.77 \\
\hline \multicolumn{5}{|l|}{ Multivariate model without interaction ( $n=51$ ) } \\
\hline m'l'OR positive neurons per $(x 40)$ field & 1.15 & $1.05,1.27$ & 0.005 & 0.76 \\
\hline Age at surgery & 0.87 & $0.76,0.99$ & 0.036 & \\
\hline \multicolumn{5}{|l|}{ Final Model with interaction $(n=51)$} \\
\hline mIYOR positive neurons per $(x 40)$ field & a & is & 0.002 & 0.83 \\
\hline ml'OR positive neurons per $(x 40)$ field * age at surgery & a & u & 0.010 & \\
\hline
\end{tabular}

${ }^{\mathrm{a}} \mathrm{OR}$ and $95 \% \mathrm{CI}$ 's are not appropriate when an interaction term is present.

AUR, area under the ROC curve.

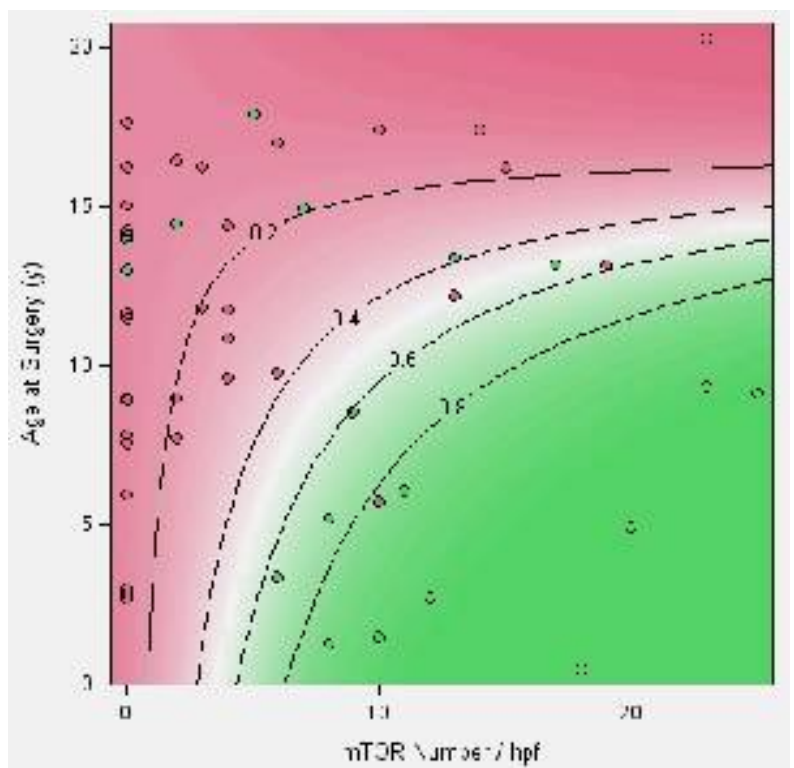

Figure. Contour plot showing the probability of a seizure-free outcome (green) vs. non-seizure-free outcome (red) as a function of number of mTOR positive neurons per (x40) microscopic field and age at surgery.

\subsection{0}

ABNORMAL SUBSURFACE CISTERNS IN SMALL NEURONS OF HUMAN EPILEPTIC HYPOTHALAMIC HAMARTOMA: AN ULTRASTRUCTURAL STUDY John Beggs ${ }^{1}$, J. Wu ${ }^{2}$, S. W. Coons ${ }^{1}$ and J. Kerrigan ${ }^{2,3}$ $\left({ }^{1}\right.$ Neuropathology, Barrow Neurological Institute, Phoenix, AZ; ${ }^{2}$ Neurology, Barrow Neurological Institute, Phoenix, AZ and

${ }^{3}$ Pediatrric Neurology, Phoenix Childrens Hospital, Phoenix, AZ)

Rationale: Hypothalamic hamartomas $(\mathrm{HH})$ are intrinsically epileptogenic for gelastic seizures, although the basic cellular mechanisms remain unknown. HH tissue consists of clusters of small interneuron-like neurons, and smaller numbers of large projectiontype neurons, each with specific electrophysiological features, which together suggest a model for increased neuronal excitation. We have hypothesized that there is also increased synchrony within small neuron clusters, and that this synchrony results from abnormal nonsynaptic cellular interactions. We predicted that evidence for abnormal non-synaptic connectivity would be apparent with thinsection electron microscopy. Here, we have examined the ultrastructure of subsurface cisterns (SSC), which occur immediately beneath the plasma membrane of neuron cell bodies. Normally, these micro-regions of the plasma membrane possess differences in ionic conductance and channel expression relative to other regions of the soma membrane, and almost universally have intimate contact with adjacent astrocytic processes. SSCs also appear to have a role in intracellular signaling pathways as stores of intracellular calcium. Abnormalities of SSC have been linked to experimental epilepsy models.

Methods: Surgically-resected HH tissue was utilized following informed consent under approved protocols. Thin (0.08um) sections from six patients were examined with a Philips CM 100 EM fitted with a digital camera. Single sections and 3-D reconstruction of serial sections of small $\mathrm{HH}$ neurons were used for analysis.

Results: Most small neurons exhibited one or more SSCs, confined to the soma and proximal dendrites. HH SSCs appeared as discs composed of two collapsed or fused membranes of endoplasmic reticulum (ER) cisterns. The lumen of the cistern was essentially obliterated except at their lateral ends where a small canal remained. SSCs were 0.5 to $1.5 \mathrm{um}$ in length, and always ran parallel with the neuronal plasma membrane. Dense granular material $15-30 \mathrm{~nm}$ in thickness always connected SSCs to the plasma membrane. The neuronal plasma membrane domains overlying SSCs were significantly more electron dense compared to adjacent regions. Reconstruction of serial sections showed that only two of 24 SSCs were in close proximity to symmetrical (putative inhibitory) synapses. Abnormalities included absent or reduced coverage of the $\mathrm{SSC}$-associated plasma membrane by astrocytic processes. A wide diversity of SSC/glia morphology was evident.

Conclusions: SSCs of small HH neurons show abnormal ultrastructural features, with a lack of the expected relationship between the neuron and surrounding glia. SSCs are important regional sub-domains of membrane ionic conductance and sites of intracellular signaling. Abnormalities of SSC function may contribute to altered excitability or synchrony of $\mathrm{HH}$ tissue. This is the first study to implicate glial mechanisms as a possible component of seizure genesis within $\mathrm{HH}$ tissue. 


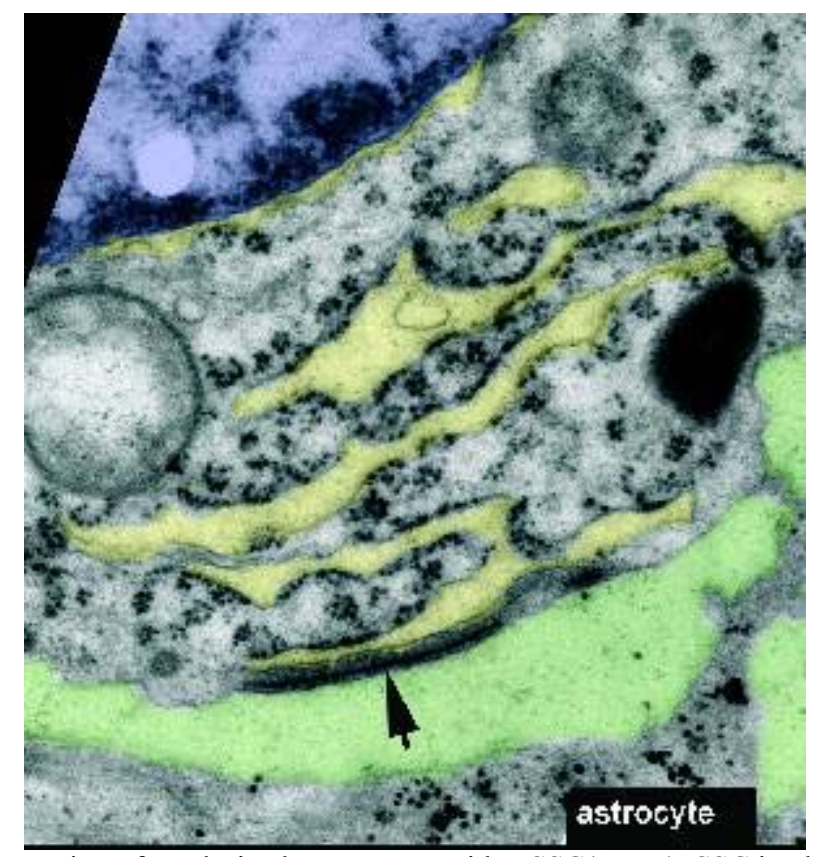

Portion of a colorized HH neuron with a SSC(arrow). SSC in close proximity to endoplasmic reticulum(ER,yellow). Overlying the plasma membrane is a widened extracellular space(ES)(green).Nucleus is blue. Astrocyte surrounds the enlarged ES. 3-D reconstruction (not shown) shows that the SSC has a continuity with the nuclear envelope canal via the ER.

\subsection{1}

\section{EXPRESSION OF HMGB1 AND TOLL-LIKE RECEPTORS IN THE HIPPOCAMPUS OF CASES OF HUMAN INTRACTABLE TEMPORAL LOBE EPILEPSY}

Madoka Nakajima, H. Sugano, T. Higo, Y. Iimura, H. Nakanishi and H. Arai (Neurosurgery, Juntendo University, School of Medicine, Tokyo, Japan)

Rationale: Inflammation of the brain has received widespread attention as an important factor in acquired epileptogenicity, but the effect of specific inflammation mediators on neuron excitability has not yet been clarified. Recently, Maroso et al. reported that epileptogenic pathways are activated when high-mobility group box1 (HMGB1) released from neurons and glia bind to toll-like receptor 4 (TLR4), a receptor that plays an important role in natural immunity. Therefore, to clarify inflammation mediator expression in intractable temporal lobe epilepsy patients, we used the hippocampus specimens of operated epilepsy patients to perform immunostaining for HMGB1, receptors TLR-2 and -4 , and the receptor for advanced glycation end products (RAGE), investigating the expression of each of them

Methods: This study targeted 19 patients who underwent resection surgery of the hippocampus or temporal lobe as treatment for intractable epilepsy at the Juntendo School of Medicine, Department of Neurosurgery from November 2004 through March 2012. Three specimens, including the patients of the same age range of 42 years, were used as controls. Immunostaining was performed for TLR2, TLR4, HMGB1, and RAGE receptors, and results were comparatively investigated.

Results: Strong immune reactions for HMGB1, TLR2, and RAGE were observed in the pyramidal neurons, astrocytes, and microglia of epilepsy patients' cortical specimens. Meanwhile, in control cortexes, HMGB1 was detected in ubiquitous glia and neuron nuclei, but we did not observe TLR4 or RAGE expression.

Conclusions: This study suggests that expression of HMGB1 and TLR4 increases in the epileptogenic site of the human brain and that HMGB1-TLR4 pathway also plays an important part in human epileptogenis. It was found that in human hippocampal epilepsy,
HMGB1 has prolonged action in localized areas and TLR4 becomes involved. This may lead to hippocampal sclerosis and atrophy.

\subsection{2 \\ THE INTERPLAY BETWEEN KYNURENINE PATHWAY ENZYMES AND THE DRUG RESISTANCE-ASSOCIATED MAJOR VAULT PROTEIN IN TUBEROUS SCLEROSIS COMPLEX: A REPORT OF 25 SURGICAL CASES} Carlos Eduardo Batista, H. T. Chugani, E. Asano, D. Cukovic, S. Sood, A. Dombkowski and D. C. Chugani (Wayne State University, Detroit, MI)

Rationale: Tuberous sclerosis complex (TSC) is a multisystemic disease associated with seizures and inflammation in the brain. We have shown that epileptogenic tubers have increased tryptophan uptake and evidence that this uptake is related to increased tryptophan metabolism by the kynurenine pathway (KP). Products of the KP include the neurotoxic metabolite quinolinic acid, and the end product of the KP is nicotinamide adenine dinucleotide (NAD), a substrate for many cell reactions including poly(ADP-ribosyl)ation. Recently, epileptogenic tubers were found to have increased expression of a protein associated with drug resistance called major vault protein (MVP), which constitutes the majority of the ribonucleoprotein vault particle. The vault particle also contains a poly(ADP-ribose) polymerase termed vPARP. Here we investigate whether there is association between MVP and the KP in epileptogenic tubers. We localized the presence of MVP and the ratelimiting enzyme of the KP, indoleamine 2,3-dioxygenase (IDO), in epileptogenic tubers. Then we studied whether there is an association between the expression of MVP and IDO and the downstream KP enzyme 3-hydroxyanthranilate-3,4-dioxygenase (HAO), which leads to the production of the pro-convulsant metabolite quinolinic acid. Methods: Immunocytochemistry for IDO and MVP $(\mathrm{N}=8)$ and western blots for IDO (isoforms 1 and 2), HAO, and MVP $(\mathrm{N}=25)$ were performed on surgical specimens from patients with TSC who underwent epilepsy surgery for whom epileptogenic tuber and relatively normal tissue areas were available. Actin was used as endogenous control.

Results: IDO expression was present in dysplastic cells and neuropil (Figure 1A and 1B), while some cells did not stain (Figure 1C). The perivascular tissue also showed positive immunoreaction (Figure 1D). MVP demonstrated similar pattern, staining dysplastic cells and neuropil (Figure 1E and 1F). Similarly to IDO, some dysplastic cells were not stained (Figure 1G). The vascular bed was also positive (Figure 1H). Epileptogenic tubers had higher expression of MVP, IDO2 and HAO compared to control areas (all $\mathrm{p}<0.01$ ), but not IDO1 $(\mathrm{p}=0.25)$. Expression of MVP correlated with HAO in the epileptogenic tissue $(\mathrm{r}=0.7, \mathrm{p}<0.001)$ and in the control area $(\mathrm{r}=0.8$, $\mathrm{p}<0.001$ ) (Figure 2A and 2B). A trend was observed between expression of MVP and IDO1 in the epileptogenic tissue $(r=0.4$, $\mathrm{p}=0.086$ ), while expression of MVP in the control tissues correlated with IDO1 ( $\mathrm{r}=0.44, \mathrm{p}=0.028)$.

Conclusions: Coordinate expression of KP enzymes and MVP may be due to common regulatory mechanisms such as IFN- $\gamma /$ Jak-STAT signaling related to inflammation in TSC. Alternatively, increased demand for NAD due to vPARP activity of the vault particle may drive KP activity including the formation of quinolinic acid. Further study of the interplay between MVP and the KP may help better understand how lesions in TSC become epileptogenic and drug resistant, providing further targets for pharmacological treatment. 


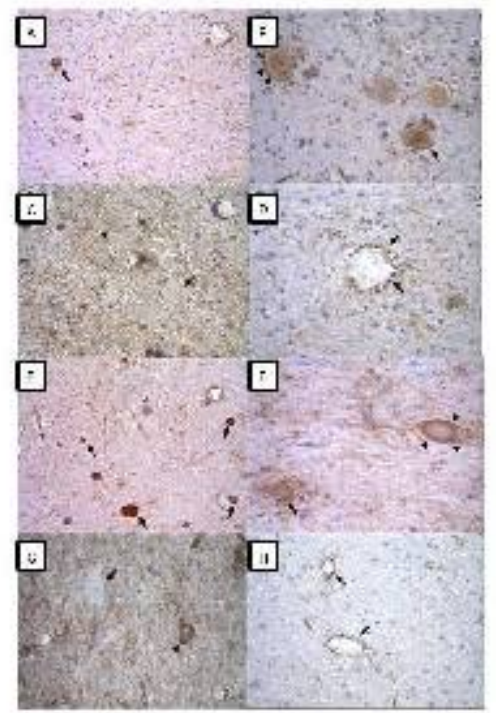

Higure 1
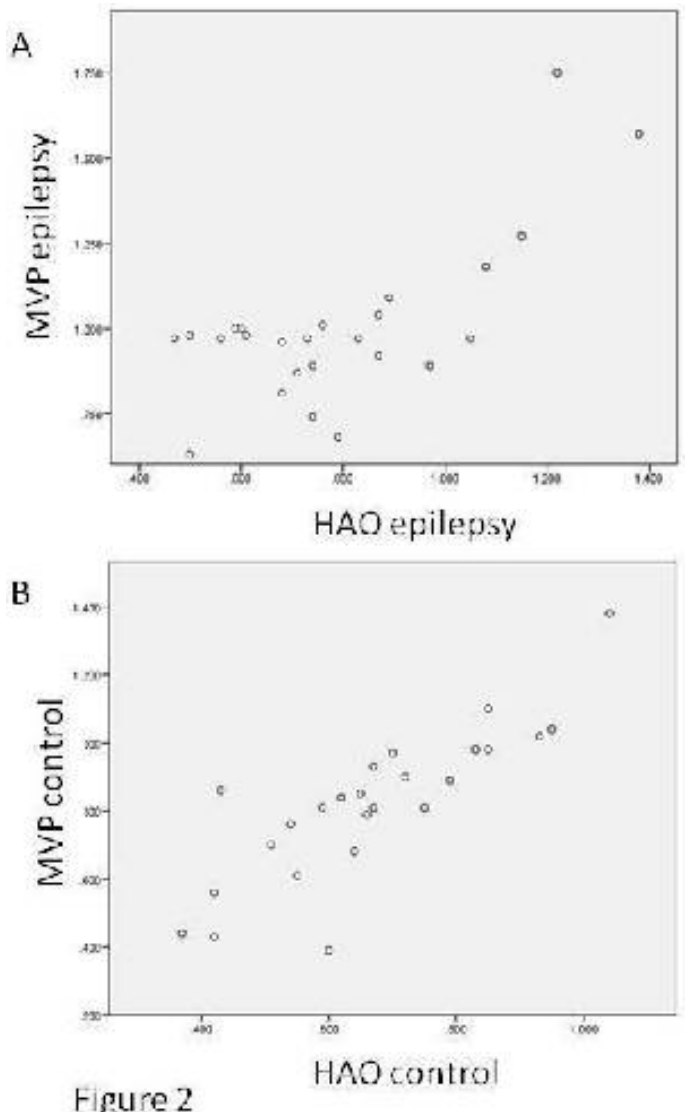

\footnotetext{
3.333

GRANULE CELL DISPERSION IS ASSOCIATED WITH MEMORY IMPAIRMENT IN RIGHT MESIAL TEMPORAL LOBE EPILEPSY

Rafael S. Neves ${ }^{1}$, I. S. Tudesco, ${ }^{1,2}$, A. P. Jardim ${ }^{1}$, L. O. Caboclo ${ }^{1}$, C. L. Lancellotti ${ }^{3,4}$, T. F. Marinho ${ }^{1}$, M. M. Marinho ${ }^{1}$, R. S. Centeno ${ }^{1}$, A. P. Hamad ${ }^{1}$, E. A. Cavalheiro ${ }^{5}$, C. A. Scorza ${ }^{5}$ and E. M. Yacubian ${ }^{1}$ ( ${ }^{1}$ Department of Neurology and Neurosurgery, Universidade Federal de São Paulo, Escola Paulista de Medicina, São Paulo, Brazil; ${ }^{2}$ Department of Psychobiology, Universidade Federal de São Paulo, Escola Paulista de Medicina, São Paulo, Brazil; ${ }^{3}$ Department of Pathology, Santa Casa de São Paulo, São Paulo, Brazil; ${ }^{4}$ Department of Pathology, Associação Fundo de Incentivo à Pesquisa (AFIP),
}

Medical Diagnosis, São Paulo, Brazil and ${ }^{5}$ Department of Neurology and Neuroscience, Experimental Neurology,, Universidade Federal de São Paulo, Escola Paulista de Medicina, São Paulo, Brazil)

Rationale: GCD (granule cell dispersion) is a common phenomenon occurring in almost $50 \%$ of mesial temporal sclerosis (MTS) specimens. Little is known about its pathogenic mechanisms and clinical significance. Hippocampus plays a crucial role in learning and memory. Seizure frequency, duration of epilepsy, antiepileptic drugs, and interictal epileptiform discharges, have been implicated in the memory dysfunction in mesial temporal lobe epilepsy (MTLE) patients. Histopathological investigation of surgical specimens may also help understand memory impairment in these patients. Human studies and animal models have correlated the degree of hippocampal and dentate gyrus (DG) cell loss with memory decline. We studied the occurrence of GCD in refractory MTLE-MTS patients and investigated the relationship between this abnormality and memory performance, clinical characteristics, patterns of MTS, as well as surgical outcome.

Methods: Fifty-four patients (27 men), all right-handed, with medically refractory MTLE and unilateral MTS (27 right and 27 left) on MRI were included in this study. Right and left MTLE patients were separated in GCD and no-GCD groups. All patients had extensive presurgical evaluation including high-resolution MRI and prolonged noninvasive video-EEG recording, and when concordant data was obtained in this evaluation, a surgical resection was performed. Logical Memory I and II and the Rey Auditory Verbal Learning Test, were used to assess verbal memory. Visual Reproduction I and II and Rey-Osterrieth complex figure test, were employed to assess visuospatial memory. Measurements of width of granule cell layer (GCL) and neuronal cells count were performed on ImageJ software. The average width of GCL was measured in each surgical case on NeuN stained section. Neuronal cell counts in all epilepsy tissue and control specimens were analyzed according to Blumcke et al. (2007) Values of neuronal cell counts from each hippocampal subfield were transformed into z-scores. Student's t test was used to analyze the importance of clinical data; $p$ values $<0.05$ were considered as statistical significant.

Results: GCD was observed in 28 cases (51.9\%): 14 in right and 14 in left MTLE. Clinical variables, different patterns of MTS and postoperative outcome were similar between GCD and no-GCD groups in right and left MTLE. Visuospatial memory was significantly affected in right MTLE GCD group when compared to the no-GCD group. However, in left MTLE-MTS group presence of GCD was not associated with memory performance.

Conclusions: GCD was not correlated with clinical variables, MTS type and seizure outcome. The occurrence of GCD was associated with visuospatial memory deficit in right MTLE patients. However, the presence of GCD was not associated with memory performance in left GCD group. The present findings emphasize the importance of performing histopathology evaluation as part of surgery epilepsy protocol, and its contribution in the understanding of memory performance in TLE patients. However, the role of GCD in memory function is yet to be precisely defined.

\subsection{4 \\ PROBABILITY FOR AURAS CORRELATES WITH IMPAIRED CHLORIDE REGULATION IN HUMAN EPILEPTOGENIC NEURONES}

Rudolf A. Deisz ${ }^{1}$, T. N. Lehmann ${ }^{2}$, P. Horn ${ }^{3}$ and C. Dehnicke ${ }^{4}$ ( ${ }^{1}$ Institute for Cell biology and Neurobiology, Charité Universitätsmedizin Berlin, Berlin, Germany; ${ }^{2}$ Neurosurgery Clinic, Helios Hospital, Bad Saarow, Germany; ${ }^{3}$ Neurosurgery Department, Charité Universitätsmedizin Berlin, Berlin, Germany and ${ }^{4}$ Epilepsy Center Berlin -Brandenburg, Königin Elisabeth Hospital, Berlin, Germany) 
Rationale: The normal function of $\mathrm{GABA}_{\mathrm{A}}$ receptor-mediated inhibition crucially depends upon the chloride gradient across the membrane. The gradient is governed by the operation of cation coupled chloride transporters, which are altered in human epilepsy. To evaluate links between impaired transporters and the severity of seizure activity, we quantified neuronal properties and correlated them with different clinical parameters including type and frequency of seizures.

Methods: Experiments were approved by the Ethics Committee and patients provided informed consent to use the tissue for research. Human neocortical tissues from epilepsy surgery were investigated in vitro with intracellular sharp microelectrode recordings using standard procedures. The electrodes were filled with either $1 \mathrm{M}$ potassium acetate or $1 \mathrm{M} \mathrm{KCl}$. Synaptic properties were evaluated with input-output relationships. The kinetics of chloride extrusion was determined from changes of the peak amplitudes of IPSP $_{\mathrm{A}}$ following iontophoretic chloride injections. We related the cellular data, averaged for each resection, to clinical parameters, including duration of epilepsy, the documented annual numbers of auras, complex partial seizures (CPS) and grand maux (GM). The clinical data were segregated as follows: duration of epilepsy $</>15 \mathrm{yrs}$, auras $<>>52$ p.a.; CPS $<>>52$ p.a. and GM $<>>1$ p.a..

Results: The synaptic responses of human neurons in epileptogenic tissues elicited with high SI were significantly larger $(20.0 \pm 6.3 \mathrm{mV}$, 129 neurons $(\mathrm{n}))$ than in neurons from control tissues $(13.3 \pm 5.1 \mathrm{mV}$, $\mathrm{n}=12 ; \mathrm{p}<0.01$ ) when recorded with KAc-filled electrodes. The maximal amplitudes differed between tissues with a different history of auras (few auras: $17.3 \pm 11.8 \mathrm{mV}, 28$ resections (r); many auras: $20.6 \pm 8.5, \mathrm{r}=27, \mathrm{p}<0.05)$. Recordings with $\mathrm{KCl}$-filled microelectrodes yielded even larger synaptic responses $26.7 \pm 7.1 \mathrm{mV}(\mathrm{r}=48 ; \mathrm{p}<0.01$ vs. KAc). Reversal potentials of isolated $\operatorname{IPSP}_{\mathrm{A}}\left(\mathrm{E}_{\mathrm{IPSP}}\right)$ averaged $61.5 \pm 8.1 \mathrm{mV}$ with KAc electrodes $(\mathrm{n}=50)$ and $-59.7 \pm 7.8 \mathrm{mV}(\mathrm{n}=53)$ with $\mathrm{KCl}$ electrodes. The average tau of $\mathrm{IPSP}_{\mathrm{A}}$ recovery was $19.0 \pm 9.6 \mathrm{~s}(\mathrm{n}=63$; rat neurons: $7.2 \pm 2.4 \mathrm{~s})$. Neurons with larger taus of chloride extrusion exhibited less negative reversal potentials. The tau of recovery (few auras: $16.4 \pm 4.6 \mathrm{~s}, \mathrm{r}=20$; many auras: $24.2 \pm 10.1 \mathrm{~s}$, $\mathrm{r}=15 ; \mathrm{p}<0.05$ ) and $\mathrm{E}_{\mathrm{IPSP}}$ (few auras: $-61.3 \pm 7.0, \mathrm{r}=16$; many auras: $55.3 \pm 7.7, \mathrm{r}=13 ; \mathrm{p}<0.05)$ were significantly different between these two clinical groups. Segregation of neuronal data according to the other clinical data revealed no significant differences for duration of epilepsy, number of CPS or number of GM.

Conclusions: Neurons in human epileptogenic cortex exhibit larger synaptic responses partly mediated by a depolarizing chloride gradient, which is caused by a reduced chloride extrusion. Tissues with a history many auras have a significantly reduced chloride extrusion, indicating that the probability for auras is increasing with worsening of chloride homeostasis. The more generalized activities require additional mechanisms including reductions of $\mathrm{GABA}_{\mathrm{B}}$ receptors and decreases of $\mathrm{I}_{\mathrm{H}}$.

\subsection{5 \\ NEUROPATHOLOGY IN PROLONGED REFRACTORY STATUS EPILEPTICUS}

Mathew Pulicken ${ }^{1}$, S. Reynolds ${ }^{2}$, J. Szaflarski ${ }^{3}$, L. Hirsch ${ }^{4}$ and R. Kilbride ${ }^{1}\left({ }^{1}\right.$ Massachusetts General Hospital, Boston, MA; ${ }^{2}$ Columbia New York Presbyterian, New York, NY; ${ }^{3}$ University of Cincinnati, Cincinnati, $\mathrm{OH}$ and ${ }^{4}$ Yale University, New Haven, CT)

Rationale: Status Epilepticus (SE) is a commonly encountered medical emergency, and a significant percentage of patients prove refractory to first- and second-line anticonvulsant therapies. Prolonged Refractory SE (PRSE) is status epilepticus that persists despite $\geq 7$ days of continuous anesthetic infusion, and represents a rarer subset of SE, but is not infrequent in Neuro Intensive Care Units. We examined the pathology in a subset of our PRSE patients through biopsy and autopsy specimens.

Methods: Three academic centers systematically identified patients with PRSE (excluding cardiac arrest) by review of records from 2001 to 2010. A subset of the study population who underwent biopsy and autopsy were included in the study and samples were examined by a certified Neuropathologist at each of the centers.

Results: Sixty-three patients, 26 male and 37 females (age 14 to 86 yr), were identified with PRSE (SE duration 8 to 169 days). We reviewed the histo-pathological findings in 7 biopsies performed to establish a cause for PRSE. 3 out of the 7 samples established a diagnosis including radiation necrosis, gliotic brain with infract at site of brain metastatic lesion resection and atypical meningioma. Autopsies were performed on 6 out of 21 patients who died secondary to PRSE. 3 out of 6 samples provided an explanatory cause for their PRSE including sub acute bilateral occipital ischemia with acute to subacute global ischemia, acute ischemia and vasculitic changes secondary to Behcet's Disease (diagnosis made on multiple organ pathology evaluation)

Conclusions: PRSE remains a clinically challenging entity. Biospy during PRSE was helpful, but in less than $50 \%$ of cases. More extensive neuro-pathological studies with autopsy specimens could help with establishing various etiologies of PRSE and guide in alternative and optimal treatment modalities. A larger study sample with multi-center cooperation would help in understanding the pathology of this severe form of SE.

\subsection{6 \\ MALFORMATION OF CORTICAL DEVELOPMENT ASSOCIATED WITH EARLY ONSET OF MEDICALLY INTRACTABLE EPILEPSY IN STURGE-WEBER SYNDROME}

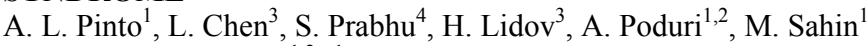
and Masanori Takeoka ${ }^{1,2}\left({ }^{1}\right.$ Department of Neurology, Children's Hospital Boston, Boston, MA; ${ }^{2}$ Division of Epilepsy and Clinical Neurophysiology, Children's Hospital Boston, Boston, MA; ${ }^{3}$ Department of Pathology, Children's Hospital Boston, Boston, MA and ${ }^{4}$ Department of Radiology, Children's Hospital Boston, Boston, MA)

Rationale: Severe Sturge-Weber Syndrome (SWS) cases may present in early life with intractable seizures; these cases may be associated with developmental disorganization of the cortex, but the frequency and significance of this association remains unclear. In this study we compared the clinical features, neuroimaging and neuropathological findings in such severe cases of SWS who required surgical treatment for intractable epilepsy.

Methods: We retrospectively reviewed the clinical features, preoperative magnetic resonance imaging (MRI) studies, and pathological findings of all SWS patients who underwent excisional surgery for intractable epilepsy at Boston Children's Hospital between 1994 and 2011.

Results: Eleven patients (male/female $=4 / 7$ ) with SWS were identified who underwent surgery for intractable epilepsy (mean age $13+/-6.2$ months), including hemispherectomy $(\mathrm{n}=10)$ and focal cortical resection $(n=1)$. Mean age at seizure onset was $15+/-11$ weeks of age. In $50 \%(n=6)$ of patients, patients had 2 different types of seizures; 2 patients had 3 different types of seizures. Focal clonic seizures were the most common type and occurred in 9 patients; apnea as second type of seizure occurred in 4 patients. Brain MRIs were reviewed in 6 of 11 patients and had findings consistent with Sturge-Weber syndrome, in all cases. Five cases showed further evidence of accelerated myelination. Areas of poor differentiation of white and gray matter were seen in some patients on MRI, while histopathological examination revealed findings consistent with cortical dysplasia.

Conclusions: In our patients, cortical malformations were consistently identified in children with severe SWS who underwent early epilepsy surgery. Clinical presentation in this subgroup is severe, with early onset of catastrophic epilepsy, medically intractable seizures, and associated cognitive and motor deficits. Changes consistent with cortical malformation may be associated 
with the more severe early onset intractable epilepsy rather than vascular malformation changes, considering that such dysplastic changes may be more epileptogenic; further studies will be necessary to confirm such trend, and also to assess whether such dysplastic changes are developmental, or secondary to vascular and perfusion anomalies in such patients.

\subsection{7}

SYNCHRONOUS FIRING IN TWO POPULATIONS OF NEURONS IN HUMAN EPILEPTIC HYPOTHALAMIC HAMARTOMAS

Peter N. Steinmetz ${ }^{1,2}$, S. Wait ${ }^{2}$, G. P. Lekovic ${ }^{3}$, H. L. Rekate ${ }^{4}$ and J. F. Kerrigan ${ }^{1,5}\left({ }^{1}\right.$ Comprehensive Epilepsy Center, Barrow

Neurological Institute, Phoenix, AZ; ${ }^{2}$ Department of Neurosurgery, Barrow Neurological Institute, Phoenix, AZ; ${ }^{3}$ House Ear Institute, University of Southern California School of Medicine, Los Angeles, $\mathrm{CA} ;{ }^{4}$ Cushing Neuroscience Institute, North Shore University Hospital, Great Neck, NY and ${ }^{5}$ Division of Pediatric Neurology, Barrow Neurological Institute at Phoenix Children's Hospital, Phoenix, AZ)

Rationale: Human hypothalamic hamartomas $(\mathrm{HH})$ are intrinsically epileptogenic for treatment-resistant galactic seizures. However, the basic cellular mechanisms responsible for the epileptogenicity of $\mathrm{HH}$ tissue are unknown. Microelectrode patch-clamp recordings from $\mathrm{HH}$ neurons in resected slice preparations show that small $\mathrm{HH}$ neurons demonstrate intrinsic pacemaker-like activity. We hypothesized that spontaneous firing of single neurons is present in-situ, and that microwire recordings in $\mathrm{HH}$ tissue prior to surgical resection would demonstrate firing behavior consistent with a functional network. Methods: Patients undergoing transventricular endoscopic resection of $\mathrm{HH}$ for treatment-resistant epilepsy were included. Single neuron recordings from bundled microwires (total of 9 contacts) were obtained from $\mathrm{HH}$ tissue immediately prior to surgical resection in 14 patients. Spontaneous activity was recorded for two or three 5-minute epochs under steady-state general anesthesia. Off-line analysis included cluster analysis of single neuron activity, and probability analysis of firing relationships between different neurons.

Results: A total of 222 neurons were identified in 14 patients (mean 6 neurons per recording epoch). Cluster analysis of single neuron behavior identified two distinct populations on the basis of mean firing rate $(\mathrm{FR})$ and burst density $(\mathrm{Bisi}=\#$ interspike intervals $>10 \mathrm{~ms}$ / \# interspike intervals $<10 \mathrm{~ms}$ ) :

1. A Low FR group with median $F R=0.85$ spikes/s and median Bisi $=0.018$, and

2: A High FR group with median 16.3 spikes/s, median Bisi=0.19. Analysis of spontaneous firing behavior between single neurons showed that 417 of 813 pairs of neurons $(51 \%)$ had a significant $(p<0.05)$ degree of cross-correlation within $+-2 \mathrm{~ms}$ of zero delay. Pairs of High FR neurons were significantly $(p<0.001)$ more likely to be correlated.

Conclusions: $\mathrm{HH}$ tissue in situ contains neurons with are spontaneously active. The activity of single neurons is diverse but distributes into at least two electrophysiological phenoytpes. Synchronous firing between pairs of neurons suggests that $\mathrm{HH}$ neurons exist within local networks that may contribute to epileptogenic firing.

\subsection{8 \\ DETECTION OF HUMAN HERPERSVIRUS-7 IN 305 PATIENTS WITH INTRACTABLE EPILEPSY}

Jin-Mei Li, C. Huang and D. Zhou (Department of Neurology, West China Hospital, Chengdu, China)

Rationale: Human herpesvirus-7 (HHV-7) is a $\beta$-herpesvirus isolated from peripheral blood CD4+ T cells from healthy individual in 1990. Most closely related to HHV-6 and cytomegalovirus, HHV-7 infection is ubiquitous and primary infection occurs during childhood and persistent long time. HHV-7 DNA also has been detected in CSF and normal brain tissue but not detected frequently than HHV-6. Previous studies have provided the evidence that CMV and HHV-6 are responsible for neurological diseases including encephalitis, Guillain-Barre syndrome, multiple sclerosis and seizures etc. A few previous studies reported HHV-7 definition in immunocompetent or allograft patients and showed that HHV-7 might be involved in immunologic or inflammatory process of CNS disease. However, small cases lead litter information is available about intractable epilepsy associated with HHV-7 infection till now.

Methods: Brain tissues of 305 consecutive cases with intractable epilepsy from 2009 to 2012 were analyzed for neuropathology and for HHV-7 KR4 antigen by immunohistochemistry. 21 samples as control group were obtained from patients with brain trauma or stroke.

Results: The 305 patients included 201 males (65.9\%) and 104 females $(34.1 \%)$. The mean age was $23.88 \pm 6.23$ years (range 18 to 45). HHV-7 KR4 protein was detected in samples from 27 out of 305 $(8.9 \%)$ patients with intractable epilepsy and in 0 of 21 control samples. The most prominent etiologic factors for HHV-7 infection were hippocampus sclerois (11 out of 69 cases), scar lesion ( 8 out of 58 cases) and vascular malformation (4 out of 52 cases).

Conclusions: These findings firstly suggest a possible association between HHV-7 positivity and intractable epilepsy especially hippocampus sclerosis. This study will provide us with additional insight to the underlying pathogenesis of intractable epilepsy.

\subsection{9 \\ PRESENTATION, DIAGNOSIS AND TREATMENT OF BILATERAL RASMUSSEN'S ENCEPHALITIS IN A 12 YEAR OLD FEMALE}

Katrina Peariso $^{1}$, S. M. Standridge ${ }^{1}$, B. E. Hallinan ${ }^{1}$, J. L. Leach ${ }^{2}$, L. Miles $^{3}$, F. T. Mangano ${ }^{4}$ and H. M. Greiner ${ }^{1}\left({ }^{1}\right.$ Neurology, Cincinnati Children's Hospital Medical Center, Cincinnati, OH; ${ }^{2}$ Radiology, Cincinnati Children's Hospital Medical Center, Cincinnati, OH; ${ }^{3}$ Pathology, Cincinnati Children's Hospital Medical Center, Cincinnati, $\mathrm{OH}$ and ${ }^{4}$ Neurosurgery, Cincinnati Children's Hospital Medical Center, Cincinnati, $\mathrm{OH})$

Rationale: To describe the diagnosis and management of a 12 year old female who presented with new onset seizures and a brain MRI demonstrating atrophy of the left cerebral and right cerebellar hemispheres. Initially, eletrophysiologic studies suggested a left posterior frontal-parietal focus. Approximately six months into her course, the seizure semiology changed to involve frequent left face, arm and leg clonic movements and epilepsia partialis continua (EPC) of the left upper extremity. Additionally, the EEG and PET imaging studies demonstrated a right parasagittal seizure focus.

Methods: Chart review of the clinical course, electrophysiologic, imaging, laboratory evaluations and treatment of a patient presenting to Cincinnati Children's Hospital Medical Center with intractable epilepsy, subsequently diagnosed with bilateral Rasmussen's encephalitis.

Results: An extensive evaluation for paraneoplastic, mitochondrial and genetic epilepsy syndromes was unrevealing. The cerebrospinal fluid studies showed normal glucose and cell counts but were positive for seven oligoclonal bands not found in her serum. The initial brain MRI showed left cerebral and right cerebellar atrophy with T2 hyperintensity in the left parietal region. Over the course of six months, an additional T2 hyperintensity was noted in the right frontal lobe. On biopsy evaluation seven months after her initial seizure, bilateral chronic encephalitis was demonstrated. This patient's clinical EPC with the biopsy confirmation of a T-cell encephalitis with activated microglia and reactive astrogliosis, supports the diagnosis of bilateral Rasmussen encephalitis. She was treated with immunomodulatory therapy with improvement in her seizure frequency and motor function. At this time, surgical intervention has been declined. 
Conclusions: Rasmussen's encephalitis can be a challenging disgnosis. We have described a rare case of bilateral Rasmussen's encephalitis highlighting the diagnostic challenges and the treatment dilemmas that arise in an adolescent presenting with bilateral inflammatory lesions of Rasmussen's encephalitis.

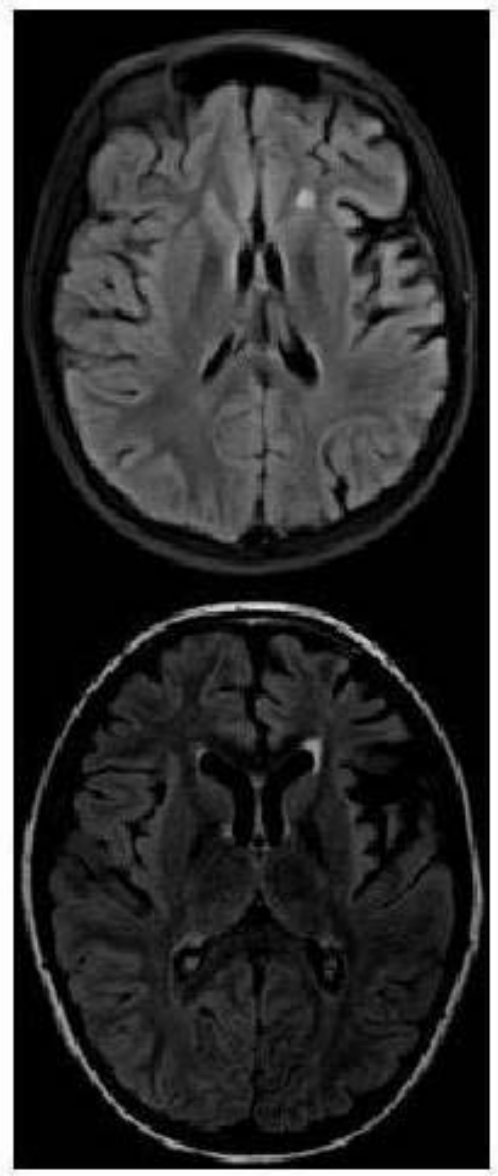

Axial FLAIR sequences from July 2011 (top) and January 2012 demonstrating significant left hemispheric atrophy over the 7 month period.

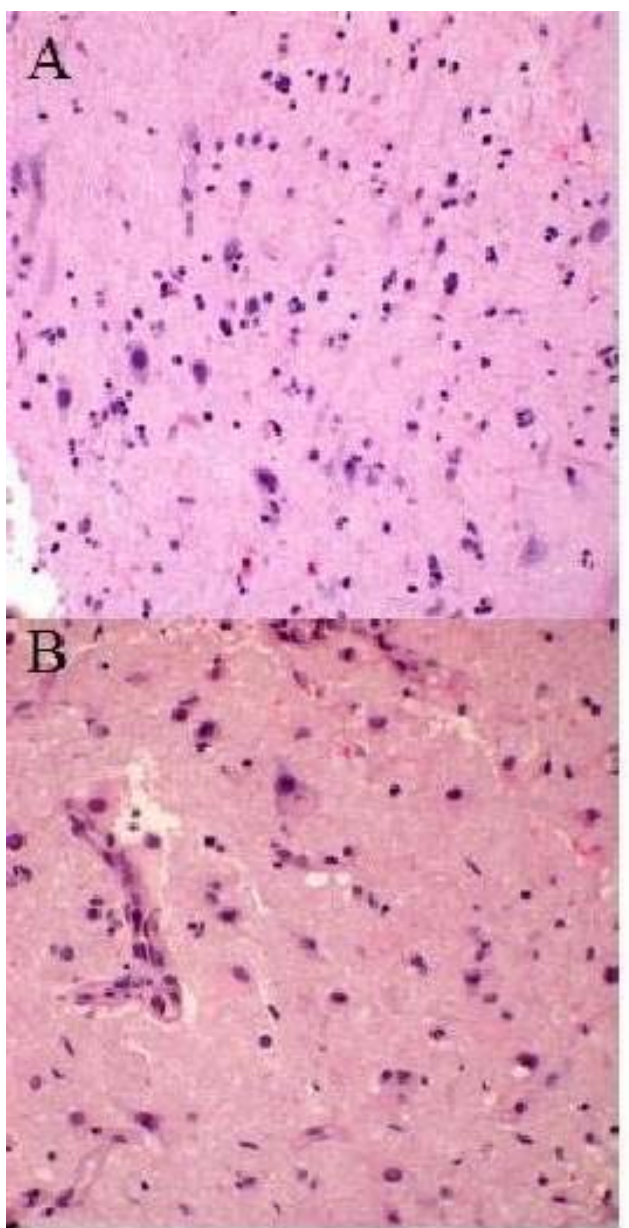

H\&E stained sections from the (A) left frontal and (B) right pre-motor cortex demonstrating neurons with reactive changes including red neurons, neurons with chromatolysis and diffuse lymphocytic infiltration.

\subsection{0}

SMALL AND LARGE NEURONS FROM HUMAN EPILEPTIC HYPOTHALAMIC HAMARTOMA: GOLGI ANALYSIS OF SURGICALLY-RESECTED TISSUE

J. F. Kerrigan ${ }^{1,2}$, A. Parsons ${ }^{1}$, K. Simeone ${ }^{3}$, J. Wu ${ }^{1}$, J. Beggs ${ }^{1}$ and $\mathrm{S}$. Coons ${ }^{1}\left({ }^{1}\right.$ Barrow Neurological Institute, Phoenix, AZ; ${ }^{2}$ Phoenix Children's Hospital, Phoenix, AZ and ${ }^{3}$ Creighton University School of Medicine, Omaha, NE)

Rationale: Hypothalamic hamartomas $(\mathrm{HH})$ are congenital tumors of the ventral hypothalamus resulting in treatment-resistant epilepsy. $\mathrm{HH}$ are intrinsically epileptogenic for gelastic seizures.

Electrophysiological features specific to Small and Large $\mathrm{HH}$ neurons have been identified, but the structure of these neurons is largely unexplored. We wished to study the microanatomical features of Small and Large HH neurons in order to gain further insights into their function within the epileptic network.

Methods: Surgically-resected HH tissue from 13 patients (54\% male; median age 5.3 years) was fixed, sectioned, and stained utilizing a modified Golgi-Cox method [FD NeuroTechnologies, Ellicott City, MD, USA]. Two-dimensional representations of selected cells were obtained with bright-field microscopy and a camera lucida drawing tube and analyzed for morphological features, including Sholl analysis. Neurons were assigned to the Small or Large type guided by our prior experience with single-cell microinjections subsequent to patch-clamp electrophysiological recordings. 
Results: Not unexpectedly, Small $(n=50)$ and Large $(n=10) \mathrm{HH}$ neurons differ in the cross-sectional area of their soma (mean 115 versus $345 \mu \mathrm{m} 2 ; \mathrm{p}<0.0001)$. Small neurons have universally round soma, while Large neurons are markedly pleomorphic and typically include at least one thick proximal dendrite. The two populations diverge significantly with respect their dendritic fields as shown by Sholl Analysis. Small neurons have dendrites that are thin, minimally branched and spineless ( $\mathrm{p}=0.0002$ ). $90 \%$ of Small neurons have dendrites that extend no more than $100 \mathrm{um}$ from the soma. Dendritic beading is common in both types of cells.

Conclusions: Small HH neurons are abundant, spontaneously-firing GABAergic neurons that occur in cellular clusters. Golgi staining of individual Small $\mathrm{HH}$ neurons shows simple and spatially limited dendritic fields consistent with an interneuron phenotype. Their network interactions may be largely within the local cluster. While Large HH neurons are morphologically consistent with excitatory projection-type neurons, they are much less stereotyped, suggesting that there is more diversity within the Large $\mathrm{HH}$ neuron subgroup than previously appreciated.

\section{Neuropathology of Epilepsy: Animal Studies}

\subsection{1 \\ INFLAMMATORY AND NEUROPLASTIC CHANGES IN THE HIPPOCAMPUS 24 HOURS AFTER LATERAL FLUID PERCUSSION INJURY \\ Lee Shapiro $^{1,3}$, M. J. Ruch ${ }^{1}$, s. Mukherjee ${ }^{2}$ and S. Zeitouni ${ }^{1}$ \\ ( ${ }^{1}$ Surgery, Texas A \& M University HSC COM, Temple, TX; ${ }^{2}$ Neurosurgery, Scott \& White Hospital, Temple, TX and ${ }^{3}$ CTVHCS, Temple, TX)}

Rationale: Traumatic brain injury (TBI) is a devastating injury that involves neuroplasticity and neuroinflammation. Life-long co-morbid outcomes include increased seizure susceptibility, epilepsy, cognitive dysfunction and emotional disturbances in addition to injury-related neuromotor deficits. Interneuron loss and/or alterations have been reported at 7 days after FPI and in humans. Because dysfunction and/or loss of interneurons are involved in seizures, determining when neuroplastic changes begin to affect this population of neurons is vital to our mechanistic understanding of TBI and the posttraumatic consequences. Recent evidence further demonstrates that neuroinflammatory cascades are initiated following TBI. Inhibiting this neuroinflammation may improve functional outcomes and limit epileptic activity. We have previously shown that multiple inflammatory cytokines are elevated in the cortex 24 hours after a lateral fluid percussion injury. Considering that the hippocampus is known to be affected by TBI and is also frequently implicated in seizures, understanding the temporal sequence of inflammation and interneuron changes is important for developing more efficacious therapeutic interventions. Thus, we chose to examine neuroinflammation, glial cell activation and interneuron changes in the hipoocampus at $24 \mathrm{hrs}$ after a fluid percussion injury.

Methods: Male Sprague-Dawley rats $(250-300 \mathrm{~g}, \mathrm{n}=12)$ were subjected to a lateral FPI and inflammatory mechanisms were assessed at 24 hours after injury in the hippocampus (as previously described, Mukherjee et. al 2011). Astrocytes were stained with GFAP as described previously (Arisi et al., 2011). Astrocytes were traced in 3-D using the Neurolucida program. Stereological techniques were used to count parvalbumin labeled interneurons in the pyramidal layer of CA1, CA3, as well as the hilus and granule cell layers

Results: Process length, amount of process endings and amount of process bifurcations were examined. We found that at the 20 and 30 micron circles, the ipsilateral FPI rats have greater length relative to ipsilateral controls. No differences were detected in the contralateral hemisphere. . Analysis of process bifurcations revealed significantly more bifurcations in ipsilateral FPI animals within the first 10 microns from the cell body. No significant differences were found at this 24 hour time point for parvalbumin-labeled cells. Cytokine analysis revealed numerous significant differences in the ipsi- and contra-lateral hemispheres.

Conclusions: The finding of numerous cytokine alterations in the hippocampus at 24 hours after FPI, indicates relatively rapid inflammation in the hippocampus following an FPI initiated in the cortex. Morphological analysis of astrocytes in the hippocampus supports this idea. Although interneurons labeled with parvalbumin are not significantly decreased at 24 hours after FPI, such cell loss is well documented at 7 days after injury. Thus, these cells have not yet died in significant numbers, indicating a possible therapeutic window.

\subsection{2 \\ METHAMPHETAMINE ONLY INDUCES NEURONAL NECROSIS IN MICE WITH ELECTROGRAPHIC SEIZURE DISCHARGES \\ Denson G. Fujikawa ${ }^{1,2}$, E. S. Pais ${ }^{3}$ and E. R. Aviles, Jr. ${ }^{3}\left({ }^{1}\right.$ Neurology, VA Greater Los Angeles Healthcare System, North Hills, CA; ${ }^{2}$ Neurology, David Geffen School of Medicine, Los Angeles, CA and ${ }^{3}$ Research \& Development, VA Greater Los Angeles Healthcare System, North Hills, CA)}

Rationale: The purpose of our study was (1) to characterize the morphology of methamphetamine (METH)-induced neuronal death; (2) to determine the regional distribution and severity of METHinduced neuronal death; and (3) to determine the role played, if any, by METH-induced electrographic seizure discharges.

Methods: Anesthetized C57BL/6 male mice (25 g when given $\mathrm{METH})$ underwent skull-screw implantation for EEG recording one week prior to receiving $40 \mathrm{mg} / \mathrm{kg}$ of free-base METH s.c. or i.p. in METH-treated mice or saline in 6 controls. They had EEGs, behavior and rectal temperatures monitored for at least $4 \mathrm{~h}$, or until their monitored parameters returned to baseline. Twenty-four $\mathrm{h}$ after METH injection they were euthanized and underwent transcardiac brain perfusion-fixation with $4 \%$ phosphate-buffered paraformaldehyde, after which brains were removed, embedded in paraffin, sectioned at 6- $\mu \mathrm{m}$ thickness, stained with hematoxylin and eosin and examined. The data conformed to a Poisson distribution rather than a normal curve, so an analysis of deviance, with post-hoc comparisons under the Poisson model, was performed.

Results: Fifty-eight per cent ( 7 of 12) had repetitive electrographic seizure discharges (RESDs). The time from METH injection to the appearance of RESDs was $47.8 \pm 15.2 \mathrm{~min}$, the duration of RESDs was $8.53 \pm 4.21 \mathrm{~min}$, and the total time that RESDs were present was $321 \pm 124$ min (mean \pm SEM, $n=7)$. Only $43 \%$ of mice with RESDs ( 3 of 7) had the clonic forelimb movements characteristic of seizures induced in rodents (stage 3 behavioral seizures in the Racine classification). Sixty per cent of mice without RESDs ( 3 of 5) had clonic forelimb movements; none of the 3 had evidence of neuronal damage. One of the 5 mice without RESDs had a few acidophilic neurons in dorsal CA3a. Five of 7 mice with RESDs had acidophilic neurons (the light-microscopic appearance of necrotic neurons by electron microscopy) in the hippocampal CA1-CA3 regions and hilus, amygdala, piriform cortex and entorhinal cortex, and the overall mean damage scores were significantly greater than those for both the METH group without RESDs $(0.69 \pm 0.06$ vs. $0.01 \pm 0.01$, p $<0.001)$ and controls $(0.69 \pm 0.06$ vs $0.00 \pm 0.00, \mathrm{p}<0.001)$. The damage scores for each brain region were also significantly greater than those for both the METH group without RESDs and controls ( $p$ $<0.001$ for each comparison). Maximum rectal temperatures did not differ significantly in mice with and without RESDs following METH administration. Spearman correlation analysis showed that Tmax was not correlated with either the maximum damage score or the number of brain regions damaged $(\mathrm{p}=0.324$ and 0.381 respectively).

Conclusions: Our findings indicate that in mice EEG recording to document RESDs is mandatory to document seizure activity and that 
clonic forelimb movements should not be used as an indicator of seizure activity. Moreover, METH-treated mice with RESDs had acidophilic neurons in many of the same brain regions as in generalized seizures, and METH-induced neuronal necrosis only occurred in mice with RESDs.

\subsection{3 \\ GLYCEMIC MODULATION AGGRAVATES STATUS EPILEPTICUS-INDUCED HIPPOCAMPAL DAMAGE IN EXCITOTOXIN-RESISTANT MICE}

P. Elyse Schauwecker (University of Southern California, Los Angeles, CA)

Rationale: Inbred strains of mice differ in their susceptibility to excitotoxin-induced cell death, but the genetic basis of individual variation is unknown. We were interested in identifying the relationship between glycemic index and susceptibility to seizureinduced excitotoxic cell death and establishing whether modulation of glycemic index could modify the susceptibility to epilepsy. As a first step, we wanted to determine if glucose administration following kainate-induced status could reduce seizure-induced cell death in mice susceptible to excitotoxin-induced cell loss (FVB/N). Secondly, we wanted to determine if modulation of the glycemic index, in models of hypo- or hyperglycemia, could affect seizure susceptibility and its neuropathological consequences following kainate administration in a mouse strain previously found to be resistant to seizure-induced cell death (C57BL/6).

Methods: C57BL/6 mice were purchased from Jackson Laboratories and injected with insulin (hypoglycemia), glucose (non-ketotic hyperglycemia) or streptozotocin (STZ; diabetic hyperglycemia) to modify their glycemic status. C57BL/6 mice with modified glycemic status or normoglycemic FVB/N mice, purchased from Jackson Laboratories, received one subcutaneous injection of kainic acid. Following kainate injections, mice were monitored continuously for 4 $\mathrm{h}$ for the onset of locomotor activity, behavioral manifestations of limbic seizure episodes, and scored for seizure activity as defined previously (Racine, 1972). Brains from animals in each age group were processed for light microscopic histopathologic evaluation seven days following kainate administration to evaluate the severity of seizure-induced brain damage.

Results: Kainate administration to FVB/N mice resulted in a 2-fold reduction in blood glucose, which could be restored by glucose infusion following KA-induced status epilepticus. As well, glucose infusion post status epilepticus, also significantly reduced the extent of seizure-induced damage throughout the hippocampus. While hypoglycemia was without effect on kainate-induced seizure activity, it substantially increased the extent of seizure-induced cell death in C57BL/6 mice. In contrast, both diabetic hyperglycemia and nonketotic hyperglycemia modulated seizure sensitivity and aggravated the extent of seizure-induced cell death in C57BL/6 mice postkainate

Conclusions: We found that glycemic control could rescue hippocampal cells from seizure-induced excitotoxic cell death in an excitotoxin-susceptible mouse strain, FVB/N. As well, the results presented here illustrate that hyper- or hypoglycemia additively increased the extent of seizure-induced cell death in an excitotoxinresistant mouse strain, C57BL/6. The ability of glucose dysregulation to elicit a phenotypic switch from excitotoxin resistant to susceptible after kainate administration implicates glucose dysfunction as a key event in the pathogenesis of seizure-induced excitotoxic cell death

\subsection{4}

CROSS-SECTIONAL STUDY TO EVALUATE THE ROLE OF BRAIN INFLAMMATION IN THE DISEASE ONTOGENESIS AND PROGRESSION OF TEMPORAL LOBE EPILEPSY

Halima Amhaoul ${ }^{1}$, J. Hamaide ${ }^{1}$, K. Szewczyk ${ }^{1}$, K. Van Den Eynde ${ }^{1}$, E. Geerts ${ }^{2}$, D. Van Dam², P. P. De Deyn ${ }^{2}$, I. Pintelon ${ }^{3}$, S. Staelens ${ }^{4}$,
S. Kumar-Singh ${ }^{1}$ and S. Dedeurwaerdere ${ }^{1}{ }^{1}$ Medicine and Health Sciences, University of Antwerp, Wilrijk, Belgium; ${ }^{2}$ Biomedical Sciences, University of Antwerp, Wilrijk, Belgium; ${ }^{3}$ Veterinary Sciences, University of Antwerp, Wilrijk, Belgium and ${ }^{4}$ Nuclear Medicine, University of Antwerp, Wilrijk, Belgium)

Rationale: Recently, brain inflammation has been recognized as a key factor in the pathology of various types of epilepsy, including temporal lobe epilepsy (TLE). However, its ambiguous role is not completely understood. The aim of the present study was to determine the spatial and temporal profile of microglial activation and cell loss during the disease ontogenesis and the chronic phase and to link this profile to the occurrence of spontaneous recurrent seizures (SRS) in the kainic acid-induced status epilepticus model (KASE)

Methods: SE was induced in male Wistar rats by low-dose subcutaneous injection of kainic acid $(n=29)$ while controls received saline $(n=21)$. Different cohorts were sacrificed at $2 d, 7 d, 2 w$ and $12 \mathrm{w}$ after $\mathrm{SE}$ ( $\mathrm{n}=6-9$ per treatment group). Microglial activation (Ox42) was determined in cortex, hippocampus, amygdala, piriform cortex, cerebellum and pons.Cell loss (cresyl violet) was scored in the hippocampus. Chronic epileptic animals (12w post SE) underwent $100 \mathrm{~h}$ of continuous video-EEG to quantify SRS.

Results: At $2 \mathrm{~d}$ post SE, the severity of the SE correlated with cell loss in the hippocampus $(\mathrm{P}<0.05)$. Significant hippocampal cell loss was also seen at $7 \mathrm{~d}, 2 \mathrm{w}$ and $12 \mathrm{w}$ post $\mathrm{SE}$, with a maximum of cell loss at $2 \mathrm{w}$. At all time points investigated, KASE animals displayed Ox42 immunoreactivity. A significant upregulation of activated microglia peaked at the early phase of epileptogenesis $(7 d \& 2 w$ post $\mathrm{SE})$ in the entire hippocampus, amygdala and piriform cortex. The Ox42 immunoreactivity was attenuated by $12 \mathrm{w}$ post SE compared to $2 \mathrm{w}$ post $\mathrm{SE}$, but still significantly higher than controls in CA1 $(\mathrm{P}<0.05)$, the amygdala $(\mathrm{P}<0.01)$ and piriform cortex $(\mathrm{P}<0.05)$. During the chronic phase the animals showed SRS and interictal spikes. A positive correlation between the latency of the last SRS to sacrification and the Ox42 immunoreactivity score in CA1 was found $(\mathrm{P}<0.05)$.

Conclusions: Microglial activation is present during both disease ontogenesis and the chronic phase in regions that are important in seizure generation. Firstly,we showed that acute cell loss was a consequence of the primary insult, namely SE. Secondly, we hypothesize that these acute neurodegenerative events activate scanning microglia in the area. In turn, these activated microglia probably attract other microglia from the vicinity to the affected regions with a peak in microglia activation and cell clearance $7 \mathrm{~d}$ to $2 \mathrm{w}$. This process probably needs time and could therefore explain the delay in microglial activation. During the chronic phase cell loss and microglial activation was less pronounced, which may indicate that once microglia cleared the neuronal debris they returned to inactivated status. Finally, the current $\mathrm{Ox} 42$ results showed that more brain inflammation in CA1 was associated with a longer latency since the last seizure, which might suggest a neuroprotective effect of microglia during the chronic epileptic phase. Nevertheless, this immature hypothesis needs confirmation with more data.

\subsection{5 \\ QUANTIFICATION OF HETEROTOPIC NEURONS IN BABOONS WITH GENERALIZED INTERICTAL EPILEPTIC DISCHARGES}

Clyde F. Phelix ${ }^{1}$ and C. A. Szabo ${ }^{2}\left({ }^{1}\right.$ Biology Department, University of Texas at San Antonio, San Antonio, TX and ${ }^{2}$ South Texas Comprehensive Epilepsy Center, University of Texas Health Science Center San Antonio, San Antonio, TX)

Rationale: There is mounting evidence of cortical developmental abnormalities from morphometric MRI studies in people with idiopathic generalized epilepsies (IGE). These findings were also reflected in the baboon model for IGE. In this preliminary study, we 
attempted to compare stereoscopically quantified heterotopic neurons in the molecular layer, cortical layer VI and subcortical white matter in baboons that were photosensitive to baboons with normal scalp EEG studies.

Methods: Ten adult baboon (Papio hamadryas anubis and hybrids), ages 19 to 24 years old, were used in this study. Six baboons had generalized interictal epileptic discharges (IED+) on scalp EEG (three were photosensitive), while the four control (CTL) group had normal studies. None of the baboons had a history of seizures. Based upon prior MRI morphometric studies in baboons with IEDs on scalp EEG, three cortical sulci were selected for histopathological sampling: dorsal intraparietal (IPS), ventral lunate (LS), and anterior cingulate sulci (CGS). Postmortem samples of $1 \mathrm{~cm} 3$, which included both banks of adjacent gyri and the underlying white matter (WM), were collected within 30 minutes. Formalin-fixed paraffin embedded sections were stained with anti-NeuN primary antibody using indirect immunohistochemistry and diaminbenzidine as the chromogen. The sections were counterstained with hemotoxylin. Standard-sized rectangular areas of layers 1 and 6 and the subjacent WM were used to perform total cell counts of NeuN-positive neurons. Quantification was performed without knowledge of the baboons' EEg findings. Neuron densities of each sulcus and area were compared between control and epileptic animals with Student's t-test; $\mathrm{p}<0.05$.

Results: Density of NeuN neurons ranged from 22.2 to 668.7 per square millimeter with the lowest in the WM and highest in layer 6 . There were no remarkable differences of density within layers across sulci. Only one sulcus (CGS) displayed a statistically significant $(p<0.05)$ difference between control and epileptic groups.

Conclusions: This preliminary study, shows that there are differences in neuronal counts between PS and CTL baboons, particularly in the frontal subcortical white matter. This finding is consistent with earlier histopathological studies in patients with juvenile myoclonic epilepsy. The increase of subcortical neurons may represent markers of abnormal cortical development in PS baboons. Additional baboons need to be studied to confirm these findings, including baboons with clinical seizures, with the ultimate goal of characterizing the heterotopic neurons, many of which may be subplate neurons.

\subsection{6 \\ TRPC6 EXPRESSION CHANGES IN A MOUSE MODEL OF TEMPORAL LOBE EPILEPSY}

Isabella Ferando ${ }^{1,3}$, M. R. Kelley ${ }^{4}$ and I. Mody ${ }^{1,2}\left({ }^{1}\right.$ Neurology, David Geffen School of Medicine UCLA, Los Angeles, CA; ${ }^{2}$ Physiology, David Geffen School of Medicine UCLA, Los Angeles, CA; ${ }^{3}$ MCIP graduate program, UCLA, Los Angeles, CA and ${ }^{4}$ Neuroscience Undergraduate Program, UCLA, Los Angeles, CA)

Rationale: TRPC6 is a Ca2+-permeable non-selective cation channel expressed in the molecular layer of the dentate gyrus (DGML) in the hippocampus. TRPC6 activation has been linked to dendritic growth and seems to be a key player in the formation of excitatory synapses. Temporal lobe epilepsy (TLE) involves functional changes to the dentate gyrus, including mossy fiber sprouting, hilar cell loss, interneuronal loss, and aberrant granule cell proliferation and development. With this study we therefore sought to determine how TRPC6 expression is affected in the pilocarpine mouse model of TLE.

Methods: Age matched male mice were injected i.p. with $300 \mathrm{mg} / \mathrm{kg}$ pilocarpine following injection of $1 \mathrm{mg} / \mathrm{kg}$ scopolamine. Mice were kept in status epilepticus for 2 hours, and then administered $10 \mathrm{mg} / \mathrm{kg}$ diazepam. Mice were intracardially perfused with $4 \%$ PFA and brains sectioned at $35 \mu \mathrm{m}$, then stained with a primary antibody specific for TRPC6 (Alomone Labs).

Results: Optical density of bright field images was assessed with ImageJ and different groups compared with one-way ANOVA. TRPC6 expression levels and pattern were thus determined. Brains were processed at different time points after pilocarpine induced status epilepticus: 1 day, 7 days, and 8 weeks. Our findings show a significant TRPC6 staining reduction in the DGML of the 7 day group, with no changes detected at 1 day or after 8 weeks. Epileptogenesis was confirmed in the 8-week group by the presence of mossy fiber sprouting. Mossy fiber boutons were labeled for zinc transporter 3 and sprouting was detected in the inner DGML. Neuronal cell loss was confirmed with NeuN stain.

Conclusions: These results provide evidence regarding TRPC6 modulation during epileptogenesis of TLE and open further questions on the functional consequences of its reduction. Due to its association with excitatory synapses, TRPC6 down regulation may be a neuroprotective mechanism during the epileptogenic process. Drug based activation or inhibition of TRPC6 could lead to future clinical applications in TLE.

\subsection{7 \\ CONTRIBUTION OF ENHANCED INNATE IMMUNE RECEPTOR SIGNALING TO EARLY DENTATE EXCITABILITY AFTER CONCUSSIVE BRAIN INJURY}

Y. Li ${ }^{1}$, A. Korgaonkar ${ }^{1}$, J. Wang ${ }^{1}$, E. Townes-Anderson ${ }^{1}$, S. Elkabes ${ }^{2}$ and Viji Santhakumar ${ }^{1,3}$ ( ${ }^{1}$ Neurology and Neurosicences, University of Medicine and Dentistry, Newark, NJ; ${ }^{2}$ Neurological Surgery, University of Medicine and Dentistry, Newark, NJ and

${ }^{3}$ Pharmacology and Physiology, University of Medicine and Dentistry, Newark, NJ)

Rationale: Over a million people sustain traumatic brain injury (TBI) in the USA each year. Patients sustaining brain injury have an increased risk for developing epilepsy. Closed head injury causes an acute and sterile inflammatory response including the activation of innate immune receptors and microglia. Here, we propose that increases in toll-like receptor (TLR) signaling following brain injury can modulate neuronal activity and augment post-traumatic dentate network excitability.

Methods: Young adult Wistar rats (25-27 days) were subject to moderate (2 atm) lateral fluid percussion injury (FPI) or sham injury using standard methods (Gupta et al., 2012). Western blot and immunohistochemical analyses were used to examine post-traumatic changes in the expression of TLR2 and TLR4 in the ipsilateral hippocampus $4 \mathrm{hr}, 24 \mathrm{hr}$, and 7 days after FPI. Field potential recordings were performed on acute hippocampal slices prepared 3-7 days after injury. Network activity was evoked using a bipolar stimulation electrode placed in the perforant path and recordings were obtained in the dentate granule cell layer. In each slice, the granule cell population spike amplitude in control ACSF was compared to responses recorded following a 2 hour incubation in either control ACSF or $1 \mu \mathrm{M}$ LPS-RS, a selective TLR4 antagonist. Results: Western blots revealed that, when compared to shamcontrols, the expression of TLR2 and TLR4 in the hippocampus was increased 4 and 24 hours after FPI but not 7 days post-injury. In preliminary immunostaining studies, both the intensity of labeling and number of cells expressing TLR2 and TLR4 were increased in hippocampal sections obtained 4 hours after FPI . While TLR2 was expressed in GFAP-labeled astrocytes, TLR4 was expressed in NeuN-positive neuronal profiles in dentate gyrus, CA1 and CA3. Large TLR4 labeled neurons were observed in the dentate hilus and stratum radiatum of CA1 and CA3, suggesting that certain classes of GABAergic interneurons may express TLR4. In slices from FPI rats, LPS-RS decreased the amplitude of the granule cell population spike evoked by a $4 \mathrm{~mA}$ stimulus, to $30 \%$ of the amplitude in ACSF (amplitude in $\mathrm{mV}$, FPI in ACSF: $1.29 \pm 0.27$, FPI in LSP-RS: $0.38 \pm$ $0.11 \mathrm{~mA}$, t-test, $\mathrm{p}<0.005, \mathrm{n}=15$ ). The reduction of evoked population spike amplitude by LPS-RS was specific to slices from FPI rats and was not present in slices from sham-controls incubated in LPS-RS (amplitude in $\mathrm{mV}$, sham in ACSF: $0.08 \pm 0.06$, sham in LPS-RS: $0.06 \pm 0.06$, t-test, $\mathrm{p}>0.05, \mathrm{n}=8$ ) or in slices incubated in ACSF. Conclusions: These data demonstrate an early and transient increase in the expression of the innate immune receptors TLR2 and TLR4 in 
the post-traumatic hippocampus. The distribution of TLR4-labeled profiles suggests regional and cell-type specific increase TLR2 and TLR4 after brain injury. Physiological studies indicate that TLR4 signaling contributes to the early increase in dentate excitability after TBI. These findings pave the way for elucidating the role of innate immune receptor signaling in the enhanced risk for epilepsy following brain injury.

\subsection{8}

\section{NEURONAL DEGENERATION INDUCED BY STATUS} EPILEPTICUS IN SEPTAL NUCLEI OF IMMATURE RATS R. Druga ${ }^{1,2}$, Pavel Mares ${ }^{1}$ and H. Kubova ${ }^{1}$ ( ${ }^{1}$ Institute of Physiology Academy of Sciences, Prague 4, Czech Republic and ${ }^{2}$ Anatomy, Charles University, 1st and 2nd Faculty of Medicine, Prague, Czech Republic)

Rationale: The septum (septum verum) is a prominent telencephalic structure, anatomically and functionally heterogenous which operates in the transmission of signals from the brain stem to the hippocampus and other cortical areas. The glutamatergic projections from the hippocampus and subiculum dominate among the afferent connections of the lateral septum. The efferent connections arise mainly in the medial septal region and terminate in all fields of the hippocampus. The prominent part of the septohippocampal projection is cholinergic.

Experimental data about neuronal damage within the septum after status epilepticus

(SE) are sparse and only adult animals were analyzed. Systematic study and developmental data about neuronal damage of the septum in immature animals after SE are missing.

Methods: Experiments were carried out in male Wistar rat pups 12, 15, 18, 21 and 25 days old. Lithium-pilocarpine model of SE was used. Lithium chloride ( $3 \mathrm{mmol} / \mathrm{kg}$ i.p.) was injected 24 hours before pilocarpine $(40 \mathrm{mg} / \mathrm{kg}$, i.p.). Control animals were treated with $\mathrm{LiCl}$ and saline. Only animals exhibiting convulsive status epilepticus (SE) were included in this study. The rats survived for 4, 8, 12, 24, 48 hours and 1 week after SE. Four to five animals were processed in each survival interval. The animals were perfused under an overdose of urethane anesthesia with PBS followed by 4\% paraformaldehyde in PBS. Coronal sections ( $40 \mu \mathrm{m}$ thick) were processed with cresyl violet or with a fluorescent stain Fluoro-Jade B (FJB) used for detection of degenerated neurons. Sections were examined microscopically with an epifluorescence microscope using a filter system suitable for visualizing fluorescein or FITC.

Results: No or only isolated degenerated neurons were found mainly in the lateral region of 12-day-old and 15-day-old rat pups. In 18-dayold and older pups degenerated neurons were distributed in lateral septal region with prevalence to intermediate a ventral nucleus and to posterior half of the region. Since P18 neuronal damage was found in all survival intervals and reached peak at 24 and $48 \mathrm{~h}$ after SE. No FJB-positive cells were present in medial septal region in all age categories and survival intervals. Moderate to large number of degenerated neurons was evident in the bed nucleus of the stria terminalis.

Conclusions: Lithium-pilocarpine model of SE in immature rats resulted in degeneration of neurons in the lateral septal region with prevalence in its posterior half. Consistent neuronal damage was found in 18-day-old and older animals. Damage increased with survival interval and reached peak at 24 and $48 \mathrm{~h}$ after SE. No degenerating neurons were found in the medial septal region. This study was supported by Projects No. P304/12/G069 of the Grant Agency and No. ME08045 and LH11015 of the Ministry of Education of the Czech Republic.

\subsection{9}

NEURONAL MIGRATION ARREST IN A JUVENILE MYOCLONIC EPILEPSY K-O MICE

Jesus Machado-Salas ${ }^{1}$, M. Tanaka ${ }^{1}$, M. AvilaCosta ${ }^{2}$, J. Espinosa ${ }^{2}, \mathrm{~K}$. Yamakawa ${ }^{3}$ and A. Delgado-Escueta ${ }^{1}$ ( ${ }^{1}$ Epilepsy Center, UCLA/VA GLA, Los Angeles, CA; ${ }^{2}$ Neurociencia FESIZTACALA, UNAM MEXICO, Mexico D,F., Mexico and ${ }^{3}$ NEUROBIOLOGY RIKEN, RIKEN, Saitama, Japan)

Rationale: Juvenile myoclonic epilepsy (JME) is the most frequent of the idiopathic generalized epilepsies. Absences, Myoclonias and generalized seizures are the prominent neurological manifestations shown by the otherwise normal JME patients. Despite the prominent clinical and electroencephalographic features of the JME the neuropathological findings have been meager. Nevertheless, some recent morphometric studies have shown some cortical thickness abnormalities in JME patients. Nonetheless, these findings have not been corroborated by other authors. Our epilepsy consortium and other authors have identified myoclonin as an important enzyme playing a putative fundamental role on neuronal physiology and, perhaps also during early neurodevelopment .

We have developed a myoclonin-deficient k-o mice as a JME laboratory model (ehfc1

$-/-)$.

Methods: Expermental and wild mice were periodically sacrificed and perfused through their hearts. Their brains were carefully removed and processed with several neurohistological methods. For the very first time we present the results of our neuromorphological observations

Results: 1) Gross macroscopic appearence of the encephalon of myoclonin-deficient mice is normal.

2) There is prominent supratentorial ventriculomegaly.

3) At the fissura choroidea level the choroid plexus appears smaller and out of its usual position.

4)Several cortical and subcortical regions show disorganized cytoarchitectural patterns.

5) Hippocampus shows a globose appearence.

6)Our preliminary scanning electron microscopy showed a prominent morphological disturbance and number decrement of the pendymal cells ciliae.

Conclusions: These are the first observations of nerumorpholigical abnormalities observed in a JME laboratory model. We shall discuss the possible implications of these prominent changes in the physiopathogeny of JME.

\section{Epidemiology:}

\subsection{0}

CLINICAL FACTORS ASSOCIATED WITH SEIZURE REMISSION IN ADULTS WITH IDIOPATHIC GENERALIZED EPILEPSY

X. R. Liu ${ }^{1,2}$, Jonathan Dykeman ${ }^{1}$, Y. Aghakhani ${ }^{1}$, M. W. Lowerison ${ }^{1}$, N. Jette ${ }^{1}$, N. Pillay ${ }^{1}$, P. Federico ${ }^{1}$, W. Murphy ${ }^{1}$, A. Hanson ${ }^{1}$, B. Klassen ${ }^{1}$ and S. Wiebe ${ }^{1}\left({ }^{1}\right.$ University of Calgary, Calgary, AB, Canada and ${ }^{2}$ Institute of Neuroscience and the Second Affiliated Hospital of Guangzhou Medical University, Guangzhou, China)

Rationale: Research on seizure remission (SR) in patients with idiopathic generalized epilepsy (IGE) focuses mostly on children. We aimed to investigate the clinical factors associated with SR among adults with IGE.

Methods: We captured data on all consecutive patients during their first encounter in a tertiary outpatient epilepsy program serving a large geographic region. We included all adults with a probable diagnosis of IGE as judged by epileptologists. SR was defined as seizure freedom during the previous year. Variables of interest included age, age of onset, family history of seizures, having ever been referred to an epilepsy-monitoring unit (EMU), number of 
antiepileptic drugs (AEDs) ever tried (0-2 or 3 or more), number of seizure types $(1,2$, or 3 or more), having ever been exposed to carbamazepine, oxcarbazepine, or phenytoin (CBZ/OXC/PHT), and experiencing generalized tonic-clonic seizures (GTCS), myoclonic seizures (MS) and/or absence seizures (AS). Logistic regression was used to investigate the association between the variables of interest and SR, with statistical significance as $p<0.05$. Interactions between age of onset, number of AEDs, and number and types of seizures were explored using two models, one involving the number and the other involving the specific types of seizures.

Results: Among 1588 consecutive patients, 174 (10.96\%) aged $>18$ years were diagnosed with probable IGE. Their age (mean $+/-$ standard deviation) was $33.6+/-15.0$ years and age of onset was $18.94+/-14.4$ years. Fifty-one (30.2\%) patients were in SR. Sixtynine (58.5\%) patients not in SR and $28(54.9 \%)$ in SR had ever tried CBZ/OXC/PHT and 33 (28.0\%) not in SR and 10 (19.6\%) in SR had tried more than two AEDs. Nineteen (16.2\%) patients not in SR and $10(19.6 \%)$ in SR had a family history of seizures and $16(13.6 \%)$ not in SR and $4(7.8 \%)$ in SR had been referred to the EMU. Of patients not in SR, 61 (51.7\%) had one seizure type, 35 (29.7\%) had two types, $18(15.25)$ had three types, $98(83.0 \%)$ had GTCS, $23(19.5 \%)$ had MS, and $33(28.0 \%)$ had AS. Of patients in SR, $34(66.7 \%)$ had one seizure type, $13(25.5 \%)$ had two types, $3(5.9 \%)$ had three types, $42(82.4 \%)$ had GTCS, $8(15.7 \%)$ had MS, and $5(9.8 \%)$ had AS. None of the interactions were significant in either model $(\mathrm{p}>0.05)$. Most factors were not significantly associated with SR ( $p>0.05)$. However, patients with $>3$ seizure types were significantly less likely to be in SR compared to those experiencing one seizure type (OR 0.27 [95\% CI:0.075-0.97]). Analyses by specific seizure types showed that patients with AS were significantly less likely to be in SR (OR 0.23 [95\%CI:0.081-0.65]).

Conclusions: The proportion of patients with IGE not achieving SR was high $(69.8 \%)$ in our population, perhaps reflecting the focus on adults seen in tertiary care, or an evolving diagnosis. In this population, only the number and types of seizures were significantly associated with SR. AS are typically treatment responsive in children, their persistence into adulthood may be a marker of non-remission.

\subsection{1}

\section{FACTORS ASSOCIATED WITH SEIZURE REMISSION IN ADULT IDIOPATHIC GENERALIZED EPILEPSY: A SYSTEMATIC REVIEW}

M. W. Lowerison ${ }^{1}$, Jonathan Dykeman ${ }^{1}$, K. M. Fiest ${ }^{1}$, Y. Aghakhani ${ }^{1}$, S. Wiebe ${ }^{1}$ and X. R. Liu ${ }^{1,2}\left({ }^{1}\right.$ University of Calgary, Calgary, AB, Canada and ${ }^{2}$ Institute of Neuroscience and the Second Affiliated Hospital of Guangzhou Medical University, Guangzhou, China)

Rationale: Idiopathic generalized epilepsies (IGE) are generally viewed as having favorable outcomes, but seizures often persist despite medical therapy. We aimed to describe the evidence surrounding the factors associated with achieving seizure remission (SR) in adults.

Methods: Medline and Embase were searched using sub-headings and keywords related to IGE, remission, seizure freedom, and refractoriness in adults. Two independent reviewers screened abstracts and full-text articles and abstracted data from eligible studies. Included studies were original research reporting on factors associated with SR in adults with IGE. Analyses were done in three phases to 1) assess sample characteristics, and 2) assess the factors associated with SR.

Results: Of 3601 abstracts, 211 were reviewed in full-text, and 35 meet eligibility criteria. The median number of patients across the studies was 90.5 (range: 17-962). Sixteen studies reported on patients with IGE, thirteen with juvenile myoclonic epilepsy (JME), four on juvenile absence epilepsy (JAE), and two on absence epilepsy. SR ranged from $22.4 \%$ to $65 \%$ across the studies.

In five studies, aspects of absence seizures were significantly associated with a lack of SR. Patients who experienced absence seizures were significantly less likely to achieve $S R(p=0.001)$. In two studies the pattern of absence seizures (sporadic vs. daily/monthly and pyknoleptic vs. not) was significantly associated with a lack of SR ( $\mathrm{p}=0.004$ and $\mathrm{p}=0.04)$. In another, absence seizures persisting $>12$ years were associated with a lack of $S R(p<0.05)$. Finally, JAE had significantly worse seizure prognosis than other IGE syndromes $(\mathrm{p}=0.02)$.

Psychiatric comorbidity was associated with SR. Three studies found significantly lower prevalence of psychiatric disorders in patients who achieved SR $(19 \%, 21.9 \%$, and $19.5 \%$ respectively) compared to patients who had not achieved SR $(58.3 \%, 75 \%$, and $40.5 \%$ respectively). Another study reported fewer psychiatric conditions among patients with good seizure control compared to those with moderate/poor seizure control ( $25 \%$ vs. $4 \%, \mathrm{p}=.029)$. Lastly, one study found a significant association between use of two or more psychotropic drugs and a lack of SR.

The number of seizure types was associated with SR; $62.5 \%$ and $72 \%$ of patients with poorly controlled seizures had $>3$ seizure types, compared to $23.3 \%$ and $30.0 \%$ in the well-controlled groups. In three studies age of onset was significantly associated with SR. Patients with earlier age of onset $(<5,<11$, and mean 12.6 years $)$ were significantly less like to achieve SR in adulthood $(\mathrm{p}<0.05)$. Combination therapy (two studies) and generalized tonic-clonic seizures (three studies) were also associated with a lack of SR. Conclusions: Important factors associated with SR in adult IGE have been identified by this review. The next important step is to evaluate the use of these factors prospectively in their ability to predict seizure remission followed by approaches to alter these risk factors to potentially influence seizure outcomes.

\subsection{2 \\ WTIHDRAWN}

\subsection{3}

ARE THERE RACIAL DIFFERENCES IN PERCEPTIONS OF EPILEPSY CARE

Candice S. Waked ${ }^{1}$, D. W. Loring ${ }^{1}$, R. D. Bautista ${ }^{2}$ and K. J. Meador ${ }^{1}$ $\left({ }^{1}\right.$ Neurology, Emory University, Atlanta, GA and ${ }^{2}$ Neurology, University of Florida- SHANDS Jacksonville, Jacksonville, FL)

Rationale: To discern if medication non-compliance, depression, negative beliefs about medicine, disability, and poor quality of life are associated with a specific patient population. If a specific population can be identified, targeted treatment or counseling could potentially be implemented.

Methods: We conducted face-to-face survey-interviews of patients who were seen at the out-patient clinic of the Comprehensive Epilepsy Program at the University of Florida, Shands Jacksonville. The patients considered for inclusion must have an age greater than 17 and a history of at least two unprovoked seizures with a diagnosis of epilepsy. Patients that had non-epileptic spells, aphasia or cognitive impairment were exluded from the study. The principal investigator and research assistant reviewed each chart in detail and identified patients eligible for inclusion. Patients were asked to enroll in this study during the course of their regularly scheduled clinic visit. Informed consent was performed. This study was approved by the University of Florida institutional IRB.

Individual patient information obtained included: age, gender, race, marital status, educational level, annual household income, work status, and driving status. Surveys administered included: the Neurological Institute Disorders Depression Inventory for Epilepsy (NIDDIE), Quality of Life in Epilepsy-10 inventory (QOLIE-10), Beliefs about Medicine Questionnaire (BMQ), Sheehan Disability Scale (SDS), Morisky Medication Adherence Questionnaire (MMAQ), and Wide Range Achievement Test-3 (WRAT3). All patient information and survey results were recorded, de-identified, and stored on a secure institutional server. 
Results: 77 patients were included in this study: $75 \%$ women and $31 \%$ African American. The median patient age was 41 with a median disease duration of 22.1 and a median age of onset of 18.5 years. 35 patients were disabled and 28 patients were driving. There were no significant differences between race (Table 1) or gender (Table 2) in the scores of the NIDDIE, QOLIE-10, BMQ, SDS, MMAQ, or WRAT3.

Conclusions: Our study indicates that there are no differences in medication non-compliance, depression, beliefs about medicine, disability, and quality of life in our patients with respect to either race or gender. This suggests that each race and sex is equally impacted by epilepsy, thus no specific patient group was identified for targeted pre-emptive specific therapy. Thus, all patients with epilepsy, regardless of race or gender, need to be screened for major comorbidities commonly seen in epilepsy.

Questionnaire results by race

\begin{tabular}{|c|c|c|c|}
\hline BMQ-S & White & 5.8 & \multirow{2}{*}{$\begin{array}{c}\mathrm{P} \\
0.413\end{array}$} \\
\hline & Black & 4.6 & \\
\hline \multirow[t]{2}{*}{ BMQ-G } & White & 19.6 & \multirow{2}{*}{$\begin{array}{c}\mathrm{P} \\
0.604\end{array}$} \\
\hline & Black & 20.3 & \\
\hline \multirow[t]{2}{*}{ Shechan } & Whitc & 13.2 & \multirow{2}{*}{$\begin{array}{c}\mathrm{P} \\
0.766\end{array}$} \\
\hline & Black & 12.4 & \\
\hline \multirow[t]{2}{*}{ MMAQ } & White & 4.3 & \multirow{2}{*}{$\underset{0.536}{\mathrm{P}}$} \\
\hline & Black & 3.8 & \\
\hline \multirow[t]{2}{*}{ QOLIE-10 } & White & 34.8 & \multirow{2}{*}{$\begin{array}{c}\mathrm{P} \\
0.995\end{array}$} \\
\hline & Black & 34.8 & \\
\hline \multirow[t]{2}{*}{ NIDDT: } & White & 14.3 & \multirow{2}{*}{$\begin{array}{c}\mathrm{P} \\
0.194\end{array}$} \\
\hline & Black & 12.7 & \\
\hline \multirow[t]{2}{*}{ WRAT-3 } & Whitc & 43.77 & \multirow{2}{*}{$\stackrel{\mathrm{P}}{0.639}$} \\
\hline & Black & 42.83 & \\
\hline
\end{tabular}

Questionnaire results by sex

\begin{tabular}{|c|c|c|c|}
\hline \multirow{2}{*}{ BMQ-S } & Male & 7.44 & \multirow{2}{*}{$\begin{array}{c}\text { P Value } \\
0.069\end{array}$} \\
\hline & remale & 4.69 & \\
\hline \multirow[t]{2}{*}{ BMQ-G } & & & \multirow{2}{*}{$\begin{array}{c}\text { P'value } \\
0.969\end{array}$} \\
\hline & Female & 29.95 & \\
\hline \multirow[t]{2}{*}{ Shcehan } & Male & 14.08 & \multirow{2}{*}{$\begin{array}{c}\text { P value } \\
0.652\end{array}$} \\
\hline & Female & 12.86 & \\
\hline \multirow[t]{2}{*}{ MMAQ } & Male & 5.00 & \multirow{2}{*}{$\begin{array}{c}\text { P value } \\
0.114\end{array}$} \\
\hline & Female & 3.81 & \\
\hline \multirow[t]{2}{*}{ QOLIE-10 } & Male & 32.88 & \multirow{2}{*}{$\begin{array}{c}\text { P value } \\
0.153\end{array}$} \\
\hline & Female & 35.67 & \\
\hline \multirow[t]{2}{*}{ NIDDगT: } & Male & 14.76 & \multirow{2}{*}{$\begin{array}{c}\text { P value } \\
0.344\end{array}$} \\
\hline & Гcmale & 13.48 & \\
\hline \multirow[t]{2}{*}{ Wrat-3 } & Malc & 43,58 & \multirow{2}{*}{$\begin{array}{c}p \text { value } \\
0.951\end{array}$} \\
\hline & Temale & 43.45 & \\
\hline
\end{tabular}

\subsection{4}

\section{PREVALENCE OF NON-EPILEPTIC EVENTS IN CHILDREN WITH AND WITHOUT EPILEPSY ADMITTED TO A LEVEL \\ 4 EPILEPSY CENTER}

Angel W. Hernandez, L. Bailey, C. Johnson, M. S. Perry and S. I. Malik (The Jane and John Justin Neuroscience Center, Cook Children's Health Care System, Fort Worth, TX)

Rationale: Non-epileptic events are a common finding in pediatric patients admitted to an epilepsy monitoring unit (EMU), but few reports characterizing prevalence and patient characteristics are available in the literature. We evaluate the prevalence and characteristics of patients with non-epileptic events admitted to the EMU.

Methods: A random sample of patients admitted to the EMU for continuous video EEG at Cook Children's Medical Center from January 2006-September 2011 was retrospectively reviewed and data collected, including age, gender, and type of non-epileptic event. The type of non-epileptic event was characterized as motor manifestations, mental status change, apparent loss of consciousness, and isolated staring.

Results: Three hundred and sixty-two of 12,844 (2.8\%) patients admitted to the EMU during the time period under study were randomly reviewed. Ninety-nine $(27.4 \%)$ patients experienced one or more non-epileptic events and 23 (23\%) had both epileptic and nonepileptic events. Demographics for each group are presented in Table 1. Amongst patients with nonepileptic events, semiologies with motor manifestations were observed more often $(\chi 2=55.121, \mathrm{p}<.001)$, occurring in $42.4 \%$ of the patients. Staring events occurred in $21.2 \%$, mental status changes $8.1 \%$, dizziness $7.1 \%$, unclassified manifestations $9.1 \%$ or any combination thereof $12.1 \%$.

Conclusions: The prevalence of non-epileptic events in children admitted to our EMU appears to be similar to those reported in adult EMUs. When non-epileptic events are present, they are more often characterized by motor manifestations. Gender and age do not appear to be predictors of non-epileptic event occurrence.

Table 1

\begin{tabular}{|c|ccc|}
\hline & Non-epileptic events present $(\mathrm{n}=99)$ & Non-epileptic events absent $(\mathrm{n}=263)$ & P-Value \\
\hline Age $(\mathrm{y}, \mathrm{SI})$ & $8.68(5.13)$ & $8.18(5.77)$ & NS \\
\hline Gicnder $(\mathrm{M}: \mathrm{\Gamma})$ & $47: 52$ & $132: 131$ & NS \\
\hline
\end{tabular}

\subsection{5 \\ NEW ONSET AFEBRILE SEIZURES: FREQUENCY OF SERUM ELECTROLYTES AND GLUCOSE ABNORMALITIES IN CHILDREN}

Dewi Frances T. Depositario-Cabacar, T. Chang, H. Burton and W. D. Gaillard (Children's National Medical Center, Burtonsville, MD)

Rationale: Though several studies have examined electrolyte and glucose abnormalities in mixed groups of pediatric and adult patients with febrile or non-febrile, recurrent or new onset seizures, there has not been a comprehensive prospective evaluation done in a large pediatric population. We have prospectively evaluated the frequency of electrolyte and glucose abnormalities in the immediate evaluation of children with new onset afebrile seizure.

Methods: Prospective data were obtained from a mandated critical care pathway. One thousand four hundred sixty five patients presented with new onset seizures between 2001 and 2008.

Electrolytes abnormalities were screened for different age groups $(0$ $<6$ months, 6-24 months and $>2$ years to 18 years) and predefined set values were assigned for each of the electrolytes and glucose results.

Results: From our database, 1465 patients presented with new onset afebrile seizures $(0<6$ months of age $=222 ; 6-24$ months $=347 ;>2$ years $=896)$. Those with significant results are mentioned.

Of the 1465 patients, $25 \%(n=374)$ had abnormal sodium $(\mathrm{Na})$. Of the $25 \%$ abnormalites, $4.2 \%$ had severe hyponatremia. Twenty one percent of the total abnormal $\mathrm{Na}$ at 0 to $<6$ months of age have severe hyponatremia. Fifty five percent $(n=209)$ had mild hyponatremia with no significant difference in age groups. Out of the $1465,17 \%(n=257)$ had abnormal potassium. Mild hyperkalemia was seen in $62 \%(n=161)$ of the 257 patients. Of the total abnormal potassium at 0 to $<6$ months $(n=85), 89 \%$ had mild hyperkalemia. Mild hypokalemia was seen in $29 \%(n=75)$ of the 257 patients. Minimal patients had severe hyper or hypokalemia. Five percent $(\mathrm{n}=80)$ had abnormal calcium. Mild hypocalcemia occurred more at age $>2$ years (63\% at a total of 24 abnormal) and 
mild hypercalcemia was seen in greater frequency at the 0 to $<6$ months $(74 \%$ at a total of 31 patients). Five percent $(n=85)$ had abnormal phosphate values while eight percent had abnormal chloride values.

For glucose, $22 \%(\mathrm{n}=326)$ had abnormal glucose with hypoglycemia seen more in $0-<6$ months and hyperglycemia seen more in $>2$ years old.

Conclusions: Based on the data obtained, severe hyponatremia, mild hyprekalemia, mild hypercalcemia and mild hypoglycemia were seen more in the children younger than 6 months. Mild hyperkalemia and hyperglycemia were seen in children older than 2 years although this may be secondary to some hemolysis during blood draw and stress during the seizures respectively. Children younger than 6 months were more predisposed to laboratory abnormalities.

\subsection{6}

CLINICAL AND EEG FINDINGS IN IDIOPATHIC (GENETIC) GENERALIZED EPILEPSIES: ARE THERE ANY DISTINCTIVE FEATURES TO DISTINGUISH SYNDROMES? Ali Akbar Asadi-Pooya ${ }^{1,2}$, M. Emami ${ }^{1}$ and M. Sperling ${ }^{2}\left({ }^{1}\right.$ Neurology, Shiraz University of Medical Sciences, Shiraz, Islamic Republic of Iran and ${ }^{2}$ Neurology, Thomas Jefferson University, Philadelphia, PA)

Rationale: Epidemiologic data concerning idiopathic (genetic) generalized epilepsies (IGEs) are scarce or often unreliable. In the current study, we specifically analyzed the syndromes if IGE based on their demographic, clinical, and EEG findings.

Methods: In this retrospective study, all patients with a clinical diagnosis of IGE were recruited in the outpatient epilepsy clinic at Shiraz University of Medical Sciences, from September 2008 through May 2011. Demographic variables and relevant clinical and EEG variables were summarized descriptively. Statistical analyses were performed using Chi square and Fisher's Exact tests.

Results: 2190 patients with epilepsy were registered at our epilepsy clinic and 442 patients (20.2\%) were diagnosed as having IGE. Age of seizure onset was $12 \pm 7$ years. Juvenile myoclonic epilepsy was the most frequent syndrome, followed by epilepsy with generalized tonic-clonic seizures only and juvenile absence epilepsy. Epilepsy risk factors, physical examination, MRI and even EEG could not differentiate various syndromes.

Conclusions: IGE encompasses several different syndromes, which are diagnosed by strict clinical features. The key element in making the correct diagnosis is obtaining a detailed clinical history. An abnormal physical examination, if present, is often irrelevant and should not deter from the correct diagnosis. MRI or laboratory tests in a patient with seizures, in whom you are suspecting an IGE based on a detailed history, are not necessary. Even EEG has limitations and atypical findings in a patient with typical history for an IGE should not deter from the correct diagnosis.

Recognition of the presence of absences or myoclonic seizures has important implications on the choice of antiepileptic drugs, but distinguishing various syndromes from each another is not always easy; sometimes they have overlapping features. This is particularly true with regards to JME and JAE. In order to prevent any confusion in classification, we recommend the term juvenile idiopathic generalized epilepsy to replace these currents terms (JAE and JME) in adolescents with both seizure types (absence and myoclonus) with or without GTCSs, while retaining JAE for patients with absences with or without GTCSs and no myoclonic seizures and retaining JME for adolescents with myoclonic seizures with or without GTCSs and no absences.

\subsection{7}

PREVALENCE OF DRUG RESISTANT EPILEPSY IN A CANADIAN REFERRAL CENTER

Lizbeth Hernandez Ronquillo $^{1}$, J. F. Tellez Zenteno ${ }^{1}$, S. M. Buckley ${ }^{1}$ and R. Sahagun ${ }^{2}\left({ }^{1}\right.$ Medicine, University of Saskatchewan, Saskatoon, SK, Canada and ${ }^{2}$ Neurology, IMSS, Nayarit, Mexico)

Rationale: Drug resistant epilepsy (DRE) affects a significant proportion of people with epilepsy. Studies have reported rates ranging from 16 to $60 \%$ depending of the center. Studies have explored the prevalence of DRE using different definitions. In this study we analyzed the prevalence of DRE in a Canadian center using four definitions, including the most recent definition provided by the ILAE.

Methods: We randomly selected two hundred and fifty charts from 700 available at the epilepsy clinic of the University Hospital in the province of Saskatchewan, Canada (1 million population). Two independent chart reviewers underwent training regarding the application of the four definitions used in this study. The reviewers performed a pilot with 20 charts. The reviewers did not know the patients and the evolution of the cases over time. We used the definition of DRE published by Kwan and Brodie, Berg, Camfield and the one provided by the ILAE. The reviewers abstracted demographic data, diagnostic of epilepsy, ILAE classification parameters, evolution and used medications. After the extraction of the necessary clinical information, the four definitions were applied. We measure the prevalence of DRE using the four mentioned definitions.

Results: Of the 250 charts of patient with epilepsy $53 \%$ were males, the age ranged between 17 and 83 years (mean $37 \pm 15.6$ ). With respect to classification of epilepsy, 59\% had partial onset epilepsy and $23 \%$ were classified as idiopathic. For epileptic syndromes, $52 \%$ were symptomatic, $24 \%$ were unknown, $23.5 \%$ were idiopathic and one was cryptogenic. The prevalence of drug resistant epilepsy using the definition of Berg was $27.5 \%$, Kwan and Brodie 33.5\%, Camfield $36.3 \%$ and for the definition of the ILAE was $32.4 \%$. There was no statistical difference between the rates provided by the four definitions ( $p>0.05$ ). Using only the definition provided by the ILAE, the prevalence of DRE was $33.8 \%$ in patients with partial epilepsy vs. $30.4 \%$ in patients with generalized epilepsy $(p=0.57)$. The prevalence of DRE in patients with symptomatic epilepsy was $37.7 \%$ $(p=0.063)$, idiopathic $35.6 \%(p=0.47)$, unknown $16.7 \%$ and for cryptogenic only one patient was in this category and also had DRE. More information will be displayed in the meeting.

Conclusions: This is the first study of prevalence of DRE in a Canadian center. The prevalence of DRE was high, probably reflecting a reference pattern, being the only center to assess complex patients in the province of Saskatchewan. We found that the prevalence of DRE showed similar rates between focal and generalized epilepsy. No differences were seen between idiopathic and cryptogenic epilepsy. The four definitions showed similar rates, supporting their use in clinical studies.

\subsection{8 \\ THE ROLE OF PARENTAL CONSANGUINITY IN EPILEPSY: UPDATES FROM EPILEPSY REGISTRY} Fawzi A. Babtain ${ }^{1,2}$, M. Velmurugan ${ }^{2}$ and N. Al Otaibi ${ }^{3}$ ( ${ }^{1}$ Department of internal Medicine, King Khalid University, Abha, Saudi Arabia; ${ }^{2}$ Division Of Neurology, Aseer Centrsl Hospital, Abha, Saudi Arabia and ${ }^{3}$ Division Of Neurology, King Abdulaziz National Guard Hospital, Al Hufof, Saudi Arabia)

Rationale: The association between consanguinity and epilepsy has been evaluated in the past, and was found to be non-significant in children with epilepsy (Daoud et al; 2003), yet the data is scarce regarding this association in adults with epilepsy, for which this study was conducted to evaluate the impact of consanguinity on epilepsy. 
Methods: This is a six months data collected from the ongoing epilepsy registry in Neurology service, Aseer central hospital, Abha, Saudi Arabia between December 2011 to May 2012. Detailed Patient's demographics were collected for the registry, including the file number, age, gender, age at disease onset, epilepsy risk factors (including family history of epilepsy), brain MRI and routine EEG findings, and current anti-epileptics patient is on. Epilepsy was classified in those patients based on clinical, radiological and electrophysiological data available at time of evaluation. History of parenteral consanguinity (defined as a union between a couple related as second cousin or closer) was obtained for all patients.

Results: 420 patients with epilepsy were included until the time of the study. There were 205 men (49\%), the mean age at presentation was 27 years (range; 13-85), and the mean age at disease onset was 18.7 years. partial epilepsy was seen in $47 \%$ of the patients, generalized epilepsy in $40 \%$ and only $13 \%$ of the patients had unclassified epilepsy. Consanguinity was seen in 123 patients (29\%), and family history of epilepsy was documented in 113 patients $(27 \%)$. Patients were divided into two groups, based on the presence or absence of parental consanguinity, and further analysis of these groups (table 1) revealed no difference of the distribution of epilepsy classification across the groups (Figure 1) ( $\mathrm{p}$ value $>0.05$ ). The mean age at presentation and at the disease onset was non-significantly different. Finally, the MRI and EEG findings were also nondiscriminating among the groups.

Conclusions: Our data showed lack of association between parental consanguinity of first and second-degree relatives and epilepsy classifications in the adult population with epilepsy, particularly with primary generalized epilepsy. There were no other differences observed regarding age at diagnosis or disease onset, and no MRI or EEG findings seen significantly in association to consanguinity. Despite the fact that a high rate of consanguinity is documented in our population, with its known impact on other hereditary diseases, consanguinity has no impact on epilepsy in our population, which still could be explained by heterogeneous etiologies leading to epilepsy. Further studies will be required to assess this association, preferably from areas with high rates of consanguinity.

Demographics of the patients

\begin{tabular}{|c|ccc|}
\hline & $\begin{array}{c}\text { Parental Consanguinity } \\
(\mathrm{N}=123)\end{array}$ & $\begin{array}{c}\text { No parental consanguinity } \\
(\mathrm{N}=299)\end{array}$ & P value \\
\hline Men $(\%)$ & 45 & 50 & NS \\
Mean age at presentation & 27 years & 28 ycars & NS \\
Mcan agc at discasc onset & 24 ycars & 19 ycars & NS \\
\hline Abnormal Brain MRI $(\%)$ & 26 & 28 & NS \\
\hline Abnormal EEG $(\%)$ & 76 & 74 & SN \\
\hline
\end{tabular}

NS $=$ Non-significant

MRI = Magnetic Resonance Imaging

$\mathrm{EEG}=$ Electroencephalogram

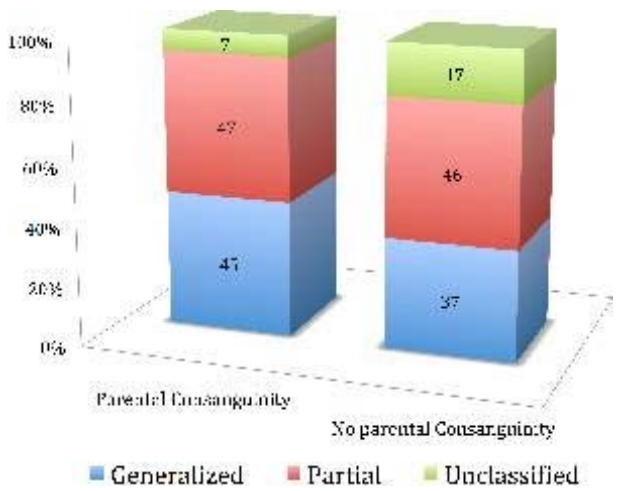

Monday, December 3, 2012

\author{
Investigators' Workshop \\ It Takes Two to Tango: Dance of Neuronal Ion Channels and \\ Their Auxiliary Subunits \\ 3:45 p.m.-5:15 p.m.
}

Geoff Abbott $^{2}$, Lori L. Isom ${ }^{1}$, Yoav Noam ${ }^{3}$ and Tallie Z. Baram ${ }^{2}$ ( ${ }^{1}$ Pharmacology, UC-Irvine, Irvine, CA; ${ }^{2}$ Pharmacology; Integrative Phyisology, University of Michigan, Ann Arbor, MI and ${ }^{3}$ Pediatrics; Anatomy/Neurobiology, UC-Irvine, Irvine, CA)

Summary: The abundance, subcellular localization and surface expression of ion channels are highly regulated, and contribute to intrinsic neuronal behavior, as well as to network responses. In addition, pathological alterations in these parameters contribute to altered excitability in the epileptic brain. In recent years, novel and exciting data have been emerging regarding the involvement of channel-interacting (auxiliary) proteins in the mechanisms of turnover, trafficking and membrane expression of a number of neuronal ion channels.

The goal of this session is to discuss new developments in our understanding of the molecular complexes that control ion channel trafficking, surface-expression and function. The session will focus on proteins that regulate several key ion channels, and their potential relevance to epilepsy and the epileptogenic process.

1. Geoff Abbot will discuss the role of potassium channels beta subunits (KCNEs) in neuronal excitability

2. Yoav Noam will discuss novel data on the involvement of the actin-binding protein Filamin A in ion channel trafficking and surface expression.

3. Lori L. Isom will discuss novel roles for sodium channels beta subunits, involved in familial forms of epilepsy.

Whereas pore-forming subunits of ion channels and receptor have received enormous (and highly justified) attention. The roles of 'beta' or interacting subunits in the normal function of ion channels are supported by discoveries of epilepsy-related mutations in beta subunits. Thus, the exquisite interactions among components of ion channel complexes are emerging as an important topic of study over the coming years.

\section{Monday, December 3, 2012}

\section{Pediatric Epilepsy Hightlights Session} 4:00 p.m.-5:30 p.m.

For abstract content, please refer to the following poster numbers listed below in Poster Session 1 on Saturday, December 1, 2012.

$1.137,1.153,1.154,1.185,1.223,1.247,1.277,1.280,1.322,1.332$ 


\section{Monday, December 3, 2012}

Platform A

4:00 p.m.-6:15 p.m.

\begin{abstract}
A.01
MATERNAL FOLIC ACID EXPOSURE DURING GESTATION INCREASES SEIZURE SUSCEPTIBILITY IN OFFSPRING

Michael A. Colicos ${ }^{1}$, F. Girotto ${ }^{1,3}$, L. Scott ${ }^{1}$, Y. Avchalumov ${ }^{1}$, J. Harris $^{1}$, R. Tobias ${ }^{2}$, C. Drummond-Main ${ }^{2}$, L. Bello-Espinosa ${ }^{2}$, J. M. $\mathrm{Rho}^{2}$, J. Davidsen ${ }^{3}$ and C. G. Teskey ${ }^{4}\left({ }^{1}\right.$ Department of Physiology \& Pharmacology, University of Calgary, Calgary, AB, Canada; ${ }^{2}$ Pediatric Neurology and the Alberta Children's Hospital Research Institute, University of Calgary, Calgary, $\mathrm{AB}$, Canada; ${ }^{3}$ Department of Physics and Astronomy, University of Calgary, Calgary, AB, Canada and ${ }^{4}$ Department of Cell Biology \& Anatomy, University of Calgary, Calgary, AB, Canada)
\end{abstract}

Rationale: Maternal folic acid (FA) supplementation is essential to reduce the risk of neural tube defects. However since the neural tube closes at day 27 during human gestation, FA administration after this point may have little benefit, and could even be detrimental. Clinical observations suggest that large doses of FA during the entire pregnancy may correlate with a higher incidence of epilepsy in offspring. FA and congeners are based on pteroic acid conjugated to L-glutamate, the major excitatory neurotransmitter in the brain. Given the tight link between neuronal activity and synaptic development, we hypothesized that the continued presence of FA during neuronal development might alter the delicate balance of normal network formation and function.

Methods: We administered therapeutic doses of FA (4.0 mg PO daily) during pregnancy to rats and analyzed the seizure threshold of their offspring (i.e., latency to first behavioral seizure following an IP injection of $15 \mathrm{mg} / \mathrm{kg}$ kainic acid). In vitro, we analyzed network activity of cultured rat hippocampal neurons grown on silicon wafers, reared in the presence of the functional metabolite of FA (4Hfolate at $50 \mathrm{nM}$ ). In these experiments, we used a novel assay, performed by stimulating the entire network using photoconductive stimulation (PMID: 17406269) in a single train (30 seconds, $60 \mathrm{~Hz})$ and monitoring the network's ability to subsequently stabilize its activity patterns. Additionally, we investigated electrophysiological changes in the AMPA to NMDA receptor ratio, and quantified protein expression levels of glutamate receptor subunits in 4 Hfolate reared neurons.

Results: Offspring from pregnant rats which were administered FA during pregnancy had a $42 \%$ decrease in their seizure threshold $(p=0.02)$. Acute application of 4 Hfolate directly to neurons induced hyper-excitability and bursting behavior. Cultured neurons allowed to develop in the presence of 4Hfolate for 3 weeks had reduced capacity to stabilize their network dynamics after a burst of high frequency activity in comparison to control cultures $(\sim 2.5 \mathrm{x}, \mathrm{n}=17, \mathrm{p}<0.05)$. Compared to controls, we found that 4 Hfolate-reared cultures exhibited a higher AMPA/NMDA receptor ratio $(1.24 \mathrm{x}, \mathrm{p}<0.05)$, as well as an increase in mEPSC frequency $(1.37 \mathrm{x}, \mathrm{p}<0.05)$. Finally, we found that protein expression of the AMPA receptor subunit GluR1 was increased by 4 Hfolate in comparison to controls $(\sim 2 \mathrm{x}, \mathrm{n}=5$, $\mathrm{p}<0.05$ ).

Conclusions: The most parsimonious interpretation of these data is that, by virtue of its relationship to the excitatory neurotransmitter Lglutamate, FA - at chronic high levels during neuronal development causes an increase in neuronal activity, which in turn accelerates neuronal connectivity and leads to a hyper-excitable network. Our results suggest that continued maternal FA supplementation after the period of organogenesis should be carefully reassessed, as it could potentially lead to a more epileptogenic state.

\section{A.02}

A MOUSE MODEL OF EARLY ONSET EPILEPTIC ENCEPHALOPATHY REVEALS A CELLULAR DEFECT AND SUGGESTS A TARGETED THERAPEUTIC INTERVENTION

Bryan T. Leaw ${ }^{1}$, C. A. Reid ${ }^{1,2}$, K. L. Richards ${ }^{1}$, V. Wimmer ${ }^{1}$, J. W. Low $^{1}$, E. Thomas ${ }^{1,2}$, E. L. Hill ${ }^{1}$, H. Lerche ${ }^{4,5}$, I. Scheffer ${ }^{1,3}$, S. Berkovic $^{3}$ and S. Petrou ${ }^{1,2}\left({ }^{1}\right.$ Florey Neuroscience Institutes, The University of Melbourne, Parkville, VIC, Australia; ${ }^{2}$ The Centre for Neuroscience, The University of Melbourne, Parkville, VIC, Australia; ${ }^{3}$ Department of Medicine, Austin Health, The University of Melbourne, Heidelberg West, VIC, Australia; ${ }^{4}$ Department of Neurology and Epilpetology, Hertie Institute of Clinical Brain Research, University Hopistal, Tübingen, Germany and ${ }^{5}$ Neurological Clinic and Institute of Applied Physiology, University of Ulm, Germany)

Rationale: Early onset epileptic encephalopathies (EOEE), such as Dravet syndrome, are a severe group of epilepsies with developmental regression for which multiple genetic causes are emerging. Previously EOEE has been reported in a SCN1B functional null patient and to further elucidate the pathogenic mechanisms we investigated a $\operatorname{Scn} 1 b(\mathrm{C} 121 \mathrm{~W})$ homozygous mouse model and examined behavioural and neuronal phenotypes.

Methods: Thermal seizure susceptibility was determined by placing P14-17 mice in a chamber heated to $41^{\circ} \mathrm{C}$, and the time to first tonicclonic seizure was recorded. Gait was assessed using Digigait (Mouse Specifics Inc., Boston, MA, USA) when mice were P17. Whole-cell patch clamp was used to compare neuronal properties in homozygous and control subicular pyramidal neurons from P14-16 mice. Current clamp mode was used to measure input-output relationships, and cellular passive properties were also recorded. Neuronal tracing and analysis was performed using the Neurolucida and Neurolucida Explorer software package (MBF Biosciences, Willison, VT). Results: Homozygous mice exhibited heightened susceptibility to thermally triggered seizures, possessed abnormal gait, died prematurely at around P21 and, similar to human Dravet patients, were responsive to stiripentol and unresponsive to lamotrigine. Neurons from homozygous mice had left-shifted action potential (AP) input-output curves $(n=10, p<0.05)$, suggesting increased excitability. While the voltage threshold for AP firing was unchanged, neurons from homozygous mice had a significantly higher input resistance $\left(\mathrm{R}_{\text {in }}\right)$ (WT 131.92 $\pm 8.45 \mathrm{M} \Omega, \mathrm{n}=25$; Hom $216.98 \pm 17.8 \mathrm{M} \Omega, \mathrm{n}=18$ ), which could readily account for this hyperexcitability. Neuron morphological analysis revealed that the average distance to the first dendritic branch point was significantly smaller in homozygous animals compared with WT (WT 25.64 \pm 3.35 microns, $n=10$; Hom 13.99 \pm 1.41 microns, $n=11 ; \mathrm{p}<0.01$ ). Membrane capacitance values recorded during whole-cell recordings were significantly reduced in homozygous $(95.48 \pm 5.95 \mathrm{pF}, \mathrm{n}=18)$ compared to WT $(116.5 \pm 7.26 \mathrm{pF}, \mathrm{n}=25)$ neurons. We used retigabine, a voltage-gated potassium channel activator, as a way to reduce $R_{\text {in }}$ to test the idea of a disease mechanism specific therapy. Retigabine effectively lowered $\mathrm{R}_{\text {in }}$ in pyramidal neurons from homozygous mice and significantly decreased AP firing $(n=4, p<0.05)$. Importantly, retigabine treated mice displayed a profound decrease in sensitivity to thermally triggered seizures $(n=9, p<0.05)$.

Conclusions: These data suggest that altered membrane passive properties enhance membrane excitability in neurons from $\operatorname{Scn} 1 b(\mathrm{C} 121 \mathrm{~W})$ homozygous mice, presumably as a consequence of altered neuronal morphology, demonstrating a new disease mechanism in genetic epilepsy. Further, drugs that decrease input resistance, and thereby specifically target the functional deficit, dampen hyperexcitability of homozygous neurons and reduce thermal seizure susceptibility in mice. These findings highlight the potential therapeutic utility in targeting disease specific mechanisms in epilepsy. 


\section{A.03}

WIN55, 212 ATTENUATES NMDA BUT NOT KAINATE INTRA-HIPPOCAMPAL SEIZURES VIA MGLUR1 $\alpha$ AND CB1 RECEPTORS IN DEVELOPMENT

Linda K. Friedman and V. Rudenko (New York College of Osteopathic Medicine, Old Westbury, NY)

Rationale: In adult rats, the synthetic cannabinoid agonist, WIN55212, suppresses spontaneous seizure activity and IPSCs of DHPG-sensitive hippocampal neurons. Anticonvulsant effects were attributed to reducing pre-synaptic release of glutamate via G-protein coupled $\mathrm{CB} 1$ receptors. In postnatal $(\mathrm{P})$ day 20 rats, we found that kainate (KA)-induced epileptic activity is inversely related to WIN55212 dosage after peripheral injection. Higher doses were proconvulsant and injurious whereas lower doses were anticonvulsant and neuroprotective.

Methods: To determine whether the hippocampus was responsible for this inverse relationship on the seizure threshold at P20, stereotaxic microinfusions of WIN55212 $(0.5 \mu \mathrm{g})$ were delivered to the CA1 followed by NMDA $(0.1 \mu \mathrm{g})$ or KA $(0.3 \mu \mathrm{g})$ in the presence and absence of mGluR1 $\alpha$, mGluR5, and CB1 receptor antagonists, LY367385, MPEP or AM251, respectively. Electroencephalography (EEG) recordings from the CA1 were simultaneously determined with a bipolar/cannula assembly. CB1 and NMDA receptor proteins were examined.

Results: Intrahippocampal WIN 55,212 alone caused rapid sedation and low amplitude synchronous oscillations in the EEG. NMDA (50 $\mathrm{nM}$ ) produced early spikes within seconds and late spike activity and seizure behavior after $20 \mathrm{~min}$. In the presence of WIN55212, NMDAinduced spikes and epileptic currents were delayed and attenuated. Blockade of mGluR1 $\alpha$ receptors with LY367385 or CB1 receptors with AM251 (but not MPEP) prevented the attenuation. In contrast, microinfusion of a saturating concentration of KA led to increased periodic oscillations containing high frequency spikes in the presence and absence of WIN55212, LY367385 or AM251.

Immunohistochemistry revealed a dose-dependent reduction in CB1 receptor expression with increasing concentrations of WIN55212 in presence or absence of KA seizures. There were steady levels of mGluR $1 \alpha$ receptors, and variable distribution of NR1 and the NMDA $\mathrm{C} 2$ terminal splice variant patterns.

Conclusions: Differential responses of NMDA and KA on seizure threshold subsequent to WIN55212 application suggests selective cooperation between NMDA and mGluR receptors such that NMDA is expected to co-activate mGluR group I receptors to synergistically activate release of endocannabinoids to further attenuate EEG spiking. Unexpectedly, mGluR $1 \alpha$ subunits appear required for the inhibitory actions of WIN5521 on NMDA currents and that KA receptor activation is not coupled to the cannabinoid/mGluR pathway. CB1 receptor re-distributions caused by seizures may also impart different action on regulatory mechanisms of transmitter release to control the seizure threshold and associated brain injury.

\section{A.04 \\ HIPPOCAMPAL DEEP BRAIN STIMULATION HAS ANTIEPILEPTOGENIC POTENTIAL}

Bregt Van Nieuwenhuyse ${ }^{1}$, R. Raedt ${ }^{1}, K$. Vonck ${ }^{1}$, A. Meurs ${ }^{1}$, W. Wadman $^{2}$ and P. Boon ${ }^{1}\left({ }^{1}\right.$ Ghent University-Neurology-LCEN3, Ghent, Belgium and ${ }^{2}$ University of Amsterdam-Neurobiology-SILS, Amsterdam, Netherlands)

Rationale: Hippocampal deep brain stimulation (DBS) is an experimental therapy for patients with pharmacoresistant temporal lobe epilepsy (TLE). No studies have investigated the antiepileptogenic potential of hippocampal DBS. In this animal experimental study, we evaluated the effect of hippocampal DBS on the development of spontaneous seizures in the systemic kainic acid rat model.
Methods: Rats were implanted with a quadripolar DBS/EEGregistration electrode in the right hippocampus and a bipolar EEG recording electrode in the left hippocampus. 24 hours after kainic acid (KA) induced status epilepticus (SE), one group $(n=6)$ was subjected to short term DBS (ST-DBS) (Poisson Distributed Stimulation, $130 \mathrm{PPS}, 100 \mu \mathrm{s}$ PW, $100 \mu \mathrm{A}$ ) of 1 week, a second group $(\mathrm{n}=7)$ was subjected to long term DBS (LT-DBS) (PDS, 130 PPS, $100 \mu \mathrm{s}$ PW, $100-400 \mu \mathrm{A})$ of 10 weeks. A control group $(\mathrm{n}=9)$ received sham stimulation (SHAM). EEG was recorded continuously during 14 weeks in the LT-DBS and SHAM group. In the ST-DBS group, EEG was recorded continuously during 8 weeks.

Results: The gradual increase in mean daily seizure frequency following SE was significantly lower in the LT-DBS group compared to the SHAM group $(p<0.01)$. While seizure frequency increased from $0.1 \pm 0.1$ seizures per day in week 1 to $10.3 \pm 1.3$ seizures per day in week 10 in the SHAM group, this increase was not observed in the LT-DBS group $(0.5 \pm 0.1$ seizures per day in week 1 versus $1.2 \pm$ 0.4 seizures per day in week 10 ). When DBS was stopped in the LTDBS group seizure frequency remained low $(4.14 \pm 1$ seizures per day in week 14), while in the SHAM group it continued to increase $(26.6 \pm 3.1$ seizures per day in week 14). In the ST-DBS group there was no significant difference in mean daily seizure frequency increase compared to the SHAM group.

Conclusions: Long term hippocampal DBS reduces the gradual increase in seizure frequency in a rat model for temporal lobe epilepsy. These findings strongly support the hypothesis that hippocampal DBS affects epileptogenesis.

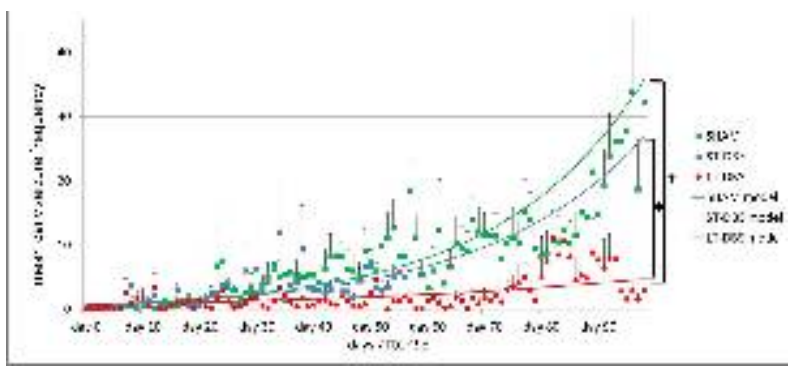

\section{A.05 \\ MODULATING THE ANTIEPILEPTIC HIPPOCAMPAL THETA RHYTHM VIA OPTOGENETIC NEUROMODULATION OF THE MEDIAL SEPTUM} Nealen Laxpati ${ }^{1,2}$, J. Tung ${ }^{2,3}$, J. Newman ${ }^{2}$, R. Zeller-Townson ${ }^{2}$, C. A. Gutekunst ${ }^{1}$ and R. E. Gross ${ }^{1,2}\left({ }^{1}\right.$ Department of Neurosurgery, Emory University School of Medicine, Atlanta, GA; ${ }^{2}$ Coulter Department of Biomedical Engineering, Georgia Institute of Technology, Atlanta, GA and ${ }^{3}$ The Medical College of Georgia, Georgia Health Sciences University, Augusta, GA)

Rationale: The septohippocampal axis - connecting the medial septum (MS) and hippocampus - has been described as a pacemaker for the generation and maintenance of the hippocampal theta rhythm. Theta, in turn, is associated with the prevention or cessation of epileptic activity. Modulating hippocampal theta could prove a viable method of controlling seizures. Glutamatergic neurons of the MS have been proposed as a theta pacemaker due to their direct projections to hippocampal pyramidal neurons and tendency to fire at theta frequencies. To functionally investigate these connections in vivo, we explored cell-type specific optogenetic activation of the MS and the resulting oscillatory local field potential (LFP) activity in the dorsal hippocampus.

Methods: Sprague-Dawley rats were infected in the MS with AAV2CaMKII $\alpha-C h R 2$, targeting glutamatergic neurons; AAV5-hSyn$\mathrm{ChR} 2$, non-specifically expressing in all neurons; or a control virus. After two weeks, each rat was anesthetized and implanted with an optical fiber targeting the MS and a 16-channel microwire multielectrode array (Tucker-Davis Technologies) simultaneously 
targeting CA3 and CA1 of the hippocampus. Animals underwent 473 $\mathrm{nm}$ blue laser stimulation across numerous parameters, including frequencies from $7-42 \mathrm{~Hz}$ and pulse widths of $1-10 \mathrm{~ms}$. Electrode and optical fiber location was confirmed histologically and cellspecific transgene expression was immunohistochemically determined. Recorded electrophysiological data was spectrographically analyzed using custom-written and Chronux Matlab scripts.

Results: Optogenetic control of cell-type specific neurons of the MS in awake and behaving rats drove activity locally as well as in the dorsal hippocampus. At beta $(15-35 \mathrm{~Hz})$ and gamma $(40+\mathrm{Hz})$ frequencies, increase in stimulus frequency-specific power in the hippocampal LFP was observed with either neuronal target, but not in ChR2-negative controls. Notably, frequency-specific power generated in CaMKII $\alpha$-ChR2 rats was less than that generated by hSyn-ChR2 rats. In anesthetized animals (reduced theta state), only hSyn-ChR2 rats generated corresponding hippocampal LFP oscillations with theta stimulation. In awake and behaving nonepileptic animals, neither hSyn-ChR2 rats nor CaMKII $\alpha-C h R 2$ stimulation could increase theta power, but hSyn-ChR2 rats could phase-lock extant hippocampal theta oscillations to the optical stimulus.

Conclusions: We have developed a system for optogenetic stimulation and multielectrode recording, and used it to begin dissecting the neural circuitry of the septohippocampal axis. We demonstrate support for the hypothesis that the medial septum functionally modulates hippocampal activity. Furthermore, our results suggests that the glutamatergic neurons of the medial septum are not the drivers of theta activity, due to their inability to modulate the hippocampal theta rhythm in awake, behaving animals, and must play some other role in the septohippocampal axis.

\section{A.06 \\ HFO CORRELATES OF CORTICO-CORTICAL EVOKED POTENTIALS REVEAL ALTERED EXCITABILITY IN THE HUMAN EPILEPTIC FOCUS}

Katsuya Kobayashi ${ }^{1}$, R. Matsumoto ${ }^{1}$, M. Matsuhashi ${ }^{2}$, K. Usami ${ }^{1}$, A. Shimotake ${ }^{1}$, T. Kunieda ${ }^{3}$, N. Mikuni ${ }^{4}$, S. Miyamoto ${ }^{3}$, H. Fukuyama ${ }^{2}$, R. Takahashi ${ }^{1}$ and A. Ikeda ${ }^{1}\left({ }^{1}\right.$ Department of Neurology, Kyoto University Graduate School of Medicine, Kyoto, Japan; ${ }^{2}$ Human Brain Research Center, Kyoto University Graduate School of Medicine, Kyoto, Japan; ${ }^{3}$ Department of Neurosurgery, Kyoto University Graduate School of Medicine, Kyoto, Japan and ${ }^{4}$ Department of Neurosurgery, Sapporo Medical University, Sapporo, Japan)

Rationale: Pathological high frequency oscillations (HFOs), ripple (R:80-250 Hz) and fast ripple (FR: $>250 \mathrm{~Hz}$ ), have been recently recorded with macroelectrodes as a possible biomarker of epileptogenesis. However, analysis of spontaneous interictal HFOs needs long sampling time and skillful technique. We have focused on the early responses (cortico-cortical evoked potentials: CCEPs) to $1 \mathrm{~Hz}$ single-pulse cortical stimulation in order to trace cortico-cortial networks involved in brain functions and seizure propagation. By using CCEP [early negative (N1) and late negative (N2) components] as a dynamic measure of cortical excitability, we also have investigated modulation of cortical excitability at and around the focus (Matsumoto et al., 2005; Iwasaki et al., 2010). Our objective is to clarify the HFO correlates of CCEPs and its relation to human epileptogenesis.

Methods: We employed 8 patients with intractable partial epilepsy who underwent invasive presurgical evaluation with chronic implantation of subdural electrodes (IRB \#443). Repetitive singlepulse electrical stimulation at $1 \mathrm{~Hz}$ was applied to 1) the seizure onset zone (SOZ) and 2) the normal cortex of the same cortical architecture (nSOZ) in each patient. After carefully removing epochs mixed with spikes, CCEPs were recorded from the surrounding cortices within the SOZ (in case of 1) and nSOZ (in case of 2) by off-line averaging time-locked to the stimulus onset. We applied short-time Fourier transform as time-frequency analysis to obtain induced responses at the timing of N1 (HFO-N1) and N2 (HFO-N2). We then calculated the logarithmic power spectra of HFO-N1 and HFO-N2 and analyzed power change in reference to the baseline activity for the following 3 frequency bands - below R $(<\mathrm{R}), \mathrm{R}$, and FR. Repeated measures ANOVA was adopted with logarithmic power values in each frequency band $(<\mathrm{R}, \mathrm{R}, \mathrm{FR})$ as a within-group factor and with the aforementioned 4 groups (SOZ-HFO-N1/N2, nSOZ-HFO-N1/N2) as a between-group factor.

Results: A total of 16 stimulus sites in $\mathrm{SOZ}$ and 16 sites in $\mathrm{nSOZ}$ showed clear CCEP responses. In both groups across the 3 frequency bands, HFO-N1 showed the power increase in all stimulus sites and HFO-N2 showed the power decrease or no change in all but 1 stimulus site at SOZ. Significant interaction was observed between the 4 groups. Post-hoc analyses showed significant interactions between HFO-N1 and HFO-N2 for both SOZ and nSOZ groups. HFO-N1 power increase was statistically larger in SOZ group than in $\mathrm{nSOZ}$ group in all the frequency bands.

Conclusions: The present study demonstrated that 1) HFO-N1 and HFO-N2 behaviors were different and 2) increase of HFO-N1 was more evident at the SOZ than at the nSOZ. Recently, Alarcon et al. (2012), by using microelectrodes, showed that neuronal responses to single-pulse electrical stimulation consist of brief synchronized burst firing and following long inhibition that likely corresponds to CCEP $\mathrm{N} 1$ and N2, respectively. Taken together, HFO counterparts of CCEPs could be a surrogate marker of abnormally enhanced cortical excitability in human epileptic focus.

\section{A.07 \\ HOMEOSTATIC SYNAPTIC COMPENSATION FOLLOWING INTERNEURON LOSS AND RESCUE BY MGE PROGENITOR CELL TRANSPLANTATION IN EPILEPTIC DLX1-/- MICE \\ MacKenzie A. Howard ${ }^{1}$, J. Rubenstein ${ }^{2}$ and S. C. Baraban ${ }^{1}$ ( ${ }^{1}$ Neurological Surgery, University of California, San Francisco, San Francisco, CA and ${ }^{2}$ Psychiatry, University of California, San Francisco, San Francisco, CA)}

Rationale: DLX proteins are transcription factors important for the normal development of GABAergic interneurons. DLX1 mutant mice lose a subset of cortical and hippocampal somatostatin-, NPY-, VIP-, and calretinin-positive interneurons die at $\sim 1$ month of age. Selective interneuron loss is accompanied by a decrease in inhibitory synaptic transmission onto cortical and hippocampal pyramidal neurons, and late-onset of epilepsy (Cobos et al 2005). Excitatory synaptic transmission onto surviving interneurons also undergoes changes following interneuron death (Jones et al 2011). Spontaneous seizures become apparent at a low frequency in some mice as young as 2 months and in most mice by 6 months (Cobos et al 2005). To further investigate hippocampal circuit changes triggered by selective interneuron loss, and test a cell therapy approach to reverse these deficits a series of electrophysiological, anatomical and transplantation experiments were performed.

Methods: Voltage- and current clamp recordings in acute hippocampal slices. Embryonic dissection and postnatal transplantation of interneuron progenitors isolated from the medial ganglionic eminence (MGE) of GFP+ donor embryos. Immunohistochemistry for GAD67, NeuN, parvalbumin, somatostatin, NPY and calretinin.

Results: We report homeostatic compensation in excitatory connections between pyramidal neurons of DLX1-/- mice following interneuron loss. Specifically, decreased glutamatergic synaptic connections between hippocampal CA3 and CA1 pyramidal neurons (assessed in voltage-clamp recordings of miniature and evoked EPSCs), and decreased excitability of CA1 pyramidal neurons (assessed in current-clamp recordings of input resistance and rheobase). Next, we generated new interneurons by transplanting 
MGE progenitors into hippocampi of P2 DLX1-/- and wild-type mice. At 30 days after transplantation, MGE cell grafts generated GABAergic interneurons that functionally integrated and restored inhibitory synaptic transmission in DLX1-/- mice to WT levels. MGE-derived interneurons stained positively for interneuron-specific antibodies, as described (Alvarez-Dolado et al. 2006). Interestingly, DLX1-/- mice receiving MGE transplants exhibited normal levels of excitatory synaptic activity between pyramidal neurons, and normal pyramidal neuron excitability.

Conclusions: Our results suggest that changes in inhibitory synaptic activity, or interneuron number, elicit homeostatic compensation in the excitatory circuitry of the hippocampus in vivo. Furthermore, circuit deficits caused by DLX1 deficiency and subsequent interneuron loss can be corrected by MGE progenitor cell transplant.

\section{A.08 \\ THE PRECLINICAL ANTICONVULSANT PROFILE OF THE NOVEL INVESTIGATIONAL DRUG TONABERSAT}

S. V. Elrod ${ }^{1}$, H. S. White ${ }^{2}$, P. R. Blower ${ }^{3}$ and M. B. Halvorsen ${ }^{1}$

${ }^{1}$ Upsher-Smith Laboratories, Inc., Maple Grove, MN;

${ }^{2}$ Anticonvulsant Drug Development Program, University of Utah, Salt Lake City, UT and ${ }^{3}$ Biophar Consulting Ltd, Essex, United Kingdom)

Rationale: Tonabersat (TBR) is one of a series of novel benzoylamino benzopyran compounds initially developed for the treatment of migraine and epilepsy. This group of compounds was designed by replacing the 2-pyrrolidinone group of cromakalim with a fluorobenzoylamino group, which introduces anti-seizure activity whilst totally removing potassium channel activator activity. The binding site for these compounds has been primarily detected in the brain, with minimal binding observed in peripheral tissues (eg, liver, heart, kidney). Further, this binding site is distinct from other CNS pharmacologic agents including anti-migraine compounds and antiepileptic drugs (AEDs). Carabersat (CBS), a close analogue of TBR, with a lower affinity for the binding site, demonstrated efficacy in a clinical proof-of-concept study as an adjunctive therapy in the treatment of partial onset seizures. Therefore, TBR was submitted to the National Institute of Neurological Disorders and Stroke Anticonvulsant Screening Project (ASP) for evaluation of its antiseizure properties.

Methods: The ASP evaluated TBR for toxicity and anti-seizure properties. In vivo rodent models of generalized tonic-clonic seizures (maximal electroshock [MES]), generalized myoclonic seizures (subcutaneous administration of metrazol), generalized clonic seizures (subcutaneous picrotoxin and bicuculline), focal seizures with secondary generalization (6 Hz), audiogenic seizures (Frings), hippocampal kindled seizures and pharmacoresistant seizures with secondary generalization (lamotrigine [LTG]-resistant kindled) were employed to evaluate the anti-seizure profile of TBR. Toxicity was assessed with the minimal motor impairment scale or rotarod test. The median effective and toxic dose $\left(\mathrm{ED}_{50}\right.$ and $\mathrm{TD}_{50}$, respectively) at the time of peak anti-seizure effect was calculated using probit analysis. In models where TBR demonstrated efficacy, therapeutic index $\left(\mathrm{TI}=\mathrm{TD}_{50} / \mathrm{ED}_{50}\right)$ was calculated.

Results: TBR demonstrated a potent oral anti-seizure profile. TBR blocked tonic extension seizures in the MES model with an $\mathrm{ED}_{50}=$ $2.1 \mathrm{mg} / \mathrm{kg}$ and a TI $>240$. This effect was consistent with data observed in the threshold tonic extension model where a dose of TBR $(10 \mathrm{mg} / \mathrm{kg})$ was found to elevate tonic hindlimb extension threshold in rats $>1000 \%$. Fully expressed seizures in hippocampal- or LTGresistant kindled rats after supra-maximal stimulation were not inhibited by TBR. Although TBR did not protect against limbic or generalized myoclonic seizures, it was effective against audiogenic seizures with a TI of $>2000$. Further, TBR demonstrated low toxicity with a $\mathrm{TD}_{50}>250 \mathrm{mg} / \mathrm{kg}$ (mice) and $>500 \mathrm{mg} / \mathrm{kg}$ (rats).

Conclusions: TBR displayed an anti-seizure profile similar to that of other AEDs (eg, carbamazepine and LTG) but was distinguished by a higher TI. TBR has the ability to prevent seizure spread in models of generalized epilepsy, and therefore may be effective as a treatment for generalized tonic-clonic, complex partial seizures secondarily generalized, and/or partial onset seizures.

\section{A.09 \\ SOLUTE CARRIER TRANSPORTERS IN PHARMACORESISTANT EPILEPSY: AN INTEGRATIVE IN SILICO AND EX VIVO ANALYSIS}

Nasir Mirza ${ }^{1}$, O. Vasieva ${ }^{2}$, R. Appleton ${ }^{3}$, S. Burn ${ }^{4}$, D. du Plessis ${ }^{5}$, J.

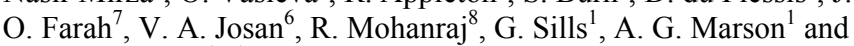
M. Pirmohamed ${ }^{1}\left({ }^{1}\right.$ Molecular and Clinical Pharmacology, University of Liverpool, Liverpool, United Kingdom; ${ }^{2}$ Institute of Integrative Biology, University of Liverpool, Liverpool, United Kingdom; ${ }^{3}$ Roald Dahl EEG Department, Alder Hey Children's NHS Foundation Trust, Liverpool, United Kingdom; ${ }^{4}$ Department of Paediatric Neurosurgery, Alder Hey Children's NHS Foundation Trust, Liverpool, United Kingdom; ${ }^{5}$ Neuropathology Unit, Salford Royal Hospitals NHS Foundation Trust, Salford, United Kingdom; ${ }^{6}$ Department of Neurosurgery, Salford Royal Hospitals NHS Foundation Trust, Salford, United Kingdom; ${ }^{7}$ Department of Neurosurgery, Walton Centre NHS Foundation Trust, Liverpool, United Kingdom and ${ }^{8}$ Department of Neurology, Salford Royal Hospitals NHS Foundation Trust, Salford, United Kingdom)

Rationale: According to the multidrug transporter hypothesis, pharmacoresistance in epilepsy results from impaired drug penetration into the epileptic focus secondary to localized dysregulation of drug transporters. Solute carrier transporters (SLCs) are known to mediate chemoresistance in a number of diseases but have not been systematically studied in epilepsy. As SLCs are primarily influx transporters, our aim was to identify the SLCs most significantly downregulated in the pharmacoresistant epileptic hippocampus by the initial use of an in silico strategy and then to confirm this by ex vivo analysis of human brain tissue.

Methods: We extracted data relating to SLCs from (1) our previously published integrative analysis of microarray studies on brain tissue from epilepsy surgery (Hum Mol Genet 2011;20:4381-94), and (2) a comprehensive review of published literature on epilepsy pharmacoresistance, and integrated all the data using "convergent functional genomics $(\mathrm{CFG})$ ' - a validated technique for prioritizing genes involved in complex diseases by collating evidence using a pre-defined scoring system. To identify SLCs which have not been studied in epilepsy pharmacoresistance but could potentially be involved, we employed a computational gene prioritization tool called Endeavour, using SLCs with the highest CFG-scores as the training genes. We validated this computationally prioritized gene list by (1) prioritizing the same candidate genes using a robust independent training set comprising the 10 most consistently downregulated genes from the aforementioned integrative analysis and, then, demonstrating significant rank order similarity with the original list, and (2) prioritizing the same candidate genes using a training set comprising 10 randomly chosen genes from the integrative analysis and demonstrating no significant rank order similarity with the original list (see figure). In parallel with our bioinformatics analysis, we used a custom Agilent oligonucletoide microarray containing exon probes for all known SLCs to analyse 24 hippocampal samples obtained from surgery for pharmacoresistant mesial temporal lobe epilepsy and 24 hippocampal samples from normal post-mortem controls. The whole-transcript amplification protocol of exon arrays allows more accurate measurement of gene expression than standard microarrays.

Results: Our exon array identified 15 SLCs significantly (FDR $<0.05$ ) downregulated in the epileptic hippocampus by 1.5 fold or more (see table). There was a highly significant overlap between the genes identified by our in silico and ex vivo strategies: $\mathrm{p}<1.5 \times 10^{-7}$ (hypergeometric distribution) for the overlap between the top 30 bioinformatically-identified genes and the 15 experimentallyidentified genes. 
Conclusions: The most significantly downregulated SLCs in the pharmacoresistant epileptic human hippocampus were identified using a robust in silico methodology and confirmed ex vivo. Our analysis shows the power of stringently applied bioinformatic techniques. The role of these SLCs in the epileptic hippocampus will need to be defined through functional studies.

Table: Solute carrier transporter genes found to be significantly downregulated in the pharmacoresistant human hippocampus by gene-level expression profiling of an exon array. Genes also identified using our bioinformatic strategy are shown in bold italics.

$\mathrm{FDR}=$ false discovery rate.

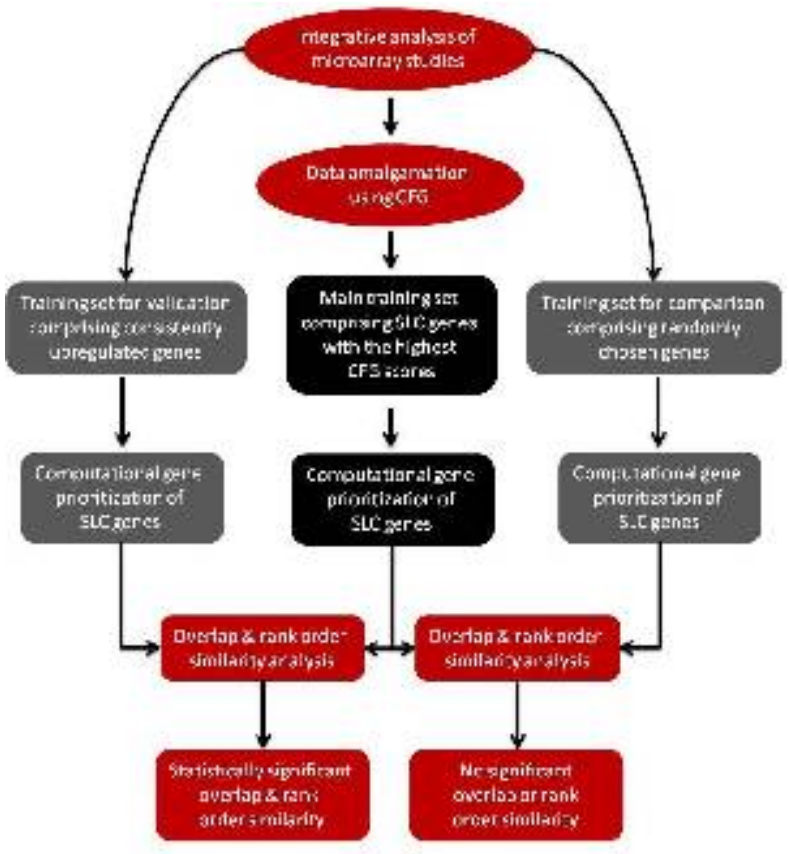

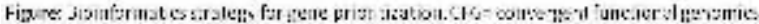
Bioinformatics strategy for gene prioritization.

\section{Monday, December 3, 2012}

Platform B

4:00 p.m.-6:15 p.m.

\section{B.01 \\ SMALLER SUBCORTICAL VOLUMES IN PATIENTS WITH IDIOPATHIC GENERALISED EPILEPSY AND THEIR FIRST DEGREE RELATIVES USING FIRST ANALYSIS}

Fahmida Chowdhury ${ }^{1}$, J. O'Muircheartaigh ${ }^{2}$, G. J. Barker ${ }^{2}$ and M. P. Richardson ${ }^{1}{ }^{1}$ Clinical Neuroscience, IOP, King's College London, London, United Kingdom and ${ }^{2}$ Neuroimaging, IOP, King's College London, London, United Kingdom)

Rationale: Idiopathic generalised epilepsies (IGE) have a complex inheritance pattern. Endophenotypes are subclinical phenotypes that have a simpler genetic basis than the overall phenotype, and may represent a genetic liability to develop symptoms. Characterising such traits could be of considerable use both for understanding seizure pathology, and identifying individuals at increased risk. Although structural imaging is qualitatively normal in IGE, quantitative imaging studies have reported focal abnormalities in patients with IGE compared to controls with reduced volumes of the subcortical nuclei such as thalamus, caudate, globus pallidus and putamen (Epilepsia 2005 46(10):1642-5, J. Neurol. Neurosurg. Psychiatry 2006 77(4):489-94, Neurology 2006 67(4):683-6). There are no published studies of imaging in relatives of patients with IGE. In this study we sought to identify whether patients with IGE and their first degree relatives had smaller subcortical nuclei, using FIRST (FSL 4.14, FMRIB http://www.fmrib.ox.ac.uk/fsl/first/), (J. Magn Reson. Imaging 2011 33(4):817-21, Neuroimage 2011 56(3):907-22 ) an automated model based segmentation/registration tool which uses a training data set of manually segmented images to investigate shape and volume of subcortical grey matter nuclei (thalamus, putamen, globus pallidus, hippocampus, caudate and amygdala).

Methods: 30 patients with IGE, 36 unaffected first degree relatives and 40 controls were scanned using a $3 \mathrm{~T}$ scanner. A 3-d isotropic high resolution inversion recovery prepared spoiled gradient-recalled echo (SPGR) scan was obtained in the coronal plane $(1.1 \mathrm{~mm} 3$ voxels, TE $2.8 \mathrm{~ms}$, TR $7 \mathrm{~ms}$, Inversion time $450 \mathrm{~ms}$ ). Each axially reoriented coronal SPGR image was processed using FIRST. FIRST gives outputs of total volume for each subcortical structure. ANOVA was used to investigate group differences between the three groups with age and total brain volume (SPM 8, VBM toolbox) entered as covariates. In order to correct for multiple comparisons, a Bonferroni correction factor of 8 was used. For those measures where it appeared that patients and relatives both differed in a similar way from controls, a 2-tailed test for linear trends was carried out.

Results: Patients and relatives showed significantly lower volumes of right thalamus and right caudate compared with controls (right thalamus: $F=5.51$, df 2:101, $\mathrm{p}=0.005$, right caudate: $\mathrm{F}=4.95$, df 2:101, $\mathrm{p}=0.006)$. See Figure 1. There were significant linear trends for right thalamus $(p=0.038)$, right caudate $(p=0.006)$, left caudate $(p=0.006)$, right $(\mathrm{p}=0.012)$ and left putamen $(\mathrm{p}=0.011)$ with relatives falling between patients and controls for all these regions.

Conclusions: Our data support the hypothesis that IGE is associated with reduced volumes of subcortical nuclei, consistent with the theory that abnormal cortico-subcortical connections underlie the pathophysiology of IGE. Our data also provides evidence that such changes are found in clinically unaffected relatives of patients with IGE, suggesting that such abnormalities are a heritable endophenotype.

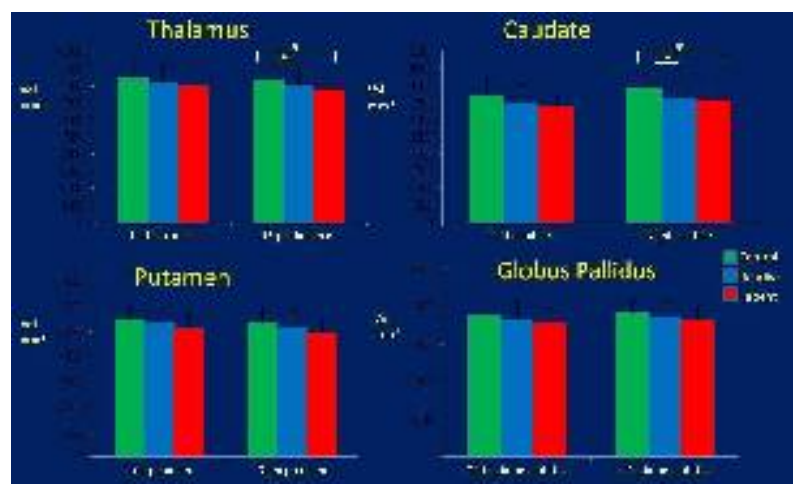

Figure 1: Bar plots showing subcortical volumes by group for each region for controls (green), patients (red) and relatives (blue). Error bars show standard deviations and significant differences are highlighted with stars

B.02

REDUCTION OF STRUCTURAL HUB REGIONS IN NEWONSET PEDIATRIC EPILEPSY

Leonardo Bonilha ${ }^{1}$, J. J. Lin ${ }^{3}$, A. Tabesh ${ }^{1}$, K. Dabbs ${ }^{2}$, D. Hsu ${ }^{2}$, C. E. Stafstrom $^{2}$ and B. Hermann ${ }^{2}\left({ }^{1}\right.$ Medical University of South Carolina, Charleston, SC; ${ }^{2}$ University of Wisconsin School of Medicine and Public Health, Madison, WI and ${ }^{3}$ University of California, Irvine, CA) 
Rationale: The organization of the brain is nonrandom and highly economical. Balancing the cost and efficiency of this system requires a network connecting highly modular communities with topographically important central nodes or hubs. A highly promising but largely untested theory suggests that epilepsy affects complex brain networks, leading to abnormal systems rearrangement with implications for epileptogenesis and cognitive development. In this study, we aimed to evaluate the role of regional brain structures within the brain network of pediatric patients with new-onset epilepsy. We hypothesized that pediatric epilepsy is associated with a disruption of the normal pattern of structural network organization.

Methods: We studied a group of 67 subjects: 28 healthy controls (mean age $13.3 \pm 3.28$ years, 11 males), 39 children with new-onset epilepsy ( 21 LRE $(11.6 \pm 2.68$ years, 12 males $)$ and 18 IGE $(15 \pm 3.3$ years, 7 males $))$. Patients and controls were similar in age $(p=0.6)$ and gender distribution $(\mathrm{p}=0.9)$. Subjects underwent MRI scanning yielding SPGR images (1.5 Tesla GE Signa MR scanner, TR = 24 $\mathrm{ms}, \mathrm{TE}=5 \mathrm{~ms}$, flip angle $=40^{\circ}$, Slice thickness $\left.1.5 \mathrm{~mm}\right)$, which underwent automatic volumetric segmentation utilizing FreeSurfer. Data from 171 regions of interest were utilized for the construction of group-wise adjacency matrices (171x171 entries representing the partial correlation between the volumes from each pair of regions, controlled for age). Graphical metric properties (Betweenness Centrality and Clustering Coefficient) were calculated through the use of the Brain Connectivity Toolbox. Betweenness centrality represents the fraction of all the shortest paths that run through a node, and nodes with high betweenness centrality have high influence on the network and function as hubs. Clustering coefficient is a measure of "cliquishness" of a local community and represents the number of triangular connections between neighborhoods of 3 nodes. The regional properties were classified according to their distribution in percentiles ranging from 0 to 80 th percentile, and the classified data was plotted according to the stereotaxic coordinates of the structural region on interest.

Results: We observed a significant rearrangement of the regional network role of structures of interest between patients and controls. The results are summarized in Figure 1. Patients with epilepsy demonstrated a significant decrement in betweenness centrality within paracentral regions, with a concurrent segregation of these regions, as demonstrated by the diffuse lack of decrement in clustering coefficient.

Conclusions: Children with new-onset epilepsy have a decreased number of hub regions while the local neighborhoods remained highly connected. These findings suggest that the normal developmental process is disrupted in pediatric epilepsy resulting in highly segregated and poorly integrated brain networks. Thus pediatric epilepsy is not only associated with regional structural changes, but also a global network restructuring, which may have direct impact on epileptogenesis, treatment and global cognitive development.

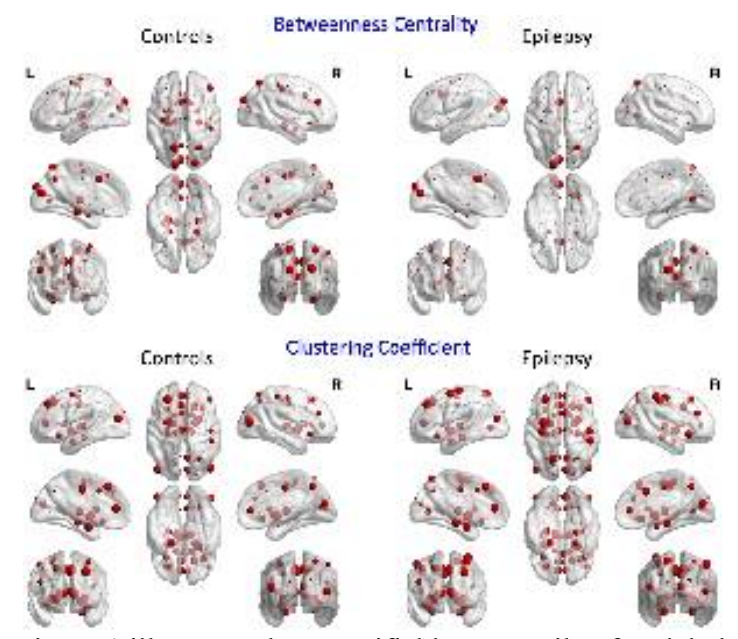

Figure 1 illustrates the quantifiable percentile of nodal clustering coefficient and betweenness centrality of regions of interest. The size of the sphere is proportional to the percentile in which the corresponding region falls in comparison with all other nodes in the network, ranging from 0.2 to 0.8 , in 0.2 intervals (e.g., 0.2 if the region's clustering coefficient falls between the $20 \%$ and $40 \%$ percentile of the distribution of regional clustering coefficients of the network).

\section{B.03 \\ HIPPOCAMPAL ABNORMALITY IN COMMUNITY-BASED NON REFRACTORY FOCAL EPILEPSY: A QUANTITATIVE T2 AND VOLUME STUDY \\ G. D. Jackson ${ }^{1,2}$, Heath R. Pardoe ${ }^{1}$, S. U. Schuele ${ }^{3}$, R. K. Fulbright ${ }^{4}$ and A. T. Berg ${ }^{5}\left({ }^{1}\right.$ Brain Research Insitute, Florey Neuroscience Institutes, Melbourne, VIC, Australia; ${ }^{2}$ Department of Medicine, University of Melbourne, Melbourne, VIC, Australia; ${ }^{3}$ Department of Neurology, Northwestern University, Chicago, IL; ${ }^{4}$ Department of Radiology, Yale School of Medicine, New Haven, CT and ${ }^{5}$ Department of Pediatrics, Ann \& Robert H. Lurie Children's Hospital of Chicago, Chicago, IL)}

Rationale: T2 relaxometry can detect hippocampal sclerosis with high sensitivity, and show minor changes associated with seizures or other pathology $[1,2]$. In a community cohort of subjects with nonsyndromic focal epilepsies, we previously observed an excess of bilaterally small $(<2 \mathrm{SD})$ and large $(>2 \mathrm{SD})$ hippocampi relative to controls. Here we examine whether these size anomalies are associated with increased hippocampal T2.

Methods: 65 focal epilepsy cases (age $23.1 \pm 3.2$ years) and 36 healthy controls (age $23.48 \pm 3.27$ years) were included. Focal epilepsy patients were classified as 'complicated' $(n=14)$ when there was an associated lesion or neurodisability, or 'noncomplicated' $(\mathrm{n}=$ 51 ) in which there was no identified lesion. Clinical information was assessed in each case for indicators suggesting temporal lobe epilepsy. T2-weighted multi-spin echo MRI was acquired using a 1.5T Siemens Sonata MRI scanner. 16 echo times were acquired from $\mathrm{TE}=22 \mathrm{~ms}$ to $352 \mathrm{~ms}$, incrementally increased by $22 \mathrm{~ms}$. TR = $3000 \mathrm{~ms}$, in-plane resolution $=0.44 \times 0.44 \mathrm{~mm}$. Slices were $5 \mathrm{~mm}$ thick with a $5 \mathrm{~mm}$ slice gap. 8 slices were acquired per participant, covering the length of the hippocampus. Hippocampal T2 relaxation time was assessed in the hippocampal body. Hippocampal volume was assessed using automated hippocampal segmentation of whole brain T1 MRI acquired in the same imaging session, using Freesurfer 5.1. Hippocampal volumes were corrected for brain volume and categorised into three groups by ranking the volumes and taking the lower 25th percentile (low volume), 25th to 75th percentile (normal volume) and upper 25 th percentile (high volume).

Results: Relative to controls, T2 was increased in the complicated focal group; $2.9 \mathrm{~ms}$ left $\mathrm{p}<0.05,1.6 \mathrm{~ms}$ right $\mathrm{p}>0.05$ (see Table 1 for hippocampal T2 and volumes). There were no general T2 increases in the uncomplicated group, however subgroup analysis suggests a relationship between clinical indication of TLE and increased hippocampal T2; $1.64 \mathrm{~ms}$ right $\mathrm{p}=0.05,1.2 \mathrm{~ms}$ left $\mathrm{p}>$ 0.05 . Further, there was no significant relationship between hippocampal volume and hippocampal $\mathrm{T} 2$ increases in either of the focal subgroups.

Conclusions: Hippocampal T2 increases are limited to complicated focal epilepsy. Subtle hippocampal T2 abnormalities are independent of volumetric abnormalities, suggesting that these two measurements index different epilepsy-related neurological changes in non-mesial temporal lobe epilepsy. The classic MRI-based markers of mesial temporal lobe epilepsy, high T2 in combination with hippocampal atrophy, are specific to hippocampal sclerosis and relatively rare in the population.

References: [1] Scott et al, Brain (2003) 126(9): 1968 - 1974 [2] Van Paesschen et al Ann Neurol (1997) 41(1): 41 - 51 Funding: NIH-NINDS R37-NS31146, NHMRC program grant 628952 (Australia) 
Hippocampal T2 and volumes in community-based focal epilepsy and healthy controls

\begin{tabular}{|c|cc|cc|}
\hline & $\begin{array}{c}\text { Left Hipp T2 } \\
(\mathrm{ms})\end{array}$ & $\begin{array}{c}\text { Right Hipp T2 } \\
(\mathrm{ms})\end{array}$ & $\begin{array}{c}\text { Left Hipp Vol } \\
\left(\mathrm{mm}^{\wedge} 3\right)\end{array}$ & $\begin{array}{c}\text { Right Hipp Vol } \\
\left(\mathrm{mm}^{\wedge} 3\right)\end{array}$ \\
\hline Controls & $97.8 \pm 3.6$ & $97.3 \pm 3.7$ & $4242.3 \pm 263.4$ & $4269.3 \pm 287.2$ \\
$\begin{array}{c}\text { Complicatcd Focal } \\
\text { Uncomplicated } \\
\text { Focal }\end{array}$ & $100.7 \pm 5.3^{*}$ & $98.9 \pm 7.0$ & $4181.1 \pm 732.1$ & $4106.3 \pm 420.2$ \\
\hline
\end{tabular}

$* \mathrm{p}<0.05$

\section{B.04 \\ ARE PROLONGED FEBRILE SEIZURES ASSOCIATED WITH LONG-TERM CHANGES IN HIPPOCAMPUS? A QUANTITATIVE HIPPOCAMPAL VOLUMETRIC \\ ANALYSIS}

Suresh Pujar ${ }^{1,2}$, M. Martinos ${ }^{1}$, B. Neville ${ }^{1,2}$, C. Clark ${ }^{1}$, R. Scott ${ }^{1,3}$ and R. Chin ${ }^{1,4}\left({ }^{1}\right.$ Neurosciences Unit, UCL Institute of Child Health, London, United Kingdom; ${ }^{2}$ Young Epilepsy, Lingfield, United Kingdom; ${ }^{3}$ Dartmouth Medical School, Hanover, NH and ${ }^{4}$ The University of Edinburgh, Edinburgh, United Kingdom)

Rationale: There is a long-standing hypothesis that prolonged febrile seizures (PFS) cause mesial temporal sclerosis (MTS), a common pathology seen in temporal lobe epilepsy (TLE). Human and animal data suggest PFS may result in transient hippocampal changes, but whether PFS results in any long-term hippocampal changes or MTS is uncertain.

In an ongoing follow-up study, we are investigating a prospectively ascertained population-based cohort of children to determine their outcomes within 10 years after an episode of convulsive status epilepticus. Here, we report preliminary quantitative hippocampal volumetry results in a subgroup that had PFS.

Methods: All enrolled children were invited to have brain MR Imaging (MRI) on a Siemens $1.5 \mathrm{~T}$ scanner. In addition to conventional sequences, T1-weighted images were acquired using a three-dimensional fast low angle shot (3D FLASH) sequence providing $1 \mathrm{~mm}^{3}$ isometric voxels for volumetry.

Hippocampal volumetry was performed on 3D FLASH images using FSL version 4.1.9. The images were reformatted in tilted coronal plane perpendicular to the long axis of hippocampus and each hippocampal slice was measured on each side. Hippocampal volumetry was performed blind to clinical information and using conventional hippocampal boundaries. The hippocampal volume (HV) was measured for each side and right-left asymmetry calculated using asymmetry index (AI= (HV Right- HV Left) / 0.5x (HV Right + HV Left)). Nonparametric statistical tests were used to compare results between PFS group and controls in SPSS version 19.

Results: Hippocampal volumetry was performed on 19 children with PFS (9 male, mean age 9.6 (range 7.5 to 13.1 ) years) and 5 controls ( 2 male, mean age 9.2 (range 7.6 to 11.9 ) years). Mean interval between PFS and MRI study was 8.15 (range 6.7 to 9.6) years. The right hippocampus was larger than the left in all controls and in $14(74 \%)$ of the PFS group. There was no significant difference in the average HV between PFS group (median $3151 \mathrm{~mm}^{3}$, range 2255 to 3638 ), and controls (median $3109 \mathrm{~mm}^{3}$, range 2505 to 3176 ), or in their AI (median 0.069 , range 0.0 to 0.28 for PFS; median 0.068 , range 0.04 to 0.11 for controls). However, the AI for 6 children in the PFS group (31\%) were more than the highest value in the control group. None of these children with a high AI had any reported neurological or neurocognitive impairments.

Conclusions: Our data suggests that although there are no group differences in hippocampal volumes between children who had PFS and controls, a subgroup of PFS children will demonstrate hippocampal asymmetry within 10 years after PFS. Examination of factors that may identify such a group and longer term follow up are needed.
B.05

MULTIREGIONAL NETWORK OF MRI STRUCTURAL AND FUNCTIONAL CONNECTIVITY CHANGES IN LEFT TLE Victoria L. Morgan ${ }^{1}$, M. J. Holmes ${ }^{1}$, X. Yang ${ }^{4}$, B. A. Landman ${ }^{4}$, Z. Ding $^{1}$, H. Kang ${ }^{3}$, H. H. Sonmezturk ${ }^{2}$ and B. W. Abou-Khalil ${ }^{2}$ $\left({ }^{1}\right.$ Radiology, Vanderbilt University, Nashville, TN; ${ }^{2}$ Neurology, Vanderbilt University, Nashville, TN; ${ }^{3}$ Biostatisitics, Vanderbilt University, Nashville, TN and ${ }^{4}$ Electrical Engineering, Vanderbilt University, Nashville, TN)

Rationale: Structural atrophy indicated by decreases in gray matter compared to controls has been detected across the brain in temporal lobe epilepsy (TLE) using MRI. TLE has also been associated with changes in functional connectivity using resting functional MRI. We hypothesized that widespread networks affected by TLE would include regions where gray matter decreases and functional connectivity are linearly related. Our goal was to identify individual brain regions where gray matter decreases correlated with functional connectivity in a group of left TLE patients, and to determine whether any of these regions formed synchronous multiregional networks unique to left TLE.

Methods: In a group of 15 electrographically determined left TLE patients (35 $\pm 10 \mathrm{yrs}, 10 \mathrm{~F}, 1$ left-handed), we performed 3T MRI imaging including T1-weighted structural (ultra-fast gradient echo, 1 $\mathrm{mm} 3)$ and resting functional scans $(3.75 \times 3.75 \times 5 \mathrm{~mm}, \mathrm{TE}=35 \mathrm{~ms}$, $\mathrm{TR}=2 \mathrm{~s}, 200$ dynamics). The T1-weighted images were used to perform optimized voxel-based morphometry [1] to quantify voxelwise gray matter concentration (GMC). The functional MRI images were used to calculate voxel-wise functional connectivity (FC) maps to the left hippocampus (LH) and to the left thalamus (LThal) using the general linear model [2]. Regions of linear correlation between the GMC and FC map to the LH were found using biological parametric mapping (BPM) [3], a linear modeling method for comparing two image sets. The BPM was repeated to find linear correlations between the GMC and FC map to the LThal. Finally, we looked for FC connections among these regions that were correlated with each other to form multiregional networks. To do this we assumed that the LH to LThal connection was the hub of the network and determined which other connections were correlated with it across the group. We also compared the FC of the same connections in 15 healthy controls.

Results: Twenty regions had GMC correlated with FC to either the LH or LThal. We found 5 of these connections correlated with the connection LH - LThal (conn A) across the patient group (Figure 1). These connections include: the LH and the anterior cerebellum (conn B), LH and the right temporal lobe (conn C), LThal and the midcingulate gyrus (conn D), LThal and the left postcentral gyrus (conn E) and LThal and the right postcentral gyrus (conn F). This means that in patients where the strength of connection A was high, the strength of these other connections were also high; and those in which the strength of connection A was low, these others were low. This relationship was not found in controls.

Conclusions: We performed voxel-wise regression to determine regions of correlated GMC decreases and FC in left TLE. From these regions we identified a multiregional network of synchronous connections unique to the patient group which may reflect seizure propagation in left TLE.

NIH T32 EB001628, R01 NS055822 and UL1 RR024975-01

[1] Ashburner J. 2000. NeuroImage 11(6):805-821. [2] Morgan VL. 2012 Epilepsia 52(9):1741-1749. [3] Casanova R. 2007. NeuroImage 34(1):137-143. 
Table 1. Intercorrelations of multiregional network in left TLE

\begin{tabular}{|c|ccc|ccc|}
\hline & A & B & C & D & E & F \\
\hline A & I & r $=0.64$ & $\mathrm{r}=-0.55$ & $\mathrm{r}=-0.52$ & $\mathrm{r}=-0.51^{*}$ & $\mathrm{r}=-0.52$ \\
\hline B & & 1 & & $\mathrm{r}=-0.69$ & $\mathrm{r}=0-50^{* *}$ & $\mathrm{r}=-0.53$ \\
C & & & 1 & & & $\mathrm{r}=0.54$ \\
D & & & & 1 & $\mathrm{r}=0.75$ & $\mathrm{r}=0.66$ \\
\hline E & & & & & 1 & $\mathrm{r}=0.52$ \\
\hline F & & & & & $\mathrm{r}$ \\
\hline
\end{tabular}

Pearson correlation values for each pair of connections shown in Figure 1 across group. For all correlations, the $p$-value is $p<0.05$ or smaller except in two cases $(* \mathrm{p}=0.052$ and $* * \mathrm{p}=0.059)$.

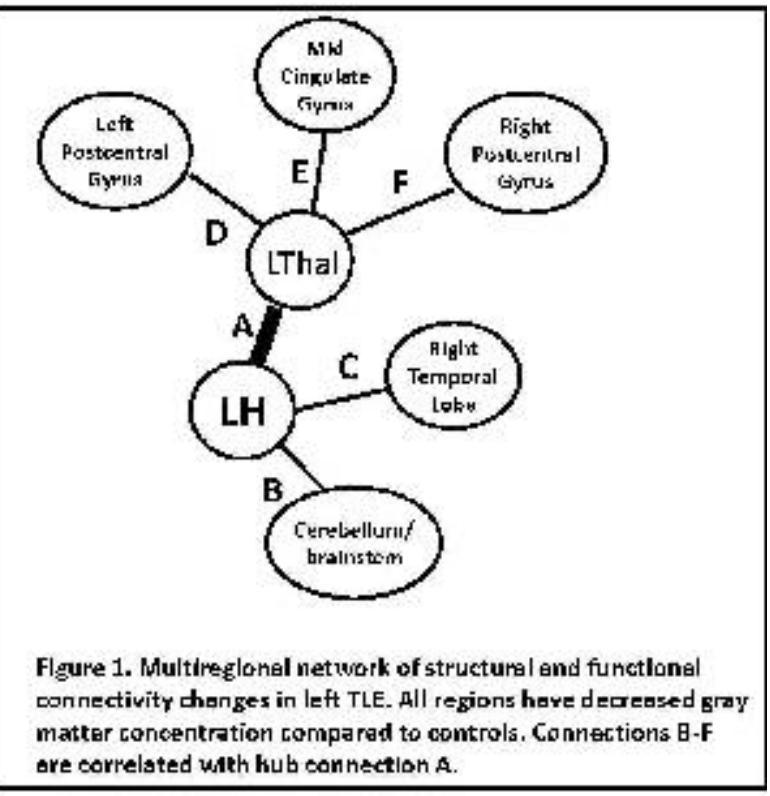

B.06

MESIAL TEMPORAL LOBE ICTAL-NETWORK PROPAGATION IS LATERALITY DEPENDENT

David Jones $^{1}$, B. H. Brinkmann ${ }^{1}$, D. B. Burkholder ${ }^{1}$, V. Sulc ${ }^{1}$, B. P. Mullan $^{2}$, K. M. Welker ${ }^{3}$, E. L. So ${ }^{1}$, S. Stead ${ }^{1}$ and G. A. Worrell ${ }^{1}$ $\left({ }^{1}\right.$ Neurology, Mayo Clinic, Rochester, MN; ${ }^{2}$ Nuclear Medicine, Mayo Clinic, Rochester, $\mathrm{MN}$ and ${ }^{3}$ Radiology, Mayo Clinic, Rochester, MN)

Rationale: Temporal lobe epilepsy (TLE) is the most common form of medically intractable epilepsy, with the most common pathologic finding being mesial temporal sclerosis (MTS). Lateralization of the epileptogenic focus is critical to successful epilepsy surgery. Whether clinically meaningful differences exist in the epileptic networks involved in complex partial seizures which is dependent on laterality of the hemisphere of origin is currently unknown.

Methods: This study compares the ictal SPECT perfusion patterns in subjects with lateralized TLE who had pathologically confirmed MTS and Engel class I postsurgical outcome to ensure correct lateralization ( $n=20$ left TLE, $n=18$ right TLE). A SPM analysis of ictal SPECT images was used to isolate hypoperfusion differences between the right and left TLE. We used these laterality of seizurefocus-dependent hypoperfusion differences to create an anterior-toposterior hypoperfusion asymmetry index (API) and evaluated lateralization accuracy in various support vector machine (SVM) classifier algorithms in ictal, interictal, and subtraction SPECT analyses.

In order to characterize the networks involved in the hypoperfusion differences observed, we used task-free fMRI to investigate the functional anatomy of the anterior and posterior limbic networks (ALN and PLN) of normal controls. We then evaluated whether normal limbic network connectivity was predictive of ictal SPECT perfusion in TLE.

Results: We observed significant differences in perfusion between right and left MTS (FDR $p<0.001)$. This consists of greater frontal lobe hypoperfusion (Figure 1a) associated with thalamus, midbrain, and brainstem hyperperfusion in left MTS and a greater parietal lobe hypoperfusion (Figure 1b) associated with basal ganglia and right middle temporal hyperperfusion in right MTS.

The accuracy of various SVM classifier models indicate that API adds lateralizing value to traditional ictal and interictal measures of right-left hyperperfusion asymmetry, but not to ictal-interictal subtraction analyses.

The normal functional anatomy of the ALN and PLN (Figure 1c) is predictive of the ictal SPECT perfusion changes seen in MTS. PLN connectivity was most predicative of ictal perfusion in left MTS $(r=$ $3.8, \mathrm{p}=3.1 \mathrm{E}-05$ ) (Figure 2a) while ALN connectivity was most predictive of ictal perfusion in right MTS $(r=3.8, p=3.3 \mathrm{E}-05)$ (Figure 2b).

Conclusions: There are differences in the large-scale epileptic network effects of TLE depending on the laterality of origin, which are clinically meaningful. We also found that limbic network connectivity was predictive of ictal SPECT perfusion patterns. Rightsided foci preferentially affect anterior limbic circuitry, while leftsided foci preferentially affect posterior limbic circuitry.

Understanding the laterality dependent differences in epileptic networks will not only facilitate clinical localization, but will also have important implications for targeted surgical interventions aimed at indirectly modifying epileptic networks (e.g. vagal nerve stimulation and deep brain stimulation).
A.

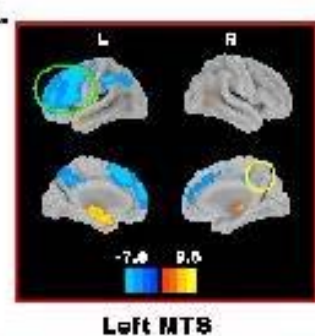

c.

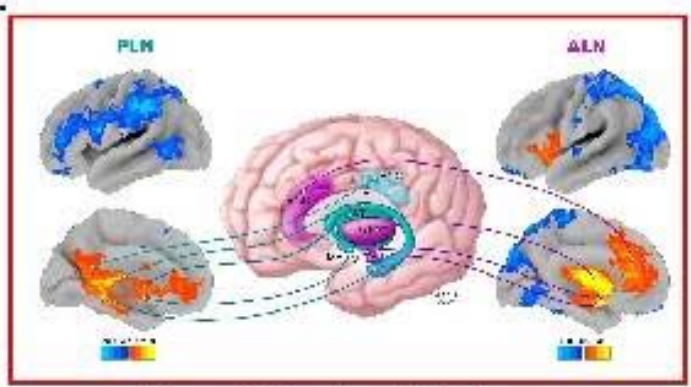

Normal Functional Anatomy

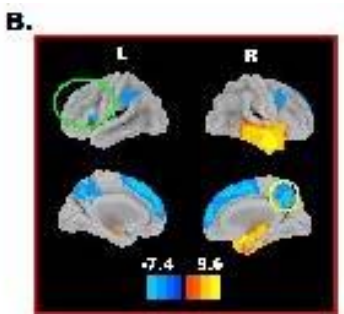

Right HTs
Figure 1: Right (A) and left (B) TLE differences in ictal SPECT perfusion are highlighted in the frontal lobe (green circle) and parietal lobe (yellow circle). The normal functional anatomy (C) of the PLN and ALN is displayed for comparison (correlations in orange-yellow and anticorrelations in blue).
A.

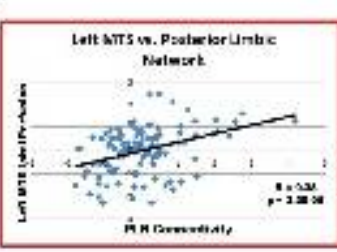

B.

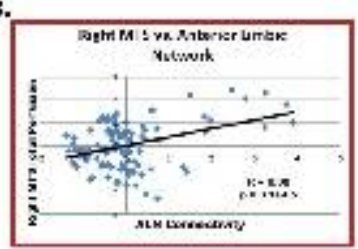

Figure 2: PLN connectivity is predictive of ictal perfusion in left MTS (A), while ALN connectivity is predictive of ictal perfusion in right MTS (B). 


\section{B.07}

\section{LENNOX GASTAUT SYNDROME: A SECONDARY NETWORK EPILEPSY}

John S. ARCHER ${ }^{1,2}$, M. Stagnitti ${ }^{1}$, R. Masterton ${ }^{2}$, D. Abbott ${ }^{2}$ and G. Jackson ${ }^{2,3}\left({ }^{1}\right.$ The University of Melbourne, Melbourne, VIC, Australia; ${ }^{2}$ Florey Neuroscience Institutes, Melbourne, VIC, Australia and ${ }^{3}$ Austin Health, Melbourne, VIC, Australia)

Rationale: Lennox-Gastaut syndrome (LGS) is a severe epilepsy phenotype with diverse aetiologies, including cortical lesions of varying type and location. Characteristic interictal EEG features are slow spike-and wave (SSW) and generalised paroxysmal fast activity (GPFA). Tonic seizures are a key clinical feature. The shared electroclinical characteristics suggest common cerebral networks are involved. We sought to reveal these networks and the contribution of lesions to the interictal activity of LGS using EEG-fMRI with time course analysis.

Methods: i) Clinical - Six subjects (32-42 yrs), with intractable epilepsy of 'Lennox-Gastaut Phenotype', were identified from a larger EEG-fMRI study of 'secondary generalised epilepsy' (NHMRC project grant 628725; Melbourne, Australia). Inclusion criteria were 1) interictal EEG with SSW and GPFA; 2) tonic seizures on video-EEG monitoring or history; 3 ) lesion on structural MRI (table 1).

ii) fMRI - Up to 60 minutes of fMRI data were acquired during continuous EEG (in-house hardware/software); 3T GE Signa LX; EPI, TR $3.2 \mathrm{sec}$, TE $40 \mathrm{~ms}, 3.4 \mathrm{~mm} 3$ voxels. fMRI data were preprocessed and analyzed using SPM8 and the iBrain Analysis Toolbox. The timing of events on the study EEG were used in an event-related fMRI analysis. One-tailed t tests created maps of regional BOLD signal change, associated with each subject's SSW and GPFA. A second-level group analysis revealed regions of consistent BOLD signal change.

iii) Time Course - Several spherical regions of interest (ROIs) were selected by four different Methods: a) Attentional and REST areas from previously published co-ordinates (Fox et al. PNAS 2005); b) Cortical and subcortical regions of maximal signal change on the GPFA group activation map; c) primary cortical structures identified anatomically; d) within the lesion.

Results: GPFA - Robust increases in fMRI activity are seen in frontal and parietal association cortical areas, thalamus and pons. Time course analysis shows simultaneous activity increases in both 'attentional' and 'REST' networks, a highly unusual pattern. SSW - Mixed increased and decreased activity, with maximal increases in association cortex and thalamus, and reduced activity in posterior cingulate, precuneus and primary cortical areas. There is pre-event rise in fMRI activity across the network, followed by prominent post-event reduction.

Lesion - activity increases with both GPFA and SSW.

Conclusions: The epileptiform activity of LGS recruits association cortical areas, pons and thalamus. Simultaneous activity increases in 'attention' and 'REST' areas during GPFA suggest a failure of the normal anti-correlated behaviour of these networks. Pre-SSW rises of fMRI activity in association cortex, suggest that SSW occurrence is dependent on rising activity in this network.

Increased fMRI activity was present in lesions during generalised epileptiform activity, confirming the lesion is part of the abnormal network. Subsequent seizure freedom ( $>1$ year) in the 3 subjects $(2$, 3 and 5) who proceeded to lesionectomy confirms the role of lesions in establishing and maintaining this abnormal network behaviour.
Subject details

\begin{tabular}{|c|c|c|c|c|c|c|c|}
\hline \multirow[t]{2}{*}{ II) } & \multirow[t]{2}{*}{ Age } & \multirow[t]{2}{*}{ Sex } & \multirow[t]{2}{*}{$\begin{array}{l}\text { Seizure onset } \\
\text { age }\end{array}$} & \multirow[t]{2}{*}{$\begin{array}{l}\text { Seizure } \\
\text { types }\end{array}$} & \multicolumn{2}{|c|}{$\begin{array}{c}\text { In scanner events } \\
\text { Number (dur.n } \\
{[\mathrm{scc}] \text { ) }}\end{array}$} & \multirow[t]{2}{*}{ MRI abnormality } \\
\hline & & & & & GPFA & ssw & \\
\hline 1 & 33 & $\mathrm{~F}$ & $4 \mathrm{yrs}$ & Tonic, GTC & $55(83)$ & - & Bilateral parietal double cortex \\
\hline 2 & 38 & $\mathrm{M}$ & $8 \mathrm{yrs}$ & Tonic, GTC & $6(6.2)$ & $69(45)$ & L frontal balloon cell dysplasia \\
\hline 3 & 32 & $\mathrm{~F}$ & 3 mths & Tonic, CPS & $41(61)$ & - & $\begin{array}{l}\mathrm{R} \text { parietal gliosis - infantile } \\
\text { meningitis }\end{array}$ \\
\hline 4 & 42 & M & $7 \mathrm{yrs}$ & Tomic, (jTC & $17(37)$ & $302(48)$ & $\begin{array}{c}\text { Right frontal transmantlc } \\
\text { dysplasia }\end{array}$ \\
\hline 5 & 38 & $\mathrm{M}$ & $4 \mathrm{yrs}$ & Tonic, CPS & $7(20)$ & $560(224)$ & $\begin{array}{l}\text { R lat. temp. gliosis ?perinatal } \\
\text { infarct }\end{array}$ \\
\hline 6 & 40 & $\Gamma$ & $?$ & Tonic & $25(30)$ & - & Complex R parictal dysplasia \\
\hline
\end{tabular}

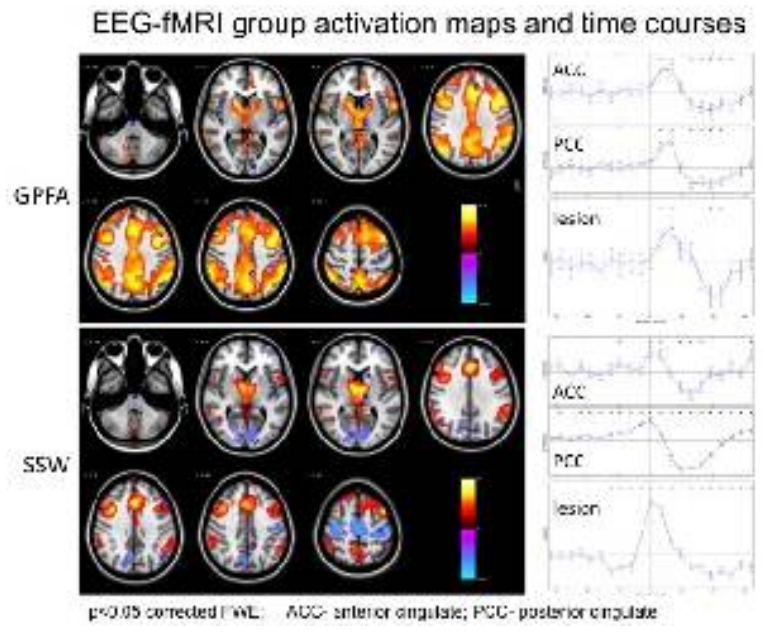

Left panel group activation maps for GPFA (top) and SSW (bottom). Right panel time course of fMRI signal from select regions, $3 \mathrm{sec}$ time bins from $30 \mathrm{sec}$ pre/post epileptiform discharge (vertical line)

\section{B.08 \\ MALFORMATIONS OF CORTICAL DEVELOPMENT: DETECTION OF METABOLIC ABNORMALITIES OUTSIDE THE VISIBLE LESIONS WITH PHOSPHORUS-31 MR SPECTROSCOPY}

Celi S. Andrade ${ }^{1}$, M. C. Otaduy ${ }^{1}$, E. J. Park ${ }^{1}$, K. D. Valente ${ }^{1}$, M. H. Tsunemi ${ }^{2}$ and C. C. Leite ${ }^{1}\left({ }^{1}\right.$ Clinics Hospital - University of Sao Paulo, Sao Paulo, Brazil and ${ }^{2}$ Universidade Estadual Paulista Julio de Mesquita Filho, Botucatu, Brazil)

Rationale: Malformations of cortical development (MCD) are a heterogeneous group of lesions that share in common a strong association with epilepsy. Although the boundless evidences of structural and metabolic abnormalities in the aberrant lesions of $\mathrm{MCD}$, recent studies have started looking into the associated abnormalities in the normal appearing parenchyma (NAP) of these patients. Intracellular $\mathrm{pH}$ is an important parameter of brain bionergetics and electrophysiological status in neurons and glia, and there is a large body of evidence pointing to neurometabolic disturbances as key factors in epileptogenesis. The goal of this study was to evaluate the intracellular hydrogen-ion potential $(\mathrm{pH})$ in the NAP of patients with MCD and epilepsy.

Methods: Three-dimensional phosphorus magnetic resonance spectroscopy (31P-MRS) at 3.0 Tesla was performed in $37 \mathrm{MCD}$ patients and in 31 matched-control subjects. The patients were assigned into three main MCD subgroups:

hemimegalencephaly/cortical dysplasia $(\mathrm{n}=10)$; heterotopia $(\mathrm{n}=$ $14)$; schizencephaly/polymicrogyria $(\mathrm{n}=13)$. Voxels $(12.5 \mathrm{~cm} 3)$ were selected in five homologous regions containing NAP: right 
putamen; left putamen; frontoparietal parasagittal cortex; right centrum semiovale; and left centrum semiovale. Robust methods of quantification were applied, and the intracellular $\mathrm{pH}$ was calculated with the chemical shifts of inorganic phosphate $(\mathrm{Pi})$ relative to phosphocreatine (PCr).

Results: In comparison to controls and assuming a $p$ value $<0.05$, MCD patients presented acidosis in the frontoparietal parasagittal cortex, right centrum semiovale, and left centrum semiovale (Table 1). Dunnet and Dunn tests demonstrated that the differences in $\mathrm{pH}$ values remained statistically significant in all MCD subgroups (Figure 1).

Conclusions: Hydrogen ions play a remarkable role in the maintenance of electrophysiological status and in the regulation of several mitochondrial reactions. The present study demonstrates widespread acidosis in the NAP, and reinforces the idea that MCD visible lesions are only the tip of the iceberg. This issue may be of particular importance in patients who are candidates for lesion resection. The NAP could be a secondary target for medical interventions, and understanding the biochemical abnormalities that occur beyond the visible lesions may facilitate this approach.

Table 1 - Comparisons of $\mathrm{pH}$ values between MCD patients and control subjects in five cerebral regions with normal appearing parenchyma

\begin{tabular}{|cc|c|ccc|}
\hline & \multicolumn{2}{c|}{ Palients } & \multicolumn{2}{c|}{ Conlrols } \\
\hline Region & Mean & SD & Mean & SD & $\mathrm{p}$-value \\
Right putamen & 6.914 & 0.033 & 7.007 & 0.046 & $\mathrm{p}=0.621$ \\
I.cft putamen & 7.011 & 0.040 & 6.995 & 0.043 & $\mathrm{p}=0.170$ \\
\hline Frontoparictal parasagittal cortex & 6.985 & 0.022 & 7.087 & 0.048 & $\mathrm{p}<0.001 *$ \\
\hline Right centrum semiovale & 7.004 & 0.029 & 7.096 & 0.042 & $\mathrm{p}<0.001$ \\
\hline Left centrum semiovale & 6.995 & 0.030 & 7.088 & 0.045 & $\mathrm{p}<0.001$ \\
\hline
\end{tabular}

Note: *p-value obtained with Mann-Whitney test, other p-values obtained with Student's t test. $\mathrm{pH}=$ hydrogen-ion potential, $\mathrm{SD}=$ standard deviation.

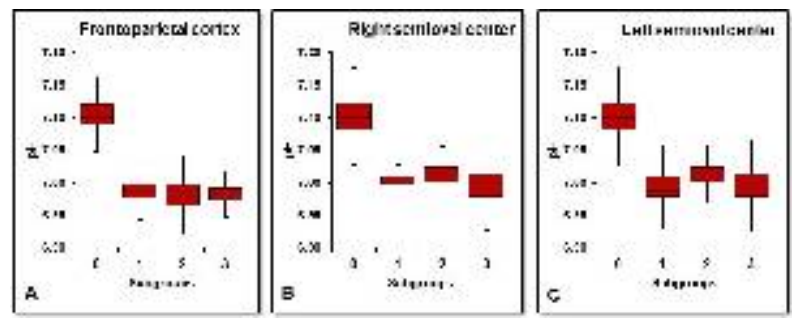

Figure 1: Box-plot graphics. Comparisons of mean hydrogen-ion potential $(\mathrm{pH})$ values in the normal appearing parenchyma in the following regions: A) Frontoparietal parasagittal cortex, B) Right centrum semiovale, C) Left centrum semiovale. $0=$ control-group; 1 $=$ hemimegalencephaly or cortical dysplasia; $2=$ heterotopia; $3=$ schizencephaly or polymicrogyria. In all these regions, Dunnet and Dunn tests demonstrated statistically significant differences $(\mathrm{p}<$ 0.05 ) between the MCD subgroups compared to the control-group.

\section{B.09}

\section{T MR SPECTROSCOPIC IMAGING FOR LOCALIZATION-} RELATED EPILEPSY

Jullie W. Pan ${ }^{1}$, Y. Zhang ${ }^{1}$, R. B. Duckrow ${ }^{1}$, S. R. Resor Jr ${ }^{2}$, J. Gerrard $^{1}$, L. Hirsch ${ }^{1}$, H. P. Hetherington ${ }^{1}$ and D. D. Spencer ${ }^{1}\left({ }^{1}\right.$ Yale University School of Medicine, New Haven, CT and ${ }^{2}$ Neurology, Columbia University School of Medicine, New York City, NY)

Rationale: With the success that surgical approaches can provide for localization related epilepsy, accurate seizure localization remains important. MR spectroscopy has had success in earlier studies in MTLE. With anticipated improvements in data quality at ultra-high field MR, we report on the implementation of high resolution 7T MR spectroscopic imaging (MRSI) in 23 patients studied over a 1.5year period.
Methods: Clinical: 23 localization-related epilepsy (LRE) patients were studied from the Yale and Columbia Comprehensive Epilepsy Centers over a $\sim 1.5 y e a r$ period. Since "whole brain" studies were not routinely done, the regions of MRSI study were defined from the clinical assessment. We evaluated the added MRSI data as how it agreed (or not) with consensus localization PRIOR to any invasive procedures. The pre-surgical evaluation $(n=23)$ used an A-B-C classification: MRSI concordant with consensus (group A); overlaps with consensus (group B); or discordant identifying a different lobe or lateralization (group C). As 10/23 patients thus far have proceeded to surgery ( 7 intracranial monitoring, 10 therapy), the MRSI localization can also be evaluated AFTER invasive localization. The post-surgical evaluation $(\mathrm{n}=10)$ used a two group AA-BB

classification: MRSI concordant with surgical consensus (group AA), or overlaps with surgical consensus (group BB). None of the surgical patients were discordant. MR: All data were acquired with a head only actively shielded 7T magnet (Agilent) with an 8-channel transceiver. Targeted regions of interest were performed using multislice IR gradient echo acquisitions (TR/TE 3s/8ms). Localized B0 and B1 shimming was performed as previously described. MRSI data were acquired using moderate TR/TE sequences $(1.5 \mathrm{~s} / 40 \mathrm{~ms})$, performed over $10 \mathrm{~mm}$ target slices $192 \times 192 \mathrm{~mm}$ FOV with $24 \times 24$ phase encoding to give a nominal voxel of $0.64 \mathrm{cc}$. Spectra were fit with Gaussian lineshapes to generate ratios of NAA/Ch and NAA/Cr. Anatomical images used a two-point T1 acquisition to enable tissue segmentation, with abnormal voxels determined by evaluation of $\mathrm{NAA} / \mathrm{Cr}$ and $\mathrm{NAA} / \mathrm{Ch}$ values.

Results: Based on the pre-surgical consensus, 21/23 required intracranial monitoring for more precise localization. MRSI classified $8 / 23$ patients as A, $10 / 23$ as B and $5 / 23$ as C (Table). 4/8 of the group A patients had an overt MRI or PET lesion (e.g., hippocampal atrophy). For the invasive analysis, 6/10 classified as AA, 4/10 classified as BB. Outcome durations are short in this initial analysis; however so far, $6 / 6$ in the AA group have good outcome (ILAE $<=$ III) while only $1 / 4$ in the BB group has good outcome.

Conclusions: Thus far, MRSI has generally identified a region of metabolic abnormality or part of the seizure network. In MTLE, the abnormalities involve the hippocampi and insula asymmetrically. Neocortical TLE (not dual pathology) typically did not show extensive hippocampal abnormality. This ongoing work argues that MRSI can help define metabolically injured or involved regions in LRE to provide more localization information in patients requiring intracranial monitoring.

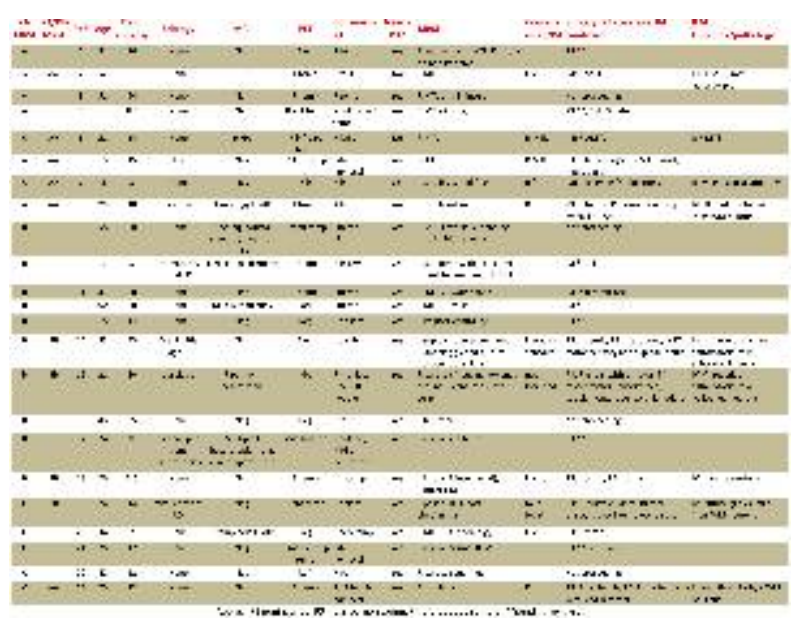


Monday, December 3, 2012

Platform C

4:00 p.m.-6:15 p.m.

\section{C.01}

POSTASPHYXIAL NEONATAL SEIZURE BURDEN IS STRONGLY RELATED TO THE SEVERITY AND PATTERN OF BRAIN INJURY

Perumpillichira J. Cherian ${ }^{1}$, R. M. Swarte ${ }^{2}$, M. Lequin ${ }^{3}$, P. Govaert ${ }^{2}$, W. F. Arts ${ }^{4}$ and G. H. Visser ${ }^{1}\left({ }^{1}\right.$ Neurology, section of Clinical Neurophsyiology, Erasmus MC, University Medical Center, Rotterdam, Netherlands; ${ }^{2}$ Pediatrics, section of Neonatology, Erasmus MC-Sophia, University Medical Center, Rotterdam, Netherlands; ${ }^{3}$ Pediatric Radiology, Erasmus MC-Sophia, University Medical Center, Rotterdam, Netherlands and ${ }^{4}$ Neurology,section of Child Neurology, Erasmus MC-Sophia, University Medical Center, Rotterdam, Netherlands)

Rationale: Postasphyxial neonatal seizures often respond poorly to antiepileptic drug (AED) therapy. The factors associated with higher seizure burdens and AED refractoriness in neonates with hypoxic ischemic encephalopathy (HIE) are poorly understood. Whether electrographic seizures independently predict poor outcome is a controversial issue. This study aims to assess the relationships between postasphyxial electrographic seizure characteristics, EEG background, and clinical outcome through detailed analysis of continuous EEG monitoring (cEEG) data. With this we expect to refine prognostication and also to better guide clinicians during treatment of postasphyxial seizures

Methods: EEG background and seizure characteristics were studied in 42 (out of 119 with HIE) consecutive term neonates with postasphyxial seizures using video-EEG for $\geq 24$ hours, starting $<24 \mathrm{~h}$ post partum. Electrographic seizures were aggressively treated with AED according to protocol. An eight-grade EEG background score was developed. MRI brain done in 39 were compared with those from a control group of 36 neonates with HIE who had cEEG during the same period and no seizures recorded. For statistical analyses, patients with seizures were grouped into those with mild to moderate (group I, grades 1-5, $\mathrm{n}=20$ ) and severe (group II, grades 6-8, $\mathrm{n}=22$ ) abnormalities of EEG background. Survivors were followed for at least two years.

Results: Increasing total seizure number (rho 0.73, $\mathrm{p}<0.001$ ) and total seizure burden in minutes (rho $0.41, \mathrm{p}<0.01$ ) were strongly correlated with worsening EEG background. The median seizure burden was significantly increased in EEG group II [109.3 (range 17.5-468.7) versus 44.6 (range 5.1-370.9) minutes, $\mathrm{p}=0.03$ ] as was the number of neonates showing injury to thalamus and basal ganglia on MRI $(16 / 22$ versus $3 / 20, p<0.001)$, when compared to EEG group I. Logistic regression analysis showed that EEG background strongly predicted $(\mathrm{p}=0.003)$ poor outcome, defined as death or severe disability. Neither total seizure number, burden, nor MRI confirmation of lesions of thalami and basal ganglia significantly added to this. Compared to the seizure group, significantly more patients in the non-seizure group had relative sparing of the cerebral cortex on MRI ( $10 / 36$ versus $3 / 39, \mathrm{p}=0.03)$.

Conclusions: In neonates with HIE, higher electrographic seizure burdens and AED refractoriness occur in the context of more severe brain injury as reflected by the EEG background and MRI abnormalities. Relative sparing of cerebral cortex is seen in neonates with HIE and no seizures. In future studies of treatment of postasphyxial neonatal seizures, patient stratification according severity of underlying encephalopathy and brain injury pattern is indicated.

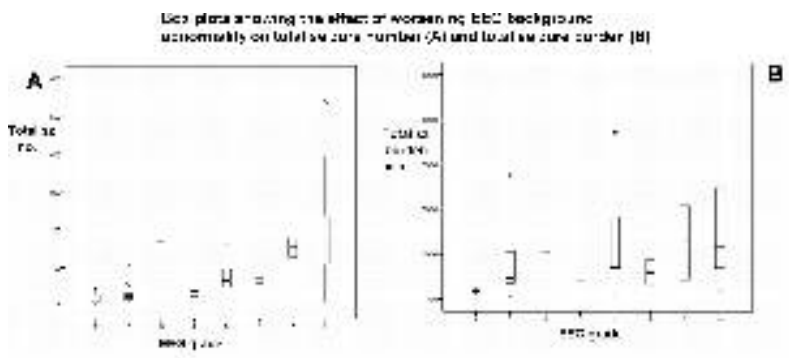

Box plots showing the effect of worsening EEG background abnormality on A. total seizure number, B. total seizure burden (cumulative duration of seizures) in minutes. Grade 1 denotes the mildest EEG abnormality and 8, the most severe abnormality

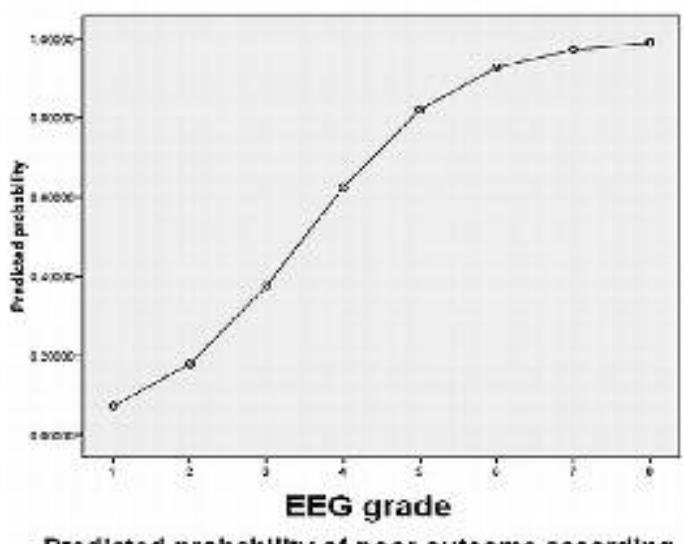

Predleted probablity of poor outcome according to severity of EEG background abnormality

Predicted probability of severely abnormal outcome according to severity of EEG background abnormality. Grade 1 denotes the mildest abnormality and 8 , the most severe abnormality. Poor outcome was defined as death or severe handicap

\section{C.02}

\section{ARE HIGH FREQUENCY OSCILLATIONS ASSOCIATED WITH ALTERED FUNCTIONAL NETWORK CHARACTERISTICS?}

Eric G. van Diessen ${ }^{1}$, J. I. Hanemaaijer ${ }^{1}$, J. Jacobs ${ }^{2}$, R. Zelmann ${ }^{2}$, F. E. Jansen ${ }^{1}$, C. J. Stam ${ }^{3}$, J. Gotman ${ }^{2}$ and M. Zijlmans ${ }^{1}{ }^{1}$ Neurology \& Neurosurgery, UMC Utrecht, Utrecht, Netherlands; ${ }^{2}$ Montreal Neurological Institute, McGill University, Montreal, QC, Canada and ${ }^{3}$ Clinical Neurophysiology, VU medical center, Amsterdam, Netherlands)

Rationale: The epileptic brain is increasingly associated with pathological functional networks. Furthermore, the epileptogenic focus can be reliable identified by the presence of high frequency oscillations (HFOs) in the intracranial electroencephalogram (iEEG). In this study, we investigated whether HFOs spatially coincide with altered functional network characteristics derived from iEEG. Methods: Twelve patients with temporal lobe epilepsy (TLE) underwent bilateral depth electrode EEG recordings. We assessed epileptic network properties by computing phase lag index (PLI) as a measure for functional connectivity, and eigenvector centrality measure (ECM) as a measure for centrality of each electrode in the theta frequency band. We compared PLI and ECM between hemispheres and iEEG electrodes and correlated the left-right mirror electrodes HFO rate difference to PLI and ECM differences (Spearman's rho correlation).

Results: A higher PLI was observed in left compared to the right hemisphere, regardless of lateralization of seizure onset zone. No differences were found between affected and non-affected hemisphere. Further analysis revealed a negative correlation between the difference in HFOs (fast ripples with a frequency of 250-500 Hz) 
and the difference in PLI $(r=-0.8 ; \mathrm{p}<0.01)$ and ECM ( $\mathrm{r}=-0.8 ; \mathrm{p}$ $<0.01)$.

Conclusions: Both PLI and ECM are decreased in electrodes with HFOs. These results revealed that the epileptogenic focus, identified by the presence of fast ripples, is associated with an altered functional network characteristics. High fast ripples rates are associated with low connectivity measures. Possibly, iEEG electrodes with HFOs are functionally isolated from their neighbouring channels.

\section{C.03 \\ HIGH-FREQUENCY ACTIVITY AND THETA COUPLING DURING PARTIAL EPILEPTIC SEIZURES}

Christophe C. Jouny, U. Malinowska, M. Cervenka and G. K. Bergey (Johns Hopkins School of Medicine, Baltimore, MD)

Rationale: The amplitude of gamma oscillations has been shown to be coupled to the phase of theta oscillations in humans and in animals. The strength of the coupling is modulated by the various states of the brain, including performing mental and/or memory tasks. We investigate here the strength of the coupling between theta and the high-frequency activity during the onset and ictal period of partial complex seizures in humans to assess if the mechanisms responsible for the coupling are maintained during ictal events, and to evaluate the potential of the coupling strength to be used as an indicator of upcoming ictal events.

Methods: Data include 331 partial onset seizures recorded from 45 consecutive patients with either mesial temporal, neocortical temporal or extra-temporal onset seizures. Data for each channel closest to the focus was first filtered using a zero phase-lag filter to prevent phase distortion. Instant phase for the theta band $(4-8 \mathrm{~Hz})$ was calculated using Hilbert transform and local minima in the instantaneous phase were used to determined theta troughs. Higher frequency bands were filtered, and instantaneous amplitudes were calculated using Hilbert transform. Average preictal and ictal periods were calculated over a 20 -second window taken 40 seconds before and respectively 20 seconds after the onset. Using a +/- 500ms window centered on each trough, the average theta wave and spectral analysis were computed over all troughs.

Results: Due to the occurrence of the ictal event, the average theta wave is significantly larger during the ictal period (Figure 1 - lower). Although there is indication of high-frequency modulation during the preictal periods, during the ictal period the high amplitude theta waves clearly modulated the high-frequency activity in a large range of frequency (Figure 1 - upper). Evidence of the coupling is not apparent in all seizures as not all seizure onsets have a theta component.

Conclusions: There is strong indication that the high-frequency theta coupling observed during cognitive tasks is also present during ictal events. Ictal events with high amplitude theta waves, especially during the early phase of the seizures show that the high-frequency activity in the cortical areas is very strongly modulated by the pattern of the theta waves. That modulation is stronger during ictal events than during preictal periods. Previous reports of gamma-theta coupling often highlight the possibility of memory consolidation and learning being linked to that modulation, but the mechanisms behind the coupling observed here may be different.

Funded by NIH NS075020
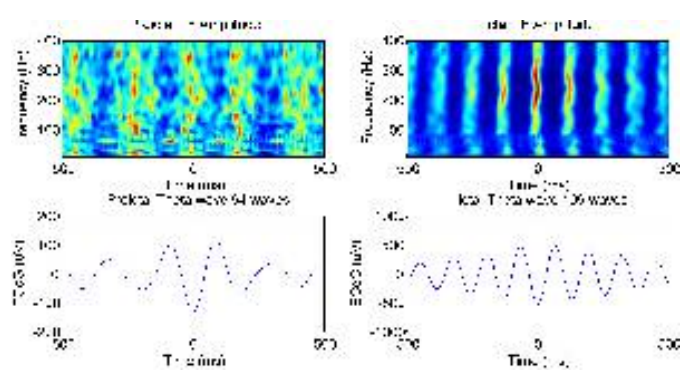

Figure 1. High-frequency and theta coupling during the preictal and ictal period of a complex partial seizure in a patient with mesial temporal onset epilepsy. Lower panels show the average waves obtained by aligning the troughs of the consecutives theta waves over the preictal window (left) and ictal window (right). Upper panels show the corresponding average signal power for all frequency bands calculated with the same alignment of theta wave troughs.

\section{C.04}

CORRELATION OF OSTEOPOROSIS DEVELOPMENT AND CHRONIC CEREBRAL HYPERACTIVITY IN EPILEPSY Brenda Y. Wu ${ }^{1,2}$, L. Lee ${ }^{2}$, J. Grimes ${ }^{3}$, A. Nanduri ${ }^{2}$ and M. Wagner ${ }^{2,1}$ ( ${ }^{1}$ Neurology, UMDNJ-Robert Wood Johnson Medical School, New Brunswick, NJ; ${ }^{2}$ Pharmacy Practice \& Administration, Ernest Mario School of Pharmacy of Rutgers University, Piscataway, NJ and ${ }^{3}$ Internal Medicine, UMDNJ-Robert Wood Johnson Medical School, New Brunswick, NJ)

Rationale: Patients with epilepsy (PWE) are predisposed to a 2-fold higher risk of fractures due to multiple factors associated with their condition. Previous studies revealed higher prevalence of osteoporosis in PWE than general population. The development of osteoporosis or osteopenia in PWE might be caused by multiple factors including adverse effects of long-term use of antiepileptics (AED) and vitamin D deficiency. However, the effects of chronic cerebral hyperactivity in PWE, has never been well described. Fibromyalgia and osteoarthritis are now considered as conditions resulted from CNS hyperactivity. We hence studied the relationship of osteoporosis development and chronic cerebral activation in PWE diagnosed with localization-related epilepsy.

Methods: Retrospective chart review of 340 subjects at age 21-72, men and women, followed at UMDNJ-Robert Wood Johnson UMG epilepsy clinic of one physician (Dr. Brenda Wu) were performed. Results of Dual-Energy X-Ray Absorptiometry (DXA) scan, seizure history, seizure lateralization and control levels as well as overall neuropsychiatric status (insomnia, headaches, anxiety and mood swings, memory lapses) were reviewed. Only 85 subjects (45 women) had DXA scan performed at bilateral femur (hip) and the rest of $75 \%$ subjects only had one side femur examined. Bone mineral density of bilateral femoral neck from DXA were studied with correlation of the confirmed seizure onset side per EEG/semiology.

Results: About 79\% (67/85) subjects had lower BMD at the femoral neck on the affected side (contralateral), correlated to the seizure onset side (example, worse at the left hip in subjects with right frontal seizure onset). Two third of subjects reported that their seizures were relatively well controlled but half of them admitted of having ongoing neuropsychiatric symptoms. Three subjects showed inconsistent lateralization to their typical seizure onset side but more than one epileptic focus were suspected.

Conclusions: 1 . Central activation may be a cause in osteoporosis independent to AED therapy and may cause systemic focal deficits including low BMD.

2. Like many other systemic illnesses, osteopenia/osteoporosis could be somatic presentation of uncontrolled CNS hyperactivity and could be reversible with appropriate interventions.

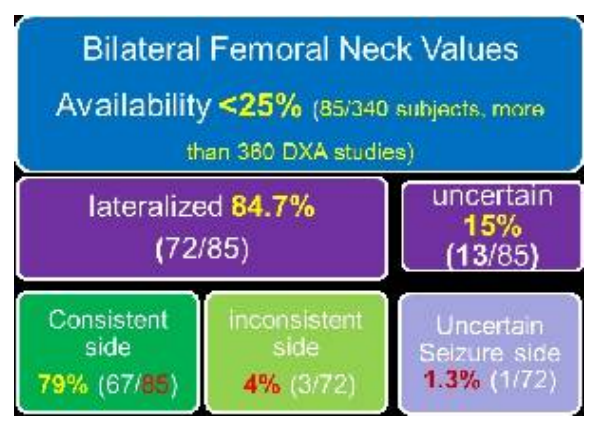




\section{C.05}

DO ANTIDEPRESSANTS HAVE AN IMPACT IN SEIZURE FREQUENCY AND PSYCHIATRIC SYMPTOMS IN PATIENTS WITH EPILEPSY?

Ramses Ribot, B. Ouyang and A. M. Kanner (Rush University

Medical Center, Chicago, IL)

Rationale: Depression is the most common psychiatric co-morbidity in patients with epilepsy (PWE) with a lifetime prevalence of approximately $30 \%$. Concern for the pro-convulsive properties of certain antidepressants has led to under-treating depression and anxiety disorders in these patients. Data from animal models and open trials of selective serotonin re-uptake inhibitors (SSRI's) in patients with treatment resistance epilepsy have suggested a potential anticonvulsant effect of these medications. The primary aim of this study was to establish if SSRIs and serotonin-norepinephrine reuptake inhibitors (SNRIs) were associated with an improvement in seizure frequency in PWE. The secondary aim was to determine whether there was a correlation between improvement in psychiatric symptoms and seizure frequency.

Methods: This was a retrospective observational study of 100 consecutive PWE started on SSRIs or SNRIs for the treatment of depressive and/or anxiety disorders. Seizure frequency was recorded from medical records six months prior to introduction of antidepressants and three and six months after initiating therapy. 16 patients were excluded from the analysis due to changes in antiepileptic drug regimen/doses $(n=10)$, or procedures $(n=6)$ at the time of introduction of the antidepressant. Final analysis using dichotomized outcome of seizure frequency $(<1 /$ month and $\geq$ $1 /$ month) was conducted in 84 patients. Responder rate was calculated in patients with a seizure frequency $\geq 1 /$ month. Changes in mood and anxiety data was obtained from the clinical assessment by AMK at three and six months after treatment and were divided into two categories: unchanged and improvement/remission. 13 patients were excluded due to insufficient data.

Results: Among the 84 patients, 40 patients had a seizure frequency $\geq 1 /$ month. Eleven of these patients $(27.5 \%)$ had a reduction in seizure frequency and $28(70 \%)$ remained unchanged. Two patients had an increment in their seizure frequency from $1 /$ month to $2-$ $3 /$ month. 44 patients had a seizure frequency $<1 /$ month which remained unchanged in all. None of the patients discontinued antidepressants before six months. Responder rate was $40 \%(16 / 40)$. Six month after the start of therapy, improvement and/or remission of psychiatric symptoms occurred in $61 / 71$ patients $(86 \%)$. The relation between changes in psychiatric symptoms and seizure frequency appear in Table 1.

Conclusions: SSRIs and SNRIs do not appear to worsen seizure frequency in PWE. In patients with frequent seizures SSRIs and SNRIs appear to have an antiepileptic effect. These drugs appear to yield good therapeutic response independently of seizure frequency.These data need to be replicated in prospective double blind placebo controlled trials.

Table 1.

\begin{tabular}{|c|c|c|}
\hline \multirow[b]{3}{*}{$\begin{array}{l}\text { Patients with a baseline scizure frequency } \\
<1 / \text { month who remained }<1 / \text { month }(n=35)\end{array}$} & \multicolumn{2}{|c|}{$\begin{array}{l}\text { Psychiatric symptoms six months after initiating } \\
\text { treatement with SSRIs and SNRIs }\end{array}$} \\
\hline & Unchnaged & Inprovement/Remission \\
\hline & $6(17.1 \%)$ & $29(82.9 \%)$ \\
\hline $\begin{array}{l}\text { Patients with a baseline seizure frequency } \geq \\
1 / \text { month who remained with a seizure } \\
\text { frequency } \geq 1 / \text { month }(\mathrm{n}=25)\end{array}$ & $2(8 \%)$ & $23(92 \%)$ \\
\hline $\begin{array}{l}\text { Patients with a baseline seizure frequency } \geq \\
1 / \text { month who had in improvement in scizurc } \\
\text { frcquency to }<1 / \text { month }(\mathrm{n}=11)\end{array}$ & $2(18.1 \%)$ & $9(81.9 \%)$ \\
\hline
\end{tabular}

C.06

PREVENTING DEPRESSION IN EPILEPSY: PROJECT UPLIFT

Nancy J. Thompson ${ }^{1}$, A. Patel ${ }^{1}$, L. M. Selwa ${ }^{2}$, C. E. Begley ${ }^{3}$, R. T. Fraser $^{4}$, E. Johnson ${ }^{4}$ and S. Stoll ${ }^{2}\left({ }^{1}\right.$ Department of Behavioral Sciences and Health Education, Rollins School of Public Health of Emory University, Atlanta, GA; ${ }^{2}$ Health Behavior and Health Education, University of Michigan, Ann Arbor, MI; ${ }^{3}$ Management, Policy, and Community Health, The University of Texas Health Science Center, Houston, TX and ${ }^{4}$ Neurology, Neurological Surgery, $\&$ Rehabilitation Medicine, University of Washington, Seattle, WA)

Rationale: Depression is a common psychiatric disorder among people with epilepsy. It has more strongly predicted reduced quality of life than seizure frequency, although individuals with epilepsy and depression also perceive increased levels of seizure severity. A review of studies reported the rate of depression in people with epilepsy is between $32 \%$ and $48 \%$.

Project UPLIFT was originally developed as a home-based treatment for depression in people with epilepsy, funded by the Centers for Disease Control and Prevention. The UPLIFT materials include modules for eight sessions based upon Mindfulness-based Cognitive Therapy for Depression, and are designed for group delivery by telephone or Web. A pilot effectiveness study found they significantly reduced depressive symptoms more than a treatment-asusual (TAU) waitlist condition.

The goals of this multi-site project, funded by the National Center for Minority Health and Health Disparities, were to revise the Project UPLIFT materials for use in prevention (rather than treatment) of depression, and estimate their effectiveness in reducing the risk of depression in people with epilepsy.

Methods: Persons diagnosed with epilepsy for at least 3 months were recruited through epilepsy clinics at Emory University, University of Michigan, University of Texas-Houston, and University of Washington. Each state's target enrollment was 42 people. Investigators modified the language and activities for use in prevention; two focus groups of people with epilepsy assisted in reevaluating the revised intervention, before it was tested. Supervised by a licensed psychologist, two co-facilitators led each intervention group: a graduate student in public health and a person with epilepsy. The outcomes of the preventive intervention were assessed using a cross-over design. The 130 consenting participants were randomized to intervention or TAU; those assigned to UPLIFT who required a specific mode of delivery were assigned to that mode and the remainder were assigned to equalize the groups. UPLIFT groups received the program immediately (some by telephone conference call, and some by Web) and TAU groups received the program later. Data, collected at baseline and about 8 and 20 weeks afterward, included measures of depression, knowledge and skills, self-efficacy, self-compassion, satisfaction with life, and quality of life.

Results: The intervention was effective. None $(0 \%)$ of 52 in UPLIFT group developed Major Depressive Disorder compared to $6(11 \%)$ of 56 in TAU $(p=0.028)$. A repeated measures ANOVA found that Beck Depression scores decreased more in UPLIFT than in TAU $\left(F_{1,102}=9.46, p=0.003\right)$, as did number of seizures $\left(F_{1,105}=4.06, p=\right.$ 0.046). Knowledge and Skills $\left(\mathrm{F}_{1,105}=4.09, \mathrm{p}=0.046\right)$ and Satisfaction with Life $\left(\mathrm{F}_{1,106}=6.93, \mathrm{p}=0.010\right)$ increased more in UPLIFT than TAU.

Conclusions: UPLIFT prevented depression at relatively low cost, while reducing seizures and increasing life satisfaction. In addition, distance delivery reduced access problems, reducing health disparities for those who were mobility-limited or located in rural areas. 
C.07

LONG-TERM POST-OPERATIVE SEIZURE OUTCOME AFTER RESECTIVE SURGERY FOR EPILEPSY

Vibhangini S. Wasade ${ }^{1}$, R. Tahir ${ }^{2}$, L. Schultz ${ }^{3}$, B. J. Smith ${ }^{4}$, K. Elisevich ${ }^{4}$, J. Schwalb ${ }^{1}$ and M. Spanaki ${ }^{1}\left({ }^{1}\right.$ Henry Ford

Comprehensive Epilepsy Program, Henry Ford Health System, Detroit, MI; ${ }^{2}$ Neurosciences, Henry Ford Health System, Detroit, MI; ${ }^{3}$ Public Health Services, Henry Ford Health System, Detroit, MI and ${ }^{4}$ Neurosciences, Spectrum Health System, Grand Rapids, MI)

Rationale: Resective surgery is considered a successful treatment option for refractory localization related epilepsy. Most studies have reported seizure outcomes of 2-5 years post-surgery and a few up to 10 years. Our aim was to assess post-surgical outcomes that exceeds 10 years of follow up,and correlate the outcomes to the pathology and the side of resection.

Methods: After obtaining IRB approval, the Henry Ford Health System Corporate Data Store was used to identify patients who had surgical resection for localization related epilepsy from 1993 to 2011. Demographics, age at epilepsy onset and surgery, seizure frequency before surgery, pathology, and number of antiepileptic drugs (AEDs) before and after surgery was gathered from electronic medical records. Phone surveys were conducted by research assistants in 05/2012 to determine the patients' current seizure frequency. Surgical outcomes were based on Engel's classification (class I and II = favorable outcome). To assess the differences, chi-square tests, two sample t-tests and Wilcoxon two sample tests were used.

Results: A total of 470 patients who had resective epilepsy surgery for localization related epilepsy were identified, 44 (9\%) of which deceased since the time of their surgery. Of the remaining 426 patients, $202(47 \%)$ were contacted. These patients had a mean age at the time of the survey of $46.6 \pm 13.3$ years, mean age at epilepsy onset $16.3 \pm 14.1$ years and mean age at epilepsy surgery $36.8 \pm 13.1$ years. The mean delay between epilepsy onset and surgery was $20.8 \pm 13.8$ years, and follow up was obtained at $10.0 \pm 5.2(24 \%$ patients had follow up of 15 years or longer). Mean number of AEDs before surgery were $2.1 \pm 0.9$ and after surgery were $2.1 \pm 0.8(\mathrm{p}=0.073)$. Of the 202 patients surveyed, Engel's classification was available for 200. Fifty six patients (28\%) were seizure free and 146 patients (73\%) had a favorable outcome (Class I and II) (table 1).

Resection was performed in 94 patients (47\%) after scalp EEG monitoring (sEEG) (phase I) and 106 patients (53\%) after extraoperative electrocorticography (eECoG) (phase II). Statistical significance was noted in favorable outcomes when surgery was performed after sEEG ( $83 \%$ vs $64 \%$ ) and in temporal region ( $77 \%$ vs $46 \%$ ). Favorable outcomes were not significantly different based on pathology, and number of AEDs before and after surgery. Most importantly, the favorable outcomes, as well as seizure freedom rates remained stable over the long-term follow-up period after surgery [ 42 patients: $<5$ years $(76 \%, 40 \%), 59$ patients: $5-10$ years $(63 \%, 20 \%)$, 50 patients: $10-15$ years $(78 \%, 30 \%)$, and 49 patients: $>15$ years $(78 \%, 24 \%)]$.

Conclusions: Our study shows that a significant number of patients achieve favorable seizure outcomes $(73 \%)$ or seizure freedom $(28 \%)$ after resective epilepsy surgery, and demonstrates that the seizure outcomes remain stable over more than 15 years post surgery, irrespective of the pathology or the side of resection. Additional assessment of psychosocial outcomes are necessary to better help establish overall post-surgical outcomes after resective surgery for epilepsy.

Table I: Long term post-surgical outcomes (Demographics, location, side of surgery, pathology)

\begin{tabular}{|c|c|c|c|c|c|c|}
\hline Variable & Response & $\mathrm{N}$ & $\begin{array}{l}\text { \#with lavorable } \\
\text { outcome (\%) }\end{array}$ & p-value & $\begin{array}{c}\text { \#Seizure liree } \\
(\%)\end{array}$ & $\mathrm{p}$-value \\
\hline \multirow{2}{*}{ Gender } & Females & 108 & $81(75 \%)$ & \multirow{2}{*}{0.490} & $33(31 \%)$ & \multirow{2}{*}{0.383} \\
\hline & Males & 92 & $65(71 \%)$ & & $23(25 \%)$ & \\
\hline \multirow{3}{*}{ Race } & Caucalsian & 159 & $111(70 \%)$ & \multirow{3}{*}{0.138} & $40(25 \%)$ & \multirow{3}{*}{0.060} \\
\hline & African American & 15 & $12(80 \%)$ & & $5(33 \%)$ & \\
\hline & Other & 8 & $8(100 \%)$ & & $5(63 \%)$ & \\
\hline \multirow{2}{*}{ Phase } & 1-surgery after sEEG & 94 & $78(83 \%)$ & \multirow{2}{*}{0.003} & $36(38 \%)$ & \multirow{2}{*}{0.002} \\
\hline & Il-surgery after eECoG & 106 & $68(64 \%)$ & & $20(19 \%)$ & \\
\hline \multirow{2}{*}{ I.ocation } & Temporal resection & 172 & $133(77 \%)$ & \multirow{2}{*}{$<0.001$} & $52(30 \%)$ & \multirow{2}{*}{0.081} \\
\hline & Fixtra-temporal resection & 28 & $13(46 \%)$ & & $4(14 \%)$ & \\
\hline \multirow{3}{*}{ Side } & Right & 109 & $84(77 \%)$ & \multirow{3}{*}{0.111} & $33(30 \%)$ & \multirow{3}{*}{0.626} \\
\hline & Lelit & 90 & $62(69 \%)$ & & $23(26 \%)$ & \\
\hline & Bilateral & 1 & $0(0 \%)$ & & $0(0 \%)$ & \\
\hline \multirow{7}{*}{ Pathology } & Hippocampal Sclerosis & 53 & $38(72 \%)$ & \multirow{7}{*}{0.916} & $20(38 \%)$ & \multirow{7}{*}{0.285} \\
\hline & Gliosis & 26 & $18(69 \%)$ & & $5(19 \%)$ & \\
\hline & Focal cortical dysplasia & 18 & $14(78 \%)$ & & $7(39 \%)$ & \\
\hline & $\begin{array}{l}\text { Tumor (benign and } \\
\text { malignant) }\end{array}$ & 9 & $6(67 \%)$ & & $1(11 \%)$ & \\
\hline & $\begin{array}{c}\text { Neuronal mixed glial } \\
\text { tumors }\end{array}$ & 7 & $6(86 \%)$ & & $3(43 \%)$ & \\
\hline & Vascular malformation & 5 & $3(60 \%)$ & & $1(20 \%)$ & \\
\hline & Other & 80 & $61(76 \%)$ & & $19(24 \%)$ & \\
\hline
\end{tabular}

P-values $<0.05$ were considered statistically significant.

\section{C.08 \\ ELECTROCORTICOGRAPHY OF FACE AND PLACE SPECIFICITY DURING VISUAL NAMING}

Christopher Conner, C. Kadipasaoglu, T. Pieters and N. Tandon (Neurosurgery, UT Houston, Houston, TX)

Rationale: The ventral and lateral occipitotemporal cortex, known as the 'ventral stream', activates in a category specific manner during visual object recognition. Studies of the ventral stream have focused predominantly on the specificity of neural responses to three major categories: faces, places, and common objects. The regional selectivity for each of these categories has been studied using fMRI, but data from electrophysiology is sparse due to the location. Methods: Thirteen patients diagnosed with temporal lobe epilepsy were scheduled for implantation of subdural electrodes (SDEs) for clinical localization of seizure focus sites. Subjects were presented images of faces and places during concurrent collection of electrocorticography $(\mathrm{ECoG})$ from lingual, fusiform, inferior temporal, and parahippocampal gyrii. ECoG data were decomposed into seven frequency bands: delta $(0-4 \mathrm{~Hz})$, theta (4-8), alpha (813), beta (13-30), low gamma (30-60), mid gamma (60-120) and high gamma (120-240). Percent change was calculated over prestimulus baseline.

Results: In all SDEs, power increases in the gamma frequency bands were most strongly locked to task performance. Right hemisphere electrodes showed greater increases in gamma power during face naming tasks than place naming tasks, while left hemisphere SDEs had greater activation during place naming. Further, medial SDEs in the left hemisphere were more active for places while lateral SDEs were more active for faces.

Conclusions: These results collaborate findings from similar fMRI studies. However, the temporal resolution and signal-to-noise ratio afforded by SDEs is greater than that of neuroimaging. This allows for validation of current models of lexical access and precise mapping of category specificity in naming. 

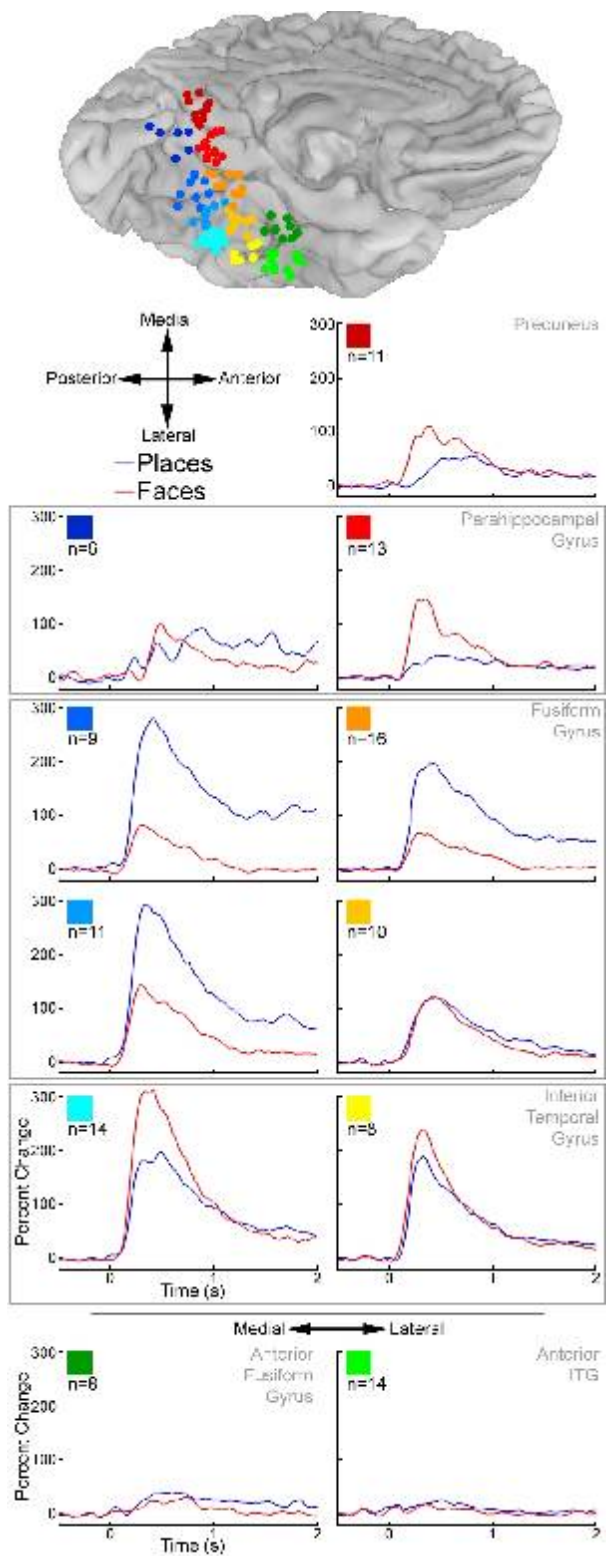

\section{C.09}

RELATIVE RISKS OF SPONTANEOUS FETAL LOSS IN UNINTENDED VERSUS INTENDED PREGNANCIES IN WOMEN WITH EPILEPSY: INTERIM ANALYSIS OF THE EPILEPSY BIRTH CONTROL REGISTRY

Andrew G. Herzog ${ }^{1}$, A. R. Davis ${ }^{2}$, W. A. Hauser ${ }^{3}$, K. E. Cahill ${ }^{1}$, K. M. Fowler ${ }^{1}$, A. N. Saporta ${ }^{1}$ and H. B. Mandle ${ }^{1}\left({ }^{1}\right.$ Harvard

Neuroendocrine Unit, Beth Israel Deaconess Medical Center, Boston, MA; ${ }^{2}$ Obstetrics and Gynecology, Columbia College of Physicians and Surgeons, New York, NY and ${ }^{3}$ Neurology and Epidemiology, Columbia College of Physicians and Surgeons, New York, NY)

Rationale: Since women with epilepsy (WWE) have higher rates of unintended pregnancies and unintended pregnancies may be associated with more adverse reproductive outcomes in the general population, this investigation assessed whether pregnancy outcomes (live births, induced abortions and spontaneous fetal loss) differed between unintended and intended pregnancies in WWE.

Methods: The Epilepsy Birth Control Registry (EBCR) is a webbased survey and educational site that gathers demographic, epilepsy, antiepileptic drug (AED), contraceptive and reproductive data from WWE in the community. These retrospective data come from the first 550 WWE, 18-47 years of age, who completed the survey. We determined frequencies, rates and relative risks (RR) of live births, induced abortions and spontaneous fetal loss for unintended versus intended pregnancies. Spontaneous fetal loss is reported as a proportion of the non-aborted as well as the total number of pregnancies. Univariate ANOVA was carried out to determine whether pregnancy planning, pregnancy before or after the development of seizures, use of AEDs, maternal age and age of seizure onset were significant factors in determining live birth versus spontaneous fetal loss in non-aborted pregnancies.

Results: 205 WWE reported 451 pregnancies of which 365 (80.9\%) followed the development of seizures. Of the 348 for which we have planning and outcome data, $238(68.4 \%)$ were unintended and 110 $(31.6 \%)$ were intended. The unintended pregnancies resulted in higher rates of induced abortion [53/238 (22.3\%) v 2/110 (1.8\%); $\chi 2$ $=22.133, \mathrm{df}=1, \mathrm{p}<.0001]$ and spontaneous fetal loss as a proportion of non-aborted pregnancies [55/185 (29.7\%) v 18/108 (16.7\%); $\chi 2=$ $6.220, \mathrm{df}=1, \mathrm{p}=.0126]$ than did intended pregnancies (Table 1). The relative risk of spontaneous fetal loss as a proportion of nonaborted pregnancies was significantly greater for unintended than intended pregnancies [RR $=1.784$ (95\%CI: 1.108-2.872); $\mathrm{p}=.0173$ ]. Pregnancy planning was the only significant factor related to pregnancy outcome, i.e. live birth versus spontaneous fetal loss, $(\mathrm{p}=$ $.002)$. Of note, spontaneous fetal loss rates relative to non-aborted pregnancies did not differ significantly between pregnancies that occurred before $(20 / 72=27.8 \%)$ versus after $(73 / 293=24.9 \%)$ the development of seizures nor between pregnancies exposed to AEDs $(44 / 136=32.4 \%)$ or no AEDs $(7 / 25=28.0 \%)$.

Conclusions: Interim analysis of the EBCR survey data raises the possibility that unintended pregnancies in WWE may be associated with significantly more spontaneous fetal loss, as well as induced abortions, than intended pregnancies. Pregnancy planning was the only statistically significant factor in pregnancy outcomes. The findings highlight the importance of developing guidelines for safe and effective contraception for pregnancy planning in WWE.

Supported in part by the Epilepsy Foundation

Relative Risks of Live Birth, Induced Abortion and Spontaneous Fetal Loss in Unintended Versus Intended Pregnancies in Women with Epilepsy

\begin{tabular}{|c|c|c|c|}
\hline & $\begin{array}{c}\text { Unintended } \\
\text { Pregnancies } \\
(\mathrm{N}=238)\end{array}$ & $\begin{array}{c}\text { Intended } \\
\text { Pregnancies } \\
(\mathrm{N}=110)\end{array}$ & $\begin{array}{c}\text { Relative Risks ol Outcomes in Unintended v } \\
\text { Intended Pregnancies (95\% CI) }\end{array}$ \\
\hline Live Birth & $130(54.6 \%)$ & $90(81.8 \%)$ & $\begin{array}{c}6.34(.552-729) \\
\mathrm{p}<.0001\end{array}$ \\
\hline Abortion & $53(22.3 \%)$ & $2(1.8 \%)$ & $\begin{array}{c}12.025(2.985-48.444) \\
\mathrm{p}=.0005\end{array}$ \\
$\begin{array}{c}\text { Spontancous } \\
\text { Fetal Loss }\end{array}$ & $55(23.1 \%)$ & $18(16.4 \%)$ & $\begin{array}{c}1.401(.865-2.268) \\
\mathrm{p}=.1714\end{array}$ \\
\hline $\begin{array}{c}\text { Spontaneous } \\
\text { Fetal Loss/ } \\
\text { Non-Abortcd } \\
\text { Pregnancics }\end{array}$ & $55 / 185(29.7 \%)$ & $18 / 108(16.7 \%)$ & $\begin{array}{c}1.784(1.108-2.872) \\
\mathrm{p}=.0173\end{array}$ \\
\hline
\end{tabular}

\section{Monday, December 3, 2012}

\author{
Pediatric State of the Art Symposium \\ 6:30 p.m.-9:00 p.m.
}

WHAT FEBSTAT TELLS US ABOUT FEBRILE STATUS EPILEPTICUS (FSE), HS AND TLE

Shlomo Shinnar (Neurology, Pediatrics and Epidemiology and Population Health, Montefiore Medical Center, Albert Einstein College of Medicine, Bronx, NY) 
Summary: While brief febrile seizures (FS) are thought to be benign, prolonged FS may be less so. There is accumulating human data the febrile status epilepticus (FSE) may be associated with hippocampal injury, and subsequent hippocampal sclerosis (HS) and temporal lobe epilepsy (TLE). Several prior smaller studies and case reports have suggested a potential causal association though the large FS epidemiological studies have minimized the role of prolonged FS in causing HS and TLE and have attributed both the FSE and the ensuing epilepsy to pre-existing pathology.

The FEBSTAT study prospectively recruited 199 children with FSE. Median seizure duration was $>1$ hour. In addition there is a pilot cohort of 23 children with FSE recruited at Duke with similar criteria. Finally, there is a control cohort of 96 children with a first simple FS recruited as part of a separate study at Columbia who had imaging at baseline and one year. On MR imaging within 72 hours, approximately $12 \%$ of the FEBSTAT cohort had abnormal, hippocampal T2 signal, a finding not present in simple FS controls. In addition, there was a higher prevalence of developmental abnormalities of the hippocampus and temporal lobe, in particular HIMAL, in FSE cases compared with controls. Quantitative volumetric studies demonstrate that as a group, the children with FSE have smaller hippocampi than the controls. Initial EEGs frequently show focal temporal slowing or attenuation which is associated with hippocampal T2 abnormalities. Approximately one third of cases were associated with acute or reactivated Herpesvirus 6 or 7 infections.

On follow-up MRIs at one year, those with initial abnormal T2 hippocampal signal demonstrated hippocampal volume loss and persistent T2 signal consistent with HS though the majority of these children have not developed epilepsy to date. In addition, on analysis, the hippocampi of children with FSE whose initial MRIs were normal did not grow as well as those of children with simple FS. The focal temporal slowing or attenuation was present in $20 \%$ at follow-up. Children with FSE have a higher prevalence of pre-existing, often very subtle, hippocampal abnormalities that may predispose them to FSE. However, once FSE occurs, it results in diminished hippocampal growth and sometimes frank HS. Further follow-up and cognitive testing of this cohort is in progress. As the children were very young at the time of FSE with median age of 15 months, detailed memory testing will have to await the 5 year evaluations which are in progress. Ultimately, the FEBSTAT study will address the relationship between FSE and subsequent HS and TLE as well as the associated neurocognitive deficits. Genetic and genomic specimens are being collected and may also shed further light on the process. The EEG and MRI abnormalities may serve as potential biomarkers as well as targets for potential trials of novel antiepileptogenic agents. FSE remains an extremely promising model in both the human and animal to both study and prevent epileptogenesis following the insult.

Supported by Grant NS 43209 from NINDS

\section{Tuesday, December 4, 2012}

Scientific Symposium
8:30 a.m.-10:00 a.m.

STEREOTACTIC ELECTROENCEPHALOGRAPHY (SEEG) IN THE PRE-SURGICAL INVESTIGATION OF REFRACTORY FOCAL EPILEPSY

Hans Luders ${ }^{1}$, Giorgio LoRusso ${ }^{2}$, Jonathan P. Miller ${ }^{3}$, Jorge Gonzalez-Martinez ${ }^{4}$, Philippe Kahane ${ }^{5}$ and Samden Lhatoo ${ }^{1}$ ( ${ }^{1}$ Department of Neurology, University Hospitals, Case Medical Center, Cleveland, OH; ${ }^{2}$ "Claudio Munari" Center for Epilepsy Surgery, Milan, Italy; ${ }^{3}$ Department of Neurosurgery, University Hospitals, Case Medical Center, Cleveland, $\mathrm{OH} ;{ }^{4}$ Department of Neurosurgery, Cleveland Clinic, Cleveland, $\mathrm{OH}$ and ${ }^{5}$ Neurology Department, University Hospitals, Grenoble, France)

Summary: A significant proportion of patients with refractory focal epilepsy who are being evaluated for resective surgery require invasive evaluations with subdural or depth electrode studies in order to better delineate the most likely epileptogenic zone. In most parts of the world that have the expertise and infrastructure to carry out intracranial electrode studies, the preferred method is subdural grid insertion with or without limited, non-stereotactic depth electrodes. A handful of centers in Europe and North America employ the use of stereotactically implanted multiple depth electrodes (stereotactic electroencephalography or sEEG) which have both advantages and potential limitations. Many centers have noted an increasing complexity of surgical cases presenting for presurgical evaluations, for example, patients who are MRI lesion negative, or who have dual or multiple epileptogenic pathologies. Such patients may be studied best using sEEG.

The European approach and the North-American approach to sEEG will be discussed including details of the methodology used for insertion of depth electrodes (Stereoencephalography in the Presurgical Evaluation of Focal Epilepsy: A Retrospective Analysis of 215 Procedures. M. Cossu et al. Neurosurgery 57: 706-718, 2005). The relative advantages and disadvantages of depth vs. subdural electrodes will be presented. Special techniques used to define the epileptogenic zone with depth electrodes and the results of electrical stimulation of depth electrodes for mapping of cortical eloquent areas will also be discussed (The Bancaud and Talairach view on the epileptogenic zone: a working hypothesis. P. Kahane et al. Epileptic Disord 8(Suppl.2): S16-26, 2006). 